\title{
ISOLOK VALVE ACCEPTANCE TESTING FOR DWPF SME SAMPLING PROCESS
}

K.R. Hera

C.J. Coleman

M.A. Jones

B.J. Wiedenman

T.B. Edwards

November 2011

Research \& Development Engineering Section Energy Security and Engineering Directorate Savannah River National Laboratory Aiken, SC 29808 


\section{DISCLAIMER}

\section{Contract No:}

This document was prepared in conjunction with work accomplished under Contract No. DE-AC09-08SR22470 with the U.S. Department of Energy.

\section{Disclaimer:}

This work was prepared under an agreement with and funded by the U.S. Government. Neither the U.S. Government or its employees, nor any of its contractors, subcontractors or their employees, makes any express or implied: 1 . warranty or assumes any legal liability for the accuracy, completeness, or for the use of results of such use of any information, product, or process disclosed; or 2. representation that such use or results of such use would not infringe privately owned rights; or 3. endorsement of recommendation of any specifically identified commercial product, process, or service. Any views and opinions of authors expressed in this work do not necessarily state or reflect those of the United States Government, or its contractors, or subcontractors.

\begin{tabular}{|c|}
\hline Printed in the United States of America \\
Prepared For \\
U.S. Department of Energy \\
\hline
\end{tabular}




\section{REVIEWS AND APPROVALS}

\section{AUTHOR(S):}

\begin{tabular}{ll}
\hline K.R. Hera, SRNL, Mechanical Systems \& Custom Equipment Dev. Date
\end{tabular}

\begin{tabular}{ll}
\hline C.J. Coleman, SRNL, Spectroscopy and Separations & Date
\end{tabular}

M.A. Jones, SRNL, Spectroscopy and Separations $\quad$ Date

\begin{tabular}{ll}
\hline B.J. Wiedenman, SRNL, Spectroscopy and Separations & Date
\end{tabular}

T.B. Edwards, SRNL, Applied Computational Engineering and Statistics $\quad$ Date

TECHNICAL REVIEWER(S):

R.L. Walker, SRNL, Applied Computational Engineering and Statistics $\quad$ Date

A.D. Marzolf, SRNL, Mechanical Systems \& Custom Equipment Dev. Date

\section{APPROVERS}

\begin{tabular}{lc}
\hline P.L. Lee, Manager, SRNL, Applied Computational Engineering and Statistics & Date
\end{tabular}

L.T. Reid, Manager, SRNL, Mechanical Systems \& Custom Equipment Dev. $\quad$ Date

S.L. Tibrea, Manager, SRNL, Research \& Development Engineering $\quad$ Date

\begin{tabular}{lc}
\hline J.E. Occhipinti, Manager, LWO, WS Engineering & Date
\end{tabular} 


\section{EXECUTIVE SUMMARY}

Evaluation of the Defense Waste Processing Facility (DWPF) Chemical Process Cell (CPC) cycle time identified several opportunities to improve the CPC processing time. Of the opportunities, a focus area related to optimizing the equipment and efficiency of the sample turnaround time for the DWPF Analytical Laboratory was identified. The Mechanical Systems \& Custom Equipment Development (MS\&CED) Section of the Savannah River National Laboratory (SRNL) evaluated the possibility of using an Isolok ${ }^{\circledR}$ sampling valve as an alternative to the Hydragard ${ }^{\circledR}$ valve for taking process samples. Previous viability testing was conducted with favorable results using the Isolok sampler and is reported in SRNL-STI-201000749 (1). This task has the potential to improve operability, reduce maintenance time and decrease CPC cycle time. This report summarizes the results from acceptance testing which was requested in Task Technical Request (TTR) HLW-DWPF-TTR-2010-0036 (2) and which was conducted as outlined in Task Technical and Quality Assurance Plan (TTQAP) SRNL-RP-2011-00145 (3).

The objective of this study was to qualify the Isolok for use in sampling the Slurry Mix Evaporator (SME) tank at the DWPF. The purpose of SME sampling is to obtain representative samples of the contents of the SME tank. The obtained samples are vitrified, and the compositions of the vitrified samples are measured analytically with the resulting measured glass compositions being assessed for acceptability through the use of DWPF's Product Composition Control System (PCCS). For the acceptability decision process to be reliably conducted, the contents of the SME need to be well-mixed and the samples need to be representative. Therefore RW-0333P QA requirements apply to this task.

The primary metric used in making comparisons between two sampling systems (e.g., low Coliwasa versus high Coliwasa or HFR 3mL Isolok versus combined Coliwasa) was the \% difference between the averages of the two sets of measurements. Table A provides the percent differences between the mean of the screened measurements of the samples from the indicated sampling mechanism and the mean of the corresponding screened measurements from the Coliwasa samples for the reportable elements. Entries with \% differences (in absolute value) less than $5 \%$ are shaded green. The vast majority of the entries in this table are shaded, and the \% differences of the Isolok were comparable to \% differences of the Hydragard, which was the primary method by which the performance of the Isolok was assessed for this study. Specifically, while the Hydragard had 8 entries with \% differences greater than 5\%, the Isolok had only 3.

Table A. Percent Differences for Screened Oxide Measurements for All Test Phases

\begin{tabular}{|c|c|c|c|c|c|c|c|c|c|c|}
\hline Test Phase/Sampler & $\mathrm{Al}_{2} \mathbf{O}_{3}$ & $\mathbf{B}_{2} \mathbf{O}_{3}$ & $\mathrm{CaO}$ & $\mathrm{Fe}_{2} \mathrm{O}_{3}$ & $\mathrm{Li}_{2} \mathrm{O}$ & MgO & MnO & $\mathrm{Na}_{2} \mathrm{O}$ & $\mathrm{NiO}$ & $\mathrm{SiO}_{2}$ \\
\hline 1st/Hydragard - HFR & 3.3 & -0.7 & 3.2 & 2.4 & 0.4 & 1.7 & 2.1 & 0.8 & not reportable & -0.9 \\
\hline 1st/Hydragard-LFR & 3 & 3.8 & 4 & 2.8 & 2.8 & 4.2 & 3.3 & 2.4 & not reportable & 0.5 \\
\hline 1st/Isolok-HFR-12mL & -0.2 & 2.8 & 0.3 & \begin{tabular}{|c|}
-0.9 \\
\end{tabular} & -0.1 & \begin{tabular}{|c|}
-0.9 \\
\end{tabular} & -0.8 & -2.9 & not reportable & 1.4 \\
\hline 1st/Isolok-HFR-3mL & -1.5 & 1.5 & \begin{tabular}{|c|}
-0.5 \\
\end{tabular} & \begin{tabular}{|c|}
-0.6 \\
\end{tabular} & 2 & -0.8 & -0.7 & -3 & not reportable & 2 \\
\hline 1st/Isolok-LFR-12mL & 2.8 & 4.9 & 4.6 & 3.4 & 0.8 & 3.7 & 3.9 & 2.2 & not reportable & 0.8 \\
\hline 1st/Isolok-LFR-3mL & 1.5 & -3.4 & 1.7 & 1.6 & -0.1 & 1.6 & 2 & -0.5 & not reportable & 0 \\
\hline 2nd/Hydragard - HFR & 4.5 & -5.4 & 2.1 & 5.8 & -2.3 & not reportable & 4.2 & -0.9 & 5.8 & -2.2 \\
\hline 2nd/Hydragard-LFR & 1.4 & -2.9 & 0.3 & 0.9 & -0.2 & not reportable & 0 & 0.1 & -0.6 & -0.8 \\
\hline 2nd/Isolok-HFR-12mL & -0.4 & -5.2 & -1.3 & -0.2 & 0.7 & not reportable & -2 & -0.4 & -1.8 & 1.2 \\
\hline 2nd/Isolok-HFR-3mL & -0.3 & -1.1 & -1.8 & 0.1 & 1.9 & not reportable & 0.5 & \begin{tabular}{|c|}
-0.7 \\
\end{tabular} & 1.2 & 1.3 \\
\hline 2nd/Isolok-LFR-12mL & 2.1 & -4.7 & 1.3 & 1.9 & 0.7 & not reportable & 1.7 & 1.3 & 1 & -2 \\
\hline 2nd/Isolok-LFR-3mL & 3.6 & -2.4 & 2.3 & 3.3 & 0.2 & not reportable & 4.6 & 0.4 & 3.1 & 1 \\
\hline 3rd/Hydragard - HFR & 4.7 & -5.1 & 5.1 & 5.1 & -2.2 & 5.5 & 5.2 & 0 & not reportable & -1.9 \\
\hline 3rd/Hydragard-LFR & 2 & -1.8 & 2.2 & 1.5 & -2.5 & 1.3 & 0.9 & 3 & not reportable & -2.3 \\
\hline 3rd/Isolok-HFR-12mL & -2.3 & -1.4 & -3.1 & -2.8 & 1 & -3.6 & -3.7 & -0.3 & not reportable & -0.6 \\
\hline 3rd/Isolok-HFR-3mL & -0.7 & -2.1 & -0.8 & -1.5 & 1.8 & -2.1 & -2.1 & 1.5 & not reportable & 1.1 \\
\hline 3rd/Isolok-LFR-12mL & -2.9 & -0.8 & -3.1 & -4.4 & 4.1 & -6.1 & -5.8 & 1.9 & not reportable & 0.9 \\
\hline 3rd/Isolok-LFR-3mL & -1.2 & \begin{tabular}{|c|}
-0.9 \\
\end{tabular} & -2.7 & -1.6 & -0.1 & -2.5 & -2.5 & -0.5 & not reportable & 0.6 \\
\hline
\end{tabular}

Based on the acceptance testing data and discussions with the DWPF Laboratory improvement team, SRNL recommends proceeding with the design and installation of the Isolok system for the SME sampling process. Based on the success of the Isolok testing to date, SRNL recommends testing to acquire a smaller 1-1.5 mL sample size. A smaller sample size used in conjunction with the cesium carbonate fusion would allow the elimination of the grinding step for the glass, thus further reducing analytical cycle time. 


\section{TABLE OF CONTENTS}

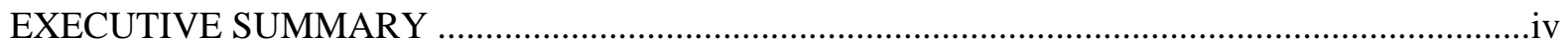

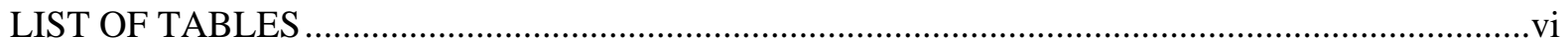

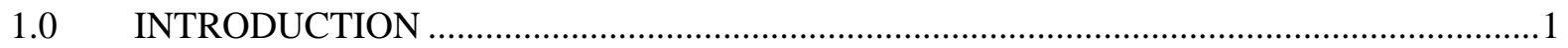

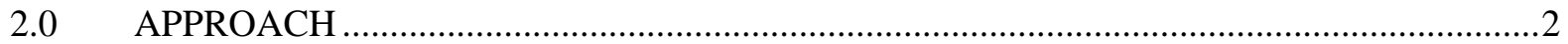

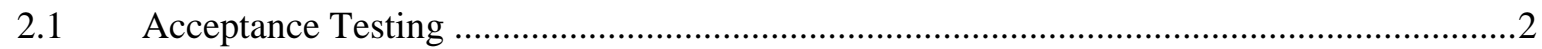

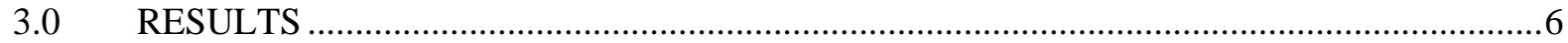

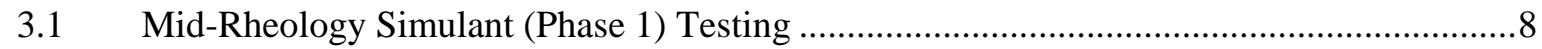

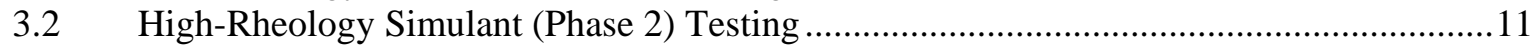

3.3 Low-Rheology Simulant (Phase 3) Testing..................................................................13

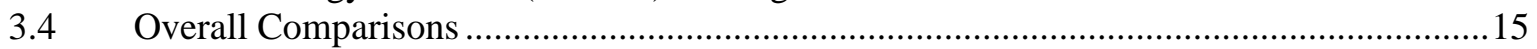

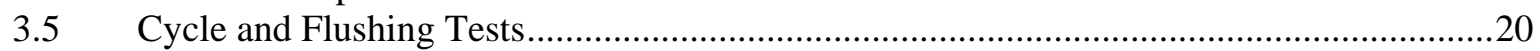

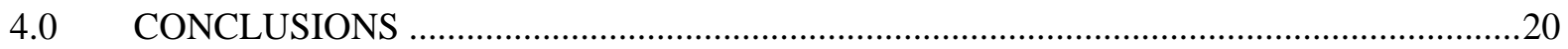

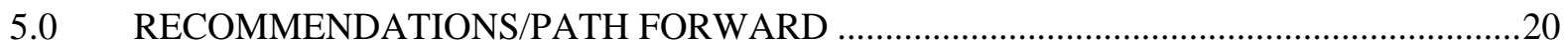

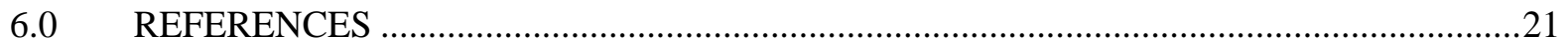




\section{LIST OF FIGURES}

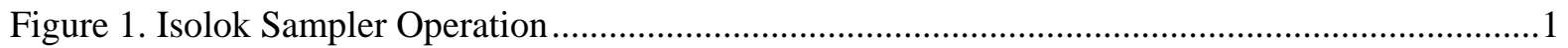

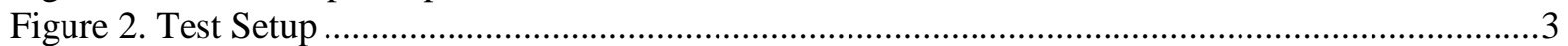

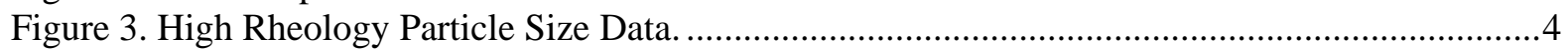

Figure 4. Mid and Low Rheology Particle Size Data. ....................................................................5

Figure 5. \% Differences for Screen Measurements of Major Oxides. ...............................................17

Figure 6. Sludge and Frit Components versus wt\% dried solids. .....................................................19

\section{LIST OF TABLES}

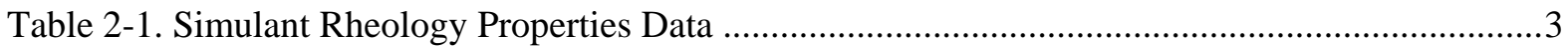

Table 2-2. High Rheology Simulant Elemental Composition ...............................................................6

Table 2-3. Mid \& Low Rheology Simulant Elemental Composition ..................................................6

Table 3-1. \% Differences for the Screened Density and Solids Measurements from Phase 1

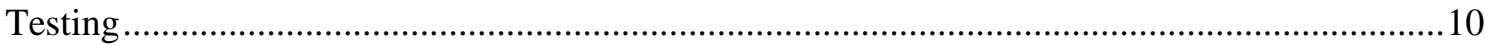

Table 3-2. \% Differences for Screened Oxide Measurements from Phase 1 Testing ...........................11

Table 3-3. \% Differences for the Screened Density and Solids Measurements from Phase 2

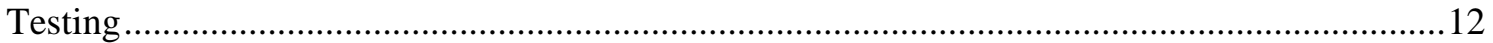

Table 3-4. \% Differences for Screened Oxide Measurements from Phase 2 Testing ...........................13

Table 3-5. \% Differences for the Screened Density and Solids Measurements from Phase 3

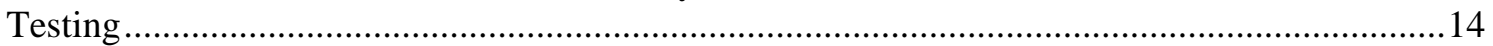

Table 3-6. \% Differences for Screened Oxide Measurements from Phase 3 Testing ...........................15

Table 3-7. \% Differences for Screened Oxide Measurements for All Test Phases ..............................17

Table 3-8. \% Relative Bias Bounds for Screened Oxide Measurements for All Test Phases...............18 


\subsection{INTRODUCTION}

Evaluation of the Defense Waste Processing Facility (DWPF) Chemical Process Cell (CPC) cycle time identified several opportunities to improve the CPC processing time. Of the opportunities, a focus area related to optimizing the equipment and efficiency of the sample turnaround time for DWPF Analytical Laboratory was identified. The Mechanical Systems \& Custom Equipment Development (MS\&CED) Section of the Savannah River National Laboratory (SRNL) evaluated the possibility of using an Isolok ${ }^{\circledR}$ sampling valve as an alternative to the Hydragard ${ }^{\circledR}$ valve for taking process samples. Previous viability testing was conducted with favorable results using the Isolok sampler and reported in SRNL-STI-2010-00749 (1). This task has the potential to improve operability, reduce maintenance time and decrease CPC cycle time. This report summarizes the results from acceptance testing which was requested in Task Technical Request (TTR) HLWDWPF-TTR-2010-0036 (2) and which was conducted as outlined in Task Technical and Quality Assurance Plan (TTQAP) SRNL-RP-2011-00145 (3). The Isolok to be tested is the same model which was tested, qualified, and installed in the Sludge Receipt Adjustment Tank (SRAT) sample system. RW-0333P QA requirements apply to this task.

This task was to qualify the Isolok sampler for use in the DWPF Slurry Mix Evaporator (SME) sampling process. The Hydragard, which is the current baseline sampling method, was used for comparison to the Isolok sampling data. The Isolok sampler is an air powered grab sampler used to "pull" a sample volume from a process line. The operation of the sampler is shown in Figure 1. The image on the left shows the Isolok's spool extended into the process line and the image on the right shows the sampler retracted and then dispensing the liquid into the sampling container.
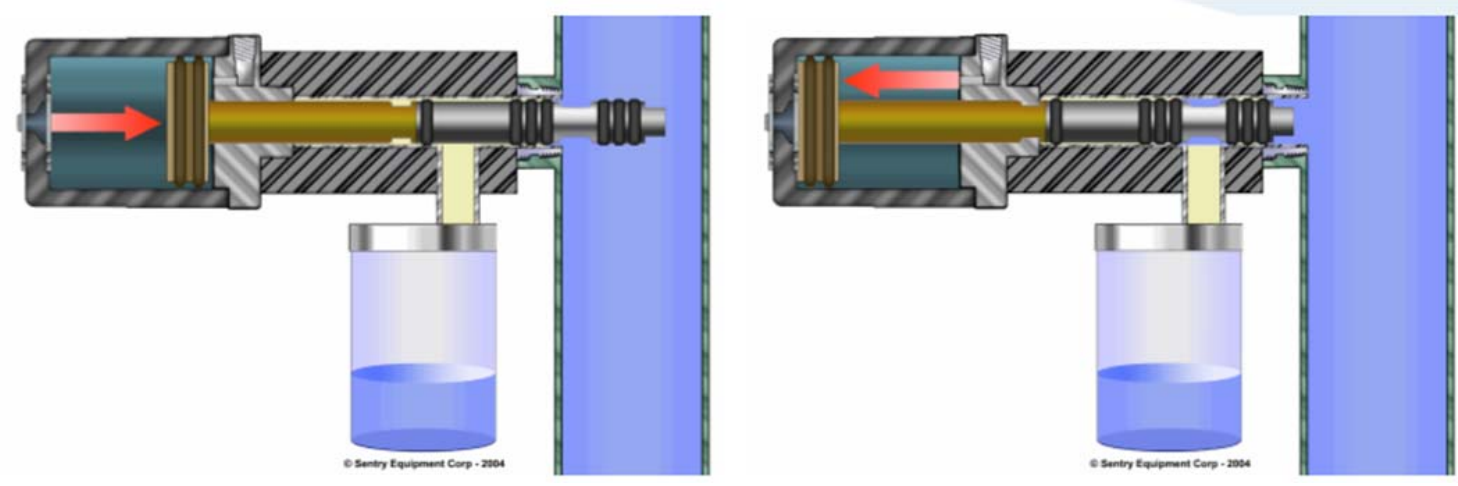

Figure 1. Isolok Sampler Operation

To determine tank homogeneity, a Coliwasa sampler was used to grab samples at a high and low location within the mixing tank. Data from the two locations were compared to determine if the contents of the tank were well mixed. The Coliwasa sampler is a tube with a stopper at the bottom and is designed to obtain grab samples from specific locations within the drum contents. A position paper (4) was issued to address the prototypic flow loop issues and simulant selections. A statistically designed plan (5) was issued to address the total number of samples each sampler needed to pull, to provide the random order in which samples were pulled and to group samples for elemental analysis. The TTR required that the Isolok sampler perform as well as the Hydragard sampler during these tests to ensure the acceptability of the Isolok sampler for use in the DWPF sampling cells. Procedure \#L9.4-5015 was used to document the sample parameters and process steps. Completed procedures are located in R\&D Engineering job folder 23269. 


\subsection{APPROACH}

\subsection{Acceptance Testing}

This acceptance testing was conducted in Building 723-A using a combined simulant from SRNL 22L SME runs, the same simulant from 22L runs but diluted, and Sludge Batch 6H (SB6H) melter feed simulant. Figure 2 below shows the piping setup for the test. All simulants were pumped from a 1/6 scale SME vessel through a mockup flow loop of the SME process piping. Drawing EES23269-R1-005 is an assembly drawing of the acceptance test piping setup. The Isolok was positioned in series below a Hydragard sampler. A drum mixer with dual 9" propellers was used to mix the simulant and ensure simulant homogeneity. The simulant was mixed for approximately 30 minutes prior to sampling and continued to mix while the samples were taken during the acceptance testing.

Rheology data for the three simulants tested are shown in Table 2-1, particle size data are provided in Figure3 and Figure 4, and the measured compositions of the simulants are given in Table 2-2 and Table 2-3. For the tests, the $3 \mathrm{~mL}$ Isolok samples were pulled in platinum crucibles, $12 \mathrm{~mL}$ Isolok and Hydragard samples in p-nut vials and the Coliwasa samples in $60 \mathrm{~mL}$ plastic bottles. Additional samples for density measurements were pulled in p-nut vials of known weight and volume. The samples were dried at $110^{\circ} \mathrm{C}$ and vitrified at $1050{ }^{\circ} \mathrm{C}$ to obtain glass wafers that were cracked to produce glass shards. The weight percent (wt\%) dried solids and the wt\% vitrified solids were recorded and are included in the measurements evaluated as part of this study. The shards were dissolved by fusing them at $1050{ }^{\circ} \mathrm{C}$ with a mixture of cesium carbonate and germanium dioxide per ADS-2502 procedure (6) and detailed in lab notebook SRNL-NB-2011-00016 page 27 (7). The fusion residue was dissolved with sequential additions of a dilute hydrogen peroxide solution and a dilute nitric acid solution. The solutions were analyzed for elemental composition by inductively coupled plasma-optical emission spectroscopy (ICP-OES) techniques.

A Coliwasa sampler was used to take dip samples at two locations (high and low) in the tank. The Coliwasa sample measurements were used to ensure homogeneity of the simulant material within the mixing tank and to serve as a basis for comparison for both Hydragard and Isolok sampling systems. During the sampling, the Coliwasa sampler was closed as it was inserted and positioned into the tank, and then the sampler was opened to remove a sample at the proper tank location.

In addition to testing the Isolok's ability to pull a representative sample, flush and cycling tests were also requested in the TTR. Flush water testing was conducted at the end of the first test to determine the amount of strokes required to clean the inner surfaces of the Isolok. Water was pumped through the Isolok flush ports, and the Isolok was cycled in and out to flush the needle and overflow port. After completion of the flushing, the system was dismantled and visually inspected to verify the Isolok was clean.

After the completion of the third test, the acceptance test system was aligned and setup for cycle testing. For this test the Isolok was actuated for two seconds in the stream then three seconds retracted. This was repeated for a number of cycles; then stopped for 1 minute and the Isolok needle inspected for leakage at the needle. If the Isolok was beginning to fail, drips of liquid would drop from the Isolok needle. These steps were repeated until a point was reached that represented an acceptable life span for the Isolok. The Isolok controller was programmed to count the number of cycles, and that number was then recorded into the laboratory notebook (8). The low rheology simulant was used for this test. 


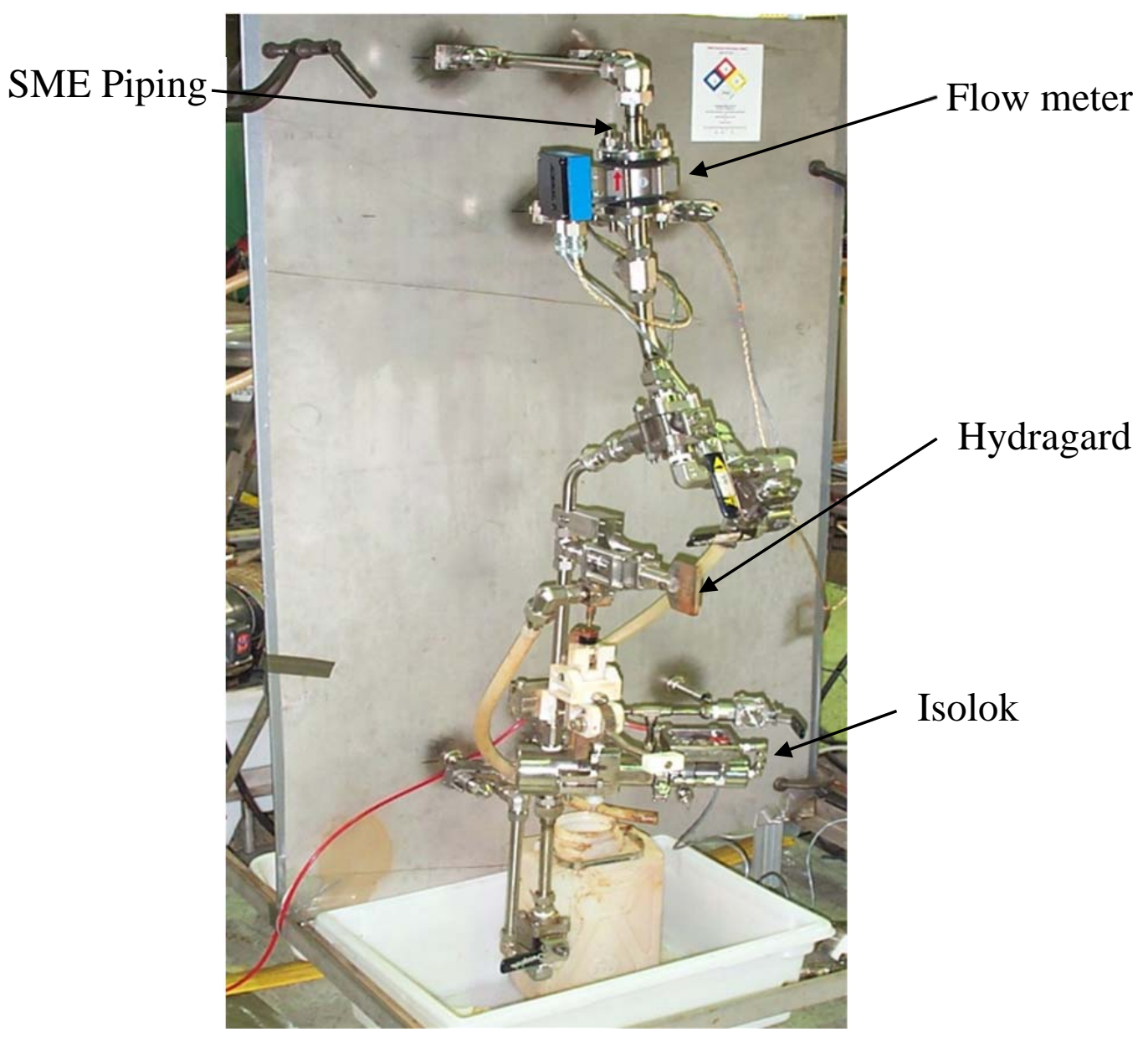

Figure 2. Test Setup

Table 2-1. Simulant Rheology Properties Data

\begin{tabular}{|r|c|l|}
\hline \multicolumn{3}{|c|}{ Low Rheology Simulant } \\
\hline Total Solids & 31.7 & $\mathrm{Wt} \%$ \\
\hline Insoluble Solids & 27.35 & $\mathrm{Wt} \%$ \\
\hline $\mathrm{pH}$ & 8.47 & \\
\hline Yield Stress (Up Curve) & 3.15 & Pascal \\
\hline Consistency (Up Curve) & 9.8 & $\mathrm{cP}$ \\
\hline Density & 1.2186 & $\mathrm{~g} / \mathrm{mL}$ \\
\hline
\end{tabular}

\begin{tabular}{|r|c|l|}
\hline \multicolumn{3}{|c|}{ Mid Rheology Simulant } \\
\hline Total Solids & 36.75 & $\mathrm{Wt} \%$ \\
\hline Insoluble Solids & 31.75 & $\mathrm{Wt} \%$ \\
\hline $\mathrm{pH}$ & 8.37 & \\
\hline $\begin{array}{c}\text { Yield Stress (Up } \\
\text { Curve) }\end{array}$ & 5 & Pascal \\
\hline Consistency (Up Curve) & 17 & $\mathrm{cP}$ \\
\hline Density & 1.27 & $\mathrm{~g} / \mathrm{mL}$ \\
\hline
\end{tabular}

\begin{tabular}{|r|c|l|}
\hline \multicolumn{3}{|c|}{ High Rheology Simulant } \\
\hline Total Solids & 41.8 & $\mathrm{Wt} \%$ \\
\hline Insoluble Solids & 30.7 & $\mathrm{Wt} \%$ \\
\hline $\mathrm{pH}$ & 3.46 & \\
\hline Yield Stress (Up Curve) & 23 & $\mathrm{Pascal}$ \\
\hline Consistency (Up Curve) & 51 & $\mathrm{cP}$ \\
\hline Density & 1.233 & $\mathrm{~g} / \mathrm{mL}$ \\
\hline
\end{tabular}




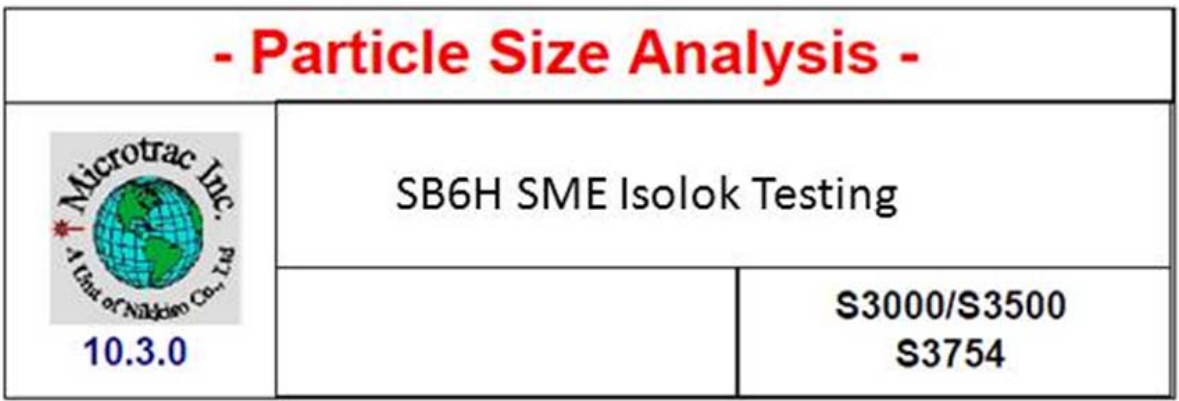

\begin{tabular}{|c|c|}
\hline Data Item & Value \\
\hline MV(um): & 140.9 \\
\hline MN(um): & 0.391 \\
\hline MA(um): & 3.94 \\
\hline CS: & 1.521 \\
\hline SD: & 85.15 \\
\hline & \\
\hline
\end{tabular}

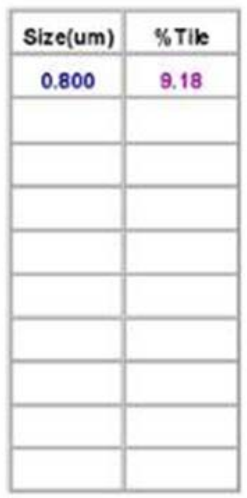

\begin{tabular}{|c|c|}
\hline$\%$ Tile & Size(um) \\
\hline 10.00 & 0.928 \\
\hline 16.00 & 42.30 \\
\hline 25.00 & 99.11 \\
\hline 40.00 & 135.2 \\
\hline 50.00 & 152.0 \\
\hline 60.00 & 167.5 \\
\hline 70.00 & 183.8 \\
\hline 75.00 & 192.8 \\
\hline 90.00 & 231.7 \\
\hline 95.00 & 258.0 \\
\hline
\end{tabular}

\begin{tabular}{|c|c|c|}
\hline Dia & Vol\% & Width \\
\hline 164.1 & 84.5 & 108.00 \\
\hline 0.656 & 15.5 & 1.84 \\
\hline & & \\
\hline & & \\
\hline & & \\
\hline & & \\
\hline
\end{tabular}

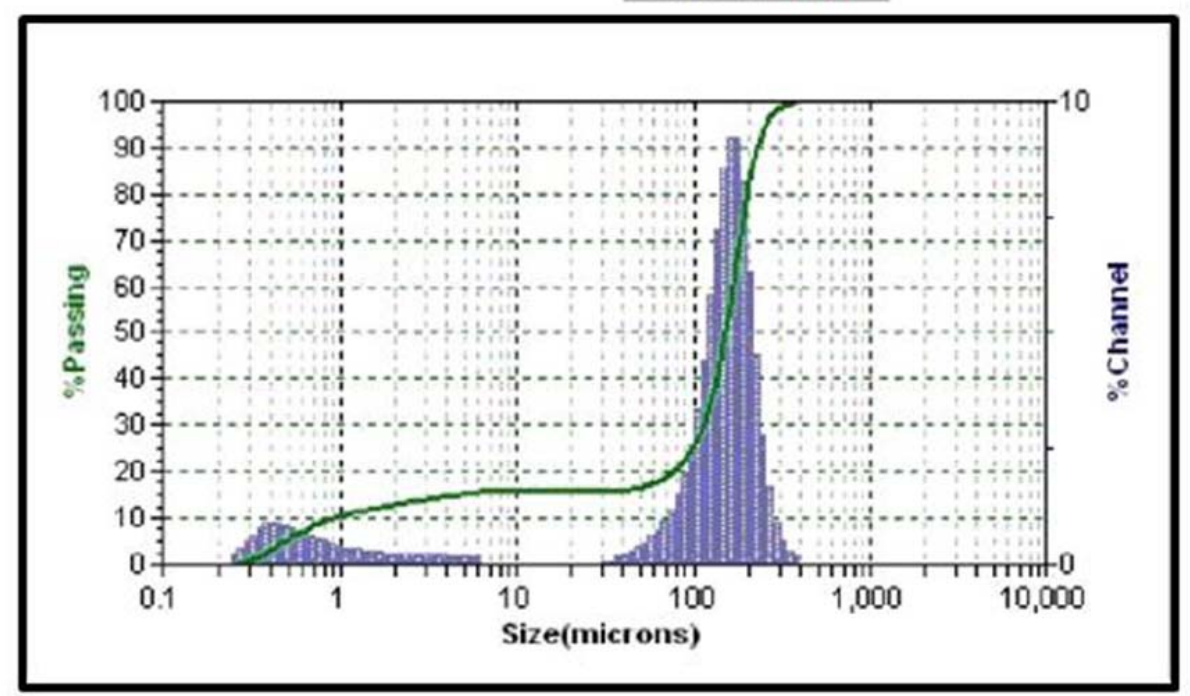

Figure 3. High Rheology Particle Size Data. 


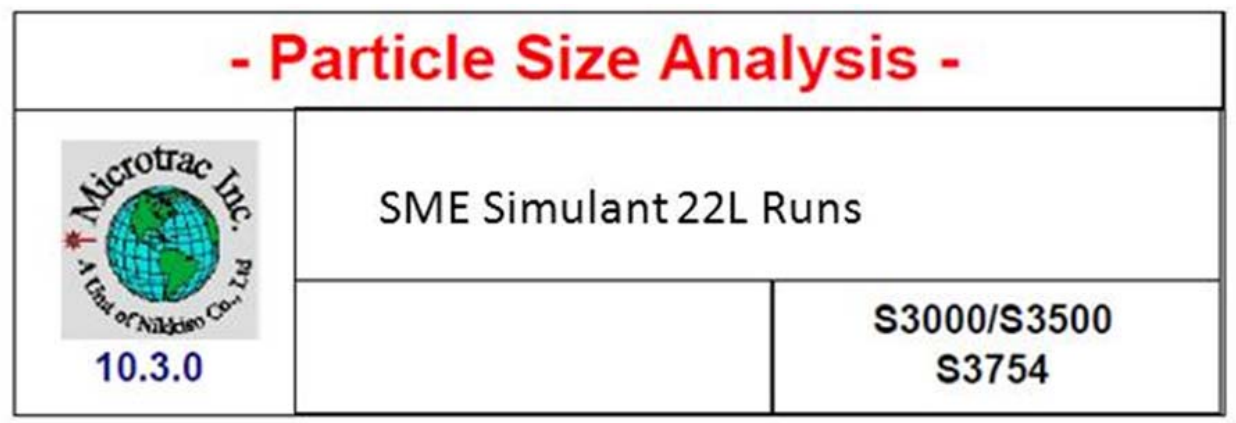

\begin{tabular}{|c|c|}
\hline Data Item & Value \\
\hline MV(um): & 120.6 \\
\hline MN(um): & 0.648 \\
\hline MA(um): & 5.81 \\
\hline CS: & 1.033 \\
\hline SD: & 96.59 \\
\hline & \\
\hline
\end{tabular}

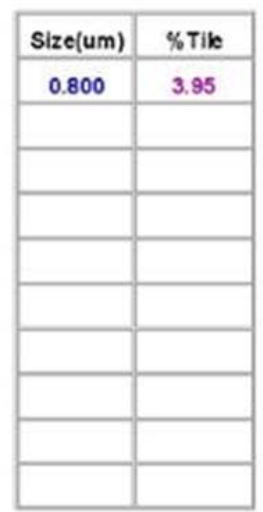

\begin{tabular}{|c|c|}
\hline$\%$ Tile & Size(um) \\
\hline 10.00 & 1.417 \\
\hline 16.00 & 3.01 \\
\hline 25.00 & 58.36 \\
\hline 40.00 & 111.6 \\
\hline 50.00 & 130.3 \\
\hline 60.00 & 147.1 \\
\hline 70.00 & 164.8 \\
\hline 75.00 & 174.6 \\
\hline 90.00 & 216.6 \\
\hline 95.00 & 245.6 \\
\hline
\end{tabular}

\begin{tabular}{|c|c|c|}
\hline Dia & Vol\% & Width \\
\hline 143.7 & 84.0 & 121.50 \\
\hline 1.184 & 16.0 & 1.35 \\
\hline & & \\
\hline & & \\
\hline & & \\
\hline & & \\
\hline
\end{tabular}

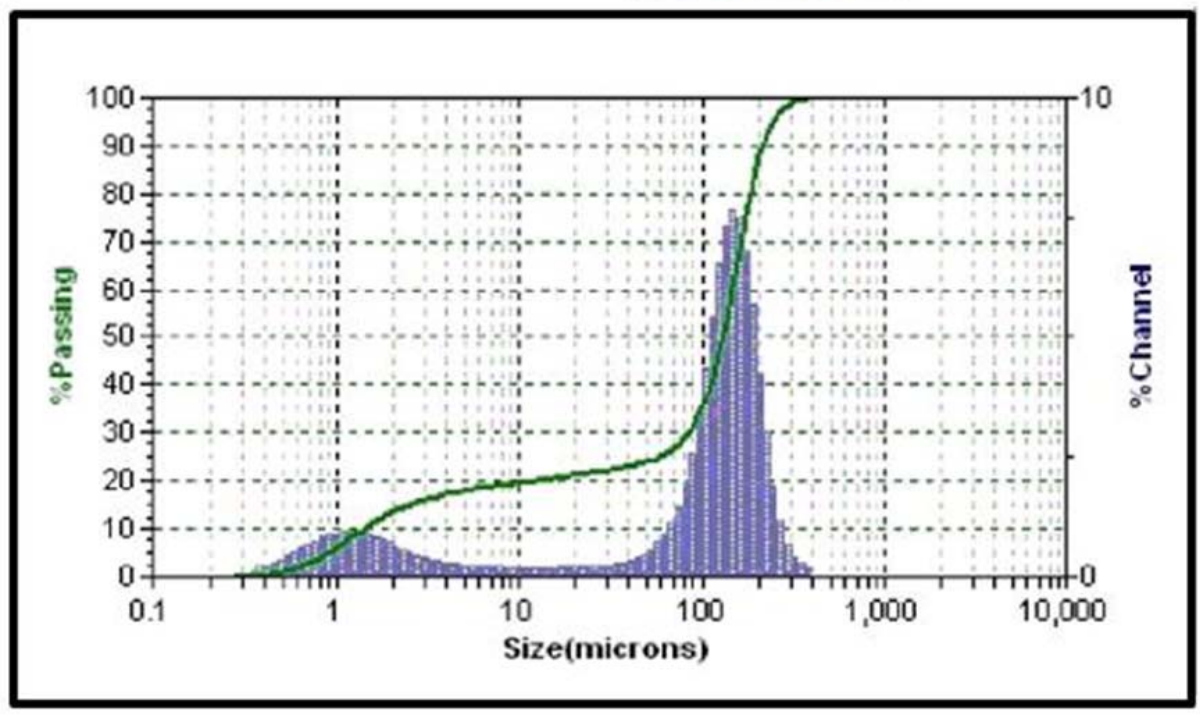

Figure 4. Mid and Low Rheology Particle Size Data. 


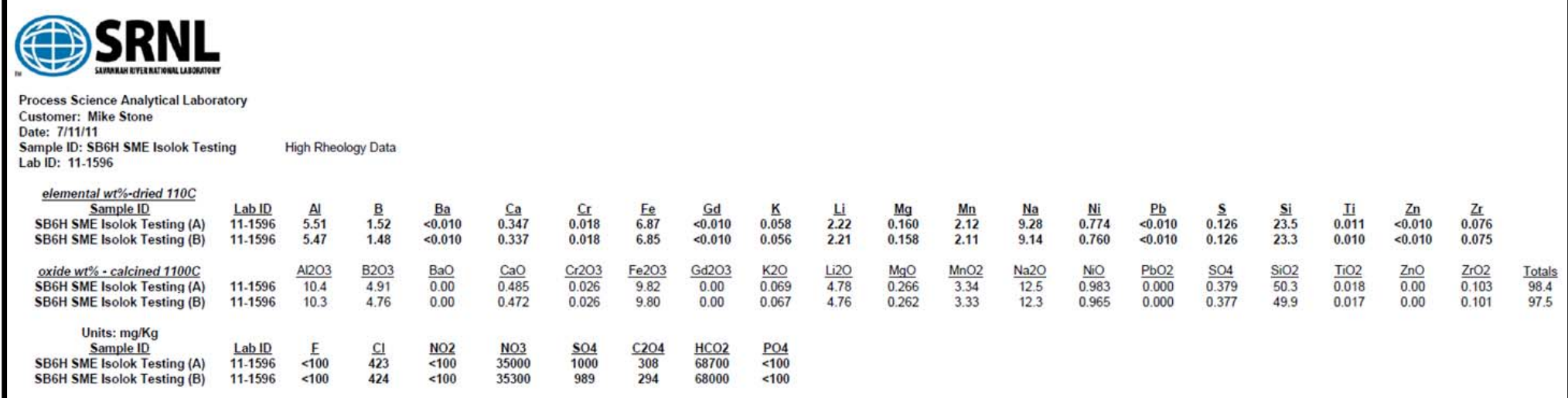

Table 2-3. Mid \& Low Rheology Simulant Elemental Composition

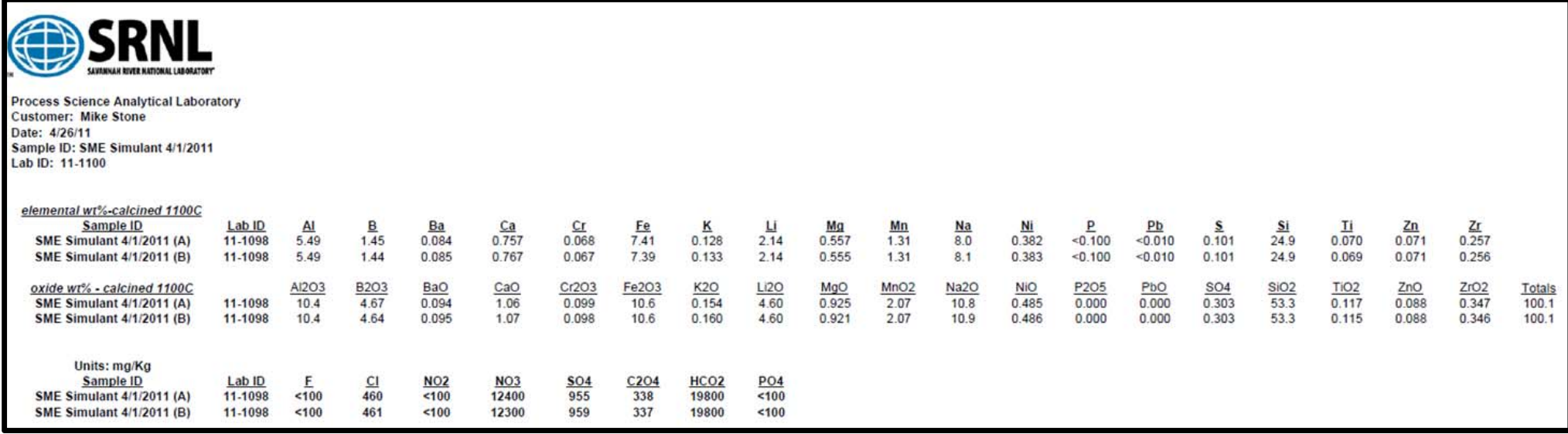

\subsection{RESULTS}

The objective of this study was to qualify the Isolok for use in sampling the SME for acceptability decisions at the DWPF. The purpose of SME sampling is to obtain representative samples of the contents of the SME tank. The samples are vitrified, and the compositions of the vitrified samples are measured analytically with the resulting measured glass compositions being assessed for acceptability through the use of DWPF's Product Composition Control System (PCCS). For the acceptability decision process to be reliably conducted, the contents of the SME need to be wellmixed and the samples need to be representative.

A well-mixed tank was also of critical importance for each phase of the testing conducted at 723-A. The question of the tank being well-mixed for one of the test phases was addressed using the Coliwasa samples, specifically, by comparing the samples taken at the low location in the tank to those taken at the high location in the tank during the test phase. The samples for the low location were considered to be the base-line (BL) samples as these comparisons were conducted. After the contents of the tank were established to be well-mixed, both sets (those taken from the low location in the tank and those taken from the high location) of Coliwasa samples were used to "represent" the contents of the tank and, thus, become the BL for other comparisons. That is, the performance 
of each of the other sampling mechanisms was assessed based upon how well its samples compared to those from the Coliwasa.

What types of comparisons were made between the BL measurements of an analyte of interest and the measurements from a sampling mechanism in question? There were two: (1) a comparison of the variances of the two sets of measurements and (2) a comparison of the means of the two sets of measurements. Tests for the statistical significance of the differences determined for the variances of the measurements and for the means were conducted as part of the investigations of this study. A difference between the means of the two sets of measurements represents a bias between the sampling mechanism and the BL. While some of these biases may be found to be statistically significant, their practical impact was also assessed as part of the conclusions of this study.

Information provided in DWPF's Waste Form Qualification Report, specifically, in Table 2 of WSRC-IM-91-116-2, Revision 4 (9) was used to facilitate the assessment of the comparisons conducted as part of this testing. The information provided in this table indicated that, in general, biases up to $\sim 4.6 \%$ on a relative basis had been determined for the errors in the reported chemical composition for slurry sampling during DWPF's Startup Test Program. Thus, in evaluating the performance of the Hydragard and Isolok sampler relative to the Coliwasa, the goal was that the biases seen in this testing would be less than $5 \%$ for the elements at concentrations in the simulant that would be considered reportable by the DWPF. From the discussion in Section 4 of WSRC-IM91-116-2, Revision 4 (9), an element at a concentration of $0.5 \mathrm{wt} \%$ or more is considered to be reportable.

The sampler currently utilized to support the SME acceptability process at DWPF is the Hydragard sampler, and the performance of the Isolok sampler for the testing conducted here was judged as acceptable if its performance was comparable to the performance of the Hydragard during the testing. The performance of the Hydragard was quantified for each test phase using statistical comparisons of the measurements of its samples to the measurements of the Coliwasa samples for analytes of interest. Similarly, the performance of the Isolok was quantified for each test phase using statistical comparisons of the measurements of its samples to the measurements of the Coliwasa samples for the analytes of interest. With these results in hand, the relative performance of the Isolok to that of the Hydragard was evaluated.

Assessments of the performance of the Hydragard and Isolok relative to the Coliwasa BL were made for the testing system under two different flow rates for the sample loop piping: a low flow rate ( 4 to 5 gallons per minute) and a high flow rate (9 to 10 gallons per minute). In addition, while the Hydragard system was used to provide sample volumes of approximately $12 \mathrm{~mL}$, the Isolok system was used to yield $3 \mathrm{~mL}$ and $12 \mathrm{~mL}$ sample volumes. The $3 \mathrm{~mL}$ samples were taken in platinum crucibles and the $12 \mathrm{~mL}$ samples were taken in p-nut vials (an insufficient number of crucibles was available to dispense all of the Isolok samples directly into crucibles, hence the need to take the $12 \mathrm{~mL}$ Isolok samples in p-nut vials). The Hydragard samples were taken in p-nut vials as is done now in the DWPF. Evaluations of the performance of the Hydragard samples, of the 3 $\mathrm{mL}$ Isolok samples, and of the $12 \mathrm{~mL}$ Isolok samples were conducted. Note that the $3 \mathrm{~mL}$ Isolok system was not used to provide measurements of density, one of the analytes of interest in this study, since the measurement protocol for density currently relies on the use of p-nut vials.

JMP Version 7.0.2 from SAS Institute, Inc. (10) was used to support this investigation and to make the comparisons between the samplers. In comparing two samplers, JMP was used to make a statistical comparison between the means of the two sets of measurements. In the discussion that follows the difference in the means and the percent difference relative to the mean of the BL set of measurements are presented. The statistical significance of the difference in the means was 
assessed based upon the standard deviations of the two sets of measurements. The JMP results include Levene's test which provides a comparison of the standard deviations of the two sets of measurements. Specifically, this test was used to assess the statistical significance of the difference between the variability of the two sets. If the p-value from Levene's test is $5 \%$ or smaller, the null hypothesis of equal variances for the two sets of measurements was rejected in favor of the alternative hypothesis of a difference in the variances.

The outcome of Levene's test provided guidance as to which of two t-tests was used to assess the statistical significance of the difference in the means for the two sets of measurements. Specifically, each set of JMP results included a t-test for a significant difference in the means under the assumption of equal variances for the two sets of measurements and a t-test for a significant difference in the means under the assumption of unequal variances. Both of these t-tests are presented as part of each set of JMP results, and the outcome from Levene's test was used to select the appropriate t-test. If the p-value for the selected t-test was $5 \%$ or smaller, then there was a statistically significant difference between the means of the two sets of measurements at the 5\% significance level.

Regardless of the statistical significance of the difference in the means, a bound on the difference was determined (with greater than 95\% confidence) from the 95\% confidence interval for the difference in the means from the selected t-test. Confidence intervals for the difference in the means were provided by JMP for both t-tests. The values of the endpoints of each interval were determined at 95\% confidence, and the larger endpoint-value (in absolute value) provides a bound on the difference in the means of the two sets of measurements with at least $95 \%$ confidence.

Some comments regarding the analytes of this study are provided. The elemental compositions of the vitrified samples were determined by ICP-OES and reported in $\mu$ g of element per $g$ of glass. As these elemental measurements were converted to wt\% oxide measurements (using the appropriate gravimetric factors), any elemental concentration value below the detection limit of the analytical process was reduced by half during the conversion. Also, please note that the values of two ratios, specifically, the ratio of $\mathrm{Al}_{2} \mathrm{O}_{3}$ to $\mathrm{B}_{2} \mathrm{O}_{3}$ (i.e., $\mathrm{Al} / \mathrm{B}$ ) and the ratio of $\mathrm{Fe}_{2} \mathrm{O}_{3}$ to $\mathrm{Li}_{2} \mathrm{O}$ (i.e., $\mathrm{Fe} / \mathrm{Li}$ ) were included as analytes in these investigations. These ratios helped track the relationship between the sludge and frit components of the SME simulant for the samplers. And, finally, the sum of oxides for each vitrified sample was determined and included as an analyte for this study even though this analyte is more of a reflection of the analytical process than of the sampling.

The statistical comparisons and significance tests outlined in the paragraphs above were repeatedly and consistently applied in making comparisons between samplers using the measurements generated by the three test phases. The results from these comparisons and tests are presented in sections 3.1 through 3.3 for each phase of testing. An effort was made to provide a consistent format for presenting the investigations into the measurements for each test phase. Section 3.4 provides a summary of the results across all test phases, and, finally, Section 3.5 provides results from the cycle testing of the Isolok.

\subsection{Mid-Rheology Simulant (Phase 1) Testing}

A mid-rheology simulant was used for Phase 1 of this testing. The density measurements, wt\% dried solids, wt\% vitrified solids, and elemental measurements (in two parts) for the Phase 1 testing are provided in Tables A1, A2, and A3, respectively, in Appendix A. In addition to the elemental measurement of the simulant, the two parts of Table A3 provide measurements of samples of the 
Analytical Reference Glass -1 (ARG-1) that were included in the analytical plan (5). ${ }^{f}$ The elemental concentrations were converted to oxide concentrations yielding the measurements in Table A4 of Appendix A. Table A5 in Appendix A provides the reference composition of the Phase 1 SME simulant.

To provide a general overview of the measurements from this phase of testing, plots of the density, solids, and oxide measurements are presented in Exhibit A1 in Appendix A. The groupings for these measurements include the two flow rates, and the sample IDs are also shown.

The JMP results for the statistical comparisons of these measurements as outlined above for each of the analytes of interest are provided in Exhibit A2 of Appendix A. Comparisons between the low and high located Coliwasa samples were conducted as well as comparisons between the collection of Coliwasa samples and samples from the other sampling mechanisms (at low and high flow rates for both the Hydragard and Isolok and using 3-and 12-mL sample volumes for the Isolok as described above).

A plot of the two sets of measurements is provided for each analyte as part of the JMP results in Exhibit A2. Superimposed on each set of measurements is a box plot. These boxplots served as the basis for screening the data for potential outliers. An immediate concern, as one works with real data, is the potential for the presence of one or more outliers in the data and the possible impact of such outliers on the conclusions being made about the data. Using the boxplots, those measurements for an analyte that are beyond the distances given by:

upper quartile $+1.5^{*}$ (interquartile range) and lower quartile $-1.5 *$ (interquartile range),

(based upon the quartiles computed from the set of measurements with the interquartile range being the difference between the 1st quartile and 3rd quartile) were considered as questionable points (i.e., potential outliers). These are the points that fall beyond the "whiskers" (the lines extended from the boxes) of the boxplots. Some points were considered as potential outliers based upon this approach, and these were excluded from the screened data for an analyte in the analyses below. The statistical comparisons were repeated for those situations where potential outliers were identified, and the JMP results from these investigations for the screened measurements are provided in Exhibit A3 of Appendix A.

A summary of the results from the JMP output of Exhibit A2 and Exhibit A3 is provided in Table A6, Table A7, Table A8, and Table A9 in Appendix A. The format for each of these tables is very similar. The means of the measurements for each set of samples is given, the difference between the sampler in question and the BL is given, the \% difference (relative to the BL mean) is given, a column used to flag a statistically significant difference (i.e., a bias at the $5 \%$ significance level) between the means is provided, the sample standard deviation for the measurements for the sampler in question is given, the sample standard deviation of the BL samples is given, a column used to flag a statistically significant difference (at the $5 \%$ significance level) between the variances is provided, a bound on the bias (at a 95\% confidence level) is given, the bound is also provided expressed as a percentage of the BL sample mean, the number of observations (n sampler) for the sampler in question is given, and, finally, the number of observations for the BL sampler (n BL) is given.

Table A6 provides the results of the comparisons for density, wt $\%$ dried solids, and wt $\%$ vitrified solids for all of the measurements and for the screened (scrnd) measurements. The \% differences

\footnotetext{
${ }^{f}$ A statistical evaluation of the ARG-1 results is to be provided in a separate report: SRNL-STI-2011-00711.
} 
for the means of the high- and low-located Coliwasa samples for density, wt\% dried solids, and wt $\%$ vitrified solids were $0.9 \%, 0.9 \%$, and $1.0 \%$, respectively. While the differences in the solids means were statistically significant at the $5 \%$ level, the bounds (at $95 \%$ confidence) on these differences and on the difference in the means of the density measurements were all less than $2 \%$. The results from these comparisons for density and solids between the high- and low-located Coliwasa samples suggested no issues with the tank being well-mixed. Comparisons for density and for solids between the Coliwasa (using the collection of both the high- and low-located samples as BL) and the other sampling mechanisms shown in Table A6 yielded \% differences (in absolute value) of no more than $1.1 \%$ for density, $1.5 \%$ for dried solids, and $2.6 \%$ for vitrified solids. Over all of these results, the performance of the Isolok sampler was comparable to that of the Hydragard. See Table 3-1 for a summary of the \% differences.

Table 3-1. \% Differences for the Screened Density and Solids Measurements from Phase 1 Testing

\begin{tabular}{|c|c|c|c|}
\hline Comparison & Density & wt \% dried solids & wt \% vitrified solids \\
\hline Coliwasa High Location vs Coliwasa Low Location (BL) & $0.9 \%$ & $0.9 \%$ & $1.0 \%$ \\
\hline Hydragard (HF) vs Coliwasa & $0.3 \%$ & $-1.1 \%$ & $-2.6 \%$ \\
\hline Hydragard (LF) vs Coliwasa & $0.7 \%$ & $-1.0 \%$ & $-2.1 \%$ \\
\hline Isolok (12; LF) vs Coliwasa & $-0.2 \%$ & $-1.5 \%$ & $-2.2 \%$ \\
\hline Isolok (12; HF) vs Coliwasa & $0.8 \%$ & $0.2 \%$ & $-0.1 \%$ \\
\hline Isolok (3; LF) vs Coliwasa & na & $-1.4 \%$ & $-2.1 \%$ \\
\hline Isolok (3; HF) vs Coliwasa & na & $0.6 \%$ & $0.1 \%$ \\
\hline
\end{tabular}

Table A7 provides comparisons of the oxide measurements from the low-located and high-located Coliwasa samples for all of the data and for the screened data. The analytical plan stipulated that the Coliwasa samples that were prepared (using the cesium carbonate fusion dissolution method) were to be measured in each of six different analytical blocks. The oxide measurements were averaged over the six analytical blocks to provide the data for comparing the high- and low-located Coliwasa results. All of the \% differences (in absolute value) were less than 5\% except for $\mathrm{Cr}_{2} \mathrm{O}_{3}$ (5.5\% for all of the data) and for the $\mathrm{Fe} / \mathrm{Li}(5.0 \%$ for the screened data). The results from these comparisons for the oxide measurements between the high- and low-located Coliwasa samples suggested no issues with the tank being well-mixed.

Tables A8 and A9 provide comparisons between the Coliwasa measurements and the other sampler mechanisms arranged by analytical block for all of the measurements and for the screened measurements, respectively. For each ICP-OES block, measurements were made of the Coliwasa samples (both the high- and low-located samples) and of a set of samples from a sampler mechanism in question. The JMP comparisons were conducted using the ICP-OES analytical blocks for grouping the measurements to be compared. For example, analytical block 1 involved the Coliwasa samples and the $3 \mathrm{~mL}$ samples from the Isolok under low flow rate conditions. Table A10 in Appendix A provides the same information as Table A9, but with the results grouped by analyte and sorted by the size of the bound (from the largest to the smallest) on the bias between the two sampling systems. The entries for the \% bias and for \% relative bias bound that are less than $5 \%$ are shaded in green in this table. See Table 3-2 for a summary of the \% differences determined for the oxide measurements from Phase 1 . There are very few values for the \% differences that are $5 \%$ or more. 
Table 3-2. \% Differences for Screened Oxide Measurements from Phase 1 Testing

\begin{tabular}{|c|c|c|c|c|c|c|c|}
\hline Oxide & $\begin{array}{c}\text { High vs Low } \\
\text { Coliwasa }\end{array}$ & $\begin{array}{c}\text { Isolok- } \\
\text { LFR-3mL }\end{array}$ & $\begin{array}{c}\text { Hydragard } \\
\text { - HFR }\end{array}$ & $\begin{array}{c}\text { Isolok- } \\
\text { LFR-12mL }\end{array}$ & $\begin{array}{c}\text { Isolok- } \\
\text { HFR-12mL }\end{array}$ & $\begin{array}{c}\text { Isolok- } \\
\text { HFR-3mL }\end{array}$ & $\begin{array}{c}\text { Hydragard- } \\
\text { LFR }\end{array}$ \\
\hline $\mathrm{Al} / \mathrm{B}$ & $-1.2 \%$ & $5.9 \%$ & $4.9 \%$ & $-0.3 \%$ & $-2.4 \%$ & $-2.1 \%$ & $0.4 \%$ \\
\hline $\mathrm{Al}_{2} \mathrm{O}_{3}$ & $-3.6 \%$ & $1.5 \%$ & $3.3 \%$ & $2.8 \%$ & $-0.2 \%$ & $-1.5 \%$ & $3.0 \%$ \\
\hline $\mathrm{B}_{2} \mathrm{O}_{3}$ & $-1.5 \%$ & $-3.4 \%$ & $-0.7 \%$ & $4.9 \%$ & $2.8 \%$ & $1.5 \%$ & $3.8 \%$ \\
\hline $\mathrm{BaO}$ & $-3.1 \%$ & $2.2 \%$ & $2.1 \%$ & $4.0 \%$ & $0.6 \%$ & $-0.6 \%$ & $2.9 \%$ \\
\hline $\mathrm{CaO}$ & $-4.3 \%$ & $1.7 \%$ & $3.2 \%$ & $4.6 \%$ & $0.3 \%$ & $-0.5 \%$ & $4.0 \%$ \\
\hline $\mathrm{Cr}_{2} \mathrm{O}_{3}$ & $-5.5 \%$ & $-1.9 \%$ & $1.2 \%$ & $2.2 \%$ & $0.2 \%$ & $-3.8 \%$ & $-0.4 \%$ \\
\hline $\mathrm{CuO}$ & $-2.4 \%$ & $1.3 \%$ & $0.1 \%$ & $4.3 \%$ & $7.2 \%$ & $1.2 \%$ & $1.3 \%$ \\
\hline $\mathrm{Fe} / \mathrm{Li}$ & $-5.0 \%$ & $2.2 \%$ & $1.8 \%$ & $1.4 \%$ & $-1.4 \%$ & $-3.4 \%$ & $0.6 \%$ \\
\hline $\mathrm{Fe} \mathrm{O}_{3}$ & $-3.5 \%$ & $1.6 \%$ & $2.4 \%$ & $3.4 \%$ & $-0.9 \%$ & $-0.6 \%$ & $2.8 \%$ \\
\hline $\mathrm{K}_{2} \mathrm{O}$ & $-1.1 \%$ & $1.5 \%$ & $0.4 \%$ & $4.2 \%$ & $5.4 \%$ & $1.2 \%$ & $1.9 \%$ \\
\hline $\mathrm{Li} \mathrm{O}_{2} \mathrm{O}$ & $0.4 \%$ & $-0.1 \%$ & $0.4 \%$ & $0.8 \%$ & $-0.1 \%$ & $2.0 \%$ & $2.8 \%$ \\
\hline $\mathrm{MgO}$ & $-3.3 \%$ & $1.6 \%$ & $1.7 \%$ & $3.7 \%$ & $-0.9 \%$ & $-0.8 \%$ & $4.2 \%$ \\
\hline $\mathrm{MnO}^{\mathrm{NaO} O} \%$ & $-3.6 \%$ & $2.0 \%$ & $2.1 \%$ & $3.9 \%$ & $-0.8 \%$ & $-0.7 \%$ & $3.3 \%$ \\
\hline $\mathrm{Na}_{2} \mathrm{O}$ & $-1.9 \%$ & $-0.5 \%$ & $0.8 \%$ & $2.2 \%$ & $-2.9 \%$ & $-3.0 \%$ & $2.4 \%$ \\
\hline $\mathrm{NiO}_{\mathrm{SiO}}$ & $-3.8 \%$ & $1.7 \%$ & $2.8 \%$ & $3.5 \%$ & $-1.7 \%$ & $-1.3 \%$ & $2.5 \%$ \\
\hline $\mathrm{TiO}_{2}$ & $2.2 \%$ & $0.0 \%$ & $-0.9 \%$ & $0.8 \%$ & $1.4 \%$ & $2.0 \%$ & $0.5 \%$ \\
\hline $\mathrm{ZnO}$ & $1.4 \%$ & $-0.6 \%$ & $-1.0 \%$ & $0.4 \%$ & $0.0 \%$ & $2.0 \%$ & $0.3 \%$ \\
\hline $\mathrm{ZrO} 2$ & $-4.2 \%$ & $0.8 \%$ & $2.7 \%$ & $4.8 \%$ & $0.1 \%$ & $-0.6 \%$ & $3.3 \%$ \\
\hline $\mathrm{Sum}$ & $-1.8 \%$ & $0.7 \%$ & $1.7 \%$ & $2.3 \%$ & $-0.7 \%$ & $0.2 \%$ & $2.0 \%$ \\
\hline
\end{tabular}

\subsection{High-Rheology Simulant (Phase 2) Testing}

The investigations into the measurements of the Phase 2, high-rheology, testing followed the same pattern as that conducted for the Phase 1 results. The density measurements, wt $\%$ dried solids, wt $\%$ vitrified solids, and elemental measurements (in two parts) for the Phase 2 testing are provided in Tables B1, B2, and B3, respectively, in Appendix B. In addition to the oxide measurement of the simulant, the two parts of Table B3 provide measurements of the ARG-1 samples that were included in the analytical plan (5). ${ }^{f}$ The elemental concentrations were converted to oxide concentrations yielding the measurements in Table B4 of Appendix B. Table B5 in Appendix B provides the reference composition of the Phase 2 SME simulant.

To provide a general overview of the measurements from this phase of testing, plots of the density, solids, and oxide measurements are presented in Exhibit B1 in Appendix B. The groupings for these measurements include the two flow rates, and the sample IDs are also shown.

The JMP results for the statistical comparisons of these measurements as outlined above for each of the analytes of interest are provided in Exhibit B2 of Appendix B. As for the Phase 1 results, comparisons between the low and high located Coliwasa samples are conducted as well as comparisons between the collection of Coliwasa samples and samples from the other sampling mechanisms (at low and high flow rates for both the Hydragard and Isolok and using 3-and 12-mL sample volumes for the Isolok as described above). Box plots are included in the JMP results, and these are used to conduct a screening process for outliers as was done for the Phase 1 results.

A summary of the results from the JMP output is provided in Table B6, Table B7, Table B8, and Table B9 in Appendix B. The format for each of these tables is similar the tables prepared for the Phase 1 results. Table B6 provides the results of the comparisons for density, wt $\%$ dried solids, and

\footnotetext{
${ }^{f}$ A statistical evaluation of the ARG-1 results is to be provided in a separate report: SRNL-STI-2011-00711.
} 
wt\% vitrified solids for all of the measurements and for the screened (scrnd) measurements. The \% differences for the means of the high- and low-located Coliwasa samples for density, wt $\%$ dried solids, and wt $\%$ vitrified solids are $-0.2 \%,-0.3 \%$, and $-0.4 \%$, respectively. While the differences in the solids means are statistically significant at the $5 \%$ level, the bounds (at $95 \%$ confidence) on these differences and on the difference in the means of the density measurements are all less than $2 \%$. The results from these comparisons for density and solids between the high- and low-located Coliwasa samples suggested no issues with the tank being well-mixed. Comparisons for density and for solids between the Coliwasa (using the collection of both the high- and low-located samples as BL) and the other sampling mechanisms shown in Table B6 yields \% differences (in absolute value) of no more than $0.7 \%$ for density, $2.4 \%$ for dried solids, and $1.8 \%$ for vitrified solids. Over all of these results, the performance of the Isolok sampler is comparable to that of the Hydragard. See Table 3-3 for a complete listing of these \% differences.

Table 3-3. \% Differences for the Screened Density and Solids Measurements from Phase 2 Testing

\begin{tabular}{|c|c|c|c|}
\hline Comparison & Density & wt\% dried solids & wt \% vitrified solids \\
\hline Coliwasa High Location vs Coliwasa Low Location (BL) & $-0.2 \%$ & $-0.3 \%$ & $-0.4 \%$ \\
\hline Hydragard (HF) vs Coliwasa & $0.0 \%$ & $-1.2 \%$ & $-1.6 \%$ \\
\hline Hydragard (LF) vs Coliwasa & $0.4 \%$ & $-0.2 \%$ & $0.0 \%$ \\
\hline Isolok (12; LF) vs Coliwasa & $-0.4 \%$ & $0.8 \%$ & $-0.4 \%$ \\
\hline Isolok (12; HF) vs Coliwasa & $0.5 \%$ & $1.9 \%$ & $1.5 \%$ \\
\hline Isolok (3; LF) vs Coliwasa & na & $-0.1 \%$ & $-0.9 \%$ \\
\hline Isolok (3; HF) vs Coliwasa & na & $2.3 \%$ & $1.7 \%$ \\
\hline
\end{tabular}

Table B7 provides comparisons of the oxide measurements from the low-located and high-located Coliwasa samples for all of the data and for the screened data. The analytical plan stipulated that the Coliwasa samples that were prepared (using the cesium carbonate fusion dissolution method) were to be measured in each of six different analytical blocks. The oxide measurements were averaged over the six analytical blocks to provide the data for comparing the high- and low-located Coliwasa results. All of the \% differences (in absolute value) are less than $5 \%$ except for $\mathrm{TiO}_{2}$ (6.6\% for the screened) and for the $\mathrm{ZnO}$ (10.9\% for all the data). The results from these comparisons for the oxide measurements between the high- and low-located Coliwasa samples suggested no issues with the tank being well-mixed.

Tables B8 and B9 provide comparisons between the Coliwasa measurements and the other sampler mechanisms arranged by analytical block for all of the measurements and for the screened measurements, respectively. For each ICP-OES block, measurements were made of the Coliwasa samples (both the high- and low-located samples) and of a set of samples from a sampler mechanism in question. The JMP comparisons were conducted using the ICP-OES analytical blocks for grouping the measurements to be compared. For example, analytical block 1 involved the Coliwasa samples and the $3 \mathrm{~mL}$ samples from the Isolok under low flow rate conditions. Table B10 in Appendix B provides the same information as Table B9, but with the results grouped by analyte and sorted by the size of the bound (from the largest to the smallest) on the bias between the two sampling systems. The entries for the \% bias and for \% relative bias bound that are less than 5\% are shaded in green in this table. See Table 3-4 for a summary of the \% differences determined for the oxide measurements from Phase 1 . There are very few values for the \% differences that are $5 \%$ or more. While there are a few large entries in this table, these entries are associated with oxides that are at very low concentrations (less than $0.25 \mathrm{wt} \%$ ) in the Phase 2 simulant. 
Table 3-4. \% Differences for Screened Oxide Measurements from Phase 2 Testing

\begin{tabular}{|c|c|c|c|c|c|c|c|}
\hline Oxide & $\begin{array}{c}\text { High vs Low } \\
\text { Coliwasa }\end{array}$ & $\begin{array}{c}\text { Isolok- } \\
\text { LFR-3mL }\end{array}$ & $\begin{array}{c}\text { Hydragard } \\
\text { - HFR }\end{array}$ & $\begin{array}{c}\text { Isolok- } \\
\text { LFR-12mL }\end{array}$ & $\begin{array}{c}\text { Isolok- } \\
\text { HFR-12mL }\end{array}$ & $\begin{array}{c}\text { Isolok- } \\
\text { HFR-3mL }\end{array}$ & $\begin{array}{c}\text { Hydragard- } \\
\text { LFR }\end{array}$ \\
\hline $\mathrm{Al} / \mathrm{B}$ & $-0.3 \%$ & $6.9 \%$ & $9.5 \%$ & $6.0 \%$ & $4.6 \%$ & $0.6 \%$ & $5.0 \%$ \\
\hline $\mathrm{Al}_{2} \mathrm{O}_{3}$ & $-1.8 \%$ & $3.6 \%$ & $4.5 \%$ & $2.1 \%$ & $-0.4 \%$ & $-0.3 \%$ & $1.4 \%$ \\
\hline $\mathrm{B}_{2} \mathrm{O}_{3}$ & $-1.5 \%$ & $-2.4 \%$ & $-5.4 \%$ & $-4.7 \%$ & $-5.2 \%$ & $-1.1 \%$ & $-2.9 \%$ \\
\hline $\mathrm{BaO}$ & $0.3 \%$ & $0.0 \%$ & $2.3 \%$ & $201.3 \%$ & $132.1 \%$ & $1.8 \%$ & $113.5 \%$ \\
\hline $\mathrm{CaO}$ & $-1.6 \%$ & $2.3 \%$ & $2.1 \%$ & $1.3 \%$ & $-1.3 \%$ & $-1.8 \%$ & $0.3 \%$ \\
\hline $\mathrm{Cr}_{2} \mathrm{O}_{3}$ & $2.2 \%$ & $3.2 \%$ & $19.3 \%$ & $1.9 \%$ & $32.0 \%$ & $14.4 \%$ & $-6.6 \%$ \\
\hline $\mathrm{CuO}$ & $-3.8 \%$ & $-3.9 \%$ & $-0.1 \%$ & $-2.5 \%$ & $-6.3 \%$ & $-5.2 \%$ & $-1.1 \%$ \\
\hline $\mathrm{Fe} / \mathrm{Li}$ & $-2.3 \%$ & $3.5 \%$ & $7.8 \%$ & $0.4 \%$ & $-0.9 \%$ & $-1.2 \%$ & $0.3 \%$ \\
\hline $\mathrm{Fe}_{2} \mathrm{O}_{3}$ & $-1.8 \%$ & $3.3 \%$ & $5.8 \%$ & $1.9 \%$ & $-0.2 \%$ & $0.1 \%$ & $0.9 \%$ \\
\hline $\mathrm{K}_{2} \mathrm{O}$ & $0.9 \%$ & $-3.0 \%$ & $-1.5 \%$ & $-0.9 \%$ & $-3.7 \%$ & $-0.1 \%$ & $-0.6 \%$ \\
\hline $\mathrm{Li}_{2} \mathrm{O}$ & $0.5 \%$ & $0.2 \%$ & $-2.3 \%$ & $0.7 \%$ & $0.7 \%$ & $1.9 \%$ & $-0.2 \%$ \\
\hline $\mathrm{MgO}$ & $-2.2 \%$ & $4.1 \%$ & $2.9 \%$ & $1.5 \%$ & $-1.6 \%$ & $-0.6 \%$ & $0.6 \%$ \\
\hline $\mathrm{MnO}$ & $-1.6 \%$ & $4.6 \%$ & $4.2 \%$ & $1.7 \%$ & $-2.0 \%$ & $0.5 \%$ & $0.0 \%$ \\
\hline $\mathrm{Na}_{2} \mathrm{O}$ & $-1.3 \%$ & $0.4 \%$ & $-0.9 \%$ & $1.3 \%$ & $-0.4 \%$ & $-0.7 \%$ & $0.1 \%$ \\
\hline $\mathrm{NiO}$ & $-1.2 \%$ & $3.1 \%$ & $5.8 \%$ & $1.0 \%$ & $-1.8 \%$ & $1.2 \%$ & $-0.6 \%$ \\
\hline $\mathrm{SiO}_{2}$ & $0.0 \%$ & $1.0 \%$ & $-2.2 \%$ & $-2.0 \%$ & $1.2 \%$ & $1.3 \%$ & $-0.8 \%$ \\
\hline $\mathrm{TiO}_{2}$ & $6.6 \%$ & $-3.7 \%$ & $9.7 \%$ & $-1.4 \%$ & $-1.4 \%$ & $1.5 \%$ & $-1.6 \%$ \\
\hline $\mathrm{ZnO}$ & $0.4 \%$ & $-3.0 \%$ & $-1.0 \%$ & $-0.6 \%$ & $-3.6 \%$ & $-1.1 \%$ & $-1.0 \%$ \\
\hline $\mathrm{ZrO}_{2}$ & $0.1 \%$ & $4.0 \%$ & $4.6 \%$ & $0.6 \%$ & $0.1 \%$ & $-1.1 \%$ & $0.4 \%$ \\
\hline Sum & $-0.7 \%$ & $1.1 \%$ & $-0.3 \%$ & $0.0 \%$ & $0.1 \%$ & $0.7 \%$ & $-0.6 \%$ \\
\hline
\end{tabular}

\subsection{Low-Rheology Simulant (Phase 3) Testing}

The investigations into the measurements of the Phase 3, low-rheology, testing followed the same pattern as that conducted for the results from the other two phases. The density measurements, wt $\%$ dried solids, wt\% vitrified solids, and elemental measurements (in two parts) for the Phase 3 testing are provided in Tables C1, C2, and C3, respectively, in Appendix C. In addition to the oxide measurement of the simulant, the two parts of Table C3 provide measurements of the ARG-1 samples that were included in the analytical plan (5). ${ }^{f}$ The elemental concentrations were converted to oxide concentrations yielding the measurements in Table C4 of Appendix C. Table C5 in Appendix C provides the reference composition of the Phase 3 SME simulant.

To provide a general overview of the measurements from this phase of testing, plots of the density, solids, and oxide measurements are presented in Exhibit C1 in Appendix C. The groupings for these measurements include the two flow rates, and the sample IDs are also shown.

The JMP results for the statistical comparisons of these measurements as outlined above for each of the analytes of interest are provided in Exhibit C2 of Appendix C. As for the results for the earlier phases, comparisons between the low and high located Coliwasa samples were conducted as well as comparisons between the collection of Coliwasa samples and samples from the other sampling mechanisms (at low and high flow rates for both the Hydragard and Isolok and using 3-and 12-mL sample volumes for the Isolok as described above). Box plots are included in the JMP results, and these are used to conduct a screening process for outliers as was done for the previous phases.

\footnotetext{
${ }^{f}$ A statistical evaluation of the ARG-1 results is to be provided in a separate report: SRNL-STI-2011-00711.
} 
A summary of the results from the JMP output is provided in Table C6, Table C7, Table C8, and Table C9 in Appendix C. The format for each of these tables is similar the tables prepared for the results from the earlier phases. Table C6 provides the results of the comparisons for density, wt\% dried solids, and wt\% vitrified solids for all of the measurements and for the screened (scrnd) measurements. The \% differences for the means of the high- and low-located Coliwasa samples for density, wt $\%$ dried solids, and wt $\%$ vitrified solids were $-0.7 \%,-0.2 \%$, and $-0.2 \%$, respectively. The bounds (at 95\% confidence) on these differences and on the difference in the means of the density measurements are all no more than $1.5 \%$. The results from these comparisons for density and solids between the high- and low-located Coliwasa samples suggested no issues with the tank being well-mixed. Comparisons for density and for solids between the Coliwasa (using the collection of both the high- and low-located samples as BL) and the other sampling mechanisms shown in Table C6 yielded \% differences (in absolute value) of no more than $0.4 \%$ for density, $2.6 \%$ for dried solids, and 3.2\% for vitrified solids. Over all of these results, the performance of the Isolok sampler was comparable to that of the Hydragard. See Table 3-5 for a complete listing of these \% differences.

\section{Table 3-5. \% Differences for the Screened Density and Solids Measurements from Phase 3 Testing}

\begin{tabular}{|c|c|c|c|}
\hline Comparison & Density & wt \% dried solids & wt $\%$ vitrified solids \\
\hline Coliwasa High Location vs Coliwasa Low Location (BL) & $-0.7 \%$ & $-0.2 \%$ & $-0.2 \%$ \\
\hline Hydragard (HF) vs Coliwasa & $0.0 \%$ & $-2.6 \%$ & $-3.2 \%$ \\
\hline Hydragard (LF) vs Coliwasa & $-0.4 \%$ & $-2.2 \%$ & $-3.0 \%$ \\
\hline Isolok (12; LF) vs Coliwasa & $0.2 \%$ & $0.1 \%$ & $0.0 \%$ \\
\hline Isolok (12; HF) vs Coliwasa & $-0.4 \%$ & $-0.1 \%$ & $0.0 \%$ \\
\hline Isolok (3; LF) vs Coliwasa & na & $0.2 \%$ & $0.2 \%$ \\
\hline Isolok (3; HF) vs Coliwasa & na & $0.0 \%$ & $0.6 \%$ \\
\hline
\end{tabular}

Table C7 provides comparisons of the oxide measurements from the low-located and high-located Coliwasa samples for all of the data and for the screened data. The analytical plan stipulated that the Coliwasa samples that were prepared (using the cesium carbonate fusion dissolution method) were to be measured in each of six different analytical blocks. The oxide measurements were averaged over the six analytical blocks to provide the data for comparing the high- and low-located Coliwasa results. All of the \% differences (in absolute value) are less than $5 \%$ except for $\mathrm{Cr}_{2} \mathrm{O}_{3}$ (-6.2\% for all of the data) and for the $\mathrm{K}_{2} \mathrm{O}(-5.0 \%$ for the screened data). The results from these comparisons for the oxide measurements between the high- and low-located Coliwasa samples suggested no issues with the tank being well-mixed.

Tables C8 and C9 provide comparisons between the Coliwasa measurements and the other sampler mechanisms arranged by analytical block for all of the measurements and for the screened measurements, respectively. For each ICP-OES block, measurements were made of the Coliwasa samples (both the high- and low-located samples) and of a set of samples from a sampler mechanism in question. The JMP comparisons were conducted using the ICP-OES analytical blocks for grouping the measurements to be compared. For example, analytical block 1 involved the Coliwasa samples and the $3 \mathrm{~mL}$ samples from the Isolok under low flow rate conditions. Table C10 in Appendix C provides the same information as Table C9, but with the results grouped by analyte and sorted by the size of the bound (from the largest to the smallest) on the bias between the two sampling systems. The entries for the \% bias and for \% relative bias bound that are less than $5 \%$ are shaded in green in this table. See Table 3-6 for a summary of the \% differences determined for the oxide measurements from Phase 3 . There are very few values for the \% 
differences that are 5\% or more with the Hydragard results for the high flow rate testing being above the $5 \%$ value most often.

Table 3-6. \% Differences for Screened Oxide Measurements from Phase 3 Testing

\begin{tabular}{|c|c|c|c|c|c|c|c|}
\hline Oxide & $\begin{array}{c}\text { High vs Low } \\
\text { Coliwasa }\end{array}$ & $\begin{array}{c}\text { Isolok- } \\
\text { LFR-3mL }\end{array}$ & $\begin{array}{c}\text { Hydragard } \\
\text { - HFR }\end{array}$ & $\begin{array}{c}\text { Isolok- } \\
\text { LFR-12mL }\end{array}$ & $\begin{array}{c}\text { Isolok- } \\
\text { HFR-12mL }\end{array}$ & $\begin{array}{c}\text { Isolok- } \\
\text { HFR-3mL }\end{array}$ & $\begin{array}{c}\text { Hydragard- } \\
\text { LFR }\end{array}$ \\
\hline $\mathrm{Al} / \mathrm{B}$ & $-0.7 \%$ & $-0.1 \%$ & $8.9 \%$ & $-1.7 \%$ & $0.6 \%$ & $1.4 \%$ & $3.8 \%$ \\
\hline $\mathrm{Al}_{2} \mathrm{O}_{3}$ & $0.1 \%$ & $-1.2 \%$ & $4.7 \%$ & $-2.9 \%$ & $-2.3 \%$ & $-0.7 \%$ & $2.0 \%$ \\
\hline $\mathrm{B}_{2} \mathrm{O}_{3}$ & $0.9 \%$ & $-0.9 \%$ & $-5.1 \%$ & $-0.8 \%$ & $-1.4 \%$ & $-2.1 \%$ & $-1.8 \%$ \\
\hline $\mathrm{BaO}$ & $-0.4 \%$ & $-2.4 \%$ & $4.2 \%$ & $-4.0 \%$ & $-2.7 \%$ & $-0.5 \%$ & $1.4 \%$ \\
\hline $\mathrm{CaO}$ & $0.3 \%$ & $-2.7 \%$ & $5.1 \%$ & $-3.1 \%$ & $-3.1 \%$ & $-0.8 \%$ & $2.2 \%$ \\
\hline $\mathrm{Cr}_{2} \mathrm{O}_{3}$ & $-6.2 \%$ & $-14.3 \%$ & $8.3 \%$ & $-3.8 \%$ & $10.3 \%$ & $-5.2 \%$ & $1.8 \%$ \\
\hline $\mathrm{CuO}$ & $-2.2 \%$ & $5.3 \%$ & $1.9 \%$ & $6.0 \%$ & $4.0 \%$ & $5.2 \%$ & $-4.3 \%$ \\
\hline $\mathrm{Fe} / \mathrm{Li}$ & $-1.2 \%$ & $-1.0 \%$ & $7.5 \%$ & $-8.0 \%$ & $-2.0 \%$ & $-3.2 \%$ & $4.1 \%$ \\
\hline $\mathrm{Fe}_{2} \mathrm{O}_{3}$ & $-0.4 \%$ & $-1.6 \%$ & $5.1 \%$ & $-4.4 \%$ & $-2.8 \%$ & $-1.5 \%$ & $1.5 \%$ \\
\hline $\mathrm{K}_{2} \mathrm{O}$ & $-5.0 \%$ & $1.5 \%$ & $-1.1 \%$ & $1.4 \%$ & $-0.9 \%$ & $1.9 \%$ & $-0.5 \%$ \\
\hline $\mathrm{Li}_{2} \mathrm{O}$ & $1.8 \%$ & $-0.1 \%$ & $-2.2 \%$ & $4.1 \%$ & $1.0 \%$ & $1.8 \%$ & $-2.5 \%$ \\
\hline $\mathrm{MgO}$ & $0.4 \%$ & $-2.5 \%$ & $5.5 \%$ & $-6.1 \%$ & $-3.6 \%$ & $-2.1 \%$ & $1.3 \%$ \\
\hline $\mathrm{MnO}$ & $-0.1 \%$ & $-2.5 \%$ & $5.2 \%$ & $-5.8 \%$ & $-3.7 \%$ & $-2.1 \%$ & $0.9 \%$ \\
\hline $\mathrm{Na}_{2} \mathrm{O}$ & $0.6 \%$ & $-0.5 \%$ & $0.0 \%$ & $1.9 \%$ & $-0.3 \%$ & $1.5 \%$ & $3.0 \%$ \\
\hline $\mathrm{NiO}$ & $-0.3 \%$ & $-2.4 \%$ & $5.0 \%$ & $-2.8 \%$ & $-0.2 \%$ & $-1.9 \%$ & $2.8 \%$ \\
\hline $\mathrm{SiO}_{2}$ & $-0.1 \%$ & $0.6 \%$ & $-1.9 \%$ & $0.9 \%$ & $-0.6 \%$ & $1.1 \%$ & $-2.3 \%$ \\
\hline $\mathrm{TiO}_{2}$ & $0.4 \%$ & $2.0 \%$ & $-1.7 \%$ & $0.5 \%$ & $0.7 \%$ & $2.0 \%$ & $-2.5 \%$ \\
\hline $\mathrm{ZnO}$ & $0.2 \%$ & $-2.8 \%$ & $4.9 \%$ & $-4.6 \%$ & $-3.2 \%$ & $-0.9 \%$ & $2.2 \%$ \\
\hline $\mathrm{ZrO}_{2}$ & $-0.4 \%$ & $-0.6 \%$ & $1.2 \%$ & $-3.5 \%$ & $-2.8 \%$ & $0.1 \%$ & $-3.2 \%$ \\
\hline Sum & $0.2 \%$ & $-0.2 \%$ & $0.1 \%$ & $-0.3 \%$ & $-0.3 \%$ & $0.5 \%$ & $-0.6 \%$ \\
\hline
\end{tabular}

\subsection{Overall Comparisons}

The results from each phase of this testing were reviewed in turn with DWPF personnel as the study progressed; the intent of the review was to identify any problems as early as possible so that a decision could be made on the merit of continuing the testing if an unsuccessful outcome had been seen during the early test phases. However, as shown by the results of the comparisons presented in the previous three sections, there were no major issues identified as the study progressed through the three phases of testing. In this section, a more global view of the results is presented to confirm that no pattern evolved in the outcomes of the comparisons among the samplers that raised concerns regarding the ability of the samplers to provide a representative sample of a well-mixed tank.

The primary metric used in making comparisons between two sampling systems (e.g., low Coliwasa versus. high Coliwasa or HFR 3mL Isolok versus combined Coliwasa) was the \% difference between the averages of the two sets of measurements. And as indicated in the discussion above, the goal was that the biases seen in this testing would be less than $5 \%$ for the elements at concentrations in the simulant that would be considered reportable by the DWPF. Table 3-7 provides the \% differences between the mean of the screened measurements of the samples from the indicated sampling mechanism and the mean of the corresponding screened measurements from the Coliwasa samples for those oxides whose elemental concentrations were at least $0.5 \mathrm{wt} \%$ in the simulant tested. Entries with \% differences (in absolute value) less than 5\% are shaded. The vast majority of the entries in this table are shaded, and, once again, the \% differences of the Isolok were comparable to \% differences of the Hydragard, which was the primary method by which the 
performance of the Isolok was assessed for this study. Specifically, note that while the Hydragard has 8 entries in Table 3-7 with \% differences greater than 5\%, the Isolok has only 3.

Figure 5 provides additional help in the overall assessment of the test results for the reportable elements. Figure 5 provides a plot of the \% relative differences for the sets of Hydragard and Isolok samples grouped by oxide for the major oxides. Once again, the vast majority of these \% differences (in absolute value) were less than $5 \%$ with a few of the Hydragard and a few of the Isolok differences falling beyond $+/-5 \%$. Thus, no problems were seen in the performance of the Isolok relative to the performance of the Hydragard during this testing. Also, there were no issues associated with flow rate and no issues associated with the use of a $3 \mathrm{~mL}$ sample volume versus a $12 \mathrm{~mL}$ sample volume for the Isolok. 
Table 3-7. \% Differences for Screened Oxide Measurements for All Test Phases

\begin{tabular}{|c|c|c|c|c|c|c|c|c|c|c|}
\hline Test Phase/Sampler & $\mathbf{A l}_{2} \mathbf{O}_{3}$ & $\mathbf{B}_{2} \mathbf{O}_{3}$ & $\mathrm{CaO}$ & $\mathrm{Fe}_{2} \mathbf{O}_{3}$ & $\mathbf{L i}_{2} \mathbf{O}$ & MgO & MnO & $\mathrm{Na}_{2} \mathrm{O}$ & $\mathrm{NiO}$ & $\mathrm{SiO}_{2}$ \\
\hline 1st/Hydragard - HFR & 3.3 & -0.7 & 3.2 & 2.4 & 0.4 & 1.7 & 2.1 & 0.8 & not reportable & -0.9 \\
\hline 1st/Hydragard-LFR & 3 & 3.8 & 4 & 2.8 & 2.8 & 4.2 & 3.3 & 2.4 & not reportable & 0.5 \\
\hline 1st/Isolok-HFR-12mL & -0.2 & 2.8 & 0.3 & -0.9 & -0.1 & -0.9 & -0.8 & -2.9 & not reportable & 1.4 \\
\hline 1st/Isolok-HFR-3mL & -1.5 & 1.5 & -0.5 & -0.6 & 2 & -0.8 & -0.7 & -3 & not reportable & 2 \\
\hline 1st/Isolok-LFR-12mL & 2.8 & 4.9 & 4.6 & 3.4 & 0.8 & 3.7 & 3.9 & 2.2 & not reportable & 0.8 \\
\hline 1st/Isolok-LFR-3mL & 1.5 & -3.4 & 1.7 & 1.6 & -0.1 & 1.6 & 2 & -0.5 & not reportable & 0 \\
\hline 2nd/Hydragard - HFR & 4.5 & -5.4 & 2.1 & 5.8 & -2.3 & not reportable & 4.2 & -0.9 & 5.8 & -2.2 \\
\hline 2nd/Hydragard-LFR & 1.4 & -2.9 & 0.3 & 0.9 & -0.2 & not reportable & 0 & 0.1 & -0.6 & -0.8 \\
\hline 2nd/Isolok-HFR-12mL & -0.4 & -5.2 & -1.3 & -0.2 & 0.7 & not reportable & -2 & -0.4 & -1.8 & 1.2 \\
\hline 2nd/Isolok-HFR-3mL & -0.3 & -1.1 & -1.8 & 0.1 & 1.9 & not reportable & 0.5 & -0.7 & 1.2 & 1.3 \\
\hline 2nd/Isolok-LFR-12mL & 2.1 & -4.7 & 1.3 & 1.9 & 0.7 & not reportable & 1.7 & 1.3 & 1 & -2 \\
\hline 2nd/Isolok-LFR-3mL & 3.6 & -2.4 & 2.3 & 3.3 & 0.2 & not reportable & 4.6 & 0.4 & 3.1 & 1 \\
\hline 3rd/Hydragard - HFR & 4.7 & -5.1 & 5.1 & 5.1 & -2.2 & 5.5 & 5.2 & 0 & not reportable & -1.9 \\
\hline 3rd/Hydragard-LFR & 2 & -1.8 & 2.2 & 1.5 & -2.5 & 1.3 & 0.9 & 3 & not reportable & -2.3 \\
\hline 3rd/Isolok-HFR-12mL & -2.3 & -1.4 & -3.1 & -2.8 & 1 & -3.6 & -3.7 & -0.3 & not reportable & -0.6 \\
\hline 3rd/Isolok-HFR-3mL & -0.7 & -2.1 & -0.8 & -1.5 & 1.8 & -2.1 & -2.1 & 1.5 & not reportable & 1.1 \\
\hline 3rd/Isolok-LFR-12mL & -2.9 & -0.8 & -3.1 & -4.4 & 4.1 & -6.1 & -5.8 & 1.9 & not reportable & 0.9 \\
\hline 3rd/Isolok-LFR-3mL & -1.2 & -0.9 & -2.7 & -1.6 & -0.1 & -2.5 & -2.5 & -0.5 & not reportable & 0.6 \\
\hline
\end{tabular}
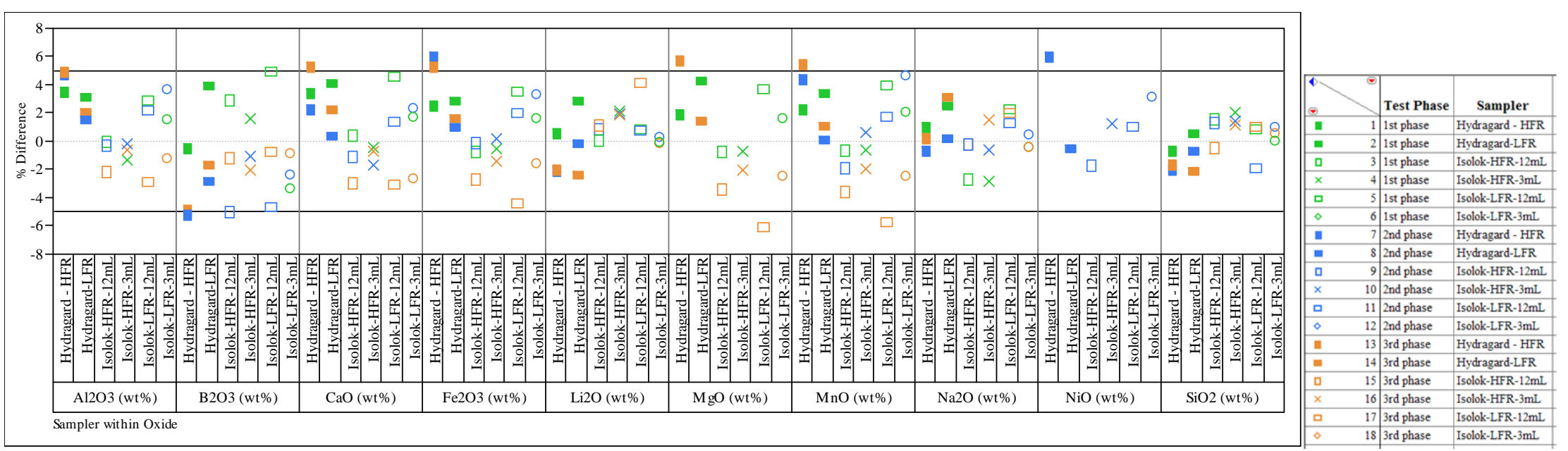

Figure 5. \% Differences for Screen Measurements of Major Oxides. 
One other metric considered as part of the evaluation of a sampler mechanism during the course of these three phases of testing was the bound on the \% bias of the sampling mechanism relative to the Coliwasa. As described in the discussion above, these bounds were taken from the JMP analyses and were determined at a 95\% confidence level. Tables A10, B10, and C10 provide a summary of these values in a manner that facilitates comparisons between the performance of the Isolok and that of the Hydragard for this metric. The values of these bounds from these tables are summarized in Table 3-8 for the reportable elements. The entries with values less than $5 \%$ are shaded in green. Most all of these bounding values are less than $10 \%$, and they indicate that, relative to this metric, the performance of the Isolok was comparable to that of the Hydragard for this testing.

Table 3-8. \% Relative Bias Bounds for Screened Oxide Measurements for All Test Phases

\begin{tabular}{|l|l|r|r|r|r|r|r|r|r|r|r|}
\hline $\begin{array}{c}\text { Test } \\
\text { Phase }\end{array}$ & \multicolumn{1}{|c|}{ Sampler } & $\mathbf{A l}_{\mathbf{2}} \mathbf{O}_{\mathbf{3}}$ & $\mathbf{B}_{\mathbf{2}} \mathbf{O}_{\mathbf{3}}$ & $\mathbf{C a O}$ & $\mathbf{F e}_{\mathbf{2}} \mathbf{O}_{\mathbf{3}}$ & $\mathbf{L i}_{\mathbf{2}} \mathbf{O}$ & $\mathbf{M g O}^{f}$ & $\mathbf{M n O}$ & $\mathbf{N a}_{\mathbf{2}} \mathbf{O}$ & $\mathbf{N i O}$ & $\mathbf{S i O}_{\mathbf{2}}$ \\
\hline 1st phase & Hydragard - HFR & 5.2 & 2.4 & 5.4 & 4.8 & 1.8 & 4.3 & 5.0 & 3.4 & $\mathrm{nr}$ & 2.6 \\
\hline 1st phase & Hydragard-LFR & 6.2 & 6.6 & 7.6 & 6.0 & 5.7 & 7.4 & 7.0 & 5.5 & $\mathrm{nr}$ & 2.9 \\
\hline 1st phase & Isolok-HFR-12mL & 2.7 & 5.2 & 3.6 & 4.2 & 1.7 & 4.3 & 4.4 & 5.5 & $\mathrm{nr}$ & 3.5 \\
\hline 1st phase & Isolok-HFR-3mL & 4.1 & 4.3 & 3.4 & 2.7 & 5.7 & 2.5 & 3.0 & 6.1 & $\mathrm{nr}$ & 4.1 \\
\hline 1st phase & Isolok-LFR-12mL & 6.1 & 7.8 & 8.0 & 6.8 & 4.4 & 7.3 & 7.8 & 5.7 & $\mathrm{nr}$ & 2.7 \\
\hline 1st phase & Isolok-LFR-3mL & 3.8 & 5.5 & 4.1 & 4.0 & 2.1 & 3.6 & 4.8 & 4.3 & $\mathrm{nr}$ & 1.4 \\
\hline 2nd phase & Hydragard - HFR & 6.1 & 7.8 & 5.1 & 8.1 & 4.8 & $\mathrm{nr}$ & 9.0 & 3.0 & 13.0 & 3.5 \\
\hline 2nd phase & Hydragard-LFR & 3.1 & 6.0 & 2.6 & 2.8 & 2.0 & $\mathrm{nr}$ & 2.9 & 2.5 & 5.7 & 2.2 \\
\hline 2nd phase & Isolok-HFR-12mL & 2.0 & 7.9 & 4.1 & 2.4 & 2.7 & $\mathrm{nr}$ & 5.1 & 2.2 & 6.2 & 2.3 \\
\hline 2nd phase & Isolok-HFR-3mL & 1.7 & 3.5 & 3.8 & 1.4 & 3.7 & $\mathrm{nr}$ & 3.1 & 2.8 & 5.1 & 2.8 \\
\hline 2nd phase & Isolok-LFR-12mL & 3.5 & 7.9 & 3.3 & 3.5 & 3.4 & $\mathrm{nr}$ & 5.1 & 3.9 & 6.3 & 3.5 \\
\hline 2nd phase & Isolok-LFR-3mL & 6.3 & 5.2 & 5.0 & 5.1 & 2.5 & $\mathrm{nr}$ & 8.0 & 3.2 & 8.5 & 1.9 \\
\hline 3rd phase & Hydragard - HFR & 5.8 & 8.3 & 7.6 & 6.5 & 4.1 & 7.4 & 7.1 & 1.5 & $\mathrm{nr}$ & 3.1 \\
\hline 3rd phase & Hydragard-LFR & 3.9 & 4.7 & 5.8 & 3.7 & 4.4 & 4.5 & 3.6 & 5.2 & $\mathrm{nr}$ & 3.6 \\
\hline 3rd phase & Isolok-HFR-12mL & 4.3 & 4.8 & 5.4 & 5.0 & 2.6 & 6.4 & 6.6 & 4.1 & $\mathrm{nr}$ & 2.9 \\
\hline 3rd phase & Isolok-HFR-3mL & 1.8 & 5.8 & 2.8 & 2.8 & 3.6 & 3.3 & 3.2 & 3.7 & $\mathrm{nr}$ & 1.9 \\
\hline 3rd phase & Isolok-LFR-12mL & 5.9 & 4.2 & 5.7 & 8.0 & 7.4 & 10.9 & 10.7 & 4.3 & $\mathrm{nr}$ & 2.3 \\
\hline 3rd phase & Isolok-LFR-3mL & 2.3 & 2.6 & 4.2 & 2.6 & 2.2 & 3.5 & 3.6 & 3.1 & $\mathrm{nr}$ & 1.7 \\
\hline
\end{tabular}

Figure 6 was prepared as an additional check on the overall performance of the sampling mechanisms. The plots provided in this figure offer an opportunity to investigate an important aspect of sampling SME material. Since this material consisted of sludge components and heavier frit components, the question arises: Was there an indication that samples that have higher dried solids contained more frit and less sludge, and vice versa: with lower dried solids was there a tendency toward less frit and more sludge? Figure 6 plots values for $\mathrm{Al}_{2} \mathrm{O}_{3}$ (sludge), $\mathrm{B}_{2} \mathrm{O}_{3}$ (frit), $\mathrm{Fe}_{2} \mathrm{O}_{3}$ (sludge), and $\mathrm{SiO}_{2}$ (frit) versus values for wt\% dried solids for the three phases of this testing with different colors representing the different sampling mechanisms (see the legend). Note that the most obvious clustering and greatest tendency for trending is seen for the Hydragard sampler during the low-rheology (phase 3) testing. While this was not considered to be so severe as to indicate a failure of the Hydragard, it does reveal the need for this type of investigation into the performance of samplers of SME material. Also, note that this behavior was not seen in performance of the Isolok sampler.

\footnotetext{
${ }^{f} \mathrm{nr}$ - not reportable
} 


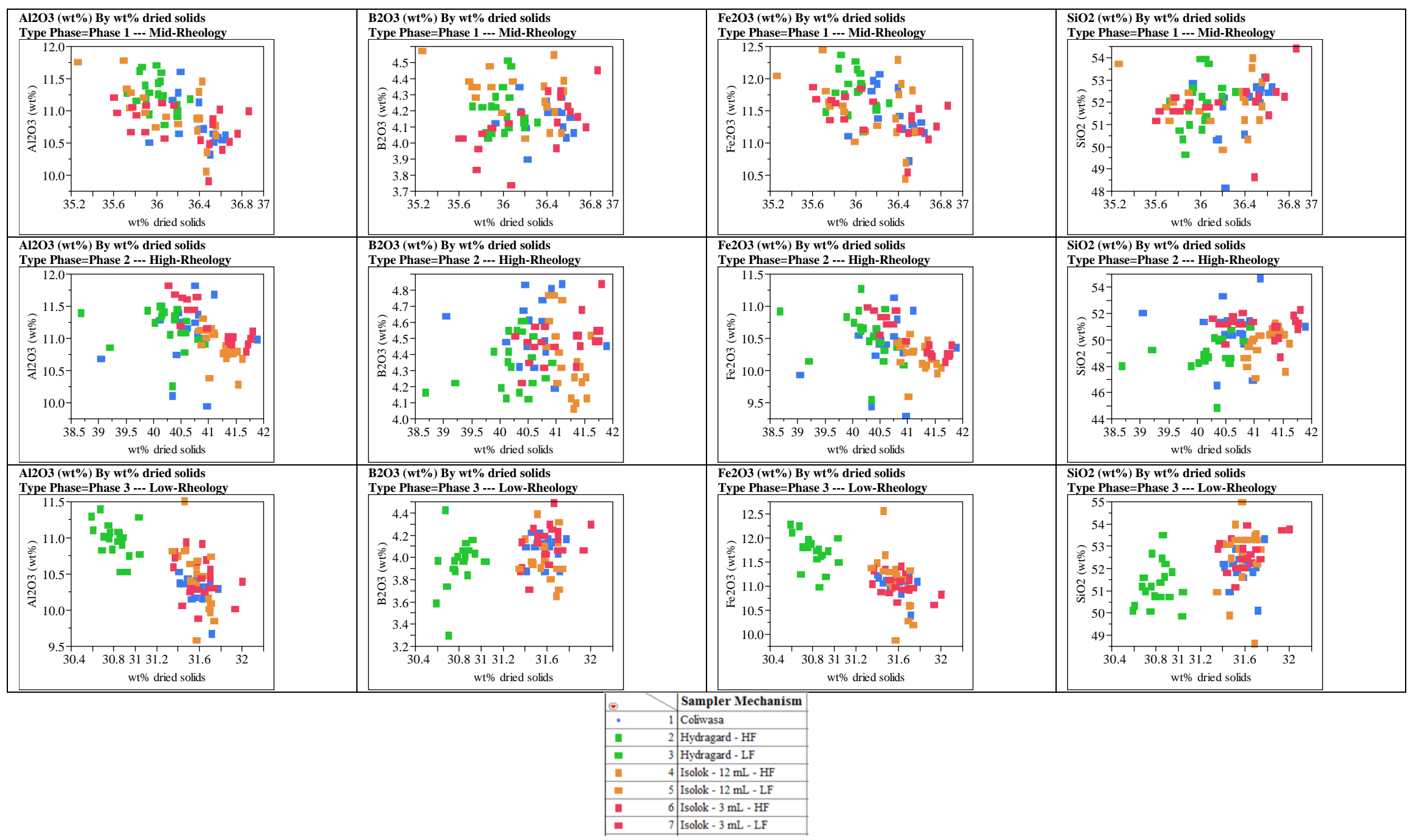

Figure 6. Sludge and Frit Components versus wt \% dried solids. 


\subsection{Cycle and Flushing Tests}

The Isolok sampler was cycled a total of 10,000 times during the test. This does not include the 540 strokes for the three simulant tests or any of the strokes used for flushing the system. The testing was stopped since there was no wear indicated by the Isolok. The total cycles correspond to approximately one year of facility operation if for each SME sample run, thirty-five $12 \mathrm{~mL}$ samples (4 strokes with the 3mL Isolok) are pulled, and the SME is sampled six times per month for twelve months.

Results for the flushing show that extending and then retracting the Isolok 30 times as water is pumped through the Isolok will visually clean the inner spool of any simulant. The Isolok was cycled 10 times by holding the spool extended and then retracting for 2 seconds each time, and then it was visually inspected to determine if further flushing was required. After three sets of 10 cycles the Isolok was cleaned of any simulant.

\subsection{CONCLUSIONS}

A total of three tests were performed for the Isolok acceptance testing. Simulant rheology ranged from low, medium and high yield stress and consistency values. Data from the acceptance testing show that the Isolok sampler typically pulls samples with bias errors less than $5 \%$ and that the performance of the Isolok in providing representative samples during this testing was comparable to that of the Hydragard sampler. Wear test of the Viton O-rings located on the Isolok inner spool show that it can with-stand the abrasiveness of the frit and last for at least 1 years of operation (10,000 cycles). Flushing of the Isolok showed that cycling it 30 times as flush water is pumped through the flush ports is sufficient to clean the internal spool. Visual verification could also be used by watching the water as it is flushed and letting it run until the water becomes clear.

\subsection{RECOMMENDATIONS/PATH FORWARD}

Based on the acceptance testing data and discussions with the DWPF Laboratory improvement team, SRNL recommends proceeding with the design and installation of the Isolok system for the SME sampling process. Based on the success of the Isolok testing to date, SRNL recommends testing to acquire a smaller 1-1.5 mL sample size. A smaller sample size used in conjunction with the cesium carbonate fusion would allow the elimination of the grinding step for the glass, thus further reducing analytical cycle time. 


\subsection{REFERENCES}

1. Hera, Kevin R. Isolok Valve Viability Testing for DWPF SME Sampling Process. Savannah River Site, Aiken, SC, 29808 : s.n., 2011. SRNL-STI-2010-00749.

2. Bovan, Perry L. SME Isolok Acceptance Testing. Savannah River Site, Aiken, SC, 29808 : s.n., 2010. HLW-DWPF-TTR-2010-0036.

3. Hera, Kevin R. Task Technical and Quality Assurance Plan for SME Isolok Acceptance Testing Requirements. Savannah River Site, Aiken, SC 29808 : s.n., 2011. SRNL-RP-2011-00145.

4. Stone, Mike E. Position on Isolok SME Testing Programs. Savannah River Site, Aiken, SC, 29809 : s.n., 2010. SRNL-L3100-2011-00044.

5. Edwards, Thomas B. Sampling and Analytical Plan Supporting the SME Acceptance Testing of the Isolok Sampler. Savannah River Site, Aiken, SC, 29808 : s.n., 2011. SRNL-RP-2011-00294.

6. Alkali Fusion Dissolutions of Sludge and Glass for Elemental and Anion Analysis (U). Savannah River Site, Aiken, SC, 29808 : s.n., 2008. L16.1 ADS-2502 Rev. 6.

7. Coleman, Charles J. Lab Notebook. 2011. SRNL-NB-2011-00016 Pg 27.

8. Hera, Kevin R. Isolok Valve Testing. 2008. WSRC-NB-2008-00007.

9. Ray, J.W., A.V. Staub, S.L. Marra, C.J. Coleman, and M.J. Plodinec. Reporting the Chemical Composition of the DWPF Product (U). Aiken, SC : Washington Savannah River Company, 2006. WSRC-IM-91-116-2, Revision 4.

10. SAS, Institute. JMP Version 7.0.2. Cary NC : s.n., 1989-2007. 


\section{Appendix A.}

Supplemental Tables and Exhibits for Mid-Rheology (Phase 1) Simulant Testing 
Table A1. Density Measurements for Phase 1

\begin{tabular}{|c|c|c|c|c|}
\hline Sampling Mechanism & Flow Rate (LF/HF) & Sampler w Location & Sample ID & Sample Density (g/mL) \\
\hline Coliwasa & LF & Coliwasa Low & DCL01 & 1.2911 \\
\hline Coliwasa & LF & Coliwasa Low & DCL02 & 1.2717 \\
\hline Coliwasa & LF & Coliwasa Low & DCL03 & 1.2900 \\
\hline Coliwasa & LF & Coliwasa Low & DCL04 & 1.2668 \\
\hline Coliwasa & $\mathrm{HF}$ & Coliwasa Low & DCL05 & 1.2909 \\
\hline Coliwasa & HF & Coliwasa Low & DCL06 & 1.3028 \\
\hline Coliwasa & HF & Coliwasa Low & DCL07 & 1.3082 \\
\hline Coliwasa & HF & Coliwasa Low & DCL08 & 1.2844 \\
\hline Coliwasa & LF & Coliwasa High & DCH01 & 1.3000 \\
\hline Coliwasa & LF & Coliwasa High & DCH02 & 1.3160 \\
\hline Coliwasa & LF & Coliwasa High & DCH03 & 1.2883 \\
\hline Coliwasa & LF & Coliwasa High & DCH04 & 1.3039 \\
\hline Coliwasa & $\mathrm{HF}$ & Coliwasa High & DCH05 & 1.2982 \\
\hline Coliwasa & $\mathrm{HF}$ & Coliwasa High & DCH06 & 1.2928 \\
\hline Coliwasa & HF & Coliwasa High & DCH07 & 1.2962 \\
\hline Coliwasa & $\mathrm{HF}$ & Coliwasa High & DCH08 & 1.2987 \\
\hline Hydragard & LF & Hydragard & DLHY01 & 1.3065 \\
\hline Hydragard & LF & Hydragard & DLHY02 & 1.2982 \\
\hline Hydragard & LF & Hydragard & DLHY03 & 1.3016 \\
\hline Hydragard & LF & Hydragard & DLHY04 & 1.3089 \\
\hline Hydragard & LF & Hydragard & DLHY05 & 1.3114 \\
\hline Hydragard & LF & Hydragard & DLHY06 & 1.2997 \\
\hline Hydragard & LF & Hydragard & DLHY07 & 1.2891 \\
\hline Hydragard & LF & Hydragard & DLHY08 & 1.3072 \\
\hline Hydragard & LF & Hydragard & DLHY09 & 1.3085 \\
\hline Hydragard & LF & Hydragard & DLHY10 & 1.3022 \\
\hline Hydragard & $\mathrm{HF}$ & Hydragard & DHHY01 & 1.2989 \\
\hline Hydragard & $\mathrm{HF}$ & Hydragard & DHHY02 & 1.3189 \\
\hline Hydragard & $\mathrm{HF}$ & Hydragard & DHHY03 & 1.2842 \\
\hline Hydragard & $\mathrm{HF}$ & Hydragard & DHHY04 & 1.2943 \\
\hline Hydragard & $\mathrm{HF}$ & Hydragard & DHHY05 & 1.2954 \\
\hline Hydragard & $\mathrm{HF}$ & Hydragard & DHHY06 & 1.4033 \\
\hline Hydragard & $\mathrm{HF}$ & Hydragard & DHHY07 & 1.2912 \\
\hline Hydragard & $\mathrm{HF}$ & Hydragard & DHHY08 & 1.3047 \\
\hline Hydragard & $\mathrm{HF}$ & Hydragard & DHHY09 & 1.2876 \\
\hline Hydragard & $\mathrm{HF}$ & Hydragard & DHHY10 & 1.3030 \\
\hline Isolok & LF & Isolok & DLIB01 & 1.2810 \\
\hline Isolok & LF & Isolok & DLIB02 & 1.2908 \\
\hline Isolok & LF & Isolok & DLIB03 & 1.3007 \\
\hline Isolok & LF & Isolok & DLIB04 & 1.2580 \\
\hline Isolok & LF & Isolok & DLIB05 & 1.2877 \\
\hline Isolok & LF & Isolok & DLIB06 & 1.2980 \\
\hline Isolok & LF & Isolok & DLIB07 & 1.2887 \\
\hline Isolok & LF & Isolok & DLIB08 & 1.2993 \\
\hline Isolok & LF & Isolok & DLIB09 & 1.2839 \\
\hline Isolok & LF & Isolok & DLIB10 & 1.2892 \\
\hline Isolok & $\mathrm{HF}$ & Isolok & DHIB01 & 1.2995 \\
\hline Isolok & $\mathrm{HF}$ & Isolok & DHIB02 & 1.3285 \\
\hline Isolok & $\mathrm{HF}$ & Isolok & DHIB03 & 1.3112 \\
\hline Isolok & $\mathrm{HF}$ & Isolok & DHIB04 & 1.2932 \\
\hline Isolok & $\mathrm{HF}$ & Isolok & DHIB05 & 1.2948 \\
\hline Isolok & $\mathrm{HF}$ & Isolok & DHIB06 & 1.3079 \\
\hline Isolok & $\mathrm{HF}$ & Isolok & DHIB07 & 1.2857 \\
\hline Isolok & $\mathrm{HF}$ & Isolok & DHIB08 & 1.3191 \\
\hline Isolok & $\mathrm{HF}$ & Isolok & DHIB09 & 1.2954 \\
\hline Isolok & $\mathrm{HF}$ & Isolok & DHIB10 & 1.3025 \\
\hline
\end{tabular}


Table A2. Solids Measurements for Phase 1

\begin{tabular}{|c|c|c|c|c|c|}
\hline Sampling Mechanism & Flow Rate (LF/HF) & Sampler w Location & Sample ID & wt \% dried solids & wt \% vitrified solids \\
\hline Coliwasa & LF & \begin{tabular}{|l|} 
Coliwasa Low \\
\end{tabular} & \begin{tabular}{|l|l} 
CL01 \\
\end{tabular} & 36.2 & 30.88 \\
\hline Coliwasa & LF & Coliwasa Low & CL02 & 36.14 & 30.79 \\
\hline Coliwasa & LF & Coliwasa Low & CL03 & 36.21 & 30.74 \\
\hline Coliwasa & LF & Coliwasa Low & CL04 & 35.92 & 30.51 \\
\hline Coliwasa & HF & Coliwasa Low & CL05 & 36.2 & 30.8 \\
\hline Coliwasa & $\mathrm{HF}$ & Coliwasa Low & \begin{tabular}{|l|} 
CL06 \\
\end{tabular} & 36.39 & 31.01 \\
\hline Coliwasa & $\mathrm{HF}$ & Coliwasa Low & CL07 & 36.5 & 31.11 \\
\hline Coliwasa & HF & Coliwasa Low & CL08 & 36.16 & 30.76 \\
\hline Coliwasa & LF & Coliwasa High & $\mathrm{CH} 01$ & 36.52 & 31.17 \\
\hline Coliwasa & LF & Coliwasa High & CH02 & 36.4 & 31.05 \\
\hline Coliwasa & LF & Coliwasa High & $\mathrm{CH} 03$ & 36.6 & 31.18 \\
\hline Coliwasa & LF & Coliwasa High & CH04 & 36.52 & 31.11 \\
\hline Coliwasa & HF & Coliwasa High & CH05 & 36.57 & 31.23 \\
\hline Coliwasa & HF & Coliwasa High & CH06 & 36.54 & 31.15 \\
\hline Coliwasa & HF & Coliwasa High & $\mathrm{CH} 07$ & 36.64 & 31.22 \\
\hline Coliwasa & HF & Coliwasa High & CH08 & 36.44 & 31.02 \\
\hline Hydragard & LF & Hydragard & LHY01 & 36.05 & 30.41 \\
\hline Hydragard & LF & Hydragard & LHY02 & 36.06 & 30.39 \\
\hline Hydragard & LF & Hydragard & LHY03 & 35.92 & 30.25 \\
\hline Hydragard & LF & Hydragard & LHY04 & 35.8 & 30.16 \\
\hline Hydragard & LF & Hydragard & LHY05 & 36.04 & 30.39 \\
\hline Hydragard & LF & Hydragard & LHY06 & 36.03 & 30.38 \\
\hline Hydragard & LF & Hydragard & LHY07 & 35.85 & 30.19 \\
\hline Hydragard & LF & Hydragard & LHY08 & 36.18 & 30.48 \\
\hline Hydragard & LF & Hydragard & LHY09 & 35.99 & 30.2 \\
\hline Hydragard & LF & Hydragard & LHY10 & 36.18 & 30.47 \\
\hline Hydragard & HF & Hydragard & HHY01 & 35.84 & 30.04 \\
\hline Hydragard & HF & Hydragard & HHY02 & 35.89 & 30.13 \\
\hline Hydragard & HF & Hydragard & HHY03 & 36.01 & 30.26 \\
\hline Hydragard & HF & Hydragard & HHY04 & 35.72 & 29.89 \\
\hline Hydragard & HF & Hydragard & HHY05 & 35.9 & 30.07 \\
\hline Hydragard & HF & Hydragard & HHY06 & 36.31 & 30.58 \\
\hline Hydragard & HF & Hydragard & HHY07 & 36.01 & 30.18 \\
\hline Hydragard & HF & Hydragard & HHY08 & 36.19 & 30.44 \\
\hline Hydragard & HF & Hydragard & HHY09 & 35.93 & 30.09 \\
\hline Hydragard & HF & Hydragard & HHY10 & 36.03 & 30.2 \\
\hline Isolok (3 mL) & LF & Isolok (3 mL) & LIS01 & 35.89 & 30.45 \\
\hline Isolok (3 mL) & LF & Isolok (3 mL) & LIS02 & 35.62 & 30.16 \\
\hline Isolok (3 mL) & LF & Isolok (3 mL) & LIS03 & 35.75 & 30.32 \\
\hline Isolok (3 mL) & LF & Isolok (3 mL) & LIS04 & 36.06 & 30.52 \\
\hline Isolok (3 mL) & LF & Isolok (3 mL) & LIS05 & 35.88 & 30.43 \\
\hline Isolok (3 mL) & LF & Isolok (3 mL) & LIS06 & 35.59 & 30.08 \\
\hline Isolok (3 mL) & LF & Isolok (3 mL) & LIS07 & 35.76 & 30.13 \\
\hline Isolok (3 mL) & LF & Isolok (3 mL) & LIS08 & 35.8 & 30.2 \\
\hline Isolok (3 mL) & LF & Isolok (3 mL) & LIS09 & 36.15 & 30.6 \\
\hline Isolok (3 mL) & LF & Isolok (3 mL) & LIS10 & 36.04 & 30.54 \\
\hline Isolok (3 mL) & HF & Isolok (3 mL) & HIS01 & 36.41 & 30.86 \\
\hline Isolok (3 mL) & HF & Isolok (3 mL) & HIS02 & 36.68 & 31.06 \\
\hline Isolok (3 mL) & HF & Isolok (3 mL) & HIS03 & 36.75 & 31.2 \\
\hline Isolok (3 mL) & HF & Isolok (3 mL) & HIS04 & 36.48 & 30.93 \\
\hline Isolok (3 mL) & HF & Isolok (3 mL) & HIS05 & 36.49 & 31 \\
\hline Isolok (3 mL) & HF & Isolok (3 mL) & HIS06 & 36.52 & 30.96 \\
\hline Isolok (3 mL) & HF & Isolok (3 mL) & HIS07 & 36.86 & 31.21 \\
\hline Isolok (3 mL) & HF & Isolok (3 mL) & HIS08 & 36.59 & 31.03 \\
\hline Isolok (3 mL) & HF & Isolok (3 mL) & HIS09 & 36.52 & 31.02 \\
\hline Isolok (3 mL) & HF & Isolok (3 mL) & HIS10 & 36.61 & 31.02 \\
\hline Isolok (12 mL) & LF & Isolok $(12 \mathrm{~mL})$ & LIB01 & 35.25 & 29.83 \\
\hline Isolok (12 mL) & LF & Isolok (12 mL) & LIB02 & 35.87 & 30.38 \\
\hline Isolok (12 mL) & LF & Isolok $(12 \mathrm{~mL})$ & LIB03 & 35.98 & 30.47 \\
\hline Isolok (12 mL) & LF & Isolok $(12 \mathrm{~mL})$ & LIB04 & 35.88 & 30.38 \\
\hline Isolok (12 mL) & LF & Isolok (12 mL) & LIB05 & 35.68 & 30.18 \\
\hline Isolok (12 mL) & LF & Isolok $(12 \mathrm{~mL})$ & LIB06 & 35.71 & 30.12 \\
\hline Isolok (12 mL) & LF & Isolok $(12 \mathrm{~mL})$ & LIB07 & 35.74 & 30.22 \\
\hline Isolok (12 mL) & LF & Isolok (12 mL) & LIB08 & 35.75 & 30.23 \\
\hline
\end{tabular}


SRNL-STI-2011-00693

Revision 0

Table A2. Solids Measurements for Phase 1

\begin{tabular}{|l|l|l|l|r|r|}
\hline Sampling Mechanism & Flow Rate (LF/HF) & Sampler w Location & Sample ID & wt\% dried solids & wt\% vitrified solids \\
\hline Isolok $(12 \mathrm{~mL})$ & LF & Isolok $(12 \mathrm{~mL})$ & LIB09 & 36.08 & 30.5 \\
\hline Isolok $(12 \mathrm{~mL})$ & LF & Isolok $(12 \mathrm{~mL})$ & LIB10 & 36.19 & 30.64 \\
\hline Isolok $(12 \mathrm{~mL})$ & HF & Isolok $(12 \mathrm{~mL})$ & HIB01 & 36.38 & 30.9 \\
\hline Isolok $(12 \mathrm{~mL})$ & HF & Isolok $(12 \mathrm{~mL})$ & HIB02 & 36.37 & 30.88 \\
\hline Isolok $(12 \mathrm{~mL})$ & HF & Isolok $(12 \mathrm{~mL})$ & HIB03 & 36.42 & 36.4 \\
\hline Isolok $(12 \mathrm{~mL})$ & HF & Isolok $(12 \mathrm{~mL})$ & HIB04 & 36.53 & 30.91 \\
\hline Isolok $(12 \mathrm{~mL})$ & HF & Isolok $(12 \mathrm{~mL})$ & HIB05 & 36.55 & 31.09 \\
\hline Isolok $(12 \mathrm{~mL})$ & HF & Isolok $(12 \mathrm{~mL})$ & HIB06 & 36.55 & 31.07 \\
\hline Isolok $(12 \mathrm{~mL})$ & HF & Isolok $(12 \mathrm{~mL})$ & HIB07 & 36.46 & 31.02 \\
\hline Isolok $(12 \mathrm{~mL})$ & HF & Isolok $(12 \mathrm{~mL})$ & HIB08 & 36.39 & 30.84 \\
\hline Isolok $(12 \mathrm{~mL})$ & HF & Isolok $(12 \mathrm{~mL})$ & HIB09 & 36.47 & 30.84 \\
\hline Isolok $(12 \mathrm{~mL})$ & HF & Isolok $(12 \mathrm{~mL})$ & HIB10 & & 30.9 \\
\hline
\end{tabular}


Table A3. Phase 1 Elemental Concentration Measurements (part 1)

\begin{tabular}{|c|c|c|c|c|c|c|c|c|c|c|c|c|c|c|c|c|c|c|c|}
\hline $\begin{array}{c}\text { Type of } \\
\text { Material }\end{array}$ & Sampler & $\begin{array}{l}\text { Flow } \\
\text { Rate }\end{array}$ & \begin{tabular}{|c|} 
Sample \\
Order \\
\end{tabular} & $\begin{array}{c}\text { Preparation } \\
\text { Block }\end{array}$ & $\begin{array}{c}\text { Preparation } \\
\text { Sequence }\end{array}$ & $\begin{array}{c}\text { Analytical } \\
\text { Block }\end{array}$ & $\begin{array}{c}\text { Analytical } \\
\text { Sequence } \\
\end{array}$ & $\begin{array}{c}\text { Sample } \\
\text { ID }\end{array}$ & $\begin{array}{c}\mathrm{Al} \\
(\mathrm{ug} / \mathrm{g})\end{array}$ & $\begin{array}{c}\text { B } \\
(\mathrm{ug} / \mathrm{g})\end{array}$ & $\begin{array}{c}\mathrm{Ba} \\
(\mathrm{ug} / \mathrm{g})\end{array}$ & $\begin{array}{c}\mathrm{Ca} \\
(\mathrm{ug} / \mathrm{g})\end{array}$ & $\begin{array}{c}\mathrm{Ce} \\
\text { (ug/g) }\end{array}$ & $\begin{array}{c}\mathrm{Cr} \\
(\mathrm{ug} / \mathrm{g})\end{array}$ & $\begin{array}{c}\mathrm{Cu} \\
(\mathrm{ug} / \mathrm{g})\end{array}$ & 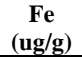 & $\begin{array}{c}\mathbf{K} \\
(\mathbf{u g} / \mathrm{g})\end{array}$ & $\begin{array}{c}\mathrm{La} \\
(\mathrm{ug} / \mathrm{g})\end{array}$ & \begin{tabular}{|c|}
$\mathbf{L i}$ \\
$(\mathrm{ug} / \mathrm{g})$
\end{tabular} \\
\hline ARG-1 & None & \begin{tabular}{|l|}
0 \\
\end{tabular} & $\begin{array}{r}0 \\
\end{array}$ & \begin{tabular}{|r|}
1 \\
\end{tabular} & \begin{tabular}{|r|}
1 \\
\end{tabular} & \begin{tabular}{r|}
1 \\
\end{tabular} & \begin{tabular}{|r|}
1 \\
\end{tabular} & \begin{tabular}{|l|} 
ARG-1B11 \\
\end{tabular} & 24000 & 25500 & 804 & 10400 & $<437$ & 663 & $<318$ & 97600 & 22700 & $<182$ & 14800 \\
\hline SME Simulant & Isolok - $3 \mathrm{~mL}$ & LF & 18 & 1 & 9 & 1 & 2 & \begin{tabular}{|l|l|} 
LIS03 \\
\end{tabular} & 56400 & 11900 & 939 & 8570 & $<564$ & 718 & $<410$ & 79400 & $<2770$ & $<235$ & 22000 \\
\hline SME Simulant & Isolok - $3 \mathrm{~mL}$ & LF & 8 & 1 & 27 & 1 & 3 & LIS01 & 58600 & 12700 & 953 & 8900 & $<530$ & 750 & $<386$ & 81900 & $<2610$ & $<221$ & 22200 \\
\hline SME Simulant & \begin{tabular}{|l|} 
Coliwasa \\
\end{tabular} & LF & 20 & 1 & 2 & 1 & 4 & CL02 & 59100 & 13500 & 956 & 9030 & $<546$ & 718 & $<397$ & 82500 & $<2690$ & $<227$ & 22100 \\
\hline SME Simulant & \begin{tabular}{|l|} 
Coliwasa \\
\end{tabular} & LF & 38 & 1 & 16 & 1 & 5 & CH03 & 55800 & 12900 & 919 & 8810 & $<569$ & 712 & $<414$ & 79100 & $<2800$ & $<237$ & 22300 \\
\hline SME Simulant & \begin{tabular}{|l|} 
Coliwasa \\
\end{tabular} & LF & 63 & 1 & 13 & 1 & 6 & CH04 & 56300 & 13300 & 927 & 8480 & $<562$ & 715 & $<409$ & 78900 & $<2760$ & $<234$ & 21900 \\
\hline SME Simulant & Isolok - $3 \mathrm{~mL}$ & LF & 44 & 1 & 3 & 1 & 7 & LIS07 & 58500 & 12300 & 938 & 8660 & $<553$ & 681 & $<402$ & 81600 & $<2720$ & $<230$ & 21900 \\
\hline SME Simulant & \begin{tabular}{|l|} 
Coliwasa \\
\end{tabular} & $\mathrm{HF}$ & 23 & 1 & 28 & 1 & 8 & CH06 & 56000 & 12700 & 906 & 8490 & $<523$ & 719 & $<381$ & 78400 & $<2570$ & $<218$ & 22900 \\
\hline SME Simulant & \begin{tabular}{|l|} 
Coliwasa \\
\end{tabular} & LF & 21 & 1 & 14 & 1 & 9 & $\mathrm{CH} 02$ & 56700 & 13000 & 924 & 8440 & $<577$ & 722 & $<419$ & 79800 & $<2830$ & $<240$ & 21800 \\
\hline SME Simulant & \begin{tabular}{|l|l|} 
Coliwasa \\
\end{tabular} & $\mathrm{HF}$ & 3 & 1 & 20 & 1 & 10 & CL05 & 58400 & 12800 & 932 & 8690 & $<498$ & 744 & $<362$ & 82500 & $<2450$ & $<207$ & 21600 \\
\hline SME Simulant & Isolok - $3 \mathrm{~mL}$ & LF & 12 & 1 & 25 & 1 & 11 & LIS02 & 58000 & 12500 & 940 & 8700 & $<519$ & 702 & $<377$ & 81700 & $<2550$ & $<216$ & 21600 \\
\hline SME Simulant & Coliwasa & LF & 40 & 1 & 19 & 1 & 12 & CL03 & 61400 & 12100 & 964 & 8960 & $<592$ & 777 & $<430$ & 84400 & $<2910$ & $<246$ & 20400 \\
\hline SME Simulant & \begin{tabular}{|l|} 
Coliwasa \\
\end{tabular} & $\mathrm{HF}$ & 18 & 1 & 26 & 1 & 13 & CL06 & 58800 & 13200 & 960 & 8830 & $<589$ & 809 & $<428$ & 82800 & $<2900$ & $<245$ & 21800 \\
\hline SME Simulant & \begin{tabular}{|l} 
Coliwasa \\
\end{tabular} & $\mathrm{HF}$ & 1 & 1 & 22 & 1 & 14 & $\begin{array}{ll}\text { CH05 } \\
\end{array}$ & 55900 & 12500 & 910 & 8370 & $<588$ & 712 & $<427$ & 78100 & $<2890$ & $<245$ & 21400 \\
\hline ARG-1 & None & 0 & 0 & 1 & 15 & 1 & 15 & \begin{tabular}{|l|} 
ARG-1B12 \\
\end{tabular} & 24100 & 25200 & 809 & 10400 & $<445$ & 656 & $<324$ & 97500 & 22100 & $<185$ & 14800 \\
\hline SME Simulant & \begin{tabular}{|l|} 
Coliwasa \\
\end{tabular} & $\mathrm{HF}$ & 36 & 1 & 21 & 1 & 16 & \begin{tabular}{|l|} 
CL07 \\
\end{tabular} & 54500 & 13000 & 908 & 7920 & $<562$ & 692 & $<409$ & 74800 & $<2670$ & $<234$ & 24000 \\
\hline SME Simulant & Isolok - $3 \mathrm{~mL}$ & LF & 31 & 1 & 24 & 1 & 17 & LIS05 & 56400 & 12700 & 927 & 8550 & $<591$ & 704 & $<430$ & 79500 & $<2910$ & $<246$ & 21700 \\
\hline SME Simulant & Isolok - $3 \mathrm{~mL}$ & LF & 58 & 1 & \begin{tabular}{l|l}
4 \\
\end{tabular} & 1 & 18 & LIS10 & 58800 & 12800 & 959 & 8900 & $<554$ & 739 & $<403$ & 82800 & $<2720$ & $<231$ & 21500 \\
\hline SME Simulant & \begin{tabular}{|l} 
Coliwasa \\
\end{tabular} & $\mathrm{HF}$ & 63 & 1 & 5 & 1 & 19 & CL08 & 58600 & 13000 & 975 & 8920 & $<508$ & 749 & $<369$ & 83600 & $<2500$ & $<211$ & 22500 \\
\hline SME Simulant & Isolok - $3 \mathrm{~mL}$ & LF & 25 & 1 & 7 & 1 & 20 & LIS04 & 55900 & 11600 & 906 & 8460 & $<551$ & 668 & $<401$ & 78300 & $<2710$ & $<229$ & 22300 \\
\hline SME Simulant & Isolok - $3 \mathrm{~mL}$ & LF & 42 & 1 & 11 & 1 & 21 & LIS06 & 59300 & 12500 & 971 & 8930 & $<556$ & 719 & $<405$ & 83000 & $<2730$ & $<232$ & 21600 \\
\hline SME Simulant & Coliwasa & $\mathrm{HF}$ & 65 & 1 & 6 & 1 & 22 & CH08 & 56600 & 13000 & 905 & 8360 & $<555$ & 707 & $<404$ & 78300 & $<2730$ & $<231$ & 21800 \\
\hline SME Simulant & \begin{tabular}{|l|} 
Coliwasa \\
\end{tabular} & $\mathrm{HF}$ & 34 & 1 & 18 & 1 & 23 & CH07 & 56100 & 12600 & 922 & 8550 & $<515$ & 697 & $<374$ & 77900 & $<2530$ & $<214$ & 21300 \\
\hline SME Simulant & \begin{tabular}{|l|} 
Coliwasa \\
\end{tabular} & LF & 1 & 1 & 12 & 1 & 24 & CH01 & 55600 & 13000 & 905 & 8450 & $<511$ & 691 & $<372$ & 78000 & $<2510$ & $<213$ & 22100 \\
\hline SME Simulant & Isolok - $3 \mathrm{~mL}$ & LF & 54 & 1 & 17 & 1 & 25 & LIS09 & 58600 & 13000 & 948 & 8860 & $<587$ & 699 & $<427$ & 81400 & $<2880$ & $<244$ & 21300 \\
\hline SME Simulant & \begin{tabular}{|l|} 
Coliwasa \\
\end{tabular} & LF & 4 & 1 & 10 & 1 & 26 & CL01 & 56300 & 12700 & 916 & 8630 & $<572$ & 716 & $<416$ & 79600 & $<2810$ & $<238$ & 22600 \\
\hline SME Simulant & Isolok - $3 \mathrm{~mL}$ & LF & 49 & 1 & 8 & 1 & 27 & LIS08 & 57800 & 12600 & 952 & 8790 & $<577$ & 688 & $<420$ & 81200 & $<2840$ & $<240$ & 22400 \\
\hline SME Simulant & Coliwasa & LF & 65 & 1 & 23 & 1 & 28 & CL04 & 55600 & 12800 & 902 & 8410 & $<553$ & 738 & $<402$ & 77600 & $<2720$ & $<230$ & 21600 \\
\hline ARG-1 & \begin{tabular}{|l|} 
None \\
\end{tabular} & 0 & 0 & 1 & 29 & 1 & 29 & ARG-1B13 & 24900 & 24400 & 820 & 10600 & $<556$ & 651 & $<404$ & 98000 & 21800 & $<231$ & 14900 \\
\hline ARG-1 & None & 0 & 0 & 2 & 1 & 2 & 1 & ARG-1B21 & 24700 & 24100 & 810 & 10400 & $<381$ & 686 & $<277$ & 98000 & 22300 & $<159$ & 15100 \\
\hline SME Simulant & \begin{tabular}{|l|} 
Hydragard \\
\end{tabular} & $\mathrm{HF}$ & 11 & 2 & 3 & 2 & 2 & HHY02 & 60200 & 13100 & 944 & 9030 & $<561$ & 736 & $<408$ & 82100 & $<2760$ & $<233$ & 22200 \\
\hline SME Simulant & \begin{tabular}{|l|} 
Coliwasa \\
\end{tabular} & $\mathrm{HF}$ & 36 & 1 & 21 & 2 & 3 & CL07 & 55500 & 12800 & 885 & 8110 & $<562$ & 715 & $<409$ & 75100 & $<2760$ & $<234$ & 24500 \\
\hline SME Simulant & \begin{tabular}{|l|} 
Hydragard \\
\end{tabular} & $\mathrm{HF}$ & 24 & 2 & 2 & 2 & 4 & HHY04 & 59600 & 13100 & 949 & 8920 & $<607$ & 761 & $<442$ & 80200 & $<2990$ & $<253$ & 22300 \\
\hline SME Simulant & \begin{tabular}{|l|} 
Coliwasa \\
\end{tabular} & $\mathrm{HF}$ & 1 & 1 & 22 & 2 & 5 & CH05 & 56800 & 12600 & 926 & 8400 & $<588$ & 772 & $<427$ & 78100 & $<2890$ & $<245$ & 21700 \\
\hline SME Simulant & \begin{tabular}{|l|} 
Coliwasa \\
\end{tabular} & $\mathrm{HF}$ & 3 & 1 & 20 & 2 & 6 & CL05 & 59600 & 12800 & 966 & 8910 & 533 & 785 & $<362$ & 83200 & $<2450$ & $<207$ & 22200 \\
\hline SME Simulant & \begin{tabular}{|l|} 
Coliwasa \\
\end{tabular} & $\mathrm{HF}$ & 23 & 1 & 28 & 2 & 7 & CH06 & 57000 & 12800 & 930 & 8530 & $<523$ & 761 & $<381$ & 78900 & $<2570$ & $<218$ & 22700 \\
\hline SME Simulant & \begin{tabular}{|l} 
Coliwasa \\
\end{tabular} & LF & 1 & 1 & 12 & 2 & 8 & CH01 & 56400 & 13000 & 925 & 8570 & $<511$ & 733 & $<372$ & 78800 & $<2510$ & $<213$ & 22500 \\
\hline SME Simulant & \begin{tabular}{|l|} 
Coliwasa \\
\end{tabular} & LF & 20 & 1 & 2 & 2 & 9 & CL02 & 60700 & 13400 & 992 & 8980 & $<546$ & 751 & $<397$ & 83400 & $<2690$ & $<227$ & 22000 \\
\hline SME Simulant & $\begin{array}{l}\text { Coliwasa } \\
\end{array}$ & $\mathrm{HF}$ & 63 & 1 & 5 & 2 & 10 & CL08 & 59200 & 13100 & 961 & 8920 & $<508$ & 800 & $<369$ & 84100 & $<2500$ & $<211$ & 22900 \\
\hline SME Simulant & $\begin{array}{l}\text { Coliwasa } \\
\end{array}$ & $\mathrm{HF}$ & 65 & 1 & 6 & 2 & 11 & CH08 & 57200 & 13200 & 920 & 8410 & $<555$ & 718 & $<404$ & 78500 & $<2730$ & $<231$ & 22000 \\
\hline SME Simulant & Hydragard & $\mathrm{HF}$ & 45 & 2 & 7 & 2 & 12 & HHY07 & 59500 & 12600 & 953 & 8810 & $<490$ & 792 & $<356$ & 82400 & $<2410$ & $<204$ & 21900 \\
\hline SME Simulant & \begin{tabular}{|l|} 
Coliwasa \\
\end{tabular} & LF & 4 & 1 & 10 & 2 & 13 & CL01 & 57900 & 13000 & 959 & 8720 & $<572$ & 762 & $<416$ & 80500 & $<2810$ & $<238$ & 22800 \\
\hline SME Simulant & \begin{tabular}{|l|} 
Coliwasa \\
\end{tabular} & $\mathrm{HF}$ & 18 & 1 & 26 & 2 & 14 & CL06 & 59600 & 13200 & 987 & 8940 & $<589$ & 869 & $<428$ & 82800 & $<2800$ & $<245$ & 22100 \\
\hline ARG-1 & None & 0 & 0 & 2 & 12 & 2 & 15 & ARG-1B22 & 25400 & 23500 & 820 & 10600 & $<402$ & 665 & $<292$ & 98300 & 20300 & $<167$ & 15100 \\
\hline SME Simulant & Coliwasa & LF & 65 & 1 & 23 & 2 & 16 & CL04 & 57000 & 12700 & 947 & 8600 & $<553$ & 760 & $<402$ & 78200 & $<2720$ & $<230$ & 21800 \\
\hline SME Simulant & \begin{tabular}{|l} 
Coliwasa \\
\end{tabular} & LF & 40 & 1 & 19 & 2 & 17 & CL03 & 63500 & 11800 & 1000 & 9010 & $<592$ & 777 & $<430$ & 84700 & $<2910$ & $<246$ & 20900 \\
\hline SME Simulant & \begin{tabular}{|l|} 
Coliwasa \\
\end{tabular} & LF & 38 & 1 & 16 & 2 & 18 & CH03 & 56700 & 12500 & 931 & 8650 & $<569$ & 724 & $<414$ & 78800 & $<2800$ & $<237$ & 22600 \\
\hline SME Simulant & Hydragard & $\mathrm{HF}$ & 57 & 2 & \begin{tabular}{l|l}
6 \\
\end{tabular} & $2 \mid$ & 19 & HHY09 & 57800 & 13300 & 948 & 8820 & $<494$ & 768 & $<359$ & 79800 & $<2430$ & $<205$ & 22400 \\
\hline
\end{tabular}


Table A3. Phase 1 Elemental Concentration Measurements (part 1)

\begin{tabular}{|c|c|c|c|c|c|c|c|c|c|c|c|c|c|c|c|c|c|c|c|}
\hline $\begin{array}{c}\text { Type of } \\
\text { Material } \\
\end{array}$ & Sampler & $\begin{array}{l}\text { Flow } \\
\text { Rate }\end{array}$ & \begin{tabular}{|c|} 
Sample \\
Order \\
\end{tabular} & $\begin{array}{c}\text { Preparation } \\
\text { Block }\end{array}$ & $\begin{array}{c}\text { Preparation } \\
\text { Sequence } \\
\end{array}$ & $\begin{array}{c}\text { Analytical } \\
\text { Block }\end{array}$ & $\begin{array}{c}\text { Analytical } \\
\text { Sequence } \\
\end{array}$ & \begin{tabular}{|c} 
Sample \\
ID
\end{tabular} & $\begin{array}{c}\mathrm{Al} \\
(\mathrm{ug} / \mathrm{g})\end{array}$ & $\begin{array}{c}\text { B } \\
(\mathrm{ug} / \mathrm{g})\end{array}$ & $\begin{array}{c}\mathrm{Ba} \\
(\mathrm{ug} / \mathrm{g})\end{array}$ & $\begin{array}{c}\mathrm{Ca} \\
(\mathrm{ug} / \mathrm{g})\end{array}$ & $\begin{array}{c}\mathrm{Ce} \\
\text { (ug/g) }\end{array}$ & $\begin{array}{c}\mathrm{Cr} \\
(\mathrm{ug} / \mathrm{g})\end{array}$ & $\begin{array}{c}\mathrm{Cu} \\
(\mathrm{ug} / \mathrm{g})\end{array}$ & $\begin{array}{c}\text { Fe } \\
(\mathrm{ug} / \mathrm{g})\end{array}$ & $\begin{array}{c}\mathbf{K} \\
(\mathbf{u g} / \mathrm{g})\end{array}$ & $\begin{array}{c}\mathrm{La} \\
(\mathrm{ug} / \mathrm{g})\end{array}$ & \begin{tabular}{|c|}
$\mathbf{L i}$ \\
$(\mathrm{ug} / \mathrm{g})$
\end{tabular} \\
\hline SME Simulant & Coliwasa & LF & $\begin{array}{r}21 \\
\end{array}$ & \begin{tabular}{|r|}
1 \\
\end{tabular} & \begin{tabular}{|r|}
14 \\
\end{tabular} & \begin{tabular}{r|}
2 \\
\end{tabular} & \begin{tabular}{|r|}
20 \\
\end{tabular} & $\begin{array}{ll}\mathrm{CH} 02 \\
\end{array}$ & 58100 & 13000 & 947 & 8560 & $<577$ & 746 & $<419$ & 80100 & $<2830$ & $<240$ & 22500 \\
\hline SME Simulant & Hydragard & $\mathrm{HF}$ & 6 & 2 & 4 & 2 & 21 & HHY01 & 61400 & 12600 & 1010 & 9280 & $<596$ & 759 & $<434$ & 84900 & $<2930$ & $<248$ & 21600 \\
\hline SME Simulant & Hydragard & $\mathrm{HF}$ & 19 & 2 & 10 & 2 & 22 & HHY03 & 60400 & 12600 & 993 & 9200 & $<567$ & 767 & $<412$ & 84800 & $<2790$ & $<236$ & 22100 \\
\hline SME Simulant & Hydragard & $\mathrm{HF}$ & 50 & 2 & 5 & 2 & 23 & HHY08 & 58600 & 12700 & 965 & 8880 & 646 & 749 & $<415$ & 80900 & $<2810$ & $<238$ & 22300 \\
\hline SME Simulant & \begin{tabular}{|l|} 
Hydragard \\
\end{tabular} & $\mathrm{HF}$ & 37 & 2 & 9 & 2 & 24 & HHY06 & 59100 & 12800 & 964 & 8690 & $<577$ & 758 & $<420$ & 81100 & $<2840$ & $<240$ & 22300 \\
\hline SME Simulant & Coliwasa & $\mathrm{HF}$ & 34 & 1 & 18 & 2 & 25 & CH07 & 57000 & 12900 & 943 & 8560 & $<515$ & 738 & $<374$ & 78800 & $<2530$ & $<214$ & 21700 \\
\hline SME Simulant & \begin{tabular}{|l|} 
Coliwasa \\
\end{tabular} & LF & 63 & 1 & 13 & 2 & 26 & $\begin{array}{ll}\mathrm{CH} 04 \\
\end{array}$ & 57100 & 12900 & 946 & 8600 & $<562$ & 726 & $<409$ & 78800 & $<2760$ & $<234$ & 22400 \\
\hline SME Simulant & Hydragard & $\mathrm{HF}$ & 32 & 2 & 8 & 2 & 27 & HHY05 & 59100 & 12600 & 962 & 8660 & $<545$ & 771 & $<396$ & 81900 & $<2680$ & $<227$ & 22600 \\
\hline SME Simulant & Hydragard & $\mathrm{HF}$ & 61 & 2 & 11 & 2 & 28 & HHY10 & 60500 & 12900 & 991 & 9020 & 565 & 739 & $<370$ & 82500 & $<2500$ & $<212$ & 22300 \\
\hline ARG-1 & \begin{tabular}{|l|} 
None \\
\end{tabular} & 0 & 0 & 2 & 23 & 2 & 29 & ARG-1B23 & 25100 & 24300 & 838 & 10600 & $<501$ & 679 & $<365$ & 97900 & 21100 & $<209$ & 15100 \\
\hline ARG-1 & None & 0 & 0 & 3 & 1 & 3 & 1 & ARG-1B31 & 24800 & 26300 & 828 & 10500 & $<557$ & 714 & $<343$ & 98100 & 22100 & $<196$ & 15100 \\
\hline SME Simulant & Isolok - $12 \mathrm{~mL}$ & LF & 11 & 3 & 4 & 3 & 2 & LIB02 & 59300 & 13900 & 948 & 8890 & $<672$ & 753 & $<414$ & 81000 & $<2800$ & $<237$ & 21900 \\
\hline SME Simulant & \begin{tabular}{|l|} 
Coliwasa \\
\end{tabular} & $\mathrm{HF}$ & 36 & 1 & 21 & 3 & 3 & CL07 & 54900 & 13200 & 874 & 7950 & $<664$ & 681 & $<409$ & 73900 & $<2760$ & $<234$ & 24100 \\
\hline SME Simulant & Isolok - $12 \mathrm{~mL}$ & LF & 26 & 3 & 8 & 3 & 4 & LIB04 & 58000 & 13500 & 964 & 8890 & $<652$ & 741 & $<401$ & 80300 & $<2710$ & $<229$ & 22600 \\
\hline SME Simulant & \begin{tabular}{|l|} 
Coliwasa \\
\end{tabular} & $\mathrm{HF}$ & 1 & 1 & 22 & 3 & 5 & CH05 & 54800 & 12300 & 879 & 8100 & $<694$ & 695 & $<427$ & 74700 & $<2890$ & $<245$ & 20800 \\
\hline SME Simulant & \begin{tabular}{|l|} 
Coliwasa \\
\end{tabular} & $\mathrm{HF}$ & 3 & 1 & 20 & 3 & 6 & CL05 & 59700 & 13400 & 956 & 8930 & $<589$ & 790 & $<362$ & 82500 & $<2450$ & $<207$ & 22000 \\
\hline SME Simulant & \begin{tabular}{|l|} 
Coliwasa \\
\end{tabular} & $\mathrm{HF}$ & 23 & 1 & 28 & 3 & 7 & CH06 & 58300 & 12500 & 925 & 8560 & $<618$ & 750 & $<381$ & 78300 & $<2570$ & $<218$ & 22600 \\
\hline SME Simulant & $\begin{array}{l}\text { Coliwasa } \\
\end{array}$ & LF & 1 & 1 & 12 & 3 & 8 & CH01 & 57700 & 12900 & 924 & 8710 & $<604$ & 717 & $<372$ & 78300 & $<2510$ & $<213$ & 22400 \\
\hline SME Simulant & \begin{tabular}{|l|} 
Coliwasa \\
\end{tabular} & LF & 20 & 1 & 2 & 3 & 9 & CL02 & 60900 & 13600 & 972 & 9010 & $<646$ & 746 & $<397$ & 82500 & $<2690$ & $<227$ & 21700 \\
\hline SME Simulant & \begin{tabular}{|l} 
Coliwasa \\
\end{tabular} & $\mathrm{HF}$ & 63 & 1 & 5 & 3 & 10 & CL08 & 60000 & 13000 & 965 & 9030 & $<600$ & 790 & $<369$ & 83700 & $<2500$ & $<211$ & 22800 \\
\hline SME Simulant & \begin{tabular}{|l|} 
Coliwasa \\
\end{tabular} & $\mathrm{HF}$ & 65 & 1 & 6 & 3 & 11 & CH08 & 57300 & 13500 & 915 & 8400 & $<656$ & 707 & $<404$ & 77900 & $<2730$ & $<231$ & 21900 \\
\hline SME Simulant & Isolok - $12 \mathrm{~mL}$ & LF & 45 & 3 & 7 & 3 & 12 & LIB07 & 58400 & 13300 & 950 & 8990 & $<682$ & 757 & $<419$ & 81400 & 14600 & $<240$ & 21800 \\
\hline SME Simulant & \begin{tabular}{|l|} 
Coliwasa \\
\end{tabular} & LF & 4 & 1 & 10 & 3 & 13 & CL01 & 58100 & 12800 & 942 & 8720 & $<676$ & 745 & $<416$ & 79200 & $<2810$ & $<238$ & 22400 \\
\hline SME Simulant & \begin{tabular}{|l|} 
Coliwasa \\
\end{tabular} & $\mathrm{HF}$ & 18 & 1 & 26 & 3 & 14 & CL06 & 60000 & 13400 & 976 & 8970 & $<696$ & 833 & $<428$ & 82200 & $<2900$ & $<245$ & 21800 \\
\hline ARG-1 & \begin{tabular}{|l|} 
None \\
\end{tabular} & 0 & 0 & 3 & 12 & 3 & 15 & ARG-1B32 & 25000 & 24700 & 811 & 10500 & $<575$ & 654 & $<354$ & 97200 & 21000 & $<202$ & 14900 \\
\hline SME Simulant & $\begin{array}{l}\text { Coliwasa } \\
\end{array}$ & LF & 65 & 1 & 23 & 3 & 16 & $\begin{array}{ll}\text { CL04 } \\
\end{array}$ & 56400 & 13200 & 911 & 8420 & $<654$ & 772 & $<402$ & 76600 & $<2720$ & $<230$ & 21300 \\
\hline SME Simulant & \begin{tabular}{|l|} 
Coliwasa \\
\end{tabular} & LF & 40 & 1 & 19 & 3 & 17 & CL03 & 61300 & 12000 & 944 & 8580 & $<699$ & 771 & $<430$ & 81400 & $<2910$ & $<246$ & 20100 \\
\hline SME Simulant & Coliwasa & LF & 38 & 1 & 16 & 3 & 18 & $\mathrm{CH} 03$ & 57300 & 13100 & 931 & 8670 & $<672$ & 736 & $<414$ & 78900 & $<2800$ & $<237$ & 22500 \\
\hline SME Simulant & Isolok - $12 \mathrm{~mL}$ & LF & 56 & 3 & 11 & 3 & 19 & LIB09 & 57700 & 13600 & 939 & 8680 & $<700$ & 754 & $<431$ & 78200 & $<2910$ & $<246$ & 21600 \\
\hline SME Simulant & \begin{tabular}{|l|} 
Coliwasa \\
\end{tabular} & LF & 21 & 1 & 14 & 3 & 20 & $\mathrm{CH} 02$ & 53800 & 12600 & 868 & 7980 & $<682$ & 699 & $<419$ & 74100 & $<2830$ & $<240$ & 20600 \\
\hline SME Simulant & Isolok - $12 \mathrm{~mL}$ & LF & 5 & 3 & 5 & 3 & 21 & LIB01 & 62200 & 14200 & 992 & 9290 & $<695$ & 772 & $<428$ & 84200 & $<2890$ & $<245$ & 22900 \\
\hline SME Simulant & Isolok - $12 \mathrm{~mL}$ & LF & 23 & 3 & 10 & 3 & 22 & LIB03 & 56800 & 13000 & 923 & 8360 & $<715$ & 721 & $<440$ & 77000 & $<2970$ & $<252$ & 20800 \\
\hline SME Simulant & Isolok - $12 \mathrm{~mL}$ & LF & 50 & 3 & 2 & 3 & 23 & LIB08 & 59700 & 13500 & 977 & 8920 & $<710$ & 776 & $<437$ & 80900 & $<2950$ & $<250$ & 22100 \\
\hline SME Simulant & Isolok - $12 \mathrm{~mL}$ & LF & 41 & 3 & 9 & 3 & 24 & LIB06 & 60000 & 13500 & 981 & 9380 & $<687$ & 805 & $<423$ & 82500 & $<2860$ & $<242$ & 22400 \\
\hline SME Simulant & Coliwasa & $\mathrm{HF}$ & 34 & 1 & 18 & 3 & 25 & CH07 & 55900 & 12400 & 905 & 8420 & $<608$ & 702 & $<374$ & 76500 & $<2530$ & $<214$ & 20900 \\
\hline SME Simulant & \begin{tabular}{|l|} 
Coliwasa \\
\end{tabular} & LF & 63 & 1 & 13 & 3 & 26 & CH04 & 53900 & 13000 & 874 & 8080 & $<664$ & 704 & $<409$ & 74300 & $<2760$ & $<234$ & 21000 \\
\hline SME Simulant & Isolok - $12 \mathrm{~mL}$ & LF & 32 & 3 & 3 & 3 & 27 & LIB05 & 62300 & 13600 & 1010 & 9320 & $<629$ & 769 & $<387$ & 87000 & $<2620$ & $<222$ & 23000 \\
\hline SME Simulant & Isolok - $12 \mathrm{~mL}$ & LF & 60 & 3 & 6 & 3 & 28 & \begin{tabular}{|l|} 
LIB10 \\
\end{tabular} & 57100 & 12500 & 915 & 8510 & $<649$ & 710 & $<399$ & 78800 & $<2700$ & $<228$ & 20800 \\
\hline ARG-1 & None & 0 & 0 & 3 & 23 & 3 & 29 & \begin{tabular}{|l|l|} 
ARG-1B33 \\
\end{tabular} & 25700 & 25600 & 834 & 10700 & $<508$ & 690 & $<313$ & 100000 & 22800 & $<179$ & 15400 \\
\hline ARG-1 & None & 0 & 0 & 4 & 1 & 4 & 1 & ARG-1B41 & 24600 & 24800 & 809 & 10400 & $<482$ & 659 & $<351$ & 98000 & 22800 & $<201$ & 15000 \\
\hline SME Simulant & Isolok - $12 \mathrm{~mL}$ & $\mathrm{HF}$ & 13 & 4 & 11 & 4 & 2 & HIB02 & 57500 & 13500 & 939 & 8720 & $<539$ & 721 & $<392$ & 79500 & $<2650$ & $<224$ & 22100 \\
\hline SME Simulant & \begin{tabular}{|l|} 
Coliwasa \\
\end{tabular} & $\mathrm{HF}$ & 36 & 1 & 21 & 4 & 3 & CL07 & 55000 & 12700 & 877 & 8050 & $<562$ & 679 & $<409$ & 75500 & $<2760$ & $<234$ & 24900 \\
\hline SME Simulant & Isolok - $12 \mathrm{~mL}$ & $\mathrm{HF}$ & 25 & 4 & 4 & 4 & 4 & HIB04 & 57500 & 13100 & 949 & 8650 & $<605$ & 775 & $<440$ & 82100 & 3170 & $<252$ & 22200 \\
\hline SME Simulant & \begin{tabular}{|l|} 
Coliwasa \\
\end{tabular} & $\mathrm{HF}$ & 1 & 1 & 22 & 4 & 5 & CH05 & 56800 & 12500 & 914 & 8340 & $<588$ & 730 & $<427$ & 78700 & $<2890$ & $<245$ & 21500 \\
\hline SME Simulant & Coliwasa & $\mathrm{HF}$ & 3 & 1 & 20 & 4 & 6 & CL05 & 59100 & 13100 & 953 & 8830 & $<498$ & 779 & $<362$ & 83600 & $<2450$ & $<207$ & 22500 \\
\hline SME Simulant & \begin{tabular}{|l} 
Coliwasa \\
\end{tabular} & $\mathrm{HF}$ & 23 & 1 & 28 & 4 & 7 & CH06 & 56500 & 12500 & 916 & 8470 & $<523$ & 726 & $<381$ & 79100 & $<2590$ & $<218$ & 22900 \\
\hline SME Simulant & \begin{tabular}{|l|} 
Coliwasa \\
\end{tabular} & LF & 1 & 1 & 12 & 4 & 8 & CH01 & 56200 & 13100 & 920 & 8560 & $<511$ & 698 & $<372$ & 78500 & $<2510$ & $<213$ & 22600 \\
\hline SME Simulant & \begin{tabular}{|l|} 
Coliwasa \\
\end{tabular} & LF & 20 & 1 & 2 & 4 & 9 & CL02 & 59800 & 13300 & 966 & 8920 & $<546$ & 730 & $<397$ & 83300 & $<2690$ & $<227$ & 21900 \\
\hline
\end{tabular}


Table A3. Phase 1 Elemental Concentration Measurements (part 1)

\begin{tabular}{|c|c|c|c|c|c|c|c|c|c|c|c|c|c|c|c|c|c|c|c|}
\hline $\begin{array}{c}\text { Type of } \\
\text { Material } \\
\end{array}$ & Sampler & $\begin{array}{l}\text { Flow } \\
\text { Rate }\end{array}$ & \begin{tabular}{|c|} 
Sample \\
Order \\
\end{tabular} & $\begin{array}{c}\text { Preparation } \\
\text { Block }\end{array}$ & $\begin{array}{c}\text { Preparation } \\
\text { Sequence } \\
\end{array}$ & \begin{tabular}{|c|}
$\begin{array}{c}\text { Analytical } \\
\text { Block }\end{array}$ \\
\end{tabular} & \begin{tabular}{|c|} 
Analytical \\
Sequence \\
\end{tabular} & \begin{tabular}{|c} 
Sample \\
ID
\end{tabular} & $\begin{array}{c}\mathrm{Al} \\
(\mathrm{ug} / \mathrm{g})\end{array}$ & $\begin{array}{c}\text { B } \\
(\mathrm{ug} / \mathrm{g})\end{array}$ & $\begin{array}{c}\mathrm{Ba} \\
(\mathrm{ug} / \mathrm{g})\end{array}$ & $\begin{array}{c}\mathrm{Ca} \\
(\mathrm{ug} / \mathrm{g})\end{array}$ & $\begin{array}{c}\mathrm{Ce} \\
(\mathrm{ug} / \mathrm{g})\end{array}$ & $\begin{array}{c}\mathbf{C r} \\
(\mathrm{ug} / \mathrm{g})\end{array}$ & $\begin{array}{c}\mathrm{Cu} \\
\text { (ug/g) }\end{array}$ & 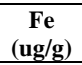 & $\begin{array}{c}\mathbf{K} \\
(\mathrm{ug} / \mathrm{g})\end{array}$ & 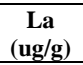 & \begin{tabular}{|c|}
$\mathbf{L i}$ \\
$(\mathrm{ug} / \mathrm{g})$
\end{tabular} \\
\hline SME Simulant & $\begin{array}{l}\text { Coliwasa } \\
\end{array}$ & $\mathrm{HF}$ & $\begin{array}{r}63 \\
\end{array}$ & \begin{tabular}{|r|}
1 \\
\end{tabular} & \begin{tabular}{|r|}
5 \\
\end{tabular} & \begin{tabular}{|r|}
4 \\
\end{tabular} & \begin{tabular}{|r|}
10 \\
\end{tabular} & $\begin{array}{ll}\text { CL08 } \\
\end{array}$ & 58400 & 12700 & 940 & 8710 & $<508$ & 751 & $<369$ & 84000 & $<2500$ & $<211$ & 22700 \\
\hline SME Simulant & \begin{tabular}{|l|} 
Coliwasa \\
\end{tabular} & $\mathrm{HF}$ & 65 & 1 & 6 & 4 & 11 & CH08 & 56400 & 13200 & 903 & 8180 & $<555$ & 694 & $<404$ & 78800 & $<2730$ & $<231$ & 21800 \\
\hline SME Simulant & Isolok - $12 \mathrm{~mL}$ & $\mathrm{HF}$ & 46 & 4 & 2 & 4 & 12 & HIB07 & 56900 & 13400 & 921 & 8570 & $<614$ & 723 & $<447$ & 78100 & $<3020$ & $<256$ & 21500 \\
\hline SME Simulant & \begin{tabular}{|l|} 
Coliwasa \\
\end{tabular} & LF & 4 & 1 & 10 & 4 & 13 & CL01 & 57200 & 13000 & 938 & 8620 & $<572$ & 747 & $<416$ & 80400 & $<2810$ & $<238$ & 22400 \\
\hline SME Simulant & $\begin{array}{l}\text { Coliwasa } \\
\end{array}$ & $\mathrm{HF}$ & 18 & 1 & 26 & 4 & 14 & CL06 & 58500 & 12900 & 960 & 8920 & $<589$ & 816 & $<428$ & 82800 & $<2900$ & $<245$ & 21700 \\
\hline ARG-1 & None & 0 & 0 & 4 & 7 & 4 & 15 & ARG-1B42 & 24300 & 25100 & 804 & 10300 & $<469$ & 659 & $<341$ & 97700 & 23700 & $<195$ & 14900 \\
\hline SME Simulant & $\begin{array}{l}\text { Coliwasa } \\
\end{array}$ & LF & 65 & 1 & 23 & 4 & 16 & $\begin{array}{ll}\text { CL04 } \\
\end{array}$ & 56400 & 13100 & 908 & 8410 & $<553$ & 752 & $<402$ & 78000 & $<2720$ & $<230$ & 21900 \\
\hline SME Simulant & \begin{tabular}{|l|} 
Coliwasa \\
\end{tabular} & LF & 40 & 1 & 19 & 4 & 17 & CL03 & 62200 & 12100 & 967 & 9040 & $<592$ & 776 & $<430$ & 84300 & $<2910$ & $<246$ & 20600 \\
\hline SME Simulant & Coliwasa & LF & 38 & 1 & 16 & 4 & 18 & $\mathrm{CH} 03$ & 56100 & 12600 & 916 & 8470 & $<569$ & 689 & $<414$ & 79100 & $<2800$ & $<237$ & 22500 \\
\hline SME Simulant & Isolok - $12 \mathrm{~mL}$ & $\mathrm{HF}$ & 56 & 4 & 9 & 4 & 19 & HIB09 & 59700 & 13100 & 1000 & 9190 & $<588$ & 790 & $<427$ & 85900 & $<2890$ & $<245$ & 22000 \\
\hline SME Simulant & \begin{tabular}{|l|} 
Coliwasa \\
\end{tabular} & LF & 21 & 1 & 14 & 4 & 20 & $\mathrm{CH} 02$ & 56900 & 13000 & 921 & 8310 & $<577$ & 714 & $<419$ & 79700 & 3290 & $<240$ & 22000 \\
\hline SME Simulant & Isolok - $12 \mathrm{~mL}$ & $\mathrm{HF}$ & 7 & 4 & 12 & 4 & 21 & HIB01 & 56500 & 13200 & 911 & 8240 & $<599$ & 732 & $<436$ & 78000 & $<2950$ & $<249$ & 22500 \\
\hline SME Simulant & Isolok - $12 \mathrm{~mL}$ & $\mathrm{HF}$ & 21 & 4 & 6 & 4 & 22 & HIB03 & 60500 & 12600 & 983 & 9330 & $<578$ & 784 & $<420$ & 83200 & $<2840$ & $<241$ & 22000 \\
\hline SME Simulant & Isolok - $12 \mathrm{~mL}$ & $\mathrm{HF}$ & 49 & 4 & 5 & 4 & 23 & HIB08 & 53100 & 14100 & 900 & 8120 & $<609$ & 667 & $<443$ & 72900 & $<3000$ & $<254$ & 23600 \\
\hline SME Simulant & Isolok - $12 \mathrm{~mL}$ & $\mathrm{HF}$ & 38 & 4 & 8 & 4 & 24 & HIB06 & 56100 & 13600 & 906 & 8400 & $<497$ & 709 & $<362$ & 78000 & $<2450$ & $<207$ & 22700 \\
\hline SME Simulant & \begin{tabular}{|l|} 
Coliwasa \\
\end{tabular} & $\mathrm{HF}$ & 34 & 1 & 18 & 4 & 25 & CH07 & 56600 & 12900 & 921 & 8540 & $<515$ & 699 & $<374$ & 78500 & $<2530$ & $<214$ & 22000 \\
\hline SME Simulant & \begin{tabular}{|l|} 
Coliwasa \\
\end{tabular} & LF & 63 & 1 & 13 & 4 & 26 & CH04 & 56700 & 13100 & 925 & 8550 & $<562$ & 720 & $<409$ & 79100 & $<2760$ & $<234$ & 22600 \\
\hline SME Simulant & Isolok - $12 \mathrm{~mL}$ & $\mathrm{HF}$ & 33 & 4 & 10 & 4 & 27 & HIB05 & 57400 & 12600 & 930 & 8520 & $<604$ & 761 & $<439$ & 82500 & $<2970$ & $<251$ & 22200 \\
\hline SME Simulant & Isolok - $12 \mathrm{~mL}$ & $\mathrm{HF}$ & 58 & 4 & 3 & 4 & 28 & HIB10 & 54700 & 13000 & 896 & 8070 & $<584$ & 668 & $<424$ & 74700 & $<2870$ & $<243$ & 22500 \\
\hline ARG-1 & None & 0 & 0 & 4 & 13 & 4 & 29 & \begin{tabular}{|l|l} 
ARG-1B43 \\
\end{tabular} & 24700 & 24400 & 810 & 10200 & $<486$ & 644 & $<354$ & 97900 & 21900 & $<202$ & 15000 \\
\hline ARG-1 & None & 0 & 0 & 2 & 1 & 5 & 1 & ARG-1B21 & 24400 & 24100 & 797 & 10500 & $<381$ & 662 & $<277$ & 96800 & 22800 & $<159$ & 15200 \\
\hline SME Simulant & Isolok - $3 \mathrm{~mL}$ & $\mathrm{HF}$ & 20 & 2 & 16 & 5 & 2 & HIS03 & 56200 & 12700 & 924 & 8500 & $<637$ & 713 & $<463$ & 78600 & $<3130$ & $<265$ & 22800 \\
\hline SME Simulant & Isolok - $3 \mathrm{~mL}$ & $\mathrm{HF}$ & 9 & 2 & 21 & 5 & 3 & HIS01 & 55700 & 13400 & 912 & 8390 & $<510$ & 676 & $<371$ & 78400 & $<2510$ & $<212$ & 22500 \\
\hline SME Simulant & \begin{tabular}{|l|} 
Coliwasa \\
\end{tabular} & LF & 20 & 1 & 2 & 5 & 4 & CL02 & 58500 & 13000 & 954 & 8830 & $<546$ & 720 & $<397$ & 81500 & $<2690$ & $<227$ & 21400 \\
\hline SME Simulant & \begin{tabular}{|l|} 
Coliwasa \\
\end{tabular} & LF & 38 & 1 & 16 & 5 & 5 & $\mathrm{CH} 03$ & 57900 & 13000 & 945 & 9040 & $<569$ & 723 & $<414$ & 81400 & $<2800$ & $<237$ & 23500 \\
\hline SME Simulant & \begin{tabular}{|l|} 
Coliwasa \\
\end{tabular} & LF & 63 & 1 & 13 & 5 & 6 & CH04 & 54000 & 12500 & 882 & 8220 & $<562$ & 698 & $<409$ & 75600 & $<2760$ & $<234$ & 21300 \\
\hline SME Simulant & Isolok - $3 \mathrm{~mL}$ & $\mathrm{HF}$ & 44 & 2 & 18 & 5 & 7 & HIS07 & 58100 & 13800 & 931 & 8840 & $<580$ & 730 & $<422$ & 80900 & $<2850$ & $<241$ & 22700 \\
\hline SME Simulant & Coliwasa & $\mathrm{HF}$ & 23 & 1 & 28 & 5 & 8 & CH06 & 54800 & 12200 & 883 & 8270 & $<523$ & 722 & $<381$ & 76200 & $<2570$ & $<218$ & 21900 \\
\hline SME Simulant & \begin{tabular}{|l|} 
Coliwasa \\
\end{tabular} & LF & 21 & 1 & 14 & 5 & 9 & $\mathrm{CH} 02$ & 58500 & 13000 & 944 & 8740 & $<577$ & 744 & $<419$ & 82000 & $<2830$ & $<240$ & 22600 \\
\hline SME Simulant & \begin{tabular}{|l|} 
Coliwasa \\
\end{tabular} & $\mathrm{HF}$ & 3 & 1 & 20 & 5 & 10 & CL05 & 56600 & 12500 & 910 & 8680 & $<498$ & 750 & $<362$ & 80000 & $<2450$ & $<207$ & 21600 \\
\hline SME Simulant & Isolok - $3 \mathrm{~mL}$ & $\mathrm{HF}$ & 14 & 2 & 15 & 5 & 11 & HIS02 & 55600 & 12900 & 895 & 8430 & $<544$ & 700 & $<395$ & 77200 & $<2670$ & $<226$ & 22100 \\
\hline SME Simulant & \begin{tabular}{|l|} 
Coliwasa \\
\end{tabular} & LF & 40 & 1 & 19 & 5 & 12 & CL03 & 60800 & 12000 & 951 & 8640 & $<592$ & 777 & $<430$ & 83300 & $<2910$ & $<246$ & 20100 \\
\hline SME Simulant & \begin{tabular}{|l} 
Coliwasa \\
\end{tabular} & $\mathrm{HF}$ & 18 & 1 & 26 & 5 & 13 & CL06 & 58000 & 13200 & 952 & 9000 & $<589$ & 810 & $<428$ & 81900 & $<2900$ & $<245$ & 21300 \\
\hline SME Simulant & \begin{tabular}{|l|} 
Coliwasa \\
\end{tabular} & $\mathrm{HF}$ & 1 & 1 & 22 & 5 & 14 & $\begin{array}{ll}\mathrm{CH} 05 \\
\end{array}$ & 55800 & 12600 & 904 & 7990 & $<588$ & 721 & $<427$ & 77400 & $<2890$ & $<245$ & 21100 \\
\hline ARG-1 & None & 0 & 0 & 2 & 12 & 5 & 15 & ARG-1B22 & 22600 & 22700 & 743 & 9770 & $<402$ & 613 & $<292$ & 90000 & 20700 & $<167$ & 14100 \\
\hline SME Simulant & \begin{tabular}{|l|l|} 
Coliwasa \\
\end{tabular} & $\mathrm{HF}$ & 36 & 1 & 21 & 5 & 16 & \begin{tabular}{|l|l} 
CL07 \\
\end{tabular} & 54100 & 12800 & 886 & 8270 & $<562$ & 690 & $<409$ & 74300 & $<2760$ & $<234$ & 24800 \\
\hline SME Simulant & Isolok - $3 \mathrm{~mL}$ & $\mathrm{HF}$ & 31 & 2 & 19 & 5 & 17 & HIS05 & 55400 & 12800 & 917 & 8470 & $<571$ & 691 & $<415$ & 77900 & $<2810$ & $<238$ & 22000 \\
\hline SME Simulant & Isolok - $3 \mathrm{~mL}$ & $\mathrm{HF}$ & 60 & 2 & 22 & 5 & 18 & HIS10 & 54900 & 13000 & 912 & 8650 & $<530$ & 709 & $<386$ & 78100 & $<2610$ & $<221$ & 22100 \\
\hline SME Simulant & \begin{tabular}{|l|} 
Coliwasa \\
\end{tabular} & $\mathrm{HF}$ & 63 & 1 & 5 & 5 & 19 & CL08 & 58300 & 12900 & 939 & 9070 & $<508$ & 753 & $<369$ & 83000 & $<2500$ & $<211$ & 22900 \\
\hline SME Simulant & Isolok - $3 \mathrm{~mL}$ & $\mathrm{HF}$ & 26 & 2 & 14 & 5 & 20 & HIS04 & 52300 & 12300 & 866 & 8070 & $<550$ & 669 & $<400$ & 73600 & $<2710$ & $<229$ & 20900 \\
\hline SME Simulant & Isolok - $3 \mathrm{~mL}$ & $\mathrm{HF}$ & 35 & 2 & 20 & 5 & 21 & HIS06 & 57000 & 13400 & 915 & 8810 & $<529$ & 724 & $<384$ & 79400 & $<2600$ & $<220$ & 23300 \\
\hline SME Simulant & \begin{tabular}{|l|} 
Coliwasa \\
\end{tabular} & $\mathrm{HF}$ & 65 & 1 & 6 & 5 & 22 & CH08 & 56500 & 13500 & 907 & 8420 & $<555$ & 701 & $<404$ & 78300 & $<2730$ & $<231$ & 21900 \\
\hline SME Simulant & \begin{tabular}{|l|} 
Coliwasa \\
\end{tabular} & $\mathrm{HF}$ & 34 & 1 & 18 & 5 & 23 & CH07 & 56500 & 13200 & 930 & 8610 & $<515$ & 727 & $<374$ & 78500 & $<2530$ & $<214$ & 21600 \\
\hline SME Simulant & \begin{tabular}{|l|} 
Coliwasa \\
\end{tabular} & LF & 1 & 1 & 12 & 5 & 24 & CH01 & 55200 & 12800 & 891 & 8400 & $<511$ & 686 & $<372$ & 76900 & $<2510$ & $<213$ & 21700 \\
\hline SME Simulant & Isolok - $3 \mathrm{~mL}$ & $\mathrm{HF}$ & 54 & 2 & 17 & 5 & 25 & HISO9 & 57400 & 13300 & 917 & 8900 & $<580$ & 717 & $<422$ & 79700 & $<2850$ & $<241$ & 22700 \\
\hline SME Simulant & \begin{tabular}{|l|} 
Coliwasa \\
\end{tabular} & LF & 4 & 1 & 10 & 5 & 26 & CL01 & 58300 & 13300 & 960 & 8880 & $<572$ & 753 & $<416$ & 81900 & $<2810$ & $<238$ & 23000 \\
\hline SME Simulant & Isolok - $3 \mathrm{~mL}$ & $\mathrm{HF}$ & 48 & 2 & 13 & 5 & 27 & HIS08 & 58200 & 13100 & 933 & 8660 & $<545$ & 730 & $<397$ & 80500 & $<2680$ & $<227$ & 22000 \\
\hline SME Simulant & \begin{tabular}{|l|} 
Coliwasa \\
\end{tabular} & LF & 65 & 1 & 23 & 5 & 28 & CL04 & 56700 & 13300 & 924 & 8810 & $<553$ & 763 & $<402$ & 78800 & $<2720$ & $<230$ & 21600 \\
\hline
\end{tabular}


Table A3. Phase 1 Elemental Concentration Measurements (part 1)

\begin{tabular}{|c|c|c|c|c|c|c|c|c|c|c|c|c|c|c|c|c|c|c|c|}
\hline $\begin{array}{l}\text { Type of } \\
\text { Material }\end{array}$ & Sampler & $\begin{array}{l}\text { Flow } \\
\text { Rate }\end{array}$ & $\begin{array}{c}\text { Sample } \\
\text { Order }\end{array}$ & $\begin{array}{c}\text { Preparation } \\
\text { Block }\end{array}$ & \begin{tabular}{|c|} 
Preparation \\
Sequence
\end{tabular} & $\begin{array}{c}\text { Analytical } \\
\text { Block }\end{array}$ & \begin{tabular}{|c|} 
Analytical \\
Sequence
\end{tabular} & $\begin{array}{c}\text { Sample } \\
\text { ID }\end{array}$ & $\begin{array}{c}\mathrm{Al} \\
(\mathrm{ug} / \mathrm{g})\end{array}$ & $\begin{array}{c}\mathrm{B} \\
(\mathrm{ug} / \mathrm{g})\end{array}$ & $\begin{array}{c}\text { Ba } \\
\text { (ug/g) }\end{array}$ & $\begin{array}{c}\text { Ca } \\
\text { (ug/g) }\end{array}$ & $\begin{array}{c}\mathrm{Ce} \\
(\mathrm{ug} / \mathrm{g})\end{array}$ & $\begin{array}{c}\mathrm{Cr} \\
(\mathrm{ug} / \mathrm{g})\end{array}$ & $\begin{array}{c}\mathrm{Cu} \\
(\mathrm{ug} / \mathrm{g})\end{array}$ & $\begin{array}{c}\mathrm{Fe} \\
(\mathrm{ug} / \mathrm{g})\end{array}$ & $\begin{array}{c}\mathrm{K} \\
(\mathrm{ug} / \mathrm{g})\end{array}$ & $\begin{array}{c}\text { La } \\
\text { (ug/g) }\end{array}$ & $\begin{array}{c}\mathbf{L i} \\
(\mathrm{ug} / \mathrm{g})\end{array}$ \\
\hline \begin{tabular}{|l|} 
ARG-1 \\
\end{tabular} & None & 0 & 0 & 2 & 23 & 5 & \begin{tabular}{|r|}
29 \\
\end{tabular} & ARG-1B23 & 24500 & 24000 & 812 & 10300 & $<501$ & 661 & $<365$ & 98000 & 22200 & $<209$ & 14900 \\
\hline ARG-1 & \begin{tabular}{|l|} 
None \\
\end{tabular} & 0 & 0 & 3 & 1 & 6 & 1 & ARG-1B31 & 24500 & 24700 & 797 & 10300 & $<471$ & 656 & $<343$ & 97700 & 22400 & $<196$ & 14700 \\
\hline SME Simulant & Hydragard & LF & 15 & 3 & 21 & 6 & 2 & \begin{tabular}{|l|} 
LHY03 \\
\end{tabular} & 59700 & 13100 & 953 & 9100 & $<555$ & 717 & $<404$ & 83300 & $<2730$ & $<231$ & 23400 \\
\hline SME Simulant & Hydragard & LF & 6 & 3 & 18 & 6 & 3 & LHY01 & 59500 & 13300 & 957 & 8830 & $<594$ & 725 & $<432$ & 83100 & $<2920$ & $<247$ & 21700 \\
\hline SME Simulant & \begin{tabular}{|l|} 
Coliwasa \\
\end{tabular} & LF & 20 & 1 & 2 & 6 & 4 & CL02 & 60000 & 13300 & 959 & 9090 & $<546$ & 728 & $<397$ & 83400 & $<2690$ & $<227$ & 22300 \\
\hline SME Simulant & Coliwasa & LF & 38 & 1 & 16 & 6 & 5 & $\mathrm{CH} 03$ & 57000 & 12800 & 928 & 8700 & $<569$ & 719 & $<414$ & 79900 & $<2800$ & $<237$ & 22700 \\
\hline SME Simulant & \begin{tabular}{|l|} 
Coliwasa \\
\end{tabular} & LF & 63 & 1 & 13 & 6 & 6 & CH04 & 56400 & 12900 & 917 & 8480 & $<562$ & 715 & $<409$ & 79000 & $<2760$ & $<234$ & 21800 \\
\hline SME Simulant & \begin{tabular}{|l|} 
Hydragard \\
\end{tabular} & LF & 47 & 3 & 13 & 6 & 7 & LHY07 & 61800 & 12500 & 971 & 9240 & $<609$ & 757 & $<443$ & 86500 & $<3000$ & $<254$ & 22200 \\
\hline SME Simulant & \begin{tabular}{|l|} 
Coliwasa \\
\end{tabular} & $\mathrm{HF}$ & 23 & 1 & 28 & 6 & 8 & CH06 & 57100 & 12300 & 911 & 8480 & $<523$ & 722 & $<381$ & 79700 & $<2570$ & $<218$ & 22300 \\
\hline SME Simulant & Coliwasa & LF & 21 & 1 & 14 & 6 & 9 & CH02 & 54400 & 12100 & 867 & 8030 & $<577$ & 681 & $<419$ & 76400 & $<2830$ & $<240$ & 20700 \\
\hline SME Simulant & \begin{tabular}{|l|} 
Coliwasa \\
\end{tabular} & HF & 3 & 1 & 20 & 6 & 10 & CL05 & 60400 & 13000 & 972 & 9100 & $<498$ & 773 & 380 & 85500 & $<2450$ & $<207$ & 22700 \\
\hline SME Simulant & Hydragard & LF & 10 & 3 & 20 & 6 & 11 & LHY02 & 57000 & 13900 & 893 & 8230 & $<599$ & 682 & $<436$ & 78100 & $<2950$ & $<249$ & 23100 \\
\hline SME Simulant & \begin{tabular}{|l|} 
Coliwasa \\
\end{tabular} & LF & 40 & 1 & 19 & 6 & 12 & CL03 & 63700 & 12100 & 984 & 8990 & $<592$ & 790 & $<430$ & 86800 & $<2910$ & $<246$ & 20900 \\
\hline SME Simulant & Coliwasa & $\mathrm{HF}$ & 18 & 1 & 26 & 6 & 13 & CL06 & 58600 & 12500 & 947 & 8910 & $<589$ & 795 & $<428$ & 82600 & $<2900$ & $<245$ & 21500 \\
\hline SME Simulant & Coliwasa & $\mathrm{HF}$ & 1 & 1 & 22 & 6 & 14 & CH05 & 57500 & 12500 & 926 & 8480 & $<588$ & 738 & $<427$ & 80000 & $<2890$ & $<245$ & 21800 \\
\hline \begin{tabular}{|l|} 
ARG-1 \\
\end{tabular} & None & 0 & 0 & 3 & 12 & 6 & 15 & ARG-1B32 & 24100 & 24700 & 783 & 10200 & $<486$ & 653 & $<354$ & 95600 & 21800 & $<202$ & 14400 \\
\hline SME Simulant & \begin{tabular}{|l|} 
Coliwasa \\
\end{tabular} & $\mathrm{HF}$ & 36 & 1 & 21 & 6 & 16 & $\begin{array}{ll}\text { CL07 } \\
\end{array}$ & 55300 & 12400 & 879 & 8050 & $<562$ & 670 & $<409$ & 75900 & $<2760$ & $<234$ & 24400 \\
\hline SME Simulant & Hydragard & LF & 30 & 3 & 14 & 6 & 17 & LHY05 & 59200 & 12700 & 949 & 8870 & $<530$ & 754 & $<386$ & 83400 & $<2610$ & $<221$ & 22000 \\
\hline SME Simulant & Hydragard & LF & 59 & 3 & 17 & 6 & 18 & LHY10 & 58200 & 12900 & 939 & 8990 & $<570$ & 691 & $<415$ & 80900 & $<2800$ & $<237$ & 23300 \\
\hline SME Simulant & \begin{tabular}{|l|} 
Coliwasa \\
\end{tabular} & HF & 63 & 1 & 5 & 6 & 19 & CL08 & 60500 & 13000 & 957 & 8930 & $<508$ & 766 & $<369$ & 85700 & $<2500$ & $<211$ & 22500 \\
\hline SME Simulant & \begin{tabular}{|l|} 
Hydragard \\
\end{tabular} & LF & 28 & 3 & 16 & 6 & 20 & LHY04 & 59000 & 13100 & 942 & 8940 & $<559$ & 716 & $<407$ & 82400 & $<2750$ & $<233$ & 22300 \\
\hline SME Simulant & Hydragard & LF & 37 & 3 & 15 & 6 & 21 & LHY06 & 61300 & 14000 & 978 & 9290 & $<575$ & 746 & $<418$ & 84500 & $<2830$ & $<239$ & 23500 \\
\hline SME Simulant & \begin{tabular}{|l|} 
Coliwasa \\
\end{tabular} & HF & 65 & 1 & 6 & 6 & 22 & CH08 & 55100 & 12600 & 875 & 8070 & $<555$ & 663 & $<404$ & 76600 & $<2730$ & $<231$ & 21400 \\
\hline SME Simulant & Coliwasa & HF & 34 & 1 & 18 & 6 & 23 & CH07 & 58700 & 13200 & 955 & 9000 & $<515$ & 742 & $<374$ & 81900 & $<2530$ & $<214$ & 23200 \\
\hline SME Simulant & \begin{tabular}{|l|} 
Coliwasa \\
\end{tabular} & LF & 1 & 1 & 12 & 6 & 24 & CH01 & 55300 & 12600 & 887 & 8410 & $<511$ & 678 & $<372$ & 76800 & $<2510$ & $<213$ & 22000 \\
\hline SME Simulant & Hydragard & LF & 53 & 3 & 19 & 6 & 25 & LHY09 & 61900 & 13500 & 1010 & 9610 & $<513$ & 772 & $<373$ & 85800 & $<2520$ & $<214$ & 23800 \\
\hline SME Simulant & \begin{tabular}{|l|} 
Coliwasa \\
\end{tabular} & LF & 4 & 1 & 10 & 6 & 26 & CL01 & 57300 & 12700 & 924 & 8810 & $<572$ & 727 & $<416$ & 80400 & $<2810$ & $<238$ & 23100 \\
\hline SME Simulant & Hydragard & LF & 48 & 3 & 22 & 6 & 27 & LHY08 & 57700 & 12800 & 934 & 8730 & $<511$ & 703 & $<372$ & 80400 & $<2510$ & $<213$ & 21600 \\
\hline SME Simulant & \begin{tabular}{|l|} 
Coliwasa \\
\end{tabular} & LF & 65 & 1 & 23 & 6 & 28 & CL04 & 57300 & 13200 & 920 & 8620 & $<553$ & 760 & $<402$ & 79000 & $<2720$ & $<230$ & 22200 \\
\hline ARG-1 & None & 0 & 0 & 3 & 23 & 6 & 29 & ARG-1B33 & 23200 & 24000 & 762 & 9910 & $<430$ & 618 & $<313$ & 92300 & 21900 & $<179$ & 14500 \\
\hline
\end{tabular}


Table A3. Phase 1 Elemental Concentration Measurements (part 2)

\begin{tabular}{|c|c|c|c|c|c|c|c|c|c|c|c|c|c|c|c|c|c|c|c|}
\hline $\begin{array}{c}\text { Type of } \\
\text { Material }\end{array}$ & Sampler & $\begin{array}{l}\text { Flow } \\
\text { Rate }\end{array}$ & $\begin{array}{c}\text { Sample } \\
\text { Order }\end{array}$ & $\begin{array}{c}\text { Preparation } \\
\text { Block }\end{array}$ & $\begin{array}{c}\text { Preparation } \\
\text { Sequence }\end{array}$ & $\begin{array}{c}\text { Analytical } \\
\text { Block }\end{array}$ & $\begin{array}{c}\text { Analytical } \\
\text { Sequence }\end{array}$ & Sample ID & $\begin{array}{c}\begin{array}{c}\text { Mg } \\
\text { (ug/g) }\end{array} \\
\end{array}$ & $\begin{array}{c}\begin{array}{c}\text { Mn } \\
(\mathbf{u g} / \mathrm{g})\end{array} \\
\end{array}$ & $\begin{array}{c}\mathrm{Na} \\
(\mathrm{ug} / \mathrm{g})\end{array}$ & $\begin{array}{c}\mathbf{N i} \\
(\mathrm{ug} / \mathrm{g})\end{array}$ & \begin{tabular}{|c|}
$\mathbf{P}$ \\
$(\mathrm{ug} / \mathrm{g})$
\end{tabular} & \begin{tabular}{|c|}
$\mathbf{P b}$ \\
(ug/g)
\end{tabular} & \begin{tabular}{|c}
$\mathrm{S}$ \\
$(\mathrm{ug} / \mathrm{g})$
\end{tabular} & \begin{tabular}{|c|}
$\mathrm{Si}$ \\
$(\mathrm{ug} / \mathrm{g})$
\end{tabular} & \begin{tabular}{|c}
$\mathrm{Ti}$ \\
$(\mathrm{ug} / \mathrm{g})$
\end{tabular} & $\begin{array}{c}\mathrm{Zn} \\
(\mathrm{ug} / \mathrm{g})\end{array}$ & $\begin{array}{c}\mathbf{Z r} \\
(\mathrm{ug} / \mathrm{g})\end{array}$ \\
\hline ARG-1 & None & 0 & 0 & 1 & 1 & 1 & 1 & \begin{tabular}{|l} 
ARG-1B11 \\
\end{tabular} & 5170 & 14300 & 85500 & 8130 & 848 & \begin{tabular}{|c|}
$<813$ \\
\end{tabular} & $<649$ & 226000 & 6920 & 194 & 985 \\
\hline SME Simulant & Isolok - $3 \mathrm{~mL}$ & LF & 18 & 1 & 9 & 1 & 2 & \begin{tabular}{|l|} 
LIS03 \\
\end{tabular} & 6420 & 13500 & 89000 & 3590 & $<417$ & $<1050$ & 855 & 241000 & 486 & 832 & 2710 \\
\hline SME Simulant & Isolok - $3 \mathrm{~mL}$ & LF & 8 & 1 & 27 & 1 & 3 & LIS01 & 6490 & 13900 & 78600 & 3640 & $<392$ & $<985$ & 1190 & 243000 & 496 & 825 & 2820 \\
\hline SME Simulant & $\begin{array}{l}\text { Coliwasa } \\
\end{array}$ & LF & 20 & 1 & 2 & 1 & 4 & CL02 & 6640 & 14000 & 84000 & 3650 & $<404$ & $<1020$ & 1680 & 235000 & 486 & 828 & 2800 \\
\hline SME Simulant & Coliwasa & LF & 38 & 1 & 16 & 1 & 5 & CH03 & 6370 & 13600 & 83900 & 3560 & $<421$ & $<1060$ & 1420 & 245000 & 509 & 816 & 2730 \\
\hline SME Simulant & Coliwasa & LF & 63 & 1 & 13 & 1 & 6 & CH04 & 6310 & 13400 & 83800 & 3520 & $<415$ & $<1040$ & 1540 & 243000 & 511 & 817 & 2720 \\
\hline SME Simulant & Isolok - $3 \mathrm{~mL}$ & LF & 44 & 1 & 3 & 1 & 7 & LIS07 & 6510 & 14000 & 80900 & 3560 & $<409$ & $<1030$ & $<821$ & 244000 & 488 & 804 & 2770 \\
\hline SME Simulant & Coliwasa & $\mathrm{HF}$ & 23 & 1 & 28 & 1 & 8 & $\mathrm{CH} 06$ & 6280 & 13300 & 80700 & 3490 & $<387$ & $<972$ & 1070 & 246000 & 511 & 782 & 2780 \\
\hline SME Simulant & Coliwasa & LF & 21 & 1 & 14 & 1 & 9 & $\mathrm{CH} 02$ & 6370 & 13500 & 82900 & 3560 & $<426$ & $<1070$ & 1430 & 245000 & 497 & 804 & 2750 \\
\hline SME Simulant & Coliwasa & $\mathrm{HF}$ & 3 & 1 & 20 & 1 & 10 & CL05 & 6600 & 14100 & 82100 & 3750 & $<368$ & $<926$ & 1590 & \begin{tabular}{|l|}
239000 \\
\end{tabular} & 486 & 825 & 2790 \\
\hline SME Simulant & Isolok - $3 \mathrm{~mL}$ & LF & 12 & 1 & 25 & 1 & 11 & LIS02 & 6530 & 13800 & 83100 & 3630 & $<384$ & $<964$ & 1160 & 241000 & 489 & 828 & 2800 \\
\hline SME Simulant & Coliwasa & LF & 40 & 1 & 19 & 1 & 12 & CL03 & 6690 & 14100 & 86900 & 3670 & $<437$ & $<1100$ & 1290 & 225000 & 458 & 860 & 2800 \\
\hline SME Simulant & Coliwasa & $\mathrm{HF}$ & 18 & 1 & 26 & 1 & 13 & CL06 & 6620 & 14200 & 88600 & 3720 & $<436$ & $<1090$ & 1730 & 236000 & 500 & 833 & 2800 \\
\hline SME Simulant & Coliwasa & $\mathrm{HF}$ & 1 & 1 & 22 & 1 & 14 & $\begin{array}{l}\mathrm{CH} 05 \\
\end{array}$ & 6220 & 13200 & 82300 & 3490 & $<434$ & $<1090$ & 1410 & 248000 & 479 & 795 & 2720 \\
\hline ARG-1 & None & 0 & 0 & 1 & 15 & 1 & 15 & \begin{tabular}{|l|} 
ARG-1B12 \\
\end{tabular} & 5180 & 14400 & 86100 & 8140 & \begin{tabular}{|l|}
881 \\
\end{tabular} & $\begin{array}{l}<827 \\
\end{array}$ & 764 & 226000 & 6900 & 198 & 985 \\
\hline SME Simulant & Coliwasa & $\mathrm{HF}$ & 36 & 1 & 21 & 1 & 16 & $\begin{array}{ll}\text { CL07 } \\
\end{array}$ & 5910 & 12400 & 84200 & 3080 & $<415$ & $<1040$ & 1610 & 246000 & 499 & 783 & 2640 \\
\hline SME Simulant & Isolok - $3 \mathrm{~mL}$ & LF & 31 & 1 & 24 & 1 & 17 & LIS05 & 6380 & 13500 & 88200 & 3520 & $<437$ & $<1100$ & 1250 & 242000 & 474 & 800 & 2720 \\
\hline SME Simulant & Isolok - $3 \mathrm{~mL}$ & LF & 58 & 1 & 4 & 1 & 18 & LIS10 & 6610 & 14100 & 78600 & 3670 & $<410$ & $<1030$ & 952 & 242000 & 500 & 829 & 2800 \\
\hline SME Simulant & Coliwasa & $\mathrm{HF}$ & 63 & 1 & 5 & 1 & 19 & CL08 & 6650 & 14500 & 84800 & 4040 & $<376$ & 1270 & 1640 & 235000 & 462 & 882 & 2720 \\
\hline SME Simulant & Isolok - $3 \mathrm{~mL}$ & LF & 25 & 1 & 7 & 1 & 20 & LIS04 & 6310 & 13300 & 88600 & 3460 & $<407$ & $<1020$ & 1180 & 242000 & 486 & 790 & 2670 \\
\hline SME Simulant & Isolok - $3 \mathrm{~mL}$ & LF & 42 & 1 & 11 & 1 & 21 & LIS06 & 6610 & 14200 & 84500 & 3710 & $<411$ & $<1030$ & 843 & 239000 & 491 & 843 & 2800 \\
\hline SME Simulant & Coliwasa & $\mathrm{HF}$ & 65 & 1 & 6 & 1 & 22 & $\mathrm{CH} 08$ & 6230 & 13200 & 86700 & 3440 & $<411$ & $<1030$ & 1460 & 244000 & 490 & 785 & 2760 \\
\hline SME Simulant & Coliwasa & $\mathrm{HF}$ & 34 & 1 & 18 & 1 & 23 & CH07 & 6250 & 13300 & 79600 & 3450 & $<381$ & $<<957$ & 1440 & \begin{tabular}{|l|}
246000 \\
\end{tabular} & 478 & 801 & 2760 \\
\hline SME Simulant & Coliwasa & LF & 1 & 1 & 12 & 1 & 24 & CH01 & 6270 & 13300 & 83300 & 3460 & $<378$ & $<949$ & 1360 & \begin{tabular}{|l|}
245000 \\
\end{tabular} & 502 & 795 & 2730 \\
\hline SME Simulant & Isolok - $3 \mathrm{~mL}$ & LF & 54 & 1 & 17 & 1 & 25 & LIS09 & 6430 & 14000 & 78000 & 3670 & $<434$ & $<1090$ & 1520 & 243000 & 498 & 818 & 2820 \\
\hline SME Simulant & Coliwasa & LF & 4 & 1 & 10 & 1 & 26 & CL01 & 6410 & 13600 & 80700 & 3580 & $<423$ & $<1060$ & 1190 & 244000 & 501 & 809 & 2750 \\
\hline SME Simulant & Isolok - $3 \mathrm{~mL}$ & LF & 49 & 1 & 8 & 1 & 27 & LIS08 & 6540 & 14000 & 81200 & 3630 & $<427$ & $<1070$ & 1240 & \begin{tabular}{|l|}
241000 \\
\end{tabular} & 482 & 816 & 2770 \\
\hline SME Simulant & Coliwasa & LF & 65 & 1 & 23 & 1 & 28 & $\begin{array}{l}\text { CL04 } \\
\end{array}$ & 6250 & 13200 & 80700 & 3340 & $<409$ & $<1030$ & 1380 & 247000 & 499 & 771 & 2750 \\
\hline ARG-1 & None & 0 & 0 & 1 & 29 & 1 & 29 & \begin{tabular}{|l} 
ARG-1B13 \\
\end{tabular} & 5210 & 14400 & 86700 & 8060 & 876 & $<1030$ & $<825$ & 226000 & 6940 & 191 & 1010 \\
\hline ARG-1 & None & 0 & 0 & 2 & 1 & 2 & 1 & \begin{tabular}{|l|} 
ARG-1B21 \\
\end{tabular} & 5240 & 14600 & 86300 & 8390 & 902 & $<>709$ & $<566$ & 227000 & 6940 & 193 & 1000 \\
\hline SME Simulant & Hydragard & $\mathrm{HF}$ & 11 & 2 & 3 & 2 & 2 & $\begin{array}{l}\text { HHY02 } \\
\end{array}$ & 6660 & 14100 & 85900 & 3730 & $<415$ & $<1040$ & 1290 & 241000 & 495 & 827 & 2740 \\
\hline SME Simulant & Coliwasa & $\mathrm{HF}$ & 36 & 1 & 21 & 2 & 3 & CL07 & 6000 & 12600 & 84600 & 3080 & $<415$ & $<1040$ & 1290 & 246000 & 499 & 760 & 2650 \\
\hline SME Simulant & Hydragard & $\mathrm{HF}$ & 24 & 2 & 2 & 2 & 4 & HHY04 & 6380 & 13700 & 84600 & 3680 & \begin{tabular}{|l|}
466 \\
\end{tabular} & $<1130$ & 1350 & 243000 & 524 & 834 & 2780 \\
\hline SME Simulant & Coliwasa & $\mathrm{HF}$ & 1 & 1 & 22 & 2 & 5 & $\mathrm{CH} 05$ & 6270 & 13400 & 81800 & 3540 & $<434$ & $<1090$ & 1160 & \begin{tabular}{|l|}
248000 \\
\end{tabular} & 502 & 795 & 2710 \\
\hline SME Simulant & Coliwasa & $\mathrm{HF}$ & 3 & 1 & 20 & 2 & 6 & CL05 & 6710 & 14500 & 82200 & 3860 & $<368$ & 1070 & 1210 & 242000 & 490 & 825 & 2780 \\
\hline SME Simulant & Coliwasa & $\mathrm{HF}$ & 23 & 1 & 28 & 2 & 7 & CH06 & 6370 & 13600 & 81000 & 3590 & $<387$ & $<972$ & 1000 & 247000 & 497 & 814 & 2760 \\
\hline SME Simulant & Coliwasa & LF & 1 & 1 & 12 & 2 & 8 & CH01 & 6390 & 13700 & 82900 & 3520 & $<3$ & $<949$ & 1290 & \begin{tabular}{|l|}
247000 \\
\end{tabular} & 485 & 805 & 2720 \\
\hline SME Simulant & Coliwasa & LF & 20 & 1 & 2 & 2 & 9 & CL02 & 6770 & 14400 & 84900 & 3760 & $<404$ & $<1020$ & 1450 & 238000 & 493 & 850 & 2840 \\
\hline SME Simulant & Coliwasa & $\mathrm{HF}$ & 63 & 1 & 5 & 2 & 10 & CL08 & 6740 & 14700 & 85100 & 4050 & $<376$ & $<944$ & 1570 & 237000 & 489 & 862 & 2750 \\
\hline SME Simulant & Coliwasa & $\mathrm{HF}$ & 65 & 1 & 6 & 2 & 11 & CH08 & 6320 & 13300 & 87100 & 3520 & $<411$ & $<1030$ & 1180 & \begin{tabular}{|l|}
244000 \\
\end{tabular} & 486 & 808 & 2740 \\
\hline SME Simulant & Hydragard & $\mathrm{HF}$ & 45 & 2 & 7 & 2 & 12 & HHY07 & 6440 & 14000 & 80300 & 3960 & $<362$ & $<910$ & 1270 & 244000 & 508 & 841 & 2810 \\
\hline SME Simulant & Coliwasa & LF & 4 & 1 & 10 & 2 & 13 & CL01 & 6540 & 14000 & 81000 & 3680 & $<423$ & $<1060$ & 1100 & \begin{tabular}{|l|}
247000 \\
\end{tabular} & 504 & 832 & 2750 \\
\hline SME Simulant & Coliwasa & $\mathrm{HF}$ & 18 & 1 & 26 & 2 & 14 & CL06 & 6700 & 14300 & 89000 & 3820 & $<436$ & $\begin{array}{l}<1090 \\
\end{array}$ & 1800 & 237000 & 492 & 869 & 2750 \\
\hline ARG-1 & None & 0 & 0 & 2 & 12 & 2 & 15 & \begin{tabular}{|l} 
ARG-1B22 \\
\end{tabular} & 5280 & 14700 & 86800 & 8090 & \begin{tabular}{|l|}
917 \\
\end{tabular} & 1170 & $<596$ & 229000 & 6950 & 187 & 1000 \\
\hline SME Simulant & Coliwasa & LF & 65 & 1 & 23 & 2 & 16 & \begin{tabular}{|l|} 
CL04 \\
\end{tabular} & 6340 & 13500 & 81000 & 3430 & $<409$ & 1640 & 1510 & 248000 & 484 & 805 & 2680 \\
\hline SME Simulant & Coliwasa & LF & 40 & 1 & 19 & 2 & 17 & CL03 & 6780 & 14500 & 88200 & 3730 & $<437$ & 1570 & 1140 & 228000 & 446 & 884 & 2810 \\
\hline SME Simulant & Coliwasa & LF & 38 & 1 & 16 & 2 & 18 & $\mathrm{CH} 03$ & 6410 & 13700 & 84500 & 3540 & $<421$ & $<1060$ & 954 & 245000 & 506 & 816 & 2680 \\
\hline SME Simulant & Hydragard & $\mathrm{HF}$ & 57 & 2 & 6 & 2 & 19 & HHY09 & 6440 & 13800 & 83200 & 3620 & $<365$ & $<<918$ & 1340 & 245000 & 499 & 838 & 2760 \\
\hline
\end{tabular}


Table A3. Phase 1 Elemental Concentration Measurements (part 2)

\begin{tabular}{|c|c|c|c|c|c|c|c|c|c|c|c|c|c|c|c|c|c|c|c|}
\hline $\begin{array}{c}\text { Type of } \\
\text { Material } \\
\end{array}$ & Sampler & $\begin{array}{l}\text { Flow } \\
\text { Rate }\end{array}$ & $\begin{array}{c}\text { Sample } \\
\text { Order }\end{array}$ & $\begin{array}{c}\text { Preparation } \\
\text { Block }\end{array}$ & $\begin{array}{c}\text { Preparation } \\
\text { Sequence }\end{array}$ & $\begin{array}{c}\text { Analytical } \\
\text { Block }\end{array}$ & $\begin{array}{c}\text { Analytical } \\
\text { Sequence }\end{array}$ & Sample ID & $\begin{array}{c}\begin{array}{c}\text { Mg } \\
\text { (ug/g) }\end{array} \\
\end{array}$ & $\begin{array}{c}\begin{array}{c}\text { Mn } \\
(\mathbf{u g} / \mathrm{g})\end{array} \\
\end{array}$ & $\begin{array}{c}\mathrm{Na} \\
(\mathrm{ug} / \mathrm{g})\end{array}$ & $\left.\begin{array}{c}\mathbf{N i} \\
(\mathbf{u g} / \mathrm{g})\end{array}\right)$ & \begin{tabular}{|c|}
$\begin{array}{r}\text { P } \\
(\mathbf{u g} / \mathrm{g})\end{array}$ \\
\end{tabular} & \begin{tabular}{|c|}
$\mathbf{P b}$ \\
$(\mathbf{u g} / \mathrm{g})$
\end{tabular} & \begin{tabular}{|c|}
$\mathrm{S}$ \\
$(\mathrm{ug} / \mathrm{g})$
\end{tabular} & \begin{tabular}{|c|}
$\mathrm{Si}$ \\
$(\mathrm{ug} / \mathrm{g})$
\end{tabular} & \begin{tabular}{|c}
$\mathrm{Ti}$ \\
$(\mathrm{ug} / \mathrm{g})$
\end{tabular} & $\begin{array}{c}\mathrm{Zn} \\
(\mathrm{ug} / \mathrm{g})\end{array}$ & $\begin{array}{c}\mathrm{Zr} \\
(\mathrm{ug} / \mathrm{g})\end{array}$ \\
\hline SME Simulant & Coliwasa & LF & 21 & 1 & 14 & 2 & 20 & $\mathrm{CHO2}$ & 6470 & 13800 & 84100 & 3650 & $<426$ & $<1070$ & 1280 & 246000 & 493 & 827 & 2730 \\
\hline SME Simulant & Hydragard & $\mathrm{HF}$ & 6 & 2 & 4 & 2 & 21 & HHY01 & 6890 & 14700 & 87900 & 3710 & $<441$ & 1130 & 1230 & 235000 & 494 & 868 & 2840 \\
\hline SME Simulant & Hydragard & $\mathrm{HF}$ & 19 & 2 & 10 & 2 & 22 & HHY03 & 6840 & 14700 & 84400 & 3900 & $<419$ & 1250 & 1530 & 237000 & 473 & 870 & 2740 \\
\hline SME Simulant & Hydragard & $\mathrm{HF}$ & 50 & 2 & 5 & 2 & 23 & HHY08 & 6470 & 13800 & 84800 & 3620 & $<422$ & 1210 & 1650 & 244000 & 480 & 842 & 2780 \\
\hline SME Simulant & Hydragard & $\mathrm{HF}$ & 37 & 2 & 9 & 2 & 24 & HHY06 & 6490 & 13900 & 80700 & 3720 & $<427$ & 1530 & 1480 & 245000 & 493 & 828 & 2750 \\
\hline SME Simulant & Coliwasa & $\mathrm{HF}$ & 34 & 1 & 18 & 2 & 25 & CH07 & 6380 & 13600 & 80500 & 3580 & $<381$ & 1040 & 1340 & 249000 & 496 & 811 & 2750 \\
\hline SME Simulant & Coliwasa & LF & 63 & 1 & 13 & 2 & 26 & CH04 & 6390 & 13600 & 84600 & 3510 & $<415$ & 1260 & 1570 & 244000 & 507 & 817 & 2710 \\
\hline SME Simulant & Hydragard & $\mathrm{HF}$ & 32 & 2 & 8 & 2 & 27 & HHY05 & 6580 & 14200 & 88000 & 3840 & $<403$ & 1370 & 1100 & 238000 & 473 & 859 & 2760 \\
\hline SME Simulant & Hydragard & $\mathrm{HF}$ & 61 & 2 & 11 & 2 & 28 & HHY10 & 6630 & 14200 & 85800 & 3760 & $<376$ & 1620 & 1810 & 239000 & 462 & 852 & 2830 \\
\hline ARG-1 & None & 0 & 0 & 2 & 23 & 2 & 29 & \begin{tabular}{|l|} 
ARG-1B23 \\
\end{tabular} & 5240 & 14700 & 87200 & 8290 & \begin{tabular}{|l|}
952 \\
\end{tabular} & $<932$ & $<745$ & \begin{tabular}{|l|}
227000 \\
\end{tabular} & 6960 & 192 & 983 \\
\hline ARG-1 & None & 0 & 0 & 3 & 1 & 3 & 1 & \begin{tabular}{|l|} 
ARG-1B31 \\
\end{tabular} & 5250 & 14700 & 86600 & 8420 & 876 & $<876$ & $<700$ & 229000 & 7000 & 190 & 980 \\
\hline SME Simulant & Isolok - $12 \mathrm{~mL}$ & LF & 11 & 3 & 4 & 3 & 2 & \begin{tabular}{|l|} 
LIB02 \\
\end{tabular} & 6560 & 14000 & 82800 & 3600 & $<421$ & $<1060$ & 850 & 242000 & 506 & 839 & 2780 \\
\hline SME Simulant & Coliwasa & $\mathrm{HF}$ & 36 & 1 & 21 & 3 & 3 & CL07 & 5910 & 12300 & 82900 & 3000 & $<415$ & $<1040$ & 1100 & 243000 & 499 & 760 & 2630 \\
\hline SME Simulant & Isolok - $12 \mathrm{~mL}$ & LF & 26 & 3 & 8 & 3 & 4 & LIB04 & 6610 & 14000 & 81700 & 3670 & $<408$ & $<1020$ & 1180 & 245000 & 501 & 847 & 2700 \\
\hline SME Simulant & Coliwasa & $\mathrm{HF}$ & 1 & 1 & 22 & 3 & 5 & $\mathrm{CH} 05$ & 6010 & 12800 & 77800 & 3340 & $<434$ & $<1090$ & $<872$ & \begin{tabular}{|l|}
239000 \\
\end{tabular} & 487 & 760 & 2620 \\
\hline SME Simulant & Coliwasa & $\mathrm{HF}$ & 3 & 1 & 20 & 3 & 6 & CL05 & 6660 & 14300 & 81400 & 3860 & $<368$ & $<926$ & 1420 & 241000 & 503 & 845 & 2790 \\
\hline SME Simulant & Coliwasa & $\mathrm{HF}$ & 23 & 1 & 28 & 3 & 7 & CH06 & 6370 & 13600 & 80600 & 3570 & $<387$ & 1440 & 1480 & 247000 & 518 & 814 & 2730 \\
\hline SME Simulant & Coliwasa & LF & 1 & 1 & 12 & 3 & 8 & CH01 & 6380 & 13600 & 82400 & 3480 & $<378$ & 1620 & 1550 & 247000 & 506 & 815 & 2690 \\
\hline SME Simulant & Coliwasa & LF & 20 & 1 & 2 & 3 & 9 & CL02 & 6710 & 14300 & 84100 & 3700 & $<404$ & $<1020$ & 1510 & 235000 & 508 & 839 & 2800 \\
\hline SME Simulant & Coliwasa & $\mathrm{HF}$ & 63 & 1 & 5 & 3 & 10 & CL08 & 6740 & 14700 & 84500 & 4020 & $<376$ & 1120 & 1450 & \begin{tabular}{|l|}
237000 \\
\end{tabular} & 482 & 862 & 2750 \\
\hline SME Simulant & Coliwasa & $\mathrm{HF}$ & 65 & 1 & 6 & 3 & 11 & CH08 & 6260 & 13300 & 85800 & 3500 & $<411$ & $<1030$ & 1550 & \begin{tabular}{|l|}
243000 \\
\end{tabular} & 494 & 797 & 2750 \\
\hline SME Simulant & Isolok - $12 \mathrm{~mL}$ & LF & 45 & 3 & 7 & 3 & 12 & LIB07 & 6650 & 14200 & 116000 & 3680 & 3740 & $<1070$ & 5090 & 243000 & 489 & 827 & 2770 \\
\hline SME Simulant & $\begin{array}{l}\text { Coliwasa } \\
\end{array}$ & LF & 4 & 1 & 10 & 3 & 13 & CL01 & 6430 & 13800 & \begin{tabular}{|l|}
79800 \\
\end{tabular} & 3650 & $<423$ & 1440 & 1730 & \begin{tabular}{|l|}
244000 \\
\end{tabular} & 508 & 832 & 2700 \\
\hline SME Simulant & Coliwasa & $\mathrm{HF}$ & 18 & 1 & 26 & 3 & 14 & \begin{tabular}{|l|} 
CL06 \\
\end{tabular} & 6640 & 14200 & 87300 & 3780 & $<436$ & 1450 & 1840 & 237000 & 500 & 857 & 2770 \\
\hline ARG-1 & None & 0 & 0 & 3 & 12 & 3 & 15 & \begin{tabular}{|l} 
ARG-1B32 \\
\end{tabular} & 5220 & 14600 & 85900 & 8090 & 934 & 1870 & 806 & 226000 & 6890 & 187 & 963 \\
\hline SME Simulant & Coliwasa & LF & 65 & 1 & 23 & 3 & 16 & \begin{tabular}{|l|} 
CL04 \\
\end{tabular} & 6230 & 13300 & 79000 & 3440 & $<409$ & $<1030$ & 1120 & 244000 & 503 & 783 & 2670 \\
\hline SME Simulant & Coliwasa & LF & 40 & 1 & 19 & 3 & 17 & CL03 & 6510 & 13900 & 84100 & 3590 & $<437$ & $<1100$ & 1310 & \begin{tabular}{|l|}
219000 \\
\end{tabular} & 454 & 837 & 2720 \\
\hline SME Simulant & Coliwasa & LF & 38 & 1 & 16 & 3 & 18 & $\mathrm{CH} 03$ & 6410 & 13700 & 83500 & 3600 & $<421$ & $<1060$ & 885 & 244000 & 517 & 804 & 2720 \\
\hline SME Simulant & Isolok - $12 \mathrm{~mL}$ & LF & 56 & 3 & 11 & 3 & 19 & LIB09 & 6300 & 13600 & 83800 & 3590 & $<438$ & 1230 & 1440 & 239000 & 514 & 825 & 2690 \\
\hline SME Simulant & $\begin{array}{l}\text { Coliwasa } \\
\end{array}$ & LF & 21 & 1 & 14 & 3 & 20 & CH02 & 5960 & 12700 & 76800 & 3370 & $<426$ & $<1070$ & 874 & 227000 & 478 & 746 & 2600 \\
\hline SME Simulant & Isolok - $12 \mathrm{~mL}$ & LF & 5 & 3 & 5 & 3 & 21 & LIB01 & 6770 & 14600 & 88900 & 3800 & $<435$ & $<1090$ & 1220 & 251000 & 535 & 879 & 2930 \\
\hline SME Simulant & Isolok - $12 \mathrm{~mL}$ & LF & 23 & 3 & 10 & 3 & 22 & LIB03 & 6160 & 13300 & 77800 & 3560 & $<447$ & 1880 & 1420 & 241000 & 477 & 807 & 2680 \\
\hline SME Simulant & Isolok - $12 \mathrm{~mL}$ & LF & 50 & 3 & 2 & 3 & 23 & LIB08 & 6500 & 14000 & 86900 & 3680 & $<444$ & 2440 & 1500 & 244000 & 473 & 861 & 2780 \\
\hline SME Simulant & Isolok - $12 \mathrm{~mL}$ & LF & 41 & 3 & 9 & 3 & 24 & LIB06 & 6730 & 14400 & 83800 & 3700 & $<430$ & 1300 & 1570 & \begin{tabular}{|l|}
239000 \\
\end{tabular} & 470 & 858 & 2740 \\
\hline SME Simulant & Coliwasa & $\mathrm{HF}$ & 34 & 1 & 18 & 3 & 25 & $\mathrm{CH} 07$ & 6180 & 13300 & 77300 & 3410 & $<381$ & 1360 & 1460 & 242000 & 478 & 780 & 2640 \\
\hline SME Simulant & Coliwasa & LF & 63 & 1 & 13 & 3 & 26 & CH04 & 6000 & 12800 & 79300 & 3340 & $<415$ & $<1040$ & 1020 & \begin{tabular}{|l|}
230000 \\
\end{tabular} & 454 & 760 & 2550 \\
\hline SME Simulant & Isolok - $12 \mathrm{~mL}$ & LF & 32 & 3 & 3 & 3 & 27 & LIB05 & 7110 & 15100 & 85800 & 3850 & $<394$ & 1160 & 1240 & \begin{tabular}{|l|}
242000 \\
\end{tabular} & 484 & 893 & 2830 \\
\hline SME Simulant & Isolok - $12 \mathrm{~mL}$ & LF & 60 & 3 & 6 & 3 & 28 & LIB10 & 6300 & 13500 & 79700 & 3500 & $<406$ & 1150 & 1280 & 233000 & 499 & 810 & 2670 \\
\hline ARG-1 & None & 0 & 0 & 3 & 23 & 3 & 29 & \begin{tabular}{|l} 
ARG-1B33 \\
\end{tabular} & 5350 & 15000 & 88400 & 8350 & 877 & $<799$ & $<638$ & 234000 & 7090 & 182 & 1030 \\
\hline ARG-1 & None & 0 & 0 & 4 & 1 & 4 & 1 & \begin{tabular}{|l|} 
ARG-1B41 \\
\end{tabular} & 5210 & 14500 & 85000 & 7970 & 798 & $<897$ & $<716$ & \begin{tabular}{|l|}
225000 \\
\end{tabular} & 6910 & 185 & 973 \\
\hline SME Simulant & Isolok - $12 \mathrm{~mL}$ & $\mathrm{HF}$ & 13 & 4 & 11 & 4 & 2 & HIB02 & 6400 & 13500 & 79000 & 3350 & $<398$ & $<1000$ & 1130 & 245000 & 497 & 813 & 2700 \\
\hline SME Simulant & Coliwasa & $\mathrm{HF}$ & 36 & 1 & 21 & 4 & 3 & CL07 & 5940 & 12400 & 82800 & 2980 & $<415$ & $<1040$ & 1660 & \begin{tabular}{|l|}
244000 \\
\end{tabular} & 512 & 764 & 2640 \\
\hline SME Simulant & Isolok - $12 \mathrm{~mL}$ & $\mathrm{HF}$ & 25 & 4 & 4 & 4 & 4 & HIB04 & 6460 & 13900 & 84100 & 3900 & 546 & $<1120$ & 1840 & 243000 & 495 & 835 & 2730 \\
\hline SME Simulant & Coliwasa & $\mathrm{HF}$ & 1 & 1 & 22 & 4 & 5 & CH05 & 6210 & 13200 & 80500 & 3470 & $<434$ & $<1090$ & 1260 & 246000 & 513 & 799 & 2640 \\
\hline SME Simulant & Coliwasa & $\mathrm{HF}$ & 3 & 1 & 20 & 4 & 6 & CL05 & 6670 & 14300 & 81100 & 3800 & $<368$ & $<926$ & 1350 & 239000 & 494 & 846 & 2740 \\
\hline SME Simulant & Coliwasa & $\mathrm{HF}$ & 23 & 1 & 28 & 4 & 7 & CH06 & 6320 & 13400 & 79400 & 3450 & $<387$ & $<972$ & 1510 & \begin{tabular}{|l|}
244000 \\
\end{tabular} & 505 & 803 & 2720 \\
\hline SME Simulant & Coliwasa & LF & 1 & 1 & 12 & 4 & 8 & $\mathrm{CH} 01$ & 6330 & 13400 & 81300 & 3450 & $<378$ & $<949$ & 1300 & 244000 & 498 & 802 & 2650 \\
\hline SME Simulant & Coliwasa & LF & 20 & 1 & 2 & 4 & 9 & CL02 & 6690 & 14200 & 83300 & 3630 & $<404$ & $<1020$ & 1460 & 234000 & 482 & 838 & 2770 \\
\hline
\end{tabular}


Table A3. Phase 1 Elemental Concentration Measurements (part 2)

\begin{tabular}{|c|c|c|c|c|c|c|c|c|c|c|c|c|c|c|c|c|c|c|c|}
\hline $\begin{array}{c}\text { Type of } \\
\text { Material } \\
\end{array}$ & Sampler & $\begin{array}{l}\text { Flow } \\
\text { Rate }\end{array}$ & $\begin{array}{c}\text { Sample } \\
\text { Order }\end{array}$ & $\begin{array}{c}\text { Preparation } \\
\text { Block }\end{array}$ & $\begin{array}{c}\text { Preparation } \\
\text { Sequence }\end{array}$ & $\begin{array}{c}\text { Analytical } \\
\text { Block }\end{array}$ & $\begin{array}{c}\text { Analytical } \\
\text { Sequence }\end{array}$ & Sample ID & $\begin{array}{c}\begin{array}{c}\text { Mg } \\
\text { (ug/g) }\end{array} \\
\end{array}$ & $\begin{array}{c}\begin{array}{c}\text { Mn } \\
(\mathbf{u g} / \mathrm{g})\end{array} \\
\end{array}$ & $\begin{array}{c}\mathrm{Na} \\
(\mathrm{ug} / \mathrm{g})\end{array}$ & $\left.\begin{array}{c}\mathbf{N i} \\
(\mathbf{u g} / \mathrm{g})\end{array}\right)$ & \begin{tabular}{|c|}
$\begin{array}{r}\text { P } \\
(\mathbf{u g} / \mathrm{g})\end{array}$ \\
\end{tabular} & \begin{tabular}{|c|}
$\mathbf{P b}$ \\
(ug/g)
\end{tabular} & $\begin{array}{c}\mathrm{S} \\
\text { (ug/g) }\end{array}$ & \begin{tabular}{|c|}
$\mathrm{Si}$ \\
$(\mathrm{ug} / \mathrm{g})$
\end{tabular} & \begin{tabular}{|c|}
$\mathrm{Ti}$ \\
$(\mathrm{ug} / \mathrm{g})$
\end{tabular} & $\begin{array}{c}\mathrm{Zn} \\
(\mathrm{ug} / \mathrm{g})\end{array}$ & $\begin{array}{c}\mathrm{Zr} \\
(\mathrm{ug} / \mathrm{g})\end{array}$ \\
\hline SME Simulant & Coliwasa & $\mathrm{HF}$ & 63 & 1 & 5 & 4 & 10 & CL08 & 6660 & 14500 & 82800 & 3900 & $<376$ & $<944$ & 1580 & 233000 & 493 & 840 & 2700 \\
\hline SME Simulant & Coliwasa & $\mathrm{HF}$ & 65 & 1 & 6 & 4 & 11 & CH08 & 6250 & 13200 & 85200 & 3390 & $<411$ & $<1030$ & 1230 & 242000 & 501 & 792 & 2740 \\
\hline SME Simulant & Isolok - $12 \mathrm{~mL}$ & $\mathrm{HF}$ & 46 & 4 & 2 & 4 & 12 & HIB07 & 6200 & 13300 & 76300 & 3360 & $<454$ & $<1140$ & 1360 & 247000 & 490 & 808 & 2700 \\
\hline SME Simulant & $\begin{array}{l}\text { Coliwasa } \\
\end{array}$ & LF & 4 & 1 & 10 & 4 & 13 & CL01 & 6450 & 13700 & 79500 & 3640 & $<423$ & $<1060$ & 1090 & 244000 & 501 & 832 & 2720 \\
\hline SME Simulant & Coliwasa & $\mathrm{HF}$ & 18 & 1 & 26 & 4 & 14 & CL06 & 6580 & 14000 & 86800 & 3650 & $<436$ & $<1090$ & 1440 & 234000 & 479 & 834 & 2690 \\
\hline ARG-1 & None & 0 & 0 & 4 & 7 & 4 & 15 & \begin{tabular}{|l} 
ARG-1B42 \\
\end{tabular} & 5190 & 14400 & 85000 & 7910 & 847 & $<872$ & $<696$ & 225000 & 6930 & 198 & 982 \\
\hline SME Simulant & Coliwasa & LF & 65 & 1 & 23 & 4 & 16 & \begin{tabular}{|l|} 
CL04 \\
\end{tabular} & 6260 & 13200 & 78900 & 3330 & $<409$ & $<1030$ & 1300 & 245000 & 506 & 779 & 2700 \\
\hline SME Simulant & Coliwasa & LF & 40 & 1 & 19 & 4 & 17 & CL03 & 6660 & 14200 & 85700 & 3640 & $<437$ & $<1100$ & 1480 & \begin{tabular}{|l|}
223000 \\
\end{tabular} & 482 & 870 & 2700 \\
\hline SME Simulant & Coliwasa & LF & 38 & 1 & 16 & 4 & 18 & $\mathrm{CH} 03$ & 6350 & 13500 & 82300 & 3460 & $<421$ & $<1060$ & 1120 & 243000 & 517 & 797 & 2690 \\
\hline SME Simulant & Isolok - $12 \mathrm{~mL}$ & $\mathrm{HF}$ & 56 & 4 & 9 & 4 & 19 & HIB09 & 6940 & 14700 & 74700 & 3740 & $<434$ & $<1090$ & 1130 & \begin{tabular}{|l|}
239000 \\
\end{tabular} & 494 & 878 & 2690 \\
\hline SME Simulant & Coliwasa & LF & 21 & 1 & 14 & 4 & 20 & $\mathrm{CH} 02$ & 6370 & 13500 & 81500 & 3510 & $<426$ & $<1070$ & 1190 & 241000 & 506 & 797 & 2680 \\
\hline SME Simulant & Isolok - $12 \mathrm{~mL}$ & $\mathrm{HF}$ & 7 & 4 & 12 & 4 & 21 & HIB01 & 6190 & 13200 & 83600 & 3410 & $<443$ & $<1110$ & 978 & 244000 & 512 & 781 & 2730 \\
\hline SME Simulant & Isolok - $12 \mathrm{~mL}$ & $\mathrm{HF}$ & 21 & 4 & 6 & 4 & 22 & HIB03 & 6630 & 14100 & 79600 & 3590 & $<427$ & $<1070$ & 1090 & 235000 & 499 & 840 & 2760 \\
\hline SME Simulant & Isolok - $12 \mathrm{~mL}$ & $\mathrm{HF}$ & 49 & 4 & 5 & 4 & 23 & HIB08 & 5830 & 12400 & 78200 & 3130 & $<451$ & $<1130$ & $<905$ & 250000 & 512 & 788 & 2540 \\
\hline SME Simulant & Isolok - $12 \mathrm{~mL}$ & $\mathrm{HF}$ & 38 & 4 & 8 & 4 & 24 & HIB06 & 6300 & 13300 & 82000 & 3400 & $<368$ & $<925$ & 1150 & \begin{tabular}{|l|}
242000 \\
\end{tabular} & 498 & 800 & 2650 \\
\hline SME Simulant & Coliwasa & $\mathrm{HF}$ & 34 & 1 & 18 & 4 & 25 & CH07 & 6280 & 13400 & 78800 & 3420 & $<381$ & $<957$ & 1440 & 245000 & 500 & 786 & 2740 \\
\hline SME Simulant & $\begin{array}{l}\text { Coliwasa } \\
\end{array}$ & LF & 63 & 1 & 13 & 4 & 26 & CH04 & 6320 & 13400 & 83200 & 3380 & $<415$ & $<1040$ & 1270 & 241000 & 493 & 792 & 2660 \\
\hline SME Simulant & Isolok - $12 \mathrm{~mL}$ & $\mathrm{HF}$ & 33 & 4 & 10 & 4 & 27 & HIB05 & 6490 & 13900 & 80800 & 3730 & $<447$ & $<1120$ & 1360 & 239000 & 495 & 819 & 2630 \\
\hline SME Simulant & Isolok - $12 \mathrm{~mL}$ & $\mathrm{HF}$ & 58 & 4 & 3 & 4 & 28 & \begin{tabular}{|l|} 
HIB10 \\
\end{tabular} & 5930 & 12600 & 79000 & 3190 & $<432$ & $<1080$ & 1170 & 252000 & 498 & 755 & 2660 \\
\hline ARG-1 & None & 0 & 0 & 4 & 13 & 4 & 29 & \begin{tabular}{|l|} 
ARG-1B43 \\
\end{tabular} & 5180 & 14400 & 85500 & 7900 & 765 & $<904$ & $<722$ & 225000 & 6930 & 190 & 981 \\
\hline ARG-1 & None & 0 & 0 & 2 & 1 & 5 & 1 & \begin{tabular}{|l|} 
ARG-1B21 \\
\end{tabular} & 5110 & 14200 & 85000 & 7980 & 755 & $<709$ & $<566$ & \begin{tabular}{|l|}
223000 \\
\end{tabular} & 6840 & 196 & 976 \\
\hline SME Simulant & Isolok - $3 \mathrm{~mL}$ & $\mathrm{HF}$ & 20 & 2 & 16 & 5 & 2 & HIS03 & 6160 & 13200 & 80000 & 3520 & $<471$ & $<1180$ & $<945$ & 244000 & 508 & 805 & 2650 \\
\hline SME Simulant & Isolok - $3 \mathrm{~mL}$ & $\mathrm{HF}$ & 9 & 2 & 21 & 5 & 3 & HISO1 & 6200 & 13200 & 85400 & 3490 & $<377$ & $<949$ & $<758$ & \begin{tabular}{|l|}
245000 \\
\end{tabular} & 503 & 798 & 2690 \\
\hline SME Simulant & Coliwasa & LF & 20 & 1 & 2 & 5 & 4 & CL02 & 6480 & 13800 & 82400 & 3570 & $<404$ & $<1020$ & 1420 & \begin{tabular}{|l|}
230000 \\
\end{tabular} & 474 & 818 & 2660 \\
\hline SME Simulant & Coliwasa & LF & 38 & 1 & 16 & 5 & 5 & CH03 & 6480 & 13900 & 85800 & 3590 & $<421$ & $<1060$ & 1320 & 251000 & 523 & 820 & 2740 \\
\hline SME Simulant & Coliwasa & LF & 63 & 1 & 13 & 5 & 6 & CH04 & 6010 & 12800 & 79900 & 3290 & $<415$ & $<1040$ & 1400 & 231000 & 473 & 760 & 2540 \\
\hline SME Simulant & Isolok - $3 \mathrm{~mL}$ & $\mathrm{HF}$ & 44 & 2 & 18 & 5 & 7 & HIS07 & 6310 & 13500 & 81600 & 3540 & $<429$ & $<1080$ & 919 & 254000 & 513 & 809 & 2780 \\
\hline SME Simulant & Coliwasa & $\mathrm{HF}$ & 23 & 1 & 28 & 5 & 8 & $\mathrm{CH} 06$ & 6060 & 13000 & 78100 & 3350 & $<387$ & $<972$ & 1120 & 237000 & 488 & 763 & 2620 \\
\hline SME Simulant & Coliwasa & LF & 21 & 1 & 14 & 5 & 9 & $\mathrm{CH} 02$ & 6460 & 13700 & 84500 & 3600 & $<426$ & $<1070$ & 1230 & 250000 & 503 & 820 & 2740 \\
\hline SME Simulant & $\begin{array}{l}\text { Coliwasa } \\
\end{array}$ & $\mathrm{HF}$ & 3 & 1 & 20 & 5 & 10 & CL05 & 6320 & 13600 & 78400 & 3630 & $<368$ & $<926$ & 1110 & 230000 & 475 & 808 & 2600 \\
\hline SME Simulant & Isolok - $3 \mathrm{~mL}$ & $\mathrm{HF}$ & 14 & 2 & 15 & 5 & 11 & HIS02 & 6110 & 13100 & 73800 & 3370 & $<402$ & $<1010$ & 979 & 245000 & 502 & 777 & 2700 \\
\hline SME Simulant & Coliwasa & LF & 40 & 1 & 19 & 5 & 12 & CL03 & 6500 & 13900 & 85300 & 3610 & $<437$ & $<1100$ & 1170 & 221000 & 468 & 856 & 2670 \\
\hline SME Simulant & Coliwasa & $\mathrm{HF}$ & 18 & 1 & 26 & 5 & 13 & CL06 & 6470 & 13800 & 86800 & 3660 & $<436$ & $<1090$ & 1640 & \begin{tabular}{|l|}
232000 \\
\end{tabular} & 484 & 841 & 2690 \\
\hline SME Simulant & Coliwasa & $\mathrm{HF}$ & 1 & 1 & 22 & 5 & 14 & \begin{tabular}{|l}
$\mathrm{CH} 05$ \\
\end{tabular} & 6110 & 13000 & 80800 & 3410 & $<434$ & $<1090$ & 1040 & \begin{tabular}{|l|}
245000 \\
\end{tabular} & 486 & 782 & 2650 \\
\hline ARG-1 & None & 0 & 0 & 2 & 12 & 5 & 15 & \begin{tabular}{|l} 
ARG-1B22 \\
\end{tabular} & 4760 & 13200 & 79700 & 7360 & 686 & $<746$ & $<596$ & 209000 & 6370 & 180 & 905 \\
\hline SME Simulant & Coliwasa & $\mathrm{HF}$ & 36 & 1 & 21 & 5 & 16 & $\begin{array}{ll}\text { CL07 } \\
\end{array}$ & 5850 & 12200 & 84000 & 3010 & $<415$ & $<1040$ & 1230 & \begin{tabular}{|l|}
242000 \\
\end{tabular} & 518 & 767 & 2570 \\
\hline SME Simulant & Isolok - $3 \mathrm{~mL}$ & $\mathrm{HF}$ & 31 & 2 & 19 & 5 & 17 & HISO5 & 6180 & 13200 & 77000 & 3430 & $<4$ & $<1060$ & $<847$ & \begin{tabular}{|l|}
243000 \\
\end{tabular} & 495 & 802 & 2600 \\
\hline SME Simulant & Isolok - $3 \mathrm{~mL}$ & $\mathrm{HF}$ & 60 & 2 & 22 & 5 & 18 & HIS10 & 6390 & 13500 & 82100 & 3380 & $<392$ & $<985$ & 1070 & 240000 & 503 & 798 & 2570 \\
\hline SME Simulant & Coliwasa & $\mathrm{HF}$ & 63 & 1 & 5 & 5 & 19 & CL08 & 6560 & 14300 & 84000 & 3890 & $<376$ & $<944$ & 1160 & 234000 & 486 & 828 & 2680 \\
\hline SME Simulant & Isolok - $3 \mathrm{~mL}$ & $\mathrm{HF}$ & 26 & 2 & 14 & 5 & 20 & HISO4 & 5880 & 12500 & 76500 & 3270 & $<407$ & $<1020$ & $<817$ & \begin{tabular}{|l|}
227000 \\
\end{tabular} & 483 & 749 & 2540 \\
\hline SME Simulant & Isolok - $3 \mathrm{~mL}$ & $\mathrm{HF}$ & 35 & 2 & 20 & 5 & 21 & HISO6 & 6280 & 13300 & 84500 & 3450 & $<391$ & $<983$ & 1340 & 244000 & 496 & 790 & 2730 \\
\hline SME Simulant & Coliwasa & $\mathrm{HF}$ & 65 & 1 & 6 & 5 & 22 & CH08 & 6180 & 13100 & 87000 & 3430 & $<411$ & $<1030$ & 1380 & \begin{tabular}{|l|}
243000 \\
\end{tabular} & 517 & 781 & 2700 \\
\hline SME Simulant & Coliwasa & $\mathrm{HF}$ & 34 & 1 & 18 & 5 & 23 & CH07 & 6250 & 13300 & 80100 & 3490 & $<381$ & $<957$ & 1280 & \begin{tabular}{|l|}
247000 \\
\end{tabular} & 504 & 791 & 2720 \\
\hline SME Simulant & Coliwasa & LF & 1 & 1 & 12 & 5 & 24 & CH01 & 6100 & 13000 & 81100 & 3360 & $<378$ & $<949$ & 1290 & 238000 & 473 & 778 & 2570 \\
\hline SME Simulant & Isolok - $3 \mathrm{~mL}$ & $\mathrm{HF}$ & 54 & 2 & 17 & 5 & 25 & HIS09 & 6260 & 13400 & 81300 & 3520 & $<429$ & $<1080$ & $<861$ & 244000 & 522 & 864 & 2740 \\
\hline SME Simulant & Coliwasa & LF & 4 & 1 & 10 & 5 & 26 & CL01 & 6540 & 14000 & 82800 & 3720 & $<423$ & $<1060$ & 1190 & \begin{tabular}{|l|}
250000 \\
\end{tabular} & 505 & 849 & 2780 \\
\hline SME Simulant & Isolok - $3 \mathrm{~mL}$ & $\mathrm{HF}$ & 48 & 2 & 13 & 5 & 27 & HIS08 & 6270 & 13500 & 79900 & 3580 & $<403$ & $<1010$ & 842 & 248000 & 493 & 820 & 2710 \\
\hline SME Simulant & Coliwasa & LF & 65 & 1 & 23 & 5 & 28 & CL04 & 6270 & 13300 & 81400 & 3420 & $<409$ & $<1030$ & 1320 & 249000 & 492 & 807 & 2720 \\
\hline
\end{tabular}


Table A3. Phase 1 Elemental Concentration Measurements (part 2)

\begin{tabular}{|c|c|c|c|c|c|c|c|c|c|c|c|c|c|c|c|c|c|c|c|}
\hline $\begin{array}{r}\text { Type of } \\
\text { Material } \\
\end{array}$ & Sampler & $\begin{array}{l}\text { Flow } \\
\text { Rate }\end{array}$ & $\begin{array}{c}\text { Sample } \\
\text { Order }\end{array}$ & $\begin{array}{c}\text { Preparation } \\
\text { Block }\end{array}$ & $\begin{array}{c}\text { Preparation } \\
\text { Sequence } \\
\end{array}$ & $\begin{array}{c}\text { Analytical } \\
\text { Block } \\
\end{array}$ & $\begin{array}{c}\text { Analytical } \\
\text { Sequence }\end{array}$ & Sample ID & $\begin{array}{c}\mathrm{Mg} \\
\text { (ug/g) }\end{array}$ & $\begin{array}{c}\text { Mn } \\
\text { (ug/g) }\end{array}$ & $\begin{array}{c}\mathrm{Na} \\
(\mathrm{ug} / \mathrm{g})\end{array}$ & $\begin{array}{c}\mathbf{N i} \\
(\mathrm{ug} / \mathrm{g})\end{array}$ & $\begin{array}{c}\mathbf{P} \\
(\mathrm{ug} / \mathrm{g})\end{array}$ & \begin{tabular}{|c|}
$\mathbf{P b}$ \\
(ug/g)
\end{tabular} & \begin{tabular}{|c}
$\mathrm{S}$ \\
$(\mathrm{ug} / \mathrm{g})$
\end{tabular} & \begin{tabular}{|c|c}
$\mathrm{Si}$ \\
$(\mathrm{ug} / \mathrm{g})$
\end{tabular} & $\begin{array}{c}\mathbf{T i} \\
\text { (ug/g) }\end{array}$ & $\begin{array}{c}\begin{array}{c}\text { Zn } \\
\text { (ug/g) }\end{array} \\
\end{array}$ & $\begin{array}{c}\mathrm{Zr} \\
(\mathrm{ug} / \mathrm{g})\end{array}$ \\
\hline ARG-1 & None & 0 & 0 & 2 & 23 & 5 & 29 & ARG-1B23 & 5150 & 14400 & 86900 & 7980 & 697 & $<932$ & $<745$ & 226000 & 6920 & 204 & 974 \\
\hline ARG-1 & None & 0 & 0 & 3 & 1 & 6 & 1 & \begin{tabular}{|l|} 
ARG-1B31 \\
\end{tabular} & 5050 & 14100 & 85400 & 7960 & 673 & $<876$ & $<700$ & 224000 & 6760 & 192 & 959 \\
\hline SME Simulant & Hydragard & LF & 15 & 3 & 21 & 6 & 2 & \begin{tabular}{|l|} 
LHY03 \\
\end{tabular} & 6570 & 14000 & 85800 & 3630 & $<411$ & $<1030$ & 919 & 246000 & 485 & 829 & 2740 \\
\hline SME Simulant & Hydragard & LF & 6 & 3 & 18 & 6 & 3 & LHY01 & 6490 & 13800 & 83800 & 3660 & $<439$ & $<1100$ & 1260 & 243000 & 493 & 844 & 2730 \\
\hline SME Simulant & Coliwasa & LF & 20 & 1 & 2 & 6 & 4 & CL02 & 6570 & 14000 & 84600 & 3680 & $<404$ & $<1020$ & 1290 & \begin{tabular}{|l|}
237000 \\
\end{tabular} & 469 & 840 & 2720 \\
\hline SME Simulant & Coliwasa & LF & 38 & 1 & 16 & 6 & 5 & $\mathrm{CH} 03$ & 6260 & 13300 & 84000 & 3600 & $<421$ & $<1060$ & 1030 & 246000 & 493 & 824 & 2660 \\
\hline SME Simulant & $\begin{array}{l}\text { Coliwasa } \\
\end{array}$ & LF & 63 & 1 & 13 & 6 & 6 & $\mathrm{CH} 04$ & 6200 & 13200 & 83700 & 3450 & $<415$ & $<1040$ & 1390 & \begin{tabular}{|l|}
243000 \\
\end{tabular} & 486 & 798 & 2630 \\
\hline SME Simulant & Hydragard & LF & 47 & 3 & 13 & 6 & 7 & LHY07 & 6800 & 14400 & 94700 & 3730 & $<451$ & $<1130$ & 1160 & 232000 & 454 & 881 & 2700 \\
\hline SME Simulant & Coliwasa & $\mathrm{HF}$ & 23 & 1 & 28 & 6 & 8 & CH06 & 6230 & 13300 & 81300 & 3500 & $<387$ & $<972$ & 1010 & 248000 & 489 & 802 & 2690 \\
\hline SME Simulant & Coliwasa & LF & 21 & 1 & 14 & 6 & 9 & $\mathrm{CH} 02$ & 5950 & 12600 & 79200 & 3350 & $<426$ & $<1070$ & 986 & 233000 & 462 & 758 & 2530 \\
\hline SME Simulant & Coliwasa & $\mathrm{HF}$ & 3 & 1 & 20 & 6 & 10 & CL05 & 6670 & 14300 & 84600 & 3900 & $<368$ & $<926$ & 1250 & 246000 & 478 & 872 & 2770 \\
\hline SME Simulant & Hydragard & LF & 10 & 3 & 20 & 6 & 11 & LHY02 & 5900 & 12800 & 85200 & 3500 & $<443$ & $<1110$ & 1370 & 251000 & 503 & 785 & 2670 \\
\hline SME Simulant & $\begin{array}{l}\text { Coliwasa } \\
\end{array}$ & LF & 40 & 1 & 19 & 6 & 12 & CL03 & 6700 & 14300 & 89600 & 3770 & $<437$ & $<1100$ & 1280 & 231000 & 466 & 883 & 2770 \\
\hline SME Simulant & Coliwasa & $\mathrm{HF}$ & 18 & 1 & 26 & 6 & 13 & CL06 & 6430 & 13700 & 88400 & 3700 & $<436$ & $<1090$ & 1550 & 235000 & 474 & 840 & 2680 \\
\hline SME Simulant & Coliwasa & $\mathrm{HF}$ & 1 & 1 & 22 & 6 & 14 & \begin{tabular}{|l}
$\mathrm{CH} 05$ \\
\end{tabular} & 6210 & 13200 & 84000 & 3550 & $<434$ & $<1090$ & 1200 & 253000 & 485 & 808 & 2650 \\
\hline ARG-1 & None & 0 & 0 & 3 & 12 & 6 & 15 & \begin{tabular}{|l} 
ARG-1B32 \\
\end{tabular} & 4960 & 13800 & 84700 & 7840 & 720 & $<<904$ & $<722$ & 221000 & 6620 & 187 & 971 \\
\hline SME Simulant & $\begin{array}{l}\text { Coliwasa } \\
\end{array}$ & $\mathrm{HF}$ & 36 & 1 & 21 & 6 & 16 & \begin{tabular}{|l|} 
CL07 \\
\end{tabular} & 5840 & 12200 & 85700 & 3030 & $<415$ & $<1040$ & 1310 & 246000 & 480 & 771 & 2560 \\
\hline SME Simulant & Hydragard & LF & 30 & 3 & 14 & 6 & 17 & LHY05 & 6500 & 13900 & 83400 & 3770 & $<392$ & $<985$ & 1580 & 240000 & 462 & 846 & 2690 \\
\hline SME Simulant & Hydragard & LF & 59 & 3 & 17 & 6 & 18 & LHY10 & 6380 & 13600 & 83700 & 3500 & $<422$ & $<1060$ & 1560 & 246000 & 477 & 829 & 2720 \\
\hline SME Simulant & $\begin{array}{l}\text { Coliwasa } \\
\end{array}$ & $\mathrm{HF}$ & 63 & 1 & 5 & 6 & 19 & CL08 & 6640 & 14400 & 86800 & 4040 & $<376$ & $<944$ & 1340 & 240000 & 480 & 878 & 2690 \\
\hline SME Simulant & Hydragard & LF & 28 & 3 & 16 & 6 & 20 & LHY04 & 6430 & 13800 & 84400 & 3630 & $<414$ & $<1040$ & 1460 & 237000 & 471 & 843 & 2660 \\
\hline SME Simulant & Hydragard & LF & 37 & 3 & 15 & 6 & 21 & LHY06 & 6600 & 14000 & 91100 & 3710 & $<425$ & $<1070$ & 1140 & 252000 & 503 & 877 & 2820 \\
\hline SME Simulant & $\begin{array}{l}\text { Coliwasa } \\
\end{array}$ & $\mathrm{HF}$ & 65 & 1 & 6 & 6 & 22 & CH08 & 5930 & 12500 & 85000 & 3320 & $<411$ & $<1030$ & 1180 & \begin{tabular}{|l|}
237000 \\
\end{tabular} & 478 & 762 & 2620 \\
\hline SME Simulant & Coliwasa & $\mathrm{HF}$ & 34 & 1 & 18 & 6 & 23 & CH07 & 6400 & 13700 & 83500 & 3600 & $<381$ & $<<957$ & 1080 & 258000 & 506 & 830 & 2780 \\
\hline SME Simulant & $\begin{array}{l}\text { Coliwasa } \\
\end{array}$ & LF & 1 & 1 & 12 & 6 & 24 & CH01 & 6050 & 12800 & 81900 & 3370 & $<378$ & $<949$ & 940 & 240000 & 476 & 777 & 2590 \\
\hline SME Simulant & Hydragard & LF & 53 & 3 & 19 & 6 & 25 & LHY09 & 6820 & 14500 & 87100 & 3790 & $<380$ & $<954$ & 1370 & 252000 & 502 & 868 & 2870 \\
\hline SME Simulant & Coliwasa & LF & 4 & 1 & 10 & 6 & 26 & CL01 & 6300 & 13500 & 81800 & 3610 & $<423$ & $<1060$ & 1060 & 246000 & 494 & 806 & 2770 \\
\hline SME Simulant & Hydragard & LF & 48 & 3 & 22 & 6 & 27 & LHY08 & 6350 & 13500 & 82500 & 3520 & $\mid<378$ & $<950$ & 1250 & 243000 & 481 & 812 & 2710 \\
\hline SME Simulant & $\begin{array}{l}\text { Coliwasa } \\
\end{array}$ & LF & 65 & 1 & 23 & 6 & 28 & CL04 & 6200 & 13200 & 82300 & 3400 & $<409$ & $<1030$ & 986 & 250000 & 494 & 788 & 2750 \\
\hline ARG-1 & None & 0 & 0 & 3 & 23 & 6 & 29 & \begin{tabular}{|l|} 
ARG-1B33 \\
\end{tabular} & 4790 & 13400 & 82500 & 7650 & 649 & $<799$ & $<638$ & 214000 & 6420 & 178 & 949 \\
\hline
\end{tabular}


Table A4. Phase 1 Oxide Concentration Measurements (part 1)

\begin{tabular}{|c|c|c|c|c|c|c|c|c|c|c|c|c|c|c|c|c|c|}
\hline $\begin{array}{l}\text { Type of } \\
\text { Material }\end{array}$ & Sampler Details & $\begin{array}{l}\text { Flow } \\
\text { Rate }\end{array}$ & $\begin{array}{c}\text { Sample } \\
\text { Order }\end{array}$ & $\begin{array}{c}\text { Preparation } \\
\text { Block }\end{array}$ & $\begin{array}{c}\text { Preparation } \\
\text { Sequence }\end{array}$ & $\begin{array}{c}\text { Analytical } \\
\text { Block }\end{array}$ & $\begin{array}{c}\text { Analytical } \\
\text { Sequence }\end{array}$ & Sample ID & $\begin{array}{l}\text { Al2O3 } \\
\text { (wt\%) }\end{array}$ & $\begin{array}{l}\text { B2O3 } \\
\text { (wt\%) }\end{array}$ & $\begin{array}{c}\mathrm{BaO} \\
\text { (wt\%) }\end{array}$ & $\begin{array}{c}\mathrm{CaO} \\
\text { (wt\%) }\end{array}$ & $\begin{array}{l}\text { Cr2O3 } \\
\text { (wt\%) }\end{array}$ & $\begin{array}{c}\mathrm{CuO} \\
\text { (wt\%) }\end{array}$ & $\begin{array}{l}\text { Fe2O3 } \\
\text { (wt\%) }\end{array}$ & $\begin{array}{c}\text { K2O } \\
\text { (wt\%) }\end{array}$ & $\begin{array}{l}\text { Li2O } \\
\text { (wt\%) }\end{array}$ \\
\hline ARG-1 & None & 0 & 0 & 1 & 1 & 1 & 1 & ARG-1B11 & 4.535 & 8.211 & 0.090 & 1.455 & 0.097 & 0.020 & 13.954 & 2.734 & 3.186 \\
\hline SME Simulant & Isolok - $3 \mathrm{~mL}$ & LF & 18 & 1 & 9 & 1 & 2 & LIS03 & 10.657 & 3.832 & 0.105 & 1.199 & 0.105 & 0.026 & 11.352 & 0.167 & 4.736 \\
\hline SME Simulant & Isolok - $3 \mathrm{~mL}$ & LF & 8 & 1 & 27 & 1 & 3 & LIS01 & 11.072 & 4.089 & 0.106 & 1.245 & 0.110 & 0.024 & 11.709 & 0.157 & 4.779 \\
\hline SME Simulant & Coliwasa - low & LF & 20 & 1 & 2 & 1 & 4 & CL02 & 11.167 & 4.347 & 0.107 & 1.263 & 0.105 & 0.025 & 11.795 & 0.162 & 4.758 \\
\hline SME Simulant & \begin{tabular}{|l|} 
Coliwasa - high \\
\end{tabular} & LF & 38 & 1 & 16 & 1 & 5 & CH03 & 10.543 & 4.154 & 0.103 & 1.233 & 0.104 & 0.026 & 11.309 & 0.169 & 4.801 \\
\hline SME Simulant & Coliwasa - high & LF & 63 & 1 & 13 & 1 & 6 & CH04 & 10.638 & 4.282 & 0.103 & 1.187 & 0.105 & 0.026 & 11.280 & 0.166 & 4.715 \\
\hline SME Simulant & \begin{tabular}{|l} 
Isolok - $3 \mathrm{~mL}$ \\
\end{tabular} & LF & 44 & 1 & 3 & 1 & 7 & LIS07 & 11.054 & 3.960 & 0.105 & 1.212 & 0.100 & 0.025 & 11.666 & 0.164 & 4.715 \\
\hline SME Simulant & \begin{tabular}{|l|} 
Coliwasa - high \\
\end{tabular} & $\mathrm{HF}$ & 23 & 1 & 28 & 1 & 8 & CH06 & 10.581 & 4.089 & 0.101 & 1.188 & 0.105 & 0.024 & 11.209 & 0.155 & 4.930 \\
\hline SME Simulant & \begin{tabular}{|l|} 
Coliwasa - high \\
\end{tabular} & LF & 21 & 1 & 14 & 1 & 9 & $\mathrm{CH} 02$ & 10.713 & 4.186 & 0.103 & 1.181 & 0.106 & 0.026 & 11.409 & 0.170 & 4.693 \\
\hline SME Simulant & Coliwasa - low & $\mathrm{HF}$ & 3 & 1 & 20 & 1 & 10 & CL05 & 11.035 & 4.121 & 0.104 & 1.216 & 0.109 & 0.023 & 11.795 & 0.148 & 4.650 \\
\hline SME Simulant & Isolok - $3 \mathrm{~mL}$ & LF & 12 & 1 & 25 & 1 & 11 & LIS02 & 10.959 & 4.025 & 0.105 & 1.217 & 0.103 & 0.024 & 11.681 & 0.154 & 4.650 \\
\hline SME Simulant & Coliwasa - low & LF & 40 & 1 & 19 & 1 & 12 & CL03 & 11.602 & 3.896 & 0.108 & 1.254 & 0.114 & 0.027 & 12.067 & 0.175 & 4.392 \\
\hline SME Simulant & Coliwasa - low & $\mathrm{HF}$ & 18 & 1 & 26 & 1 & 13 & CL06 & 11.110 & 4.250 & 0.107 & 1.235 & 0.118 & 0.027 & 11.838 & 0.175 & 4.693 \\
\hline SME Simulant & \begin{tabular}{|l|} 
Coliwasa - high \\
\end{tabular} & $\mathrm{HF}$ & 1 & 1 & 22 & 1 & 14 & CH05 & 10.562 & 4.025 & 0.102 & 1.171 & 0.104 & 0.027 & 11.166 & 0.174 & 4.607 \\
\hline ARG-1 & None & 0 & 0 & 1 & 15 & 1 & 15 & ARG-1B12 & 4.554 & 8.114 & 0.090 & 1.455 & 0.096 & 0.020 & 13.940 & 2.662 & 3.186 \\
\hline SME Simulant & Coliwasa - low & $\mathrm{HF}$ & 36 & 1 & 21 & 1 & 16 & CL07 & 10.298 & 4.186 & 0.101 & 1.108 & 0.101 & 0.026 & 10.694 & 0.161 & 5.167 \\
\hline SME Simulant & Isolok - $3 \mathrm{~mL}$ & LF & 31 & 1 & 24 & 1 & 17 & LIS05 & 10.657 & 4.089 & 0.103 & 1.196 & 0.103 & 0.027 & 11.366 & 0.175 & 4.672 \\
\hline SME Simulant & Isolok - $3 \mathrm{~mL}$ & LF & 58 & 1 & 4 & 1 & 18 & LIS10 & 11.110 & 4.121 & 0.107 & 1.245 & 0.108 & 0.025 & 11.838 & 0.164 & 4.629 \\
\hline SME Simulant & Coliwasa - low & $\mathrm{HF}$ & 63 & 1 & 5 & 1 & 19 & CL08 & 11.072 & 4.186 & 0.109 & 1.248 & 0.109 & 0.023 & 11.952 & 0.151 & 4.844 \\
\hline SME Simulant & Isolok - $3 \mathrm{~mL}$ & LF & 25 & 1 & 7 & 1 & 20 & LIS04 & 10.562 & 3.735 & 0.101 & 1.184 & 0.098 & 0.025 & 11.195 & 0.163 & 4.801 \\
\hline SME Simulant & Isolok - 3 mL & LF & 42 & 1 & 11 & 1 & 21 & LIS06 & 11.205 & 4.025 & 0.108 & 1.249 & 0.105 & 0.025 & 11.867 & 0.164 & 4.650 \\
\hline SME Simulant & \begin{tabular}{|l|} 
Coliwasa - high \\
\end{tabular} & $\mathrm{HF}$ & 65 & 1 & 6 & 1 & 22 & CH08 & 10.695 & 4.186 & 0.101 & 1.170 & 0.103 & 0.025 & 11.195 & 0.164 & 4.693 \\
\hline SME Simulant & \begin{tabular}{|l|} 
Coliwasa - high \\
\end{tabular} & $\mathrm{HF}$ & 34 & 1 & 18 & 1 & 23 & CH07 & 10.600 & 4.057 & 0.103 & 1.196 & 0.102 & 0.023 & 11.137 & 0.152 & 4.586 \\
\hline SME Simulant & \begin{tabular}{|l} 
Coliwasa - high \\
\end{tabular} & LF & 1 & 1 & 12 & 1 & 24 & CH01 & 10.506 & 4.186 & 0.101 & 1.182 & 0.101 & 0.023 & 11.152 & 0.151 & 4.758 \\
\hline SME Simulant & Isolok - $3 \mathrm{~mL}$ & LF & 54 & 1 & 17 & 1 & 25 & LIS09 & 11.072 & 4.186 & 0.106 & 1.240 & 0.102 & 0.027 & 11.638 & 0.173 & 4.586 \\
\hline SME Simulant & Coliwasa - low & LF & 4 & 1 & 10 & 1 & 26 & CL01 & 10.638 & 4.089 & 0.102 & 1.208 & 0.105 & 0.026 & 11.380 & 0.169 & 4.866 \\
\hline SME Simulant & Isolok - $3 \mathrm{~mL}$ & LF & 49 & 1 & 8 & 1 & 27 & LIS08 & 10.921 & 4.057 & 0.106 & 1.230 & 0.101 & 0.026 & 11.609 & 0.171 & 4.822 \\
\hline SME Simulant & Coliwasa - low & LF & 65 & 1 & 23 & 1 & 28 & CL04 & 10.506 & 4.121 & 0.101 & 1.177 & 0.108 & 0.025 & 11.094 & 0.164 & 4.650 \\
\hline ARG-1 & None & 0 & 0 & 1 & 29 & 1 & 29 & ARG-1B13 & 4.705 & 7.857 & 0.092 & 1.483 & 0.095 & 0.025 & 14.011 & 2.626 & 3.208 \\
\hline ARG-1 & \begin{tabular}{|l|} 
None \\
\end{tabular} & 0 & 0 & 2 & 1 & 2 & 1 & ARG-1B21 & 4.667 & 7.760 & 0.090 & 1.455 & 0.100 & 0.017 & 14.011 & 2.686 & 3.251 \\
\hline SME Simulant & Hydragard & $\mathrm{HF}$ & 11 & 2 & 3 & 2 & 2 & HHY02 & 11.375 & 4.218 & 0.105 & 1.263 & 0.108 & 0.026 & 11.738 & 0.166 & 4.779 \\
\hline SME Simulant & Coliwasa - low & $\mathrm{HF}$ & 36 & 1 & 21 & 2 & 3 & CL07 & 10.487 & 4.121 & 0.099 & 1.135 & 0.105 & 0.026 & 10.737 & 0.166 & 5.275 \\
\hline SME Simulant & Hydragard & $\mathrm{HF}$ & 24 & 2 & 2 & 2 & 4 & HHY04 & 11.261 & 4.218 & 0.106 & 1.248 & 0.111 & 0.028 & 11.466 & 0.180 & 4.801 \\
\hline SME Simulant & Coliwasa - high & $\mathrm{HF}$ & 1 & 1 & 22 & 2 & 5 & CH05 & 10.732 & 4.057 & 0.103 & 1.175 & 0.113 & 0.027 & 11.166 & 0.174 & 4.672 \\
\hline SME Simulant & \begin{tabular}{|l|} 
Coliwasa - low \\
\end{tabular} & $\mathrm{HF}$ & 3 & 1 & 20 & 2 & 6 & CL05 & 11.261 & 4.121 & 0.108 & 1.247 & 0.115 & 0.023 & 11.895 & 0.148 & 4.779 \\
\hline SME Simulant & \begin{tabular}{|l|} 
Coliwasa - high \\
\end{tabular} & $\mathrm{HF}$ & 23 & 1 & 28 & 2 & 7 & CH06 & 10.770 & 4.121 & 0.104 & 1.194 & 0.111 & 0.024 & 11.280 & 0.155 & 4.887 \\
\hline SME Simulant & \begin{tabular}{|l|} 
Coliwasa - high \\
\end{tabular} & LF & 1 & 1 & 12 & 2 & 8 & CH01 & 10.657 & 4.186 & 0.103 & 1.199 & 0.107 & 0.023 & 11.266 & 0.151 & 4.844 \\
\hline SME Simulant & \begin{tabular}{|l|} 
Coliwasa - low \\
\end{tabular} & LF & 20 & 1 & 2 & 2 & 9 & CL02 & 11.469 & 4.315 & 0.111 & 1.256 & 0.110 & 0.025 & 11.924 & 0.162 & 4.736 \\
\hline SME Simulant & Coliwasa - low & $\mathrm{HF}$ & 63 & 1 & 5 & 2 & 10 & CL08 & 11.186 & 4.218 & 0.107 & 1.248 & 0.117 & 0.023 & 12.024 & 0.151 & 4.930 \\
\hline SME Simulant & \begin{tabular}{|l|} 
Coliwasa - high \\
\end{tabular} & $\mathrm{HF}$ & 65 & 1 & 6 & 2 & 11 & CH08 & 10.808 & 4.250 & 0.103 & 1.177 & 0.105 & 0.025 & 11.223 & 0.164 & 4.736 \\
\hline SME Simulant & \begin{tabular}{|l} 
Hydragard \\
\end{tabular} & $\mathrm{HF}$ & 45 & 2 & 7 & 2 & 12 & HHY07 & 11.243 & 4.057 & 0.106 & 1.233 & 0.116 & 0.022 & 11.781 & 0.145 & 4.715 \\
\hline SME Simulant & Coliwasa - low & LF & 4 & 1 & 10 & 2 & 13 & CL01 & 10.940 & 4.186 & 0.107 & 1.220 & 0.111 & 0.026 & 11.509 & 0.169 & 4.909 \\
\hline SME Simulant & Coliwasa - low & $\mathrm{HF}$ & 18 & 1 & 26 & 2 & 14 & CL06 & 11.261 & 4.250 & 0.110 & 1.251 & 0.127 & 0.027 & 11.838 & 0.169 & 4.758 \\
\hline ARG-1 & None & 0 & 0 & 2 & 12 & 2 & 15 & ARG-1B22 & 4.799 & 7.567 & 0.092 & 1.483 & 0.097 & 0.018 & 14.054 & 2.445 & 3.251 \\
\hline SME Simulant & Coliwasa - low & LF & 65 & 1 & 23 & 2 & 16 & CL04 & 10.770 & 4.089 & 0.106 & 1.203 & 0.111 & 0.025 & 11.180 & 0.164 & 4.693 \\
\hline SME Simulant & Coliwasa - low & LF & 40 & 1 & 19 & 2 & 17 & CL03 & 11.998 & 3.799 & 0.112 & 1.261 & 0.114 & 0.027 & 12.110 & 0.175 & 4.500 \\
\hline SME Simulant & \begin{tabular}{|l|} 
Coliwasa - high \\
\end{tabular} & LF & 38 & 1 & 16 & 2 & 18 & CH03 & 10.713 & 4.025 & 0.104 & 1.210 & 0.106 & 0.026 & 11.266 & 0.169 & 4.866 \\
\hline SME Simulant & \begin{tabular}{|l} 
Hydragard \\
\end{tabular} & $\mathrm{HF}$ & 57 & 2 & 6 & 2 & 19 & HHY09 & 10.921 & 4.282 & 0.106 & 1.234 & 0.112 & 0.022 & 11.409 & 0.146 & 4.822 \\
\hline
\end{tabular}


Table A4. Phase 1 Oxide Concentration Measurements (part 1)

\begin{tabular}{|c|c|c|c|c|c|c|c|c|c|c|c|c|c|c|c|c|c|}
\hline $\begin{array}{c}\text { Type of } \\
\text { Material }\end{array}$ & Sampler Details & $\begin{array}{l}\text { Flow } \\
\text { Rate }\end{array}$ & $\begin{array}{c}\text { Sample } \\
\text { Order }\end{array}$ & $\begin{array}{c}\text { Preparation } \\
\text { Block }\end{array}$ & $\begin{array}{c}\text { Preparation } \\
\text { Sequence }\end{array}$ & $\begin{array}{c}\text { Analytical } \\
\text { Block }\end{array}$ & $\begin{array}{l}\text { Analytical } \\
\text { Sequence }\end{array}$ & Sample ID & $\begin{array}{l}\text { Al2O3 } \\
\text { (wt\%) }\end{array}$ & $\begin{array}{l}\text { B2O3 } \\
\text { (wt\%) }\end{array}$ & $\begin{array}{c}\mathrm{BaO} \\
\text { (wt\%) }\end{array}$ & $\begin{array}{c}\mathrm{CaO} \\
\text { (wt\%) }\end{array}$ & $\begin{array}{l}\text { Cr2O3 } \\
\text { (wt\%) }\end{array}$ & $\begin{array}{c}\mathrm{CuO} \\
\text { (wt\%) }\end{array}$ & $\begin{array}{l}\text { Fe2O3 } \\
\text { (wt\%) }\end{array}$ & $\begin{array}{c}\text { K2O } \\
\text { (wt\%) }\end{array}$ & $\begin{array}{l}\text { Li2O } \\
\text { (wt\%) }\end{array}$ \\
\hline SME Simulant & Coliwasa - high & LF & 21 & 1 & 14 & 2 & 20 & CH02 & 10.978 & 4.186 & 0.106 & 1.198 & 0.109 & 0.026 & 11.452 & 0.170 & 4.844 \\
\hline SME Simulant & Hydragard & $\mathrm{HF}$ & 6 & 2 & 4 & 2 & 21 & HHY01 & 11.602 & 4.057 & 0.113 & 1.298 & 0.111 & 0.027 & 12.138 & 0.176 & 4.650 \\
\hline SME Simulant & Hydragard & $\mathrm{HF}$ & 19 & 2 & 10 & 2 & 22 & HHY03 & 11.413 & 4.057 & 0.111 & 1.287 & 0.112 & 0.026 & 12.124 & 0.168 & 4.758 \\
\hline SME Simulant & Hydragard & $\mathrm{HF}$ & 50 & 2 & 5 & 2 & 23 & HHY08 & 11.072 & 4.089 & 0.108 & 1.242 & 0.109 & 0.026 & 11.566 & 0.169 & 4.801 \\
\hline SME Simulant & Hydragard & $\mathrm{HF}$ & 37 & 2 & 9 & 2 & 24 & HHY06 & 11.167 & 4.121 & 0.108 & 1.216 & 0.111 & 0.026 & 11.595 & 0.171 & 4.801 \\
\hline SME Simulant & Coliwasa - high & $\mathrm{HF}$ & 34 & 1 & 18 & 2 & 25 & CH07 & 10.770 & 4.154 & 0.105 & 1.198 & 0.108 & 0.023 & 11.266 & 0.152 & 4.672 \\
\hline SME Simulant & \begin{tabular}{|l|} 
Coliwasa - high \\
\end{tabular} & LF & 63 & 1 & 13 & 2 & 26 & CH04 & 10.789 & 4.154 & 0.106 & 1.203 & 0.106 & 0.026 & 11.266 & 0.166 & 4.822 \\
\hline SME Simulant & Hydragard & $\mathrm{HF}$ & 32 & 2 & 8 & 2 & 27 & HHY05 & 11.167 & 4.057 & 0.107 & 1.212 & 0.113 & 0.025 & 11.709 & 0.161 & 4.866 \\
\hline SME Simulant & Hydragard & $\mathrm{HF}$ & 61 & 2 & 11 & 2 & 28 & HHY10 & 11.431 & 4.154 & 0.111 & 1.262 & 0.108 & 0.023 & 11.795 & 0.151 & 4.801 \\
\hline ARG-1 & None & 0 & 0 & 2 & 23 & 2 & 29 & ARG-1B23 & 4.743 & 7.824 & 0.094 & 1.483 & 0.099 & 0.023 & 13.997 & 2.542 & 3.251 \\
\hline ARG-1 & None & 0 & 0 & 3 & 1 & 3 & 1 & ARG-1B31 & 4.686 & 8.468 & 0.092 & 1.469 & 0.104 & 0.021 & 14.025 & 2.662 & 3.251 \\
\hline SME Simulant & Isolok - $12 \mathrm{~mL}$ & LF & 11 & 3 & 4 & 3 & 2 & LIB02 & 11.205 & 4.476 & 0.106 & 1.244 & 0.110 & 0.026 & 11.581 & 0.169 & 4.715 \\
\hline SME Simulant & Coliwasa - low & $\mathrm{HF}$ & 36 & 1 & 21 & 3 & 3 & CL07 & 10.373 & 4.250 & 0.098 & 1.112 & 0.100 & 0.026 & 10.565 & 0.166 & 5.188 \\
\hline SME Simulant & Isolok - $12 \mathrm{~mL}$ & LF & 26 & 3 & 8 & 3 & 4 & LIB04 & 10.959 & 4.347 & 0.108 & 1.244 & 0.108 & 0.025 & 11.480 & 0.163 & 4.866 \\
\hline SME Simulant & \begin{tabular}{|l|} 
Coliwasa - high \\
\end{tabular} & $\mathrm{HF}$ & 1 & 1 & 22 & 3 & 5 & CH05 & 10.354 & 3.960 & 0.098 & 1.133 & 0.102 & 0.027 & 10.680 & 0.174 & 4.478 \\
\hline SME Simulant & \begin{tabular}{|l|l|} 
Coliwasa - low \\
\end{tabular} & $\mathrm{HF}$ & 3 & 1 & 20 & 3 & 6 & CL05 & 11.280 & 4.315 & 0.107 & 1.249 & 0.115 & 0.023 & 11.795 & 0.148 & 4.736 \\
\hline SME Simulant & \begin{tabular}{|l|} 
Coliwasa - high \\
\end{tabular} & $\mathrm{HF}$ & 23 & 1 & 28 & 3 & 7 & CH06 & 11.016 & 4.025 & 0.103 & 1.198 & 0.110 & 0.024 & 11.195 & 0.155 & 4.866 \\
\hline SME Simulant & \begin{tabular}{|l|} 
Coliwasa - high \\
\end{tabular} & LF & 1 & 1 & 12 & 3 & 8 & CH01 & 10.902 & 4.154 & 0.103 & 1.219 & 0.105 & 0.023 & 11.195 & 0.151 & 4.822 \\
\hline SME Simulant & \begin{tabular}{|l|} 
Coliwasa - low \\
\end{tabular} & LF & 20 & 1 & 2 & 3 & 9 & CL02 & 11.507 & 4.379 & 0.109 & 1.261 & 0.109 & 0.025 & 11.795 & 0.162 & 4.672 \\
\hline SME Simulant & Coliwasa - low & $\mathrm{HF}$ & 63 & 1 & 5 & 3 & 10 & CL08 & 11.337 & 4.186 & 0.108 & 1.263 & 0.115 & 0.023 & 11.967 & 0.151 & 4.909 \\
\hline SME Simulant & \begin{tabular}{|l|} 
Coliwasa - high \\
\end{tabular} & $\mathrm{HF}$ & 65 & 1 & 6 & 3 & 11 & CH08 & 10.827 & 4.347 & 0.102 & 1.175 & 0.103 & 0.025 & 11.137 & 0.164 & 4.715 \\
\hline SME Simulant & \begin{tabular}{|l|} 
Isolok - $12 \mathrm{~mL}$ \\
\end{tabular} & LF & 45 & 3 & 7 & 3 & 12 & LIB07 & 11.035 & 4.282 & 0.106 & 1.258 & 0.111 & 0.026 & 11.638 & 1.759 & 4.693 \\
\hline SME Simulant & Coliwasa - low & LF & 4 & 1 & 10 & 3 & 13 & CL01 & 10.978 & 4.121 & 0.105 & 1.220 & 0.109 & 0.026 & 11.323 & 0.169 & 4.822 \\
\hline SME Simulant & Coliwasa - low & $\mathrm{HF}$ & 18 & 1 & 26 & 3 & 14 & CL06 & 11.337 & 4.315 & 0.109 & 1.255 & 0.122 & 0.027 & 11.752 & 0.175 & 4.693 \\
\hline ARG-1 & None & 0 & 0 & 3 & 12 & 3 & 15 & ARG-1B32 & 4.724 & 7.953 & 0.091 & 1.469 & 0.096 & 0.022 & 13.897 & 2.530 & 3.208 \\
\hline SME Simulant & Coliwasa - low & LF & 65 & 1 & 23 & 3 & 16 & CL04 & 10.657 & 4.250 & 0.102 & 1.178 & 0.113 & 0.025 & 10.952 & 0.164 & 4.586 \\
\hline SME Simulant & Coliwasa - low & LF & 40 & 1 & 19 & 3 & 17 & CL03 & 11.583 & 3.864 & 0.105 & 1.201 & 0.113 & 0.027 & 11.638 & 0.175 & 4.327 \\
\hline SME Simulant & \begin{tabular}{|l|} 
Coliwasa - high \\
\end{tabular} & LF & 38 & 1 & 16 & 3 & 18 & CH03 & 10.827 & 4.218 & 0.104 & 1.213 & 0.108 & 0.026 & 11.280 & 0.169 & 4.844 \\
\hline SME Simulant & \begin{tabular}{|l|} 
Isolok - $12 \mathrm{~mL}$ \\
\end{tabular} & LF & 56 & 3 & 11 & 3 & 19 & LIB09 & 10.902 & 4.379 & 0.105 & 1.215 & 0.110 & 0.027 & 11.180 & 0.175 & 4.650 \\
\hline SME Simulant & \begin{tabular}{|l|} 
Coliwasa - high \\
\end{tabular} & LF & 21 & 1 & 14 & 3 & 20 & CH02 & 10.166 & 4.057 & 0.097 & 1.117 & 0.102 & 0.026 & 10.594 & 0.170 & 4.435 \\
\hline SME Simulant & \begin{tabular}{|l|l|l} 
Isolok - $12 \mathrm{~mL}$ \\
\end{tabular} & $\mathrm{LF}$ & 5 & 3 & 5 & 3 & 21 & LIB01 & 11.753 & 4.572 & 0.111 & 1.300 & 0.113 & 0.027 & 12.038 & 0.174 & 4.930 \\
\hline SME Simulant & Isolok - $12 \mathrm{~mL}$ & LF & 23 & 3 & 10 & 3 & 22 & LIB03 & 10.732 & 4.186 & 0.103 & 1.170 & 0.105 & 0.028 & 11.009 & 0.179 & 4.478 \\
\hline SME Simulant & Isolok - $12 \mathrm{~mL}$ & LF & 50 & 3 & 2 & 3 & 23 & $\begin{array}{ll}\text { LIB08 } \\
\end{array}$ & 11.280 & 4.347 & 0.109 & 1.248 & 0.113 & 0.027 & 11.566 & 0.178 & 4.758 \\
\hline SME Simulant & Isolok - $12 \mathrm{~mL}$ & LF & 41 & 3 & 9 & 3 & 24 & LIB06 & 11.337 & 4.347 & 0.110 & 1.312 & 0.118 & 0.026 & 11.795 & 0.172 & 4.822 \\
\hline SME Simulant & \begin{tabular}{|l|} 
Coliwasa - high \\
\end{tabular} & $\mathrm{HF}$ & 34 & 1 & 18 & 3 & 25 & CH07 & 10.562 & 3.993 & 0.101 & 1.178 & 0.103 & 0.023 & 10.937 & 0.152 & 4.500 \\
\hline SME Simulant & \begin{tabular}{|l|} 
Coliwasa - high \\
\end{tabular} & LF & 63 & 1 & 13 & 3 & 26 & CH04 & 10.184 & 4.186 & 0.098 & 1.131 & 0.103 & 0.026 & 10.623 & 0.166 & 4.521 \\
\hline SME Simulant & \begin{tabular}{|l|l|l} 
Isolok - $12 \mathrm{~mL}$ \\
\end{tabular} & LF & 32 & 3 & 3 & 3 & 27 & LIB05 & 11.772 & 4.379 & 0.113 & 1.304 & 0.112 & 0.024 & 12.438 & 0.158 & 4.952 \\
\hline SME Simulant & Isolok $-12 \mathrm{~mL}$ & LF & 60 & 3 & 6 & 3 & 28 & LIB10 & 10.789 & 4.025 & 0.102 & 1.191 & 0.104 & 0.025 & 11.266 & 0.163 & 4.478 \\
\hline ARG-1 & None & 0 & 0 & 3 & 23 & 3 & 29 & ARG-1B33 & 4.856 & 8.243 & 0.093 & 1.497 & 0.101 & 0.020 & 14.297 & 2.746 & 3.315 \\
\hline ARG-1 & None & 0 & 0 & 4 & 1 & 4 & 1 & ARG-1B41 & 4.648 & 7.985 & 0.090 & 1.455 & 0.096 & 0.022 & 14.011 & 2.746 & 3.229 \\
\hline SME Simulant & Isolok - $12 \mathrm{~mL}$ & $\mathrm{HF}$ & 13 & 4 & 11 & 4 & 2 & HIB02 & 10.865 & 4.347 & 0.105 & 1.220 & 0.105 & 0.025 & 11.366 & 0.160 & 4.758 \\
\hline SME Simulant & Coliwasa - low & $\mathrm{HF}$ & 36 & 1 & 21 & 4 & 3 & CL07 & 10.392 & 4.089 & 0.098 & 1.126 & 0.099 & 0.026 & 10.794 & 0.166 & 5.361 \\
\hline SME Simulant & Isolok - $12 \mathrm{~mL}$ & $\mathrm{HF}$ & 25 & 4 & 4 & 4 & 4 & HIB04 & 10.865 & 4.218 & 0.106 & 1.210 & 0.113 & 0.028 & 11.738 & 0.382 & 4.779 \\
\hline SME Simulant & Coliwasa - high & $\mathrm{HF}$ & 1 & 1 & 22 & 4 & 5 & CH05 & 10.732 & 4.025 & 0.102 & 1.167 & 0.107 & 0.027 & 11.252 & 0.174 & 4.629 \\
\hline SME Simulant & \begin{tabular}{|l|} 
Coliwasa - low \\
\end{tabular} & $\mathrm{HF}$ & 3 & 1 & 20 & 4 & 6 & CL05 & 11.167 & 4.218 & 0.106 & 1.235 & 0.114 & 0.023 & 11.952 & 0.148 & 4.844 \\
\hline SME Simulant & \begin{tabular}{|l|} 
Coliwasa - high \\
\end{tabular} & $\mathrm{HF}$ & 23 & 1 & 28 & 4 & 7 & CH06 & 10.676 & 4.025 & 0.102 & 1.185 & 0.106 & 0.024 & 11.309 & 0.156 & 4.930 \\
\hline SME Simulant & \begin{tabular}{|l|} 
Coliwasa - high \\
\end{tabular} & LF & 1 & 1 & 12 & 4 & 8 & CH01 & 10.619 & 4.218 & 0.103 & 1.198 & 0.102 & 0.023 & 11.223 & 0.151 & 4.866 \\
\hline SME Simulant & Coliwasa - low & LF & 20 & 1 & 2 & 4 & 9 & CL02 & 11.299 & 4.282 & 0.108 & 1.248 & 0.107 & 0.025 & 11.909 & 0.162 & 4.715 \\
\hline
\end{tabular}


Table A4. Phase 1 Oxide Concentration Measurements (part 1)

\begin{tabular}{|c|c|c|c|c|c|c|c|c|c|c|c|c|c|c|c|c|c|}
\hline $\begin{array}{c}\text { Type of } \\
\text { Material }\end{array}$ & Sampler Details & $\begin{array}{l}\text { Flow } \\
\text { Rate }\end{array}$ & $\begin{array}{c}\text { Sample } \\
\text { Order }\end{array}$ & $\begin{array}{c}\text { Preparation } \\
\text { Block }\end{array}$ & $\begin{array}{c}\text { Preparation } \\
\text { Sequence }\end{array}$ & $\begin{array}{c}\text { Analytical } \\
\text { Block }\end{array}$ & $\begin{array}{l}\text { Analytical } \\
\text { Sequence }\end{array}$ & Sample ID & $\begin{array}{l}\text { Al2O3 } \\
\text { (wt\%) }\end{array}$ & $\begin{array}{l}\text { B2O3 } \\
\text { (wt\%) }\end{array}$ & $\begin{array}{c}\mathrm{BaO} \\
\text { (wt\%) }\end{array}$ & $\begin{array}{c}\mathrm{CaO} \\
\text { (wt\%) }\end{array}$ & $\begin{array}{l}\text { Cr2O3 } \\
\text { (wt\%) }\end{array}$ & $\begin{array}{c}\mathrm{CuO} \\
\text { (wt\%) }\end{array}$ & $\begin{array}{l}\text { Fe2O3 } \\
\text { (wt\%) }\end{array}$ & $\begin{array}{c}\text { K2O } \\
\text { (wt\%) }\end{array}$ & $\begin{array}{l}\text { Li2O } \\
\text { (wt\%) }\end{array}$ \\
\hline SME Simulant & Coliwasa - low & $\mathrm{HF}$ & 63 & 1 & 5 & 4 & 10 & CL08 & 11.035 & 4.089 & 0.105 & 1.219 & 0.110 & 0.023 & 12.009 & 0.151 & 4.887 \\
\hline SME Simulant & Coliwasa - high & $\mathrm{HF}$ & 65 & 1 & 6 & 4 & 11 & CH08 & 10.657 & 4.250 & 0.101 & 1.145 & 0.101 & 0.025 & 11.266 & 0.164 & 4.693 \\
\hline SME Simulant & \begin{tabular}{|l|l|l|l} 
Isolok - $12 \mathrm{~mL}$ \\
\end{tabular} & $\mathrm{HF}$ & 46 & 4 & 2 & 4 & 12 & HIB07 & 10.751 & 4.315 & 0.103 & 1.199 & 0.106 & 0.028 & 11.166 & 0.182 & 4.629 \\
\hline SME Simulant & Coliwasa - low & LF & 4 & 1 & 10 & 4 & 13 & CL01 & 10.808 & 4.186 & 0.105 & 1.206 & 0.109 & 0.026 & 11.495 & 0.169 & 4.822 \\
\hline SME Simulant & Coliwasa - low & $\mathrm{HF}$ & 18 & 1 & 26 & 4 & 14 & CL06 & 11.054 & 4.154 & 0.107 & 1.248 & 0.119 & 0.027 & 11.838 & 0.175 & 4.672 \\
\hline ARG-1 & None & 0 & 0 & 4 & 7 & 4 & 15 & ARG-1B42 & 4.591 & 8.082 & 0.090 & 1.441 & 0.096 & 0.021 & 13.968 & 2.855 & 3.208 \\
\hline SME Simulant & Coliwasa - low & LF & 65 & 1 & 23 & 4 & 16 & CL04 & 10.657 & 4.218 & 0.101 & 1.177 & 0.110 & 0.025 & 11.152 & 0.164 & 4.715 \\
\hline SME Simulant & Coliwasa - low & LF & 40 & 1 & 19 & 4 & 17 & CL03 & 11.753 & 3.896 & 0.108 & 1.265 & 0.113 & 0.027 & 12.052 & 0.175 & 4.435 \\
\hline SME Simulant & \begin{tabular}{|l|} 
Coliwasa - high \\
\end{tabular} & LF & 38 & 1 & 16 & 4 & 18 & CH03 & 10.600 & 4.057 & 0.102 & 1.185 & 0.101 & 0.026 & 11.309 & 0.169 & 4.844 \\
\hline SME Simulant & Isolok - $12 \mathrm{~mL}$ & $\mathrm{HF}$ & 56 & 4 & 9 & 4 & 19 & HIB09 & 11.280 & 4.218 & 0.112 & 1.286 & 0.115 & 0.027 & 12.281 & 0.174 & 4.736 \\
\hline SME Simulant & \begin{tabular}{|l} 
Coliwasa - high \\
\end{tabular} & $\mathrm{LF}$ & 21 & 1 & 14 & 4 & 20 & CH02 & 10.751 & 4.186 & 0.103 & 1.163 & 0.104 & 0.026 & 11.395 & 0.396 & 4.736 \\
\hline SME Simulant & Isolok - $12 \mathrm{~mL}$ & $\mathrm{HF}$ & 7 & 4 & 12 & 4 & 21 & HIB01 & 10.676 & 4.250 & 0.102 & 1.153 & 0.107 & 0.027 & 11.152 & 0.178 & 4.844 \\
\hline SME Simulant & Isolok $-12 \mathrm{~mL}$ & $\mathrm{HF}$ & 21 & 4 & 6 & 4 & 22 & HIB03 & 11.431 & 4.057 & 0.110 & 1.305 & 0.115 & 0.026 & 11.895 & 0.171 & 4.736 \\
\hline SME Simulant & Isolok - $12 \mathrm{~mL}$ & $\mathrm{HF}$ & 49 & 4 & 5 & 4 & 23 & HIB08 & 10.033 & 4.540 & 0.100 & 1.136 & 0.097 & 0.028 & 10.423 & 0.181 & 5.081 \\
\hline SME Simulant & Isolok - $12 \mathrm{~mL}$ & $\mathrm{HF}$ & 38 & 4 & 8 & 4 & 24 & HIB06 & 10.600 & 4.379 & 0.101 & 1.175 & 0.104 & 0.023 & 11.152 & 0.148 & 4.887 \\
\hline SME Simulant & \begin{tabular}{|l|} 
Coliwasa - high \\
\end{tabular} & $\mathrm{HF}$ & 34 & 1 & 18 & 4 & 25 & CH07 & 10.695 & 4.154 & 0.103 & 1.195 & 0.102 & 0.023 & 11.223 & 0.152 & 4.736 \\
\hline SME Simulant & \begin{tabular}{|l|} 
Coliwasa - high \\
\end{tabular} & LF & 63 & 1 & 13 & 4 & 26 & CH04 & 10.713 & 4.218 & 0.103 & 1.196 & 0.105 & 0.026 & 11.309 & 0.166 & 4.866 \\
\hline SME Simulant & Isolok - $12 \mathrm{~mL}$ & $\mathrm{HF}$ & 33 & 4 & 10 & 4 & 27 & HIB05 & 10.846 & 4.057 & 0.104 & 1.192 & 0.111 & 0.027 & 11.795 & 0.179 & 4.779 \\
\hline SME Simulant & Isolok - $12 \mathrm{~mL}$ & $\mathrm{HF}$ & 58 & 4 & 3 & 4 & 28 & HIB10 & 10.336 & 4.186 & 0.100 & 1.129 & 0.098 & 0.027 & 10.680 & 0.173 & 4.844 \\
\hline ARG-1 & None & 0 & 0 & 4 & 13 & 4 & 29 & ARG-1B43 & 4.667 & 7.857 & 0.090 & 1.427 & 0.094 & 0.022 & 13.997 & 2.638 & 3.229 \\
\hline ARG-1 & \begin{tabular}{|l|} 
None \\
\end{tabular} & 0 & 0 & 2 & 1 & 5 & 1 & ARG-1B21 & 4.610 & 7.760 & 0.089 & 1.469 & 0.097 & 0.017 & 13.839 & 2.746 & 3.272 \\
\hline SME Simulant & Isolok - $3 \mathrm{~mL}$ & $\mathrm{HF}$ & 20 & 2 & 16 & 5 & 2 & HIS03 & 10.619 & 4.089 & 0.103 & 1.189 & 0.104 & 0.029 & 11.237 & 0.189 & 4.909 \\
\hline SME Simulant & Isolok - $3 \mathrm{~mL}$ & $\mathrm{HF}$ & 9 & 2 & 21 & 5 & 3 & HIS01 & 10.525 & 4.315 & 0.102 & 1.174 & 0.099 & 0.023 & 11.209 & 0.151 & 4.844 \\
\hline SME Simulant & Coliwasa - low & LF & 20 & 1 & $\frac{2}{2}$ & 5 & 4 & CL02 & 11.054 & 4.186 & 0.107 & 1.235 & 0.105 & 0.025 & 11.652 & 0.162 & 4.607 \\
\hline SME Simulant & Coliwasa - high & LF & 38 & 1 & 16 & 5 & 5 & $\mathrm{CH} 03$ & 10.940 & 4.186 & 0.106 & 1.265 & 0.106 & 0.026 & 11.638 & 0.169 & 5.059 \\
\hline SME Simulant & \begin{tabular}{|l|} 
Coliwasa - high \\
\end{tabular} & LF & 63 & 1 & 13 & 5 & 6 & $\mathrm{CH} 04$ & 10.203 & 4.025 & 0.098 & 1.150 & 0.102 & 0.026 & 10.809 & 0.166 & 4.586 \\
\hline SME Simulant & Isolok - $3 \mathrm{~mL}$ & $\mathrm{HF}$ & 44 & 2 & 18 & 5 & 7 & HIS07 & 10.978 & 4.443 & 0.104 & 1.237 & 0.107 & 0.026 & 11.566 & 0.172 & 4.887 \\
\hline SME Simulant & \begin{tabular}{|l|} 
Coliwasa - high \\
\end{tabular} & $\mathrm{HF}$ & 23 & 1 & 28 & 5 & 8 & CH06 & 10.354 & 3.928 & 0.099 & 1.157 & 0.106 & 0.024 & 10.894 & 0.155 & 4.715 \\
\hline SME Simulant & \begin{tabular}{|l|} 
Coliwasa - high \\
\end{tabular} & LF & 21 & 1 & 14 & 5 & 9 & CH02 & 11.054 & 4.186 & 0.105 & 1.223 & 0.109 & 0.026 & 11.724 & 0.170 & 4.866 \\
\hline SME Simulant & \begin{tabular}{|l|} 
Coliwasa - low \\
\end{tabular} & $\mathrm{HF}$ & 3 & 1 & 20 & 5 & 10 & CL05 & 10.695 & 4.025 & 0.102 & 1.215 & 0.110 & 0.023 & 11.438 & 0.148 & 4.650 \\
\hline SME Simulant & Isolok - $3 \mathrm{~mL}$ & $\mathrm{HF}$ & 14 & 2 & 15 & 5 & 11 & HISO2 & 10.506 & 4.154 & 0.100 & 1.180 & 0.102 & 0.025 & 11.037 & 0.161 & 4.758 \\
\hline SME Simulant & Coliwasa - low & LF & 40 & 1 & 19 & 5 & 12 & CL03 & 11.488 & 3.864 & 0.106 & 1.209 & 0.114 & 0.027 & 11.909 & 0.175 & 4.327 \\
\hline SME Simulant & Coliwasa - low & $\mathrm{HF}$ & 18 & 1 & 26 & 5 & 13 & CL06 & 10.959 & 4.250 & 0.106 & 1.259 & 0.118 & 0.027 & 11.709 & 0.175 & 4.586 \\
\hline SME Simulant & \begin{tabular}{|l|l} 
Coliwasa - high \\
\end{tabular} & $\mathrm{HF}$ & 1 & 1 & 22 & 5 & 14 & CH05 & 10.543 & 4.057 & 0.101 & 1.118 & 0.105 & 0.027 & 11.066 & 0.174 & 4.543 \\
\hline ARG-1 & \begin{tabular}{|l|} 
None \\
\end{tabular} & 0 & 0 & 2 & 12 & 5 & 15 & ARG-1B22 & 4.270 & 7.309 & 0.083 & 1.367 & 0.090 & 0.018 & 12.867 & 2.494 & 3.036 \\
\hline SME Simulant & Coliwasa - low & $\mathrm{HF}$ & 36 & 1 & 21 & 5 & 16 & CL07 & 10.222 & 4.121 & 0.099 & 1.157 & 0.101 & 0.026 & 10.623 & 0.166 & 5.339 \\
\hline SME Simulant & Isolok - 3 mL & $\mathrm{HF}$ & 31 & 2 & 19 & 5 & 17 & HIS05 & 10.468 & 4.121 & 0.102 & 1.185 & 0.101 & 0.026 & 11.137 & 0.169 & 4.736 \\
\hline SME Simulant & Isolok - $3 \mathrm{~mL}$ & $\mathrm{HF}$ & 60 & 2 & 22 & 5 & 18 & HIS10 & 10.373 & 4.186 & 0.102 & 1.210 & 0.104 & 0.024 & 11.166 & 0.157 & 4.758 \\
\hline SME Simulant & Coliwasa - low & $\mathrm{HF}$ & 63 & 1 & 5 & 5 & 19 & CL08 & 11.016 & 4.154 & 0.105 & 1.269 & 0.110 & 0.023 & 11.867 & 0.151 & 4.930 \\
\hline SME Simulant & Isolok - $3 \mathrm{~mL}$ & $\mathrm{HF}$ & 26 & 2 & 14 & 5 & 20 & HISO4 & 9.882 & 3.960 & 0.097 & 1.129 & 0.098 & 0.025 & 10.523 & 0.163 & 4.500 \\
\hline SME Simulant & Isolok - $3 \mathrm{~mL}$ & $\mathrm{HF}$ & 35 & 2 & 20 & 5 & 21 & HIS06 & 10.770 & 4.315 & 0.102 & 1.233 & 0.106 & 0.024 & 11.352 & 0.157 & 5.016 \\
\hline SME Simulant & \begin{tabular}{|l|} 
Coliwasa - high \\
\end{tabular} & $\mathrm{HF}$ & 65 & 1 & 6 & 5 & 22 & CH08 & 10.676 & 4.347 & 0.101 & 1.178 & 0.102 & 0.025 & 11.195 & 0.164 & 4.715 \\
\hline SME Simulant & Coliwasa - high & $\mathrm{HF}$ & 34 & 1 & 18 & 5 & 23 & CH07 & 10.676 & 4.250 & 0.104 & 1.205 & 0.106 & 0.023 & 11.223 & 0.152 & 4.650 \\
\hline SME Simulant & Coliwasa - high & LF & 1 & 1 & 12 & 5 & 24 & $\mathrm{CH} 01$ & 10.430 & 4.121 & 0.099 & 1.175 & 0.100 & 0.023 & 10.994 & 0.151 & 4.672 \\
\hline SME Simulant & Isolok - $3 \mathrm{~mL}$ & $\mathrm{HF}$ & 54 & 2 & 17 & 5 & 25 & HIS09 & 10.846 & 4.282 & 0.102 & 1.245 & 0.105 & 0.026 & 11.395 & 0.172 & 4.887 \\
\hline SME Simulant & Coliwasa - low & LF & 4 & 1 & 10 & 5 & 26 & CL01 & 11.016 & 4.282 & 0.107 & 1.242 & 0.110 & 0.026 & 11.709 & 0.169 & 4.952 \\
\hline SME Simulant & Isolok - $3 \mathrm{~mL}$ & $\mathrm{HF}$ & 48 & 2 & 13 & 5 & 27 & HIS08 & 10.997 & 4.218 & 0.104 & 1.212 & 0.107 & 0.025 & 11.509 & 0.161 & 4.736 \\
\hline SME Simulant & Coliwasa - low & LF & 65 & 1 & 23 & 5 & 28 & CL04 & 10.713 & 4.282 & 0.103 & 1.233 & 0.112 & 0.025 & 11.266 & 0.164 & 4.650 \\
\hline
\end{tabular}


Table A4. Phase 1 Oxide Concentration Measurements (part 1)

\begin{tabular}{|c|c|c|c|c|c|c|c|c|c|c|c|c|c|c|c|c|c|}
\hline $\begin{array}{c}\text { Type of } \\
\text { Material }\end{array}$ & Sampler Details & $\begin{array}{l}\text { Flow } \\
\text { Rate }\end{array}$ & $\begin{array}{c}\text { Sample } \\
\text { Order }\end{array}$ & $\begin{array}{l}\text { Preparation } \\
\text { Block }\end{array}$ & $\begin{array}{c}\text { Preparation } \\
\text { Sequence }\end{array}$ & $\begin{array}{c}\text { Analytical } \\
\text { Block }\end{array}$ & $\begin{array}{l}\text { Analytical } \\
\text { Sequence }\end{array}$ & Sample ID & $\begin{array}{l}\text { Al2O3 } \\
\text { (wt\%) }\end{array}$ & $\begin{array}{l}\text { B2O3 } \\
\text { (wt\%) }\end{array}$ & $\begin{array}{c}\text { BaO } \\
\text { (wt\%) }\end{array}$ & $\begin{array}{c}\mathrm{CaO} \\
\text { (wt\%) }\end{array}$ & $\begin{array}{l}\text { Cr2O3 } \\
\text { (wt\%) }\end{array}$ & $\begin{array}{c}\mathrm{CuO} \\
\text { (wt\%) }\end{array}$ & $\begin{array}{l}\text { Fe2O3 } \\
\text { (wt \%) }\end{array}$ & $\begin{array}{c}\text { K2O } \\
\text { (wt\%) }\end{array}$ & $\begin{array}{l}\text { Li2O } \\
\text { (wt\%) }\end{array}$ \\
\hline ARG-1 & None & 0 & 0 & 2 & 23 & 5 & 29 & ARG-1B23 & 4.629 & 7.728 & 0.091 & 1.441 & 0.097 & 0.023 & 14.011 & 2.674 & 3.208 \\
\hline ARG-1 & None & 0 & 0 & 3 & 1 & 6 & 1 & ARG-1B31 & 4.629 & 7.953 & 0.089 & 1.441 & 0.096 & 0.021 & 13.968 & 2.698 & 3.165 \\
\hline SME Simulant & Hydragard & LF & 15 & 3 & 21 & 6 & 2 & LHY03 & 11.280 & 4.218 & 0.106 & 1.273 & 0.105 & 0.025 & 11.909 & 0.164 & 5.038 \\
\hline SME Simulant & Hydragard & $\mathrm{LF}$ & 6 & 3 & 18 & 6 & 3 & LHY01 & 11.243 & 4.282 & 0.107 & 1.235 & 0.106 & 0.027 & 11.881 & 0.176 & 4.672 \\
\hline SME Simulant & Coliwasa - low & LF & 20 & 1 & 2 & 6 & 4 & CL02 & 11.337 & 4.282 & 0.107 & 1.272 & 0.106 & 0.025 & 11.924 & 0.162 & 4.801 \\
\hline SME Simulant & Coliwasa - high & LF & 38 & 1 & 16 & 6 & 5 & $\mathrm{CH} 03$ & 10.770 & 4.121 & 0.104 & 1.217 & 0.105 & 0.026 & 11.423 & 0.169 & 4.887 \\
\hline SME Simulant & \begin{tabular}{|l|} 
Coliwasa - high \\
\end{tabular} & LF & 63 & 1 & 13 & 6 & 6 & CH04 & 10.657 & 4.154 & 0.102 & 1.187 & 0.105 & 0.026 & 11.295 & 0.166 & 4.693 \\
\hline SME Simulant & \begin{tabular}{|l} 
Hydragard \\
\end{tabular} & LF & 47 & 3 & 13 & 6 & 7 & LHY07 & 11.677 & 4.025 & 0.108 & 1.293 & 0.111 & 0.028 & 12.367 & 0.181 & 4.779 \\
\hline SME Simulant & Coliwasa - high & $\mathrm{HF}$ & 23 & 1 & 28 & 6 & 8 & CH06 & 10.789 & 3.960 & 0.102 & 1.187 & 0.106 & 0.024 & 11.395 & 0.155 & 4.801 \\
\hline SME Simulant & \begin{tabular}{|l|} 
Coliwasa - high \\
\end{tabular} & $\mathrm{LF}$ & 21 & 1 & 14 & 6 & 9 & $\mathrm{CH} 02$ & 10.279 & 3.896 & 0.097 & 1.124 & 0.100 & 0.026 & 10.923 & 0.170 & 4.457 \\
\hline SME Simulant & Coliwasa - low & $\mathrm{HF}$ & 3 & 1 & 20 & 6 & 10 & CL05 & 11.413 & 4.186 & 0.109 & 1.273 & 0.113 & 0.048 & 12.224 & 0.148 & 4.887 \\
\hline SME Simulant & Hydragard & LF & 10 & 3 & 20 & 6 & 11 & LHY02 & 10.770 & 4.476 & 0.100 & 1.152 & 0.100 & 0.027 & 11.166 & 0.178 & 4.973 \\
\hline SME Simulant & Coliwasa - low & LF & 40 & 1 & 19 & 6 & 12 & CL03 & 12.036 & 3.896 & 0.110 & 1.258 & 0.115 & 0.027 & 12.410 & 0.175 & 4.500 \\
\hline SME Simulant & Coliwasa - low & $\mathrm{HF}$ & 18 & 1 & 26 & 6 & 13 & CL06 & 11.072 & 4.025 & 0.106 & 1.247 & 0.116 & 0.027 & 11.809 & 0.175 & 4.629 \\
\hline SME Simulant & \begin{tabular}{|l|} 
Coliwasa - high \\
\end{tabular} & $\mathrm{HF}$ & 1 & 1 & 22 & 6 & 14 & $\mathrm{CH} 05$ & 10.865 & 4.025 & 0.103 & 1.187 & 0.108 & 0.027 & 11.438 & 0.174 & 4.693 \\
\hline ARG-1 & \begin{tabular}{|l} 
None \\
\end{tabular} & 0 & 0 & 3 & 12 & 6 & 15 & ARG-1B32 & 4.554 & 7.953 & 0.087 & 1.427 & 0.095 & 0.022 & 13.668 & 2.626 & 3.100 \\
\hline SME Simulant & Coliwasa - low & $\mathrm{HF}$ & 36 & 1 & 21 & 6 & 16 & CL07 & 10.449 & 3.993 & 0.098 & 1.126 & 0.098 & 0.026 & 10.851 & 0.166 & 5.253 \\
\hline SME Simulant & Hydragard & LF & 30 & 3 & 14 & 6 & 17 & LHY05 & 11.186 & 4.089 & 0.106 & 1.241 & 0.110 & 0.024 & 11.924 & 0.157 & 4.736 \\
\hline SME Simulant & Hydragard & LF & 59 & 3 & 17 & 6 & 18 & LHY10 & 10.997 & 4.154 & 0.105 & 1.258 & 0.101 & 0.026 & 11.566 & 0.169 & 5.016 \\
\hline SME Simulant & Coliwasa - low & $\mathrm{HF}$ & 63 & 1 & 5 & 6 & 19 & CL08 & 11.431 & 4.186 & 0.107 & 1.249 & 0.112 & 0.023 & 12.253 & 0.151 & 4.844 \\
\hline SME Simulant & Hydragard & LF & 28 & 3 & 16 & 6 & 20 & LHY04 & 11.148 & 4.218 & 0.105 & 1.251 & 0.105 & 0.025 & 11.781 & 0.166 & 4.801 \\
\hline SME Simulant & Hydragard & LF & 37 & 3 & 15 & 6 & 21 & LHY06 & 11.583 & 4.508 & 0.109 & 1.300 & 0.109 & 0.026 & 12.081 & 0.170 & 5.059 \\
\hline SME Simulant & \begin{tabular}{|l} 
Coliwasa - high \\
\end{tabular} & $\mathrm{HF}$ & 65 & 1 & 6 & 6 & 22 & CH08 & 10.411 & 4.057 & 0.098 & 1.129 & 0.097 & 0.025 & 10.952 & 0.164 & 4.607 \\
\hline SME Simulant & \begin{tabular}{|l} 
Coliwasa - high \\
\end{tabular} & $\mathrm{HF}$ & 34 & 1 & 18 & 6 & 23 & CH07 & 11.091 & 4.250 & 0.107 & 1.259 & 0.108 & 0.023 & 11.709 & 0.152 & 4.995 \\
\hline SME Simulant & Coliwasa - high & LF & 1 & 1 & 12 & 6 & 24 & CH01 & 10.449 & 4.057 & 0.099 & 1.177 & 0.099 & 0.023 & 10.980 & 0.151 & 4.736 \\
\hline SME Simulant & Hydragard & LF & 53 & 3 & 19 & 6 & 25 & LHY09 & 11.696 & 4.347 & 0.113 & 1.345 & 0.113 & 0.023 & 12.267 & 0.152 & 5.124 \\
\hline SME Simulant & Coliwasa - low & LF & 4 & 1 & 10 & 6 & 26 & CL01 & 10.827 & 4.089 & 0.103 & 1.233 & 0.106 & 0.026 & 11.495 & 0.169 & 4.973 \\
\hline SME Simulant & Hydragard & LF & 48 & 3 & 22 & 6 & 27 & LHY08 & 10.902 & 4.121 & 0.104 & 1.222 & 0.103 & 0.023 & 11.495 & 0.151 & 4.650 \\
\hline SME Simulant & Coliwasa - low & LF & 65 & 1 & 23 & 6 & 28 & CL04 & 10.827 & 4.250 & 0.103 & 1.206 & 0.111 & 0.025 & 11.295 & 0.164 & 4.779 \\
\hline ARG-1 & None & 0 & 0 & 3 & 23 & 6 & 29 & ARG-1B33 & 4.384 & 7.728 & 0.085 & 1.387 & 0.090 & 0.020 & 13.196 & 2.638 & 3.122 \\
\hline
\end{tabular}


Table A4. Phase 1 Oxide Concentration Measurements (part 2)

\begin{tabular}{|c|c|c|c|c|c|c|c|c|c|c|c|c|c|c|c|c|c|}
\hline \begin{tabular}{|l|} 
Type of \\
Material \\
\end{tabular} & Sampler Details & $\begin{array}{l}\text { Flow } \\
\text { Rate }\end{array}$ & $\begin{array}{r}\text { Sample } \\
\text { Order }\end{array}$ & $\begin{array}{r}\text { Preparation } \\
\text { Block } \\
\end{array}$ & $\begin{array}{r}\text { Preparation } \\
\text { Sequence } \\
\end{array}$ & $\begin{array}{r}\text { Analytical } \\
\text { Block } \\
\end{array}$ & $\begin{array}{r}\text { Analytical } \\
\text { Sequence } \\
\end{array}$ & Sample ID & $\begin{array}{r}\text { MgO } \\
\text { (wt\%) }\end{array}$ & $\begin{array}{r}\text { MnO } \\
\text { (wt\%) } \\
\end{array}$ & $\begin{array}{l}\text { Na2O } \\
\text { (wt\%) }\end{array}$ & $\begin{array}{r}\mathrm{NiO} \\
(\mathrm{wt} \%)\end{array}$ & $\begin{array}{r}\mathrm{SiO2} \\
(\mathrm{wt} \%) \\
\end{array}$ & $\begin{array}{r}\text { TiO2 } \\
\text { (wt\%) } \\
\end{array}$ & $\begin{array}{r}\mathrm{ZnO} \\
\text { (wt\%) }\end{array}$ & $\begin{array}{r}\text { ZrO2 } \\
\text { (wt\%) } \\
\end{array}$ & $\begin{array}{r}\text { Sum } \\
\text { (wt\%) }\end{array}$ \\
\hline ARG-1 & None & 0 & 0 & 1 & 1 & 1 & 1 & ARG-1B11 & 0.857 & 1.846 & 11.525 & 1.035 & 48.348 & 1.154 & 0.024 & 0.133 & 99.2052 \\
\hline SME Simulant & Isolok - $3 \mathrm{~mL}$ & LF & 18 & 1 & 9 & 1 & 2 & LIS03 & 1.065 & 1.743 & 11.997 & 0.457 & 51.557 & 0.081 & 0.104 & 0.366 & 99.5477 \\
\hline SME Simulant & Isolok - $3 \mathrm{~mL}$ & LF & 8 & 1 & 27 & 1 & 3 & LIS01 & 1.076 & 1.795 & 10.595 & 0.463 & 51.985 & 0.083 & 0.103 & 0.381 & 99.7739 \\
\hline SME Simulant & Coliwasa - low & $\mathrm{LF}$ & 20 & 1 & 2 & 1 & 4 & CL02 & 1.101 & 1.808 & 11.323 & 0.464 & 50.274 & 0.081 & 0.103 & 0.378 & 99.2611 \\
\hline SME Simulant & Coliwasa - high & LF & 38 & 1 & 16 & 1 & 5 & $\mathrm{CH} 03$ & 1.056 & 1.756 & 11.310 & 0.453 & 52.413 & 0.085 & 0.102 & 0.369 & 99.9841 \\
\hline SME Simulant & Coliwasa - high & LF & 63 & 1 & 13 & 1 & 6 & $\mathrm{CH} 04$ & 1.046 & 1.730 & 11.296 & 0.448 & 51.985 & 0.085 & 0.102 & 0.367 & 99.5620 \\
\hline SME Simulant & Isolok - $3 \mathrm{~mL}$ & LF & 44 & 1 & 3 & 1 & 7 & LIS07 & 1.080 & 1.808 & 10.905 & 0.453 & 52.199 & 0.081 & 0.100 & 0.374 & 100.0003 \\
\hline SME Simulant & $\begin{array}{l}\text { Coliwasa - high } \\
\end{array}$ & $\mathrm{HF}$ & 23 & 1 & 28 & 1 & 8 & CH06 & 1.041 & 1.717 & 10.878 & 0.444 & 52.627 & 0.085 & 0.097 & 0.376 & \begin{tabular}{|l|l|}
99.6483 \\
\end{tabular} \\
\hline SME Simulant & Coliwasa - high & LF & 21 & 1 & 14 & 1 & 9 & $\mathrm{CH} 02$ & 1.056 & 1.743 & 11.175 & 0.453 & 52.413 & 0.083 & 0.100 & 0.371 & 99.9826 \\
\hline SME Simulant & Coliwasa - low & $\mathrm{HF}$ & 3 & 1 & 20 & 1 & 10 & CL05 & 1.094 & 1.821 & 11.067 & 0.477 & 51.129 & 0.081 & 0.103 & 0.377 & 99.3496 \\
\hline SME Simulant & Isolok - $3 \mathrm{~mL}$ & LF & 12 & 1 & 25 & 1 & 11 & LIS02 & 1.083 & 1.782 & 11.202 & 0.462 & 51.557 & 0.082 & 0.103 & 0.378 & 99.5654 \\
\hline SME Simulant & Coliwasa - low & LF & 40 & 1 & 19 & 1 & 12 & CL03 & 1.109 & 1.821 & 11.714 & 0.467 & 48.134 & 0.076 & 0.107 & 0.378 & 97.4403 \\
\hline SME Simulant & Coliwasa - low & $\mathrm{HF}$ & 18 & 1 & 26 & 1 & 13 & CL06 & 1.098 & 1.834 & 11.943 & 0.473 & 50.487 & 0.083 & 0.104 & 0.378 & 99.9549 \\
\hline SME Simulant & Coliwasa - high & $\mathrm{HF}$ & 1 & 1 & 22 & 1 & 14 & CH05 & 1.031 & 1.704 & 11.094 & 0.444 & 53.055 & 0.080 & 0.099 & 0.367 & 99.8128 \\
\hline ARG-1 & None & 0 & 0 & 1 & 15 & 1 & 15 & ARG-1B12 & 0.859 & 1.859 & 11.606 & 1.036 & 48.348 & 1.151 & 0.025 & 0.133 & 99.1348 \\
\hline SME Simulant & Coliwasa - low & $\mathrm{HF}$ & 36 & 1 & 21 & 1 & 16 & CL07 & 0.980 & 1.601 & 11.350 & 0.392 & 52.627 & 0.083 & 0.097 & 0.357 & 99.3292 \\
\hline SME Simulant & Isolok - $3 \mathrm{~mL}$ & $\mathrm{LF}$ & 31 & 1 & 24 & 1 & 17 & LIS05 & 1.058 & 1.743 & 11.889 & 0.448 & 51.771 & 0.079 & 0.100 & 0.367 & 99.8444 \\
\hline SME Simulant & Isolok - $3 \mathrm{~mL}$ & LF & 58 & 1 & 4 & 1 & 18 & LIS10 & 1.096 & 1.821 & 10.595 & 0.467 & 51.771 & 0.083 & 0.103 & 0.378 & 99.6627 \\
\hline SME Simulant & Coliwasa - low & $\mathrm{HF}$ & 63 & 1 & 5 & 1 & 19 & CL08 & 1.103 & 1.872 & 11.431 & 0.514 & 50.274 & 0.077 & 0.110 & 0.367 & 99.4427 \\
\hline SME Simulant & Isolok - $3 \mathrm{~mL}$ & LF & 25 & 1 & 7 & 1 & 20 & LISO4 & 1.046 & 1.717 & 11.943 & 0.440 & 51.771 & 0.081 & 0.098 & 0.361 & 99.3221 \\
\hline SME Simulant & Isolok - $3 \mathrm{~mL}$ & LF & 42 & 1 & 11 & 1 & 21 & LIS06 & 1.096 & 1.834 & 11.391 & 0.472 & 51.129 & 0.082 & 0.105 & 0.378 & 99.8858 \\
\hline SME Simulant & Coliwasa - high & $\mathrm{HF}$ & 65 & 1 & 6 & 1 & 22 & $\mathrm{CH} 08$ & 1.033 & 1.704 & 11.687 & 0.438 & 52.199 & 0.082 & 0.098 & 0.373 & 99.9457 \\
\hline SME Simulant & $\begin{array}{l}\text { Coliwasa - high } \\
\end{array}$ & $\mathrm{HF}$ & 34 & 1 & 18 & 1 & 23 & $\mathrm{CH} 07$ & 1.036 & 1.717 & 10.730 & 0.439 & 52.627 & 0.080 & 0.100 & 0.373 & 99.0590 \\
\hline SME Simulant & Coliwasa - high & LF & 1 & 1 & 12 & 1 & 24 & $\mathrm{CH} 01$ & 1.040 & 1.717 & 11.229 & 0.440 & 52.413 & 0.084 & 0.099 & 0.369 & 99.5504 \\
\hline SME Simulant & \begin{tabular}{|l|} 
Isolok - $3 \mathrm{~mL}$ \\
\end{tabular} & LF & 54 & 1 & 17 & 1 & 25 & LIS09 & 1.066 & 1.808 & 10.514 & 0.467 & 51.985 & 0.083 & 0.102 & 0.381 & 99.5358 \\
\hline SME Simulant & Coliwasa - low & LF & 4 & 1 & 10 & 1 & 26 & CL01 & 1.063 & 1.756 & 10.878 & 0.456 & 52.199 & 0.084 & 0.101 & 0.371 & 99.4904 \\
\hline SME Simulant & Isolok - $3 \mathrm{~mL}$ & LF & 49 & 1 & 8 & 1 & 27 & LIS08 & 1.085 & 1.808 & 10.946 & 0.462 & 51.557 & 0.080 & 0.102 & 0.374 & 99.4573 \\
\hline SME Simulant & Coliwasa - low & LF & 65 & 1 & 23 & 1 & 28 & CL04 & 1.036 & 1.704 & 10.878 & 0.425 & 52.841 & 0.083 & 0.096 & 0.371 & 99.3817 \\
\hline ARG-1 & None & 0 & 0 & 1 & 29 & 1 & 29 & \begin{tabular}{|l} 
ARG-1B13 \\
\end{tabular} & 0.864 & 1.859 & 11.687 & 1.026 & 48.348 & 1.158 & 0.024 & 0.136 & 99.2035 \\
\hline ARG-1 & \begin{tabular}{|l} 
None \\
\end{tabular} & 0 & 0 & 2 & 1 & 2 & 1 & ARG-1B21 & 0.869 & 1.885 & 11.633 & 1.068 & 48.562 & 1.158 & 0.024 & 0.135 & \begin{tabular}{|l|l}
99.3722 \\
\end{tabular} \\
\hline SME Simulant & Hydragard & $\mathrm{HF}$ & 11 & 2 & 3 & 2 & 2 & HHY02 & 1.104 & 1.821 & 11.579 & 0.475 & 51.557 & 0.083 & 0.103 & 0.370 & 100.8701 \\
\hline SME Simulant & Coliwasa - low & $\mathrm{HF}$ & 36 & 1 & 21 & 2 & 3 & CL07 & 0.995 & 1.627 & 11.404 & 0.392 & 52.627 & 0.083 & 0.095 & 0.358 & 99.7302 \\
\hline SME Simulant & Hydragard & $\mathrm{HF}$ & 24 & 2 & 2 & 2 & 4 & HHY04 & 1.058 & 1.769 & 11.404 & 0.468 & 51.985 & 0.087 & 0.104 & 0.376 & 100.6707 \\
\hline \begin{tabular}{|l} 
SME Simulant \\
\end{tabular} & Coliwasa - high & $\mathrm{HF}$ & 1 & 1 & 22 & 2 & 5 & CH05 & 1.040 & 1.730 & 11.027 & 0.450 & 53.055 & 0.084 & 0.099 & 0.366 & 100.0700 \\
\hline SME Simulant & Coliwasa - low & $\mathrm{HF}$ & 3 & 1 & 20 & 2 & 6 & CL05 & 1.113 & 1.872 & 11.081 & 0.491 & 51.771 & 0.082 & 0.103 & 0.376 & 100.5846 \\
\hline SME Simulant & $\begin{array}{l}\text { Coliwasa - high } \\
\end{array}$ & $\mathrm{HF}$ & 23 & 1 & 28 & 2 & 7 & $\mathrm{CH} 06$ & 1.056 & 1.756 & 10.919 & 0.457 & 52.841 & 0.083 & 0.101 & 0.373 & 100.2320 \\
\hline SME Simulant & Coliwasa - high & LF & 1 & 1 & 12 & 2 & 8 & $\mathrm{CH} 01$ & 1.060 & 1.769 & 11.175 & 0.448 & 52.841 & 0.081 & 0.100 & 0.367 & 100.3774 \\
\hline SME Simulant & Coliwasa - low & LF & 20 & 1 & 2 & 2 & 9 & CL02 & 1.123 & 1.859 & 11.445 & 0.478 & 50.915 & 0.082 & 0.106 & 0.384 & 100.4999 \\
\hline SME Simulant & Coliwasa - low & $\mathrm{HF}$ & 63 & 1 & 5 & 2 & 10 & CL08 & 1.118 & 1.898 & 11.471 & 0.515 & 50.701 & 0.082 & 0.107 & 0.371 & 100.2682 \\
\hline SME Simulant & $\begin{array}{l}\text { Coliwasa - high } \\
\end{array}$ & $\mathrm{HF}$ & 65 & 1 & 6 & 2 & 11 & CH08 & 1.048 & 1.717 & 11.741 & 0.448 & 52.199 & 0.081 & 0.101 & 0.370 & 100.2969 \\
\hline SME Simulant & Hydragard & $\mathrm{HF}$ & 45 & 2 & 7 & 2 & 12 & HHY07 & 1.068 & 1.808 & 10.824 & 0.504 & 52.199 & 0.085 & 0.105 & 0.380 & 100.3894 \\
\hline SME Simulant & Coliwasa - low & LF & 4 & 1 & 10 & 2 & 13 & CL01 & 1.085 & 1.808 & 10.919 & 0.468 & 52.841 & 0.084 & 0.104 & 0.371 & 100.8567 \\
\hline \begin{tabular}{|l} 
SME Simulant \\
\end{tabular} & Coliwasa - low & $\mathrm{HF}$ & 18 & 1 & 26 & 2 & 14 & CL06 & 1.111 & 1.846 & 11.997 & 0.486 & 50.701 & 0.082 & 0.108 & 0.371 & 100.4949 \\
\hline ARG-1 & None & 0 & 0 & 2 & 12 & 2 & 15 & ARG-1B22 & 0.876 & 1.898 & 11.701 & 1.029 & 48.990 & 1.159 & 0.023 & 0.135 & \begin{tabular}{|l|}
99.6178 \\
\end{tabular} \\
\hline SME Simulant & Coliwasa - low & LF & 65 & 1 & 23 & 2 & 16 & CL04 & 1.051 & 1.743 & 10.919 & 0.436 & 53.055 & 0.081 & 0.100 & 0.362 & 100.0895 \\
\hline SME Simulant & Coliwasa - low & LF & 40 & 1 & 19 & 2 & 17 & CL03 & 1.124 & 1.872 & 11.889 & 0.475 & 48.776 & 0.074 & 0.110 & 0.380 & 98.7956 \\
\hline SME Simulant & Coliwasa - high & LF & 38 & 1 & 16 & 2 & 18 & CH03 & 1.063 & 1.769 & 11.391 & 0.450 & 52.413 & 0.084 & 0.102 & 0.362 & 100.1184 \\
\hline SME Simulant & Hydragard & $\mathrm{HF}$ & 57 & 2 & 6 & 2 & 19 & HHY09 & 1.068 & 1.782 & 11.215 & 0.461 & 52.413 & 0.083 & 0.104 & 0.373 & 100.5553 \\
\hline
\end{tabular}


Table A4. Phase 1 Oxide Concentration Measurements (part 2)

\begin{tabular}{|c|c|c|c|c|c|c|c|c|c|c|c|c|c|c|c|c|c|}
\hline $\begin{array}{l}\text { Type of } \\
\text { Material } \\
\end{array}$ & Sampler Details & $\begin{array}{l}\text { Flow } \\
\text { Rate } \\
\end{array}$ & $\begin{array}{r}\begin{array}{r}\text { Sample } \\
\text { Order }\end{array} \\
\end{array}$ & $\begin{array}{r}\text { Preparation } \\
\text { Block } \\
\end{array}$ & $\begin{array}{r}\text { Preparation } \\
\text { Sequence } \\
\end{array}$ & $\begin{array}{r}\text { Analytical } \\
\text { Block } \\
\end{array}$ & $\begin{array}{r}\text { Analytical } \\
\text { Sequence } \\
\end{array}$ & Sample ID & $\begin{array}{r}\text { MgO } \\
\text { (wt\%) }\end{array}$ & $\begin{array}{r}\mathrm{MnO} \\
(\mathrm{wt} \%)\end{array}$ & $\begin{array}{r}\mathrm{Na2O} \\
\text { (wt\%) }\end{array}$ & $\begin{array}{r}\mathrm{NiO} \\
(\mathrm{wt} \%)\end{array}$ & $\begin{array}{r}\mathrm{SiO2} \\
(\mathrm{wt} \%) \\
\end{array}$ & $\begin{array}{r}\begin{array}{r}\mathrm{TiO2} \\
(\mathrm{wt} \%)\end{array} \\
\end{array}$ & $\begin{array}{r}\mathrm{ZnO} \\
(\mathrm{wt} \%)\end{array}$ & $\begin{array}{r}\mathrm{ZrO2} \\
(\mathrm{wt} \%)\end{array}$ & $\begin{array}{r}\begin{array}{r}\text { Sum } \\
\text { (wt \%) }\end{array} \\
\end{array}$ \\
\hline SME Simulant & Coliwasa - high & LF & 21 & $\begin{array}{r}1 \\
\end{array}$ & $\begin{array}{r}14 \\
\end{array}$ & 2 & 20 & $\mathrm{CH} 02$ & 1.073 & 1.782 & 11.337 & 0.464 & 52.627 & 0.082 & 0.103 & 0.369 & 100.9056 \\
\hline SME Simulant & Hydragard & $\mathrm{HF}$ & 6 & 2 & 4 & 2 & 21 & HHY01 & 1.143 & \begin{tabular}{ll|}
1.898 \\
\end{tabular} & 11.849 & 0.472 & 50.274 & 0.082 & 0.108 & 0.384 & 100.3821 \\
\hline SME Simulant & Hydragard & $\mathrm{HF}$ & 19 & 2 & 10 & 2 & 22 & HHY03 & 1.134 & 1.898 & 11.377 & 0.496 & 50.701 & 0.079 & 0.108 & 0.370 & 100.2199 \\
\hline SME Simulant & Hydragard & $\mathrm{HF}$ & 50 & 2 & 5 & 2 & 23 & HHY08 & 1.073 & 1.782 & 11.431 & 0.461 & 52.199 & 0.080 & 0.105 & 0.376 & 100.6897 \\
\hline SME Simulant & Hydragard & $\mathrm{HF}$ & 37 & 2 & 9 & 2 & 24 & HHY06 & 1.076 & 1.795 & 10.878 & 0.473 & 52.413 & 0.082 & 0.103 & 0.371 & 100.5083 \\
\hline SME Simulant & Coliwasa - high & $\mathrm{HF}$ & 34 & 1 & 18 & 2 & 25 & CH07 & 1.058 & 1.756 & 10.851 & 0.456 & 53.269 & 0.083 & 0.101 & 0.371 & 100.3930 \\
\hline SME Simulant & Coliwasa - high & LF & 63 & 1 & 13 & 2 & 26 & \begin{tabular}{|l|l|}
$\mathrm{CH} 04$ \\
\end{tabular} & 1.060 & \begin{tabular}{ll|}
1.756 \\
\end{tabular} & 11.404 & 0.447 & 52.199 & 0.085 & $\begin{array}{ll}0.102 \\
\end{array}$ & 0.366 & 100.0558 \\
\hline SME Simulant & Hydragard & $\mathrm{HF}$ & 32 & 2 & 8 & 2 & 27 & HHY05 & 1.091 & 1.834 & 11.862 & 0.489 & 50.915 & 0.079 & $\begin{array}{ll}0.107 \\
\end{array}$ & 0.373 & 100.1665 \\
\hline SME Simulant & Hydragard & $\mathrm{HF}$ & 61 & 2 & 11 & 2 & 28 & HHY10 & 1.099 & 1.834 & 11.566 & 0.478 & 51.129 & 0.077 & 0.106 & 0.382 & 100.5075 \\
\hline ARG-1 & $\begin{array}{l}\text { None } \\
\end{array}$ & 0 & 0 & 2 & 23 & 2 & 29 & ARG-1B23 & 0.869 & 1.898 & 11.755 & 1.055 & 48.562 & 1.161 & 0.024 & 0.133 & \begin{tabular}{|l|}
99.5114 \\
\end{tabular} \\
\hline ARG-1 & None & 0 & 0 & 3 & 1 & 3 & 1 & ARG-1B31 & 0.871 & 1.898 & 11.674 & 1.071 & 48.990 & 1.168 & 0.024 & 0.132 & 100.6075 \\
\hline SME Simulant & Isolok - $12 \mathrm{~mL}$ & LF & 11 & 3 & 4 & 3 & 2 & LIB02 & 1.088 & 1.808 & 11.161 & 0.458 & 51.771 & 0.084 & 0.104 & 0.376 & 100.4807 \\
\hline SME Simulant & Coliwasa - low & $\mathrm{HF}$ & 36 & 1 & 21 & 3 & 3 & CL07 & 0.980 & 1.588 & 11.175 & 0.382 & 51.985 & 0.083 & 0.095 & 0.355 & 98.5219 \\
\hline SME Simulant & Isolok - $12 \mathrm{~mL}$ & LF & 26 & 3 & 8 & 3 & 4 & LIB04 & 1.096 & 1.808 & 11.013 & 0.467 & 52.413 & 0.084 & 0.105 & 0.365 & 100.6507 \\
\hline SME Simulant & Coliwasa - high & $\mathrm{HF}$ & 1 & 1 & 22 & 3 & 5 & $\mathrm{CH} 05$ & 0.997 & 1.653 & 10.487 & 0.425 & 51.129 & 0.081 & 0.095 & 0.354 & \begin{tabular}{|l|l|}
96.2275 \\
\end{tabular} \\
\hline SME Simulant & Coliwasa - low & $\mathrm{HF}$ & 3 & 1 & 20 & 3 & 6 & CL05 & 1.104 & 1.846 & 10.973 & 0.491 & 51.557 & 0.084 & 0.105 & 0.377 & 100.3061 \\
\hline SME Simulant & Coliwasa - high & $\mathrm{HF}$ & 23 & 1 & 28 & 3 & 7 & CH06 & 1.056 & 1.756 & 10.865 & 0.454 & 52.841 & 0.086 & \begin{tabular}{ll|}
0.101 \\
\end{tabular} & 0.369 & 100.2188 \\
\hline SME Simulant & Coliwasa - high & LF & 1 & 1 & 12 & 3 & 8 & $\mathrm{CH} 01$ & 1.058 & 1.756 & 11.108 & 0.443 & 52.841 & 0.084 & 0.101 & 0.363 & 100.4286 \\
\hline SME Simulant & Coliwasa - low & LF & 20 & 1 & 2 & 3 & 9 & CL02 & 1.113 & 1.846 & 11.337 & 0.471 & 50.274 & 0.085 & 0.104 & 0.378 & 99.6256 \\
\hline SME Simulant & Coliwasa - low & $\mathrm{HF}$ & 63 & 1 & 5 & 3 & 10 & CL08 & 1.118 & \begin{tabular}{ll|}
1.898 \\
\end{tabular} & 11.391 & 0.512 & 50.701 & 0.080 & 0.107 & 0.371 & 100.2369 \\
\hline SME Simulant & Coliwasa - high & $\mathrm{HF}$ & 65 & 1 & 6 & 3 & 11 & CH08 & 1.038 & 1.717 & 11.566 & 0.445 & 51.985 & 0.082 & $\begin{array}{ll}0.099 \\
\end{array}$ & 0.371 & \begin{tabular}{|l|}
99.9011 \\
\end{tabular} \\
\hline SME Simulant & Isolok - $12 \mathrm{~mL}$ & LF & 45 & 3 & 7 & 3 & 12 & LIB07 & 1.103 & 1.834 & 15.637 & 0.468 & 51.985 & 0.082 & 0.103 & 0.374 & 106.4928 \\
\hline SME Simulant & Coliwasa - low & LF & 4 & 1 & 10 & 3 & 13 & CL01 & 1.066 & 1.782 & 10.757 & 0.464 & 52.199 & 0.085 & 0.104 & 0.365 & \begin{tabular}{|l|l}
99.6962 \\
\end{tabular} \\
\hline SME Simulant & Coliwasa - low & $\mathrm{HF}$ & 18 & 1 & 26 & 3 & 14 & CL06 & 1.101 & 1.834 & 11.768 & 0.481 & 50.701 & 0.083 & 0.107 & 0.374 & 100.2337 \\
\hline ARG-1 & None & 0 & 0 & 3 & 12 & 3 & 15 & ARG-1B32 & 0.866 & 1.885 & 11.579 & 1.029 & 48.348 & 1.149 & 0.023 & 0.130 & \begin{tabular}{|l|l|}
98.9989 \\
\end{tabular} \\
\hline SME Simulant & Coliwasa - low & LF & 65 & 1 & 23 & 3 & 16 & \begin{tabular}{|l|l} 
CL04 \\
\end{tabular} & 1.033 & 1.717 & 10.649 & 0.438 & 52.199 & 0.084 & 0.097 & 0.361 & 98.6042 \\
\hline SME Simulant & Coliwasa - low & LF & 40 & 1 & 19 & 3 & 17 & CL03 & 1.080 & 1.795 & 11.337 & 0.457 & 46.851 & 0.076 & $\begin{array}{ll}0.104 \\
\end{array}$ & 0.367 & \begin{tabular}{|l|l}
95.0982 \\
\end{tabular} \\
\hline SME Simulant & Coliwasa - high & LF & 38 & 1 & 16 & 3 & 18 & $\mathrm{CH} 03$ & 1.063 & 1.769 & 11.256 & 0.458 & 52.199 & 0.086 & 0.100 & 0.367 & 100.0869 \\
\hline SME Simulant & Isolok - $12 \mathrm{~mL}$ & LF & 56 & 3 & 11 & 3 & 19 & LIB09 & 1.045 & 1.756 & 11.296 & 0.457 & 51.129 & 0.086 & 0.103 & 0.363 & \begin{tabular}{|l|l|}
98.9787 \\
\end{tabular} \\
\hline SME Simulant & Coliwasa - high & LF & 21 & 1 & 14 & 3 & 20 & $\mathrm{CH} 02$ & $\begin{array}{ll}0.988 \\
\end{array}$ & 1.640 & 10.353 & 0.429 & 48.562 & 0.080 & 0.093 & 0.351 & 93.2595 \\
\hline SME Simulant & Isolok - $12 \mathrm{~mL}$ & LF & 5 & 3 & 5 & 3 & 21 & LIB01 & 1.123 & 1.885 & 11.984 & 0.484 & 53.696 & 0.089 & 0.109 & 0.396 & 104.7834 \\
\hline SME Simulant & Isolok - $12 \mathrm{~mL}$ & LF & 23 & 3 & 10 & 3 & 22 & LIB03 & 1.022 & \begin{tabular}{ll|l}
1.717 \\
\end{tabular} & 10.487 & 0.453 & 51.557 & 0.080 & 0.100 & 0.362 & \begin{tabular}{|l|l|}
97.7680 \\
\end{tabular} \\
\hline SME Simulant & Isolok - $12 \mathrm{~mL}$ & $\mathrm{LF}$ & 50 & 3 & 2 & 3 & 23 & LIB08 & $\begin{array}{ll}1.078 \\
\end{array}$ & 1.808 & 11.714 & 0.468 & 52.199 & $\begin{array}{ll}0.079 \\
\end{array}$ & 0.107 & 0.376 & 101.4555 \\
\hline SME Simulant & Isolok - $12 \mathrm{~mL}$ & LF & 41 & 3 & 9 & 3 & 24 & LIB06 & 1.116 & 1.859 & 11.296 & 0.471 & 51.129 & 0.078 & 0.107 & 0.370 & 100.4668 \\
\hline SME Simulant & Coliwasa - high & $\mathrm{HF}$ & 34 & 1 & 18 & 3 & 25 & CH07 & 1.025 & 1.717 & 10.420 & 0.434 & 51.771 & 0.080 & 0.097 & 0.357 & 97.4499 \\
\hline SME Simulant & Coliwasa - high & LF & 63 & 1 & 13 & 3 & 26 & $\mathrm{CH} 04$ & 0.995 & 1.653 & 10.690 & 0.425 & 49.204 & 0.076 & 0.095 & 0.344 & \begin{tabular}{|l|l}
94.5180 \\
\end{tabular} \\
\hline SME Simulant & Isolok - $12 \mathrm{~mL}$ & LF & 32 & 3 & 3 & 3 & 27 & LIB05 & 1.179 & 1.950 & 11.566 & 0.490 & 51.771 & 0.081 & 0.111 & 0.382 & 102.7817 \\
\hline SME Simulant & Isolok - $12 \mathrm{~mL}$ & LF & 60 & 3 & 6 & 3 & 28 & \begin{tabular}{|l|} 
LIB10 \\
\end{tabular} & 1.045 & 1.743 & 10.744 & 0.445 & 49.846 & 0.083 & 0.101 & 0.361 & \begin{tabular}{|l|}
96.5094 \\
\end{tabular} \\
\hline ARG-1 & None & 0 & 0 & 3 & 23 & 3 & 29 & ARG-1B33 & 0.887 & 1.937 & 11.916 & 1.063 & 50.060 & 1.183 & 0.023 & 0.139 & 102.3755 \\
\hline ARG-1 & None & 0 & 0 & 4 & 1 & 4 & 1 & ARG-1B41 & 0.864 & 1.872 & 11.458 & 1.014 & 48.134 & 1.153 & 0.023 & 0.131 & \begin{tabular}{|l|l|}
98.9339 \\
\end{tabular} \\
\hline SME Simulant & Isolok - $12 \mathrm{~mL}$ & $\mathrm{HF}$ & 13 & 4 & 11 & 4 & 2 & HIB02 & 1.061 & 1.743 & 10.649 & 0.426 & 52.413 & 0.083 & 0.101 & 0.365 & 99.7916 \\
\hline SME Simulant & Coliwasa - low & $\mathrm{HF}$ & 36 & 1 & 21 & 4 & 3 & CL07 & 0.985 & 1.601 & 11.161 & 0.379 & 52.199 & 0.085 & 0.095 & 0.357 & 99.0146 \\
\hline SME Simulant & Isolok - $12 \mathrm{~mL}$ & $\mathrm{HF}$ & 25 & 4 & 4 & 4 & 4 & HIB04 & 1.071 & 1.795 & 11.337 & 0.496 & 51.985 & 0.083 & 0.104 & 0.369 & 100.6782 \\
\hline SME Simulant & Coliwasa - high & $\mathrm{HF}$ & 1 & 1 & 22 & 4 & 5 & CH05 & 1.030 & 1.704 & 10.851 & 0.442 & 52.627 & 0.086 & 0.099 & 0.357 & \begin{tabular}{|l}
99.4097 \\
\end{tabular} \\
\hline SME Simulant & Coliwasa - low & $\mathrm{HF}$ & 3 & 1 & 20 & 4 & 6 & CL05 & 1.106 & 1.846 & 10.932 & 0.484 & 51.129 & 0.082 & 0.105 & 0.370 & 99.8627 \\
\hline SME Simulant & Coliwasa - high & $\mathrm{HF}$ & 23 & 1 & 28 & 4 & 7 & CH06 & 1.048 & 1.730 & 10.703 & 0.439 & 52.199 & 0.084 & 0.100 & 0.367 & 99.1839 \\
\hline SME Simulant & Coliwasa - high & LF & 1 & 1 & 12 & 4 & 8 & $\mathrm{CH} 01$ & 1.050 & 1.730 & 10.959 & 0.439 & 52.199 & 0.083 & 0.100 & 0.358 & 99.4206 \\
\hline SME Simulant & Coliwasa - low & $\mathrm{LF}$ & 20 & 1 & 2 & 4 & 9 & CL02 & 1.109 & 1.834 & 11.229 & 0.462 & 50.060 & 0.080 & $\begin{array}{ll}0.104 \\
\end{array}$ & 0.374 & 99.1076 \\
\hline
\end{tabular}


Table A4. Phase 1 Oxide Concentration Measurements (part 2)

\begin{tabular}{|c|c|c|c|c|c|c|c|c|c|c|c|c|c|c|c|c|c|}
\hline \begin{tabular}{l|} 
Type of \\
Material \\
\end{tabular} & Sampler Details & \begin{tabular}{|l|} 
Flow \\
Rate \\
\end{tabular} & $\begin{array}{r}\text { Sample } \\
\text { Order }\end{array}$ & $\begin{array}{r}\text { Preparation } \\
\text { Block } \\
\end{array}$ & $\begin{array}{r}\text { Preparation } \\
\text { Sequence } \\
\end{array}$ & $\begin{array}{r}\text { Analytical } \\
\text { Block } \\
\end{array}$ & $\begin{array}{r}\text { Analytical } \\
\text { Sequence }\end{array}$ & Sample ID & $\begin{array}{r}\text { MgO } \\
\text { (wt\%) }\end{array}$ & $\begin{array}{r}\text { MnO } \\
(w t \%)\end{array}$ & $\begin{array}{r}\text { Na2O } \\
(w t \%)\end{array}$ & \begin{tabular}{|r|}
$\mathrm{NiO}$ \\
$(\mathrm{wt} \%)$
\end{tabular} & $\begin{array}{r}\mathrm{SiO} 2 \\
(\mathrm{wt} \%)\end{array}$ & $\begin{array}{r}\text { TiO2 } \\
(\mathrm{wt} \%)\end{array}$ & $\begin{array}{r}\mathrm{ZnO} \\
(\mathrm{wt} \%)\end{array}$ & $\begin{array}{r}\text { ZrO2 } \\
\text { (wt\%) } \\
\end{array}$ & $\begin{array}{r}\text { Sum } \\
\text { (wt\%) }\end{array}$ \\
\hline SME Simulant & Coliwasa - low & $\mathrm{HF}$ & 63 & 1 & 5 & 4 & 10 & CL08 & 1.104 & 1.872 & 11.161 & 0.496 & 49.846 & $\begin{array}{l}0.082 \\
\end{array}$ & 0.105 & 0.365 & 98.6592 \\
\hline SME Simulant & Coliwasa - high & $\mathrm{HF}$ & 65 & 1 & 6 & 4 & 11 & CH08 & 1.036 & 1.704 & 11.485 & 0.431 & 51.771 & 0.084 & 0.099 & 0.370 & 99.3834 \\
\hline SME Simulant & Isolok - $12 \mathrm{~mL}$ & $\mathrm{HF}$ & 46 & 4 & 2 & 4 & 12 & HIB07 & 1.028 & 1.717 & 10.285 & 0.428 & 52.841 & 0.082 & 0.101 & 0.365 & 99.3241 \\
\hline SME Simulant & Coliwasa - low & LF & 4 & 1 & 10 & 4 & 13 & CL01 & 1.070 & 1.769 & 10.717 & 0.463 & 52.199 & 0.084 & 0.104 & 0.367 & 99.6982 \\
\hline SME Simulant & Coliwasa - low & $\mathrm{HF}$ & 18 & 1 & 26 & 4 & 14 & \begin{tabular}{|l|l|} 
CL06 \\
\end{tabular} & 1.091 & 1.808 & 11.701 & 0.464 & 50.060 & 0.080 & 0.104 & 0.363 & 99.0636 \\
\hline ARG-1 & None & 0 & 0 & 4 & 7 & 4 & 15 & ARG-1B42 & 0.861 & 1.859 & 11.458 & 1.007 & 48.134 & 1.156 & 0.025 & 0.133 & 98.9849 \\
\hline SME Simulant & Coliwasa - low & LF & 65 & 1 & 23 & 4 & 16 & \begin{tabular}{|l|} 
CL04 \\
\end{tabular} & 1.038 & 1.704 & 10.636 & 0.424 & 52.413 & 0.084 & 0.097 & 0.365 & 99.0792 \\
\hline SME Simulant & Coliwasa - low & LF & 40 & 1 & 19 & \begin{tabular}{c|c}
4 \\
\end{tabular} & 17 & CL03 & 1.104 & 1.834 & \begin{tabular}{ll|}
11.552 \\
\end{tabular} & 0.463 & \begin{tabular}{ll|}
47.706 \\
\end{tabular} & 0.080 & 0.108 & 0.365 & \begin{tabular}{|l|l|}
97.0378 \\
\end{tabular} \\
\hline SME Simulant & Coliwasa - high & LF & 38 & 1 & 16 & 4 & 18 & $\mathrm{CH} 03$ & 1.053 & 1.743 & 11.094 & 0.440 & 51.985 & 0.086 & 0.099 & 0.363 & 99.2570 \\
\hline SME Simulant & \begin{tabular}{|l|l} 
Isolok - $12 \mathrm{~mL}$ \\
\end{tabular} & $\mathrm{HF}$ & 56 & 4 & 9 & 4 & 19 & HIB09 & 1.151 & 1.898 & 10.070 & 0.476 & 51.129 & 0.082 & 0.109 & 0.363 & 99.5084 \\
\hline SME Simulant & Coliwasa - high & LF & 21 & 1 & 14 & 4 & 20 & $\mathrm{CH} 02$ & 1.056 & 1.743 & 10.986 & 0.447 & 51.557 & 0.084 & 0.099 & 0.362 & 99.1957 \\
\hline SME Simulant & Isolok - $12 \mathrm{~mL}$ & $\mathrm{HF}$ & 7 & 4 & 12 & 4 & 21 & HIB01 & 1.026 & 1.704 & 11.269 & 0.434 & 52.199 & 0.085 & 0.097 & 0.369 & 99.6726 \\
\hline SME Simulant & Isolok - $12 \mathrm{~mL}$ & $\mathrm{HF}$ & 21 & 4 & 6 & 4 & 22 & HIB03 & 1.099 & 1.821 & 10.730 & 0.457 & 50.274 & 0.083 & 0.105 & 0.373 & 98.7883 \\
\hline SME Simulant & Isolok - $12 \mathrm{~mL}$ & $\mathrm{HF}$ & 49 & 4 & 5 & 4 & 23 & HIB08 & 0.967 & 1.601 & 10.541 & 0.398 & 53.483 & 0.085 & 0.098 & 0.343 & 99.1358 \\
\hline SME Simulant & Isolok - $12 \mathrm{~mL}$ & $\mathrm{HF}$ & 38 & 4 & 8 & 4 & 24 & HIB06 & 1.045 & 1.717 & 11.054 & 0.433 & 51.771 & 0.083 & 0.100 & 0.358 & 99.1282 \\
\hline SME Simulant & Coliwasa - high & $\mathrm{HF}$ & 34 & 1 & 18 & 4 & 25 & $\mathrm{CH} 07$ & 1.041 & 1.730 & 10.622 & 0.435 & 52.413 & 0.083 & 0.098 & 0.370 & 99.1767 \\
\hline SME Simulant & Coliwasa - high & LF & 63 & 1 & 13 & 4 & 26 & $\mathrm{CH} 04$ & 1.048 & 1.730 & 11.215 & 0.430 & 51.557 & 0.082 & 0.099 & 0.359 & 99.2237 \\
\hline SME Simulant & Isolok - $12 \mathrm{~mL}$ & $\mathrm{HF}$ & 33 & 4 & 10 & 4 & 27 & HIB05 & 1.076 & 1.795 & 10.892 & 0.475 & 51.129 & 0.083 & 0.102 & 0.355 & 98.9973 \\
\hline SME Simulant & Isolok - $12 \mathrm{~mL}$ & $\mathrm{HF}$ & 58 & 4 & 3 & 4 & 28 & HIB10 & 0.983 & 1.627 & 10.649 & 0.406 & 53.910 & 0.083 & 0.094 & 0.359 & 99.6837 \\
\hline ARG-1 & None & 0 & 0 & 4 & 13 & 4 & 29 & ARG-1B43 & 0.859 & 1.859 & 11.525 & 1.005 & 48.134 & 1.156 & 0.024 & 0.133 & 98.7171 \\
\hline ARG-1 & \begin{tabular}{|l|} 
None \\
\end{tabular} & 0 & 0 & 2 & 1 & 5 & 1 & ARG-1B21 & 0.847 & 1.834 & \begin{tabular}{l|l}
11.458 \\
\end{tabular} & 1.015 & \begin{tabular}{l|l}
47.706 \\
\end{tabular} & 1.141 & 0.024 & 0.132 & 98.0589 \\
\hline SME Simulant & Isolok - $3 \mathrm{~mL}$ & $\mathrm{HF}$ & 20 & 2 & 16 & 5 & 2 & HISO3 & 1.022 & 1.704 & 10.784 & 0.448 & 52.199 & 0.085 & 0.100 & 0.358 & 99.1682 \\
\hline SME Simulant & Isolok - $3 \mathrm{~mL}$ & $\mathrm{HF}$ & 9 & 2 & 21 & 5 & 3 & HIS01 & 1.028 & 1.704 & 11.512 & 0.444 & 52.413 & 0.084 & 0.099 & 0.363 & 100.0890 \\
\hline SME Simulant & Coliwasa - low & LF & 20 & 1 & 2 & 5 & 4 & CL02 & 1.075 & 1.782 & 11.108 & 0.454 & 49.204 & 0.079 & 0.102 & 0.359 & \begin{tabular}{|l|}
97.2952 \\
\end{tabular} \\
\hline SME Simulant & Coliwasa - high & LF & 38 & 1 & 16 & 5 & 5 & CH03 & 1.075 & 1.795 & 11.566 & 0.457 & 53.696 & 0.087 & 0.102 & 0.370 & 102.6416 \\
\hline SME Simulant & Coliwasa - high & LF & 63 & 1 & 13 & 5 & 6 & $\mathrm{CH} 04$ & 0.997 & 1.653 & 10.771 & 0.419 & 49.418 & 0.079 & 0.095 & 0.343 & \begin{tabular}{|l|}
94.9378 \\
\end{tabular} \\
\hline SME Simulant & Isolok - $3 \mathrm{~mL}$ & $\mathrm{HF}$ & 44 & 2 & 18 & 5 & 7 & HIS07 & 1.046 & \begin{tabular}{|c|}
1.743 \\
\end{tabular} & 11.000 & 0.450 & 54.338 & 0.086 & 0.101 & 0.376 & 102.6601 \\
\hline SME Simulant & Coliwasa - high & $\mathrm{HF}$ & 23 & 1 & 28 & 5 & 8 & $\mathrm{CH} 06$ & 1.005 & 1.679 & 10.528 & 0.426 & 50.701 & 0.081 & 0.095 & 0.354 & \begin{tabular}{|l|}
96.3011 \\
\end{tabular} \\
\hline SME Simulant & \begin{tabular}{|l|} 
Coliwasa - high \\
\end{tabular} & LF & 21 & 1 & 14 & 5 & 9 & CH02 & 1.071 & 1.769 & 11.391 & 0.458 & 53.483 & 0.084 & 0.102 & 0.370 & 102.1898 \\
\hline SME Simulant & Coliwasa - low & $\mathrm{HF}$ & 3 & 1 & 20 & 5 & 10 & CL05 & 1.048 & 1.756 & 10.568 & 0.462 & 49.204 & 0.079 & 0.101 & 0.351 & \begin{tabular}{|l|}
95.9725 \\
\end{tabular} \\
\hline SME Simulant & Isolok - $3 \mathrm{~mL}$ & $\mathrm{HF}$ & 14 & 2 & 15 & 5 & 11 & HIS02 & 1.013 & 1.691 & 9.948 & 0.429 & 52.413 & 0.084 & 0.097 & 0.365 & 98.0616 \\
\hline SME Simulant & Coliwasa - low & LF & 40 & 1 & 19 & 5 & 12 & CL03 & 1.078 & 1.795 & 11.498 & 0.459 & 47.279 & 0.078 & 0.107 & 0.361 & 95.8739 \\
\hline SME Simulant & Coliwasa - low & $\mathrm{HF}$ & 18 & 1 & 26 & 5 & 13 & CL06 & 1.073 & 1.782 & 11.701 & 0.466 & 49.632 & 0.081 & 0.105 & 0.363 & 98.3914 \\
\hline SME Simulant & Coliwasa - high & HF & 1 & 1 & 22 & 5 & 14 & $\begin{array}{l}\text { CH05 } \\
\end{array}$ & 1.013 & 1.679 & 10.892 & 0.434 & 52.413 & 0.081 & 0.097 & 0.358 & 98.7008 \\
\hline ARG-1 & \begin{tabular}{|l|} 
None \\
\end{tabular} & 0 & 0 & 2 & 12 & 5 & 15 & ARG-1B22 & 0.789 & 1.704 & 10.744 & 0.937 & 44.711 & 1.063 & 0.022 & 0.122 & 91.6261 \\
\hline SME Simulant & Coliwasa - low & $\mathrm{HF}$ & 36 & 1 & 21 & 5 & 16 & \begin{tabular}{|l|} 
CL07 \\
\end{tabular} & 0.970 & 1.575 & 11.323 & 0.383 & 51.771 & 0.086 & 0.095 & 0.347 & 98.4060 \\
\hline SME Simulant & Isolok - $3 \mathrm{~mL}$ & $\mathrm{HF}$ & 31 & 2 & 19 & 5 & 17 & HIS05 & 1.025 & 1.704 & 10.380 & 0.436 & 51.985 & 0.083 & 0.100 & 0.351 & 98.1106 \\
\hline SME Simulant & Isolok - $3 \mathrm{~mL}$ & $\mathrm{HF}$ & 60 & 2 & 22 & 5 & 18 & HIS10 & 1.060 & 1.743 & 11.067 & 0.430 & 51.343 & 0.084 & 0.099 & 0.347 & 98.2538 \\
\hline SME Simulant & Coliwasa - low & $\mathrm{HF}$ & 63 & 1 & 5 & 5 & 19 & CL08 & 1.088 & 1.846 & 11.323 & 0.495 & 50.060 & 0.081 & 0.103 & 0.362 & 98.9820 \\
\hline SME Simulant & Isolok - $3 \mathrm{~mL}$ & $\mathrm{HF}$ & 26 & 2 & 14 & 5 & 20 & HIS04 & 0.975 & 1.614 & 10.312 & 0.416 & 48.562 & 0.081 & 0.093 & 0.343 & 92.7730 \\
\hline SME Simulant & Isolok - $3 \mathrm{~mL}$ & $\mathrm{HF}$ & 35 & 2 & 20 & 5 & 21 & HIS06 & 1.041 & 1.717 & 11.391 & 0.439 & 52.199 & 0.083 & 0.098 & 0.369 & 100.4113 \\
\hline SME Simulant & \begin{tabular}{|l|} 
Coliwasa - high \\
\end{tabular} & $\mathrm{HF}$ & 65 & 1 & 6 & 5 & 22 & CH08 & 1.025 & 1.691 & 11.728 & 0.436 & 51.985 & 0.086 & $\begin{array}{ll}0.097 \\
\end{array}$ & 0.365 & 99.9170 \\
\hline SME Simulant & Coliwasa - high & $\mathrm{HF}$ & 34 & 1 & 18 & 5 & 23 & CH07 & 1.036 & 1.717 & 10.797 & 0.444 & 52.841 & 0.084 & 0.098 & 0.367 & 99.7759 \\
\hline SME Simulant & \begin{tabular}{|l|} 
Coliwasa - high \\
\end{tabular} & LF & 1 & 1 & 12 & 5 & 24 & CH01 & 1.012 & 1.679 & 10.932 & 0.428 & 50.915 & 0.079 & 0.097 & 0.347 & 97.2554 \\
\hline SME Simulant & Isolok - $3 \mathrm{~mL}$ & $\mathrm{HF}$ & 54 & 2 & 17 & 5 & 25 & HIS09 & 1.038 & 1.730 & 10.959 & 0.448 & 52.199 & 0.087 & 0.108 & 0.370 & 99.9996 \\
\hline SME Simulant & Coliwasa - low & LF & 4 & 1 & 10 & 5 & 26 & CL01 & 1.085 & 1.808 & 11.161 & 0.473 & \begin{tabular}{|l|}
53.483 \\
\end{tabular} & 0.084 & 0.106 & 0.376 & 102.1891 \\
\hline SME Simulant & Isolok - $3 \mathrm{~mL}$ & $\mathrm{HF}$ & 48 & 2 & 13 & 5 & 27 & HIS08 & 1.040 & 1.743 & 10.771 & 0.456 & 53.055 & 0.082 & 0.102 & 0.366 & 100.6832 \\
\hline SME Simulant & Coliwasa - low & LF & \begin{tabular}{l|l}
65 \\
\end{tabular} & 1 & 23 & 5 & 28 & CL04 & 1.040 & 1.717 & \begin{tabular}{l|l|}
10.973 \\
\end{tabular} & 0.435 & \begin{tabular}{l|l}
53.269 \\
\end{tabular} & 0.082 & 0.100 & 0.367 & 100.5321 \\
\hline
\end{tabular}


Table A4. Phase 1 Oxide Concentration Measurements (part 2)

\begin{tabular}{|c|c|c|c|c|c|c|c|c|c|c|c|c|c|c|c|c|c|}
\hline \begin{tabular}{|l|} 
Type of \\
Material \\
\end{tabular} & Sampler Details & $\begin{array}{l}\text { Flow } \\
\text { Rate }\end{array}$ & $\begin{array}{r}\text { Sample } \\
\text { Order }\end{array}$ & $\begin{array}{r}\text { Preparation } \\
\text { Block } \\
\end{array}$ & $\begin{array}{r}\text { Preparation } \\
\text { Sequence } \\
\end{array}$ & $\begin{array}{r}\text { Analytical } \\
\text { Block } \\
\end{array}$ & $\begin{array}{r}\text { Analytical } \\
\text { Sequence } \\
\end{array}$ & Sample ID & $\begin{array}{r}\text { MgO } \\
\text { (wt\%) }\end{array}$ & $\begin{array}{r}\text { MnO } \\
(w t \%) \\
\end{array}$ & $\begin{array}{l}\text { Na2O } \\
\text { (wt\%) }\end{array}$ & $\begin{array}{r}\mathrm{NiO} \\
(\mathrm{wt} \%)\end{array}$ & $\begin{array}{r}\mathrm{SiO2} \\
(\mathrm{wt} \%) \\
\end{array}$ & $\begin{array}{r}\text { TiO2 } \\
\text { (wt\%) } \\
\end{array}$ & $\begin{array}{r}\mathrm{ZnO} \\
\text { (wt\%) }\end{array}$ & $\begin{array}{r}\text { ZrO2 } \\
\text { (wt\%) }\end{array}$ & $\begin{array}{r}\text { Sum } \\
\text { (wt\%) }\end{array}$ \\
\hline ARG-1 & None & 0 & 0 & 2 & 23 & 5 & 29 & ARG-1B23 & 0.854 & 1.859 & 11.714 & 1.015 & 48.348 & 1.154 & 0.025 & 0.132 & 99.0037 \\
\hline ARG-1 & None & 0 & 0 & 3 & 1 & 6 & 1 & ARG-1B31 & 0.837 & 1.821 & 11.512 & 1.013 & 47.920 & 1.128 & 0.024 & 0.130 & 98.4454 \\
\hline SME Simulant & Hydragard & LF & 15 & 3 & 21 & 6 & 2 & LHY03 & 1.090 & 1.808 & 11.566 & 0.462 & 52.627 & 0.081 & 0.103 & 0.370 & 102.2257 \\
\hline SME Simulant & Hydragard & LF & 6 & 3 & 18 & 6 & 3 & LHY01 & 1.076 & 1.782 & 11.296 & 0.466 & 51.985 & 0.082 & 0.105 & 0.369 & 100.8899 \\
\hline SME Simulant & Coliwasa - low & LF & 20 & 1 & 2 & 6 & 4 & CL02 & 1.090 & 1.808 & 11.404 & 0.468 & 50.701 & 0.078 & 0.105 & 0.367 & 100.0375 \\
\hline SME Simulant & Coliwasa - high & LF & 38 & 1 & 16 & 6 & 5 & $\mathrm{CH} 03$ & 1.038 & 1.717 & 11.323 & 0.458 & 52.627 & 0.082 & 0.103 & 0.359 & 100.5302 \\
\hline SME Simulant & Coliwasa - high & LF & 63 & 1 & 13 & 6 & 6 & CH04 & 1.028 & 1.704 & 11.283 & 0.439 & 51.985 & 0.081 & 0.099 & 0.355 & \begin{tabular}{|l|}
99.3586 \\
\end{tabular} \\
\hline SME Simulant & $\begin{array}{l}\text { Hydragard } \\
\end{array}$ & LF & 47 & 3 & 13 & 6 & 7 & LHY07 & 1.128 & 1.859 & 12.766 & 0.475 & 49.632 & 0.076 & 0.110 & 0.365 & 100.9777 \\
\hline SME Simulant & Coliwasa - high & $\mathrm{HF}$ & 23 & 1 & 28 & 6 & 8 & CH06 & 1.033 & 1.717 & 10.959 & 0.445 & 53.055 & 0.082 & 0.100 & 0.363 & 100.2720 \\
\hline SME Simulant & $\begin{array}{l}\text { Coliwasa - high } \\
\end{array}$ & LF & 21 & 1 & 14 & 6 & 9 & $\mathrm{CH} 02$ & 0.987 & 1.627 & 10.676 & 0.426 & 49.846 & 0.077 & 0.094 & 0.342 & \begin{tabular}{|l|}
95.1458 \\
\end{tabular} \\
\hline SME Simulant & Coliwasa - low & $\mathrm{HF}$ & 3 & 1 & 20 & 6 & 10 & CL05 & 1.106 & 1.846 & 11.404 & 0.496 & 52.627 & 0.080 & 0.109 & 0.374 & 102.4415 \\
\hline SME Simulant & Hydragard & LF & 10 & 3 & 20 & 6 & 11 & LHY02 & 0.978 & 1.653 & 11.485 & 0.445 & 53.696 & 0.084 & 0.098 & 0.361 & 101.7410 \\
\hline SME Simulant & Coliwasa - low & LF & 40 & 1 & 19 & 6 & 12 & CL03 & 1.111 & 1.846 & 12.078 & 0.480 & 49.418 & 0.078 & 0.110 & 0.374 & 100.0219 \\
\hline SME Simulant & Coliwasa - low & $\mathrm{HF}$ & 18 & 1 & 26 & 6 & 13 & CL06 & 1.066 & 1.769 & 11.916 & 0.471 & 50.274 & $\begin{array}{ll}0.079 \\
\end{array}$ & 0.105 & 0.362 & \begin{tabular}{|l|l|}
99.2470 \\
\end{tabular} \\
\hline SME Simulant & $\begin{array}{l}\text { Coliwasa - high } \\
\end{array}$ & $\mathrm{HF}$ & 1 & 1 & 22 & 6 & 14 & CH05 & 1.030 & 1.704 & 11.323 & 0.452 & 54.124 & 0.081 & 0.101 & 0.358 & 101.7918 \\
\hline ARG-1 & None & 0 & 0 & 3 & 12 & 6 & 15 & ARG-1B32 & 0.823 & 1.782 & 11.418 & 0.998 & 47.279 & 1.104 & 0.023 & 0.131 & \begin{tabular}{|l|l|}
97.0899 \\
\end{tabular} \\
\hline SME Simulant & Coliwasa - low & $\mathrm{HF}$ & 36 & 1 & 21 & 6 & 16 & $\begin{array}{ll}\text { CL07 } \\
\end{array}$ & 0.968 & 1.575 & 11.552 & 0.386 & 52.627 & 0.080 & 0.096 & 0.346 & 99.6906 \\
\hline SME Simulant & Hydragard & LF & 30 & 3 & 14 & 6 & 17 & LHY05 & 1.078 & 1.795 & 11.242 & 0.480 & 51.343 & 0.077 & 0.105 & 0.363 & 100.0575 \\
\hline SME Simulant & Hydragard & LF & 59 & 3 & 17 & 6 & 18 & LHY10 & 1.058 & 1.756 & 11.283 & 0.445 & 52.627 & 0.080 & 0.103 & 0.367 & 101.1105 \\
\hline SME Simulant & Coliwasa - low & $\mathrm{HF}$ & 63 & 1 & 5 & 6 & 19 & CL08 & 1.101 & 1.859 & 11.701 & 0.514 & 51.343 & 0.080 & 0.109 & 0.363 & 101.4270 \\
\hline SME Simulant & Hydragard & LF & 28 & 3 & 16 & 6 & 20 & LHY04 & 1.066 & 1.782 & 11.377 & 0.462 & 50.701 & 0.079 & 0.105 & 0.359 & \begin{tabular}{|l}
99.5310 \\
\end{tabular} \\
\hline SME Simulant & Hydragard & LF & 37 & 3 & 15 & 6 & 21 & LHY06 & 1.094 & 1.808 & 12.280 & 0.472 & 53.910 & 0.084 & 0.109 & 0.381 & 105.0844 \\
\hline SME Simulant & $\begin{array}{l}\text { Coliwasa - high } \\
\end{array}$ & $\mathrm{HF}$ & 65 & 1 & 6 & 6 & 22 & CH08 & 0.983 & 1.614 & 11.458 & 0.422 & 50.701 & 0.080 & 0.095 & 0.354 & 97.2481 \\
\hline SME Simulant & Coliwasa - high & $\mathrm{HF}$ & 34 & 1 & 18 & 6 & 23 & CH07 & 1.061 & 1.769 & 11.256 & 0.458 & 55.194 & 0.084 & 0.103 & 0.376 & 103.9971 \\
\hline SME Simulant & Coliwasa - high & LF & 1 & 1 & 12 & 6 & 24 & CH01 & 1.003 & 1.653 & 11.040 & 0.429 & 51.343 & 0.079 & 0.097 & 0.350 & 97.7659 \\
\hline SME Simulant & Hydragard & LF & 53 & 3 & 19 & 6 & 25 & LHY09 & 1.131 & 1.872 & 11.741 & 0.482 & 53.910 & 0.084 & 0.108 & 0.388 & 104.8953 \\
\hline SME Simulant & Coliwasa - low & LF & 4 & 1 & 10 & 6 & 26 & CL01 & 1.045 & 1.743 & 11.027 & 0.459 & 52.627 & 0.082 & 0.100 & 0.374 & 100.4790 \\
\hline SME Simulant & Hydragard & LF & 48 & 3 & 22 & 6 & 27 & LHY08 & 1.053 & 1.743 & 11.121 & 0.448 & 51.985 & 0.080 & 0.101 & 0.366 & \begin{tabular}{|l}
99.6694 \\
\end{tabular} \\
\hline SME Simulant & Coliwasa - low & LF & 65 & 1 & 23 & 6 & 28 & CL04 & 1.028 & 1.704 & 11.094 & 0.433 & 53.483 & 0.082 & 0.098 & 0.371 & 101.0537 \\
\hline ARG-1 & None & 0 & 0 & 3 & 23 & 6 & 29 & ARG-1B33 & 0.794 & 1.730 & 11.121 & 0.973 & 45.781 & \begin{tabular}{ll|}
1.071 \\
\end{tabular} & 0.022 & $\begin{array}{ll}0.128 \\
\end{array}$ & \begin{tabular}{|l|}
94.2701 \\
\end{tabular} \\
\hline
\end{tabular}


Table A5. Reference Calcine Oxide Concentrations for Mid-Rheology SME Simulant (Phase 1 Testing)

\begin{tabular}{|c|c|}
\hline Oxide & Targeted wt \% \\
\hline $\mathrm{Al} 2 \mathrm{O} 3$ (wt\%) & 10.869 \\
\hline B2O3 (wt\%) & 4.259 \\
\hline $\mathrm{BaO}(\mathrm{wt} \%)$ & 0.0919 \\
\hline $\mathrm{CaO}(\mathrm{wt} \%)$ & 1.1462 \\
\hline Cr2O3 (wt\%) & 0.0998 \\
\hline $\mathrm{CuO}(\mathrm{wt} \%)$ & 0.0504 \\
\hline $\mathrm{Fe} 2 \mathrm{O} 3(\mathrm{wt} \%)$ & 11.462 \\
\hline K2O (wt\%) & 0.1591 \\
\hline Li2O (wt\%) & 4.674 \\
\hline $\mathrm{MgO}(\mathrm{wt} \%)$ & 1.014 \\
\hline $\mathrm{MnO}(\mathrm{wt} \%)$ & 1.779 \\
\hline $\mathrm{Na} 2 \mathrm{O}(\mathrm{wt} \%)$ & 11.659 \\
\hline $\mathrm{NiO}(\mathrm{wt} \%)$ & 0.41 \\
\hline $\mathrm{SiO} 2(\mathrm{wt} \%)$ & 50.985 \\
\hline TiO2 (wt\%) & 0.0711 \\
\hline $\mathrm{ZnO}(\mathrm{wt} \%)$ & 0.0958 \\
\hline ZrO2 (wt\%) & 0.3547 \\
\hline Sum of Oxides (wt\%) & 99.553 \\
\hline
\end{tabular}

Shaded rows indicate oxides that are at reportable concentrations and that are considered critical in the comparisons conducted as part of this study. 
Table A6. Comparisons for Density and Solids from Mid-Rheology (Phase 1) Testing

Density

Sampler Mean BL Mean Difference Difference Statistically

Statistically $\quad \mathrm{g} / \mathrm{mL} \quad \%$ of BL

\begin{tabular}{|c|c|c|c|c|c|c|c|c|c|c|c|c|}
\hline & $(\mathrm{g} / \mathrm{mL})$ & $(\mathrm{g} / \mathrm{mL})$ & $(\mathrm{g} / \mathrm{mL})$ & $(\%)$ & Significant Bias & Sampler Std Dev & BL Std Dev & Variances & Bias (95\%) & Bias $(95 \%)$ & n sampler & $\mathrm{nBL}$ \\
\hline Coliwasa High Location vs Coliwasa Low Location (BL) & 1.29927 & 1.28822 & 0.011 & $0.9 \%$ & no & 0.0082421 & 0.0140021 & no & 0.02337 & $1.8 \%$ & 8 & 8 \\
\hline Hydragard (LF) vs Coliwasa & 1.30334 & 1.29374 & 0.010 & $0.7 \%$ & yes & 0.0066311 & 0.0124792 & no & 0.018477 & $1.4 \%$ & 10 & 16 \\
\hline Hydragard (HF) vs Coliwasa & 1.30816 & 1.29374 & 0.014 & $1.1 \%$ & no & 0.0348372 & 0.0124792 & no & 0.03397 & $2.6 \%$ & 10 & 16 \\
\hline Hydragard (HF-scrnd) vs Coliwasa & 1.29759 & 1.29374 & 0.004 & $0.3 \%$ & no & 0.0104389 & 0.0124792 & no & 0.01403 & $1.1 \%$ & 9 & 16 \\
\hline Isolok (LF) vs Coliwasa & 1.28772 & 1.29374 & -0.006 & $-0.5 \%$ & no & 0.0123206 & 0.0124792 & no & 0.01635 & $1.3 \%$ & 10 & 16 \\
\hline Isolok (LF - scrnd) vs Coliwasa & 1.29103 & 1.29374 & -0.003 & $-0.2 \%$ & no & 0.0069123 & 0.0124792 & no & 0.01209 & $0.9 \%$ & 9 & 16 \\
\hline Isolok (HF) vs Coliwasa & 1.30378 & 1.29374 & 0.010 & $0.8 \%$ & no & 0.0130293 & 0.0124792 & no & 0.0206 & $1.6 \%$ & 10 & 16 \\
\hline
\end{tabular}

Dried wt\% Solids

\begin{tabular}{|l|l|l|l|c|c|c|c|} 
& & & Statistically & $\mathrm{g} / \mathrm{mL}$ & $\%$ of BL \\
\hline Sampler Mean & BL Mean & Difference & Difference & Statistically & Different & Bound on & Bound on \\
\hline
\end{tabular}

on $\quad$ Bound on

\begin{tabular}{|c|c|c|c|c|c|c|c|c|c|c|c|c|}
\hline & & & & & & & & & & & & \\
\hline & $(\mathrm{g} / \mathrm{mL})$ & $(\mathrm{g} / \mathrm{mL})$ & $(\mathrm{g} / \mathrm{mL})$ & $(\%)$ & Significant Bias & Sampler Std Dev & BL Std Dev & Variances & Bias (95\%) & Bias (95\%) & n sampler & $\mathrm{nBL}$ \\
\hline Coliwasa High Location vs Coliwasa Low Location (BL) & 36.5288 & 36.2150 & 0.314 & $0.9 \%$ & yes & 0.079181 & 0.172378 & no & 0.45759 & $1.3 \%$ & 8 & 8 \\
\hline Hydragard (HF) vs Coliwasa & 35.9830 & 36.3719 & -0.389 & $-1.1 \%$ & yes & 0.170428 & 0.207468 & no & 0.55062 & $1.5 \%$ & 10 & 16 \\
\hline Hydragard (LF) vs Coliwasa & 36.0100 & 36.3719 & -0.362 & $-1.0 \%$ & yes & 0.125344 & 0.207468 & yes & 0.4966 & $1.4 \%$ & 10 & 16 \\
\hline Isolok (12; LF) vs Coliwasa & 35.8130 & 36.3719 & -0.559 & $-1.5 \%$ & yes & 0.258974 & 0.207468 & no & 0.74869 & $2.1 \%$ & 10 & 16 \\
\hline Isolok (12; HF) vs Coliwasa & 36.4520 & 36.3719 & 0.080 & $0.2 \%$ & no & 0.070836 & 0.207468 & yes & 0.19799 & $0.5 \%$ & 10 & 16 \\
\hline Isolok (3; LF) vs Coliwasa & 35.8540 & 36.3719 & -0.518 & $-1.4 \%$ & yes & 0.186679 & 0.207468 & no & 0.68421 & $1.9 \%$ & 10 & 16 \\
\hline Isolok (3; HF) vs Coliwasa & 36.5910 & 36.3719 & 0.219 & $0.6 \%$ & yes & 0.137957 & 0.207468 & no & 0.372623 & $1.0 \%$ & 10 & 16 \\
\hline
\end{tabular}

Vitrified wt\% Solids

Sampler Mean BL Mean Difference Difference Statistically

Statistically $\quad \mathrm{g} / \mathrm{mL} \quad \%$ of BL

Different Bound on Bound on

\begin{tabular}{|c|c|c|c|c|c|c|c|c|c|c|c|c|}
\hline & Sampler Mean & BL Mean & Differenc & Difference & Statistically & & & Different & Bound on & Bound on & & \\
\hline & $(\mathrm{g} / \mathrm{mL})$ & $(\mathrm{g} / \mathrm{mL})$ & $(\mathrm{g} / \mathrm{mL})$ & $(\%)$ & Significant Bias & Sampler Std Dev & BL Std Der & Variances & Bias (95\%) & Bias $(95 \%)$ & n sampler & $\mathrm{nBL}$ \\
\hline Coliwasa High Location vs Coliwasa Low Location (BL) & 31.1413 & 30.8250 & 0.316 & $1.0 \%$ & yes & 0.076052 & 0.1811659 & no & 0.46559 & $1.5 \%$ & 8 & 8 \\
\hline Hydragard (HF) vs Coliwasa & 30.1880 & 30.9831 & -0.795 & $-2.6 \%$ & yes & 0.200044 & 0.211588 & no & 0.96762 & $3.1 \%$ & 10 & 16 \\
\hline Hydragard (LF) vs Coliwasa & 30.3320 & 30.9831 & -0.651 & $-2.1 \%$ & yes & 0.120167 & 0.211588 & no & 0.80317 & $2.6 \%$ & 10 & 16 \\
\hline Isolok (12; LF) vs Coliwasa & 30.2950 & 30.9831 & -0.688 & $-2.2 \%$ & yes & 0.229988 & 0.211588 & no & 0.87005 & $2.8 \%$ & 10 & 16 \\
\hline Isolok (12; HF) vs Coliwasa & 30.9380 & 30.9831 & -0.045 & $-0.1 \%$ & no & 0.090406 & 0.211588 & yes & 0.16985 & $0.5 \%$ & 10 & 16 \\
\hline Isolok (3; LF) vs Coliwasa & 30.3430 & 30.9831 & -0.640 & $-2.1 \%$ & yes & 0.189798 & 0.211588 & no & 0.80959 & $2.6 \%$ & 10 & 16 \\
\hline Isolok (3; HF) vs Coliwasa & 31.0290 & 30.9831 & 0.046 & $0.1 \%$ & no & 0.109082 & 0.211588 & yes & 0.17638 & $0.6 \%$ & 10 & 16 \\
\hline
\end{tabular}


Table A7. Oxide Comparions between the Low and High Coliwasa Samples from the Mid-Rheology (Phase 1) Testing

\begin{tabular}{|c|c|c|c|c|c|c|c|c|c|c|c|c|c|}
\hline Analytical Block & Oxide & \begin{tabular}{|c} 
Mean(Measured \\
wt\%) Coliwasa - low \\
location
\end{tabular} & $\begin{array}{c}\text { Mean(Measured } \\
\text { wt\%) Coliwasa - } \\
\text { high location }\end{array}$ & Diff & \%Diff & Sig. Diff & Bias Bnd & $\begin{array}{c}\% \text { Rel } \\
\text { Bias Bnd } \\
\end{array}$ & \begin{tabular}{|c|}
$\begin{array}{c}\text { Std Dev(Measured } \\
\text { wt\%) Coliwasa - low } \\
\text { location }\end{array}$ \\
\end{tabular} & \begin{tabular}{|c|} 
Std \\
Dev(Measured \\
wt\%) Coliwasa \\
high location \\
\end{tabular} & Sig. Diff & $\mathrm{n}$ low & $\mathrm{n}$ high \\
\hline avg & $\mathrm{Al} / \mathrm{B}$ & 2.668 & 2.585 & -0.083 & \begin{tabular}{|l|l|}
$-3.1 \%$ \\
\end{tabular} & No & 0.2102 & \begin{tabular}{|l|}
7.88 \\
\end{tabular} & 0.1600 & 0.0535 & No & 8 & 8 \\
\hline avg & Al2O3 (wt\%) & 11.054 & 10.657 & -0.397 & $-3.6 \%$ & Yes & 0.7446 & 6.74 & 0.4117 & 0.0698 & Yes & 8 & 8 \\
\hline avg & B2O3 (wt\%) & 4.150 & 4.125 & -0.025 & $-0.6 \%$ & No & 0.1338 & 3.22 & 0.1243 & 0.0721 & No & 8 & 8 \\
\hline avg & $\mathrm{BaO}(\mathrm{wt} \%)$ & 0.105 & 0.102 & -0.003 & $-3.1 \%$ & Yes & 0.0059 & 5.64 & 0.0032 & 0.0010 & Yes & 8 & 8 \\
\hline avg & $\mathrm{CaO}(\mathrm{wt} \%)$ & 1.222 & 1.183 & -0.039 & $-3.2 \%$ & Yes & 0.0758 & 6.20 & 0.0430 & 0.0217 & No & 8 & 8 \\
\hline avg & $\mathrm{Cr} 2 \mathrm{O} 3(\mathrm{wt} \%)$ & 0.111 & 0.105 & -0.006 & $-5.5 \%$ & Yes & 0.0106 & 9.56 & 0.0057 & 0.0018 & No & 8 & 8 \\
\hline avg & $\mathrm{CuO}(\mathrm{wt} \%)$ & 0.026 & 0.025 & -0.001 & $-2.4 \%$ & No & 0.0020 & 7.91 & 0.0013 & 0.0013 & No & 8 & 8 \\
\hline avg & $\mathrm{Fe} / \mathrm{Li}$ & 2.435 & 2.366 & -0.068 & $-2.8 \%$ & No & 0.2208 & 9.07 & 0.1971 & 0.0408 & No & 8 & 8 \\
\hline avg & Fe2O3 (wt $\%)$ & 11.610 & 11.201 & -0.409 & $-3.5 \%$ & Yes & 0.7990 & 6.88 & 0.4646 & 0.0893 & Yes & 8 & 8 \\
\hline avg & K2O (wt\%) & 0.163 & 0.168 & 0.004 & $2.5 \%$ & No & 0.0199 & 12.18 & 0.0100 & 0.0183 & No & 8 & 8 \\
\hline avg & Li2O (wt\%) & 4.785 & 4.735 & -0.050 & $-1.0 \%$ & No & 0.2497 & 5.22 & 0.2448 & 0.0963 & No & 8 & 8 \\
\hline avg & $\mathrm{MgO}(\mathrm{wt} \%)$ & 1.072 & 1.037 & -0.035 & $-3.3 \%$ & No & 0.0723 & 6.74 & 0.0437 & 0.0109 & Yes & 8 & 8 \\
\hline avg & $\mathrm{MnO}(\mathrm{wt} \%)$ & 1.782 & 1.718 & -0.064 & $-3.6 \%$ & No & 0.1385 & 7.78 & 0.0888 & 0.0218 & Yes & 8 & 8 \\
\hline avg & $\mathrm{Na2O}(\mathrm{wt} \%)$ & 11.292 & 11.080 & -0.212 & $-1.9 \%$ & No & 0.5530 & 4.90 & 0.3546 & 0.2758 & No & 8 & 8 \\
\hline avg & $\mathrm{NiO}(\mathrm{wt} \%)$ & 0.460 & 0.442 & -0.018 & $-3.8 \%$ & No & 0.0485 & 10.55 & 0.0368 & 0.0059 & Yes & 8 & 8 \\
\hline avg & SiO2 (wt\%) & 51.009 & 52.132 & 1.123 & $2.2 \%$ & No & 2.4407 & 4.78 & 1.6023 & 0.6723 & No & 8 & 8 \\
\hline avg & Sum of Oxides (wt\%) & 99.393 & 99.253 & -0.139 & $-0.1 \%$ & No & 1.0470 & 1.05 & 0.9004 & 0.7885 & No & 8 & 8 \\
\hline avg & TiO2 (wt\%) & 0.081 & 0.083 & 0.001 & $1.4 \%$ & No & 0.0030 & 3.71 & 0.0021 & 0.0013 & No & 8 & 8 \\
\hline avg & $\mathrm{ZnO}(\mathrm{wt} \%)$ & 0.103 & 0.099 & -0.004 & $-3.9 \%$ & Yes & 0.0076 & 7.36 & 0.0042 & 0.0010 & Yes & 8 & 8 \\
\hline avg & ZrO2 (wt\%) & 0.368 & 0.363 & -0.005 & $-1.2 \%$ & No & 0.0105 & 2.86 & 0.0063 & 0.0047 & No & 8 & 8 \\
\hline
\end{tabular}

\begin{tabular}{|c|c|c|c|c|c|c|c|c|c|c|c|c|c|}
\hline \multicolumn{14}{|l|}{ Screened Data } \\
\hline Analytical Block & Oxide & $\begin{array}{c}\text { Mean(Measured } \\
\text { wt\%) Coliwasa - low } \\
\text { location }\end{array}$ & $\begin{array}{l}\text { Mean(Measured } \\
\text { wt\%) Coliwasa - } \\
\text { high location }\end{array}$ & Diff & $\%$ Diff & Siq. Diff & Bias Bnd & $\begin{array}{c}\% \text { Rel } \\
\text { Bias Bnd }\end{array}$ & $\begin{array}{l}\text { Std Dev(Measured } \\
\text { wt\%) Coliwasa - low } \\
\text { location }\end{array}$ & $\begin{array}{c}\text { Std } \\
\text { Dev(Measured } \\
\text { wt\%) Coliwasa - } \\
\text { hivhtion }\end{array}$ & Sig Diff & Dlow & high \\
\hline avg & Al/B & \begin{tabular}{|l}
2.615 \\
\end{tabular} & 2.585 & -0.030 & $-1.2 \%$ & No & 0.0943 & \begin{tabular}{|l|}
3.60 \\
\end{tabular} & 0.0640 & 0.0535 & No & 7 & 8 \\
\hline$\frac{a v g}{\text { avg }}$ & Al2O3 (wt\%) & 11.054 & 10.657 & -0.397 & $-3.6 \%$ & Yes & 0.7446 & 6.74 & 0.4117 & 0.0698 & Yes & 8 & $\frac{0}{8}$ \\
\hline avg & B2O3 (wt\%) & 4.190 & 4.125 & -0.065 & $-1.5 \%$ & No & 0.1374 & 3.28 & 0.0551 & 0.0721 & No & 7 & 8 \\
\hline avg & $\mathrm{BaO}(\mathrm{wt} \%)$ & 0.105 & 0.102 & -0.003 & $-3.1 \%$ & Yes & 0.0059 & 5.64 & 0.0032 & 0.0010 & Yes & 8 & 8 \\
\hline$\frac{a v y}{\text { avg }}$ & $\mathrm{CaO}(\mathrm{wt} \%)$ & 1.236 & 1.183 & -0.053 & \begin{tabular}{|l|}
$-4.3 \%$ \\
\end{tabular} & Yes & 0.0767 & 6.04 & 0.0210 & 0.0217 & No & $\frac{0}{7}$ & $\frac{0}{8}$ \\
\hline avg & $\mathrm{Cr} 2 \mathrm{O} 3$ (wt\%) & 0.111 & 0.105 & -0.006 & $-5.5 \%$ & Yes & 0.0106 & 9.56 & 0.0057 & 0.0018 & No & 8 & 8 \\
\hline avg & CuO (wt \%) & 0.026 & 0.025 & -0.001 & $-2.4 \%$ & No & 0.0020 & 7.91 & 0.0013 & 0.0013 & No & $\frac{0}{8}$ & 8 \\
\hline avg & $\mathrm{Fe} / \mathrm{Li}$ & 2.492 & 2.366 & -0.125 & $-5.0 \%$ & Yes & 0.2208 & 8.86 & 0.1222 & 0.0408 & No & $\frac{0}{7}$ & 8 \\
\hline avg & $\mathrm{Fe} 2 \mathrm{O} 3(\mathrm{wt} \%)$ & 11.610 & 11.201 & -0.409 & $-3.5 \%$ & Yes & 0.7990 & 6.88 & 0.4646 & 0.0893 & Yes & 8 & 8 \\
\hline avg & $\mathrm{K} 2 \mathrm{O}(\mathrm{wt} \%)$ & 0.163 & 0.162 & -0.002 & $-1.1 \%$ & No & 0.0124 & 7.56 & 0.0100 & 0.0089 & No & 8 & 7 \\
\hline avg & Li2O (wt\%) & 4.717 & 4.735 & 0.018 & $0.4 \%$ & No & 0.1644 & 3.49 & 0.1621 & 0.0963 & No & 7 & 8 \\
\hline avg & $\mathrm{MgO}(\mathrm{wt} \%)$ & 1.072 & 1.037 & -0.035 & $-3.3 \%$ & No & 0.0723 & 6.74 & 0.0437 & 0.0109 & Yes & 8 & 8 \\
\hline avg & $\mathrm{MnO}(\mathrm{wt} \%)$ & 1.782 & 1.718 & -0.064 & $-3.6 \%$ & No & 0.1385 & 7.78 & 0.0888 & 0.0218 & Yes & 8 & 8 \\
\hline$\frac{a v y}{\text { avg }}$ & $\mathrm{Na} 2 \mathrm{O}(\mathrm{wt} \%)$ & $\frac{1.10 \mathrm{~L}}{11.292}$ & $\frac{1.110}{11.080}$ & -0.004 & \begin{tabular}{|l|}
$-1.9 \%$ \\
$-1.9 \%$ \\
\end{tabular} & No & 0.15530 & 4.90 & 0.0000 & 0.2758 & No & $\frac{0}{8}$ & $\frac{0}{8}$ \\
\hline avg & $\mathrm{NiO}(\mathrm{wt} \%)$ & 0.460 & 0.442 & -0.018 & \begin{tabular}{|l|}
$-3.8 \%$ \\
\end{tabular} & No & 0.0485 & 10.55 & 0.0368 & 0.0059 & Yes & $\frac{0}{8}$ & 8 \\
\hline avg & $\mathrm{SiO} 2(\mathrm{wt} \%)$ & 51.009 & 52.132 & 1.123 & $2.2 \%$ & No & 2.4407 & 4.78 & 1.6023 & 0.6723 & No & 8 & 8 \\
\hline avg & Sum of Oxides (wt\%) & 99.681 & 99.253 & \begin{tabular}{|c|c|c|}
-0.427 \\
\end{tabular} & $-0.4 \%$ & No & 1.1471 & 1.15 & 0.4155 & 0.7885 & No & $\frac{0}{7}$ & 8 \\
\hline avg & \begin{tabular}{|l|}
$\mathrm{TiO} 2(\mathrm{wt} \%)$ \\
\end{tabular} & 0.081 & 0.083 & 0.001 & $1.4 \%$ & No & 0.0030 & 3.71 & 0.0021 & 0.0013 & No & 8 & 8 \\
\hline$\frac{a v y}{\text { avg }}$ & $\mathrm{ZnO}(\mathrm{wt} \%)$ & 0.103 & 0.099 & -0.004 & $\frac{.1 .4 \%}{-4.2 \%}$ & Yes & 0.0079 & $\frac{.11}{7.64}$ & 0.0042 & 0.0005 & Yes & $\frac{0}{8}$ & $\frac{0}{7}$ \\
\hline avg & ZrO2 (wt\%) & 0.370 & 0.363 & -0.007 & $-1.8 \%$ & Yes & 0.0108 & 2.93 & 0.0026 & 0.0047 & Yes & 7 & 8 \\
\hline
\end{tabular}


Table A8. Oxide Comparions between Samplers for the Mid-Rheology (Phase 1) Testing

\begin{tabular}{|c|c|c|c|c|c|c|c|c|c|c|c|c|c|c|}
\hline $\begin{array}{c}\text { Analytical } \\
\text { Block }\end{array}$ & Sampler & Oxide & $\begin{array}{c}\text { Mean(Measured } \\
\text { wt\%) Coliwasa } \\
\text { (low+high) }\end{array}$ & $\begin{array}{c}\text { Mean(Measured } \\
\text { wt\%) Other } \\
\text { Sampler }\end{array}$ & Difference & $\%$ Diff & $\begin{array}{l}\text { Sig. } \\
\text { Diff }\end{array}$ & $\begin{array}{l}\text { Bias } \\
\text { Bnd }\end{array}$ & $\begin{array}{l}\text { \% Rel } \\
\text { Bias } \\
\text { Bnd }\end{array}$ & $\begin{array}{c}\text { Std } \\
\text { Dev(Measured } \\
\text { wt\%) } \\
\text { Coliwasa } \\
\text { (low+high) }\end{array}$ & $\begin{array}{c}\text { Std } \\
\text { Dev(Measured } \\
\text { wt\%) Other } \\
\text { Sampler }\end{array}$ & $\begin{array}{l}\text { Sig. } \\
\text { Diff }\end{array}$ & $\begin{array}{c}\text { n } \\
\text { Col }\end{array}$ & $\begin{array}{c}\text { n } \\
\text { Other }\end{array}$ \\
\hline 1 & Isolok-LFR-3mL & $\mathrm{Al} / \mathrm{B}$ & 2.598 & 2.725 & 0.128 & $4.9 \%$ & Yes & 0.2121 & 8.17 & 0.1166 & 0.0702 & No & 16 & 10 \\
\hline 1 & Isolok-LFR-3mL & $\mathrm{Al2O}(\mathrm{wt} \%)$ & 10.767 & 10.927 & 0.160 & $1.5 \%$ & No & 0.4089 & 3.80 & 0.3358 & 0.2234 & No & 16 & 10 \\
\hline 1 & Isolok-LFR-3mL & B2O3 (wt\%) & 4.148 & 4.012 & -0.136 & $-3.3 \%$ & Yes & 0.2345 & 5.65 & 0.1066 & 0.1368 & No & 16 & 10 \\
\hline 1 & Isolok-LFR-3mL & $\mathrm{BaO}$ (wt\%) & 0.103 & 0.105 & 0.002 & $1.8 \%$ & No & 0.0039 & 3.73 & 0.0027 & 0.0020 & No & 16 & 10 \\
\hline 1 & Isolok-LFR-3mL & $\mathrm{CaO}(\mathrm{wt} \%)$ & 1.201 & 1.222 & 0.021 & $1.7 \%$ & No & 0.0495 & 4.12 & 0.0397 & 0.0236 & No & 16 & 10 \\
\hline 1 & Isolok-LFR-3mL & Cr2O3 (wt\%) & 0.106 & 0.103 & -0.003 & $-2.7 \%$ & No & 0.0064 & 6.02 & 0.0046 & 0.0037 & No & 16 & 10 \\
\hline 1 & Isolok-LFR-3mL & $\mathrm{CuO}$ (wt\%) & 0.025 & 0.025 & 0.000 & $1.3 \%$ & No & 0.0014 & 5.58 & 0.0014 & 0.0010 & No & 16 & 10 \\
\hline 1 & Isolok-LFR-3mL & $\mathrm{Fe} / \mathrm{Li}$ & 2.411 & 2.465 & 0.054 & $2.2 \%$ & No & 0.1544 & 6.40 & 0.1414 & 0.0750 & No & 16 & 10 \\
\hline 1 & Isolok-LFR-3mL & Fe2O3 (wt\%) & 11.405 & 11.592 & 0.188 & $1.6 \%$ & No & 0.4594 & 4.03 & 0.3770 & 0.2191 & No & 16 & 10 \\
\hline 1 & Isolok-LFR-3mL & K2O (wt\%) & 0.163 & 0.165 & 0.002 & $1.5 \%$ & No & 0.0094 & 5.74 & 0.0092 & 0.0068 & No & 16 & 10 \\
\hline 1 & Isolok-LFR-3mL & Li2O (wt\%) & 4.738 & 4.704 & -0.034 & $-0.7 \%$ & No & 0.1528 & 3.22 & 0.1703 & 0.0794 & No & 16 & 10 \\
\hline 1 & Isolok-LFR-3mL & $\mathrm{MgO}$ (wt\%) & 1.058 & 1.075 & 0.017 & $1.6 \%$ & No & 0.0382 & 3.61 & 0.0351 & 0.0162 & Yes & 16 & 10 \\
\hline 1 & Isolok-LFR-3mL & $\mathrm{MnO}(\mathrm{wt} \%)$ & 1.750 & 1.786 & 0.035 & $2.0 \%$ & No & 0.0836 & 4.78 & 0.0670 & 0.0385 & No & 16 & 10 \\
\hline 1 & Isolok-LFR-3mL & $\mathrm{Na} 2 \mathrm{O}$ (wt\%) & 11.249 & 11.198 & -0.051 & $-0.5 \%$ & No & 0.4892 & 4.35 & 0.3356 & 0.5824 & Yes & 16 & 10 \\
\hline 1 & Isolok-LFR-3mL & $\mathrm{NiO}$ (wt\%) & 0.452 & 0.459 & 0.007 & $1.6 \%$ & No & 0.0253 & 5.61 & 0.0262 & 0.0097 & No & 16 & 10 \\
\hline 1 & Isolok-LFR-3mL & $\mathrm{SiO} 2(\mathrm{wt} \%)$ & 51.731 & 51.728 & -0.003 & $0.0 \%$ & No & 0.7290 & 1.41 & 1.3262 & 0.2992 & Yes & 16 & 10 \\
\hline 1 & Isolok-LFR-3mL & Sum (wt\%) & 99.450 & 99.660 & 0.210 & $0.2 \%$ & No & 0.6234 & 0.63 & 0.6067 & 0.2124 & No & 16 & 10 \\
\hline 1 & Isolok-LFR-3mL & $\mathrm{TiO} 2$ (wt\%) & 0.082 & 0.082 & 0.000 & $-0.6 \%$ & No & 0.0021 & 2.55 & 0.0027 & 0.0013 & Yes & 16 & 10 \\
\hline 1 & Isolok-LFR-3mL & $\mathrm{ZnO}$ (wt\%) & 0.101 & 0.102 & 0.001 & $0.8 \%$ & No & 0.0035 & 3.44 & 0.0037 & 0.0020 & No & 16 & 10 \\
\hline 1 & Isolok-LFR-3mL & $\mathrm{ZrO} 2$ (wt\%) & 0.371 & 0.374 & 0.002 & $0.7 \%$ & No & 0.0075 & 2.03 & 0.0056 & 0.0069 & No & 16 & 10 \\
\hline 2 & Hydragard - HFR & $\mathrm{Al} / \mathrm{B}$ & 2.654 & 2.728 & 0.074 & $2.8 \%$ & No & 0.1778 & 6.70 & 0.1433 & 0.0845 & No & 16 & 10 \\
\hline 2 & Hydragard - HFR & Al2O3 (wt\%) & 10.975 & 11.265 & 0.291 & $2.6 \%$ & Yes & 0.5596 & 5.10 & 0.3792 & 0.1971 & No & 16 & 10 \\
\hline 2 & Hydragard - HFR & B2O3 (wt\%) & 4.140 & 4.131 & -0.008 & $-0.2 \%$ & No & 0.0966 & 2.33 & 0.1176 & 0.0832 & No & 16 & 10 \\
\hline 2 & Hydragard - HFR & $\mathrm{BaO}(\mathrm{wt} \%)$ & 0.106 & 0.108 & 0.002 & $2.1 \%$ & No & 0.0048 & 4.51 & 0.0033 & 0.0025 & No & 16 & 10 \\
\hline 2 & Hydragard - HFR & $\mathrm{CaO}(\mathrm{wt} \%)$ & 1.211 & 1.250 & 0.039 & $3.2 \%$ & Yes & 0.0657 & 5.43 & 0.0347 & 0.0285 & No & 16 & 10 \\
\hline 2 & Hydragard - HFR & Cr2O3 (wt\%) & 0.111 & 0.111 & 0.000 & $0.2 \%$ & No & 0.0041 & 3.71 & 0.0056 & 0.0024 & No & 16 & 10 \\
\hline 2 & Hydragard - HFR & $\mathrm{CuO}(\mathrm{wt} \%)$ & 0.025 & 0.025 & 0.000 & $0.1 \%$ & No & 0.0014 & 5.42 & 0.0014 & 0.0019 & No & 16 & 10 \\
\hline 2 & Hydragard - HFR & $\mathrm{Fe} / \mathrm{Li}$ & 2.388 & 2.455 & 0.067 & $2.8 \%$ & No & 0.1651 & 6.91 & 0.1360 & 0.0768 & No & 16 & 10 \\
\hline 2 & Hydragard - HFR & Fe2O3 (wt\%) & 11.463 & 11.732 & 0.270 & $2.4 \%$ & No & 0.5518 & 4.81 & 0.3843 & 0.2465 & No & 16 & 10 \\
\hline 2 & Hydragard - HFR & K2O (wt\%) & 0.163 & 0.163 & 0.001 & $0.4 \%$ & No & 0.0092 & 5.62 & 0.0088 & 0.0123 & No & 16 & 10 \\
\hline 2 & Hydragard - HFR & Li2O (wt\%) & 4.808 & 4.779 & -0.028 & $-0.6 \%$ & No & 0.1417 & 2.95 & 0.1662 & 0.0600 & No & 16 & 10 \\
\hline 2 & Hydragard - HFR & MgO (wt\%) & 1.074 & 1.091 & 0.018 & $1.7 \%$ & No & 0.0458 & 4.27 & 0.0361 & 0.0288 & No & 16 & 10 \\
\hline 2 & Hydragard - HFR & $\mathrm{MnO}(\mathrm{wt} \%)$ & 1.785 & 1.822 & 0.037 & $2.1 \%$ & No & 0.0889 & 4.98 & 0.0708 & 0.0457 & No & 16 & 10 \\
\hline 2 & Hydragard - HFR & $\mathrm{Na} 2 \mathrm{O}$ (wt\%) & 11.311 & 11.399 & 0.088 & $0.8 \%$ & No & 0.3822 & 3.38 & 0.3543 & 0.3518 & No & 16 & 10 \\
\hline 2 & Hydragard - HFR & $\mathrm{NiO}$ (wt\%) & 0.460 & 0.478 & 0.018 & $3.8 \%$ & No & 0.0370 & 8.04 & 0.0274 & 0.0145 & No & 16 & 10 \\
\hline 2 & Hydragard - HFR & $\mathrm{SiO} 2(\mathrm{wt} \%)$ & 52.052 & 51.579 & -0.473 & $-0.9 \%$ & No & 1.3620 & 2.62 & 1.2095 & 0.7776 & No & 16 & 10 \\
\hline 2 & Hydragard - HFR & Sum (wt\%) & 100.236 & 100.496 & 0.260 & $0.3 \%$ & No & 0.5996 & 0.60 & 0.4877 & 0.2163 & No & 16 & 10 \\
\hline 2 & Hydragard - HFR & TiO2 (wt\%) & 0.082 & 0.082 & 0.000 & $-0.3 \%$ & No & 0.0025 & 3.05 & 0.0024 & 0.0031 & No & 16 & 10 \\
\hline 2 & Hydragard - HFR & $\mathrm{ZnO}$ (wt\%) & 0.103 & 0.105 & 0.003 & $2.7 \%$ & Yes & 0.0055 & 5.32 & 0.0038 & 0.0019 & No & 16 & 10 \\
\hline
\end{tabular}


Table A8. Oxide Comparions between Samplers for the Mid-Rheology (Phase 1) Testing

\begin{tabular}{|c|c|c|c|c|c|c|c|c|c|c|c|c|c|c|}
\hline $\begin{array}{c}\text { Analytical } \\
\text { Block }\end{array}$ & Sampler & Oxide & $\begin{array}{c}\text { Mean(Measured } \\
\text { wt\%) Coliwasa } \\
\text { (low+high) }\end{array}$ & $\begin{array}{c}\text { Mean(Measured } \\
\text { wt\%) Other } \\
\text { Sampler }\end{array}$ & Difference & $\%$ Diff & $\begin{array}{l}\text { Sig. } \\
\text { Diff }\end{array}$ & $\begin{array}{l}\text { Bias } \\
\text { Bnd }\end{array}$ & $\begin{array}{l}\text { \% Rel } \\
\text { Bias } \\
\text { Bnd }\end{array}$ & $\begin{array}{c}\text { Std } \\
\text { Dev(Measured } \\
\text { wt\%) } \\
\text { Coliwasa } \\
\text { (low+high) }\end{array}$ & $\begin{array}{c}\text { Std } \\
\text { Dev(Measured } \\
\text { wt\%) Other } \\
\text { Sampler }\end{array}$ & $\begin{array}{l}\text { Sig. } \\
\text { Diff }\end{array}$ & $\begin{array}{c}\text { n } \\
\text { Col }\end{array}$ & $\begin{array}{c}\text { n } \\
\text { Other }\end{array}$ \\
\hline 2 & Hydragard - HFR & $\mathrm{ZrO} 2$ (wt\%) & 0.370 & 0.375 & 0.006 & $1.5 \%$ & Yes & 0.0105 & 2.83 & 0.0065 & 0.0049 & No & 16 & 10 \\
\hline 3 & Isolok-LFR-12mL & $\mathrm{Al} / \mathrm{B}$ & 2.613 & 2.580 & -0.033 & $-1.3 \%$ & No & 0.1290 & 4.94 & 0.1363 & 0.0672 & No & 16 & 10 \\
\hline 3 & Isolok-LFR-12mL & Al2O3 (wt\%) & 10.868 & 11.176 & 0.308 & $2.8 \%$ & No & 0.6640 & 6.11 & 0.4601 & 0.3672 & No & 16 & 10 \\
\hline 3 & Isolok-LFR-12mL & B2O3 (wt\%) & 4.164 & 4.334 & 0.170 & $4.1 \%$ & Yes & 0.2949 & 7.08 & 0.1500 & 0.1496 & No & 16 & 10 \\
\hline 3 & Isolok-LFR-12mL & $\mathrm{BaO}$ (wt\%) & 0.103 & 0.107 & 0.004 & $4.0 \%$ & Yes & 0.0073 & 7.10 & 0.0040 & 0.0011 & No & 16 & 10 \\
\hline 3 & Isolok-LFR-12mL & $\mathrm{CaO}(\mathrm{wt} \%)$ & 1.194 & 1.249 & 0.055 & $4.6 \%$ & Yes & 0.0960 & 8.04 & 0.0510 & 0.0479 & No & 16 & 10 \\
\hline 3 & Isolok-LFR-12mL & Cr2O3 (wt\%) & 0.108 & 0.110 & 0.002 & $2.2 \%$ & No & 0.0069 & 6.40 & 0.0062 & 0.0013 & No & 16 & 10 \\
\hline 3 & Isolok-LFR-12mL & $\mathrm{CuO}(\mathrm{wt} \%)$ & 0.025 & 0.026 & 0.001 & $4.3 \%$ & No & 0.0022 & 8.57 & 0.0014 & 0.0011 & No & 16 & 10 \\
\hline 3 & Isolok-LFR-12mL & $\mathrm{Fe} / \mathrm{Li}$ & 2.393 & 2.450 & 0.058 & $2.4 \%$ & No & 0.1506 & 6.29 & 0.1367 & 0.0470 & No & 16 & 10 \\
\hline 3 & Isolok-LFR-12mL & Fe2O3 (wt\%) & 11.214 & 11.599 & 0.385 & $3.4 \%$ & Yes & 0.7611 & 6.79 & 0.4704 & 0.4200 & No & 16 & 10 \\
\hline 3 & $\begin{array}{l}\text { Isolok-LFR-12mL } \\
\end{array}$ & K2O (wt\%) & 0.163 & 0.329 & 0.166 & $101.5 \%$ & No & 0.5251 & 321.72 & 0.0092 & 0.5024 & Yes & 16 & 10 \\
\hline 3 & Isolok-LFR-12mL & Li2O (wt\%) & 4.695 & 4.734 & 0.040 & $0.8 \%$ & No & 0.2057 & 4.38 & 0.2169 & 0.1672 & No & 16 & 10 \\
\hline 3 & Isolok-LFR-12mL & $\mathrm{MgO}(\mathrm{wt} \%)$ & 1.051 & 1.089 & 0.038 & $3.7 \%$ & Yes & 0.0763 & 7.26 & 0.0455 & 0.0457 & No & 16 & 10 \\
\hline 3 & Isolok-LFR-12mL & $\mathrm{MnO}$ (wt\%) & 1.748 & 1.817 & 0.069 & $3.9 \%$ & Yes & 0.1356 & 7.76 & 0.0862 & 0.0697 & No & 16 & 10 \\
\hline 3 & Isolok-LFR-12mL & $\mathrm{Na} 2 \mathrm{O}$ (wt\%) & 11.008 & 11.690 & 0.682 & $6.2 \%$ & No & 1.4743 & 13.39 & 0.4252 & 1.4556 & No & 16 & 10 \\
\hline 3 & Isolok-LFR-12mL & $\mathrm{NiO}$ (wt\%) & 0.451 & 0.466 & 0.016 & $3.5 \%$ & No & 0.0369 & 8.18 & 0.0306 & 0.0136 & No & 16 & 10 \\
\hline 3 & Isolok-LFR-12mL & $\mathrm{SiO} 2(\mathrm{wt} \%)$ & 51.062 & 51.750 & 0.687 & $1.3 \%$ & No & 1.8869 & 3.70 & 1.6529 & 0.9955 & No & 16 & 10 \\
\hline 3 & Isolok-LFR-12mL & Sum (wt\%) & 98.401 & 101.037 & 2.636 & $2.7 \%$ & Yes & 4.8331 & 4.91 & 2.3685 & 3.0410 & No & 16 & 10 \\
\hline 3 & Isolok-LFR-12mL & $\mathrm{TiO} 2$ (wt\%) & 0.082 & 0.083 & 0.000 & $0.4 \%$ & No & 0.0031 & 3.71 & 0.0033 & 0.0034 & No & 16 & 10 \\
\hline 3 & Isolok-LFR-12mL & $\mathrm{ZnO}$ (wt\%) & 0.100 & 0.105 & 0.005 & $4.8 \%$ & Yes & 0.0084 & 8.40 & 0.0047 & 0.0035 & No & 16 & 10 \\
\hline 3 & Isolok-LFR-12mL & $\mathrm{ZrO} 2$ (wt\%) & 0.364 & 0.372 & 0.008 & $2.3 \%$ & No & 0.0167 & 4.60 & 0.0097 & 0.0108 & No & 16 & 10 \\
\hline 4 & Isolok-HFR-12mL & $\mathrm{Al} / \mathrm{B}$ & 2.622 & 2.534 & -0.088 & $-3.3 \%$ & No & 0.2036 & 7.77 & 0.1211 & 0.1656 & No & 16 & 10 \\
\hline 4 & Isolok-HFR-12mL & $\mathrm{Al} 2 \mathrm{O} 3$ (wt\%) & 10.851 & 10.768 & -0.082 & $-0.8 \%$ & No & 0.3852 & 3.55 & 0.3358 & 0.4071 & No & 16 & 10 \\
\hline 4 & Isolok-HFR-12mL & $\mathrm{B} 2 \mathrm{O} 3(\mathrm{wt} \%)$ & 4.142 & 4.257 & 0.115 & $2.8 \%$ & Yes & 0.2161 & 5.22 & 0.1031 & 0.1470 & No & 16 & 10 \\
\hline 4 & Isolok-HFR-12mL & $\mathrm{BaO}(\mathrm{wt} \%)$ & 0.104 & 0.104 & 0.001 & $0.6 \%$ & No & 0.0033 & 3.21 & 0.0028 & 0.0039 & No & 16 & 10 \\
\hline 4 & Isolok-HFR-12mL & $\mathrm{CaO}(\mathrm{wt} \%)$ & 1.197 & 1.201 & 0.003 & $0.3 \%$ & No & 0.0425 & 3.55 & 0.0386 & 0.0587 & No & 16 & 10 \\
\hline 4 & Isolok-HFR-12mL & Cr2O3 (wt\%) & 0.107 & 0.107 & 0.000 & $0.2 \%$ & No & 0.0052 & 4.82 & 0.0055 & 0.0065 & No & 16 & 10 \\
\hline 4 & Isolok-HFR-12mL & $\mathrm{CuO}(\mathrm{wt} \%)$ & 0.025 & 0.026 & 0.001 & $5.5 \%$ & Yes & 0.0026 & 10.56 & 0.0014 & 0.0017 & No & 16 & 10 \\
\hline 4 & Isolok-HFR-12mL & $\mathrm{Fe} / \mathrm{Li}$ & 2.396 & 2.367 & -0.029 & $-1.2 \%$ & No & 0.1552 & 6.48 & 0.1472 & 0.1598 & No & 16 & 10 \\
\hline 4 & Isolok-HFR-12mL & $\mathrm{Fe} 2 \mathrm{O} 3(\mathrm{wt} \%)$ & 11.468 & 11.365 & -0.103 & $-0.9 \%$ & No & 0.4822 & 4.20 & 0.3692 & 0.5708 & No & 16 & 10 \\
\hline 4 & Isolok-HFR-12mL & $\mathrm{K} 2 \mathrm{O}(\mathrm{wt} \%)$ & 0.177 & 0.193 & 0.015 & $8.6 \%$ & No & 0.0670 & 37.78 & 0.0591 & 0.0673 & No & 16 & 10 \\
\hline 4 & Isolok-HFR-12mL & Li2O (wt\%) & 4.797 & 4.807 & 0.011 & $0.2 \%$ & No & 0.1520 & 3.17 & 0.1940 & 0.1201 & No & 16 & 10 \\
\hline 4 & Isolok-HFR-12mL & $\mathrm{MgO}(\mathrm{wt} \%)$ & 1.061 & 1.051 & -0.010 & $-0.9 \%$ & No & 0.0455 & 4.29 & 0.0346 & 0.0541 & No & 16 & 10 \\
\hline 4 & Isolok-HFR-12mL & $\mathrm{MnO}(\mathrm{wt} \%)$ & 1.755 & 1.742 & -0.013 & $-0.8 \%$ & No & 0.0770 & 4.39 & 0.0690 & 0.0893 & No & 16 & 10 \\
\hline 4 & Isolok-HFR-12mL & $\mathrm{Na} 2 \mathrm{O}$ (wt\%) & 11.063 & 10.748 & -0.315 & $-2.9 \%$ & Yes & 0.6128 & 5.54 & 0.3266 & 0.4041 & No & 16 & 10 \\
\hline 4 & Isolok-HFR-12mL & $\mathrm{NiO}$ (wt\%) & 0.446 & 0.443 & -0.003 & $-0.7 \%$ & No & 0.0274 & 6.13 & 0.0268 & 0.0320 & No & 16 & 10 \\
\hline 4 & Isolok-HFR-12mL & $\mathrm{SiO} 2(w t \%)$ & 51.370 & 52.113 & 0.743 & $1.4 \%$ & No & 1.7839 & 3.47 & 1.3254 & 1.1148 & No & 16 & 10 \\
\hline 4 & Isolok-HFR-12mL & Sum (wt\%) & 99.111 & 99.471 & 0.360 & $0.4 \%$ & No & 0.8499 & 0.86 & 0.6183 & 0.5364 & No & 16 & 10 \\
\hline 4 & Isolok-HFR-12mL & $\mathrm{TiO} 2$ (wt\%) & 0.083 & 0.083 & 0.000 & $0.0 \%$ & No & 0.0014 & 1.69 & 0.0019 & 0.0012 & No & 16 & 10 \\
\hline
\end{tabular}


SRNL-STI-2011-00693

Revision 0

Table A8. Oxide Comparions between Samplers for the Mid-Rheology (Phase 1) Testing

\begin{tabular}{|c|c|c|c|c|c|c|c|c|c|c|c|c|c|c|}
\hline $\begin{array}{c}\text { Analytical } \\
\text { Block }\end{array}$ & Sampler & Oxide & $\begin{array}{c}\text { Mean(Measured } \\
\text { wt\%) Coliwasa } \\
\text { (low+high) }\end{array}$ & $\begin{array}{l}\text { Mean(Measured } \\
\text { wt\%) Other } \\
\text { Sampler }\end{array}$ & Difference & \%Diff & $\begin{array}{l}\text { Sig. } \\
\text { Diff }\end{array}$ & $\begin{array}{l}\text { Bias } \\
\text { Bnd }\end{array}$ & $\begin{array}{l}\text { \% Rel } \\
\text { Bias } \\
\text { Bnd }\end{array}$ & $\begin{array}{c}\text { Std } \\
\text { Dev(Measured } \\
\text { wt\%) } \\
\text { Coliwasa } \\
\text { (low+high) }\end{array}$ & $\begin{array}{c}\text { Std } \\
\text { Dev(Measured } \\
\text { wt\%) Other } \\
\text { Sampler }\end{array}$ & $\begin{array}{l}\text { Sig. } \\
\text { Diff }\end{array}$ & $\underset{\text { Col }}{\mathbf{n}}$ & $\begin{array}{c}\text { n } \\
\text { Other }\end{array}$ \\
\hline 4 & Isolok-HFR-12mL & $\mathrm{ZnO}$ (wt\%) & 0.101 & 0.101 & 0.000 & $0.1 \%$ & No & 0.0033 & 3.31 & 0.0036 & 0.0043 & No & 16 & 10 \\
\hline 4 & Isolok-HFR-12mL & ZrO2 (wt\%) & 0.365 & 0.362 & -0.003 & $-0.7 \%$ & No & 0.0082 & 2.25 & 0.0052 & 0.0085 & No & 16 & 10 \\
\hline 5 & Isolok-HFR-3mL & $\mathrm{Al} / \mathrm{B}$ & 2.599 & 2.518 & -0.080 & $-3.1 \%$ & No & 0.1631 & 6.28 & 0.1191 & 0.0537 & No & 16 & 10 \\
\hline 5 & Isolok-HFR-3mL & Al2O3 (wt\%) & 10.752 & 10.596 & -0.156 & $-1.5 \%$ & No & 0.4420 & 4.11 & 0.3507 & 0.3315 & No & 16 & 10 \\
\hline 5 & Isolok-HFR-3mL & B2O3 (wt\%) & 4.146 & 4.208 & 0.062 & $1.5 \%$ & No & 0.1794 & 4.33 & 0.1340 & 0.1375 & No & 16 & 10 \\
\hline 5 & Isolok-HFR-3mL & $\mathrm{BaO}(\mathrm{wt} \%)$ & 0.103 & 0.102 & -0.001 & $-1.1 \%$ & No & 0.0033 & 3.21 & 0.0031 & 0.0022 & Yes & 16 & 10 \\
\hline 5 & Isolok-HFR-3mL & $\mathrm{CaO}(\mathrm{wt} \%)$ & 1.206 & 1.199 & -0.006 & $-0.5 \%$ & No & 0.0411 & 3.41 & 0.0454 & 0.0352 & No & 16 & 10 \\
\hline 5 & Isolok-HFR-3mL & Cr2O3 (wt\%) & 0.107 & 0.103 & -0.004 & $-3.8 \%$ & Yes & 0.0077 & 7.13 & 0.0049 & 0.0032 & No & 16 & 10 \\
\hline 5 & Isolok-HFR-3mL & $\mathrm{CuO}(\mathrm{wt} \%)$ & 0.025 & 0.025 & 0.000 & $1.2 \%$ & No & 0.0015 & 6.14 & 0.0014 & 0.0016 & No & 16 & 10 \\
\hline 5 & Isolok-HFR-3mL & $\mathrm{Fe} / \mathrm{Li}$ & 2.402 & 2.335 & -0.067 & $-2.8 \%$ & No & 0.1717 & 7.15 & 0.1554 & 0.0452 & No & 16 & 10 \\
\hline 5 & Isolok-HFR-3mL & Fe2O3 (wt\%) & 11.357 & 11.213 & -0.144 & $-1.3 \%$ & No & 0.4490 & 3.95 & 0.4035 & 0.2946 & No & 16 & 10 \\
\hline 5 & Isolok-HFR-3mL & K2O (wt\%) & 0.163 & 0.165 & 0.002 & $1.2 \%$ & No & 0.0101 & 6.16 & 0.0092 & 0.0106 & No & 16 & 10 \\
\hline 5 & Isolok-HFR-3mL & Li2O (wt\%) & 4.740 & 4.803 & 0.063 & $1.3 \%$ & No & 0.2359 & 4.98 & 0.2398 & 0.1404 & No & 16 & 10 \\
\hline 5 & Isolok-HFR-3mL & $\mathrm{MgO}(\mathrm{wt} \%)$ & 1.043 & 1.029 & -0.014 & $-1.4 \%$ & No & 0.0384 & 3.68 & 0.0364 & 0.0232 & Yes & 16 & 10 \\
\hline 5 & Isolok-HFR-3mL & $\mathrm{MnO}(\mathrm{wt} \%)$ & 1.733 & 1.710 & -0.023 & $-1.3 \%$ & No & 0.0675 & 3.89 & 0.0708 & 0.0386 & Yes & 16 & 10 \\
\hline 5 & Isolok-HFR-3mL & $\mathrm{Na} 2 \mathrm{O}(\mathrm{wt} \%)$ & 11.141 & 10.812 & -0.329 & $-3.0 \%$ & No & 0.6819 & 6.12 & 0.3820 & 0.4867 & No & 16 & 10 \\
\hline 5 & Isolok-HFR-3mL & $\mathrm{NiO}$ (wt\%) & 0.446 & 0.440 & -0.006 & $-1.3 \%$ & No & 0.0241 & 5.41 & 0.0260 & 0.0120 & No & 16 & 10 \\
\hline 5 & Isolok-HFR-3mL & $\mathrm{SiO} 2(\mathrm{wt} \%)$ & 51.210 & 52.071 & 0.861 & $1.7 \%$ & No & 2.3405 & 4.57 & 1.9433 & 1.4621 & No & 16 & 10 \\
\hline 5 & Isolok-HFR-3mL & Sum (wt\%) & 98.710 & 99.021 & 0.311 & $0.3 \%$ & No & 2.3698 & 2.40 & 2.3880 & 2.6127 & No & 16 & 10 \\
\hline 5 & $\begin{array}{l}\text { Isolok-HFR-3mL } \\
\end{array}$ & $\mathrm{TiO} 2$ (wt\%) & 0.082 & 0.084 & 0.002 & $2.0 \%$ & No & 0.0036 & 4.40 & 0.0030 & 0.0018 & Yes & 16 & 10 \\
\hline 5 & Isolok-HFR-3mL & $\mathrm{ZnO}(\mathrm{wt} \%)$ & 0.100 & 0.100 & 0.000 & $-0.4 \%$ & No & 0.0035 & 3.53 & 0.0038 & 0.0037 & No & 16 & 10 \\
\hline 5 & Isolok-HFR-3mL & $\mathrm{ZrO} 2$ (wt\%) & 0.360 & 0.361 & 0.001 & $0.2 \%$ & No & 0.0090 & 2.49 & 0.0094 & 0.0106 & No & 16 & 10 \\
\hline 6 & $\begin{array}{l}\text { Hydragard-LFR } \\
\end{array}$ & $\mathrm{Al} / \mathrm{B}$ & 2.672 & 2.654 & -0.018 & $-0.7 \%$ & No & 0.1239 & 4.64 & 0.1292 & 0.1243 & No & 16 & 10 \\
\hline 6 & Hydragard-LFR & $\mathrm{Al} 2 \mathrm{O} 3$ (wt\%) & 10.919 & 11.248 & 0.329 & $3.0 \%$ & No & 0.6749 & 6.18 & 0.4633 & 0.3202 & No & 16 & 10 \\
\hline 6 & Hydragard-LFR & B2O3 (wt\%) & 4.089 & 4.244 & 0.155 & $3.8 \%$ & Yes & 0.2692 & 6.58 & 0.1222 & 0.1605 & No & 16 & 10 \\
\hline 6 & Hydragard-LFR & $\mathrm{BaO}(\mathrm{wt} \%)$ & 0.103 & 0.106 & 0.003 & $2.9 \%$ & No & 0.0062 & 5.97 & 0.0040 & 0.0034 & No & 16 & 10 \\
\hline 6 & Hydragard-LFR & $\mathrm{CaO}$ (wt\%) & 1.208 & 1.257 & 0.049 & $4.0 \%$ & Yes & 0.0917 & 7.59 & 0.0515 & 0.0519 & No & 16 & 10 \\
\hline 6 & Hydragard-LFR & Cr2O3 (wt\%) & 0.107 & 0.106 & 0.000 & $-0.4 \%$ & No & 0.0050 & 4.68 & 0.0060 & 0.0044 & No & 16 & 10 \\
\hline 6 & Hydragard-LFR & $\mathrm{CuO}(\mathrm{wt} \%)$ & 0.027 & 0.026 & -0.001 & $-4.0 \%$ & No & 0.0049 & 18.47 & 0.0057 & 0.0016 & No & 16 & 10 \\
\hline 6 & Hydragard-LFR & $\mathrm{Fe} / \mathrm{Li}$ & 2.413 & 2.427 & 0.014 & $0.6 \%$ & No & 0.1250 & 5.18 & 0.1466 & 0.1077 & No & 16 & 10 \\
\hline 6 & Hydragard-LFR & Fe2O3 (wt\%) & 11.523 & 11.844 & 0.320 & $2.8 \%$ & No & 0.6939 & 6.02 & 0.4939 & 0.3626 & No & 16 & 10 \\
\hline 6 & Hydragard-LFR & K2O (wt\%) & 0.163 & 0.166 & 0.003 & $1.9 \%$ & No & 0.0112 & 6.86 & 0.0092 & 0.0104 & No & 16 & 10 \\
\hline 6 & Hydragard-LFR & Li2O (wt\%) & 4.783 & 4.885 & 0.101 & $2.1 \%$ & No & 0.2594 & 5.42 & 0.1980 & 0.1753 & No & 16 & 10 \\
\hline 6 & Hydragard-LFR & $\mathrm{MgO}(\mathrm{wt} \%)$ & 1.042 & 1.075 & 0.033 & $3.1 \%$ & No & 0.0693 & 6.65 & 0.0444 & 0.0431 & No & 16 & 10 \\
\hline 6 & Hydragard-LFR & $\mathrm{MnO}$ (wt\%) & 1.729 & 1.786 & 0.057 & $3.3 \%$ & No & 0.1215 & 7.03 & 0.0854 & 0.0618 & No & 16 & 10 \\
\hline 6 & Hydragard-LFR & $\mathrm{Na} 2 \mathrm{O}$ (wt\%) & 11.343 & 11.616 & 0.272 & $2.4 \%$ & No & 0.6274 & 5.53 & 0.3577 & 0.5221 & No & 16 & 10 \\
\hline 6 & Hydragard-LFR & $\mathrm{NiO}$ (wt\%) & 0.452 & 0.464 & 0.011 & $2.5 \%$ & No & 0.0330 & 7.29 & 0.0310 & 0.0138 & No & 16 & 10 \\
\hline 6 & Hydragard-LFR & $\mathrm{SiO} 2$ (wt\%) & 51.998 & 52.242 & 0.243 & $0.5 \%$ & No & 1.5168 & 2.92 & 1.5942 & 1.4183 & No & 16 & 10 \\
\hline 6 & Hydragard-LFR & Sum (wt\%) & 100.032 & 101.618 & 1.586 & $1.6 \%$ & No & 3.2932 & 3.29 & 2.0987 & 1.9700 & No & 16 & 10 \\
\hline
\end{tabular}


SRNL-STI-2011-00693

Revision 0

Table A8. Oxide Comparions between Samplers for the Mid-Rheology (Phase 1) Testing

\begin{tabular}{|c|c|c|c|c|c|c|c|c|c|c|c|c|c|c|}
\hline $\begin{array}{c}\text { Analytical } \\
\text { Block }\end{array}$ & Sampler & Oxide & $\begin{array}{c}\text { Mean(Measured } \\
\text { wt\%) Coliwasa } \\
\text { (low+high) }\end{array}$ & $\begin{array}{c}\text { Mean(Measured } \\
\text { wt\%) Other } \\
\text { Sampler }\end{array}$ & Difference & $\%$ Diff & $\begin{array}{l}\text { Sig. } \\
\text { Diff }\end{array}$ & $\begin{array}{l}\text { Bias } \\
\text { Bnd }\end{array}$ & $\begin{array}{l}\% \text { Rel } \\
\text { Bias } \\
\text { Bnd }\end{array}$ & $\begin{array}{c}\text { Std } \\
\text { Dev(Measured } \\
\text { wt\%) } \\
\text { Coliwasa } \\
\text { (low+high) }\end{array}$ & $\begin{array}{c}\text { Std } \\
\text { Dev(Measured } \\
\text { wt\%) Other } \\
\text { Sampler }\end{array}$ & $\begin{array}{l}\text { Sig. } \\
\text { Diff }\end{array}$ & $\begin{array}{c}\text { n } \\
\text { Col }\end{array}$ & $\begin{array}{c}\text { n } \\
\text { Other }\end{array}$ \\
\hline 6 & Hydragard-LFR & TiO2 (wt\%) & 0.080 & 0.081 & 0.000 & $0.3 \%$ & No & 0.0022 & 2.69 & 0.0019 & 0.0029 & No & 16 & 10 \\
\hline 6 & Hydragard-LFR & $\mathrm{ZnO}(\mathrm{wt} \%)$ & 0.101 & 0.105 & 0.003 & $3.3 \%$ & No & 0.0071 & 7.00 & 0.0050 & 0.0037 & No & 16 & 10 \\
\hline 6 & Hydragard-LFR & ZrO2 (wt\%) & 0.362 & 0.369 & 0.007 & $2.0 \%$ & No & 0.0154 & 4.26 & 0.0107 & 0.0089 & No & 16 & 10 \\
\hline
\end{tabular}


SRNL-STI-2011-00693

Revision 0

Table A9. Oxide Comparions between Samplers for the Mid-Rheology (Phase 1) Testing (screened data)

\begin{tabular}{|c|c|c|c|c|c|c|c|c|c|c|c|c|c|c|}
\hline $\begin{array}{c}\text { Analytical } \\
\text { Block }\end{array}$ & Sampler & Oxide & $\begin{array}{c}\text { Mean(Measured } \\
\text { wt\%) Coliwasa } \\
\text { (low+high) }\end{array}$ & $\begin{array}{c}\text { Mean(Measured } \\
\text { wt\%) Other } \\
\text { Sampler } \\
\end{array}$ & Diff & \%Diff & $\begin{array}{l}\text { Sig. } \\
\text { Diff }\end{array}$ & $\begin{array}{l}\text { Bias } \\
\text { Bnd } \\
\end{array}$ & $\begin{array}{c}\% \\
\text { Rel } \\
\text { Bias } \\
\text { Bnd } \\
\end{array}$ & $\begin{array}{c}\text { Std } \\
\text { Dev(Measured } \\
\text { wt\%) Coliwasa } \\
\text { (low+high) } \\
\end{array}$ & $\begin{array}{c}\text { Std } \\
\text { Dev(Measured } \\
\text { wt\%) Other } \\
\text { Sampler } \\
\end{array}$ & $\begin{array}{l}\text { Sig. } \\
\text { Diff }\end{array}$ & $\begin{array}{c}\text { n } \\
\text { Col }\end{array}$ & $\begin{array}{c}\text { n } \\
\text { Other }\end{array}$ \\
\hline 1 & Isolok-LFR-3mL & $\mathrm{Al} / \mathrm{B}$ & 2.572 & 2.725 & 0.153 & $5.9 \%$ & Yes & 0.2069 & 8.04 & 0.0597 & 0.0702 & No & 15 & 10 \\
\hline 1 & Isolok-LFR-3mL & $\mathrm{Al2O} 3(\mathrm{wt} \%)$ & 10.767 & 10.927 & 0.160 & $1.5 \%$ & No & 0.4089 & 3.80 & 0.3358 & 0.2234 & No & 16 & 10 \\
\hline 1 & Isolok-LFR-3mL & B2O3 (wt\%) & 4.151 & 4.012 & -0.139 & $-3.4 \%$ & Yes & 0.2283 & 5.50 & 0.0720 & 0.1368 & No & 14 & 10 \\
\hline 1 & Isolok-LFR-3mL & $\mathrm{BaO}(\mathrm{wt} \%)$ & 0.103 & 0.106 & 0.002 & $2.2 \%$ & Yes & 0.0043 & 4.14 & 0.0027 & 0.0015 & No & 16 & 9 \\
\hline 1 & Isolok-LFR-3mL & $\mathrm{CaO}$ (wt\%) & 1.201 & 1.222 & 0.021 & $1.7 \%$ & No & 0.0495 & 4.12 & 0.0397 & 0.0236 & No & 16 & 10 \\
\hline 1 & Isolok-LFR-3mL & Cr2O3 (wt\%) & 0.105 & 0.103 & -0.002 & $-1.9 \%$ & No & 0.0050 & 4.73 & 0.0034 & 0.0037 & No & 15 & 10 \\
\hline 1 & Isolok-LFR-3mL & $\mathrm{CuO}(\mathrm{wt} \%)$ & 0.025 & 0.025 & 0.000 & $1.3 \%$ & No & 0.0014 & 5.58 & 0.0014 & 0.0010 & No & 16 & 10 \\
\hline 1 & Isolok-LFR-3mL & $\mathrm{Fe} / \mathrm{Li}$ & 2.412 & 2.465 & 0.053 & $2.2 \%$ & No & 0.1171 & 4.86 & 0.0734 & 0.0750 & No & 14 & 10 \\
\hline 1 & Isolok-LFR-3mL & Fe2O3 (wt\%) & 11.405 & 11.592 & 0.188 & $1.6 \%$ & No & 0.4594 & 4.03 & 0.3770 & 0.2191 & No & 16 & 10 \\
\hline 1 & Isolok-LFR-3mL & K2O (wt\%) & 0.163 & 0.165 & 0.002 & $1.5 \%$ & No & 0.0094 & 5.74 & 0.0092 & 0.0068 & No & 16 & 10 \\
\hline 1 & Isolok-LFR-3mL & Li2O (wt\%) & 4.709 & 4.704 & -0.005 & $-0.1 \%$ & No & 0.1007 & 2.14 & 0.1306 & 0.0794 & No & 15 & 10 \\
\hline 1 & Isolok-LFR-3mL & $\mathrm{MgO}(\mathrm{wt} \%)$ & 1.058 & 1.075 & 0.017 & $1.6 \%$ & No & 0.0382 & 3.61 & 0.0351 & 0.0162 & Yes & 16 & 10 \\
\hline 1 & Isolok-LFR-3mL & $\mathrm{MnO}(\mathrm{wt} \%)$ & 1.750 & 1.786 & 0.035 & $2.0 \%$ & No & 0.0836 & 4.78 & 0.0670 & 0.0385 & No & 16 & 10 \\
\hline 1 & Isolok-LFR-3mL & $\mathrm{Na} 2 \mathrm{O}(\mathrm{wt} \%)$ & 11.249 & 11.198 & -0.051 & $-0.5 \%$ & No & 0.4892 & 4.35 & 0.3356 & 0.5824 & Yes & 16 & 10 \\
\hline 1 & Isolok-LFR-3mL & $\mathrm{NiO}(\mathrm{wt} \%)$ & 0.452 & 0.459 & 0.008 & $1.7 \%$ & No & 0.0187 & 4.14 & 0.0148 & 0.0097 & No & 14 & 10 \\
\hline 1 & Isolok-LFR-3mL & $\mathrm{SiO} 2(\mathrm{wt} \%)$ & 51.731 & 51.728 & -0.003 & $0.0 \%$ & No & 0.7290 & 1.41 & 1.3262 & 0.2992 & Yes & 16 & 10 \\
\hline 1 & Isolok-LFR-3mL & Sum (wt\%) & 99.584 & 99.660 & 0.076 & $0.1 \%$ & No & 0.3001 & 0.30 & 0.2946 & 0.2124 & No & 15 & 10 \\
\hline 1 & Isolok-LFR-3mL & TiO2 (wt\%) & 0.082 & 0.082 & 0.000 & $-0.6 \%$ & No & 0.0021 & 2.55 & 0.0027 & 0.0013 & Yes & 16 & 10 \\
\hline 1 & Isolok-LFR-3mL & $\mathrm{ZnO}(\mathrm{wt} \%)$ & 0.101 & 0.102 & 0.001 & $0.8 \%$ & No & 0.0035 & 3.44 & 0.0037 & 0.0020 & No & 16 & 10 \\
\hline 1 & Isolok-LFR-3mL & $\mathrm{ZrO} 2$ (wt\%) & 0.371 & 0.374 & 0.002 & $0.7 \%$ & No & 0.0075 & 2.03 & 0.0056 & 0.0069 & No & 16 & 10 \\
\hline 2 & Hydragard - HFR & $\mathrm{Al} / \mathrm{B}$ & 2.620 & 2.748 & 0.128 & $4.9 \%$ & Yes & 0.1755 & 6.70 & 0.0514 & 0.0603 & No & 15 & 9 \\
\hline 2 & Hydragard - HFR & $\mathrm{Al} 2 \mathrm{O} 3(\mathrm{wt} \%)$ & 10.906 & 11.265 & 0.359 & $3.3 \%$ & Yes & 0.5665 & 5.19 & 0.2723 & 0.1971 & No & 15 & 10 \\
\hline 2 & Hydragard - HFR & B2O3 (wt\%) & 4.162 & 4.131 & -0.031 & $-0.7 \%$ & No & 0.0985 & 2.37 & 0.0774 & 0.0832 & No & 15 & 10 \\
\hline 2 & Hydragard - HFR & $\mathrm{BaO}(\mathrm{wt} \%)$ & 0.106 & 0.108 & 0.002 & $2.1 \%$ & No & 0.0048 & 4.51 & 0.0033 & 0.0025 & No & 16 & 10 \\
\hline 2 & Hydragard - HFR & $\mathrm{CaO}(\mathrm{wt} \%)$ & 1.211 & 1.250 & 0.039 & $3.2 \%$ & Yes & 0.0657 & 5.43 & 0.0347 & 0.0285 & No & 16 & 10 \\
\hline 2 & Hydragard - HFR & Cr2O3 (wt\%) & 0.110 & 0.111 & 0.001 & $1.2 \%$ & No & 0.0041 & 3.70 & 0.0038 & 0.0024 & No & 15 & 10 \\
\hline 2 & Hydragard - HFR & $\mathrm{CuO}$ (wt\%) & 0.025 & 0.025 & 0.000 & $0.1 \%$ & No & 0.0014 & 5.42 & 0.0014 & 0.0019 & No & 16 & 10 \\
\hline 2 & Hydragard - HFR & $\mathrm{Fe} / \mathrm{Li}$ & 2.411 & 2.455 & 0.044 & $1.8 \%$ & No & 0.1223 & 5.07 & 0.1017 & 0.0768 & No & 15 & 10 \\
\hline 2 & Hydragard - HFR & Fe2O3 (wt\%) & 11.463 & 11.732 & 0.270 & $2.4 \%$ & No & 0.5518 & 4.81 & 0.3843 & 0.2465 & No & 16 & 10 \\
\hline 2 & Hydragard - HFR & $\mathrm{K} 2 \mathrm{O}(\mathrm{wt} \%)$ & 0.163 & 0.163 & 0.001 & $0.4 \%$ & No & 0.0092 & 5.62 & 0.0088 & 0.0123 & No & 16 & 10 \\
\hline 2 & Hydragard - HFR & $\mathrm{Li} 2 \mathrm{O}$ (wt\%) & 4.777 & 4.794 & 0.017 & $0.4 \%$ & No & 0.0852 & 1.78 & 0.1139 & 0.0417 & Yes & 14 & 10 \\
\hline 2 & Hydragard - HFR & $\mathrm{MgO}(\mathrm{wt} \%)$ & 1.074 & 1.091 & 0.018 & $1.7 \%$ & No & 0.0458 & 4.27 & 0.0361 & 0.0288 & No & 16 & 10 \\
\hline 2 & Hydragard - HFR & $\mathrm{MnO}(\mathrm{wt} \%)$ & 1.785 & 1.822 & 0.037 & $2.1 \%$ & No & 0.0889 & 4.98 & 0.0708 & 0.0457 & No & 16 & 10 \\
\hline 2 & Hydragard - HFR & $\mathrm{Na} 2 \mathrm{O}$ (wt\%) & 11.311 & 11.399 & 0.088 & $0.8 \%$ & No & 0.3822 & 3.38 & 0.3543 & 0.3518 & No & 16 & 10 \\
\hline 2 & Hydragard - HFR & $\mathrm{NiO}$ (wt\%) & 0.465 & 0.478 & 0.013 & $2.8 \%$ & No & 0.0289 & 6.21 & 0.0212 & 0.0145 & No & 15 & 10 \\
\hline 2 & Hydragard - HFR & $\mathrm{SiO} 2(\mathrm{wt} \%)$ & 52.052 & 51.579 & -0.473 & $-0.9 \%$ & No & 1.3620 & 2.62 & 1.2095 & 0.7776 & No & 16 & 10 \\
\hline 2 & Hydragard - HFR & Sum (wt\%) & 100.332 & 100.496 & 0.164 & $0.2 \%$ & No & 0.3991 & 0.40 & 0.3112 & 0.2163 & No & 15 & 10 \\
\hline 2 & Hydragard - HFR & $\mathrm{TiO} 2(\mathrm{wt} \%)$ & 0.083 & 0.082 & -0.001 & $-1.0 \%$ & No & 0.0031 & 3.71 & 0.0013 & 0.0031 & Yes & 15 & 10 \\
\hline 2 & Hydragard - HFR & $\mathrm{ZnO}$ (wt\%) & 0.103 & 0.105 & 0.003 & $2.7 \%$ & Yes & 0.0055 & 5.32 & 0.0038 & 0.0019 & No & 16 & 10 \\
\hline 2 & Hydragard - HFR & $\mathrm{ZrO} 2$ (wt\%) & 0.369 & 0.375 & 0.006 & $1.7 \%$ & Yes & 0.0109 & 2.96 & 0.0055 & 0.0049 & No & 15 & 10 \\
\hline
\end{tabular}


Table A9. Oxide Comparions between Samplers for the Mid-Rheology (Phase 1) Testing (screened data)

\begin{tabular}{|c|c|c|c|c|c|c|c|c|c|c|c|c|c|c|}
\hline $\begin{array}{c}\text { Analytical } \\
\text { Block }\end{array}$ & Sampler & Oxide & $\begin{array}{c}\text { Mean(Measured } \\
\text { wt\%) Coliwasa } \\
\text { (low+high) }\end{array}$ & $\begin{array}{l}\text { Mean(Measured } \\
\text { wt\%) Other } \\
\text { Sampler }\end{array}$ & Diff & \%Diff & $\begin{array}{l}\text { Sig. } \\
\text { Diff }\end{array}$ & $\begin{array}{l}\text { Bias } \\
\text { Bnd }\end{array}$ & $\begin{array}{c}\% \\
\text { Rel } \\
\text { Bias } \\
\text { Bnd }\end{array}$ & $\begin{array}{c}\text { Std } \\
\text { Dev(Measured } \\
\text { wt\%) Coliwasa } \\
\text { (low+high) }\end{array}$ & $\begin{array}{c}\text { Std } \\
\text { Dev(Measured } \\
\text { wt\%) Other } \\
\text { Sampler }\end{array}$ & $\begin{array}{l}\text { Sig. } \\
\text { Diff }\end{array}$ & $\stackrel{\mathbf{n}}{\mathrm{Col}}$ & $\begin{array}{c}\text { n } \\
\text { Other }\end{array}$ \\
\hline 3 & Isolok-LFR-12mL & $\mathrm{Al} / \mathrm{B}$ & 2.587 & 2.580 & -0.007 & $-0.3 \%$ & No & 0.0781 & 3.02 & 0.0928 & 0.0672 & No & 15 & 10 \\
\hline 3 & Isolok-LFR-12mL & $\mathrm{Al} 2 \mathrm{O} 3$ (wt\%) & 10.868 & 11.176 & 0.308 & $2.8 \%$ & No & 0.6640 & 6.11 & 0.4601 & 0.3672 & No & 16 & 10 \\
\hline 3 & Isolok-LFR-12mL & $\mathrm{B} 2 \mathrm{O} 3(\mathrm{wt} \%)$ & 4.164 & 4.368 & 0.205 & $4.9 \%$ & Yes & 0.3228 & 7.75 & 0.1500 & 0.1092 & No & 16 & 9 \\
\hline 3 & Isolok-LFR-12mL & $\mathrm{BaO}(\mathrm{wt} \%)$ & 0.103 & 0.107 & 0.004 & $4.0 \%$ & Yes & 0.0073 & 7.10 & 0.0040 & 0.0011 & No & 16 & 10 \\
\hline 3 & Isolok-LFR-12mL & $\mathrm{CaO}(\mathrm{wt} \%)$ & 1.194 & 1.249 & 0.055 & $4.6 \%$ & Yes & 0.0960 & 8.04 & 0.0510 & 0.0479 & No & 16 & 10 \\
\hline 3 & Isolok-LFR-12mL & Cr2O3 (wt\%) & 0.108 & 0.110 & 0.002 & $2.2 \%$ & No & 0.0069 & 6.40 & 0.0062 & 0.0013 & No & 16 & 10 \\
\hline 3 & Isolok-LFR-12mL & $\mathrm{CuO}(\mathrm{wt} \%)$ & 0.025 & 0.026 & 0.001 & $4.3 \%$ & No & 0.0022 & 8.57 & 0.0014 & 0.0011 & No & 16 & 10 \\
\hline 3 & Isolok-LFR-12mL & $\mathrm{Fe} / \mathrm{Li}$ & 2.417 & 2.450 & 0.034 & $1.4 \%$ & No & 0.1053 & 4.36 & 0.1017 & 0.0470 & No & 15 & 10 \\
\hline 3 & Isolok-LFR-12mL & Fe2O3 (wt\%) & 11.214 & 11.599 & 0.385 & $3.4 \%$ & Yes & 0.7611 & 6.79 & 0.4704 & 0.4200 & No & 16 & 10 \\
\hline 3 & Isolok-LFR-12mL & $\mathrm{K} 2 \mathrm{O}(\mathrm{wt} \%)$ & 0.163 & 0.170 & 0.007 & $4.2 \%$ & No & 0.0143 & 8.74 & 0.0092 & 0.0074 & No & 16 & 9 \\
\hline 3 & Isolok-LFR-12mL & Li2O (wt\%) & 4.695 & 4.734 & 0.040 & $0.8 \%$ & No & 0.2057 & 4.38 & 0.2169 & 0.1672 & No & 16 & 10 \\
\hline 3 & Isolok-LFR-12mL & $\mathrm{MgO}(\mathrm{wt} \%)$ & 1.051 & 1.089 & 0.038 & $3.7 \%$ & Yes & 0.0763 & 7.26 & 0.0455 & 0.0457 & No & 16 & 10 \\
\hline 3 & Isolok-LFR-12mL & $\mathrm{MnO}(\mathrm{wt} \%)$ & 1.748 & 1.817 & 0.069 & $3.9 \%$ & Yes & 0.1356 & 7.76 & 0.0862 & 0.0697 & No & 16 & 10 \\
\hline 3 & Isolok-LFR-12mL & Na2O (wt\%) & 11.008 & 11.251 & 0.243 & $2.2 \%$ & No & 0.6232 & 5.66 & 0.4252 & 0.4690 & No & 16 & 9 \\
\hline 3 & Isolok-LFR-12mL & $\mathrm{NiO}(\mathrm{wt} \%)$ & 0.451 & 0.466 & 0.016 & $3.5 \%$ & No & 0.0369 & 8.18 & 0.0306 & 0.0136 & No & 16 & 10 \\
\hline 3 & Isolok-LFR-12mL & $\mathrm{SiO} 2$ (wt\%) & 51.343 & 51.750 & 0.406 & $0.8 \%$ & No & 1.3866 & 2.70 & 1.2553 & 0.9955 & No & 15 & 10 \\
\hline 3 & Isolok-LFR-12mL & Sum (wt\%) & 98.401 & 101.037 & 2.636 & $2.7 \%$ & Yes & 4.8331 & 4.91 & 2.3685 & 3.0410 & No & 16 & 10 \\
\hline 3 & Isolok-LFR-12mL & $\mathrm{TiO} 2$ (wt\%) & 0.082 & 0.083 & 0.000 & $0.4 \%$ & No & 0.0031 & 3.71 & 0.0033 & 0.0034 & No & 16 & 10 \\
\hline 3 & Isolok-LFR-12mL & $\mathrm{ZnO}(\mathrm{wt} \%)$ & 0.100 & 0.105 & 0.005 & $4.8 \%$ & Yes & 0.0084 & 8.40 & 0.0047 & 0.0035 & No & 16 & 10 \\
\hline 3 & Isolok-LFR-12mL & $\mathrm{ZrO} 2$ (wt\%) & 0.364 & 0.372 & 0.008 & $2.3 \%$ & No & 0.0167 & 4.60 & 0.0097 & 0.0108 & No & 16 & 10 \\
\hline 4 & Isolok-HFR-12mL & $\mathrm{Al} / \mathrm{B}$ & 2.596 & 2.534 & -0.061 & $-2.4 \%$ & No & 0.1823 & 7.02 & 0.0621 & 0.1656 & Yes & 15 & 10 \\
\hline 4 & Isolok-HFR-12mL & $\mathrm{Al2O}$ (wt\%) & 10.790 & 10.768 & -0.022 & $-0.2 \%$ & No & 0.2899 & 2.69 & 0.2424 & 0.4071 & No & 15 & 10 \\
\hline 4 & Isolok-HFR-12mL & $\mathrm{B} 2 \mathrm{O} 3(\mathrm{wt} \%)$ & 4.142 & 4.257 & 0.115 & $2.8 \%$ & Yes & 0.2161 & 5.22 & 0.1031 & 0.1470 & No & 16 & 10 \\
\hline 4 & Isolok-HFR-12mL & $\mathrm{BaO}(\mathrm{wt} \%)$ & 0.104 & 0.104 & 0.001 & $0.6 \%$ & No & 0.0033 & 3.21 & 0.0028 & 0.0039 & No & 16 & 10 \\
\hline 4 & Isolok-HFR-12mL & $\mathrm{CaO}(\mathrm{wt} \%)$ & 1.197 & 1.201 & 0.003 & $0.3 \%$ & No & 0.0425 & 3.55 & 0.0386 & 0.0587 & No & 16 & 10 \\
\hline 4 & Isolok-HFR-12mL & Cr2O3 (wt\%) & 0.107 & 0.107 & 0.000 & $0.2 \%$ & No & 0.0052 & 4.82 & 0.0055 & 0.0065 & No & 16 & 10 \\
\hline 4 & Isolok-HFR-12mL & $\mathrm{CuO}(\mathrm{wt} \%)$ & 0.025 & 0.027 & 0.002 & $7.2 \%$ & Yes & 0.0029 & 11.69 & 0.0014 & 0.0011 & No & 16 & 9 \\
\hline 4 & Isolok-HFR-12mL & $\mathrm{Fe} / \mathrm{Li}$ & 2.400 & 2.367 & -0.033 & $-1.4 \%$ & No & 0.1518 & 6.32 & 0.0760 & 0.1598 & Yes & 14 & 10 \\
\hline 4 & Isolok-HFR-12mL & Fe2O3 (wt\%) & 11.468 & 11.365 & -0.103 & $-0.9 \%$ & No & 0.4822 & 4.20 & 0.3692 & 0.5708 & No & 16 & 10 \\
\hline 4 & Isolok-HFR-12mL & $\mathrm{K} 2 \mathrm{O}(\mathrm{wt} \%)$ & 0.163 & 0.172 & 0.009 & $5.4 \%$ & Yes & 0.0175 & 10.76 & 0.0093 & 0.0112 & No & 15 & 9 \\
\hline 4 & Isolok-HFR-12mL & $\mathrm{Li} 2 \mathrm{O}$ (wt\%) & 4.783 & 4.777 & -0.005 & $-0.1 \%$ & No & 0.0830 & 1.73 & 0.0932 & 0.0765 & No & 14 & 9 \\
\hline 4 & Isolok-HFR-12mL & $\mathrm{MgO}(\mathrm{wt} \%)$ & 1.061 & 1.051 & -0.010 & $-0.9 \%$ & No & 0.0455 & 4.29 & 0.0346 & 0.0541 & No & 16 & 10 \\
\hline 4 & Isolok-HFR-12mL & $\mathrm{MnO}(\mathrm{wt} \%)$ & 1.755 & 1.742 & -0.013 & $-0.8 \%$ & No & 0.0770 & 4.39 & 0.0690 & 0.0893 & No & 16 & 10 \\
\hline 4 & Isolok-HFR-12mL & $\mathrm{Na} 2 \mathrm{O}$ (wt\%) & 11.063 & 10.748 & -0.315 & $-2.9 \%$ & Yes & 0.6128 & 5.54 & 0.3266 & 0.4041 & No & 16 & 10 \\
\hline 4 & Isolok-HFR-12mL & $\mathrm{NiO}(\mathrm{wt} \%)$ & 0.451 & 0.443 & -0.008 & $-1.7 \%$ & No & 0.0295 & 6.55 & 0.0208 & 0.0320 & No & 15 & 10 \\
\hline 4 & Isolok-HFR-12mL & $\mathrm{SiO} 2(\mathrm{wt} \%)$ & 51.370 & 52.113 & 0.743 & $1.4 \%$ & No & 1.7839 & 3.47 & 1.3254 & 1.1148 & No & 16 & 10 \\
\hline 4 & Isolok-HFR-12mL & Sum (wt\%) & 99.249 & 99.337 & 0.088 & $0.1 \%$ & No & 0.3591 & 0.36 & 0.2867 & 0.3481 & No & 15 & 9 \\
\hline 4 & Isolok-HFR-12mL & $\mathrm{TiO} 2$ (wt\%) & 0.083 & 0.083 & 0.000 & $0.0 \%$ & No & 0.0014 & 1.69 & 0.0019 & 0.0012 & No & 16 & 10 \\
\hline 4 & Isolok-HFR-12mL & $\mathrm{ZnO}(\mathrm{wt} \%)$ & 0.101 & 0.101 & 0.000 & $0.1 \%$ & No & 0.0033 & 3.31 & 0.0036 & 0.0043 & No & 16 & 10 \\
\hline 4 & Isolok-HFR-12mL & $\mathrm{ZrO} 2$ (wt\%) & 0.365 & 0.362 & -0.003 & $-0.7 \%$ & No & 0.0082 & 2.25 & 0.0052 & 0.0085 & No & 16 & 10 \\
\hline
\end{tabular}


Table A9. Oxide Comparions between Samplers for the Mid-Rheology (Phase 1) Testing (screened data)

\begin{tabular}{|c|c|c|c|c|c|c|c|c|c|c|c|c|c|c|}
\hline $\begin{array}{l}\text { Analytical } \\
\text { Block }\end{array}$ & Sampler & Oxide & $\begin{array}{c}\text { Mean(Measured } \\
\text { wt \%) Coliwasa } \\
\text { (low+high) }\end{array}$ & $\begin{array}{l}\text { Mean(Measured } \\
\text { wt\%) Other } \\
\text { Sampler }\end{array}$ & Diff & \%Diff & $\begin{array}{l}\text { Sig. } \\
\text { Diff }\end{array}$ & $\begin{array}{l}\text { Bias } \\
\text { Bnd }\end{array}$ & $\begin{array}{c}\% \\
\text { Rel } \\
\text { Bias } \\
\text { Bnd }\end{array}$ & $\begin{array}{c}\text { Std } \\
\text { Dev(Measured } \\
\text { wt\%) Coliwasa } \\
\text { (low+high) }\end{array}$ & $\begin{array}{c}\text { Std } \\
\text { Dev(Measured } \\
\text { wt\%) Other } \\
\text { Sampler }\end{array}$ & $\begin{array}{l}\text { Sig. } \\
\text { Diff }\end{array}$ & $\stackrel{\mathbf{n}}{\mathrm{Col}}$ & $\begin{array}{c}\mathbf{n} \\
\text { Other }\end{array}$ \\
\hline 5 & Isolok-HFR-3mL & $\mathrm{Al} / \mathrm{B}$ & 2.574 & 2.518 & -0.055 & $-2.1 \%$ & Yes & 0.1078 & 4.19 & 0.0672 & 0.0537 & No & 15 & 10 \\
\hline 5 & Isolok-HFR-3mL & $\mathrm{Al} 2 \mathrm{O} 3(\mathrm{wt} \%)$ & 10.752 & 10.596 & -0.156 & $-1.5 \%$ & No & 0.4420 & 4.11 & 0.3507 & 0.3315 & No & 16 & 10 \\
\hline 5 & Isolok-HFR-3mL & B2O3 (wt\%) & 4.146 & 4.208 & 0.062 & $1.5 \%$ & No & 0.1794 & 4.33 & 0.1340 & 0.1375 & No & 16 & 10 \\
\hline 5 & Isolok-HFR-3mL & $\mathrm{BaO}(\mathrm{wt} \%)$ & 0.103 & 0.102 & -0.001 & $-0.6 \%$ & No & 0.0024 & 2.36 & 0.0031 & 0.0013 & Yes & 16 & 9 \\
\hline 5 & Isolok-HFR-3mL & $\mathrm{CaO}(\mathrm{wt} \%)$ & 1.206 & 1.199 & -0.006 & $-0.5 \%$ & No & 0.0411 & 3.41 & 0.0454 & 0.0352 & No & 16 & 10 \\
\hline 5 & Isolok-HFR-3mL & Cr2O3 (wt\%) & 0.107 & 0.103 & -0.004 & $-3.8 \%$ & Yes & 0.0077 & 7.13 & 0.0049 & 0.0032 & No & 16 & 10 \\
\hline 5 & Isolok-HFR-3mL & $\mathrm{CuO}(\mathrm{wt} \%)$ & 0.025 & 0.025 & 0.000 & $1.2 \%$ & No & 0.0015 & 6.14 & 0.0014 & 0.0016 & No & 16 & 10 \\
\hline 5 & Isolok-HFR-3mL & $\mathrm{Fe} / \mathrm{Li}$ & 2.407 & 2.325 & -0.082 & $-3.4 \%$ & Yes & 0.1359 & 5.65 & 0.0729 & 0.0324 & No & 14 & 9 \\
\hline 5 & Isolok-HFR-3mL & Fe2O3 (wt\%) & 11.357 & 11.290 & -0.067 & $-0.6 \%$ & No & 0.3096 & 2.73 & 0.4035 & 0.1771 & Yes & 16 & 9 \\
\hline 5 & Isolok-HFR-3mL & K2O (wt\%) & 0.163 & 0.165 & 0.002 & $1.2 \%$ & No & 0.0101 & 6.16 & 0.0092 & 0.0106 & No & 16 & 10 \\
\hline 5 & Isolok-HFR-3mL & Li2O (wt\%) & 4.740 & 4.837 & 0.096 & $2.0 \%$ & No & 0.2704 & 5.70 & 0.2398 & 0.0969 & No & 16 & 9 \\
\hline 5 & Isolok-HFR-3mL & $\mathrm{MgO}(\mathrm{wt} \%)$ & 1.043 & 1.035 & -0.008 & $-0.8 \%$ & No & 0.0259 & 2.48 & 0.0364 & 0.0142 & Yes & 16 & 9 \\
\hline 5 & Isolok-HFR-3mL & $\mathrm{MnO}(\mathrm{wt} \%)$ & 1.733 & 1.720 & -0.012 & $-0.7 \%$ & No & 0.0521 & 3.01 & 0.0708 & 0.0202 & Yes & 16 & 9 \\
\hline 5 & Isolok-HFR-3mL & $\mathrm{Na} 2 \mathrm{O}$ (wt\%) & 11.141 & 10.812 & -0.329 & $-3.0 \%$ & No & 0.6819 & 6.12 & 0.3820 & 0.4867 & No & 16 & 10 \\
\hline 5 & Isolok-HFR-3mL & $\mathrm{NiO}(\mathrm{wt} \%)$ & 0.446 & 0.440 & -0.006 & $-1.3 \%$ & No & 0.0241 & 5.41 & 0.0260 & 0.0120 & No & 16 & 10 \\
\hline 5 & Isolok-HFR-3mL & $\mathrm{SiO} 2(\mathrm{wt} \%)$ & 51.210 & 52.226 & 1.016 & $2.0 \%$ & No & 2.0956 & 4.09 & 1.9433 & 0.4775 & Yes & 16 & 8 \\
\hline 5 & Isolok-HFR-3mL & Sum (wt\%) & 98.710 & 99.715 & 1.005 & $1.0 \%$ & No & 2.8345 & 2.87 & 2.3880 & 1.5025 & No & 16 & 9 \\
\hline 5 & Isolok-HFR-3mL & $\mathrm{TiO} 2$ (wt\%) & 0.082 & 0.084 & 0.002 & $2.0 \%$ & No & 0.0036 & 4.40 & 0.0030 & 0.0018 & Yes & 16 & 10 \\
\hline 5 & Isolok-HFR-3mL & $\mathrm{ZnO}(\mathrm{wt} \%)$ & 0.100 & 0.100 & -0.001 & $-0.6 \%$ & No & 0.0029 & 2.86 & 0.0038 & 0.0016 & Yes & 16 & 8 \\
\hline 5 & Isolok-HFR-3mL & $\mathrm{ZrO} 2$ (wt\%) & 0.360 & 0.361 & 0.001 & $0.2 \%$ & No & 0.0090 & 2.49 & 0.0094 & 0.0106 & No & 16 & 10 \\
\hline 6 & Hydragard-LFR & $\mathrm{Al} / \mathrm{B}$ & 2.644 & 2.654 & 0.010 & $0.4 \%$ & No & 0.0664 & 2.51 & 0.0679 & 0.0487 & No & 15 & 8 \\
\hline 6 & Hydragard-LFR & $\mathrm{Al} 2 \mathrm{O} 3(\mathrm{wt} \%)$ & 10.919 & 11.248 & 0.329 & $3.0 \%$ & No & 0.6749 & 6.18 & 0.4633 & 0.3202 & No & 16 & 10 \\
\hline 6 & Hydragard-LFR & B2O3 (wt\%) & 4.089 & 4.244 & 0.155 & $3.8 \%$ & Yes & 0.2692 & 6.58 & 0.1222 & 0.1605 & No & 16 & 10 \\
\hline 6 & Hydragard-LFR & $\mathrm{BaO}(\mathrm{wt} \%)$ & 0.103 & 0.106 & 0.003 & $2.9 \%$ & No & 0.0062 & 5.97 & 0.0040 & 0.0034 & No & 16 & 10 \\
\hline 6 & Hydragard-LFR & $\mathrm{CaO}$ (wt\%) & 1.208 & 1.257 & 0.049 & $4.0 \%$ & Yes & 0.0917 & 7.59 & 0.0515 & 0.0519 & No & 16 & 10 \\
\hline 6 & Hydragard-LFR & Cr2O3 (wt\%) & 0.107 & 0.106 & 0.000 & $-0.4 \%$ & No & 0.0050 & 4.68 & 0.0060 & 0.0044 & No & 16 & 10 \\
\hline 6 & Hydragard-LFR & $\mathrm{CuO}(\mathrm{wt} \%)$ & 0.025 & 0.026 & 0.000 & $1.3 \%$ & No & 0.0015 & 6.02 & 0.0013 & 0.0016 & No & 15 & 10 \\
\hline 6 & Hydragard-LFR & $\mathrm{Fe} / \mathrm{Li}$ & 2.413 & 2.427 & 0.014 & $0.6 \%$ & No & 0.0930 & 3.86 & 0.0797 & 0.1077 & No & 14 & 10 \\
\hline 6 & Hydragard-LFR & Fe2O3 (wt\%) & 11.523 & 11.844 & 0.320 & $2.8 \%$ & No & 0.6939 & 6.02 & 0.4939 & 0.3626 & No & 16 & 10 \\
\hline 6 & Hydragard-LFR & $\mathrm{K} 2 \mathrm{O}$ (wt\%) & 0.163 & 0.166 & 0.003 & $1.9 \%$ & No & 0.0112 & 6.86 & 0.0092 & 0.0104 & No & 16 & 10 \\
\hline 6 & Hydragard-LFR & $\mathrm{Li} 2 \mathrm{O}(\mathrm{wt} \%)$ & 4.752 & 4.885 & 0.133 & $2.8 \%$ & No & 0.2725 & 5.73 & 0.1587 & 0.1753 & No & 15 & 10 \\
\hline 6 & Hydragard-LFR & $\mathrm{MgO}(\mathrm{wt} \%)$ & 1.042 & 1.086 & 0.044 & $4.2 \%$ & Yes & 0.0776 & 7.44 & 0.0444 & 0.0280 & No & 16 & 9 \\
\hline 6 & Hydragard-LFR & $\mathrm{MnO}(\mathrm{wt} \%)$ & 1.729 & 1.786 & 0.057 & $3.3 \%$ & No & 0.1215 & 7.03 & 0.0854 & 0.0618 & No & 16 & 10 \\
\hline 6 & Hydragard-LFR & $\mathrm{Na} 2 \mathrm{O}$ (wt\%) & 11.343 & 11.616 & 0.272 & $2.4 \%$ & No & 0.6274 & 5.53 & 0.3577 & 0.5221 & No & 16 & 10 \\
\hline 6 & Hydragard-LFR & $\mathrm{NiO}(\mathrm{wt} \%)$ & 0.452 & 0.464 & 0.011 & $2.5 \%$ & No & 0.0330 & 7.29 & 0.0310 & 0.0138 & No & 16 & 10 \\
\hline 6 & Hydragard-LFR & $\mathrm{SiO} 2(\mathrm{wt} \%)$ & 51.998 & 52.242 & 0.243 & $0.5 \%$ & No & 1.5168 & 2.92 & 1.5942 & 1.4183 & No & 16 & 10 \\
\hline 6 & Hydragard-LFR & Sum (wt\%) & 100.357 & 101.618 & 1.261 & $1.3 \%$ & No & 2.7912 & 2.78 & 1.7030 & 1.9700 & No & 15 & 10 \\
\hline 6 & Hydragard-LFR & TiO2 (wt\%) & 0.080 & 0.081 & 0.000 & $0.3 \%$ & No & 0.0022 & 2.69 & 0.0019 & 0.0029 & No & 16 & 10 \\
\hline 6 & Hydragard-LFR & $\mathrm{ZnO}(\mathrm{wt} \%)$ & 0.101 & 0.105 & 0.003 & $3.3 \%$ & No & 0.0071 & 7.00 & 0.0050 & 0.0037 & No & 16 & 10 \\
\hline 6 & Hydragard-LFR & $\mathrm{ZrO} 2(\mathrm{wt} \%)$ & 0.362 & 0.369 & 0.007 & $2.0 \%$ & No & 0.0154 & 4.26 & 0.0107 & 0.0089 & No & 16 & 10 \\
\hline
\end{tabular}


SRNL-STI-2011-00693

Revision 0

Table A10. Phase 1 (Mid-Rheology) Sampler Comparisons Grouped by Analytical Block (screened data)

\begin{tabular}{|c|c|c|c|c|c|c|c|c|c|c|c|c|c|c|}
\hline $\begin{array}{c}\text { Analytical } \\
\text { Block }\end{array}$ & Sampler & Oxide & $\begin{array}{c}\text { Mean } \\
\text { (Measured } \\
\text { wt\%) Coliwasa } \\
\text { (low+high) }\end{array}$ & $\begin{array}{c}\text { Mean } \\
\text { (Measured } \\
\text { wt\%) Other } \\
\text { Sampler }\end{array}$ & Difference & \%Diff & $\begin{array}{l}\text { Sig. } \\
\text { Diff }\end{array}$ & $\begin{array}{l}\text { Bias } \\
\text { Bnd }\end{array}$ & $\begin{array}{c}\% \\
\text { Rel } \\
\text { Bias } \\
\text { Bnd } \\
\end{array}$ & $\begin{array}{c}\text { Std Dev } \\
\text { (Measured } \\
\text { wt\%) Coliwasa } \\
\text { (low+high) }\end{array}$ & $\begin{array}{c}\text { Std Dev } \\
\text { (Measured } \\
\text { wt\%) Other } \\
\text { Sampler }\end{array}$ & $\begin{array}{l}\text { Sig. } \\
\text { Diff }\end{array}$ & $\begin{array}{c}\mathrm{n} \\
\text { Col }\end{array}$ & $\begin{array}{c}\text { n } \\
\text { Other }\end{array}$ \\
\hline 1 & Isolok-LFR-3mL & $\mathrm{Al} / \mathrm{B}$ & 2.572 & 2.725 & 0.153 & $5.9 \%$ & Yes & 0.2069 & 8.04 & 0.0597 & 0.0702 & No & 15 & 10 \\
\hline 4 & Isolok-HFR-12mL & $\mathrm{Al} / \mathrm{B}$ & 2.596 & 2.534 & -0.061 & $-2.4 \%$ & No & 0.1823 & 7.02 & 0.0621 & 0.1656 & Yes & 15 & 10 \\
\hline 2 & Hydragard - HFR & $\mathrm{Al} / \mathrm{B}$ & 2.620 & 2.748 & 0.128 & $4.9 \%$ & Yes & 0.1755 & 6.70 & 0.0514 & 0.0603 & No & 15 & 9 \\
\hline 5 & Isolok-HFR-3mL & $\mathrm{Al} / \mathrm{B}$ & 2.574 & 2.518 & -0.055 & $-2.1 \%$ & Yes & 0.1078 & 4.19 & 0.0672 & 0.0537 & No & 15 & 10 \\
\hline 3 & Isolok-LFR-12mL & $\mathrm{Al} / \mathrm{B}$ & 2.587 & 2.580 & -0.007 & $-0.3 \%$ & No & 0.0781 & 3.02 & 0.0928 & 0.0672 & No & 15 & 10 \\
\hline 6 & Hydragard-LFR & $\mathrm{Al} / \mathrm{B}$ & 2.644 & 2.654 & 0.010 & $0.4 \%$ & No & 0.0664 & 2.51 & 0.0679 & 0.0487 & No & 15 & 8 \\
\hline 6 & Hydragard-LFR & Al2O3 (wt\%) & 10.919 & 11.248 & 0.329 & $3.0 \%$ & No & 0.6749 & 6.18 & 0.4633 & 0.3202 & No & 16 & 10 \\
\hline 3 & Isolok-LFR-12mL & Al2O3 (wt\%) & 10.868 & 11.176 & 0.308 & $2.8 \%$ & No & 0.6640 & 6.11 & 0.4601 & 0.3672 & No & 16 & 10 \\
\hline 2 & Hydragard - HFR & Al2O3 (wt\%) & 10.906 & 11.265 & 0.359 & $3.3 \%$ & Yes & 0.5665 & 5.19 & 0.2723 & 0.1971 & No & 15 & 10 \\
\hline 5 & Isolok-HFR-3mL & Al2O3 (wt\%) & 10.752 & 10.596 & -0.156 & $-1.5 \%$ & No & 0.4420 & 4.11 & 0.3507 & 0.3315 & No & 16 & 10 \\
\hline 1 & Isolok-LFR-3mL & Al2O3 (wt\%) & 10.767 & 10.927 & 0.160 & $1.5 \%$ & No & 0.4089 & 3.80 & 0.3358 & 0.2234 & No & 16 & 10 \\
\hline 4 & Isolok-HFR-12mL & Al2O3 (wt\%) & 10.790 & 10.768 & -0.022 & $-0.2 \%$ & No & 0.2899 & 2.69 & 0.2424 & 0.4071 & No & 15 & 10 \\
\hline 3 & Isolok-LFR-12mL & B2O3 (wt\%) & 4.164 & 4.368 & 0.205 & $4.9 \%$ & Yes & 0.3228 & 7.75 & 0.1500 & 0.1092 & No & 16 & 9 \\
\hline 6 & Hydragard-LFR & $\mathrm{B} 2 \mathrm{O} 3(\mathrm{wt} \%)$ & 4.089 & 4.244 & 0.155 & $3.8 \%$ & Yes & 0.2692 & 6.58 & 0.1222 & 0.1605 & No & 16 & 10 \\
\hline 1 & Isolok-LFR-3mL & B2O3 (wt\%) & 4.151 & 4.012 & -0.139 & $-3.4 \%$ & Yes & 0.2283 & 5.50 & 0.0720 & 0.1368 & No & 14 & 10 \\
\hline 4 & Isolok-HFR-12mL & B2O3 (wt\%) & 4.142 & 4.257 & 0.115 & $2.8 \%$ & Yes & 0.2161 & 5.22 & 0.1031 & 0.1470 & No & 16 & 10 \\
\hline 5 & Isolok-HFR-3mL & B2O3 (wt\%) & 4.146 & 4.208 & 0.062 & $1.5 \%$ & No & 0.1794 & 4.33 & 0.1340 & 0.1375 & No & 16 & 10 \\
\hline 2 & Hydragard - HFR & $\mathrm{B} 2 \mathrm{O} 3(\mathrm{wt} \%)$ & 4.162 & 4.131 & -0.031 & $-0.7 \%$ & No & 0.0985 & 2.37 & 0.0774 & 0.0832 & No & 15 & 10 \\
\hline 3 & Isolok-LFR-12mL & $\mathrm{BaO}(\mathrm{wt} \%)$ & 0.103 & 0.107 & 0.004 & $4.0 \%$ & Yes & 0.0073 & 7.10 & 0.0040 & 0.0011 & No & 16 & 10 \\
\hline 6 & Hydragard-LFR & $\mathrm{BaO}$ (wt\%) & 0.103 & 0.106 & 0.003 & $2.9 \%$ & No & 0.0062 & 5.97 & 0.0040 & 0.0034 & No & 16 & 10 \\
\hline 2 & Hydragard - HFR & $\mathrm{BaO}(\mathrm{wt} \%)$ & 0.106 & 0.108 & 0.002 & $2.1 \%$ & No & 0.0048 & 4.51 & 0.0033 & 0.0025 & No & 16 & 10 \\
\hline 1 & Isolok-LFR-3mL & $\mathrm{BaO}(\mathrm{wt} \%)$ & 0.103 & 0.106 & 0.002 & $2.2 \%$ & Yes & 0.0043 & 4.14 & 0.0027 & 0.0015 & No & 16 & 9 \\
\hline 4 & Isolok-HFR-12mL & $\mathrm{BaO}(\mathrm{wt} \%)$ & 0.104 & 0.104 & 0.001 & $0.6 \%$ & No & 0.0033 & 3.21 & 0.0028 & 0.0039 & No & 16 & 10 \\
\hline 5 & Isolok-HFR-3mL & $\mathrm{BaO}(\mathrm{wt} \%)$ & 0.103 & 0.102 & -0.001 & $-0.6 \%$ & No & 0.0024 & 2.36 & 0.0031 & 0.0013 & Yes & 16 & 9 \\
\hline 3 & Isolok-LFR-12mL & $\mathrm{CaO}(\mathrm{wt} \%)$ & 1.194 & 1.249 & 0.055 & $4.6 \%$ & Yes & 0.0960 & 8.04 & 0.0510 & 0.0479 & No & 16 & 10 \\
\hline 6 & Hydragard-LFR & $\mathrm{CaO}(\mathrm{wt} \%)$ & 1.208 & 1.257 & 0.049 & $4.0 \%$ & Yes & 0.0917 & 7.59 & 0.0515 & 0.0519 & No & 16 & 10 \\
\hline 2 & Hydragard - HFR & $\mathrm{CaO}$ (wt\%) & 1.211 & 1.250 & 0.039 & $3.2 \%$ & Yes & 0.0657 & 5.43 & 0.0347 & 0.0285 & No & 16 & 10 \\
\hline 1 & Isolok-LFR-3mL & $\mathrm{CaO}(\mathrm{wt} \%)$ & 1.201 & 1.222 & 0.021 & $1.7 \%$ & No & 0.0495 & 4.12 & 0.0397 & 0.0236 & No & 16 & 10 \\
\hline 4 & Isolok-HFR-12mL & $\mathrm{CaO}(\mathrm{wt} \%)$ & 1.197 & 1.201 & 0.003 & $0.3 \%$ & No & 0.0425 & 3.55 & 0.0386 & 0.0587 & No & 16 & 10 \\
\hline 5 & Isolok-HFR-3mL & $\mathrm{CaO}(\mathrm{wt} \%)$ & 1.206 & 1.199 & -0.006 & $-0.5 \%$ & No & 0.0411 & 3.41 & 0.0454 & 0.0352 & No & 16 & 10 \\
\hline 5 & Isolok-HFR-3mL & Cr2O3 (wt\%) & 0.107 & 0.103 & -0.004 & $-3.8 \%$ & Yes & 0.0077 & 7.13 & 0.0049 & 0.0032 & No & 16 & 10 \\
\hline 3 & Isolok-LFR-12mL & Cr2O3 (wt\%) & 0.108 & 0.110 & 0.002 & $2.2 \%$ & No & 0.0069 & 6.40 & 0.0062 & 0.0013 & No & 16 & 10 \\
\hline 4 & Isolok-HFR-12mL & Cr2O3 (wt\%) & 0.107 & 0.107 & 0.000 & $0.2 \%$ & No & 0.0052 & 4.82 & 0.0055 & 0.0065 & No & 16 & 10 \\
\hline 1 & Isolok-LFR-3mL & Cr2O3 (wt\%) & 0.105 & 0.103 & -0.002 & $-1.9 \%$ & No & 0.0050 & 4.73 & 0.0034 & 0.0037 & No & 15 & 10 \\
\hline 6 & Hydragard-LFR & Cr2O3 (wt\%) & 0.107 & 0.106 & 0.000 & $-0.4 \%$ & No & 0.0050 & 4.68 & 0.0060 & 0.0044 & No & 16 & 10 \\
\hline 2 & Hydragard - HFR & Cr2O3 (wt\%) & 0.110 & 0.111 & 0.001 & $1.2 \%$ & No & 0.0041 & 3.70 & 0.0038 & 0.0024 & No & 15 & 10 \\
\hline 4 & Isolok-HFR-12mL & $\mathrm{CuO}(\mathrm{wt} \%)$ & 0.025 & 0.027 & 0.002 & $7.2 \%$ & Yes & 0.0029 & 11.69 & 0.0014 & 0.0011 & No & 16 & 9 \\
\hline 3 & Isolok-LFR-12mL & $\mathrm{CuO}$ (wt\%) & 0.025 & 0.026 & 0.001 & $4.3 \%$ & No & 0.0022 & 8.57 & 0.0014 & 0.0011 & No & 16 & 10 \\
\hline 5 & Isolok-HFR-3mL & $\mathrm{CuO}(\mathrm{wt} \%)$ & 0.025 & 0.025 & 0.000 & $1.2 \%$ & No & 0.0015 & 6.14 & 0.0014 & 0.0016 & No & 16 & 10 \\
\hline 6 & Hydragard-LFR & $\mathrm{CuO}$ (wt\%) & 0.025 & 0.026 & 0.000 & $1.3 \%$ & No & 0.0015 & 6.02 & 0.0013 & 0.0016 & No & 15 & 10 \\
\hline
\end{tabular}


SRNL-STI-2011-00693

Revision 0

Table A10. Phase 1 (Mid-Rheology) Sampler Comparisons Grouped by Analytical Block (screened data)

\begin{tabular}{|c|c|c|c|c|c|c|c|c|c|c|c|c|c|c|}
\hline $\begin{array}{c}\text { Analytical } \\
\text { Block }\end{array}$ & Sampler & Oxide & $\begin{array}{c}\text { Mean } \\
\text { (Measured } \\
\text { wt\%) Coliwasa } \\
\text { (low+high) }\end{array}$ & $\begin{array}{c}\text { Mean } \\
\text { (Measured } \\
\text { wt\%) Other } \\
\text { Sampler } \\
\end{array}$ & Difference & $\%$ Diff & $\begin{array}{l}\text { Sig. } \\
\text { Diff }\end{array}$ & $\begin{array}{l}\text { Bias } \\
\text { Bnd }\end{array}$ & $\begin{array}{c}\% \\
\text { Rel } \\
\text { Bias } \\
\text { Bnd } \\
\end{array}$ & $\begin{array}{c}\text { Std Dev } \\
\text { (Measured } \\
\text { wt\%) Coliwasa } \\
\text { (low+high) }\end{array}$ & $\begin{array}{c}\text { Std Dev } \\
\text { (Measured } \\
\text { wt\%) Other } \\
\text { Sampler } \\
\end{array}$ & $\begin{array}{l}\text { Sig. } \\
\text { Diff }\end{array}$ & $\begin{array}{c}n \\
\text { Col }\end{array}$ & $\begin{array}{c}\text { n } \\
\text { Other }\end{array}$ \\
\hline 1 & Isolok-LFR-3mL & $\mathrm{CuO}(\mathrm{wt} \%)$ & 0.025 & 0.025 & 0.000 & $1.3 \%$ & No & 0.0014 & 5.58 & 0.0014 & 0.0010 & No & 16 & 10 \\
\hline 2 & Hydragard - HFR & $\mathrm{CuO}(\mathrm{wt} \%)$ & 0.025 & 0.025 & 0.000 & $0.1 \%$ & No & 0.0014 & 5.42 & 0.0014 & 0.0019 & No & 16 & 10 \\
\hline 4 & Isolok-HFR-12mL & $\mathrm{Fe} / \mathrm{Li}$ & 2.400 & 2.367 & -0.033 & $-1.4 \%$ & No & 0.1518 & 6.32 & 0.0760 & 0.1598 & Yes & 14 & 10 \\
\hline 5 & Isolok-HFR-3mL & $\mathrm{Fe} / \mathrm{Li}$ & 2.407 & 2.325 & -0.082 & $-3.4 \%$ & Yes & 0.1359 & 5.65 & 0.0729 & 0.0324 & No & 14 & 9 \\
\hline 2 & Hydragard - HFR & $\mathrm{Fe} / \mathrm{Li}$ & 2.411 & 2.455 & 0.044 & $1.8 \%$ & No & 0.1223 & 5.07 & 0.1017 & 0.0768 & No & 15 & 10 \\
\hline 1 & Isolok-LFR-3mL & $\mathrm{Fe} / \mathrm{Li}$ & 2.412 & 2.465 & 0.053 & $2.2 \%$ & No & 0.1171 & 4.86 & 0.0734 & 0.0750 & No & 14 & 10 \\
\hline 3 & Isolok-LFR-12mL & $\mathrm{Fe} / \mathrm{Li}$ & 2.417 & 2.450 & 0.034 & $1.4 \%$ & No & 0.1053 & 4.36 & 0.1017 & 0.0470 & No & 15 & 10 \\
\hline 6 & Hydragard-LFR & $\mathrm{Fe} / \mathrm{Li}$ & 2.413 & 2.427 & 0.014 & $0.6 \%$ & No & 0.0930 & 3.86 & 0.0797 & 0.1077 & No & 14 & 10 \\
\hline 3 & Isolok-LFR-12mL & Fe2O3 (wt\%) & 11.214 & 11.599 & 0.385 & $3.4 \%$ & Yes & 0.7611 & 6.79 & 0.4704 & 0.4200 & No & 16 & 10 \\
\hline 6 & Hydragard-LFR & Fe2O3 (wt\%) & 11.523 & 11.844 & 0.320 & $2.8 \%$ & No & 0.6939 & 6.02 & 0.4939 & 0.3626 & No & 16 & 10 \\
\hline 2 & Hydragard - HFR & Fe2O3 (wt\%) & 11.463 & 11.732 & 0.270 & $2.4 \%$ & No & 0.5518 & 4.81 & 0.3843 & 0.2465 & No & 16 & 10 \\
\hline 4 & Isolok-HFR-12mL & $\mathrm{Fe} 2 \mathrm{O} 3$ (wt\%) & 11.468 & 11.365 & -0.103 & $-0.9 \%$ & No & 0.4822 & 4.20 & 0.3692 & 0.5708 & No & 16 & 10 \\
\hline 1 & Isolok-LFR-3mL & Fe2O3 (wt\%) & 11.405 & 11.592 & 0.188 & $1.6 \%$ & No & 0.4594 & 4.03 & 0.3770 & 0.2191 & No & 16 & 10 \\
\hline 5 & Isolok-HFR-3mL & Fe2O3 (wt\%) & 11.357 & 11.290 & -0.067 & $-0.6 \%$ & No & 0.3096 & 2.73 & 0.4035 & 0.1771 & Yes & 16 & 9 \\
\hline 4 & Isolok-HFR-12mL & $\mathrm{K} 2 \mathrm{O}(\mathrm{wt} \%)$ & 0.163 & 0.172 & 0.009 & $5.4 \%$ & Yes & 0.0175 & 10.76 & 0.0093 & 0.0112 & No & 15 & 9 \\
\hline 3 & Isolok-LFR-12mL & K2O (wt\%) & 0.163 & 0.170 & 0.007 & $4.2 \%$ & No & 0.0143 & 8.74 & 0.0092 & 0.0074 & No & 16 & 9 \\
\hline 6 & Hydragard-LFR & K2O (wt\%) & 0.163 & 0.166 & 0.003 & $1.9 \%$ & No & 0.0112 & 6.86 & 0.0092 & 0.0104 & No & 16 & 10 \\
\hline 5 & Isolok-HFR-3mL & K2O (wt\%) & 0.163 & 0.165 & 0.002 & $1.2 \%$ & No & 0.0101 & 6.16 & 0.0092 & 0.0106 & No & 16 & 10 \\
\hline 1 & Isolok-LFR-3mL & K2O (wt\%) & 0.163 & 0.165 & 0.002 & $1.5 \%$ & No & 0.0094 & 5.74 & 0.0092 & 0.0068 & No & 16 & 10 \\
\hline 2 & Hydragard - HFR & $\mathrm{K} 2 \mathrm{O}$ (wt\%) & 0.163 & 0.163 & 0.001 & $0.4 \%$ & No & 0.0092 & 5.62 & 0.0088 & 0.0123 & No & 16 & 10 \\
\hline 6 & Hydragard-LFR & Li2O (wt\%) & 4.752 & 4.885 & 0.133 & $2.8 \%$ & No & 0.2725 & 5.73 & 0.1587 & 0.1753 & No & 15 & 10 \\
\hline 5 & Isolok-HFR-3mL & Li2O (wt\%) & 4.740 & 4.837 & 0.096 & $2.0 \%$ & No & 0.2704 & 5.70 & 0.2398 & 0.0969 & No & 16 & 9 \\
\hline 3 & Isolok-LFR-12mL & Li2O (wt\%) & 4.695 & 4.734 & 0.040 & $0.8 \%$ & No & 0.2057 & 4.38 & 0.2169 & 0.1672 & No & 16 & 10 \\
\hline 1 & Isolok-LFR-3mL & $\mathrm{Li} 2 \mathrm{O}(\mathrm{wt} \%)$ & 4.709 & 4.704 & -0.005 & $-0.1 \%$ & No & 0.1007 & 2.14 & 0.1306 & 0.0794 & No & 15 & 10 \\
\hline 2 & Hydragard - HFR & Li2O (wt\%) & 4.777 & 4.794 & 0.017 & $0.4 \%$ & No & 0.0852 & 1.78 & 0.1139 & 0.0417 & Yes & 14 & 10 \\
\hline 4 & Isolok-HFR-12mL & Li2O (wt\%) & 4.783 & 4.777 & -0.005 & $-0.1 \%$ & No & 0.0830 & 1.73 & 0.0932 & 0.0765 & No & 14 & 9 \\
\hline 6 & Hydragard-LFR & MgO (wt\%) & 1.042 & 1.086 & 0.044 & $4.2 \%$ & Yes & 0.0776 & 7.44 & 0.0444 & 0.0280 & No & 16 & 9 \\
\hline 3 & Isolok-LFR-12mL & $\mathrm{MgO}(\mathrm{wt} \%)$ & 1.051 & 1.089 & 0.038 & $3.7 \%$ & Yes & 0.0763 & 7.26 & 0.0455 & 0.0457 & No & 16 & 10 \\
\hline 4 & Isolok-HFR-12mL & $\mathrm{MgO}(\mathrm{wt} \%)$ & 1.061 & 1.051 & -0.010 & $-0.9 \%$ & No & 0.0455 & 4.29 & 0.0346 & 0.0541 & No & 16 & 10 \\
\hline 2 & Hydragard - HFR & MgO (wt\%) & 1.074 & 1.091 & 0.018 & $1.7 \%$ & No & 0.0458 & 4.27 & 0.0361 & 0.0288 & No & 16 & 10 \\
\hline 1 & Isolok-LFR-3mL & $\mathrm{MgO}(\mathrm{wt} \%)$ & 1.058 & 1.075 & 0.017 & $1.6 \%$ & No & 0.0382 & 3.61 & 0.0351 & 0.0162 & Yes & 16 & 10 \\
\hline 5 & Isolok-HFR-3mL & $\mathrm{MgO}(\mathrm{wt} \%)$ & 1.043 & 1.035 & -0.008 & $-0.8 \%$ & No & 0.0259 & 2.48 & 0.0364 & 0.0142 & Yes & 16 & 9 \\
\hline 3 & Isolok-LFR-12mL & $\mathrm{MnO}(\mathrm{wt} \%)$ & 1.748 & 1.817 & 0.069 & $3.9 \%$ & Yes & 0.1356 & 7.76 & 0.0862 & 0.0697 & No & 16 & 10 \\
\hline 6 & Hydragard-LFR & $\mathrm{MnO}(\mathrm{wt} \%)$ & 1.729 & 1.786 & 0.057 & $3.3 \%$ & No & 0.1215 & 7.03 & 0.0854 & 0.0618 & No & 16 & 10 \\
\hline 2 & Hydragard - HFR & $\mathrm{MnO}(\mathrm{wt} \%)$ & 1.785 & 1.822 & 0.037 & $2.1 \%$ & No & 0.0889 & 4.98 & 0.0708 & 0.0457 & No & 16 & 10 \\
\hline 1 & Isolok-LFR-3mL & $\mathrm{MnO}(\mathrm{wt} \%)$ & 1.750 & 1.786 & 0.035 & $2.0 \%$ & No & 0.0836 & 4.78 & 0.0670 & 0.0385 & No & 16 & 10 \\
\hline 4 & Isolok-HFR-12mL & $\mathrm{MnO}(\mathrm{wt} \%)$ & 1.755 & 1.742 & -0.013 & $-0.8 \%$ & No & 0.0770 & 4.39 & 0.0690 & 0.0893 & No & 16 & 10 \\
\hline 5 & Isolok-HFR-3mL & $\mathrm{MnO}(\mathrm{wt} \%)$ & 1.733 & 1.720 & -0.012 & $-0.7 \%$ & No & 0.0521 & 3.01 & 0.0708 & 0.0202 & Yes & 16 & 9 \\
\hline 5 & Isolok-HFR-3mL & $\mathrm{Na} 2 \mathrm{O}$ (wt\%) & 11.141 & 10.812 & -0.329 & $-3.0 \%$ & No & 0.6819 & 6.12 & 0.3820 & 0.4867 & No & 16 & 10 \\
\hline 3 & Isolok-LFR-12mL & $\mathrm{Na} 2 \mathrm{O}$ (wt\%) & 11.008 & 11.251 & 0.243 & $2.2 \%$ & No & 0.6232 & 5.66 & 0.4252 & 0.4690 & No & 16 & 9 \\
\hline 4 & Isolok-HFR-12mL & $\mathrm{Na} 2 \mathrm{O}$ (wt\%) & 11.063 & 10.748 & -0.315 & $-2.9 \%$ & Yes & 0.6128 & 5.54 & 0.3266 & 0.4041 & No & 16 & 10 \\
\hline 6 & Hydragard-LFR & $\mathrm{Na} 2 \mathrm{O}(\mathrm{wt} \%)$ & 11.343 & 11.616 & 0.272 & $2.4 \%$ & No & 0.6274 & 5.53 & 0.3577 & 0.5221 & No & 16 & 10 \\
\hline
\end{tabular}

A-32 
SRNL-STI-2011-00693

Revision 0

Table A10. Phase 1 (Mid-Rheology) Sampler Comparisons Grouped by Analytical Block (screened data)

\begin{tabular}{|c|c|c|c|c|c|c|c|c|c|c|c|c|c|c|}
\hline $\begin{array}{c}\text { Analytical } \\
\text { Block }\end{array}$ & Sampler & Oxide & $\begin{array}{c}\text { Mean } \\
\text { (Measured } \\
\text { wt\%) Coliwasa } \\
\text { (low+high) }\end{array}$ & $\begin{array}{c}\text { Mean } \\
\text { (Measured } \\
\text { wt\%) Other } \\
\text { Sampler } \\
\end{array}$ & Difference & $\%$ Diff & $\begin{array}{l}\text { Sig. } \\
\text { Diff }\end{array}$ & $\begin{array}{l}\text { Bias } \\
\text { Bnd }\end{array}$ & $\begin{array}{c}\% \\
\text { Rel } \\
\text { Bias } \\
\text { Bnd } \\
\end{array}$ & $\begin{array}{c}\text { Std Dev } \\
\text { (Measured } \\
\text { wt\%) Coliwasa } \\
\text { (low+high) }\end{array}$ & $\begin{array}{c}\text { Std Dev } \\
\text { (Measured } \\
\text { wt\%) Other } \\
\text { Sampler } \\
\end{array}$ & $\begin{array}{l}\text { Sig. } \\
\text { Diff }\end{array}$ & $\begin{array}{c}n \\
\text { Col }\end{array}$ & $\begin{array}{c}\text { n } \\
\text { Other }\end{array}$ \\
\hline 1 & Isolok-LFR-3mL & $\mathrm{Na} 2 \mathrm{O}$ (wt\%) & 11.249 & 11.198 & -0.051 & $-0.5 \%$ & No & 0.4892 & 4.35 & 0.3356 & 0.5824 & Yes & 16 & 10 \\
\hline 2 & Hydragard - HFR & $\mathrm{Na} 2 \mathrm{O}$ (wt\%) & 11.311 & 11.399 & 0.088 & $0.8 \%$ & No & 0.3822 & 3.38 & 0.3543 & 0.3518 & No & 16 & 10 \\
\hline 3 & Isolok-LFR-12mL & $\mathrm{NiO}(\mathrm{wt} \%)$ & 0.451 & 0.466 & 0.016 & $3.5 \%$ & No & 0.0369 & 8.18 & 0.0306 & 0.0136 & No & 16 & 10 \\
\hline 6 & Hydragard-LFR & $\mathrm{NiO}(\mathrm{wt} \%)$ & 0.452 & 0.464 & 0.011 & $2.5 \%$ & No & 0.0330 & 7.29 & 0.0310 & 0.0138 & No & 16 & 10 \\
\hline 4 & Isolok-HFR-12mL & $\mathrm{NiO}(\mathrm{wt} \%)$ & 0.451 & 0.443 & -0.008 & $-1.7 \%$ & No & 0.0295 & 6.55 & 0.0208 & 0.0320 & No & 15 & 10 \\
\hline 2 & Hydragard - HFR & $\mathrm{NiO}$ (wt\%) & 0.465 & 0.478 & 0.013 & $2.8 \%$ & No & 0.0289 & 6.21 & 0.0212 & 0.0145 & No & 15 & 10 \\
\hline 5 & Isolok-HFR-3mL & $\mathrm{NiO}(\mathrm{wt} \%)$ & 0.446 & 0.440 & -0.006 & $-1.3 \%$ & No & 0.0241 & 5.41 & 0.0260 & 0.0120 & No & 16 & 10 \\
\hline 1 & Isolok-LFR-3mL & $\mathrm{NiO}(\mathrm{wt} \%)$ & 0.452 & 0.459 & 0.008 & $1.7 \%$ & No & 0.0187 & 4.14 & 0.0148 & 0.0097 & No & 14 & 10 \\
\hline 5 & Isolok-HFR-3mL & SiO2 (wt\%) & 51.210 & 52.226 & 1.016 & $2.0 \%$ & No & 2.0956 & 4.09 & 1.9433 & 0.4775 & Yes & 16 & 8 \\
\hline 4 & Isolok-HFR-12mL & SiO2 (wt\%) & 51.370 & 52.113 & 0.743 & $1.4 \%$ & No & 1.7839 & 3.47 & 1.3254 & 1.1148 & No & 16 & 10 \\
\hline 6 & Hydragard-LFR & $\mathrm{SiO} 2$ (wt\%) & 51.998 & 52.242 & 0.243 & $0.5 \%$ & No & 1.5168 & 2.92 & 1.5942 & 1.4183 & No & 16 & 10 \\
\hline 3 & Isolok-LFR-12mL & $\mathrm{SiO} 2(\mathrm{wt} \%)$ & 51.343 & 51.750 & 0.406 & $0.8 \%$ & No & 1.3866 & 2.70 & 1.2553 & 0.9955 & No & 15 & 10 \\
\hline 2 & Hydragard - HFR & $\mathrm{SiO} 2$ (wt\%) & 52.052 & 51.579 & -0.473 & $-0.9 \%$ & No & 1.3620 & 2.62 & 1.2095 & 0.7776 & No & 16 & 10 \\
\hline 1 & Isolok-LFR-3mL & SiO2 (wt\%) & 51.731 & 51.728 & -0.003 & $0.0 \%$ & No & 0.7290 & 1.41 & 1.3262 & 0.2992 & Yes & 16 & 10 \\
\hline 3 & Isolok-LFR-12mL & Sum (wt\%) & 98.401 & 101.037 & 2.636 & $2.7 \%$ & Yes & 4.8331 & 4.91 & 2.3685 & 3.0410 & No & 16 & 10 \\
\hline 5 & Isolok-HFR-3mL & Sum (wt\%) & 98.710 & 99.715 & 1.005 & $1.0 \%$ & No & 2.8345 & 2.87 & 2.3880 & 1.5025 & No & 16 & 9 \\
\hline 6 & Hydragard-LFR & Sum (wt\%) & 100.357 & 101.618 & 1.261 & $1.3 \%$ & No & 2.7912 & 2.78 & 1.7030 & 1.9700 & No & 15 & 10 \\
\hline 2 & Hydragard - HFR & Sum (wt\%) & 100.332 & 100.496 & 0.164 & $0.2 \%$ & No & 0.3991 & 0.40 & 0.3112 & 0.2163 & No & 15 & 10 \\
\hline 4 & Isolok-HFR-12mL & Sum (wt\%) & 99.249 & 99.337 & 0.088 & $0.1 \%$ & No & 0.3591 & 0.36 & 0.2867 & 0.3481 & No & 15 & 9 \\
\hline 1 & Isolok-LFR-3mL & Sum (wt\%) & 99.584 & 99.660 & 0.076 & $0.1 \%$ & No & 0.3001 & 0.30 & 0.2946 & 0.2124 & No & 15 & 10 \\
\hline 5 & Isolok-HFR-3mL & TiO2 (wt\%) & 0.082 & 0.084 & 0.002 & $2.0 \%$ & No & 0.0036 & 4.40 & 0.0030 & 0.0018 & Yes & 16 & 10 \\
\hline 3 & Isolok-LFR-12mL & TiO2 (wt\%) & 0.082 & 0.083 & 0.000 & $0.4 \%$ & No & 0.0031 & 3.71 & 0.0033 & 0.0034 & No & 16 & 10 \\
\hline 2 & Hydragard - HFR & TiO2 (wt\%) & 0.083 & 0.082 & -0.001 & $-1.0 \%$ & No & 0.0031 & 3.71 & 0.0013 & 0.0031 & Yes & 15 & 10 \\
\hline 6 & Hydragard-LFR & $\mathrm{TiO} 2(\mathrm{wt} \%)$ & 0.080 & 0.081 & 0.000 & $0.3 \%$ & No & 0.0022 & 2.69 & 0.0019 & 0.0029 & No & 16 & 10 \\
\hline 1 & Isolok-LFR-3mL & TiO2 (wt\%) & 0.082 & 0.082 & 0.000 & $-0.6 \%$ & No & 0.0021 & 2.55 & 0.0027 & 0.0013 & Yes & 16 & 10 \\
\hline 4 & Isolok-HFR-12mL & TiO2 (wt\%) & 0.083 & 0.083 & 0.000 & $0.0 \%$ & No & 0.0014 & 1.69 & 0.0019 & 0.0012 & No & 16 & 10 \\
\hline 3 & Isolok-LFR-12mL & $\mathrm{ZnO}(\mathrm{wt} \%)$ & 0.100 & 0.105 & 0.005 & $4.8 \%$ & Yes & 0.0084 & 8.40 & 0.0047 & 0.0035 & No & 16 & 10 \\
\hline 6 & Hydragard-LFR & $\mathrm{ZnO}(\mathrm{wt} \%)$ & 0.101 & 0.105 & 0.003 & $3.3 \%$ & No & 0.0071 & 7.00 & 0.0050 & 0.0037 & No & 16 & 10 \\
\hline 2 & Hydragard - HFR & $\mathrm{ZnO}(\mathrm{wt} \%)$ & 0.103 & 0.105 & 0.003 & $2.7 \%$ & Yes & 0.0055 & 5.32 & 0.0038 & 0.0019 & No & 16 & 10 \\
\hline 1 & Isolok-LFR-3mL & $\mathrm{ZnO}(\mathrm{wt} \%)$ & 0.101 & 0.102 & 0.001 & $0.8 \%$ & No & 0.0035 & 3.44 & 0.0037 & 0.0020 & No & 16 & 10 \\
\hline 4 & Isolok-HFR-12mL & $\mathrm{ZnO}$ (wt\%) & 0.101 & 0.101 & 0.000 & $0.1 \%$ & No & 0.0033 & 3.31 & 0.0036 & 0.0043 & No & 16 & 10 \\
\hline 5 & Isolok-HFR-3mL & $\mathrm{ZnO}(\mathrm{wt} \%)$ & 0.100 & 0.100 & -0.001 & $-0.6 \%$ & No & 0.0029 & 2.86 & 0.0038 & 0.0016 & Yes & 16 & 8 \\
\hline 3 & Isolok-LFR-12mL & ZrO2 (wt\%) & 0.364 & 0.372 & 0.008 & $2.3 \%$ & No & 0.0167 & 4.60 & 0.0097 & 0.0108 & No & 16 & 10 \\
\hline 6 & Hydragard-LFR & ZrO2 (wt\%) & 0.362 & 0.369 & 0.007 & $2.0 \%$ & No & 0.0154 & 4.26 & 0.0107 & 0.0089 & No & 16 & 10 \\
\hline 2 & Hydragard - HFR & ZrO2 (wt\%) & 0.369 & 0.375 & 0.006 & $1.7 \%$ & Yes & 0.0109 & 2.96 & 0.0055 & 0.0049 & No & 15 & 10 \\
\hline 5 & Isolok-HFR-3mL & ZrO2 (wt\%) & 0.360 & 0.361 & 0.001 & $0.2 \%$ & No & 0.0090 & 2.49 & 0.0094 & 0.0106 & No & 16 & 10 \\
\hline 4 & Isolok-HFR-12mL & ZrO2 (wt\%) & 0.365 & 0.362 & -0.003 & $-0.7 \%$ & No & 0.0082 & 2.25 & 0.0052 & 0.0085 & No & 16 & 10 \\
\hline 1 & Isolok-LFR-3mL & ZrO2 (wt\%) & 0.371 & 0.374 & 0.002 & $0.7 \%$ & No & 0.0075 & 2.03 & 0.0056 & 0.0069 & No & 16 & 10 \\
\hline
\end{tabular}




\section{Exhibit A1. Density, Solids, and Oxide Concentration Measurments for the Mid-Rheology (Phase 1) Testing}
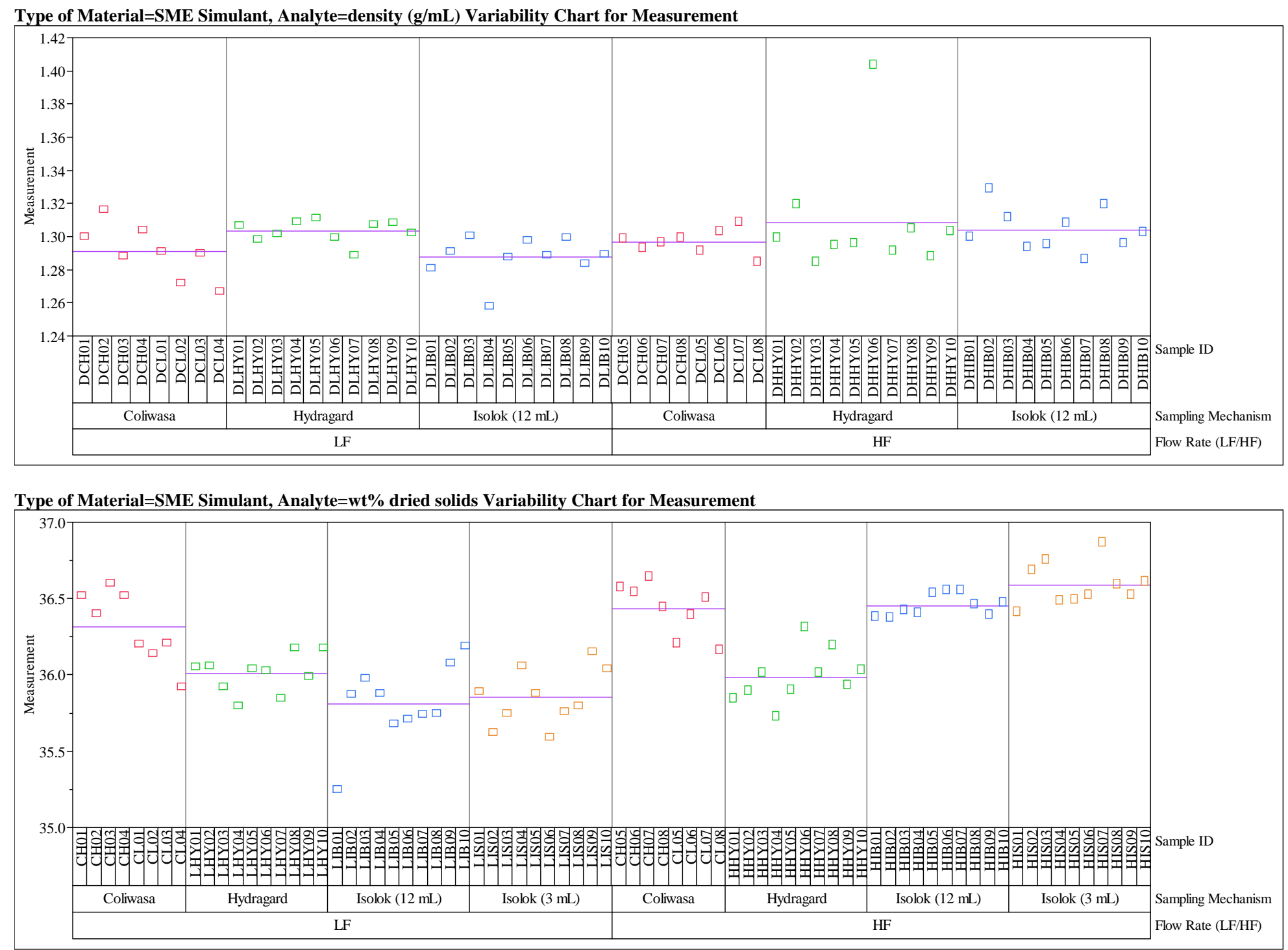
Exhibit A1. Density, Solids, and Oxide Concentration Measurments for the Mid-Rheology (Phase 1) Testing

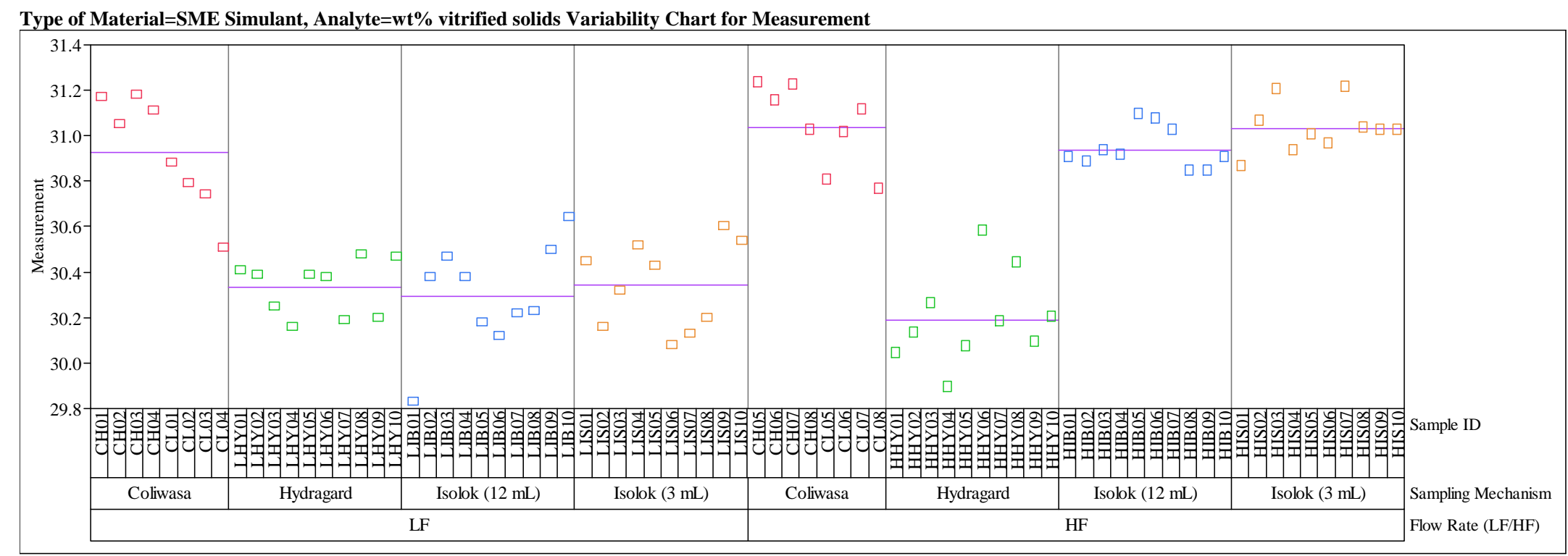

Type of Material=SME Simulant, Analyte=Al/B

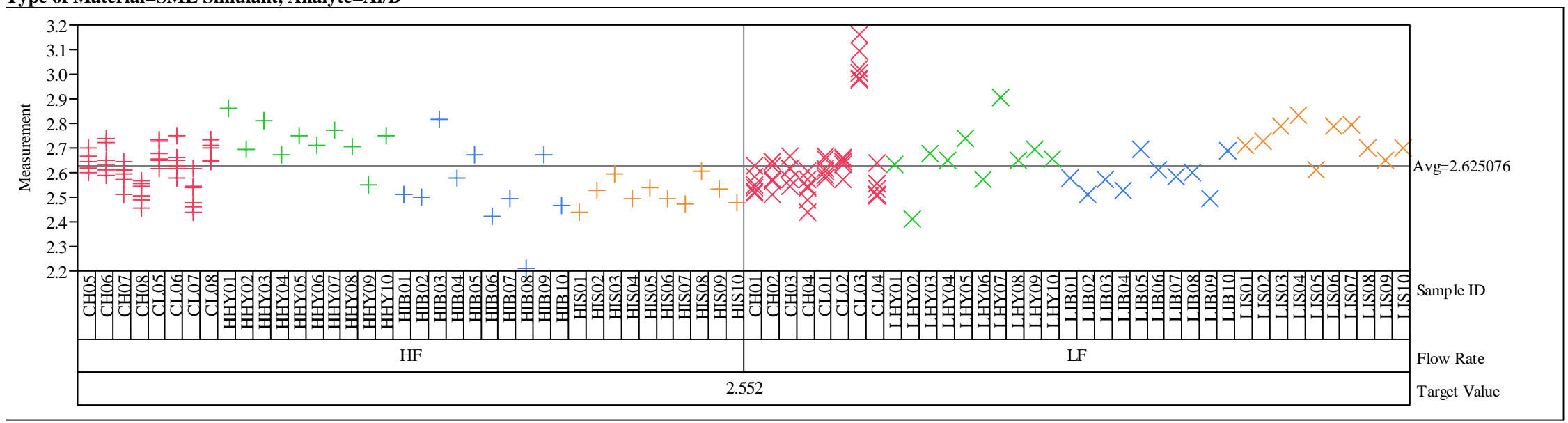




\section{Exhibit A1. Density, Solids, and Oxide Concentration Measurments for the Mid-Rheology (Phase 1) Testing}

Type of Material=SME Simulant, Analyte=Al2O3 (wt \%)

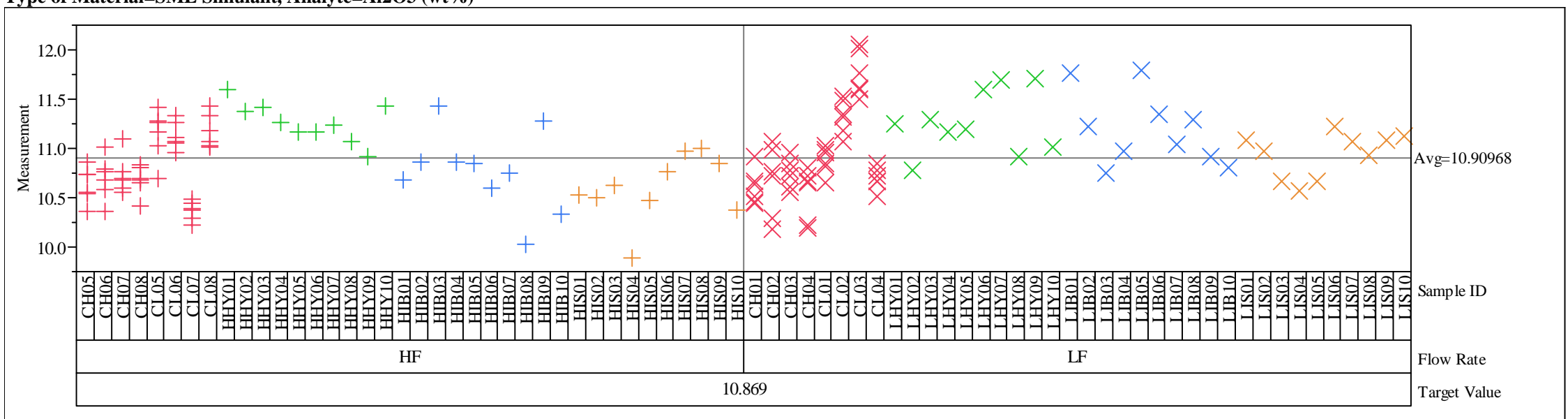

Type of Material=SME Simulant, Analyte=B2O3 (wt \%)

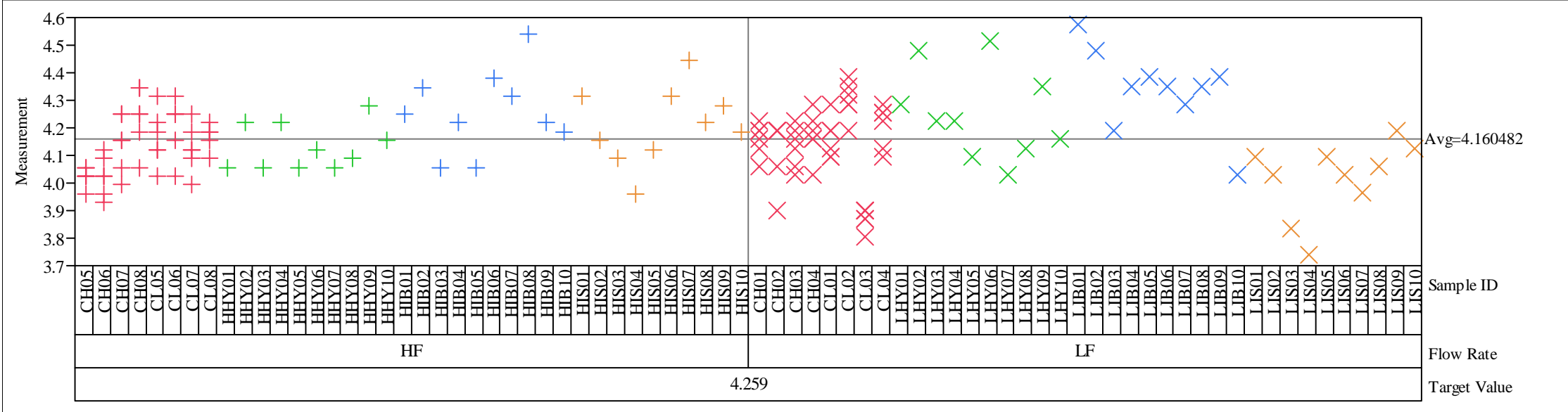




\section{Exhibit A1. Density, Solids, and Oxide Concentration Measurments for the Mid-Rheology (Phase 1) Testing}

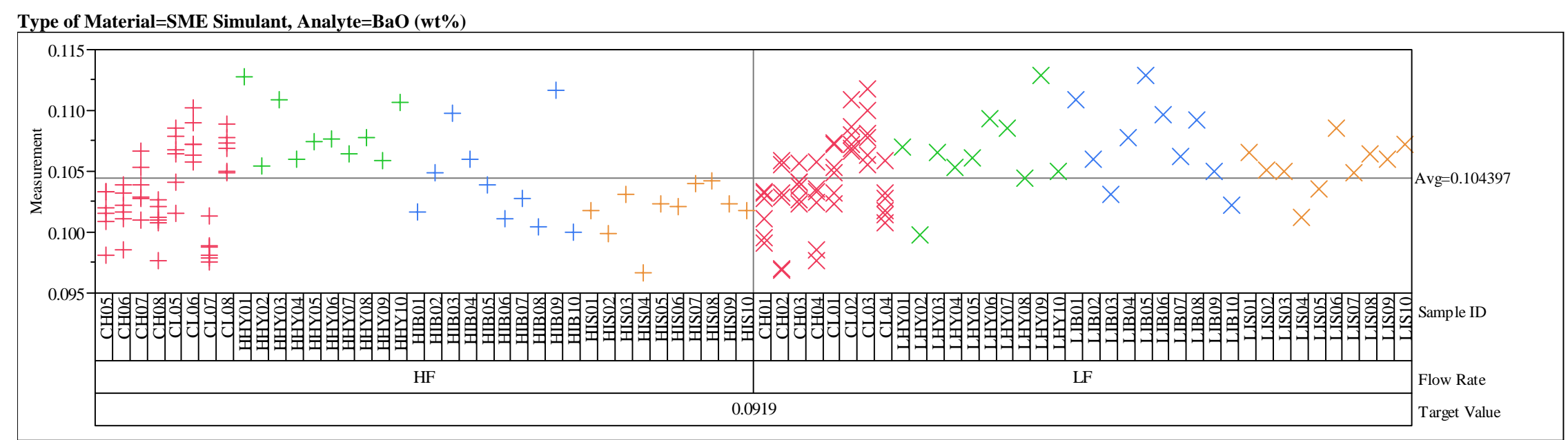

Type of Material=SME Simulant, Analyte=CaO (wt\%)

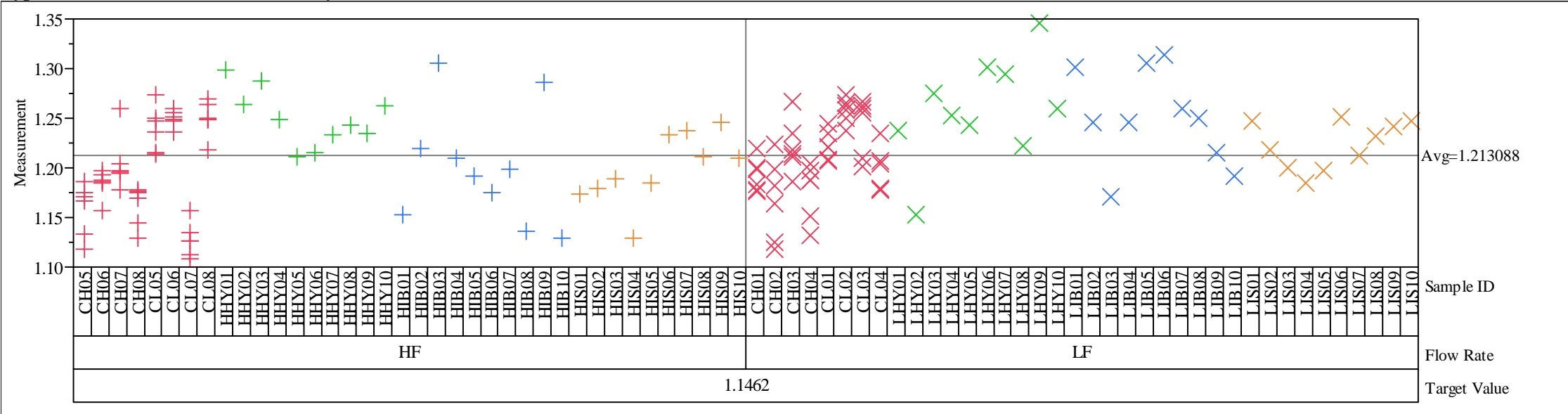




\section{Exhibit A1. Density, Solids, and Oxide Concentration Measurments for the Mid-Rheology (Phase 1) Testing}

Type of Material=SME Simulant, Analyte=Cr2O3 (wt\%)

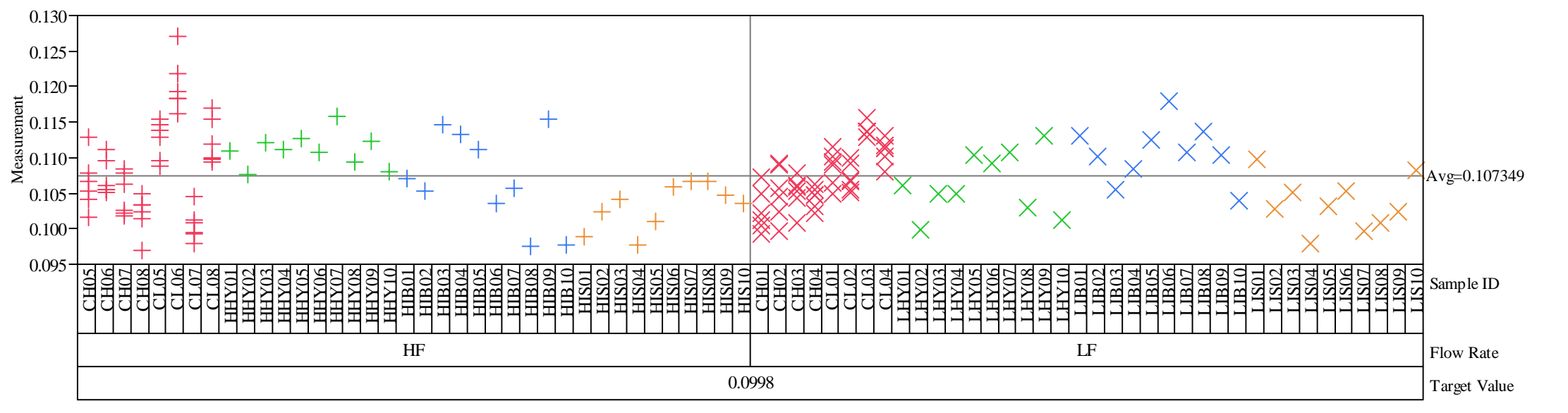

Type of Material=SME Simulant, Analyte=CuO (wt\%)

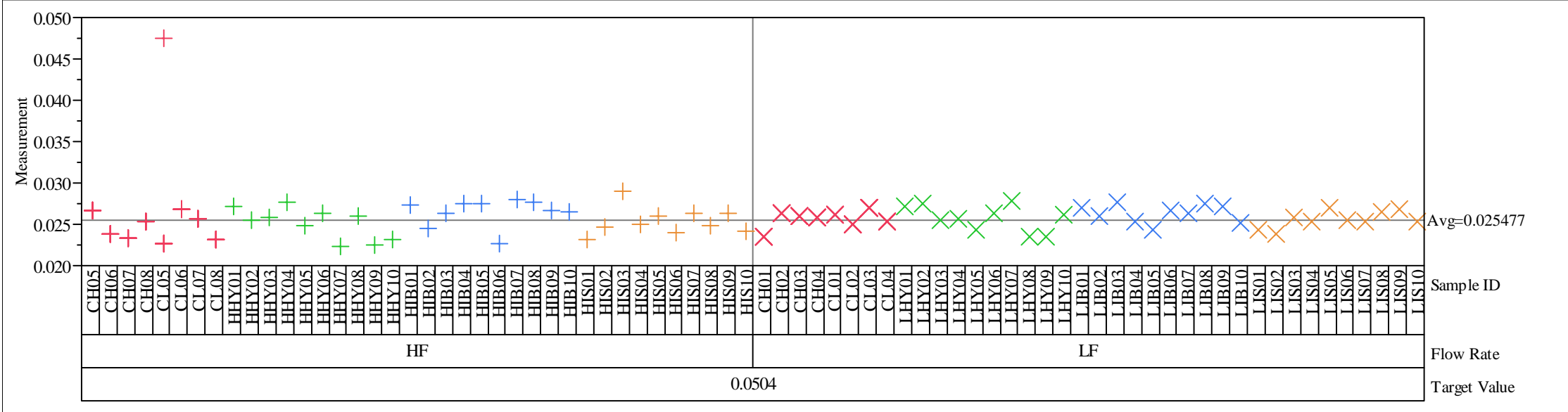




\section{Exhibit A1. Density, Solids, and Oxide Concentration Measurments for the Mid-Rheology (Phase 1) Testing}

Type of Material=SME Simulant, Analyte $=\mathrm{Fe} / \mathrm{Li}$

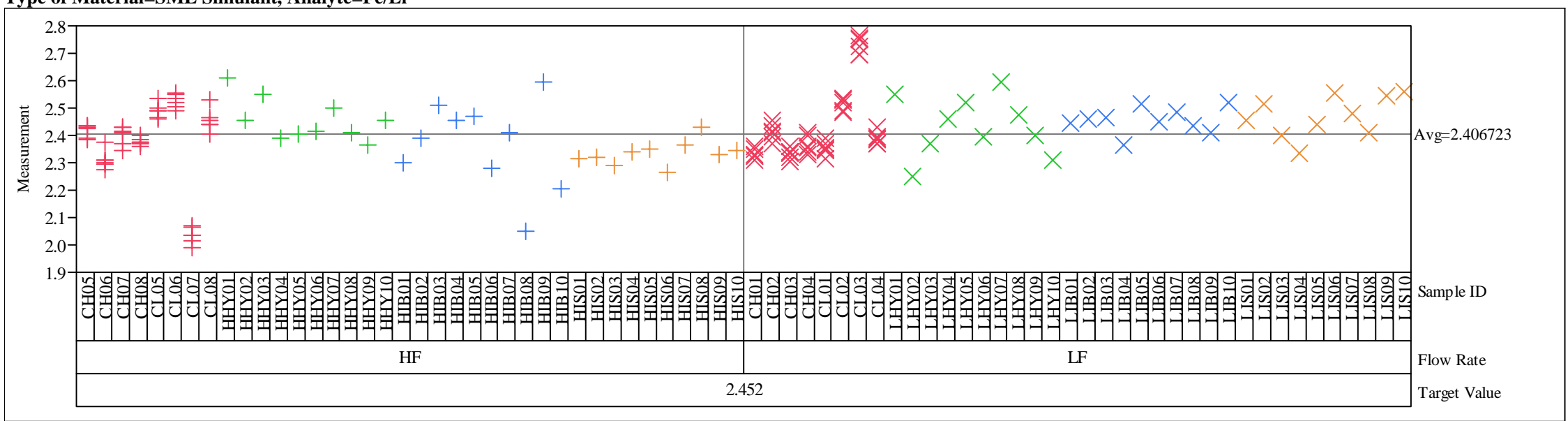

Type of Material=SME Simulant, Analyte=Fe2O3 (wt\%)

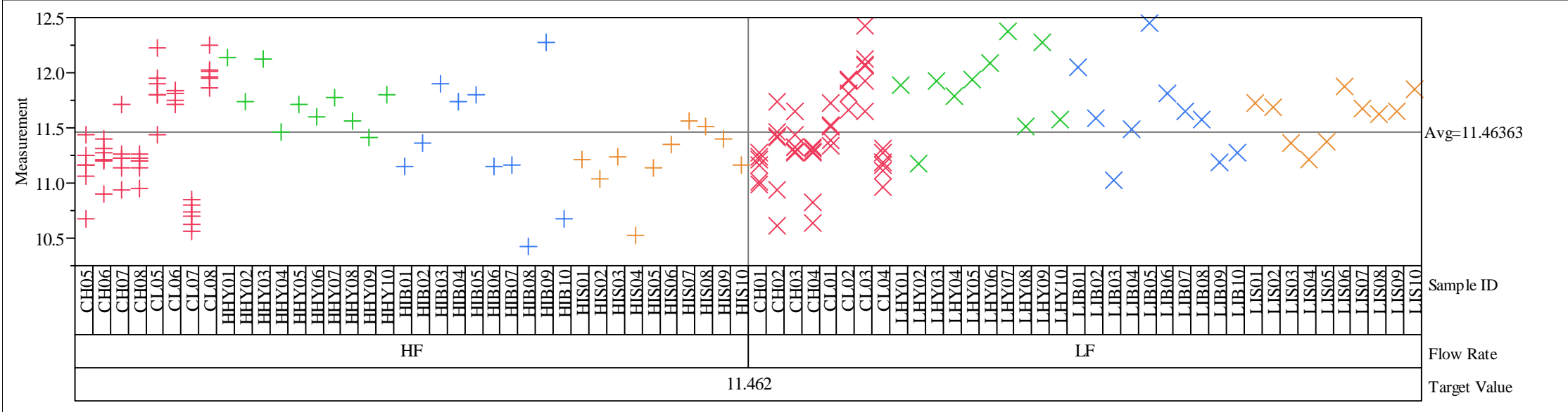




\section{Exhibit A1. Density, Solids, and Oxide Concentration Measurments for the Mid-Rheology (Phase 1) Testing}

Type of Material=SME Simulant, Analyte=K2O (wt\%)

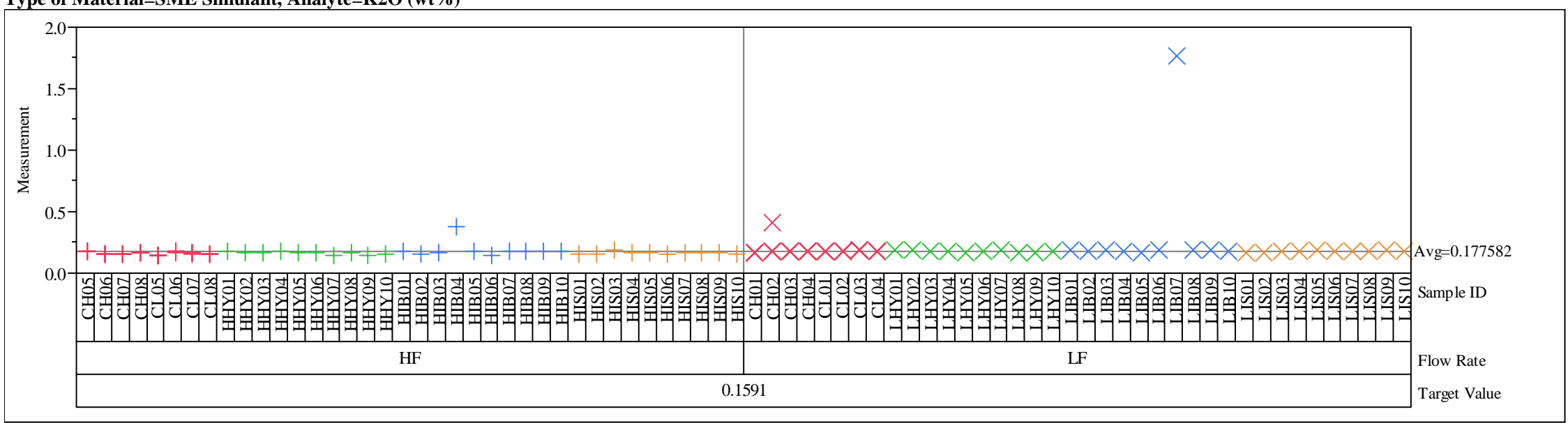

Type of Material=SME Simulant, Analyte=Li2O (wt\%)

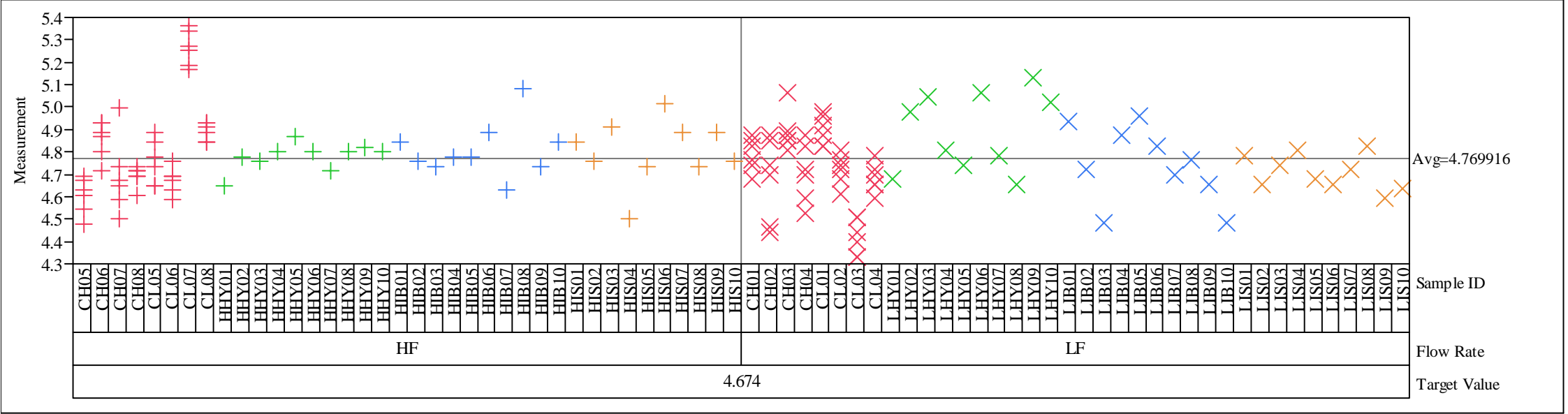




\section{Exhibit A1. Density, Solids, and Oxide Concentration Measurments for the Mid-Rheology (Phase 1) Testing}

Type of Material=SME Simulant, Analyte=MgO (wt \%)

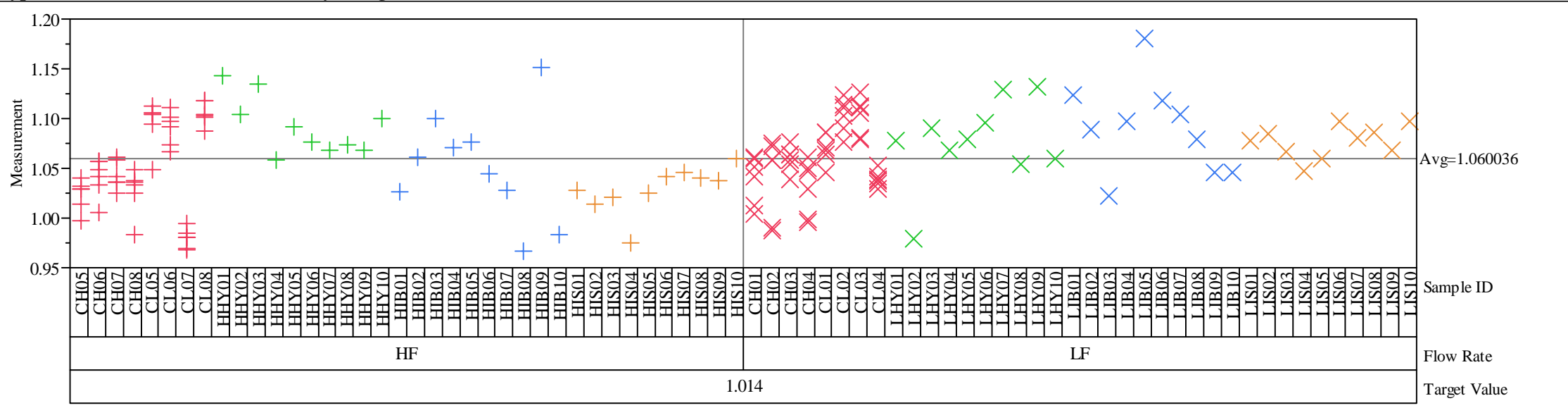

Type of Material=SME Simulant, Analyte=MnO (wt\%)

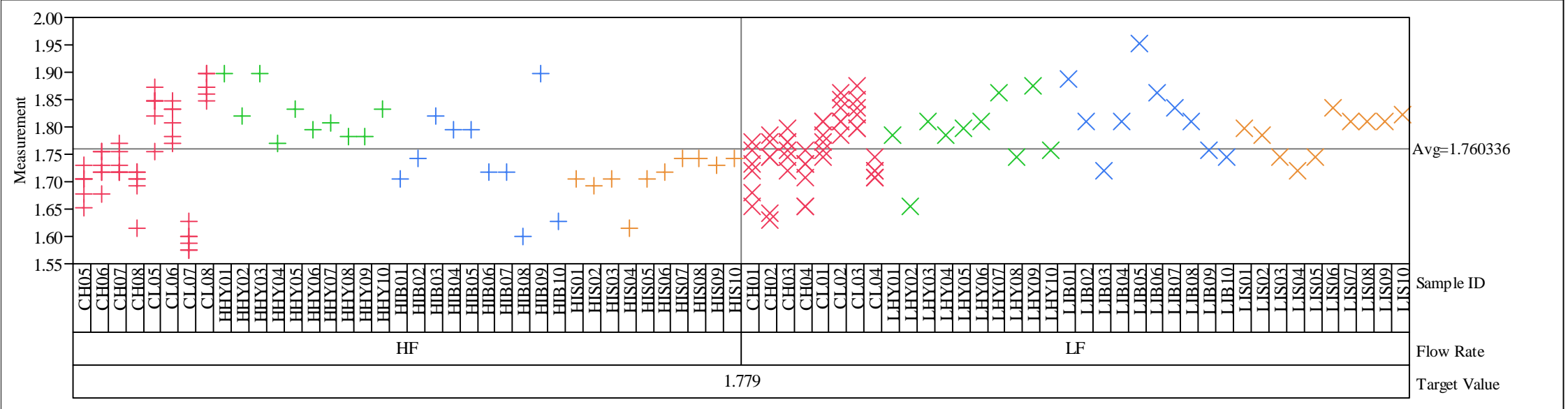




\section{Exhibit A1. Density, Solids, and Oxide Concentration Measurments for the Mid-Rheology (Phase 1) Testing}

Type of Material=SME Simulant, Analyte=Na2O (wt \%)

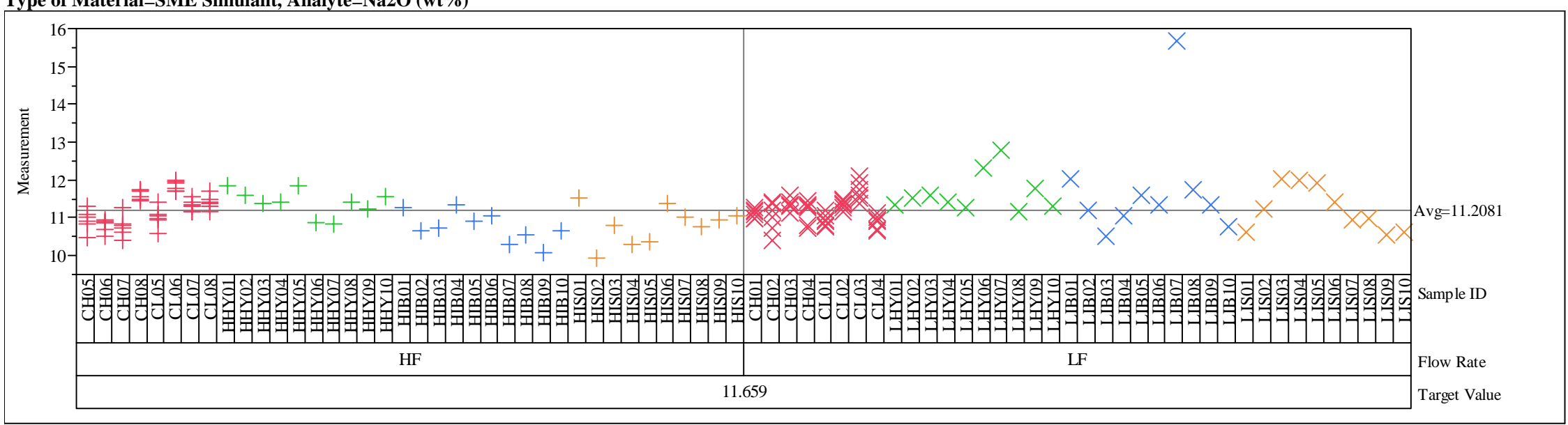

Type of Material=SME Simulant, Analyte=NiO (wt\%)

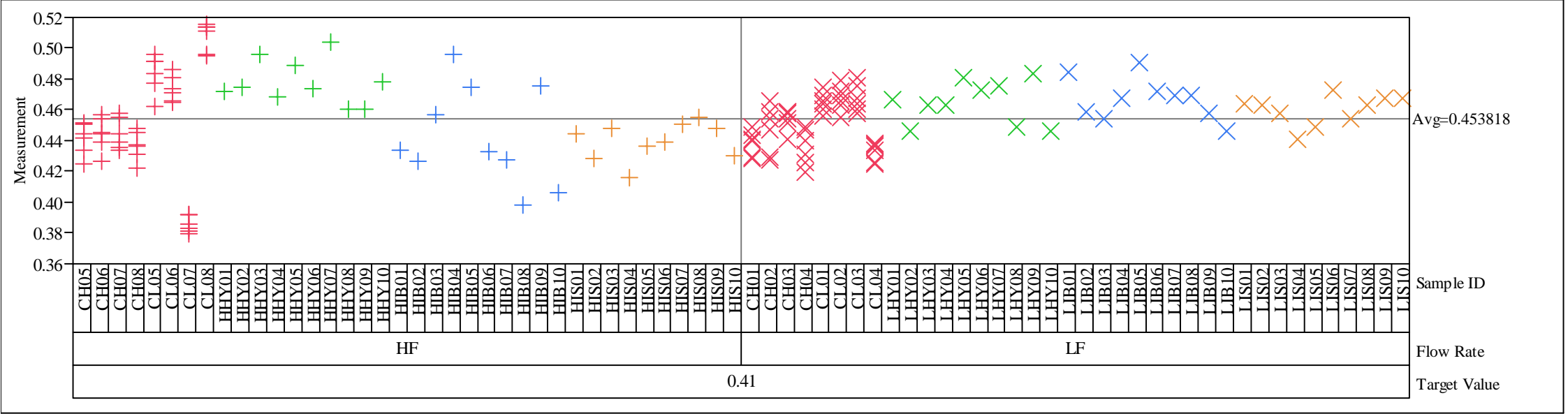




\section{Exhibit A1. Density, Solids, and Oxide Concentration Measurments for the Mid-Rheology (Phase 1) Testing}

Type of Material=SME Simulant, Analyte=SiO2 (wt \%)

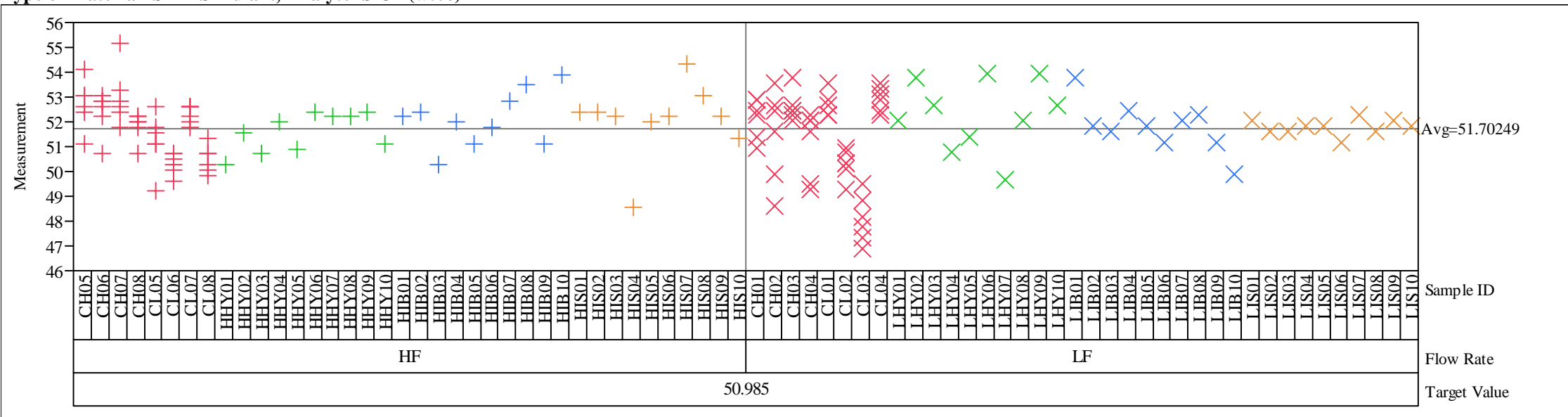

Type of Material=SME Simulant, Analyte=Sum of Oxides (wt\%)

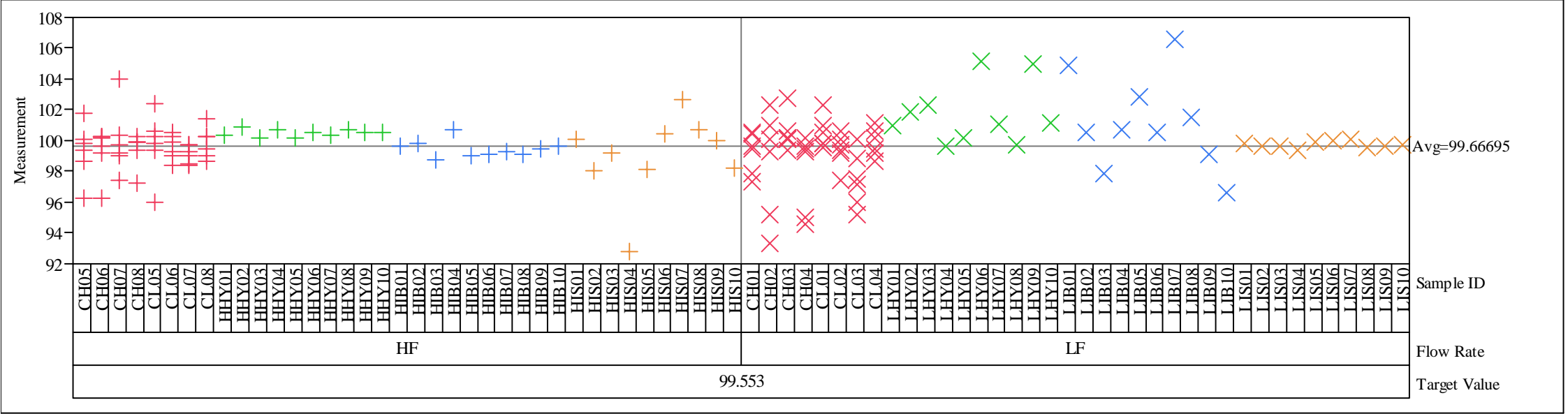




\section{Exhibit A1. Density, Solids, and Oxide Concentration Measurments for the Mid-Rheology (Phase 1) Testing}

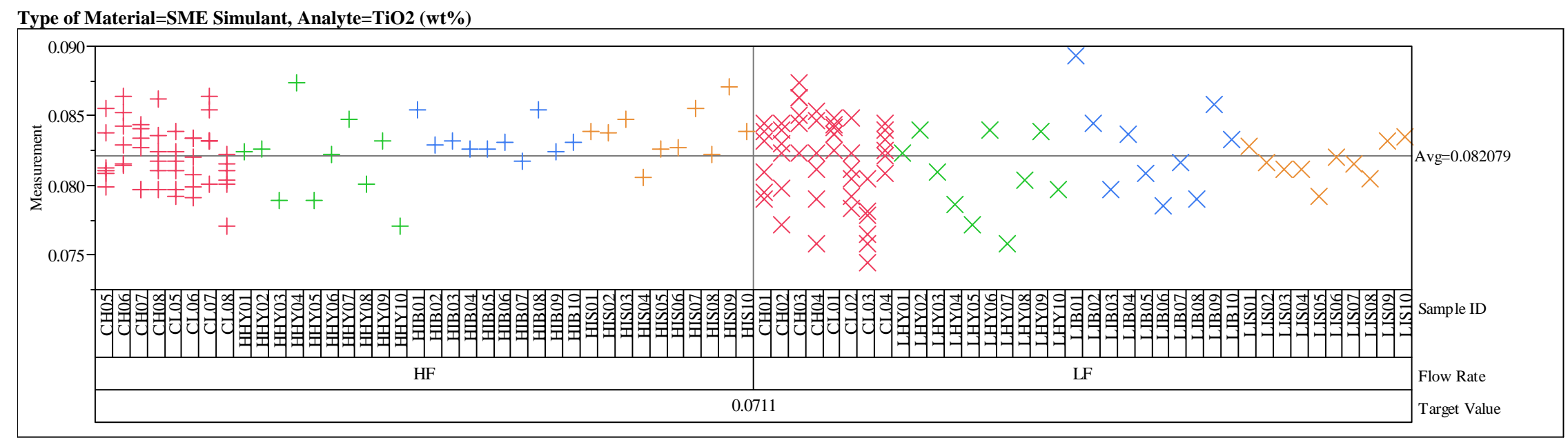

Type of Material=SME Simulant, Analyte=ZnO (wt \%)

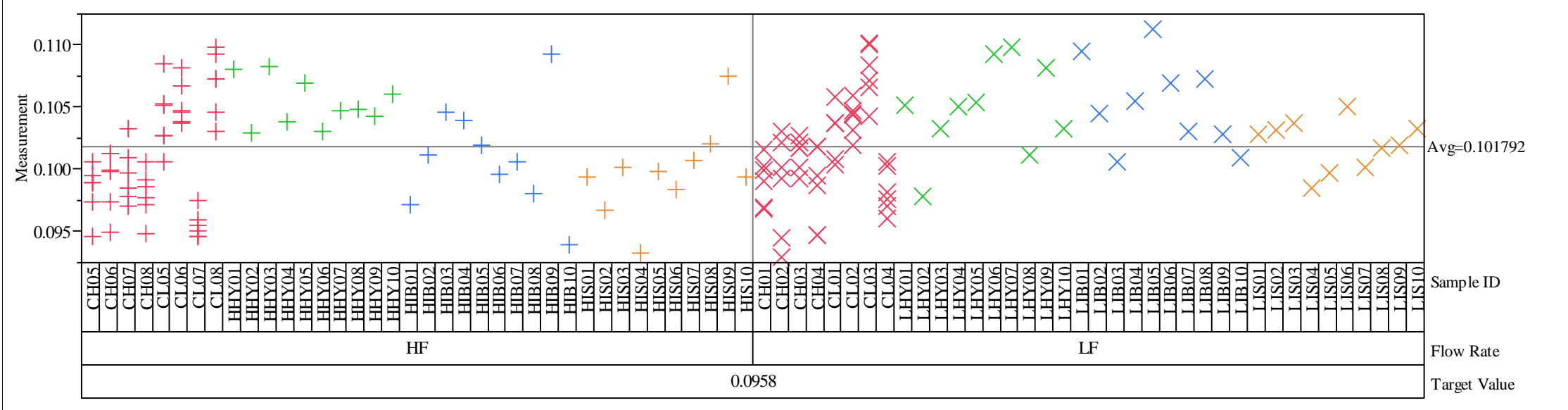




\section{Exhibit A1. Density, Solids, and Oxide Concentration Measurments for the Mid-Rheology (Phase 1) Testing}

Type of Material=SME Simulant, Analyte=ZrO2 (wt \%)

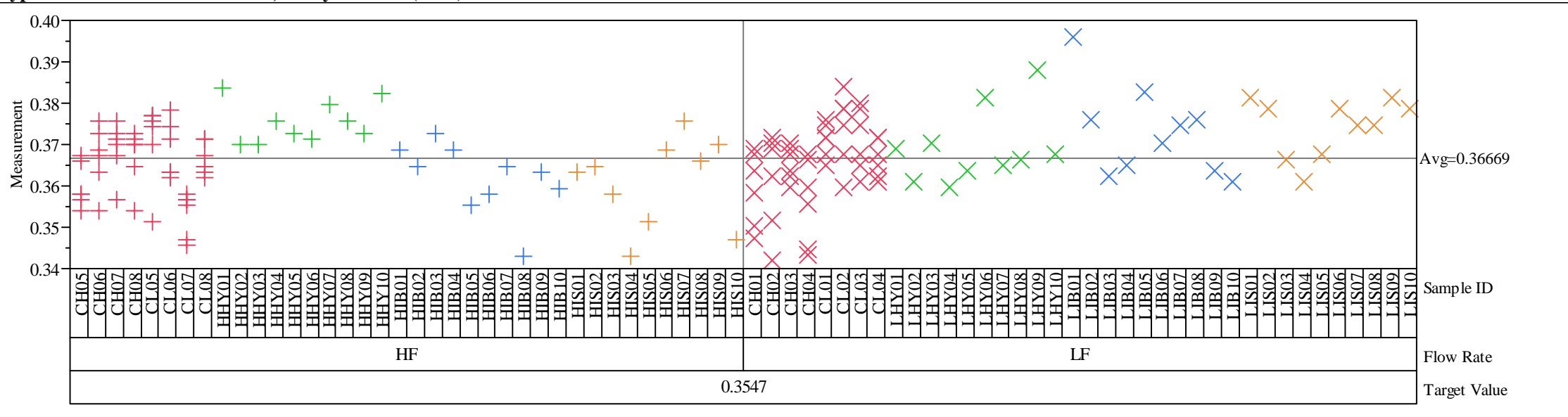




\section{Exhibit A2. Statistical Comparisons for Mid-Rheology (Phase 1) Testing}

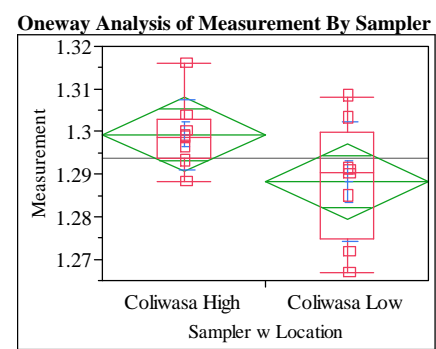

Oneway Anova

Summary of Fit

Rsquare

Adj Rsquare

Root Mean Square Error $\quad 0.152413$

Mean of Response $\quad 0.011489$

Observations (or Sum Wgts) $\quad 16$

t Test

Coliwasa Low-Coliwasa High

Assuming equal variances

Difference $\quad-0.01105$ t Ratio -1.92284

$\begin{array}{lrr}\text { Std Err Dif } \quad 0.00574 \text { DF } & 14\end{array}$

Upper CL Dif $\quad 0.00127$ Prob $>|t| \quad 0.0751$

Lower CL Dif -0.02337 Prob $>t \quad 0.9625$

$\begin{array}{lrl}\text { Confidence } & 0.95 \text { Prob }<\mathrm{t} & 0.0375\end{array}$

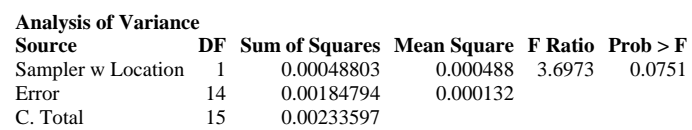

Means for Oneway Anova

Level Number Mean Std Error Lower 95\% Upper 95\%

$\begin{array}{lrrrrr}\text { Coliwasa High } & 8 & 1.29927 & 0.00406 & 1.2906 & 1.3080\end{array}$

$\begin{array}{llllll}\text { Coliwasa Low } & 8 & 1.28822 & 0.00406 & 1.2795 & 1.2969\end{array}$

Std Error uses a pooled estimate of error variance

Means and Std Deviations

Level Number Mean Std Dev Std Err Mean Lower 95\% Upper 95\%

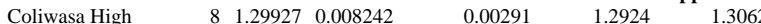

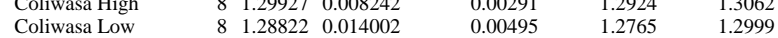

t Test

Coliwasa Low-Coliwasa High

Assuming unequal variances

Difference $\quad-0.01105$ t Ratio -1.92284

Std Err Dif $\quad 0.00574$ DF $\quad 11.33094$

Upper CL Dif 0.00155 Prob $>|t| \quad 0.0800$

Lower CL Dif -0.02364 Prob $>t \quad 0.9600$

$\begin{array}{lrl}\text { Confidence } & 0.95 \text { Prob }<\mathrm{t} & 0.0400\end{array}$

Tests that the Variances are Equal

Level Count Std Dev MeanAbsDif to Mean MeanAbsDif to Median

$\begin{array}{lllll} & & & \\ \text { Coliwasa High } & 8 & 0.0082421 & 0.0055373 & 0.0054055\end{array}$

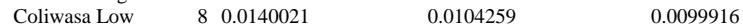

Test F Ratio DFNum DFDen p-Value

\begin{tabular}{lrrrr} 
O'Brien[.5] & 1.8785 & 1 & 14 & 0.192 \\
\hline
\end{tabular}

$\begin{array}{lllll}\text { Brown-Forsythe } & 1.3710 & 1 & 14 & 0.1921 \\ & 1.8257 & 1 & 14 & 0.1981\end{array}$

$\begin{array}{lllll}\text { Levene } & 1.8257 & 1 & 14 & 0.198\end{array}$

$\begin{array}{lllll}\text { Bartlett } & 1.7549 & 1 & ; & 0.1853 \\ \text { F Test 2-sided } & 2.8861 & 7 & 7 & 0.1854\end{array}$

Welch Anova testing Means Equal, allowing Std Devs Not Equal

F Ratio DFNum DFDen Prob $>$ F

$\begin{array}{rrrr}3.6973 & 1 & 11.331 & 0.0800\end{array}$

t Test

1.9228

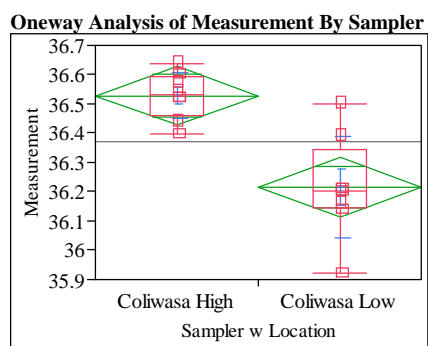

Oneway Anova

Summary of Fit

Rsquare

Adj Rsquare

Root Mean Square Error

Mean of Response

Observations (or Sum Wgts)

0.609866

0.581999

0.134134

36.37188

t Test

Coliwasa Low-Coliwasa High

Assuming equal variances

$\begin{array}{lrr}\text { Difference } & -0.31375 \text { t Ratio } & -4.67815 \\ \text { Std Err Dif } & 0.06707 & 14\end{array}$

Upper CL Dif -0.16991 Prob $>|t| 0.0004$

$\begin{array}{lll}\text { Lower CL Dif }-0.45759 & \text { Prob }>t & 0.9998\end{array}$

$\begin{array}{lrl}\text { Confidence } & 0.95 \text { Prob }<\mathrm{t} & 0.0002\end{array}$

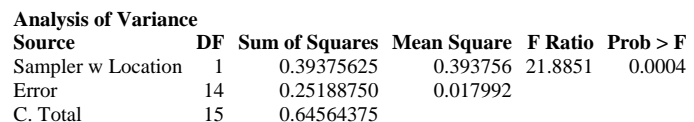

Means for Oneway Anova

Level Number Mean Std Error Lower 95\% Upper 95\%

$\begin{array}{lrrrrr}\text { Coliwasa High } & 8 & 36.5288 & 0.04742 & 36.427 & 36.630\end{array}$

$\begin{array}{llllll}\text { Coliwasa Low } & 8 & 36.2150 & 0.04742 & 36.113 & 36.317\end{array}$

Std Error uses a pooled estimate of error variance

Means and Std Deviations

Level Number Mean Std Dev Std Err Mean Lower 95\% Upper 95\%

$\begin{array}{lllllll}\text { Coliwasa High } & 8 & 36.5288 & 0.079181 & 0.02799 & 36.463 & 36.595\end{array}$

$\begin{array}{lllllll}\text { Coliwasa Low } & 8 & 36.2150 & 0.172378 & 0.06094 & 36.071 & 36.359\end{array}$

Test

Coliwasa Low-Coliwasa High

Assuming unequal variances

$\begin{array}{lrlr}\text { Difference } & -0.31375 & \text { t Ratio } & -4.67815 \\ \text { Std Err Dif } & 0.06707 & \text { DF } & 9.828061 \\ \text { Upper CL Dif } & -0.16396 & \text { Prob }>|t| & 0.0009 \\ \text { Lower CL Dif } & -0.46354 & \text { Prob }>\text { t } & 0.9995 \\ \text { Confidence } & 0.95 & \text { Prob }<\mathrm{t} & 0.0005\end{array}$

Tests that the Variances are Equal

Level Count Std Dev MeanAbsDif to Mean MeanAbsDif to Median

$\begin{array}{lllll}\text { Coliwasa High } & 8 & 0.0791811 & 0.0587500 & 0.0587500\end{array}$

$\begin{array}{lllll}\text { Coliwasa Low } & 8 & 0.1723783 & 0.1150000 & 0.1100000\end{array}$

Test F Ratio DFNum DFDen p-Value

$\begin{array}{lrrrr}\text { O'Brien[.5] } & 2.0041 & 1 & 14 & 0.1787\end{array}$

$\begin{array}{lllll}\text { Brown-Forsythe } & 1.1377 & 1 & 14 & 0.3042\end{array}$

$\begin{array}{lllll}\text { Levene } & 1.4956 & 1 & 14 & 0.2415\end{array}$

$\begin{array}{lllll}\text { Bartlett } & 3.6097 & 1 & \text {; } & 0.0574 \\ \text { F Test 2-sided } & 4.7394 & 7 & 7 & 0.0573\end{array}$

Welch Anova testing Means Equal, allowing Std Devs Not Equal

F Ratio DFNum DFDen Prob $>$ F

$21.8851 \quad 1 \quad 9.8281 \quad 0.0009$

t Test

4.6782 


\section{Exhibit A2. Statistical Comparisons for Mid-Rheology (Phase 1) Testing}

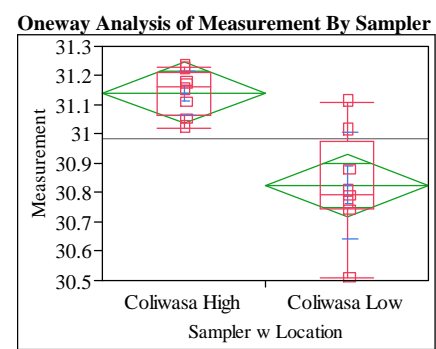

Hydragard - Low Flow Rate

Oneway Analysis of Measurement By Sampling Mechanism Analyte $=$ density $(\mathrm{g} / \mathrm{mL})$

Oneway Anova

Summary of Fit

$\begin{array}{lr}\text { Adj Rsquare } & 0.56685 \\ \text { Root Mean Square Error } \quad 0.139255\end{array}$

0.595726

Mean of Response 30.98313

Observations (or Sum Wgts)

t Test

Coliwasa Low-Coliwasa High

Assuming equal variances

Difference $\quad-0.31625$ t Ratio $\quad-4.54203$

Std Err Dif $\quad 0.06963$ DF 14

Upper CL Dif -0.16691 Prob $>|t| \quad 0.0005$

Lower CL Dif -0.46559 Prob $>\mathrm{t} \quad 0.9998$

$\begin{array}{lrr}\text { Confidence } & 0.95 \text { Prob }<\mathrm{t} & 0.0002\end{array}$

$\begin{array}{lrrrrr}\text { Analysis of Variance } & & & & & \\ \text { Source } & \text { DF } & \text { Sum of Squares } & \text { Mean Square } & \text { F Ratio } & \text { Prob > F } \\ \text { Sampler w Location } & 1 & 0.40005625 & 0.400056 & 20.6300 & 0.0005 \\ \text { Error } & 14 & 0.27148750 & 0.019392 & & \\ \text { C. Total } & 15 & 0.67154375 & & & \end{array}$

Means for Oneway Anova

Level Number Mean Std Error Lower 95\% Upper 95\%

$\begin{array}{lrrrrr}\text { Coliwasa High } & 8 & 31.1413 & 0.04923 & 31.036 & 31.247 \\ \text { Coliwasa Low } & 8 & 30.8250 & 0.04923 & 30.719 & 30.931\end{array}$

Std Error uses a pooled estimate of error variance

Means and Std Deviations

Level Number Mean Std Dev Std Err Mean Lower 95\% Upper 95\%

$\begin{array}{lrrrrrr}\text { Coliwasa High } & 8 & 31.1413 & 0.076052 & 0.02689 & 31.078 & 31.205\end{array}$

$\begin{array}{lllllll}\text { Coliwasa Low } & 8 & 30.8250 & 0.181659 & 0.06423 & 30.673 & 30.977\end{array}$

Test

Coliwasa Low-Coliwasa High

Assuming unequal variances

Difference $\quad-0.31625$ t Ratio $\quad-4.54203$

Std Err Dif $\quad 0.06963$ DF $\quad 9.380655$

Upper CL Dif -0.15971 Prob $>|t| \quad 0.0013$

Lower CL Dif -0.47279 Prob $>t \quad 0.9994$

$\begin{array}{lrl}\text { Confidence } & 0.95 \text { Prob }<\mathrm{t} & 0.0006\end{array}$

Tests that the Variances are Equal

Level Count Std Dev MeanAbsDif to Mean MeanAbsDif to Median

$\begin{array}{lllll} & & & \text { Std } & \\ \text { Coliwasa High } & 8 & 0.0760521 & 0.0609375 & 0.0587500\end{array}$

$\begin{array}{lllll}\text { Coliwasa Low } & 8 & 0.1816590 & 0.1312500 & 0.1250000\end{array}$

Test F Ratio DFNum DFDen p-Value

$\begin{array}{lrrrr}\text { O'Brien[.5] } & 2.4139 & 1 & 14 & 0.1426\end{array}$

$\begin{array}{lllll}\text { Brown-Forsythe } & 1.9070 & 1 & 14 & 0.1489 \\ & 2.6629 & 1 & 14 & 0.1250\end{array}$

$\begin{array}{lllll}\text { Levene } & 2.6629 & 1 & 14 & 0.1250\end{array}$

$\begin{array}{lllll}\text { Bartlett } & 4.4304 & 1 & \text {; } & 0.0353 \\ \text { F Test 2-sided } & 5.7055 & 7 & 7 & 0.0351\end{array}$

Welch Anova testing Means Equal, allowing Std Devs Not Equal

F Ratio DFNum DFDen Prob $>$ F

$\begin{array}{rrrrr}20.6300 & 1 & 9.3807 & 0.0013\end{array}$

t Test

4.5420

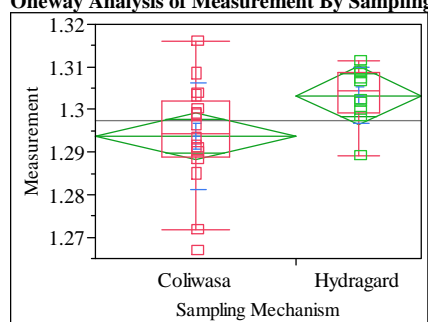

Oneway Anova

Summary of Fit

Adj Rsquare

0.137427

0.010669

Mean of Response 1.297435

t Test

Hydragard-Coliwasa

Assuming equal variances

Difference $\quad 0.009600$ t Ratio $\quad 2.232274$

Std Err Dif $\quad 0.004301$ DF 24

Upper CL Dif 0.018477 Prob $>|t| \quad 0.0352$

Lower CL Dif 0.000724 Prob $>t \quad 0.0176$

$\begin{array}{lrl}\text { Confidence } & 0.95 \text { Prob }<\mathrm{t} & 0.9824\end{array}$

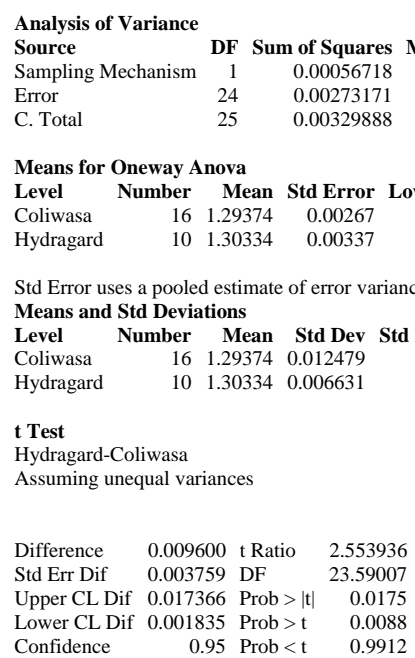

Tests that the Variances are Equal

Level Count Std Dev MeanAbsDif to Mean MeanAbsDif to Median

$\begin{array}{lrrrr}\text { Coliwasa } & 16 & 0.0124792 & 0.0092521 & 0.0092521\end{array}$

$\begin{array}{lllll}\text { Coliwasa } & 16 & 0.0124792 & 0.0092521 & 0.0092521 \\ \text { Hydragard } & 10 & 0.0066311 & 0.0051731 & 0.0051731\end{array}$

Test F Ratio DFNum DFDen p-Value

$\begin{array}{lrrrr}\text { O'Brien[.5] } & 1.9470 & 1 & 24 & 0.1757\end{array}$

$\begin{array}{lllll}\text { Brown-Forsythe } & 2.2067 & 1 & 24 & 0.1504\end{array}$

$\begin{array}{lllll}\text { Levene } & 2.2451 & 1 & 24 & 0.1471\end{array}$

$\begin{array}{lrrrr}\text { Bartlett } & 3.6899 & 1 & \text {. } & 0.0547 \\ \text { F Test 2-sided } & 3.5417 & 15 & 9 & 0.0609\end{array}$

Welch Anova testing Means Equal, allowing Std Devs Not Equal

F Ratio DFNum DFDen Prob $>$ F

$\begin{array}{rrrr}6.5226 & 1 & 23.59 & 0.0175\end{array}$

t Test

2.5539 


\section{Exhibit A2. Statistical Comparisons for Mid-Rheology (Phase 1) Testing}

Hydragard - Low Flow Rate Oneway Analysis of Measurement By Sampling Mechanism Analyte=wt \% dried solids

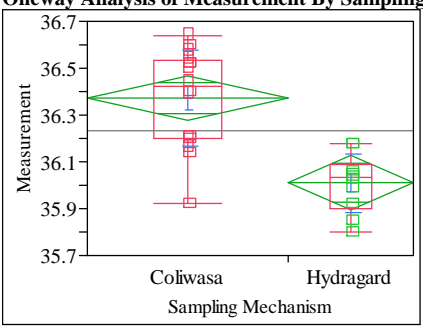

Oneway Anova

Summary of Fit

Adj Rsquare

$\quad 0.18109$

Observations (or Sum Wgts) $\quad 26$

t Test

Hydragard-Coliwasa

Assuming equal variances

Difference $\quad-0.36188$ t Ratio $\quad-4.95722$

Std Err Dif $\quad 0.07300$ DF $\quad 24$

Upper CL Dif -0.21121 Prob $>|t| \quad<.0001$

Lower CL Dif -0.51254 Prob $>t \quad 1.0000$

$\begin{array}{lrl}\text { Confidence } & 0.95 \text { Prob }<\mathrm{t} & <.0001\end{array}$

Analysis of Variance

$\begin{array}{lrrrrr}\text { Source } & \text { DF } & \text { Sum of Squares } & \text { Mean Square } & \text { F Ratio } & \text { Prob > F } \\ \text { Sampling Mechanism } & 1 & 0.8058678 & 0.805868 & 24.5740 & <.0001\end{array}$

$\begin{array}{llll}\text { Error } & 24 & 0.7870437 & 0.032793\end{array}$

$\begin{array}{lll}\text { C. Total } & 25 & 1.5929115\end{array}$

Means for Oneway Anova

Level Number Mean Std Error Lower 95\% Upper 95\%

$\begin{array}{lrrrrr}\text { Coliwasa } & 16 & 36.3719 & 0.04527 & 36.278 & 36.465\end{array}$

$\begin{array}{llllll}\text { Hydragard } & 10 & 36.0100 & 0.05727 & 35.892 & 36.128\end{array}$

Std Error uses a pooled estimate of error variance

Means and Std Deviations

Level Number Mean Std Dev Std Err Mean Lower 95\% Upper 95\%

$\begin{array}{lrrrrrr}\text { Coliwasa } & 16 & 36.3719 & 0.207468 & 0.05187 & 36.261 & 36.482\end{array}$

$\begin{array}{lllllll}\text { Hydragard } & 10 & 36.0100 & 0.125344 & 0.03964 & 35.920 & 36.400\end{array}$

Test

Hydragard-Coliwasa

Assuming unequal variances

Difference $\quad-0.36188$ t Ratio $\quad-5.54355$

Std Err Dif $\quad 0.06528$ DF 23.99593

Upper CL Dif -0.22715 Prob $>|t| r .0001$

Lower CL Dif -0.49660 Prob $>t \quad 1.0000$

$\begin{array}{lrl}\text { Confidence } & 0.95 \text { Prob }<\mathrm{t} & <.0001\end{array}$

Tests that the Variances are Equal

Level Count Std Dev MeanAbsDif to Mean MeanAbsDif to Median

Coliwasa $\quad 16 \quad 0.2074679$

$\begin{array}{lllll}\text { Hydragard } & 10 & 0.1253440 & 0.1751562 & 0.1693750 \\ & & & 0.0960000 & 0.0920000\end{array}$

Test F Ratio DFNum DFDen p-Value

$\begin{array}{lrrrr}\text { O'Brien[.5] } & 2.4347 & 1 & 24 & 0.1318\end{array}$

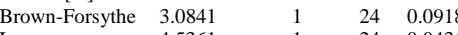

$\begin{array}{lllll}\text { Levene } & 4.5361 & 1 & 24 & 0.0436\end{array}$

$\begin{array}{lrrrr}\text { Bartlett } & 2.4329 & 1 & \text {. } & 0.1188\end{array}$

Welch Anova testing Means Equal, allowing Std Devs Not Equal

F Ratio DFNum DFDen Prob $>$ F

$\begin{array}{rrrr}30.7309 & 1 & 23.996 & <.0001\end{array}$

t Test
5.5435
Hydragard - Low Flow Rate

Oneway Analysis of Measurement By Sampling Mechanism Analyte=wt $\%$ vitrified solids

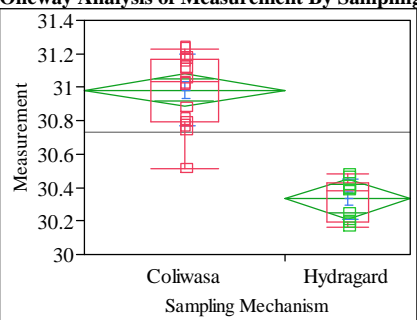

Oneway Anova

Summary of Fit

Adj Rsquare

0.182746

Mean of Response 30.73269

Observations (or Sum Wgts) $\quad 26$

Test

Hydragard-Coliwasa

Assuming equal variances

$\begin{array}{lrlr}\text { Difference } & -0.65113 & \mathrm{t} \text { Ratio } & -8.83874 \\ \text { Std Err Dif } & 0.07367 & \text { DF } & 24 \\ \text { Upper CL Dif } & -0.49908 & \text { Prob }>|t| & <.0001 \\ \text { Lower CL Dif } & -0.80317 & \text { Prob }>\mathrm{t} & 1.0000 \\ \text { Confidence } & 0.95 & \text { Prob }<\mathrm{t} & <.0001\end{array}$

Analysis of Variance

Source DF Sum of Squares Mean Square F Ratio Prob > F

Sampling Mechanism $\quad 1 \quad 2.6090078$

$\begin{array}{lll}24 & 0.8015037\end{array}$

$2.6090178 .1234<.000$

C. Total $25 \quad 3.4105115$

Means for Oneway Anova

Level Number Mean Std Error Lower 95\% Upper 95\%

$\begin{array}{lrrrrr}\text { Coliwasa } & 16 & 30.9831 & 0.04569 & 30.889 & 31.077\end{array}$

$\begin{array}{llllll}\text { Hydragard } & 10 & 30.3320 & 0.05779 & 30.213 & 30.45\end{array}$

Std Error uses a pooled estimate of error variance

Means and Std Deviations

Level Number Mean Std Dev Std Err Mean Lower 95\% Upper 95\%

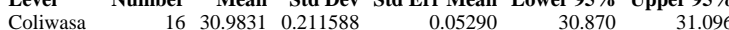

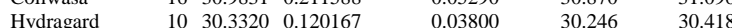

Test

Hydragard-Coliwasa

Assuming unequal variances

$\begin{array}{lrlr}\text { Difference } & -0.65113 & \text { t Ratio } & -9.9971 \\ \text { Std Err Dif } & 0.06513 & \text { DF } & 23.87802 \\ \text { Upper CL Dif } & -0.51666 \text { Prob }>|t| & <.0001 \\ \text { Lower CL Dif } & -0.78559 & \text { Prob }>\text { t } & 1.0000 \\ \text { Confidence } & 0.95 & \text { Prob }<\mathrm{t} & <.0001\end{array}$

Tests that the Variances are Equal

Level Count Std Dev MeanAbsDif to Mean MeanAbsDif to Median

$\begin{array}{lllll}\text { Coliwasa } & 16 & 0.2115882 & 0.1773437 & 0.1693750\end{array}$

$\begin{array}{lllll}\text { Hydragard } & 10 & 0.1201666 & 0.1056000 & 0.0960000\end{array}$

Test F Ratio DFNum DFDen p-Value

$\begin{array}{lrrrr}\text { O'Brien[.5] } & 2.6258 & 1 & 24 & 0.1182\end{array}$

$\begin{array}{lllll}\text { Brown-Forsythe } & 2.4734 & 1 & 24 & 0.1289\end{array}$

$\begin{array}{lllll}\text { Levene } & 4.0701 & 1 & 24 & 0.0550\end{array}$

$\begin{array}{lrrrr}\text { Bartlett } & 3.0128 & 1 & \text {. } & 0.0826 \\ \text { F Test 2-sided } & 3.1004 & 15 & 9 & 0.0913\end{array}$

Welch Anova testing Means Equal, allowing Std Devs Not Equal

F Ratio DFNum DFDen Prob $>$ F

$\begin{array}{lll}99.9420 & 1 & 23.878<0001\end{array}$

t Test

9.997 


\section{Exhibit A2. Statistical Comparisons for Mid-Rheology (Phase 1) Testing}

Hydragard - High Flow Rate

Oneway Analysis of Measurement By Sampling Mechanism Analyte=density (g/mL)

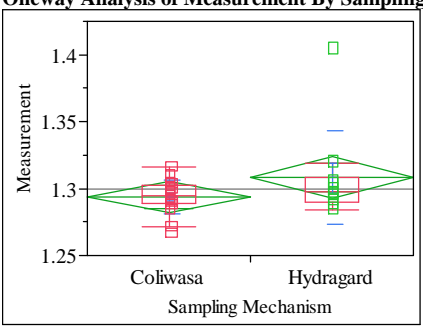

Oneway Anova

Summary of Fit

Adj Rsquare

0.087973

\begin{tabular}{ll}
0.023504 \\
\hline
\end{tabular}

Observations (or Sum Wgts) $\quad 26$

t Test

Hydragard-Coliwasa

Assuming equal variances

Difference $\quad 0.01442$ t Ratio 1.521512

Std Err Dif $\quad 0.00947$ DF $\quad 24$

Upper CL Dif 0.03397 Prob $>|t| \quad 0.1412$

Lower CL Dif -0.00514 Prob $>$ t $\quad 0.0706$

$\begin{array}{lrl}\text { Confidence } & 0.95 \text { Prob }<\mathrm{t} & 0.9294\end{array}$

Analysis of Variance

$\begin{array}{lrrrrr}\text { Source } & \text { DF } & \text { Sum of Squares } & \text { Mean Square } & \text { F Ratio } & \text { Prob }>\text { F } \\ \text { Sampling Mechanism } & 1 & 0.00127890 & 0.001279 & 2.3150 & 0.1412\end{array}$

$\begin{array}{llll}\text { Error } & 24 & 0.01325862 & 0.000552\end{array}$

$25 \quad 0.01453752$

Means for Oneway Anova

Level Number Mean Std Error Lower 95\% Upper 95\%

$\begin{array}{lrrrrr}\text { Coliwasa } & 16 & 1.29374 & 0.00588 & 1.2816 & 1.3059\end{array}$

$\begin{array}{llllll}\text { Hydragard } & 10 & 1.30816 & 0.00743 & 1.2928 & 1.3235\end{array}$

Std Error uses a pooled estimate of error variance

Means and Std Deviations

Level Number Mean Std Dev Std Err Mean Lower 95\% Upper 95\%

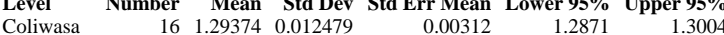

$\begin{array}{lllllll}\text { Hydragard } & 10 & 1.30816 & 0.034837 & 0.01102 & 1.2832 & 1.3331\end{array}$

tTest

Hydragard-Coliwasa

Assuming unequal variances

$\begin{array}{lrlr}\text { Difference } & 0.01442 & \text { t Ratio } & 1.259073 \\ \text { Std Err Dif } & 0.01145 & \text { DF } & 10.4611 \\ \text { Upper CL Dif } & 0.03978 \text { Prob }>|t| & 0.2354 \\ \text { Lower CL Dif } & -0.01094 & \text { Prob }>t & 0.1177 \\ \text { Confidence } & 0.95 & \text { Prob }<\mathrm{t} & 0.8823\end{array}$

Tests that the Variances are Equal

Level Count Std Dev MeanAbsDif to Mean MeanAbsDif to Median

$\begin{array}{llll}\text { Coliwasa } & 16 & 0.0124792\end{array}$

$\begin{array}{lllll}\text { Hydragard } & 10 & 0.0348372 & 0.0092521 & 0.0092521 \\ & & & 0.0211733 & 0.0176025\end{array}$

Test F Ratio DFNum DFDen p-Value

$\begin{array}{lrrrr}\text { O'Brien[.5] } & 1.6653 & 1 & 24 & 0.2092\end{array}$

Brown-Forsythe $1.0291 \quad 1 \quad 24 \quad 0.3205$

$\begin{array}{lrlll}\text { Levene } & 2.8342 & 1 & 24 & 0.1052\end{array}$

$\begin{array}{lrrrr}\text { Bartlett } & 11.3932 & 1 & \text {. } & 0.0007\end{array}$

Welch Anova testing Means Equal, allowing Std Devs Not Equal

F Ratio DFNum DFDen Prob $>$ F

$\begin{array}{rrrr}1.5853 & 1 & 10.461 & 0.2354\end{array}$

t Test

1.2591
Hydragard - High Flow Rate

Oneway Analysis of Measurement By Sampling Mechanism Analyte $=w$ t $\%$ dried solids

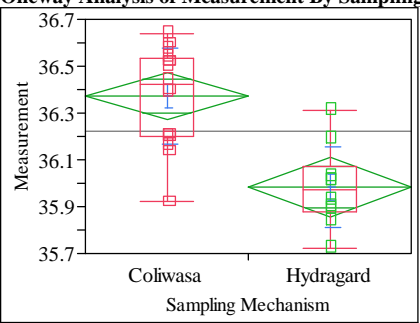

Oneway Anova

Summary of Fit

Adj Rsquare

0.506409

\begin{tabular}{ll}
0.485842 \\
\hline
\end{tabular}

$\begin{array}{lr}\text { Mean of Response } & 36.22231 \\ \text { Observations (or Sum Wgts) } & 26\end{array}$

t Test

Hydragard-Coliwasa

Assuming equal variances

$\begin{array}{lrlr}\text { Difference } & -0.38887 & \text { t Ratio } & -4.96218 \\ \text { Std Err Dif } & 0.07837 & \text { DF } & 24 \\ \text { Upper CL Dif } & -0.22713 & \text { Prob }>|t| & <.0001 \\ \text { Lower CL Dif } & -0.55062 & \text { Prob }>t & 1.0000 \\ \text { Confidence } & 0.95 & \text { Prob }<\mathrm{t} & <.0001\end{array}$

Analysis of Variance

$\begin{array}{lrrrrr}\text { Source } & \text { DF } & \text { Sum of Squares } & \text { Mean Square } & \text { F Ratio } & \text { Prob }>\text { F }\end{array}$

$\begin{array}{llll}\text { Error } & 24 & 0.9070538 & 0.037794\end{array}$

$\begin{array}{lll}\text { C. Total } & 25 & 1.8376615\end{array}$

Means for Oneway Anova

Level Number Mean Std Error Lower 95\% Upper 95\%

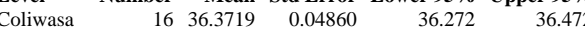

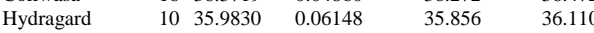

Std Error uses a pooled estimate of error variance

Means and Std Deviations

Level Number Mean Std Dev Std Err Mean Lower 95\% Upper 95\%

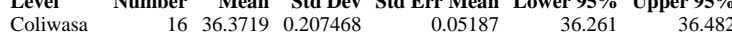

$\begin{array}{lllllll}\text { Hydragard } & 10 & 35.9830 & 0.170428 & 0.05389 & 35.861 & 36.105\end{array}$

T Test

Hydragard-Coliwasa

Assuming unequal variances

$\begin{array}{lrlr}\text { Difference } & -0.38887 & \mathrm{t} \text { Ratio } & -5.199 \\ \text { Std Err Dif } & 0.07480 & \text { DF } & 22.04528 \\ \text { Upper CL Dif } & -0.23377 & \text { Prob }>|\mathrm{t}| & <.0001 \\ \text { Lower CL Dif } & -0.54398 & \text { Prob }>\mathrm{t} & 1.0000 \\ \text { Confidence } & 0.95 & \text { Prob }<\mathrm{t} & <.0001\end{array}$

Tests that the Variances are Equal

Level Count Std Dev MeanAbsDif to Mean MeanAbsDif to Median

$\begin{array}{lllll}\text { Coliwasa } & 16 & 0.2074679 & 0.1751562 & 0.1693750\end{array}$

$\begin{array}{lllll}\text { Coliwasa } & 16 & 0.2074679 & 0.1751562 & 0.1693750 \\ \text { Hydragard } & 10 & 0.1704276 & 0.1270000 & 0.1270000\end{array}$

Test F Ratio DFNum DFDen p-Value

$\begin{array}{lrrrr}\text { O'Brien[.5] } & 0.4950 & 1 & 24 & 0.4885\end{array}$

$\begin{array}{lllll}\text { Brown-Forsythe } & 0.8147 & 1 & 24 & 0.3757\end{array}$

$\begin{array}{lllll}\text { Levene } & 1.3437 & 1 & 24 & 0.2578\end{array}$

$\begin{array}{lrrrr}\text { Bartlett } & 0.4006 & 1 & \text {. } & 0.5268 \\ \text { F Test 2-sided } & 1.4819 & 15 & 9 & 0.5597\end{array}$

Welch Anova testing Means Equal, allowing Std Devs Not Equal

F Ratio DFNum DFDen Prob $>$ F

$27.0296 r \begin{array}{lll}2 & 2.045 & <.0001\end{array}$

t Test

5.1990 
SRNL-STI-2011-00693

Revision 0

\section{Exhibit A2. Statistical Comparisons for Mid-Rheology (Phase 1) Testing}

Hydragard - High Flow Rate

Oneway Analysis of Measurement By Sampling Mechanism Analyte=wt \% vitrified solids

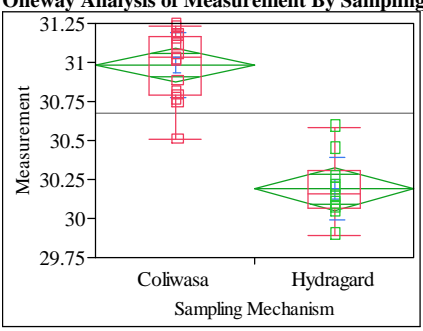

Oneway Anova

Summary of Fit

Adj Rsquare

0.781669

are Error $\quad 0.207335$

Mean of Response

Observations (or Sum Wgts) $\quad 26$

Test

Hydragard-Coliwasa

Assuming equal variances

Difference $\quad-0.79513$ t Ratio $\quad-9.51342$

Std Err Dif $\quad 0.08358$ DF 24

Upper CL Dif -0.62263 Prob $>|t| r .0001$

Lower CL Dif -0.96762 Prob $>t \quad 1.0000$

$\begin{array}{lrl}\text { Confidence } & 0.95 \text { Prob }<\mathrm{t} & <.0001\end{array}$

Analysis of Variance

$\begin{array}{lrrrrr}\text { Source } & \text { DF } & \text { Sum of Squares } & \text { Mean Square } & \text { F Ratio } & \text { Prob }>\text { F } \\ \text { Sampling Mechanism } & 1 & 3.8906078 & 3.89061 & 90.5052 & <.0001\end{array}$

$\begin{array}{llll}\text { Error } & 24 & 1.0317037 & 0.04299\end{array}$

$\begin{array}{lll}\text { C. Total } & 25 & 4.9223115\end{array}$

Means for Oneway Anova

Level Number Mean Std Error Lower 95\% Upper 95\%

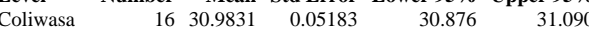

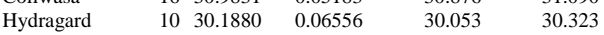

Std Error uses a pooled estimate of error variance

Means and Std Deviations

Level Number Mean Std Dev Std Err Mean Lower 95\% Upper 95\%

$16 \quad 30.98310 .211588$

$\begin{array}{lllllll}\text { Coliwasa } & 16 & 30.9831 & 0.211588 & 0.05290 & 30.870 & 31.096 \\ \text { Hydragard } & 10 & 30.1880 & 0.200044 & 0.06326 & 30.045 & 30.331\end{array}$

Test

Hydragard-Coliwasa

Assuming unequal variances

Difference $\quad-0.79513$ t Ratio $\quad-9.64239$

Std Err Dif $\quad 0.08246$ DF 20.09213

Upper CL Dif -0.62316 Prob $>|t| r .0001$

Lower CL Dif -0.96709 Prob $>t \quad 1.0000$

$\begin{array}{lrl}\text { Confidence } & 0.95 \text { Prob }<\mathrm{t} & <.0001\end{array}$

Tests that the Variances are Equal

Level Count Std Dev MeanAbsDif to Mean MeanAbsDif to Median

$\begin{array}{lrrrr}\text { Coliwasa } & 16 & 0.2115882 & 0.1773437 & 0.1693750\end{array}$

$\begin{array}{lllll}\text { Coliwasa } & 16 & 0.2115882 & 0.1773437 & 0.1693750 \\ \text { Hydragard } & 10 & 0.2000444 & 0.1456000 & 0.1440000\end{array}$

Test F Ratio DFNum DFDen p-Value

$\begin{array}{lrrrr}\text { O'Brien[.5] } & 0.0401 & 1 & 24 & 0.8429\end{array}$

Brown-Forsythe $\quad 0.2268 \quad 1 \quad 24 \quad 0.6382$

$\begin{array}{lllll}\text { Levene } & 0.4702 & 1 & 24 & 0.4995\end{array}$

$\begin{array}{lrrrr}\text { Bartlett } & 0.0335 & 1 & \text {. } & 0.8547\end{array}$

Welch Anova testing Means Equal, allowing Std Devs Not Equal

F Ratio DFNum DFDen Prob $>$ F

$\begin{array}{rrrr}92.9758 & 1 & 20.092 & <.0001\end{array}$

t Test

9.6424
Isolok $12 \mathrm{~mL}$ - Low Flow Rate

Oneway Analysis of Measurement By Sampling Mechanism Analyte $=$ density $(\mathrm{g} / \mathrm{mL})$

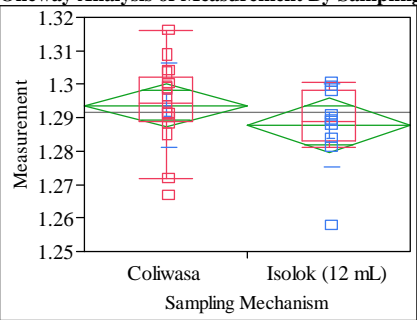

Oneway Anova

Summary of Fit

Adj Rsquare

0.01242

$\begin{array}{lr}\text { Mean of Response } & 1.291426 \\ \text { Observations (or Sum Wgts) } & 26\end{array}$

T Test

Isolok (12 mL)-Coliwasa

Assuming equal variances

$\begin{array}{lrlr}\text { Difference } & -0.00602 & \mathrm{t} \text { Ratio } & -1.20266 \\ \text { Std Err Dif } & 0.00501 & \text { DF } & 24 \\ \text { Upper CL Dif } & 0.00431 & \text { Prob }>|t| & 0.2408 \\ \text { Lower CL Dif } & -0.01635 & \text { Prob }>\mathrm{t} & 0.8796 \\ \text { Confidence } & 0.95 & \text { Prob }<\mathrm{t} & 0.1204\end{array}$

Analysis of Variance

$\begin{array}{lrrrrr}\text { Source } & \text { DF } & \text { Sum of Squares } & \text { Mean Square } & \text { F Ratio } & \text { Prob }>\text { F } \\ \text { Sampling Mechanism } & 1 & 0.00022312 & 0.000223 & 1.4464 & 0.2408 \\ \text { Error } & 24 & 0.00370215 & 0.000154 & & \\ \text { C. Total } & 25 & 0.00392527 & & & \end{array}$

Means for Oneway Anova

Level Number Mean Std Error Lower 95\% Upper 95\%

$\begin{array}{llllll}\text { Coliwasa } & 16 & 1.29374 & 0.00310 & 1.2873 & 1.3002 \\ \text { Isolok }(12 \mathrm{~mL}) & 10 & 1.28772 & 0.00393 & 1.2796 & 1.2958\end{array}$

Std Error uses a pooled estimate of error variance

Means and Std Deviations

Level Number Mean Std Dev Std Err Mean Lower 95\% Upper 95\%

\begin{tabular}{lrrrrrr} 
Coliwasa & Number & Mean & Std Dev & Std Err Mean & Lower $95 \%$ & Upper $95 \%$ \\
\hline
\end{tabular}

$\begin{array}{lllllll}\text { Isolok }(12 \mathrm{~mL}) & 10 & 1.28772 & 0.012321 & 0.00390 & 1.2789 & 1.2965\end{array}$

T Test

Isolok (12 mL)-Coliwasa

Assuming unequal variances

$\begin{array}{lrlr}\text { Difference } & -0.00602 & \mathrm{t} \text { Ratio } & -1.20637 \\ \text { Std Err Dif } & 0.00499 & \text { DF } & 19.44501 \\ \text { Upper CL Dif } & 0.00441 & \text { Prob }>|\mathrm{t}| & 0.2421 \\ \text { Lower CL Dif } & -0.01645 & \text { Prob }>\mathrm{t} & 0.8789 \\ \text { Confidence } & 0.95 & \text { Prob }<\mathrm{t} & 0.1211\end{array}$

Tests that the Variances are Equal

Level Count Std Dev MeanAbsDif to Mean MeanAbsDif to Median

$\begin{array}{lllll}\text { Coliwasa } & 16 & 0.0124792 & 0.0092521 & 0.0092521\end{array}$

$\begin{array}{lllll}\text { Isolok }(12 \mathrm{~mL}) & 10 & 0.0123206 & 0.0080711 & 0.0078740\end{array}$

Test F Ratio DFNum DFDen p-Value

$\begin{array}{lllll}\text { O'Brien[.5] } & 0.0013 & 1 & 24 & 0.9720\end{array}$

$\begin{array}{lllll}\text { Brown-Forsythe } & 0.1616 & 1 & 24 & 0.6913\end{array}$

$\begin{array}{lllll}\text { Levene } & 0.1225 & 1 & 24 & 0.7294\end{array}$

$\begin{array}{lrrrr}\text { Bartlett } & 0.0018 & 1 & \text {. } & 0.9666 \\ \text { FTest 2-sided } & 1.0259 & 15 & 9 & 1.0000\end{array}$

Welch Anova testing Means Equal, allowing Std Devs Not Equal

F Ratio DFNum DFDen Prob $>$ F

$\begin{array}{llll}1.4553 & 1 & 19.445 & 0.2421\end{array}$

t Test
1.2064 


\section{Exhibit A2. Statistical Comparisons for Mid-Rheology (Phase 1) Testing}

Isolok 12 mL - Low Flow Rate

Oneway Analysis of Measurement By Sampling Mechanism Analyte $=w t \%$ dried solids

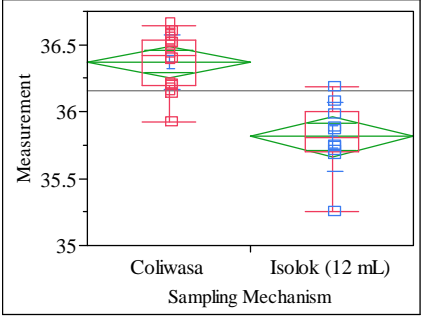

Oneway Anova

Summary of Fit

Adj Rsquare

0.589669

$\quad 0.22815$

36.15692

Observations (or Sum Wgts) $\quad 26$

TTest

Isolok (12 mL)-Coliwasa

Assuming equal variances

Difference $\quad-0.55888$ t Ratio $\quad-6.07671$

Std Err Dif $\quad 0.09197$ DF 24

Upper CL Dif -0.36906 Prob $>|t| \quad<.0001$

Lower CL Dif -0.74869 Prob $>\mathrm{t} \quad 1.0000$

$\begin{array}{lrl}\text { Confidence } & 0.95 \text { Prob }<\mathrm{t} & <.0001\end{array}$

Analysis of Variance

$\begin{array}{lrrrrr}\text { Source } & \text { DF } & \text { Sum of Squares } & \text { Mean Square } & \text { F Ratio } & \text { Prob > F } \\ \text { Sampling Mechanism } & 1 & 1.9221001 & 1.92210 & 36.9264 & <.0001 \\ \text { Error } & 24 & 1.2492538 & 0.05205 & & \\ \text { C. Total } & 25 & 3.1713538 & & & \end{array}$

Means for Oneway Anova

Level Number Mean Std Error Lower 95\% Upper 95\%

$\begin{array}{lrrrrr}\text { Coliwasa } & 16 & 36.3719 & 0.05704 & 36.254 & 36.490\end{array}$

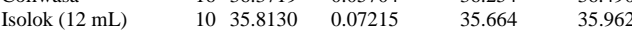

Std Error uses a pooled estimate of error variance

Means and Std Deviations

Level Number Mean Std Dev Std Err Mean Lower 95\% Upper 95\%

$\begin{array}{lrrrrrr}\text { Coliwasa } & 16 & 36.3719 & 0.207468 & 0.05187 & 36.261 & 36.482\end{array}$

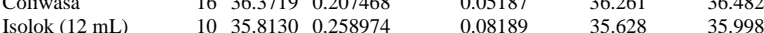

T Test

Isolok (12 mL)-Coliwasa

Assuming unequal variances

Difference $\quad-0.55888$ t Ratio $\quad-5.76529$

Std Err Dif $\quad 0.09694$ DF $\quad 16.11264$

Upper CL Dif -0.35349 Prob $>|t| r .0001$

Lower CL Dif -0.76426 Prob $>t \quad 1.0000$

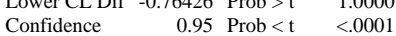

Tests that the Variances are Equal

Level Count Std Dev MeanAbsDif to Mean MeanAbsDif to Median

Coliwasa $\quad 16 \quad 0.2074679$

$\begin{array}{lllll}\text { Isolok }(12 \mathrm{~mL}) & 10 & 0.2589745 & 0.1751562 & 0.1693750 \\ & & & 0.1870000 & 0.1870000\end{array}$

Test F Ratio DFNum DFDen p-Value

$\begin{array}{lrrrr}\text { O'Brien[.5] } & 0.5065 & 1 & 24 & 0.4835\end{array}$

Brown-Forsythe $\quad 0.0960 \quad 1 \quad 24 \quad 0.7593$

$\begin{array}{lllll}\text { Levene } & 0.0507 & 1 & 24 & 0.8238\end{array}$

$\begin{array}{lllll}\text { Bartlett } & 0.5449 & 1 & 0.4604\end{array}$

Welch Anova testing Means Equal, allowing Std Devs Not Equal

F Ratio DFNum DFDen Prob $>$ F

$33.2385 \quad 1 \quad 16.113<r .0001$

t Test
5.7653
Isolok $12 \mathrm{~mL}$ - Low Flow Rate

Oneway Analysis of Measurement By Sampling Mechanism Analyte=wt $\%$ vitrified solids

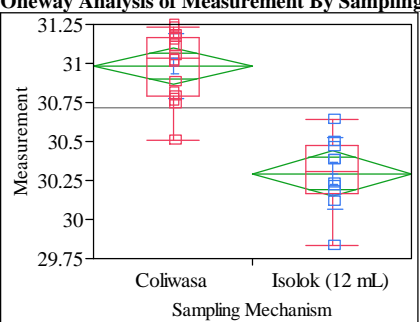

Oneway Anova

Summary of Fit

Adj Rsquare

Root Mean Square Error $\quad 0.705676$

$\begin{array}{lr}\text { Mean of Response } & 30.71846 \\ \text { Observations (or Sum Wgts) } & 26\end{array}$

t Test

Isolok (12 mL)-Coliwasa

Assuming equal variances

$\begin{array}{lrlr}\text { Difference } & -0.68813 & \mathrm{t} \text { Ratio } & -7.80642 \\ \text { Std Err Dif } & 0.08815 & \text { DF } & 24 \\ \text { Upper CL Dif } & -0.50620 & \text { Prob }>|\mathrm{t}| & <.0001 \\ \text { Lower CL Dif } & -0.87005 & \text { Prob }>\mathrm{t} & 1.0000 \\ \text { Confidence } & 0.95 & \text { Prob }<\mathrm{t} & <.0001\end{array}$

Analysis of Variance

$\begin{array}{lrrrrr}\text { Source } & \text { DF } & \text { Sum of Squares } & \text { Mean Square } & \text { F Ratio } & \text { Prob > F } \\ \text { Sampling Mechanism } & 1 & 2.9139447 & 2.91394 & 60.9403 & <.0001 \\ \text { Error } & 24 & 1.1475937 & 0.04782 & & \\ \text { C. Total } & 25 & 4.0615385 & & & \end{array}$

Means for Oneway Anova

Level Number Mean Std Error Lower 95\% Upper 95\%

$\begin{array}{llllll}\text { Coliwasa } & 16 & 30.9831 & 0.05467 & 30.870 & 31.096 \\ \text { Isolok }(12 \mathrm{~mL}) & 10 & 30.2950 & 0.06915 & 30.152 & 30.438\end{array}$

Std Error uses a pooled estimate of error variance

Means and Std Deviations

Level Number Mean Std Dev Std Err Mean Lower 95\% Upper 95\%

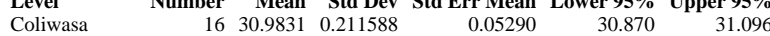

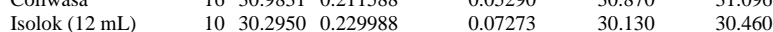

T Test

Isolok (12 mL)-Coliwasa

Assuming unequal variances

$\begin{array}{lrlr}\text { Difference } & -0.68813 & \mathrm{t} \text { Ratio } & -7.65172 \\ \text { Std Err Dif } & 0.08993 \text { DF } & 18.01561 \\ \text { Upper CL Dif } & -0.49920 \text { Prob }>|\mathrm{t}| & <.0001 \\ \text { Lower CL Dif } & -0.87705 \text { Prob }>\mathrm{t} & 1.0000 \\ \text { Confidence } & 0.95 & \text { Prob }<\mathrm{t} & <.0001\end{array}$

Tests that the Variances are Equal

Level Count Std Dev MeanAbsDif to Mean MeanAbsDif to Median

$\begin{array}{lllll}\text { Coliwasa } & 16 & 0.2115882 & 0.1773437 & 0.1693750\end{array}$

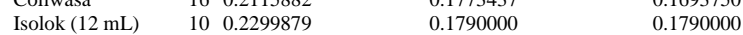

Test F Ratio DFNum DFDen p-Value

$\begin{array}{lrrrr}\text { O'Brien[.5] } & 0.0891 & 1 & 24 & 0.7679\end{array}$

$\begin{array}{lllll}\text { Brown-Forsythe } & 0.0332 & 1 & 24 & 0.8570\end{array}$

$\begin{array}{lllll}\text { Levene } & 0.0013 & 1 & 24 & 0.9721\end{array}$

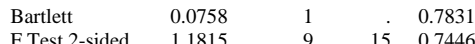

Welch Anova testing Means Equal, allowing Std Devs Not Equal

F Ratio DFNum DFDen Prob $>$ F

$\begin{array}{rrrr}58.5488 & 1 & 18.016 & <.0001\end{array}$

$\mathbf{t}$ Test
7.6517 


\section{Exhibit A2. Statistical Comparisons for Mid-Rheology (Phase 1) Testing}

Isolok $12 \mathrm{~mL}$ - High Flow Rate

Oneway Analysis of Measurement By Sampling Mechanism Analyte=density $(\mathrm{g} / \mathrm{mL})$

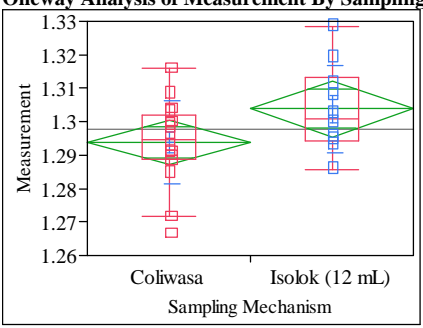

Oneway Anova

Summary of Fit

Adj Rsquare

0.138345

0.012688

Observations (or Sum Wgts) $\quad 26$

Test

Isolok (12 mL)-Coliwasa

Assuming equal variances

Difference $\quad 0.01004$ t Ratio 1.963006

Std Err Dif $\quad 0.00511$ DF 24

Upper CL Dif 0.02060 Prob $>|t| \quad 0.0613$

Lower CL Dif -0.00052 Prob $>$ t 0.0307

$\begin{array}{lrl}\text { Confidence } & 0.95 \text { Prob }<\mathrm{t} & 0.9693\end{array}$

Analysis of Variance

$\begin{array}{lrrrrr}\text { Source } & \text { DF } & \text { Sum of Squares } & \text { Mean Square } & \text { F Ratio } & \text { Prob }>\text { F } \\ \text { Sampling Mechanism } & 1 & 0.00062037 & 0.000620 & 3.8534 & 0.0613 \\ \text { Error } & 24 & 0.00386383 & 0.000161 & & \end{array}$

$25 \quad 0.00448420$

Means for Oneway Anova

Level Number Mean Std Error Lower 95\% Upper 95\%

$\begin{array}{lrrrrr}\text { Coliwasa } & 16 & 1.29374 & 0.00317 & 1.2872 & 1.3003\end{array}$

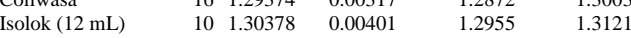

Std Error uses a pooled estimate of error variance

Means and Std Deviations

Level Number Mean Std Dev Std Err Mean Lower 95\% Upper 95\%

16 1.29374 $0.012479-0.00312$

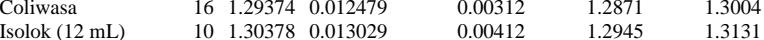

Test

Isolok (12 mL)-Coliwas

Assuming unequal variances

$\begin{array}{lrlr}\text { Difference } & 0.01004 & \text { t Ratio } & 1.94276 \\ \text { Std Err Dif } & 0.00517 & \text { DF } & 18.60844 \\ \text { Upper CL Dif } & 0.02087 & \text { Prob }>|t| & 0.0673 \\ \text { Lower CL Dif } & -0.00079 & \text { Prob }>\text { t } & 0.0337 \\ \text { Confidence } & 0.95 & \text { Prob }<\mathrm{t} & 0.9663\end{array}$

Tests that the Variances are Equal

Level Count Std Dev MeanAbsDif to Mean MeanAbsDif to Median

Coliwasa $\quad 16 \quad 0.0124792$

$\begin{array}{lllll}\text { Isolok }(12 \mathrm{~mL}) & 10 & 0.0130293 & 0.0092521 & 0.0092521 \\ & & & 0.0103153 & 0.0100603\end{array}$

Test F Ratio DFNum DFDen p-Value

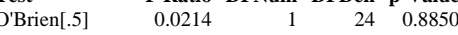

$\begin{array}{lllll}\text { Brown-Forsythe } & 0.0616 & 1 & 24 & 0.806\end{array}$

$\begin{array}{lllll}\text { Levene } & 0.1168 & 1 & 24 & 0.7356\end{array}$

$\begin{array}{lllll}\text { Bartlett } & 0.0202 & 1 & 0.8871\end{array}$

Welch Anova testing Means Equal, allowing Std Devs Not Equal

F Ratio DFNum DFDen Prob $>$ F

$\begin{array}{llll}3.7743 & 1 & 18.608 & 0.0673\end{array}$

t Test

1.9428
Isolok $12 \mathrm{~mL}$ - High Flow Rate

Oneway Analysis of Measurement By Sampling Mechanism Analyte=wt $\%$ dried solids

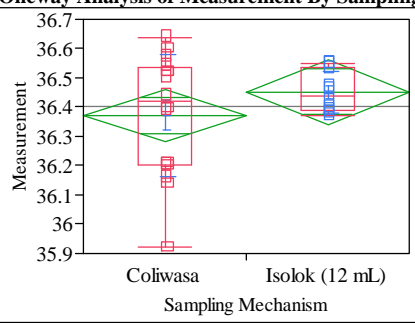

Oneway Anova

Summary of Fit

Adj Rsquare

0.169657

Mean of Response $\quad 36.40269$

t Test

Isolok (12 mL)-Coliwasa

Assuming equal variances

$\begin{array}{lrlr}\text { Difference } & 0.08012 & \mathrm{t} \text { Ratio } & 1.171574 \\ \text { Std Err Dif } & 0.06839 & \text { DF } & 24 \\ \text { Upper CL Dif } & 0.22128 & \text { Prob }>|t| & 0.2529 \\ \text { Lower CL Dif } & -0.06103 & \text { Prob }>\text { t } & 0.1264 \\ \text { Confidence } & 0.95 & \text { Prob }<\mathrm{t} & 0.8736\end{array}$

Analysis of Variance

Source DF Sum of Squares Mean Square F Ratio Prob > F

\begin{tabular}{lrrr} 
Error & 1 & 0.03950779 & 0.039508 \\
\hline
\end{tabular}

$\begin{array}{lll}\text { C. Total } & 25 & 0.73031154\end{array}$

Means for Oneway Anova

Level Number Mean Std Error Lower 95\% Upper 95\%

$\begin{array}{lrrrrr}\text { Coliwasa } & 16 & 36.3719 & 0.04241 & 36.284 & 36.459\end{array}$

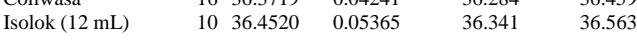

Std Error uses a pooled estimate of error variance

Means and Std Deviations

Level Number Mean Std Dev Std Err Mean Lower 95\% Upper 95\%

$\begin{array}{lrrrrrr}\text { Coliwasa } & 16 & 36.3719 & 0.207468 & 0.05187 & 36.261 & 36.482\end{array}$

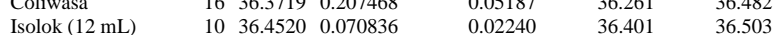

Test

Isolok $(12 \mathrm{~mL})$-Coliwasa

Assuming unequal variances

$\begin{array}{lrlr}\text { Difference } & 0.08012 & \text { t Ratio } & 1.418206 \\ \text { Std Err Dif } & 0.05650 & \text { DF } & 19.96014 \\ \text { Upper CL Dif } & 0.19799 & \text { Prob }>|t| & 0.1716 \\ \text { Lower CL Dif } & -0.03774 & \text { Prob }>t & 0.0858 \\ \text { Confidence } & 0.95 & \text { Prob }<\mathrm{t} & 0.9142\end{array}$

Tests that the Variances are Equal

Level Count Std Dev MeanAbsDif to Mean MeanAbsDif to Median

$\begin{array}{lllll}\text { Coliwasa } & 16 & 0.2074679 & 0.1751562 & 0.1693750\end{array}$

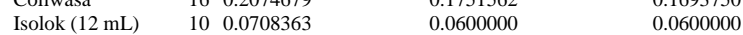

Test F Ratio DFNum DFDen p-Value

$\begin{array}{lllll}\text { O'Brien[.5] } & 5.0601 & 1 & 24 & 0.0339\end{array}$

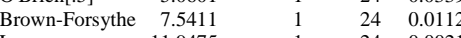

$\begin{array}{lllll}\text { Levene } & 11.9475 & 1 & 24 & 0.0021\end{array}$

$\begin{array}{lrrrr}\text { Bartlett } & 9.2650 & 1 & \text {. } & 0.0023 \\ \text { F Test 2-sided } & 8.5781 & 15 & 9 & 0.0027\end{array}$

Welch Anova testing Means Equal, allowing Std Devs Not Equal

F Ratio DFNum DFDen Prob $>$ F

$2.0113 \quad 1 \quad 19.96 \quad 0.1716$

$\mathbf{t}$ Test
1.4182 
SRNL-STI-2011-00693

Revision 0

\section{Exhibit A2. Statistical Comparisons for Mid-Rheology (Phase 1) Testing}

Isolok $12 \mathrm{~mL}$ - High Flow Rate

Oneway Analysis of Measurement By Sampling Mechanism Analyte $=w t \%$ vitrified solids

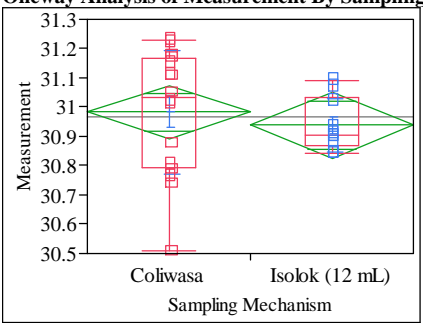

Oneway Anova

Summary of Fit

Adj Rsquare

$-0.02444$

$\quad 0.176199$

(osponse

Observations (or Sum Wgts) $\quad 26$

Test

Isolok (12 mL)-Coliwasa

Assuming equal variances

Difference $\quad-0.04513$ t Ratio -0.63531

Std Err Dif $\quad 0.07103$ DF 24

Upper CL Dif 0.10147 Prob $>|t| r \mid r .5312$

Lower CL Dif -0.19172 Prob $>\mathrm{t} \quad 0.7344$

$\begin{array}{lrl}\text { Confidence } & 0.95 \text { Prob }<\mathrm{t} & 0.2656\end{array}$

Analysis of Variance

$\begin{array}{lrrrrr}\text { Source } & \text { DF } & \text { Sum of Squares } & \text { Mean Square } & \text { F Ratio } & \text { Prob }>\text { F } \\ \text { Sampling Mechanism } & 1 & 0.01253087 & 0.012531 & 0.4036 & 0.5312 \\ \text { Error } & 24 & 0.74510375 & 0.031046 & & \end{array}$

$25 \quad 0.75763462$

Means for Oneway Anova

Level Number Mean Std Error Lower 95\% Upper 95\%

$\begin{array}{lrrrrr}\text { Coliwasa } & 16 & 30.9831 & 0.04405 & 30.892 & 31.074\end{array}$

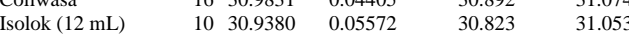

Std Error uses a pooled estimate of error variance

Means and Std Deviations

Level Number Mean Std Dev Std Err Mean Lower 95\% Upper 95\%

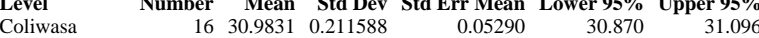

$\begin{array}{lllllll}\text { Coliwasa } & 16 & 30.9831 & 0.211588 & 0.05290 & 30.870 & 31.096 \\ \text { Isolok (12 mL) } & 10 & 30.9380 & 0.090406 & 0.02859 & 30.873 & 31.003\end{array}$

Test

Isolok (12 mL)-Coliwasa

Assuming unequal variances

Difference $\quad-0.04513$ t Ratio $\quad-0.75048$

Std Err Dif $\quad 0.06013$ DF 21.92506

Upper CL Dif 0.07960 Prob $>|t| \quad 0.4609$

Lower CL Dif -0.16985 Prob $>t \quad 0.7695$

$\begin{array}{lrl}\text { Confidence } & 0.95 \text { Prob }<\mathrm{t} & 0.2305\end{array}$

Tests that the Variances are Equal

Level Count Std Dev MeanAbsDif to Mean MeanAbsDif to Median

Coliwasa $\quad 16 \quad 0.2115882$

$\begin{array}{lllll}\text { Coliwasa } & 16 & 0.2115882 & 0.1773437 & 0.1693750 \\ \text { Isolok }(12 \mathrm{~mL}) & 10 & 0.0904065 & 0.0732000 & 0.0660000\end{array}$

Test F Ratio DFNum DFDen p-Value

$\begin{array}{lrrrr}\text { O'Brien[.5] } & 3.8548 & 1 & 24 & 0.0613\end{array}$

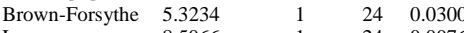

$\begin{array}{lllll}\text { Levene } & 8.5066 & 1 & 24 & 0.0076\end{array}$

$\begin{array}{lrrrr}\text { Bartlett } & 6.2375 & 1 & \text {. } & 0.0125 \\ \text { F Test 2-sided } & 5.4775 & 15 & 9 & 0.0141\end{array}$

Welch Anova testing Means Equal, allowing Std Devs Not Equal

F Ratio DFNum DFDen Prob $>$ F

$\begin{array}{lrrr}0.5632 & 1 & 21.925 & 0.4609\end{array}$

t Test
0.7505
Isolok $3 \mathrm{~mL}$ - Low Flow Rate

Oneway Analysis of Measurement By Sampling Mechanism Analyte $=w$ t $\%$ dried solids

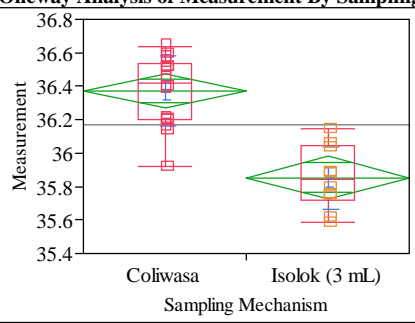

Oneway Anova

Summary of Fit

Adj Rsquare

0.199925

Mean of Response $\quad 36.17269$

t Test

Isolok (3 mL)-Coliwasa

Assuming equal variances

$\begin{array}{lrlr}\text { Difference } & -0.51788 & \mathrm{t} \text { Ratio } & -6.42585 \\ \text { Std Err Dif } & 0.08059 & \text { DF } & 24 \\ \text { Upper CL Dif } & -0.35154 & \text { Prob }>|t| & <.0001 \\ \text { Lower CL Dif } & -0.68421 & \text { Prob }>t & 1.0000 \\ \text { Confidence } & 0.95 & \text { Prob }<t & <.0001\end{array}$

Analysis of Variance

$\begin{array}{lrrrrr}\text { Source } & \text { DF } & \text { Sum of Squares } & \text { Mean Square } & \text { F Ratio } & \text { Prob }>\text { F } \\ \text { Sampling Mechanism } & 1 & 1.6504278 & 1.65043 & 41.2915 & <.0001\end{array}$

$\begin{array}{llll}\text { Error } & 24 & 0.9592837 & 0.03997\end{array}$

$\begin{array}{lll}\text { C. Total } & 25 & 2.6097115\end{array}$

Means for Oneway Anova

Level Number Mean Std Error Lower 95\% Upper 95\%

$\begin{array}{llrrrr}\text { Coliwasa } & 16 & 36.3719 & 0.04998 & 36.269 & 36.475 \\ \text { Isolok }(3 \mathrm{~mL}) & 10 & 35.8540 & 0.06322 & 35.724 & 35.984\end{array}$

Std Error uses a pooled estimate of error variance

Means and Std Deviations

Level Number Mean Std Dev Std Err Mean Lower 95\% Upper 95\%

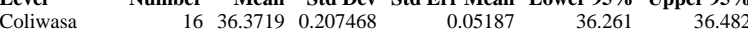

$\begin{array}{lllllll}\text { Isolok }(3 \mathrm{~mL}) & 10 & 35.8540 & 0.186679 & 0.05903 & 35.720 & 35.988\end{array}$

TTest

Isolok (3 mL)-Coliwasa

Assuming unequal variances

$\begin{array}{lrlr}\text { Difference } & -0.51788 & \mathrm{t} \text { Ratio } & -6.59028 \\ \text { Std Err Dif } & 0.07858 \text { DF } & 20.81578 \\ \text { Upper CL Dif } & -0.35437 & \text { Prob }>|\mathrm{t}| & <.0001 \\ \text { Lower CL Dif } & -0.68138 & \text { Prob }>\mathrm{t} & 1.0000 \\ \text { Confidence } & 0.95 & \text { Prob }<\mathrm{t} & <.0001\end{array}$

Tests that the Variances are Equal

Level Count Std Dev MeanAbsDif to Mean MeanAbsDif to Median

$\begin{array}{lllll}\text { Coliwasa } & 16 & 0.2074679 & 0.1751562 & 0.1693750\end{array}$

$\begin{array}{lllll}\text { Isolok }(3 \mathrm{~mL}) & 10 & 0.1866786 & 0.1500000 & 0.1500000\end{array}$

Test F Ratio DFNum DFDen p-Value

$\begin{array}{lrrrr}\text { O'Brien[.5] } & 0.1821 & 1 & 24 & 0.6734\end{array}$

$\begin{array}{lllll}\text { Brown-Forsythe } & 0.1764 & 1 & 24 & 0.6782\end{array}$

$\begin{array}{lllll}\text { Levene } & 0.3840 & 1 & 24 & 0.5413\end{array}$

$\begin{array}{lrrrr}\text { Bartlett } & 0.1177 & 1 & \text {. } & 0.7315 \\ \text { F Test 2-sided } & 1.2351 & 15 & 9 & 0.7694\end{array}$

Welch Anova testing Means Equal, allowing Std Devs Not Equal

F Ratio DFNum DFDen Prob $>$ F

$\begin{array}{rrrr}43.4318 & 1 & 20.816 & <.0001\end{array}$

t Test

6.5903 


\section{Exhibit A2. Statistical Comparisons for Mid-Rheology (Phase 1) Testing}

Isolok 3 mL - Low Flow Rate

Oneway Analysis of Measurement By Sampling Mechanism Analyte=wt $\%$ vitrified solids

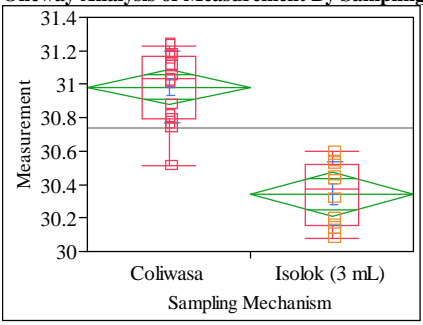

Oneway Anova

Summary of Fit

Adj Rsquare

0.716903

Mean of Response

0.705107

$\quad 0.20369$

Test

Isolok (3 mL)-Coliwasa

Assuming equal variances

Difference $\quad-0.64013$ t Ratio $\quad-7.79593$

Std Err Dif $\quad 0.08211$ DF 24

$\begin{array}{rrr}\text { Upper CL Dif } & -0.47066 \text { Prob }>|t| & <.0001\end{array}$

Lower CL Dif -0.80959 Prob $>\mathrm{t} \quad 1.0000$

$\begin{array}{lrl}\text { Confidence } & 0.95 \text { Prob }<\mathrm{t} & <.0001\end{array}$

Analysis of Variance

$\begin{array}{lrrrrr}\text { Source } & \text { DF } & \text { Sum of Squares } & \text { Mean Square } & \text { F Ratio } & \text { Prob > F } \\ \text { Sampling Mechanism } & 1 & 2.5216001 & 2.52160 & 60.7765 & <.0001 \\ \text { Error } & 24 & 0.9957537 & 0.04149 & & \\ \text { C. Total } & 25 & 3.5173538 & & & \end{array}$

Means for Oneway Anova

Level Number Mean Std Error Lower 95\% Upper 95\%

$\begin{array}{lrrrrr}\text { Coliwasa } & 16 & 30.9831 & 0.05092 & 30.878 & 31.088\end{array}$

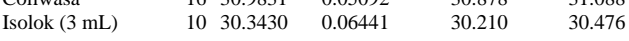

Std Error uses a pooled estimate of error variance

Means and Std Deviations

Level Number Mean Std Dev Std Err Mean Lower 95\% Upper 95\%

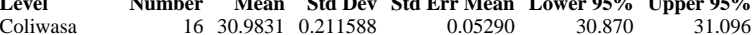

$\begin{array}{lllllll}\text { Coliwasa } & 16 & 30.9831 & 0.211588 & 0.05290 & 30.870 & 31.096 \\ \text { Isolok (3 mL) } & 10 & 30.3430 & 0.189798 & 0.06002 & 30.207 & 30.479\end{array}$

Test

Isolok (3 mL)-Coliwasa

Assuming unequal variances

Difference $\quad-0.64013$ t Ratio $\quad-8.00129$

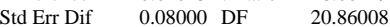

$\begin{array}{rrr}\text { Upper CL Dif } & -0.47368 \text { Prob }>|t| & <.0001\end{array}$

Lower CL Dif -0.80657 Prob $>t \quad 1.0000$

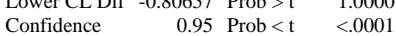

Tests that the Variances are Equal

Level Count Std Dev MeanAbsDif to Mean MeanAbsDif to Median

$\begin{array}{lllll}\text { Coliwasa } & 16 & 0.2115882 & 0.1773437 & 0.1693750\end{array}$

$\begin{array}{lllll}\text { Coliwasa } & 16 & 0.2115882 & 0.1773437 & 0.1693750 \\ \text { Isolok }(3 \mathrm{~mL}) & 10 & 0.1897981 & 0.1650000 & 0.1650000\end{array}$

Test F Ratio DFNum DFDen p-Value

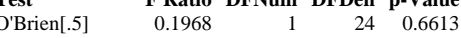

$\begin{array}{lllll}\text { Brown-Forsythe } & 0.0089 & 1 & 24 & 0.9256\end{array}$

$\begin{array}{lllll}\text { Levene } & 0.1022 & 1 & 24 & 0.7520\end{array}$

$\begin{array}{lrrrr}\text { Bartlett } & 0.1246 & 1 & \text {. } & 0.7241\end{array}$

Welch Anova testing Means Equal, allowing Std Devs Not Equal

F Ratio DFNum DFDen Prob $>$ F

$\begin{array}{rrrr}64.0207 & 1 & 20.86 & <.0001\end{array}$

t Test

8.0013
Isolok $3 \mathrm{~mL}$ - High Flow Rate

Oneway Analysis of Measurement By Sampling Mechanism Analyte $=w t \%$ dried solids

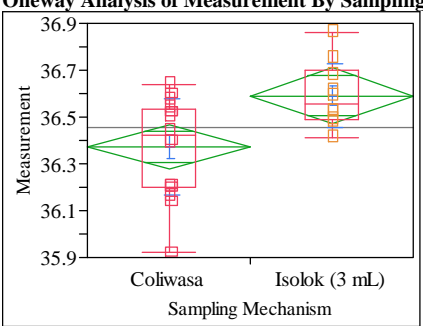

Oneway Anova

Summary of Fit

Adj Rsquare

0.184496

Mean of Response $\quad 36.45615$

t Test

Isolok (3 mL)-Coliwasa

Assuming equal variances

Difference $\quad 0.219125$ t Ratio 2.946303

Std Err Dif $\quad 0.074373$ DF 24

Upper CL Dif 0.372623 Prob $>|t| \quad 0.0070$

Lower CL Dif 0.065627 Prob $>$ t 0.0035

$\begin{array}{lrl}\text { Confidence } & 0.95 \mathrm{Prob}<\mathrm{t} & 0.9965\end{array}$

Analysis of Variance

$\begin{array}{lrrrrr}\text { Source } & \text { DF } & \text { Sum of Squares } & \text { Mean Square } & \text { F Ratio } & \text { Prob }>\text { F } \\ \text { Sampling Mechanism } & 1 & 0.2954816 & 0.295482 & 8.6807 & 0.0070 \\ \text { Error } & 24 & 0.8169338 & 0.034039 & & \\ \text { C. Total } & 25 & 1.1124154 & & & \end{array}$

$25 \quad 1.1124154$

Means for Oneway Anova

Level Number Mean Std Error Lower 95\% Upper 95\%

$\begin{array}{lrrrrr}\text { Coliwasa } & 16 & 36.3719 & 0.04612 & 36.277 & 36.467 \\ \text { Isolok }(3 \mathrm{~mL}) & 10 & 36.5910 & 0.05834 & 36.471 & 36.711\end{array}$

Std Error uses a pooled estimate of error variance

Means and Std Deviations

Level Number Mean Std Dev Std Err Mean Lower 95\% Upper 95\%

$\begin{array}{lrrrrrr}\text { Coliwasa } & 16 & 36.3719 & 0.207468 & 0.05187 & 36.261 & 36.482\end{array}$

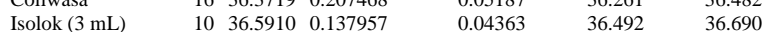

Test

Isolok (3 mL)-Coliwasa

Assuming unequal variances

$\begin{array}{lrlr}\text { Difference } & 0.219125 & \text { t Ratio } & 3.233141 \\ \text { Std Err Dif } & 0.067775 & \text { DF } & 23.84258 \\ \text { Upper CL Dif } & 0.359054 & \text { Prob }>|t| & 0.0036 \\ \text { Lower CL Dif } & 0.079196 & \text { Prob }>t & 0.0018 \\ \text { Confidence } & 0.95 & \text { Prob }<t & 0.9982\end{array}$

Tests that the Variances are Equal

Level Count Std Dev MeanAbsDif to Mean MeanAbsDif to Median

$\begin{array}{lllll}\text { Coliwasa } & 16 & 0.2074679 & 0.1751562 & 0.1693750\end{array}$

$\begin{array}{lllll}\text { Isolok }(3 \mathrm{~mL}) & 10 & 0.1379573 & 0.1072000 & 0.1070000\end{array}$

Test F Ratio DFNum DFDen p-Value

$\begin{array}{lrrrr}\text { O'Brien[.5] } & 1.7682 & 1 & 24 & 0.1961\end{array}$

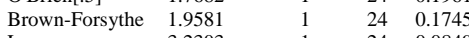

$\begin{array}{lllll}\text { Levene } & 3.2303 & 1 & 24 & 0.0849\end{array}$

$\begin{array}{lrrrr}\text { Bartlett } & 1.6376 & 1 & \text {. } & 0.2007 \\ \text { F Test 2-sided } & 2.2616 & 15 & 9 & 0.2182\end{array}$

Welch Anova testing Means Equal, allowing Std Devs Not Equal

F Ratio DFNum DFDen Prob $>$ F

$\begin{array}{rrrr}10.4532 & 1 & 23.843 & 0.0036\end{array}$

t Test

3.2331 


\section{Exhibit A2. Statistical Comparisons for Mid-Rheology (Phase 1) Testing}

Isolok $3 \mathrm{~mL}$ - High Flow Rate

Oneway Analysis of Measurement By Sampling Mechanism Analyte=wt \% vitrified solids

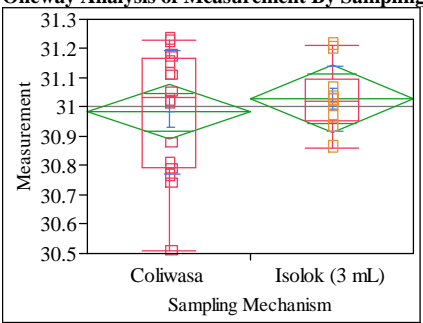

Oneway Anova

Summary of Fit

Adj Rsquare

\begin{tabular}{lr}
0.18012 \\
\hline 1.00077
\end{tabular}

31.00077

Test

Isolok (3 mL)-Coliwasa

Assuming equal variances

Difference $\quad 0.04588$ t Ratio $\quad 0.631813$

Std Err Dif $\quad 0.07261$ DF $\quad 24$

Upper CL Dif 0.19573 Prob $>|t| r 0.5335$

$\begin{array}{lrl}\text { Lower CL Dif }-0.10398 \text { Prob }>t & 0.2667\end{array}$

$\begin{array}{lrl}\text { Confidence } & 0.95 \text { Prob }<\mathrm{t} & 0.7333\end{array}$

Analysis of Variance

$\begin{array}{lrrrrr}\text { Source } & \text { DF } & \text { Sum of Squares } & \text { Mean Square } & \text { F Ratio } & \text { Prob }>\text { F } \\ \text { Sampling Mechanism } & 1 & 0.01295087 & 0.012951 & 0.3992 & 0.5335 \\ \text { Error } & 24 & 0.77863375 & 0.032443 & & \end{array}$

$25 \quad 0.79158462$

Means for Oneway Anova

Level Number Mean Std Error Lower 95\% Upper 95\%

$\begin{array}{lrrrrr}\text { Coliwasa } & 16 & 30.9831 & 0.04503 & 30.890 & 31.076\end{array}$

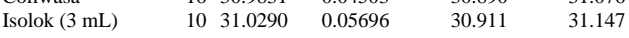

Std Error uses a pooled estimate of error variance

Means and Std Deviations

Level Number Mean Std Dev Std Err Mean Lower 95\% Upper 95\%

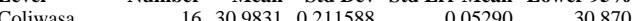

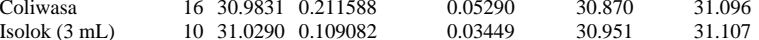

Test

Isolok (3 mL)-Coliwasa

Assuming unequal variances

$\begin{array}{lrlr}\text { Difference } & 0.04588 & \mathrm{t} \text { Ratio } & 0.726439 \\ \text { Std Err Dif } & 0.06315 & \text { DF } & 23.41336 \\ \text { Upper CL Dif } & 0.17638 & \text { Prob }>|t| & 0.4748 \\ \text { Lower CL Dif } & -0.08463 & \text { Prob }>\mathrm{t} & 0.2374 \\ \text { Confidence } & 0.95 & \text { Prob }<\mathrm{t} & 0.7626\end{array}$

Tests that the Variances are Equal

Level Count Std Dev MeanAbsDif to Mean MeanAbsDif to Median

$\begin{array}{lllll}\text { Coliwasa } & 16 & 0.2115882 & 0.1773437 & 0.1693750\end{array}$

$\begin{array}{lllll}\text { Coliwasa } & 16 & 0.2115882 & 0.1773437 & 0.1693750 \\ \text { Isolok }(3 \mathrm{~mL}) & 10 & 0.1090820 & 0.0768000 & 0.0750000\end{array}$

Test F Ratio DFNum DFDen p-Value

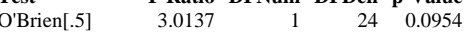

$\begin{array}{lllll}\text { Brown-Forsythe } & 4.2814 & 1 & 24 & 0.0495\end{array}$

$\begin{array}{lllll}\text { Levene } & 6.8982 & 1 & 24 & 0.0148\end{array}$

$\begin{array}{lrrrr}\text { Bartlett } & 4.0146 & 1 & \text {. } & 0.0451\end{array}$

Welch Anova testing Means Equal, allowing Std Devs Not Equal

F Ratio DFNum DFDen Prob $>$ F

$\begin{array}{rrrr}0.5277 & 1 & 23.413 & 0.4748\end{array}$

t Test
0.7264
Oneway Analysis of Mean(Measurement) By Sampler Details Analyte=Al/B, Target=2.552

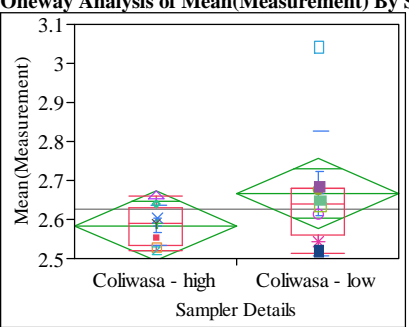

Oneway Anova

Summary of Fi

Rsquare

Root Mean Square Error $\quad 0.1186$

Mean of Response 2.626169

Observations (or Sum Wgts) 16

Test

Coliwasa - low-Coliwasa - high

Assuming equal variances

Difference $\quad 0.08297$ t Ratio 1.399218

Upper CL Dif 0.21016 Prob $>|t| \quad 0.1835$

$\begin{array}{lll}\text { Lower CL Dif }-0.04421 \text { Prob }>\text { t } & 0.0918\end{array}$

$\begin{array}{lrl}\text { Confidence } & 0.95 \text { Prob }<\mathrm{t} & 0.9082\end{array}$

Analysis of Variance

$\begin{array}{lllrr}\text { Source } & \text { DF Sum of Squares Mean Square } & \text { F Ratio Prob }>\text { F }\end{array}$

$\begin{array}{lrrr}\text { Error } & 1 & 0.02753837 & 0.027538 \\ \text { C. } & 14 & 0.19692270 & 0.014066\end{array}$

$\begin{array}{lll}\text { C. Total } & 15 & 0.22446107\end{array}$

Means for Oneway Anova

Level Number Mean Std Error Lower 95\% Upper 95\%

$\begin{array}{lrrrrr}\text { Coliwasa - high } & 8 & 2.58468 & 0.04193 & 2.4947 & 2.6746\end{array}$

$\begin{array}{llllll}\text { Coliwasa - low } & 8 & 2.58468 & 0.04193 & 2.4947 & 2.6746 \\ & & & & \end{array}$

Std Error uses a pooled estimate of error variance

Means and Std Deviations

Level Number Mean Std Dev Std Err Mean Lower 95\% Upper 95\%

$\begin{array}{lllllll}\text { Coliwasa - high } & 8 & 2.58468 & 0.050463 & 0.01784 & 2.5425 & 2.6269\end{array}$

$\begin{array}{lllllll}\text { Coliwasa - low } & 8 & 2.66766 & 0.159954 & 0.05655 & 2.5339 & 2.8014\end{array}$

Test

Coliwasa - low-Coliwasa - high

Assuming unequal variances

$\begin{array}{lrlr}\text { Difference } & 0.08297 & \mathrm{t} \text { Ratio } & 1.399218 \\ \text { Std Err Dif } & 0.05930 & \text { DF } & 8.379761 \\ \text { Upper CL Dif } & 0.21865 \text { Prob }>|\mathrm{t}| & 0.1977 \\ \text { Lower CL Dif } & -0.05270 & \text { Prob }>\mathrm{t} & 0.0988 \\ \text { Confidence } & 0.95 & \text { Prob }<\mathrm{t} & 0.9012\end{array}$

Tests that the Variances are Equal

Level Count Std Dev MeanAbsDif to Mean MeanAbsDif to Median

$\begin{array}{lllll}\text { Coliwasa - high } & 8 & 0.0504631 & 0.0394613 & 0.0383357\end{array}$

$\begin{array}{lllll}\text { Coliwasa - low } & 8 & 0.1599540 & 0.0973599 & 0.0921859\end{array}$

Test F Ratio DFNum DFDen p-Value

$\begin{array}{lrrrr}\text { O'Brien[.5] } & 1.2833 & 1 & 14 & 0.2763\end{array}$

$\begin{array}{llllll}\text { Brown-Forsythe } & 1.3077 & 1 & 14 & 0.2720\end{array}$

$\begin{array}{llllll}\text { Levene } & 1.7281 & 1 & 14 & 0.2098\end{array}$

$\begin{array}{lrrrr}\text { Bartlett } & 7.2570 & 1 & \text {; } & 0.0071 \\ \text { F Test 2-sided } & 10.0472 & 7 & 7 & 0.0069\end{array}$

Welch Anova testing Means Equal, allowing Std Devs Not Equal

F Ratio DFNum DFDen Prob $>$ F

$1.9578 \quad 1 \quad 8.3798 \quad 0.1977$

t Test

1.3992 


\section{Exhibit A2. Statistical Comparisons for Mid-Rheology (Phase 1) Testing}

Oneway Analysis of Mean(Measurement) By Sampler Details Analyte=Al2O3 (wt\%), Target $=10.869$

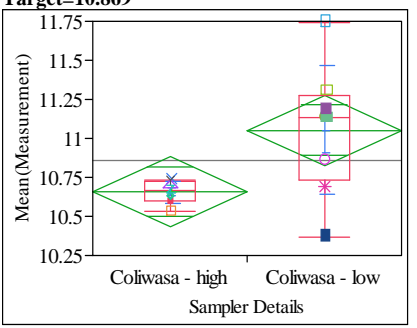

Oneway Anova

Summary of Fit

0.337188

Root Mean Square Error $\quad 0.297366$

Mean of Response

10.85518

Test

Coliwasa - low-Coliwasa - high

Assuming equal variances

Difference $\quad 0.396795$ t Ratio 2.668733

Std Err Dif $\quad 0.148683$ DF $\quad 14$

Upper CL Dif 0.715688 Prob $>|t| \quad 0.0183$

Lower CL Dif 0.077902 Prob $>\mathrm{t} \quad 0.0092$

$\begin{array}{lrl}\text { Confidence } & 0.95 \text { Prob }<\mathrm{t} & 0.9908\end{array}$

Analysis of Variance

Source DF Sum of Squares Mean Square F Ratio Prob > F

$\begin{array}{llllll}\text { Sampler Details } & 1 & 0.6297851 & 0.629785 & 7.1221 & 0.0183\end{array}$

$\begin{array}{llll}\text { Error } & 14 & 1.2379704 & 0.088426\end{array}$

$\begin{array}{lll}\text { C. Total } & 15 & 1.8677555\end{array}$

Means for Oneway Anova

Level Number Mean Std Error Lower 95\% Upper 95\%

$\begin{array}{lrrrrr}\text { Coliwasa - high } & 8 & 10.6568 & 0.10513 & 10.431 & 10.882\end{array}$

$\begin{array}{llllll}\text { Coliwasa- low } & 8 & 11.0536 & 0.10513 & 10.828 & 11.279\end{array}$

Std Error uses a pooled estimate of error variance

Means and Std Deviations

Level Number Mean Std Dev Std Err Mean Lower 95\% Upper 95\%

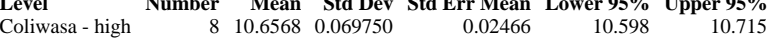

$\begin{array}{lllllll}\text { Coliwasa - low } & 8 & 11.0536 & 0.414714 & 0.02466 & 10.598 & 10.715 \\ & & & & & & \end{array}$

Test

Coliwasa - low-Coliwasa - high

Assuming unequal variances

Difference $\quad 0.396795$ t Ratio 2.668733

Std Err Dif $\quad 0.148683$ DF $\quad 7.39571$

Upper CL Dif 0.744597 Prob $>|t| \quad 0.0305$

Lower CL Dif 0.048993 Prob $>t \quad 0.0152$

$\begin{array}{lrl}\text { Confidence } & 0.95 \text { Prob }<\mathrm{t} & 0.9848\end{array}$

Tests that the Variances are Equal

Level Count Std Dev MeanAbsDif to Mean MeanAbsDif to Median

Coliwasa - high $\quad 8 \quad 0.0697504$

$\begin{array}{lllll}\text { Coliwasa - high } & 8 & 0.0697504 & 0.0535358 & 0.0535358 \\ \text { Coliwasa - low } & 8 & 0.4147141 & 0.3086183 & 0.2889360\end{array}$

Test F Ratio DFNum DFDen p-Value

$\begin{array}{lrrrr}\text { O'Brien[.5] } & 3.5588 & 1 & 14 & 0.0801\end{array}$

$\begin{array}{lllll}\text { Brown-Forsythe } & 5.1389 & 1 & 14 & 0.0398\end{array}$

$\begin{array}{lllll}\text { Levene } & 8.0422 & 1 & 14 & 0.0132\end{array}$

$\begin{array}{lllll}\text { Bartlett } & 14.6009 & 1 & \text {. } & 0.0001\end{array}$

Welch Anova testing Means Equal, allowing Std Devs Not Equal

F Ratio DFNum DFDen Prob $>$ F

$\begin{array}{lrrr}7.1221 & 1 & 7.3957 & 0.0305\end{array}$

t Test

2.6687
Oneway Analysis of Mean(Measurement) By Sampler Details Analyte=B2O3 (wt\%), Target $=\mathbf{4} .259$

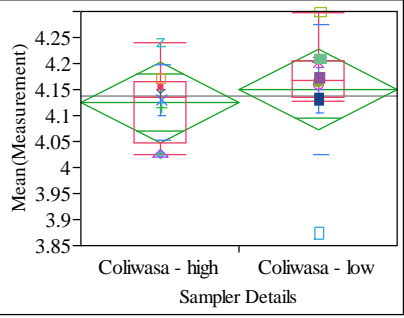

Oneway Anova

Summary of Fit

Adj Rsquare

0.016767

\begin{tabular}{ll}
-0.05346 \\
\hline
\end{tabular}

$\begin{array}{lr} & 4.137236 \\ \text { Observations (or Sum Wgts) } & 16\end{array}$

t Test

Coliwasa - low-Coliwasa - high

Assuming equal variances

$\begin{array}{lrlr}\text { Difference } & 0.02482 & \text { t Ratio } & 0.488616 \\ \text { Std Err Dif } & 0.05080 & \text { DF } & 14 \\ \text { Upper CL Dif } & 0.13377 & \text { Prob }>|t| & 0.6327 \\ \text { Lower CL Dif } & -0.08413 & \text { Prob }>\text { t } & 0.3163 \\ \text { Confidence } & 0.95 & \text { Prob }<\mathrm{t} & 0.6837\end{array}$

Analysis of Variance

$\begin{array}{lrrrrr}\text { Source } & \text { DF } & \text { Sum of Squares } & \text { Mean Square } & \text { F Ratio } & \text { Prob > F } \\ \text { Sampler Details } & 1 & 0.00246414 & 0.002464 & 0.2387 & 0.6327\end{array}$

$\begin{array}{llll}\text { Error } & 14 & 0.14449700 & 0.010321\end{array}$

$\begin{array}{lll}\text { C. Total } & 15 & 0.14696114\end{array}$

Means for Oneway Anova

Level Number Mean Std Error Lower 95\% Upper 95\%

$\begin{array}{lrrrrr}\text { Coliwasa - high } & 8 & 4.12483 & 0.03592 & 4.0478 & 4.2019\end{array}$

$\begin{array}{llllll}\text { Coliwasa - low } & 8 & 4.14965 & 0.03592 & 4.0726 & 4.2267\end{array}$

Std Error uses a pooled estimate of error variance

Means and Std Deviations

Level Number Mean Std Dev Std Err Mean Lower 95\% Upper 95\%

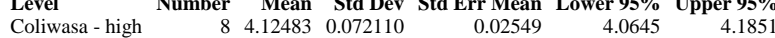

$\begin{array}{lllllll}\text { Coliwasa - low } & 8 & 4.14965 & 0.124268 & 0.04394 & 4.0458 & 4.2535\end{array}$

Test

Coliwasa - low-Coliwasa - high

Assuming unequal variances

$\begin{array}{lrlr}\text { Difference } & 0.02482 & \mathrm{t} \text { Ratio } & 0.488616 \\ \text { Std Err Dif } & 0.05080 & \text { DF } & 11.23402 \\ \text { Upper CL Dif } & 0.13634 & \text { Prob }>|\mathrm{t}| & 0.6345 \\ \text { Lower CL Dif } & -0.08670 & \text { Prob }>\mathrm{t} & 0.3173 \\ \text { Confidence } & 0.95 & \text { Prob }<\mathrm{t} & 0.6827\end{array}$

Tests that the Variances are Equal

Level Count Std Dev MeanAbsDif to Mean MeanAbsDif to Median

$\begin{array}{lrrrr}\text { Coliwasa - high } & 8 & 0.0721098 & 0.0521557 & 0.0516526\end{array}$

$\begin{array}{lllll}\text { Coliwasa - low } & 8 & 0.1242683 & 0.0758018 & 0.0697645\end{array}$

Test F Ratio DFNum DFDen p-Value

$\begin{array}{lrrrr}\text { O'Brien[.5] } & 0.7040 & 1 & 14 & 0.4155\end{array}$

$\begin{array}{lllll}\text { Brown-Forsythe } & 0.2100 & 1 & 14 & 0.6538\end{array}$

$\begin{array}{lllll}\text { Levene } & 0.4079 & 1 & 14 & 0.5334\end{array}$

$\begin{array}{lllll}\text { Bartlett } & 1.8466 & 1 & \text {; } & 0.1742 \\ \text { F Test 2-sided } & 2.9698 & 7 & 7 & 0.1743\end{array}$

Welch Anova testing Means Equal, allowing Std Devs Not Equal

F Ratio DFNum DFDen Prob $>$ F

$\begin{array}{llll}0.2387 & 1 & 11.234 & 0.6345\end{array}$

t Test

0.4886 


\section{Exhibit A2. Statistical Comparisons for Mid-Rheology (Phase 1) Testing}

Oneway Analysis of Mean(Measurement) By Sampler Details Analyte=BaO (wt\%), Target $=\mathbf{0 . 0 9 1 9}$

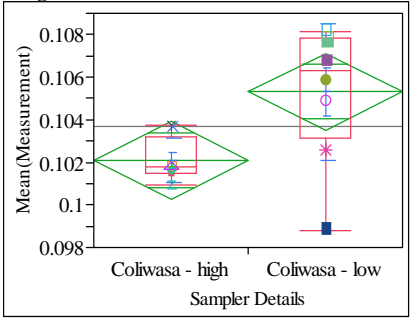

Oneway Anova

Summary of Fit

Adj Rsquare

Root Mean Square Error $\quad 0.002387$

Mean of Response $\quad 0.10370$ 0.103709

t Test

Coliwasa - low-Coliwasa - high

Assuming equal variances

Difference $\quad 0.003215$ t Ratio $\quad 2.692917$

$\begin{array}{lrlr}\text { Std Err Dif } & 0.001194 & \text { DF } & 14 \\ \text { Upper CL Dif } & 0.005775 & \text { Prob }>|t| & 0.0175\end{array}$

Lower CL Dif 0.000654 Prob $>t \quad 0.0087$

$\begin{array}{lrl}\text { Confidence } & 0.95 \text { Prob }<\mathrm{t} & 0.9913\end{array}$

Analysis of Variance

Source DF Sum of Squares Mean Square F Ratio Prob $>$ F

\begin{tabular}{lrrr} 
Error & 14 & 0.00004133 & 0.000041 \\
\hline
\end{tabular}

$\begin{array}{lll}\text { C. Total } & 15 & 0.00012113\end{array}$

Means for Oneway Anova

Level Number Mean Std Error Lower 95\% Upper 95\%

$\begin{array}{lrrrrr}\text { Coliwasa - high } & 8 & 0.102102 & 0.00084 & 0.10029 & 0.10391\end{array}$

$\begin{array}{llllll}\text { Coliwasa - low } & 8 & 0.105316 & 0.00084 & 0.10351 & 0.10713\end{array}$

Std Error uses a pooled estimate of error variance

Means and Std Deviations

Level Number Mean Std Dev Std Err Mean Lower 95\% Upper 95\%

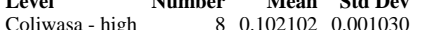

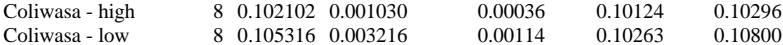

t Test

Coliwasa - low-Coliwasa - high

Assuming unequal variances

Difference $\quad 0.003215$ t Ratio $\quad 2.692917$

Std Err Dif $\quad 0.001194$ DF $\quad 8.420492$

Upper CL Dif 0.005944 Prob $>|t| \quad 0.0262$

Lower CL Dif 0.000486 Prob $>t \quad 0.013$

$\begin{array}{lrl}\text { Confidence } & 0.95 \text { Prob }<\mathrm{t} & 0.9869\end{array}$

Tests that the Variances are Equal

Level Count Std Dev MeanAbsDif to Mean MeanAbsDif to Median

Coliwasa - high $\quad 8 \quad 0.0010296$

$\begin{array}{lllll}\text { Coliwasa - low } & 8 & 0.0032155 & 0.0008013 & 0.0006536 \\ & & & \end{array}$

Test F Ratio DFNum DFDen p-Value

$\begin{array}{lrrrr}\text { O'Brien[.5] } & 2.3120 & 1 & 14 & 0.1506\end{array}$

$\begin{array}{lllll}\text { Brown-Forsythe } & 3.3946 & 1 & 14 & 0.0867\end{array}$

$\begin{array}{lllll}\text { Levene } & 5.1916 & 1 & 14 & 0.0389\end{array}$

$\begin{array}{lllll}\text { Bartlett } & 7.0986 & 1 & \text {. } & 0.0077\end{array}$

Welch Anova testing Means Equal, allowing Std Devs Not Equal

F Ratio DFNum DFDen Prob $>$ F

$\begin{array}{llll}7.2518 & 1 & 8.4205 & 0.0262\end{array}$

t Test

2.6929
Oneway Analysis of Mean(Measurement) By Sampler Details Analyte=CaO (wt \%), Target $=1.1462$

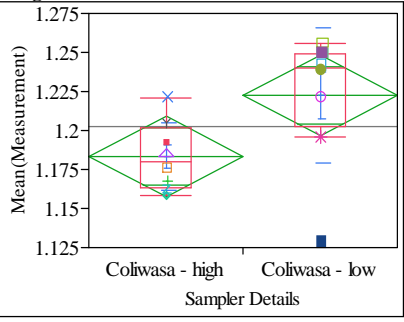

Oneway Anova

Summary of Fit

Adj Rsquare

0.274876

Root Mean Square Error $\quad 0.034063$

$\begin{array}{lr}\text { Mean of Response } & 1.202846 \\ \text { Observations (or Sum Wgts) } & 16\end{array}$

t Test

Coliwasa - low-Coliwasa - high

Assuming equal variances

Difference 0.039236 t Ratio 2.3037

Std Err Dif $\quad 0.017032$ DF $\quad 14$

Upper CL Dif 0.075765 Prob $>|t| 0.0371$

Lower CL Dif 0.002707 Prob $>t \quad 0.0185$

$\begin{array}{lrl}\text { Confidence } & 0.95 \text { Prob }<\mathrm{t} & 0.9815\end{array}$

Analysis of Variance

$\begin{array}{lrrrrr}\text { Source } & \text { DF } & \text { Sum of Squares } & \text { Mean Square } & \text { F Ratio } & \text { Prob }>\text { F } \\ \text { Sampler Details } & 1 & 0.00615782 & 0.006158 & 5.3070 & 0.0371\end{array}$

$\begin{array}{llll}\text { Error } & 14 & 0.01624440 & 0.001160\end{array}$

$\begin{array}{lll}\text { C. Total } & 15 & 0.02240222\end{array}$

Means for Oneway Anova

Level Number Mean Std Error Lower 95\% Upper 95\%

$\begin{array}{lrrrrr}\text { Coliwasa - high } & 8 & 1.18323 & 0.01204 & 1.1574 & 1.209\end{array}$

$\begin{array}{llllll}\text { Coliwasa - low } & 8 & 1.22246 & 0.01204 & 1.1966 & 1.2483\end{array}$

Std Error uses a pooled estimate of error variance

Means and Std Deviations

Level Number Mean Std Dev Std Err Mean Lower 95\% Upper 95\%

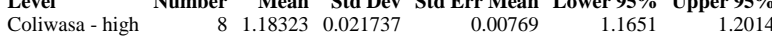

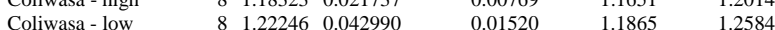

Test

Coliwasa - low-Coliwasa - high

Assuming unequal variances

$\begin{array}{lrlr}\text { Difference } & 0.039236 & \text { t Ratio } & 2.3037 \\ \text { Std Err Dif } & 0.017032 & \text { DF } & 10.35953 \\ \text { Upper CL Dif } & 0.077007 & \text { Prob }>|t| & 0.0431 \\ \text { Lower CL Dif } & 0.001465 & \text { Prob }>t & 0.0216 \\ \text { Confidence } & 0.95 & \text { Prob }<\mathrm{t} & 0.9784\end{array}$

Tests that the Variances are Equal

Level Count Std Dev MeanAbsDif to Mean MeanAbsDif to Median

$\begin{array}{lrrrr}\text { Coliwasa - high } & 8 & 0.0217366 & 0.0172859 & 0.0172859\end{array}$

$\begin{array}{lllll}\text { Coliwasa - low } & 8 & 0.0429901 & 0.0306877 & 0.0264973\end{array}$

Test F Ratio DFNum DFDen p-Value

$\begin{array}{lllll}\text { O'Brien[.5] } & 1.0668 & 1 & 14 & 0.3192\end{array}$

$\begin{array}{lllll}\text { Brown-Forsythe } & 0.4392 & 1 & 14 & 0.5183\end{array}$

$\begin{array}{lllll}\text { Levene } & 1.5914 & 1 & 14 & 0.2277\end{array}$

$\begin{array}{lllll}\text { Bartlett } & 2.8287 & 1 & \text {; } & 0.0926 \\ \text { F Test 2-sided } & 3.9116 & 7 & 7 & 0.0925\end{array}$

Welch Anova testing Means Equal, allowing Std Devs Not Equal

F Ratio DFNum DFDen Prob $>$ F

$\begin{array}{llll}5.3070 & 1 & 10.36 & 0.0431\end{array}$

t Test

2.3037 


\section{Exhibit A2. Statistical Comparisons for Mid-Rheology (Phase 1) Testing}

Oneway Analysis of Mean(Measurement) By Sampler Details Analyte=Cr2O3 (wt\%), Target $=0.0998$

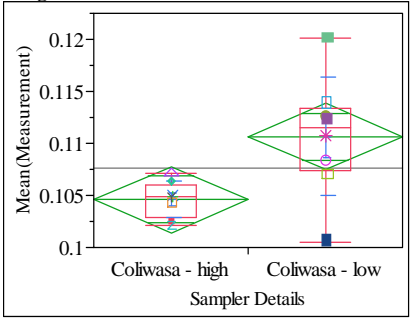

Oneway Anova

Summary of Fit

Adj Rsquare $\quad 0.372373$

Root Mean Square Error $\quad 0.004209$

Mean of Response

Observations (or Sum Wgts) $\quad 16$

t Test

Coliwasa - low-Coliwasa - high

Assuming equal variances

Difference $\quad 0.006066$ t Ratio 2.882055

Std Err Dif $\quad 0.002105$ DF $\quad 14$

Upper CL Dif 0.010580 Prob $>|t| \quad 0.0121$

Lower CL Dif 0.001552 Prob $>t \quad 0.0060$

$\begin{array}{lrl}\text { Confidence } & 0.95 \text { Prob }<\mathrm{t} & 0.9940\end{array}$

Analysis of Variance

Source DF Sum of Squares Mean Square F Ratio Prob > F

$\begin{array}{lllllll}\text { Sampler Details } & 1 & 0.00014717 & 0.000147 & 8.3062 & 0.0121\end{array}$

$\begin{array}{llll}\text { Error } & 14 & 0.00024805 & 0.000018\end{array}$

$\begin{array}{lll}\text { C. Total } & 15 & 0.00039522\end{array}$

Means for Oneway Anova

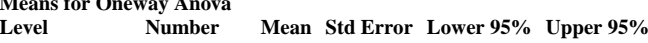

$\begin{array}{lrrrrr}\text { Coliwasa - high } & 8 & 0.104605 & 0.00149 & 0.10141 & 0.10780\end{array}$

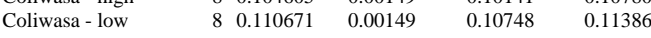

Std Error uses a pooled estimate of error variance

Means and Std Deviations

Level Number Mean Std Dev Std Err Mean Lower 95\% Upper 95\%

$\begin{array}{lrrrrrr} & \text { Number } & \text { Mean } & \text { Std Dev } & \text { Std Err Mean } & \text { Lower 95\% } & \text { Upper 95\% } \\ \text { Coliwasa - high } & 8 & 0.104605 & 0.001757 & 0.00062 & 0.10314 & 0.10607\end{array}$

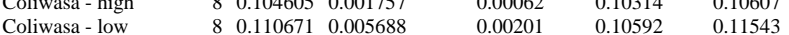

t Test

Coliwasa - low-Coliwasa - high

Assuming unequal variances

Difference $\quad 0.006066$ t Ratio 2.882055

Std Err Dif $\quad 0.002105$ DF $\quad 8.323794$

Upper CL Dif 0.010886 Prob $>|t| \quad 0.0196$

Lower CL Dif 0.001245 Prob $>t \quad 0.0098$

$\begin{array}{lrl}\text { Confidence } & 0.95 \text { Prob }<\mathrm{t} & 0.9902\end{array}$

Tests that the Variances are Equal

Level Count Std Dev MeanAbsDif to Mean MeanAbsDif to Median

Coliwasa - high $\quad 8 \quad 0.0017569$

$\begin{array}{lllll}\text { Coliwasa - low } & 8 & 0.0056876 & 0.0012873 & 0.0012332 \\ & & & \end{array}$

Test F Ratio DFNum DFDen p-Value

$\begin{array}{lrrrr}\text { O'Brien[.5] } & 2.4877 & 1 & 14 & 0.1371\end{array}$

$\begin{array}{lllll}\text { Brown-Forsythe } & 3.7875 & 1 & 14 & 0.0720\end{array}$

$\begin{array}{lllll}\text { Levene } & 3.9403 & 1 & 14 & 0.0671\end{array}$

$\begin{array}{lrrrr}\text { Bartlett } & 7.4837 & 1 & \text {. } & 0.0062\end{array}$

Welch Anova testing Means Equal, allowing Std Devs Not Equal

F Ratio DFNum DFDen Prob $>$ F

$\begin{array}{rrrr}8.3062 & 1 & 8.3238 & 0.0196\end{array}$

t Test

2.8821
Oneway Analysis of Mean(Measurement) By Sampler Details Analyte $=\mathrm{CuO}(\mathrm{wt} \%)$, Target $\mathbf{0 . 0 5 0 4}$

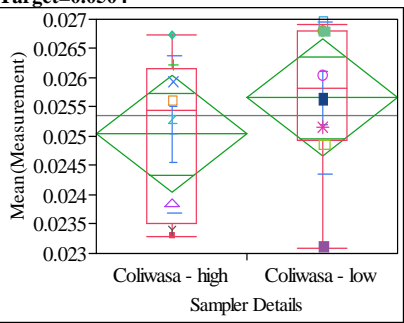

Oneway Anova

Summary of Fit

Adj Rsquare

0.001319

0.025346

t Test

Coliwasa - low-Coliwasa - high

Assuming equal variances

$\begin{array}{lrlr}\text { Difference } & 0.00062 & \mathrm{t} \text { Ratio } & 0.94132 \\ \text { Std Err Dif } & 0.00066 & \text { DF } & 14 \\ \text { Upper CL Dif } & 0.00203 & \text { Prob }>|t| & 0.3625 \\ \text { Lower CL Dif } & -0.00079 & \text { Prob }>t & 0.1813 \\ \text { Confidence } & 0.95 & \text { Prob }<\mathrm{t} & 0.8187\end{array}$

Analysis of Variance

Source DF Sum of Squares Mean Square F Ratio Prob $>$ F

$\begin{array}{lrrr}\text { Error } & 1 & 0.00000154 & 1.541 \mathrm{e}-6 \\ \text { C. } & 14 & 0.00002435 & 1.7391 \mathrm{e}-6\end{array}$

$\begin{array}{lll}\text { C. Total } & 15 & 0.00002589\end{array}$

Means for Oneway Anova

Level Number Mean Std Error Lower 95\% Upper 95\%

$\begin{array}{lrrrrr}\text { Coliwasa - high } & 8 & 0.025036 & 0.00047 & 0.02404 & 0.02604\end{array}$

$\begin{array}{llllll}\text { Coliwasa - low } & 8 & 0.025657 & 0.00047 & 0.02466 & 0.02666\end{array}$

Std Error uses a pooled estimate of error variance

Means and Std Deviations

Level Number Mean Std Dev Std Err Mean Lower 95\% Upper 95\%

$\begin{array}{lrrrrrr} & \text { Number } & \text { Mean } & \text { Std Dev } & \text { Std Err Mean } & \text { Lower } 95 \% & \text { Upper } 95 \% \\ \text { Coliwasa - high } & 8 & 0.025036 & 0.001339 & 0.00047 & 0.02392 & 0.02616\end{array}$

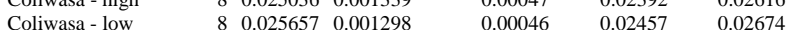

Test

Coliwasa - low-Coliwasa - high

Assuming unequal variances

$\begin{array}{lrlr}\text { Difference } & 0.00062 & \mathrm{t} \text { Ratio } & 0.94132 \\ \text { Std Err Dif } & 0.00066 & \text { DF } & 13.98652 \\ \text { Upper CL Dif } & 0.00204 & \text { Prob }>|\mathrm{t}| & 0.3625 \\ \text { Lower CL Dif } & -0.00079 & \text { Prob }>\mathrm{t} & 0.1813 \\ \text { Confidence } & 0.95 & \text { Prob }<\mathrm{t} & 0.8187\end{array}$

Tests that the Variances are Equal

Level Count Std Dev MeanAbsDif to Mean MeanAbsDif to Median

$\begin{array}{lllll}\text { Coliwasa - high } & 8 & 0.0013391 & 0.0011423 & 0.0010797\end{array}$

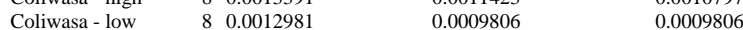

Test F Ratio DFNum DFDen p-Value

$\begin{array}{lrrrr}\text { O'Brien[.5] } & 0.0102 & 1 & 14 & 0.9209\end{array}$

$\begin{array}{lllll}\text { Brown-Forsythe } & 0.0621 & 1 & 14 & 0.8069\end{array}$

$\begin{array}{lllll}\text { Levene } & 0.2355 & 1 & 14 & 0.6350\end{array}$

$\begin{array}{lllll}\text { Bartlett } & 0.0063 & 1 & \text {. } & 0.9367 \\ \text { F Test 2-sided } & 1.0641 & 7 & 7 & 0.9368\end{array}$

Welch Anova testing Means Equal, allowing Std Devs Not Equal

F Ratio DFNum DFDen Prob $>$ F

$\begin{array}{rrrr}0.8861 & 1 & 13.987 & 0.3625\end{array}$

t Test

0.9413 


\section{Exhibit A2. Statistical Comparisons for Mid-Rheology (Phase 1) Testing}

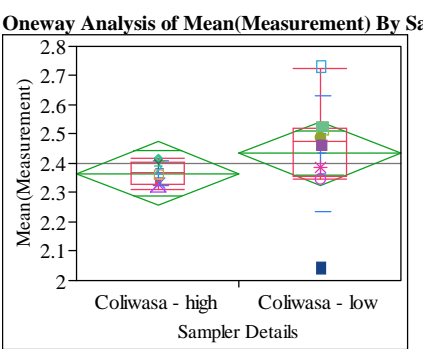

Oneway Analysis of Mean(Measurement) By Sampler Details Analyte=Fe2O3 (wt\%), Target=11.462

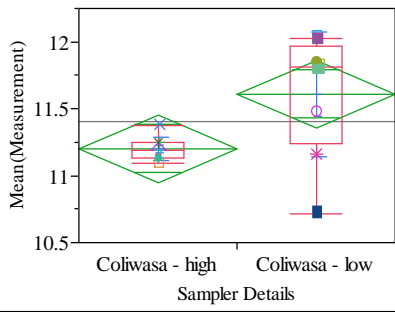

Oneway Anova

Summary of Fit

Rsquare $\quad 0.061505$

Adj Rsquare $\quad-0.00553$

2.400486

Observations (or Sum Wgts)

Test

Coliwasa - low-Coliwasa - high

Assuming equal variances

Difference $\quad 0.06817$ t Ratio $\quad 0.957863$

Std Err Dif $\quad 0.07117$ DF 14

Upper CL Dif 0.22081 Prob $>|t| \quad 0.3544$

Lower CL Dif -0.08447 Prob $>t \quad 0.1772$

$\begin{array}{lll}\text { Confidence } & 0.95 \text { Prob }<\mathrm{t} \quad 0.8228\end{array}$

$\begin{array}{lrrrrr}\begin{array}{l}\text { Analysis of Variance } \\ \text { Source }\end{array} & \text { DF } & \text { Sum of Squares } & \text { Mean Square } & \text { F Ratio } & \text { Prob }>\text { F } \\ \text { Sampler Details } & 1 & 0.01858820 & 0.018588 & 0.9175 & 0.3544 \\ \text { Error } & 14 & 0.28363438 & 0.020260 & & \\ \text { C. Total } & 15 & 0.30222258 & & & \end{array}$

Means for Oneway Anova

Level Number Mean Std Error Lower 95\% Upper 95\%

$\begin{array}{lrrrrr}\text { Coliwasa - high } & 8 & 2.36640 & 0.05032 & 2.2585 & 2.4743\end{array}$

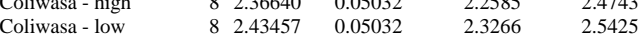

Std Error uses a pooled estimate of error variance

Means and Std Deviations

Level Number Mean Std Dev Std Err Mean Lower 95\% Upper 95\%

$\begin{array}{llllllr}\text { Coliwasa - high } & 8 & 2.36640 & 0.040814 & 0.01443 & 2.3323 & 2.4005\end{array}$

$\begin{array}{lllllll}\text { Coliwasa - low } & 8 & 2.43457 & 0.197113 & 0.06969 & 2.2698 & 2.5994\end{array}$

Test

Coliwasa - low-Coliwasa - high

Assuming unequal variances

Difference $\quad 0.06817$ t Ratio $\quad 0.957863$

Std Err Dif $\quad 0.07117$ DF $\quad 7.599124$

Upper CL Dif 0.23380 Prob $>|t| \quad 0.3676$

Lower CL Dif -0.09746 Prob $>\mathrm{t} \quad 0.1838$

$\begin{array}{lrl}\text { Confidence } & 0.95 \text { Prob }<\mathrm{t} & 0.8162\end{array}$

Tests that the Variances are Equal

Level Count Std Dev MeanAbsDif to Mean MeanAbsDif to Median

$\begin{array}{lllll}\text { Coliwasa - high } & 8 & 0.0408139 & 0.0344145 & 0.0344145\end{array}$

Test F Ratio DFNum DFDen p-Value

$\begin{array}{lrrrr}\text { Test } & \text { F Ratio } & \text { DFNum } & \text { DFDen } & \text { p-Value } \\ \text { O'Brien[.5] } & 2.1397 & 1 & 14 & 0.1656 \\ \text { Brown-Forsythe } & 3.2083 & 1 & 14 & 0.0949 \\ \text { Levene } & 4.2376 & 1 & 14 & 0.0587 \\ \text { Bartlett } & 12.0682 & 1 & \text {. } & 0.0005 \\ \text { F Test 2-sided } & 23.3246 & 7 & 7 & 0.0005\end{array}$

Welch Anova testing Means Equal, allowing Std Devs Not Equal

F Ratio DFNum DFDen Prob > F

$\begin{array}{lrrr}0.9175 & 1 & 7.5991 & 0.3676\end{array}$

t Test
0.9579

Oneway Anova

Summary of Fit

Adj Rsquare

0.249167

0.334528

Mean of Response 11.40498

Observations (or Sum Wgts) 16

Test

Coliwasa - low-Coliwasa - high

Assuming equal variances

$\begin{array}{lrlr}\text { Difference } & 0.408954 & \text { t Ratio } & 2.444957 \\ \text { Std Err Dif } & 0.167264 & \text { DF } & 14 \\ \text { Upper CL Dif } & 0.767700 & \text { Prob }>|t| & 0.0283 \\ \text { Lower CL Dif } & 0.050208 & \text { Prob }>t & 0.0142 \\ \text { Confidence } & 0.95 & \text { Prob }<\mathrm{t} & 0.9858\end{array}$

Analysis of Variance

$\begin{array}{lrrrrr}\text { Source } & \text { DF } & \text { Sum of Squares } & \text { Mean Square } & \text { F Ratio } & \text { Prob }>\text { F } \\ \text { Sampler Details } & 1 & 0.6689727 & 0.668973 & 5.9778 & 0.0283\end{array}$

$\begin{array}{llll}\text { Error } & 14 & 1.5667292 & 0.111909\end{array}$

$\begin{array}{lll}\text { C. Total } & 15 & 2.2357019\end{array}$

Means for Oneway Anova

Level Number Mean Std Error Lower 95\% Upper 95\%

$\begin{array}{lrrrrr}\text { Coliwasa - high } & 8 & 11.2005 & 0.11827 & 10.947 & 11.454\end{array}$

$\begin{array}{llllll}\text { Coliwasa - low } & 8 & 11.6095 & 0.11827 & 11.356 & 11.863\end{array}$

Std Error uses a pooled estimate of error variance

Means and Std Deviations

Level Number Mean Std Dev Std Err Mean Lower 95\% Upper 95\%

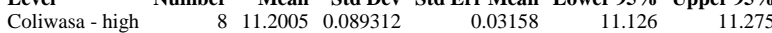

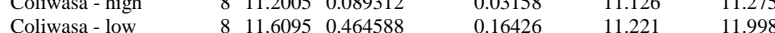

Test

Coliwasa - low-Coliwasa - high

Assuming unequal variances

$\begin{array}{lrlr}\text { Difference } & 0.408954 & \text { t Ratio } & 2.444957 \\ \text { Std Err Dif } & 0.167264 & \text { DF } & 7.516677 \\ \text { Upper CL Dif } & 0.799026 & \text { Prob }>|t| & 0.0421 \\ \text { Lower CL Dif } & 0.018882 & \text { Prob }>t & 0.0211 \\ \text { Confidence } & 0.95 & \text { Prob }<\text { t } & 0.9789\end{array}$

Tests that the Variances are Equal

Level Count Std Dev MeanAbsDif to Mean MeanAbsDif to Median

$\begin{array}{lrrrr}\text { Coliwasa - high } & 8 & 0.0893120 & 0.0702936 & 0.0702936\end{array}$

$\begin{array}{lllll}\text { Coliwasa - low } & 8 & 0.4645878 & 0.3689668 & 0.3219804\end{array}$

Test F Ratio DFNum DFDen p-Value

$\begin{array}{lrrrr}\text { O'Brien[.5] } & 3.3259 & 1 & 14 & 0.0896\end{array}$

$\begin{array}{lllll}\text { Brown-Forsythe } & 3.4172 & 1 & 14 & 0.0857\end{array}$

$\begin{array}{lllll}\text { Levene } & 11.4025 & 1 & 14 & 0.0045\end{array}$

$\begin{array}{lllll}\text { Bartlett } & 12.9642 & 1 & \text {. } & 0.0003 \\ \text { F Test 2-sided } & 27.0593 & 7 & 7 & 0.0003\end{array}$

Welch Anova testing Means Equal, allowing Std Devs Not Equal

F Ratio DFNum DFDen Prob $>$ F

$\begin{array}{lrrr}5.9778 & 1 & 7.5167 & 0.0421\end{array}$

t Test

2.4450 


\section{Exhibit A2. Statistical Comparisons for Mid-Rheology (Phase 1) Testing}

Oneway Analysis of Mean(Measurement) By Sampler Details Analyte=K2O (wt \%), Target $=\mathbf{0 . 1 5 9 1}$

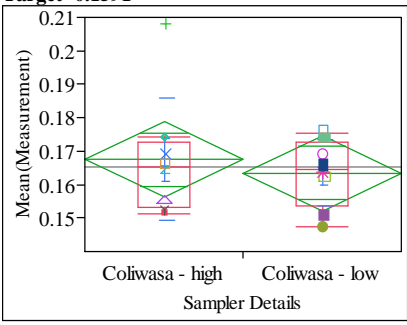

Oneway Anova

Summary of Fit

Adj Rsquare

0.021174

-0.04874
0.014775

Observations (or Sum Wgts) $\quad 16$

t Test

Coliwasa - low-Coliwasa - high

Assuming equal variances

Difference $\quad-0.00407$ t Ratio $\quad-0.55032$

Std Err Dif $\quad 0.00739$ DF $\quad 14$

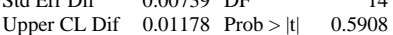

Lower CL Dif -0.01991 Prob $>t \quad 0.7046$

$\begin{array}{lrl}\text { Confidence } & 0.95 \text { Prob }<\mathrm{t} \quad 0.2954\end{array}$

Analysis of Variance

Source DF Sum of Squares Mean Square F Ratio Prob > F

$\begin{array}{llllll}\text { Sampler Details } & 1 & 0.00006611 & 0.000066 & 0.3028 & 0.5908\end{array}$

$\begin{array}{llll}\text { Error } & 14 & 0.00305628 & 0.000218\end{array}$

$\begin{array}{lll}\text { C. Total } & 15 & 0.00312240\end{array}$

Means for Oneway Anova

Level Number Mean Std Error Lower 95\% Upper 95\%

$\begin{array}{lrrrrr}\text { Coliwasa - high } & 8 & 0.167502 & 0.00522 & 0.15630 & 0.17871\end{array}$

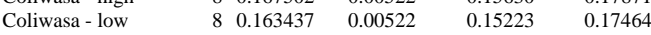

Std Error uses a pooled estimate of error variance

Means and Std Deviations

Level Number Mean Std Dev Std Err Mean Lower 95\% Upper 95\%

$\begin{array}{lrrrrrr} & \text { Number } & \text { Mean } & \text { Std Dev } & \text { Std Err Mean } & \text { Lower 95\% } & \text { Upper 95\% } \\ \text { Coliwasa - high } & 8 & 0.167502 & 0.018341 & 0.00648 & 0.15217 & 0.18284\end{array}$

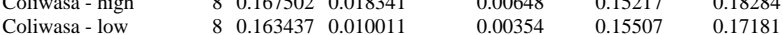

Test

Coliwasa - low-Coliwasa - high

Assuming unequal variances

Difference $\quad-0.00407$ t Ratio $\quad-0.55032$

Std Err Dif $\quad 0.00739$ DF $\quad 10.83055$

Upper CL Dif 0.01223 Prob $>|t| r \mid r .5933$

Lower CL Dif -0.02036 Prob $>t \quad 0.7034$

$\begin{array}{lrl}\text { Confidence } & 0.95 \text { Prob }<\mathrm{t} & 0.2966\end{array}$

Tests that the Variances are Equal

Level Count Std Dev MeanAbsDif to Mean MeanAbsDif to Median

Coliwasa - high $\quad 8 \quad 0.0183412$

$0.0120742-10070$

Test F Ratio DFNum DFDen p-Value

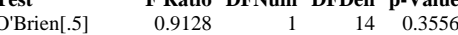

Brown-Forsythe $\quad 0.6707 \quad 1 \quad 14 \quad 0.4265$

$\begin{array}{lllll}\text { Levene } & 0.8027 & 1 & 14 & 0.3854\end{array}$

$\begin{array}{lllll}\text { Bartlett } & 2.2619 & 1 & \text {. } & 0.1326\end{array}$

Welch Anova testing Means Equal, allowing Std Devs Not Equal

F Ratio DFNum DFDen Prob $>$ F

$\begin{array}{llll}0.3028 & 1 & 10.831 & 0.5933\end{array}$

t Test

0.5503
Oneway Analysis of Mean(Measurement) By Sampler Details Analyte=Li2O (wt\%), Target $=4.674$

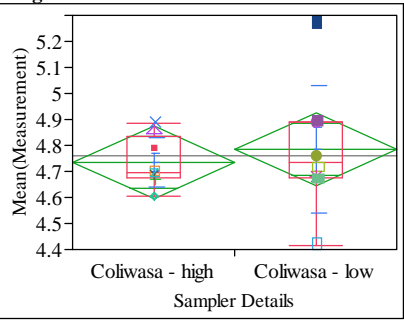

Oneway Anova

Summary of Fit

Adj Rsquare

0.020416

\begin{tabular}{ll}
-0.04955 \\
\hline
\end{tabular}

4.760152

t Test

Coliwasa - low-Coliwasa - high

Assuming equal variances

$\begin{array}{lrlr}\text { Difference } & 0.05023 & \mathrm{t} \text { Ratio } & 0.540164 \\ \text { Std Err Dif } & 0.09300 & \text { DF } & 14 \\ \text { Upper CL Dif } & 0.24970 & \text { Prob }>|\mathrm{t}| & 0.5976 \\ \text { Lower CL Dif } & -0.14923 & \text { Prob }>\mathrm{t} & 0.2988 \\ \text { Confidence } & 0.95 & \text { Prob }<\mathrm{t} & 0.7012\end{array}$

Analysis of Variance

Source DF Sum of Squares Mean Square F Ratio Prob > F

$\begin{array}{llllll}\text { Sampler Details } & 1 & 0.01009395 & 0.010094 & 0.2918 & 0.5976\end{array}$

$\begin{array}{llll}\text { Error } & 14 & 0.48432628 & 0.034595\end{array}$

$\begin{array}{lll}\text { C. Total } & 15 & 0.49442023\end{array}$

Means for Oneway Anova

Level Number Mean Std Error Lower 95\% Upper 95\%

\begin{tabular}{lrrrrr} 
Coliwasa - high & 8 & 4.73503 & 0.06576 & 4.5940 & 4.876 \\
\hline & 8 & 4.78527 & 0.06576 & 4.6442 & 4.9263
\end{tabular}

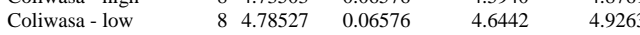

Std Error uses a pooled estimate of error variance

Means and Std Deviations

Level Number Mean Std Dev Std Err Mean Lower 95\% Upper 95\%

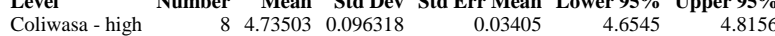

$\begin{array}{lllllll}\text { Coliwasa - low } & 8 & 4.78527 & 0.244770 & 0.08654 & 4.5806 & 4.9899\end{array}$

Test

Coliwasa - low-Coliwasa - high

Assuming unequal variances

$\begin{array}{lrlr}\text { Difference } & 0.05023 & \text { t Ratio } & 0.540164 \\ \text { Std Err Dif } & 0.09300 & \text { DF } & 9.117058 \\ \text { Upper CL Dif } & 0.26020 & \text { Prob }>|t| & 0.6020 \\ \text { Lower CL Dif } & -0.15973 & \text { Prob }>\text { t } & 0.3010 \\ \text { Confidence } & 0.95 & \text { Prob }<\mathrm{t} & 0.6990\end{array}$

Tests that the Variances are Equal

Level Count Std Dev MeanAbsDif to Mean MeanAbsDif to Median

$\begin{array}{lrrrr}\text { Coliwasa - high } & 8 & 0.0963176 & 0.0790518 & 0.0704178\end{array}$

$\begin{array}{lllll}\text { Coliwasa - low } & 8 & 0.2447701 & 0.1723441 & 0.1655042\end{array}$

Test F Ratio DFNum DFDen p-Value

$\begin{array}{lrrrr}\text { O'Brien[.5] } & 1.8496 & 1 & 14 & 0.1953\end{array}$

$\begin{array}{lllll}\text { Brown-Forsythe } & 1.9757 & 1 & 14 & 0.1816\end{array}$

$\begin{array}{lllll}\text { Levene } & 2.4777 & 1 & 14 & 0.1378\end{array}$

$\begin{array}{lllll}\text { Bartlett } & 5.0109 & 1 & \text {. } & 0.0252 \\ \text { F Test 2-sided } & 6.4581 & 7 & 7 & 0.0250\end{array}$

Welch Anova testing Means Equal, allowing Std Devs Not Equal

F Ratio DFNum DFDen Prob $>$ F

$\begin{array}{lrrr}0.2918 & 1 & 9.1171 & 0.6020\end{array}$

t Test

0.5402 


\section{Exhibit A2. Statistical Comparisons for Mid-Rheology (Phase 1) Testing}

Oneway Analysis of Mean(Measurement) By Sampler Details Analyte=MgO (wt\%), Target $=1.014$

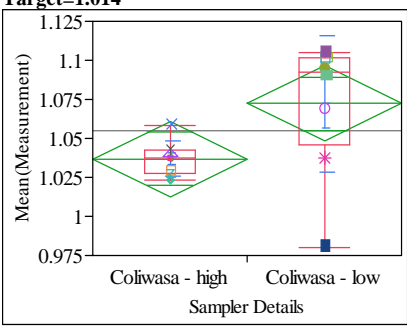

Oneway Anova

Summary of Fit

Adj Rsquare $\quad 0.26081$

Root Mean Square Error $\quad 0.031865$

Observations (or Sum Wgts) 1.054765

t Test

Coliwasa - low-Coliwasa - high

Assuming equal variances

Difference $\quad 0.035412$ t Ratio 2.22257

Std Err Dif 0.015933 DF $\quad 14$

Upper CL Dif 0.069584 Prob $>|t| \quad 0.0432$

Lower CL Dif 0.001239 Prob $>t \quad 0.0216$

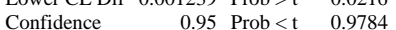

Analysis of Variance

Source DF Sum of Squares Mean Square F Ratio Prob > F

$\begin{array}{lrrr}\text { Sampler Details } & 1 & 0.00501593 & 0.005016 \\ \text { Error } & 14 & 0.01421571 & 0.001015\end{array}$

$\begin{array}{lll}\text { C. Total } & 15 & 0.01923164\end{array}$

Means for Oneway Anova

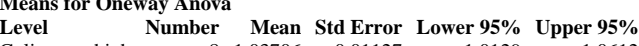

$\begin{array}{lrrrrr}\text { Coliwasa - high } & 8 & 1.03706 & 0.01127 & 1.0129 & 1.0612\end{array}$

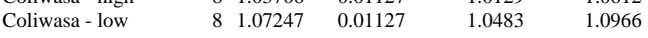

Std Error uses a pooled estimate of error variance

Means and Std Deviations

Level Number Mean Std Dev Std Err Mean Lower 95\% Upper 95\%

$\begin{array}{lrrrrrr}\text { Coliwasa - high } & 8 & 1.03706 & 0.010898 & 0.00385 & 1.0279 & 1.0462\end{array}$

$\begin{array}{lllllll}\text { Coliwasa - low } & 8 & 1.07247 & 0.043727 & 0.01546 & 1.0359 & 1.0469\end{array}$

t Test

Coliwasa - low-Coliwasa - high

Assuming unequal variances

Difference $\quad 0.03541$ t Ratio $\quad 2.22257$

Std Err Dif $\quad 0.01593$ DF $\quad 7.866265$

Upper CL Dif 0.07226 Prob $>|t| \quad 0.0575$

Lower CL Dif -0.00144 Prob $>t \quad 0.0288$

$\begin{array}{lrlr}\text { Lower CL Dif } & -0.00144 & \text { Prob }>t & 0.0288 \\ \text { Confidence } & 0.95 & \text { Prob }<\mathrm{t} & 0.9712\end{array}$

Tests that the Variances are Equal

Level Count Std Dev MeanAbsDif to Mean MeanAbsDif to Median

Coliwasa - high $\quad 8 \quad 0.0108980$

$\begin{array}{lllll}\text { Coliwasa - low } & 8 & 0.0437270 & 0.0078769 & 0.0078769 \\ & & & \end{array}$

Test F Ratio DFNum DFDen p-Value

$\begin{array}{lrrrr}\text { O'Brien[.5] } & 2.1000 & 1 & 14 & 0.1693\end{array}$

$\begin{array}{lllll}\text { Brown-Forsythe } & 2.2267 & 1 & 14 & 0.1578\end{array}$

$\begin{array}{lllll}\text { Levene } & 6.7543 & 1 & 14 & 0.0210\end{array}$

$\begin{array}{lrlll}\text { Bartlett } & 9.8849 & 1 & \text {. } & 0.0017 \\ & 16.0992 & 7 & 7 & 0.0016\end{array}$

Welch Anova testing Means Equal, allowing Std Devs Not Equal

F Ratio DFNum DFDen Prob $>$ F

$4.9398 \quad 1 \quad 7.8663 \quad 0.0575$

t Test

2.2226
Oneway Analysis of Mean(Measurement) By Sampler Details Analyte=MnO (wt\%), Target $=1.779$

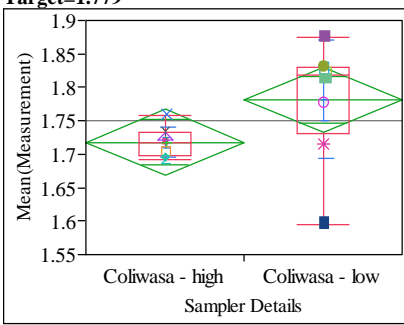

Oneway Anova

Summary of Fit

Adj Rsquare

$\quad 0.064636$

$\begin{array}{r}1.74998 \\ \hline\end{array}$

t Test

Coliwasa - low-Coliwasa - high

Assuming equal variances

$\begin{array}{lrlr}\text { Difference } & 0.06375 & \mathrm{t} \text { Ratio } & 1.972663 \\ \text { Std Err Dif } & 0.03232 & \text { DF } & 14 \\ \text { Upper CL Dif } & 0.13307 & \text { Prob }>|\mathrm{t}| & 0.0686 \\ \text { Lower CL Dif } & -0.00556 & \text { Prob }>\mathrm{t} & 0.0343 \\ \text { Confidence } & 0.95 & \text { Prob }<\mathrm{t} & 0.9657\end{array}$

Analysis of Variance

Source DF Sum of Squares Mean Square F Ratio Prob > F

Sampler Details $\quad 1 \quad 0.01625778 \quad 0.016258 \quad 3.8914 \quad 0.0686$

$\begin{array}{llll}\text { Error } & 14 & 0.05849026 & 0.004178\end{array}$

$\begin{array}{lll}\text { C. Total } & 15 & 0.07474804\end{array}$

Means for Oneway Anova

Level $\quad$ Number Mean Std Error Lower 95\% Upper 95\%

$\begin{array}{lrrrrr}\text { Coliwasa - high } & 8 & 1.71810 & 0.02285 & 1.6691 & 1.767 \\ & 8 & 1.78186 & 0.02285 & 1.7328 & 1.8309\end{array}$

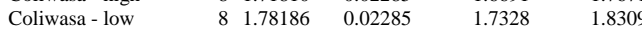

Std Error uses a pooled estimate of error variance

Means and Std Deviations

Level Number Mean Std Dev Std Err Mean Lower 95\% Upper 95\%

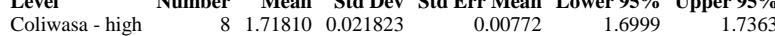

$\begin{array}{lllllll}\text { Coliwasa - low } & 8 & 1.78186 & 0.088767 & 0.03138 & 1.7076 & 1.8561\end{array}$

Test

Coliwasa - low-Coliwasa - high

Assuming unequal variances

$\begin{array}{lrlr}\text { Difference } & 0.06375 & \mathrm{t} \text { Ratio } & 1.972663 \\ \text { Std Err Dif } & 0.03232 & \text { DF } & 7.84312 \\ \text { Upper CL Dif } & 0.13854 & \text { Prob }>|\mathrm{t}| & 0.0847 \\ \text { Lower CL Dif } & -0.01103 & \text { Prob }>\mathrm{t} & 0.0424 \\ \text { Confidence } & 0.95 & \text { Prob }<\mathrm{t} & 0.9576\end{array}$

Tests that the Variances are Equal

Level Count Std Dev MeanAbsDif to Mean MeanAbsDif to Median

$\begin{array}{lllll}\text { Coliwasa - high } & 8 & 0.0218234 & 0.0160727 & 0.0158710\end{array}$

$\begin{array}{lllll}\text { Coliwasa - low } & 8 & 0.0887665 & 0.0645600 & 0.0570280\end{array}$

Test F Ratio DFNum DFDen p-Value

$\begin{array}{lllll}\text { O'Brien[.5] } & 2.0914 & 1 & 14 & 0.1701\end{array}$

$\begin{array}{lllll}\text { Brown-Forsythe } & 2.3393 & 1 & 14 & 0.1484\end{array}$

$\begin{array}{lllll}\text { Levene } & 5.7045 & 1 & 14 & 0.0316\end{array}$

$\begin{array}{lllll}\text { Bartlett } & 10.0426 & 1 & \text {. } & 0.0015 \\ \text { F Test 2-sided } & 16.5445 & 7 & 7 & 0.0015\end{array}$

Welch Anova testing Means Equal, allowing Std Devs Not Equal

F Ratio DFNum DFDen Prob $>$ F

$\begin{array}{llll}3.8914 & 1 & 7.8431 & 0.0847\end{array}$

t Test

1.972 
SRNL-STI-2011-00693

Revision 0

\section{Exhibit A2. Statistical Comparisons for Mid-Rheology (Phase 1) Testing}

Oneway Analysis of Mean(Measurement) By Sampler Details Analyte=Na2O (wt\%), Target $=11.659$

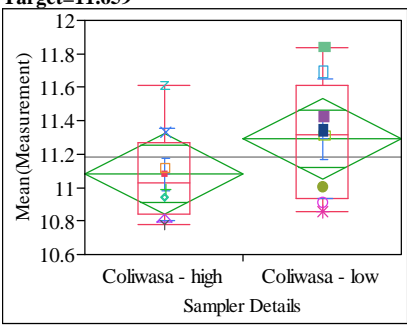

Oneway Anova

Summary of Fit

Adj Rsquare $\quad 0.113182$

Root Mean Square Error $\quad 0.317661$

Mean of Response 11.18587

11.1858

Test

Coliwasa - low-Coliwasa - high

Assuming equal variances

Difference $\quad 0.21231$ t Ratio 1.336707

Std Err Dif $\quad 0.15883$ DF $\quad 14$

Upper CL Dif 0.55297 Prob $>|t| \quad 0.2026$

Lower CL Dif -0.12835 Prob $>\mathrm{t} \quad 0.1013$

$\begin{array}{lrl}\text { Confidence } & 0.95 \text { Prob }<\mathrm{t} & 0.8987\end{array}$

Analysis of Variance

$\begin{array}{lrrrrr}\text { Source } & \text { DF } & \text { Sum of Squares } & \text { Mean Square } & \text { F Ratio } & \text { Prob > F } \\ \text { Sampler Details } & 1 & 0.1803021 & 0.180302 & 1.7868 & 0.2026\end{array}$

$\begin{array}{llll}\text { Error } & 14 & 1.4127214 & 0.100909\end{array}$

$\begin{array}{lll}\text { C. Total } & 15 & 1.5930235\end{array}$

Means for Oneway Anova

Level Number Mean Std Error Lower 95\% Upper 95\%

$\begin{array}{lrrrrr}\text { Coliwasa - high } & 8 & 11.0797 & 0.11231 & 10.839 & 11.32\end{array}$

$\begin{array}{llllll}\text { Coliwasa - low } & 8 & 11.2920 & 0.11231 & 11.051 & 11.533\end{array}$

Std Error uses a pooled estimate of error variance

Means and Std Deviations

Level Number Mean Std Dev Std Err Mean Lower 95\% Upper 95\%

$\begin{array}{lrrrrrr} & \text { Number } & \text { Mean } & \text { Std Dev } & \text { Std Err Mean } & \text { Lower 95\% } & \text { Upper 95\% } \\ \text { Coliwasa - high } & 8 & 11.0797 & 0.275811 & 0.09751 & 10.849 & 11.310\end{array}$

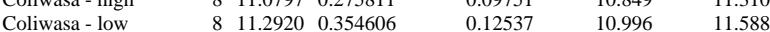

t Test

Coliwasa - low-Coliwasa - high

Assuming unequal variances

Difference $\quad 0.21231$ t Ratio 1.336707

Std Err Dif $\quad 0.15883$ DF $\quad 13.2003$

Upper CL Dif 0.55491 Prob $>|t| \quad 0.2039$

Lower CL Dif -0.13029 Prob $>t \quad 0.1020$

$\begin{array}{lrl}\text { Confidence } & 0.95 \text { Prob }<\mathrm{t} & 0.8980\end{array}$

Tests that the Variances are Equal

Level Count Std Dev MeanAbsDif to Mean MeanAbsDif to Median

$\begin{array}{lllll}\text { Coliwasa - high } & 8 & 0.2758109 & 0.2011469 & 0.1996725\end{array}$

$\begin{array}{lllll}\text { Coliwasa - high } & 8 & 0.2758109 & 0.2011469 & 0.1996725 \\ \text { Coliwasa - low } & 8 & 0.3546064 & 0.2759890 & 0.2721275\end{array}$

Test F Ratio DFNum DFDen p-Value

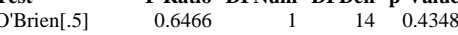

Brown-Forsythe $\quad 0.5586 \quad 1 \quad 14 \quad 0.4672$

$\begin{array}{lllll}\text { Levene } & 0.6539 & 1 & 14 & 0.4322\end{array}$

$\begin{array}{lllll}\text { Bartlett } & 0.4083 & 1 & \text {. } & 0.5228 \\ & 1.6530 & 7 & 7 & 0.5232\end{array}$

Welch Anova testing Means Equal, allowing Std Devs Not Equal

F Ratio DFNum DFDen Prob $>$ F

$\begin{array}{rrrr}1.7868 & 1 & 13.2 & 0.2039\end{array}$

t Test

1.3367
Oneway Analysis of Mean(Measurement) By Sampler Details Analyte=NiO (wt\%), Target $=0.41$

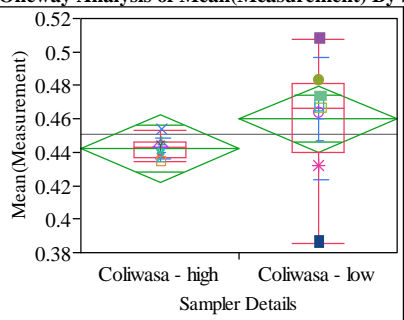

Oneway Anova

Summary of Fit

Adj Rsquare

Root Mean Square Error $\quad 0.050935$

Mean of Response $\quad 0.451088$

Observations (or Sum Wgts) 16

Test

Coliwasa - low-Coliwasa - high

Assuming equal variances

Difference $\quad 0.01768$ t Ratio 1.343512

Std Err Dif $\quad 0.01316$ DF 14

Upper CL Dif 0.04591 Prob $>|t| \quad 0.2005$

Lower CL Dif -0.01055 Prob $>t \quad 0.1002$

$\begin{array}{lrl}\text { Confidence } & 0.95 \text { Prob }<\mathrm{t} & 0.8998\end{array}$

Analysis of Variance

$\begin{array}{lrrrr}\text { Source } & \text { DF Sum of Squares } & \text { Mean Square } & \text { F Ratio } & \text { Prob }>\text { F } \\ \text { Sampler } & & \end{array}$

$\begin{array}{lrrr}\text { Error } & 1 & 0.00125068 & 0.001251 \\ \text { C. } & 14 & 0.00970040 & 0.000693\end{array}$

$\begin{array}{lll}\text { C. Total } & 15 & 0.01095107\end{array}$

Means for Oneway Anova

Level Number Mean Std Error Lower 95\% Upper 95\%

$\begin{array}{lrrrrr}\text { Coliwasa - high } & 8 & 0.442247 & 0.00931 & 0.42229 & 0.46221\end{array}$

$\begin{array}{llllll}\text { Coliwasa - low } & 8 & 0.459929 & 0.00931 & 0.43997 & 0.47989\end{array}$

Std Error uses a pooled estimate of error variance

Means and Std Deviations

Level Number Mean Std Dev Std Err Mean Lower 95\% Upper 95\%

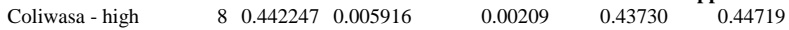

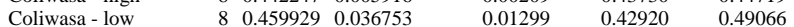

Test

Coliwasa - low-Coliwasa - high

Assuming unequal variances

$\begin{array}{lrlr}\text { Difference } & 0.01768 \text { t Ratio } & 1.343512 \\ \text { Std Err Dif } & 0.01316 \text { DF } & 7.36255 \\ \text { Upper CL Dif } & 0.04850 \text { Prob }>|t| & 0.2190 \\ \text { Lower CL Dif } & -0.01313 \text { Prob }>\text { t } & 0.1095 \\ \text { Confidence } & 0.95 \text { Prob }<\mathrm{t} & 0.8905\end{array}$

Tests that the Variances are Equal

Level Count Std Dev MeanAbsDif to Mean MeanAbsDif to Median

$\begin{array}{lllll}\text { Coliwasa - high } & 8 & 0.0059164 & 0.0046658 & 0.0046658\end{array}$

$\begin{array}{lllll}\text { Coliwasa - low } & 8 & 0.0367528 & 0.0256223 & 0.0229845\end{array}$

Test F Ratio DFNum DFDen p-Value

$\begin{array}{lrrrr}\text { O'Brien[.5] } & 2.4698 & 1 & 14 & 0.1384\end{array}$

$\begin{array}{lllll}\text { Brown-Forsythe } & 3.3225 & 1 & 14 & 0.1384 \\ & 5.7540 & 1 & 14 & 0.0309\end{array}$

$\begin{array}{lllll}\text { Levene } & 5.7540 & 1 & 14 & 0.0309\end{array}$

$\begin{array}{lllll}\text { Bartlett } & 15.1433 & 1 & ; & <.0001 \\ \text { F Test 2-sided } & 38.5894 & 7 & 7 & <.0001\end{array}$

Welch Anova testing Means Equal, allowing Std Devs Not Equal

F Ratio DFNum DFDen Prob $>$ F

$1.8050 \quad 1 \quad 7.3626 \quad 0.2190$

t Test

.3435 


\section{Exhibit A2. Statistical Comparisons for Mid-Rheology (Phase 1) Testing}

Oneway Analysis of Mean(Measurement) By Sampler Details Analyte=SiO2 (wt\%), Target $\mathbf{5 0 . 9 8 5}$

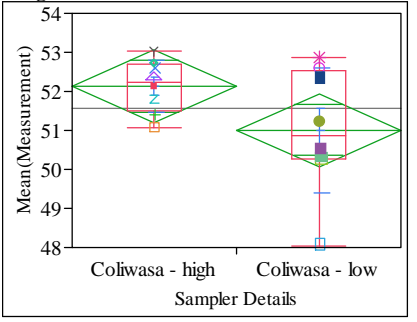

Oneway Anova

Summary of Fit

Adj Rsquare 0.192728

Root Mean Square Error $\quad 1.228666$

Observations (or Sum Wgts) 51.570 .

Test

Coliwasa - low-Coliwasa - high

Assuming equal variances

Difference $\quad-1.1231$ t Ratio -1.8282

Std Err Dif $\quad 0.6143$ DF $\quad 14$

Upper CL Dif 0.1945 Prob $>|t| \quad 0.0889$

Lower CL Dif -2.4407 Prob $>t \quad 0.9555$

$\begin{array}{lrr}\text { Confidence } & 0.95 \text { Prob }<\mathrm{t} & 0.0445\end{array}$

Analysis of Variance

$\begin{array}{lrrrrr}\text { Source } & \text { DF } & \text { Sum of Squares } & \text { Mean Square } & \text { F Ratio } & \text { Prob }>\text { F } \\ \text { Sampler Details } & 1 & 5.045706 & 5.04571 & 3.3424 & 0.0889\end{array}$

$\begin{array}{llrr}\text { Error } & 14 & 21.134696 & 1.50962\end{array}$

C. Total $\quad 15 \quad 26.180402$

Means for Oneway Anova

Level Number Mean Std Error Lower 95\% Upper 95\%

$\begin{array}{lrrrrr}\text { Coliwasa - high } & 8 & 52.1321 & 0.43440 & 51.200 & 53.064\end{array}$

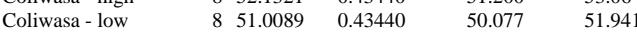

Std Error uses a pooled estimate of error variance

Means and Std Deviations

Level Number Mean Std Dev Std Err Mean Lower 95\% Upper 95\%

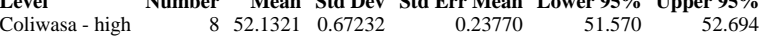

$\begin{array}{lllllll}\text { Coliwasa - low } & 8 & 51.0089 & 1.60226 & 0.56648 & 49.669 & 52.348\end{array}$

t Test

Coliwasa - low-Coliwasa - high

Assuming unequal variances

$\begin{array}{lrlr}\text { Difference } & -1.1231 & \text { t Ratio } & -1.82821 \\ \text { Std Err Dif } & 0.6143 & \text { DF } & 9.390836 \\ \text { Upper CL Dif } & 0.2578 & \text { Prob }>|t| & 0.0994 \\ \text { Lower CL Dif } & -2.5041 & \text { Prob }>\text { t } & 0.9503 \\ \text { Confidence } & 0.95 & \text { Prob }<\mathrm{t} & 0.0497\end{array}$

Tests that the Variances are Equal

Level Count Std Dev MeanAbsDif to Mean MeanAbsDif to Median

Coliwasa - high $\quad 8 \quad 0.672315$

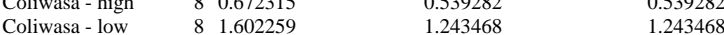

Test F Ratio DFNum DFDen p-Value

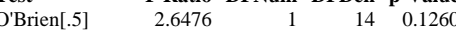

$\begin{array}{lllll}\text { Brown-Forsythe } & 4.1474 & 1 & 14 & 0.0611\end{array}$

$\begin{array}{lllll}\text { Levene } & 4.3131 & 1 & 14 & 0.0567\end{array}$

$\begin{array}{lllll}\text { Bartlett } & 4.4096 & 1 & \text {. } & 0.0357 \\ \text { F Test 2-sided } & 5.6796 & 7 & 7 & 0.0355\end{array}$

Welch Anova testing Means Equal, allowing Std Devs Not Equal

F Ratio DFNum DFDen Prob $>$ F

$\begin{array}{lrrr}3.3424 & 1 & 9.3908 & 0.0994\end{array}$

t Test

1.8282
Oneway Analysis of Mean(Measurement) By Sampler Details Analyte=Sum of Oxides (wt \%), Target $=99.553$

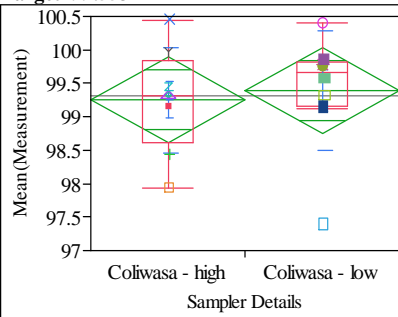

Oneway Anova

Summary of Fit

Adj Rsquare

$\quad 0.846315$

99.32313

t Test

Coliwasa - low-Coliwasa - high

Assuming equal variances

$\begin{array}{lrlr}\text { Difference } & 0.1394 & \mathrm{t} \text { Ratio } & 0.329446 \\ \text { Std Err Dif } & 0.4232 & \text { DF } & 14 \\ \text { Upper CL Dif } & 1.0470 & \text { Prob }>|t| & 0.7467 \\ \text { Lower CL Dif } & -0.7682 & \text { Prob }>t & 0.3733 \\ \text { Confidence } & 0.95 & \text { Prob }<t & 0.6267\end{array}$

Analysis of Variance

Source DF Sum of Squares Mean Square F Ratio Prob $>$ F

$\begin{array}{lrrrrr}\text { Sampler Details } & 1 & 0.077738 & 0.077738 & 0.1085 & 0.7467\end{array}$

$\begin{array}{llll}\text { Error } & 14 & 10.027487 & 0.716249\end{array}$

$\begin{array}{lll}\text { C. Total } & 15 & 10.105225\end{array}$

Means for Oneway Anova

Level Number Mean Std Error Lower 95\% Upper 95\%

$\begin{array}{lrrrrr}\text { Coliwasa - high } & 8 & 99.2534 & 0.29922 & 98.612 & 99.90\end{array}$

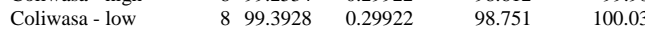

Std Error uses a pooled estimate of error variance

Means and Std Deviations

Level Number Mean Std Dev Std Err Mean Lower 95\% Upper 95\%

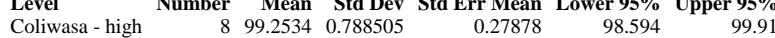

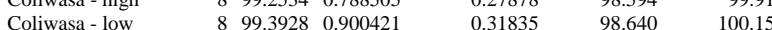

Test

Coliwasa - low-Coliwasa - high

Assuming unequal variances

$\begin{array}{lrlr}\text { Difference } & 0.1394 & \mathrm{t} \text { Ratio } & 0.329446 \\ \text { Std Err Dif } & 0.4232 & \text { DF } & 13.76042 \\ \text { Upper CL Dif } & 1.0485 \text { Prob }>|t| & 0.7468 \\ \text { Lower CL Dif } & -0.7697 & \text { Prob }>\mathrm{t} & 0.3734 \\ \text { Confidence } & 0.95 & \text { Prob }<\mathrm{t} & 0.6266\end{array}$

Tests that the Variances are Equal

Level Count Std Dev MeanAbsDif to Mean MeanAbsDif to Median

$\begin{array}{lrrrr}\text { Coliwasa - high } & 8 & 0.7885049 & 0.5595168 & 0.5455333\end{array}$

$\begin{array}{lllll}\text { Coliwasa - low } & 8 & 0.9004211 & 0.5951568 & 0.5522997\end{array}$

Test F Ratio DFNum DFDen p-Value

$\begin{array}{lllll}\text { O'Brien[.5] } & 0.0776 & 1 & 14 & 0.7846\end{array}$

Brown-Forsythe $\quad 0.0004 \quad 1 \quad 14 \quad 0.9835$

$\begin{array}{lllll}\text { Levene } & 0.0152 & 1 & 14 & 0.9037\end{array}$

$\begin{array}{lllll}\text { Bartlett } & 0.1148 & 1 & \text {. } & 0.7348 \\ \text { F Test 2-sided } & 1.3040 & 7 & 7 & 0.7351\end{array}$

Welch Anova testing Means Equal, allowing Std Devs Not Equal

F Ratio DFNum DFDen Prob $>$ F

$\begin{array}{rrrr}0.1085 & 1 & 13.76 & 0.7468\end{array}$

t Test

0.3294 


\section{Exhibit A2. Statistical Comparisons for Mid-Rheology (Phase 1) Testing}

Oneway Analysis of Mean(Measurement) By Sampler Details Analyte=TiO2 (wt\%), Target $=\mathbf{0 . 0 7 1 1}$

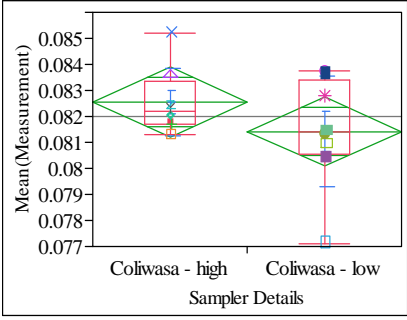

Oneway Anova

Summary of Fit

0.105318

Root Mean Square Error $\quad 0.001759$

Mean of Response $\quad 0.081987$

Observations (or Sum Wgts) $\quad 16$

t Test

Coliwasa - low-Coliwasa - high

Assuming equal variances

Difference $\quad-0.00113$ t Ratio $\quad-1.28375$

Std Err Dif $\quad 0.00088$ DF 14

Upper CL Dif 0.00076 Prob $>|t| \quad 0.2201$

Lower CL Dif -0.00302 Prob $>t \quad 0.8900$

$\begin{array}{lrl}\text { Confidence } & 0.95 \text { Prob }<\mathrm{t} & 0.1100\end{array}$

Analysis of Variance

Source DF Sum of Squares Mean Square F Ratio Prob > F

Sampler Details $\quad 1 \quad 0.00000510 \quad 5.102 \mathrm{e}-6 \quad 1.6480 \quad 0.2201$

$\begin{array}{llll}\text { Error } & 14 & 0.00004334 & 3.0958 \mathrm{e}-6\end{array}$

$\begin{array}{lll}\text { C. Total } & 15 & 0.00004844\end{array}$

Means for Oneway Anova

Level $\quad$ Number Mean Std Error Lower 95\% Upper 95\%

$\begin{array}{lrrrrr}\text { Coliwasa - high } & 8 & 0.082552 & 0.00062 & 0.08122 & 0.08389\end{array}$

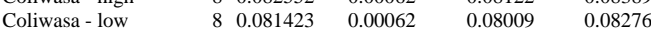

Std Error uses a pooled estimate of error variance

Means and Std Deviations

Level Number Mean Std Dev Std Err Mean Lower 95\% Upper 95\%

Covel

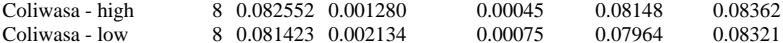

t Test

Coliwasa - low-Coliwasa - high

Assuming unequal variances

Difference $\quad-0.00113$ t Ratio -1.28375

Std Err Dif $\quad 0.00088$ DF $\quad 11.46272$

Upper CL Dif 0.00080 Prob $>|t| r \mid r .2246$

$\begin{array}{lll}\text { Lower CL Dif }-0.00306 \text { Prob }>t & 0.8877\end{array}$

$\begin{array}{lrl}\text { Confidence } & 0.95 \text { Prob }<\mathrm{t} & 0.1123\end{array}$

Tests that the Variances are Equal

Level Count Std Dev MeanAbsDif to Mean MeanAbsDif to Median

Coliwasa - high $\quad 8 \quad 0.0012804$

$\begin{array}{lllll}\text { Coliwasa - low } & 8 & 0.0021336 & 0.00014699 & 0.0014699\end{array}$

Test F Ratio DFNum DFDen p-Value

$\begin{array}{lllll}\text { O'Brien[.5] } & 0.9948 & 1 & 14 & 0.3355\end{array}$

Brown-Forsythe $\quad 0.9999 \quad 1 \quad 14 \quad 0.3343$

$\begin{array}{lllll}\text { Levene } & 0.8498 & 1 & 14 & 0.3722\end{array}$

$\begin{array}{lllll}\text { Bartlett } & 1.6346 & 1 & \text {. } & 0.2011\end{array}$

Welch Anova testing Means Equal, allowing Std Devs Not Equal

F Ratio DFNum DFDen Prob $>$ F

$\begin{array}{llrr}1.6480 & 1 & 11.463 & 0.2246\end{array}$

t Test

1.2837
Oneway Analysis of Mean(Measurement) By Sampler Details Analyte=ZnO (wt\%), Target $=0.0958$

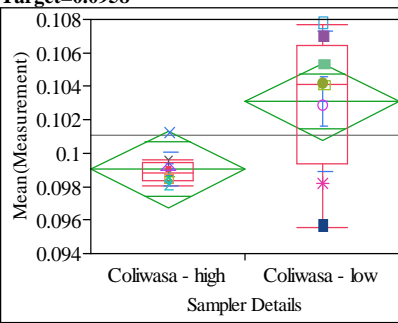

Oneway Anova

Summary of Fit

Adj Rsquare

0.335132

0.287641
0.00305

0.101054

Test

Coliwasa - low-Coliwasa - high

Assuming equal variances

$\begin{array}{lrlr}\text { Difference } & 0.004051 & \text { t Ratio } & 2.656465 \\ \text { Std Err Dif } & 0.001525 & \text { DF } & 14 \\ \text { Upper CL Dif } & 0.007321 & \text { Prob }>|t| & 0.0188 \\ \text { Lower CL Dif } & 0.000780 & \text { Prob }>t & 0.0094 \\ \text { Confidence } & 0.95 & \text { Prob }<t & 0.9906\end{array}$

Analysis of Varianc

Source DF Sum of Squares Mean Square F Ratio Prob $>$ F

$\begin{array}{llllll}\text { Sampler Details } & 1 & 0.00006564 & 0.000066 & 7.0568 & 0.0188\end{array}$

Error $\quad 14 \quad 0.00013021 \quad 9.301 \mathrm{e}-6$

$\begin{array}{lll}\text { C. Total } & 15 & 0.00019585\end{array}$

Means for Oneway Anova

Level Number Mean Std Error Lower 95\% Upper 95\%

$\begin{array}{lrrrrr}\text { Coliwasa - high } & 8 & 0.099029 & 0.00108 & 0.09672 & 0.10134\end{array}$

$\begin{array}{llllll}\text { Coliwasa - low } & 8 & 0.103080 & 0.00108 & 0.10077 & 0.10539\end{array}$

Std Error uses a pooled estimate of error variance

Means and Std Deviations

Level Number Mean Std Dev Std Err Mean Lower 95\% Upper 95\%

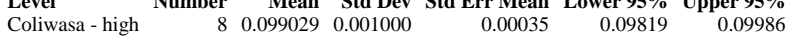

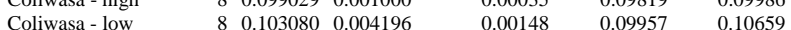

Test

Coliwasa - low-Coliwasa - high

Assuming unequal variances

$\begin{array}{lrlr}\text { Difference } & 0.004051 & \text { t Ratio } & 2.656465 \\ \text { Std Err Dif } & 0.001525 & \text { DF } & 7.792623 \\ \text { Upper CL Dif } & 0.007584 & \text { Prob }>|t| & 0.0296 \\ \text { Lower CL Dif } & 0.000518 & \text { Prob }>t & 0.0148 \\ \text { Confidence } & 0.95 & \text { Prob }<\text { t } & 0.9852\end{array}$

Tests that the Variances are Equal

Level Count Std Dev MeanAbsDif to Mean MeanAbsDif to Median

$\begin{array}{lrrrr}\text { Coliwasa - high } & 8 & 0.0009999 & 0.0006963 & 0.0006898\end{array}$

$\begin{array}{lllll}\text { Coliwasa - low } & 8 & 0.0041955 & 0.0031509 & 0.0029201\end{array}$

Test F Ratio DFNum DFDen p-Value

$\begin{array}{lllll}\text { O'Brien[.5] } & 3.8663 & 1 & 14 & 0.0694\end{array}$

$\begin{array}{lllll}\text { Brown-Forsythe } & 4.1744 & 1 & 14 & 0.0603\end{array}$

$\begin{array}{lllll}\text { Levene } & 7.1925 & 1 & 14 & 0.0179\end{array}$

$\begin{array}{lllll}\text { Bartlett } & 10.4039 & 1 & \text {; } & 0.0013 \\ \text { F Test 2-sided } & 17.6061 & 7 & 7 & 0.0012\end{array}$

Welch Anova testing Means Equal, allowing Std Devs Not Equal

F Ratio DFNum DFDen Prob $>$ F

$\begin{array}{rrrr}7.0568 & 1 & 7.7926 & 0.0296\end{array}$

t Test
2.6565 


\section{Exhibit A2. Statistical Comparisons for Mid-Rheology (Phase 1) Testing}

Oneway Analysis of Mean(Measurement) By Sampler Details Analyte=ZrO2 (wt \%), Target $\mathbf{0 . 3 5 4 7}$

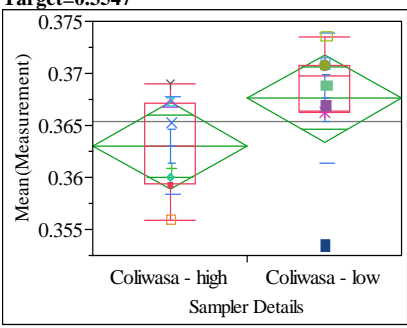

Oneway Anova

Summary of Fit

Adj Rsquare $\quad 0.164259$

Root Mean Square Error $\quad 0.005531$

Mean of Response $\quad 0.36532$

Observations (or Sum Wgts) 0.36532

t Test

Coliwasa - low-Coliwasa - high

Assuming equal variances

Difference $\quad 0.00459$ t Ratio 1.658793

Std Err Dif $\quad 0.00277$ DF $\quad 14$

Upper CL Dif 0.01052 Prob $>|t| \quad 0.1194$

Lower CL Dif -0.00134 Prob $>t \quad 0.0597$

$\begin{array}{lrl}\text { Confidence } & 0.95 \text { Prob }<\mathrm{t} & 0.9403\end{array}$

Analysis of Variance

Source DF Sum of Squares Mean Square F Ratio Prob > F

Sampler Details $\quad 1 \quad 0.00008417 \quad 0.000084 \quad 2.7516 \quad 0.1194$

$\begin{array}{llll}\text { Error } & 14 & 0.00042823 & 0.000031\end{array}$

$\begin{array}{lll}\text { C. Total } & 15 & 0.00051240\end{array}$

Means for Oneway Anova

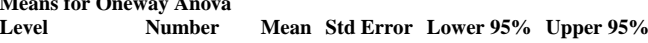

$\begin{array}{lrrrrr}\text { Coliwasa - high } & 8 & 0.363028 & 0.00196 & 0.35883 & 0.36722\end{array}$

$\begin{array}{llllll}\text { Coliwasa - low } & 8 & 0.367615 & 0.00196 & 0.36342 & 0.37181\end{array}$

Std Error uses a pooled estimate of error variance

Means and Std Deviations

Level Number Mean Std Dev Std Err Mean Lower 95\% Upper 95\%

$\begin{array}{lrrrrrr} & \text { Number } & \text { Mean } & \text { Std Dev } & \text { Std Err Mean } & \text { Lower 95\% } & \text { Upper 95\% } \\ \text { Coliwasa - high } & 8 & 0.363028 & 0.004670 & 0.00165 & 0.35912 & 0.36693\end{array}$

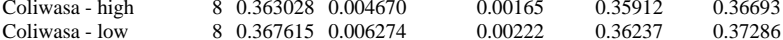

t Test

Coliwasa - low-Coliwasa - high

Assuming unequal variances

Difference $\quad 0.00459$ t Ratio 1.658793

Std Err Dif $\quad 0.00277$ DF 12.93455

Upper CL Dif 0.01056 Prob $>|t| \quad 0.1212$

Lower CL Dif -0.00139 Prob $>t \quad 0.0606$

$\begin{array}{lrl}\text { Confidence } & 0.95 \text { Prob }<\mathrm{t} & 0.9394\end{array}$

Tests that the Variances are Equal

Level Count Std Dev MeanAbsDif to Mean MeanAbsDif to Median

Coliwasa - high $\quad 8 \quad 0.0046700$

8 Coliwasa - low $0.0062743-2$

Test F Ratio DFNum DFDen p-Value

$\begin{array}{lrrrr}\text { O'Brien[.5] } & 0.3079 & 1 & 14 & 0.5877\end{array}$

$\begin{array}{lllll}\text { Brown-Forsythe } & 0.0101 & 1 & 14 & 0.9214\end{array}$

$\begin{array}{lllll}\text { Levene } & 0.0029 & 1 & 14 & 0.9575\end{array}$

$\begin{array}{lllll}\text { Bartlett } & 0.5616 & 1 & \text {. } & 0.4536\end{array}$

Welch Anova testing Means Equal, allowing Std Devs Not Equal

F Ratio DFNum DFDen Prob $>$ F

$\begin{array}{llll}2.7516 & 1 & 12.935 & 0.1212\end{array}$

t Test

1.6588
Oneway Analysis of Measurement By Sampler Mechanism Type of Material=SME Simulant, Analytical Block $=1$, Analyte $=A \mathrm{l} / \mathrm{B}$, Target Value $=2.552$

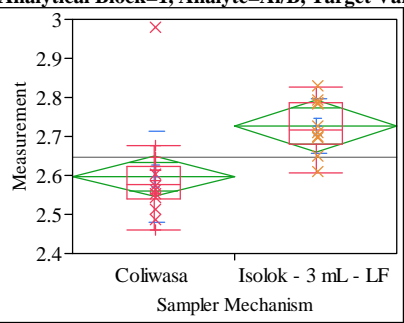

Oneway Anova

Summary of Fit

Adj Rsquare

0.101709

Observations (or Sum Wgts) 2.6468

Test

Isolok - $3 \mathrm{~mL}$ - LF-Coliwasa

Assuming equal variances

Difference $\quad 0.127525$ t Ratio 3.110335

Std Err Dif $\quad 0.041000$ DF 24

Upper CL Dif 0.212146 Prob $>|t| \quad 0.0048$

Lower CL Dif 0.042904 Prob $>t \quad 0.0024$

$\begin{array}{lr}\text { Confidence } & 0.95 \text { Prob }<\mathrm{t} \quad 0.9976\end{array}$

Analysis of Variance

$\begin{array}{lrrrrr}\text { Source } & \text { DF } & \text { Sum of Squares } & \text { Mean Square } & \text { F Ratio } & \text { Prob }>\text { F } \\ \text { Sampler Mechanism } & 1 & 0.10007762 & 0.100078 & 9.6742 & 0.0048\end{array}$

$\begin{array}{llll}\text { Error } & 24 & 0.24827556 & 0.010345\end{array}$

$\begin{array}{lll}\text { C. Total } & 25 & 0.34835318\end{array}$

Means for Oneway Anova

Level Number Mean Std Error Lower 95\% Upper 95\%

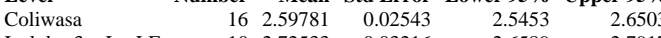

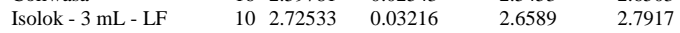

Std Error uses a pooled estimate of error variance

Means and Std Deviations

Level Number Mean Std Dev Std Err Mean Lower 95\% Upper 95\%

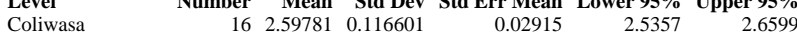

$\begin{array}{lllllll}\text { Isolok - 3 mL - LF } & 10 & 2.72533 & 0.070188 & 0.02220 & 2.6751 & 2.7755\end{array}$

Test

Isolok - $3 \mathrm{~mL}$ - LF-Coliwasa

Assuming unequal variances

$\begin{array}{lrlr}\text { Difference } & 0.127525 & \text { t Ratio } & 3.480626 \\ \text { Std Err Dif } & 0.036639 & \text { DF } & 23.99343 \\ \text { Upper CL Dif } & 0.203144 & \text { Prob }>|t| & 0.0019 \\ \text { Lower CL Dif } & 0.051906 & \text { Prob }>t & 0.0010 \\ \text { Confidence } & 0.95 & \text { Prob }<t & 0.9990\end{array}$

Tests that the Variances are Equal

Level Count Std Dev MeanAbsDif to Mean MeanAbsDif to Median

$\begin{array}{lllll}\text { Coliwasa } & 16 & 0.1166014 & 0.0710113 & 0.0697292\end{array}$

$\begin{array}{lllll}\text { Isolok - } 3 \mathrm{~mL} \text { - LF } & 10 & 0.0701882 & 0.0565213 & 0.0560235\end{array}$

Test F Ratio DFNum DFDen p-Value

$\begin{array}{lrrrr}\text { O'Brien[.5] } & 0.4756 & 1 & 24 & 0.4970\end{array}$

$\begin{array}{lllll}\text { Brown-Forsythe } & 0.4756 & 1 & 24 & 0.4970 \\ & 0.1897 & 1 & 24 & 0.6670\end{array}$

$\begin{array}{lllll}\text { Levene } & 0.2286 & 1 & 24 & 0.6369\end{array}$

$\begin{array}{lrrrr}\text { Bartlett } & 2.4658 & 1 & \text {. } & 0.1163 \\ \text { F Test 2-sided } & 2.7598 & 15 & 9 & 0.1279\end{array}$

Welch Anova testing Means Equal, allowing Std Devs Not Equal

F Ratio DFNum DFDen Prob $>$ F

$\begin{array}{llll}12.1148 & 1 & 23.993 & 0.0019\end{array}$

t Test

3.4806 


\section{Exhibit A2. Statistical Comparisons for Mid-Rheology (Phase 1) Testing}

Oneway Analysis of Measurement By Sampler Mechanism Type of Material=SME Simulant, Analytical Block=1, Analyte=Al2O3 (wt \%), Target Value=10.869

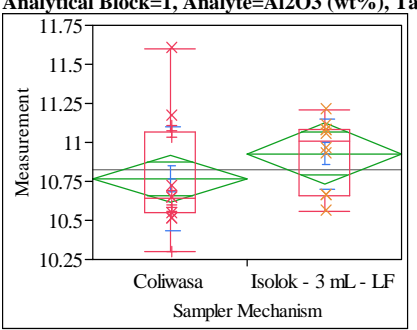

Oneway Anova

Summary of Fit

Adj Rsquare

0.03004

Error $\quad 0.29867$

Mean of Response

Observations (or Sum Wgts) $\quad 26$

Test

Isolok - $3 \mathrm{~mL}$ - LF-Coliwasa

Assuming equal variances

Difference $\quad 0.16037$ t Ratio 1.332013

Std Err Dif $\quad 0.12040$ DF 24

Upper CL Dif 0.40886 Prob $>|t| \quad 0.1954$

Lower CL Dif -0.08812 Prob $>\mathrm{t} \quad 0.0977$

$\begin{array}{lrl}\text { Confidence } & 0.95 \text { Prob }<\mathrm{t} & 0.9023\end{array}$

Analysis of Variance

$\begin{array}{lrrrrr}\text { Source } & \text { DF } & \text { Sum of Squares } & \text { Mean Square } & \text { F Ratio } & \text { Prob }>\text { F } \\ \text { Sampler Mechanism } & 1 & 0.1582705 & 0.158271 & 1.7743 & 0.1954\end{array}$

$\begin{array}{llll}\text { Error } & 24 & 2.1408900 & 0.089204\end{array}$

$\begin{array}{lll}\text { C. Total } & 25 & 2.2991605\end{array}$

Means for Oneway Anova

$\begin{array}{lrrrrr}\text { Level } & \text { Number } & \text { Mean } & \text { Std Error } & \text { Lower 95\% } & \text { Upper 95\% } \\ \text { Coliwasa } & 16 & 10.7666 & 0.07467 & 10.613 & 10.921\end{array}$

$\begin{array}{lrrrrr}\text { Coliwasa } & 16 & 10.7666 & 0.07467 & 10.613 & 10.921 \\ \text { Isolok - 3 mL - LF } & 10 & 10.9270 & 0.09445 & 10.732 & 11.122\end{array}$

Std Error uses a pooled estimate of error variance

Means and Std Deviations

Level Number Mean Std Dev Std Err Mean Lower 95\% Upper 95\%

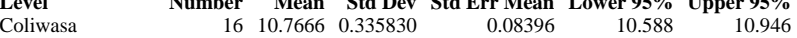

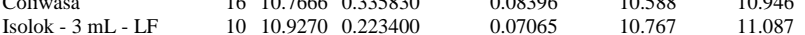

Test

Isolok - $3 \mathrm{~mL}$ - LF-Coliwasa

Assuming unequal variances

Difference $\quad 0.16037$ t Ratio $\quad 1.461574$

Std Err Dif $\quad 0.10973$ DF 23.84106

Upper CL Dif $\quad 0.38691$ Prob $>|t| r|r| r 6$

$\begin{array}{llll}\text { Lower CL Dif }-0.06617 \text { Prob }>t & 0.0785\end{array}$

$\begin{array}{lrl} & \\ \text { Confidence } & 0.95 \text { Prob }<\mathrm{t} & 0.9215\end{array}$

Tests that the Variances are Equal

Level Count Std Dev MeanAbsDif to Mean MeanAbsDif to Median

Coliwasa $\quad 16 \quad 0.3358295$

$\begin{array}{lllll}\text { Coliwasa } & 16 & 0.3358295 & 0.2691061 & 0.2373684 \\ \text { Isolok - } 3 \mathrm{~mL} \text { - LF } & 10 & 0.2234000 & 0.1821478 & 0.1757235\end{array}$

Test F Ratio DFNum DFDen p-Value

$\begin{array}{lrrrr}\text { O'Brien[.5] } & 1.0781 & 1 & 24 & 0.3095\end{array}$

$\begin{array}{lllll}\text { Brown-Forsythe } & 0.4459 & 1 & 24 & 0.5107\end{array}$

$\begin{array}{lllll}\text { Levene } & 1.7171 & 1 & 24 & 0.2025\end{array}$

$\begin{array}{lrrrr}\text { Bartlett } & 1.6347 & 1 & \text {. } & 0.2011\end{array}$

Welch Anova testing Means Equal, allowing Std Devs Not Equal

F Ratio DFNum DFDen Prob $>$ F

$\begin{array}{rrrr}2.1362 & 1 & 23.841 & 0.1569\end{array}$

t Test

1.4616
Oneway Analysis of Measurement By Sampler Mechanism Type of Material=SME Simulant, Analytical Block $=1$, Analyte $=\mathrm{B} 2 \mathrm{O} 3(\mathrm{wt} \%)$, Target Value $=4.259$

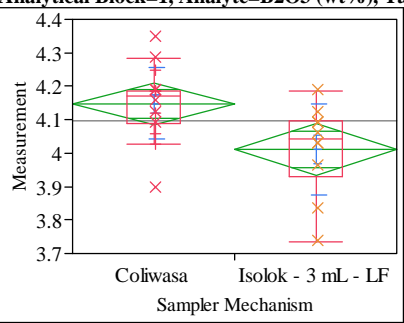

Oneway Anova

Summary of Fit

Adj Rsquare 0.118824
4.095465

Mean of Response

Observations (or Sum Wgts)

Isolok - $3 \mathrm{~mL}$ - LF-Coliwasa

Assuming equal variances

$\begin{array}{lrlr}\text { Difference } & -0.13564 & \mathrm{t} \text { Ratio } & -2.83174 \\ \text { Std Err Dif } & 0.04790 & \text { DF } & 24 \\ \text { Upper CL Dif } & -0.03678 & \text { Prob }>|t| & 0.0092 \\ \text { Lower CL Dif } & -0.23450 & \text { Prob }>\mathrm{t} & 0.9954 \\ \text { Confidence } & 0.95 & \text { Prob }<\mathrm{t} & 0.0046\end{array}$

Analysis of Variance

Source DF Sum of Squares Mean Square F Ratio Prob $>$ F

Sampler Mechanism $\quad 1 \quad 0.11321689$

$\begin{array}{ll}24 & 0.33885715\end{array}$

0.01411

Means for Oneway Anova

Level Number Mean Std Error Lower 95\% Upper 95\%

$\begin{array}{lrrrrr}\text { Coliwasa } & 16 & 4.14763 & 0.02971 & 4.0863 & 4.2089\end{array}$

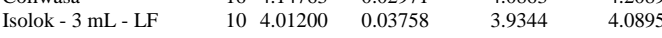

Std Error uses a pooled estimate of error variance

Means and Std Deviations

Level Number Mean Std Dev Std Err Mean Lower 95\% Upper 95\%

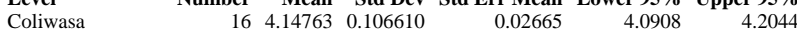

$\begin{array}{lllllll}\text { Isolok - 3 mL - LF } & 10 & 4.01200 & 0.136777 & 0.04325 & 3.9142 & 4.1098\end{array}$

Test

Isolok - $3 \mathrm{~mL}$ - LF-Coliwasa

Assuming unequal variances

$\begin{array}{lrlr}\text { Difference } & -0.13564 & \mathrm{t} \text { Ratio } & -2.66978 \\ \text { Std Err Dif } & 0.05081 & \text { DF } & 15.76823 \\ \text { Upper CL Dif } & -0.02781 & \text { Prob }>|t| & 0.0169 \\ \text { Lower CL Dif } & -0.24347 & \text { Prob }>\mathrm{t} & 0.9915 \\ \text { Confidence } & 0.95 & \text { Prob }<\mathrm{t} & 0.0085\end{array}$

Tests that the Variances are Equal

Level Count Std Dev MeanAbsDif to Mean MeanAbsDif to Median

$\begin{array}{lllll}\text { Coliwasa } & 16 & 0.1066098 & 0.0792397 & 0.0784851\end{array}$

$\begin{array}{llll}\text { Isolok - } 3 \mathrm{~mL} \text { - LF } & 10 & 0.1367773 & 0.1017488\end{array}$

Test F Ratio DFNum DFDen p-Value

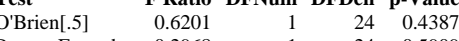

$\begin{array}{lllll}\text { Brown-Forsythe } & 0.2968 & 1 & 24 & 0.5909\end{array}$

$\begin{array}{lllll}\text { Levene } & 0.5548 & 1 & 24 & 0.4636\end{array}$

$\begin{array}{lllll}\text { Bartlett } & 0.6898 & 1 & 0.4062 \\ \text { F Test 2-sided } & 1.6460 & 9 & 15 & 0.3781\end{array}$

Welch Anova testing Means Equal, allowing Std Devs Not Equal

F Ratio DFNum DFDen Prob $>$ F

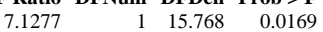

t Test

2.6698 


\section{Exhibit A2. Statistical Comparisons for Mid-Rheology (Phase 1) Testing}

Oneway Analysis of Measurement By Sampler Mechanism Type of Material=SME Simulant, Analytical Block=1, Analyte $=\mathrm{BaO}($ wt $\%)$, Target Value $=0.0919$

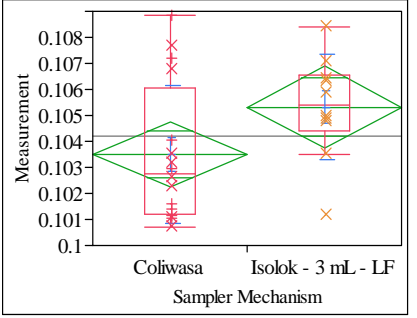

Oneway Anova

Summary of Fit

Adj Rsquare

0.002439

Observations (or Sum Wgts) $\quad 26$

tTest

Isolok - $3 \mathrm{~mL}$ - LF-Coliwasa

Assuming equal variances

Difference $\quad 0.00183$ t Ratio 1.858259

$\begin{array}{lrlr}\text { Std Err Dif } & 0.00098 & \text { DF } & 24 \\ \text { Upper CL Dif } & 0.00386 & \text { Prob }>|t| & 0.0754\end{array}$

Lower CL Dif -0.00020 Prob $>t \quad 0.0377$

$\begin{array}{lrl}\text { Confidence } & 0.95 \text { Prob }<\mathrm{t} & 0.9623\end{array}$

Analysis of Variance

$\begin{array}{lrrrrr}\text { Source } & \text { DF } & \text { Sum of Squares } & \text { Mean Square } & \text { F Ratio } & \text { Prob > F } \\ \text { Sampler Mechanism } & 1 & 0.00002054 & 0.000021 & 3.4531 & 0.0754\end{array}$

$\begin{array}{llll}\text { Error } & 24 & 0.00014275 & 5.948 \mathrm{e}-6\end{array}$

$\begin{array}{lll}\text { C. Total } & 25 & 0.00016328\end{array}$

Means for Oneway Anova

Level $\quad$ Number Mean Std Error Lower 95\% Upper 95\%

$\begin{array}{lrrrrr}\text { Coliwasa } & 16 & 0.103493 & 0.00061 & 0.10223 & 0.10475\end{array}$

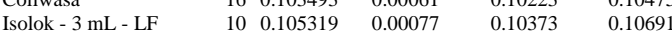

Std Error uses a pooled estimate of error variance

Means and Std Deviations

Level Number Mean Std Dev Std Err Mean Lower 95\% Upper 95\%

$\begin{array}{lrrrrrr}\text { Level } & \text { Number } & \text { Mean } & \text { Std Dev } & \text { Std Err Mean } & \text { Lower 95\% } & \text { Upper 95\% } \\ \text { Coliwasa } & 16 & 0.103493 & 0.002663 & 0.00067 & 0.10207 & 0.10491\end{array}$

$\begin{array}{lllllll}\text { Coliwasa } & 16 & 0.103493 & 0.002663 & 0.00067 & 0.10207 & 0.10491 \\ \text { Isolok - 3 mL - LF } & 10 & 0.105319 & 0.002011 & 0.00064 & 0.10388 & 0.10676\end{array}$

tTest

Isolok - $3 \mathrm{~mL}$ - LF-Coliwasa

Assuming unequal variances

Difference $\quad 0.00183$ t Ratio 1.984374

Std Err Dif $\quad 0.00092$ DF 22.97603

Upper CL Dif 0.00373 Prob $>|t| r \mid r .0593$

$\begin{array}{llll}\text { Lower CL Dif } & -7.77 \mathrm{e}-5 \text { Prob }>\mathrm{t} & 0.0296\end{array}$

$\begin{array}{lrll} & \\ \text { Confidence } & 0.95 & \text { Prob }<\mathrm{t} & 0.9704\end{array}$

Tests that the Variances are Equal

Level Count Std Dev MeanAbsDif to Mean MeanAbsDif to Median

Coliwasa $\quad 16 \quad 0.0026626$

$\begin{array}{lllll}\text { Coliwasa } & 16 & 0.0026626 & 0.0021266 & 0.0020167 \\ \text { Isolok - } 3 \mathrm{~mL} \text { - LF } & 10 & 0.0020111 & 0.0014849 & 0.0014849\end{array}$

Test F Ratio DFNum DFDen p-Value

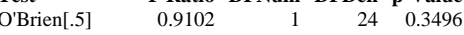

Brown-Forsythe $\quad 0.6530 \quad 1 \quad 24 \quad 0.4270$

$\begin{array}{lllll}\text { Levene } & 1.2579 & 1 & 24 & 0.2731\end{array}$

$\begin{array}{lrrrr}\text { Bartlett } & 0.8001 & 1 & \text {. } & 0.3711 \\ \text { F Test 2-sided } & 1.7529 & 15 & 9 & 0.3980\end{array}$

Welch Anova testing Means Equal, allowing Std Devs Not Equal

F Ratio DFNum DFDen Prob $>$ F

$\begin{array}{rrrr}3.9377 & 1 & 22.976 & 0.0593\end{array}$

t Test

1.9844
Oneway Analysis of Measurement By Sampler Mechanism Type of Material=SME Simulant, Analytical Block $=1$, Analyte $=\mathrm{CaO}(\mathrm{wt} \%)$, Target Value $=1.1462$

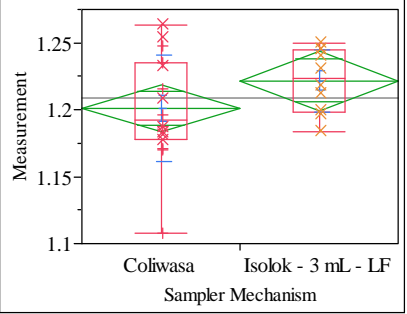

Oneway Anova

Summary of Fi

Adj Rsquare

0.034507

Mean of Response $\quad 1.209016$

Observations (or Sum Wgts) $\quad 26$

T Test

Isolok - $3 \mathrm{~mL}$ - LF-Coliwasa

Assuming equal variances

Difference $\quad 0.02074$ t Ratio 1.491203

Std Err Dif $\quad 0.01391$ DF 24

Upper CL Dif 0.04945 Prob $>|t| \quad 0.1489$

$\begin{array}{llll}\text { Lower CL Dif }-0.00797 & \text { Prob }>t & 0.0745\end{array}$

$\begin{array}{lrl}\text { Confidence } & 0.95 \text { Prob }<\mathrm{t} & 0.9255\end{array}$

Analysis of Variance

$\begin{array}{lrrrrr}\text { Source } & \text { DF } & \text { Sum of Squares } & \text { Mean Square } & \text { F Ratio } & \text { Prob }>\text { F } \\ \text { Sampler Mechanism } & 1 & 0.00264786 & 0.002648 & 2.2237 & 0.1489\end{array}$

$\begin{array}{llll}\text { Error } & 24 & 0.02857812 & 0.001191\end{array}$

$\begin{array}{lll}\text { C. Total } & 25 & 0.03122598\end{array}$

Means for Oneway Anova

Level Number Mean Std Error Lower 95\% Upper 95\%

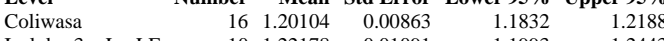

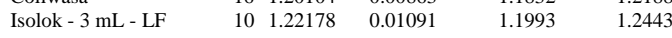

Std Error uses a pooled estimate of error variance

Means and Std Deviations

Level Number Mean Std Dev Std Err Mean Lower 95\% Upper 95\%

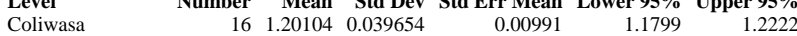

$\begin{array}{lllllll}\text { Isolok - } 3 \text { mL - LF } & 10 & 1.22178 & 0.023550 & 0.00991 & 1.1799 & 1.2222 \\ & & & & & & \end{array}$

TTest

Isolok - $3 \mathrm{~mL}$ - LF-Coliwasa

Assuming unequal variances

$\begin{array}{lrlr}\text { Difference } & 0.02074 & \mathrm{t} \text { Ratio } & 1.67295 \\ \text { Std Err Dif } & 0.01240 & \text { DF } & 23.97922 \\ \text { Upper CL Dif } & 0.04633 \text { Prob }>|\mathrm{t}| & 0.1073 \\ \text { Lower CL Dif } & -0.00485 \text { Prob }>\mathrm{t} & 0.0537 \\ \text { Confidence } & 0.95 & \text { Prob }<\mathrm{t} & 0.9463\end{array}$

Tests that the Variances are Equal

Level Count Std Dev MeanAbsDif to Mean MeanAbsDif to Median

$\begin{array}{lllll}\text { Coliwasa } & 16 & 0.0396540 & 0.0311978 & 0.0306075\end{array}$

$\begin{array}{llll}\text { Isolok - } 3 \mathrm{~mL} \text { - LF } & 10 & 0.0235502 & 0.0201485\end{array}$

Test F Ratio DFNum DFDen p-Value

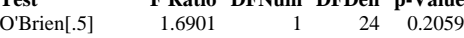

Brown-Forsythe $\begin{array}{llll}1.4910 & 1 & 24 & 0.2339\end{array}$

$\begin{array}{lllll}\text { Levene } & 2.0156 & 1 & 24 & 0.1685\end{array}$

$\begin{array}{lrrrr}\text { Bartlett } & 2.5886 & 1 & \text {. } & 0.1076\end{array}$

Welch Anova testing Means Equal, allowing Std Devs Not Equal

F Ratio DFNum DFDen Prob $>$ F

$\begin{array}{rrrr}2.7988 & 1 & 23.979 & 0.1073\end{array}$

t Test

1.6729 


\section{Exhibit A2. Statistical Comparisons for Mid-Rheology (Phase 1) Testing}

Oneway Analysis of Measurement By Sampler Mechanism Type of Material=SME Simulant, Analytical Block=1, Analyte $=\mathrm{Cr} 2 \mathrm{O} 3(\mathrm{wt} \%)$, Target Value $=0.0998$

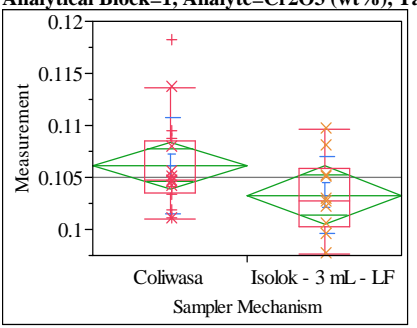

Oneway Anova

Summary of Fit

Adj Rsquare

0.100342

Root Mean Squarc

Mean of Response

0.062856

Observations (or Sum Wgts) $\quad 26$

Test

Isolok - $3 \mathrm{~mL}$ - LF-Coliwasa

Assuming equal variances

Difference $\quad-0.00282$ t Ratio -1.63609

Std Err Dif $\quad 0.00173$ DF 24

Upper CL Dif 0.00074 Prob $>|t| r \mid r .1149$

Lower CL Dif -0.00639 Prob $>\mathrm{t} \quad 0.9426$

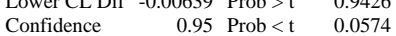

Analysis of Variance

$\begin{array}{lrrrrr}\text { Source } & \text { DF } & \text { Sum of Squares } & \text { Mean Square } & \text { F Ratio } & \text { Prob }>\text { F } \\ \text { Sampler Mechanism } & 1 & 0.00004910 & 0.000049 & 2.6768 & 0.1149\end{array}$

$\begin{array}{lrrr}\text { Error } & 24 & 0.00044019 & 0.000018\end{array}$

$\begin{array}{lll}\text { C. Total } & 25 & 0.00048928\end{array}$

Means for Oneway Anova

Level $\quad$ Number Mean Std Error Lower 95\% Upper 95\%

$\begin{array}{lrrrrr}\text { Coliwasa } & 16 & 0.106130 & 0.00107 & 0.10392 & 0.10834\end{array}$

$\begin{array}{llllll}\text { Isolok - } 3 \text { mL - LF } & 10 & 0.103306 & 0.00135 & 0.10051 & 0.10610\end{array}$

Std Error uses a pooled estimate of error variance

Means and Std Deviations

Level Number Mean Std Dev Std Err Mean Lower 95\% Upper 95\%

160.1061300 .004593

$\begin{array}{lllllll}\text { Coliwasa } & 16 & 0.106130 & 0.004593 & 0.00115 & 0.10368 & 0.10858 \\ \text { Isolok - 3 mL - LF } & 10 & 0.103306 & 0.003708 & 0.00117 & 0.10065 & 0.10596\end{array}$

Test

Isolok - $3 \mathrm{~mL}$ - LF-Coliwasa

Assuming unequal variances

Difference $\quad-0.00282$ t Ratio $\quad-1.721$

Std Err Dif $\quad 0.00164$ DF 22.25556

Upper CL Dif $\begin{array}{rrr}0.00058 & \text { Prob }>|t| & 0.0991\end{array}$

Lower CL Dif -0.00623 Prob $>t \quad 0.9504$

$\begin{array}{lrl}\text { Confidence } & 0.95 \text { Prob }<\mathrm{t} & 0.0496\end{array}$

Tests that the Variances are Equal

Level Count Std Dev MeanAbsDif to Mean MeanAbsDif to Median

Coliwasa $\quad 16 \quad 0.0045929$

$\begin{array}{lllll} & 16 & 0.0045929 & 0.0034051 & 0.0030511 \\ \text { Isolok - } 3 \mathrm{~mL} \text { - LF } & 10 & 0.0037084 & 0.0028881 & 0.0028063\end{array}$

Test F Ratio DFNum DFDen p-Value

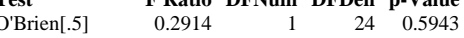

Brown-Forsythe $\quad 0.0363 \quad 1 \quad 240.8506$

$\begin{array}{lllll}\text { Levene } & 0.2305 & 1 & 24 & 0.6355\end{array}$

$\begin{array}{lrrrr}\text { Bartlett } & 0.4722 & 1 & \text {. } & 0.4920 \\ \text { F Test 2-sided } & 1.5339 & 15 & 9 & 0.5237\end{array}$

Welch Anova testing Means Equal, allowing Std Devs Not Equal

F Ratio DFNum DFDen Prob $>$ F

$\begin{array}{llll}2.9618 & 1 & 22.256 & 0.0991\end{array}$

t Test
1.7210
Oneway Analysis of Measurement By Sampler Mechanism Type of Material=SME Simulant, Analytical Block $=1$, Analyte $=\mathrm{CuO}(\mathrm{wt} \%)$, Target Value $=0.0504$

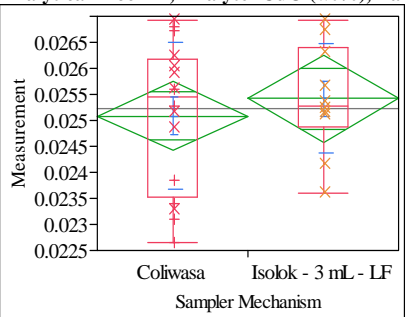

Oneway Anova

Summary of Fit

Adj Rsquare

Error $\quad 0.001287$

Mean of Response $\quad 0.025214$

Observations (or Sum Wgts) $\quad 26$

T Test

Isolok - $3 \mathrm{~mL}$ - LF-Coliwasa

Assuming equal variances

Difference $\quad 0.00033$ t Ratio 0.637712

$\begin{array}{lrlr}\text { Std Err Dif } & 0.00052 & \text { DF } & 24 \\ \text { Upper CL Dif } & 0.00140 & \text { Prob }>|t| & 0.5297\end{array}$

$\begin{array}{lll}\text { Lower CL Dif }-0.00074 \text { Prob }>t & 0.2648\end{array}$

$\begin{array}{lrl}\text { Confidence } & 0.95 \text { Prob }<\mathrm{t} & 0.7352\end{array}$

Analysis of Variance

$\begin{array}{lrrrrr}\text { Source } & \text { DF } & \text { Sum of Squares } & \text { Mean Square } & \text { F Ratio } & \text { Prob > F } \\ \text { Sampler Mechanism } & 1 & 0.00000067 & 6.74 \mathrm{e}-7 & 0.4067 & 0.5297\end{array}$

$\begin{array}{llll}\text { Error } & 24 & 0.00003978 & 1.6573 \mathrm{e}-6\end{array}$

$\begin{array}{lll}\text { C. Total } & 25 & 0.00004045\end{array}$

Means for Oneway Anova

Level Number Mean Std Error Lower 95\% Upper 95\%

$\begin{array}{llllrr}\text { Coliwasa } & 16 & 0.025087 & 0.00032 & 0.02442 & 0.02575\end{array}$

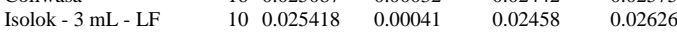

Std Error uses a pooled estimate of error variance

Means and Std Deviations

Level Number Mean Std Dev Std Err Mean Lower 95\% Upper 95\%

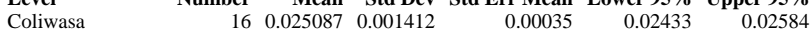

$\begin{array}{lllllll}\text { Isolok - } 3 \mathrm{~mL} \text { - LF } & 10 & 0.025418 & 0.001048 & 0.00033 & 0.02467 & 0.02617\end{array}$

Test

Isolok - $3 \mathrm{~mL}$ - LF-Coliwasa

Assuming unequal variances

$\begin{array}{lrlr}\text { Difference } & 0.00033 & \text { t Ratio } & 0.683686 \\ \text { Std Err Dif } & 0.00048 \text { DF } & 23.1405 \\ \text { Upper CL Dif } & 0.00133 \text { Prob }>|t| & 0.5010 \\ \text { Lower CL Dif } & -0.00067 & \text { Prob }>t & 0.2505 \\ \text { Confidence } & 0.95 & \text { Prob }<\mathrm{t} & 0.7495\end{array}$

Tests that the Variances are Equal

Level Count Std Dev MeanAbsDif to Mean MeanAbsDif to Median

$\begin{array}{lllll}\text { Coliwasa } & 16 & 0.0014119 & 0.0011726 & 0.0011384\end{array}$

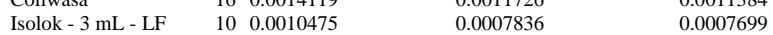

Test F Ratio DFNum DFDen p-Value

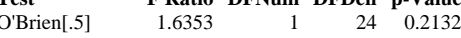

Brown-Forsythe $\quad 1.3095 \quad 1 \quad 24 \quad 0.2638$

$\begin{array}{lllll}\text { Levene } & 1.9203 & 1 & 24 & 0.1786\end{array}$

$\begin{array}{lrrrr}\text { Bartlett } & 0.9012 & 1 & \text {. } & 0.3425 \\ \text { F Test 2-sided } & 1.8166 & 15 & 9 & 0.3680\end{array}$

Welch Anova testing Means Equal, allowing Std Devs Not Equal

F Ratio DFNum DFDen Prob $>$ F

$\begin{array}{lrrr}0.4674 & 1 & 23.141 & 0.5010\end{array}$

t Test

0.6837 


\section{Exhibit A2. Statistical Comparisons for Mid-Rheology (Phase 1) Testing}

Oneway Analysis of Measurement By Sampler Mechanism Type of Material=SME Simulant, Analytical Block=1, Analyte $=\mathrm{Fe} / \mathrm{Li}$, Target Value $=2.452$

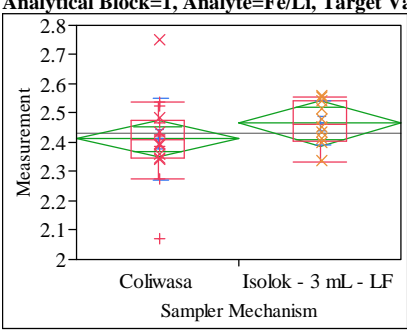

Oneway Anova

Summary of Fit

Adj Rsquare 0.048507

Root Mean Square Error $\quad 0.12084$

Mean of Response Eror 2.432034

$\begin{array}{lr}\text { Mean of Response } & 26 \\ \text { Observations (or Sum Wgts) }\end{array}$

t Test

Isolok - $3 \mathrm{~mL}$ - LF-Coliwasa

Assuming equal variances

Difference $\quad 0.05388$ t Ratio 1.106122

Std Err Dif $\quad 0.04871$ DF 24

Upper CL Dif 0.15442 Prob $>|t| \quad 0.2796$

Lower CL Dif -0.04666 Prob $>\mathrm{t} \quad 0.1398$

$\begin{array}{lrl}\text { Confidence } & 0.95 \text { Prob }<\mathrm{t} & 0.8602\end{array}$

Analysis of Variance

$\begin{array}{lrrrrr}\text { Source } & \text { DF } & \text { Sum of Squares } & \text { Mean Square } & \text { F Ratio } & \text { Prob }>\text { F } \\ \text { Sampler Mechanism } & 1 & 0.01786727 & 0.017867 & 1.2235 & 0.2796\end{array}$

$\begin{array}{llll}\text { Error } & 24 & 0.35048041 & 0.014603\end{array}$

$\begin{array}{lll}\text { C. Total } & 25 & 0.36834768\end{array}$

Means for Oneway Anova

Level $\quad$ Number Mean Std Error Lower 95\% Upper 95\%

$\begin{array}{lrrrrr}\text { Coliwasa } & 16 & 2.41131 & 0.03021 & 2.3490 & 2.4737\end{array}$

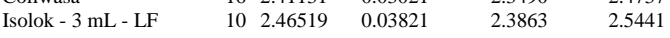

Std Error uses a pooled estimate of error variance

Means and Std Deviations

Level Number Mean Std Dev Std Err Mean Lower 95\% Upper 95\%

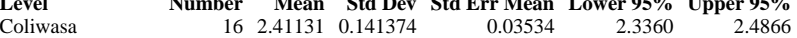

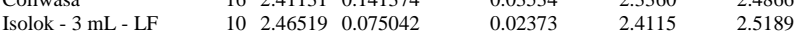

Test

Isolok - $3 \mathrm{~mL}$ - LF-Coliwasa

Assuming unequal variances

Difference $\quad 0.05388$ t Ratio $\quad 1.265731$

Std Err Dif $\quad 0.04257$ DF 23.58436

Upper CL Dif 0.14183 Prob $>|t| r \mid r .2180$

Lower CL Dif -0.03406 Prob $>t \quad 0.1090$

$\begin{array}{lrl}\text { Confidence } & 0.95 \text { Prob }<\mathrm{t} & 0.8910\end{array}$

Tests that the Variances are Equal

Level Count Std Dev MeanAbsDif to Mean MeanAbsDif to Median

Coliwasa $\quad 16 \quad 0.1413738$

$\begin{array}{lllll}\text { Isolok - } 3 \mathrm{~mL} \text { - LF } & 10 & 0.0750424 & 0.0931734 & 0.0931734 \\ & & & 0.0614741\end{array}$

Test F Ratio DFNum DFDen p-Value

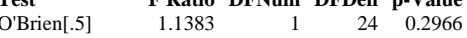

$\begin{array}{lllll}\text { Brown-Forsythe } & 0.8527 & 1 & 24 & 0.3650\end{array}$

$\begin{array}{lllll}\text { Levene } & 0.8540 & 1 & 24 & 0.3646\end{array}$

$\begin{array}{lrrrr}\text { Bartlett } & 3.7011 & 1 & \text {. } & 0.0544\end{array}$

Welch Anova testing Means Equal, allowing Std Devs Not Equal

F Ratio DFNum DFDen Prob > F

$\begin{array}{llrr}1.6021 & 1 & 23.584 & 0.2180\end{array}$

$\mathbf{t}$ Test
1.2657
Oneway Analysis of Measurement By Sampler Mechanism Type of Material=SME Simulant, Analytical Block=1, Analyte=Fe2O3 (wt \%), Target Value=11.462

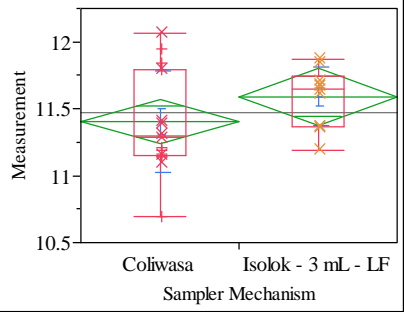

Oneway Anova

Summary of Fit

Adj Rsquare

$\quad 0.326844$

Mean of Response 11.47664

Observations (or Sum Wgts) $\quad 26$

T Test

Isolok - $3 \mathrm{~mL}$ - LF-Coliwasa

Assuming equal variances

$\begin{array}{lrlr}\text { Difference } & 0.18747 & \mathrm{t} \text { Ratio } & 1.422863 \\ \text { Std Err Dif } & 0.13176 & \text { DF } & 24 \\ \text { Upper CL Dif } & 0.45940 & \text { Prob }>|t| & 0.1676 \\ \text { Lower CL Dif } & -0.08446 & \text { Prob }>t & 0.0838 \\ \text { Confidence } & 0.95 & \text { Prob }<\mathrm{t} & 0.9162\end{array}$

Analysis of Variance

Source DF Sum of Squares Mean Square F Ratio Prob > F

Sampler Mechanism $\quad 1 \quad 0.2162756$

$\begin{array}{ll}24 & 2.5638497\end{array}$

$\begin{array}{lll}0.216276 & 2.0245 & 0.1676\end{array}$

C. Total

Means for Oneway Anova

Level Number Mean Std Error Lower 95\% Upper 95\%

$\begin{array}{lrrrrr}\text { Coliwasa } & 16 & 11.4045 & 0.08171 & 11.236 & 11.573\end{array}$

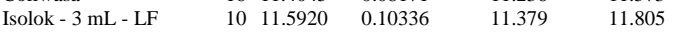

Std Error uses a pooled estimate of error variance

Means and Std Deviations

Level Number Mean Std Dev Std Err Mean Lower 95\% Upper 95\%

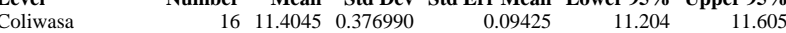

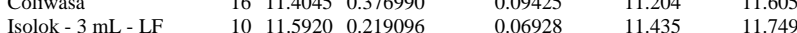

Test

Isolok - $3 \mathrm{~mL}$ - LF-Coliwasa

Assuming unequal variances

$\begin{array}{lrlr}\text { Difference } & 0.18747 & \text { t Ratio } & 1.602659 \\ \text { Std Err Dif } & 0.11697 & \text { DF } & 23.94031 \\ \text { Upper CL Dif } & 0.42892 & \text { Prob }>|t| & 0.1221 \\ \text { Lower CL Dif } & -0.05398 & \text { Prob }>t & 0.0611 \\ \text { Confidence } & 0.95 & \text { Prob }<\mathrm{t} & 0.9389\end{array}$

Tests that the Variances are Equal

Level Count Std Dev MeanAbsDif to Mean MeanAbsDif to Median

$\begin{array}{lllll}\text { Coliwasa } & 16 & 0.3769899 & 0.3035879 & 0.2886207\end{array}$

Isolok - $3 \mathrm{~mL}$ - LF $\quad 10 \quad 0.2190963 \quad 0.1727078 \quad 0.1601264$

Test F Ratio DFNum DFDen p-Value

$\begin{array}{lrrrr}\text { O'Brien[.5] } & 2.7845 & 1 & 24 & 0.1082\end{array}$

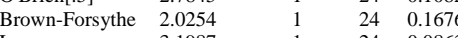

$\begin{array}{lllll}\text { Levene } & 3.1987 & 1 & 24 & 0.0863\end{array}$

$\begin{array}{lrrrr}\text { Bartlett } & 2.7908 & 1 & \text {. } & 0.0948 \\ \text { F Test 2-sided } & 2.9607 & 15 & 9 & 0.1045\end{array}$

Welch Anova testing Means Equal, allowing Std Devs Not Equal

F Ratio DFNum DFDen Prob $>$ F

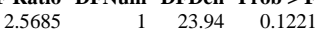

t Test

1.602 


\section{Exhibit A2. Statistical Comparisons for Mid-Rheology (Phase 1) Testing}

Oneway Analysis of Measurement By Sampler Mechanism Type of Material=SME Simulant, Analytical Block=1, Analyte $=$ K2O (wt \%), Target Value $=\mathbf{0 . 1 5 9 1}$

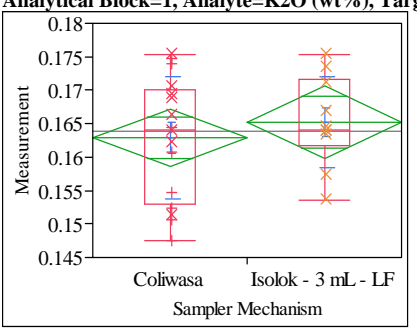

Oneway Anova

Summary of Fit

Adj Rsquare $\quad 0.020432$

Root Mean Square Error $\quad 0.008368$

0.163802

$\begin{array}{lr}\text { Mean of Response } & 0.163802 \\ \text { Observations (or Sum Wgts) } & 26\end{array}$

t Test

Isolok - $3 \mathrm{~mL}$ - LF-Coliwasa

Assuming equal variances

Difference $\quad 0.00239$ t Ratio 0.707535

Std Err Dif $\quad 0.00337$ DF 24

Upper CL Dif 0.00935 Prob $>|t| \quad 0.4860$

$\begin{array}{lll}\text { Lower CL Dif }-0.00458 \text { Prob }>t & 0.2430\end{array}$

$\begin{array}{lrl}\text { Confidence } & 0.95 \text { Prob }<\mathrm{t} & 0.7570\end{array}$

Analysis of Variance

$\begin{array}{lrrrrr}\text { Source } & \text { DF } & \text { Sum of Squares } & \text { Mean Square } & \text { F Ratio } & \text { Prob }>\text { F } \\ \text { Sampler Mechanism } & 1 & 0.00003505 & 0.000035 & 0.5006 & 0.4860\end{array}$

$\begin{array}{lrrr}\text { Error } & 24 & 0.00168045 & 0.000070\end{array}$

$\begin{array}{lll}\text { C. Total } & 25 & 0.00171550\end{array}$

Means for Oneway Anova

Level $\quad$ Number Mean Std Error Lower 95\% Upper 95\%

$\begin{array}{lrrrrr}\text { Coliwasa } & 16 & 0.162885 & 0.00209 & 0.15857 & 0.16720\end{array}$

$\begin{array}{llllll}\text { Isolok - } 3 \text { mL - LF } & 10 & 0.165271 & 0.00265 & 0.15981 & 0.17073\end{array}$

Std Error uses a pooled estimate of error variance

Means and Std Deviations

Level Number Mean Std Dev Std Err Mean Lower 95\% Upper 95\%

Leliwas 0.1628850 .009190

$\begin{array}{lllllll}\text { Coliwasa } & 16 & 0.162885 & 0.009190 & 0.00230 & 0.15799 & 0.16778 \\ \text { Isolok - 3 mL - LF } & 10 & 0.165271 & 0.006780 & 0.00214 & 0.16042 & 0.17012\end{array}$

Test

Isolok - $3 \mathrm{~mL}$ - LF-Coliwasa

Assuming unequal variances

Difference $\quad 0.00239$ t Ratio $\quad 0.759484$

Std Err Dif $\quad 0.00314$ DF 23.18976

Upper CL Dif 0.00888 Prob $>|t| r 0.4552$

$\begin{array}{lll}\text { Lower CL Dif }-0.00411 \text { Prob }>t & 0.2276\end{array}$

$\begin{array}{lrll} & \\ \text { Confidence } & 0.95 \text { Prob }<\mathrm{t} & 0.7724\end{array}$

Tests that the Variances are Equal

Level Count Std Dev MeanAbsDif to Mean MeanAbsDif to Median

Coliwasa $\quad 16 \quad 0.0091897$

$\begin{array}{lllll} & \\ \text { Isolok - } 3 \mathrm{~mL} \text { - LF } & 10 & 0.0067799 & 0.0076087 & 0.0051075\end{array}$

Test F Ratio DFNum DFDen p-Value

$\begin{array}{lrrrr}\text { O'Brien[.5] } & \text { F Ratio } & \text { DFNum } & \text { DFDen } & \text { p-Value } \\ & 1.7561 & 1 & 24 & 0.1976\end{array}$

$\begin{array}{lllll}\text { Brown-Forsythe } & 1.6689 & 1 & 24 & 0.2087\end{array}$

$\begin{array}{lllll}\text { Levene } & 1.8731 & 1 & 24 & 0.1838\end{array}$

$\begin{array}{lrrrr}\text { Bartlett } & 0.9343 & 1 & \text {. } & 0.3337\end{array}$

Welch Anova testing Means Equal, allowing Std Devs Not Equal

F Ratio DFNum DFDen Prob $>$ F

$\begin{array}{lrrr}0.5768 & 1 & 23.19 & 0.4552\end{array}$

t Test

0.7595
Oneway Analysis of Measurement By Sampler Mechanism Type of Material=SME Simulant, Analytical Block $=1$, Analyte $=$ Li2O (wt \%), Target Value $=4.674$

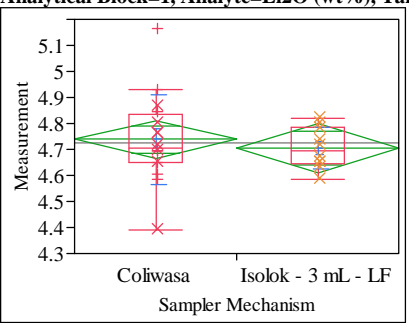

Oneway Anova

Summary of Fit

Adj Rsquare

$\quad 0.143174$

4.724787

t Test

Isolok - $3 \mathrm{~mL}$ - LF-Coliwasa

Assuming equal variances

Difference $\quad-0.03364$ t Ratio $\quad-0.58284$

Std Err Dif $\quad 0.05772$ DF $\quad 24$

Upper CL Dif 0.08548 Prob $>|\mathrm{t}| \quad 0.5654$

$\begin{array}{lll}\text { Lower CL Dif }-0.15276 & \text { Prob }>t & 0.7173\end{array}$

$\begin{array}{lrl}\text { Confidence } & 0.95 \text { Prob }<\mathrm{t} \quad 0.2827\end{array}$

Analysis of Variance

$\begin{array}{lrrrrr}\text { Source } & \text { DF } & \text { Sum of Squares } & \text { Mean Square } & \text { F Ratio } & \text { Prob }>\text { F } \\ \text { Sampler Mechanism } & 1 & 0.00696361 & 0.006964 & 0.3397 & 0.5654\end{array}$

$\begin{array}{llll}\text { Error } & 24 & 0.49197399 & 0.020499\end{array}$

$\begin{array}{lll}\text { C. Total } & 25 & 0.49893760\end{array}$

Means for Oneway Anova

Level Number Mean Std Error Lower 95\% Upper 95\%

$\begin{array}{lrrrrr}\text { Coliwasa } & 16 & 4.73773 & 0.03579 & 4.6639 & 4.8116 \\ \text { Isolok - 3 mL - LF } & 10 & 4.70409 & 0.04528 & 4.6106 & 4.7975\end{array}$

Std Error uses a pooled estimate of error variance

Means and Std Deviations

Level Number Mean Std Dev Std Err Mean Lower 95\% Upper 95\%

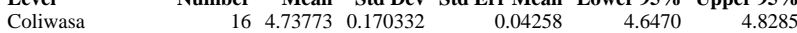

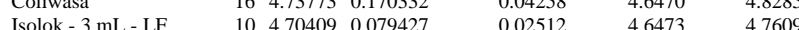

Test

Isolok - $3 \mathrm{~mL}$ - LF-Coliwasa

Assuming unequal variances

$\begin{array}{lrlr}\text { Difference } & -0.03364 & \mathrm{t} \text { Ratio } & -0.68042 \\ \text { Std Err Dif } & 0.04944 & \text { DF } & 22.67798 \\ \text { Upper CL Dif } & 0.06871 & \text { Prob }>|\mathrm{t}| & 0.5031 \\ \text { Lower CL Dif } & -0.13599 & \text { Prob }>\mathrm{t} & 0.7484 \\ \text { Confidence } & 0.95 & \text { Prob }<\mathrm{t} & 0.2516\end{array}$

Tests that the Variances are Equal

Level Count Std Dev MeanAbsDif to Mean MeanAbsDif to Median

$\begin{array}{lllll}\text { Coliwasa } & 16 & 0.1703321 & 0.1199233 & 0.1170639\end{array}$

$\begin{array}{lllll}\text { Isolok - } 3 \mathrm{~mL} \text { - LF } & 10 & 0.0794275 & 0.0667399 & 0.0667399\end{array}$

Test F Ratio DFNum DFDen p-Value

$\begin{array}{lrrrr}\text { O'Brien[.5] } & 1.5824 & 1 & 24 & 0.2205\end{array}$

$\begin{array}{lllll}\text { Brown-Forsythe } & 1.5117 & 1 & 24 & 0.2308\end{array}$

$\begin{array}{lllll}\text { Levene } & 1.9222 & 1 & 24 & 0.1784\end{array}$

$\begin{array}{lrrrr}\text { Bartlett } & 5.1612 & 1 & \text {. } & 0.0231 \\ \text { F Test 2-sided } & 4.5989 & 15 & 9 & 0.0260\end{array}$

Welch Anova testing Means Equal, allowing Std Devs Not Equal

F Ratio DFNum DFDen Prob $>$ F

$\begin{array}{lrrr}0.4630 & 1 & 22.678 & 0.5031\end{array}$

t Test

0.6804 


\section{Exhibit A2. Statistical Comparisons for Mid-Rheology (Phase 1) Testing}

Oneway Analysis of Measurement By Sampler Mechanism Type of Material=SME Simulant, Analytical Block=1, Analyte $=\mathrm{MgO}($ wt \%), Target Value=1.014

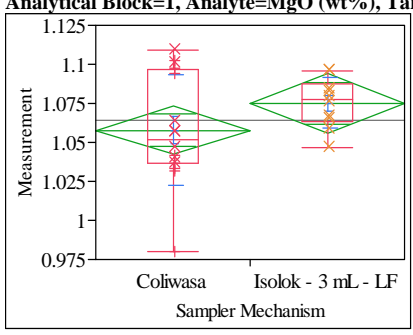

Oneway Anova

Summary of Fit

Adj Rsquare

$\quad 0.029496$

1.064501

Observations (or Sum Wgts) $\quad 26$

t Test

Isolok - $3 \mathrm{~mL}$ - LF-Coliwasa

Assuming equal variances

Difference $\quad 0.01718$ t Ratio 1.445226

Std Err Dif $\quad 0.01189$ DF 24

Upper CL Dif 0.04172 Prob $>|t| \quad 0.1613$

Lower CL Dif -0.00736 Prob $>\mathrm{t} \quad 0.0807$

$\begin{array}{lrl}\text { Confidence } & 0.95 \text { Prob }<\mathrm{t} & 0.9193\end{array}$

Analysis of Variance

$\begin{array}{lrrrrr}\text { Source } & \text { DF } & \text { Sum of Squares } & \text { Mean Square } & \text { F Ratio } & \text { Prob }>\text { F } \\ \text { Sampler Mechanism } & 1 & 0.00181720 & 0.001817 & 2.0887 & 0.1613\end{array}$

$\begin{array}{llll}\text { Error } & 24 & 0.02088054 & 0.000870\end{array}$

$\begin{array}{lll}\text { C. Total } & 25 & 0.02269774\end{array}$

Means for Oneway Anova

Level Number Mean Std Error Lower 95\% Upper 95\%

$\begin{array}{lrrrrr}\text { Coliwasa } & 16 & 1.05789 & 0.00737 & 1.0427 & 1.0731\end{array}$

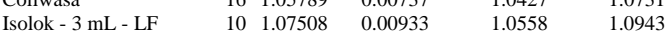

Std Error uses a pooled estimate of error variance

Means and Std Deviations

Level Number Mean Std Dev Std Err Mean Lower 95\% Upper 95\%

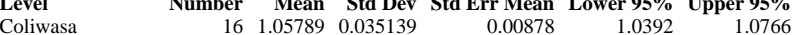

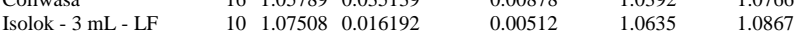

Test

Isolok - $3 \mathrm{~mL}$ - LF-Coliwasa

Assuming unequal variances

Difference $\quad 0.01718$ t Ratio 1.690012

Std Err Dif $\quad 0.01017$ DF 22.58007

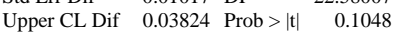

$\begin{array}{lll}\text { Lower CL Dif }-0.00387 \text { Prob }>t & 0.0524\end{array}$

$\begin{array}{lrl} & \\ \text { Confidence } & 0.95 \text { Prob }<\mathrm{t} & 0.9476\end{array}$

Tests that the Variances are Equal

Level Count Std Dev MeanAbsDif to Mean MeanAbsDif to Median

Coliwasa $\quad 16 \quad 0.0351386$

$\begin{array}{lllll}\text { Isolok - } 3 \mathrm{~mL} \text { - LF } & 10 & 0.0161924 & 0.0130011 & 0.0127689\end{array}$

Test F Ratio DFNum DFDen p-Value

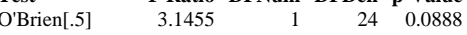

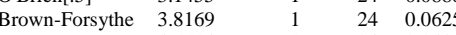

$\begin{array}{lllll}\text { Levene } & 4.5514 & 1 & 24 & 0.0433\end{array}$

$\begin{array}{lrrrr}\text { Bartlett } & 5.3031 & 1 & \text {. } & 0.0213\end{array}$

Welch Anova testing Means Equal, allowing Std Devs Not Equal

F Ratio DFNum DFDen Prob $>$ F

$\begin{array}{rrrr}2.8561 & 1 & 22.58 & 0.1048\end{array}$

t Test

1.6900
Oneway Analysis of Measurement By Sampler Mechanism Type of Material=SME Simulant, Analytical Block $=1$, Analyte $=\mathrm{MnO}(\mathrm{wt} \%)$, Target Value $=1.779$

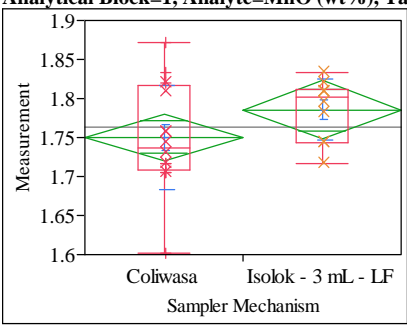

Oneway Anova

Summary of Fit

Adj Rsquare

0.086987
0.048945

0.057987

1.763978

t Test

Isolok - $3 \mathrm{~mL}$ - LF-Coliwasa

Assuming equal variances

Difference $\quad 0.03535$ t Ratio 1.512145

Std Err Dif $\quad 0.02338$ DF 24

Upper CL Dif 0.08359 Prob $>|t| \quad 0.1436$

$\begin{array}{lrll}\text { Upper CL Dif } & 0.08359 & \text { Prob }>|t| & 0.1436 \\ \text { Lower CL Dif } & -0.01290 & \text { Prob }>t & 0.0718\end{array}$

$\begin{array}{lrl}\text { Confidence } & 0.95 \text { Prob }<\mathrm{t} & 0.9282\end{array}$

Analysis of Variance

$\begin{array}{lrrrrr}\text { Source } & \text { DF } & \text { Sum of Squares } & \text { Mean Square } & \text { F Ratio } & \text { Prob }>\text { F } \\ \text { Sampler Mechanism } & 1 & 0.00768851 & 0.007689 & 2.2866 & 0.1436\end{array}$

$\begin{array}{llll}\text { Error } & 24 & 0.08069861 & 0.003362\end{array}$

$\begin{array}{lll}\text { C. Total } & 25 & 0.08838711\end{array}$

Means for Oneway Anova

Level Number Mean Std Error Lower 95\% Upper 95\%

$\begin{array}{lrrrrr}\text { Coliwasa } & 16 & 1.75038 & 0.01450 & 1.7205 & 1.7803 \\ \text { Isolok - 3 mL - LF } & 10 & 1.78573 & 0.01834 & 1.7479 & 1.8236\end{array}$

Std Error uses a pooled estimate of error variance

Means and Std Deviations

Level Number Mean Std Dev Std Err Mean Lower 95\% Upper 95\%

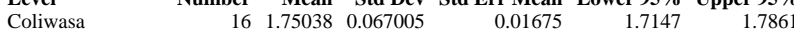

$\begin{array}{lllllll}\text { Coliwasa } & 16 & 1.75038 & 0.067005 & 0.01675 & 1.7147 & 1.7861 \\ \text { Isolok - 3 mL - LF } & 10 & 1.78573 & 0.038520 & 0.01218 & 1.7582 & 1.8133\end{array}$

TTest

Isolok - $3 \mathrm{~mL}$ - LF-Coliwasa

Assuming unequal variances

$\begin{array}{lrlr}\text { Difference } & 0.03535 & \mathrm{t} \text { Ratio } & 1.706586 \\ \text { Std Err Dif } & 0.02071 & \text { DF } & 23.91354 \\ \text { Upper CL Dif } & 0.07810 & \text { Prob }>|t| & 0.1009 \\ \text { Lower CL Dif } & -0.00741 & \text { Prob }>t & 0.0504 \\ \text { Confidence } & 0.95 & \text { Prob }<\mathrm{t} & 0.9496\end{array}$

Tests that the Variances are Equal

Level Count Std Dev MeanAbsDif to Mean MeanAbsDif to Median

$\begin{array}{lllll}\text { Coliwasa } & 16 & 0.0670047 & 0.0517489 & 0.0508410\end{array}$

$\begin{array}{llll}\text { Isolok - } 3 \mathrm{~mL} \text { - LF } & 10 & 0.0385202 & 0.0315053\end{array}$

Test F Ratio DFNum DFDen p-Value

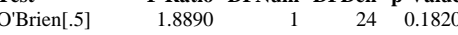

$\begin{array}{lllll}\text { Brown-Forsythe } & 1.8374 & 1 & 24 & 0.1879\end{array}$

$\begin{array}{lllll}\text { Levene } & 2.1674 & 1 & 24 & 0.1540\end{array}$

$\begin{array}{lrrrr}\text { Bartlett } & 2.8947 & 1 & \text {. } & 0.0889\end{array}$

Welch Anova testing Means Equal, allowing Std Devs Not Equal

F Ratio DFNum DFDen Prob $>$ F

$\begin{array}{rrrr}2.9124 & 1 & 23.914 & 0.1009\end{array}$

t Test
1.7066 


\section{Exhibit A2. Statistical Comparisons for Mid-Rheology (Phase 1) Testing}

Oneway Analysis of Measurement By Sampler Mechanism Type of Material=SME Simulant, Analytical Block=1, Analyte=Na2O (wt\%), Target Value=11.659

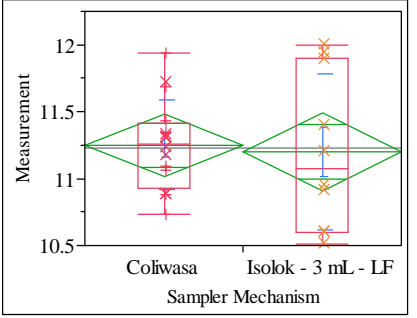

Oneway Anova

Summary of Fit

Adj Rsquare

0.003394

Root Mean Squ

Mean of Response

$-0.03813$

11.22936

t Test

Isolok - $3 \mathrm{~mL}$ - LF-Coliwasa

Assuming equal variances

Difference $\quad-0.05122$ t Ratio $\quad-0.28587$

Std Err Dif $\quad 0.17918$ DF $\quad 24$

Upper CL Dif 0.31859 Prob $>|t| r \mid r .7774$

$\begin{array}{lll}\text { Lower CL Dif }-0.42104 \text { Prob }>t & 0.6113\end{array}$

$\begin{array}{lrl}\text { Confidence } & 0.95 \text { Prob }<\mathrm{t} & 0.3887\end{array}$

Analysis of Variance

$\begin{array}{lrrrrr}\text { Source } & \text { DF } & \text { Sum of Squares } & \text { Mean Square } & \text { F Ratio } & \text { Prob }>\text { F } \\ \text { Sampler Mechanism } & 1 & 0.0161471 & 0.016147 & 0.0817 & 0.7774\end{array}$

$\begin{array}{llll}\text { Error } & 24 & 4.7419328 & 0.197581\end{array}$

$\begin{array}{lll}\text { C. Total } & 25 & 4.7580798\end{array}$

Means for Oneway Anova

$\begin{array}{lrrrrr}\text { Level } & \text { Number } & \text { Mean } & \text { Std Error } & \text { Lower 95\% } & \text { Upper 95\% } \\ \text { Coliwasa } & 16 & 11.2491 & 0.11113 & 11.020 & 11.478\end{array}$

$\begin{array}{lrrrrr}\text { Coliwasa } & 16 & 11.2491 & 0.11113 & 11.020 & 11.478 \\ \text { Isolok - 3 mL - LF } & 10 & 11.1978 & 0.14056 & 10.908 & 11.488\end{array}$

Std Error uses a pooled estimate of error variance

Means and Std Deviations

Level Number Mean Std Dev Std Err Mean Lower 95\% Upper 95\%

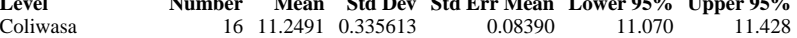

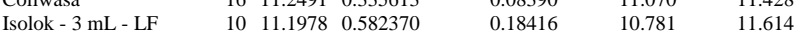

Test

Isolok - $3 \mathrm{~mL}$ - LF-Coliwasa

Assuming unequal variances

Difference $\quad-0.05122$ t Ratio $\quad-0.25312$

Std Err Dif $\quad 0.20237$ DF $\quad 12.79327$

$\begin{array}{lrr}\text { Upper CL Dif } & 0.38670 & \text { Prob }>|t| \\ & 0.8042\end{array}$

Lower CL Dif -0.48915 Prob $>t \quad 0.5979$

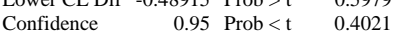

Tests that the Variances are Equal

Level Count Std Dev MeanAbsDif to Mean MeanAbsDif to Median

$\begin{array}{llll}\text { Coliwasa } & 16 & 0.3356132\end{array}$

$\begin{array}{lllll}\text { Coliwasa } & 16 & 0.3356132 & 0.2578050 & 0.2578050 \\ \text { Isolok - } 3 \mathrm{~mL} \text { - LF } & 10 & 0.5823696 & 0.4866280 & 0.4866280\end{array}$

Test F Ratio DFNum DFDen p-Value

$\begin{array}{lrrrr}\text { O'Brien[.5] } & 7.3012 & 1 & 24 & 0.0124 \\ \text { Brown-Forsythe } & 5.2708 & 1 & 24 & 0.0307\end{array}$

$\begin{array}{lllll}\text { Levene } & 5.9012 & 1 & 24 & 0.0230\end{array}$

$\begin{array}{lllll}\text { Bartlett } & 3.4122 & 1 & .0 .0647\end{array}$

Welch Anova testing Means Equal, allowing Std Devs Not Equal

F Ratio DFNum DFDen Prob $>$ F

$\begin{array}{lrrr}0.0641 & 1 & 12.793 & 0.8042\end{array}$

t Test
Oneway Analysis of Measurement By Sampler Mechanism Type of Material=SME Simulant, Analytical Block $=1$, Analyte $=\mathrm{NiO}($ wt $\%)$, Target Value $=0.41$

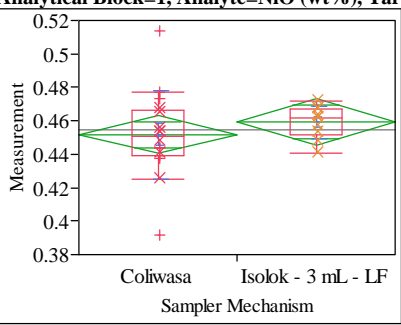

Oneway Anova

Summary of Fit

Adj Rsquare

$\quad 0.021579$

Mean of Response 0.454576

Observations (or Sum Wgts) $\quad 26$

T Test

Isolok - $3 \mathrm{~mL}$ - LF-Coliwasa

Assuming equal variances

Difference $\quad 0.00738$ t Ratio $\quad 0.848461$

$\begin{array}{lll}\text { Std Err Dif } \quad 0.00870 \text { DF } & 24\end{array}$

Upper CL Dif 0.02533 Prob $>|t| \quad 0.4046$

$\begin{array}{lll}\text { Lower CL Dif }-0.01057 \text { Prob }>t & 0.2023\end{array}$

$\begin{array}{lrl}\text { Confidence } & 0.95 \text { Prob }<\mathrm{t} & 0.7977\end{array}$

Analysis of Variance

$\begin{array}{lrrrrr}\text { Source } & \text { DF } & \text { Sum of Squares } & \text { Mean Square } & \text { F Ratio } & \text { Prob }>\text { F } \\ \text { Sampler Mechanism } & 1 & 0.00033521 & 0.000335 & 0.7199 & 0.4046\end{array}$

$\begin{array}{llll}\text { Error } & 24 & 0.01117546 & 0.000466\end{array}$

$\begin{array}{lll}\text { C. Total } & 25 & 0.01151067\end{array}$

Means for Oneway Anova

Level Number Mean Std Error Lower 95\% Upper 95\%

$\begin{array}{llllrr}\text { Coliwasa } & 16 & 0.451738 & 0.00539 & 0.44060 & 0.46287\end{array}$

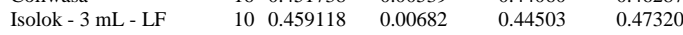

Std Error uses a pooled estimate of error variance

Means and Std Deviations

Level Number Mean Std Dev Std Err Mean Lower 95\% Upper 95\%

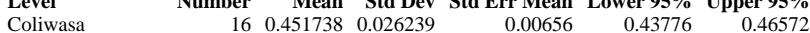

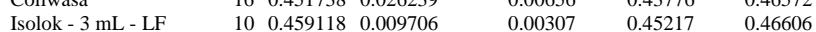

Test

Isolok - $3 \mathrm{~mL}$ - LF-Coliwasa

Assuming unequal variances

$\begin{array}{lrlr}\text { Difference } & 0.00738 & \mathrm{t} \text { Ratio } & 1.01907 \\ \text { Std Err Dif } & 0.00724 & \text { DF } & 20.63798 \\ \text { Upper CL Dif } & 0.02246 & \text { Prob }>|\mathrm{t}| & 0.3200 \\ \text { Lower CL Dif } & -0.00770 & \text { Prob }>\mathrm{t} & 0.1600 \\ \text { Confidence } & 0.95 & \text { Prob }<\mathrm{t} & 0.8400\end{array}$

Tests that the Variances are Equal

Level Count Std Dev MeanAbsDif to Mean MeanAbsDif to Median

$\begin{array}{lllll}\text { Coliwasa } & 16 & 0.0262394 & 0.0179741 & 0.0179741\end{array}$

$\begin{array}{llll}\text { Isolok - 3 mL - LF } & 10 & 0.0097059 & 0.0076859\end{array}$

Test F Ratio DFNum DFDen p-Value

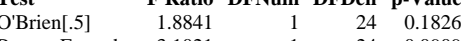

$\begin{array}{lllll}\text { Brown-Forsythe } & 3.1021 & 1 & 24 & 0.0909\end{array}$

$\begin{array}{lllll}\text { Levene } & 2.8866 & 1 & 24 & 0.1022\end{array}$

$\begin{array}{lrrrr}\text { Bartlett } & 8.1454 & 1 & \text {. } & 0.0043 \\ \text { F Test 2-sided } & 7.3086 & 15 & 9 & 0.0049\end{array}$

Welch Anova testing Means Equal, allowing Std Devs Not Equal

F Ratio DFNum DFDen Prob $>$ F

$\begin{array}{rrrr}1.0385 & 1 & 20.638 & 0.3200\end{array}$

t Test

1.019 


\section{Exhibit A2. Statistical Comparisons for Mid-Rheology (Phase 1) Testing}

Oneway Analysis of Measurement By Sampler Mechanism Type of Material=SME Simulant, Analytical Block=1, Analyte=SiO2 (wt \%), Target Value=50.985

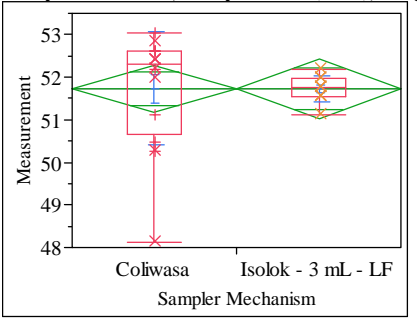

Oneway Anova

Summary of Fit

Adj Rsquare

$-0.04166$

1.064322

Observations (or Sum Wgts) $\quad 26$

t Test

Isolok - $3 \mathrm{~mL}$ - LF-Coliwasa

Assuming equal variances

Difference $\quad-0.00267$ t Ratio $\quad-0.00623$

Std Err Dif $\quad 0.42904$ DF $\quad 24$

$\begin{array}{rrr}\text { Upper CL Dif } & 0.88282 & \text { Prob }>|t| \\ & 0.9951\end{array}$

$\begin{array}{lll}\text { Lower CL Dif }-0.88817 \text { Prob }>t & 0.5025\end{array}$

$\begin{array}{lrr}\text { Confidence } & 0.95 \text { Prob }<\mathrm{t} & 0.4975\end{array}$

Analysis of Variance

$\begin{array}{lrrrrr}\text { Source } & \text { DF } & \text { Sum of Squares } & \text { Mean Square } & \text { F Ratio } & \text { Prob }>\text { F } \\ \text { Sampler Mechanism } & 1 & 0.000044 & 0.000044 & 0.0000 & 0.9951\end{array}$

$\begin{array}{lrrr}\text { Error } & 24 & 27.186747 & 1.13278\end{array}$

27.186791

Means for Oneway Anova

$\begin{array}{lrrrrr}\text { Level } & \text { Number } & \text { Mean } & \text { Std Error } & \text { Lower 95\% } & \text { Upper 95\% } \\ \text { Coliwasa } & 16 & 51.7309 & 0.26608 & 51.182 & 52.280\end{array}$

$\begin{array}{llllll}\text { Isolok - 3 mL - LF } & 16 & 51.7309 & 0.26608 & 51.182 & 52.280 \\ & 10 & 51.7283 & 0.33657 & 51.034 & 52.423\end{array}$

Std Error uses a pooled estimate of error variance

Means and Std Deviations

Level Number Mean Std Dev Std Err Mean Lower 95\% Upper 95\%

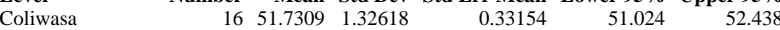

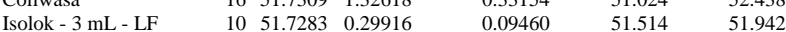

Test

Isolok - $3 \mathrm{~mL}$ - LF-Coliwasa

Assuming unequal variances

Difference $\quad-0.00267$ t Ratio $\quad-0.00776$

Std Err Dif $\quad 0.34478$ DF $\quad 17.35033$

$\begin{array}{lrr}\text { Upper CL Dif } & 0.72363 \text { Prob }>|t| & 0.9939\end{array}$

Lower CL Dif -0.72897 Prob $>t \quad 0.5030$

$\begin{array}{lrl}\text { Confidence } & 0.95 \text { Prob }<\mathrm{t} & 0.4970\end{array}$

Tests that the Variances are Equal

Level Count Std Dev MeanAbsDif to Mean MeanAbsDif to Median

$\begin{array}{lrrrr}\text { Coliwasa } & 16 & 1.326179 & 1.044580 & 0.8958319\end{array}$

$\begin{array}{lllll}\text { Coliwasa } & 16 & 1.326179 & 1.044580 & 0.8958319 \\ \text { Isolok - 3 mL - LF } & 10 & 0.299162 & 0.222487 & 0.2139300\end{array}$

Test F Ratio DFNum DFDen p-Value

$\begin{array}{lrrrr}\text { O'Brien[.5] } & 2.3612 & 1 & 24 & 0.1375 \\ \text { Brown-Forsythe } & 3.5776 & 1 & 24 & 0.0707\end{array}$

$\begin{array}{lllll}\text { Levene } & 10.8103 & 1 & 24 & 0.0031\end{array}$

$\begin{array}{lrrrr}\text { Bartlett } & 15.5400 & 1 & \text {. } & <.0001\end{array}$

Welch Anova testing Means Equal, allowing Std Devs Not Equal

F Ratio DFNum DFDen Prob $>$ F

$\begin{array}{lllr}0.0001 & 1 & 17.35 & 0.9939\end{array}$

t Test

0.0078
Oneway Analysis of Measurement By Sampler Mechanism Type of Material=SME Simulant, Analytical Block $=1$, Analyte $=$ Sum of Oxides ( $w$ t $\%)$, Target Value $=99.553$

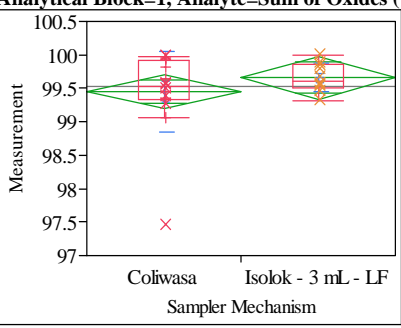

Oneway Anova

Summary of Fit

Adj Rsquare

$\quad 0.49698$

Mean of Response 99.5304

Observations (or Sum Wgts) $\quad 26$

T Test

Isolok - $3 \mathrm{~mL}$ - LF-Coliwasa

Assuming equal variances

Difference $\quad 0.20987$ t Ratio $\quad 1.047565$

Std Err Dif $\quad 0.20034$ DF 24

Upper CL Dif 0.62335 Prob $>|t| \quad 0.3053$

Lower CL Dif -0.20361 Prob $>\mathrm{t} \quad 0.1526$

$\begin{array}{lrl}\text { Confidence } & 0.95 \text { Prob }<\mathrm{t} & 0.8474\end{array}$

Analysis of Variance

$\begin{array}{lrrrrr}\text { Source } & \text { DF } & \text { Sum of Squares } & \text { Mean Square } & \text { F Ratio } & \text { Prob }>\text { F } \\ \text { Sampler Mechanism } & 1 & 0.2710437 & 0.271044 & 1.0974 & 0.3053\end{array}$

$\begin{array}{llll}\text { Error } & 24 & 5.9277347 & 0.246989\end{array}$

$\begin{array}{lll}\text { C. Total } & 25 & 6.1987784\end{array}$

Means for Oneway Anova

Level Number Mean Std Error Lower 95\% Upper 95\%

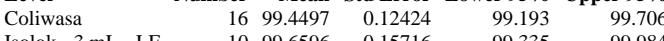

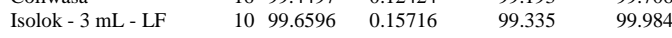

Std Error uses a pooled estimate of error variance

Means and Std Deviations

Level Number Mean Std Dev Std Err Mean Lower 95\% Upper 95\%

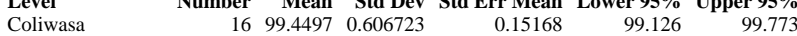

$\begin{array}{lllllll}\text { Isolok - 3 mL - LF } & 10 & 99.6596 & 0.212403 & 0.06717 & 99.508 & 99.811\end{array}$

Test

Isolok - $3 \mathrm{~mL}$ - LF-Coliwasa

Assuming unequal variances

$\begin{array}{lrlr}\text { Difference } & 0.20987 & \mathrm{t} \text { Ratio } & 1.265125 \\ \text { Std Err Dif } & 0.16589 & \text { DF } & 20.1671 \\ \text { Upper CL Dif } & 0.55572 & \text { Prob }>|\mathrm{t}| & 0.2203 \\ \text { Lower CL Dif } & -0.13598 & \text { Prob }>\mathrm{t} & 0.1101 \\ \text { Confidence } & 0.95 & \text { Prob }<\mathrm{t} & 0.8899\end{array}$

Tests that the Variances are Equal

Level Count Std Dev MeanAbsDif to Mean MeanAbsDif to Median

$\begin{array}{lllll}\text { Coliwasa } & 16 & 0.6067234 & 0.3605186 & 0.3554267\end{array}$

$\begin{array}{lllll}\text { Isolok - } 3 \mathrm{~mL} \text { - LF } & 10 & 0.2124031 & 0.1738794 & 0.1738794\end{array}$

Test F Ratio DFNum DFDen p-Value

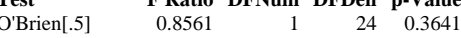

Brown-Forsythe $\quad 1.3139 \quad 1124 \quad 0.2630$

$\begin{array}{lllll}\text { Levene } & 1.4509 & 1 & 24 & 0.2401\end{array}$

$\begin{array}{lrrrr}\text { Bartlett } & 8.9111 & 1 & \text {. } & 0.0028 \\ \text { F Test 2-sided } & 8.1594 & 15 & 9 & 0.0032\end{array}$

Welch Anova testing Means Equal, allowing Std Devs Not Equal

F Ratio DFNum DFDen Prob $>$ F

$\begin{array}{rrrr}1.6005 & 1 & 20.167 & 0.2203\end{array}$

t Test

1.265 


\section{Exhibit A2. Statistical Comparisons for Mid-Rheology (Phase 1) Testing}

Oneway Analysis of Measurement By Sampler Mechanism Type of Material=SME Simulant, Analytical Block $=1$, Analyte $=$ TiO2 $($ wt $\%)$, Target Value $=0.0711$

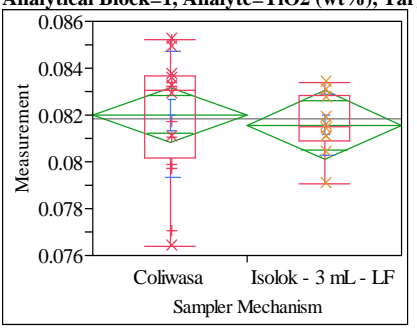

Oneway Anova

Summary of Fit

Adj Rsquare

-0.03082
0.081847

0.081847

t Test

Isolok - $3 \mathrm{~mL}$ - LF-Coliwasa

Assuming equal variances

Difference $\quad-0.00046$ t Ratio $\quad-0.50252$

Std Err Dif $\quad 0.00091$ DF 24

Upper CL Dif 0.00143 Prob $>|t| r \mid r .6199$

Lower CL Dif -0.00234 Prob $>\mathrm{t} \quad 0.6901$

$\begin{array}{lrl}\text { Confidence } & 0.95 \text { Prob }<\mathrm{t} & 0.3099\end{array}$

Analysis of Variance

$\begin{array}{lrrrrr}\text { Source } & \text { DF } & \text { Sum of Squares } & \text { Mean Square } & \text { F Ratio } & \text { Prob }>\text { F } \\ \text { Sampler Mechanism } & 1 & 0.00000129 & 1.2948 \mathrm{e}-6 & 0.2525 & 0.6199\end{array}$

$\begin{array}{llll}\text { Error } & 24 & 0.00012306 & 5.1274 \mathrm{e}-6\end{array}$

$\begin{array}{lll}\text { C. Total } & 25 & 0.00012435\end{array}$

Means for Oneway Anova

Level $\quad$ Number Mean Std Error Lower 95\% Upper 95\%

$\begin{array}{lrrrrr}\text { Coliwasa } & 16 & 0.082024 & 0.00057 & 0.08086 & 0.08319\end{array}$

$\begin{array}{llllll}\text { Isolok - } 3 \text { mL - LF } & 10 & 0.081565 & 0.00072 & 0.08009 & 0.08304\end{array}$

Std Error uses a pooled estimate of error variance

Means and Std Deviations

Level Number Mean Std Dev Std Err Mean Lower 95\% Upper 95\%

160.0820240 .002681

$\begin{array}{lllllll}\text { Coliwasa } & 16 & 0.082024 & 0.002681 & 0.00067 & 0.08060 & 0.08345 \\ \text { Isolok - } 3 \text { mL - LF } & 10 & 0.081565 & 0.001302 & 0.00041 & 0.08063 & 0.08250\end{array}$

Test

Isolok - $3 \mathrm{~mL}$ - LF-Coliwasa

Assuming unequal variances

Difference $\quad-0.00046$ t Ratio $\quad-0.5832$

Std Err Dif $\quad 0.00079$ DF 22.99606

Upper CL Dif $\quad 0.00117$ Prob $>|t| r \mid r .5654$

Lower CL Dif -0.00209 Prob $>t \quad 0.7173$

$\begin{array}{lrl} & \\ \text { Confidence } & 0.95 \text { Prob }<\mathrm{t} & 0.2827\end{array}$

Tests that the Variances are Equal

Level Count Std Dev MeanAbsDif to Mean MeanAbsDif to Median

Coliwasa $\quad 16 \quad 0.0026809$

$\begin{array}{lllll}\text { Coliwasa } & 16 & 0.0026809 & 0.0021528 & 0.0020433 \\ \text { Isolok - } 3 \mathrm{~mL} \text { - LF } & 10 & 0.0013016 & 0.0009674 & 0.0009674\end{array}$

Test F Ratio DFNum DFDen p-Value

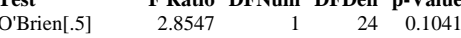

Brown-Forsythe $2.6563 \quad 1 \quad 24 \quad 0.1162$

$\begin{array}{lllll}\text { Levene } & 5.2469 & 1 & 24 & 0.031\end{array}$

\begin{tabular}{lrrrr} 
Bartlett & 4.6885 & 1 & . & 0.0304 \\
\hline
\end{tabular}

Welch Anova testing Means Equal, allowing Std Devs Not Equal

F Ratio DFNum DFDen Prob $>$ F

$\begin{array}{llll}0.3401 & 1 & 22.996 & 0.5654\end{array}$

t Test
Oneway Analysis of Measurement By Sampler Mechanism Type of Material=SME Simulant, Analytical Block $=1$, Analyte $=\mathrm{ZnO}($ wt $\%)$, Target Value $=0.0958$

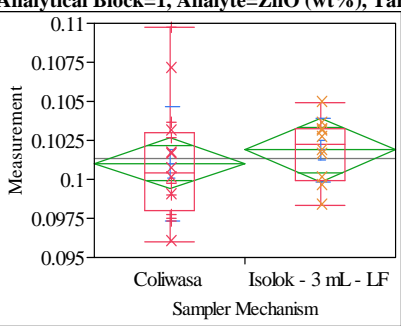

Oneway Anova

Summary of Fit

Adj Rsquare

Error $\quad 0.003156$

Observations (or Sum Wgts)

26

Test

Isolok - $3 \mathrm{~mL}$ - LF-Coliwasa

Assuming equal variances

Difference $\quad 0.00086$ t Ratio $\quad 0.672656$

Std Err Dif $\quad 0.00127$ DF $\quad 24$

Upper CL Dif 0.00348 Prob $>|t| \quad 0.5076$

Lower CL Dif -0.00177 Prob $>t \quad 0.2538$

$\begin{array}{lrl}\text { Confidence } & 0.95 \text { Prob }<\mathrm{t} & 0.7462\end{array}$

Analysis of Variance

$\begin{array}{lrrrrr}\text { Source } & \text { DF } & \text { Sum of Squares } & \text { Mean Square } & \text { F Ratio } & \text { Prob }>\text { F } \\ \text { Sampler Mechanism } & 1 & 0.00000451 & 4.507 \mathrm{e}-6 & 0.4525 & 0.5076\end{array}$

$\begin{array}{llll}\text { Error } & 24 & 0.00023906 & 9.961 \mathrm{e}-6\end{array}$

$\begin{array}{lll}\text { C. Total } & 25 & 0.00024357\end{array}$

Means for Oneway Anova

Level Number Mean Std Error Lower 95\% Upper 95\%

$\begin{array}{llllrr}\text { Coliwasa } & 16 & 0.101031 & 0.00079 & 0.09940 & 0.10266\end{array}$

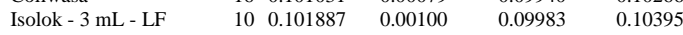

Std Error uses a pooled estimate of error variance

Means and Std Deviations

Level Number Mean Std Dev Std Err Mean Lower 95\% Upper 95\%

$\begin{array}{lrrrrrr}\text { Coliwasa } & 16 & 0.101031 & 0.003669 & 0.00092 & 0.09908 & 0.10299\end{array}$

$\begin{array}{lllllll}\text { Isolok - } 3 \mathrm{~mL} \text { - LF } & 10 & 0.101887 & 0.002031 & 0.00064 & 0.10043 & 0.10334\end{array}$

Isolok - $3 \mathrm{~mL}$ - LF-Coliwasa

Assuming unequal variances

$\begin{array}{lrlr}\text { Difference } & 0.00086 & \mathrm{t} \text { Ratio } & 0.764235 \\ \text { Std Err Dif } & 0.00112 & \text { DF } & 23.78559 \\ \text { Upper CL Dif } & 0.00317 & \text { Prob }>|\mathrm{t}| & 0.4522 \\ \text { Lower CL Dif } & -0.00146 & \text { Prob }>\mathrm{t} & 0.2261 \\ \text { Confidence } & 0.95 & \text { Prob }<\mathrm{t} & 0.7739\end{array}$

Tests that the Variances are Equal

Level Count Std Dev MeanAbsDif to Mean MeanAbsDif to Median

$\begin{array}{lllll}\text { Coliwasa } & 16 & 0.0036691 & 0.0027950 & 0.0027541\end{array}$

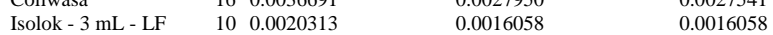

Test F Ratio DFNum DFDen p-Value

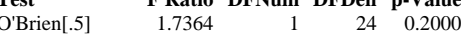

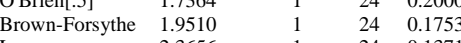

$\begin{array}{lllll}\text { Levene } & 2.3656 & 1 & 24 & 0.1371\end{array}$

$\begin{array}{lrrrr}\text { Bartlett } & 3.2662 & 1 & \text {. } & 0.0707 \\ \text { F Test 2-sided } & 3.2627 & 15 & 9 & 0.0783\end{array}$

Welch Anova testing Means Equal, allowing Std Devs Not Equal

F Ratio DFNum DFDen Prob $>$ F

$\begin{array}{rrrr}0.5841 & 1 & 23.786 & 0.4522\end{array}$

t Test
0.7642 


\section{Exhibit A2. Statistical Comparisons for Mid-Rheology (Phase 1) Testing}

Oneway Analysis of Measurement By Sampler Mechanism Type of Material=SME Simulant, Analytical Block=1, Analyte $=\mathrm{ZrO} 2$ (wt \%), Target Value $=0.3547$

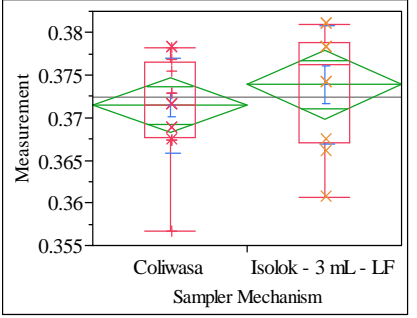

Oneway Anova

Summary of Fit

Adj Rsquare $\quad 0.038675$

Root Mean Square Error $\quad 0.006138$

Mean of Response $\quad 0.372405$

$\begin{array}{lr}\text { Mean of Response } & 0.372405 \\ \text { Observations (or Sum Wgts) } & 26\end{array}$

t Test

Isolok - $3 \mathrm{~mL}$ - LF-Coliwasa

Assuming equal variances

Difference $\quad 0.00243$ t Ratio 0.98262

Std Err Dif $\quad 0.00247$ DF 24

Upper CL Dif 0.00754 Prob $>|t| \quad 0.3356$

Lower CL Dif -0.00268 Prob $>\mathrm{t} \quad 0.1678$

$\begin{array}{lrl}\text { Confidence } & 0.95 \text { Prob }<\mathrm{t} & 0.8322\end{array}$

Analysis of Variance

$\begin{array}{lrrrrr}\text { Source } & \text { DF } & \text { Sum of Squares } & \text { Mean Square } & \text { F Ratio } & \text { Prob > F } \\ \text { Sampler Mechanism } & 1 & 0.00003638 & 0.000036 & 0.9655 & 0.3356\end{array}$

$\begin{array}{llll}\text { Error } & 24 & 0.00090430 & 0.000038\end{array}$

$\begin{array}{lll}\text { C. Total } & 25 & 0.00094068\end{array}$

Means for Oneway Anova

$\begin{array}{lrrrrr}\text { Level } & \text { Number } & \text { Mean } & \text { Std Error } & \text { Lower 95\% } & \text { Upper 95\% } \\ \text { Coliwasa } & 16 & 0.371470 & 0.00153 & 0.36830 & 0.37464\end{array}$

$\begin{array}{lrrrrr}\text { Coliwasa } & 16 & 0.371470 & 0.00153 & 0.36830 & 0.37464 \\ \text { Isolok - 3 mL - LF } & 10 & 0.373901 & 0.00194 & 0.36990 & 0.37791\end{array}$

Std Error uses a pooled estimate of error variance

Means and Std Deviations

Level Number Mean Std Dev Std Err Mean Lower 95\% Upper 95\%

$\begin{array}{lrrrrrr}\text { Leliwasa } & \text { Number } & \text { Mean } & \text { Std Dev } & \text { Std Err Mean } & \text { Lower 95\% } & \text { Upper 95\% } \\ \text { Colite } & 0.371470 & 0.005602 & 0.00140 & 0.36848 & 0.37446\end{array}$

$\begin{array}{lllllll}\text { Coliwasa } & 16 & 0.371470 & 0.005602 & 0.00140 & 0.36848 & 0.37446 \\ \text { Isolok - 3 mL - LF } & 10 & 0.373901 & 0.006941 & 0.00219 & 0.36894 & 0.37887\end{array}$

Test

Isolok - $3 \mathrm{~mL}$ - LF-Coliwasa

Assuming unequal variances

Difference $\quad 0.00243$ t Ratio 0.933884

Std Err Dif $\quad 0.00260$ DF 16.20924

Upper CL Dif 0.00794 Prob $>|t| \quad 0.3641$

Lower CL Dif -0.00308 Prob $>t \quad 0.1820$

$\begin{array}{lrl}\text { Confidence } & 0.95 \text { Prob }<\mathrm{t} & 0.8180\end{array}$

Tests that the Variances are Equal

Level Count Std Dev MeanAbsDif to Mean MeanAbsDif to Median

Coliwasa $\quad 16 \quad 0.0056022$

$\begin{array}{lllll}\text { Isolok - } 3 \mathrm{~mL} \text { - LF } & 10 & 0.0069405 & 0.0040524 & 0.0055113\end{array}$

Test F Ratio DFNum DFDen p-Value

$\begin{array}{lrrrr} & \text { F Ratio } & \text { DFNum } & \text { DFDen } & \text { p-Value } \\ \text { O'Brien[.5] } & 0.4881 & 1 & 24 & 0.4915\end{array}$

$\begin{array}{lllll}\text { Brown-Forsythe } & 0.6698 & 1 & 24 & 0.4212\end{array}$

$\begin{array}{lllll}\text { Levene } & 0.9305 & 1 & 24 & 0.344\end{array}$

$\begin{array}{lllll}\text { Bartlett } & 0.5082 & 1 & . & 0.4759\end{array}$

Test 2-sided 1.5349

F Ratio DFNum DFDen Prob > F

$\begin{array}{rrrr}0.8721 & 1 & 16.209 & 0.3641\end{array}$

t Test

0.9339
Oneway Analysis of Measurement By Sampler Mechanism Type of Material=SME Simulant, Analytical Block $=2$, Analyte $=$ Al $/ B$, Target Value $=2.552$

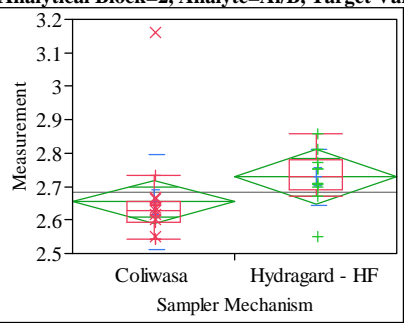

Oneway Anova

Summary of Fit

Adj Rsquare

0.124529

Mean of Response 2.682538

t Test

Hydragard - HF-Coliwasa

Assuming equal variances

Difference $\quad 0.07422$ t Ratio 1.478587

$\begin{array}{lll}\text { Std Err Dif } \quad 0.05020 & \text { DF }\end{array}$

Upper CL Dif 0.17783 Prob $>|t| \quad 0.1523$

$\begin{array}{lll}\text { Lower CL Dif }-0.02938 \text { Prob }>t & 0.0761\end{array}$

$\begin{array}{lll}\text { Confidence } & 0.95 \text { Prob }<\mathrm{t} \quad 0.9239\end{array}$

Analysis of Variance

$\begin{array}{lrrrrr}\text { Source } & \text { DF } & \text { Sum of Squares } & \text { Mean Square } & \text { F Ratio } & \text { Prob }>\text { F } \\ \text { Sampler Mechanism } & 1 & 0.03390294 & 0.033903 & 2.1862 & 0.1523\end{array}$

$\begin{array}{llll}\text { Error } & 24 & 0.37218165 & 0.015508\end{array}$

$\begin{array}{lll}\text { C. Total } & 25 & 0.40608459\end{array}$

Means for Oneway Anova

Level Number Mean Std Error Lower 95\% Upper 95\%

$\begin{array}{lllllr}\text { Coliwasa } & 16 & 2.65399 & 0.03113 & 2.5897 & 2.7182 \\ \text { Hydragard - HF } & 10 & 2.72821 & 0.03938 & 2.6469 & 2.8095\end{array}$

Std Error uses a pooled estimate of error variance

Means and Std Deviations

Level Number Mean Std Dev Std Err Mean Lower 95\% Upper 95\%

$\begin{array}{lrrrrrr}\text { Coliwasa } & 16 & 2.65399 & 0.143267 & 0.03582 & 2.5776 & 2.7303\end{array}$

$\begin{array}{lllllll}\text { Hydragard - HF } & 10 & 2.72821 & 0.084525 & 0.02673 & 2.6677 & 2.7887\end{array}$

Test

Hydragard - HF-Coliwasa

Assuming unequal variances

$\begin{array}{lrlr}\text { Difference } & 0.07422 & \mathrm{t} \text { Ratio } & 1.660829 \\ \text { Std Err Dif } & 0.04469 & \text { DF } & 23.96943 \\ \text { Upper CL Dif } & 0.16647 & \text { Prob }>|\mathrm{t}| & 0.1098 \\ \text { Lower CL Dif } & -0.01802 & \text { Prob }>\mathrm{t} & 0.0549 \\ \text { Confidence } & 0.95 & \text { Prob }<\mathrm{t} & 0.9451\end{array}$

Tests that the Variances are Equal

Level Count Std Dev MeanAbsDif to Mean MeanAbsDif to Median

$\begin{array}{lllll}\text { Coliwasa } & 16 & 0.1432669 & 0.0742898 & 0.0698653\end{array}$

$\begin{array}{lllll}\text { Hydragard - HF } & 10 & 0.0845252 & 0.0614414 & 0.0614414\end{array}$

Test F Ratio DFNum DFDen p-Value

$\begin{array}{lrrrr}\text { O'Brien[.5] } & 0.3603 & 1 & 24 & 0.5540\end{array}$

$\begin{array}{lllll}\text { Brown-Forsythe } & 0.0392 & 1 & 24 & 0.8446\end{array}$

$\begin{array}{lllll}\text { Levene } & 0.0991 & 1 & 24 & 0.7557\end{array}$

$\begin{array}{lrrrr}\text { Bartlett } & 2.6496 & 1 & \text {. } & 0.1036 \\ \text { F Test 2-sided } & 2.8729 & 15 & 9 & 0.1140\end{array}$

Welch Anova testing Means Equal, allowing Std Devs Not Equal

F Ratio DFNum DFDen Prob $>$ F

$\begin{array}{rrrr}2.7584 & 1 & 23.969 & 0.1098\end{array}$

t Test

1.6608 


\section{Exhibit A2. Statistical Comparisons for Mid-Rheology (Phase 1) Testing}

Oneway Analysis of Measurement By Sampler Mechanism Type of Material=SME Simulant, Analytical Block=2, Analyte=Al2O3 (wt \%), Target Value=10.869

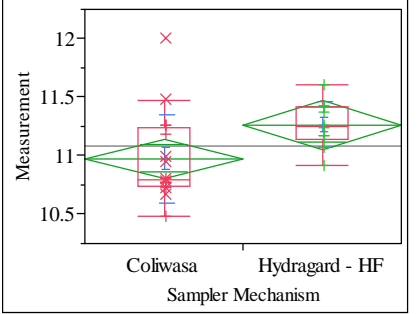

Oneway Anova

Summary of Fit

0.171884

Root Mean Square Error $\quad 0.323155$

$\begin{array}{lr}\text { Mean of Response } & 11.08628 \\ \text { Observations (or Sum Wgts) } & 26\end{array}$

Test

Hydragard - HF-Coliwasa

Assuming equal variances

Difference $\quad 0.290747$ t Ratio $\quad 2.231913$

Std Err Dif $\quad 0.130268$ DF 24

Upper CL Dif 0.559607 Prob $>|t| \quad 0.0352$

Lower CL Dif 0.021887 Prob $>t \quad 0.0176$

$\begin{array}{lrl}\text { Confidence } & 0.95 \text { Prob }<\mathrm{t} & 0.9824\end{array}$

Analysis of Variance

$\begin{array}{lrrrrr}\text { Source } & \text { DF } & \text { Sum of Squares } & \text { Mean Square } & \text { F Ratio } & \text { Prob }>\text { F } \\ \text { Sampler Mechanism } & 1 & 0.5202074 & 0.520207 & 4.9814 & 0.0352\end{array}$

$\begin{array}{llll}\text { Error } & 24 & 2.5063010 & 0.104429\end{array}$

3.0265084

Means for Oneway Anova

Level Number Mean Std Error Lower 95\% Upper 95\%

$\begin{array}{lrrrrr}\text { Coliwasa } & 16 & 10.9745 & 0.08079 & 10.808 & 11.14\end{array}$

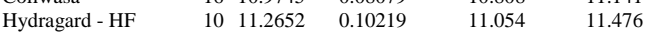

Std Error uses a pooled estimate of error variance

Means and Std Deviations

Level Number Mean Std Dev Std Err Mean Lower 95\% Upper 95\%

$16 \quad 10.9745 \quad 0.379171$

$\begin{array}{lllllll}\text { Coliwasa } & 16 & 10.9745 & 0.379171 & 0.09479 & 10.772 & 11.176 \\ \text { Hydragard - HF } & 10 & 11.2652 & 0.197129 & 0.06234 & 11.124 & 11.406\end{array}$

Test

Hydragard - HF-Coliwasa

Assuming unequal variances

Difference $\quad 0.290747$ t Ratio $\quad 2.5627$

Std Err Dif $\quad 0.113453$ DF $\quad 23.46504$

Upper CL Dif 0.525186 Prob $>|t| r \mid 0.0172$

Lower CL Dif 0.056308 Prob $>t \quad 0.0086$

$\begin{array}{lrl}\text { Confidence } & 0.95 \mathrm{Prob}<\mathrm{t} & 0.9914\end{array}$

Tests that the Variances are Equal

Level Count Std Dev MeanAbsDif to Mean MeanAbsDif to Median

Coliwasa $\quad 16 \quad 0.3791713$

$\begin{array}{lllll}\text { Hydragard - HF } & 10 & 0.1971288 & 0.1519158 & 0.1511600\end{array}$

Test F Ratio DFNum DFDen p-Value

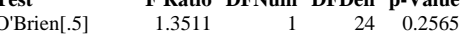

Brown-Forsythe $1.1163 \quad 1 \quad 24 \quad 0.3012$

$\begin{array}{lllll}\text { Levene } & 2.9153 & 1 & 24 & 0.1006\end{array}$

$\begin{array}{lrrrr}\text { Bartlett } & 3.9233 & 1 & \text {. } & 0.0476\end{array}$

Welch Anova testing Means Equal, allowing Std Devs Not Equal

F Ratio DFNum DFDen Prob > F

$\begin{array}{rrrr}6.5674 & 1 & 23.465 & 0.0172\end{array}$

t Test
Oneway Analysis of Measurement By Sampler Mechanism Type of Material=SME Simulant, Analytical Block=2, Analyte $=\mathrm{B} 2 \mathrm{O} 3(\mathrm{wt} \%)$, Target Value $=4.259$

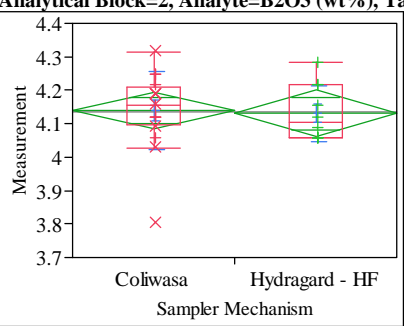

Oneway Anova

Summary of Fit

Adj Rsquare

0.001628

$-0.03997$

0.105988

Mean of Response

4.136333

Observations (or Sum Wgts)

Test

Hydragard - HF-Coliwasa

Assuming equal variances

Difference $\quad-0.00845$ t Ratio $\quad-0.19783$

Std Err Dif $\quad 0.04272$ DF $\quad 24$

Upper CL Dif 0.07973 Prob $>|t| \quad 0.8448$

Lower CL Dif -0.09663 Prob $>t \quad 0.5776$

$\begin{array}{lrl}\text { Confidence } & 0.95 \text { Prob }<\mathrm{t} \quad 0.4224\end{array}$

Analysis of Variance

Source DF Sum of Squares Mean Square F Ratio Prob > F

$\begin{array}{llll}\text { Error } & 24 & 0.26960054 & 0.011233\end{array}$

$\begin{array}{lll}\text { C. Total } & 25 & 0.27004017\end{array}$

Means for Oneway Anova

Level Number Mean Std Error Lower 95\% Upper 95\%

$\begin{array}{lrrrrr}\text { Coliwasa } & 16 & 4.13958 & 0.02650 & 4.0849 & 4.1943\end{array}$

$\begin{array}{llllll}\text { Hydragard - HF } & 10 & 4.13113 & 0.03352 & 4.0620 & 4.2003\end{array}$

Std Error uses a pooled estimate of error variance

Means and Std Deviations

Level Number Mean Std Dev Std Err Mean Lower 95\% Upper 95\%

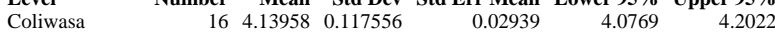

$\begin{array}{lllllll}\text { Hydragard - HF } & 10 & 4.13113 & 0.083207 & 0.02631 & 4.0716 & 4.1907\end{array}$

T Test

Hydragard - HF-Coliwasa

Assuming unequal variances

$\begin{array}{lrlr}\text { Difference } & -0.00845 & \mathrm{t} \text { Ratio } & -0.21427 \\ \text { Std Err Dif } & 0.03945 & \text { DF } & 23.50944 \\ \text { Upper CL Dif } & 0.07305 & \text { Prob }>|\mathrm{t}| & 0.8322 \\ \text { Lower CL Dif } & -0.08996 & \text { Prob }>\mathrm{t} & 0.5839 \\ \text { Confidence } & 0.95 & \text { Prob }<\mathrm{t} & 0.4161\end{array}$

Tests that the Variances are Equal

Level Count Std Dev MeanAbsDif to Mean MeanAbsDif to Median

$\begin{array}{lllll}\text { Coliwasa } & 16 & 0.1175558 & 0.0802459 & 0.0784851\end{array}$

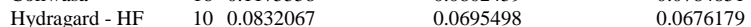

Test F Ratio DFNum DFDen p-Value

$\begin{array}{lrrrr}\text { O'Brien[.5] } & 0.4553 & 1 & 24 & 0.5063\end{array}$

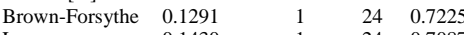

$\begin{array}{lllll}\text { Levene } & 0.1430 & 1 & 24 & 0.7087\end{array}$

$\begin{array}{lrrrr}\text { Bartlett } & 1.1940 & 1 & \text {. } & 0.2745 \\ \text { F Test 2-sided } & 1.9960 & 15 & 9 & 0.2966\end{array}$

Welch Anova testing Means Equal, allowing Std Devs Not Equal

F Ratio DFNum DFDen Prob $>$ F

$\begin{array}{lllr}0.0459 & 1 & 23.509 & 0.8322\end{array}$

t Test

0.2143 


\section{Exhibit A2. Statistical Comparisons for Mid-Rheology (Phase 1) Testing}

Oneway Analysis of Measurement By Sampler Mechanism Type of Material=SME Simulant, Analytical Block=2, Analyte=BaO (wt\%), Target Value=0.0919

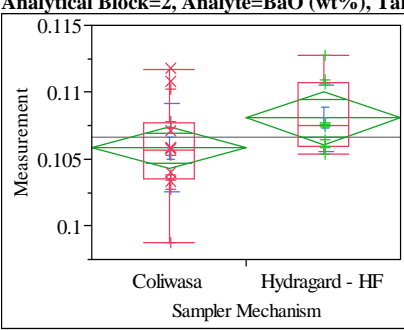

Oneway Anova

Summary of Fit

Adj Rsquare

0.122875

Root Mean Squarc

Mean of Response

0.086329

Observations (or Sum Wgts) $\quad 26$

t Test

Hydragard - HF-Coliwasa

Assuming equal variances

Difference $\quad 0.00224$ t Ratio 1.833613

Std Err Dif $\quad 0.00122$ DF 24

$\begin{array}{rrr}\text { Upper CL Dif } & 0.00477 & \text { Prob }>|t| \\ 0.0791\end{array}$

Lower CL Dif -0.00028 Prob $>\mathrm{t} \quad 0.0396$

$\begin{array}{lrl}\text { Confidence } & 0.95 \text { Prob }<\mathrm{t} & 0.9604\end{array}$

Analysis of Variance

$\begin{array}{lrrrrr}\text { Source } & \text { DF } & \text { Sum of Squares } & \text { Mean Square } & \text { F Ratio } & \text { Prob }>\text { F } \\ \text { Sampler Mechanism } & 1 & 0.00003095 & 0.000031 & 3.3621 & 0.0791\end{array}$

$\begin{array}{llll}\text { Error } & 24 & 0.00022096 & 9.207 \mathrm{e}-6\end{array}$

$\begin{array}{lll}\text { C. Total } & 25 & 0.00025191\end{array}$

$\begin{array}{lrrrrr}\text { Means for } & \text { Oneway Anova } & & & & \\ \text { Level } & \text { Number } & \text { Mean } & \text { Std Error } & \text { Lower 95\% } & \text { Upper 95\% } \\ \text { Coliwasa } & 16 & 0.105823 & 0.00076 & 0.10426 & 0.10739\end{array}$

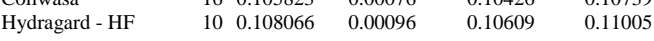

Std Error uses a pooled estimate of error variance

Means and Std Deviations

Level Number Mean Std Dev Std Err Mean Lower 95\% Upper 95\%

Coliwasa 160.1058230 .003310

$\begin{array}{lllllll}\text { Coliwasa } & 16 & 0.105823 & 0.003310 & 0.00083 & 0.10406 & 0.10759 \\ \text { Hydragard - HF } & 10 & 0.108066 & 0.002508 & 0.00079 & 0.10627 & 0.10986\end{array}$

t Test

Hydragard - HF-Coliwasa

Assuming unequal variances

Difference $\quad 0.00224$ t Ratio 1.956712

Std Err Dif $\quad 0.00115$ DF 22.94631

Upper CL Dif $\quad 0.00461$ Prob $>|t| r \mid r .0627$

Lower CL Dif -0.00013 Prob $>t \quad 0.0313$

$\begin{array}{lrl} & \\ \text { Confidence } & 0.95 \text { Prob }<\mathrm{t} & 0.9687\end{array}$

Tests that the Variances are Equal

Level Count Std Dev MeanAbsDif to Mean MeanAbsDif to Median

Coliwasa $\quad 16 \quad 0.0033101$

$\begin{array}{lllll}\text { Hydragard - HF } & 10 & 0.0025079 & 0.0024860 & 0.0024633 \\ & & & 0.0020164 & 0.0018646\end{array}$

Test F Ratio DFNum DFDen p-Value

$\begin{array}{lrrrr}\text { O'Brien[.5] } & 0.7543 & 1 & 24 & 0.3937\end{array}$

Brown-Forsythe $\quad 0.5727 \quad 1 \quad 24 \quad 0.4566$

$\begin{array}{lllll}\text { Levene } & 0.3999 & 1 & 24 & 0.5331\end{array}$

$\begin{array}{lrrrr}\text { Bartlett } & 0.7831 & 1 & \text {. } & 0.3762 \\ \text { F Test 2-sided } & 1.7421 & 15 & 9 & 0.4033\end{array}$

Welch Anova testing Means Equal, allowing Std Devs Not Equal

F Ratio DFNum DFDen Prob > F

$\begin{array}{llll}3.8287 & 1 & 22.946 & 0.0627\end{array}$

t Test
1.9567
Oneway Analysis of Measurement By Sampler Mechanism Type of Material=SME Simulant, Analytical Block $=2$, Analyte $=\mathrm{CaO}($ wt $\%)$, Target Value $=1.1462$

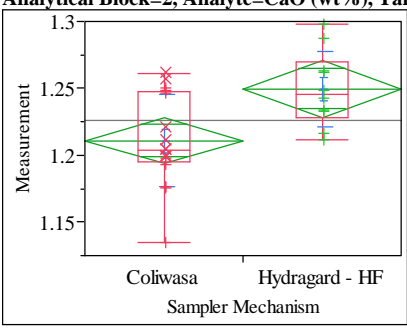

Oneway Anova

Summary of Fi

Adj Rsquare

0.266741

0.236189

Mean of Response 1.225807

Mean or Response 1.225807

t Test

Hydragard - HF-Coliwasa

Assuming equal variances

Difference $\quad 0.038705$ t Ratio 2.954759

Std Err Dif $\quad 0.013099$ DF 24

Upper CL Dif 0.065741 Prob $>|t| \quad 0.0069$

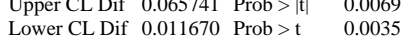

$\begin{array}{lrl}\text { Confidence } & 0.95 \text { Prob }<\mathrm{t} & 0.9965\end{array}$

Analysis of Variance

$\begin{array}{lrrrrr}\text { Source } & \text { DF } & \text { Sum of Squares } & \text { Mean Square } & \text { F Ratio } & \text { Prob }>\text { F } \\ \text { Sampler Mechanism } & 1 & 0.00921911 & 0.009219 & 8.7306 & 0.0069 \\ \text { Error } & 24 & 0.02534289 & 0.001056 & & \end{array}$

C. Total $-25-0.0$

Means for Oneway Anova

Level Number Mean Std Error Lower 95\% Upper 95\%

$\begin{array}{lrrrrr}\text { Coliwasa } & 16 & 1.21092 & 0.00812 & 1.1942 & 1.2277 \\ \text { Hydragard - HF } & 10 & 1.24963 & 0.01028 & 1.2284 & 1.2708\end{array}$

Std Error uses a pooled estimate of error variance

Means and Std Deviations

Level Number Mean Std Dev Std Err Mean Lower 95\% Upper 95\%

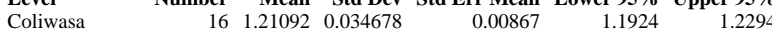

$\begin{array}{lllllll}\text { Hydragard - HF } & 10 & 1.24963 & 0.028488 & 0.00901 & 1.2292 & 1.2700\end{array}$

T Test

Hydragard - HF-Coliwasa

Assuming unequal variances

$\begin{array}{lrlr}\text { Difference } & 0.038705 & \text { t Ratio } & 3.095746 \\ \text { Std Err Dif } & 0.012503 & \text { DF } & 22.04472 \\ \text { Upper CL Dif } & 0.064631 & \text { Prob }>|t| & 0.0053 \\ \text { Lower CL Dif } & 0.012779 & \text { Prob }>t & 0.0026 \\ \text { Confidence } & 0.95 & \text { Prob }<\text { t } & 0.9974\end{array}$

Tests that the Variances are Equal

Level Count Std Dev MeanAbsDif to Mean MeanAbsDif to Median

$\begin{array}{lllll}\text { Coliwasa } & 16 & 0.0346782 & 0.0271751 & 0.0261475\end{array}$

$\begin{array}{lllll}\text { Hydragard - HF } & 10 & 0.0284882 & 0.0225551 & 0.0222473\end{array}$

Test F Ratio DFNum DFDen p-Value

$\begin{array}{lrrrr}\text { O'Brien[.5] } & 0.4651 & 1 & 24 & 0.5018\end{array}$

$\begin{array}{lllll}\text { Brown-Forsythe } & 0.2126 & 1 & 24 & 0.6489\end{array}$

$\begin{array}{lllll}\text { Levene } & 0.3735 & 1 & 24 & 0.5468\end{array}$

$\begin{array}{lrrrr}\text { Bartlett } & 0.4004 & 1 & \text {. } & 0.5269 \\ \text { F Test 2-sided } & 1.4818 & 15 & 9 & 0.5598\end{array}$

Welch Anova testing Means Equal, allowing Std Devs Not Equal

F Ratio DFNum DFDen Prob $>$ F

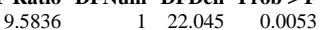

t Test

3.095 


\section{Exhibit A2. Statistical Comparisons for Mid-Rheology (Phase 1) Testing}

Oneway Analysis of Measurement By Sampler Mechanism Type of Material=SME Simulant, Analytical Block $=2$, Analyte $=\mathrm{Cr} 2 \mathrm{O} 3(\mathrm{wt} \%)$, Target Value $=\mathbf{0 . 0 9 9 8}$

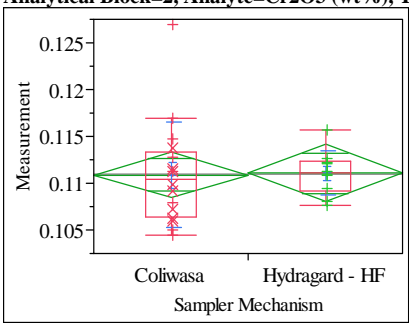

Oneway Anova

Summary of Fit

Adj Rsquare

0.000516

Root Mean Squarc

Mean of Response

$-0.04113$

re Error $\quad 0.004684$

t Test

Hydragard - HF-Coliwasa

Assuming equal variances

Difference $\quad 0.00021$ t Ratio 0.111284

Std Err Dif $\quad 0.00189$ DF 24

Upper CL Dif 0.00411 Prob $>|t| \quad 0.9123$

Lower CL Dif -0.00369 Prob $>\mathrm{t} \quad 0.4562$

$\begin{array}{lrl}\text { Confidence } & 0.95 \text { Prob }<\mathrm{t} & 0.5438\end{array}$

Analysis of Variance

$\begin{array}{lrrrrr}\text { Source } & \text { DF } & \text { Sum of Squares } & \text { Mean Square } & \text { F Ratio } & \text { Prob }>\text { F } \\ \text { Sampler Mechanism } & 1 & 0.00000027 & 2.717 \mathrm{e}-7 & 0.0124 & 0.9123\end{array}$

$\begin{array}{llll}\text { Error } & 24 & 0.00052646 & 0.000022\end{array}$

$\begin{array}{lll}\text { C. Total } & 25 & 0.00052673\end{array}$

Means for Oneway Anova

Level Number Mean Std Error Lower 95\% Upper 95\%

$\begin{array}{lrrrrr}\text { Coliwasa } & 16 & 0.110871 & 0.00117 & 0.10845 & 0.11329\end{array}$

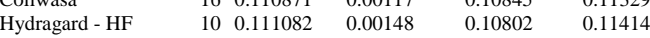

Std Error uses a pooled estimate of error variance

Means and Std Deviations

Level Number Mean Std Dev Std Err Mean Lower 95\% Upper 95\%

$\begin{array}{llllllll}\text { Coliwasa } & 16 & 0.110871 & 0.005628 & 0.00141 & 0.10787 & 0.11387\end{array}$

$\begin{array}{lllllll}\text { Hydragard - HF } & 10 & 0.111082 & 0.002388 & 0.00076 & 0.10937 & 0.11279\end{array}$

t Test

Hydragard - HF-Coliwasa

Assuming unequal variances

Difference $\quad 0.00021$ t Ratio $\quad 0.131575$

Std Err Dif $\quad 0.00160$ DF $\quad 21.86172$

Upper CL Dif 0.00352 Prob $>|t| r \mid r .8965$

Lower CL Dif -0.00310 Prob $>t \quad 0.4483$

$\begin{array}{lrl} & \\ \text { Confidence } & 0.95 \text { Prob }<\mathrm{t} & 0.5517\end{array}$

Tests that the Variances are Equal

Level Count Std Dev MeanAbsDif to Mean MeanAbsDif to Median

Coliwasa $\quad 16 \quad 0.0056282$

Hydragard - HF $10 \quad 0.0023878$

Test F Ratio DFNum DFDen p-Value

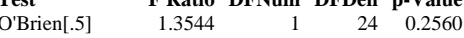

Brown-Forsythe $3.0211 \quad 1 \quad 24 \quad 0.0950$

$\begin{array}{lllll}\text { Levene } & 3.0608 & 1 & 24 & 0.0930\end{array}$

$\begin{array}{lrrrr}\text { Bartlett } & 6.3277 & 1 & \text {. } & 0.0119 \\ \text { F Test 2-sided } & 5.5559 & 15 & 9 & 0.0134\end{array}$

Welch Anova testing Means Equal, allowing Std Devs Not Equal

F Ratio DFNum DFDen Prob $>$ F

$\begin{array}{llll}0.0173 & 1 & 21.862 & 0.8965\end{array}$

t Test

0.1316
Oneway Analysis of Measurement By Sampler Mechanism Type of Material=SME Simulant, Analytical Block $=2$, Analyte $=\mathrm{CuO}(\mathrm{wt} \%)$, Target Value $=0.0504$

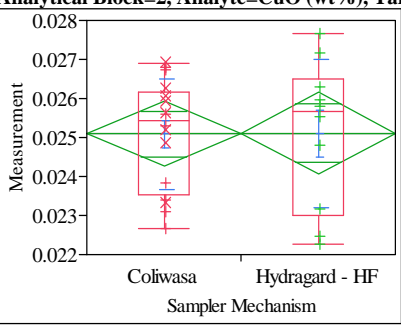

Oneway Anova

Summary of Fit

Adj Rsquare

0.001611

0.025096

Test

Hydragard - HF-Coliwasa

Assuming equal variances

Difference 2.425e-5 t Ratio 0.037342

Std Err Dif $\quad 0.00065$ DF 24

Upper CL Dif 0.00136 Prob $>|t| \quad 0.9705$

Lower CL Dif -0.00132 Prob $>t \quad 0.4853$

$\begin{array}{lrl}\text { Confidence } & 0.95 \text { Prob }<\mathrm{t} & 0.5147\end{array}$

Analysis of Variance

$\begin{array}{lrrrrr}\text { Source } & \text { DF } & \text { Sum of Squares } & \text { Mean Square } & \text { F Ratio } & \text { Prob }>\text { F } \\ \text { Sampler Mechanism } & 1 & 3.61993 \mathrm{e}-9 & 3.6199 \mathrm{e}-9 & 0.0014 & 0.9705\end{array}$

$\begin{array}{llll}\text { Error } & 24 & 0.00006230 & 2.596 \mathrm{e}-6\end{array}$

$\begin{array}{lll}\text { C. Total } & 25 & 0.00006231\end{array}$

Means for Oneway Anova

Level Number Mean Std Error Lower 95\% Upper 95\%

$\begin{array}{lrrrrr}\text { Coliwasa } & 16 & 0.025087 & 0.00040 & 0.02426 & 0.02592\end{array}$

$\begin{array}{llllll}\text { Hydragard - HF } & 10 & 0.025111 & 0.00051 & 0.02406 & 0.02616\end{array}$

Std Error uses a pooled estimate of error variance

Means and Std Deviations

Level Number Mean Std Dev Std Err Mean Lower 95\% Upper 95\%

$\begin{array}{lrrrrrr}\text { Coliwasa } & 16 & 0.025087 & 0.001412 & 0.00035 & 0.02433 & 0.02584\end{array}$

$\begin{array}{lllllll}\text { Hydragard - HF } & 10 & 0.025111 & 0.001897 & 0.00060 & 0.02375 & 0.02647\end{array}$

T Test

Hydragard - HF-Coliwasa

Assuming unequal variances

$\begin{array}{lrlr}\text { Difference } & 2.425 \mathrm{e}-5 & \mathrm{t} \text { Ratio } & 0.03484 \\ \text { Std Err Dif } & 0.00070 & \text { DF } & 15.21311 \\ \text { Upper CL Dif } & 0.00151 & \text { Prob }>|\mathrm{t}| & 0.9727 \\ \text { Lower CL Dif } & -0.00146 & \text { Prob }>\mathrm{t} & 0.4863 \\ \text { Confidence } & 0.95 & \text { Prob }<\mathrm{t} & 0.5137\end{array}$

Tests that the Variances are Equal

Level Count Std Dev MeanAbsDif to Mean MeanAbsDif to Median

$\begin{array}{lllll}\text { Coliwasa } & 16 & 0.0014119 & 0.0011726 & 0.0011384\end{array}$

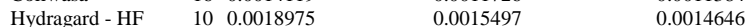

Test F Ratio DFNum DFDen p-Value

$\begin{array}{lrrrr}\text { O'Brien[.5] } & 2.1891 & 1 & 24 & 0.1520\end{array}$

$\begin{array}{lllll}\text { Brown-Forsythe } & 2.1891 & 1 & 24 & 0.1520 \\ & 0.6243 & 1 & 24 & 0.4372\end{array}$

$\begin{array}{lllll}\text { Levene } & 1.2897 & 1 & 24 & 0.2673\end{array}$

$\begin{array}{lllll}\text { Bartlett } & 0.9745 & 1 & \text {. } & 0.3236 \\ \text { F Test 2-sided } & 1.8062 & 9 & 15 & 0.2992\end{array}$

Welch Anova testing Means Equal, allowing Std Devs Not Equal

F Ratio DFNum DFDen Prob $>$ F

$\begin{array}{lllr}0.0012 & 1 & 15.213 & 0.9727\end{array}$

t Test

0.0348 


\section{Exhibit A2. Statistical Comparisons for Mid-Rheology (Phase 1) Testing}

Oneway Analysis of Measurement By Sampler Mechanism Type of Material=SME Simulant, Analytical Block $=2$, Analyte $=\mathrm{Fe} / \mathrm{Li}$, Target Value $=2.452$

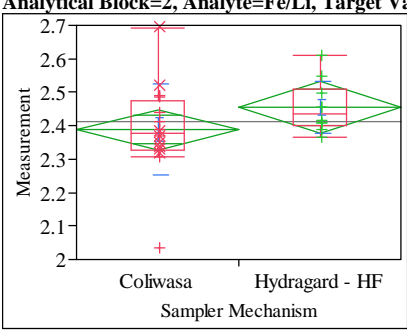

Oneway Anova

Summary of Fit

Adj Rsquare $\quad 0.07819$

Root Mean Square Error $\quad 0.117325$

Mean of Response E.413936

2.413936
Observations (or Sum Wgts)

Test

Hydragard - HF-Coliwasa

Assuming equal variances

Difference $\quad 0.06748$ t Ratio 1.426795

Std Err Dif $\quad 0.04730$ DF $\quad 24$

Upper CL Dif 0.16509 Prob $>|t| r \mid r .1665$

Lower CL Dif -0.03013 Prob $>\mathrm{t} \quad 0.0833$

$\begin{array}{lrl}\text { Confidence } & 0.95 \text { Prob }<\mathrm{t} & 0.9167\end{array}$

Analysis of Variance

$\begin{array}{lrrrrr}\text { Source } & \text { DF } & \text { Sum of Squares } & \text { Mean Square } & \text { F Ratio } & \text { Prob }>\text { F } \\ \text { Sampler Mechanism } & 1 & 0.02802216 & 0.028022 & 2.0357 & 0.1665\end{array}$

$\begin{array}{llll}\text { Error } & 24 & 0.02802216 & 0.028022 \\ \text { C. } & 24 & 0.33036159 & 0.013765\end{array}$

$\begin{array}{lll}\text { C. Total } & 25 & 0.35838374\end{array}$

Means for Oneway Anova

Level Number Mean Std Error Lower 95\% Upper 95\%

$\begin{array}{lrrrrr}\text { Coliwasa } & 16 & 2.38798 & 0.02933 & 2.3274 & 2.4485\end{array}$

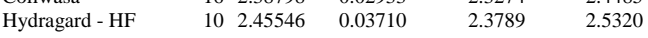

Std Error uses a pooled estimate of error variance

Means and Std Deviations

Level Number Mean Std Dev Std Err Mean Lower 95\% Upper 95\%

$\begin{array}{lrrrrrr}\text { Coliwasa } & 16 & 2.38798 & 0.135952 & 0.03399 & 2.3155 & 2.4604\end{array}$

$\begin{array}{lllllll}\text { Hydragard - HF } & 10 & 2.45546 & 0.076825 & 0.02429 & 2.4005 & 2.5104\end{array}$

Test

Hydragard - HF-Coliwasa

Assuming unequal variances

$\begin{array}{lrlr}\text { Difference } & 0.06748 & \mathrm{t} \text { Ratio } & 1.615221 \\ \text { Std Err Dif } & 0.04178 \text { DF } & 23.86176 \\ \text { Upper CL Dif } & 0.15373 \text { Prob }>|t| & 0.1194 \\ \text { Lower CL Dif } & -0.01877 & \text { Prob }>\mathrm{t} & 0.0597 \\ \text { Confidence } & 0.95 & \text { Prob }<\mathrm{t} & 0.9403\end{array}$

Tests that the Variances are Equal

Level Count Std Dev MeanAbsDif to Mean MeanAbsDif to Median

Coliwasa $\quad 16 \quad 0.1359518$

$\begin{array}{lllll}\text { Hydragard - HF } & 10 & 0.0768246 & 0.0584781 & 0.0880401 \\ & & & \end{array}$

Test F Ratio DFNum DFDen p-Value

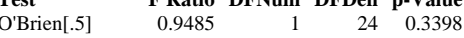

$\begin{array}{lllll}\text { Brown-Forsythe } & 0.7232 & 1 & 24 & 0.4035\end{array}$

$\begin{array}{lllll}\text { Levene } & 0.7966 & 1 & 24 & 0.3810\end{array}$

$\begin{array}{lrrrr}\text { Bartlett } & 3.0619 & 1 & \text {. } & 0.0801\end{array}$

Welch Anova testing Means Equal, allowing Std Devs Not Equal

F Ratio DFNum DFDen Prob $>$ F

$\begin{array}{lrrr}2.6089 & 1 & 23.862 & 0.1194\end{array}$

t Test

1.6152
Oneway Analysis of Measurement By Sampler Mechanism Type of Material=SME Simulant, Analytical Block=2, Analyte $=$ Fe2O3 (wt $\%$ ), Target Value $=11.462$

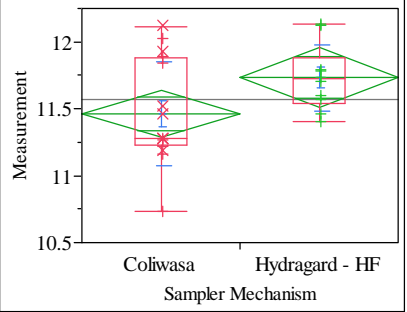

Oneway Anova

Summary of Fit

Adj Rsquare

0.139274

0.10341
0.339251

Mean or Response 11.56627

t Test

Hydragard - HF-Coliwasa

Assuming equal variances

Difference $\quad 0.26950$ t Ratio 1.970643

Std Err Dif $\quad 0.13676$ DF 24

Upper CL Dif 0.55175 Prob $>|t| \quad 0.0604$

Lower CL Dif -0.01275 Prob $>t \quad 0.0302$

$\begin{array}{lrl}\text { Confidence } & 0.95 \text { Prob }<\mathrm{t} & 0.9698\end{array}$

Analysis of Variance

$\begin{array}{lrrrrr}\text { Source } & \text { DF } & \text { Sum of Squares } & \text { Mean Square } & \text { F Ratio } & \text { Prob }>\text { F } \\ \text { Sampler Mechanism } & 1 & 0.4469502 & 0.446950 & 3.8834 & 0.0604\end{array}$

$\begin{array}{llll}\text { Error } & 24 & 2.7621958 & 0.115091\end{array}$

$\begin{array}{lll}\text { C. Total } & 25 & 3.2091461\end{array}$

Means for Oneway Anova

Level Number Mean Std Error Lower 95\% Upper 95\%

$\begin{array}{lrrrrr}\text { Coliwasa } & 16 & 11.4626 & 0.08481 & 11.288 & 11.638\end{array}$

$\begin{array}{lllll}\text { Hydragard - HF } \quad 10 & 11.7321 & 0.10728 & 11.511 & 11.954\end{array}$

Std Error uses a pooled estimate of error variance

Means and Std Deviations

Level Number Mean Std Dev Std Err Mean Lower 95\% Upper 95\%

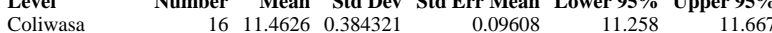

$\begin{array}{lllllll}\text { Hydragard - HF } & 10 & 11.7321 & 0.246455 & 0.07794 & 11.556 & 11.908\end{array}$

T Test

Hydragard - HF-Coliwasa

Assuming unequal variances

$\begin{array}{lrlr}\text { Difference } & 0.269498 \text { t Ratio } & 2.178382 \\ \text { Std Err Dif } & 0.123715 & \text { DF } & 23.9512 \\ \text { Upper CL Dif } & 0.524861 & \text { Prob }>|t| & 0.0395 \\ \text { Lower CL Dif } & 0.014136 & \text { Prob }>t & 0.0197 \\ \text { Confidence } & 0.95 & \text { Prob }<\mathrm{t} & 0.9803\end{array}$

Tests that the Variances are Equal

Level Count Std Dev MeanAbsDif to Mean MeanAbsDif to Median

$\begin{array}{lllll}\text { Coliwasa } & 16 & 0.3843208 & 0.3154276 & 0.2913014\end{array}$

$\begin{array}{lllll}\text { Hydragard - HF } & 10 & 0.2464546 & 0.1830016 & 0.1830016\end{array}$

Test F Ratio DFNum DFDen p-Value

$\begin{array}{lrrrr}\text { O'Brien[.5] } & 2.2188 & 1 & 24 & 0.1494\end{array}$

$\begin{array}{lllll}\text { Brown-Forsythe } & 1.0534 & 1 & 24 & 0.3150\end{array}$

$\begin{array}{lllll}\text { Levene } & 3.1004 & 1 & 24 & 0.0910\end{array}$

$\begin{array}{lrrrr}\text { Bartlett } & 1.9228 & 1 & \text {. } & 0.1655 \\ \text { F Test 2-sided } & 2.4317 & 15 & 9 & 0.1807\end{array}$

Welch Anova testing Means Equal, allowing Std Devs Not Equal

F Ratio DFNum DFDen Prob $>$ F

$\begin{array}{llll}4.7453 & 1 & 23.951 & 0.0395\end{array}$

t Test
2.1784 


\section{Exhibit A2. Statistical Comparisons for Mid-Rheology (Phase 1) Testing}

Oneway Analysis of Measurement By Sampler Mechanism Type of Material=SME Simulant, Analytical Block=2, Analyte $=$ K2O (wt \%), Target Value $=\mathbf{0 . 1 5 9 1}$

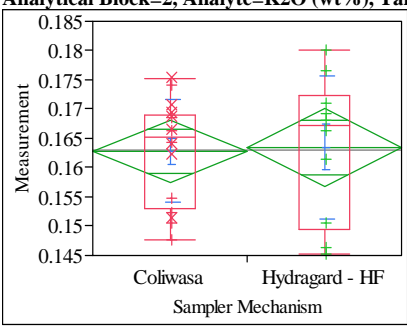

Oneway Anova

Summary of Fit

Adj Rsquare

$\quad 0.01027$

Observations (or Sum Wgts) $\quad 26$

t Test

Hydragard - HF-Coliwasa

Assuming equal variances

Difference $\quad 0.00062$ t Ratio 0.149119

Std Err Dif $\quad 0.00414$ DF 24

Upper CL Dif 0.00916 Prob $>|t| \quad 0.8827$

Lower CL Dif -0.00793 Prob $>t \quad 0.4414$

$\begin{array}{lrl}\text { Confidence } & 0.95 \text { Prob }<\mathrm{t} & 0.5586\end{array}$

Analysis of Variance

$\begin{array}{lrrrrr}\text { Source } & \text { DF } & \text { Sum of Squares } & \text { Mean Square } & \text { F Ratio } & \text { Prob }>\text { F } \\ \text { Sampler Mechanism } & 1 & 0.00000235 & 2.345 \mathrm{e}-6 & 0.0222 & 0.8827\end{array}$

$\begin{array}{llll}\text { Error } & 24 & 0.00253143 & 0.000105\end{array}$

$\begin{array}{lll}\text { C. Total } & 25 & 0.00253378\end{array}$

Means for Oneway Anova

Level $\quad$ Number Mean Std Error Lower 95\% Upper 95\%

\begin{tabular}{lrrrrr} 
Coliwasa & 16 & 0.162847 & 0.00257 & 0.15755 & 0.16815 \\
\hline
\end{tabular}

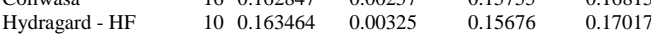

Std Error uses a pooled estimate of error variance

Means and Std Deviations

Level Number Mean Std Dev Std Err Mean Lower 95\% Upper 95\%

Coliwasa 160.1628470 .008824

$\begin{array}{lllllll}\text { Coliwasa } & 16 & 0.162847 & 0.008824 & 0.00221 & 0.15814 & 0.16755 \\ \text { Hydragard - HF } & 10 & 0.163464 & 0.012308 & 0.00389 & 0.15466 & 0.17227\end{array}$

t Test

Hydragard - HF-Coliwasa

Assuming unequal variances

Difference $\quad 0.00062$ t Ratio $\quad 0.137991$

Std Err Dif $\quad 0.00447$ DF $\quad 14.79527$

Upper CL Dif $\quad 0.01016$ Prob $>|t| r \mid r .8921$

Lower CL Dif -0.00893 Prob $>t \quad 0.4461$

$\begin{array}{lrll} & \\ \text { Confidence } & 0.95 & \text { Prob }<\mathrm{t} & 0.5539\end{array}$

Tests that the Variances are Equal

$\begin{array}{lrrrr}\text { Level } & \text { Count } & \text { Std Dev } & \text { MeanAbsDif to Mean } & \text { MeanAbsDif to Median } \\ \text { Coliwasa } & 16 & 0.0088243 & 0.0073217 & 0.0070017\end{array}$

$\begin{array}{lllll}\text { Hydragard - HF } & 10 & 0.0123082 & 0.0073217 & 0.0070017 \\ & & & 0.0100705 & 0.0095163\end{array}$

Test F Ratio DFNum DFDen p-Value

$\begin{array}{lrrrr}\text { O'Brien[.5] } & 2.6790 & 1 & 24 & 0.1147\end{array}$

$\begin{array}{lllll}\text { Brown-Forsythe } & 0.8686 & 1 & 24 & 0.3606\end{array}$

$\begin{array}{lllll}\text { Levene } & 1.6919 & 1 & 24 & 0.2057\end{array}$

$\begin{array}{lllll}\text { Bartlett } & 1.2376 & 1 & 0.2659\end{array}$

Welch Anova testing Means Equal, allowing Std Devs Not Equal

F Ratio DFNum DFDen Prob $>$ F

$\begin{array}{lrrr}0.0190 & 1 & 14.795 & 0.8921\end{array}$

t Test

0.1380
Oneway Analysis of Measurement By Sampler Mechanism Type of Material=SME Simulant, Analytical Block $=2$, Analyte $=\mathrm{Li} 2 \mathrm{O}(\mathrm{wt} \%)$, Target Value $=4.674$

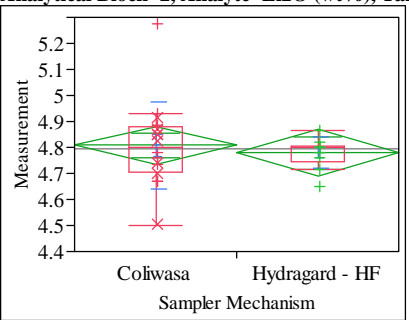

Oneway Anova

Summary of Fit

Adj Rsquare

$\quad 0.136405$

4.796827

t Test

Hydragard - HF-Coliwasa

Assuming equal variances

Difference $\quad-0.02826$ t Ratio $\quad-0.51389$

Std Err Dif $\quad 0.05499$ DF 24

Upper CL Dif 0.08523 Prob $>|t| \quad 0.6120$

Lower CL Dif -0.14174 Prob $>t \quad 0.6940$

$\begin{array}{lrl}\text { Confidence } & 0.95 \text { Prob }<\mathrm{t} & 0.3060\end{array}$

Analysis of Variance

$\begin{array}{lrrrrr}\text { Source } & \text { DF } & \text { Sum of Squares } & \text { Mean Square } & \text { F Ratio } & \text { Prob }>\text { F } \\ \text { Sampler Mechanism } & 1 & 0.00491352 & 0.004914 & 0.2641 & 0.6120\end{array}$

$\begin{array}{llll}\text { Error } & 24 & 0.44655120 & 0.018606\end{array}$

$\begin{array}{lll}\text { C. Total } & 25 & 0.45146472\end{array}$

Means for Oneway Anova

Level Number Mean Std Error Lower 95\% Upper 95\%

$\begin{array}{lrrrrr}\text { Coliwasa } & 16 & 4.80769 & 0.03410 & 4.7373 & 4.8781\end{array}$

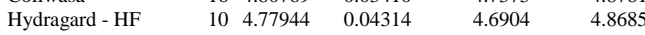

Std Error uses a pooled estimate of error variance

Means and Std Deviations

Level Number Mean Std Dev Std Err Mean Lower 95\% Upper 95\%

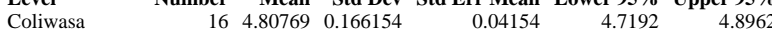

$\begin{array}{lllllll}\text { Hydragard - HF } & 10 & 4.77944 & 0.060042 & 0.01899 & 4.7365 & 4.8224\end{array}$

Test

Hydragard - HF-Coliwasa

Assuming unequal variances

$\begin{array}{lrlr}\text { Difference } & -0.02826 & \mathrm{t} \text { Ratio } & -0.61869 \\ \text { Std Err Dif } & 0.04567 & \text { DF } & 20.43592 \\ \text { Upper CL Dif } & 0.06688 & \text { Prob }>|\mathrm{t}| & 0.5430 \\ \text { Lower CL Dif } & -0.12340 & \text { Prob }>\mathrm{t} & 0.7285 \\ \text { Confidence } & 0.95 & \text { Prob }<\mathrm{t} & 0.2715\end{array}$

Tests that the Variances are Equal

Level Count Std Dev MeanAbsDif to Mean MeanAbsDif to Median

$\begin{array}{lllll}\text { Coliwasa } & 16 & 0.1661538 & 0.1143728 & 0.1143728\end{array}$

$\begin{array}{lllll}\text { Hydragard - HF } & 10 & 0.0600415 & 0.0430580 & 0.0387522\end{array}$

Test F Ratio DFNum DFDen p-Value

$\begin{array}{lrrrr}\text { O'Brien[.5] } & 1.4706 & 1 & 24 & 0.2370\end{array}$

Brown-Forsythe $\begin{array}{llll}3.7109 & 1 & 24 & 0.0660\end{array}$

$\begin{array}{lllll}\text { Levene } & 3.4343 & 1 & 24 & 0.0762\end{array}$

$\begin{array}{lrrrr}\text { Bartlett } & 8.4679 & 1 & \text {. } & 0.0036 \\ \text { F Test 2-sided } & 7.6580 & 15 & 9 & 0.0041\end{array}$

Welch Anova testing Means Equal, allowing Std Devs Not Equal

F Ratio DFNum DFDen Prob $>$ F

$\begin{array}{llll}0.3828 & 1 & 20.436 & 0.5430\end{array}$

t Test

0.6187 


\section{Exhibit A2. Statistical Comparisons for Mid-Rheology (Phase 1) Testing}

Oneway Analysis of Measurement By Sampler Mechanism Type of Material=SME Simulant, Analytical Block=2, Analyte $=$ MgO (wt \%), Target Value $=1.014$

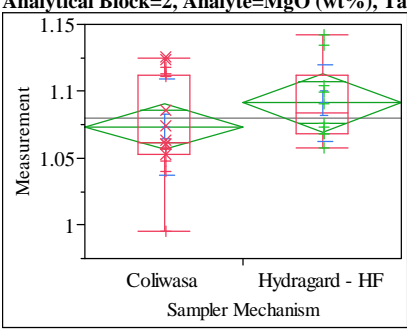

Oneway Anova

Summary of Fit

Adj Rsquare $\quad 0.068479$

Root Mean Square Error $\quad 0.033526$

1.080446

$\begin{array}{lr}\text { Mean of Response } & 1.080446 \\ \text { Observations (or Sum Wgts) }\end{array}$

Test

Hydragard - HF-Coliwasa

Assuming equal variances

Difference $\quad 0.01795$ t Ratio 1.328271

Std Err Dif $\quad 0.01351$ DF 24

Upper CL Dif 0.04584 Prob $>|t| \quad 0.1966$

Lower CL Dif -0.00994 Prob $>t \quad 0.0983$

$\begin{array}{lrl}\text { Confidence } & 0.95 \text { Prob }<\mathrm{t} & 0.9017\end{array}$

Analysis of Variance

$\begin{array}{lrrrrr}\text { Source } & \text { DF } & \text { Sum of Squares } & \text { Mean Square } & \text { F Ratio } & \text { Prob }>\text { F } \\ \text { Sampler Mechanism } & 1 & 0.00198303 & 0.001983 & 1.7643 & 0.1966\end{array}$

$\begin{array}{llll}\text { Error } & 24 & 0.02697531 & 0.001124\end{array}$

250.02895834

Means for Oneway Anova

Level Number Mean Std Error Lower 95\% Upper 95\%

$\begin{array}{lrrrrr}\text { Coliwasa } & 16 & 1.07354 & 0.00838 & 1.0562 & 1.0908\end{array}$

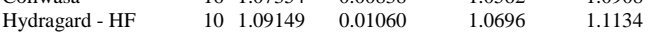

Std Error uses a pooled estimate of error variance

Means and Std Deviations

Level Number Mean Std Dev Std Err Mean Lower 95\% Upper 95\%

161.073540 .036052

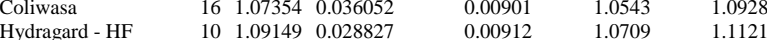

t Test

Hydragard - HF-Coliwasa

Assuming unequal variances

$\begin{array}{lrlr}\text { Difference } & 0.01795 & \mathrm{t} \text { Ratio } & 1.400324 \\ \text { Std Err Dif } & 0.01282 & \text { DF } & 22.37038 \\ \text { Upper CL Dif } & 0.04451 & \text { Prob }>|\mathrm{t}| & 0.1751 \\ \text { Lower CL Dif } & -0.00861 & \text { Prob }>\mathrm{t} & 0.0876 \\ \text { Confidence } & 0.95 & \text { Prob }<\mathrm{t} & 0.9124\end{array}$

Tests that the Variances are Equal

Level Count Std Dev MeanAbsDif to Mean MeanAbsDif to Median

Coliwasa $\quad 16 \quad 0.0360523$

$\begin{array}{lllll}\text { Hydragard - HF } & 10 & 0.0288267 & 0.0229509 & 0.0228845\end{array}$

Test F Ratio DFNum DFDen p-Value

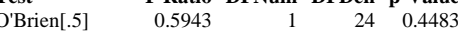

$\begin{array}{lllll}\text { Brown-Forsythe } & 0.2577 & 1 & 24 & 0.6163\end{array}$

$\begin{array}{lllll}\text { Levene } & 0.6458 & 1 & 24 & 0.4295\end{array}$

$\begin{array}{lrrrr}\text { Bartlett } & 0.5151 & 1 & \text {. } & 0.4730\end{array}$

Welch Anova testing Means Equal, allowing Std Devs Not Equal

F Ratio DFNum DFDen Prob $>$ F

$\begin{array}{lrrr}1.9609 & 1 & 22.37 & 0.1751\end{array}$

t Test

1.4003
Oneway Analysis of Measurement By Sampler Mechanism Type of Material=SME Simulant, Analytical Block $=2$, Analyte $=\mathrm{MnO}(\mathrm{wt} \%)$, Target Value $=1.779$

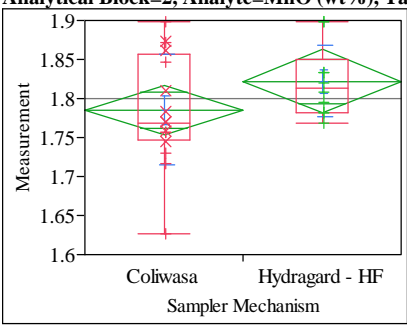

Oneway Anova

Summary of Fit

Adj Rsquare

0.081423

Reot 0.043149

1.799238
Observations (or Sum Wgts)

t Test

Hydragard - HF-Coliwasa

Assuming equal variances

Difference $\quad 0.03680$ t Ratio 1.458555

Std Err Dif $\quad 0.02523$ DF 24

Upper CL Dif 0.08887 Prob $>|t| \quad 0.1577$

$\begin{array}{llll}\text { Lower CL Dif }-0.01527 & \text { Prob }>t & 0.0788\end{array}$

$\begin{array}{lll}\text { Confidence } & 0.95 \text { Prob }<\mathrm{t} \quad 0.9212\end{array}$

Analysis of Variance

$\begin{array}{lrrrrr}\text { Source } & \text { DF } & \text { Sum of Squares } & \text { Mean Square } & \text { F Ratio } & \text { Prob }>\text { F } \\ \text { Sampler Mechanism } & 1 & 0.00833342 & 0.008333 & 2.1274 & 0.1577\end{array}$

$\begin{array}{llll}\text { Error } & 24 & 0.09401326 & 0.003917\end{array}$

$\begin{array}{lll}\text { C. Total } & 25 & 0.10234669\end{array}$

Means for Oneway Anova

Level Number Mean Std Error Lower 95\% Upper 95\%

$\begin{array}{lrrrrr}\text { Coliwasa } & 16 & 1.78508 & 0.01565 & 1.7528 & 1.8174 \\ \text { Hydragard - HF } & 10 & 1.82188 & 0.01979 & 1.7810 & 1.8627\end{array}$

Std Error uses a pooled estimate of error variance

Means and Std Deviations

Level Number Mean Std Dev Std Err Mean Lower 95\% Upper 95\%

$\begin{array}{lrrrrrr}\text { Coliwasa } & 16 & 1.78508 & 0.070800 & 0.01770 & 1.7474 & 1.8228\end{array}$

$\begin{array}{lllllll}\text { Hydragard - HF } & 10 & 1.82188 & 0.045732 & 0.01446 & 1.7892 & 1.8546\end{array}$

T Test

Hydragard - HF-Coliwasa

Assuming unequal variances

$\begin{array}{lrlr}\text { Difference } & 0.03680 & \mathrm{t} \text { Ratio } & 1.609987 \\ \text { Std Err Dif } & 0.02286 & \text { DF } & 23.93453 \\ \text { Upper CL Dif } & 0.08398 & \text { Prob }>|t| & 0.1205 \\ \text { Lower CL Dif } & -0.01038 & \text { Prob }>\mathrm{t} & 0.0603 \\ \text { Confidence } & 0.95 & \text { Prob }<\mathrm{t} & 0.9397\end{array}$

Tests that the Variances are Equal

Level Count Std Dev MeanAbsDif to Mean MeanAbsDif to Median

$\begin{array}{lllll}\text { Coliwasa } & 16 & 0.0708005 & 0.0556830 & 0.0532620\end{array}$

$\begin{array}{lllll}\text { Hydragard - HF } & 10 & 0.0457319 & 0.0351206 & 0.0348624\end{array}$

Test F Ratio DFNum DFDen p-Value

$\begin{array}{lrrrr}\text { O'Brien[.5] } & 1.5114 & 1 & 24 & 0.2308\end{array}$

Brown-Forsythe $\quad \begin{array}{llll}1.2125 & 1 & 24 & 0.2818\end{array}$

$\begin{array}{lllll}\text { Levene } & 1.9472 & 1 & 24 & 0.1757\end{array}$

$\begin{array}{lrrrr}\text { Bartlett } & 1.8644 & 1 & \text {. } & 0.1721 \\ \text { F Test 2-sided } & 2.3968 & 15 & 9 & 0.1878\end{array}$

Welch Anova testing Means Equal, allowing Std Devs Not Equal

F Ratio DFNum DFDen Prob $>$ F

$\begin{array}{rrrr}2.5921 & 1 & 23.935 & 0.1205\end{array}$

t Test

1.6100 


\section{Exhibit A2. Statistical Comparisons for Mid-Rheology (Phase 1) Testing}

Oneway Analysis of Measurement By Sampler Mechanism Type of Material=SME Simulant, Analytical Block=2, Analyte=Na2O (wt\%), Target Value=11.659

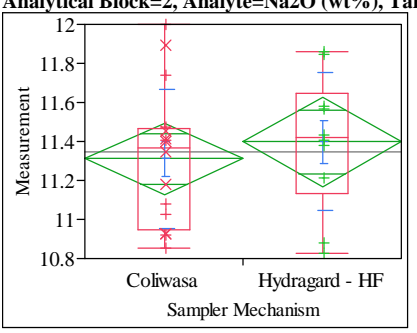

Oneway Anova

Summary of Fit

Adj Rsquare $\quad 0.015694$

Root Mean Square Error $\quad 0.353399$

$\begin{array}{lr}\text { Mean of Response } & 11.34446 \\ \text { Observations (or Sum Wgts) } & 26\end{array}$

Test

Hydragard - HF-Coliwasa

Assuming equal variances

Difference $\quad 0.08813$ t Ratio 0.6186

Std Err Dif $\quad 0.14246$ DF 24

Upper CL Dif 0.38215 Prob $>|t| 0.5420$

Lower CL Dif -0.20590 Prob $>t \quad 0.2710$

$\begin{array}{lrl} & & \\ \text { Confidence } & 0.95 \text { Prob }<\mathrm{t} & 0.7290\end{array}$

Analysis of Variance

$\begin{array}{lrrrrr}\text { Source } & \text { DF } & \text { Sum of Squares } & \text { Mean Square } & \text { F Ratio } & \text { Prob > F } \\ \text { Sampler Mechanism } & 1 & 0.0477914 & 0.047791 & 0.3827 & 0.5420 \\ \text { Error } & 24 & 2.9973744 & 0.124891 & & \end{array}$

$\begin{array}{lll}\text { C. Total } & 25 & 3.0451658\end{array}$

Means for Oneway Anova

Level Number Mean Std Error Lower 95\% Upper 95\%

$\begin{array}{lrrrrr}\text { Coliwasa } & 16 & 11.3106 & 0.08835 & 11.128 & 11.493\end{array}$

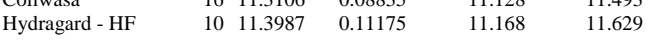

Std Error uses a pooled estimate of error variance

Means and Std Deviations

Level Number Mean Std Dev Std Err Mean Lower 95\% Upper 95\%

$16 \quad 11.31060 .354346$

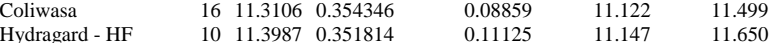

t Test

Hydragard - HF-Coliwasa

Assuming unequal variances

$\begin{array}{lrlr}\text { Difference } & 0.08813 \text { t Ratio } & 0.619667 \\ \text { Std Err Dif } & 0.14221 & \text { DF } & 19.36073 \\ \text { Upper CL Dif } & 0.38541 & \text { Prob }>|t| & 0.5427 \\ \text { Lower CL Dif } & -0.20916 & \text { Prob }>\text { t } & 0.2714 \\ \text { Confidence } & 0.95 & \text { Prob }<\mathrm{t} & 0.7286\end{array}$

Tests that the Variances are Equal

Level Count Std Dev MeanAbsDif to Mean MeanAbsDif to Median

Coliwasa $\quad 16 \quad 0.3543461$

$\begin{array}{lllll}\text { Hydragard - HF } & 10 & 0.3518139 & 0.2855022 & 0.2822375 \\ & & & \end{array}$

Test F Ratio DFNum DFDen p-Value

$\begin{array}{lrrrr}\text { O'Brien[.5] } & 0.0009 & 1 & 24 & 0.9767\end{array}$

$\begin{array}{lllll}\text { Brown-Forsythe } & 0.0735 & 1 & 24 & 0.7886\end{array}$

$\begin{array}{lllll}\text { Levene } & 0.0952 & 1 & 24 & 0.7604\end{array}$

$\begin{array}{llrrr}\text { Bartlett } & 0.0006 & 1 & \text {. } & 0.9812\end{array}$

Welch Anova testing Means Equal, allowing Std Devs Not Equal

F Ratio DFNum DFDen Prob > F

$\begin{array}{llll}0.3840 & 1 & 19.361 & 0.5427\end{array}$

t Test

0.6197
Oneway Analysis of Measurement By Sampler Mechanism Type of Material=SME Simulant, Analytical Block $=2$, Analyte $=\mathrm{NiO}(\mathrm{wt} \%)$, Target Value $=0.41$

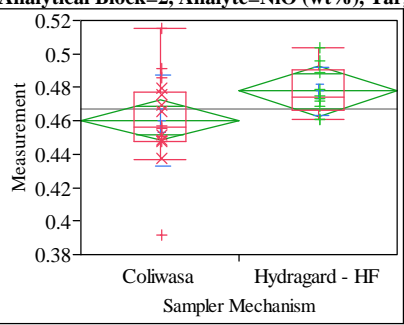

Oneway Anova

Summary of Fit

Adj Rsquare

0.126022

\begin{tabular}{ll}
0.089606 \\
\hline
\end{tabular}

0.46691

0.46691
26

Test

Hydragard - HF-Coliwasa

Assuming equal variances

Difference $\quad 0.01753$ t Ratio 1.860277

Std Err Dif $\quad 0.00942$ DF 24

$\begin{array}{lrl}\text { Upper CL Dif } 0.03698 & \text { Prob }>|t| & 0.0751\end{array}$

Lower CL Dif -0.00192 Prob $>\mathrm{t} \quad 0.0376$

$\begin{array}{lrl}\text { Confidence } & 0.95 \text { Prob }<\mathrm{t} & 0.9624\end{array}$

Analysis of Variance

$\begin{array}{lrrrrr}\text { Source } & \text { DF } & \text { Sum of Squares } & \text { Mean Square } & \text { F Ratio } & \text { Prob }>\text { F }\end{array}$

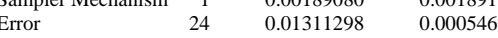

$\begin{array}{lll}\text { C. Total } & 25 & 0.01500378\end{array}$

Means for Oneway Anova

Level Number Mean Std Error Lower 95\% Upper 95\%

$\begin{array}{lrlrrr}\text { Coliwasa } & 16 & 0.460168 & 0.00584 & 0.44811 & 0.47223\end{array}$

$\begin{array}{llllll}\text { Hydragard - HF } & 10 & 0.477697 & 0.00739 & 0.46244 & 0.49295\end{array}$

Std Error uses a pooled estimate of error variance

Means and Std Deviations

Level Number Mean Std Dev Std Err Mean Lower 95\% Upper 95\%

$\begin{array}{lrrrrrr}\text { Coliwasa } & 16 & 0.460168 & 0.027363 & 0.00684 & 0.44559 & 0.47475\end{array}$

$\begin{array}{lllllll}\text { Hydragard - HF } & 10 & 0.477697 & 0.014462 & 0.00457 & 0.46735 & 0.48804\end{array}$

Test

Hydragard - HF-Coliwasa

Assuming unequal variances

$\begin{array}{lrlr}\text { Difference } & 0.017529 & \text { t Ratio } & 2.130238 \\ \text { Std Err Dif } & 0.008229 & \text { DF } & 23.56049 \\ \text { Upper CL Dif } & 0.034528 & \text { Prob }>|t| & 0.0438 \\ \text { Lower CL Dif } & 0.000529 & \text { Prob }>t & 0.0219 \\ \text { Confidence } & 0.95 & \text { Prob }<\text { t } & 0.9781\end{array}$

Tests that the Variances are Equal

Level Count Std Dev MeanAbsDif to Mean MeanAbsDif to Median

$\begin{array}{lllll}\text { Coliwasa } & 16 & 0.0273627 & 0.0196641 & 0.0192466\end{array}$

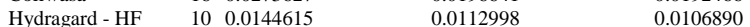

Test F Ratio DFNum DFDen p-Value

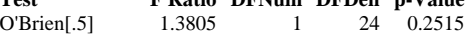

$\begin{array}{lllll}\text { Brown-Forsythe } & 1.6814 & 1 & 24 & 0.2071\end{array}$

$\begin{array}{lllll}\text { Levene } & 1.8290 & 1 & 24 & 0.1888\end{array}$

$\begin{array}{lrrrr}\text { Bartlett } & 3.7470 & 1 & \text {. } & 0.0529\end{array}$

Welch Anova testing Means Equal, allowing Std Devs Not Equal

F Ratio DFNum DFDen Prob $>$ F

$\begin{array}{llll}4.5379 & 1 & 23.56 & 0.0438\end{array}$

t Test

2.1302 


\section{Exhibit A2. Statistical Comparisons for Mid-Rheology (Phase 1) Testing}

Oneway Analysis of Measurement By Sampler Mechanism Type of Material=SME Simulant, Analytical Block=2, Analyte $=\mathrm{SiO} 2$ (wt \%), Target Value $=\mathbf{5 0 . 9 8 5}$

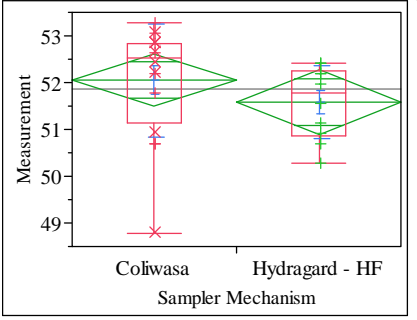

Oneway Anova

Summary of Fit

Adj Rsquare

Root Mean Square Error $\quad 1.068167$

51.8698

t Test

Hydragard - HF-Coliwasa

Assuming equal variances

Difference $\quad-0.4733$ t Ratio $\quad-1.09923$

Std Err Dif $\quad 0.4306$ DF 24

Upper CL Dif 0.4154 Prob $>|t| \quad 0.2826$

Lower CL Dif -1.3620 Prob $>t \quad 0.8587$

$\begin{array}{lrl}\text { Confidence } & 0.95 \text { Prob }<\mathrm{t} \quad 0.1413\end{array}$

Analysis of Variance

$\begin{array}{lrrrrr}\text { Source } & \text { DF } & \text { Sum of Squares } & \text { Mean Square } & \text { F Ratio } & \text { Prob }>\text { F } \\ \text { Sampler Mechanism } & 1 & 1.378658 & 1.37866 & 1.2083 & 0.2826\end{array}$

$\begin{array}{llll}\text { Error } & 24 & 27.383541 & 1.14098\end{array}$

28.762199

Means for Oneway Anova

Level Number Mean Std Error Lower 95\% Upper 95\%

$\begin{array}{lrrrrr}\text { Coliwasa } & 16 & 52.0518 & 0.26704 & 51.501 & 52.603\end{array}$

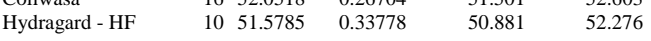

Std Error uses a pooled estimate of error variance

Means and Std Deviations

Level Number Mean Std Dev Std Err Mean Lower 95\% Upper 95\%

Coliwasa $\quad 16 \quad 52.0518 \quad 1.20946 \quad 0.30237 \quad 51.407$

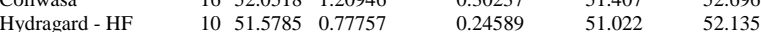

Test

Hydragard - HF-Coliwasa

Assuming unequal variances

$\begin{array}{lrlr}\text { Difference } & -0.4733 & \text { t Ratio } & -1.21449 \\ \text { Std Err Dif } & 0.3897 & \text { DF } & 23.94561 \\ \text { Upper CL Dif } & 0.3311 & \text { Prob }>|t| & 0.2364 \\ \text { Lower CL Dif } & -1.2778 & \text { Prob }>\text { t } & 0.8818 \\ \text { Confidence } & 0.95 & \text { Prob }<\mathrm{t} & 0.1182\end{array}$

Tests that the Variances are Equal

Level Count Std Dev MeanAbsDif to Mean MeanAbsDif to Median

Coliwasa $\quad 16 \quad 1.209462$

$\begin{array}{lllll}\text { Hydragard - HF } & 10 & 0.777573 & 0.9242445 & 0.8423494 \\ & & & 0.6631830 & 0.6631830\end{array}$

Test F Ratio DFNum DFDen p-Value

$\begin{array}{lrrrr}\text { O'Brien[.5] } & 0.8793 & 1 & 24 & 0.3577\end{array}$

$\begin{array}{lllll}\text { Brown-Forsythe } & 0.3057 & 1 & 24 & 0.585\end{array}$

$\begin{array}{lllll}\text { Levene } & 1.0803 & 1 & 24 & 0.3090\end{array}$

$\begin{array}{lrrrr}\text { Bartlett } & 1.9022 & 1 & \text {. } & 0.1678\end{array}$

Welch Anova testing Means Equal, allowing Std Devs Not Equal

F Ratio DFNum DFDen Prob $>$ F

$\begin{array}{rrrr}1.4750 & 1 & 23.946 & 0.2364\end{array}$

$\mathbf{t}$ Test
1.2145
Oneway Analysis of Measurement By Sampler Mechanism Type of Material=SME Simulant, Analytical Block $=2$, Analyte $=$ Sum of Oxides (wt \%), Target Value $=99.553$

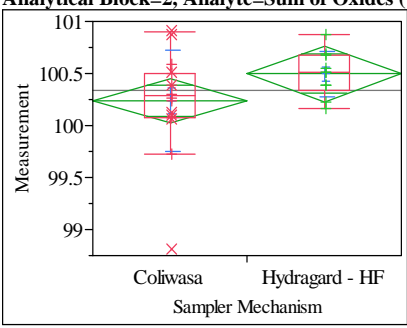

\section{Oneway Anova}

Summary of Fit

Adj Rsquare

0.407634

100.3357

t Test

Hydragard - HF-Coliwasa

Assuming equal variances

Difference $\quad 0.26041$ t Ratio 1.584769

Std Err Dif $\quad 0.16432$ DF 24

Upper CL Dif 0.59956 Prob $>|t| \quad 0.1261$

$\begin{array}{rrr}\text { Lower CL Dif }-0.07873 \text { Prob }>t & 0.0631\end{array}$

$\begin{array}{lrl}\text { Confidence } & 0.95 \text { Prob }<\mathrm{t} & 0.9369\end{array}$

Analysis of Variance

Source DF Sum of Squares Mean Square F Ratio Prob > F

Sampler Mechanism $\quad 1 \quad 0.4173240$

$24 \quad 3.9879754$

$0.417324 \quad 2.5115 \quad 0.1261$

C. Total

Means for Oneway Anova

Level Number Mean Std Error Lower 95\% Upper 95\%

$\begin{array}{lrrrrr}\text { Coliwasa } & 16 & 100.236 & 0.10191 & 100.03 & 100.45\end{array}$

$\begin{array}{llllll}\text { Hydragard - HF } & 10 & 100.496 & 0.12891 & 100.23 & 100.76\end{array}$

Std Error uses a pooled estimate of error variance

Means and Std Deviations

Level Number Mean Std Dev Std Err Mean Lower 95\% Upper 95\%

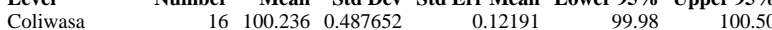

$\begin{array}{lllllrr}\text { Hydragard - HF } & 10 & 100.496 & 0.216257 & 0.06839 & 100.34 & 100.65\end{array}$

T Test

Hydragard - HF-Coliwasa

Assuming unequal variances

$\begin{array}{lrlr}\text { Difference } & 0.26041 & \mathrm{t} \text { Ratio } & 1.862973 \\ \text { Std Err Dif } & 0.13978 & \text { DF } & 22.25281 \\ \text { Upper CL Dif } & 0.55012 & \text { Prob }>|\mathrm{t}| & 0.0757 \\ \text { Lower CL Dif } & -0.02929 & \text { Prob }>\mathrm{t} & 0.0379 \\ \text { Confidence } & 0.95 & \text { Prob }<\mathrm{t} & 0.9621\end{array}$

Tests that the Variances are Equal

Level Count Std Dev MeanAbsDif to Mean MeanAbsDif to Median

$\begin{array}{lllll}\text { Coliwasa } & 16 & 0.4876524 & 0.3196610 & 0.3155839\end{array}$

$\begin{array}{lllll}\text { Hydragard - HF } & 10 & 0.2162567 & 0.1651795 & 0.1628642\end{array}$

Test F Ratio DFNum DFDen p-Value

$\begin{array}{lrrrr}\text { O'Brien[.5] } & 1.1118 & 1 & 24 & 0.3022\end{array}$

$\begin{array}{lllll}\text { Brown-Forsythe } & 1.5904 & 1 & 24 & 0.2194\end{array}$

$\begin{array}{lllll}\text { Levene } & 1.6944 & 1 & 24 & 0.2054\end{array}$

$\begin{array}{lrrrr}\text { Bartlett } & 5.7714 & 1 & \text {. } & 0.0163 \\ \text { F Test 2-sided } & 5.0849 & 15 & 9 & 0.0184\end{array}$

Welch Anova testing Means Equal, allowing Std Devs Not Equal

F Ratio DFNum DFDen Prob $>$ F

$\begin{array}{rrrr}3.4707 & 1 & 22.253 & 0.0757\end{array}$

t Test

1.8630 


\section{Exhibit A2. Statistical Comparisons for Mid-Rheology (Phase 1) Testing}

Oneway Analysis of Measurement By Sampler Mechanism Type of Material=SME Simulant, Analytical Block=2, Analyte $=$ TiO2 (wt \%), Target Value $=0.0711$

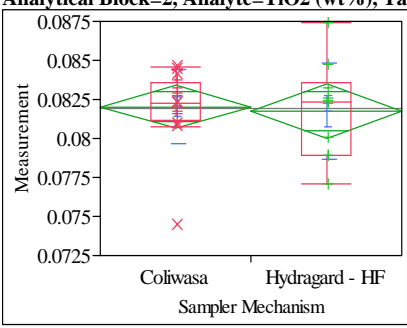

Oneway Anova

Summary of Fit

Adj Rsquare

Error $\quad 0.002661$

Observations (or Sum Wgts) $\quad 26$

t Test

Hydragard - HF-Coliwasa

Assuming equal variances

Difference $\quad-0.00029$ t Ratio $\quad-0.26631$

Std Err Dif $\quad 0.00107$ DF $\quad 24$

Upper CL Dif 0.00193 Prob $>|t| r \mid r .7923$

Lower CL Dif -0.00250 Prob $>t \quad 0.6039$

$\begin{array}{lrl}\text { Confidence } & 0.95 \text { Prob }<\mathrm{t} & 0.3961\end{array}$

Analysis of Variance

$\begin{array}{lrrrrr}\text { Source } & \text { DF } & \text { Sum of Squares } & \text { Mean Square } & \text { F Ratio } & \text { Prob }>\text { F } \\ \text { Sampler Mechanism } & 1 & 0.00000050 & 5.0211 \mathrm{e}-7 & 0.0709 & 0.7923\end{array}$

$\begin{array}{llll}\text { Error } & 24 & 0.00016992 & 7.08 \mathrm{e}-6\end{array}$

$\begin{array}{lll}\text { C. Total } & 25 & 0.00017042\end{array}$

$\begin{array}{lrrrrr}\text { Means for Oneway Anova } & & & & \\ \text { Level } & \text { Number } & \text { Mean } & \text { Std Error } & \text { Lower 95\% } & \text { Upper 95\% } \\ \text { Coliwasa } & 16 & 0.082034 & 0.00067 & 0.08066 & 0.08341\end{array}$

$\begin{array}{llllll}\text { Hydragard - HF } & 10 & 0.081749 & 0.00084 & 0.08001 & 0.08349\end{array}$

Std Error uses a pooled estimate of error variance

Means and Std Deviations

Level Number Mean Std Dev Std Err Mean Lower 95\% Upper 95\%

Coliwasa 160.0820340 .002372

$\begin{array}{lllllll}\text { Coliwasa } & 16 & 0.082034 & 0.002372 & 0.00059 & 0.08077 & 0.08330 \\ \text { Hydragard - HF } & 10 & 0.081749 & 0.003082 & 0.00097 & 0.07954 & 0.08395\end{array}$

t Test

Hydragard - HF-Coliwasa

Assuming unequal variances

Difference $\quad-0.00029$ t Ratio $\quad-0.25036$

Std Err Dif $\quad 0.00114$ DF $\quad 15.61492$

Upper CL Dif 0.00214 Prob $>|t| r 0.8056$

Lower CL Dif -0.00271 Prob $>t \quad 0.5972$

$\begin{array}{lrl} & \\ \text { Confidence } & 0.95 \text { Prob }<\mathrm{t} & 0.4028\end{array}$

Tests that the Variances are Equal

Level Count Std Dev MeanAbsDif to Mean MeanAbsDif to Median

Coliwasa $16 \quad 0.0023724$

Hydragard - HF $\quad 100.0030821$

Test F Ratio DFNum DFDen p-Value

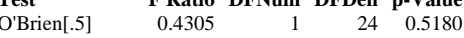

Brown-Forsythe $\quad \begin{array}{llll}1.2920 & 1 & 24 & 0.2669\end{array}$

$\begin{array}{lllll}\text { Levene } & 1.6937 & 1 & 24 & 0.2055\end{array}$

$\begin{array}{lllll}\text { Bartlett } & 0.7617 & 1 & 0.3828\end{array}$

Welch Anova testing Means Equal, allowing Std Devs Not Equal

F Ratio DFNum DFDen Prob $>$ F

$\begin{array}{rrrr}0.0627 & 1 & 15.615 & 0.8056\end{array}$

t Test
0.2504
Oneway Analysis of Measurement By Sampler Mechanism Type of Material=SME Simulant, Analytical Block $=2$, Analyte $=\mathrm{ZnO}(\mathrm{wt} \%)$, Target Value $=0.0958$

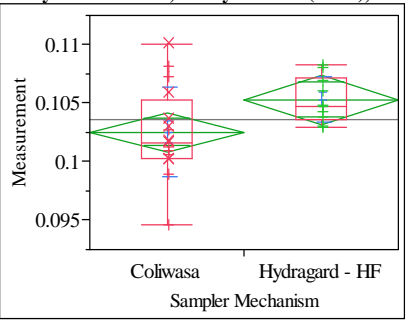

Oneway Anova

Summary of Fit

Adj Rsquare

0.003245

0.103601

t Test

Hydragard - HF-Coliwasa

Assuming equal variances

Difference $\quad 0.002757$ t Ratio 2.10753

Std Err Dif $\quad 0.001308$ DF 24

Upper CL Dif 0.005457 Prob $>|t| \quad 0.0457$

$\begin{array}{lll}\text { Lower CL Dif } 0.000057 \text { Prob }>t & 0.0229\end{array}$

$\begin{array}{lrl}\text { Confidence } & 0.95 \text { Prob }<\mathrm{t} & 0.9771\end{array}$

Analysis of Variance

$\begin{array}{lrrrrr}\text { Source } & \text { DF } & \text { Sum of Squares } & \text { Mean Square } & \text { F Ratio } & \text { Prob > F } \\ \text { Sampler Mechanism } & 1 & 0.00004678 & 0.000047 & 4.4417 & 0.0457 \\ \text { Error } & 24 & 0.00025279 & 0.000011 & & \\ \text { C. Total } & 25 & 0.00029957 & & & \end{array}$

Means for Oneway Anova

Level Number Mean Std Error Lower 95\% Upper 95\%

$\begin{array}{lrrrrr}\text { Coliwasa } & 16 & 0.102540 & 0.00081 & 0.10087 & 0.10421\end{array}$

$\begin{array}{llllll}\text { Hydragard - HF } & 10 & 0.105298 & 0.00103 & 0.10318 & 0.10742\end{array}$

Std Error uses a pooled estimate of error variance

Means and Std Deviations

Level Number Mean Std Dev Std Err Mean Lower 95\% Upper 95\%

$\begin{array}{lrrrrrr}\text { Coliwasa } & 16 & 0.102540 & 0.003819 & 0.00095 & 0.10051 & 0.10458\end{array}$

$\begin{array}{lllllll}\text { Hydragard - HF } & 10 & 0.105298 & 0.001946 & 0.00062 & 0.10391 & 0.10669\end{array}$

Test

Hydragard - HF-Coliwasa

Assuming unequal variances

$\begin{array}{lrlr}\text { Difference } & 0.002757 & \text { t Ratio } & 2.427702 \\ \text { Std Err Dif } & 0.001136 & \text { DF } & 23.33856 \\ \text { Upper CL Dif } & 0.005105 & \text { Prob }>|t| & 0.0233 \\ \text { Lower CL Dif } & 0.000410 & \text { Prob }>t & 0.0117 \\ \text { Confidence } & 0.95 & \text { Prob }<t & 0.9883\end{array}$

Tests that the Variances are Equal

Level Count Std Dev MeanAbsDif to Mean MeanAbsDif to Median

$\begin{array}{lllll}\text { Coliwasa } & 16 & 0.0038185 & 0.0028436 & 0.0027386\end{array}$

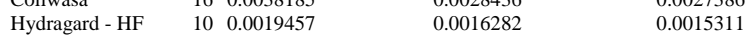

Test F Ratio DFNum DFDen p-Value

$\begin{array}{lrrrr}\text { O'Brien[.5] } & 2.3015 & 1 & 24 & 0.1423\end{array}$

$\begin{array}{lllll}\text { Brown-Forsythe } & 1.7174 & 1 & 24 & 0.2024\end{array}$

$\begin{array}{lllll}\text { Levene } & 2.2514 & 1 & 24 & 0.1465\end{array}$

$\begin{array}{lrrrr}\text { Bartlett } & 4.1428 & 1 & \text {. } & 0.0418 \\ \text { F Test 2-sided } & 3.8515 & 15 & 9 & 0.0467\end{array}$

Welch Anova testing Means Equal, allowing Std Devs Not Equal

F Ratio DFNum DFDen Prob $>$ F

$\begin{array}{rrrr}5.8937 & 1 & 23.339 & 0.0233\end{array}$

t Test
2.4277 


\section{Exhibit A2. Statistical Comparisons for Mid-Rheology (Phase 1) Testing}

Oneway Analysis of Measurement By Sampler Mechanism Type of Material=SME Simulant, Analytical Block $=2$, Analyte $=\mathrm{ZrO} 2$ (wt $\%)$, Target Value $=0.3547$

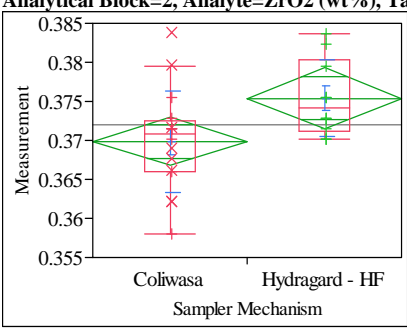

Oneway Anova

Summary of Fit

Adj Rsquare

0.180331

Root Mean Squarc

0.146178

Mean of Response

0.005961

Observations (or Sum Wgts) $\quad 26$

t Test

Hydragard - HF-Coliwasa

Assuming equal variances

Difference $\quad 0.005521$ t Ratio 2.297848

Std Err Dif $\quad 0.002403$ DF 24

Upper CL Dif 0.010481 Prob $>|t| \quad 0.0306$

Lower CL Dif 0.000562 Prob $>t \quad 0.0153$

$\begin{array}{lrl}\text { Confidence } & 0.95 \text { Prob }<\mathrm{t} & 0.9847\end{array}$

Analysis of Variance

$\begin{array}{lrrrrr}\text { Source } & \text { DF } & \text { Sum of Squares } & \text { Mean Square } & \text { F Ratio } & \text { Prob }>\text { F } \\ \text { Sampler Mechanism } & 1 & 0.00018760 & 0.000188 & 5.2801 & 0.0306\end{array}$

$\begin{array}{llll}\text { Error } & 24 & 0.00085273 & 0.000036\end{array}$

$\begin{array}{lll}\text { C. Total } & 25 & 0.00104034\end{array}$

Means for Oneway Anova

Level Number Mean Std Error Lower 95\% Upper 95\%

$\begin{array}{lrrrrr}\text { Coliwasa } & 16 & 0.369866 & 0.00149 & 0.36679 & 0.37294\end{array}$

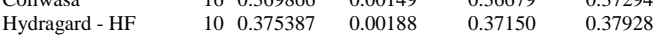

Std Error uses a pooled estimate of error variance

Means and Std Deviations

Level Number Mean Std Dev Std Err Mean Lower 95\% Upper 95\%

$\begin{array}{llllllll}\text { Coliwasa } & 16 & 0.369866 & 0.006510 & 0.00163 & 0.36640 & 0.37334\end{array}$

$\begin{array}{lllllll}\text { Hydragard - HF } & 10 & 0.375387 & 0.004910 & 0.00155 & 0.37188 & 0.37890\end{array}$

Test

Hydragard - HF-Coliwasa

Assuming unequal variances

Difference $\quad 0.005521$ t Ratio $\quad 2.454641$

Std Err Dif $\quad 0.002249$ DF 22.99079

Upper CL Dif 0.010175 Prob $>|t| \quad 0.0221$

Lower CL Dif 0.000868 Prob $>t \quad 0.0110$

$\begin{array}{lrl}\text { Confidence } & 0.95 \text { Prob }<\mathrm{t} \quad 0.9890\end{array}$

Tests that the Variances are Equal

Level Count Std Dev MeanAbsDif to Mean MeanAbsDif to Median

Coliwasa $\quad 16 \quad 0.0065104$

$\begin{array}{lllll}\text { Hydragard - HF } & 10 & 0.0049098 & 0.0039173 & 0.0039173\end{array}$

Test F Ratio DFNum DFDen p-Value

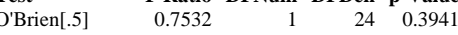

$\begin{array}{lllll}\text { Brown-Forsythe } & 0.3310 & 1 & 24 & 0.5704\end{array}$

$\begin{array}{lllll}\text { Levene } & 0.3915 & 1 & 24 & 0.5374\end{array}$

$\begin{array}{lrrrr}\text { Bartlett } & 0.8086 & 1 & \text {. } & 0.3685 \\ & 1.7583 & 15 & 9 & 0.3953\end{array}$

Welch Anova testing Means Equal, allowing Std Devs Not Equal

F Ratio DFNum DFDen Prob $>$ F

$\begin{array}{rrrr}6.0253 & 1 & 22.991 & 0.0221\end{array}$

t Test
2.4546
Oneway Analysis of Measurement By Sampler Mechanism Type of Material=SME Simulant, Analytical Block $=3$, Analyte $=\mathrm{Al} / \mathrm{B}$, Target Value $=2.552$

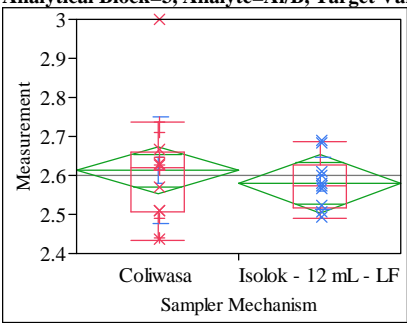

Oneway Anova

Summary of Fit

Adj Rsquare

Root Mean Square Error $\quad 0.115318$

Mean of Response 2.60009

2.60009

Test

Isolok - $12 \mathrm{~mL}$ - LF-Coliwasa

Assuming equal variances

$\begin{array}{lrlr}\text { Difference } & -0.03309 & \mathrm{t} \text { Ratio } & -0.71181 \\ \text { Std Err Dif } & 0.04649 & \text { DF } & 24 \\ \text { Upper CL Dif } & 0.06285 & \text { Prob }>|t| & 0.4834 \\ \text { Lower CL Dif } & -0.12903 & \text { Prob }>\mathrm{t} & 0.7583 \\ \text { Confidence } & 0.95 & \text { Prob }<\mathrm{t} & 0.2417\end{array}$

Analysis of Variance

$\begin{array}{lrrrrr}\text { Source } & \text { DF } & \text { Sum of Squares } & \text { Mean Square } & \text { F Ratio } & \text { Prob }>\text { F } \\ \text { Sampler Mechanism } & 1 & 0.00673796 & 0.006738 & 0.5067 & 0.4834\end{array}$

$\begin{array}{llll}\text { Error } & 24 & 0.31916011 & 0.013298\end{array}$

$\begin{array}{lll}\text { C. Total } & 25 & 0.32589807\end{array}$

Means for Oneway Anova

Level Number Mean Std Error Lower 95\% Upper 95\%

$\begin{array}{lrrrrr}\text { Coliwasa } & 16 & 2.61282 & 0.02883 & 2.5533 & 2.6723 \\ \text { Isolok - 12 mL - LF } & 10 & 2.57973 & 0.03647 & 2.5045 & 2.6550\end{array}$

Std Error uses a pooled estimate of error variance

Means and Std Deviations

Level Number Mean Std Dev Std Err Mean Lower 95\% Upper 95\%

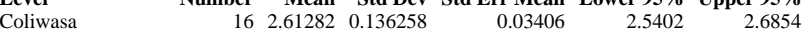

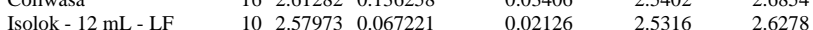

Test

Isolok - $12 \mathrm{~mL}$ - LF-Coliwasa

Assuming unequal variances

$\begin{array}{lrlr}\text { Difference } & -0.03309 & \text { t Ratio } & -0.82409 \\ \text { Std Err Dif } & 0.04015 & \text { DF } & 23.11498 \\ \text { Upper CL Dif } & 0.04995 & \text { Prob }>|t| & 0.4183 \\ \text { Lower CL Dif } & -0.11613 & \text { Prob }>\text { t } & 0.7908 \\ \text { Confidence } & 0.95 & \text { Prob }<\mathrm{t} & 0.2092\end{array}$

Tests that the Variances are Equal

Level Count Std Dev MeanAbsDif to Mean MeanAbsDif to Median

$\begin{array}{lllll}\text { Coliwasa } & 16 & 0.1362576 & 0.0915971 & 0.0911937\end{array}$

Isolok - $12 \mathrm{~mL}$ - LF $\quad 100.0672209$

Test F Ratio DFNum DFDen p-Value

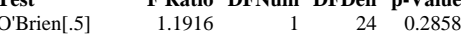

$\begin{array}{lllll} & 1.1916 & 1 & 24 & 0.2858 \\ \text { Brown-Forsythe } & 1.5456 & 1 & 24 & 0.2258\end{array}$

$\begin{array}{lllll}\text { Levene } & 1.5586 & 1 & 24 & 0.2239\end{array}$

$\begin{array}{lrrrr}\text { Bartlett } & 4.5049 & 1 & \text {. } & 0.0338 \\ & 4.1088 & 15 & 9 & 0.0378\end{array}$

Welch Anova testing Means Equal, allowing Std Devs Not Equal

F Ratio DFNum DFDen Prob $>$ F

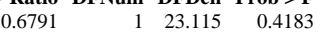

t Test

0.8241 


\section{Exhibit A2. Statistical Comparisons for Mid-Rheology (Phase 1) Testing}

Oneway Analysis of Measurement By Sampler Mechanism Type of Material=SME Simulant, Analytical Block=3, Analyte $=\mathrm{Al} 2 \mathrm{O} 3(\mathrm{wt} \%)$, Target Value $=10.869$

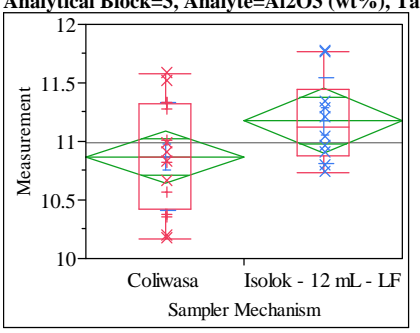

Oneway Anova

Summary of Fit

0.117567

Root Mean Square Error $\quad 0.427596$

Mean of Response

$\begin{array}{lr}\text { Mean of Response } & 10.98672 \\ \text { Observations (or Sum Wgts) } & 26\end{array}$

t Test

Isolok - $12 \mathrm{~mL}$ - LF-Coliwasa

Assuming equal variances

Difference $\quad 0.30822$ t Ratio 1.788162

Std Err Dif $\quad 0.17237$ DF $\quad 24$

Upper CL Dif 0.66398 Prob $>|t| \quad 0.0864$

Lower CL Dif -0.04753 Prob $>t \quad 0.0432$

$\begin{array}{lrl}\text { Confidence } & 0.95 \text { Prob }<\mathrm{t} & 0.9568\end{array}$

Analysis of Variance

$\begin{array}{lrrrrr}\text { Source } & \text { DF } & \text { Sum of Squares } & \text { Mean Square } & \text { F Ratio } & \text { Prob > F } \\ \text { Sampler Mechanism } & 1 & 0.5846305 & 0.584631 & 3.1975 & 0.0864\end{array}$

$\begin{array}{llll}\text { Error } & 24 & 4.3881231 & 0.182838\end{array}$

4.9727536

Means for Oneway Anova

Level Number Mean Std Error Lower 95\% Upper 95\%

$\begin{array}{lrrrrr}\text { Coliwasa } & 16 & 10.8682 & 0.10690 & 10.648 & 11.089\end{array}$

$\begin{array}{llllll}\text { Coliwasa } & 16 & 10.8682 & 0.10690 & 10.648 & 11.089 \\ \text { Isolok - 12 mL - LF } & 10 & 11.1764 & 0.13522 & 10.897 & 11.455\end{array}$

Std Error uses a pooled estimate of error variance

Means and Std Deviations

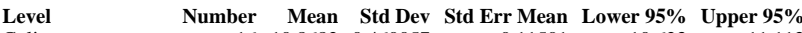

\begin{tabular}{lrrrrrr} 
Coliwasa & Number & Mean & Std Dev & Std Err Mean & Lower 95\% & Upper 95\% \\
\hline & 16 & 10.8682 & 0.460057 & 0.11501 & 10.623 & 11.113
\end{tabular}

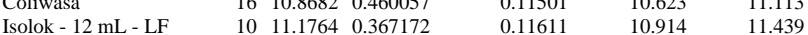

Test

Isolok - $12 \mathrm{~mL}$ - LF-Coliwas

Assuming unequal variances

$\begin{array}{lrlr}\text { Difference } & 0.30822 & \mathrm{t} \text { Ratio } & 1.885959 \\ \text { Std Err Dif } & 0.16343 \text { DF } & 22.39184 \\ \text { Upper CL Dif } & 0.64682 & \text { Prob }>|\mathrm{t}| & 0.0723 \\ \text { Lower CL Dif } & -0.03037 & \text { Prob }>\mathrm{t} & 0.0362 \\ \text { Confidence } & 0.95 & \text { Prob }<\mathrm{t} & 0.9638\end{array}$

Tests that the Variances are Equal

Level Count Std Dev MeanAbsDif to Mean MeanAbsDif to Median

$\begin{array}{lrrrr}\text { Coliwasa } & 16 & 0.4600570 & 0.3743572 & 0.3743572\end{array}$

$\begin{array}{lllll}\text { Coliwasa } & 16 & 0.4600570 & 0.3743572 & 0.3743572 \\ \text { Isolok - } 12 \mathrm{~mL} \text { - LF } & 10 & 0.3671718 & 0.2928725 & 0.2928725\end{array}$

Test F Ratio DFNum DFDen p-Value

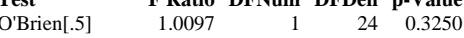

$\begin{array}{lllll}\text { Brown-Forsythe } & 0.7427 & 1 & 24 & 0.3973\end{array}$

$\begin{array}{lllll}\text { Levene } & 0.7613 & 1 & 24 & 0.3916\end{array}$

$\begin{array}{lrrrr}\text { Bartlett } & 0.5234 & 1 & \text {. } & 0.4694\end{array}$

Welch Anova testing Means Equal, allowing Std Devs Not Equal

F Ratio DFNum DFDen Prob $>$ F

$\begin{array}{rrrr}3.5568 & 1 & 22.392 & 0.0723\end{array}$

t Test

1.8860
Oneway Analysis of Measurement By Sampler Mechanism Type of Material=SME Simulant, Analytical Block $=3$, Analyte $=\mathrm{B} 2 \mathrm{O} 3(\mathrm{wt} \%)$, Target Value $=4.259$

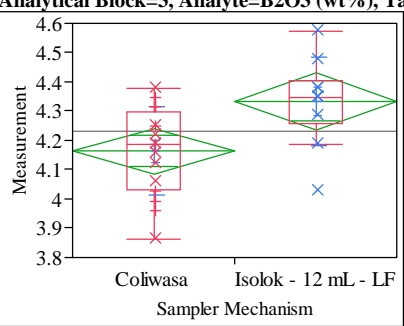

Oneway Anova

Summary of Fit

Adj Rsquare

$\quad 0.149855$

Mean of Response 4.229215

Observations (or Sum Wgts) $\quad 26$

T Test

Isolok - $12 \mathrm{~mL}$ - LF-Coliwasa

Assuming equal variances

Difference $\quad 0.170252$ t Ratio 2.818354

Std Err Dif $\quad 0.060408$ DF 24

Upper CL Dif 0.294929 Prob $>|t| \quad 0.0095$

Lower CL Dif 0.045575 Prob $>t \quad 0.0048$

$\begin{array}{lll}\text { Confidence } & 0.95 \mathrm{Prob}<\mathrm{t} & 0.9952\end{array}$

Analysis of Variance

$\begin{array}{lrrrrr}\text { Source } & \text { DF } & \text { Sum of Squares } & \text { Mean Square } & \text { F Ratio } & \text { Prob }>\text { F } \\ \text { Sampler Mechanism } & 1 & 0.17837425 & 0.178374 & 7.9431 & 0.0095\end{array}$

$\begin{array}{llll}\text { Error } & 24 & 0.53895484 & 0.022456\end{array}$

$\begin{array}{lll}\text { C. Total } & 25 & 0.71732909\end{array}$

Means for Oneway Anova

Level Number Mean Std Error Lower 95\% Upper 95\%

$\begin{array}{lrrrrr}\text { Coliwasa } & 16 & 4.16373 & 0.03746 & 4.0864 & 4.2411\end{array}$

$\begin{array}{llllll}\text { Coliwasa } & 16 & 4.16373 & 0.03746 & 4.0864 & 4.2411 \\ \text { Isolok - 12 mL - LF } & 10 & 4.33399 & 0.04739 & 4.2362 & 4.4318\end{array}$

Std Error uses a pooled estimate of error variance

Means and Std Deviations

Level Number Mean Std Dev Std Err Mean Lower 95\% Upper 95\%

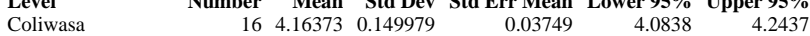

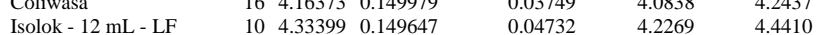

Test

Isolok - $12 \mathrm{~mL}$ - LF-Coliwasa

Assuming unequal variances

Difference $\quad 0.170252$ t Ratio 2.819854

Std Err Dif $\quad 0.060376$ DF $\quad 19.28636$

Upper CL Dif 0.296494 Prob $>|t| 0.0108$

Lower CL Dif 0.044010 Prob $>$ t 0.0054

$\begin{array}{lrl}\text { Confidence } & 0.95 \text { Prob }<\mathrm{t} & 0.9946\end{array}$

Tests that the Variances are Equal

Level Count Std Dev MeanAbsDif to Mean MeanAbsDif to Median

$\begin{array}{lllll}\text { Coliwasa } & 16 & 0.1499790 & 0.1215009 & 0.1187338\end{array}$

$\begin{array}{lllll}\text { Isolok - } 12 \mathrm{~mL} \text { - LF } & 10 & 0.1496474 & 0.1017488 & 0.0965970\end{array}$

Test F Ratio DFNum DFDen p-Value

$\begin{array}{lrrrr}\text { O'Brien[.5] } & 0.0001 & 1 & 24 & 0.9937\end{array}$

$\begin{array}{lllll}\text { Brown-Forsythe } & 0.3152 & 1 & 24 & 0.5797\end{array}$

$\begin{array}{lllll}\text { Levene } & 0.2892 & 1 & 24 & 0.5957\end{array}$

$\begin{array}{lrrrr}\text { Bartlett } & 0.0001 & 1 & \text {. } & 0.9942 \\ \text { F Test 2-sided } & 1.0044 & 15 & 9 & 1.0000\end{array}$

Welch Anova testing Means Equal, allowing Std Devs Not Equal

F Ratio DFNum DFDen Prob $>$ F

$\begin{array}{lrrr}7.9516 & 1 & 19.286 & 0.0108\end{array}$

t Test
2.8199 


\section{Exhibit A2. Statistical Comparisons for Mid-Rheology (Phase 1) Testing}

Oneway Analysis of Measurement By Sampler Mechanism Type of Material=SME Simulant, Analytical Block $=3$, Analyte $=\mathrm{BaO}($ wt $\%)$, Target Value $=\mathbf{0 . 0 9 1 9}$

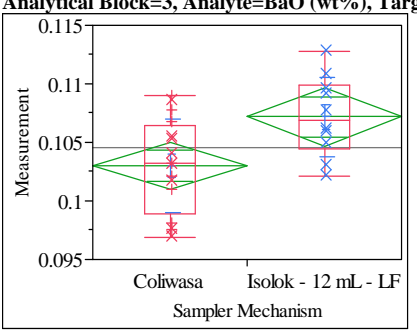

Oneway Anova

Summary of Fit

Adj Rsquare $\quad 0.237292$

Root Mean Square Error $\quad 0.003785$

Mean of Response $\quad 0.104607$

Observations (or Sum Wgts) $\quad 26$

t Test

Isolok - $12 \mathrm{~mL}$ - LF-Coliwasa

Assuming equal variances

Difference $\quad 0.004169$ t Ratio 2.732547

Std Err Dif $\quad 0.001526$ DF 24

$\begin{array}{lrl}\text { Upper CL Dif } & 0.007317 & \text { Prob }>|t| \\ & 0.0116\end{array}$

Lower CL Dif 0.001020 Prob $>t \quad 0.0058$

$\begin{array}{lrl}\text { Confidence } & 0.95 \text { Prob }<\mathrm{t} & 0.9942\end{array}$

Analysis of Variance

$\begin{array}{lrrrrr}\text { Source } & \text { DF } & \text { Sum of Squares } & \text { Mean Square } & \text { F Ratio } & \text { Prob }>\text { F } \\ \text { Sampler Mechanism } & 1 & 0.00010694 & 0.000107 & 7.4668 & 0.0116\end{array}$

$\begin{array}{llll}\text { Error } & 24 & 0.00034374 & 0.000014\end{array}$

$\begin{array}{lll}\text { C. Total } & 25 & 0.00045068\end{array}$

Means for Oneway Anova

$\begin{array}{lrrrrr}\text { Level } & \text { Number } & \text { Mean } & \text { Std Error } & \text { Lower 95\% } & \text { Upper 95\% } \\ \text { Coliwasa } & 16 & 0.103004 & 0.00095 & 0.10105 & 0.10496\end{array}$

$\begin{array}{lrrrrr}\text { Coliwasa } & 16 & 0.103004 & 0.00095 & 0.10105 & 0.10496 \\ \text { Isolok - 12 mL - LF } & 10 & 0.107173 & 0.00120 & 0.10470 & 0.10964\end{array}$

Std Error uses a pooled estimate of error variance

Means and Std Deviations

$\begin{array}{lrrrrrr}\text { Level } & \text { Number } & \text { Mean } & \text { Std Dev } & \text { Std Err Mean } & \text { Lower 95\% } & \text { Upper 95\% } \\ \text { Coliwasa } & 16 & 0.103004 & 0.003997 & 0.00100 & 0.10087 & 0.10513\end{array}$

$\begin{array}{lllllll}\text { Isolok - } 12 \mathrm{~mL} \text { - LF } & 10 & 0.107173 & 0.003401 & 0.00108 & 0.10474 & 0.10961\end{array}$

TTest

Isolok - $12 \mathrm{~mL}$ - LF-Coliwas

Assuming unequal variances

Difference $\quad 0.004169$ t Ratio 2.839755

Std Err Dif $\quad 0.001468$ DF 21.59371

Upper CL Dif 0.007216 Prob $>|t| \quad 0.0096$

Lower CL Dif 0.001121 Prob $>t \quad 0.0048$

$\begin{array}{lrl}\text { Confidence } & 0.95 \text { Prob }<\mathrm{t} & 0.9952\end{array}$

Tests that the Variances are Equal

Level Count Std Dev MeanAbsDif to Mean MeanAbsDif to Median

$\begin{array}{lrrrr}\text { Coliwasa } & 16 & 0.0039972 & 0.0032370 & 0.0032169\end{array}$

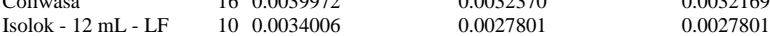

Test F Ratio DFNum DFDen p-Value

$\begin{array}{lrrrr}\text { O'Brien[.5] } & 0.5544 & 1 & 24 & 0.4637\end{array}$

Brown-Forsythe $\quad 0.2745 \quad 1 \quad 24 \quad 0.605$

$\begin{array}{lllll}\text { Levene } & 0.3120 & 1 & 24 & 0.5816\end{array}$

$\begin{array}{lrrrr}\text { Bartlett } & 0.2727 & 1 & \text {. } & 0.6015 \\ & 1.3817 & 15 & 9 & 0.6366\end{array}$

Welch Anova testing Means Equal, allowing Std Devs Not Equal

F Ratio DFNum DFDen Prob $>$ F

$\begin{array}{llll}8.0642 & 1 & 21.594 & 0.0096\end{array}$

t Test

2.8398
Oneway Analysis of Measurement By Sampler Mechanism Type of Material=SME Simulant, Analytical Block $=3$, Analyte $=\mathrm{CaO}(\mathrm{wt} \%)$, Target Value $=1.1462$

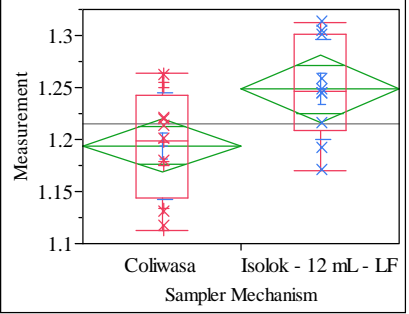

Oneway Anova

Summary of Fi

Adj Rsquare

are Error $\quad 0.049856$

$\begin{array}{lr}\text { Mean of Response } & 1.214936 \\ \text { Observations (or Sum Wgts) } & 26\end{array}$

Test

Isolok - $12 \mathrm{~mL}$ - LF-Coliwasa

Assuming equal variances

Difference $\quad 0.054551$ t Ratio 2.714296

Std Err Dif $\quad 0.020098$ DF 24

Upper CL Dif 0.096031 Prob $>|t| \quad 0.0121$

Lower CL Dif 0.013072 Prob $>t \quad 0.0061$

$\begin{array}{lrl}\text { Confidence } & 0.95 \mathrm{Prob}<\mathrm{t} & 0.9939\end{array}$

Analysis of Variance

Source DF Sum of Squares Mean Square F Ratio Prob > F

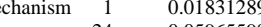

\begin{tabular}{lll}
24 & 0.05965598 \\
\hline
\end{tabular}

0.002486

Means for Oneway Anova

Level $\quad$ Number Mean Std Error Lower 95\% Upper 95\%

$\begin{array}{llllrr}\text { Coliwasa } & 16 & 1.19395 & 0.01246 & 1.1682 & 1.2197\end{array}$

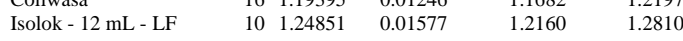

Std Error uses a pooled estimate of error variance

Means and Std Deviations

Level Number Mean Std Dev Std Err Mean Lower 95\% Upper 95\%

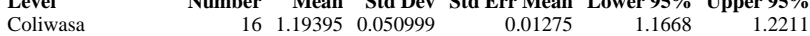

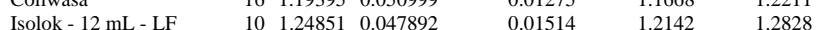

Test

Isolok - $12 \mathrm{~mL}$ - LF-Coliwasa

Assuming unequal variances

$\begin{array}{lrlr}\text { Difference } & 0.054551 & \text { t Ratio } & 2.75554 \\ \text { Std Err Dif } & 0.019797 & \text { DF } & 20.19216 \\ \text { Upper CL Dif } & 0.095822 & \text { Prob }>|t| & 0.0121 \\ \text { Lower CL Dif } & 0.013281 & \text { Prob }>t & 0.0061 \\ \text { Confidence } & 0.95 & \text { Prob }<\mathrm{t} & 0.9939\end{array}$

Tests that the Variances are Equal

Level Count Std Dev MeanAbsDif to Mean MeanAbsDif to Median

$\begin{array}{lllll}\text { Coliwasa } & 16 & 0.0509988 & 0.0416590 & 0.0411890\end{array}$

Isolok - $12 \mathrm{~mL}$ - LF $\quad 10 \quad 0.0478920 \quad 0.0360434 \quad 0.0359594$

Test F Ratio DFNum DFDen p-Value

$\begin{array}{lrrrr}\text { O'Brien[.5] } & 0.0878 & 1 & 24 & 0.7695\end{array}$

Brown-Forsythe $\quad 0.2012 \quad 1 \quad 24 \quad 0.6577$

$\begin{array}{lllll}\text { Levene } & 0.2465 & 1 & 24 & 0.6241\end{array}$

$\begin{array}{llrrr}\text { Bartlett } & 0.0420 & 1 & \text {. } & 0.8375 \\ \text { F Test 2-sided } & 1.1339 & 15 & 9 & 0.8768\end{array}$

Welch Anova testing Means Equal, allowing Std Devs Not Equal

F Ratio DFNum DFDen Prob $>$ F

$\begin{array}{lrrr}7.5930 & 1 & 20.192 & 0.0121\end{array}$

t Test
2.7555 


\section{Exhibit A2. Statistical Comparisons for Mid-Rheology (Phase 1) Testing}

Oneway Analysis of Measurement By Sampler Mechanism Type of Material=SME Simulant, Analytical Block $=3$, Analyte $=\mathrm{Cr} 2 \mathrm{O} 3(\mathrm{wt} \%)$, Target Value $=\mathbf{0 . 0 9 9 8}$

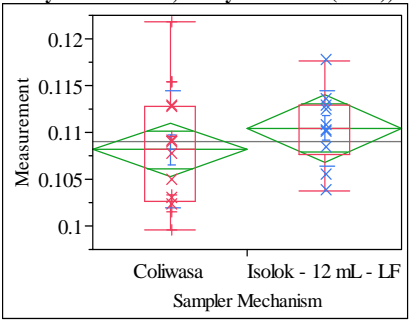

Oneway Anova

Summary of Fit

Adj Rsquare

0.043689

Root Mean Squarc

Mean of Response

0.003842

Observations (or Sum Wgts) $\quad 26$

Test

Isolok - $12 \mathrm{~mL}$ - LF-Coliwasa

Assuming equal variances

Difference $\quad 0.00233$ t Ratio 1.047107

Std Err Dif $\quad 0.00222$ DF 24

Upper CL Dif 0.00692 Prob $>|t| r 0.3055$

Lower CL Dif -0.00226 Prob $>t \quad 0.1527$

$\begin{array}{lrl}\text { Confidence } & 0.95 \text { Prob }<\mathrm{t} & 0.8473\end{array}$

Analysis of Variance

$\begin{array}{lrrrrr}\text { Source } & \text { DF } & \text { Sum of Squares } & \text { Mean Square } & \text { F Ratio } & \text { Prob > F } \\ \text { Sampler Mechanism } & 1 & 0.00003334 & 0.000033 & 1.0964 & 0.3055\end{array}$

$\begin{array}{llll}\text { Error } & 24 & 0.00072978 & 0.000030\end{array}$

C. Total $\quad 25 \quad 0.00076312$

Means for Oneway Anova

$\begin{array}{lrrrrr}\text { Means for Oneway Anova } & & & & \\ \text { Level } & \text { Number } & \text { Mean } & \text { Std Error } & \text { Lower 95\% } & \text { Upper 95\% } \\ \text { Coliwasa } & 16 & 0.108140 & 0.00138 & 0.10529 & 0.11099\end{array}$

$\begin{array}{lrrrrr}\text { Coliwasa } & 16 & 0.108140 & 0.00138 & 0.10529 & 0.11099 \\ \text { Isolok - 12 mL - LF } & 10 & 0.110468 & 0.00174 & 0.10687 & 0.11407\end{array}$

Std Error uses a pooled estimate of error variance

Means and Std Deviations

Level Number Mean Std Dev Std Err Mean Lower 95\% Upper 95\%

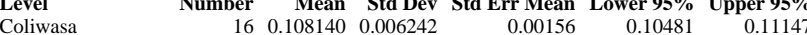

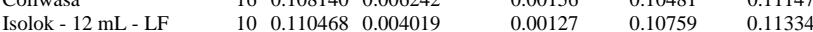

TTest

Isolok - $12 \mathrm{~mL}$ - LF-Coliwas

Assuming unequal variances

$\begin{array}{lrlr}\text { Difference } & 0.00233 & \text { t Ratio } & 1.156539 \\ \text { Std Err Dif } & 0.00201 & \text { DF } & 23.942 \\ \text { Upper CL Dif } & 0.00648 & \text { Prob }>|t| & 0.2589 \\ \text { Lower CL Dif } & -0.00183 \text { Prob }>\text { t } & 0.1294 \\ \text { Confidence } & 0.95 & \text { Prob }<t & 0.8706\end{array}$

Tests that the Variances are Equal

Level Count Std Dev MeanAbsDif to Mean MeanAbsDif to Median

$\begin{array}{lrrrr}\text { Coliwasa } & 16 & 0.0062418 & 0.0050791 & 0.0050791\end{array}$

$\begin{array}{lllll}\text { Coliwasa } & 16 & 0.0062418 & 0.0050791 & 0.0050791 \\ \text { Isolok - } 12 \mathrm{~mL} \text { - LF } & 10 & 0.0040192 & 0.0029232 & 0.0029232\end{array}$

Test F Ratio DFNum DFDen p-Value

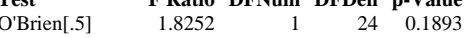

$\begin{array}{lllll}\text { Brown-Forsythe } & 2.9622 & 1 & 24 & 0.098\end{array}$

$\begin{array}{lllll}\text { Levene } & 2.9641 & 1 & 24 & 0.0980\end{array}$

$\begin{array}{lrrrr}\text { Bartlett } & 1.8894 & 1 & \text {. } & 0.1693 \\ & 2.4118 & 15 & 9 & 0.1847\end{array}$

Welch Anova testing Means Equal, allowing Std Devs Not Equal

F Ratio DFNum DFDen Prob > F

$\begin{array}{lrrr}1.3376 & 1 & 23.942 & 0.2589\end{array}$

t Test

1.1565
Oneway Analysis of Measurement By Sampler Mechanism Type of Material=SME Simulant, Analytical Block $=3$, Analyte $=\mathrm{CuO}($ wt $\%)$, Target Value $=0.0504$

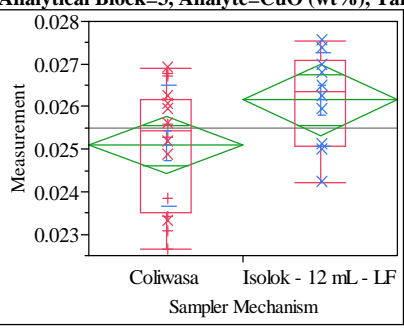

Oneway Anova

Summary of Fit

Adj Rsquare

0.001303

Mean of Response $\quad 0.025498$

Observations (or Sum Wgts) $\quad 26$

\section{Test}

Isolok - $12 \mathrm{~mL}$ - LF-Coliwasa

Assuming equal variances

Difference $\quad 0.00107$ t Ratio $\quad 2.03636$

Std Err Dif $\quad 0.00053$ DF 24

Upper CL Dif 0.00215 Prob $>|t| \quad 0.0529$

Lower CL Dif $-1.45 \mathrm{e}-5$ Prob $>\mathrm{t} \quad 0.0264$

$\begin{array}{lrl}\text { Confidence } & 0.95 \text { Prob }<\mathrm{t} & 0.9736\end{array}$

Analysis of Variance

$\begin{array}{lrrrrr}\text { Source } & \text { DF } & \text { Sum of Squares } & \text { Mean Square } & \text { F Ratio } & \text { Prob }>\text { F } \\ \text { Sampler Mechanism } & 1 & 0.00000704 & 7.039 \mathrm{e}-6 & 4.1468 & 0.0529\end{array}$

$\begin{array}{llll}\text { Error } & 24 & 0.00004074 & 1.6975 \mathrm{e}-6\end{array}$

$\begin{array}{lll}\text { C. Total } & 25 & 0.00004778\end{array}$

Means for Oneway Anova

Level Number Mean Std Error Lower 95\% Upper 95\%

$\begin{array}{lllrrr}\text { Coliwasa } & 16 & 0.025087 & 0.00033 & 0.02441 & 0.02576\end{array}$

Isolok - $12 \mathrm{~mL}$ - LF $\quad \begin{array}{lllll}10 & 0.026156 & 0.00041 & 0.02531 & 0.02701\end{array}$

Std Error uses a pooled estimate of error variance

Means and Std Deviations

Level Number Mean Std Dev Std Err Mean Lower 95\% Upper 95\%

$\begin{array}{lllllrr}\text { Coliwasa } & 16 & 0.025087 & 0.001412 & 0.00035 & 0.02433 & 0.02584\end{array}$

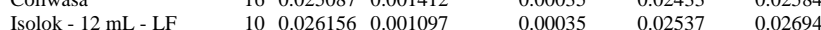

Isolok - $12 \mathrm{~mL}$ - LF-Coliwasa

Assuming unequal variances

$\begin{array}{lrlr}\text { Difference } & 0.001070 & \text { t Ratio } & 2.160636 \\ \text { Std Err Dif } & 0.000495 & \text { DF } & 22.68546 \\ \text { Upper CL Dif } & 0.002094 & \text { Prob }>|t| & 0.0415 \\ \text { Lower CL Dif } & 0.000045 & \text { Prob }>t & 0.0208 \\ \text { Confidence } & 0.95 & \text { Prob }<\text { t } & 0.9792\end{array}$

Tests that the Variances are Equal

Level Count Std Dev MeanAbsDif to Mean MeanAbsDif to Median

$\begin{array}{lllll}\text { Coliwasa } & 16 & 0.0014119 & 0.0011726 & 0.001138\end{array}$

$\begin{array}{lllll}\text { Isolok - } 12 \mathrm{~mL} \text { - LF } & 10 & 0.0010974 & 0.0008838 & 0.0008700\end{array}$

Test F Ratio DFNum DFDen p-Value

$\begin{array}{lrrrr}\text { O'Brien[.5] } & 1.2802 & 1 & 24 & 0.2690\end{array}$

Brown-Forsythe $\quad 0.7167 \quad 1 \quad 24 \quad 0.4056$

$\begin{array}{lllll}\text { Levene } & 1.1272 & 1 & 24 & 0.2989\end{array}$

$\begin{array}{lrrrr}\text { Bartlett } & 0.6492 & 1 & \text {. } & 0.4204 \\ \text { F Test 2-sided } & 1.6551 & 15 & 9 & 0.4494\end{array}$

Welch Anova testing Means Equal, allowing Std Devs Not Equal

F Ratio DFNum DFDen Prob $>$ F

$\begin{array}{llll}4.6683 & 1 & 22.685 & 0.0415\end{array}$

t Test

2.1606 


\section{Exhibit A2. Statistical Comparisons for Mid-Rheology (Phase 1) Testing}

Oneway Analysis of Measurement By Sampler Mechanism Type of Material=SME Simulant, Analytical Block $=3$, Analyte $=\mathrm{Fe} / \mathrm{Li}$, Target Value $=2.452$

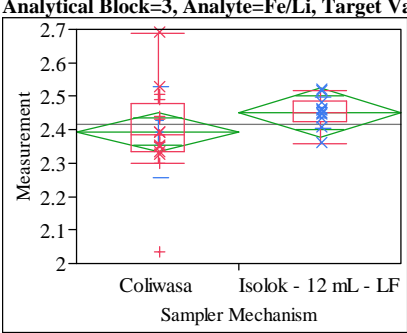

Oneway Anova

Summary of Fit

Adj Rsquare $\quad 0.063599$

Root Mean Square Error $\quad 0.11185$

Mean of Response Eror 2.415005

Observations (or Sum Wgts) $\quad 26$

t Test

Isolok - $12 \mathrm{~mL}$ - LF-Coliwasa

Assuming equal variances

Difference $\quad 0.05757$ t Ratio 1.276735

Std Err Dif $\quad 0.04509$ DF 24

Upper CL Dif 0.15062 Prob $>|t| \quad 0.2139$

Lower CL Dif -0.03549 Prob $>\mathrm{t} \quad 0.1070$

$\begin{array}{lrl}\text { Confidence } & 0.95 \text { Prob }<\mathrm{t} & 0.8930\end{array}$

Analysis of Variance

$\begin{array}{lrrrrr}\text { Source } & \text { DF } & \text { Sum of Squares } & \text { Mean Square } & \text { F Ratio } & \text { Prob > F } \\ \text { Sampler Mechanism } & 1 & 0.02039286 & 0.020393 & 1.6301 & 0.2139\end{array}$

$\begin{array}{llll}\text { Error } & 24 & 0.30025351 & 0.012511\end{array}$

C. Total $\quad 25 \quad 0.32064637$

Means for Oneway Anova

Level $\quad$ Number Mean Std Error Lower 95\% Upper 95\%

$\begin{array}{lrrrrr}\text { Coliwasa } & 16 & 2.39286 & 0.02796 & 2.3352 & 2.4506 \\ \text { Isolok - 12 mL - LF } & 10 & 2.45043 & 0.03537 & 2.3774 & 2.5234\end{array}$

Std Error uses a pooled estimate of error variance

Means and Std Deviations

Level Number Mean Std Dev Std Err Mean Lower 95\% Upper 95\%

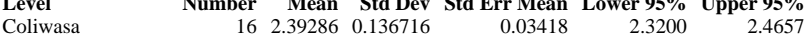

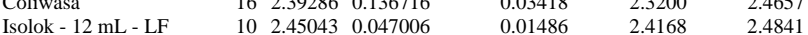

TTest

Isolok - $12 \mathrm{~mL}$ - LF-Coliwas

Assuming unequal variances

$\begin{array}{lrlr}\text { Difference } & 0.05757 & \mathrm{t} \text { Ratio } & 1.544509 \\ \text { Std Err Dif } & 0.03727 & \text { DF } & 20.01741 \\ \text { Upper CL Dif } & 0.13531 & \text { Prob }>|t| & 0.1381 \\ \text { Lower CL Dif } & -0.02018 & \text { Prob }>\mathrm{t} & 0.0691 \\ \text { Confidence } & 0.95 & \text { Prob }<\mathrm{t} & 0.9309\end{array}$

Tests that the Variances are Equal

Level Count Std Dev MeanAbsDif to Mean MeanAbsDif to Median

$\begin{array}{lrrrr}\text { Coliwasa } & 16 & 0.1367156 & 0.0899789 & 0.0888823\end{array}$

$\begin{array}{lllll}\text { Coliwasa } & 16 & 0.1367156 & 0.0899789 & 0.0888823 \\ \text { Isolok - 12 mL - LF } & 10 & 0.0470062 & 0.0339728 & 0.0339728\end{array}$

Test F Ratio DFNum DFDen p-Value

O'Brien[.5] $\quad \begin{array}{rrrr}\text { 1.6665 } & 1 & 24 & 0.2090\end{array}$

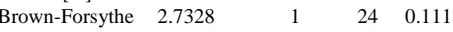

$\begin{array}{lllll}\text { Levene } & 2.9104 & 1 & 24 & 0.1009\end{array}$

$\begin{array}{lrrrr}\text { Bartlett } & 9.1659 & 1 & \text {. } & 0.0025\end{array}$

Welch Anova testing Means Equal, allowing Std Devs Not Equal

F Ratio DFNum DFDen Prob $>$ F

$\begin{array}{rrrr}2.3855 & 1 & 20.017 & 0.1381\end{array}$

t Test

1.5445
Oneway Analysis of Measurement By Sampler Mechanism Type of Material=SME Simulant, Analytical Block $=3$, Analyte $=\mathrm{Fe} 2 \mathrm{O} 3(\mathrm{wt} \%)$, Target Value $=11.462$

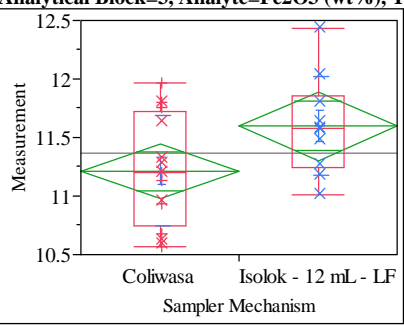

Oneway Anova

Summary of Fi

Adj Rsquare

Root Mean Square Error $\quad 0.452147$

$\begin{array}{lr}\text { Mean of Response } & 11.36227 \\ \text { Observations (or Sum Wgts) } & 26\end{array}$

Test

Isolok - $12 \mathrm{~mL}$ - LF-Coliwasa

Assuming equal variances

Difference $\quad 0.384947$ t Ratio $\quad 2.112001$

Std Err Dif $\quad 0.182266$ DF 24

Upper CL Dif 0.761126 Prob $>|t| \quad 0.0453$

Lower CL Dif 0.008768 Prob $>t \quad 0.0226$

$\begin{array}{lrl}\text { Confidence } & 0.95 \text { Prob }<\mathrm{t} & 0.9774\end{array}$

Analysis of Variance

Source DF Sum of Squares Mean Square F Ratio Prob > F

\begin{tabular}{llll} 
Error & 1 & 0.9119014 & 0.911901 \\
\hline
\end{tabular}

$\begin{array}{lll}\text { C. Total } & 25 & 5.8183894\end{array}$

Means for Oneway Anova

Level Number Mean Std Error Lower 95\% Upper 95\%

$\begin{array}{lrrrrr}\text { Coliwasa } & 16 & 11.2142 & 0.11304 & 10.981 & 11.448\end{array}$

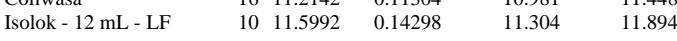

Std Error uses a pooled estimate of error variance

Means and Std Deviations

Level Number Mean Std Dev Std Err Mean Lower 95\% Upper 95\%

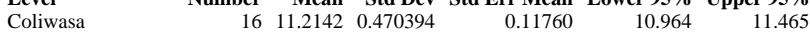

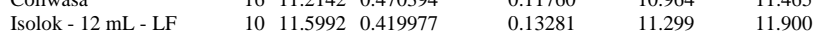

Test

Isolok - $12 \mathrm{~mL}$ - LF-Coliwasa

Assuming unequal variances

$\begin{array}{lrlr}\text { Difference } & 0.384947 & \text { t Ratio } & 2.17005 \\ \text { Std Err Dif } & 0.177391 & \text { DF } & 20.92701 \\ \text { Upper CL Dif } & 0.753929 & \text { Prob }>|t| & 0.0417 \\ \text { Lower CL Dif } & 0.015964 & \text { Prob }>t & 0.0208 \\ \text { Confidence } & 0.95 & \text { Prob }<\mathrm{t} & 0.9792\end{array}$

Tests that the Variances are Equal

Level Count Std Dev MeanAbsDif to Mean MeanAbsDif to Median

$\begin{array}{lllll}\text { Coliwasa } & 16 & 0.4703945 & 0.3813278 & 0.3788705\end{array}$

$\begin{array}{lllll}\text { Isolok - } 12 \mathrm{~mL} \text { - LF } & 10 & 0.4199767 & 0.3025245 & 0.2988073\end{array}$

Test F Ratio DFNum DFDen p-Value

$\begin{array}{lrrrr}\text { O'Brien[.5] } & 0.2376 & 1 & 24 & 0.6304\end{array}$

$\begin{array}{lllll}\text { Brown-Forsythe } & 0.5473 & 1 & 24 & 0.466\end{array}$

$\begin{array}{lllll}\text { Levene } & 0.5509 & 1 & 24 & 0.4651\end{array}$

$\begin{array}{lrrrr}\text { Bartlett } & 0.1355 & 1 & \text {. } & 0.7128 \\ \text { F Test 2-sided } & 1.2545 & 15 & 9 & 0.7504\end{array}$

Welch Anova testing Means Equal, allowing Std Devs Not Equal

F Ratio DFNum DFDen Prob $>$ F

$\begin{array}{rrrr}4.7091 & 1 & 20.927 & 0.0417\end{array}$

t Test

2.1700 


\section{Exhibit A2. Statistical Comparisons for Mid-Rheology (Phase 1) Testing}

Oneway Analysis of Measurement By Sampler Mechanism Type of Material=SME Simulant, Analytical Block=3, Analyte $=$ K2O (wt \%), Target Value $=\mathbf{0 . 1 5 9 1}$

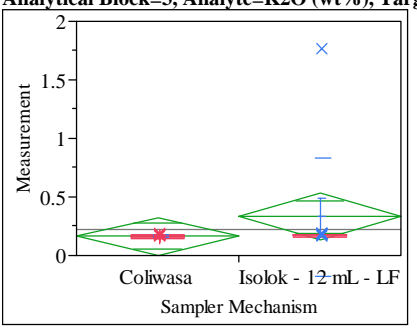

Oneway Anova

Summary of Fit

Adj Rsquare

0.030396

0.30776

Mean of Response

Observations (or Sum Wgts) 0.226951

TTest

Isolok - $12 \mathrm{~mL}$ - LF-Coliwasa

Assuming equal variances

Difference $\quad 0.16569$ t Ratio 1.335565

Std Err Dif $\quad 0.12406$ DF 24

Upper CL Dif 0.42174 Prob $>|t| \quad 0.1942$

Lower CL Dif -0.09036 Prob $>t \quad 0.0971$

$\begin{array}{lrl}\text { Confidence } & 0.95 \text { Prob }<\mathrm{t} & 0.9029\end{array}$

Analysis of Variance

$\begin{array}{lrrrrr}\text { Source } & \text { DF } & \text { Sum of Squares } & \text { Mean Square } & \text { F Ratio } & \text { Prob > F } \\ \text { Sampler Mechanism } & 1 & 0.1689482 & 0.168948 & 1.7837 & 0.1942\end{array}$

$\begin{array}{llll}\text { Error } & 24 & 2.2731856 & 0.094716\end{array}$

$\begin{array}{lll}\text { C. Total } & 25 & 2.4421338\end{array}$

Means for Oneway Anova

$\begin{array}{lrrrrr}\text { Level } & \text { Number } & \text { Mean } & \text { Std Error } & \text { Lower 95\% } & \text { Upper 95\% } \\ \text { Coliwasa } & 16 & 0.163223 & 0.07694 & 0.00443 & 0.32202\end{array}$

$\begin{array}{lrrrrr}\text { Coliwasa } & 16 & 0.163223 & 0.07694 & 0.00443 & 0.32202 \\ \text { Isolok - 12 mL - LF } & 10 & 0.328916 & 0.09732 & 0.12805 & 0.52978\end{array}$

Std Error uses a pooled estimate of error variance

Means and Std Deviations

Level Number Mean Std Dev Std Err Mean Lower 95\% Upper 95\%

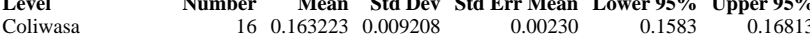

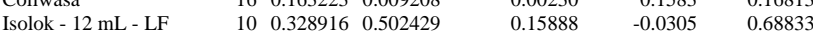

TTest

Isolok - $12 \mathrm{~mL}$ - LF-Coliwas

Assuming unequal variances

$\begin{array}{lrlr}\text { Difference } & 0.16569 & \mathrm{t} \text { Ratio } & 1.042757 \\ \text { Std Err Dif } & 0.15890 & \text { DF } & 9.003779 \\ \text { Upper CL Dif } & 0.52512 & \text { Prob }>|t| & 0.3242 \\ \text { Lower CL Dif } & -0.19374 & \text { Prob }>\mathrm{t} & 0.1621 \\ \text { Confidence } & 0.95 & \text { Prob }<\mathrm{t} & 0.8379\end{array}$

Tests that the Variances are Equal

Level Count Std Dev MeanAbsDif to Mean MeanAbsDif to Median

$\begin{array}{lrrrr}\text { Coliwasa } & 16 & 0.0092082 & 0.0076040 & 0.0073782\end{array}$

$\begin{array}{lllll}\text { Coliwasa } & 16 & 0.0092082 & 0.0076040 & 0.0073782 \\ \text { Isolok - } 12 \mathrm{~mL} \text { - LF } & 10 & 0.5024290 & 0.2859600 & 0.1640063\end{array}$

Test F Ratio DFNum DFDen p-Value

$\begin{array}{lrrrr}\text { O'Brien[.5] } & 1.8393 & 1 & 24 & 0.1877\end{array}$

$\begin{array}{lllll}\text { Brown-Forsythe } & 1.6132 & 1 & 24 & 0.216\end{array}$

$\begin{array}{lllll}\text { Levene } & 7.8675 & 1 & 24 & 0.0098\end{array}$

$\begin{array}{lrrrr}\text { Bartlett } & 92.2682 & 1 & <.0001\end{array}$

Welch Anova testing Means Equal, allowing Std Devs Not Equal

F Ratio DFNum DFDen Prob $>$ F

$\begin{array}{rrrr}1.0873 & 1 & 9.0038 & 0.3242\end{array}$

t Test

1.0428
Oneway Analysis of Measurement By Sampler Mechanism Type of Material=SME Simulant, Analytical Block $=3$, Analyte $=\mathrm{Li2O}(\mathrm{wt} \%)$, Target Value $=4.674$

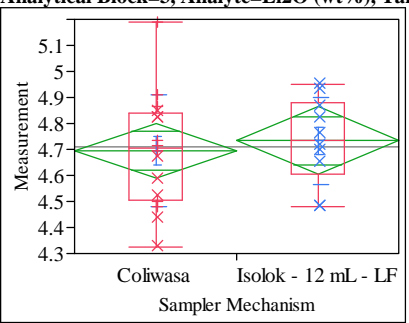

Oneway Anova

Summary of Fit

Adj Rsquare

0.199741

4.709883
26

t Test

Isolok - $12 \mathrm{~mL}$ - LF-Coliwasa

Assuming equal variances

Difference $\quad 0.03956$ t Ratio $\quad 0.491313$

Std Err Dif $\quad 0.08052$ DF 24

Upper CL Dif 0.20574 Prob $>|t| \quad 0.6277$

Lower CL Dif -0.12662 Prob $>t \quad 0.3138$

$\begin{array}{lrl}\text { Confidence } & 0.95 \text { Prob }<\mathrm{t} \quad 0.6862\end{array}$

Analysis of Variance

$\begin{array}{lrrrrr}\text { Source } & \text { DF } & \text { Sum of Squares } & \text { Mean Square } & \text { F Ratio } & \text { Prob > F } \\ \text { Sampler Mechanism } & 1 & 0.00963050 & 0.009631 & 0.2414 & 0.6277 \\ \text { Error } & 24 & 0.95751122 & 0.039896 & & \\ \text { C. Total } & 25 & 0.96714173 & & & \end{array}$

Means for Oneway Anova

Level Number Mean Std Error Lower 95\% Upper 95\%

$\begin{array}{lrrrrr}\text { Coliwasa } & 16 & 4.69467 & 0.04994 & 4.5916 & 4.7977\end{array}$

$\begin{array}{lllll}\text { Isolok - } 12 \mathrm{~mL} \text { - LF } & 10 & 4.73423 & 0.06316 & 4.6039\end{array}$

Std Error uses a pooled estimate of error variance

Means and Std Deviations

Level Number Mean Std Dev Std Err Mean Lower 95\% Upper 95\%

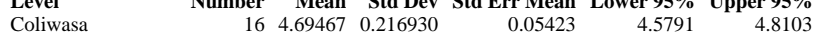

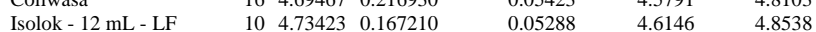

Test

Isolok - $12 \mathrm{~mL}$ - LF-Coliwasa

Assuming unequal variances

$\begin{array}{lrlr}\text { Difference } & 0.03956 & \mathrm{t} \text { Ratio } & 0.522283 \\ \text { Std Err Dif } & 0.07574 & \text { DF } & 22.77367 \\ \text { Upper CL Dif } & 0.19633 \text { Prob }>|\mathrm{t}| & 0.6065 \\ \text { Lower CL Dif } & -0.11721 & \text { Prob }>\mathrm{t} & 0.3033 \\ \text { Confidence } & 0.95 & \text { Prob }<\mathrm{t} & 0.6967\end{array}$

Tests that the Variances are Equal

Level Count Std Dev MeanAbsDif to Mean MeanAbsDif to Median

$\begin{array}{lllll}\text { Coliwasa } & 16 & 0.2169298 & 0.1681953 & 0.1681953\end{array}$

$\begin{array}{llll}\text { Isolok - } 12 \mathrm{~mL} \text { - LF } & 10 & 0.1672101 & 0.1313269\end{array}$

Test F Ratio DFNum DFDen p-Value

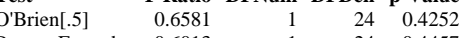

$\begin{array}{lllll}\text { Brown-Forsythe } & 0.6013 & 1 & 24 & 0.4457\end{array}$

$\begin{array}{lllll}\text { Levene } & 0.6039 & 1 & 24 & 0.4447\end{array}$

$\begin{array}{lrrrr}\text { Bartlett } & 0.6918 & 1 & \text {. } & 0.4056 \\ \text { F Test 2-sided } & 1.6831 & 15 & 9 & 0.4340\end{array}$

Welch Anova testing Means Equal, allowing Std Devs Not Equal

F Ratio DFNum DFDen Prob $>$ F

$\begin{array}{lrrr}0.2728 & 1 & 22.774 & 0.6065\end{array}$

t Test
0.5223 


\section{Exhibit A2. Statistical Comparisons for Mid-Rheology (Phase 1) Testing}

Oneway Analysis of Measurement By Sampler Mechanism Type of Material=SME Simulant, Analytical Block=3, Analyte $=\mathrm{MgO}(\mathrm{wt} \%)$, Target Value=1.014

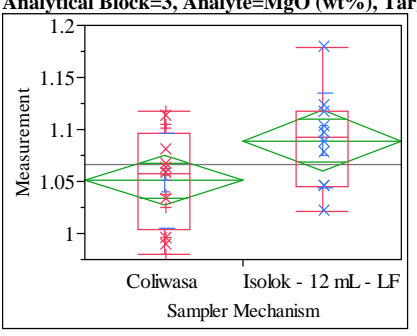

Oneway Anova

Summary of Fit

Adj Rsquare

0.153724

0.118463

0.045611

$\begin{array}{lr}\text { Mean of Response } & 1.065713 \\ \text { Observations (or Sum Wgts) } & 26\end{array}$

t Test

Isolok - $12 \mathrm{~mL}$ - LF-Coliwasa

Assuming equal variances

Difference $\quad 0.038390$ t Ratio 2.087955

Std Err Dif $\quad 0.018386$ DF 24

Upper CL Dif 0.076337 Prob $>|t| \quad 0.0476$

Lower CL Dif 0.000442 Prob $>t \quad 0.0238$

$\begin{array}{lrl}\text { Confidence } & 0.95 \text { Prob }<\mathrm{t} & 0.9762\end{array}$

Analysis of Variance

$\begin{array}{lrrrrr}\text { Source } & \text { DF } & \text { Sum of Squares } & \text { Mean Square } & \text { F Ratio } & \text { Prob }>\text { F } \\ \text { Sampler Mechanism } & 1 & 0.00906932 & 0.009069 & 4.3596 & 0.0476\end{array}$

$\begin{array}{llll}\text { Error } & 24 & 0.04992798 & 0.002080\end{array}$

$\begin{array}{lll}\text { C. Total } & 25 & 0.05899730\end{array}$

Means for Oneway Anova

Level Number Mean Std Error Lower 95\% Upper 95\%

$\begin{array}{lrrrrr}\text { Coliwasa } & 16 & 1.05095 & 0.01140 & 1.0274 & 1.0745 \\ \text { Isolok - 12 mL - LF } & 10 & 1.08934 & 0.01442 & 1.0596 & 1.1191\end{array}$

Std Error uses a pooled estimate of error variance

Means and Std Deviations

Level Number Mean Std Dev Std Err Mean Lower 95\% Upper 95\%

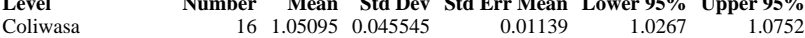

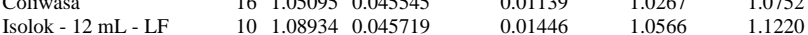

TTest

Isolok - $12 \mathrm{~mL}$ - LF-Coliwas

Assuming unequal variances

$\begin{array}{lrlr}\text { Difference } & 0.03839 & \text { t Ratio } & 2.086044 \\ \text { Std Err Dif } & 0.01840 \text { DF } & 19.19606 \\ \text { Upper CL Dif } & 0.07688 & \text { Prob }>|t| & 0.0506 \\ \text { Lower CL Dif } & -0.00010 & \text { Prob }>\text { t } & 0.0253 \\ \text { Confidence } & 0.95 & \text { Prob }<\mathrm{t} & 0.9747\end{array}$

Tests that the Variances are Equal

Level Count Std Dev MeanAbsDif to Mean MeanAbsDif to Median

$\begin{array}{lrrrr}\text { Coliwasa } & 16 & 0.0455454 & 0.0375709 & 0.0368972\end{array}$

$\begin{array}{lllll}\text { Coliwasa } & 16 & 0.0455454 & 0.0375709 & 0.0368972 \\ \text { Isolok - } 12 \mathrm{~mL} \text { - LF } & 10 & 0.0457192 & 0.0339952 & 0.0339952\end{array}$

Test F Ratio DFNum DFDen p-Value

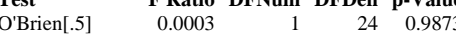

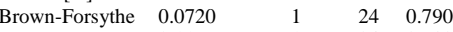

$\begin{array}{lllll}\text { Levene } & 0.1196 & 1 & 24 & 0.7325\end{array}$

$\begin{array}{lllll}\text { Bartlett } & 0.0002 & 1 & . & 0.9900\end{array}$

Welch Anova testing Means Equal, allowing Std Devs Not Equat

F Ratio DFNum DFDen Prob $>$ F

$\begin{array}{rrrr}4.3516 & 1 & 19.196 & 0.0506\end{array}$

t Test

2.0860
Oneway Analysis of Measurement By Sampler Mechanism Type of Material=SME Simulant, Analytical Block $=3$, Analyte $=\mathrm{MnO}(\mathrm{wt} \%)$, Target Value $=1.779$

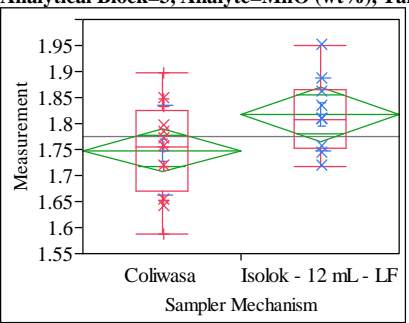

Oneway Anova

Summary of Fit

Adj Rsquare

0.122926

\begin{tabular}{rr}
0.08037 \\
\hline
\end{tabular}

$\begin{array}{lr}\text { Mean of Response } & 1.774407 \\ \text { Observations (or Sum Wgts) } & 26\end{array}$

t Test

Isolok - $12 \mathrm{~mL}$ - LF-Coliwasa

Assuming equal variances

Difference $\quad 0.068756$ t Ratio 2.122228

Std Err Dif $\quad 0.032398$ DF 24

Upper CL Dif 0.135623 Prob $>|t| \quad 0.0443$

$\begin{array}{llll}\text { Upper CL Dif } & 0.135623 & \text { Prob }>|t| & 0.0443 \\ \text { Lower CL Dif } & 0.001890 & \text { Prob }>t & 0.0222\end{array}$

$\begin{array}{lrr}\text { Confidence } & 0.95 \text { Prob }<\mathrm{t} & 0.9778\end{array}$

Analysis of Variance

$\begin{array}{lrrrrr}\text { Source } & \text { DF } & \text { Sum of Squares } & \text { Mean Square } & \text { F Ratio } & \text { Prob > F } \\ \text { Sampler Mechanism } & 1 & 0.02909195 & 0.029092 & 4.5039 & 0.0443 \\ \text { Error } & 24 & 0.15502435 & 0.006459 & & \\ \text { C. Total } & 25 & 0.18411631 & & & \end{array}$

Means for Oneway Anova

Level Number Mean Std Error Lower 95\% Upper 95\%

$\begin{array}{lrrrrr}\text { Coliwasa } & 16 & 1.74796 & 0.02009 & 1.7065 & 1.7894 \\ \text { Isolok - 12 mL - LF } & 10 & 1.81672 & 0.02542 & 1.7643 & 1.8692\end{array}$

Std Error uses a pooled estimate of error variance

Means and Std Deviations

Level Number Mean Std Dev Std Err Mean Lower 95\% Upper 95\%

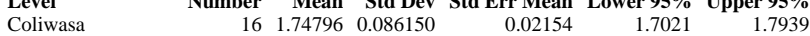

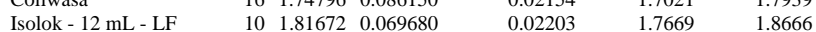

Test

Isolok - $12 \mathrm{~mL}$ - LF-Coliwasa

Assuming unequal variances

$\begin{array}{lrlr}\text { Difference } & 0.068756 & \text { t Ratio } & 2.231471 \\ \text { Std Err Dif } & 0.030812 & \text { DF } & 22.23476 \\ \text { Upper CL Dif } & 0.132618 & \text { Prob }>|t| & 0.0360 \\ \text { Lower CL Dif } & 0.004895 & \text { Prob }>t & 0.0180 \\ \text { Confidence } & 0.95 & \text { Prob }<t & 0.9820\end{array}$

Tests that the Variances are Equal

Level Count Std Dev MeanAbsDif to Mean MeanAbsDif to Median

$\begin{array}{lllll}\text { Coliwasa } & 16 & 0.0861499 & 0.0687967 & 0.0677880\end{array}$

$\begin{array}{llll}\text { Isolok - } 12 \mathrm{~mL} \text { - LF } & 10 & 0.0696796 & 0.0521645\end{array}$

Test F Ratio DFNum DFDen p-Value

$\begin{array}{lrrrr}\text { O'Brien[.5] } & 0.6300 & 1 & 24 & 0.4351\end{array}$

$\begin{array}{lllll}\text { Brown-Forsythe } & 0.7737 & 1 & 24 & 0.3878\end{array}$

$\begin{array}{lllll}\text { Levene } & 0.7844 & 1 & 24 & 0.3846\end{array}$

$\begin{array}{lrrrr}\text { Bartlett } & 0.4647 & 1 & \text {. } & 0.4954 \\ \text { F Test 2-sided } & 1.5286 & 15 & 9 & 0.5273\end{array}$

Welch Anova testing Means Equal, allowing Std Devs Not Equal

F Ratio DFNum DFDen Prob $>$ F

$\begin{array}{llll}4.9795 & 1 & 22.235 & 0.0360\end{array}$

t Test

2.2315 


\section{Exhibit A2. Statistical Comparisons for Mid-Rheology (Phase 1) Testing}

Oneway Analysis of Measurement By Sampler Mechanism Type of Material=SME Simulant, Analytical Block=3, Analyte $=\mathrm{Na2O}(\mathrm{wt} \%)$, Target Value=11.659

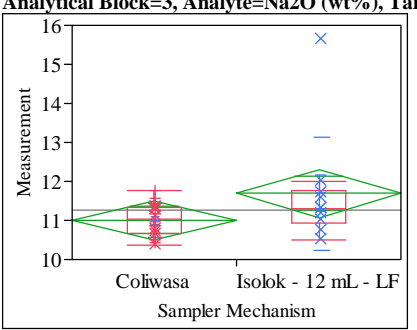

Oneway Anova

Summary of Fit

Adj Rsquare $\quad 0.116077$

Root Mean Square Error $\quad 0.952637$

$\begin{array}{lr}\text { Mean of Response } & 11.27032 \\ \text { Observations (or Sum Wgts) } & 26\end{array}$

Test

Isolok - $12 \mathrm{~mL}$ - LF-Coliwasa

Assuming equal variances

Difference $\quad 0.6818$ t Ratio 1.775299

Std Err Dif $\quad 0.3840$ DF 24

Upper CL Dif 1.4743 Prob $>|t| \quad 0.0885$

$\begin{array}{llll}\text { Lower CL Dif }-0.1108 \text { Prob }>t & 0.0443\end{array}$

$\begin{array}{lrl}\text { Confidence } & 0.95 \text { Prob }<\mathrm{t} \quad 0.9557\end{array}$

Analysis of Variance

$\begin{array}{lrrrrr}\text { Source } & \text { DF } & \text { Sum of Squares } & \text { Mean Square } & \text { F Ratio } & \text { Prob }>\text { F } \\ \text { Sampler Mechanism } & 1 & 2.860212 & 2.86021 & 3.1517 & 0.0885\end{array}$

$\begin{array}{llll}\text { Error } & 24 & 21.780417 & 0.90752\end{array}$

24.640629

Means for Oneway Anova

Level Number Mean Std Error Lower 95\% Upper 95\%

$\begin{array}{lrrrrr}\text { Coliwasa } & 16 & 11.0081 & 0.23816 & 10.517 & 11.500\end{array}$

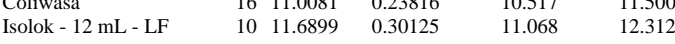

Std Error uses a pooled estimate of error variance

Means and Std Deviations

Level Number Mean Std Dev Std Err Mean Lower 95\% Upper 95\%

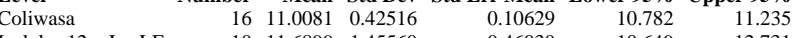

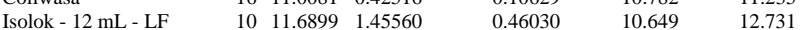

TTest

Isolok - $12 \mathrm{~mL}$ - LF-Coliwas

Assuming unequal variances

Difference $\quad 0.6818$ t Ratio 1.443121

Std Err Dif $\quad 0.4724$ DF 9.968373

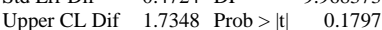

Lower CL Dif -0.3713 Prob $>t \quad 0.0898$

$\begin{array}{lrl}\text { Confidence } & 0.95 \text { Prob }<\mathrm{t} & 0.9102\end{array}$

Tests that the Variances are Equal

Level Count Std Dev MeanAbsDif to Mean MeanAbsDif to Median

$\begin{array}{lllll}\text { Coliwasa } & 16 & 0.425162 & 0.3589050 & 0.3589050\end{array}$

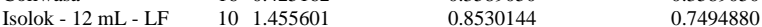

Test F Ratio DFNum DFDen p-Value

$\begin{array}{lllll}\text { O'Brien[.5] } & 1.8959 & 1 & 24 & 0.1812 \\ \text { Brown-Forsythe } & 1.4381 & 1 & 24 & 0.2422\end{array}$

$\begin{array}{lllll}\text { Levene } & 2.8979 & 1 & 24 & 0.1016\end{array}$

\begin{tabular}{lllll} 
Bartlett & 15.8528 & 1 & $<.0001$ \\
\hline & 11.7213 & 9 & 15 & $<.0001$
\end{tabular}

Welch Anova testing Means Equal, allowing Std Devs Not Equal

F Ratio DFNum DFDen Prob > F

$\begin{array}{rrrr}2.0826 & 1 & 9.9684 & 0.1797\end{array}$

t Test

1.4431
Oneway Analysis of Measurement By Sampler Mechanism Type of Material=SME Simulant, Analytical Block $=3$, Analyte $=\mathrm{NiO}(\mathrm{wt} \%)$, Target Value $=\mathbf{0 . 4 1}$

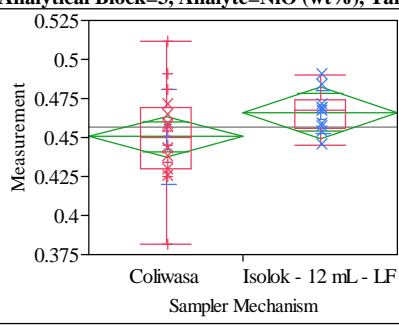

Oneway Anova

Summary of Fit

Adj Rsquare

0.025581

Mean of Response 0.456534

t Test

Isolok - $12 \mathrm{~mL}$ - LF-Coliwasa

Assuming equal variances

Difference $\quad 0.01557$ t Ratio $\quad 1.510106$

Std Err Dif $\quad 0.01031$ DF $\quad 24$

Upper CL Dif 0.03686 Prob $>|t| \quad 0.1441$

$\begin{array}{lll}\text { Lower CL Dif }-0.00571 \text { Prob }>t & 0.0720\end{array}$

$\begin{array}{lrl}\text { Confidence } & 0.95 \text { Prob }<\mathrm{t} & 0.9280\end{array}$

Analysis of Variance

$\begin{array}{lrrrrr}\text { Source } & \text { DF } & \text { Sum of Squares } & \text { Mean Square } & \text { F Ratio } & \text { Prob }>\text { F } \\ \text { Sampler Mechanism } & 1 & 0.00149227 & 0.001492 & 2.2804 & 0.1441\end{array}$

$\begin{array}{llll}\text { Error } & 24 & 0.01570523 & 0.000654\end{array}$

$\begin{array}{lll}\text { C. Total } & 25 & 0.01719750\end{array}$

Means for Oneway Anova

Level $\quad$ Number Mean Std Error Lower 95\% Upper 95\%

$\begin{array}{llllrr}\text { Coliwasa } & 16 & 0.450545 & 0.00640 & 0.43735 & 0.46374\end{array}$

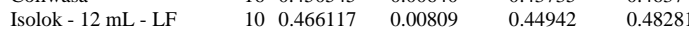

Std Error uses a pooled estimate of error variance

Means and Std Deviations

Level Number Mean Std Dev Std Err Mean Lower 95\% Upper 95\%

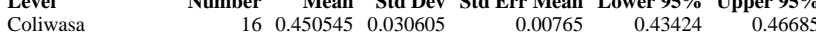

$\begin{array}{lllllll}\text { Isolok - } 12 \mathrm{~mL} \text { - LF } & 10 & 0.466117 & 0.013561 & 0.00429 & 0.45642 & 0.47582\end{array}$

Isolok - $12 \mathrm{~mL}$ - LF-Coliwasa

Assuming unequal variances

$\begin{array}{lrlr}\text { Difference } & 0.01557 & \mathrm{t} \text { Ratio } & 1.775406 \\ \text { Std Err Dif } & 0.00877 & \text { DF } & 22.24537 \\ \text { Upper CL Dif } & 0.03375 & \text { Prob }>|\mathrm{t}| & 0.0895 \\ \text { Lower CL Dif } & -0.00261 & \text { Prob }>\mathrm{t} & 0.0448 \\ \text { Confidence } & 0.95 & \text { Prob }<\mathrm{t} & 0.9552\end{array}$

Tests that the Variances are Equal

Level Count Std Dev MeanAbsDif to Mean MeanAbsDif to Median

$\begin{array}{lllll}\text { Coliwasa } & 16 & 0.0306052 & 0.0229845 & 0.0229845\end{array}$

$\begin{array}{llll}\text { Isolok - } 12 \mathrm{~mL} \text { - LF } & 10 & 0.0135607 & 0.0102309\end{array}$

Test F Ratio DFNum DFDen p-Value

$\begin{array}{lrrrr}\text { O'Brien[.5] } & 2.3401 & 1 & 24 & 0.1392\end{array}$

$\begin{array}{lllll}\text { Brown-Forsythe } & 3.9370 & 1 & 24 & 0.0588\end{array}$

$\begin{array}{lllll}\text { Levene } & 3.8710 & 1 & 24 & 0.0608\end{array}$

$\begin{array}{lrrrr}\text { Bartlett } & 5.7820 & 1 & \text {. } & 0.0162 \\ \text { F Test 2-sided } & 5.0936 & 15 & 9 & 0.0183\end{array}$

Welch Anova testing Means Equal, allowing Std Devs Not Equal

F Ratio DFNum DFDen Prob $>$ F

$\begin{array}{lrrr}3.1521 & 1 & 22.245 & 0.0895\end{array}$

t Test
1.7754 


\section{Exhibit A2. Statistical Comparisons for Mid-Rheology (Phase 1) Testing}

Oneway Analysis of Measurement By Sampler Mechanism Type of Material=SME Simulant, Analytical Block=3, Analyte=SiO2 (wt \%), Target Value=50.985

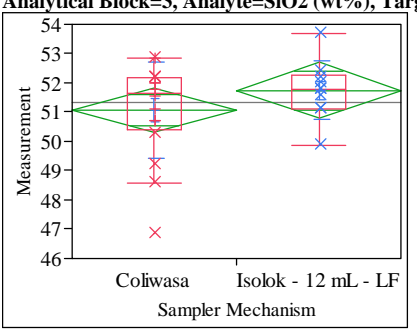

Oneway Anova

Summary of Fit

0.055041

Root Mean Square Error 1.441938

$\begin{array}{lr}\text { Mean of Response } & 51.32674 \\ \text { Observations (or Sum Wgts) } & 26\end{array}$

Test

Isolok - $12 \mathrm{~mL}$ - LF-Coliwasa

Assuming equal variances

Difference $\quad 0.6873$ t Ratio 1.182338

$\begin{array}{lrlr}\text { Std Err Dif } & 0.5813 & \text { DF } & 24 \\ \text { Upper CL Dif } & 1.8869 & \text { Prob }>|t| & 0.2487\end{array}$

Lower CL Dif -0.5124 Prob $>$ t 0.1243

$\begin{array}{lrl}\text { Confidence } & 0.95 \text { Prob }<\mathrm{t} & 0.8757\end{array}$

Analysis of Variance

$\begin{array}{lrrrrr}\text { Source } & \text { DF } & \text { Sum of Squares } & \text { Mean Square } & \text { F Ratio } & \text { Prob }>\text { F } \\ \text { Sampler Mechanism } & 1 & 2.906540 & 2.90654 & 1.3979 & 0.2487\end{array}$

$\begin{array}{lrrr}\text { Error } & 24 & 49.900435 & 2.07918\end{array}$

$\begin{array}{lll}\text { C. Total } & 25 & 52.806975\end{array}$

Means for Oneway Anova

Level Number Mean Std Error Lower 95\% Upper 95\%

$\begin{array}{lrrrrr}\text { Coliwasa } & 16 & 51.0624 & 0.36048 & 50.318 & 51.806 \\ \text { Isolok - 12 mL - LF } & 10 & 51.7497 & 0.45598 & 50.809 & 52.691\end{array}$

Std Error uses a pooled estimate of error variance

Means and Std Deviations

Level Number Mean Std Dev Std Err Mean Lower 95\% Upper 95\%

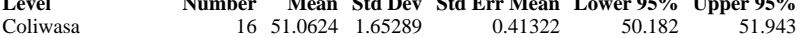

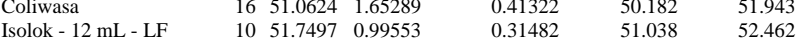

tTest

Isolok - $12 \mathrm{~mL}$ - LF-Coliwas

Assuming unequal variances

Difference $\quad 0.6873$ t Ratio 1.322953

Std Err Dif $\quad 0.5195$ DF 23.99386

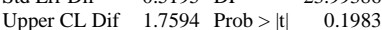

Lower CL Dif -0.3849 Prob $>$ t 0.0992

$\begin{array}{lrr}\text { Confidence } & -0.95 \text { Prob }<\mathrm{t} & 0.9008\end{array}$

Tests that the Variances are Equal

Level Count Std Dev MeanAbsDif to Mean MeanAbsDif to Median

$\begin{array}{lrrrr}\text { Coliwasa } & 16 & 1.652889 & 1.260181 & 1.189986\end{array}$

$\begin{array}{lllll}\text { Coliwasa } & 16 & 1.652889 & 1.260181 & 1.189986 \\ \text { Isolok - 12 mL - LF } & 10 & 0.995535 & 0.667462 & 0.663183\end{array}$

Test F Ratio DFNum DFDen p-Value

$\begin{array}{lrrrr}\text { O'Brien[.5] } & 1.1799 & 1 & 24 & 0.2882 \\ \text { Brown-Forsythe } & 1.4310 & 1 & 24 & 0.2433\end{array}$

$\begin{array}{lllll}\text { Levene } & 2.5896 & 1 & 24 & 0.1206\end{array}$

$\begin{array}{lrrrr}\text { Bartlett } & 2.4606 & 1 & \text {. } & 0.1167\end{array}$

Welch Anova testing Means Equal, allowing Std Devs Not Equal

F Ratio DFNum DFDen Prob $>$ F

$\begin{array}{rrrr}1.7502 & 1 & 23.994 & 0.1983\end{array}$

t Test
Oneway Analysis of Measurement By Sampler Mechanism Type of Material=SME Simulant, Analytical Block $=3$, Analyte $=$ Sum of Oxides (wt $\%$ ), Target Value $=99.553$

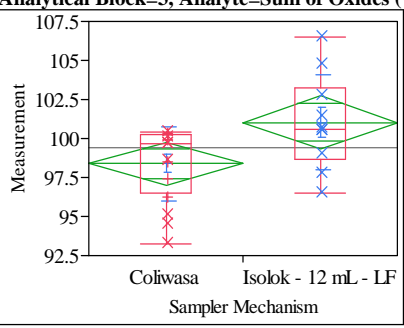

Oneway Anova

Summary of Fit

Adj Rsquare

2.640836
99.41464

Mean of Response

Observations (or Sum Wgts)

Test

Isolok - $12 \mathrm{~mL}$ - LF-Coliwasa

Assuming equal variances

Difference $\quad 2.63594$ t Ratio 2.476093

Std Err Dif 1.06455 DF 24

Upper CL Dif 4.83307 Prob $>|t| \quad 0.0207$

Lower CL Dif 0.43880 Prob $>$ t 0.0104

$\begin{array}{lrl}\text { Confidence } & 0.95 \text { Prob }<\mathrm{t} \quad 0.9896\end{array}$

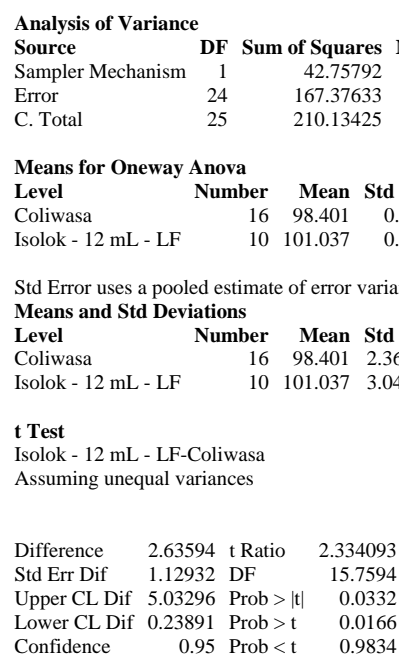

Tests that the Variances are Equal

Level Count Std Dev MeanAbsDif to Mean MeanAbsDif to Median

$\begin{array}{lllll}\text { Coliwasa } & 16 & 2.368543 & 1.931375 & 1.737718\end{array}$

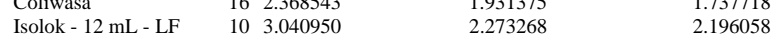

Test F Ratio DFNum DFDen p-Value

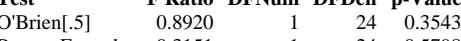

$\begin{array}{lllll}\text { Brown-Forsythe } & 0.3151 & 1 & 24 & 0.5798\end{array}$

$\begin{array}{lllll}\text { Levene } & 0.3082 & 1 & 24 & 0.5839\end{array}$

$\begin{array}{lllll}\text { Bartlett } & 0.6938 & 1 & \text {. } & 0.4049 \\ \text { F Test 2-sided } & 1.6484 & 9 & 15 & 0.3768\end{array}$

Welch Anova testing Means Equal, allowing Std Devs Not Equal

F Ratio DFNum DFDen Prob $>$ F

$\begin{array}{rrrr}5.4480 & 1 & 15.759 & 0.0332\end{array}$

t Test
2.3341 


\section{Exhibit A2. Statistical Comparisons for Mid-Rheology (Phase 1) Testing}

Oneway Analysis of Measurement By Sampler Mechanism Type of Material=SME Simulant, Analytical Block=3, Analyte $=$ TiO2 (wt \%), Target Value $=0.0711$

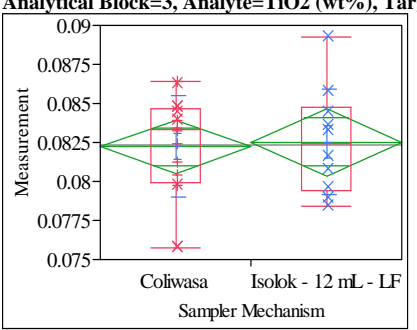

Oneway Anova

Summary of Fit

Adj Rsquare $\quad 0.001956$

Root Mean Square Error $\quad 0.003315$

Mean of Response $\quad 0.08235$

$\begin{array}{lr}\text { Observations (or Sum Wgts) } & 26\end{array}$

Test

Isolok - $12 \mathrm{~mL}$ - LF-Coliwasa

Assuming equal variances

Difference $\quad 0.00029$ t Ratio $\quad 0.216887$

Std Err Dif $\quad 0.00134$ DF 24

Upper CL Dif 0.00305 Prob $>|t| \quad 0.8301$

$\begin{array}{lll}\text { Lower CL Dif }-0.00247 \text { Prob }>t & 0.4151\end{array}$

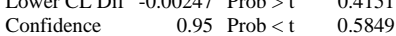

Analysis of Variance

$\begin{array}{lrrrrr}\text { Source } & \text { DF } & \text { Sum of Squares } & \text { Mean Square } & \text { F Ratio } & \text { Prob > F } \\ \text { Sampler Mechanism } & 1 & 0.00000052 & 5.169 \mathrm{e}-7 & 0.0470 & 0.8301\end{array}$

$\begin{array}{llll}\text { Error } & 24 & 0.00026371 & 0.000011\end{array}$

$\begin{array}{lll}\text { C. Total } & 25 & 0.00026423\end{array}$

Means for Oneway Anova

$\begin{array}{lrrrrr}\text { Level } & \text { Number } & \text { Mean } & \text { Std Error } & \text { Lower 95\% } & \text { Upper 95\% } \\ \text { Coliwasa } & 16 & 0.082243 & 0.00083 & 0.08053 & 0.08395\end{array}$

$\begin{array}{lrrrrr}\text { Coliwasa } & 16 & 0.082243 & 0.00083 & 0.08053 & 0.08395 \\ \text { Isolok - 12 mL - LF } & 10 & 0.082533 & 0.00105 & 0.08037 & 0.08470\end{array}$

Std Error uses a pooled estimate of error variance

Means and Std Deviations

Level Number Mean Std Dev Std Err Mean Lower 95\% Upper 95\%

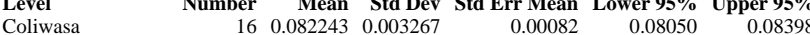

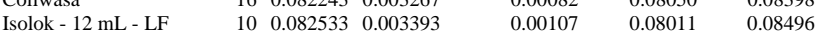

TTest

Isolok - $12 \mathrm{~mL}$ - LF-Coliwas

Assuming unequal variances

Difference $\quad 0.00029$ t Ratio $\quad 0.214929$

Std Err Dif $\quad 0.00135$ DF $\quad 18.68886$

Upper CL Dif 0.00312 Prob $>|t| \quad 0.8322$

Lower CL Dif -0.00254 Prob $>t \quad 0.4161$

$\begin{array}{lrl}\text { Confidence } & 0.95 \text { Prob }<\mathrm{t} & 0.5839\end{array}$

Tests that the Variances are Equal

Level Count Std Dev MeanAbsDif to Mean MeanAbsDif to Median

$\begin{array}{lrrrr}\text { Coliwasa } & 16 & 0.0032672 & 0.0026141 & 0.0024707\end{array}$

$\begin{array}{lllll}\text { Coliwasa } & 16 & 0.0032672 & 0.0026141 & 0.0024707 \\ \text { Isolok - } 12 \mathrm{~mL} \text { - LF } & 10 & 0.0033928 & 0.0027022 & 0.0027022\end{array}$

Test F Ratio DFNum DFDen p-Value

$\begin{array}{lrrrr}\text { O'Brien[.5] } & 0.0183 & 1 & 24 & 0.8934 \\ \text { Brown-Forsythe } & 0.0709 & 1 & 24 & 0.7924\end{array}$

$\begin{array}{lllll}\text { Levene } & 0.0141 & 1 & 24 & 0.9065\end{array}$

$\begin{array}{lllll}\text { Bartlett } & 0.0154 & 1 & & 0.9012\end{array}$

Welch Anova testing Means Equal, allowing Std Devs Not Equal

F Ratio DFNum DFDen Prob $>$ F

$\begin{array}{rrrr}0.0462 & 1 & 18.689 & 0.8322\end{array}$

t Test
0.2149
Oneway Analysis of Measurement By Sampler Mechanism Type of Material=SME Simulant, Analytical Block $=3$, Analyte $=\mathrm{ZnO}($ wt $\%)$, Target Value $=\mathbf{0 . 0 9 5 8}$

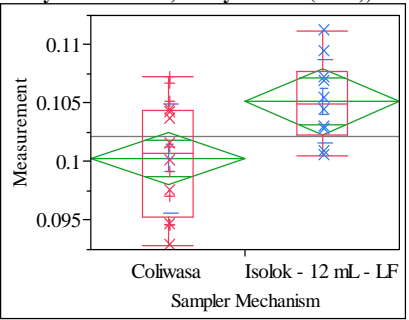

Oneway Anova

Summary of Fit

Adj Rsquare

0.004303

Mean of Response $\quad 0.102155$

\section{Test}

Isolok - $12 \mathrm{~mL}$ - LF-Coliwasa

Assuming equal variances

Difference $\quad 0.004844$ t Ratio 2.792316

Std Err Dif $\quad 0.001735$ DF 24

Upper CL Dif 0.008424 Prob $>|t| \quad 0.0101$

Lower CL Dif 0.001264 Prob $>t \quad 0.0051$

$\begin{array}{lll}\text { Confidence } & 0.95 \mathrm{Prob}<\mathrm{t} & 0.9949\end{array}$

Analysis of Variance

$\begin{array}{lrrrrr}\text { Source } & \text { DF } & \text { Sum of Squares } & \text { Mean Square } & \text { F Ratio } & \text { Prob }>\text { F } \\ \text { Sampler Mechanism } & 1 & 0.00014439 & 0.000144 & 7.7970 & 0.0101\end{array}$

$\begin{array}{llll}\text { Error } & 24 & 0.00044443 & 0.000019\end{array}$

$\begin{array}{lll}\text { C. Total } & 25 & 0.00058882\end{array}$

Means for Oneway Anova

Level Number Mean Std Error Lower 95\% Upper 95\%

\begin{tabular}{lrrrrr} 
Coliwasa & 16 & 0.100292 & 0.00108 & 0.09807 & 0.1025 \\
\hline
\end{tabular}

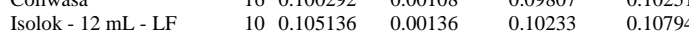

Std Error uses a pooled estimate of error variance

Means and Std Deviations

Level Number Mean Std Dev Std Err Mean Lower 95\% Upper 95\%

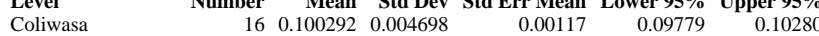

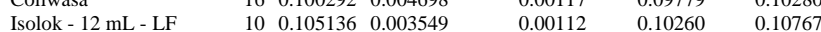

Test

Isolok - $12 \mathrm{~mL}$ - LF-Coliwasa

Assuming unequal variances

$\begin{array}{lrlr}\text { Difference } & 0.004844 & \text { t Ratio } & 2.981683 \\ \text { Std Err Dif } & 0.001625 & \text { DF } & 22.97399 \\ \text { Upper CL Dif } & 0.008205 & \text { Prob }>|t| & 0.0067 \\ \text { Lower CL Dif } & 0.001483 & \text { Prob }>t & 0.0033 \\ \text { Confidence } & 0.95 & \text { Prob }<t & 0.9967\end{array}$

Tests that the Variances are Equal

Level Count Std Dev MeanAbsDif to Mean MeanAbsDif to Median

$\begin{array}{lllll}\text { Coliwasa } & 16 & 0.0046980 & 0.0039756 & 0.0039756\end{array}$

$\begin{array}{llll}\text { Isolok - } 12 \mathrm{~mL} \text { - LF } & 10 & 0.0035492 & 0.0028630\end{array}$

Test F Ratio DFNum DFDen p-Value

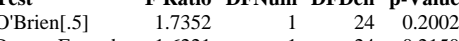

Brown-Forsythe $1.6221 \quad 1 \quad 24 \quad 0.2150$

$\begin{array}{lllll}\text { Levene } & 1.6683 & 1 & 24 & 0.2088\end{array}$

$\begin{array}{lrrrr}\text { Bartlett } & 0.7989 & 1 & \text {. } & 0.3714\end{array}$

Welch Anova testing Means Equal, allowing Std Devs Not Equal

F Ratio DFNum DFDen Prob > F

$\begin{array}{rrrr}8.8904 & 1 & 22.974 & 0.0067\end{array}$

t Test

2.9817 


\section{Exhibit A2. Statistical Comparisons for Mid-Rheology (Phase 1) Testing}

Oneway Analysis of Measurement By Sampler Mechanism Type of Material=SME Simulant, Analytical Block $=3$, Analyte $=\mathrm{ZrO} 2(\mathrm{wt} \%)$, Target Value $=0.3547$

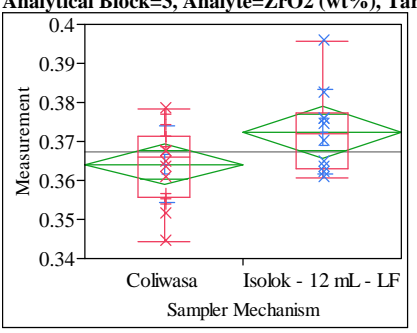

Oneway Anova

Summary of Fit

0.145806

Root Mean Square Error $\quad 0.01016$

$\begin{array}{lr}\text { Mean of Response } & 0.367314 \\ \text { Observations (or Sum Wgts) } & 26\end{array}$

t Test

Isolok - $12 \mathrm{~mL}$ - LF-Coliwasa

Assuming equal variances

Difference $\quad 0.00829$ t Ratio $\quad 2.024019$

Std Err Dif $\quad 0.00410$ DF 24

Upper CL Dif 0.01674 Prob $>|t| \quad 0.0542$

Lower CL Dif -0.00016 Prob $>\mathrm{t} \quad 0.0271$

$\begin{array}{lrl}\text { Confidence } & 0.95 \text { Prob }<\mathrm{t} & 0.9729\end{array}$

Analysis of Variance

$\begin{array}{lrrrrr}\text { Source } & \text { DF } & \text { Sum of Squares } & \text { Mean Square } & \text { F Ratio } & \text { Prob > F } \\ \text { Sampler Mechanism } & 1 & 0.00042297 & 0.000423 & 4.0967 & 0.0542\end{array}$

$\begin{array}{llll}\text { Error } & 24 & 0.00247796 & 0.000103\end{array}$

$\begin{array}{lll}\text { C. Total } & 25 & 0.00290093\end{array}$

Means for Oneway Anova

$\begin{array}{lrrrrr}\text { Level } & \text { Number } & \text { Mean } & \text { Std Error } & \text { Lower 95\% } & \text { Upper 95\% } \\ \text { Coliwasa } & 16 & 0.364125 & 0.00254 & 0.35888 & 0.36937\end{array}$

$\begin{array}{lrrrrr}\text { Coliwasa } & 16 & 0.364125 & 0.00254 & 0.35888 & 0.36937 \\ \text { Isolok - 12 mL - LF } & 10 & 0.372416 & 0.00321 & 0.36578 & 0.37905\end{array}$

Std Error uses a pooled estimate of error variance

Means and Std Deviations

Level Number Mean Std Dev Std Err Mean Lower 95\% Upper 95\%

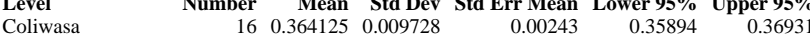

$\begin{array}{lllllll}\text { Coliwasa } & 16 & 0.364125 & 0.009728 & 0.00243 & 0.35894 & 0.36931 \\ \text { Isolok - 12 mL - LF } & 10 & 0.372416 & 0.010845 & 0.00343 & 0.36466 & 0.38017\end{array}$

Test

Isolok - $12 \mathrm{~mL}$ - LF-Coliwas

Assuming unequal variances

$\begin{array}{lrlr}\text { Difference } & 0.00829 & \text { t Ratio } & 1.971958 \\ \text { Std Err Dif } & 0.00420 & \text { DF } & 17.64993 \\ \text { Upper CL Dif } & 0.01714 & \text { Prob }>|t| & 0.0645 \\ \text { Lower CL Dif } & -0.00055 & \text { Prob }>\text { t } & 0.0322 \\ \text { Confidence } & 0.95 & \text { Prob }<\mathrm{t} & 0.9678\end{array}$

Tests that the Variances are Equal

Level Count Std Dev MeanAbsDif to Mean MeanAbsDif to Median

$\begin{array}{lrrrr}\text { Coliwasa } & 16 & 0.0097279 & 0.0079254 & 0.0078515\end{array}$

$\begin{array}{lllll}\text { Isolok - } 12 \mathrm{~mL} \text { - LF } & 10 & 0.0108448 & 0.0082399 & 0.0082399\end{array}$

Test F Ratio DFNum DFDen p-Value

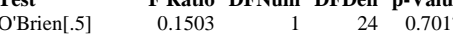

$\begin{array}{lllll}\text { Brown-Forsythe } & 0.0255 & 1 & 24 & 0.8745\end{array}$

$\begin{array}{lllll}\text { Levene } & 0.0184 & 1 & 24 & 0.8932\end{array}$

$\begin{array}{lllll}\text { Bartlett } & 0.1292 & 1 & & 0.7192\end{array}$

Welch Anova testing Means Equal, allowing Std Devs Not Equat

F Ratio DFNum DFDen Prob $>$ F

$\begin{array}{lrrr}3.8886 & 1 & 17.65 & 0.0645\end{array}$

t Test

1.9720
Oneway Analysis of Measurement By Sampler Mechanism Type of Material=SME Simulant, Analytical Block $=4$, Analyte $=A \mathrm{l} / \mathrm{B}$, Target Value $=2.552$

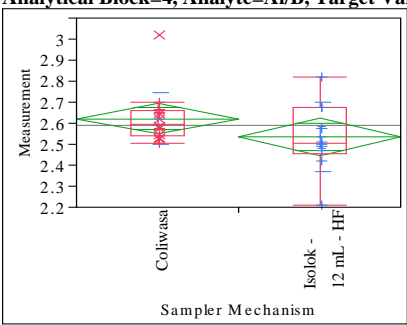

Oneway Anova

Summary of Fit

Adj Rsquare

0.054001

$\quad 0.139483$

Mean of Response 2.588273

26

T Test

Isolok - $12 \mathrm{~mL}$ - HF-Coliwasa

Assuming equal variances

Difference $\quad-0.08760$ t Ratio $\quad-1.55791$

Std Err Dif $\quad 0.05623$ DF 24

Upper CL Dif 0.02845 Prob $>|t| \quad 0.1323$

Lower CL Dif -0.20364 Prob $>t \quad 0.9338$

$\begin{array}{lll}\text { Confidence } & 0.95 \text { Prob }<\mathrm{t} \quad 0.0662\end{array}$

Analysis of Variance

$\begin{array}{lrrrrr}\text { Source } & \text { DF } & \text { Sum of Squares } & \text { Mean Square } & \text { F Ratio } & \text { Prob }>\text { F } \\ \text { Sampler Mechanism } & 1 & 0.04722023 & 0.047220 & 2.4271 & 0.1323\end{array}$

$\begin{array}{llll}\text { Error } & 24 & 0.46693034 & 0.019455\end{array}$

$\begin{array}{lll}\text { C. Total } & 25 & 0.51415057\end{array}$

Means for Oneway Anova

Level Number Mean Std Error Lower 95\% Upper 95\%

$\begin{array}{lrrrrr}\text { Coliwasa } & 16 & 2.62196 & 0.03487 & 2.5500 & 2.6939 \\ \text { Isolok - 12 mL - HF } & 10 & 2.53437 & 0.04411 & 2.4433 & 2.6254\end{array}$

Std Error uses a pooled estimate of error variance

Means and Std Deviations

Level Number Mean Std Dev Std Err Mean Lower 95\% Upper 95\%

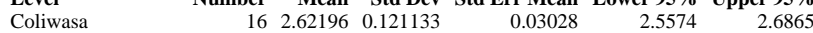

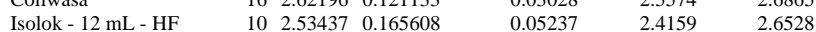

T Test

Isolok - $12 \mathrm{~mL}$ - HF-Coliwasa

Assuming unequal variances

$\begin{array}{lrlr}\text { Difference } & -0.08760 & \mathrm{t} \text { Ratio } & -1.44801 \\ \text { Std Err Dif } & 0.06050 & \text { DF } & 15.01768 \\ \text { Upper CL Dif } & 0.04133 & \text { Prob }>|t| & 0.1682 \\ \text { Lower CL Dif } & -0.21653 & \text { Prob }>\mathrm{t} & 0.9159 \\ \text { Confidence } & 0.95 & \text { Prob }<\mathrm{t} & 0.0841\end{array}$

Tests that the Variances are Equal

Level Count Std Dev MeanAbsDif to Mean MeanAbsDif to Median

$\begin{array}{lrrrr}\text { Coliwasa } & 16 & 0.1211327 & 0.0784021 & 0.0772495\end{array}$

$\begin{array}{lllll}\text { Coliwasa } & 16 & 0.1211327 & 0.0784021 & 0.0772495 \\ \text { Isolok - } 12 \mathrm{~mL} \text { - HF } & 10 & 0.1656077 & 0.1207008 & 0.1161805\end{array}$

Test F Ratio DFNum DFDen p-Value

$\begin{array}{lrrrr}\text { O'Brien[.5] } & 0.5487 & 1 & 24 & 0.4660\end{array}$

$\begin{array}{lllll}\text { Brown-Forsythe } & 0.8798 & 1 & 24 & 0.3576\end{array}$

$\begin{array}{lllll}\text { Levene } & 1.1855 & 1 & 24 & 0.2870\end{array}$

$\begin{array}{lllll}\text { Bartlett } & 1.0918 & 1 & 0.2961 \\ \text { F Test 2-sided } & 1.8691 & 9 & 15 & 0.2731\end{array}$

Welch Anova testing Means Equal, allowing Std Devs Not Equal

F Ratio DFNum DFDen Prob > F

$\begin{array}{rrrr}2.0967 & 1 & 15.018 & 0.1682\end{array}$

t Test
1.4480 


\section{Exhibit A2. Statistical Comparisons for Mid-Rheology (Phase 1) Testing}

Oneway Analysis of Measurement By Sampler Mechanism Type of Material=SME Simulant, Analytical Block=4, Analyte $=\mathrm{Al} 2 \mathrm{O} 3(\mathrm{wt} \%)$, Target Value $=10.869$

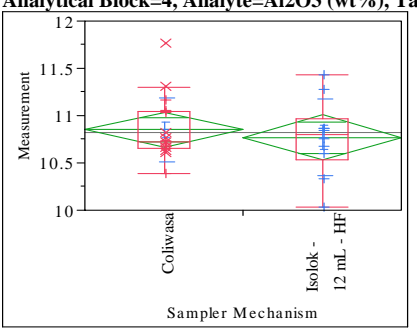

Oneway Anova

Summary of Fit

Adj Rsquare 0.012895

Root Mean Square Error $\quad 0.364146$

Mean of Response

$\begin{array}{lr}\text { Observations (or Sum Wgts) } & 26\end{array}$

TTest

Isolok - $12 \mathrm{~mL}$ - HF-Coliwasa

Assuming equal variances

Difference $\quad-0.08219$ t Ratio $\quad-0.55993$

Std Err Dif $\quad 0.14679$ DF 24

$\begin{array}{rr}\text { Upper CL Dif } 0.22077 \text { Prob }>|t| & 0.5807\end{array}$

Lower CL Dif -0.38516 Prob $>\mathrm{t} \quad 0.7096$

$\begin{array}{lrl}\text { Confidence } & 0.95 \text { Prob }<\mathrm{t} & 0.2904\end{array}$

Analysis of Variance

$\begin{array}{lrrrrr}\text { Source } & \text { DF } & \text { Sum of Squares } & \text { Mean Square } & \text { F Ratio } & \text { Prob > F } \\ \text { Sampler Mechanism } & 1 & 0.0415737 & 0.041574 & 0.3135 & 0.5807\end{array}$

$\begin{array}{llll}\text { Error } & 24 & 3.1824497 & 0.132602\end{array}$

$\begin{array}{lll}\text { C. Total } & 25 & 3.2240234\end{array}$

Means for Oneway Anova

Level Number Mean Std Error Lower 95\% Upper 95\%

$\begin{array}{lrrrrr}\text { Coliwasa } & 16 & 10.8505 & 0.09104 & 10.663 & 11.038\end{array}$

$\begin{array}{llllll}\text { Coliwasa } & 16 & 10.8505 & 0.09104 & 10.663 & 11.038 \\ \text { Isolok - 12 mL - HF } & 10 & 10.7683 & 0.11515 & 10.531 & 11.006\end{array}$

Std Error uses a pooled estimate of error variance

Means and Std Deviations

Level Number Mean Std Dev Std Err Mean Lower 95\% Upper 95\%

\begin{tabular}{lrrrrrr} 
Coliwasa & Number & Mean & Std Dev & Std Err Mean & Lower $95 \%$ & Upper 95\% \\
\hline & 16 & 10.8505 & 0.335779 & 0.08394 & 10.672 & 11.029
\end{tabular}

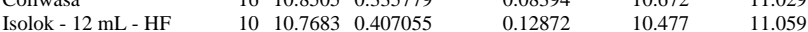

Test

Isolok - $12 \mathrm{~mL}$ - HF-Coliwasa

Assuming unequal variances

$\begin{array}{lrlr}\text { Difference } & -0.08219 & \mathrm{t} \text { Ratio } & -0.53485 \\ \text { Std Err Dif } & 0.15368 & \text { DF } & 16.49311 \\ \text { Upper CL Dif } & 0.24279 & \text { Prob }>|\mathrm{t}| & 0.5999 \\ \text { Lower CL Dif } & -0.40718 & \text { Prob }>\mathrm{t} & 0.7001 \\ \text { Confidence } & 0.95 & \text { Prob }<\mathrm{t} & 0.2999\end{array}$

Tests that the Variances are Equal

Level Count Std Dev MeanAbsDif to Mean MeanAbsDif to Median

$\begin{array}{lrrrr}\text { Coliwasa } & 16 & 0.3357786 & 0.2568539 & 0.2243781\end{array}$

$\begin{array}{lllll}\text { Coliwasa } & 16 & 0.3357786 & 0.2568539 & 0.2243781 \\ \text { Isolok - } 12 \mathrm{~mL} \text { - HF } & 10 & 0.4070546 & 0.2890935 & 0.2890935\end{array}$

Test F Ratio DFNum DFDen p-Value

$\begin{array}{lrrrr}\text { O'Brien[.5] } & 0.3348 & 1 & 24 & 0.5682\end{array}$

Brown-Forsythe $\quad 0.3416 \quad 1 \quad 24 \quad 0.5643$

$\begin{array}{lllll}\text { Levene } & 0.1189 & 1 & 24 & 0.7332\end{array}$

$\begin{array}{lllll}\text { Bartlett } & 0.4094 & 1 & . & 0.5223\end{array}$

Welch Anova testing Means Equal, allowing Std Devs Not Equal

F Ratio DFNum DFDen Prob $>$ F

$\begin{array}{llll}0.2861 & 1 & 16.493 & 0.5999\end{array}$

t Test
0.5349
Oneway Analysis of Measurement By Sampler Mechanism Type of Material=SME Simulant, Analytical Block $=4$, Analyte $=\mathrm{B} 2 \mathrm{O} 3(\mathrm{wt} \%)$, Target Value $=4.259$

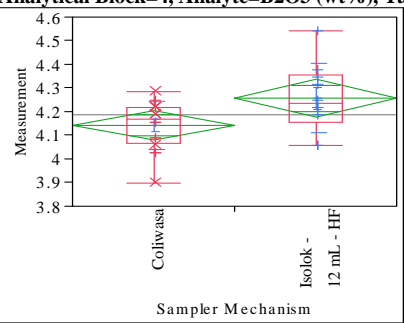

Oneway Anova

Summary of Fit

Adj Rsquare

0.153398

$\quad 0.121433$

4.18587

Test

Isolok - $12 \mathrm{~mL}$ - HF-Coliwasa

Assuming equal variances

Difference $\quad 0.115111$ t Ratio 2.351554

Std Err Dif $\quad 0.048951$ DF 24

Upper CL Dif 0.216142 Prob $>|t| \quad 0.0272$

Lower CL Dif 0.014081 Prob $>t \quad 0.0136$

$\begin{array}{lrl}\text { Confidence } & 0.95 \text { Prob }<\mathrm{t} & 0.9864\end{array}$

Analysis of Variance

$\begin{array}{lrrrrr}\text { Source } & \text { DF } & \text { Sum of Squares } & \text { Mean Square } & \text { F Ratio } & \text { Prob }>\text { F } \\ \text { Sampler Mechanism } & 1 & 0.08154240 & 0.081542 & 5.5298 & 0.0272\end{array}$

$\begin{array}{llll}\text { Error } & 24 & 0.35390335 & 0.014746\end{array}$

$\begin{array}{lll}\text { C. Total } & 25 & 0.43544575\end{array}$

Means for Oneway Anova

Level Number Mean Std Error Lower 95\% Upper 95\%

$\begin{array}{lrrrrr}\text { Coliwasa } & 16 & 4.14160 & 0.03036 & 4.0789 & 4.2043\end{array}$

$\begin{array}{llllll}\text { Isolok - } 12 \text { mL - HF } & 10 & 4.25671 & 0.03840 & 4.1775 & 4.3360\end{array}$

Std Error uses a pooled estimate of error variance

Means and Std Deviations

Level Number Mean Std Dev Std Err Mean Lower 95\% Upper 95\%

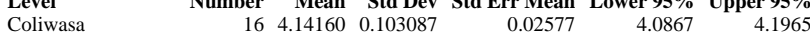

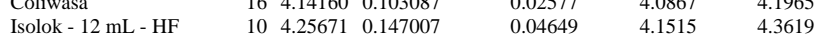

Test

Isolok - $12 \mathrm{~mL}$ - HF-Coliwasa

Assuming unequal variances

Difference $\quad 0.115111$ t Ratio 2.165645

Std Err Dif $\quad 0.053153$ DF $\quad 14.55715$

Upper CL Dif 0.228706 Prob $>|t| r \mid 0.0474$

Lower CL Dif 0.001517 Prob $>\mathrm{t} \quad 0.0237$

$\begin{array}{lrl}\text { Confidence } & 0.95 \text { Prob }<\mathrm{t} & 0.9763\end{array}$

Tests that the Variances are Equal

Level Count Std Dev MeanAbsDif to Mean MeanAbsDif to Median

$\begin{array}{lllll}\text { Coliwasa } & 16 & 0.1030871 & 0.0835162 & 0.0804975\end{array}$

$\begin{array}{llll}\text { Isolok - } 12 \mathrm{~mL} \text { - HF } & 10 & 0.1470068 & 0.1107646\end{array}$

Test F Ratio DFNum DFDen p-Value

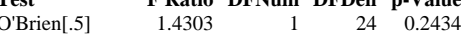

$\begin{array}{lllll}\text { Brown-Forsythe } & 0.8372 & 1 & 24 & 0.3693\end{array}$

$\begin{array}{lllll}\text { Levene } & 0.9167 & 1 & 24 & 0.3479\end{array}$

$\begin{array}{lllll}\text { Bartlett } & 1.4096 & 1 & 0.2351 \\ \text { F Test 2-sided } & 2.0336 & 9 & 15 & 0.2156\end{array}$

Welch Anova testing Means Equal, allowing Std Devs Not Equal

F Ratio DFNum DFDen Prob > F

$\begin{array}{rrrr}4.6900 & 1 & 14.557 & 0.0474\end{array}$

t Test

2.1656 


\section{Exhibit A2. Statistical Comparisons for Mid-Rheology (Phase 1) Testing}

Oneway Analysis of Measurement By Sampler Mechanism Type of Material=SME Simulant, Analytical Block=4, Analyte $=\mathrm{BaO}($ wt $\%)$, Target Value $=0.0919$

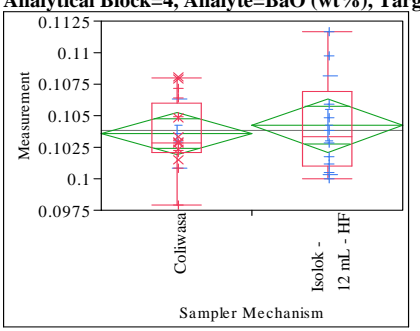

Oneway Anova

Summary of Fit

Adj Rsquare $\quad-0.009739$

Root Mean Square Error $\quad 0.003242$

Mean of Response 0.103835

Observations (or Sum Wgts) $\quad 26$

Test

Isolok - $12 \mathrm{~mL}$ - HF-Coliwasa

Assuming equal variances

Difference $\quad 0.00064$ t Ratio 0.485831

Std Err Dif $\quad 0.00131$ DF 24

Upper CL Dif 0.00333 Prob $>|t| r 0.6315$

Lower CL Dif -0.00206 Prob $>\mathrm{t} \quad 0.3157$

$\begin{array}{lrl}\text { Confidence } & 0.95 \text { Prob }<\mathrm{t} & 0.6843\end{array}$

Analysis of Variance

$\begin{array}{lrrrrr}\text { Source } & \text { DF } & \text { Sum of Squares } & \text { Mean Square } & \text { F Ratio } & \text { Prob }>\text { F } \\ \text { Sampler Mechanism } & 1 & 0.00000248 & 2.481 \mathrm{e}-6 & 0.2360 & 0.6315\end{array}$

$\begin{array}{llll}\text { Error } & 24 & 0.00025232 & 0.000011\end{array}$

$\begin{array}{lll}\text { C. Total } & 25 & 0.00025480\end{array}$

Means for Oneway Anova

$\begin{array}{lrrrrr}\text { Level } & \text { Number } & \text { Mean } & \text { Std Error } & \text { Lower 95\% } & \text { Upper 95\% } \\ \text { Coliwasa } & 16 & 0.103590 & 0.00081 & 0.10192 & 0.10526\end{array}$

$\begin{array}{lrrrrr}\text { Coliwasa } & 16 & 0.103590 & 0.00081 & 0.10192 & 0.10526 \\ \text { Isolok - 12 mL - HF } & 10 & 0.104225 & 0.00103 & 0.10211 & 0.10634\end{array}$

Std Error uses a pooled estimate of error variance

Means and Std Deviations

Level Number Mean Std Dev Std Err Mean Lower 95\% Upper 95\%

$\begin{array}{lrrrrrr}\text { Leviwasa } & \text { Number } & \text { Mean } & \text { Std Dev } & \text { Std Err Mean } & \text { Lower 95\% } & \text { Upper 95\% } \\ \text { Co } & 16 & 0.103590 & 0.002756 & 0.00069 & 0.10212 & 0.10506\end{array}$

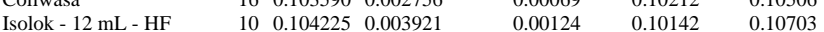

TTest

Isolok - $12 \mathrm{~mL}$ - HF-Coliwasa

Assuming unequal variances

$\begin{array}{lrlr}\text { Difference } & 0.00064 & \text { t Ratio } & 0.447629 \\ \text { Std Err Dif } & 0.00142 & \text { DF } & 14.57992 \\ \text { Upper CL Dif } & 0.00367 & \text { Prob }>|t| & 0.6610 \\ \text { Lower CL Dif } & -0.00240 & \text { Prob }>\text { t } & 0.3305 \\ \text { Confidence } & 0.95 & \text { Prob }<\mathrm{t} & 0.6695\end{array}$

Tests that the Variances are Equal

Level Count Std Dev MeanAbsDif to Mean MeanAbsDif to Median

$\begin{array}{lrrrr}\text { Coliwasa } & 16 & 0.0027558 & 0.0021929 & 0.0020585\end{array}$

$\begin{array}{lllll}\text { Coliwasa } & 16 & 0.0027558 & 0.0021929 & 0.0020585 \\ \text { Isolok - } 12 \mathrm{~mL} \text { - HF } & 10 & 0.0039215 & 0.0030592 & 0.0029811\end{array}$

Test F Ratio DFNum DFDen p-Value

$\begin{array}{lrrrr}\text { O'Brien[.5] } & \text { F Ratio } & \text { DFNum } & \text { DFDen } & \text { p-Value } \\ & 1.6906 & 1 & 24 & 0.2059\end{array}$

Brown-Forsythe $1.1134 \quad 1 \quad 24 \quad 0.3018$

$\begin{array}{lllll}\text { Levene } & 1.3553 & 1 & 24 & 0.2558\end{array}$

$\begin{array}{lllll}\text { Bartlett } & 1.3924 & 1 & 0.2380\end{array}$

Welch Anova testing Means Equal, allowing Std Devs Not Equal

F Ratio DFNum DFDen Prob $>$ F

$\begin{array}{rrrr}0.2004 & 1 & 14.58 & 0.6610\end{array}$

t Test

0.4476
Oneway Analysis of Measurement By Sampler Mechanism Type of Material=SME Simulant, Analytical Block $=4$, Analyte $=\mathrm{CaO}($ wt $\%)$, Target Value $=1.1462$

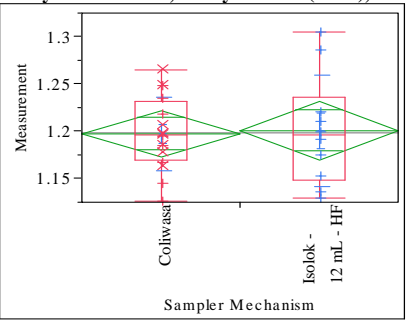

Oneway Anova

Summary of Fit

Adj Rsquare

0.001245
-0.04037

-0.047152
1.19863

1.19863

t Test

Isolok - $12 \mathrm{~mL}$ - HF-Coliwasa

Assuming equal variances

$\begin{array}{lrlr}\text { Difference } & 0.00329 & \mathrm{t} \text { Ratio } & 0.172988 \\ \text { Std Err Dif } & 0.01901 & \text { DF } & 24 \\ \text { Upper CL Dif } & 0.04252 & \text { Prob }>|t| & 0.8641 \\ \text { Lower CL Dif } & -0.03594 & \text { Prob }>t & 0.4321 \\ \text { Confidence } & 0.95 & \text { Prob }<t & 0.5679\end{array}$

Analysis of Variance

$\begin{array}{lrrrrr}\text { Source } & \text { DF } & \text { Sum of Squares } & \text { Mean Square } & \text { F Ratio } & \text { Prob > F } \\ \text { Sampler Mechanism } & 1 & 0.00006653 & 0.000067 & 0.0299 & 0.8641 \\ \text { Error } & 24 & 0.05336053 & 0.002223 & & \end{array}$

$25 \quad 0.05342706$

Means for Oneway Anova

Level Number Mean Std Error Lower 95\% Upper 95\%

$\begin{array}{lrrrrr}\text { Coliwasa } & 16 & 1.19737 & 0.01179 & 1.1730 & 1.2217 \\ \text { Isolok - 12 mL - HF } & 10 & 1.20065 & 0.01491 & 1.1699 & 1.2314\end{array}$

Std Error uses a pooled estimate of error variance

Means and Std Deviations

Level Number Mean Std Dev Std Err Mean Lower 95\% Upper 95\%

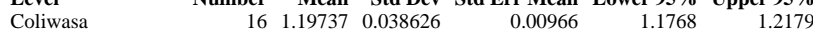

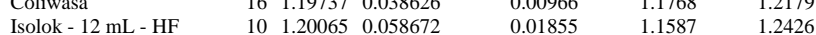

T Test

Isolok - $12 \mathrm{~mL}$ - HF-Coliwasa

Assuming unequal variances

$\begin{array}{lrlr}\text { Difference } & 0.00329 & \mathrm{t} \text { Ratio } & 0.157205 \\ \text { Std Err Dif } & 0.02092 & \text { DF } & 13.92322 \\ \text { Upper CL Dif } & 0.04817 & \text { Prob }>|\mathrm{t}| & 0.8773 \\ \text { Lower CL Dif } & -0.04160 & \text { Prob }>\mathrm{t} & 0.4387 \\ \text { Confidence } & 0.95 & \text { Prob }<\mathrm{t} & 0.5613\end{array}$

Tests that the Variances are Equal

Level Count Std Dev MeanAbsDif to Mean MeanAbsDif to Median

$\begin{array}{lrrrr}\text { Coliwasa } & 16 & 0.0386257 & 0.0296893 & 0.0295581\end{array}$

$\begin{array}{llll}\text { Isolok - } 12 \mathrm{~mL} \text { - HF } & 10 & 0.0586717 & 0.0438229\end{array}$

Test F Ratio DFNum DFDen p-Value

$\begin{array}{lrrrr}\text { O'Brien[.5] } & 2.4845 & 1 & 24 & 0.1281\end{array}$

$\begin{array}{lllll}\text { Brown-Forsythe } & 1.3866 & 1 & 24 & 0.2505\end{array}$

$\begin{array}{lllll}\text { Levene } & 1.4713 & 1 & 24 & 0.2369\end{array}$

$\begin{array}{lllll}\text { Bartlett } & 1.9608 & 1 & \text {. } & 0.1614 \\ \text { F Test 2-sided } & 2.3073 & 9 & 15 & 0.1466\end{array}$

Welch Anova testing Means Equal, allowing Std Devs Not Equal

F Ratio DFNum DFDen Prob $>$ F

$\begin{array}{rrrr}0.0247 & 1 & 13.923 & 0.8773\end{array}$

$\mathbf{t}$ Test
0.1572 


\section{Exhibit A2. Statistical Comparisons for Mid-Rheology (Phase 1) Testing}

Oneway Analysis of Measurement By Sampler Mechanism Type of Material=SME Simulant, Analytical Block $=4$, Analyte $=\mathrm{Cr} 2 \mathrm{O} 3(\mathrm{wt} \%)$, Target Value $=0.0998$

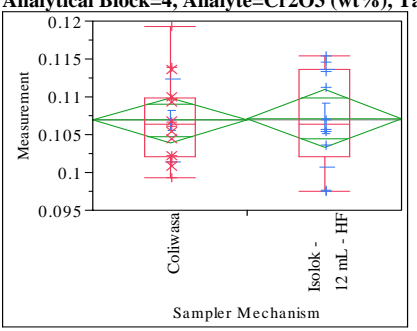

Oneway Anova

Summary of Fit

Adj Rsquare

0.000485

-0.04116
0.00588

0.106978

Observations (or Sum Wgts) $\quad 26$

TTest

Isolok - $12 \mathrm{~mL}$ - HF-Coliwasa

Assuming equal variances

Difference $\quad 0.00026$ t Ratio $\quad 0.107918$

Std Err Dif $\quad 0.00237$ DF 24

Upper CL Dif 0.00515 Prob $>|t| \quad 0.9150$

Lower CL Dif -0.00464 Prob $>t \quad 0.4575$

$\begin{array}{lrl}\text { Confidence } & 0.95 \text { Prob }<\mathrm{t} & 0.5425\end{array}$

Analysis of Variance

$\begin{array}{lrrrrr}\text { Source } & \text { DF } & \text { Sum of Squares } & \text { Mean Square } & \text { F Ratio } & \text { Prob }>\text { F } \\ \text { Sampler Mechanism } & 1 & 0.00000040 & 4.026 \mathrm{e}-7 & 0.0116 & 0.9150\end{array}$

$\begin{array}{llll}\text { Error } & 24 & 0.00082966 & 0.000035\end{array}$

$\begin{array}{lll}\text { C. Total } & 25 & 0.00083007\end{array}$

Means for Oneway Anova

$\begin{array}{lrrrrr}\text { Level } & \text { Number } & \text { Mean } & \text { Std Error } & \text { Lower 95\% } & \text { Upper 95\% } \\ \text { Coliwasa } & 16 & 0.106880 & 0.00147 & 0.10385 & 0.10991\end{array}$

$\begin{array}{lrrrrr}\text { Coliwasa } & 16 & 0.106880 & 0.00147 & 0.10385 & 0.10991 \\ \text { Isolok - 12 mL - HF } & 10 & 0.107135 & 0.00186 & 0.10330 & 0.11097\end{array}$

Std Error uses a pooled estimate of error variance

Means and Std Deviations

Level Number Mean Std Dev Std Err Mean Lower 95\% Upper 95\%

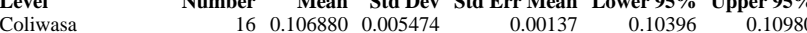

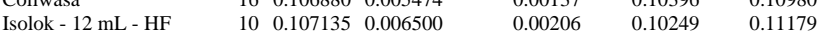

TTest

Isolok - $12 \mathrm{~mL}$ - HF-Coliwasa

Assuming unequal variances

$\begin{array}{lrlr}\text { Difference } & 0.00026 & \mathrm{t} \text { Ratio } & 0.103583 \\ \text { Std Err Dif } & 0.00247 & \text { DF } & 16.76903 \\ \text { Upper CL Dif } & 0.00547 & \text { Prob }>|t| & 0.9187 \\ \text { Lower CL Dif } & -0.00496 & \text { Prob }>\mathrm{t} & 0.4594 \\ \text { Confidence } & 0.95 & \text { Prob }<\mathrm{t} & 0.5406\end{array}$

Tests that the Variances are Equal

Level Count Std Dev MeanAbsDif to Mean MeanAbsDif to Median

$\begin{array}{lllll}\text { Coliwasa } & 16 & 0.0054736 & 0.0042660 & 0.0042204\end{array}$

$\begin{array}{lllll}\text { Coliwasa } & 16 & 0.0054736 & 0.0042660 & 0.0042204 \\ \text { Isolok - } 12 \mathrm{~mL} \text { - HF } & 10 & 0.0065001 & 0.0052033 & 0.0051741\end{array}$

Test F Ratio DFNum DFDen p-Value

$\begin{array}{lrrrr}\text { O'Brien[.5] } & 0.5081 & 1 & 24 & 0.4828\end{array}$

Brown-Forsythe $\quad 0.4680 \quad 1 \quad 24 \quad 0.5005$

$\begin{array}{lllll}\text { Levene } & 0.4846 & 1 & 24 & 0.4930\end{array}$

$\begin{array}{lllll}\text { Bartlett } & 0.3256 & 1 & .5682\end{array}$

Welch Anova testing Means Equal, allowing Std Devs Not Equal

F Ratio DFNum DFDen Prob $>$ F

$\begin{array}{rrrr}0.0107 & 1 & 16.769 & 0.9187\end{array}$

t Test

0.1036
Oneway Analysis of Measurement By Sampler Mechanism Type of Material=SME Simulant, Analytical Block $=4$, Analyte $=\mathrm{CuO}(\mathrm{wt} \%)$, Target Value $=0.0504$

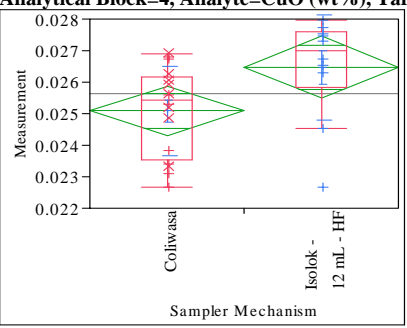

Oneway Anova

Summary of Fit

Adj Rsquare

0.177577

Root Mean Square Error $\quad 0.001513$

$\begin{array}{lr}\text { Mean of Response } & 0.025621 \\ \text { Observations (or Sum Wgts) } & 26\end{array}$

Test

Isolok - $12 \mathrm{~mL}$ - HF-Coliwasa

Assuming equal variances

Difference $\quad 0.001389$ t Ratio $\quad 2.276416$

Std Err Dif $\quad 0.000610$ DF 24

Upper CL Dif 0.002648 Prob $>|t| \quad 0.0320$

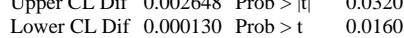

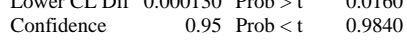

Analysis of Variance

$\begin{array}{lrrrrr}\text { Source } & \text { DF } & \text { Sum of Squares } & \text { Mean Square } & \text { F Ratio } & \text { Prob }>\text { F } \\ \text { Sampler Mechanism } & 1 & 0.00001187 & 0.000012 & 5.1821 & 0.0320\end{array}$

Error $\quad 24 \quad 0.00005496 \quad 2.29 \mathrm{e}-6$

$\begin{array}{lll}\text { C. Total } & 25 & 0.00006683\end{array}$

Means for Oneway Anova

Level $\quad$ Number Mean Std Error Lower 95\% Upper 95\%

$\begin{array}{lrrrrr}\text { Coliwasa } & 16 & 0.025087 & 0.00038 & 0.02431 & 0.02587\end{array}$

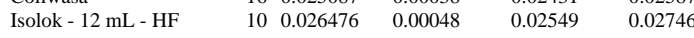

Std Error uses a pooled estimate of error variance

Means and Std Deviations

Level Number Mean Std Dev Std Err Mean Lower 95\% Upper 95\%

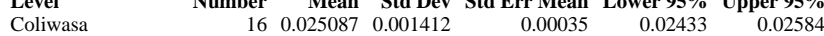

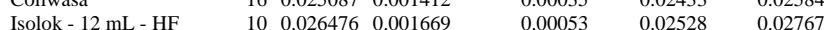

Test

Isolok - $12 \mathrm{~mL}$ - HF-Coliwasa

Assuming unequal variances

$\begin{array}{lrlr}\text { Difference } & 0.001389 & \text { t Ratio } & 2.18736 \\ \text { Std Err Dif } & 0.000635 & \text { DF } & 16.83234 \\ \text { Upper CL Dif } & 0.002729 & \text { Prob }>|t| & 0.0431 \\ \text { Lower CL Dif } & 0.000048 & \text { Prob }>t & 0.0216 \\ \text { Confidence } & 0.95 & \text { Prob }<t & 0.9784\end{array}$

Tests that the Variances are Equal

Level Count Std Dev MeanAbsDif to Mean MeanAbsDif to Median

$\begin{array}{lllll}\text { Coliwasa } & 16 & 0.0014119 & 0.0011726 & 0.0011384\end{array}$

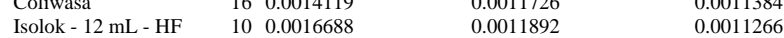

Test F Ratio DFNum DFDen p-Value

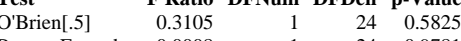

$\begin{array}{lllll}\text { Brown-Forsythe } & 0.0008 & 1 & 24 & 0.9781\end{array}$

$\begin{array}{lllll}\text { Levene } & 0.0022 & 1 & 24 & 0.9632\end{array}$

$\begin{array}{lllll}\text { Bartlett } & 0.3080 & 1 & 0.5789\end{array}$

Welch Anova testing Means Equal, allowing Std Devs Not Equal

F Ratio DFNum DFDen Prob $>$ F

$\begin{array}{rrrr}4.7845 & 1 & 16.832 & 0.0431\end{array}$

t Test

2.1874 


\section{Exhibit A2. Statistical Comparisons for Mid-Rheology (Phase 1) Testing}

Oneway Analysis of Measurement By Sampler Mechanism Type of Material=SME Simulant, Analytical Block $=4$, Analyte $=\mathrm{Fe} / \mathrm{Li}$, Target Value $=2.452$

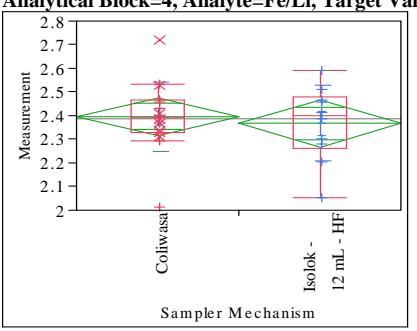

Oneway Anova

Summary of Fit

Adj Rsquare $\quad 0.009066$

Root Mean Square Error $\quad 0.152046$

$\begin{array}{lr}\text { Mean of Response } & 2.384618 \\ \text { Observations (or Sum Wgts) } & 26\end{array}$

TTest

Isolok - $12 \mathrm{~mL}$ - HF-Coliwasa

Assuming equal variances

Difference $\quad-0.02872$ t Ratio $\quad-0.46859$

Std Err Dif $\quad 0.06129$ DF 24

Upper CL Dif 0.09778 Prob $>|t| r \mid r .6436$

Lower CL Dif -0.15522 Prob $>t \quad 0.6782$
Con

$\begin{array}{lrl}\text { Confidence } & 0.95 \text { Prob }<\mathrm{t} & 0.3218\end{array}$

Analysis of Variance

$\begin{array}{lrrrrr}\text { Source } & \text { DF } & \text { Sum of Squares } & \text { Mean Square } & \text { F Ratio } & \text { Prob > F } \\ \text { Sampler Mechanism } & 1 & 0.00507625 & 0.005076 & 0.2196 & 0.6436\end{array}$

$\begin{array}{lrrr}\text { Error } & 24 & 0.55483237 & 0.023118\end{array}$

$\begin{array}{lll}\text { C. Total } & 25 & 0.55990863\end{array}$

Means for Oneway Anova

Level $\quad$ Number Mean Std Error Lower 95\% Upper 95\%

$\begin{array}{lrrrrr}\text { Coliwasa } & 16 & 2.39566 & 0.03801 & 2.3172 & 2.4741\end{array}$

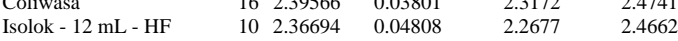

Std Error uses a pooled estimate of error variance

Means and Std Deviations

Level Number Mean Std Dev Std Err Mean Lower 95\% Upper 95\%

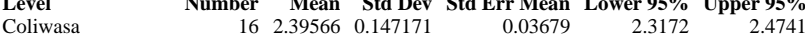

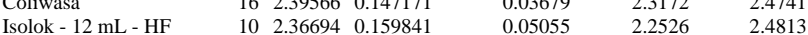

Test

Isolok - $12 \mathrm{~mL}$ - HF-Coliwasa

Assuming unequal variances

Difference $\quad-0.02872$ t Ratio $\quad-0.4594$

$\begin{array}{lll}\text { Std Err Dif } \quad 0.06252 \text { DF } & 18.02732\end{array}$

Upper CL Dif 0.10261 Prob $>|t| \quad 0.6514$

Lower CL Dif -0.16005 Prob $>t \quad 0.6743$

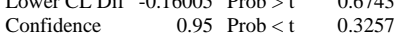

Tests that the Variances are Equal

Level Count Std Dev MeanAbsDif to Mean MeanAbsDif to Median

$\begin{array}{lrrrr}\text { Coliwasa } & 16 & 0.1471712 & 0.0967494 & 0.0967494\end{array}$

$\begin{array}{lllll}\text { Coliwasa } & 16 & 0.1471712 & 0.0967494 & 0.0967494 \\ \text { Isolok - 12 mL - HF } & 10 & 0.1598408 & 0.1255358 & 0.1211468\end{array}$

Test F Ratio DFNum DFDen p-Value

$\begin{array}{lrrrr} & \text { F Ratio } & \text { DFNum } & \text { DFDen } & \text { p-Value } \\ \text { O'Brien[.5] } & 0.0506 & 1 & 24 & 0.8240\end{array}$

$\begin{array}{lllll}\text { Brown-Forsythe } & 0.3258 & 1 & 24 & 0.5735\end{array}$

$\begin{array}{lllll}\text { Levene } & 0.4945 & 1 & 24 & 0.4887\end{array}$

$\begin{array}{lllll}\text { Bartlett } & 0.0743 & 1 & . & 0.7851\end{array}$

Welch Anova testing Means Equal, allowing Std Devs Not Equat

F Ratio DFNum DFDen Prob $>$ F

$\begin{array}{rrrr}0.2110 & 1 & 18.027 & 0.6514\end{array}$

t Test

0.4594
Oneway Analysis of Measurement By Sampler Mechanism Type of Material=SME Simulant, Analytical Block $=4$, Analyte $=\mathrm{Fe} 2 \mathrm{O} 3(\mathrm{wt} \%)$, Target Value $=11.462$

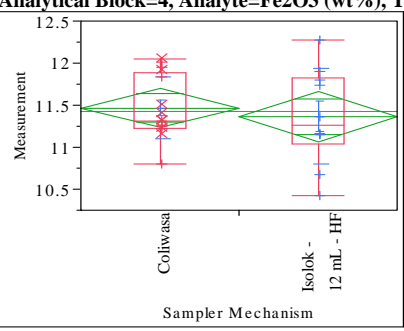

Oneway Anova

Summary of Fit

Adj Rsquare $\quad 0.01302$

Root Mean Square Error $\quad 0.455383$

Mean of Response $\quad 11.42825$

Observations (or Sum Wgts) $\quad 26$

T Test

Isolok - $12 \mathrm{~mL}$ - HF-Coliwasa

Assuming equal variances

Difference $\quad-0.10330$ t Ratio $\quad-0.5627$

Std Err Dif $\quad 0.18357$ DF 24

Upper CL Dif 0.27558 Prob $>|t| \quad 0.5789$

$\begin{array}{llll}\text { Lower CL Dif }-0.48217 \text { Prob }>t & 0.7106\end{array}$

$\begin{array}{lrl}\text { Confidence } & 0.95 \text { Prob }<\mathrm{t} & 0.2894\end{array}$

Analysis of Variance

$\begin{array}{lrrrrr}\text { Source } & \text { DF } & \text { Sum of Squares } & \text { Mean Square } & \text { F Ratio } & \text { Prob > F } \\ \text { Sampler Mechanism } & 1 & 0.0656617 & 0.065662 & 0.3166 & 0.5789 \\ \text { Error } & 24 & 4.9769665 & 0.207374 & & \end{array}$

$\begin{array}{lll}\text { C. Total } & 25 & 5.0426283\end{array}$

Means for Oneway Anova

Level Number Mean Std Error Lower 95\% Upper 95\%

$\begin{array}{lrrrrr}\text { Coliwasa } & 16 & 11.4680 & 0.11385 & 11.233 & 11.703 \\ \text { Isolok - 12 mL - HF } & 10 & 11.3647 & 0.14400 & 11.067 & 11.662\end{array}$

Std Error uses a pooled estimate of error variance

Means and Std Deviations

Level Number Mean Std Dev Std Err Mean Lower 95\% Upper 95\%

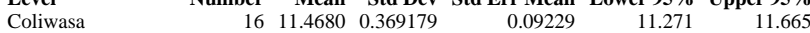

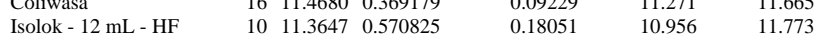

Test

Isolok - $12 \mathrm{~mL}$ - HF-Coliwasa

Assuming unequal variances

$\begin{array}{lrlr}\text { Difference } & -0.10330 & \mathrm{t} \text { Ratio } & -0.50951 \\ \text { Std Err Dif } & 0.20274 & \text { DF } & 13.75666 \\ \text { Upper CL Dif } & 0.33226 & \text { Prob }>|\mathrm{t}| & 0.6185 \\ \text { Lower CL Dif } & -0.53885 & \text { Prob }>\mathrm{t} & 0.6908 \\ \text { Confidence } & 0.95 & \text { Prob }<\mathrm{t} & 0.3092\end{array}$

Tests that the Variances are Equal

Level Count Std Dev MeanAbsDif to Mean MeanAbsDif to Median

$\begin{array}{lllll}\text { Coliwasa } & 16 & 0.3691793 & 0.3060452 & 0.2770044\end{array}$

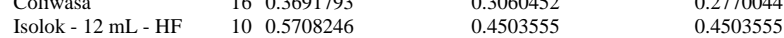

Test F Ratio DFNum DFDen p-Value

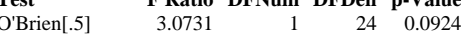

Brown-Forsythe $1.9964 \quad 1 \quad 24 \quad 0.1705$

$\begin{array}{lllll}\text { Levene } & 2.1210 & 1 & 24 & 0.1583\end{array}$

$\begin{array}{lllll}\text { Bartlett } & 2.1320 & 1 & \text {. } & 0.1443\end{array}$

Welch Anova testing Means Equal, allowing Std Devs Not Equal

F Ratio DFNum DFDen Prob $>$ F

$\begin{array}{rrrr}0.2596 & 1 & 13.757 & 0.6185\end{array}$

$\mathbf{t}$ Test
0.5095 


\section{Exhibit A2. Statistical Comparisons for Mid-Rheology (Phase 1) Testing}

Oneway Analysis of Measurement By Sampler Mechanism Type of Material=SME Simulant, Analytical Block $=4$, Analyte $=$ K2O $($ wt $\%)$, Target Value $=0.1591$

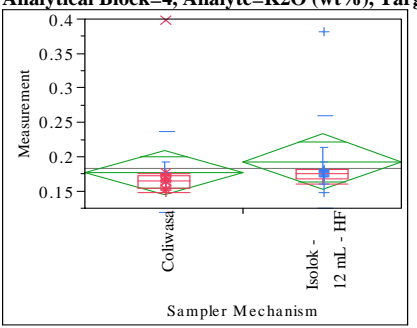

Oneway Anova

Summary of Fit

Adj Rsquare

0.015041

-0.026
-0.06286

0.062286

Observations (or Sum Wgts) $\quad 26$

Test

Isolok - $12 \mathrm{~mL}$ - HF-Coliwasa

Assuming equal variances

Difference $\quad 0.01520$ t Ratio 0.605399

Std Err Dif $\quad 0.02511$ DF 24

Upper CL Dif 0.06702 Prob $>|t| r 0.5506$

Lower CL Dif -0.03662 Prob $>$ t 0.2753

$\begin{array}{lrl}\text { Confidence } & 0.95 \text { Prob }<\mathrm{t} & 0.7247\end{array}$

Analysis of Variance

$\begin{array}{lrrrrr}\text { Source } & \text { DF } & \text { Sum of Squares } & \text { Mean Square } & \text { F Ratio } & \text { Prob }>\text { F } \\ \text { Sampler Mechanism } & 1 & 0.00142189 & 0.001422 & 0.3665 & 0.5506\end{array}$

$\begin{array}{llll}\text { Error } & 24 & 0.09310935 & 0.003880\end{array}$

$\begin{array}{lll}\text { C. Total } & 25 & 0.09453123\end{array}$

Means for Oneway Anova

$\begin{array}{lrrrrr}\text { Means for Oneway Anova } & & & & \\ \text { Level } & \text { Number } & \text { Mean } & \text { Std Error } & \text { Lower 95\% } & \text { Upper 95\% } \\ \text { Coliwasa } & 16 & 0.177415 & 0.01557 & 0.14528 & 0.20955\end{array}$

$\begin{array}{lrrrrr}\text { Coliwasa } & 16 & 0.177415 & 0.01557 & 0.14528 & 0.20955 \\ \text { Isolok - 12 mL - HF } & 10 & 0.192616 & 0.01970 & 0.15196 & 0.23327\end{array}$

Std Error uses a pooled estimate of error variance

Means and Std Deviations

Level Number Mean Std Dev Std Err Mean Lower 95\% Upper 95\%

\begin{tabular}{lrrrrrr} 
Coliwasa & Number & Mean & Std Dev & Std Err Mean & Lower 95\% & Upper 95\% \\
\hline & 16 & 0.177415 & 0.059053 & 0.01476 & 0.14595 & 0.20888
\end{tabular}

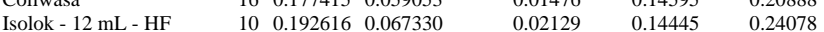

Test

Isolok - $12 \mathrm{~mL}$ - HF-Coliwasa

Assuming unequal variances

$\begin{array}{lrlr}\text { Difference } & 0.01520 & \mathrm{t} \text { Ratio } & 0.586684 \\ \text { Std Err Dif } & 0.02591 \text { DF } & 17.33083 \\ \text { Upper CL Dif } & 0.06978 \text { Prob }>|\mathrm{t}| & 0.5650 \\ \text { Lower CL Dif } & -0.03938 & \text { Prob }>\mathrm{t} & 0.2825 \\ \text { Confidence } & 0.95 & \text { Prob }<\mathrm{t} & 0.7175\end{array}$

Tests that the Variances are Equal

Level Count Std Dev MeanAbsDif to Mean MeanAbsDif to Median

$\begin{array}{lrrrr}\text { Coliwasa } & 16 & 0.0590532 & 0.0273623 & 0.0214193\end{array}$

$\begin{array}{lllll}\text { Coliwasa } & 16 & 0.0590532 & 0.0273623 & 0.0214193 \\ \text { Isolok - } 12 \mathrm{~mL} \text { - HF } & 10 & 0.0673301 & 0.0378485 & 0.0275853\end{array}$

Test F Ratio DFNum DFDen p-Value

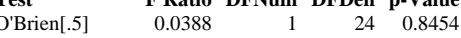

Brown-Forsythe $\quad 0.0674 \quad 1 \quad 24 \quad 0.7974$

$\begin{array}{lllll}\text { Levene } & 0.2431 & 1 & 24 & 0.6265\end{array}$

$\begin{array}{lllll}\text { Bartlett } & 0.1887 & 1 & & 0.6640\end{array}$

Welch Anova testing Means Equal, allowing Std Devs Not Equal

F Ratio DFNum DFDen Prob $>$ F

$\begin{array}{rrrr}0.3442 & 1 & 17.331 & 0.5650\end{array}$

t Test
0.5867
Oneway Analysis of Measurement By Sampler Mechanism Type of Material=SME Simulant, Analytical Block $=4$, Analyte $=\mathrm{Li} 2 \mathrm{O}(\mathrm{wt} \%)$, Target Value $=4.674$

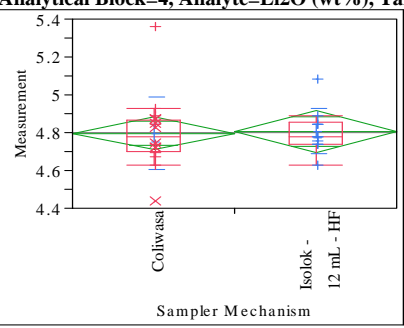

Oneway Anova

Summary of Fit

Adj Rsquare

0.000975

\begin{tabular}{ll}
-0.04065 \\
\hline
\end{tabular}

Mean of Response

4.800967

Test

Isolok - $12 \mathrm{~mL}$ - HF-Coliwasa

Assuming equal variances

$\begin{array}{lrlr}\text { Difference } & 0.01050 & \text { t Ratio } & 0.153045 \\ \text { Std Err Dif } & 0.06858 & \text { DF } & 24 \\ \text { Upper CL Dif } & 0.15203 & \text { Prob }>|t| & 0.8796 \\ \text { Lower CL Dif } & -0.13104 & \text { Prob }>t & 0.4398 \\ \text { Confidence } & 0.95 & \text { Prob }<\mathrm{t} & 0.5602\end{array}$

Analysis of Variance

$\begin{array}{lrrrr}\text { Source } & \text { DF Sum of Squares } & \text { Mean Square } & \text { F Ratio Prob }>\text { F }\end{array}$

24 0.00067787

$\begin{array}{lll}\text { C. Total } & 25 & 0.69524676\end{array}$

Means for Oneway Anova

Level Number Mean Std Error Lower 95\% Upper 95\%

$\begin{array}{lrrrrr}\text { Coliwasa } & 16 & 4.79693 & 0.04253 & 4.7092 & 4.8847\end{array}$

$\begin{array}{lllll}\text { Isolok - } 12 \text { mL - HF } & 10 & 4.80743 & 0.05380 & 4.6964\end{array}$

Std Error uses a pooled estimate of error variance

Means and Std Deviations

Level Number Mean Std Dev Std Err Mean Lower 95\% Upper 95\%

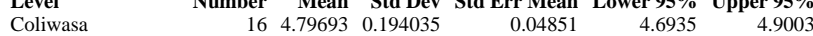

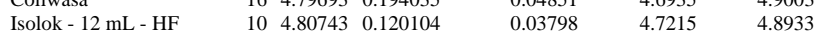

Test

Isolok - $12 \mathrm{~mL}$ - HF-Coliwasa

Assuming unequal variances

$\begin{array}{lrlr}\text { Difference } & 0.01050 & \mathrm{t} \text { Ratio } & 0.170356 \\ \text { Std Err Dif } & 0.06161 & \text { DF } & 23.99739 \\ \text { Upper CL Dif } & 0.13765 & \text { Prob }>|t| & 0.8662 \\ \text { Lower CL Dif } & -0.11666 & \text { Prob }>\text { t } & 0.4331 \\ \text { Confidence } & 0.95 & \text { Prob }<\mathrm{t} & 0.5669\end{array}$

Tests that the Variances are Equal

Level Count Std Dev MeanAbsDif to Mean MeanAbsDif to Median

$\begin{array}{lllll}\text { Coliwasa } & 16 & 0.1940349 & 0.1305196 & 0.1305196\end{array}$

$\begin{array}{llll}\text { Isolok - } 12 \mathrm{~mL} \text { - HF } & 10 & 0.1201045 & 0.0852548\end{array}$

Test F Ratio DFNum DFDen p-Value

$\begin{array}{lrrrr}\text { O'Brien[.5] } & 0.6174 & 1 & 24 & 0.4397\end{array}$

$\begin{array}{lllll}\text { Brown-Forsythe } & 1.0291 & 1 & 24 & 0.3205\end{array}$

$\begin{array}{lllll}\text { Levene } & 0.8663 & 1 & 24 & 0.3613\end{array}$

$\begin{array}{lrrrr}\text { Bartlett } & 2.2195 & 1 & \text {. } & 0.1363 \\ \text { F Test 2-sided } & 2.6100 & 15 & 9 & 0.1493\end{array}$

Welch Anova testing Means Equal, allowing Std Devs Not Equal

F Ratio DFNum DFDen Prob $>$ F

$\begin{array}{llll}0.0290 & 1 & 23.997 & 0.8662\end{array}$

t Test

0.1704 


\section{Exhibit A2. Statistical Comparisons for Mid-Rheology (Phase 1) Testing}

Oneway Analysis of Measurement By Sampler Mechanism Type of Material=SME Simulant, Analytical Block=4, Analyte $=\mathrm{MgO}(\mathrm{wt} \%)$, Target Value $=1.014$

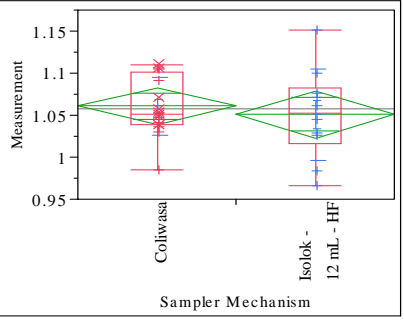

Oneway Anova

Summary of Fit

Adj Rsquare $\quad 0.013252$

Root Mean Square Error $\quad 0.042932$

Mean of Response

Observations (or Sum Wgts) $\quad 26$

Test

Isolok - $12 \mathrm{~mL}$ - HF-Coliwasa

Assuming equal variances

Difference $\quad-0.00983$ t Ratio $\quad-0.56773$

Std Err Dif $\quad 0.01731$ DF 24

Upper CL Dif 0.02589 Prob $>|t| r \mid r .5755$

$\begin{array}{lll}\text { Lower CL Dif }-0.04554 \text { Prob }>t & 0.7123\end{array}$

$\begin{array}{lrl}\text { Confidence } & 0.95 \text { Prob }<\mathrm{t} & 0.2877\end{array}$

Analysis of Variance

$\begin{array}{lrrrrr}\text { Source } & \text { DF } & \text { Sum of Squares } & \text { Mean Square } & \text { F Ratio } & \text { Prob }>\text { F } \\ \text { Sampler Mechanism } & 1 & 0.00059409 & 0.000594 & 0.3223 & 0.5755\end{array}$

$\begin{array}{llll}\text { Error } & 24 & 0.04423543 & 0.001843\end{array}$

$\begin{array}{lll}\text { C. Total } & 25 & 0.04482951\end{array}$

Means for Oneway Anova

Level Number Mean Std Error Lower 95\% Upper 95\%

$\begin{array}{lrrrrr}\text { Coliwasa } & 16 & 1.06069 & 0.01073 & 1.0385 & 1.0828\end{array}$

$\begin{array}{llllll}\text { Isolok - 12 mL - HF } & 10 & 1.05086 & 0.01358 & 1.0228 & 1.0789\end{array}$

Std Error uses a pooled estimate of error variance

Means and Std Deviations

Level Number Mean Std Dev Std Err Mean Lower 95\% Upper 95\%

$\begin{array}{lrrrrrr}\text { Coliwasa } & \text { Number } & \text { Mean } & \text { Std Dev } & \text { Std Err Mean } & \text { Lower 95\% } & \text { Upper 95\% } \\ \text { 16 } & 1.06069 & 0.034578 & 0.00864 & 1.0423 & 1.0791\end{array}$

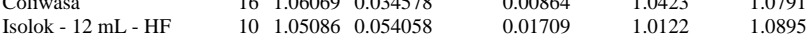

TTest

Isolok - $12 \mathrm{~mL}$ - HF-Coliwasa

Assuming unequal variances

Difference $\quad-0.00983$ t Ratio $\quad-0.51291$

Std Err Dif $\quad 0.01916$ DF $\quad 13.65556$

Upper CL Dif 0.03136 Prob $>|t| \quad 0.6162$

Lower CL Dif -0.05101 Prob $>t \quad 0.6919$

$\begin{array}{lrl}\text { Confidence } & 0.95 \text { Prob }<\mathrm{t} & 0.3081\end{array}$

Tests that the Variances are Equal

Level Count Std Dev MeanAbsDif to Mean MeanAbsDif to Median

$\begin{array}{lllll}\text { Coliwasa } & 16 & 0.0345780 & 0.0276211 & 0.0261182\end{array}$

$\begin{array}{lllll}\text { Coliwasa } & 16 & 0.0345780 & 0.0276211 & 0.0261182 \\ \text { Isolok - } 12 \mathrm{~mL} \text { - HF } & 10 & 0.0540585 & 0.0409600 & 0.0409600\end{array}$

Test F Ratio DFNum DFDen p-Value

$\begin{array}{lrrrr}\text { O'Brien[.5] } & 2.3105 & 1 & 24 & 0.1416 \\ \text { Brown-Forsythe } & 1.8092 & 1 & 24 & 0.1912\end{array}$

$\begin{array}{lllll}\text { Levene } & 1.7230 & 1 & 24 & 0.2017\end{array}$

$\begin{array}{lllll}\text { Bartlett } & 2.2420 & 1 & 0.1343\end{array}$

Welch Anova testing Means Equal, allowing Std Devs Not Equal

F Ratio DFNum DFDen Prob $>$ F

$\begin{array}{rrrr}0.2631 & 1 & 13.656 & 0.6162\end{array}$

t Test

0.5129
Oneway Analysis of Measurement By Sampler Mechanism Type of Material=SME Simulant, Analytical Block $=4$, Analyte $=\mathrm{MnO}(\mathrm{wt} \%)$, Target Value $=1.779$

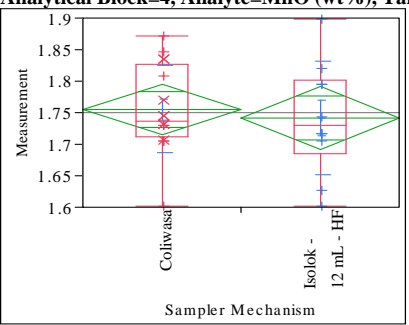

Oneway Anova

Summary of Fit

Adj Rsquare

0.007645
-0.0337

\begin{tabular}{ll}
0.077285 \\
\hline
\end{tabular}

1.750073

t Test

Isolok - $12 \mathrm{~mL}$ - HF-Coliwasa

Assuming equal variances

$\begin{array}{lrlr}\text { Difference } & -0.01340 & \mathrm{t} \text { Ratio } & -0.42999 \\ \text { Std Err Dif } & 0.03115 & \mathrm{DF} & 24 \\ \text { Upper CL Dif } & 0.05090 & \text { Prob }>|t| & 0.6710 \\ \text { Lower CL Dif } & -0.07770 & \text { Prob }>\mathrm{t} & 0.6645 \\ \text { Confidence } & 0.95 & \text { Prob }<\mathrm{t} & 0.3355\end{array}$

Analysis of Variance

$\begin{array}{lrrrrr}\text { Source } & \text { DF } & \text { Sum of Squares } & \text { Mean Square } & \text { F Ratio } & \text { Prob > F } \\ \text { Sampler Mechanism } & 1 & 0.00110436 & 0.001104 & 0.1849 & 0.6710\end{array}$

$\begin{array}{lrrr}\text { Error } & 24 & 0.14335189 & 0.005973\end{array}$

$\begin{array}{lll}\text { C. Total } & 25 & 0.14445625\end{array}$

Means for Oneway Anova

Level Number Mean Std Error Lower 95\% Upper 95\%

$\begin{array}{lrrrrr}\text { Coliwasa } & 16 & 1.75523 & 0.01932 & 1.7153 & 1.7951\end{array}$

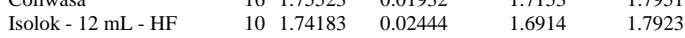

Std Error uses a pooled estimate of error variance

Means and Std Deviations

Level Number Mean Std Dev Std Err Mean Lower 95\% Upper 95\%

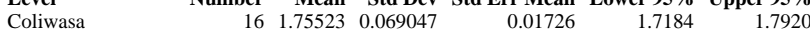

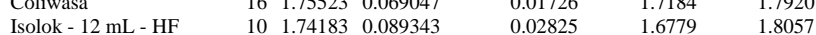

Test

Isolok - $12 \mathrm{~mL}$ - HF-Coliwasa

Assuming unequal variances

$\begin{array}{lrlr}\text { Difference } & -0.01340 & \mathrm{t} \text { Ratio } & -0.40461 \\ \text { Std Err Dif } & 0.03311 & \text { DF } & 15.66376 \\ \text { Upper CL Dif } & 0.05691 & \text { Prob }>|t| & 0.6912 \\ \text { Lower CL Dif } & -0.08371 & \text { Prob }>\mathrm{t} & 0.6544 \\ \text { Confidence } & 0.95 & \text { Prob }<\mathrm{t} & 0.3456\end{array}$

Tests that the Variances are Equal

Level Count Std Dev MeanAbsDif to Mean MeanAbsDif to Median

$\begin{array}{lllll}\text { Coliwasa } & 16 & 0.0690470 & 0.0538673 & 0.0508410\end{array}$

$\begin{array}{llll}\text { Isolok - } 12 \mathrm{~mL} \text { - HF } & 10 & 0.0893430 & 0.0684336\end{array}$

Test F Ratio DFNum DFDen p-Value

$\begin{array}{lrrrr}\text { O'Brien[.5] } & 0.8875 & 1 & 24 & 0.3555\end{array}$

$\begin{array}{lllll}\text { Brown-Forsythe } & 0.7370 & 1 & 24 & 0.399\end{array}$

$\begin{array}{lllll}\text { Levene } & 0.6256 & 1 & 24 & 0.4367\end{array}$

$\begin{array}{lllll}\text { Bartlett } & 0.7383 & 1 & \text {. } & 0.3902 \\ \text { F Test 2-sided } & 1.6743 & 9 & 15 & 0.3628\end{array}$

Welch Anova testing Means Equal, allowing Std Devs Not Equal

F Ratio DFNum DFDen Prob $>$ F

$\begin{array}{rrrr}0.1637 & 1 & 15.664 & 0.6912\end{array}$

t Test
0.4046 


\section{Exhibit A2. Statistical Comparisons for Mid-Rheology (Phase 1) Testing}

Oneway Analysis of Measurement By Sampler Mechanism Type of Material=SME Simulant, Analytical Block=4, Analyte $=\mathrm{Na2O}(\mathrm{wt} \%)$, Target Value=11.659

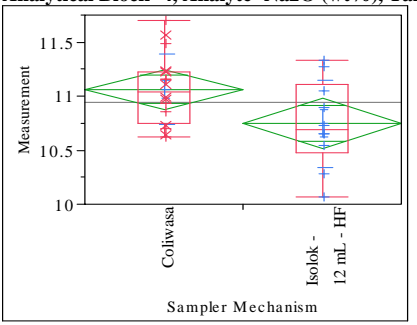

Oneway Anova

Summary of Fit

Rsquare

Adj Rsquare 0.16616

Root Mean Square Error $\quad 0.357618$

$\begin{array}{lr}\text { Mean of Response } & 10.94161 \\ \text { Observations (or Sum Wgts) } & 26\end{array}$

TTest

Isolok - $12 \mathrm{~mL}$ - HF-Coliwasa

Assuming equal variances

Difference $\quad-0.31526$ t Ratio $\quad-2.1869$

Std Err Dif $\quad 0.14416$ DF 24

Upper CL Dif -0.01773 Prob $>|t| \quad 0.0387$

Lower CL Dif -0.61280 Prob $>t \quad 0.9806$

$\begin{array}{lrl}\text { Confidence } & 0.95 \text { Prob }<\mathrm{t} \quad 0.0194\end{array}$

Analysis of Variance

$\begin{array}{lrrrrr}\text { Source } & \text { DF } & \text { Sum of Squares } & \text { Mean Square } & \text { F Ratio } & \text { Prob > F } \\ \text { Sampler Mechanism } & 1 & 0.6116374 & 0.611637 & 4.7825 & 0.0387\end{array}$

$\begin{array}{llll}\text { Error } & 24 & 3.0693680 & 0.127890\end{array}$

3.6810054

Means for Oneway Anova

Level Number Mean Std Error Lower 95\% Upper 95\%

$\begin{array}{lrrrrr}\text { Coliwasa } & 16 & 11.0629 & 0.08940 & 10.878 & 11.247\end{array}$

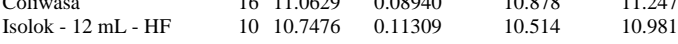

Std Error uses a pooled estimate of error variance

Means and Std Deviations

Level Number Mean Std Dev Std Err Mean Lower 95\% Upper 95\%

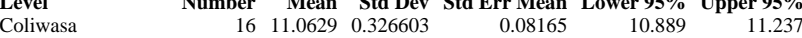

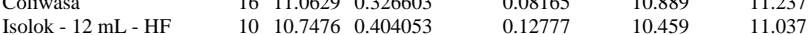

Test

Isolok - $12 \mathrm{~mL}$ - HF-Coliwasa

Assuming unequal variances

Difference $\quad-0.31526$ t Ratio $\quad-2.07912$

Std Err Dif $\quad 0.15163$ DF $\quad 16.22765$

Upper CL Dif 0.00582 Prob $>|t| r \mid 0.0538$

Lower CL Dif -0.63635 Prob $>t \quad 0.9731$

$\begin{array}{lrl}\text { Confidence } & 0.95 \text { Prob }<\mathrm{t} & 0.0269\end{array}$

Tests that the Variances are Equal

Level Count Std Dev MeanAbsDif to Mean MeanAbsDif to Median

$\begin{array}{lllll}\text { Coliwasa } & 16 & 0.3266027 & 0.2620175 & 0.2620175\end{array}$

$\begin{array}{lllll}\text { Coliwasa } & 16 & 0.3266027 & 0.2620175 & 0.2620175 \\ \text { Isolok - } 12 \mathrm{~mL} \text { - HF } & 10 & 0.4040529 & 0.3121968 & 0.3086920\end{array}$

Test F Ratio DFNum DFDen p-Value

\begin{tabular}{lrrrr} 
& F Ratio & DFNum & DFDen & p-Value \\
O'Brien[.5] & 0.8031 & 1 & 24 & 0.379 \\
\hline
\end{tabular}

$\begin{array}{lllll}\text { Brown-Forsythe } & 0.3036 & 1 & 24 & 0.5867\end{array}$

$\begin{array}{lllll}\text { Levene } & 0.3733 & 1 & 24 & 0.5470\end{array}$

$\begin{array}{lllll}\text { Bartlett } & 0.5014 & 1 & . & 0.4789 \\ & 1.5305 & 9 & 15 & 0.4479\end{array}$

Welch Anova testing Means Equal, allowing Std Devs Not Equal

F Ratio DFNum DFDen Prob $>$ F

$\begin{array}{rrrr}4.3227 & 1 & 16.228 & 0.0538\end{array}$

t Test

2.0791
Oneway Analysis of Measurement By Sampler Mechanism Type of Material=SME Simulant, Analytical Block $=4$, Analyte $=\mathrm{NiO}($ wt $\%)$, Target Value $=0.41$

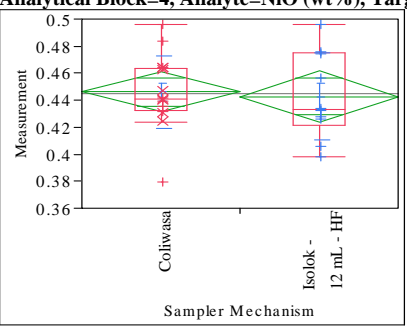

Oneway Anova

Summary of Fit

Adj Rsquare

Error $\quad 0.028873$ 0.444886

t Test

Isolok - $12 \mathrm{~mL}$ - HF-Coliwasa

Assuming equal variances

$\begin{array}{lrlr}\text { Difference } & -0.00334 & \mathrm{t} \text { Ratio } & -0.28699 \\ \text { Std Err Dif } & 0.01164 & \text { DF } & 24 \\ \text { Upper CL Dif } & 0.02068 & \text { Prob }>|t| & 0.7766 \\ \text { Lower CL Dif } & -0.02736 & \text { Prob }>\mathrm{t} & 0.6117 \\ \text { Confidence } & 0.95 & \text { Prob }<\mathrm{t} & 0.3883\end{array}$

Analysis of Variance

Source DF Sum of Squares Mean Square F Ratio Prob > F

\begin{tabular}{lrrr} 
Error & 1 & 0.00006866 & 0.000069 \\
\hline
\end{tabular}

$\begin{array}{lll}\text { C. Total } & 25 & 0.02007579\end{array}$

Means for Oneway Anova

Level Number Mean Std Error Lower 95\% Upper 95\%

$\begin{array}{lrrrrr}\text { Coliwasa } & 16 & 0.446170 & 0.00722 & 0.43127 & 0.46107\end{array}$

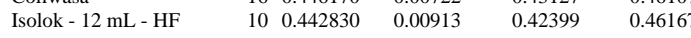

Std Error uses a pooled estimate of error variance

Means and Std Deviations

Level Number Mean Std Dev Std Err Mean Lower 95\% Upper 95\%

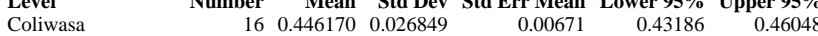

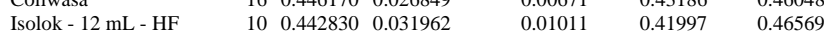

Test

Isolok - $12 \mathrm{~mL}$ - HF-Coliwasa

Assuming unequal variances

$\begin{array}{lrlr}\text { Difference } & -0.00334 & \mathrm{t} \text { Ratio } & -0.27531 \\ \text { Std Err Dif } & 0.01213 & \text { DF } & 16.73591 \\ \text { Upper CL Dif } & 0.02229 & \text { Prob }>|t| & 0.7865 \\ \text { Lower CL Dif } & -0.02897 & \text { Prob }>\mathrm{t} & 0.6068 \\ \text { Confidence } & 0.95 & \text { Prob }<\mathrm{t} & 0.3932\end{array}$

Tests that the Variances are Equal

Level Count Std Dev MeanAbsDif to Mean MeanAbsDif to Median

$\begin{array}{lllll}\text { Coliwasa } & 16 & 0.0268489 & 0.0195050 & 0.0189284\end{array}$

$\begin{array}{lllll}\text { Isolok - } 12 \mathrm{~mL} \text { - HF } & 10 & 0.0319620 & 0.0264680 & 0.0246865\end{array}$

Test F Ratio DFNum DFDen p-Value

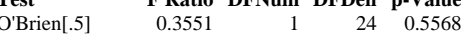

$\begin{array}{lllll}\text { Brown-Forsythe } & 0.5137 & 1 & 24 & 0.4805\end{array}$

$\begin{array}{lllll}\text { Levene } & 1.0356 & 1 & 24 & 0.3190\end{array}$

$\begin{array}{lllll}\text { Bartlett } & 0.3351 & 1 & \text {. } & 0.5627 \\ \text { FTest 2-sided } & 1.4171 & 9 & 15 & 0.5290\end{array}$

Welch Anova testing Means Equal, allowing Std Devs Not Equal

F Ratio DFNum DFDen Prob $>$ F

$\begin{array}{lrrr}0.0758 & 1 & 16.736 & 0.7865\end{array}$

$\mathbf{t}$ Test
0.2753 


\section{Exhibit A2. Statistical Comparisons for Mid-Rheology (Phase 1) Testing}

Oneway Analysis of Measurement By Sampler Mechanism Type of Material=SME Simulant, Analytical Block=4, Analyte $=\mathrm{SiO} 2($ wt $\%)$, Target Value=50.985

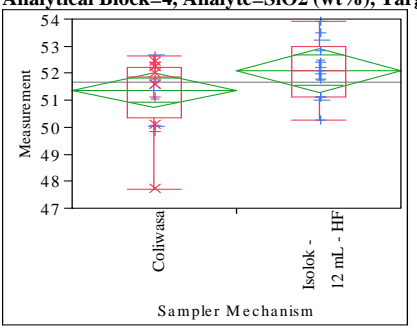

Oneway Anova

Summary of Fit

Adj Rsquare $\quad 0.08308$

Root Mean Square Error $\quad 1.25058$

51.65587

Observations (or Sum Wgts) $\quad 26$

TTest

Isolok - $12 \mathrm{~mL}$ - HF-Coliwasa

Assuming equal variances

Difference $\quad 0.7434$ t Ratio 1.474644

Std Err Dif $\quad 0.5041$ DF 24

Upper CL Dif 1.7839 Prob $>|t| \quad 0.1533$

Lower CL Dif -0.2971 Prob $>t \quad 0.0767$

$\begin{array}{lrl}\text { Confidence } & 0.95 \text { Prob }<\mathrm{t} & 0.9233\end{array}$

Analysis of Variance

$\begin{array}{lrrrrr}\text { Source } & \text { DF } & \text { Sum of Squares } & \text { Mean Square } & \text { F Ratio } & \text { Prob }>\text { F } \\ \text { Sampler Mechanism } & 1 & 3.400945 & 3.40095 & 2.1746 & 0.1533\end{array}$

$\begin{array}{lrrr}\text { Error } & 24 & 37.535022 & 1.56396\end{array}$

$\begin{array}{lll}\text { C. Total } & 25 & 40.935967\end{array}$

Means for Oneway Anova

$\begin{array}{lrrrrr}\text { Level } & \text { Number } & \text { Mean } & \text { Std Error } & \text { Lower 95\% } & \text { Upper 95\% } \\ \text { Coliwasa } & 16 & 51.3699 & 0.31265 & 50.725 & 52.015\end{array}$

$\begin{array}{lrrrrr}\text { Coliwasa } & 16 & 51.3699 & 0.31265 & 50.725 & 52.015 \\ \text { Isolok - 12 mL - HF } & 10 & 52.1133 & 0.39547 & 51.297 & 52.930\end{array}$

Std Error uses a pooled estimate of error variance

Means and Std Deviations

Level Number Mean Std Dev Std Err Mean Lower 95\% Upper 95\%

\begin{tabular}{lrrrrrr} 
Coliwasa & Number & Mean & Std Dev & Std Err Mean & Lower $95 \%$ & Upper 95\% \\
\hline & 16 & 51.3699 & 1.32539 & 0.33135 & 50.664 & 52.076
\end{tabular}

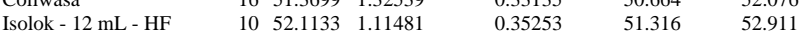

Test

Isolok - $12 \mathrm{~mL}$ - HF-Coliwasa

Assuming unequal variances

$\begin{array}{lrlr}\text { Difference } & 0.7434 & \text { t Ratio } & 1.536571 \\ \text { Std Err Dif } & 0.4838 & \text { DF } & 21.7437 \\ \text { Upper CL Dif } & 1.7475 & \text { Prob }>|t| & 0.1388 \\ \text { Lower CL Dif } & -0.2606 & \text { Prob }>t & 0.0694 \\ \text { Confidence } & 0.95 & \text { Prob }<\mathrm{t} & 0.9306\end{array}$

Tests that the Variances are Equal

Level Count Std Dev MeanAbsDif to Mean MeanAbsDif to Median

$\begin{array}{lllll}\text { Coliwasa } & 16 & 1.325388 & 1.006140 & 0.9092025\end{array}$

$\begin{array}{lllll}\text { Coliwasa } & 16 & 1.325388 & 1.006140 & 0.9092025 \\ \text { Isolok - 12 mL - HF } & 10 & 1.114810 & 0.855720 & 0.8557200\end{array}$

Test F Ratio DFNum DFDen p-Value

$\begin{array}{lrrrr}\text { O'Brien[.5] } & 0.1856 & 1 & 24 & 0.6705 \\ \text { Brown-Forsythe } & 0.0200 & 1 & 24 & 0.8887\end{array}$

$\begin{array}{lllll}\text { Levene } & 0.2384 & 1 & 24 & 0.6298\end{array}$

$\begin{array}{lrrrr}\text { Bartlett } & 0.3117 & 1 & \text {. } & 0.5767\end{array}$

Welch Anova testing Means Equal, allowing Std Devs Not Equal

F Ratio DFNum DFDen Prob $>$ F

$\begin{array}{llll}2.3611 & 1 & 21.744 & 0.1388\end{array}$

$\mathbf{t}$ Test
1.5366
Oneway Analysis of Measurement By Sampler Mechanism Type of Material=SME Simulant, Analytical Block $=4$, Analyte $=$ Sum of Oxides ( $w t \%)$, Target Value $=99.553$

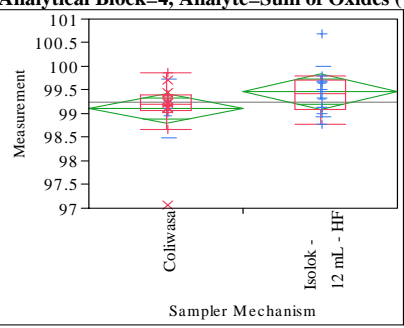

Oneway Anova

Summary of Fit

Adj Rsquare

0.588922

Mean of Response 99.24931

Observations (or Sum Wgts) $\quad 26$

T Test

Isolok - $12 \mathrm{~mL}$ - HF-Coliwasa

Assuming equal variances

Difference $\quad 0.35995$ t Ratio 1.516188

Std Err Dif $\quad 0.23740$ DF $\quad 24$

Upper CL Dif 0.84992 Prob $>|t| \quad 0.1425$

$\begin{array}{rrr}\text { Lower CL Dif }-0.13003 \text { Prob }>t & 0.0713\end{array}$

$\begin{array}{lrl}\text { Confidence } & 0.95 \text { Prob }<\mathrm{t} & 0.9287\end{array}$

Analysis of Variance

Source DF Sum of Squares Mean Square F Ratio Prob $>$ F

$\begin{array}{llll}\text { Error } & 24 & 8.3239076 & 0.346829\end{array}$

$\begin{array}{lll}\text { C. Total } & 25 & 9.1212081\end{array}$

Means for Oneway Anova

Level Number Mean Std Error Lower 95\% Upper 95\%

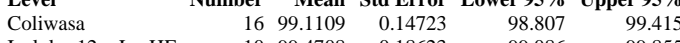

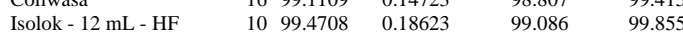

Std Error uses a pooled estimate of error variance

Means and Std Deviations

Level Number Mean Std Dev Std Err Mean Lower 95\% Upper 95\%

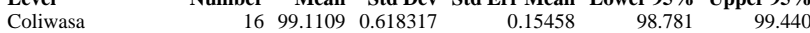

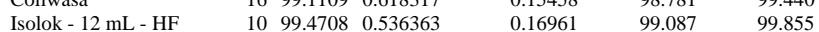

Test

Isolok - $12 \mathrm{~mL}$ - HF-Coliwasa

Assuming unequal variances

$\begin{array}{lrlr}\text { Difference } & 0.35995 & \mathrm{t} \text { Ratio } & 1.568497 \\ \text { Std Err Dif } & 0.22948 & \text { DF } & 21.3303 \\ \text { Upper CL Dif } & 0.83674 & \text { Prob }>|t| & 0.1315 \\ \text { Lower CL Dif } & -0.11684 & \text { Prob }>\mathrm{t} & 0.0657 \\ \text { Confidence } & 0.95 & \text { Prob }<\mathrm{t} & 0.9343\end{array}$

Tests that the Variances are Equal

Level Count Std Dev MeanAbsDif to Mean MeanAbsDif to Median

$\begin{array}{lllll}\text { Coliwasa } & 16 & 0.6183171 & 0.3378880 & 0.3205276\end{array}$

$\begin{array}{llll}\text { Isolok - } 12 \mathrm{~mL} \text { - HF } & 10 & 0.5363629 & 0.3960690\end{array}$

Test F Ratio DFNum DFDen p-Value

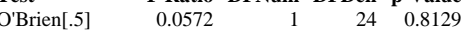

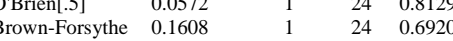

$\begin{array}{lllll}\text { Levene } & 0.1014 & 1 & 24 & 0.7529\end{array}$

$\begin{array}{lrrrr}\text { Bartlett } & 0.2119 & 1 & \text {. } & 0.6453\end{array}$

Welch Anova testing Means Equal, allowing Std Devs Not Equal

F Ratio DFNum DFDen Prob $>$ F

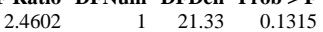

t Test

1.5685 


\section{Exhibit A2. Statistical Comparisons for Mid-Rheology (Phase 1) Testing}

Oneway Analysis of Measurement By Sampler Mechanism Type of Material=SME Simulant, Analytical Block=4, Analyte $=$ TiO2 $($ wt $\%)$, Target Value $=0.0711$

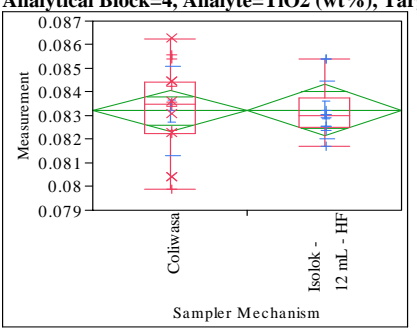

Oneway Anova

Summary of Fit

Adj Rsquare $\quad 4.023 e-5$

Root Mean Square Error $\quad 0.001664$

Mean of Response

$\begin{array}{lr}\text { Observations (or Sum Wgts) } & 26\end{array}$

TTest

Isolok - $12 \mathrm{~mL}$ - HF-Coliwasa

Assuming equal variances

Difference $\quad 2.085 \mathrm{e}-5$ t Ratio $\quad 0.031075$

Std Err Dif $\quad 0.00067$ DF 24

Upper CL Dif 0.00141 Prob $>|t| \quad 0.9755$

Lower CL Dif -0.00136 Prob $>t \quad 0.4877$

$\begin{array}{lrl}\text { Confidence } & 0.95 \text { Prob }<\mathrm{t} & 0.5123\end{array}$

Analysis of Variance

$\begin{array}{lrrrrr}\text { Source } & \text { DF } & \text { Sum of Squares } & \text { Mean Square } & \text { F Ratio } & \text { Prob > F } \\ \text { Sampler Mechanism } & 1 & 2.67522 \mathrm{e}-9 & 2.6752 \mathrm{e}-9 & 0.0010 & 0.9755\end{array}$

$\begin{array}{llll}\text { Error } & 24 & 0.00006649 & 2.7703 \mathrm{e}-6\end{array}$

$\begin{array}{lll}\text { C. Total } & 25 & 0.00006649\end{array}$

Means for Oneway Anova

$\begin{array}{lrrrrr}\text { Level } & \text { Number } & \text { Mean } & \text { Std Error } & \text { Lower 95\% } & \text { Upper 95\% } \\ \text { Leliwasa } & 16 & 0.083212 & 0.00042 & 0.08235 & 0.08407\end{array}$

$\begin{array}{lrrrrr}\text { Coliwasa } & 16 & 0.083212 & 0.00042 & 0.08235 & 0.08407 \\ \text { Isolok - 12 mL - HF } & 10 & 0.083233 & 0.00053 & 0.08215 & 0.08432\end{array}$

Std Error uses a pooled estimate of error variance

Means and Std Deviations

Level Number Mean Std Dev Std Err Mean Lower 95\% Upper 95\%

$\begin{array}{lrrrrrr}\text { Leviwasa } & \text { Number } & \text { Mean } & \text { Std Dev } & \text { Std Err Mean } & \text { Lower } 95 \% & \text { Upper 95\% } \\ \text { Coliwa } & 0.083212 & 0.001881 & 0.00047 & 0.08221 & 0.08421\end{array}$

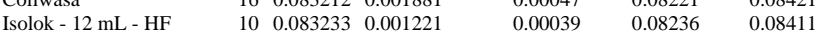

Test

Isolok - $12 \mathrm{~mL}$ - HF-Coliwasa

Assuming unequal variances

Difference 2.085e-5 t Ratio 0.03427

Std Err Dif $\quad 0.00061$ DF 23.9226

Upper CL Dif 0.00128 Prob $>|t| r \mid \begin{array}{ll}23.922 \\ 0.9729\end{array}$

Lower CL Dif -0.00124 Prob $>t \quad 0.4865$

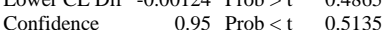

Tests that the Variances are Equal

Level Count Std Dev MeanAbsDif to Mean MeanAbsDif to Median

$\begin{array}{lrrrr}\text { Coliwasa } & 16 & 0.0018811 & 0.0014830 & 0.0014595\end{array}$

$\begin{array}{lllll}\text { Coliwasa } & 16 & 0.0018811 & 0.0014830 & 0.0014595 \\ \text { Isolok - } 12 \mathrm{~mL} \text { - HF } & 10 & 0.0012207 & 0.0008674 & 0.0008006\end{array}$

Test F Ratio DFNum DFDen p-Value

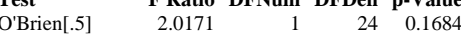

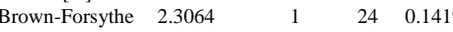

$\begin{array}{lllll}\text { Levene } & 2.3538 & 1 & 24 & 0.138\end{array}$

$\begin{array}{lrrrr}\text { Bartlett } & 1.8275 & 1 & \text {. } & 0.1764\end{array}$

Welch Anova testing Means Equal, allowing Std Devs Not Equal

F Ratio DFNum DFDen Prob > F

$\begin{array}{rrrr}0.0012 & 1 & 23.923 & 0.9729\end{array}$

t Test

0.0343
Oneway Analysis of Measurement By Sampler Mechanism Type of Material=SME Simulant, Analytical Block $=4$, Analyte $=\mathrm{ZnO}(\mathrm{wt} \%)$, Target Value $=\mathbf{0 . 0 9 5 8}$

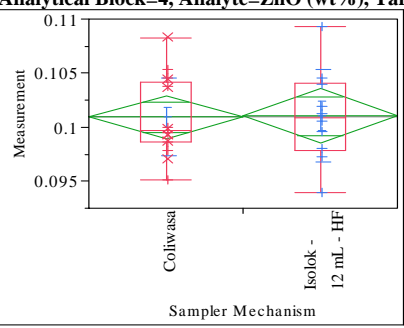

Oneway Anova

Summary of Fit

Adj Rsquare 0.003868
0.100963

Mean of Response

Observations (or Sum Wgts)

Test

Isolok - $12 \mathrm{~mL}$ - HF-Coliwasa

Assuming equal variances

Difference $\quad 0.00013$ t Ratio 0.080838

Std Err Dif $\quad 0.00156$ DF 24

Upper CL Dif 0.00334 Prob $>|t| \quad 0.9362$

Lower CL Dif -0.00309 Prob $>\mathrm{t} \quad 0.4681$

$\begin{array}{lrl}\text { Confidence } & 0.95 \text { Prob }<\mathrm{t} & 0.5319\end{array}$

Analysis of Variance

$\begin{array}{lrrrrr}\text { Source } & \text { DF } & \text { Sum of Squares } & \text { Mean Square } & \text { F Ratio } & \text { Prob }>\text { F } \\ \text { Sampler Mechanism } & 1 & 9.77543 e-8 & 9.775 e-8 & 0.0065 & 0.9362\end{array}$

$\begin{array}{llll}\text { Error } & 24 & 0.00035902 & 0.000015\end{array}$

$\begin{array}{lll}\text { C. Total } & 25 & 0.00035912\end{array}$

Means for Oneway Anova

Level $\quad$ Number Mean Std Error Lower 95\% Upper 95\%

$\begin{array}{lllllr}\text { Coliwasa } & 16 & 0.100914 & 0.00097 & 0.09892 & 0.1029\end{array}$

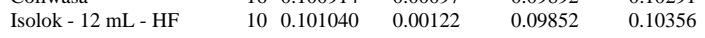

Std Error uses a pooled estimate of error variance

Means and Std Deviations

Level Number Mean Std Dev Std Err Mean Lower 95\% Upper 95\%

$\begin{array}{lllllrr}\text { Coliwasa } & 16 & 0.100914 & 0.003594 & 0.00090 & 0.09900 & 0.10283\end{array}$

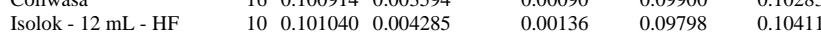

Test

Isolok - $12 \mathrm{~mL}$ - HF-Coliwasa

Assuming unequal variances

$\begin{array}{lrlr}\text { Difference } & 0.00013 & \mathrm{t} \text { Ratio } & 0.077519 \\ \text { Std Err Dif } & 0.00163 & \text { DF } & 16.71636 \\ \text { Upper CL Dif } & 0.00356 & \text { Prob }>|t| & 0.9391 \\ \text { Lower CL Dif } & -0.00331 & \text { Prob }>\mathrm{t} & 0.4696 \\ \text { Confidence } & 0.95 & \text { Prob }<\mathrm{t} & 0.5304\end{array}$

Tests that the Variances are Equal

Level Count Std Dev MeanAbsDif to Mean MeanAbsDif to Median

$\begin{array}{lllll}\text { Coliwasa } & 16 & 0.0035942 & 0.0030478 & 0.0027930\end{array}$

$\begin{array}{llll}\text { Isolok - } 12 \mathrm{~mL} \text { - HF } & 10 & 0.0042849 & 0.0031493\end{array}$

Test F Ratio DFNum DFDen p-Value

$\begin{array}{lrrrr}\text { O'Brien[.5] } & 0.4172 & 1 & 24 & 0.5245\end{array}$

$\begin{array}{lllll}\text { Brown-Forsythe } & 0.1165 & 1 & 24 & 0.7358\end{array}$

$\begin{array}{lllll}\text { Levene } & 0.0137 & 1 & 24 & 0.9078\end{array}$

$\begin{array}{lllll}\text { Bartlett } & 0.3407 & 1 & 0.5594 \\ \text { F Test 2-sided } & 1.4213 & 9 & 15 & 0.5258\end{array}$

Welch Anova testing Means Equal, allowing Std Devs Not Equal

F Ratio DFNum DFDen Prob $>$ F

$\begin{array}{lrrr}0.0060 & 1 & 16.716 & 0.9391\end{array}$

t Test

0.0775 


\section{Exhibit A2. Statistical Comparisons for Mid-Rheology (Phase 1) Testing}

Oneway Analysis of Measurement By Sampler Mechanism Type of Material=SME Simulant, Analytical Block $=4$, Analyte $=\mathrm{ZrO2}(\mathrm{wt} \%)$, Target Value $=\mathbf{0 . 3 5 4 7}$

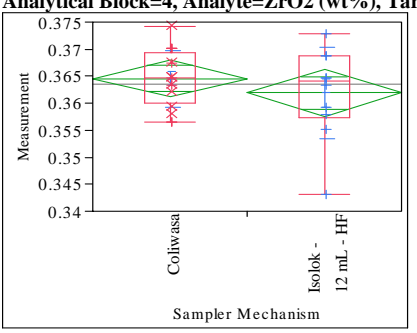

Oneway Anova

Summary of Fit

Adj Rsquare $\quad 0.039698$

Root Mean Square Error $\quad 0.006644$

$\begin{array}{lr}\text { Mean of Response } & 0.363521 \\ \text { Observations (or Sum Wgts) } & 26\end{array}$

Test

Isolok - $12 \mathrm{~mL}$ - HF-Coliwasa

Assuming equal variances

Difference $\quad-0.00267$ t Ratio $\quad-0.99607$

Std Err Dif $\quad 0.00268$ DF 24

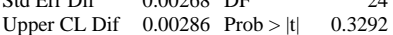

Lower CL Dif -0.00820 Prob $>\mathrm{t} \quad 0.8354$

$\begin{array}{lrr}\text { Confidence } & 0.95 \text { Prob }<\mathrm{t} & 0.1646\end{array}$

Analysis of Variance

$\begin{array}{lrrrrr}\text { Source } & \text { DF } & \text { Sum of Squares } & \text { Mean Square } & \text { F Ratio } & \text { Prob }>\text { F } \\ \text { Sampler Mechanism } & 1 & 0.00004380 & 0.000044 & 0.9922 & 0.3292\end{array}$

$\begin{array}{lrrr}\text { Error } & 24 & 0.00105949 & 0.000044\end{array}$

$\begin{array}{lll}\text { C. Total } & 25 & 0.00110329\end{array}$

Means for Oneway Anova

$\begin{array}{lrrrrr}\text { Level } & \text { Number } & \text { Mean } & \text { Std Error } & \text { Lower 95\% } & \text { Upper 95\% } \\ \text { Coliwasa } & 16 & 0.364547 & 0.00166 & 0.36112 & 0.36798\end{array}$

$\begin{array}{llllll}\text { Isolok - 12 mL - HF } & 16 & 0.364547 & 0.00166 & 0.36112 & 0.36798 \\ & 10 & 0.361879 & 0.00210 & 0.35754 & 0.36622\end{array}$

Std Error uses a pooled estimate of error variance

Means and Std Deviations

Level Number Mean Std Dev Std Err Mean Lower 95\% Upper 95\%

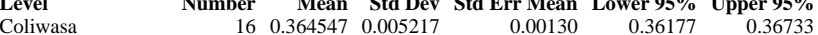

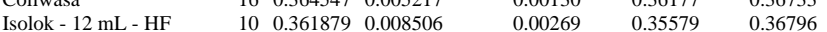

Test

Isolok - $12 \mathrm{~mL}$ - HF-Coliwasa

Assuming unequal variances

$\begin{array}{lrlr}\text { Difference } & -0.00267 & \text { t Ratio } & -0.89241 \\ \text { Std Err Dif } & 0.00299 & \text { DF } & 13.28851 \\ \text { Upper CL Dif } & 0.00378 & \text { Prob }>|t| & 0.3880 \\ \text { Lower CL Dif } & -0.00911 & \text { Prob }>\text { t } & 0.8060 \\ \text { Confidence } & 0.95 & \text { Prob }<\mathrm{t} & 0.1940\end{array}$

Tests that the Variances are Equal

Level Count Std Dev MeanAbsDif to Mean MeanAbsDif to Median

$\begin{array}{lllll}\text { Coliwasa } & 16 & 0.0052171 & 0.0040735 & 0.0040524\end{array}$

Isolok - $12 \mathrm{~mL}$ - HF $\quad 100.0085063 \quad 0.0063758 \quad 0.0060786$

Test F Ratio DFNum DFDen p-Value

$\begin{array}{lrrrr} & \text { F Ratio } & \text { DFNum } & \text { DFDen } & \text { p-Value } \\ \text { O'Brien[.5] } & 1.9045 & 1 & 24 & 0.1803\end{array}$

Brown-Forsythe $\quad 1.2781 \quad 1 \quad 24 \quad 0.269$

$\begin{array}{lllll}\text { Levene } & 2.0203 & 1 & 24 & 0.168\end{array}$

$\begin{array}{lllll}\text { Bartlett } & 2.6852 & 1 & 0.1013\end{array}$

Welch Anova testing Means Equal, allowing Std Devs Not Equal

F Ratio DFNum DFDen Prob $>$ F

$\begin{array}{rrrr}0.7964 & 1 & 13.289 & 0.3880\end{array}$

t Test

0.8924
Oneway Analysis of Measurement By Sampler Mechanism Type of Material=SME Simulant, Analytical Block $=5$, Analyte $=\mathrm{Al} / \mathrm{B}$, Target Value $=2.552$

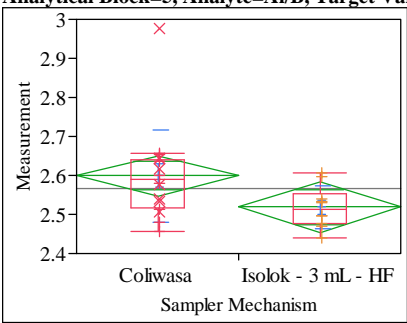

Oneway Anova

Summary of Fit

Adj Rsquare

0.099752

Observations (or Sum Wgts) 2.567809

t Test

Isolok - $3 \mathrm{~mL}$ - HF-Coliwasa

Assuming equal variances

Difference $\quad-0.08014$ t Ratio -1.99305

Std Err Dif $\quad 0.04021$ DF 24

Upper CL Dif 0.00285 Prob $>|t| \quad 0.0577$

$\begin{array}{lll}\text { Lower CL Dif }-0.16314 \text { Prob }>t & 0.9711\end{array}$

$\begin{array}{lrl}\text { Confidence } & 0.95 \text { Prob }<\mathrm{t} & 0.0289\end{array}$

Analysis of Variance

$\begin{array}{lrrrrr}\text { Source } & \text { DF } & \text { Sum of Squares } & \text { Mean Square } & \text { F Ratio } & \text { Prob }>\text { F } \\ \text { Sampler Mechanism } & 1 & 0.03952563 & 0.039526 & 3.9722 & 0.0577\end{array}$

$\begin{array}{llll}\text { Error } & 24 & 0.23881173 & 0.009950\end{array}$

$\begin{array}{lll}\text { C. Total } & 25 & 0.27833736\end{array}$

Means for Oneway Anova

Level Number Mean Std Error Lower 95\% Upper 95\%

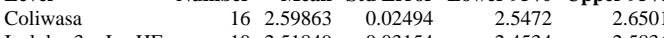

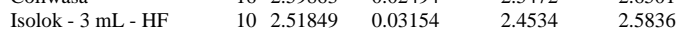

Std Error uses a pooled estimate of error variance

Means and Std Deviations

Level Number Mean Std Dev Std Err Mean Lower 95\% Upper 95\%

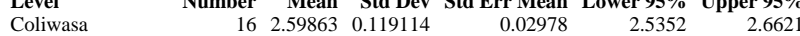

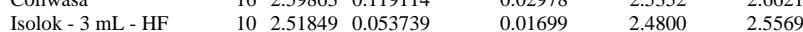

TTest

Isolok - $3 \mathrm{~mL}$ - HF-Coliwasa

Assuming unequal variances

$\begin{array}{lrlr}\text { Difference } & -0.08014 & \mathrm{t} \text { Ratio } & -2.33747 \\ \text { Std Err Dif } & 0.03429 & \text { DF } & 22.40125 \\ \text { Upper CL Dif } & -0.00911 & \text { Prob }>|\mathrm{t}| & 0.0287 \\ \text { Lower CL Dif } & -0.15117 & \text { Prob }>\mathrm{t} & 0.9856 \\ \text { Confidence } & 0.95 & \text { Prob }<\mathrm{t} & 0.0144\end{array}$

Tests that the Variances are Equal

Level Count Std Dev MeanAbsDif to Mean MeanAbsDif to Median

$\begin{array}{lllll}\text { Coliwasa } & 16 & 0.1191135 & 0.0778695 & 0.0778695\end{array}$

$\begin{array}{lllll}\text { Isolok - 3 mL - HF } & 10 & 0.0537394 & 0.0426169 & 0.0426169\end{array}$

Test F Ratio DFNum DFDen p-Value

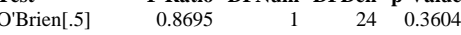

Brown-Forsythe $\begin{array}{llll}1.4617 & 1 & 24 & 0.2384\end{array}$

$\begin{array}{lllll}\text { Levene } & 1.4846 & 1 & 24 & 0.2349\end{array}$

$\begin{array}{lrrrr}\text { Bartlett } & 5.5599 & 1 & \text {. } & 0.0184 \\ \text { F Test 2-sided } & 4.9129 & 15 & 9 & 0.0207\end{array}$

Welch Anova testing Means Equal, allowing Std Devs Not Equal

F Ratio DFNum DFDen Prob $>$ F

$\begin{array}{lrrr}5.4638 & 1 & 22.401 & 0.0287\end{array}$

t Test
2.3375 


\section{Exhibit A2. Statistical Comparisons for Mid-Rheology (Phase 1) Testing}

Oneway Analysis of Measurement By Sampler Mechanism Type of Material=SME Simulant, Analytical Block $=5$, Analyte $=\mathrm{Al} 2 \mathrm{O} 3(\mathrm{wt} \%)$, Target Value $=10.869$

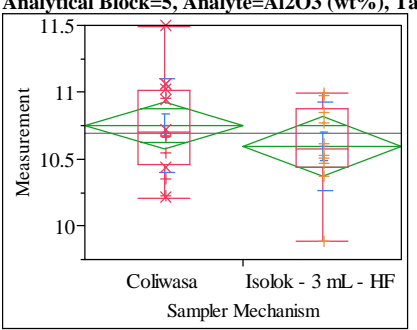

Oneway Anova

Summary of Fit

Adj Rsquare 0.050273

Root Mean Square Error $\quad 0.343603$

$\begin{array}{lr}\text { Mean of Response } & 10.69239 \\ \text { Observations (or Sum Wgts) } & 26\end{array}$

Test

Isolok - $3 \mathrm{~mL}$ - HF-Coliwasa

Assuming equal variances

Difference $\quad-0.15612$ t Ratio -1.12713

Std Err Dif $\quad 0.13851$ DF $\quad 24$

Upper CL Dif 0.12975 Prob $>|t| \quad 0.2708$

Lower CL Dif -0.44199 Prob $>\mathrm{t} \quad 0.8646$

$\begin{array}{lrl}\text { Confidence } & 0.95 \text { Prob }<\mathrm{t} & 0.1354\end{array}$

Analysis of Variance

$\begin{array}{lrrrrr}\text { Source } & \text { DF } & \text { Sum of Squares } & \text { Mean Square } & \text { F Ratio } & \text { Prob }>\text { F } \\ \text { Sampler Mechanism } & 1 & 0.1499904 & 0.149990 & 1.2704 & 0.2708\end{array}$

$\begin{array}{llll}\text { Error } & 24 & 2.8335108 & 0.118063\end{array}$

$\begin{array}{lll}\text { C. Total } & 25 & 2.9835011\end{array}$

Means for Oneway Anova

$\begin{array}{lrrrrr}\text { Level } & \text { Number } & \text { Mean } & \text { Std Error } & \text { Lower 95\% } & \text { Upper 95\% } \\ \text { Coliwasa } & 16 & 10.7524 & 0.08590 & 10.575 & 10.930\end{array}$

$\begin{array}{lrrrrr}\text { Coliwasa } & 16 & 10.7524 & 0.08590 & 10.575 & 10.930 \\ \text { Isolok - 3 mL - HF } & 10 & 10.5963 & 0.10866 & 10.372 & 10.821\end{array}$

Std Error uses a pooled estimate of error variance

Means and Std Deviations

Level Number Mean Std Dev Std Err Mean Lower 95\% Upper 95\%

$\begin{array}{llrrrrr}\text { Coliwasa } & 16 & 10.7524 & 0.350686 & 0.08767 & 10.566 & 10.939\end{array}$

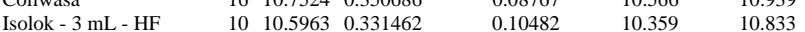

Test

Isolok - $3 \mathrm{~mL}$ - HF-Coliwasa

Assuming unequal variances

Difference $\quad-0.15612$ t Ratio $\quad-1.14249$

Std Err Dif $\quad 0.13665$ DF $\quad 20.09618$

Upper CL Dif 0.12884 Prob $>|t| \quad 0.2667$

Lower CL Dif -0.44108 Prob $>t \quad 0.8667$

$\begin{array}{lrl}\text { Confidence } & 0.95 \text { Prob }<\mathrm{t} & 0.1333\end{array}$

Tests that the Variances are Equal

Level Count Std Dev MeanAbsDif to Mean MeanAbsDif to Median

Coliwasa $\quad 16 \quad 0.3506855$

$\begin{array}{lllll}\text { Isolok - } 3 \mathrm{~mL} \text { - HF } & 10 & 0.3314623 & 0.2823917 & 0.24756350\end{array}$

Test F Ratio DFNum DFDen p-Value

$\begin{array}{lrrrr}\text { O'Brien[.5] } & 0.0368 & 1 & 24 & 0.8495\end{array}$

Brown-Forsythe $\quad 0.1442 \quad 1 \quad 24 \quad 0.7075$

$\begin{array}{lllll}\text { Levene } & 0.2091 & 1 & 24 & 0.6516\end{array}$

$\begin{array}{lrrrr}\text { Bartlett } & 0.0339 & 1 & \text {. } & 0.8540\end{array}$

Welch Anova testing Means Equal, allowing Std Devs Not Equal

F Ratio DFNum DFDen Prob > F

$\begin{array}{lllr}1.3053 & 1 & 20.096 & 0.2667\end{array}$

t Test

1.1425
Oneway Analysis of Measurement By Sampler Mechanism Type of Material=SME Simulant, Analytical Block $=5$, Analyte $=\mathrm{B} 2 \mathrm{O} 3(\mathrm{wt} \%)$, Target Value $=4.259$

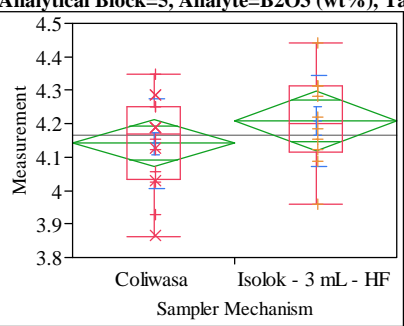

Oneway Anova

Summary of Fit

Adj Rsquare

0.135314

Observations (or Sum Wgts) $\quad 26$

Test

Isolok - $3 \mathrm{~mL}$ - HF-Coliwasa

Assuming equal variances

Difference $\quad 0.06681$ t Ratio 1.224873

Std Err Dif $\quad 0.05455$ DF $\quad 24$

Upper CL Dif 0.17939 Prob $>|t| \quad 0.2325$

$\begin{array}{llll}\text { Lower CL Dif }-0.04577 \text { Prob }>t & 0.1163\end{array}$

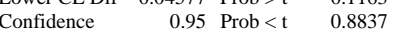

Analysis of Variance

$\begin{array}{lrrrrr}\text { Source } & \text { DF } & \text { Sum of Squares } & \text { Mean Square } & \text { F Ratio } & \text { Prob }>\text { F } \\ \text { Sampler Mechanism } & 1 & 0.02747057 & 0.027471 & 1.5003 & 0.2325\end{array}$

$\begin{array}{llll}\text { Error } & 24 & 0.43943734 & 0.018310\end{array}$

$\begin{array}{lll}\text { C. Total } & 25 & 0.46690790\end{array}$

Means for Oneway Anova

Level Number Mean Std Error Lower 95\% Upper 95\%

$\begin{array}{lrrrrr}\text { Coliwasa } & 16 & 4.14160 & 0.03383 & 4.0718 & 4.2114\end{array}$

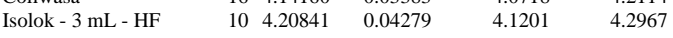

Std Error uses a pooled estimate of error variance

Means and Std Deviations

Level Number Mean Std Dev Std Err Mean Lower 95\% Upper 95\%

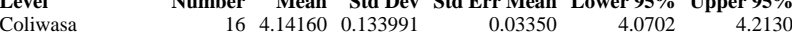

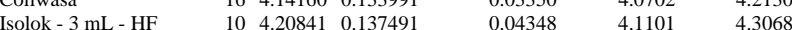

Test

Isolok - $3 \mathrm{~mL}$ - HF-Coliwasa

Assuming unequal variances

$\begin{array}{lrlr}\text { Difference } & 0.06681 & \mathrm{t} \text { Ratio } & 1.217302 \\ \text { Std Err Dif } & 0.05489 & \text { DF } & 18.86694 \\ \text { Upper CL Dif } & 0.18175 & \text { Prob }>|\mathrm{t}| & 0.2385 \\ \text { Lower CL Dif } & -0.04812 & \text { Prob }>\mathrm{t} & 0.1192 \\ \text { Confidence } & 0.95 & \text { Prob }<\mathrm{t} & 0.8808\end{array}$

Tests that the Variances are Equal

Level Count Std Dev MeanAbsDif to Mean MeanAbsDif to Median

$\begin{array}{lllll}\text { Coliwasa } & 16 & 0.1339907 & 0.1061561 & 0.1046467\end{array}$

$\begin{array}{lllll}\text { Isolok - } 3 \mathrm{~mL} \text { - HF } & 10 & 0.1374914 & 0.1062567 & 0.1062567\end{array}$

Test F Ratio DFNum DFDen p-Value

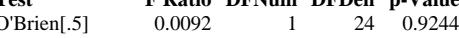

Brown-Forsythe $\quad 0.0023 \quad 1 \quad 24 \quad 0.9619$

$\begin{array}{lllll}\text { Levene } & 0.0000 & 1 & 24 & 0.9975\end{array}$

$\begin{array}{lllll}\text { Bartlett } & 0.0072 & 1 & 0.9324 \\ \text { F Test 2-sided } & 1.0529 & 9 & 15 & 0.8925\end{array}$

Welch Anova testing Means Equal, allowing Std Devs Not Equal

F Ratio DFNum DFDen Prob $>$ F

$\begin{array}{rrrr}1.4818 & 1 & 18.867 & 0.2385\end{array}$

t Test

1.2173 


\section{Exhibit A2. Statistical Comparisons for Mid-Rheology (Phase 1) Testing}

Oneway Analysis of Measurement By Sampler Mechanism Type of Material=SME Simulant, Analytical Block $=5$, Analyte $=\mathrm{BaO}($ wt $\%)$, Target Value $=0.0919$

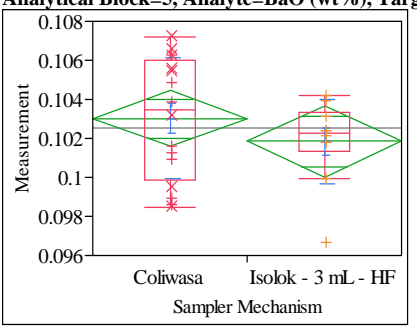

Oneway Anova

Summary of Fit

Adj Rsquare $\quad 0.042539$

Root Mean Square Error $\quad 0.002796$

Mean of Response

$\begin{array}{lr}\text { Mean of Response } & 0.102563 \\ \text { Observations (or Sum Wgts) } & 26\end{array}$

TTest

Isolok - $3 \mathrm{~mL}$ - HF-Coliwasa

Assuming equal variances

Difference $\quad-0.00116$ t Ratio $\quad-1.03261$

Std Err Dif $\quad 0.00113$ DF 24

$\begin{array}{rrr}\text { Upper CL Dif } & 0.00116 & \text { Prob }>|t| \\ & 0.3121\end{array}$

Lower CL Dif -0.00349 Prob $>\mathrm{t} \quad 0.8440$

$\begin{array}{lrl}\text { Confidence } & 0.95 \text { Prob }<\mathrm{t} & 0.1560\end{array}$

Analysis of Variance

$\begin{array}{lrrrrr}\text { Source } & \text { DF } & \text { Sum of Squares } & \text { Mean Square } & \text { F Ratio } & \text { Prob > F } \\ \text { Sampler Mechanism } & 1 & 0.00000834 & 8.3371 \mathrm{e}-6 & 1.0663 & 0.3121\end{array}$

$\begin{array}{llll}\text { Error } & 24 & 0.00018765 & 7.8188 \mathrm{e}-6\end{array}$

$\begin{array}{lll}\text { C. Total } & 25 & 0.00019599\end{array}$

Means for Oneway Anova

Level $\quad$ Number Mean Std Error Lower 95\% Upper 95\%

$\begin{array}{lrrrrr}\text { Coliwasa } & 16 & 0.103011 & 0.00070 & 0.10157 & 0.10445\end{array}$

$\begin{array}{llllll}\text { Isolok - } 3 \mathrm{~mL}-\mathrm{HF} & 10 & 0.101847 & 0.00088 & 0.10002 & 0.10367\end{array}$

Std Error uses a pooled estimate of error variance

Means and Std Deviations

Level Number Mean Std Dev Std Err Mean Lower 95\% Upper 95\%

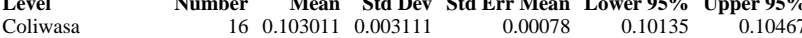

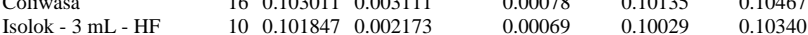

Test

Isolok - $3 \mathrm{~mL}$ - HF-Coliwasa

Assuming unequal variances

Difference $\quad-0.00116$ t Ratio $\quad-1.12155$

Std Err Dif $\quad 0.00104$ DF 23.59437

Upper CL Dif 0.00098 Prob $>|t| r \mid r .2733$

Lower CL Dif -0.00331 Prob $>t \quad 0.8633$

$\begin{array}{lrl}\text { Confidence } & 0.95 \text { Prob }<\mathrm{t} & 0.1367\end{array}$

Tests that the Variances are Equal

Level Count Std Dev MeanAbsDif to Mean MeanAbsDif to Median

Coliwasa $16 \quad 0.0031107$

$\begin{array}{llll}\text { Isolok - } 3 \mathrm{~mL} \text { - HF } & 10 & 0.0021731 & 0.0014247\end{array}$

Test F Ratio DFNum DFDen p-Value

$\begin{array}{lrrrr}\text { O'Brien[.5] } & 2.1419 & 1 & 24 & 0.1563 \\ \text { Brown-Forsythe } & 4.6553 & 1 & 24 & 0.0412\end{array}$

$\begin{array}{lllll}\text { Levene } & 5.1737 & 1 & 24 & 0.0322\end{array}$

$\begin{array}{lrrrr}\text { Bartlett } & 1.2818 & 1 & \text {. } & 0.2576\end{array}$

Welch Anova testing Means Equal, allowing Std Devs Not Equal

F Ratio DFNum DFDen Prob > F

$\begin{array}{lllr}1.2579 & 1 & 23.594 & 0.2733\end{array}$

t Test

1.1216
Oneway Analysis of Measurement By Sampler Mechanism Type of Material=SME Simulant, Analytical Block $=5$, Analyte $=\mathrm{CaO}(\mathrm{wt} \%)$, Target Value $=1.1462$

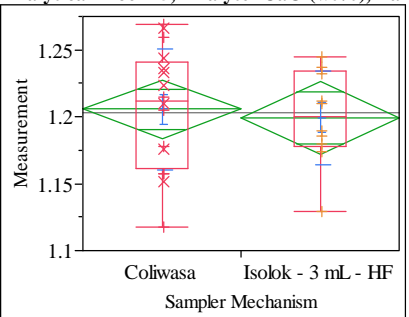

Oneway Anova

Summary of Fit

Adj Rsquare

0.041884

$\begin{array}{lr}\text { Mean of Response } & 1.203258 \\ \text { Observations (or Sum Wgts) } & 26\end{array}$

T Test

Isolok - $3 \mathrm{~mL}$ - HF-Coliwasa

Assuming equal variances

Difference $\quad-0.00628$ t Ratio $\quad-0.37189$

Std Err Dif $\quad 0.01688$ DF $\quad 24$

Upper CL Dif 0.02857 Prob $>|t| r \mid 0.7132$

$\begin{array}{llll}\text { Lower CL Dif }-0.04113 \text { Prob }>t & 0.6434\end{array}$

$\begin{array}{lrl}\text { Confidence } & 0.95 \text { Prob }<\mathrm{t} & 0.3566\end{array}$

Analysis of Variance

$\begin{array}{lrrrrr}\text { Source } & \text { DF } & \text { Sum of Squares } & \text { Mean Square } & \text { F Ratio } & \text { Prob }>\text { F } \\ \text { Sampler Mechanism } & 1 & 0.00024261 & 0.000243 & 0.1383 & 0.7132\end{array}$

$\begin{array}{llll}\text { Error } & 24 & 0.04210172 & 0.001754\end{array}$

$\begin{array}{lll}\text { C. Total } & 25 & 0.04234433\end{array}$

Means for Oneway Anova

Level Number Mean Std Error Lower 95\% Upper 95\%

$\begin{array}{lrrrrr}\text { Coliwasa } & 16 & 1.20567 & 0.01047 & 1.1841 & 1.2273 \\ \text { Isolok - 3 mL - HF } & 10 & 1.19939 & 0.01324 & 1.1721 & 1.2267\end{array}$

Std Error uses a pooled estimate of error variance

Means and Std Deviations

Level Number Mean Std Dev Std Err Mean Lower 95\% Upper 95\%

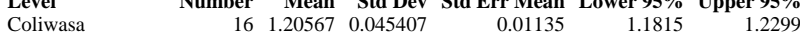

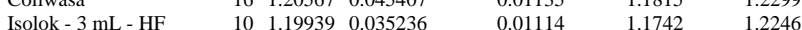

TTest

Isolok - $3 \mathrm{~mL}$ - HF-Coliwasa

Assuming unequal variances

$\begin{array}{lrlr}\text { Difference } & -0.00628 & \mathrm{t} \text { Ratio } & -0.39473 \\ \text { Std Err Dif } & 0.01591 & \text { DF } & 22.70337 \\ \text { Upper CL Dif } & 0.02665 \text { Prob }>|\mathrm{t}| & 0.6967 \\ \text { Lower CL Dif } & -0.03921 & \text { Prob }>\mathrm{t} & 0.6516 \\ \text { Confidence } & 0.95 & \text { Prob }<\mathrm{t} & 0.3484\end{array}$

Tests that the Variances are Equal

Level Count Std Dev MeanAbsDif to Mean MeanAbsDif to Median

$\begin{array}{lllll}\text { Coliwasa } & 16 & 0.0454075 & 0.0373958 & 0.0369913\end{array}$

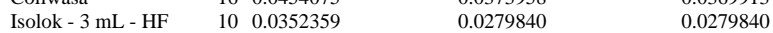

Test F Ratio DFNum DFDen p-Value

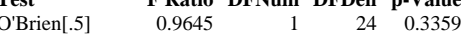

$\begin{array}{lllll}\text { Brown-Forsythe } & 0.9245 & 1 & 24 & 0.3359 \\ & 1.0998 & 1 & 24 & 0.3459\end{array}$

$\begin{array}{lllll}\text { Levene } & 1.0998 & 1 & 24 & 0.3048\end{array}$

$\begin{array}{lrrrr}\text { Bartlett } & 0.6576 & 1 & \text {. } & 0.4174 \\ \text { F Test 2-sided } & 1.6607 & 15 & 9 & 0.4463\end{array}$

Welch Anova testing Means Equal, allowing Std Devs Not Equal

F Ratio DFNum DFDen Prob $>$ F

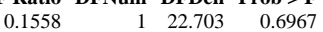

t Test

0.3947 


\section{Exhibit A2. Statistical Comparisons for Mid-Rheology (Phase 1) Testing}

Oneway Analysis of Measurement By Sampler Mechanism Type of Material=SME Simulant, Analytical Block $=5$, Analyte $=\mathrm{Cr} 2 \mathrm{O} 3(\mathrm{wt} \%)$, Target Value $=0.0998$

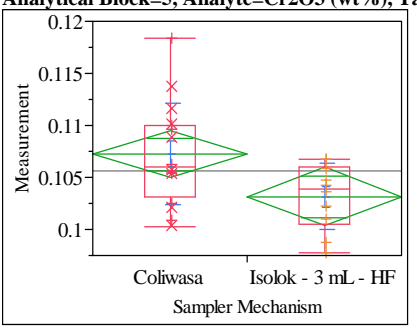

Oneway Anova

Summary of Fit

Adj Rsquare

0.149608

0.004327

Mean of Response $\quad 0.105668$

Observations (or Sum Wgts) $\quad 26$

Test

Isolok - $3 \mathrm{~mL}$ - HF-Coliwas

Assuming equal variances

Difference $\quad-0.00405$ t Ratio -2.3234

Std Err Dif $\quad 0.00174$ DF 24

Upper CL Dif -0.00045 Prob $>|t| \quad 0.0290$

Lower CL Dif -0.00765 Prob $>\mathrm{t} \quad 0.9855$

$\begin{array}{lrl}\text { Confidence } & 0.95 \text { Prob }<\mathrm{t} \quad 0.0145\end{array}$

Analysis of Variance

$\begin{array}{lrrrrr}\text { Source } & \text { DF } & \text { Sum of Squares } & \text { Mean Square } & \text { F Ratio } & \text { Prob }>\text { F } \\ \text { Sampler Mechanism } & 1 & 0.00010105 & 0.000101 & 5.3982 & 0.0290\end{array}$

$\begin{array}{llll}\text { Error } & 24 & 0.00044927 & 0.000019\end{array}$

$\begin{array}{lll}\text { C. Total } & 25 & 0.00055032\end{array}$

$\begin{array}{lrrrr}\text { Means for Oneway Anova } & & & & \\ \text { Level } & \text { Number } & \text { Mean Std Error } & \text { Lower 95\% } & \text { Upper 95\% }\end{array}$

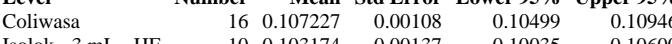

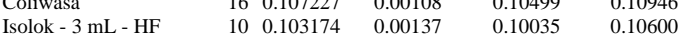

Std Error uses a pooled estimate of error variance

Means and Std Deviations

Level Number Mean Std Dev Std Err Mean Lower 95\% Upper 95\%

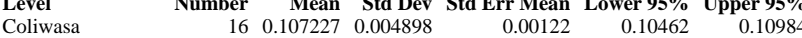

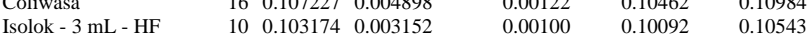

TTest

Isolok - $3 \mathrm{~mL}$ - HF-Coliwasa

Assuming unequal variances

Difference $\quad-0.00405$ t Ratio $\quad-2.56658$

Std Err Dif $\quad 0.00158$ DF 23.94366

Upper CL Dif -0.00079 Prob $>|t| \quad 0.0170$

Lower CL Dif -0.00731 Prob $>t \quad 0.9915$

$\begin{array}{lrl}\text { Confidence } & 0.95 \text { Prob }<\mathrm{t} \quad 0.0085\end{array}$

Tests that the Variances are Equal

Level Count Std Dev MeanAbsDif to Mean MeanAbsDif to Median

$\begin{array}{lllll}\text { Coliwasa } & 16 & 0.0048981 & 0.0039212 & 0.0038002\end{array}$

$\begin{array}{lllll}\text { Isolok - } 3 \mathrm{~mL} \text { - HF } & 10 & 0.0031517 & 0.0025607 & 0.0024701\end{array}$

Test F Ratio DFNum DFDen p-Value

\begin{tabular}{lrrrr} 
O'Brien[.5] & F Ratio & DFNum & DFDen & p-Value \\
\hline & 1.5170 & 1 & 24 & 0.2300
\end{tabular}

Brown-Forsythe $1.3884 \quad 11 \quad 24 \quad 0.2502$

$\begin{array}{lllll}\text { Levene } & 1.9854 & 1 & 24 & 0.1716\end{array}$

$\begin{array}{lrrrr}\text { Bartlett } & 1.8952 & 1 & \text {. } & 0.1686\end{array}$

Welch Anova testing Means Equal, allowing Std Devs Not Equal

F Ratio DFNum DFDen Prob > F

$\begin{array}{rrrr}6.5874 & 1 & 23.944 & 0.0170\end{array}$

t Test
Oneway Analysis of Measurement By Sampler Mechanism Type of Material=SME Simulant, Analytical Block $=5$, Analyte $=\mathrm{CuO}(\mathrm{wt} \%)$, Target Value $=0.0504$

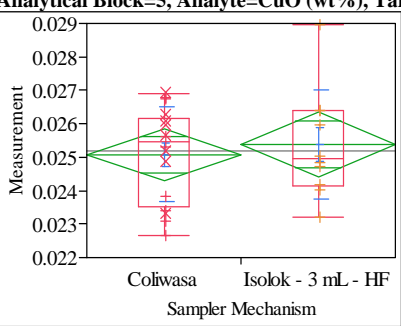

Oneway Anova

Summary of Fi

Adj Rsquare

\begin{tabular}{ll}
-0.03157 \\
\hline
\end{tabular}

$\quad 0.001502$

0.0252

Test

Isolok - $3 \mathrm{~mL}$ - HF-Coliwasa

Assuming equal variances

$\begin{array}{lrlr}\text { Difference } & 0.00029 & \mathrm{t} \text { Ratio } & 0.484599 \\ \text { Std Err Dif } & 0.00061 & \text { DF } & 24 \\ \text { Upper CL Dif } & 0.00154 & \text { Prob }>|t| & 0.6324 \\ \text { Lower CL Dif } & -0.00096 & \text { Prob }>t & 0.3162 \\ \text { Confidence } & 0.95 & \text { Prob }<\mathrm{t} & 0.6838\end{array}$

Analysis of Variance

$\begin{array}{lrrrrr}\text { Source } & \text { DF } & \text { Sum of Squares } & \text { Mean Square } & \text { F Ratio } & \text { Prob }>\text { F } \\ \text { Sampler Mechanism } & 1 & 0.00000053 & 5.2971 \mathrm{e}-7 & 0.2348 & 0.6324\end{array}$

$\begin{array}{llll}\text { Error } & 24 & 0.00005414 & 2.2557 \mathrm{e}-6\end{array}$

$\begin{array}{lll}\text { C. Total } & 25 & 0.00005467\end{array}$

Means for Oneway Anova

Level Number Mean Std Error Lower 95\% Upper 95\%

$\begin{array}{lllllr}\text { Coliwasa } & 16 & 0.025087 & 0.00038 & 0.02431 & 0.02586\end{array}$

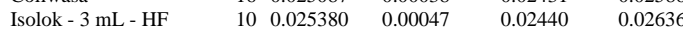

Std Error uses a pooled estimate of error variance

Means and Std Deviations

Level Number Mean Std Dev Std Err Mean Lower 95\% Upper 95\%

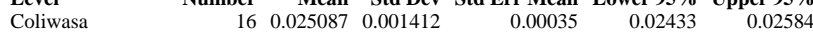

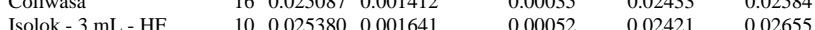

Test

Isolok - $3 \mathrm{~mL}$ - HF-Coliwasa

Assuming unequal variances

$\begin{array}{lrlr}\text { Difference } & 0.00029 & \text { t Ratio } & 0.467488 \\ \text { Std Err Dif } & 0.00063 & \text { DF } & 17.06273 \\ \text { Upper CL Dif } & 0.00162 & \text { Prob }>|t| & 0.6461 \\ \text { Lower CL Dif } & -0.00103 & \text { Prob }>t & 0.3230 \\ \text { Confidence } & 0.95 & \text { Prob }<\mathrm{t} & 0.6770\end{array}$

Tests that the Variances are Equal

Level Count Std Dev MeanAbsDif to Mean MeanAbsDif to Median

$\begin{array}{lllll}\text { Coliwasa } & 16 & 0.0014119 & 0.0011726 & 0.0011384\end{array}$

$\begin{array}{llll}\text { Isolok - } 3 \mathrm{~mL} \text { - HF } & 10 & 0.0016410 & 0.0012518\end{array}$

Test F Ratio DFNum DFDen p-Value

$\begin{array}{lrrrr}\text { O'Brien[.5] } & 0.2902 & 1 & 24 & 0.5951\end{array}$

$\begin{array}{lllll}\text { Brown-Forsythe } & 0.0126 & 1 & 24 & 0.9116\end{array}$

$\begin{array}{lllll}\text { Levene } & 0.0563 & 1 & 24 & 0.8145\end{array}$

$\begin{array}{lllll}\text { Bartlett } & 0.2487 & 1 & \text {. } & 0.6180 \\ \text { FTest 2-sided } & 1.3509 & 9 & 15 & 0.5828\end{array}$

Welch Anova testing Means Equal, allowing Std Devs Not Equal

F Ratio DFNum DFDen Prob $>$ F

$\begin{array}{llll}0.2185 & 1 & 17.063 & 0.6461\end{array}$

t Test

0.4675 


\section{Exhibit A2. Statistical Comparisons for Mid-Rheology (Phase 1) Testing}

Oneway Analysis of Measurement By Sampler Mechanism Type of Material=SME Simulant, Analytical Block $=5$, Analyte $=\mathrm{Fe} / \mathrm{Li}$, Target Value $=\mathbf{2 . 4 5 2}$

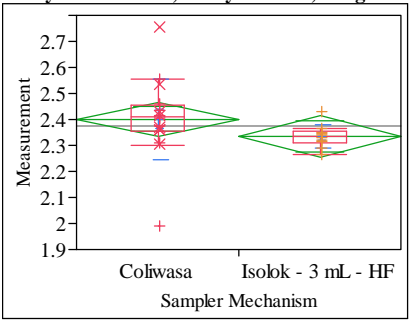

Oneway Anova

Summary of Fit

Adj Rsquare $\quad 0.06754$

Root Mean Square Error $\quad 0.125919$

Mean of Response

$\begin{array}{lr}\text { Mean of Response } & 2.3763 \\ \text { Observations (or Sum Wgts) } & 26\end{array}$

tTest

Isolok - $3 \mathrm{~mL}$ - HF-Coliwasa

Assuming equal variances

Difference $\quad-0.06692$ t Ratio -1.31847

Std Err Dif $\quad 0.05076$ DF 24

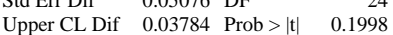

Lower CL Dif -0.17169 Prob $>t \quad 0.9001$

$\begin{array}{lrl}\text { Confidence } & 0.95 \text { Prob }<\mathrm{t} & 0.0999\end{array}$

Analysis of Variance

$\begin{array}{lrrrrr}\text { Source } & \text { DF } & \text { Sum of Squares } & \text { Mean Square } & \text { F Ratio } & \text { Prob > F } \\ \text { Sampler Mechanism } & 1 & 0.02756278 & 0.027563 & 1.7384 & 0.1998\end{array}$

$\begin{array}{llll}\text { Error } & 24 & 0.38053222 & 0.015856\end{array}$

$\begin{array}{lll}\text { C. Total } & 25 & 0.40809500\end{array}$

Means for Oneway Anova

Level Number Mean Std Error Lower 95\% Upper 95\%

$\begin{array}{lrrrrr}\text { Coliwasa } & 16 & 2.40204 & 0.03148 & 2.3371 & 2.4670\end{array}$

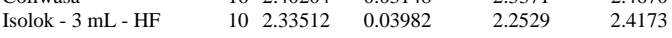

Std Error uses a pooled estimate of error variance

Means and Std Deviations

Level Number Mean Std Dev Std Err Mean Lower 95\% Upper 95\%

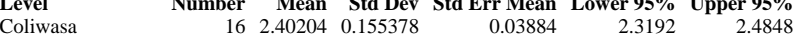

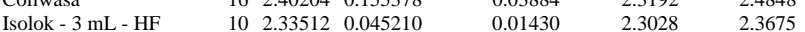

Test

Isolok - $3 \mathrm{~mL}$ - HF-Coliwasa

Assuming unequal variances

Difference $\quad-0.06692$ t Ratio $\quad-1.61686$

Std Err Dif $\quad 0.04139$ DF $\quad 18.76511$

Upper CL Dif 0.01978 Prob $>|t| \quad 0.1226$

Lower CL Dif -0.15363 Prob $>t \quad 0.9387$

$\begin{array}{lrl}\text { Confidence } & 0.95 \text { Prob }<\mathrm{t} & 0.0613\end{array}$

Tests that the Variances are Equal

Level Count Std Dev MeanAbsDif to Mean MeanAbsDif to Median

Coliwasa $\quad 16 \quad 0.1553784$

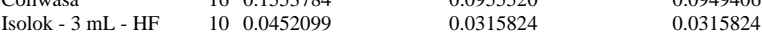

Test F Ratio DFNum DFDen p-Value

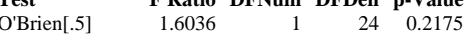

Brown-Forsythe $2.6124 \quad 1 \quad 24 \quad 0.119$

$\begin{array}{lllll}\text { Levene } & 2.6924 & 1 & 24 & 0.1139\end{array}$

$\begin{array}{lrrrr}\text { Bartlett } & 11.6044 & 1 & \text {. } & 0.0007\end{array}$

Welch Anova testing Means Equal, allowing Std Devs Not Equal

F Ratio DFNum DFDen Prob $>$ F

$\begin{array}{rrrr}2.6142 & 1 & 18.765 & 0.1226\end{array}$

t Test

1.6169
Oneway Analysis of Measurement By Sampler Mechanism Type of Material=SME Simulant, Analytical Block $=5$, Analyte $=$ Fe2O3 (wt $\%$ ), Target Value $=11.462$

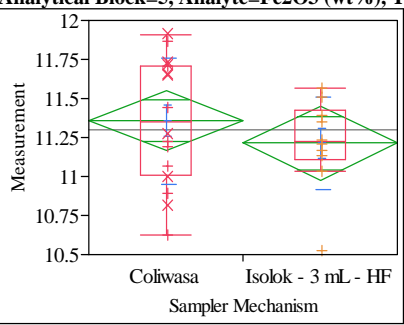

Oneway Anova

Summary of Fit

Adj Rsquare

0.366482

Observations (or Sum Wgts) $\quad 26$

Isolok - $3 \mathrm{~mL}$ - HF-Coliwasa

Assuming equal variances

$\begin{array}{lrlr}\text { Difference } & -0.14404 & \mathrm{t} \text { Ratio } & -0.97501 \\ \text { Std Err Dif } & 0.14773 & \text { DF } & 24 \\ \text { Upper CL Dif } & 0.16087 & \text { Prob }>|t| & 0.3393 \\ \text { Lower CL Dif } & -0.44895 & \text { Prob }>\text { t } & 0.8304 \\ \text { Confidence } & 0.95 & \text { Prob }<\mathrm{t} & 0.1696\end{array}$

Analysis of Variance

$\begin{array}{lrrrrr}\text { Source } & \text { DF } & \text { Sum of Squares } & \text { Mean Square } & \text { F Ratio } & \text { Prob }>\text { F } \\ \text { Sampler Mechanism } & 1 & 0.1276811 & 0.127681 & 0.9506 & 0.3393\end{array}$

$\begin{array}{llll}\text { Error } & 24 & 3.2234237 & 0.134309\end{array}$

$\begin{array}{lll}\text { C. Total } & 25 & 3.3511048\end{array}$

Means for Oneway Anova

Level Number Mean Std Error Lower 95\% Upper 95\%

$\begin{array}{lrrrrr}\text { Coliwasa } & 16 & 11.3572 & 0.09162 & 11.168 & 11.546\end{array}$

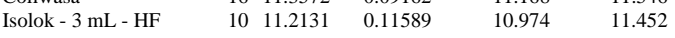

Std Error uses a pooled estimate of error variance

Means and Std Deviations

Level Number Mean Std Dev Std Err Mean Lower 95\% Upper 95\%

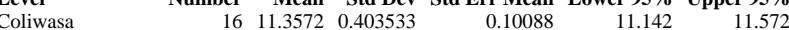

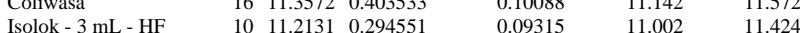

TTest

Isolok - $3 \mathrm{~mL}$ - HF-Coliwasa

Assuming unequal variances

$\begin{array}{lrlr}\text { Difference } & -0.14404 & \mathrm{t} \text { Ratio } & -1.04905 \\ \text { Std Err Dif } & 0.13731 \text { DF } & 23.27928 \\ \text { Upper CL Dif } & 0.13981 & \text { Prob }>|\mathrm{t}| & 0.3049 \\ \text { Lower CL Dif } & -0.42790 & \text { Prob }>\mathrm{t} & 0.8475 \\ \text { Confidence } & 0.95 & \text { Prob }<\mathrm{t} & 0.1525\end{array}$

Tests that the Variances are Equal

Level Count Std Dev MeanAbsDif to Mean MeanAbsDif to Median

$\begin{array}{lllll}\text { Coliwasa } & 16 & 0.4035327 & 0.3484894 & 0.3484894\end{array}$

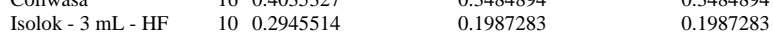

Test F Ratio DFNum DFDen p-Value

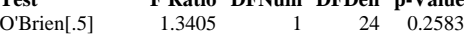

$\begin{array}{lllll}\text { Brown-Forsythe } & 3.7352 & 1 & 24 & 0.0652\end{array}$

$\begin{array}{lllll}\text { Levene } & 3.7413 & 1 & 24 & 0.0650\end{array}$

$\begin{array}{lrrrr}\text { Bartlett } & 0.9984 & 1 & \text {. } & 0.3177 \\ \text { F Test 2-sided } & 1.8769 & 15 & 9 & 0.3420\end{array}$

Welch Anova testing Means Equal, allowing Std Devs Not Equal

F Ratio DFNum DFDen Prob $>$ F

$\begin{array}{llrr}1.1005 & 1 & 23.279 & 0.3049\end{array}$

t Test

1.0490 


\section{Exhibit A2. Statistical Comparisons for Mid-Rheology (Phase 1) Testing}

Oneway Analysis of Measurement By Sampler Mechanism Type of Material=SME Simulant, Analytical Block=5, Analyte $=$ K2O (wt \%), Target Value $=\mathbf{0 . 1 5 9 1}$

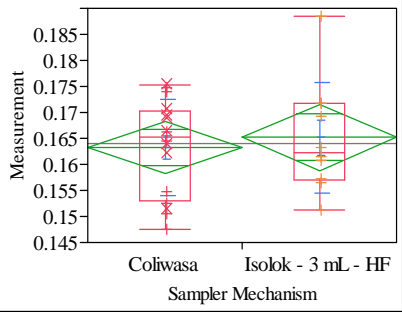

Oneway Anova

Summary of Fit

Adj Rsquare

0.009895

$-0.03136$

Error $\quad 0.009763$

Observations (or Sum Wgts) $\quad 26$

t Test

Isolok - $3 \mathrm{~mL}$ - HF-Coliwasa

Assuming equal variances

Difference $\quad 0.00193$ t Ratio 0.48974

Std Err Dif $\quad 0.00394$ DF $\quad 24$

Upper CL Dif 0.01005 Prob $>|t| \quad 0.6288$

Lower CL Dif -0.00620 Prob $>\mathrm{t} \quad 0.3144$

$\begin{array}{lrl}\text { Confidence } & 0.95 \text { Prob }<\mathrm{t} & 0.6856\end{array}$

Analysis of Variance

$\begin{array}{lrrrrr}\text { Source } & \text { DF } & \text { Sum of Squares } & \text { Mean Square } & \text { F Ratio } & \text { Prob > F } \\ \text { Sampler Mechanism } & 1 & 0.00002286 & 0.000023 & 0.2398 & 0.6288\end{array}$

$\begin{array}{llll}\text { Error } & 24 & 0.00228745 & 0.000095\end{array}$

$\begin{array}{lll}\text { C. Total } & 25 & 0.00231031\end{array}$

Means for Oneway Anova

$\begin{array}{lrrrrr}\text { Level } & \text { Number } & \text { Mean } & \text { Std Error } & \text { Lower 95\% } & \text { Upper 95\% } \\ \text { Coliwasa } & 16 & 0.163223 & 0.00244 & 0.15819 & 0.16826\end{array}$

$\begin{array}{lrrrrr}\text { Coliwasa } & 16 & 0.163223 & 0.00244 & 0.15819 & 0.16826 \\ \text { Isolok - 3 mL - HF } & 10 & 0.165151 & 0.00309 & 0.15878 & 0.17152\end{array}$

Std Error uses a pooled estimate of error variance

Means and Std Deviations

Level Number Mean Std Dev Std Err Mean Lower 95\% Upper 95\%

\begin{tabular}{lrrrrrr} 
Coliwasa & Number & Mean & Std Dev & Std Err Mean & Lower 95\% & Upper 95\% \\
\hline & 16 & 0.163223 & 0.009208 & 0.00230 & 0.15832 & 0.16813
\end{tabular}

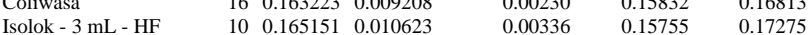

TTest

Isolok - $3 \mathrm{~mL}$ - HF-Coliwasa

Assuming unequal variances

Difference $\quad 0.00193$ t Ratio 0.473283

Std Err Dif $\quad 0.00407$ DF $\quad 17.16649$

Upper CL Dif 0.01051 Prob $>|t| \quad 0.6420$

Lower CL Dif -0.00666 Prob $>t \quad 0.3210$

$\begin{array}{lrl} & \\ \text { Confidence } & 0.95 \text { Prob }<\mathrm{t} & 0.6790\end{array}$

Tests that the Variances are Equal

Level Count Std Dev MeanAbsDif to Mean MeanAbsDif to Median

$\begin{array}{lllll}\text { Coliwasa } & 16 & 0.0092082 & 0.0076040 & 0.0073782\end{array}$

$\begin{array}{lllll}\text { Isolok - } 3 \mathrm{~mL} \text { - HF } & 10 & 0.0106228 & 0.0076040 & 0.0073782 \\ & & & 0.0080949 & 0.0077094\end{array}$

Test F Ratio DFNum DFDen p-Value

$\begin{array}{lrrrr}\text { O'Brien[.5] } & 0.2619 & 1 & 24 & 0.6135\end{array}$

$\begin{array}{lllll}\text { Brown-Forsythe } & 0.0167 & 1 & 24 & 0.898\end{array}$

$\begin{array}{lllll}\text { Levene } & 0.0503 & 1 & 24 & 0.8244\end{array}$

$\begin{array}{lllll}\text { Bartlett } & 0.2244 & 1 & \text {. } & 0.6357\end{array}$

Test 2-sided 1.3309

F Ratio DFNum DFDen Prob $>$ F

$\begin{array}{rrrr}0.2240 & 1 & 17.166 & 0.6420\end{array}$

t Test

0.4733
Oneway Analysis of Measurement By Sampler Mechanism Type of Material=SME Simulant, Analytical Block $=5$, Analyte $=\mathrm{Li} 2 \mathrm{O}(\mathrm{wt} \%)$, Target Value $=4.674$

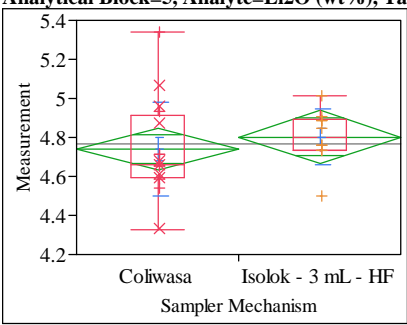

Oneway Anova

Summary of Fit

Adj Rsquare

$\quad 0.208145$

Observations (or Sum Wgts) $\quad 26$

T Test

Isolok - $3 \mathrm{~mL}$ - HF-Coliwasa

Assuming equal variances

$\begin{array}{lrlr}\text { Difference } & 0.06270 & \mathrm{t} \text { Ratio } & 0.747304 \\ \text { Std Err Dif } & 0.08391 & \text { DF } & 24 \\ \text { Upper CL Dif } & 0.23588 & \text { Prob }>|t| & 0.4621 \\ \text { Lower CL Dif } & -0.11047 & \text { Prob }>t & 0.2311 \\ \text { Confidence } & 0.95 & \text { Prob }<t & 0.7689\end{array}$

Analysis of Variance

$\begin{array}{lrrrrr}\text { Source } & \text { DF } & \text { Sum of Squares } & \text { Mean Square } & \text { F Ratio } & \text { Prob }>\text { F } \\ \text { Sampler Mechanism } & 1 & 0.0241950 & 0.024195 & 0.5585 & 0.4621\end{array}$

$\begin{array}{llll}\text { Error } & 24 & 1.0397821 & 0.043324\end{array}$

$\begin{array}{lll}\text { C. Total } & 25 & 1.0639771\end{array}$

Means for Oneway Anova

Level Number Mean Std Error Lower 95\% Upper 95\%

$\begin{array}{lrrrrr}\text { Coliwasa } & 16 & 4.74042 & 0.05204 & 4.6330 & 4.8478 \\ \text { Isolok - 3 mL - HF } & 10 & 4.80312 & 0.06582 & 4.6673 & 4.9390\end{array}$

Std Error uses a pooled estimate of error variance

Means and Std Deviations

Level Number Mean Std Dev Std Err Mean Lower 95\% Upper 95\%

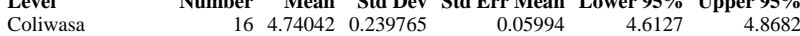

$\begin{array}{lllllll}\text { Isolok - } 3 \text { mL - HF } & 10 & 4.80312 & 0.140425 & 0.04441 & 4.7027 & 4.9036\end{array}$

TTest

Isolok - $3 \mathrm{~mL}$ - HF-Coliwasa

Assuming unequal variances

$\begin{array}{lrlr}\text { Difference } & 0.06270 & \mathrm{t} \text { Ratio } & 0.840547 \\ \text { Std Err Dif } & 0.07460 & \text { DF } & 23.95642 \\ \text { Upper CL Dif } & 0.21668 & \text { Prob }>|t| & 0.4089 \\ \text { Lower CL Dif } & -0.09127 & \text { Prob }>\mathrm{t} & 0.2045 \\ \text { Confidence } & 0.95 & \text { Prob }<\mathrm{t} & 0.7955\end{array}$

Tests that the Variances are Equal

Level Count Std Dev MeanAbsDif to Mean MeanAbsDif to Median

$\begin{array}{lllll}\text { Coliwasa } & 16 & 0.2397650 & 0.1804736 & 0.1655042\end{array}$

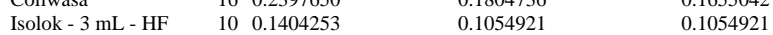

Test F Ratio DFNum DFDen p-Value

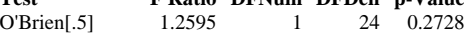

Brown-Forsythe $\quad 0.8998 \quad 11 \quad 24 \quad 0.3523$

$\begin{array}{lllll}\text { Levene } & 2.0384 & 1 & 24 & 0.1663\end{array}$

$\begin{array}{lrrrr}\text { Bartlett } & 2.7180 & 1 & \text {. } & 0.0992 \\ \text { F Test 2-sided } & 2.9153 & 15 & 9 & 0.1093\end{array}$

Welch Anova testing Means Equal, allowing Std Devs Not Equal

F Ratio DFNum DFDen Prob $>$ F

$\begin{array}{rrrr}0.7065 & 1 & 23.956 & 0.4089\end{array}$

t Test

0.8405 


\section{Exhibit A2. Statistical Comparisons for Mid-Rheology (Phase 1) Testing}

Oneway Analysis of Measurement By Sampler Mechanism Type of Material=SME Simulant, Analytical Block=5, Analyte $=\mathrm{MgO}(\mathrm{wt} \%)$, Target Value=1.014

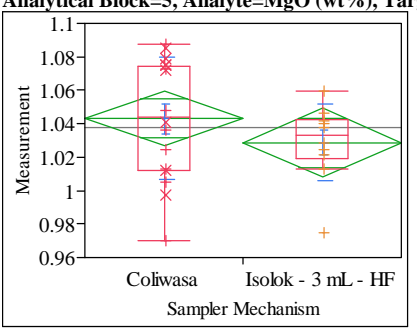

Oneway Anova

Summary of Fit

Adj Rsquare

0.048298

0.008644
0.032056

$\begin{array}{lr}\text { Mean of Response } & 1.037586 \\ \text { Observations (or Sum Wgts) } & 26\end{array}$

t Test

Isolok - $3 \mathrm{~mL}$ - HF-Coliwasa

Assuming equal variances

Difference $\quad-0.01426$ t Ratio $\quad-1.10362$

Std Err Dif $\quad 0.01292$ DF 24

Upper CL Dif 0.01241 Prob $>|t| r \mid \begin{array}{r}24 \\ 0.2807\end{array}$

Lower CL Dif -0.04093 Prob $>\mathrm{t} \quad 0.8596$

$\begin{array}{lrl}\text { Confidence } & 0.95 \text { Prob }<\mathrm{t} & 0.1404\end{array}$

Analysis of Variance

$\begin{array}{lrrrrr}\text { Source } & \text { DF } & \text { Sum of Squares } & \text { Mean Square } & \text { F Ratio } & \text { Prob }>\text { F } \\ \text { Sampler Mechanism } & 1 & 0.00125161 & 0.001252 & 1.2180 & 0.2807\end{array}$

$\begin{array}{llll}\text { Error } & 24 & 0.02466273 & 0.001028\end{array}$

$\begin{array}{lll}\text { C. Total } & 25 & 0.02591434\end{array}$

Means for Oneway Anova

Level Number Mean Std Error Lower 95\% Upper 95\%

$\begin{array}{lrrrrr}\text { Coliwasa } & 16 & 1.04307 & 0.00801 & 1.0265 & 1.0596\end{array}$

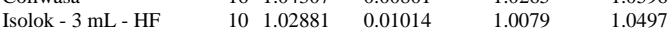

Std Error uses a pooled estimate of error variance

Means and Std Deviations

Level Number Mean Std Dev Std Err Mean Lower 95\% Upper 95\%

$\begin{array}{lrrrrr}\text { Coliwasa } & 16 & 1.04307 & 0.036367 & 0.00909 & 1.0237\end{array}$

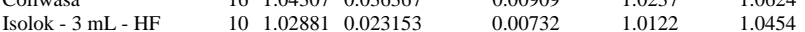

Test

Isolok - $3 \mathrm{~mL}$ - HF-Coliwasa

Assuming unequal variances

Difference $\quad-0.01426$ t Ratio $\quad-1.22171$

Std Err Dif $\quad 0.01167$ DF $\quad 23.9654$

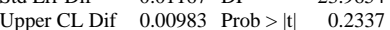

Lower CL Dif -0.03836 Prob $>t \quad 0.8832$

$\begin{array}{lrl}\text { Confidence } & 0.95 \text { Prob }<\mathrm{t} & 0.1168\end{array}$

Tests that the Variances are Equal

Level Count Std Dev MeanAbsDif to Mean MeanAbsDif to Median

Coliwasa $\quad 16 \quad 0.0363668$

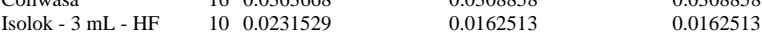

Test F Ratio DFNum DFDen p-Value

$\begin{array}{lrrrr}\text { O'Brien[.5] } & 2.3124 & 1 & 24 & 0.1414 \\ \text { Brown-Forsythe } & 4.5476 & 1 & 24 & 0.0434\end{array}$

$\begin{array}{lllll}\text { Levene } & 4.6800 & 1 & 24 & 0.0407\end{array}$

$\begin{array}{lrrrr}\text { Bartlett } & 1.9820 & 1 & \text {. } & 0.1592\end{array}$

Welch Anova testing Means Equal, allowing Std Devs Not Equal

F Ratio DFNum DFDen Prob > F

$\begin{array}{rrrr}1.4926 & 1 & 23.965 & 0.2337\end{array}$

t Test

1.2217
Oneway Analysis of Measurement By Sampler Mechanism Type of Material=SME Simulant, Analytical Block $=5$, Analyte $=\mathrm{MnO}(\mathrm{wt} \%)$, Target Value $=1.779$

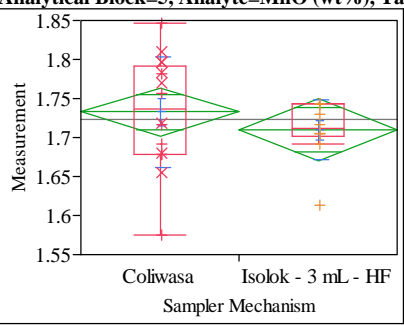

Oneway Anova

Summary of Fit

Adj Rsquare

0.035718

Root Mean Square Error $\quad-0.00446$

1.723752

t Test

Isolok - $3 \mathrm{~mL}$ - HF-Coliwasa

Assuming equal variances

Difference $\quad-0.02308$ t Ratio -0.94285

Std Err Dif $\quad 0.02448$ DF 24

Upper CL Dif 0.02744 Prob $>|t| \quad 0.3552$

Lower CL Dif -0.07360 Prob $>t \quad 0.8224$

$\begin{array}{lrl}\text { Confidence } & 0.95 \text { Prob }<\mathrm{t} & 0.1776\end{array}$

Analysis of Variance

$\begin{array}{lrrrrr}\text { Source } & \text { DF } & \text { Sum of Squares } & \text { Mean Square } & \text { F Ratio } & \text { Prob }>\text { F } \\ \text { Sampler Mechanism } & 1 & 0.00327813 & 0.003278 & 0.8890 & 0.3552 \\ \text { Error } & 24 & 0.08850109 & 0.003688 & & \end{array}$

Means for Oneway Anova

Level Number Mean Std Error Lower 95\% Upper 95\%

$\begin{array}{lrrrrr}\text { Coliwasa } & 16 & 1.73263 & 0.01518 & 1.7013 & 1.7640\end{array}$

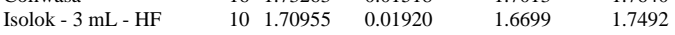

Std Error uses a pooled estimate of error variance

Means and Std Deviations

Level Number Mean Std Dev Std Err Mean Lower 95\% Upper 95\%

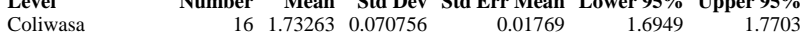

$\begin{array}{lllllll}\text { Isolok - } 3 \mathrm{~mL} \text { - HF } & 10 & 1.70955 & 0.038592 & 0.01220 & 1.6819 & 1.7372\end{array}$

Test

Isolok - $3 \mathrm{~mL}$ - HF-Coliwasa

Assuming unequal variances

$\begin{array}{lrlr}\text { Difference } & -0.02308 & \mathrm{t} \text { Ratio } & -1.07397 \\ \text { Std Err Dif } & 0.02149 & \text { DF } & 23.72088 \\ \text { Upper CL Dif } & 0.02130 & \text { Prob }>|t| & 0.2936 \\ \text { Lower CL Dif } & -0.06746 & \text { Prob }>\mathrm{t} & 0.8532 \\ \text { Confidence } & 0.95 & \text { Prob }<\mathrm{t} & 0.1468\end{array}$

Tests that the Variances are Equal

Level Count Std Dev MeanAbsDif to Mean MeanAbsDif to Median

$\begin{array}{lllll}\text { Coliwasa } & 16 & 0.0707563 & 0.0589110 & 0.0589110\end{array}$

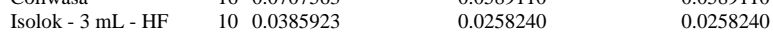

Test F Ratio DFNum DFDen p-Value

$\begin{array}{lrrrr}\text { O'Brien[.5] } & 2.2943 & 1 & 24 & 0.1429\end{array}$

$\begin{array}{lllll}\text { Brown-Forsythe } & 6.0827 & 1 & 24 & 0.0212\end{array}$

$\begin{array}{lllll}\text { Levene } & 6.1468 & 1 & 24 & 0.0206\end{array}$

$\begin{array}{lrrrr}\text { Bartlett } & 3.4179 & 1 & \text {. } & 0.0645 \\ \text { F Test 2-sided } & 3.3615 & 15 & 9 & 0.0715\end{array}$

Welch Anova testing Means Equal, allowing Std Devs Not Equal

F Ratio DFNum DFDen Prob $>$ F

$\begin{array}{lrrr}1.1534 & 1 & 23.721 & 0.2936\end{array}$

t Test

1.0740 


\section{Exhibit A2. Statistical Comparisons for Mid-Rheology (Phase 1) Testing}

Oneway Analysis of Measurement By Sampler Mechanism Type of Material=SME Simulant, Analytical Block=5, Analyte= $\mathrm{Na2O}(\mathrm{wt} \%)$, Target Value=11.659

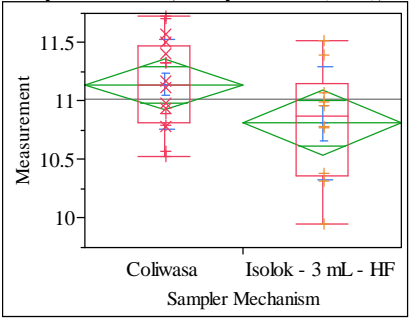

Oneway Anova

Summary of Fit

Adj Rsquare $\quad 0.133506$

Root Mean Square Error $\quad 0.424307$

$\begin{array}{lr}\text { Mean of Response } & 11.01472 \\ \text { Observations (or Sum Wgts) } & 26\end{array}$

tTest

Isolok - $3 \mathrm{~mL}$ - HF-Coliwasa

Assuming equal variances

Difference $\quad-0.32891$ t Ratio $\quad-1.92297$

Std Err Dif $\quad 0.17104$ DF 24

Upper CL Dif 0.02410 Prob $>|t| \quad 0.0664$

Lower CL Dif -0.68193 Prob $>\mathrm{t} \quad 0.9668$

$\begin{array}{lrl}\text { Confidence } & 0.95 \text { Prob }<\mathrm{t} & 0.0332\end{array}$

Analysis of Variance

$\begin{array}{lrrrrr}\text { Source } & \text { DF } & \text { Sum of Squares } & \text { Mean Square } & \text { F Ratio } & \text { Prob > F } \\ \text { Sampler Mechanism } & 1 & 0.6657422 & 0.665742 & 3.6978 & 0.0664\end{array}$

$\begin{array}{llll}\text { Error } & 1 & 0.357422 & 0.665742 \\ \text { C. } & 24 & 4.3208734 & 0.180036\end{array}$

$\begin{array}{lll}\text { C. Total } & 25 & 4.9866156\end{array}$

Means for Oneway Anova

Level Number Mean Std Error Lower 95\% Upper 95\%

$\begin{array}{lrrrrr}\text { Coliwasa } & 16 & 11.1412 & 0.10608 & 10.922 & 11.360\end{array}$

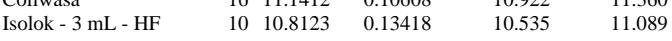

Std Error uses a pooled estimate of error variance

Means and Std Deviations

Level Number Mean Std Dev Std Err Mean Lower 95\% Upper 95\%

$\begin{array}{lrrrrr}\text { Coliwasa } & 16 & 11.1412 & 0.382002 & 0.09550 & 10.938\end{array}$

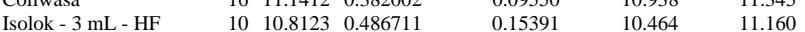

Test

Isolok - $3 \mathrm{~mL}$ - HF-Coliwasa

Assuming unequal variances

Difference $\quad-0.32891$ t Ratio $\quad-1.81586$

Std Err Dif $\quad 0.18113$ DF $\quad 15.85416$

Upper CL Dif 0.05536 Prob $>|t| r \mid r .0884$

Lower CL Dif -0.71318 Prob $>t \quad 0.9558$

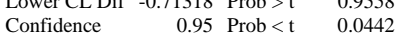

Tests that the Variances are Equal

Level Count Std Dev MeanAbsDif to Mean MeanAbsDif to Median

Coliwasa $\quad 16 \quad 0.3820020$

$\begin{array}{lllll}\text { Coliwasa } & 16 & 0.3820020 & 0.3201500 & 0.3201500 \\ \text { Isolok - } 3 \mathrm{~mL} \text { - HF } & 10 & 0.4867112 & 0.3733960 & 0.3733960\end{array}$

Test F Ratio DFNum DFDen p-Value

\begin{tabular}{lrrrr} 
O'Brien[.5] & F Ratio & DFNum & DFDen & p-Value \\
\hline & 1.1159 & 1 & 24 & 0.3013
\end{tabular}

$\begin{array}{lllll}\text { Brown-Forsythe } & 0.3166 & 1 & 24 & 0.5789\end{array}$

$\begin{array}{lllll}\text { Levene } & 0.3254 & 1 & 24 & 0.5737\end{array}$

$\begin{array}{lllll}\text { Bartlett } & 0.6515 & 1 & 0.4196\end{array}$

Welch Anova testing Means Equal, allowing Std Devs Not Equal

F Ratio DFNum DFDen Prob $>$ F

$\begin{array}{rrrr}3.2973 & 1 & 15.854 & 0.0884\end{array}$

t Test

1.8159
Oneway Analysis of Measurement By Sampler Mechanism Type of Material=SME Simulant, Analytical Block $=5$, Analyte $=\mathrm{NiO}($ wt $\%)$, Target Value $=0.41$

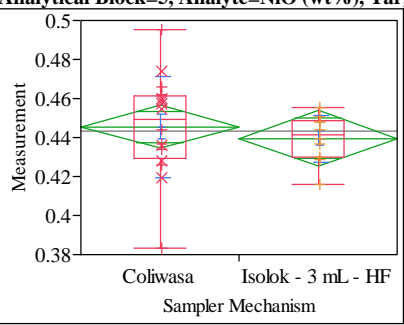

Oneway Anova

Summary of Fit

Adj Rsquare

$-0.02204$

0.021796

Mean of Response 0.443319

t Test

Isolok - $3 \mathrm{~mL}$ - HF-Coliwasa

Assuming equal variances

Difference $\quad-0.00596$ t Ratio $\quad-0.6789$

Std Err Dif $\quad 0.00879$ DF $\quad 24$

Upper CL Dif 0.01217 Prob $>|t| \begin{array}{rl}24 & 0.5037\end{array}$

Lower CL Dif -0.02410 Prob $>t \quad 0.7482$

$\begin{array}{lrl}\text { Confidence } & 0.95 \text { Prob }<\mathrm{t} \quad 0.2518\end{array}$

Analysis of Variance

$\begin{array}{lrrrrr}\text { Source } & \text { DF } & \text { Sum of Squares } & \text { Mean Square } & \text { F Ratio } & \text { Prob }>\text { F } \\ \text { Sampler Mechanism } & 1 & 0.00021895 & 0.000219 & 0.4609 & 0.5037\end{array}$

$\begin{array}{llll}\text { Error } & 24 & 0.01140108 & 0.000475\end{array}$

$\begin{array}{lll}\text { C. Total } & 25 & 0.01162003\end{array}$

Means for Oneway Anova

Level Number Mean Std Error Lower 95\% Upper 95\%

$\begin{array}{lrrrrr}\text { Coliwasa } & 16 & 0.445614 & 0.00545 & 0.43437 & 0.45686\end{array}$

$\begin{array}{llllll}\text { Isolok - 3 mL - HF } & 10 & 0.439649 & 0.00689 & 0.42542 & 0.45387\end{array}$

Std Error uses a pooled estimate of error variance

Means and Std Deviations

Level Number Mean Std Dev Std Err Mean Lower 95\% Upper 95\%

$\begin{array}{lrrrrrr}\text { Coliwasa } & 16 & 0.445614 & 0.025951 & 0.00649 & 0.43179 & 0.45944\end{array}$

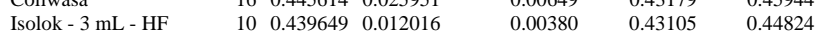

Test

Isolok - $3 \mathrm{~mL}$ - HF-Coliwasa

Assuming unequal variances

$\begin{array}{lrlr}\text { Difference } & -0.00596 & \mathrm{t} \text { Ratio } & -0.79335 \\ \text { Std Err Dif } & 0.00752 & \text { DF } & 22.61989 \\ \text { Upper CL Dif } & 0.00960 & \text { Prob }>|\mathrm{t}| & 0.4358 \\ \text { Lower CL Dif } & -0.02153 & \text { Prob }>\mathrm{t} & 0.7821 \\ \text { Confidence } & 0.95 & \text { Prob }<\mathrm{t} & 0.2179\end{array}$

Tests that the Variances are Equal

Level Count Std Dev MeanAbsDif to Mean MeanAbsDif to Median

$\begin{array}{lllll}\text { Coliwasa } & 16 & 0.0259508 & 0.0199623 & 0.0199623\end{array}$

$\begin{array}{lllll}\text { Isolok - 3 mL - HF } & 10 & 0.0120160 & 0.0095437 & 0.0095437\end{array}$

Test F Ratio DFNum DFDen p-Value

$\begin{array}{lrrrr}\text { O'Brien[.5] } & 2.0013 & 1 & 24 & 0.1700\end{array}$

Brown-Forsythe $3.6806 \quad 1 \quad 24 \quad 0.0670$

$\begin{array}{lllll}\text { Levene } & 3.8965 & 1 & 24 & 0.0600\end{array}$

$\begin{array}{lrrrr}\text { Bartlett } & 5.2455 & 1 & \text {. } & 0.0220 \\ \text { F Test 2-sided } & 4.6643 & 15 & 9 & 0.0247\end{array}$

Welch Anova testing Means Equal, allowing Std Devs Not Equal

F Ratio DFNum DFDen Prob $>$ F

$\begin{array}{rrrr}0.6294 & 1 & 22.62 & 0.4358\end{array}$

t Test
0.7934 


\section{Exhibit A2. Statistical Comparisons for Mid-Rheology (Phase 1) Testing}

Oneway Analysis of Measurement By Sampler Mechanism Type of Material=SME Simulant, Analytical Block $=5$, Analyte $=\mathrm{SiO} 2$ (wt $\%)$, Target Value $=50.985$

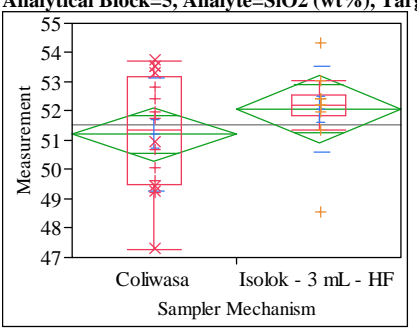

Oneway Anova

Summary of Fit

Adj Rsquare $\quad 0.056715$

Root Mean Square Error $\quad 1.778189$

Mean of Response $\quad 51.54067$

Observations (or Sum Wgts) $\quad 26$

t Test

Isolok - $3 \mathrm{~mL}$ - HF-Coliwasa

Assuming equal variances

Difference $\quad 0.8611$ t Ratio 1.201249

Std Err Dif $\quad 0.7168$ DF 24

Upper CL Dif 2.3405 Prob $>|t| \quad 0.2414$

Lower CL Dif -0.6184 Prob $>\mathrm{t} \quad 0.1207$

$\begin{array}{lrl}\text { Confidence } & 0.95 \text { Prob }<\mathrm{t} & 0.8793\end{array}$

Analysis of Variance

$\begin{array}{lrrrrr}\text { Source } & \text { DF } & \text { Sum of Squares } & \text { Mean Square } & \text { F Ratio } & \text { Prob }>\text { F } \\ \text { Sampler Mechanism } & 1 & 4.562699 & 4.56270 & 1.4430 & 0.2414\end{array}$

$\begin{array}{lrrr}\text { Error } & 24 & 75.886967 & 3.16196\end{array}$

$\begin{array}{lll}\text { C. Total } & 25 & 80.449666\end{array}$

Means for Oneway Anova

$\begin{array}{lrrrrr}\text { Level } & \text { Number } & \text { Mean } & \text { Std Error } & \text { Lower 95\% } & \text { Upper 95\% } \\ \text { Coliwasa } & 16 & 51.2095 & 0.44455 & 50.292 & 52.127\end{array}$

$\begin{array}{lrrrrr}\text { Coliwasa } & 16 & 51.2095 & 0.44455 & 50.292 & 52.127 \\ \text { Isolok - 3 mL - HF } & 10 & 52.0706 & 0.56231 & 50.910 & 53.231\end{array}$

Std Error uses a pooled estimate of error variance

Means and Std Deviations

Level Number Mean Std Dev Std Err Mean Lower 95\% Upper 95\%

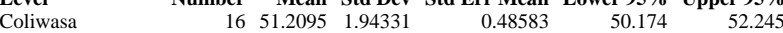

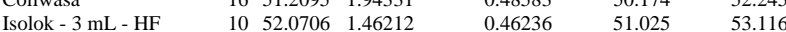

TTest

Isolok - $3 \mathrm{~mL}$ - HF-Coliwasa

Assuming unequal variances

Difference $\quad 0.8611$ t Ratio $\quad 1.28388$

Std Err Dif $\quad 0.6707$ DF $\quad 23.01288$

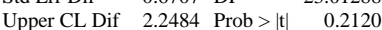

Lower CL Dif -0.5263 Prob $>t \quad 0.1060$

$\begin{array}{lrl}\text { Confidence } & 0.95 \text { Prob }<\mathrm{t} & 0.8940\end{array}$

Tests that the Variances are Equal

Level Count Std Dev MeanAbsDif to Mean MeanAbsDif to Median

$\begin{array}{lllll}\text { Coliwasa } & 16 & 1.943312 & 1.657958 & 1.657958\end{array}$

$\begin{array}{lllll}\text { Isolok - } 3 \mathrm{~mL} \text { - HF } & 10 & 1.462116 & 0.864277 & 0.812934\end{array}$

Test F Ratio DFNum DFDen p-Value

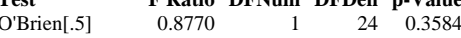

$\begin{array}{lllll}\text { Brown-Forsythe } & 4.0959 & 1 & 24 & 0.0543\end{array}$

$\begin{array}{lllll}\text { Levene } & 3.8073 & 1 & 24 & 0.0628\end{array}$

$\begin{array}{lrrrr}\text { Bartlett } & 0.8216 & 1 & \text {. } & 0.3647\end{array}$

Welch Anova testing Means Equal, allowing Std Devs Not Equal

F Ratio DFNum DFDen Prob $>$ F

$\begin{array}{rrrr}1.6483 & 1 & 23.013 & 0.2120\end{array}$

t Test
1.2839
Oneway Analysis of Measurement By Sampler Mechanism Type of Material=SME Simulant, Analytical Block $=5$, Analyte $=$ Sum of Oxides (wt $\%$ ), Target Value $=99.553$

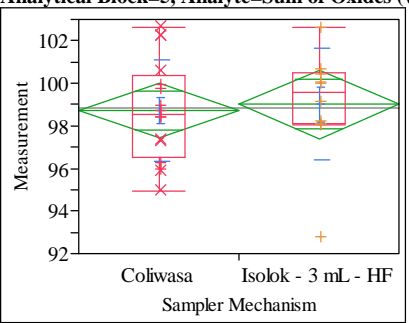

Oneway Anova

Summary of Fit

Adj Rsquare

-0.03747647
98.82969

Mean of Response

Observations (or Sum Wgts)

26

t Test

Isolok - $3 \mathrm{~mL}$ - HF-Coliwasa

Assuming equal variances

Difference $\quad 0.3109$ t Ratio $\quad 0.311694$

Std Err Dif $\quad 0.9976$ DF 24

Upper CL Dif 2.3698 Prob $>|t| \quad 0.7580$

Lower CL Dif -1.7479 Prob $>t \quad 0.3790$

$\begin{array}{lrl}\text { Confidence } & 0.95 \text { Prob }<\mathrm{t} \quad 0.6210\end{array}$

Analysis of Variance

$\begin{array}{lrrrrr}\text { Source } & \text { DF } & \text { Sum of Squares } & \text { Mean Square } & \text { F Ratio } & \text { Prob > F } \\ \text { Sampler Mechanism } & 1 & 0.59495 & 0.59495 & 0.0972 & 0.7580\end{array}$

$\begin{array}{llll}\text { Error } & 24 & 146.97303 & 6.12388\end{array}$

$\begin{array}{lll}\text { C. Total } & 25 & 147.56798\end{array}$

Means for Oneway Anova

Level Number Mean Std Error Lower 95\% Upper 95\%

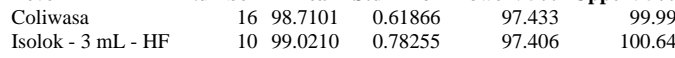

Std Error uses a pooled estimate of error variance

Means and Std Deviations

Level Number Mean Std Dev Std Err Mean Lower 95\% Upper 95\%

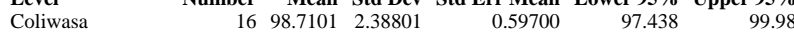

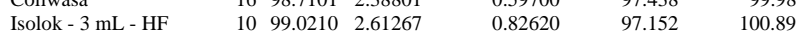

TTest

Isolok - $3 \mathrm{~mL}$ - HF-Coliwasa

Assuming unequal variances

$\begin{array}{lrlr}\text { Difference } & 0.3109 & \mathrm{t} \text { Ratio } & 0.30504 \\ \text { Std Err Dif } & 1.0193 \text { DF } & 17.92069 \\ \text { Upper CL Dif } & 2.4531 \text { Prob }>|\mathrm{t}| & 0.7638 \\ \text { Lower CL Dif } & -1.8313 \text { Prob }>\mathrm{t} & 0.3819 \\ \text { Confidence } & 0.95 & \text { Prob }<\mathrm{t} & 0.6181\end{array}$

Tests that the Variances are Equal

Level Count Std Dev MeanAbsDif to Mean MeanAbsDif to Median

$\begin{array}{lrrrr}\text { Coliwasa } & 16 & 2.388008 & 1.907102 & 1.905940 \\ \text { Isolok - 3 mL - HF } & 10 & 2.612667 & 1.777035 & 1.747612\end{array}$

Test F Ratio DFNum DFDen p-Value

$\begin{array}{lrrrr}\text { O'Brien[.5] } & 0.0771 & 1 & 24 & 0.7837\end{array}$

$\begin{array}{lllll}\text { Brown-Forsythe } & 0.0598 & 1 & 24 & 0.8088\end{array}$

$\begin{array}{lllll}\text { Levene } & 0.0437 & 1 & 24 & 0.8362\end{array}$

$\begin{array}{lllll}\text { Bartlett } & 0.0882 & 1 & \text {. } & 0.7665 \\ \text { F } & & 1 & 15 & 0.7282\end{array}$

Welch Anova testing Means Equal, allowing Std Devs Not Equal

F Ratio DFNum DFDen Prob > F

$\begin{array}{rrrr}0.0930 & 1 & 17.921 & 0.7638\end{array}$

$\mathbf{t}$ Test
0.3050 


\section{Exhibit A2. Statistical Comparisons for Mid-Rheology (Phase 1) Testing}

Oneway Analysis of Measurement By Sampler Mechanism Type of Material=SME Simulant, Analytical Block $=5$, Analyte $=$ TiO2 $($ wt $\%)$, Target Value $=0.0711$

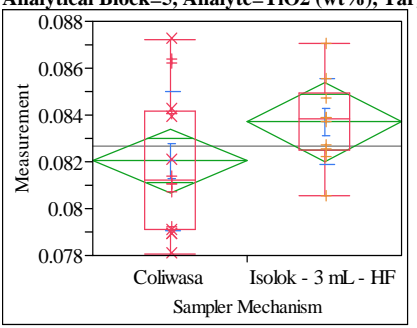

Oneway Anova

Summary of Fit

0.095054

Root Mean Square Error $\quad 0.002603$

Mean of Response

$\begin{array}{lr}\text { Mean of Response } & 0.082675 \\ \text { Observations (or Sum Wgts) } & 26\end{array}$

tTest

Isolok - $3 \mathrm{~mL}$ - HF-Coliwasa

Assuming equal variances

Difference $\quad 0.00167$ t Ratio 1.587739

Std Err Dif $\quad 0.00105$ DF 24

Upper CL Dif 0.00383 Prob $>|t| r \mid r .1254$

$\begin{array}{lll}\text { Lower CL Dif }-0.00050 \text { Prob }>t & 0.0627\end{array}$

$\begin{array}{lrl}\text { Confidence } & 0.95 \text { Prob }<\mathrm{t} & 0.9373\end{array}$

Analysis of Variance

$\begin{array}{lrrrrr}\text { Source } & \text { DF } & \text { Sum of Squares } & \text { Mean Square } & \text { F Ratio } & \text { Prob > F } \\ \text { Sampler Mechanism } & 1 & 0.00001708 & 0.000017 & 2.5209 & 0.1254\end{array}$

$\begin{array}{llll}\text { Error } & 24 & 0.00016259 & 6.775 \mathrm{e}-6\end{array}$

$\begin{array}{lll}\text { C. Total } & 25 & 0.00017967\end{array}$

Means for Oneway Anova

Level Number Mean Std Error Lower 95\% Upper 95\%

$\begin{array}{lrrrrr}\text { Coliwasa } & 16 & 0.082034 & 0.00065 & 0.08069 & 0.08338\end{array}$

$\begin{array}{llllll} & \\ \text { Isolok - } 3 \mathrm{~mL} \text { - HF } & 10 & 0.083700 & 0.00082 & 0.08200 & 0.08540\end{array}$

Std Error uses a pooled estimate of error variance

Means and Std Deviations

Level Number Mean Std Dev Std Err Mean Lower 95\% Upper 95\%

$\begin{array}{lrrrrrr}\text { Coliwasa } & 16 & 0.082034 & 0.002971 & 0.00074 & 0.08045 & 0.08362\end{array}$

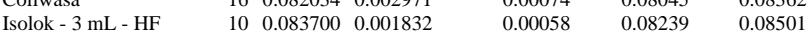

Test

Isolok - $3 \mathrm{~mL}$ - HF-Coliwasa

Assuming unequal variances

$\begin{array}{lrlr}\text { Difference } & 0.00167 & \text { t Ratio } & 1.768626 \\ \text { Std Err Dif } & 0.00094 & \text { DF } & 23.99891 \\ \text { Upper CL Dif } & 0.00361 & \text { Prob }>|t| & 0.0897 \\ \text { Lower CL Dif } & -0.00028 & \text { Prob }>\text { t } & 0.0448 \\ \text { Confidence } & 0.95 & \text { Prob }<\mathrm{t} & 0.9552\end{array}$

Tests that the Variances are Equal

Level Count Std Dev MeanAbsDif to Mean MeanAbsDif to Median

Coliwasa $\quad 16 \quad 0.0029709$

$\begin{array}{lllll}\text { Isolok - } 3 \mathrm{~mL} \text { - HF } & 10 & 0.0018319 & 0.0024877 & 0.0024082 \\ & & & 0.0013411 & 0.0013344\end{array}$

Test F Ratio DFNum DFDen p-Value

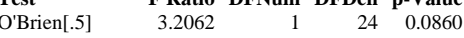

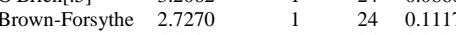

$\begin{array}{lllll}\text { Levene } & 4.2586 & 1 & 24 & 0.0500\end{array}$

$\begin{array}{lrrrr}\text { Bartlett } & 2.2524 & 1 & \text {. } & 0.1334 \\ \text { F Test 2-sided } & 2.6299 & 15 & 9 & 0.1462\end{array}$

Welch Anova testing Means Equal, allowing Std Devs Not Equal

F Ratio DFNum DFDen Prob $>$ F

$\begin{array}{rrrr}3.1280 & 1 & 23.999 & 0.0897\end{array}$

t Test
1.7686
Oneway Analysis of Measurement By Sampler Mechanism Type of Material=SME Simulant, Analytical Block $=5$, Analyte $=\mathrm{ZnO}$ (wt \%), Target Value $=0.0958$

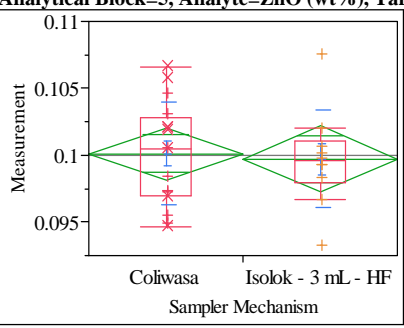

Oneway Anova

Summary of Fit

Adj Rsquare

$\quad 0.003776$

0.099972

Test

Isolok - $3 \mathrm{~mL}$ - HF-Coliwasa

Assuming equal variances

$\begin{array}{lrlr}\text { Difference } & -0.00039 & \mathrm{t} \text { Ratio } & -0.25456 \\ \text { Std Err Dif } & 0.00152 & \text { DF } & 24 \\ \text { Upper CL Dif } & 0.00275 & \text { Prob }>|\mathrm{t}| & 0.8012 \\ \text { Lower CL Dif } & -0.00353 & \text { Prob }>\mathrm{t} & 0.5994 \\ \text { Confidence } & 0.95 & \text { Prob }<\mathrm{t} & 0.4006\end{array}$

Analysis of Variance

$\begin{array}{lrrrrr}\text { Source } & \text { DF } & \text { Sum of Squares } & \text { Mean Square } & \text { F Ratio } & \text { Prob }>\text { F } \\ \text { Sampler Mechanism } & 1 & 0.00000092 & 9.238 \mathrm{e}-7 & 0.0648 & 0.8012\end{array}$

$\begin{array}{llll}\text { Error } & 24 & 0.00034212 & 0.000014\end{array}$

$\begin{array}{lll}\text { C. Total } & 25 & 0.00034304\end{array}$

Means for Oneway Anova

Level Number Mean Std Error Lower 95\% Upper 95\%

$\begin{array}{llllll}\text { Coliwasa } & 16 & 0.100121 & 0.00094 & 0.09817 & 0.10207\end{array}$

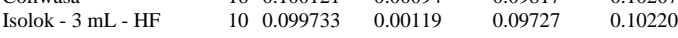

Std Error uses a pooled estimate of error variance

Means and Std Deviations

Level Number Mean Std Dev Std Err Mean Lower 95\% Upper 95\%

$\begin{array}{lrrrrrr}\text { Coliwasa } & 16 & 0.100121 & 0.003837 & 0.00096 & 0.09808 & 0.10217\end{array}$

$\begin{array}{lllllll}\text { Coliwasa } & 16 & 0.100121 & 0.003837 & 0.00096 & 0.09808 & 0.10217 \\ \text { Isolok - } 3 \mathrm{~mL}-\mathrm{HF} & 10 & 0.099733 & 0.003672 & 0.00116 & 0.09711 & 0.10236\end{array}$

Test

Isolok - $3 \mathrm{~mL}$ - HF-Coliwasa

Assuming unequal variances

$\begin{array}{lrlr}\text { Difference } & -0.00039 & \mathrm{t} \text { Ratio } & -0.25727 \\ \text { Std Err Dif } & 0.00151 & \text { DF } & 19.91191 \\ \text { Upper CL Dif } & 0.00275 & \text { Prob }>|t| & 0.7996 \\ \text { Lower CL Dif } & -0.00353 & \text { Prob }>\mathrm{t} & 0.6002 \\ \text { Confidence } & 0.95 & \text { Prob }<\mathrm{t} & 0.3998\end{array}$

Tests that the Variances are Equal

Level Count Std Dev MeanAbsDif to Mean MeanAbsDif to Median

$\begin{array}{lllll}\text { Coliwasa } & 16 & 0.0038367 & 0.0032394 & 0.0031976\end{array}$

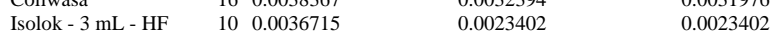

Test F Ratio DFNum DFDen p-Value

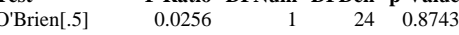

Brown-Forsythe $\quad 0.8582 \quad 1 \quad 24 \quad 0.3635$

$\begin{array}{lllll}\text { Levene } & 0.9996 & 1 & 24 & 0.3274\end{array}$

$\begin{array}{lrrrr}\text { Bartlett } & 0.0207 & 1 & \text {. } & 0.8857\end{array}$

Welch Anova testing Means Equal, allowing Std Devs Not Equal

F Ratio DFNum DFDen Prob $>$ F

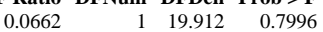

t Test

0.2573 


\section{Exhibit A2. Statistical Comparisons for Mid-Rheology (Phase 1) Testing}

Oneway Analysis of Measurement By Sampler Mechanism Type of Material=SME Simulant, Analytical Block $=5$, Analyte $=\mathrm{ZrO2}(\mathrm{wt} \%)$, Target Value $=0.3547$

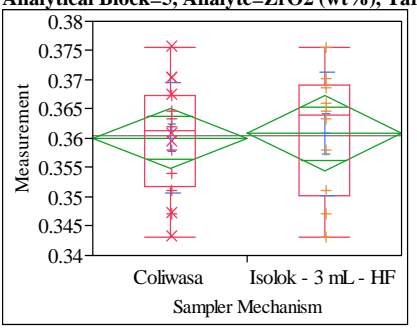

Oneway Anova

Summary of Fit

Adj Rsquare

0.001382

Root Mean Squarc

Mean of Response

$-0.04023$

Observations (or Sum Wgts) $\quad 26$

t Test

Isolok - $3 \mathrm{~mL}$ - HF-Coliwas

Assuming equal variances

Difference $\quad 0.00073$ t Ratio 0.182236

Std Err Dif $\quad 0.00398$ DF 24

Upper CL Dif 0.00895 Prob $>|t| \quad 0.8569$

$\begin{array}{lll}\text { Lower CL Dif }-0.00750 \text { Prob }>t & 0.4285\end{array}$

$\begin{array}{lrl}\text { Confidence } & 0.95 \text { Prob }<\mathrm{t} & 0.5715\end{array}$

Analysis of Variance

$\begin{array}{lrrrrr}\text { Source } & \text { DF } & \text { Sum of Squares } & \text { Mean Square } & \text { F Ratio } & \text { Prob }>\text { F } \\ \text { Sampler Mechanism } & 1 & 0.00000324 & 3.244 \mathrm{e}-6 & 0.0332 & 0.8569\end{array}$

$\begin{array}{lrrr}\text { Error } & 1 & 0.0000324 & 3.244 e-6 \\ & 24 & 0.00234439 & 0.000098\end{array}$

$\begin{array}{lll}\text { C. Total } & 25 & 0.00234764\end{array}$

Means for Oneway Anova

Level $\quad$ Number Mean Std Error Lower 95\% Upper 95\%

$\begin{array}{lrrrrr}\text { Coliwasa } & 16 & 0.360073 & 0.00247 & 0.35497 & 0.36517\end{array}$

$\begin{array}{llllll}\text { Isolok - } 3 \text { mL - HF } & 10 & 0.360799 & 0.00313 & 0.35435 & 0.36725\end{array}$

Std Error uses a pooled estimate of error variance

Means and Std Deviations

Level Number Mean Std Dev Std Err Mean Lower 95\% Upper 95\%

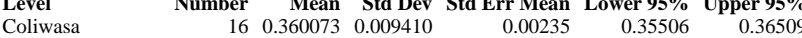

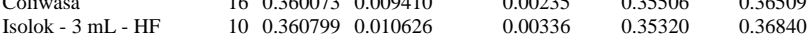

TTest

Isolok - $3 \mathrm{~mL}$ - HF-Coliwasa

Assuming unequal variances

Difference $\quad 0.00073$ t Ratio $\quad 0.177008$

Std Err Dif $\quad 0.00410$ DF $\quad 17.46739$

Upper CL Dif $\quad 0.00936$ Prob $>|t| r \begin{array}{r}17.46739 \\ 0.8615\end{array}$

Lower CL Dif -0.00791 Prob $>\mathrm{t} \quad 0.4308$

$\begin{array}{lrl}\text { Confidence } & 0.95 \text { Prob }<\mathrm{t} & 0.5692\end{array}$

Tests that the Variances are Equal

Level Count Std Dev MeanAbsDif to Mean MeanAbsDif to Median

Coliwasa $\quad 16 \quad 0.0094101$

$\begin{array}{lllll}\text { Isolok - } 3 \mathrm{~mL} \text { - HF } & 10 & 0.0106257 & 0.0087532 & 0.0082399\end{array}$

Test F Ratio DFNum DFDen p-Value

$\begin{array}{lrrrr}\text { O'Brien[.5] } & 0.3105 & 1 & 24 & 0.5825\end{array}$

$\begin{array}{lllll}\text { Brown-Forsythe } & 0.0872 & 1 & 24 & 0.7703\end{array}$

$\begin{array}{lllll}\text { Levene } & 0.3053 & 1 & 24 & 0.5857\end{array}$

$\begin{array}{lllll}\text { Bartlett } & 0.1617 & 1 & \text {. } & 0.6876\end{array}$

Welch Anova testing Means Equal, allowing Std Devs Not Equal

F Ratio DFNum DFDen Prob $>$ F

$\begin{array}{rrrr}0.0313 & 1 & 17.467 & 0.8615\end{array}$

t Test

0.1770
Oneway Analysis of Measurement By Sampler Mechanism Type of Material=SME Simulant, Analytical Block $=6$, Analyte $=A \mathrm{l} / \mathrm{B}$, Target Value $=2.552$

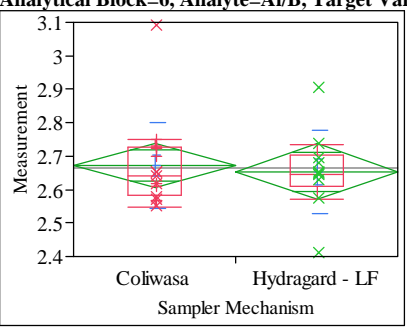

Oneway Anova

Summary of Fit

Adj Rsquare

Square Error $\quad 0.127381$

2.664893

t Test

Hydragard - LF-Coliwasa

Assuming equal variances

Difference $\quad-0.01797$ t Ratio $\quad-0.34987$

Std Err Dif $\quad 0.05135$ DF 24

Upper CL Dif 0.08801 Prob $>|t| r \mid \begin{array}{r}24 \\ 0.7295\end{array}$

$\begin{array}{lll}\text { Lower CL Dif }-0.12394 \text { Prob }>t & 0.6353\end{array}$

$\begin{array}{lrl}\text { Confidence } & 0.95 \text { Prob }<\mathrm{t} & 0.3647\end{array}$

Analysis of Variance

$\begin{array}{lrrrrr}\text { Source } & \text { DF } & \text { Sum of Squares } & \text { Mean Square } & \text { F Ratio } & \text { Prob > F } \\ \text { Sampler Mechanism } & 1 & 0.00198618 & 0.001986 & 0.1224 & 0.7295 \\ \text { Error } & 24 & 0.38942422 & 0.016226 & & \\ \text { C. Total } & 25 & 0.39141040 & & & \end{array}$

Means for Oneway Anova

Level Number Mean Std Error Lower 95\% Upper 95\%

$\begin{array}{lrrrrr}\text { Coliwasa } & 16 & 2.67180 & 0.03185 & 2.6061 & 2.7375 \\ \text { Hydragard - LF } & 10 & 2.65384 & 0.04028 & 2.5707 & 2.7370\end{array}$

Std Error uses a pooled estimate of error variance

Means and Std Deviations

Level Number Mean Std Dev Std Err Mean Lower 95\% Upper 95\%

\begin{tabular}{lrrrrr} 
Coliwasa & 16 & 2.67180 & 0.129198 & 0.03230 & 2.6030 \\
\hline
\end{tabular}

$\begin{array}{lllllll}\text { Hydragard - LF } & 10 & 2.65384 & 0.124295 & 0.03931 & 2.5649 & 2.7428\end{array}$

Test

Hydragard - LF-Coliwasa

Assuming unequal variances

$\begin{array}{lrlr}\text { Difference } & -0.01797 & \mathrm{t} \text { Ratio } & -0.35313 \\ \text { Std Err Dif } & 0.05087 & \text { DF } & 19.83273 \\ \text { Upper CL Dif } & 0.08821 & \text { Prob }>|\mathrm{t}| & 0.7277 \\ \text { Lower CL Dif } & -0.12414 & \text { Prob }>\mathrm{t} & 0.6361 \\ \text { Confidence } & 0.95 & \text { Prob }<\mathrm{t} & 0.3639\end{array}$

Tests that the Variances are Equal

Level Count Std Dev MeanAbsDif to Mean MeanAbsDif to Median

$\begin{array}{lllll}\text { Coliwasa } & 16 & 0.1291977 & 0.0863064 & 0.0802212\end{array}$

$\begin{array}{lllll}\text { Hydragard - LF } & 10 & 0.1242952 & 0.0863064 & 0.0772512\end{array}$

Test F Ratio DFNum DFDen p-Value

$\begin{array}{lrrrr}\text { O'Brien[.5] } & 0.0056 & 1 & 24 & 0.9412\end{array}$

$\begin{array}{lllll}\text { Brown-Forsythe } & 0.0109 & 1 & 24 & 0.9177\end{array}$

$\begin{array}{lllll}\text { Levene } & 0.0575 & 1 & 24 & 0.8125\end{array}$

$\begin{array}{lrrrr}\text { Bartlett } & 0.0160 & 1 & \text {. } & 0.8994 \\ \text { F Test 2-sided } & 1.0804 & 15 & 9 & 0.9392\end{array}$

Welch Anova testing Means Equal, allowing Std Devs Not Equal

F Ratio DFNum DFDen Prob $>$ F

$\begin{array}{rrrr}0.1247 & 1 & 19.833 & 0.7277\end{array}$

t Test

0.3531 


\section{Exhibit A2. Statistical Comparisons for Mid-Rheology (Phase 1) Testing}

Oneway Analysis of Measurement By Sampler Mechanism Type of Material=SME Simulant, Analytical Block=6, Analyte=Al2O3 (wt \%), Target Value=10.869

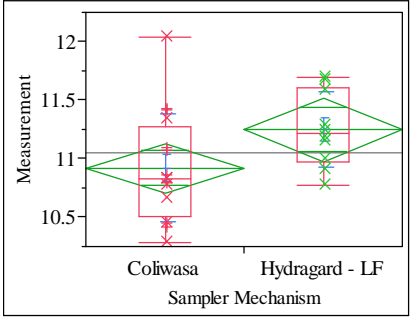

Oneway Anova

Summary of Fit

Adj Rsquare

0.138712

0.102825
0.415437

$\begin{array}{lr}\text { Mean of Response } & 11.04558 \\ \text { Observations (or Sum Wgts) } & 26\end{array}$

Test

Hydragard - LF-Coliwasa

Assuming equal variances

Difference $\quad 0.32925$ t Ratio 1.966021

Std Err Dif $\quad 0.16747$ DF $\quad 24$

Upper CL Dif 0.67488 Prob $>|t| r 0.0610$

Lower CL Dif -0.01639 Prob $>\mathrm{t} \quad 0.0305$

$\begin{array}{lrl}\text { Confidence } & 0.95 \text { Prob }<\mathrm{t} & 0.9695\end{array}$

Analysis of Variance

$\begin{array}{lrrrrr}\text { Source } & \text { DF } & \text { Sum of Squares } & \text { Mean Square } & \text { F Ratio } & \text { Prob > F } \\ \text { Sampler Mechanism } & 1 & 0.6670924 & 0.667092 & 3.8652 & 0.0610\end{array}$

$\begin{array}{llll}\text { Error } & 24 & 4.1421044 & 0.172588\end{array}$

4.8091968

Means for Oneway Anova

Level Number Mean Std Error Lower 95\% Upper 95\%

$\begin{array}{lrrrrr}\text { Coliwasa } & 16 & 10.9189 & 0.10386 & 10.705 & 11.133\end{array}$

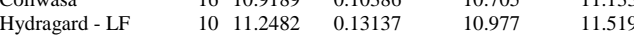

Std Error uses a pooled estimate of error variance

Means and Std Deviations

Level Number Mean Std Dev Std Err Mean Lower 95\% Upper 95\%

$\begin{array}{lrrrrrr}\text { Coliwasa } & 16 & 10.9189 & 0.463287 & 0.11582 & 10.672 & 11.166\end{array}$

$\begin{array}{lllllll}\text { Coliwasa } & 16 & 10.9189 & 0.463287 & 0.11582 & 10.672 & 11.166 \\ \text { Hydragard - LF } & 10 & 11.2482 & 0.320170 & 0.10125 & 11.019 & 11.477\end{array}$

t Test

Hydragard - LF-Coliwasa

Assuming unequal variances

Difference $\quad 0.329245$ t Ratio $\quad 2.140234$

Std Err Dif $\quad 0.153836$ DF 23.6586

Upper CL Dif 0.646990 Prob $>|t| r 0.0429$

Lower CL Dif 0.011501 Prob $>t \quad 0.0214$

$\begin{array}{lrl}\text { Confidence } & 0.95 \text { Prob }<\mathrm{t} & 0.9786\end{array}$

Tests that the Variances are Equal

Level Count Std Dev MeanAbsDif to Mean MeanAbsDif to Median

Coliwasa $\quad 16 \quad 0.4632873$

$\begin{array}{lllll}\text { Hydragard - LF } & 10 & 0.3201698 & 0.2486582 & 0.2475245\end{array}$

Test F Ratio DFNum DFDen p-Value

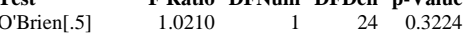

Brown-Forsythe $\quad 0.6934 \quad 1 \quad 24 \quad 0.4132$

$\begin{array}{lllll}\text { Levene } & 1.2116 & 1 & 24 & 0.2819\end{array}$

$\begin{array}{lrrrr}\text { Bartlett } & 1.3566 & 1 & \text {. } & 0.2441\end{array}$

Welch Anova testing Means Equal, allowing Std Devs Not Equal

F Ratio DFNum DFDen Prob $>$ F

$\begin{array}{lrrr}4.5806 & 1 & 23.659 & 0.0429\end{array}$

t Test

$$
123.659 \quad 0.0429
$$

2.1402
Oneway Analysis of Measurement By Sampler Mechanism Type of Material=SME Simulant, Analytical Block $=6$, Analyte $=\mathrm{B} 2 \mathrm{O} 3(\mathrm{wt} \%)$, Target Value $=4.259$

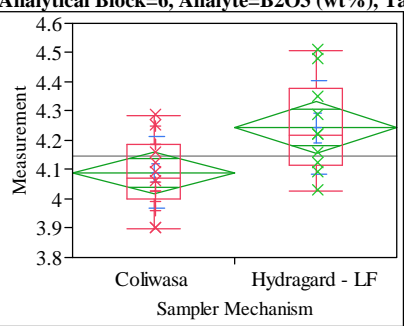

Oneway Anova

Summary of Fit

Adj Rsquare

0.137805
4.148717

ean of Response

Observations (or Sum Wgts)

Test

Hydragard - LF-Coliwasa

Assuming equal variances

Difference $\quad 0.154555$ t Ratio $\quad 2.782219$

Std Err Dif $\quad 0.055551$ DF 24

Upper CL Dif 0.269207 Prob $>|t| \quad 0.0103$

Lower CL Dif 0.039903 Prob $>t \quad 0.0052$

$\begin{array}{lrl}\text { Confidence } & 0.95 \text { Prob }<\mathrm{t} & 0.9948\end{array}$

Analysis of Variance

$\begin{array}{lrrrrr}\text { Source } & \text { DF } & \text { Sum of Squares } & \text { Mean Square } & \text { F Ratio } & \text { Prob }>\text { F } \\ \text { Sampler Mechanism } & 1 & 0.14699883 & 0.146999 & 7.7407 & 0.0103 \\ \text { Error } & 24 & 0.45576655 & 0.018990 & & \end{array}$

C. Total

Means for Oneway Anova

Level Number Mean Std Error Lower 95\% Upper 95\%

$\begin{array}{lrrrrr}\text { Coliwasa } & 16 & 4.08927 & 0.03445 & 4.0182 & 4.1604\end{array}$

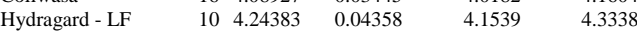

Std Error uses a pooled estimate of error variance

Means and Std Deviations

Level Number Mean Std Dev Std Err Mean Lower 95\% Upper 95\%

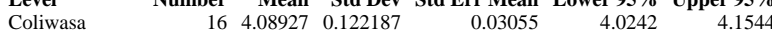

$\begin{array}{lllllll}\text { Hydragard - LF } & 10 & 4.24383 & 0.160493 & 0.05075 & 4.1290 & 4.3586\end{array}$

T Test

Hydragard - LF-Coliwasa

Assuming unequal variances

$\begin{array}{lrlr}\text { Difference } & 0.154555 & \text { t Ratio } & 2.609141 \\ \text { Std Err Dif } & 0.059236 & \text { DF } & 15.48258 \\ \text { Upper CL Dif } & 0.280472 & \text { Prob }>|t| & 0.0194 \\ \text { Lower CL Dif } & 0.028638 & \text { Prob }>t & 0.0097 \\ \text { Confidence } & 0.95 & \text { Prob }<\mathrm{t} & 0.9903\end{array}$

Tests that the Variances are Equal

Level Count Std Dev MeanAbsDif to Mean MeanAbsDif to Median

$\begin{array}{lllll}\text { Coliwasa } & 16 & 0.1221866 & 0.1006219 & 0.1006219\end{array}$

$\begin{array}{lllll}\text { Hydragard - LF } & 10 & 0.1604933 & 0.1275080 & 0.1223562\end{array}$

Test F Ratio DFNum DFDen p-Value

$\begin{array}{lrrrr}\text { O'Brien[.5] } & 1.5046 & 1 & 24 & 0.2319\end{array}$

$\begin{array}{lllll}\text { Brown-Forsythe } & 0.4506 & 1 & 24 & 0.5085\end{array}$

$\begin{array}{lllll}\text { Levene } & 0.8138 & 1 & 24 & 0.3760\end{array}$

$\begin{array}{lllll}\text { Bartlett } & 0.8278 & 1 & \text {. } & 0.3629 \\ \text { F Test 2-sided } & 1.7253 & 9 & 15 & 0.3367\end{array}$

Welch Anova testing Means Equal, allowing Std Devs Not Equal

F Ratio DFNum DFDen Prob $>$ F

$\begin{array}{llrr}6.8076 & 1 & 15.483 & 0.0194\end{array}$

t Test

2.609 


\section{Exhibit A2. Statistical Comparisons for Mid-Rheology (Phase 1) Testing}

Oneway Analysis of Measurement By Sampler Mechanism Type of Material=SME Simulant, Analytical Block=6, Analyte=BaO (wt\%), Target Value=0.0919

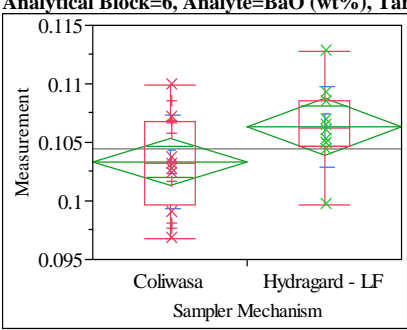

Oneway Anova

Summary of Fit

Adj Rsquare

0.141251

Root Mean Squ

Mean of Response

0.10547

Observations (or Sum Wgts) $\quad 26$

t Test

Hydragard - LF-Coliwasa

Assuming equal variances

Difference $\quad 0.00303$ t Ratio 1.986865

Std Err Dif $\quad 0.00152$ DF 24

Upper CL Dif 0.00617 Prob $>|t| r 0.0585$

Lower CL Dif -0.00012 Prob $>t \quad 0.0292$

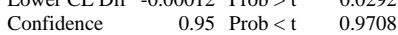

Analysis of Variance

$\begin{array}{lrrrrr}\text { Source } & \text { DF } & \text { Sum of Squares } & \text { Mean Square } & \text { F Ratio } & \text { Prob }>\text { F } \\ \text { Sampler Mechanism } & 1 & 0.00005634 & 0.000056 & 3.9476 & 0.0585\end{array}$

$\begin{array}{llll}\text { Error } & 24 & 0.00034251 & 0.000014\end{array}$

$\begin{array}{lll}\text { C. Total } & 25 & 0.00039885\end{array}$

Means for Oneway Anova

Level Number Mean Std Error Lower 95\% Upper 95\%

\begin{tabular}{lrrrrr} 
Coliwasa & 16 & 0.103332 & 0.00094 & 0.10138 & 0.10528 \\
\hline
\end{tabular}

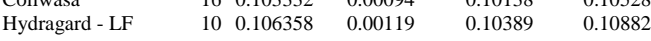

Std Error uses a pooled estimate of error variance

Means and Std Deviations

Level Number Mean Std Dev Std Err Mean Lower 95\% Upper 95\%

Novel 0.1033320 .003971

$\begin{array}{lllllll}\text { Coliwasa } & 16 & 0.103332 & 0.003971 & 0.00099 & 0.10122 & 0.10545 \\ \text { Hydragard - LF } & 10 & 0.106358 & 0.003432 & 0.00109 & 0.10390 & 0.10881\end{array}$

t Test

Hydragard - LF-Coliwasa

Assuming unequal variances

Difference $\quad 0.00303$ t Ratio $\quad 2.057065$

Std Err Dif $\quad 0.00147$ DF $\quad 21.37711$

Upper CL Dif 0.00608 Prob $>|t| \quad 0.0521$

Lower CL Dif -0.00003 Prob $>t \quad 0.0260$

$\begin{array}{lrl}\text { Confidence } & 0.95 \text { Prob }<\mathrm{t} & 0.9740\end{array}$

Tests that the Variances are Equal

$\begin{array}{lrrrr}\text { Level } & \text { Count } & \text { Std Dev } & \text { MeanAbsDif to Mean } & \text { MeanAbsDif to Median } \\ \text { Coliwasa } & 16 & 0.0039705 & 0.0031262 & 0.0031262\end{array}$

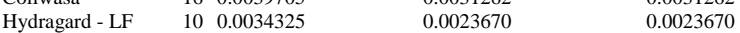

Test F Ratio DFNum DFDen p-Value

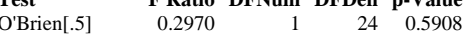

$\begin{array}{lllll}\text { Brown-Forsythe } & 0.6524 & 1 & 24 & 0.4272\end{array}$

$\begin{array}{lllll}\text { Levene } & 0.6543 & 1 & 24 & 0.4265\end{array}$

$\begin{array}{lrrrr}\text { Bartlett } & 0.2220 & 1 & \text {. } & 0.6375\end{array}$

Welch Anova testing Means Equal, allowing Std Devs Not Equal

F Ratio DFNum DFDen Prob $>$ F

$\begin{array}{llll}4.2315 & 1 & 21.377 & 0.0521\end{array}$

t Test

$$
121.377 \quad 0.0521
$$

2.0571
Oneway Analysis of Measurement By Sampler Mechanism Type of Material=SME Simulant, Analytical Block=6, Analyte $=\mathrm{CaO}($ wt $\%)$, Target Value $=1.1462$

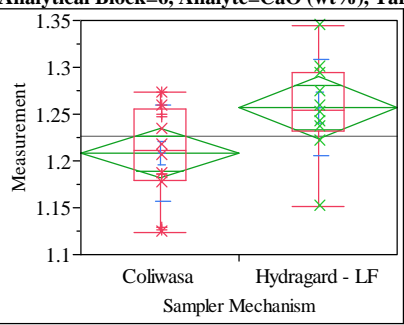

Oneway Anova

Summary of Fit

Adj Rsquare

0.051621

1.226883

Observations (or Sum Wgts) $\quad 26$

Test

Hydragard - LF-Coliwasa

Assuming equal variances

Difference $\quad 0.048780$ t Ratio 2.344171

Std Err Dif $\quad 0.020809$ DF 24

Upper CL Dif 0.091727 Prob $>|t| \quad 0.0277$

Lower CL Dif 0.005832 Prob $>t \quad 0.0138$

$\begin{array}{lrl}\text { Confidence } & 0.95 \text { Prob }<\mathrm{t} & 0.9862\end{array}$

Analysis of Variance

$\begin{array}{lrrrrr}\text { Source } & \text { DF } & \text { Sum of Squares } & \text { Mean Square } & \text { F Ratio } & \text { Prob > F } \\ \text { Sampler Mechanism } & 1 & 0.01464277 & 0.014643 & 5.4951 & 0.0277 \\ \text { Error } & 24 & 0.06395228 & 0.002665 & & \end{array}$

$\begin{array}{ll}\text { Total } & 25-0.07859505\end{array}$

Means for Oneway Anova

Level Number Mean Std Error Lower 95\% Upper 95\%

$\begin{array}{lrrrrr}\text { Coliwasa } & 16 & 1.20812 & 0.01291 & 1.1815 & 1.2348\end{array}$

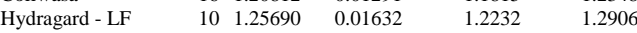

Std Error uses a pooled estimate of error variance

Means and Std Deviations

Level Number Mean Std Dev Std Err Mean Lower 95\% Upper 95\%

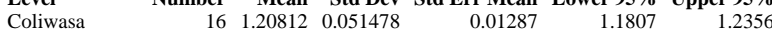

$\begin{array}{lllllll}\text { Hydragard - LF } & 10 & 1.25690 & 0.051857 & 0.01640 & 1.2198 & 1.2940\end{array}$

T Test

Hydragard - LF-Coliwasa

Assuming unequal variances

$\begin{array}{lrlr}\text { Difference } & 0.048780 & \text { t Ratio } & 2.340048 \\ \text { Std Err Dif } & 0.020846 & \text { DF } & 19.14336 \\ \text { Upper CL Dif } & 0.092388 & \text { Prob }>|t| & 0.0303 \\ \text { Lower CL Dif } & 0.005171 & \text { Prob }>t & 0.0151 \\ \text { Confidence } & 0.95 & \text { Prob }<\text { t } & 0.9849\end{array}$

Tests that the Variances are Equal

Level Count Std Dev MeanAbsDif to Mean MeanAbsDif to Median

$\begin{array}{lllll}\text { Coliwasa } & 16 & 0.0514783 & 0.0429380 & 0.0429380\end{array}$

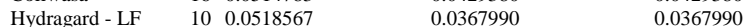

Test F Ratio DFNum DFDen p-Value

$\begin{array}{lrrrr}\text { O'Brien[.5] } & 0.0008 & 1 & 24 & 0.9781\end{array}$

$\begin{array}{lllll}\text { Brown-Forsythe } & 0.0008 & 1 & 24 & 0.9781 \\ & 0.2628 & 1 & 24 & 0.6129\end{array}$

$\begin{array}{lllll}\text { Levene } & 0.2662 & 1 & 24 & 0.6106\end{array}$

$\begin{array}{lllll}\text { Bartlett } & 0.0006 & 1 & 0.9808\end{array}$

Welch Anova testing Means Equal, allowing Std Devs Not Equal

F Ratio DFNum DFDen Prob > F

$\begin{array}{lllr}5.4758 & 1 & 19.143 & 0.0303\end{array}$

t Test

2.3400 


\section{Exhibit A2. Statistical Comparisons for Mid-Rheology (Phase 1) Testing}

Oneway Analysis of Measurement By Sampler Mechanism Type of Material=SME Simulant, Analytical Block $=6$, Analyte $=\mathrm{Cr} 2 \mathrm{O} 3(\mathrm{wt} \%)$, Target Value $=\mathbf{0 . 0 9 9 8}$

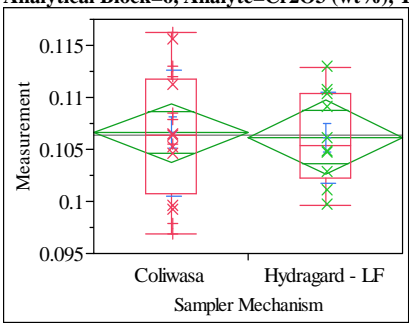

Oneway Anova

Summary of Fit

Adj Rsquare

0.001515

Root Mean Squarc

Mean of Response

$-0.04009$

0.005486

0.106416

t Test

Hydragard - LF-Coliwasa

Assuming equal variances

Difference $\quad-0.00042$ t Ratio -0.19083

$\begin{array}{lrlr}\text { Std Err Dif } & 0.00221 & \text { DF } & 24 \\ \text { Upper CL Dif } & 0.00414 & \text { Prob }>|t| & 0.8503\end{array}$

Lower CL Dif -0.00499 Prob $>t \quad 0.5749$

$\begin{array}{lrl}\text { Confidence } & 0.95 \text { Prob }<\mathrm{t} & 0.4251\end{array}$

Analysis of Variance

$\begin{array}{lrrrrr}\text { Source } & \text { DF } & \text { Sum of Squares } & \text { Mean Square } & \text { F Ratio } & \text { Prob }>\text { F } \\ \text { Sampler Mechanism } & 1 & 0.00000110 & 1.096 \mathrm{e}-6 & 0.0364 & 0.8503\end{array}$

$\begin{array}{llll}\text { Error } & 24 & 0.00072241 & 0.000030\end{array}$

$\begin{array}{lll}\text { C. Total } & 25 & 0.00072351\end{array}$

Means for Oneway Anova

Level Number Mean Std Error Lower 95\% Upper 95\%

$\begin{array}{lrrrrr}\text { Coliwasa } & 16 & 0.106578 & 0.00137 & 0.10375 & 0.10941\end{array}$

$\begin{array}{llllll}\text { Hydragard - LF } & 10 & 0.106156 & 0.00173 & 0.10258 & 0.10974\end{array}$

Std Error uses a pooled estimate of error variance

Means and Std Deviations

Level Number Mean Std Dev Std Err Mean Lower 95\% Upper 95\%

$\begin{array}{lrrrrrr}\text { Coliwasa } & \text { Number } & \text { Mean } & \text { Std Dev } & \text { Std Err Mean } & \text { Lower } 95 \% & \text { Upper 95\% } \\ \text { Co. } & 16 & 0.106578 & 0.006048 & 0.00151 & 0.10336 & 0.10980\end{array}$

$\begin{array}{lllllll}\text { Coliwasa } & 16 & 0.106578 & 0.006048 & 0.00151 & 0.10336 & 0.10980 \\ \text { Hydragard - LF } & 10 & 0.106156 & 0.004393 & 0.00139 & 0.10301 & 0.10930\end{array}$

t Test

Hydragard - LF-Coliwasa

Assuming unequal variances

Difference $\quad-0.00042$ t Ratio $\quad-0.20553$

Std Err Dif $\quad 0.00205$ DF 23.31805

Upper CL Dif 0.00382 Prob $>|t| r \mid r .8389$

Lower CL Dif -0.00467 Prob $>\mathrm{t} \quad 0.5805$

$\begin{array}{lrl}\text { Confidence } & 0.95 \text { Prob }<\mathrm{t} & 0.4195\end{array}$

Tests that the Variances are Equal

Level Count Std Dev MeanAbsDif to Mean MeanAbsDif to Median

Coliwasa $\quad 16 \quad 0.0060481$

$\begin{array}{lllll}\text { Hydragard - LF } & 10 & 0.0043935 & 0.0036189 & 0.0035809\end{array}$

Test F Ratio DFNum DFDen p-Value

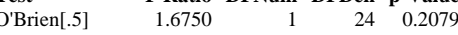

$\begin{array}{lllll}\text { Brown-Forsythe } & 0.7883 & 1 & 24 & 0.3834\end{array}$

$\begin{array}{lllll}\text { Levene } & 0.8090 & 1 & 24 & 0.3773\end{array}$

$\begin{array}{lrrrr}\text { Bartlett } & 1.0280 & 1 & \text {. } & 0.3106\end{array}$

Welch Anova testing Means Equal, allowing Std Devs Not Equal

F Ratio DFNum DFDen Prob $>$ F

$\begin{array}{llll}0.0422 & 1 & 23.318 & 0.8389\end{array}$

t Test
0.2055
Oneway Analysis of Measurement By Sampler Mechanism Type of Material=SME Simulant, Analytical Block $=6$, Analyte $=\mathrm{CuO}(\mathrm{wt} \%)$, Target Value $=0.0504$

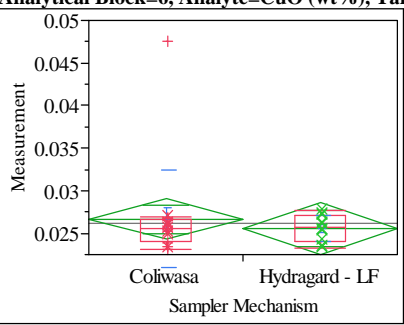

Oneway Anova

Summary of Fit

Adj Rsquare

$-0.02757$

$\quad 0.004624$

Mean of Response 0.026232

Test

Hydragard - LF-Coliwasa

Assuming equal variances

$\begin{array}{lrlr}\text { Difference } & -0.00107 & \mathrm{t} \text { Ratio } & -0.57376 \\ \text { Std Err Dif } & 0.00186 & \text { DF } & 24 \\ \text { Upper CL Dif } & 0.00278 & \text { Prob }>|t| & 0.5715 \\ \text { Lower CL Dif } & -0.00492 & \text { Prob }>\mathrm{t} & 0.7143 \\ \text { Confidence } & 0.95 & \text { Prob }<\mathrm{t} & 0.2857\end{array}$

Analysis of Variance

Source DF Sum of Squares Mean Square F Ratio Prob > F

Sampler Mechanism $\quad 1 \quad 0.00000704$

$\begin{array}{ll}24 & 0.00051317\end{array}$

$\begin{array}{lll}7.039 \mathrm{e}-6 & 0.3292 & 0.5715\end{array}$

$\begin{array}{lll}\text { C. Total } & 25 & 0.00052021\end{array}$

Means for Oneway Anova

Level Number Mean Std Error Lower 95\% Upper 95\%

$\begin{array}{lrrrrr}\text { Coliwasa } & 16 & 0.026644 & 0.00116 & 0.02426 & 0.02903\end{array}$

$\begin{array}{llllll}\text { Hydragard - LF } & 10 & 0.025574 & 0.00146 & 0.02256 & 0.02859\end{array}$

Std Error uses a pooled estimate of error variance

Means and Std Deviations

Level Number Mean Std Dev Std Err Mean Lower 95\% Upper 95\%

$\begin{array}{lrrrrrr}\text { Coliwasa } & 16 & 0.026644 & 0.005719 & 0.00143 & 0.02360 & 0.02969\end{array}$

$\begin{array}{lllllll}\text { Hydragard - LF } & 10 & 0.025574 & 0.001582 & 0.00050 & 0.02444 & 0.02671\end{array}$

T Test

Hydragard - LF-Coliwasa

Assuming unequal variances

$\begin{array}{lrlr}\text { Difference } & -0.00107 & \text { t Ratio } & -0.70603 \\ \text { Std Err Dif } & 0.00151 & \text { DF } & 18.43845 \\ \text { Upper CL Dif } & 0.00211 & \text { Prob }>|t| & 0.4890 \\ \text { Lower CL Dif } & -0.00425 & \text { Prob }>t & 0.7555 \\ \text { Confidence } & 0.95 & \text { Prob }<\mathrm{t} & 0.2445\end{array}$

Tests that the Variances are Equal

Level Count Std Dev MeanAbsDif to Mean MeanAbsDif to Median

$\begin{array}{lllll}\text { Coliwasa } & 16 & 0.0057192 & 0.0026777 & 0.0023276\end{array}$

$\begin{array}{llll}\text { Hydragard - LF } & 10 & 0.0015824 & 0.0012643\end{array}$

Test F Ratio DFNum DFDen p-Value

$\begin{array}{lllll}\text { O'Brien[.5] } & 0.6231 & 1 & 24 & 0.4376\end{array}$

$\begin{array}{lllll}\text { Brown-Forsythe } & 0.3899 & 1 & 24 & 0.5382\end{array}$

$\begin{array}{lllll}\text { Levene } & 0.7714 & 1 & 24 & 0.3885\end{array}$

$\begin{array}{lrrrr}\text { Bartlett } & 12.3649 & 1 & \text {. } & 0.0004 \\ \text { F Test 2-sided } & 13.0632 & 15 & 9 & 0.0005\end{array}$

Welch Anova testing Means Equal, allowing Std Devs Not Equal

F Ratio DFNum DFDen Prob $>$ F

$\begin{array}{rrrr}0.4985 & 1 & 18.438 & 0.4890\end{array}$

t Test
0.7060 


\section{Exhibit A2. Statistical Comparisons for Mid-Rheology (Phase 1) Testing}

Oneway Analysis of Measurement By Sampler Mechanism Type of Material=SME Simulant, Analytical Block $=6$, Analyte $=\mathrm{Fe} / \mathrm{Li}$, Target Value $=2.452$

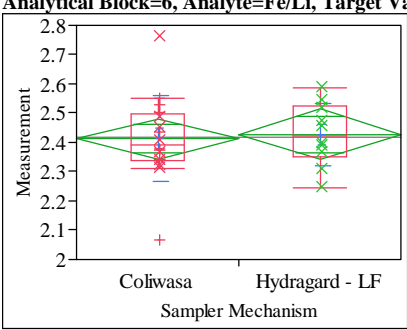

Oneway Anova

Summary of Fit

Adj Rsquare

$\quad 0.13334$

2.418443

t Test

Hydragard - LF-Coliwasa

Assuming equal variances

Difference $\quad 0.01401$ t Ratio $\quad 0.260728$

Std Err Dif $\quad 0.05375$ DF $\quad 24$

Upper CL Dif 0.12495 Prob $>|t| \quad 0.7965$

Lower CL Dif -0.09692 Prob $>\mathrm{t} \quad 0.3983$

$\begin{array}{lrl}\text { Confidence } & 0.95 \text { Prob }<\mathrm{t} & 0.6017\end{array}$

Analysis of Variance

$\begin{array}{lrrrrr}\text { Source } & \text { DF } & \text { Sum of Squares } & \text { Mean Square } & \text { F Ratio } & \text { Prob }>\text { F } \\ \text { Sampler Mechanism } & 1 & 0.00120865 & 0.001209 & 0.0680 & 0.7965\end{array}$

$\begin{array}{llll}\text { Error } & 24 & 0.42671118 & 0.017780\end{array}$

$\begin{array}{lll}\text { C. Total } & 25 & 0.42791983\end{array}$

Means for Oneway Anova

Level Number Mean Std Error Lower 95\% Upper 95\%

$\begin{array}{lrrrrr}\text { Coliwasa } & 16 & 2.41305 & 0.03334 & 2.3443 & 2.4819\end{array}$

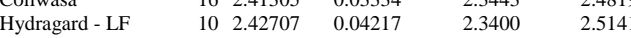

Std Error uses a pooled estimate of error variance

Means and Std Deviations

Level Number Mean Std Dev Std Err Mean Lower 95\% Upper 95\%

$\begin{array}{lrrrrrr}\text { Coliwasa } & 16 & 2.41305 & 0.146566 & 0.03664 & 2.3350 & 2.4912\end{array}$

$\begin{array}{lllllll}\text { Coliwasa } & 16 & 2.41305 & 0.146566 & 0.03664 & 2.3350 & 2.4912 \\ \text { Hydragard - LF } & 10 & 2.42707 & 0.107749 & 0.03407 & 2.3500 & 2.5041\end{array}$

t Test

Hydragard - LF-Coliwasa

Assuming unequal variances

$\begin{array}{lrlr}\text { Difference } & 0.01401 & \mathrm{t} \text { Ratio } & 0.280089 \\ \text { Std Err Dif } & 0.05004 & \text { DF } & 23.22002 \\ \text { Upper CL Dif } & 0.11747 & \text { Prob }>|t| & 0.7819 \\ \text { Lower CL Dif } & -0.08944 & \text { Prob }>t & 0.3909 \\ \text { Confidence } & 0.95 & \text { Prob }<\mathrm{t} & 0.6091\end{array}$

Tests that the Variances are Equal

Level Count Std Dev MeanAbsDif to Mean MeanAbsDif to Median

Coliwasa $\quad 16 \quad 0.1465658$

$\begin{array}{lllll}\text { Hydragard - LF } & 10 & 0.1077488 & 0.1025282 & 0.1017131 \\ & & & 0.0876864 & 0.0876864\end{array}$

Test F Ratio DFNum DFDen p-Value

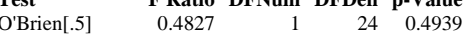

$\begin{array}{lllll}\text { Brown-Forsythe } & 0.1516 & 1 & 24 & 0.7004\end{array}$

$\begin{array}{lllll}\text { Levene } & 0.1791 & 1 & 24 & 0.6759\end{array}$

\begin{tabular}{lrrrr} 
Bartlett & 0.9554 & 1 & . & 0.3284 \\
\hline & 1.8503 & 15 & 9 & 0.3532
\end{tabular}

Welch Anova testing Means Equal, allowing Std Devs Not Equal

F Ratio DFNum DFDen Prob $>$ F

$\begin{array}{llll}0.0784 & 1 & 23.22 & 0.7819\end{array}$

t Test

0.2801
Oneway Analysis of Measurement By Sampler Mechanism Type of Material=SME Simulant, Analytical Block $=6$, Analyte $=\mathrm{Fe} 2 \mathrm{O} 3(\mathrm{wt} \%)$, Target Value $=11.462$

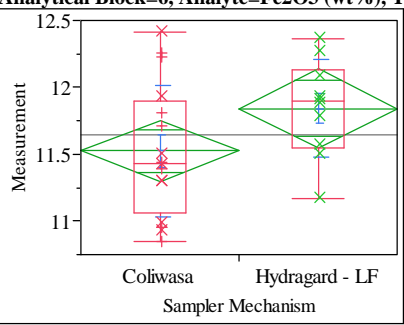

Oneway Anova

Summary of Fi

Adj Rsquare

$\quad 0.449147$

Mean of Response 11.64656

Observations (or Sum Wgts) $\quad 26$

t Test

Hydragard - LF-Coliwasa

Assuming equal variances

$\begin{array}{lrlr}\text { Difference } & 0.32025 & \mathrm{t} \text { Ratio } & 1.768795 \\ \text { Std Err Dif } & 0.18106 & \text { DF } & 24 \\ \text { Upper CL Dif } & 0.69394 & \text { Prob }>|t| & 0.0896 \\ \text { Lower CL Dif } & -0.05343 & \text { Prob }>\text { t } & 0.0448 \\ \text { Confidence } & 0.95 & \text { Prob }<\mathrm{t} & 0.9552\end{array}$

Analysis of Variance

$\begin{array}{lrrrrr}\text { Source } & \text { DF } & \text { Sum of Squares } & \text { Mean Square } & \text { F Ratio } & \text { Prob }>\text { F } \\ \text { Sampler Mechanism } & 1 & 0.6311499 & 0.631150 & 3.1286 & 0.0896\end{array}$

$\begin{array}{llll}\text { Error } & 24 & 4.8415999 & 0.201733\end{array}$

$\begin{array}{lll}\text { C. Total } & 25 & 5.4727497\end{array}$

Means for Oneway Anova

Level Number Mean Std Error Lower 95\% Upper 95\%

\begin{tabular}{lrrrrr} 
Coliwasa & 16 & 11.5234 & 0.11229 & 11.292 & 11.75 \\
\hline
\end{tabular}

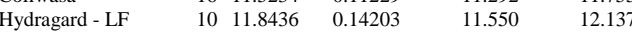

Std Error uses a pooled estimate of error variance

Means and Std Deviations

Level Number Mean Std Dev Std Err Mean Lower 95\% Upper 95\%

$\begin{array}{lrrrrrr}\text { Coliwasa } & 16 & 11.5234 & 0.493857 & 0.12346 & 11.260 & 11.787\end{array}$

$\begin{array}{lllllll}\text { Hydragard - LF } & 10 & 11.8436 & 0.362579 & 0.11466 & 11.584 & 12.103\end{array}$

T Test

Hydragard - LF-Coliwasa

Assuming unequal variances

$\begin{array}{lrlr}\text { Difference } & 0.32025 & \mathrm{t} \text { Ratio } & 1.900692 \\ \text { Std Err Dif } & 0.16849 \text { DF } & 23.23126 \\ \text { Upper CL Dif } & 0.66861 & \text { Prob }>|t| & 0.0698 \\ \text { Lower CL Dif } & -0.02811 & \text { Prob }>t & 0.0349 \\ \text { Confidence } & 0.95 & \text { Prob }<\mathrm{t} & 0.9651\end{array}$

Tests that the Variances are Equal

Level Count Std Dev MeanAbsDif to Mean MeanAbsDif to Median

$\begin{array}{lllll}\text { Coliwasa } & 16 & 0.4938574 & 0.3985289 & 0.3842319\end{array}$

$\begin{array}{lllll}\text { Hydragard - LF } & 10 & 0.3625792 & 0.2733586 & 0.2659242\end{array}$

Test F Ratio DFNum DFDen p-Value

$\begin{array}{lrrrr}\text { O'Brien[.5] } & 1.3876 & 1 & 24 & 0.2504\end{array}$

Brown-Forsythe $\begin{array}{llll}1.0672 & 1 & 24 & 0.3119\end{array}$

$\begin{array}{lllll}\text { Levene } & 1.4899 & 1 & 24 & 0.2341\end{array}$

$\begin{array}{lrrrr}\text { Bartlett } & 0.9634 & 1 & \text {. } & 0.3263 \\ \text { F Test 2-sided } & 1.8552 & 15 & 9 & 0.3511\end{array}$

Welch Anova testing Means Equal, allowing Std Devs Not Equal

F Ratio DFNum DFDen Prob $>$ F

$\begin{array}{lrrr}3.6126 & 1 & 23.231 & 0.0698\end{array}$

t Test

1.9007 


\section{Exhibit A2. Statistical Comparisons for Mid-Rheology (Phase 1) Testing}

Oneway Analysis of Measurement By Sampler Mechanism Type of Material=SME Simulant, Analytical Block=6, Analyte $=$ K2O (wt \%), Target Value $=0.1591$

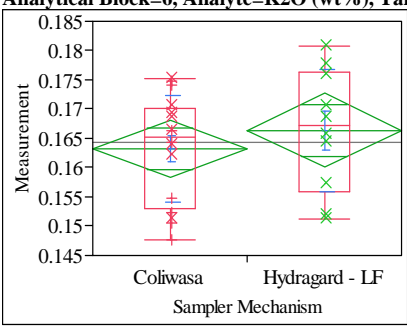

Oneway Anova

Summary of Fit

Adj Rsquare

Error $\quad 0.00968$

0.164428

t Test

Hydragard - LF-Coliwasa

Assuming equal variances

Difference $\quad 0.00313$ t Ratio $\quad 0.802602$

Std Err Dif $\quad 0.00390$ DF $\quad 24$

Upper CL Dif 0.01119 Prob $>|t| \quad 0.4301$

Lower CL Dif -0.00492 Prob $>\mathrm{t} \quad 0.2150$

$\begin{array}{lrl}\text { Confidence } & 0.95 \text { Prob }<\mathrm{t} & 0.7850\end{array}$

Analysis of Variance

$\begin{array}{lrrrrr}\text { Source } & \text { DF } & \text { Sum of Squares } & \text { Mean Square } & \text { F Ratio } & \text { Prob }>\text { F } \\ \text { Sampler Mechanism } & 1 & 0.00006036 & 0.000060 & 0.6442 & 0.4301\end{array}$

$\begin{array}{llll}\text { Error } & 24 & 0.00224900 & 0.000094\end{array}$

$\begin{array}{lll}\text { C. Total } & 25 & 0.00230936\end{array}$

Means for Oneway Anova

Level Number Mean Std Error Lower 95\% Upper 95\%

$\begin{array}{lrrrrr}\text { Coliwasa } & 16 & 0.163223 & 0.00242 & 0.15823 & 0.16822\end{array}$

$\begin{array}{llllll}\text { Hydragard - LF } & 10 & 0.166355 & 0.00306 & 0.16004 & 0.17267\end{array}$

Std Error uses a pooled estimate of error variance

Means and Std Deviations

Level Number Mean Std Dev Std Err Mean Lower 95\% Upper 95\%

Num 0.1632230 .009208

$\begin{array}{lllllll}\text { Coliwasa } & 16 & 0.163223 & 0.009208 & 0.00230 & 0.15832 & 0.16813 \\ \text { Hydragard - LF } & 10 & 0.166355 & 0.010420 & 0.00330 & 0.15890 & 0.17381\end{array}$

t Test

Hydragard - LF-Coliwasa

Assuming unequal variances

Difference $\quad 0.00313$ t Ratio $\quad 0.779187$

Std Err Dif $\quad 0.00402$ DF $\quad 17.43741$

Upper CL Dif 0.01160 Prob $>|t| \quad 0.4463$

$\begin{array}{lll}\text { Lower CL Dif }-0.00533 \text { Prob }>t & 0.2232\end{array}$

$\begin{array}{lrl}\text { Confidence } & 0.95 \text { Prob }<\mathrm{t} & 0.7768\end{array}$

Tests that the Variances are Equal

$\begin{array}{lrrrr}\text { Level } & \text { Count } & \text { Std Dev } & \text { MeanAbsDif to Mean } & \text { MeanAbsDif to Median } \\ \text { Coliwasa } & 16 & 0.0092082 & 0.0076040 & 0.0073782\end{array}$

$\begin{array}{lllll}\text { Hydragard - LF } & 10 & 0.0104198 & 0.0076040 & 0.0073782 \\ & & & 0.0083117 & 0.0083117\end{array}$

Test F Ratio DFNum DFDen p-Value

$\begin{array}{lrrrr}\text { O'Brien[.5] } & 0.4037 & 1 & 24 & 0.5312\end{array}$

$\begin{array}{lllll}\text { Brown-Forsythe } & 0.1684 & 1 & 24 & 0.6852\end{array}$

$\begin{array}{lllll}\text { Levene } & 0.1169 & 1 & 24 & 0.7354\end{array}$

$\begin{array}{lllll}\text { Bartlett } & 0.1675 & 1 & 0.6824\end{array}$

Welch Anova testing Means Equal, allowing Std Devs Not Equal

F Ratio DFNum DFDen Prob $>$ F

$\begin{array}{llll}0.6071 & 1 & 17.437 & 0.4463\end{array}$

t Test
0.7792
Oneway Analysis of Measurement By Sampler Mechanism Type of Material=SME Simulant, Analytical Block $=6$, Analyte $=\mathrm{Li} 2 \mathrm{O}(\mathrm{wt} \%)$, Target Value $=4.674$

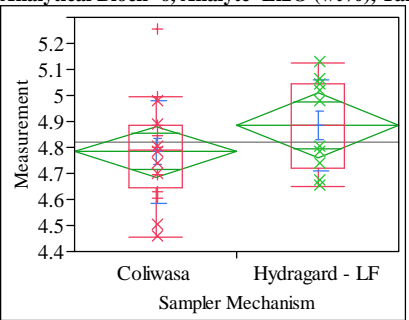

Oneway Anova

Summary of Fit

Adj Rsquare

0.1898

Mean of Response 4.822496

Observations (or Sum Wgts) $\quad 26$

T Test

Hydragard - LF-Coliwasa

Assuming equal variances

$\begin{array}{lrlr}\text { Difference } & 0.10146 & \mathrm{t} \text { Ratio } & 1.326025 \\ \text { Std Err Dif } & 0.07651 & \text { DF } & 24 \\ \text { Upper CL Dif } & 0.25937 & \text { Prob }>|t| & 0.1973 \\ \text { Lower CL Dif } & -0.05646 & \text { Prob }>t & 0.0987 \\ \text { Confidence } & 0.95 & \text { Prob }<\mathrm{t} & 0.9013\end{array}$

Analysis of Variance

$\begin{array}{lrrrrr}\text { Source } & \text { DF } & \text { Sum of Squares } & \text { Mean Square } & \text { F Ratio } & \text { Prob }>\text { F } \\ \text { Sampler Mechanism } & 1 & 0.06334277 & 0.063343 & 1.7583 & 0.1973\end{array}$

$\begin{array}{llll}\text { Error } & 24 & 0.86457990 & 0.036024\end{array}$

$\begin{array}{lll}\text { C. Total } & 25 & 0.92792268\end{array}$

Means for Oneway Anova

Level Number Mean Std Error Lower 95\% Upper 95\%

$\begin{array}{lrrrrr}\text { Coliwasa } & 16 & 4.78347 & 0.04745 & 4.6855 & 4.8814\end{array}$

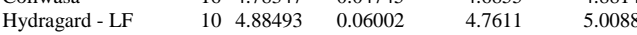

Std Error uses a pooled estimate of error variance

Means and Std Deviations

Level Number Mean Std Dev Std Err Mean Lower 95\% Upper 95\%

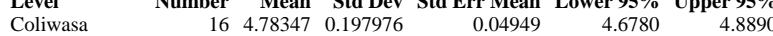

$\begin{array}{lllllll}\text { Hydragard - LF } & 10 & 4.88493 & 0.175329 & 0.05544 & 4.7595 & 5.0104\end{array}$

T Test

Hydragard - LF-Coliwasa

Assuming unequal variances

$\begin{array}{lrlr}\text { Difference } & 0.10146 & \mathrm{t} \text { Ratio } & 1.365089 \\ \text { Std Err Dif } & 0.07432 & \text { DF } & 21.04193 \\ \text { Upper CL Dif } & 0.25600 & \text { Prob }>|t| & 0.1866 \\ \text { Lower CL Dif } & -0.05309 & \text { Prob }>t & 0.0933 \\ \text { Confidence } & 0.95 & \text { Prob }<\mathrm{t} & 0.9067\end{array}$

Tests that the Variances are Equal

Level Count Std Dev MeanAbsDif to Mean MeanAbsDif to Median

$\begin{array}{lllll}\text { Coliwasa } & 16 & 0.1979761 & 0.1466663 & 0.1466663\end{array}$

$\begin{array}{lllll}\text { Hydragard - LF } & 10 & 0.1753289 & 0.1571617 & 0.1571617\end{array}$

Test F Ratio DFNum DFDen p-Value

$\begin{array}{lrrrr}\text { O'Brien[.5] } & 0.1607 & 1 & 24 & 0.6920\end{array}$

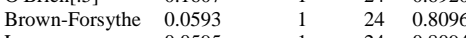

$\begin{array}{lllll}\text { Levene } & 0.0595 & 1 & 24 & 0.8094\end{array}$

$\begin{array}{lrrrr}\text { Bartlett } & 0.1553 & 1 & \text {. } & 0.6935 \\ \text { F Test 2-sided } & 1.2750 & 15 & 9 & 0.7307\end{array}$

Welch Anova testing Means Equal, allowing Std Devs Not Equal

F Ratio DFNum DFDen Prob $>$ F

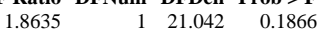

t Test

1.365 


\section{Exhibit A2. Statistical Comparisons for Mid-Rheology (Phase 1) Testing}

Oneway Analysis of Measurement By Sampler Mechanism Type of Material=SME Simulant, Analytical Block=6, Analyte $=\mathrm{MgO}($ wt \%), Target Value $=1.014$

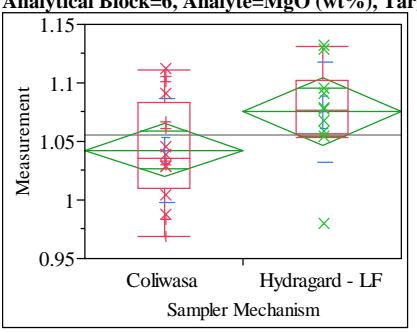

Oneway Anova

Summary of Fit

$\begin{array}{ll}\text { Rsquare } & 0.125033 \\ \text { Adj Rsquare } & 0.088576\end{array}$

Root Mean Square Error $\quad 0.043927$

\begin{tabular}{lr} 
Observations (or Sum Wgts) & 1.055061 \\
\hline
\end{tabular}

Test

Hydragard - LF-Coliwasa

Assuming equal variances

Difference $\quad 0.03279$ t Ratio $\quad 1.851921$

Std Err Dif $\quad 0.01771$ DF 24

Upper CL Dif 0.06934 Prob $>|t| \quad 0.0764$

Lower CL Dif -0.00375 Prob $>t \quad 0.0382$

$\begin{array}{lrl}\text { Confidence } & 0.95 \text { Prob }<\mathrm{t} & 0.9618\end{array}$

Analysis of Variance

$\begin{array}{lrrrrr}\text { Source } & \text { DF } & \text { Sum of Squares } & \text { Mean Square } & \text { F Ratio } & \text { Prob > F } \\ \text { Sampler Mechanism } & 1 & 0.00661768 & 0.006618 & 3.4296 & 0.0764\end{array}$

$\begin{array}{llll}\text { Error } & 24 & 0.04630972 & 0.001930\end{array}$

$\begin{array}{lll}\text { C. Total } & 25 & 0.05292740\end{array}$

Means for Oneway Anova

Level Number Mean Std Error Lower 95\% Upper 95\%

$\begin{array}{lrrrrr}\text { Coliwasa } & 16 & 1.04245 & 0.01098 & 1.0198 & 1.065\end{array}$

$\begin{array}{llllll}\text { Hydragard - LF } & 10 & 1.07524 & 0.01389 & 1.0466 & 1.1039\end{array}$

Std Error uses a pooled estimate of error variance

Means and Std Deviations

Level Number Mean Std Dev Std Err Mean Lower 95\% Upper 95\%

$\begin{array}{lllllll}\text { Coliwasa } & 16 & 1.04245 & 0.044443 & 0.01111 & 1.0188 & 1.0661 \\ \text { Hydragard - LF } & 10 & 1.07524 & 0.043053 & 0.01361 & 1.0444 & 1.1060\end{array}$

t Test

Hydragard - LF-Coliwasa

Assuming unequal variances

$\begin{array}{lrlr}\text { Difference } & 0.03279 & \mathrm{t} \text { Ratio } & 1.866105 \\ \text { Std Err Dif } & 0.01757 & \text { DF } & 19.72924 \\ \text { Upper CL Dif } & 0.06948 & \text { Prob }>|\mathrm{t}| & 0.0770 \\ \text { Lower CL Dif } & -0.00390 & \text { Prob }>\mathrm{t} & 0.0385 \\ \text { Confidence } & 0.95 & \text { Prob }<\mathrm{t} & 0.9615\end{array}$

Tests that the Variances are Equal

Level Count Std Dev MeanAbsDif to Mean MeanAbsDif to Median

Coliwasa $\quad 16 \quad 0.0444427$

$\begin{array}{lllll}\text { Hydragard - LF } & 10 & 0.0430534 & 0.0353684 & 0.0348243 \\ & & & 0.0288544\end{array}$

Test F Ratio DFNum DFDen p-Value

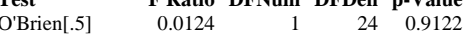

$\begin{array}{lllll}\text { Brown-Forsythe } & 0.2719 & 1 & 24 & 0.6069\end{array}$

$\begin{array}{lllll}\text { Levene } & 0.3299 & 1 & 24 & 0.5711\end{array}$

$\begin{array}{lrrrr}\text { Bartlett } & 0.0108 & 1 & \text {. } & 0.9172 \\ \text { F Test 2-sided } & 1.0656 & 15 & 9 & 0.9571\end{array}$

Welch Anova testing Means Equal, allowing Std Devs Not Equal

F Ratio DFNum DFDen Prob $>$ F

$\begin{array}{rrrr}3.4823 & 1 & 19.729 & 0.0770\end{array}$

t Test

1.8661
Oneway Analysis of Measurement By Sampler Mechanism Type of Material=SME Simulant, Analytical Block $=6$, Analyte $=\mathrm{MnO}(\mathrm{wt} \%)$, Target Value $=1.779$

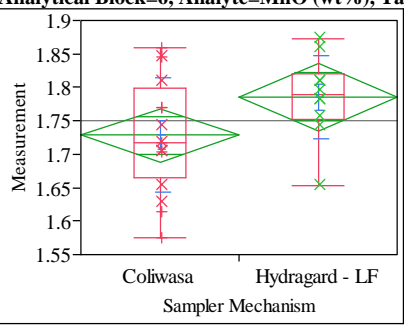

Oneway Anova

Summary of Fit

Adj Rsquare

0.122662

0.086106
0.077376

$\begin{array}{lr} & 1.750569 \\ \text { Observations (or Sum Wgts) } & 26\end{array}$

Test

Hydragard - LF-Coliwasa

Assuming equal variances

$\begin{array}{lrlr}\text { Difference } & 0.05714 & \mathrm{t} \text { Ratio } & 1.831793 \\ \text { Std Err Dif } & 0.03119 & \text { DF } & 24 \\ \text { Upper CL Dif } & 0.12151 & \text { Prob }>|t| & 0.0794 \\ \text { Lower CL Dif } & -0.00724 & \text { Prob }>t & 0.0397 \\ \text { Confidence } & 0.95 & \text { Prob }<\mathrm{t} & 0.9603\end{array}$

Analysis of Variance

Source DF Sum of Squares Mean Square F Ratio Prob $>$ F

$\begin{array}{lrrr}\text { Error } & 1 & 0.02008909 & 0.020089 \\ & 24 & 0.14368741 & 0.00598\end{array}$

$\begin{array}{lll}\text { C. Total } & 25 & 0.16377650\end{array}$

Means for Oneway Anova

Level Number Mean Std Error Lower 95\% Upper 95\%

$\begin{array}{lrrrrr}\text { Coliwasa } & 16 & 1.72859 & 0.01934 & 1.6887 & 1.7685 \\ \text { Hydragard - LF } & 10 & 1.78573 & 0.02447 & 1.7352 & 1.8362\end{array}$

Std Error uses a pooled estimate of error variance

Means and Std Deviations

Level Number Mean Std Dev Std Err Mean Lower 95\% Upper 95\%

$\begin{array}{lrrrrr}\text { Coliwasa } & 16 & 1.72859 & 0.085372 & 0.02134 & 1.6831\end{array}$

$\begin{array}{lllllll}\text { Hydragard - LF } & 10 & 1.78573 & 0.061789 & 0.01954 & 1.7415 & 1.8299\end{array}$

T Test

Hydragard - LF-Coliwasa

Assuming unequal variances

$\begin{array}{lrlr}\text { Difference } & 0.05714 & \mathrm{t} \text { Ratio } & 1.974524 \\ \text { Std Err Dif } & 0.02894 & \text { DF } & 23.34696 \\ \text { Upper CL Dif } & 0.11695 & \text { Prob }>|t| & 0.0603 \\ \text { Lower CL Dif } & -0.00267 & \text { Prob }>t & 0.0301 \\ \text { Confidence } & 0.95 & \text { Prob }<\mathrm{t} & 0.9699\end{array}$

Tests that the Variances are Equal

Level Count Std Dev MeanAbsDif to Mean MeanAbsDif to Median

$\begin{array}{lllll}\text { Coliwasa } & 16 & 0.0853723 & 0.0675863 & 0.0661740\end{array}$

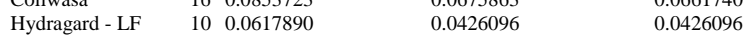

Test F Ratio DFNum DFDen p-Value

$\begin{array}{lrrrr}\text { O'Brien[.5] } & 1.2497 & 1 & 24 & 0.2747\end{array}$

$\begin{array}{lllll}\text { Brown-Forsythe } & 1.4244 & 1 & 24 & 0.2443\end{array}$

$\begin{array}{lllll}\text { Levene } & 1.7568 & 1 & 24 & 0.1975\end{array}$

$\begin{array}{lrrrr}\text { Bartlett } & 1.0508 & 1 & \text {. } & 0.3053 \\ \text { F Test 2-sided } & 1.9090 & 15 & 9 & 0.3290\end{array}$

Welch Anova testing Means Equal, allowing Std Devs Not Equal

F Ratio DFNum DFDen Prob $>$ F

$\begin{array}{lrrr}3.8987 & 1 & 23.347 & 0.0603\end{array}$

t Test

1.974 


\section{Exhibit A2. Statistical Comparisons for Mid-Rheology (Phase 1) Testing}

Oneway Analysis of Measurement By Sampler Mechanism Type of Material=SME Simulant, Analytical Block=6, Analyte=Na2O (wt\%), Target Value=11.659

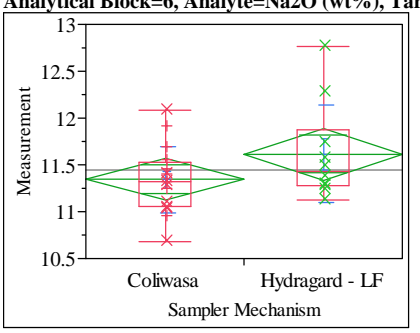

Oneway Anova

Summary of Fit

Rsquare

Adj Rsquare

0.094495

Root Mean Squarc

Mean of Response

0.056765

are Error $\quad 0.426826$

11.44815

t Test

Hydragard - LF-Coliwasa

Assuming equal variances

Difference $\quad 0.27230$ t Ratio 1.582572

Std Err Dif $\quad 0.17206$ DF 24

Upper CL Dif 0.62741 Prob $>|t| r 0.1266$

Lower CL Dif -0.08282 Prob $>\mathrm{t} \quad 0.0633$

$\begin{array}{lrl}\text { Confidence } & 0.95 \text { Prob }<\mathrm{t} & 0.9367\end{array}$

Analysis of Variance

$\begin{array}{lrrrrr}\text { Source } & \text { DF } & \text { Sum of Squares } & \text { Mean Square } & \text { F Ratio } & \text { Prob }>\text { F } \\ \text { Sampler Mechanism } & 1 & 0.4562776 & 0.456278 & 2.5045 & 0.1266 \\ \text { Error } & 24 & 4.3723338 & 0.182181 & & \end{array}$

$25 \quad 4.8286114$

Means for Oneway Anova

Level Number Mean Std Error Lower 95\% Upper 95\%

$\begin{array}{lrrrrr}\text { Coliwasa } & 16 & 11.3434 & 0.10671 & 11.123 & 11.564\end{array}$

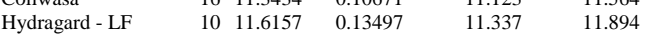

Std Error uses a pooled estimate of error variance

Means and Std Deviations

Level Number Mean Std Dev Std Err Mean Lower 95\% Upper 95\%

Coliwasa

$\begin{array}{lllllll}\text { Coliwasa } & 16 & 11.3434 & 0.357699 & 0.08942 & 11.153 & 11.534 \\ \text { Hydragard - LF } & 10 & 11.6157 & 0.522080 & 0.16510 & 11.242 & 11.989\end{array}$

t Test

Hydragard - LF-Coliwasa

Assuming unequal variances

Difference $\quad 0.27230$ t Ratio $\quad 1.45024$

Std Err Dif $\quad 0.18776$ DF $\quad 14.31627$

Upper CL Dif $\quad 0.67417$ Prob $>|t| \quad 0.1685$

Lower CL Dif -0.12957 Prob $>t \quad 0.0843$

$\begin{array}{lrl}\text { Confidence } & 0.95 \text { Prob }<\mathrm{t} & 0.9157\end{array}$

Tests that the Variances are Equal

Level Count Std Dev MeanAbsDif to Mean MeanAbsDif to Median

Coliwasa $\quad 16 \quad 0.3576987$

$\begin{array}{lllll}\text { Hydragard - LF } & 10 & 0.5220801 & 0.3879544 & 0.3518280\end{array}$

Test F Ratio DFNum DFDen p-Value

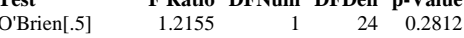

$\begin{array}{lllll}\text { Brown-Forsythe } & 0.5077 & 1 & 24 & 0.4830\end{array}$

$\begin{array}{lllll}\text { Levene } & 1.2995 & 1 & 24 & 0.2656\end{array}$

$\begin{array}{lllll}\text { Bartlett } & 1.6019 & 1 & 0.2056\end{array}$

Welch Anova testing Means Equal, allowing Std Devs Not Equal

F Ratio DFNum DFDen Prob $>$ F

$\begin{array}{rrrr}2.1032 & 1 & 14.316 & 0.1685\end{array}$

t Test

1.4502
Oneway Analysis of Measurement By Sampler Mechanism Type of Material=SME Simulant, Analytical Block $=6$, Analyte $=\mathrm{NiO}(\mathrm{wt} \%)$, Target Value $=0.41$

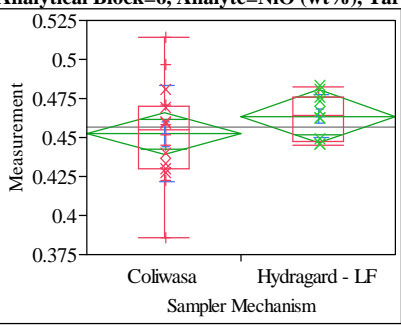

Oneway Anova

Summary of Fit

Adj Rsquare

0.025921

0.456681
Observations (or Sum Wgts)

t Test

Hydragard - LF-Coliwasa

Assuming equal variances

$\begin{array}{lrlr}\text { Difference } & 0.01140 & \text { t Ratio } & 1.091477 \\ \text { Std Err Dif } & 0.01045 & \text { DF } & 24 \\ \text { Upper CL Dif } & 0.03297 & \text { Prob }>|t| & 0.2859 \\ \text { Lower CL Dif } & -0.01016 & \text { Prob }>\text { t } & 0.1429 \\ \text { Confidence } & 0.95 & \text { Prob }<\mathrm{t} & 0.8571\end{array}$

Analysis of Variance

$\begin{array}{lrrrrr}\text { Source } & \text { DF } & \text { Sum of Squares } & \text { Mean Square } & \text { F Ratio } & \text { Prob > F } \\ \text { Sampler Mechanism } & 1 & 0.00080042 & 0.000800 & 1.1913 & 0.2859\end{array}$

$\begin{array}{llll}\text { Error } & 24 & 0.01612510 & 0.000672\end{array}$

$\begin{array}{lll}\text { C. Total } & 25 & 0.01692553\end{array}$

Means for Oneway Anova

Level Number Mean Std Error Lower 95\% Upper 95\%

$\begin{array}{lrrrrr}\text { Coliwasa } & 16 & 0.452294 & 0.00648 & 0.43892 & 0.46567\end{array}$

$\begin{array}{llllll}\text { Hydragard - LF } & 10 & 0.463699 & 0.00820 & 0.44678 & 0.48062\end{array}$

Std Error uses a pooled estimate of error variance

Means and Std Deviations

Level Number Mean Std Dev Std Err Mean Lower 95\% Upper 95\%

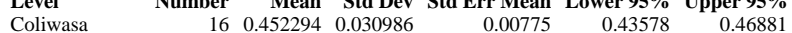

$\begin{array}{lllllll}\text { Hydragard - LF } & 10 & 0.463699 & 0.013838 & 0.00438 & 0.45380 & 0.47360\end{array}$

Test

Hydragard - LF-Coliwasa

Assuming unequal variances

$\begin{array}{lrlr}\text { Difference } & 0.01140 & \mathrm{t} \text { Ratio } & 1.281866 \\ \text { Std Err Dif } & 0.00890 & \text { DF } & 22.3141 \\ \text { Upper CL Dif } & 0.02984 & \text { Prob }>|\mathrm{t}| & 0.2130 \\ \text { Lower CL Dif } & -0.00703 & \text { Prob }>\mathrm{t} & 0.1065 \\ \text { Confidence } & 0.95 & \text { Prob }<\mathrm{t} & 0.8935\end{array}$

Tests that the Variances are Equal

Level Count Std Dev MeanAbsDif to Mean MeanAbsDif to Median

$\begin{array}{lllll}\text { Coliwasa } & 16 & 0.0309855 & 0.0233027 & 0.0233027\end{array}$

$\begin{array}{lllll}\text { Hydragard - LF } & 10 & 0.0138385 & 0.0111980 & 0.0111980\end{array}$

Test F Ratio DFNum DFDen p-Value

$\begin{array}{lrrrr}\text { O'Brien[.5] } & 2.5625 & 1 & 24 & 0.1225\end{array}$

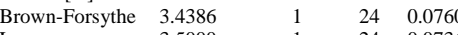

$\begin{array}{lllll}\text { Levene } & 3.5000 & 1 & 24 & 0.0736\end{array}$

$\begin{array}{lrrrr}\text { Bartlett } & 5.6842 & 1 & \text {. } & 0.0171 \\ \text { F Test 2-sided } & 5.0135 & 15 & 9 & 0.0193\end{array}$

Welch Anova testing Means Equal, allowing Std Devs Not Equal

F Ratio DFNum DFDen Prob $>$ F

$\begin{array}{llrr}1.6432 & 1 & 22.314 & 0.2130\end{array}$

t Test

1.2819 


\section{Exhibit A2. Statistical Comparisons for Mid-Rheology (Phase 1) Testing}

Oneway Analysis of Measurement By Sampler Mechanism Type of Material=SME Simulant, Analytical Block=6, Analyte=SiO2 (wt \%), Target Value=50.985

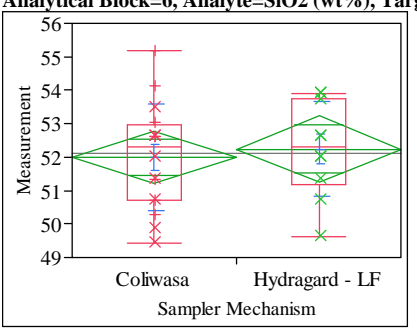

Oneway Anova

Summary of Fit

$\begin{array}{lr}\text { Rsquare } & 0.00644 \\ \text { Adj Rsquare } & -0.03496\end{array}$

Root Mean Square Error $\quad 1.530595$

52.09196

$\begin{array}{lr}\text { Mean of Response } & 52.09196 \\ \text { Observations (or Sum Wgts) }\end{array}$

Test

Hydragard - LF-Coliwasa

Assuming equal variances

Difference $\quad 0.2433$ t Ratio 0.394399

Std Err Dif $\quad 0.6170$ DF 24

Upper CL Dif 1.5168 Prob $>|t| \quad 0.6968$

Lower CL Dif -1.0301 Prob $>t \quad 0.3484$

$\begin{array}{lrl}\text { Confidence } & 0.95 \text { Prob }<\mathrm{t} & 0.6516\end{array}$

Analysis of Variance

$\begin{array}{lrrrrr}\text { Source } & \text { DF } & \text { Sum of Squares } & \text { Mean Square } & \text { F Ratio } & \text { Prob }>\text { F } \\ \text { Sampler Mechanism } & 1 & 0.364412 & 0.36441 & 0.1556 & 0.6968\end{array}$

$\begin{array}{llr}\text { Error } & 24 & 56.225302\end{array}$

$\begin{array}{lll}\text { C. Total } & 25 & 56.589715\end{array}$

Means for Oneway Anova

Level Number Mean Std Error Lower 95\% Upper 95\%

$\begin{array}{lrrrrr}\text { Coliwasa } & 16 & 51.9984 & 0.38265 & 51.209 & 52.788\end{array}$

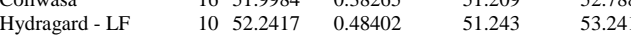

Std Error uses a pooled estimate of error variance

Means and Std Deviations

Level Number Mean Std Dev Std Err Mean Lower 95\% Upper 95\%

$\begin{array}{lrrrrrr}\text { Coliwasa } & \text { Numb } & 51.9984 & 1.59416 & 0.39854 & 51.149 & 52.848 \\ \text { Hydragard - LF } & 10 & 52.2417 & 1.41833 & 0.44852 & 51.227 & 53.256\end{array}$

t Test

Hydragard - LF-Coliwasa

Assuming unequal variances

$\begin{array}{lrlr}\text { Difference } & 0.2433 & \text { t Ratio } & 0.405575 \\ \text { Std Err Dif } & 0.6000 & \text { DF } & 20.97662 \\ \text { Upper CL Dif } & 1.4912 & \text { Prob }>|t| & 0.6892 \\ \text { Lower CL Dif } & -1.0045 & \text { Prob }>\text { t } & 0.3446 \\ \text { Confidence } & 0.95 & \text { Prob }<\mathrm{t} & 0.6554\end{array}$

Tests that the Variances are Equal

Level Count Std Dev MeanAbsDif to Mean MeanAbsDif to Median

Coliwasa $\quad 16 \quad 1.594161$

$\begin{array}{lllll}\text { Hydragard - LF } & 16 & 1.594161 & 1.296951 & 1.296951 \\ & 10 & 1.418334 & 1.112436 & 1.112436\end{array}$

Test F Ratio DFNum DFDen p-Value

$\begin{array}{lrrrr}\text { O'Brien[.5] } & 0.2034 & 1 & 24 & 0.6560\end{array}$

$\begin{array}{lllll}\text { Brown-Forsythe } & 0.2719 & 1 & 24 & 0.6068\end{array}$

$\begin{array}{lllll}\text { Levene } & 0.2969 & 1 & 24 & 0.5909\end{array}$

$\begin{array}{lrrrr}\text { Bartlett } & 0.1439 & 1 & \text {. } & 0.7045 \\ & 1.2633 & 15 & 9 & 0.7419\end{array}$

Welch Anova testing Means Equal, allowing Std Devs Not Equal

F Ratio DFNum DFDen Prob $>$ F

$\begin{array}{rrrr}0.1645 & 1 & 20.977 & 0.6892\end{array}$

t Test
0.4056
Oneway Analysis of Measurement By Sampler Mechanism Type of Material=SME Simulant, Analytical Block $=6$, Analyte $=$ Sum of Oxides (wt \%), Target Value $=99.553$

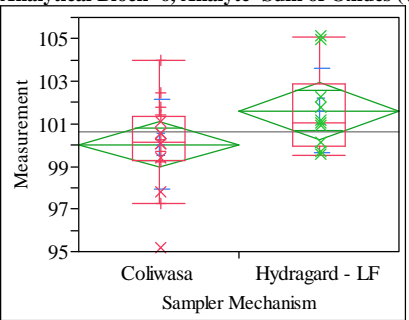

Oneway Anova

Summary of Fit

Adj Rsquare

2.051343

Mean of Response

Observations (or Sum Wgts)

26

Test

Hydragard - LF-Coliwasa

Assuming equal variances

$\begin{array}{lrlr}\text { Difference } & 1.5865 & \mathrm{t} \text { Ratio } & 1.91856 \\ \text { Std Err Dif } & 0.8269 & \text { DF } & 24 \\ \text { Upper CL Dif } & 3.2932 & \text { Prob }>|t| & 0.0670 \\ \text { Lower CL Dif } & -0.1202 & \text { Prob }>\mathrm{t} & 0.0335 \\ \text { Confidence } & 0.95 & \text { Prob }<\mathrm{t} & 0.9665\end{array}$

Analysis of Variance

$\begin{array}{lrrrrr}\text { Source } & \text { DF } & \text { Sum of Squares } & \text { Mean Square } & \text { F Ratio } & \text { Prob }>\text { F } \\ \text { Sampler Mechanism } & 1 & 15.48914 & 15.4891 & 3.6809 & 0.0670 \\ \text { Error } & 24 & 100.99224 & 4.2080 & & \end{array}$

C. Total

Means for Oneway Anova

Level $\quad$ Number Mean Std Error Lower 95\% Upper 95\%

$\begin{array}{lrrrrr}\text { Coliwasa } & 16 & 100.032 & 0.51284 & 98.97 & 101.09\end{array}$

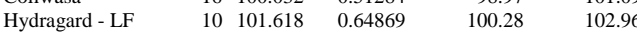

Std Error uses a pooled estimate of error variance

Means and Std Deviations

Level Number Mean Std Dev Std Err Mean Lower 95\% Upper 95\%

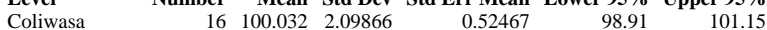

$\begin{array}{llllrrr}\text { Hydragard - LF } & 10 & 101.618 & 1.96996 & 0.52295 & 100.21 & 103.03\end{array}$

T Test

Hydragard - LF-Coliwasa

Assuming unequal variances

$\begin{array}{lrlr}\text { Difference } & 1.5865 & \mathrm{t} \text { Ratio } & 1.947916 \\ \text { Std Err Dif } & 0.8145 \text { DF } & 20.19861 \\ \text { Upper CL Dif } & 3.2844 \text { Prob }>|t| & 0.0655 \\ \text { Lower CL Dif } & -0.1114 & \text { Prob }>\mathrm{t} & 0.0327 \\ \text { Confidence } & 0.95 & \text { Prob }<\mathrm{t} & 0.9673\end{array}$

Tests that the Variances are Equal

Level Count Std Dev MeanAbsDif to Mean MeanAbsDif to Median

$\begin{array}{lllll}\text { Coliwasa } & 16 & 2.098661 & 1.468017 & 1.467296\end{array}$

$\begin{array}{lllll}\text { Coliwasa } & 16 & 2.098661 & 1.468017 & 1.467296 \\ \text { Hydragard - LF } & 10 & 1.969956 & 1.494689 & 1.393149\end{array}$

Test F Ratio DFNum DFDen p-Value

$\begin{array}{lrrrr}\text { O'Brien[.5] } & 0.0376 & 1 & 24 & 0.8479\end{array}$

Brown-Forsythe $\begin{array}{llll}0.0160 & 1 & 24 & 0.9003\end{array}$

$\begin{array}{lllll}\text { Levene } & 0.0024 & 1 & 24 & 0.9615\end{array}$

$\begin{array}{lrrrr}\text { Bartlett } & 0.0426 & 1 & \text {. } & 0.8364 \\ \text { F Test 2-sided } & 1.1349 & 15 & 9 & 0.8757\end{array}$

Welch Anova testing Means Equal, allowing Std Devs Not Equal

F Ratio DFNum DFDen Prob $>$ F

$\begin{array}{rrrr}3.7944 & 1 & 20.199 & 0.0655\end{array}$

t Test

1.9479 


\section{Exhibit A2. Statistical Comparisons for Mid-Rheology (Phase 1) Testing}

Oneway Analysis of Measurement By Sampler Mechanism Type of Material=SME Simulant, Analytical Block=6, Analyte $=$ TiO2 $($ wt $\%)$, Target Value $=0.0711$

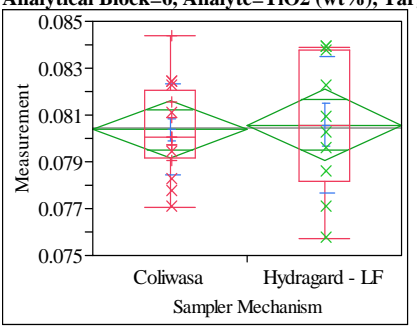

Oneway Anova

Summary of Fit

Adj Rsquare

0.001938

Root Mean Squarc

Mean of Response

$-0.03965$

Observations (or Sum Wgts) $\quad 26$

t Test

Hydragard - LF-Coliwasa

Assuming equal variances

Difference $\quad 0.00020$ t Ratio $\quad 0.215902$

Std Err Dif $\quad 0.00095$ DF 24

Upper CL Dif 0.00216 Prob $>|t| r 0.8309$

Lower CL Dif -0.00175 Prob $>t \quad 0.4154$

$\begin{array}{lrl}\text { Confidence } & 0.95 \text { Prob }<\mathrm{t} & 0.5846\end{array}$

Analysis of Variance

$\begin{array}{lrrrrr}\text { Source } & \text { DF } & \text { Sum of Squares } & \text { Mean Square } & \text { F Ratio } & \text { Prob > F } \\ \text { Sampler Mechanism } & 1 & 0.00000026 & 2.5693 \mathrm{e}-7 & 0.0466 & 0.8309\end{array}$

$\begin{array}{llll}\text { Error } & 24 & 0.00013229 & 5.5119 \mathrm{e}-6\end{array}$

$\begin{array}{lll}\text { C. Total } & 25 & 0.00013254\end{array}$

Means for Oneway Anova

Level Number Mean Std Error Lower 95\% Upper 95\%

$\begin{array}{lrrrrr}\text { Coliwasa } & 16 & 0.080377 & 0.00059 & 0.07917 & 0.08159\end{array}$

$\begin{array}{llllll}\text { Hydragard - LF } & 10 & 0.080581 & 0.00074 & 0.07905 & 0.08211\end{array}$

Std Error uses a pooled estimate of error variance

Means and Std Deviations

Level Number Mean Std Dev Std Err Mean Lower 95\% Upper 95\%

Level Number 0.0803770 .001937

$\begin{array}{lllllll}\text { Coliwasa } & 16 & 0.080377 & 0.001937 & 0.00048 & 0.07934 & 0.08141 \\ \text { Hydragard - LF } & 10 & 0.080581 & 0.002906 & 0.00092 & 0.07850 & 0.08266\end{array}$

t Test

Hydragard - LF-Coliwasa

Assuming unequal variances

Difference $\quad 0.00020$ t Ratio $\quad 0.196724$

Std Err Dif $\quad 0.00104$ DF $\quad 14.04632$

Upper CL Dif 0.00243 Prob $>|t| \quad 0.8469$

Lower CL Dif -0.00202 Prob $>t \quad 0.4234$

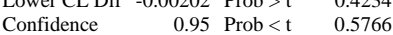

Tests that the Variances are Equal

Level Count Std Dev MeanAbsDif to Mean MeanAbsDif to Median

Coliwasa $\quad 16 \quad 0.0019374$

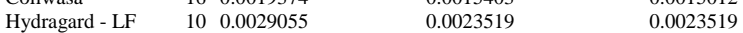

Test F Ratio DFNum DFDen p-Value

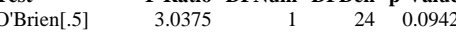

$\begin{array}{lllll}\text { Brown-Forsythe } & 2.5159 & 1 & 24 & 0.1258\end{array}$

$\begin{array}{lllll}\text { Levene } & 2.4936 & 1 & 24 & 0.1274\end{array}$

$\begin{array}{lllll}\text { Bartlett } & 1.8419 & 1 & 0.1747\end{array}$

Welch Anova testing Means Equal, allowing

Welch Anova testing Means Equal, allowing Std Devs Not Equal

F Ratio DFNum DFDen Prob $>$ F

$\begin{array}{rrrr}0.0387 & 1 & 14.046 & 0.8469\end{array}$

t Test
0.1967
Oneway Analysis of Measurement By Sampler Mechanism Type of Material=SME Simulant, Analytical Block $=6$, Analyte $=\mathrm{ZnO}($ wt $\%)$, Target Value $=\mathbf{0 . 0 9 5 8}$

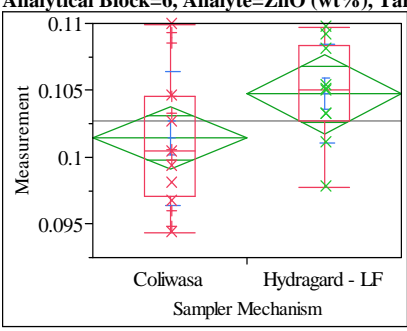

Oneway Anova

Summary of Fit

Adj Rsquare

0.004551

$\begin{array}{lr}\text { Mean of Response } & 0.102701 \\ \text { Observations (or Sum Wgts) } & 26\end{array}$

t Test

Hydragard - LF-Coliwasa

Assuming equal variances

Difference $\quad 0.00331$ t Ratio 1.804044

Std Err Dif $\quad 0.00183$ DF 24

$\begin{array}{lll}\text { Upper CL Dif } 0.00710 & \text { Prob }>|t| & 0.0838\end{array}$

Lower CL Dif -0.00048 Prob $>$ t 0.0419

$\begin{array}{lll}\text { Confidence } & 0.95 \text { Prob }<\mathrm{t} & 0.9581\end{array}$

Analysis of Variance

$\begin{array}{lrrrrr}\text { Source } & \text { DF } & \text { Sum of Squares } & \text { Mean Square } & \text { F Ratio } & \text { Prob }>\text { F } \\ \text { Sampler Mechanism } & 1 & 0.00006741 & 0.000067 & 3.2546 & 0.0838\end{array}$

$\begin{array}{llll}\text { Error } & 24 & 0.00049707 & 0.000021\end{array}$

$\begin{array}{lll}\text { C. Total } & 25 & 0.00056448\end{array}$

Means for Oneway Anova

Level $\quad$ Number Mean Std Error Lower 95\% Upper 95\%

$\begin{array}{lrrrrr}\text { Coliwasa } & 16 & 0.101428 & 0.00114 & 0.09908 & 0.10378\end{array}$

$\begin{array}{llllll}\text { Hydragard - LF } & 10 & 0.104737 & 0.00144 & 0.10177 & 0.10771\end{array}$

Std Error uses a pooled estimate of error variance

Means and Std Deviations

Level Number Mean Std Dev Std Err Mean Lower 95\% Upper 95\%

\begin{tabular}{lrrrrrr} 
Coliwasa & Number & Mean & Std Dev & Std Err Mean & Lower $95 \%$ & Upper $95 \%$ \\
\hline
\end{tabular}

$\begin{array}{lllllll}\text { Hydragard - LF } & 10 & 0.104737 & 0.003694 & 0.00117 & 0.10209 & 0.10738\end{array}$

Test

Hydragard - LF-Coliwasa

Assuming unequal variances

$\begin{array}{lrlr}\text { Difference } & 0.00331 & \mathrm{t} \text { Ratio } & 1.935461 \\ \text { Std Err Dif } & 0.00171 & \text { DF } & 23.16858 \\ \text { Upper CL Dif } & 0.00685 & \text { Prob }>|\mathrm{t}| & 0.0652 \\ \text { Lower CL Dif } & -0.00023 & \text { Prob }>\mathrm{t} & 0.0326 \\ \text { Confidence } & 0.95 & \text { Prob }<\mathrm{t} & 0.9674\end{array}$

Tests that the Variances are Equal

Level Count Std Dev MeanAbsDif to Mean MeanAbsDif to Median

$\begin{array}{lllll}\text { Coliwasa } & 16 & 0.0049950 & 0.0040971 & 0.0039911\end{array}$

$\begin{array}{lllll}\text { Hydragard - LF } & 10 & 0.0036942 & 0.0027535 & 0.0027137\end{array}$

Test F Ratio DFNum DFDen p-Value

$\begin{array}{lrrrr}\text { O'Brien[.5] } & 1.3493 & 1 & 24 & 0.2568\end{array}$

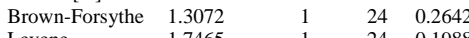

$\begin{array}{lllll}\text { Levene } & 1.7465 & 1 & 24 & 0.1988\end{array}$

$\begin{array}{lrrrr}\text { Bartlett } & 0.9199 & 1 & \text {. } & 0.3375 \\ \text { F Test 2-sided } & 1.8283 & 15 & 9 & 0.3628\end{array}$

Welch Anova testing Means Equal, allowing Std Devs Not Equal

F Ratio DFNum DFDen Prob $>$ F

$\begin{array}{rrrr}3.7460 & 1 & 23.169 & 0.0652\end{array}$

t Test

1.9355 


\section{Exhibit A2. Statistical Comparisons for Mid-Rheology (Phase 1) Testing}

Oneway Analysis of Measurement By Sampler Mechanism Type of Material=SME Simulant, Analytical Block $=6$, Analyte $=\mathrm{ZrO} 2$ (wt $\%)$, Target Value $=0.3547$

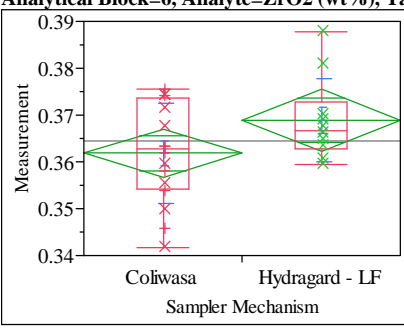

Oneway Anova

Summary of Fit

Adj Rsquare $\quad 0.07542$

Root Mean Square Error $\quad 0.010043$

Mean of Response

Mean of Response 0.36456

t Test

Hydragard - LF-Coliwasa

Assuming equal variances

Difference $\quad 0.00706$ t Ratio 1.74336

$\begin{array}{lrr}\text { Std Err Dif } & 0.00405 \text { DF } & 24\end{array}$

Upper CL Dif 0.01541 Prob $>|t| \quad 0.094$

$\begin{array}{lrrr}\text { Lower CL Dif } & -0.00130 & \text { Prob }>t & 0.0470 \\ \text { Confidence } & 0.95 & \text { Prob }<\mathrm{t} & 0.9530\end{array}$

Analysis of Variance

Source DF Sum of Squares Mean Square F Ratio Prob $>$ F

$\begin{array}{lrrr} & & 0.00030655 & 0.000307 \\ \text { Error } & 24 & 0.00242069 & 0.000101\end{array}$

$\begin{array}{lll}\text { C. Total } & 25 & 0.00272724\end{array}$

Means for Oneway Anova

Level Number Mean Std Error Lower 95\% Upper 95\%

$\begin{array}{lrrrrr}\text { Coliwasa } & 16 & 0.361846 & 0.00251 & 0.35666 & 0.36703\end{array}$

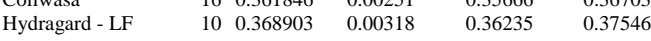

Std Error uses a pooled estimate of error variance

Means and Std Deviations

Level Number Mean Std Dev Std Err Mean Lower 95\% Upper 95\%

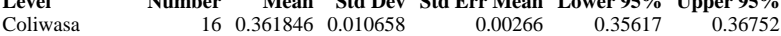

$\begin{array}{lllllll}\text { Coliwasa } & 16 & 0.361846 & 0.010658 & 0.00266 & 0.35617 & 0.36752 \\ \text { Hydragard - LF } & 10 & 0.368903 & 0.008925 & 0.00282 & 0.36252 & 0.37529\end{array}$

t Test

Hydragard - LF-Coliwasa

Assuming unequal variances

$\begin{array}{lrlr}\text { Difference } & 0.00706 & \text { t Ratio } & 1.81843 \\ \text { Std Err Dif } & 0.00388 & \text { DF } & 21.80082 \\ \text { Upper CL Dif } & 0.01511 & \text { Prob }>|t| & 0.0828 \\ \text { Lower CL Dif } & -0.00100 & \text { Prob }>\text { t } & 0.0414 \\ \text { Confidence } & 0.95 & \text { Prob }<\mathrm{t} & 0.9586\end{array}$

Tests that the Variances are Equal

Level Count Std Dev MeanAbsDif to Mean MeanAbsDif to Median

$\begin{array}{lllll}\text { Coliwasa } & 16 & 0.0106576 & 0.0086325 & 0.0086114\end{array}$

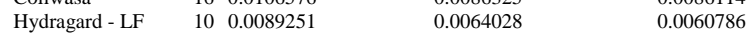

Test F Ratio DFNum DFDen p-Value

$\begin{array}{lllll}\text { O'Brien[.5] } & 0.4467 & 1 & 24 & 0.5103\end{array}$

$\begin{array}{lllll}\text { Brown-Forsythe } & 1.0267 & 1 & 24 & 0.321\end{array}$

$\begin{array}{lllll}\text { Levene } & 0.8971 & 1 & 24 & 0.3530\end{array}$

$\begin{array}{lrrrr}\text { Bartlett } & 0.3274 & 1 & \text {. } & 0.5672 \\ \text { FTest 2-sided } & 1.4259 & 15 & 9 & 0.6013\end{array}$

Welch Anova testing Means Equal, allowing Std Devs Not Equal

F Ratio DFNum DFDen Prob > F

$\begin{array}{lrrr}3.3067 & 1 & 21.801 & 0.0828\end{array}$

t Test

1.8184 


\section{Exhibit A3. Statistical Comparisons for Mid-Rheology (Phase 1) Testing (screened data)}

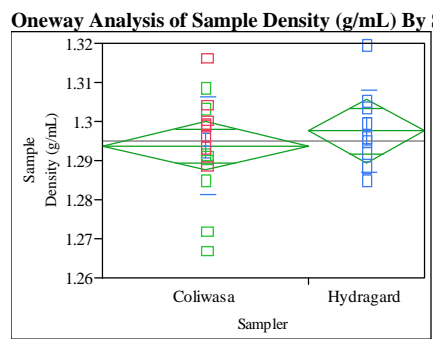

Oneway Anova

Summary of Fit

Rsquare

Adj Rsquare

Root Mean Square

Mean of Response

25

t Test

Hydragard-Coliwasa

Assuming equal variances

Difference $\quad 0.00385$ t Ratio $\quad 0.782071$

Std Err Dif 0.00492 DF

Upper CL Dif 0.01403 Prob $>|t| \quad 0.4422$

$\begin{array}{lll}\text { Lower CL Dif }-0.00633 \text { Prob }>t & 0.2211\end{array}$

$\begin{array}{lll}\text { Confidence } & 0.95 \text { Prob }<\mathrm{t} & 0.7789\end{array}$

Analysis of Variance

Source DF Sum of Squares Mean Square F Ratio Prob $>$ F

$\begin{array}{lrrrrr}\text { Sampler } & 1 & 0.00008530 & 0.000085 & 0.6116 & 0.4422\end{array}$

$\begin{array}{llll}\text { Error } & 23 & 0.00320773 & 0.000139\end{array}$

C. Total $24 \quad 0.00329304$

Means for Oneway Anova

Level Number Mean Std Error Lower 95\% Upper 95\%

$\begin{array}{lllllr}\text { Coliwasa } & 16 & 1.29374 & 0.00295 & 1.2876 & 1.2998\end{array}$

$\begin{array}{lrrrrr}\text { Hydragard } & 9 & 1.29759 & 0.00394 & 1.2894 & 1.3057\end{array}$

Std Error uses a pooled estimate of error variance

Means and Std Deviations

Level Number Mean Std Dev Std Err Mean Lower 95\% Upper 95\%

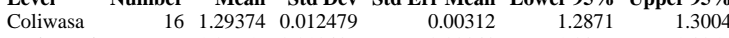

$\begin{array}{lllllll}\text { Hydragard } & 9 & 1.29759 & 0.010439 & 0.00348 & 1.2896 & 1.3056\end{array}$

t Test

Hydragard-Coliwasa

Assuming unequal variances

Difference $\quad 0.00385$ t Ratio 0.823443

$\begin{array}{lll}\text { Std Err Dif } \quad 0.00467 & \text { DF } & 19.35951\end{array}$

Upper CL Dif 0.01362 Prob $>|t| \quad 0.4203$

Lower CL Dif -0.00592 Prob $>\mathrm{t} \quad 0.2101$

$\begin{array}{lll}\text { Confidence } & 0.95 \text { Prob }<\mathrm{t} \quad 0.7899\end{array}$

Tests that the Variances are Equal

Level Count Std Dev MeanAbsDif to Mean MeanAbsDif to Median

$\begin{array}{lrrrr}\text { Coliwasa } & 16 & 0.0124792 & 0.0092521 & 0.0092521\end{array}$

$\begin{array}{lrrrr}\text { Hydragard } & 9 & 0.0104389 & 0.0092521 & 0.0092521 \\ & & & \end{array}$

Test F Ratio DFNum DFDen p-Value

$\begin{array}{lllll}\text { O'Brien[.5] } & 0.2524 & 1 & 23 & 0.6202\end{array}$

$\begin{array}{lllll}\text { Brown-Forsythe } & 0.2360 & 1 & 23 & 0.6317\end{array}$

$\begin{array}{lllll}\text { Levene } & 0.2120 & 1 & 23 & 0.6496\end{array}$

$\begin{array}{lllll}\text { Bartlett } & 0.3044 & 1 & \text {. } & 0.5812\end{array}$

$\begin{array}{lllll}\text { F Test 2-sided } & 1.4291 & 15 & 8 & 0.6248\end{array}$

Welch Anova testing Means Equal, allowing Std Devs Not Equal

F Ratio DFNum DFDen Prob $>$ F

$\begin{array}{llll}0.6781 & 1 & 19.36 & 0.4203\end{array}$

t Test
0.8234

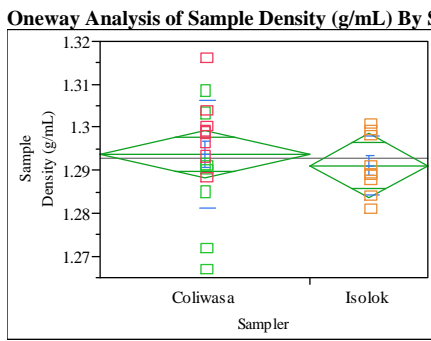

Oneway Anova

Summary of Fit

Rsquare

Adj Rsquare

Root Mean Square Error $\quad-0.02743$

0.010871

Observations (or Sum Wgts) \begin{tabular}{rr}
1.292765 \\
\hline
\end{tabular}

t Test

Isolok-Coliwasa

Assuming equal variances

$\begin{array}{lrlr}\text { Difference } & -0.00271 & \mathrm{t} \text { Ratio } & -0.59935 \\ \text { Std Err Dif } & 0.00453 & \mathrm{DF} & 23 \\ \text { Upper CL Dif } & 0.00666 & \text { Prob }>|\mathrm{t}| & 0.5548 \\ \text { Lower CL Dif } & -0.01209 & \text { Prob }>\mathrm{t} & 0.7226 \\ \text { Confidence } & 0.95 & \text { Prob }<\mathrm{t} & 0.2774\end{array}$

Analysis of Variance

Source DF Sum of Squares Mean Square F Ratio Prob $>$ F

$\begin{array}{lrrrrr}\text { Sampler } & 1 & 0.00004245 & 0.000042 & 0.3592 & 0.5548\end{array}$

$\begin{array}{llll}\text { Error } 23 & 0.00271821 & 0.000118\end{array}$

$\begin{array}{lll}\text { C. Total } 24 & 24 & 0.00276067\end{array}$

Means for Oneway Anova

Level Number Mean Std Error Lower 95\% Upper 95\%

$\begin{array}{llllll}\text { Coliwasa } & 16 & 1.29374 & 0.00272 & 1.2881 & 1.2994\end{array}$

$\begin{array}{llllll}\text { Isolok } & 9 & 1.29103 & 0.00362 & 1.2835 & 1.2985\end{array}$

Std Error uses a pooled estimate of error variance

Means and Std Deviations

Level Number Mean Std Dev Std Err Mean Lower 95\% Upper 95\%

$\begin{array}{lrrrrrr}\text { Coliwasa } & 16 & 1.29374 & 0.012479 & 0.00312 & 1.2871 & 1.3004 \\ \text { Isolok } & 9 & 1.29103 & 0.006912 & 0.00230 & 1.2857 & 1.2963\end{array}$

t Test

Isolok-Coliwasa

Assuming unequal variances

Difference $\quad-0.00271$ t Ratio $\quad-0.7$

Std Err Dif $\quad 0.00388$ DF 22.99735

Upper CL Dif 0.00531 Prob $>|t| \quad 0.4910$

Lower CL Dif -0.01074 Prob $>t \quad 0.7545$

$\begin{array}{lll}\text { Confidence } & 0.95 \text { Prob }<\mathrm{t} \quad 0.2455\end{array}$

Tests that the Variances are Equal

$\begin{array}{lrrrr}\text { Level } & \text { Count } & \text { Std Dev } & \text { MeanAbsDif to Mean } & \text { MeanAbsDif to Median } \\ \text { Coliwasa } & 16 & 0.0124792 & 0.0092521 & 0.0092521\end{array}$

$\begin{array}{lrrrr}\text { Coliwasa } & 16 & 0.0124792 & 0.0092521 & 0.0092521 \\ \text { Isolok } & 9 & 0.0069123 & 0.0055343 & 0.0053329\end{array}$

Test F Ratio DFNum DFDen p-Value

\begin{tabular}{rrrr}
1.6792 & 1 & 23 & 0.2079 \\
\hline
\end{tabular}

Brown-Forsythe $1.7962 \quad 1 \quad 23 \quad 0.1933$

$\begin{array}{lllll}\text { Levene } & 1.7066 & 1 & 23 & 0.2043\end{array}$

$\begin{array}{lrrrr}\text { Bartlett } & 2.9603 & 1 & \text {. } & 0.0853 \\ \text { F Test 2-sided } & 3.2593 & 15 & 8 & 0.0966\end{array}$

Welch Anova testing Means Equal, allowing Std Devs Not Equal

F Ratio DFNum DFDen Prob $>$ F

t Test 
Exhibit A3. Statistical Comparisons for Mid-Rheology (Phase 1) Testing (screened data)

Oneway Analysis of Measurement By Sampler Analytical Block=1, Analyte=Al/B, Target Value $=2.552$

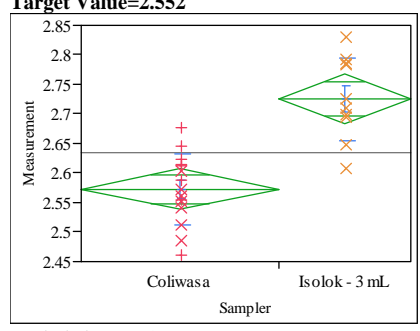

Excluded Rows 1

Oneway Anova

Rsquare $\quad 0.597847$

Adj Rsquare $\quad 0.580362$

Root Mean Square Error $\quad 0.064031$

$\begin{array}{lr}\text { Mean of Response } & 2.633619 \\ \text { Observations (or Sum Wgts) } & 25\end{array}$

t Test

Isolok - $3 \mathrm{~mL}$-Coliwasa

Assuming equal variances

Difference $\quad 0.152854$ t Ratio $\quad 5.847411$

$\begin{array}{lrrr}\text { Std Err Dif } & 0.026140 \text { DF } & 23 \\ \text { Upper CL Dif } & 0.206930 \text { Prob }>|t| & <.0001\end{array}$

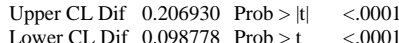

$\begin{array}{lrl}\text { Lower CL Dif } & 0.098778 \text { Prob }>t & <.0001 \\ \text { Confidence } & 0.95 \text { Prob }<\mathrm{t} & 1.0000\end{array}$

Analysis of Variance

Source DF Sum of Squares Mean Square F Ratio Prob $>$ F

$\begin{array}{lrrrrr}\text { Sampler } & 1 & 0.14018639 & 0.140186 & 34.1922 & <.0001\end{array}$

$\begin{array}{lll}\text { Error } \quad 23 & 0.09429887\end{array}$

0.004100

C. Total $24-0.23448526$

Means for Oneway Anova

Level Number Mean Std Error Lower 95\% Upper 95\%

$\begin{array}{lrrrrr}\text { Coliwasa } & 15 & 2.57248 & 0.01653 & 2.5383 & 2.6067\end{array}$

$\begin{array}{llllll}\text { Coliwasa } & 15 & 2.57248 & 0.01653 & 2.5383 & 2.6067 \\ \text { Isolok - } 3 \mathrm{~mL} & 10 & 2.72533 & 0.02025 & 2.6834 & 2.7672\end{array}$

Std Error uses a pooled estimate of error variance

Means and Std Deviations

Level Number Mean Std Dev Std Err Mean

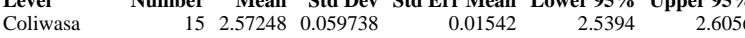

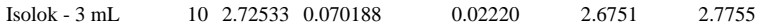

t Test

Isolok - 3 mL-Coliwasa

Assuming unequal variances

Difference $\quad 0.152854$ t Ratio $\quad 5.655258$

Std Err Dif $\quad 0.027029$ DF 17.21133

Upper CL Dif 0.209826 Prob $>|t|<.0001$

Lower CL Dif 0.095882 Prob $>t \quad<.0001$

Confidence $\quad 0.95$ Prob $<\mathrm{t} \quad 1.0000$

Tests that the Variances are Equal

Level Count Std Dev MeanAbsDif to Mean MeanAbsDif to Median

$\begin{array}{lrrrr}\text { Coliwasa } & 15 & 0.0597384 & 0.0473600 & 0.0477612\end{array}$

$\begin{array}{lllll}\text { Isolok }-3 \mathrm{~mL} & 10 & 0.0701882 & 0.0565213 & 0.0560235\end{array}$

Test F Ratio DFNum DFDen p-Value

$\begin{array}{lrrrr}\text { O'Brien[.5] } & 0.4478 & 1 & 23 & 0.5100\end{array}$

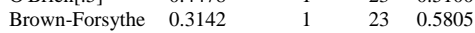

$\begin{array}{lllll}\text { Levene } & 0.4035 & 1 & 23 & 0.5316\end{array}$

$\begin{array}{lllll}\text { Bartlett } & 0.2774 & 1 & \text {. } & 0.5984\end{array}$

$\begin{array}{lllll}\text { F Test 2-sided } & 1.3805 & 9 & 14 & 0.5679\end{array}$

Welch Anova testing Means Equal, allowing Std Devs Not Equal

F Ratio DFNum DFDen Prob $>$ F

$\begin{array}{llll}31.9819 & 1 & 17.211 & <.0001\end{array}$

t Test
5.6553
Oneway Analysis of Measurement By Sampler Analytical Block=1, Analyte=B2O3 (wt \%), Target Value $=4.259$

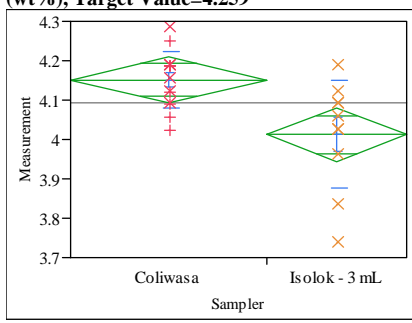

Excluded Rows 2

Oneway Anova

Rsquare $\quad 0.324683$

Adj Rsquare $\quad 0.293987$

Root Mean Square Error $\quad 0.103504$

$\begin{array}{lr}\text { Mean of Response } & 4.093298 \\ \text { Observations (or Sum Wgts) } & 24\end{array}$

t Test

Isolok - 3 mL-Coliwasa

Assuming equal variances

Difference $\quad-0.13938$ t Ratio $\quad-3.25228$

$\begin{array}{lrr}\text { Std Err Dif } & 0.04285 \text { DF } & 22 \\ \text { Upper CL } & 0.0037\end{array}$

Upper CL Dif -0.05050 Prob $>|t| \quad 0.0037$

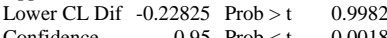

Confidence $\quad 0.95$ Prob $<\mathrm{t} \quad 0.0018$

Analysis of Variance

Source DF Sum of Squares Mean Square F Ratio Prob $>$ F

$\begin{array}{lrrrrr}\text { Sampler } & 1 & 0.11331587 & 0.113316 & 10.5773 & 0.0037\end{array}$

$\begin{array}{llll}\text { Error } & 22 & 0.23568872 & 0.010713\end{array}$

C. Total $23 \quad 0.34900459$

Means for Oneway Anova

Level Number Mean Std Error Lower 95\% Upper 95\%

$\begin{array}{lrrrrr}\text { Coliwasa } & 14 & 4.15137 & 0.02766 & 4.0940 & 4.2087\end{array}$

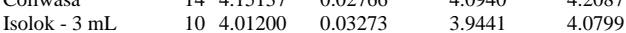

Std Error uses a pooled estimate of error variance

Means and Std Deviations

Level Number Mean Std Dev Std Err Mean Lower 95\% Upper 95\%

$\begin{array}{lllllll}\text { Coliwasa } & 14 & 4.15137 & 0.071960 & 0.01923 & 4.1098 & 4.1929 \\ \text { Isolok - 3 mL } & 10 & 4.01200 & 0.136777 & 0.04325 & 3.9142 & 4.1098\end{array}$

t Test

Isolok - 3 mL-Coliwasa

Assuming unequal variances

$\begin{array}{lrlr}\text { Difference } & -0.13938 & \text { t Ratio } & -2.94441 \\ \text { Std Err Dif } & 0.04734 & \text { DF } & 12.57034 \\ \text { Upper CL Dif } & -0.03676 & \text { Prob }>|t| & 0.0118 \\ \text { Lower CL Dif } & -0.24199 & \text { Prob }>t & 0.9941 \\ \text { Confidence } & 0.95 & \text { Prob }<t & 0.0059\end{array}$

Tests that the Variances are Equal

Level Count Std Dev MeanAbsDif to Mean MeanAbsDif to Median

$\begin{array}{lrrrr}\text { Coliwasa } & 14 & 0.0719596 & 0.0578268 & 0.0574982 \\ \text { Isolok - 3 mL } & 10 & 0.1367773 & 0.1017488 & 0.0965970\end{array}$

Test F Ratio DFNum DFDen p-Value

O'Brien[.5] 2.9885

$\begin{array}{lllll}\text { O'Brien[.5] } & 2.9885 & 1 & 22 & 0.0979 \\ \text { Brown-Forsythe } & 1.7960 & 1 & 22 & 0.1939 \\ \text { Levene }\end{array}$

$\begin{array}{lllll}\text { Levene } & 2.9008 & 1 & 22 & 0.1026\end{array}$

$\begin{array}{lrrrr}\text { Bartlett } & 4.2327 & 1 & 0.0397 \\ \text { F Test 2-sided } & 3.6129 & 9 & 13 & 0.0361\end{array}$

Welch Anova testing Means Equal, allowing Std Devs Not Equal

F Ratio DFNum DFDen Prob $>$ F

$\begin{array}{llll}8.6695 & 1 & 12.57 & 0.0118\end{array}$

t Test 
Exhibit A3. Statistical Comparisons for Mid-Rheology (Phase 1) Testing (screened data)

Oneway Analysis of Measurement By Sampler Analytical Block=1, Analyte=BaO (wt \%), Target Value $=0.0919$

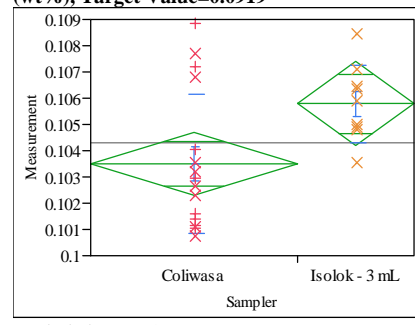

Excluded Rows 1

Oneway Anova

Rsquare $\quad 0.196495$

Adj Rsquare $\quad 0.16156$

Root Mean Square Error $\quad 0.002317$

Mean of Response $\quad 0.104317$

Observations (or Sum Wgts)

t Test

Isolok - $3 \mathrm{~mL}$-Coliwasa

Assuming equal variances

Difference $\quad 0.002290$ t Ratio $\quad 2.371621$

$\begin{array}{lrlr}\text { Std Err Dif } & 0.000965 \text { DF } & 23 \\ \text { Upper CL } & 0.004287 \text { Prob }>|t| & 0.0265\end{array}$

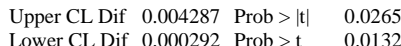

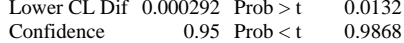

Analysis of Variance

Source DF Sum of Squares Mean Square F Ratio Prob $>$ F

$\begin{array}{llllll}\text { Sampler } & 1 & 0.00003020 & 0.000030 & 5.6246 & 0.0265\end{array}$

$\begin{array}{llll}\text { Error } & 23 & 0.00012347 & 5.368 \mathrm{e}-6\end{array}$

$\begin{array}{lll}\text { C. Total } & 24 & 0.00015367\end{array}$

Means for Oneway Anova

Level Number Mean Std Error Lower 95\% Upper 95\%

$\begin{array}{lrrrrr}\text { Level } & \text { Number } & \text { Mean } & \text { Std Error } & \text { Lower 95\% } & \text { Upper 95\% } \\ \text { Coliwasa } & 16 & 0.103493 & 0.00058 & 0.10229 & 0.10469\end{array}$

$\begin{array}{lrrrrr}\text { Coliwasa } & 16 & 0.103493 & 0.00058 & 0.10229 & 0.10469 \\ \text { Isolok - 3 mL } & 9 & 0.105782 & 0.00077 & 0.10418 & 0.10738\end{array}$

Std Error uses a pooled estimate of error variance

Means and Std Deviations

Level Number Mean Std Dev

$\begin{array}{llll}\text { Coliwasa } & 16 & 0.103493 & 0.002663\end{array}$

Isolok - 3

$\begin{array}{llll}9 & 0.105782 & 0.001463\end{array}$

$\mathrm{mL}$

t Test

Isolok - $3 \mathrm{~mL}-$ Coliwasa

Assuming unequal variances

$\begin{array}{lll}\text { Difference } & 0.002290 \text { t Ratio } 2.774453\end{array}$

Std Err Dif $\quad 0.000825$ DF 22.99976

Upper CL Dif 0.003997 Prob $>|t| 0.0108$

Lower CL Dif 0.000582 Prob $>t \quad 0.0054$

$\begin{array}{lrl}\text { Confidence } & 0.95 \text { Prob }<\mathrm{t} \quad 0.9946\end{array}$

Tests that the Variances are Equal

Level Count Std Dev MeanAbsDif to Mean MeanAbsDif to Median

$\begin{array}{lrrrr}\text { Coliwasa } & 16 & 0.0026626 & 0.0021266 & 0.0020167\end{array}$

$\begin{array}{lrrrr}\text { Isolok - } 3 \mathrm{~mL} & 9 & 0.0014633 & 0.0011358 & 0.0011785\end{array}$

$\begin{array}{lrrrr}\text { Test } & \text { F Ratio } & \text { DFNum } & \text { DFDen } & \text { p-Value } \\ \text { O'Brien[.5] } & 2.7488 & 1 & 23 & 0.1109 \\ \text { Brown-Forsythe } & 1.7107 & 1 & 23 & 0.2038 \\ \text { Levene } & 3.2918 & 1 & 23 & 0.0827 \\ \text { Bartlett } & 3.0318 & 1 & . & 0.0816 \\ \text { F Test 2-sided } & 3.3108 & 15 & 8 & 0.0925\end{array}$

Welch Anova testing Means Equal, allowing Std Devs Not Equal

F Ratio DFNum DFDen Prob > F

$\begin{array}{llll}7.6976 & 1 & 23 & 0.0108\end{array}$

t Test

2.7745
Oneway Analysis of Measurement By Sampler Analytical Block=1, Analyte=Cr2O3 (wt $\%)$, Target Value $=0.0998$

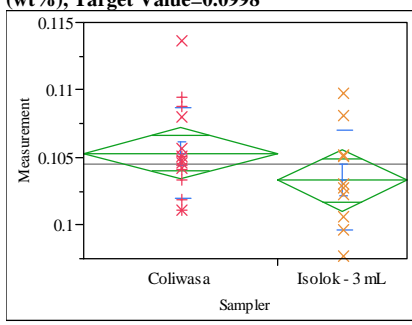

Excluded Rows 1

Oneway Anova

Rsquare $\quad 0.07923$

Adj Rsquare $\quad 0.039196$

Root Mean Square Error $\quad 0.003512$

$\begin{array}{lr}\text { Mean of Response } & 0.104516 \\ \text { Observations (or Sum Wgts) } & 25\end{array}$

t Test

Isolok - 3 mL-Coliwasa

Assuming equal variances

Difference $\quad-0.00202$ t Ratio $\quad-1.4068$

$\begin{array}{lrlr}\text { Std Err Dif } & 0.00143 & \text { DF } & 23 \\ \text { Upper CL Dif } & 0.00095 & \text { Prob }>|t| & 0.1729\end{array}$

$\begin{array}{lrll}\text { Upper CL Dif } & 0.00095 & \text { Prob }>|t| & 0.1729 \\ & & 0.9136\end{array}$

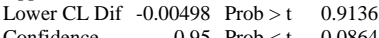

Confidence

Analysis of Variance

Source DF Sum of Squares Mean Square F Ratio Prob $>$ F

$\begin{array}{lrrrrr}\text { Sampler } & 1 & 0.00002441 & 0.000024 & 1.9791 & 0.1729\end{array}$

$\begin{array}{lll}\text { Error } & 23 & 0.00028368 \\ \text { C. Total } & 24 & 0.00030809\end{array}$

Means for Oneway Anova

Level Number Mean Std Error Lower 95\% Upper 95\%

$\begin{array}{lrrrrr}\text { Level } & \text { Number } & \text { Mean } & \text { Std Error } & \text { Lower 95\% } & \text { Upper 95\% } \\ \text { Coliwasa } & 15 & 0.105323 & 0.00091 & 0.10345 & 0.10720\end{array}$

$\begin{array}{llllll}\text { Isolok - } 3 \mathrm{~mL} & 10 & 0.103306 & 0.00111 & 0.10101 & 0.10560\end{array}$

Std Error uses a pooled estimate of error variance

Means and Std Deviations

Upper Level Number Mean Std Dev

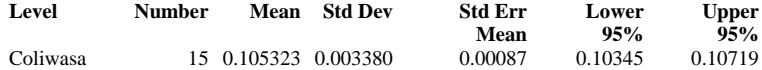

$\begin{array}{lll}0.00087 & 0.10345 & 0.10719 \\ 0.00117 & 0.10065 & 0.10596\end{array}$

$\mathrm{mL}$

Test

Isolok - $3 \mathrm{~mL}$-Coliwasa

Assuming unequal variances

$\begin{array}{lrlr}\text { Difference } & -0.00202 & \text { t Ratio } & -1.37987 \\ \text { Std Err Dif } & 0.00146 & \text { DF } & 18.14933 \\ \text { Upper CL Dif } & 0.00105 & \text { Prob }>|t| & 0.1844 \\ \text { Lower CL Dif } & -0.00509 & \text { Prob }>\text { t } & 0.9078 \\ \text { Confidence } & 0.95 & \text { Prob }<\mathrm{t} & 0.0922\end{array}$

Tests that the Variances are Equal

Level Count Std Dev MeanAbsDif to Mean MeanAbsDif to Median

$\begin{array}{lllll} & 15 & 0.0033797 & 0.0024750 & 0.0023580\end{array}$

Test F Ratio DFNum DFDen p-Value

$\begin{array}{lrrrr}\text { O'Brien[.5] } & 0.0960 & 1 & 23 & 0.7595\end{array}$

Brown-Forsythe $\begin{array}{llll}0.2110 & 1 & 23 & 0.6503\end{array}$

$\begin{array}{lllll}\text { Levene } & 0.2173 & 1 & 23 & 0.6455\end{array}$

$\begin{array}{lllll}\text { Bartlett } & 0.0913 & 1 & \text {. } & 0.7626\end{array}$

Welch Anova testing Means Equal, allowing Std Devs Not Equal

F Ratio DFNum DFDen Prob $>$ F

$\begin{array}{rrrr}1.9040 & 1 & 18.149 & 0.1844\end{array}$

t Test

1.3799 
Exhibit A3. Statistical Comparisons for Mid-Rheology (Phase 1) Testing (screened data)

Oneway Analysis of Measurement By Sampler Analytical Block=1, Analyte=Fe/Li, Target Value $=\mathbf{2 . 4 5 2}$

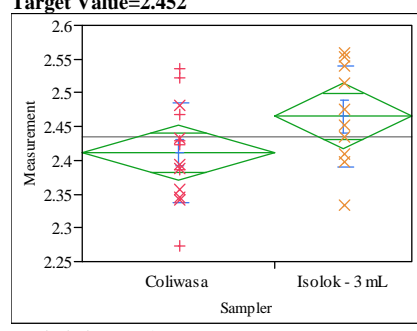

Excluded Rows 2

Oneway Anova

Rsquare $\quad 0.121425$

Adj Rsquare $\quad 0.08149$

Root Mean Square Error $\quad 0.074097$

Mean of Response 2.433987

Observations (or Sum Wgts)

t Test

Isolok - $3 \mathrm{~mL}-$ Coliwasa

Assuming equal variances

Difference $\quad 0.05350$ t Ratio $\quad 1.743718$

$\begin{array}{lrlr}\text { Std Err Dif } & 0.03068 \text { DF } & 22 \\ \text { Upper C } & \text { Drob }>|\mathrm{t}| & 0.0952\end{array}$

Upper CL Dif 0.11712 Prob $>|t| \quad 0.0952$

$\begin{array}{lrl}\text { Lower CL Dif }-0.01013 \text { Prob }>t & 0.0476\end{array}$

Confidence

Analysis of Variance

Source DF Sum of Squares Mean Square F Ratio Prob $>$ F

$\begin{array}{lrrrrr}\text { Sampler } & 1 & 0.01669385 & 0.016694 & 3.0406 & 0.0952\end{array}$

$\begin{array}{lll}\text { Error } & 22 & 0.12078884 \\ \text { C. Total } & 23 & 0.13748269\end{array}$

Means for Oneway Anova

Level Number Mean Std Error Lower 95\% Upper 95\%

$\begin{array}{lrrrrr}\text { Level } & \text { Number } & \text { Mean } & \text { Std Error } & \text { Lower 95\% } & \text { Upper 95\% } \\ \text { Coliwasa } & 14 & 2.41170 & 0.01980 & 2.3706 & 2.4528\end{array}$

$\begin{array}{llllll}\text { Coliwasa } & 14 & 2.41170 & 0.01980 & 2.3706 & 2.4528 \\ \text { Isolok - } 3 \mathrm{~mL} & 10 & 2.46519 & 0.02343 & 2.4166 & 2.5138\end{array}$

Std Error uses a pooled estimate of error variance

Means and Std Deviation

Level Number

$\begin{array}{lrrrrrr}\text { Coliwasa } & \text { Number } & \text { Mean } & \text { Std Dev } & \text { Std Err Mean } & \text { Lower 95\% } & \text { Upper 95\% } \\ \text { 14 } & 2.41170 & 0.073436 & 0.01963 & 2.3693 & 2.4541\end{array}$

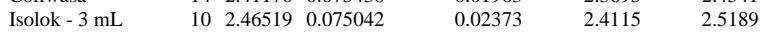

t Test
Isolok - 3 mL-Coliwasa

Assuming unequal variances

Difference $\quad 0.05350$ t Ratio 1.737155

$\begin{array}{lll}\text { Std Err Dif } \quad 0.03080 \text { DF } & 19.27869\end{array}$

Upper CL Dif 0.11789 Prob $>|t| 0.0983$

Lower CL Dif -0.01090 Prob $>t \quad 0.0492$

$\begin{array}{lll}\text { Confidence } & 0.95 \text { Prob }<\mathrm{t} \quad 0.9508\end{array}$

Tests that the Variances are Equal

Level Count Std Dev MeanAbsDif to Mean MeanAbsDif to Median

$\begin{array}{lrrrr}\text { Level } & \text { Count } & \text { Std Dev } & \text { MeanAbsDif to Mean } & \text { MeanAbsDif to Median } \\ \text { Coliwasa } & 14 & 0.0734358 & 0.0580729 & 0.0580729\end{array}$

$\begin{array}{lllll}\text { Isolok - } 3 \mathrm{~mL} & 10 & 0.0750424 & 0.0614741 & 0.0614741\end{array}$

Test F Ratio DFNum DFDen p-Value

$\begin{array}{lrrrr}\text { O'Brien[.5] } & 0.0074 & 1 & 22 & 0.9320\end{array}$

$\begin{array}{lllll}\text { O'Brien[.5] } & 0.0074 & 1 & 22 & 0.9320 \\ \text { Brown-Forsythe } & 0.0412 & 1 & 22 & 0.8411\end{array}$

$\begin{array}{lllll}\text { Brown-Forsythe } & 0.0412 & 1 & 22 & 0.8411 \\ \text { Levene } & 0.0415 & 1 & 22 & 0.8405\end{array}$

$\begin{array}{lllll}\text { Levene } & 0.0415 & 1 & 22 & 0.8405 \\ \text { Bartlett } & 0.0048 & 1 & & 0.9450\end{array}$

$\begin{array}{lllll}\text { F Test 2-sided } & 1.0442 & 9 & 13 & 0.9150\end{array}$

Welch Anova testing Means Equal, allowing Std Devs Not Equal

F Ratio DFNum DFDen Prob $>$ F

$\begin{array}{llll}3.0177 & 1 & 19.279 & 0.0983\end{array}$

$\mathbf{t}$ Test
1.7372
Oneway Analysis of Measurement By Sampler Analytical Block=1, Analyte=Li2O (wt \%), Target Value $=4.674$

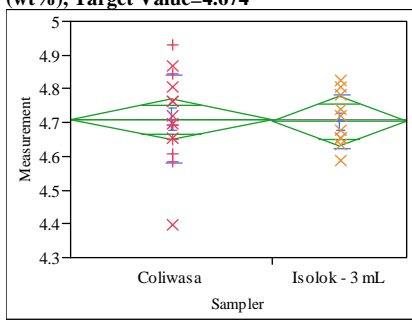

Excluded Rows 1

Oneway Anova

Rsquare $\quad 0.000512$

Adj Rsquare $\quad-0.04294$

Root Mean Square Error $\quad 0.113338$

$\begin{array}{lr}\text { Mean of Response } & 4.707101 \\ \text { Observations (or Sum Wgts) } & 25\end{array}$

t Test

Isolok - 3 mL-Coliwasa

Assuming equal variances

Difference $\quad-0.00502$ t Ratio -0.10857

$\begin{array}{lrlr}\text { Std Err Dif } & 0.04627 & \text { DF } & 23 \\ \text { Up } & & 0.9145\end{array}$

Upper CL Dif 0.09069 Prob $>|t| \quad 0.9145$

Lower CL Dif -0.10074 Prob $>$ t $\quad 0.5428$

Confidence $\quad 0.95$ Prob $<\mathrm{t} \quad 0.4572$

Analysis of Variance

Source DF Sum of Squares Mean Square F Ratio Prob $>$ F

$\begin{array}{lrrrrr}\text { Sampler } & 1 & 0.00015141 & 0.000151 & 0.0118 & 0.9145\end{array}$

Error $23 \quad 0.29544897$

$\begin{array}{lll}\text { C. Total } & 24 & 0.29560038\end{array}$

Means for Oneway Anova

Level Number Mean Std Error Lower 95\% Upper 95\%

$\begin{array}{lrrrrr}\text { Coliwasa } & \text { Number } & \text { Mean } & \text { Std Error } & \text { Lower 95\% } & \text { Upper 95\% } \\ \text { (15 } & 4.70911 & 0.02926 & 4.6486 & 4.7696\end{array}$

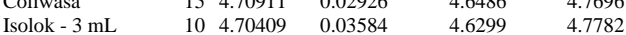

Std Error uses a pooled estimate of error variance

Means and Std Deviations

Level Number Mean Std Dev Std Err Mean Lower 95\% Upper 95\%

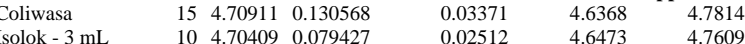

t Test

Isolok - 3 mL-Coliwasa

Assuming unequal variances

$\begin{array}{lrlr}\text { Difference } & -0.00502 & \text { t Ratio } & -0.11949 \\ \text { Std Err Dif } & 0.04204 & \text { DF } & 22.88659 \\ \text { Upper CL Dif } & 0.08197 & \text { Prob }>|t| & 0.9059 \\ \text { Lower CL Dif } & -0.09201 & \text { Prob }>\text { t } & 0.5470 \\ \text { Confidence } & 0.95 & \text { Prob }<t & 0.4530\end{array}$

Tests that the Variances are Equal

Level Count Std Dev MeanAbsDif to Mean MeanAbsDif to Median

$\begin{array}{lrrrr}\text { Coliwasa } & 15 & 0.1305676 & 0.0943449 & 0.0932923 \\ \text { Isolok - 3 mL } & 10 & 0.0794275 & 0.0667399 & 0.0667399\end{array}$

Test F Ratio DFNum DFDen p-Value

$\begin{array}{lrl}\text { Test } & \text { F Ratio DFNum DFDen p-Value } \\ \text { O'Brien[.5] } & 1.2407 & 1\end{array}$

$\begin{array}{lllll}\text { O'Brien[.5] } & 1.2407 & 1 & 23 & 0.2768 \\ \text { Brown-Forsythe } & 0.7768 & 1 & 23 & 0.3872\end{array}$

$\begin{array}{lllll}\text { Brown-Forsythe } & 0.7768 & 1 & 23 & 0.3872 \\ \text { Levene } & 0.8958 & 1 & 23 & 0.3537\end{array}$

$\begin{array}{llrrr}\text { Levene } & 0.8958 & 1 & 23 & 0.3537 \\ \text { Bartlett } & 2.3293 & 1 & . & 0.1270\end{array}$

$\begin{array}{lrrrr}\text { F Test 2-sided } & 2.7023 & 14 & 9 & 0.1270\end{array}$

Welch Anova testing Means Equal, allowing Std Devs Not Equal

F Ratio DFNum DFDen Prob $>$ F

$\begin{array}{lllll}0.0143 & 1 & 22.887 & 0.9059\end{array}$

t Test 
Exhibit A3. Statistical Comparisons for Mid-Rheology (Phase 1) Testing (screened data)

Oneway Analysis of Measurement By Sampler Analytical Block=1, Analyte=NiO (wt \%), Target Value $=0.41$

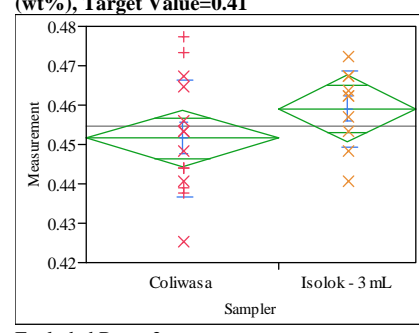

Excluded Rows 2

Oneway Anova

Summary of Fit

Rsquare

Adj Rsquare

0.082496

uare Error -0.040791

Observations (or Sum Wgts) 24

t Test

Isolok - $3 \mathrm{~mL}$-Coliwasa

Assuming equal variances

Difference $\quad 0.00756$ t Ratio 1.406445

$\begin{array}{lrr}\text { Std Err Dif } & 0.00538 \text { DF } & 22 \\ \text { Uper CL Dif } & 0.01871 \text { Prob }>|t| & 0.1736\end{array}$

Upper CL Dif 0.01871 Prob $>|t| \quad 0.1736$

$\begin{array}{lll}\text { Lower CL Dif }-0.00359 \text { Prob }>t & 0.0868\end{array}$

$\begin{array}{lll}\text { Confidence } & 0.95 \text { Prob }<\mathrm{t} \quad 0.9132\end{array}$

Analysis of Variance

Source DF Sum of Squares Mean Square F Ratio Prob $>$ F

$\begin{array}{lrrrrr}\text { Sampler } & 1 & 0.00033360 & 0.000334 & 1.9781 & 0.1736\end{array}$

$\begin{array}{lll}\text { Error } & 22 & 0.00371022 \\ \text { C. Total } & 23 & 0.00404382\end{array}$

Means for Oneway Anova

Level Number Mean Std Error Lower 95\% Upper 95\%

$\begin{array}{lrrrrr}\text { Level } & \text { Number } & \text { Mean } & \text { Std Error } & \text { Lower } 95 \% & \text { Upper 95\% } \\ \text { Coliwasa } & 14 & 0.451556 & 0.00347 & 0.44436 & 0.45875\end{array}$

$\begin{array}{llllll}\text { Coliwasa } & 14 & 0.451556 & 0.00347 & 0.44436 & 0.45875 \\ \text { Isolok - 3 mL } & 10 & 0.459118 & 0.00411 & 0.45060 & 0.46763\end{array}$

Std Error uses a pooled estimate of error variance

Means and Std Deviations

Level Number Mean Std Dev

$\begin{array}{lrllrr} & & & \text { Mean } & \mathbf{9 5 \%} \\ \text { Coliwasa } & 14 & 0.451556 & 0.014839 & 0.00397 & 0.44299 \\ \text { Isolok - 3 } & 10 & 0.459118 & 0.009706 & 0.00307 & 0.45217\end{array}$

$\mathrm{mL}$

t Test

Isolok - $3 \mathrm{~mL}$-Coliwasa

Assuming unequal variances

Difference $\quad 0.00756$ t Ratio 1.508005

Std Err Dif $\quad 0.00501$ DF 21.89229

Upper CL Dif 0.01797 Prob $>|t| \quad 0.1458$

$\begin{array}{lll}\text { Lower CL Dif }-0.00284 \text { Prob }>t & 0.0729\end{array}$

$\begin{array}{lrl}\text { Confidence } & 0.95 \text { Prob }<\mathrm{t} \quad 0.9271\end{array}$

Tests that the Variances are Equal

Level Count Std Dev MeanAbsDif to Mean MeanAbsDif to Median

$\begin{array}{lllll}\text { Coliwasa } & 14 & 0.0148386 & 0.0118161 & 0.0118161\end{array}$

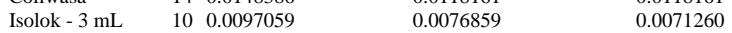

$\begin{array}{lrrrr}\text { Test } & \text { F Ratio } & \text { DFNum } & \text { DFDen } & \text { p-Value } \\ \text { O'Brien[.5] } & 1.8888 & 1 & 22 & 0.1832 \\ \text { Brown-Forsythe } & 2.1023 & 1 & 22 & 0.1612 \\ \text { Levene } & 1.8794 & 1 & 22 & 0.1842 \\ \text { Bartlett } & 1.6940 & 1 & . & 0.1931 \\ \text { F Test 2-sided } & 2.3373 & 13 & 9 & 0.2059\end{array}$

Welch Anova testing Means Equal, allowing Std Devs Not Equal

F Ratio DFNum DFDen Prob > F

$\begin{array}{rrrr}2.2741 & 1 & 21.892 & 0.1458\end{array}$

t Test

1.5080
Oneway Analysis of Measurement By Sampler Analytical Block=1, Analyte=Sum of Oxides (wt \%), Target Value $=99.553$

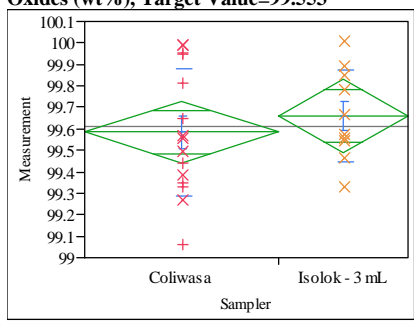

Excluded Rows 1

Oneway Anova

Rsquare $\quad 0.020884$

Adj Rsquare $\quad-0.02169$

Root Mean Square Error $\quad 0.265472$

$\begin{array}{lr}\text { Mean of Response } & 99.61401 \\ \text { Observations (or Sum Wgts) } & 25\end{array}$

t Test

Isolok - 3 mL-Coliwasa

Assuming equal variances

$\begin{array}{lrlr}\text { Difference } & 0.07591 & \text { t Ratio } & 0.700407 \\ \text { Std Err Dif } & 0.10838 \text { DF } & 23 \\ \text { Upper CL Dif } & 0.30011 & \text { Prob }>|t| & 0.4907 \\ \text { Lower CL Dif } & -0.14829 & \text { Prob }>t & 0.2453 \\ \text { Confidence } & 0.95 & \text { Prob }<t & 0.7547\end{array}$

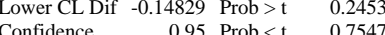

Analysis of Variance

Source DF Sum of Squares Mean Square F Ratio Prob > F

$\begin{array}{lrrrrr}\text { Sompler } & 1 & 0.0345732 & 0.034573 & 0.4906 & 0.4907\end{array}$

Error $\quad 23 \quad 1.6209394$

$\begin{array}{lll}\text { C. Total } 24 & 24.6555126\end{array}$

Means for Oneway Anova

Level Number Mean Std Error Lower 95\% Upper 95\%

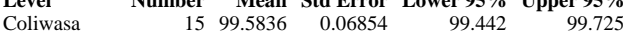

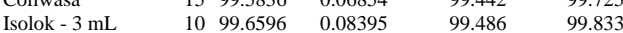

Std Error uses a pooled estimate of error variance

Means and Std Deviations

Upper Level Number Mean Std Dev Std Err Mean Lower 95\% Upper 95\%

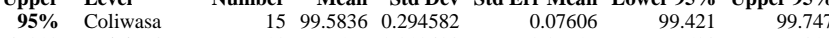
0.46606

t Test

Isolok - 3 mL-Coliwas

Assuming unequal variances

Difference $\quad 0.07591$ t Ratio $\quad 0.748073$

Std Err Dif $\quad 0.10147$ DF 22.79004

Upper CL Dif 0.28593 Prob $>|t| \quad 0.462$

Lower CL Dif -0.13411 Prob $>t \quad 0.2310$

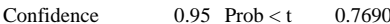

Tests that the Variances are Equal

Level Count Std Dev MeanAbsDif to Mean MeanAbsDif to Median

$\begin{array}{lrrrr}\text { Coliwasa } & 15 & 0.2945825 & 0.2435547 & 0.2392251 \\ \text { Isolok - } 3 \mathrm{~mL} & 10 & 0.2124031 & 0.1738794 & 0.1738794\end{array}$

Test F Ratio DFNum DFDen p-Value

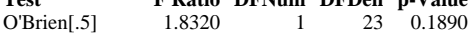

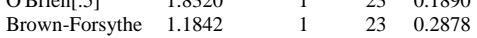

$\begin{array}{lllll}\text { Brown-Forsythe } & 1.1842 & 1 & 23 & 0.2878 \\ \text { Levene } & 1.5623 & 1 & 23 & 0.2239\end{array}$

$\begin{array}{lllll}\text { Levene } & 1.5623 & 1 & 23 & 0.2239 \\ \text { Bartlett } & 1.0523 & 1 & & 0.3050\end{array}$

$\begin{array}{llrrr}\text { F Test 2-sided } & 1.9235 & 14 & \dot{9} & 0.3259\end{array}$

Welch Anova testing Means Equal, allowing Std Devs Not Equal

F Ratio DFNum DFDen Prob > F

$\begin{array}{llll}0.5596 & 1 & 22.79 & 0.4621\end{array}$

t Test 


\section{Exhibit A3. Statistical Comparisons for Mid-Rheology (Phase 1) Testing (screened data)}

Oneway Analysis of Measurement By Sampler Analytical Block=2, Analyte=Al/B, Target Value $=2.552$

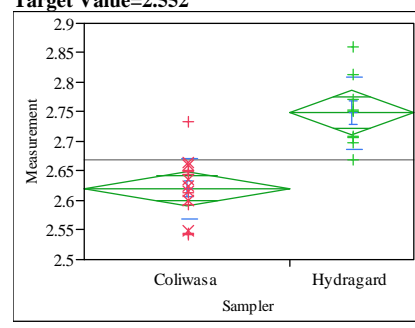

Excluded Rows 2

Oneway Anova

Summary of Fit

Rsquare

Adj Rsquare

0.580596

0.561532

Root Mean Square Error $\quad 0.054835$

Mean of Response 2.668245

Observations (or Sum Wgts) 24

t Test

Hydragard-Coliwasa

Assuming equal variances

Difference $\quad 0.127592$ t Ratio $\quad 5.518637$

$\begin{array}{llll}\text { Std Err Dif } & 0.023120 & \text { DF } & 22 \\ \text { Upper CL } & 0.175541 & \text { Prob }>|t| & <.0001\end{array}$

$\begin{array}{lll}\text { Upper CL Dif } & 0.175541 \text { Prob }>|t| & <.0001 \\ \text { Lower } & & 2.0001\end{array}$

$\begin{array}{lrl}\text { Lower CL Dif } & 0.079644 \text { Prob }>t & <.0001 \\ \text { Confidence } & 0.95 \text { Prob }<\mathrm{t} & 1.0000\end{array}$

Analysis of Variance

Source DF Sum of Squares Mean Square F Ratio Prob $>$ F

$\begin{array}{lrrrrr}\text { Sampler } & 1 & 0.09157384 & 0.091574 & 30.4553 & <.0001\end{array}$

$\begin{array}{llll}\text { Error } & 22 & 0.06615010 & 0.003007\end{array}$

C. Total $23 \quad 0.15772394$

Means for Oneway Anova

Level Number Mean Std Error Lower 95\% Upper 95\%

$\begin{array}{lrrrrr}\text { Coliwasa } & 15 & 2.62040 & 0.01416 & 2.5910 & 2.6498 \\ \text { Hydragard } & 9 & 2.74799 & 0.01828 & 2.7101 & 2.7859\end{array}$

Std Error uses a pooled estimate of error variance

Means and Std Deviations

Level Number Mean Std Dev Std Err Mean Lower 95\% Upper 95\%

$\begin{array}{lrrrrrr}\text { Coliwasa } & 15 & 2.62040 & 0.051440 & 0.01328 & 2.5919 & 2.6489 \\ \text { Hydragard } & 9 & 2.74799 & 0.060317 & 0.02011 & 2.7016 & 2.7944\end{array}$

t Test

Hydragard-Coliwasa

Assuming unequal variances

Difference $\quad 0.127592$ t Ratio $\quad 5.295038$

Std Err Dif $\quad 0.024097$ DF 14.88571

Upper CL Dif 0.178987 Prob $>|t| \quad<.0001$

Lower CL Dif 0.076197 Prob $>t \quad<.0001$

$\begin{array}{lll}\text { Confidence } & 0.95 \text { Prob }<\mathrm{t} \quad 1.0000\end{array}$

Tests that the Variances are Equal

Level Count Std Dev MeanAbsDif to Mean MeanAbsDif to Median

$\begin{array}{lrrrr}\text { Coliwasa } & 15 & 0.0514398 & 0.0389884 & 0.0394414 \\ \text { Hydragard } & 9 & 0.0603173 & 0.0462958 & 0.0458710\end{array}$

Test $\quad$ F Ratio DFNum DFDen p-Value

$\begin{array}{lrrrr}\text { O'Brien[.5] } & 0.3033 & 1 & 22 & 0.5874\end{array}$

$\begin{array}{lllll}\text { O'Brien[.5] } & 0.3033 & 1 & 22 & 0.5874 \\ \text { Brown-Forsythe } & 0.2115 & 1 & 22 & 0.6501 \\ \text { Levene } & 0.2747 & 1 & 22 & 0.6055\end{array}$

$\begin{array}{lllll}\text { Levene } & 0.2747 & 1 & 22 & 0.6055 \\ \text { Bartlett } & 0.2520 & 1 & & 0.6157\end{array}$

$\begin{array}{lllll}\text { Bartlett } & 0.2520 & 1 & & 0.6157 \\ \text { F Test 2-sided } & 1.3749 & 8 & 14 & 0.5754\end{array}$

Welch Anova testing Means Equal, allowing Std Devs Not Equal

F Ratio DFNum DFDen Prob $>$ F

$\begin{array}{llll}28.0374 & 1 & 14.886 & <.0001\end{array}$

t Test
Oneway Analysis of Measurement By Sampler Analytical Block=2, Analyte=Al2O3 (wt \%), Target Value $=10.869$

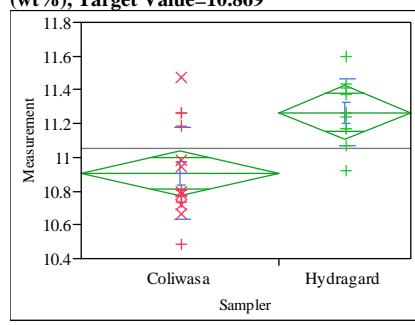

Excluded Rows 1

Oneway Anova

Summary of Fit

Rsquare

$\begin{array}{lr}0.35778 \\ \text { Adj Rsquare } & 0.329857\end{array}$

Root Mean Square Error $\quad 0.245667$

Mean of Response $\quad 11.0498$

Observations (or Sum Wgts) 25

t Test

Hydragard-Coliwasa

Assuming equal variances

Difference $\quad 0.359005$ t Ratio 3.579563

Std Err Dif $\quad 0.100293$ DF 23

Upper CL Dif 0.566477 Prob $>|t| \quad 0.0016$

Lower CL Dif 0.151533 Prob $>t \quad 0.0008$

Confidence $\quad 0.95$ Prob $<\mathrm{t} \quad 0.9992$

Analysis of Variance

Source DF Sum of Squares Mean Square F Ratio Prob $>$ F

$\begin{array}{lrrrrr}\text { Sampler } & 1 & 0.7733075 & 0.773308 & 12.8133 & 0.0016\end{array}$

Error $23 \quad 1.388097$

C. Total $24 \quad 2.1614053$

Means for Oneway Anova

Level Number Mean Std Error Lower 95\% Upper 95\%

$\begin{array}{lrrrrr}\text { Coliwasa } & 15 & 10.9062 & 0.06343 & 10.775 & 11.037\end{array}$

$\begin{array}{llllll}\text { Hydragard } & 10 & 11.2652 & 0.07769 & 11.104 & 11.426\end{array}$

Std Error uses a pooled estimate of error variance

Means and Std Deviations

Level Number Mean Std Dev Std Err Mean Lower 95\% Upper 95\%

$\begin{array}{lrrrrrr}\text { Coliwasa } & 15 & 10.9062 & 0.272339 & 0.07032 & 10.755 & 11.057\end{array}$

$\begin{array}{lllllll}\text { Hydragard } & 10 & 11.2652 & 0.197129 & 0.06234 & 11.124 & 11.406\end{array}$

t Test

Hydragard-Coliwasa

Assuming unequal variances

Difference $\quad 0.359005$ t Ratio $\quad 3.820381$

Std Err Dif $\quad 0.093971$ DF 22.77272

Upper CL Dif 0.553506 Prob $>|\mathrm{t}| \quad 0.0009$

Lower CL Dif 0.164504 Prob $>t \quad 0.0004$

Confidence $\quad 0.95$ Prob $<\mathrm{t} \quad 0.9996$

Tests that the Variances are Equal

$\begin{array}{lrrrr}\text { Level } & \text { Count } & \text { Std Dev } & \text { MeanAbsDif to Mean } & \text { MeanAbsDif to Median } \\ \text { Coliwasa } & 15 & 0.2723391 & 0.2211975 & 0.2015467\end{array}$

$\begin{array}{lllll}\text { Hydragard } & 15 & 0.2723391 & 0.2211975 & 0.2015467 \\ & 10 & 0.1971288 & 0.1519158 & 0.1511600\end{array}$

Test F Ratio DFNum DFDen p-Value

$\begin{array}{lllll}\text { O'Brien[.5] } & 1.1140 & 1 & 23 & 0.3022\end{array}$

$\begin{array}{lllll}\text { Brown-Forsythe } & 0.4621 & 1 & 23 & 0.5034\end{array}$

$\begin{array}{lllll}\text { Levene } & 1.5645 & 1 & 23 & 0.2236\end{array}$

$\begin{array}{llrrr}\text { Bartlett } & 1.0284 & 1 & . & 0.3105\end{array}$

$\begin{array}{lllll}\text { F Test 2-sided } & 1.9086 & 14 & 9 & 0.3317\end{array}$

Welch Anova testing Means Equal, allowing Std Devs Not Equal

F Ratio DFNum DFDen Prob $>$ F

$\begin{array}{llll}14.5953 & 1 & 22.773 & 0.0009\end{array}$

t Test 
Exhibit A3. Statistical Comparisons for Mid-Rheology (Phase 1) Testing (screened data)

Oneway Analysis of Measurement By Sampler Analytical Block=2, Analyte=B2O3 (wt \%), Target Value $=4.259$

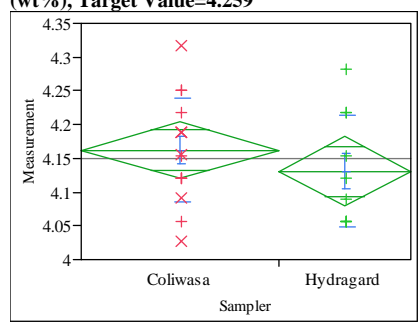

Excluded Rows 1

Oneway Anova

Rsquare $\quad 0.038234$

Adj Rsquare $\quad-0.00358$

Root Mean Square Error $\quad 0.079733$

$\begin{array}{lr}\text { Mean of Response } & 4.149807 \\ \text { Observations (or Sum Wgts) } & 25\end{array}$

t Test

Hydragard-Coliwasa

Assuming equal variances

Difference $\quad-0.03113$ t Ratio $\quad-0.95621$

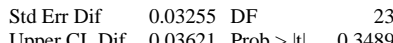

Upper CL Dif 0.03621 Prob $>|t| \quad 0.3489$

Lower CL Dif -0.09846 Prob $>t \quad 0.8255$

$\begin{array}{lll}\text { Confidence } & 0.95 \text { Prob }<\mathrm{t} \quad 0.1745\end{array}$

Analysis of Variance

Source DF Sum of Squares Mean Square F Ratio Prob $>$ F

$\begin{array}{llllll}\text { Sampler } & 1 & 0.00581286 & 0.005813 & 0.9143 & 0.3489\end{array}$

$\begin{array}{lll}\text { Error } & 23 & 0.14621992\end{array}$ 0.006357

C. Total $24 \quad 0.15203277$

Level $\quad$ Std Error Lower 95\% Upper 95\%

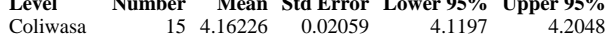

$\begin{array}{llllll}\text { Coliwasa } & 15 & 4.16226 & 0.02059 & 4.1197 & 4.2048 \\ \text { Hydragard } & 10 & 4.13113 & 0.02521 & 4.0790 & 4.1833\end{array}$

Std Error uses a pooled estimate of error variance

Means and Std Deviation

Level Number Mean Std Dev Std Err Mean Lower 95\% Upper 95\%

$\begin{array}{lllllll}\text { Coliwasa } & 15 & 4.16226 & 0.077418 & 0.01999 & 4.1194 & 4.2051 \\ \text { Hydragard } & 10 & 4.13113 & 0.083207 & 0.02631 & 4.0716 & 4.1907\end{array}$

t Test

Hydragard-Coliwasa

Assuming unequal variances

Difference $\quad-0.03113$ t Ratio $\quad-0.94195$

Std Err Dif $\quad 0.03304$ DF 18.43809

Upper CL Dif 0.03818 Prob $>|t| \quad 0.3584$

Lower CL Dif -0.10043 Prob $>t \quad 0.8208$

$\begin{array}{lll}\text { Confidence } & 0.95 \text { Prob }<\mathrm{t} \quad 0.1792\end{array}$

Tests that the Variances are Equal

Level Count Std Dev MeanAbsDif to Mean MeanAbsDif to Median

$\begin{array}{lrrrr}\text { Coliwasa } & 15 & 0.0774180 & 0.0606772 & 0.0601048 \\ \text { Hydragard } & 10 & 0.0832067 & 0.0695498 & 0.0676179\end{array}$

Test $\quad$ F Ratio DFNum DFDen p-Value

$\begin{array}{lrrrr}\text { O'Brien[.5] } & 0.0847 & 1 & 23 & 0.7736\end{array}$

$\begin{array}{lllll}\text { OBrien[.5] } & 0.0847 & 1 & 23 & 0.7736 \\ \text { Brown-Forsythe } & 0.1441 & 1 & 23 & 0.7077\end{array}$

$\begin{array}{lllll}\text { Brown-Forsythe } & 0.1441 & 1 & 23 & 0.7077 \\ \text { Levene } & 0.2549 & 1 & 23 & 0.6185\end{array}$

$\begin{array}{lllll}\text { Levene } & 0.2549 & 1 & 23 & 0.6185 \\ \text { Bartlett } & 0.0550 & 1 & . & 0.8146\end{array}$

$\begin{array}{lllll}\text { F Test 2-sided } & 1.1551 & 9 & 14 & 0.7803\end{array}$

Welch Anova testing Means Equal, allowing Std Devs Not Equal

F Ratio DFNum DFDen Prob > F

$\begin{array}{llll}0.8873 & 1 & 18.438 & 0.3584\end{array}$

t Test
0.9419
Oneway Analysis of Measurement By Sampler Analytical Block=2, Analyte $=\mathrm{Cr} 2 \mathrm{O} 3$ (wt \%), Target Value $=0.0998$

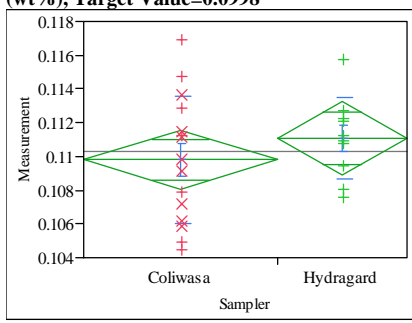

Excluded Rows 1

Oneway Anova

Summary of Fit

Rsquare $\quad 0.038403$

Adj Rsquare $\quad-0.00341$

Root Mean Square Error $\quad 0.003287$

$\begin{array}{lr}\text { Mean of Response } & 0.11031 \\ \text { Observations (or Sum Wgts) } & 25\end{array}$

t Test

Hydragard-Coliwasa

Assuming equal variances

Difference $\quad 0.00129$ t Ratio 0.958408

$\begin{array}{lrlr}\text { Std Err Dif } & 0.00134 & \text { DF } & 23 \\ \text { Upper CL } & 0.00406 & \text { Prob }>|t| & 0.3478\end{array}$

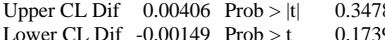

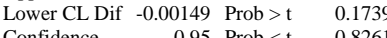

Confidence

Analysis of Variance

Source DF Sum of Squares Mean Square F Ratio Prob $>$ F

$\begin{array}{lrrrrr}\text { Sampler } & 1 & 0.00000993 & 0.00001 & 0.9185 & 0.3478\end{array}$

Error $23 \quad 0.0002485$

$\begin{array}{lll}\text { C. Total } 24 & 0.00025847\end{array}$

Means for Oneway Anova

Level Number Mean Std Error Lower 95\% Upper 95\%

\begin{tabular}{lrrrrr} 
Coliwasa & 15 & 0.109795 & 0.00085 & 0.10804 & 0.11155 \\
\hline
\end{tabular}

$\begin{array}{llllll}\text { Hydragard } & 10 & 0.111082 & 0.00104 & 0.10893 & 0.11323\end{array}$

Std Error uses a pooled estimate of error variance

Means and Std Deviation

Level Number Mean Std Dev Std Err Mean Lower 95\% Upper 95\%

$\begin{array}{lllllll}\text { Coliwasa } & 15 & 0.109795 & 0.003753 & 0.00097 & 0.10772 & 0.11187\end{array}$

$\begin{array}{lllllll}\text { Hydragard } & 10 & 0.111082 & 0.002388 & 0.00076 & 0.10937 & 0.11279\end{array}$

t Test

Hydragard-Coliwasa

Assuming unequal variances

$\begin{array}{lrlr}\text { Difference } & 0.00129 & \text { t Ratio } & 1.046932 \\ \text { Std Err Dif } & 0.00123 & \text { DF } & 22.98227 \\ \text { Upper CL Dif } & 0.00383 & \text { Prob }>|t| & 0.3060 \\ \text { Lower CL Dif } & -0.00126 & \text { Prob }>t & 0.1530 \\ \text { Confidence } & 0.95 & \text { Prob }<\mathrm{t} & 0.8470\end{array}$

Tests that the Variances are Equal

$\begin{array}{lrrrr}\text { Level } & \text { Count } & \text { Std Dev } & \text { MeanAbsDif to Mean } & \text { MeanAbsDif to Median } \\ \text { Coliwasa } & 15 & 0.0037534 & 0.0030908 & 0.0031376\end{array}$

$\begin{array}{lllll}\text { Hydragard } & 10 & 0.0023878 & 0.0017247 & 0.0017247\end{array}$

Test F Ratio DFNum DFDen p-Value

$\begin{array}{lllll}\text { O'Brien[.5] } & 2.4059 & 1 & 23 & 0.1345\end{array}$

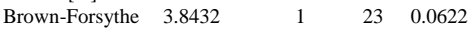

$\begin{array}{lllll}\text { Levene } & 3.4111 & 1 & 23 & 0.0777\end{array}$

$\begin{array}{lrrrr}\text { Bartlett } & 1.9514 & 1 & \text {. } & 0.1624 \\ \text { F Test 2-sided } & 2.4709 & 14 & 9 & 0.1756\end{array}$

Welch Anova testing Means Equal, allowing Std Devs Not Equal

F Ratio DFNum DFDen Prob > F

$\begin{array}{llll}1.0961 & 1 & 22.982 & 0.3060\end{array}$

t Test 
Exhibit A3. Statistical Comparisons for Mid-Rheology (Phase 1) Testing (screened data)

Oneway Analysis of Measurement By Sampler Analytical Block=2, Analyte=Fe/Li, Value=2.452

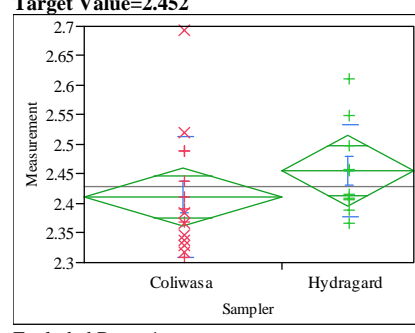

Excluded Rows 1

Oneway Anova

Summary of Fit

Rsquare

Adj Rsquare

0.055411

0.014342

Root Mean Square Error $\quad 0.092764$

Mean of Response 2.429069

Observations (or Sum Wg

25

t Test

Hydragard-Coliwasa

Assuming equal variances

Difference $\quad 0.04399$ t Ratio 1.161556

$\begin{array}{lrlr}\text { Std Err Dif } & 0.03787 & \text { DF } & 23 \\ \text { Upper CL } & 0.12233 & \text { Prob }>|t| & 0.2573\end{array}$

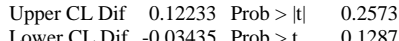

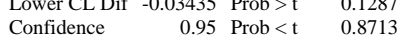

Analysis of Variance

Source DF Sum of Squares Mean Square F Ratio Prob $>$ F

$\begin{array}{lrrrrr}\text { Sampler } & 1 & 0.01161019 & 0.011610 & 1.3492 & 0.2573\end{array}$

$\begin{array}{llll}\text { Error } & 23 & 0.19791887 & 0.008605\end{array}$

C. Total $24 \quad 0.20952906$

Means for Oneway Anova

Level Number Mean Std Error Lower 95\% Upper 95\%

$\begin{array}{lrrrrr}\text { Coliwasa } & 15 & 2.41147 & 0.02395 & 2.3619 & 2.4610\end{array}$

$\begin{array}{llllll}\text { Coliwasa } & 15 & 2.41147 & 0.02395 & 2.3619 & 2.4610 \\ \text { Hydragard } & 10 & 2.45546 & 0.02933 & 2.3948 & 2.5161\end{array}$

Std Error uses a pooled estimate of error variance

Means and Std Deviations

Level Number Mean Std Dev Std Err Mean Lower 95\% Upper 95\%

$\begin{array}{llllllr}\text { Coliwasa } & 15 & 2.41147 & 0.101700 & 0.02626 & 2.3552 & 2.4678 \\ \text { Hydragard } & 10 & 2.45546 & 0.076825 & 0.02429 & 2.4005 & 2.5104\end{array}$

t Test

Hydragard-Coliwasa

Assuming unequal variances

Difference $\quad 0.04399$ t Ratio $\quad 1.22966$

$\begin{array}{lll}\text { Std Err Dif } \quad 0.03577 \text { DF } 22.53782 & \end{array}$

Upper CL Dif 0.11808 Prob $>|t| \quad 0.2315$

Lower CL Dif -0.03010 Prob $>t \quad 0.1158$

Confidence $\quad 0.95$ Prob $<\mathrm{t} \quad 0.8842$

Tests that the Variances are Equal

Level Count Std Dev MeanAbsDif to Mean MeanAbsDif to Median

$\begin{array}{lrrrr}\text { Coliwasa } & 15 & 0.1017001 & 0.0756127 & 0.0713339 \\ \text { Hydragard } & 10 & 0.0768246 & 0.0584781 & 0.0584781\end{array}$

Test F Ratio DFNum DFDen p-Value

$\begin{array}{lrrrr}\text { O'Brien[.5] } & 0.3812 & 1 & 23 & 0.5430\end{array}$

$\begin{array}{lllll}\text { OBrien[.5] } & 0.3812 & 1 & 23 & 0.5430 \\ \text { Brown-Forsythe } & 0.2186 & 1 & 23 & 0.6445\end{array}$

$\begin{array}{lllll}\text { Brown-Forsythe } & 0.2186 & 1 & 23 & 0.6445 \\ \text { Levene } & 0.5197 & 1 & 23 & 0.4782\end{array}$

$\begin{array}{lllll}\text { Levene } & 0.5197 & 1 & 23 & 0.4782 \\ \text { Bartlett } & 0.7822 & 1 & . & 0.3765\end{array}$

$\begin{array}{lllll}\text { F Test 2-sided } & 1.7524 & 14 & 9 & 0.4765 \\ \end{array}$

Welch Anova testing Means Equal, allowing Std Devs Not Equal

F Ratio DFNum DFDen Prob $>$ F

$\begin{array}{llll}1.5121 & 1 & 22.538 & 0.2315\end{array}$

$\mathbf{t}$ Test
1.2297
Oneway Analysis of Measurement By Sampler Analytical Block=2, Analyte=Li2O (wt \%), Target Value $=4.674$

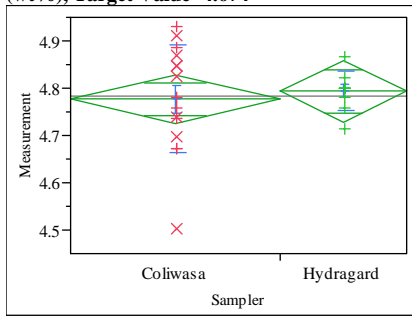

Excluded Rows 2

Oneway Anova

Rsquare $\quad 0.008464$

Adj Rsquare $\quad-0.03661$

Root Mean Square Error $\quad 0.094261$

$\begin{array}{lr}\text { Mean of Response } & 4.783026 \\ \text { Observations (or Sum Wgts) } & 24\end{array}$

t Test

Hydragard-Coliwasa

Assuming equal variances

Difference $\quad 0.01722$ t Ratio 0.433355

$\begin{array}{lrlr}\text { Std Err Dif } & 0.03974 & \text { DF } & 22 \\ \text { Upper CL } & 0.09965 & \text { Prob }>|t| & 0.6690\end{array}$

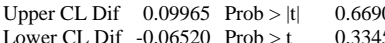

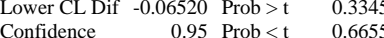

Analysis of Variance

Source DF Sum of Squares Mean Square F Ratio Prob $>$ F

$\begin{array}{lrrrrr}\text { Sampler } & 1 & 0.00166859 & 0.001669 & 0.1878 & 0.6690\end{array}$

$\begin{array}{lll}\text { Error } & 22 & 0.19547249 \\ \text { C. Total } & 23 & 0.19714108\end{array}$

Means for Oneway Anova

Level Number Mean Std Error Lower 95\% Upper 95\%

$\begin{array}{lrrrrr}\text { Coliwasa } & 15 & 4.77657 & 0.02434 & 4.7261 & 4.8270\end{array}$

$\begin{array}{lrrrrr}\text { Hydragard } & 9 & 4.79379 & 0.03142 & 4.7286 & 4.8590\end{array}$

Std Error uses a pooled estimate of error variance

Means and Std Deviation

Level Number Mean Std Dev Std Err Mean Lower 95\% Upper 95\%

$\begin{array}{lrrrrrr}\text { Coliwasa } & 15 & 4.77657 & 0.113882 & 0.02940 & 4.7135 & 4.8396\end{array}$

$\begin{array}{lllllll}\text { Hydragard } & 9 & 4.79379 & 0.041691 & 0.01390 & 4.7617 & 4.8258\end{array}$

t Test

Hydragard-Coliwasa

Assuming unequal variances

Difference $\quad 0.01722$ t Ratio 0.529573

Std Err Dif $\quad 0.03252$ DF 19.27023

Upper CL Dif 0.08523 Prob $>|t| \quad 0.6025$

Lower CL Dif -0.05078 Prob $>\mathrm{t} \quad 0.3012$

$\begin{array}{lll}\text { Confidence } & 0.95 \text { Prob }<\mathrm{t} \quad 0.6988\end{array}$

Tests that the Variances are Equal

$\begin{array}{lrrrr}\text { Level } & \text { Count } & \text { Std Dev } & \text { MeanAbsDif to Mean } & \text { MeanAbsDif to Median } \\ \text { Coliwasa } & 15 & 0.1138820 & 0.0891779 & 0.0904218\end{array}$

$\begin{array}{lrrrr}\text { Hydragard } & 15 & 0.1138820 & 0.0891779 & 0.0904218 \\ & 9 & 0.0416907 & 0.0287053 & 0.0263132\end{array}$

Test F Ratio DFNum DFDen p-Value

$\begin{array}{lllll}\text { O'Brien[.5] } & 2.4451 & 1 & 22 & 0.1322\end{array}$

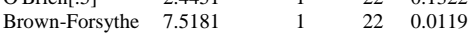

$\begin{array}{lllll}\text { Levene } & 6.5809 & 1 & 22 & 0.0176\end{array}$

$\begin{array}{lllll}\text { Bartlett } & 7.3862 & 1 & 22 & 0.0176 \\ \text { F Test } & 7.4616 & 14 & 8 & 0.0066\end{array}$

$\begin{array}{lrrrr}\text { F Test 2-sided } & 7.4616 & 14 & 8 & 0.0066\end{array}$

Welch Anova testing Means Equal, allowing Std Devs Not Equal

F Ratio DFNum DFDen Prob $>$ F

$\begin{array}{llll}0.2804 & 1 & 19.27 & 0.6025\end{array}$

t Test 
Exhibit A3. Statistical Comparisons for Mid-Rheology (Phase 1) Testing (screened data)

Oneway Analysis of Measurement By Sampler Analytical Block=2, Analyte=NiO (wt \%), Target Value $=0.41$

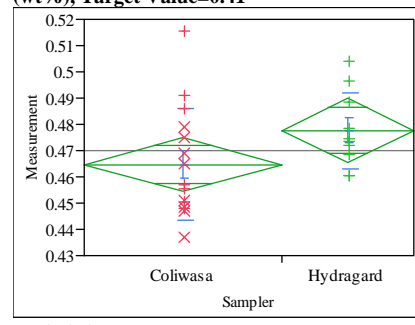

Excluded Rows 1

Oneway Anova

Rsquare $\quad 0.110386$

Adj Rsquare

0.071708

Root Mean Square Error $\quad 0.01882$

$\begin{array}{lr}\text { Mean of Response } & 0.469909 \\ \text { Observations (or Sum Wgts) } & 25\end{array}$

Observations (or Sum Wg

t Test

Hydragard-Coliwasa

Assuming equal variances

Difference $\quad 0.01298$ t Ratio 1.689356

$\begin{array}{lrlr}\text { Std Err Dif } & 0.00768 & \text { DF } & 23 \\ \text { Upper CL Dif } & 0.02887 & \text { Prob }>|t| & 0.1047\end{array}$

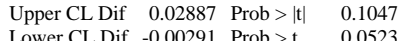

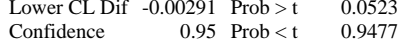

Analysis of Variance

$\begin{array}{llllll}\text { Sampler } & 1 & 0.00101080 & 0.001011 & 2.8539 & 0.1047\end{array}$

$\begin{array}{llll}\text { Error } & 23 & 0.00814615 & 0.000354\end{array}$

C. Total $24 \quad 0.00915696$

Means for Oneway Anova

Level Number Mean Std Error Lower 95\% Upper 95\%

$\begin{array}{lrrrrr}\text { Coliwasa } & 15 & 0.464717 & 0.00486 & 0.45466 & 0.47477\end{array}$

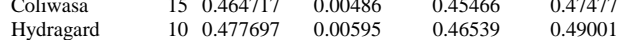

Std Error uses a pooled estimate of error variance

Level Number Mean Std Dev Std Err Mean Lower 95\% Upper 95\%

$\begin{array}{lllllll}\text { Coliwasa } & 15 & 0.464717 & 0.021152 & 0.00546 & 0.45300 & 0.47643 \\ \text { Hydragard } & 10 & 0.477697 & 0.014462 & 0.00457 & 0.46735 & 0.48804\end{array}$ 0.00457
Source DF Sum of Squares Mean Square F Ratio Prob $>$ F

Means and Std Deviations

Oneway Analysis of Measurement By Sampler Analytical Block=2, Analyte=Sum of Oxides (wt \%), Target Value $=99.553$

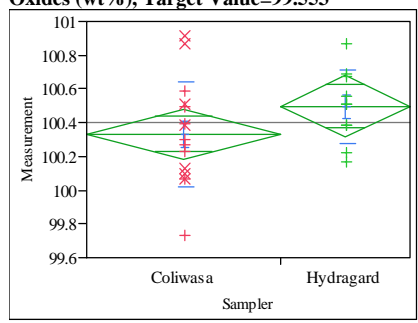

Excluded Rows 1

Oneway Anova

Summary of Fit

Rsquare

Adj Rsquare $\quad 0.043829$

Root Mean Square Error $\quad 0.277911$

$\begin{array}{lr}\text { Mean of Response } & 100.3973 \\ \text { Observations (or Sum Wgts) } & 25\end{array}$

t Test

Hydragard-Coliwasa

Assuming equal variances

Difference $\quad 0.16442$ t Ratio 1.449179

$\begin{array}{lrlr}\text { Std Err Dif } & 0.11346 & \text { DF } & 23 \\ \text { Upper CL } & 0.39912 & \text { Prob }>|t| & 0.1608\end{array}$

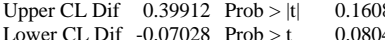

$\begin{array}{lrrr}\text { Lower CL Dif } & -0.07028 \text { Prob }>t & 0.0804 \\ \text { Confidence } & 0.95 & \text { Prob }<t & 0.9196\end{array}$

Analysis of Variance

Source DF Sum of Squares Mean Square F Ratio Prob > F

$\begin{array}{lrrrrr}\text { Sampler } & 1 & 0.1622017 & 0.162202 & 2.1001 & 0.1608\end{array}$

Error $23 \quad 1.7763933$

C. Total $24 \quad 1.9385950$

Means for Oneway Anova

Level Number Mean Std Error Lower 95\% Upper 95\%

$\begin{array}{lrrrrr}\text { Coliwasa } & 15 & 100.332 & 0.07176 & 100.18 & 100.48\end{array}$

$\begin{array}{llllll}\text { Hydragard } & 10 & 100.496 & 0.08788 & 100.31 & 100.68\end{array}$

Std Error uses a pooled estimate of error variance

Means and Std Deviation

Level Number Mean Std Dev Std Err Mean Lower 95\% Upper 95\%

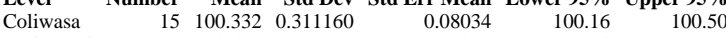

$\begin{array}{lllllll}\text { Hydragard } & 10 & 100.496 & 0.216257 & 0.06839 & 100.34 & 100.65\end{array}$

t Test

Hydragard-Coliwasa

Assuming unequal variances

Hydragard-Coliwasa
Assuming unequal variances

Difference $\quad 0.01298$ t Ratio 1.822111

Std Err Dif $\quad 0.00712$ DF 22.95809

Upper CL Dif 0.02772 Prob $>|t| \quad 0.0815$

Lower CL Dif -0.00176 Prob $>\mathrm{t} \quad 0.0407$

Confidence $\quad 0.95$ Prob $<\mathrm{t} \quad 0.9593$

Tests that the Variances are Equal

Level Count Std Dev MeanAbsDif to Mean MeanAbsDif to Median

$\begin{array}{lrrrr}\text { Coliwasa } & 15 & 0.0211524 & 0.0167631 & 0.0162880 \\ \text { Hydragard } & 10 & 0.0144615 & 0.0112998 & 0.0106890\end{array}$

Test F Ratio DFNum DFDen p-Value

$\begin{array}{lrrrr}\text { O'Brien[.5] } & 1.0228 & 1 & 23 & 0.3224\end{array}$

$\begin{array}{lllll}\text { OBrien[.5] } & 1.0228 & 1 & 23 & 0.3224 \\ \text { Brown-Forsythe } & 1.0563 & 1 & 23 & 0.3148\end{array}$

$\begin{array}{lllll}\text { Brown-Forsythe } & 1.0563 & 1 & 23 & 0.3148 \\ \text { Levene } & 1.5518 & 1 & 23 & 0.2254\end{array}$

$\begin{array}{lllll}\text { Levene } & 1.5518 & 1 & 23 & 0.2254 \\ \text { Bartlett } & 1.4045 & 1 & . & 0.2360\end{array}$

$\begin{array}{llrrr}\text { F Test 2-sided } & 2.1394 & 14 & 9 & 0.2534\end{array}$

Welch Anova testing Means Equal, allowing Std Devs Not Equal

F Ratio DFNum DFDen Prob $>$ F

$\begin{array}{llll}3.3201 & 1 & 22.958 & 0.0815\end{array}$

t Test
1.8221 $\begin{array}{lrlr}\text { Difference } & 0.16442 & \text { t Ratio } & 1.558392 \\ \text { Std Err Dif } & 0.10551 & \text { DF } & 22.92001 \\ \text { Upper CL Dif } & 0.38272 & \text { Prob }>|t| & 0.1328 \\ \text { Lower CL Dif } & -0.05388 & \text { Prob }>t & 0.0664 \\ \text { Confidence } & 0.95 & \text { Prob }<\mathrm{t} & 0.9336\end{array}$

Tests that the Variances are Equal

$\begin{array}{lrrrr}\text { Level } & \text { Count } & \text { Std Dev } & \text { MeanAbsDif to Mean } & \text { MeanAbsDif to Median } \\ \text { Coliwasa } & 15 & 0.3111603 & 0.2388519 & 0.2384541\end{array}$

$\begin{array}{lllll}\text { Hydragard } & 10 & 0.2162567 & 0.1651795 & 0.1628642\end{array}$

Test F Ratio DFNum DFDen p-Value

$\begin{array}{lllll}\text { O'Brien[.5] } & 1.1660 & 1 & 23 & 0.2914\end{array}$

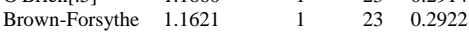

$\begin{array}{lllll}\text { Levene } & 1.1562 & 1 & 23 & 0.2934\end{array}$

$\begin{array}{lllll}\text { Bartlett } & 1.2910 & 1 & . & 0.2559\end{array}$

$\begin{array}{lllll}\text { F Test 2-sided } & 2.0703 & 14 & 9 & 0.2744\end{array}$

Welch Anova testing Means Equal, allowing Std Devs Not Equal

F Ratio DFNum DFDen Prob $>$ F

$\begin{array}{llll}2.4286 & 1 & 22.92 & 0.1328\end{array}$

t Test 
Exhibit A3. Statistical Comparisons for Mid-Rheology (Phase 1) Testing (screened data)

Oneway Analysis of Measurement By Sampler Analytical Block=2, Analyte=TiO2 $(w t \%)$, Target Value $=\mathbf{0 . 0 7 1 1}$

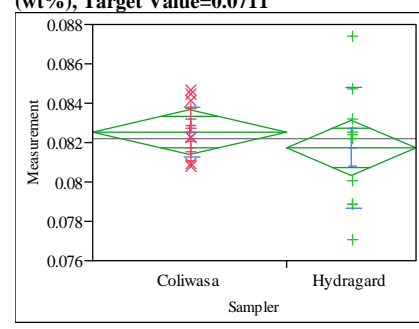

Excluded Rows 1

Oneway Anova

Summary of Fit

Rsquare

Adj Rsquare

0.034039

$-0.00796$

Root Mean Square Error $\quad 0.002163$

Mean of Response 0.082226

Observations (or Sum Wgts)

t Test

Hydragard-Coliwasa

Assuming equal variances

Difference $\quad-0.00080$ t Ratio $\quad-0.90028$

$\begin{array}{lrrr}\text { Lower CL Dif } & -0.00262 & \text { Prob }>t & 0.8113 \\ \text { Confidence } & 0.95 & \text { Prob }<\mathrm{t} & 0.1887\end{array}$

Analysis of Variance

Source DF Sum of Squares Mean Square F Ratio Prob $>$ F

$\begin{array}{lrrrrr}\text { Sampler } & 1 & 0.00000379 & 3.7929 \mathrm{e}-6 & 0.8105 & 0.3773\end{array}$

$\begin{array}{lll}\text { C. Total } 24 & 24 & 0.00011143\end{array}$

Means for Oneway Anova

Level Number Mean Std Error Lower 95\% Upper 95\%

$\begin{array}{lrrrrr}\text { Coliwasa } & 15 & 0.082544 & 0.00056 & 0.08139 & 0.08370\end{array}$

$\begin{array}{llllll}\text { Coliwasa } & 15 & 0.082544 & 0.00056 & 0.08139 & 0.08370 \\ \text { Hydragard } & 10 & 0.081749 & 0.00068 & 0.08033 & 0.08316\end{array}$

Std Error uses a pooled estimate of error variance

Means and Std Deviation

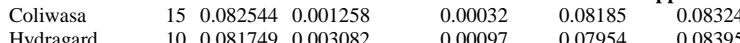
0.00097 $\begin{array}{lrlr}\text { Std Err Dif } & 0.00088 & \text { DF } & 23 \\ \text { Upper CL Dif } & 0.00103 & \text { Prob }>|t| & 0.3773\end{array}$

Upper CL Dif 0.00103 Prob $>|t| \quad 0.3773$

$\begin{array}{llll}\text { Error } & 23 & 0.00010763 & 4.6797 \mathrm{e}-6\end{array}$

Level Number Mean Std Dev Std Err Mean Lower 95\% Upper 95\%

$\begin{array}{llll}\text { Hydragard } & 10 & 0.081749 & 0.003082\end{array}$

Oneway Analysis of Measurement By Sampler Analytical Block=2, Analyte=ZrO2 (wt $\%)$, Target Value $=\mathbf{0 . 3 5 4 7}$

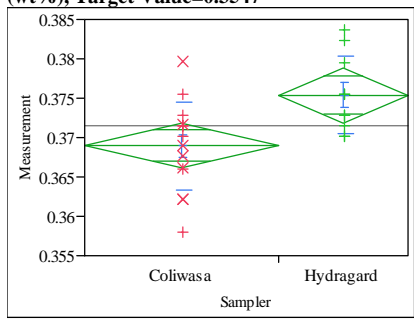

Excluded Rows 1

Oneway Anova

Summary of Fit

Adj Rsquare $\quad 0.245093$

Root Mean Square Error $\quad 0.005319$

$\begin{array}{lr}\text { Mean of Response } & 0.371524 \\ \text { Observations (or Sum Wgts) } & 25\end{array}$

t Test

Hydragard-Coliwasa

Assuming equal variances

Difference $\quad 0.006439$ t Ratio 2.965127

Std Err Dif $\quad 0.002172$ DF 23

Upper CL Dif 0.010931 Prob $>|t| \quad 0.0069$

Lower CL Dif 0.001947 Prob $>t \quad 0.0035$

$\begin{array}{lll}\text { Confidence } & 0.95 \text { Prob }<\mathrm{t} \quad 0.9965\end{array}$

Analysis of Variance

Source DF Sum of Squares Mean Square F Ratio Prob > F

$\begin{array}{lrrrrr}\text { Sampler } & 1 & 0.00024875 & 0.000249 & 8.7920 & 0.0069\end{array}$

$\begin{array}{lll}\text { Error } & 23 & 0.00065073\end{array}$

C. Total $24 \quad 0.00089948$

Means for Oneway Anova

$\begin{array}{lrrrrr}\text { Level } & \text { Number } & \text { Mean } & \text { Std Error } & \text { Lower 95\% } & \text { Upper 95\% } \\ \text { Coliwasa } & 15 & 0.368949 & 0.00137 & 0.36611 & 0.37179\end{array}$

$\begin{array}{llllll}\text { Hydragard } & 10 & 0.375387 & 0.00168 & 0.37191 & 0.37887\end{array}$

Std Error uses a pooled estimate of error variance

Means and Std Deviations

Level Number Mean Std Dev Std Err Mean Lower 95\% Upper 95\%

$\begin{array}{lllllll}\text { Coliwasa } & 15 & 0.368949 & 0.005566 & 0.00144 & 0.36587 & 0.37203\end{array}$

$\begin{array}{lllllll}\text { Hydragard } & 10 & 0.375387 & 0.004910 & 0.00155 & 0.37188 & 0.37890\end{array}$

t Test

Hydragard-Coliwas

Assuming unequal variances

Hydragard-Coliwasa
Assuming unequal variances

Difference $\quad-0.00080$ t Ratio $\quad-0.77394$

Std Err Dif $\quad 0.00103$ DF $\quad 11.02121$

Upper CL Dif 0.00147 Prob $>|t| \quad 0.4552$

Lower CL Dif -0.00306 Prob $>\mathrm{t} \quad 0.7724$

Confidence $\quad 0.95$ Prob $<\mathrm{t} \quad 0.2276$

Tests that the Variances are Equal

Level Count Std Dev MeanAbsDif to Mean MeanAbsDif to Median

$\begin{array}{lrrrr}\text { Coliwasa } & 15 & 0.0012575 & 0.0010438 & 0.0010230 \\ \text { Hydragard } & 10 & 0.0030821 & 0.0024153 & 0.0023185\end{array}$

Test $\quad$ Ratio DFNum DFDen p-Value

$\begin{array}{lrrrr}\text { O'Brien[.5] } & 6.0608 & 1 & 23 & 0.0217\end{array}$

$\begin{array}{lllll}\text { O'Brien[.5] } & 6.0608 & 1 & 23 & 0.0217 \\ \text { Brown-Forsythe } & 5.4049 & 1 & 23 & 0.0293\end{array}$

$\begin{array}{lllll}\text { Levene } & 7.8761 & 1 & 23 & 0.0100 \\ \text { Bartlett } & 8.4269 & 1 & & 0.0037\end{array}$

$\begin{array}{lllll}\text { Bartlett } & 8.4269 & 1 & & 0.0037 \\ \text { F Test 2-sided } & 6.0071 & 9 & 14 & 0.0032\end{array}$

Welch Anova testing Means Equal, allowing Std Devs Not Equal

F Ratio DFNum DFDen Prob $>$ F

$\begin{array}{llll}0.5990 & 1 & 11.021 & 0.4552\end{array}$

t Test
0.7739
Difference $\quad 0.006439$ t Ratio $\quad 3.043341$

Std Err Dif $\quad 0.002116$ DF 21.0815

Upper CL Dif 0.010838 Prob $>|t| 0.0062$

Lower CL Dif 0.002040 Prob $>t \quad 0.0031$

$\begin{array}{lll}\text { Confidence } & 0.95 \text { Prob }<\mathrm{t} \quad 0.9969\end{array}$

Tests that the Variances are Equal

$\begin{array}{lrrrr}\text { Level } & \text { Count } & \text { Std Dev } & \text { MeanAbsDif to Mean } & \text { MeanAbsDif to Median } \\ \text { Coliwasa } & 15 & 0.0055664 & 0.0043106 & 0.0043226\end{array}$

$\begin{array}{lllll}\text { Hydragard } & 15 & 0.0055664 & 0.0043106 & 0.0043226 \\ & 10 & 0.0049098 & 0.0039173 & 0.0039173\end{array}$

Test F Ratio DFNum DFDen p-Value

$\begin{array}{lllll}\text { O'Brien[.5] } & 0.1978 & 1 & 23 & 0.6607\end{array}$

$\begin{array}{lllll}\text { Brown-Forsythe } & 0.0892 & 1 & 23 & 0.7679\end{array}$

$\begin{array}{lllll}\text { Levene } & 0.0976 & 1 & 23 & 0.7575\end{array}$

$\begin{array}{llrrr}\text { Bartlett } & 0.1616 & 1 & . & 0.6877\end{array}$

$\begin{array}{lllll}\text { F Test 2-sided } & 1.2854 & 14 & 9 & 0.7204\end{array}$

Welch Anova testing Means Equal, allowing Std Devs Not Equal

F Ratio DFNum DFDen Prob $>$ F

$\begin{array}{llll}9.2619 & 1 & 21.082 & 0.0062\end{array}$

t Test 
Exhibit A3. Statistical Comparisons for Mid-Rheology (Phase 1) Testing (screened data)

Oneway Analysis of Measurement By Sampler Analytical Block=3, Analyte=Al/B, Target Value $=2.552$

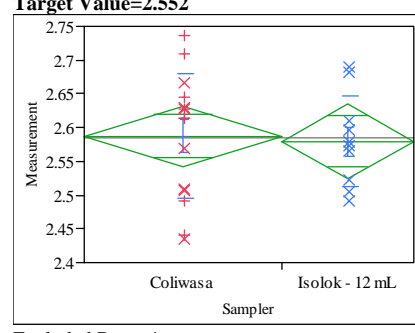

Excluded Rows 1

Oneway Anova

Rsquare $\quad 0.002052$

Adj Rsquare $\quad-0.04134$

Root Mean Square Error $\quad 0.083711$

$\begin{array}{lr}\text { Mean of Response } & 2.584187 \\ \text { Observations (or Sum Wgts) } & 25\end{array}$

Observations (or Sum Wgs)

t Test

Isolok - $12 \mathrm{~mL}$-Coliwasa

Assuming equal variances

Difference $\quad-0.00743$ t Ratio -0.21749

$\begin{array}{lrlr}\text { Std Err Dif } & 0.03417 & \text { DF } & 23 \\ \text { Upper CL } & 0.06326 & \text { Prob }>|t| & 0.8297\end{array}$

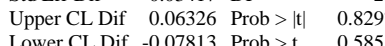

$\begin{array}{lrrr}\text { Lower CL Dif } & -0.07813 & \text { Prob }>t & 0.5851 \\ \text { Confidence } & 0.95 & \text { Prob }<t & 0.4149\end{array}$

Analysis of Variance

Source DF Sum of Squares Mean Square F Ratio Prob $>$ F

$\begin{array}{lrrrrr}\text { Sampler } & 1 & 0.00033147 & 0.000331 & 0.0473 & 0.8297\end{array}$

$\begin{array}{lll}\text { Error } & 23 & 0.16117424 \\ \text { C. Total } & 24 & 0.16150571\end{array}$

Means for Oneway Anova

Level Number Mean Std Error Lower 95\% Upper 95\%

$\begin{array}{lrrrrr}\text { Level } & \text { Number } & \text { Mean } & \text { Std Error } & \text { Lower 95\% } & \text { Upper 95\% } \\ \text { Coliwasa } & 15 & 2.58716 & 0.02161 & 2.5424 & 2.6319\end{array}$

$\begin{array}{llllll}\text { Coliwasa } & 15 & 2.58716 & 0.02161 & 2.5424 & 2.6319 \\ \text { Isolok - 12 mL } & 10 & 2.57973 & 0.02647 & 2.5250 & 2.6345\end{array}$

Std Error uses a pooled estimate of error variance

Means and Std Deviations

Level Number Mean Std Dev

Coliwasa

$\begin{array}{lll}15 & 2.58716 & 0.092777\end{array}$

Isolok - 12

$\begin{array}{lll}10 & 2.57973 & 0.067221\end{array}$

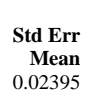

$0.02395 \quad 2.5358$

$\mathrm{mL}$

t Test

Isolok - $12 \mathrm{~mL}$-Coliwasa

Assuming unequal variances

$\begin{array}{lll}\text { Difference } & -0.00743 \text { t Ratio } \quad-0.23208\end{array}$

Std Err Dif $\quad 0.03203$ DF 22.76826

Upper CL Dif 0.05886 Prob $>|t| \quad 0.8186$

Lower CL Dif -0.07372 Prob $>t \quad 0.5907$

$\begin{array}{lrl}\text { Confidence } & 0.95 \text { Prob }<\mathrm{t} \quad 0.4093\end{array}$

Tests that the Variances are Equal

Level Count Std Dev MeanAbsDif to Mean MeanAbsDif to Median

$\begin{array}{lllll}\text { Coliwasa } & 15 & 0.0927771 & 0.0771781 & 0.0717276\end{array}$

$\begin{array}{lllll}\text { Isolok - } 12 \mathrm{~mL} & 10 & 0.0672209 & 0.0505934 & 0.0499902\end{array}$

Test F Ratio DFNum DFDen p-Value

$\begin{array}{lrrrr}\text { O'Brien[.5] } & 1.5354 & 1 & 23 & 0.2278\end{array}$

$\begin{array}{lllll}\text { Brown-Forsythe } & 0.9241 & 1 & 23 & 0.3464\end{array}$

$\begin{array}{lllll}\text { Levene } & 2.1097 & 1 & 23 & 0.1599\end{array}$

$\begin{array}{lrrrr}\text { Bartlett } & 1.0225 & 1 & \text {. } & 0.3119 \\ \text { F Test 2-sided } & 1.9049 & 14 & 9 & 0.3331\end{array}$

Welch Anova testing Means Equal, allowing Std Devs Not Equal

F Ratio DFNum DFDen Prob > F

$\begin{array}{rrrr}0.0539 & 1 & 22.768 & 0.8186\end{array}$

t Tes

0.2321
Oneway Analysis of Measurement By Sampler Analytical Block=3, Analyte=B2O3 (wt \%), Target Value $=4.259$

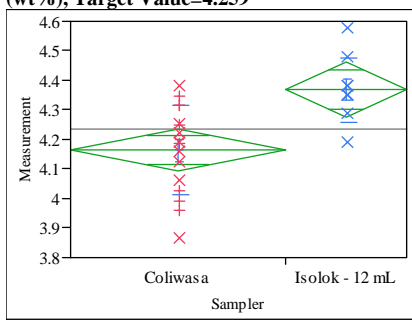

Excluded Rows 1

Oneway Anova

Rsquare $\quad 0.357788$

$\begin{array}{ll}\text { Adj Rsquare } & 0.329866 \\ \text { Root Mean Square Error } & 0.137175\end{array}$

$\begin{array}{ll}\text { Root Mean Square Error } & 0.137175 \\ \text { Mean of Response } & 4.237388\end{array}$

Observations (or Sum Wgts) 25

t Test

Isolok - $12 \mathrm{~mL}$-Coliwasa

Assuming equal variances

Difference $\quad 0.204598$ t Ratio $\quad 3.579629$

$\begin{array}{lrrr}\text { Std Err Dif } & 0.057156 \text { DF } & 23 \\ \text { Upper CL } & 0.322834 \text { Prob }>|t| & 0.0016\end{array}$

Upper CL Dif 0.322834 Prob $>|t| \quad 0.0016$

$\begin{array}{lrrr}\text { Lower CL Dif } & 0.086361 & \text { Prob }>t & 0.0008 \\ \text { Confidence } & 0.95 & \text { Prob }<t & 0.9992\end{array}$

Analysis of Variance

Source DF Sum of Squares Mean Square F Ratio Prob $>$ F

$\begin{array}{lrrrrr}\text { Sampler } & 1 & 0.24111513 & 0.241115 & 12.8137 & 0.0016\end{array}$

$\begin{array}{lll}\text { Error } & 23 & 0.43278901 \\ \text { C. Total } & 24 & 0.67390414\end{array}$

Means for Oneway Anova

Level Number Mean Std Error Lower 95\% Upper 95\%

$\begin{array}{lrrrrr}\text { Coliwasa } & 16 & 4.16373 & 0.03429 & 4.0928 & 4.2347\end{array}$

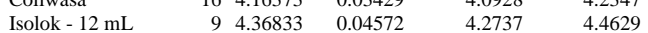

Std Error uses a pooled estimate of error variance

Means and Std Deviations

upper Level Number Mean Std Dev

Tests that the Variances are Equal

Level Count Std Dev MeanAbsDif to Mean MeanAbsDif to Median

$\begin{array}{lrrrr}\text { Coliwasa } & 16 & 0.1499790 & 0.1215009 & 0.1187338 \\ \text { Isolok - 12 mL } & 9 & 0.1091921 & 0.0739384 & 0.0715533\end{array}$

Test F Ratio DFNum DFDen p-Value

$\begin{array}{lrrrr}\text { O'Brien[.5] } & 1.1160 & 1 & 23 & 0.3018\end{array}$

$\begin{array}{lllll}\text { Brown-Forsythe } & 1.7033 & 1 & 23 & 0.2048\end{array}$

$\begin{array}{lllll}\text { Levene } & 2.0334 & 1 & 23 & 0.1673\end{array}$

$\begin{array}{lrrrr}\text { Bartlett } & 0.9273 & 1 & \text {. } & 0.3356 \\ \text { F Test 2-sided } & 1.8866 & 15 & 8 & 0.3674\end{array}$

Welch Anova testing Means Equal, allowing Std Devs Not Equal

F Ratio DFNum DFDen Prob $>$ F

$\begin{array}{llll}15.3299 & 1 & 21.235 & 0.0008\end{array}$

t Test

3.9153 


\section{Exhibit A3. Statistical Comparisons for Mid-Rheology (Phase 1) Testing (screened data)}

Oneway Analysis of Measurement By Sampler Analytical Block=3, Analyte=Fe/Li Ane

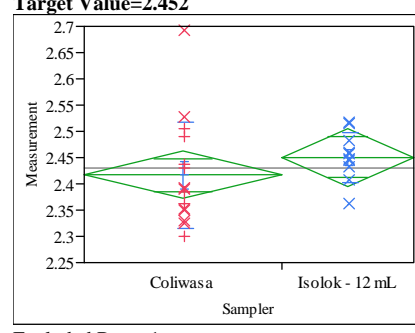

Excluded Rows 1

Oneway Anova

Summary of Fit

Rsquare

Adj Rsquare

0.039958

$-0.00178$

Root Mean Square Error $\quad 0.084613$

Mean of Response 2.430151

Observations (or Sum Wg

t Test

Isolok - 12 mL-Coliwasa

Assuming equal variances

Difference $\quad 0.03380$ t Ratio $\quad 0.978409$

$\begin{array}{lrrr}\text { Std Err Dif } & 0.03454 \text { DF } & 23 \\ \text { Upper CL Dif } & 0.10525 \text { Prob }>|t| & 0.3381\end{array}$

Upper CL Dif 0.10525 Prob $>|t| \quad 0.3381$

$\begin{array}{lrl} & \text { Lower CL Dif }-0.03766 \text { Prob }>t & 0.1690 \\ \text { Confidence } & 0.95 \text { Prob }<t \quad 0.8310\end{array}$

Analysis of Variance

Source DF Sum of Squares Mean Square F Ratio Prob $>$ F

$\begin{array}{lrllll}\text { Sampler } & 1 & 0.00685348 & 0.006853 & 0.9573 & 0.3381\end{array}$

$\begin{array}{lll}\text { Error } & 23 & 0.16466378 \\ \text { C. Total } & 24 & 0.17151726\end{array}$

Means for Oneway Anova

Level Number Mean Std Error Lower 95\% Upper 95\%

$\begin{array}{lrrrrr}\text { Coliwasa } & 15 & 2.41663 & 0.02185 & 2.3714 & 2.4618\end{array}$

$\begin{array}{llllll}\text { Coliwasa } & 15 & 2.41663 & 0.02185 & 2.3714 & 2.4618 \\ \text { Isolok - 12 mL } & 10 & 2.45043 & 0.02676 & 2.3951 & 2.5058\end{array}$

Std Error uses a pooled estimate of error variance

Means and Std Deviations

Level Number Mean Std Dev

Coliwasa

$\begin{array}{lll}15 & 2.41663 & 0.101692\end{array}$

Isolok - 12

$\begin{array}{lll}10 & 2.45043 & 0.047006\end{array}$

$\begin{array}{rr}\text { Std Err } & \text { Lower } \\ \text { Mean } & \mathbf{9 5 \%} \\ 0.02626 & 2.3603 \\ 0.01486 & 2.4168\end{array}$

t Test

Isolok - $12 \mathrm{~mL}$-Coliwas

Assuming unequal variances

Difference $\quad 0.03380$ t Ratio 1.120133

Std Err Dif $\quad 0.03017$ DF 21.04876

Upper CL Dif 0.09654 Prob $>|t| \quad 0.2753$

Lower CL Dif -0.02894 Prob $>t \quad 0.1376$

$\begin{array}{lll}\text { Confidence } & 0.95 \text { Prob }<\mathrm{t} \quad 0.8624\end{array}$

Tests that the Variances are Equal

Level Count Std Dev MeanAbsDif to Mean MeanAbsDif to Median

$\begin{array}{lllll}\text { Coliwasa } & 15 & 0.1016920 & 0.0769624 & 0.0713874\end{array}$

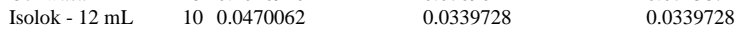

Test $\quad$ F Ratio DFNum DFDen p-Value

$\begin{array}{lrrrr}\text { O'Brien[.5] } & 1.5322 & 1 & 23 & 0.2283\end{array}$

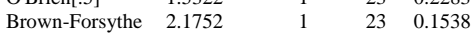

$\begin{array}{lllll}\text { Levene } & 3.9680 & 1 & 23 & 0.0584\end{array}$

$\begin{array}{lrrrr}\text { Bartlett } & 5.1916 & 1 & \text {. } & 0.0227 \\ \text { F Test 2-sided } & 4.6802 & 14 & 9 & 0.0252\end{array}$

Welch Anova testing Means Equal, allowing Std Devs Not Equal

F Ratio DFNum DFDen Prob $>$ F

$\begin{array}{rrrr}1.2547 & 1 & 21.049 & 0.2753\end{array}$

t Tes

1.1201
Oneway Analysis of Measurement By Sampler Analytical Block=3, Analyte=K2O (wt \%), Target Value $=\mathbf{0 . 1 5 9 1}$

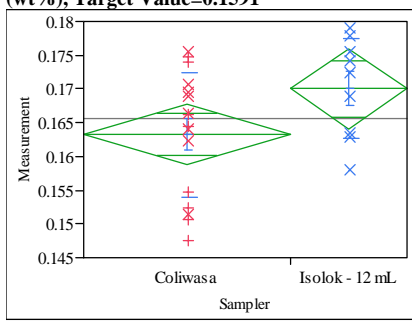

Excluded Rows 1

Oneway Anova

Rsquare $\quad 0.135655$

Adj Rsquare $\quad 0.098075$

Root Mean Square Error $\quad 0.008623$

$\begin{array}{lr}\text { Mean of Response } & 0.165681 \\ \text { Observations (or Sum Wgts) } & 25\end{array}$

t Test

Isolok - $12 \mathrm{~mL}$-Coliwasa

Assuming equal variances

Difference $\quad 0.00683$ t Ratio 1.899932

$\begin{array}{lrr}\text { Std Err Dif } & 0.00359 \text { DF } & 23 \\ \text { Upper CL Dif } & 0.01426 \text { Prob }>|t| & 0.0701\end{array}$

$\begin{array}{lrll}\text { Upper CL Dif } & 0.01426 & \text { Prob }>|t| & 0.070 \\ \text { Lower CL Dif } & -0.00061 & \text { Prob }>t & 0.0350\end{array}$

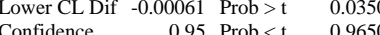

Analysis of Variance

Source DF Sum of Squares Mean Square F Ratio Prob $>$ F

$\begin{array}{lrrrrr}\text { Sampler } & 1 & 0.00026839 & 0.000268 & 3.6097 & 0.0701\end{array}$

Error $23 \quad 0.00171008$

C. Total $24 \quad 0.00197846$

Means for Oneway Anova

Level Number Mean Std Error Lower 95\% Upper 95\%

$\begin{array}{lrrrrr}\text { Coliwasa } & 16 & 0.163223 & 0.00216 & 0.15876 & 0.16768\end{array}$

$\begin{array}{lrllll}\text { Isolok - } 12 \mathrm{~mL} & 9 & 0.170049 & 0.00287 & 0.16410 & 0.17600\end{array}$

Std Error uses a pooled estimate of error variance

Means and Std Deviations

\begin{tabular}{|c|c|c|c|c|c|c|}
\hline $\begin{array}{r}\text { Upper } \\
95 \%\end{array}$ & Level & Number & Mean & Std Dev & $\begin{array}{r}\text { Std Err } \\
\text { Mean }\end{array}$ & $\begin{array}{r}\text { Lower } \\
95 \%\end{array}$ \\
\hline 2.4729 & Coliwasa & 16 & .163223 & 0.009208 & 0.00230 & 0.15832 \\
\hline
\end{tabular}

$\begin{array}{lrrrrrr}\text { Coliwasa } & 16 & 0.163223 & 0.009208 & 0.00230 & 0.15832 & 0.16813 \\ \text { Isolok - 12 } & 9 & 0.170049 & 0.007401 & 0.00247 & 0.16436 & 0.17574\end{array}$

$\mathrm{mL}$

Test

Isolok - $12 \mathrm{~mL}$-Coliwasa

Assuming unequal variances

Difference $\quad 0.00683$ t Ratio 2.022968

Std Err Dif $\quad 0.00337$ DF 19.93555

Upper CL Dif 0.01387 Prob $>|t| \quad 0.0567$

Lower CL Dif -0.00021 Prob $>t \quad 0.0283$

$\begin{array}{llll}\text { Confidence } & 0.95 \text { Prob }<\mathrm{t} \quad 0.9717\end{array}$

Tests that the Variances are Equal

Level Count Std Dev MeanAbsDif to Mean MeanAbsDif to Median

$\begin{array}{lrrrr}\text { Coliwasa } & 16 & 0.0092082 & 0.0076040 & 0.0073782 \\ \text { Isolok - 12 mL } & 9 & 0.0074012 & 0.0062015 & 0.0061568\end{array}$

Test F Ratio DFNum DFDen p-Value

$\begin{array}{lrrrr}\text { O'Brien[.5] } & 0.9609 & 1 & 23 & 0.3371\end{array}$

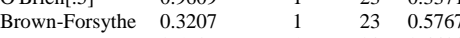

$\begin{array}{lllll}\text { Levene } & 0.5939 & 1 & 23 & 0.4488\end{array}$

$\begin{array}{lrrrr}\text { Bartlett } & 0.4511 & 1 & . & 0.5018\end{array}$

Welch Anova testing Means Equal, allowing Std Devs Not Equal

F Ratio DFNum DFDen Prob > F

$\begin{array}{lllr}4.0924 & 1 & 19.936 & 0.0567\end{array}$

t Test

2.0230 
Exhibit A3. Statistical Comparisons for Mid-Rheology (Phase 1) Testing (screened data)

Oneway Analysis of Measurement By Sampler Analytical Block=3, Analyte=Na2O (wt \%), Target Value=11.659

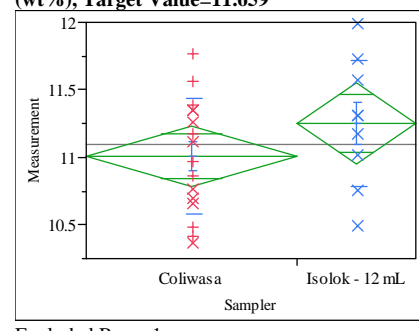

Excluded Rows 1

Oneway Anova

Rsquare $\quad 0.070802$

Adj Rsquare $\quad 0.030402$

Root Mean Square Error $\quad 0.440904$

\begin{tabular}{lr} 
Mean of Response & 11.09566 \\
\hline
\end{tabular}

t Test

Isolok - 12 mL-Coliwasa

Assuming equal variances

Difference $\quad 0.24320$ t Ratio 1.323834

$\begin{array}{lrlr}\text { Std Err Dif } & 0.18371 \text { DF } & 23 \\ \text { Upper CL } & 0.62323 \text { Prob }>|t| & 0.1986\end{array}$

$\begin{array}{rrr}\text { Upper CL Dif } 0.62323 & \text { Prob }>|t| & 0.1986 \\ \text { Lower CL Dif }-0.13683 & \text { Prob }>t & 0.0993\end{array}$

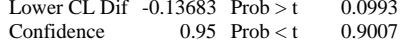

Analysis of Variance

Source DF Sum of Squares Mean Square F Ratio Prob $>$ F

$\begin{array}{llllll}\text { Sampler } & 1 & 0.3406870 & 0.340687 & 1.7525 & 0.1986\end{array}$

$\begin{array}{lll}\text { Error } & 23 & 4.4711207 \\ \text { C. Total } & 24 & 4.8118077\end{array}$

Means for Oneway Anova

Level Number Mean Std Error Lower 95\% Upper 95\%

$\begin{array}{lrrrrr}\text { Coliwasa } & 16 & 11.0081 & 0.11023 & 10.780 & 11.236 \\ \text { Isolok - 12 mL } & 9 & 11.2513 & 0.14697 & 10.947 & 11.555\end{array}$

Std Error uses a pooled estimate of error variance

Means and Std Deviations

Level Number Mean Std Dev

$\begin{array}{lrll}\text { Coliwasa } & 16 & 11.0081 & 0.425162 \\ \text { Isolok - 12 } & 9 & 11.2513 & 0.468999\end{array}$

Isolok - 12

$\mathrm{mL}$

$\begin{array}{rr}\text { Std Err } & \text { Lower } \\ \text { Mean } & \mathbf{9 5 \%} \\ 0.10629 & 10.782 \\ 0.15633 & 10.891\end{array}$

t Test

Isolok - $12 \mathrm{~mL}$-Coliwas

Assuming unequal variances

Difference $\quad 0.24320$ t Ratio 1.286481

Std Err Dif $\quad 0.18904$ DF 15.35563

Upper CL Dif 0.64533 Prob $>|t| \quad 0.2173$

Lower CL Dif -0.15893 Prob $>t \quad 0.1087$

$\begin{array}{lrl}\text { Confidence } & 0.95 \text { Prob }<\mathrm{t} \quad 0.8913\end{array}$

Tests that the Variances are Equal

Level Count Std Dev MeanAbsDif to Mean MeanAbsDif to Median $\begin{array}{lllll}\text { Coliwasa } & 16 & 0.4251617 & 0.3589050 & 0.3589050\end{array}$

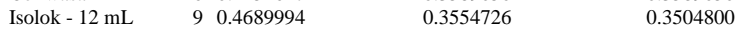

Test F Ratio DFNum DFDen p-Value

$\begin{array}{lrrrr}\text { O'Brien[.5] } & 0.1819 & 1 & 23 & 0.6737\end{array}$

Brown-Forsythe $\begin{array}{llll}0.0070 & 1 & 23 & 0.9339\end{array}$

$\begin{array}{llll}0.0012 & 1 & 23 & 0.9724\end{array}$

$\begin{array}{lllll}\text { Bartlett } & 0.0975 & 1 & . & 0.7548 \\ \text { F Test 2-sided } & 1.2168 & 8 & 15 & 0.7067\end{array}$

Welch Anova testing Means Equal, allowing Std Devs Not Equal

F Ratio DFNum DFDen Prob > F

$\begin{array}{rrrr}1.6550 & 1 & 15.356 & 0.2173\end{array}$

t Tes

1.2865
Oneway Analysis of Measurement By Sampler Analytical Block=3, Analyte=SiO2 (wt \%), Target Value $=\mathbf{5 0 . 9 8 5}$

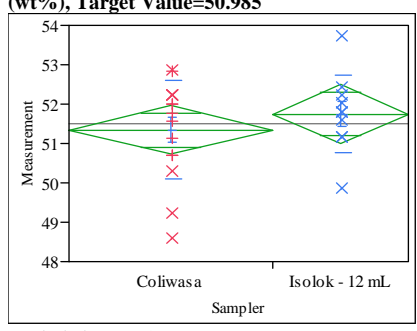

Excluded Rows 1

Oneway Anova

Rsquare $\quad 0.031007$

Adj Rsquare $\quad-0.01112$

Root Mean Square Error $\quad 1.160566$

$\begin{array}{lr}\text { Mean of Response } & 51.50579 \\ \text { Observations (or Sum Wgts) } & 25\end{array}$

t Test

Isolok - $12 \mathrm{~mL}$-Coliwasa

Assuming equal variances

Difference $\quad 0.4065$ t Ratio $\quad 0.857889$

$\begin{array}{lrlr}\text { Std Err Dif } & 0.4738 \text { DF } & 23 \\ \text { Upper CL } & 1.3866 & \text { Prob }>|t| & 0.3998\end{array}$

$\begin{array}{lrl}\text { Upper CL Dif } 1.3866 \text { Prob }>|t| & 0.3998 \\ \text { Lower CL Dif }-0.5737 \text { Prob }>t & 0.1999\end{array}$

$\begin{array}{lrr}\text { Lower CL Dif } & -0.5737 \text { Prob }>t & 0.1999 \\ \text { Confidence } & 0.95 \text { Prob }<\mathrm{t} & 0.8001\end{array}$

Analysis of Variance

Source DF Sum of Squares Mean Square F Ratio Prob $>$ F

$\begin{array}{lrrrrr}\text { Sompler } & 1 & 0.991293 & 0.99129 & 0.7360 & 0.3998\end{array}$

$\begin{array}{llll}\text { Error } & 23 & 30.979036 & 1.34691\end{array}$

C. Total $24 \quad 31.970328$

Means for Oneway Anova

Level Number Mean Std Error Lower 95\% Upper 95\%

$\begin{array}{lrrrrr}\text { Coliwasa } & 15 & 51.3432 & 0.29966 & 50.723 & 51.963\end{array}$

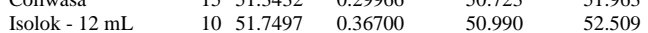

Std Error uses a pooled estimate of error variance

Means and Std Deviations

Upper Level Number Mean Std Dev

$1551.3432 \quad 1.25525$

$\begin{array}{llll}\text { Coliwasa } & 15 & 51.3432 & 1.25525 \\ \text { Isolok - } 12 & 10 & 51.7497 & 0.99553\end{array}$

$\mathrm{mL}$

t Test

Isolok - $12 \mathrm{~mL}$-Coliwasa

Assuming unequal variances

$\begin{array}{lrlr}\text { Difference } & 0.4065 \text { t Ratio } & 0.899596 \\ \text { Std Err Dif } & 0.4518 \text { DF } & 22.17459 \\ \text { Upper CL Dif } & 1.3431 \text { Prob }>|t| & 0.3780 \\ \text { Lower CL Dif } & -0.5301 & \text { Prob }>\text { t } & 0.1890 \\ \text { Confidence } & 0.95 & \text { Prob }<\text { t } & 0.8110\end{array}$

Tests that the Variances are Equal

Level Count Std Dev MeanAbsDif to Mean MeanAbsDif to Median $\begin{array}{lrrrr}\text { Coliwasa } & 15 & 1.255253 & 0.9983400 & 0.9555540 \\ \text { Isolok - } 12 \mathrm{~mL} & 10 & 0.995535 & 0.6674616 & 0.6631830\end{array}$

$\begin{array}{lrrrr}\text { Test } & \text { F Ratio } & \text { DFNum } & \text { DFDen } & \text { p-Value } \\ \text { O'Brien[.5] } & 0.4501 & 1 & 23 & 0.5090 \\ \text { Brown-Forsythe } & 0.7523 & 1 & 23 & 0.3947 \\ \text { Levene } & 1.3054 & 1 & 23 & 0.2650 \\ \text { Bartlett } & 0.5399 & 1 & . & 0.4625 \\ \text { F Test 2-sided } & 1.5898 & 14 & 9 & 0.4895\end{array}$

Welch Anova testing Means Equal, allowing Std Devs Not Equal

$\begin{array}{rrrr}\text { F Ratio } & \text { DFNum } & \text { DFDen } & \text { Prob }>\text { F } \\ 0.8093 & 1 & 22.175 & 0.3780 \\ & & & \\ \text { t Test } & & & \\ 0.8996 & & & \end{array}$


Exhibit A3. Statistical Comparisons for Mid-Rheology (Phase 1) Testing (screened data)

Oneway Analysis of Measurement By Sampler Analytical Block=4, Analyte=Al/B,

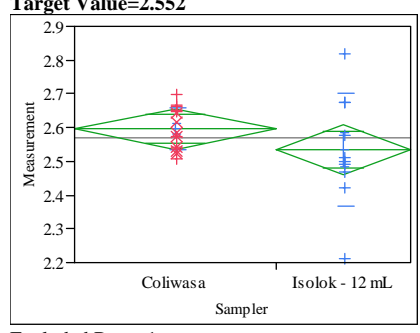

Excluded Rows 1

Oneway Anova

Summary of Fit

Rsquare

Adj Rsquare

0.11437

Observations (or Sum Wgts) 25

t Test

Isolok - 12 mL-Coliwasa

Assuming equal variances

Difference $\quad-0.06129$ t Ratio $\quad-1.31269$

$\begin{array}{lrr}\text { Std Err Dif } & 0.04669 \text { DF } & 23 \\ \text { Uper CL } & 0.03530 \text { Prob }>|t| & 0.2022\end{array}$

Upper CL Dif 0.03530 Prob $>|t| \quad 0.2022$

Lower CL Dif -0.15788 Prob $>t \quad 0.8989$

Confidence Target Value $=\mathbf{2 . 5 5 2}$

Root Mean Square Error $\quad 0.02925$

Oneway Analysis of Measurement By Sampler Analytical Block=4, Analyte=Al2O3 (wt \%), Target Value $=\mathbf{1 0 . 8 6 9}$

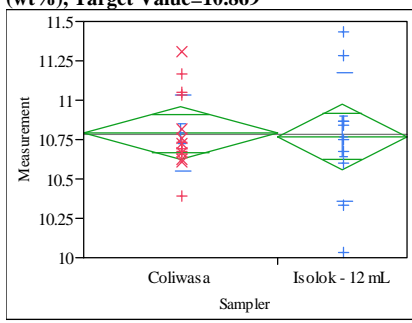

Excluded Rows 1

Oneway Anova

Summary of Fit

Rsquare

Root Mean Square Error $\quad 0.317199$

$\begin{array}{lr}\text { Mean of Response } & 10.78149 \\ \text { Observations (or Sum Wgts) } & 25\end{array}$

t Test

Isolok - $12 \mathrm{~mL}$-Coliwasa

Assuming equal variances

Difference $\quad-0.02204$ t Ratio $\quad-0.17023$

$\begin{array}{lrr}\text { Std Err Dif } & 0.12950 \text { DF } & 23 \\ \text { Upp } & & 0.8663\end{array}$

Upper CL Dif 0.24584 Prob $>|t| \quad 0.8663$

Lower CL Dif -0.28993 Prob $>\mathrm{t} \quad 0.5668$

$\begin{array}{lll}\text { Confidence } & 0.95 \text { Prob }<\mathrm{t} \quad 0.4332\end{array}$

Analysis of Variance

Source DF Sum of Squares Mean Square F Ratio Prob > F

$\begin{array}{lrrrrr}\text { Sampler } & 1 & 0.0029157 & 0.002916 & 0.0290 & 0.8663\end{array}$

$\begin{array}{lll}\text { Error } & 23 & 2.3141508 \\ \text { C. Total } & 24 & 2.3170665\end{array}$

Means for Oneway Anova

Level Number Mean Std Error Lower 95\% Upper 95\%

$\begin{array}{lrrrrr}\text { Coliwasa } & 15 & 10.7903 & 0.08190 & 10.621 & 10.960\end{array}$

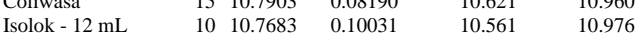

Std Error uses a pooled estimate of error variance

Means and Std Deviations

Std Error uses a pooled estimate of error variance

Means and Std Deviations

Level Number Mean Std Dev

$\begin{array}{lrrrrr} & & & & \text { Mean } & \mathbf{9 5 \%} \\ \text { Coliwasa } & 15 & 2.59566 & 0.062120 & 0.01604 & 2.5613 \\ \text { Isolok - 12 } & 10 & 2.53437 & 0.165608 & 0.05237 & 2.4159\end{array}$

0.05237

$\mathrm{mL}$

$\begin{array}{lll}15 & 10.7903 & 0.242444\end{array}$

Test

Isolok - $12 \mathrm{~mL}$-Coliwasa

Assuming unequal variances

Difference $\quad-0.02204$ t Ratio $\quad-0.15401$

Std Err Dif $\quad 0.14314$ DF $\quad 13.28275$

Upper CL Dif 0.28651 Prob $>|t| \quad 0.8799$

$\begin{array}{lll}\text { Lower CL Dif }-0.33060 \text { Prob }>t & 0.5600\end{array}$

$\begin{array}{lrl}\text { Confidence } & 0.95 \text { Prob }<\mathrm{t} \quad 0.4400\end{array}$

Tests that the Variances are Equal

Level Count Std Dev MeanAbsDif to Mean MeanAbsDif to Median $\begin{array}{lllll}\text { Coliwasa } & 15 & 0.2424443 & 0.1881102 & 0.1713147\end{array}$

$\begin{array}{lllll}\text { Isolok }-12 \mathrm{~mL} & 10 & 0.4070546 & 0.2890935 & 0.2890935\end{array}$

Test F Ratio DFNum DFDen p-Value

$\begin{array}{lrrrr}\text { O'Brien[.5] } & 2.5859 & 1 & 23 & 0.1215\end{array}$

$\begin{array}{lllll}\text { Brown-Forsythe } & 1.6848 & 1 & 23 & 0.2072\end{array}$

$\begin{array}{lllll}\text { Levene } & 1.4851 & 1 & 23 & 0.2353\end{array}$

$\begin{array}{lllll}\text { Bartlett } & 2.9012 & 1 & . & 0.0885\end{array}$

$\begin{array}{lllll}\text { F Test 2-sided } & 2.8189 & 9 & 14 & 0.0804\end{array}$

Welch Anova testing Means Equal, allowing Std Devs Not Equal

F Ratio DFNum DFDen Prob $>$ F

$\begin{array}{rrrr}0.0237 & 1 & 13.283 & 0.8799\end{array}$

t Test

0.1540 
Exhibit A3. Statistical Comparisons for Mid-Rheology (Phase 1) Testing (screened data)

Oneway Analysis of Measurement By Sampler Analytical Block=4, Analyte $=\mathrm{CuO}$ (wt \%), Target Value $=\mathbf{0 . 0 5 0}$

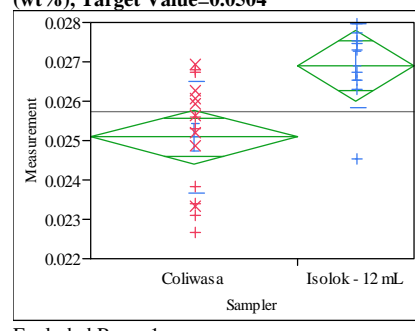

Excluded Rows 1

Oneway Anova

Summary of Fit

Rsquare

Adj Rsquare

0.328109

0.298896

Root Mean Square Error $\quad 0.001298$

$\begin{array}{lr}\text { Mean of Response } & 0.02574 \\ \text { Observations (or Sum Wgts) } & 25\end{array}$

25

t Test

Isolok - 12 mL-Coliwasa

Assuming equal variances

Difference $\quad 0.001813$ t Ratio 3.351376

$\begin{array}{lrlr}\text { Std Err Dif } & 0.000541 & \text { DF } & 23 \\ \text { Upper CL } & 0.002932 & \text { Prob }>|t| & 0.0028\end{array}$

$\begin{array}{lrll}\text { Upper CL Dif } & 0.002932 & \text { Prob }>|t| & 0.0028 \\ \text { Lower CL Dif } & 0.000694 & \text { Prob }>t & 0.0014\end{array}$

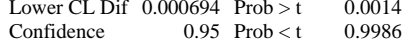

Analysis of Variance

Source DF Sum of Squares Mean Square F Ratio Prob $>$ F

$\begin{array}{llllll}\text { Sampler } & 1 & 0.00001893 & 0.000019 & 11.2317 & 0.0028\end{array}$

Error $23 \quad 0.00003877 \quad 1.686 \mathrm{e}-6$

C. Total $24 \quad 0.00005770$

Means for Oneway Anova

$\begin{array}{lrrrrr}\text { Level } & \text { Number } & \text { Mean } & \text { Std Error } & \text { Lower 95\% } & \text { Upper 95\% } \\ \text { Coliwasa } & 16 & 0.025087 & 0.00032 & 0.02442 & 0.02576\end{array}$ $\begin{array}{lrrrrr}\text { Isolok - } 12 \mathrm{~mL} & 9 & 0.026900 & 0.00043 & 0.02600 & 0.02780\end{array}$

Std Error uses a pooled estimate of error variance

Means and Std Deviations

Level Number Mean Std Dev

$\begin{array}{lllll}\text { Coliwasa } & 16 & 0.025087 & 0.001412\end{array}$

Isolok - 12

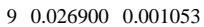

$\mathrm{mL}$

t Test

Isolok - $12 \mathrm{~mL}$-Coliwas

Assuming unequal variances

Difference $\quad 0.001813$ t Ratio 3.642348

Std Err Dif $\quad 0.000498$ DF 20.94215

Upper CL Dif 0.002848 Prob $>|t| \quad 0.0015$

Lower CL Dif 0.000778 Prob $>t \quad 0.0008$

Confidence $\quad 0.95$ Prob $<\mathrm{t} \quad 0.9992$

Tests that the Variances are Equal

Level Count Std Dev MeanAbsDif to Mean MeanAbsDif to Median

$\begin{array}{lrrrr}\text { Coliwasa } & 16 & 0.0014119 & 0.0011726 & 0.0011384\end{array}$

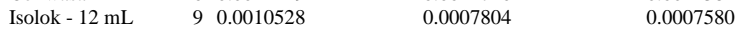

Test $\quad$ F Ratio DFNum DFDen p-Value

$\begin{array}{lrrrr}\text { O'Brien[.5] } & 1.1347 & 1 & 23 & 0.2978\end{array}$

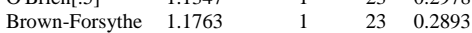

$\begin{array}{lllll}\text { Levene } & 1.8050 & 1 & 23 & 0.1922\end{array}$

$\begin{array}{lllll}\text { Bartlett } & 0.7979 & 1 & . & 0.3717\end{array}$

$\begin{array}{lllll}\text { F Test 2-sided } & 1.7983 & 15 & 8 & 0.4057\end{array}$

Welch Anova testing Means Equal, allowing Std Devs Not Equal

F Ratio DFNum DFDen Prob $>$ F

$\begin{array}{rrrr}13.2667 & 1 & 20.942 & 0.0015\end{array}$

t Tes

3.6423

0.02433
Oneway Analysis of Measurement By Sampler Analytical Block=4, Analyte=Fe/Li, Target Value $=\mathbf{2 . 4 5 2}$

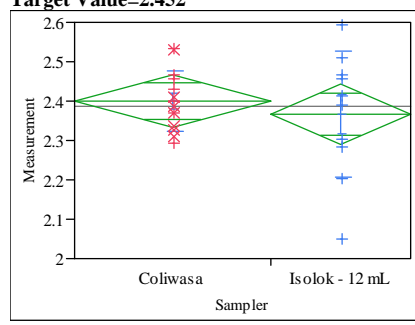

Excluded Rows 2

Oneway Anova

Rsquare $\quad 0.020429$

Adj Rsquare $\quad-0.0241$

Root Mean Square Error $\quad 0.117736$

$\begin{array}{lr}\text { Mean of Response } & 2.386205 \\ \text { Observations (or Sum Wgts) } & 24\end{array}$

t Test

Isolok - $12 \mathrm{~mL}$-Coliwasa

Assuming equal variances

Difference $\quad-0.03302$ t Ratio -0.67735

$\begin{array}{lrr}\text { Std Err Dif } & 0.04875 \text { DF } & 22 \\ \text { Upper } & \text { DL } & -0.5052\end{array}$

$\begin{array}{lrlr}\text { Upper CL Dif } & 0.06808 \text { Prob }>|t| & 0.5052 \\ \text { Lower CL Dif } & -0.13411 & \text { Prob }>t & 0.7474\end{array}$

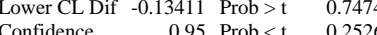

Analysis of Variance

Source DF Sum of Squares Mean Square F Ratio Prob $>$ F

$\begin{array}{lrrrrr}\text { Sampler } & 1 & 0.00635990 & 0.006360 & 0.4588 & 0.5052\end{array}$

Error $22 \quad 0.30495848$

$\begin{array}{lll}\text { C. Total } & 23 & 0.31131837\end{array}$

Means for Oneway Anova

Level Number Mean Std Error Lower 95\% Upper 95\%

$\begin{array}{lllrrr}\text { Coliwasa } & 14 & 2.39996 & 0.03147 & 2.3347 & 2.4652\end{array}$

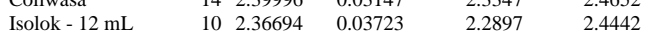

Std Error uses a pooled estimate of error variance

Means and Std Deviations

Lower Upper Level Number Mean Std Dev

$\begin{array}{lrr}\text { Mean } & \mathbf{9 5 \%} & \mathbf{9 5 \%} \\ & & \end{array}$

0.02584 Coliwasa

Isolok - 12

$\begin{array}{llll}14 & 2.39996 & 0.075964\end{array}$

$\begin{array}{lll}10 & 2.36694 & 0.159841\end{array}$

$\begin{array}{rrr}\text { Std Err } & \text { Lower } & \text { Upper } \\ \text { Mean } & \mathbf{9 5 \%} & \mathbf{9 5 \%} \\ 0.02030 & 2.3561 & 2.4438 \\ 0.05055 & 2.2526 & 2.4813\end{array}$

$\mathrm{mL}$

Test

Isolok - $12 \mathrm{~mL}$-Coliwasa

Assuming unequal variances

Difference $\quad-0.03302$ t Ratio $\quad-0.60618$

Std Err Dif $\quad 0.05447$ DF $\quad 11.92331$

Upper CL Dif 0.08575 Prob $>|t| \quad 0.5558$

Lower CL Dif -0.15179 Prob $>t \quad 0.7221$

$\begin{array}{lrr}\text { Confidence } & 0.95 \text { Prob }<\mathrm{t} \quad 0.2779\end{array}$

Tests that the Variances are Equal

Level Count Std Dev MeanAbsDif to Mean MeanAbsDif to Median

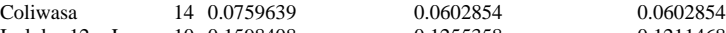

$\begin{array}{lllll}\text { Isolok }-12 \mathrm{~mL} & 10 & 0.1598408 & 0.1255358 & 0.1211468\end{array}$

Test F Ratio DFNum DFDen p-Value

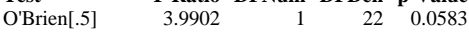

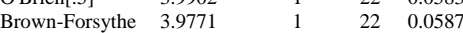

$\begin{array}{lllll}\text { Levene } & 5.6630 & 1 & 22 & 0.0264\end{array}$

$\begin{array}{lllll}\text { Bartlett } & 5.6224 & 1 & . & 0.0177\end{array}$

$\begin{array}{lllll}\text { F Test 2-sided } & 4.4275 & 9 & 13 & 0.0159\end{array}$

Welch Anova testing Means Equal, allowing Std Devs Not Equal

F Ratio DFNum DFDen Prob $>$ F

$\begin{array}{rrrr}0.3675 & 1 & 11.923 & 0.5558\end{array}$

t Test

0.6062 
Exhibit A3. Statistical Comparisons for Mid-Rheology (Phase 1) Testing (screened data)

Oneway Analysis of Measurement By Sampler Analytical Block=4, Analyte=K2O (wt \%), Target Value $=\mathbf{0 . 1 5 9}$

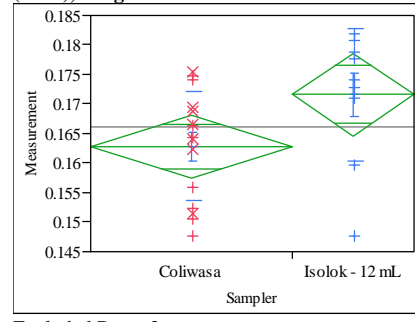

Excluded Rows 2

Oneway Anova

Summary of Fit

Rsquare

Adj Rsquare

0.163836

0.125829

Root Mean Square Error $\quad 0.010015$

$\begin{array}{lr}\text { Mean of Response } & 0.166109 \\ \text { Observations (or Sum Wgts) } & 24\end{array}$

Test

Isolok - $12 \mathrm{~mL}$-Coliwasa

Assuming equal variances

Difference $\quad 0.008767$ t Ratio 2.076205

$\begin{array}{lrlr}\text { Std Err Dif } & 0.004223 \text { DF } & 22 \\ \text { Upper CL } & \end{array}$

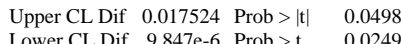

$\begin{array}{lrrr}\text { Lower CL Dif } & 9.847 \mathrm{e}-6 & \text { Prob }>t & 0.0249 \\ \text { Confidence } & 0.95 & \text { Prob }<\mathrm{t} & 0.9751\end{array}$

Analysis of Variance

Source DF Sum of Squares Mean Square F Ratio Prob $>$ F

$\begin{array}{lrrrrr}\text { Sampler } & 1 & 0.00043232 & 0.000432 & 4.3106 & 0.0498\end{array}$

$\begin{array}{lll}\text { Error } & 22 & 0.00220642 \\ \text { C. Total } & 23 & 0.00263874\end{array}$

Means for Oneway Anova

Level Number Mean Std Error Lower 95\% Upper 95\%

$\begin{array}{lrrrrr}\text { Coliwasa } & 15 & 0.162822 & 0.00259 & 0.15746 & 0.16818\end{array}$

$\begin{array}{lrrrrr}\text { Coliwasa } & 15 & 0.162822 & 0.00259 & 0.15746 & 0.16818 \\ \text { Isolok - 12 mL } & 9 & 0.171589 & 0.00334 & 0.16467 & 0.17851\end{array}$

Std Error uses a pooled estimate of error variance

Means and Std Deviations

Level Number Mean Std Dev

$\begin{array}{lrll}\text { Coliwasa } & 15 & 0.162822 & 0.009252 \\ \text { Isolok - } 12 & 9 & 0.171589 & 0.011225\end{array}$

$\begin{array}{llll}9 & 0.171589 & 0.011225\end{array}$

$\begin{array}{rr}\begin{array}{rr}\text { Std Err } \\ \text { Mean }\end{array} & \begin{array}{r}\text { Lower } \\ \mathbf{9 5 \%}\end{array} \\ 0.00239 & 0.15770 \\ 0.00374 & 0.16296\end{array}$

$\mathrm{mL}$

t Test

Isolok - $12 \mathrm{~mL}$-Coliwas

Assuming unequal variances

Difference $\quad 0.00877$ t Ratio 1.974811

Std Err Dif $\quad 0.00444$ DF 14.47584

Upper CL Dif 0.01826 Prob $>|t| \quad 0.0677$

Lower CL Dif -0.00073 Prob $>t \quad 0.0338$

$\begin{array}{llll}\text { Confidence } & 0.95 \text { Prob }<\mathrm{t} & 0.9662\end{array}$

Tests that the Variances are Equal

Level Count Std Dev MeanAbsDif to Mean MeanAbsDif to Median

$\begin{array}{lllll}\text { Coliwasa } & 15 & 0.0092518 & 0.0076291 & 0.0074284\end{array}$

$\begin{array}{lllll}\text { Isolok - } 12 \mathrm{~mL} & 9 & 0.0112254 & 0.0081199 & 0.0076961\end{array}$

Test F Ratio DFNum DFDen p-Value

$\begin{array}{lrrrr}\text { O'Brien[.5] } & 0.4239 & 1 & 22 & 0.5217\end{array}$

Brown-Forsythe $\quad 0.0094 \quad 11220.9236$

$\begin{array}{llll}0.0403 & 1 & 22 & 0.8428\end{array}$

$\begin{array}{lllll}\text { Bartlett } & 0.3733 & 1 & .5 & 0.5412 \\ \text { F Test 2-sided } & 1.4722 & 8 & 14 & 0.5033\end{array}$

Welch Anova testing Means Equal, allowing Std Devs Not Equal

F Ratio DFNum DFDen Prob $>$ F

$\begin{array}{llll}3.8999 & 1 & 14.476 & 0.0677\end{array}$

t Tes

1.9748
Oneway Analysis of Measurement By Sampler Analytical Block=4, Analyte=Li2O (wt \%), Target Value $=4.674$

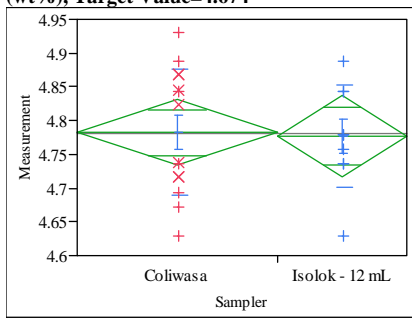

Excluded Rows 3

Oneway Anova

Rsquare $\quad 0.001024$

$\begin{array}{ll}\text { Adj Rsquare } & -0.04655 \\ \text { Root Mean Square Error } & 0.087212\end{array}$

$\begin{array}{ll}\text { Root Mean Square Error } & 0.087212 \\ \text { Mean of Response } & 4.780374\end{array}$

Observations (or Sum Wgts) 23

t Test

Isolok - $12 \mathrm{~mL}$-Coliwasa

Assuming equal variances

Difference $\quad-0.00547$ t Ratio $\quad-0.14674$

$\begin{array}{lrr}\text { Std Err Dif } & 0.03726 \text { DF } & 21 \\ \text { Upe } & & 0.8847\end{array}$

Upper CL Dif 0.07202 Prob $>|t| \quad 0.8847$

Lower CL Dif -0.08296 Prob $>$ t 0.5576

Confidence $\quad 0.95$ Prob $<\mathrm{t} \quad 0.4424$

Analysis of Variance

Source DF Sum of Squares Mean Square F Ratio Prob $>$ F

$\begin{array}{lrrrrr}\text { Sampler } & 1 & 0.00016378 & 0.000164 & 0.0215 & 0.8847\end{array}$

$\begin{array}{lll}\text { Error } & 21 & 0.15972283 \\ \text { C. Total } & 22 & 0.15988660\end{array}$

Means for Oneway Anova

Level Number Mean Std Error Lower 95\% Upper 95\%

$\begin{array}{lrrrrr}\text { Coliwasa } & 14 & 4.78251 & 0.02331 & 4.7340 & 4.8310\end{array}$

$\begin{array}{lrrrrr}\text { Isolok - } 12 \mathrm{~mL} & 9 & 4.77705 & 0.02907 & 4.7166 & 4.8375\end{array}$

Std Error uses a pooled estimate of error variance

Means and Std Deviations

Upper Level Number Mean Std Dev

$\begin{array}{rllll}\mathbf{9 5 \%} & & & \\ 0.16795 & \text { Coliwasa } & 14 & 4.78251 & 0.093216\end{array}$

Isolok - 12

$\begin{array}{llll}9 & 4.77705 & 0.076454\end{array}$

$\begin{array}{rrr}\text { Std Err } & \text { Lower } & \text { Upper } \\ \text { Mean } & \mathbf{9 5 \%} & \mathbf{9 5 \%} \\ 0.02491 & 4.7287 & 4.8363\end{array}$

$\begin{array}{llr}0.02491 & 4.7287 & 4.8363 \\ 0.02548 & 4.7183 & 4.8358\end{array}$

$\mathrm{mL}$

Test

Isolok - $12 \mathrm{~mL}$-Coliwasa

Assuming unequal variances

Difference $\quad-0.00547$ t Ratio $\quad-0.15342$

Std Err Dif $\quad 0.03564$ DF $\quad 19.58792$

Upper CL Dif 0.06897 Prob $>|t| \quad 0.8796$

Lower CL Dif -0.07991 Prob $>t \quad 0.5602$

$\begin{array}{lrl}\text { Confidence } & 0.95 \text { Prob }<\mathrm{t} & 0.4398\end{array}$

Tests that the Variances are Equal

Level Count Std Dev MeanAbsDif to Mean MeanAbsDif to Median

$\begin{array}{lllll}\text { Coliwasa } & 14 & 0.0932165 & 0.0830404 & 0.0830404\end{array}$

$\begin{array}{lrlll}\text { Isolok - } 12 \mathrm{~mL} & 9 & 0.0764541 & 0.0552843 & 0.0550186\end{array}$

Test F Ratio DFNum DFDen p-Value

$\begin{array}{lrrrr}\text { O'Brien[.5] } & 0.6524 & 1 & 21 & 0.4283\end{array}$

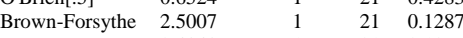

$\begin{array}{lllll}\text { Levene } & 2.4843 & 1 & 21 & 0.1299\end{array}$

$\begin{array}{lrrrr}\text { Bartlett } & 0.3567 & 1 & . & 0.5503\end{array}$

Welch Anova testing Means Equal, allowing Std Devs Not Equal

F Ratio DFNum DFDen Prob > F

$\begin{array}{llll}0.0235 & 1 & 19.588 & 0.8796\end{array}$

t Test

0.1534 
Exhibit A3. Statistical Comparisons for Mid-Rheology (Phase 1) Testing (screened data)

Oneway Analysis of Measurement By Sampler Analytical Block=4, Analyte=NiO (wt \%), Target Value $=0.41$

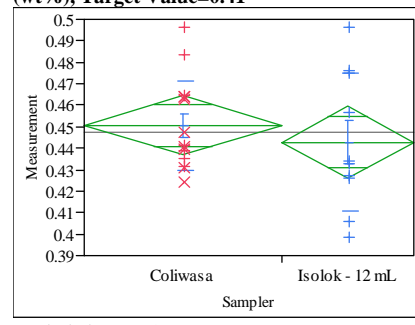

Excluded Rows 1

Oneway Anova

Rsquare $\quad 0.023444$

Adj Rsquare $\quad-0.01901$

Root Mean Square Error $\quad 0.025728$

$\begin{array}{lr}\text { Mean of Response } & 0.447513 \\ \text { Observations (or Sum Wgts) } & 25\end{array}$

Observations (or Sum Wgs)

t Test

Isolok - $12 \mathrm{~mL}$-Coliwasa

Assuming equal variances

Difference $\quad-0.00780$ t Ratio -0.74307

$\begin{array}{lrrr}\text { Std Err Dif } & 0.01050 \text { DF } & 23 \\ \text { Uper } & \text { D } & -0.74307\end{array}$

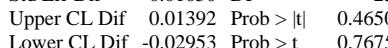

$\begin{array}{lrr}\text { Lower CL Dif } & -0.02953 \text { Prob }>t & 0.7675 \\ \text { Confidence } & 0.95 \text { Prob }<\mathrm{t} & 0.2325\end{array}$

Analysis of Variance

Source DF Sum of Squares Mean Square F Ratio Prob $>$ F

$\begin{array}{lrrrrr}\text { Sampler } & 1 & 0.00036548 & 0.000365 & 0.5522 & 0.4650\end{array}$

$\begin{array}{llll}\text { Error } & 23 & 0.01522382 & 0.000662\end{array}$

$\begin{array}{lll}\text { C. Total } \quad 24 & 0.01558929\end{array}$

Means for Oneway Anova

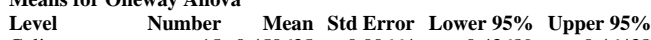

$\begin{array}{lrrrrr}\text { Level } & \text { Number } & \text { Mean } & \text { Std Error } & \text { Lower 95\% } & \text { Upper 95\% } \\ \text { Coliwasa } & 15 & 0.450635 & 0.00664 & 0.43689 & 0.46438\end{array}$ $\begin{array}{llllll}\text { Coliwasa } & 15 & 0.450635 & 0.00664 & 0.43689 & 0.46438 \\ \text { Isolok - 12 mL } & 10 & 0.442830 & 0.00814 & 0.42600 & 0.45966\end{array}$

Std Error uses a pooled estimate of error variance

Means and Std Deviations

Level Number Mean Std Dev

$\begin{array}{llll}\text { Coliwasa } & 15 & 0.450635 & 0.020753 \\ \text { Isolok - } 12 & 10 & 0.442830 & 0.031962\end{array}$

$10 \begin{array}{lll}10.442830 & 0.031962\end{array}$ 0.45966

$\mathrm{mL}$

$\begin{array}{rr}\text { Std Err } & \text { Lower } \\ \text { Mean } & \mathbf{9 5 \%} \\ 0.00536 & 0.43914 \\ 0.01011 & 0.41997\end{array}$

t Test

Isolok - $12 \mathrm{~mL}$-Coliwasa

Assuming unequal variances

Difference $\quad-0.00780$ t Ratio $\quad-0.68224$

Std Err Dif $\quad 0.01144$ DF 14.05631

Upper CL Dif 0.01672 Prob $>|t| \quad 0.5062$

$\begin{array}{llll}\text { Lower CL Dif } & -0.03233 & \text { Prob }>t & 0.7469\end{array}$

$\begin{array}{lrl}\text { Confidence } & 0.95 \text { Prob }<\mathrm{t} & 0.2531\end{array}$

Tests that the Variances are Equal

Level Count Std Dev MeanAbsDif to Mean MeanAbsDif to Median $\begin{array}{lllll}\text { Coliwasa } & 15 & 0.0207531 & 0.0171703 & 0.0161183\end{array}$

$\begin{array}{lllll}\text { Isolok - } 12 \mathrm{~mL} & 10 & 0.0319620 & 0.0264680 & 0.0246865\end{array}$

Test F Ratio DFNum DFDen p-Value

$\begin{array}{lrrrr}\text { O'Brien[.5] } & 2.9852 & 1 & 23 & 0.0974\end{array}$

$\begin{array}{lllll}\text { Brown-Forsythe } & 1.3726 & 1 & 23 & 0.2534\end{array}$

$\begin{array}{lllll}\text { Levene } & 3.1427 & 1 & 23 & 0.0895\end{array}$

$\begin{array}{lllll}\text { Bartlett } & 2.0170 & 1 & & 0.1556 \\ \text { F Test 2-sided } & 2.3719 & 9 & 14 & 0.1429\end{array}$

Welch Anova testing Means Equal, allowing Std Devs Not Equal

F Ratio DFNum DFDen Prob > F

$\begin{array}{rrrr}0.4654 & 1 & 14.056 & 0.5062\end{array}$

t Test

0.6822
Oneway Analysis of Measurement By Sampler Analytical Block=4, Analyte=Sum of Oxides (wt \%), Target Value $=99.553$

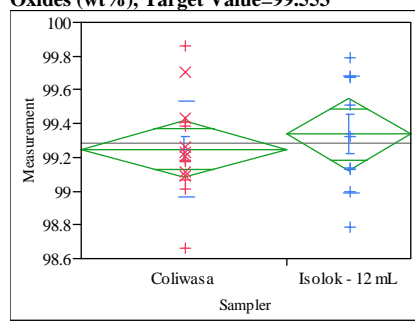

Excluded Rows 2

Oneway Anova

Summary of Fit

Rsquare $\quad 0.019949$

Adj Rsquare $\quad-0.0246$

Root Mean Square Error $\quad 0.31045$

$\begin{array}{lr}\text { Mean of Response } & 99.28192 \\ \text { Observations (or Sum Wgts) } & 24\end{array}$

t Test

Isolok - $12 \mathrm{~mL}$-Coliwasa

Assuming equal variances

Difference $\quad 0.08760$ t Ratio $\quad 0.669193$

$\begin{array}{lrlr}\text { Std Err Dif } & 0.13090 & \text { DF } & 22 \\ \text { Upper CL } & 0.35906 & \text { Prob }>|t| & 0.5103\end{array}$

$\begin{array}{rrrr}\text { Upper CL Dif } & 0.35906 & \text { Prob }>|t| & 0.5103 \\ \text { Lower CL Dif } & -0.18387 & \text { Prob }>t & 0.2552\end{array}$

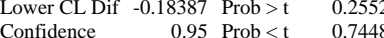

Analysis of Variance

Source DF Sum of Squares Mean Square F Ratio Prob $>$ F

$\begin{array}{lrrrrr}\text { Sampler } & 1 & 0.0431605 & 0.043161 & 0.4478 & 0.5103\end{array}$

$\begin{array}{lll}\text { Error } & 22 & 2.1203454 \\ \text { C. Total } & 23 & 2.1635060\end{array}$

Means for Oneway Anova

Level Number Mean Std Error Lower 95\% Upper 95\%

$\begin{array}{lrrrrr}\text { Coliwasa } & 15 & 99.2491 & 0.08016 & 99.083 & 99.415\end{array}$

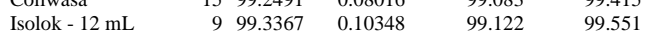

Std Error uses a pooled estimate of error variance

Means and Std Deviations

Upper Level Number Mean Std Dev

$95 \%$

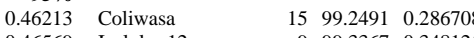

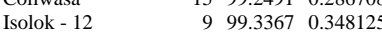

$\mathrm{mL}$

Test

Isolok - 12 mL-Coliwasa

Assuming unequal variances

Difference $\quad 0.08760$ t Ratio $\quad 0.636394$

Std Err Dif $\quad 0.13764$ DF $\quad 14.46724$

Upper CL Dif 0.38192 Prob $>|t| \quad 0.5345$

Lower CL Dif -0.20673 Prob $>\mathrm{t} \quad 0.2672$

$\begin{array}{lrl}\text { Confidence } & 0.95 \text { Prob }<\mathrm{t} \quad 0.7328\end{array}$

Tests that the Variances are Equal

Level Count Std Dev MeanAbsDif to Mean MeanAbsDif to Median

$\begin{array}{lrrrr}\text { Coliwasa } & 15 & 0.2867075 & 0.2049800 & 0.1988269\end{array}$

$\begin{array}{lrrrr}\text { Isolok - } 12 \mathrm{~mL} & 9 & 0.3481251 & 0.2910221 & 0.3101023\end{array}$

Test F Ratio DFNum DFDen p-Value

$\begin{array}{lrrrr}\text { O'Brien[.5] } & 0.4916 & 1 & 22 & 0.4906\end{array}$

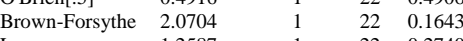

$\begin{array}{lllll}\text { Levene } & 1.2587 & 1 & 22 & 0.2740\end{array}$

$\begin{array}{lllrl}\text { Bartlett } & 0.3762 & 1 & . & 0.5397 \\ \text { F Test 2-sided } & 1.4743 & 8 & 14 & 0.5018\end{array}$

Welch Anova testing Means Equal, allowing Std Devs Not Equal

F Ratio DFNum DFDen Prob $>$ F

$\begin{array}{rrrr}0.4050 & 1 & 14.467 & 0.5345\end{array}$

t Test

0.6364 
Exhibit A3. Statistical Comparisons for Mid-Rheology (Phase 1) Testing (screened data)

Oneway Analysis of Measurement By Sampler Analytical Block=5, Analyte=Al/B, Target Value $=2.552$

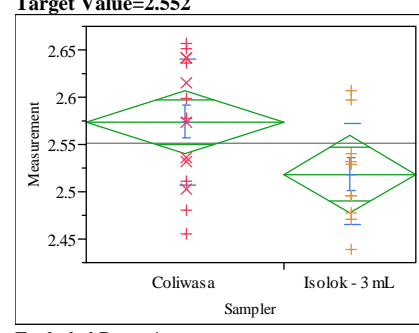

Excluded Rows 1

Oneway Anova

Rsquare $\quad 0.170035$

Adj Rsquare $\quad 0.13395$

Root Mean Square Error $\quad 0.062256$

$\begin{array}{lr}\text { Mean of Response } & 2.551593 \\ \text { Observations (or Sum Wgts) } & 25\end{array}$

t Test

Isolok - $3 \mathrm{~mL}$-Coliwasa

Assuming equal variances

Difference $\quad-0.05517$ t Ratio $\quad-2.17072$

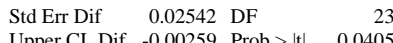

$\begin{array}{lrll}\text { Upper CL Dif } & -0.00259 & \text { Prob }>|t| & 0.0405 \\ \text { Lower CL Dif }-0.10775 & \text { Prob }>t & 0.9797\end{array}$

$\begin{array}{lrll}\text { Lower CL Dif } & -0.10775 & \text { Prob }>t & 0.975 \\ \text { Confidence } & 0.95 & \text { Prob }<\mathrm{t} & 0.0203\end{array}$

Analysis of Variance

Source DF Sum of Squares Mean Square F Ratio Prob $>$ F

$\begin{array}{lrrrrr}\text { Sampler } & 1 & 0.01826288 & 0.018263 & 4.7120 & 0.0405\end{array}$

$\begin{array}{llll}\text { Error } & 23 & 0.08914360 & 0.003876\end{array}$

$\begin{array}{lll}\text { C. Total } \quad 24 & 0.10740648\end{array}$

Means for Oneway Anova

Level Number Mean Std Error Lower 95\% Upper 95\%

$\begin{array}{lrrrrr}\text { Coliwasa } & 15 & 2.57366 & 0.01607 & 2.5404 & 2.6069\end{array}$

$\begin{array}{llllll}\text { Coliwasa } & 15 & 2.57366 & 0.01607 & 2.5404 & 2.6069 \\ \text { Isolok - } 3 \mathrm{~mL} & 10 & 2.51849 & 0.01969 & 2.4778 & 2.5592\end{array}$

Std Error uses a pooled estimate of error variance

Means and Std Deviations

Level Number Mean Std Dev Std Err Mean Lower 95\% Upper 95\%

$\begin{array}{lllllll}\text { Coliwasa } & 15 & 2.57366 & 0.067163 & 0.01734 & 2.5365 & 2.6109\end{array}$

$\begin{array}{lllllll}\text { Isolok - } 3 \mathrm{~mL} & 10 & 2.51849 & 0.053739 & 0.01699 & 2.4800 & 2.5569\end{array}$

t Test

Isolok - 3 mL-Coliwasa

Assuming unequal variances

Difference $\quad-0.05517$ t Ratio $\quad-2.27227$

Std Err Dif $\quad 0.02428$ DF 22.09847

Upper CL Dif -0.00483 Prob $>|t| \quad 0.0331$

Lower CL Dif -0.10551 Prob $>t \quad 0.9834$

Confidence $\quad 0.95$ Prob $<\mathrm{t} \quad 0.0166$

Tests that the Variances are Equal

Level Count Std Dev MeanAbsDif to Mean MeanAbsDif to Median

$\begin{array}{lrrrr}\text { Coliwasa } & 15 & 0.0671631 & 0.0570620 & 0.0571529\end{array}$

$\begin{array}{lllll}\text { Isolok - } 3 \mathrm{~mL} & 10 & 0.0537394 & 0.0426169 & 0.0426169\end{array}$

Test F Ratio DFNum DFDen p-Value

$\begin{array}{lrrrr}\text { O'Brien[.5] } & 1.0343 & 1 & 23 & 0.3197\end{array}$

$\begin{array}{lllll}\text { OBrien[.5] } & 1.0343 & 1 & 23 & 0.3197 \\ \text { Brown-Forsythe } & 1.2845 & 1 & 23 & 0.2687\end{array}$

$\begin{array}{lllll}\text { Brown-Forsythe } & 1.2845 & 1 & 23 & 0.2687 \\ \text { Levene } & 1.3006 & 1 & 23 & 0.2658\end{array}$

$\begin{array}{lllll}\text { Levene } & 1.3006 & 1 & 23 & 0.2658 \\ \text { Bartlett } & 0.5004 & 1 & & 0.4793\end{array}$

$\begin{array}{lllll}\text { F Test 2-sided } & 1.5620 & 14 & 9 & 0.5069\end{array}$

Welch Anova testing Means Equal, allowing Std Devs Not Equal

F Ratio DFNum DFDen Prob $>$ F

$\begin{array}{llll}5.1632 & 1 & 22.098 & 0.0331\end{array}$

$\mathbf{t}$ Test
2.2723
Oneway Analysis of Measurement By Sampler Analytical Block=5, Analyte=BaO (wt \%), Target Value $=0.0919$

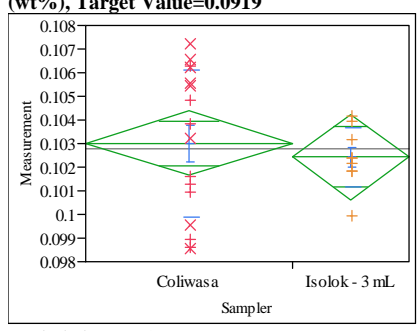

Excluded Rows 1

Oneway Anova

Rsquare $\quad 0.012559$

Adj Rsquare $\quad-0.03037$

Root Mean Square Error $\quad 0.002622$

$\begin{array}{lr}\text { Mean of Response } & 0.102798 \\ \text { Observations (or Sum Wgts) } & 25\end{array}$

t Test

Isolok - 3 mL-Coliwasa

Assuming equal variances

Difference $\quad-0.00059$ t Ratio $\quad-0.54085$

$\begin{array}{lrlr}\text { Std Err Dif } & 0.00109 & \text { DF } & 23 \\ \text { Upper CL } & 0.00167 & \text { Prob }>|t| & 0.5938\end{array}$

Upper CL Dif 0.00167 Prob $>|t| \quad 0.5938$

Lower CL Dif -0.00285 Prob $>t \quad 0.7031$

Confidence

Analysis of Variance

Source DF Sum of Squares Mean Square F Ratio Prob $>$ F

$\begin{array}{lrrrrr}\text { Sampler } & 1 & 0.00000201 & 2.0106 \mathrm{e}-6 & 0.2925 & 0.5938\end{array}$

$\begin{array}{lll}\text { Error } & 23 & 0.00015809 \\ \text { C. Total } & 24 & 0.00016010\end{array}$

Means for Oneway Anova

Level Number Mean Std Error Lower 95\% Upper 95\%

$\begin{array}{lrrrrr}\text { Level } & \text { Number } & \text { Mean } & \text { Std Error } & \text { Lower 95\% } & \text { Upper 95\% } \\ \text { Coliwasa } & 16 & 0.103011 & 0.00066 & 0.10166 & 0.10437\end{array}$

$\begin{array}{lrrrrr}\text { Isolok - } 3 \mathrm{~mL} & 9 & 0.102420 & 0.00087 & 0.10061 & 0.10423\end{array}$

Std Error uses a pooled estimate of error variance

Means and Std Deviations

Level Number Mean Std Dev

$\begin{array}{lllll}\text { Coliwasa } & 16 & 0.103011 & 0.003111\end{array}$

$\begin{array}{llll}\text { Isolok - } 3 & 9 & 0.102420 & 0.001272\end{array}$

$\begin{array}{rrr}\text { Std Err } & \text { Lower } & \text { Upper } \\ \text { Mean } & \mathbf{9 5 \%} & \mathbf{9 5 \%} \\ 0.00078 & 0.10135 & 0.10467 \\ 0.00042 & 0.10144 & 0.10340\end{array}$

$\mathrm{mL}$

tTest

Isolok - 3 mL-Coliwasa

Assuming unequal variances

Difference $\quad-0.00059$ t Ratio $\quad-0.66705$

Std Err Dif $\quad 0.00089$ DF 21.65403

Upper CL Dif 0.00125 Prob $>|t| 0.5118$

Lower CL Dif -0.00243 Prob $>t \quad 0.7441$

$\begin{array}{lrl}\text { Confidence } & 0.95 \text { Prob }<\mathrm{t} & 0.2559\end{array}$

Tests that the Variances are Equal

Level Count Std Dev MeanAbsDif to Mean MeanAbsDif to Median

$\begin{array}{lrrrr}\text { Coliwasa } & 16 & 0.0031107 & 0.0027267 & 0.0027075 \\ \text { Isolok - } 3 \mathrm{~mL} & 9 & 0.0012718 & 0.0008932 & 0.0008808\end{array}$

Test F Ratio DFNum DFDen p-Value

$\begin{array}{lrrrr}\text { O'Brien[.5] } & 9.3752 & 1 & 23 & 0.0055\end{array}$

$\begin{array}{llll}\text { Brown-Forsythe } 11.7432 & 1 & 23 & 0.0023\end{array}$

$\begin{array}{lllll}\text { Levene } & 13.9400 & 1 & 23 & 0.0011\end{array}$

$\begin{array}{lrrrr}\text { Bartlett } & 6.1405 & 1 & & 0.0132 \\ \text { F Test 2-sided } & 5.9827 & 15 & 8 & 0.0154\end{array}$

Welch Anova testing Means Equal, allowing Std Devs Not Equal

F Ratio DFNum DFDen Prob $>$ F

$\begin{array}{lrrr}0.4450 & 1 & 21.654 & 0.5118\end{array}$

t Test

0.6670 
Exhibit A3. Statistical Comparisons for Mid-Rheology (Phase 1) Testing (screened data)

Oneway Analysis of Measurement By Sampler Analytical Block=5, Analyte=Fe/Li, Target Value $=2.452$

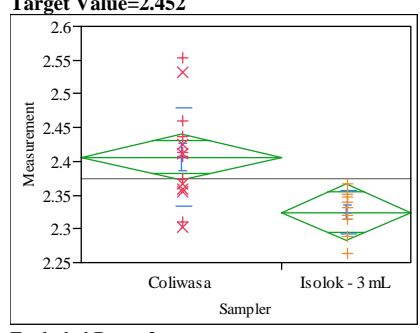

Excluded Rows 3

Oneway Anova

Summary of Fit

Rsquare

Adj Rsquare

Root Mean Square Error $\quad 0.289243$

Observations (or Sum Wgts) 23

t Test

Isolok - 3 mL-Coliwasa

Assuming equal variances

Difference $\quad-0.08192$ t Ratio $\quad-3.15482$

$\begin{array}{lrlr}\text { Std Err Dif } & 0.02597 & \text { DF } & 21 \\ \text { Uper CL } & -0.02792 & \text { Prob }>|t| & 0.0048\end{array}$

Upper CL Dif -0.02792 Prob $>|t| \quad 0.0048$

Lower CL Dif -0.13591 Prob $>t \quad 0.9976$

Analysis of Variance

Source DF Sum of Squares Mean Square F Ratio Prob $>$ F

$\begin{array}{llllll}\text { Sampler } & 1 & 0.03676027 & 0.036760 & 9.9529 & 0.0048\end{array}$

$\begin{array}{llll}\text { Error } & 21 & 0.07756186 & 0.003693\end{array}$

C. Total $22 \quad 0.11432213$

Means for Oneway Anova

Level Number Mean Std Error Lower 95\% Upper 95\%

$\begin{array}{lrrrrr}\text { Coliwasa } & 14 & 2.40650 & 0.01624 & 2.3727 & 2.4403\end{array}$

$\begin{array}{lrrrrr}\text { Coliwasa } & 14 & 2.40650 & 0.01624 & 2.3727 & 2.4403 \\ \text { Isolok - } 3 \mathrm{~mL} & 9 & 2.32458 & 0.02026 & 2.2825 & 2.3667\end{array}$

Std Error uses a pooled estimate of error variance

Means and Std Deviation

Level Number Mean Std Dev Std Err Mean Lower 95\% Upper 95\%

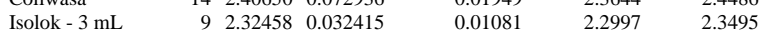

t Test

Isolok - 3 mL-Coliwasa

Assuming unequal variances

Difference $\quad-0.08192$ t Ratio $\quad-3.67543$

Std Err Dif $\quad 0.02229$ DF 19.26113

Upper CL Dif -0.03531 Prob $>|t| \quad 0.0016$

Lower CL Dif -0.12852 Prob $>t \quad 0.9992$

$\begin{array}{lll}\text { Confidence } & 0.95 \text { Prob }<\mathrm{t} \quad 0.0008\end{array}$

Tests that the Variances are Equal

Level Count Std Dev MeanAbsDif to Mean MeanAbsDif to Median

$\begin{array}{lrrrr}\text { Coliwasa } & 14 & 0.0729361 & 0.0540963 & 0.0540341 \\ \text { Isolok - } 3 \mathrm{~mL} & 9 & 0.0324155 & 0.0249451 & 0.0249414\end{array}$

F Ratio DFNum DFDen p-Value

O'Brien[5] F D PFim DFDen p-Value

$\begin{array}{lllll}\text { O'Brien[.5] } & 2.7626 & 1 & 21 & 0.1113 \\ \text { Brown-Forsythe } & 3.0793 & 1 & 21 & 0.0939\end{array}$

$\begin{array}{llrrr}\text { Levene } & 3.1545 & 1 & 21 & 0.0902 \\ \text { Bartlett } & 5.0532 & 1 & . & 0.0246\end{array}$

$\begin{array}{lllll}\text { F Test 2-sided } & 5.0627 & 13 & \dot{8} & 0.0246 \\ \end{array}$

Welch Anova testing Means Equal, allowing Std Devs Not Equal

F Ratio DFNum DFDen Prob $>$ F

$\begin{array}{llll}13.5088 & 1 & 19.261 & 0.0016\end{array}$

$\mathbf{t}$ Test
3.6754
Oneway Analysis of Measurement By Sampler Analytical Block=5, Analyte=Fe2O3 (wt \%), Target Value $=11.462$

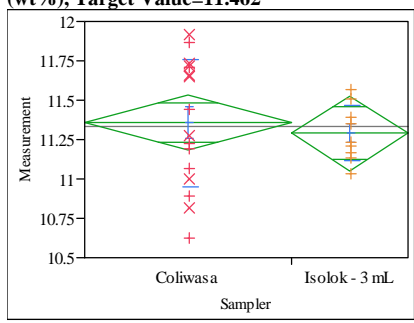

Excluded Rows 1

Oneway Anova

Summary of Fit

Rsquare $\quad 0.009597$

Adj Rsquare $\quad-0.03346$

Root Mean Square Error $\quad 0.342217$

$\begin{array}{lr}\text { Mean of Response } & 11.33295 \\ \text { Observations (or Sum Wgts) } & 25\end{array}$

t Test

Isolok - 3 mL-Coliwasa

Assuming equal variances

Difference $\quad-0.06732$ t Ratio $\quad-0.47209$

$\begin{array}{lrr}\text { Std Err Dif } & 0.14259 \text { DF } & 23 \\ \text { Upp } & & 0.6413\end{array}$

Upper CL Dif 0.22766 Prob $>|t| \quad 0.6413$

Lower CL Dif -0.36229 Prob $>$ t 0.6793

Confidence $\quad 0.95$ Prob $<\mathrm{t} \quad 0.3207$

Analysis of Variance

Source DF Sum of Squares Mean Square F Ratio Prob $>$ F

$\begin{array}{lrrrrr}\text { Sampler } & 1 & 0.0261004 & 0.026100 & 0.2229 & 0.6413\end{array}$

Error $23 \quad 2.6935876$

C. Total $24 \quad 2.7196879$

Means for Oneway Anova

Level Number Mean Std Error Lower 95\% Upper 95\%

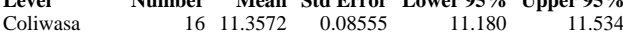

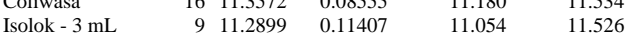

Std Error uses a pooled estimate of error variance

Means and Std Deviations

Level Number Mean Std Dev Std Err Mean Lower 95\% Upper 95\%

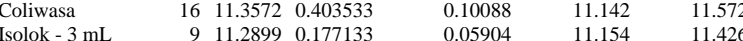

t Test

Isolok - 3 mL-Coliwasa

Assuming unequal variances

$\begin{array}{lrlr}\text { Difference } & -0.06732 & \text { t Ratio } & -0.57588 \\ \text { Std Err Dif } & 0.11689 & \text { DF } & 22.16087 \\ \text { Upper CL Dif } & 0.17500 & \text { Prob }>|t| & 0.5705 \\ \text { Lower CL Dif } & -0.30963 & \text { Prob }>t & 0.7147 \\ \text { Confidence } & 0.95 & \text { Prob }<t & 0.2853\end{array}$

Tests that the Variances are Equal

Level Count Std Dev MeanAbsDif to Mean MeanAbsDif to Median

$\begin{array}{lrrrr}\text { Coliwasa } & 16 & 0.4035327 & 0.3484894 & 0.3484894 \\ \text { Isolok - 3 mL } & 9 & 0.1771328 & 0.1472061 & 0.1445586\end{array}$

Test F Ratio DFNum DFDen p-Value

$\begin{array}{lrrrr}\text { O'Brien[.5] } & \text { F Ratio } & \text { DFNum } & \text { DFDen } & \text { p-Value } \\ \text { O'0419 } & 1 & 23 & 0.0219\end{array}$

$\begin{array}{lllll}\text { O'Brien[.5] } & 6.0419 & 1 & 23 & 0.0219 \\ \text { Brown-Forsythe } & 9.3635 & 1 & 23 & 0.0055\end{array}$

$\begin{array}{lllll}\text { Brown-Forsythe } & 9.3635 & 1 & 23 & 0.0055 \\ \text { Levene } & 9.6633 & 1 & 23 & 0.0049\end{array}$

$\begin{array}{llrrr}\text { Levene } & 9.6633 & 1 & 23 & 0.0049 \\ \text { Bartlett } & 5.3291 & 1 & . & 0.0210\end{array}$

$\begin{array}{lrrrr}\text { F Test 2-sided } & 5.1899 & 1 & \dot{1} & 0.0210 \\ \end{array}$

Welch Anova testing Means Equal, allowing Std Devs Not Equal

F Ratio DFNum DFDen Prob $>$ F

$\begin{array}{llll}0.3316 & 1 & 22.161 & 0.5705\end{array}$

t Test 


\section{Exhibit A3. Statistical Comparisons for Mid-Rheology (Phase 1) Testing (screened data)}

Oneway Analysis of Measurement By Sampler Analytical Block=5, Analyte=Li2O (wt \%), Target Value $=4.674$

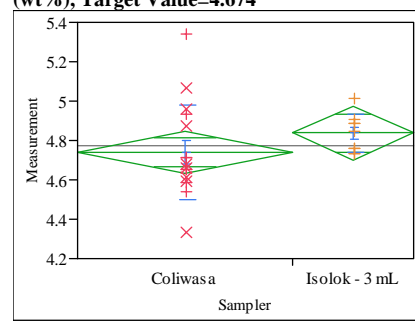

Excluded Rows 1

Oneway Anova

Summary of Fit

Rsquare

Adj Rsquare

0.054052

0.012923

0.201882

$\begin{array}{lr}\text { Mean of Response } & 4.775132 \\ \text { Observations (or Sum Wgts) } & 25\end{array}$

t Test

Isolok - $3 \mathrm{~mL}$-Coliwasa

Assuming equal variances

Difference $\quad 0.09643$ t Ratio 1.146396

$\begin{array}{lrlr}\text { Std Err Dif } & 0.08412 \text { DF } & 23 \\ \text { Upper CL Dif } & 0.27044 \text { Prob }>|t| & 0.2634\end{array}$

$\begin{array}{rrr}\text { Upper CL Dif } & 0.27044 \text { Prob }>|t| & 0.2634 \\ \text { Lower CL Dif }-0.07758 \text { Prob }>t & 0.1317\end{array}$

$\begin{array}{lrl}\text { Confidence } & 0.95 \text { Prob }<\mathrm{t} & 0.8683\end{array}$

Analysis of Variance

Source DF Sum of Squares Mean Square F Ratio Prob $>$ F

$\begin{array}{lrrrrr}\text { Sampler } & 1 & 0.05356297 & 0.053563 & 1.3142 & 0.2634\end{array}$

$\begin{array}{lll}\text { Error } & 23 & 0.93739541 \\ \text { C. Total } & 24 & 0.99095838\end{array}$

Means for Oneway Anova

Level Number Mean Std Error Lower 95\% Upper 95\%

$\begin{array}{lrrrrr}\text { Coliwasa } & \text { Number } & \text { Mean } & \text { Std Error } & \text { Lower 95\% } & \text { Uper 95\% } \\ \text { Isolok - 3 mL } & 9 & 4.83685 & 0.05047 & 4.6360 & 4.8448 \\ & & & & 4.66729 & 4.9761\end{array}$

Std Error uses a pooled estimate of error variance

Means and Std Deviations

Level Number Mean Std Dev Std Err Mean Lower 95\% Upper 95\%

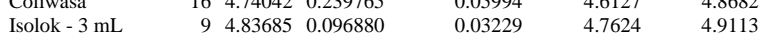

t Test

Isolok - 3 mL-Coliwasa

Assuming unequal variances

Difference $\quad 0.09643$ t Ratio 1.416308

Std Err Dif $\quad 0.06809$ DF 21.56487

Upper CL Dif 0.23780 Prob $>|t| \quad 0.1710$

Lower CL Dif -0.04494 Prob $>t \quad 0.0855$

$\begin{array}{lll}\text { Confidence } & 0.95 \text { Prob }<\mathrm{t} \quad 0.9145\end{array}$

Tests that the Variances are Equal

Level Count Std Dev MeanAbsDif to Mean MeanAbsDif to Median

$\begin{array}{lrrrr}\text { Coliwasa } & 16 & 0.2397650 & 0.1804736 & 0.1655042 \\ \text { Isolok - } 3 \mathrm{~mL} & 9 & 0.0968805 & 0.0797370 & 0.0837239\end{array}$

Test Fatio DFNum DFDen p-Value

O'Brien[.5] F Ratio DFNum DFDen p-Value

$\begin{array}{lllll}\text { O'Brien[.5] } & 1.9447 & 1 & 23 & 0.1765 \\ \text { Brown-Forsythe } & 1.6432 & 1 & 23 & 0.2127\end{array}$

$\begin{array}{lllll}\text { Levene } & 3.7444 & 1 & 23 & 0.0654 \\ \text { Bartlett } & 6.2779 & 1 & & 0.0122\end{array}$

$\begin{array}{lllll}\text { F Test 2-sided } & 6.1249 & 15 & 8 & 0.0142\end{array}$

Welch Anova testing Means Equal, allowing Std Devs Not Equal

F Ratio DFNum DFDen Prob $>$ F

$\begin{array}{llll}2.0059 & 1 & 21.565 & 0.1710\end{array}$

$\mathbf{t}$ Test
1.4163
Oneway Analysis of Measurement By Sampler Analytical Block=5, Analyte=MgO (wt \%), Target Value $=1.014$

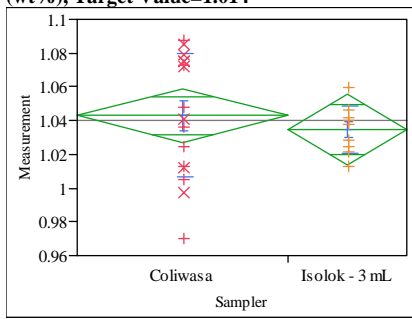

Excluded Rows 1

Oneway Anova

Rsquare $\quad 0.018122$

Adj Rsquare $\quad-0.02457$

Root Mean Square Error $\quad 0.030542$

$\begin{array}{lr}\text { Mean of Response } & 1.040086 \\ \text { Observations (or Sum Wgts) } & 25\end{array}$

t Test

Isolok - 3 mL-Coliwasa

Assuming equal variances

Difference $\quad-0.00829$ t Ratio $\quad-0.65154$

$\begin{array}{lrrr}\text { Std Err Dif } & 0.01273 \text { DF } & 23 \\ \text { Upper } & \text { DL } & -0.5212\end{array}$

$\begin{array}{lrll}\text { Upper CL Dif } & 0.01803 & \text { Prob }>|t| & 0.5212 \\ \text { Lower CL Dif } & -0.03462 & \text { Prob }>t & 0.7394\end{array}$

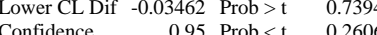

Analysis of Variance

Source DF Sum of Squares Mean Square F Ratio Prob $>$ F

$\begin{array}{lrrrrr}\text { Sampler } & 1 & 0.00039599 & 0.000396 & 0.4245 & 0.5212\end{array}$

Error $\quad 23 \quad 0.02145518$

$\begin{array}{lll}\text { C. Total } 24 & 0.02185117\end{array}$

Means for Oneway Anova

Level Number Mean Std Error Lower 95\% Upper 95\%

$\begin{array}{lrrrrr}\text { Coliwasa } & 16 & 1.04307 & 0.00764 & 1.0273 & 1.0589\end{array}$

$\begin{array}{lrrrrr}\text { Isolok - } 3 \mathrm{~mL} & 9 & 1.03478 & 0.01018 & 1.0137 & 1.0558\end{array}$

Std Error uses a pooled estimate of error variance

Means and Std Deviations

Level Number Mean Std Dev Std Err Mean Lower 95\% Upper 95\%

$\begin{array}{lllllll}\text { Coliwasa } & 16 & 1.04307 & 0.036367 & 0.00909 & 1.0237 & 1.0624\end{array}$

$\begin{array}{lllllll}\text { Isolok - } 3 \mathrm{~mL} & 9 & 1.03478 & 0.014217 & 0.00474 & 1.0239 & 1.0457\end{array}$

t Test

Isolok - 3 mL-Coliwasa

Assuming unequal variances

Difference $\quad-0.00829$ t Ratio $\quad-0.80872$

Std Err Dif $\quad 0.01025$ DF 21.30878

Upper CL Dif 0.01301 Prob $>|t| \quad 0.4276$

Lower CL Dif -0.02959 Prob $>t \quad 0.7862$

$\begin{array}{lll}\text { Confidence } & 0.95 \text { Prob }<\mathrm{t} \quad 0.2138\end{array}$

Tests that the Variances are Equal

Level Count Std Dev MeanAbsDif to Mean MeanAbsDif to Media

$\begin{array}{lrrrr}\text { Coliwasa } & 16 & 0.0363668 & 0.0308858 & 0.0308858 \\ \text { Isolok - 3 mL } & 9 & 0.0142170 & 0.0114238 & 0.0112396\end{array}$

Test F Ratio DFNum DFDen p-Value

$\begin{array}{lrrrr}\text { O'Brien[.5] } & 5.5196 & 1 & 23 & 0.0278\end{array}$

$\begin{array}{lllll}\text { Brown-Forsythe } & 9.8943 & 1 & 23 & 0.0278 \\ \text { B Brien[.5] } & 1 & 23 & 0.0045\end{array}$

$\begin{array}{lrrrr}\text { Brown-Forsythe } & 9.8943 & 1 & 23 & 0.0045 \\ \text { Levene } & 10.0005 & 1 & 23 & 0.0044\end{array}$

$\begin{array}{lrrrr}\text { Levene } & 10.0005 & 1 & 23 & 0.0044 \\ \text { Bartlett } & 6.6691 & 1 & . & 0.0098\end{array}$

$\begin{array}{lrrrr}\text { F Test 2-sided } & 6.5433 & 15 & 8 & 0.0114\end{array}$

Welch Anova testing Means Equal, allowing Std Devs Not Equal

F Ratio DFNum DFDen Prob $>$ F

$\begin{array}{llll}0.6540 & 1 & 21.309 & 0.4276\end{array}$

t Test 
Exhibit A3. Statistical Comparisons for Mid-Rheology (Phase 1) Testing (screened data)

Oneway Analysis of Measurement By Sampler Analytical Block=5, Analyte=MnO (wt \%), Target Value $=1.779$

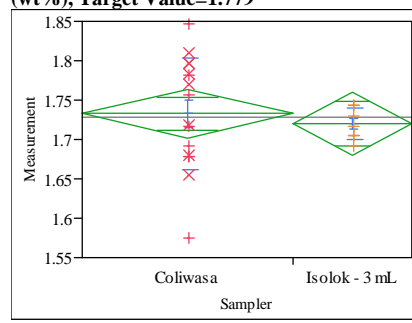

Excluded Rows 1

Oneway Anova

Summary of Fit

Rsquare

Adj Rsquare

Root Mean Square Error $\quad-0.0317$

ror $\quad 0.058368$

Observations (or Sum Wgts) $r .728142$

t Test

Isolok - $3 \mathrm{~mL}-$-Coliwasa

Assuming equal variances

Difference $\quad-0.01246$ t Ratio $\quad-0.51249$

$\begin{array}{lrlr}\text { Std Err Dif } & 0.02432 & \text { DF } & 23 \\ \text { Uper CL } & 0.03785 & \text { Prob }>|t| & 0.6132\end{array}$

$\begin{array}{rrr}\text { Upper CL Dif } & 0.03785 \text { Prob }>|t| & 0.6132 \\ \text { Cow } & & \end{array}$

$\begin{array}{lrrr}\text { Lower CL Dif } & -0.06277 & \text { Prob }>t & 0.633 \\ \text { Confidence } & 0.95 & \text { Prob }<\mathrm{t} & 0.3066\end{array}$

Analysis of Variance

Source DF Sum of Squares Mean Square F Ratio Prob $>$ F

$\begin{array}{llllll}\text { Sampler } & 1 & 0.00089478 & 0.000895 & 0.2626 & 0.6132\end{array}$

$\begin{array}{lll}\text { Error } & 23 & 0.07835712 \\ \text { C. Total } & 24 & 0.07925190\end{array}$

Means for Oneway Anova

Level Number Mean Std Error Lower 95\% Upper 95\%

\begin{tabular}{lrrrrr} 
Coliwasa & 16 & 1.73263 & 0.01459 & 1.7024 & 1.7628 \\
\hline
\end{tabular}

$\begin{array}{lrrrrr}\text { Coliwasa } & 16 & 1.73263 & 0.01459 & 1.7024 & 1.7628 \\ \text { Isolok - } 3 \mathrm{~mL} & 9 & 1.72017 & 0.01946 & 1.6799 & 1.7604\end{array}$

Std Error uses a pooled estimate of error variance

Means and Std Deviation

Level Number Mean Std Dev Std Err Mean Lower $95 \%$ Upper $95 \%$

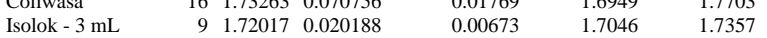

t Test

Isolok - 3 mL-Coliwasa

Assuming unequal variances

Difference $\quad-0.01246$ t Ratio $\quad-0.65855$

Std Err Dif $\quad 0.01893$ DF 18.91294

Upper CL Dif 0.02716 Prob $>|t| \quad 0.5181$

Lower CL Dif -0.05209 Prob $>t \quad 0.7409$

$\begin{array}{lll}\text { Confidence } & 0.95 \text { Prob }<\mathrm{t} \quad 0.2591\end{array}$

Tests that the Variances are Equal

Level Count Std Dev MeanAbsDif to Mean MeanAbsDif to Median

$\begin{array}{lrrrr}\text { Coliwasa } & 16 & 0.0707563 & 0.0589110 & 0.0589110 \\ \text { Isolok - } 3 \mathrm{~mL} & 9 & 0.0201875 & 0.0175348 & 0.0186507\end{array}$

F Ratio DFNum DFDen p-Value

O'Brien[.5] F Ratio DFNum DFDen p-Value

$\begin{array}{lrrrr}\text { O'Brien[.5] } & 4.0015 & 1 & 23 & 0.0574 \\ \text { Brown-Forsythe } & 10.6306 & 1 & 23 & 0.0034\end{array}$

$\begin{array}{lllll}\text { Levene } & 11.3053 & 1 & 23 & 0.0027 \\ \text { Bartlett } & 10.6853 & 1 & & 0.0011\end{array}$

$\begin{array}{lrrrr}\text { F Test 2-sided } & 12.2847 & 15 & 8 & 0.0013\end{array}$

Welch Anova testing Means Equal, allowing Std Devs Not Equal

F Ratio DFNum DFDen Prob $>$ F

$\begin{array}{llll}0.4337 & 1 & 18.913 & 0.5181\end{array}$

$\mathbf{t}$ Test
0.6586
Oneway Analysis of Measurement By Sampler Analytical Block=5, Analyte=SiO2 (wt \%), Target Value $=\mathbf{5 0 . 9 8 5}$

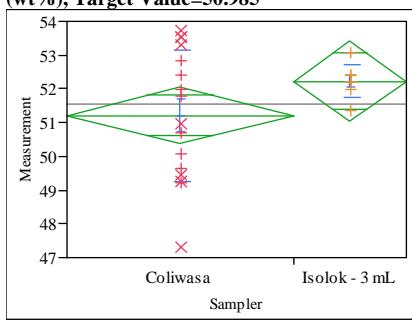

Excluded Rows 2

Oneway Anova

Rsquare $\quad 0.086387$

$\begin{array}{ll}\text { Adj Rsquare } & 0.044859 \\ \text { Root Mean Square Error } & 1.627086\end{array}$

$\begin{array}{ll}\text { Root Mean Square Error } & 1.627086 \\ \text { Mean of Response } & 51.54822\end{array}$

Observations (or Sum Wgts) 24

t Test

Isolok - $3 \mathrm{~mL}$-Coliwasa

Assuming equal variances

Difference $\quad 1.0162$ t Ratio 1.442295

$\begin{array}{lrlr}\text { Std Err Dif } & 0.7045 \text { DF } & 22 \\ \text { Upper } & \end{array}$

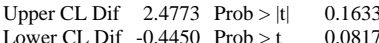

$\begin{array}{lll}\text { Confidence } & 0.95 \text { Prob }<\mathrm{t} \quad 0.9183\end{array}$

Analysis of Variance

Source DF Sum of Squares Mean Square F Ratio Prob $>$ F

$\begin{array}{lrrrrr}\text { Sampler } & 1 & 5.507181 & 5.50718 & 2.0802 & 0.1633\end{array}$

$\begin{array}{llll}\text { Error } & 22 & 58.243013 & 2.64741\end{array}$

C. Total $23 \quad 63.750194$

Means for Oneway Anova

Level Number Mean Std Error Lower 95\% Upper 95\%

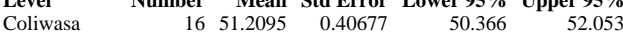

$\begin{array}{lrrrrr}\text { Isolok - } 3 \mathrm{~mL} & 8 & 52.2257 & 0.57526 & 51.033 & 53.419\end{array}$

Std Error uses a pooled estimate of error variance

Means and Std Deviations

Level Number Mean Std Dev Std Err Mean Lower 95\% Upper 95\%

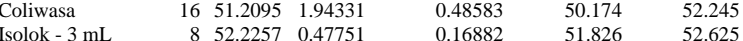

t Test

Isolok - 3 mL-Coliwasa

Assuming unequal variances

$\begin{array}{lrlr}\text { Difference } & 1.0162 \text { t Ratio } & 1.975729 \\ \text { Std Err Dif } & 0.5143 \text { DF } & 18.27048 \\ \text { Upper CL Dif } & 2.0956 \text { Prob }>|t| & 0.0635 \\ \text { Lower CL Dif } & -0.0632 & \text { Prob }>\text { t } & 0.0317 \\ \text { Confidence } & 0.95 & \text { Prob }<\mathrm{t} & 0.9683\end{array}$

Tests that the Variances are Equal

Level Count Std Dev MeanAbsDif to Mean MeanAbsDif to Median

$\begin{array}{lrrrr}\text { Coliwasa } & 16 & 1.943312 & 1.657958 & 1.657958 \\ \text { Isolok - } 3 \mathrm{~mL} & 8 & 0.477507 & 0.300839 & 0.294154\end{array}$

Test F Ratio DFNum DFDen p-Value

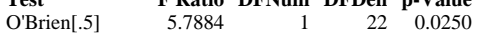

$\begin{array}{lrrrr}\text { Brown-Forsythe } & 15.7441 & 1 & 22 & 0.0007\end{array}$

$\begin{array}{lllll}\text { Brown-Forsythe } & 15.7441 & 1 & 22 & 0.0007 \\ \text { Levene } & 15.9628 & 1 & 22 & 0.0006\end{array}$

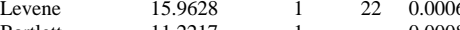

$\begin{array}{lrrrr}\text { Bartlett } & 11.2217 & 1 & & 0.0008 \\ \text { F Test 2-sided } & 16.5625 & 15 & 7 & 0.0010\end{array}$

Welch Anova testing Means Equal, allowing Std Devs Not Equal

F Ratio DFNum DFDen Prob $>$ F

$\begin{array}{llll}3.9035 & 1 & 18.27 & 0.0635\end{array}$

t Test 
Exhibit A3. Statistical Comparisons for Mid-Rheology (Phase 1) Testing (screened data)

Oneway Analysis of Measurement By Sampler Analytical Block=5, Analyte=Sum of Oxides (wt\%), Target Value $=99.553$

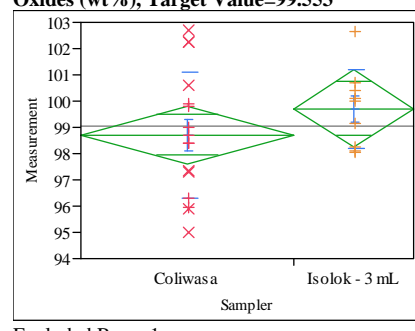

Excluded Rows 1

Oneway Anova

Summary of Fit

Rsquare

Adj Rsquare

Root Mean Square Error $\quad 2.122319$

Mean of Response 99.07196

Observations (or Sum Wg

t Test

Isolok - $3 \mathrm{~mL}$-Coliwasa

Assuming equal variances

Difference $\quad 1.0052$ t Ratio $\quad 1.136674$

Std Err Dif $\quad 0.8843$ DF 23

Upper CL Dif 2.8345 Prob $>|t| \quad 0.2674$

Lower CL Dif -0.8242 Prob $>t \quad 0.1337$

Confidence $\quad 0.95$ Prob $<\mathrm{t} \quad 0.8663$

Analysis of Variance

Source DF Sum of Squares Mean Square F Ratio Prob $>$ F

$\begin{array}{lrrrrr}\text { Sampler } & 1 & 5.81960 & 5.81960 & 1.2920 & 0.2674\end{array}$

$\begin{array}{llll}\text { Error } & 23 & 103.59749 & 4.50424 \\ \text { C. Total } & 24 & 109.41709 & \end{array}$

Means for Oneway Anova

$\begin{array}{lrrrrr}\text { Level } & \text { Number } & \text { Mean } & \text { Std Error } & \text { Lower 95\% } & \text { Upper 95\% } \\ \text { Coliwasa } & 16 & 98.7101 & 0.53058 & 97.613 & 99.81\end{array}$

$\begin{array}{lrrrrr}\text { Coliwasa } & 16 & 98.7101 & 0.53058 & 97.613 & 99.81 \\ \text { Isolok - } 3 \mathrm{~mL} & 9 & 99.7153 & 0.70744 & 98.252 & 101.18\end{array}$

Std Error uses a pooled estimate of error variance

Means and Std Deviations

Level Number Mean Std Dev Std Err Mean Lower 95\% Upper 95\%

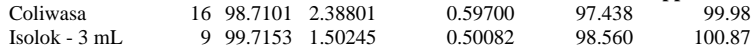

t Test

Isolok - $3 \mathrm{~mL}$-Coliwasa

Assuming unequal variances

Difference $\quad 1.0052$ t Ratio 1.289911

Std Err Dif $\quad 0.7792$ DF 22.57659

Upper CL Dif 2.6188 Prob $>|t| 0.2101$

Lower CL Dif -0.6085 Prob $>t \quad 0.1051$

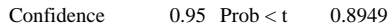

Tests that the Variances are Equal

Level Count Std Dev MeanAbsDif to Mean MeanAbsDif to Median

$\begin{array}{lrrrr} & \text { Count } & \text { Std Dev } & \text { MeanAbsDif to Mean } & \text { MeanAbsDif to Median } \\ \text { Coliwasa } & 16 & 2.388008 & 1.907102 & 1.905940 \\ \text { Isolok - 3 mL } & 9 & 1.502445 & 1.170428 & 1.148759\end{array}$

Test F Ratio DFNum DFDen p-Value

$\begin{array}{lrrrr}\text { O'Brien[.5] } & 2.4552 & 1 & 23 & 0.1308\end{array}$

$\begin{array}{lllll}\text { OBrien[.5] } & 2.4552 & 1 & 23 & 0.1308 \\ \text { Brown-Forsythe } & 2.1871 & 1 & 23 & 0.1527\end{array}$

$\begin{array}{lllll}\text { Brown-Forsythe } & 2.1871 & 1 & 23 & 0.1527 \\ \text { Levene } & 2.1737 & 1 & 23 & 0.1539\end{array}$

$\begin{array}{lllll}\text { Levene } & 2.1737 & 1 & 23 & 0.1539 \\ \text { Bartlett } & 1.8946 & 1 & . & 0.1687\end{array}$

$\begin{array}{lllll}\text { F Test 2-sided } & 2.5262 & 15 & \dot{8} & 0.1881\end{array}$

Welch Anova testing Means Equal, allowing Std Devs Not Equal

F Ratio DFNum DFDen Prob $>$ F

$\begin{array}{llll}1.6639 & 1 & 22.577 & 0.2101\end{array}$

$\mathbf{t}$ Test
1.2899
Oneway Analysis of Measurement By Sampler Analytical Block=5, Analyte=ZnO (wt \%), Target Value $=0.0958$

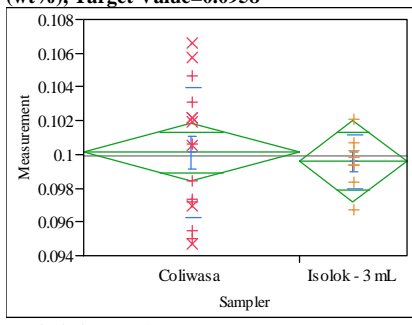

Excluded Rows 2

Oneway Anova

Rsquare $\quad 0.006775$

Adj Rsquare $\quad-0.03837$

Root Mean Square Error $\quad 0.003293$

$\begin{array}{lr}\text { Mean of Response } & 0.099937 \\ \text { Observations (or Sum Wgts) } & 24\end{array}$

t Test

Isolok - 3 mL-Coliwasa

Assuming equal variances

Difference $\quad-0.00055$ t Ratio $\quad-0.38738$

$\begin{array}{lrlr}\text { Std Err Dif } & 0.00143 \text { DF } & 22 \\ \text { Upper CL Dif } & 0.00240 \text { Prob }>|t| & 0.7022\end{array}$

Upper CL Dif 0.00240 Prob $>|t| \quad 0.7022$

Lower CL Dif -0.00351 Prob $>t \quad 0.6489$

Confidence $\quad 0.95$ Prob $<\mathrm{t} \quad 0.3511$

Analysis of Variance

Source DF Sum of Squares Mean Square F Ratio Prob $>$ F

$\begin{array}{lrrrrr}\text { Sampler } & 1 & 0.00000163 & 1.627 \mathrm{e}-6 & 0.1501 & 0.7022\end{array}$

Error $22 \quad 0.00023857$

$\begin{array}{lll}\text { C. Total } & 23 & 0.00024020\end{array}$

Means for Oneway Anova

Level Number Mean Std Error Lower 95\% Upper 95\%

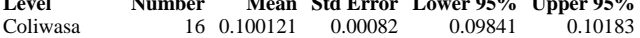

$\begin{array}{lrrrrr}\text { Isolok - } 3 \mathrm{~mL} & 8 & 0.099568 & 0.00116 & 0.09715 & 0.10198\end{array}$

Std Error uses a pooled estimate of error variance

Means and Std Deviations

Level Number Mean Std Dev

$\begin{array}{llll}\text { Coliwasa } & 16 & 0.100121 & 0.003837\end{array}$

$\begin{array}{llll}\text { Isolok - } 3 & 8 & 0.099568 & 0.001593\end{array}$

$\begin{array}{rrr}\text { Std Err } & \text { Lower } & \text { Upper } \\ \text { Mean } & \mathbf{9 5 \%} & \mathbf{9 5 \%} \\ 0.00096 & 0.09808 & 0.10217 \\ 0.00056 & 0.09824 & 0.10090\end{array}$

Isolok - $3 \mathrm{~mL}$-Coliwasa

Assuming unequal variances

Difference $\quad-0.00055$ t Ratio $\quad-0.49659$

Std Err Dif $\quad 0.00111$ DF 21.62048

Upper CL Dif 0.00176 Prob $>|t| \quad 0.6245$

Lower CL Dif -0.00286 Prob $>t \quad 0.6878$

$\begin{array}{lrl}\text { Confidence } & 0.95 \text { Prob }<\mathrm{t} & 0.3122\end{array}$

Tests that the Variances are Equal

Level Count Std Dev MeanAbsDif to Mean MeanAbsDif to Median

$\begin{array}{lllll}\text { Coliwasa } & 16 & 0.0038367 & 0.0032394 & 0.0031976\end{array}$

$\begin{array}{lllll}\text { Isolok - } 3 \mathrm{~mL} & 8 & 0.0015933 & 0.0011359 & 0.0011359\end{array}$

Test F Ratio DFNum DFDen p-Value

$\begin{array}{lrrrr}\text { O'Brien[.5] } & 5.4788 & 1 & 22 & 0.0287\end{array}$

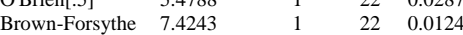

$\begin{array}{lllll}\text { Levene } & 8.6029 & 1 & 22 & 0.0077\end{array}$

$\begin{array}{lrrrr}\text { Bartlett } & 5.2906 & 1 & . & 0.0214\end{array}$

$\begin{array}{lllll}\text { F Test 2-sided } & 5.7981 & 15 & 7 & 0.0256\end{array}$

Welch Anova testing Means Equal, allowing Std Devs Not Equal

F Ratio DFNum DFDen Prob $>$ F

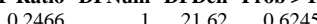

t Test

0.4966 
Exhibit A3. Statistical Comparisons for Mid-Rheology (Phase 1) Testing (screened data)

Oneway Analysis of Measurement By Sampler Analytical Block=6, Analyte=Al/B, Target Value $=2.552$

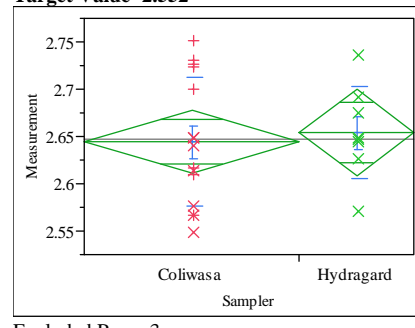

Excluded Rows 3

Oneway Anova

Rsquare $\quad 0.006236$

Adj Rsquare

$-0.04109$

Root Mean Square Error $\quad 0.062133$

$\begin{array}{lr}\text { Mean of Response } & 2.647405 \\ \text { Observations (or Sum Wgts) } & 23\end{array}$

t Test

Hydragard-Coliwasa

Assuming equal variances

Difference $\quad 0.00987$ t Ratio $\quad 0.363004$

$\begin{array}{lrlr}\text { Std Err Dif } & 0.02720 & \text { DF } & 21 \\ \text { Upper CL Dif } & 0.06644 & \text { Prob }>|t| & 0.7202\end{array}$

$\begin{array}{lll}\text { Upper CL Dif } 0.06644 \text { Prob }>|t| & 0.7202 \\ \text { Low } & \end{array}$

$\begin{array}{lrrr}\text { Lower CL Dif } & -0.04669 & \text { Prob }>t & 0.3601 \\ \text { Confidence } & 0.95 & \text { Prob }<\mathrm{t} & 0.6399\end{array}$

Analysis of Variance

Source DF Sum of Squares Mean Square F Ratio Prob $>$ F

$\begin{array}{llllll}\text { Sampler } & 1 & 0.00050870 & 0.000509 & 0.1318 & 0.7202\end{array}$

Error $21 \quad 0.08106964$

0.003860

Means for Oneway Anova

Level Number Mean Std Error Lower 95\% Upper 95\%

$\begin{array}{lrrrrr}\text { Coliwasa } & 15 & 2.64397 & 0.01604 & 2.6106 & 2.6773\end{array}$

$\begin{array}{lrrrrr}\text { Coliwasa } & 15 & 2.64397 & 0.01604 & 2.6106 & 2.6773 \\ \text { Hydragard } & 8 & 2.65384 & 0.02197 & 2.6082 & 2.6995\end{array}$

Std Error uses a pooled estimate of error variance

Means and Std Deviations

Level Number Mean Std Dev Std Err Mean Lower 95\% Upper 95\%

$\begin{array}{lrrrrrr}\text { Coliwasa } & 15 & 2.64397 & 0.067858 & 0.01752 & 2.6064 & 2.6815 \\ \text { Hydragard } & 8 & 2.65384 & 0.048703 & 0.01722 & 2.6131 & 2.6946\end{array}$

t Test

t Test
Hydragard-Coliwasa

Hydragard-Coliwasa
Assuming unequal variances

Difference $\quad 0.00987$ t Ratio $\quad 0.401953$

Std Err Dif $\quad 0.02457$ DF 18.87967

Upper CL Dif 0.06131 Prob $>|t| 0.6922$

Lower CL Dif -0.04156 Prob $>t \quad 0.3461$

$\begin{array}{lll}\text { Confidence } & 0.95 \text { Prob }<\mathrm{t} \quad 0.6539\end{array}$

Tests that the Variances are Equal

Level Count Std Dev MeanAbsDif to Mean MeanAbsDif to Median

$\begin{array}{lrrrr}\text { Coliwasa } & 15 & 0.0678580 & 0.0558814 & 0.0561037 \\ \text { Hydragard } & 8 & 0.0487027 & 0.0347092 & 0.0331258\end{array}$

Test F Ratio DFNum DFDen p-Value

$\begin{array}{lrrrr}\text { O'Brien[.5] } & 1.6144 & 1 & 21 & 0.2178\end{array}$

$\begin{array}{lllll}\text { OBrien[.5] } & 1.6144 & 1 & 21 & 0.2178 \\ \text { Brown-Forsythe } & 2.2172 & 1 & 21 & 0.1513\end{array}$

$\begin{array}{lllll}\text { Brown-Forsythe } & 2.2172 & 1 & 21 & 0.1513 \\ \text { Levene } & 1.9972 & 1 & 21 & 0.1722\end{array}$

$\begin{array}{llrrr}\text { Levene } & 1.9972 & 1 & 21 & 0.1722 \\ \text { Bartlett } & 0.8918 & 1 & . & 0.3450\end{array}$

$\begin{array}{lllll}\text { F Test 2-sided } & 1.9413 & 14 & 7 & 0.3825\end{array}$

Welch Anova testing Means Equal, allowing Std Devs Not Equal

F Ratio DFNum DFDen Prob > F

$\begin{array}{llll}0.1616 & 1 & 18.88 & 0.6922\end{array}$

t Test
0.4020
Oneway Analysis of Measurement By Sampler Analytical Block=6, Analyte=CuO (wt \%), Target Value $=\mathbf{0 . 0 5 0 4}$

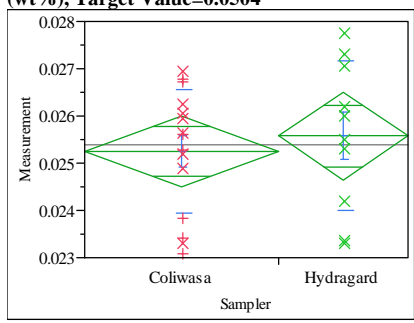

Excluded Rows 1

Oneway Anova

Summary of Fit

Rsquare $\quad 0.013588$

Adj Rsquare $\quad-0.0293$

Root Mean Square Error $\quad 0.001416$

$\begin{array}{lr}\text { Mean of Response } & 0.025379 \\ \text { Observations (or Sum Wgts) } & 25\end{array}$

t Test

Hydragard-Coliwasa

Assuming equal variances

Difference $\quad 0.00033$ t Ratio 0.562869

$\begin{array}{lrrr}\text { Std Err Dif } & 0.00058 \text { DF } & 23 \\ \text { Upper CL } & \text { Dif } & 0.00152 \text { Prob }>|\mathrm{t}| & 0.5790\end{array}$

$\begin{array}{rrrr}\text { Upper CL Dif } & 0.00152 & \text { Prob }>|t| & 0.5790 \\ \text { Lower CL Dif } & -0.00087 & \text { Prob }>t & 0.2895\end{array}$

$\begin{array}{lrll}\text { Lower CL Dif } & -0.00087 & \text { Prob }>t & 0.2895 \\ \text { Conf } & \text { Prob }<t & 0.7105\end{array}$

Analysis of Variance

Source DF Sum of Squares Mean Square F Ratio Prob $>$ F

$\begin{array}{lrrrrr}\text { Sampler } & 1 & 0.00000064 & 6.3558 \mathrm{e}-7 & 0.3168 & 0.5790\end{array}$

$\begin{array}{llll}\text { Error } & 23 & 0.00004614 & 2.0061 \mathrm{e}-6\end{array}$

C. Total $24 \quad 0.00004678$

Means for Oneway Anova

Level Number Mean Std Error Lower 95\% Upper 95\%

$\begin{array}{lrrrrr}\text { Level } & \text { Number } & \text { Mean } & \text { Std Error } & \text { Lower 95\% } & \text { Upper 95\% } \\ \text { Coliwasa } & 15 & 0.025249 & 0.00037 & 0.02449 & 0.02601\end{array}$

$\begin{array}{llllll}\text { Hydragard } & 10 & 0.025574 & 0.00045 & 0.02465 & 0.02650\end{array}$

Std Error uses a pooled estimate of error variance

Means and Std Deviations

Level Number Mean Std Dev Std Err Mean Lower 95\% Upper 95\%

$\begin{array}{lrrrrrr}\text { Coliwasa } & 15 & 0.025249 & 0.001298 & 0.00034 & 0.02453 & 0.02597\end{array}$

$\begin{array}{llllllll}\text { Hydragard } & 10 & 0.025574 & 0.001582 & 0.00050 & 0.02444 & 0.02671\end{array}$

t Test

Hydragard-Coliwasa

Assuming unequal variances

$\begin{array}{lrlr}\text { Difference } & 0.00033 & \text { t Ratio } & 0.540352 \\ \text { Std Err Dif } & 0.00060 \text { DF } & 16.72732 \\ \text { Upper CL Dif } & 0.00160 & \text { Prob }>|t| & 0.5961 \\ \text { Lower CL Dif } & -0.00095 & \text { Prob }>t & 0.2980 \\ \text { Confidence } & 0.95 & \text { Prob }<\mathrm{t} & 0.7020\end{array}$

Tests that the Variances are Equal

$\begin{array}{lrrrr}\text { Level } & \text { Count } & \text { Std Dev } & \text { MeanAbsDif to Mean } & \text { MeanAbsDif to Median } \\ \text { Coliwasa } & 15 & 0.0012985 & 0.0010465 & 0.0010181\end{array}$

$\begin{array}{lllll}\text { Hydragard } & 10 & 0.0015824 & 0.0012643 & 0.0012643\end{array}$

Test F Ratio DFNum DFDen p-Value

$\begin{array}{lllll}\text { O'Brien[.5] } & 0.9369 & 1 & 23 & 0.3432\end{array}$

$\begin{array}{lllll}\text { Brown-Forsythe } & 0.5016 & 1 & 23 & 0.4859\end{array}$

$\begin{array}{lllll}\text { Levene } & 0.4769 & 1 & 23 & 0.4967\end{array}$

$\begin{array}{lllll}\text { Bartlett } & 0.4186 & 1 & 0.5176 \\ \text { F Test 2-sided } & 1.4850 & 9 & 14 & 0.4892\end{array}$

Welch Anova testing Means Equal, allowing Std Devs Not Equal

F Ratio DFNum DFDen Prob $>$ F

$\begin{array}{llll}0.2920 & 1 & 16.727 & 0.5961\end{array}$

t Test 
Exhibit A3. Statistical Comparisons for Mid-Rheology (Phase 1) Testing (screened data)

Oneway Analysis of Measurement By Sampler Analytical Block=6, Analyte=Fe/Li, Target Value $=2.452$

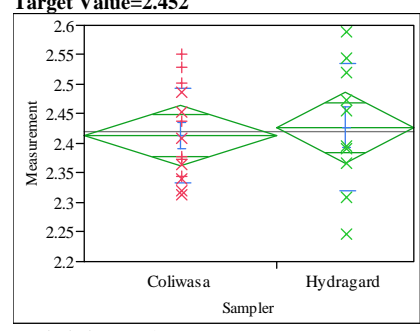

Excluded Rows 2

Oneway Anova

Rsquare $\quad 0.005941$

Adj Rsquare

Root Mean Square Error $\quad 0.092217$

Mean of Response 2.418991

Observations (or Sum Wgts) 24

t Test

Hydragard-Coliwasa

Assuming equal variances

Difference $\quad 0.01384$ t Ratio $\quad 0.362604$

$\begin{array}{lrlr}\text { Std Err Dif } & 0.03818 \text { DF } & 22 \\ \text { Upper CL Dif } & 0.09303 \text { Prob }>|t| & 0.7204\end{array}$

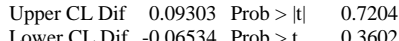

$\begin{array}{lrll}\text { Lower CL Dif } & -0.06534 & \text { Prob }>t & 0.3602 \\ \text { Confidence } & 0.95 & \text { Prob }<\mathrm{t} & 0.6398\end{array}$

Analysis of Variance

Source DF Sum of Squares Mean Square F Ratio Prob $>$ F

$\begin{array}{lrrrrr}\text { Sampler } & 1 & 0.00111811 & 0.001118 & 0.1315 & 0.7204\end{array}$

$\begin{array}{llll}\text { Error } & 22 & 0.18708623 & 0.008504\end{array}$

C. Total $23 \quad 0.18820434$

Means for Oneway Anova

Level Number Mean Std Error Lower 95\% Upper 95\%

$\begin{array}{lrrrrr}\text { Coliwasa } & 14 & 2.41322 & 0.02465 & 2.3621 & 2.4643\end{array}$

$\begin{array}{llllll}\text { Coliwasa } & 14 & 2.41322 & 0.02465 & 2.3621 & 2.4643 \\ \text { Hydragard } & 10 & 2.42707 & 0.02916 & 2.3666 & 2.4875\end{array}$

Std Error uses a pooled estimate of error variance

Means and Std Deviations

Level Number Mean Std Dev Std Err Mean Lower 95\% Upper 95\%

$\begin{array}{llllllr}\text { Coliwasa } & 14 & 2.41322 & 0.079710 & 0.02130 & 2.3672 & 2.4592 \\ \text { Hydragard } & 10 & 2.42707 & 0.107749 & 0.03407 & 2.3500 & 2.5041\end{array}$

t Test

Hydragard-Coliwasa

Hydragard-Coliwasa
Assuming unequal variances

Difference $\quad 0.01384$ t Ratio 0.344526

Std Err Dif $\quad 0.04018$ DF 15.74583

Upper CL Dif 0.09914 Prob $>|t| \quad 0.7350$

Lower CL Dif -0.07146 Prob $>t \quad 0.3675$

Confidence $\quad 0.95$ Prob $<\mathrm{t} \quad 0.6325$

Tests that the Variances are Equal

Level Count Std Dev MeanAbsDif to Mean MeanAbsDif to Median

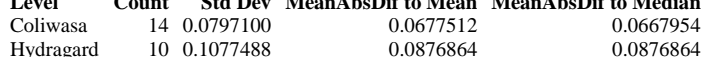

Test F Ratio DFNum DFDen p-Value

$\begin{array}{lrrrr}\text { O'Brien[.5] } & 1.6796 & 1 & 22 & 0.2084\end{array}$

$\begin{array}{lllll}\text { OBBrien[.5] } & 1.6796 & 1 & 22 & 0.2084 \\ \text { Brown-Forsythe } & 1.0321 & 1 & 22 & 0.3207\end{array}$

$\begin{array}{lllll}\text { Brown-Forsythe } & 1.0321 & 1 & 22 & 0.3207 \\ \text { Levene } & 1.1103 & 1 & 22 & 0.3034\end{array}$

$\begin{array}{lllrr}\text { Levene } & 1.1103 & 1 & 22 & 0.3034 \\ \text { Bartlett } & 0.9427 & 1 & . & 0.3316\end{array}$

$\begin{array}{lllll}\text { F Test 2-sided } & 1.8273 & 9 & 13 & 0.3131\end{array}$

Welch Anova testing Means Equal, allowing Std Devs Not Equal

F Ratio DFNum DFDen Prob > F

$\begin{array}{llll}0.1187 & 1 & 15.746 & 0.7350\end{array}$

t Test
0.3445
Oneway Analysis of Measurement By Sampler Analytical Block=6, Analyte=Li2O (wt \%), Target Value $=4.674$

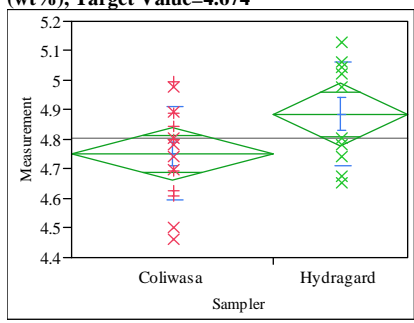

Excluded Rows 1

Oneway Anova

Rsquare $\quad 0.143863$

Adj Rsquare $\quad 0.10664$

Root Mean Square Error $\quad 0.165418$

$\begin{array}{lr}\text { Mean of Response } & 4.805273 \\ \text { Observations (or Sum Wgts) } & 25\end{array}$

t Test

Hydragard-Coliwasa

Assuming equal variances

Difference $\quad 0.13276$ t Ratio 1.965924

$\begin{array}{lrlr}\text { Std Err Dif } & 0.06753 & \text { DF } & 23 \\ \text { Upper CL Dif } & 0.27246 & \text { Prob }>|t| & 0.0615\end{array}$

$\begin{array}{rrrr}\text { Upper CL Dif } & 0.27246 & \text { Prob }>|t| & 0.0615 \\ \text { Lower CL Dif } & -0.00694 & \text { Prob }>t & 0.0307\end{array}$

$\begin{array}{lrll}\text { Lower CL Dif } & -0.00694 & \text { Prob }>t & 0.0307 \\ \text { Confidence } & 0.95 & \text { Prob }<t & 0.9693\end{array}$

Analysis of Variance

Source DF Sum of Squares Mean Square F Ratio Prob $>$ F

$\begin{array}{lrrrrr}\text { Sampler } & 1 & 0.10575476 & 0.105755 & 3.8649 & 0.0615\end{array}$

$\begin{array}{lll}\text { Error } & 23 & 0.62935282 \\ \text { C. Total } & 24 & 0.73510758\end{array}$

Means for Oneway Anova

Level Number Mean Std Error Lower 95\% Upper 95\%

$\begin{array}{lrrrrr}\text { Coliwasa } & 15 & 4.75217 & 0.04271 & 4.6638 & 4.8405\end{array}$

$\begin{array}{llllll}\text { Coliwasa } & 15 & 4.75217 & 0.04271 & 4.6638 & 4.8405 \\ \text { Hydragard } & 10 & 4.88493 & 0.05231 & 4.7767 & 4.9931\end{array}$

Std Error uses a pooled estimate of error variance

Means and Std Deviations

Level Number Mean Std Dev Std Err Mean Lower 95\% Upper 95\%

$\begin{array}{lrrrrrr}\text { Coliwasa } & 15 & 4.75217 & 0.158721 & 0.04098 & 4.6643 & 4.8401\end{array}$

$\begin{array}{lllllll}\text { Hydragard } & 10 & 4.88493 & 0.175329 & 0.05544 & 4.7595 & 5.0104\end{array}$

t Test

Hydragard-Coliwasa

Assuming unequal variances

$\begin{array}{lrlr}\text { Difference } & 0.13276 & \text { t Ratio } & 1.925606 \\ \text { Std Err Dif } & 0.06895 & \text { DF } & 18.05595 \\ \text { Upper CL Dif } & 0.27758 & \text { Prob }>|t| & 0.0701 \\ \text { Lower CL Dif } & -0.01206 & \text { Prob }>\mathrm{t} & 0.0350 \\ \text { Confidence } & 0.95 & \text { Prob }<\mathrm{t} & 0.9650\end{array}$

Tests that the Variances are Equal

$\begin{array}{lrrrr}\text { Level } & \text { Count } & \text { Std Dev } & \text { MeanAbsDif to Mean } & \text { MeanAbsDif to Median } \\ \text { Coliwasa } & 15 & 0.1587205 & 0.1266862 & 0.1263035\end{array}$

$\begin{array}{lllll}\text { Hydragard } & 10 & 0.1753289 & 0.1266862 & 0.1263035 \\ & & & 0.1571617 & 0.1571617\end{array}$

Test F Ratio DFNum DFDen p-Value

$\begin{array}{lllll}\text { O'Brien[.5] } & 0.2398 & 1 & 23 & 0.6290\end{array}$

$\begin{array}{lllll}\text { Brown-Forsythe } & 0.8493 & 1 & 23 & 0.3663\end{array}$

$\begin{array}{lllll}\text { Levene } & 0.9051 & 1 & 23 & 0.3513\end{array}$

$\begin{array}{lllll}\text { Bartlett } & 0.1050 & 1 & . & 0.7459\end{array}$

$\begin{array}{lllll}\text { F Test 2-sided } & 1.2202 & 9 & 14 & 0.7125\end{array}$

Welch Anova testing Means Equal, allowing Std Devs Not Equal

F Ratio DFNum DFDen Prob $>$ F

$\begin{array}{llll}3.7080 & 1 & 18.056 & 0.0701\end{array}$

t Test 
Exhibit A3. Statistical Comparisons for Mid-Rheology (Phase 1) Testing (screened data)

Oneway Analysis of Measurement By Sampler Analytical Block=6, Analyte=MgO (wt \%), Target Value $=1.014$

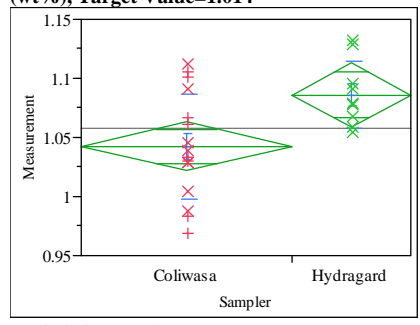

Excluded Rows 1

Oneway Anova

Rsquare $\quad 0.23339$

Adj Rsquare $\quad 0.20006$

Root Mean Square Error $\quad 0.039502$

\begin{tabular}{lr} 
Mean of Response & 1.058128 \\
\hline
\end{tabular}

Observations (or Sum Wgts) 25

t Test

Hydragard-Coliwasa

Assuming equal variances

Difference $\quad 0.043553$ t Ratio 2.646173

$\begin{array}{lrlr}\text { Std Err Dif } & 0.016459 \text { DF } & 23 \\ \text { Upper CL } & 0.077601 \text { Prob }>|t| & 0.0144\end{array}$

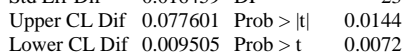

$\begin{array}{lrl}\text { Confidence } & 0.95 \text { Prob }<\mathrm{t} & 0.9928\end{array}$

Analysis of Variance

Source DF Sum of Squares Mean Square F Ratio Prob $>$ F

$\begin{array}{lrrrrr}\text { Sampler } & 1 & 0.01092614 & 0.010926 & 7.0022 & 0.0144\end{array}$

$\begin{array}{llll}\text { Error } & 23 & 0.03588872 & 0.001560\end{array}$

C. Total $24 \quad 0.04681486$

Means for Oneway Anova

Level Number Mean Std Error Lower 95\% Upper 95\%

$\begin{array}{lrrrrr}\text { Coliwasa } & 16 & 1.04245 & 0.00988 & 1.0220 & 1.0629\end{array}$

$\begin{array}{lrrrrr}\text { Coliwasa } & 16 & 1.04245 & 0.00988 & 1.0220 & 1.0629 \\ \text { Hydragard } & 9 & 1.08600 & 0.01317 & 1.0588 & 1.1132\end{array}$

Std Error uses a pooled estimate of error variance

Means and Std Deviations

Level Number Mean Std Dev Std Err Mean Lower 95\% Upper 95\%

$\begin{array}{lrrrrrr}\text { Coliwasa } & 16 & 1.04245 & 0.044443 & 0.01111 & 1.0188 & 1.0661 \\ \text { Hydragard } & 9 & 1.08600 & 0.027976 & 0.00933 & 1.0645 & 1.1075\end{array}$

\section{t Test}

Hydragard-Coliwasa

Hydragard-Coliwasa
Assuming unequal variances

Difference $\quad 0.043553$ t Ratio 3.002537

Std Err Dif $\quad 0.014506$ DF 22.57338

Upper CL Dif 0.073592 Prob $>|t| 0.0064$

Lower CL Dif 0.013515 Prob $>$ t 0.0032

$\begin{array}{lrl}\text { Confidence } & 0.95 \text { Prob }<\mathrm{t} \quad 0.9968\end{array}$

Tests that the Variances are Equal

Level Count Std Dev MeanAbsDif to Mean MeanAbsDif to Median

$\begin{array}{lrrrr}\text { Coliwasa } & 16 & 0.0444427 & 0.0353684 & 0.0348243 \\ \text { Hydragard } & 9 & 0.0279762 & 0.0219059 & 0.0211894\end{array}$

Test F Ratio DFNum DFDen p-Value

$\begin{array}{lrrrr}\text { O'Brien[.5] } & 2.5385 & 1 & 23 & 0.1248\end{array}$

$\begin{array}{lllll}\text { OBBrien[.5] } & 2.5385 & 1 & 23 & 0.1248 \\ \text { Brown-Forsythe } & 1.7864 & 1 & 23 & 0.1944\end{array}$

$\begin{array}{lllll}\text { Brown-Forsythe } & 1.7864 & 1 & 23 & 0.1944 \\ \text { Levene } & 2.0779 & 1 & 23 & 0.1629\end{array}$

$\begin{array}{llrrr}\text { Levene } & 2.0779 & 1 & 23 & 0.1629 \\ \text { Bartlett } & 1.8906 & 1 & . & 0.1691\end{array}$

$\begin{array}{lllll}\text { F Test 2-sided } & 2.5236 & 15 & 8 & 0.1886\end{array}$

Welch Anova testing Means Equal, allowing Std Devs Not Equal

F Ratio DFNum DFDen Prob $>$ F

$\begin{array}{llll}9.0152 & 1 & 22.573 & 0.0064\end{array}$

t Test
3.0025
Oneway Analysis of Measurement By Sampler Analytical Block=6, Analyte=Sum of Oxides (wt \%), Target Value $=99.553$

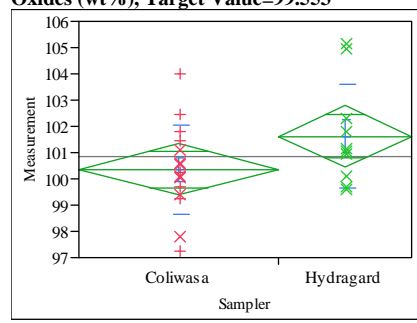

Excluded Rows 1

Oneway Anova

Rsquare $\quad 0.112117$

Adj Rsquare $\quad 0.073513$

Root Mean Square Error $\quad 1.812142$

$\begin{array}{lr}\text { Mean of Response } & 100.8618 \\ \text { Observations (or Sum Wgts) } & 25\end{array}$

t Test

Hydragard-Coliwasa

Assuming equal variances

Difference $\quad 1.2608$ t Ratio 1.704201

$\begin{array}{lrlr}\text { Std Err Dif } & 0.7398 & \text { DF } & 23 \\ \text { Upper CL } & 2.7912 & \text { Prob }>|t| & 0.1018\end{array}$

$\begin{array}{lrll}\text { Upper CL Dif } & 2.7912 & \text { Prob }>|t| & 0.1018 \\ \text { Lower CL Dif } & -0.2696 & \text { Prob }>t & 0.0509\end{array}$

$\begin{array}{lrll}\text { Lower CL Dif } & -0.2696 & \text { Prob }>t & 0.0509 \\ \text { Confidence } & 0.95 & \text { Prob }<\mathrm{t} & 0.9491\end{array}$

Analysis of Variance

Source DF Sum of Squares Mean Square F Ratio Prob $>$ F

$\begin{array}{lrrrrr}\text { Sampler } & 1 & 9.537315 & 9.53731 & 2.9043 & 0.1018\end{array}$

$\begin{array}{llll}\text { Error } & 23 & 75.528775 & 3.28386\end{array}$

C. Total $24 \quad 85.066090$

Means for Oneway Anova

Level Number Mean Std Error Lower 95\% Upper 95\%

$\begin{array}{lrrrrr}\text { Coliwasa } & 15 & 100.357 & 0.46789 & 99.39 & 101.33\end{array}$

$\begin{array}{lllrrr}\text { Hydragard } & 10 & 101.618 & 0.57305 & 100.43 & 102.80\end{array}$

Std Error uses a pooled estimate of error variance

Means and Std Deviations

Level Number Mean Std Dev Std Err Mean Lower 95\% Upper 95\%

$\begin{array}{lrrrrrr}\text { Coliwasa } & 15 & 100.357 & 1.70299 & 0.43971 & 99.41 & 101.30\end{array}$

$\begin{array}{lllllll}\text { Hydragard } & 10 & 101.618 & 1.96996 & 0.62295 & 100.21 & 103.03\end{array}$

t Test

Hydragard-Coliwasa

Assuming unequal variances

$\begin{array}{lrlr}\text { Difference } & 1.2608 & \mathrm{t} \text { Ratio } & 1.65346 \\ \text { Std Err Dif } & 0.7625 \text { DF } & 17.42186 \\ \text { Upper CL Dif } & 2.8666 & \text { Prob }>|\mathrm{t}| & 0.1161 \\ \text { Lower CL Dif } & -0.3450 & \text { Prob }>\mathrm{t} & 0.0581 \\ \text { Confidence } & 0.95 & \text { Prob }<\mathrm{t} & 0.9419\end{array}$

Tests that the Variances are Equal

$\begin{array}{lrrrr}\text { Level } & \text { Count } & \text { Std Dev } & \text { MeanAbsDif to Mean } & \text { MeanAbsDif to Median } \\ \text { Coliwasa } & 15 & 1.702985 & 1.229066 & 1.237171 \\ \text { Hydragard } & 10 & 1.969956 & 1.494689 & 1.393149\end{array}$

Test F Ratio DFNum DFDen p-Value

$\begin{array}{lllll}\text { O'Brien[.5] } & 0.2484 & 1 & 23 & 0.6230\end{array}$

$\begin{array}{lllll}\text { Brown-Forsythe } & 0.0917 & 1 & 23 & 0.7647\end{array}$

$\begin{array}{lllll}\text { Levene } & 0.3189 & 1 & 23 & 0.5777\end{array}$

$\begin{array}{lllll}\text { Bartlett } & 0.2260 & 1 & . & 0.6345\end{array}$

$\begin{array}{lllll}\text { F Test 2-sided } & 1.3381 & 9 & 14 & 0.6031\end{array}$

Welch Anova testing Means Equal, allowing Std Devs Not Equal

F Ratio DFNum DFDen Prob > F

$\begin{array}{llll}2.7339 & 1 & 17.422 & 0.1161\end{array}$

t Test
1.6535 
Appendix B.

Supplemental Tables and Exhibits for High-Rheology (Phase 2) Simulant Testing 
Table B1. Density Measurements for Phase 2

\begin{tabular}{|c|c|c|c|c|}
\hline Sampling Mechanism & Flow Rate (LF/HF) & Sampler w Location & Sample ID & Sample Density (g/mL) \\
\hline Coliwasa & LF & Coliwasa Low & DCL01 & 1.3383 \\
\hline Coliwasa & $\mathrm{LF}$ & Coliwasa Low & \begin{tabular}{|l|} 
DCL02 \\
\end{tabular} & 1.3471 \\
\hline Coliwasa & $\mathrm{LF}$ & Coliwasa Low & DCL03 & 1.3291 \\
\hline Coliwasa & LF & Coliwasa Low & DCL04 & 1.3499 \\
\hline Coliwasa & $\mathrm{HF}$ & Coliwasa Low & \begin{tabular}{|l|l} 
DCL05 \\
\end{tabular} & 1.3416 \\
\hline Coliwasa & $\mathrm{HF}$ & Coliwasa Low & DCL06 & 1.3406 \\
\hline Coliwasa & $\mathrm{HF}$ & Coliwasa Low & \begin{tabular}{|l|l} 
DCL07 \\
\end{tabular} & 1.3285 \\
\hline Coliwasa & $\mathrm{HF}$ & Coliwasa Low & DCL08 & 1.3366 \\
\hline Coliwasa & $\mathrm{LF}$ & Coliwasa High & DCH01 & 1.3291 \\
\hline Coliwasa & LF & Coliwasa High & DCH02 & 1.3437 \\
\hline Coliwasa & $\mathrm{LF}$ & Coliwasa High & DCH03 & 1.3519 \\
\hline Coliwasa & $\mathrm{LF}$ & Coliwasa High & DCH04 & 1.3510 \\
\hline Coliwasa & $\mathrm{HF}$ & Coliwasa High & DCH05 & 1.3306 \\
\hline Coliwasa & $\mathrm{HF}$ & Coliwasa High & DCH06 & 1.3380 \\
\hline Coliwasa & $\mathrm{HF}$ & Coliwasa High & DCH07 & 1.3357 \\
\hline Coliwasa & $\mathrm{HF}$ & Coliwasa High & \begin{tabular}{|l|} 
DCH08 \\
\end{tabular} & 1.3121 \\
\hline Hydragard & LF & Hydragard & DLHY01 & 1.3890 \\
\hline Hydragard & $\mathrm{LF}$ & Hydragard & DLHY02 & 1.3403 \\
\hline Hydragard & $\mathrm{LF}$ & Hydragard & DLHY03 & 1.3422 \\
\hline Hydragard & $\mathrm{LF}$ & Hydragard & DLHY04 & 1.3220 \\
\hline Hydragard & $\mathrm{LF}$ & Hydragard & DLHY05 & 1.3345 \\
\hline Hydragard & LF & Hydragard & DLHY06 & 1.3315 \\
\hline Hydragard & LF & Hydragard & DLHY07 & 1.3524 \\
\hline Hydragard & $\mathrm{LF}$ & Hydragard & DLHY08 & 1.3463 \\
\hline Hydragard & LF & Hydragard & DLHY09 & 1.3653 \\
\hline Hydragard & LF & Hydragard & DLHY10 & 1.3481 \\
\hline Hydragard & $\mathrm{HF}$ & \begin{tabular}{|l} 
Hydragard \\
\end{tabular} & DHHY01 & 1.3578 \\
\hline Hydragard & $\mathrm{HF}$ & Hydragard & DHHY02 & 1.3462 \\
\hline Hydragard & $\mathrm{HF}$ & Hydragard & DHHY03 & 1.3374 \\
\hline Hydragard & $\mathrm{HF}$ & Hydragard & DHHY04 & 1.3304 \\
\hline Hydragard & $\mathrm{HF}$ & Hydragard & DHHY05 & 1.3216 \\
\hline Hydragard & $\mathrm{HF}$ & Hydragard & DHHY06 & 1.3556 \\
\hline Hydragard & $\mathrm{HF}$ & Hydragard & DHHY07 & 1.3352 \\
\hline Hydragard & $\mathrm{HF}$ & Hydragard & DHHY08 & 1.3244 \\
\hline Hydragard & $\mathrm{HF}$ & Hydragard & DHHY09 & 1.3279 \\
\hline Hydragard & $\mathrm{HF}$ & Hydragard & DHHY10 & 1.3349 \\
\hline Isolok & $\mathrm{LF}$ & Isolok & DLIB01 & 1.3238 \\
\hline Isolok & LF & Isolok & DLIB02 & 1.3515 \\
\hline Isolok & LF & Isolok & DLIB03 & 1.3342 \\
\hline Isolok & LF & Isolok & DLIB04 & 1.3234 \\
\hline Isolok & LF & Isolok & DLIB05 & 1.3303 \\
\hline Isolok & $\mathrm{LF}$ & Isolok & DLIB06 & 1.3183 \\
\hline Isolok & $\mathrm{LF}$ & Isolok & DLIB07 & 1.3505 \\
\hline Isolok & LF & Isolok & \begin{tabular}{|l|} 
DLIB08 \\
\end{tabular} & 1.3331 \\
\hline Isolok & LF & Isolok & DLIB09 & 1.3402 \\
\hline Isolok & $\mathrm{LF}$ & Isolok & DLIB10 & 1.3166 \\
\hline Isolok & $\mathrm{HF}$ & Isolok & \begin{tabular}{|l|} 
DHIB01 \\
\end{tabular} & 1.3681 \\
\hline Isolok & $\mathrm{HF}$ & Isolok & \begin{tabular}{|l} 
DHIB02 \\
\end{tabular} & 1.3666 \\
\hline Isolok & $\mathrm{HF}$ & Isolok & DHIB03 & 1.3242 \\
\hline Isolok & $\mathrm{HF}$ & Isolok & DHIB04 & 1.3467 \\
\hline Isolok & $\mathrm{HF}$ & Isolok & DHIB05 & 1.3410 \\
\hline Isolok & $\mathrm{HF}$ & Isolok & DHIB06 & 1.3770 \\
\hline Isolok & $\mathrm{HF}$ & Isolok & DHIB07 & 1.3264 \\
\hline Isolok & $\mathrm{HF}$ & Isolok & DHIB08 & 1.3191 \\
\hline Isolok & $\mathrm{HF}$ & Isolok & DHIB09 & 1.3323 \\
\hline Isolok & $\mathrm{HF}$ & Isolok & DHIB10 & 1.3484 \\
\hline
\end{tabular}


SRNL-STI-2011-00693

Revision 0

Table B2. Solids Measurements for Phase 2

\begin{tabular}{|c|c|c|c|c|c|}
\hline $\begin{array}{l}\text { Sampling } \\
\text { Mechanism }\end{array}$ & \begin{tabular}{|l|}
$\begin{array}{l}\text { Flow Rate } \\
\text { (LF/HF) }\end{array}$ \\
\end{tabular} & \begin{tabular}{|l|}
$\begin{array}{l}\text { Sampler w } \\
\text { Location }\end{array}$ \\
\end{tabular} & Sample ID & wt\% dried solids & wt \% vitrified solids \\
\hline Coliwasa & LF & Coliwasa Low & CL01 & 40.73 & 33.14 \\
\hline Coliwasa & LF & Coliwasa Low & CL02 & 40.73 & 32.94 \\
\hline Coliwasa & LF & Coliwasa Low & CL03 & 40.42 & 32.74 \\
\hline Coliwasa & LF & Coliwasa Low & CL04 & 40.58 & 32.90 \\
\hline Coliwasa & HF & Coliwasa Low & CL05 & 40.51 & 32.73 \\
\hline Coliwasa & HF & Coliwasa Low & CL06 & 40.34 & 32.70 \\
\hline Coliwasa & HF & Coliwasa Low & CL07 & 40.79 & 33.01 \\
\hline Coliwasa & HF & Coliwasa Low & CL08 & 41.88 & 33.88 \\
\hline Coliwasa & LF & Coliwasa High & CH01 & 39.03 & 31.57 \\
\hline Coliwasa & LF & Coliwasa High & $\mathrm{CH} 02$ & 40.39 & 32.57 \\
\hline Coliwasa & LF & Coliwasa High & $\mathrm{CH} 03$ & 40.95 & 33.19 \\
\hline Coliwasa & LF & Coliwasa High & $\mathrm{CH} 04$ & 40.09 & 32.45 \\
\hline Coliwasa & $\mathrm{HF}$ & Coliwasa High & CH05 & 40.91 & 33.15 \\
\hline Coliwasa & HF & Coliwasa High & CH06 & 40.81 & 33.09 \\
\hline Coliwasa & HF & Coliwasa High & $\mathrm{CH} 07$ & 41.09 & 33.30 \\
\hline Coliwasa & HF & Coliwasa High & CH08 & 40.47 & 32.71 \\
\hline Hydragard & LF & Hydragard & LHY01 & 40.37 & 32.74 \\
\hline Hydragard & LF & Hydragard & LHY02 & 40.36 & 32.72 \\
\hline Hydragard & LF & Hydragard & LHY03 & 40.41 & 32.77 \\
\hline Hydragard & LF & Hydragard & LHY04 & 40.54 & 32.87 \\
\hline Hydragard & LF & Hydragard & LHY05 & 40.57 & 32.94 \\
\hline Hydragard & LF & Hydragard & LHY06 & 39.19 & 31.84 \\
\hline Hydragard & LF & Hydragard & LHY07 & 40.48 & 32.89 \\
\hline Hydragard & LF & Hydragard & LHY08 & 40.55 & 32.85 \\
\hline Hydragard & LF & Hydragard & LHY09 & 40.90 & 33.19 \\
\hline Hydragard & LF & Hydragard & LHY10 & 40.79 & 33.12 \\
\hline Hydragard & $\mathrm{HF}$ & Hydragard & HHY01 & 39.88 & 32.09 \\
\hline Hydragard & $\mathrm{HF}$ & Hydragard & HHY02 & 40.15 & 32.40 \\
\hline Hydragard & $\mathrm{HF}$ & Hydragard & HHY03 & 38.67 & 32.24 \\
\hline Hydragard & $\mathrm{HF}$ & Hydragard & HHY04 & 40.11 & 32.37 \\
\hline Hydragard & $\mathrm{HF}$ & Hydragard & HHY05 & 40.02 & 32.33 \\
\hline Hydragard & $\mathrm{HF}$ & Hydragard & HHY06 & 40.15 & 32.44 \\
\hline Hydragard & $\mathrm{HF}$ & Hydragard & HHY07 & 40.34 & 32.62 \\
\hline Hydragard & $\mathrm{HF}$ & Hydragard & HHY08 & 40.30 & 32.52 \\
\hline Hydragard & HF & Hydragard & HHY09 & 40.13 & 32.41 \\
\hline Hydragard & HF & Hydragard & HHY10 & 40.19 & 32.44 \\
\hline Isolok (3 mL) & LF & Isolok (3 mL) & LIS01 & 40.60 & 32.70 \\
\hline Isolok (3 mL) & LF & Isolok (3 mL) & LIS02 & 40.47 & 32.58 \\
\hline Isolok (3 mL) & LF & Isolok (3 mL) & LIS03 & 40.25 & 32.35 \\
\hline Isolok (3 mL) & LF & Isolok (3 mL) & LIS04 & 40.50 & 32.54 \\
\hline Isolok (3 mL) & LF & Isolok (3 mL) & LIS05 & 40.37 & 32.49 \\
\hline Isolok (3 mL) & LF & Isolok (3 mL) & LIS06 & 40.78 & 32.77 \\
\hline Isolok (3 mL) & LF & Isolok (3 mL) & LIS07 & 40.58 & 32.58 \\
\hline Isolok (3 mL) & LF & Isolok (3 mL) & LIS08 & 40.76 & 32.60 \\
\hline Isolok (3 mL) & LF & Isolok (3 mL) & LIS09 & 40.72 & 32.64 \\
\hline Isolok (3 mL) & LF & Isolok (3 mL) & LIS10 & 40.95 & 32.91 \\
\hline Isolok (3 mL) & $\mathrm{HF}$ & Isolok (3 mL) & HIS01 & 41.80 & 33.63 \\
\hline Isolok (3 mL) & $\mathrm{HF}$ & Isolok (3 mL) & HIS02 & 41.39 & 33.35 \\
\hline Isolok (3 mL) & HF & Isolok (3 mL) & HIS03 & 41.71 & 33.78 \\
\hline Isolok (3 mL) & HF & Isolok (3 mL) & HIS04 & 41.34 & 33.23 \\
\hline Isolok (3 mL) & $\mathrm{HF}$ & Isolok (3 mL) & HIS05 & 41.77 & 33.62 \\
\hline Isolok (3 mL) & HF & Isolok (3 mL) & HIS06 & 41.42 & 33.36 \\
\hline Isolok (3 mL) & HF & Isolok (3 mL) & HIS07 & 41.45 & 33.41 \\
\hline Isolok (3 mL) & $\mathrm{HF}$ & Isolok (3 mL) & HIS08 & 41.35 & 33.16 \\
\hline Isolok (3 mL) & HF & Isolok (3 mL) & HIS09 & 41.75 & 33.56 \\
\hline Isolok (3 mL) & $\mathrm{HF}$ & Isolok (3 mL) & HIS10 & 41.68 & 33.58 \\
\hline Isolok (12 mL) & LF & Isolok (12 mL) & LIB01 & 40.85 & 32.70 \\
\hline Isolok (12 mL) & LF & Isolok (12 mL) & LIB02 & 40.85 & 32.69 \\
\hline Isolok (12 mL) & LF & Isolok (12 mL) & LIB03 & 40.80 & 32.63 \\
\hline Isolok (12 mL) & LF & Isolok (12 mL) & LIB04 & 40.93 & 32.72 \\
\hline Isolok (12 mL) & LF & Isolok (12 mL) & LIB05 & 40.87 & 32.69 \\
\hline Isolok (12 mL) & LF & Isolok (12 mL) & LIB06 & 40.99 & 32.81 \\
\hline Isolok $(12 \mathrm{~mL})$ & LF & Isolok (12 mL) & LIB07 & 41.08 & 32.93 \\
\hline
\end{tabular}


SRNL-STI-2011-00693

Revision 0

Table B2. Solids Measurements for Phase 2

\begin{tabular}{|c|c|c|c|c|c|}
\hline $\begin{array}{l}\text { Sampling } \\
\text { Mechanism }\end{array}$ & $\begin{array}{l}\text { Flow Rate } \\
\text { (LF/HF) } \\
\end{array}$ & $\begin{array}{l}\text { Sampler w } \\
\text { Location } \\
\end{array}$ & Sample ID & wt\% dried solids & wt $\%$ vitrified solids \\
\hline Isolok (12 mL) & LF & Isolok (12 mL) & LIB08 & 40.96 & 32.75 \\
\hline Isolok (12 mL) & LF & Isolok (12 mL) & LIB09 & 41.06 & 32.82 \\
\hline Isolok (12 mL) & LF & Isolok (12 mL) & LIB10 & 41.03 & 32.83 \\
\hline Isolok (12 mL) & $\mathrm{HF}$ & Isolok (12 mL) & HIB01 & 41.52 & 33.45 \\
\hline Isolok (12 mL) & $\mathrm{HF}$ & Isolok (12 mL) & HIB02 & 41.60 & 33.56 \\
\hline Isolok (12 mL) & $\mathrm{HF}$ & Isolok (12 mL) & HIB03 & 41.26 & 33.23 \\
\hline Isolok (12 mL) & $\mathrm{HF}$ & Isolok (12 mL) & HIB04 & 41.42 & 33.33 \\
\hline Isolok (12 mL) & HF & Isolok (12 mL) & HIB05 & 41.31 & 33.28 \\
\hline Isolok (12 mL) & $\mathrm{HF}$ & Isolok (12 mL) & HIB06 & 41.36 & 33.58 \\
\hline Isolok (12 mL) & HF & Isolok (12 mL) & HIB07 & 41.53 & 33.45 \\
\hline Isolok (12 mL) & $\mathrm{HF}$ & Isolok (12 mL) & HIB08 & 41.30 & 33.26 \\
\hline Isolok (12 mL) & HF & Isolok (12 mL) & HIB09 & 41.44 & 33.38 \\
\hline Isolok (12 mL) & $\mathrm{HF}$ & Isolok (12 mL) & HIB10 & 41.35 & 33.33 \\
\hline
\end{tabular}


Table B3. Phase 2 Elemental Concentration Measurements (part 1)

\begin{tabular}{|c|c|c|c|c|c|c|c|c|c|c|c|c|c|c|c|c|c|c|c|}
\hline $\begin{array}{c}\text { Type of } \\
\text { Material }\end{array}$ & Sampler & $\begin{array}{l}\text { Flow } \\
\text { Rate }\end{array}$ & $\begin{array}{c}\text { Sample } \\
\text { Order }\end{array}$ & \begin{tabular}{|c|}
$\begin{array}{c}\text { Preparation } \\
\text { Block }\end{array}$ \\
\end{tabular} & \begin{tabular}{|c|} 
Preparation \\
Sequence \\
\end{tabular} & \begin{tabular}{|c|}
$\begin{array}{c}\text { Analytical } \\
\text { Block }\end{array}$ \\
\end{tabular} & \begin{tabular}{|c|} 
Analytical \\
Sequence \\
\end{tabular} & Sample ID & Al (ug/g) & B (ug/g) & $\begin{array}{c}\begin{array}{c}\text { Ba } \\
\text { (ug/g) }\end{array} \\
\left(\mathrm{u} /{ }^{2}\right.\end{array}$ & $\begin{array}{c}\begin{array}{c}\text { Ca } \\
\text { (ug/g) }\end{array} \\
\left(\mathrm{u} /{ }^{2}\right.\end{array}$ & $\begin{array}{c}\mathrm{Ce} \\
\text { (ug/g) }\end{array}$ & $\begin{array}{c}\mathrm{Cr} \\
\text { (ug/g) }\end{array}$ & $\begin{array}{c}\mathrm{Cu} \\
(\mathrm{ug} / \mathrm{g})\end{array}$ & $\mathrm{Fe}(\mathrm{ug} / \mathrm{g})$ & K (ug/g) & $\begin{array}{c}\mathbf{L a} \\
\text { (ug/g) }\end{array}$ & $\mathrm{Li}$ (ug/g) \\
\hline ARG-1 & None & 0 & 0 & $\begin{array}{l}1 \\
\end{array}$ & 1 & \begin{tabular}{|l|}
1 \\
\end{tabular} & \begin{tabular}{|l|}
1 \\
\end{tabular} & ARG-1B11 & 25200 & 24800 & 894 & 10500 & $<593$ & 681 & 75.5 & 100000 & 23100 & $<123$ & 14800 \\
\hline SME Simulant & Isolok - $3 \mathrm{~mL}$ & LF & 18 & 1 & 9 & 1 & 2 & LIS03 & 62500 & 14000 & 46.8 & 4540 & $<564$ & 153 & 804 & 76800 & $<2770$ & $<117$ & 23500 \\
\hline SME Simulant & Isolok - $3 \mathrm{~mL}$ & LF & 8 & 1 & 27 & 1 & 3 & LIS01 & 60500 & 14200 & 48.7 & 4410 & $<574$ & 159 & 774 & 74900 & $<2820$ & $<119$ & 23500 \\
\hline SME Simulant & Coliwasa & LF & 20 & 1 & 2 & 1 & 4 & CL02 & 62500 & 14700 & 50.2 & 4690 & $<559$ & 128 & 837 & 77800 & $<2750$ & $<116$ & 25100 \\
\hline SME Simulant & Coliwasa & LF & 38 & 1 & 16 & 1 & 5 & $\mathrm{CH} 03$ & 52600 & 13000 & 45.2 & 4020 & $<589$ & 132 & 707 & 65000 & $<2890$ & $<122$ & 22200 \\
\hline SME Simulant & $\begin{array}{l}\text { Coliwasa } \\
\end{array}$ & LF & 63 & 1 & 13 & 1 & 6 & CH04 & 59700 & 13900 & 57.6 & 4240 & $<581$ & 124 & 801 & 73700 & $<2860$ & $<121$ & 22800 \\
\hline SME Simulant & Isolok - $3 \mathrm{~mL}$ & LF & 44 & 1 & 3 & 1 & 7 & LIS07 & 61400 & 13800 & 52.3 & 4470 & $<522$ & 148 & 721 & 75700 & $<2570$ & $<109$ & 23700 \\
\hline SME Simulant & Coliwasa & $\mathrm{HF}$ & 23 & 1 & 28 & 1 & 8 & CH06 & 60100 & 13700 & 47.7 & 4430 & $<530$ & 150 & 769 & 75400 & $<2610$ & $<110$ & 23700 \\
\hline SME Simulant & Coliwasa & LF & 21 & 1 & 14 & 1 & 9 & $\mathrm{CH} 02$ & 56800 & 14500 & 45.7 & 3790 & $<558$ & 155 & 641 & 71500 & $<2740$ & $<116$ & 23200 \\
\hline SME Simulant & Coliwasa & $\mathrm{HF}$ & 3 & 1 & 20 & 1 & 10 & CL05 & 58900 & 14300 & 43.2 & 4240 & $<547$ & 136 & 723 & 73100 & $<2690$ & $<114$ & 23200 \\
\hline SME Simulant & Isolok - $3 \mathrm{~mL}$ & LF & 12 & 1 & 25 & 1 & 11 & LIS02 & 59200 & 13900 & 45.2 & 4290 & $<553$ & 139 & 640 & 73800 & $<2720$ & $<115$ & 23800 \\
\hline SME Simulant & Coliwasa & LF & 40 & 1 & 19 & 1 & 12 & CL03 & 60300 & 15000 & 69.4 & 4360 & $<593$ & 127 & 819 & 73800 & $<2910$ & $<123$ & 24200 \\
\hline SME Simulant & Coliwasa & $\mathrm{HF}$ & 18 & 1 & 26 & 1 & 13 & CL06 & 53400 & 13400 & 43.3 & 3970 & $<542$ & 123 & 741 & 65900 & $<2660$ & $<113$ & 21800 \\
\hline SME Simulant & Coliwasa & $\mathrm{HF}$ & 1 & 1 & 22 & 1 & 14 & CH05 & 58100 & 14900 & 53.7 & 4450 & $<603$ & 130 & 764 & 71800 & $<2970$ & $<126$ & 24900 \\
\hline ARG-1 & None & 0 & 0 & 1 & 15 & 1 & 15 & ARG-1B12 & 25300 & 24900 & 888 & 10600 & $<498$ & 687 & $<36.2$ & 102000 & 23100 & $<104$ & 15000 \\
\hline SME Simulant & $\begin{array}{l}\text { Coliwasa } \\
\end{array}$ & $\mathrm{HF}$ & 36 & 1 & 21 & 1 & 16 & CL07 & 58500 & 13900 & 45.3 & 4290 & $<554$ & 126 & 775 & 73000 & $<2720$ & $<115$ & 22800 \\
\hline SME Simulant & Isolok - $3 \mathrm{~mL}$ & LF & 31 & 1 & 24 & 1 & 17 & LIS05 & 61800 & 13100 & 55.3 & 4390 & $<589$ & 115 & 760 & 76400 & $<2890$ & $<122$ & 23400 \\
\hline SME Simulant & Isolok - $3 \mathrm{~mL}$ & LF & 58 & 1 & 4 & 1 & 18 & LIS10 & 59000 & 13800 & 45.6 & 4170 & $<524$ & 108 & 725 & 73100 & $<2580$ & $<109$ & 24000 \\
\hline SME Simulant & $\begin{array}{l}\text { Coliwasa } \\
\end{array}$ & $\mathrm{HF}$ & 63 & 1 & 5 & 1 & 19 & CL08 & 58000 & 13800 & 46.1 & 4310 & $<563$ & 131 & 747 & 72300 & $<2770$ & $<117$ & 23800 \\
\hline SME Simulant & Isolok - $3 \mathrm{~mL}$ & LF & 25 & 1 & 7 & 1 & 20 & LIS04 & 61500 & 13500 & 52.3 & 5360 & $<575$ & 152 & 814 & 75700 & $<2820$ & $<120$ & 23700 \\
\hline SME Simulant & Isolok - $3 \mathrm{~mL}$ & LF & 42 & 1 & 11 & 1 & 21 & LIS06 & 61600 & 14200 & 60.3 & 4430 & $<574$ & 169 & 749 & 76400 & $<2820$ & $<119$ & 24600 \\
\hline SME Simulant & Coliwasa & $\mathrm{HF}$ & 65 & 1 & 6 & 1 & 22 & CH08 & 59400 & 13800 & 50.5 & 4570 & $<549$ & 143 & 835 & 72700 & $<2700$ & $<114$ & 24200 \\
\hline SME Simulant & Coliwasa & $\mathrm{HF}$ & 34 & 1 & 18 & 1 & 23 & CH07 & 61700 & 15000 & 56.6 & 4520 & $<602$ & 169 & 823 & 76300 & $<2960$ & $<125$ & 25000 \\
\hline SME Simulant & Coliwasa & LF & 1 & 1 & 12 & 1 & 24 & CH01 & 56500 & 14400 & 48.2 & 4240 & $<575$ & 120 & 698 & 69400 & $<2830$ & $<120$ & 24600 \\
\hline SME Simulant & Isolok - $3 \mathrm{~mL}$ & LF & 54 & 1 & 17 & 1 & 25 & LIS09 & 60500 & 13900 & 51.1 & 4410 & $<496$ & 108 & 710 & 74900 & $<2440$ & $<103$ & 23800 \\
\hline SME Simulant & $\begin{array}{l}\text { Coliwasa } \\
\end{array}$ & LF & 4 & 1 & 10 & 1 & 26 & $\begin{array}{ll}\text { CL01 } \\
\end{array}$ & 59500 & 14300 & 48.1 & 4200 & $<553$ & 135 & 818 & 73600 & $<2720$ & $<115$ & 23000 \\
\hline SME Simulant & Isolok - $3 \mathrm{~mL}$ & LF & 49 & 1 & 8 & 1 & 27 & LIS08 & 61600 & 13400 & $\begin{array}{ll}47.8 \\
\end{array}$ & 4460 & $<544$ & 113 & 690 & 76400 & $<2670$ & $<113$ & 23800 \\
\hline SME Simulant & Coliwasa & LF & 65 & 1 & 23 & 1 & 28 & CL04 & 59000 & 13400 & 57.9 & 4330 & $<597$ & 123 & 795 & 72700 & $<2930$ & $<124$ & 23600 \\
\hline ARG-1 & None & 0 & 0 & 1 & 29 & 1 & 29 & ARG-1B13 & 23000 & 21900 & 803 & 9750 & $<578$ & 616 & 49 & 92000 & 19600 & $<120$ & 13500 \\
\hline ARG-1 & None & 0 & 0 & 2 & 1 & 2 & 1 & ARG-1B21 & 23100 & 23700 & 812 & 9440 & $<544$ & 627 & $<39.6$ & 92600 & 22200 & $<113$ & 14000 \\
\hline SME Simulant & Hydragard & $\mathrm{HF}$ & 11 & 2 & 3 & 2 & 2 & HHY02 & 60100 & 13700 & 50.5 & 4230 & $<538$ & 156 & 745 & 74100 & $<2640$ & $<112$ & 24200 \\
\hline SME Simulant & $\begin{array}{l}\text { Coliwasa } \\
\end{array}$ & $\mathrm{HF}$ & 36 & 1 & 21 & 2 & 3 & $\begin{array}{l}\text { CL07 } \\
\end{array}$ & 58000 & 14000 & 42 & 4160 & $<554$ & 122 & 806 & 71900 & $<2720$ & $<115$ & 23700 \\
\hline SME Simulant & Hydragard & $\mathrm{HF}$ & 24 & 2 & 2 & 2 & 4 & HHY04 & 60700 & 12800 & 61.8 & 4090 & $<588$ & 162 & 727 & 76300 & $<2890$ & $<122$ & 22100 \\
\hline SME Simulant & Coliwasa & $\mathrm{HF}$ & 1 & 1 & 22 & 2 & 5 & CH05 & 56900 & 14400 & 48.2 & 4120 & $<603$ & 140 & 813 & 70200 & $<2970$ & $<126$ & 24800 \\
\hline SME Simulant & Coliwasa & $\mathrm{HF}$ & 3 & 1 & 20 & 2 & 6 & CL05 & 58700 & 14700 & 49.8 & 4090 & $<547$ & 132 & 758 & 72600 & $<2690$ & $<114$ & 24100 \\
\hline SME Simulant & $\begin{array}{l}\text { Coliwasa } \\
\end{array}$ & $\mathrm{HF}$ & 23 & 1 & 28 & 2 & 7 & CH06 & 59300 & 14000 & 51.4 & 4140 & $<530$ & 134 & 728 & 74600 & $<2610$ & $<110$ & 23500 \\
\hline SME Simulant & $\begin{array}{l}\text { Coliwasa } \\
\end{array}$ & LF & 1 & 1 & 12 & 2 & 8 & CH01 & 55800 & 14300 & 45.4 & 4000 & $<575$ & 115 & 751 & 68700 & $<2830$ & $<120$ & 24800 \\
\hline SME Simulant & $\begin{array}{l}\text { Coliwasa } \\
\end{array}$ & LF & 20 & 1 & 2 & 2 & 9 & CL02 & 59500 & 14300 & 46.3 & 4240 & $<559$ & 118 & 759 & 74500 & $<2750$ & $<116$ & 24000 \\
\hline SME Simulant & $\begin{array}{l}\text { Coliwasa } \\
\end{array}$ & $\mathrm{HF}$ & 63 & 1 & 5 & 2 & 10 & $\begin{array}{ll}\text { CL08 } \\
\end{array}$ & 56800 & 14000 & 51.2 & 3970 & $<563$ & 137 & 736 & 70800 & $<2770$ & $<117$ & 23700 \\
\hline SME Simulant & $\begin{array}{l}\text { Coliwasa } \\
\end{array}$ & $\mathrm{HF}$ & 65 & 1 & 6 & 2 & 11 & CH08 & 58100 & 13800 & 47.2 & 4230 & $<549$ & 125 & 750 & 71300 & $<2700$ & $<114$ & 23600 \\
\hline SME Simulant & Hydragard & $\mathrm{HF}$ & 45 & 2 & 7 & 2 & 12 & HHY07 & 54200 & 12900 & 43.9 & 3790 & $<579$ & 153 & 678 & 66600 & $<2840$ & $<120$ & 21800 \\
\hline SME Simulant & Coliwasa & LF & 4 & 1 & 10 & 2 & 13 & CL01 & 59100 & 14700 & 43.6 & 4090 & $<553$ & 137 & 782 & 73400 & $<2720$ & $<115$ & 24200 \\
\hline SME Simulant & Coliwasa & $\mathrm{HF}$ & 18 & 1 & 26 & 2 & 14 & CL06 & 57900 & 14600 & 50.2 & 4180 & $<592$ & 135 & 796 & 71500 & $<2910$ & $<123$ & 25100 \\
\hline ARG-1 & None & 0 & 0 & 2 & 12 & 2 & 15 & ARG-1B22 & 24200 & 25200 & 874 & 10200 & $<553$ & 646 & $<40.2$ & 97500 & 23300 & $<115$ & 14800 \\
\hline SME Simulant & Coliwasa & LF & 65 & 1 & 23 & 2 & 16 & CL04 & 58000 & 13600 & 58.5 & 4050 & $<597$ & 119 & 788 & 71800 & $<2930$ & $<124$ & 23600 \\
\hline SME Simulant & Coliwasa & $\begin{array}{l}\mathrm{LF} \\
\end{array}$ & 40 & 1 & 19 & 2 & 17 & CL03 & 56800 & 14200 & 55.7 & 3880 & $<593$ & 115 & 748 & 69600 & $<2910$ & $<123$ & 23200 \\
\hline SME Simulant & $\begin{array}{l}\text { Coliwasa } \\
\end{array}$ & LF & 38 & 1 & 16 & 2 & 18 & CH03 & 56800 & 14100 & 52.9 & 4200 & $<589$ & 120 & 769 & 70300 & $<2890$ & $<122$ & 25300 \\
\hline SME Simulant & Hydragard & $\mathrm{HF}$ & 57 & 2 & 6 & 2 & 19 & HHY09 & 59900 & 13500 & 45.3 & 4140 & $<498$ & 145 & 760 & 74500 & $<2450$ & $<104$ & 23300 \\
\hline
\end{tabular}


Table B3. Phase 2 Elemental Concentration Measurements (part 1)

\begin{tabular}{|c|c|c|c|c|c|c|c|c|c|c|c|c|c|c|c|c|c|c|c|}
\hline $\begin{array}{c}\text { Type of } \\
\text { Material } \\
\end{array}$ & Sampler & $\begin{array}{l}\text { Flow } \\
\text { Rate }\end{array}$ & $\begin{array}{c}\text { Sample } \\
\text { Order }\end{array}$ & \begin{tabular}{|c|}
$\begin{array}{c}\text { Preparation } \\
\text { Block }\end{array}$ \\
\end{tabular} & \begin{tabular}{|c|} 
Preparation \\
Sequence \\
\end{tabular} & \begin{tabular}{|c|}
$\begin{array}{c}\text { Analytical } \\
\text { Block }\end{array}$ \\
\end{tabular} & \begin{tabular}{|c|} 
Analytical \\
Sequence \\
\end{tabular} & Sample ID & Al (ug/g) & B (ug/g) & $\begin{array}{c}\mathrm{Ba} \\
(\mathrm{ug} / \mathrm{g})\end{array}$ & $\begin{array}{c}\text { Ca } \\
\text { (ug/g) }\end{array}$ & $\begin{array}{c}\mathrm{Ce} \\
\text { (ug/g) }\end{array}$ & $\begin{array}{c}\mathrm{Cr} \\
\text { (ug/g) }\end{array}$ & $\begin{array}{c}\mathrm{Cu} \\
(\mathrm{ug} / \mathrm{g})\end{array}$ & $\mathrm{Fe}(\mathrm{ug} / \mathrm{g})$ & K (ug/g) & $\begin{array}{c}\mathbf{L a} \\
(\mathrm{ug} / \mathrm{g})\end{array}$ & $\mathrm{Li}$ (ug/g) \\
\hline SME Simulant & Coliwasa & LF & 21 & $\begin{array}{l}1 \\
\end{array}$ & 14 & \begin{tabular}{|l|}
2 \\
\end{tabular} & \begin{tabular}{|l|}
20 \\
\end{tabular} & CH02 & 55300 & 14500 & 42.3 & 3510 & $<558$ & 151 & 682 & 69300 & $<2740$ & $<116$ & 23500 \\
\hline SME Simulant & Hydragard & $\mathrm{HF}$ & 6 & 2 & 4 & 2 & 21 & HHY01 & 60400 & 13700 & 54.2 & 4320 & $<590$ & 166 & 763 & 75600 & $<2900$ & $<123$ & 23500 \\
\hline SME Simulant & Hydragard & $\mathrm{HF}$ & 19 & 2 & 10 & 2 & 22 & HHY03 & 60200 & 12900 & 52.9 & 4180 & $<575$ & 160 & 763 & 76200 & $<2820$ & $<120$ & 23300 \\
\hline SME Simulant & Hydragard & $\mathrm{HF}$ & 50 & 2 & 5 & 2 & 23 & HHY08 & 58400 & 14100 & 55 & 3990 & $<586$ & 148 & 749 & 73000 & $<2880$ & $<122$ & 23200 \\
\hline SME Simulant & Hydragard & $\mathrm{HF}$ & 37 & 2 & 9 & 2 & 24 & HHY06 & 60700 & 14100 & 49.3 & 4260 & $<574$ & 195 & 786 & 78700 & $<2820$ & $<119$ & 23300 \\
\hline SME Simulant & $\begin{array}{l}\text { Coliwasa } \\
\end{array}$ & $\mathrm{HF}$ & 34 & 1 & 18 & 2 & 25 & CH07 & 54300 & 13600 & 51.1 & 3830 & $<602$ & 121 & 728 & 67200 & $<2960$ & $<125$ & 22900 \\
\hline SME Simulant & $\begin{array}{l}\text { Coliwasa } \\
\end{array}$ & LF & 63 & 1 & 13 & 2 & 26 & CH04 & 57600 & 14100 & 55.8 & 3990 & $<581$ & 102 & 715 & 71000 & $<2860$ & $<121$ & 23900 \\
\hline SME Simulant & Hydragard & $\mathrm{HF}$ & 32 & 2 & 8 & 2 & 27 & HHY05 & 59400 & 13000 & 48.4 & 4260 & $<544$ & 133 & 754 & 75000 & $<2680$ & $<113$ & 23500 \\
\hline SME Simulant & Hydragard & $\mathrm{HF}$ & 61 & 2 & 11 & 2 & 28 & HHY10 & 60300 & 13400 & 44.8 & 4370 & $<541$ & 134 & 829 & 74400 & 2880 & $<112$ & 24500 \\
\hline ARG-1 & None & 0 & 0 & 2 & 23 & 2 & 29 & ARG-1B23 & 24400 & 25300 & 872 & 10300 & $<572$ & 645 & $<41.6$ & 97600 & 24400 & $<119$ & 15100 \\
\hline ARG-1 & None & 0 & 0 & 3 & 1 & 3 & 1 & ARG-1B31 & 23300 & 24800 & 763 & 9880 & $<559$ & 640 & 54.2 & 93000 & 21700 & $<116$ & 14200 \\
\hline SME Simulant & Isolok - $12 \mathrm{~mL}$ & LF & 11 & 3 & 4 & 3 & 2 & LIB02 & 57600 & 14800 & 105 & 4120 & $<570$ & 127 & 721 & 70800 & $<2800$ & $<119$ & 24500 \\
\hline SME Simulant & Coliwasa & $\mathrm{HF}$ & 36 & 1 & 21 & 3 & 3 & CL07 & 57900 & 14600 & 35.8 & 4220 & $<554$ & 134 & 768 & 71800 & $<2720$ & $<115$ & 23300 \\
\hline SME Simulant & Isolok - $12 \mathrm{~mL}$ & LF & 26 & 3 & 8 & 3 & 4 & LIB04 & 58200 & 14800 & 100 & 4270 & $<564$ & 116 & 738 & 72700 & $<2770$ & $<117$ & 24700 \\
\hline SME Simulant & \begin{tabular}{|l|} 
Coliwasa \\
\end{tabular} & $\mathrm{HF}$ & 1 & 1 & 22 & 3 & 5 & CH05 & 57000 & 15100 & 39 & 4410 & $<603$ & 148 & 724 & 70300 & $<2970$ & $<126$ & 25200 \\
\hline SME Simulant & Coliwasa & $\mathrm{HF}$ & 3 & 1 & 20 & 3 & 6 & CL05 & 58900 & 15600 & 39.8 & 4310 & $<547$ & 142 & 723 & 72900 & $<2690$ & $<114$ & 25000 \\
\hline SME Simulant & Coliwasa & $\mathrm{HF}$ & 23 & 1 & 28 & 3 & 7 & CH06 & 59600 & 14400 & 35.3 & 4300 & $<530$ & 156 & 746 & 74600 & $<2610$ & $<110$ & 23400 \\
\hline SME Simulant & Coliwasa & LF & 1 & 1 & 12 & 3 & 8 & CH01 & 55800 & 14700 & 32.5 & 4070 & $<575$ & 132 & 722 & 68400 & $<2830$ & $<120$ & 24000 \\
\hline SME Simulant & Coliwasa & LF & 20 & 1 & 2 & 3 & 9 & CL02 & 59500 & 14800 & 31.6 & 4370 & $<559$ & 160 & 764 & 74000 & $<2750$ & $<116$ & 23800 \\
\hline SME Simulant & Coliwasa & $\mathrm{HF}$ & 63 & 1 & 5 & 3 & 10 & CL08 & 56500 & 14900 & 30.7 & 4040 & $<563$ & 145 & 760 & 70000 & $<2770$ & $<117$ & 22900 \\
\hline SME Simulant & Coliwasa & $\mathrm{HF}$ & 65 & 1 & 6 & 3 & 11 & CH08 & 55300 & 13700 & 27.8 & 4120 & $<549$ & 133 & 756 & 67800 & $<2700$ & $<114$ & 22300 \\
\hline SME Simulant & Isolok - $12 \mathrm{~mL}$ & LF & 45 & 3 & 7 & 3 & 12 & LIB07 & 58500 & 14700 & 110 & 4270 & $<571$ & 169 & 807 & 72000 & $<2810$ & $<119$ & 24600 \\
\hline SME Simulant & \begin{tabular}{|l|} 
Coliwasa \\
\end{tabular} & LF & 4 & 1 & 10 & 3 & 13 & CL01 & 58800 & 15200 & 31.3 & 4260 & $<553$ & 132 & 779 & 72700 & $<2720$ & $<115$ & 24600 \\
\hline SME Simulant & Coliwasa & HF & 18 & 1 & 26 & 3 & 14 & CL06 & 57000 & 14900 & 31.1 & 4230 & $<592$ & 135 & 792 & 70100 & $<2910$ & $<123$ & 24100 \\
\hline ARG-1 & None & 0 & 0 & 3 & 12 & 3 & 15 & ARG-1B32 & 24400 & 25600 & 882 & 10600 & $<560$ & 655 & 71.3 & 97900 & 24100 & $<116$ & 14900 \\
\hline SME Simulant & Coliwasa & LF & 65 & 1 & 23 & 3 & 16 & CL04 & 58000 & 15000 & 35 & 4300 & $<597$ & 121 & 800 & 71800 & $<2930$ & $<124$ & 24100 \\
\hline SME Simulant & Coliwasa & LF & 40 & 1 & 19 & 3 & 17 & CL03 & 57200 & 14900 & 38.3 & 4140 & $<593$ & 141 & 803 & 70300 & $<2910$ & $<123$ & 23200 \\
\hline SME Simulant & Coliwasa & LF & 38 & 1 & 16 & 3 & 18 & $\mathrm{CH} 03$ & 56900 & 14700 & 34.5 & 4290 & $<589$ & 135 & 773 & 70100 & $<2890$ & $<122$ & 24800 \\
\hline SME Simulant & Isolok - $12 \mathrm{~mL}$ & LF & 56 & 3 & 11 & 3 & 19 & LIB09 & 58700 & 13400 & 104 & 4230 & $<555$ & 149 & 732 & 71900 & $<2730$ & $<115$ & 23700 \\
\hline SME Simulant & $\begin{array}{l}\text { Coliwasa } \\
\end{array}$ & LF & 21 & 1 & 14 & 3 & 20 & CH02 & 55600 & 14900 & 31.6 & 3750 & $<558$ & 152 & 688 & 69600 & $<2740$ & $<116$ & 24500 \\
\hline SME Simulant & Isolok - $12 \mathrm{~mL}$ & LF & 5 & 3 & 5 & 3 & 21 & LIB01 & 58900 & 14300 & 93.6 & 4380 & $<558$ & 142 & 728 & 72500 & $<2740$ & $<116$ & 23800 \\
\hline SME Simulant & Isolok - $12 \mathrm{~mL}$ & LF & 23 & 3 & 10 & 3 & 22 & LIB03 & 58800 & 13800 & 94.5 & 4270 & $<571$ & 118 & 752 & 72900 & $<2810$ & $<119$ & 23800 \\
\hline SME Simulant & Isolok - $12 \mathrm{~mL}$ & LF & 50 & 3 & 2 & 3 & 23 & LIB08 & 58800 & 14000 & 114 & 4290 & $<562$ & 174 & 735 & 71900 & $<2760$ & $<117$ & 23800 \\
\hline SME Simulant & Isolok - $12 \mathrm{~mL}$ & LF & 41 & 3 & 9 & 3 & 24 & LIB06 & 54900 & 13100 & 97 & 4220 & $<572$ & 156 & 751 & 67100 & $<2810$ & $<119$ & 22900 \\
\hline SME Simulant & \begin{tabular}{|l|} 
Coliwasa \\
\end{tabular} & $\mathrm{HF}$ & 34 & 1 & 18 & 3 & 25 & CH07 & 57500 & 14500 & 36.5 & 4240 & $<602$ & 124 & 766 & 70500 & $<2960$ & $<125$ & 24900 \\
\hline SME Simulant & \begin{tabular}{|l|} 
Coliwasa \\
\end{tabular} & LF & 63 & 1 & 13 & 3 & 26 & CH04 & 58400 & 14400 & 39.9 & 4110 & $<581$ & 125 & 723 & 71900 & $<2860$ & $<121$ & 23200 \\
\hline SME Simulant & Isolok - $12 \mathrm{~mL}$ & LF & 32 & 3 & 3 & 3 & 27 & LIB05 & 59800 & 14800 & 112 & 4410 & $<578$ & 114 & 717 & 73100 & $<2840$ & $<120$ & 24400 \\
\hline SME Simulant & Isolok - $12 \mathrm{~mL}$ & LF & 60 & 3 & 6 & 3 & 28 & LIB10 & 59000 & 13700 & 107 & 4370 & $<567$ & 145 & 753 & 73900 & $<2790$ & $<118$ & 25100 \\
\hline ARG-1 & \begin{tabular}{|l|} 
None \\
\end{tabular} & 0 & 0 & 3 & 23 & 3 & 29 & ARG-1B33 & 25100 & 25700 & 880 & 10600 & $<577$ & 676 & $<41.9$ & 99500 & 24200 & $<120$ & 15300 \\
\hline ARG-1 & None & 0 & 0 & 4 & 1 & 4 & 1 & ARG-1B41 & 23400 & 23900 & 778 & 9940 & $<525$ & 643 & $<38.2$ & 93600 & 20900 & $<109$ & 14000 \\
\hline SME Simulant & Isolok - $12 \mathrm{~mL}$ & $\mathrm{HF}$ & 13 & 4 & 11 & 4 & 2 & HIB02 & 56400 & 14000 & 122 & 4160 & $<563$ & 210 & 695 & 70100 & $<2770$ & $<117$ & 23700 \\
\hline SME Simulant & \begin{tabular}{|l|} 
Coliwasa \\
\end{tabular} & $\mathrm{HF}$ & 36 & 1 & 21 & 4 & 3 & CL07 & 58000 & 14200 & 50.9 & 4260 & $<554$ & 140 & 758 & 72100 & $<2720$ & $<115$ & 23100 \\
\hline SME Simulant & Isolok - $12 \mathrm{~mL}$ & $\mathrm{HF}$ & 25 & 4 & 4 & 4 & 4 & HIB04 & 57300 & 13400 & 144 & 4030 & $<588$ & 199 & 675 & 71200 & $<2890$ & $<122$ & 23900 \\
\hline SME Simulant & Coliwasa & $\mathrm{HF}$ & 1 & 1 & 22 & 4 & 5 & CH05 & 57300 & 14400 & 53.7 & 4280 & $<603$ & 146 & 719 & 70400 & $<2970$ & $<126$ & 24200 \\
\hline SME Simulant & $\begin{array}{l}\text { Coliwasa } \\
\end{array}$ & $\mathrm{HF}$ & 3 & 1 & 20 & 4 & 6 & CL05 & 58800 & 14600 & 47.6 & 4280 & $<547$ & 138 & 687 & 72900 & $<2690$ & $<114$ & 23900 \\
\hline SME Simulant & Coliwasa & $\mathrm{HF}$ & 23 & 1 & 28 & 4 & 7 & CH06 & 59300 & 13900 & 49.8 & 4350 & $<530$ & 149 & 744 & 74600 & $<2610$ & $<110$ & 23700 \\
\hline SME Simulant & Coliwasa & LF & 1 & 1 & 12 & 4 & 8 & $\mathrm{CH} 01$ & 55400 & 13800 & 48.8 & 4110 & $<575$ & 129 & 712 & 68000 & $<2830$ & $<120$ & 24100 \\
\hline SME Simulant & Coliwasa & LF & 20 & 1 & 2 & 4 & 9 & CL02 & 59300 & 13900 & 47.4 & 4430 & $<559$ & 117 & 782 & 74000 & $<2750$ & $<116$ & 23900 \\
\hline
\end{tabular}


Table B3. Phase 2 Elemental Concentration Measurements (part 1)

\begin{tabular}{|c|c|c|c|c|c|c|c|c|c|c|c|c|c|c|c|c|c|c|c|}
\hline $\begin{array}{c}\text { Type of } \\
\text { Material } \\
\end{array}$ & Sampler & $\begin{array}{l}\text { Flow } \\
\text { Rate }\end{array}$ & $\begin{array}{c}\text { Sample } \\
\text { Order }\end{array}$ & \begin{tabular}{|c|}
$\begin{array}{c}\text { Preparation } \\
\text { Block }\end{array}$ \\
\end{tabular} & $\begin{array}{c}\text { Preparation } \\
\text { Sequence }\end{array}$ & \begin{tabular}{|c|}
$\begin{array}{c}\text { Analytical } \\
\text { Block }\end{array}$ \\
\end{tabular} & \begin{tabular}{|c|} 
Analytical \\
Sequence \\
\end{tabular} & Sample ID & $\mathrm{Al}(\mathbf{u g} / \mathrm{g})$ & B (ug/g) & $\begin{array}{c}\text { Ba } \\
(\mathrm{ug} / \mathrm{g})\end{array}$ & $\begin{array}{c}\text { Ca } \\
\text { (ug/g) }\end{array}$ & $\begin{array}{c}\mathrm{Ce} \\
\text { (ug/g) }\end{array}$ & $\begin{array}{c}\mathbf{C r} \\
\text { (ug/g) }\end{array}$ & $\begin{array}{c}\mathrm{Cu} \\
(\mathrm{ug} / \mathrm{g})\end{array}$ & Fe (ug/g) & K (ug/g) & 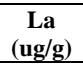 & $\mathrm{Li}$ (ug/g) \\
\hline SME Simulant & Coliwasa & $\mathrm{HF}$ & 63 & 1 & 5 & \begin{tabular}{|l|}
4 \\
\end{tabular} & \begin{tabular}{|l|}
10 \\
\end{tabular} & CL08 & 56100 & 13600 & 48.4 & 4030 & $<563$ & 139 & 714 & 69800 & $<2770$ & $<117$ & 22500 \\
\hline SME Simulant & $\begin{array}{l}\text { Coliwasa } \\
\end{array}$ & $\mathrm{HF}$ & 65 & 1 & 6 & 4 & 11 & CH08 & 57500 & 13400 & 47.8 & 4390 & $<549$ & 152 & 768 & 71000 & $<2700$ & $<114$ & 23500 \\
\hline SME Simulant & Isolok - $12 \mathrm{~mL}$ & $\mathrm{HF}$ & 46 & 4 & 2 & 4 & 12 & HIB07 & 54300 & 12800 & 117 & 3950 & $<576$ & 170 & 669 & 69500 & $<2830$ & $<120$ & 22300 \\
\hline SME Simulant & \begin{tabular}{|l|} 
Coliwasa \\
\end{tabular} & LF & 4 & 1 & 10 & 4 & 13 & CL01 & 58800 & 14300 & 45.8 & 4240 & $<553$ & 133 & 747 & 73400 & $<2720$ & $<115$ & 23700 \\
\hline SME Simulant & Coliwasa & $\mathrm{HF}$ & 18 & 1 & 26 & 4 & 14 & CL06 & 57700 & 13900 & 46.1 & 4260 & $<592$ & 140 & 749 & 71100 & $<2910$ & $<123$ & 23700 \\
\hline ARG-1 & None & 0 & 0 & 4 & 7 & 4 & 15 & ARG-1B42 & 24000 & 23700 & 867 & 10400 & $<587$ & 656 & $<42.7$ & 96800 & 22200 & $<122$ & 14400 \\
\hline SME Simulant & Coliwasa & $\mathrm{LF}$ & 65 & 1 & 23 & 4 & 16 & CL04 & 57700 & 13500 & 51.9 & 4280 & $<597$ & 133 & 755 & 71600 & $<2930$ & $<124$ & 23700 \\
\hline SME Simulant & Coliwasa & LF & 40 & 1 & 19 & 4 & 17 & $\begin{array}{ll}\text { CL03 } \\
\end{array}$ & 56600 & 14000 & 58.1 & 4100 & $<593$ & 138 & 784 & 69800 & $<2910$ & $<123$ & 23000 \\
\hline SME Simulant & Coliwasa & LF & 38 & 1 & 16 & 4 & 18 & $\mathrm{CH} 03$ & 56700 & 14000 & 55.9 & 4170 & $<589$ & 123 & 735 & 70200 & $<2890$ & $<122$ & 23200 \\
\hline SME Simulant & \begin{tabular}{|l} 
Isolok - $12 \mathrm{~mL}$ \\
\end{tabular} & $\mathrm{HF}$ & 56 & 4 & 9 & 4 & 19 & HIB09 & 56900 & 13100 & 114 & 4240 & $<519$ & 167 & 759 & 71100 & $<2550$ & $<108$ & 23500 \\
\hline SME Simulant & \begin{tabular}{|l|} 
Coliwasa \\
\end{tabular} & LF & 21 & 1 & 14 & 4 & 20 & CH02 & 55200 & 14100 & 47.4 & 3870 & $<558$ & 139 & 657 & 69700 & $<2740$ & $<116$ & 24000 \\
\hline SME Simulant & Isolok - $12 \mathrm{~mL}$ & $\mathrm{HF}$ & 7 & 4 & 12 & 4 & 21 & HIB01 & 57000 & 13200 & 119 & 4150 & $<536$ & 195 & 676 & 70600 & $<2630$ & $<111$ & 23900 \\
\hline SME Simulant & Isolok - $12 \mathrm{~mL}$ & $\mathrm{HF}$ & 21 & 4 & 6 & 4 & 22 & HIB03 & 56900 & 12800 & 110 & 4200 & $<505$ & 177 & 694 & 70600 & $<2480$ & $<105$ & 24200 \\
\hline SME Simulant & Isolok - $12 \mathrm{~mL}$ & $\mathrm{HF}$ & 49 & 4 & 5 & 4 & 23 & HIB08 & 57500 & 12600 & 122 & 4310 & $<544$ & 175 & 725 & 72400 & $<2670$ & $<113$ & 24300 \\
\hline SME Simulant & Isolok - $12 \mathrm{~mL}$ & $\mathrm{HF}$ & 38 & 4 & 8 & 4 & 24 & HIB06 & 57800 & 13500 & 128 & 4310 & $<588$ & 197 & 685 & 73100 & $<2890$ & $<122$ & 24300 \\
\hline SME Simulant & \begin{tabular}{|l|} 
Coliwasa \\
\end{tabular} & $\mathrm{HF}$ & 34 & 1 & 18 & 4 & 25 & CH07 & 53700 & 12700 & 52.3 & 3980 & $<602$ & 131 & 660 & 66700 & $<2960$ & $<125$ & 22700 \\
\hline SME Simulant & \begin{tabular}{|l|} 
Coliwasa \\
\end{tabular} & LF & 63 & 1 & 13 & 4 & 26 & CH04 & 57900 & 13300 & 59.9 & 4120 & $<581$ & 131 & 724 & 72100 & $<2860$ & $<121$ & 22800 \\
\hline SME Simulant & \begin{tabular}{|l} 
Isolok - $12 \mathrm{~mL}$ \\
\end{tabular} & $\mathrm{HF}$ & 33 & 4 & 10 & 4 & 27 & HIB05 & 56500 & 13200 & 124 & 4040 & $<561$ & 167 & 675 & 70100 & $<2760$ & $<117$ & 23400 \\
\hline SME Simulant & Isolok - $12 \mathrm{~mL}$ & $\mathrm{HF}$ & 58 & 4 & 3 & 4 & 28 & HIB10 & 56800 & 12700 & 104 & 4050 & $<528$ & 156 & 668 & 70400 & $<2590$ & $<110$ & 22900 \\
\hline ARG-1 & None & 0 & 0 & 4 & 13 & 4 & 29 & ARG-1B43 & 24600 & 24200 & 819 & 10400 & $<584$ & 704 & $<42.4$ & 98900 & 22700 & $<121$ & 14700 \\
\hline ARG-1 & \begin{tabular}{|l|} 
None \\
\end{tabular} & 0 & 0 & 2 & 1 & 5 & 1 & ARG-1B21 & 24200 & 25100 & 872 & 10200 & $<544$ & 654 & 62.6 & 97300 & 22200 & $<113$ & 14600 \\
\hline SME Simulant & \begin{tabular}{|l} 
Isolok - $3 \mathrm{~mL}$ \\
\end{tabular} & $\mathrm{HF}$ & 20 & 2 & 16 & 5 & 2 & HIS03 & 57600 & 14100 & 39.4 & 4040 & $<527$ & 147 & 709 & 71600 & $<2590$ & $<110$ & 23700 \\
\hline SME Simulant & Isolok - $3 \mathrm{~mL}$ & $\mathrm{HF}$ & 9 & 2 & 21 & 5 & 3 & HIS01 & 58700 & 15000 & 40 & 4070 & $<582$ & 127 & 702 & 72600 & $<2860$ & $<121$ & 24600 \\
\hline SME Simulant & Coliwasa & LF & 20 & 1 & 2 & 5 & 4 & CL02 & 57700 & 13400 & 40.6 & 4160 & $<559$ & 115 & 729 & 71400 & $<2750$ & $<116$ & 22800 \\
\hline SME Simulant & Coliwasa & LF & 38 & 1 & 16 & 5 & 5 & $\mathrm{CH} 03$ & 56200 & 13700 & 42.8 & 4070 & $<589$ & 117 & 748 & 68800 & $<2890$ & $<122$ & 23400 \\
\hline SME Simulant & $\begin{array}{l}\text { Coliwasa } \\
\end{array}$ & LF & 63 & 1 & 13 & 5 & 6 & CH04 & 58800 & 13800 & 42.3 & 4040 & $<581$ & 128 & 782 & 72700 & $<2860$ & $<121$ & 22500 \\
\hline SME Simulant & Isolok - $3 \mathrm{~mL}$ & $\mathrm{HF}$ & 44 & 2 & 18 & 5 & 7 & HIS07 & 58200 & 14500 & 42.8 & 4030 & $<572$ & 165 & 747 & 71300 & $<2810$ & $<119$ & 23700 \\
\hline SME Simulant & Coliwasa & $\mathrm{HF}$ & 23 & 1 & 28 & 5 & 8 & CH06 & 60100 & 14200 & 40.7 & 4270 & $<530$ & 149 & 727 & 75100 & $<2610$ & $<110$ & 23400 \\
\hline SME Simulant & Coliwasa & LF & 21 & 1 & 14 & 5 & 9 & $\mathrm{CH} 02$ & 55900 & 14500 & 40.6 & 3720 & $<558$ & 142 & 673 & 69700 & $<2740$ & $<116$ & 24100 \\
\hline SME Simulant & $\begin{array}{l}\text { Coliwasa } \\
\end{array}$ & $\mathrm{HF}$ & 3 & 1 & 20 & 5 & 10 & $\begin{array}{ll}\text { CL05 } \\
\end{array}$ & 58000 & 14700 & 39.8 & 4110 & $<547$ & 133 & 724 & 71800 & $<2690$ & $<114$ & 23500 \\
\hline SME Simulant & Isolok - $3 \mathrm{~mL}$ & $\mathrm{HF}$ & 14 & 2 & 15 & 5 & 11 & HIS02 & 57700 & 14000 & 45.6 & 4130 & $<565$ & 149 & 733 & 72600 & $<2780$ & $<118$ & 24300 \\
\hline SME Simulant & $\begin{array}{l}\text { Coliwasa } \\
\end{array}$ & LF & 40 & 1 & 19 & 5 & 12 & $\begin{array}{ll}\text { CL03 } \\
\end{array}$ & 58100 & 14600 & 45.5 & 4070 & $<593$ & 126 & 834 & 71000 & $<2910$ & $<123$ & 23100 \\
\hline SME Simulant & Coliwasa & $\mathrm{HF}$ & 18 & 1 & 26 & 5 & 13 & $\begin{array}{ll}\text { CL06 } \\
\end{array}$ & 57900 & 14600 & 40.6 & 4230 & $<592$ & 130 & 756 & 71000 & $<2910$ & $<123$ & 24100 \\
\hline SME Simulant & Coliwasa & $\mathrm{HF}$ & 1 & 1 & 22 & 5 & 14 & CH05 & 57200 & 14400 & 45.1 & 4220 & $<603$ & 141 & 778 & 70200 & $<2970$ & $<126$ & 24100 \\
\hline ARG-1 & None & 0 & 0 & 2 & 12 & 5 & 15 & ARG-1B22 & 24700 & 25200 & 884 & 10100 & $<553$ & 655 & $<40.2$ & 98400 & 22600 & $<115$ & 14700 \\
\hline SME Simulant & Coliwasa & $\mathrm{HF}$ & 36 & 1 & 21 & 5 & 16 & CL07 & 58100 & 14000 & 44.8 & 4190 & $<554$ & 132 & 792 & 71700 & $<2720$ & $<115$ & 23200 \\
\hline SME Simulant & Isolok - $3 \mathrm{~mL}$ & $\mathrm{HF}$ & 31 & 2 & 19 & 5 & 17 & HIS05 & 58100 & 14100 & 41 & 4310 & $<580$ & 132 & 765 & 71600 & $<2850$ & $<121$ & 24600 \\
\hline SME Simulant & Isolok - $3 \mathrm{~mL}$ & $\mathrm{HF}$ & 60 & 2 & 22 & 5 & 18 & HIS10 & 57000 & 13900 & 43.4 & 3990 & $<581$ & 135 & 669 & 70700 & $<2850$ & $<121$ & 24000 \\
\hline SME Simulant & Coliwasa & $\mathrm{HF}$ & 63 & 1 & 5 & 5 & 19 & $\begin{array}{ll}\text { CL08 } \\
\end{array}$ & 57300 & 13900 & 41 & 4090 & $<563$ & 153 & 768 & 70800 & $<2770$ & $<117$ & 23400 \\
\hline SME Simulant & Isolok - $3 \mathrm{~mL}$ & $\mathrm{HF}$ & 26 & 2 & 14 & 5 & 20 & HIS04 & 58200 & 14000 & 43.7 & 4150 & $<570$ & 151 & 733 & 71800 & $<2800$ & $<119$ & 23500 \\
\hline SME Simulant & Isolok - $3 \mathrm{~mL}$ & $\mathrm{HF}$ & 35 & 2 & 20 & 5 & 21 & HIS06 & 57600 & 13800 & 43.4 & 4150 & $<565$ & 177 & 718 & 71600 & $<2780$ & $<118$ & 23900 \\
\hline SME Simulant & Coliwasa & $\mathrm{HF}$ & 65 & 1 & 6 & 5 & 22 & CH08 & 59700 & 14300 & 40 & 4410 & $<549$ & 140 & 814 & 72700 & $<2700$ & $<114$ & 23900 \\
\hline SME Simulant & Coliwasa & $\mathrm{HF}$ & 34 & 1 & 18 & 5 & 23 & CH07 & 58700 & 14200 & 43.8 & 4200 & $<602$ & 121 & 749 & 72100 & $<2960$ & $<125$ & 24200 \\
\hline SME Simulant & Coliwasa & LF & 1 & 1 & 12 & 5 & 24 & CH01 & 53200 & 13300 & 39.5 & 3820 & $<575$ & 120 & 681 & 64900 & $<2830$ & $<120$ & 22800 \\
\hline SME Simulant & Isolok - $3 \mathrm{~mL}$ & $\mathrm{HF}$ & 54 & 2 & 17 & 5 & 25 & HIS09 & 58100 & 13900 & 47.2 & 4090 & $<598$ & 158 & 725 & 71400 & $<2940$ & $<125$ & 23700 \\
\hline SME Simulant & Coliwasa & LF & 4 & 1 & 10 & 5 & 26 & $\begin{array}{ll}\text { CL01 } \\
\end{array}$ & 59400 & 14500 & 44.7 & 4140 & $<553$ & 132 & 820 & 73300 & $<2720$ & $<115$ & 23400 \\
\hline SME Simulant & Isolok - $3 \mathrm{~mL}$ & $\mathrm{HF}$ & 48 & 2 & 13 & 5 & 27 & HIS08 & 57900 & 13400 & 43.7 & 4030 & $<515$ & 159 & 701 & 71800 & $<2530$ & $<107$ & 23300 \\
\hline SME Simulant & Coliwasa & LF & 65 & 1 & 23 & 5 & 28 & CL04 & 58200 & 13900 & 44.6 & 4230 & $<597$ & 118 & 781 & 71800 & $<2930$ & $<124$ & 23800 \\
\hline
\end{tabular}


Table B3. Phase 2 Elemental Concentration Measurements (part 1)

\begin{tabular}{|c|c|c|c|c|c|c|c|c|c|c|c|c|c|c|c|c|c|c|c|}
\hline $\begin{array}{l}\text { Type of } \\
\text { Material }\end{array}$ & Sampler & $\begin{array}{l}\text { Flow } \\
\text { Rate }\end{array}$ & $\begin{array}{l}\text { Sample } \\
\text { Order }\end{array}$ & $\begin{array}{c}\text { Preparation } \\
\text { Block }\end{array}$ & $\begin{array}{c}\text { Preparation } \\
\text { Sequence }\end{array}$ & $\begin{array}{c}\text { Analytical } \\
\text { Block }\end{array}$ & $\begin{array}{c}\text { Analytical } \\
\text { Sequence }\end{array}$ & Sample ID & $\mathrm{Al}(\mathrm{ug} / \mathrm{g})$ & B (ug/g) & $\begin{array}{c}\text { Ba } \\
\text { (ug/g) }\end{array}$ & $\begin{array}{c}\mathrm{Ca} \\
\text { (ug/g) }\end{array}$ & $\begin{array}{c}\mathrm{Ce} \\
(\mathrm{ug} / \mathrm{g})\end{array}$ & $\begin{array}{c}\mathrm{Cr} \\
\text { (ug/g) }\end{array}$ & $\begin{array}{c}\mathrm{Cu} \\
\text { (ug/g) }\end{array}$ & $\mathrm{Fe}(\mathrm{ug} / \mathrm{g})$ & K (ug/g) & $\begin{array}{c}\mathrm{La} \\
\text { (ug/g) }\end{array}$ & Li (ug/g) \\
\hline ARG-1 & None & 0 & 0 & 2 & 23 & 5 & 29 & \begin{tabular}{|l|} 
ARG-1B23 \\
\end{tabular} & 23500 & 23100 & 834 & 9640 & $<572$ & 626 & $<41.6$ & 93400 & 21200 & $<119$ & 14000 \\
\hline ARG-1 & None & 0 & 0 & 3 & 1 & 6 & 1 & ARG-1B31 & 24500 & 24900 & 821 & 10300 & $<557$ & 664 & $<40.5$ & 97900 & 22900 & $<116$ & 14600 \\
\hline SME Simulant & Hydragard & LF & 15 & 3 & 21 & 6 & 2 & LHY03 & 60600 & 14300 & 41.2 & 4330 & $<551$ & 122 & 782 & 74500 & $<2710$ & $<115$ & 24200 \\
\hline SME Simulant & Hydragard & LF & 6 & 3 & 18 & 6 & 3 & LHY01 & 60100 & 14300 & 98.1 & 4430 & $<528$ & 127 & 803 & 73700 & $<2590$ & $<110$ & 24100 \\
\hline SME Simulant & Coliwasa & LF & 20 & 1 & 2 & 6 & 4 & CL02 & 60200 & 14300 & 36.1 & 4410 & $<559$ & 125 & 798 & 75000 & $<2750$ & $<116$ & 24000 \\
\hline SME Simulant & Coliwasa & LF & 38 & 1 & 16 & 6 & 5 & $\mathrm{CH} 03$ & 56600 & 14100 & 46.4 & 4200 & $<589$ & 131 & 780 & 70300 & $<2890$ & $<122$ & 23800 \\
\hline SME Simulant & Coliwasa & LF & 63 & 1 & 13 & 6 & 6 & CH04 & 58800 & 13900 & 47 & 4200 & $<581$ & 125 & 787 & 73000 & $<2860$ & $<121$ & 23400 \\
\hline SME Simulant & Hydragard & LF & 47 & 3 & 13 & 6 & 7 & LHY07 & 58600 & 12800 & 105 & 4260 & $<579$ & 154 & 746 & 72900 & $<2840$ & $<120$ & 23300 \\
\hline SME Simulant & Coliwasa & $\mathrm{HF}$ & 23 & 1 & 28 & 6 & 8 & CH06 & 56000 & 13200 & 35.3 & 4040 & $<530$ & 132 & 760 & 70500 & $<2610$ & $<110$ & 21800 \\
\hline SME Simulant & Coliwasa & LF & 21 & 1 & 14 & 6 & 9 & CH02 & 55900 & 14500 & 39.4 & 3750 & $<558$ & 146 & 727 & 70500 & $<2740$ & $<116$ & 24400 \\
\hline SME Simulant & $\begin{array}{l}\text { Coliwasa } \\
\end{array}$ & $\mathrm{HF}$ & 3 & 1 & 20 & 6 & 10 & CL05 & 57900 & 14600 & 42 & 4160 & $<547$ & 132 & 737 & 72000 & $<2690$ & $<114$ & 23300 \\
\hline SME Simulant & Hydragard & LF & 10 & 3 & 20 & 6 & 11 & LHY02 & 59700 & 14100 & 47.6 & 4250 & $<574$ & 105 & 741 & 73500 & $<2820$ & $<119$ & 23400 \\
\hline SME Simulant & Coliwasa & LF & 40 & 1 & 19 & 6 & 12 & CL03 & 57900 & 14500 & 44.3 & 4120 & $<593$ & 134 & 836 & 71200 & $<2910$ & $<123$ & 23300 \\
\hline SME Simulant & Coliwasa & $\mathrm{HF}$ & 18 & 1 & 26 & 6 & 13 & CL06 & 58200 & 14100 & 43 & 4280 & $<592$ & 128 & 771 & 72000 & $<2910$ & $<123$ & 23700 \\
\hline SME Simulant & Coliwasa & $\mathrm{HF}$ & 1 & 1 & 22 & 6 & 14 & CH05 & 57400 & 14400 & 41.4 & 4240 & $<603$ & 136 & 764 & 71000 & $<2970$ & $<126$ & 23700 \\
\hline ARG-1 & None & 0 & 0 & 3 & 12 & 6 & 15 & ARG-1B32 & 24700 & 24400 & 873 & 10300 & $<560$ & 668 & $<40.7$ & 98500 & 23100 & $<116$ & 14700 \\
\hline SME Simulant & $\begin{array}{l}\text { Coliwasa } \\
\end{array}$ & $\mathrm{HF}$ & 36 & 1 & 21 & 6 & 16 & CL07 & 58000 & 13700 & 40.3 & 4230 & $<554$ & 132 & 716 & 72500 & $<2720$ & $<115$ & 23000 \\
\hline SME Simulant & Hydragard & LF & 30 & 3 & 14 & 6 & 17 & LHY05 & 59600 & 14000 & 42.4 & 3980 & $<567$ & 122 & 725 & 76600 & $<2790$ & $<118$ & 22800 \\
\hline SME Simulant & Hydragard & LF & 59 & 3 & 17 & 6 & 18 & LHY10 & 58100 & 13200 & 105 & 4360 & $<541$ & 116 & 776 & 72400 & $<2660$ & $<112$ & 24400 \\
\hline SME Simulant & Coliwasa & $\mathrm{HF}$ & 63 & 1 & 5 & 6 & 19 & CL08 & 57100 & 13900 & 39.8 & 4220 & $<563$ & 141 & 741 & 71300 & $<2770$ & $<117$ & 23800 \\
\hline SME Simulant & Hydragard & LF & 28 & 3 & 16 & 6 & 20 & LHY04 & 57000 & 13600 & 113 & 4170 & $<590$ & 129 & 791 & 70900 & $<2900$ & $<123$ & 23500 \\
\hline SME Simulant & Hydragard & LF & 37 & 3 & 15 & 6 & 21 & LHY06 & 57400 & 13100 & 114 & 4320 & $<590$ & 165 & 750 & 70900 & $<2900$ & $<123$ & 23600 \\
\hline SME Simulant & $\begin{array}{l}\text { Coliwasa } \\
\end{array}$ & $\mathrm{HF}$ & 65 & 1 & 6 & 6 & 22 & CH08 & 59100 & 13800 & 43.3 & 4470 & $<549$ & 159 & 784 & 73500 & $<2700$ & $<114$ & 24000 \\
\hline SME Simulant & Coliwasa & $\mathrm{HF}$ & 34 & 1 & 18 & 6 & 23 & CH07 & 58600 & 14100 & 42.6 & 4280 & $<602$ & 137 & 778 & 72900 & $<2960$ & $<125$ & 24300 \\
\hline SME Simulant & $\begin{array}{l}\text { Coliwasa } \\
\end{array}$ & LF & 1 & 1 & 12 & 6 & 24 & CH01 & 53300 & 12900 & 39.5 & 3850 & $<575$ & 113 & 640 & 65500 & $<2830$ & $<120$ & 22400 \\
\hline SME Simulant & Hydragard & LF & 53 & 3 & 19 & 6 & 25 & LHY09 & 57700 & 13500 & 119 & 4200 & $<577$ & 127 & 759 & 70500 & $<2840$ & $<120$ & 23400 \\
\hline SME Simulant & Coliwasa & LF & 4 & 1 & 10 & 6 & 26 & CL01 & 59200 & 14500 & 48 & 4200 & $<553$ & 145 & 790 & 74300 & $<2720$ & $<115$ & 23900 \\
\hline SME Simulant & Hydragard & LF & 48 & 3 & 22 & 6 & 27 & LHY08 & 58600 & 13100 & 119 & 4160 & $<585$ & 123 & 735 & 72000 & $<2870$ & $<122$ & 23100 \\
\hline SME Simulant & Coliwasa & LF & 65 & 1 & 23 & 6 & 28 & CL04 & 58100 & 13700 & 49.4 & 4220 & $<597$ & 125 & 769 & 71900 & $<2930$ & $<124$ & 23500 \\
\hline ARG-1 & None & 0 & 0 & 3 & 23 & 6 & 29 & ARG-1B33 & 23400 & 22700 & 807 & 9870 & $<577$ & 631 & $<41.9$ & 92700 & 21500 & $<120$ & 14000 \\
\hline
\end{tabular}


Table B3. Phase 2 Elemental Concentration Measurements (part 2)

\begin{tabular}{|c|c|c|c|c|c|c|c|c|c|c|c|c|c|c|c|c|c|c|c|}
\hline $\begin{array}{c}\text { Type of } \\
\text { Material }\end{array}$ & Sampler & Flow Rate & $\begin{array}{c}\text { Sample } \\
\text { Order }\end{array}$ & $\begin{array}{c}\text { Preparation } \\
\text { Block }\end{array}$ & $\begin{array}{c}\text { Preparation } \\
\text { Sequence }\end{array}$ & \begin{tabular}{|c|}
$\begin{array}{c}\text { Analytical } \\
\text { Block }\end{array}$ \\
\end{tabular} & $\begin{array}{c}\text { Analytical } \\
\text { Sequence } \\
\end{array}$ & Sample ID & Mg (ug/g) & $\begin{array}{c}\text { Mn } \\
\text { (ug/g) }\end{array}$ & $\begin{array}{c}\mathrm{Na} \\
\text { (ug/g) }\end{array}$ & $\mathrm{Ni}(\mathrm{ug} / \mathrm{g})$ & \begin{tabular}{|c|}
$\mathbf{P}$ \\
$(\mathrm{ug} / \mathrm{g})$
\end{tabular} & 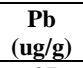 & $\begin{array}{c}\mathrm{S} \\
(\mathrm{ug} / \mathrm{g})\end{array}$ & Si (ug/g) & $\begin{array}{c}\begin{array}{c}\mathbf{T i} \\
(\mathrm{ug} / \mathrm{g})\end{array} \\
\end{array}$ & \begin{tabular}{|c|}
$\mathbf{Z n}$ \\
$(\mathbf{u g} / \mathbf{g})$
\end{tabular} & \begin{tabular}{|c|}
$\mathrm{Zr}$ \\
$(\mathrm{ug} / \mathrm{g})$
\end{tabular} \\
\hline ARG-1 & None & 0 & 0 & 1 & 1 & 1 & 1 & ARG-1B11 & 5160 & 14300 & 87700 & 8090 & \begin{tabular}{|l|}
807 \\
\end{tabular} & $<276$ & $<881$ & 231000 & 6940 & 212 & \begin{tabular}{|l|}
966 \\
\end{tabular} \\
\hline SME Simulant & Isolok - $3 \mathrm{~mL}$ & LF & 18 & 1 & 9 & 1 & 2 & LIS03 & 2020 & 23400 & 102000 & 10100 & $<209$ & 471 & 1170 & 241000 & 108 & $<94.6$ & 829 \\
\hline SME Simulant & Isolok - $3 \mathrm{~mL}$ & LF & 8 & 1 & 27 & 1 & 3 & LIS01 & 1910 & 22200 & 99700 & 9470 & $<212$ & 363 & $<852$ & 242000 & 98.7 & $<96.2$ & 833 \\
\hline SME Simulant & Coliwasa & LF & 20 & 1 & 2 & 1 & 4 & $\begin{array}{l}\text { CL02 } \\
\end{array}$ & 2050 & 24200 & 107000 & 11100 & $<207$ & 602 & 1790 & 240000 & 104 & $<93.7$ & 825 \\
\hline SME Simulant & $\begin{array}{l}\text { Coliwasa } \\
\end{array}$ & LF & 38 & 1 & 16 & 1 & 5 & CH03 & 1730 & 20300 & 93600 & 9060 & $<218$ & $<273$ & 1480 & 219000 & 124 & $<98.7$ & 688 \\
\hline SME Simulant & Coliwasa & LF & 63 & 1 & 13 & 1 & 6 & $\mathrm{CH} 04$ & 1830 & 21200 & 103000 & 9250 & $<215$ & 795 & 1860 & 240000 & 105 & $<97.4$ & 796 \\
\hline SME Simulant & Isolok - $3 \mathrm{~mL}$ & LF & 44 & 1 & 3 & 1 & 7 & LIS07 & 1980 & 23100 & 103000 & 9990 & \begin{tabular}{|c|}
$<193$ \\
\end{tabular} & 554 & $<775$ & 240000 & 95.8 & $<87.6$ & 812 \\
\hline SME Simulant & $\begin{array}{l}\text { Coliwasa } \\
\end{array}$ & $\mathrm{HF}$ & 23 & 1 & 28 & 1 & 8 & CH06 & 1940 & 23100 & 102000 & 10700 & \begin{tabular}{|c|}
$<196$ \\
\end{tabular} & 821 & 1600 & 232000 & 86 & $\begin{array}{c}<88.9 \\
\end{array}$ & 794 \\
\hline SME Simulant & Coliwasa & LF & 21 & 1 & 14 & 1 & 9 & $\mathrm{CH} 02$ & 1680 & 19600 & 93600 & 9000 & $<206$ & 656 & 1700 & 240000 & 96.7 & $<93.5$ & 778 \\
\hline SME Simulant & Coliwasa & $\mathrm{HF}$ & 3 & 1 & 20 & 1 & 10 & CL05 & 1850 & 21400 & 103000 & 8980 & $<202$ & 680 & 1620 & 240000 & 96.8 & $<91.8$ & 791 \\
\hline SME Simulant & Isolok - $3 \mathrm{~mL}$ & LF & 12 & 1 & 25 & 1 & 11 & LIS02 & 1890 & 22300 & 101000 & 9740 & $<204$ & 636 & 896 & 232000 & 83.8 & $<92.7$ & 773 \\
\hline SME Simulant & Coliwasa & LF & 40 & 1 & 19 & 1 & 12 & CL03 & 1880 & 21300 & 108000 & 9030 & $<219$ & 1240 & 1950 & 249000 & 84.5 & $<99.4$ & 776 \\
\hline SME Simulant & Coliwasa & $\mathrm{HF}$ & 18 & 1 & 26 & 1 & 13 & CL06 & 1750 & 20100 & 92200 & 8440 & $<200$ & 776 & 1340 & 217000 & 90.5 & $<90.9$ & 728 \\
\hline SME Simulant & Coliwasa & $\mathrm{HF}$ & 1 & 1 & 22 & 1 & 14 & CH05 & 1900 & 22100 & 105000 & 9470 & \begin{tabular}{|l|}
$<223$ \\
\end{tabular} & 1040 & 1870 & 240000 & 100 & $<101$ & 786 \\
\hline ARG-1 & None & 0 & 0 & 1 & 15 & 1 & 15 & ARG-1B12 & 5260 & 14600 & 89300 & 8150 & \begin{tabular}{|l|}
597 \\
\end{tabular} & 719 & 988 & 236000 & 7040 & 208 & 973 \\
\hline SME Simulant & Coliwasa & $\mathrm{HF}$ & 36 & 1 & 21 & 1 & 16 & CL07 & 1900 & 22200 & 99200 & 9520 & $<205$ & 935 & 1540 & 235000 & 90.6 & $<92.9$ & 778 \\
\hline SME Simulant & Isolok - $3 \mathrm{~mL}$ & LF & 31 & 1 & 24 & 1 & 17 & LIS05 & 1960 & 22900 & 102000 & 9810 & $<218$ & 1200 & $<874$ & 239000 & 96.3 & $<98.7$ & 784 \\
\hline SME Simulant & Isolok - $3 \mathrm{~mL}$ & LF & 58 & 1 & 4 & 1 & 18 & LIS10 & 1850 & 21600 & 99500 & 9070 & $<194$ & 573 & 976 & 240000 & 89 & $<87.9$ & 797 \\
\hline SME Simulant & Coliwasa & $\mathrm{HF}$ & 63 & 1 & 5 & 1 & 19 & CL08 & 1850 & 21500 & 104000 & 9310 & $<208$ & 893 & 1410 & 238000 & 94.8 & $<94.5$ & 739 \\
\hline SME Simulant & Isolok - $3 \mathrm{~mL}$ & LF & 25 & 1 & 7 & 1 & 20 & LIS04 & 1960 & 22600 & 104000 & 9630 & $<212$ & 1080 & 1010 & 239000 & 92 & $<96.4$ & 780 \\
\hline SME Simulant & Isolok - $3 \mathrm{~mL}$ & LF & 42 & 1 & 11 & 1 & 21 & LIS06 & 1970 & 23100 & 104000 & 10100 & \begin{tabular}{|l|}
$<212$ \\
\end{tabular} & 1030 & 870 & 243000 & 93.9 & $<96.2$ & 804 \\
\hline SME Simulant & Coliwasa & $\mathrm{HF}$ & 65 & 1 & 6 & 1 & 22 & CH08 & 1960 & 23200 & 106000 & 10100 & 208 & 879 & 1580 & 235000 & 88.7 & $<92.1$ & 779 \\
\hline SME Simulant & $\begin{array}{l}\text { Coliwasa } \\
\end{array}$ & $\mathrm{HF}$ & 34 & 1 & 18 & 1 & 23 & CH07 & 1980 & 23000 & 108000 & 9970 & $<223$ & 1420 & 1300 & 255000 & 105 & $<101$ & 804 \\
\hline SME Simulant & Coliwasa & LF & 1 & 1 & 12 & 1 & 24 & CH01 & 1820 & 20700 & 103000 & 8630 & $<213$ & 1190 & 1210 & 243000 & 83.3 & $<96.4$ & 772 \\
\hline SME Simulant & Isolok - $3 \mathrm{~mL}$ & LF & 54 & 1 & 17 & 1 & 25 & LIS09 & 1940 & 22900 & 102000 & 9990 & $<183$ & 1100 & 1070 & 239000 & 81.9 & $<83.2$ & 808 \\
\hline SME Simulant & Coliwasa & LF & 4 & 1 & 10 & 1 & 26 & CL01 & 1850 & 21400 & 98700 & 9390 & $<204$ & 1450 & 1710 & 236000 & 95.3 & $<92.7$ & 781 \\
\hline SME Simulant & Isolok - $3 \mathrm{~mL}$ & LF & 49 & 1 & 8 & 1 & 27 & LIS08 & 1980 & 23300 & 104000 & 10100 & $<201$ & 1430 & $<807$ & 239000 & 79.4 & $<91.1$ & 805 \\
\hline SME Simulant & Coliwasa & LF & 65 & 1 & 23 & 1 & 28 & CL04 & 1940 & 22600 & 101000 & 10100 & $<221$ & 1520 & 1780 & 235000 & 81.2 & $<100$ & 737 \\
\hline ARG-1 & None & 0 & 0 & 1 & 29 & 1 & 29 & ARG-1B13 & 4730 & 13100 & 80700 & 7420 & \begin{tabular}{|l|}
700 \\
\end{tabular} & 937 & $<858$ & 211000 & 6340 & 183 & 867 \\
\hline ARG-1 & None & 0 & 0 & 2 & 1 & 2 & 1 & ARG-1B21 & 4820 & 13500 & 79700 & 7580 & \begin{tabular}{|l|}
577 \\
\end{tabular} & $<253$ & $<808$ & 210000 & 6570 & 201 & 962 \\
\hline SME Simulant & Hydragard & $\mathrm{HF}$ & 11 & 2 & 3 & 2 & 2 & HHY02 & 1930 & 22800 & 96500 & 9560 & $\mid<199$ & $<250$ & 1430 & 228000 & 108 & $<90.1$ & 906 \\
\hline SME Simulant & Coliwasa & $\mathrm{HF}$ & 36 & 1 & 21 & 2 & 3 & CL07 & 1890 & 22300 & 99300 & 9600 & $<205$ & $<257$ & 1360 & 231000 & 102 & $<92.9$ & 863 \\
\hline SME Simulant & Hydragard & $\mathrm{HF}$ & 24 & 2 & 2 & 2 & 4 & HHY04 & 1880 & 22400 & 98400 & 10000 & \begin{tabular}{|l|}
$<217$ \\
\end{tabular} & 662 & 1770 & 228000 & 110 & $<98.6$ & 857 \\
\hline SME Simulant & Coliwasa & $\mathrm{HF}$ & 1 & 1 & 22 & 2 & 5 & CH05 & 1880 & 22000 & 100000 & 9180 & $<223$ & $<280$ & 1660 & 233000 & 94.7 & $<101$ & 873 \\
\hline SME Simulant & Coliwasa & $\mathrm{HF}$ & 3 & 1 & 20 & 2 & 6 & CL05 & 1860 & 21700 & 103000 & 9190 & $<202$ & 270 & 1600 & 238000 & 103 & $<91.8$ & 881 \\
\hline SME Simulant & $\begin{array}{l}\text { Coliwasa } \\
\end{array}$ & $\mathrm{HF}$ & 23 & 1 & 28 & 2 & 7 & CH06 & 1930 & 23400 & 97000 & 10700 & $<196$ & 335 & 1780 & 229000 & 109 & $<88.9$ & 855 \\
\hline SME Simulant & Coliwasa & LF & 1 & 1 & 12 & 2 & 8 & CH01 & 1810 & 20800 & 98700 & 8300 & $<213$ & $<267$ & 1300 & 240000 & 106 & $<96.4$ & 846 \\
\hline SME Simulant & Coliwasa & LF & 20 & 1 & 2 & 2 & 9 & CL02 & 1980 & 23600 & 98800 & 10700 & $<207$ & $<260$ & 1860 & 228000 & 111 & $<93.7$ & 878 \\
\hline SME Simulant & Coliwasa & $\mathrm{HF}$ & 63 & 1 & 5 & 2 & 10 & CL08 & 1820 & 21400 & 100000 & 9400 & $<208$ & $<262$ & 1490 & 233000 & 110 & $<94.5$ & 839 \\
\hline SME Simulant & Coliwasa & $\mathrm{HF}$ & 65 & 1 & 6 & 2 & 11 & CH08 & 1930 & 23000 & 99000 & 9990 & $\mid<203$ & $<255$ & 1480 & 230000 & 104 & $<92.1$ & 857 \\
\hline SME Simulant & Hydragard & $\mathrm{HF}$ & 45 & 2 & 7 & 2 & 12 & HHY07 & 1720 & 19800 & 91700 & 8110 & $<214$ & $<269$ & 1440 & 209000 & 101 & $<97$ & 801 \\
\hline SME Simulant & Coliwasa & LF & 4 & 1 & 10 & 2 & 13 & CL01 & 1860 & 21900 & 100000 & 9480 & $<204$ & 326 & 1710 & 236000 & 109 & $<92.7$ & 851 \\
\hline SME Simulant & Coliwasa & $\mathrm{HF}$ & 18 & 1 & 26 & 2 & 14 & CL06 & 1910 & 22200 & 101000 & 9150 & $<219$ & 333 & 1670 & 235000 & 97.9 & $<99.2$ & 847 \\
\hline ARG-1 & None & 0 & 0 & 2 & 12 & 2 & 15 & ARG-1B22 & 5070 & 14200 & 85000 & 8020 & \begin{tabular}{|l|}
649 \\
\end{tabular} & 488 & $<821$ & 222000 & 6930 & 216 & 1010 \\
\hline SME Simulant & Coliwasa & LF & 65 & 1 & 23 & 2 & 16 & CL04 & 1930 & 22800 & 96600 & 10000 & $<221$ & 445 & 1570 & 229000 & 97.2 & $<100$ & 836 \\
\hline SME Simulant & Coliwasa & LF & 40 & 1 & 19 & 2 & 17 & CL03 & 1790 & 20500 & 99500 & 8520 & \begin{tabular}{|l|}
$<219$ \\
\end{tabular} & $<275$ & 1620 & 233000 & 112 & $<99.4$ & 834 \\
\hline SME Simulant & Coliwasa & LF & 38 & 1 & 16 & 2 & 18 & CH03 & 1870 & 22300 & 104000 & 9770 & \begin{tabular}{|c|}
$<218$ \\
\end{tabular} & $<273$ & 1170 & 234000 & 134 & $<98.7$ & 825 \\
\hline SME Simulant & Hydragard & $\mathrm{HF}$ & 57 & 2 & 6 & 2 & 19 & HHY09 & 1910 & 22800 & 98100 & 9780 & \begin{tabular}{|l|}
$<184$ \\
\end{tabular} & $<231$ & 1580 & 226000 & 109 & $<83.5$ & 873 \\
\hline
\end{tabular}


Table B3. Phase 2 Elemental Concentration Measurements (part 2)

\begin{tabular}{|c|c|c|c|c|c|c|c|c|c|c|c|c|c|c|c|c|c|c|c|}
\hline $\begin{array}{c}\text { Type of } \\
\text { Material }\end{array}$ & Sampler & Flow Rate & $\begin{array}{c}\text { Sample } \\
\text { Order }\end{array}$ & $\begin{array}{c}\text { Preparation } \\
\text { Block }\end{array}$ & $\begin{array}{c}\text { Preparation } \\
\text { Sequence }\end{array}$ & \begin{tabular}{|c|}
$\begin{array}{c}\text { Analytical } \\
\text { Block }\end{array}$ \\
\end{tabular} & $\begin{array}{c}\text { Analytical } \\
\text { Sequence } \\
\end{array}$ & Sample ID & Mg (ug/g) & $\begin{array}{c}\text { Mn } \\
\text { (ug/g) }\end{array}$ & $\begin{array}{c}\mathrm{Na} \\
\text { (ug/g) }\end{array}$ & $\mathrm{Ni}(\mathrm{ug} / \mathrm{g})$ & \begin{tabular}{|c|}
$\mathbf{P}$ \\
$(\mathrm{ug} / \mathrm{g})$
\end{tabular} & 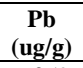 & $\begin{array}{c}\mathrm{S} \\
(\mathrm{ug} / \mathrm{g})\end{array}$ & $\mathrm{Si}(\mathrm{ug} / \mathrm{g})$ & $\begin{array}{c}\begin{array}{c}T i \\
\text { (ug/g) }\end{array} \\
(\mathrm{u}\end{array}$ & \begin{tabular}{|c|}
$\mathbf{Z n}$ \\
$(\mathbf{u g} / \mathbf{g})$
\end{tabular} & \begin{tabular}{|c|}
$\mathrm{Zr}$ \\
$(\mathrm{ug} / \mathrm{g})$
\end{tabular} \\
\hline SME Simulant & $\begin{array}{l}\text { Coliwasa } \\
\end{array}$ & LF & 21 & 1 & 14 & 2 & 20 & CH02 & 1640 & 19500 & 90200 & 8850 & $<206$ & $<259$ & 1470 & 232000 & 111 & $<93.5$ & \begin{tabular}{|l|}
858 \\
\end{tabular} \\
\hline SME Simulant & Hydragard & $\mathrm{HF}$ & 6 & 2 & 4 & 2 & 21 & HHY01 & 1980 & 23700 & 98700 & 10400 & $<218$ & $<274$ & 1460 & 224000 & 117 & $<98.9$ & 913 \\
\hline SME Simulant & Hydragard & $\mathrm{HF}$ & 19 & 2 & 10 & 2 & 22 & HHY03 & 1950 & 23700 & 99200 & 11000 & $<212$ & $<267$ & 1590 & 224000 & 115 & $<96.4$ & 896 \\
\hline SME Simulant & Hydragard & $\mathrm{HF}$ & 50 & 2 & 5 & 2 & 23 & HHY08 & 1780 & 20400 & 105000 & 8820 & $<216$ & $<272$ & 1730 & 234000 & 121 & $<98.2$ & 881 \\
\hline SME Simulant & Hydragard & $\mathrm{HF}$ & 37 & 2 & 9 & 2 & 24 & HHY06 & 1970 & 24200 & 99800 & 11600 & $\mid<212$ & $<267$ & 1660 & 229000 & 118 & $<96.2$ & 898 \\
\hline SME Simulant & Coliwasa & $\mathrm{HF}$ & 34 & 1 & 18 & 2 & 25 & CH07 & 1760 & 20700 & 94500 & 8820 & $<223$ & $<280$ & 1290 & 225000 & 131 & $<101$ & 826 \\
\hline SME Simulant & Coliwasa & LF & 63 & 1 & 13 & 2 & 26 & CH04 & 1790 & 20900 & 104000 & 9130 & $<215$ & $<270$ & 1340 & 231000 & 131 & $<97.4$ & 873 \\
\hline SME Simulant & Hydragard & $\mathrm{HF}$ & 32 & 2 & 8 & 2 & 27 & HHY05 & 1950 & 23800 & 100000 & 10400 & $<201$ & $<253$ & 1700 & 225000 & 130 & $<91.3$ & 891 \\
\hline SME Simulant & Hydragard & $\mathrm{HF}$ & 61 & 2 & 11 & 2 & 28 & HHY10 & 2000 & 23600 & 99600 & 10000 & $<200$ & $<251$ & 1870 & 227000 & 124 & $<90.6$ & 911 \\
\hline ARG-1 & None & 0 & 0 & 2 & 23 & 2 & 29 & ARG-1B23 & 5080 & 14300 & 86400 & 7970 & \begin{tabular}{|l|}
694 \\
\end{tabular} & $<266$ & $<849$ & 223000 & 7030 & 203 & 1060 \\
\hline ARG-1 & None & 0 & 0 & 3 & 1 & 3 & 1 & ARG-1B31 & 4870 & 13700 & 80500 & 7580 & 808 & $<260$ & $<830$ & 212000 & 6600 & 178 & 916 \\
\hline SME Simulant & Isolok - $12 \mathrm{~mL}$ & LF & 11 & 3 & 4 & 3 & 2 & LIB02 & 1810 & 21000 & 100000 & 8430 & 220 & $<265$ & 1120 & 224000 & 108 & $<95.5$ & 814 \\
\hline SME Simulant & Coliwasa & $\mathrm{HF}$ & 36 & 1 & 21 & 3 & 3 & CL07 & 1900 & 22500 & 97700 & 9460 & $<205$ & $<257$ & 1130 & 231000 & 111 & $<92.9$ & 825 \\
\hline SME Simulant & Isolok - $12 \mathrm{~mL}$ & LF & 26 & 3 & 8 & 3 & 4 & LIB04 & 1880 & 22200 & 102000 & 9770 & \begin{tabular}{|c|}
$<209$ \\
\end{tabular} & $<262$ & 1010 & 233000 & 110 & $<94.6$ & 834 \\
\hline SME Simulant & Coliwasa & $\mathrm{HF}$ & 1 & 1 & 22 & 3 & 5 & CH05 & 1900 & 22200 & 102000 & 9090 & $<223$ & $<280$ & 1180 & 234000 & 103 & $<101$ & 856 \\
\hline SME Simulant & Coliwasa & $\mathrm{HF}$ & 3 & 1 & 20 & 3 & 6 & CL05 & 1880 & 21900 & 106000 & 9210 & \begin{tabular}{|l|}
$<202$ \\
\end{tabular} & $<254$ & 1130 & 241000 & 106 & $<91.8$ & 854 \\
\hline SME Simulant & Coliwasa & $\mathrm{HF}$ & 23 & 1 & 28 & 3 & 7 & CH06 & 1940 & 23500 & 96700 & 10700 & $<196$ & $<246$ & 1310 & 231000 & 105 & $<88.9$ & 863 \\
\hline SME Simulant & $\begin{array}{l}\text { Coliwasa } \\
\end{array}$ & LF & 1 & 1 & 12 & 3 & 8 & CH01 & 1820 & 21000 & 96000 & 8290 & 286 & $<267$ & $<854$ & 241000 & 108 & $<96.4$ & 825 \\
\hline SME Simulant & Coliwasa & LF & 20 & 1 & 2 & 3 & 9 & CL02 & 1980 & 23600 & 97500 & 10500 & $<207$ & $<260$ & 1380 & 228000 & 111 & $<93.7$ & 838 \\
\hline SME Simulant & Coliwasa & $\mathrm{HF}$ & 63 & 1 & 5 & 3 & 10 & CL08 & 1820 & 21400 & 97100 & 9280 & $<208$ & $<262$ & 1050 & 231000 & 111 & $<94.5$ & 811 \\
\hline SME Simulant & Coliwasa & $\mathrm{HF}$ & 65 & 1 & 6 & 3 & 11 & CH08 & 1850 & 22100 & 93500 & 9400 & \begin{tabular}{|l|}
234 \\
\end{tabular} & $<255$ & 1050 & 220000 & 103 & $<92.1$ & 825 \\
\hline SME Simulant & Isolok - $12 \mathrm{~mL}$ & LF & 45 & 3 & 7 & 3 & 12 & LIB07 & 1900 & 22100 & 101000 & 9260 & $<211$ & $<265$ & 1120 & 235000 & 123 & $<95.7$ & 864 \\
\hline SME Simulant & $\begin{array}{l}\text { Coliwasa } \\
\end{array}$ & LF & 4 & 1 & 10 & 3 & 13 & CL01 & 1850 & 21800 & 101000 & 9330 & \begin{tabular}{|l|}
275 \\
\end{tabular} & $<257$ & 1090 & 235000 & 115 & $<92.7$ & 866 \\
\hline SME Simulant & Coliwasa & $\mathrm{HF}$ & 18 & 1 & 26 & 3 & 14 & CL06 & 1890 & 22000 & 97700 & 8900 & $<219$ & $<275$ & 1050 & 232000 & 111 & $<99.2$ & 841 \\
\hline ARG-1 & None & 0 & 0 & 3 & 12 & 3 & 15 & ARG-1B32 & 5140 & 14400 & 86100 & 7880 & \begin{tabular}{|l|}
854 \\
\end{tabular} & $<260$ & $<831$ & 226000 & 6980 & 200 & 1030 \\
\hline SME Simulant & Coliwasa & LF & 65 & 1 & 23 & 3 & 16 & CL04 & 1940 & 23000 & 99100 & 10000 & $<221$ & $<277$ & 1270 & 231000 & 107 & $<100$ & 802 \\
\hline SME Simulant & Coliwasa & LF & 40 & 1 & 19 & 3 & 17 & CL03 & 1810 & 20800 & 99600 & 8460 & \begin{tabular}{|l|}
$<219$ \\
\end{tabular} & $<275$ & 1490 & 237000 & 119 & $<99.4$ & 825 \\
\hline SME Simulant & Coliwasa & LF & 38 & 1 & 16 & 3 & 18 & CH03 & 1890 & 22500 & 101000 & 9690 & $<218$ & $<273$ & 1030 & 235000 & 144 & 281 & 823 \\
\hline SME Simulant & Isolok - $12 \mathrm{~mL}$ & LF & 56 & 3 & 11 & 3 & 19 & LIB09 & 1870 & 21400 & 101000 & 8590 & 233 & $<258$ & 1090 & 235000 & 122 & $<93$ & 874 \\
\hline SME Simulant & Coliwasa & LF & 21 & 1 & 14 & 3 & 20 & CH02 & 1660 & 19600 & 93900 & 8760 & $<206$ & $<259$ & 1050 & 234000 & 111 & $<93.5$ & 819 \\
\hline SME Simulant & Isolok - $12 \mathrm{~mL}$ & LF & 5 & 3 & 5 & 3 & 21 & LIB01 & 1970 & 22900 & 98000 & 9690 & $<206$ & $<259$ & 1020 & 227000 & 114 & $<93.6$ & 856 \\
\hline SME Simulant & Isolok - $12 \mathrm{~mL}$ & LF & 23 & 3 & 10 & 3 & 22 & LIB03 & 1930 & 22700 & 98900 & 9530 & $<211$ & $<265$ & 1120 & 227000 & 113 & $<95.7$ & 820 \\
\hline SME Simulant & Isolok - $12 \mathrm{~mL}$ & LF & 50 & 3 & 2 & 3 & 23 & LIB08 & 1930 & 22700 & 97400 & 9090 & \begin{tabular}{|l|}
$<208$ \\
\end{tabular} & $<261$ & 1320 & 234000 & 106 & $<94.3$ & 849 \\
\hline SME Simulant & Isolok - $12 \mathrm{~mL}$ & LF & 41 & 3 & 9 & 3 & 24 & LIB06 & 1880 & 22100 & 94700 & 8910 & $<211$ & $<266$ & 886 & 220000 & 98.3 & $<95.9$ & 780 \\
\hline SME Simulant & $\begin{array}{l}\text { Coliwasa } \\
\end{array}$ & $\mathrm{HF}$ & 34 & 1 & 18 & 3 & 25 & CH07 & 1860 & 21800 & 103000 & 9160 & $<223$ & $<280$ & 997 & 237000 & 127 & $<101$ & 822 \\
\hline SME Simulant & $\begin{array}{l}\text { Coliwasa } \\
\end{array}$ & LF & 63 & 1 & 13 & 3 & 26 & CH04 & 1820 & 21300 & 100000 & 9110 & \begin{tabular}{|l|}
222 \\
\end{tabular} & $<270$ & $<863$ & 235000 & 117 & $<97.4$ & 838 \\
\hline SME Simulant & Isolok - $12 \mathrm{~mL}$ & LF & 32 & 3 & 3 & 3 & 27 & LIB05 & 1950 & 22800 & 104000 & 9550 & $<214$ & $<269$ & 1570 & 231000 & 104 & $<96.9$ & 857 \\
\hline SME Simulant & Isolok - $12 \mathrm{~mL}$ & LF & 60 & 3 & 6 & 3 & 28 & LIB10 & 1930 & 23100 & 104000 & 10500 & $<210$ & $<264$ & 1240 & 230000 & 95.8 & $<95.1$ & 834 \\
\hline ARG-1 & None & 0 & 0 & 3 & 23 & 3 & 29 & ARG-1B33 & 5230 & 14700 & 87500 & 8060 & \begin{tabular}{|l|}
868 \\
\end{tabular} & $<268$ & $<856$ & 229000 & 7080 & 210 & 986 \\
\hline ARG-1 & None & 0 & 0 & 4 & 1 & 4 & 1 & ARG-1B41 & 4810 & 13400 & 80500 & 7710 & 684 & $<244$ & $<779$ & 215000 & 6640 & 192 & 931 \\
\hline SME Simulant & Isolok - $12 \mathrm{~mL}$ & $\mathrm{HF}$ & 13 & 4 & 11 & 4 & 2 & HIB02 & 1840 & 21600 & 96500 & 9610 & $<208$ & $<262$ & 1550 & 232000 & 99.8 & $<94.5$ & 792 \\
\hline SME Simulant & \begin{tabular}{|l|} 
Coliwasa \\
\end{tabular} & $\mathrm{HF}$ & 36 & 1 & 21 & 4 & 3 & CL07 & 1880 & 22000 & 99000 & 9710 & $<205$ & 314 & 1700 & 232000 & 99.1 & $<92.9$ & 814 \\
\hline SME Simulant & Isolok - $12 \mathrm{~mL}$ & $\mathrm{HF}$ & 25 & 4 & 4 & 4 & 4 & HIB04 & 1770 & 20700 & 96700 & 9190 & $<217$ & $<273$ & 1360 & 238000 & 99.2 & $<98.6$ & 794 \\
\hline SME Simulant & \begin{tabular}{|l|} 
Coliwasa \\
\end{tabular} & $\mathrm{HF}$ & 1 & 1 & 22 & 4 & 5 & CH05 & 1860 & 21700 & 99400 & 9220 & $<223$ & $<280$ & 1340 & 236000 & 95.1 & $<101$ & 813 \\
\hline SME Simulant & Coliwasa & $\mathrm{HF}$ & 3 & 1 & 20 & 4 & 6 & CL05 & 1850 & 21400 & 103000 & 9310 & $<202$ & $<254$ & 1480 & 241000 & 104 & $<91.8$ & 841 \\
\hline SME Simulant & Coliwasa & $\mathrm{HF}$ & 23 & 1 & 28 & 4 & 7 & CH06 & 1900 & 22900 & 97600 & 10700 & \begin{tabular}{|c|}
$<196$ \\
\end{tabular} & $<246$ & 1790 & 230000 & 117 & $<88.9$ & 833 \\
\hline SME Simulant & Coliwasa & LF & 1 & 1 & 12 & 4 & 8 & CH01 & 1780 & 20400 & 98100 & 8250 & \begin{tabular}{|c|}
$<213$ \\
\end{tabular} & $<267$ & 1230 & 239000 & 121 & $<96.4$ & 829 \\
\hline SME Simulant & Coliwasa & LF & 20 & 1 & 2 & 4 & 9 & CL02 & 1940 & 23100 & 99600 & 10600 & \begin{tabular}{|l|}
$<207$ \\
\end{tabular} & $<260$ & 1720 & 229000 & 118 & $<93.7$ & 851 \\
\hline
\end{tabular}


Table B3. Phase 2 Elemental Concentration Measurements (part 2)

\begin{tabular}{|c|c|c|c|c|c|c|c|c|c|c|c|c|c|c|c|c|c|c|c|}
\hline $\begin{array}{c}\text { Type of } \\
\text { Material }\end{array}$ & Sampler & Flow Rate & $\begin{array}{c}\text { Sample } \\
\text { Order }\end{array}$ & $\begin{array}{c}\text { Preparation } \\
\text { Block }\end{array}$ & $\begin{array}{c}\text { Preparation } \\
\text { Sequence }\end{array}$ & $\begin{array}{c}\text { Analytical } \\
\text { Block }\end{array}$ & $\begin{array}{c}\text { Analytical } \\
\text { Sequence }\end{array}$ & Sample ID & $\mathbf{M g}$ (ug/g) & $\begin{array}{c}\begin{array}{c}\text { Mn } \\
\text { (ug/g) }\end{array} \\
\text { (ung }\end{array}$ & $\begin{array}{c}\mathrm{Na} \\
(\mathrm{ug} / \mathrm{g})\end{array}$ & $\mathrm{Ni}(\mathrm{ug} / \mathrm{g})$ & \begin{tabular}{|c|}
$\mathbf{P}$ \\
$(\mathrm{ug} / \mathrm{g})$
\end{tabular} & 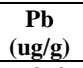 & $\begin{array}{c}\mathrm{S} \\
(\mathrm{ug} / \mathrm{g})\end{array}$ & Si (ug/g) & 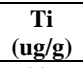 & 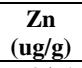 & $\left.\begin{array}{|c|}\mathbf{Z r} \\
(\mathrm{ug} / \mathrm{g})\end{array}\right)$ \\
\hline SME Simulant & Coliwasa & $\mathrm{HF}$ & 63 & 1 & 5 & 4 & 10 & $\begin{array}{ll}\text { CL08 } \\
\end{array}$ & 1780 & 20900 & 96800 & 9120 & $<208$ & $<262$ & 1420 & 231000 & 116 & $<94.5$ & \begin{tabular}{|l|}
799 \\
\end{tabular} \\
\hline SME Simulant & Coliwasa & $\mathrm{HF}$ & 65 & 1 & 6 & 4 & 11 & CH08 & 1900 & 22500 & 98200 & 9910 & \begin{tabular}{|c|}
$<203$ \\
\end{tabular} & $<255$ & 1490 & 230000 & 115 & $<92.1$ & 837 \\
\hline SME Simulant & Isolok - $12 \mathrm{~mL}$ & $\mathrm{HF}$ & 46 & 4 & 2 & 4 & 12 & HIB07 & 1770 & 20900 & 90700 & 9380 & $<213$ & $<268$ & 1590 & 222000 & 115 & $<96.6$ & 782 \\
\hline SME Simulant & \begin{tabular}{|l|} 
Coliwasa \\
\end{tabular} & LF & 4 & 1 & 10 & 4 & 13 & CL01 & 1830 & 21400 & 99400 & 9430 & $<204$ & $<257$ & 1580 & 236000 & 123 & $<92.7$ & 836 \\
\hline SME Simulant & Coliwasa & $\mathrm{HF}$ & 18 & 1 & 26 & 4 & 14 & CL06 & 1880 & 21700 & 97500 & 9050 & $<219$ & $<275$ & 1590 & 235000 & 103 & $<99.2$ & 819 \\
\hline ARG-1 & None & 0 & 0 & 4 & 7 & 4 & 15 & ARG-1B42 & 4980 & 13900 & 84100 & 7920 & \begin{tabular}{|l|}
589 \\
\end{tabular} & $<273$ & $<871$ & 222000 & 6890 & 217 & 960 \\
\hline SME Simulant & Coliwasa & LF & 65 & 1 & 23 & 4 & 16 & CL04 & 1900 & 22400 & 100000 & 9870 & \begin{tabular}{l|}
$<221$ \\
\end{tabular} & $<277$ & 1330 & 230000 & 124 & $<100$ & 795 \\
\hline SME Simulant & Coliwasa & LF & 40 & 1 & 19 & 4 & 17 & CL03 & 1780 & 20200 & 100000 & 8600 & $<219$ & $<275$ & 1820 & 236000 & 116 & $<99.4$ & 792 \\
\hline SME Simulant & Coliwasa & LF & 38 & 1 & 16 & 4 & 18 & $\mathrm{CH} 03$ & 1860 & 22000 & 96100 & 9700 & $<218$ & $<273$ & 1420 & 236000 & 157 & $<98.7$ & 812 \\
\hline SME Simulant & Isolok - $12 \mathrm{~mL}$ & $\mathrm{HF}$ & 56 & 4 & 9 & 4 & 19 & HIB09 & 1830 & 21400 & 96000 & 9270 & $<192$ & $<241$ & 1290 & 236000 & 126 & $<87$ & 830 \\
\hline SME Simulant & \begin{tabular}{|l|} 
Coliwasa \\
\end{tabular} & LF & 21 & 1 & 14 & 4 & 20 & CH02 & 1630 & 19200 & 94100 & 8750 & $<206$ & $<259$ & 1520 & 234000 & 119 & $<93.5$ & 819 \\
\hline SME Simulant & Isolok - $12 \mathrm{~mL}$ & $\mathrm{HF}$ & 7 & 4 & 12 & 4 & 21 & HIB01 & 1790 & 20400 & 98100 & 8560 & $<198$ & $<249$ & 1260 & 235000 & 127 & $<89.8$ & 846 \\
\hline SME Simulant & Isolok - $12 \mathrm{~mL}$ & $\mathrm{HF}$ & 21 & 4 & 6 & 4 & 22 & HIB03 & 1810 & 21100 & 99400 & 9250 & $<187$ & $<235$ & 1040 & 235000 & 112 & $<84.7$ & 855 \\
\hline SME Simulant & Isolok - $12 \mathrm{~mL}$ & $\mathrm{HF}$ & 49 & 4 & 5 & 4 & 23 & HIB08 & 1870 & 21900 & 101000 & 9450 & \begin{tabular}{l|}
$<201$ \\
\end{tabular} & $<253$ & 1600 & 237000 & 114 & $<91.2$ & 841 \\
\hline SME Simulant & Isolok - $12 \mathrm{~mL}$ & $\mathrm{HF}$ & 38 & 4 & 8 & 4 & 24 & HIB06 & 1810 & 21000 & 100000 & 9310 & \begin{tabular}{l|}
$<<217$ \\
\end{tabular} & $<273$ & 1830 & 239000 & 118 & $<98.6$ & 842 \\
\hline SME Simulant & $\begin{array}{l}\text { Coliwasa } \\
\end{array}$ & $\mathrm{HF}$ & 34 & 1 & 18 & 4 & 25 & CH07 & 1720 & 20200 & 95100 & 8650 & $<223$ & $<280$ & 1220 & 223000 & 128 & $<101$ & 779 \\
\hline SME Simulant & Coliwasa & LF & 63 & 1 & 13 & 4 & 26 & CH04 & 1790 & 20800 & 101000 & 9060 & \begin{tabular}{l|}
$<215$ \\
\end{tabular} & $<270$ & 1530 & 235000 & 116 & $<97.4$ & 825 \\
\hline SME Simulant & Isolok - $12 \mathrm{~mL}$ & $\mathrm{HF}$ & 33 & 4 & 10 & 4 & 27 & HIB05 & 1740 & 20300 & 98500 & 9030 & $<207$ & $<261$ & 1370 & 237000 & 116 & $<94$ & 796 \\
\hline SME Simulant & Isolok - $12 \mathrm{~mL}$ & $\mathrm{HF}$ & 58 & 4 & 3 & 4 & 28 & HIB10 & 1780 & 20600 & 96300 & 8930 & $<195$ & $<245$ & 1460 & 236000 & 99.7 & $<88.5$ & 810 \\
\hline ARG-1 & None & 0 & 0 & 4 & 13 & 4 & 29 & ARG-1B43 & 5080 & 14200 & 86000 & 7990 & \begin{tabular}{|l|}
749 \\
\end{tabular} & $<271$ & $<867$ & 228000 & 6990 & 179 & 1030 \\
\hline ARG-1 & None & 0 & 0 & 2 & 1 & 5 & 1 & ARG-1B21 & 5050 & 14100 & 84400 & 7970 & 851 & $<253$ & $<808$ & 223000 & 6910 & 206 & 986 \\
\hline SME Simulant & Isolok - $3 \mathrm{~mL}$ & $\mathrm{HF}$ & 20 & 2 & 16 & 5 & 2 & HISO3 & 1840 & 21500 & 95700 & 9370 & 201 & 270 & $<782$ & 240000 & 98.2 & $<88.3$ & 829 \\
\hline SME Simulant & Isolok - $3 \mathrm{~mL}$ & $\mathrm{HF}$ & 9 & 2 & 21 & 5 & 3 & HIS01 & 1850 & 21600 & 98600 & 9560 & \begin{tabular}{l|}
$<215$ \\
\end{tabular} & 341 & $<864$ & 244000 & 98 & $<97.6$ & 820 \\
\hline SME Simulant & Coliwasa & LF & 20 & 1 & 2 & 5 & 4 & CL02 & 1880 & 22300 & 93000 & 10000 & \begin{tabular}{|l|}
240 \\
\end{tabular} & $<260$ & 1660 & 220000 & 98.9 & $<93.7$ & 802 \\
\hline SME Simulant & Coliwasa & LF & 38 & 1 & 16 & 5 & 5 & $\mathrm{CH} 03$ & 1840 & 21600 & 96000 & 9510 & $<218$ & $<273$ & 1430 & 230000 & 130 & $<98.7$ & 788 \\
\hline SME Simulant & Coliwasa & LF & 63 & 1 & 13 & 5 & 6 & CH04 & 1820 & 21100 & 98700 & 9150 & $<215$ & $<270$ & 1420 & 236000 & 107 & $<97.4$ & 850 \\
\hline SME Simulant & Isolok - 3 mL & $\mathrm{HF}$ & 44 & 2 & 18 & 5 & 7 & HIS07 & 1850 & 21800 & 97100 & 9470 & $<212$ & 348 & $<850$ & 227000 & 100 & $<95.9$ & 783 \\
\hline SME Simulant & Coliwasa & $\mathrm{HF}$ & 23 & 1 & 28 & 5 & 8 & CH06 & 1930 & 23200 & 97800 & 10700 & $<196$ & $<246$ & 1690 & 231000 & 100 & $<88.9$ & 851 \\
\hline SME Simulant & Coliwasa & LF & 21 & 1 & 14 & 5 & 9 & CH02 & 1650 & 19300 & 94300 & 8780 & $<206$ & $<259$ & 1420 & 234000 & 98.2 & $<93.5$ & 824 \\
\hline SME Simulant & Coliwasa & $\mathrm{HF}$ & 3 & 1 & 20 & 5 & 10 & CL05 & 1840 & 21200 & 102000 & 9070 & $<202$ & $<254$ & 1630 & 236000 & 102 & $<91.8$ & 818 \\
\hline SME Simulant & Isolok - $3 \mathrm{~mL}$ & $\mathrm{HF}$ & 14 & 2 & 15 & 5 & 11 & HIS02 & 1840 & 21600 & 99200 & 9450 & $<209$ & 440 & $<839$ & 238000 & 96.7 & $<94.7$ & 815 \\
\hline SME Simulant & Coliwasa & LF & 40 & 1 & 19 & 5 & 12 & CL03 & 1830 & 20600 & 100000 & 8740 & \begin{tabular}{|l|}
300 \\
\end{tabular} & $<275$ & 1460 & 239000 & 102 & $<99.4$ & 816 \\
\hline SME Simulant & Coliwasa & $\mathrm{HF}$ & 18 & 1 & 26 & 5 & 13 & CL06 & 1890 & 21900 & 99200 & 9090 & $<219$ & $<275$ & 1090 & 235000 & 92 & $<99.2$ & 831 \\
\hline SME Simulant & Coliwasa & $\mathrm{HF}$ & 1 & 1 & 22 & 5 & 14 & CH05 & 1870 & 21800 & 99100 & 9110 & \begin{tabular}{l|}
$<223$ \\
\end{tabular} & 367 & 1390 & 235000 & 95 & $<101$ & 830 \\
\hline ARG-1 & None & 0 & 0 & 2 & 12 & 5 & 15 & ARG-1B22 & 5090 & 14100 & 85000 & 8040 & \begin{tabular}{|l|}
815 \\
\end{tabular} & 367 & 841 & 226000 & 6930 & 203 & 1010 \\
\hline SME Simulant & Coliwasa & $\mathrm{HF}$ & 36 & 1 & 21 & 5 & 16 & CL07 & 1880 & 22000 & 98900 & 9590 & $<205$ & 267 & 1260 & 230000 & 91.2 & $<92.9$ & 803 \\
\hline SME Simulant & Isolok - $3 \mathrm{~mL}$ & $\mathrm{HF}$ & 31 & 2 & 19 & 5 & 17 & HIS05 & 1910 & 22400 & 100000 & 9780 & \begin{tabular}{|l|}
$<214$ \\
\end{tabular} & $<269$ & 1070 & 239000 & 94.9 & $<97.2$ & 813 \\
\hline SME Simulant & Isolok - $3 \mathrm{~mL}$ & $\mathrm{HF}$ & 60 & 2 & 22 & 5 & 18 & HIS10 & 1780 & 20800 & 96700 & 9100 & $<215$ & $<270$ & 911 & 242000 & 99.8 & $<97.4$ & 820 \\
\hline SME Simulant & Coliwasa & $\mathrm{HF}$ & 63 & 1 & 5 & 5 & 19 & CL08 & 1820 & 21300 & 99800 & 9420 & $<208$ & $<262$ & 1680 & 234000 & 84.2 & $<94.5$ & 811 \\
\hline SME Simulant & Isolok - $3 \mathrm{~mL}$ & $\mathrm{HF}$ & 26 & 2 & 14 & 5 & 20 & HIS04 & 1870 & 21900 & 97100 & 9470 & \begin{tabular}{|l|}
$<211$ \\
\end{tabular} & 313 & $<846$ & 238000 & 100 & $<95.5$ & 823 \\
\hline SME Simulant & Isolok - $3 \mathrm{~mL}$ & $\mathrm{HF}$ & 35 & 2 & 20 & 5 & 21 & HIS06 & 1860 & 21900 & 98300 & 9690 & 216 & 358 & 1100 & 234000 & 100 & $<94.7$ & 804 \\
\hline SME Simulant & Coliwasa & $\mathrm{HF}$ & 65 & 1 & 6 & 5 & 22 & CH08 & 1970 & 23300 & 102000 & 10200 & \begin{tabular}{|l|}
269 \\
\end{tabular} & 337 & 1490 & 236000 & 98.8 & $<92.1$ & 845 \\
\hline SME Simulant & Coliwasa & $\mathrm{HF}$ & 34 & 1 & 18 & 5 & 23 & CH07 & 1880 & 21900 & 101000 & 9380 & $<2$ & $<280$ & 1160 & 242000 & 102 & $<101$ & 822 \\
\hline SME Simulant & Coliwasa & LF & 1 & 1 & 12 & 5 & 24 & CH01 & 1710 & 19500 & 92800 & 7920 & $<213$ & $<267$ & 1180 & 228000 & 93.6 & $<96.4$ & 766 \\
\hline SME Simulant & Isolok - $3 \mathrm{~mL}$ & $\mathrm{HF}$ & 54 & 2 & 17 & 5 & 25 & HIS09 & 1880 & 22000 & 96900 & 9420 & $<221$ & 677 & 1010 & 237000 & 93.1 & $<100$ & 793 \\
\hline SME Simulant & Coliwasa & LF & 4 & 1 & 10 & 5 & 26 & CL01 & 1840 & 21500 & 98000 & 9490 & \begin{tabular}{|l|}
254 \\
\end{tabular} & 476 & 1490 & 235000 & 90.5 & $<92.7$ & 834 \\
\hline SME Simulant & Isolok - $3 \mathrm{~mL}$ & $\mathrm{HF}$ & 48 & 2 & 13 & 5 & 27 & HIS08 & 1850 & 21800 & 95700 & 9650 & $<190$ & 551 & 1290 & 233000 & 82.7 & $<86.3$ & 792 \\
\hline SME Simulant & Coliwasa & LF & 65 & 1 & 23 & 5 & 28 & CL04 & 1920 & 22600 & 99300 & 10000 & $<221$ & 472 & 1620 & 231000 & 91.1 & $<100$ & 800 \\
\hline
\end{tabular}


Table B3. Phase 2 Elemental Concentration Measurements (part 2)

\begin{tabular}{|c|c|c|c|c|c|c|c|c|c|c|c|c|c|c|c|c|c|c|c|}
\hline $\begin{array}{c}\text { Type of } \\
\text { Material } \\
\end{array}$ & Sampler & Flow Rate & $\begin{array}{c}\text { Sample } \\
\text { Order }\end{array}$ & $\begin{array}{c}\begin{array}{c}\text { Preparation } \\
\text { Block }\end{array} \\
\end{array}$ & $\begin{array}{c}\text { Preparation } \\
\text { Sequence }\end{array}$ & \begin{tabular}{|c|c|}
$\begin{array}{c}\text { Analytical } \\
\text { Block }\end{array}$ \\
\end{tabular} & $\begin{array}{c}\text { Analytical } \\
\text { Sequence }\end{array}$ & Sample ID & Mg (ug/g) & $\begin{array}{c}\begin{array}{c}\text { Mn } \\
\text { (ug/g) }\end{array} \\
\end{array}$ & $\begin{array}{c}\mathrm{Na} \\
(\mathrm{ug} / \mathrm{g})\end{array}$ & $\mathrm{Ni}(\mathrm{ug} / \mathrm{g})$ & \begin{tabular}{|c|}
$\mathbf{P}$ \\
$(\mathrm{ug} / \mathrm{g})$
\end{tabular} & $\begin{array}{c}\begin{array}{c}\mathbf{P b} \\
(\mathrm{ug} / \mathrm{g})\end{array} \\
\end{array}$ & $\begin{array}{c}\mathrm{S} \\
(\mathrm{ug} / \mathrm{g})\end{array}$ & $\mathrm{Si}(\mathbf{u g} / \mathbf{g})$ & $\begin{array}{c}\mathbf{T i} \\
(\mathbf{u g} / \mathrm{g})\end{array}$ & \begin{tabular}{|c}
$\begin{array}{c}\mathrm{Zn} \\
(\mathrm{ug} / \mathrm{g})\end{array}$ \\
\end{tabular} & \begin{tabular}{|c|}
$\mathbf{Z r}$ \\
$(\mathbf{u g} / \mathrm{g})$
\end{tabular} \\
\hline ARG-1 & None & 0 & 0 & 2 & 23 & \begin{tabular}{|l}
5 \\
\end{tabular} & 29 & ARG-1B23 & 4830 & 13400 & 81100 & 7610 & \begin{tabular}{|l|}
716 \\
\end{tabular} & $<266$ & $<849$ & 214000 & 6550 & 209 & 931 \\
\hline ARG-1 & None & 0 & 0 & 3 & 1 & 6 & 1 & ARG-1B31 & 5060 & 14100 & 84200 & 7890 & 720 & $<259$ & $<827$ & 222000 & 6930 & 188 & 983 \\
\hline SME Simulant & Hydragard & LF & 15 & 3 & 21 & 6 & 2 & LHY03 & 1930 & 22300 & 98900 & 9460 & $<204$ & $<256$ & 1500 & 234000 & 105 & $<92.5$ & 841 \\
\hline SME Simulant & Hydragard & LF & 6 & 3 & 18 & 6 & 3 & LHY01 & 1970 & 22800 & 99000 & 9370 & $<195$ & 298 & 1420 & 233000 & 97 & $<88.5$ & 843 \\
\hline SME Simulant & Coliwasa & LF & 20 & 1 & 2 & 6 & 4 & CL02 & 1980 & 23500 & 98200 & 10600 & $<207$ & 378 & 1640 & 229000 & 94.9 & $<93.7$ & 831 \\
\hline SME Simulant & Coliwasa & LF & 38 & 1 & 16 & 6 & 5 & CH03 & 1880 & 22100 & 96400 & 9640 & $<218$ & 325 & 1030 & 233000 & 115 & $<98.7$ & 800 \\
\hline SME Simulant & $\begin{array}{l}\text { Coliwasa } \\
\end{array}$ & LF & 63 & 1 & 13 & 6 & 6 & CH04 & 1830 & 21200 & 102000 & 9130 & $\begin{array}{l}<215 \\
\end{array}$ & 587 & 1110 & 235000 & 110 & $<97.4$ & 823 \\
\hline SME Simulant & Hydragard & LF & 47 & 3 & 13 & 6 & 7 & LHY07 & 1890 & 22000 & 94700 & 9560 & $<214$ & 441 & 1550 & 227000 & 104 & $<<97$ & 807 \\
\hline SME Simulant & Coliwasa & $\mathrm{HF}$ & 23 & 1 & 28 & 6 & 8 & CH06 & 1820 & 21800 & 90800 & 10000 & $<196$ & $<246$ & 1380 & 216000 & 95.5 & $<88.9$ & 781 \\
\hline SME Simulant & Coliwasa & LF & 21 & 1 & 14 & 6 & 9 & CH02 & 1660 & 19400 & 93600 & 8890 & $<206$ & $<259$ & 1230 & 234000 & 101 & $<93.5$ & 823 \\
\hline SME Simulant & Coliwasa & $\mathrm{HF}$ & 3 & 1 & 20 & 6 & 10 & CL05 & 1840 & 21200 & 99400 & 8900 & $<202$ & 401 & 1500 & 235000 & 98.9 & $<91.8$ & 821 \\
\hline SME Simulant & Hydragard & LF & 10 & 3 & 20 & 6 & 11 & LHY02 & 1850 & 21300 & 101000 & 8720 & $<212$ & 763 & 1480 & 233000 & 81.8 & $<96.3$ & 802 \\
\hline SME Simulant & $\begin{array}{l}\text { Coliwasa } \\
\end{array}$ & LF & 40 & 1 & 19 & 6 & 12 & CL03 & 1820 & 20700 & 99300 & 8640 & $<219$ & $<275$ & 1400 & 238000 & 104 & $<99.4$ & 827 \\
\hline SME Simulant & Coliwasa & $\mathrm{HF}$ & 18 & 1 & 26 & 6 & 13 & CL06 & 1920 & 22100 & 96100 & 8930 & $<219$ & 462 & 1280 & 235000 & 90.2 & $<99.2$ & 820 \\
\hline SME Simulant & Coliwasa & $\mathrm{HF}$ & 1 & 1 & 22 & 6 & 14 & CH05 & 1890 & 22000 & 95800 & 9150 & $<223$ & 295 & 1400 & 236000 & 87.2 & $<101$ & 835 \\
\hline ARG-1 & None & 0 & 0 & 3 & 12 & 6 & 15 & ARG-1B32 & 5110 & 14100 & 84400 & 7930 & \begin{tabular}{|l|}
702 \\
\end{tabular} & 284 & $<831$ & 224000 & 6940 & 213 & 969 \\
\hline SME Simulant & $\begin{array}{l}\text { Coliwasa } \\
\end{array}$ & $\mathrm{HF}$ & 36 & 1 & 21 & 6 & 16 & CL07 & 1890 & 22100 & 97100 & 9570 & $<205$ & 270 & 1150 & 229000 & 97.4 & $<92.9$ & 807 \\
\hline SME Simulant & Hydragard & LF & 30 & 3 & 14 & 6 & 17 & LHY05 & 1770 & 20800 & 101000 & 9290 & $<210$ & 321 & 1360 & 237000 & 97.7 & $<<95$ & 835 \\
\hline SME Simulant & Hydragard & LF & 59 & 3 & 17 & 6 & 18 & LHY10 & 1910 & 22600 & 98700 & 9950 & $<200$ & $<251$ & 1510 & 233000 & 101 & $<90.6$ & 812 \\
\hline SME Simulant & $\begin{array}{l}\text { Coliwasa } \\
\end{array}$ & $\mathrm{HF}$ & 63 & 1 & 5 & 6 & 19 & CL08 & 1830 & 21400 & 101000 & 9340 & $<208$ & 267 & 1410 & 233000 & 104 & $<94.5$ & 814 \\
\hline SME Simulant & Hydragard & LF & 28 & 3 & 16 & 6 & 20 & LHY04 & 1820 & 21000 & 96900 & 8850 & $<218$ & 354 & 1440 & 225000 & 105 & 161 & 793 \\
\hline SME Simulant & Hydragard & LF & 37 & 3 & 15 & 6 & 21 & LHY06 & 1930 & 22400 & 96200 & 9470 & $<218$ & 301 & 1150 & 230000 & 94.5 & $<98.9$ & 796 \\
\hline SME Simulant & Coliwasa & $\mathrm{HF}$ & 65 & 1 & 6 & 6 & 22 & CH08 & 1980 & 23400 & 101000 & 10100 & $<203$ & 361 & 1500 & 235000 & 93.3 & $<92.1$ & 817 \\
\hline SME Simulant & Coliwasa & $\mathrm{HF}$ & 34 & 1 & 18 & 6 & 23 & $\begin{array}{l}\text { CH07 } \\
\end{array}$ & 1890 & 22000 & 100000 & 9370 & $<223$ & 609 & 1250 & 239000 & 110 & $<101$ & 794 \\
\hline SME Simulant & $\begin{array}{l}\text { Coliwasa } \\
\end{array}$ & LF & 1 & 1 & 12 & 6 & 24 & CH01 & 1720 & 19700 & 89500 & 7840 & $<213$ & 507 & $<854$ & 228000 & 77.3 & $<96.4$ & 768 \\
\hline SME Simulant & Hydragard & LF & 53 & 3 & 19 & 6 & 25 & LHY09 & 1860 & 21200 & 98900 & 8770 & $<213$ & 869 & 1510 & 238000 & 78.2 & $<96.8$ & 799 \\
\hline SME Simulant & Coliwasa & LF & 4 & 1 & 10 & 6 & 26 & CL01 & 1860 & 21600 & 98800 & 9530 & $<204$ & 929 & 1510 & 235000 & 89.4 & $<92.7$ & 825 \\
\hline SME Simulant & Hydragard & LF & 48 & 3 & 22 & 6 & 27 & LHY08 & 1900 & 22300 & 92900 & 9390 & $<216$ & 796 & 1320 & 227000 & 91 & $<98$ & 800 \\
\hline SME Simulant & Coliwasa & LF & 65 & 1 & 23 & 6 & 28 & CL04 & 1930 & 22600 & 96700 & 9840 & $<221$ & 1170 & 1340 & 230000 & 84.5 & $<100$ & 763 \\
\hline ARG-1 & None & 0 & 0 & 3 & 23 & 6 & 29 & ARG-1B33 & 4810 & 13400 & 79700 & 7500 & \begin{tabular}{|l|}
570 \\
\end{tabular} & 363 & $<856$ & 211000 & 6530 & 202 & 896 \\
\hline
\end{tabular}


Table B4. Phase 2 Oxide Concentration Measurements (part 1)

Revision 0

\begin{tabular}{|c|c|c|c|c|c|c|c|c|c|c|c|c|c|c|c|c|c|}
\hline $\begin{array}{l}\text { Type of } \\
\text { Material }\end{array}$ & Sampler Details & \begin{tabular}{|l|} 
Flow \\
Rate
\end{tabular} & $\begin{array}{r}\text { Sample } \\
\text { Order }\end{array}$ & $\begin{array}{r}\text { Preparation } \\
\text { Block }\end{array}$ & $\begin{array}{r}\text { Preparation } \\
\text { Sequence }\end{array}$ & $\begin{array}{r}\text { Analytical } \\
\text { Block }\end{array}$ & $\begin{array}{r}\text { Analytical } \\
\text { Sequence }\end{array}$ & Sample ID & $\begin{array}{l}\text { Al2O3 } \\
\text { (wt\%) }\end{array}$ & $\begin{array}{r}\mathrm{B2O} 3 \\
\text { (wt\%) }\end{array}$ & $\begin{array}{r}\text { BaO } \\
\text { (wt\%) }\end{array}$ & $\begin{array}{r}\mathrm{CaO} \\
\text { (wt\%) }\end{array}$ & $\begin{array}{r}\text { Cr2O3 } \\
\text { (wt\%) }\end{array}$ & $\begin{array}{r}\text { CuO } \\
\text { (wt\%) }\end{array}$ & $\begin{array}{l}\mathrm{Fe2O3} \\
\text { (wt\%) }\end{array}$ & $\begin{array}{r}\text { K2O } \\
\text { (wt\%) }\end{array}$ & $\begin{array}{r}\text { Li2O } \\
\text { (wt\%) }\end{array}$ \\
\hline ARG-1 & None & 0 & 0 & 1 & 1 & 1 & 1 & \begin{tabular}{|l|} 
ARG-1B11 \\
\end{tabular} & 4.762 & 7.985 & 0.100 & 1.469 & 0.100 & 0.009 & 14.297 & 2.783 & 3.186 \\
\hline SME Simulant & Isolok - 3 mL & LF & 18 & 1 & 9 & 1 & 2 & \begin{tabular}{|l|} 
LIS03 \\
\end{tabular} & 11.809 & 4.508 & 0.005 & 0.635 & 0.022 & 0.101 & 10.980 & \begin{tabular}{l|l|}
0.167 \\
\end{tabular} & 5.059 \\
\hline SME Simulant & Isolok - $3 \mathrm{~mL}$ & LF & 8 & 1 & 27 & 1 & 3 & LIS01 & 11.431 & 4.572 & 0.005 & 0.617 & 0.023 & 0.097 & 10.708 & 0.170 & 5.059 \\
\hline SME Simulant & Coliwasa - low & LF & 20 & 1 & 2 & 1 & 4 & \begin{tabular}{|l|} 
CL02 \\
\end{tabular} & 11.809 & 4.733 & 0.006 & 0.656 & 0.019 & 0.105 & 11.123 & 0.166 & 5.404 \\
\hline SME Simulant & Coliwasa - high & LF & 38 & 1 & 16 & 1 & 5 & \begin{tabular}{|l|} 
CH03 \\
\end{tabular} & \begin{tabular}{r|}
9.939 \\
\end{tabular} & 4.186 & 0.005 & 0.562 & 0.019 & 0.089 & 9.293 & 0.174 & 4.779 \\
\hline SME Simulant & Coliwasa - high & LF & 63 & 1 & 13 & 1 & 6 & CH04 & 11.280 & 4.476 & 0.006 & 0.593 & 0.018 & 0.100 & 10.537 & 0.172 & 4.909 \\
\hline SME Simulant & Isolok - 3 mL & LF & 44 & 1 & 3 & 1 & 7 & LIS07 & 11.602 & 4.443 & 0.006 & 0.625 & 0.022 & 0.090 & 10.823 & 0.155 & 5.102 \\
\hline SME Simulant & Coliwasa - high & $\mathrm{HF}$ & 23 & 1 & 28 & 1 & 8 & CH06 & $\begin{array}{ll}11.356 \\
\end{array}$ & 4.411 & 0.005 & 0.620 & 0.022 & 0.096 & 10.780 & 0.157 & 5.102 \\
\hline SME Simulant & Coliwasa - high & LF & 21 & 1 & 14 & 1 & 9 & CH02 & 10.732 & 4.669 & 0.005 & 0.530 & 0.023 & 0.080 & 10.222 & 0.165 & 4.995 \\
\hline SME Simulant & Coliwasa - low & HF & 3 & 1 & 20 & 1 & 10 & \begin{tabular}{|l|} 
CL05 \\
\end{tabular} & 11.129 & 4.604 & 0.005 & 0.593 & 0.020 & 0.091 & 10.451 & 0.162 & 4.995 \\
\hline SME Simulant & Isolok - $3 \mathrm{~mL}$ & LF & 12 & 1 & 25 & 1 & 11 & LIS02 & 11.186 & 4.476 & 0.005 & 0.600 & 0.020 & 0.080 & 10.551 & 0.164 & 5.124 \\
\hline SME Simulant & Coliwasa - low & LF & 40 & 1 & 19 & 1 & 12 & CL03 & 11.394 & 4.830 & 0.008 & 0.610 & 0.019 & 0.103 & 10.551 & 0.175 & 5.210 \\
\hline SME Simulant & Coliwasa - low & $\mathrm{HF}$ & 18 & 1 & 26 & 1 & 13 & CL06 & 10.090 & 4.315 & 0.005 & 0.555 & 0.018 & 0.093 & 9.422 & 0.160 & 4.693 \\
\hline SME Simulant & Coliwasa - high & $\mathrm{HF}$ & 1 & 1 & 22 & 1 & 14 & \begin{tabular}{|l|}
$\mathrm{CH} 05$ \\
\end{tabular} & 10.978 & 4.798 & 0.006 & 0.623 & 0.019 & 0.096 & 10.265 & 0.179 & 5.361 \\
\hline ARG-1 & None & 0 & 0 & 1 & 15 & 1 & 15 & \begin{tabular}{|l|} 
ARG-1B12 \\
\end{tabular} & \begin{tabular}{|l|}
4.780 \\
\end{tabular} & 8.018 & 0.099 & 1.483 & 0.100 & 0.002 & 14.583 & 2.783 & 3.229 \\
\hline SME Simulant & Coliwasa - low & $\mathrm{HF}$ & 36 & 1 & 21 & 1 & 16 & \begin{tabular}{|l|} 
CL07 \\
\end{tabular} & \begin{tabular}{l|}
11.054 \\
\end{tabular} & 4.476 & 0.005 & 0.600 & 0.018 & 0.097 & 10.437 & 0.164 & 4.909 \\
\hline SME Simulant & Isolok - $3 \mathrm{~mL}$ & LF & 31 & 1 & 24 & 1 & 17 & LIS05 & 11.677 & 4.218 & 0.006 & 0.614 & $\begin{array}{ll}0.017 \\
\end{array}$ & 0.095 & 10.923 & 0.174 & 5.038 \\
\hline SME Simulant & Isolok - $3 \mathrm{~mL}$ & LF & 58 & 1 & 4 & 1 & 18 & \begin{tabular}{|l|} 
LIS10 \\
\end{tabular} & $\begin{array}{l}11.148 \\
\end{array}$ & 4.443 & 0.005 & 0.583 & 0.016 & 0.091 & 10.451 & 0.155 & 5.167 \\
\hline SME Simulant & Coliwasa - low & $\mathrm{HF}$ & 63 & 1 & 5 & 1 & 19 & CL08 & 10.959 & 4.443 & 0.005 & 0.603 & 0.019 & 0.094 & 10.337 & 0.167 & 5.124 \\
\hline SME Simulant & Isolok - $3 \mathrm{~mL}$ & LF & 25 & 1 & 7 & 1 & 20 & LIS04 & 11.620 & 4.347 & 0.006 & 0.750 & 0.022 & 0.102 & 10.823 & 0.170 & 5.102 \\
\hline SME Simulant & Isolok - 3 mL & LF & 42 & 1 & 11 & 1 & 21 & LIS06 & 11.639 & 4.572 & 0.007 & 0.620 & 0.025 & 0.094 & 10.923 & 0.170 & 5.296 \\
\hline SME Simulant & Coliwasa - high & $\mathrm{HF}$ & 65 & 1 & 6 & 1 & 22 & CH08 & 11.224 & 4.443 & 0.006 & 0.639 & 0.021 & 0.105 & 10.394 & 0.163 & 5.210 \\
\hline SME Simulant & Coliwasa - high & HF & 34 & 1 & 18 & 1 & 23 & \begin{tabular}{|l|} 
CH07 \\
\end{tabular} & 11.658 & 4.830 & 0.006 & 0.632 & 0.025 & 0.103 & 10.909 & 0.178 & 5.382 \\
\hline SME Simulant & Coliwasa - high & LF & 1 & 1 & 12 & 1 & 24 & \begin{tabular}{|l|} 
CH01 \\
\end{tabular} & $\begin{array}{l}10.676 \\
\end{array}$ & 4.637 & 0.005 & 0.593 & 0.018 & 0.087 & 9.922 & 0.170 & 5.296 \\
\hline SME Simulant & Isolok - $3 \mathrm{~mL}$ & LF & 54 & 1 & 17 & 1 & 25 & LIS09 & 11.431 & 4.476 & 0.006 & 0.617 & 0.016 & 0.089 & 10.708 & 0.147 & 5.124 \\
\hline SME Simulant & Coliwasa - low & LF & 4 & 1 & 10 & 1 & 26 & CL01 & 11.243 & 4.604 & 0.005 & 0.588 & 0.020 & 0.102 & 10.523 & 0.164 & 4.952 \\
\hline SME Simulant & Isolok - $3 \mathrm{~mL}$ & LF & 49 & 1 & 8 & 1 & 27 & \begin{tabular}{|l|} 
LIS08 \\
\end{tabular} & $\begin{array}{l}11.639 \\
\end{array}$ & 4.315 & 0.005 & 0.624 & 0.017 & 0.086 & 10.923 & 0.161 & 5.124 \\
\hline SME Simulant & Coliwasa - low & LF & 65 & 1 & 23 & 1 & 28 & CL04 & 11.148 & 4.315 & 0.006 & 0.606 & 0.018 & 0.100 & 10.394 & 0.176 & 5.081 \\
\hline ARG-1 & \begin{tabular}{|l|} 
None \\
\end{tabular} & 0 & 0 & 1 & 29 & 1 & 29 & \begin{tabular}{|l|} 
ARG-1B13 \\
\end{tabular} & 4.346 & 7.052 & 0.090 & 1.364 & 0.090 & 0.006 & 13.153 & 2.361 & 2.906 \\
\hline ARG-1 & None & 0 & 0 & 2 & 1 & 2 & 1 & \begin{tabular}{|l|} 
ARG-1B21 \\
\end{tabular} & 4.365 & 7.631 & 0.091 & 1.321 & 0.092 & 0.002 & 13.239 & 2.674 & 3.014 \\
\hline SME Simulant & \begin{tabular}{|l|} 
Hydragard \\
\end{tabular} & $\mathrm{HF}$ & 11 & 2 & 3 & 2 & 2 & $\begin{array}{l}\text { HHY02 } \\
\end{array}$ & \begin{tabular}{l|}
11.356 \\
\end{tabular} & 4.411 & 0.006 & 0.592 & 0.023 & 0.093 & 10.594 & 0.159 & 5.210 \\
\hline SME Simulant & Coliwasa - low & $\mathrm{HF}$ & 36 & 1 & 21 & 2 & 3 & \begin{tabular}{|l|} 
CL07 \\
\end{tabular} & 10.959 & 4.508 & 0.005 & 0.582 & 0.018 & 0.101 & 10.280 & 0.164 & 5.102 \\
\hline SME Simulant & Hydragard & $\mathrm{HF}$ & 24 & 2 & 2 & 2 & 4 & \begin{tabular}{|l|} 
HHY04 \\
\end{tabular} & 11.469 & 4.121 & 0.007 & 0.572 & 0.024 & 0.091 & 10.909 & 0.174 & 4.758 \\
\hline SME Simulant & Coliwasa - high & $\mathrm{HF}$ & 1 & 1 & 22 & 2 & 5 & \begin{tabular}{|l|}
$\mathrm{CH} 05$ \\
\end{tabular} & \begin{tabular}{l|}
10.751 \\
\end{tabular} & 4.637 & 0.005 & 0.576 & 0.020 & 0.102 & 10.036 & 0.179 & 5.339 \\
\hline SME Simulant & Coliwasa - low & $\mathrm{HF}$ & 3 & 1 & 20 & 2 & 6 & CL05 & 11.091 & 4.733 & 0.006 & 0.572 & 0.019 & 0.095 & 10.380 & 0.162 & 5.188 \\
\hline SME Simulant & Coliwasa - high & $\mathrm{HF}$ & 23 & 1 & 28 & 2 & 7 & \begin{tabular}{|l|} 
CH06 \\
\end{tabular} & $\begin{array}{l}11.205 \\
\end{array}$ & 4.508 & 0.006 & 0.579 & 0.020 & 0.091 & 10.666 & 0.157 & 5.059 \\
\hline SME Simulant & Coliwasa - high & LF & 1 & 1 & 12 & 2 & 8 & \begin{tabular}{|l|} 
CH01 \\
\end{tabular} & 10.543 & 4.604 & 0.005 & 0.560 & 0.017 & 0.094 & $\begin{array}{r}9.822 \\
\end{array}$ & 0.170 & 5.339 \\
\hline SME Simulant & Coliwasa - low & LF & 20 & 1 & 2 & 2 & 9 & CL02 & 11.243 & 4.604 & 0.005 & 0.593 & 0.017 & 0.095 & 10.651 & 0.166 & 5.167 \\
\hline SME Simulant & Coliwasa - low & $\mathrm{HF}$ & 63 & 1 & 5 & 2 & 10 & \begin{tabular}{|l|} 
CL08 \\
\end{tabular} & 10.732 & 4.508 & 0.006 & 0.555 & 0.020 & 0.092 & 10.122 & 0.167 & 5.102 \\
\hline SME Simulant & Coliwasa - high & $\mathrm{HF}$ & 65 & 1 & 6 & 2 & 11 & \begin{tabular}{|l|}
$\mathrm{CH} 08$ \\
\end{tabular} & $\begin{array}{l}10.978 \\
\end{array}$ & 4.443 & 0.005 & 0.592 & 0.018 & 0.094 & 10.194 & 0.163 & 5.081 \\
\hline SME Simulant & Hydragard & $\mathrm{HF}$ & 45 & 2 & 7 & 2 & 12 & HHY07 & 10.241 & 4.154 & 0.005 & 0.530 & 0.022 & 0.085 & 9.522 & 0.171 & 4.693 \\
\hline SME Simulant & Coliwasa - low & LF & 4 & 1 & 10 & 2 & 13 & \begin{tabular}{|l|} 
CL01 \\
\end{tabular} & $\begin{array}{l}11.167 \\
\end{array}$ & 4.733 & 0.005 & 0.572 & 0.020 & 0.098 & 10.494 & 0.164 & 5.210 \\
\hline SME Simulant & Coliwasa - low & $\mathrm{HF}$ & 18 & 1 & 26 & 2 & 14 & \begin{tabular}{|l|} 
CL06 \\
\end{tabular} & 10.940 & 4.701 & 0.006 & 0.585 & 0.020 & 0.100 & 10.222 & 0.175 & 5.404 \\
\hline ARG-1 & \begin{tabular}{|l|} 
None \\
\end{tabular} & 0 & 0 & 2 & 12 & 2 & 15 & \begin{tabular}{|l|} 
ARG-1B22 \\
\end{tabular} & \begin{tabular}{r|}
4.573 \\
\end{tabular} & 8.114 & 0.098 & 1.427 & 0.094 & 0.003 & 13.940 & 2.807 & 3.186 \\
\hline SME Simulant & Coliwasa - low & LF & 65 & 1 & 23 & 2 & 16 & \begin{tabular}{|l|} 
CL04 \\
\end{tabular} & 10.959 & 4.379 & 0.007 & 0.567 & 0.017 & 0.099 & 10.265 & 0.176 & 5.081 \\
\hline SME Simulant & Coliwasa - low & LF & 40 & $\frac{1}{1}$ & 19 & 2 & 17 & \begin{tabular}{|l|} 
CL03 \\
\end{tabular} & 10.732 & 4.572 & 0.006 & 0.543 & 0.017 & 0.094 & 9.951 & 0.175 & 4.995 \\
\hline SME Simulant & \begin{tabular}{|l|} 
Coliwasa - high \\
\end{tabular} & LF & 38 & 1 & 16 & 2 & 18 & \begin{tabular}{|l|}
$\mathrm{CH} 03$ \\
\end{tabular} & $\begin{array}{l}10.732 \\
\end{array}$ & 4.540 & 0.006 & 0.588 & 0.018 & 0.096 & 10.051 & 0.174 & 5.447 \\
\hline SME Simulant & Hydragard & $\mathrm{HF}$ & 57 & 2 & 6 & 2 & 19 & \begin{tabular}{|l|} 
HHY09 \\
\end{tabular} & 11.318 & 4.347 & 0.005 & 0.579 & 0.021 & 0.095 & 10.651 & 0.148 & 5.016 \\
\hline
\end{tabular}


Table B4. Phase 2 Oxide Concentration Measurements (part 1)

Revision 0

\begin{tabular}{|c|c|c|c|c|c|c|c|c|c|c|c|c|c|c|c|c|c|}
\hline $\begin{array}{l}\text { Type of } \\
\text { Material }\end{array}$ & Sampler Details & \begin{tabular}{|l|} 
Flow \\
Rate
\end{tabular} & $\begin{array}{r}\text { Sample } \\
\text { Order }\end{array}$ & $\begin{array}{r}\text { Preparation } \\
\text { Block }\end{array}$ & $\begin{array}{r}\text { Preparation } \\
\text { Sequence }\end{array}$ & $\begin{array}{r}\text { Analytical } \\
\text { Block }\end{array}$ & $\begin{array}{r}\text { Analytical } \\
\text { Sequence }\end{array}$ & Sample ID & $\begin{array}{l}\text { Al2O3 } \\
\text { (wt\%) }\end{array}$ & $\begin{array}{r}\text { B2O3 } \\
\text { (wt\%) }\end{array}$ & $\begin{array}{r}\text { BaO } \\
(\text { wt \%) }\end{array}$ & $\begin{array}{r}\mathrm{CaO} \\
(\mathrm{wt} \%) \\
\end{array}$ & $\begin{array}{l}\text { Cr2O3 } \\
\text { (wt\%) }\end{array}$ & $\begin{array}{r}\mathrm{CuO} \\
(\mathrm{wt} \%)\end{array}$ & $\begin{array}{l}\text { Fe2O3 } \\
\text { (wt\%) }\end{array}$ & $\begin{array}{r}\text { K2O } \\
\text { (wt\%) }\end{array}$ & $\begin{array}{r}\text { Li2O } \\
\text { (wt\%) }\end{array}$ \\
\hline SME Simulant & Coliwasa - high & LF & 21 & 1 & 14 & 2 & 20 & CH02 & 10.449 & 4.669 & 0.005 & 0.491 & 0.022 & 0.085 & 9.908 & 0.165 & 5.059 \\
\hline SME Simulant & Hydragard & $\mathrm{HF}$ & 6 & 2 & 4 & 2 & 21 & \begin{tabular}{|l|} 
HHY01 \\
\end{tabular} & $\begin{array}{ll}11.413 \\
\end{array}$ & 4.411 & 0.006 & 0.604 & 0.024 & 0.096 & 10.809 & 0.175 & 5.059 \\
\hline SME Simulant & Hydragard & $\mathrm{HF}$ & 19 & 2 & 10 & 2 & 22 & HHY03 & 11.375 & 4.154 & 0.006 & 0.585 & 0.023 & 0.096 & 10.894 & 0.170 & 5.016 \\
\hline SME Simulant & \begin{tabular}{|l|} 
Hydragard \\
\end{tabular} & HF & 50 & 2 & 5 & 2 & 23 & \begin{tabular}{|l|} 
HHY08 \\
\end{tabular} & $\begin{array}{l}11.035 \\
\end{array}$ & 4.540 & 0.006 & 0.558 & 0.022 & 0.094 & 10.437 & 0.173 & 4.995 \\
\hline SME Simulant & \begin{tabular}{|l|} 
Hydragard \\
\end{tabular} & $\mathrm{HF}$ & 37 & 2 & 9 & 2 & 24 & \begin{tabular}{|l|} 
HHY06 \\
\end{tabular} & $\begin{array}{ll}11.469 \\
\end{array}$ & 4.540 & 0.006 & 0.596 & 0.029 & 0.098 & 11.252 & 0.170 & 5.016 \\
\hline SME Simulant & Coliwasa - high & $\mathrm{HF}$ & 34 & 1 & 18 & 2 & 25 & CH07 & 10.260 & 4.379 & 0.006 & 0.536 & 0.018 & 0.091 & 9.608 & 0.178 & 4.930 \\
\hline SME Simulant & Coliwasa - high & LF & 63 & 1 & 13 & 2 & 26 & \begin{tabular}{|l|}
$\mathrm{CH} 04$ \\
\end{tabular} & 10.884 & 4.540 & 0.006 & 0.558 & 0.015 & 0.090 & 10.151 & 0.172 & 5.145 \\
\hline SME Simulant & Hydragard & $\mathrm{HF}$ & 32 & 2 & 8 & 2 & 27 & \begin{tabular}{|l|} 
HHY05 \\
\end{tabular} & 11.224 & 4.186 & 0.005 & 0.596 & 0.019 & 0.094 & 10.723 & 0.161 & 5.059 \\
\hline SME Simulant & Hydragard & $\mathrm{HF}$ & 61 & 2 & 11 & 2 & 28 & HHY10 & 11.394 & 4.315 & 0.005 & 0.611 & 0.020 & 0.104 & 10.637 & 0.347 & 5.275 \\
\hline ARG-1 & \begin{tabular}{|l|} 
None \\
\end{tabular} & 0 & 0 & 2 & 23 & 2 & 29 & \begin{tabular}{|l|} 
ARG-1B23 \\
\end{tabular} & \begin{tabular}{|l|}
4.610 \\
\end{tabular} & 8.146 & 0.097 & 1.441 & 0.094 & 0.003 & 13.954 & 2.939 & 3.251 \\
\hline ARG-1 & None & 0 & 0 & 3 & 1 & 3 & 1 & \begin{tabular}{|l|} 
ARG-1B31 \\
\end{tabular} & 4.403 & 7.985 & 0.085 & 1.382 & 0.094 & 0.007 & 13.296 & 2.614 & 3.057 \\
\hline SME Simulant & Isolok - $12 \mathrm{~mL}$ & LF & 11 & 3 & 4 & 3 & 2 & LIB02 & 10.884 & 4.765 & 0.012 & 0.576 & 0.019 & 0.090 & 10.122 & 0.169 & 5.275 \\
\hline SME Simulant & Coliwasa - low & $\mathrm{HF}$ & 36 & 1 & 21 & 3 & 3 & CL07 & 10.940 & 4.701 & 0.004 & 0.590 & 0.020 & 0.096 & 10.265 & 0.164 & 5.016 \\
\hline SME Simulant & Isolok - $12 \mathrm{~mL}$ & LF & 26 & 3 & 8 & 3 & 4 & LIB04 & 10.997 & 4.765 & 0.011 & 0.597 & 0.017 & 0.092 & 10.394 & 0.167 & 5.318 \\
\hline SME Simulant & Coliwasa - high & $\mathrm{HF}$ & 1 & 1 & 22 & 3 & 5 & CH05 & 10.770 & 4.862 & 0.004 & 0.617 & 0.022 & 0.091 & 10.051 & 0.179 & 5.425 \\
\hline SME Simulant & Coliwasa - low & $\mathrm{HF}$ & 3 & 1 & 20 & 3 & 6 & CL05 & 11.129 & 5.023 & 0.004 & 0.603 & 0.021 & 0.091 & 10.423 & 0.162 & 5.382 \\
\hline SME Simulant & Coliwasa - high & HF & 23 & 1 & 28 & 3 & 7 & \begin{tabular}{|l|} 
CH06 \\
\end{tabular} & 11.261 & 4.637 & 0.004 & 0.602 & 0.023 & 0.093 & 10.666 & 0.157 & 5.038 \\
\hline SME Simulant & Coliwasa - high & LF & 1 & 1 & 12 & 3 & 8 & \begin{tabular}{|l|} 
CH01 \\
\end{tabular} & \begin{tabular}{l|}
10.543 \\
\end{tabular} & 4.733 & 0.004 & 0.569 & 0.019 & 0.090 & $\begin{array}{r}9.779 \\
\end{array}$ & 0.170 & 5.167 \\
\hline SME Simulant & Coliwasa - low & LF & 20 & 1 & 2 & 3 & 9 & CL02 & 11.243 & 4.765 & 0.004 & 0.611 & 0.023 & 0.096 & 10.580 & 0.166 & 5.124 \\
\hline SME Simulant & Coliwasa - low & $\mathrm{HF}$ & 63 & 1 & 5 & 3 & 10 & \begin{tabular}{|l|} 
CL08 \\
\end{tabular} & 10.676 & 4.798 & 0.003 & 0.565 & 0.021 & 0.095 & 10.008 & 0.167 & 4.930 \\
\hline SME Simulant & Coliwasa - high & $\mathrm{HF}$ & 65 & 1 & 6 & 3 & 11 & CH08 & 10.449 & 4.411 & 0.003 & 0.576 & 0.019 & 0.095 & 9.693 & 0.163 & 4.801 \\
\hline SME Simulant & Isolok - $12 \mathrm{~mL}$ & LF & 45 & 3 & 7 & 3 & 12 & LIB07 & 11.054 & 4.733 & 0.012 & 0.597 & 0.025 & 0.101 & 10.294 & 0.169 & 5.296 \\
\hline SME Simulant & Coliwasa - low & LF & 4 & 1 & 10 & 3 & 13 & \begin{tabular}{|l|} 
CL01 \\
\end{tabular} & 11.110 & 4.894 & 0.003 & 0.596 & 0.019 & 0.098 & 10.394 & 0.164 & 5.296 \\
\hline SME Simulant & Coliwasa - low & $\mathrm{HF}$ & 18 & 1 & 26 & 3 & 14 & \begin{tabular}{|l|} 
CL06 \\
\end{tabular} & 10.770 & 4.798 & 0.003 & 0.592 & 0.020 & 0.099 & 10.022 & 0.175 & 5.188 \\
\hline ARG-1 & None & 0 & 0 & 3 & 12 & 3 & 15 & \begin{tabular}{|l|} 
ARG-1B32 \\
\end{tabular} & 4.610 & 8.243 & 0.098 & 1.483 & 0.096 & 0.009 & 13.997 & 2.903 & 3.208 \\
\hline SME Simulant & Coliwasa - low & LF & 65 & 1 & 23 & 3 & 16 & \begin{tabular}{|l|} 
CL04 \\
\end{tabular} & 10.959 & 4.830 & 0.004 & 0.602 & 0.018 & 0.100 & 10.265 & 0.176 & 5.188 \\
\hline SME Simulant & Coliwasa - low & LF & 40 & 1 & 19 & 3 & 17 & \begin{tabular}{|l|} 
CL03 \\
\end{tabular} & 10.808 & 4.798 & 0.004 & 0.579 & 0.021 & 0.101 & 10.051 & 0.175 & 4.995 \\
\hline SME Simulant & Coliwasa - high & LF & 38 & 1 & 16 & 3 & 18 & $\mathrm{CH} 03$ & 10.751 & 4.733 & 0.004 & 0.600 & 0.020 & 0.097 & 10.022 & 0.174 & 5.339 \\
\hline SME Simulant & Isolok - $12 \mathrm{~mL}$ & LF & 56 & 3 & 11 & 3 & 19 & LIB09 & 11.091 & 4.315 & 0.012 & 0.592 & 0.022 & 0.092 & 10.280 & 0.164 & 5.102 \\
\hline SME Simulant & Coliwasa - high & LF & 21 & 1 & 14 & 3 & 20 & CH02 & 10.506 & 4.798 & 0.004 & 0.525 & 0.022 & 0.086 & 9.951 & 0.165 & 5.275 \\
\hline SME Simulant & Isolok - $12 \mathrm{~mL}$ & LF & 5 & 3 & 5 & 3 & 21 & LIB01 & $\begin{array}{l}11.129 \\
\end{array}$ & 4.604 & 0.010 & 0.613 & 0.021 & 0.091 & 10.365 & 0.165 & 5.124 \\
\hline SME Simulant & Isolok - $12 \mathrm{~mL}$ & LF & 23 & 3 & 10 & 3 & 22 & LIB03 & 11.110 & 4.443 & 0.011 & 0.597 & 0.017 & 0.094 & 10.423 & 0.169 & 5.124 \\
\hline SME Simulant & Isolok - $12 \mathrm{~mL}$ & LF & 50 & 3 & 2 & 3 & 23 & LIB08 & 11.110 & 4.508 & 0.013 & 0.600 & 0.025 & 0.092 & 10.280 & 0.166 & 5.124 \\
\hline SME Simulant & Isolok - $12 \mathrm{~mL}$ & LF & 41 & 3 & 9 & 3 & 24 & LIB06 & $\begin{array}{ll}10.373 \\
\end{array}$ & 4.218 & 0.011 & 0.590 & 0.023 & 0.094 & 9.593 & 0.169 & 4.930 \\
\hline SME Simulant & Coliwasa - high & $\mathrm{HF}$ & 34 & 1 & 18 & 3 & 25 & CH07 & 10.865 & 4.669 & 0.004 & 0.593 & 0.018 & 0.096 & 10.079 & 0.178 & 5.361 \\
\hline SME Simulant & \begin{tabular}{|l|} 
Coliwasa - high \\
\end{tabular} & LF & 63 & 1 & 13 & 3 & 26 & \begin{tabular}{|l|} 
CH04 \\
\end{tabular} & $\begin{array}{l}11.035 \\
\end{array}$ & 4.637 & 0.004 & 0.575 & 0.018 & 0.091 & 10.280 & 0.172 & 4.995 \\
\hline SME Simulant & \begin{tabular}{|l|} 
Isolok - $12 \mathrm{~mL}$ \\
\end{tabular} & LF & 32 & 3 & 3 & 3 & 27 & LIB05 & 11.299 & 4.765 & 0.013 & 0.617 & 0.017 & 0.090 & 10.451 & 0.171 & 5.253 \\
\hline SME Simulant & Isolok - $12 \mathrm{~mL}$ & LF & 60 & 3 & 6 & 3 & 28 & \begin{tabular}{|l|} 
LIB10 \\
\end{tabular} & 11.148 & 4.411 & 0.012 & 0.611 & 0.021 & 0.094 & 10.565 & 0.168 & 5.404 \\
\hline ARG-1 & None & 0 & 0 & 3 & 23 & 3 & 29 & \begin{tabular}{|l|} 
ARG-1B33 \\
\end{tabular} & 4.743 & 8.275 & 0.098 & 1.483 & 0.099 & 0.003 & 14.226 & 2.915 & 3.294 \\
\hline ARG-1 & None & 0 & 0 & 4 & 1 & 4 & 1 & \begin{tabular}{|l|} 
ARG-1B41 \\
\end{tabular} & 4.421 & 7.696 & 0.087 & 1.391 & 0.094 & 0.002 & 13.382 & 2.518 & 3.014 \\
\hline SME Simulant & Isolok - $12 \mathrm{~mL}$ & HF & 13 & 4 & 11 & 4 & 2 & HIB02 & 10.657 & 4.508 & 0.014 & 0.582 & 0.031 & 0.087 & 10.022 & 0.167 & 5.102 \\
\hline SME Simulant & Coliwasa - low & HF & 36 & 1 & 21 & 4 & 3 & \begin{tabular}{|l|l|} 
CL07 \\
\end{tabular} & 10.959 & 4.572 & 0.006 & 0.596 & 0.020 & 0.095 & 10.308 & 0.164 & 4.973 \\
\hline SME Simulant & Isolok - $12 \mathrm{~mL}$ & $\mathrm{HF}$ & 25 & 4 & 4 & 4 & 4 & HIB04 & 10.827 & 4.315 & 0.016 & 0.564 & 0.029 & 0.084 & 10.179 & 0.174 & 5.145 \\
\hline SME Simulant & Coliwasa - high & HF & 1 & 1 & 22 & 4 & 5 & CH05 & 10.827 & 4.637 & 0.006 & 0.599 & 0.021 & 0.090 & 10.065 & 0.179 & 5.210 \\
\hline SME Simulant & Coliwasa - low & $\mathrm{HF}$ & 3 & 1 & 20 & 4 & 6 & \begin{tabular}{|l|} 
CL05 \\
\end{tabular} & 11.110 & 4.701 & 0.005 & 0.599 & 0.020 & 0.086 & 10.423 & 0.162 & 5.145 \\
\hline SME Simulant & Coliwasa - high & $\mathrm{HF}$ & 23 & $\frac{1}{1}$ & 28 & 4 & 7 & \begin{tabular}{|l|} 
CH06 \\
\end{tabular} & 11.205 & 4.476 & 0.006 & 0.609 & 0.022 & 0.093 & 10.666 & 0.157 & 5.102 \\
\hline SME Simulant & \begin{tabular}{|l|} 
Coliwasa - high \\
\end{tabular} & LF & 1 & 1 & 12 & 4 & 8 & CH01 & $\begin{array}{ll}10.468 \\
\end{array}$ & 4.443 & 0.005 & 0.575 & 0.019 & 0.089 & 9.722 & 0.170 & 5.188 \\
\hline SME Simulant & Coliwasa - low & LF & 20 & 1 & 2 & 4 & 9 & CL02 & 11.205 & 4.476 & 0.005 & 0.620 & 0.017 & 0.098 & 10.580 & 0.166 & 5.145 \\
\hline
\end{tabular}


Table B4. Phase 2 Oxide Concentration Measurements (part 1)

Revision 0

\begin{tabular}{|c|c|c|c|c|c|c|c|c|c|c|c|c|c|c|c|c|c|}
\hline $\begin{array}{l}\text { Type of } \\
\text { Material }\end{array}$ & Sampler Details & \begin{tabular}{|l|} 
Flow \\
Rate
\end{tabular} & $\begin{array}{r}\text { Sample } \\
\text { Order }\end{array}$ & $\begin{array}{r}\begin{array}{r}\text { Preparation } \\
\text { Block }\end{array} \\
\end{array}$ & $\begin{array}{r}\text { Preparation } \\
\text { Sequence }\end{array}$ & $\begin{array}{r}\text { Analytical } \\
\text { Block }\end{array}$ & $\begin{array}{r}\text { Analytical } \\
\text { Sequence }\end{array}$ & Sample ID & $\begin{array}{l}\text { Al2O3 } \\
\text { (wt\%) }\end{array}$ & $\begin{array}{r}\mathrm{B} 2 \mathrm{O} 3 \\
\text { (wt\%) }\end{array}$ & $\begin{array}{r}\text { BaO } \\
\text { (wt\%) }\end{array}$ & $\begin{array}{r}\mathrm{CaO} \\
\text { (wt\%) }\end{array}$ & $\begin{array}{l}\text { Cr2O3 } \\
\text { (wt\%) }\end{array}$ & $\begin{array}{r}\mathrm{CuO} \\
\text { (wt\%) }\end{array}$ & $\begin{array}{l}\text { Fe2O3 } \\
\text { (wt\%) }\end{array}$ & $\begin{array}{r}\mathrm{K} 2 \mathrm{O} \\
\text { (wt\%) }\end{array}$ & $\begin{array}{r}\text { Li2O } \\
\text { (wt\%) }\end{array}$ \\
\hline SME Simulant & Coliwasa - low & $\mathrm{HF}$ & 63 & 1 & 5 & 4 & 10 & \begin{tabular}{|l|} 
CL08 \\
\end{tabular} & 10.600 & 4.379 & 0.005 & 0.564 & 0.020 & 0.089 & 9.979 & $\begin{array}{l}0.167 \\
\end{array}$ & 4.844 \\
\hline SME Simulant & Coliwasa - high & $\mathrm{HF}$ & 65 & 1 & 6 & 4 & 11 & CH08 & $\begin{array}{l}10.865 \\
\end{array}$ & 4.315 & 0.005 & 0.614 & 0.022 & 0.096 & 10.151 & 0.163 & 5.059 \\
\hline SME Simulant & Isolok - $12 \mathrm{~mL}$ & HF & 46 & 4 & 2 & 4 & 12 & HIB07 & 10.260 & 4.121 & 0.013 & 0.553 & 0.025 & 0.084 & 9.936 & 0.170 & 4.801 \\
\hline SME Simulant & Coliwasa - low & LF & 4 & 1 & 10 & 4 & 13 & CL01 & 11.110 & 4.604 & 0.005 & 0.593 & 0.019 & 0.094 & 10.494 & 0.164 & 5.102 \\
\hline SME Simulant & Coliwasa - low & \begin{tabular}{|l|l}
$\mathrm{HF}$ \\
\end{tabular} & 18 & 1 & 26 & 4 & 14 & \begin{tabular}{|l|} 
CL06 \\
\end{tabular} & 10.902 & 4.476 & 0.005 & 0.596 & 0.020 & 0.094 & 10.165 & 0.175 & 5.102 \\
\hline ARG-1 & \begin{tabular}{|l|} 
None \\
\end{tabular} & 0 & 0 & 4 & 7 & 4 & 15 & \begin{tabular}{|l|} 
ARG-1B42 \\
\end{tabular} & 4.535 & 7.631 & 0.097 & 1.455 & 0.096 & 0.003 & 13.839 & 2.674 & 3.100 \\
\hline SME Simulant & Coliwasa - low & LF & 65 & 1 & 23 & 4 & 16 & \begin{tabular}{|l|l|} 
CL04 \\
\end{tabular} & 10.902 & 4.347 & 0.006 & 0.599 & 0.019 & 0.095 & 10.237 & 0.176 & 5.102 \\
\hline SME Simulant & Coliwasa - low & \begin{tabular}{|l|l|}
$L F$ \\
\end{tabular} & 40 & $\frac{1}{1}$ & 19 & 4 & 17 & \begin{tabular}{|l|} 
CL03 \\
\end{tabular} & 10.695 & 4.508 & 0.006 & 0.574 & 0.020 & $\begin{array}{l}0.098 \\
\end{array}$ & 9.979 & 0.175 & 4.952 \\
\hline SME Simulant & Coliwasa - high & LF & 38 & 1 & 16 & 4 & 18 & CH03 & 10.713 & 4.508 & 0.006 & 0.583 & 0.018 & 0.092 & 10.036 & 0.174 & 4.995 \\
\hline SME Simulant & Isolok - $12 \mathrm{~mL}$ & HF & 56 & 4 & 9 & 4 & 19 & \begin{tabular}{|l|} 
HIB09 \\
\end{tabular} & \begin{tabular}{l|}
10.751 \\
\end{tabular} & 4.218 & 0.013 & 0.593 & 0.024 & 0.095 & 10.165 & 0.154 & 5.059 \\
\hline SME Simulant & Coliwasa - high & LF & 21 & 1 & 14 & 4 & 20 & CH02 & 10.430 & 4.540 & 0.005 & 0.541 & 0.020 & 0.082 & $\begin{array}{r}9.965 \\
\end{array}$ & 0.165 & 5.167 \\
\hline SME Simulant & Isolok - $12 \mathrm{~mL}$ & HF & 7 & 4 & 12 & 4 & 21 & HIB01 & 10.770 & 4.250 & 0.013 & 0.581 & 0.029 & 0.085 & 10.094 & 0.158 & 5.145 \\
\hline SME Simulant & Isolok - $12 \mathrm{~mL}$ & $\mathrm{HF}$ & 21 & 4 & 6 & 4 & 22 & HIB03 & 10.751 & 4.121 & 0.012 & 0.588 & 0.026 & 0.087 & 10.094 & 0.149 & 5.210 \\
\hline SME Simulant & Isolok - $12 \mathrm{~mL}$ & $\mathrm{HF}$ & 49 & 4 & 5 & 4 & 23 & \begin{tabular}{|l|l|} 
HIB08 \\
\end{tabular} & 10.865 & 4.057 & 0.014 & 0.603 & 0.026 & 0.091 & 10.351 & $\begin{array}{l}0.161 \\
0.171\end{array}$ & 5.232 \\
\hline SME Simulant & Isolok - $12 \mathrm{~mL}$ & HF & 38 & 4 & 8 & 4 & 24 & HIB06 & 10.921 & 4.347 & 0.014 & 0.603 & 0.029 & 0.086 & 10.451 & 0.174 & 5.232 \\
\hline SME Simulant & Coliwasa - high & HF & 34 & 1 & 18 & 4 & 25 & CH07 & \begin{tabular}{l|}
10.147 \\
\end{tabular} & 4.089 & 0.006 & 0.557 & 0.019 & 0.083 & 9.536 & 0.178 & 4.887 \\
\hline SME Simulant & Coliwasa - high & LF & 63 & 1 & 13 & 4 & 26 & \begin{tabular}{|l|} 
CH04 \\
\end{tabular} & 10.940 & 4.282 & 0.007 & 0.576 & 0.019 & 0.091 & 10.308 & 0.172 & 4.909 \\
\hline SME Simulant & Isolok - $12 \mathrm{~mL}$ & \begin{tabular}{|l|}
$\mathrm{HF}$ \\
\end{tabular} & 33 & 4 & 10 & 4 & 27 & \begin{tabular}{|l|} 
HIB05 \\
\end{tabular} & 10.676 & 4.250 & 0.014 & 0.565 & 0.024 & 0.084 & 10.022 & 0.166 & 5.038 \\
\hline SME Simulant & Isolok - $12 \mathrm{~mL}$ & $\mathrm{HF}$ & 58 & 4 & 3 & 4 & 28 & HIB10 & 10.732 & 4.089 & 0.012 & 0.567 & 0.023 & 0.084 & 10.065 & 0.156 & 4.930 \\
\hline ARG-1 & None & \begin{tabular}{|l|l|}
0 \\
\end{tabular} & 0 & 4 & 13 & 4 & 29 & \begin{tabular}{|l|} 
ARG-1B43 \\
\end{tabular} & \begin{tabular}{|l|}
4.648 \\
\end{tabular} & 7.792 & 0.091 & 1.455 & 0.103 & 0.003 & 14.140 & 2.734 & 3.165 \\
\hline ARG-1 & None & $\mid 0$ & 0 & 2 & 1 & 5 & 1 & \begin{tabular}{|l|} 
ARG-1B21 \\
\end{tabular} & 4.573 & 8.082 & $\begin{array}{l}0.097 \\
\end{array}$ & $\begin{array}{l}1.427 \\
\end{array}$ & $\begin{array}{l}0.096 \\
\end{array}$ & 0.008 & 13.911 & 2.674 & 3.143 \\
\hline SME Simulant & Isolok - $3 \mathrm{~mL}$ & HF & 20 & 2 & \begin{tabular}{|c|}
16 \\
\end{tabular} & 5 & 2 & HIS03 & 10.884 & 4.540 & 0.004 & 0.565 & 0.021 & 0.089 & 10.237 & 0.156 & 5.102 \\
\hline SME Simulant & Isolok - 3 mL & HF & 9 & 2 & 21 & 5 & 3 & HIS01 & 11.091 & 4.830 & 0.004 & 0.569 & 0.019 & 0.088 & 10.380 & 0.172 & 5.296 \\
\hline SME Simulant & Coliwasa - low & LF & 20 & 1 & 2 & 5 & 4 & \begin{tabular}{|l|l|} 
CL02 \\
\end{tabular} & 10.902 & 4.315 & 0.005 & 0.582 & $\begin{array}{l}0.017 \\
\end{array}$ & 0.091 & 10.208 & 0.166 & 4.909 \\
\hline SME Simulant & Coliwasa - high & LF & 38 & 1 & 16 & 5 & 5 & \begin{tabular}{|l|} 
CH03 \\
\end{tabular} & 10.619 & 4.411 & 0.005 & 0.569 & 0.017 & 0.094 & 9.836 & 0.174 & 5.038 \\
\hline SME Simulant & Coliwasa - high & \begin{tabular}{|l|} 
LF \\
\end{tabular} & 63 & 1 & 13 & 5 & 6 & \begin{tabular}{|l|} 
CH04 \\
\end{tabular} & 11.110 & 4.443 & 0.005 & 0.565 & 0.019 & 0.098 & 10.394 & 0.172 & 4.844 \\
\hline SME Simulant & Isolok - $3 \mathrm{~mL}$ & \begin{tabular}{|l} 
\\
$\mathrm{HF}$
\end{tabular} & 44 & $\frac{1}{2}$ & 18 & 5 & 7 & \begin{tabular}{|l|} 
HIS07 \\
\end{tabular} & $\begin{array}{l}1.997 \\
10.997\end{array}$ & 4.669 & 0.005 & 0.564 & $\begin{array}{ll}0.024 \\
\end{array}$ & 0.094 & 10.194 & 0.169 & 5.102 \\
\hline SME Simulant & Coliwasa - high & $\mathrm{HF}$ & 23 & 1 & 28 & 5 & 8 & CH06 & 11.356 & 4.572 & 0.005 & 0.597 & 0.022 & 0.091 & 10.737 & 0.157 & 5.038 \\
\hline SME Simulant & Coliwasa - high & LF & 21 & 1 & 14 & 5 & 9 & \begin{tabular}{|l|} 
CH02 \\
\end{tabular} & 10.562 & 4.669 & 0.005 & 0.521 & 0.021 & 0.084 & 9.965 & 0.165 & 5.188 \\
\hline SME Simulant & Coliwasa - low & HF & 3 & 1 & 20 & 5 & 10 & \begin{tabular}{|l|} 
CL05 \\
\end{tabular} & $\begin{array}{l}10.959 \\
\end{array}$ & 4.733 & 0.004 & 0.575 & 0.019 & 0.091 & 10.265 & 0.162 & 5.059 \\
\hline SME Simulant & Isolok - $3 \mathrm{~mL}$ & HF & 14 & 2 & 15 & 5 & 11 & \begin{tabular}{|l|} 
HISO2 \\
\end{tabular} & 10.902 & 4.508 & 0.005 & 0.578 & 0.022 & 0.092 & 10.380 & 0.167 & 5.232 \\
\hline SME Simulant & Coliwasa - low & LF & 40 & 1 & 19 & 5 & 12 & CL03 & 10.978 & 4.701 & 0.005 & 0.569 & 0.018 & 0.104 & 10.151 & 0.175 & 4.973 \\
\hline SME Simulant & Coliwasa - low & \begin{tabular}{|l|l} 
HF \\
\end{tabular} & 18 & $\frac{1}{1}$ & 26 & 5 & $\mid 13$ & \begin{tabular}{|l|} 
CL06 \\
\end{tabular} & 10.940 & 4.701 & 0.005 & 0.592 & 0.019 & 0.095 & 10.151 & 0.175 & 5.188 \\
\hline SME Simulant & Coliwasa - high & $\mathrm{HF}$ & 1 & $\frac{1}{1}$ & 22 & 5 & 14 & \begin{tabular}{|l|} 
CH05 \\
\end{tabular} & $\begin{array}{l}10.808 \\
\end{array}$ & 4.637 & 0.005 & 0.590 & $\begin{array}{l}0.021 \\
\end{array}$ & 0.097 & 10.036 & 0.179 & 5.188 \\
\hline ARG-1 & \begin{tabular}{|l} 
None \\
\end{tabular} & 0 & 0 & 2 & 12 & 5 & 15 & \begin{tabular}{|l} 
ARG-1B22 \\
\end{tabular} & \begin{tabular}{|l|}
4.667 \\
\end{tabular} & 8.114 & 0.099 & 1.413 & 0.096 & 0.003 & 14.068 & 2.722 & 3.165 \\
\hline SME Simulant & Coliwasa - low & HF & 36 & 1 & 21 & 5 & 16 & \begin{tabular}{|l|} 
CL07 \\
\end{tabular} & 10.978 & 4.508 & 0.005 & 0.586 & 0.019 & 0.099 & 10.251 & 0.164 & 4.995 \\
\hline SME Simulant & Isolok - $3 \mathrm{~mL}$ & HF & 31 & 2 & 19 & 5 & 17 & HIS05 & 10.978 & 4.540 & 0.005 & 0.603 & 0.019 & 0.096 & 10.237 & 0.172 & 5.296 \\
\hline SME Simulant & Isolok - $3 \mathrm{~mL}$ & $\mathrm{HF}$ & 60 & 2 & 22 & 5 & 18 & HIS10 & 10.770 & 4.476 & 0.005 & 0.558 & 0.020 & 0.084 & 10.108 & 0.172 & 5.167 \\
\hline SME Simulant & Coliwasa - low & $\mathrm{HF}$ & 63 & 1 & 5 & 5 & 19 & \begin{tabular}{ll|} 
CL08 \\
\end{tabular} & 10.827 & 4.476 & 0.005 & 0.572 & 0.022 & 0.096 & 10.122 & 0.167 & 5.038 \\
\hline SME Simulant & Isolok - $3 \mathrm{~mL}$ & $\mathrm{HF}$ & 26 & $\frac{1}{2}$ & 14 & 5 & 20 & \begin{tabular}{|l|} 
HISOS \\
\end{tabular} & $\begin{array}{l}10.997 \\
\end{array}$ & 4.508 & 0.005 & 0.581 & $\begin{array}{l}0.022 \\
\end{array}$ & 0.092 & 10.265 & 0.169 & 5.059 \\
\hline SME Simulant & Isolok - $3 \mathrm{~mL}$ & $\mathrm{HF}$ & 35 & 2 & 20 & 5 & 21 & \begin{tabular}{|l|} 
HISO6 \\
\end{tabular} & 10.884 & 4.443 & 0.005 & 0.581 & 0.026 & 0.090 & 10.237 & 0.167 & 5.145 \\
\hline SME Simulant & Coliwasa - high & HF & 65 & 1 & 6 & 5 & 22 & \begin{tabular}{ll|} 
CH08 \\
\end{tabular} & 11.280 & 4.604 & 0.004 & 0.617 & 0.020 & 0.102 & 10.394 & 0.163 & 5.145 \\
\hline SME Simulant & Coliwasa - high & $\mathrm{HF}$ & 34 & 1 & 18 & 5 & 23 & \begin{tabular}{|l|} 
CH07 \\
\end{tabular} & $\begin{array}{l}11.091 \\
\end{array}$ & 4.572 & 0.005 & 0.588 & 0.018 & 0.094 & 10.308 & 0.178 & 5.210 \\
\hline SME Simulant & Coliwasa - high & LF & 1 & 1 & 12 & 5 & 24 & \begin{tabular}{|l|} 
CH01 \\
\end{tabular} & 10.052 & 4.282 & 0.004 & 0.534 & 0.018 & 0.085 & $\begin{array}{l}9.279 \\
\end{array}$ & 0.170 & 4.909 \\
\hline SME Simulant & Isolok - $3 \mathrm{~mL}$ & $\mathrm{HF}$ & 54 & 2 & 17 & 5 & 25 & \begin{tabular}{|l|} 
HISO9 \\
\end{tabular} & $\begin{array}{l}10.978 \\
\end{array}$ & 4.476 & 0.005 & 0.572 & 0.023 & 0.091 & 10.208 & 0.177 & 5.102 \\
\hline SME Simulant & Coliwasa - low & LF & 4 & 1 & 10 & 5 & 26 & \begin{tabular}{|l|} 
CL01 \\
\end{tabular} & 11.224 & 4.669 & 0.005 & 0.579 & $\begin{array}{l}0.019 \\
\end{array}$ & 0.103 & 10.480 & 0.164 & 5.038 \\
\hline SME Simulant & Isolok - $3 \mathrm{~mL}$ & \begin{tabular}{|l|l}
$\mathrm{HF}$ \\
\end{tabular} & 48 & $\frac{1}{2}$ & 13 & 5 & 27 & \begin{tabular}{|l|} 
HIS08 \\
\end{tabular} & 10.940 & 4.315 & 0.005 & 0.564 & 0.023 & 0.088 & 10.265 & 0.152 & 5.016 \\
\hline SME Simulant & Coliwasa - low & LF & 65 & 1 & 23 & 5 & 28 & \begin{tabular}{|l|l|} 
CL04 \\
\end{tabular} & $\begin{array}{l}10.997 \\
\end{array}$ & 4.476 & 0.005 & 0.592 & $\begin{array}{l}0.017 \\
\end{array}$ & \begin{tabular}{|l|l|}
0.098 \\
\end{tabular} & 10.265 & 0.176 & 5.124 \\
\hline
\end{tabular}


Table B4. Phase 2 Oxide Concentration Measurements (part 1)

\begin{tabular}{|c|c|c|c|c|c|c|c|c|c|c|c|c|c|c|c|c|c|}
\hline $\begin{array}{l}\text { Type of } \\
\text { Material }\end{array}$ & Sampler Details & $\begin{array}{l}\text { Flow } \\
\text { Rate }\end{array}$ & $\begin{array}{r}\text { Sample } \\
\text { Order }\end{array}$ & $\begin{array}{r}\text { Preparation } \\
\text { Block }\end{array}$ & $\begin{array}{r}\text { Preparation } \\
\text { Sequence }\end{array}$ & $\begin{array}{r}\text { Analytical } \\
\text { Block }\end{array}$ & $\begin{array}{r}\text { Analytical } \\
\text { Sequence }\end{array}$ & Sample ID & $\begin{array}{l}\text { Al2O3 } \\
\text { (wt\%) }\end{array}$ & $\begin{array}{r}\mathrm{B2O} 3 \\
\text { (wt\%) }\end{array}$ & $\begin{array}{r}\text { BaO } \\
\text { (wt\%) }\end{array}$ & $\begin{array}{r}\mathrm{CaO} \\
\text { (wt\%) }\end{array}$ & $\begin{array}{l}\text { Cr2O3 } \\
\text { (wt\%) }\end{array}$ & $\begin{array}{r}\mathrm{CuO} \\
\text { (wt\%) }\end{array}$ & $\begin{array}{l}\text { Fe2O3 } \\
\text { (wt\%) }\end{array}$ & $\begin{array}{r}\mathrm{K} 20 \\
\text { (wt\%) }\end{array}$ & $\begin{array}{r}\text { Li2O } \\
\text { (wt\%) }\end{array}$ \\
\hline ARG-1 & None & 0 & 0 & 2 & 23 & 5 & 29 & \begin{tabular}{|l|} 
ARG-1B23 \\
\end{tabular} & 4.440 & 7.438 & 0.093 & 1.349 & 0.091 & 0.003 & 13.353 & 2.554 & 3.014 \\
\hline ARG-1 & None & 0 & 0 & 3 & 1 & 6 & 1 & ARG-1B31 & 4.629 & 8.018 & 0.092 & 1.441 & 0.097 & 0.003 & 13.997 & 2.759 & 3.143 \\
\hline SME Simulant & Hydragard & LF & 15 & 3 & 21 & 6 & 2 & LHY03 & 11.450 & 4.604 & 0.005 & 0.606 & 0.018 & 0.098 & 10.651 & 0.163 & 5.210 \\
\hline SME Simulant & Hydragard & LF & 6 & 3 & 18 & 6 & 3 & LHY01 & 11.356 & 4.604 & 0.011 & 0.620 & 0.019 & 0.101 & 10.537 & 0.156 & 5.188 \\
\hline SME Simulant & Coliwasa - low & LF & 20 & 1 & 2 & 6 & 4 & CL02 & 11.375 & 4.604 & 0.004 & 0.617 & 0.018 & 0.100 & 10.723 & 0.166 & 5.167 \\
\hline SME Simulant & Coliwasa - high & LF & 38 & 1 & 16 & 6 & 5 & $\mathrm{CH} 03$ & 10.695 & 4.540 & 0.005 & 0.588 & 0.019 & 0.098 & 10.051 & 0.174 & 5.124 \\
\hline SME Simulant & Coliwasa - high & LF & 63 & 1 & 13 & 6 & 6 & CH04 & 11.110 & 4.476 & 0.005 & 0.588 & 0.018 & 0.099 & 10.437 & 0.172 & 5.038 \\
\hline SME Simulant & Hydragard & LF & 47 & 3 & 13 & 6 & 7 & LHY07 & 11.072 & 4.121 & 0.012 & 0.596 & 0.023 & 0.093 & 10.423 & 0.171 & 5.016 \\
\hline SME Simulant & Coliwasa - high & $\mathrm{HF}$ & 23 & 1 & 28 & 6 & 8 & CH06 & 10.581 & 4.250 & 0.004 & 0.565 & 0.019 & 0.095 & 10.079 & 0.157 & 4.693 \\
\hline SME Simulant & Coliwasa - high & LF & 21 & 1 & 14 & 6 & 9 & $\mathrm{CH} 02$ & 10.562 & 4.669 & 0.004 & 0.525 & 0.021 & 0.091 & 10.079 & 0.165 & 5.253 \\
\hline SME Simulant & Coliwasa - low & $\mathrm{HF}$ & 3 & 1 & 20 & 6 & 10 & CL05 & 10.940 & 4.701 & 0.005 & 0.582 & 0.019 & 0.092 & 10.294 & 0.162 & 5.016 \\
\hline SME Simulant & Hydragard & LF & 10 & 3 & 20 & 6 & 11 & LHY02 & 11.280 & 4.540 & 0.005 & 0.595 & 0.015 & 0.093 & 10.508 & 0.170 & 5.038 \\
\hline SME Simulant & Coliwasa - low & LF & 40 & 1 & 19 & 6 & 12 & CL03 & 10.940 & 4.669 & 0.005 & 0.576 & 0.020 & 0.105 & 10.179 & 0.175 & 5.016 \\
\hline SME Simulant & Coliwasa - low & $\mathrm{HF}$ & 18 & 1 & 26 & 6 & 13 & CL06 & 10.997 & 4.540 & 0.005 & 0.599 & 0.019 & 0.097 & 10.294 & 0.175 & 5.102 \\
\hline SME Simulant & Coliwasa - high & $\mathrm{HF}$ & 1 & 1 & 22 & 6 & 14 & \begin{tabular}{|l} 
CH05 \\
\end{tabular} & 10.846 & 4.637 & 0.005 & 0.593 & 0.020 & 0.096 & 10.151 & 0.179 & 5.102 \\
\hline ARG-1 & None & 0 & 0 & 3 & 12 & 6 & 15 & \begin{tabular}{|l|} 
ARG-1B32 \\
\end{tabular} & 4.667 & 7.857 & 0.097 & 1.441 & 0.098 & 0.003 & 14.083 & 2.783 & 3.165 \\
\hline SME Simulant & Coliwasa - low & $\mathrm{HF}$ & 36 & 1 & 21 & 6 & 16 & $\begin{array}{ll}\text { CL07 } \\
\end{array}$ & 10.959 & 4.411 & 0.004 & 0.592 & 0.019 & 0.090 & 10.365 & 0.164 & 4.952 \\
\hline SME Simulant & Hydragard & LF & 30 & 3 & 14 & 6 & 17 & LHY05 & 11.261 & 4.508 & 0.005 & 0.557 & 0.018 & 0.091 & 10.952 & 0.168 & 4.909 \\
\hline SME Simulant & Hydragard & LF & 59 & 3 & 17 & 6 & 18 & LHY10 & 10.978 & 4.250 & 0.012 & 0.610 & 0.017 & 0.097 & 10.351 & 0.160 & 5.253 \\
\hline SME Simulant & Coliwasa - low & $\mathrm{HF}$ & 63 & 1 & 5 & 6 & 19 & CL08 & 10.789 & 4.476 & 0.004 & 0.590 & 0.021 & 0.093 & 10.194 & 0.167 & 5.124 \\
\hline SME Simulant & Hydragard & LF & 28 & 3 & 16 & 6 & 20 & LHY04 & 10.770 & \begin{tabular}{ll|}
4.379 \\
\end{tabular} & 0.013 & 0.583 & 0.019 & 0.099 & 10.137 & 0.175 & 5.059 \\
\hline SME Simulant & Hydragard & LF & 37 & 3 & 15 & 6 & 21 & LHY06 & 10.846 & 4.218 & 0.013 & 0.604 & 0.024 & 0.094 & 10.137 & 0.175 & 5.081 \\
\hline SME Simulant & Coliwasa - high & $\mathrm{HF}$ & 65 & 1 & 6 & 6 & 22 & $\begin{array}{ll}\mathrm{CH} 08 \\
\end{array}$ & 11.167 & 4.443 & 0.005 & 0.625 & 0.023 & 0.098 & 10.508 & 0.163 & 5.167 \\
\hline SME Simulant & Coliwasa - high & $\mathrm{HF}$ & 34 & 1 & 18 & 6 & 23 & CH07 & 11.072 & 4.540 & 0.005 & 0.599 & 0.020 & 0.097 & 10.423 & 0.178 & 5.232 \\
\hline SME Simulant & Coliwasa - high & LF & 1 & 1 & 12 & 6 & 24 & CH01 & 10.071 & 4.154 & 0.004 & 0.539 & 0.017 & 0.080 & 9.365 & 0.170 & 4.822 \\
\hline SME Simulant & Hydragard & LF & 53 & 3 & 19 & 6 & 25 & LHY09 & 10.902 & 4.347 & 0.013 & 0.588 & 0.019 & 0.095 & 10.079 & 0.171 & 5.038 \\
\hline SME Simulant & Coliwasa - low & LF & 4 & 1 & 10 & 6 & 26 & \begin{tabular}{|l|} 
CL01 \\
\end{tabular} & 11.186 & 4.669 & 0.005 & 0.588 & 0.021 & 0.099 & 10.623 & 0.164 & 5.145 \\
\hline SME Simulant & Hydragard & LF & 48 & 3 & 22 & 6 & 27 & LHY08 & 11.072 & 4.218 & 0.013 & 0.582 & 0.018 & 0.092 & 10.294 & 0.173 & 4.973 \\
\hline SME Simulant & Coliwasa - low & LF & 65 & 1 & 23 & 6 & 28 & CL04 & 10.978 & 4.411 & 0.006 & 0.590 & 0.018 & 0.096 & 10.280 & 0.176 & 5.059 \\
\hline ARG-1 & None & 0 & 0 & 3 & 23 & 6 & 29 & ARG-1B33 & 4.421 & 7.309 & 0.090 & 1.381 & 0.092 & 0.003 & 13.253 & 2.590 & 3.014 \\
\hline
\end{tabular}


Table B4. Phase 2 Oxide Concentration Measurements (part 2)

\begin{tabular}{|c|c|c|c|c|c|c|c|c|c|c|c|c|c|c|c|c|c|}
\hline $\begin{array}{l}\text { Type of } \\
\text { Material }\end{array}$ & \begin{tabular}{|l|} 
Sampler \\
Details
\end{tabular} & \begin{tabular}{|l} 
Flow \\
Rate
\end{tabular} & $\begin{array}{r}\text { Sample } \\
\text { Order }\end{array}$ & $\begin{array}{r}\text { Preparation } \\
\text { Block }\end{array}$ & $\begin{array}{r}\text { Preparation } \\
\text { Sequence }\end{array}$ & $\begin{array}{r}\text { Analytical } \\
\text { Block }\end{array}$ & $\begin{array}{r}\text { Analytical } \\
\text { Sequence }\end{array}$ & Sample ID & $\begin{array}{r}\text { MgO } \\
\text { (wt\%) }\end{array}$ & $\begin{array}{r}\text { MnO } \\
\text { (wt\%) }\end{array}$ & \begin{tabular}{|r|} 
Na2O \\
(wt\%)
\end{tabular} & $\begin{array}{r}\mathrm{NiO} \\
\text { (wt\%) }\end{array}$ & $\begin{array}{r}\mathrm{SiO2} \\
\text { (wt\%) }\end{array}$ & $\begin{array}{r}\text { TiO2 } \\
\text { (wt\%) }\end{array}$ & \begin{tabular}{|r|}
$\mathrm{ZnO}$ \\
(wt\%)
\end{tabular} & $\begin{array}{r}\mathrm{ZrO2} \\
\text { (wt\%) }\end{array}$ & $\begin{array}{c}\text { Sum of } \\
\text { Oxides } \\
\text { (wt\%) }\end{array}$ \\
\hline ARG-1 & None & 0 & 0 & 1 & 1 & 1 & 1 & ARG-1B11 & 0.856 & 1.846 & 11.822 & 1.029 & 49.418 & 1.158 & 0.026 & 0.130 & 100.977 \\
\hline SME Simulant & Isolok - $3 \mathrm{~mL}$ & LF & 18 & 1 & 9 & 1 & 2 & $\begin{array}{l}\text { LIS03 } \\
\end{array}$ & 0.335 & 3.021 & 13.750 & 1.285 & 51.557 & $\begin{array}{ll}0.018 \\
\end{array}$ & 0.006 & 0.112 & 103.371 \\
\hline SME Simulant & Isolok - $3 \mathrm{~mL}$ & LF & 8 & 1 & 27 & 1 & 3 & LIS01 & 0.317 & 2.866 & 13.440 & 1.205 & 51.771 & 0.016 & 0.006 & 0.113 & 102.418 \\
\hline SME Simulant & Coliwasa - low & LF & 20 & 1 & 2 & 1 & 4 & CL02 & 0.340 & 3.125 & 14.424 & 1.412 & 51.343 & 0.017 & 0.006 & 0.111 & 104.799 \\
\hline SME Simulant & Coliwasa - high & LF & 38 & 1 & 16 & 1 & 5 & $\mathrm{CH} 03$ & 0.287 & 2.621 & 12.617 & 1.153 & 46.851 & 0.021 & 0.006 & 0.093 & 92.695 \\
\hline SME Simulant & Coliwasa - high & LF & 63 & 1 & 13 & 1 & 6 & CH04 & 0.303 & 2.737 & 13.884 & 1.177 & 51.343 & 0.018 & 0.006 & 0.108 & 101.668 \\
\hline SME Simulant & Isolok - $3 \mathrm{~mL}$ & LF & 44 & 1 & 3 & 1 & 7 & LIS07 & 0.328 & \begin{tabular}{|l|l|}
2.983 \\
\end{tabular} & 13.884 & 1.271 & 51.343 & $\begin{array}{ll}0.016 \\
\end{array}$ & 0.005 & 0.110 & 102.809 \\
\hline SME Simulant & Coliwasa - high & $\mathrm{HF}$ & 23 & 1 & 28 & 1 & 8 & CH06 & 0.322 & 2.983 & 13.750 & 1.362 & 49.632 & 0.014 & 0.006 & 0.107 & 100.724 \\
\hline SME Simulant & Coliwasa - high & LF & 21 & 1 & 14 & 1 & 9 & $\mathrm{CH} 02$ & 0.279 & 2.531 & 12.617 & 1.145 & 51.343 & 0.016 & 0.006 & 0.105 & 99.464 \\
\hline SME Simulant & Coliwasa - low & $\mathrm{HF}$ & 3 & 1 & 20 & 1 & 10 & CL05 & 0.307 & 2.763 & 13.884 & 1.143 & 51.343 & 0.016 & 0.006 & 0.107 & 101.619 \\
\hline SME Simulant & Isolok - 3 mL & LF & 12 & 1 & 25 & 1 & 11 & LIS02 & 0.313 & 2.879 & 13.615 & 1.239 & 49.632 & 0.014 & 0.006 & 0.104 & 100.009 \\
\hline SME Simulant & Coliwasa - low & LF & 40 & 1 & 19 & 1 & 12 & CL03 & 0.312 & 2.750 & 14.558 & 1.149 & 53.269 & 0.014 & 0.006 & 0.105 & 105.062 \\
\hline SME Simulant & Coliwasa - low & $\mathrm{HF}$ & 18 & 1 & 26 & 1 & 13 & CL06 & 0.290 & 2.595 & 12.429 & 1.074 & 46.423 & $\begin{array}{ll}0.015 \\
\end{array}$ & 0.006 & 0.098 & 92.281 \\
\hline SME Simulant & Coliwasa - high & $\mathrm{HF}$ & 1 & 1 & 22 & 1 & 14 & CH05 & 0.315 & 2.854 & 14.154 & 1.205 & 51.343 & 0.017 & 0.006 & 0.106 & 102.324 \\
\hline ARG-1 & None & 0 & 0 & 1 & 15 & 1 & 15 & ARG-1B12 & 0.872 & 1.885 & 12.038 & 1.037 & 50.487 & 1.174 & 0.026 & 0.131 & 102.729 \\
\hline SME Simulant & Coliwasa - low & $\mathrm{HF}$ & 36 & 1 & 21 & 1 & 16 & CL07 & 0.315 & 2.866 & 13.372 & 1.211 & 50.274 & 0.015 & 0.006 & 0.105 & 99.924 \\
\hline SME Simulant & Isolok - 3 mL & LF & 31 & 1 & 24 & 1 & 17 & LIS05 & 0.325 & 2.957 & 13.750 & 1.248 & 51.129 & 0.016 & 0.006 & 0.106 & 102.299 \\
\hline SME Simulant & Isolok - 3 mL & LF & 58 & 1 & 4 & 1 & 18 & LIS10 & 0.307 & 2.789 & 13.413 & 1.154 & 51.343 & 0.015 & 0.005 & 0.108 & 101.194 \\
\hline SME Simulant & Coliwasa - low & $\mathrm{HF}$ & 63 & 1 & 5 & 1 & 19 & CL08 & 0.307 & 2.776 & 14.019 & 1.185 & 50.915 & 0.016 & 0.006 & 0.100 & 101.075 \\
\hline SME Simulant & Isolok - $3 \mathrm{~mL}$ & LF & 25 & 1 & 7 & 1 & 20 & LIS04 & 0.325 & 2.918 & 14.019 & 1.225 & 51.129 & $\begin{array}{ll}0.015 \\
\end{array}$ & 0.006 & 0.105 & 102.686 \\
\hline SME Simulant & Isolok - 3 mL & LF & 42 & 1 & 11 & 1 & 21 & LIS06 & 0.327 & 2.983 & 14.019 & 1.285 & 51.985 & 0.016 & 0.006 & 0.109 & 104.075 \\
\hline SME Simulant & Coliwasa - high & $\mathrm{HF}$ & 65 & 1 & 6 & 1 & 22 & CH08 & 0.325 & 2.996 & 14.289 & 1.285 & 50.274 & 0.015 & 0.006 & 0.105 & 101.498 \\
\hline SME Simulant & Coliwasa - high & HF & 34 & 1 & 18 & 1 & 23 & CH07 & 0.328 & 2.970 & 14.558 & 1.269 & 54.552 & 0.018 & 0.006 & 0.109 & 107.533 \\
\hline SME Simulant & Coliwasa - high & LF & 1 & 1 & 12 & 1 & 24 & CH01 & 0.302 & 2.673 & 13.884 & 1.098 & 51.985 & 0.014 & 0.006 & 0.104 & 101.471 \\
\hline SME Simulant & Isolok - 3 mL & LF & 54 & 1 & 17 & 1 & 25 & LIS09 & 0.322 & 2.957 & 13.750 & 1.271 & 51.129 & 0.014 & 0.005 & 0.109 & 102.171 \\
\hline SME Simulant & Coliwasa - low & LF & \begin{tabular}{l|}
4 \\
\end{tabular} & 1 & 10 & 1 & 26 & CL01 & 0.307 & 2.763 & 13.305 & 1.195 & 50.487 & $\begin{array}{ll}0.016 \\
\end{array}$ & 0.006 & 0.105 & 100.384 \\
\hline SME Simulant & Isolok - 3 mL & LF & 49 & 1 & 8 & 1 & 27 & LIS08 & 0.328 & 3.008 & 14.019 & 1.285 & 51.129 & 0.013 & 0.006 & 0.109 & 102.792 \\
\hline SME Simulant & Coliwasa - low & LF & 65 & 1 & 23 & 1 & 28 & CL04 & 0.322 & 2.918 & 13.615 & 1.285 & 50.274 & 0.014 & 0.006 & 0.100 & 100.376 \\
\hline ARG-1 & None & 0 & 0 & 1 & 29 & 1 & 29 & \begin{tabular}{|l} 
ARG-1B13 \\
\end{tabular} & 0.784 & 1.691 & 10.878 & 0.944 & 45.139 & 1.058 & $\begin{array}{ll}0.023 \\
\end{array}$ & 0.117 & 92.003 \\
\hline ARG-1 & None & 0 & 0 & 2 & 1 & 2 & 1 & ARG-1B21 & 0.799 & 1.743 & 10.744 & 0.965 & 44.925 & 1.096 & 0.025 & 0.130 & 92.856 \\
\hline SME Simulant & Hydragard & HF & 11 & 2 & 3 & 2 & 2 & HHY02 & 0.320 & 2.944 & 13.008 & 1.217 & 48.776 & 0.018 & 0.006 & 0.122 & 98.855 \\
\hline SME Simulant & Coliwasa - low & HF & 36 & 1 & 21 & 2 & 3 & \begin{tabular}{|l|} 
CL07 \\
\end{tabular} & 0.313 & 2.879 & 13.386 & 1.222 & 49.418 & 0.017 & 0.006 & 0.117 & 99.075 \\
\hline SME Simulant & Hydragard & $\mathrm{HF}$ & 24 & 2 & 2 & 2 & 4 & HHY04 & 0.312 & 2.892 & 13.264 & 1.273 & 48.776 & 0.018 & 0.006 & 0.116 & 98.782 \\
\hline SME Simulant & Coliwasa - high & HF & 1 & 1 & 22 & 2 & 5 & CH05 & 0.312 & 2.841 & 13.480 & 1.168 & 49.846 & 0.016 & 0.006 & 0.118 & 99.433 \\
\hline SME Simulant & Coliwasa - low & $\mathrm{HF}$ & 3 & 1 & 20 & 2 & 6 & CL05 & 0.308 & 2.802 & 13.884 & 1.169 & 50.915 & 0.017 & 0.006 & 0.119 & 101.468 \\
\hline SME Simulant & Coliwasa - high & HF & 23 & 1 & 28 & 2 & 7 & CH06 & 0.320 & 3.021 & 13.076 & 1.362 & 48.990 & 0.018 & 0.006 & 0.115 & 99.198 \\
\hline SME Simulant & Coliwasa - high & LF & 1 & 1 & 12 & 2 & 8 & CH01 & 0.300 & 2.686 & $\begin{array}{l}13.305 \\
\end{array}$ & 1.056 & 51.343 & 0.018 & 0.006 & 0.114 & 99.983 \\
\hline SME Simulant & Coliwasa - low & LF & 20 & 1 & 2 & 2 & 9 & CL02 & 0.328 & 3.047 & 13.318 & 1.362 & 48.776 & 0.019 & 0.006 & 0.119 & 99.516 \\
\hline SME Simulant & Coliwasa - low & $\mathrm{HF}$ & 63 & 1 & 5 & 2 & 10 & CL08 & 0.302 & 2.763 & 13.480 & 1.196 & 49.846 & $\begin{array}{ll}0.018 \\
\end{array}$ & 0.006 & $\begin{array}{ll}0.113 \\
\end{array}$ & 99.029 \\
\hline SME Simulant & Coliwasa - high & HF & 65 & 1 & 6 & 2 & 11 & CH08 & 0.320 & 2.970 & 13.345 & 1.271 & 49.204 & 0.017 & 0.006 & 0.116 & 98.817 \\
\hline SME Simulant & Hydragard & $\mathrm{HF}$ & 45 & 2 & 7 & 2 & 12 & HHY07 & 0.285 & 2.557 & 12.361 & 1.032 & 44.711 & 0.017 & 0.006 & 0.108 & 90.501 \\
\hline SME Simulant & Coliwasa - low & LF & 4 & 1 & 10 & 2 & 13 & CL01 & 0.308 & 2.828 & 13.480 & 1.206 & 50.487 & $\begin{array}{ll}0.018 \\
\end{array}$ & 0.006 & 0.115 & 100.912 \\
\hline SME Simulant & Coliwasa - low & $\mathrm{HF}$ & 18 & 1 & 26 & 2 & 14 & CL06 & 0.317 & 2.866 & 13.615 & 1.164 & 50.274 & 0.016 & 0.006 & 0.114 & 100.525 \\
\hline ARG-1 & None & 0 & 0 & 2 & 12 & 2 & 15 & ARG-1B22 & 0.841 & 1.834 & 11.458 & 1.021 & 47.492 & 1.156 & 0.027 & 0.136 & 98.206 \\
\hline SME Simulant & Coliwasa - low & LF & 65 & 1 & 23 & 2 & 16 & CL04 & 0.320 & 2.944 & 13.022 & 1.273 & 48.990 & 0.016 & 0.006 & 0.113 & 98.233 \\
\hline SME Simulant & Coliwasa - low & LF & 40 & 1 & 19 & 2 & 17 & CL03 & 0.297 & 2.647 & 13.413 & 1.084 & 49.846 & 0.019 & 0.006 & 0.113 & 98.509 \\
\hline SME Simulant & Coliwasa - high & LF & 38 & 1 & 16 & 2 & 18 & CH03 & 0.310 & 2.879 & 14.019 & 1.243 & 50.060 & 0.022 & 0.006 & 0.111 & 100.303 \\
\hline
\end{tabular}


Table B4. Phase 2 Oxide Concentration Measurements (part 2)

\begin{tabular}{|c|c|c|c|c|c|c|c|c|c|c|c|c|c|c|c|c|c|}
\hline $\begin{array}{l}\text { Type of } \\
\text { Material }\end{array}$ & \begin{tabular}{|l|} 
Sampler \\
Details
\end{tabular} & \begin{tabular}{|l} 
Flow \\
Rate
\end{tabular} & $\begin{array}{r}\text { Sample } \\
\text { Order }\end{array}$ & $\begin{array}{r}\text { Preparation } \\
\text { Block }\end{array}$ & $\begin{array}{r}\text { Preparation } \\
\text { Sequence }\end{array}$ & $\begin{array}{r}\text { Analytical } \\
\text { Block }\end{array}$ & $\begin{array}{r}\text { Analytical } \\
\text { Sequence }\end{array}$ & Sample ID & $\begin{array}{r}\text { MgO } \\
\text { (wt\%) }\end{array}$ & \begin{tabular}{|r|} 
MnO \\
(wt\%)
\end{tabular} & \begin{tabular}{|r|} 
Na2O \\
(wt\%)
\end{tabular} & $\begin{array}{r}\mathrm{NiO} \\
\text { (wt\%) }\end{array}$ & $\begin{array}{r}\mathrm{SiO2} \\
\text { (wt\%) }\end{array}$ & $\begin{array}{r}\text { TiO2 } \\
\text { (wt\%) }\end{array}$ & \begin{tabular}{|r|}
$\mathrm{ZnO}$ \\
(wt\%)
\end{tabular} & $\begin{array}{r}\mathrm{ZrO2} \\
\text { (wt\%) }\end{array}$ & $\begin{array}{l}\text { Sum of } \\
\text { Oxides } \\
\text { (wt\%) }\end{array}$ \\
\hline SME Simulant & Hydragard & $\mathrm{HF}$ & 57 & 2 & 6 & 2 & 19 & HHY09 & \begin{tabular}{ll|}
0.317 \\
\end{tabular} & 2.944 & 13.224 & 1.245 & 48.348 & $\begin{array}{ll}0.018 \\
\end{array}$ & 0.005 & 0.118 & 98.399 \\
\hline SME Simulant & Coliwasa - high & LF & 21 & 1 & 14 & 2 & 20 & $\mathrm{CH} 02$ & 0.272 & 2.518 & 12.159 & 1.126 & 49.632 & 0.019 & 0.006 & 0.116 & 96.700 \\
\hline SME Simulant & Hydragard & $\mathrm{HF}$ & 6 & 2 & 4 & 2 & 21 & HHY01 & 0.328 & 3.060 & 13.305 & 1.323 & 47.920 & 0.020 & 0.006 & 0.123 & 98.683 \\
\hline SME Simulant & \begin{tabular}{|l|} 
Hydragard \\
\end{tabular} & $\mathrm{HF}$ & 19 & 2 & 10 & 2 & 22 & HHY03 & 0.323 & 3.060 & 13.372 & 1.400 & 47.920 & 0.019 & 0.006 & 0.121 & 98.541 \\
\hline SME Simulant & \begin{tabular}{|l|} 
Hydragard \\
\end{tabular} & $\mathrm{HF}$ & 50 & 2 & 5 & 2 & 23 & HHY08 & 0.295 & 2.634 & 14.154 & 1.122 & 50.060 & 0.020 & 0.006 & 0.119 & 100.270 \\
\hline SME Simulant & Hydragard & $\mathrm{HF}$ & 37 & 2 & 9 & 2 & 24 & HHY06 & 0.327 & 3.125 & 13.453 & 1.476 & 48.990 & 0.020 & 0.006 & 0.121 & 100.693 \\
\hline SME Simulant & Coliwasa - high & $\mathrm{HF}$ & 34 & 1 & 18 & 2 & 25 & $\begin{array}{ll}\mathrm{CH} 07 \\
\end{array}$ & 0.292 & 2.673 & 12.739 & 1.122 & 48.134 & $\begin{array}{ll}0.022 \\
\end{array}$ & 0.006 & 0.112 & 95.105 \\
\hline SME Simulant & Coliwasa - high & LF & 63 & 1 & 13 & 2 & 26 & CH04 & 0.297 & 2.699 & 14.019 & 1.162 & 49.418 & 0.022 & 0.006 & 0.118 & 99.301 \\
\hline SME Simulant & Hydragard & $\mathrm{HF}$ & 32 & 2 & 8 & 2 & 27 & HHY05 & 0.323 & 3.073 & 13.480 & 1.323 & 48.134 & 0.022 & 0.006 & 0.120 & 98.550 \\
\hline SME Simulant & \begin{tabular}{|l|} 
Hydragard \\
\end{tabular} & $\mathrm{HF}$ & 61 & 2 & 11 & 2 & 28 & HHY10 & 0.332 & 3.047 & 13.426 & 1.273 & 48.562 & 0.021 & 0.006 & 0.123 & 99.496 \\
\hline ARG-1 & \begin{tabular}{|l|} 
None \\
\end{tabular} & 0 & 0 & 2 & 23 & 2 & 29 & ARG-1B23 & 0.842 & 1.846 & 11.647 & 1.014 & 47.706 & 1.173 & 0.025 & 0.143 & 98.933 \\
\hline ARG-1 & \begin{tabular}{|l|} 
None \\
\end{tabular} & 0 & 0 & 3 & 1 & 3 & 1 & ARG-1B31 & 0.808 & 1.769 & 10.851 & 0.965 & 45.353 & 1.101 & 0.022 & 0.124 & 93.916 \\
\hline SME Simulant & Isolok - $12 \mathrm{~mL}$ & LF & 11 & 3 & 4 & 3 & 2 & LIB02 & 0.300 & 2.712 & 13.480 & 1.073 & 47.920 & 0.018 & 0.006 & 0.110 & 97.530 \\
\hline SME Simulant & Coliwasa - low & $\mathrm{HF}$ & 36 & 1 & 21 & 3 & 3 & CL07 & 0.315 & 2.905 & 13.170 & 1.204 & 49.418 & 0.019 & 0.006 & 0.111 & 98.944 \\
\hline SME Simulant & Isolok - $12 \mathrm{~mL}$ & LF & 26 & 3 & 8 & 3 & 4 & LIB04 & 0.312 & 2.866 & 13.750 & 1.243 & 49.846 & 0.018 & 0.006 & 0.113 & 100.512 \\
\hline SME Simulant & Coliwasa - high & $\mathrm{HF}$ & 1 & 1 & 22 & 3 & 5 & CH05 & 0.315 & 2.866 & 13.750 & 1.157 & 50.060 & 0.017 & 0.006 & 0.116 & 100.307 \\
\hline SME Simulant & Coliwasa - low & $\mathrm{HF}$ & 3 & 1 & 20 & 3 & 6 & CL05 & 0.312 & 2.828 & 14.289 & 1.172 & 51.557 & 0.018 & 0.006 & 0.115 & 103.134 \\
\hline SME Simulant & Coliwasa - high & $\mathrm{HF}$ & 23 & 1 & 28 & 3 & 7 & CH06 & 0.322 & 3.034 & $\begin{array}{l}13.035 \\
\end{array}$ & 1.362 & 49.418 & 0.018 & 0.006 & 0.117 & 99.791 \\
\hline SME Simulant & Coliwasa - high & LF & 1 & 1 & 12 & 3 & 8 & $\mathrm{CH} 01$ & 0.302 & 2.712 & 12.941 & 1.055 & 51.557 & 0.018 & 0.006 & 0.111 & 99.778 \\
\hline SME Simulant & Coliwasa - low & LF & 20 & 1 & 2 & 3 & 9 & CL02 & 0.328 & 3.047 & 13.143 & 1.336 & 48.776 & 0.019 & 0.006 & $\begin{array}{ll}0.113 \\
\end{array}$ & 99.380 \\
\hline SME Simulant & Coliwasa - low & $\mathrm{HF}$ & 63 & 1 & 5 & 3 & 10 & \begin{tabular}{|l|} 
CL08 \\
\end{tabular} & 0.302 & 2.763 & 13.089 & 1.181 & 49.418 & 0.019 & 0.006 & 0.110 & 98.150 \\
\hline SME Simulant & Coliwasa - high & $\mathrm{HF}$ & 65 & 1 & 6 & 3 & 11 & CH08 & 0.307 & 2.854 & 12.604 & 1.196 & 47.065 & 0.017 & 0.006 & 0.111 & 94.370 \\
\hline SME Simulant & Isolok - $12 \mathrm{~mL}$ & LF & 45 & 3 & 7 & 3 & 12 & $\begin{array}{ll}\text { LIB07 } \\
\end{array}$ & 0.315 & 2.854 & 13.615 & 1.178 & 50.274 & 0.021 & 0.006 & 0.117 & 100.660 \\
\hline SME Simulant & Coliwasa - low & LF & 4 & 1 & 10 & 3 & 13 & CL01 & 0.307 & 2.815 & 13.615 & 1.187 & 50.274 & 0.019 & 0.006 & 0.117 & 100.914 \\
\hline SME Simulant & Coliwasa - low & $\mathrm{HF}$ & 18 & 1 & 26 & 3 & 14 & CL06 & 0.313 & 2.841 & 13.170 & 1.133 & 49.632 & 0.019 & 0.006 & 0.114 & 98.895 \\
\hline ARG-1 & None & 0 & 0 & 3 & 12 & 3 & 15 & ARG-1B32 & 0.852 & 1.859 & 11.606 & 1.003 & 48.348 & 1.164 & 0.025 & 0.139 & 99.644 \\
\hline SME Simulant & Coliwasa - low & LF & 65 & 1 & 23 & 3 & 16 & CL04 & 0.322 & 2.970 & 13.359 & 1.273 & 49.418 & 0.018 & 0.006 & 0.108 & 99.615 \\
\hline SME Simulant & Coliwasa - low & LF & 40 & 1 & 19 & 3 & 17 & CL03 & 0.300 & 2.686 & 13.426 & 1.077 & 50.701 & 0.020 & 0.006 & 0.111 & 99.858 \\
\hline SME Simulant & Coliwasa - high & LF & 38 & 1 & 16 & 3 & 18 & $\mathrm{CH} 03$ & 0.313 & 2.905 & 13.615 & 1.233 & 50.274 & 0.024 & 0.035 & $\begin{array}{ll}0.111 \\
\end{array}$ & 100.251 \\
\hline SME Simulant & Isolok - $12 \mathrm{~mL}$ & LF & 56 & 3 & 11 & 3 & 19 & LIB09 & 0.310 & 2.763 & 13.615 & 1.093 & 50.274 & 0.020 & 0.006 & 0.118 & 99.868 \\
\hline SME Simulant & Coliwasa - high & LF & 21 & 1 & 14 & 3 & 20 & CH02 & 0.275 & 2.531 & 12.658 & 1.115 & 50.060 & 0.019 & 0.006 & 0.111 & 98.103 \\
\hline SME Simulant & Isolok - $12 \mathrm{~mL}$ & LF & 5 & 3 & 5 & 3 & 21 & \begin{tabular}{|l|} 
LIB01 \\
\end{tabular} & 0.327 & 2.957 & 13.210 & 1.233 & 48.562 & 0.019 & 0.006 & 0.116 & 98.553 \\
\hline SME Simulant & Isolok - $12 \mathrm{~mL}$ & LF & 23 & 3 & 10 & 3 & 22 & \begin{tabular}{|l|l|} 
LIB03 \\
\end{tabular} & 0.320 & 2.931 & 13.332 & 1.213 & 48.562 & 0.019 & 0.006 & 0.111 & 98.482 \\
\hline SME Simulant & Isolok - $12 \mathrm{~mL}$ & LF & 50 & 3 & 2 & 3 & 23 & LIB08 & 0.320 & 2.931 & 13.130 & 1.157 & 50.060 & 0.018 & 0.006 & 0.115 & 99.653 \\
\hline SME Simulant & Isolok - $12 \mathrm{~mL}$ & LF & 41 & 3 & 9 & 3 & 24 & LIB06 & 0.312 & 2.854 & 12.766 & 1.134 & 47.065 & 0.016 & 0.006 & 0.105 & 94.259 \\
\hline SME Simulant & Coliwasa - high & $\mathrm{HF}$ & 34 & 1 & 18 & 3 & 25 & CH07 & 0.308 & 2.815 & 13.884 & 1.166 & 50.701 & 0.021 & 0.006 & 0.111 & 100.876 \\
\hline SME Simulant & Coliwasa - high & LF & 63 & 1 & 13 & 3 & 26 & CH04 & 0.302 & 2.750 & 13.480 & 1.159 & 50.274 & 0.020 & 0.006 & 0.113 & 99.910 \\
\hline SME Simulant & Isolok - $12 \mathrm{~mL}$ & LF & 32 & 3 & 3 & 3 & 27 & LIB05 & 0.323 & 2.944 & 14.019 & 1.215 & 49.418 & 0.017 & 0.006 & 0.116 & 100.735 \\
\hline SME Simulant & Isolok - $12 \mathrm{~mL}$ & LF & 60 & 3 & 6 & 3 & 28 & LIB10 & 0.320 & \begin{tabular}{|l|l|}
2.983 \\
\end{tabular} & 14.019 & 1.336 & 49.204 & $\begin{array}{ll}0.016 \\
\end{array}$ & 0.006 & $\begin{array}{ll}0.113 \\
\end{array}$ & 100.432 \\
\hline ARG-1 & None & 0 & 0 & 3 & 23 & 3 & 29 & ARG-1B33 & 0.867 & $\begin{array}{ll}1.898 \\
\end{array}$ & \begin{tabular}{ll|}
11.795 \\
\end{tabular} & 1.026 & 48.990 & 1.181 & 0.026 & 0.133 & 101.051 \\
\hline ARG-1 & None & 0 & 0 & 4 & 1 & 4 & 1 & ARG-1B41 & 0.798 & 1.730 & 10.851 & 0.981 & 45.995 & 1.108 & 0.024 & 0.126 & 94.217 \\
\hline SME Simulant & Isolok - $12 \mathrm{~mL}$ & $\mathrm{HF}$ & 13 & 4 & 11 & 4 & 2 & HIB02 & 0.305 & 2.789 & 13.008 & 1.223 & 49.632 & 0.017 & 0.006 & 0.107 & 98.256 \\
\hline SME Simulant & Coliwasa - low & HF & 36 & 1 & 21 & 4 & 3 & CL07 & 0.312 & 2.841 & 13.345 & 1.236 & 49.632 & 0.017 & 0.006 & 0.110 & 99.191 \\
\hline SME Simulant & Isolok - $12 \mathrm{~mL}$ & $\mathrm{HF}$ & 25 & 4 & 4 & 4 & 4 & HIB04 & 0.294 & 2.673 & 13.035 & 1.169 & 50.915 & 0.017 & 0.006 & 0.107 & 99.550 \\
\hline SME Simulant & Coliwasa - high & HF & 1 & 1 & 22 & 4 & 5 & CH05 & 0.308 & 2.802 & 13.399 & 1.173 & 50.487 & 0.016 & 0.006 & 0.110 & 99.936 \\
\hline SME Simulant & Coliwasa - low & $\mathrm{HF}$ & 3 & 1 & 20 & 4 & 6 & CL05 & 0.307 & 2.763 & 13.884 & 1.185 & 51.557 & 0.017 & 0.006 & 0.114 & 102.084 \\
\hline SME Simulant & Coliwasa - high & HF & 23 & 1 & 28 & 4 & 7 & CH06 & 0.315 & 2.957 & 13.156 & 1.362 & 49.204 & 0.020 & 0.006 & 0.113 & 99.466 \\
\hline
\end{tabular}


Table B4. Phase 2 Oxide Concentration Measurements (part 2)

\begin{tabular}{|c|c|c|c|c|c|c|c|c|c|c|c|c|c|c|c|c|c|}
\hline $\begin{array}{l}\text { Type of } \\
\text { Material }\end{array}$ & \begin{tabular}{|l|} 
Sampler \\
Details
\end{tabular} & $\begin{array}{l}\text { Flow } \\
\text { Rate }\end{array}$ & $\begin{array}{r}\text { Sample } \\
\text { Order }\end{array}$ & $\begin{array}{r}\text { Preparation } \\
\text { Block }\end{array}$ & $\begin{array}{r}\text { Preparation } \\
\text { Sequence }\end{array}$ & $\begin{array}{r}\text { Analytical } \\
\text { Block }\end{array}$ & $\begin{array}{r}\text { Analytical } \\
\text { Sequence }\end{array}$ & Sample ID & $\begin{array}{r}\text { MgO } \\
\text { (wt\%) }\end{array}$ & $\begin{array}{r}\text { MnO } \\
\text { (wt\%) }\end{array}$ & $\begin{array}{r}\text { Na2O } \\
\text { (wt\%) }\end{array}$ & $\begin{array}{r}\mathrm{NiO} \\
\text { (wt\%) }\end{array}$ & $\begin{array}{r}\mathrm{SiO2} \\
\text { (wt\%) }\end{array}$ & $\begin{array}{r}\text { TiO2 } \\
\text { (wt\%) }\end{array}$ & \begin{tabular}{|r|}
$\mathrm{ZnO}$ \\
(wt\%)
\end{tabular} & $\begin{array}{r}\mathrm{ZrO2} \\
\text { (wt\%) }\end{array}$ & $\begin{array}{l}\text { Sum of } \\
\text { Oxides } \\
\text { (wt\%) }\end{array}$ \\
\hline SME Simulant & Coliwasa - high & LF & 1 & 1 & 12 & 4 & 8 & CH01 & 0.295 & 2.634 & 13.224 & 1.050 & 51.129 & 0.020 & 0.006 & 0.112 & 99.151 \\
\hline SME Simulant & Coliwasa - low & LF & 20 & 1 & 2 & 4 & 9 & CL02 & 0.322 & 2.983 & 13.426 & 1.349 & 48.990 & 0.020 & 0.006 & 0.115 & 99.521 \\
\hline SME Simulant & Coliwasa - low & $\mathrm{HF}$ & 63 & 1 & 5 & 4 & 10 & CL08 & 0.295 & 2.699 & 13.049 & 1.161 & 49.418 & 0.019 & 0.006 & 0.108 & 97.402 \\
\hline SME Simulant & Coliwasa - high & $\mathrm{HF}$ & 65 & 1 & 6 & 4 & 11 & CH08 & 0.315 & 2.905 & 13.237 & 1.261 & 49.204 & 0.019 & 0.006 & 0.113 & 98.351 \\
\hline SME Simulant & Isolok - $12 \mathrm{~mL}$ & $\mathrm{HF}$ & 46 & 4 & 2 & 4 & 12 & \begin{tabular}{|l|} 
HIB07 \\
\end{tabular} & 0.294 & 2.699 & 12.226 & 1.194 & 47.492 & 0.019 & 0.006 & 0.106 & 93.999 \\
\hline SME Simulant & Coliwasa - low & LF & 4 & 1 & 10 & 4 & 13 & CL01 & 0.303 & 2.763 & 13.399 & 1.200 & 50.487 & 0.021 & 0.006 & 0.113 & 100.479 \\
\hline SME Simulant & Coliwasa - low & $\mathrm{HF}$ & 18 & 1 & 26 & 4 & 14 & CL06 & 0.312 & 2.802 & 13.143 & 1.152 & 50.274 & 0.017 & 0.006 & 0.111 & 99.352 \\
\hline ARG-1 & None & 0 & 0 & 4 & 7 & 4 & 15 & ARG-1B42 & 0.826 & 1.795 & 11.337 & 1.008 & 47.492 & 1.149 & 0.027 & 0.130 & 97.194 \\
\hline SME Simulant & Coliwasa - low & LF & 65 & 1 & 23 & 4 & 16 & CL04 & 0.315 & 2.892 & 13.480 & 1.256 & 49.204 & 0.021 & 0.006 & 0.107 & 98.765 \\
\hline SME Simulant & Coliwasa - low & LF & 40 & 1 & 19 & 4 & 17 & CL03 & 0.295 & 2.608 & 13.480 & 1.094 & 50.487 & 0.019 & 0.006 & 0.107 & 99.105 \\
\hline SME Simulant & Coliwasa - high & LF & 38 & 1 & 16 & 4 & 18 & $\mathrm{CH} 03$ & 0.308 & 2.841 & 12.954 & 1.234 & 50.487 & 0.026 & 0.006 & 0.110 & 99.093 \\
\hline SME Simulant & Isolok - $12 \mathrm{~mL}$ & $\mathrm{HF}$ & 56 & 4 & 9 & 4 & 19 & HIB09 & 0.303 & 2.763 & 12.941 & 1.180 & 50.487 & 0.021 & 0.005 & 0.112 & 98.886 \\
\hline SME Simulant & Coliwasa - high & LF & 21 & 1 & 14 & 4 & 20 & CH02 & 0.270 & 2.479 & 12.685 & 1.113 & 50.060 & 0.020 & 0.006 & 0.111 & 97.660 \\
\hline SME Simulant & Isolok - $12 \mathrm{~mL}$ & $\mathrm{HF}$ & 7 & 4 & 12 & 4 & 21 & HIB01 & 0.297 & 2.634 & 13.224 & 1.089 & 50.274 & 0.021 & 0.006 & 0.114 & 98.784 \\
\hline SME Simulant & Isolok - $12 \mathrm{~mL}$ & $\mathrm{HF}$ & 21 & 4 & 6 & 4 & 22 & HIB03 & 0.300 & 2.724 & 13.399 & 1.177 & 50.274 & 0.019 & 0.005 & 0.115 & 99.052 \\
\hline SME Simulant & Isolok - $12 \mathrm{~mL}$ & $\mathrm{HF}$ & 49 & 4 & 5 & 4 & 23 & HIB08 & 0.310 & 2.828 & 13.615 & 1.203 & 50.701 & 0.019 & 0.006 & 0.114 & 100.193 \\
\hline SME Simulant & Isolok - $12 \mathrm{~mL}$ & HF & 38 & 4 & 8 & 4 & 24 & HIB06 & 0.300 & 2.712 & 13.480 & 1.185 & 51.129 & 0.020 & 0.006 & 0.114 & 100.802 \\
\hline SME Simulant & Coliwasa - high & HF & 34 & 1 & 18 & 4 & 25 & CH07 & 0.285 & 2.608 & 12.819 & 1.101 & 47.706 & 0.021 & 0.006 & 0.105 & 94.155 \\
\hline SME Simulant & Coliwasa - high & LF & 63 & 1 & 13 & 4 & 26 & CH04 & 0.297 & 2.686 & 13.615 & 1.153 & 50.274 & 0.019 & 0.006 & 0.111 & 99.465 \\
\hline SME Simulant & Isolok - $12 \mathrm{~mL}$ & $\mathrm{HF}$ & 33 & 4 & 10 & 4 & 27 & HIB05 & 0.289 & 2.621 & 13.278 & 1.149 & 50.701 & $\begin{array}{ll}0.019 \\
\end{array}$ & 0.006 & 0.108 & 99.011 \\
\hline SME Simulant & Isolok - $12 \mathrm{~mL}$ & $\mathrm{HF}$ & 58 & 4 & 3 & 4 & 28 & HIB10 & 0.295 & 2.660 & 12.981 & 1.136 & 50.487 & 0.017 & 0.006 & 0.109 & 98.349 \\
\hline ARG-1 & None & 0 & 0 & 4 & 13 & 4 & 29 & ARG-1B43 & 0.842 & 1.834 & 11.593 & 1.017 & 48.776 & 1.166 & 0.022 & 0.139 & 99.520 \\
\hline ARG-1 & \begin{tabular}{|l|} 
None \\
\end{tabular} & 0 & 0 & 2 & 1 & 5 & 1 & \begin{tabular}{|l|} 
ARG-1B21 \\
\end{tabular} & 0.837 & 1.821 & 11.377 & 1.014 & 47.706 & 1.153 & 0.026 & 0.133 & 98.078 \\
\hline SME Simulant & Isolok - $3 \mathrm{~mL}$ & HF & 20 & 2 & 16 & 5 & 2 & HIS03 & 0.305 & 2.776 & 12.900 & 1.192 & 51.343 & 0.016 & 0.005 & 0.112 & 100.249 \\
\hline SME Simulant & Isolok - 3 mL & $\mathrm{HF}$ & 9 & 2 & 21 & 5 & 3 & HIS01 & 0.307 & 2.789 & 13.291 & 1.217 & 52.199 & 0.016 & 0.006 & 0.111 & 102.385 \\
\hline SME Simulant & Coliwasa - low & LF & 20 & 1 & 2 & 5 & 4 & CL02 & 0.312 & 2.879 & 12.536 & 1.273 & 47.065 & $\begin{array}{ll}0.016 \\
\end{array}$ & 0.006 & 0.108 & 95.389 \\
\hline SME Simulant & Coliwasa - high & LF & 38 & 1 & 16 & 5 & 5 & CH03 & 0.305 & 2.789 & 12.941 & 1.210 & 49.204 & 0.022 & 0.006 & 0.106 & 97.347 \\
\hline SME Simulant & Coliwasa - high & LF & 63 & 1 & 13 & 5 & 6 & CH04 & 0.302 & 2.724 & 13.305 & 1.164 & 50.487 & 0.018 & 0.006 & 0.115 & 99.772 \\
\hline SME Simulant & Isolok - $3 \mathrm{~mL}$ & $\mathrm{HF}$ & 44 & 2 & 18 & 5 & 7 & HIS07 & 0.307 & 2.815 & 13.089 & 1.205 & 48.562 & 0.017 & 0.006 & 0.106 & 97.924 \\
\hline SME Simulant & Coliwasa - high & $\mathrm{HF}$ & 23 & 1 & 28 & 5 & 8 & CH06 & 0.320 & 2.996 & 13.183 & 1.362 & 49.418 & 0.017 & 0.006 & 0.115 & 99.991 \\
\hline SME Simulant & Coliwasa - high & LF & 21 & 1 & 14 & 5 & 9 & $\mathrm{CH} 02$ & 0.274 & 2.492 & 12.712 & 1.117 & 50.060 & 0.016 & 0.006 & 0.111 & 97.967 \\
\hline SME Simulant & Coliwasa - low & $\mathrm{HF}$ & 3 & 1 & 20 & 5 & 10 & CL05 & 0.305 & 2.737 & 13.750 & 1.154 & 50.487 & 0.017 & 0.006 & 0.110 & 100.435 \\
\hline SME Simulant & Isolok - 3 mL & $\mathrm{HF}$ & 14 & 2 & 15 & 5 & 11 & HIS02 & 0.305 & 2.789 & 13.372 & 1.203 & 50.915 & 0.016 & 0.006 & 0.110 & 100.602 \\
\hline SME Simulant & Coliwasa - low & LF & 40 & 1 & 19 & 5 & 12 & CL03 & 0.303 & 2.660 & 13.480 & 1.112 & 51.129 & 0.017 & 0.006 & 0.110 & 100.494 \\
\hline SME Simulant & Coliwasa - low & $\mathrm{HF}$ & 18 & 1 & 26 & 5 & 13 & CL06 & 0.313 & 2.828 & 13.372 & 1.157 & 50.274 & 0.015 & 0.006 & 0.112 & 99.943 \\
\hline SME Simulant & Coliwasa - high & $\mathrm{HF}$ & 1 & 1 & 22 & 5 & 14 & CH05 & 0.310 & 2.815 & 13.359 & 1.159 & 50.274 & 0.016 & 0.006 & 0.112 & 99.613 \\
\hline ARG-1 & None & 0 & 0 & 2 & 12 & 5 & 15 & \begin{tabular}{|l|} 
ARG-1B22 \\
\end{tabular} & 0.844 & 1.821 & 11.458 & 1.023 & 48.348 & 1.156 & 0.025 & 0.136 & 99.158 \\
\hline SME Simulant & Coliwasa - low & $\mathrm{HF}$ & 36 & 1 & 21 & 5 & 16 & CL07 & 0.312 & 2.841 & 13.332 & 1.220 & 49.204 & 0.015 & 0.006 & 0.108 & 98.643 \\
\hline SME Simulant & Isolok - $3 \mathrm{~mL}$ & $\mathrm{HF}$ & 31 & 2 & 19 & 5 & 17 & HIS05 & $\begin{array}{ll}0.317 \\
\end{array}$ & 2.892 & 13.480 & 1.245 & 51.129 & $\begin{array}{ll}0.016 \\
\end{array}$ & 0.006 & 0.110 & 101.140 \\
\hline SME Simulant & Isolok - 3 mL & $\mathrm{HF}$ & 60 & 2 & 22 & 5 & 18 & HIS10 & 0.295 & 2.686 & 13.035 & 1.158 & 51.771 & 0.017 & 0.006 & 0.111 & 100.438 \\
\hline SME Simulant & Coliwasa - low & $\mathrm{HF}$ & 63 & 1 & 5 & 5 & 19 & CL08 & 0.302 & 2.750 & 13.453 & 1.199 & 50.060 & 0.014 & 0.006 & 0.110 & 99.218 \\
\hline SME Simulant & Isolok - 3 mL & $\mathrm{HF}$ & 26 & 2 & 14 & 5 & 20 & HIS04 & 0.310 & 2.828 & 13.089 & 1.205 & 50.915 & 0.017 & 0.006 & $\begin{array}{ll}0.111 \\
\end{array}$ & 100.178 \\
\hline SME Simulant & Isolok - 3 mL & $\mathrm{HF}$ & 35 & 2 & 20 & 5 & 21 & HIS06 & 0.308 & 2.828 & 13.251 & 1.233 & 50.060 & 0.017 & 0.006 & 0.109 & 99.389 \\
\hline SME Simulant & Coliwasa - high & $\mathrm{HF}$ & 65 & 1 & 6 & 5 & 22 & CH08 & 0.327 & 3.008 & 13.750 & 1.298 & 50.487 & 0.016 & 0.006 & 0.114 & 101.337 \\
\hline SME Simulant & Coliwasa - high & $\mathrm{HF}$ & 34 & 1 & 18 & 5 & 23 & CH07 & 0.312 & 2.828 & 13.615 & 1.194 & 51.771 & 0.017 & 0.006 & 0.111 & 101.917 \\
\hline SME Simulant & Coliwasa - high & LF & 1 & 1 & 12 & 5 & 24 & CH01 & 0.284 & 2.518 & 12.509 & 1.008 & 48.776 & 0.016 & 0.006 & 0.103 & 94.554 \\
\hline SME Simulant & Isolok - 3 mL & $\mathrm{HF}$ & 54 & 2 & 17 & 5 & 25 & HIS09 & 0.312 & 2.841 & 13.062 & 1.199 & 50.701 & 0.016 & 0.006 & 0.107 & 99.876 \\
\hline
\end{tabular}


Revision 0

Table B4. Phase 2 Oxide Concentration Measurements (part 2)

\begin{tabular}{|c|c|c|c|c|c|c|c|c|c|c|c|c|c|c|c|c|c|}
\hline $\begin{array}{l}\text { Type of } \\
\text { Material }\end{array}$ & \begin{tabular}{|l|} 
Sampler \\
Details
\end{tabular} & \begin{tabular}{|l} 
Flow \\
Rate
\end{tabular} & $\begin{array}{r}\text { Sample } \\
\text { Order }\end{array}$ & $\begin{array}{r}\text { Preparation } \\
\text { Block }\end{array}$ & $\begin{array}{r}\text { Preparation } \\
\text { Sequence }\end{array}$ & $\begin{array}{r}\text { Analytical } \\
\text { Block }\end{array}$ & $\begin{array}{r}\text { Analytical } \\
\text { Sequence }\end{array}$ & Sample ID & $\begin{array}{r}\text { MgO } \\
\text { (wt\%) }\end{array}$ & $\begin{array}{r}\text { MnO } \\
\text { (wt\%) }\end{array}$ & $\begin{array}{r}\mathrm{Na2O} \\
\text { (wt\%) }\end{array}$ & $\begin{array}{r}\mathrm{NiO} \\
\text { (wt\%) }\end{array}$ & $\begin{array}{r}\mathrm{SiO2} \\
\text { (wt\%) }\end{array}$ & $\begin{array}{r}\mathrm{TiO2} \\
\text { (wt\%) }\end{array}$ & $\begin{array}{r}\mathrm{ZnO} \\
\text { (wt\%) }\end{array}$ & $\begin{array}{r}\mathrm{ZrO2} \\
\text { (wt\%) }\end{array}$ & $\begin{array}{c}\text { Sum of } \\
\text { Oxides } \\
\text { (wt\%) }\end{array}$ \\
\hline SME Simulant & Coliwasa - low & LF & 4 & 1 & 10 & 5 & 26 & CL01 & 0.305 & 2.776 & 13.210 & 1.208 & 50.274 & 0.015 & 0.006 & 0.113 & 100.186 \\
\hline SME Simulant & Isolok - 3 mL & $\mathrm{HF}$ & 48 & 2 & 13 & 5 & 27 & HIS08 & 0.307 & 2.815 & 12.900 & 1.228 & 49.846 & 0.014 & 0.005 & 0.107 & 98.590 \\
\hline SME Simulant & Coliwasa - low & LF & 65 & 1 & 23 & 5 & 28 & CL04 & 0.318 & 2.918 & 13.386 & 1.273 & 49.418 & 0.015 & 0.006 & 0.108 & 99.192 \\
\hline ARG-1 & None & 0 & 0 & 2 & 23 & 5 & 29 & ARG-1B23 & 0.801 & 1.730 & 10.932 & $\begin{array}{ll}0.968 \\
\end{array}$ & 45.781 & \begin{tabular}{ll|}
1.093 \\
\end{tabular} & $\begin{array}{ll}0.026 \\
\end{array}$ & $\begin{array}{ll}0.126 \\
\end{array}$ & 93.793 \\
\hline ARG-1 & None & 0 & 0 & 3 & 1 & 6 & 1 & ARG-1B31 & 0.839 & 1.821 & \begin{tabular}{ll|}
11.350 \\
\end{tabular} & 1.004 & 47.492 & 1.156 & 0.023 & $\begin{array}{ll}0.133 \\
\end{array}$ & 97.996 \\
\hline SME Simulant & Hydragard & LF & 15 & 3 & 21 & 6 & 2 & LHY03 & 0.320 & 2.879 & 13.332 & 1.204 & 50.060 & 0.018 & 0.006 & 0.114 & 100.737 \\
\hline SME Simulant & \begin{tabular}{|l|} 
Hydragard \\
\end{tabular} & LF & 6 & 3 & 18 & 6 & 3 & LHY01 & 0.327 & 2.944 & 13.345 & 1.192 & 49.846 & $\begin{array}{ll}0.016 \\
\end{array}$ & 0.006 & 0.114 & 100.381 \\
\hline SME Simulant & Coliwasa - low & LF & 20 & 1 & 2 & 6 & 4 & CL02 & 0.328 & 3.034 & $\begin{array}{l}13.237 \\
\end{array}$ & 1.349 & 48.990 & 0.016 & 0.006 & 0.112 & 99.847 \\
\hline SME Simulant & Coliwasa - high & LF & 38 & 1 & 16 & 6 & 5 & $\mathrm{CH} 03$ & 0.312 & 2.854 & 12.995 & 1.227 & 49.846 & 0.019 & 0.006 & 0.108 & 98.659 \\
\hline SME Simulant & Coliwasa - high & LF & 63 & 1 & 13 & 6 & 6 & \begin{tabular}{|l|l|}
$\mathrm{CH} 04$ \\
\end{tabular} & 0.303 & 2.737 & 13.750 & 1.162 & 50.274 & $\begin{array}{ll}0.018 \\
\end{array}$ & 0.006 & \begin{tabular}{ll|}
0.111 \\
\end{tabular} & 100.304 \\
\hline SME Simulant & Hydragard & LF & 47 & 3 & 13 & 6 & 7 & LHY07 & 0.313 & 2.841 & $\begin{array}{ll}12.766 \\
\end{array}$ & 1.217 & 48.562 & $\begin{array}{l}0.017 \\
\end{array}$ & 0.006 & $\begin{array}{ll}0.109 \\
\end{array}$ & 97.358 \\
\hline SME Simulant & Coliwasa - high & $\mathrm{HF}$ & 23 & 1 & 28 & 6 & 8 & CH06 & 0.302 & 2.815 & 12.240 & 1.273 & 46.209 & $\begin{array}{ll}0.016 \\
\end{array}$ & 0.006 & $\begin{array}{ll}0.105 \\
\end{array}$ & 93.410 \\
\hline SME Simulant & Coliwasa - high & LF & 21 & 1 & 14 & 6 & 9 & CH02 & 0.275 & 2.505 & 12.617 & 1.131 & 50.060 & 0.017 & 0.006 & 0.111 & 98.092 \\
\hline SME Simulant & Coliwasa - low & HF & 3 & 1 & 20 & 6 & 10 & CL05 & 0.305 & 2.737 & 13.399 & 1.133 & 50.274 & 0.016 & 0.006 & 0.111 & 99.792 \\
\hline SME Simulant & Hydragard & LF & 10 & 3 & 20 & 6 & 11 & LHY02 & 0.307 & 2.750 & 13.615 & 1.110 & 49.846 & 0.014 & 0.006 & 0.108 & 100.000 \\
\hline SME Simulant & Coliwasa - low & LF & 40 & 1 & 19 & 6 & 12 & CL03 & 0.302 & 2.673 & 13.386 & 1.099 & 50.915 & 0.017 & 0.006 & 0.112 & 100.196 \\
\hline SME Simulant & Coliwasa - low & $\mathrm{HF}$ & 18 & 1 & 26 & 6 & 13 & CL06 & 0.318 & 2.854 & 12.954 & 1.136 & 50.274 & $\begin{array}{ll}0.015 \\
\end{array}$ & 0.006 & \begin{tabular}{ll|}
0.111 \\
\end{tabular} & 99.495 \\
\hline SME Simulant & Coliwasa - high & HF & 1 & 1 & 22 & 6 & 14 & CH05 & 0.313 & 2.841 & 12.914 & 1.164 & \begin{tabular}{|l}
50.487 \\
\end{tabular} & 0.015 & 0.006 & $\begin{array}{ll}0.113 \\
\end{array}$ & 99.481 \\
\hline ARG-1 & None & 0 & 0 & 3 & 12 & 6 & 15 & ARG-1B32 & 0.847 & 1.821 & 11.377 & 1.009 & 47.920 & 1.158 & 0.027 & 0.131 & 98.482 \\
\hline SME Simulant & Coliwasa - low & $\mathrm{HF}$ & 36 & 1 & 21 & 6 & 16 & \begin{tabular}{|l|l|} 
CL07 \\
\end{tabular} & 0.313 & 2.854 & 13.089 & 1.218 & 48.990 & 0.016 & 0.006 & 0.109 & 98.151 \\
\hline SME Simulant & Hydragard & LF & 30 & 3 & 14 & 6 & 17 & LHY05 & 0.294 & 2.686 & 13.615 & 1.182 & 50.701 & 0.016 & 0.006 & 0.113 & 101.080 \\
\hline SME Simulant & Hydragard & LF & 59 & 3 & 17 & 6 & 18 & LHY10 & 0.317 & 2.918 & 13.305 & 1.266 & 49.846 & 0.017 & 0.006 & 0.110 & 99.512 \\
\hline SME Simulant & Coliwasa - low & $\mathrm{HF}$ & 63 & 1 & 5 & 6 & 19 & \begin{tabular}{|l|l|} 
CL08 \\
\end{tabular} & 0.303 & 2.763 & $\begin{array}{ll}13.615 \\
\end{array}$ & 1.189 & 49.846 & $\begin{array}{l}0.017 \\
\end{array}$ & 0.006 & 0.110 & $\begin{array}{l}99.306 \\
\end{array}$ \\
\hline SME Simulant & Hydragard & LF & 28 & 3 & 16 & 6 & 20 & LHY04 & 0.302 & 2.712 & $\begin{array}{l}13.062 \\
\end{array}$ & 1.126 & 48.134 & $\begin{array}{ll}0.018 \\
\end{array}$ & 0.020 & 0.107 & 96.714 \\
\hline SME Simulant & Hydragard & LF & 37 & 3 & 15 & 6 & 21 & LHY06 & 0.320 & 2.892 & 12.968 & 1.205 & 49.204 & 0.016 & 0.006 & 0.108 & 97.910 \\
\hline SME Simulant & Coliwasa - high & $\mathrm{HF}$ & 65 & 1 & 6 & 6 & 22 & CH08 & 0.328 & 3.021 & 13.615 & 1.285 & 50.274 & $\begin{array}{ll}0.016 \\
\end{array}$ & 0.006 & 0.110 & 100.855 \\
\hline SME Simulant & Coliwasa - high & $\mathrm{HF}$ & 34 & 1 & 18 & 6 & 23 & CH07 & 0.313 & 2.841 & 13.480 & 1.192 & 51.129 & 0.018 & 0.006 & 0.107 & 101.253 \\
\hline SME Simulant & Coliwasa - high & LF & 1 & 1 & 12 & 6 & 24 & CH01 & 0.285 & 2.544 & 12.065 & 0.998 & 48.776 & 0.013 & 0.006 & 0.104 & 94.012 \\
\hline SME Simulant & Hydragard & LF & 53 & 3 & 19 & 6 & 25 & LHY09 & 0.308 & 2.737 & 13.332 & 1.116 & 50.915 & $\begin{array}{ll}0.013 \\
\end{array}$ & 0.006 & 0.108 & $\begin{array}{l}99.788 \\
\end{array}$ \\
\hline SME Simulant & Coliwasa - low & LF & \begin{tabular}{|l|}
4 \\
\end{tabular} & 1 & 10 & 6 & 26 & \begin{tabular}{|l|} 
CL01 \\
\end{tabular} & 0.308 & 2.789 & $\begin{array}{ll}13.318 \\
\end{array}$ & 1.213 & 50.274 & 0.015 & 0.006 & 0.111 & 100.534 \\
\hline SME Simulant & Hydragard & LF & 48 & 3 & 22 & 6 & 27 & LHY08 & 0.315 & 2.879 & 12.523 & 1.195 & 48.562 & 0.015 & 0.006 & 0.108 & 97.039 \\
\hline SME Simulant & Coliwasa - low & LF & 65 & 1 & 23 & 6 & 28 & \begin{tabular}{|l|l|} 
CL04 \\
\end{tabular} & 0.320 & 2.918 & 13.035 & 1.252 & 49.204 & 0.014 & 0.006 & 0.103 & 98.468 \\
\hline ARG-1 & None & 0 & 0 & 3 & 23 & 6 & 29 & ARG-1B33 & $\begin{array}{ll}0.798 \\
\end{array}$ & 1.730 & \begin{tabular}{|l|}
10.744 \\
\end{tabular} & \begin{tabular}{ll|}
0.954 \\
\end{tabular} & 45.139 & 1.089 & 0.025 & 0.121 & 92.754 \\
\hline
\end{tabular}


Table B5. Reference Calcine Oxide Concentrations for High-Rheology SME Simulant (Phase 2 Testing)

\begin{tabular}{|c|c|}
\hline Oxide & Targeted wt\% \\
\hline $\mathrm{Al2O} 3$ (wt\%) & 10.934 \\
\hline $\mathrm{B} 2 \mathrm{O}$ (wt\%) & 5.281 \\
\hline $\mathrm{BaO}$ (wt\%) & 0 \\
\hline $\mathrm{CaO}$ (wt\%) & 0.543 \\
\hline $\mathrm{Cr} 2 \mathrm{O} 3$ (wt\%) & 0.015 \\
\hline $\mathrm{CuO}$ (wt\%) & 0.103 \\
\hline $\mathrm{Fe} 2 \mathrm{O} 3$ (wt\%) & 9.931 \\
\hline $\mathrm{K} 2 \mathrm{O}$ (wt\%) & 0.076 \\
\hline $\mathrm{Li} 2 \mathrm{O}$ (wt\%) & 4.988 \\
\hline $\mathrm{MgO}$ (wt\%) & 0.286 \\
\hline $\mathrm{MnO}$ (wt\%) & 2.918 \\
\hline $\mathrm{Na} 2 \mathrm{O}$ (wt\%) & 13.318 \\
\hline $\mathrm{NiO}$ (wt\%) & 1.033 \\
\hline $\mathrm{SiO} 2$ (wt\%) & 50.183 \\
\hline $\mathrm{TiO} 2$ (wt\%) & 0.013 \\
\hline $\mathrm{ZnO}$ (wt\%) & 0 \\
\hline $\mathrm{ZrO} 2$ (wt\%) & 0.111 \\
\hline Sum of Oxides (wt\%) & 99.73 \\
\hline
\end{tabular}

Shaded rows indicate oxides that are at reportable concentrations and that are considered critical in the comparisons conducted as part of this study. 
Table B6. Comparisons for Density and Solids from the High-Rheology (Phase 2) Testing

Density

Sampler Mean BL Mean Difference Difference Statistically

Statistically $\quad \mathrm{g} / \mathrm{mL} \quad \%$ of BL

Different Bound on Bound on

\begin{tabular}{|c|c|c|c|c|c|c|c|c|c|c|c|c|}
\hline & & & & & & & & & & & & \\
\hline & $(\mathrm{g} / \mathrm{mL})$ & $(\mathrm{g} / \mathrm{mL})$ & $(\mathrm{g} / \mathrm{mL})$ & $(\%)$ & Significant Bias & Sampler Std Dev & BL Std Dev & Variances & Bias $(95 \%)$ & Bias (95\%) & n sampler & $n$ BL \\
\hline Coliwasa High Location vs Coliwasa Low Location (BL) & 1.33651 & 1.33896 & -0.002 & $-0.2 \%$ & \begin{tabular}{|l|l} 
no \\
\end{tabular} & 0.013022 & 0.007637 & no & 0.0139 & $1.0 \%$ & 8 & 8 \\
\hline Hydraga & 1.34716 & 1.33774 & 0.009 & $0.7 \%$ & no & 0.018912 & 0.01039 & no & 0.02124 & $1.6 \%$ & 10 & 16 \\
\hline Hydragard (LF) vs & 1.34251 & 1.33774 & 0.005 & $0.4 \%$ & no & 0.012619 & 0.01039 & no & 0.01444 & $1.1 \%$ & 9 & 16 \\
\hline Hydragard (HF) vs Coliwasa & 1.33714 & 1.33774 & -0.001 & $0.0 \%$ & no & 0.012442 & 0.01039 & no & 0.00992 & $0.7 \%$ & 10 & 16 \\
\hline Isolok (LF) vs Coliwasa & 1.33219 & 1.33774 & -0.006 & $-0.4 \%$ & no & 0.012312 & 0.01039 & no & 0.01482 & $1.1 \%$ & 10 & 16 \\
\hline Isolok (HF) vs Coliwasa & 1.34498 & 1.33774 & 0.007 & $0.5 \%$ & no & 0.020176 & 0.01039 & yes & 0.02225 & $1.7 \%$ & 10 & 16 \\
\hline
\end{tabular}

Dried wt\% Solids

Sampler Mean BL Mean Difference Difference Statistically

Statistically $\quad(\mathrm{wt} \%) \quad \%$ of BL Different Bound on Bound on

$\begin{array}{llllllllll}(\mathrm{wt} \%) & (\mathrm{wt} \%) & (\mathrm{wt} \%) & (\%) & \text { Significant Bias Sampler Std Dev BL Std Dev Variances Bias (95\%) Bias (95\%) n sampler } & \mathrm{n} \text { BL }\end{array}$

\begin{tabular}{|c|c|c|c|c|c|c|c|c|c|c|c|c|}
\hline & (wt\%) & $(\mathrm{wt} \%)$ & $(\mathrm{wt} \%)$ & $(\%)$ & Significant Bias & Sampler Std Dev & BL Std Dev & Variances & Bias (95\%) & Bias (95\%) & n sampler & nBL \\
\hline Coliwasa High Location vs Coliwasa Low Location (BL) & 40.4675 & 40.7475 & -0.280 & $-0.7 \%$ & no & 0.670645 & 0.484436 & no & 0.90735 & $2.2 \%$ & 8 & 8 \\
\hline Coliwasa High Location vs Coliwasa Low Location (BL) - scrnd & 40.4675 & 40.5857 & -0.118 & $-0.3 \%$ & no & 0.670645 & 0.171742 & no & 0.6837 & $1.7 \%$ & 8 & 7 \\
\hline Hydragard (LF) vs Coliwasa & 40.4160 & 40.6075 & -0.192 & $-0.5 \%$ & no & 0.464715 & 0.583364 & no & 0.64237 & $1.6 \%$ & 10 & 16 \\
\hline Hydragard (LF) vs Coliwasa ---- scrnd & 40.5522 & 40.6293 & -0.077 & $-0.2 \%$ & no & 0.184917 & 0.276001 & no & 0.29503 & $0.7 \%$ & 9 & 14 \\
\hline Hydragard (HF) vs Coliwasa & 39.9940 & 40.6075 & -0.614 & $-1.5 \%$ & yes & 0.482935 & 0.583364 & no & 1.0693 & $2.6 \%$ & 10 & 16 \\
\hline Hydragard (HF) vs Coliwasa ---- scrnd & 40.1411 & 40.6293 & -0.488 & $-1.2 \%$ & yes & 0.137518 & 0.276001 & yes & 0.66927 & $1.6 \%$ & 9 & 14 \\
\hline Isolok (12; LF) vs Coliwasa & 40.9420 & 40.6075 & 0.334 & $0.8 \%$ & no & 0.097616 & 0.583364 & yes & 0.650016 & $1.6 \%$ & 10 & 16 \\
\hline Isolok (12; LF) vs Coliwasa ---- scrnd & 40.9420 & 40.6293 & 0.313 & $0.8 \%$ & yes & 0.097616 & 0.276001 & yes & 0.481279 & $1.2 \%$ & 10 & 14 \\
\hline Isolok (12; HF) vs Coliwasa & 41.4090 & 40.6075 & 0.801 & $2.0 \%$ & yes & 0.112689 & 0.583364 & no & 1.18947 & $2.9 \%$ & 10 & 16 \\
\hline Isolok (12; HF) vs Coliwasa ----- scrnd & 41.4090 & 40.6293 & 0.780 & $1.9 \%$ & yes & 0.112689 & 0.276001 & yes & 0.9516 & $2.3 \%$ & 10 & 14 \\
\hline Isolok (3; LF) vs Coliwasa & 40.5980 & 40.6075 & -0.010 & $0.0 \%$ & no & 0.210175 & 0.583364 & no & 0.40786 & $1.0 \%$ & 10 & 16 \\
\hline Isolok (3; LF) vs Coliwasa ---- scrnd & 40.5980 & 40.6293 & -0.031 & $-0.1 \%$ & no & 0.210175 & 0.276001 & no & 0.24695 & $0.6 \%$ & 10 & 14 \\
\hline Isolok (3; HF) vs Coliwasa & 41.5660 & 40.6075 & 0.959 & $2.4 \%$ & yes & 0.190741 & 0.583364 & no & 1.35432 & $3.3 \%$ & 10 & 16 \\
\hline Isolok (3; HF) vs Coliwasa ---- scrnd & 41.5660 & 40.6293 & 0.937 & $2.3 \%$ & yes & 0.190741 & 0.276001 & no & 1.14686 & $2.8 \%$ & 10 & 14 \\
\hline
\end{tabular}

Vitrified wt\% Solids

\begin{tabular}{l|l}
0.190741 & 0.27600 \\
\hline
\end{tabular}

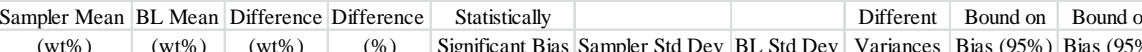

Statistically (wt\%) \% of BL

Different Bound on Bound

Coliwasa High Location vs Coliwasa Low Location (BL)

\begin{tabular}{|c|c|c|c|c|c|c|c|c|c|c|c|c|}
\hline & & & & & & & & & & & i sampler & \\
\hline Coliwasa High Location vs Coliwasa Low Location (BL) & 32.7538 & 33.0050 & -0.251 & $-0.8 \%$ & no & 0.571713 & 0.385079 & no & 0.77395 & $2.3 \%$ & 8 & 8 \\
\hline Coliwasa High Location vs Coliwasa Low Location (BL) - scrnd & 32.7538 & 32.8800 & -0.126 & $-0.4 \%$ & no & 0.571713 & 0.164823 & yes & 0.61089 & $1.9 \%$ & 8 & 7 \\
\hline Hydragard (LF) vs Coliwasa & 32.7930 & 32.8794 & -0.086 & $-0.3 \%$ & no & 0.368422 & 0.488432 & no & 0.45845 & $1.4 \%$ & 10 & 16 \\
\hline Hydragard (LF) vs Coliwasa --- scrnd & 32.8989 & 32.9014 & -0.003 & $0.0 \%$ & no & 0.162976 & 0.256601 & no & 0.20295 & $0.6 \%$ & 9 & 14 \\
\hline Hydragard (HF) vs Coliwasa & 32.3860 & 32.8794 & -0.493 & $-1.5 \%$ & yes & 0.145922 & 0.488432 & yes & 0.76664 & $2.3 \%$ & 10 & 16 \\
\hline Hydragard (HF) vs Coliwasa ---- scrnd & 32.3860 & 32.9014 & -0.515 & $-1.6 \%$ & yes & 0.145922 & 0.256601 & yes & 0.68724 & $2.1 \%$ & 10 & 14 \\
\hline Isolok (12; LF) vs Coliwasa & 32.7570 & 32.8794 & -0.122 & $-0.4 \%$ & no & 0.089325 & 0.488432 & yes & 0.38733 & $1.2 \%$ & 10 & 16 \\
\hline Isolok (12; LF) vs Coliwasa ---- scrnd & 32.7570 & 32.9014 & -0.144 & $-0.4 \%$ & no & 0.089325 & 0.256601 & yes & 0.30086 & $0.9 \%$ & 10 & 14 \\
\hline Isolok (12; HF) vs Coliwasa & 33.3850 & 32.8794 & 0.506 & $1.5 \%$ & yes & 0.121952 & 0.488432 & yes & 0.774822 & $2.4 \%$ & 10 & 16 \\
\hline Isolok (12; HF) vs Coliwasa ---- scrnd & 33.3850 & 32.9014 & 0.484 & $1.5 \%$ & yes & 0.121952 & 0.256601 & yes & 0.647864 & $2.0 \%$ & 10 & 14 \\
\hline Isolok (3; LF) vs Coliwasa & 32.6160 & 32.8794 & -0.263 & $-0.8 \%$ & no & 0.153854 & 0.488432 & no & 0.59406 & $1.8 \%$ & 10 & 16 \\
\hline Isolok (3; LF) vs Coliwasa ----- scrnd & 32.6160 & 32.9014 & -0.285 & $-0.9 \%$ & yes & 0.153854 & 0.256601 & yes & 0.46004 & $1.4 \%$ & 10 & 14 \\
\hline Isolok (3; HF) vs Coliwasa & 33.4680 & 32.8794 & 0.589 & $1.8 \%$ & yes & 0.196684 & 0.488432 & no & 0.925153 & $2.8 \%$ & 10 & 16 \\
\hline Isolok (3; HF) vs Coliwasa ---- scrnd & 33.4680 & 32.9014 & 0.567 & $1.7 \%$ & yes & 0.196684 & 0.256601 & no & 0.767457 & $2.3 \%$ & 10 & 14 \\
\hline
\end{tabular}


Table B7. Oxide Comparisons between the Low and High Colowasa Samples for the High-Rheology (Phase 2) Testing

\begin{tabular}{|c|c|c|c|c|c|c|c|c|c|c|c|c|c|c|}
\hline $\begin{array}{c}\text { Type of } \\
\text { Material }\end{array}$ & Test Phase & $\begin{array}{l}\text { Analytical } \\
\text { Block }\end{array}$ & Oxide & $\begin{array}{c}\text { Mean(Measured } \\
\text { wt\%) Coliwasa - low } \\
\text { location }\end{array}$ & $\begin{array}{l}\text { Mean(Measured } \\
\text { wt\%) Coliwasa - } \\
\text { high location }\end{array}$ & $\%$ Diff & Sig. Diff & Bias Bnd & $\begin{array}{c}\% \text { Rel } \\
\text { Bias Bnd }\end{array}$ & $\begin{array}{c}\text { Std Dev(Measured } \\
\text { wt\%) Coliwasa - low } \\
\text { location }\end{array}$ & $\begin{array}{c}\text { Std } \\
\text { Dev(Measured } \\
\text { wt\%) Coliwasa - } \\
\text { high location }\end{array}$ & Sig. Diff & $\mathrm{n}$ low & $\mathrm{n}$ high \\
\hline SME Simulant & 2nd phase & avg & $\mathrm{Al} / \mathrm{B}$ & \begin{tabular}{|c|}
2.393 \\
\end{tabular} & 2.386 & $-0.3 \%$ & \begin{tabular}{|l|} 
No \\
Non
\end{tabular} & 0.0853 & 3.57 & \begin{tabular}{|l|}
0.0552 \\
\end{tabular} & 0.0886 & No & 8 & 8 \\
\hline SME Simulant & 2nd phase & avg & Al2O3 (wt\%) & 10.995 & 10.800 & $-1.8 \%$ & No & 0.4439 & 4.04 & 0.1831 & 0.2733 & No & 8 & 8 \\
\hline SME Simulant & 2nd phase & avg & B2O3 (wt \%) & 4.600 & 4.530 & $-1.5 \%$ & No & 0.1761 & 3.83 & 0.1003 & 0.0979 & No & 8 & 8 \\
\hline SME Simulant & 2nd phase & avg & $\mathrm{BaO}(\mathrm{wt} \%)$ & 0.005 & 0.005 & $0.3 \%$ & No & 0.0004 & 8.58 & 0.0004 & 0.0003 & No & 8 & 8 \\
\hline SME Simulant & 2nd phase & avg & $\mathrm{CaO}(\mathrm{wt} \%)$ & 0.588 & 0.579 & $-1.6 \%$ & No & 0.0322 & 5.47 & 0.0120 & 0.0275 & No & 8 & 8 \\
\hline SME Simulant & 2nd phase & avg & $\mathrm{Cr} 2 \mathrm{O} 3$ (wt $\%)$ & 0.019 & 0.020 & $2.2 \%$ & No & 0.0018 & 9.13 & 0.0008 & 0.0015 & Yes & 8 & 8 \\
\hline SME Simulant & 2nd phase & avg & CuO (wt\%) & 0.096 & 0.093 & $-3.8 \%$ & No & 0.0077 & 7.96 & 0.0031 & 0.0043 & No & 8 & 8 \\
\hline SME Simulant & 2nd phase & avg & $\mathrm{Fe} / \mathrm{Li}$ & 2.028 & 1.982 & $-2.3 \%$ & No & 0.1167 & 5.76 & 0.0334 & 0.0824 & Yes & 8 & 8 \\
\hline SME Simulant & 2nd phase & avg & $\mathrm{Fe} 2 \mathrm{O} 3(\mathrm{wt} \%)$ & 10.305 & 10.120 & $-1.8 \%$ & No & 0.4514 & 4.38 & 0.2012 & 0.2890 & No & 8 & 8 \\
\hline SME Simulant & 2nd phase & avg & $\mathrm{K} 2 \mathrm{O}(\mathrm{wt} \%)$ & 0.168 & 0.170 & $0.9 \%$ & No & 0.0088 & 5.20 & 0.0057 & 0.0077 & No & 8 & 8 \\
\hline SME Simulant & 2nd phase & avg & Li2O (wt\%) & 5.084 & 5.111 & $0.5 \%$ & No & 0.1126 & 2.22 & 0.0602 & 0.0943 & No & $\frac{8}{8}$ & $\frac{8}{8}$ \\
\hline SME Simulant & 2nd phase & avg & $\mathrm{MgO}(\mathrm{wt} \%)$ & 0.311 & 0.304 & $-2.2 \%$ & No & 0.0199 & 6.39 & 0.0086 & 0.0146 & No & 8 & 8 \\
\hline SME Simulant & 2nd phase & avg & $\mathrm{MnO}(\mathrm{wt} \%)$ & 2.824 & 2.778 & $-1.6 \%$ & No & 0.1905 & 6.75 & 0.1093 & 0.1565 & No & 8 & 8 \\
\hline SME Simulant & 2nd phase & avg & $\mathrm{Na} 2 \mathrm{O}$ (wt\%) & 13.421 & 13.250 & $-1.3 \%$ & No & 0.4961 & 3.70 & 0.2255 & 0.3642 & No & 8 & 8 \\
\hline SME Simulant & 2nd phase & avg & $\mathrm{NiO}(\mathrm{wt} \%)$ & 1.202 & 1.188 & $-1.2 \%$ & No & 0.1046 & 8.70 & 0.0775 & 0.0910 & No & 8 & 8 \\
\hline SME Simulant & 2nd phase & avg & $\mathrm{SiO} 2(\mathrm{wt} \%)$ & 49.975 & 49.988 & $0.0 \%$ & No & 0.7896 & 1.58 & 0.7706 & 0.6738 & No & 8 & 8 \\
\hline SME Simulant & 2nd phase & avg & Sum of Oxides (wt\%) & 99.726 & 99.072 & $-0.7 \%$ & No & 1.7222 & 1.73 & 1.0263 & 0.9636 & No & 8 & 8 \\
\hline SME Simulant & 2nd phase & avg & TiO2 (wt\%) & 0.017 & 0.018 & $6.6 \%$ & No & 0.0029 & 17.13 & 0.0006 & 0.0021 & Yes & 8 & 8 \\
\hline SME Simulant & 2nd phase & avg & $\mathrm{ZnO}(\mathrm{wt} \%)$ & 0.006 & 0.007 & $10.9 \%$ & No & 0.0020 & 33.87 & 0.0002 & 0.0018 & No & 8 & 8 \\
\hline SME Simulant & 2nd phase & avg & $\mathrm{ZrO} 2(\mathrm{wt} \%)$ & 0.110 & 0.110 & $0.1 \%$ & No & 0.0025 & 2.27 & 0.0023 & 0.0022 & No & 8 & 8 \\
\hline
\end{tabular}

\begin{tabular}{|c|c|c|c|c|c|c|c|c|c|c|c|c|c|c|}
\hline \multicolumn{15}{|l|}{ Screened Data } \\
\hline $\begin{array}{l}\text { Type of } \\
\text { Material }\end{array}$ & Test Phase & $\begin{array}{c}\text { Analytical } \\
\text { Block }\end{array}$ & Oxide & $\begin{array}{c}\text { Mean(Measured } \\
\text { wt\%) Coliwasa - low } \\
\text { location }\end{array}$ & $\begin{array}{c}\text { Mean(Measured } \\
\text { wt\%) Coliwasa - } \\
\text { high location }\end{array}$ & $\%$ Diff & Sig. Diff & Bias Bnd & \begin{tabular}{|c|}
$\%$ Rel \\
Bias Bnd
\end{tabular} & $\begin{array}{c}\text { Std Dev(Measured } \\
\text { wt\%) Coliwasa - low } \\
\text { location }\end{array}$ & $\begin{array}{c}\text { Std } \\
\text { Dev(Measured } \\
\text { wt\%) Coliwasa - } \\
\text { high location }\end{array}$ & Sig. Diff & $\mathrm{n}$ low & $\mathrm{n}$ high \\
\hline SME Simulant & 2nd phase & avg & $\mathrm{Al} / \mathrm{B}$ & \begin{tabular}{|l|}
2.393 \\
\end{tabular} & 2.386 & $-0.3 \%$ & \begin{tabular}{|l|} 
No \\
\end{tabular} & 0.0853 & \begin{tabular}{|l|}
3.57 \\
\end{tabular} & \begin{tabular}{|l|}
0.0552 \\
\end{tabular} & 0.0886 & No & 8 & 8 \\
\hline SME Simulant & 2nd phase & avg & Al2O3 (wt\%) & 10.995 & 10.800 & $-1.8 \%$ & No & 0.4439 & 4.04 & 0.1831 & 0.2733 & No & 8 & 8 \\
\hline SME Simulant & 2nd phase & avg & B2O3 (wt\%) & 4.600 & 4.530 & $-1.5 \%$ & No & 0.1761 & 3.83 & 0.1003 & 0.0979 & No & 8 & 8 \\
\hline SME Simulant & 2nd phase & avg & $\mathrm{BaO}(\mathrm{wt} \%)$ & 0.005 & 0.005 & $0.3 \%$ & No & 0.0004 & 8.58 & 0.0004 & 0.0003 & No & 8 & 8 \\
\hline SME Simulant & 2nd phase & avg & $\mathrm{CaO}(\mathrm{wt} \%)$ & 0.588 & 0.579 & $-1.6 \%$ & No & 0.0322 & 5.47 & 0.0120 & 0.0275 & No & 8 & 8 \\
\hline SME Simulant & 2nd phase & avg & $\mathrm{Cr} 2 \mathrm{O} 3(\mathrm{wt} \%)$ & 0.019 & 0.020 & $2.2 \%$ & No & 0.0018 & 9.13 & 0.0008 & 0.0015 & Yes & 8 & 8 \\
\hline SME Simulant & 2nd phase & avg & CuO (wt\%) & 0.096 & 0.093 & $-3.8 \%$ & No & 0.0077 & 7.96 & 0.0031 & 0.0043 & No & 8 & 8 \\
\hline SME Simulant & 2nd phase & avg & $\mathrm{Fe} / \mathrm{Li}$ & 2.028 & 1.982 & $-2.3 \%$ & No & 0.1167 & 5.76 & 0.0334 & 0.0824 & Yes & 8 & 8 \\
\hline SME Simulant & 2nd phase & avg & $\mathrm{Fe} 2 \mathrm{O} 3(\mathrm{wt} \%)$ & 10.305 & 10.120 & $-1.8 \%$ & No & 0.4514 & 4.38 & 0.2012 & 0.2890 & No & 8 & 8 \\
\hline SME Simulant & 2nd phase & avg & $\mathrm{K} 2 \mathrm{O}(\mathrm{wt} \%)$ & 0.168 & 0.170 & $0.9 \%$ & No & 0.0088 & 5.20 & 0.0057 & 0.0077 & No & 8 & 8 \\
\hline SME Simulant & 2nd phase & avg & Li2O (wt\%) & 5.084 & 5.111 & $0.5 \%$ & No & 0.1126 & 2.22 & 0.0602 & 0.0943 & No & 8 & 8 \\
\hline SME Simulant & 2nd phase & avg & $\mathrm{MgO}(\mathrm{wt} \%)$ & 0.311 & 0.304 & $-2.2 \%$ & No & 0.0199 & 6.39 & 0.0086 & 0.0146 & No & 8 & 8 \\
\hline SME Simulant & 2nd phase & avg & $\mathrm{MnO}(\mathrm{wt} \%)$ & 2.824 & 2.778 & $-1.6 \%$ & No & 0.1905 & 6.75 & 0.1093 & 0.1565 & No & 8 & 8 \\
\hline SME Simulant & 2nd phase & avg & $\mathrm{Na2O}$ (wt\%) & 13.421 & 13.250 & $-1.3 \%$ & No & 0.4961 & 3.70 & 0.2255 & 0.3642 & No & 8 & 8 \\
\hline SME Simulant & 2nd phase & avg & $\mathrm{NiO}(\mathrm{wt} \%)$ & 1.202 & 1.188 & $-1.2 \%$ & No & 0.1046 & 8.70 & 0.0775 & 0.0910 & No & 8 & 8 \\
\hline SME Simulant & 2nd phase & avg & $\mathrm{SiO} 2(\mathrm{wt} \%)$ & 49.975 & 49.988 & $0.0 \%$ & No & 0.7896 & 1.58 & 0.7706 & 0.6738 & No & 8 & 8 \\
\hline SME Simulant & 2nd phase & avg & Sum of Oxides (wt $\%)$ & 99.726 & 99.072 & $-0.7 \%$ & No & 1.7222 & 1.73 & 1.0263 & 0.9636 & No & 8 & 8 \\
\hline SME Simulant & 2nd phase & avg & $\mathrm{TiO} 2(\mathrm{wt} \%)$ & 0.017 & 0.018 & $6.6 \%$ & No & 0.0029 & 17.13 & 0.0006 & 0.0021 & Yes & 8 & 8 \\
\hline SME Simulant & 2nd phase & avg & $\mathrm{ZnO}(\mathrm{wt} \%)$ & 0.006 & 0.006 & $0.4 \%$ & No & 0.0003 & 5.06 & 0.0002 & 0.0003 & No & 8 & 7 \\
\hline SME Simulant & 2nd phase & avg & $\mathrm{ZrO2}(\mathrm{wt} \%)$ & 0.110 & 0.110 & $0.1 \%$ & No & 0.0025 & 2.27 & 0.0023 & 0.0022 & No & 8 & 8 \\
\hline
\end{tabular}


Table B8. Oxide Comparisons Between Samplers for the High-Rheology (Phase 2) Testing

\begin{tabular}{|c|c|c|c|c|c|c|c|c|c|c|c|c|c|}
\hline $\begin{array}{c}\text { Analytical } \\
\text { Block }\end{array}$ & Sampler & Oxide & $\begin{array}{c}\text { Mean(Measured } \\
\text { wt\%) Coliwasa } \\
\text { (low+high) }\end{array}$ & $\begin{array}{c}\text { Mean(Measured } \\
\text { wt\%) Other } \\
\text { Sampler }\end{array}$ & $\%$ Diff & $\begin{array}{l}\text { Sig. } \\
\text { Diff }\end{array}$ & $\begin{array}{l}\text { Bias } \\
\text { Bnd }\end{array}$ & $\begin{array}{l}\% \text { Rel } \\
\text { Bias } \\
\text { Bnd }\end{array}$ & $\begin{array}{c}\text { Std } \\
\text { Dev(Measured } \\
\text { wt\%) Coliwasa } \\
\text { (low+high) }\end{array}$ & $\begin{array}{c}\text { Std } \\
\text { Dev(Measured } \\
\text { wt\%) Other } \\
\text { Sampler }\end{array}$ & $\begin{array}{l}\text { Sig. } \\
\text { Diff }\end{array}$ & $\underset{\text { Col }}{\text { n }}$ & $\begin{array}{c}\text { n } \\
\text { Other }\end{array}$ \\
\hline 1 & Isolok-LFR-3mL & $\mathrm{Al} / \mathrm{B}$ & 2.429 & 2.598 & $6.9 \%$ & Yes & 0.248 & 10.19 & 0.096 & 0.093 & No & 16 & 10 \\
\hline 1 & Isolok-LFR-3mL & Al2O3 (wt\%) & 11.042 & 11.518 & $4.3 \%$ & Yes & 0.821 & 7.44 & 0.497 & 0.216 & No & 16 & 10 \\
\hline 1 & Isolok-LFR-3mL & $\mathrm{B} 2 \mathrm{O} 3(\mathrm{wt} \%)$ & 4.548 & 4.437 & $-2.4 \%$ & No & 0.236 & 5.20 & 0.196 & 0.113 & Yes & 16 & 10 \\
\hline 1 & Isolok-LFR-3mL & $\mathrm{BaO}(\mathrm{wt} \%)$ & 0.006 & 0.006 & $0.0 \%$ & No & 0.001 & 10.28 & 0.001 & 0.001 & No & 16 & 10 \\
\hline 1 & Isolok-LFR-3mL & $\mathrm{CaO}(\mathrm{wt} \%)$ & 0.600 & 0.629 & $4.7 \%$ & No & 0.059 & 9.90 & 0.032 & 0.045 & No & 16 & 10 \\
\hline 1 & Isolok-LFR-3mL & Cr2O3 (wt\%) & 0.020 & 0.020 & $1.4 \%$ & No & 0.003 & 14.40 & 0.002 & 0.003 & Yes & 16 & 10 \\
\hline 1 & Isolok-LFR-3mL & $\mathrm{CuO}(\mathrm{wt} \%)$ & 0.096 & 0.092 & $-3.9 \%$ & No & 0.009 & 9.80 & 0.007 & 0.007 & No & 16 & 10 \\
\hline 1 & Isolok-LFR-3mL & $\mathrm{Fe} / \mathrm{Li}$ & 2.035 & 2.106 & $3.5 \%$ & Yes & 0.128 & 6.29 & 0.078 & 0.048 & No & 16 & 10 \\
\hline 1 & Isolok-LFR-3mL & Fe2O3 (wt\%) & 10.347 & 10.781 & $4.2 \%$ & Yes & 0.760 & 7.34 & 0.477 & 0.175 & No & 16 & 10 \\
\hline 1 & Isolok-LFR-3mL & K2O (wt\%) & 0.168 & 0.163 & $-3.0 \%$ & No & 0.011 & 6.75 & 0.007 & 0.009 & No & 16 & 10 \\
\hline 1 & Isolok-LFR-3mL & Li2O (wt\%) & 5.088 & 5.120 & $0.6 \%$ & No & 0.154 & 3.02 & 0.214 & 0.073 & Yes & 16 & 10 \\
\hline 1 & Isolok-LFR-3mL & $\mathrm{MgO}(\mathrm{wt} \%)$ & 0.310 & 0.323 & $4.1 \%$ & Yes & 0.024 & 7.76 & 0.016 & 0.008 & No & 16 & 10 \\
\hline 1 & Isolok-LFR-3mL & $\mathrm{MnO}$ (wt\%) & 2.808 & 2.936 & $4.6 \%$ & Yes & 0.226 & 8.04 & 0.163 & 0.073 & Yes & 16 & 10 \\
\hline 1 & Isolok-LFR-3mL & Na2O (wt\%) & 13.710 & 13.766 & $0.4 \%$ & No & 0.442 & 3.22 & 0.682 & 0.226 & Yes & 16 & 10 \\
\hline 1 & Isolok-LFR-3mL & $\mathrm{NiO}$ (wt\%) & 1.209 & 1.247 & $3.1 \%$ & No & 0.102 & 8.45 & 0.092 & 0.043 & No & 16 & 10 \\
\hline 1 & Isolok-LFR-3mL & $\mathrm{SiO} 2$ (wt\%) & 26.732 & 27.845 & $4.2 \%$ & No & 1.613 & 6.03 & 1.220 & 0.525 & Yes & 16 & 10 \\
\hline 1 & Isolok-LFR-3mL & Sum (wt\%) & 100.806 & 102.382 & $1.6 \%$ & No & 4.190 & 4.16 & 3.877 & 1.125 & No & 16 & 10 \\
\hline 1 & Isolok-LFR-3mL & TiO2 (wt\%) & 0.016 & 0.015 & $-3.7 \%$ & No & 0.002 & 12.44 & 0.002 & 0.001 & No & 16 & 10 \\
\hline 1 & Isolok-LFR-3mL & $\mathrm{ZnO}(\mathrm{wt} \%)$ & 0.006 & 0.006 & $-3.0 \%$ & No & 0.000 & 6.74 & 0.000 & 0.000 & No & 16 & 10 \\
\hline 1 & Isolok-LFR-3mL & $\mathrm{ZrO} 2$ (wt\%) & 0.104 & 0.108 & $4.0 \%$ & Yes & 0.007 & 7.10 & 0.005 & 0.003 & No & 16 & 10 \\
\hline 2 & Hydragard - HFR & $\mathrm{Al} / \mathrm{B}$ & 2.378 & 2.603 & $9.5 \%$ & Yes & 0.300 & 12.64 & 0.073 & 0.112 & No & 16 & 10 \\
\hline 2 & Hydragard - HFR & Al2O3 (wt\%) & 10.852 & 11.229 & $3.5 \%$ & Yes & 0.640 & 5.89 & 0.276 & 0.370 & No & 16 & 10 \\
\hline 2 & Hydragard - HFR & B2O3 (wt\%) & 4.566 & 4.318 & $-5.4 \%$ & Yes & 0.358 & 7.84 & 0.112 & 0.159 & No & 16 & 10 \\
\hline 2 & Hydragard - HFR & $\mathrm{BaO}(\mathrm{wt} \%)$ & 0.006 & 0.006 & $2.3 \%$ & No & 0.001 & 10.86 & 0.001 & 0.001 & No & 16 & 10 \\
\hline 2 & Hydragard - HFR & $\mathrm{CaO}(\mathrm{wt} \%)$ & 0.566 & 0.582 & $3.0 \%$ & No & 0.038 & 6.70 & 0.026 & 0.024 & No & 16 & 10 \\
\hline 2 & Hydragard - HFR & Cr2O3 (wt\%) & 0.018 & 0.023 & $22.7 \%$ & Yes & 0.006 & 32.36 & 0.002 & 0.003 & No & 16 & 10 \\
\hline 2 & Hydragard - HFR & $\mathrm{CuO}(\mathrm{wt} \%)$ & 0.095 & 0.095 & $-0.2 \%$ & No & 0.004 & 4.19 & 0.004 & 0.005 & No & 16 & 10 \\
\hline 2 & Hydragard - HFR & $\mathrm{Fe} / \mathrm{Li}$ & 1.971 & 2.126 & $7.8 \%$ & Yes & 0.222 & 11.27 & 0.075 & 0.091 & No & 16 & 10 \\
\hline 2 & Hydragard - HFR & $\mathrm{Fe} 2 \mathrm{O} 3(\mathrm{wt} \%)$ & 10.175 & 10.643 & $4.6 \%$ & Yes & 0.766 & 7.53 & 0.287 & 0.452 & No & 16 & 10 \\
\hline 2 & Hydragard - HFR & K2O (wt\%) & $\begin{array}{l}0.169 \\
\end{array}$ & 0.185 & $9.2 \%$ & No & 0.057 & 33.57 & 0.007 & 0.058 & Yes & 16 & 10 \\
\hline 2 & Hydragard - HFR & $\mathrm{Li} 2 \mathrm{O}$ (wt\%) & 5.166 & 5.010 & $-3.0 \%$ & Yes & 0.288 & 5.58 & 0.148 & 0.176 & No & 16 & 10 \\
\hline 2 & Hydragard - HFR & $\mathrm{MgO}(\mathrm{wt} \%)$ & 0.307 & 0.316 & $2.9 \%$ & No & 0.021 & 6.78 & 0.014 & 0.015 & No & 16 & 10 \\
\hline 2 & Hydragard - HFR & $\mathrm{MnO}$ (wt\%) & 2.816 & 2.934 & $4.2 \%$ & No & 0.254 & 9.03 & 0.146 & 0.193 & No & 16 & 10 \\
\hline 2 & Hydragard - HFR & $\mathrm{Na} 2 \mathrm{O}$ (wt\%) & 13.359 & 13.305 & $-0.4 \%$ & No & 0.436 & 3.26 & 0.468 & 0.445 & No & 16 & 10 \\
\hline 2 & Hydragard - HFR & $\mathrm{NiO}$ (wt\%) & 1.199 & 1.268 & $5.8 \%$ & No & 0.156 & 13.02 & 0.088 & 0.128 & No & 16 & 10 \\
\hline 2 & Hydragard - HFR & $\mathrm{SiO} 2$ (wt\%) & 49.699 & 48.220 & $-3.0 \%$ & Yes & 2.366 & 4.76 & 0.818 & 1.383 & No & 16 & 10 \\
\hline 2 & Hydragard - HFR & Sum (wt\%) & 99.132 & 98.277 & $-0.9 \%$ & No & 2.628 & 2.65 & 1.557 & 2.842 & No & 16 & 10 \\
\hline 2 & Hydragard - HFR & TiO2 (wt\%) & 0.018 & 0.019 & $4.7 \%$ & No & 0.002 & 12.84 & 0.002 & 0.001 & No & 16 & 10 \\
\hline 2 & Hydragard - HFR & $\mathrm{ZnO}(\mathrm{wt} \%)$ & 0.006 & 0.006 & $-1.8 \%$ & No & 0.000 & 5.53 & 0.000 & 0.000 & No & 16 & 10 \\
\hline 2 & Hydragard - HFR & $\mathrm{ZrO} 2(\mathrm{wt} \%)$ & 0.115 & 0.119 & $3.5 \%$ & Yes & 0.007 & 5.96 & 0.002 & 0.005 & No & 16 & 10 \\
\hline
\end{tabular}


Table B8. Oxide Comparisons Between Samplers for the High-Rheology (Phase 2) Testing

\begin{tabular}{|c|c|c|c|c|c|c|c|c|c|c|c|c|c|}
\hline $\begin{array}{c}\text { Analytical } \\
\text { Block }\end{array}$ & Sampler & Oxide & $\begin{array}{c}\text { Mean(Measured } \\
\text { wt\%) Coliwasa } \\
\text { (low+high) }\end{array}$ & $\begin{array}{c}\text { Mean(Measured } \\
\text { wt\%) Other } \\
\text { Sampler }\end{array}$ & $\%$ Diff & $\begin{array}{l}\text { Sig. } \\
\text { Diff }\end{array}$ & $\begin{array}{l}\text { Bias } \\
\text { Bnd }\end{array}$ & $\begin{array}{l}\% \text { Rel } \\
\text { Bias } \\
\text { Bnd }\end{array}$ & $\begin{array}{c}\text { Std } \\
\text { Dev(Measured } \\
\text { wt\%) Coliwasa } \\
\text { (low+high) }\end{array}$ & $\begin{array}{c}\text { Std } \\
\text { Dev(Measured } \\
\text { wt\%) Other } \\
\text { Sampler }\end{array}$ & $\begin{array}{l}\text { Sig. } \\
\text { Diff }\end{array}$ & $\underset{\text { Col }}{\text { n }}$ & $\begin{array}{c}\text { n } \\
\text { Other }\end{array}$ \\
\hline 3 & Isolok-LFR-12mL & $\mathrm{Al} / \mathrm{B}$ & 2.286 & 2.424 & $6.0 \%$ & Yes & 0.206 & 9.00 & 0.071 & 0.097 & No & 16 & 10 \\
\hline 3 & Isolok-LFR-12mL & Al2O3 (wt\%) & 10.863 & 11.020 & $1.4 \%$ & No & 0.365 & 3.36 & 0.251 & 0.251 & No & 16 & 10 \\
\hline 3 & Isolok-LFR-12mL & $\mathrm{B} 2 \mathrm{O} 3(\mathrm{wt} \%)$ & 4.755 & 4.553 & $-4.3 \%$ & Yes & 0.358 & 7.54 & 0.135 & 0.204 & Yes & 16 & 10 \\
\hline 3 & Isolok-LFR-12mL & $\mathrm{BaO}(\mathrm{wt} \%)$ & 0.004 & 0.012 & $201.3 \%$ & Yes & 0.008 & 216.95 & 0.000 & 0.001 & Yes & 16 & 10 \\
\hline 3 & Isolok-LFR-12mL & $\mathrm{CaO}(\mathrm{wt} \%)$ & 0.587 & 0.599 & $2.0 \%$ & No & 0.028 & 4.75 & 0.022 & 0.012 & No & 16 & 10 \\
\hline 3 & Isolok-LFR-12mL & Cr2O3 (wt\%) & 0.020 & 0.021 & $1.9 \%$ & No & 0.003 & 13.54 & 0.002 & 0.003 & Yes & 16 & 10 \\
\hline 3 & Isolok-LFR-12mL & $\mathrm{CuO}(\mathrm{wt} \%)$ & 0.095 & 0.093 & $-1.6 \%$ & No & 0.005 & 4.90 & 0.004 & 0.003 & No & 16 & 10 \\
\hline 3 & Isolok-LFR-12mL & $\mathrm{Fe} / \mathrm{Li}$ & 1.972 & 1.979 & $0.4 \%$ & No & 0.056 & 2.85 & 0.081 & 0.040 & Yes & 16 & 10 \\
\hline 3 & Isolok-LFR-12mL & Fe2O3 (wt\%) & 10.158 & 10.277 & $1.2 \%$ & No & 0.353 & 3.47 & 0.270 & 0.268 & No & 16 & 10 \\
\hline 3 & Isolok-LFR-12mL & K2O (wt\%) & 0.169 & 0.168 & $-0.9 \%$ & No & 0.005 & 3.08 & 0.007 & 0.002 & Yes & 16 & 10 \\
\hline 3 & Isolok-LFR-12mL & Li2O (wt\%) & 5.158 & 5.195 & $0.7 \%$ & No & 0.177 & 3.42 & 0.182 & 0.138 & No & 16 & 10 \\
\hline 3 & Isolok-LFR-12mL & $\mathrm{MgO}(\mathrm{wt} \%)$ & 0.309 & 0.316 & $2.2 \%$ & No & 0.016 & 5.12 & 0.012 & 0.008 & No & 16 & 10 \\
\hline 3 & Isolok-LFR-12mL & $\mathrm{MnO}$ (wt\%) & 2.833 & 2.879 & $1.7 \%$ & No & 0.144 & 5.08 & 0.131 & 0.088 & No & 16 & 10 \\
\hline 3 & Isolok-LFR-12mL & Na2O (wt\%) & 13.327 & 13.493 & $1.3 \%$ & No & 0.522 & 3.92 & 0.444 & 0.397 & No & 16 & 10 \\
\hline 3 & Isolok-LFR-12mL & $\mathrm{NiO}$ (wt\%) & 1.188 & 1.187 & $0.0 \%$ & No & 0.068 & 5.69 & 0.083 & 0.078 & No & 16 & 10 \\
\hline 3 & Isolok-LFR-12mL & $\mathrm{SiO} 2$ (wt\%) & 49.913 & 49.118 & $-1.6 \%$ & No & 1.693 & 3.39 & 1.082 & 1.078 & No & 16 & 10 \\
\hline 3 & Isolok-LFR-12mL & Sum (wt\%) & 99.517 & 99.068 & $-0.5 \%$ & No & 2.023 & 2.03 & 1.818 & 2.009 & No & 16 & 10 \\
\hline 3 & Isolok-LFR-12mL & TiO2 (wt\%) & 0.019 & 0.018 & $-3.2 \%$ & No & 0.002 & 10.50 & 0.002 & 0.002 & No & 16 & 10 \\
\hline 3 & Isolok-LFR-12mL & $\mathrm{ZnO}(\mathrm{wt} \%)$ & 0.008 & 0.006 & $-23.8 \%$ & No & 0.007 & 85.37 & 0.007 & 0.000 & No & 16 & 10 \\
\hline 3 & Isolok-LFR-12mL & $\mathrm{ZrO} 2$ (wt\%) & 0.113 & 0.113 & $0.6 \%$ & No & 0.003 & 2.84 & 0.003 & 0.004 & No & 16 & 10 \\
\hline 4 & Isolok-HFR-12mL & $\mathrm{Al} / \mathrm{B}$ & 2.427 & 2.538 & $4.6 \%$ & Yes & 0.177 & 7.31 & 0.076 & 0.086 & No & 16 & 10 \\
\hline 4 & Isolok-HFR-12mL & Al2O3 (wt\%) & 10.817 & 10.721 & $-0.9 \%$ & No & 0.312 & 2.89 & 0.297 & 0.181 & No & 16 & 10 \\
\hline 4 & Isolok-HFR-12mL & B2O3 (wt\%) & 4.460 & 4.228 & $-5.2 \%$ & Yes & 0.354 & 7.94 & 0.151 & 0.138 & No & 16 & 10 \\
\hline 4 & Isolok-HFR-12mL & $\mathrm{BaO}(\mathrm{wt} \%)$ & 0.006 & 0.013 & $137.3 \%$ & Yes & 0.009 & 152.96 & 0.000 & 0.001 & Yes & 16 & 10 \\
\hline 4 & Isolok-HFR-12mL & $\mathrm{CaO}(\mathrm{wt} \%)$ & 0.587 & 0.580 & $-1.3 \%$ & No & 0.024 & 4.09 & 0.021 & 0.017 & No & 16 & 10 \\
\hline 4 & Isolok-HFR-12mL & Cr2O3 (wt\%) & 0.020 & 0.026 & $33.2 \%$ & Yes & 0.009 & 42.83 & 0.001 & 0.003 & Yes & 16 & 10 \\
\hline 4 & Isolok-HFR-12mL & $\mathrm{CuO}(\mathrm{wt} \%)$ & 0.091 & 0.087 & $-5.3 \%$ & Yes & 0.009 & 9.32 & 0.005 & 0.004 & No & 16 & 10 \\
\hline 4 & Isolok-HFR-12mL & $\mathrm{Fe} / \mathrm{Li}$ & 2.011 & 1.993 & $-0.9 \%$ & No & 0.065 & 3.21 & 0.064 & 0.039 & No & 16 & 10 \\
\hline 4 & Isolok-HFR-12mL & $\mathrm{Fe} 2 \mathrm{O} 3(\mathrm{wt} \%)$ & 10.163 & 10.138 & $-0.2 \%$ & No & 0.240 & 2.36 & 0.302 & 0.157 & No & 16 & 10 \\
\hline 4 & Isolok-HFR-12mL & K2O (wt\%) & 0.169 & 0.163 & $-3.7 \%$ & Yes & 0.012 & 7.38 & 0.007 & 0.009 & No & 16 & 10 \\
\hline 4 & Isolok-HFR-12mL & Li2O (wt\%) & 5.055 & 5.089 & $0.7 \%$ & No & 0.137 & 2.72 & 0.114 & 0.139 & No & 16 & 10 \\
\hline 4 & Isolok-HFR-12mL & $\mathrm{MgO}(\mathrm{wt} \%)$ & 0.303 & 0.299 & $-1.6 \%$ & No & 0.014 & 4.64 & 0.013 & 0.006 & No & 16 & 10 \\
\hline 4 & Isolok-HFR-12mL & $\mathrm{MnO}$ (wt\%) & 2.766 & 2.710 & $-2.0 \%$ & No & 0.141 & 5.09 & 0.140 & 0.067 & Yes & 16 & 10 \\
\hline 4 & Isolok-HFR-12mL & $\mathrm{Na} 2 \mathrm{O}$ (wt\%) & 13.269 & 13.119 & $-1.1 \%$ & No & 0.431 & 3.24 & 0.303 & 0.388 & No & 16 & 10 \\
\hline 4 & Isolok-HFR-12mL & $\mathrm{NiO}(\mathrm{wt} \%)$ & 1.192 & 1.170 & $-1.8 \%$ & No & 0.074 & 6.18 & 0.087 & 0.038 & Yes & 16 & 10 \\
\hline 4 & Isolok-HFR-12mL & $\mathrm{SiO} 2$ (wt\%) & 49.913 & 50.209 & $0.6 \%$ & No & 1.112 & 2.23 & 0.943 & 1.039 & No & 16 & 10 \\
\hline 4 & Isolok-HFR-12mL & Sum (wt\%) & 98.949 & 98.688 & $-0.3 \%$ & No & 1.699 & 1.72 & 1.668 & 1.827 & No & 16 & 10 \\
\hline 4 & Isolok-HFR-12mL & TiO2 (wt\%) & 0.020 & 0.019 & $-3.7 \%$ & No & 0.002 & 12.76 & 0.002 & 0.002 & No & 16 & 10 \\
\hline 4 & Isolok-HFR-12mL & $\mathrm{ZnO}(\mathrm{wt} \%)$ & 0.006 & 0.006 & $-3.6 \%$ & No & 0.000 & 7.21 & 0.000 & 0.000 & No & 16 & 10 \\
\hline 4 & Isolok-HFR-12mL & $\mathrm{ZrO} 2(\mathrm{wt} \%)$ & 0.111 & 0.111 & $0.1 \%$ & No & 0.003 & 2.36 & 0.003 & 0.004 & No & 16 & 10 \\
\hline
\end{tabular}


SRNL-STI-2011-00693

Revision 0

Table B8. Oxide Comparisons Between Samplers for the High-Rheology (Phase 2) Testing

\begin{tabular}{|c|c|c|c|c|c|c|c|c|c|c|c|c|c|}
\hline $\begin{array}{c}\text { Analytical } \\
\text { Block }\end{array}$ & Sampler & Oxide & $\begin{array}{c}\text { Mean(Measured } \\
\text { wt\%) Coliwasa } \\
(\text { low+high) }\end{array}$ & $\begin{array}{c}\text { Mean(Measured } \\
\text { wt\%) Other } \\
\text { Sampler }\end{array}$ & $\%$ Diff & $\begin{array}{l}\text { Sig. } \\
\text { Diff }\end{array}$ & $\begin{array}{l}\text { Bias } \\
\text { Bnd } \\
\end{array}$ & $\begin{array}{l}\% \text { Rel } \\
\text { Bias } \\
\text { Bnd }\end{array}$ & $\begin{array}{c}\text { Std } \\
\text { Dev(Measured } \\
\text { wt \%) Coliwasa } \\
(\text { low+high) } \\
\end{array}$ & $\begin{array}{c}\text { Std } \\
\text { Dev(Measured } \\
\text { wt\%) Other } \\
\text { Sampler } \\
\end{array}$ & $\begin{array}{l}\text { Sig. } \\
\text { Diff }\end{array}$ & $\begin{array}{c}\mathbf{n} \\
\text { Col }\end{array}$ & $\begin{array}{c}\mathbf{n} \\
\text { Other }\end{array}$ \\
\hline 5 & Isolok-HFR-3mL & $\mathrm{Al} / \mathrm{B}$ & 2.402 & 2.417 & $0.6 \%$ & No & 0.074 & 3.08 & 0.075 & 0.063 & No & 16 & 10 \\
\hline 5 & Isolok-HFR-3mL & Al2O3 (wt\%) & 10.918 & 10.942 & $0.2 \%$ & No & 0.199 & 1.82 & 0.315 & 0.087 & Yes & 16 & 10 \\
\hline 5 & Isolok-HFR-3mL & B2O3 (wt\%) & 4.548 & 4.530 & $-0.4 \%$ & No & 0.133 & 2.92 & 0.139 & 0.137 & No & 16 & 10 \\
\hline 5 & Isolok-HFR-3mL & $\mathrm{BaO}$ (wt\%) & 0.005 & 0.005 & $1.8 \%$ & No & 0.000 & 6.14 & 0.000 & 0.000 & No & 16 & 10 \\
\hline 5 & Isolok-HFR-3mL & $\mathrm{CaO}(\mathrm{wt} \%)$ & 0.577 & 0.574 & $-0.6 \%$ & No & 0.021 & 3.58 & 0.023 & 0.013 & No & 16 & 10 \\
\hline 5 & Isolok-HFR-3mL & Cr2O3 (wt\%) & 0.019 & 0.022 & $14.4 \%$ & Yes & 0.004 & 22.83 & 0.002 & 0.002 & No & 16 & 10 \\
\hline 5 & Isolok-HFR-3mL & $\mathrm{CuO}(\mathrm{wt} \%)$ & 0.095 & 0.090 & $-5.2 \%$ & Yes & 0.009 & 9.58 & 0.006 & 0.003 & No & 16 & 10 \\
\hline 5 & Isolok-HFR-3mL & $\mathrm{Fe} / \mathrm{Li}$ & 2.014 & 1.990 & $-1.2 \%$ & No & 0.068 & 3.37 & 0.074 & 0.034 & Yes & 16 & 10 \\
\hline 5 & Isolok-HFR-3mL & Fe2O3 (wt\%) & 10.178 & 10.251 & $0.7 \%$ & No & 0.250 & 2.45 & 0.319 & 0.081 & Yes & 16 & 10 \\
\hline 5 & Isolok-HFR-3mL & K2O (wt\%) & 0.169 & 0.167 & $-1.1 \%$ & No & 0.008 & 4.56 & 0.007 & 0.008 & No & 16 & 10 \\
\hline 5 & Isolok-HFR-3mL & Li2O (wt\%) & 5.055 & 5.152 & $1.9 \%$ & Yes & 0.185 & 3.66 & 0.112 & 0.096 & No & 16 & 10 \\
\hline 5 & Isolok-HFR-3mL & $\mathrm{MgO}(\mathrm{wt} \%)$ & 0.306 & 0.307 & $0.3 \%$ & No & 0.010 & 3.20 & 0.013 & 0.006 & No & 16 & 10 \\
\hline 5 & Isolok-HFR-3mL & $\mathrm{MnO}(\mathrm{wt} \%)$ & 2.785 & 2.806 & $0.7 \%$ & No & 0.119 & 4.27 & 0.143 & 0.053 & Yes & 16 & 10 \\
\hline 5 & Isolok-HFR-3mL & $\mathrm{Na} 2 \mathrm{O}$ (wt\%) & 13.243 & 13.147 & $-0.7 \%$ & No & 0.369 & 2.78 & 0.386 & 0.195 & No & 16 & 10 \\
\hline 5 & Isolok-HFR-3mL & $\mathrm{NiO}$ (wt\%) & 1.194 & 1.208 & $1.2 \%$ & No & 0.032 & 2.71 & 0.084 & 0.024 & Yes & 16 & 10 \\
\hline 5 & Isolok-HFR-3mL & SiO2 (wt\%) & 49.899 & 50.744 & $1.7 \%$ & No & 1.728 & 3.46 & 1.072 & 1.042 & No & 16 & 10 \\
\hline 5 & Isolok-HFR-3mL & Sum (wt\%) & 99.125 & 100.077 & $1.0 \%$ & No & 2.407 & 2.43 & 1.985 & 1.259 & No & 16 & 10 \\
\hline 5 & Isolok-HFR-3mL & $\mathrm{TiO} 2(\mathrm{wt} \%)$ & 0.016 & 0.016 & $-2.2 \%$ & No & 0.002 & 9.55 & 0.002 & 0.001 & No & 16 & 10 \\
\hline 5 & Isolok-HFR-3mL & $\mathrm{ZnO}(\mathrm{wt} \%)$ & 0.006 & 0.006 & $-1.1 \%$ & No & 0.000 & 4.53 & 0.000 & 0.000 & No & 16 & 10 \\
\hline 5 & Isolok-HFR-3mL & $\mathrm{ZrO} 2$ (wt\%) & 0.111 & 0.109 & $-1.1 \%$ & No & 0.004 & 3.18 & 0.003 & 0.002 & No & 16 & 10 \\
\hline 6 & Hydragard-LFR & $\mathrm{Al} / \mathrm{B}$ & 2.415 & 2.537 & $5.0 \%$ & Yes & 0.183 & 7.58 & 0.073 & 0.076 & No & 16 & 10 \\
\hline 6 & Hydragard-LFR & $\mathrm{Al} 2 \mathrm{O} 3(\mathrm{wt} \%)$ & 10.892 & 11.099 & $1.9 \%$ & No & 0.442 & 4.06 & 0.309 & 0.230 & No & 16 & 10 \\
\hline 6 & Hydragard-LFR & B2O3 (wt\%) & 4.512 & 4.379 & $-2.9 \%$ & No & 0.269 & 5.96 & 0.155 & 0.176 & No & 16 & 10 \\
\hline 6 & Hydragard-LFR & $\mathrm{BaO}(\mathrm{wt} \%)$ & 0.005 & 0.010 & $113.5 \%$ & Yes & 0.008 & 169.14 & 0.000 & 0.004 & Yes & 16 & 10 \\
\hline 6 & Hydragard-LFR & $\mathrm{CaO}(\mathrm{wt} \%)$ & 0.585 & 0.594 & $1.6 \%$ & No & 0.028 & 4.82 & 0.025 & 0.018 & No & 16 & 10 \\
\hline 6 & Hydragard-LFR & Cr2O3 (wt\%) & 0.020 & 0.019 & $-3.6 \%$ & No & 0.002 & 12.12 & 0.002 & 0.003 & No & 16 & 10 \\
\hline 6 & Hydragard-LFR & $\mathrm{CuO}(\mathrm{wt} \%)$ & 0.095 & 0.095 & $0.0 \%$ & No & 0.004 & 4.20 & 0.005 & 0.003 & No & 16 & 10 \\
\hline 6 & Hydragard-LFR & $\mathrm{Fe} / \mathrm{Li}$ & 2.026 & 2.051 & $1.3 \%$ & No & 0.080 & 3.93 & 0.059 & 0.074 & No & 16 & 10 \\
\hline 6 & Hydragard-LFR & Fe2O3 (wt\%) & 10.253 & 10.407 & $1.5 \%$ & No & 0.398 & 3.88 & 0.307 & 0.269 & No & 16 & 10 \\
\hline 6 & Hydragard-LFR & K2O (wt\%) & 0.169 & 0.168 & $-0.6 \%$ & No & 0.007 & 3.86 & 0.007 & 0.006 & No & 16 & 10 \\
\hline 6 & Hydragard-LFR & Li2O (wt\%) & 5.063 & 5.077 & $0.3 \%$ & No & 0.124 & 2.44 & 0.145 & 0.109 & No & 16 & 10 \\
\hline 6 & Hydragard-LFR & $\mathrm{MgO}(\mathrm{wt} \%)$ & 0.308 & 0.312 & $1.3 \%$ & No & 0.014 & 4.68 & 0.014 & 0.010 & No & 16 & 10 \\
\hline 6 & Hydragard-LFR & $\mathrm{MnO}(\mathrm{wt} \%)$ & 2.799 & 2.824 & $0.9 \%$ & No & 0.131 & 4.68 & 0.143 & 0.094 & No & 16 & 10 \\
\hline 6 & Hydragard-LFR & $\mathrm{Na} 2 \mathrm{O}$ (wt\%) & 13.107 & 13.186 & $0.6 \%$ & No & 0.443 & 3.38 & 0.480 & 0.354 & No & 16 & 10 \\
\hline 6 & Hydragard-LFR & $\mathrm{NiO}(\mathrm{wt} \%)$ & 1.189 & 1.181 & $-0.6 \%$ & No & 0.068 & 5.70 & 0.083 & 0.050 & No & 16 & 10 \\
\hline 6 & Hydragard-LFR & $\mathrm{SiO} 2(\mathrm{wt} \%)$ & 49.739 & 49.568 & $-0.3 \%$ & No & 1.070 & 2.15 & 1.161 & 0.930 & No & 16 & 10 \\
\hline 6 & Hydragard-LFR & Sum (wt\%) & 98.866 & 99.052 & $0.2 \%$ & No & 1.865 & 1.89 & 2.217 & 1.635 & No & 16 & 10 \\
\hline 6 & Hydragard-LFR & TiO2 (wt\%) & 0.016 & 0.016 & $-1.6 \%$ & No & 0.002 & 10.01 & 0.002 & 0.002 & No & 16 & 10 \\
\hline 6 & Hydragard-LFR & $\mathrm{ZnO}$ (wt\%) & 0.006 & 0.007 & $22.7 \%$ & No & 0.005 & 76.46 & 0.000 & 0.004 & Yes & 16 & 10 \\
\hline 6 & Hydragard-LFR & $\mathrm{ZrO} 2$ (wt\%) & 0.109 & 0.110 & $0.4 \%$ & No & 0.003 & 2.61 & 0.003 & 0.003 & No & 16 & 10 \\
\hline
\end{tabular}


SRNL-STI-2011-00693

Revision 0

Table B9. Oxide Comparisons Between Samplers for the High-Rheology (Phase 2) Testing (screened data)

\begin{tabular}{|c|c|c|c|c|c|c|c|c|c|c|c|c|c|}
\hline $\begin{array}{c}\text { Analytical } \\
\text { Block }\end{array}$ & Sampler & Oxide & $\begin{array}{c}\text { Mean(Measured } \\
\text { wt\%) Coliwasa } \\
\text { (low+high) }\end{array}$ & $\begin{array}{c}\text { Mean(Measured } \\
\text { wt\%) Other } \\
\text { Sampler }\end{array}$ & $\%$ Diff & $\begin{array}{l}\text { Sig. } \\
\text { Diff }\end{array}$ & $\begin{array}{l}\text { Bias } \\
\text { Bnd } \\
\end{array}$ & $\begin{array}{l}\text { \% Rel } \\
\text { Bias } \\
\text { Bnd } \\
\end{array}$ & $\begin{array}{c}\text { Std } \\
\text { Dev(Measured } \\
\text { wt\%) Coliwasa } \\
(\text { low+high) } \\
\end{array}$ & $\begin{array}{c}\text { Std } \\
\text { Dev(Measured } \\
\text { wt\%) Other } \\
\text { Sampler } \\
\end{array}$ & $\begin{array}{l}\text { Sig. } \\
\text { Diff }\end{array}$ & $\begin{array}{c}\text { n } \\
\text { Col }\end{array}$ & $\begin{array}{c}\text { n } \\
\text { Other }\end{array}$ \\
\hline 1 & Isolok-LFR-3mL & $\mathrm{Al} / \mathrm{B}$ & 2.429 & 2.598 & $6.9 \%$ & Yes & 0.24756 & 10.19 & 0.096 & 0.093 & No & 16 & 10 \\
\hline 1 & Isolok-LFR-3mL & $\mathrm{Al} 2 \mathrm{O} 3$ (wt\%) & 11.115 & 11.518 & $3.6 \%$ & Yes & 0.69912 & 6.29 & 0.415 & 0.216 & No & 15 & 10 \\
\hline 1 & Isolok-LFR-3mL & B2O3 (wt\%) & 4.548 & 4.437 & $-2.4 \%$ & No & 0.23636 & 5.20 & 0.196 & 0.113 & Yes & 16 & 10 \\
\hline 1 & Isolok-LFR-3mL & $\mathrm{BaO}$ (wt\%) & 0.006 & 0.006 & $0.0 \%$ & No & 0.00058 & 10.28 & 0.001 & 0.001 & No & 16 & 10 \\
\hline 1 & Isolok-LFR-3mL & $\mathrm{CaO}$ (wt\%) & 0.605 & 0.619 & $2.3 \%$ & No & 0.03043 & 5.03 & 0.027 & 0.010 & Yes & 15 & 8 \\
\hline 1 & Isolok-LFR-3mL & Cr2O3 (wt\%) & 0.019 & 0.020 & $3.2 \%$ & No & 0.00311 & 16.10 & 0.001 & 0.003 & Yes & 15 & 10 \\
\hline 1 & Isolok-LFR-3mL & $\mathrm{CuO}(\mathrm{wt} \%)$ & 0.096 & 0.092 & $-3.9 \%$ & No & 0.00943 & 9.80 & 0.007 & 0.007 & No & 16 & 10 \\
\hline 1 & Isolok-LFR-3mL & $\mathrm{Fe} / \mathrm{Li}$ & 2.035 & 2.106 & $3.5 \%$ & Yes & 0.12805 & 6.29 & 0.078 & 0.048 & No & 16 & 10 \\
\hline 1 & Isolok-LFR-3mL & Fe2O3 (wt\%) & 10.440 & 10.781 & $3.3 \%$ & Yes & 0.5322 & 5.10 & 0.246 & 0.175 & No & 13 & 10 \\
\hline 1 & Isolok-LFR-3mL & K2O (wt\%) & 0.168 & 0.163 & $-3.0 \%$ & No & 0.01136 & 6.75 & 0.007 & 0.009 & No & 16 & 10 \\
\hline 1 & Isolok-LFR-3mL & Li2O (wt\%) & 5.088 & 5.100 & $0.2 \%$ & No & 0.12893 & 2.53 & 0.214 & 0.041 & Yes & 16 & 9 \\
\hline 1 & Isolok-LFR-3mL & $\mathrm{MgO}(\mathrm{wt} \%)$ & 0.310 & 0.323 & $4.1 \%$ & Yes & 0.02405 & 7.76 & 0.016 & 0.008 & No & 16 & 10 \\
\hline 1 & Isolok-LFR-3mL & $\mathrm{MnO}(\mathrm{wt} \%)$ & 2.808 & 2.936 & $4.6 \%$ & Yes & 0.22562 & 8.04 & 0.163 & 0.073 & Yes & 16 & 10 \\
\hline 1 & Isolok-LFR-3mL & $\mathrm{Na} 2 \mathrm{O}$ (wt\%) & 13.710 & 13.766 & $0.4 \%$ & No & 0.4421 & 3.22 & 0.682 & 0.226 & Yes & 16 & 10 \\
\hline 1 & Isolok-LFR-3mL & $\mathrm{NiO}(\mathrm{wt} \%)$ & 1.209 & 1.247 & $3.1 \%$ & No & 0.10219 & 8.45 & 0.092 & 0.043 & No & 16 & 10 \\
\hline 1 & Isolok-LFR-3mL & SiO2 (wt\%) & 50.880 & 51.391 & $1.0 \%$ & No & 0.98359 & 1.93 & 0.682 & 0.317 & Yes & 12 & 9 \\
\hline 1 & Isolok-LFR-3mL & Sum (wt\%) & 101.568 & 102.646 & $1.1 \%$ & No & 2.3453 & 2.31 & 1.687 & 0.800 & No & 13 & 9 \\
\hline 1 & Isolok-LFR-3mL & TiO2 (wt\%) & 0.016 & 0.015 & $-3.7 \%$ & No & 0.00198 & 12.44 & 0.002 & 0.001 & No & 16 & 10 \\
\hline 1 & Isolok-LFR-3mL & $\mathrm{ZnO}$ (wt\%) & 0.006 & 0.006 & $-3.0 \%$ & No & 0.0004 & 6.74 & 0.000 & 0.000 & No & 16 & 10 \\
\hline 1 & Isolok-LFR-3mL & ZrO2 (wt\%) & 0.104 & 0.108 & $4.0 \%$ & Yes & 0.0074 & 7.10 & 0.005 & 0.003 & No & 16 & 10 \\
\hline 2 & Hydragard - HFR & $\mathrm{Al} / \mathrm{B}$ & 2.3775 & 2.6031 & $9.5 \%$ & Yes & 0.30041 & 12.64 & 0.073 & 0.112 & No & 16 & 10 \\
\hline 2 & Hydragard - HFR & $\mathrm{Al} 2 \mathrm{O} 3(\mathrm{wt} \%)$ & 10.8516 & 11.3391 & $4.5 \%$ & Yes & 0.65871 & 6.07 & 0.276 & 0.137 & Yes & 16 & 9 \\
\hline 2 & Hydragard - HFR & B2O3 (wt\%) & 4.5662 & 4.3179 & $-5.4 \%$ & Yes & 0.35788 & 7.84 & 0.112 & 0.159 & No & 16 & 10 \\
\hline 2 & Hydragard - HFR & $\mathrm{BaO}(\mathrm{wt} \%)$ & 0.0055 & 0.0057 & $2.3 \%$ & No & 0.0006 & 10.86 & 0.001 & 0.001 & No & 16 & 10 \\
\hline 2 & Hydragard - HFR & $\mathrm{CaO}(\mathrm{wt} \%)$ & 0.5706 & 0.5825 & $2.1 \%$ & No & 0.02897 & 5.08 & 0.017 & 0.024 & No & 15 & 10 \\
\hline 2 & Hydragard - HFR & Cr2O3 (wt\%) & 0.0185 & 0.0220 & $19.3 \%$ & Yes & 0.00508 & 27.49 & 0.002 & 0.002 & No & 16 & 9 \\
\hline 2 & Hydragard - HFR & $\mathrm{CuO}(\mathrm{wt} \%)$ & 0.0947 & 0.0946 & $-0.1 \%$ & No & 0.00355 & 3.75 & 0.004 & 0.002 & No & 16 & 8 \\
\hline 2 & Hydragard - HFR & $\mathrm{Fe} / \mathrm{Li}$ & 1.9711 & 2.1255 & $7.8 \%$ & Yes & 0.22218 & 11.27 & 0.075 & 0.091 & No & 16 & 10 \\
\hline 2 & Hydragard - HFR & Fe2O3 (wt\%) & 10.1750 & 10.7672 & $5.8 \%$ & Yes & 0.8254 & 8.11 & 0.287 & 0.236 & No & 16 & 9 \\
\hline 2 & Hydragard - HFR & K2O (wt\%) & 0.1692 & 0.1668 & $-1.5 \%$ & No & 0.00902 & 5.33 & 0.007 & 0.009 & No & 16 & 9 \\
\hline 2 & Hydragard - HFR & Li2O (wt\%) & 5.1656 & 5.0450 & $-2.3 \%$ & No & 0.24728 & 4.79 & 0.148 & 0.145 & No & 16 & 9 \\
\hline 2 & Hydragard - HFR & $\mathrm{MgO}(\mathrm{wt} \%)$ & 0.3073 & 0.3162 & $2.9 \%$ & No & 0.02083 & 6.78 & 0.014 & 0.015 & No & 16 & 10 \\
\hline 2 & Hydragard - HFR & $\mathrm{MnO}(\mathrm{wt} \%)$ & 2.8164 & 2.9336 & $4.2 \%$ & No & 0.25446 & 9.03 & 0.146 & 0.193 & No & 16 & 10 \\
\hline 2 & Hydragard - HFR & $\mathrm{Na} 2 \mathrm{O}$ (wt\%) & 13.4387 & 13.3166 & $-0.9 \%$ & No & 0.39713 & 2.96 & 0.353 & 0.154 & No & 15 & 8 \\
\hline 2 & Hydragard - HFR & $\mathrm{NiO}(\mathrm{wt} \%)$ & 1.1992 & 1.2683 & $5.8 \%$ & No & 0.15608 & 13.02 & 0.088 & 0.128 & No & 16 & 10 \\
\hline 2 & Hydragard - HFR & SiO2 (wt\%) & 49.6986 & 48.6097 & $-2.2 \%$ & Yes & 1.7516 & 3.52 & 0.818 & 0.666 & No & 16 & 9 \\
\hline 2 & Hydragard - HFR & Sum (wt\%) & 99.4002 & 99.1409 & $-0.3 \%$ & No & 1.1834 & 1.19 & 1.167 & 0.828 & No & 15 & 9 \\
\hline 2 & Hydragard - HFR & TiO2 (wt\%) & 0.0175 & 0.0192 & $9.7 \%$ & Yes & 0.00274 & 15.61 & 0.001 & 0.001 & No & 13 & 10 \\
\hline 2 & Hydragard - HFR & $\mathrm{ZnO}$ (wt\%) & 0.0060 & 0.0059 & $-1.8 \%$ & No & 0.00033 & 5.53 & 0.000 & 0.000 & No & 16 & 10 \\
\hline 2 & Hydragard - HFR & $\mathrm{ZrO} 2$ (wt\%) & 0.1152 & 0.1205 & $4.6 \%$ & Yes & 0.0074 & 6.42 & 0.002 & 0.003 & No & 16 & 9 \\
\hline
\end{tabular}


SRNL-STI-2011-00693

Revision 0

Table B9. Oxide Comparisons Between Samplers for the High-Rheology (Phase 2) Testing (screened data)

\begin{tabular}{|c|c|c|c|c|c|c|c|c|c|c|c|c|c|}
\hline $\begin{array}{c}\text { Analytical } \\
\text { Block }\end{array}$ & Sampler & Oxide & $\begin{array}{c}\text { Mean(Measured } \\
\text { wt\%) Coliwasa } \\
\text { (low+high) }\end{array}$ & $\begin{array}{c}\text { Mean(Measured } \\
\text { wt\%) Other } \\
\text { Sampler }\end{array}$ & $\%$ Diff & $\begin{array}{l}\text { Sig. } \\
\text { Diff }\end{array}$ & $\begin{array}{l}\text { Bias } \\
\text { Bnd } \\
\end{array}$ & $\begin{array}{l}\text { \% Rel } \\
\text { Bias } \\
\text { Bnd } \\
\end{array}$ & $\begin{array}{c}\text { Std } \\
\text { Dev(Measured } \\
\text { wt\%) Coliwasa } \\
(\text { low+high) } \\
\end{array}$ & $\begin{array}{c}\text { Std } \\
\text { Dev(Measured } \\
\text { wt\%) Other } \\
\text { Sampler } \\
\end{array}$ & $\begin{array}{l}\text { Sig. } \\
\text { Diff }\end{array}$ & $\begin{array}{c}\text { n } \\
\text { Col }\end{array}$ & $\begin{array}{c}\text { n } \\
\text { Other }\end{array}$ \\
\hline 3 & Isolok-LFR-12mL & $\mathrm{Al} / \mathrm{B}$ & 2.286 & 2.424 & $6.0 \%$ & Yes & 0.20575 & 9.00 & 0.071 & 0.097 & No & 16 & 10 \\
\hline 3 & Isolok-LFR-12mL & $\mathrm{Al} 2 \mathrm{O} 3$ (wt\%) & 10.863 & 11.091 & $2.1 \%$ & Yes & 0.37978 & 3.50 & 0.251 & 0.113 & Yes & 16 & 9 \\
\hline 3 & Isolok-LFR-12mL & B2O3 (wt\%) & 4.778 & 4.553 & $-4.7 \%$ & Yes & 0.37705 & 7.89 & 0.102 & 0.204 & Yes & 15 & 10 \\
\hline 3 & Isolok-LFR-12mL & $\mathrm{BaO}(\mathrm{wt} \%)$ & 0.004 & 0.012 & $201.3 \%$ & Yes & 0.00834 & 216.95 & 0.000 & 0.001 & Yes & 16 & 10 \\
\hline 3 & Isolok-LFR-12mL & $\mathrm{CaO}$ (wt\%) & 0.591 & 0.599 & $1.3 \%$ & No & 0.01976 & 3.34 & 0.015 & 0.012 & No & 15 & 10 \\
\hline 3 & Isolok-LFR-12mL & Cr2O3 (wt\%) & 0.020 & 0.021 & $1.9 \%$ & Yes & 0.00233 & 11.52 & 0.002 & 0.003 & No & 16 & 10 \\
\hline 3 & Isolok-LFR-12mL & $\mathrm{CuO}(\mathrm{wt} \%)$ & 0.095 & 0.092 & $-2.5 \%$ & No & 0.00479 & 5.07 & 0.004 & 0.002 & Yes & 16 & 9 \\
\hline 3 & $\begin{array}{l}\text { Isolok-LFR-12mL } \\
\end{array}$ & $\mathrm{Fe} / \mathrm{Li}$ & 1.972 & 1.979 & $0.4 \%$ & No & 0.05619 & 2.85 & 0.081 & 0.040 & Yes & 16 & 10 \\
\hline 3 & Isolok-LFR-12mL & Fe2O3 (wt\%) & 10.158 & 10.353 & $1.9 \%$ & No & 0.35938 & 3.54 & 0.270 & 0.127 & Yes & 16 & 9 \\
\hline 3 & Isolok-LFR-12mL & K2O (wt\%) & 0.169 & 0.168 & $-0.9 \%$ & No & 0.00521 & 3.08 & 0.007 & 0.002 & Yes & 16 & 10 \\
\hline 3 & Isolok-LFR-12mL & Li2O (wt\%) & 5.158 & 5.195 & $0.7 \%$ & No & 0.17654 & 3.42 & 0.182 & 0.138 & No & 16 & 10 \\
\hline 3 & Isolok-LFR-12mL & $\mathrm{MgO}(\mathrm{wt} \%)$ & 0.311 & 0.316 & $1.5 \%$ & No & 0.01158 & 3.72 & 0.008 & 0.008 & No & 15 & 10 \\
\hline 3 & Isolok-LFR-12mL & $\mathrm{MnO}(\mathrm{wt} \%)$ & 2.833 & 2.879 & $1.7 \%$ & No & 0.14388 & 5.08 & 0.131 & 0.088 & No & 16 & 10 \\
\hline 3 & Isolok-LFR-12mL & $\mathrm{Na} 2 \mathrm{O}$ (wt\%) & 13.327 & 13.493 & $1.3 \%$ & No & 0.52207 & 3.92 & 0.444 & 0.397 & No & 16 & 10 \\
\hline 3 & Isolok-LFR-12mL & $\mathrm{NiO}(\mathrm{wt} \%)$ & 1.176 & 1.187 & $1.0 \%$ & No & 0.07368 & 6.26 & 0.071 & 0.078 & No & 15 & 10 \\
\hline 3 & Isolok-LFR-12mL & $\mathrm{SiO} 2(\mathrm{wt} \%)$ & 50.102 & 49.118 & $-2.0 \%$ & Yes & 1.7587 & 3.51 & 0.797 & 1.078 & No & 15 & 10 \\
\hline 3 & Isolok-LFR-12mL & Sum (wt\%) & 99.627 & 99.603 & $0.0 \%$ & No & 0.90081 & 0.90 & 0.869 & 1.153 & No & 14 & 9 \\
\hline 3 & Isolok-LFR-12mL & TiO2 (wt\%) & 0.019 & 0.018 & $-1.4 \%$ & No & 0.00133 & 7.18 & 0.001 & 0.002 & No & 15 & 10 \\
\hline 3 & Isolok-LFR-12mL & $\mathrm{ZnO}$ (wt\%) & 0.006 & 0.006 & $-0.6 \%$ & No & 0.00018 & 3.02 & 0.000 & 0.000 & Yes & 15 & 10 \\
\hline 3 & Isolok-LFR-12mL & ZrO2 (wt\%) & 0.113 & 0.113 & $0.6 \%$ & No & 0.0032 & 2.84 & 0.003 & 0.004 & No & 16 & 10 \\
\hline 4 & Isolok-HFR-12mL & $\mathrm{Al} / \mathrm{B}$ & 2.427 & 2.538 & $4.6 \%$ & Yes & 0.17747 & 7.31 & 0.076 & 0.086 & No & 16 & 10 \\
\hline 4 & Isolok-HFR-12mL & $\mathrm{Al} 2 \mathrm{O} 3(\mathrm{wt} \%)$ & 10.817 & 10.772 & $-0.4 \%$ & No & 0.21143 & 1.95 & 0.297 & 0.086 & Yes & 16 & 9 \\
\hline 4 & Isolok-HFR-12mL & B2O3 (wt\%) & 4.460 & 4.228 & $-5.2 \%$ & Yes & 0.35388 & 7.94 & 0.151 & 0.138 & No & 16 & 10 \\
\hline 4 & Isolok-HFR-12mL & $\mathrm{BaO}(\mathrm{wt} \%)$ & 0.006 & 0.013 & $132.1 \%$ & Yes & 0.00802 & 141.63 & 0.000 & 0.001 & No & 16 & 9 \\
\hline 4 & Isolok-HFR-12mL & $\mathrm{CaO}(\mathrm{wt} \%)$ & 0.587 & 0.580 & $-1.3 \%$ & No & 0.02401 & 4.09 & 0.021 & 0.017 & No & 16 & 10 \\
\hline 4 & Isolok-HFR-12mL & Cr2O3 (wt\%) & 0.020 & 0.026 & $32.0 \%$ & No & 0.00797 & 39.69 & 0.001 & 0.003 & No & 15 & 10 \\
\hline 4 & Isolok-HFR-12mL & $\mathrm{CuO}(\mathrm{wt} \%)$ & 0.091 & 0.086 & $-6.3 \%$ & Yes & 0.00873 & 9.54 & 0.005 & 0.002 & Yes & 16 & 9 \\
\hline 4 & Isolok-HFR-12mL & $\mathrm{Fe} / \mathrm{Li}$ & 2.011 & 1.993 & $-0.9 \%$ & No & 0.06463 & 3.21 & 0.064 & 0.039 & No & 16 & 10 \\
\hline 4 & Isolok-HFR-12mL & Fe2O3 (wt\%) & 10.163 & 10.138 & $-0.2 \%$ & No & 0.23974 & 2.36 & 0.302 & 0.157 & No & 16 & 10 \\
\hline 4 & Isolok-HFR-12mL & K2O (wt\%) & 0.169 & 0.163 & $-3.7 \%$ & Yes & 0.01249 & 7.38 & 0.007 & 0.009 & No & 16 & 10 \\
\hline 4 & Isolok-HFR-12mL & Li2O (wt\%) & 5.055 & 5.089 & $0.7 \%$ & No & 0.13726 & 2.72 & 0.114 & 0.139 & No & 16 & 10 \\
\hline 4 & $\begin{array}{l}\text { Isolok-HFR-12mL } \\
\end{array}$ & $\mathrm{MgO}(\mathrm{wt} \%)$ & 0.303 & 0.299 & $-1.6 \%$ & No & 0.01409 & 4.64 & 0.013 & 0.006 & No & 16 & 10 \\
\hline 4 & $\begin{array}{l}\text { Isolok-HFR-12mL } \\
\end{array}$ & $\mathrm{MnO}(\mathrm{wt} \%)$ & 2.766 & 2.710 & $-2.0 \%$ & No & 0.14082 & 5.09 & 0.140 & 0.067 & Yes & 16 & 10 \\
\hline 4 & Isolok-HFR-12mL & $\mathrm{Na} 2 \mathrm{O}$ (wt\%) & 13.269 & 13.218 & $-0.4 \%$ & No & 0.29491 & 2.22 & 0.303 & 0.243 & No & 16 & 9 \\
\hline 4 & $\begin{array}{l}\text { Isolok-HFR-12mL } \\
\end{array}$ & $\mathrm{NiO}(\mathrm{wt} \%)$ & 1.192 & 1.170 & $-1.8 \%$ & No & 0.07365 & 6.18 & 0.087 & 0.038 & Yes & 16 & 10 \\
\hline 4 & Isolok-HFR-12mL & SiO2 (wt\%) & 49.913 & 50.511 & $1.2 \%$ & No & 1.17157 & 2.35 & 0.943 & 0.434 & Yes & 16 & 9 \\
\hline 4 & Isolok-HFR-12mL & Sum (wt\%) & 99.067 & 99.209 & $0.1 \%$ & No & 0.87682 & 0.89 & 0.820 & 0.838 & No & 14 & 9 \\
\hline 4 & Isolok-HFR-12mL & TiO2 (wt\%) & 0.019 & 0.019 & $-1.4 \%$ & No & 0.00166 & 8.71 & 0.002 & 0.002 & No & 15 & 10 \\
\hline 4 & Isolok-HFR-12mL & $\mathrm{ZnO}(\mathrm{wt} \%)$ & 0.006 & 0.006 & $-3.6 \%$ & No & 0.00043 & 7.21 & 0.000 & 0.000 & No & 16 & 10 \\
\hline 4 & Isolok-HFR-12mL & ZrO2 (wt\%) & 0.111 & 0.111 & $0.1 \%$ & No & 0.00261 & 2.36 & 0.003 & 0.004 & No & 16 & 10 \\
\hline
\end{tabular}


SRNL-STI-2011-00693

Revision 0

Table B9. Oxide Comparisons Between Samplers for the High-Rheology (Phase 2) Testing (screened data)

\begin{tabular}{|c|c|c|c|c|c|c|c|c|c|c|c|c|c|}
\hline $\begin{array}{c}\text { Analytical } \\
\text { Block }\end{array}$ & Sampler & Oxide & $\begin{array}{c}\text { Mean(Measured } \\
\text { wt\%) Coliwasa } \\
\text { (low+high) }\end{array}$ & $\begin{array}{l}\text { Mean(Measured } \\
\text { wt\%) Other } \\
\text { Sampler }\end{array}$ & $\%$ Diff & $\begin{array}{l}\text { Sig. } \\
\text { Diff }\end{array}$ & $\begin{array}{l}\text { Bias } \\
\text { Bnd }\end{array}$ & $\begin{array}{l}\text { \% Rel } \\
\text { Bias } \\
\text { Bnd }\end{array}$ & $\begin{array}{c}\text { Std } \\
\text { Dev(Measured } \\
\text { wt\%) Coliwasa } \\
\text { (low+high) }\end{array}$ & $\begin{array}{c}\text { Std } \\
\text { Dev(Measured } \\
\text { wt\%) Other } \\
\text { Sampler }\end{array}$ & $\begin{array}{l}\text { Sig. } \\
\text { Diff }\end{array}$ & $\stackrel{\text { n }}{\text { Col }}$ & $\begin{array}{c}\text { n } \\
\text { Other }\end{array}$ \\
\hline 5 & Isolok-HFR-3mL & $\mathrm{Al} / \mathrm{B}$ & 2.402 & 2.417 & $0.6 \%$ & No & 0.07407 & 3.08 & 0.075 & 0.063 & No & 16 & 10 \\
\hline 5 & Isolok-HFR-3mL & Al2O3 (wt\%) & 10.975 & 10.942 & $-0.3 \%$ & No & 0.18639 & 1.70 & 0.221 & 0.087 & No & 15 & 10 \\
\hline 5 & Isolok-HFR-3mL & B2O3 (wt\%) & 4.548 & 4.497 & $-1.1 \%$ & No & 0.15878 & 3.49 & 0.139 & 0.094 & No & 16 & 9 \\
\hline 5 & Isolok-HFR-3mL & $\mathrm{BaO}(\mathrm{wt} \%)$ & 0.005 & 0.005 & $1.8 \%$ & No & 0.00029 & 6.14 & 0.000 & 0.000 & No & 16 & 10 \\
\hline 5 & Isolok-HFR-3mL & $\mathrm{CaO}(\mathrm{wt} \%)$ & 0.584 & 0.574 & $-1.8 \%$ & No & 0.02197 & 3.76 & 0.014 & 0.013 & No & 14 & 10 \\
\hline 5 & Isolok-HFR-3mL & Cr2O3 (wt\%) & 0.019 & 0.022 & $14.4 \%$ & Yes & 0.00437 & 22.83 & 0.002 & 0.002 & No & 16 & 10 \\
\hline 5 & Isolok-HFR-3mL & $\mathrm{CuO}(\mathrm{wt} \%)$ & 0.095 & 0.090 & $-5.2 \%$ & Yes & 0.00911 & 9.58 & 0.006 & 0.003 & No & 16 & 10 \\
\hline 5 & Isolok-HFR-3mL & $\mathrm{Fe} / \mathrm{Li}$ & 2.014 & 1.990 & $-1.2 \%$ & No & 0.06793 & 3.37 & 0.074 & 0.034 & Yes & 16 & 10 \\
\hline 5 & Isolok-HFR-3mL & Fe2O3 (wt\%) & 10.238 & 10.251 & $0.1 \%$ & No & 0.14309 & 1.40 & 0.219 & 0.081 & Yes & 15 & 10 \\
\hline 5 & Isolok-HFR-3mL & K2O (wt\%) & 0.169 & 0.169 & $-0.1 \%$ & No & 0.00569 & 3.36 & 0.007 & 0.006 & No & 16 & 9 \\
\hline 5 & Isolok-HFR-3mL & Li2O (wt\%) & 5.055 & 5.152 & $1.9 \%$ & Yes & 0.18516 & 3.66 & 0.112 & 0.096 & No & 16 & 10 \\
\hline 5 & Isolok-HFR-3mL & $\mathrm{MgO}(\mathrm{wt} \%)$ & 0.310 & 0.309 & $-0.6 \%$ & No & 0.00741 & 2.39 & 0.007 & 0.004 & No & 14 & 9 \\
\hline 5 & Isolok-HFR-3mL & $\mathrm{MnO}(\mathrm{wt} \%)$ & 2.804 & 2.819 & $0.5 \%$ & No & 0.08663 & 3.09 & 0.125 & 0.035 & Yes & 15 & 9 \\
\hline 5 & Isolok-HFR-3mL & $\mathrm{Na2O}$ (wt\%) & 13.243 & 13.147 & $-0.7 \%$ & No & 0.36851 & 2.78 & 0.386 & 0.195 & No & 16 & 10 \\
\hline 5 & Isolok-HFR-3mL & $\mathrm{NiO}(\mathrm{wt} \%)$ & 1.194 & 1.208 & $1.2 \%$ & No & 0.06082 & 5.09 & 0.084 & 0.024 & Yes & 16 & 10 \\
\hline 5 & Isolok-HFR-3mL & $\mathrm{SiO} 2$ (wt\%) & 50.088 & 50.744 & $1.3 \%$ & No & 1.4126 & 2.82 & 0.788 & 1.042 & No & 15 & 10 \\
\hline 5 & Isolok-HFR-3mL & Sum (wt\%) & 99.430 & 100.077 & $0.7 \%$ & No & 1.9057 & 1.92 & 1.621 & 1.259 & No & 15 & 10 \\
\hline 5 & Isolok-HFR-3mL & $\mathrm{TiO} 2(\mathrm{wt} \%)$ & 0.016 & 0.016 & $1.5 \%$ & No & 0.00085 & 5.28 & 0.001 & 0.000 & Yes & 15 & 9 \\
\hline 5 & Isolok-HFR-3mL & $\mathrm{ZnO}$ (wt\%) & 0.006 & 0.006 & $-1.1 \%$ & No & 0.00027 & 4.53 & 0.000 & 0.000 & No & 16 & 10 \\
\hline 5 & Isolok-HFR-3mL & ZrO2 (wt\%) & 0.111 & 0.109 & $-1.1 \%$ & No & 0.00351 & 3.18 & 0.003 & 0.002 & No & 16 & 10 \\
\hline 6 & Hydragard-LFR & $\mathrm{Al} / \mathrm{B}$ & 2.415 & 2.537 & $5.0 \%$ & Yes & 0.18303 & 7.58 & 0.0728 & 0.0760 & No & 16 & 10 \\
\hline 6 & Hydragard-LFR & Al2O3 (wt\%) & 10.947 & 11.099 & $1.4 \%$ & No & 0.34459 & 3.15 & 0.2260 & 0.2299 & No & 15 & 10 \\
\hline 6 & Hydragard-LFR & B2O3 (wt\%) & 4.512 & 4.379 & $-2.9 \%$ & No & 0.26873 & 5.96 & 0.1550 & 0.1764 & No & 16 & 10 \\
\hline 6 & Hydragard-LFR & $\mathrm{BaO}(\mathrm{wt} \%)$ & 0.005 & 0.010 & $113.5 \%$ & Yes & 0.008 & 169.14 & 0.0004 & 0.0037 & Yes & 16 & 10 \\
\hline 6 & Hydragard-LFR & $\mathrm{CaO}(\mathrm{wt} \%)$ & 0.592 & 0.594 & $0.3 \%$ & No & 0.01569 & 2.65 & 0.0151 & 0.0178 & No & 14 & 10 \\
\hline 6 & Hydragard-LFR & $\mathrm{Cr} 2 \mathrm{O} 3(\mathrm{wt} \%)$ & 0.020 & 0.018 & $-6.6 \%$ & No & 0.00274 & 14.01 & 0.0016 & 0.0019 & No & 16 & 9 \\
\hline 6 & Hydragard-LFR & $\mathrm{CuO}(\mathrm{wt} \%)$ & 0.096 & 0.095 & $-1.1 \%$ & No & 0.0041 & 4.26 & 0.0038 & 0.0032 & No & 15 & 10 \\
\hline 6 & Hydragard-LFR & $\mathrm{Fe} / \mathrm{Li}$ & 2.026 & 2.031 & $0.3 \%$ & No & 0.05157 & 2.55 & 0.0593 & 0.0411 & No & 16 & 9 \\
\hline 6 & Hydragard-LFR & Fe2O3 (wt\%) & 10.312 & 10.407 & $0.9 \%$ & No & 0.28959 & 2.81 & 0.2018 & 0.2694 & No & 15 & 10 \\
\hline 6 & Hydragard-LFR & K2O (wt\%) & 0.169 & 0.168 & $-0.6 \%$ & No & 0.00654 & 3.86 & 0.0067 & 0.0063 & No & 16 & 10 \\
\hline 6 & Hydragard-LFR & Li2O (wt\%) & 5.088 & 5.077 & $-0.2 \%$ & No & 0.10411 & 2.05 & 0.1100 & 0.1092 & No & 15 & 10 \\
\hline 6 & Hydragard-LFR & $\mathrm{MgO}(\mathrm{wt} \%)$ & 0.310 & 0.312 & $0.6 \%$ & No & 0.01081 & 3.48 & 0.0111 & 0.0098 & No & 15 & 10 \\
\hline 6 & Hydragard-LFR & $\mathrm{MnO}(\mathrm{wt} \%)$ & 2.823 & 2.824 & $0.0 \%$ & No & 0.08047 & 2.85 & 0.0884 & 0.0937 & No & 13 & 10 \\
\hline 6 & Hydragard-LFR & $\mathrm{Na} 2 \mathrm{O}$ (wt\%) & 13.176 & 13.186 & $0.1 \%$ & No & 0.33555 & 2.55 & 0.4045 & 0.3542 & No & 15 & 10 \\
\hline 6 & Hydragard-LFR & $\mathrm{NiO}$ (wt\%) & 1.189 & 1.181 & $-0.6 \%$ & No & 0.06781 & 5.70 & 0.0832 & 0.0497 & No & 16 & 10 \\
\hline 6 & Hydragard-LFR & SiO2 (wt\%) & 49.974 & 49.568 & $-0.8 \%$ & No & 1.0822 & 2.17 & 0.7040 & 0.9300 & No & 15 & 10 \\
\hline 6 & Hydragard-LFR & Sum (wt\%) & 99.602 & 99.052 & $-0.6 \%$ & No & 1.7993 & 1.81 & 0.9915 & 1.6350 & Yes & 14 & 10 \\
\hline 6 & Hydragard-LFR & TiO2 (wt\%) & 0.016 & 0.016 & $-1.6 \%$ & No & 0.00162 & 10.01 & 0.0017 & 0.0016 & No & 16 & 10 \\
\hline 6 & Hydragard-LFR & $\mathrm{ZnO}$ (wt\%) & 0.006 & 0.006 & $-1.0 \%$ & No & 0.00026 & 4.36 & 0.0002 & 0.0002 & No & 16 & 9 \\
\hline 6 & Hydragard-LFR & ZrO2 (wt\%) & 0.109 & 0.110 & $0.4 \%$ & No & 0.00285 & 2.61 & 0.0030 & 0.0026 & No & 16 & 10 \\
\hline
\end{tabular}


SRNL-STI-2011-00693

Revision 0

Table B10. Phase 2 (High-Rheology) Sampler Comparions Grouped by Analytical Block (screened data)

\begin{tabular}{|c|c|c|c|c|c|c|c|c|c|c|c|c|c|c|}
\hline $\begin{array}{c}\text { Analytical } \\
\text { Block }\end{array}$ & Sampler & Oxide & $\begin{array}{c}\text { Mean(Measured } \\
\text { wt\%) Coliwasa } \\
\text { (low+high) }\end{array}$ & $\begin{array}{c}\text { Mean(Measured } \\
\text { wt\%) Other } \\
\text { Sampler } \\
\end{array}$ & Diff & $\%$ Diff & $\begin{array}{l}\text { Sig. } \\
\text { Diff }\end{array}$ & $\begin{array}{l}\text { Bias } \\
\text { Bnd }\end{array}$ & $\begin{array}{l}\text { \% Rel } \\
\text { Bias } \\
\text { Bnd } \\
\end{array}$ & $\begin{array}{c}\text { Std Dev(Measured } \\
\text { wt\%) Coliwasa } \\
\text { (low+high) }\end{array}$ & $\begin{array}{c}\text { Std Dev(Measured } \\
\text { wt\%) Other } \\
\text { Sampler } \\
\end{array}$ & $\begin{array}{l}\text { Sig. } \\
\text { Diff }\end{array}$ & $\begin{array}{c}\mathbf{n} \\
\text { Col } \\
\end{array}$ & $\begin{array}{c}\text { n } \\
\text { Other }\end{array}$ \\
\hline 2 & Hydragard - HFR & $\mathrm{Al} / \mathrm{B}$ & 2.378 & 2.603 & 0.226 & $9.5 \%$ & Yes & 0.300 & 12.64 & 0.073 & 0.112 & No & 16 & 10 \\
\hline 1 & Isolok-LFR-3mL & $\mathrm{Al} / \mathrm{B}$ & 2.429 & 2.598 & 0.168 & $6.9 \%$ & Yes & 0.248 & 10.19 & 0.096 & 0.093 & No & 16 & 10 \\
\hline 3 & Isolok-LFR-12mL & $\mathrm{Al} / \mathrm{B}$ & 2.286 & 2.424 & 0.138 & $6.0 \%$ & Yes & 0.206 & 9.00 & 0.071 & 0.097 & No & 16 & 10 \\
\hline 6 & Hydragard-LFR & $\mathrm{Al} / \mathrm{B}$ & 2.415 & 2.537 & 0.121 & $5.0 \%$ & Yes & 0.183 & 7.58 & 0.073 & 0.076 & No & 16 & 10 \\
\hline 4 & Isolok-HFR-12mL & $\mathrm{Al} / \mathrm{B}$ & 2.427 & 2.538 & 0.111 & $4.6 \%$ & Yes & 0.177 & 7.31 & 0.076 & 0.086 & No & 16 & 10 \\
\hline 5 & Isolok-HFR-3mL & $\mathrm{Al} / \mathrm{B}$ & 2.402 & 2.417 & 0.015 & $0.6 \%$ & No & 0.074 & 3.08 & 0.075 & 0.063 & No & 16 & 10 \\
\hline 1 & Isolok-LFR-3mL & Al2O3 (wt\%) & 11.115 & 11.518 & 0.403 & $3.6 \%$ & Yes & 0.699 & 6.29 & 0.415 & 0.216 & No & 15 & 10 \\
\hline 2 & Hydragard - HFR & $\mathrm{Al} 2 \mathrm{O} 3(\mathrm{wt} \%)$ & 10.852 & 11.339 & 0.487 & $4.5 \%$ & Yes & 0.659 & 6.07 & 0.276 & 0.137 & Yes & 16 & 9 \\
\hline 3 & Isolok-LFR-12mL & $\mathrm{Al} 2 \mathrm{O} 3(\mathrm{wt} \%)$ & 10.863 & 11.091 & 0.228 & $2.1 \%$ & Yes & 0.380 & 3.50 & 0.251 & 0.113 & Yes & 16 & 9 \\
\hline 6 & Hydragard-LFR & Al2O3 (wt\%) & 10.947 & 11.099 & 0.152 & $1.4 \%$ & No & 0.345 & 3.15 & 0.226 & 0.230 & No & 15 & 10 \\
\hline 4 & Isolok-HFR-12mL & $\mathrm{Al} 2 \mathrm{O} 3(\mathrm{wt} \%)$ & 10.817 & 10.772 & -0.045 & $-0.4 \%$ & No & 0.211 & 1.95 & 0.297 & 0.086 & Yes & 16 & 9 \\
\hline 5 & Isolok-HFR-3mL & $\mathrm{Al} 2 \mathrm{O} 3(\mathrm{wt} \%)$ & 10.975 & 10.942 & -0.033 & $-0.3 \%$ & No & 0.186 & 1.70 & 0.221 & 0.087 & No & 15 & 10 \\
\hline 4 & Isolok-HFR-12mL & $\mathrm{B} 2 \mathrm{O} 3(\mathrm{wt} \%)$ & 4.460 & 4.228 & -0.232 & $-5.2 \%$ & Yes & 0.354 & 7.94 & 0.151 & 0.138 & No & 16 & 10 \\
\hline 3 & Isolok-LFR-12mL & B2O3 (wt\%) & 4.778 & 4.553 & -0.225 & $-4.7 \%$ & Yes & 0.377 & 7.89 & 0.102 & 0.204 & Yes & 15 & 10 \\
\hline 2 & Hydragard - HFR & B2O3 (wt\%) & 4.566 & 4.318 & -0.248 & $-5.4 \%$ & Yes & 0.358 & 7.84 & 0.112 & 0.159 & No & 16 & 10 \\
\hline 6 & Hydragard-LFR & B2O3 (wt\%) & 4.512 & 4.379 & -0.133 & $-2.9 \%$ & No & 0.269 & 5.96 & 0.155 & 0.176 & No & 16 & 10 \\
\hline 1 & Isolok-LFR-3mL & B2O3 (wt\%) & 4.548 & 4.437 & -0.111 & $-2.4 \%$ & No & 0.236 & 5.20 & 0.196 & 0.113 & Yes & 16 & 10 \\
\hline 5 & Isolok-HFR-3mL & B2O3 (wt\%) & 4.548 & 4.497 & -0.051 & $-1.1 \%$ & No & 0.159 & 3.49 & 0.139 & 0.094 & No & 16 & 9 \\
\hline 3 & Isolok-LFR-12mL & $\mathrm{BaO}(\mathrm{wt} \%)$ & 0.004 & 0.012 & 0.008 & $201.3 \%$ & Yes & 0.008 & 216.95 & 0.000 & 0.001 & Yes & 16 & 10 \\
\hline 6 & Hydragard-LFR & $\mathrm{BaO}(\mathrm{wt} \%)$ & 0.005 & 0.010 & 0.005 & $113.5 \%$ & Yes & 0.008 & 169.14 & 0.000 & 0.004 & Yes & 16 & 10 \\
\hline 4 & Isolok-HFR-12mL & $\mathrm{BaO}(\mathrm{wt} \%)$ & 0.006 & 0.013 & 0.007 & $132.1 \%$ & Yes & 0.008 & 141.63 & 0.000 & 0.001 & No & 16 & 9 \\
\hline 2 & Hydragard - HFR & $\mathrm{BaO}$ (wt\%) & 0.006 & 0.006 & 0.000 & $2.3 \%$ & No & 0.001 & 10.86 & 0.001 & 0.001 & No & 16 & 10 \\
\hline 1 & Isolok-LFR-3mL & $\mathrm{BaO}(\mathrm{wt} \%)$ & 0.006 & 0.006 & 0.000 & $0.0 \%$ & No & 0.001 & 10.28 & 0.001 & 0.001 & No & 16 & 10 \\
\hline 5 & Isolok-HFR-3mL & $\mathrm{BaO}(\mathrm{wt} \%)$ & 0.005 & 0.005 & 0.000 & $1.8 \%$ & No & 0.000 & 6.14 & 0.000 & 0.000 & No & 16 & 10 \\
\hline 2 & Hydragard - HFR & $\mathrm{CaO}(\mathrm{wt} \%)$ & 0.571 & 0.582 & 0.012 & $2.1 \%$ & No & 0.029 & 5.08 & 0.017 & 0.024 & No & 15 & 10 \\
\hline 1 & Isolok-LFR-3mL & $\mathrm{CaO}$ (wt\%) & 0.605 & 0.619 & 0.014 & $2.3 \%$ & No & 0.030 & 5.03 & 0.027 & 0.010 & Yes & 15 & 8 \\
\hline 4 & Isolok-HFR-12mL & $\mathrm{CaO}(\mathrm{wt} \%)$ & 0.587 & 0.580 & -0.007 & $-1.3 \%$ & No & 0.024 & 4.09 & 0.021 & 0.017 & No & 16 & 10 \\
\hline 5 & Isolok-HFR-3mL & $\mathrm{CaO}$ (wt\%) & 0.584 & 0.574 & -0.010 & $-1.8 \%$ & No & 0.022 & 3.76 & 0.014 & 0.013 & No & 14 & 10 \\
\hline 3 & Isolok-LFR-12mL & $\mathrm{CaO}$ (wt\%) & 0.591 & 0.599 & 0.008 & $1.3 \%$ & No & 0.020 & 3.34 & 0.015 & 0.012 & No & 15 & 10 \\
\hline 6 & Hydragard-LFR & $\mathrm{CaO}(\mathrm{wt} \%)$ & 0.592 & 0.594 & 0.002 & $0.3 \%$ & No & 0.016 & 2.65 & 0.015 & 0.018 & No & 14 & 10 \\
\hline 4 & Isolok-HFR-12mL & Cr2O3 (wt\%) & 0.020 & 0.026 & 0.006 & $32.0 \%$ & No & 0.008 & 39.69 & 0.001 & 0.003 & No & 15 & 10 \\
\hline 2 & Hydragard - HFR & Cr2O3 (wt\%) & 0.018 & 0.022 & 0.004 & $19.3 \%$ & Yes & 0.005 & 27.49 & 0.002 & 0.002 & No & 16 & 9 \\
\hline 5 & Isolok-HFR-3mL & Cr2O3 (wt\%) & 0.019 & 0.022 & 0.003 & $14.4 \%$ & Yes & 0.004 & 22.83 & 0.002 & 0.002 & No & 16 & 10 \\
\hline 1 & Isolok-LFR-3mL & Cr2O3 (wt\%) & 0.019 & 0.020 & 0.001 & $3.2 \%$ & No & 0.003 & 16.10 & 0.001 & 0.003 & Yes & 15 & 10 \\
\hline 6 & Hydragard-LFR & Cr2O3 (wt\%) & 0.020 & 0.018 & -0.001 & $-6.6 \%$ & No & 0.003 & 14.01 & 0.002 & 0.002 & No & 16 & 9 \\
\hline 3 & Isolok-LFR-12mL & Cr2O3 (wt\%) & 0.020 & 0.021 & 0.000 & $1.9 \%$ & Yes & 0.002 & 11.52 & 0.002 & 0.003 & No & 16 & 10 \\
\hline 1 & Isolok-LFR-3mL & $\mathrm{CuO}(\mathrm{wt} \%)$ & 0.096 & 0.092 & -0.004 & $-3.9 \%$ & No & 0.009 & 9.80 & 0.007 & 0.007 & No & 16 & 10 \\
\hline 5 & Isolok-HFR-3mL & $\mathrm{CuO}(\mathrm{wt} \%)$ & 0.095 & 0.090 & -0.005 & $-5.2 \%$ & Yes & 0.009 & 9.58 & 0.006 & 0.003 & No & 16 & 10 \\
\hline 4 & Isolok-HFR-12mL & $\mathrm{CuO}(\mathrm{wt} \%)$ & 0.091 & 0.086 & -0.006 & $-6.3 \%$ & Yes & 0.009 & 9.54 & 0.005 & 0.002 & Yes & 16 & 9 \\
\hline 3 & Isolok-LFR-12mL & $\mathrm{CuO}(\mathrm{wt} \%)$ & 0.095 & 0.092 & -0.002 & $-2.5 \%$ & No & 0.005 & 5.07 & 0.004 & 0.002 & Yes & 16 & 9 \\
\hline 6 & Hydragard-LFR & $\mathrm{CuO}(\mathrm{wt} \%)$ & 0.096 & 0.095 & -0.001 & $-1.1 \%$ & No & 0.004 & 4.26 & 0.004 & 0.003 & No & 15 & 10 \\
\hline
\end{tabular}


SRNL-STI-2011-00693

Revision 0

Table B10. Phase 2 (High-Rheology) Sampler Comparions Grouped by Analytical Block (screened data)

\begin{tabular}{|c|c|c|c|c|c|c|c|c|c|c|c|c|c|c|}
\hline $\begin{array}{c}\text { Analytical } \\
\text { Block } \\
2\end{array}$ & $\begin{array}{c}\text { Sampler } \\
\text { Hydragard - HFR }\end{array}$ & $\begin{array}{c}\text { Oxide } \\
\mathrm{CuO} \text { (wt\%) }\end{array}$ & $\begin{array}{c}\text { Mean(Measured } \\
\text { wt\%) Coliwasa } \\
\text { (low+high) } \\
0.095 \\
\end{array}$ & $\begin{array}{c}\text { Mean(Measured } \\
\text { wt\%) Other } \\
\text { Sampler } \\
0.095 \\
\end{array}$ & $\begin{array}{c}\text { Diff } \\
0.000\end{array}$ & $\begin{array}{c}\text { \% Diff } \\
-0.1 \% \\
\end{array}$ & $\begin{array}{l}\text { Sig. } \\
\text { Diff } \\
\text { No }\end{array}$ & $\begin{array}{c}\text { Bias } \\
\text { Bnd } \\
0.004 \\
\end{array}$ & 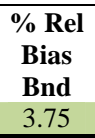 & $\begin{array}{c}\text { Std Dev(Measured } \\
\text { wt\%) Coliwasa } \\
\text { (low+high) } \\
0.004 \\
\end{array}$ & $\begin{array}{c}\text { Std Dev(Measured } \\
\text { wt\%) Other } \\
\text { Sampler } \\
0.002 \\
\end{array}$ & $\begin{array}{l}\text { Sig. } \\
\text { Diff } \\
\text { No }\end{array}$ & $\begin{array}{c}\mathbf{n} \\
\text { Col } \\
16 \\
\end{array}$ & $\begin{array}{c}\text { n } \\
\text { Other } \\
8 \\
\end{array}$ \\
\hline 2 & Hydragard - HFR & $\mathrm{Fe} / \mathrm{Li}$ & 1.971 & 2.126 & 0.154 & $7.8 \%$ & Yes & 0.222 & 11.27 & 0.075 & 0.091 & No & 16 & 10 \\
\hline 1 & Isolok-LFR-3mL & $\mathrm{Fe} / \mathrm{Li}$ & 2.035 & 2.106 & 0.071 & $3.5 \%$ & Yes & 0.128 & 6.29 & 0.078 & 0.048 & No & 16 & 10 \\
\hline 5 & Isolok-HFR-3mL & $\mathrm{Fe} / \mathrm{Li}$ & 2.014 & 1.990 & -0.024 & $-1.2 \%$ & No & 0.068 & 3.37 & 0.074 & 0.034 & Yes & 16 & 10 \\
\hline 4 & Isolok-HFR-12mL & $\mathrm{Fe} / \mathrm{Li}$ & 2.011 & 1.993 & -0.018 & $-0.9 \%$ & No & 0.065 & 3.21 & 0.064 & 0.039 & No & 16 & 10 \\
\hline 3 & Isolok-LFR-12mL & $\mathrm{Fe} / \mathrm{Li}$ & 1.972 & 1.979 & 0.007 & $0.4 \%$ & No & 0.056 & 2.85 & 0.081 & 0.040 & Yes & 16 & 10 \\
\hline 6 & Hydragard-LFR & $\mathrm{Fe} / \mathrm{Li}$ & 2.026 & 2.031 & 0.005 & $0.3 \%$ & No & 0.052 & 2.55 & 0.059 & 0.041 & No & 16 & 9 \\
\hline 2 & Hydragard - HFR & Fe2O3 (wt\%) & 10.175 & 10.767 & 0.592 & $5.8 \%$ & Yes & 0.825 & 8.11 & 0.287 & 0.236 & No & 16 & 9 \\
\hline 1 & Isolok-LFR-3mL & Fe2O3 (wt\%) & 10.440 & 10.781 & 0.341 & $3.3 \%$ & Yes & 0.532 & 5.10 & 0.246 & 0.175 & No & 13 & 10 \\
\hline 3 & Isolok-LFR-12mL & Fe2O3 (wt\%) & 10.158 & 10.353 & 0.195 & $1.9 \%$ & No & 0.359 & 3.54 & 0.270 & 0.127 & Yes & 16 & 9 \\
\hline 6 & Hydragard-LFR & Fe2O3 (wt\%) & 10.312 & 10.407 & 0.095 & $0.9 \%$ & No & 0.290 & 2.81 & 0.202 & 0.269 & No & 15 & 10 \\
\hline 4 & Isolok-HFR-12mL & Fe2O3 (wt\%) & 10.163 & 10.138 & -0.025 & $-0.2 \%$ & No & 0.240 & 2.36 & 0.302 & 0.157 & No & 16 & 10 \\
\hline 5 & Isolok-HFR-3mL & Fe2O3 (wt\%) & 10.238 & 10.251 & 0.013 & $0.1 \%$ & No & 0.143 & 1.40 & 0.219 & 0.081 & Yes & 15 & 10 \\
\hline 4 & Isolok-HFR-12mL & $\mathrm{K} 2 \mathrm{O}(\mathrm{wt} \%)$ & 0.169 & 0.163 & -0.006 & $-3.7 \%$ & Yes & 0.012 & 7.38 & 0.007 & 0.009 & No & 16 & 10 \\
\hline 1 & Isolok-LFR-3mL & $\mathrm{K} 2 \mathrm{O}(\mathrm{wt} \%)$ & 0.168 & 0.163 & -0.005 & $-3.0 \%$ & No & 0.011 & 6.75 & 0.007 & 0.009 & No & 16 & 10 \\
\hline 2 & Hydragard - HFR & K2O (wt\%) & 0.169 & 0.167 & -0.002 & $-1.5 \%$ & No & 0.009 & 5.33 & 0.007 & 0.009 & No & 16 & 9 \\
\hline 6 & Hydragard-LFR & K2O (wt\%) & 0.169 & 0.168 & -0.001 & $-0.6 \%$ & No & 0.007 & 3.86 & 0.007 & 0.006 & No & 16 & 10 \\
\hline 5 & Isolok-HFR-3mL & K2O (wt\%) & 0.169 & 0.169 & 0.000 & $-0.1 \%$ & No & 0.006 & 3.36 & 0.007 & 0.006 & No & 16 & 9 \\
\hline 3 & Isolok-LFR-12mL & $\mathrm{K} 2 \mathrm{O}(\mathrm{wt} \%)$ & 0.169 & 0.168 & -0.001 & $-0.9 \%$ & No & 0.005 & 3.08 & 0.007 & 0.002 & Yes & 16 & 10 \\
\hline 2 & Hydragard - HFR & Li2O (wt\%) & 5.166 & 5.045 & -0.121 & $-2.3 \%$ & No & 0.247 & 4.79 & 0.148 & 0.145 & No & 16 & 9 \\
\hline 5 & Isolok-HFR-3mL & $\mathrm{Li} 2 \mathrm{O}$ (wt\%) & 5.055 & 5.152 & 0.097 & $1.9 \%$ & Yes & 0.185 & 3.66 & 0.112 & 0.096 & No & 16 & 10 \\
\hline 3 & Isolok-LFR-12mL & Li2O (wt\%) & 5.158 & 5.195 & 0.037 & $0.7 \%$ & No & 0.177 & 3.42 & 0.182 & 0.138 & No & 16 & 10 \\
\hline 4 & Isolok-HFR-12mL & Li2O (wt\%) & 5.055 & 5.089 & 0.034 & $0.7 \%$ & No & 0.137 & 2.72 & 0.114 & 0.139 & No & 16 & 10 \\
\hline 1 & Isolok-LFR-3mL & Li2O (wt\%) & 5.088 & 5.100 & 0.012 & $0.2 \%$ & No & 0.129 & 2.53 & 0.214 & 0.041 & Yes & 16 & 9 \\
\hline 6 & Hydragard-LFR & $\mathrm{Li} 2 \mathrm{O}$ (wt\%) & 5.088 & 5.077 & -0.011 & $-0.2 \%$ & No & 0.104 & 2.05 & 0.110 & 0.109 & No & 15 & 10 \\
\hline 1 & Isolok-LFR-3mL & $\mathrm{MgO}(\mathrm{wt} \%)$ & 0.310 & 0.323 & 0.013 & $4.1 \%$ & Yes & 0.024 & 7.76 & 0.016 & 0.008 & No & 16 & 10 \\
\hline 2 & Hydragard - HFR & $\mathrm{MgO}(\mathrm{wt} \%)$ & 0.307 & 0.316 & 0.009 & $2.9 \%$ & No & 0.021 & 6.78 & 0.014 & 0.015 & No & 16 & 10 \\
\hline 4 & Isolok-HFR-12mL & $\mathrm{MgO}(\mathrm{wt} \%)$ & 0.303 & 0.299 & -0.005 & $-1.6 \%$ & No & 0.014 & 4.64 & 0.013 & 0.006 & No & 16 & 10 \\
\hline 3 & Isolok-LFR-12mL & $\mathrm{MgO}(\mathrm{wt} \%)$ & 0.311 & 0.316 & 0.005 & $1.5 \%$ & No & 0.012 & 3.72 & 0.008 & 0.008 & No & 15 & 10 \\
\hline 6 & Hydragard-LFR & $\mathrm{MgO}(\mathrm{wt} \%)$ & 0.310 & 0.312 & 0.002 & $0.6 \%$ & No & 0.011 & 3.48 & 0.011 & 0.010 & No & 15 & 10 \\
\hline 5 & Isolok-HFR-3mL & $\mathrm{MgO}(\mathrm{wt} \%)$ & 0.310 & 0.309 & -0.002 & $-0.6 \%$ & No & 0.007 & 2.39 & 0.007 & 0.004 & No & 14 & 9 \\
\hline 2 & Hydragard - HFR & $\mathrm{MnO}(\mathrm{wt} \%)$ & 2.816 & 2.934 & 0.117 & $4.2 \%$ & No & 0.254 & 9.03 & 0.146 & 0.193 & No & 16 & 10 \\
\hline 1 & Isolok-LFR-3mL & $\mathrm{MnO}(\mathrm{wt} \%)$ & 2.808 & 2.936 & 0.129 & $4.6 \%$ & Yes & 0.226 & 8.04 & 0.163 & 0.073 & Yes & 16 & 10 \\
\hline 4 & Isolok-HFR-12mL & $\mathrm{MnO}(\mathrm{wt} \%)$ & 2.766 & 2.710 & -0.056 & $-2.0 \%$ & No & 0.141 & 5.09 & 0.140 & 0.067 & Yes & 16 & 10 \\
\hline 3 & Isolok-LFR-12mL & $\mathrm{MnO}(\mathrm{wt} \%)$ & 2.833 & 2.879 & 0.047 & $1.7 \%$ & No & 0.144 & 5.08 & 0.131 & 0.088 & No & 16 & 10 \\
\hline 5 & Isolok-HFR-3mL & $\mathrm{MnO}(\mathrm{wt} \%)$ & 2.804 & 2.819 & 0.015 & $0.5 \%$ & No & 0.087 & 3.09 & 0.125 & 0.035 & Yes & 15 & 9 \\
\hline 6 & Hydragard-LFR & $\mathrm{MnO}(\mathrm{wt} \%)$ & 2.823 & 2.824 & 0.001 & $0.0 \%$ & No & 0.080 & 2.85 & 0.088 & 0.094 & No & 13 & 10 \\
\hline 3 & Isolok-LFR-12mL & $\mathrm{Na} 2 \mathrm{O}$ (wt\%) & 13.327 & 13.493 & 0.167 & $1.3 \%$ & No & 0.522 & 3.92 & 0.444 & 0.397 & No & 16 & 10 \\
\hline 1 & Isolok-LFR-3mL & $\mathrm{Na2O}$ (wt\%) & 13.710 & 13.766 & 0.056 & $0.4 \%$ & No & 0.442 & 3.22 & 0.682 & 0.226 & Yes & 16 & 10 \\
\hline 2 & Hydragard - HFR & Na2O (wt\%) & 13.439 & 13.317 & -0.122 & $-0.9 \%$ & No & 0.397 & 2.96 & 0.353 & 0.154 & No & 15 & 8 \\
\hline 5 & Isolok-HFR-3mL & $\mathrm{Na} 2 \mathrm{O}$ (wt\%) & 13.243 & 13.147 & -0.096 & $-0.7 \%$ & No & 0.369 & 2.78 & 0.386 & 0.195 & No & 16 & 10 \\
\hline
\end{tabular}


SRNL-STI-2011-00693

Revision 0

Table B10. Phase 2 (High-Rheology) Sampler Comparions Grouped by Analytical Block (screened data)

\begin{tabular}{|c|c|c|c|c|c|c|c|c|c|c|c|c|c|c|}
\hline $\begin{array}{c}\text { Analytical } \\
\text { Block } \\
6\end{array}$ & $\begin{array}{c}\text { Sampler } \\
\text { Hydragard-LFR }\end{array}$ & $\begin{array}{c}\text { Oxide } \\
\text { Na2O (wt\%) }\end{array}$ & $\begin{array}{c}\text { Mean(Measured } \\
\text { wt\%) Coliwasa } \\
\text { (low+high) } \\
13.176\end{array}$ & $\begin{array}{c}\text { Mean(Measured } \\
\text { wt\%) Other } \\
\text { Sampler } \\
13.186\end{array}$ & $\begin{array}{c}\text { Diff } \\
0.010\end{array}$ & $\begin{array}{c}\text { \% Diff } \\
0.1 \% \\
\end{array}$ & $\begin{array}{l}\text { Sig. } \\
\text { Diff } \\
\text { No }\end{array}$ & $\begin{array}{c}\text { Bias } \\
\text { Bnd } \\
0.336 \\
\end{array}$ & $\begin{array}{l}\text { \% Rel } \\
\text { Bias } \\
\text { Bnd } \\
2.55 \\
\end{array}$ & $\begin{array}{c}\text { Std Dev(Measured } \\
\text { wt\%) Coliwasa } \\
\text { (low+high) } \\
0.405 \\
\end{array}$ & $\begin{array}{c}\text { Std Dev(Measured } \\
\text { wt\%) Other } \\
\text { Sampler } \\
0.354 \\
\end{array}$ & $\begin{array}{l}\text { Sig. } \\
\text { Diff } \\
\text { No }\end{array}$ & $\begin{array}{c}\mathbf{n} \\
\text { Col } \\
15 \\
\end{array}$ & $\begin{array}{c}\mathbf{n} \\
\text { Other } \\
10 \\
\end{array}$ \\
\hline 4 & Isolok-HFR-12mL & $\mathrm{Na} 2 \mathrm{O}(\mathrm{wt} \%)$ & 13.269 & 13.218 & -0.051 & $-0.4 \%$ & No & 0.295 & 2.22 & 0.303 & 0.243 & No & 16 & 9 \\
\hline 2 & Hydragard - HFR & $\mathrm{NiO}(\mathrm{wt} \%)$ & 1.199 & 1.268 & 0.069 & $5.8 \%$ & No & 0.156 & 13.02 & 0.088 & 0.128 & No & 16 & 10 \\
\hline 1 & Isolok-LFR-3mL & $\mathrm{NiO}(\mathrm{wt} \%)$ & 1.209 & 1.247 & 0.038 & $3.1 \%$ & No & 0.102 & 8.45 & 0.092 & 0.043 & No & 16 & 10 \\
\hline 3 & Isolok-LFR-12mL & $\mathrm{NiO}$ (wt\%) & 1.176 & 1.187 & 0.011 & $1.0 \%$ & No & 0.074 & 6.26 & 0.071 & 0.078 & No & 15 & 10 \\
\hline 4 & Isolok-HFR-12mL & $\mathrm{NiO}(\mathrm{wt} \%)$ & 1.192 & 1.170 & -0.022 & $-1.8 \%$ & No & 0.074 & 6.18 & 0.087 & 0.038 & Yes & 16 & 10 \\
\hline 6 & Hydragard-LFR & $\mathrm{NiO}$ (wt\%) & 1.189 & 1.181 & -0.007 & $-0.6 \%$ & No & 0.068 & 5.70 & 0.083 & 0.050 & No & 16 & 10 \\
\hline 5 & Isolok-HFR-3mL & $\mathrm{NiO}(\mathrm{wt} \%)$ & 1.194 & 1.208 & 0.014 & $1.2 \%$ & No & 0.061 & 5.09 & 0.084 & 0.024 & Yes & 16 & 10 \\
\hline 2 & Hydragard - HFR & $\mathrm{SiO} 2$ (wt\%) & 49.699 & 48.610 & -1.089 & $-2.2 \%$ & Yes & 1.752 & 3.52 & 0.818 & 0.666 & No & 16 & 9 \\
\hline 3 & Isolok-LFR-12mL & $\mathrm{SiO} 2$ (wt\%) & 50.102 & 49.118 & -0.984 & $-2.0 \%$ & Yes & 1.759 & 3.51 & 0.797 & 1.078 & No & 15 & 10 \\
\hline 5 & Isolok-HFR-3mL & $\mathrm{SiO} 2$ (wt\%) & 50.088 & 50.744 & 0.656 & $1.3 \%$ & No & 1.413 & 2.82 & 0.788 & 1.042 & No & 15 & 10 \\
\hline 4 & Isolok-HFR-12mL & $\mathrm{SiO} 2(\mathrm{wt} \%)$ & 49.913 & 50.511 & 0.599 & $1.2 \%$ & No & 1.172 & 2.35 & 0.943 & 0.434 & Yes & 16 & 9 \\
\hline 6 & Hydragard-LFR & SiO2 (wt\%) & 49.974 & 49.568 & -0.406 & $-0.8 \%$ & No & 1.082 & 2.17 & 0.704 & 0.930 & No & 15 & 10 \\
\hline 1 & Isolok-LFR-3mL & $\mathrm{SiO} 2(\mathrm{wt} \%)$ & 50.880 & 51.391 & 0.511 & $1.0 \%$ & No & 0.984 & 1.93 & 0.682 & 0.317 & Yes & 12 & 9 \\
\hline 1 & Isolok-LFR-3mL & Sum (wt\%) & 101.568 & 102.646 & 1.078 & $1.1 \%$ & No & 2.345 & 2.31 & 1.687 & 0.800 & No & 13 & 9 \\
\hline 5 & Isolok-HFR-3mL & Sum (wt\%) & 99.430 & 100.077 & 0.647 & $0.7 \%$ & No & 1.906 & 1.92 & 1.621 & 1.259 & No & 15 & 10 \\
\hline 6 & Hydragard-LFR & Sum (wt\%) & 99.602 & 99.052 & -0.551 & $-0.6 \%$ & No & 1.799 & 1.81 & 0.991 & 1.635 & Yes & 14 & 10 \\
\hline 2 & Hydragard - HFR & Sum (wt\%) & 99.400 & 99.141 & -0.259 & $-0.3 \%$ & No & 1.183 & 1.19 & 1.167 & 0.828 & No & 15 & 9 \\
\hline 3 & Isolok-LFR-12mL & Sum (wt\%) & 99.627 & 99.603 & -0.024 & $0.0 \%$ & No & 0.901 & 0.90 & 0.869 & 1.153 & No & 14 & 9 \\
\hline 4 & Isolok-HFR-12mL & Sum (wt\%) & 99.067 & 99.209 & 0.142 & $0.1 \%$ & No & 0.877 & 0.89 & 0.820 & 0.838 & No & 14 & 9 \\
\hline 2 & Hydragard - HFR & TiO2 (wt\%) & 0.018 & 0.019 & 0.002 & $9.7 \%$ & Yes & 0.003 & 15.61 & 0.001 & 0.001 & No & 13 & 10 \\
\hline 1 & Isolok-LFR-3mL & TiO2 (wt \%) & 0.016 & 0.015 & -0.001 & $-3.7 \%$ & No & 0.002 & 12.44 & 0.002 & 0.001 & No & 16 & 10 \\
\hline 6 & Hydragard-LFR & TiO2 (wt\%) & 0.016 & 0.016 & 0.000 & $-1.6 \%$ & No & 0.002 & 10.01 & 0.002 & 0.002 & No & 16 & 10 \\
\hline 4 & Isolok-HFR-12mL & TiO2 (wt\%) & 0.019 & 0.019 & 0.000 & $-1.4 \%$ & No & 0.002 & 8.71 & 0.002 & 0.002 & No & 15 & 10 \\
\hline 3 & Isolok-LFR-12mL & TiO2 (wt\%) & 0.019 & 0.018 & 0.000 & $-1.4 \%$ & No & 0.001 & 7.18 & 0.001 & 0.002 & No & 15 & 10 \\
\hline 5 & Isolok-HFR-3mL & TiO2 (wt\%) & 0.016 & 0.016 & 0.000 & $1.5 \%$ & No & 0.001 & 5.28 & 0.001 & 0.000 & Yes & 15 & 9 \\
\hline 4 & Isolok-HFR-12mL & $\mathrm{ZnO}(\mathrm{wt} \%)$ & 0.006 & 0.006 & 0.000 & $-3.6 \%$ & No & 0.000 & 7.21 & 0.000 & 0.000 & No & 16 & 10 \\
\hline 1 & Isolok-LFR-3mL & $\mathrm{ZnO}(\mathrm{wt} \%)$ & 0.006 & 0.006 & 0.000 & $-3.0 \%$ & No & 0.000 & 6.74 & 0.000 & 0.000 & No & 16 & 10 \\
\hline 2 & Hydragard - HFR & $\mathrm{ZnO}(\mathrm{wt} \%)$ & 0.006 & 0.006 & 0.000 & $-1.8 \%$ & No & 0.000 & 5.53 & 0.000 & 0.000 & No & 16 & 10 \\
\hline 5 & Isolok-HFR-3mL & $\mathrm{ZnO}$ (wt\%) & 0.006 & 0.006 & 0.000 & $-1.1 \%$ & No & 0.000 & 4.53 & 0.000 & 0.000 & No & 16 & 10 \\
\hline 6 & Hydragard-LFR & $\mathrm{ZnO}(\mathrm{wt} \%)$ & 0.006 & 0.006 & 0.000 & $-1.0 \%$ & No & 0.000 & 4.36 & 0.000 & 0.000 & No & 16 & 9 \\
\hline 3 & Isolok-LFR-12mL & $\mathrm{ZnO}(\mathrm{wt} \%)$ & 0.006 & 0.006 & 0.000 & $-0.6 \%$ & No & 0.000 & 3.02 & 0.000 & 0.000 & Yes & 15 & 10 \\
\hline 1 & Isolok-LFR-3mL & $\mathrm{ZrO} 2$ (wt\%) & 0.104 & 0.108 & 0.004 & $4.0 \%$ & Yes & 0.007 & 7.10 & 0.005 & 0.003 & No & 16 & 10 \\
\hline 2 & Hydragard - HFR & $\mathrm{ZrO} 2$ (wt\%) & 0.115 & 0.120 & 0.005 & $4.6 \%$ & Yes & 0.007 & 6.42 & 0.002 & 0.003 & No & 16 & 9 \\
\hline 5 & Isolok-HFR-3mL & $\mathrm{ZrO} 2$ (wt\%) & 0.111 & 0.109 & -0.001 & $-1.1 \%$ & No & 0.004 & 3.18 & 0.003 & 0.002 & No & 16 & 10 \\
\hline 3 & Isolok-LFR-12mL & $\mathrm{ZrO} 2$ (wt\%) & 0.113 & 0.113 & 0.001 & $0.6 \%$ & No & 0.003 & 2.84 & 0.003 & 0.004 & No & 16 & 10 \\
\hline 6 & Hydragard-LFR & $\mathrm{ZrO} 2$ (wt\%) & 0.109 & 0.110 & 0.000 & $0.4 \%$ & No & 0.003 & 2.61 & 0.003 & 0.003 & No & 16 & 10 \\
\hline 4 & Isolok-HFR-12mL & $\mathrm{ZrO} 2$ (wt\%) & 0.111 & 0.111 & 0.000 & $0.1 \%$ & No & 0.003 & 2.36 & 0.003 & 0.004 & No & 16 & 10 \\
\hline
\end{tabular}




\section{Exhibit B1. Density, Weight Percent Solids, and Oxide Measurements from the High-Rheology (Phase 2) Testing}

Variability Gauge Analyte=density $(\mathrm{g} / \mathrm{mL})$

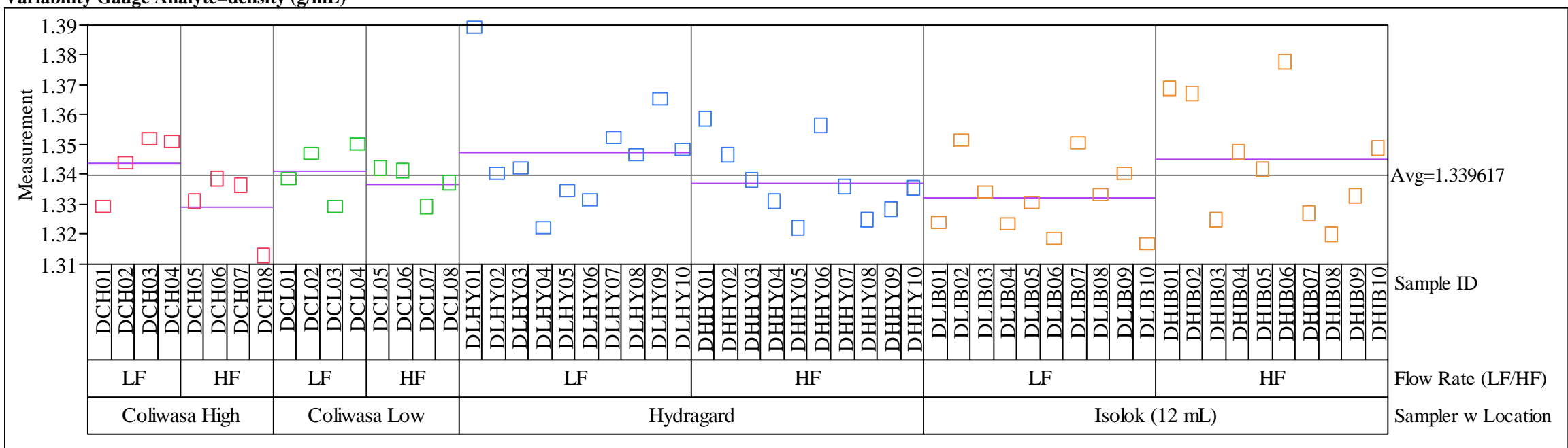

Variability Gauge Analyte $=$ wt $\%$ dried solids

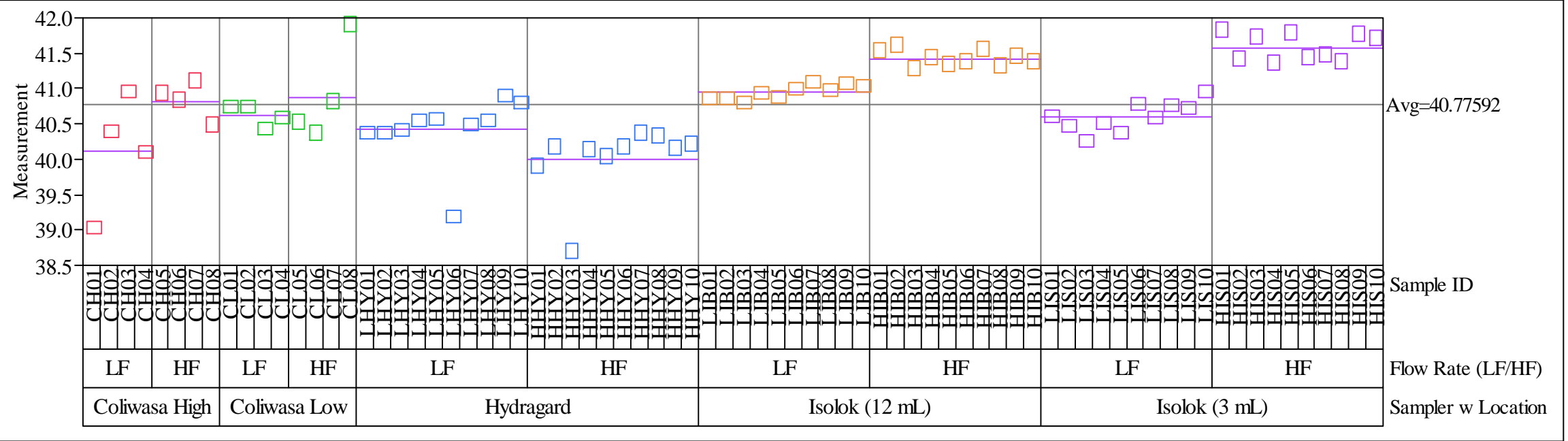




\section{Exhibit B1. Density, Weight Percent Solids, and Oxide Measurements from the High-Rheology (Phase 2) Testing}

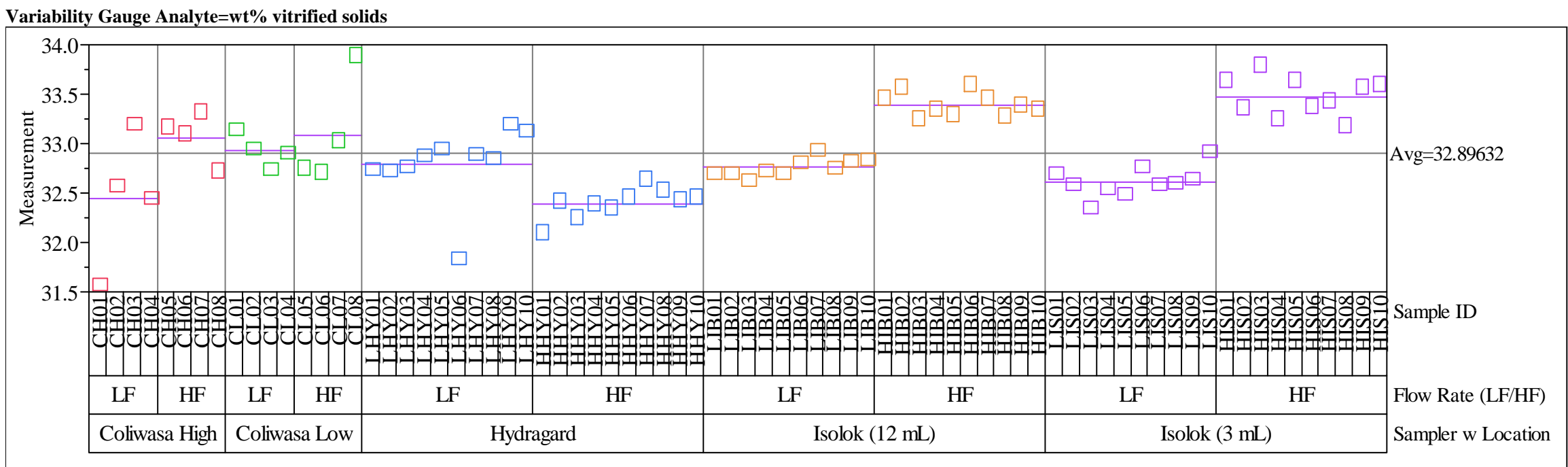

Variability Gauge Type of Material=SME Simulant, Analyte=Al/B

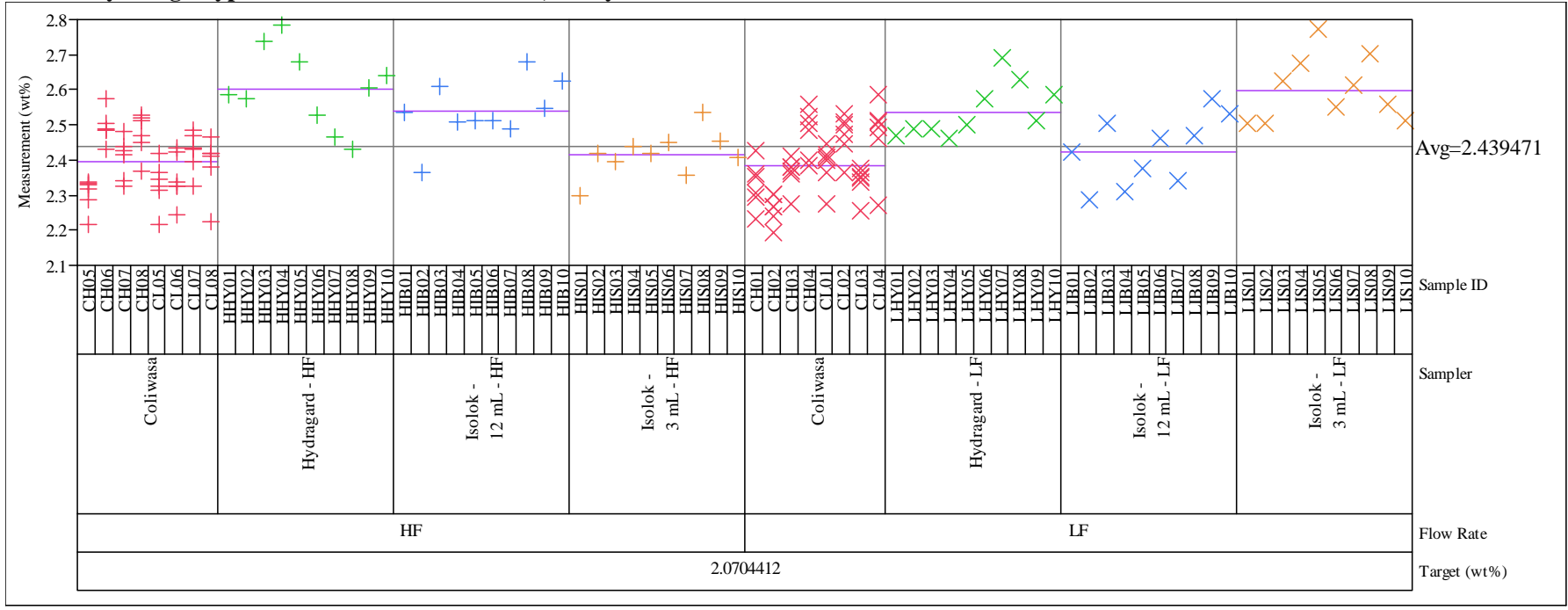




\section{Exhibit B1. Density, Weight Percent Solids, and Oxide Measurements from the High-Rheology (Phase 2) Testing}

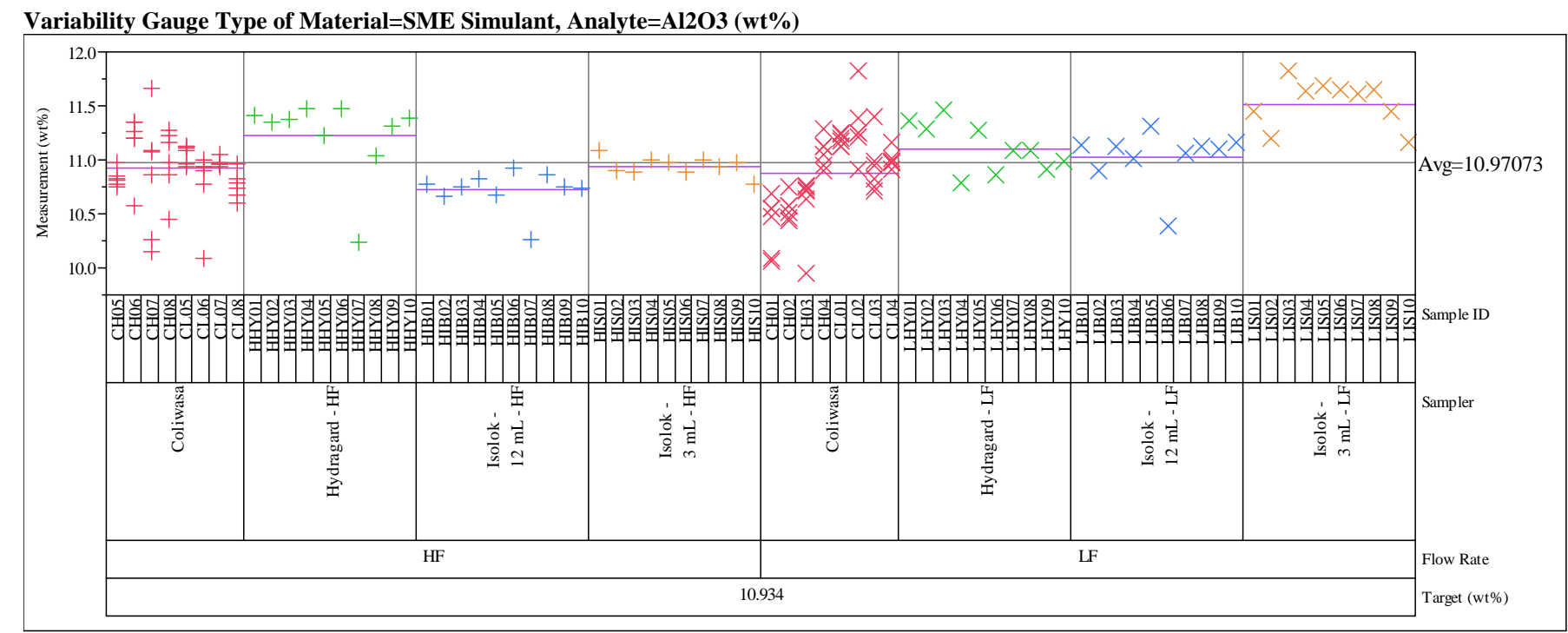

Variability Gauge Type of Material=SME Simulant, Analyte=B2O3 (wt\%)

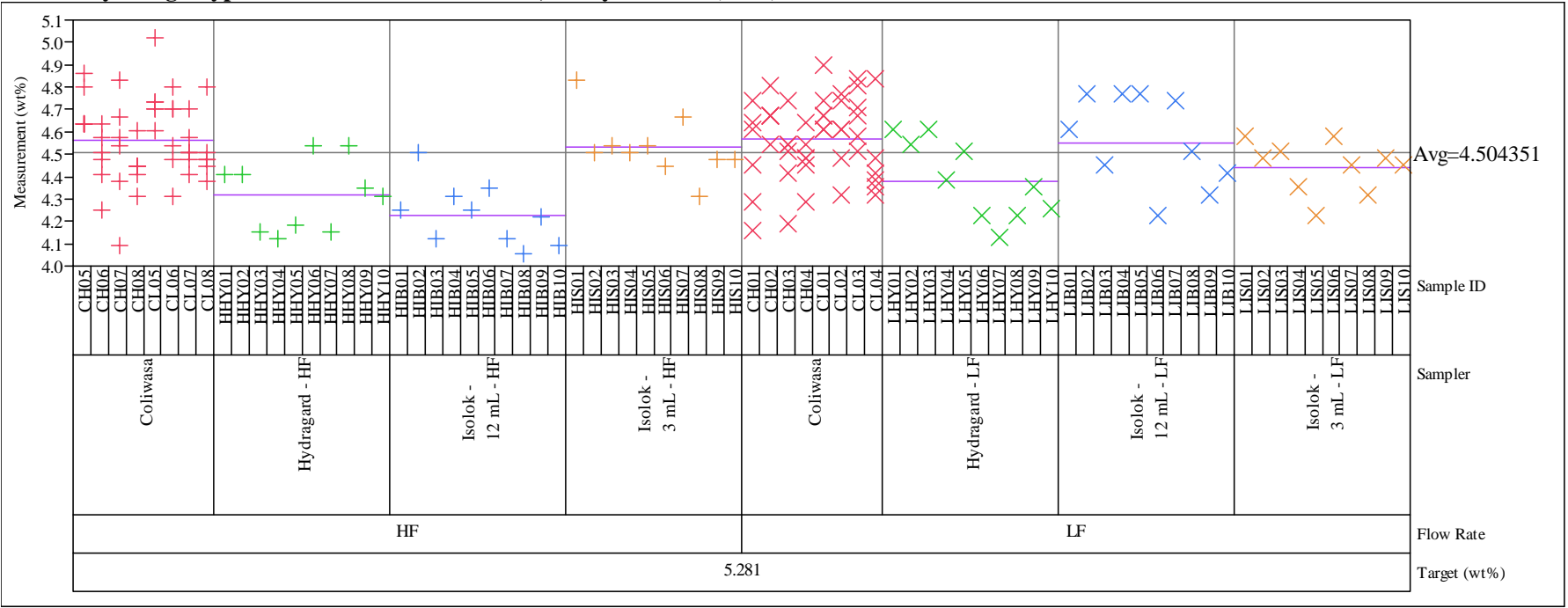




\section{Exhibit B1. Density, Weight Percent Solids, and Oxide Measurements from the High-Rheology (Phase 2) Testing}

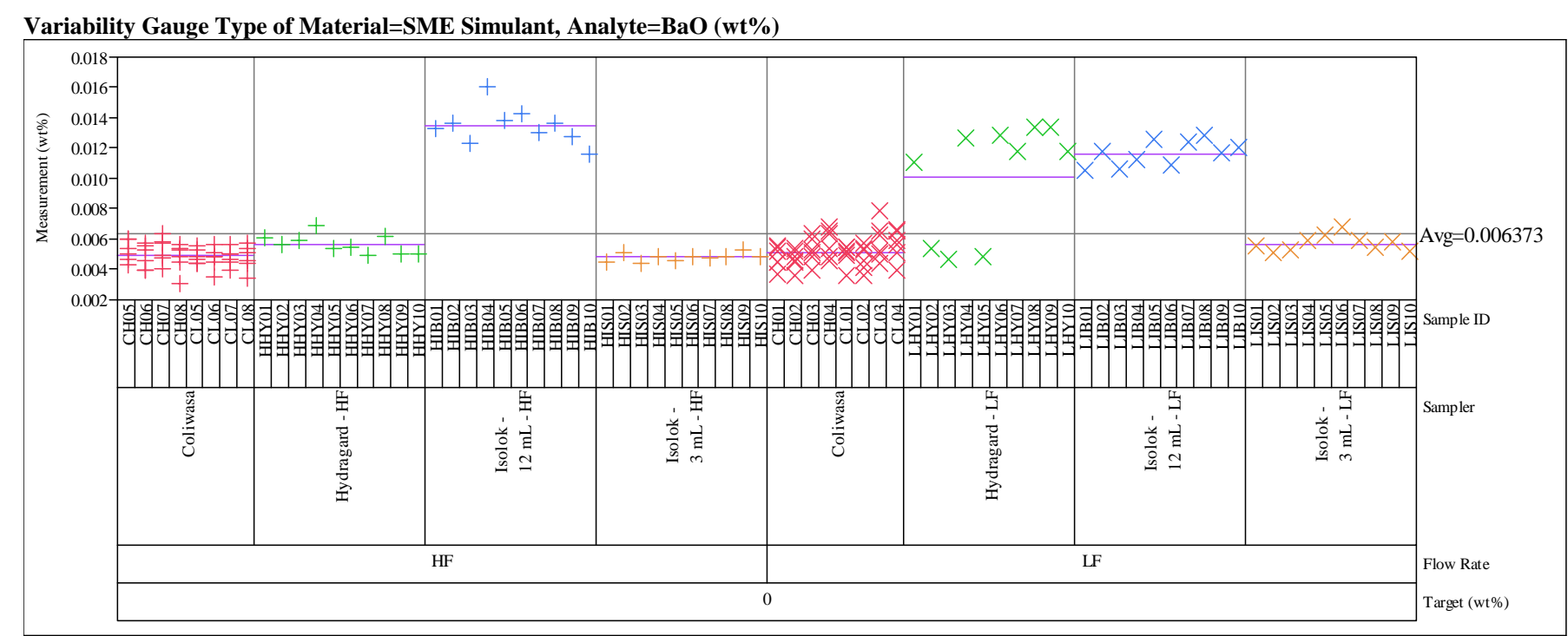

Variability Gauge Type of Material=SME Simulant, Analyte=CaO (wt \%)

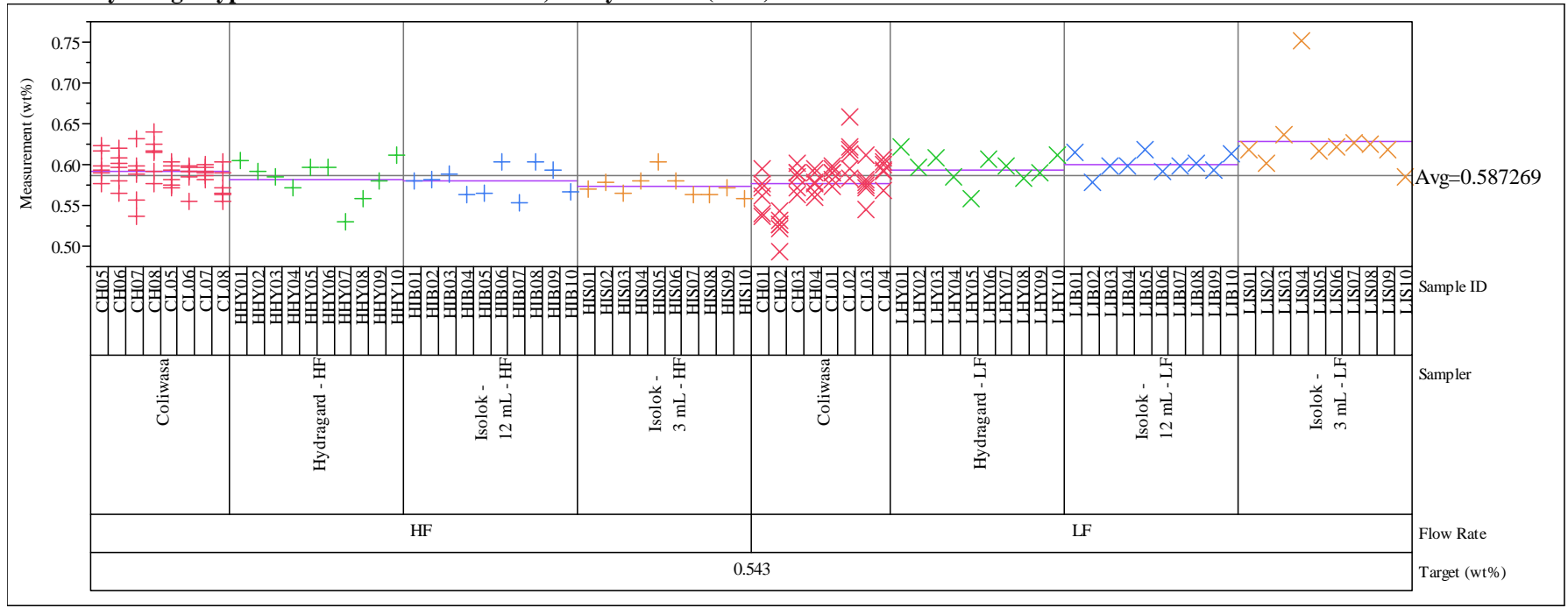




\section{Exhibit B1. Density, Weight Percent Solids, and Oxide Measurements from the High-Rheology (Phase 2) Testing}

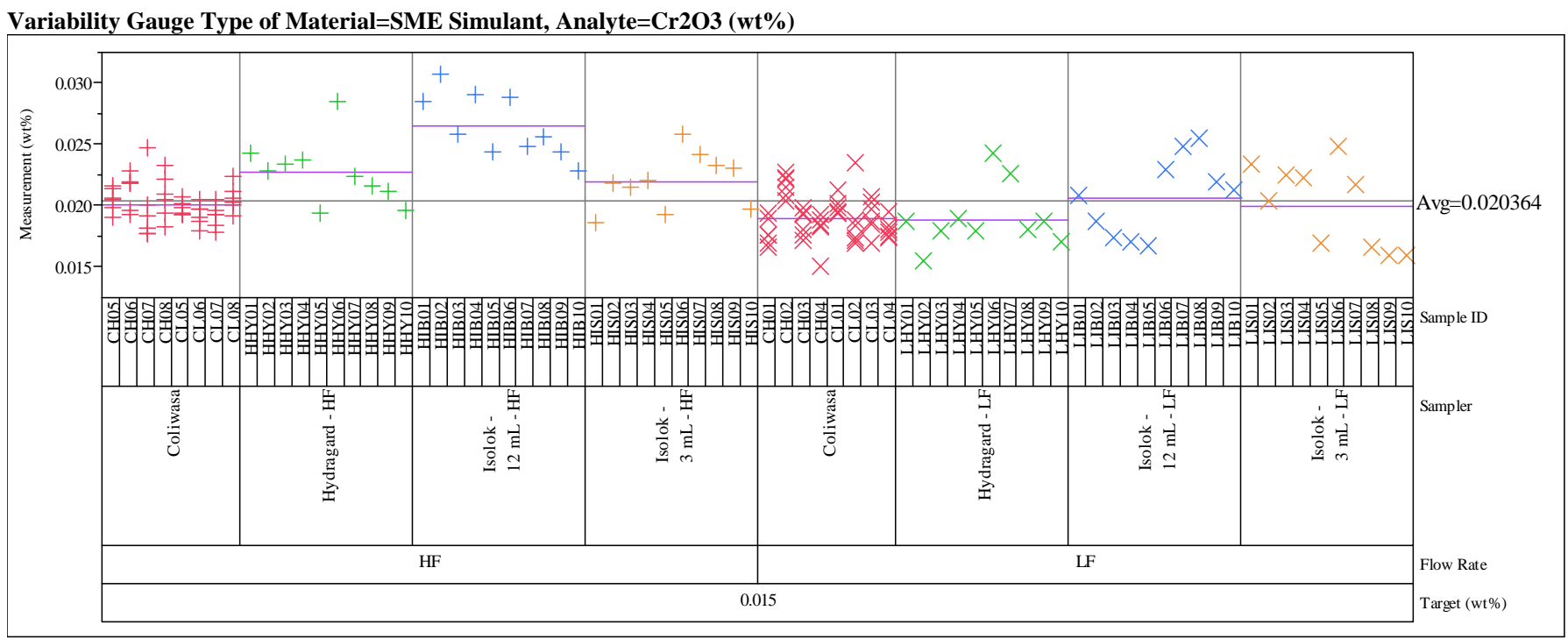

Variability Gauge Type of Material=SME Simulant, Analyte=CuO (wt \%)

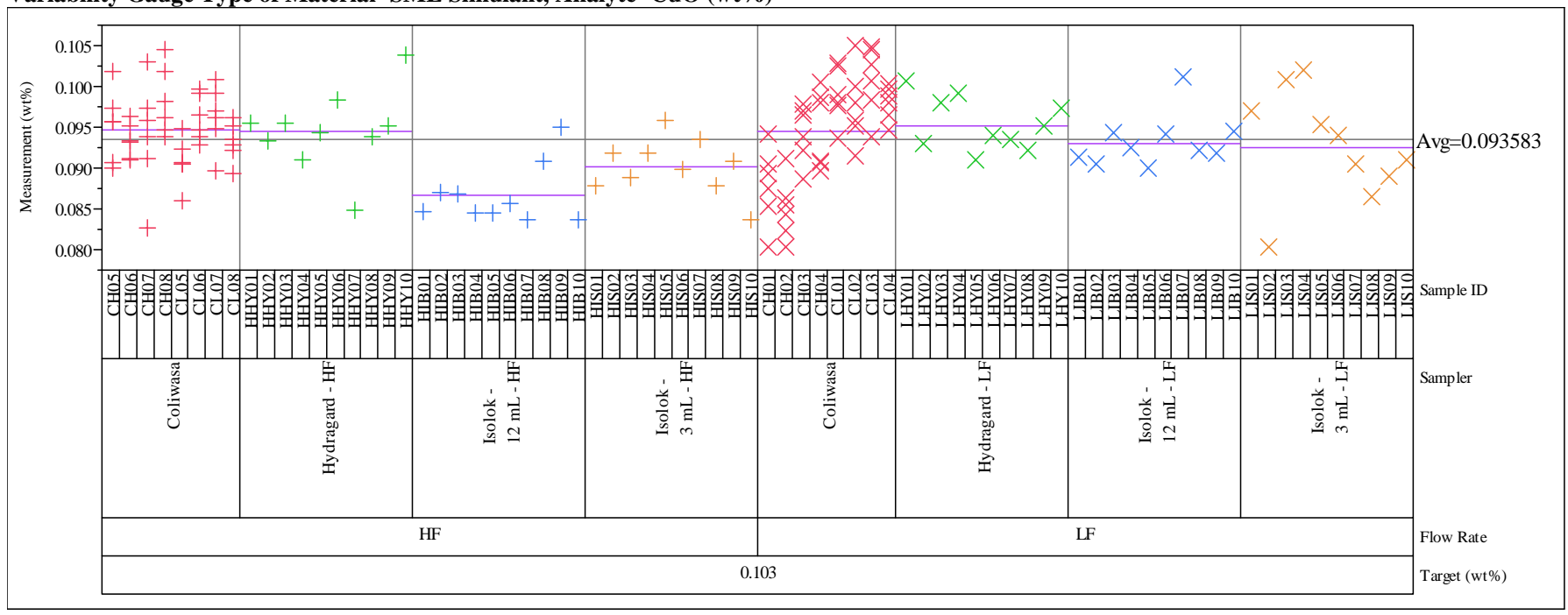




\section{Exhibit B1. Density, Weight Percent Solids, and Oxide Measurements from the High-Rheology (Phase 2) Testing}

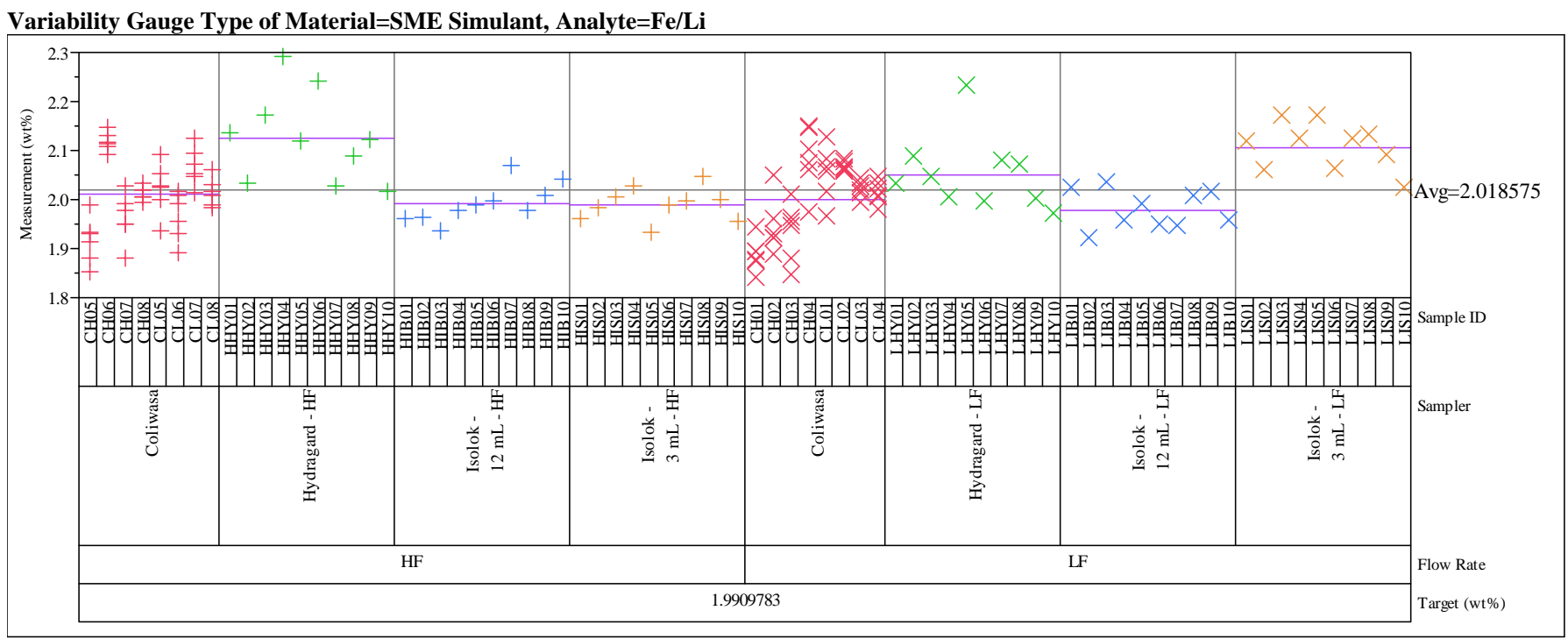

Variability Gauge Type of Material=SME Simulant, Analyte=Fe2O3 (wt\%)

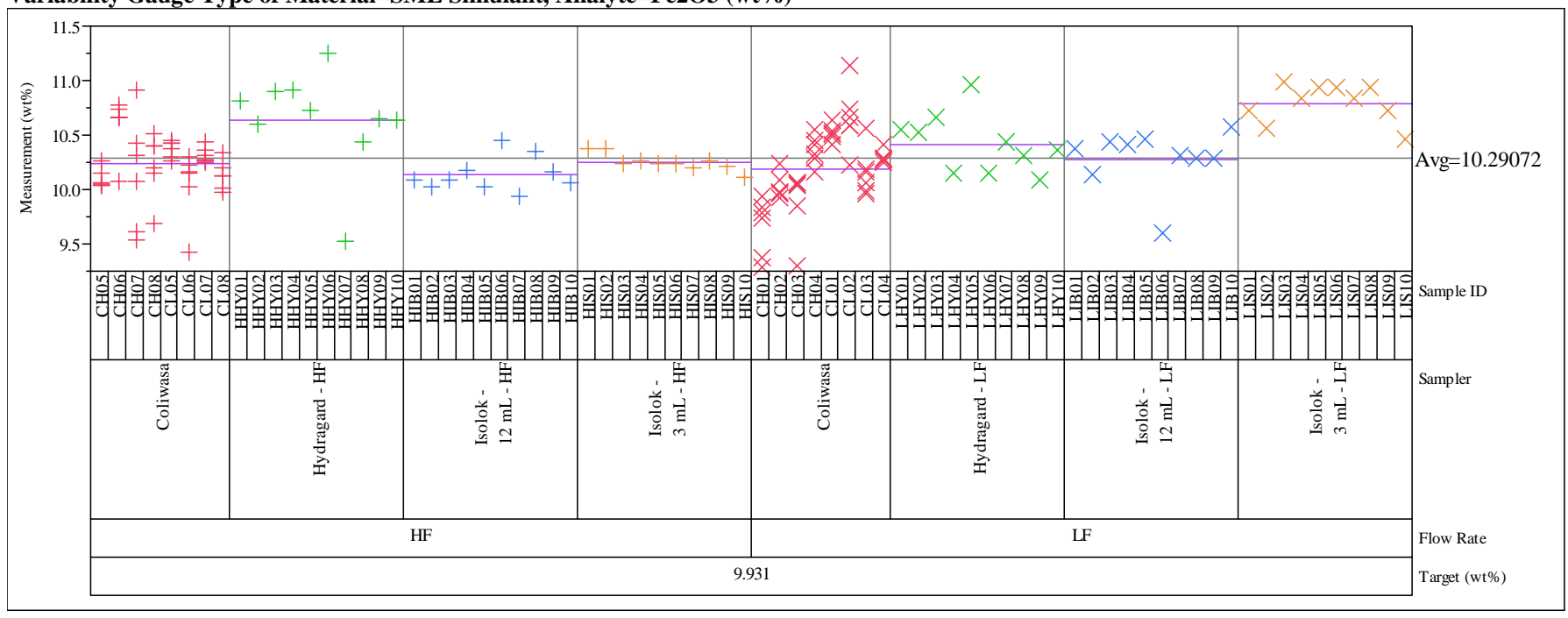




\section{Exhibit B1. Density, Weight Percent Solids, and Oxide Measurements from the High-Rheology (Phase 2) Testing}

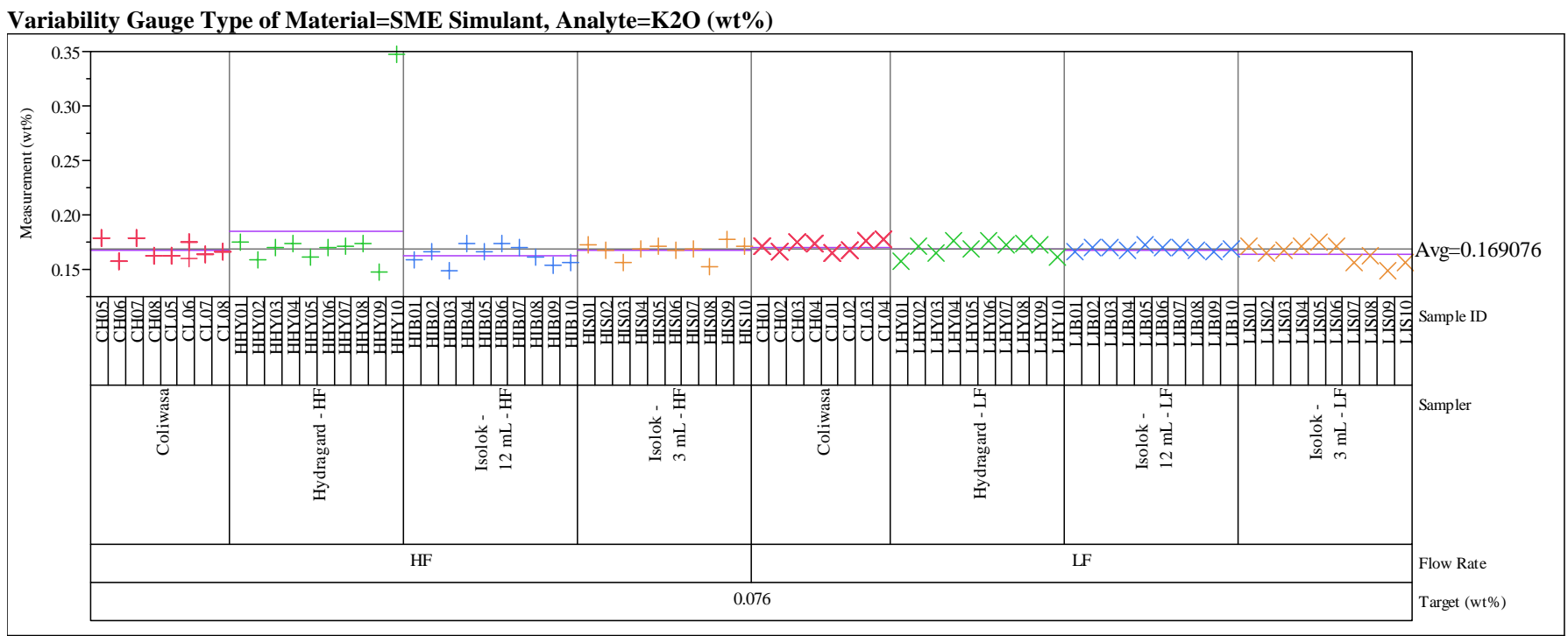

Variability Gauge Type of Material=SME Simulant, Analyte=Li2O (wt \%)

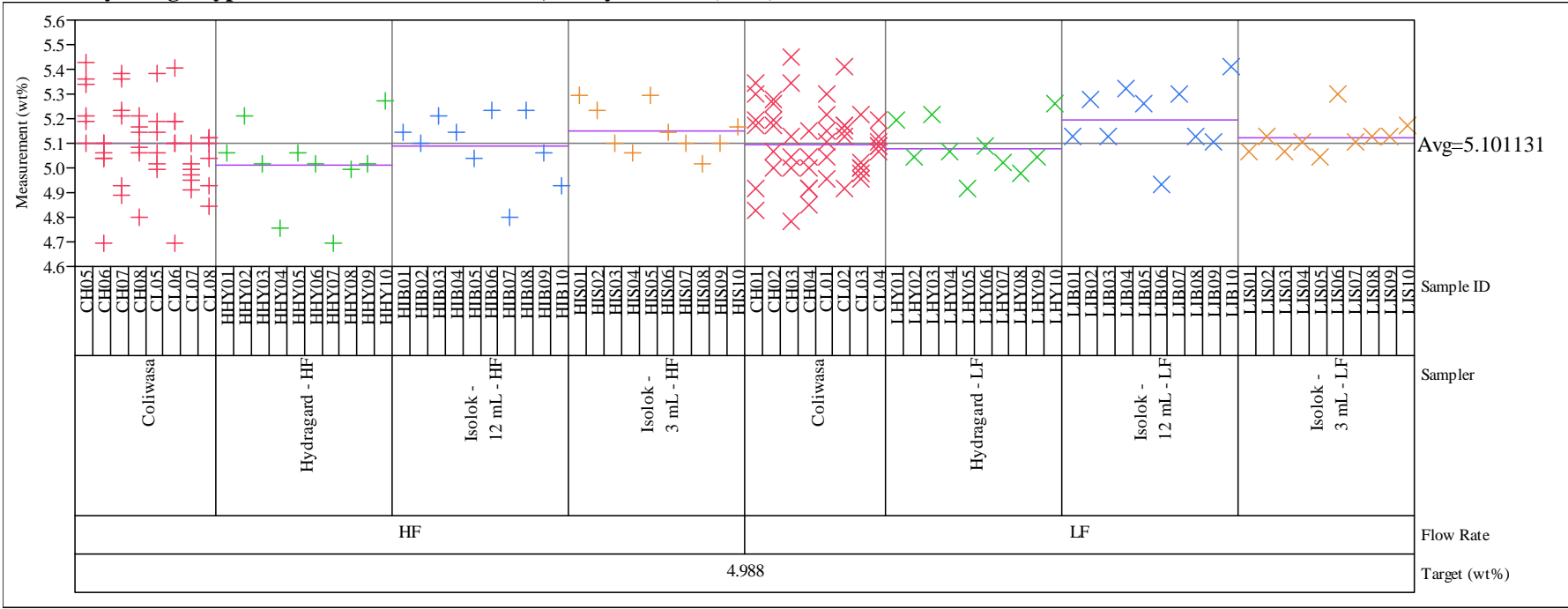




\section{Exhibit B1. Density, Weight Percent Solids, and Oxide Measurements from the High-Rheology (Phase 2) Testing}

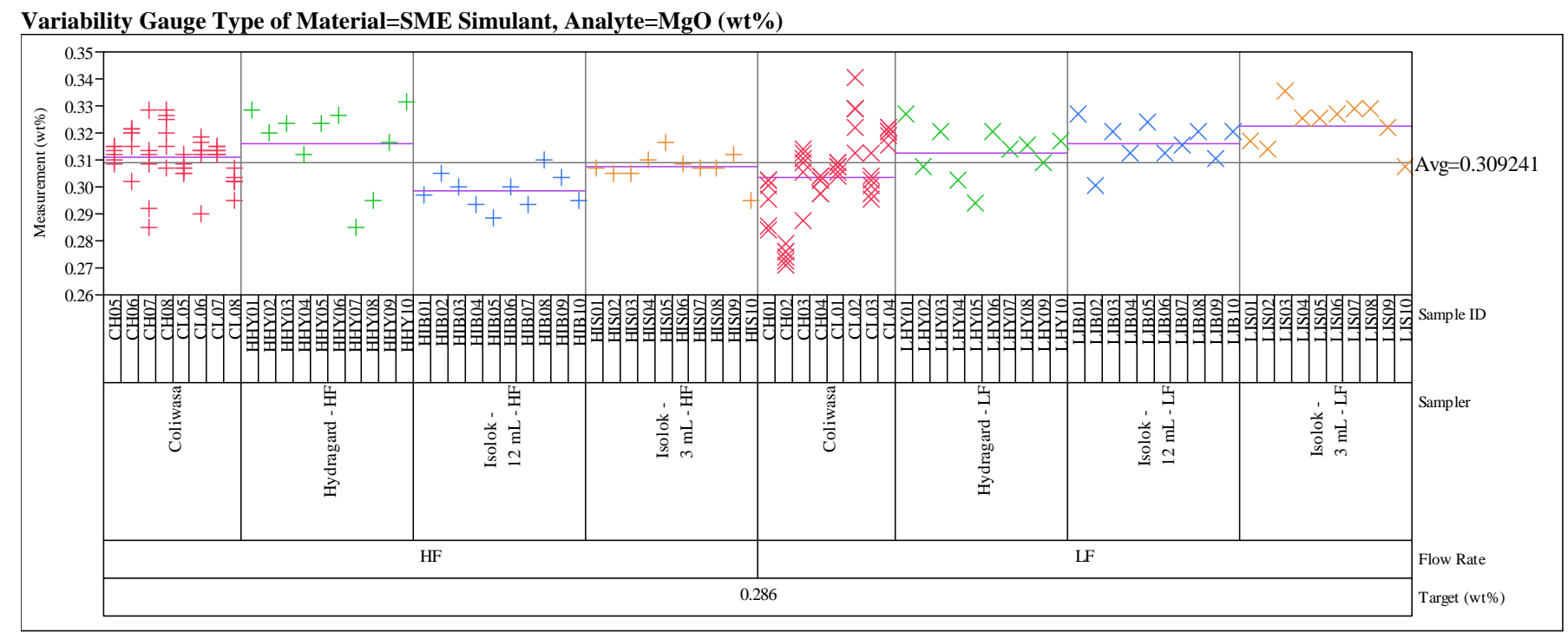

Variability Gauge Type of Material=SME Simulant, Analyte=MnO (wt\%)

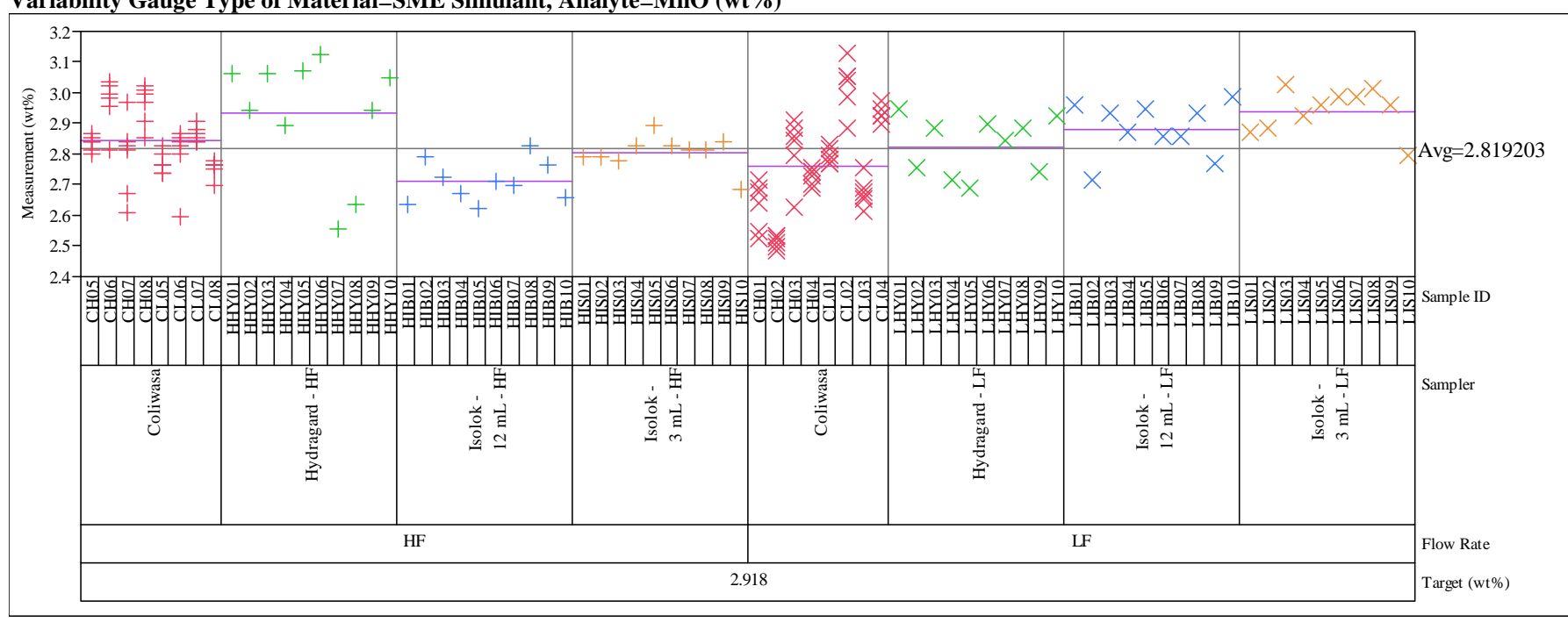




\section{Exhibit B1. Density, Weight Percent Solids, and Oxide Measurements from the High-Rheology (Phase 2) Testing}
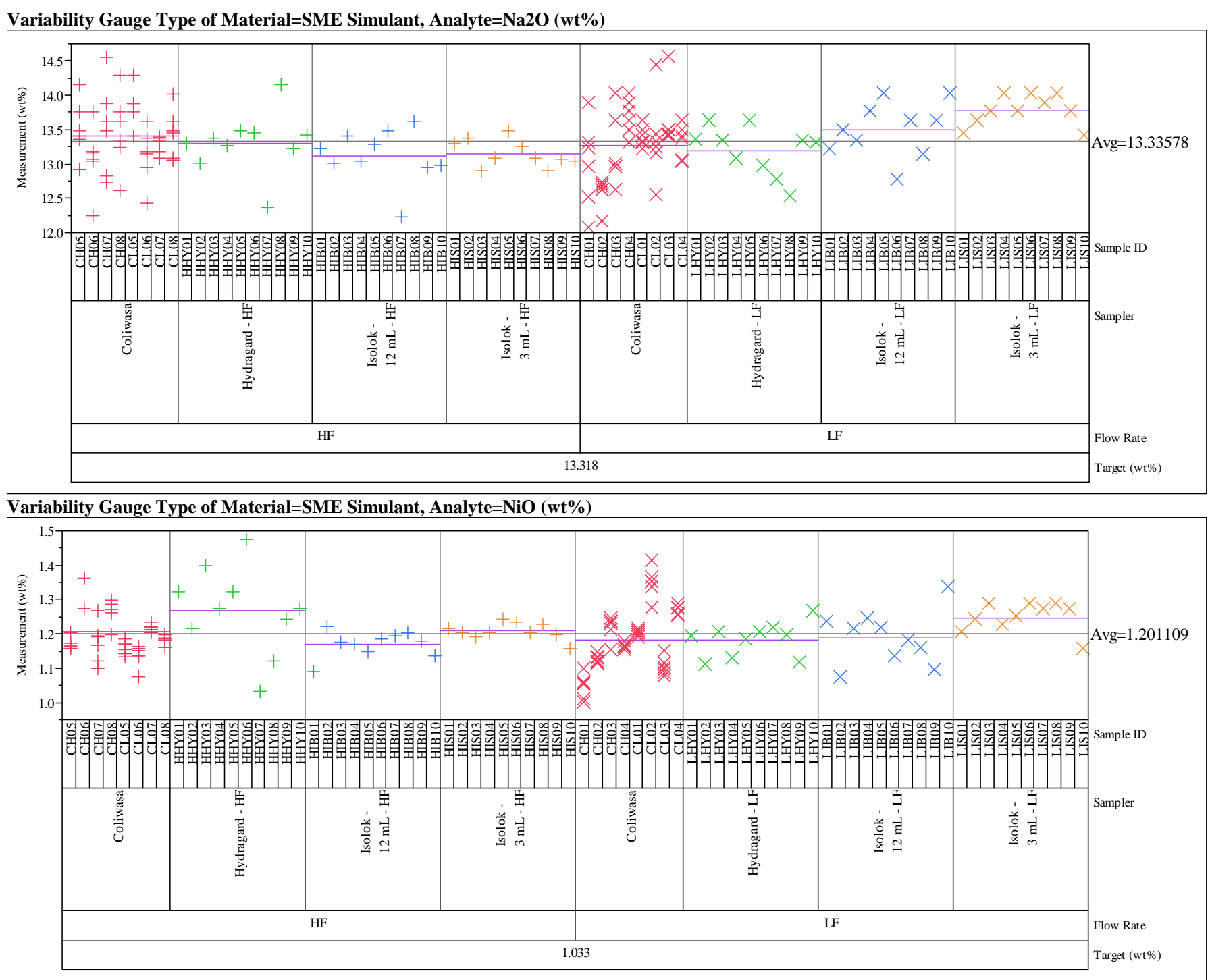


\section{Exhibit B1. Density, Weight Percent Solids, and Oxide Measurements from the High-Rheology (Phase 2) Testing}

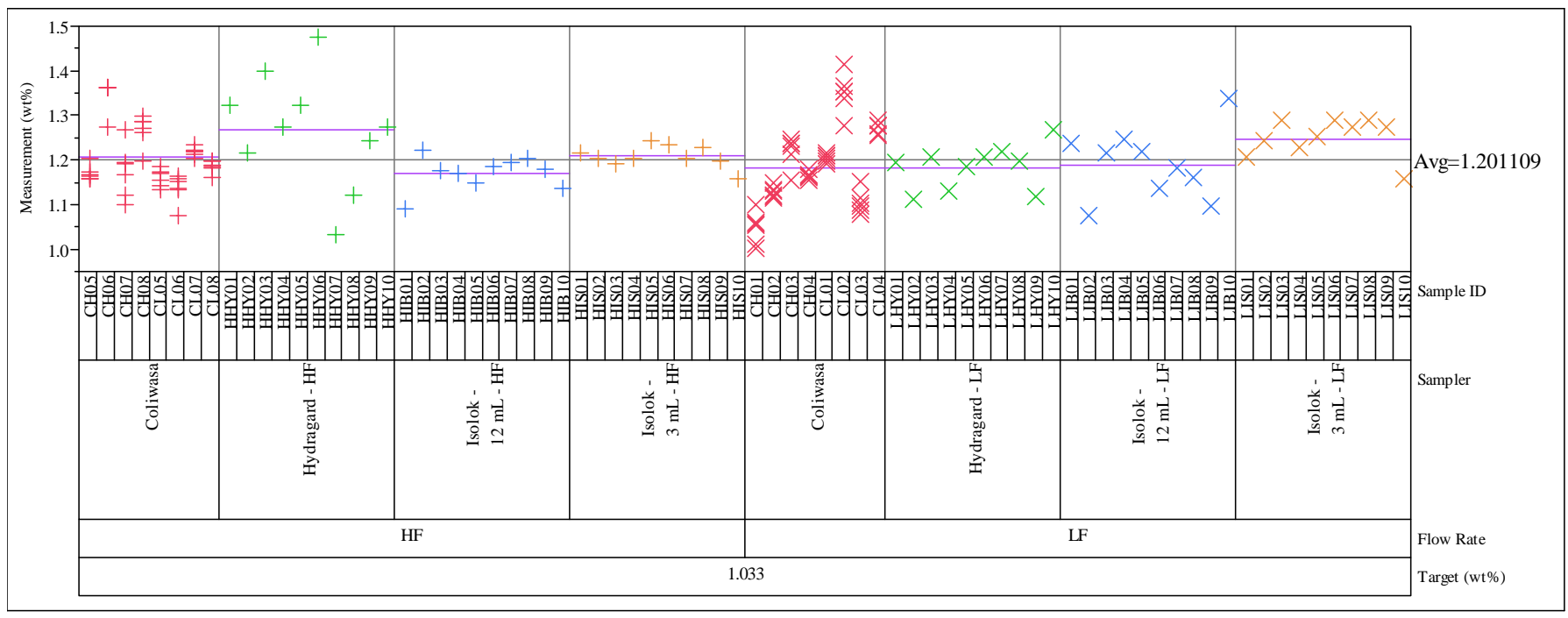

Variability Gauge Type of Material=SME Simulant, Analyte=SiO2 (wt \%)

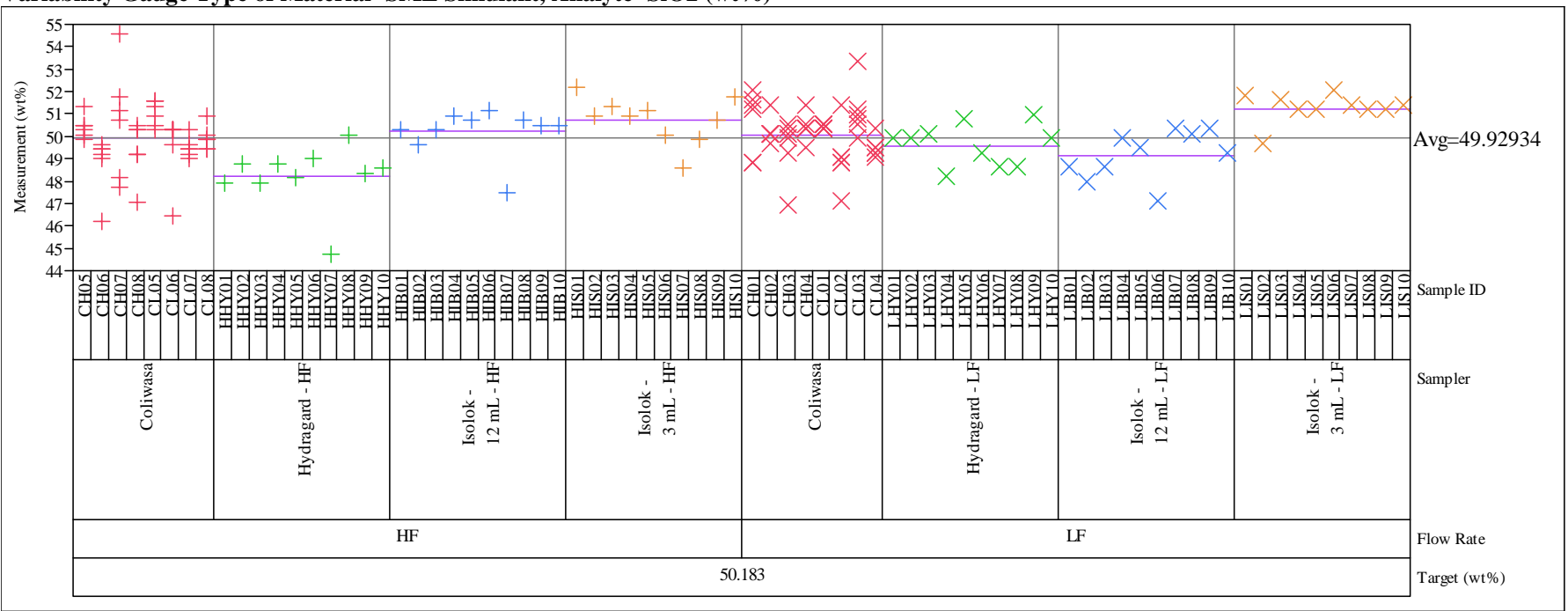




\section{Exhibit B1. Density, Weight Percent Solids, and Oxide Measurements from the High-Rheology (Phase 2) Testing}

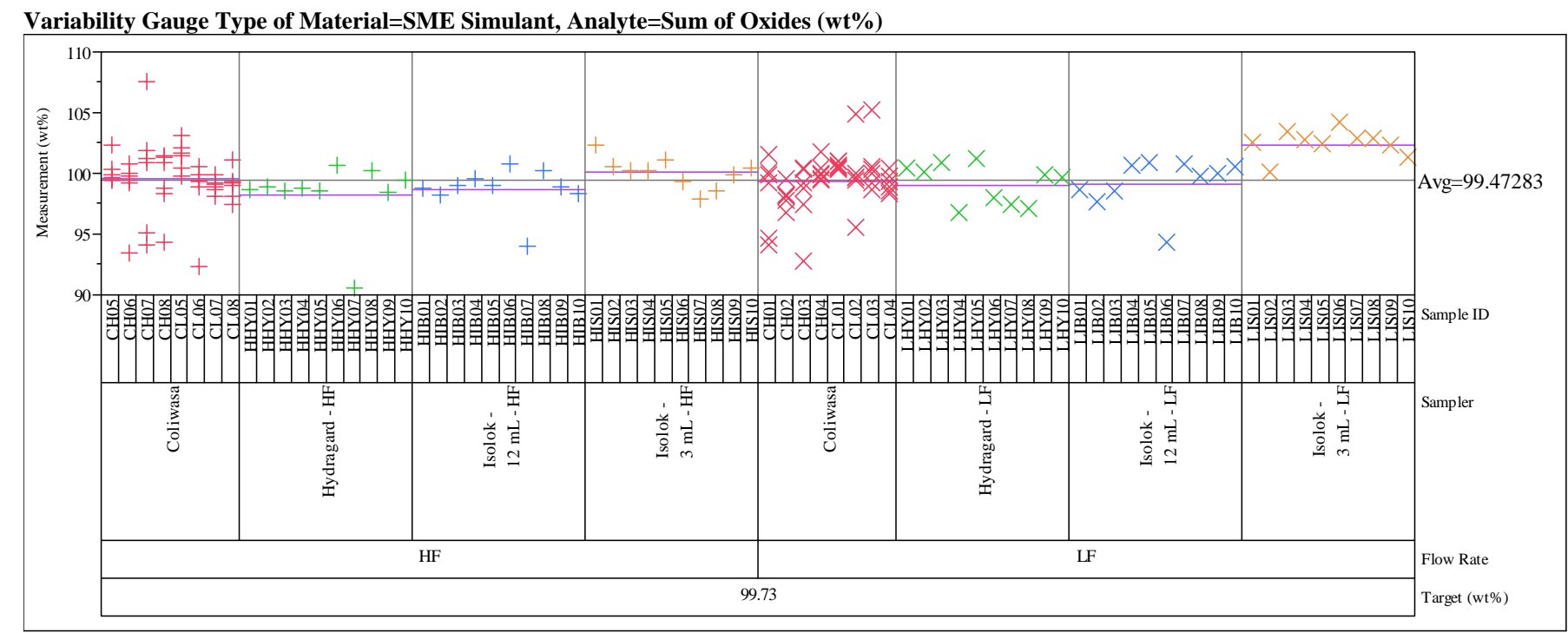

Variability Gauge Type of Material=SME Simulant, Analyte=TiO2 (wt\%)

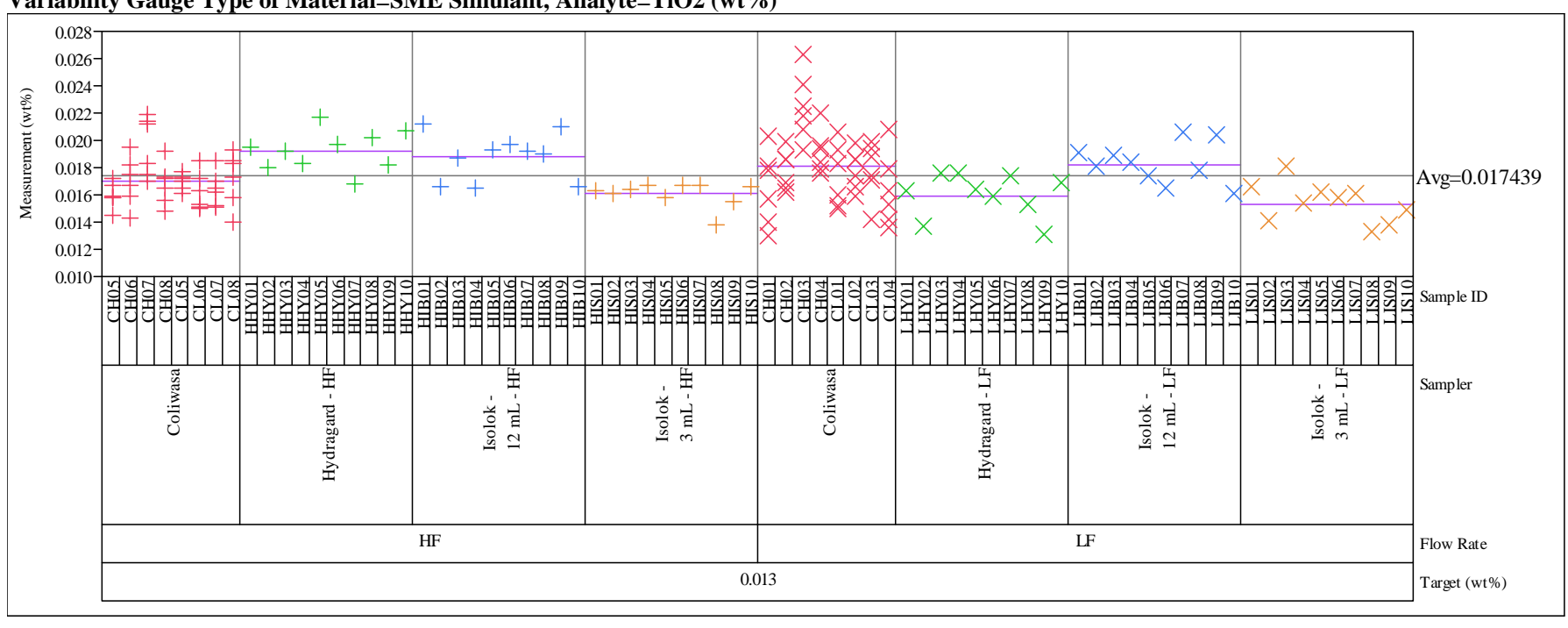


Exhibit B1. Density, Weight Percent Solids, and Oxide Measurements from the High-Rheology (Phase 2) Testing

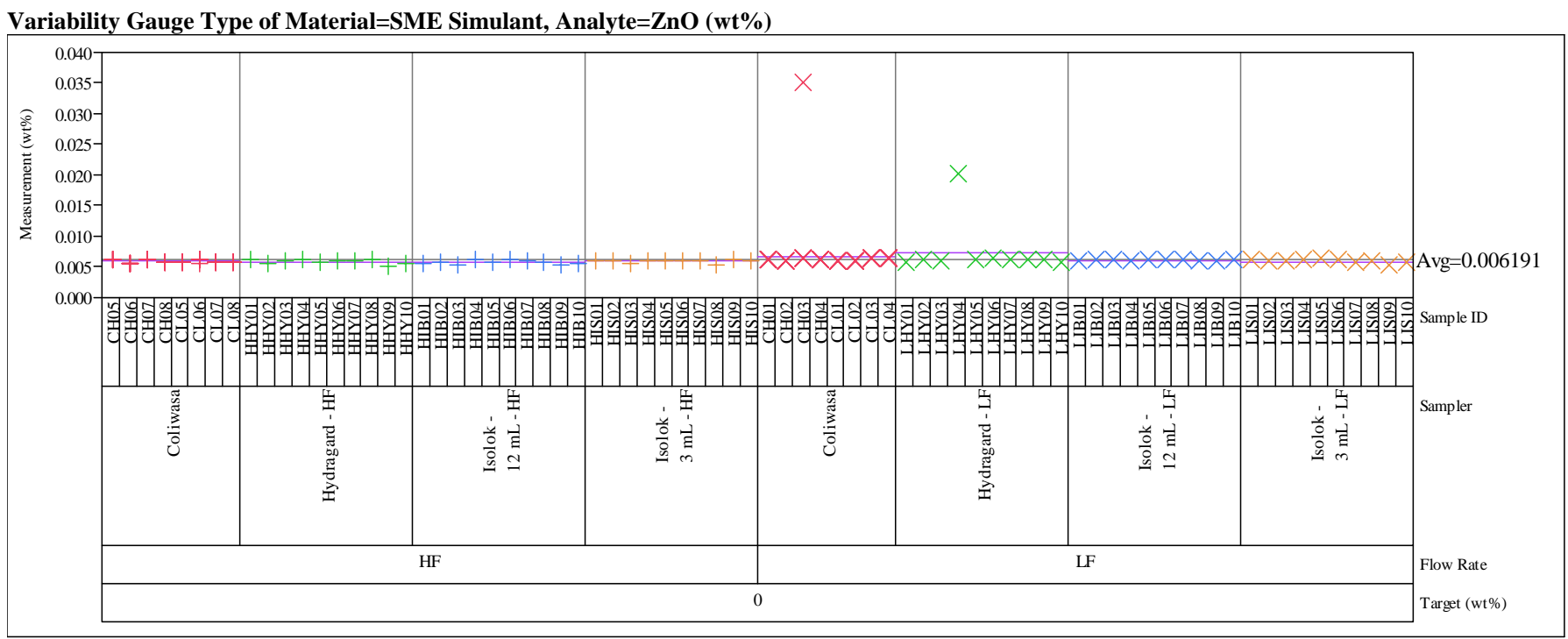

Variability Gauge Type of Material=SME Simulant, Analyte=ZrO2 (wt \%)

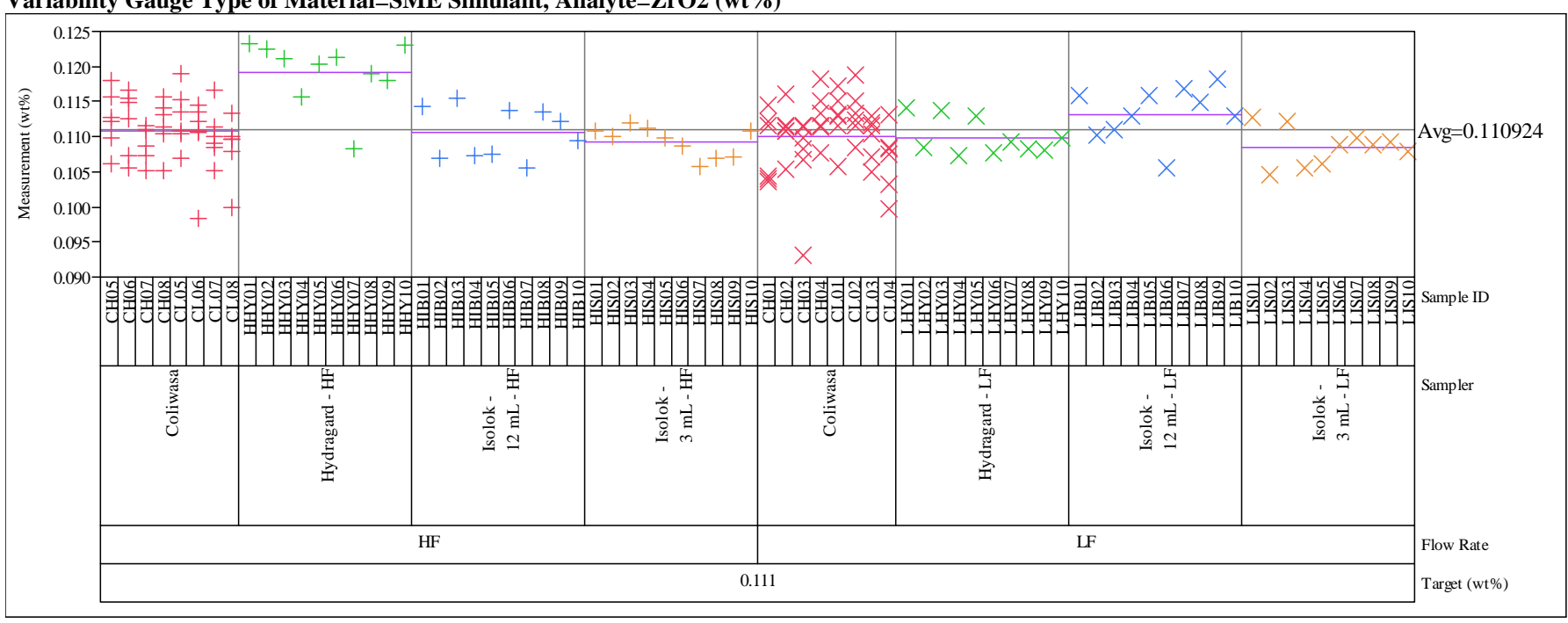


SRNL-STI-2011-00693

Revision 0

Exhibit B2. Statistical Comparisons for High-Rheology (Phase 2) Simulant Testing

Oneway Analysis of Measurement By Sampler w Location Analyte=density $(\mathrm{g} / \mathrm{mL})$

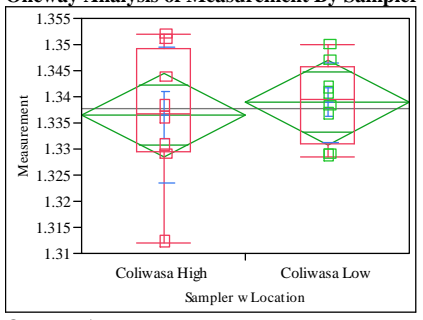

Oneway Anova

Summary of Fit

Root Mean Square Error $\quad-0.0555$

Mean of Res

\begin{tabular}{lr} 
Observations (or Sum Wgts) & 1.337738 \\
\hline
\end{tabular}

t Test

Coliwasa Low-Coliwasa High

Assuming equal variances

Difference $\quad 0.00245$ t Ratio $\quad 0.459027$

$\begin{array}{lll}\text { Std Err Dif } \quad 0.00534 \text { DF } & 14\end{array}$

Upper CL Dif $\quad 0.01390$ Prob $>|t| \quad 0.6533$

Lower CL Dif -0.00900 Prob $>t \quad 0.3266$

$\begin{array}{lll}\text { Confidence } & 0.95 \text { Prob }<\mathrm{t} \quad 0.6734\end{array}$

\begin{abstract}
$\begin{array}{lrrrrr}\text { Analysis of Variance } & & & & \\ \text { Source } & \text { DF } & \text { Sum of Squares } & \text { Mean Square } & \text { F Ratio } & \text { Prob > F } \\ \text { Sampler w Location } & 1 & 0.00002401 & 0.000024 & 0.2107 & 0.6533\end{array}$
$\begin{array}{llllll}\text { Sampler w Location } & 1 & 0.00002401 & 0.000024 & 0.2107 & 0.6533\end{array}$

$\begin{array}{llll}\text { Error } & 14 & 0.00159531 & 0.000114\end{array}$

$\begin{array}{lll}\text { C. Total } & 15 & 0.00161932\end{array}$

Means for Oneway Anova

Level Number Mean Std Error Lower 95\% Upper 95\%

$\begin{array}{llllll} & 8 & 1.33651 & 0.00377 & 1.3284 & 1.3446\end{array}$
\end{abstract}

Std Error uses a pooled estimate of error variance

Means and Std Deviations

Level Number Mean Std Dev Std Err Mean Lower 95\% Upper 95\%

$\begin{array}{lrrrrrr}\text { Coliwasa High } & 8 & 1.33651 & 0.013022 & 0.00460 & 1.3256 & 1.3474 \\ \text { Coliwasa Low } & 8 & 1.33896 & 0.007637 & 0.00270 & 1.3326 & 1.3453\end{array}$

t Test

Coliwasa Low-Coliwasa High

Assuming unequal variances

Difference $\quad 0.00245$ t Ratio $\quad 0.459027$

Std Err Dif $\quad 0.00534$ DF 11.30546

Upper CL Dif 0.01416 Prob $>|t| \quad 0.6549$

$\begin{array}{lll}\text { Lower CL Dif }-0.00926 \text { Prob }>t & 0.3275\end{array}$

$\begin{array}{lll}\text { Confidence } & 0.95 \text { Prob }<\mathrm{t} \quad 0.6725\end{array}$

Tests that the Variances are Equal

Level Count Std Dev MeanAbsDif to Mean MeanAbsDif to Median

$\begin{array}{lrrrr} & \text { Count } & \text { Std Dev } & \text { MeanAbsDif to Mean } & \text { MeanAbsDif to Median } \\ \text { Coliwasa High } & 8 & 0.0130223 & 0.0096375 & 0.0096375\end{array}$

$\begin{array}{lllll}\text { Coliwasa Low } & 8 & 0.0076367 & 0.0058375 & 0.0096375 \\ & & & \end{array}$

Test F Ratio DFNum DFDen p-Value

$\begin{array}{lllll}\text { O'Brien[.5] } & 1.4805 & 1 & 14 & 0.2438\end{array}$

$\begin{array}{lllll}\text { Brown-Forsythe } & 1.3883 & 1 & 14 & 0.2583\end{array}$

$\begin{array}{lllll}\text { Levene } & 1.3951 & 1 & 14 & 0.2572\end{array}$

$\begin{array}{lllll}\text { Levene } & 1.3951 & 1 & 14 & 0.2572 \\ \text { Bartlett } & 1.7787 & 1 & & 0.1823\end{array}$

$\begin{array}{lllll}\text { Bartlett } & 1.7787 & 1 & \text {. } & 0.1823 \\ \text { F Test 2-sided } & 2.9078 & 7 & 7 & 0.1824\end{array}$

Welch Anova testing Means Equal, allowing Std Devs Not Equal

$\begin{array}{rrrr}\text { F Ratio } & \text { DFNum } & \text { DFDen } & \text { Prob }>\text { F } \\ 0.2107 & 1 & 11.305 & 0.6549\end{array}$

t Test
0.4590
Oneway Analysis of Measurement By Sampler w Location Analyte $=w t \%$ dried solids

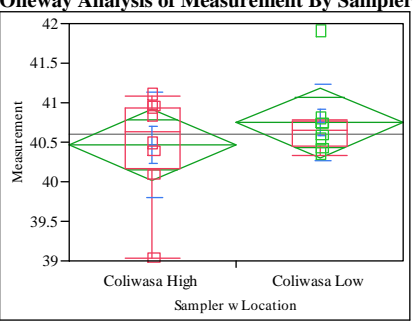

way Anova

Summary of Fit

Rsquare

Adj Rsquare

0.061434

oot Mean Squ

$-0.00561$

0.584997

Mean of Response

40.6075

Observations (or Sum Wgts)

Test

Coliwasa Low-Coliwasa High

Assuming equal variances

Difference $\quad 0.28000$ t Ratio $\quad 0.95727$

Std Err Dif $\quad 0.29250$ DF $\quad 14$

Upper CL Dif 0.90735 Prob $>|t| \quad 0.3547$

Lower CL Dif -0.34735 Prob $>t \quad 0.1773$

$\begin{array}{lll}\text { Confidence } & 0.95 \text { Prob }<\mathrm{t} \quad 0.8227\end{array}$

Analysis of Variance

$\begin{array}{lrrrrr}\text { Source } & \text { DF } & \text { Sum of Squares } & \text { Mean Square } & \text { F Ratio } & \text { Prob > F } \\ \text { Sampler w Location } & 1 & 0.3136000 & 0.313600 & 0.9164 & 0.3547\end{array}$

$\begin{array}{lrrr}\text { Sampler w Location } & 1 & 0.3136000 & 0.313600 \\ \text { Error } & 14 & 4.7911000 & 0.342221\end{array}$

C. Tota

$15 \quad 5.1047000$

Means for Oneway Anova

Level Number Mean Std Error Lower 95\% Upper 95\%

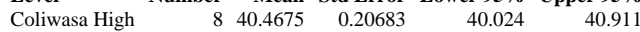

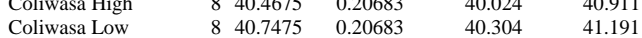

Std Error uses a pooled estimate of error variance

Means and Std Deviations

Level Number Mean Std Dev Std Err Mean Lower 95\% Upper 95\%

$\begin{array}{lrrrrrr}\text { Coliwasa High } & 8 & 40.4675 & 0.670645 & 0.23711 & 39.907 & 41.028 \\ \text { Coliwasa Low } & 8 & 40.7475 & 0.484436 & 0.17127 & 40.343 & 41.152\end{array}$

t Test

Coliwasa Low-Coliwasa High

Assuming unequal variances

Difference $\quad 0.28000$ t Ratio $\quad 0.95727$

$\begin{array}{lll}\text { Std Err Dif } \quad 0.29250 \text { DF } 12.74172 & \end{array}$

$\begin{array}{lll}\text { Upper CL Dif } & 0.91321 \quad \text { Prob }>|t| & 0.3562\end{array}$

Lower CL Dif -0.35321 Prob $>t \quad 0.1781$

$\begin{array}{lrl}\text { Confidence } & 0.95 \text { Prob }<\mathrm{t} & 0.8219\end{array}$

Tests that the Variances are Equal

$\begin{array}{lrrrr}\text { Level } & \text { Count } & \text { Std Dev } & \text { MeanAbsDif to Mean } & \text { MeanAbsDif to Median } \\ \text { Coliwasa High } & 8 & 0.6706447 & 0.4731250 & 0.4725000\end{array}$

$\begin{array}{lllll}\text { Coliwasa Low } & 8 & 0.4844363 & 0.2937500 & 0.2850000\end{array}$

Test F Ratio DFNum DFDen p-Value

$\begin{array}{lrrrr}\text { O'Brien[.5] } & 0.3628 & 1 & 14 & 0.5566\end{array}$

\begin{tabular}{lllll} 
Brown-Forsythe & 0.7396 & 1 & 14 & 0.5566 \\
\hline & 0.7800 & 1 & 14 & 0.3043
\end{tabular}

$\begin{array}{lllll}\text { Brown-Forsythe } & 0.7396 & 1 & 14 & 0.4043 \\ \text { Levene } & 0.7800 & 1 & 14 & 0.3921\end{array}$

$\begin{array}{lllll}\text { Levene } & 0.7800 & 1 & 14 & 0.3921 \\ \text { Bartlett } & 0.6793 & 1 & . & 0.4098\end{array}$

$\begin{array}{lllll}\text { F Test 2-sided } & 1.9165 & 7 & 7 & 0.4102\end{array}$

Welch Anova testing Means Equal, allowing Std Devs Not Equal

F Ratio DFNum DFDen Prob $>$ F

$\begin{array}{llll}0.9164 & 1 & 12.742 & 0.3562\end{array}$

t Test
0.9573 
SRNL-STI-2011-00693

Revision 0

Exhibit B2. Statistical Comparisons for High-Rheology (Phase 2) Simulant Testing

Oneway Analysis of Measurement By Sampler $w$ Location Analyte=wt $\%$ vitrified solids

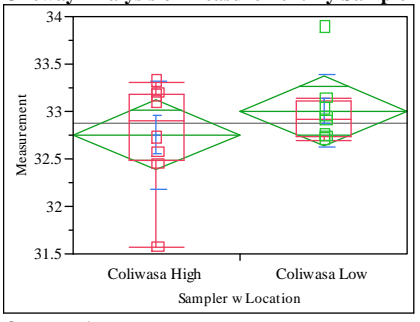

Oneway Anova

Rsquare $\quad 0.070562$

Adj Rsquare $\quad 0.004174$

Root Mean Square Error $\quad 0.487412$

$\begin{array}{lr}\text { Mean of Response } & 32.87938 \\ \text { Observations (or Sum Wgts) } & 16\end{array}$

t Test

Coliwasa Low-Coliwasa High

Assuming equal variances

Difference $\quad 0.25125$ t Ratio 1.030955

$\begin{array}{lrrr}\text { Std Err Dif } & 0.24371 & \text { DF } & 14 \\ \text { Upper CL Dif } & 0.77395 & \text { Prob }>|t| & 0.3200\end{array}$

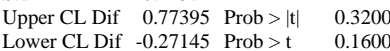

$\begin{array}{lrl} & \\ \text { Confidence } & 0.95 \text { Prob }<\mathrm{t} & 0.8400\end{array}$

$\begin{array}{lrrrrr}\text { Analysis of Variance } & & & & \\ \text { Source } & \text { DF } & \text { Sum of Squares } & \text { Mean Square } & \text { F Ratio } & \text { Prob > F } \\ \text { Sampler w Location } & 1 & 0.2525062 & 0.252506 & 1.0629 & 0.3200 \\ \text { Error } & 14 & 3.3259875 & 0.237571 & & \\ \text { C. Total } & 15 & 3.5784937 & & & \end{array}$

Means for Oneway Anova

Level Number Mean Std Error Lower 95\% Upper 95\%

$\begin{array}{lrrrrr}\text { Level } & \text { Number } & \text { Mean } & \text { Std Error } & \text { Lower 95\% } & \text { Upper 95\% } \\ \text { Coliwasa High } & 8 & 32.7538 & 0.17233 & 32.384 & 33.123\end{array}$

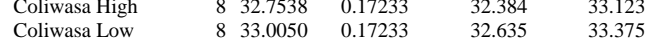

Std Error uses a pooled estimate of error variance

Means and Std Deviations

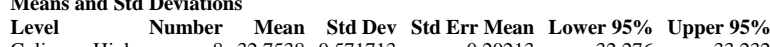

$\begin{array}{lrrrrrr}\text { Coliwasa High } & 8 & 32.7538 & 0.571713 & 0.20213 & 32.276 & 33.232 \\ \text { Coliwasa Low } & 8 & 33.0050 & 0.385079 & 0.13615 & 32.683 & 33.327\end{array}$

t Test

Coliwasa Low-Coliwasa High

Assuming unequal variances

Difference $\quad 0.25125$ t Ratio 1.030955

Std Err Dif $\quad 0.24371$ DF 12.26731

Upper CL Dif 0.78096 Prob $>|t| 0.3225$

Lower CL Dif -0.27846 Prob $>t \quad 0.1612$

$\begin{array}{lrl}\text { Confidence } & 0.95 \text { Prob }<\mathrm{t} \quad 0.8388\end{array}$

Tests that the Variances are Equal

$\begin{array}{lrrrr}\text { Level } & \text { Count } & \text { Std Dev } & \text { MeanAbsDif to Mean } & \text { MeanAbsDif to Median } \\ \text { Coliwasa High } & 8 & 0.5717127 & 0.4287500 & 0.4287500\end{array}$

$\begin{array}{lllll}\text { Coliwasa Low } & 8 & 0.3850788 & 0.2537500 & 0.4287500 \\ & & \end{array}$

$\begin{array}{llll}\text { Test } & \text { F Ratio DFNum DFDen p-Value }\end{array}$

$\begin{array}{lllll}\text { O'Brien[.5] } & 0.5965 & 1 & 14 & 0.4527\end{array}$

$\begin{array}{lllll}\text { Brown-Forsythe } & 1.2543 & 1 & 14 & 0.2816 \\ \text { Levene } & 1.2796 & 1 & 14 & 0.2770\end{array}$

$\begin{array}{lllrl}\text { Bartlett } & 0.9948 & 1 & . & 0.3186\end{array}$

$\begin{array}{lllll}\text { F Test 2-sided } & 2.2042 & 7 & 7 & 0.3189\end{array}$

Welch Anova testing Means Equal, allowing Std Devs Not Equal

F Ratio DFNum DFDen Prob $>$ F

$\begin{array}{llll}1.0629 & 1 & 12.267 & 0.3225\end{array}$

t Test

1.0310
Hydragard at Low Flow Rate

Oneway Analysis of Measurement By Sampling Mechanism Analyte $=$ density $(\mathrm{g} / \mathrm{mL})$

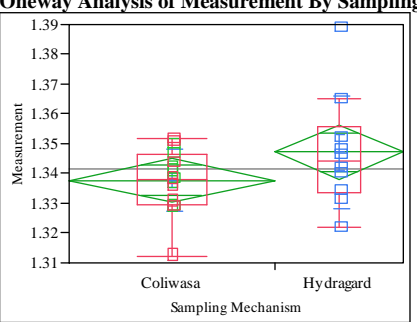

Oneway Anova

Summary of Fit

Rsquare

Adj Rsquare

Root Mean Square Error $\quad 0.014198$

Mean of Response $\quad 1.341362$

t Test

Hydragard-Coliwasa

Assuming equal variances

Difference $\quad 0.00942$ t Ratio 1.646271

$\begin{array}{lllr}\text { Std Err Dif } & 0.00572 & \text { DF } & 24 \\ \text { Upper CL Dif } & 0.02124 & \text { Prob }>|t| & 0.1127\end{array}$

$\begin{array}{lrll}\text { Upper CL Dif } & 0.02124 & \text { Prob }>|t| & 0.1127 \\ \text { Lower CL Dif } & -0.00239 & \text { Prob }>t & 0.0564\end{array}$

$\begin{array}{lll}\text { Confidence } & 0.95 \text { Prob }<\mathrm{t} & 0.9436\end{array}$

Analysis of Variance

$\begin{array}{lrrrrr}\text { Source } & \text { DF } & \text { Sum of Squares } & \text { Mean Square } & \text { F Ratio } & \text { Prob }>\text { F } \\ \text { Sampling Mechanism } & 1 & 0.00054636 & 0.000546 & 2.7102 & 0.1127\end{array}$

$\begin{array}{rrrrr}1 & 0.00054636 & 0.000546 & 2.7102 & 0.1127 \\ 24 & 0.00483824 & 0.000202 & & \end{array}$

C. Total

0.00538460

Means for Oneway Anova

Level Number Mean Std Error Lower 95\% Upper 95\%

$\begin{array}{lrrrrr}\text { Level } & \text { Number } & \text { Mean } & \text { Std Error } & \text { Lower 95\% } & \text { Upper 95\% } \\ \text { Coliwasa } & 16 & 1.33774 & 0.00355 & 1.3304 & 1.3451\end{array}$

$\begin{array}{llllll}\text { Coliwasa } & 16 & 1.33774 & 0.00355 & 1.3304 & 1.3451 \\ \text { Hydragard } & 10 & 1.34716 & 0.00449 & 1.3379 & 1.3564\end{array}$

Std Error uses a pooled estimate of error variance

Means and Std Deviations

Level Number Mean Std Dev Std Err Mean Lower 95\% Upper 95\%

$\begin{array}{llllllr}\text { Coliwasa } & 16 & 1.33774 & 0.010390 & 0.00260 & 1.3322 & 1.3433 \\ \text { Hydragard } & 10 & 1.34716 & 0.018912 & 0.00598 & 1.3336 & 1.3607\end{array}$

Hydraga

0.00598

t Test

Hydragard-Coliwasa

Assuming unequal variances

Difference $\quad 0.00942$ t Ratio 1.445125

Std Err Dif $\quad 0.00652$ DF 12.45011

Upper CL Dif 0.02357 Prob $>|t| \quad 0.1731$

Lower CL Dif -0.00473 Prob $>t \quad 0.0866$

$\begin{array}{lll}\text { Confidence } & 0.95 \text { Prob }<\mathrm{t} \quad 0.9134\end{array}$

Tests that the Variances are Equal

$\begin{array}{lrrrr}\text { Level } & \text { Count } & \text { Std Dev } & \text { MeanAbsDif to Mean } & \text { MeanAbsDif to Median } \\ \text { Coliwasa } & 16 & 0.0103901 & 0.0078078 & 0.0077750 \\ \text { Hydragard } & 10 & 0.0189119 & 0.0132320 & 0.0130600\end{array}$

Test F Ratio DFNum DFDen p-Value

$\begin{array}{lllll}\text { O'Brien[.5] } & 2.2250 & 1 & 24 & 0.1488\end{array}$

Brown-Forsythe $\begin{array}{llll}1.8312 & 1 & 24 & 0.1886\end{array}$

$\begin{array}{lllll}\text { Levene } & 2.0575 & 1 & 24 & 0.1644\end{array}$

$\begin{array}{lllrl}\text { Bartlett } & 4.0256 & 1 & & 0.0448 \\ \text { F Test 2-sided } & 3.3130 & 9 & 15 & 0.0395\end{array}$

Welch Anova testing Means Equal, allowing Std Devs Not Equal

F Ratio DFNum DFDen Prob $>$ F

$\begin{array}{llll}2.0884 & 1 & 12.45 & 0.1731\end{array}$

t Test

1.4451 
SRNL-STI-2011-00693

Revision 0

\section{Exhibit B2. Statistical Comparisons for High-Rheology (Phase 2) Simulant Testing}

Hydragard at Low Flow Rate

Oneway Analysis of Measurement By Sampling Mechanism Analyte $=w t \%$ dried solids

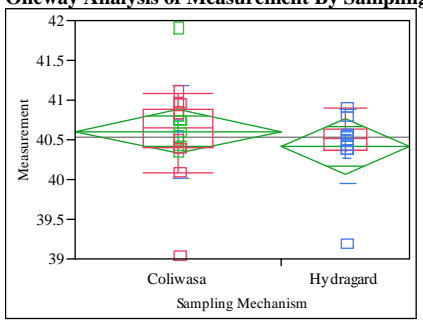

Rsquare $\quad 0.031025$

Adj Rsquare

$-0.00935$

Root Mean Square Error $\quad 0.541923$

Mean of Response $\quad 40.53385$

Observations (or Sum Wgts) 26

Test

Hydragard-Coliwasa

Assuming equal variances

Difference $\quad-0.19150$ t Ratio $\quad-0.8766$

$\begin{array}{lrlr}\text { Std Err Dif } & 0.21846 \text { DF } & 24 \\ \text { Upper CL Dif } & 0.25937 \text { Prob }>|t| & 0.3894\end{array}$

$\begin{array}{lrlr}\text { Upper CL Dif } & 0.25937 & \text { Prob }>|t| & 0.3894 \\ \text { Lower CL Dif } & -0.64237 & \text { Prob }>t & 0.8053\end{array}$

$\begin{array}{lrl}\text { Confidence } & 0.95 \text { Prob }<\mathrm{t} & 0.1947\end{array}$

Analysis of Variance

$\begin{array}{lrrrrr}\text { Analysis of Variance } & & & & & \\ \text { Source } & \text { DF } & \text { Sum of Squares } & \text { Mean Square } & \text { F Ratio } & \text { Prob }>\text { F } \\ \text { Sampling Mechanism } & 1 & 0.2256754 & 0.225675 & 0.7684 & 0.3894 \\ \text { Error } & 24 & 7.0483400 & 0.293681 & & \\ \text { C. Total } & 25 & 7.2740154 & & & \end{array}$

(1)

Means for Oneway Anova

$\begin{array}{lrrrrr}\text { Level } & \text { Number } & \text { Mean } & \text { Std Error } & \text { Lower 95\% } & \text { Upper 95\% } \\ \text { Coliwasa } & 16 & 40.6075 & 0.13548 & 40.328 & 40.887\end{array}$

$\begin{array}{llllll}\text { Coliwasa } & 16 & 40.6075 & 0.13548 & 40.328 & 40.887 \\ \text { Hydragard } & 10 & 40.4160 & 0.17137 & 40.062 & 40.770\end{array}$

Std Error uses a pooled estimate of error variance

Means and Std Deviations

Level Number Mean Std Dev Std Err Mean Lower 95\% Upper 95\%

$\begin{array}{lllllll}\text { Coliwasa } & 16 & 40.6075 & 0.583364 & 0.14584 & 40.297 & 40.918\end{array}$

$\begin{array}{lllllll}\text { Hydragard } & 10 & 40.4160 & 0.464715 & 0.14696 & 40.084 & 40.748\end{array}$

t Test

Hydragard-Coliwasa

Assuming unequal variances

Difference $\quad-0.19150$ t Ratio $\quad-0.92494$

Std Err Dif $\quad 0.20704$ DF 22.41336

Upper CL Dif 0.23742 Prob $>|t| \quad 0.3648$

Lower CL Dif -0.62042 Prob $>t \quad 0.8176$

$\begin{array}{lll}\text { Confidence } & 0.95 \text { Prob }<\mathrm{t} \quad 0.1824\end{array}$

Tests that the Variances are Equal

$\begin{array}{lrrrr}\text { Level } & \text { Count } & \text { Std Dev } & \text { MeanAbsDif to Mean } & \text { MeanAbsDif to Median } \\ \text { Coliwasa } & 16 & 0.5833638 & 0.3787500 & 0.3787500\end{array}$

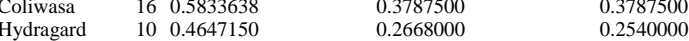

Test F Ratio DFNum DFDen p-Value

$\begin{array}{lllll}\text { O'Brien[.5] } & 0.1953 & 1 & 24 & 0.6625\end{array}$

$\begin{array}{lllll}\text { Brown-Forsythe } & 0.5431 & 1 & 24 & 0.4683\end{array}$

$\begin{array}{lllll}\text { Levene } & 0.4580 & 1 & 24 & 0.5050\end{array}$

$\begin{array}{lrrrr}\text { Bartlett } & 0.5319 & 1 & \text {. } & 0.4658 \\ \text { F Test 2-sided } & 1.5758 & 15 & 9 & 0.4966\end{array}$

Welch Anova testing Means Equal, allowing Std Devs Not Equal

F Ratio DFNum DFDen Prob $>$ F

$\begin{array}{llll}0.8555 & 1 & 22.413 & 0.3648\end{array}$

t Test

0.9249
Hydragard at Low Flow Rate

Oneway Analysis of Measurement By Sampling Mechanism Analyte=wt \% vitrified solids

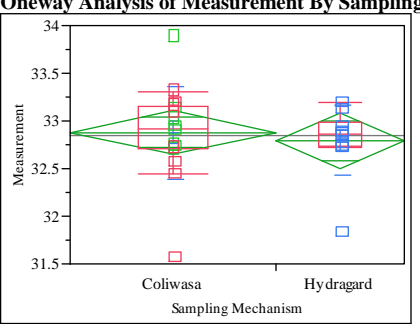

Oneway Anova

Summary of Fit

Rsquare

Adj Rsquare

Mean of Response

Observations (or Sum Wgts) r 26

t Test

Hydragard-Coliwasa

Assuming equal variances

Difference $\quad-0.08637$ t Ratio $\quad-0.47912$

$\begin{array}{lrr}\text { Std Err Dif } & 0.18028 \text { DF } & 24 \\ \text { Uper CL Dif } & 0.28570 \text { Prob }>|t| & 0.6362\end{array}$

Lower CL Dif -0.45845 Prob $>t \quad 0.6819$

$\begin{array}{lll}\text { Confidence } \quad 0.95 \text { Prob }<\mathrm{t} \quad 0.3181 & 0.0819\end{array}$

Analysis of Variance

$\begin{array}{lrrrrr}\text { Source } & \text { DF } & \text { Sum of Squares } & \text { Mean Square } & \text { F Ratio } & \text { Prob > F } \\ \text { Sampling Mechanism } & 1 & 0.0459116 & 0.045912 & 0.2296 & 0.6362\end{array}$

Error

$\begin{array}{lll}24 & 4.8001038 & 0.200004 \\ 25 & 4.8460154\end{array}$

Means for Oneway Anova

Level Number Mean Std Error Lower 95\% Upper 95\%

$\begin{array}{lrrrrr}\text { Level } & \text { Number } & \text { Mean } & \text { Std Error } & \text { Lower 95\% } & \text { Upper 95\% } \\ \text { Coliwasa } & 16 & 32.8794 & 0.11180 & 32.649 & 33.110\end{array}$

$\begin{array}{llllll}\text { Coliwasa } & 16 & 32.8794 & 0.11180 & 32.649 & 33.110 \\ \text { Hydragard } & 10 & 32.7930 & 0.14142 & 32.501 & 33.085\end{array}$

Std Error uses a pooled estimate of error variance

Means and Std Deviations

Level Number Mean Std Dev Std Err Mean Lower 95\% Upper 95\%

$\begin{array}{lllllll}\text { Coliwasa } & 16 & 32.8794 & 0.488432 & 0.12211 & 32.619 & 33.140 \\ \text { Hydragard } & 10 & 32.7930 & 0.36842 & 0.11651 & 32.529 & 33.057\end{array}$

t Test

Hydragard-Coliwasa

Assuming unequal variances

$\begin{array}{lrlr}\text { Difference } & -0.08637 & \text { t Ratio } & -0.51179 \\ \text { Std Err Dif } & 0.16877 \text { DF } & 22.98887 \\ \text { Upper CL Dif } & 0.26276 \text { Prob }>|t| r & 0.6137 \\ \text { Lower CL Dif } & -0.43551 & \text { Prob }>\text { t } & 0.6932\end{array}$

Confidence $\quad 0.95$ Prob $<\mathrm{t} \quad 0.3068$

Tests that the Variances are Equal

$\begin{array}{lrrrr}\text { Level } & \text { Count } & \text { Std Dev } & \text { MeanAbsDif to Mean } & \text { MeanAbsDif to Median } \\ \text { Coliwasa } & 16 & 0.4884324 & 0.3357031 & 0.3331250 \\ \text { Hydragard } & 10 & 0.3684216 & 0.2204000 & 0.2090000\end{array}$

$\begin{array}{lrrrr}\text { Test } & \text { F Ratio } & \text { DFNum } & \text { DFDen } & \text { p-Value } \\ \text { O'Brien[.5] } & 0.3156 & 1 & 24 & 0.5794 \\ \text { Brown-Forsythe } & 0.8558 & 1 & 24 & 0.3641 \\ \text { Levene } & 0.7819 & 1 & 24 & 0.3853 \\ \text { Bartlett } & 0.8075 & 1 & \text {. } & 0.3689 \\ \text { F Test 2-sided } & 1.7576 & 15 & 9 & 0.3957\end{array}$

Welch Anova testing Means Equal, allowing Std Devs Not Equal

F Ratio DFNum DFDen Prob $>$ F

$\begin{array}{llll}0.2619 & 1 & 22.989 & 0.6137\end{array}$

t Test

0.5118 
SRNL-STI-2011-00693

Revision 0

Exhibit B2. Statistical Comparisons for High-Rheology (Phase 2) Simulant Testing

Hydragard at High Flow Rate

Oneway Analysis of Measurement By Sampling Mechanism Analyte $=$ density $(\mathrm{g} / \mathrm{mL})$

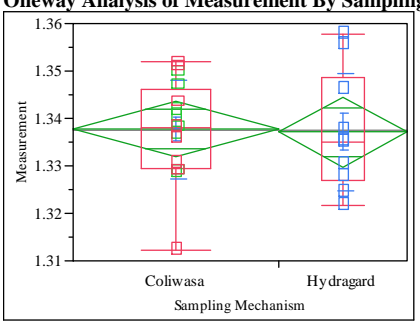

Summary of Fit

Adj Rsquare

oot Mean Square Error $\quad-0.0409$

Mean of Response $\quad 0.011204$

Observations (or Sum Wgts)

Test

Hydragard-Coliwasa

Assuming equal variances

Difference $\quad-0.00060$ t Ratio $\quad-0.1323$

$\begin{array}{lrrr}\text { Std Err Dif } & 0.00452 & \text { DF } & 24 \\ \text { Upper Cl } & 0.00872 \text { Prob }>|t| & 0.8959\end{array}$

$\begin{array}{rrrr}\text { Upper CL Dif } & 0.00872 & \text { Prob }>|t| & 0.8959 \\ \text { Lower CLL Dif } & -0.00992 & \text { Prob }>t & 0.5521\end{array}$

$\begin{array}{lrl}\text { Confidence } & 0.95 \text { Prob }<\mathrm{t} \quad 0.4479\end{array}$

Analysis of Variance

$\begin{array}{lrrrrr}\text { Analysis of Variance } & & & & & \\ \text { Source } & \text { DF } & \text { Sum of Squares } & \text { Mean Square } & \text { F Ratio } & \text { Prob }>\text { F } \\ \text { Sampling Mechanism } & 1 & 0.00000220 & 2.197 \mathrm{e}-6 & 0.0175 & 0.8959 \\ \text { Error } & 24 & 0.00301246 & 0.000126 & & \\ \text { C. Total } & 25 & 0.00301466 & & & \end{array}$

(1)

Means for Oneway Anova

Level Number Mean Std Error Lower 95\% Upper 95\%

\begin{tabular}{lrrrrr} 
Level & Number & Mean & Std Error & Lower 95\% & Upper 95\% \\
Coliwasa & 16 & 1.33774 & 0.00280 & 1.3320 & 1.3435 \\
\hline
\end{tabular}

$\begin{array}{llllll}\text { Coliwasa } & 16 & 1.33774 & 0.00280 & 1.3320 & 1.3435 \\ \text { Hydragard } & 10 & 1.33714 & 0.00354 & 1.3298 & 1.3445\end{array}$

Std Error uses a pooled estimate of error variance

Means and Std Deviations

Level Number Mean Std Dev Std Err Mean Lower 95\% Upper 95\%

$\begin{array}{lllllll}\text { Coliwasa } & 16 & 1.33774 & 0.010390 & 0.00260 & 1.3322 & 1.3433\end{array}$

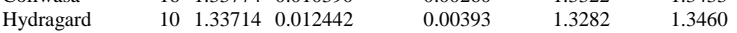

t Test

Hydragard-Coliwasa

Assuming unequal variances

Difference $\quad-0.00060$ t Ratio $\quad-0.12674$

Std Err Dif $\quad 0.00471$ DF 16.65696

Upper CL Dif 0.00936 Prob $>|t| \quad 0.9007$

Lower CL Dif -0.01056 Prob $>t \quad 0.5497$

$\begin{array}{lll}\text { Confidence } & 0.95 \text { Prob }<\mathrm{t} \quad 0.4503\end{array}$

Tests that the Variances are Equal

$\begin{array}{lrrrr}\text { Level } & \text { Count } & \text { Std Dev } & \text { MeanAbsDif to Mean } & \text { MeanAbsDif to Median } \\ \text { Coliwasa } & 16 & 0.0103901 & 0.0078078\end{array}$

$\begin{array}{lllll}\text { Coliwasa } & 16 & 0.0103901 & 0.0078078 & 0.0077750 \\ \text { Hydragard } & 10 & 0.0124416 & 0.0096880 & 0.0093000\end{array}$

Test F Ratio DFNum DFDen p-Value

$\begin{array}{lllll}\text { O'Brien[.5] } & 0.4244 & 1 & 24 & 0.5209\end{array}$

$\begin{array}{lllll}\text { Brown-Forsythe } & 0.2800 & 1 & 24 & 0.6016\end{array}$

$\begin{array}{lllll}\text { Levene } & 0.4753 & 1 & 24 & 0.4972\end{array}$

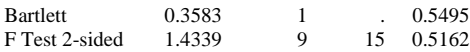

Welch Anova testing Means Equal, allowing Std Devs Not Equal

F Ratio DFNum DFDen Prob $>$ F

$\begin{array}{rrrr}0.0161 & 1 & 16.657 & 0.9007\end{array}$

t Test

0.1267
Hydragard at High Flow Rate

Oneway Analysis of Measurement By Sampling Mechanism Analyte $=w t \%$ dried solids

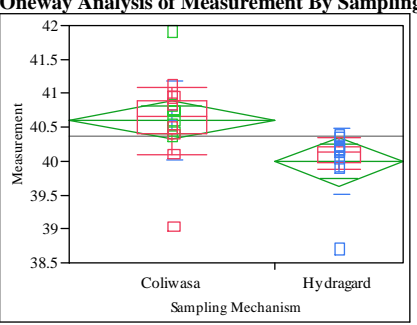

Oneway Anova

Summary of Fit

Rsquare

Adj Rsquare

Root Mean Square Error $\quad 0.547865$

Mean of Response $\quad 40.37154$

t Test

Hydragard-Coliwasa

Assuming equal variances

$\begin{array}{lrlr}\text { Difference } & -0.6135 & \mathrm{t} \text { Ratio } & -2.77789 \\ \text { Std Err Dif } & 0.2209 & \text { DF } & 24 \\ \text { Upper CL Dif } & -0.1577 & \text { Prob }>|t| & 0.0105 \\ \text { Lower CL Dif } & -1.0693 & \text { Prob }>\mathrm{t} & 0.9948 \\ \text { Confidence } & 0.95 & \text { Prob }<\mathrm{t} & 0.0052\end{array}$

Analysis of Variance

$\begin{array}{lrrrrr}\text { Source } & \text { DF } & \text { Sum of Squares } & \text { Mean Square } & \text { F Ratio } & \text { Prob }>\text { F } \\ \text { Sampling Mechanism } & 1 & 2.3161985 & 2.31620 & 7.7167 & 0.0105\end{array}$

$\begin{array}{rrrrr}1 & 2.3161985 & 2.31620 & 7.7167 & 0.0105 \\ 24 & 7.2037400 & 0.30016 & & \end{array}$

C. Total

9.5199385

Means for Oneway Anova

Level Number Mean Std Error Lower 95\% Upper 95\%

$\begin{array}{lrrrrr}\text { Level } & \text { Number } & \text { Mean } & \text { Std Error } & \text { Lower 95\% } & \text { Upper 95\% } \\ \text { Coliwasa } & 16 & 40.6075 & 0.13697 & 40.325 & 40.890\end{array}$

$\begin{array}{llllll}\text { Coliwasa } & 16 & 40.6075 & 0.13697 & 40.325 & 40.890 \\ \text { Hydragard } & 10 & 39.9940 & 0.17325 & 39.636 & 40.352\end{array}$

Std Error uses a pooled estimate of error variance

Means and Std Deviations

Level Number Mean Std Dev Std Err Mean Lower 95\% Upper 95\%

$\begin{array}{lllllll}\text { Coliwasa } & 16 & 40.6075 & 0.583364 & 0.14584 & 40.297 & 40.918\end{array}$

$\begin{array}{lllllll}\text { Hydragard } & 10 & 39.9940 & 0.482935 & 0.15272 & 39.649 & 40.339\end{array}$

t Test

Hydragard-Coliwasa

Assuming unequal variances

Difference $\quad-0.6135$ t Ratio -2.90526

Std Err Dif $\quad 0.2112$ DF 21.9482

Upper CL Dif -0.1755 Prob $>|t| 0.0082$

Lower CL Dif -1.0515 Prob $>t \quad 0.9959$

Confidence $\quad 0.95$ Prob $<\mathrm{t} \quad 0.0041$

Tests that the Variances are Equal

$\begin{array}{lrrrr}\text { Level } & \text { Count } & \text { Std Dev } & \text { MeanAbsDif to Mean } & \text { MeanAbsDif to Median } \\ \text { Coliwasa } & 16 & 0.5833638 & 0.3787500 & 0.3787500 \\ \text { Hydragard } & 10 & 0.4829355 & 0.2876000 & 0.2320000\end{array}$

$\begin{array}{lrrrr}\text { Test } & \text { F Ratio } & \text { DFNum } & \text { DFDen } & \text { p-Value } \\ \text { O'Brien[.5] } & 0.1337 & 1 & 24 & 0.7179 \\ \text { Brown-Forsythe } & 0.6885 & 1 & 24 & 0.4148 \\ \text { Levene } & 0.3007 & 1 & 24 & 0.5885 \\ \text { Bartlett } & 0.3703 & 1 & \text {. } & 0.5428 \\ \text { F Test 2-sided } & 1.4592 & 15 & 9 & 0.5762\end{array}$

Welch Anova testing Means Equal, allowing Std Devs Not Equal

F Ratio DFNum DFDen Prob $>$ F

$\begin{array}{llll}8.4405 & 1 & 21.948 & 0.0082\end{array}$

t Test

2.9053 
SRNL-STI-2011-00693

Revision 0

\section{Exhibit B2. Statistical Comparisons for High-Rheology (Phase 2) Simulant Testing}

Hydragard at High Flow Rate Oneway Analysis of Measurement By Sampling Mechanism Analyte=wt \% vitrified solids

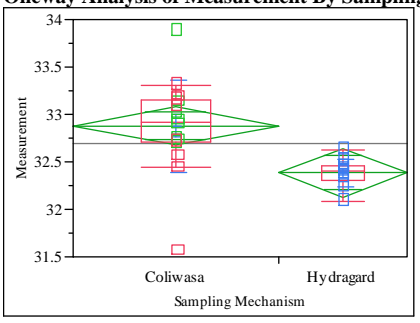

Summary of Fit

Adj Rsquare

Root Mean Square Error $\quad 0.25452$

Observations (or Sum Wgts) 26

Test

Hydragard-Coliwasa

Assuming equal variances

Difference $\quad-0.49338$ t Ratio $\quad-3.088$

$\begin{array}{lrlr}\text { Std Err Dif } & 0.15977 \text { DF } & 24 \\ & & \end{array}$

Upper CL Dif -0.16362 Prob $>|t| 0.0050$

$\begin{array}{lrll}\text { Lower CL Dif } & -0.82313 & \text { Prob }>t & 0.9975 \\ \text { Confidence } & 0.95 & \text { Prob }<\mathrm{t} & 0.0025\end{array}$

Analysis of Variance

$\begin{array}{lrrrrr}\text { Analysis of Variance } & & & & & \\ \text { Source } & \text { DF } & \text { Sum of Squares } & \text { Mean Square } & \text { F Ratio } & \text { Prob }>\text { F } \\ \text { Sampling Mechanism } & 1 & 1.4979624 & 1.49796 & 9.5358 & 0.0050 \\ \text { Error } & 24 & 3.7701337 & 0.15709 & & \\ \text { C. Total } & 25 & 5.2680962 & & & \end{array}$

Means for Oneway Anova

Level Number Mean Std Error Lower 95\% Upper 95\%

Level Number Mean Std Error Lower 95\% Upper 95\%

$\begin{array}{llllll}\text { Coliwasa } & 16 & 32.8794 & 0.09909 & 32.675 & 33.084 \\ \text { Hydragard } & 10 & 32.3860 & 0.12534 & 32.127 & 32.645\end{array}$

Std Error uses a pooled estimate of error variance

Means and Std Deviations

Level Number Mean Std Dev Std Err Mean Lower 95\% Upper 95\%

$\begin{array}{lllllll}\text { Coliwasa } & 16 & 32.8794 & 0.488432 & 0.12211 & 32.619 & 33.140\end{array}$

$\begin{array}{lllllll}\text { Hydragard } & 10 & 32.3860 & 0.145922 & 0.04614 & 32.282 & 32.490\end{array}$

t Test

Hydragard-Coliwasa

Assuming unequal variances

Difference $\quad-0.49338$ t Ratio $\quad-3.7796$

Std Err Dif $\quad 0.13054$ DF 18.94618

Upper CL Dif -0.22011 Prob $>|t| \quad 0.0013$

Lower CL Dif -0.76664 Prob $>t \quad 0.9994$

Confidence $\quad 0.95$ Prob $<\mathrm{t} \quad 0.0006$

Tests that the Variances are Equal

Level Count Std Dev MeanAbsDif to Mean MeanAbsDif to Median

$\begin{array}{lllll}\text { Coliwasa } & 16 & 0.4884324 & 0.3357031 & 0.3331250 \\ \text { Hydragard } & 10 & 0.1459224 & 0.1028000 & 0.1000000\end{array}$

Test F Ratio DFNum DFDen p-Value

$\begin{array}{lllll}\text { O'Brien[.5] } & 1.7581 & 1 & 24 & 0.1973\end{array}$

Brown-Forsythe $\quad 4.1706 \quad 1 \quad 24 \quad 0.0523$

$\begin{array}{lllll}\text { Levene } & 4.3042 & 1 & 24 & 0.0489\end{array}$

$\begin{array}{lrrrr}\text { Bartlett } & 11.2096 & 1 & & 0.0008 \\ \text { F Test 2-sided } & 11.2038 & 15 & 9 & 0.0009\end{array}$

Welch Anova testing Means Equal, allowing Std Devs Not Equal

F Ratio DFNum DFDen Prob $>$ F

$14.2854 \quad 1 \quad 18.946 \quad 0.0013$

t Test

3.7796
Isolok at Low Flow Rate

Oneway Analysis of Measurement By Sampling Mechanism Analyte $=$ density $(\mathrm{g} / \mathrm{mL})$

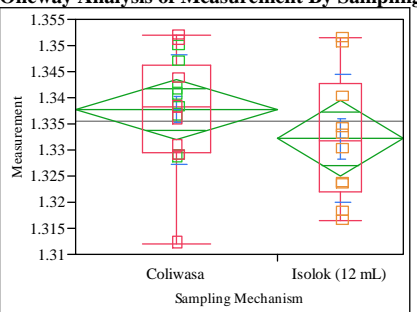

Oneway Anova

Summary of Fit

Rsquare

Adj Rsquare

Root Mean Square Error $\quad 0.01115$

Mean of Response 1.335604
26

t Test

Isolok $(12 \mathrm{~mL})$-Coliwasa

Assuming equal variances

Difference $\quad-0.00555$ t Ratio -1.23424

$\begin{array}{lrlr}\text { Std Err Dif } & 0.00449 \text { DF } & 24 \\ \text { Upper CL Dif } & 0.00373 & \text { Prob }>|t| & 0.2291\end{array}$

$\begin{array}{lrlr}\text { Upper CL Dif } & 0.00373 & \text { Prob }>|t| & 0.2291 \\ \text { Lower CL Dif } & -0.01482 & \text { Prob }>t & 0.8855\end{array}$

$\begin{array}{lrl}\text { Confidence } & 0.95 \text { Prob }<\mathrm{t} & 0.1145\end{array}$

Analysis of Variance

Source DF Sum of Squares Mean Square F Ratio Prob > F

$\begin{array}{lrllll}\text { Sampling Mechanism } & 1 & 0.00018938 & 0.000189 & 1.5233 & 0.2291\end{array}$

Error

$\begin{array}{lll}24 & 0.00298369 & 0.000124 \\ 25 & 0.00317307 & \end{array}$

Means for Oneway Anova

Level Number Mean Std Error Lower 95\% Upper 95\%

$\begin{array}{lrrrrr}\text { Coliwasa } & 16 & 1.33774 & 0.00279 & 1.3320 & 1.3435 \\ \text { Isolok }(12 \mathrm{~mL}) & 10 & 1.33219 & 0.00353 & 1.3249 & 1.3395\end{array}$

Std Error uses a pooled estimate of error variance

Means and Std Deviations

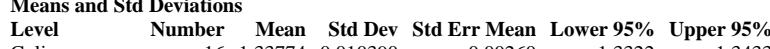

$\begin{array}{lrrrrrr}\text { Coliwasa } & 16 & 1.33774 & 0.010390 & 0.00260 & 1.3322 & 1.3433 \\ \text { Isolok }(12 \mathrm{~mL}) & 10 & 1.33219 & 0.012312 & 0.00389 & 1.3234 & 1.3410\end{array}$

t Test

Isolok (12 mL)-Coliwasa

Assuming unequal variances

$\begin{array}{lrlr}\text { Difference } & -0.00555 & \text { t Ratio } & -1.18524 \\ \text { Std Err Dif } & 0.00468 \text { DF } & 16.79766 \\ \text { Upper CL Dif } & 0.00434 & \text { Prob }>|t| & 0.2524 \\ \text { Lower CL Dif } & -0.01543 & \text { Prob }>\mathrm{t} & 0.8738 \\ \text { Confidence } & 0.95 & \text { Prob }<\mathrm{t} & 0.1262\end{array}$

Tests that the Variances are Equal

\begin{tabular}{|c|c|c|c|c|c|}
\hline Level & Count & Std Dev & MeanAl & bsDif to Mean & MeanAbsDif to Median \\
\hline Coliwasa & 16 & 0.0103901 & & 0.0078078 & 0.0077750 \\
\hline Isolok (12 mL) & 10 & 0.0123125 & & 0.0097100 & 0.0097100 \\
\hline Test & F Ratio & o DFNum & DFDen & p-Value & \\
\hline O'Brien[.5] & 0.3886 & $6 \quad 1$ & 24 & 0.5389 & \\
\hline Brown-Forsythe & 0.5126 & 1 & 24 & 0.4809 & \\
\hline Levene & 0.5016 & 1 & 24 & 0.4856 & \\
\hline Bartlett & 0.3176 & 1 & & 0.5731 & \\
\hline F Test 2-sided & 1.4043 & 9 & 15 & 0.5391 & \\
\hline
\end{tabular}

Welch Anova testing Means Equal, allowing Std Devs Not Equal

F Ratio DFNum DFDen Prob $>$ F

$\begin{array}{llll}1.4048 & 1 & 16.798 & 0.2524\end{array}$

t Test

1.1852 
SRNL-STI-2011-00693

Revision 0

\section{Exhibit B2. Statistical Comparisons for High-Rheology (Phase 2) Simulant Testing}

Isolok at Low Flow Rate

Oneway Analysis of Measurement By Sampling Mechanism Analyte $=w t \%$ dried solids

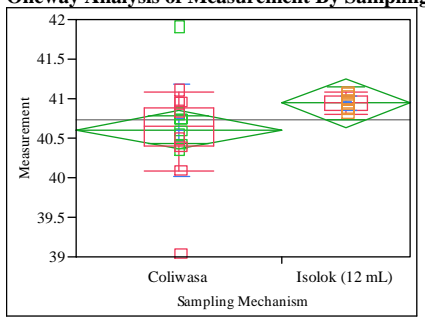

Oneway Anova

Summary of Fit

0.117121
0.080334

Root Mean Square Error $\quad 0.465047$

Mean of Response 40.73615

Observations (or Sum Wgts) $\quad 26$

t Test

Isolok (12 mL)-Coliwasa

Assuming equal variances

Difference $\quad 0.33450$ t Ratio 1.784318

$\begin{array}{lrr}\text { Std Err Dif } & 0.18747 \text { DF } & 24 \\ \text { Dpp } & & \end{array}$

Upper CL Dif 0.72141 Prob $>|t| \quad 0.0870$

$\begin{array}{lrll}\text { Lower CL Dif } & -0.05241 & \text { Prob }>t & 0.0435 \\ \text { Confidence } & 0.95 & \text { Prob }<t & 0.9565\end{array}$

$\begin{array}{lrrrrr}\begin{array}{l}\text { Analysis of Variance } \\ \text { Source }\end{array} & \text { DF } & \text { Sum of Squares } & \text { Mean Square } & \text { F Ratio } & \text { Prob > F } \\ \text { Sampling Mechanism } & 1 & 0.6885554 & 0.688555 & 3.1838 & 0.0870 \\ \text { Error } & 24 & 5.1904600 & 0.216269 & & \\ \text { C. Total } & 25 & 5.8790154 & & & \end{array}$

Means for Oneway Anova

$\begin{array}{lrrrrr}\text { Level } & \text { Number } & \text { Mean } & \text { Std Error } & \text { Lower 95\% } & \text { Upper 95\% } \\ \text { Coliwasa } & 16 & 40.6075 & 0.11626 & 40.368 & 40.847\end{array}$

$\begin{array}{llllll}\text { Coliwasa } & 16 & 40.6075 & 0.11626 & 40.368 & 40.847 \\ \text { Isolok }(12 \mathrm{~mL}) & 10 & 40.9420 & 0.14706 & 40.638 & 41.246\end{array}$

Std Error uses a pooled estimate of error variance

Means and Std Deviations

Level Number Mean Std Dev Std Err Mean Lower 95\% Upper 95\%

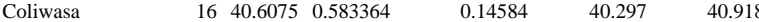

$\begin{array}{lllllll}\text { Isolok }(12 \mathrm{~mL}) & 10 & 40.9420 & 0.097616 & 0.03087 & 40.872 & 41.012\end{array}$

t Test

Isolok (12 mL)-Coliwasa

Assuming unequal variances

Difference $\quad 0.334500$ t Ratio 2.243882

Std Err Dif $\quad 0.149072$ DF $\quad 16.31953$

Upper CL Dif 0.650016 Prob $>|t| \quad 0.0390$

Lower CL Dif 0.018984 Prob $>$ t 0.0195

$\begin{array}{lll}\text { Confidence } & 0.95 \text { Prob }<\mathrm{t} \quad 0.9805\end{array}$

Tests that the Variances are Equal

Level Count Std Dev MeanAbsDif to Mean MeanAbsDif to Median

$\begin{array}{llll}16 & 0.5833638 & 0.3787500 & 0.3787500\end{array}$

$\begin{array}{lllll}\text { Isolok }(12 \mathrm{~mL}) & 10 & 0.0976160 & 0.0820000 & 0.0820000\end{array}$

Test F Ratio DFNum DFDen p-Value

$\begin{array}{lrrrr}\text { O'Brien[.5] } & 1.8022 & 1 & 24 & 0.1920\end{array}$

$\begin{array}{lllll}\text { Brown-Forsythe } & 4.5409 & 1 & 24 & 0.0435\end{array}$

$\begin{array}{lllll}\text { Levene } & 4.5990 & 1 & 24 & 0.0423\end{array}$

$\begin{array}{lrrrr}\text { Bartlett } & 20.3752 & 1 & & <.0001 \\ \text { F Test 2-sided } & 35.7139 & 15 & 9 & <.0001\end{array}$

Welch Anova testing Means Equal, allowing Std Devs Not Equal

F Ratio DFNum DFDen Prob $>$ F

$5.0350 \quad 16.32 \quad 0.0390$

t Test

2.2439
Isolok at Low Flow Rate

Oneway Analysis of Measurement By Sampling Mechanism Analyte $=w t \%$ vitrified solids

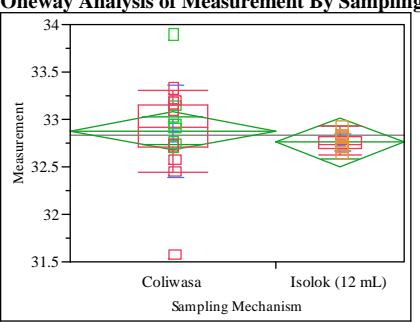

Oneway Anova

Summary of Fit

Adj Rsquare

Mean of Response $\quad 32.8323$

Observations (or Sum Wgts)

t Test

Isolok (12 mL)-Coliwasa

Assuming equal variances

$\begin{array}{lrlr}\text { Difference } & -0.12237 \text { t Ratio } & -0.77841 \\ \text { Std Err Dif } & 0.15721 & \text { DF } & 24\end{array}$

Upper CL Dif 0.20209 Prob $>|t| \quad 0.4439$

Lower CL Dif -0.44684 Prob $>t \quad 0.7780$

0.95 Prob $<\mathrm{t}-0.2220$

Analysis of Variance

$\begin{array}{lrrrrr}\text { Source } & \text { DF } & \text { Sum of Squares } & \text { Mean Square } & \text { F Ratio } & \text { Prob }>\text { F } \\ \text { Sampling Mechanism } & 1 & 0.0921578 & 0.092158 & 0.6059 & 0.4439 \\ \text { Error } & 24 & 3.6503038 & 0.152096 & & \end{array}$

24
25
2

Teans for Oneway Anova

Means for Oneway Anova
Level Number Mean Std Error Lower 95\% Upper 95\%

$\begin{array}{lrrrrr}\text { Level } & \text { Number } & \text { Mean } & \text { Std Error } & \text { Lower 95\% } & \text { Upper 95\% } \\ \text { Coliwasa } & 16 & 32.8794 & 0.09750 & 32.678 & 33.081\end{array}$

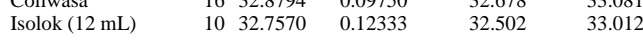

Std Error uses a pooled estimate of error variance

Means and Std Deviations

Level Number Mean Std Dev Std Err Mean Lower 95\% Upper 95\%

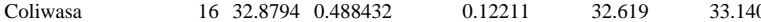

$\begin{array}{llllll}\text { Isolok }(12 \mathrm{~mL}) & 10 & 32.7570 & 0.089325 & 0.02825 & 32.693\end{array}$

Test

Isolok (12 mL)-Coliwas

Assuming unequal variances

Difference $\quad-0.12237$ t Ratio $\quad-0.9764$

$\begin{array}{lll}\text { Std Err Dif } & 0.12533 \text { DF } 16.56924\end{array}$

Upper CL Dif 0.14258 Prob $>|t| \quad 0.3429$

Lower CL Dif -0.38733 Prob $>\mathrm{t} \quad 0.8285$

$\begin{array}{lll}\text { Confidence } & 0.95 \text { Prob }<\mathrm{t} \quad 0.1715\end{array}$

Tests that the Variances are Equal

Level Count Std Dev MeanAbsDif to Mean MeanAbsDif to Median

$\begin{array}{lllll}\text { Coliwasa } & 16 & 0.4884324 & 0.3357031 & 0.3331250\end{array}$

$\begin{array}{llll}\text { Isolok }(12 \mathrm{~mL}) & 10 & 0.0893246 & 0.0724000-\end{array}$

Test F Ratio DFNum DFDen p-Value

$\begin{array}{lrrrr}\text { O'Brien[.5] } & 1.9852 & 1 & 24 & 0.1717\end{array}$

$\begin{array}{lllll}\text { Brown-Forsythe } & 5.4688 & 1 & 24 & 0.0280\end{array}$

$\begin{array}{lllll}\text { Levene } & 5.7052 & 1 & 24 & 0.0251\end{array}$

$\begin{array}{lrrrr}\text { Bartlett } & 18.9190 & 1 & \cdot & <.0001 \\ \text { F Test 2-sided } & 29.8997 & 15 & 9 & <.0001\end{array}$

Welch Anova testing Means Equal, allowing Std Devs Not Equal

F Ratio DFNum DFDen Prob $>$ F

$0.9534 \quad 1 \quad 16.569 \quad 0.3429$

t Test

0.976 
SRNL-STI-2011-00693

Revision 0

Exhibit B2. Statistical Comparisons for High-Rheology (Phase 2) Simulant Testing

Isolok at High Flow Rate

Oneway Analysis of Measurement By Sampling Mechanism Analyte $=$ density $(\mathrm{g} / \mathrm{mL})$

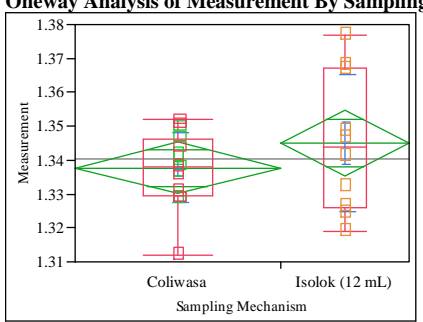

Oneway Anova

Summary of Fit

Adj Rsquare

Root Mean Square Error $\quad 0.01837$

Mean of Response $\quad 1.340524$

Observations (or Sum Wgts) 26

t Test

Isolok $(12 \mathrm{~mL})$-Coliwasa

Assuming equal variances

Difference $\quad 0.00725$ t Ratio 1.211541

$\begin{array}{lrr}\text { Std Err Dif } & 0.00598 \text { DF } & 24 \\ & & \end{array}$

Upper CL Dif 0.01959 Prob $>|t| \quad 0.2375$

$\begin{array}{lrll}\text { Lower CL Dif } & -0.00510 & \text { Prob }>t & 0.1187 \\ \text { Confidence } & 0.95 & \text { Prob }<t & 0.8813\end{array}$

$\begin{array}{lrrrrr}\text { Analysis of Variance } & & & & & \\ \text { Source } & \text { DF } & \text { Sum of Squares } & \text { Mean Square } & \text { F Ratio } & \text { Prob > F } \\ \text { Sampling Mechanism } & 1 & 0.00032310 & 0.000323 & 1.4678 & 0.2375 \\ \text { Error } & 24 & 0.00528286 & 0.000220 & & \\ \text { C. Total } & 25 & 0.00560596 & & & \end{array}$

Means for Oneway Anova

$\begin{array}{lclll}\text { Means for Oneway Anova } & & & \\ \text { Level } & \text { Number } & \text { Mean Std Error Lower 95\% Upper 95\% }\end{array}$

$\begin{array}{lrrrrr}\text { Level } & \text { Number } & \text { Mean } & \text { Std Error } & \text { Lower 95\% } & \text { Upper 95\% } \\ \text { Coliwasa } & 16 & 1.33774 & 0.00371 & 1.3301 & 1.3454\end{array}$

$\begin{array}{llllll}\text { Isolok }(12 \mathrm{~mL}) & 10 & 1.34498 & 0.00469 & 1.3353 & 1.3547\end{array}$

Std Error uses a pooled estimate of error variance

Means and Std Deviations

Level Number Mean Std Dev Std Err Mean Lower 95\% Upper 95\%

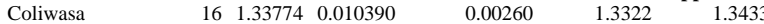

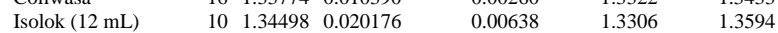

\section{t Test}

Isolok (12 mL)-Coliwasa

Assuming unequal variances

Difference $\quad 0.00725$ t Ratio 1.051868

$\begin{array}{lrr}\text { Std Err Dif } \quad 0.00689 \text { DF } & 12.03248\end{array}$

Upper CL Dif 0.02225 Prob $>|t| \quad 0.3135$

Lower CL Dif -0.00776 Prob $>t \quad 0.1568$

Confidence $\quad 0.95$ Prob $<\mathrm{t} \quad 0.8432$

Tests that the Variances are Equal

Level Count Std Dev MeanAbsDif to Mean MeanAbsDif to Median

$\begin{array}{lllll}\text { Coliwasa } & 16 & 0.0103901 & 0.0078078 & 0.0077750 \\ \text { solok }(12 \mathrm{~mL}) & 10 & 0.0201757 & 0.0163766 & 0.0163766\end{array}$

Test F Ratio DFNum DFDen p-Value

$\begin{array}{lrrrr}\text { O'Brien[.5] } & 7.1075 & 1 & 24 & 0.0135\end{array}$

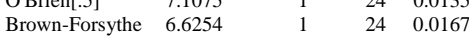

$\begin{array}{lllll}\text { Levene } & 6.6710 & 1 & 24 & 0.0163\end{array}$

$\begin{array}{lllll}\text { Bartlett } & 4.9301 & 1 & \text {. } & 0.0264 \\ \text { F Test 2-sided } & 3.7707 & 9 & 15 & 0.0230\end{array}$

Welch Anova testing Means Equal, allowing Std Devs Not Equal

F Ratio DFNum DFDen Prob $>$ F

$\begin{array}{lllr}1.1064 & 1 & 12.032 & 0.3135\end{array}$

t Test
1.0519
Isolok at High Flow Rate

Oneway Analysis of Measurement By Sampling Mechanism Analyte $=w t \%$ dried solids

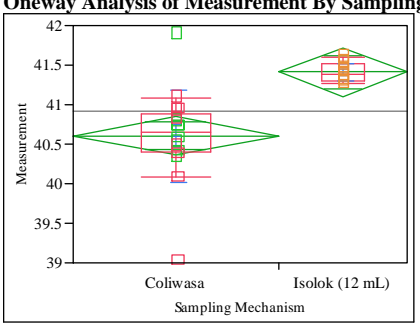

Oneway Anova

Summary of Fit

Rsquare

Adj Rsquare

Mean of Response $\quad 40.91577$

Observations (or Sum Wgts)

26

\section{t Test}

Isolok (12 mL)-Coliwasa

Assuming equal variances

Difference $\quad 0.80150$ t Ratio $\quad 4.263725$

Std Err Dif $\quad 0.18798$ DF 24

Upper CL Dif 1.18947 Prob $>|t| \quad 0.0003$

Lower CL Dif 0.41353 Prob $>t \quad 0.0001$

$\begin{array}{lll}\text { Confidence } & 0.95 \text { Prob }<\mathrm{t} \quad 0.9999\end{array}$

Analysis of Variance

$\begin{array}{lrrrrr}\text { Analysis of Variance } & \text { DF } & \text { Sum of Squares } & \text { Mean Square } & \text { F Ratio } & \text { Prob > F } \\ \text { Source } & 1 & 3.9532446 & 3.95324 & 18.1794 & 0.0003 \\ \text { Sampling Mechanism } & 24 & 5.2189900 & 0.21746 & & \\ \text { Error } & 2 & 9.1722346 & & & \end{array}$

24
25

Ceans for Oneway Anova

$\begin{array}{llll}\text { Means for Oneway Anova } & & \\ \text { Level } & \text { Number } & \text { Mean Std Error } & \text { Lower 95\% Upper 95\% }\end{array}$

$\begin{array}{lrrrrr}\text { Level } & \text { Number } & \text { Mean } & \text { Std Error } & \text { Lower 95\% } & \text { Upper 95\% } \\ \text { Coliwasa } & 16 & 40.6075 & 0.11658 & 40.367 & 40.848\end{array}$

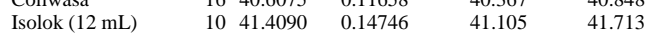

Std Error uses a pooled estimate of error variance

Means and Std Deviations

Level Number Mean Std Dev Std Err Mean Lower 95\% Upper 95\%

$\begin{array}{lllllll}\text { Coliwasa } & 16 & 40.6075 & 0.583364 & 0.14584 & 40.297 & 40.918\end{array}$

$\begin{array}{llllll}\text { Isolok }(12 \mathrm{~mL}) & 10 & 41.4090 & 0.112689 & 0.03564 & 41.328\end{array}$

t Test

Isolok (12 mL)-Coliwasa

Assuming unequal variances

Difference $\quad 0.80150$ t Ratio $\quad 5.338653$

Std Err Dif $\quad 0.15013$ DF 16.74512

Upper CL Dif 1.11862 Prob $>|t|<.0001$

Lower CL Dif 0.48438 Prob $>t \quad<.0001$

Confidence $\quad 0.95$ Prob $<\mathrm{t} \quad 1.0000$

Tests that the Variances are Equal

Level Count Std Dev MeanAbsDif to Mean MeanAbsDif to Median

$\begin{array}{lllll}\text { Coliwasa } & 16 & 0.5833638 & 0.3787500 & 0.3787500\end{array}$

$\begin{array}{llll}\text { Isolok }(12 \mathrm{~mL}) \quad 10 & 0.1126893 & 0.0930000 & 0.0930000\end{array}$

Test F Ratio DFNum DFDen p-Value

$\begin{array}{lrrrr}\text { O'Brien[.5] } & 1.7677 & 1 & 24 & 0.1962\end{array}$

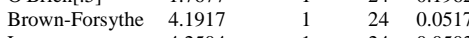

$\begin{array}{lrrrr}\text { Levene } & 4.2504 & 1 & 24 & 0.0502\end{array}$

$\begin{array}{lrrrr}\text { Bartlett } & 18.0285 & 1 & \cdot & <.0001 \\ \text { F Test 2-sided } & 26.7987 & 15 & 9 & <.0001\end{array}$

Welch Anova testing Means Equal, allowing Std Devs Not Equal

F Ratio DFNum DFDen Prob $>$ F

$28.5012 \quad 1 \quad 16.745<0001$

t Test

5.338 
SRNL-STI-2011-00693

Revision 0

Exhibit B2. Statistical Comparisons for High-Rheology (Phase 2) Simulant Testing

Isolok at High Flow Rate

Oneway Analysis of Measurement By Sampling Mechanism Analyte $=w t \%$ vitrified solids

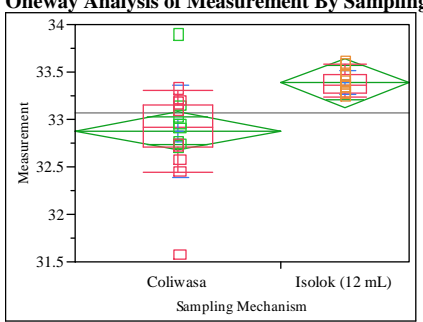

Oneway Anova

Summary of Fit

Adj Rsquare

Root Mean Square Error $\quad 0.268387$

Mean of Response

Observations (or Sum Wgts)

t Test

Isolok (12 mL)-Coliwasa

Assuming equal variances

Difference $\quad 0.505625$ t Ratio $\quad 3.189212$

$\begin{array}{lrr}\text { Std Err Dif } & 0.158542 \text { DF } & 24 \\ & & \end{array}$

Upper CL Dif 0.832840 Prob $>|t| \quad 0.0039$

Lower CL Dif 0.178410 Prob $>t \quad 0.0020$

Confidence $\quad 0.95$ Prob $<\mathrm{t} \quad 0.9980$

$\begin{array}{lrrrrr}\text { Analysis of Variance } & & & & & \\ \text { Source } & \text { DF } & \text { Sum of Squares } & \text { Mean Square } & \text { F Ratio } & \text { Prob > F } \\ \text { Sampling Mechanism } & 1 & 1.5732716 & 1.57327 & 10.1711 & 0.0039 \\ \text { Error } & 24 & 3.7123437 & 0.15468 & & \\ \text { C. Total } & 25 & 5.2856154 & & & \end{array}$

Means for Oneway Anova

$\begin{array}{lllll}\text { Means for Oneway Anova } & & & \\ \text { Level Number } & \text { Mean Std Error Lower 95\% Upper 95\% }\end{array}$

$\begin{array}{lrrrrr}\text { Level } & \text { Number } & \text { Mean } & \text { Std Error } & \text { Lower 95\% } & \text { Upper 95\% } \\ \text { Coliwasa } & 16 & 32.8794 & 0.09832 & 32.676 & 33.082\end{array}$

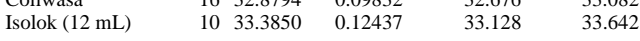

Std Error uses a pooled estimate of error variance

Means and Std Deviations

Level Number Mean Std Dev Std Err Mean Lower 95\% Upper 95\%

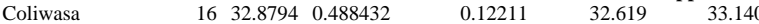

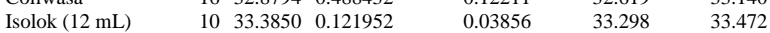

t Test

Isolok (12 mL)-Coliwasa

Assuming unequal variances

Difference $\quad 0.505625$ t Ratio 3.948555

Std Err Dif $\quad 0.128053$ DF $\quad 17.84565$

Upper CL Dif 0.774822 Prob $>|t| \quad 0.0010$

Lower CL Dif 0.236428 Prob $>t \quad 0.0005$

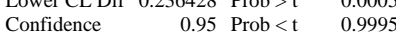

Tests that the Variances are Equal

Level Count Std Dev MeanAbsDif to Mean MeanAbsDif to Median

$\begin{array}{lllll}\text { Coliwasa } & 16 & 0.4884324 & 0.3357031 & 0.3331250\end{array}$

Test F Ratio DFNum DFDen p-Value

$\begin{array}{lrrrr}\text { O'Brien[.5] } & 1.8677 & 1 & 24 & 0.1844\end{array}$

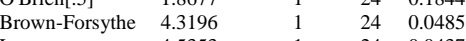

$\begin{array}{lrrrr}\text { Levene } & 4.5353 & 1 & 24 & 0.0437\end{array}$

$\begin{array}{lrrrr}\text { Bartlett } & 13.9449 & 1 & \text {. } & 0.0002 \\ \text { F Test 2-sided } & 16.0411 & 15 & 9 & 0.0002\end{array}$

Welch Anova testing Means Equal, allowing Std Devs Not Equal

F Ratio DFNum DFDen Prob $>$ F

$\begin{array}{rrrr}15.5911 & 1 & 17.846 & 0.0010\end{array}$

t Test

3.9486
Isolok at Low Flow Rate

Oneway Analysis of Measurement By Sampling Mechanism Analyte $=w t \%$ dried solids

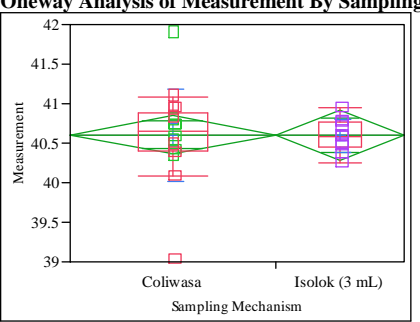

Oneway Anova

Summary of Fit

Rsquare

Adj Rsquare

Root Mean Square Error $\quad-0.04156$

Mean of Response $\quad 40.60385$

Observations (or Sum Wgts)

26

\section{t Test}

Isolok (3 mL)-Coliwasa

Assuming equal variances

$\begin{array}{lrlr}\text { Difference } & -0.00950 & \text { t Ratio } & -0.04922 \\ \text { Std Err Dif } & 0.19302 & \text { DF } & 24 \\ \text { Upper CL Dif } & 0.38886 & \text { Prob }>|t| & 0.9612 \\ \text { Lower CL Dif } & -0.40786 & \text { Prob }>\text { t } & 0.5194 \\ \text { Confidence } & 0.95 & \text { Prob }<\mathrm{t} & 0.4806\end{array}$

Lower CL Dif -0.40786 Prob $>\mathrm{t} \quad 0.5194$

Analysis of Variance

$\begin{array}{lrrrrr}\text { Analysis of Variance } & & & & & \\ \text { Source } & \text { DF } & \text { Sum of Squares } & \text { Mean Square } & \text { F Ratio } & \text { Prob }>\text { F } \\ \text { Sampling Mechanism } & 1 & 0.0005554 & 0.000555 & 0.0024 & 0.9612 \\ \text { Error } & 24 & 5.5022600 & 0.229261 & & \\ \text { C. Total } & 25 & 5.5028154 & & & \end{array}$

Means for Oneway Anova
Level Number Mean Std Error Lower 95\% Upper 95\%

$\begin{array}{lrrrrr}\text { Level } & \text { Number } & \text { Mean } & \text { Std Error } & \text { Lower 95\% } & \text { Upper 95\% } \\ \text { Coliwasa } & 16 & 40.6075 & 0.11970 & 40.360 & 40.855\end{array}$

$\begin{array}{llllll}\text { Isolok }(3 \mathrm{~mL}) & 10 & 40.5980 & 0.15141 & 40.285 & 40.911\end{array}$

Std Error uses a pooled estimate of error variance

Means and Std Deviations

Level Number Mean Std Dev Std Err Mean Lower 95\% Upper 95\%

$\begin{array}{lllllll}\text { Coliwasa } & 16 & 40.6075 & 0.583364 & 0.14584 & 40.297 & 40.918\end{array}$

$\begin{array}{llllll}\text { Isolok }(3 \mathrm{~mL}) & 10 & 40.5980 & 0.210175 & 0.06646 & 40.448\end{array}$

t Test

Isolok (3 mL)-Coliwasa

Assuming unequal variances

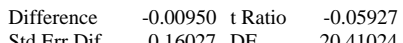

$\begin{array}{lll}\text { Std Err Dif } \quad 0.16027 & \text { DF } & 20.41024\end{array}$

Upper CL Dif 0.32439 Prob $>|t| 0.9533$

Lower CL Dif -0.34339 Prob $>\mathrm{t} \quad 0.5233$

$\begin{array}{lll}\text { Confidence } & 0.95 \text { Prob }<\mathrm{t} \quad 0.4767\end{array}$

Tests that the Variances are Equal

$\begin{array}{lrrr}\text { Level Count } & \text { Std Dev MeanAbsDif to Mean MeanAbsDif to Median }\end{array}$

$\begin{array}{lllll}\text { Isolok }(3 \mathrm{~mL}) & 16 & 0.5833638 & 0.3787500 & 0.3787500\end{array}$

Test F Ratio DFNum DFDen p-Value

$\begin{array}{lrrrr}\text { O'Brien[.5] } & 1.4403 & 1 & 24 & 0.2418\end{array}$

$\begin{array}{lllll}\text { Brown-Forsythe } & 2.2896 & 1 & 24 & 0.1433\end{array}$

$\begin{array}{lllll}\text { Levene } & 2.3183 & 1 & 24 & 0.1409\end{array}$

$\begin{array}{lrrrr}\text { Bartlett } & 8.5095 & 1 & \text {. } & 0.0035 \\ \text { F Test 2-sided } & 7.7040 & 15 & 9 & 0.0040\end{array}$

Welch Anova testing Means Equal, allowing Std Devs Not Equal

F Ratio DFNum DFDen Prob $>$ F

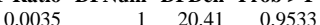

t Test

0.0593 
SRNL-STI-2011-00693

Revision 0

\section{Exhibit B2. Statistical Comparisons for High-Rheology (Phase 2) Simulant Testing}

Isolok at Low Flow Rate

Oneway Analysis of Measurement By Sampling Mechanism Analyte=wt \% vitrified solids

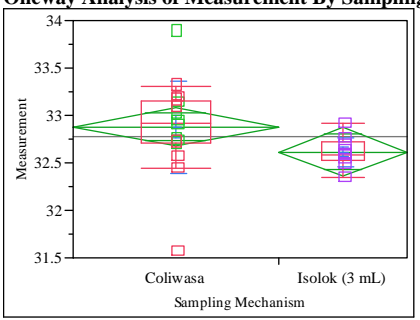

Oneway Anova

Summary of Fit

Adj Rsquare

Root Mean Square Error $\quad 0.063742$

Mean of Response $\quad 0.397468$

Observations (or Sum Wgts)

Test

Isolok (3 mL)-Coliwasa

Assuming equal variances

Difference $\quad-0.26337$ t Ratio $\quad-1.64379$

$\begin{array}{lrr}\text { Std Err Dif } & 0.16022 \text { DF } & 24 \\ \end{array}$

Upper CL Dif 0.06731 Prob $>|\mathrm{t}| \quad 0.1133$

$\begin{array}{lrrr}\text { Lower CL Dif } & -0.59406 & \text { Prob }>t & 0.9434 \\ \text { Confidence } & 0.95 & \text { Prob }<\mathrm{t} & 0.0566\end{array}$

$\begin{array}{lrrrrr}\begin{array}{lrrrr}\text { Analysis of Variance } \\ \text { Source }\end{array} & & & & & \\ \text { Sampling Mechanism } & 1 & \text { Sum of Squares } & \text { Mean Square } & \text { F Ratio } & \text { Prob > F } \\ \text { Error } & 24 & 3.4268701 & 0.426870 & 2.7020 & 0.1133 \\ \text { C. Total } & 25 & 4.2184038 & 0.157981 & & \\ & & & & & \\ \end{array}$

Teans for Oneway Anova

$\begin{array}{llll}\text { Means for Oneway Anova } & & \\ \text { Level Number Mean Std Error Lower 95\% Upper 95\% }\end{array}$

$\begin{array}{lrrrrr}\text { Level } & \text { Number } & \text { Mean } & \text { Std Error } & \text { Lower 95\% } & \text { Upper 95\% } \\ \text { Coliwasa } & 16 & 32.8794 & 0.09937 & 32.674 & 33.084\end{array}$

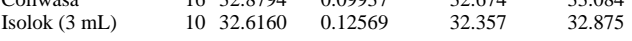

Std Error uses a pooled estimate of error variance

Means and Std Deviations

Level Number Mean Std Dev Std Err Mean Lower 95\% Upper 95\%

$\begin{array}{lllllll} & 16 & 32.8794 & 0.488432 & 0.12211 & 32.619 & 33.140\end{array}$

t Test

Isolok (3 mL)-Coliwasa

Assuming unequal variances

Difference $\quad-0.26337$ t Ratio $\quad-2.00371$

Std Err Dif $\quad 0.13144$ DF 19.32881

Upper CL Dif 0.01142 Prob $>|t| \quad 0.0593$

Lower CL Dif -0.53817 Prob $>\mathrm{t} \quad 0.9703$

Confidence $\quad 0.95$ Prob $<\mathrm{t} \quad 0.0297$

Tests that the Variances are Equal

Level Count Std Dev MeanAbsDif to Mean MeanAbsDif to Median

$\begin{array}{lllll} & 16 & 0.4884324 & 0.3357031 & 0.3331250\end{array}$

Test F Ratio DFNum DFDen p-Value

$\begin{array}{lrrrr}\text { O'Brien[.5] } & 1.7193 & 1 & 24 & 0.2022\end{array}$

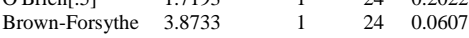

$\begin{array}{lrrrr}\text { Levene } & 3.9920 & 1 & 24 & 0.0572\end{array}$

$\begin{array}{lrrrr}\text { Bartlett } & 10.4282 & 1 & \text {. } & 0.0012 \\ \text { F Test 2-sided } & 10.0784 & 15 & 9 & 0.0014\end{array}$

Welch Anova testing Means Equal, allowing Std Devs Not Equal

F Ratio DFNum DFDen Prob > F

$\begin{array}{llll}4.0148 & 1 & 19.329 & 0.0593\end{array}$

t Test

2.0037
Isolok at High Flow Rate

Oneway Analysis of Measurement By Sampling Mechanism Analyte $=w t \%$ dried solids

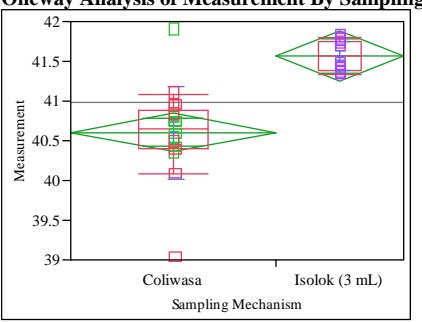

Oneway Anova

Summary of Fit

Adj Rsquare

Mean of Response $\quad 40.97615$

Observations (or Sum Wgts)

\section{t Test}

Isolok (3 mL)-Coliwasa

Assuming equal variances

Difference $\quad 0.95850$ t Ratio $\quad 4.997877$

$\begin{array}{lrr}\text { Std Err Dif } & 0.19178 \text { DF } & 24\end{array}$

Upper CL Dif 1.35432 Prob $>|t| \quad<.0001$

Lower CL Dif 0.56268 Prob $>t \quad<.0001$

Confidence $\quad 0.95$ Prob $<\mathrm{t} \quad 1.0000$

Analysis of Variance

$\begin{array}{lrrrrr}\text { Source } & \text { DF } & \text { Sum of Squares } & \text { Mean Square } & \text { F Ratio } & \text { Prob }>\text { F } \\ \text { Sampling Mechanism } & 1 & 5.653675 & 5.65368 & 24.9788 & <.0001 \\ \text { Error } & 24 & 5.432140 & 0.22634 & & \\ \text { C. Total } & 25 & 11.085815 & & & \end{array}$

Means for Oneway Anova

Level Number Mean Std Error Lower 95\% Upper 95\%

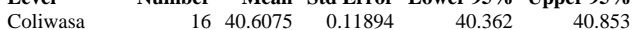

$\begin{array}{llllll}\text { Isolok }(3 \mathrm{~mL}) & 10 & 41.5660 & 0.15045 & 41.255 & 41.877\end{array}$

Std Error uses a pooled estimate of error variance

Means and Std Deviations

Level Number Mean Std Dev Std Err Mean Lower 95\% Upper 95\%

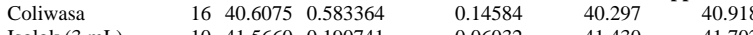

$\begin{array}{lllllll}\text { Isolok }(3 \mathrm{~mL}) & 10 & 41.5660 & 0.190741 & 0.06032 & 41.430 & 41.702\end{array}$

t Test

Isolok (3 mL)-Coliwasa

Assuming unequal variances

Difference $\quad 0.95850$ t Ratio $\quad 6.073295$

Std Err Dif $\quad 0.15782$ DF 19.61399

Upper CL Dif 1.28813 Prob $>|t|<.0001$

Lower CL Dif 0.62887 Prob $>t \quad<.0001$

Confidence $\quad 0.95$ Prob $<\mathrm{t} \quad 1.0000$

Tests that the Variances are Equal

Level Count Std Dev MeanAbsDif to Mean MeanAbsDif to Median

$\begin{array}{lllll}\text { Isolok }(3 \mathrm{~mL}) & 16 & 0.5833638 & 0.3787500 & 0.3787500\end{array}$

Test F Ratio DFNum DFDen p-Value

$\begin{array}{lrrrr}\text { O'Brien[.5] } & 1.5211 & 1 & 24 & 0.2294\end{array}$

$\begin{array}{lllll}\text { Brown-Forsythe } & 2.1204 & 1 & 24 & 0.1583\end{array}$

$\begin{array}{lllll}\text { Levene } & 2.1475 & 1 & 24 & 0.1558\end{array}$

$\begin{array}{lrrrr}\text { Bartlett } & 9.8856 & 1 & & 0.0017 \\ \text { F Test 2-sided } & 9.3538 & 15 & 9 & 0.0019\end{array}$

Welch Anova testing Means Equal, allowing Std Devs Not Equal

F Ratio DFNum DFDen Prob $>$ F

$36.8849 \quad 1 \quad 19.614<0001$

t Test

6.0733 
SRNL-STI-2011-00693

Revision 0

Exhibit B2. Statistical Comparisons for High-Rheology (Phase 2) Simulant Testing

Isolok at High Flow Rate

Oneway Analysis of Measurement By Sampling Mechanism Analyte $=w t \%$ vitrified solids

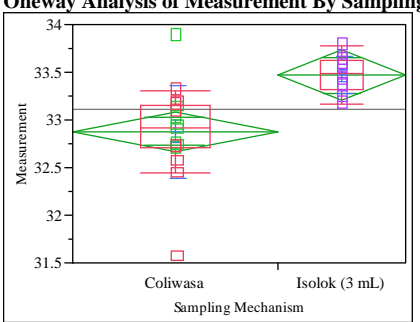

Oneway Anova

Summary of Fit

Root Mean Square Error $\quad 0.324909$

Mean of Response 33.10577

Observations (or Sum Wgts) 26

t Test

Isolok (3 mL)-Coliwasa

Assuming equal variances

Difference $\quad 0.588625$ t Ratio 3.609993

Std Err Dif 0.163054 DF

Upper CL Dif 0.925153 Prob $>|t| \quad 0.0014$

Lower CL Dif 0.252097 Prob $>t \quad 0.0007$

$\begin{array}{lll}\text { Confidence } & 0.95 \text { Prob }<\mathrm{t} \quad 0.9993\end{array}$

$\begin{array}{lrrrrr}\text { Analysis of Variance } & & & & & \\ \text { Source } & \text { DF } & \text { Sum of Squares } & \text { Mean Square } & \text { F Ratio } & \text { Prob }>\text { F } \\ \text { Sampling Mechanism } & 1 & 2.1321809 & 2.13218 & 13.0320 & 0.0014 \\ \text { Error } & 24 & 3.9266537 & 0.16361 & & \\ \text { C. Total } & 25 & 6.0588346 & & & \end{array}$

Means for Oneway Anova

$\begin{array}{llll}\text { Means for Oneway Anova } & & \\ \text { Level } & \text { Number Mean Std Error Lower 95\% Upper 95\% }\end{array}$

$\begin{array}{lrrrrr}\text { Level } & \text { Number } & \text { Mean } & \text { Std Error } & \text { Lower 95\% } & \text { Upper 95\% } \\ \text { Coliwasa } & 16 & 32.8794 & 0.10112 & 32.671 & 33.088\end{array}$

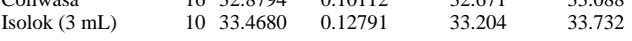

Std Error uses a pooled estimate of error variance

Means and Std Deviations

Level Number Mean Std Dev Std Err Mean Lower 95\% Upper 95\%

$\begin{array}{lllllll}\text { Coliwasa } & 16 & 32.8794 & 0.488432 & 0.12211 & 32.619 & 33.140\end{array}$

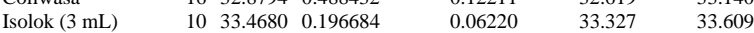

t Test

Isolok (3 mL)-Coliwasa

Assuming unequal variances

Difference $\quad 0.588625$ t Ratio 4.295408

Std Err Dif $\quad 0.137036$ DF 21.39305

Upper CL Dif 0.873288 Prob $>|t| 0.0003$

Lower CL Dif 0.303962 Prob $>t-10$

$\begin{array}{lrl}\text { Confidence } & 0.95 \text { Prob }<\mathrm{t} \quad 0.9998\end{array}$

Tests that the Variances are Equal

Level Count Std Dev MeanAbsDif to Mean MeanAbsDif to Median

$\begin{array}{lllll} & 16 & 0.4884324 & 0.3357031 & 0.3331250 \\ \text { Isolok }(3 \mathrm{~mL}) & 10 & 0.1966836 & 0.1660000 & 0.1660000\end{array}$

Test F Ratio DFNum DFDen p-Value

$\begin{array}{lrrrr}\text { O'Brien[.5] } & 1.4861 & 1 & 24 & 0.2347\end{array}$

$\begin{array}{lllll}\text { Brown-Forsythe } & 2.1656 & 1 & 24 & 0.1541\end{array}$

$\begin{array}{lllll}\text { Levene } & 2.3017 & 1 & 24 & 0.1423\end{array}$

$\begin{array}{lrrrr}\text { Bartlett } & 7.0033 & 1 & \text {. } & 0.0081 \\ \text { F Test 2-sided } & 6.1670 & 15 & 9 & 0.0092\end{array}$

Welch Anova testing Means Equal, allowing Std Devs Not Equal

F Ratio DFNum DFDen Prob > F

$\begin{array}{rrrr}18.4505 & 1 & 21.393 & 0.0003\end{array}$

t Test
Oneway Analysis of Measurement (wt \%) By Sampler Type of Material=SME Simulant, Analytical Block $=1$, Analyte $=A \mathrm{~A} / \mathrm{B}$, Target $(\mathrm{wt} \%)=2.0704412$

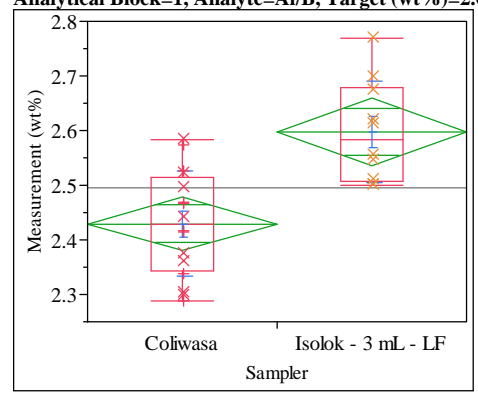

Oneway Anova

Summary of Fit

Adj Rsquare $\quad 0.446219$

Root Mean Square Error $\quad 0.095043$

Reot $\quad 0.095043$

$\begin{array}{lr}\text { Mean of Response } & 2.494118 \\ \text { Observations (or Sum Wgts) } & 26\end{array}$

t Test

Isolok - $3 \mathrm{~mL}$ - LF-Coliwasa

Assuming equal variances

Difference $\quad 0.168483$ t Ratio 4.397545

Std Err Dif $\quad 0.038313$ DF 24

$\begin{array}{lrlr}\text { Std Err Dif } & 0.038313 & \text { DF } & 24 \\ \text { Upper CL Dif } & 0.247557 & \text { Prob }>|t| & 0.0002\end{array}$

$\begin{array}{lll}\text { Lower CL Dif } 0.089409 \text { Prob }>t & <.0001\end{array}$

$\begin{array}{lrr}\text { Confidence } & 0.95 \text { Prob }<\mathrm{t} & 0.9999\end{array}$

Analysis of Variance

Source DF Sum of Squares Mean Square F Ratio Prob $>$ F

$\begin{array}{lrrrrr}\text { Sampler } & 1 & 0.17468607 & 0.174686 & 19.3384 & 0.0002\end{array}$

$\begin{array}{llll}\text { Error } & 24 & 0.21679481 & 0.009033\end{array}$

$\begin{array}{lll}\text { C. Total } 25 & 0.39148088\end{array}$

Means for Oneway Anova

Level Number Mean Std Error Lower 95\% Upper 95\%

$\begin{array}{lrrrrr}\text { Coliwasa } & 16 & 2.42932 & 0.02376 & 2.3803 & 2.4784 \\ \text { Isolok - 3 mL - LF } & 10 & 2.59780 & 0.03006 & 2.5358 & 2.6598\end{array}$

Std Error uses a pooled estimate of error variance

Means and Std Deviations

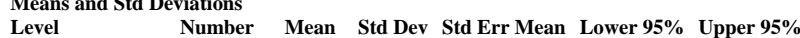

$\begin{array}{lrrrrrr}\text { Level } & \text { Number } & \text { Mean } & \text { Std Dev } & \text { Std Err Mean } & \text { Lower 95\% } & \text { Upper 95\% } \\ \text { Coliwasa } & 16 & 2.42932 & 0.096476 & 0.02412 & 2.3779 & 2.4807\end{array}$

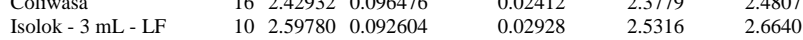

t Test

Isolok - $3 \mathrm{~mL}$ - LF-Coliwasa

Assuming unequal variances

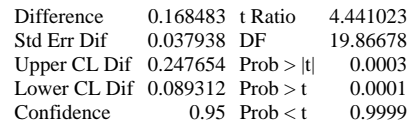

Confidence $\quad 0.95$ Prob $<$ t 0.0909

Tests that the Variances are Equal

$\begin{array}{lrrrr}\text { Level } & \text { Count } & \text { Std Dev } & \text { MeanAbsDif to Mean } & \text { MeanAbsDif to Median } \\ \text { Coliwasa } & 16 & 0.0964763 & 0.0803064 & 0.0803064\end{array}$

$\begin{array}{lllll}\text { Isolok - 3 mL - LF } & 10 & 0.0926040 & 0.0703064 & 0.0803064 \\ & & & 0.0761823\end{array}$

Test F Ratio DFNum DFDen p-Value

$\begin{array}{lrrrr}\text { O'Brien[.5] } & 0.0370 & 1 & 24 & 0.8490\end{array}$

$\begin{array}{lllll}\text { Brown-Forsythe } & 0.0434 & 1 & 24 & 0.8368\end{array}$

$\begin{array}{lllll}\text { Levene } & 0.0452 & 1 & 24 & 0.8334\end{array}$

$\begin{array}{lrrrr}\text { Bartlett } & 0.0179 & 1 & \text {. } & 0.8935 \\ \text { F Test 2-sided } & 1.0854 & 15 & 9 & 0.9332\end{array}$

Welch Anova testing Means Equal, allowing Std Devs Not Equal

F Ratio DFNum DFDen Prob $>$ F

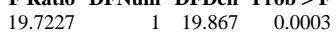

t Test

4.4410 


\section{Exhibit B2. Statistical Comparisons for High-Rheology (Phase 2) Simulant Testing}

Oneway Analysis of Measurement (wt \%) By Sampler Type of Material=SME Simulant, Analytical Block $=1$, Analyte $=$ Al2O3 $(w t \%)$, Target $(w t \%)=10.934$

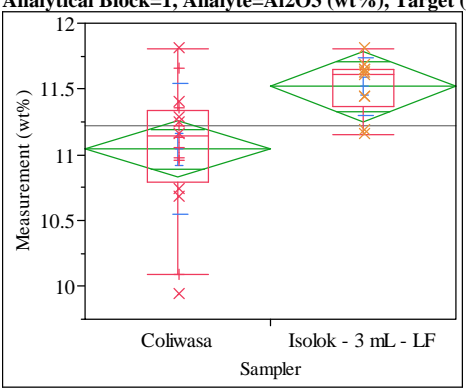

Oneway Anova

Summary of Fit

Adj Rsquare

\begin{tabular}{rr}
0.41451 \\
\hline
\end{tabular}

Observations (or Sum Wgts)

t Test

Isolok - $3 \mathrm{~mL}$ - LF-Coliwasa

Assuming equal variances

Difference $\quad 0.476626$ t Ratio $\quad 2.852439$

Std Err Dif $\quad 0.167094$ DF 24

Upper CL Dif 0.821492 Prob $>|t| \quad 0.0088$

Lower CL Dif 0.131761 Prob $>t \quad 0.0044$

$\begin{array}{lrl}\text { Confidence } & 0.95 \text { Prob }<\mathrm{t} \quad 0.9956\end{array}$

Analysis of Variance

Source DF Sum of Squares Mean Square F Ratio Prob $>$ F

$\begin{array}{llllll}\text { Sampler } & 1 & 1.3979859 & 1.39799 & 8.1364 & 0.0088\end{array}$

$\begin{array}{llll}\text { Error } & 24 & 4.1236464 & 0.17182\end{array}$

C. Total $25 \quad 5.5216322$

Means for Oneway Anova

Level Number Mean Std Error Lower 95\% Upper 95\%

$\begin{array}{lrrrrr}\text { Coliwasa } & 16 & 11.0418 & 0.10363 & 10.828 & 11.256\end{array}$

$\begin{array}{llllll}\text { Isolok - 3 mL - LF } & 10 & 11.5184 & 0.13108 & 11.248 & 11.789\end{array}$

Std Error uses a pooled estimate of error variance

Means and Std Deviations

Level Number Mean Std Dev Std Err Mean Lower 95\% Upper 95\%

$\begin{array}{lllllll}16 & 11.0418 & 0.496948 & 0.12424 & 10.777 & 11.307\end{array}$

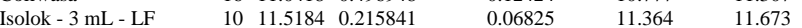

t Test

Isolok - $3 \mathrm{~mL}$ - LF-Coliwasa

Assuming unequal variances

Difference $\quad 0.476626$ t Ratio $\quad 3.362402$

Std Err Dif $\quad 0.141752$ DF 22.07038

Upper CL Dif 0.770547 Prob $>|t| \quad 0.0028$

Lower CL Dif 0.182706 Prob $>t \quad 0.0014$

$\begin{array}{lrl}\text { Confidence } & 0.95 \text { Prob }<\mathrm{t} \quad 0.9986\end{array}$

Tests that the Variances are Equa

Level Count Std Dev MeanAbsDif to Mean MeanAbsDif to Median

$\begin{array}{lllll}\text { Coliwasa } & 16 & 0.4969481 & 0.3595955 & 0.3471956\end{array}$

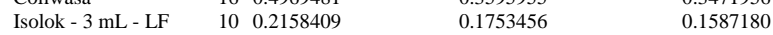

Test F Ratio DFNum DFDen p-Value

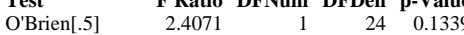

$\begin{array}{lllll}\text { Brown-Forsythe } & 2.4071 & 1 & 24 & 0.1339 \\ & 2.4080 & 1 & 24 & 0.1338\end{array}$

$\begin{array}{lllll}\text { Levene } & 2.8696 & 1 & 24 & 0.1032\end{array}$

$\begin{array}{lrrrr}\text { Bartlett } & 6.0307 & 1 & \text {. } & 0.0141 \\ \text { F Test 2-sided } & 5.3010 & 15 & 9 & 0.0159\end{array}$

Welch Anova testing Means Equal, allowing Std Devs Not Equal

F Ratio DFNum DFDen Prob $>$ F

$\begin{array}{rrrr}11.3057 & 1 & 22.07 & 0.0028\end{array}$

t Test

3.3624
Oneway Analysis of Measurement (wt \%) By Sampler Type of Material=SME Simulant, Analytical Block $=1$, Analyte $=\mathrm{B} 2 \mathrm{O} 3(\mathrm{wt} \%)$, Target $(\mathrm{wt} \%)=5.281$

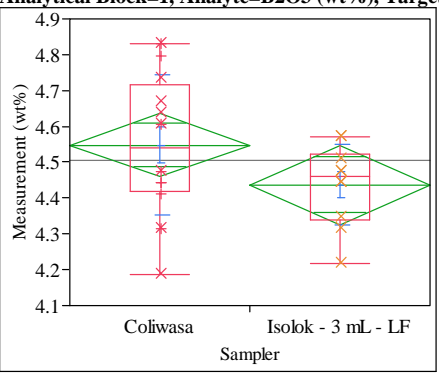

Oneway Anova

Summary of Fit

Adj Rsquare $\quad 0.099002$

0.06146
0.169695

0.169695

Mean of Respons 4.505383
26

t Test

Isolok - $3 \mathrm{~mL}$ - LF-Coliwasa

Assuming equal variances

$\begin{array}{lrlr}\text { Difference } & -0.11109 & \text { t Ratio } & -1.62392 \\ \text { Std Err Dif } & 0.06841 & \text { DF } & 24 \\ \text { Upper CL Dif } & 0.03010 & \text { Prob }>|t| & 0.1175 \\ \text { Lower CL Dif } & -0.25227 & \text { Prob }>t & 0.9413 \\ \text { Confidence } & 0.95 & \text { Prob }<\mathrm{t} & 0.0587\end{array}$

Analysis of Variance

Source DF Sum of Squares Mean Square F Ratio Prob $>$ F

$\begin{array}{lrrrrr}\text { Sampler } & 1 & 0.07593983 & 0.075940 & 2.6371 & 0.1175\end{array}$

$\begin{array}{llll}\text { Error } & 24 & 0.69111462 & 0.028796\end{array}$

C. Total $25 \quad 0.76705444$

Means for Oneway Anova

Level Number Mean Std Error Lower 95\% Upper 95\%

$\begin{array}{lllllr}\text { Coliwasa } & 16 & 4.54811 & 0.04242 & 4.4606 & 4.6357 \\ \text { Isolok - 3 mL - LF } & 10 & 4.43702 & 0.05366 & 4.3263 & 4.5478\end{array}$

Std Error uses a pooled estimate of error variance

Means and Std Deviations

$\begin{array}{lllll}\text { Means and Std Deviations } & & & \\ \text { Level } & \text { Number } & \text { Mean } & \text { Std Dev Std Err Mean Lower 95\% Upper 95\% }\end{array}$

$\begin{array}{lrrrrrr}\text { Coliwasa } & 16 & 4.54811 & 0.195859 & 0.04896 & 4.4437 & 4.6525\end{array}$

$\begin{array}{lllllll}\text { Coliwasa } & 16 & 4.54811 & 0.195859 & 0.04896 & 4.4437 & 4.6525 \\ \text { Isolok - 3 mL - LF } & 10 & 4.43702 & 0.113384 & 0.03586 & 4.3559 & 4.5181\end{array}$

t Test

Isolok - $3 \mathrm{~mL}$ - LF-Coliwasa

Assuming unequal variances

$\begin{array}{lrlr}\text { Difference } & -0.11109 & \text { t Ratio } & -1.83043 \\ \text { Std Err Dif } & 0.06069 & \text { DF } & 23.93124 \\ \text { Upper CL Dif } & 0.01419 & \text { Prob }>|t| & 0.0797 \\ \text { Lower CL Dif } & -0.23636 & \text { Prob }>t & 0.9602 \\ \text { Confidence } & 0.95 & \text { Prob }<\mathrm{t} & 0.0398\end{array}$

Tests that the Variances are Equal

Level Count Std Dev MeanAbsDif to Mean MeanAbsDif to Median

$\begin{array}{lrrrr}\text { Coliwasa } & 16 & 0.1958589 & 0.1650199 & 0.1650199\end{array}$

$\begin{array}{lllll}\text { Isolok - } 3 \mathrm{~mL} \text { - LF } & 10 & 0.1133844 & 0.1650199 & 0.1650199 \\ & & & 0.0862933 & 0.0837174\end{array}$

Test F Ratio DFNum DFDen p-Value

$\begin{array}{lllll}\text { O'Brien[.5] } & 3.3808 & 1 & 24 & 0.0784\end{array}$

$\begin{array}{lllll}\text { Brown-Forsythe } & 5.0995 & 1 & 24 & 0.0333\end{array}$

$\begin{array}{lllll}\text { Levene } & 5.0589 & 1 & 24 & 0.0339 \\ \text { Batlett } & 2.8279 & 1 & & 0.0926\end{array}$

$\begin{array}{lllll}\text { F Test 2-sided } & 2.9839 & 15 & 9 & 0.1022\end{array}$

Welch Anova testing Means Equal, allowing Std Devs Not Equal

F Ratio DFNum DFDen Prob $>$ F

$\begin{array}{rrrr}3.3505 & 1 & 23.931 & 0.0797\end{array}$

t Test

1.8304 


\section{Exhibit B2. Statistical Comparisons for High-Rheology (Phase 2) Simulant Testing}

Oneway Analysis of Measurement (wt\%) By Sampler Type of Material=SME Simulant, Analytical Block=1, Analyte $=\mathrm{BaO}(\mathrm{wt} \%)$, Target $(\mathrm{wt} \%)=0$

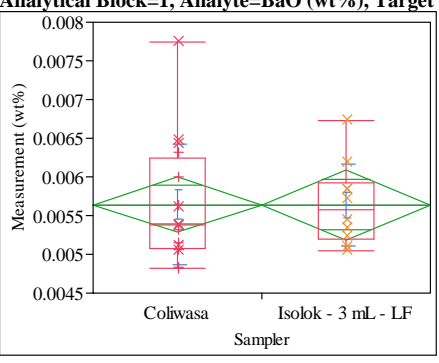

Oneway Anova

Summary of Fi

Adj Rsquare

Root Mean Square Eror

Mean of Response

0.000696 0.005643

Observations (or Sum Wgts) $\quad 26$

t Test

Isolok - $3 \mathrm{~mL}$ - LF-Coliwasa

Assuming equal variances

Difference $\quad-4.19 \mathrm{e}-7$ t Ratio $\quad-0.00149$

Std Err Dif $\quad 0.00028$ DF $\quad 24$

Upper CL Dif 0.00058 Prob $>|t| r \mid r .9988$

$\begin{array}{lll}\text { Lower CL Dif }-0.00058 \text { Prob }>t & 0.5006\end{array}$

Confidence $\quad 0.95$ Prob $<\mathrm{t} \quad 0.4994$

Analysis of Variance

Source DF Sum of Squares Mean Square F Ratio Prob $>$ F

$\begin{array}{lrrrrr}\text { Sampler } & 1 & 1.0788 \mathrm{e}-12 & 1.079 \mathrm{e}-12 & 0.0000 & 0.9988\end{array}$

$\begin{array}{lrrr}\text { Sampler } & 1 & 1.0788 \mathrm{e}-12 & 1.079 \mathrm{e}-12 \\ \text { Error } & 24 & 0.00001164 & 4.8495 \mathrm{e}-7\end{array}$

$\begin{array}{lll}\text { C. Total } & 25 & 0.00001164\end{array}$

Means for Oneway Anova

Level Number Mean Std Error Lower 95\% Upper 95\%

$\begin{array}{llllll} & 16 & 0.005643 & 0.00017 & 0.00528 & 0.00600 \\ \text { Isolok - 3 mL - LF } & 10 & 0.005643 & 0.00022 & 0.00519 & 0.00610\end{array}$

Std Error uses a pooled estimate of error variance

Means and Std Deviations

Level Number Mean Std Dev Std Err Mean Lower 95\% Upper 95\%

$\begin{array}{llllllr}\text { Coliwasa } & 16 & 0.005643 & 0.000780 & 0.00019 & 0.00523 & 0.00606 \\ \text { Isolok - 3 mL - LF } & 10 & 0.005643 & 0.000529 & 0.00017 & 0.00526 & 0.00602\end{array}$

t Test

Isolok - $3 \mathrm{~mL}$ - LF-Coliwasa

Assuming unequal variances

Difference $\quad-4.19 \mathrm{e}-7$ t Ratio $\quad-0.00163$

Std Err Dif $\quad 0.00026$ DF 23.75286

Upper CL Dif 0.00053 Prob $>|t| r|r| r 98$

Lower CL Dif -0.00053 Prob $>t \quad 0.5006$

$\begin{array}{lll}\text { Confidence } & 0.95 \text { Prob }<\mathrm{t} \quad 0.4994\end{array}$

Tests that the Variances are Equal

Level Count Std Dev MeanAbsDif to Mean MeanAbsDif to Median

$\begin{array}{lrrrr}\text { Coliwasa } & 16 & 0.0007797 & 0.0005929 & 0.0005548\end{array}$

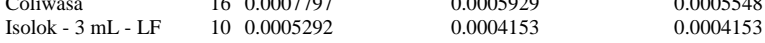

Test F Ratio DFNum DFDen p-Value

$\begin{array}{lrrrr}\text { O'Brien[.5] } & 0.7190 & 1 & 24 & 0.4048\end{array}$

Brown-Forsythe $\quad 0.4643 \quad 1 \quad 24 \quad 0.5021$

$\begin{array}{lllll}\text { Levene } & 1.0853 & 1 & 24 & 0.3079\end{array}$

$\begin{array}{lrrrr}1.4843 & 1 & 0.223\end{array}$

Welch Anova testing Means Equal, allowing Std Devs Not Equal

F Ratio DFNum DFDen Prob $>$ F

$\begin{array}{lllll}0.0000 & 1 & 23.753 & 0.9987\end{array}$

t Test

0.0016
Oneway Analysis of Measurement (wt\%) By Sampler Type of Material=SME Simulant, Analytical Block $=1$, Analyte $=\mathrm{CaO}(\mathrm{wt} \%)$, Target $(\mathrm{wt} \%)=0.543$

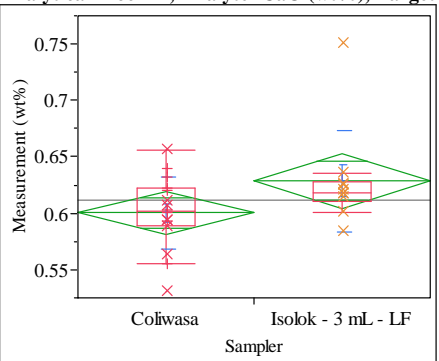

Oneway Anova

Summary of Fit

Adj Rsquare

0.037389

Mean of Response 0.611235

Observations (or Sum Wgts)

t Test

Isolok - $3 \mathrm{~mL}$ - LF-Coliwasa

Assuming equal variances

Difference $\quad 0.02832$ t Ratio 1.878745

$\begin{array}{llr}\text { Std Err Dif } \quad 0.01507 \text { DF } & 24\end{array}$

Upper CL Dif 0.05942 Prob $>|t| \quad 0.0725$

$\begin{array}{lll}\text { Lower CL Dif }-0.00279 \text { Prob }>t \quad 0.0362 & 0.9638\end{array}$

$\begin{array}{lrl}\text { Confidence } & 0.95 \text { Prob }<\mathrm{t} & 0.9638\end{array}$

Analysis of Variance

Source DF Sum of Squares Mean Square F Ratio Prob $>$ F

$\begin{array}{llllll}\text { Sampler } & 1 & 0.00493424 & 0.004934 & 3.5297 & 0.0725\end{array}$

$\begin{array}{llll}\text { Error } & 24 & 0.03355022 & 0.001398\end{array}$

C. Total $25 \quad 0.03848445$

Means for Oneway Anova

Level Number Mean Std Error Lower 95\% Upper 95\%

$\begin{array}{lrrrrr}\text { Coliwasa } & 16 & 0.600344 & 0.00935 & 0.58105 & 0.61964\end{array}$

$\begin{array}{llllll}\text { Isolok - 3 mL - LF } & 16 & 0.600344 & 0.00935 & 0.58105 & 0.61964 \\ & 10 & 0.628661 & 0.01182 & 0.60426 & 0.65306\end{array}$

Std Error uses a pooled estimate of error variance

Means and Std Deviations

Level Number Mean Std Dev Std Err Mean Lower 95\% Upper 95\%

$\begin{array}{lrrrrrr}\text { Coliwasa } & 16 & 0.600344 & 0.031994 & 0.00800 & 0.58330 & 0.61739\end{array}$

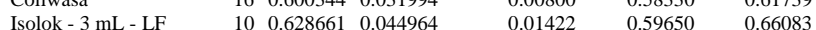

tTest

Isolok - $3 \mathrm{~mL}$ - LF-Coliwasa

Assuming unequal variances

$\begin{array}{lrlr}\text { Difference } & 0.02832 & \mathrm{t} \text { Ratio } & 1.735692 \\ \text { Std Err Dif } & 0.01631 & \text { DF } & 14.71329 \\ \text { Upper CL Dif } & 0.06315 & \text { Prob }>|t| & 0.1035 \\ \text { Lower CL Dif } & -0.00652 & \text { Prob }>\mathrm{t} & 0.0518 \\ \text { Confidence } & 0.95 & \text { Prob }<\mathrm{t} & 0.9482\end{array}$

Tests that the Variances are Equal

Level Count Std Dev MeanAbsDif to Mean MeanAbsDif to Median

$\begin{array}{lllll}\text { Coliwasa } & 16 & 0.0319944 & 0.0233491 & 0.0233491\end{array}$

$\begin{array}{lllll}\text { Isolok - } 3 \mathrm{~mL} \text { - LF } & 10 & 0.0449637 & 0.0255774 & 0.0222473\end{array}$

Test F Ratio DFNum DFDen p-Value

$\begin{array}{lrrrr}\text { O'Brien[.5] } & 0.4938 & 1 & 24 & 0.4890\end{array}$

$\begin{array}{lllll}\text { Brown-Forsythe } & 0.0086 & 1 & 24 & 0.9271\end{array}$

$\begin{array}{lllll}\text { Levene } & 0.0401 & 1 & 24 & 0.8430\end{array}$

$\begin{array}{lllrl}\text { Bartlett } & 1.2949 & 1 & \text {. } & 0.2551 \\ \text { F Test 2-sided } & 1.9750 & 9 & 15 & 0.2344\end{array}$

Welch Anova testing Means Equal, allowing Std Devs Not Equal

F Ratio DFNum DFDen Prob $>$ F

$\begin{array}{rrrr}3.0126 & 1 & 14.713 & 0.1035\end{array}$

t Test

1.7357 
SRNL-STI-2011-00693

Revision 0

\section{Exhibit B2. Statistical Comparisons for High-Rheology (Phase 2) Simulant Testing}

Oneway Analysis of Measurement (wt \%) By Sampler Type of Material=SME Simulant, Analytical Block $=1$, Analyte $=$ Cr2O3 $(w t \%)$, Target $(w t \%)=0.015$

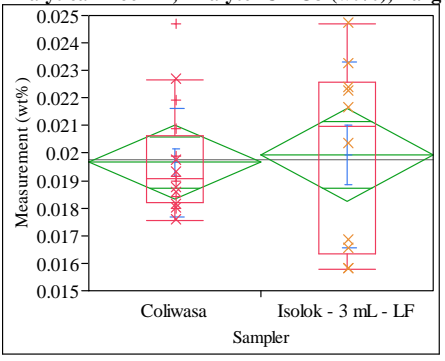

Oneway Anova

Summary of Fit

Rsquare

Adj Rsquare

Root Mean Square Error $\quad 0.002596$

Mean of Response 0.019765

Observations (or Sum Wgts)

Test

Isolok - $3 \mathrm{~mL}$ - LF-Coliwas

Assuming equal variances

Difference $\quad 0.00028$ t Ratio $\quad 0.265348$

$\begin{array}{lrr}\text { Std Err Dif } & 0.00105 \text { DF } & 24 \\ \text { Dpper } & & \\ \text { D Dif } & 0.00244 \text { Prob }>|t| & 0.7930\end{array}$

Upper CL Dif 0.00244 Prob $>|t| \quad 0.7930$

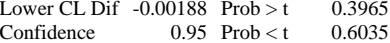

Analysis of Variance

Source DF Sum of Squares Mean Square F Ratio Prob $>$ F

$\begin{array}{lrrrrr}\text { Sampler } & 1 & 0.00000047 & 4.7458 \mathrm{e}-7 & 0.0704 & 0.7930\end{array}$

$\begin{array}{llll}\text { Error } & 24 & 0.00016177 & 6.7403 \mathrm{e}-6\end{array}$

C. Total $25 \quad 0.00016224$

Means for Oneway Anova

Level Number Mean Std Error Lower 95\% Upper 95\%

$\begin{array}{lrrrrr}\text { Coliwasa } & 16 & 0.019659 & 0.00065 & 0.01832 & 0.02100\end{array}$

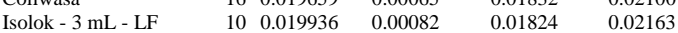

Std Error uses a pooled estimate of error variance

Means and Std Deviations

$\begin{array}{lrrrrrr}\text { Level } & \text { Number } & \text { Mean } & \text { Std Dev } & \text { Std Err Mean } & \text { Lower 95\% } & \text { Upper 95\% }\end{array}$

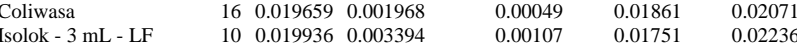

t Test

Isolok - $3 \mathrm{~mL}$ - LF-Coliwasa

Assuming unequal variances

Difference $\quad 0.00028$ t Ratio $\quad 0.235216$

Std Err Dif $\quad 0.00118$ DF $\quad 12.84094$

Upper CL Dif 0.00283 Prob $>|t| 0.8178$

Lower CL Dif -0.00228 Prob $>t \quad 0.4089$

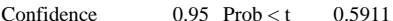

Tests that the Variances are Equal

$\begin{array}{lrrrr}\text { Level } & \text { Count } & \text { Std Dev } & \text { MeanAbsDif to Mean } & \text { MeanAbsDif to Median } \\ \text { Coliwasa } & 16 & 0.0019682 & 0.0014799 & 0.0013703\end{array}$

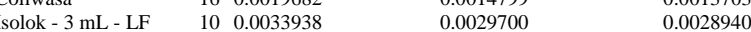

Test F Ratio DFNum DFDen p-Value

O'Brien[.5]

$\begin{array}{lllll}\text { O'Brien[.5] } & 6.3563 & 1 & 24 & 0.0187 \\ \text { Brown-Forsythe } & 5.3400 & 1 & 24 & 0.0297\end{array}$

$\begin{array}{lllll}\text { Levene } & 8.5135 & 1 & 24 & 0.0075 \\ \text { Bartlett } & 3.3347 & 1 & & 0.0678\end{array}$

$\begin{array}{lllll}\text { F Test 2-sided } & 2.9733 & 9 & 15 & 0.0604\end{array}$

Welch Anova testing Means Equal, allowing Std Devs Not Equal

F Ratio DFNum DFDen Prob $>$ F

$\begin{array}{llll}0.0553 & 1 & 12.841 & 0.8178\end{array}$

t Test

0.2352
Oneway Analysis of Measurement (wt\%) By Sampler Type of Material=SME Simulant, Analytical Block $=1$, Analyte $=\mathrm{CuO}(\mathrm{wt} \%)$, Target $(\mathrm{wt} \%)=0.103$

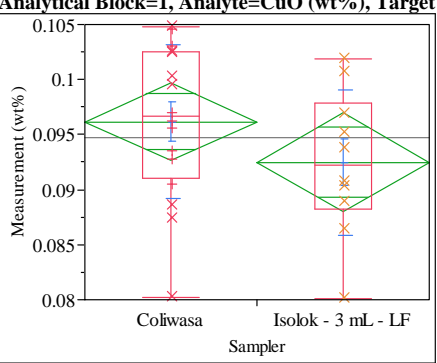

Oneway Anova

Summary of Fit

Rsquare

Adj Rsquare

Root Mean Square Error $\quad 0.006875$

Mean of Response 0.094752

Test

Isolok - $3 \mathrm{~mL}$ - LF-Coliwasa

Assuming equal variances

Difference $\quad-0.00371$ t Ratio -1.3376

$\begin{array}{lrr}\text { Std Err Dif } & 0.00277 \text { DF } & 24 \\ \text { Upper CL Dif } & 0.00201 \quad \text { Prob }>|t| & 0.1936\end{array}$

$\begin{array}{lrll}\text { Upper CL Dif } 0.00201 & \text { Prob }>|t| & 0.1936 \\ \text { Lower CL Dif }-0.00943 & \text { Prob }>t & 0.9032\end{array}$

$\begin{array}{lrl}\text { Confidence } & 0.95 \text { Prob }<\mathrm{t} & 0.0968\end{array}$

Analysis of Variance

ource DF Sum of Squares Mean Square F Ratio Prob > F

$\begin{array}{lrrrrr}\text { Sampler } & 1 & 0.00008456 & 0.000085 & 1.7893 & 0.1936\end{array}$

$\begin{array}{llll}\text { Error } & 24 & 0.00113422 & 0.000047\end{array}$

C. Total $25 \quad 0.00121878$

Means for Oneway Anova

Level Number Mean Std Error Lower 95\% Upper 95\%

$\begin{array}{lrrrrr}\text { Level } & \text { Number } & \text { Mean } & \text { Std Error } & \text { Lower } \mathbf{9 5 \%} & \text { Upper 95\% } \\ \text { Coliwasa } & 16 & 0.096177 & 0.00172 & 0.09263 & 0.09972\end{array}$

$\begin{array}{llllll}\text { Isolok - 3 mL - LF } & 10 & 0.092470 & 0.00217 & 0.08798 & 0.09696\end{array}$

Std Error uses a pooled estimate of error variance

Means and Std Deviations

Level Number Mean Std Dev Std Err Mean Lower 95\% Upper 95\%

$\begin{array}{lllllll}\text { Coliwasa } & 16 & 0.096177 & 0.007022 & 0.00176 & 0.09244 & 0.09992\end{array}$

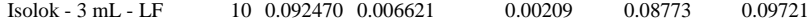

\section{t Test}

Isolok - 3 mL - LF-Coliwasa

Assuming unequal variances

$\begin{array}{lll}\text { Difference } & -0.00371 \text { t Ratio } & -1.35666\end{array}$

Std Err Dif $\quad 0.00273$ DF 20.13263

Upper CL Dif 0.00199 Prob $>|t| \quad 0.1899$

Lower CL Dif -0.00940 Prob $>\mathrm{t} \quad 0.9050$

Confidence $\quad 0.95$ Prob $<\mathrm{t} \quad 0.0950$

Tests that the Variances are Equal

$\begin{array}{lrrrr}\text { Level } & \text { Count } & \text { Std Dev } & \text { MeanAbsDif to Mean } & \text { MeanAbsDif to Median } \\ \text { Coliwasa } & 16 & 0.0070222 & 0.0055891 & 0.0055783\end{array}$

Isolok - $3 \mathrm{~mL}$ - LF $\quad 10 \quad 0.0066210 \quad 0.0051950 \quad 0.0051950$

Test F Ratio DFNum DFDen p-Value

O'Brien[.5] 0.0441

$\begin{array}{lllll}\text { OBrien[.5] } & 0.0441 & 1 & 24 & 0.8355 \\ \text { Brown-Forsythe } & 0.0586 & 1 & 24 & 0.8108\end{array}$

Brown-Forsythe $0.0586-1-24 \quad 0.8108$

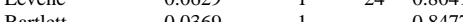

$\begin{array}{lrrrr}\text { Bartlett } & 0.0369 & 1 & \dot{0} & 0.8477 \\ \text { F Test 2-sided } & 1.1249 & 15 & 9 & 0.8871\end{array}$

Welch Anova testing Means Equal, allowing Std Devs Not Equal

F Ratio DFNum DFDen Prob $>$ F

1.8405

t Test

1.3567 


\section{Exhibit B2. Statistical Comparisons for High-Rheology (Phase 2) Simulant Testing}

Oneway Analysis of Measurement (wt \%) By Sampler Type of Material=SME Simulant, Analytical Block $=1$, Analyte $=\mathrm{Fe} / \mathrm{Li}$, Target $(\mathrm{wt} \%)=1.99097835$

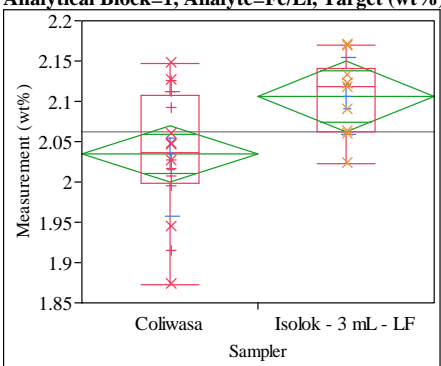

Oneway Anova

Summary of Fit

Adj Rsquare

0.22036

0.187875

$\begin{array}{lr}\text { Mean of Response } & 2.062369 \\ \text { Observations (or Sum Wgts) } & 26\end{array}$

t Test

Isolok - $3 \mathrm{~mL}$ - LF-Coliwasa

Assuming equal variances

Difference $\quad 0.071437$ t Ratio 2.6045

$\begin{array}{lrlr}\text { Std Err Dif } & 0.027428 & \text { DF } & 24 \\ \text { Upper CL Dif } & 0.128047 & \text { Prob }>|t| & 0.0155\end{array}$

Upper CL Dif 0.128047 Prob $>|t| 0.0155$

$\begin{array}{lrll}\text { Lower CL Dif } & 0.014828 & \text { Prob }>t & 0.0078 \\ \text { Confidence } & 0.95 & \text { Prob }<\mathrm{t} & 0.9922\end{array}$

Analysis of Variance

Source DF Sum of Squares Mean Square F Ratio Prob $>$ F

$\begin{array}{llllll}\text { Sampler } & 1 & 0.03140473 & 0.031405 & 6.7834 & 0.0155\end{array}$

$\begin{array}{llll}\text { Error } & 24 & 0.11111113 & 0.004630\end{array}$

C. Total $25 \quad 0.14251586$

Means for Oneway Anova

$\begin{array}{lrrrrr}\text { Level } & \text { Number } & \text { Mean } & \text { Std Error } & \text { Lower 95\% } & \text { Upper 95\% } \\ \text { Coliwasa } & 16 & 2.03489 & 0.01701 & 1.9998 & 2.0700\end{array}$

$\begin{array}{lrrrrr}\text { Coliwasa } & 16 & 2.03489 & 0.01701 & 1.9998 & 2.0700 \\ \text { Isolok - 3 mL - LF } & 10 & 2.10633 & 0.02152 & 2.0619 & 2.1507\end{array}$

Std Error uses a pooled estimate of error variance

Means and Std Deviations

Level $\quad$ Number Mean Std Dev Std Err Mean Lower 95\% Upper 95\%

Coliwasa $16 \quad 2.034890 .077713$

$\begin{array}{lllllll}\text { Coliwasa } & 16 & 2.03489 & 0.077713 & 0.01943 & 1.9935 & 2.0763 \\ \text { Isolok - 3 mL - LF } & 10 & 2.10633 & 0.047750 & 0.01510 & 2.0722 & 2.1405\end{array}$

Test

Isolok - $3 \mathrm{~mL}$ - LF-Coliwasa

Assuming unequal variances

Difference $\quad 0.071437$ t Ratio 2.903208

Std Err Dif $\quad 0.024606$ DF 23.99974

Upper CL Dif 0.122222 Prob $>|t| 0.0078$

Lower CL Dif 0.020652 Prob $>t \quad 0.0039$

$\begin{array}{lrl}\text { Confidence } & 0.95 \text { Prob }<\mathrm{t} & 0.9961\end{array}$

Tests that the Variances are Equal

Level Count Std Dev MeanAbsDif to Mean MeanAbsDif to Median

$\begin{array}{lllll}\text { Coliwasa } & 16 & 0.0777132 & 0.0593291 & 0.0593291\end{array}$

$\begin{array}{lllll}\text { Isolok - } 3 \mathrm{~mL} \text { - LF } & 10 & 0.0477504 & 0.0382198 & 0.0361695\end{array}$

Test F Ratio DFNum DFDen p-Value

$\begin{array}{lrrrr}\text { O'Brien[.5] } & 2.0420 & 1 & 24 & 0.1659\end{array}$

Brown-Forsythe $1.8292 \quad 11 \quad 24 \quad 0.1888$

$\begin{array}{lllll}\text { Levene } & 1.6378 & 1 & 24 & 0.2129\end{array}$

$\begin{array}{lrrrr}\text { Bartlett } & 2.2834 & 1 & \text {. } & 0.1308 \\ \text { F Test 2-sided } & 2.6487 & 15 & 9 & 0.1434\end{array}$

Welch Anova testing Means Equal, allowing Std Devs Not Equal

F Ratio DFNum DFDen Prob $>$ F

$\begin{array}{rrrr}8.4286 & 1 & 24 & 0.0078\end{array}$

t Test

2.9032
Oneway Analysis of Measurement (wt \%) By Sampler Type of Material=SME Simulant, Analytical Block=1, Analyte=Fe2O3 (wt \%), Target $($ wt $\%)=9.931$

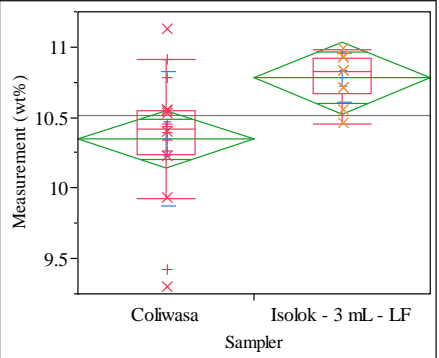

Oneway Anova

Summary of Fit

Adj Rsquare 0.391712

Mean of Response

Observations (or Sum Wgts)

t Test

Isolok - $3 \mathrm{~mL}$ - LF-Coliwasa

Assuming equal variances

Difference $\quad 0.433914$ t Ratio 2.747955

Std Err Dif $\quad 0.157904$ DF 24

Upper CL Dif 0.759812 Prob $>|t| \quad 0.0112$

Lower CL Dif 0.108015 Prob $>t \quad 0.0056$

$\begin{array}{lrl}\text { Confidence } & 0.95 \text { Prob }<\mathrm{t} & 0.9944\end{array}$

Analysis of Variance

Source DF Sum of Squares Mean Square F Ratio Prob $>$ F

$\begin{array}{lllllll}\text { Sampler } & 1 & 1.1586543 & 1.15865 & 7.5513 & 0.0112\end{array}$

$\begin{array}{llll}\text { Error } & 24 & 3.6825258 & 0.15344\end{array}$

C. Total $25 \quad 4.8411800$

Means for Oneway Anova

Level Number Mean Std Error Lower 95\% Upper 95\%

$\begin{array}{lrrrrr}\text { Coliwasa } & 16 & 10.3475 & 0.09793 & 10.145 & 10.550\end{array}$

$\begin{array}{llllll}\text { Isolok - 3 mL - LF } & 16 & 10.3475 & 0.09793 & 10.145 & 10.550 \\ & 10 & 10.7814 & 0.12387 & 10.526 & 11.037\end{array}$

Std Error uses a pooled estimate of error variance

Means and Std Deviations

Level Number Mean Std Dev Std Err Mean Lower 95\% Upper 95\%

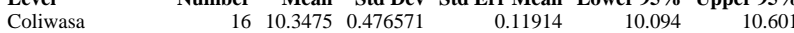

$\begin{array}{lllllll}\text { Isolok - 3 mL - LF } & 10 & 10.7814 & 0.175030 & 0.05535 & 10.656 & 10.907\end{array}$

t Test

Isolok - $3 \mathrm{~mL}$ - LF-Coliwasa

Assuming unequal variances

$\begin{array}{lrlr}\text { Difference } & 0.433914 & \text { t Ratio } & 3.302943 \\ \text { Std Err Dif } & 0.131372 & \text { DF } & 20.57595 \\ \text { Upper CL Dif } & 0.707460 & \text { Prob }>|t| & 0.0035 \\ \text { Lower CL Dif } & 0.160368 & \text { Prob }>t & 0.0017 \\ \text { Confidence } & 0.95 & \text { Prob }<t & 0.9983\end{array}$

Tests that the Variances are Equal

Level Count Std Dev MeanAbsDif to Mean MeanAbsDif to Median

$\begin{array}{lllll}\text { Coliwasa } & 16 & 0.4765714 & 0.3279374 & 0.3163211\end{array}$

$\begin{array}{lllll}\text { Isolok - } 3 \mathrm{~mL} \text { - LF } & 10 & 0.1750304 & 0.1412544 & 0.1329621\end{array}$

Test F Ratio DFNum DFDen p-Value

$\begin{array}{lrrrr}\text { O'Brien[.5] } & 2.6105 & 1 & 24 & 0.1192\end{array}$

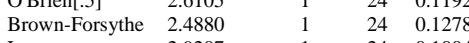

$\begin{array}{lllll}\text { Levene } & 2.9207 & 1 & 24 & 0.1004\end{array}$

$\begin{array}{lrrrr}\text { Bartlett } & 8.2435 & 1 & \text {. } & 0.0041 \\ \text { F Test 2-sided } & 7.4136 & 15 & 9 & 0.0047\end{array}$

Welch Anova testing Means Equal, allowing Std Devs Not Equal

F Ratio DFNum DFDen Prob $>$ F

$\begin{array}{rrrr}10.9094 & 1 & 20.576 & 0.0035\end{array}$

t Test

3.3029 
SRNL-STI-2011-00693

Revision 0

\section{Exhibit B2. Statistical Comparisons for High-Rheology (Phase 2) Simulant Testing}

Oneway Analysis of Measurement (wt\%) By Sampler Type of Material=SME Simulant, Analytical Block=1, Analyte $=$ K2O (wt \%), Target $(w t \%)=0.076$

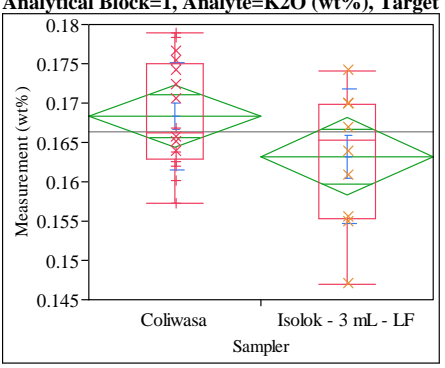

Oneway Anova

Summary of Fit

Rsquare

Adj Rsquare

Root Mean Square Error $\quad 0.007545$

Mean of Response $\quad 0.166351$

Observations (or Sum Wgts)

Test

Isolok - $3 \mathrm{~mL}$ - LF-Coliwas

Assuming equal variances

Difference $\quad-0.00508$ t Ratio -1.67092

$\begin{array}{lrr}\text { Std Err Dif } & 0.00304 \text { DF } & 24 \\ \text { Upper CL Dif } & 0.00120 \text { Prob }>|t| & 0.1077\end{array}$

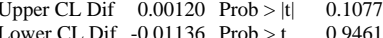

$\begin{array}{lrl}\text { Confidence } & 0.95 \text { Prob }<\mathrm{t} & 0.0539\end{array}$

Analysis of Variance

Source DF Sum of Squares Mean Square F Ratio Prob $>$ F

$\begin{array}{lrrrrr}\text { Sampler } & 1 & 0.00015893 & 0.000159 & 2.7920 & 0.1077\end{array}$

$\begin{array}{llll}\text { Error } & 24 & 0.00136615 & 0.00005\end{array}$

C. Total $25 \quad 0.00152508$

Means for Oneway Anova

Level Number Mean Std Error Lower 95\% Upper 95\%

$\begin{array}{lrrrrr}\text { Coliwasa } & 16 & 0.168305 & 0.00189 & 0.16441 & 0.17220\end{array}$

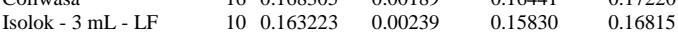

Std Error uses a pooled estimate of error variance

Means and Std Deviations

Level Number Mean Std Dev Std Err Mean Lower 95\% Upper 95\%

$\begin{array}{lllllll}\text { Coliwasa } & 16 & 0.168305 & 0.006835 & 0.00171 & 0.16466 & 0.17195\end{array}$

$\begin{array}{lllllll}\text { Isolok - } 3 \mathrm{~mL} \text { - LF } & 10 & 0.163223 & 0.008598 & 0.00272 & 0.15707 & 0.16937\end{array}$

t Test

Isolok - $3 \mathrm{~mL}$ - LF-Coliwasa

Assuming unequal variances

Difference $\quad-0.00508$ t Ratio $\quad-1.5825$

Std Err Dif $\quad 0.00321$ DF 16.01539

Upper CL Dif 0.00173 Prob $>|t| \quad 0.1331$

Lower CL Dif -0.01189 Prob $>t \quad 0.9335$

Confidence $\quad 0.95$ Prob $<\mathrm{t} \quad 0.0665$

Tests that the Variances are Equal

$\begin{array}{lrrrr}\text { Level } & \text { Count } & \text { Std Dev } & \text { MeanAbsDif to Mean } & \text { MeanAbsDif to Median } \\ \text { Coliwasa } & 16 & 0.0068354 & 0.0059430 & 0.0057595\end{array}$

$\begin{array}{llll}\text { Isolok - } 3 \mathrm{~mL} \text { - LF } & 10 & 0.0085979 & 0.0069867\end{array}$

$\begin{array}{lrrrr}\text { Test } & \text { F Ratio } & \text { DFNum } & \text { DFDen } & \text { p-Value } \\ \text { O'Brien[.5] } & 1.0538 & 1 & 24 & 0.3149 \\ \text { Brown-Forsythe } & 0.3793 & 1 & 24 & 0.5438 \\ \text { Levene } & 0.5141 & 1 & 24 & 0.4803 \\ \text { Bartlett } & 0.5836 & 1 & . & 0.4449 \\ \text { F Test 2-sided } & 1.5822 & 9 & 15 & 0.4152\end{array}$

Welch Anova testing Means Equal, allowing Std Devs Not Equal

F Ratio DFNum DFDen Prob $>$ F

$\begin{array}{llll}2.5043 & 1 & 16.015 & 0.1331\end{array}$

t Test

1.5825
Oneway Analysis of Measurement (wt \%) By Sampler Type of Material=SME Simulant, Analytical Block=1, Analyte $=\mathrm{Li} 2 \mathrm{O}(\mathrm{wt} \%)$, Target $(\mathrm{wt} \%)=4.988$

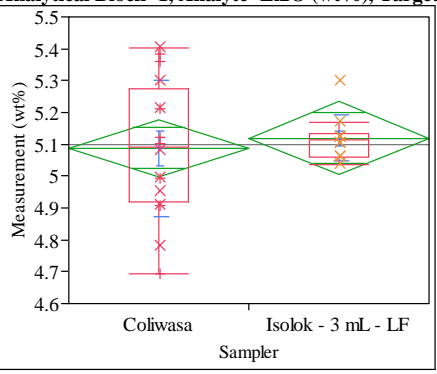

Oneway Anova

Summary of Fit

Rsquare $\quad 0.008517$

Adj Rsquare $\quad-0.03279$

Root Mean Square Error $\quad 0.17496$

Mean of Response 5.099889

Observations (or Sum Wgts) $\quad 26$

t Test

Isolok - $3 \mathrm{~mL}$ - LF-Coliwasa

Assuming equal variances

$\begin{array}{lrlr}\text { Difference } & 0.03202 & \text { t Ratio } & 0.454063 \\ \text { Std Err Dif } & 0.07053 \text { DF } & 24 \\ \text { Upper CL Dif } & 0.17759 & \text { Prob }>|t| & 0.6539 \\ \text { Lower CL Dif } & -0.11354 & \text { Prob }>t & 0.3269 \\ \text { Confidence } & 0.95 & \text { Prob }<\mathrm{t} & 0.6731\end{array}$

Analysis of Variance

Source DF Sum of Squares Mean Square F Ratio Prob $>$ F

$\begin{array}{lrrrrr}\text { Sampler } & 1 & 0.00631115 & 0.006311 & 0.2062 & 0.6539\end{array}$

$\begin{array}{llll}\text { Error } & 24 & 0.73466146 & 0.030611\end{array}$

C. Total $25 \quad 0.74097261$

Means for Oneway Anov

Level Number Mean Std Error Lower 95\% Upper 95\%

\begin{tabular}{lllllr} 
Coliwasa & 16 & 5.08757 & 0.04374 & 4.9973 & 5.1778 \\
\hline
\end{tabular}

Stertimate of error variance

Means and Std Deviations

$\begin{array}{lllll}\text { Means and Std Deviations } & & & \\ \text { Level } & \text { Number } & \text { Mean } & \text { Std Dev Std Err Mean Lower 95\% Upper 95\% }\end{array}$

$\begin{array}{lrrrrrr}\text { Level } & \text { Number } & \text { Mean } & \text { Std Dev } & \text { Std Err Mean } & \text { Lower 95\% } & \text { Upper 95\% } \\ \text { Coliwasa } & 16 & 5.08757 & 0.213954 & 0.05349 & 4.9736 & 5.2016\end{array}$

$\begin{array}{lllllll}\text { Isolok - 3 mL - LF } & 16 & 5.08757 & 0.213954 & 0.05349 & 4.9736 & 5.2016 \\ & 10 & 5.11960 & 0.073044 & 0.02310 & 5.0673 & 5.1718\end{array}$

t Test

Isolok - $3 \mathrm{~mL}$ - LF-Coliwasa

Assuming unequal variances

Difference $\quad 0.03202$ t Ratio 0.549654

Std Err Dif $\quad 0.05826$ DF 19.95935

Upper CL Dif 0.15357 Prob $>|t| r 0.5887$

Lower CL Dif -0.08953 Prob $>t \quad 0.2943$

$\begin{array}{lll}\text { Confidence } & 0.95 \text { Prob }<\mathrm{t} \quad 0.7057\end{array}$

Tests that the Variances are Equal

Level Count Std Dev MeanAbsDif to Mean MeanAbsDif to Median

$\begin{array}{lrrrr}\text { Coliwasa } & 16 & 0.2139537 & 0.1735776 & 0.1735776\end{array}$

$\begin{array}{lllll}\text { Isolok - } 3 \mathrm{~mL} \text { - LF } & 10 & 0.0730437 & 0.0473638 & 0.1735776 \\ & & & \end{array}$

Test F Ratio DFNum DFDen p-Value

$\begin{array}{lllll}\text { O'Brien[.5] } & 6.0654 & 1 & 24 & 0.0213\end{array}$

Brown-Forsythe $10.1920 \quad 1 \quad 24 \quad 0.0039$

$\begin{array}{lllll}\text { Levene } & 10.2221 & 1 & 24 & 0.0039\end{array}$

$\begin{array}{lllll}\text { Bartlett } & 9.2664 & 1 & 0.0023\end{array}$

$\begin{array}{lllll}\text { F Test 2-sided } & 8.5798 & 15 & 9 & 0.0027\end{array}$

Welch Anova testing Means Equal, allowing Std Devs Not Equal

F Ratio DFNum DFDen Prob $>$ F

$\begin{array}{rrrr}0.3021 & 1 & 19.959 & 0.5887\end{array}$

t Test 


\section{Exhibit B2. Statistical Comparisons for High-Rheology (Phase 2) Simulant Testing}

Oneway Analysis of Measurement (wt \%) By Sampler Type of Material=SME Simulant, Analytical Block=1, Analyte $=\mathrm{MgO}(\mathrm{wt} \%)$, Target $(\mathrm{wt} \%)=\mathbf{0 . 2 8 6}$

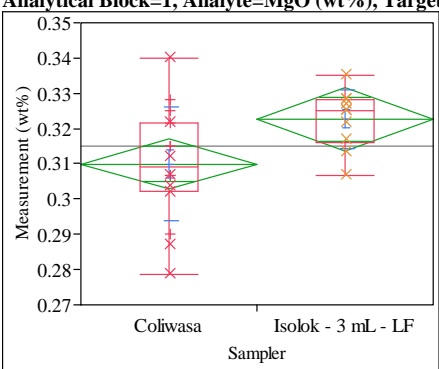

Oneway Anova

Summary of Fit

Adj Rsquare

0.148165

Error $\quad 0.01363$

0.314886

Test

Isolok - $3 \mathrm{~mL}$ - LF-Coliwasa

Assuming equal variances

Difference $\quad 0.012707$ t Ratio $\quad 2.312665$

Std Err Dif $\quad 0.005494$ DF 24

Upper CL Dif 0.024047 Prob $>|t| \quad 0.0296$

Lower CL Dif 0.001367 Prob $>t \quad 0.0148$

$\begin{array}{lrl}\text { Confidence } & 0.95 \text { Prob }<\mathrm{t} \quad 0.9852\end{array}$

Analysis of Variance

Source DF Sum of Squares Mean Square F Ratio Prob > F

$\begin{array}{llllll}\text { Sampler } & 1 & 0.00099361 & 0.000994 & 5.3484 & 0.0296\end{array}$

$\begin{array}{llll}\text { Error } & 24 & 0.00445861 & 0.000186\end{array}$

C. Total $25 \quad 0.00545222$

Means for Oneway Anova

Level $\quad$ Number Mean Std Error Lower 95\% Upper 95\%

$\begin{array}{lrrrrr}\text { Coliwasa } & 16 & 0.309998 & 0.00341 & 0.30297 & 0.31703\end{array}$

$\begin{array}{llllll}\text { Isolok - } 3 \text { mL - LF } & 10 & 0.322705 & 0.00431 & 0.31381 & 0.33160\end{array}$

Std Error uses a pooled estimate of error variance

Means and Std Deviations

Level Number Mean Std Dev Std Err Mean Lower 95\% Upper 95\%

Coliwasa $16 \quad 0.3099980 .016003$

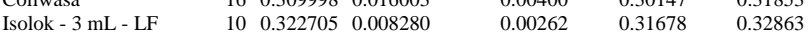

TTest

Isolok - $3 \mathrm{~mL}$ - LF-Coliwasa

Assuming unequal variances

Difference $\quad 0.012707$ t Ratio 2.657467

Std Err Dif $\quad 0.004782$ DF 23.43602

Upper CL Dif 0.022588 Prob $>|t| r \mid r .0139$

Lower CL Dif 0.002826 Prob $>t \quad 0.0070$

$\begin{array}{lrl}\text { Confidence } & 0.95 \text { Prob }<\mathrm{t} & 0.9930\end{array}$

Tests that the Variances are Equal

Level Count Std Dev MeanAbsDif to Mean MeanAbsDif to Median

$\begin{array}{lllll}\text { Coliwasa } & 16 & 0.0160032 & 0.0123336 & 0.0123336\end{array}$

$\begin{array}{lllll}\text { Isolok - } 3 \mathrm{~mL} \text { - LF } & 10 & 0.0082804 & 0.0064342 & 0.0059699\end{array}$

Test F Ratio DFNum DFDen p-Value

$\begin{array}{lrrrr}\text { O'Brien[.5] } & 2.7066 & 1 & 24 & 0.1130\end{array}$

Brown-Forsythe $3.4560 \quad 1 \quad 24 \quad 0.0753$

$\begin{array}{lllll}\text { Levene } & 3.1912 & 1 & 24 & 0.0867\end{array}$

$\begin{array}{lrrrr}\text { Bartlett } & 3.9749 & 1 & \text {. } & 0.0462 \\ \text { F Test 2-sided } & 3.7351 & 15 & 9 & 0.0515\end{array}$

Welch Anova testing Means Equal, allowing Std Devs Not Equal

F Ratio DFNum DFDen Prob $>$ F

$\begin{array}{rrrr}7.0621 & 1 & 23.436 & 0.0139\end{array}$

t Test

$$
123.436 \quad 0.0139
$$

2.6575
Oneway Analysis of Measurement (wt \%) By Sampler Type of Material=SME Simulant, Analytical Block $=1$, Analyte $=\mathrm{MnO}(\mathrm{wt} \%)$, Target $(\mathrm{wt} \%)=2.918$

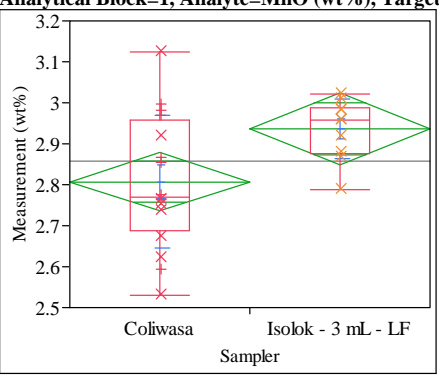

Oneway Anova

Summary of Fit

Rsquare

Root Mean Square Error

Root Mean sc

$\begin{array}{lr}\text { Mean of Response } & 2.857028 \\ \text { Observations (or Sum Wgts) } & 26\end{array}$

t Test

Isolok - $3 \mathrm{~mL}$ - LF-Coliwasa

Assuming equal variances

Difference $\quad 0.128636$ t Ratio 2.340329

Std Err Dif $\quad 0.054965$ DF $\quad 24$

Upper CL Dif 0.242078 Prob $>|t| \quad 0.0279$

Lower CL Dif 0.015194 Prob $>t \quad 0.0140$

$\begin{array}{lrl}\text { Confidence } & 0.95 \text { Prob }<\mathrm{t} \quad 0.9860\end{array}$

Analysis of Variance

Source DF Sum of Squares Mean Square F Ratio Prob $>$ F

$\begin{array}{lrrrrr}\text { Sampler } & 1 & 0.10182873 & 0.101829 & 5.4771 & 0.0279\end{array}$

$\begin{array}{lrll}\text { Error } & 24 & 0.44619830 & 0.018592\end{array}$

C. Total $25 \quad 0.54802704$

Means for Oneway Anova

$\begin{array}{lrrrrr}\text { Level } & \text { Number } & \text { Mean } & \text { Std Error } & \text { Lower 95\% } & \text { Upper 95\% } \\ \text { Coliwasa } & 16 & 2.80755 & 0.03409 & 2.7372 & 2.8779\end{array}$

$\begin{array}{lllll}\text { Isolok - } 3 \mathrm{~mL} \text { - LF } & 10 & 2.93619 & 0.04312 & 2.8472\end{array}$

Std Error uses a pooled estimate of error variance

Means and Std Deviations

Level Number Mean Std Dev Std Err Mean Lower 95\% Upper 95\%

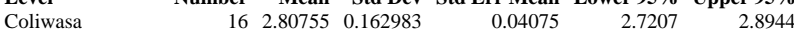

$\begin{array}{lllllll}\text { Isolok - 3 mL - LF } & 16 & 2.80755 & 0.162983 & 0.04075 & 2.7207 & 2.8944 \\ & 10 & 2.93619 & 0.072838 & 0.02303 & 2.8841 & 2.9883\end{array}$

t Test

Isolok - $3 \mathrm{~mL}$ - LF-Coliwasa

Assuming unequal variances

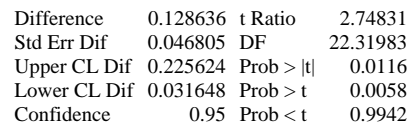

$\begin{array}{lrl}\text { Confidence } & 0.95 \text { Prob }<\mathrm{t} & 0.9942\end{array}$

Tests that the Variances are Equal

Level Count Std Dev MeanAbsDif to Mean MeanAbsDif to Median

$\begin{array}{lrrrr}\text { Coliwasa } & 16 & 0.1629826 & 0.1322471 & 0.1283130\end{array}$

$\begin{array}{lllll}\text { Isolok - 3 mL - LF } & 10 & 0.0728381 & 0.0583622 & 0.0542304\end{array}$

Test F Ratio DFNum DFDen p-Value

$\begin{array}{lllll}\text { O'Brien[.5] } & 4.1612 & 1 & 24 & 0.0525\end{array}$

Brown-Forsythe $4.4870 \quad 1 \quad 24 \quad 0.0447$

$\begin{array}{lllll}\text { Levene } & 6.0937 & 1 & 24 & 0.0211\end{array}$

$\begin{array}{lllll}\text { F Test 2-sided } \quad 5.0069 & 15 & 9 & 0.0194\end{array}$

Welch Anova testing Means Equal, allowing Std Devs Not Equal

F Ratio DFNum DFDen Prob $>$ F

$\begin{array}{llrr}7.5532 & 1 & 22.32 & 0.0116\end{array}$

t Test 


\section{Exhibit B2. Statistical Comparisons for High-Rheology (Phase 2) Simulant Testing}

Oneway Analysis of Measurement (wt \%) By Sampler Type of Material=SME Simulant, Analytical Block=1, Analyte $=\mathrm{Na2O}(\mathrm{wt} \%)$, Target $(\mathrm{wt} \%)=13.318$

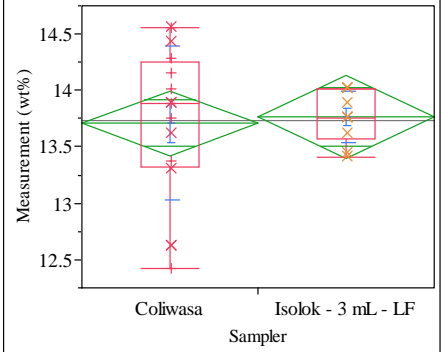

Oneway Anova

Summary of Fit

Adj Rsquare

$-0.039$

0.557091

13.7314

Test

Isolok - $3 \mathrm{~mL}$ - LF-Coliwasa

Assuming equal variances

Difference $\quad 0.05577$ t Ratio $\quad 0.248356$

Std Err Dif $\quad 0.22457$ DF 24

Upper CL Dif 0.51926 Prob $>|t| \quad 0.8060$

Lower CL Dif -0.40772 Prob $>t \quad 0.4030$

$\begin{array}{lrl}\text { Confidence } & 0.95 \text { Prob }<\mathrm{t} \quad 0.5970\end{array}$

Analysis of Variance

Source DF Sum of Squares Mean Square F Ratio Prob $>$ F

$\begin{array}{llllll}\text { Sampler } & 1 & 0.0191427 & 0.019143 & 0.0617 & 0.8060\end{array}$

$\begin{array}{llll}\text { Error } & 24 & 7.4484070 & 0.310350\end{array}$

C. Total $25 \quad 7.4675496$

Means for Oneway Anova

Level Number Mean Std Error Lower 95\% Upper 95\%

$\begin{array}{lrrrrr}\text { Coliwasa } & 16 & 13.7100 & 0.13927 & 13.423 & 13.997\end{array}$

$\begin{array}{llllll} & 16 & 13.7100 & 0.13927 & 13.423 & 13.997 \\ \text { Isolok - 3 mL - LF } & 10 & 13.7658 & 0.17617 & 13.402 & 14.129\end{array}$

Std Error uses a pooled estimate of error variance

Means and Std Deviations

Level Number Mean Std Dev Std Err Mean Lower 95\% Upper 95\%

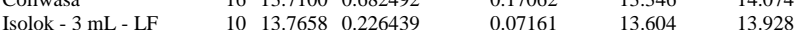

tTest

Isolok - $3 \mathrm{~mL}$ - LF-Coliwasa

Assuming unequal variances

$\begin{array}{lrlr}\text { Difference } & 0.05577 & \text { t Ratio } & 0.301414 \\ \text { Std Err Dif } & 0.18504 & \text { DF } & 19.72911 \\ \text { Upper CL Dif } & 0.44210 & \text { Prob }>|t| & 0.7663 \\ \text { Lower CL Dif } & -0.33055 & \text { Prob }>\text { t } & 0.3831 \\ \text { Confidence } & 0.95 & \text { Prob }<\mathrm{t} & 0.6169\end{array}$

Tests that the Variances are Equal

Level Count Std Dev MeanAbsDif to Mean MeanAbsDif to Median

$\begin{array}{lllll}\text { Coliwasa } & 16 & 0.6824923 & 0.5381469 & 0.5113975\end{array}$

$\begin{array}{lllll} & 16 & 0.6824923 & 0.5381469 & 0.5113975 \\ \text { Isolok - } 3 \mathrm{~mL} \text { - LF } & 10 & 0.2264390 & 0.1757792 & 0.1725440\end{array}$

Test F Ratio DFNum DFDen p-Value

$\begin{array}{lrrrr}\text { O'Brien[.5] } & 5.1765 & 1 & 24 & 0.032\end{array}$

$\begin{array}{lllll}\text { Brown-Forsythe } & 4.9076 & 1 & 24 & 0.0365\end{array}$

$\begin{array}{lllll}\text { Levene } & 7.7395 & 1 & 24 & 0.0104\end{array}$

$\begin{array}{lrrrr}\text { Bartlett } & 9.6749 & 1 & \text {. } & 0.0019 \\ \text { F Test 2-sided } & 9.0843 & 15 & 9 & 0.0021\end{array}$

Welch Anova testing Means Equal, allowing Std Devs Not Equal

F Ratio DFNum DFDen Prob $>$ F

$\begin{array}{lrrr}0.0909 & 1 & 19.729 & 0.7663\end{array}$

t Test

0.3014
Oneway Analysis of Measurement (wt\%) By Sampler Type of Material=SME Simulant, Analytical Block=1, Analyte $=\mathrm{NiO}(\mathrm{wt} \%)$, Target $(\mathrm{wt} \%)=1.033$

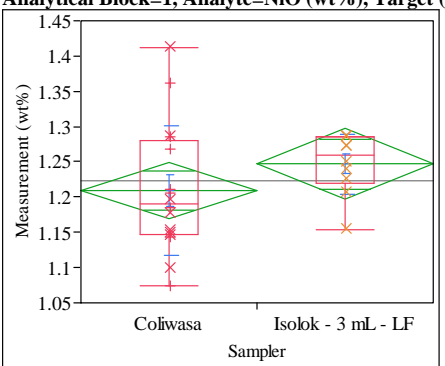

Oneway Anova

Summary of Fit

Adj Rsquare

Root Mean Square Error $\quad 0.07742$

Mean of Response

Observations (or Sum Wgts) $\quad 26$

Test

Isolok - $3 \mathrm{~mL}$ - LF-Coliwasa

Assuming equal variances

$\begin{array}{lrlr}\text { Difference } & 0.03778 & \text { t Ratio } & 1.210403 \\ \text { Std Err Dif } & 0.03121 & \text { DF } & 24 \\ \text { Upper CL Dif } & 0.10219 & \text { Prob }>|t| & 0.2379 \\ \text { Lower CL Dif } & -0.02664 & \text { Prob }>\text { t } & 0.1190 \\ \text { Confidence } & 0.95 & \text { Prob }<\mathrm{t} & 0.8810\end{array}$

Analysis of Variance

Source DF Sum of Squares Mean Square F Ratio Prob $>$ F

$\begin{array}{lrllll}\text { Sampler } & 1 & 0.00878232 & 0.008782 & 1.4651 & 0.2379\end{array}$

$\begin{array}{llll}\text { Error } & 24 & 0.14386677 & 0.005994\end{array}$

C. Total $25 \quad 0.15264909$

Means for Oneway Anova

Level Number Mean Std Error Lower 95\% Upper 95\%

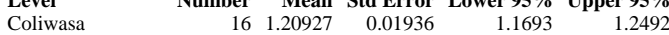

$\begin{array}{llllll}\text { Isolok - 3 mL - LF } & 16 & 1.20927 & 0.01936 & 1.1693 & 1.2492 \\ & 10 & 1.24705 & 0.02448 & 1.1965 & 1.2976\end{array}$

Std Error uses a pooled estimate of error variance

Means and Std Deviations

Level Number Mean Std Dev Std Err Mean Lower 95\% Upper 95\%

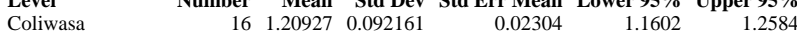

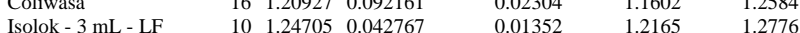

t Test

Isolok - $3 \mathrm{~mL}$ - LF-Coliwasa

Assuming unequal variances

$\begin{array}{lrlr}\text { Difference } & 0.03778 & \mathrm{t} \text { Ratio } & 1.414019 \\ \text { Std Err Dif } & 0.02672 & \text { DF } & 22.63804 \\ \text { Upper CL Dif } & 0.09309 & \text { Prob }>|\mathrm{t}| & 0.1710 \\ \text { Lower CL Dif } & -0.01754 & \text { Prob }>\mathrm{t} & 0.0855 \\ \text { Confidence } & 0.95 & \text { Prob }<\mathrm{t} & 0.9145\end{array}$

Tests that the Variances are Equal

Level Count Std Dev MeanAbsDif to Mean MeanAbsDif to Median

$\begin{array}{lllll}\text { Coliwasa } & 16 & 0.0921612 & 0.0711208 & 0.0687945\end{array}$

$\begin{array}{lllll}\text { Isolok - } 3 \mathrm{~mL} \text { - LF } & 10 & 0.0427673 & 0.0328305 & 0.0325760\end{array}$

Test F Ratio DFNum DFDen p-Value

$\begin{array}{lrrrr}\text { O'Brien[.5] } & 2.7966 & 1 & 24 & 0.1075\end{array}$

Brown-Forsythe $2.9703 \quad 1 \quad 24 \quad 0.0977$

$\begin{array}{lllll}\text { Levene } & 4.1516 & 1 & 24 & 0.0528\end{array}$

$\begin{array}{lrrrr}\text { Bartlett } & 5.2192 & 1 & \text {. } & 0.0223 \\ \text { F Test 2-sided } & 4.6438 & 15 & 9 & 0.0251\end{array}$

Welch Anova testing Means Equal, allowing Std Devs Not Equal

F Ratio DFNum DFDen Prob $>$ F

$\begin{array}{rrrr}1.9995 & 1 & 22.638 & 0.1710\end{array}$

t Test

1.4140 
SRNL-STI-2011-00693

Revision 0

Exhibit B2. Statistical Comparisons for High-Rheology (Phase 2) Simulant Testing

Oneway Analysis of Measurement (wt \%) By Sampler Type of Material=SME Simulant, Analytical Block=1, Analyte $=\mathrm{SiO} 2(\mathrm{wt} \%)$, Target $(\mathrm{wt} \%)=50.183$

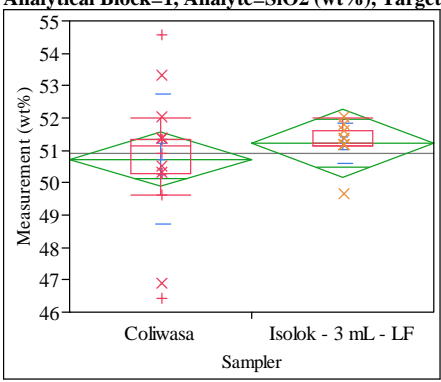

Oneway Anova

Summary of Fit

Rsquare

Adj Rsquare

$\quad 1.62815$

Observations (or Sum Wgts) $\quad 26$

t Test

Isolok - $3 \mathrm{~mL}$ - LF-Coliwasa

Assuming equal variances

Difference $\quad 0.4867$ t Ratio $\quad 0.741531$

$\begin{array}{lrlr}\text { Std Err Dif } & 0.6563 \text { DF } & 24 \\ \text { Upper CL Dif } & 1.8413 \text { Prob }>|t| & 0.4656\end{array}$

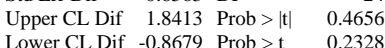

$\begin{array}{lrll}\text { Lower CL Dif } & -0.8679 & \text { Prob }>t & 0.2328 \\ \text { Confidence } & 0.95 & \text { Prob }<t & 0.7672\end{array}$

Analysis of Variance

Source DF Sum of Squares Mean Square F Ratio Prob $>$ F

$\begin{array}{lrrrrr}\text { Sampler } & 1 & 1.457649 & 1.45765 & 0.5499 & 0.4656\end{array}$

$\begin{array}{llll}\text { Error } & 24 & 63.621667 & 2.65090\end{array}$

C. Total $25 \quad 65.079316$

Means for Oneway Anova

Level Number Mean Std Error Lower 95\% Upper 95\%

$\begin{array}{llllll}\text { Coliwasa } & 16 & 50.7282 & 0.40704 & 49.888 & 51.568\end{array}$

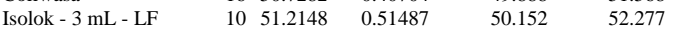

Std Error uses a pooled estimate of error variance

Means and Std Deviations

Level $\quad$ Number Mean Std Dev Std Err Mean Lower 95\% Upper 95\%

$\begin{array}{lrrrrrr}\text { Coliwasa } & 16 & 50.7282 & 2.00056 & 0.50014 & 49.662 & 51.794 \\ \text { Isolok - 3 mL - LF } & 10 & 51.2148 & 0.63141 & 0.19967 & 50.763 & 51.667\end{array}$

t Test

Isolok - $3 \mathrm{~mL}$ - LF-Coliwasa

Assuming unequal variances

Difference $\quad 0.4867$ t Ratio $\quad 0.903751$

Std Err Dif $\quad 0.5385$ DF 19.34349

Upper CL Dif 1.6125 Prob $>|t| r \mid 0.3772$

Lower CL Dif -0.6391 Prob $>t \quad 0.1886$

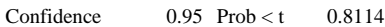

Tests that the Variances are Equal

$\begin{array}{lrrrr}\text { Level } & \text { Count } & \text { Std Dev } & \text { MeanAbsDif to Mean } & \text { MeanAbsDif to Median } \\ \text { Coliwasa } & 16 & 2.000560 & 1.360461 & 1.337063 \\ \text { Isolok - 3 mL - LF } & 10 & 0.631406 & 0.385074 & 0.385074\end{array}$

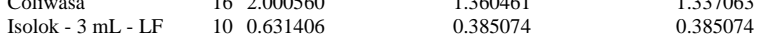

Test F Ratio DFNum DFDen p-Value

$\begin{array}{lllll}\text { O'Brien[.5] } & 2.5724 & 1 & 24 & 0.1218\end{array}$

$\begin{array}{lllll}\text { Brown-Forsythe } & 3.7063 & 1 & 24 & 0.0661\end{array}$

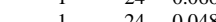

$\begin{array}{lrrrr}\text { Levene } & 4.3201 & 1 & 24 & 0.0485 \\ \text { Bartlett } & 10.3995 & 1 & & 0.0013\end{array}$

$\begin{array}{lrrrr}\text { Bartlett } & 10.3995 & 1 & & 0.0013 \\ \text { F Test 2-sided } & 10.0389 & 15 & 9 & 0.0014\end{array}$

Welch Anova testing Means Equal, allowing Std Devs Not Equal

F Ratio DFNum DFDen Prob > F

$\begin{array}{llll}0.8168 & 1 & 19.343 & 0.3772\end{array}$

t Test
0.9038
Oneway Analysis of Measurement (wt \%) By Sampler Type of Material=SME Simulant, Analytical Block=1, Analyte $=$ Sum of Oxides $(w t \%)$, Target $(w t \%)=99.73$

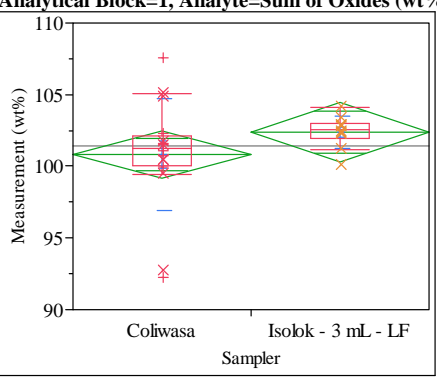

Oneway Anova

Summary of Fit

Rsquare $\quad 0.060647$

0.021507

Root Mean Square Error $\quad 3.141203$

Mean of Response 101.4124

Observations (or Sum Wgts)

t Test

Isolok - $3 \mathrm{~mL}$ - LF-Coliwasa

Assuming equal variances

Difference $\quad 1.5762$ t Ratio 1.244787

Std Err Dif $\quad 1.2663$ DF 24

Upper CL Dif 4.1897 Prob $>|t| \quad 0.2252$

Lower CL Dif -1.0372 Prob $>t \quad 0.1126$

Confidence $\quad 0.95$ Prob $<\mathrm{t} \quad 0.8874$

Analysis of Variance

Source DF Sum of Squares Mean Square F Ratio Prob $>$ F

$\begin{array}{lrrrrr}\text { Sampler } & 1 & 15.28910 & 15.2891 & 1.5495 & 0.2252\end{array}$

$\begin{array}{lrrr}\text { Sampler } & 1 & 15.28910 & 15.2891 \\ \text { Error } & 24 & 236.81169 & 9.8672\end{array}$

$\begin{array}{lll}\text { C. Total } & 25 & 252.10079\end{array}$

Means for Oneway Anova

Level Number Mean Std Error Lower 95\% Upper 95\%

$\begin{array}{llrrrr}\text { Coliwasa } & 16 & 100.806 & 0.78530 & 99.19 & 102.43 \\ \text { Isolok } 3 \text { mL - LF } & 10 & 102.382 & 0.99334 & 100.33 & 104.43\end{array}$

Std Error uses a pooled estimate of error variance

Means and Std Deviations

$\begin{array}{lllll}\text { Means and Std Deviations } & & & \\ \text { Level } & \text { Number } & \text { Mean Std Dev Std Err Mean Lower 95\% Upper 95\% }\end{array}$

$\begin{array}{lrrrrrr}\text { Level } & \text { Number } & \text { Mean } & \text { Std Dev } & \text { Std Err Mean } & \text { Lower 95\% } & \text { Upper 95\% } \\ \text { Coliwasa } & 16 & 100.806 & 3.87668 & 0.96917 & 98.74 & 102.87\end{array}$

$\begin{array}{llllrrr} & 16 & 100.806 & 3.87668 & 0.96917 & 98.74 & 102.87 \\ \text { Isolok - 3 mL - LF } & 10 & 102.382 & 1.12455 & 0.35561 & 101.58 & 103.19\end{array}$

t Test

Isolok - $3 \mathrm{~mL}$ - LF-Coliwasa

Assuming unequal variances

$\begin{array}{lrlr}\text { Difference } & 1.5762 & \text { t Ratio } & 1.526824 \\ \text { Std Err Dif } & 1.0324 & \text { DF } & 18.74466 \\ \text { Upper CL Dif } & 3.7390 \text { Prob }>|t| & 0.1435 \\ \text { Lower CL Dif } & -0.5865 & \text { Prob }>\text { t } & 0.0718 \\ \text { Confidence } & 0.95 \text { Prob }<\mathrm{t} & 0.9282\end{array}$

Tests that the Variances are Equal

Level Count Std Dev MeanAbsDif to Mean MeanAbsDif to Median

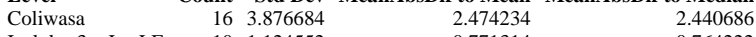

\begin{tabular}{|c|c|}
\hline Isolok - $3 \mathrm{~mL}$ - LF & \\
\hline
\end{tabular}

Test F Ratio DFNum DFDen p-Value

$\begin{array}{lrrrr}\text { O'Brien[.5] } & 2.5166 & 1 & 24 & 0.1257\end{array}$

$\begin{array}{lllll}\text { Brown-Forsythe } & 2.9771 & 1 & 24 & 0.0973\end{array}$

$\begin{array}{lrrrr}\text { Levene } & 3.2224 & 1 & 24 & 0.0852\end{array}$

$\begin{array}{lrrrr}\text { F Test 2-sided } & 11.8840 & 15 & 9 & 0.0007\end{array}$

Welch Anova testing Means Equal, allowing Std Devs Not Equal

F Ratio DFNum DFDen Prob $>$ F

$\begin{array}{rrrr}2.3312 & 1 & 18.745 & 0.1435\end{array}$

t Test 
SRNL-STI-2011-00693

Revision 0

\section{Exhibit B2. Statistical Comparisons for High-Rheology (Phase 2) Simulant Testing}

Oneway Analysis of Measurement (wt \%) By Sampler Type of Material=SME Simulant, Analytical Block=1, Analyte $=$ TiO2 $(w t \%)$, Target $(w t \%)=0.013$

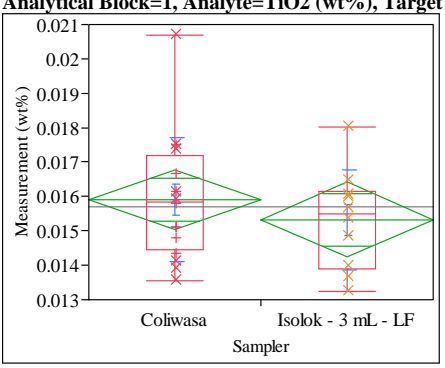

Oneway Anova

Summary of Fit

Rsquare

Adj Rsquare

Root Mean Square Error $\quad-0.00989$

Mean of Response $\quad 0.015687$

Observations (or Sum Wgts)

Test

Isolok - $3 \mathrm{~mL}$ - LF-Coliwas

Assuming equal variances

Difference $\quad-0.00059$ t Ratio $\quad-0.86907$

$\begin{array}{lrlr}\text { Std Err Dif } & 0.00068 \text { DF } & 24 \\ \text { Upper CL Dif } & 0.00081 & \text { Prob }>|t| & 0.3934\end{array}$

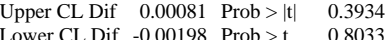

$\begin{array}{lll}\text { Confidence } & 0.95 \text { Prob }<\mathrm{t} \quad 0.1967\end{array}$

Analysis of Variance

Source DF Sum of Squares Mean Square F Ratio Prob $>$ F

$\begin{array}{lrrrrr}\text { Sampler } & 1 & 0.00000212 & 2.1214 \mathrm{e}-6 & 0.7553 & 0.3934\end{array}$

$\begin{array}{llll}\text { Error } & 24 & 0.00006741 & 2.8087 \mathrm{e}-6\end{array}$

C. Total $25 \quad 0.00006953$

Means for Oneway Anova

Level Number Mean Std Error Lower 95\% Upper 95\%

$\begin{array}{lrrrrr}\text { Level } & \text { Number } & \text { Mean } & \text { Std Error } & \text { Lower 95\% } & \text { Upper 95\% } \\ \text { Coliwasa } & 16 & 0.015913 & 0.00042 & 0.01505 & 0.01678\end{array}$

$\begin{array}{llllll}\text { Coliwasa } & 16 & 0.015913 & 0.00042 & 0.01505 & 0.01678 \\ \text { Isolok - 3 mL - LF } & 10 & 0.015326 & 0.00053 & 0.01423 & 0.01642\end{array}$

Std Error uses a pooled estimate of error variance

Means and Std Deviations

Level Number Mean Std Dev Std Err Mean Lower 95\% Upper 95\%

$\begin{array}{llllllr}\text { Coliwasa } & 16 & 0.015913 & 0.001801 & 0.00045 & 0.01495 & 0.01687\end{array}$

$\begin{array}{lllllll}\text { Isolok - } 3 \mathrm{~mL} \text { - LF } & 10 & 0.015326 & 0.001444 & 0.00046 & 0.01429 & 0.01636\end{array}$

t Test

Isolok - $3 \mathrm{~mL}$ - LF-Coliwasa

Assuming unequal variances

Difference $\quad-0.00059$ t Ratio $\quad-0.91566$

Std Err Dif $\quad 0.00064$ DF 22.33961

Upper CL Dif 0.00074 Prob $>|t| \quad 0.3696$

Lower CL Dif -0.00192 Prob $>t \quad 0.8152$

$\begin{array}{lrr}\text { Confidence } & 0.95 \text { Prob }<\mathrm{t} \quad 0.1848\end{array}$

Tests that the Variances are Equal

$\begin{array}{lrrrr}\text { Level } & \text { Count } & \text { Std Dev } & \text { MeanAbsDif to Mean } & \text { MeanAbsDif to Median } \\ \text { Coliwasa } & 16 & 0.0018009 & 0.0013281 & 0.0013261\end{array}$

$\begin{array}{lllll} & 16 & 0.0018009 & 0.0013281 & 0.0013261 \\ \text { Isolok - } 3 \mathrm{~mL}-\mathrm{LF} & 10 & 0.0014438 & 0.0011149 & 0.0011109\end{array}$

Test F Ratio DFNum DFDen p-Value

Test F Ratio DFNum DFDen p-Value

$\begin{array}{lllll}\text { O'Brien[.5] } & 0.3179 & 1 & 24 & 0.5781 \\ \text { Brown-Forsythe } & 0.2504 & 1 & 24 & 0.6213\end{array}$

$\begin{array}{lllll}\text { Brown-Forsythe } & 0.2504 & 1 & 24 & 0.6213 \\ \text { Levene } & 0.2510 & 1 & 24 & 0.6209\end{array}$

$\begin{array}{lllll}\text { Levene } & 0.2510 & 1 & 24 & 0.6209 \\ \text { Bartlett } & 0.5033 & 1 & & 0.4781\end{array}$

$\begin{array}{lllll}\text { F Test 2-sided } & 1.5559 & 15 & 9 & 0.5093\end{array}$

Welch Anova testing Means Equal, allowing Std Devs Not Equal

F Ratio DFNum DFDen Prob $>$ F

$\begin{array}{llll}0.8384 & 1 & 22.34 & 0.3696\end{array}$

t Test

0.9157
Oneway Analysis of Measurement (wt \%) By Sampler Type of Material=SME Simulant, Analytical Block $=1$, Analyte $=\mathrm{ZnO}(\mathrm{wt} \%)$, Target $(\mathrm{wt} \%)=0$

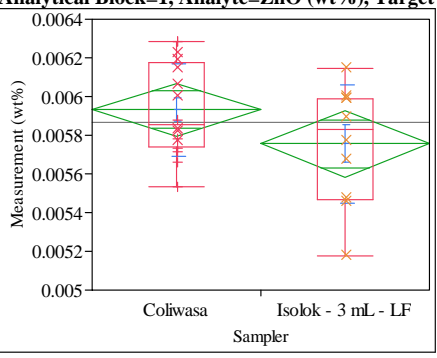

Oneway Anova

Summary of Fit

$\begin{array}{lr}\text { Rsquare } & 0.101376 \\ \text { Adj Rsquare } & 0.063933 \\ \text { Root Mean Square Error } & 0.000267 \\ \text { Mean of Response } & 0.005864 \\ \text { Observations (or Sum Wgts) } & 26\end{array}$

t Test

Isolok - $3 \mathrm{~mL}$ - LF-Coliwasa

Assuming equal variances

Difference $\quad-0.00018$ t Ratio $\quad-1.64545$

Std Err Dif $\quad 0.00011$ DF 24

Upper CL Dif 0.000045 Prob $>|t| \quad 0.1129$

$\begin{array}{lll}\text { Lower CL Dif }-0.00040 \text { Prob }>t & 0.9435\end{array}$

Confidence $\quad 0.95$ Prob $<\mathrm{t} \quad 0.0565$

Analysis of Variance

Source DF Sum of Squares Mean Square F Ratio Prob $>$ F

$\begin{array}{lrrrrr}\text { Source } & \text { DF } & \text { Sum of Squares } & \text { Mean Square } & \text { F Ratio } & \text { Prob }>\text { F } \\ \text { Sampler } & 1 & 1.93122 \mathrm{e}-7 & 1.9312 \mathrm{e}-7 & 2.7075 & 0.1129\end{array}$

$\begin{array}{llll}\text { Error } & 24 & 1.71189 \mathrm{e}-6 & 7.1329 \mathrm{e}-8\end{array}$

$\begin{array}{lll}\text { Error } & 24 & 1.71189 \mathrm{e}-6 \\ \text { C. Total } & 25 & 1.90501 \mathrm{e}-6\end{array}$

Means for Oneway Anova

Level Number Mean Std Error Lower 95\% Upper 95\%

$\begin{array}{llllll} & 16 & 0.005932 & 6.68 \mathrm{e}-5 & 0.00579 & 0.00607 \\ \text { Isolok - } 3 \mathrm{~mL}-\mathrm{LF} & 10 & 0.005755 & 8.45 \mathrm{e}-5 & 0.00558 & 0.00593\end{array}$

Std Error uses a pooled estimate of error variance

Means and Std Deviations

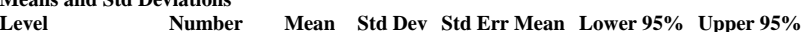

$\begin{array}{lrrrrrr}\text { Coliwasa } & 16 & 0.005932 & 0.000240 & 0.00006 & 0.00580 & 0.00606\end{array}$

$\begin{array}{lrrrrrr}\text { Isolok - 3 mL - LF } & 16 & 0.005932 & 0.000240 & 0.00006 & 0.00580 & 0.00606 \\ & 10 & 0.005755 & 0.000307 & 0.0001 & 0.00554 & 0.00597\end{array}$

t Test

Isolok - $3 \mathrm{~mL}$ - LF-Coliwasa

Assuming unequal variances

Difference $\quad-0.00018$ t Ratio -1.55213

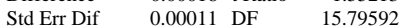

Upper CL Dif 0.000065 Prob $>|t| r .1404$

$\begin{array}{lll}\text { Lower CL Dif }-0.00042 \text { Prob }>t & 0.9298\end{array}$

$\begin{array}{lll}\text { Confidence } & 0.95 \text { Prob }<\mathrm{t} \quad 0.0702\end{array}$

Tests that the Variances are Equal

Level Count Std Dev MeanAbsDif to Mean MeanAbsDif to Median

$\begin{array}{lrrrr}\text { Coliwasa } & 16 & 0.0002399 & 0.0002082 & 0.0002019\end{array}$

$\begin{array}{lllll}\text { Isolok - } 3 \mathrm{~mL} \text { - LF } & 10 & 0.0003071 & 0.0002082 & 0.0002495\end{array}$

Test F Ratio DFNum DFDen p-Value

$\begin{array}{lllll}\text { O'Brien[.5] } & 1.2293 & 1 & 24 & 0.2785\end{array}$

$\begin{array}{lllll}\text { Brown-Forsythe } & 0.4914 & 1 & 24 & 0.4900\end{array}$

$\begin{array}{lllll}\text { Levene } & 0.6355 & 1 & 24 & 0.4332\end{array}$

$\begin{array}{lllll}\text { Bartlett } & 0.6773 & 1 & 0.4105\end{array}$

$\begin{array}{lllll}\text { F Test 2-sided } & 1.6387 & 9 & 15 & 0.3822\end{array}$

Welch Anova testing Means Equal, allowing Std Devs Not Equal

F Ratio DFNum DFDen Prob $>$ F

$\begin{array}{llll}2.4091 & 1 & 15.796 & 0.1404\end{array}$

t Test

1.5521 


\section{Exhibit B2. Statistical Comparisons for High-Rheology (Phase 2) Simulant Testing}

Oneway Analysis of Measurement (wt \%) By Sampler Type of Material=SME Simulant, Analytical Block=1, Analyte $=\mathrm{ZrO} 2(\mathrm{wt} \%)$, Target $(\mathrm{wt} \%)=0.111$

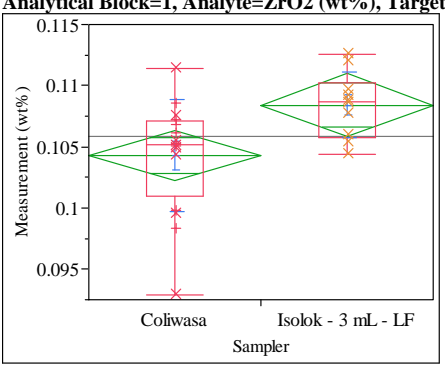

Oneway Anova

Summary of Fit

Rsquare

Adj Rsquare

Root Mean Square Error $\quad 0.003946$

Mean of Response $\quad 0.105866$

Observations (or Sum Wgts)

26

Test

Isolok - $3 \mathrm{~mL}$ - LF-Coliwas

Assuming equal variances

Difference $\quad 0.004120$ t Ratio 2.589799

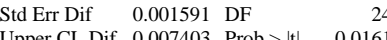

$\begin{array}{llll}\text { Upper CL Dif } & 0.007403 & \text { Prob }>|t| & 0.016 \\ \text { Cower CL Dif } & 0.000837 & \text { Prob }>t & 0.0080\end{array}$

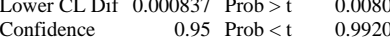

Analysis of Variance

Source DF Sum of Squares Mean Square F Ratio Prob > F

$\begin{array}{lrrrrr}\text { Sampler } & 1 & 0.00010445 & 0.000104 & 6.7071 & 0.0161\end{array}$

$\begin{array}{llll}\text { Error } & 24 & 0.00037377 & 0.000016\end{array}$

C. Total $25 \quad 0.00047823$

Means for Oneway Anova

Level Number Mean Std Error Lower 95\% Upper 95\%

$\begin{array}{lrrrrr}\text { Coliwasa } & 16 & 0.104282 & 0.00099 & 0.10225 & 0.10632\end{array}$

$\begin{array}{llllll}\text { Isolok - } 3 \mathrm{~mL} \text { - LF } & 10 & 0.108402 & 0.00125 & 0.10583 & 0.11098\end{array}$

Std Error uses a pooled estimate of error variance

Means and Std Deviations

Level Number Mean Std Dev Std Err Mean Lower 95\% Upper 95\%

$\begin{array}{lllllll}\text { Coliwasa } & 16 & 0.104282 & 0.004544 & 0.00114 & 0.10186 & 0.10670\end{array}$

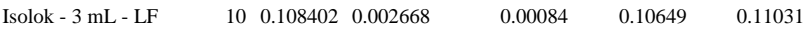

t Test

Isolok - $3 \mathrm{~mL}$ - LF-Coliwasa

Assuming unequal variances

Difference $\quad 0.004120$ t Ratio 2.911629

Std Err Dif $\quad 0.001415$ DF 23.96099

Upper CL Dif 0.007041 Prob $>|t| \quad 0.0077$

Lower CL Dif 0.001199 Prob $>t \quad 0.0038$

$\begin{array}{lrl}\text { Confidence } & 0.95 \text { Prob }<\mathrm{t} \quad 0.9962\end{array}$

Tests that the Variances are Equal

$\begin{array}{lrrrr}\text { Level } & \text { Count } & \text { Std Dev } & \text { MeanAbsDif to Mean } & \text { MeanAbsDif to Median } \\ \text { Coliwasa } & 16 & 0.0045440 & 0.0033095 & 0.0030393\end{array}$

$\begin{array}{lllll} & 16 & 0.0045440 & 0.0033095 & 0.0030393 \\ \text { Isolok - } 3 \mathrm{~mL}-\mathrm{LF} & 10 & 0.0026678 & 0.0020532 & 0.0020127\end{array}$

Test F Ratio DFNum DFDen p-Value

Test F [5] DFNum DFDen p-Value

$\begin{array}{lllll}\text { O'Brien[.5] } & 1.3485 & 1 & 24 & 0.2570 \\ \text { Brown-Forsythe } & 0.7840 & 1 & 24 & 0.3847\end{array}$

$\begin{array}{lllll}\text { Brown-Forsythe } & 0.7840 & 1 & 24 & 0.3847 \\ \text { Levene } & 1.4905 & 1 & 24 & 0.2340\end{array}$

$\begin{array}{lllll}\text { Levene } & 1.4905 & 1 & 24 & 0.2340 \\ \text { Bartlett } & 2.6952 & 1 & . & 0.1007\end{array}$

$\begin{array}{lrrrr}\text { F Test 2-sided } & 2.9011 & 15 & 9 & 0.1109\end{array}$

Welch Anova testing Means Equal, allowing Std Devs Not Equal

F Ratio DFNum DFDen Prob $>$ F

$\begin{array}{llll}8.4776 & 1 & 23.961 & 0.0077\end{array}$

t Test

2.9116
Oneway Analysis of Measurement (wt \%) By Sampler Type of Material=SME Simulant, Analytical Block $=2$, Analyte $=$ Al $/ B$, Target $(w t \%)=2.0704412$

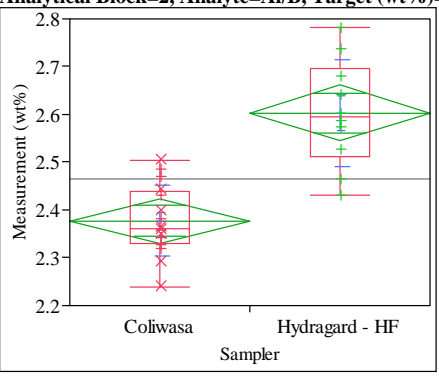

Oneway Anova

Summary of Fit

Rot Mean Square Error

Mean of Response 2.464264

t Test

Hydragard - HF-Coliwasa

Assuming equal variances

$\begin{array}{lrlr}\text { Difference } & 0.225573 & \text { t Ratio } & 6.221199 \\ \text { Std Err Dif } & 0.036259 & \text { DF } & 24 \\ \text { Upper CL Dif } & 0.300408 & \text { Prob }>|t| & <.0001 \\ \text { Lower CL Dif } & 0.150739 & \text { Prob }>t & <.0001 \\ \text { Confidence } & 0.95 & \text { Prob }<t & 1.0000\end{array}$

Analysis of Variance

Source DF Sum of Squares Mean Square F Ratio Prob $>$ F

$\begin{array}{lrrrrr}\text { Sampler } & 1 & 0.31312805 & 0.313128 & 38.7033 & <.0001\end{array}$

$\begin{array}{llll}\text { Error } & 24 & 0.19417129 & 0.008090\end{array}$

C. Total $25 \quad 0.50729934$

Means for Oneway Anova

Level Number Mean Std Error Lower 95\% Upper 95\%

$\begin{array}{llllll}\text { Coliwasa } & 16 & 2.37751 & 0.02249 & 2.3311 & 2.4239\end{array}$

Std Error uses a pooled estimate of error variance

Means and Std Deviations

$\begin{array}{llllll}\text { Means and Std Deviations } & & & & \\ \text { Level } & \text { Number Mean Std Dev Std Err Mean Lower 95\% Upper 95\% }\end{array}$

$\begin{array}{lrrrrrr}\text { Level } & \text { Number } & \text { Mean } & \text { Std Dev } & \text { Std Err Mean } & \text { Lower 95\% } & \text { Upper 95\% } \\ \text { Coliwasa } & 16 & 2.37751 & 0.073175 & 0.01829 & 2.3385 & 2.4165\end{array}$

$\begin{array}{lllllll}\text { Hydragard - HF } & 16 & 2.37751 & 0.073175 & 0.01829 & 2.3385 & 2.4165 \\ & 10 & 2.60308 & 0.112474 & 0.03557 & 2.5226 & 2.6835\end{array}$

t Test

Hydragard - HF-Coliwasa

Assuming unequal variances

Difference $\quad 0.225573$ t Ratio $\quad 5.639866$

Std Err Dif $\quad 0.039996$ DF 13.81166

Upper CL Dif 0.311467 Prob $>|t| r .0001$

Lower CL Dif 0.139680 Prob $>t \quad<.0001$

$\begin{array}{lll}\text { Confidence } & 0.95 \text { Prob }<\mathrm{t} \quad 1.0000\end{array}$

Tests that the Variances are Equal

Level Count Std Dev MeanAbsDif to Mean MeanAbsDif to Median

$\begin{array}{lrrrr}\text { Coliwasa } & 16 & 0.0731745 & 0.0583840 & 0.0566865\end{array}$

$\begin{array}{lllll}\text { Hydragard - HF } & 10 & 0.1124740 & 0.0863295 & 0.0566855\end{array}$

Test F Ratio DFNum DFDen p-Value

$\begin{array}{lllll}\text { O'Brien[.5] } & 3.0090 & 1 & 24 & 0.0956\end{array}$

$\begin{array}{lllll}\text { Brown-Forsythe } & 1.7811 & 1 & 24 & 0.1945 \\ & 1.7716 & 1 & 24 & 0.1957\end{array}$

$\begin{array}{lllll}\text { Levene } & 1.7716 & 1 & 24 & 0.1957\end{array}$

$\begin{array}{lllll}\text { F Test 2-sided } & 2.3626 & 9 & 15 & 0.1358\end{array}$

Welch Anova testing Means Equal, allowing Std Devs Not Equal

F Ratio DFNum DFDen Prob $>$ F

$\begin{array}{rrrr}31.8081 & 1 & 13.812 & <.0001\end{array}$

t Test 


\section{Exhibit B2. Statistical Comparisons for High-Rheology (Phase 2) Simulant Testing}

Oneway Analysis of Measurement (wt \%) By Sampler Type of Material=SME Simulant, Analytical Block $=2$, Analyte $=\mathrm{Al} 2 \mathrm{O} 3(\mathrm{wt} \%)$, Target $(\mathrm{wt} \%)=10.934$

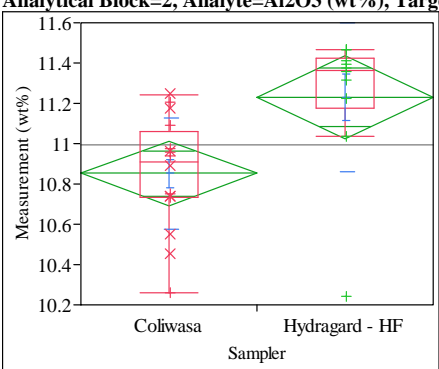

Oneway Anova

Summary of Fit

Adj Rsquare

\begin{tabular}{ll}
0.314748 \\
\hline
\end{tabular} 10.99689

Observations (or Sum Wgts) $\quad 26$

Test

Hydragard - HF-Coliwasa

Assuming equal variances

Difference $\quad 0.377664$ t Ratio 2.976565

Std Err Dif $\quad 0.126879$ DF $\quad 24$

Upper CL Dif 0.639529 Prob $>|t| \quad 0.0066$

Lower CL Dif 0.115798 Prob $>t \quad 0.0033$

$\begin{array}{lrl}\text { Confidence } & 0.95 \text { Prob }<\mathrm{t} \quad 0.9967\end{array}$

Analysis of Variance

Source DF Sum of Squares Mean Square F Ratio Prob $>$ F

$\begin{array}{llllll}\text { Sampler } & 1 & 0.8777228 & 0.877723 & 8.8599 & 0.0066\end{array}$

$\begin{array}{llll}\text { Error } \quad 24 & 2.3775949 & 0.099066\end{array}$

C. Total $25 \quad 3.2553177$

Means for Oneway Anova

Level Number Mean Std Error Lower 95\% Upper 95\%

$\begin{array}{lrrrrr}\text { Coliwasa } & 16 & 10.8516 & 0.07869 & 10.689 & 11.014\end{array}$

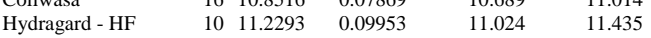

Std Error uses a pooled estimate of error variance

Means and Std Deviations

Level Number Mean Std Dev Std Err Mean Lower 95\% Upper 95\%

Coliwasa $10.8516 \quad 0.275955$

$\begin{array}{lllllll}\text { Hydragard - HF } & 10 & 11.2293 & 0.370484 & 0.11716 & 10.964 & 11.494\end{array}$

Test

Hydragard - HF-Coliwasa

Assuming unequal variances

Difference $\quad 0.377664$ t Ratio 2.777742

Std Err Dif $\quad 0.135961$ DF $\quad 15.22525$

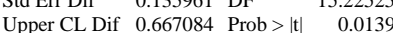

Lower CL Dif 0.088243 Prob $>t \quad 0.0070$

$\begin{array}{lrl}\text { Confidence } & 0.95 \text { Prob }<\mathrm{t} & 0.9930\end{array}$

Tests that the Variances are Equal

Level Count Std Dev MeanAbsDif to Mean MeanAbsDif to Median

Coliwasa $\quad 16 \quad 0.2759549$

$\begin{array}{lllll}\text { Hydragard - HF } & 10 & 0.3704844 & 0.22376991 & 0.2161116 \\ & & & \end{array}$

Test F Ratio DFNum DFDen p-Value

$\begin{array}{lrrrr}\text { O'Brien[.5] } & 0.4276 & 1 & 24 & 0.5194\end{array}$

Brown-Forsythe $\quad 0.0456 \quad 1 \quad 24 \quad 0.8326$

$\begin{array}{lllll}\text { Levene } & 0.0441 & 1 & 24 & 0.8354\end{array}$

$\begin{array}{lllll}\text { Bartlett } & 0.9675 & 1 & 0.3253 \\ \text { F Test 2-sided } & 1.8025 & 9 & 15 & 0.3009\end{array}$

Welch Anova testing Means Equal, allowing Std Devs Not Equal

F Ratio DFNum DFDen Prob $>$ F

$\begin{array}{llll}7.7159 & 1 & 15.225 & 0.0139\end{array}$

t Test

$$
\begin{array}{lrr}
1 & 15.225 & 0.0139
\end{array}
$$

2.7777
Oneway Analysis of Measurement (wt\%) By Sampler Type of Material=SME Simulant, Analytical Block=2, Analyte $=\mathrm{B} 2 \mathrm{O} 3(\mathrm{wt} \%)$, Target $(\mathrm{wt} \%)=5.281$

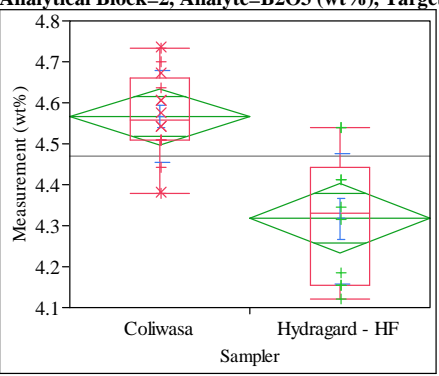

Oneway Anova

Summary of Fit

A

0.13167

Mean of Response 4.470707
26

Test

Hydragard - HF-Coliwasa

Assuming equal variances

Difference -0.24833 t Ratio -4.67864

$\begin{array}{llll}\text { Std Err Dif } & 0.05308 \text { DF } & 24\end{array}$

Upper CL Dif -0.13879 Prob $>|t| \quad<.0001$

Lower CL Dif -0.35788 Prob $>$ t 1.0000

$\begin{array}{lrl}\text { Confidence } & 0.95 \text { Prob }<\mathrm{t} & <.0001\end{array}$

Analysis of Variance

Source DF Sum of Squares Mean Square F Ratio Prob $>$ F

$\begin{array}{lllllll}\text { Sampler } & 1 & 0.37950872 & 0.379509 & 21.8896 & <.0001\end{array}$

$\begin{array}{llll}\text { Error } & 24 & 0.41609693 & 0.017337\end{array}$

C. Total $25 \quad 0.79560565$

Means for Oneway Anova

Level Number Mean Std Error Lower 95\% Upper 95\%

$\begin{array}{llllll}\text { Coliwasa } & 16 & 4.56622 & 0.03292 & 4.4983 & 4.6342 \\ \text { Hydragard - HF } & 10 & 4.31789 & 0.04164 & 4.2319 & 4.4038\end{array}$

Std Error uses a pooled estimate of error variance

$\begin{array}{llllll}\begin{array}{l}\text { Means and Std Deviations } \\ \text { Level }\end{array} \text { Number } & \text { Mean } & \text { Std Dev Std Err Mean Lower 95\% } & \text { Upper 95\% }\end{array}$

$\begin{array}{lrrrrrr}\text { Level } & \text { Number } & \text { Mean } & \text { Std Dev } & \text { Std Err Mean } & \text { Lower 95\% } & \text { Upper 95\% } \\ \text { Coliwasa } & 16 & 4.56622 & 0.112293 & 0.02807 & 4.5064 & 4.6261\end{array}$

$\begin{array}{lllllll}\text { Coliwasa } & 16 & 4.56622 & 0.112293 & 0.02807 & 4.5064 & 4.6261 \\ \text { Hydragard - HF } & 10 & 4.31789 & 0.158798 & 0.05022 & 4.2043 & 4.4315\end{array}$

t Test

Hydragard - HF-Coliwasa

Assuming unequal variances

Difference $\quad-0.24833$ t Ratio $\quad-4.31656$

Std Err Dif $\quad 0.05753$ DF $\quad 14.64637$

Upper CL Dif -0.12545 Prob $>|t| \quad 0.0006$

Lower CL Dif -0.37122 Prob $>$ t 0.9997

$\begin{array}{lll}\text { Confidence } & 0.95 \text { Prob }<\mathrm{t} \quad 0.0003\end{array}$

Tests that the Variances are Equal

Level Count Std Dev MeanAbsDif to Mean MeanAbsDif to Median

$\begin{array}{lrrrr}\text { Coliwasa } & 16 & 0.1122933 & 0.0905597 & 0.0905597\end{array}$

$\begin{array}{lllll}\text { Hydragard - HF } & 10 & 0.1587976 & 0.1320159 & 0.1320159\end{array}$

Test F Ratio DFNum DFDen p-Value

$\begin{array}{lllll}\text { O'Brien[.5] } & 3.1672 & 1 & 24 & 0.0878\end{array}$

$\begin{array}{lllll}\text { Brown-Forsythe } & 2.2290 & 1 & 24 & 0.1485\end{array}$

$\begin{array}{lllll}\text { Levene } & 2.2950 & 1 & 24 & 0.1428\end{array}$

$\begin{array}{lllll} & \text { F Test 2-sided } & 1.3432 & 1 & 0.2465\end{array}$

Welch Anova testing Means Equal, allowing Std Devs Not Equal

F Ratio DFNum DFDen Prob $>$ F

$\begin{array}{rrrr}18.6327 & 1 & 14.646 & 0.0006\end{array}$

t Test 


\section{Exhibit B2. Statistical Comparisons for High-Rheology (Phase 2) Simulant Testing}

Oneway Analysis of Measurement (wt \%) By Sampler Type of Material=SME Simulant, Analytical Block $=2$, Analyte $=\mathrm{BaO}(\mathrm{wt} \%)$, Target $(\mathrm{wt} \%)=0$

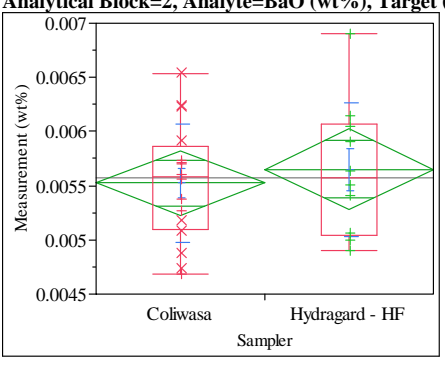

Oneway Anova

Summary of Fit

$\begin{array}{lr}\text { Rsquare } & 0.01236 \\ \text { Adj Rsquare } & -0.02879 \\ \text { Root Mean Square Error } & 0.000574 \\ \text { Mean of Response } & 0.005573 \\ \text { Observations (or Sum Wgts) } & 26\end{array}$

t Test

Hydragard - HF-Coliwasa

Assuming equal variances

Difference $\quad 0.00013$ t Ratio $\quad 0.548044$

$\begin{array}{lll}\text { Std Err Dif } \quad 0.00023 \mathrm{DF} & 24\end{array}$

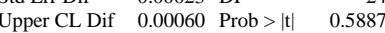

Lower CL Dif -0.00035 Prob $>t \quad 0.2944$

$\begin{array}{lrl}\text { Confidence } & 0.95 \text { Prob }<\mathrm{t} \quad 0.7056\end{array}$

Analysis of Variance

Source DF Sum of Squares Mean Square F Ratio Prob $>$ F

$\begin{array}{lrrrrr}\text { Source } & \text { DF } & \text { Sum of Squares } & \text { Mean Square } & \text { F Ratio } & \text { Prob }>\text { F } \\ \text { Sampler } & 1 & 9.88225 \mathrm{e}-8 & 9.8822 \mathrm{e}-8 & 0.3004 & 0.5887\end{array}$

$\begin{array}{lrrr}\text { Sampler } & 1 & 9.88225 \mathrm{e}-8 & 9.8822 \mathrm{e}-8 \\ \text { Error } & 24 & 7.89652 \mathrm{e}-6 & 3.2902 \mathrm{e}-7\end{array}$

$\begin{array}{lll}\text { C. Total } 25 & 7.99535 \mathrm{e}-6\end{array}$

Means for Oneway Anova

$\begin{array}{llrrrr}\text { Level Number } & \text { Mean } & \text { Std Error } & \text { Lower 95\% } & \text { Upper 95\% }\end{array}$

$\begin{array}{llllll}\text { Hydragard - HF } & 16 & 0.005524 & 0.00014 & 0.00523 & 0.00582 \\ & 10 & 0.005651 & 0.00018 & 0.00528 & 0.00602\end{array}$

Std Error uses a pooled estimate of error variance

Means and Std Deviations

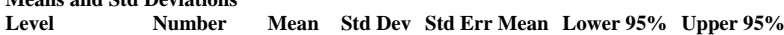

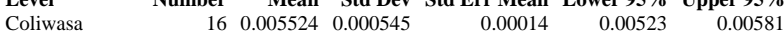

$\begin{array}{lllllll}\text { Hydragard - HF } & 10 & 0.005651 & 0.000619 & 0.00020 & 0.00521 & 0.00609\end{array}$

t Test

Hydragard - HF-Coliwasa

Assuming unequal variances

Difference $\quad 0.00013$ t Ratio $\quad 0.531656$

Std Err Dif $\quad 0.00024$ DF 17.39274

Upper CL Dif 0.00063 Prob $>|t| r 0.6017$

Lower CL Dif -0.00038 Prob $>t \quad 0.3008$
C

$\begin{array}{lll}\text { Confidence } & 0.95 \text { Prob }<\mathrm{t} \quad 0.6992\end{array}$

Tests that the Variances are Equal

Level Count Std Dev MeanAbsDif to Mean MeanAbsDif to Median

$\begin{array}{lrrrr}\text { Coliwasa } & 16 & 0.0005449 & 0.0004372 & 0.0004326 \\ \text { Hydragard - HF } & 10 & 0.0006185 & 0.0004792 & 0.0004767\end{array}$

Test F Ratio DFNum DFDen p-Value

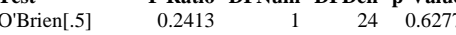

$\begin{array}{lllll}\text { Brown-Forsythe } & 0.1046 & 1 & 24 & 0.7492\end{array}$

$\begin{array}{lllll}\text { Levene } & 0.1026 & 1 & 24 & 0.7515\end{array}$

$\begin{array}{lllll}\text { Bartlett } & 0.1762 & 1 & 0.6746\end{array}$

Welch Anova testing Means Equal, allowing Std Devs Not Equal

F Ratio DFNum DFDen Prob $>$ F

$\begin{array}{llll}0.2827 & 1 & 17.393 & 0.6017\end{array}$

t Test
Oneway Analysis of Measurement (wt\%) By Sampler Type of Material=SME Simulant, Analytical Block $=2$, Analyte $=\mathrm{CaO}(\mathrm{wt} \%)$, Target $(\mathrm{wt} \%)=0.543$

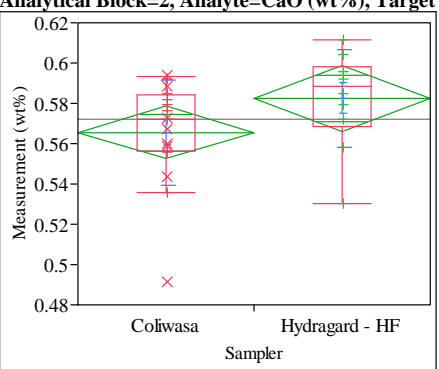

Oneway Anova

Summary of Fit

Adj Rsquare

$\quad 0.025268$

Mean of Response 0.57211

t Test

Hydragard - HF-Coliwasa

Assuming equal variances

Difference $\quad 0.01686$ t Ratio 1.655257

Std Err Dif $\quad 0.01019$ DF 24

Upper CL Dif 0.03788 Prob $>|t| 0.1109$

Lower CL Dif -0.00416 Prob $>t \quad 0.0554$

$\begin{array}{lrl}\text { Confidence } & 0.95 \text { Prob }<\mathrm{t} & 0.9446\end{array}$

Analysis of Variance

Source DF Sum of Squares Mean Square F Ratio Prob $>$ F

$\begin{array}{llllll}\text { Sampler } & 1 & 0.00174936 & 0.001749 & 2.7399 & 0.1109\end{array}$

$\begin{array}{llll}\text { Error } & 24 & 0.01532359 & 0.000638\end{array}$

C. Total $25 \quad 0.01707295$

Means for Oneway Anova

Level Number Mean Std Error Lower 95\% Upper 95\%

$\begin{array}{lrrrrr}\text { Coliwasa } & 16 & 0.565627 & 0.00632 & 0.55259 & 0.57866\end{array}$

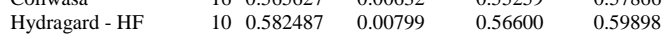

Std Error uses a pooled estimate of error variance

Means and Std Deviations

Level Number Mean Std Dev Std Err Mean Lower 95\% Upper 95\%

$\begin{array}{lrrrrrr}\text { Coliwasa } & 16 & 0.565627 & 0.025969 & 0.00649 & 0.55179 & 0.57946\end{array}$

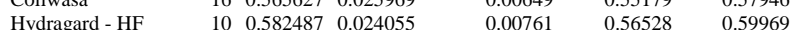

Test

Hydragard - HF-Coliwasa

Assuming unequal variances

$\begin{array}{lrlr}\text { Difference } & 0.01686 & \mathrm{t} \text { Ratio } & 1.685919 \\ \text { Std Err Dif } & 0.01000 & \text { DF } & 20.39409 \\ \text { Upper CL Dif } & 0.03770 & \text { Prob }>|t| & 0.1071 \\ \text { Lower CL Dif } & -0.00397 & \text { Prob }>\mathrm{t} & 0.0535 \\ \text { Confidence } & 0.95 & \text { Prob }<\mathrm{t} & 0.9465\end{array}$

Tests that the Variances are Equal

Level Count Std Dev MeanAbsDif to Mean MeanAbsDif to Median

$\begin{array}{lllll}\text { Coliwasa } & 16 & 0.0259689 & 0.0188018 & 0.0178398\end{array}$

$\begin{array}{lllll}\text { Hydragard - HF } & 10 & 0.0240551 & 0.0179657 & 0.0174900\end{array}$

Test F Ratio DFNum DFDen p-Value

$\begin{array}{lrrrr}\text { O'Brien[.5] } & 0.0326 & 1 & 24 & 0.8581\end{array}$

$\begin{array}{lllll}\text { Brown-Forsythe } & 0.0022 & 1 & 24 & 0.9630\end{array}$

$\begin{array}{lllll}\text { Levene } & 0.0160 & 1 & 24 & 0.9003\end{array}$

$\begin{array}{lrrrr}\text { Bartlett } & 0.0622 & 1 & \text {. } & 0.8030 \\ \text { F Test 2-sided } & 1.1654 & 15 & 9 & 0.8420\end{array}$

Welch Anova testing Means Equal, allowing Std Devs Not Equal

F Ratio DFNum DFDen Prob $>$ F

$2.8423 \quad 1 \quad 20.394 \quad 0.1071$

t Test

1.6859 
SRNL-STI-2011-00693

Revision 0

\section{Exhibit B2. Statistical Comparisons for High-Rheology (Phase 2) Simulant Testing}

Oneway Analysis of Measurement (wt \%) By Sampler Type of Material=SME Simulant, Analytical Block $=2$, Analyte $=$ Cr2O3 $(w t \%)$, Target $(w t \%)=0.015$

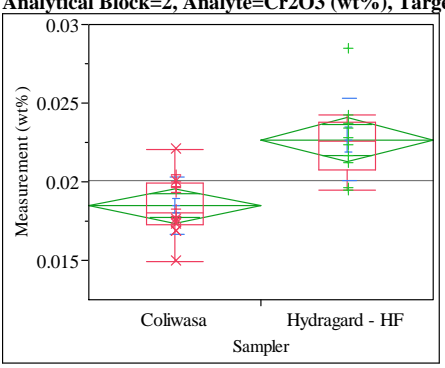

Oneway Anova

Summary of Fit

Rsquare

Adj Rsquare

Root Mean Square Error $\quad 0.002136$

$\begin{array}{lr}\text { Mean of Response } & 0.020097 \\ \text { Observations (or Sum Wgts) } & 26\end{array}$

Test

Hydragard - HF-Coliwasa

Assuming equal variances

Difference $\quad 0.004204$ t Ratio $\quad 4.883381$

$\begin{array}{lrr}\text { Std Err Dif } & 0.000861 \text { DF } & 24 \\ \text { Dpper } & & <.0001\end{array}$

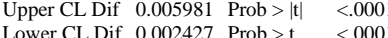

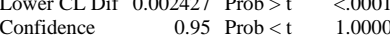

Analysis of Variance

Source DF Sum of Squares Mean Square F Ratio Prob $>$ F

$\begin{array}{lrrrrr}\text { Sampler } & 1 & 0.00010876 & 0.000109 & 23.8474 & <.0001\end{array}$

$\begin{array}{llll}\text { Error } & 24 & 0.00010945 & 4.561 \mathrm{e}-6\end{array}$

C. Total $25 \quad 0.0002182$

Means for Oneway Anova

Level Number Mean Std Error Lower 95\% Upper 95\%

$\begin{array}{lrrrrr}\text { Level } & \text { Number } & \text { Mean } & \text { Std Error } & \text { Lower 95\% } & \text { Upper 95\% } \\ \text { Coliwasa } & 16 & 0.018480 & 0.00053 & 0.01738 & 0.01958\end{array}$

$\begin{array}{llllll}\text { Hydragard - HF } & 10 & 0.022684 & 0.00068 & 0.02129 & 0.02408\end{array}$

Std Error uses a pooled estimate of error variance

Means and Std Deviations

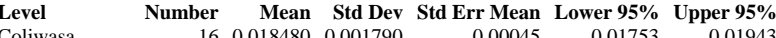

Coliwasa

$\begin{array}{llllll}16 & 0.018480 & 0.001790 & 0.00045 & 0.01753 & 0.01943 \\ 10 & 0.022684 & 0.002612 & 0.00083 & 0.02082 & 0.02455\end{array}$

t Test

Hydragard - HF-Coliwasa

Assuming unequal variances

Difference $\quad 0.004204$ t Ratio $\quad 4.475407$

Std Err Dif $\quad 0.000939$ DF $\quad 14.3202$

Upper CL Dif 0.006214 Prob $>|t| \quad 0.0005$

Lower CL Dif 0.002193 Prob $>t \quad 0.0002$

$\begin{array}{lll}\text { Confidence } & 0.95 \text { Prob }<\mathrm{t} \quad 0.9998\end{array}$

Tests that the Variances are Equal

Level Count Std Dev MeanAbsDif to Mean MeanAbsDif to Median

$\begin{array}{lllll}\text { Coliwasa } & 16 & 0.0017901 & 0.0014787 & 0.0014525 \\ \text { Hydragard - HF } & 10 & 0.0026117 & 0.0018416 & 0.0018416\end{array}$

Fest Ratio DFNum DFDen p-Value

Test F Ratio DFNum DFDen p-Value

$\begin{array}{lllll}\text { O'Brien[.5] } & 1.1629 & 1 & 24 & 0.2916 \\ \text { Brown-Forsythe } & 0.4988 & 1 & 24 & 0.4868\end{array}$

$\begin{array}{lllll}\text { Levene } & 0.4796 & 1 & 24 & 0.4952 \\ \text { Bartlett } & 1.5986 & 1 & & 0.2061\end{array}$

$\begin{array}{lllll}\text { F Test 2-sided } & 2.1287 & 9 & 15 & 0.1883\end{array}$

Welch Anova testing Means Equal, allowing Std Devs Not Equal

F Ratio DFNum DFDen Prob $>$ F

$\begin{array}{llll}20.0293 & 1 & 14.32 & 0.0005\end{array}$

t Test

4.4754
Oneway Analysis of Measurement (wt\%) By Sampler Type of Material=SME Simulant, Analytical Block $=2$, Analyte $=\mathrm{CuO}(\mathrm{wt} \%)$, Target $(\mathrm{wt} \%)=0.103$

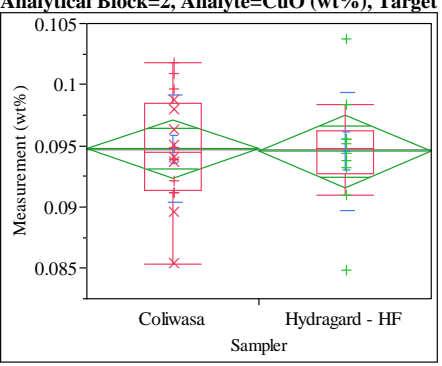

Oneway Anova

Summary of Fit

Rsquare

$\begin{array}{ll}\text { Adj Rsquare } & -0.04127 \\ \text { Root Mean Square Error } & 0.004564\end{array}$

0.000385

Mean of Response $\quad 0.09467$

Observations (or Sum Wgts)

26

\section{t Test}

Hydragard - HF-Coliwasa

Assuming equal variances

Difference $\quad-0.00018$ t Ratio $\quad-0.096$

Std Err Dif $\quad 0.00184$ DF

Upper CL Dif 0.00362 Prob $>|t| 0.9242$

Lower CL Dif -0.00397 Prob $>t \quad 0.5379$

Confidence 0.95 Prob $<\mathrm{t} \quad 0.4621$

Analysis of Variance

ource DF Sum of Squares Mean Square F Ratio Prob $>$ F

$\begin{array}{lrrrrr}\text { Sampler } & 1 & 0.00000019 & 1.924 \mathrm{e}-7 & 0.0092 & 0.9242\end{array}$

$\begin{array}{llll}\text { Error } & 24 & 0.00050001 & 0.000021\end{array}$

C. Total $25 \quad 0.00050020$

Means for Oneway Anova

Level Number Mean Std Error Lower 95\% Upper 95\%

$\begin{array}{lrrrrr}\text { Level } & \text { Number } & \text { Mean } & \text { Std Error } & \text { Lower 95\% } & \text { Upper 95\% } \\ \text { Coliwasa } & 16 & 0.094738 & 0.00114 & 0.09238 & 0.09709\end{array}$

$\begin{array}{llllll}\text { Hydragard - HF } & 10 & 0.094561 & 0.00144 & 0.09158 & 0.09754\end{array}$

Std Error uses a pooled estimate of error variance

Means and Std Deviations

Level Number Mean Std Dev Std Err Mean Lower 95\% Upper 95\%

$\begin{array}{lllllll}\text { Coliwasa } & 16 & 0.094738 & 0.004393 & 0.00110 & 0.09240 & 0.09708\end{array}$

$\begin{array}{lllllll}\text { Hydragard - HF } & 10 & 0.094561 & 0.004837 & 0.00153 & 0.09110 & 0.09802\end{array}$

Test

Hydragard - HF-Coliwasa

Assuming unequal variances

$\begin{array}{lrlr}\text { Difference } & -0.00018 & \text { t Ratio } & -0.0939 \\ \text { Std Err Dif } & 0.00188 \text { DF } & 17.82695 \\ \text { Upper CL Dif } & 0.00378 \text { Prob }>|t| & 0.9262 \\ \text { Lower CL Dif } & -0.00414 & \text { Prob }>\text { t } & 0.5369 \\ \text { Confidence } & 0.95 & \text { Prob }<\mathrm{t} & 0.4631\end{array}$

Tests that the Variances are Equal

Level Count Std Dev MeanAbsDif to Mean MeanAbsDif to Median

$\begin{array}{lrrrr} & 16 & 0.0043927 & 0.0033877 & 0.0033877 \\ \text { Hydragard - HF } & 10 & 0.0048371 & 0.0031045 & 0.0031045\end{array}$

Test F Ratio DFNum DFDen p-Value

$\begin{array}{lllll}\text { O'Brien[.5] } & 0.0923 & 1 & 24 & 0.7638\end{array}$

$\begin{array}{lllll}\text { Brown-Forsythe } & 0.0534 & 1 & 24 & 0.8192\end{array}$

$\begin{array}{lllll}\text { Brown-Forsythe } & 0.0534 & 1 & 24 & 0.8192 \\ \text { Levene } & 0.0538 & 1 & 24 & 0.8185\end{array}$

$\begin{array}{lllll}\text { Levene } & 0.0538 & 1 & 24 & 0.8185 \\ \text { Bartlett } & 0.1015 & 1 & & 0.7501\end{array}$

$\begin{array}{lllll}\text { F Test 2-sided } & 1.2126 & 9 & 15 & 0.7121\end{array}$

Welch Anova testing Means Equal, allowing Std Devs Not Equal

F Ratio DFNum DFDen Prob $>$ F

$\begin{array}{llll}0.0088 & 1 & 17.827 & 0.9262\end{array}$

t Test

0.0939 


\section{Exhibit B2. Statistical Comparisons for High-Rheology (Phase 2) Simulant Testing}

Oneway Analysis of Measurement (wt \%) By Sampler Type of Material=SME Simulant, Analytical Block=2, Analyte=Fe/Li, Target $(\mathrm{wt} \%)=1.99097835$

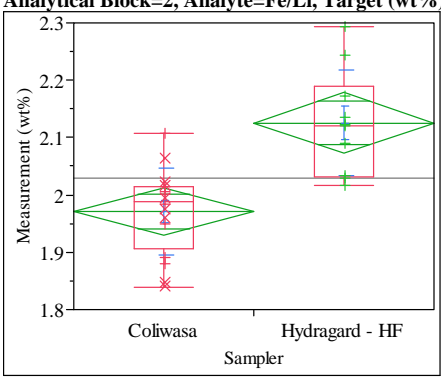

Oneway Anova

Summary of Fit

Adj Rsquare $\quad 0.47936$

0.457666

\begin{tabular}{lr} 
Root Mean Square Error $\quad 0.081477$ \\
Mean of Response & 2.0305 \\
\hline
\end{tabular}

$\begin{array}{lr}\text { Mean of Response } & 2.0305 \\ \text { Observations (or Sum Wgts) } & 26\end{array}$

Test

Hydragard - HF-Coliwasa

Assuming equal variances

Difference $\quad 0.154394$ t Ratio $\quad 4.700752$

Std Err Dif $\quad 0.032845$ DF 24

Upper CL Dif 0.222182 Prob $>|t|<.0001$

Lower CL Dif 0.086606 Prob $>t \quad<.0001$

$\begin{array}{lrl} & \\ \text { Confidence } & 0.95 \text { Prob }<\mathrm{t} & 1.0000\end{array}$

Analysis of Variance

Source DF Sum of Squares Mean Square F Ratio Prob $>$ F

$\begin{array}{lrrrrr}\text { Sampler } & 1 & 0.14669233 & 0.146692 & 22.0971 & <.0001\end{array}$

$\begin{array}{llll}\text { Error } & 24 & 0.15932503 & 0.006639\end{array}$

C. Total $25 \quad 0.30601736$

Means for Oneway Anova

Level Number Mean Std Error Lower 95\% Upper 95\%

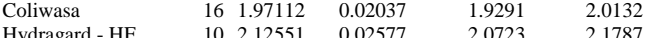

Std Error uses a pooled estimate of error variance

Means and Std Deviations

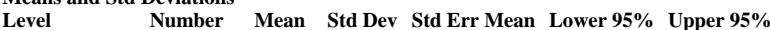

$\begin{array}{lrrrrrr}\text { Coliwasa } & 16 & 1.97112 & 0.074949 & 0.01874 & 1.9312 & 2.0111\end{array}$

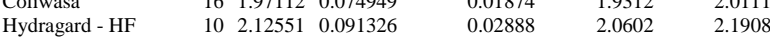

t Test

Hydragard - HF-Coliwasa

Assuming unequal variances

Difference $\quad 0.154394$ t Ratio $\quad 4.484836$

Std Err Dif $\quad 0.034426$ DF 16.42542

Upper CL Dif $\begin{array}{llr}0.227220 & \text { Prob }>|t| & 0.0004\end{array}$

Lower CL Dif 0.081568 Prob $>t \quad 0.0002$

$\begin{array}{lll}\text { Confidence } & 0.95 \text { Prob }<\mathrm{t} \quad 0.9998\end{array}$

Tests that the Variances are Equal

Level Count Std Dev MeanAbsDif to Mean MeanAbsDif to Median

$\begin{array}{lrrrr}\text { Coliwasa } & 16 & 0.0749492 & 0.0579101 & 0.0561107\end{array}$

$\begin{array}{lllll}\text { Hydragard - HF } & 10 & 0.0913262 & 0.0683811 & 0.0561107 \\ & & \end{array}$

Test F Ratio DFNum DFDen p-Value

$\begin{array}{lrrrr}\text { O'Brien[.5] } & 0.6116 & 1 & 24 & 0.4418\end{array}$

$\begin{array}{lllll}\text { Brown-Forsythe } & 0.3065 & 1 & 24 & 0.5850\end{array}$

$\begin{array}{lllll}\text { Levene } & 0.2749 & 1 & 24 & 0.6049\end{array}$

$\begin{array}{llll}0.4318 & 1 & 0.511\end{array}$

Welch Anova testing Means Equal, allowing Std Devs Not Equal

F Ratio DFNum DFDen Prob $>$ F

$\begin{array}{rrrr}20.1138 & 1 & 16.425 & 0.0004\end{array}$

t Test
Oneway Analysis of Measurement (wt \%) By Sampler Type of Material=SME Simulant, Analytical Block $=2$, Analyte $=\mathrm{Fe} 2 \mathrm{O} 3(\mathrm{wt} \%)$, Target $(\mathrm{wt} \%)=9.931$

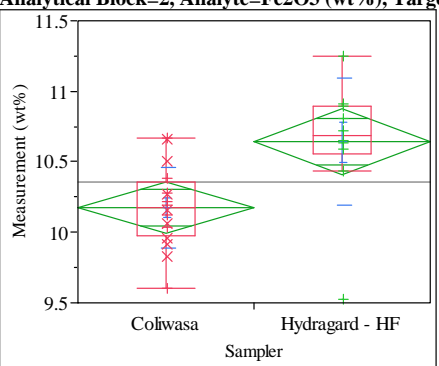

Oneway Anova

Summary of Fit

Adj Rsquare

Root Mean Square Error $\quad 0.27519$

10.35488

t Test

Hydragard - HF-Coliwasa

Assuming equal variances

Difference $\quad 0.467691$ t Ratio 3.239104

Std Err Dif $\quad 0.144389$ DF 24

Upper CL Dif 0.765695 Prob $>|t| \quad 0.0035$

$\begin{array}{lll}\text { Lower CL Dif } 0.169687 \text { Prob }>t & 0.0017\end{array}$

$\begin{array}{lrl}\text { Confidence } & 0.95 \text { Prob }<\mathrm{t} & 0.9983\end{array}$

Analysis of Variance

Source DF Sum of Squares Mean Square F Ratio Prob $>$ F

$\begin{array}{lllllll}\text { Sampler } & 1 & 1.3460585 & 1.34606 & 10.4918 & 0.0035\end{array}$

$\begin{array}{llll}\text { Error } & 24 & 3.0791118 & 0.12830\end{array}$

C. Total $25 \quad 4.4251703$

Means for Oneway Anova

Level Number Mean Std Error Lower 95\% Upper 95\%

$\begin{array}{lrrrrr}\text { Coliwasa } & 16 & 10.1750 & 0.08955 & 9.990 & 10.360\end{array}$

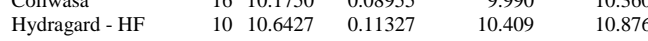

Std Error uses a pooled estimate of error variance

Means and Std Deviations

Level Number Mean Std Dev Std Err Mean Lower 95\% Upper 95\%

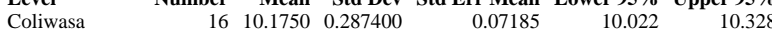

$\begin{array}{lllllll}\text { Hydragard - HF } & 10 & 10.6427 & 0.452171 & 0.14299 & 10.319 & 10.966\end{array}$

tTest

Hydragard - HF-Coliwasa

Assuming unequal variances

$\begin{array}{lrlr}\text { Difference } & 0.467691 & \text { t Ratio } & 2.922592 \\ \text { Std Err Dif } & 0.160026 & \text { DF } & 13.59848 \\ \text { Upper CL Dif } & 0.811865 & \text { Prob }>|t| & 0.0114 \\ \text { Lower CL Dif } & 0.123516 & \text { Prob }>t & 0.0057 \\ \text { Confidence } & 0.95 & \text { Prob }<t & 0.9943\end{array}$

Tests that the Variances are Equal

Level Count Std Dev MeanAbsDif to Mean MeanAbsDif to Median

$\begin{array}{lllll}\text { Coliwasa } & 16 & 0.2874002 & 0.2189228 & 0.2189228\end{array}$

$\begin{array}{lllll}\text { Hydragard - HF } & 10 & 0.4521711 & 0.2762180 & 0.2745024\end{array}$

Test F Ratio DFNum DFDen p-Value

$\begin{array}{lrrrr}\text { O'Brien[.5] } & 1.0331 & 1 & 24 & 0.3196\end{array}$

Brown-Forsythe $\begin{array}{llll}0.2890 & 1 & 24 & 0.5958\end{array}$

$\begin{array}{lllll}\text { Levene } & 0.3129 & 1 & 24 & 0.5811\end{array}$

$\begin{array}{lllll}\text { Bartlett } & 2.3063 & 1 & & 0.1288 \\ \text { F Test 2-sided } & 2.4753 & 9 & 15 & 0.1164\end{array}$

Welch Anova testing Means Equal, allowing Std Devs Not Equal

F Ratio DFNum DFDen Prob $>$ F

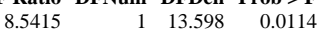

t Test

2.9226 


\section{Exhibit B2. Statistical Comparisons for High-Rheology (Phase 2) Simulant Testing}

Oneway Analysis of Measurement (wt \%) By Sampler Type of Material=SME Simulant, Analytical Block=2, Analyte $=K 20$ (wt \%), Target $(w t \%)=0.076$

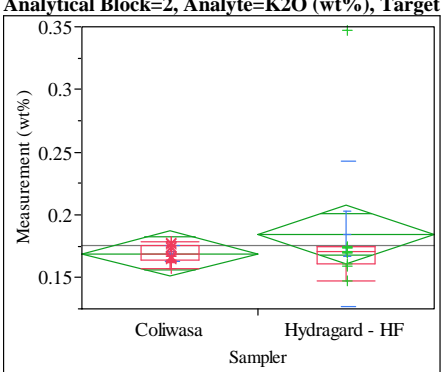

Oneway Anova

Summary of Fit

Adj Rsquare

0.006673

$\quad 0.035669$

Mean of Response

Observations (or Sum Wgts) $\quad 26$

Test

Hydragard - HF-Coliwasa

Assuming equal variances

Difference $\quad 0.01554$ t Ratio $\quad 1.080714$

Std Err Dif $\quad 0.01438$ DF 24

Upper CL Dif 0.04522 Prob $>|t| \quad 0.2906$

Lower CL Dif -0.01414 Prob $>t \quad 0.1453$

$\begin{array}{lrl}\text { Confidence } & 0.95 \text { Prob }<\mathrm{t} \quad 0.8547\end{array}$

Analysis of Variance

Source DF Sum of Squares Mean Square F Ratio Prob $>$ F

$\begin{array}{llllll}\text { Sampler } & 1 & 0.00148598 & 0.001486 & 1.1679 & 0.2906\end{array}$

$\begin{array}{llll}\text { Error } & 24 & 0.03053526 & 0.001272\end{array}$

C. Total $25 \quad 0.03202124$

Means for Oneway Anova

Level Number Mean Std Error Lower 95\% Upper 95\%

$\begin{array}{lllllr}\text { Coliwasa } & 16 & 0.169246 & 0.00892 & 0.15084 & 0.18765 \\ \text { Hydragard - HF } & 10 & 0.184786 & 0.01128 & 0.16151 & 0.20807\end{array}$

Std Error uses a pooled estimate of error variance

Means and Std Deviations

Level Number Mean Std Dev Std Err Mean Lower 95\% Upper 95\%

Lovel 160.1692460 .006682

$\begin{array}{lllllll}\text { Coliwasa } & 16 & 0.169246 & 0.006682 & 0.00167 & 0.16569 & 0.17281 \\ \text { Hydragard - HF } & 10 & 0.184786 & 0.057606 & 0.01822 & 0.14358 & 0.22599\end{array}$

t Test

Hydragard - HF-Coliwasa

Assuming unequal variances

Difference $\quad 0.01554$ t Ratio $\quad 0.849474$

Std Err Dif $\quad 0.01829$ DF $\quad 9.151601$

Upper CL Dif 0.05682 Prob $>|t| \quad 0.4173$

Lower CL Dif -0.02574 Prob $>\mathrm{t} \quad 0.2086$

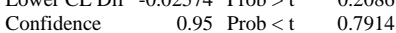

Tests that the Variances are Equal

Level Count Std Dev MeanAbsDif to Mean MeanAbsDif to Median

Coliwasa $\quad 16 \quad 0.0066816$

$\begin{array}{lllll}\text { Hydragard - HF } & 10 & 0.0576056 & 0.0324278 & 0.0232488\end{array}$

Test F Ratio DFNum DFDen p-Value

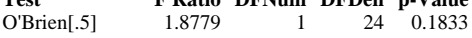

Brown-Forsythe $1.6740 \quad 1 \quad 24 \quad 0.2080$

$\begin{array}{lllll}\text { Levene } & 5.3499 & 1 & 24 & 0.0296\end{array}$

$\begin{array}{lllll}\text { Bartlett } & 39.8136 & 1 & < & <.0001 \\ \text { F Test 2-sided } & 74.3297 & 9 & 15 & <.0001\end{array}$

Welch Anova testing Means Equal, allowing Std Devs Not Equal

F Ratio DFNum DFDen Prob $>$ F

$\begin{array}{rrrr}0.7216 & 1 & 9.1516 & 0.4173\end{array}$

t Test

0.8495
Oneway Analysis of Measurement (wt \%) By Sampler Type of Material=SME Simulant, Analytical Block $=2$, Analyte $=\mathrm{Li} 2 \mathrm{O}(\mathrm{wt} \%)$, Target $(\mathrm{wt} \%)=4.988$

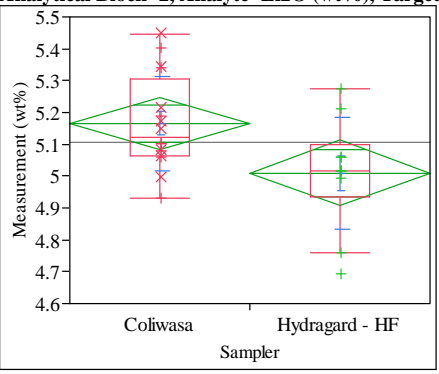

Oneway Anova

Summary of Fit

Rsquare

0.197349

0.163905

Root Mean Square Error $\quad 0.15912$

$\begin{array}{lr}\text { Mean of Response } & 5.105685 \\ \text { Observations (or Sum Wgts) } & 26\end{array}$

t Test

Hydragard - HF-Coliwasa

Assuming equal variances

Difference $\quad-0.15582$ t Ratio $\quad-2.42918$

Std Err Dif $\quad 0.06414$ DF $\quad 24$

Upper CL Dif -0.02343 Prob $>|t| \quad 0.0230$

Lower CL Dif -0.28820 Prob $>\mathrm{t} \quad 0.9885$

$\begin{array}{lll}\text { Confidence } & 0.95 \text { Prob }<\mathrm{t} \quad 0.0115\end{array}$

Analysis of Variance

Source DF Sum of Squares Mean Square F Ratio Prob $>$ F

$\begin{array}{lrrrrr}\text { Sampler } & 1 & 0.14940719 & 0.149407 & 5.9009 & 0.0230\end{array}$

$\begin{array}{llll}\text { Error } & 24 & 0.60766305 & 0.025319\end{array}$

C. Total $25 \quad 0.75707024$

Means for Oneway Anova

Level Number Mean Std Error Lower 95\% Upper 95\%

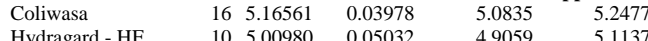

Std Error uses a pooled estimate of error variance

Means and Std Deviations

$\begin{array}{lllll}\text { Means and Std Deviations } & & & \\ \text { Level } & \text { Number Mean Std Dev Std Err Mean Lower 95\% Upper 95\% }\end{array}$

$\begin{array}{lrrrrrr}\text { Level } & \text { Number } & \text { Mean } & \text { Std Dev } & \text { Std Err Mean } & \text { Lower 95\% } & \text { Upper 95\% } \\ \text { Coliwasa } & 16 & 5.16561 & 0.148007 & 0.03700 & 5.0867 & 5.2445\end{array}$

$\begin{array}{llllllr}\text { Hydragard - HF } & 16 & 5.16561 & 0.148007 & 0.03700 & 5.0867 & 5.2445 \\ & 10 & 5.00980 & 0.176091 & 0.05568 & 4.8838 & 5.1358\end{array}$

t Test

Hydragard - HF-Coliwasa

Assuming unequal variances

Difference $\quad-0.15582$ t Ratio $\quad-2.33057$

Std Err Dif $\quad 0.06686$ DF 16.74375

Upper CL Dif -0.01459 Prob $>|t| r 0.0326$

Lower CL Dif -0.29704 Prob $>t \quad 0.9837$

$\begin{array}{lll}\text { Confidence } & 0.95 \text { Prob }<\mathrm{t} \quad 0.0163\end{array}$

Tests that the Variances are Equal

Level Count Std Dev MeanAbsDif to Mean MeanAbsDif to Median

$\begin{array}{lrrrr}\text { Coliwasa } & 16 & 0.1480070 & 0.1168957 & 0.1143728\end{array}$

$\begin{array}{lllll}\text { Hydragard - HF } & 10 & 0.1760909 & 0.1166872 & 0.1141037\end{array}$

Test F Ratio DFNum DFDen p-Value

$\begin{array}{lllll}\text { O'Brien[.5] } & 0.4269 & 1 & 24 & 0.5197\end{array}$

$\begin{array}{lllll}\text { Brown-Forsythe } & 0.0000 & 1 & 24 & 0.9953\end{array}$

$\begin{array}{lllll}\text { Levene } & 0.0000 & 1 & 24 & 0.9960\end{array}$

$\begin{array}{llll}\text { Bartlett } & 0.3328 & 1 & 0.5640\end{array}$

$\begin{array}{lllll}\text { F Test 2-sided } & 1.4155 & 9 & 15 & 0.5303\end{array}$

Welch Anova testing Means Equal, allowing Std Devs Not Equal

F Ratio DFNum DFDen Prob $>$ F

$\begin{array}{rrrr}5.4316 & 1 & 16.744 & 0.0326\end{array}$

t Test 


\section{Exhibit B2. Statistical Comparisons for High-Rheology (Phase 2) Simulant Testing}

Oneway Analysis of Measurement (wt \%) By Sampler Type of Material=SME Simulant, Analytical Block $=2$, Analyte $=\mathrm{MgO}(\mathrm{wt} \%)$, Target $(\mathrm{wt} \%)=\mathbf{0 . 2 8 6}$

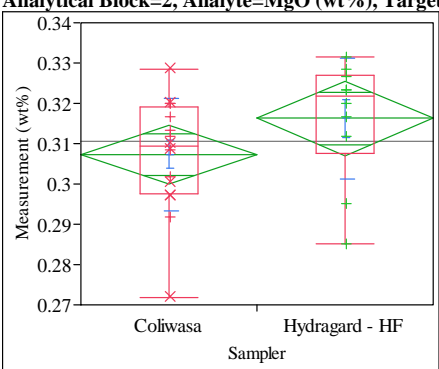

Oneway Anova

Summary of Fit

Rsquare $\quad 0.090938$

Adj Rsquare $\quad 0.05306$

Root Mean Square Error $\quad 0.014303$

$\begin{array}{lr}\text { Mean of Response } & 0.31074 \\ \text { Observations (or Sum Wgts) } & 26\end{array}$

Test

Hydragard - HF-Coliwasa

Assuming equal variances

Difference $\quad 0.00893$ t Ratio 1.549465

Std Err Dif $\quad 0.00577$ DF 24

Upper CL Dif 0.02083 Prob $>|t| \quad 0.1344$

Lower CL Dif -0.00297 Prob $>t \quad 0.0672$

$\begin{array}{lrl}\text { Confidence } & 0.95 \text { Prob }<\mathrm{t} \quad 0.9328\end{array}$

Analysis of Variance

Source DF Sum of Squares Mean Square F Ratio Prob > F

$\begin{array}{llllll}\text { Sampler } & 1 & 0.00049119 & 0.000491 & 2.4008 & 0.1344\end{array}$

$\begin{array}{llll}\text { Error } & 24 & 0.00491015 & 0.000205\end{array}$

C. Total $25 \quad 0.00540134$

Means for Oneway Anova

Level Number Mean Std Error Lower 95\% Upper 95\%

$\begin{array}{llllll}\text { Hydragard - HF } & 16 & 0.307304 & 0.00358 & 0.29992 & 0.31468 \\ & 10 & 0.316238 & 0.00452 & 0.30690 & 0.32557\end{array}$

Std Error uses a pooled estimate of error variance

Means and Std Deviations

Level Number Mean Std Dev Std Err Mean Lower 95\% Upper 95\%

Leviwasa $\quad 16 \quad 0.307304 \quad 0.013844$

$\begin{array}{lllllll}\text { Coliwasa } & 16 & 0.307304 & 0.013844 & 0.00346 & 0.29993 & 0.31468 \\ \text { Hydragard - HF } & 10 & 0.316238 & 0.015038 & 0.00476 & 0.30548 & 0.32700\end{array}$

t Test

Hydragard - HF-Coliwasa

Assuming unequal variances

$\begin{array}{lrlr}\text { Difference } & 0.00893 & \text { t Ratio } & 1.519003 \\ \text { Std Err Dif } & 0.00588 & \text { DF } & 18.02544 \\ \text { Upper CL Dif } & 0.02129 & \text { Prob }>|t| & 0.1461 \\ \text { Lower CL Dif } & -0.00342 & \text { Prob }>t & 0.0731 \\ \text { Confidence } & 0.95 & \text { Prob }<\mathrm{t} & 0.9269\end{array}$

Tests that the Variances are Equal

Level Count Std Dev MeanAbsDif to Mean MeanAbsDif to Median

Coliwasa $\quad 16 \quad 0.0138442$

$\begin{array}{lllll}\text { Hydragard - HF } & 10 & 0.0150379 & 0.0113096 & 0.0104473\end{array}$

Test F Ratio DFNum DFDen p-Value

$\begin{array}{lrrrr}\text { O'Brien[.5] } & 0.0617 & 1 & 24 & 0.8059\end{array}$

Brown-Forsythe $\quad 0.0021 \quad 1 \quad 24 \quad 0.9642$

$\begin{array}{lllll}\text { Levene } & 0.0465 & 1 & 24 & 0.8310\end{array}$

$\begin{array}{lllll}\text { Bartlett } & 0.0746 & 1 & & 0.7848 \\ \text { F Test 2-sided } & 1.1799 & 9 & 15 & 0.7463\end{array}$

Welch Anova testing Means Equal, allowing Std Devs Not Equal

F Ratio DFNum DFDen Prob $>$ F

$\begin{array}{lrrr}2.3074 & 1 & 18.025 & 0.1461\end{array}$

t Test

1.5190
Oneway Analysis of Measurement (wt \%) By Sampler Type of Material=SME Simulant, Analytical Block $=2$, Analyte $=\mathrm{MnO}(\mathrm{wt} \%)$, Target $(\mathrm{wt} \%)=2.918$

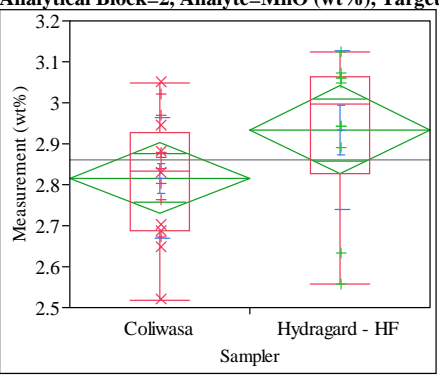

Oneway Anova

Summary of Fit

Adj Rsquare $\quad 0.114502$

0.077606

Root Mean s

$\begin{array}{lr}\text { Mean of Response } & 2.861498 \\ \text { Observations (or Sum Wgts) } & 26\end{array}$

t Test

Hydragard - HF-Coliwasa

Assuming equal variances

$\begin{array}{lrlr}\text { Difference } & 0.11718 & \text { t Ratio } & 1.761646 \\ \text { Std Err Dif } & 0.06652 & \text { DF } & 24 \\ \text { Upper CL Dif } & 0.25446 & \text { Prob }>|t| & 0.0909 \\ \text { Lower CL Dif } & -0.02010 & \text { Prob }>\mathrm{t} & 0.0454 \\ \text { Confidence } & 0.95 & \text { Prob }<\mathrm{t} & 0.9546\end{array}$

Source DF Sum of Squares Mean Square F Ratio Prob $>$ F

$\begin{array}{llllll}\text { Sampler } & 1 & 0.08449421 & 0.084494 & 3.1034 & 0.0909\end{array}$

$\begin{array}{llll}\text { Error } & 24 & 0.65343303 & 0.027226\end{array}$

C. Total $25 \quad 0.73792724$

Means for Oneway Anova

Level Number Mean Std Error Lower 95\% Upper 95\%

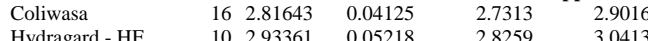

Std Error uses a pooled estimate of error variance

$\begin{array}{lllll}\begin{array}{l}\text { Means and Std Deviations } \\ \text { Level }\end{array} \text { Number } & \text { Mean } & \text { Std Dev Std Err Mean Lower 95\% Upper 95\% }\end{array}$

$\begin{array}{lrrrrrr}\text { Level } & \text { Number } & \text { Mean } & \text { Std Dev } & \text { Std Err Mean } & \text { Lower 95\% } & \text { Upper 95\% } \\ \text { Coliwasa } & 16 & 2.81643 & 0.145768 & 0.03644 & 2.7388 & 2.8941\end{array}$

$\begin{array}{lllllll}\text { Hydragard - HF } & 16 & 2.81643 & 0.145768 & 0.03644 & 2.7388 & 2.8941 \\ & 10 & 2.93361 & 0.192846 & 0.06098 & 2.7957 & 3.0716\end{array}$

t Test

Hydragard - HF-Coliwasa

Assuming unequal variances

Difference $\quad 0.11718$ t Ratio 1.649392

Std Err Dif $\quad 0.07104$ DF 15.39733

Upper CL Dif 0.26826 Prob $>|t| r 0.1193$

Lower CL Dif -0.03391 Prob $>t \quad 0.0597$

$\begin{array}{lrl}\text { Confidence } & 0.95 \text { Prob }<\mathrm{t} \quad 0.9403\end{array}$

Tests that the Variances are Equal

Level Count Std Dev MeanAbsDif to Mean MeanAbsDif to Median

$\begin{array}{lrrrr}\text { Coliwasa } & 16 & 0.1457684 & 0.1160063 & 0.1145940\end{array}$

$\begin{array}{lllll}\text { Hydragard - HF } & 10 & 0.1928461 & 0.1435814 & 0.1394496\end{array}$

Test F Ratio DFNum DFDen p-Value

$\begin{array}{lllll}\text { O'Brien[.5] } & 0.9916 & 1 & 24 & 0.3293 \\ \end{array}$

$\begin{array}{lllll}\text { Brown-Forsythe } & 0.3121 & 1 & 24 & 0.5815 \\ & 0.4822 & 1 & 24 & 0.4932\end{array}$

$\begin{array}{lllll}\text { Levene } & 0.4842 & 1 & 24 & 0.4932\end{array}$

$\begin{array}{lllll} & 0.48724 & 1 & 24 & 0.4932 \\ \text { F Test 2-sided } & 1.7502 & 9 & 15 & 0.3503\end{array}$

Welch Anova testing Means Equal, allowing Std Devs Not Equal

F Ratio DFNum DFDen Prob $>$ F

$\begin{array}{rrrr}2.7205 & 1 & 15.397 & 0.1193\end{array}$

t Test 


\section{Exhibit B2. Statistical Comparisons for High-Rheology (Phase 2) Simulant Testing}

Oneway Analysis of Measurement (wt\%) By Sampler Type of Material=SME Simulant, Analytical Block $=2$, Analyte $=\mathrm{Na2O}(\mathrm{wt} \%)$, Target $(\mathrm{wt} \%)=13.318$

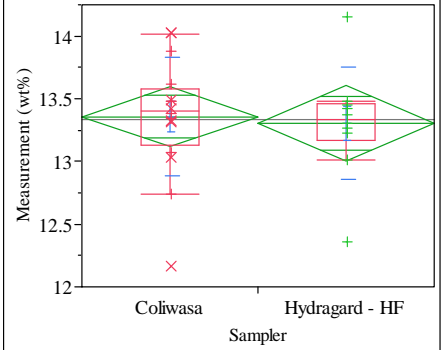

Oneway Anova

Summary of Fit

Adj Rsquare

Error $\quad 0.45936$ 13.33794

Observations (or Sum Wgts) $\quad 26$

Test

Hydragard - HF-Coliwasa

Assuming equal variances

Difference $\quad-0.05392$ t Ratio $\quad-0.29119$

Std Err Dif $\quad 0.18517$ DF $\quad 24$

Upper CL Dif 0.32826 Prob $>|t| \quad 0.7734$

Lower CL Dif -0.43610 Prob $>t \quad 0.6133$

$\begin{array}{lrl}\text { Confidence } & 0.95 \text { Prob }<\mathrm{t} & 0.3867\end{array}$

Analysis of Variance

Source DF Sum of Squares Mean Square F Ratio Prob $>$ F

$\begin{array}{llllll}\text { Sampler } & 1 & 0.0178915 & 0.017891 & 0.0848 & 0.7734\end{array}$

$\begin{array}{llll}\text { Error } \quad 24 & 5.0642688 & 0.211011\end{array}$

C. Total $25 \quad 5.0821603$

Means for Oneway Anova

Level Number Mean Std Error Lower 95\% Upper 95\%

$\begin{array}{lrrrrr}\text { Coliwasa } & 16 & 13.3587 & 0.11484 & 13.122 & 13.596\end{array}$

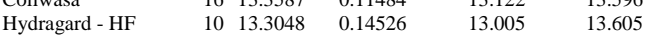

Std Error uses a pooled estimate of error variance

Means and Std Deviations

Level Number Mean Std Dev Std Err Mean Lower 95\% Upper 95\%

$0.11699-13.109-13.608$

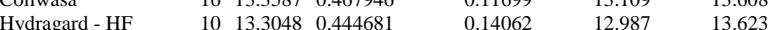

Test

Hydragard - HF-Coliwasa

Assuming unequal variances

Difference $\quad-0.05392$ t Ratio $\quad-0.29477$

Std Err Dif $\quad 0.18292$ DF $\quad 20.01623$

Upper CL Dif 0.32763 Prob $>|t| \quad 0.7712$

Lower CL Dif -0.43547 Prob $>\mathrm{t} \quad 0.6144$

$\begin{array}{lrl} & \\ \text { Confidence } & 0.95 \text { Prob }<\mathrm{t} & 0.3856\end{array}$

Tests that the Variances are Equal

Level Count Std Dev MeanAbsDif to Mean MeanAbsDif to Median

Coliwasa $\quad 16 \quad 0.4679457$

$\begin{array}{lllll}\text { Hydragard - HF } & 10 & 0.4446811 & 0.2722960 & 0.2722960\end{array}$

Test F Ratio DFNum DFDen p-Value

$\begin{array}{lrrrr}\text { O'Brien[.5] } & 0.0172 & 1 & 24 & 0.8968\end{array}$

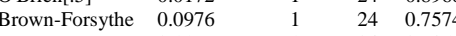

$\begin{array}{lllll}\text { Levene } & 0.1166 & 1 & 24 & 0.7357\end{array}$

$\begin{array}{lrrrr}\text { Bartlett } & 0.0277 & 1 & \dot{0} & 0.8677 \\ \text { F Test 2-sided } & 1.1074 & 15 & 9 & 0.9073\end{array}$

Welch Anova testing Means Equal, allowing Std Devs Not Equal

F Ratio DFNum DFDen Prob $>$ F

$0.0869 \quad 1 \quad 20.016 \quad 0.7712$

t Test

$$
120.016 \quad 0.7712
$$

0.2948
Oneway Analysis of Measurement (wt\%) By Sampler Type of Material=SME Simulant, Analytical Block $=2$, Analyte $=\mathrm{NiO}(w t \%)$, Target $(\mathrm{wt} \%)=1.033$

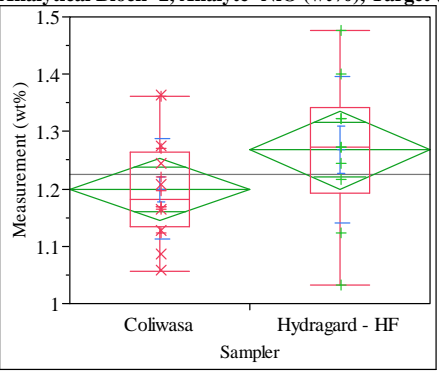

Oneway Anova

Summary of Fit

square

Adj Rsquare $\quad 0.100866$

Root Mean Square Error 0.104512

Mean of Response 1.22576

Observations (or Sum Wgts) $\quad 26$

t Test

Hydragard - HF-Coliwasa

Assuming equal variances

Difference $\quad 0.06913$ t Ratio 1.64084

Std Err Dif $\quad 0.04213$ DF $\quad 24$

Upper CL Dif 0.15608 Prob $>|t| \quad 0.1139$

Lower CL Dif -0.01782 Prob $>t \quad 0.0569$

$\begin{array}{lll}\text { Confidence } & 0.95 \text { Prob }<\mathrm{t} \quad 0.9431\end{array}$

Analysis of Variance

Source DF Sum of Squares Mean Square F Ratio Prob $>$ F

$\begin{array}{lrrrrr}\text { Sampler } & 1 & 0.02940774 & 0.029408 & 2.6924 & 0.1139\end{array}$

$\begin{array}{llll}\text { Error } & 24 & 0.26214439 & 0.010923\end{array}$

$\begin{array}{lll}\text { C. Total } & 25 & 0.29155213\end{array}$

Means for Oneway Anova

Level Number Mean Std Error Lower 95\% Upper 95\%

$\begin{array}{lllllr}\text { Coliwasa } & 16 & 1.19917 & 0.02613 & 1.1452 & 1.2531 \\ \text { Hydragard - HF } & 10 & 1.26830 & 0.03305 & 1.2001 & 1.3365\end{array}$

Std Error uses a pooled estimate of error variance

Means and Std Deviations

$\begin{array}{lllll}\text { Means and Std Deviations } & & \\ \text { Level } & \text { Number } & \text { Mean } & \text { Std Dev Std Err Mean Lower 95\% Upper 95\% }\end{array}$

$\begin{array}{lrrrrrr}\text { Level } & \text { Number } & \text { Mean } & \text { Std Dev } & \text { Std Err Mean } & \text { Lower 95\% } & \text { Upper 95\% } \\ \text { Coliwasa } & 16 & 1.19917 & 0.087631 & 0.02191 & 1.1525 & 1.2459\end{array}$

$\begin{array}{llllllr}\text { Coliwasa } & 16 & 1.19917 & 0.087631 & 0.02191 & 1.1525 & 1.2459 \\ \text { Hydragard - HF } & 10 & 1.26830 & 0.127783 & 0.04041 & 1.1769 & 1.3597\end{array}$

t Test

Hydragard - HF-Coliwasa

Assuming unequal variances

Difference $\quad 0.06913$ t Ratio $\quad 1.50393$

Std Err Dif $\quad 0.04597$ DF 14.32569

Upper CL Dif 0.16750 Prob $>|t| r 0.1543$

Lower CL Dif -0.02925 Prob $>t \quad 0.0772$

$\begin{array}{lll}\text { Confidence } & 0.95 \text { Prob }<\mathrm{t} \quad 0.9228\end{array}$

Tests that the Variances are Equal

Level Count Std Dev MeanAbsDif to Mean MeanAbsDif to Median

$\begin{array}{lrrrr}\text { Coliwasa } & 16 & 0.0876307 & 0.0679793 & 0.0676016\end{array}$

$\begin{array}{lllll}\text { Hydragard - HF } & 10 & 0.1277834 & 0.0915691 & 0.0676016 \\ \end{array}$

Test F Ratio DFNum DFDen p-Value

$\begin{array}{lllll}\text { O'Brien[.5] } & 1.6779 & 1 & 24 & 0.2075\end{array}$

$\begin{array}{lllll}\text { Brown-Forsythe } & 0.7106 & 1 & 24 & 0.4076\end{array}$

$\begin{array}{lllll}\text { Levene } & 0.7876 & 1 & 24 & 0.3836\end{array}$

$\begin{array}{llll}\text { Bartlett } & 1.5940 & 1 & 0.2068\end{array}$

$\begin{array}{lllll}\text { F Test 2-sided } & 2.1264 & 9 & 15 & 0.1889\end{array}$

Welch Anova testing Means Equal, allowing Std Devs Not Equal

F Ratio DFNum DFDen Prob > F

$\begin{array}{rrrr}2.2618 & 1 & 14.326 & 0.1543\end{array}$

t Test 
SRNL-STI-2011-00693

Revision 0

Exhibit B2. Statistical Comparisons for High-Rheology (Phase 2) Simulant Testing

Oneway Analysis of Measurement (wt\%) By Sampler Type of Material=SME Simulant, Analytical Block $=2$, Analyte $=\mathrm{SiO} 2(\mathrm{wt} \%)$, Target $(\mathrm{wt} \%)=50.183$

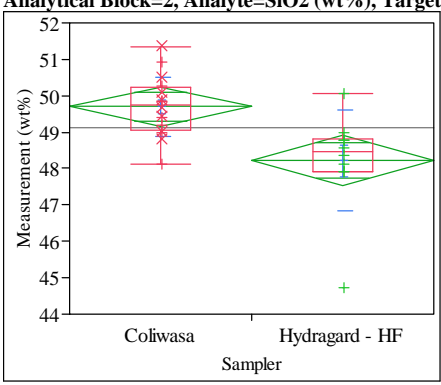

Oneway Anova

Summary of Fit

Rsquare

Adj Rsquare

0.330433

Mean of Response $\quad 49.12985$

Observations (or Sum Wgts) 26

Test

Hydragard - HF-Coliwasa

Assuming equal variances

Difference $\quad-1.4788$ t Ratio -3.44152

$\begin{array}{lrlr}\text { Std Err Dif } & 0.4297 & \text { DF } & 24 \\ \text { Upper CL } & -0.5920 & \text { Prob }>|t| & 0.002\end{array}$

$\begin{array}{rrrr}\text { Upper CL Dif } & -0.5920 & \text { Prob }>|t| & 0.0021 \\ \text { Lower CL Dif } & -2.3656 & \text { Prob }>t & 0.9989\end{array}$

$\begin{array}{lrll}\text { Lower CL Dif } & -2.3656 & \text { Prob }>t & 0.9989 \\ \text { Confidence } & 0.95 & \text { Prob }<t & 0.0011\end{array}$

Analysis of Variance

Source DF Sum of Squares Mean Square F Ratio Prob $>$ F

$\begin{array}{lrrrrr}\text { Sampler } & 1 & 13.457373 & 13.4574 & 11.8441 & 0.0021\end{array}$

$\begin{array}{llrr}\text { Error } & 24 & 27.269126 & 1.1362\end{array}$

C. Total $25 \quad 40.726499$

Means for Oneway Anova

Level Number Mean Std Error Lower 95\% Upper 95\%

$\begin{array}{llllrr}\text { Coliwasa } & 16 & 49.6986 & 0.26648 & 49.149 & 50.249\end{array}$

$\begin{array}{llllll}\text { Hydragard - HF } & 10 & 48.2198 & 0.33708 & 47.524 & 48.916\end{array}$

Std Error uses a pooled estimate of error variance

Means and Std Deviations

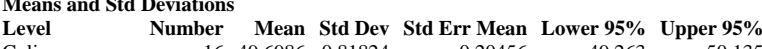

$\begin{array}{lrrrrrr}\text { Coliwasa } & \text { Number } & \text { Mean } & \text { Std Dev } & \text { Std Err Mean } & \text { Lower 95\% } & \text { Upper 95\% } \\ \text { Hydragard - HF } & 10 & 48.2198 & 1.38349 & 0.20456 & 49.263 & 50.135 \\ & & & & & 47.230 & 49.210\end{array}$

Test

Hydragard - HF-Coliwasa

Assuming unequal variances

Difference $\quad-1.4788$ t Ratio -3.06194

Std Err Dif $\quad 0.4830$ DF 12.99275

Upper CL Dif -0.4354 Prob $>|t| r 0.0091$

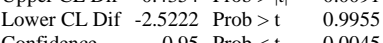

Confidence $\quad 0.95$ Prob $<\mathrm{t} \quad 0.0045$

Tests that the Variances are Equal

$\begin{array}{lrrrr}\text { Level } & \text { Count } & \text { Std Dev } & \text { MeanAbsDif to Mean } & \text { MeanAbsDif to Median } \\ \text { Coliwasa } & 16 & 0.818241 & 0.6284194 & 0.6284194\end{array}$

$\begin{array}{lrrrr}\text { Coliwasa } & 16 & 0.818241 & 0.6284194 & 0.6284194 \\ \text { Hydragard - HF } & 10 & 1.383488 & 0.8386056 & 0.8129340\end{array}$

Test $\quad$ F Ratio DFNum DFDen p-Value

$\begin{array}{lllll}\text { O'Brien[.5] } & 1.1437 & 1 & 24 & 0.2955\end{array}$

Brown-Forsythe $\quad 0.3370 \quad 1 \quad 24 \quad 0.5670$

$\begin{array}{lllll}\text { Levene } & 0.4688 & 1 & 24 & 0.5001\end{array}$

$\begin{array}{lllll}\text { Bartlett } & 3.0992 & 1 & & 0.0783 \\ \text { F Test 2-sided } & 2.8588 & 9 & 15 & 0.0699\end{array}$

Welch Anova testing Means Equal, allowing Std Devs Not Equal

F Ratio DFNum DFDen Prob $>$ F

$\begin{array}{llll}9.3755 & 1 & 12.993 & 0.0091\end{array}$

t Test
3.0619
Oneway Analysis of Measurement (wt \%) By Sampler Type of Material=SME Simulant, Analytical Block $=2$, Analyte $=$ Sum of Oxides (wt \%), Target $(w t \%)=99.73$

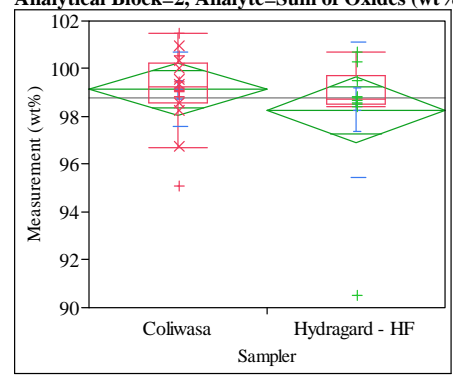

Oneway Anova

Summary of Fit

Rsquare

Adeon $\quad-0.0004$

Root Mean Sque Error 2.131604

Mean of Response 98.80298
26

t Test

Hydragard - HF-Coliwasa

Assuming equal variances
Analysis of Variance
Source DF Sum of Squares Mean Square F Ratio Prob $>$ F

$\begin{array}{lrrrrr}\text { Sampler } & 1 & 4.49763 & 4.49763 & 0.9899 & 0.3297\end{array}$

$\begin{array}{llll}\text { Error } & 24 & 109.04964 & 4.54374\end{array}$

C. Total $25-113.54727$

Means for Oneway Anova

Level Number Mean Std Error Lower 95\% Upper 95\%

$\begin{array}{llllrr}\text { Coliwasa } & 16 & 99.1318 & 0.53290 & 98.032 & 100.23 \\ \text { Hydragard - HF } & 10 & 98.2769 & 0.67407 & 96.886 & 99.67\end{array}$

Std Error uses a pooled estimate of error variance

Means and Std Deviations

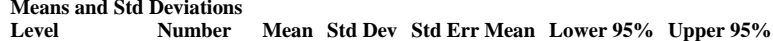

$\begin{array}{lrrrrrr}\text { Coliwasa } & 16 & 99.1318 & 1.55720 & 0.38930 & 98.302 & 99.96\end{array}$

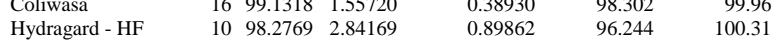

t Test

Hydragard - HF-Coliwasa

Assuming unequal variances
Difference $\quad-0.8549$ t Ratio $\quad-0.87296$

Std Err Dif $\quad 0.9793$ DF $\quad 12.4324$

1.2707 Prob $>|t| \quad 0.3992$

Lower CL Dif -2.9805 Prob $>$

Tests that the Variances are Equal

Level Count Std Dev MeanAbsDif to Mean MeanAbsDif to Median

$\begin{array}{lrrrr}\text { Coliwasa } & 16 & 1.557197 & 1.056679 & 1.048377\end{array}$

$\begin{array}{llll}\text { Hydragard - HF } & 10 & 2.841688 & 1.555220\end{array}$

Test F Ratio DFNum DFDen p-Value

$\begin{array}{lrrrr}\text { O'Brien[.5] } & 0.9922 & 1 & 24 & 0.3292\end{array}$

$\begin{array}{lllll}\text { Brown-Forsythe } & 0.1684 & 1 & 24 & 0.6852\end{array}$

$\begin{array}{lllll}\text { Levene } & 0.5479 & 1 & 24 & 0.4664\end{array}$

$\begin{array}{lllll}\text { Bartlett } & 4.0600 & 1 & 0.0439\end{array}$

$\begin{array}{lllll}\text { F Test 2-sided } & 3.3302 & 9 & 15 & 0.0387\end{array}$

Welch Anova testing Means Equal, allowing Std Devs Not Equal

F Ratio DFNum DFDen Prob $>$ F

$\begin{array}{rrrr}0.7621 & 1 & 12.432 & 0.3992\end{array}$

t Test 
SRNL-STI-2011-00693

Revision 0

\section{Exhibit B2. Statistical Comparisons for High-Rheology (Phase 2) Simulant Testing}

Oneway Analysis of Measurement (wt \%) By Sampler Type of Material=SME Simulant, Analytical Block $=2$, Analyte $=\mathrm{TiO} 2(\mathrm{wt} \%)$, Target $(\mathrm{wt} \%)=0.013$

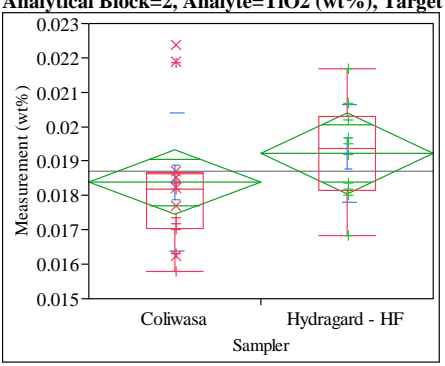

Oneway Anova

Summary of Fit

Rsquare

Adj Rsquare

Root Mean Square Error $\quad 0.01452$

Mean of Response $\quad 0.018706$

Observations (or Sum Wgts) $\quad 26$

Test

Hydragard - HF-Coliwasa

Assuming equal variances

Difference $\quad 0.00085$ t Ratio $\quad 1.169769$

$\begin{array}{lrr}\text { Std Err Dif } & 0.00073 \text { DF } & 24 \\ & & \end{array}$

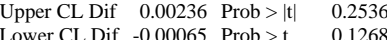

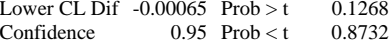

Analysis of Variance

Source DF Sum of Squares Mean Square F Ratio Prob > F

$\begin{array}{lrrrrr}\text { Sampler } & 1 & 0.00000450 & 4.497 \mathrm{e}-6 & 1.3684 & 0.2536\end{array}$

$\begin{array}{llll}\text { Error } & 24 & 0.00007887 & 3.2864 \mathrm{e}-6\end{array}$

C. Total $25 \quad 0.00008337$

Means for Oneway Anova

Level Number Mean Std Error Lower 95\% Upper 95\%

$\begin{array}{lrrrrr}\text { Coliwasa } & 16 & 0.018377 & 0.00045 & 0.01744 & 0.01931\end{array}$

$\begin{array}{llllll}\text { Hydragard - HF } & 10 & 0.019232 & 0.00057 & 0.01805 & 0.02042\end{array}$

Std Error uses a pooled estimate of error variance

Means and Std Deviations

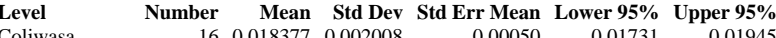

Coliwasa

$\begin{array}{llllll}16 & 0.018377 & 0.002008 & 0.00050 & 0.01731 & 0.01945 \\ 10 & 0.019232 & 0.001428 & 0.00045 & 0.01821 & 0.02025\end{array}$

t Test

Hydragard - HF-Coliwasa

Assuming unequal variances

Difference $\quad 0.00085$ t Ratio 1.265671

Std Err Dif $\quad 0.00068$ DF 23.47608

Upper CL Dif 0.00225 Prob $>|t| \quad 0.2180$

Lower CL Dif -0.00054 Prob $>t \quad 0.1090$

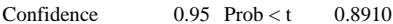

Tests that the Variances are Equal

Level Count Std Dev MeanAbsDif to Mean MeanAbsDif to Median

$\begin{array}{lrrrr}\text { Level } & 16 & 0.0020085 & 0.0014376 & 0.0014095 \\ \text { Coliwasa } & 10 & 0.0014285 & 0.0011176 & 0.0011176\end{array}$

F Ratio DFNum DFDen p-Value

Test F Ratio DFNum DFDen p-Value

$\begin{array}{lllll}\text { O'Brien[.5] } & 1.0616 & 1 & 24 & 0.3131 \\ \text { Brown-Forsythe } & 0.3560 & 1 & 24 & 0.5563\end{array}$

$\begin{array}{lllll}\text { Brown-Forsythe } & 0.3560 & 1 & 24 & 0.5563 \\ \text { Levene } & 0.4541 & 1 & 24 & 0.5069\end{array}$

$\begin{array}{lllll}\text { Levene } & 0.4541 & 1 & 24 & 0.5069 \\ \text { Bartlett } & 1.1622 & 1 & . & 0.2810\end{array}$

$\begin{array}{lrrrr}\text { F Test 2-sided } & 1.9768 & 1 & \dot{0} & 0.2810 \\ & & & \end{array}$

Welch Anova testing Means Equal, allowing Std Devs Not Equal

F Ratio DFNum DFDen Prob > F

$\begin{array}{llll}1.6019 & 1 & 23.476 & 0.2180\end{array}$

t Test

1.2657
Oneway Analysis of Measurement (wt \%) By Sampler Type of Material=SME Simulant, Analytical Block $=2$, Analyte $=\mathrm{ZnO}(\mathrm{wt} \%)$, Target $(\mathrm{wt} \%)=0$

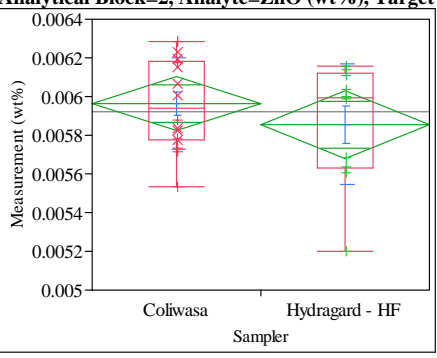

Oneway Anova

Summary of Fit

$\begin{array}{lr}\text { Rsquare } & 0.040766 \\ \text { Adj Rsquare } & 0.000798 \\ \text { Root Mean Square Error } & 0.000267 \\ \text { Mean of Response } & 0.005922 \\ \text { Observations (or Sum Wgts) } & 26\end{array}$

t Test

Hydragard - HF-Coliwasa

Assuming equal variances

Difference $\quad-0.00011$ t Ratio $\quad-1.00993$

Std Err Dif $\quad 0.00011$ DF $\quad 24$

Upper CL Dif 0.00011 Prob $>|t| \quad 0.3226$

$\begin{array}{lll}\text { Lower CL Dif }-0.00033 \text { Prob }>t & 0.8387\end{array}$

$\begin{array}{lll}\text { Confidence } & 0.95 \text { Prob }<\mathrm{t} & 0.1613\end{array}$

Analysis of Variance

Source DF Sum of Squares Mean Square F Ratio Prob > F

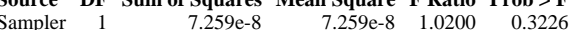

$\begin{array}{lrrr}\text { Error } & 24 & 1.70807 \mathrm{e}-6 & 7.1169 \mathrm{e}-8\end{array}$

$\begin{array}{lll}\text { Error } & 24 & 1.70807 \mathrm{e}-6 \\ \text { C. Total } & 25 & 1.78066 \mathrm{e}-6\end{array}$

Means for Oneway Anova

Level Number Mean Std Error Lower 95\% Upper 95\%

$\begin{array}{llllll}\text { Coliwasa } & 16 & 0.005964 & 6.67 \mathrm{e}-5 & 0.00583 & 0.00610 \\ \text { Hen } & 10 & 0.005856 & 8.44-5 & 0.00568 & 0.00603\end{array}$

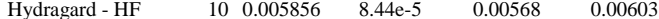

Std Error uses a pooled estimate of error variance

Means and Std Deviations

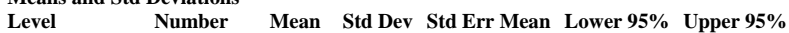

$\begin{array}{lrrrrrr}\text { Coliwasa } & 16 & 0.005964 & 0.000235 & 5.88 \mathrm{e}-5 & 0.00584 & 0.00609\end{array}$

$\begin{array}{lllllll}\text { Hydragard - HF } & 16 & 0.005964 & 0.000235 & 5.88 \mathrm{e}-5 & 0.00584 & 0.00609 \\ & 10 & 0.005856 & 0.000312 & 0.0001 & 0.00563 & 0.00608\end{array}$

t Test

Hydragard - HF-Coliwasa

Assuming unequal variances

Difference $\quad-0.00011$ t Ratio $\quad-0.9448$

Std Err Dif $\quad 0.00011$ DF 15.35441

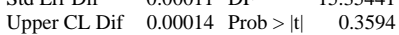

Lower CL Dif -0.00035 Prob $>t \quad 0.8203$

Confidence $\quad 0.95$ Prob $<\mathrm{t} \quad 0.1797$

Tests that the Variances are Equal

Level Count Std Dev MeanAbsDif to Mean MeanAbsDif to Median

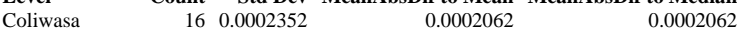

$\begin{array}{lllll}\text { Hydragard - HF } & 10 & 0.0003123 & 0.0002062 & 0.0002062 \\ & & 0.0002592 & 0.0002328\end{array}$

Test F Ratio DFNum DFDen p-Value

$\begin{array}{lllll}\text { O'Brien[.5] } & 1.1286 & 1 & 24 & 0.2986\end{array}$

Brown-Forsythe $\quad 0.1525 \quad 1124 \quad 0.6996$

$\begin{array}{lllll}\text { Levene } & 1.1643 & 1 & 24 & 0.2913\end{array}$

$\begin{array}{lllll}\text { Bartlett } & 0.8955 & 1 & 0.3440\end{array}$

$\begin{array}{lllll}\text { F Test 2-sided } & 1.7630 & 9 & 15 & 0.3187\end{array}$

Welch Anova testing Means Equal, allowing Std Devs Not Equal

F Ratio DFNum DFDen Prob $>$ F

$\begin{array}{llll}0.8927 & 1 & 15.354 & 0.3594\end{array}$

t Test

0.9448 
SRNL-STI-2011-00693

Revision 0

\section{Exhibit B2. Statistical Comparisons for High-Rheology (Phase 2) Simulant Testing}

Oneway Analysis of Measurement (wt \%) By Sampler Type of Material=SME Simulant, Analytical Block $=2$, Analyte $=\mathrm{ZrO} 2(\mathrm{wt} \%)$, Target $(\mathrm{wt} \%)=0.111$

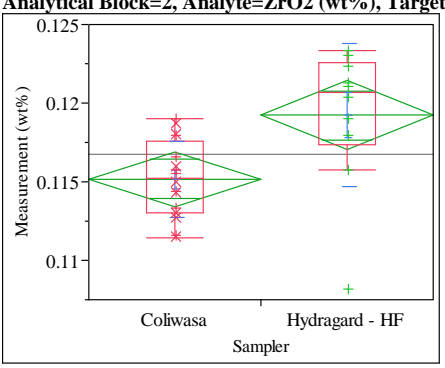

Oneway Anova

Summary of Fit

Rsquare

Adj Rsquare

0.270992

0.240617

Root Mean Square Error $\quad 0.003374$

$\begin{array}{lr}\text { Mean of Response } & 0.116735 \\ \text { Observations (or Sum Wgts) } & 26\end{array}$

Test

Hydragard - HF-Coliwasa

Assuming equal variances

Difference $\quad 0.004063$ t Ratio 2.9868

$\begin{array}{lrr}\text { Std Err Dif } & 0.001360 \text { DF } & 24 \\ & & \end{array}$

Upper CL Dif 0.006870 Prob $>|t| \quad 0.0064$

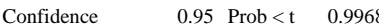

Analysis of Variance

Source DF Sum of Squares Mean Square F Ratio Prob $>$ F

$\begin{array}{lrrrrr}\text { Sampler } & 1 & 0.00010156 & 0.000102 & 8.9215 & 0.0064\end{array}$

$\begin{array}{llll}\text { Error } & 24 & 0.00027322 & 0.000011\end{array}$

C. Total $25 \quad 0.00037479$

Means for Oneway Anova

Level Number Mean Std Error Lower 95\% Upper 95\%

$\begin{array}{lrrrrr}\text { Level } & \text { Number } & \text { Mean } & \text { Std Error } & \text { Lower 95\% } & \text { Upper 95\% } \\ \text { Coliwasa } & 16 & 0.115173 & 0.00084 & 0.11343 & 0.11691\end{array}$

$\begin{array}{llllll}\text { Hydragard - HF } & 10 & 0.119235 & 0.00107 & 0.11703 & 0.12144\end{array}$

Std Error uses a pooled estimate of error variance

Means and Std Deviations

$\begin{array}{lrrrrrr}\text { Level } & \text { Number } & \text { Mean } & \text { Std Dev } & \text { Std Err Mean } & \text { Lower 95\% } & \text { Upper 95\% } \\ \text { Coliwasa } & 16 & 0.115173 & 0.002416 & 0.00060 & 0.11389 & 0.11646\end{array}$

Coliwasa

$\begin{array}{lllllll}\text { Hydragard - HF } & 10 & 0.119235 & 0.004542 & 0.00144 & 0.11599 & 0.12248\end{array}$

t Test

Hydragard - HF-Coliwasa

Assuming unequal variances

Difference $\quad 0.004063$ t Ratio 2.607397

Std Err Dif $\quad 0.001558$ DF $\quad 12.23629$

Upper CL Dif 0.007450 Prob $>|t| \quad 0.0226$

Lower CL Dif 0.000675 Prob $>t \quad 0.0113$

$\begin{array}{lll}\text { Confidence } & 0.95 \text { Prob }<\mathrm{t} \quad 0.9887\end{array}$

Tests that the Variances are Equal

Level Count Std Dev MeanAbsDif to Mean MeanAbsDif to Median $\begin{array}{lllll}\text { Coliwasa } & 16 & 0.0024163 & 0.0019755 & 0.0019755 \\ \text { Hydragard - HF } & 10 & 0.0045417 & 0.0032095 & 0.0029853\end{array}$

Test F Ratio DFNum DFDen p-Value

Test F Ratio DFNum DFDen p-Value

$\begin{array}{lllll}\text { O'Brien[.5] } & 1.8485 & 1 & 24 & 0.1866 \\ \text { Brown-Forsythe } & 1.0531 & 1 & 24 & 0.3150\end{array}$

$\begin{array}{lllll}\text { Brown-Forsythe } & 1.0531 & 1 & 24 & 0.3150\end{array}$

$\begin{array}{lllll}\text { Levene } & 2.0867 & 1 & 24 & 0.1615 \\ \text { Bartlett } & 4.4642 & 1 & & 0.0346\end{array}$

$\begin{array}{lllll}\text { F Test 2-sided } & 3.5329 & 9 & 15 & 0.0346 \\ \end{array}$

Welch Anova testing Means Equal, allowing Std Devs Not Equal

F Ratio DFNum DFDen Prob $>$ F

$\begin{array}{llll}6.7985 & 1 & 12.236 & 0.0226\end{array}$

t Test

2.6074
Oneway Analysis of Measurement (wt \%) By Sampler Type of Material=SME Simulant, Analytical Block $=3$, Analyte $=A \mathrm{~A} / \mathrm{B}$, Target $(\mathrm{wt} \%)=2.0704412$

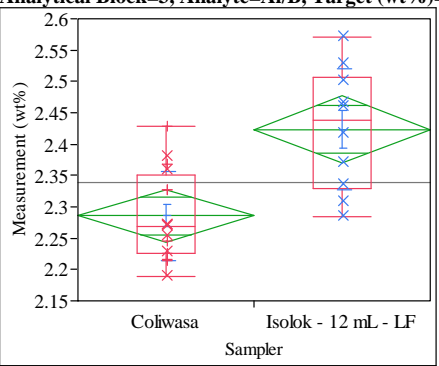

Oneway Anova

Summary of Fit

Adj Rsquare

0.39937
Root Mean Square Error $\quad 0.08151$

Mean of Response

2.338811

Test

Isolok - $12 \mathrm{~mL}$ - LF-Coliwasa

Assuming equal variances

Difference $\quad 0.137936$ t Ratio $\quad 4.197975$

Std Err Dif $\quad 0.032858$ DF 24

Upper CL Dif 0.205751 Prob $>|t| \quad 0.0003$

$\begin{array}{lll}\text { Lower CL Dif } 0.070121 & \text { Prob }>t & 0.0002\end{array}$

$\begin{array}{lrl}\text { Confidence } & 0.95 \text { Prob }<\mathrm{t} & 0.9998\end{array}$

Analysis of Variance

Source DF Sum of Squares Mean Square F Ratio Prob $>$ F

$\begin{array}{lllllll}\text { Sampler } & 1 & 0.11708511 & 0.117085 & 17.6230 & 0.0003\end{array}$

$\begin{array}{llll}\text { Error } & 24 & 0.15945320 & 0.006644\end{array}$

C. Total $25 \quad 0.27653831$

Means for Oneway Anova

Level Number Mean Std Error Lower 95\% Upper 95\%

$\begin{array}{lrrrrr}\text { Coliwasa } & 16 & 2.28576 & 0.02038 & 2.2437 & 2.3278\end{array}$

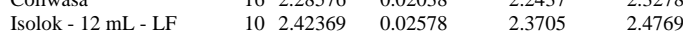

Std Error uses a pooled estimate of error variance

Means and Std Deviations

Level Number Mean Std Dev Std Err Mean Lower 95\% Upper 95\%

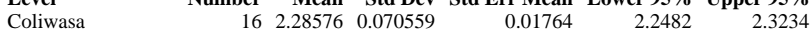

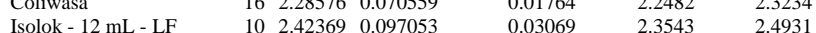

Test

Isolok - $12 \mathrm{~mL}$ - LF-Coliwasa

Assuming unequal variances

$\begin{array}{lrlr}\text { Difference } & 0.137936 & \text { t Ratio } & 3.896593 \\ \text { Std Err Dif } & 0.035399 & \text { DF } & 14.94949 \\ \text { Upper CL Dif } & 0.213410 & \text { Prob }>|t| & 0.0014 \\ \text { Lower CL Dif } & 0.062462 & \text { Prob }>t & 0.0007 \\ \text { Confidence } & 0.95 & \text { Prob }<t & 0.9993\end{array}$

Tests that the Variances are Equal

Level Count Std Dev MeanAbsDif to Mean MeanAbsDif to Median

$\begin{array}{lllll}\text { Coliwasa } & 16 & 0.0705592 & 0.0595251 & 0.0557725\end{array}$

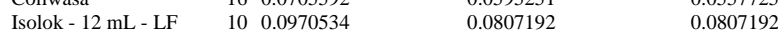

Test F Ratio DFNum DFDen p-Value

$\begin{array}{lrrrr}\text { O'Brien[.5] } & 2.3786 & 1 & 24 & 0.1361\end{array}$

Brown-Forsythe $\quad \begin{array}{llll}1.8086 & 1 & 24 & 0.1913\end{array}$

$\begin{array}{lllll}\text { Levene } & 1.7642 & 1 & 24 & 0.1966\end{array}$

$\begin{array}{lllll}\text { Bartlett } & 1.1351 & 1 & & 0.2867 \\ \text { F Test 2-sided } & 1.8920 & 9 & 15 & 0.2642\end{array}$

Welch Anova testing Means Equal, allowing Std Devs Not Equal

F Ratio DFNum DFDen Prob $>$ F

$\begin{array}{llrr}15.1834 & 1 & 14.949 & 0.0014\end{array}$

t Test

3.8966 


\section{Exhibit B2. Statistical Comparisons for High-Rheology (Phase 2) Simulant Testing}

Oneway Analysis of Measurement (wt \%) By Sampler Type of Material=SME Simulant, Analytical Block $=3$, Analyte $=\mathrm{Al} 2 \mathrm{O} 3(\mathrm{wt} \%)$, Target $(\mathrm{wt} \%)=10.934$

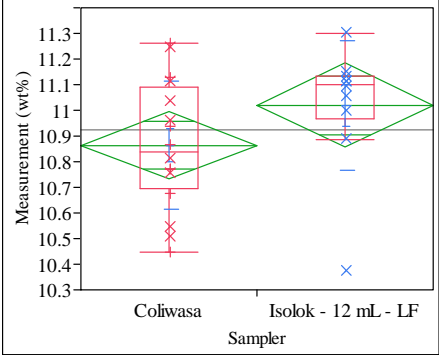

Oneway Anova

Summary of Fit

Adj Rsquare

0.05219

\begin{tabular}{rr}
0.25122 \\
\hline
\end{tabular}

10.92349

t Test

Isolok - $12 \mathrm{~mL}$ - LF-Coliwasa

Assuming equal variances

Difference $\quad 0.15612$ t Ratio 1.541621

Std Err Dif $\quad 0.10127$ DF 24

Upper CL Dif 0.36513 Prob $>|t| \quad 0.1362$

Lower CL Dif -0.05289 Prob $>t \quad 0.0681$

$\begin{array}{lrl}\text { Confidence } & 0.95 \text { Prob }<\mathrm{t} & 0.9319\end{array}$

Analysis of Variance

Source DF Sum of Squares Mean Square F Ratio Prob > F

$\begin{array}{llllll}\text { Sampler } & 1 & 0.1499904 & 0.149990 & 2.3766 & 0.1362\end{array}$

$\begin{array}{llll}\text { Error } & 24 & 1.5146751 & 0.063111\end{array}$

C. Total $25 \quad 1.6646655$

Means for Oneway Anova

Level N Number Mean Std Error Lower 95\% Upper 95\%

$\begin{array}{lrrrrr}\text { Coliwasa } & 16 & 10.8634 & 0.06280 & 10.734 & 10.993\end{array}$

$\begin{array}{llllll}\text { Coliwasa } & 16 & 10.8634 & 0.06280 & 10.734 & 10.993 \\ \text { Isolok - 12 mL - LF } & 10 & 11.0196 & 0.07944 & 10.856 & 11.184\end{array}$

Std Error uses a pooled estimate of error variance

Means and Std Deviations

Level Number Mean Std Dev Std Err Mean Lower 95\% Upper 95\%

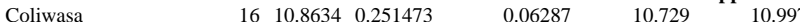

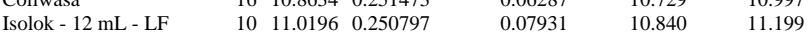

Test

Isolok - $12 \mathrm{~mL}$ - LF-Coliwas

Assuming unequal variances

$\begin{array}{lrlr}\text { Difference } & 0.15612 & \text { t Ratio } & 1.542619 \\ \text { Std Err Dif } & 0.10120 & \text { DF } & 19.29355 \\ \text { Upper CL Dif } & 0.36773 \text { Prob }>|t| & 0.1392 \\ \text { Lower CL Dif } & -0.05549 & \text { Prob }>\text { t } & 0.0696 \\ \text { Confidence } & 0.95 \text { Prob }<\mathrm{t} & 0.9304\end{array}$

Tests that the Variances are Equal

Level Count Std Dev MeanAbsDif to Mean MeanAbsDif to Median

$\begin{array}{lrrrr}\text { Coliwasa } & 16 & 0.2514733 & 0.2043022 & 0.2043022\end{array}$

$\begin{array}{llll}\text { Isolok - } 12 \mathrm{~mL} \text { - LF } & 10 & 0.2507971 & 0.1609854\end{array}$

Test F Ratio DFNum DFDen p-Value

$\begin{array}{lrrrr}\text { O'Brien[.5] } & 0.0001 & 1 & 24 & 0.9938\end{array}$

Brown-Forsythe $0.8421 \quad 1 \quad 24 \quad 0.3679$

$\begin{array}{lllll}\text { Levene } & 0.4716 & 1 & 24 & 0.4989\end{array}$

$\begin{array}{lrrrr}\text { Bartlett } & 0.0001 & 1 & \text {. } & 0.9930 \\ \text { F Test 2-sided } & 1.0054 & 15 & 9 & 1.0000\end{array}$

Welch Anova testing Means Equal, allowing Std Devs Not Equal

F Ratio DFNum DFDen Prob $>$ F

$\begin{array}{rrrr}2.3797 & 1 & 19.294 & 0.1392\end{array}$

t Test

1.5426
Oneway Analysis of Measurement (wt \%) By Sampler Type of Material=SME Simulant, Analytical Block $=3$, Analyte $=B 2 O 3(w t \%)$, Target $(w t \%)=5.281$

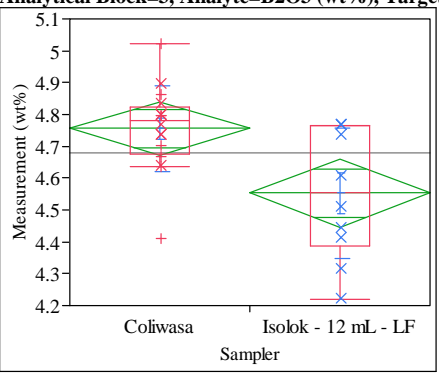

Oneway Anova

Summary of Fit

Ad Rsque

0.164162

Mean of Respons 4.677524
26

t Test

Isolok - $12 \mathrm{~mL}$ - LF-Coliwasa

Assuming equal variances

Difference $\quad-0.20245$ t Ratio $\quad-3.05929$

$\begin{array}{lll}\text { Std Err Dif } & 0.06618 \text { DF } & 24\end{array}$

Upper CL Dif -0.06587 Prob $>|t| \quad 0.0054$

Lower CL Dif -0.33903 Prob $>\mathrm{t} \quad 0.9973$

$\begin{array}{lrl}\text { Confidence } & 0.95 \text { Prob }<\mathrm{t} \quad 0.0027\end{array}$

Analysis of Variance

Source DF Sum of Squares Mean Square F Ratio Prob $>$ F

$\begin{array}{llllll}\text { Sampler } & 1 & 0.25222458 & 0.252225 & 9.3593 & 0.0054\end{array}$

$\begin{array}{lrrr}\text { Sampler } & 1 & 0.25222458 & 0.252225 \\ \text { Error } & 24 & 0.64677950 & 0.026949\end{array}$

$\begin{array}{lll}\text { Error } & 24 & 0.64677950 \\ \text { C. Total } & 25 & 0.89900407\end{array}$

Means for Oneway Anova

Level Number Mean Std Error Lower 95\% Upper 95\%

$\begin{array}{llllll}\text { Coliwasa } & 16 & 4.75539 & 0.04104 & 4.6707 & 4.8401 \\ \text { Isolok - 12 mL - LF } & 10 & 4.55294 & 0.05191 & 4.4458 & 4.6601\end{array}$

Std Error uses a pooled estimate of error variance

Means and Std Deviations

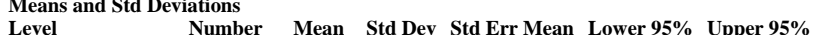

$\begin{array}{lrrrrrr}\text { Level } & \text { Number } & \text { Mean } & \text { Std Dev } & \text { Std Err Mean } & \text { Lower 95\% } & \text { Upper 95\% } \\ \text { Coliwasa } & 16 & 4.75539 & 0.134938 & 0.03373 & 4.6835 & 4.8273\end{array}$

$\begin{array}{lllllll}\text { Coliwasa } & 16 & 4.75539 & 0.134938 & 0.03373 & 4.6835 & 4.8273 \\ \text { Isolok - 12 mL - LF } & 10 & 4.55294 & 0.203757 & 0.06443 & 4.4072 & 4.6987\end{array}$

t Test

Isolok - $12 \mathrm{~mL}$ - LF-Coliwasa

Assuming unequal variances

Difference $\quad-0.20245$ t Ratio $\quad-2.78358$

Std Err Dif $\quad 0.07273$ DF $\quad 13.97996$

Upper CL Dif -0.04644 Prob $>|t| r 0.0147$

Lower CL Dif -0.35846 Prob $>\mathrm{t} \quad 0.9927$

$\begin{array}{lll}\text { Confidence } & 0.95 \text { Prob }<\mathrm{t} \quad 0.0073\end{array}$

Tests that the Variances are Equal

Level Count Std Dev MeanAbsDif to Mean MeanAbsDif to Median

$\begin{array}{lrrrr}\text { Coliwasa } & 16 & 0.1349384 & 0.0958423 & 0.0945846\end{array}$

$\begin{array}{lllll}\text { Isolok - } 12 \mathrm{~mL} \text { - LF } & 10 & 0.2037575 & 0.1738746 & 0.1738746\end{array}$

Test F Ratio DFNum DFDen p-Value

$\begin{array}{lllll}\text { O'Brien[.5] } & 2.4875 & 1 & 24 & 0.1278\end{array}$

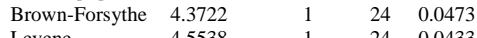

$\begin{array}{lllll}\text { Levene } & 4.5538 & 1 & 24 & 0.0433 \\ \text { Batett } & 1.9052 & 1 & & 0.1675\end{array}$

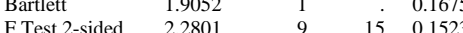

Welch Anova testing Means Equal, allowing Std Devs Not Equal

F Ratio DFNum DFDen Prob $>$ F

$\begin{array}{llrr}7.7483 & 1 & 13.98 & 0.0147\end{array}$

t Test

2.7836 
SRNL-STI-2011-00693

Revision 0

\section{Exhibit B2. Statistical Comparisons for High-Rheology (Phase 2) Simulant Testing}

Oneway Analysis of Measurement (wt \%) By Sampler Type of Material=SME Simulant, Analytical Block=3, Analyte $=\mathrm{BaO}(\mathrm{wt} \%)$, Target $(\mathrm{wt} \%)=0$

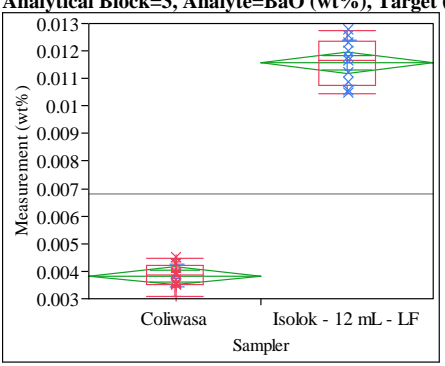

Oneway Anova

Summary of Fit

Rsquare

Adj Rsquare

Root Mean Square Error $\quad 0.000591$

Mean of Response $\quad 0.006818$

Observations (or Sum Wgts) $\quad 26$

Test

Isolok - $12 \mathrm{~mL}$ - LF-Coliwasa

Assuming equal variances

Difference $\quad 0.007736$ t Ratio $\quad 32.4617$

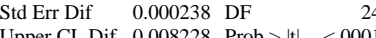

Upper CL Dif 0.008228 Prob $>|t|<.0001$

Confidence $\quad 0.95$ Prob $<\mathrm{t} \quad 1.0000$

Analysis of Variance

Source DF Sum of Squares Mean Square F Ratio Prob $>$ F

$\begin{array}{lllrrr}\text { Sampler } & 1 & 0.00036832 & 0.000368 & 1053.762 & <.0001\end{array}$

$\begin{array}{llll}\text { Error } & 24 & 0.00000839 & 3.495 \mathrm{e}-7\end{array}$

C. Total $25 \quad 0.00037670$

Means for Oneway Anova

Level Number Mean Std Error Lower 95\% Upper 95\%

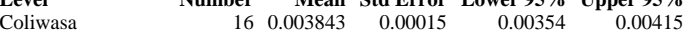

$\begin{array}{llllll}\text { Isolok - } 12 \mathrm{~mL} \text { - LF } & 10 & 0.011579 & 0.00019 & 0.01119 & 0.01197\end{array}$

Std Error uses a pooled estimate of error variance

Means and Std Deviations

Level Number Mean Std Dev Std Err Mean Lower 95\% Upper 95\%

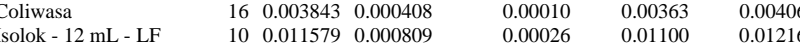

t Test

Isolok - $12 \mathrm{~mL}$ - LF-Coliwas

Assuming unequal variances

Difference $\quad 0.007736$ t Ratio 28.08834

Std Err Dif $\quad 0.000275$ DF $\quad 11.90882$

Upper CL Dif 0.008337 Prob $>|t|<.0001$

Lower CL Dif 0.007136 Prob $>t<000$

$\begin{array}{lrl}\text { Confidence } & 0.95 \text { Prob }<\mathrm{t} \quad 1.0000\end{array}$

Tests that the Variances are Equal

Level Count Std Dev MeanAbsDif to Mean MeanAbsDif to Median $\begin{array}{lllll} & 16 & 0.0004081 & 0.0003396 & 0.0003384 \\ \text { Isolok - } 12 \mathrm{~mL} \text { - LF } & 10 & 0.0008090 & 0.0006641 & 0.0006576\end{array}$

Test F Ratio DFNum DFDen p-Value

Test F Ratio DFNum DFDen p-Value

$\begin{array}{lllll}\text { O'Brien[.5] } & 9.4222 & 1 & 24 & 0.0053 \\ \text { Brown-Forsythe } & 6.4548 & 1 & 24 & 0.0179\end{array}$

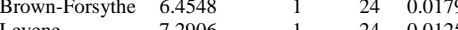

$\begin{array}{lllll}\text { Levene } & 7.2906 & 1 & 24 & 0.0125 \\ \text { Bartlett } & 5.2386 & 1 & & 0.0221\end{array}$

$\begin{array}{lllll}\text { F Test 2-sided } & 5.2386 & 1 & \dot{0} & 0.0221 \\ & 3.9309 & 9 & 15 & 0.0192\end{array}$

Welch Anova testing Means Equal, allowing Std Devs Not Equa

F Ratio DFNum DFDen Prob $>$ F

$\begin{array}{llll}788.9547 & 1 & 11.909 & <.0001\end{array}$

t Test
28.0883
Oneway Analysis of Measurement (wt \%) By Sampler Type of Material=SME Simulant, Analytical Block $=3$, Analyte $=\mathrm{CaO}(\mathrm{wt} \%)$, Target $(\mathrm{wt} \%)=0.543$

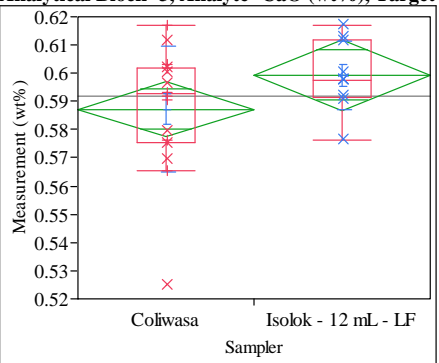

Oneway Anova

Summary of Fit

Adj Rsquare

0.090937

0.053059
Root Mean Square Error $\quad 0.019153$

Observations (or Sum Wgts) $\quad 26$

Test

Isolok - $12 \mathrm{~mL}$ - LF-Coliwasa

Assuming equal variances

Difference $\quad 0.01196$ t Ratio 1.549454

Std Err Dif $\quad 0.00772$ DF 24

Upper CL Dif 0.02790 Prob $>|t| \quad 0.1344$

$\begin{array}{lll}\text { Lower CL Dif }-0.00397 \text { Prob }>t \quad 0.0672 & 0.965\end{array}$

$\begin{array}{lrl}\text { Confidence } & 0.95 \text { Prob }<\mathrm{t} \quad 0.9328\end{array}$

Analysis of Variance

Source DF Sum of Squares Mean Square F Ratio Prob $>$ F

$\begin{array}{lllllll}\text { Sampler } & 1 & 0.00088072 & 0.000881 & 2.4008 & 0.1344\end{array}$

$\begin{array}{llll}\text { Error } & 24 & 0.00880425 & 0.000367\end{array}$

C. Total $25 \quad 0.00968497$

Means for Oneway Anova

Level Number Mean Std Error Lower 95\% Upper 95\%

$\begin{array}{lrrrrr}\text { Coliwasa } & 16 & 0.587314 & 0.00479 & 0.57743 & 0.59720\end{array}$

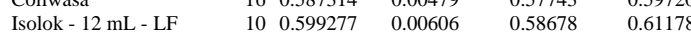

Std Error uses a pooled estimate of error variance

Means and Std Deviations

Level Number Mean Std Dev Std Err Mean Lower 95\% Upper 95\%

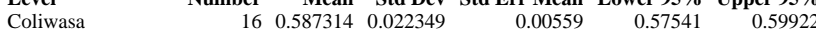

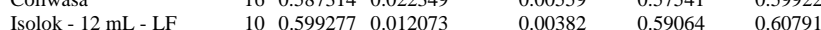

Test

Isolok - $12 \mathrm{~mL}$ - LF-Coliwasa

Assuming unequal variances

$\begin{array}{lrlr}\text { Difference } & 0.01196 & \mathrm{t} \text { Ratio } & 1.76782 \\ \text { Std Err Dif } & 0.00677 & \text { DF } & 23.67529 \\ \text { Upper CL Dif } & 0.02594 & \text { Prob }>|t| & 0.0900 \\ \text { Lower CL Dif } & -0.00201 & \text { Prob }>\text { t } & 0.0450 \\ \text { Confidence } & 0.95 & \text { Prob }<\mathrm{t} & 0.9550\end{array}$

Tests that the Variances are Equal

Level Count Std Dev MeanAbsDif to Mean MeanAbsDif to Median

$\begin{array}{lllll}\text { Coliwasa } & 16 & 0.0223493 & 0.0167030 & 0.0157410\end{array}$

$\begin{array}{llll}\text { Isolok - } 12 \mathrm{~mL} \text { - LF } & 10 & 0.0120734 & 0.0088989\end{array}$

Test F Ratio DFNum DFDen p-Value

$\begin{array}{lrrrr}\text { O'Brien[.5] } & 1.0938 & 1 & 24 & 0.3061\end{array}$

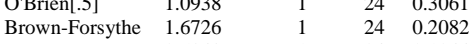

$\begin{array}{lllll}\text { Levene } & 2.5348 & 1 & 24 & 0.1244\end{array}$

$\begin{array}{lrrrr}\text { Bartlett } & 3.5170 & 1 & \text {. } & 0.0607 \\ \text { F Test 2-sided } & 3.4267 & 15 & 9 & 0.0674\end{array}$

Welch Anova testing Means Equal, allowing Std Devs Not Equal

F Ratio DFNum DFDen Prob $>$ F

$3.1252 \quad 1 \quad 23.675 \quad 0.0900$

t Test

1.7678 
SRNL-STI-2011-00693

Revision 0

\section{Exhibit B2. Statistical Comparisons for High-Rheology (Phase 2) Simulant Testing}

Oneway Analysis of Measurement (wt \%) By Sampler Type of Material=SME Simulant, Analytical Block $=3$, Analyte $=$ Cr2O3 $(w t \%)$, Target $(w t \%)=0.015$

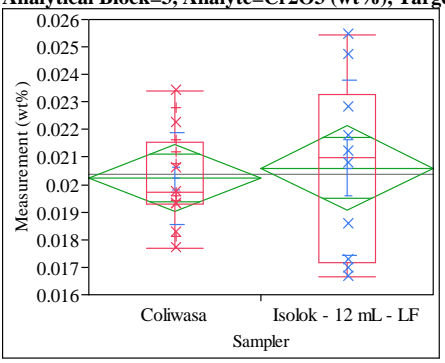

Oneway Anova

Summary of Fit

Rsquare

Adj Rsquare

Root Mean Square Error $\quad 0.002353$

Mean of Response 0.020378

Observations (or Sum Wgts) 26

t Test

Isolok - $12 \mathrm{~mL}$ - LF-Coliwas

Assuming equal variances

Difference $\quad 0.00037$ t Ratio $\quad 0.39493$

$\begin{array}{lrr}\text { Std Err Dif } & 0.00095 \text { DF } & 24 \\ & & \end{array}$

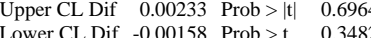

$\begin{array}{lrl}\text { Confidence } & 0.95 \text { Prob }<\mathrm{t} \quad 0.6518\end{array}$

Analysis of Variance

Source DF Sum of Squares Mean Square F Ratio Prob $>$ F

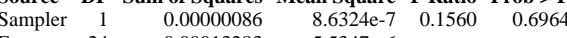

$\begin{array}{llll}\text { Error } & 24 & 0.00013283 & 5.5347 \mathrm{e}-6\end{array}$

C. Total $25 \quad 0.00013370$

Means for Oneway Anova

Level Number Mean Std Error Lower 95\% Upper 95\%

$\begin{array}{lrrrrr}\text { Coliwasa } & 16 & 0.020234 & 0.00059 & 0.01902 & 0.02145\end{array}$

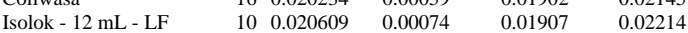

Std Error uses a pooled estimate of error variance

Means and Std Deviations

Level Number Mean Std Dev Std Err Mean Lower 95\% Upper 95\%

$\begin{array}{lllllll} & 16 & 0.020234 & 0.001671 & 0.00042 & 0.01934 & 0.02112\end{array}$

t Test

Isolok - $12 \mathrm{~mL}$ - LF-Coliwas

Assuming unequal variances

Difference $\quad 0.00037$ t Ratio $\quad 0.344037$

Std Err Dif $\quad 0.00109$ DF $\quad 12.1578$

Upper CL Dif 0.00274 Prob $>|t| 0.7367$

Lower CL Dif -0.00199 Prob $>t \quad 0.3683$

$\begin{array}{lll}\text { Confidence } & 0.95 \text { Prob }<\mathrm{t} \quad 0.6317\end{array}$

Tests that the Variances are Equal

$\begin{array}{lrrrr}\text { Level } & \text { Count } & \text { Std Dev } & \text { MeanAbsDif to Mean } & \text { MeanAbsDif to Median } \\ \text { Coliwasa } & 16 & 0.0016707 & 0.0013691\end{array}$ $\begin{array}{lllll} & 16 & 0.0016707 & 0.0013691 & 0.0013063 \\ \text { solok - } 12 \mathrm{~mL} \text { - LF } & 10 & 0.0031791 & 0.0026016 & 0.002572\end{array}$

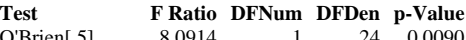

$\begin{array}{lllll}\text { O'Brien[.5] } & 8.0914 & 1 & 24 & 0.0090 \\ \text { Brown-Forsythe } & 5.2930 & 1 & 24 & 0.0304\end{array}$

$\begin{array}{lllll}\text { Brown-Forsythe } & 5.2930 & 1 & 24 & 0.0304 \\ \text { Levene } & 6.3822 & 1 & 24 & 0.0185\end{array}$

$\begin{array}{lllrl}\text { Bartlett } & 4.6377 & 1 & . & 0.0313\end{array}$

$\begin{array}{lllll}\text { F Test 2-sided } & 3.6209 & 9 & 15 & 0.0274\end{array}$

Welch Anova testing Means Equal, allowing Std Devs Not Equal

F Ratio DFNum DFDen Prob $>$ F

$\begin{array}{llll}0.1184 & 1 & 12.158 & 0.7367\end{array}$

t Test

0.3440
Oneway Analysis of Measurement (wt \%) By Sampler Type of Material=SME Simulant, Analytical Block $=3$, Analyte $=\mathrm{CuO}(\mathrm{wt} \%)$, Target $(\mathrm{wt} \%)=0.103$

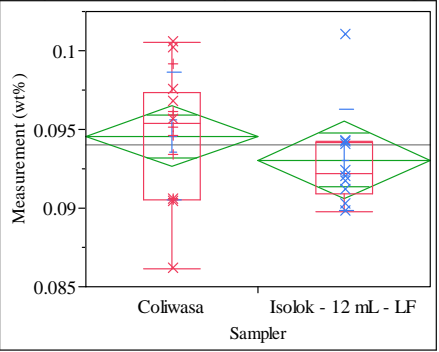

Oneway Anova

Summary of Fit

Rsquare

Adj Rsquare

Root Mean Square Error $\quad 0.00375$

Mean of Response 0.093986

Observations (or Sum Wgts) 26

\section{t Test}

Isolok - $12 \mathrm{~mL}$ - LF-Coliwasa

Assuming equal variances

Difference $\quad-0.00151$ t Ratio $\quad-0.9955$

$\begin{array}{lrlr}\text { Std Err Dif } & 0.00151 & \text { DF } & 24 \\ \text { Upper CL } & 0.00162 & \text { Prob }>|t| & 0.3294\end{array}$

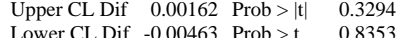

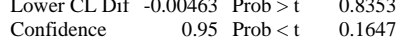

Analysis of Variance

Source DF Sum of Squares Mean Square F Ratio Prob $>$ F

$\begin{array}{lrrrrr}\text { Sampler } & 1 & 0.00001397 & 0.000014 & 0.9910 & 0.3294\end{array}$

$\begin{array}{llll}\text { Error } & 24 & 0.00033838 & 0.000014\end{array}$

C. Total $25 \quad 0.00035236$

Means for Oneway Anova

Level Number Mean Std Error Lower 95\% Upper 95\%

$\begin{array}{lrrrrr}\text { Level } & \text { Number } & \text { Mean } & \text { Std Error } & \text { Lower 95\% } & \text { Upper 95\% } \\ \text { Coliwasa } & 16 & 0.094566 & 0.00094 & 0.09263 & 0.09650\end{array}$

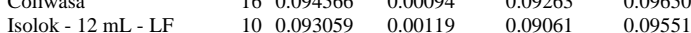

Std Error uses a pooled estimate of error variance

Means and Std Deviations

Level Number Mean Std Dev Std Err Mean Lower 95\% Upper 95\%

$\begin{array}{lllllll} & 16 & 0.094566 & 0.004045 & 0.00101 & 0.09241 & 0.09672\end{array}$

\section{Test}

Isolok - $12 \mathrm{~mL}$ - LF-Coliwas

Assuming unequal variances

$\begin{array}{lrlr}\text { Difference } & -0.00151 & \mathrm{t} \text { Ratio } & -1.05097 \\ \text { Std Err Dif } & 0.00143 & \text { DF } & 22.44054 \\ \text { Upper CL Dif } & 0.00146 & \text { Prob }>|\mathrm{t}| & 0.3045 \\ \text { Lower CL Dif } & -0.00448 & \text { Prob }>\mathrm{t} & 0.8478 \\ \text { Confidence } & 0.95 & \text { Prob }<\mathrm{t} & 0.1522\end{array}$

Tests that the Variances are Equal

$\begin{array}{lrrrr}\text { Level } & \text { Count } & \text { Std Dev } & \text { MeanAbsDif to Mean } & \text { MeanAbsDif to Median } \\ \text { Coliwasa } & 16 & 0.0040447 & 0.0032332 & 0.0031530\end{array}$

Isolok - $12 \mathrm{~mL}$ - LF $\quad 10 \quad 0.0032144 \quad 0.0022382 \quad 0.0021030$

Test F Ratio DFNum DFDen p-Value

O'Brien[.5] 0.4876 .

$\begin{array}{lllll}\text { Brown-Forsythe } & 1.0617 & 1 & 24 & 0.4917 \\ & 1.2080 & 1 & 24 & 0.3131\end{array}$

$\begin{array}{lllll}\text { Brown-Forsythe } & 1.0617 & 1 & 24 & 0.3131 \\ \text { Levene } & 1.2080 & 1 & 24 & 0.2826\end{array}$

$\begin{array}{lllll}\text { Levene } & 1.2080 & 1 & 24 & 0.2826 \\ \text { Bartlett } & 0.5427 & 1 & . & 0.4613\end{array}$

$\begin{array}{llrrr}\text { F Test 2-sided } & 1.5833 & 15 & 9 & 0.4919\end{array}$

Welch Anova testing Means Equal, allowing Std Devs Not Equal

F Ratio DFNum DFDen Prob $>$ F

$\begin{array}{llll}1.1045 & 1 & 22.441 & 0.3045\end{array}$

t Test

1.0510 


\section{Exhibit B2. Statistical Comparisons for High-Rheology (Phase 2) Simulant Testing}

Oneway Analysis of Measurement (wt\%) By Sampler Type of Material=SME Simulant, Analytical Block=3, Analyte=Fe/Li, Target $(\mathrm{wt} \%)=1.99097835$

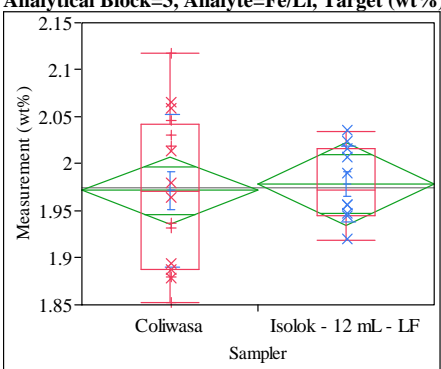

Oneway Anova

Summary of Fit

Adj Rsquare

$-0.03891$

$\quad 0.068408$

1.974291

Observations (or Sum Wgts) $\quad 26$

Test

Isolok - $12 \mathrm{~mL}$ - LF-Coliwas

Assuming equal variances

Difference $\quad 0.00696$ t Ratio $\quad 0.252534$

Std Err Dif $\quad 0.02758$ DF 24

Upper CL Dif 0.06388 Prob $>|t| \quad 0.8028$

Lower CL Dif -0.04995 Prob $>t \quad 0.4014$

$\begin{array}{lrl}\text { Confidence } & 0.95 \text { Prob }<\mathrm{t} \quad 0.5986\end{array}$

Analysis of Variance

Source DF Sum of Squares Mean Square F Ratio Prob $>$ F

$\begin{array}{llllll}\text { Sampler } & 1 & 0.00029844 & 0.000298 & 0.0638 & 0.8028\end{array}$

$\begin{array}{llll}\text { Error } & 24 & 0.11231081 & 0.004680\end{array}$

C. Total $25 \quad 0.11260924$

Means for Oneway Anova

Level N Number Mean Std Error Lower 95\% Upper 95\%

\begin{tabular}{lrrrrr} 
Coliwasa & 16 & 1.97161 & 0.01710 & 1.9363 & 2.0069 \\
\hline
\end{tabular}

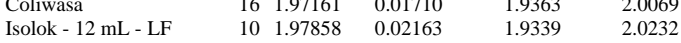

Std Error uses a pooled estimate of error variance

Means and Std Deviations

Level Number Mean Std Dev Std Err Mean Lower 95\% Upper 95\%

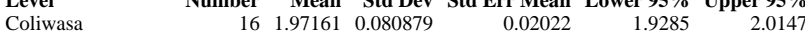

$\begin{array}{lllllll}\text { Coliwasa } & 16 & 1.97161 & 0.080879 & 0.02022 & 1.9285 & 2.0147 \\ \text { Isolok - 12 mL - LF } & 10 & 1.97858 & 0.039706 & 0.01256 & 1.9502 & 2.0070\end{array}$

t Test

Isolok - $12 \mathrm{~mL}$ - LF-Coliwas

Assuming unequal variances

Difference $\quad 0.00696$ t Ratio $\quad 0.292585$

Std Err Dif $\quad 0.02380$ DF 23.0793

Upper CL Dif 0.05619 Prob $>|t| r \mid r .7725$

Lower CL Dif -0.04226 Prob $>\mathrm{t} \quad 0.3862$

$\begin{array}{lrl} & \\ \text { Confidence } & 0.95 \text { Prob }<\mathrm{t} & 0.6138\end{array}$

Tests that the Variances are Equal

Level Count Std Dev MeanAbsDif to Mean MeanAbsDif to Median

$\begin{array}{lllll}\text { Coliwasa } & 16 & 0.0808791 & 0.0691507 & 0.0691507\end{array}$

$\begin{array}{llll}\text { Isolok - } 12 \mathrm{~mL} \text { - LF } & 10 & 0.0397064 & 0.0349048\end{array}$

Test F Ratio DFNum DFDen p-Value

$\begin{array}{lrrrr}\text { O'Brien[.5] } & 6.1244 & 1 & 24 & 0.0208\end{array}$

Brown-Forsythe $7.2099 \quad 1 \quad 24 \quad 0.0129$

$\begin{array}{lllll}\text { Levene } & 7.3338 & 1 & 24 & 0.0123\end{array}$

$\begin{array}{lrrrr}\text { Bartlett } & 4.5605 & 1 & \text {. } & 0.0327 \\ \text { F Test 2-sided } & 4.1491 & 15 & 9 & 0.0366\end{array}$

Welch Anova testing Means Equal, allowing Std Devs Not Equal

F Ratio DFNum DFDen Prob > F

$\begin{array}{rrrr}0.0856 & 1 & 23.079 & 0.7725\end{array}$

t Test

$$
123.079 \quad 0.7725
$$

0.2926
Oneway Analysis of Measurement (wt \%) By Sampler Type of Material=SME Simulant, Analytical Block=3, Analyte=Fe2O3 (wt \%), Target $(w t \%)=9.931$

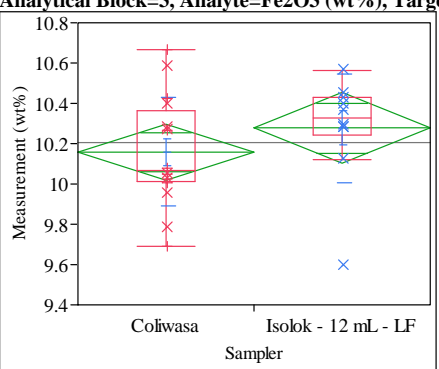

Oneway Anova

Summary of Fit

Adj Rsquare

0.269085

10.20366

Observations (or Sum Wgts) 26

Test

Isolok - $12 \mathrm{~mL}$ - LF-Coliwasa

Assuming equal variances

$\begin{array}{lrlr}\text { Difference } & 0.11867 & \mathrm{t} \text { Ratio } & 1.093973 \\ \text { Std Err Dif } & 0.10847 & \text { DF } & 24 \\ \text { Upper CL Dif } & 0.34254 & \text { Prob }>|t| & 0.2848 \\ \text { Lower CL Dif } & -0.10521 & \text { Prob }>t & 0.1424 \\ \text { Confidence } & 0.95 & \text { Prob }<\mathrm{t} & 0.8576\end{array}$

Analysis of Variance

Source DF Sum of Squares Mean Square F Ratio Prob $>$ F

$\begin{array}{llllll}\text { Sampler } & 1 & 0.0866548 & 0.086655 & 1.1968 & 0.2848\end{array}$

$\begin{array}{llll}\text { Error } & 24 & 1.7377628 & 0.072407\end{array}$

C. Total $25 \quad 1.8244176$

Means for Oneway Anova

Level Number Mean Std Error Lower 95\% Upper 95\%

$\begin{array}{lrrrrr}\text { Coliwasa } & 16 & 10.1580 & 0.06727 & 10.019 & 10.297\end{array}$

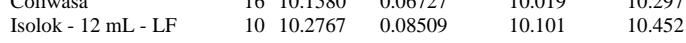

Std Error uses a pooled estimate of error variance

Means and Std Deviations

Level Number Mean Std Dev Std Err Mean Lower 95\% Upper 95\%

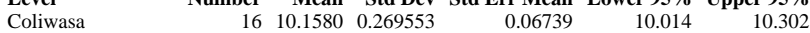

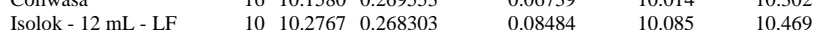

Test

Isolok - $12 \mathrm{~mL}$ - LF-Coliwas

Assuming unequal variances

$\begin{array}{lrlr}\text { Difference } & 0.11867 & \mathrm{t} \text { Ratio } & 1.095196 \\ \text { Std Err Dif } & 0.10835 & \text { DF } & 19.32287 \\ \text { Upper CL Dif } & 0.34519 & \text { Prob }>|\mathrm{t}| & 0.2869 \\ \text { Lower CL Dif } & -0.10786 & \text { Prob }>\mathrm{t} & 0.1434 \\ \text { Confidence } & 0.95 & \text { Prob }<\mathrm{t} & 0.8566\end{array}$

Tests that the Variances are Equal

Level Count Std Dev MeanAbsDif to Mean MeanAbsDif to Median

$\begin{array}{lllll}\text { Coliwasa } & 16 & 0.2695531 & 0.2207099 & 0.2108807\end{array}$

Isolok - $12 \mathrm{~mL}$ - LF $\quad 10-0.2683032$

Test F Ratio DFNum DFDen p-Value

$\begin{array}{lrrrr}\text { O'Brien[.5] } & 0.0002 & 1 & 24 & 0.9896\end{array}$

$\begin{array}{lllll}\text { Brown-Forsythe } & 0.3656 & 1 & 24 & 0.5511\end{array}$

$\begin{array}{lllll}\text { Levene } & 0.6157 & 1 & 24 & 0.4403\end{array}$

$\begin{array}{lrrrr}\text { Bartlett } & 0.0002 & 1 & \text {. } & 0.9878 \\ \text { F Test 2-sided } & 1.0093 & 15 & 9 & 1.0000\end{array}$

Welch Anova testing Means Equal, allowing Std Devs Not Equal

F Ratio DFNum DFDen Prob $>$ F

$\begin{array}{rrrr}1.1995 & 1 & 19.323 & 0.2869\end{array}$

t Test

1.0952 
SRNL-STI-2011-00693

Revision 0

\section{Exhibit B2. Statistical Comparisons for High-Rheology (Phase 2) Simulant Testing}

Oneway Analysis of Measurement (wt \%) By Sampler Type of Material=SME Simulant, Analytical Block=3, Analyte $=K 20$ (wt \%), Target $(w t \%)=0.076$

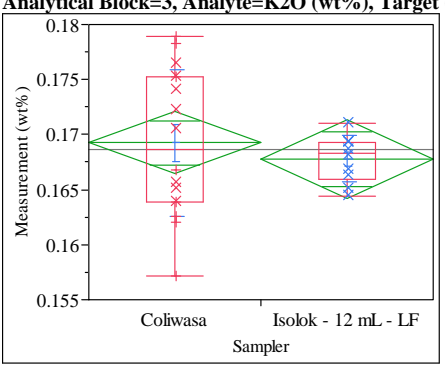

Oneway Anova

Summary of Fit

Rsquare

Adj Rsquare

Root Mean Square Error $\quad 0.005438$

Mean of Response 0.16869

Observations (or Sum Wgts) $\quad 26$

Test

Isolok - $12 \mathrm{~mL}$ - LF-Coliwasa

Assuming equal variances

Difference $\quad-0.00145$ t Ratio $\quad-0.65942$

$\begin{array}{lrr}\text { Std Err Dif } & 0.00219 \text { DF } & 24 \\ \text { Dpper } & 0.5159\end{array}$

$\begin{array}{rrrr}\text { Upper CL Dif } & 0.00308 & \text { Prob }>|t| & 0.5159 \\ \text { Cower CL Dif } & -0.00597 & \text { Prob }>t & 0.7420\end{array}$

$\begin{array}{lrl}\text { Confidence } & 0.95 \text { Prob }<\mathrm{t} \quad 0.2580\end{array}$

Analysis of Variance

Source DF Sum of Squares Mean Square F Ratio Prob > F

$\begin{array}{lrrrrr}\text { Sampler } & 1 & 0.00001286 & 0.000013 & 0.4348 & 0.5159\end{array}$

$\begin{array}{llll}\text { Error } & 24 & 0.00070971 & 0.000030\end{array}$

C. Total $25 \quad 0.0007225$

Means for Oneway Anova

Level Number Mean Std Error Lower 95\% Upper 95\%

\begin{tabular}{lrrrrr} 
Coliwasa & 16 & 0.169246 & 0.00136 & 0.16644 & 0.17205 \\
\hline
\end{tabular}

$\begin{array}{llllll}\text { Isolok - } 12 \mathrm{~mL} \text { - LF } & 10 & 0.167801 & 0.00172 & 0.16425 & 0.17135\end{array}$

Std Error uses a pooled estimate of error variance

Means and Std Deviations

Level Number Mean Std Dev Std Err Mean Lower 95\% Upper 95\%

$\begin{array}{lllllll} & 16 & 0.169246 & 0.006682 & 0.00167 & 0.16569 & 0.1728\end{array}$

t Test

Isolok - $12 \mathrm{~mL}$ - LF-Coliwas

Assuming unequal variances

Difference $\quad-0.00145$ t Ratio $\quad-0.80365$

Std Err Dif $\quad 0.00180$ DF $\quad 19.34584$

Upper CL Dif 0.00231 Prob $>|t| \quad 0.4314$

Lower CL Dif -0.00521 Prob $>t \quad 0.7843$

Confidence $\quad 0.95$ Prob $<\mathrm{t} \quad 0.2157$

Tests that the Variances are Equal

Level Count Std Dev MeanAbsDif to Mean MeanAbsDif to Median

$\begin{array}{lrrrr}\text { Coliwasa } & 16 & 0.0066816 & 0.0058724 & 0.0058724 \\ \text { Isolok - } 12 \mathrm{~mL} \text { - LF } & 10 & 0.0021095 & 0.0017346 & 0.0016864\end{array}$

Test F Ratio DFNum DFDen p-Value

$\begin{array}{lrrrr}\text { O'Brien[.5] } & 9.1424 & 1 & 24 & 0.0059\end{array}$

$\begin{array}{lrrrr}\text { O'Brien[.5] } & 9.1424 & 1 & 24 & 0.0059 \\ \text { Brown-Forsythe } & 18.7193 & 1 & 24 & 0.0002\end{array}$

$\begin{array}{lllll}\text { Brown-Forsythe } & 18.7193 & 1 & 24 & 0.0002 \\ \text { Levene } & 19.7773 & 1 & 24 & 0.0002\end{array}$

$\begin{array}{lrrrr}\text { Bartlett } & 10.3949 & 1 & . & 0.0013\end{array}$

$\begin{array}{lllll}\text { F Test 2-sided } & 10.0326 & 15 & 9 & 0.0014\end{array}$

Welch Anova testing Means Equal, allowing Std Devs Not Equal

F Ratio DFNum DFDen Prob $>$ F

$\begin{array}{llll}0.6459 & 1 & 19.346 & 0.4314\end{array}$

t Test

0.8037
Oneway Analysis of Measurement (wt\%) By Sampler Type of Material=SME Simulant, Analytical Block $=3$, Analyte $=$ Li2O $(w t \%)$, Target $(w t \%)=4.988$

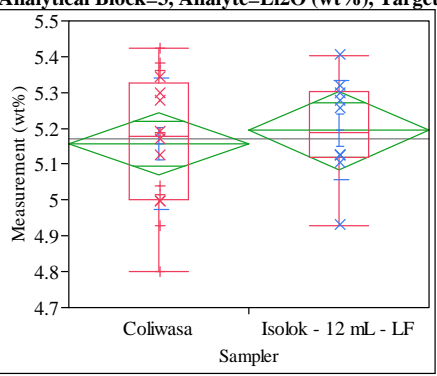

Oneway Anova

Summary of Fit

Adj Rsquare

0.02847
-0.16728

0.167228

$\begin{array}{lr}\text { Mean of Response } & 5.171928 \\ \text { Observations (or Sum Wgts) } & 26\end{array}$

t Test

Isolok - $12 \mathrm{~mL}$ - LF-Coliwasa

Assuming equal variances

$\begin{array}{lrlr}\text { Difference } & 0.03741 & \text { t Ratio } & 0.554899 \\ \text { Std Err Dif } & 0.06741 \text { DF } & 24 \\ \text { Upper CL Dif } & 0.17654 & \text { Prob }>|t| & 0.5841 \\ \text { Lower CL Dif } & -0.10172 & \text { Prob }>t & 0.2920 \\ \text { Confidence } & 0.95 & \text { Prob }<\mathrm{t} & 0.7080\end{array}$

Analysis of Variance

Source DF Sum of Squares Mean Square F Ratio Prob $>$ F

$\begin{array}{lrrrrr}\text { Sampler } & 1 & 0.00861081 & 0.008611 & 0.3079 & 0.5841\end{array}$

$\begin{array}{lrrr}\text { Eampler } & 1 & 0.00861081 & 0.008611 \\ \text { Error } & 24 & 0.67116225 & 0.027965\end{array}$

C. Total $25 \quad 0.67977306$

Means for Oneway Anova

Level Number Mean Std Error Lower 95\% Upper 95\%

$\begin{array}{lllllr}\text { Coliwasa } & 16 & 5.15754 & 0.04181 & 5.0713 & 5.2438 \\ \text { Isolok - 12 mL - LF } & 10 & 5.19495 & 0.05288 & 5.0858 & 5.3041\end{array}$

Std Error uses a pooled estimate of error variance

Means and Std Deviations

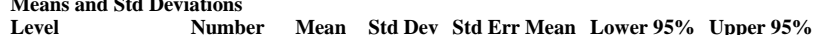

16 5.15754 0.d Dev Std Err Mean Lower 95\% Upper 95\%

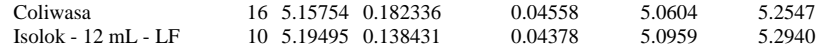

t Test

Isolok - $12 \mathrm{~mL}$ - LF-Coliwasa

Assuming unequal variances

Difference $\quad 0.03741$ t Ratio $\quad 0.59188$

Std Err Dif $\quad 0.06320$ DF 22.92623

Upper CL Dif 0.16817 Prob $>|t| r 0.5597$

Lower CL Dif -0.09336 Prob $>t \quad 0.2799$

$\begin{array}{lll}\text { Confidence } & 0.95 \text { Prob }<\mathrm{t} \quad 0.7201\end{array}$

Tests that the Variances are Equal

Level Count Std Dev MeanAbsDif to Mean MeanAbsDif to Median

$\begin{array}{lrrrr}\text { Coliwasa } & 16 & 0.1823357 & 0.1505348 & 0.1493574\end{array}$

$\begin{array}{lllll}\text { Isolok - } 12 \mathrm{~mL} \text { - LF } & 10 & 0.1384307 & 0.1141037 & 0.1141037\end{array}$

Test F Ratio DFNum DFDen p-Value

$\begin{array}{lllll}\text { O'Brien[.5] } & 1.1243 & 1 & 24 & 0.2996\end{array}$

Brown-Forsythe $\quad 0.9611 \quad 1 \quad 24 \quad 0.3367$

$\begin{array}{lllll}\text { Levene } & 1.0988 & 1 & 24 & 0.3050\end{array}$

$\begin{array}{lllll}\text { Bartlett } & 0.7719 & 1 & .0 .3796\end{array}$

$\begin{array}{lllll}\text { F Test 2-sided } & 1.7349 & 15 & 9 & 0.4069\end{array}$

Welch Anova testing Means Equal, allowing Std Devs Not Equal

F Ratio DFNum DFDen Prob $>$ F

$\begin{array}{rrrr}0.3503 & 1 & 22.926 & 0.5597\end{array}$

t Test

0.5919 
SRNL-STI-2011-00693

Revision 0

\section{Exhibit B2. Statistical Comparisons for High-Rheology (Phase 2) Simulant Testing}

Oneway Analysis of Measurement (wt \%) By Sampler Type of Material=SME Simulant, Analytical Block=3, Analyte $=\mathrm{MgO}(\mathrm{wt} \%)$, Target $(\mathrm{wt} \%)=\mathbf{0 . 2 8 6}$

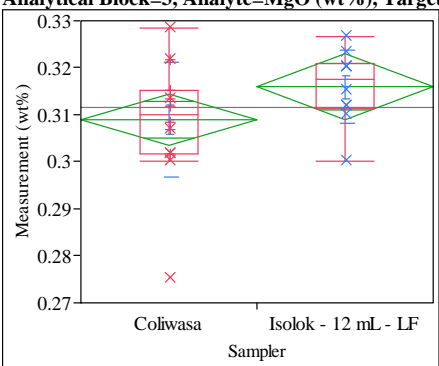

Oneway Anova

Summary of Fit

Adj Rsquare $\quad 0.09775$

Root Mean Square Error $\quad 0.010683$

Mean of Response

$\begin{array}{lr}\text { Mean of Response } & 0.311633 \\ \text { Observations (or Sum Wgts) } & 26\end{array}$

Test

Isolok - $12 \mathrm{~mL}$ - LF-Coliwasa

Assuming equal variances

Difference $\quad 0.00694$ t Ratio 1.612567

Std Err Dif $\quad 0.00431$ DF 24

Upper CL Dif 0.01583 Prob $>|t| \quad 0.1199$

Lower CL Dif -0.00194 Prob $>t \quad 0.0600$

$\begin{array}{lrl}\text { Confidence } & 0.95 \text { Prob }<\mathrm{t} \quad 0.9400\end{array}$

Analysis of Variance

Source DF Sum of Squares Mean Square F Ratio Prob > F

$\begin{array}{llllll}\text { Sampler } & 1 & 0.00029674 & 0.000297 & 2.6004 & 0.1199\end{array}$

$\begin{array}{llll}\text { Error } \quad 24 & 0.00273879 & 0.000114\end{array}$

C. Total $25 \quad 0.00303553$

Means for Oneway Anova

Level Number Mean Std Error Lower 95\% Upper 95\%

$\begin{array}{lrrrrr}\text { Coliwasa } & 16 & 0.308962 & 0.00267 & 0.30345 & 0.31447\end{array}$

$\begin{array}{llllll}\text { Isolok - 12 mL - LF } & 10 & 0.315906 & 0.00338 & 0.30893 & 0.32288\end{array}$

Std Error uses a pooled estimate of error variance

Means and Std Deviations

Level Number Mean Std Dev Std Err Mean Lower 95\% Upper 95\%

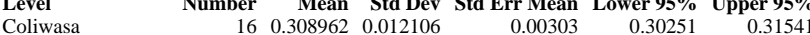

$\begin{array}{lllllll}\text { Isolok - } 12 \mathrm{~mL} \text { - LF } & 10 & 0.315906 & 0.007749 & 0.00245 & 0.31036 & 0.32145\end{array}$

Test

Isolok - $12 \mathrm{~mL}$ - LF-Coliwas

Assuming unequal variances

$\begin{array}{lrlr}\text { Difference } & 0.00694 & \text { t Ratio } & 1.783236 \\ \text { Std Err Dif } & 0.00389 & \text { DF } & 23.95519 \\ \text { Upper CL Dif } & 0.01498 & \text { Prob }>|t| & 0.0872 \\ \text { Lower CL Dif } & -0.00109 & \text { Prob }>\text { t } & 0.0436 \\ \text { Confidence } & 0.95 & \text { Prob }<\mathrm{t} & 0.9564\end{array}$

Tests that the Variances are Equal

Level Count Std Dev MeanAbsDif to Mean MeanAbsDif to Median

$\begin{array}{lllll}\text { Coliwasa } & 16 & 0.0121063 & 0.0086024 & 0.0086024\end{array}$

$\begin{array}{lllll}\text { Isolok - } 12 \mathrm{~mL} \text { - LF } & 10 & 0.0077486 & 0.0061357 & 0.0061357\end{array}$

Test F Ratio DFNum DFDen p-Value

$\begin{array}{lrrrr}\text { O'Brien[.5] } & 0.7214 & 1 & 24 & 0.4041\end{array}$

Brown-Forsythe $\quad 0.7327 \quad 1 \quad 24 \quad 0.4005$

$\begin{array}{lllll}\text { Levene } & 0.7627 & 1 & 24 & 0.3911\end{array}$

$\begin{array}{lrrrr}\text { Bartlett } & 1.9384 & 1 & \text {. } & 0.1638 \\ \text { F Test 2-sided } & 2.4410 & 15 & 9 & 0.1789\end{array}$

Welch Anova testing Means Equal, allowing Std Devs Not Equal

F Ratio DFNum DFDen Prob $>$ F

$\begin{array}{lrrr}3.1799 & 1 & 23.955 & 0.0872\end{array}$

t Test

1.7832
Oneway Analysis of Measurement (wt \%) By Sampler Type of Material=SME Simulant, Analytical Block $=3$, Analyte $=\mathrm{MnO}(\mathrm{wt} \%)$, Target $(\mathrm{wt} \%)=2.918$

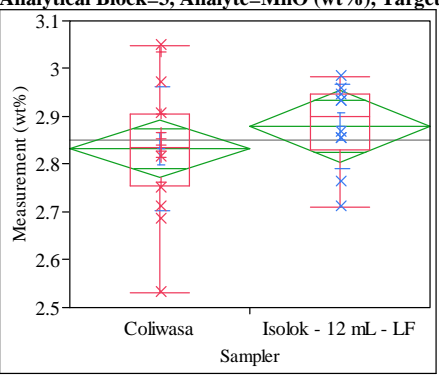

Oneway Anova

Summary of Fit

Rsqua

Adj Rsquare $\quad 0.039628$

Root

0.116678

Mean of Response

2.850572
26

t Test

Isolok - $12 \mathrm{~mL}$ - LF-Coliwasa

Assuming equal variances

$\begin{array}{lrlr}\text { Difference } & 0.04681 & \text { t Ratio } & 0.995146 \\ \text { Std Err Dif } & 0.04703 \text { DF } & 24 \\ \text { Upper CL Dif } & 0.14388 & \text { Prob }>|t| & 0.3296 \\ \text { Lower CL Dif } & -0.05027 & \text { Prob }>t & 0.1648 \\ \text { Confidence } & 0.95 & \text { Prob }<\mathrm{t} & 0.8352\end{array}$

Analysis of Variance

Source DF Sum of Squares Mean Square F Ratio Prob $>$ F

$\begin{array}{lrrrrr}\text { Sampler } & 1 & 0.01348186 & 0.013482 & 0.9903 & 0.3296\end{array}$

$\begin{array}{llll}\text { Error } & 24 & 0.32672902 & 0.013614\end{array}$

$\begin{array}{lll}\text { C. Total } & 25 & 0.34021087\end{array}$

Means for Oneway Anova

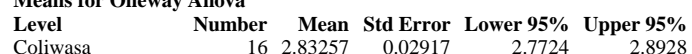

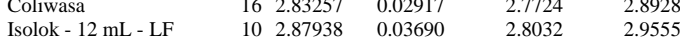

Std Error uses a pooled estimate of error variance

Means and Std Deviations

Level $\quad$ Number Mean Std Dev Std Err Mean Lower 95\% Upper 95\%

$\begin{array}{llllrrr}\text { Coliwasa } & 16 & 2.83257 & 0.130989 & 0.03275 & 2.7628 & 2.9024\end{array}$

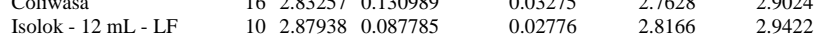

t Test

Isolok - $12 \mathrm{~mL}$ - LF-Coliwasa

Assuming unequal variances

$\begin{array}{lrlr}\text { Difference } & 0.04681 & \text { t Ratio } & 1.09028 \\ \text { Std Err Dif } & 0.04293 \text { DF } & 23.81102 \\ \text { Upper CL Dif } & 0.13545 \text { Prob }>|t| & 0.2865 \\ \text { Lower CL Dif } & -0.04183 \text { Prob }>\text { t } & 0.1433 \\ \text { Confidence } & 0.95 \text { Prob }<\mathrm{t} & 0.8567\end{array}$

Tests that the Variances are Equal

Level Count Std Dev MeanAbsDif to Mean MeanAbsDif to Median

$\begin{array}{lrrrr}\text { Coliwasa } & 16 & 0.1309895 & 0.0952260 & 0.0952260\end{array}$

$\begin{array}{lllll}\text { Isolok - } 12 \mathrm{~mL} \text { - LF } & 10 & 0.0877847 & 0.0697248 & 0.0697248\end{array}$

Test F Ratio DFNum DFDen p-Value

$\begin{array}{lllll}\text { O'Brien[.5] } & 1.0951 & 1 & 24 & 0.3058\end{array}$

$\begin{array}{lllll}\text { Brown-Forsythe } & 0.7020 & 1 & 24 & 0.4104 \\ & 0.7220 & 1 & 24 & 0.4039\end{array}$

$\begin{array}{lllll}\text { Levene } & 0.7220 & 1 & 24 & 0.4039 \\ \text { Batlett } & 1.5789 & 1 & & 0.2089\end{array}$

$\begin{array}{lllll}\text { F Test 2-sided } & 2.2266 & 15 & 9 & 0.2270\end{array}$

Welch Anova testing Means Equal, allowing Std Devs Not Equal

F Ratio DFNum DFDen Prob $>$ F

$\begin{array}{rrrr}1.1887 & 1 & 23.811 & 0.2865\end{array}$

t Test

1.0903 


\section{Exhibit B2. Statistical Comparisons for High-Rheology (Phase 2) Simulant Testing}

Oneway Analysis of Measurement ( $w t \%)$ By Sampler Type of Material=SME Simulant, Analytical Block=3, Analyte $=\mathrm{Na2O}(\mathrm{wt} \%)$, Target $(\mathrm{wt} \%)=13.318$

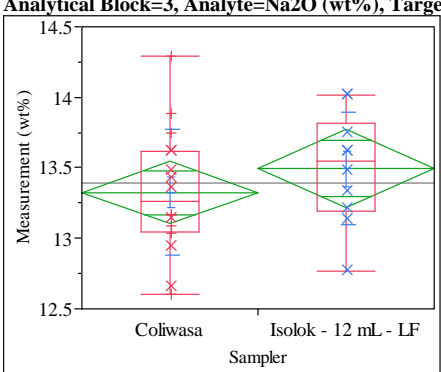

Oneway Anova

Summary of Fit

Adj Rsquare

Root Mean Square Error

Mean of Response

Observations (or Sum Wgts)

0.03766

$-0.0024$

3.39082

t Test

Isolok - $12 \mathrm{~mL}$ - LF-Coliwas

Assuming equal variances

Difference $\quad 0.16682$ t Ratio $\quad 0.969126$

Std Err Dif $\quad 0.17213$ DF 24

Upper CL Dif 0.52207 Prob $>|t| \quad 0.3421$

Lower CL Dif -0.18844 Prob $>t \quad 0.1711$

$\begin{array}{lrl}\text { Confidence } & 0.95 \text { Prob }<\mathrm{t} & 0.8289\end{array}$

Analysis of Variance

Source DF Sum of Squares Mean Square F Ratio Prob $>$ F

$\begin{array}{llllll}\text { Sampler } & 1 & 0.1712446 & 0.171245 & 0.9392 & 0.3421\end{array}$

$\begin{array}{llll}\text { Error } \quad 24 & 4.3759044 & 0.182329\end{array}$

C. Total $25 \quad 4.5471490$

Means for Oneway Anova

Level Number Mean Std Error Lower 95\% Upper 95\%

$\begin{array}{lrrrrr}\text { Coliwasa } & 16 & 13.3267 & 0.10675 & 13.106 & 13.547\end{array}$

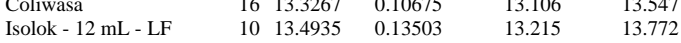

Std Error uses a pooled estimate of error variance

Means and Std Deviations

Level Number Mean Std Dev Std Err Mean Lower 95\% Upper 95\%

$\begin{array}{llrrrrr}\text { Coliwasa } & 16 & 13.3267 & 0.444169 & 0.11104 & 13.090 & 13.563\end{array}$

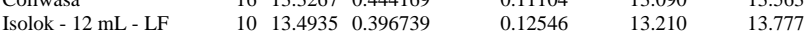

Test

Isolok - $12 \mathrm{~mL}$ - LF-Coliwas

Assuming unequal variances

Difference $\quad 0.16682$ t Ratio 0.995657

Std Err Dif $\quad 0.16754$ DF $\quad 20.92067$

Upper CL Dif 0.51532 Prob $>|t| 0.3308$

Lower CL Dif -0.18169 Prob $>t \quad 0.1654$

$\begin{array}{lrl}\text { Confidence } & 0.95 \text { Prob }<\mathrm{t} & 0.8346\end{array}$

Tests that the Variances are Equal

Level Count Std Dev MeanAbsDif to Mean MeanAbsDif to Median

$\begin{array}{lllll}\text { Coliwasa } & 16 & 0.4441689 & 0.3504800 & 0.3504800 \\ \text { Isolok - 12 mL - LF } & 10 & 0.3967387 & 0.3100400 & 0.3100400\end{array}$

Test F Ratio DFNum DFDen p-Value

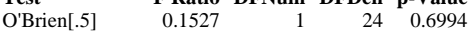

Brown-Forsythe $0.1568 \quad 1 \quad 24 \quad 0.6956$

$\begin{array}{lllll}\text { Levene } & 0.1667 & 1 & 24 & 0.6867\end{array}$

$\begin{array}{lrrrr}\text { Bartlett } & 0.1344 & 1 & \text {. } & 0.7139 \\ \text { F Test 2-sided } & 1.2534 & 15 & 9 & 0.7515\end{array}$

Welch Anova testing Means Equal, allowing Std Devs Not Equal

F Ratio DFNum DFDen Prob $>$ F

$\begin{array}{llll}0.9913 & 1 & 20.921 & 0.3308\end{array}$

t Test

$$
120.921 \quad 0.3308
$$

0.9957
Oneway Analysis of Measurement (wt \%) By Sampler Type of Material=SME Simulant, Analytical Block $=3$, Analyte $=\mathrm{NiO}(\mathrm{wt} \%)$, Target $(\mathrm{wt} \%)=1.033$

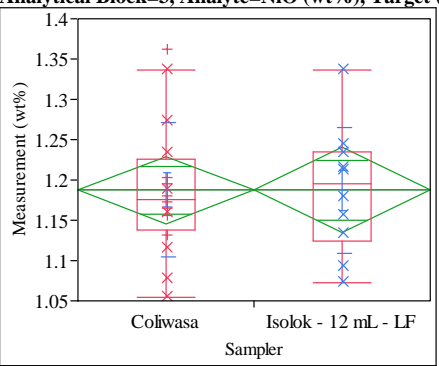

Oneway Anova

Summary of Fit

Adj Rsquare

$-0.04166$

ror $\quad 0.080994$

1.187634

Observations (or Sum Wgts) $\quad 26$

Test

Isolok - $12 \mathrm{~mL}$ - LF-Coliwasa

Assuming equal variances

Difference $\quad-0.00022$ t Ratio $\quad-0.00682$

Std Err Dif $\quad 0.03265$ DF 24

Upper CL Dif 0.06716 Prob $>|t| \quad 0.9946$

Lower CL Dif -0.06761 Prob $>t \quad 0.5027$

$\begin{array}{lrl}\text { Confidence } & 0.95 \text { Prob }<\mathrm{t} \quad 0.4973\end{array}$

Analysis of Variance

Source DF Sum of Squares Mean Square F Ratio Prob $>$ F

$\begin{array}{llllll}\text { Sampler } & 1 & 0.00000031 & 3.052 \mathrm{e}-7 & 0.0000 & 0.9946\end{array}$

$\begin{array}{llll}\text { Error } & 24 & 0.15743923 & 0.006560\end{array}$

C. Total $25 \quad 0.15743954$

Means for Oneway Anova

Level Number Mean Std Error Lower 95\% Upper 95\%

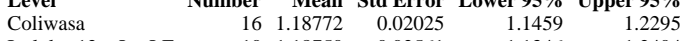

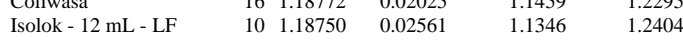

Std Error uses a pooled estimate of error variance

Means and Std Deviations

Level Number Mean Std Dev Std Err Mean Lower 95\% Upper 95\%

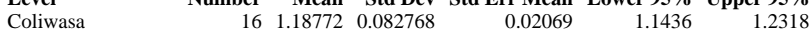

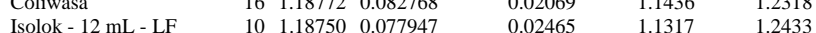

Test

Isolok - $12 \mathrm{~mL}$ - LF-Coliwas

Assuming unequal variances

$\begin{array}{lrlr}\text { Difference } & -0.00022 & \text { t Ratio } & -0.00692 \\ \text { Std Err Dif } & 0.03218 & \text { DF } & 20.15007 \\ \text { Upper CL Dif } & 0.06688 & \text { Prob }>|t| & 0.9945 \\ \text { Lower CL Dif } & -0.06732 & \text { Prob }>\text { t } & 0.5027 \\ \text { Confidence } & 0.95 & \text { Prob }<\mathrm{t} & 0.4973\end{array}$

Tests that the Variances are Equal

Level Count Std Dev MeanAbsDif to Mean MeanAbsDif to Median

$\begin{array}{lllll}\text { Coliwasa } & 16 & 0.0827678 & 0.0596087 & 0.058694\end{array}$

Isolok - $12 \mathrm{~mL}$ - LF $\quad 10 \quad 0.0779470 \quad 0.0605710 \quad 0.0605710$

Test F Ratio DFNum DFDen p-Value

$\begin{array}{lrrrr}\text { O'Brien[.5] } & 0.0397 & 1 & 24 & 0.8438\end{array}$

Brown-Forsythe $\begin{array}{llll}0.0076 & 1 & 24 & 0.9312\end{array}$

$\begin{array}{lllll}\text { Levene } & 0.0021 & 1 & 24 & 0.9635\end{array}$

$\begin{array}{lrrrr}\text { Bartlett } & 0.0383 & 1 & \text {. } & 0.8447 \\ \text { F Test 2-sided } & 1.1275 & 15 & 9 & 0.8841\end{array}$

Welch Anova testing Means Equal, allowing Std Devs Not Equal

F Ratio DFNum DFDen Prob $>$ F

$0.0000 \quad 1 \quad 20.15 \quad 0.9945$

t Test

0.0069 


\section{Exhibit B2. Statistical Comparisons for High-Rheology (Phase 2) Simulant Testing}

Oneway Analysis of Measurement (wt\%) By Sampler Type of Material=SME Simulant, Analytical Block $=3$, Analyte $=\mathrm{SiO} 2(w t \%)$, Target $(w t \%)=50.183$

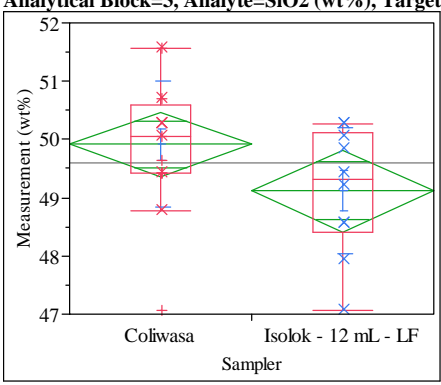

Oneway Anova

Summary of Fit

Rsquare

Adj Rsquare

0.121748

\begin{tabular}{l}
0.08015 \\
\hline
\end{tabular}

$\begin{array}{lr}\text { Mean of Response } & 49.60708 \\ \text { Observations (or Sum Wgts) } & 26\end{array}$

t Test

Isolok - $12 \mathrm{~mL}$ - LF-Coliwasa

Assuming equal variances

Difference $\quad-0.7942$ t Ratio $\quad-1.82401$

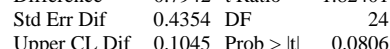

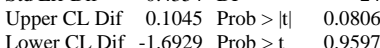

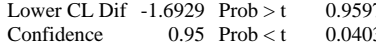

Analysis of Variance

Source DF Sum of Squares Mean Square F Ratio Prob $>$ F

$\begin{array}{lrrrrr}\text { Sampler } & 1 & 3.881709 & 3.88171 & 3.3270 & 0.0806\end{array}$

Error $24 \quad 28.001382$

1.16672

Means for Oneway Anova

Level Number Mean Std Error Lower 95\% Upper 95\%

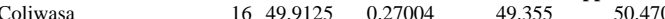

$\begin{array}{llllll}\text { Coliwasa } & 16 & 49.9125 & 0.27004 & 49.355 & 50.470 \\ \text { Isolok - 12 mL - LF } & 10 & 49.1183 & 0.34157 & 48.413 & 49.823\end{array}$

Std Error uses a pooled estimate of error variance

Means and Std Deviations

Level $\quad$ Number Mean Std Dev Std Err Mean Lower 95\% Upper 95\%

$\begin{array}{lrrrrrr}\text { Coliwasa } & 16 & 49.9125 & 1.08162 & 0.27040 & 49.336 & 50.489 \\ \text { Isolok - 12 mL - LF } & 10 & 49.1183 & 1.07770 & 0.34080 & 48.347 & 49.889\end{array}$

t Test

Isolok - $12 \mathrm{~mL}$ - LF-Coliwasa

Assuming unequal variances

Difference $\quad-0.7942$ t Ratio -1.8256

Std Err Dif $\quad 0.4350$ DF 19.30755

Upper CL Dif 0.1154 Prob $>|t| \quad 0.0834$

Lower CL Dif -1.7038 Prob $>$ t 0.9583

$\begin{array}{lll}\text { Confidence } \quad 0.95 \text { Prob }<\mathrm{t} \quad 0.0417 & \end{array}$

Tests that the Variances are Equal

$\begin{array}{lrrrr}\text { Level } & \text { Count } & \text { Std Dev } & \text { MeanAbsDif to Mean } & \text { MeanAbsDif to Median } \\ \text { Coliwasa } & 16 & 1.081617 & 0.7805102 & 0.7621256 \\ \text { Isolok - 12 mL - LF } & 10 & 1.077701 & 0.8728344 & 0.8557200\end{array}$

Test F Ratio DFNum DFDen p-Value

$\begin{array}{lllll}\text { O'Brien[.5] } & 0.0001 & 1 & 24 & 0.9918\end{array}$

$\begin{array}{lllll}\text { Brown-Forsythe } & 0.1069 & 1 & 24 & 0.7465\end{array}$

$\begin{array}{llll}0.1069 & 1 & 24 & 0.7465\end{array}$

$\begin{array}{lllrr}\text { Levene } & 0.1184 & 1 & 24 & 0.7338 \\ \text { Bartlett } & 0.0001 & 1 & . & 0.9905\end{array}$

$\begin{array}{lllll}\text { F Test 2-sided } & 1.0073 & 15 & 9 & 1.0000\end{array}$

Welch Anova testing Means Equal, allowing Std Devs Not Equal

F Ratio DFNum DFDen Prob > F

$\begin{array}{llll}3.3328 & 1 & 19.308 & 0.0834\end{array}$

t Test

1.8256
Oneway Analysis of Measurement (wt \%) By Sampler Type of Material=SME Simulant, Analytical Block $=3$, Analyte $=$ Sum of Oxides $(w t \%)$, Target $(w t \%)=99.73$

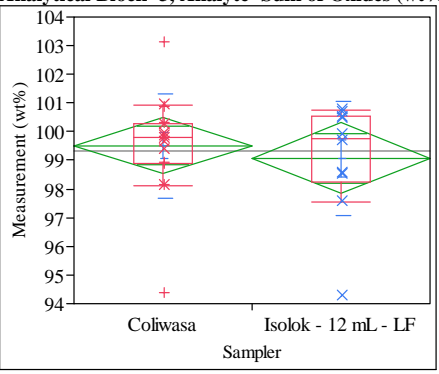

Oneway Anova

Summary of Fit

Adj Rsquare $\quad-0.02685$

Root Mean Square Error $\quad 1.891876$

Mean of Response 99.34463

t Test

Isolok - $12 \mathrm{~mL}$ - LF-Coliwasa

Assuming equal variances
Difference $\quad-0.4488$ t Ratio $\quad-0.5885$

$\begin{array}{lrlr}\text { Std Err Dif } & 0.7626 & \text { DF } & 24 \\ \text { Upper CL Dif } & 1.1252 & \text { Prob }>|t| & 0.5617\end{array}$

$\begin{array}{llll}\text { Lower CL Dif } & -2.0228 & \text { Prob }>t & 0.7192\end{array}$

$\begin{array}{lll}\text { Confidence } & 0.95 \text { Prob }<\mathrm{t} \quad 0.2808\end{array}$

Analysis of Variance

Source DF Sum of Squares Mean Square F Ratio Prob $>$ F

$\begin{array}{lrrrrr}\text { Sampler } & 1 & 1.239579 & 1.23958 & 0.3463 & 0.5617\end{array}$

$\begin{array}{lrrr}\text { Eampler } & 1 & 1.239579 & 1.23958 \\ \text { Error } & 24 & 85.900653 & 3.57919\end{array}$

$\begin{array}{lll}\text { C. Total } & 25 & 87.140232\end{array}$

Means for Oneway Anova

Level Number Mean Std Error Lower 95\% Upper 95\%

$\begin{array}{llllll}\text { Coliwasa } & 16 & 99.5172 & 0.47297 & 98.541 & 100.49 \\ \text { Isolok - 12 mL - LF } & 10 & 99.0684 & 0.59826 & 97.834 & 100.30\end{array}$

Std Error uses a pooled estimate of error variance

Means and Std Deviations

Mevel Number Mean Std Dev Std Err Mean Lower 95\% Upper 95\%

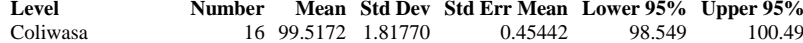

$\begin{array}{lllllll}\text { Isolok - 12 mL - LF } & 16 & 99.5172 & 1.81770 & 0.45442 & 98.549 & 100.49 \\ & 10 & 99.0684 & 2.00943 & 0.63544 & 97.631 & 100.51\end{array}$

t Test

Isolok - $12 \mathrm{~mL}$ - LF-Coliwasa

Assuming unequal variances

$\begin{array}{lrlr}\text { Difference } & -0.4488 & \mathrm{t} \text { Ratio } & -0.57451 \\ \text { Std Err Dif } & 0.7812 \text { DF } & 17.77079 \\ \text { Upper CL Dif } & 1.1940 \text { Prob }>|\mathrm{t}| & 0.5728 \\ \text { Lower CL Dif } & -2.0916 & \text { Prob }>\mathrm{t} & 0.7136 \\ \text { Confidence } & 0.95 & \text { Prob }<\mathrm{t} & 0.2864\end{array}$

Tests that the Variances are Equal

Level Count Std Dev MeanAbsDif to Mean MeanAbsDif to Median

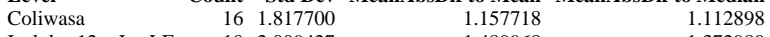

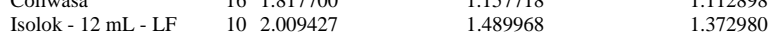

Test F Ratio DFNum DFDen p-Value

$\begin{array}{lllll}\text { O'Brien[.5] } & 0.0531 & 1 & 24 & 0.8197\end{array}$

$\begin{array}{lllll}\text { Brown-Forsythe } & 0.1879 & 1 & 24 & 0.6685\end{array}$

$\begin{array}{lllll}\text { Levene } & 0.3858 & 1 & 24 & 0.5404 \\ & 0.1099 & 1 & & 0.7403\end{array}$

$\begin{array}{lllll}\text { F Test 2-sided } & 1.2221 & 9 & 15 & 0.7025\end{array}$

Welch Anova testing Means Equal, allowing Std Devs Not Equal

F Ratio DFNum DFDen Prob $>$ F

$\begin{array}{lrrr}0.3301 & 1 & 17.771 & 0.5728\end{array}$

t Test 
SRNL-STI-2011-00693

Revision 0

\section{Exhibit B2. Statistical Comparisons for High-Rheology (Phase 2) Simulant Testing}

Oneway Analysis of Measurement (wt \%) By Sampler Type of Material=SME Simulant, Analytical Block=3, Analyte $=$ TiO2 $(w t \%)$, Target $(w t \%)=0.013$

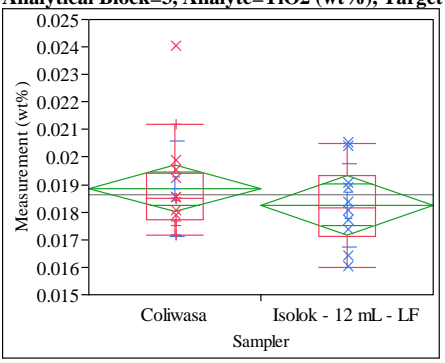

Oneway Anova

Summary of Fit

Rsquare

Adj Rsquare

Root Mean Square Error $\quad 0.001646$

Mean of Response $\quad 0.018625$

Observations (or Sum Wgts)

t Test

Isolok - $12 \mathrm{~mL}$ - LF-Coliwas

Assuming equal variances

Difference $\quad-0.00061$ t Ratio -0.9184

$\begin{array}{lrr}\text { Std Err Dif } & 0.00066 \text { DF } & 24 \\ \text { Upper CL Dif } & 0.00076 \text { Prob }>|t| & 0.3675\end{array}$

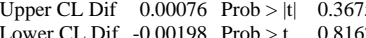

$\begin{array}{lrlll} & \text { Lower CL Dif } & -0.00198 & \text { Prob }>t & 0.8162 \\ \text { Confidence } & 0.95 & \text { Prob }<\mathrm{t} & 0.1838\end{array}$

Analysis of Variance

Source DF Sum of Squares Mean Square F Ratio Prob > F

$\begin{array}{lrrrrr}\text { Sampler } & 1 & 0.00000228 & 2.2841 \mathrm{e}-6 & 0.8435 & 0.3675\end{array}$

$\begin{array}{lllr}\text { Error } & 24 & 0.00006499 & 2.708 \mathrm{e}-6\end{array}$

C. Total $25 \quad 0.00006728$

Means for Oneway Anova

Level Number Mean Std Error Lower 95\% Upper 95\%

$\begin{array}{lrrrrr}\text { Coliwasa } & 16 & 0.018859 & 0.00041 & 0.01801 & 0.01971\end{array}$

$\begin{array}{llllll}\text { Isolok - } 12 \mathrm{~mL} \text { - LF } & 10 & 0.018250 & 0.00052 & 0.01718 & 0.01932\end{array}$

Std Error uses a pooled estimate of error variance

Means and Std Deviations

Level Number Mean Std Dev Std Err Mean Lower 95\% Upper 95\%

$\begin{array}{lllllll} & 16 & 0.018859 & 0.001726 & 0.00043 & 0.01794 & 0.01978\end{array}$

t Test

Isolok - $12 \mathrm{~mL}$ - LF-Coliwas

Assuming unequal variances

Difference $\quad-0.00061$ t Ratio -0.94955

Std Err Dif $\quad 0.00064$ DF 21.29685

Upper CL Dif 0.00072 Prob $>|t| \quad 0.3530$

Lower CL Dif -0.00194 Prob $>t \quad 0.8235$

$\begin{array}{lll}\text { Confidence } & 0.95 \text { Prob }<\mathrm{t} \quad 0.1765\end{array}$

Tests that the Variances are Equal

$\begin{array}{lrrrr}\text { Level } & \text { Count } & \text { Std Dev } & \text { MeanAbsDif to Mean } & \text { MeanAbsDif to Median } \\ \text { Coliwasa } & 16 & 0.0017264 & 0.0011819\end{array}$

$\begin{array}{lllll} & 16 & 0.0017264 & 0.0011819 & 0.0010529 \\ \text { Isolok - } 12 \mathrm{~mL} \text { - LF } & 10 & 0.0015013 & 0.0011659 & 0.0011659\end{array}$

Test F Ratio DFNum DFDen p-Value

$\begin{array}{lrrrr}\text { Test } & \text { F Ratio } & \text { DFNum } & \text { DFDen } & \text { p-Value } \\ \text { O'Brien[.5] } & 0.0929 & 1 & 24 & 0.7631 \\ \text { Brown-Forsythe } & 0.0530 & 1 & 24 & 0.8199 \\ \text { Levene } & 0.0013 & 1 & 24 & 0.9715 \\ \text { Bartlett } & 0.2048 & 1 & . & 0.6509 \\ \text { F Test 2-sided } & 1.3225 & 15 & 9 & 0.6872\end{array}$

Welch Anova testing Means Equal, allowing Std Devs Not Equal

F Ratio DFNum DFDen Prob $>$ F

$\begin{array}{llll}0.9016 & 1 & 21.297 & 0.3530\end{array}$

t Test

0.9495
Oneway Analysis of Measurement (wt \%) By Sampler Type of Material=SME Simulant, Analytical Block $=3$, Analyte $=\mathrm{ZnO}(\mathrm{wt} \%)$, Target $(\mathrm{wt} \%)=0$

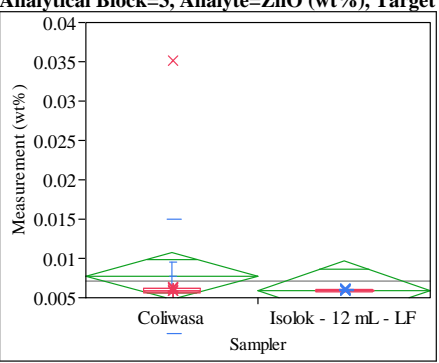

Oneway Anova

Summary of Fit

Rsquare

Rsquare

Root Mean Square Error $\quad 0.00574$

Mean of Response 0.007054

Observations (or Sum Wgts)

Test

Isolok - $12 \mathrm{~mL}$ - LF-Coliwasa

Assuming equal variances

Difference $\quad-0.00185$ t Ratio $\quad-0.80028$

$\begin{array}{lrlr}\text { Std Err Dif } & 0.00231 & \text { DF } & 24 \\ \text { Upper CL } & 0.00292 & \text { Prob }>|t| & 0.4314\end{array}$

$\begin{array}{lrll}\text { Upper CL Dif } 0.00292 & \text { Prob }>|t| & 0.4314 \\ \text { Lower CL Dif }-0.00663 & \text { Prob }>t & 0.7843\end{array}$

Confidence $\quad 0.95$ Prob $<\mathrm{t} \quad 0.2157$

Analysis of Variance

Source DF Sum of Squares Mean Square F Ratio Prob $>$ F

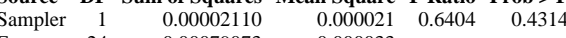

Error $24 \quad 0.00079073 \quad 0.000033$

C. Total $25 \quad 0.00081183$

Means for Oneway Anova

Level Number Mean Std Error Lower 95\% Upper 95\%

$\begin{array}{lrrrrr}\text { Level } & \text { Number } & \text { Mean } & \text { Std Error } & \text { Lower 95\% } & \text { Upper 95\% } \\ \text { Coliwasa } & 16 & 0.007766 & 0.00143 & 0.00480 & 0.01073\end{array}$

$\begin{array}{llllll}\text { Colwa - } 12 \mathrm{~mL} \text { - LF } & 16 & 0.007766 & 0.00143 & 0.00480 & 0.01073 \\ & 10 & 0.005915 & 0.00182 & 0.00217 & 0.00966\end{array}$

Std Error uses a pooled estimate of error variance

Means and Std Deviations

Level Number Mean Std Dev Std Err Mean Lower 95\% Upper 95\%

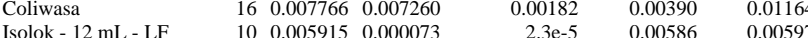

\section{t Test}

Isolok - $12 \mathrm{~mL}$ - LF-Coliwasa

Assuming unequal variances

$\begin{array}{lrlr}\text { Difference } & -0.00185 & \text { t Ratio } & -1.0201 \\ \text { Std Err Dif } & 0.00182 & \text { DF } & 15.00481 \\ \text { Upper CL Dif } & 0.00202 & \text { Prob }>|t| & 0.3238 \\ \text { Lower CL Dif } & -0.00572 & \text { Prob }>\text { t } & 0.8381 \\ \text { Confidence } & 0.95 & \text { Prob }<\mathrm{t} & 0.1619\end{array}$

Tests that the Variances are Equal

Level Count Std Dev MeanAbsDif to Mean MeanAbsDif to Median

$\begin{array}{lllll}\text { Coliwasa } & 16 & 0.0072603 & 0.0034016 & 0.0020084 \\ \text { Isolok - 12 mL - LF } & 10 & 0.0000726 & 0.0000575 & 0.0000566\end{array}$

Test $\quad$ Ratio DFNum DFDen p-Value

Test $\quad$ F Ratio DFNum DFDen p-Value

$\begin{array}{lllll}\text { O'Brien[.5] } & 0.6598 & 1 & 24 & 0.4246 \\ \text { Brown-Forsythe } & 0.7218 & 1 & 24 & 0.4040\end{array}$

$\begin{array}{lllll}\text { Brown-Forsythe } & 0.7218 & 1 & 24 & 0.4040 \\ \text { Levene } & 2.7274 & 1 & 24 & 0.1117\end{array}$

$\begin{array}{lrrrr}\text { Levene } & 2.7274 & 1 & 24 & 0.1117 \\ \text { Bartlett } & 68.4961 & 1 & . & <.0001\end{array}$

$\begin{array}{lrrrr}\text { F Test 2-sided } & 9988.2410 & 15 & \dot{9} & <.0001\end{array}$

Welch Anova testing Means Equal, allowing Std Devs Not Equal

F Ratio DFNum DFDen Prob $>$ F

$\begin{array}{llll}1.0406 & 1 & 15.005 & 0.3238\end{array}$

t Test

1.0201 


\section{Exhibit B2. Statistical Comparisons for High-Rheology (Phase 2) Simulant Testing}

Oneway Analysis of Measurement (wt \%) By Sampler Type of Material=SME Simulant, Analytical Block=3, Analyte $=\mathrm{ZrO} 2(\mathrm{wt} \%)$, Target $(\mathrm{wt} \%)=0.111$

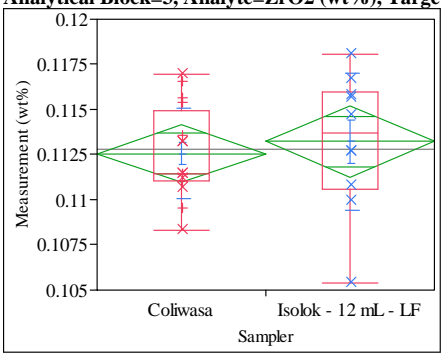

Oneway Anova

Summary of Fit

$-0.02933$

Root Mean Square Error $\quad 0.003053$

Mean of Response 0.112818

Observations (or Sum Wgts) 26

t Test

Isolok - $12 \mathrm{~mL}$ - LF-Coliwasa

Assuming equal variances

Difference $\quad 0.00066$ t Ratio $\quad 0.536373$

$\begin{array}{llll}\text { Std Err Dif } \quad 0.00123 \text { DF } & 24\end{array}$

Upper CL Dif 0.00320 Prob $>|t| \quad 0.5966$

Lower CL Dif -0.00188 Prob $>t \quad 0.2983$
C

$\begin{array}{lrl}\text { Confidence } & 0.95 \text { Prob }<\mathrm{t} \quad 0.7017\end{array}$

Analysis of Variance

Source DF Sum of Squares Mean Square F Ratio Prob $>$ F

$\begin{array}{lrrrrr}\text { Source } & \text { DF } & \text { Sum of Squares } & \text { Mean Square } & \text { F Ratio } & \text { Prob }>\text { F } \\ \text { Sampler } & 1 & 0.00000268 & 2.6823 \mathrm{e}-6 & 0.2877 & 0.5966\end{array}$

$\begin{array}{llll}\text { Error } & 24 & 0.00022376 & 9.3233 \mathrm{e}-6\end{array}$

C. Total $25 \quad 0.00022644$

Means for Oneway Anova

$\begin{array}{lrrrrr}\text { Level } & \text { Number } & \text { Mean } & \text { Std Error } & \text { Lower 95\% } & \text { Upper 95\% } \\ \text { Coliwasa } & 16 & 0.112564 & 0.00076 & 0.11099 & 0.11414\end{array}$

$\begin{array}{llllll}\text { Isolok - } 12 \mathrm{~mL} \text { - LF } & 10 & 0.113224 & 0.00097 & 0.11123 & 0.11522\end{array}$

Std Error uses a pooled estimate of error variance

Means and Std Deviations

Level Number Mean Std Dev Std Err Mean Lower 95\% Upper 95\%

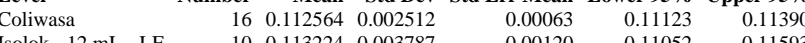

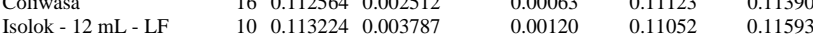

t Test

Isolok - $12 \mathrm{~mL}$ - LF-Coliwasa

Assuming unequal variances

Difference $\quad 0.00066$ t Ratio $\quad 0.488218$

Std Err Dif $\quad 0.00135$ DF 13.99756

Upper CL Dif 0.00356 Prob $>|t| \quad 0.6330$

Lower CL Dif -0.00224 Prob $>t \quad 0.3165$

$\begin{array}{lll}\text { Confidence } & 0.95 \text { Prob }<\mathrm{t} \quad 0.6835\end{array}$

Tests that the Variances are Equal

Level Count Std Dev MeanAbsDif to Mean MeanAbsDif to Median $\begin{array}{lrrrr}\text { Coliwasa } & 16 & 0.0025125 & 0.0020737 & 0.0019333\end{array}$

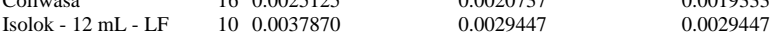

$\begin{array}{lrrrr}\text { Test } & \text { F Ratio } & \text { DFNum } & \text { DFDen } & \text { p-Value } \\ \text { O'Brien[.5] } & 1.8673 & 1 & 24 & 0.1845 \\ \text { Brown-Forsythe } & 1.5200 & 1 & 24 & 0.2296 \\ \text { Levene } & 1.6421 & 1 & 24 & 0.2123 \\ \text { Bartlett } & 1.8883 & 1 & . & 0.1694 \\ \text { F Test 2-sided } & 2.2718 & 9 & 15 & 0.1540\end{array}$

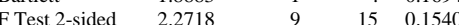

Welch Anova testing Means Equal, allowing Std Devs Not Equal

$\begin{array}{rrrr}\text { F Ratio } & \text { DFNum } & \text { DFDen } & \text { Prob }>\text { F } \\ 0.2384 & 1 & 13.998 & 0.6330\end{array}$

t Test

0.4882
Oneway Analysis of Measurement (wt\%) By Sampler Type of Material=SME Simulant, Analytical Block $=4$, Analyte $=$ Al $/ B$, Target $($ wt $\%)=2.0704412$

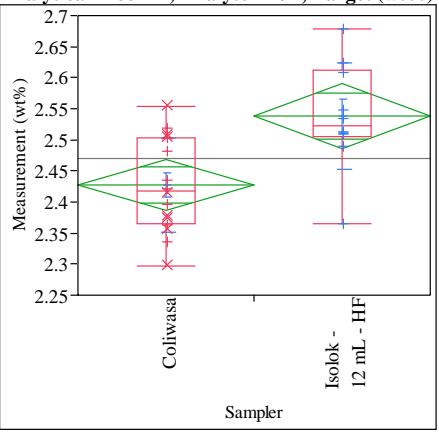

Oneway Anova

Summary of Fit

Adj Rsquare

0.329387

Root Mean Square Error $\quad 0.301445$

Mean of Response

Observations (or Sum Wgts)

Isolok - $12 \mathrm{~mL}$ - HF-Coliwasa

Assuming equal variances

Difference $\quad 0.110838$ t Ratio 3.433389

Std Err Dif $\quad 0.032282$ DF $\quad 24$

Upper CL Dif 0.177465 Prob $>|t| \quad 0.0022$

Lower CL Dif 0.044210 Prob $>t \quad 0.0011$

$\begin{array}{lrl}\text { Confidence } & 0.95 \text { Prob }<\mathrm{t} & 0.9989\end{array}$

Analysis of Variance

Source DF Sum of Squares Mean Square F Ratio Prob $>$ F

$\begin{array}{lllllll}\text { Sampler } & 1 & 0.07559991 & 0.075600 & 11.7882 & 0.0022\end{array}$

$\begin{array}{llll}\text { Error } & 24 & 0.15391698 & 0.006413\end{array}$

C. Total $25 \quad 0.22951689$

Means for Oneway Anova

$\begin{array}{lllll}\text { Level } & \text { Number } & \text { Mean Std Error Lower 95\% Upper 95\% }\end{array}$

$\begin{array}{lrrrrr}\text { Coliwasa } & 16 & 2.42725 & 0.02002 & 2.3859 & 2.4686\end{array}$

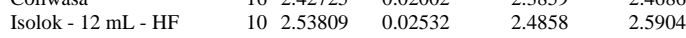

Std Error uses a pooled estimate of error variance

$\begin{array}{llllll}\text { Means and Std Deviations } & & & & \\ \text { Level } & \text { Number } & \text { Mean } & \text { Std Dev Std Err Mean Lower 95\% Upper 95\% }\end{array}$

$\begin{array}{lllllll}\text { Coliwasa } & 16 & 2.42725 & 0.076133 & 0.01903 & 2.3867 & 2.4678 \\ \text { Isolok - 12 mL - HF } & 10 & 2.53809 & 0.086264 & 0.02728 & 2.4764 & 2.5998\end{array}$

Isolok - $12 \mathrm{~mL}$ - HF-Coliwasa

Assuming unequal variances

Difference $\quad 0.110838$ t Ratio 3.332174

Std Err Dif $\quad 0.033263$ DF $\quad 17.41866$

Upper CL Dif 0.180888 Prob $>|t| r .0 .0038$

Lower CL Dif 0.040787 Prob $>$ t 0.0019

$\begin{array}{lrl}\text { Confidence } & 0.95 \text { Prob }<\mathrm{t} & 0.9981\end{array}$

Tests that the Variances are Equal

Level Count Std Dev MeanAbsDif to Mean MeanAbsDif to Median

$\begin{array}{lllll}\text { Coliwasa } & 16 & 0.0761328 & 0.0642810 & 0.0634535\end{array}$

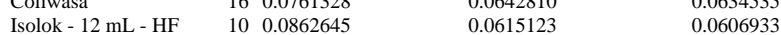

Test F Ratio DFNum DFDen p-Value

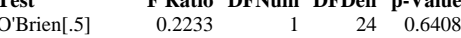

$\begin{array}{lllll}\text { Brown-Forsythe } & 0.0199 & 1 & 24 & 0.8891\end{array}$

$\begin{array}{lllll}\text { Levene } & 0.0227 & 1 & 24 & 0.8816\end{array}$

$\begin{array}{lllll}\text { Bartlett } & 0.1711 & 1 & 0.6791\end{array}$

Welch Anova testing Means Equal, allowing Std Devs Not Equal

F Ratio DFNum DFDen Prob $>$ F

$\begin{array}{lrrr}11.1034 & 1 & 17.419 & 0.0038\end{array}$

t Test

3.3322 


\section{Exhibit B2. Statistical Comparisons for High-Rheology (Phase 2) Simulant Testing}

Oneway Analysis of Measurement (wt \%) By Sampler Type of Material=SME Simulant, Analytical Block $=4$, Analyte $=\mathrm{Al} 2 \mathrm{O} 3(\mathrm{wt} \%)$, Target $(\mathrm{wt} \%)=10.934$

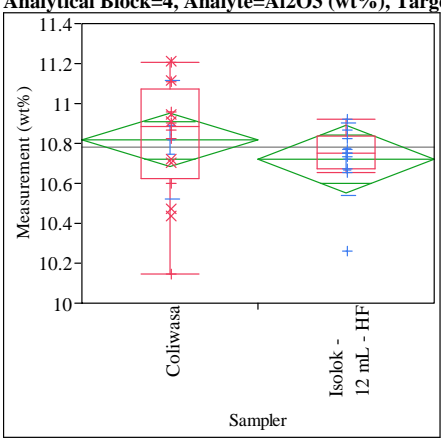

Oneway Anova

Summary of Fit

Adj Rsquare

Root Mean Square Error $\quad 0.259316$

Mean of Response

$\begin{array}{lr} & 10.78032 \\ \text { Observations (or Sum Wgts) } & 26\end{array}$

tTest

Isolok - $12 \mathrm{~mL}$ - HF-Coliwasa

Assuming equal variances

Difference $\quad-0.09636$ t Ratio $\quad-0.92185$

Std Err Dif $\quad 0.10453$ DF 24

Upper CL Dif 0.11938 Prob $>|t| \quad 0.3658$

Lower CL Dif -0.31211 Prob $>t \quad 0.8171$

$\begin{array}{lrl}\text { Confidence } & 0.95 \text { Prob }<\mathrm{t} & 0.1829\end{array}$

Analysis of Variance

Source DF Sum of Squares Mean Square F Ratio Prob $>$ F

$\begin{array}{llllll}\text { Sampler } & 1 & 0.0571453 & 0.057145 & 0.8498 & 0.3658\end{array}$

$\begin{array}{llll}\text { Error } & 24 & 1.6138778 & 0.067245\end{array}$

C. Total $25 \quad 1.6710232$

Means for Oneway Anova

Level Number Mean Std Error Lower 95\% Upper 95\%

$\begin{array}{lrrrrr}\text { Coliwasa } & 16 & 10.8174 & 0.06483 & 10.684 & 10.951\end{array}$

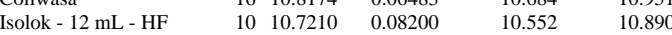

Std Error uses a pooled estimate of error variance

Means and Std Deviations

Level Number Mean Std Dev Std Err Mean Lower 95\% Upper 95\%

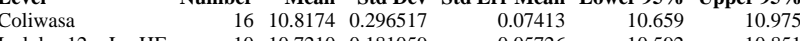

$\begin{array}{lllllll}\text { Colok - } 12 \mathrm{~mL} \text { - HF } & 10 & 10.7210 & 0.181059 & 0.05726 & 10.592 & 10.851\end{array}$

Isolok - $12 \mathrm{~mL}$ - HF-Coliwasa

Assuming unequal variances

$\begin{array}{lrlr}\text { Difference } & -0.09636 & \mathrm{t} \text { Ratio } & -1.02881 \\ \text { Std Err Dif } & 0.09367 & \text { DF } & 23.99982 \\ \text { Upper CL Dif } & 0.09695 & \text { Prob }>|t| & 0.3138 \\ \text { Lower CL Dif } & -0.28968 & \text { Prob }>\text { t } & 0.8431 \\ \text { Confidence } & 0.95 & \text { Prob }<\mathrm{t} & 0.1569\end{array}$

Tests that the Variances are Equal

Level Count Std Dev MeanAbsDif to Mean MeanAbsDif to Median

$\begin{array}{lrrrr}\text { Coliwasa } & 16 & 0.2965171 & 0.2314638 & 0.2243781\end{array}$

$\begin{array}{lllll}\text { Coliwasa } & 16 & 0.2965171 & 0.2314638 & 0.2243781 \\ \text { Isolok - } 12 \mathrm{~mL} \text { - HF } & 10 & 0.1810593 & 0.1141258 & 0.1058120\end{array}$

Test F Ratio DFNum DFDen p-Value

\begin{tabular}{lrrrr} 
O'Brien[.5] & 1.5434 & 1 & 24 & 0.226 \\
\hline
\end{tabular}

$\begin{array}{lllll}\text { Brown-Forsythe } & 2.6773 & 1 & 24 & 0.1148\end{array}$

$\begin{array}{lllll}\text { Levene } & 3.2461 & 1 & 24 & 0.0842\end{array}$

$\begin{array}{lrrrr}\text { Bartlett } & 2.3382 & 1 & \text {. } & 0.1262\end{array}$

Welch Anova testing Means Equal, allowing Std Devs Not Equal

F Ratio DFNum DFDen Prob $>$ F

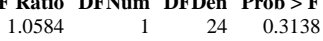

t Test

1.0288
Oneway Analysis of Measurement (wt \%) By Sampler Type of Material=SME Simulant, Analytical Block $=4$, Analyte $=\mathrm{B} 2 \mathrm{O} 3(\mathrm{wt} \%)$, Target $(\mathrm{wt} \%)=5.281$

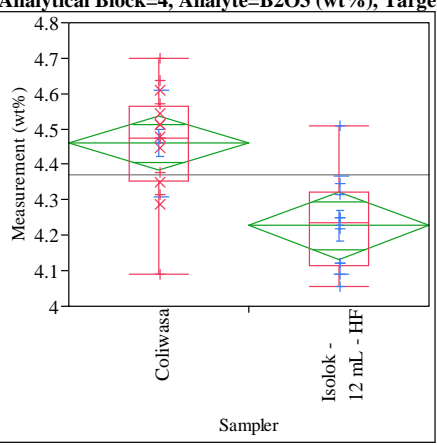

Oneway Anova

Summary of Fit

$\begin{array}{lr}\text { Rsquare } & 0.390417 \\ \text { Adj Rsquare } & 0.365017 \\ \text { Root Mean Square Error } & 0.146688 \\ \text { Mean of Response } & 4.370395 \\ \text { Observations (or Sum Wgts) } & 26\end{array}$

t Test

Isolok - $12 \mathrm{~mL}$ - HF-Coliwasa

Assuming equal variances

Difference $\quad-0.23183$ t Ratio -3.92061

$\begin{array}{lll}\text { Std Err Dif } & 0.05913 \text { DF } & 24\end{array}$

Upper CL Dif -0.10979 Prob $>|t| \quad 0.0006$

$\begin{array}{lll}\text { Lower CL Dif }-0.35388 \text { Prob }>t & 0.9997\end{array}$

Confidence $\quad 0.95$ Prob $<\mathrm{t} \quad 0.0003$

Analysis of Variance

Source DF Sum of Squares Mean Square F Ratio Prob > F

$\begin{array}{lrrrrr}\text { Source } & \text { DF } & \text { Sum of Squares } & \text { Mean Square } & \text { F Ratio } & \text { Prob }>\text { F } \\ \text { Sampler } & 1 & 0.33074737 & 0.330747 & 15.3711 & 0.0006\end{array}$

$\begin{array}{lrrr}\text { Sampler } & 1 & 0.33074737 & 0.330747 \\ \text { Error } & 24 & 0.51641793 & 0.021517\end{array}$

C. Total $25 \quad 0.84716529$

Means for Oneway Anova

Level Number Mean Std Error Lower 95\% Upper 95\%

$\begin{array}{llllll}\text { Isolok - } 12 \mathrm{~mL} \text { - HF } & 16 & 4.45956 & 0.03667 & 4.3839 & 4.5352 \\ & & 4.22773 & 0.04639 & 4.1320 & 4.3235\end{array}$

Std Error uses a pooled estimate of error variance

Means and Std Deviations

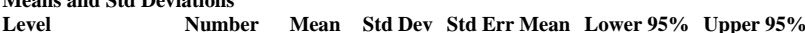

$\begin{array}{lrrrrrr}\text { Coliwasa } & 16 & 4.45956 & 0.151484 & 0.03787 & 4.3788 & 4.5403\end{array}$

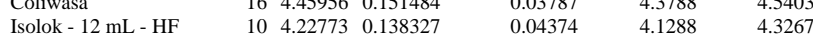

t Test

Isolok - $12 \mathrm{~mL}$ - HF-Coliwasa

Assuming unequal variances

Difference $\quad-0.23183$ t Ratio -4.00688

Std Err Dif $\quad 0.05786$ DF 20.6031

Upper CL Dif -0.11137 Prob $>|t| r 0.0007$

$\begin{array}{lll}\text { Lower CL Dif }-0.35230 & \text { Prob }>t & 0.9997\end{array}$

Confidence $\quad 0.95$ Prob $<\mathrm{t} \quad 0.0003$

Tests that the Variances are Equal

Level Count Std Dev MeanAbsDif to Mean MeanAbsDif to Median

$\begin{array}{lrrrr}\text { Coliwasa } & 16 & 0.1514837 & 0.1126965 & 0.1086716\end{array}$

$\begin{array}{lllll}\text { Isolok - } 12 \mathrm{~mL} \text { - HF } & 10 & 0.1383267 & 0.1062567 & 0.1062567\end{array}$

Test F Ratio DFNum DFDen p-Value

$\begin{array}{lllll}\text { O'Brien[.5] } & 0.0743 & 1 & 24 & 0.7875\end{array}$

$\begin{array}{lllll}\text { Brown-Forsythe } & 0.0039 & 1 & 24 & 0.9505\end{array}$

$\begin{array}{lllll}\text { Levene } & 0.0306 & 1 & 24 & 0.8627\end{array}$

$\begin{array}{lllll}\text { Bartlett } & 0.0874 & 1 & 0.7675\end{array}$

$\begin{array}{lllll}\text { F Test 2-sided } & 1.1993 & 15 & 9 & 0.8060\end{array}$

Welch Anova testing Means Equal, allowing Std Devs Not Equal

$\begin{array}{rrrr}\text { F Ratio } & \text { DFNum } & \text { DFDen } & \text { Prob }>\text { F } \\ 16.0551 & 1 & 20.603 & 0.0007\end{array}$

t Test

4.0069 


\section{Exhibit B2. Statistical Comparisons for High-Rheology (Phase 2) Simulant Testing}

Oneway Analysis of Measurement (wt \%) By Sampler Type of Material=SME Simulant, Analytical Block $=4$, Analyte $=\mathrm{BaO}(\mathrm{wt} \%)$, Target $(\mathrm{wt} \%)=0$

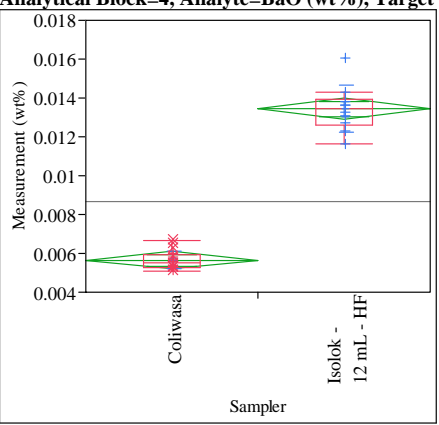

Oneway Anova

Summary of Fit

Rsquare

Adj Rsquare

Root Mean Square Error $\quad 0.955314$

Observations (or Sum Wgts) 26

Isolok - $12 \mathrm{~mL}$ - HF-Coliwasa

Assuming equal variances

Difference $\quad 0.007778$ t Ratio 23.13997

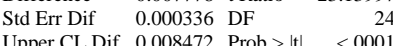

Upper CL Dif 0.008472 Prob $>|t|<\quad<.0001$

$\begin{array}{lrll}\text { Lower CL Dif } & 0.007084 & \text { Prob }>t & <.0001 \\ \text { Confidence } & 0.95 & \text { Prob }<t & 1.0000\end{array}$

Analysis of Variance

Source DF Sum of Squares Mean Square F Ratio Prob $>$ F

$\begin{array}{lrrrrr}\text { Sampler } & 1 & 0.00037227 & 0.000372 & 535.4580 & <.0001\end{array}$

$\begin{array}{llll}\text { Error } & 24 & 0.00001669 & 6.952 \mathrm{e}-7\end{array}$

C. Total $25 \quad 0.00038896$

Means for Oneway Anova

Level Number Mean Std Error Lower 95\% Upper 95\%

$\begin{array}{lllllr}\text { Coliwasa } & 16 & 0.005665 & 0.00021 & 0.00523 & 0.00610\end{array}$

Isolok - $12 \mathrm{~mL}$ - HF $\quad \begin{array}{llllll}10 & 0.013443 & 0.00026 & 0.01290 & 0.01399\end{array}$

Std Error uses a pooled estimate of error variance

Means and Std Deviations

$\begin{array}{llllll}\text { Level } & \text { Number } & \text { Mean } & \text { Std Dev Std Err Mean Lower 95\% Upper 95\% }\end{array}$

$\begin{array}{lrrrrrr}\text { Coliwasa } & 16 & 0.005665 & 0.000477 & 0.00012 & 0.00541 & 0.00592 \\ \text { Isolok - 12 mL - HF } & 10 & 0.013443 & 0.001214 & 0.00038 & 0.01257 & 0.01431\end{array}$

Isolok - $12 \mathrm{~mL}$ - HF-Coliwasa

Assuming unequal variances

Difference $\quad 0.007778$ t Ratio 19.34405

Std Err Dif $\quad 0.000402$ DF 10.76219

Upper CL Dif 0.008665 Prob $>|t|<.0001$

Lower CL Dif 0.006890 Prob $>t \quad<.0001$

$\begin{array}{lll}\text { Confidence } & 0.95 \text { Prob }<\mathrm{t} \quad 1.0000\end{array}$

Tests that the Variances are Equal

$\begin{array}{lrrrr}\text { Level } & \text { Count } & \text { Std Dev } & \text { MeanAbsDif to Mean } & \text { MeanAbsDif to Median } \\ \text { Coliwasa } & 16 & 0.0004773 & 0.0003843 & 0.0003712\end{array}$

$\begin{array}{lllll}\text { Coliwasa } & 16 & 0.0004773 & 0.0003843 & 0.0003712 \\ \text { Isolok - 12 mL - HF } & 10 & 0.0012142 & 0.0008485 & 0.0008485\end{array}$

Test F Ratio DFNum DFDen p-Value

$\begin{array}{lllll}\text { O'Brien[.5] } & 3.6168 & 1 & 24 & 0.0693\end{array}$

$\begin{array}{lllll}\text { O'Brien[.5] } & 3.6168 & 1 & 24 & 0.0693\end{array}$

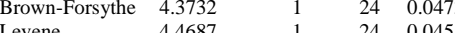

$\begin{array}{lllll}\text { Levene } & 4.4687 & 1 & 24 & 0.0451 \\ \text { Bartlett } & 9.5378 & 1 & . & 0.0020\end{array}$

$\begin{array}{lllll}\text { F Test 2-sided } & 6.4712 & 9 & 15 & 0.0017\end{array}$

Welch Anova testing Means Equal, allowing Std Devs Not Equal

F Ratio DFNum DFDen Prob $>$ F

$\begin{array}{llll}374.1922 & 1 & 10.762 & <.0001\end{array}$

t Test
Oneway Analysis of Measurement (wt\%) By Sampler Type of Material=SME Simulant, Analytical Block $=4$, Analyte $=\mathrm{CaO}(\mathrm{wt} \%)$, Target $(\mathrm{wt} \%)=0.543$

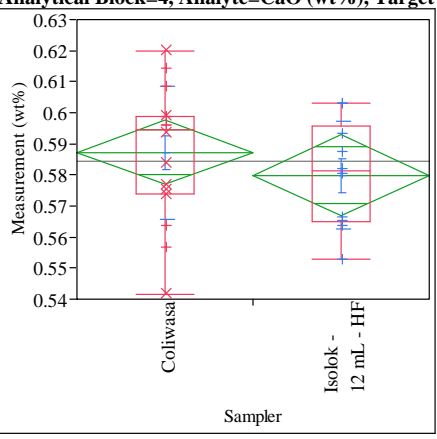

Oneway Anova

Summary of Fit

Adj Rsquare $\quad-0.00626$

Error $\quad 0.019972$

Mean of Response $\quad 0.584381$

Observations (or Sum Wgts) $\quad 26$

Isolok - $12 \mathrm{~mL}$ - HF-Coliwasa

Assuming equal variances

$\begin{array}{lrlr}\text { Difference } & -0.00740 & \text { t Ratio } & -0.91893 \\ \text { Std Err Dif } & 0.00805 & \text { DF } & 24 \\ \text { Upper CL Dif } & 0.00922 & \text { Prob }>|t| & 0.3673 \\ \text { Lower CL Dif } & -0.02401 & \text { Prob }>t & 0.8164 \\ \text { Confidence } & 0.95 & \text { Prob }<\mathrm{t} & 0.1836\end{array}$

Analysis of Variance

Source DF Sum of Squares Mean Square F Ratio Prob $>$ F

$\begin{array}{lllllll}\text { Sampler } & 1 & 0.00033683 & 0.000337 & 0.8444 & 0.3673\end{array}$

$\begin{array}{llll}\text { Error } & 24 & 0.00957313 & 0.000399\end{array}$

C. Total $25 \quad 0.00990996$

Means for Oneway Anova

Level Number Mean Std Error Lower 95\% Upper 95\%

$\begin{array}{lllrrr}\text { Coliwasa } & 16 & 0.587227 & 0.00499 & 0.57692 & 0.59753\end{array}$

Isolok - $12 \mathrm{~mL}$ - HF $\quad 10 \quad 0.579828 \quad 0.00632 \quad 0.56679 \quad 0.59286$

Std Error uses a pooled estimate of error variance

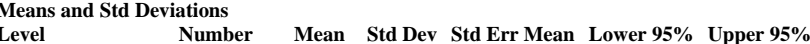

Level Number Mean Std Dev Std Err Mean Lower 95\% Upper 95\%

$\begin{array}{lllllll}\text { Coliwasa } & 16 & 0.587227 & 0.021402 & 0.00535 & 0.57582 & 0.59863 \\ \text { Isolok - 12 mL - HF } & 10 & 0.579828 & 0.017329 & 0.00548 & 0.56743 & 0.59222\end{array}$

Isolok - $12 \mathrm{~mL}$ - HF-Coliwasa

Assuming unequal variances

$\begin{array}{lrlr}\text { Difference } & -0.00740 & \mathrm{t} \text { Ratio } & -0.966 \\ \text { Std Err Dif } & 0.00766 & \text { DF } & 22.22213 \\ \text { Upper CL Dif } & 0.00848 & \text { Prob }>|t| & 0.3444 \\ \text { Lower CL Dif } & -0.02327 & \text { Prob }>\text { t } & 0.8278 \\ \text { Confidence } & 0.95 & \text { Prob }<\mathrm{t} & 0.1722\end{array}$

Tests that the Variances are Equal

Level Count Std Dev MeanAbsDif to Mean MeanAbsDif to Median

$\begin{array}{lrrrr}\text { Coliwasa } & 16 & 0.0214019 & 0.0174572 & 0.0167030\end{array}$

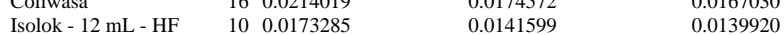

Test F Ratio DFNum DFDen p-Value

$\begin{array}{lrrrr}\text { O'Brien[.5] } & 0.5753 & 1 & 24 & 0.4555\end{array}$

$\begin{array}{lllll}\text { Brown-Forsythe } & 0.2674 & 1 & 24 & 0.6098\end{array}$

$\begin{array}{lllll}\text { Levene } & 0.5965 & 1 & 24 & 0.4475\end{array}$

$\begin{array}{lrrrr}\text { Bartlett } & 0.4602 & 1 & \text {. } & 0.4975 \\ \text { F Test 2-sided } & 1.5254 & 15 & 9 & 0.5294\end{array}$

Welch Anova testing Means Equal, allowing Std Devs Not Equal

F Ratio DFNum DFDen Prob $>$ F

$\begin{array}{rrrr}0.9332 & 1 & 22.222 & 0.3444\end{array}$

t Test

0.9660 
SRNL-STI-2011-00693

Revision 0

\section{Exhibit B2. Statistical Comparisons for High-Rheology (Phase 2) Simulant Testing}

Oneway Analysis of Measurement (wt \%) By Sampler Type of Material=SME Simulant, Analytical Block $=4$, Analyte $=$ Cr2O3 $(w t \%)$, Target $(w t \%)=0.015$

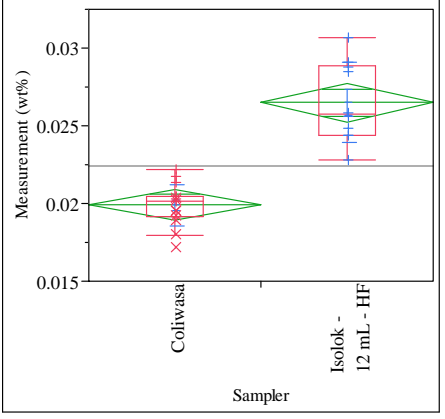

Oneway Anova

Summary of Fit

Rsquare

Adj Rsquare

Root Mean Square Error $\quad 0.001895$

$\begin{array}{lr}\text { Mean of Response } & 0.022436 \\ \text { Observations (or Sum Wgts) } & 26\end{array}$

t Test

Isolok - $12 \mathrm{~mL}$ - HF-Coliwasa

Assuming equal variances

Difference $\quad 0.006603$ t Ratio 8.641907

$\begin{array}{lrr}\text { Std Err Dif } & 0.000764 \text { DF } & 24 \\ \text { Upper CL Dif } & 0.008180 \text { Prob }>|t| & <.0001\end{array}$

Upper CL Dif 0.008180 Prob $>|t|<.0001$

$\begin{array}{lrll}\text { Lower CL Dif } & 0.005026 & \text { Prob }>t & <.0001 \\ \text { Confidence } & 0.95 & \text { Prob }<t & 1.0000\end{array}$

Analysis of Variance

Source DF Sum of Squares Mean Square F Ratio Prob $>$ F

$\begin{array}{lllrlrl}\text { Sampler } & 1 & 0.00026829 & 0.000268 & 74.6826 & <.0001\end{array}$

$\begin{array}{llll}\text { Error } & 24 & 0.00008622 & 3.592 \mathrm{e}-6\end{array}$

C. Total $25 \quad 0.00035450$

Means for Oneway Anova

Level Number Mean Std Error Lower 95\% Upper 95\%

$\begin{array}{llllll}\text { Coliwasa } & 16 & 0.019896 & 0.00047 & 0.01892 & 0.02087\end{array}$

$\begin{array}{llllll}\text { Isolok - } 12 \mathrm{~mL} \text { - HF } & 10 & 0.026499 & 0.00060 & 0.02526 & 0.02774\end{array}$

Std Error uses a pooled estimate of error variance

Means and Std Deviations

Level Number Mean Std Dev Std Err Mean Lower 95\% Upper 95\%

$\begin{array}{lrrrrrr}\text { Coliwasa } & 16 & 0.019896 & 0.001323 & 0.00033 & 0.01919 & 0.02060\end{array}$

t Test

Isolok - $12 \mathrm{~mL}$ - HF-Coliwasa

Assuming unequal variances

Difference $\quad 0.006603$ t Ratio 7.497696

Std Err Dif $\quad 0.000881$ DF 12.00664

Upper CL Dif 0.008521 Prob $>|t|<.0001$

Lower CL Dif 0.004684 Prob $>\mathrm{t} \quad<.0001$

Confidence $\quad 0.95$ Prob $<\mathrm{t} \quad 1.0000$

Tests that the Variances are Equal

$\begin{array}{lrrrr}\text { Level } & \text { Count } & \text { Std Dev } & \text { MeanAbsDif to Mean } & \text { MeanAbsDif to Median } \\ \text { Coliwasa } & 16 & 0.0013234 & 0.0010208 & 0.0009866\end{array}$

$\begin{array}{lrrrr}\text { Coliwasa } & 16 & 0.0013234 & 0.0010208 & 0.0009866 \\ \text { Isolok - 12 mL - HF } & 10 & 0.0025808 & 0.0022158 & 0.0020901\end{array}$

Test F Ratio DFNum DFDen p-Value

$\begin{array}{lrrrr}\text { O'Brien[.5] } & 7.4688 & 1 & 24 & 0.0116\end{array}$

\begin{tabular}{lllll} 
Brown-Forsythe & 5.2647 & 1 & 24 & 0.0116 \\
\hline & 1 & 24 & 0.0308
\end{tabular}

$\begin{array}{llll}\text { Brown-Forsythe } 5.2647 & 1 & 24 & 0.0308\end{array}$

$\begin{array}{lrrrr}\text { Levene } & 10.3145 & 1 & 24 & 0.0037 \\ \text { Bartlett } & 4.9928 & 1 & . & 0.0255\end{array}$

$\begin{array}{lllll}\text { F Test 2-sided } & 3.8031 & 9 & 15 & 0.0222\end{array}$

Welch Anova testing Means Equal, allowing Std Devs Not Equal

F Ratio DFNum DFDen Prob $>$ F

$\begin{array}{llll}56.2154 & 1 & 12.007 & <.0001\end{array}$

t Test
Oneway Analysis of Measurement (wt \%) By Sampler Type of Material=SME Simulant, Analytical Block $=4$, Analyte $=\mathrm{CuO}(\mathrm{wt} \%)$, Target $(\mathrm{wt} \%)=0.103$

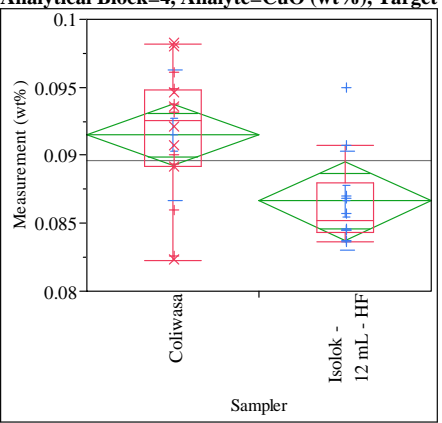

Oneway Anova

Summary of Fit

Rsquare

Adj Rsquare

Root Mean Square Error $\quad 0.004405$

Mean of Response

Observations (or Sum Wgts) $\quad 26$

t Test

Isolok - $12 \mathrm{~mL}$ - HF-Coliwasa

Assuming equal variances

Difference $\quad-0.00486$ t Ratio $\quad-2.73765$

$\begin{array}{lrlr}\text { Std Err Dif } & 0.00178 \text { DF } & 24 \\ \text { Upper CL } & & \end{array}$

$\begin{array}{llll}\text { Upper CL Dif } & -0.00120 & \text { Prob }>|t| & 0.0115 \\ \text { Lower CL Dif }-0.00853 & \text { Prob }>t & 0.9943\end{array}$

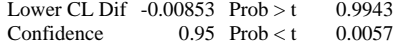

Analysis of Variance

Source DF Sum of Squares Mean Square F Ratio Prob $>$ F

$\begin{array}{lllllr}\text { Sampler } & 1 & 0.00014545 & 0.000145 & 7.4947 & 0.0115\end{array}$

$\begin{array}{lll}\text { Error } & 24 & 0.00046577 \\ \text { C. Total } & 25 & 0.00061123\end{array}$

Means for Oneway Anova

Level Number Mean Std Error Lower 95\% Upper 95\%

$\begin{array}{lrrrrr}\text { Coliwasa } & 16 & 0.091499 & 0.00110 & 0.08923 & 0.09377\end{array}$

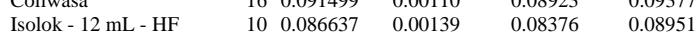

Std Error uses a pooled estimate of error variance

Means and Std Deviations

Level Number Mean Std Dev Std Err Mean Lower 95\% Upper 95\%

$\begin{array}{lllllll}\text { Coliwasa } & 16 & 0.091499 & 0.004810 & 0.00120 & 0.08894 & 0.09406\end{array}$

$\begin{array}{lllllll}\text { Isolok - } 12 \mathrm{~mL} \text { - HF } & 10 & 0.086637 & 0.003633 & 0.00115 & 0.08404 & 0.08924\end{array}$

\section{t Test}

Isolok - $12 \mathrm{~mL}$ - HF-Coliwasa

Assuming unequal variances

$\begin{array}{lrlr}\text { Difference } & -0.00486 & \mathrm{t} \text { Ratio } & -2.9235 \\ \text { Std Err Dif } & 0.00166 & \text { DF } & 22.97679 \\ \text { Upper CL Dif } & -0.00142 & \text { Prob }>|t| & 0.0076 \\ \text { Lower CL Dif } & -0.00830 & \text { Prob }>\mathrm{t} & 0.9962 \\ \text { Confidence } & 0.95 & \text { Prob }<\mathrm{t} & 0.0038\end{array}$

Tests that the Variances are Equal

$\begin{array}{lrrrr}\text { Level } & \text { Count } & \text { Std Dev } & \text { MeanAbsDif to Mean } & \text { MeanAbsDif to Median } \\ \text { Coliwasa } & 16 & 0.0048098 & 0.0038111 & 0.0037476\end{array}$

$\begin{array}{lrrrr}\text { Coliwasa } & 16 & 0.0048098 & 0.0038111 & 0.0037476 \\ \text { Isolok - 12 mL - HF } & 10 & 0.0036326 & 0.0026188 & 0.0024410\end{array}$

Test F Ratio DFNum DFDen p-Value

$\begin{array}{lllll}\text { O'Brien[.5] } & 0.7507 & 1 & 24 & 0.3948\end{array}$

Brown-Forsythe $1.1411 \quad 1124 \quad 0.2960$

Brown-Forsythe $1.1411 \quad 11-24 \quad 0.2960$

$\begin{array}{lllll}\text { Levene } & 1.2742 & 1 & 24 & 0.270 \\ & 0.8005 & 1 & \bullet & 0.3709\end{array}$

$\begin{array}{lrrrr}\text { Bartlett } & 0.8005 & 1 & \dot{0} & 0.3709 \\ \text { F Test 2-sided } & 1.7532 & 15 & 9 & 0.3978\end{array}$

Welch Anova testing Means Equal, allowing Std Devs Not Equal

F Ratio DFNum DFDen Prob $>$ F

$\begin{array}{lll}1 & 22.977 \quad 0.0076\end{array}$

t Test 


\section{Exhibit B2. Statistical Comparisons for High-Rheology (Phase 2) Simulant Testing}

Oneway Analysis of Measurement (wt\%) By Sampler Type of Material=SME Simulant, Analytical Block $=4$, Analyte $=\mathrm{Fe} / \mathrm{Li}$, Target $(\mathrm{wt} \%)=1.99097835$

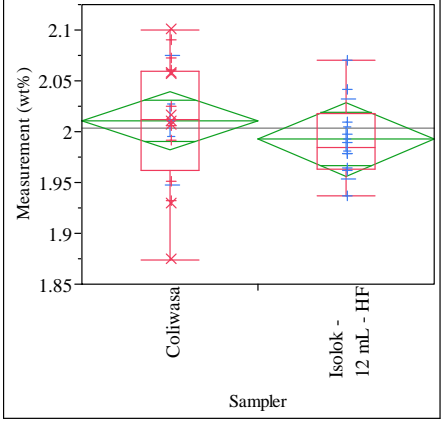

Oneway Anova

Summary of Fit

Adj Rsquare

Root Mean Square Error $\quad 0.055717$

Mean of Response 2.004018

Observations (or Sum Wgts) $\quad 26$

Test

Isolok - $12 \mathrm{~mL}$ - HF-Coliwasa

Assuming equal variances

Difference $\quad-0.01828$ t Ratio $\quad-0.8137$

Std Err Dif $\quad 0.02246$ DF $\quad 24$

Upper CL Dif 0.02808 Prob $>|t| \quad 0.4238$

Lower CL Dif -0.06463 Prob $>t \quad 0.7881$

$\begin{array}{lrl}\text { Confidence } & 0.95 \text { Prob }<\mathrm{t} & 0.2119\end{array}$

Analysis of Variance

Source DF Sum of Squares Mean Square F Ratio Prob $>$ F

$\begin{array}{llllll}\text { Sampler } & 1 & 0.00205547 & 0.002055 & 0.6621 & 0.4238\end{array}$

$\begin{array}{llll}\text { Error } & 24 & 0.07450595 & 0.003104\end{array}$

C. Total $25 \quad 0.07656142$

Means for Oneway Anova

Level Number Mean Std Error Lower 95\% Upper 95\%

$\begin{array}{lrrrrr}\text { Coliwasa } & 16 & 2.01105 & 0.01393 & 1.9823 & 2.0398\end{array}$

$\begin{array}{lllll}\text { Isolok - } 12 \mathrm{~mL} \text { - HF } & 10 & 1.99277 & 0.01762 & 1.9564\end{array}$

Std Error uses a pooled estimate of error variance

Means and Std Deviations

Level Number Mean Std Dev Std Err Mean Lower 95\% Upper 95\%

$\begin{array}{lllllll}\text { Coliwasa } & 16 & 2.01105 & 0.063586 & 0.01590 & 1.9772 & 2.0449 \\ \text { Isolok - 12 mL - HF } & 10 & 1.99277 & 0.039241 & 0.01241 & 1.9647 & 2.0208\end{array}$

Isolok - $12 \mathrm{~mL}$ - HF-Coliwasa

Assuming unequal variances

$\begin{array}{lrlr}\text { Difference } & -0.01828 & \text { t Ratio } & -0.90626 \\ \text { Std Err Dif } & 0.02017 & \text { DF } & 23.99864 \\ \text { Upper CL Dif } & 0.02335 & \text { Prob }>|t| & 0.3738 \\ \text { Lower CL Dif } & -0.05990 & \text { Prob }>\text { t } & 0.8131 \\ \text { Confidence } & 0.95 & \text { Prob }<\mathrm{t} & 0.1869\end{array}$

Tests that the Variances are Equal

Level Count Std Dev MeanAbsDif to Mean MeanAbsDif to Median

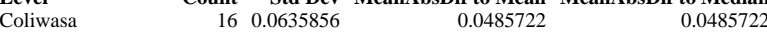

$\begin{array}{lllll}\text { Coliwasa } & 16 & 0.0635856 & 0.0485722 & 0.0485722 \\ \text { Isolok - } 12 \mathrm{~mL} \text { - HF } & 10 & 0.0392414 & 0.0294065 & 0.0287333\end{array}$

Test F Ratio DFNum DFDen p-Value

$\begin{array}{lrrrr}\text { O'Brien[.5] } & 1.9347 & 1 & 24 & 0.1770 \\ \text { Brown-Forsythe } & 1.9839 & 1 & 24 & 0.1718\end{array}$

$\begin{array}{lllll}\text { Brown-Forsythe } & 1.9839 & 1 & 24 & 0.1718 \\ & 1.9299 & 1 & 24 & 0.1775\end{array}$

$\begin{array}{lllll}\text { Levene } & 1.9299 & 1 & 24 & 0.1775 \\ \text { Bartlett } & 2.2453 & 1 & . & 0.1340\end{array}$

$\begin{array}{lllll}\text { F Test 2-sided } & 2.6256 & 15 & 9 & 0.1469\end{array}$

Welch Anova testing Means Equal, allowing Std Devs Not Equal

F Ratio DFNum DFDen Prob > F

$\begin{array}{rrrr}0.8213 & 1 & 23.999 & 0.3738\end{array}$

t Test

0.9063
Oneway Analysis of Measurement (wt\%) By Sampler Type of Material=SME Simulant, Analytical Block=4, Analyte=Fe2O3 (wt \%), Target $($ wt $\%)=9.931$

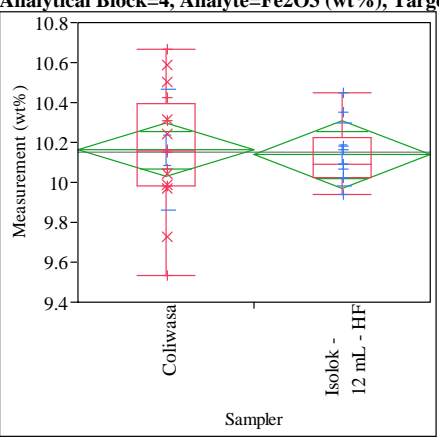

Oneway Anova

Summary of Fit

Adj Rsquare

$-0.03908$

Re $\quad 0.257654$

10.15362

Test

Isolok - $12 \mathrm{~mL}$ - HF-Coliwasa

Assuming equal variances

$\begin{array}{lrlr}\text { Difference } & -0.02538 & \mathrm{t} \text { Ratio } & -0.24433 \\ \text { Std Err Dif } & 0.10386 & \text { DF } & 24 \\ \text { Upper CL Dif } & 0.18899 & \text { Prob }>|t| & 0.8091 \\ \text { Lower CL Dif } & -0.23974 & \text { Prob }>\mathrm{t} & 0.5955 \\ \text { Confidence } & 0.95 & \text { Prob }<\mathrm{t} & 0.4045\end{array}$

Analysis of Variance

Source DF Sum of Squares Mean Square F Ratio Prob $>$ F

$\begin{array}{llllll}\text { Sampler } & 1 & 0.0039631 & 0.003963 & 0.0597 & 0.8091\end{array}$

$\begin{array}{llll}\text { Error } & 24 & 1.5932593 & 0.066386\end{array}$

C. Total $25 \quad 1.5972224$

Means for Oneway Anova

Level Number Mean Std Error Lower 95\% Upper 95\%

$\begin{array}{lrrrrr}\text { Coliwasa } & 16 & 10.1634 & 0.06441 & 10.030 & 10.296\end{array}$

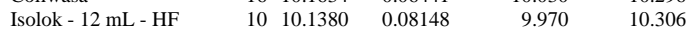

Std Error uses a pooled estimate of error variance

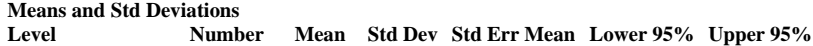

$\begin{array}{lrrrrrr}\text { Level } & \text { Number } & \text { Mean } & \text { Std Dev } & \text { Std Err Mean } & \text { Lower 95\% } & \text { Upper 95\% } \\ \text { Coliwasa } & 16 & 10.1634 & 0.302289 & 0.07557 & 10.002 & 10.324\end{array}$

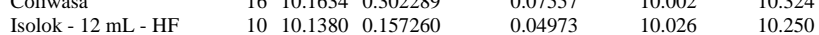

Isolok - $12 \mathrm{~mL}$ - HF-Coliwasa

Assuming unequal variances

$\begin{array}{lrlr}\text { Difference } & -0.02538 & \mathrm{t} \text { Ratio } & -0.28051 \\ \text { Std Err Dif } & 0.09047 & \text { DF } & 23.46892 \\ \text { Upper CL Dif } & 0.16156 & \text { Prob }>|\mathrm{t}| & 0.7815 \\ \text { Lower CL Dif } & -0.21232 & \text { Prob }>\mathrm{t} & 0.6092 \\ \text { Confidence } & 0.95 & \text { Prob }<\mathrm{t} & 0.3908\end{array}$

Tests that the Variances are Equal

Level Count Std Dev MeanAbsDif to Mean MeanAbsDif to Median

$\begin{array}{lllll}\text { Coliwasa } & 16 & 0.3022894 & 0.2341134 & 0.2341134\end{array}$

Isolok - $12 \mathrm{~mL}$ - HF $\quad 10 \quad 0.1572598 \quad 0.1189510 \quad 0.1100869$

Test F Ratio DFNum DFDen p-Value

$\begin{array}{lrrrr}\text { O'Brien[.5] } & 2.6795 & 1 & 24 & 0.1147\end{array}$

$\begin{array}{lllll}\text { Brown-Forsythe } & 3.6928 & 1 & 24 & 0.0666\end{array}$

$\begin{array}{lllll}\text { Levene } & 3.4076 & 1 & 24 & 0.0773\end{array}$

$\begin{array}{lrrrr}\text { Bartlett } & 3.9163 & 1 & \text {. } & 0.0478\end{array}$

Welch Anova testing Means Equal, allowing Std Devs Not Equal

F Ratio DFNum DFDen Prob $>$ F

$\begin{array}{rrrr}0.0787 & 1 & 23.469 & 0.7815\end{array}$

t Test

0.2805 


\section{Exhibit B2. Statistical Comparisons for High-Rheology (Phase 2) Simulant Testing}

Oneway Analysis of Measurement (wt\%) By Sampler Type of Material=SME Simulant, Analytical Block $=4$, Analyte $=\mathrm{K} 2 \mathrm{O}(\mathrm{wt} \%)$, Target $(\mathrm{wt} \%)=0.076$

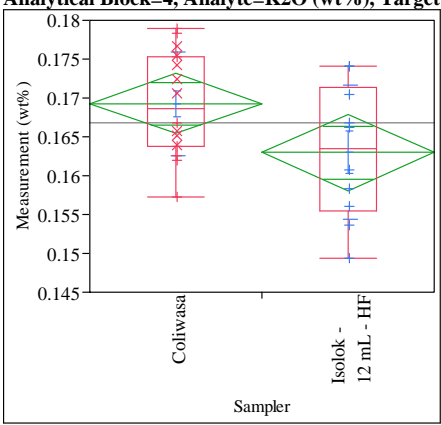

Oneway Anova

Summary of Fit

Rsquare

Adj Rsquare

Root Mean Square Error $\quad 0.007485$

Mean of Response 0.166837

Observations (or Sum Wgts) 26

t Test

Isolok - $12 \mathrm{~mL}$ - HF-Coliwasa

Assuming equal variances

Difference $\quad-0.00626$ t Ratio $\quad-2.07604$

\begin{tabular}{lrlr} 
Std Err Dif & 0.00302 & DF & 24 \\
\hline & &
\end{tabular}

$\begin{array}{lrll}\text { Upper CL Dif } & -3.66 \mathrm{e}-5 & \text { Prob }>|\mathrm{t}| & 0.0488 \\ \text { Lower CL Dif } & -0.01249 & \text { Prob }>t & 0.9756\end{array}$

$\begin{array}{lrl}\text { Confidence } & 0.95 \text { Prob }<\mathrm{t} \quad 0.0244\end{array}$

Analysis of Variance

Source DF Sum of Squares Mean Square F Ratio Prob $>$ F

$\begin{array}{llllll}\text { Sampler } & 1 & 0.00024146 & 0.000241 & 4.3100 & 0.0488\end{array}$

Error $24 \quad 0.0013445$

C. Total $25 \quad 0.00158601$

Means for Oneway Anova

Level Number Mean Std Error Lower 95\% Upper 95\%

$\begin{array}{lllllr}\text { Coliwasa } & 16 & 0.169246 & 0.00187 & 0.16538 & 0.17311\end{array}$

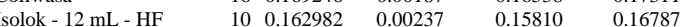

Std Error uses a pooled estimate of error variance

Means and Std Deviations

Level Number Mean Std Dev Std Err Mean Lower 95\% Upper 95\%

$\begin{array}{llllllr}\text { Coliwasa } & 16 & 0.169246 & 0.006682 & 0.00167 & 0.16569 & 0.17281 \\ \text { Isolok - 12 mL - HF } & 10 & 0.162982 & 0.008660 & 0.00274 & 0.15679 & 0.16918\end{array}$

t Test

Isolok - $12 \mathrm{~mL}$ - HF-Coliwasa

Assuming unequal variances

Difference $\quad-0.00626$ t Ratio -1.9528

Std Err Dif $\quad 0.00321$ DF 15.64423

Upper CL Dif 0.00055 Prob $>|t| \quad 0.0690$

Lower CL Dif -0.01308 Prob $>t \quad 0.9655$

Confidence $\quad 0.95$ Prob $<\mathrm{t} \quad 0.0345$

Tests that the Variances are Equal

$\begin{array}{lrrrr}\text { Level } & \text { Count } & \text { Std Dev } & \text { MeanAbsDif to Mean } & \text { MeanAbsDif to Median } \\ \text { Coliwasa } & 16 & 0.0066816 & 0.0058724 & 0.0058724\end{array}$

$\begin{array}{lllll}\text { Coliwasa } & 16 & 0.0066816 & 0.0058724 & 0.0058724 \\ \text { Isolok - 12 mL - HF } & 10 & 0.0086595 & 0.0073481 & 0.0073481\end{array}$

Test F Ratio DFNum DFDen p-Value

O'Brien[.5] $\quad 1.8909 \quad-1 \quad 24 \quad 0.1818$

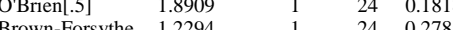

Brown-Forsythe $1.2294 \quad 11 \quad 24 \quad 0.2785$

$\begin{array}{lllll}\text { Levene } & 1.2719 & 1 & 24 & 0.2706 \\ \text { Bartlett } & 0.7476 & 1 & . & 0.3872\end{array}$

$\begin{array}{lllll}\text { F Test 2-sided } & 1.6797 & 9 & 15 & 0.3599\end{array}$

Welch Anova testing Means Equal, allowing Std Devs Not Equal

F Ratio DFNum DFDen Prob $>$ F

$\begin{array}{llll}3.8134 & 1 & 15.644 & 0.0690\end{array}$

$\mathbf{t}$ Test
1.9528
Oneway Analysis of Measurement (wt \%) By Sampler Type of Material=SME Simulant, Analytical Block $=4$, Analyte $=\mathrm{Li} 2 \mathrm{O}(\mathrm{wt} \%)$, Target $(\mathrm{wt} \%)=4.988$

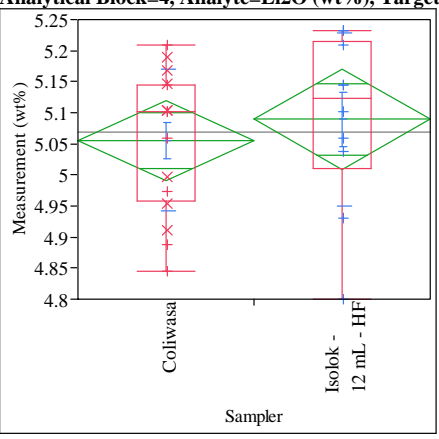

Oneway Anova

Summary of Fit

Adj Rsquare

0.019138

Root Mean Square Error $\quad-0.02173$

Mean of Response $\quad 0.123896$

$\begin{array}{lr}\text { Mean of Response } & 5.068423 \\ \text { Observations (or Sum Wgts) } & 26\end{array}$

Test

Isolok - $12 \mathrm{~mL}$ - HF-Coliwasa

Assuming equal variances

$\begin{array}{lrlr}\text { Difference } & 0.03418 & \text { t Ratio } & 0.684311 \\ \text { Std Err Dif } & 0.04994 \text { DF } & 24 \\ \text { Upper CL Dif } & 0.13726 & \text { Prob }>|t| & 0.5003 \\ \text { Lower CL Dif } & -0.06890 & \text { Prob }>t & 0.2502 \\ \text { Confidence } & 0.95 & \text { Prob }<\mathrm{t} & 0.7498\end{array}$

Analysis of Variance

Source DF Sum of Squares Mean Square F Ratio Prob $>$ F

$\begin{array}{llllll}\text { Sampler } & 1 & 0.00718823 & 0.007188 & 0.4683 & 0.5003\end{array}$

$\begin{array}{llll}\text { Error } & 24 & 0.36840547 & 0.015350\end{array}$

C. Total $25 \quad 0.37559369$

Means for Oneway Anova

Level Number Mean Std Error Lower 95\% Upper 95\%

$\begin{array}{lrrrrr}\text { Coliwasa } & 16 & 5.05528 & 0.03097 & 4.9914 & 5.1192\end{array}$

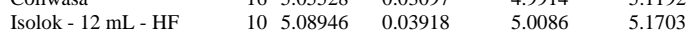

Std Error uses a pooled estimate of error variance

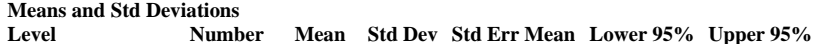

Level Number Mean Std Dev Std Err Mean Lower 95\% Upper 95\%

$\begin{array}{lllllll}\text { Coliwasa } & 16 & 5.05528 & 0.113980 & 0.02850 & 4.9945 & 5.1160 \\ \text { Isolok - 12 mL - HF } & 10 & 5.08946 & 0.138858 & 0.04391 & 4.9901 & 5.1888\end{array}$

Isolok - $12 \mathrm{~mL}$ - HF-Coliwasa

Assuming unequal variances

$\begin{array}{lrlr}\text { Difference } & 0.03418 \text { t Ratio } & 0.65291 \\ \text { Std Err Dif } & 0.05235 & \text { DF } & 16.42806 \\ \text { Upper CL Dif } & 0.14491 & \text { Prob }>|t| & 0.5228 \\ \text { Lower CL Dif } & -0.07656 & \text { Prob }>t & 0.2614 \\ \text { Confidence } & 0.95 & \text { Prob }<\mathrm{t} & 0.7386\end{array}$

Tests that the Variances are Equal

Level Count Std Dev MeanAbsDif to Mean MeanAbsDif to Median

$\begin{array}{lrrrr}\text { Coliwasa } & 16 & 0.1139801 & 0.0965441 & 0.0901527\end{array}$

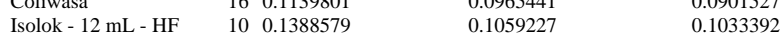

Test F Ratio DFNum DFDen p-Value

$\begin{array}{lrrrr}\text { O'Brien[.5] } & 0.5528 & 1 & 24 & 0.4644\end{array}$

$\begin{array}{lllll}\text { Brown-Forsythe } & 0.1435 & 1 & 24 & 0.7081\end{array}$

$\begin{array}{lllll}\text { Levene } & 0.1213 & 1 & 24 & 0.7306\end{array}$

$\begin{array}{lllll}\text { Bartlett } & 0.4309 & 1 & \text {. } & 0.5116 \\ \text { F Test 2-sided } & 1.4842 & 9 & 15 & 0.4795\end{array}$

Welch Anova testing Means Equal, allowing Std Devs Not Equal

F Ratio DFNum DFDen Prob $>$ F

$\begin{array}{rrrr}0.4263 & 1 & 16.428 & 0.5228\end{array}$

t Test

0.6529 


\section{Exhibit B2. Statistical Comparisons for High-Rheology (Phase 2) Simulant Testing}

Oneway Analysis of Measurement (wt \%) By Sampler Type of Material=SME Simulant, Analytical Block $=4$, Analyte $=\mathrm{MgO}(\mathrm{wt} \%)$, Target $(\mathrm{wt} \%)=\mathbf{0 . 2 8 6}$

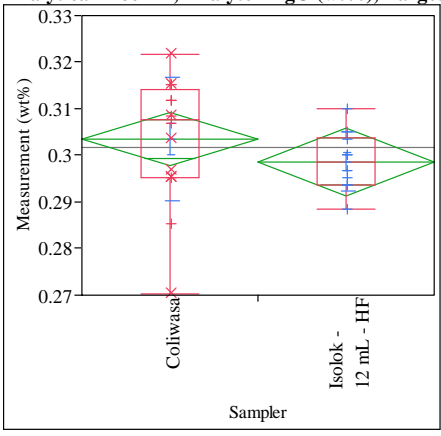

Oneway Anova

Summary of Fit

0.005679

$\quad 0.01116$

Mean of Response 0.301619

Observations (or Sum Wgts) $\quad 26$

Test

Isolok - $12 \mathrm{~mL}$ - HF-Coliwasa

Assuming equal variances

Difference $\quad-0.00481$ t Ratio $\quad-1.06901$

Std Err Dif $\quad 0.00450$ DF $\quad 24$

Upper CL Dif 0.00448 Prob $>|t| \quad 0.2957$

Lower CL Dif -0.01409 Prob $>\mathrm{t} \quad 0.8522$

$\begin{array}{lrl}\text { Confidence } & 0.95 \text { Prob }<\mathrm{t} & 0.1478\end{array}$

Analysis of Variance

Source DF Sum of Squares Mean Square F Ratio Prob $>$ F

$\begin{array}{llllll}\text { Sampler } & 1 & 0.00014232 & 0.000142 & 1.1428 & 0.2957\end{array}$

$\begin{array}{llll}\text { Error } & 24 & 0.00298893 & 0.000125\end{array}$

C. Total $25 \quad 0.00313125$

Means for Oneway Anova

$\begin{array}{lrrrrr}\text { Level } & \text { Number } & \text { Mean } & \text { Std Error } & \text { Lower 95\% } & \text { Upper 95\% } \\ \text { Coliwasa } & 16 & 0.303469 & 0.00279 & 0.29771 & 0.30923\end{array}$

$\begin{array}{lrrrrr}\text { Coliwasa } & 16 & 0.303469 & 0.00279 & 0.29771 & 0.30923 \\ \text { Isolok - 12 mL - HF } & 10 & 0.298660 & 0.00353 & 0.29138 & 0.30594\end{array}$

Std Error uses a pooled estimate of error variance

Means and Std Deviations

Level Number Mean Std Dev Std Err Mean Lower 95\% Upper 95\%

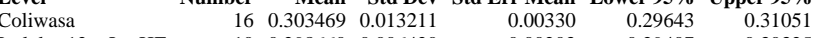

$\begin{array}{lllllll}\text { Isolok - } 12 \mathrm{~mL} \text { - HF } & 10 & 0.298660 & 0.006420 & 0.00203 & 0.29407 & 0.30325\end{array}$

Isolok - $12 \mathrm{~mL}$ - HF-Coliwasa

Assuming unequal variances

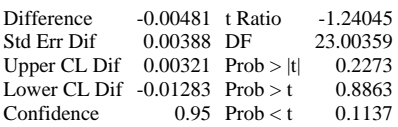

Tests that the Variances are Equal

$\begin{array}{lrrrr}\text { Level } & \text { Count } & \text { Std Dev } & \text { MeanAbsDif to Mean } & \text { MeanAbsDif to Median } \\ \text { Coliwasa } & 16 & 0.0132110 & 0.0103644 & 0.0099498\end{array}$

$\begin{array}{lllll}\text { Coliwasa } & 16 & 0.0132110 & 0.0103644 & 0.0099498 \\ \text { Isolok - } 12 \mathrm{~mL}-\mathrm{HF} & 10 & 0.0064202 & 0.0051407 & 0.0051407\end{array}$

Test F Ratio DFNum DFDen p-Value

$\begin{array}{lrrrr}\text { Test } & \text { F Ratio } & \text { DFNum } & \text { DFDen } & \text { p-Value } \\ \text { O'Brien[.5] } & 1.9469 & 1 & 24 & 0.1757 \\ \text { Brown-Forsythe } & 2.4123 & 1 & 24 & 0.1335 \\ \text { Levene } & 4.0062 & 1 & 24 & 0.0568 \\ \text { Bartlett } & 4.6770 & 1 & . & 0.0306 \\ \text { F Test 2-sided } & 4.2342 & 15 & 9 & 0.0342\end{array}$

$\begin{array}{llll} & & & \end{array}$

Welch Anova testing Means Equal, allowing Std Devs Not Equal

F Ratio DFNum DFDen Prob $>$ F

$\begin{array}{rrrr}1.5387 & 1 & 23.004 & 0.2273\end{array}$

t Test

1.2405
Oneway Analysis of Measurement (wt\%) By Sampler Type of Material=SME Simulant, Analytical Block $=4$, Analyte $=\mathrm{MnO}(\mathrm{wt} \%)$, Target $(\mathrm{wt} \%)=2.918$

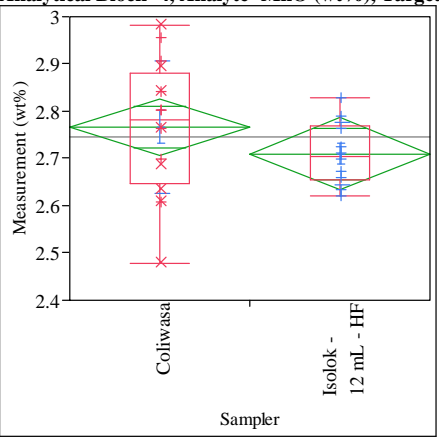

Oneway Anova

Summary of Fit

$\begin{array}{lr}\text { Rsquare } & 0.05497 \\ \text { Adj Rsquare } & 0.015594 \\ \text { Root Mean Square Error } & 0.117926 \\ \text { Mean of Response } & 2.744793 \\ \text { Observations (or Sum Wgts) } & 26\end{array}$

t Test

Isolok - $12 \mathrm{~mL}$ - HF-Coliwasa

Assuming equal variances

Difference $\quad-0.05617$ t Ratio $\quad-1.18154$

$\begin{array}{lll}\text { Std Err Dif } & 0.04754 \text { DF } & 24\end{array}$

Upper CL Dif 0.04195 Prob $>|t| \quad 0.2490$

$\begin{array}{lll}\text { Lower CL Dif }-0.15428 \text { Prob }>t & 0.8755\end{array}$

$\begin{array}{lrl}\text { Confidence } & 0.95 \text { Prob }<\mathrm{t} & 0.1245\end{array}$

Analysis of Variance

Source DF Sum of Squares Mean Square F Ratio Prob $>$ F

$\begin{array}{lrrrrr}\text { Sampler } & 1 & 0.01941387 & 0.019414 & 1.3960 & 0.2490\end{array}$

$\begin{array}{lrrr}\text { Error } & 24 & 0.33375626 & 0.013907\end{array}$

C. Total $25 \quad 0.35317013$

Means for Oneway Anova

Level Number Mean Std Error Lower 95\% Upper 95\%

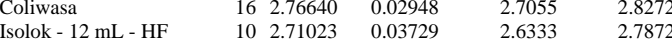

Std Error uses a pooled estimate of error variance

Means and Std Deviations

Level Number Mean Std Dev Std Err Mean Lower 95\% Upper 95\%

$\begin{array}{lrrrrrr}\text { Coliwasa } & 16 & 2.76640 & 0.139744 & 0.03494 & 2.6919 & 2.8409\end{array}$

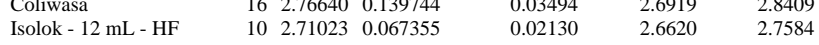

t Test

Isolok - $12 \mathrm{~mL}$ - HF-Coliwasa

Assuming unequal variances

Difference $\quad-0.05617$ t Ratio -1.37271

Std Err Dif $\quad 0.04092$ DF 22.9408

Upper CL Dif $\quad 0.02849$ Prob $>|t| r 0.183$

Lower CL Dif -0.14082 Prob $>t \quad 0.9084$

Confidence $\quad 0.95$ Prob $<\mathrm{t} \quad 0.0916$

Tests that the Variances are Equal

Level Count Std Dev MeanAbsDif to Mean MeanAbsDif to Median

$\begin{array}{lrrrr}\text { Coliwasa } & 16 & 0.1397442 & 0.1113660 & 0.1113660\end{array}$

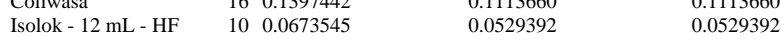

Test F Ratio DFNum DFDen p-Value

$\begin{array}{lllll}\text { O'Brien[.5] } & 3.6449 & 1 & 24 & 0.0683\end{array}$

Brown-Forsythe $\quad 4.5126 \quad 1 \quad 24 \quad 0.0441$

$\begin{array}{lllll}\text { Levene } & 4.6991 & 1 & 24 & 0.0403\end{array}$

$\begin{array}{lllll}\text { Bartlett } & 4.7723 & 1 & 0.0289\end{array}$

$\begin{array}{lllll}\text { F Test 2-sided } & 4.3046 & 15 & 9 & 0.0324\end{array}$

Welch Anova testing Means Equal, allowing Std Devs Not Equal

F Ratio DFNum DFDen Prob $>$ F

$\begin{array}{llll}1.8843 & 1 & 22.941 & 0.1831\end{array}$

t Test

1.3727 


\section{Exhibit B2. Statistical Comparisons for High-Rheology (Phase 2) Simulant Testing}

Oneway Analysis of Measurement (wt \%) By Sampler Type of Material=SME Simulant, Analytical Block $=4$, Analyte $=\mathrm{Na} 2 \mathrm{O}(\mathrm{wt} \%)$, Target $(\mathrm{wt} \%)=13.318$

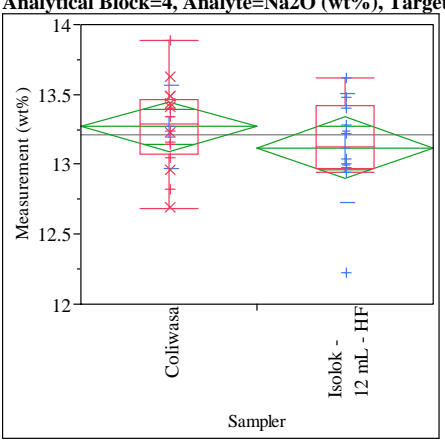

Oneway Anova

Summary of Fit

0.048112

Root Mean Square Error $\quad 0.337393$

Mean of Response

Observations (or Sum Wgts) $\quad 26$

Test

Isolok - $12 \mathrm{~mL}$ - HF-Coliwasa

Assuming equal variances

Difference $\quad-0.14980$ t Ratio $\quad-1.10138$

$\begin{array}{lrlr}\text { Std Err Dif } & 0.13601 & \text { DF } & 24 \\ \text { Upper CL Dif } & 0.13091 & \text { Prob }>|t| & 0.2817\end{array}$

Lower CL Dif -0.43050 Prob $>\mathrm{t} \quad 0.8592$

$\begin{array}{lrl}\text { Confidence } & 0.95 \text { Prob }<\mathrm{t} & 0.1408\end{array}$

Analysis of Variance

Source DF Sum of Squares Mean Square F Ratio Prob $>$ F

$\begin{array}{llllll}\text { Sampler } & 1 & 0.1380861 & 0.138086 & 1.2130 & 0.2817\end{array}$

$\begin{array}{llll}\text { Error } & 24 & 2.7320227 & 0.113834\end{array}$

C. Total $25 \quad 2.8701088$

Means for Oneway Anova

Level Number Mean Std Error Lower 95\% Upper 95\%

$\begin{array}{lrrrrr}\text { Coliwasa } & 16 & 13.2685 & 0.08435 & 13.094 & 13.443\end{array}$

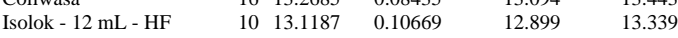

Std Error uses a pooled estimate of error variance

Means and Std Deviations

Level Number Mean Std Dev Std Err Mean Lower 95\% Upper 95\%

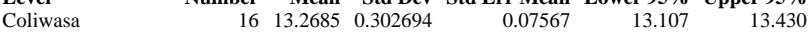

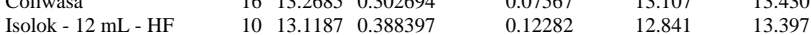

Isolok - $12 \mathrm{~mL}$ - HF-Coliwasa

Assuming unequal variances

Difference $\quad-0.14980$ t Ratio $\quad-1.03836$

Std Err Dif $\quad 0.14426$ DF $\quad 15.76668$

Upper CL Dif 0.15639 Prob $>|t| \quad 0.3148$

Lower CL Dif -0.45599 Prob $>t \quad 0.8426$

$\begin{array}{lrl}\text { Confidence } & 0.95 \text { Prob }<\mathrm{t} & 0.1574\end{array}$

Tests that the Variances are Equal

Level Count Std Dev MeanAbsDif to Mean MeanAbsDif to Median

$\begin{array}{lrrrr}\text { Coliwasa } & 16 & 0.3026940 & 0.2350575 & 0.2350575\end{array}$

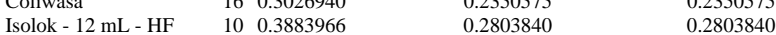

Test F Ratio DFNum DFDen p-Value

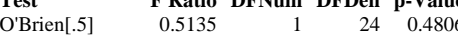

$\begin{array}{lllll}\text { Brown-Forsythe } & 0.2832 & 1 & 24 & 0.5995\end{array}$

$\begin{array}{lllll}\text { Levene } & 0.2858 & 1 & 24 & 0.5979\end{array}$

$\begin{array}{lllll}\text { Bartlett } & 0.6905 & 1 & 0.4060\end{array}$

Welch Anova testing Means Equal, allowing Std Devs Not Equal

F Ratio DFNum DFDen Prob $>$ F

$\begin{array}{rrrr}1.0782 & 1 & 15.767 & 0.3148\end{array}$

t Test

1.0384
Oneway Analysis of Measurement (wt \%) By Sampler Type of Material=SME Simulant, Analytical Block $=4$, Analyte $=\mathrm{NiO}(\mathrm{wt} \%)$, Target $(\mathrm{wt} \%)=1.033$

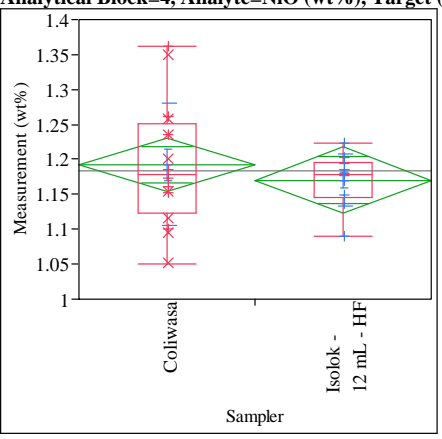

Oneway Anova

Summary of Fit

Adj Rsquare

0.022735

Root Mean Square Error $\quad-0.01798$

Mean of Response Eror

$\begin{array}{lr}\text { Mean of Response } & 1.183963 \\ \text { Observations (or Sum Wgts) } & 26\end{array}$

Test

Isolok - $12 \mathrm{~mL}$ - HF-Coliwasa

Assuming equal variances

$\begin{array}{lrlr}\text { Difference } & -0.02197 & \text { t Ratio } & -0.74722 \\ \text { Std Err Dif } & 0.02940 & \text { DF } & 24 \\ \text { Upper CL Dif } & 0.03871 & \text { Prob }>|t| & 0.4622 \\ \text { Lower CL Dif } & -0.08264 & \text { Prob }>t & 0.7689 \\ \text { Confidence } & 0.95 & \text { Prob }<\mathrm{t} & 0.2311\end{array}$

Analysis of Variance

Source DF Sum of Squares Mean Square F Ratio Prob $>$ F

$\begin{array}{llllll}\text { Sampler } & 1 & 0.00296941 & 0.002969 & 0.5583 & 0.4622\end{array}$

$\begin{array}{llll}\text { Error } & 24 & 0.12764036 & 0.005318\end{array}$

C. Total $25 \quad 0.13060977$

Means for Oneway Anova

$\begin{array}{lllll}\text { Level } & \text { Number Mean Std Error Lower 95\% Upper 95\% }\end{array}$

$\begin{array}{lrrrrr}\text { Coliwasa } & 16 & 1.19241 & 0.01823 & 1.1548 & 1.2300\end{array}$

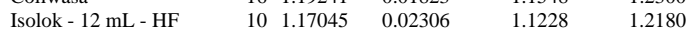

Std Error uses a pooled estimate of error variance

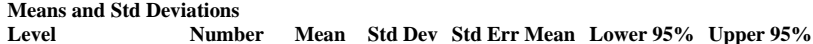

$\begin{array}{lrrrrrr}\text { Level } & \text { Number } & \text { Mean } & \text { Std Dev } & \text { Std Err Mean } & \text { Lower 95\% } & \text { Upper 95\% } \\ \text { Coliwasa } & 16 & 1.19241 & 0.087477 & 0.02187 & 1.1458 & 1.2390\end{array}$

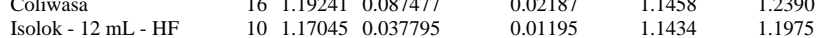

Isolok - $12 \mathrm{~mL}$ - HF-Coliwasa

Assuming unequal variances

$\begin{array}{lrlr}\text { Difference } & -0.02197 & \mathrm{t} \text { Ratio } & -0.88141 \\ \text { Std Err Dif } & 0.02492 & \text { DF } & 22.02391 \\ \text { Upper CL Dif } & 0.02972 & \text { Prob }>|t| & 0.3876 \\ \text { Lower CL Dif } & -0.07365 & \text { Prob }>\text { t } & 0.8062 \\ \text { Confidence } & 0.95 & \text { Prob }<\mathrm{t} & 0.1938\end{array}$

Tests that the Variances are Equal

Level Count Std Dev MeanAbsDif to Mean MeanAbsDif to Median

$\begin{array}{lllll}\text { Coliwasa } & 16 & 0.0874773 & 0.0688045 & 0.0678402\end{array}$

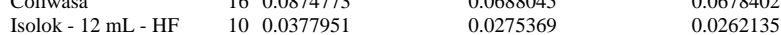

Test F Ratio DFNum DFDen p-Value

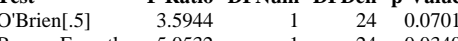

Brown-Forsythe $5.0532 \quad 1 \quad 24 \quad 0.0340$

$\begin{array}{lllll}\text { Levene } & 5.6761 & 1 & 24 & 0.0255\end{array}$

$\begin{array}{lrrrr}\text { Bartlett } & 6.0968 & 1 & \text {. } & 0.0135 \\ \text { F Test 2-sided } & 5.3570 & 15 & 9 & 0.0153\end{array}$

Welch Anova testing Means Equal, allowing Std Devs Not Equal

F Ratio DFNum DFDen Prob > F

$\begin{array}{rrrr}0.7769 & 1 & 22.024 & 0.3876\end{array}$

t Test

0.8814 
SRNL-STI-2011-00693

Revision 0

Exhibit B2. Statistical Comparisons for High-Rheology (Phase 2) Simulant Testing

Oneway Analysis of Measurement (wt \%) By Sampler Type of Material=SME Simulant, Analytical Block $=4$, Analyte $=\mathrm{SiO} 2(\mathrm{wt} \%)$, Target $(\mathrm{wt} \%)=50.183$

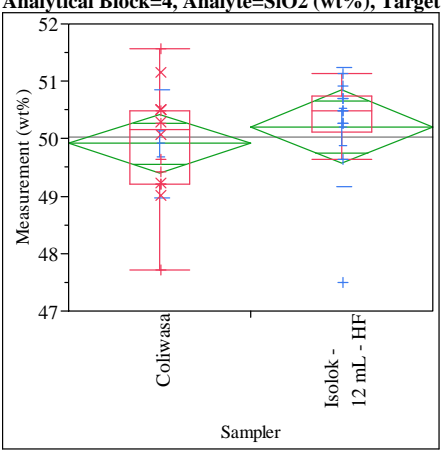

Oneway Anova

Summary of Fit

Rsquare

$\begin{array}{ll}\text { Rsquare } & 0.022987 \\ \text { Adj Rsquare } & -0.01772\end{array}$

Mean of Response 50.02671

Observations (or Sum Wgts) 26

Isolok - $12 \mathrm{~mL}$ - HF-Coliwasa

Assuming equal variances

Difference $\quad 0.2968$ t Ratio 0.751443

$\begin{array}{lrr}\text { Std Err Dif } & 0.3950 \text { DF } & 24 \\ & & \end{array}$

Upper CL Dif 1.1121 Prob $>|t| \quad 0.4597$

$\begin{array}{lrr}\text { Lower CL Dif } & -0.5184 \text { Prob }>t \quad 0.2298 \\ \text { Confidence } & 0.95 \text { Prob }<\text { t } & 0.7702\end{array}$

Analysis of Variance

Source DF Sum of Squares Mean Square F Ratio Prob $>$ F

$\begin{array}{lrrrrr}\text { Sampler } & 1 & 0.542196 & 0.542196 & 0.5647 & 0.4597\end{array}$

$\begin{array}{llll}\text { Error } & 24 & 23.044920 & 0.960205\end{array}$

C. Total $25 \quad 23.587115$

Means for Oneway Anova

Level Number Mean Std Error Lower 95\% Upper 95\%

$\begin{array}{lrrrrr}\text { Coliwasa } & 16 & 49.9125 & 0.24498 & 49.407 & 50.418\end{array}$

$\begin{array}{llllll}\text { Coliwasa } & 16 & 49.9125 & 0.24498 & 49.407 & 50.418 \\ \text { Isolok - 12 mL - HF } & 10 & 50.2094 & 0.30987 & 49.570 & 50.849\end{array}$

Std Error uses a pooled estimate of error variance

Means and Std Deviations

$\begin{array}{lrrrrrr}\text { Level } & \text { Number } & \text { Mean } & \text { Std Dev } & \text { Std Err Mean } & \text { Lower 95\% } & \text { Upper 95\% } \\ \text { Coliwasa } & 16 & 49.9125 & 0.94297 & 0.23574 & 49.410 & 50.415\end{array}$

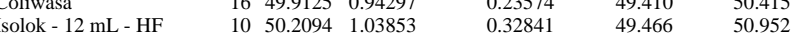

Test

Isolok - $12 \mathrm{~mL}$ - HF-Coliwasa

Assuming unequal variances

Difference $\quad 0.2968$ t Ratio $\quad 0.734241$

Std Err Dif $\quad 0.4043$ DF $\quad 17.8248$

Upper CL Dif 1.1468 Prob $>|t| \quad 0.4724$

Lower CL Dif -0.5531 Prob $>t \quad 0.2362$

Confidence $\quad 0.95$ Prob $<\mathrm{t} \quad 0.7638$

Tests that the Variances are Equal

$\begin{array}{lrrrr}\text { Level } & \text { Count } & \text { Std Dev } & \text { MeanAbsDif to Mean } & \text { MeanAbsDif to Median } \\ \text { Coliwasa } & 16 & 0.942972 & 0.7537690 & 0.7353844\end{array}$

Isolok - $12 \mathrm{~mL}$ - HF $\quad 101.038534 \quad 0.6589044 \quad 0.5776110$

Test F Ratio DFNum DFDen p-Value

O'Brien[-5] $\quad 0.0564$

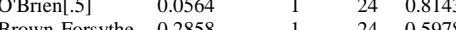

$\begin{array}{lllll}\text { Brown-Forsythe } & 0.2858 & 1 & 24 & 0.5978\end{array}$

$\begin{array}{lllll}\text { Levene } & 0.1383 & 1 & 24 & 0.7133 \\ \text { Bartlett } & 0.1018 & 1 & . & 0.7497\end{array}$

$\begin{array}{lllll}\text { F Test 2-sided } & 1.2130 & 9 & 15 & 0.7118\end{array}$

Welch Anova testing Means Equal, allowing Std Devs Not Equal

F Ratio DFNum DFDen Prob $>$ F

$\begin{array}{llll}0.5391 & 1 & 17.825 & 0.4724\end{array}$

t Test
0.7342
Oneway Analysis of Measurement (wt \%) By Sampler Type of Material=SME Simulant, Analytical Block $=4$, Analyte $=$ Sum of Oxides $(w t \%)$, Target $(w t \%)=99.73$

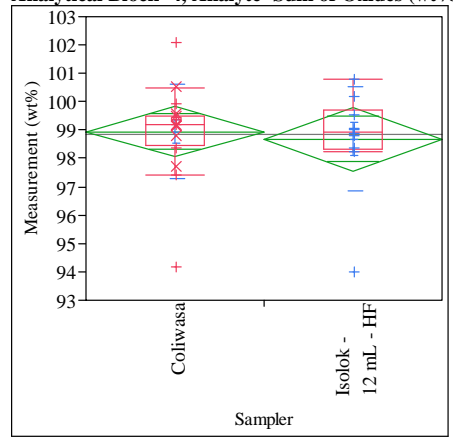

Oneway Anova

Summary of Fit

$\begin{array}{lr}\text { Rsquare } & 0.005775 \\ \text { Adj Rsquare } & -0.03565 \\ \text { Root Mean Square Error } & 1.72958 \\ \text { Mean of Response } & 98.84839 \\ \text { Observations (or Sum Wgts) } & 26\end{array}$

t Test

Isolok - $12 \mathrm{~mL}$ - HF-Coliwasa

Assuming equal variances

Difference $\quad-0.2603$ t Ratio $\quad-0.37338$

Std Err Dif $\quad 0.6972$ DF 24

Upper CL Dif 1.1787 Prob $>|t| \quad 0.712$

Lower CL Dif -1.6993 Prob $>t \quad 0.6439$

Confidence $\quad 0.95$ Prob $<\mathrm{t} \quad 0.3561$

Analysis of Variance

Source DF Sum of Squares Mean Square F Ratio Prob $>$ F

$\begin{array}{lrrrrr}\text { Sampler } & 1 & 0.417040 & 0.41704 & 0.1394 & 0.7121\end{array}$

$\begin{array}{lrrr}\text { Error } & 24 & 71.794700 & 2.99145\end{array}$

C. Total $25 \quad 72.211740$

Means for Oneway Anova

Level Number Mean Std Error Lower 95\% Upper 95\%

$\begin{array}{llllll}\text { Isolok - } 12 \mathrm{~mL} \text { - HF } & 16 & 98.9485 & 0.43239 & 98.056 & 99.841 \\ & & 98.6882 & 0.54694 & 97.559 & 99.817\end{array}$

Std Error uses a pooled estimate of error variance

Means and Std Deviations

Level r Number Mean Std Dev Std Err Mean Lower 95\% Upper 95\%

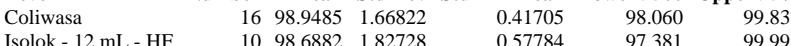

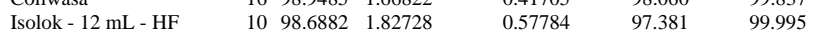

t Test

Isolok - $12 \mathrm{~mL}$ - HF-Coliwasa

Assuming unequal variances

$\begin{array}{lrlr}\text { Difference } & -0.2603 & \text { t Ratio } & -0.36531 \\ \text { Std Err Dif } & 0.7126 & \text { DF } & 17.90389 \\ \text { Upper CL Dif } & 1.2374 & \text { Prob }>|t| & 0.7192 \\ \text { Lower CL Dif } & -1.7581 & \text { Prob }>t & 0.6404 \\ \text { Confidence } & 0.95 & \text { Prob }<\mathrm{t} & 0.3596\end{array}$

Tests that the Variances are Equal

Level Count Std Dev MeanAbsDif to Mean MeanAbsDif to Median

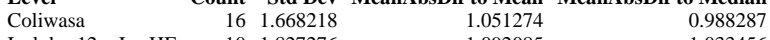

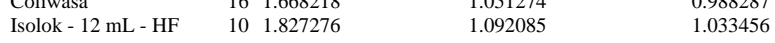

Test F Ratio DFNum DFDen p-Value

$\begin{array}{lllll}\text { O'Brien[.5] } & 0.0371 & 1 & 24 & 0.8490\end{array}$

$\begin{array}{lllll}\text { Brown-Forsythe } & 0.0064 & 1 & 24 & 0.9368\end{array}$

$\begin{array}{lllll}\text { Levene } & 0.0058 & 1 & 24 & 0.9398\end{array}$

$\begin{array}{lllll}\text { Bartlett } & 0.0905 & 1 & 0.7635\end{array}$

$\begin{array}{lllll}\text { F Test 2-sided } & 1.1998 & 9 & 15 & 0.7253\end{array}$

Welch Anova testing Means Equal, allowing Std Devs Not Equal

$\begin{array}{rrrr}\text { F Ratio } & \text { DFNum } & \text { DFDen } & \text { Prob }>\text { F } \\ 0.1334 & 1 & 17.904 & 0.7192\end{array}$

t Test

0.3653 
SRNL-STI-2011-00693

Revision 0

\section{Exhibit B2. Statistical Comparisons for High-Rheology (Phase 2) Simulant Testing}

Oneway Analysis of Measurement (wt \%) By Sampler Type of Material=SME Simulant, Analytical Block=4, Analyte $=$ TiO2 $(w t \%)$, Target $(w t \%)=0.013$

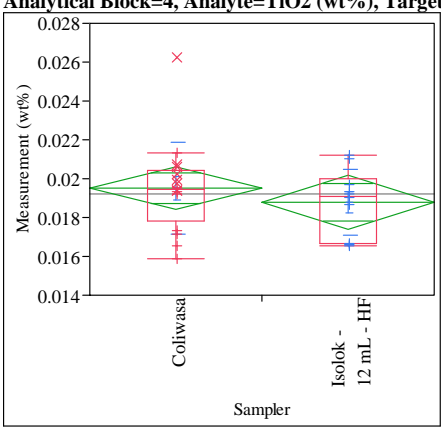

Oneway Anova

Summary of Fit

Rsquare

Adj Rsquare

Root Mean Square Error $\quad 0.002138$

Mean of Response $\quad 0.019233$

Observations (or Sum Wgts) 26

Isolok - $12 \mathrm{~mL}$ - HF-Coliwasa

Assuming equal variances

Difference $\quad-0.00071$ t Ratio $\quad-0.82819$

$\begin{array}{lll}\text { Std Err Dif } \quad 0.00086 \text { DF } & 24\end{array}$

Upper CL Dif 0.00107 Prob $>|t| \quad 0.4157$

Lower CL Dif -0.00249 Prob $>\mathrm{t} \quad 0.7921$

$\begin{array}{lll}\text { Confidence } & 0.95 \text { Prob }<\mathrm{t} \quad 0.2079\end{array}$

Analysis of Variance

Source DF Sum of Squares Mean Square F Ratio Prob > F

Sampler $\begin{array}{rrrrr}1 & 0.00000314 & 3.1364 \mathrm{e}-6 & 0.6859 & 0.4157\end{array}$

$\begin{array}{llll}\text { Error } & 24 & 0.00010974 & 4.5727 \mathrm{e}-6\end{array}$

C. Total $25 \quad 0.00011288$

Means for Oneway Anova

Level Number Mean Std Error Lower 95\% Upper 95\%

$\begin{array}{lllllr}\text { Coliwasa } & 16 & 0.019507 & 0.00053 & 0.01840 & 0.02061\end{array}$

$\begin{array}{llllll}\text { Isolok - } 12 \mathrm{~mL} \text { - HF } & 10 & 0.018793 & 0.00068 & 0.01740 & 0.02019\end{array}$

Std Error uses a pooled estimate of error variance

Means and Std Deviations

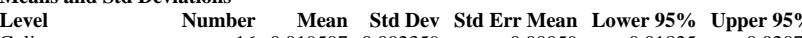

$\begin{array}{llllllr}\text { Coliwasa } & 16 & 0.019507 & 0.002359 & 0.00059 & 0.01825 & 0.02076 \\ \text { Isolok - 12 mL - HF } & 10 & 0.018793 & 0.001710 & 0.00054 & 0.01757 & 0.02002\end{array}$

t Test

Isolok - $12 \mathrm{~mL}$ - HF-Coliwasa

Assuming unequal variances

Difference $\quad-0.00071$ t Ratio $\quad-0.89242$

Std Err Dif $\quad 0.00080$ DF 23.33491

Upper CL Dif 0.00094 Prob $>|t| 0.3813$

Lower CL Dif -0.00237 Prob $>\mathrm{t} \quad 0.8094$

Confidence $\quad 0.95$ Prob $<\mathrm{t} \quad 0.1906$

Tests that the Variances are Equal

$\begin{array}{lrrrr}\text { Level } & \text { Count } & \text { Std Dev } & \text { MeanAbsDif to Mean } & \text { MeanAbsDif to Median } \\ \text { Coliwasa } & 16 & 0.0023585 & 0.0014887 & 0.0014887\end{array}$

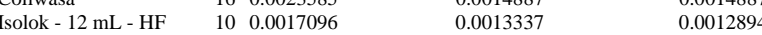

Test F Ratio DFNum DFDen p-Value

$1-24 \quad 0.5163$

$\begin{array}{lllll} & 0.4341 & 1 & 24 & 0.5163\end{array}$

Brown-Forsythe $\quad 0.1000 \quad 1 \quad 24 \quad 0.7546$

$\begin{array}{lllll}\text { Levene } & 0.0628 & 1 & 24 & 0.8043 \\ \text { Bartlett } & 1.0412 & 1 & . & 0.3075\end{array}$

$\begin{array}{lrrrr}\text { F Test 2-sided } & 1.9031 & 15 & \dot{9} & 0.3313\end{array}$

Welch Anova testing Means Equal, allowing Std Devs Not Equal

F Ratio DFNum DFDen Prob $>$ F

$\begin{array}{llll}0.7964 & 1 & 23.335 & 0.3813\end{array}$

t Test
Oneway Analysis of Measurement (wt \%) By Sampler Type of Material=SME Simulant, Analytical Block $=4$, Analyte $=\mathrm{ZnO}(\mathrm{wt} \%)$, Target $(\mathrm{wt} \%)=0$

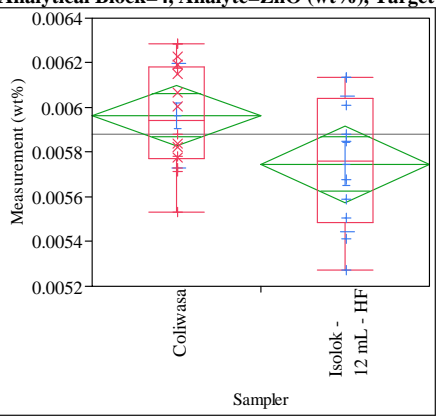

Oneway Anova

Summary of Fit

0.11256

Error $\quad 0.000263$

Mean of Response 0.00588

Test

Isolok - $12 \mathrm{~mL}$ - HF-Coliwasa

Assuming equal variances

Difference $\quad-0.00022$ t Ratio $\quad-2.04229$

Std Err Dif $\quad 0.00011$ DF 24

Upper CL Dif $2.288 \mathrm{e}-6$ Prob $>|\mathrm{t}| \quad 0.0523$

Lower CL Dif -0.00043 Prob $>$ t 0.9739

$\begin{array}{lrl}\text { Confidence } & 0.95 \mathrm{Prob}<\mathrm{t} & 0.0261\end{array}$

Analysis of Variance

Source DF Sum of Squares Mean Square F Ratio Prob $>$ F

$\begin{array}{rrrrrr}\text { Source } & \text { DF } & \text { Sum of Squares } & \text { Mean Square } & \text { F Ratio } & \text { Prob }>\mathbf{F} \\ \text { Sampler } & 1 & 2.87869 \mathrm{e}-7 & 2.8787 \mathrm{e}-7 & 4.1709 & 0.0523\end{array}$

$\begin{array}{lrll}\text { Sampler } & 1 & 2.87869 \mathrm{e}-7 & 2.8787 \mathrm{e}-7 \\ \text { Error } & 24 & 1.65643 \mathrm{e}-6 & 6.9018 \mathrm{e}-8\end{array}$

$\begin{array}{lll}\text { Error } & 24 & 1.65643 \mathrm{e}-6 \\ \text { C. Total } & 25 & 1.94429 \mathrm{e}-6\end{array}$

Means for Oneway Anova

Level Number Mean Std Error Lower 95\% Upper 95\%

$\begin{array}{llllll}\text { Isolok - } 12 \text { mL - HF } & 16 & 0.005964 & 6.57 \mathrm{e}-5 & 0.00583 & 0.00610 \\ & 10 & 0.005748 & 8.31 \mathrm{e}-5 & 0.00558 & 0.00592\end{array}$

Std Error uses a pooled estimate of error variance

Means and Std Deviations

Level Number Mean Std Dev Std Err Mean Lower 95\% Upper 95\%

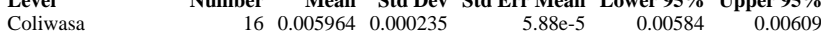

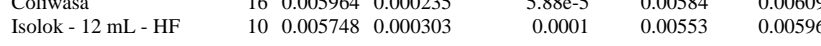

t Test

Isolok - $12 \mathrm{~mL}$ - HF-Coliwasa

Assuming unequal variances

Difference $\quad-0.00022$ t Ratio $\quad-1.92372$

Std Err Dif $\quad 0.00011$ DF 15.71872

Upper CL Dif $2.24 \mathrm{e}-5$ Prob $>|\mathrm{t}| \quad 0.0727$

Lower CL Dif -0.00045 Prob $>t \quad 0.9637$

$\begin{array}{lll}\text { Confidence } & 0.95 \text { Prob }<\mathrm{t} \quad 0.0363\end{array}$

Tests that the Variances are Equal

Level Count Std Dev MeanAbsDif to Mean MeanAbsDif to Median

$\begin{array}{lrrrr}\text { Coliwasa } & 16 & 0.0002352 & 0.0002062 & 0.0002062\end{array}$

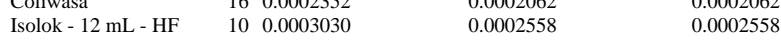

Test F Ratio DFNum DFDen p-Value

$\begin{array}{lllll}\text { O'Brien[.5] } & 1.7731 & 1 & 24 & 0.1955\end{array}$

Brown-Forsythe $1.0924 \quad 1 \quad 24 \quad 0.3063$

$\begin{array}{lllll}\text { Levene } & 1.1302 & 1 & 24 & 0.2983\end{array}$

$\begin{array}{lllll}\text { Bartlett } & 0.7125 & 1 & 0.3986\end{array}$

$\begin{array}{lllll}\text { Fatlett } & 0.7125 & 1 & & 0.3986\end{array}$

Welch Anova testing Means Equal, allowing Std Devs Not Equal

$\begin{array}{rrrr}\text { F Ratio } & \text { DFNum } & \text { DFDen } & \text { Prob }>\text { F } \\ 3.7007 & 1 & 15.719 & 0.0727\end{array}$

t Test 


\section{Exhibit B2. Statistical Comparisons for High-Rheology (Phase 2) Simulant Testing}

Oneway Analysis of Measurement (wt \%) By Sampler Type of Material=SME Simulant, Analytical Block=4, Analyte $=\mathrm{ZrO} 2(\mathrm{wt} \%)$, Target $(\mathrm{wt} \%)=0.111$

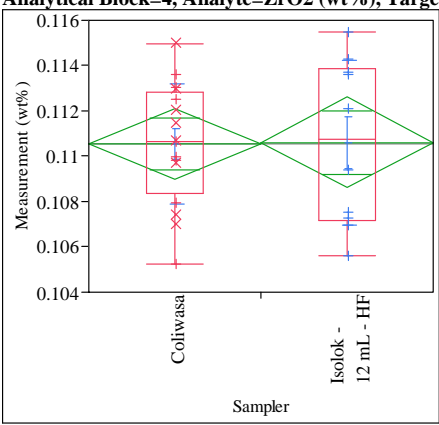

Oneway Anova

Summary of Fit

Rsquare

Adj Rsquare

Root Mean Square Error $\quad-0.0415$

Mean of Response

Observations (or Sum Wgts) $\quad 26$

t Test

Isolok - $12 \mathrm{~mL}$ - HF-Coliwasa

Assuming equal variances

Difference $\quad 5.741 \mathrm{e}-5$ t Ratio $\quad 0.04647$

$\begin{array}{lrr}\text { Std Err Dif } & 0.00124 \text { DF } & 24 \\ & & \end{array}$

Upper CL Dif 0.00261 Prob $>|t| 0.9633$

$\begin{array}{lrll}\text { Lower CL Dif } & -0.00249 & \text { Prob }>t & 0.4817 \\ \text { Confidence } & 0.95 & \text { Prob }<\mathrm{t} & 0.5183\end{array}$

Analysis of Variance

Source DF Sum of Squares Mean Square F Ratio Prob $>$ F

$\begin{array}{llllll}\text { Sampler } & 1 & 2.02818 \mathrm{e}-8 & 2.0282 \mathrm{e}-8 & 0.0022 & 0.9633\end{array}$

$\begin{array}{llll}\text { Error } & 24 & 0.00022541 & 9.3919 \mathrm{e}-6\end{array}$

C. Total $25 \quad 0.00022543$

Means for Oneway Anova

Level Number Mean Std Error Lower 95\% Upper 95\%

$\begin{array}{lrrrrr}\text { Coliwasa } & 16 & 0.110546 & 0.00077 & 0.10896 & 0.11213\end{array}$

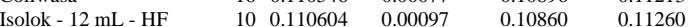

Std Error uses a pooled estimate of error variance

Means and Std Deviations

Level Number Mean Std Dev Std Err Mean Lower 95\% Upper 95\%

$\begin{array}{lllllrr}\text { Coliwasa } & 16 & 0.110546 & 0.002670 & 0.00067 & 0.10912 & 0.11197 \\ \text { Isolok - 12 mL - HF } & 10 & 0.110604 & 0.003628 & 0.00115 & 0.10801 & 0.11320\end{array}$

t Test

Isolok - $12 \mathrm{~mL}$ - HF-Coliwasa

Assuming unequal variances

Difference $\quad 5.741 \mathrm{e}-5$ t Ratio $\quad 0.043252$

Std Err Dif $\quad 0.00133$ DF 15.08896

Upper CL Dif 0.00289 Prob $>|t| 0.9661$

$\begin{array}{lrl}\text { Lower CL Dif } & -0.00277 \text { Prob }>t & 0.4830\end{array}$

$\begin{array}{lrl}\text { Confidence } & 0.95 \text { Prob }<\mathrm{t} \quad 0.5170\end{array}$

Tests that the Variances are Equal

$\begin{array}{lrrrr}\text { Level } & \text { Count } & \text { Std Dev } & \text { MeanAbsDif to Mean } & \text { MeanAbsDif to Median } \\ \text { Coliwasa } & 16 & 0.0026703 & 0.0021043 & 0.0020937\end{array}$

$\begin{array}{lrrrr}\text { Coliwasa } & 16 & 0.0026703 & 0.0021043 & 0.0020937 \\ \text { Isolok - 12 mL - HF } & 10 & 0.0036278 & 0.0032419 & 0.0032419\end{array}$

Test F Ratio DFNum DFDen p-Value

O'Brien[.5] 2.8212

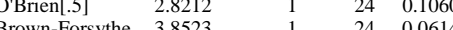

Brown-Forsythe $3.8523 \quad 1 \quad 24 \quad 0.0614$

$\begin{array}{lllll}\text { Levene } & 3.8648 & 1 & 24 & 0.0610 \\ \text { Bartlett } & 1.0479 & 1 & . & 0.3060\end{array}$

$\begin{array}{lllll}\text { F Test 2-sided } & 1.8458 & 9 & 15 & 0.2825\end{array}$

Welch Anova testing Means Equal, allowing Std Devs Not Equal

F Ratio DFNum DFDen Prob $>$ F

$\begin{array}{llll}0.0019 & 1 & 15.089 & 0.9661\end{array}$

t Test
Oneway Analysis of Measurement (wt \%) By Sampler Type of Material=SME Simulant, Analytical Block $=5$, Analyte $=A \mathrm{I} / \mathrm{B}$, Target $(\mathrm{wt} \%)=2.0704412$

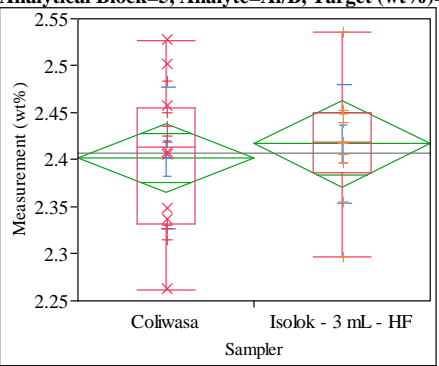

Oneway Anova

Summary of Fit

Adj Rsquare

0.011712

-0.02947
Root Mean Square Error $\quad 0.070742$

Mean of Response 2.40756

Observations (or Sum Wgts) $\quad 26$

t Test

Isolok - $3 \mathrm{~mL}$ - HF-Coliwasa

Assuming equal variances

Difference $\quad 0.01521$ t Ratio 0.533319

$\begin{array}{llr}\text { Std Err Dif } \quad 0.02852 \text { DF } & 24\end{array}$

Upper CL Dif 0.07407 Prob $>|t| 0.5987$

$\begin{array}{lll}\text { Lower CL Dif }-0.04365 \text { Prob }>t & 0.2994\end{array}$

$\begin{array}{lrl}\text { Confidence } & 0.95 \text { Prob }<\mathrm{t} \quad 0.7006\end{array}$

Analysis of Variance

Source DF Sum of Squares Mean Square F Ratio Prob $>$ F

$\begin{array}{lllllll}\text { Sampler } & 1 & 0.00142341 & 0.001423 & 0.2844 & 0.5987\end{array}$

$\begin{array}{llll}\text { Error } & 24 & 0.12010695 & 0.005004\end{array}$

C. Total $25 \quad 0.12153037$

Means for Oneway Anova

Level Number Mean Std Error Lower 95\% Upper 95\%

$\begin{array}{lrrrrr}\text { Coliwasa } & 16 & 2.40171 & 0.01769 & 2.3652 & 2.4382\end{array}$

$\begin{array}{llllll}\text { Isolok - 3 mL - HF } & 16 & 2.40171 & 0.01769 & 2.3652 & 2.4382 \\ & 10 & 2.41692 & 0.02237 & 2.3707 & 2.4631\end{array}$

Std Error uses a pooled estimate of error variance

Means and Std Deviations

Level Number Mean Std Dev Std Err Mean Lower 95\% Upper 95\%

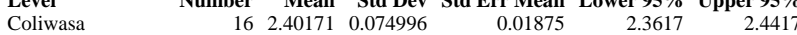

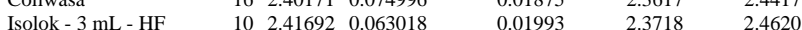

tTest

Isolok - $3 \mathrm{~mL}$ - HF-Coliwasa

Assuming unequal variances

$\begin{array}{lrlr}\text { Difference } & 0.01521 & \text { t Ratio } & 0.555842 \\ \text { Std Err Dif } & 0.02736 \text { DF } & 21.7565 \\ \text { Upper CL Dif } & 0.07199 & \text { Prob }>|t| & 0.5840 \\ \text { Lower CL Dif } & -0.04157 & \text { Prob }>\text { t } & 0.2920 \\ \text { Confidence } & 0.95 & \text { Prob }<\mathrm{t} & 0.7080\end{array}$

Tests that the Variances are Equal

Level Count Std Dev MeanAbsDif to Mean MeanAbsDif to Median

$\begin{array}{lllll}\text { Coliwasa } & 16 & 0.0749956 & 0.0614966 & 0.0605266\end{array}$

Isolok - $3 \mathrm{~mL}$ - HF $\quad 10 \quad 0.0630184$

Test F Ratio DFNum DFDen p-Value

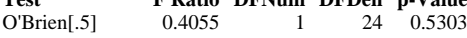

Brown-Forsythe $1.0798 \quad 1124 \quad 0.3091$

$\begin{array}{lllll}\text { Levene } & 1.2880 & 1 & 24 & 0.2676\end{array}$

$\begin{array}{lrrrr}\text { Bartlett } & 0.3152 & 1 & \text {. } & 0.5745 \\ \text { F Test 2-sided } & 1.4162 & 15 & 9 & 0.6089\end{array}$

Welch Anova testing Means Equal, allowing Std Devs Not Equal

F Ratio DFNum DFDen Prob $>$ F

$0.3090 \quad 1 \quad 21.756 \quad 0.5840$

t Test

0.5558 
SRNL-STI-2011-00693

Revision 0

\section{Exhibit B2. Statistical Comparisons for High-Rheology (Phase 2) Simulant Testing}

Oneway Analysis of Measurement (wt \%) By Sampler Type of Material=SME Simulant, Analytical Block=5, Analyte=Al2O3 (wt \%), Target $($ wt $\%)=10.934$

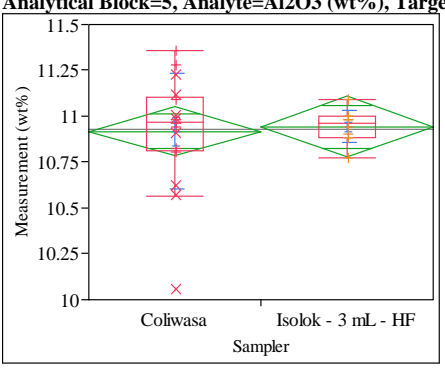

Oneway Anova

Summary of Fit

Rsquare

Adj Rsquare

Root Mean Square Error $\quad 0.254479$

Mean of Response 10.92712

Observations (or Sum Wgts)

Test

Isolok - $3 \mathrm{~mL}$ - HF-Coliwasa

Assuming equal variances

Difference $\quad 0.02433$ t Ratio $\quad 0.237146$

$\begin{array}{lrrr}\text { Std Err Dif } & 0.10258 \text { DF } & 24 \\ \text { Dpper CL Dif } & 0.23605 \text { Prob }>|t| & 0.8146\end{array}$

$\begin{array}{rrr}\text { Upper CL Dif } & 0.23605 \text { Prob }>|t| & 0.8146 \\ \text { Cower } & & \end{array}$

$\begin{array}{lrl}\text { Confidence } & 0.95 \text { Prob }<\mathrm{t} & 0.5927\end{array}$

Analysis of Variance

Source DF Sum of Squares Mean Square F Ratio Prob > F

$\begin{array}{lrrrrr}\text { Sampler } & 1 & 0.0036420 & 0.003642 & 0.0562 & 0.8146\end{array}$

$\begin{array}{llll}\text { Error } & 24 & 1.5542330 & 0.064760\end{array}$

C. Total $25 \quad 1.5578750$

Means for Oneway Anova

Level Number Mean Std Error Lower 95\% Upper 95\%

$\begin{array}{lrrrrr}\text { Coliwasa } & \text { Number } & \text { Mean } & \text { Std Error } & \text { Lower 95\% } & \text { Upper 95\% } \\ \text { 16 } & 10.9178 & 0.06362 & 10.786 & 11.049\end{array}$

$\begin{array}{llllll}\text { Coliwasa } & 16 & 10.9178 & 0.06362 & 10.786 & 11.049 \\ \text { Isolok - 3 mL - HF } & 10 & 10.9421 & 0.08047 & 10.776 & 11.108\end{array}$

Std Error uses a pooled estimate of error variance

Means and Std Deviations

Level Number Mean Std Dev Std Err Mean Lower 95\% Upper 95\%

$\begin{array}{llllllr}\text { Coliwasa } & 16 & 10.9178 & 0.314681 & 0.07867 & 10.750 & 11.085 \\ \text { Isolok - 3 mL - HF } & 10 & 10.9421 & 0.087477 & 0.02766 & 10.880 & 11.005\end{array}$

t Test

Isolok - $3 \mathrm{~mL}$ - HF-Coliwasa

Assuming unequal variances

Difference $\quad 0.02433$ t Ratio $\quad 0.291722$

Std Err Dif $\quad 0.08339$ DF $\quad 18.468$

Upper CL Dif 0.19921 Prob $>|t| 0.7738$

Lower CL Dif -0.15056 Prob $>t \quad 0.3869$

$\begin{array}{lrl}\text { Confidence } & 0.95 \text { Prob }<\mathrm{t} \quad 0.6131\end{array}$

Tests that the Variances are Equal

$\begin{array}{lrrrr}\text { Level } & \text { Count } & \text { Std Dev } & \text { MeanAbsDif to Mean } & \text { MeanAbsDif to Median } \\ \text { Coliwasa } & 16 & 0.3146812 & 0.2169973 & 0.2090259\end{array}$

Isolok - $3 \mathrm{~mL}$ - HF $\quad 10 \quad 0.0874766 \quad 0.0661325 \quad 0.0661325$

$\begin{array}{lrrrr}\text { Test } & \text { F Ratio DFNum DFDen p-Value } & \\ \text { O'Brien[5] } & 1.9655 & 1 & 0.1737\end{array}$

$\begin{array}{lllll}\text { O'Brien[.5] } & 1.9655 & 1 & 24 & 0.1737 \\ \text { Brown-Forsythe } & 3.5247 & 1 & 24 & 0.0727\end{array}$

$\begin{array}{lllll}\text { Brown-Forsythe } & 3.5247 & 1 & 24 & 0.0727 \\ \text { Levene } & 4.4400 & 1 & 24 & 0.0457\end{array}$

$\begin{array}{lrrrr}\text { Bartlett } & 12.2933 & 1 & . & 0.0005\end{array}$

$\begin{array}{lllll}\text { F Test 2-sided } & 12.9407 & 15 & 9 & 0.0005\end{array}$

Welch Anova testing Means Equal, allowing Std Devs Not Equal

F Ratio DFNum DFDen Prob $>$ F

$\begin{array}{llll}0.0851 & 1 & 18.468 & 0.7738\end{array}$

t Test

0.2917
Oneway Analysis of Measurement (wt \%) By Sampler Type of Material=SME Simulant, Analytical Block $=5$, Analyte $=\mathrm{B} 2 \mathrm{O} 3(\mathrm{wt} \%)$, Target $(\mathrm{wt} \%)=5.281$

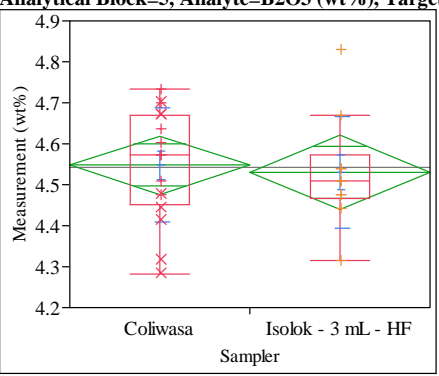

Oneway Anova

Summary of Fit

square

Adj Rsquare $\quad 0.004184$

Root Mean Square Error $\quad 0.138353$

Mean of Respor

Observations (or Sum Wgts) $\quad 26$

t Test

Isolok - $3 \mathrm{~mL}$ - HF-Coliwasa

Assuming equal variances

Difference $\quad-0.01771$ t Ratio $\quad-0.31753$

$\begin{array}{lll}\text { Std Err Dif } \quad 0.05577 \text { DF } & 24\end{array}$

Upper CL Dif 0.09740 Prob $>|t| \quad 0.7536$

Lower CL Dif -0.13282 Prob $>\mathrm{t} \quad 0.6232$

$\begin{array}{lrl}\text { Confidence } & 0.95 \text { Prob }<\mathrm{t} \quad 0.3768\end{array}$

Analysis of Variance

Source DF Sum of Squares Mean Square F Ratio Prob $>$ F

$\begin{array}{lrrrrr}\text { Sampler } & 1 & 0.00193000 & 0.001930 & 0.1008 & 0.7536\end{array}$

$\begin{array}{lrrr}\text { Sampler } & 1 & 0.00193000 & 0.001930 \\ \text { Error } & 24 & 0.45939527 & 0.019141\end{array}$

$\begin{array}{lll}\text { C. Total } & 25 & 0.46132527\end{array}$

Means for Oneway Anova

Level Number Mean Std Error Lower 95\% Upper 95\%

$\begin{array}{llllll}\text { Coliwasa } & 16 & 4.54811 & 0.03459 & 4.4767 & 4.6195 \\ \text { Isolok - 3 mL - HF } & 10 & 4.53040 & 0.04375 & 4.4401 & 4.6207\end{array}$

Std Error uses a pooled estimate of error variance

Means and Std Deviations

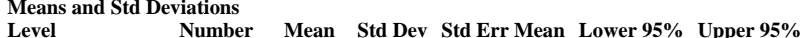

$\begin{array}{lrrrrrr}\text { Level } & \text { Number } & \text { Mean } & \text { Std Dev } & \text { Std Err Mean } & \text { Lower 95\% } & \text { Upper 95\% } \\ \text { Coliwasa } & 16 & 4.54811 & 0.138867 & 0.03472 & 4.4741 & 4.6221\end{array}$

$\begin{array}{lllllll}\text { Isolok - 3 mL - HF } & 16 & 4.54811 & 0.138867 & 0.03472 & 4.4741 & 4.6221 \\ & 10 & 4.53040 & 0.137491 & 0.04348 & 4.4320 & 4.6288\end{array}$

t Test

Isolok - $3 \mathrm{~mL}$ - HF-Coliwasa

Assuming unequal variances

Difference $\quad-0.01771$ t Ratio $\quad-0.3183$

$\begin{array}{lll}\text { Std Err Dif } \quad 0.05564 & \text { DF } & 19.40248\end{array}$

$\begin{array}{lrr}\text { Upper CL Dif } & 0.09858 \text { Prob }>|t| & 0.7537\end{array}$

Lower CL Dif -0.13400 Prob $>t \quad 0.6232$

$\begin{array}{lll}\text { Confidence } & 0.95 \text { Prob }<\mathrm{t} \quad 0.3768\end{array}$

Tests that the Variances are Equal

Level Count Std Dev MeanAbsDif to Mean MeanAbsDif to Median

$\begin{array}{lrrrr}\text { Coliwasa } & 16 & 0.1388669 & 0.1157152 & 0.1126965\end{array}$

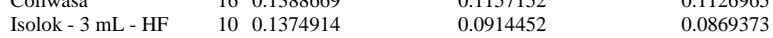

Test F Ratio DFNum DFDen p-Value

$\begin{array}{lllll}\text { O'Brien[.5] } & 0.0012 & 1 & 24 & 0.9729\end{array}$

Brown-Forsythe $\quad 0.5025 \quad 1 \quad 24 \quad 0.4852$

$\begin{array}{lllll}\text { Levene } & 0.5386 & 1 & 24 & 0.4701\end{array}$

$\begin{array}{lllll}\text { Bartlett } & 0.0011 & 1 & 0.9740\end{array}$

$\begin{array}{lllll}\text { F Test 2-sided } & 1.0201 & 15 & 9 & 1.0000\end{array}$

Welch Anova testing Means Equal, allowing Std Devs Not Equal

F Ratio DFNum DFDen Prob $>$ F

$\begin{array}{rrrr}0.1013 & 1 & 19.402 & 0.7537\end{array}$

t Test

0.3183 


\section{Exhibit B2. Statistical Comparisons for High-Rheology (Phase 2) Simulant Testing}

Oneway Analysis of Measurement (wt \%) By Sampler Type of Material=SME Simulant, Analytical Block=5, Analyte $=\mathrm{BaO}(\mathrm{wt} \%)$, Target $(\mathrm{wt} \%)=0$

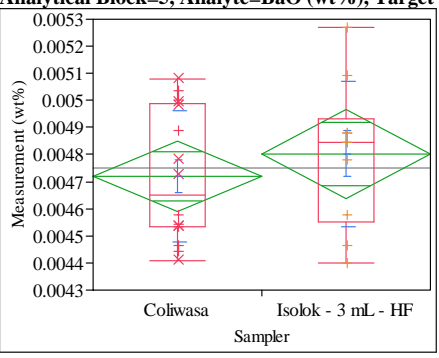

Oneway Anova

Summary of Fit

Adj Rsquare

$\begin{array}{lr}\text { Mean of Response } & 0.004752 \\ \text { Observations (or Sum Wgts) } & 26\end{array}$

t Test

Isolok - $3 \mathrm{~mL}$ - HF-Coliwasa

Assuming equal variances

Difference $\quad 8.318 \mathrm{e}-5$ t Ratio $\quad 0.820151$

$\begin{array}{lll}\text { Std Err Dif } \quad 0.00010 \text { DF } & 24\end{array}$

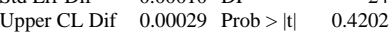

$\begin{array}{lll}\text { Lower CL Dif }-0.00013 \text { Prob }>t & 0.2101\end{array}$

Confidence $\quad 0.95$ Prob $<\mathrm{t} \quad 0.7899$

Analysis of Variance

Source DF Sum of Squares Mean Square F Ratio Prob $>$ F

$\begin{array}{lrrrrr}\text { Source } & \text { DF } & \text { Sum of Squares } & \text { Mean Square } & \text { F Ratio } & \text { Prob }>\text { F } \\ \text { Sampler } & 1 & 4.25772 \mathrm{e}-8 & 4.2577 \mathrm{e}-8 & 0.6726 & 0.4202\end{array}$

$\begin{array}{lrrr}\text { Sampler } & 1 & 4.25772 \mathrm{e}-8 & 4.2577 \mathrm{e}-8 \\ \text { Error } & 24 & 1.51915 \mathrm{e}-6 & 6.3298 \mathrm{e}-8\end{array}$

C. Total $25 \quad 1.56172 \mathrm{e}-6$

Means for Oneway Anova

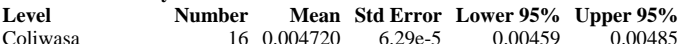

$\begin{array}{llllll}\text { Isolok - } 3 \text { mL - HF } & 16 & 0.004720 & 6.29 \mathrm{e}-5 & 0.00459 & 0.00485 \\ & 10 & 0.004803 & 0.00008 & 0.00464 & 0.00497\end{array}$

Std Error uses a pooled estimate of error variance

Means and Std Deviations

Level Number Mean Std Dev Std Err Mean Lower 95\% Upper 95\%

$\begin{array}{lrrrrrr}\text { Coliwasa } & 16 & 0.004720 & 0.000241 & 0.00006 & 0.00459 & 0.00485 \\ \text { Isolok - 3 mL - HF } & 10 & 0.004803 & 0.000268 & 8.46 e-5 & 0.00461 & 0.00499\end{array}$

t Test

Isolok - $3 \mathrm{~mL}$ - HF-Coliwasa

Assuming unequal variances

Difference $\quad 8.318 \mathrm{e}-5$ t Ratio $\quad 0.800208$

Std Err Dif $\quad 0.00010$ DF 17.73673

Upper CL Dif $\quad 0.00030$ Prob $>|t| r 0.4342$

Lower CL Dif -0.00014 Prob $>t \quad 0.2171$

Confidence $\quad 0.95$ Prob $<\mathrm{t} \quad 0.7829$

Tests that the Variances are Equal

Level Count Std Dev MeanAbsDif to Mean MeanAbsDif to Median

$\begin{array}{lrrrr}\text { Coliwasa } & 16 & 0.0002415 & 0.0002149 & 0.0002149\end{array}$

$\begin{array}{lllll}\text { Isolok - } 3 \mathrm{~mL} \text { - HF } & 10 & 0.0002676 & 0.0002149 & 0.0001983\end{array}$

Test F Ratio DFNum DFDen p-Value

$\begin{array}{lrrrr}\text { O'Brien[.5] } & 0.2572 & 1 & 24 & 0.6167\end{array}$

$\begin{array}{lllll}\text { Brown-Forsythe } & 0.1807 & 1 & 24 & 0.6745\end{array}$

$\begin{array}{lllll}\text { Levene } & 0.1057 & 1 & 24 & 0.7480\end{array}$

$\begin{array}{lllll}\text { Bartlett } & 0.1152 & 1 & . & 0.7344\end{array}$

Welch Anova testing Means Equal, allowing Std Devs Not Equal

F Ratio DFNum DFDen Prob $>$ F

$\begin{array}{llll}0.6403 & 1 & 17.737 & 0.4342\end{array}$

t Test

0.8002
Oneway Analysis of Measurement (wt \%) By Sampler Type of Material=SME Simulant, Analytical Block $=5$, Analyte $=\mathrm{CaO}(\mathrm{wt} \%)$, Target $(\mathrm{wt} \%)=0.543$

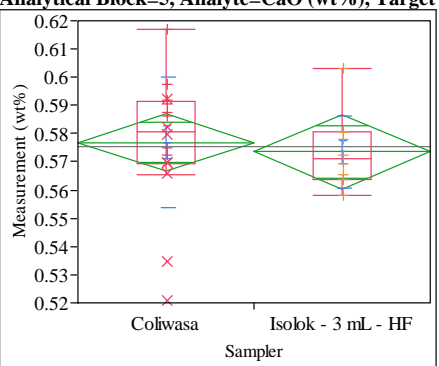

Oneway Anova

Summary of Fit

Adj Rsquare

Root Mean Square Error $\quad-0.03415$

gts)

26

t Test

Isolok - $3 \mathrm{~mL}$ - HF-Coliwasa

Assuming equal variances

$\begin{array}{lrlr}\text { Difference } & -0.00338 & \text { t Ratio } & -0.41763 \\ \text { Std Err Dif } & 0.00808 \text { DF } & 24 \\ \text { Upper CL Dif } & 0.01331 & \text { Prob }>|t| & 0.6799 \\ \text { Lower CL Dif } & -0.02006 & \text { Prob }>\text { t } & 0.6600 \\ \text { Confidence } & 0.95 & \text { Prob }<\mathrm{t} & 0.3400\end{array}$

Analysis of Variance

Source DF Sum of Squares Mean Square F Ratio Prob > F

$\begin{array}{llllll}\text { Sampler } & 1 & 0.00007012 & 0.000070 & 0.1744 & 0.6799\end{array}$

$\begin{array}{llll}\text { Error } & 24 & 0.00964851 & 0.000402\end{array}$

C. Total $25 \quad 0.00971863$

Means for Oneway Anova

Level Number Mean Std Error Lower 95\% Upper 95\%

\begin{tabular}{lrrrrr} 
Coliwasa & 16 & 0.576908 & 0.00501 & 0.56656 & 0.58725 \\
\hline
\end{tabular}

$\begin{array}{llllll}\text { Isolok - 3 mL - HF } & 10 & 0.573532 & 0.00634 & 0.56045 & 0.58662\end{array}$

Std Error uses a pooled estimate of error variance

Means and Std Deviations

Level Number Mean Std Dev Std Err Mean Lower 95\% Upper 95\%

$\begin{array}{lrrrrrr}\text { Coliwasa } & 16 & 0.576908 & 0.023311 & 0.00583 & 0.56449 & 0.58933\end{array}$

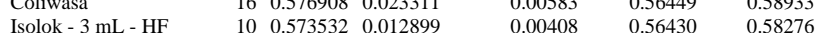

Test

Isolok - $3 \mathrm{~mL}$ - HF-Coliwasa

Assuming unequal variances

$\begin{array}{lrlr}\text { Difference } & -0.00338 & \mathrm{t} \text { Ratio } & -0.47453 \\ \text { Std Err Dif } & 0.00711 \text { DF } & 23.7836 \\ \text { Upper CL Dif } & 0.01131 & \text { Prob }>|\mathrm{t}| & 0.6394 \\ \text { Lower CL Dif } & -0.01806 & \text { Prob }>\mathrm{t} & 0.6803 \\ \text { Confidence } & 0.95 & \text { Prob }<\mathrm{t} & 0.3197\end{array}$

Tests that the Variances are Equal

Level Count Std Dev MeanAbsDif to Mean MeanAbsDif to Median

$\begin{array}{lllll}\text { Coliwasa } & 16 & 0.0233110 & 0.0164734 & 0.0161783\end{array}$

$\begin{array}{llll}\text { Isolok - } 3 \mathrm{~mL} \text { - HF } & 10 & 0.0128991 & 0.0096265\end{array}$

Test F Ratio DFNum DFDen p-Value

$\begin{array}{lrrrr}\text { O'Brien[.5] } & 1.3369 & 1 & 24 & 0.2590\end{array}$

Brown-Forsythe $1.4013 \quad 1124 \quad 0.2481$

$\begin{array}{lllll}\text { Levene } & 1.5808 & 1 & 24 & 0.2207\end{array}$

$\begin{array}{lrrrr}\text { Bartlett } & 3.2711 & 1 & \text {. } & 0.0705 \\ \text { F Test 2-sided } & 3.2659 & 15 & 9 & 0.0781\end{array}$

Welch Anova testing Means Equal, allowing Std Devs Not Equal

F Ratio DFNum DFDen Prob $>$ F

$0.2252 \quad 1 \quad 23.784 \quad 0.6394$

t Test

0.4745 
SRNL-STI-2011-00693

Revision 0

\section{Exhibit B2. Statistical Comparisons for High-Rheology (Phase 2) Simulant Testing}

Oneway Analysis of Measurement (wt \%) By Sampler Type of Material=SME Simulant, Analytical Block=5, Analyte $=\mathrm{Cr} 2 \mathrm{O} 3(\mathrm{wt} \%)$, Target $(\mathrm{wt} \%)=\mathbf{0 . 0 1 5}$

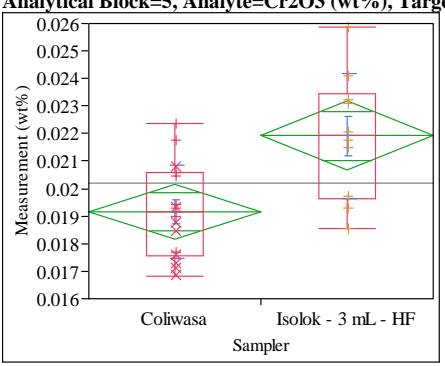

Oneway Anova

Summary of Fit

Rsquare

Adj Rsquare

Root Mean Square Error $\quad 0.001929$

Mean of Response 0.020221

Observations (or Sum Wgts)

Test

Isolok - $3 \mathrm{~mL}$ - HF-Coliwasa

Assuming equal variances

Difference $\quad 0.002768$ t Ratio $\quad 3.559301$

$\begin{array}{lrr}\text { Std Err Dif } & 0.000778 \text { DF } & 24 \\ & & \end{array}$

$\begin{array}{lll}\text { Upper CL Dif } 0.004373 & \text { Prob }>|t| & 0.0016 \\ \text { Contif } & 0.0008\end{array}$

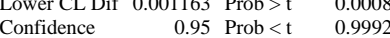

Analysis of Variance

Source DF Sum of Squares Mean Square F Ratio Prob $>$ F

$\begin{array}{lrrrrr}\text { Sampler } & 1 & 0.00004715 & 0.000047 & 12.6686 & 0.0016\end{array}$

$\begin{array}{llll}\text { Error } & 24 & 0.00008932 & 3.722 \mathrm{e}-6\end{array}$

C. Total $25 \quad 0.00013646$

Means for Oneway Anova

Level Number Mean Std Error Lower 95\% Upper 95\%

$\begin{array}{lrrrrr}\text { Coliwasa } & 16 & 0.019156 & 0.00048 & 0.01816 & 0.02015\end{array}$

$\begin{array}{llllll}\text { Isolok - } 3 \mathrm{~mL} \text { - HF } & 10 & 0.021924 & 0.00061 & 0.02066 & 0.02318\end{array}$

Std Error uses a pooled estimate of error variance

Means and Std Deviations

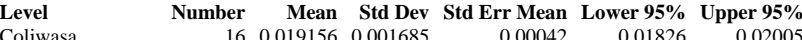

$\begin{array}{llllll}16 & 0.019156 & 0.001685 & 0.00042 & 0.01826 & 0.02005 \\ 10 & 0.021924 & 0.002279 & 0.00072 & 0.02029 & 0.02355\end{array}$

t Test

Isolok - $3 \mathrm{~mL}$ - HF-Coliwasa

Assuming unequal variances

Difference $\quad 0.002768$ t Ratio 3.315995

Std Err Dif $\quad 0.000835$ DF $\quad 15.13853$

Upper CL Dif 0.004546 Prob $>|t| \quad 0.0047$

Lower CL Dif 0.000990 Prob $>t \quad 0.0023$

Confidence $\quad 0.95$ Prob $<\mathrm{t} \quad 0.9977$

Tests that the Variances are Equal

$\begin{array}{lrrrr}\text { Level } & \text { Count } & \text { Std Dev } & \text { MeanAbsDif to Mean } & \text { MeanAbsDif to Median } \\ \text { Coliwasa } & 16 & 0.0016847 & 0.0013428 & 0.0013428\end{array}$

$\begin{array}{lllll}\text { Isolok - } 3 \mathrm{~mL} \text { - HF } & 10 & 0.0022789 & 0.0017539 & 0.0017539\end{array}$

Test F Ratio DFNum DFDen p-Value

Test [5] F Ratio DFNum DFDen p-Value

$\begin{array}{lllll}\text { O'Brien[.5] } & 1.6241 & 1 & 24 & 0.2147 \\ \text { Brown-Forsythe } & 0.8402 & 1 & 24 & 0.3685\end{array}$

$\begin{array}{lllll}\text { Brown-Forsythe } & 0.8402 & 1 & 24 & 0.3685\end{array}$

$\begin{array}{lllll}\text { Levene } & 0.8403 & 1 & 24 & 0.3684 \\ \text { Bartlett } & 1.0181 & 1 & & 0.3130\end{array}$

$\begin{array}{lllll}\text { F Test 2-sided } & 1.8298 & 9 & 15 & 0.2891\end{array}$

Welch Anova testing Means Equal, allowing Std Devs Not Equal

F Ratio DFNum DFDen Prob $>$ F

$\begin{array}{llll}10.9958 & 1 & 15.139 & 0.0047\end{array}$

t Test

3.3160
Oneway Analysis of Measurement (wt \%) By Sampler Type of Material=SME Simulant, Analytical Block $=5$, Analyte $=\mathrm{CuO}(\mathrm{wt} \%)$, Target $(\mathrm{wt} \%)=0.103$

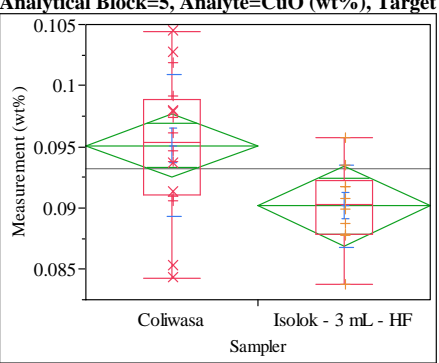

Oneway Anova

Summary of Fit

Rsquare

Adj Rsquare

Root Mean Square Error $\quad 0.005$

Mean of Response 0.093201

Test

Isolok - $3 \mathrm{~mL}$ - HF-Coliwasa

Assuming equal variances

Difference $\quad-0.00495$ t Ratio -2.45622

$\begin{array}{lrr}\text { Std Err Dif } & 0.00202 \text { DF } & 24 \\ \text { Upper CL Dif } & -0.00079 \text { Prob }>|\mathrm{t}| & 0.0217\end{array}$

$\begin{array}{lrll}\text { Upper CL Dif } & -0.00079 & \text { Prob }>|t| & 0.0217 \\ \text { Lower CL Dif }-0.00911 & \text { Prob }>t & 0.9892\end{array}$

$\begin{array}{lrr}\text { Confidence } & 0.95 \text { Prob }<\mathrm{t} & 0.0108\end{array}$

Analysis of Variance

Source DF Sum of Squares Mean Square F Ratio Prob $>$ F

$\begin{array}{lrrrrr}\text { Sampler } & 1 & 0.00015084 & 0.000151 & 6.0330 & 0.0217\end{array}$

$\begin{array}{llll}\text { Error } & 24 & 0.00060005 & 0.000025\end{array}$

C. Total $25 \quad 0.00075088$

Means for Oneway Anova

Level Number Mean Std Error Lower 95\% Upper 95\%

$\begin{array}{lrrrrr}\text { Level } & \text { Number } & \text { Mean } & \text { Std Error } & \text { Lower 95\% } & \text { Upper 95\% } \\ \text { Coliwasa } & 16 & 0.095106 & 0.00125 & 0.09253 & 0.09769\end{array}$

$\begin{array}{llllll}\text { Isolok - 3 mL - HF } & 10 & 0.090155 & 0.00158 & 0.08689 & 0.09342\end{array}$

Std Error uses a pooled estimate of error variance

Means and Std Deviations

Level Number Mean Std Dev Std Err Mean Lower 95\% Upper 95\%

$\begin{array}{lllllll}\text { Coliwasa } & 16 & 0.095106 & 0.005761 & 0.00144 & 0.09204 & 0.09818\end{array}$

$\begin{array}{lllllll}\text { Isolok - } 3 \mathrm{~mL} \text { - HF } & 10 & 0.090155 & 0.003371 & 0.00107 & 0.08774 & 0.09257\end{array}$

\section{t Test}

Isolok - $3 \mathrm{~mL}$ - HF-Coliwasa

Assuming unequal variances

$\begin{array}{lrlr}\text { Difference } & -0.00495 & \text { t Ratio } & -2.76308 \\ \text { Std Err Dif } & 0.00179 & \text { DF } & 23.95493 \\ \text { Upper CL Dif } & -0.00125 & \text { Prob }>|t| & 0.0108 \\ \text { Lower CL Dif } & -0.00865 & \text { Prob }>\text { t } & 0.9946 \\ \text { Confidence } & 0.95 & \text { Prob }<\mathrm{t} & 0.0054\end{array}$

Tests that the Variances are Equal

$\begin{array}{lrrrr}\text { Level } & \text { Count } & \text { Std Dev } & \text { MeanAbsDif to Mean } & \text { MeanAbsDif to Median } \\ \text { Coliwasa } & 16 & 0.0057605 & 0.0045534 & 0.0045534\end{array}$

$\begin{array}{llll}\text { Isolok - } 3 \mathrm{~mL} \text { - HF } & 10 & 0.0033712 & 0.0025537\end{array}$

Test F Ratio DFNum DFDen p-Value

O'Brien[.5] $2.3902-11240.1352$

$\begin{array}{lllll}\text { Brown-Forsythe } & 2.3802 & 1 & 24 & 0.1352 \\ \text { B } & 1 & 24 & 0.1023\end{array}$

$\begin{array}{lllll}\text { Brown-Forsythe } & 2.8863 & 1 & 24 & 0.1023 \\ \text { Levene } & 2.9081 & 1 & 24 & 0.1010\end{array}$

$\begin{array}{lllll}\text { Levene } & 2.9081 & 1 & 24 & 0.1010 \\ \text { Bartlett } & 2.7252 & 1 & . & 0.0988\end{array}$

$\begin{array}{lllll}\text { F Test 2-sided } & 2.9198 & 15 & 9 & 0.1089\end{array}$

Welch Anova testing Means Equal, allowing Std Devs Not Equal

F Ratio DFNum DFDen Prob $>$ F

$\begin{array}{llll}7.6346 & 1 & 23.955 & 0.0108\end{array}$

t Test

2.763 


\section{Exhibit B2. Statistical Comparisons for High-Rheology (Phase 2) Simulant Testing}

Oneway Analysis of Measurement (wt\%) By Sampler Type of Material=SME Simulant, Analytical Block=5, Analyte=Fe/Li, Target $(\mathrm{wt} \%)=1.99097835$

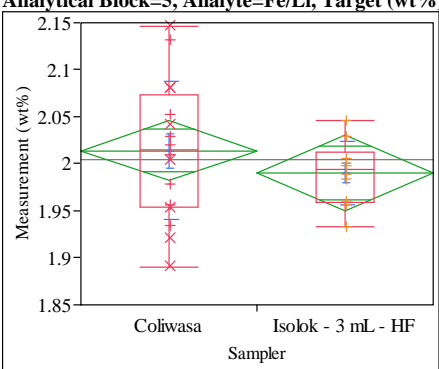

Oneway Anova

Summary of Fit

Adj Rsquare

0.036614

\begin{tabular}{ll}
-0.00353 \\
\hline
\end{tabular}

Mean of Response 2.004898

Observations (or Sum Wgts) 26

t Test

Isolok - $3 \mathrm{~mL}$ - HF-Coliwasa

Assuming equal variances

Difference $\quad-0.02379$ t Ratio $\quad-0.95506$

Std Err Dif $\quad 0.02491$ DF $\quad 24$

$\begin{array}{rrr}\text { Upper CL Dif } & 0.02762 \text { Prob }>|t| & 0.3491\end{array}$

$\begin{array}{llll}\text { Lower CL Dif }-0.07519 & \text { Prob }>t & 0.8255\end{array}$

$\begin{array}{lrr}\text { Confidence } & 0.95 \text { Prob }<\mathrm{t} & 0.1745\end{array}$

Analysis of Variance

Source DF Sum of Squares Mean Square F Ratio Prob $>$ F

$\begin{array}{llllll}\text { Sampler } & 1 & 0.00348168 & 0.003482 & 0.9121 & 0.3491\end{array}$

$\begin{array}{llll}\text { Error } & 24 & 0.09160904 & 0.003817\end{array}$

C. Total $25 \quad 0.09509072$

Means for Oneway Anova

Level Number Mean Std Error Lower 95\% Upper 95\%

$\begin{array}{llrrrr}\text { Coliwasa } & 16 & 2.01405 & 0.01545 & 1.9822 & 2.0459\end{array}$

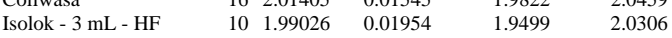

Std Error uses a pooled estimate of error variance

Means and Std Deviations

Level Number Mean Std Dev Std Err Mean Lower 95\% Upper 95\%

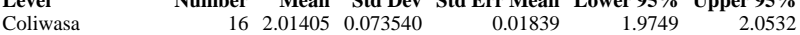

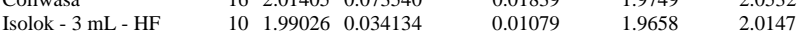

tTest

Isolok - $3 \mathrm{~mL}$ - HF-Coliwas

Assuming unequal variances

Difference $\quad-0.02379$ t Ratio $\quad-1.11568$

Std Err Dif $\quad 0.02132$ DF 22.64003

Upper CL Dif 0.02036 Prob $>|t| r \mid \begin{array}{ll}22763 \\ \text { L }\end{array}$

Lower CL Dif -0.06793 Prob $>\mathrm{t} \quad 0.8619$

$\begin{array}{lrl}\text { Confidence } & 0.95 \text { Prob }<\mathrm{t} \quad 0.1381\end{array}$

Tests that the Variances are Equal

Level Count Std Dev MeanAbsDif to Mean MeanAbsDif to Median

$\begin{array}{lllll} & 16 & 0.0735403 & 0.0583708 & 0.0583708 \\ \text { Isolok - } 3 \mathrm{~mL} \text { - HF } & 10 & 0.0341345 & 0.0257641 & 0.0257641\end{array}$

Test F Ratio DFNum DFDen p-Value

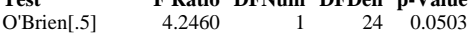

Brown-Forsythe $5.1353 \quad 1 \quad 24 \quad 0.0327$

$\begin{array}{lllll}\text { Levene } & 5.1557 & 1 & 24 & 0.0324\end{array}$

$\begin{array}{lrrrr}\text { Bartlett } & 5.2164 & 1 & \text {. } & 0.0224 \\ \text { F Test 2-sided } & 4.6416 & 15 & 9 & 0.0252\end{array}$

Welch Anova testing Means Equal, allowing Std Devs Not Equal

F Ratio DFNum DFDen Prob $>$ F

$\begin{array}{lrrr}1.2448 & 1 & 22.64 & 0.2763\end{array}$

t Test

1.1157
Oneway Analysis of Measurement (wt \%) By Sampler Type of Material=SME Simulant, Analytical Block=5, Analyte $=$ Fe2O3 (wt \%), Target $($ wt $\%)=9.931$

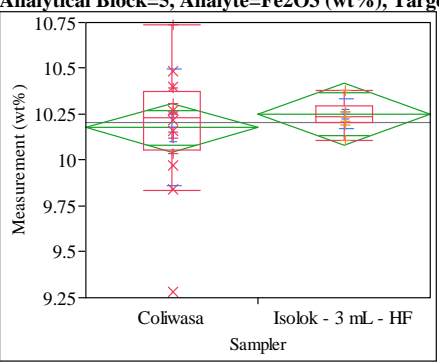

Oneway Anova

Summary of Fit

Rsquare

Adj Rsquare

Root Mean Square Error $\quad 0.257408$

Mean of Response 10.20586

Observations (or Sum Wgts)

26

Isolok - $3 \mathrm{~mL}$ - HF-Coliwasa

Assuming equal variances

Difference $\quad 0.07327$ t Ratio 0.706139

$\begin{array}{lrr}\text { Std Err Dif } & 0.10376 \text { DF } & 24 \\ & \end{array}$

$\begin{array}{lll}\text { Upper CL Dif } & 0.28743 \text { Prob }>|t| & 0.4869 \\ \text { Lower } & & \end{array}$

$\begin{array}{lrll}\text { Lower CL Dif } & -0.14089 & \text { Prob }>t & 0.2435 \\ \text { Confidence } & 0.95 & \text { Prob }<\mathrm{t} & 0.7565\end{array}$

Analysis of Variance

Source DF Sum of Squares Mean Square F Ratio Prob $>$ F

$\begin{array}{llllll}\text { Sampler } & 1 & 0.0330388 & 0.033039 & 0.4986 & 0.4869\end{array}$

$\begin{array}{llll}\text { Error } & 24 & 1.5902136 & 0.066259\end{array}$

C. Total $25 \quad 1.6232524$

Means for Oneway Anova

Level Number Mean Std Error Lower 95\% Upper 95\%

$\begin{array}{lrrrrr}\text { Level } & \text { Number } & \text { Mean } & \text { Std Error } & \text { Lower 95\% } & \text { Upper 95\% } \\ \text { Coliwasa } & 16 & 10.1777 & 0.06435 & 10.045 & 10.310\end{array}$

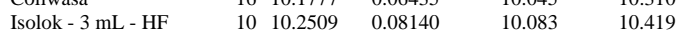

Std Error uses a pooled estimate of error variance

Means and Std Deviations

Level Number Mean Std Dev Std Err Mean Lower 95\% Upper 95\%

$\begin{array}{lllllll}\text { Coliwasa } & 16 & 10.1777 & 0.319429 & 0.07986 & 10.007 & 10.348\end{array}$

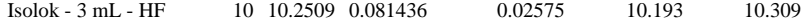

t Test

Isolok - $3 \mathrm{~mL}$ - HF-Coliwasa

Assuming unequal variances

Difference $\quad 0.07327$ t Ratio $\quad 0.873255$

Std Err Dif $\quad 0.08391$ DF $\quad 17.9583$
Up

Upper CL Dif 0.24958 Prob $>|t| \quad 0.3941$

Lower CL Dif -0.10304 Prob $>\mathrm{t} \quad 0.1970$

Confidence $\quad 0.95$ Prob $<\mathrm{t} \quad 0.8030$

Tests that the Variances are Equal

$\begin{array}{lrrrr}\text { Level } & \text { Count } & \text { Std Dev } & \text { MeanAbsDif to Mean } & \text { MeanAbsDif to Median } \\ \text { Coliwasa } & 16 & 0.3194295 & 0.2128913 & 0.2090936\end{array}$

$\begin{array}{lllll} & 16 & 0.3194295 & 0.2128913 & 0.2090936 \\ \text { Isolok - } 3 \mathrm{~mL} \text { - HF } & 10 & 0.0814357 & 0.0571880 & 0.0543286\end{array}$

Test F Ratio DFNum DFDen p-Value

O'Brien[.5] 1.7363

$\begin{array}{lllll}\text { OBrien[.5] } & 1.7363 & 1 & 24 & 0.2000 \\ \text { Brown-Forsythe } & 3.9037 & 1 & 24 & 0.0598\end{array}$

$\begin{array}{lllll}\text { Brown-Forsythe } & 3.9037 & 1 & 24 & 0.0598 \\ \text { Levene } & 4.3018 & 1 & 24 & 0.0490\end{array}$

$\begin{array}{lllll}13.3018 & 1 & 24 & 0.0490\end{array}$

$\begin{array}{lrrrr}\text { Bartlett } & 13.6211 & 1 & \dot{0} & 0.0002 \\ \text { F Test 2-sided } & 15.3858 & 15 & 9 & 0.0003\end{array}$

Welch Anova testing Means Equal, allowing Std Devs Not Equal

F Ratio DFNum DFDen Prob $>$ F

$\begin{array}{llll}0.7626 & 1 & 17.958 & 0.3941\end{array}$

t Test

0.8733 
SRNL-STI-2011-00693

Revision 0

\section{Exhibit B2. Statistical Comparisons for High-Rheology (Phase 2) Simulant Testing}

Oneway Analysis of Measurement (wt \%) By Sampler Type of Material=SME Simulant, Analytical Block $=5$, Analyte $=\mathrm{K2O}(\mathrm{wt} \%)$, Target $(\mathrm{wt} \%)=0.076$

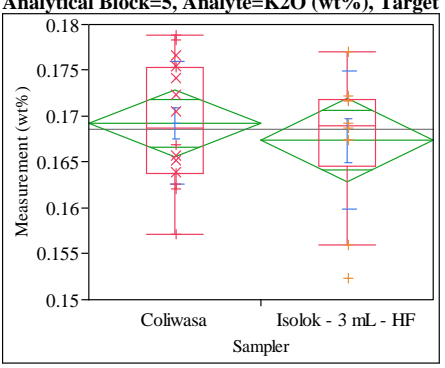

Oneway Anova

Summary of Fit

Rsquare

Adj Rsquare

0.017802

Root Mean Square Error $\quad 0.007023$

0.168528

Test

Isolok - $3 \mathrm{~mL}$ - HF-Coliwasa

Assuming equal variances

Difference $\quad-0.00187$ t Ratio $\quad-0.65954$

$\begin{array}{lrlr}\text { Std Err Dif } & 0.00283 \text { DF } & 24 \\ \text { Dpper CL Dif } & 0.00398 \text { Prob }>|t| & 0.5158\end{array}$

$\begin{array}{rrr}\text { Upper CL Dif } & 0.00398 \text { Prob }>|t| & 0.5158 \\ \text { Cower CL Dif }-0.00771 & \text { Prob }>t & 0.7421\end{array}$

$\begin{array}{lll}\text { Confidence } & 0.95 \text { Prob }<\mathrm{t} \quad 0.2579\end{array}$

Analysis of Variance

Source DF Sum of Squares Mean Square F Ratio Prob $>$ F

$\begin{array}{lrrrrr}\text { Sampler } & 1 & 0.00002145 & 0.000021 & 0.4350 & 0.5158\end{array}$

$\begin{array}{llll}\text { Error } & 24 & 0.00118367 & 0.000049\end{array}$

C. Total $25 \quad 0.00120512$

Means for Oneway Anova

Level Number Mean Std Error Lower 95\% Upper 95\%

$\begin{array}{lrrrrr}\text { Coliwasa } & 16 & 0.169246 & 0.00176 & 0.16562 & 0.17287\end{array}$

$\begin{array}{llllll}\text { Isolok - } 3 \mathrm{~mL} \text { - HF } & 10 & 0.167379 & 0.00222 & 0.16280 & 0.17196\end{array}$

Std Error uses a pooled estimate of error variance

Means and Std Deviations

Level Number Mean Std Dev Std Err Mean Lower 95\% Upper 95\% $\begin{array}{lllllll}\text { Coliwasa } & 16 & 0.169246 & 0.006682 & 0.00167 & 0.16569 & 0.17281\end{array}$ $\begin{array}{lllllll}\text { Isolok - } 3 \mathrm{~mL} \text { - HF } & 10 & 0.167379 & 0.007557 & 0.00239 & 0.16197 & 0.17279\end{array}$

Test

Isolok - $3 \mathrm{~mL}$ - HF-Coliwasa

Assuming unequal variances

Difference $\quad-0.00187$ t Ratio $\quad-0.64037$

Std Err Dif $\quad 0.00292$ DF $\quad 17.44415$

Upper CL Dif 0.00427 Prob $>|t| 0.5303$

Lower CL Dif -0.00801 Prob $>t \quad 0.7349$

$\begin{array}{lrl}\text { Confidence } & 0.95 \text { Prob }<\mathrm{t} & 0.2651\end{array}$

Tests that the Variances are Equal

$\begin{array}{lrrrr}\text { Level } & \text { Count } & \text { Std Dev } & \text { MeanAbsDif to Mean } & \text { MeanAbsDif to Median } \\ \text { Coliwasa } & 16 & 0.0066816 & 0.0058724 & 0.0058724\end{array}$

$\begin{array}{lllll}\text { Isolok - } 3 \mathrm{~mL} \text { - HF } & 10 & 0.0075572 & 0.0052761 & 0.0049991\end{array}$

Test F Ratio DFNum DFDen p-Value

$\begin{array}{lrrrr}\text { Test } & \text { F Ratio } & \text { DFNum } & \text { DFen } & \text { p-Value } \\ \text { O'Brien[.5] } & 0.2366 & 1 & 24 & 0.6311 \\ \text { Brown-Forsythe } & 0.2732 & 1 & 24 & 0.6060 \\ \text { Levene } & 0.1485 & 1 & 24 & 0.7033 \\ \text { Bartlett } & 0.1662 & 1 & & 0.6835 \\ \text { F Test 2-sided } & 1.2793 & 9 & 15 & 0.6468\end{array}$

Welch Anova testing Means Equal, allowing Std Devs Not Equal

F Ratio DFNum DFDen Prob $>$ F

$\begin{array}{llll}0.4101 & 1 & 17.444 & 0.5303\end{array}$

t Test

0.6404
Oneway Analysis of Measurement (wt \%) By Sampler Type of Material=SME Simulant, Analytical Block $=5$, Analyte $=\mathrm{Li} 2 \mathrm{O}(\mathrm{wt} \%)$, Target $(\mathrm{wt} \%)=4.988$

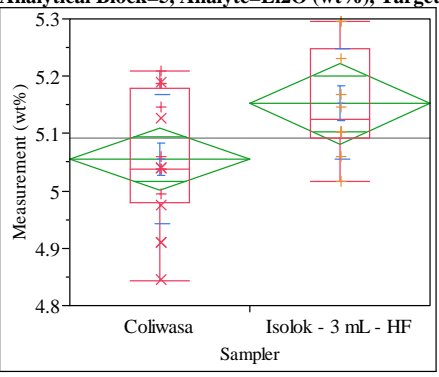

Oneway Anova

Summary of Fit

$\begin{array}{ll}\text { Rsquare } & 0.174428 \\ \text { Adj Rsquare } & 0.140029\end{array}$

0.1740029
Root Mean Square Error $\quad 0.106431$

Mean of Response

(a) 5.092437
26

t Test

Isolok - $3 \mathrm{~mL}$ - HF-Coliwasa

Assuming equal variances

Difference $\quad 0.096611$ t Ratio 2.25183

Std Err Dif $\quad 0.042904$ DF 24

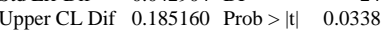

Lower CL Dif 0.008063 Prob $>$ t 0.0169

$\begin{array}{lll}\text { Confidence } & 0.95 \text { Prob }<\mathrm{t} \quad 0.9831\end{array}$

Analysis of Variance

Source DF Sum of Squares Mean Square F Ratio Prob $>$ F

$\begin{array}{lrrrrr}\text { Sampler } & 1 & 0.05743852 & 0.057439 & 5.0707 & 0.0338\end{array}$

$\begin{array}{llll}\text { Error } & 24 & 0.27185886 & 0.011327\end{array}$

C. Total $25 \quad 0.32929739$

Means for Oneway Anova

Level Number Mean Std Error Lower 95\% Upper 95\%

$\begin{array}{llllll}\text { Coliwasa } & 16 & 5.05528 & 0.02661 & 5.0004 & 5.1102 \\ \text { Isolok - 3 mL - HF } & 10 & 5.15189 & 0.03366 & 5.0824 & 5.2214\end{array}$

Std Error uses a pooled estimate of error variance

Means and Std Deviations

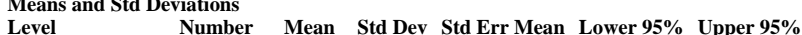

$\begin{array}{lrrrrrr}\text { Level } & \text { Number } & \text { Mean } & \text { Std Dev } & \text { Std Err Mean } & \text { Lower 95\% } & \text { Upper 95\% } \\ \text { Coliwasa } & 16 & 5.05528 & 0.112342 & 0.02809 & 4.9954 & 5.1151\end{array}$

$\begin{array}{llllllr}\text { Isolok - 3 mL - HF } & 16 & 5.05528 & 0.112342 & 0.02809 & 4.9954 & 5.1151 \\ & 10 & 5.15189 & 0.095771 & 0.03029 & 5.0834 & 5.2204\end{array}$

t Test

Isolok - $3 \mathrm{~mL}$ - HF-Coliwasa

Assuming unequal variances

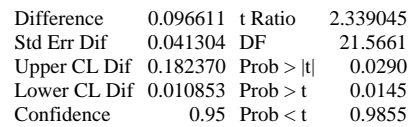

Tests that the Variances are Equal

Level Count Std Dev MeanAbsDif to Mean MeanAbsDif to Median

$\begin{array}{lrrrr}\text { Coliwasa } & 16 & 0.1123417 & 0.0896481 & 0.0874616\end{array}$

$\begin{array}{lllll}\text { Isolok - 3 mL - HF } & 10 & 0.0957711 & 0.0896481 & 0.0766432\end{array}$

Test F Ratio DFNum DFDen p-Value

$\begin{array}{lllll}\text { O'Brien[.5] } & 0.4657 & 1 & 24 & 0.5015\end{array}$

Brown-Forsythe $\quad 0.2055 \quad 1 \quad 24 \quad 0.6544$

$\begin{array}{lllll}\text { Levene } & 0.2955 & 1 & 24 & 0.5917\end{array}$

$\begin{array}{llll}\text { Bartlett } & 0.2659 & 1 & 0.6061\end{array}$

$\begin{array}{lllll}\text { F Test 2-sided } & 1.3760 & 15 & 9 & 0.6413\end{array}$

Welch Anova testing Means Equal, allowing Std Devs Not Equal

F Ratio DFNum DFDen Prob $>$ F

$\begin{array}{llll}5.4711 & 1 & 21.566 & 0.0290\end{array}$

t Test 


\section{Exhibit B2. Statistical Comparisons for High-Rheology (Phase 2) Simulant Testing}

Oneway Analysis of Measurement (wt \%) By Sampler Type of Material=SME Simulant, Analytical Block=5, Analyte $=\mathrm{MgO}(\mathrm{wt} \%)$, Target $(\mathrm{wt} \%)=\mathbf{0 . 2 8 6}$

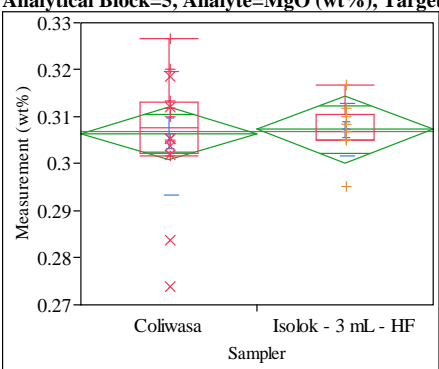

Oneway Anova

Summary of Fit

Adj Rsquare

$-0.04018$

0.010835

0.306786

26

Test

Isolok - $3 \mathrm{~mL}$ - HF-Coliwasa

Assuming equal variances

Difference $\quad 0.00081$ t Ratio 0.185093

Std Err Dif $\quad 0.00437$ DF 24

Upper CL Dif 0.00982 Prob $>|t| \quad 0.8547$

Lower CL Dif -0.00821 Prob $>t \quad 0.4274$

$\begin{array}{lrl}\text { Confidence } & 0.95 \text { Prob }<\mathrm{t} \quad 0.5726\end{array}$

Analysis of Variance

Source DF Sum of Squares Mean Square F Ratio Prob > F

$\begin{array}{llllll}\text { Sampler } & 1 & 0.00000402 & 4.022 \mathrm{e}-6 & 0.0343 & 0.8547\end{array}$

$\begin{array}{llll}\text { Error } & 24 & 0.00281744 & 0.000117\end{array}$

C. Total $25 \quad 0.00282146$

Means for Oneway Anova

Level Number Mean Std Error Lower 95\% Upper 95\%

$\begin{array}{lrrrrr}\text { Coliwasa } & 16 & 0.306475 & 0.00271 & 0.30088 & 0.31207\end{array}$

$\begin{array}{llllll}\text { Isolok - } 3 \text { mL - HF } & 10 & 0.307283 & 0.00343 & 0.30021 & 0.31435\end{array}$

Std Error uses a pooled estimate of error variance

Means and Std Deviations

Level Number Mean Std Dev Std Err Mean Lower 95\% Upper 95\%

Coliwasa 160.3064750 .013018

$\begin{array}{lllllll}\text { Coliwasa } & 16 & 0.306475 & 0.013018 & 0.00325 & 0.29954 & 0.31341 \\ \text { Isolok - 3 mL - HF } & 10 & 0.307283 & 0.005530 & 0.00175 & 0.30333 & 0.31124\end{array}$

Test

Isolok - $3 \mathrm{~mL}$ - HF-Coliwas

Assuming unequal variances

$\begin{array}{lrlr}\text { Difference } & 0.00081 & \mathrm{t} \text { Ratio } & 0.218805 \\ \text { Std Err Dif } & 0.00369 & \text { DF } & 21.87361 \\ \text { Upper CL Dif } & 0.00847 & \text { Prob }>|t| & 0.8288 \\ \text { Lower CL Dif } & -0.00686 & \text { Prob }>\text { t } & 0.4144 \\ \text { Confidence } & 0.95 & \text { Prob }<\mathrm{t} & 0.5856\end{array}$

Tests that the Variances are Equal

Level Count Std Dev MeanAbsDif to Mean MeanAbsDif to Median

Coliwasa $\quad 16 \quad 0.0130184$

$\begin{array}{lllll}\text { Isolok - } 3 \mathrm{~mL} \text { - HF } & 10 & 0.0055304 & 0.0035819 & 0.0034824\end{array}$

Test F Ratio DFNum DFDen p-Value

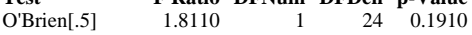

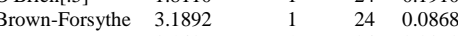

$\begin{array}{lllll}\text { Levene } & 3.1427 & 1 & 24 & 0.0890\end{array}$

$\begin{array}{lrrrr}\text { Bartlett } & 6.3108 & 1 & \text {. } & 0.0120 \\ \text { F Test 2-sided } & 5.5411 & 15 & 9 & 0.0136\end{array}$

Welch Anova testing Means Equal, allowing Std Devs Not Equal

F Ratio DFNum DFDen Prob $>$ F

$\begin{array}{llll}0.0479 & 1 & 21.874 & 0.8288\end{array}$

t Test
Oneway Analysis of Measurement (wt \%) By Sampler Type of Material=SME Simulant, Analytical Block $=5$, Analyte $=\mathrm{MnO}(\mathrm{wt} \%)$, Target $(\mathrm{wt} \%)=2.918$

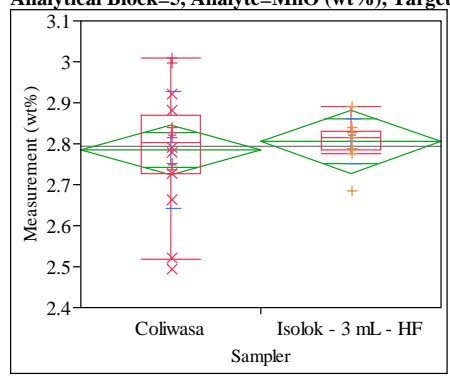

Oneway Anova

Summary of Fit

Rsquare

$\begin{array}{lr}\text { Rsquare } & 0.007915 \\ \text { Adj Rsquare } & -0.03342 \\ \text { Root Mean Square Error } & 0.118033 \\ \text { Mean of Response } & 2.792965 \\ \text { Observations (or Sum Wgts) } & 26\end{array}$

Observations (or Sum Wgts)

26

t Test

Isolok - $3 \mathrm{~mL}$ - HF-Coliwasa

Assuming equal variances

$\begin{array}{lrlr}\text { Difference } & 0.02082 & \text { t Ratio } & 0.437586 \\ \text { Std Err Dif } & 0.04758 \text { DF } & 24 \\ \text { Upper CL Dif } & 0.11902 & \text { Prob }>|t| & 0.6656 \\ \text { Lower CL Dif } & -0.07738 & \text { Prob }>\mathrm{t} & 0.3328 \\ \text { Confidence } & 0.95 & \text { Prob }<\mathrm{t} & 0.6672\end{array}$

Analysis of Variance

Source DF Sum of Squares Mean Square F Ratio Prob > F

$\begin{array}{llllll}\text { Sampler } & 1 & 0.00266768 & 0.002668 & 0.1915 & 0.6656\end{array}$

$\begin{array}{llll}\text { Error } & 24 & 0.33436270 & 0.013932\end{array}$

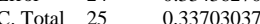

Means for Oneway Anova

Level Number Mean Std Error Lower 95\% Upper 95\%

$\begin{array}{llllll}\text { Coliwasa } & 16 & 2.78496 & 0.02951 & 2.7241 & 2.8459 \\ \text { Isolok - 3 mL - HF } & 10 & 2.80578 & 0.03733 & 2.7287 & 2.8828\end{array}$

Std Error uses a pooled estimate of error variance

Means and Std Deviations

Level $\quad$ Number Mean Std Dev Std Err Mean Lower 95\% Upper 95\%

$\begin{array}{lrrrrrr}\text { Coliwasa } & 16 & 2.78496 & 0.143451 & 0.03586 & 2.7085 & 2.8614\end{array}$

$\begin{array}{lllllll}\text { Isolok - 3 mL - HF } & 10 & 2.80578 & 0.053429 & 0.01690 & 2.7676 & 2.8440\end{array}$

t Test

Isolok - $3 \mathrm{~mL}$ - HF-Coliwasa

Assuming unequal variances

Difference $\quad 0.02082$ t Ratio 0.525198

Std Err Dif $\quad 0.03964$ DF 20.69813

Upper CL Dif 0.10334 Prob $>|t| r 0.6050$

Lower CL Dif -0.06170 Prob $>t \quad 0.3025$

$\begin{array}{lll}\text { Confidence } & 0.95 \text { Prob }<\mathrm{t} \quad 0.6975\end{array}$

Tests that the Variances are Equal

Level Count Std Dev MeanAbsDif to Mean MeanAbsDif to Median

$\begin{array}{lrrrr}\text { Coliwasa } & 16 & 0.1434506 & 0.1046074 & 0.1041030\end{array}$

$\begin{array}{lllll}\text { Isolok - 3 mL - HF } & 10 & 0.0534286 & 0.1046074 & 0.0366701\end{array}$

Test F Ratio DFNum DFDen p-Value

$\begin{array}{lllll}\text { O'Brien[.5] } & 3.2022 & 1 & 24 & 0.0862\end{array}$

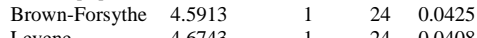

$\begin{array}{lllll}\text { Levene } & 4.6743 & 1 & 24 & 0.0408\end{array}$

$\begin{array}{lllll}\text { F Test 2-sided } & 7.2087 & 15 & 9 & 0.0052\end{array}$

Welch Anova testing Means Equal, allowing Std Devs Not Equal

F Ratio DFNum DFDen Prob $>$ F

$0.2758 \quad 120.698 \quad 0.6050$

t Test 


\section{Exhibit B2. Statistical Comparisons for High-Rheology (Phase 2) Simulant Testing}

Oneway Analysis of Measurement (wt \%) By Sampler Type of Material=SME Simulant, Analytical Block=5, Analyte $=\mathrm{Na2O}(\mathrm{wt} \%)$, Target $(\mathrm{wt} \%)=13.318$

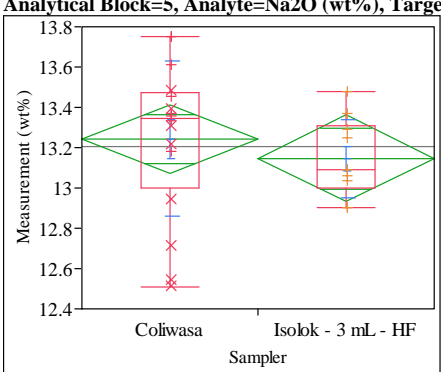

Oneway Anova

Summary of Fit

Adj Rsquare

Error $\quad 0.32729$

13.20625

Test

Isolok - $3 \mathrm{~mL}$ - HF-Coliwasa

Assuming equal variances

Difference $\quad-0.09621$ t Ratio $\quad-0.72925$

Std Err Dif $\quad 0.13193$ DF 24

Upper CL Dif 0.17609 Prob $>|t| \quad 0.4729$

Lower CL Dif -0.36851 Prob $>t \quad 0.7635$

$\begin{array}{lrl}\text { Confidence } & 0.95 \text { Prob }<\mathrm{t} & 0.2365\end{array}$

Analysis of Variance

Source DF Sum of Squares Mean Square F Ratio Prob $>$ F

$\begin{array}{llllll}\text { Sampler } & 1 & 0.0569664 & 0.056966 & 0.5318 & 0.4729\end{array}$

$\begin{array}{llll}\text { Error } & 24 & 2.5708456 & 0.107119\end{array}$

C. Total $25 \quad 2.6278119$

Means for Oneway Anova

Level Number Mean Std Error Lower 95\% Upper 95\%

$\begin{array}{lrrrrr}\text { Coliwasa } & 16 & 13.2433 & 0.08182 & 13.074 & 13.412\end{array}$

$\begin{array}{llllll}\text { Isolok - 3 mL - HF } & 16 & 13.2433 & 0.08182 & 13.074 & 13.412 \\ & 10 & 13.1470 & 0.10350 & 12.933 & 13.361\end{array}$

Std Error uses a pooled estimate of error variance

Means and Std Deviations

Level Number Mean Std Dev Std Err Mean Lower 95\% Upper 95\%

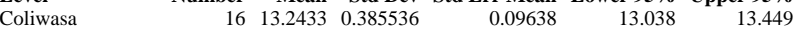

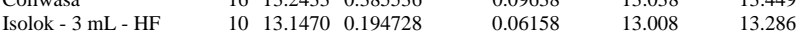

Test

Isolok - $3 \mathrm{~mL}$ - HF-Coliwas

Assuming unequal variances

Difference $\quad-0.09621$ t Ratio $\quad-0.84121$

$\begin{array}{lll}\text { Std Err Dif } \quad 0.11438 \text { DF } & 23.28\end{array}$

Upper CL Dif 0.14023 Prob $>|t| r \mid r .4088$

Lower CL Dif -0.33266 Prob $>\mathrm{t} \quad 0.7956$

$\begin{array}{lrl}\text { Confidence } & 0.95 \text { Prob }<\mathrm{t} & 0.2044\end{array}$

Tests that the Variances are Equal

Level Count Std Dev MeanAbsDif to Mean MeanAbsDif to Median

$\begin{array}{lllll}\text { Coliwasa } & 16 & 0.3855364 & 0.2959281 & 0.2771825\end{array}$

$\begin{array}{lllll}\text { Isolok - } 3 \mathrm{~mL} \text { - HF } & 10 & 0.1947278 & 0.1612208 & 0.1496280\end{array}$

Test F Ratio DFNum DFDen p-Value

O'Brien[.5] $\quad 3.0477 \quad r \quad 1 \quad 24 \quad 0.0936$

Brown-Forsythe $1.8238 \quad 1 \quad 24 \quad 0.1895$

$\begin{array}{lllll}\text { Levene } & 2.9459 & 1 & 24 & 0.0990\end{array}$

$\begin{array}{lrrrr}\text { Bartlett } & 4.2403 & 1 & \text {. } & 0.0395 \\ \text { F Test 2-sided } & 3.9199 & 15 & 9 & 0.0441\end{array}$

Welch Anova testing Means Equal, allowing Std Devs Not Equal

F Ratio DFNum DFDen Prob $>$ F

$\begin{array}{llrr}0.7076 & 1 & 23.28 & 0.4088\end{array}$

t Test

0.8412
Oneway Analysis of Measurement (wt \%) By Sampler Type of Material=SME Simulant, Analytical Block $=5$, Analyte $=\mathrm{NiO}(\mathrm{wt} \%)$, Target $(\mathrm{wt} \%)=1.033$

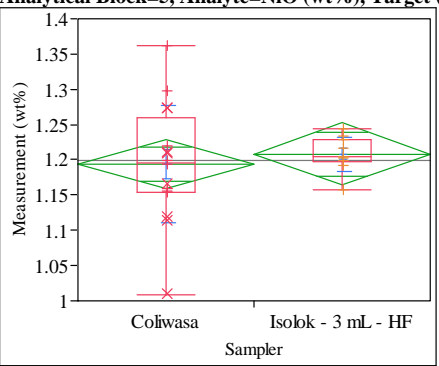

Oneway Anova

Summary of Fit

Adj Rsquare

0.0677

ean of Response

1.199625

t Test

Isolok - $3 \mathrm{~mL}$ - HF-Coliwasa

Assuming equal variances

$\begin{array}{lrlr}\text { Difference } & 0.01420 & \mathrm{t} \text { Ratio } & 0.520478 \\ \text { Std Err Dif } & 0.02729 & \text { DF } & 24 \\ \text { Upper CL Dif } & 0.07053 & \text { Prob }>|t| & 0.6075 \\ \text { Lower CL Dif } & -0.04212 & \text { Prob }>t & 0.3037 \\ \text { Confidence } & 0.95 & \text { Prob }<t & 0.6963\end{array}$

Analysis of Variance

Source DF Sum of Squares Mean Square F Ratio Prob $>$ F

$\begin{array}{llllll}\text { Sampler } & 1 & 0.00124161 & 0.001242 & 0.2709 & 0.6075\end{array}$

$\begin{array}{llll}\text { Error } & 24 & 0.10999986 & 0.004583\end{array}$

C. Total $25 \quad 0.11124147$

Means for Oneway Anova

Level Number Mean Std Error Lower 95\% Upper 95\%

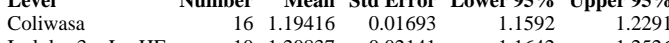

$\begin{array}{llllll}\text { Isolok - 3 mL - HF } & 16 & 1.19416 & 0.01693 & 1.1592 & 1.2291 \\ & 10 & 1.20837 & 0.02141 & 1.1642 & 1.2526\end{array}$

Std Error uses a pooled estimate of error variance

Means and Std Deviations

Level Number Mean Std Dev Std Err Mean Lower 95\% Upper 95\%

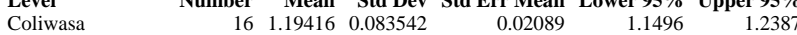

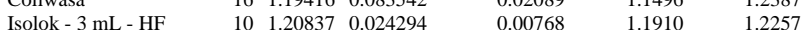

Test

Isolok - $3 \mathrm{~mL}$ - HF-Coliwasa

Assuming unequal variances

$\begin{array}{lrlr}\text { Difference } & 0.01420 & \mathrm{t} \text { Ratio } & 0.638293 \\ \text { Std Err Dif } & 0.02225 & \text { DF } & 18.76131 \\ \text { Upper CL Dif } & 0.06082 & \text { Prob }>|\mathrm{t}| & 0.5310 \\ \text { Lower CL Dif } & -0.03241 & \text { Prob }>\mathrm{t} & 0.2655 \\ \text { Confidence } & 0.95 & \text { Prob }<\mathrm{t} & 0.7345\end{array}$

Tests that the Variances are Equal

Level Count Std Dev MeanAbsDif to Mean MeanAbsDif to Median

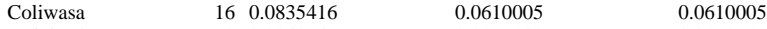

$\begin{array}{lllll}\text { Isolok - } 3 \mathrm{~mL} \text { - HF } & 10 & 0.0242941 & 0.0177132 & 0.0170515\end{array}$

Test F Ratio DFNum DFDen p-Value

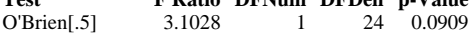

Brown-Forsythe $\quad 5.9770 \quad 1 \quad 24 \quad 0.022$

$\begin{array}{lllll}\text { Levene } & 5.8477 & 1 & 24 & 0.0236\end{array}$

$\begin{array}{lrrrr}\text { Bartlett } & 11.6129 & 1 & \text {. } & 0.0007 \\ \text { F Test 2-sided } & 11.8251 & 15 & 9 & 0.0008\end{array}$

Welch Anova testing Means Equal, allowing Std Devs Not Equal

F Ratio DFNum DFDen Prob $>$ F

$\begin{array}{rrrr}0.4074 & 1 & 18.761 & 0.5310\end{array}$

t Test

0.6383 


\section{Exhibit B2. Statistical Comparisons for High-Rheology (Phase 2) Simulant Testing}

Oneway Analysis of Measurement (wt \%) By Sampler Type of Material=SME Simulant, Analytical Block $=5$, Analyte $=\mathrm{SiO} 2(\mathrm{wt} \%)$, Target $(\mathrm{wt} \%)=50.183$

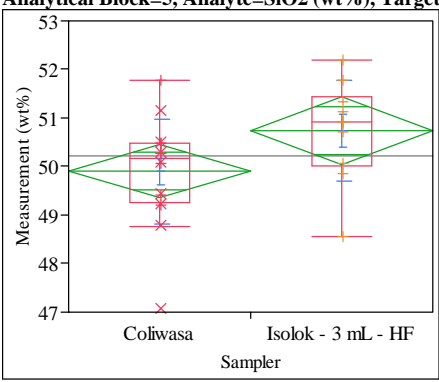

Oneway Anova

Summary of Fit

Rsquare

Adj Rsquare $\quad 0.103999$

Root Mean Square Error $\quad 1.061238$

$\begin{array}{lr}\text { Mean of Response } & 50.22418 \\ \text { Observations (or Sum Wgts) } & 26\end{array}$

t Test

Isolok - $3 \mathrm{~mL}$ - HF-Coliwasa

Assuming equal variances

Difference $\quad 0.8450$ t Ratio 1.975283

$\begin{array}{lrlr}\text { Std Err Dif } & 0.4278 \text { DF } & 24 \\ \text { Upper CL Dif } & 1.7280 & \text { Prob }>|t| & 0.0598\end{array}$

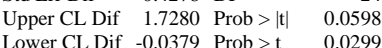

$\begin{array}{lrll}\text { Lower CL Dif } & -0.0379 & \text { Prob }>t & 0.0299 \\ \text { Confidence } & 0.95 & \text { Prob }<\mathrm{t} & 0.970\end{array}$

Analysis of Variance

Source DF Sum of Squares Mean Square F Ratio Prob $>$ F

$\begin{array}{lrrrrr}\text { Sampler } & 1 & 4.394244 & 4.39424 & 3.9017 & 0.0598\end{array}$

$\begin{array}{llrr}\text { Error } & 24 & 27.029426 & 1.12623\end{array}$

C. Total $25 \quad 31.42367$

Means for Oneway Anova

Level Number Mean Std Error Lower 95\% Upper 95\%

$\begin{array}{llrrrr}\text { Coliwasa } & 16 & 49.8992 & 0.26531 & 49.352 & 50.447\end{array}$

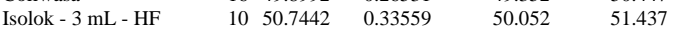

Std Error uses a pooled estimate of error variance

Means and Std Deviations

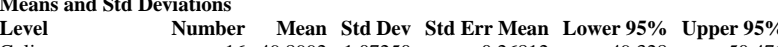

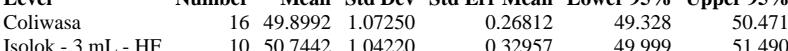

t Test

Isolok - $3 \mathrm{~mL}$ - HF-Coliwasa

Assuming unequal variances

Difference $\quad 0.8450$ t Ratio $\quad 1.98893$

Std Err Dif $\quad 0.4249$ DF 19.68279

Upper CL Dif 1.7322 Prob $>|t| 0.0608$

Lower CL Dif -0.0421 Prob $>$ t 0.0304

Confidence $\quad 0.95$ Prob $<\mathrm{t} \quad 0.9696$

Tests that the Variances are Equal

$\begin{array}{lrrrr}\text { Level } & \text { Count } & \text { Std Dev } & \text { MeanAbsDif to Mean } & \text { MeanAbsDif to Median } \\ \text { Coliwasa } & 16 & 1.072499 & 0.7888669 & 0.7487550 \\ \text { Isolok - 3 mL - HF } & 10 & 1.042200 & 0.7615908 & 0.7273620\end{array}$

Test F Ratio DFNum DFDen p-Value

$\begin{array}{lllll}\text { O'Brien[.5] } & 0.0058 & 1 & 24 & 0.9399\end{array}$

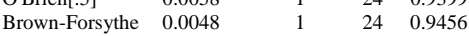

$\begin{array}{llll}0.0097 & 1 & 24 & 0.9456\end{array}$

$\begin{array}{lllll}\text { Levene } & 0.0097 & 1 & 24 & 0.9222 \\ \text { Bartlett } & 0.0088 & 1 & & 0.9253\end{array}$

$\begin{array}{lrrrr}\text { Bartlett } & 0.0088 & 1 & & 0.9253 \\ \text { F Test 2-sided } & 1.0590 & 15 & 9 & 0.9652\end{array}$

Welch Anova testing Means Equal, allowing Std Devs Not Equal

F Ratio DFNum DFDen Prob > F

$\begin{array}{llll}3.9558 & 1 & 19.683 & 0.0608\end{array}$

t Test
Oneway Analysis of Measurement (wt \%) By Sampler Type of Material=SME Simulant, Analytical Block $=5$, Analyte $=$ Sum of Oxides ( $(w t \%)$, Target $(w t \%)=99.73$

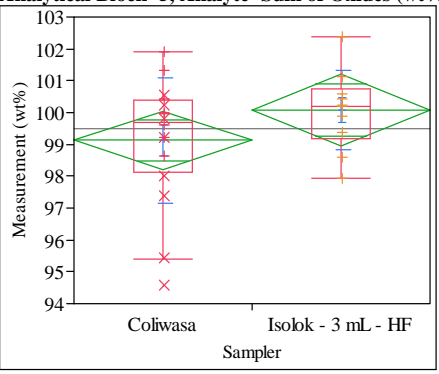

Oneway Anova

Summary of Fit

$\begin{array}{ll}\text { Rsquare } & 0.070688 \\ \text { Adj Rsquare } & 0.031967\end{array}$

Root Mean Square Error -1.748167

Mean of Response

Observations (or Sum Wgts)

1.748167

$\begin{array}{r}26 \\ \hline\end{array}$

t Test

Isolok - $3 \mathrm{~mL}$ - HF-Coliwasa

Assuming equal variances

$\begin{array}{lrlr}\text { Difference } & 0.9522 & \text { t Ratio } & 1.351135 \\ \text { Std Err Dif } & 0.7047 & \text { DF } & 24 \\ \text { Upper CL Dif } & 2.4066 \text { Prob }>|t| & 0.1893 \\ \text { Lower CL Dif } & -0.5023 & \text { Prob }>\text { t } & 0.0946 \\ \text { Confidence } & 0.95 & \text { Prob }<\mathrm{t} & 0.9054\end{array}$

Analysis of Variance

Source DF Sum of Squares Mean Square F Ratio Prob $>$ F

$\begin{array}{lrrrrr}\text { Sampler } & 1 & 5.579088 & 5.57909 & 1.8256 & 0.1893\end{array}$

$\begin{array}{llll}\text { Error } & 24 & 73.346090 & 3.05609\end{array}$

C. Total $25 \quad 78.925177$

Means for Oneway Anova

Level Number Mean Std Error Lower 95\% Upper 95\%

$\begin{array}{lrrrrr}\text { Coliwasa } & 16 & 99.125 & 0.43704 & 98.223 & 100.03 \\ \text { Isolok - 3 mL - HF } & 10 & 100.077 & 0.55282 & 98.936 & 101.22\end{array}$

Std Error uses a pooled estimate of error variance

Means and Std Deviations

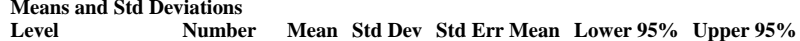

$\begin{array}{lrrrrrr}\text { Level } & \text { Number } & \text { Mean } & \text { Std Dev } & \text { Std Err Mean } & \text { Lower 95\% } & \text { Upper 95\% } \\ \text { Coliwasa } & 16 & 99.125 & 1.98464 & 0.49616 & 98.067 & 100.18\end{array}$

$\begin{array}{lrrrrrr} & 16 & 99.125 & 1.98464 & 0.49616 & 98.067 & 100.18 \\ \text { Isolok - 3 mL - HF } & 10 & 100.077 & 1.25893 & 0.39811 & 99.176 & 100.98\end{array}$

t Test

Isolok - $3 \mathrm{~mL}$ - HF-Coliwasa

Assuming unequal variances

$\begin{array}{lrlr}\text { Difference } & 0.9522 & \text { t Ratio } & 1.496788 \\ \text { Std Err Dif } & 0.6361 \text { DF } & 23.97162 \\ \text { Upper CL Dif } & 2.2652 \text { Prob }>|t| & 0.1475 \\ \text { Lower CL Dif } & -0.3608 & \text { Prob }>\text { t } & 0.0738 \\ \text { Confidence } & 0.95 \text { Prob }<\mathrm{t} & 0.9262\end{array}$

Tests that the Variances are Equal

Level Count Std Dev MeanAbsDif to Mean MeanAbsDif to Median

$\begin{array}{lrrrr}\text { Coliwasa } & 16 & 1.984640 & 1.465547 & 1.384609\end{array}$

$\begin{array}{llll}\text { Isolok - 3 mL - HF } & 10 & 1.258930 & 0.905929\end{array}$

Test F Ratio DFNum DFDen p-Value

$\begin{array}{lrrrr}\text { O'Brien[.5] } & 1.1977 & 1 & 24 & 0.2847\end{array}$

$\begin{array}{lllll}\text { Brown-Forsythe } & 0.9154 & 1 & 24 & 0.3482\end{array}$

$\begin{array}{lllll}\text { Levene } & 1.5030 & 1 & 24 & 0.2321 \\ \text { Batlett } & 2.0121 & 1 & & 0.1560\end{array}$

$\begin{array}{lllll}\text { F Test 2-sided } & 2.4852 & 15 & 9 & 0.1706\end{array}$

Welch Anova testing Means Equal, allowing Std Devs Not Equal

F Ratio DFNum DFDen Prob $>$ F

$\begin{array}{rrrr}2.2404 & 1 & 23.972 & 0.1475\end{array}$

t Test 
SRNL-STI-2011-00693

Revision 0

\section{Exhibit B2. Statistical Comparisons for High-Rheology (Phase 2) Simulant Testing}

Oneway Analysis of Measurement (wt \%) By Sampler Type of Material=SME Simulant, Analytical Block=5, Analyte $=\mathrm{TiO} 2(\mathrm{wt} \%)$, Target $(\mathrm{wt} \%)=0.013$

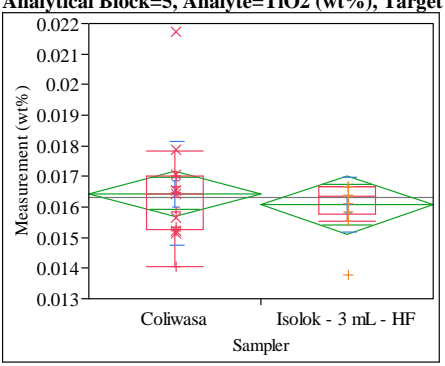

Oneway Anova

Summary of Fit

Rsquare

Adj Rsquare

Root Mean Square Error $\quad 0.00145$

Mean of Response $\quad 0.016294$

Observations (or Sum Wgts) 26

Test

Isolok - $3 \mathrm{~mL}$ - HF-Coliwas

Assuming equal variances

Difference $\quad-0.00037$ t Ratio $\quad-0.6249$

$\begin{array}{lrll}\text { Std Err Dif } & 0.00058 \text { DF } & 24 \\ \text { Upper CL Dif } & 0.00084 \text { Prob }>|t| & 0.5379\end{array}$

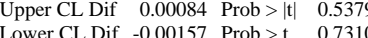

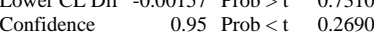

Analysis of Variance

Source DF Sum of Squares Mean Square F Ratio Prob $>$ F

$\begin{array}{lrrrrr}\text { Sampler } & 1 & 0.00000082 & 8.221 \mathrm{e}-7 & 0.3905 & 0.5379\end{array}$

$\begin{array}{llll}\text { Error } & 24 & 0.00005053 & 2.1052 \mathrm{e}-6\end{array}$

C. Total $25 \quad 0.00005135$

Means for Oneway Anova

Level Number Mean Std Error Lower 95\% Upper 95\%

$\begin{array}{lrrrrr}\text { Coliwasa } & 16 & 0.016435 & 0.00036 & 0.01569 & 0.01718\end{array}$

$\begin{array}{llllll}\text { Isolok - } 3 \mathrm{~mL} \text { - HF } & 10 & 0.016070 & 0.00046 & 0.01512 & 0.01702\end{array}$

Std Error uses a pooled estimate of error variance

Means and Std Deviations

$\begin{array}{lrrrrrr}\text { Level } & \text { Number } & \text { Mean } & \text { Std Dev } & \text { Std Err Mean } & \text { Lower 95\% } & \text { Upper 95\% } \\ \text { Coliwasa } & 16 & 0.016435 & 0.001700 & 0.00043 & 0.01553 & 0.01734\end{array}$

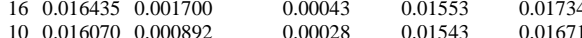

t Test

Isolok - $3 \mathrm{~mL}$ - HF-Coliwasa

Assuming unequal variances

Difference $\quad-0.00037$ t Ratio $\quad-0.71653$

Std Err Dif $\quad 0.00051$ DF 23.51511

Upper CL Dif 0.00069 Prob $>|t| \quad 0.4807$

Lower CL Dif -0.00142 Prob $>t \quad 0.7596$

$\begin{array}{lll}\text { Confidence } & 0.95 \text { Prob }<\mathrm{t} \quad 0.2404\end{array}$

Tests that the Variances are Equal

$\begin{array}{lrrrr}\text { Level } & \text { Count } & \text { Std Dev } & \text { MeanAbsDif to Mean } & \text { MeanAbsDif to Median } \\ \text { Coliwasa } & 16 & 0.0017004 & 0.0010936 & 0.0010936\end{array}$

$\begin{array}{lllll}\text { Isolok - } 3 \mathrm{~mL} \text { - HF } & 10 & 0.0008916 & 0.0006112 & 0.0005438\end{array}$

$\begin{array}{lrrrr}\text { Test } & \text { F Ratio } & \text { DFNum } & \text { DFDen } & \text { p-Value } \\ \text { O'Brien[.5] } & 0.7452 & 1 & 24 & 0.3965 \\ \text { Brown-Forsythe } & 1.5240 & 1 & 24 & 0.2290 \\ \text { Levene } & 1.2429 & 1 & 24 & 0.2759 \\ \text { Bartlett } & 3.8322 & 1 & . & 0.0503 \\ \text { F Test 2-sided } & 3.6376 & 15 & 9 & 0.0560\end{array}$

Welch Anova testing Means Equal, allowing Std Devs Not Equa

F Ratio DFNum DFDen Prob $>$ F

$\begin{array}{llll}0.5134 & 1 & 23.515 & 0.4807\end{array}$

t Test

0.7165
Oneway Analysis of Measurement (wt \%) By Sampler Type of Material=SME Simulant, Analytical Block $=5$, Analyte $=\mathrm{ZnO}(w t \%)$, Target $(w t \%)=0$

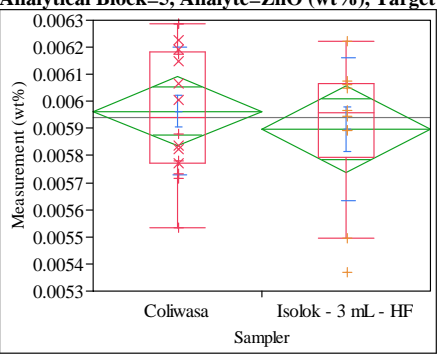

Oneway Anova

Summary of Fit

$\begin{array}{lr}\text { Rsquare } & 0.018136 \\ \text { Adj Rsquare } & -0.02277 \\ \text { Root Mean Square Error } & 0.000247 \\ \text { Mean of Response } & 0.005939 \\ \text { Observations (or Sum Wgts) } & 26\end{array}$

t Test

Isolok - $3 \mathrm{~mL}$ - HF-Coliwasa

Assuming equal variances

Difference $\quad-6.63 \mathrm{e}-5 \mathrm{t}$ Ratio $\quad-0.66582$

$\begin{array}{llll}\text { Std Err Dif } & 0.0001 \text { DF } & 24\end{array}$

Upper CL Dif $\quad 0.00014$ Prob $>|t| \quad 0.5119$

Lower CL Dif -0.00027 Prob $>t \quad 0.7441$

$\begin{array}{lrl}\text { Confidence } & 0.95 \text { Prob }<\mathrm{t} & 0.2559\end{array}$

Analysis of Variance

Source DF Sum of Squares Mean Square F Ratio Prob $>$ F

$\begin{array}{lrrrrr}\text { Source } & \text { DF } & \text { Sum of Squares } & \text { Mean Square } & \text { F Ratio } & \text { Prob }>\text { F } \\ \text { Sampler } & 1 & 2.70387 \mathrm{e}-8 & 2.7039 \mathrm{e}-8 & 0.4433 & 0.5119\end{array}$

$\begin{array}{llll}\text { Sampler } & 1 & 2.70387 \mathrm{e}-8 & 2.7039 \mathrm{e}-8 \\ \text { Error } & 24 & 1.46381 \mathrm{e}-6 & 6.0992 \mathrm{e}-8\end{array}$

$\begin{array}{lll}\text { Error } & 24 & 1.46381 \mathrm{e}-6 \\ \text { C. Total } & 25 & 1.49085 \mathrm{e}-6\end{array}$

Means for Oneway Anova

Level Number Mean Std Error Lower 95\% Upper 95\%

$\begin{array}{llllll}\text { Coliwasa } & 16 & 0.005964 & 6.17 \mathrm{e}-5 & 0.00584 & 0.00609 \\ & 10 & 0.005898 & 7.81 \mathrm{e}-5 & 0.00574 & 0.00606\end{array}$

Sterimate of error variance

Means and Std Deviations

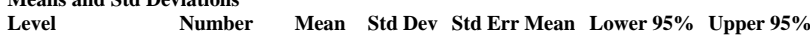

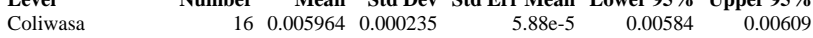

$\begin{array}{lllllll}\text { Isolok - 3 mL - HF } & 16 & 0.005964 & 0.000235 & 5.88 \mathrm{e}-5 & 0.00584 & 0.00609 \\ & 10 & 0.005898 & 0.000265 & 8.39 \mathrm{e}-5 & 0.00571 & 0.00609\end{array}$

t Test

Isolok - $3 \mathrm{~mL}$ - HF-Coliwasa

Assuming unequal variances

$\begin{array}{lrlr}\text { Difference } & -6.63 e-5 & \text { t Ratio } & -0.64687 \\ \text { Std Err Dif } & 0.00010 & \text { DF } & 17.48128 \\ \text { Upper CL Dif } & 0.00015 & \text { Prob }>|t| & 0.5261 \\ \text { Lower CL Dif } & -0.00028 & \text { Prob }>\text { t } & 0.7369 \\ \text { Confidence } & 0.95 & \text { Prob }<\mathrm{t} & 0.2631\end{array}$

Tests that the Variances are Equal

Level Count Std Dev MeanAbsDif to Mean MeanAbsDif to Median

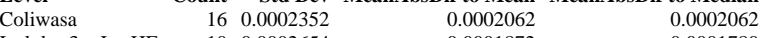

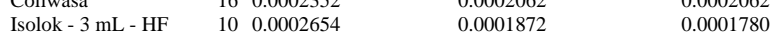

Test F Ratio DFNum DFDen p-Value

$\begin{array}{lllll}\text { O'Brien[.5] } & 0.2285 & 1 & 24 & 0.6369\end{array}$

$\begin{array}{lllll}\text { Brown-Forsythe } & 0.2298 & 1 & 24 & 0.6360\end{array}$

$\begin{array}{lllll}\text { Levene } & 0.1225 & 1 & 24 & 0.7294\end{array}$

$\begin{array}{lllll}\text { Bartlett } & 0.1591 & 1 & \text {. } & 0.6900\end{array}$

Welch Anova testing Means Equal, allowing Std Devs Not Equal

F Ratio DFNum DFDen Prob $>$ F

$\begin{array}{llll}0.4184 & 1 & 17.481 & 0.5261\end{array}$

t Test

0.6469 


\section{Exhibit B2. Statistical Comparisons for High-Rheology (Phase 2) Simulant Testing}

Oneway Analysis of Measurement (wt \%) By Sampler Type of Material=SME Simulant, Analytical Block=5, Analyte $=\mathrm{ZrO} 2(\mathrm{wt} \%)$, Target $(\mathrm{wt} \%)=0.111$

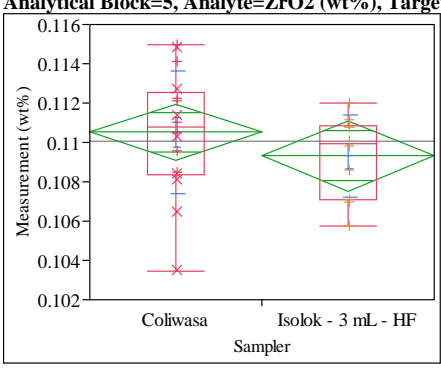

Oneway Anova

Summary of Fit

Rsquare

Adj Rsquare

Root Mean Square Error 0.00738

Mean of Response $\quad 0.110054$

Observations (or Sum Wgts)

Test

Isolok - $3 \mathrm{~mL}$ - HF-Coliwasa

Assuming equal variances

Difference $\quad-0.00121$ t Ratio $\quad-1.0889$

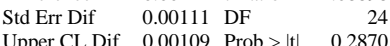

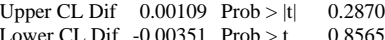

$\begin{array}{lrl}\text { Confidence } & 0.95 \text { Prob }<\mathrm{t} & 0.1435\end{array}$

Analysis of Variance

Source DF Sum of Squares Mean Square F Ratio Prob $>$ F

$\begin{array}{lrrrrr}\text { Sampler } & 1 & 0.00000907 & 9.07 \mathrm{e}-6 & 1.1859 & 0.2870\end{array}$

$\begin{array}{llll}\text { Error } & 24 & 0.00018356 & 7.6484 \mathrm{e}-6\end{array}$

C. Total $25 \quad 0.0001926$

Means for Oneway Anova

Level Number Mean Std Error Lower 95\% Upper 95\%

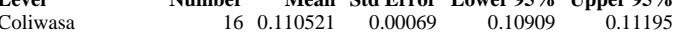

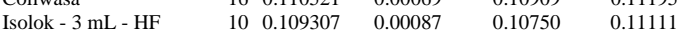

Std Error uses a pooled estimate of error variance

Means and Std Deviations

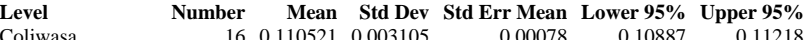

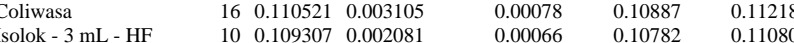

t Test

Isolok - $3 \mathrm{~mL}$ - HF-Coliwasa

Assuming unequal variances

Difference $\quad-0.00121$ t Ratio -1.19307

Std Err Dif $\quad 0.00102$ DF 23.81085

Upper CL Dif 0.00089 Prob $>|t| \quad 0.2446$

$\begin{array}{lll}\text { Lower CL Dif }-0.00332 \text { Prob }>t & 0.8777\end{array}$

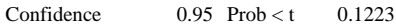

Tests that the Variances are Equal

$\begin{array}{lrrrr}\text { Level } & \text { Count } & \text { Std Dev } & \text { MeanAbsDif to Mean } & \text { MeanAbsDif to Median } \\ \text { Coliwasa } & 16 & 0.0031048 & 0.0023892 & 0.0023892\end{array}$

$\begin{array}{lllll} & 16 & 0.0031048 & 0.0023892 & 0.0023892 \\ \text { Isolok - } 3 \mathrm{~mL}-\mathrm{HF} & 10 & 0.0020808 & 0.0017506 & 0.0016480\end{array}$

Test F Ratio DFNum DFDen p-Value

$\begin{array}{lrrrr}\text { Test } & \text { F Ratio } & \text { DFNum } & \text { DFDen } & \text { p-Value } \\ \text { O'Brien[.5] } & 1.3115 & 1 & 24 & 0.2634 \\ \text { Brown-Forsythe } & 1.1555 & 1 & 24 & 0.2931 \\ \text { Levene } & 0.9781 & 1 & 24 & 0.3325 \\ \text { Bartlett } & 1.5786 & 1 & \text {. } & 0.2090 \\ \text { F Test 2-sided } & 2.2264 & 15 & 9 & 0.2271\end{array}$

Welch Anova testing Means Equal, allowing Std Devs Not Equal

F Ratio DFNum DFDen Prob $>$ F

$\begin{array}{llll}1.4234 & 1 & 23.811 & 0.2446\end{array}$

t Test

1.1931
Oneway Analysis of Measurement (wt \%) By Sampler Type of Material=SME Simulant, Analytical Block $=6$, Analyte $=$ Al $/ B$, Target $(w t \%)=2.0704412$

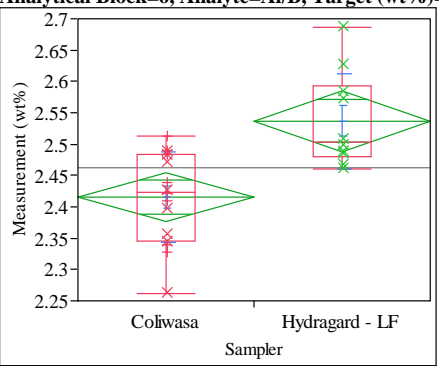

Oneway Anova

Summary of Fit

Adj Rsquare

0.383437

0.074046

Mean of Response 2.462194

Observations (or Sum Wgts) $\quad 26$

Test

Hydragard - LF-Coliwasa

Assuming equal variances

Difference $\quad 0.121420$ t Ratio $\quad 4.067844$

Std Err Dif $\quad 0.029849$ DF 24

Upper CL Dif 0.183025 Prob $>|t| \quad 0.0004$

Lower CL Dif 0.059815 Prob $>t \quad 0.0002$

$\begin{array}{lrl}\text { Confidence } & 0.95 \text { Prob }<\mathrm{t} & 0.9998\end{array}$

Analysis of Variance

Source DF Sum of Squares Mean Square F Ratio Prob $>$ F

$\begin{array}{lllllll}\text { Sampler } & 1 & 0.09072524 & 0.090725 & 16.5474 & 0.0004\end{array}$

$\begin{array}{llll}\text { Error } & 24 & 0.13158633 & 0.005483\end{array}$

C. Total $25 \quad 0.22231157$

Means for Oneway Anova

Level Number Mean Std Error Lower 95\% Upper 95\%

$\begin{array}{lrrrrr}\text { Coliwasa } & 16 & 2.41549 & 0.01851 & 2.3773 & 2.4537\end{array}$

$\begin{array}{lllll}\text { Hydragard - LF } & 10 & 2.53691 & 0.02342 & 2.4886\end{array}$

Std Error uses a pooled estimate of error variance

Means and Std Deviations

Level Number Mean Std Dev Std Err Mean Lower 95\% Upper 95\%

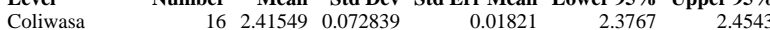

$\begin{array}{lllllll}\text { Hydragard - LF } & 10 & 2.53691 & 0.076015 & 0.02404 & 2.4825 & 2.5913\end{array}$

tTest

Hydragard - LF-Coliwasa

Assuming unequal variances

$\begin{array}{lrlr}\text { Difference } & 0.121420 & \text { t Ratio } & 4.026335 \\ \text { Std Err Dif } & 0.030156 & \text { DF } & 18.61529 \\ \text { Upper CL Dif } & 0.184627 & \text { Prob }>|t| & 0.0007 \\ \text { Lower CL Dif } & 0.058213 & \text { Prob }>t & 0.0004 \\ \text { Confidence } & 0.95 & \text { Prob }<t & 0.9996\end{array}$

Tests that the Variances are Equal

Level Count Std Dev MeanAbsDif to Mean MeanAbsDif to Median

$\begin{array}{lllll}\text { Coliwasa } & 16 & 0.0728389 & 0.0593227 & 0.0584856\end{array}$

$\begin{array}{lllll}\text { Hydragard - LF } & 10 & 0.0760145 & 0.0636069 & 0.0578460\end{array}$

Test F Ratio DFNum DFDen p-Value

$\begin{array}{lrrrr}\text { O'Brien[.5] } & 0.0294 & 1 & 24 & 0.8652\end{array}$

$\begin{array}{lllll}\text { Brown-Forsythe } & 0.0011 & 1 & 24 & 0.9740\end{array}$

$\begin{array}{lllll}\text { Levene } & 0.0778 & 1 & 24 & 0.7826\end{array}$

$\begin{array}{lllll}\text { Bartlett } & 0.0197 & 1 & & 0.8883 \\ \text { F Test 2-sided } & 1.0891 & 9 & 15 & 0.8486\end{array}$

Welch Anova testing Means Equal, allowing Std Devs Not Equal

F Ratio DFNum DFDen Prob $>$ F

$16.2114 \quad 18.615 \quad 0.0007$

t Test

4.0263 
SRNL-STI-2011-00693

Revision 0

\section{Exhibit B2. Statistical Comparisons for High-Rheology (Phase 2) Simulant Testing}

Oneway Analysis of Measurement (wt \%) By Sampler Type of Material=SME Simulant, Analytical Block=6, Analyte=Al2O3 (wt \%), Target $($ wt $\%)=10.934$

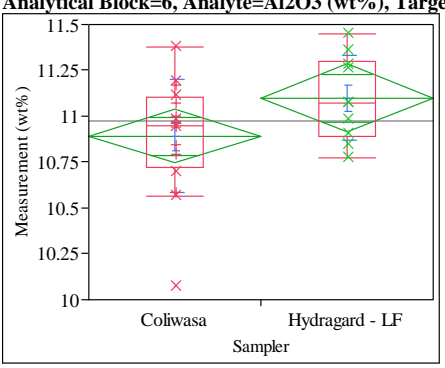

Oneway Anova

Summary of Fit

Rsquare

Adj Rsquare

Root Mean Square Error $\quad 0.282066$

Mean of Response $\quad 10.97145$

Observations (or Sum Wgts) 26

Test

Hydragard - LF-Coliwasa

Assuming equal variances

Difference $\quad 0.20714$ t Ratio $\quad 1.82171$

$\begin{array}{lrr}\text { Std Err Dif } & 0.11370 \text { DF } & 24\end{array}$

$\begin{array}{lrll}\text { Upper CL Dif } & 0.44181 & \text { Prob }>|t| & 0.0810 \\ \text { Lower CL Dif } & -0.02754 & \text { Prob }>t & 0.0405\end{array}$

$\begin{array}{lll}\text { Confidence } & 0.95 \text { Prob }<\mathrm{t} \quad 0.9595\end{array}$

Analysis of Variance

Source DF Sum of Squares Mean Square F Ratio Prob $>$ F

$\begin{array}{lrrrrr}\text { Sampler } & 1 & 0.2640339 & 0.264034 & 3.3186 & 0.0810\end{array}$

$\begin{array}{llll}\text { Error } & 24 & 1.9094689 & 0.079561\end{array}$

C. Total $25 \quad 2.1735028$

Means for Oneway Anova

Level Number Mean Std Error Lower 95\% Upper 95\%

$\begin{array}{lrrrrr}\text { Level } & \text { Number } & \text { Mean } & \text { Std Error } & \text { Lower 95\% } & \text { Upper 95\% } \\ \text { Coliwasa } & 16 & 10.8918 & 0.07052 & 10.746 & 11.037\end{array}$

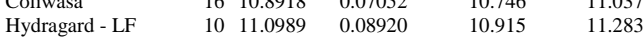

Std Error uses a pooled estimate of error variance

Means and Std Deviations

Level Number Mean Std Dev Std Err Mean Lower 95\% Upper 95\%

$\begin{array}{lllllll}\text { Coliwasa } & 16 & 10.8918 & 0.309168 & 0.07729 & 10.727 & 11.057\end{array}$

$\begin{array}{lllllll}\text { Hydragard - LF } & 10 & 11.0989 & 0.229902 & 0.07270 & 10.934 & 11.263\end{array}$

t Test

Hydragard - LF-Coliwasa

Assuming unequal variances

Difference $\quad 0.20714$ t Ratio 1.952072

Std Err Dif $\quad 0.10611$ DF 23.12048

Upper CL Dif 0.42658 Prob $>|t| \quad 0.0631$

Lower CL Dif -0.01231 Prob $>t \quad 0.0316$

$\begin{array}{lll}\text { Confidence } & 0.95 \text { Prob }<\mathrm{t} \quad 0.9684\end{array}$

Tests that the Variances are Equal

$\begin{array}{lrrrr}\text { Level } & \text { Count } & \text { Std Dev } & \text { MeanAbsDif to Mean } & \text { MeanAbsDif to Median } \\ \text { Coliwasa } & 16 & 0.3091681 & 0.2258543 & 0.2137497\end{array}$

$\begin{array}{lllll} & 16 & 0.3091681 & 0.2258543 & 0.2137497 \\ \text { Hydragard - LF } & 10 & 0.2299021 & 0.1904616 & 0.1851710\end{array}$

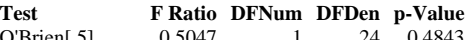

$\begin{array}{lllll}\text { O'Brien[.5] } & 0.5047 & 1 & 24 & 0.4843 \\ \text { Brown-Forsythe } & 0.1346 & 1 & 24 & 0.7169\end{array}$

$\begin{array}{lllll}\text { Brown-Forsythe } & 0.1346 & 1 & 24 & 0.7169 \\ \text { Levene } & 0.2532 & 1 & 24 & 0.6194\end{array}$

$\begin{array}{lllll}\text { Levene } & 0.2532 & 1 & 24 & 0.6194 \\ \text { Bartlett } & 0.8881 & 1 & . & 0.3460\end{array}$

$\begin{array}{lrrrr}\text { F Test 2-sided } & 1.8084 & 15 & 9 & 0.3760\end{array}$

Welch Anova testing Means Equal, allowing Std Devs Not Equal

F Ratio DFNum DFDen Prob $>$ F

$\begin{array}{llll}3.8106 & 1 & 23.12 & 0.0631\end{array}$

t Test

1.9521
Oneway Analysis of Measurement (wt \%) By Sampler Type of Material=SME Simulant, Analytical Block $=6$, Analyte $=\mathrm{B} 2 \mathrm{O} 3(\mathrm{wt} \%)$, Target $(\mathrm{wt} \%)=5.281$

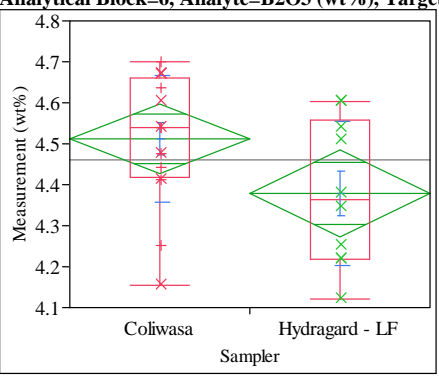

Oneway Anova

Summary of Fit

Adj Rsquare

0.109

0.163359

$\begin{array}{lr}\text { Mean of Response } & 4.4608 \\ \text { Observations (or Sum Wgts) } & 26\end{array}$

t Test

Hydragard - LF-Coliwasa

Assuming equal variances

Difference $\quad-0.13282$ t Ratio $\quad-2.01696$

$\begin{array}{lll}\text { Std Err Dif } & 0.06585 \text { DF } & 24\end{array}$

Upper CL Dif 0.00309 Prob $>|t| \quad 0.0550$

Lower CL Dif -0.26873 Prob $>\mathrm{t} \quad 0.9725$

$\begin{array}{lll}\text { Confidence } & 0.95 \text { Prob }<\mathrm{t} \quad 0.0275\end{array}$

Analysis of Variance

Source DF Sum of Squares Mean Square F Ratio Prob $>$ F

$\begin{array}{lrrrrr}\text { Sampler } & 1 & 0.10856237 & 0.108562 & 4.0681 & 0.0550\end{array}$

$\begin{array}{llll}\text { Error } & 24 & 0.64046813 & 0.026686\end{array}$

C. Total $25 \quad 0.74903050$

Means for Oneway Anova

Level Number Mean Std Error Lower 95\% Upper 95\%

$\begin{array}{llllll}\text { Coliwasa } & 16 & 4.51188 & 0.04084 & 4.4276 & 4.5962 \\ \text { Hydragard - LF } & 10 & 4.37906 & 0.05166 & 4.2724 & 4.4857\end{array}$

Std Error uses a pooled estimate of error variance

Means and Std Deviations

$\begin{array}{llllll}\text { Means and Std Deviations } & & & \\ \text { Level } & \text { Number Mean Std Dev Std Err Mean Lower 95\% Upper 95\% }\end{array}$

$\begin{array}{lrrrrrr}\text { Level } & \text { Number } & \text { Mean } & \text { Std Dev } & \text { Std Err Mean } & \text { Lower 95\% } & \text { Upper 95\% } \\ \text { Coliwasa } & 16 & 4.51188 & 0.155035 & 0.03876 & 4.4293 & 4.5945\end{array}$

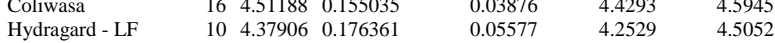

t Test

Hydragard - LF-Coliwasa

Assuming unequal variances

Difference $\quad-0.13282$ t Ratio -1.95567

Std Err Dif $\quad 0.06792$ DF 17.36301

Upper CL Dif 0.01024 Prob $>|t| \quad 0.0668$

Lower CL Dif -0.27588 Prob $>t \quad 0.9666$

$\begin{array}{lll}\text { Confidence } & 0.95 \text { Prob }<\mathrm{t} & 0.0334\end{array}$

Tests that the Variances are Equal

Level Count Std Dev MeanAbsDif to Mean MeanAbsDif to Median

$\begin{array}{lrrrr}\text { Coliwasa } & 16 & 0.1550352 & 0.1202431 & 0.1167214\end{array}$

$\begin{array}{lllll}\text { Hydragard - LF } & 10 & 0.1763612 & 0.1481154 & 0.1481154\end{array}$

Test F Ratio DFNum DFDen p-Value

$\begin{array}{lllll}\text { O'Brien[.5] } & 0.2801 & 1 & 24 & 0.6015\end{array}$

$\begin{array}{lllll}\text { Brown-Forsythe } & 0.6665 & 1 & 24 & 0.4223 \\ & 0.6047 & 1 & 24 & 0.4444\end{array}$

$\begin{array}{lllll}\text { Levene } & 0.6047 & 1 & 24 & 0.4444 \\ \text { Bartett } & 0.1822 & 1 & & 0.6695\end{array}$

$\begin{array}{lllll}\text { F Test 2-sided } & 1.2940 & 9 & 15 & 0.6331\end{array}$

Welch Anova testing Means Equal, allowing Std Devs Not Equal

F Ratio DFNum DFDen Prob $>$ F

$\begin{array}{rrrr}3.8246 & 1 & 17.363 & 0.0668\end{array}$

t Test 
SRNL-STI-2011-00693

Revision 0

\section{Exhibit B2. Statistical Comparisons for High-Rheology (Phase 2) Simulant Testing}

Oneway Analysis of Measurement (wt\%) By Sampler Type of Material=SME Simulant, Analytical Block=6, Analyte $=\mathrm{BaO}(\mathrm{wt} \%)$, Target $(\mathrm{wt} \%)=0$

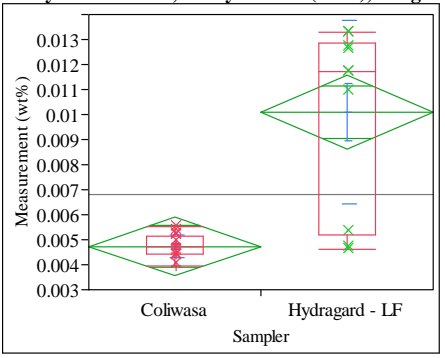

Oneway Anova

Summary of Fit

Rsquare

Adj Rsquare

Root Mean Square Error 0.570192

Mean of Response $\quad 0.006794$

Observations (or Sum Wgts) $\quad 26$

Test

Hydragard - LF-Coliwasa

Assuming equal variances

Difference $\quad 0.005367$ t Ratio $\quad 5.845126$

$\begin{array}{lrr}\text { Std Err Dif } & 0.000918 \text { DF } & 24 \\ \text { Dif } & <.0001\end{array}$

$\begin{array}{lrll}\text { Upper CL Dif } & 0.007262 & \text { Prob }>|t| & <.0001 \\ & & & 24 \\ \text { Cower CL Dif } & 0.003472 & \text { Prob }>t & <.0001\end{array}$

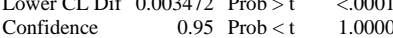

Analysis of Variance

Source DF Sum of Squares Mean Square F Ratio Prob $>$ F

$\begin{array}{lrrrrr}\text { Sampler } & 1 & 0.00017724 & 0.000177 & 34.1655 & <.0001\end{array}$

$\begin{array}{llll}\text { Error } & 24 & 0.00012451 & 5.188 \mathrm{e}-6\end{array}$

Means for Oneway Anova

Level $\quad$ Number $\quad$ Mean Std Error $\quad$ Lower 95\% Upper 95\%

$\begin{array}{lrrrrr}\text { Level } & \text { Number } & \text { Mean } & \text { Std Error } & \text { Lower 95\% } & \text { Upper 95\% } \\ \text { Coliwasa } & 16 & 0.004730 & 0.00057 & 0.00355 & 0.00590\end{array}$

$\begin{array}{llllll}\text { Coliwasa } & 16 & 0.004730 & 0.00057 & 0.00355 & 0.00590 \\ \text { Hydragard - LF } & 10 & 0.010097 & 0.00072 & 0.00861 & 0.01158\end{array}$

Std Error uses a pooled estimate of error variance

Means and Std Deviations

$\begin{array}{llrrrrr}\text { Level Number Mean } & \text { Std Dev } & \text { Std Err Mean } & \text { Lower 95\% } & \text { Upper 95\% }\end{array}$

$\begin{array}{lllllll}\text { Hydragard - LF } & 16 & 0.004730 & 0.000449 & 0.00011 & 0.00449 & 0.00497 \\ & 10 & 0.010097 & 0.003674 & 0.00116 & 0.00747 & 0.01272\end{array}$

t Test

Hydragard - LF-Coliwasa

Assuming unequal variances

Difference $\quad 0.005367$ t Ratio $\quad 4.597819$

Std Err Dif $\quad 0.001167$ DF $\quad 9.168025$

Upper CL Dif 0.008000 Prob $>|t| \quad 0.0012$

Lower CL Dif 0.002734 Prob $>t \quad 0.0006$

Confidence $\quad 0.95$ Prob $<\mathrm{t} \quad 0.9994$

Tests that the Variances are Equal

Level Count Std Dev MeanAbsDif to Mean MeanAbsDif to Median

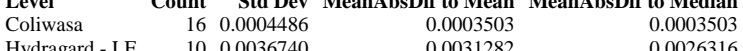

F Ratio DFNum DFDen p-Value

Test $\quad$ F Ratio DFNum DFDen p-Value

$\begin{array}{lrrrr}\text { O'Brien[.5] } & 17.0770 & 1 & 24 & 0.0004 \\ \text { Brown-Forsythe } & 9.6376 & 1 & 24 & 0.0048\end{array}$

$\begin{array}{lrrrr}\text { Brown-Forsythe } & 9.6376 & 1 & 24 & 0.0048 \\ \text { Levene } & 46.1686 & 1 & 24 & <.0001\end{array}$

$\begin{array}{lllll}\text { Levene } & 46.1686 & 1 & 24 & <.0001 \\ \text { Bartlett } & 38.3945 & 1 & & <.0001\end{array}$

$\begin{array}{lllll}\text { F Test 2-sided } & 67.3945 & 1 & \dot{0} & <.0001 \\ & 67.0756 & 9 & 15 & <.0001\end{array}$

Welch Anova testing Means Equal, allowing Std Devs Not Equal

F Ratio DFNum DFDen Prob $>$ F

$\begin{array}{llll}21.1399 & 1 & 9.168 & 0.0012\end{array}$

t Test
4.5978
Oneway Analysis of Measurement (wt \%) By Sampler Type of Material=SME Simulant, Analytical Block $=6$, Analyte $=\mathrm{CaO}(\mathrm{wt} \%)$, Target $(\mathrm{wt} \%)=0.543$

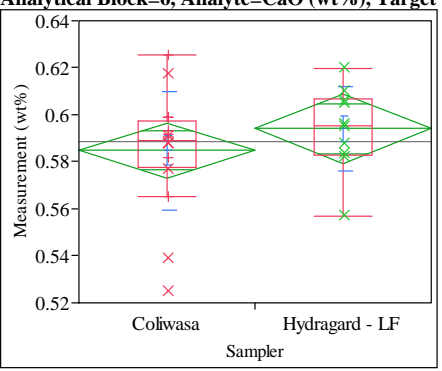

Oneway Anova

Summary of Fit

Adj Rsquare

0.022679

Mean of Response 0.588364

t Test

Hydragard - LF-Coliwasa

Assuming equal variances

Difference $\quad 0.00932$ t Ratio 1.019676

Std Err Dif $\quad 0.00914$ DF 24

Upper CL Dif $\quad 0.02819$ Prob $>|t| \quad 0.3181$

Lower CL Dif -0.00955 Prob $>t \quad 0.1590$

$\begin{array}{lrl}\text { Confidence } & 0.95 \text { Prob }<\mathrm{t} & 0.8410\end{array}$

Analysis of Variance

Source DF Sum of Squares Mean Square F Ratio Prob $>$ F

$\begin{array}{lllllll}\text { Sampler } & 1 & 0.00053479 & 0.000535 & 1.0397 & 0.3181\end{array}$

$\begin{array}{llll}\text { Error } & 24 & 0.01234434 & 0.000514\end{array}$

C. Total $25 \quad 0.01287913$

Means for Oneway Anova

Level Number Mean Std Error Lower 95\% Upper 95\%

$\begin{array}{lrrrrr}\text { Coliwasa } & 16 & 0.584778 & 0.00567 & 0.57308 & 0.59648\end{array}$

$\begin{array}{llllll}\text { Hydragard - LF } & 10 & 0.594100 & 0.00717 & 0.57930 & 0.60890\end{array}$

Std Error uses a pooled estimate of error variance

Means and Std Deviations

Level Number Mean Std Dev Std Err Mean Lower 95\% Upper 95\%

$\begin{array}{lrrrrrr}\text { Coliwasa } & 16 & 0.584778 & 0.025157 & 0.00629 & 0.57137 & 0.59818\end{array}$

$\begin{array}{lllllll}\text { Hydragard - LF } & 10 & 0.594100 & 0.017799 & 0.00563 & 0.58137 & 0.60683\end{array}$

Test

Hydragard - LF-Coliwasa

Assuming unequal variances

$\begin{array}{lrlr}\text { Difference } & 0.00932 & \mathrm{t} \text { Ratio } & 1.104509 \\ \text { Std Err Dif } & 0.00844 & \text { DF } & 23.51214 \\ \text { Upper CL Dif } & 0.02676 & \text { Prob }>|\mathrm{t}| & 0.2805 \\ \text { Lower CL Dif } & -0.00812 & \text { Prob }>\mathrm{t} & 0.1403 \\ \text { Confidence } & 0.95 & \text { Prob }<\mathrm{t} & 0.8597\end{array}$

Tests that the Variances are Equal

Level Count Std Dev MeanAbsDif to Mean MeanAbsDif to Median

$\begin{array}{lllll}\text { Coliwasa } & 16 & 0.0251569 & 0.0170855 & 0.0160033\end{array}$

$\begin{array}{lllll}\text { Hydragard - LF } & 10 & 0.0177991 & 0.0132644 & 0.0131525\end{array}$

Test F Ratio DFNum DFDen p-Value

$\begin{array}{lrrrr}\text { O'Brien[.5] } & 0.6762 & 1 & 24 & 0.4190\end{array}$

$\begin{array}{lllll}\text { Brown-Forsythe } & 0.1758 & 1 & 24 & 0.6787\end{array}$

$\begin{array}{lllll}\text { Levene } & 0.3646 & 1 & 24 & 0.5516\end{array}$

$\begin{array}{lrrrr}\text { Bartlett } & 1.1966 & 1 & & 0.2740 \\ \text { F Test 2-sided } & 1.9976 & 15 & 9 & 0.2960\end{array}$

Welch Anova testing Means Equal, allowing Std Devs Not Equal

F Ratio DFNum DFDen Prob $>$ F

$\begin{array}{rrrr}1.2199 & 1 & 23.512 & 0.2805\end{array}$

t Test

1.1045 
SRNL-STI-2011-00693

Revision 0

\section{Exhibit B2. Statistical Comparisons for High-Rheology (Phase 2) Simulant Testing}

Oneway Analysis of Measurement (wt \%) By Sampler Type of Material=SME Simulant, Analytical Block $=6$, Analyte $=$ Cr2O3 $(w t \%)$, Target $(w t \%)=0.015$

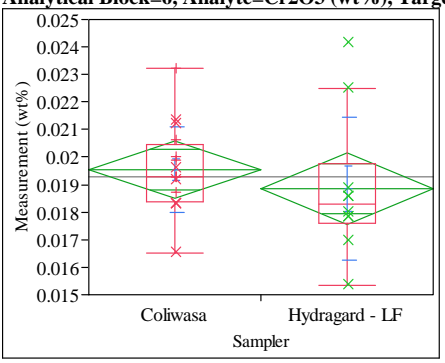

Oneway Anova

Summary of Fit

Rsquare

Adj Rsquare

Root Mean Square Error $\quad 0.002001$

$\begin{array}{lr}\text { Mean of Response } & 0.019287 \\ \text { Observations (or Sum Wgts) } & 26\end{array}$

t Test

Hydragard - LF-Coliwasa

Assuming equal variances

Difference $\quad-0.00070$ t Ratio $\quad-0.8718$

$\begin{array}{lrr}\text { Std Err Dif } & 0.00081 \text { DF } & 24 \\ \text { Upper } & \text { D } & \end{array}$

$\begin{array}{rrr}\text { Upper CL Dif } & 0.00096 \text { Prob }>|t| & 0.3919 \\ \text { Cower CL Dif }-0.00237 & \text { Prob }>t & 0.8040\end{array}$

$\begin{array}{lll}\text { Confidence } & 0.95 \text { Prob }<\mathrm{t} \quad 0.1960\end{array}$

Analysis of Variance

Source DF Sum of Squares Mean Square F Ratio Prob $>$ F

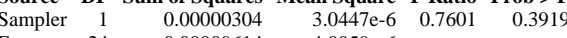

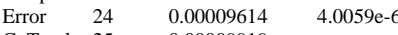

C. Total $25 \quad 0.00009919$

Means for Oneway Anova

Level Number Mean Std Error Lower 95\% Upper 95\%

$\begin{array}{lrrrrr}\text { Level } & \text { Number } & \text { Mean } & \text { Std Error } & \text { Lower 95\% } & \text { Upper 95\% } \\ \text { Coliwasa } & 16 & 0.019558 & 0.00050 & 0.01853 & 0.02059\end{array}$

$\begin{array}{llllll}\text { Hydragard - LF } & 10 & 0.018855 & 0.00063 & 0.01755 & 0.02016\end{array}$

Std Error uses a pooled estimate of error variance

Means and Std Deviations

$\begin{array}{lllllll}\text { Level Number } & \text { Mean } & \text { Std Dev } & \text { Std Err Mean Lower 95\% Upper 95\% }\end{array}$

$\begin{array}{lllllll} & 16 & 0.019558 & 0.001553 & 0.00039 & 0.01873 & 0.02039 \\ \text { Hydragard - LF } & 10 & 0.018855 & 0.002582 & 0.00082 & 0.01701 & 0.02070\end{array}$

t Test

Hydragard - LF-Coliwasa

Assuming unequal variances

Difference $\quad-0.00070$ t Ratio $\quad-0.77812$

Std Err Dif $\quad 0.00090$ DF $\quad 13.12581$

Upper CL Dif 0.00125 Prob $>|t| \quad 0.4503$

Lower CL Dif -0.00265 Prob $>t \quad 0.7749$

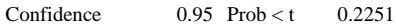

Tests that the Variances are Equal

$\begin{array}{lrrrr}\text { Level } & \text { Count } & \text { Std Dev } & \text { MeanAbsDif to Mean } & \text { MeanAbsDif to Median } \\ \text { Coliwasa } & 16 & 0.0015525 & 0.0011202 & 0.0010871\end{array}$

$\begin{array}{lllll} & 16 & 0.0015525 & 0.0011202 & 0.0010871 \\ \text { Hydragard - LF } & 10 & 0.0025817 & 0.0017832 & 0.0016662\end{array}$

Test $\quad$ F Ratio DFNum DFDen p-Value

$\begin{array}{lllll}\text { O'Brien[.5] } & 2.0400 & 1 & 24 & 0.1661 \\ \text { Brown-Forsythe } & 0.9172 & 1 & 24 & 0.3478\end{array}$

$\begin{array}{lllll}\text { Levene } & 1.4664 & 1 & 24 & 0.2377 \\ \text { Bartlett } & 2.9060 & 1 & & 0.0882\end{array}$

$\begin{array}{lllll}\text { F Test 2-sided } & 2.7652 & 1 & \dot{0} & 0.0882 \\ & & 9 & 15 & 0.0790\end{array}$

Welch Anova testing Means Equal, allowing Std Devs Not Equal

F Ratio DFNum DFDen Prob $>$ F

$\begin{array}{llll}0.6055 & 1 & 13.126 & 0.4503\end{array}$

t Test
Oneway Analysis of Measurement (wt\%) By Sampler Type of Material=SME Simulant, Analytical Block $=6$, Analyte $=\mathrm{CuO}(\mathrm{wt} \%)$, Target $(\mathrm{wt} \%)=0.103$

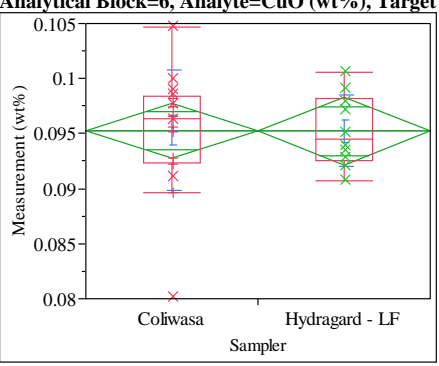

Oneway Anova

Summary of Fit

Rsquare

Adj Rsquare

Root Mean Square Error $\quad 0.004761$

$\begin{array}{lr}\text { Mean of Response } & 0.095262 \\ \text { Observations (or Sum Wgts) } & 26\end{array}$

Test

Hydragard - LF-Coliwasa

Assuming equal variances

Difference $\quad-0.00004$ t Ratio $\quad-0.0212$

Std Err Dif $\quad 0.00192$ DF 24

Upper CL Dif 0.00392 Prob $>|t| 0.9833$

Lower CL Dif -0.00400 Prob $>t \quad 0.5084$

Confidence $\quad 0.95$ Prob $<t \quad 0.4916$

Analysis of Variance

Source DF Sum of Squares Mean Square F Ratio Prob $>$ F

$\begin{array}{llllll}\text { Sampler } & 1 & 1.01855 \mathrm{e}-8 & 1.019 \mathrm{e}-8 & 0.0004 & 0.9833\end{array}$

$\begin{array}{llll}\text { Error } & 24 & 0.00054396 & 0.000023\end{array}$

C. Total $25 \quad 0.00054397$

Means for Oneway Anova

Level Number Mean Std Error Lower 95\% Upper 95\%

$\begin{array}{lrrrrr}\text { Level } & \text { Number } & \text { Mean } & \text { Std Error } & \text { Lower 95\% } & \text { Upper 95\% } \\ \text { Coliwasa } & 16 & 0.095278 & 0.00119 & 0.09282 & 0.09773\end{array}$

$\begin{array}{llllll}\text { Hydragard - LF } & 10 & 0.095237 & 0.00151 & 0.09213 & 0.09834\end{array}$

Std Error uses a pooled estimate of error variance

Means and Std Deviation

$\begin{array}{llllll}\text { Level Number Mean } & \text { Std Dev } & \text { Std Err Mean } & \text { Lower 95\% Upper 95\% }\end{array}$

$\begin{array}{lllllll}\text { Hydragard - LF } & 16 & 0.095278 & 0.005472 & 0.00137 & 0.09236 & 0.09819 \\ & 10 & 0.095237 & 0.003246 & 0.00103 & 0.09291 & 0.09756\end{array}$

Test

Hydragard - LF-Coliwasa

Assuming unequal variances

$\begin{array}{lll}\text { Difference } & -0.00004 \text { t Ratio } \quad-0.02379\end{array}$

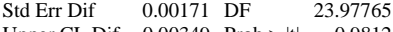

Upper CL Dif 0.00349 Prob $>|t| \quad 0.9812$

Lower CL Dif -0.00357 Prob $>\mathrm{t} \quad 0.5094$

Confidence $\quad 0.95$ Prob $<\mathrm{t} \quad 0.4906$

Tests that the Variances are Equal

Level Count Std Dev MeanAbsDif to Mean MeanAbsDif to Median

$\begin{array}{lrrrr}\text { Coliwasa } & 16 & 0.0054719 & 0.0038454 & 0.0036772 \\ \text { Hydragard - LF } & 10 & 0.0032460 & 0.0027239 & 0.0026789\end{array}$

Test F Ratio DFNum DFDen p-Value

$\begin{array}{lrrrr}\text { Test } & \text { F Ratio } & \text { DFNum } & \text { DFDen } & \text { p-Value } \\ \text { O'Brien[.5] } & 0.8916 & 1 & 24 & 0.3544\end{array}$

$\begin{array}{lllll}\text { O'Brien[.5] } & 0.8916 & 1 & 24 & 0.3544 \\ \text { Brown-Forsythe } & 0.5223 & 1 & 24 & 0.4768\end{array}$

$\begin{array}{lllll}\text { Brown-Forsythe } & 0.5223 & 1 & 24 & 0.4768 \\ \text { Levene } & 0.7966 & 1 & 24 & 0.3810\end{array}$

$\begin{array}{lllll}\text { Levene } & 0.7966 & 1 & 24 & 0.3810 \\ \text { Bartlett } & 2.5992 & 1 & . & 0.1069\end{array}$

$\begin{array}{llrrr}\text { F Test 2-sided } & 2.8417 & 15 & 9 & 0.1069\end{array}$

Welch Anova testing Means Equal, allowing Std Devs Not Equal

F Ratio DFNum DFDen Prob $>$ F

$\begin{array}{llll}0.0006 & 1 & 23.978 & 0.9812\end{array}$

t Test

0.0238 


\section{Exhibit B2. Statistical Comparisons for High-Rheology (Phase 2) Simulant Testing}

Oneway Analysis of Measurement (wt\%) By Sampler Type of Material=SME Simulant, Analytical Block=6, Analyte $=\mathrm{Fe} / \mathrm{Li}$, Target $(\mathrm{wt} \%)=1.99097835$

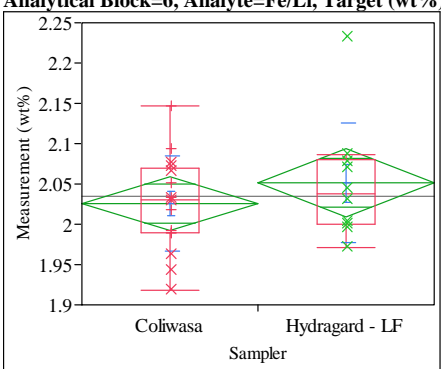

Oneway Anova

Summary of Fit

Adj Rsquare

$-0.00294$

$\quad 0.06528$ 2.035371

Observations (or Sum Wgts)

26

t Test

Hydragard - LF-Coliwasa

Assuming equal variances

Difference $\quad 0.02533$ t Ratio $\quad 0.962716$

Std Err Dif $\quad 0.02632$ DF 24

Upper CL Dif 0.07965 Prob $>|t| \quad 0.3453$

Lower CL Dif -0.02898 Prob $>t \quad 0.1726$

$\begin{array}{lrl}\text { Confidence } & 0.95 \text { Prob }<\mathrm{t} \quad 0.8274\end{array}$

Analysis of Variance

Source DF Sum of Squares Mean Square F Ratio Prob > F

$\begin{array}{llllll}\text { Sampler } & 1 & 0.00394966 & 0.003950 & 0.9268 & 0.3453\end{array}$

$\begin{array}{llll}\text { Error } & 24 & 0.10227624 & 0.004262\end{array}$

C. Total $25 \quad 0.10622590$

Means for Oneway Anova

Level $\quad$ Number Mean Std Error Lower 95\% Upper 95\%

$\begin{array}{lrrrrr}\text { Coliwasa } & 16 & 2.02563 & 0.01632 & 1.9919 & 2.0593 \\ \text { Hydragard - LF } & 10 & 2.05096 & 0.02064 & 2.0084 & 2.0936\end{array}$

Std Error uses a pooled estimate of error variance

Means and Std Deviations

Level Number Mean Std Dev Std Err Mean Lower 95\% Upper 95\%

Coliwasa $16 \quad 2.02563 \quad 0.059283$

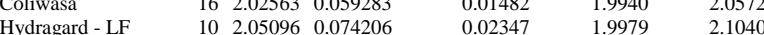

t Test

Hydragard - LF-Coliwasa

Assuming unequal variances

$\begin{array}{lrlr}\text { Difference } & 0.02533 & \text { t Ratio } & 0.912794 \\ \text { Std Err Dif } & 0.02775 \text { DF } & 16.07722 \\ \text { Upper CL Dif } & 0.08415 \text { Prob }>|t| & 0.3748 \\ \text { Lower CL Dif } & -0.03348 \text { Prob }>\text { t } & 0.1874 \\ \text { Confidence } & 0.95 \text { Prob }<\text { t } & 0.8126\end{array}$

Tests that the Variances are Equal

Level Count Std Dev MeanAbsDif to Mean MeanAbsDif to Median

Coliwasa $\quad 16 \quad 0.0592829$

$\begin{array}{lllll}\text { Hydragard - LF } & 10 & 0.0742064 & 0.0460802 & 0.0456218 \\ & & & 0.0521476 & 0.0508316\end{array}$

Test F Ratio DFNum DFDen p-Value

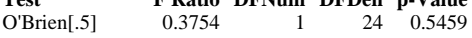

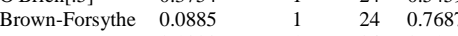

$\begin{array}{lllll}\text { Levene } & 0.1323 & 1 & 24 & 0.7193\end{array}$

$\begin{array}{lllll}\text { Bartlett } & 0.5588 & 1 & 0.4547 \\ \text { F Test 2-sided } & 1.5668 & 9 & 15 & 0.4247\end{array}$

Welch Anova testing Means Equal, allowing Std Devs Not Equal

F Ratio DFNum DFDen Prob $>$ F

$\begin{array}{rrrr}0.8332 & 1 & 16.077 & 0.3748\end{array}$

t Test

$$
1 \quad 16.077 \quad 0.3748
$$

0.9128
Oneway Analysis of Measurement (wt \%) By Sampler Type of Material=SME Simulant, Analytical Block $=6$, Analyte $=\mathrm{Fe} 2 \mathrm{O} 3(\mathrm{wt} \%)$, Target $(\mathrm{wt} \%)=9.931$

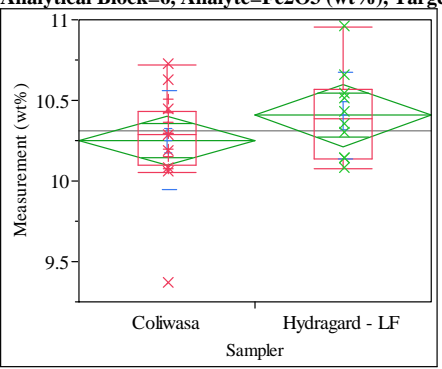

Oneway Anova

Summary of Fit

Adj Rsquare

0.293301

Mean of Response 10.31199

Observations (or Sum Wgts)

t Test

Hydragard - LF-Coliwasa

Assuming equal variances

$\begin{array}{lrlr}\text { Difference } & 0.15405 & \text { t Ratio } & 1.302931 \\ \text { Std Err Dif } & 0.11823 & \text { DF } & 24 \\ \text { Upper CL Dif } & 0.39807 & \text { Prob }>|t| & 0.2050 \\ \text { Lower CL Dif } & -0.08997 & \text { Prob }>t & 0.1025 \\ \text { Confidence } & 0.95 & \text { Prob }<\mathrm{t} & 0.8975\end{array}$

Analysis of Variance

Source DF Sum of Squares Mean Square F Ratio Prob $>$ F

$\begin{array}{llllll}\text { Sampler } & 1 & 0.1460397 & 0.146040 & 1.6976 & 0.2050\end{array}$

$\begin{array}{llll}\text { Error } & 24 & 2.0646154 & 0.086026\end{array}$

C. Total $25 \quad 2.2106551$

Means for Oneway Anova

Level Number Mean Std Error Lower 95\% Upper 95\%

$\begin{array}{llrrrr}\text { Coliwasa } & 16 & 10.2527 & 0.07333 & 10.101 & 10.404\end{array}$

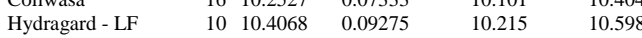

Std Error uses a pooled estimate of error variance

Means and Std Deviations

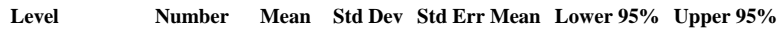

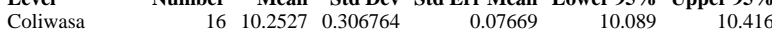

$\begin{array}{lllllll}\text { Hydragard - LF } & 10 & 10.4068 & 0.269372 & 0.08518 & 10.214 & 10.599\end{array}$

tTest

Hydragard - LF-Coliwasa

Assuming unequal variances

$\begin{array}{lrlr}\text { Difference } & 0.15405 & \text { t Ratio } & 1.344013 \\ \text { Std Err Dif } & 0.11462 & \text { DF } & 21.16127 \\ \text { Upper CL Dif } & 0.39230 & \text { Prob }>|t| & 0.1932 \\ \text { Lower CL Dif } & -0.08420 & \text { Prob }>\text { t } & 0.0966 \\ \text { Confidence } & 0.95 & \text { Prob }<\mathrm{t} & 0.9034\end{array}$

Tests that the Variances are Equal

Level Count Std Dev MeanAbsDif to Mean MeanAbsDif to Median

$\begin{array}{lllll}\text { Coliwasa } & 16 & 0.3067642 & 0.2088702 & 0.2055194\end{array}$

$\begin{array}{lllll}\text { Hydragard - LF } & 10 & 0.2693719 & 0.2073065 & 0.2073065\end{array}$

Test F Ratio DFNum DFDen p-Value

$\begin{array}{lrrrr}\text { O'Brien[.5] } & 0.0854 & 1 & 24 & 0.7726\end{array}$

Brown-Forsythe $\begin{array}{llll}0.0005 & 1 & 24 & 0.9827\end{array}$

$\begin{array}{lllll}\text { Levene } & 0.0004 & 1 & 24 & 0.9845\end{array}$

$\begin{array}{lrrrr}\text { Bartlett } & 0.1775 & 1 & \text {. } & 0.6735 \\ \text { F Test 2-sided } & 1.2969 & 15 & 9 & 0.7103\end{array}$

Welch Anova testing Means Equal, allowing Std Devs Not Equal

F Ratio DFNum DFDen Prob $>$ F

$\begin{array}{llll}1.8064 & 1 & 21.161 & 0.1932\end{array}$

t Test

1.3440 


\section{Exhibit B2. Statistical Comparisons for High-Rheology (Phase 2) Simulant Testing}

Oneway Analysis of Measurement (wt \%) By Sampler Type of Material=SME Simulant, Analytical Block=6, Analyte $=K 2 O(w t \%)$, Target $(w t \%)=0.076$

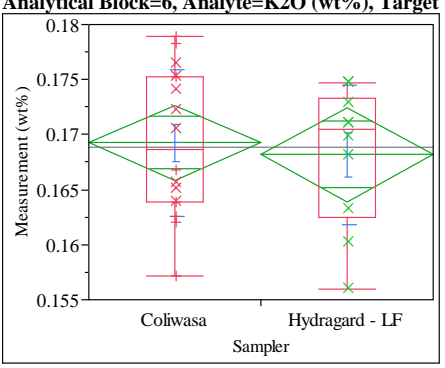

Oneway Anova

Summary of Fit

Rsquare

Adj Rsquare

Root Mean Square Error $\quad 0.006556$

Mean of Response $\quad 0.168829$

Observations (or Sum Wgts) $\quad 26$

Test

Hydragard - LF-Coliwasa

Assuming equal variances

Difference $\quad-0.00108$ t Ratio $\quad-0.41022$

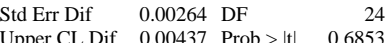

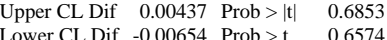

$\begin{array}{lll}\text { Confidence } & 0.95 \text { Prob }<\mathrm{t} \quad 0.3426\end{array}$

Analysis of Variance

Source DF Sum of Squares Mean Square F Ratio Prob $>$ F

$\begin{array}{lrrrrr}\text { Sampler } & 1 & 0.00000723 & 7.233 \mathrm{e}-6 & 0.1683 & 0.6853\end{array}$

Error $24 \quad 0.00103156 \quad 0.000043$

C. Total $25 \quad 0.00103879$

Means for Oneway Anov

Level Number Mean Std Error Lower 95\% Upper 95\%

$\begin{array}{lrrrrr}\text { Level } & \text { Number } & \text { Mean } & \text { Std Error } & \text { Lower 95\% } & \text { Upper 95\% } \\ \text { Coliwasa } & 16 & 0.169246 & 0.00164 & 0.16586 & 0.17263\end{array}$

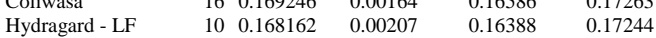

Std Error uses a pooled estimate of error variance

Means and Std Deviations

Level Number Mean Std Dev Std Err Mean Lower 95\% Upper 95\%

$\begin{array}{lllllll}\text { Coliwasa } & 16 & 0.169246 & 0.006682 & 0.00167 & 0.16569 & 0.17281\end{array}$

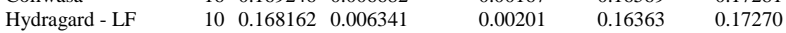

t Test

Hydragard - LF-Coliwasa

Assuming unequal variances

Difference $\quad-0.00108$ t Ratio $\quad-0.4154$

Std Err Dif $\quad 0.00261$ DF 20.03563

Upper CL Dif 0.00436 Prob $>|t| \quad 0.6823$

Lower CL Dif -0.00653 Prob $>t \quad 0.6589$

Confidence $\quad 0.95$ Prob $<\mathrm{t} \quad 0.3411$

Tests that the Variances are Equal

Level Count Std Dev MeanAbsDif to Mean MeanAbsDif to Median

$\begin{array}{lllll} & 16 & 0.0066816 & 0.0058724 & 0.0058724 \\ \text { Hydragard - LF } & 10 & 0.0063412 & 0.0050352 & 0.0046979\end{array}$

$\begin{array}{lrrrr}\text { Test } & \text { F Ratio DFNum DFDen p-Value }\end{array}$

$\begin{array}{lllll}\text { O'Brien[.5] } & 0.0576 & 1 & 24 & 0.8124 \\ \text { Brown-Forsythe } & 0.6421 & 1 & 24 & 0.4308\end{array}$

$\begin{array}{lllll}\text { Brown-Forsythe } & 0.6421 & 1 & 24 & 0.4308 \\ \text { Levene } & 0.4575 & 1 & 24 & 0.5053\end{array}$

$\begin{array}{llrrr}\text { Levene } & 0.4575 & 1 & 24 & 0.5053 \\ \text { Bartlett } & 0.0292 & 1 & . & 0.8644\end{array}$

$\begin{array}{lllll}\text { F Test 2-sided } & 1.1103 & 15 & 9 & 0.9040\end{array}$

Welch Anova testing Means Equal, allowing Std Devs Not Equal

F Ratio DFNum DFDen Prob $>$ F

$\begin{array}{llll}0.1726 & 1 & 20.036 & 0.6823\end{array}$

t Test

0.4154
Oneway Analysis of Measurement (wt\%) By Sampler Type of Material=SME Simulant, Analytical Block $=6$, Analyte $=\mathrm{Li} 2 \mathrm{O}(\mathrm{wt} \%)$, Target $(\mathrm{wt} \%)=4.988$

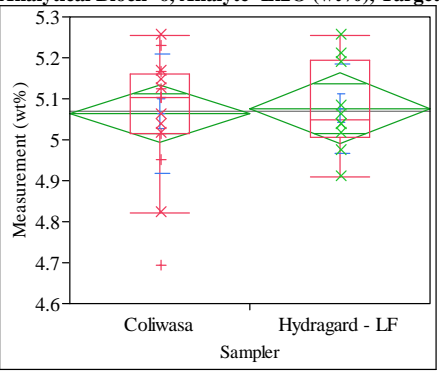

Oneway Anova

Summary of Fit

Adj Rsquare

-0.03904
0.132716

Root Mean

Mean of Response 5.068423
26

t Test

Hydragard - LF-Coliwasa

Assuming equal variances

$\begin{array}{lrlr}\text { Difference } & 0.01319 & \text { t Ratio } & 0.246478 \\ \text { Std Err Dif } & 0.05350 & \text { DF } & 24 \\ \text { Upper CL Dif } & 0.12360 & \text { Prob }>|t| & 0.8074 \\ \text { Lower CL Dif } & -0.09723 & \text { Prob }>t & 0.4037 \\ \text { Confidence } & 0.95 & \text { Prob }<\mathrm{t} & 0.5963\end{array}$

Analysis of Variance

Source DF Sum of Squares Mean Square F Ratio Prob $>$ F

$\begin{array}{lrrrrr}\text { Sampler } & 1 & 0.00107006 & 0.001070 & 0.0608 & 0.8074\end{array}$

$\begin{array}{llll}\text { Error } & 24 & 0.42272741 & 0.017614\end{array}$

C. Total $25-0.42379747$

Means for Oneway Anova

Level Number Mean Std Error Lower 95\% Upper 95\%

\begin{tabular}{llllll} 
Coliwasa & 16 & 5.06335 & 0.03318 & 4.9949 & 5.1318 \\
\hline & 10 & 5.07654 & 0.04197 & 4.9899 & 5.1632
\end{tabular}

Std Error uses a pooled estimate of error variance

Means and Std Deviations

$\begin{array}{lllll}\text { Means and Std Deviations } & & & \\ \text { Level } & \text { Number Mean Std Dev Std Err Mean Lower 95\% Upper 95\% }\end{array}$

$\begin{array}{lrrrrrr}\text { Level } & \text { Number } & \text { Mean } & \text { Std Dev } & \text { Std Err Mean } & \text { Lower 95\% } & \text { Upper 95\% } \\ \text { Coliwasa } & 16 & 5.06335 & 0.145001 & 0.03625 & 4.9861 & 5.1406\end{array}$

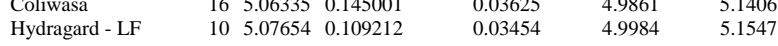

t Test

Hydragard - LF-Coliwasa

Assuming unequal variances

Difference $\quad 0.01319$ t Ratio $\quad 0.263371$

$\begin{array}{lll}\text { Std Err Dif } \quad 0.05007 & \text { DF } \quad 23.00287\end{array}$

Upper CL Dif 0.11676 Prob $>|t| r 0.7946$

Lower CL Dif -0.09039 Prob $>t \quad 0.3973$

$\begin{array}{lll}\text { Confidence } & 0.95 \mathrm{Prob}<\mathrm{t} & 0.6027\end{array}$

Tests that the Variances are Equal

Level Count Std Dev MeanAbsDif to Mean MeanAbsDif to Median

$\begin{array}{lrrrr}\text { Coliwasa } & 16 & 0.1450015 & 0.1057949 & 0.1009172\end{array}$

$\begin{array}{lllll}\text { Hydragard - LF } & 10 & 0.1092124 & 0.0852548 & 0.0818102\end{array}$

Test F Ratio DFNum DFDen p-Value

$\begin{array}{lllll}\text { O'Brien[.5] } & 0.5044 & 1 & 24 & 0.4844\end{array}$

$\begin{array}{lllll}\text { Brown-Forsythe } & 0.2396 & 1 & 24 & 0.6289 \\ & 0.3645 & 1 & 24 & 0.5517\end{array}$

$\begin{array}{lllll}\text { Levene } & 0.3645 & 1 & 24 & 0.5517 \\ \text { Bartlett } & 0.8157 & 1 & & 0.3664\end{array}$

$\begin{array}{lllll}\text { F Test 2-sided } & 1.7628 & 15 & 9 & 0.3931\end{array}$

Welch Anova testing Means Equal, allowing Std Devs Not Equal

F Ratio DFNum DFDen Prob > F

$\begin{array}{rrrr}0.0694 & 1 & 23.003 & 0.7946\end{array}$

$\mathbf{t}$ Test
0.2634 


\section{Exhibit B2. Statistical Comparisons for High-Rheology (Phase 2) Simulant Testing}

Oneway Analysis of Measurement (wt \%) By Sampler Type of Material=SME Simulant, Analytical Block=6, Analyte $=\mathrm{MgO}(\mathrm{wt} \%)$, Target $(\mathrm{wt} \%)=0.286$

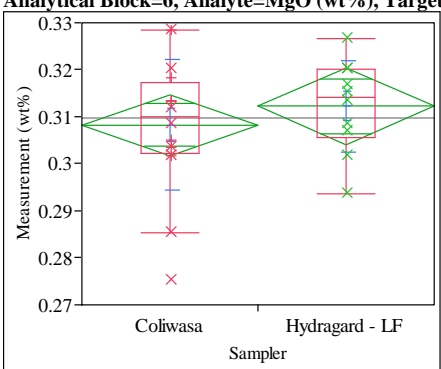

Oneway Anova

Summary of Fit

Rsquare $\quad 0.025775$

Adj Rsquare $\quad-0.01482$

Root Mean Square Error $\quad 0.012519$

Mean of Response $\quad 0.309783$

Observations (or Sum Wgts) $\quad 26$

t Test

Hydragard - LF-Coliwasa

Assuming equal variances

Difference $\quad 0.00402$ t Ratio $\quad 0.79684$

Std Err Dif $\quad 0.00505$ DF 24

Upper CL Dif 0.01444 Prob $>|t| \quad 0.4334$

$\begin{array}{lll}\text { Lower CL Dif }-0.00639 \text { Prob }>t & 0.2167\end{array}$

$\begin{array}{lrl}\text { Confidence } & 0.95 \text { Prob }<\mathrm{t} \quad 0.7833\end{array}$

Analysis of Variance

Source DF Sum of Squares Mean Square F Ratio Prob > F

$\begin{array}{llllll}\text { Sampler } & 1 & 0.00009952 & 0.000100 & 0.6350 & 0.4334\end{array}$

$\begin{array}{llll}\text { Error } & 24 & 0.00376153 & 0.000157\end{array}$

C. Total $25 \quad 0.00386105$

Means for Oneway Anova

Level Number Mean Std Error Lower 95\% Upper 95\%

160.3082370 .00313 Le 0.30178 Uper $95 \%$

$\begin{array}{lllllr}\text { Coliwasa } & 16 & 0.308237 & 0.00313 & 0.30178 & 0.31470 \\ \text { Hydragard - LF } & 10 & 0.312258 & 0.00396 & 0.30409 & 0.32043\end{array}$

Std Error uses a pooled estimate of error variance

Means and Std Deviations

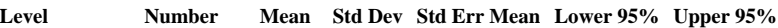

Coliwasa $160.308237 \quad 0.013899$

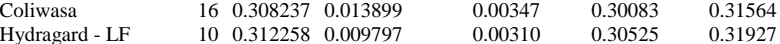

tTest

Hydragard - LF-Coliwasa

Assuming unequal variances

Difference $\quad 0.00402$ t Ratio 0.863836

Std Err Dif $\quad 0.00466$ DF 23.53756

Upper CL Dif 0.01364 Prob $>|t| r \mid r .3964$

Lower CL Dif -0.00560 Prob $>t \quad 0.1982$

$\begin{array}{lrl} & \\ \text { Confidence } & 0.95 \text { Prob }<\mathrm{t} & 0.8018\end{array}$

Tests that the Variances are Equal

Level Count Std Dev MeanAbsDif to Mean MeanAbsDif to Median

Coliwasa $\quad 16 \quad 0.0138991$

$\begin{array}{lllll}\text { Hydragard - LF } & 10 & 0.0097966 & 0.0101830 & 0.0101571 \\ & & & 0.0076945 & 0.0074623\end{array}$

Test F Ratio DFNum DFDen p-Value

$\begin{array}{lrrrr}\text { O'Brien[.5] } & 0.8007 & 1 & 24 & 0.3798\end{array}$

Brown-Forsythe $\quad 0.6503 \quad 1 \quad 24 \quad 0.4279$

$\begin{array}{lllll}\text { Levene } & 0.6055 & 1 & 24 & 0.4441\end{array}$

$\begin{array}{lrrrr}\text { Bartlett } & 1.2219 & 1 & \text {. } & 0.2690 \\ \text { F Test 2-sided } & 2.0129 & 15 & 9 & 0.2907\end{array}$

Welch Anova testing Means Equal, allowing Std Devs Not Equal

F Ratio DFNum DFDen Prob $>$ F

$\begin{array}{rrrr}0.7462 & 1 & 23.538 & 0.3964\end{array}$

t Test

$$
1 \quad 23.538 \quad 0.3964
$$

0.8638
Oneway Analysis of Measurement (wt \%) By Sampler Type of Material=SME Simulant, Analytical Block $=6$, Analyte $=\mathrm{MnO}(\mathrm{wt} \%)$, Target $(\mathrm{wt} \%)=2.918$

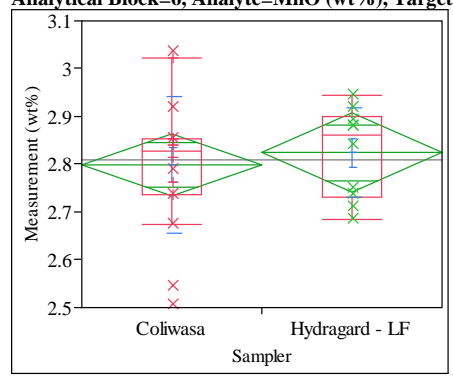

Oneway Anova

Summary of Fit

$\begin{array}{lr}\text { Rsquare } & 0.009972 \\ \text { Adj Rsquare } & -0.03128 \\ \text { Root Mean Square Error } & 0.127035 \\ \text { Mean of Response } & 2.80836 \\ \text { Observations (or Sum Wgts) } & 26\end{array}$

t Test

Hydragard - LF-Coliwasa

Assuming equal variances

$\begin{array}{lrlr}\text { Difference } & 0.02518 & \text { t Ratio } & 0.491677 \\ \text { Std Err Dif } & 0.05121 & \text { DF } & 24 \\ \text { Upper CL Dif } & 0.13087 & \text { Prob }>|t| & 0.6274 \\ \text { Lower CL Dif } & -0.08051 & \text { Prob }>t & 0.3137 \\ \text { Confidence } & 0.95 & \text { Prob }<\mathrm{t} & 0.6863\end{array}$

Analysis of Variance

Source DF Sum of Squares Mean Square F Ratio Prob $>$ F

$\begin{array}{lrrrrr}\text { Sampler } & 1 & 0.00390124 & 0.003901 & 0.2417 & 0.6274\end{array}$

$\begin{array}{llll}\text { Error } \quad 24 & 0.38730664 & 0.016138\end{array}$

C. Total $25 \quad 0.39120788$

Means for Oneway Anova

Level Number Mean Std Error Lower 95\% Upper 95\%

\begin{tabular}{llllll} 
Coliwasa & 16 & 2.79868 & 0.03176 & 2.7331 & 2.8642 \\
\hline & 10 & 2.82385 & 0.04017 & 2.7409 & 2.9068
\end{tabular}

Std Error uses a pooled estimate of error variance

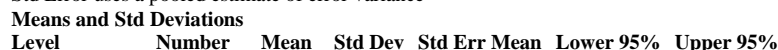

$\begin{array}{lrrrrrr}\text { Level } & \text { Number } & \text { Mean } & \text { Std Dev } & \text { Std Err Mean } & \text { Lower 95\% } & \text { Upper 95\% } \\ \text { Coliwasa } & 16 & 2.79868 & 0.143356 & 0.03584 & 2.7223 & 2.8751\end{array}$

$\begin{array}{lllllll}\text { Coliwasa } & 16 & 2.79868 & 0.143356 & 0.03584 & 2.7223 & 2.8751 \\ \text { Hydragard - LF } & 10 & 2.82385 & 0.093715 & 0.02964 & 2.7568 & 2.8909\end{array}$

t Test

Hydragard - LF-Coliwasa

Assuming unequal variances

Difference $\quad 0.02518$ t Ratio 0.541417

Std Err Dif $\quad 0.04650$ DF 23.90143

Upper CL Dif 0.12118 Prob $>|t| r 0.5932$

Lower CL Dif -0.07082 Prob $>t \quad 0.2966$

$\begin{array}{lll}\text { Confidence } & 0.95 \text { Prob }<\mathrm{t} \quad 0.7034\end{array}$

Tests that the Variances are Equal

Level Count Std Dev MeanAbsDif to Mean MeanAbsDif to Median

$\begin{array}{lrrrr}\text { Coliwasa } & 16 & 0.1433562 & 0.1053135 & 0.1032960\end{array}$

$\begin{array}{lllll}\text { Hydragard - LF } & 10 & 0.0937146 & 0.1053135 & 0.1032960 \\ & & \end{array}$

Test F Ratio DFNum DFDen p-Value

$\begin{array}{lllll}\text { O'Brien[.5] } & 1.3789 & 1 & 24 & 0.2518\end{array}$

$\begin{array}{lllll}\text { Brown-Forsythe } & 0.4908 & 1 & 24 & 0.4903\end{array}$

$\begin{array}{lllll}\text { Levene } & 0.5579 & 1 & 24 & 0.4624 \\ \text { Bartlett } & 1.7692 & 1 & & 0.1835\end{array}$

$\begin{array}{llll}\text { F Test 2-sided } \quad 2.3400 & 15 & 9 & 0.1999\end{array}$

Welch Anova testing Means Equal, allowing Std Devs Not Equal

F Ratio DFNum DFDen Prob $>$ F

$\begin{array}{rrrr}0.2931 & 1 & 23.901 & 0.5932\end{array}$

t Test 


\section{Exhibit B2. Statistical Comparisons for High-Rheology (Phase 2) Simulant Testing}

Oneway Analysis of Measurement (wt \%) By Sampler Type of Material=SME Simulant, Analytical Block $=6$, Analyte $=\mathrm{Na2O}(w t \%)$, Target $(w t \%)=13.318$

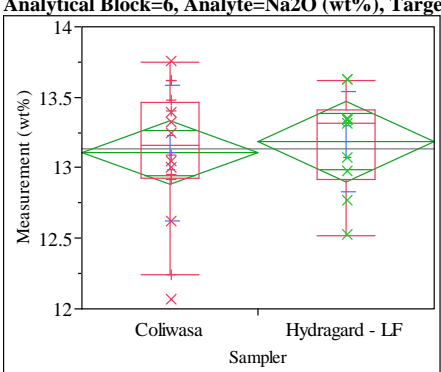

Oneway Anova

Summary of Fit

Adj Rsquare

0.008395

-0.03292
-

0.436776

13.1373

Observations (or Sum Wgts)

Hydragard - LF-Coliwasa

Assuming equal variances

Difference $\quad 0.07936$ t Ratio $\quad 0.450749$

Std Err Dif $\quad 0.17607$ DF $\quad 24$

Upper CL Dif 0.44275 Prob $>|t| \quad 0.6562$

Lower CL Dif -0.28403 Prob $>t \quad 0.3281$

$\begin{array}{lrl}\text { Confidence } & 0.95 \text { Prob }<\mathrm{t} & 0.6719\end{array}$

Analysis of Variance

Source DF Sum of Squares Mean Square F Ratio Prob $>$ F

$\begin{array}{llllll}\text { Sampler } & 1 & 0.0387604 & 0.038760 & 0.2032 & 0.6562\end{array}$

$\begin{array}{llll}\text { Error } & 24 & 4.5785638 & 0.190773\end{array}$

C. Total $25 \quad 4.6173242$

Means for Oneway Anova

Level Number Mean Std Error Lower 95\% Upper 95\%

Number 13.1068

$\begin{array}{llllll}\text { Hydragard - LF } & 16 & 13.1068 & 0.10919 & 12.881 & 13.332 \\ & 10 & 13.1861 & 0.13812 & 12.901 & 13.471\end{array}$

Std Error uses a pooled estimate of error variance

Means and Std Deviations

Level Number Mean Std Dev Std Err Mean Lower 95\% Upper 95\%

Coliwasa

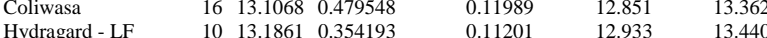

tTest

Hydragard - LF-Coliwasa

Assuming unequal variances

Difference $\quad 0.07936$ t Ratio $\quad 0.483725$

Std Err Dif $\quad 0.16407$ DF $\quad 23.18004$

Upper CL Dif 0.41862 Prob $>|t| r \mid r .6331$

Lower CL Dif -0.25989 Prob $>\mathrm{t} \quad 0.3166$

$\begin{array}{lrl}\text { Confidence } & 0.95 \text { Prob }<\mathrm{t} & 0.6834\end{array}$

Tests that the Variances are Equal

Level Count Std Dev MeanAbsDif to Mean MeanAbsDif to Median

Coliwasa $\quad 16 \quad 0.4795476$

$\begin{array}{lllll}\text { Hydragard - LF } & 10 & 0.3541933 & 0.3681725 & 0.3681725 \\ & & & 0.2852368 & 0.2615120\end{array}$

Test F Ratio DFNum DFDen p-Value

$\begin{array}{lrrrr}\text { O'Brien[.5] } & 0.8300 & 1 & 24 & 0.3713\end{array}$

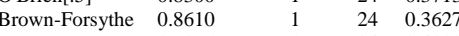

$\begin{array}{lllll}\text { Levene } & 0.6365 & 1 & 24 & 0.4328\end{array}$

$\begin{array}{lrrrr}\text { Bartlett } & 0.9277 & 1 & \text {. } & 0.3355 \\ \text { F Test 2-sided } & 1.8331 & 15 & 9 & 0.3607\end{array}$

Welch Anova testing Means Equal, allowing Std Devs Not Equal

F Ratio DFNum DFDen Prob $>$ F

$\begin{array}{llll}0.2340 & 1 & 23.18 & 0.6331\end{array}$

t Test

0.4837
Oneway Analysis of Measurement (wt \%) By Sampler Type of Material=SME Simulant, Analytical Block $=6$, Analyte $=\mathrm{NiO}(\mathrm{wt} \%)$, Target $(\mathrm{wt} \%)=1.033$

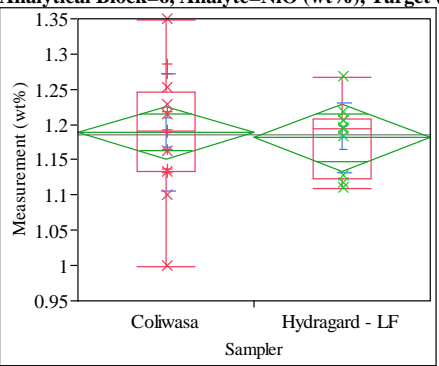

Oneway Anova

Summary of Fit

Adj Rsquare

0.072496

1.185872

Observations (or Sum Wgts) 26

t Test

Hydragard - LF-Coliwasa

Assuming equal variances

$\begin{array}{lrlr}\text { Difference } & -0.00749 & \text { t Ratio } & -0.25636 \\ \text { Std Err Dif } & 0.02922 & \text { DF } & 24 \\ \text { Upper CL Dif } & 0.05282 & \text { Prob }>|t| & 0.7999 \\ \text { Lower CL Dif } & -0.06781 & \text { Prob }>\text { t } & 0.6001 \\ \text { Confidence } & 0.95 & \text { Prob }<\mathrm{t} & 0.3999\end{array}$

Analysis of Variance

Source DF Sum of Squares Mean Square F Ratio Prob > F

$\begin{array}{llllll}\text { Sampler } & 1 & 0.00034540 & 0.000345 & 0.0657 & 0.7999\end{array}$

$\begin{array}{llll}\text { Error } & 24 & 0.12613446 & 0.005256\end{array}$

C. Total $25 \quad 0.12647986$

Means for Oneway Anova

Level Number Mean Std Error Lower 95\% Upper 95\%

$\begin{array}{lrrrrr}\text { Coliwasa } & 16 & 1.18875 & 0.01812 & 1.1513 & 1.2262\end{array}$

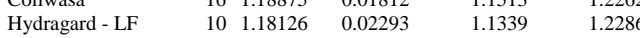

Std Error uses a pooled estimate of error variance

Means and Std Deviations

Level Number Mean Std Dev Std Err Mean Lower 95\% Upper 95\%

$\begin{array}{lrrrrr}\text { Coliwasa } & 16 & 1.18875 & 0.083217 & 0.02080 & 1.1444\end{array}$

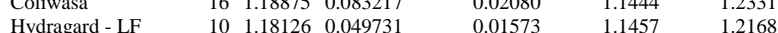

tTest

Hydragard - LF-Coliwasa

Assuming unequal variances

$\begin{array}{lrlr}\text { Difference } & -0.00749 & \text { t Ratio } & -0.28727 \\ \text { Std Err Dif } & 0.02608 \text { DF } & 23.98677 \\ \text { Upper CL Dif } & 0.04633 \text { Prob }>|t| & 0.7764 \\ \text { Lower CL Dif } & -0.06132 & \text { Prob }>\text { t } & 0.6118 \\ \text { Confidence } & 0.95 & \text { Prob }<\mathrm{t} & 0.3882\end{array}$

Tests that the Variances are Equal

Level Count Std Dev MeanAbsDif to Mean MeanAbsDif to Median

$\begin{array}{lllll}\text { Coliwasa } & 16 & 0.0832170 & 0.0622730 & 0.0622730\end{array}$

$\begin{array}{lllll}\text { Hydragard - LF } & 10 & 0.0497309 & 0.0384041 & 0.0360118\end{array}$

Test F Ratio DFNum DFDen p-Value

$\begin{array}{lrrrr}\text { O'Brien[.5] } & 1.4704 & 1 & 24 & 0.2371\end{array}$

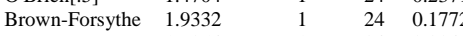

$\begin{array}{lllll}\text { Levene } & 1.7054 & 1 & 24 & 0.2040\end{array}$

$\begin{array}{lrrrr}\text { Bartlett } & 2.5315 & 1 & \text {. } & 0.1116 \\ \text { F Test 2-sided } & 2.8001 & 15 & 9 & 0.1227\end{array}$

Welch Anova testing Means Equal, allowing Std Devs Not Equal

F Ratio DFNum DFDen Prob $>$ F

$\begin{array}{rrrr}0.0825 & 1 & 23.987 & 0.7764\end{array}$

t Test

0.2873 


\section{Exhibit B2. Statistical Comparisons for High-Rheology (Phase 2) Simulant Testing}

Oneway Analysis of Measurement (wt\%) By Sampler Type of Material=SME Simulant, Analytical Block=6, Analyte $=\mathrm{SiO} 2(w t \%)$, Target $(w t \%)=50.183$

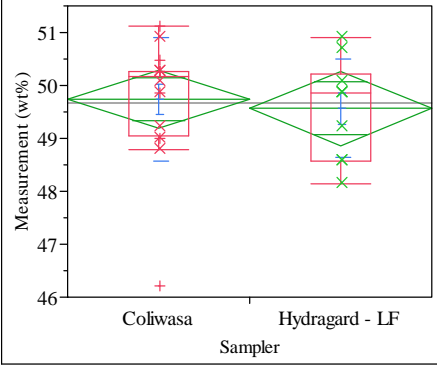

Oneway Anova

Summary of Fit

Rsquare

Adj Rsquare

Root Mean Square Error $\quad 1.080382$

$\begin{array}{lr}\text { Mean of Response } & 49.6729 \\ \text { Observations (or Sum Wgts) } & 26\end{array}$

t Test

Hydragard - LF-Coliwasa

Assuming equal variances

Difference $\quad-0.1711$ t Ratio -0.39297

$\begin{array}{lrlr}\text { Std Err Dif } & 0.4355 & \text { DF } & 24 \\ \text { Upper CL Dif } & 0.7277 \text { Prob }>|t| & 0.6978\end{array}$

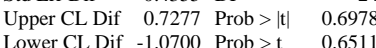

$\begin{array}{lrll}\text { Lower CL Dif } & -1.0700 & \text { Prob }>t & 0.6511 \\ \text { Confidence } & 0.95 & \text { Prob }<\mathrm{t} & 0.3489\end{array}$

Analysis of Variance

Source DF Sum of Squares Mean Square F Ratio Prob $>$ F

$\begin{array}{lrrrrr}\text { Sampler } & 1 & 0.180248 & 0.18025 & 0.1544 & 0.6978\end{array}$

$\begin{array}{llll}\text { Error } & 24 & 28.013396 & 1.16722\end{array}$

C. Total $25 \quad 28.193644$

Means for Oneway Anova

Level Number Mean Std Error Lower 95\% Upper 95\%

$\begin{array}{lllllr}\text { Coliwasa } & 16 & 49.7387 & 0.27010 & 49.181 & 50.296\end{array}$

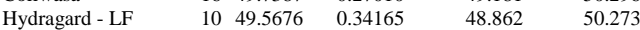

Std Error uses a pooled estimate of error variance

Means and Std Deviations

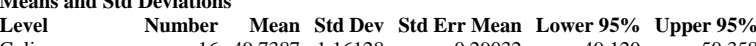

$\begin{array}{lrrrrrr}\text { Coliwasa } & \text { Nut } & 49.7387 & 1.16128 & 0.29032 & 49.120 & 50.358 \\ \text { Hydragard - LF } & 10 & 49.5676 & 0.93004 & 0.29411 & 48.902 & 50.233\end{array}$

Test

Hydragard - LF-Coliwasa

Assuming unequal variances

Difference $\quad-0.1711$ t Ratio $\quad-0.41413$

Std Err Dif $\quad 0.4133$ DF 22.3515

Upper CL Dif $\quad 0.6851$ Prob $>|t| r 0.6827$

Lower CL Dif -1.0274 Prob $>t \quad 0.6586$

$\begin{array}{lll}\text { Confidence } & 0.95 \text { Prob }<\mathrm{t} \quad 0.3414\end{array}$

Tests that the Variances are Equal

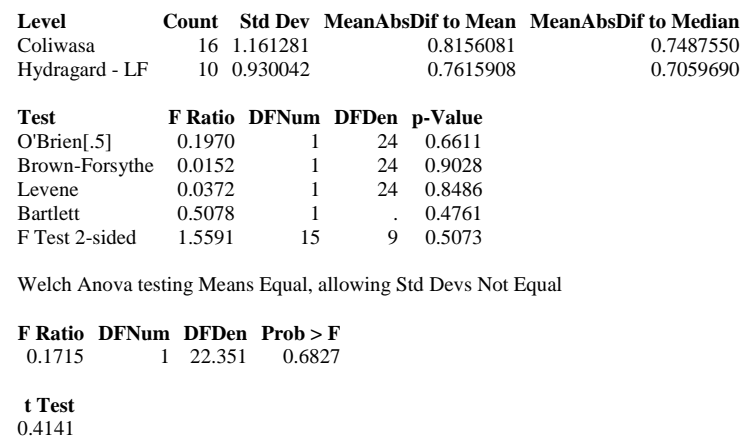

Oneway Analysis of Measurement (wt \%) By Sampler Type of Material=SME Simulant, Analytical Block $=6$, Analyte $=$ Sum of Oxides (wt $\%)$, Target $(w t \%)=99.73$

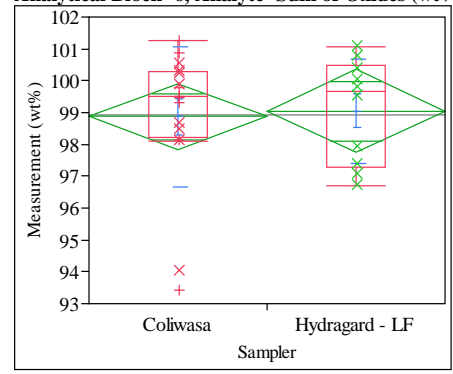

Oneway Anova

Summary of Fit

Adj Rsquare

0.002171

$\begin{array}{ll}\text { Adj Rsquare } & -0.03941 \\ \text { Root Mean Square Error } & 2.018312\end{array}$

Mean of Response

98.93749

t Test

Hydragard - LF-Coliwasa

Assuming equal variances

$\begin{array}{lrlr}\text { Difference } & 0.1859 & \text { t Ratio } & 0.228502 \\ \text { Std Err Dif } & 0.8136 \text { DF } & 24 \\ \text { Upper CL Dif } & 1.8651 & \text { Prob }>|t| & 0.8212 \\ \text { Lower CL Dif } & -1.4933 & \text { Prob }>t & 0.4106 \\ \text { Confidence } & 0.95 & \text { Prob }<t & 0.5894\end{array}$

Analysis of Variance

Source DF Sum of Squares Mean Square F Ratio Prob $>$ F

$\begin{array}{lrrrrr}\text { Sampler } & 1 & 0.212695 & 0.21270 & 0.0522 & 0.8212\end{array}$

$\begin{array}{lrrr}\text { Sampler } & 1 & 0.212695 & 0.21270 \\ \text { Error } & 24 & 97.765968 & 4.07358\end{array}$

$\begin{array}{lll}\text { Error } & 24 & 97.765968 \\ \text { C. Total } & 25 & 97.978663\end{array}$

Means for Oneway Anova

Level Number Mean Std Error Lower 95\% Upper 95\%

$\begin{array}{lrrrrr}\text { Coliwasa } & 16 & 98.8660 & 0.50458 & 97.825 & 99.91 \\ \text { Hydragard - LF } & 10 & 99.0519 & 0.63825 & 97.735 & 100.37\end{array}$

Std Erior uses a pooled estimate of error variance

Means and Std Deviations

$\begin{array}{lclll}\text { Means and Std Deviations } & & \\ \text { Level } & \text { Number Mean Std Dev Std Err Mean Lower 95\% Upper 95\% }\end{array}$

$\begin{array}{lrrrrrr}\text { Level } & \text { Number } & \text { Mean } & \text { Std Dev } & \text { Std Err Mean } & \text { Lower 95\% } & \text { Upper 95\% } \\ \text { Coliwasa } & 16 & 98.8660 & 2.21670 & 0.55417 & 97.685 & 100.05\end{array}$

$\begin{array}{lllllll}\text { Hydragard - LF } & 16 & 98.8660 & 2.21670 & 0.55417 & 97.685 & 100.05 \\ & 10 & 99.0519 & 1.63502 & 0.51704 & 97.882 & 100.22\end{array}$

t Test

Hydragard - LF-Coliwasa

Assuming unequal variances

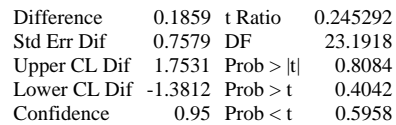

Tests that the Variances are Equal

$\begin{array}{lrrrr}\text { Coliwasa } & 16 & 2.216699 & 1.550510 & 1.418562\end{array}$

\begin{tabular}{|c|c|}
\hline Hydragard - LF & \\
\hline
\end{tabular}

Test F Ratio DFNum DFDen p-Value

$\begin{array}{lrrrr}\text { O'Brien[.5] } & 0.5065 & 1 & 24 & 0.4835\end{array}$

$\begin{array}{lllll}\text { Brown-Forsythe } & 0.0139 & 1 & 24 & 0.9072\end{array}$

$\begin{array}{lllll}\text { Levene } & 0.0490 & 1 & 24 & 0.8266 \\ & 0.9357 & 1 & & 0.3334\end{array}$

$\begin{array}{lllll}\text { F Test 2-sided } & 1.8381 & 15 & 9 & 0.3585\end{array}$

Welch Anova testing Means Equal, allowing Std Devs Not Equal

F Ratio DFNum DFDen Prob $>$ F

$\begin{array}{rrrr}0.0602 & 1 & 23.192 & 0.8084\end{array}$

t Test 
SRNL-STI-2011-00693

Revision 0

\section{Exhibit B2. Statistical Comparisons for High-Rheology (Phase 2) Simulant Testing}

Oneway Analysis of Measurement (wt \%) By Sampler Type of Material=SME Simulant, Analytical Block=6, Analyte $=\mathrm{TiO} 2(w t \%)$, Target $(w t \%)=0.013$

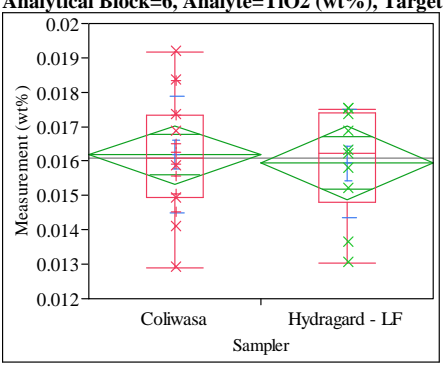

Oneway Anova

Summary of Fit

Rsquare

Adj Rsquare

Root Mean Square Error $\quad 0.001648$

Mean of Response 0.016089

Observations (or Sum Wgts) $\quad 26$

t Test

Hydragard - LF-Coliwasa

Assuming equal variances

Difference $\quad-0.00025$ t Ratio -0.3810

$\begin{array}{lrr}\text { Std Err Dif } & 0.00066 \text { DF } & 24 \\ \text { Upper } & \text { D } & \end{array}$

$\begin{array}{rrrr}\text { Upper CL Dif } & 0.00112 & \text { Prob }>|t| & 0.7065 \\ \text { Cower CL Dif } & -0.00162 & \text { Prob }>t & 0.6467\end{array}$

$\begin{array}{lrl}\text { Confidence } & 0.95 \text { Prob }<\mathrm{t} \quad 0.3533\end{array}$

Analysis of Variance

Source DF Sum of Squares Mean Square F Ratio Prob $>$ F

$\begin{array}{lrrrrr}\text { Sampler } & 1 & 0.00000039 & 3.9427 \mathrm{e}-7 & 0.1452 & 0.7065\end{array}$

$\begin{array}{llll}\text { Error } & 24 & 0.00006518 & 2.7159 \mathrm{e}-6\end{array}$

C. Total $25 \quad 0.00006558$

Means for Oneway Anova

Level Number Mean Std Error Lower 95\% Upper 95\%

$\begin{array}{lrrrrr}\text { Level } & \text { Number } & \text { Mean } & \text { Std Error } & \text { Lower 95\% } & \text { Upper 95\% } \\ \text { Coliwasa } & 16 & 0.016186 & 0.00041 & 0.01534 & 0.01704\end{array}$

$\begin{array}{llllll}\text { Coliwasa } & 16 & 0.016186 & 0.00041 & 0.01534 & 0.01704 \\ \text { Hydragard - LF } & 10 & 0.015933 & 0.00052 & 0.01486 & 0.01701\end{array}$

Std Error uses a pooled estimate of error variance

Means and Std Deviations

$\begin{array}{llrrrr}\text { Level Number Mean } & \text { Std Dev } & \text { Std Err Mean } & \text { Lower 95\% } & \text { Upper 95\% }\end{array}$

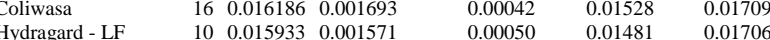

t Test

Hydragard - LF-Coliwasa

Assuming unequal variances

Difference $\quad-0.00025$ t Ratio $\quad-0.38789$

Std Err Dif $\quad 0.00065$ DF $\quad 20.36477$

Upper CL Dif 0.00111 Prob $>|t| \quad 0.7021$

Lower CL Dif -0.00161 Prob $>t \quad 0.6489$

$\begin{array}{lll}\text { Confidence } & 0.95 \text { Prob }<\mathrm{t} \quad 0.3511\end{array}$

Tests that the Variances are Equal

Level Count Std Dev MeanAbsDif to Mean MeanAbsDif to Median

$\begin{array}{lrrrr}\text { Coliwasa } & 16 & 0.0016925 & 0.0013344 & 0.0013344 \\ \text { Hydragard - LF } & 10 & 0.0015709 & 0.0012203 & 0.0011709\end{array}$

Test F Ratio DFNum DFDen p-Value

Test F Ratio DFNum DFDen p-Value

$\begin{array}{lllll}\text { O'Brien[.5] } & 0.0809 & 1 & 24 & 0.7786 \\ \text { Brown-Forsythe } & 0.1640 & 1 & 24 & 0.6891\end{array}$

$\begin{array}{lllll}\text { Brown-Forsythe } & 0.1640 & 1 & 24 & 0.6891 \\ \text { Levene } & 0.0882 & 1 & 24 & 0.7691\end{array}$

$\begin{array}{llrrr}\text { Levene } & 0.0882 & 1 & 24 & 0.7691 \\ \text { Bartlett } & 0.0590 & 1 & . & 0.8080\end{array}$

$\begin{array}{lllll}\text { F Test 2-sided } & 1.1608 & 15 & 9 & 0.8470\end{array}$

Welch Anova testing Means Equal, allowing Std Devs Not Equal

F Ratio DFNum DFDen Prob $>$ F

$\begin{array}{llll}0.1505 & 1 & 20.365 & 0.7021\end{array}$

t Test

0.3879
Oneway Analysis of Measurement (wt \%) By Sampler Type of Material=SME Simulant, Analytical Block $=6$, Analyte $=\mathrm{ZnO}(\mathrm{wt} \%)$, Target $(\mathrm{wt} \%)=0$

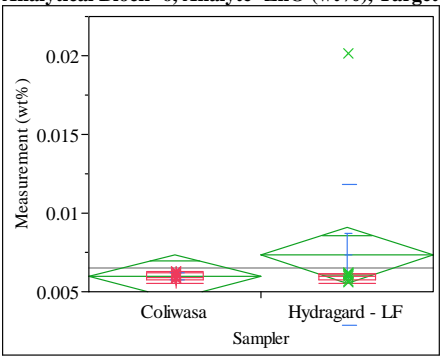

Oneway Anova

Summary of Fit

Rsquare

Adj Rsquare

Root Mean Square Error $\quad 0.002747$

Mean of Response $\quad 0.006484$

Observations (or Sum Wgts) 26

\section{t Test}

Hydragard - LF-Coliwasa

Assuming equal variances

$\begin{array}{lrlr}\text { Difference } & 0.00135 & \mathrm{t} \text { Ratio } & 1.221591 \\ \text { Std Err Dif } & 0.00111 & \text { DF } & 24 \\ \text { Upper CL Dif } & 0.00364 & \text { Prob }>|t| & 0.2337 \\ \text { Lower CL Dif } & -0.00093 & \text { Prob }>t & 0.1169 \\ \text { Confidence } & 0.95 & \text { Prob }<\mathrm{t} & 0.8831\end{array}$

Analysis of Variance

Source DF Sum of Squares Mean Square F Ratio Prob $>$ F

$\begin{array}{lrrrrr}\text { Sampler } & 1 & 0.00001126 & 0.000011 & 1.4923 & 0.2337\end{array}$

$\begin{array}{llll}\text { Error } & 24 & 0.00018112 & 7.547 \mathrm{e}-6\end{array}$

C. Total $25 \quad 0.00019238$

Means for Oneway Anova

Level Number Mean Std Error Lower 95\% Upper 95\%

$\begin{array}{lrrrrr}\text { Level } & \text { Number } & \text { Mean } & \text { Std Error } & \text { Lower 95\% } & \text { Upper 95\% } \\ \text { Coliwasa } & 16 & 0.005964 & 0.00069 & 0.00455 & 0.00738\end{array}$

$\begin{array}{llllll}\text { Hydragard - LF } & 10 & 0.007317 & 0.00087 & 0.00552 & 0.00911\end{array}$

Std Error uses a pooled estimate of error variance

Means and Std Deviation

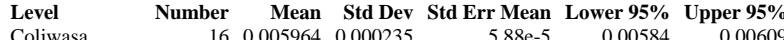

$\begin{array}{llllrrr}\text { Hydragard - LF } & 10 & 0.007317 & 0.004476 & 0.00142 & 0.00412 & 0.01052\end{array}$

t Test

Hydragard - LF-Coliwasa

Assuming unequal variances

$\begin{array}{lll}\text { Difference } & 0.00135 \text { t Ratio } & 0.954973\end{array}$

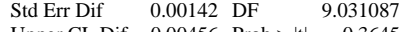

Upper CL Dif 0.00456 Prob $>|t| \quad 0.3645$

Lower CL Dif -0.00185 Prob $>\mathrm{t} \quad 0.1822$

Confidence $\quad 0.95$ Prob $<\mathrm{t} \quad 0.8178$

Tests that the Variances are Equal

Level Count Std Dev MeanAbsDif to Mean MeanAbsDif to Median

$\begin{array}{lrrrr}\text { Coliwasa } & 16 & 0.0002352 & 0.0002062 & 0.0002062 \\ \text { Hydragard - LF } & 10 & 0.0044757 & 0.0025449 & 0.0015548\end{array}$

Test F Ratio DFNum DFDen p-Value

$\begin{array}{lrrrr}\text { O'Brien[.5] } & 1.8383 & 1 & 24 & 0.1878\end{array}$

$\begin{array}{lllll}\text { O'Brien[.5] } & 1.8383 & 1 & 24 & 0.1878 \\ \text { Brown-Forsythe } & 1.5493 & 1 & 24 & 0.2253\end{array}$

$\begin{array}{lllll}\text { Brown-Forsythe } & 1.5493 & 1 & 24 & 0.2253 \\ \text { Levene } & 6.9834 & 1 & 24 & 0.0143\end{array}$

$\begin{array}{lllll}\text { Bartlett } & 62.1266 & 1 & . & <.0001\end{array}$

$\begin{array}{lllll}\text { F Test 2-sided } \quad 362.0079 & 9 & 15 & <.0001\end{array}$

Welch Anova testing Means Equal, allowing Std Devs Not Equal

F Ratio DFNum DFDen Prob $>$ F

$\begin{array}{llll}0.9120 & 1 & 9.0311 & 0.3645\end{array}$

t Test

0.9550 


\section{Exhibit B2. Statistical Comparisons for High-Rheology (Phase 2) Simulant Testing}

Oneway Analysis of Measurement (wt \%) By Sampler Type of Material=SME Simulant,

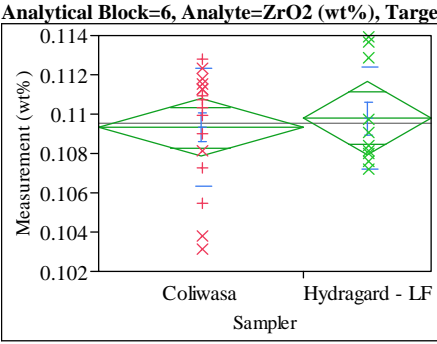

Oneway Anova

Summary of Fit

$\begin{array}{lr}\text { Rsquare } & 0.006911 \\ \text { Adj Rsquare } & -0.03447 \\ \text { Root Mean Square Error } & 0.00286 \\ \text { Mean of Response } & 0.109503 \\ \text { Observations (or Sum Wgts) } & 26\end{array}$

t Tes

Hydragard - LF-Coliwasa

Assuming equal variances

Difference $\quad 0.00047$ t Ratio $\quad 0.408684$

Std Err Dif $\quad 0.00115$

24

Upper CL Dif 0.00285 Prob $>|t| \quad 0.6864$

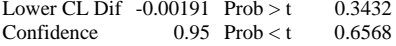

Analysis of Variance

Source DF Sum of Squares Mean Square F Ratio Prob $>$ F

$\begin{array}{llllll}\text { Sampler } & 1 & 0.00000137 & 1.3657 \mathrm{e}-6 & 0.1670 & 0.6864\end{array}$

$\begin{array}{llll}\text { Error } & 24 & 0.00019624 & 8.1768 \mathrm{e}-6\end{array}$

$\begin{array}{lll}\text { C. Total } & 25 & 0.00019761\end{array}$

Means for Oneway Anova

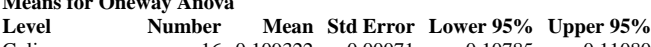

$\begin{array}{lrrrrr}\text { Coliwasa } & 16 & 0.109322 & 0.00071 & 0.10785 & 0.11080\end{array}$

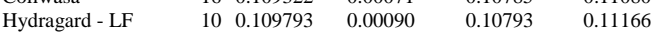

Std Error uses a pooled estimate of error variance

Means and Std Deviations

Level Number Mean Std Dev Std Err Mean Lower 95\% Upper 95\%

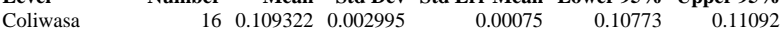

$\begin{array}{lllllll}\text { Hydragard - LF } & 10 & 0.109793 & 0.002618 & 0.00083 & 0.10792 & 0.11167\end{array}$

t Test

Hydragard - LF-Coliwasa

Assuming unequal variances

$\begin{array}{lrlr}\text { Difference } & 0.00047 & \text { t Ratio } & 0.422047 \\ \text { Std Err Dif } & 0.00112 & \text { DF } & 21.22808 \\ \text { Upper CL Dif } & 0.00279 & \text { Prob }>|t| & 0.6772 \\ \text { Lower CL Dif } & -0.00185 & \text { Prob }>\text { t } & 0.3386 \\ \text { Confidence } & 0.95 & \text { Prob }<\text { t } & 0.6614\end{array}$

Tests that the Variances are Equal

Level Count Std Dev MeanAbsDif to Mean MeanAbsDif to Median

$\begin{array}{lllll} & 16 & 0.0029953 & 0.0024124 & 0.0022035\end{array}$

$\begin{array}{lllll}\text { Hydragard - LF } & 10 & 0.0026176 & 0.0021775 & 0.0019992\end{array}$

Test F Ratio DFNum DFDen p-Value

$\begin{array}{lrrrr}\text { O'Brien[.5] } & 0.2424 & 1 & 24 & 0.6270\end{array}$

$\begin{array}{lllll}\text { Brown-Forsythe } & 0.0532 & 1 & 24 & 0.8195\end{array}$

$\begin{array}{lllll}\text { Levene } & 0.1463 & 1 & 24 & 0.7054\end{array}$

$\begin{array}{lrrrr}\text { Bartlett } & 0.1907 & 1 & . & 0.6624 \\ \text { F Test 2-sided } & 1.3094 & 15 & 9 & 0.6989\end{array}$

Welch Anova testing Means Equal, allowing Std Devs Not Equal

F Ratio DFNum DFDen Prob $>$ F

$\begin{array}{rrrr}0.1781 & 1 & 21.228 & 0.6772\end{array}$

t Test

0.4220 
SRNL-STI-2011-00693

Revision 0

Exhibit B3. Statistical Comparisons for High-Rheology (Phase 2) Simulant Testing (screened data)

Oneway Analysis of Measurement By Sampler w Location Analyte $=w t \%$ dried solids

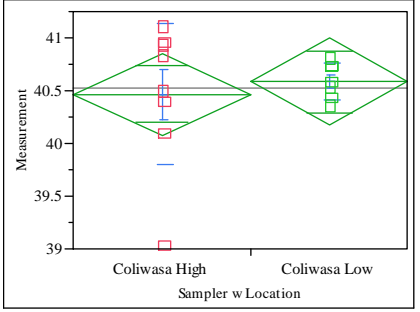

Excluded Rows

Oneway Anova

Summary of Fit

Adj Rsquare

Root Mean Square Error $\quad-0.06029$

Mean of Response $\quad 40.52267$

Observations (or Sum Wgts) 15

\section{Test}

Coliwasa Low-Coliwasa High

Assuming equal variances

Difference $\quad 0.11821$ t Ratio $\quad 0.45162$

$\begin{array}{lrr}0.26176 & \text { DF } & 13\end{array}$

Upper CL Dif 0.68370 Prob $>|t| \quad 0.6590$

$\begin{array}{lrrr}\text { Lower CL Dif } & -0.44728 & \text { Prob }>t & 0.3295 \\ \text { Confidence } & 0.95 & \text { Prob }<\mathrm{t} & 0.6705\end{array}$

$\begin{array}{lrrrrr}\begin{array}{l}\text { Analysis of Variance } \\ \text { Source }\end{array} & \text { DF } & \text { Sum of Squares } & \text { Mean Square } & \text { F Ratio } & \text { Prob > F } \\ \text { Sampler w Location } & 1 & 0.0521719 & 0.052172 & 0.2040 & 0.6590 \\ \text { Error } & 13 & 3.3253214 & 0.255794 & & \\ \text { C. Total } & 14 & 3.3774933 & & & \end{array}$

Means for Oneway Anova

$\begin{array}{lllll}\text { Means for Oneway Anova } & & \\ \text { Level Number } & \text { Mean Std Error } & \text { Lower 95\% Upper 95\% }\end{array}$

$\begin{array}{lrrrrr}\text { Level } & \text { Number } & \text { Mean } & \text { Std Error } & \text { Lower 95\% } & \text { Upper 95\% } \\ \text { Coliwasa High } & 8 & 40.4675 & 0.17881 & 40.081 & 40.854\end{array}$

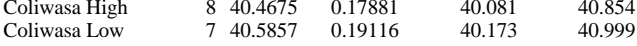

Std Error uses a pooled estimate of error variance

Means and Std Deviations

Level Number Mean Std Dev Std Err Mean Lower 95\% Upper 95\%

$\begin{array}{lllllll}\text { Coliwasa High } & 8 & 40.4675 & 0.670645 & 0.23711 & 39.907 & 41.028\end{array}$

$\begin{array}{lllllll}\text { Coliwasa Low } & 7 & 40.5857 & 0.171742 & 0.06491 & 40.427 & 40.745\end{array}$

t Test

Coliwasa Low-Coliwasa High

Assuming unequal variances

Difference $\quad 0.11821$ t Ratio $\quad 0.480871$

Std Err Dif $\quad 0.24583$ DF 8.035927

Upper CL Dif 0.68467 Prob $>|t| \quad 0.6434$

Lower CL Dif -0.44824 Prob $>t \quad 0.3217$

Confidence $\quad 0.95$ Prob $<\mathrm{t} \quad 0.6783$

Tests that the Variances are Equal

Level Count Std Dev MeanAbsDif to Mean MeanAbsDif to Median

$\begin{array}{lllll}\text { Coliwasa High } & 8 & 0.6706447 & 0.4731250 & 0.4725000\end{array}$

$\begin{array}{lllll}\text { Coliwasa Low } & 7 & 0.1717418 & 0.1408163 & 0.1500000\end{array}$

Test F Ratio DFNum DFDen p-Value

$\begin{array}{lrrrr}\text { O'Brien[.5] } & 1.6892 & 1 & 13 & 0.2163 \\ \text { Brown-Forsythe } & 3.1064 & 1 & 13 & 0.1015\end{array}$

$\begin{array}{lllll}\text { Brown-Forsythe } & 3.1064 & 1 & 13 & 0.1015 \\ \text { Levene } & 3.8399 & 1 & 13 & 0.0718\end{array}$

$\begin{array}{lrrrr}\text { Levene } & 3.8399 & 1 & 13 & 0.0718 \\ \text { Bartlett } & 8.3621 & 1 & . & 0.0038\end{array}$

$\begin{array}{lrrrr}\text { F Test 2-sided } & 15.2487 & 7 & 6 & 0.0039\end{array}$

Welch Anova testing Means Equal, allowing Std Devs Not Equal

F Ratio DFNum DFDen Prob $>$ F

$\begin{array}{llrr}0.2312 & 1 & 8.0359 & 0.6434\end{array}$

t Test

0.4809

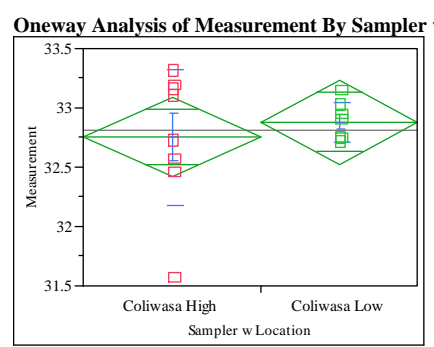

Excluded Rows

Oneway Anova

Summary of Fit

Adj Rsquare

Root Mean Square Error $\quad-0.0514$

Mean of Response $\quad 32.81267$

Observations (or Sum Wgts) 15

\section{Test}

Coliwasa Low-Coliwasa High

Assuming equal variances

$\begin{array}{lrlr}\text { Difference } & 0.12625 & \text { t Ratio } & 0.561799 \\ \text { Std Err Dif } & 0.22472 & \text { DF } & 13 \\ \text { Upper CL Dif } & 0.61174 & \text { Prob }>|t| & 0.5838 \\ \text { Lower CL Dif } & -0.35924 & \text { Prob }>t & 0.2919 \\ \text { Confidence } & 0.95 & \text { Prob }<\mathrm{t} & 0.7081\end{array}$

Analysis of Variance

$\begin{array}{lrrrrr}\text { Source } & \text { DF } & \text { Sum of Squares } & \text { Mean Square } & \text { F Ratio } & \text { Prob > F } \\ \text { Sampler w Location } & 1 & 0.0595058 & 0.059506 & 0.3156 & 0.5838 \\ \text { Error } & 13 & 2.4509875 & 0.188537 & & \\ \text { C. Total } & 14 & 2.5104933 & & & \end{array}$

Means for Oneway Anova

Level Number Mean Std Error Lower 95\% Upper 95\%

$\begin{array}{lrrrrr}\text { Level } & \text { Number } & \text { Mean } & \text { Std Error } & \text { Lower 95\% } & \text { Upper 95\% } \\ \text { Coliwasa High } & 8 & 32.7538 & 0.15352 & 32.422 & 33.085\end{array}$

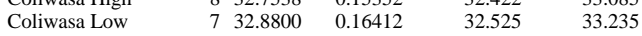

Std Error uses a pooled estimate of error variance

Means and Std Deviations

Level Number Mean Std Dev Std Err Mean Lower 95\% Upper 95\%

$\begin{array}{lllllll}\text { Coliwasa High } & 8 & 32.7538 & 0.571713 & 0.20213 & 32.276 & 33.232\end{array}$

$\begin{array}{lllllll}\text { Coliwasa Low } & 7 & 32.8800 & 0.164823 & 0.06230 & 32.728 & 33.032\end{array}$

t Test

Coliwasa Low-Coliwasa High

Assuming unequal variances

Difference $\quad 0.12625$ t Ratio 0.596889

Std Err Dif $\quad 0.21151$ DF 8.305574

Upper CL Dif 0.61089 Prob $>|t| 0.5665$

Lower CL Dif -0.35839 Prob $>\mathrm{t} \quad 0.2832$

$\begin{array}{lrl}\text { Confidence } & 0.95 \text { Prob }<\mathrm{t} \quad 0.7168\end{array}$

Tests that the Variances are Equal

Level Count Std Dev MeanAbsDif to Mean MeanAbsDif to Median

$\begin{array}{lllll}\text { Coliwasa High } & 8 & 0.5717127 & 0.4287500 & 0.4287500\end{array}$

Test F Ratio DFNum DFDen p-Value

$\begin{array}{lrrrr}\text { O'Brien[.5] } & 1.9152 & 1 & 13 & 0.1897\end{array}$

Brown-Forsythe $4.0294 \quad 1 \quad 13 \quad 0.0660$

$\begin{array}{lllll}\text { Levene } & 4.9269 & 1 & 13 & 0.0448\end{array}$

$\begin{array}{lrrrr}\text { Bartlett } & 7.2130 & 1 & . & 0.0072 \\ \text { F Test 2-sided } & 12.0315 & 7 & 6 & 0.0075\end{array}$

Welch Anova testing Means Equal, allowing Std Devs Not Equal

F Ratio DFNum DFDen Prob $>$ F

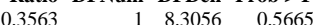

t Test
0.5969 
SRNL-STI-2011-00693

Revision 0

Exhibit B3. Statistical Comparisons for High-Rheology (Phase 2) Simulant Testing (screened data)

Hydragard at Low Flow Rate

Oneway Analysis of Measurement By Sampling Mechanism Analyte=density $(\mathrm{g} / \mathrm{mL})$

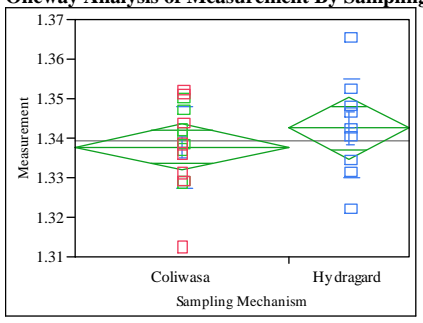

Summary of Fit

Rsquare

Adj Rsquare

Root Mean Square Error $\quad 0.011216$

Mean of Response 1.339456

Observations (or Sum Wgts) 25

Test

Hydragard-Coliwasa

Assuming equal variances

Difference $\quad 0.00477$ t Ratio 1.021497

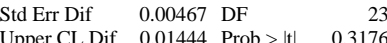

$\begin{array}{lrll}\text { Upper CL Dif } & 0.01444 \text { Prob }>|t| & 0.3176 \\ \text { Cower CL Dif } & -0.00489 & \text { Prob }>t & 0.1588\end{array}$

$\begin{array}{lrl}\text { Confidence } & 0.95 \text { Prob }<\mathrm{t} & 0.8412\end{array}$

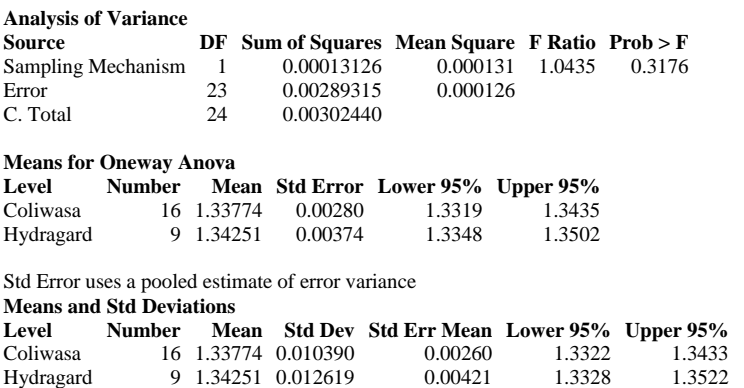

t Test

Hydragard-Coliwasa

Assuming unequal variances

Difference $\quad 0.00477$ t Ratio 0.965613

Std Err Dif $\quad 0.00494$ DF $\quad 14.16651$

Upper CL Dif 0.01536 Prob $>|t| \quad 0.3504$

Lower CL Dif -0.00582 Prob $>t \quad 0.1752$

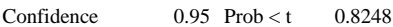

Tests that the Variances are Equal

Level Count Std Dev MeanAbsDif to Mean MeanAbsDif to Median

$\begin{array}{lrrrr}\text { Coliwasa } & 16 & 0.0103901 & 0.0078078 & 0.0077750 \\ \text { Hydragard } & 9 & 0.0126186 & 0.0093457 & 0.0095222\end{array}$

Test F Ratio DFNum DFDen p-Value

$\begin{array}{lrrrr}\text { O'Brien[.5] } & 0.3837 & 1 & 23 & 0.5417\end{array}$

$\begin{array}{lllll}\text { Brown-Forsythe } & 0.3620 & 1 & 23 & 0.5533\end{array}$

$\begin{array}{lllll}\text { Levene } & 0.2769 & 1 & 23 & 0.6038\end{array}$

$\begin{array}{lllll}\text { Bartlett } & 0.3884 & 1 & & 0.5332 \\ \text { F Test 2-sided } & 1.4750 & 8 & 15 & 0.4917\end{array}$

Welch Anova testing Means Equal, allowing Std Devs Not Equal

F Ratio DFNum DFDen Prob $>$ F

$\begin{array}{llll}0.9324 & 1 & 14.167 & 0.3504\end{array}$

t Test

0.9656
Hydragard at Low Flow Rate

Oneway Analysis of Measurement By Sampling Mechanism Analyte $=w$ t $\%$ dried solids

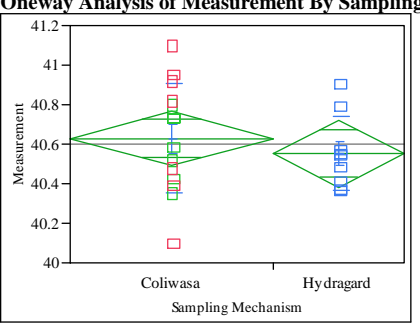

neway Anova

Summary of Fit

Adj Rsquare

Root Mean Square Error $\quad 0.245323$

Mean of Response 40.59913

Observations (or Sum Wgts)

Test

Hydragard-Coliwasa

Assuming equal variances

Difference $\quad-0.07706$ t Ratio -0.73525

$\begin{array}{lrl}\text { Std Err Dif } & 0.10481 \text { DF } & 21 \\ \text { Upper CL } & 0.14091 \text { Prob }>|t| & 0.4703\end{array}$

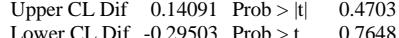

$\begin{array}{lrl}\text { Confidence } & 0.95 \text { Prob }<\mathrm{t} & 0.2352\end{array}$

Analysis of Variance

$\begin{array}{lrrrrr}\text { Source } & \text { DF } & \text { Sum of Squares } & \text { Mean Square } & \text { F Ratio } & \text { Prob }>\text { F } \\ \text { Sampling Mechanism } & 1 & 0.0325342 & 0.032534 & 0.5406 & 0.4703\end{array}$

Sampling

$\begin{array}{rrrrr}1 & 0.0325342 & 0.032534 & 0.5406 & 0.4703 \\ 21 & 1.2638484 & 0.060183 & & \\ 22 & 1.2963826 & & & \end{array}$

C. Total

1.2963826

Means for Oneway Anova

Level Number Mean Std Error Lower 95\% Upper 95\%

$\begin{array}{lrrrrr}\text { Coliwasa } & 14 & 40.6293 & 0.06557 & 40.493 & 40.766\end{array}$

$\begin{array}{lrrrrr}\text { Hydragard } & 9 & 40.5522 & 0.08177 & 40.382 & 40.722\end{array}$

Std Error uses a pooled estimate of error variance

Means and Std Deviations

Level Number Mean Std Dev Std Err Mean Lower 95\% Upper 95\%

$\begin{array}{lrlllll}\text { Coliwasa } & 14 & 40.6293 & 0.276001 & 0.07376 & 40.470 & 40.789\end{array}$

$\begin{array}{lllllll}\text { Hydragard } & 9 & 40.5522 & 0.184917 & 0.06164 & 40.410 & 40.694\end{array}$

t Test

Hydragard-Coliwasa

Assuming unequal variances

Difference $\quad-0.07706$ t Ratio $\quad-0.80168$

Std Err Dif $\quad 0.09613$ DF 20.91904

Upper CL Dif 0.12289 Prob $>|t| \quad 0.4318$

Lower CL Dif -0.27702 Prob $>t \quad 0.7841$

Confidence $\quad 0.95$ Prob $<t \quad 0.2159$

Tests that the Variances are Equal

Level Count Std Dev MeanAbsDif to Mean MeanAbsDif to Median

$\begin{array}{lrrrr}\text { Coliwasa } & 14 & 0.2760007 & 0.2292857 & 0.2292857 \\ \text { Hydragard } & 9 & 0.1849174 & 0.1340741 & 0.1333333\end{array}$

$\begin{array}{lrrrr}\text { Test } & \text { F Ratio } & \text { DFNum } & \text { DFDen } & \text { p-Value } \\ \text { O'Brien[.5] } & 1.5243 & 1 & 21 & 0.2306 \\ \text { Brown-Forsythe } & 2.7981 & 1 & 21 & 0.1092 \\ \text { Levene } & 2.8490 & 1 & 21 & 0.1062 \\ \text { Bartlett } & 1.3877 & 1 & . & 0.2388 \\ \text { F Test 2-sided } & 2.2277 & 13 & 8 & 0.2591\end{array}$

Welch Anova testing Means Equal, allowing Std Devs Not Equal

F Ratio DFNum DFDen Prob $>$ F

$\begin{array}{llll}0.6427 & 1 & 20.919 & 0.4318\end{array}$

t Test

0.8017 
SRNL-STI-2011-00693

Revision 0

Exhibit B3. Statistical Comparisons for High-Rheology (Phase 2) Simulant Testing (screened data)

Hydragard at Low Flow Rate

Oneway Analysis of Measurement By Sampling Mechanism Analyte=wt \% vitrified solids

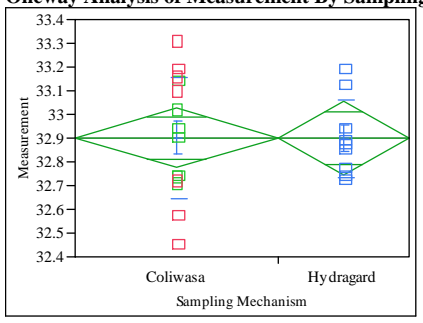

Summary of Fit

Rsquare

Adj Rsquare

oot Mean Square Error $\quad-0.04758$

Mean of Response $\quad 0.225564$

Observations (or Sum Wgts)

Test

Hydragard-Coliwasa

Assuming equal variances

Difference $\quad-0.00254$ t Ratio -0.02635

$\begin{array}{lrlr}\text { Std Err Dif } & 0.09637 & \text { DF } & 21 \\ \text { Upper CL Dif } & 0.19788 & \text { Prob }>|t| & 0.9792\end{array}$

Lower CL Dif -0.20295 Prob $>t \quad 0.5104$

$\begin{array}{lrl}\text { Confidence } & 0.95 \text { Prob }<\mathrm{t} \quad 0.4896\end{array}$

Analysis of Variance

$\begin{array}{lrrrrr}\text { Analysis of Variance } & & & & & \\ \text { Source } & \text { DF } & \text { Sum of Squares } & \text { Mean Square } & \text { F Ratio } & \text { Prob }>\text { F } \\ \text { Sampling Mechanism } & 1 & 0.0000353 & 0.000035 & 0.0007 & 0.9792 \\ \text { Error } & 21 & 1.0684603 & 0.050879 & & \\ \text { C. Total } & 22 & 1.0684957 & & & \end{array}$

Means for Oneway Anova

Level Number Mean Std Error Lower 95\% Upper 95\%

$\begin{array}{lrrrrr}\text { Level } & \text { Number } & \text { Mean } & \text { Std Error } & \text { Lower 95\% } & \text { Upper 95\% } \\ \text { Coliwasa } & 14 & 32.9014 & 0.06028 & 32.776 & 33.027\end{array}$

$\begin{array}{lrrrrr}\text { Coliwasa } & 14 & 32.9014 & 0.06028 & 32.776 & 33.027 \\ \text { Hydragard } & 9 & 32.8989 & 0.07519 & 32.743 & 33.055\end{array}$

Std Error uses a pooled estimate of error variance

Means and Std Deviations

Level Number Mean Std Dev Std Err Mean Lower 95\% Upper 95\%

$\begin{array}{lllllll}\text { Coliwasa } & 14 & 32.9014 & 0.256601 & 0.06858 & 32.753 & 33.050\end{array}$

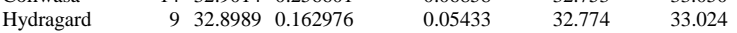

t Test

Hydragard-Coliwasa

Assuming unequal variances

Difference $\quad-0.00254$ t Ratio $\quad-0.02903$

Std Err Dif $\quad 0.08749$ DF $\quad 20.99811$

Upper CL Dif 0.17941 Prob $>|t| 0.9771$

Lower CL Dif -0.18448 Prob $>\mathrm{t} \quad 0.5114$

$\begin{array}{lll}\text { Confidence } & 0.95 \text { Prob }<\mathrm{t} \quad 0.4886\end{array}$

Tests that the Variances are Equal

$\begin{array}{lrrrr}\text { Level } & \text { Count } & \text { Std Dev } & \text { MeanAbsDif to Mean } & \text { MeanAbsDif to Median } \\ \text { Coliwasa } & 14 & 0.2566008 & 0.2157143 & 0.2157143\end{array}$

$\begin{array}{lrrrr}\text { Coliwasa } & 14 & 0.2566008 & 0.2157143 & 0.2157143 \\ \text { Hydragard } & 9 & 0.1629758 & 0.1229630 & 0.1200000\end{array}$

Test F Ratio DFNum DFDen p-Value

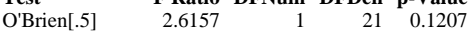

$\begin{array}{lllll}\text { Brown-Forsythe } & 3.5115 & 1 & 21 & 0.0749\end{array}$

$\begin{array}{lllll}\text { Levene } & 3.5230 & 1 & 21 & 0.0745\end{array}$

$\begin{array}{lrrrr}\text { Bartlett } & 1.7577 & 1 & \text {. } & 0.1849 \\ \text { F Test 2-sided } & 2.4790 & 13 & 8 & 0.2017\end{array}$

Welch Anova testing Means Equal, allowing Std Devs Not Equal

F Ratio DFNum DFDen Prob > F

$\begin{array}{llll}0.0008 & 1 & 20.998 & 0.9771\end{array}$

t Test

0.0290
Hydragard at High Flow Rate

Oneway Analysis of Measurement By Sampling Mechanism Analyte $=w t \%$ dried solids

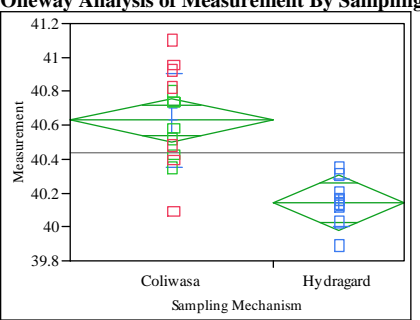

Oneway Anova

Summary of Fit

Rsquare

Adj Rsquare

Root Mean Square Error $\quad 0.23315$

Mean of Response $\quad 40.43826$

t Test

Hydragard-Coliwasa

Assuming equal variances

Difference $\quad-0.48817$ t Ratio $\quad-4.90064$

$\begin{array}{lrl}\text { Std Err Dif } & 0.09961 \text { DF } & 21 \\ \text { Upper CL } & <\text { if } & -0.28101 \\ \text { Prob }>|t| & <.0001\end{array}$

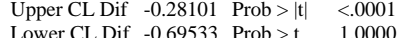

$\begin{array}{lrl}\text { Confidence } & 0.95 \text { Prob }<\mathrm{t} & <.0001\end{array}$

Analysis of Variance

$\begin{array}{lrrrrr}\text { Source } & \text { DF } & \text { Sum of Squares } & \text { Mean Square } & \text { F Ratio } & \text { Prob }>\text { F } \\ \text { Sampling Mechanism } & 1 & 1.3055487 & 1.30555 & 24.0163 & <.0001\end{array}$

$\begin{array}{rrrrr}1 & 1.3055487 & 1.30555 & 24.0163 & <.0001 \\ 21 & 1.1415817 & 0.05436 & & \\ 22 & 2.4471304 & & & \end{array}$

C. Total

Means for Oneway Anova

Level Number Mean Std Error Lower 95\% Upper 95\%

$\begin{array}{lrrrrr}\text { Coliwasa } & 14 & 40.6293 & 0.06231 & 40.500 & 40.759\end{array}$

$\begin{array}{lrrrrr}\text { Hydragard } & 9 & 40.1411 & 0.07772 & 39.979 & 40.303\end{array}$

Std Error uses a pooled estimate of error variance

Means and Std Deviations

Level Number Mean Std Dev Std Err Mean Lower 95\% Upper 95\%

$\begin{array}{lrlllll}\text { Coliwasa } & 14 & 40.6293 & 0.276001 & 0.07376 & 40.470 & 40.789\end{array}$

Hydraga

$\begin{array}{lll}40.1411 & 0.137518\end{array}$

0.04584

40.247

t Test

Hydragard-Coliwasa

Assuming unequal variances

$\begin{array}{llll}\text { Difference } & -0.48817 \text { t Ratio } & -5.62109 \\ \text { Std Err Dif } & 0.08685 & \text { DF } & 20.10663\end{array}$

$\begin{array}{lrrr}\text { Std Err Dif } & 0.08685 \text { DF } & 20.10663 \\ \text { Upper CL } & <.0001\end{array}$

$\begin{array}{lll}\text { Upper CL Dif }-0.30708 \text { Prob }>|t| & <.0001 \\ \text { Lower CL Dif }-0.66927 & \text { Prob }>t & 1.0000\end{array}$

0.95 Prob $<\mathrm{t}<0001$

Tests that the Variances are Equal

$\begin{array}{lrrrr}\text { Level } & \text { Count } & \text { Std Dev } & \text { MeanAbsDif to Mean } & \text { MeanAbsDif to Median } \\ \text { Coliwasa } & 14 & 0.2760007 & 0.2292857 & 0.2292857\end{array}$

$\begin{array}{lrrrr}\text { Hydragard } & 14 & 0.2760007 & 0.2292857 & 0.2292857 \\ & 9 & 0.1375177 & 0.0943210 & 0.0933333\end{array}$

Test F Ratio DFNum DFDen p-Value

$\begin{array}{lllll}\text { O'Brien[.5] } & 3.1035 & 1 & 21 & 0.0927\end{array}$

$\begin{array}{lllll}\text { Brown-Forsythe } & 6.3070 & 1 & 21 & 0.0203\end{array}$

$\begin{array}{lllll}\text { Levene } & 6.4379 & 1 & 21 & 0.0192\end{array}$

$\begin{array}{lrrrr}\text { Bartlett } & 3.8623 & 1 & \text {. } & 0.0494 \\ \text { F Test 2-sided } & 4.0281 & 13 & 8 & 0.0550\end{array}$

Welch Anova testing Means Equal, allowing Std Devs Not Equal

F Ratio DFNum DFDen Prob $>$ F

$\begin{array}{lll}31.5966 & 20.107<0001\end{array}$

t Test

5.6211 
SRNL-STI-2011-00693

Revision 0

Exhibit B3. Statistical Comparisons for High-Rheology (Phase 2) Simulant Testing (screened data)

Hydragard at High Flow Rate Oneway Analysis of Measurement By Sampling Mechanism Analyte=wt \% vitrified solids

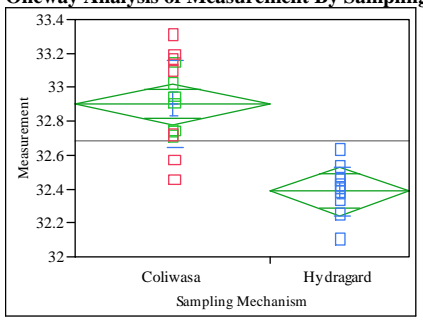

Summary of Fit

Rsquare

Adj Rsquare

0.596659

0.578325
0.218217

32.68667

Test

Hydragard-Coliwasa

Assuming equal variances

Difference $\quad-0.51543$ t Ratio $\quad-5.70477$

$\begin{array}{lrr}\text { Std Err Dif } & 0.09035 \text { DF } & 22 \\ \text { Upper CL Dif } & -0.32805 \text { Prob }>|t| & <.0001\end{array}$

$\begin{array}{rrrr}\text { Upper CL Dif }-0.32805 & \text { Prob }>|t| & <.0001 \\ \text { Cower CL Dif }-0.70280 & \text { Prob }>t & 1.0000\end{array}$

$\begin{array}{lrl}\text { Confidence } & 0.95 \text { Prob }<\mathrm{t} \quad<.0001\end{array}$

Analysis of Variance

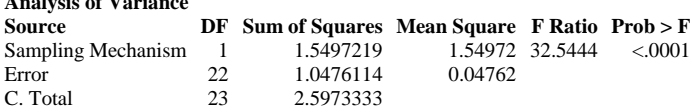

Means for Oneway Anova

$\begin{array}{lrrrrr}\text { Level } & \text { Number } & \text { Mean } & \text { Std Error } & \text { Lower 95\% } & \text { Upper 95\% } \\ \text { Coliwasa } & 14 & 32.9014 & 0.05832 & 32.780 & 33.022\end{array}$

$\begin{array}{llllll}\text { Coliwasa } & 14 & 32.9014 & 0.05832 & 32.780 & 33.022 \\ \text { Hydragard } & 10 & 32.3860 & 0.06901 & 32.243 & 32.529\end{array}$

Std Error uses a pooled estimate of error variance

Means and Std Deviations

Level Number Mean Std Dev Std Err Mean Lower 95\% Upper 95\%

$\begin{array}{llllllr}\text { Coliwasa } & 14 & 32.9014 & 0.256601 & 0.06858 & 32.753 & 33.050 \\ \text { Hydragard } & 10 & 32.3860 & 0.145922 & 0.04614 & 32.282 & 32.490\end{array}$

t Test

Hydragard-Coliwasa

Assuming unequal variances

Difference $\quad-0.51543$ t Ratio -6.23562

Std Err Dif $\quad 0.08266$ DF $\quad 21.16854$

Upper CL Dif -0.34361 Prob $>|t| \quad<.0001$

Lower CL Dif -0.68724 Prob $>t \quad 1.0000$

$\begin{array}{lll}\text { Confidence } & 0.95 \text { Prob }<\mathrm{t} \quad<.0001\end{array}$

Tests that the Variances are Equal

$\begin{array}{lrrr}\text { Level Count } & \text { Std Dev MeanAbsDif to Mean MeanAbsDif to Median } \\ \text { Coliwasa } & 14 & 0.2566008 & 0.2157143\end{array}$

$\begin{array}{lllll}\text { Coliwasa } & 14 & 0.2566008 & 0.2157143 & 0.2157143 \\ \text { Hydragard } & 10 & 0.1459224 & 0.1028000 & 0.1000000\end{array}$

$\begin{array}{lrrrr}\text { Test } & \text { F Ratio } & \text { DFNum } & \text { DFDen } & \text { p-Value } \\ \text { O'Brien[.5] } & 3.6753 & 1 & 22 & 0.0683\end{array}$

$\begin{array}{lllll}\text { O'Brien[.5] } & 3.6753 & 1 & 22 & 0.0683 \\ \text { Brown-Forsythe } & 5.6415 & 1 & 22 & 0.0267\end{array}$

$\begin{array}{lllll}\text { Brown-Forsythe } & 5.6415 & 1 & 22 & 0.0267 \\ \text { Levene } & 5.6328 & 1 & 22 & 0.0268\end{array}$

$\begin{array}{lrrrr}\text { Bartlett } & 2.8932 & 1 & . & 0.0268\end{array}$

$\begin{array}{lllll}\text { F Test 2-sided } & 3.0922 & 13 & 9 & 0.0959\end{array}$

Welch Anova testing Means Equal, allowing Std Devs Not Equal

F Ratio DFNum DFDen Prob $>$ F

$\begin{array}{llll}38.8829 & 1 & 21.169 & <.0001\end{array}$

t Test

6.2356
Isolok at Low Flow Rate

Oneway Analysis of Measurement By Sampling Mechanism Analyte $=w t \%$ dried solids

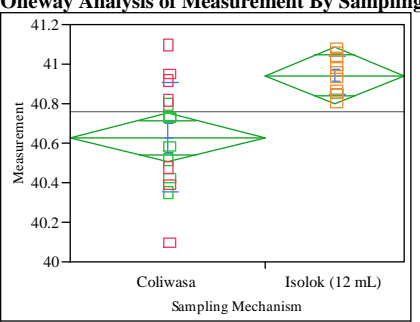

Oneway Anova

Summary of Fit

Adj Rsquare

Mean of Response $\quad 40.75958$

Observations (or Sum Wgts)

24

\section{t Test}

Isolok (12 mL)-Coliwasa

Assuming equal variances

Difference $\quad 0.312714$ t Ratio 3.415078

$\begin{array}{lrr}\text { Std Err Dif } & 0.091569 \text { DF } & 22\end{array}$

Upper CL Dif 0.502616 Prob $>|t| \quad 0.0025$

Lower CL Dif 0.122812 Prob $>t \quad 0.0012$

Confidence $\quad 0.95$ Prob $<\mathrm{t} \quad 0.9988$

Analysis of Variance

$\begin{array}{lrrrrr}\text { Source } & \text { DF } & \text { Sum of Squares } & \text { Mean Square } & \text { F Ratio } & \text { Prob }>\text { F } \\ \text { Sampling Mechanism } & 1 & 0.5704430 & 0.570443 & 11.6628 & 0.0025 \\ \text { Error } & 22 & 1.0760529 & 0.048911 & & \\ \text { C. Total } & 23 & 1.6464958 & & & \end{array}$

Means for Oneway Anova
Level
Number Mean Std Error Lower 95\% Upper 95\%

$\begin{array}{lrrrrr}\text { Level } & \text { Number } & \text { Mean } & \text { Std Error } & \text { Lower 95\% } & \text { Upper 95\% } \\ \text { Coliwasa } & 14 & 40.6293 & 0.05911 & 40.507 & 40.752\end{array}$

$\begin{array}{llllll}\text { Isolok }(12 \mathrm{~mL}) & 10 & 40.9420 & 0.06994 & 40.797 & 41.087\end{array}$

Std Error uses a pooled estimate of error variance

Means and Std Deviations

Level Number Mean Std Dev Std Err Mean Lower 95\% Upper 95\%

$\begin{array}{lllllll}\text { Coliwasa } & 14 & 40.6293 & 0.276001 & 0.07376 & 40.470 & 40.789\end{array}$

$\begin{array}{llllll}\text { Isolok }(12 \mathrm{~mL}) & 10 & 40.9420 & 0.097616 & 0.03087 & 40.872\end{array}$

t Test

Isolok (12 mL)-Coliwas

Assuming unequal variances

$\begin{array}{lll}\text { Difference } & 0.312714 \text { t Ratio } & 3.910744 \\ \text { Std }\end{array}$

$\begin{array}{lll}\text { Std Err Dif } \quad 0.079963 \text { DF } & 17.19044\end{array}$

Upper CL Dif 0.481279 Prob $>|t| \quad 0.0011$

Lower CL Dif 0.144150 Prob $>t \quad 0.0006$

$\begin{array}{lrl}\text { Confidence } & 0.95 \text { Prob }<\mathrm{t} \quad 0.9994\end{array}$

Tests that the Variances are Equal

\begin{tabular}{lrrrrr} 
Level & Count & Std Dev & MeanAbsDif to Mean & MeanAbsDif to Median \\
Coliwasa & 14 & 0.2760007 & 0.2292857 & 0.2292857 \\
Isolok (12 mL) & 10 & 0.0976160 & & 0.0820000 & 0.0820000 \\
& \multicolumn{7}{c}{} \\
Test & F Ratio & DFNum & DFDen & p-Value & \\
O'Brien[.5] & 4.9301 & 1 & 22 & 0.0370 & \\
Brown-Forsythe & 9.8668 & 1 & 22 & 0.0047 & \\
Levene & 10.2049 & 1 & 22 & 0.0042 & \\
Bartlett & 8.5551 & 1 & . & 0.0034 & \\
F Test 2-sided & 7.9943 & 13 & 9 & 0.0038 &
\end{tabular}

Welch Anova testing Means Equal, allowing Std Devs Not Equal

F Ratio DFNum DFDen Prob > F

$15.2939 \quad 1 \quad 17.19 \quad 0.0011$

t Test
3.9107 
SRNL-STI-2011-00693

Revision 0

Exhibit B3. Statistical Comparisons for High-Rheology (Phase 2) Simulant Testing (screened data)

Isolok at Low Flow Rate

Oneway Analysis of Measurement By Sampling Mechanism Analyte=wt \% vitrified solids

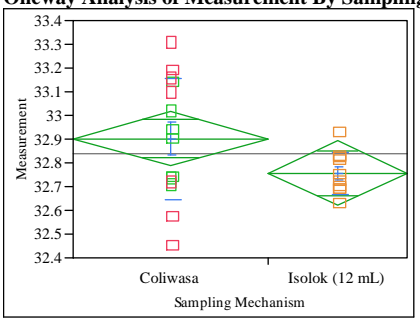

Oneway Anova

Summary of Fit

Rsquare

Adj Rsquare $\quad 0.115946$

Root Mean Square Error $\quad 0.205358$

Mean of Response 32.84125

Observations (or Sum Wgts) 24

t Test

Isolok $(12 \mathrm{~mL})$-Coliwasa

Assuming equal variances

Difference $\quad-0.14443$ t Ratio -1.69863

$\begin{array}{lrr}\text { Std Err Dif } & 0.08503 \text { DF } & 22 \\ & \end{array}$

Upper CL Dif 0.03191 Prob $>$

$\begin{array}{lrrr}\text { Lower CL Dif } & -0.32076 & \text { Prob }>t & 0.9483 \\ \text { Confidence } & 0.95 & \text { Prob }<\mathrm{t} & 0.0517\end{array}$

$\begin{array}{lrrrrr}\text { Analysis of Variance } & & & & & \\ \text { Source } & \text { DF } & \text { Sum of Squares } & \text { Mean Square } & \text { F Ratio } & \text { Prob > F } \\ \text { Sampling Mechanism } & 1 & 0.1216811 & 0.121681 & 2.8854 & 0.1035 \\ \text { Error } & 22 & 0.9277814 & 0.042172 & & \\ \text { C. Total } & 23 & 1.0494625 & & & \end{array}$

Means for Oneway Anova

$\begin{array}{lllll}\text { Means for Oneway Anova } & & & \\ \text { Level } & \text { Number } & \text { Mean Std Error } & \text { Lower 95\% Upper 95\% }\end{array}$

$\begin{array}{lrrrrr}\text { Level } & \text { Number } & \text { Mean } & \text { Std Error } & \text { Lower 95\% } & \text { Upper 95\% } \\ \text { Coliwasa } & 14 & 32.9014 & 0.05488 & 32.788 & 33.015\end{array}$

$\begin{array}{llllll}\text { Isolok }(12 \mathrm{~mL}) & 10 & 32.7570 & 0.06494 & 32.622 & 32.892\end{array}$

Std Error uses a pooled estimate of error variance

Means and Std Deviations

Level Number Mean Std Dev Std Err Mean Lower 95\% Upper 95\%

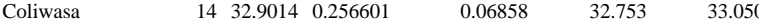

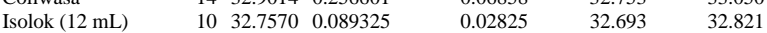

t Test

Isolok (12 mL)-Coliwasa

Assuming unequal variances

$\begin{array}{lrr}\text { Difference } & -0.14443 \text { t Ratio } & -1.94729 \\ \text { Std Err Dif } & 0.07417 \text { DF } & 17.0752\end{array}$

Upper CL Dif $\begin{array}{rr}0.07417 \text { DF } & 0.01200 \text { Prob }>|t| r .0752 \\ 0.0681\end{array}$

Lower CL Dif -0.30086 Prob $>t \quad 0.9659$

$\begin{array}{lrl} & \\ \text { Confidence } & 0.95 \text { Prob }<\mathrm{t} & 0.0341\end{array}$

Tests that the Variances are Equal

Level Count Std Dev MeanAbsDif to Mean MeanAbsDif to Median

$\begin{array}{lllll} & 14 & 0.2566008 & 0.2157143 & 0.2157143\end{array}$

Test F Ratio DFNum DFDen p-Value

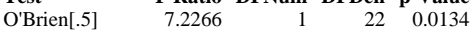

Brown-Forsythe $11.4082 \quad 1 \quad 22 \quad 0.0027$

$\begin{array}{lrrrr}\text { Levene } & 11.7720 & 1 & 22 & 0.0024\end{array}$

$\begin{array}{lrrrr}\text { Bartlett } & 8.7756 & 1 & \text {. } & 0.0031 \\ \text { F Test 2-sided } & 8.2523 & 13 & 9 & 0.0034\end{array}$

Welch Anova testing Means Equal, allowing Std Devs Not Equal

F Ratio DFNum DFDen Prob > F

$\begin{array}{rrrr}3.7919 & 1 & 17.075 & 0.0681\end{array}$

$\mathbf{t}$ Test
1.9473
Isolok at High Flow Rate

Oneway Analysis of Measurement By Sampling Mechanism Analyte $=w t \%$ dried solids

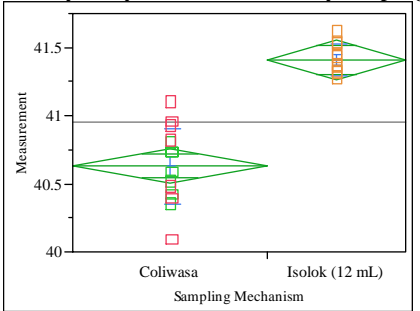

Oneway Anova

Summary of Fit

Adj Rsquare

Root Mean Square Error $\quad 0.75171$

Mean of Response

Observations (or Sum Wgts)

\section{Test}

Isolok (12 mL)-Coliwasa

Assuming equal variances

Difference $\quad 0.779714$ t Ratio 8.404388

$\begin{array}{lrr}\text { Std Err Dif } & 0.092775 \text { DF } & 22 \\ \text { Uppen }\end{array}$

Upper CL Dif 0.972117 Prob $>|t| \quad<.0001$

Lower CL Dif 0.587311 Prob $>t \quad<.0001$

Confidence $\quad 0.95$ Prob $<\mathrm{t} \quad 1.0000$

$\begin{array}{lrrrrr}\text { Analysis of Variance } & & & & & \\ \text { Source } & \text { DF } & \text { Sum of Squares } & \text { Mean Square } & \text { F Ratio } & \text { Prob > F } \\ \text { Sampling Mechanism } & 1 & 3.5464005 & 3.54640 & 70.6337 & <.0001 \\ \text { Error } & 22 & 1.1045829 & 0.05021 & & \\ \text { C. Total } & 23 & 4.6509833 & & & \end{array}$

$\begin{array}{lcccc}\text { Means for Oneway Anova } \\ \text { Level } & \text { Number } & \text { Mean Std Error } & \text { Lower } 95 \% & \text { Upper 95\% }\end{array}$

$\begin{array}{lrrrrr}\text { Level } & \text { Number } & \text { Mean } & \text { Std Error } & \text { Lower 95\% } & \text { Upper 95\% } \\ \text { Coliwasa } & 14 & 40.6293 & 0.05989 & 40.505 & 40.753\end{array}$

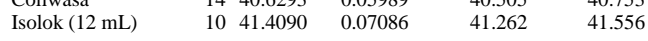

Std Error uses a pooled estimate of error variance

Means and Std Deviations

Level Number Mean Std Dev Std Err Mean Lower 95\% Upper 95\%

$\begin{array}{lllllll}\text { Coliwasa } & 14 & 40.6293 & 0.276001 & 0.07376 & 40.470 & 40.789\end{array}$

$\begin{array}{llllll}\text { Isolok }(12 \mathrm{~mL}) & 10 & 41.4090 & 0.112689 & 0.03564 & 41.328\end{array}$

t Test

Isolok (12 mL)-Coliwasa

Assuming unequal variances

$\begin{array}{lll}\text { Difference } & 0.779714 \text { t Ratio } \quad 9.517875 \\ \text { Std Err Dif } & 0.081921 & \end{array}$

Upper CL Dif 0.951600 Prob $>|t| r .0001$

Lower CL Dif 0.607829 Prob $>t$

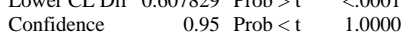

Tests that the Variances are Equal

Level Count Std Dev MeanAbsDif to Mean MeanAbsDif to Median

$\begin{array}{lllll}\text { Coliwasa } & 14 & 0.2760007 & 0.2292857 & 0.2292857\end{array}$

$\begin{array}{lllll}\text { Isolok }(12 \mathrm{~mL}) & 10 & 0.1126893 & 0.0930000 & 0.0930000\end{array}$

Test F Ratio DFNum DFDen p-Value

$\begin{array}{lrrrr}\text { O'Brien[.5] } & 4.4361 & 1 & 22 & 0.0468\end{array}$

Brown-Forsythe $8.0814 \quad 1 \quad 22 \quad 0.0095$

$\begin{array}{lllll}\text { Levene } & 8.4500 & 1 & 22 & 0.0082\end{array}$

$\begin{array}{lrrrr}\text { Bartlett } & 6.6372 & 1 & \text {. } & 0.0100 \\ \text { F Test 2-sided } & 5.9987 & 13 & 9 & 0.0110\end{array}$

Welch Anova testing Means Equal, allowing Std Devs Not Equal

F Ratio DFNum DFDen Prob $>$ F

$90.5899 \quad 1 \quad 18.334<.0001$

t Test

9.5179 
SRNL-STI-2011-00693

Revision 0

Exhibit B3. Statistical Comparisons for High-Rheology (Phase 2) Simulant Testing (screened data)

Isolok at High Flow Rate

Oneway Analysis of Measurement By Sampling Mechanism Analyte=wt $\%$ vitrified solids

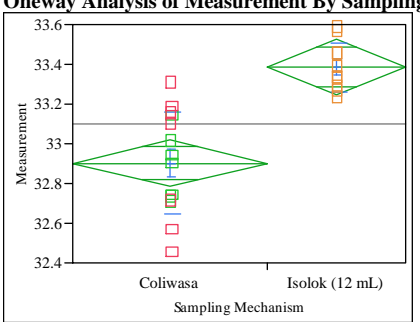

Oneway Anova

Summary of Fit

Rsquare

0.579497

Root Mean Square Error $\quad 0.212113$

Mean of Response 33.10292

Observations (or Sum Wgts) $\quad 24$

t Test

Isolok (12 mL)-Coliwasa

Assuming equal variances

Difference $\quad 0.483571$ t Ratio 5.5062

Std Err Dif $\quad 0.087823$ DF 22

Upper CL Dif 0.665705 Prob $>|t|<.0001$

$\begin{array}{lrrr}\text { Lower CL Dif } & 0.301437 & \text { Prob }>t & <.0001 \\ \text { Confidence } & 0.95 & \text { Prob }<t & 1.0000\end{array}$

$\begin{array}{lrrrrr}\text { Analysis of Variance } & & & & & \\ \text { Source } & \text { DF } & \text { Sum of Squares } & \text { Mean Square } & \text { F Ratio } & \text { Prob }>\text { F } \\ \text { Sampling Mechanism } & 1 & 1.3640744 & 1.36407 & 30.3182 & <.0001 \\ \text { Error } & 22 & 0.9898214 & 0.04499 & & \\ \text { C. Total } & 23 & 2.3538958 & & & \end{array}$

Means for Oneway Anova

$\begin{array}{lllll}\text { Means for Oneway Anova } & & & \\ \text { Level } & \text { Number } & \text { Mean Std Error Lower 95\% Upper 95\% }\end{array}$

$\begin{array}{lrrrrr}\text { Level } & \text { Number } & \text { Mean } & \text { Std Error } & \text { Lower 95\% } & \text { Upper 95\% } \\ \text { Coliwasa } & 14 & 32.9014 & 0.05669 & 32.784 & 33.019\end{array}$

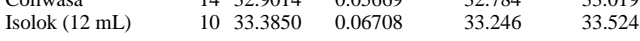

Std Error uses a pooled estimate of error variance

Means and Std Deviations

Level Number Mean Std Dev Std Err Mean Lower 95\% Upper 95\%

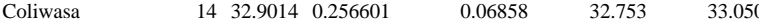

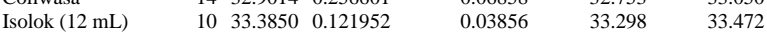

t Test

Isolok (12 mL)-Coliwasa

Assuming unequal variances

Difference $\quad 0.483571$ t Ratio 6.146142

Std Err Dif 0.078679 DF 19.67923

Upper CL Dif 0.647864 Prob $>|t|<.0001$

Lower CL Dif 0.319279 Prob $>t \quad<.0001$

$\begin{array}{lrl}\text { Confidence } & 0.95 \text { Prob }<\mathrm{t} \quad 1.0000\end{array}$

Tests that the Variances are Equal

Level Count Std Dev MeanAbsDif to Mean MeanAbsDif to Median

$\begin{array}{lllll} & 14 & 0.2566008 & 0.2157143 & 0.2157143 \\ \text { solok }(12 \mathrm{~mL}) & 10 & 0.1219517 & 0.1000000 & 0.0990000\end{array}$

Test F Ratio DFNum DFDen p-Value

$\begin{array}{lrrrr}\text { O'Brien[.5] } & 5.4986 & 1 & 22 & 0.0285\end{array}$

$\begin{array}{lllll}\text { Brown-Forsythe } & 6.8777 & 1 & 22 & 0.0155\end{array}$

$\begin{array}{lllll}\text { Levene } & 7.2090 & 1 & 22 & 0.0135\end{array}$

$\begin{array}{lrrrr}\text { Bartlett } & 4.7850 & 1 & . & 0.0287 \\ \text { F Test 2-sided } & 4.4273 & 13 & 9 & 0.0313\end{array}$

Welch Anova testing Means Equal, allowing Std Devs Not Equal

F Ratio DFNum DFDen Prob $>$ F

$\begin{array}{rrrr}37.7751 & 1 & 19.679 & <.0001\end{array}$

t Test

6.1461
Isolok at Low Flow Rate

Oneway Analysis of Measurement By Sampling Mechanism Analyte $=w t \%$ dried solids

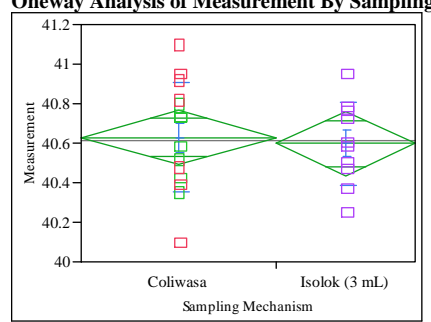

Oneway Anova

Summary of Fit

Rsquare

Adj Rsquare

Root Mean Square Error $\quad-0.04117$

Mean of Response $\quad 40.61625$

Observations (or Sum Wgts)

24

\section{t Test}

Isolok (3 mL)-Coliwasa

Assuming equal variances

$\begin{array}{lrlr}\text { Difference } & -0.03129 & \text { t Ratio } & -0.30085 \\ \text { Std Err Dif } & 0.10399 & \text { DF } & 22 \\ \text { Upper CL Dif } & 0.18438 & \text { Prob }>|t| & 0.7664 \\ \text { Lower CL Dif } & -0.24695 & \text { Prob }>\text { t } & 0.6168 \\ \text { Confidence } & 0.95 & \text { Prob }<\mathrm{t} & 0.3832\end{array}$

Lower CL Dif -0.24695 Prob $>\mathrm{t} \quad 0.6168$

Analysis of Variance

$\begin{array}{lrrrrr}\text { Source } & \text { DF } & \text { Sum of Squares } & \text { Mean Square } & \text { F Ratio } & \text { Prob }>\text { F } \\ \text { Sampling Mechanism } & 1 & 0.0057096 & 0.005710 & 0.0905 & 0.7664 \\ \text { Error } & 22 & 1.3878529 & 0.063084 & & \\ \text { C. Total } & 23 & 1.3935625 & & & \end{array}$

Means for Oneway Anova

Level Number Mean Std Error Lower 95\% Upper 95\%

$\begin{array}{lrrrrr}\text { Coliwasa } & 14 & 40.6293 & 0.06713 & 40.490 & 40.768\end{array}$

$\begin{array}{llllll}\text { Isolok }(3 \mathrm{~mL}) & 10 & 40.5980 & 0.07943 & 40.433 & 40.763\end{array}$

Std Error uses a pooled estimate of error variance

Means and Std Deviations

Level Number Mean Std Dev Std Err Mean Lower 95\% Upper 95\%

$\begin{array}{lllllll}\text { Coliwasa } & 14 & 40.6293 & 0.276001 & 0.07376 & 40.470 & 40.789\end{array}$

t Test

Isolok (3 mL)-Coliwasa

Assuming unequal variances

$\begin{array}{lrlr}\text { Difference } & -0.03129 \text { t Ratio } & -0.31509 \\ \text { Std Err Dif } & 0.09929 \text { DF } & 21.86256\end{array}$

Upper CL Dif 0.17470 Prob $>|t| \quad 0.7557$

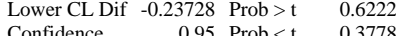

Tests that the Variances are Equal

Level Count Std Dev MeanAbsDif to Mean MeanAbsDif to Median

\begin{tabular}{llll} 
Isolok $(3 \mathrm{~mL})$ & 14 & 0.2760007 & 0.2292857 \\
\hline & 10 & 0.2101745 & 0.1640000
\end{tabular}

Test F Ratio DFNum DFDen p-Value

$\begin{array}{lrrrr}\text { O'Brien[.5] } & 0.9266 & 1 & 22 & 0.3462\end{array}$

$\begin{array}{lllll}\text { Brown-Forsythe } & 1.3926 & 1 & 22 & 0.2506\end{array}$

$\begin{array}{lllll}\text { Levene } & 1.4286 & 1 & 22 & 0.2447\end{array}$

$\begin{array}{lrrrr}\text { Bartlett } & 0.7213 & 1 & \text {. } & 0.3957 \\ \text { F Test 2-sided } & 1.7245 & 13 & 9 & 0.4167\end{array}$

Welch Anova testing Means Equal, allowing Std Devs Not Equal

F Ratio DFNum DFDen Prob $>$ F

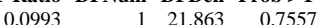

t Test

0.315 
SRNL-STI-2011-00693

Revision 0

Exhibit B3. Statistical Comparisons for High-Rheology (Phase 2) Simulant Testing (screened data)

Isolok at Low Flow Rate

Oneway Analysis of Measurement By Sampling Mechanism Analyte=wt \% vitrified solids

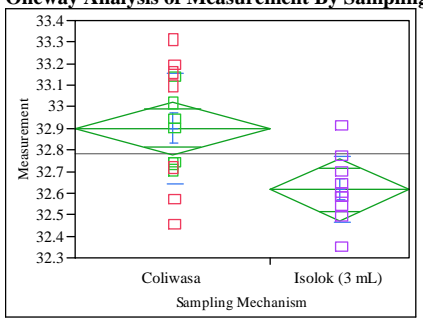

Oneway Anova

Summary of Fit

Adj Rsquare $\quad 0.307747$

Root Mean Square Error $\quad 0.220435$

Mean of Response 32.7825

Observations (or Sum Wgts) 24

t Test

Isolok (3 mL)-Coliwasa

Assuming equal variances

Difference $\quad-0.28543$ t Ratio -3.12735

$\begin{array}{lrr}\text { Std Err Dif } & 0.09127 \text { DF } & 22 \\ & \end{array}$

Upper CL Dif -0.09615 Prob $>|t| 0.0049$

$\begin{array}{lrll}\text { Lower CL Dif } & -0.47471 & \text { Prob }>t & 0.9975 \\ \text { Confidence } & 0.95 & \text { Prob }<t & 0.0025\end{array}$

$\begin{array}{lrrrrr}\text { Analysis of Variance } & & & & & \\ \text { Source } & \text { DF } & \text { Sum of Squares } & \text { Mean Square } & \text { F Ratio } & \text { Prob }>\text { F } \\ \text { Sampling Mechanism } & 1 & 0.4752386 & 0.475239 & 9.7803 & 0.0049 \\ \text { Error } & 22 & 1.0690114 & 0.048591 & & \\ \text { C. Total } & 23 & 1.5442500 & & & \end{array}$

Means for Oneway Anova

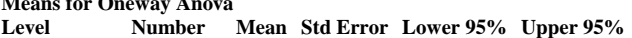

$\begin{array}{lrrrrr}\text { Level } & \text { Number } & \text { Mean } & \text { Std Error } & \text { Lower 95\% } & \text { Upper 95\% } \\ \text { Coliwasa } & 14 & 32.9014 & 0.05891 & 32.779 & 33.024\end{array}$

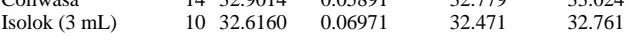

Std Error uses a pooled estimate of error variance

Means and Std Deviations

Level Number Mean Std Dev Std Err Mean Lower 95\% Upper 95\%

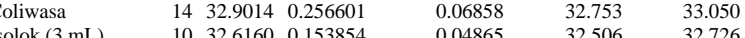

t Test

Isolok (3 mL)-Coliwasa

Assuming unequal variances

$\begin{array}{lrr}\text { Difference } & -0.28543 \text { t Ratio } & -3.39453 \\ \text { Std Err Dif } & 0.08408 \text { DF } & 21.50891\end{array}$

Upper CL Dif -0.11082 Prob $>|t| r \mid r .0027$

Lower CL Dif -0.46004 Prob $>t \quad 0.9987$

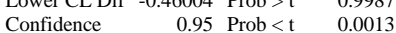

Tests that the Variances are Equal

Level Count Std Dev MeanAbsDif to Mean MeanAbsDif to Median

$\begin{array}{lllll}\text { Coliwasa } & 14 & 0.2566008 & 0.2157143 & 0.2157143\end{array}$

Test F Ratio DFNum DFDen p-Value

$\begin{array}{lrrrr}\text { O'Brien[.5] } & 3.2264 & 1 & 22 & 0.0862\end{array}$

$\begin{array}{lllll}\text { Brown-Forsythe } & 4.7647 & 1 & 22 & 0.0400\end{array}$

$\begin{array}{lllll}\text { Levene } & 4.7697 & 1 & 22 & 0.0399\end{array}$

$\begin{array}{lrrrr}\text { Bartlett } & 2.4083 & 1 & \text {. } & 0.1207 \\ \text { F Test 2-sided } & 2.7816 & 13 & 9 & 0.1296\end{array}$

Welch Anova testing Means Equal, allowing Std Devs Not Equal

F Ratio DFNum DFDen Prob > F

$\begin{array}{rrrr}11.5229 & 1 & 21.509 & 0.0027\end{array}$

t Test

3.3945
Isolok at High Flow Rate

Oneway Analysis of Measurement By Sampling Mechanism Analyte $=w t \%$ dried solids

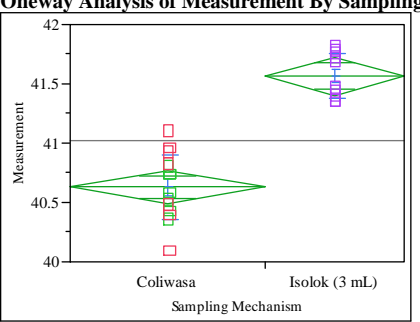

Oneway Anova

Summary of Fit

Adj Rsquare

Mean of Response $\quad 41.01958$

Observations (or Sum Wgts) 24

\section{t Test}

Isolok (3 mL)-Coliwasa

Assuming equal variances

Difference $\quad 0.93671$ t Ratio 9.244069

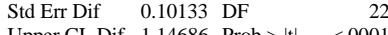

Upper CL Dif 1.14686 Prob $>|t|<.0001$

Lower CL Dif 0.72657 Prob $>\mathrm{t} \quad<.0001$

$\begin{array}{lll}\text { Confidence } & 0.95 \text { Prob }<\mathrm{t} \quad 1.0000\end{array}$

Analysis of Variance

$\begin{array}{lrrrrr}\begin{array}{l}\text { Analysis of Variance } \\ \text { Source }\end{array} & \text { DF } & \text { Sum of Squares } & \text { Mean Square } & \text { F Ratio } & \text { Prob }>\text { F } \\ \text { Sampling Mechanism } & 1 & 5.1183630 & 5.11836 & 85.4528 & <.0001 \\ \text { Error } & 22 & 1.3177329 & 0.05990 & & \\ \text { C. Total } & 23 & 6.4360958 & & & \end{array}$

Means for Oneway Anova

Level Number Mean Std Error Lower 95\% Upper 95\%

$\begin{array}{lrrrrr}\text { Coliwasa } & 14 & 40.6293 & 0.06541 & 40.494 & 40.765\end{array}$

$\begin{array}{llllll}\text { Isolok }(3 \mathrm{~mL}) & 10 & 41.5660 & 0.07739 & 41.405 & 41.727\end{array}$

Std Error uses a pooled estimate of error variance

Means and Std Deviations

Level Number Mean Std Dev Std Err Mean Lower 95\% Upper 95\%

$\begin{array}{lllllll}\text { Coliwasa } & 14 & 40.6293 & 0.276001 & 0.07376 & 40.470 & 40.789\end{array}$

t Test

Isolok (3 mL)-Coliwasa

Assuming unequal variances

Difference $\quad 0.93671$ t Ratio 9.830571

Std Err Dif $\quad 0.09529$ DF 21.99361

Upper CL Dif 1.13433 Prob $>|t| \quad<.0001$

Lower CL Dif 0.73910 Prob $>\mathrm{t} \quad<.0001$

Confidence $\quad 0.95$ Prob $<\mathrm{t} \quad 1.0000$

Tests that the Variances are Equal

Level Count Std Dev MeanAbsDif to Mean MeanAbsDif to Median

$\begin{array}{lllll}\text { Isolok }(3 \mathrm{~mL}) & 14 & 0.2760007 & 0.2292857 & 0.2292857\end{array}$

Test F Ratio DFNum DFDen p-Value

$\begin{array}{lrrrr}\text { O'Brien[.5] } & 1.7229 & 1 & 22 & 0.2028\end{array}$

$\begin{array}{lllll}\text { Brown-Forsythe } & 1.2957 & 1 & 22 & 0.2672\end{array}$

$\begin{array}{lllll}\text { Levene } & 1.3398 & 1 & 22 & 0.2595\end{array}$

$\begin{array}{lrrrr}\text { Bartlett } & 1.2996 & 1 & \text {. } & 0.2543 \\ \text { F Test 2-sided } & 2.0938 & 13 & 9 & 0.2700\end{array}$

Welch Anova testing Means Equal, allowing Std Devs Not Equal

F Ratio DFNum DFDen Prob $>$ F

$96.6401 \quad 1 \quad 21.994<.0001$

t Test

9.8306 


\section{Exhibit B3. Statistical Comparisons for High-Rheology (Phase 2) Simulant Testing (screened data)}

Isolok at High Flow Rate

Oneway Analysis of Measurement By Sampling Mechanism Analyte=wt \% vitrified solids

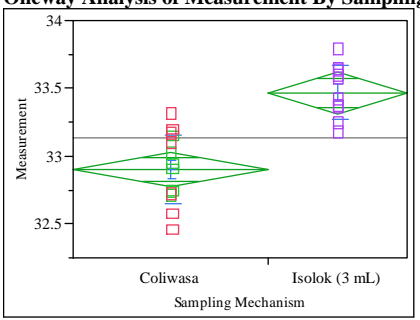

Oneway Anova

Summary of Fit

Adj Rsquare

Root Mean Square Error $\quad 0.590833$

Mean of Response $\quad \begin{array}{r}0.233951 \\ \quad 33.1375\end{array}$

Observations (or Sum Wgts)

Test

Isolok (3 mL)-Coliwasa

Assuming equal variances

Difference $\quad 0.566571$ t Ratio $\quad 5.849079$

$\begin{array}{lrr}\text { Std Err Dif } & 0.096865 \text { DF } & 22 \\ & & \end{array}$

Upper CL Dif 0.767457 Prob $>|t|<.0001$

$\begin{array}{lrrr}\text { Lower CL Dif } & 0.365686 & \text { Prob }>t & <.0001 \\ \text { Confidence } & 0.95 & \text { Prob }<t & 1.0000\end{array}$

$\begin{array}{lrrrrr}\text { Analysis of Variance } & & & & & \\ \text { Source } & \text { DF } & \text { Sum of Squares } & \text { Mean Square } & \text { F Ratio } & \text { Prob }>\text { F } \\ \text { Sampling Mechanism } & 1 & 1.8725186 & 1.87252 & 34.2117 & <.0001 \\ \text { Error } & 22 & 1.2041314 & 0.05473 & & \\ \text { C. Total } & 23 & 3.0766500 & & & \end{array}$

Means for Oneway Anova

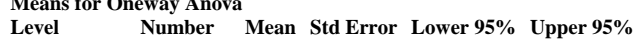

$\begin{array}{lrrrrr}\text { Level } & \text { Number } & \text { Mean } & \text { Std Error } & \text { Lower 95\% } & \text { Upper 95\% } \\ \text { Coliwasa } & 14 & 32.9014 & 0.06253 & 32.772 & 33.031\end{array}$

$\begin{array}{llllll}\text { Isolok }(3 \mathrm{~mL}) & 10 & 33.4680 & 0.07398 & 33.315 & 33.621\end{array}$

Std Error uses a pooled estimate of error variance

Means and Std Deviations

Level Number Mean Std Dev Std Err Mean Lower 95\% Upper 95\%

$\begin{array}{lllllll}\text { Coliwasa } & 14 & 32.9014 & 0.256601 & 0.06858 & 32.753 & 33.050\end{array}$

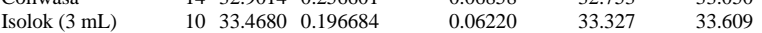

t Test

Isolok (3 mL)-Coliwasa

Assuming unequal variances

Difference $\quad 0.566571$ t Ratio 6.119615

Std Err Dif $\quad 0.092583$ DF 21.83897

Upper CL Dif 0.758659 Prob $>|t| \quad<.0001$

Lower CL Dif 0.374484 Prob $>t<<0001$

$\begin{array}{lll}\text { Confidence } & 0.95 \text { Prob }<\mathrm{t} \quad 1.0000\end{array}$

Tests that the Variances are Equal

Level Count Std Dev MeanAbsDif to Mean MeanAbsDif to Median

$\begin{array}{lllll} & 14 & 0.2566008 & 0.2157143 & 0.2157143\end{array}$

Test F Ratio DFNum DFDen p-Value

$\begin{array}{lrrrr}\text { O'Brien[.5] } & 1.2750 & 1 & 22 & 0.2710\end{array}$

$\begin{array}{lllll}\text { Brown-Forsythe } & 1.1136 & 1 & 22 & 0.3028\end{array}$

$\begin{array}{lllll}\text { Levene } & 1.1446 & 1 & 22 & 0.2963\end{array}$

$\begin{array}{lrrrr}\text { Bartlett } & 0.6880 & 1 & \text {. } & 0.4068 \\ \text { F Test 2-sided } & 1.7021 & 13 & 9 & 0.4282\end{array}$

Welch Anova testing Means Equal, allowing Std Devs Not Equal

F Ratio DFNum DFDen Prob > F

$\begin{array}{rrrr}37.4497 & 1 & 21.839 & <.0001\end{array}$

t Test

t Test
6.1196
Oneway Analysis of Measurement (wt \%) By Sampler Type of Material=SME Simulant, Analytical Block=1, Analyte=Al2O3 (wt \%), Target $(w t \%)=10.869$

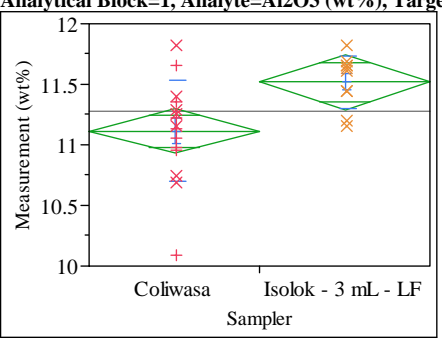

Excluded Rows

Oneway Anova

Summary of Fit

Rsquare $\quad 0.256497$

$\begin{array}{ll}\text { Adj Rsquare } & 0.224171 \\ \text { Root Mean Square Error } & 0.350524\end{array}$

$\begin{array}{ll}\text { Root Mean Square Error } & 0.350524 \\ \text { Mean of Response } & 11.27654\end{array}$

Observations (or Sum Wgts) 25

Test

Isolok - $3 \mathrm{~mL}$ - LF-Coliwasa

Assuming equal variances

Difference $\quad 0.403093$ t Ratio 2.81685

Std Err Dif $\quad 0.143101$ DF 23

Upper CL Dif 0.699120 Prob $>|t| 0.0098$

Lower CL Dif 0.107067 Prob $>t \quad 0.0049$

Confidence $\quad 0.95$ Prob $<\mathrm{t} \quad 0.9951$

Analysis of Variance

Source DF Sum of Squares Mean Square F Ratio Prob > F

$\begin{array}{lrrrrr}\text { Sampler } & 1 & 0.9749054 & 0.974905 & 7.9346 & 0.0098\end{array}$

$\begin{array}{lll}\text { Error } & 23 & 2.8259404 \\ \text { C. Total } & 24 & 3.8008458\end{array}$

Means for Oneway Anova

Level Number Mean Std Error Lower 95\% Upper 95\%

$\begin{array}{lrrrrr}\text { Coliwasa } & 15 & 11.1153 & 0.09050 & 10.928 & 11.303\end{array}$

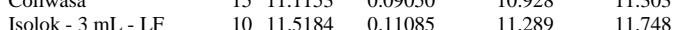

Std Error uses a pooled estimate of error variance

Means and Std Deviations

Level Number Mean Std Dev Std Err Mean Lower 95\% Upper 95\%

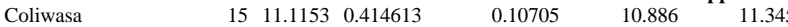

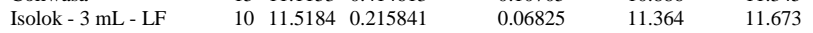

t Test

Isolok - $3 \mathrm{~mL}$ - LF-Coliwasa

Assuming unequal variances

Difference $\quad 0.403093$ t Ratio 3.174949

Std Err Dif $\quad 0.126961$ DF 22.03227

Upper CL Dif 0.666371 Prob $>|t| \quad 0.0044$

Lower CL Dif 0.139816 Prob $>t \quad 0.0022$

$\begin{array}{lrl}\text { Confidence } & 0.95 \text { Prob }<\mathrm{t} & 0.9978\end{array}$

Tests that the Variances are Equal

$\begin{array}{lrrrr}\text { Level } & \text { Count } & \text { Std Dev } & \text { MeanAbsDif to Mean } & \text { MeanAbsDif to Median } \\ \text { Coliwasa } & 15 & 0.4146130 & 0.2937543 & 0.2909830 \\ \text { Isolok - 3 mL - LF } & 10 & 0.2158409 & 0.1753456 & 0.1587180\end{array}$

Test F Ratio DFNum DFDen p-Value

$\begin{array}{lllll}\text { O'Brien[ 5] } & 1.5447 & 1 & 23 & 0.2264\end{array}$

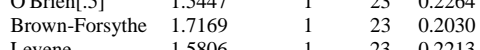

$\begin{array}{lllll}\text { Levene } & 1.5806 & 1 & 23 & 0.2213 \\ \text { Bartlett } & 3.8479 & 1 & & 0.0498\end{array}$

$\begin{array}{lllll}\text { F Test 2-sided } & 3.6899 & 14 & 9 & 0.0548\end{array}$

Welch Anova testing Means Equal, allowing Std Devs Not Equal

F Ratio DFNum DFDen Prob $>$ F

$\begin{array}{llll}10.0803 & 1 & 22.032 & 0.0044\end{array}$

t Test 


\section{Exhibit B3. Statistical Comparisons for High-Rheology (Phase 2) Simulant Testing (screened data)}

Oneway Analysis of Measurement (wt \%) By Sampler Type of Material=SME Simulant, Analytical Block $=1$, Analyte $=\mathrm{CaO}(\mathrm{wt} \%)$, Target $(\mathrm{wt} \%)=1.1462$

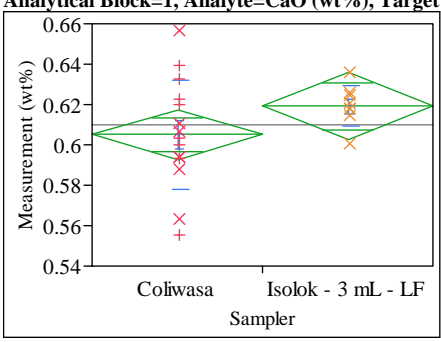

Excluded Rows

Oneway Anova

Summary of Fit

Rsquare

0.044301

Root Mean Square Error $\quad 0.022713$

$\begin{array}{lr}\text { Mean of Response } & 0.60993 \\ \text { Observations (or Sum Wgts) } & 23\end{array}$

Test

Isolok - $3 \mathrm{~mL}$ - LF-Coliwas

Assuming equal variances

Difference $\quad 0.01413$ t Ratio 1.421196

\begin{tabular}{lrr} 
Std Err Dif & 0.00994 DF & 21 \\
\hline
\end{tabular}

Upper CL Dif 0.03481 Prob $>|t| \quad 0.1699$

$\begin{array}{lrll}\text { Lower CL Dif } & -0.00655 & \text { Prob }>\mathrm{t} & 0.0850 \\ \text { Confidence } & 0.95 & \text { Prob }<\mathrm{t} & 0.9150\end{array}$

Analysis of Variance

Source DF Sum of Squares Mean Square F Ratio Prob $>$ F

$\begin{array}{llllll}\text { Sampler } & 1 & 0.00104197 & 0.001042 & 2.0198 & 0.1699\end{array}$

Error $21 \quad 0.0108334$ 0.000516

Means for Oneway Anova

Level Number Mean Std Error Lower 95\% Upper 95\%

$\begin{array}{llllrr}\text { Coliwasa } & 15 & 0.605014 & 0.00586 & 0.59282 & 0.61721\end{array}$

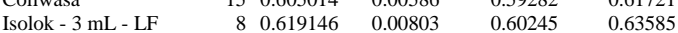

Std Error uses a pooled estimate of error variance

Means and Std Deviations

Level Number Mean Std Dev Std Err Mean Lower 95\% Upper 95\%

$\begin{array}{lrrrrrr}\text { Coliwasa } & 15 & 0.605014 & 0.026887 & 0.00694 & 0.59012 & 0.61990 \\ \text { Isolok - 3 mL - LF } & 8 & 0.619146 & 0.010090 & 0.00357 & 0.61071 & 0.62758\end{array}$

t Test

Isolok - $3 \mathrm{~mL}$ - LF-Coliwasa

Assuming unequal variances

Difference $\quad 0.01413$ t Ratio 1.810596

Std Err Dif $\quad 0.00781 \mathrm{DF} \quad 19.63186$

Upper CL Dif 0.03043 Prob $>|t| 0.0855$

Lower CL Dif -0.00217 Prob $>t \quad 0.0428$

$\begin{array}{lll}\text { Confidence } & 0.95 \text { Prob }<\mathrm{t} & 0.9572\end{array}$

Tests that the Variances are Equal

$\begin{array}{lrrrr}\text { Level } & \text { Count } & \text { Std Dev } & \text { MeanAbsDif to Mean } & \text { MeanAbsDif to Median } \\ \text { Coliwasa } & 15 & 0.0268871 & 0.0201858 & 0.0202418 \\ \text { Isolok - 3 mL - LF } & 8 & 0.0100898 & 0.0069960 & 0.0069960\end{array}$

Test F Ratio DFNum DFDen p-Value

O'Brien[.5] 2.9008

$\begin{array}{lllll}\text { O'Brien[.5] } & 2.9008 & 1 & 21 & 0.1033 \\ \text { Brown-Forsythe } & 4.4037 & 1 & 21 & 0.0481\end{array}$

Brown-Forsythe $4.4037 \quad 1 \quad 21 \quad 0.048$

$\begin{array}{lllll}\text { Levene } & 4.4022 & 1 & 21 & 0.0482 \\ \text { Bartlett } & 6.2866 & 1 & & 0.0122\end{array}$

$\begin{array}{lrrrr}\text { F Test 2-sided } & 7.1011 & 1 & 7 & 0.0122 \\ & & & \end{array}$

Welch Anova testing Means Equal, allowing Std Devs Not Equal

F Ratio DFNum DFDen Prob $>$ F

$\begin{array}{llll}3.2783 & 1 & 19.632 & 0.0855\end{array}$

$\mathbf{t}$ Test
1.8106
Oneway Analysis of Measurement (wt \%) By Sampler Type of Material=SME Simulant, Analytical Block $=1$, Analyte $=\mathrm{Cr} 2 \mathrm{O} 3(\mathrm{wt} \%)$, Target $(\mathrm{wt} \%)=\mathbf{0 . 0 9 9 8}$

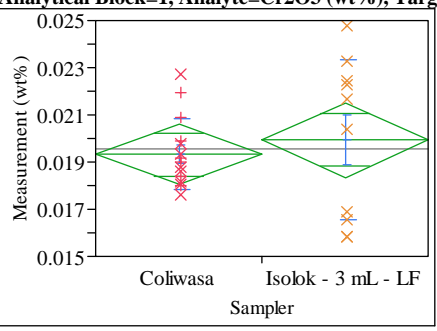

Excluded Rows

1

Oneway Anova

Summary of Fit

Rsquare $\quad 0.016515$

Adj Rsquare $\quad-0.02625$

Root Mean Square Error $\quad 0.00242$

Mean of Response $\quad 0.019568$

Observations (or Sum Wgts) $\quad 25$

t Test

Isolok - $3 \mathrm{~mL}$ - LF-Coliwasa

Assuming equal variances

Difference $\quad 0.00061$ t Ratio 0.621473

Std Err Dif $\quad 0.00099$ DF 23

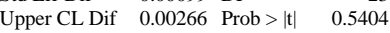

Lower CL Dif -0.00143 Prob $>t \quad 0.2702$

$\begin{array}{lll}\text { Confidence } & 0.95 \text { Prob }<\mathrm{t} & 0.7298\end{array}$

Analysis of Variance

Source DF Sum of Squares Mean Square F Ratio Prob $>$ F

$\begin{array}{lrrrrr}\text { Sampler } & 1 & 0.00000226 & 2.261 \mathrm{e}-6 & 0.3862 & 0.5404\end{array}$

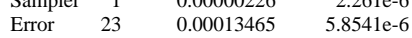

C. Total $24 \quad 0.00013691$

Means for Oneway Anova

Level Number Mean Std Error Lower 95\% Upper 95\%

$\begin{array}{lrrrrr}\text { Coliwasa } & 15 & 0.019322 & 0.00062 & 0.01803 & 0.02061 \\ \text { Isolok - 3 mL - LF } & 10 & 0.019936 & 0.00077 & 0.01835 & 0.02152\end{array}$

Std Error uses a pooled estimate of error variance

Means and Std Deviations

$\begin{array}{llllll}\text { Means and Std Deviations } & \text { Number } & \text { Mean } & \text { Std Dev Std Err Mean Lower 95\% Upper 95\% }\end{array}$

$\begin{array}{lrrrrrr}\text { Level } & \text { Number } & \text { Mean } & \text { Std Dev } & \text { Std Err Mean } & \text { Lower 95\% } & \text { Upper 95\% } \\ \text { Coliwasa } & 15 & 0.019322 & 0.001488 & 0.00038 & 0.01850 & 0.02015\end{array}$

$\begin{array}{lllllll}\text { Isolok - 3 mL - LF } & 15 & 0.019322 & 0.001488 & 0.00038 & 0.01850 & 0.02015 \\ & 10 & 0.003394 & 0.00107 & 0.01751 & 0.02236\end{array}$

t Test

Isolok - $3 \mathrm{~mL}$ - LF-Coliwasa

Assuming unequal variances

$\begin{array}{lrlr}\text { Difference } & 0.00061 & \text { t Ratio } & 0.53854 \\ \text { Std Err Dif } & 0.00114 & \text { DF } & 11.33395 \\ \text { Upper CL Dif } & 0.00311 & \text { Prob }>|t| & 0.6006 \\ \text { Lower CL Dif } & -0.00189 & \text { Prob }>\text { t } & 0.3003 \\ \text { Confidence } & 0.95 & \text { Prob }<\mathrm{t} & 0.6997\end{array}$

Tests that the Variances are Equal

Level Count Std Dev MeanAbsDif to Mean MeanAbsDif to Median

$\begin{array}{lrrrr}\text { Coliwasa } & 15 & 0.0014877 & 0.0011303 & 0.0010913\end{array}$

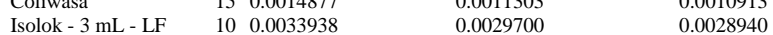

Test F Ratio DFNum DFDen p-Value

$\begin{array}{llrrr}\text { O'Brien[.5] } & 14.9636 & 1 & 23 & 0.0008\end{array}$

$\begin{array}{lllll}\text { Brown-Forsythe } & 9.8837 & 1 & 23 & 0.0045\end{array}$

$\begin{array}{lllll}\text { Levene } & 17.1241 & 1 & 23 & 0.0004\end{array}$

$\begin{array}{lllll} & 7.1938 & 1 & \text { B. } & 0.0073\end{array}$

Welch Anova testing Means Equal, allowing Std Devs Not Equal

F Ratio DFNum DFDen Prob $>$ F

$\begin{array}{lrrr}0.2900 & 1 & 11.334 & 0.6006\end{array}$

t Test 
SRNL-STI-2011-00693

Revision 0

Exhibit B3. Statistical Comparisons for High-Rheology (Phase 2) Simulant Testing (screened data)

Oneway Analysis of Measurement (wt \%) By Sampler Type of Material=SME Simulant, Analytical Block=1, Analyte=Fe2O3 (wt \%), Target $(w t \%)=11.462$

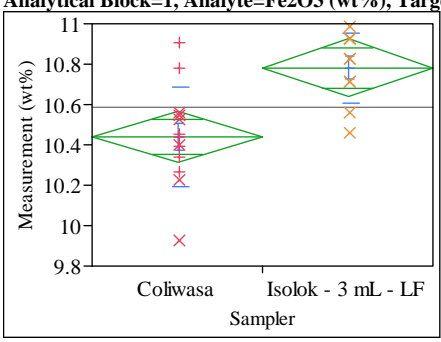

Oneway Anova

Summary of Fit

0.368082

Root Mean Square Error $\quad 0.218283$

$\begin{array}{lr}\text { Mean of Response } & 10.58848 \\ \text { Observations (or Sum Wgts) } & 23\end{array}$

Isolok - $3 \mathrm{~mL}$ - LF-Coliwas

Assuming equal variances

Difference $\quad 0.341258$ t Ratio 3.716809

$\begin{array}{lrr}\text { Std Err Dif } & 0.091815 \text { DF } & 21 \\ \text { Un } & & \end{array}$

Upper CL Dif 0.532198 Prob $>|t| \quad 0.0013$

$\begin{array}{lrll}\text { Lower CL Dif } & 0.150319 & \text { Prob }>t & 0.0006 \\ \text { Confidence } & 0.95 & \text { Prob }<\mathrm{t} & 0.9994\end{array}$

Analysis of Variance

Source DF Sum of Squares Mean Square F Ratio Prob $>$ F

$\begin{array}{lrrrrr}\text { Sampler } & 1 & 0.6582369 & 0.658237 & 13.8147 & 0.0013\end{array}$

Error $21 \quad 1.000601$ 0.047648

Means for Oneway Anova

Level Number Mean Std Error Lower 95\% Upper 95\%

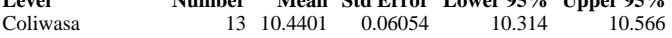

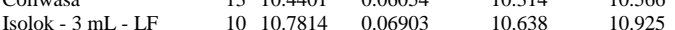

Std Error uses a pooled estimate of error variance

Means and Std Deviations

Level Number Mean Std Dev Std Err Mean Lower 95\% Upper 95\%

\begin{tabular}{lrrrrrr} 
Coliwasa & 13 & 10.4401 & 0.245778 & 0.06817 & 10.292 & 10.589 \\
\hline
\end{tabular}

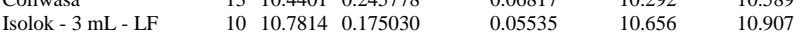

t Test

Isolok - $3 \mathrm{~mL}$ - LF-Coliwasa

Assuming unequal variances

Difference $\quad 0.341258$ t Ratio $\quad 3.886419$

Std Err Dif $\quad 0.087808$ DF 20.91668

Upper CL Dif 0.523909 Prob $>|t| 0.0009$

Lower CL Dif 0.158607 Prob $>\mathrm{t} \quad 0.0004$

$\begin{array}{lll}\text { Confidence } & 0.95 \text { Prob }<\mathrm{t} \quad 0.9996\end{array}$

Tests that the Variances are Equal

$\begin{array}{lrrrr}\text { Level } & \text { Count } & \text { Std Dev } & \text { MeanAbsDif to Mean } & \text { MeanAbsDif to Median } \\ \text { Coliwasa } & 13 & 0.2457777 & 0.1707180 & 0.1715640 \\ \text { Isolok - 3 mL - LF } & 10 & 0.1750304 & 0.1412544 & 0.1329621\end{array}$

Test F Ratio DFNum DFDen p-Value

O'Brien[.5] 0.7632

$\begin{array}{lllll}0.7632 & 1 & 21 & 0.3922 \\ \text { O'Brien[.5] } & 0.3856 & 1 & 21 & 0.5413\end{array}$

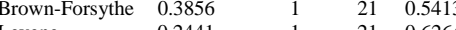

$\begin{array}{lllll}\text { Levene } & 0.2441 & 1 & 21 & 0.6264 \\ \text { Bartlett } & 1.0752 & 1 & & 0.2998\end{array}$

$\begin{array}{lrrrr}\text { F Test 2-sided } & 1.9718 & 12 & \dot{9} & 0.3138\end{array}$

Welch Anova testing Means Equal, allowing Std Devs Not Equal

F Ratio DFNum DFDen Prob $>$ F

$\begin{array}{llll}15.1043 & 1 & 20.917 & 0.0009\end{array}$

t Test

3.8864
Oneway Analysis of Measurement (wt \%) By Sampler Type of Material=SME Simulant, Analytical Block $=1$, Analyte $=\mathrm{Li} 2 \mathrm{O}(\mathrm{wt} \%)$, Target $(\mathrm{wt} \%)=4.674$

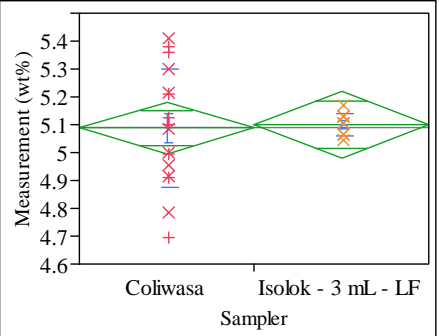

Excluded Rows

1

Oneway Anova

Summary of Fit

Rsquare $\quad 0.001265$

Adj Rsquare $\quad-0.04216$

Root Mean Square Error $\quad 0.17446$

Mean of Response 5.092039

Observations (or Sum Wgts) 25

t Test

Isolok - $3 \mathrm{~mL}$ - LF-Coliwasa

Assuming equal variances

$\begin{array}{lrlr}\text { Difference } & 0.01241 & \mathrm{t} \text { Ratio } & 0.170709 \\ \text { Std Err Dif } & 0.07269 & \text { DF } & 23 \\ \text { Upper CL Dif } & 0.16278 & \text { Prob }>|t| & 0.8659 \\ \text { Lower CL Dif } & -0.13796 & \text { Prob }>t & 0.4330 \\ \text { Confidence } & 0.95 & \text { Prob }<\mathrm{t} & 0.5670\end{array}$

Analysis of Variance

Source DF Sum of Squares Mean Square F Ratio Prob $>$ F

$\begin{array}{llllll}\text { Sampler } & 1 & 0.00088695 & 0.000887 & 0.0291 & 0.8659\end{array}$

$\begin{array}{llll}\text { Error } & 23 & 0.70003302 & 0.030436\end{array}$

C. Total $24 \quad 0.70091998$

Means for Oneway Anova

$\begin{array}{lrrrrr}\text { Level } & \text { Number } & \text { Mean } & \text { Std Error } & \text { Lower 95\% } & \text { Upper 95\% } \\ \text { Coliwasa } & 16 & 5.08757 & 0.04361 & 4.9973 & 5.1778\end{array}$

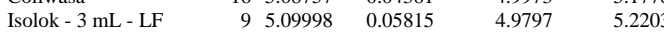

Std Error uses a pooled estimate of error variance

Means and Std Deviations

$\begin{array}{lrrrrrr}\text { Level } & \text { Number } & \text { Mean } & \text { Std Dev } & \text { Std Err Mean } & \text { Lower 95\% } & \text { Upper 95\% } \\ \text { Coliwasa } & 16 & 5.08757 & 0.213954 & 0.05349 & 4.9736 & 5.2016\end{array}$

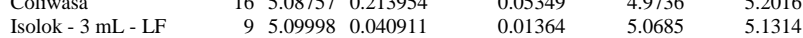

t Test

Isolok - $3 \mathrm{~mL}$ - LF-Coliwasa

Assuming unequal variances

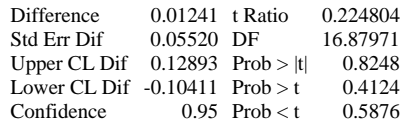

Tests that the Variances are Equal

$\begin{array}{lrrrr}\text { Level } & \text { Count } & \text { Std Dev } & \text { MeanAbsDif to Mean } & \text { MeanAbsDif to Median } \\ \text { Coliwasa } & 16 & 0.2139537 & 0.1735776 & 0.1735776\end{array}$

$\begin{array}{lrrrr}\text { Coliwasa } & 16 & 0.2139537 & 0.1735776 & 0.1735776 \\ \text { Isolok - 3 mL - LF } & 9 & 0.0409114 & 0.0318948 & 0.0310974\end{array}$

Test F Ratio DFNum DFDen p-Value

$\begin{array}{lrrrr}\text { O'Brien[.5] } & 6.6555 & 1 & 23 & 0.0167\end{array}$

$\begin{array}{lllll}\text { Brown-Forsythe } & 12.8327 & 1 & 23 & 0.0016\end{array}$

$\begin{array}{lllll}\text { Levene } & 12.7359 & 1 & 23 & 0.0016\end{array}$

$\begin{array}{lrrrr}\text { Bartlett } & 16.2785 & 1 & <.0001\end{array}$

F Test 2-sided $\quad 27.3496$

Welch Anova testing Means Equal, allowing Std Devs Not Equal

F Ratio DFNum DFDen Prob $>$ F

$\begin{array}{rrrr}0.0505 & 1 & 16.88 & 0.8248\end{array}$

t Test 


\section{Exhibit B3. Statistical Comparisons for High-Rheology (Phase 2) Simulant Testing (screened data)}

Oneway Analysis of Measurement (wt \%) By Sampler Type of Material=SME Simulant, Analytical Block=1, Analyte $=\mathrm{SiO} 2(w t \%)$, Target $(\mathrm{wt} \%)=50.985$

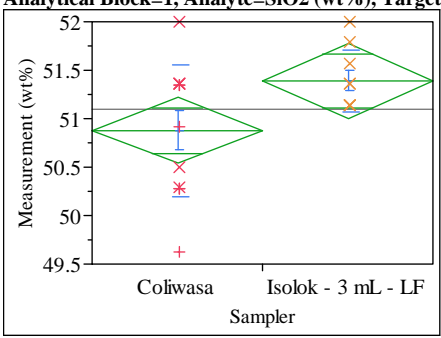

Excluded Rows

Oneway Anova

Summary of Fit

Rsquare

Adj Rsquare

Root Mean Square Error $\quad 0.557909$

Mean of Response $\quad 51.09871$

t Test

Isolok - $3 \mathrm{~mL}$ - LF-Coliwasa

Assuming equal variances

Difference $\quad 0.5111$ t Ratio 2.077334

$\begin{array}{lrr}\text { Std Err Dif } & 0.2460 \text { DF } & 19 \\ \text { Upper } & & \end{array}$

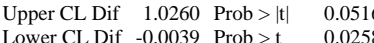

$\begin{array}{lrr}\text { Confidence } & 0.95 \text { Prob }<\mathrm{t} \quad 0.9742\end{array}$

Analysis of Variance

Source DF Sum of Squares Mean Square F Ratio Prob $>$ F

$\begin{array}{lrrrrr}\text { Sampler } & 1 & 1.3431971 & 1.34320 & 4.3153 & 0.0516\end{array}$

$\begin{array}{lll}\text { Error } & 19 & 5.9139900 \\ \text { C. Total } & 20 & 7.2571871\end{array}$

Means for Oneway Anova

Level Number Mean Std Error Lower 95\% Upper 95\%

$\begin{array}{lllllr}\text { Coliwasa } & 12 & 50.8797 & 0.16105 & 50.543 & 51.217\end{array}$

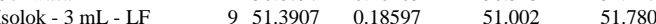

Std Error uses a pooled estimate of error variance

Means and Std Deviations

Level Number Mean Std Dev Std Err Mean Lower 95\% Upper 95\%

$\begin{array}{lrrrrrr}\text { Coliwasa } & 12 & 50.8797 & 0.681612 & 0.19676 & 50.447 & 51.313 \\ & 9 & 51.3907 & 0.316909 & 0.10564 & 51.147 & 51.634\end{array}$

t Test

Isolok - 3 mL - LF-Coliwasa

Assuming unequal variances

Difference $\quad 0.511055$ t Ratio $\quad 2.288364$

Std Err Dif $\quad 0.223328$ DF 16.38336

Upper CL Dif 0.983590 Prob $>|t| 0.0357$

Lower CL Dif 0.038520 Prob $>t \quad 0.0179$

$\begin{array}{lll}\text { Confidence } & 0.95 \text { Prob }<\mathrm{t} \quad 0.9821\end{array}$

Tests that the Variances are Equal

$\begin{array}{lrrrr}\text { Level } & \text { Count } & \text { Std Dev } & \text { MeanAbsDif to Mean } & \text { MeanAbsDif to Median } \\ \text { Coliwasa } & 12 & 0.6816118 & 0.5764225 & 0.5704800 \\ \text { Isolok - 3 mL - LF } & 9 & 0.3169086 & 0.2535467 & 0.2377000\end{array}$

Test F Ratio DFNum DFDen p-Value

O'Brien[5] $3.9440 \quad 1-190.0617$

$\begin{array}{lllll}\text { O'Brien[.5] } & 3.9440 & 1 & 19 & 0.0617\end{array}$

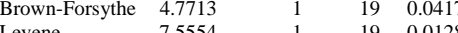

$\begin{array}{lllll}\text { Levene } & 7.5554 & 1 & 19 & 0.0128 \\ \text { Bartlett } & 4.4038 & 1 & & 0.0359\end{array}$

$\begin{array}{lrrrr}\text { F Test 2-sided } & 4.6260 & 1 & \dot{8} & 0.0358\end{array}$

Welch Anova testing Means Equal, allowing Std Devs Not Equal

F Ratio DFNum DFDen Prob $>$ F

$\begin{array}{llll}5.2366 & 1 & 16.383 & 0.0357\end{array}$

t Test
2.2884
Oneway Analysis of Measurement (wt \%) By Sampler Type of Material=SME Simulant, Analytical Block $=1$, Analyte=Sum of Oxides $(w t \%)$, Target $(w t \%)=99.553$

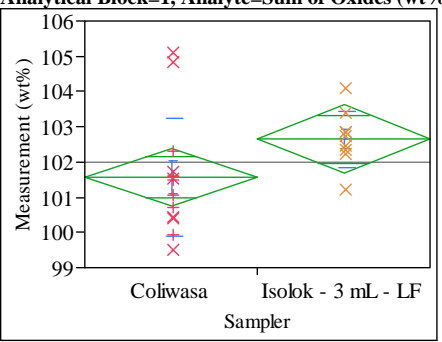

Excluded Rows

4

Oneway Anova

Summary of Fit

\begin{tabular}{lr} 
Rsquare & 0.135893 \\
Adj Rsquare & 0.092688 \\
Root Mean Square Error & 1.40135 \\
Mean of Response & 102.0092 \\
\hline Observations (or Sum Wgts) & 22
\end{tabular}

Observations (or Sum Wgts)

t Test

Isolok - $3 \mathrm{~mL}$ - LF-Coliwasa

Assuming equal variances

$\begin{array}{lrlr}\text { Difference } & 1.0777 & \text { t Ratio } & 1.773493 \\ \text { Std Err Dif } & 0.6077 \text { DF } & 20 \\ \text { Upper CL Dif } & 2.3453 \text { Prob }>|t| & 0.0914 \\ \text { Lower CL Dif } & -0.1899 & \text { Prob }>\text { t } & 0.0457 \\ \text { Confidence } & 0.95 & \text { Prob }<\mathrm{t} & 0.9543\end{array}$

Analysis of Variance

Source DF Sum of Squares Mean Square F Ratio Prob $>$ F

$\begin{array}{lrrrrr}\text { Sampler } & 1 & 6.176637 & 6.17664 & 3.1453 & 0.0914\end{array}$

$\begin{array}{llll}\text { Error } & 20 & 39.275611 & 1.96378\end{array}$

C. Total 21

$\begin{array}{lllll}\text { Means for Oneway Anova } & & & \\ \text { Level } & \text { Number } & \text { Mean Std Error } & \text { Lower 95\% } & \text { Upper 95\% }\end{array}$

$\begin{array}{lrrrrr}\text { Level } & \text { Number } & \text { Mean } & \text { Std Error } & \text { Lower 95\% } & \text { Upper 95\% } \\ \text { Coliwasa } & 13 & 101.568 & 0.38866 & 100.76 & 102.38\end{array}$

$\begin{array}{lrrrrr}\text { Coliwasa } & 13 & 101.568 & 0.38866 & 100.76 & 102.38 \\ \text { Isolok - 3 mL - LF } & 9 & 102.646 & 0.46712 & 101.67 & 103.62\end{array}$

Std Error uses a pooled estimate of error variance

Means and Std Deviations

Level Number Mean Std Dev Std Err Mean Lower 95\% Upper 95\%

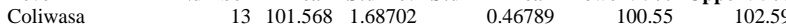

$\begin{array}{lrrrrrr}\text { Coliwasa } & 13 & 101.568 & 1.68702 & 0.46789 & 100.55 & 102.59 \\ \text { Isolok - 3 mL - LF } & 9 & 102.646 & 0.80026 & 0.26675 & 102.03 & 103.26\end{array}$

t Test

Isolok - $3 \mathrm{~mL}$ - LF-Coliwasa

Assuming unequal variances

$\begin{array}{lrlr}\text { Difference } & 1.0777 & \text { t Ratio } & 2.00094 \\ \text { Std Err Dif } & 0.5386 & \text { DF } & 18.18649 \\ \text { Upper CL Dif } & 2.2084 \text { Prob }>|t| & 0.0606 \\ \text { Lower CL Dif } & -0.0530 & \text { Prob }>\text { t } & 0.0303 \\ \text { Confidence } & 0.95 \text { Prob }<\mathrm{t} & 0.9697\end{array}$

Tests that the Variances are Equal

Level Count Std Dev MeanAbsDif to Mean MeanAbsDif to Median

$\begin{array}{lrrrr}\text { Coliwasa } & 13 & 1.687017 & 1.173894 & 1.157677 \\ \text { Isolok - 3 mL - LF } & 9 & 0.800258 & 0.556139 & 0.563487\end{array}$

Test F Ratio DFNum DFDen p-Value

$\begin{array}{lrrrr}\text { O'Brien[.5] } & 1.9502 & 1 & 20 & 0.1779\end{array}$

Brown-Forsythe $1.9615 \quad 1 \quad 200.1767$

$\begin{array}{lllll}\text { Levene } & 2.1849 & 1 & 20 & 0.1549\end{array}$

$\begin{array}{lrrrr}\text { Bartlett } & 4.2853 & 1 & \text {. } & 0.0384 \\ \text { F Test 2-sided } & 4.4440 & 12 & 8 & 0.0423\end{array}$

Welch Anova testing Means Equal, allowing Std Devs Not Equal

F Ratio DFNum DFDen Prob $>$ F

$\begin{array}{rrrr}4.0038 & 1 & 18.186 & 0.0606\end{array}$

t Test

2.0009 


\section{Exhibit B3. Statistical Comparisons for High-Rheology (Phase 2) Simulant Testing (screened data)}

Oneway Analysis of Measurement (wt \%) By Sampler Type of Material=SME Simulant, Analytical Block=2, Analyte=Al2O3 (wt \%), Target $($ wt $\%)=10.869$

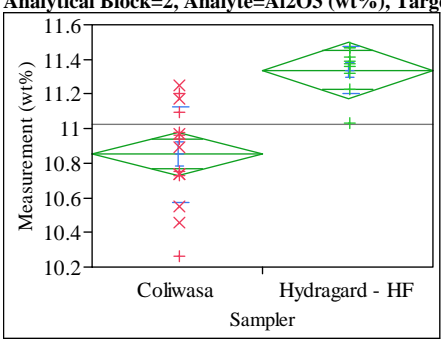

Excluded Rows

Oneway Anova

Summary of Fit

Rsquare

Adj Rsquare

Root Mean Square Error $\quad 0.237059$

Mean of Response 11.02712

t Test

Hydragard - HF-Coliwasa

Assuming equal variances

Difference $\quad 0.487465$ t Ratio $\quad 4.935119$

$\begin{array}{lrr}\text { Std Err Dif } & 0.098775 \text { DF } & 23 \\ \text { Dp } & <.0001\end{array}$

Upper CL Dif 0.691796 Prob $>|t|<.0001$

$\begin{array}{lrll}\text { Lower CL Dif } & 0.283134 & \text { Prob }>t & <.0001 \\ \text { Confidence } & 0.95 & \text { Prob }<\mathrm{t} & 1.0000\end{array}$

Analysis of Variance

$\begin{array}{lrrrrr}\text { Source } & \text { DF } & \text { Sum of Squares } & \text { Mean Square } & \text { F Ratio } & \text { Prob }>\text { F } \\ \text { Sampler } & 1 & 1.3687021 & 1.36870 & 24.3554 & <.0001\end{array}$

$\begin{array}{lll}\text { Error } & 23 & 1.2925326 \\ \text { C. Total } & 24 & 2.6612347\end{array}$

Means for Oneway Anova

Level Number Mean Std Error Lower 95\% Upper 95\%

$\begin{array}{lrrrrr}\text { Coliwasa } & 16 & 10.8516 & 0.05926 & 10.729 & 10.974\end{array}$

$\begin{array}{lrrrrr}\text { Coliwasa } & 16 & 10.8516 & 0.05926 & 10.729 & 10.974 \\ \text { Hydragard - HF } & 9 & 11.3391 & 0.07902 & 11.176 & 11.503\end{array}$

Std Error uses a pooled estimate of error variance

Means and Std Deviations

Level Number Mean Std Dev Std Err Mean Lower 95\% Upper 95\%

$\begin{array}{lllllll}\text { Coliwasa } & 16 & 10.8516 & 0.275955 & 0.06899 & 10.705 & 10.999\end{array}$

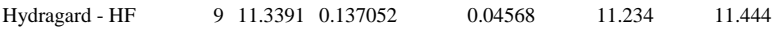

t Test

Hydragard - HF-Coliwasa

Assuming unequal variances

Difference $\quad 0.487465$ t Ratio $\quad 5.891281$

Std Err Dif $\quad 0.082743$ DF 22.8141

Upper CL Dif 0.658710 Prob $>|t|<.0001$

Lower CL Dif 0.316220 Prob $>\mathrm{t} \quad<.0001$

Confidence $\quad 0.95$ Prob $<\mathrm{t} \quad 1.0000$

Tests that the Variances are Equal

$\begin{array}{lrrrr}\text { Level } & \text { Count } & \text { Std Dev } & \text { MeanAbsDif to Mean } & \text { MeanAbsDif to Median } \\ \text { Coliwasa } & 16 & 0.2759549 & 0.2200972 & 0.2161116\end{array}$

$\begin{array}{lllll}\text { Hydragard - HF } & 9 & 0.1370521 & 0.0979741 & 0.0923756\end{array}$

Test F Ratio DFNum DFDen p-Value

O'Brien[5] 2.5912

$\begin{array}{lllll}\text { O'Brien[.5] } & 2.5912 & 1 & 23 & 0.1211 \\ \text { Brown-Forsythe } & 3.7679 & 1 & 23 & 0.0646\end{array}$

$\begin{array}{lllll}\text { Levene } & 4.5836 & 1 & 23 & 0.0431 \\ \text { Bartlett } & 4.0112 & 1 & & 0.0452\end{array}$

$\begin{array}{lrrrr}\text { F Test 2-sided } & 4.0542 & 15 & 8 & 0.0517\end{array}$

Welch Anova testing Means Equal, allowing Std Devs Not Equal

F Ratio DFNum DFDen Prob $>$ F

$\begin{array}{llll}34.7072 & 1 & 22.814 & <.0001\end{array}$

t Test
5.8913
Oneway Analysis of Measurement (wt \%) By Sampler Type of Material=SME Simulant, Analytical Block=2, Analyte $=\mathrm{CaO}(\mathrm{wt} \%)$, Target $(\mathrm{wt} \%)=1.1462$

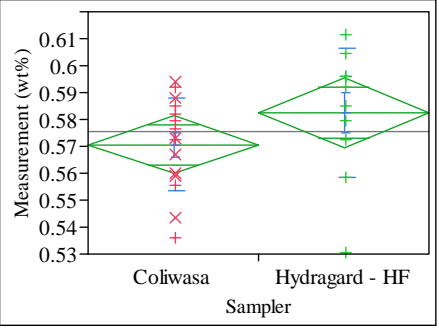

Excluded Rows

Oneway Anova

Summary of Fit

Rsquare $\quad 0.082792$

Adj Rsquare $\quad 0.042914$

Root Mean Square Error $\quad 0.020219$

Mean of Response $\quad 0.575351$

Test

Hydragard - HF-Coliwasa

Assuming equal variances

Difference $\quad 0.01189$ t Ratio 1.44087

Std Err Dif $\quad 0.00825$ DF 23

Upper CL Dif 0.02897 Prob $>|t| 0.1631$

Lower CL Dif -0.00518 Prob $>t \quad 0.0815$

$\begin{array}{lll}\text { Confidence } & 0.95 \text { Prob }<\mathrm{t} \quad 0.9185\end{array}$

Analysis of Variance

Source DF Sum of Squares Mean Square F Ratio Prob $>$ F

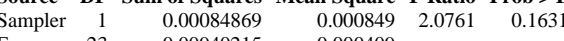

$\begin{array}{lll}\text { Error } & 23 & 0.00940215 \\ \text { C. Total } & 24 & 0.01025083\end{array}$

Means for Oneway Anova

Level Number Mean Std Error Lower 95\% Upper 95\%

$\begin{array}{lrrrrr}\text { Coliwasa } & 15 & 0.570594 & 0.00522 & 0.55979 & 0.58139\end{array}$

$\begin{array}{llllll}\text { Hydragard - HF } & 10 & 0.582487 & 0.00639 & 0.56926 & 0.59571\end{array}$

Std Error uses a pooled estimate of error variance

Means and Std Deviation

Level Number Mean Std Dev Std Err Mean Lower 95\% Upper 95\%

$\begin{array}{lllllll}\text { Coliwasa } & 15 & 0.570594 & 0.017309 & 0.00447 & 0.56101 & 0.58018\end{array}$

$\begin{array}{lllllll}\text { Hydragard - HF } & 10 & 0.582487 & 0.024055 & 0.00761 & 0.56528 & 0.59969\end{array}$

t Test

Hydragard - HF-Coliwasa

Assuming unequal variances

Difference $\quad 0.01189$ t Ratio 1.348042

Std Err Dif $\quad 0.00882$ DF 15.12667

Upper CL Dif 0.03068 Prob $>|t| \quad 0.1975$

Lower CL Dif -0.00690 Prob $>t \quad 0.0987$

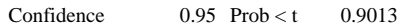

Tests that the Variances are Equal

$\begin{array}{lrrrr}\text { Level } & \text { Count } & \text { Std Dev } & \text { MeanAbsDif to Mean } & \text { MeanAbsDif to Median } \\ \text { Coliwasa } & 15 & 0.0173088 & 0.0139547 & 0.0136189\end{array}$

$\begin{array}{lllll}\text { Hydragard - HF } & 10 & 0.0240551 & 0.0179657 & 0.0174900\end{array}$

Test F Ratio DFNum DFDen p-Value

$\begin{array}{lllll}\text { O'Brien[.5] } & 1.0319 & 1 & 23 & 0.3203\end{array}$

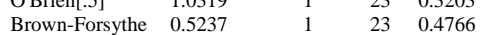

$\begin{array}{llll}\text { Brown-Forsythe } 0.5237 & 1 & 23 & 0.4766\end{array}$

$\begin{array}{lllll}\text { Levene } & 0.6824 & 1 & 23 & 0.4172 \\ \text { Bartlett } & 1.1692 & 1 & & 0.2796\end{array}$

$\begin{array}{lllll}\text { F Test 2-sided } & 1.9314 & 9 & 14 & 0.2601\end{array}$

Welch Anova testing Means Equal, allowing Std Devs Not Equal

F Ratio DFNum DFDen Prob $>$ F

$\begin{array}{llll}1.8172 & 1 & 15.127 & 0.1975\end{array}$

t Test 
SRNL-STI-2011-00693

Revision 0

Exhibit B3. Statistical Comparisons for High-Rheology (Phase 2) Simulant Testing (screened data)

Oneway Analysis of Measurement (wt \%) By Sampler Type of Material=SME Simulant, Analytical Block $=2$, Analyte $=\mathrm{Cr} 2 \mathrm{O} 3(\mathrm{wt} \%)$, Target $(\mathrm{wt} \%)=0.0998$

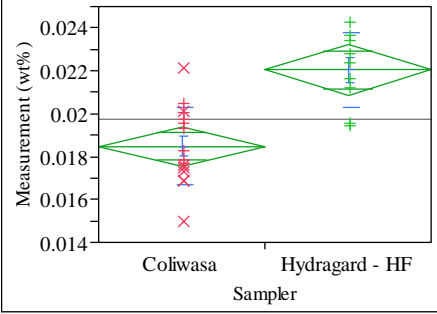

Excl
1

Oneway Anova

Summary of Fit

Rsquare $\quad 0.503617$

Adj Rsquare $\quad 0.482035$

Root Mean Square Error $\quad 0.001768$

Mean of Response $\quad 0.019761$

Observations (or Sum Wgts)

Test

Hydragard - HF-Coliwasa

Assuming equal variances

Difference $\quad 0.003558$ t Ratio 4.830648

Std Err Dif $\quad 0.000736$ DF 23

Upper CL Dif 0.005081 Prob $>|t|<.0001$

Lower CL Dif 0.002034 Prob $>t \quad<.0001$

$\begin{array}{lrl} & & \\ \text { Confidence } & 0.95 \text { Prob }<\mathrm{t} & 1.0000\end{array}$

Analysis of Variance

Source DF Sum of Squares Mean Square F Ratio Prob $>$ F

$\begin{array}{lllllll}\text { Sampler } & 1 & 0.00007290 & 0.000073 & 23.3352 & <.0001\end{array}$

$\begin{array}{lrrr}\text { Sampler } & 1 & 0.00007290 & 0.000073 \\ \text { Error } & 23 & 0.00007185 & 3.124 \mathrm{e}-6\end{array}$

C. Total $24 \quad 0.00014475$

Means for Oneway Anova

Level Number Mean Std Error Lower 95\% Upper 95\%

$\begin{array}{lrrrrr}\text { Coliwasa } & 16 & 0.018480 & 0.00044 & 0.01757 & 0.01939 \\ \text { Hydragard - HF } & 9 & 0.022038 & 0.00059 & 0.02082 & 0.02326\end{array}$

Std Error uses a pooled estimate of error variance

Means and Std Deviations

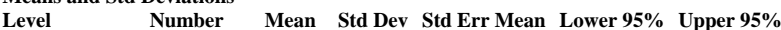

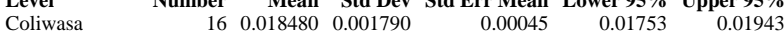

$\begin{array}{lrlllll}\text { Hydragard - HF } & 9 & 0.022038 & 0.001724 & 0.00057 & 0.02071 & 0.02336\end{array}$

t Tes

Hydragard - HF-Coliwasa

Assuming unequal variances

Difference $\quad 0.003558$ t Ratio $\quad 4.883631$

Std Err Dif $\quad 0.000728$ DF 17.25627

Upper CL Dif 0.005093 Prob $>|t| r 0.0001$

Lower CL Dif 0.002022 Prob $>t \quad<.0001$

$\begin{array}{lll}\text { Confidence } & 0.95 \text { Prob }<\mathrm{t} \quad 0.9999\end{array}$

Tests that the Variances are Equal

$\begin{array}{lrrrr}\text { Level } & \text { Count } & \text { Std Dev } & \text { MeanAbsDif to Mean } & \text { MeanAbsDif to Median } \\ \text { Coliwasa } & 16 & 0.0017901 & 0.0014787 & 0.0014525\end{array}$

$\begin{array}{lrrrrr}\text { Coliwasa } & 16 & 0.0017901 & 0.0014787 & 0.0014525 \\ \text { Hydragard - HF } & 9 & 0.0017244 & 0.0014002 & 0.0014129\end{array}$

Test F Ratio DFNum DFDen p-Value

$\begin{array}{lrrrr}\text { O'Brien[.5] } & 0.0192 & 1 & 23 & 0.8911\end{array}$

Brown-Forsythe $0.0086 \quad 1 \quad 23 \quad 0.9270$

$\begin{array}{lllll}\text { Levene } & 0.0425 & 1 & 23 & 0.8386\end{array}$

$\begin{array}{llllll}\text { Bartlett } & 0.0138 & 1 & 0.9066\end{array}$

$\begin{array}{lllll}\text { F Test 2-sided } & 1.0776 & 15 & 8 & 0.9569\end{array}$

Welch Anova testing Means Equal, allowing Std Devs Not Equal

F Ratio DFNum DFDen Prob $>$ F

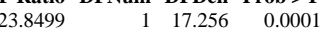

t Test

4.8836
Oneway Analysis of Measurement (wt \%) By Sampler Type of Material=SME Simulant, Analytical Block $=2$, Analyte $=\mathrm{CuO}(\mathrm{wt} \%)$, Target $(\mathrm{wt} \%)=0.0504$

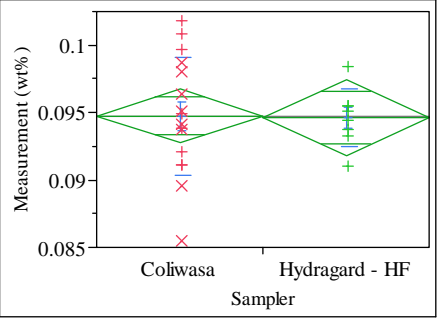

Excluded Rows

Oneway Anova

Summary of Fit

Rsquare $\quad 0.000229$

Adj Rsquare $\quad-0.04522$

Root Mean Square Error $\quad 0.003821$

Mean of Response $\quad 0.094699$

Observations (or Sum Wgts) 24

t Test

Hydragard - HF-Coliwasa

Assuming equal variances

Difference $\quad-0.00012$ t Ratio $\quad-0.07093$

$\begin{array}{lll}\text { Std Err Dif } \quad 0.00165 \text { DF } & 22\end{array}$

Upper CL Dif 0.00331 Prob $>|t| \quad 0.9441$

Lower CL Dif -0.00355 Prob $>t \quad 0.5280$

$\begin{array}{lll}\text { Confidence } & 0.95 \text { Prob }<\mathrm{t} \quad 0.4720\end{array}$

Analysis of Variance

Source DF Sum of Squares Mean Square F Ratio Prob $>$ F

$\begin{array}{lrrrrr}\text { Sampler } & 1 & 7.34533 \mathrm{e}-8 & 7.345 \mathrm{e}-8 & 0.0050 & 0.9441\end{array}$

$\begin{array}{llll}\text { Error } & 22 & 0.00032122 & 0.000015\end{array}$

C. Total $23 \quad 0.00032130$

Means for Oneway Anova

Level Number Mean Std Error Lower 95\% Upper 95\%

$\begin{array}{lrrrrr}\text { Coliwasa } & 16 & 0.094738 & 0.00096 & 0.09276 & 0.09672 \\ \text { Hydragard - HF } & 8 & 0.094620 & 0.00135 & 0.09182 & 0.09742\end{array}$

Std Error uses a pooled estimate of error variance

Means and Std Deviations

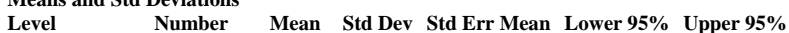

$\begin{array}{lrrrrrr}\text { Coliwasa } & 16 & 0.094738 & 0.004393 & 0.00110 & 0.09240 & 0.09708\end{array}$

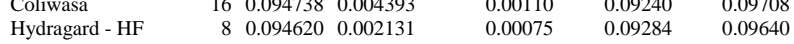

t Test

Hydragard - HF-Coliwasa

Assuming unequal variances

Difference $\quad-0.00012$ t Ratio $\quad-0.08812$

Std Err Dif $\quad 0.00133$ DF 21.99963

Upper CL Dif 0.00264 Prob $>|t| r 0.9306$

$\begin{array}{lll}\text { Lower CL Dif }-0.00288 \text { Prob }>t \quad 0.5347 & 0.4653\end{array}$

$\begin{array}{lrl}\text { Confidence } & 0.95 \text { Prob }<\mathrm{t} & 0.4653\end{array}$

Tests that the Variances are Equal

Level Count Std Dev MeanAbsDif to Mean MeanAbsDif to Median

$\begin{array}{lrrrr}\text { Coliwasa } & 16 & 0.0043927 & 0.0033877 & 0.0033877\end{array}$

$\begin{array}{lrrrr}\text { Hydragard - HF } & 8 & 0.0021311 & 0.0033877 & 0.0033877 \\ & & & \end{array}$

Test F Ratio DFNum DFDen p-Value

$\begin{array}{lrrrr}\text { O'Brien[.5] } & 2.4024 & 1 & 22 & 0.1354\end{array}$

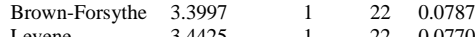

$\begin{array}{lllll}\text { Levene } & 3.4425 & 1 & 22 & 0.0770\end{array}$

$\begin{array}{lllll}\text { F Test 2-sided } & 4.2484 & 15 & \text { 7 } & 0.0517\end{array}$

Welch Anova testing Means Equal, allowing Std Devs Not Equal

F Ratio DFNum DFDen Prob > F

$\begin{array}{lrrr}0.0078 & 1 & 22 & 0.9306\end{array}$

t Test

0.088 
SRNL-STI-2011-00693

Revision 0

Exhibit B3. Statistical Comparisons for High-Rheology (Phase 2) Simulant Testing (screened data)

Oneway Analysis of Measurement (wt \%) By Sampler Type of Material=SME Simulant, Analytical Block=2, Analyte=Fe2O3 (wt \%), Target $(w t \%)=11.462$

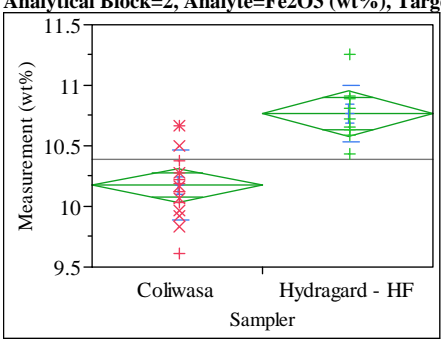

Excluded Rows

Oneway Anova

Summary of Fit

Adj Rsquare

Root Mean Square Error $\quad 0.270517$

Mean of Response $\quad 10.3882$

t Test

Hydragard - HF-Coliwasa

Assuming equal variances

Difference $\quad 0.592233$ t Ratio $\quad 5.25423$

Std Err Dif $\begin{array}{rll}0.112716 & \text { DF } & 23 \\ & & \end{array}$

Upper CL Dif 0.825403 Prob $>|t|<.0001$

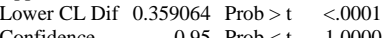

Confidence $\quad 0.95$ Prob $<\mathrm{t} \quad 1.0000$

Analysis of Variance

Source DF Sum of Squares Mean Square F Ratio Prob $>$ F

$\begin{array}{lrrrrr}\text { Sampler } & 1 & 2.0202645 & 2.02026 & 27.6069 & <.0001\end{array}$

$\begin{array}{lll}\text { Error } & 23 & 1.6831310 \\ \text { C. Total } & 24 & 3.7033955\end{array}$

Means for Oneway Anova

Level Number Mean Std Error Lower 95\% Upper 95\%

$\begin{array}{lrrrrr}\text { Coliwasa } & 16 & 10.1750 & 0.06763 & 10.035 & 10.315\end{array}$

$\begin{array}{lrrrrr}\text { Hydragard - HF } & 9 & 10.7672 & 0.09017 & 10.581 & 10.954\end{array}$

Std Error uses a pooled estimate of error variance

Means and Std Deviations

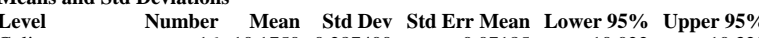

$\begin{array}{lrrrrrr}\text { Coliwasa } & 16 & 10.1750 & 0.287400 & 0.07185 & 10.022 & 10.328\end{array}$

$\begin{array}{lllllll}\text { Hydragard - HF } & 9 & 10.7672 & 0.235624 & 0.07854 & 10.586 & 10.948\end{array}$

Test

Hydragard - HF-Coliwasa

Assuming unequal variances

Difference $\quad 0.592233$ t Ratio $\quad 5.5636$

Std Err Dif $\quad 0.106448$ DF 19.65224

Upper CL Dif 0.814532 Prob $>|t| \quad<.0001$

Lower CL Dif 0.369935 Prob $>t \quad<.0001$

$\begin{array}{lll}\text { Confidence } & 0.95 \text { Prob }<\mathrm{t} \quad 1.0000\end{array}$

Tests that the Variances are Equal

Level Count Std Dev MeanAbsDif to Mean MeanAbsDif to Median

$\begin{array}{lrrrr} & \text { Count } & \text { Std Dev } & \text { MeanAbsDif to Mean } & \text { MeanAbsDif to Median } \\ \text { Coliwasa } & 16 & 0.2874002 & 0.2189228 & 0.2189228 \\ \text { Hydragard - HF } & 9 & 0.2356235 & 0.1765062 & 0.1795068\end{array}$

Test F Ratio DFNum DFDen p-Value

$\begin{array}{lrrrr}\text { O'Brien[.5] } & 0.3804 & 1 & 23 & 0.5434\end{array}$

$\begin{array}{lllll}\text { O'Brien[.5] } & 0.3804 & 1 & 23 & 0.5434 \\ \text { Brown-Forsythe } & 0.3170 & 1 & 23 & 0.5789\end{array}$

$\begin{array}{llrrr}\text { Levene } & 0.3748 & 1 & 23 & 0.5464 \\ \text { Bartlett } & 0.3749 & 1 & . & 0.5403\end{array}$

$\begin{array}{lllll}\text { F Test 2-sided } & 1.4878 & 15 & 8 & 0.5825\end{array}$

Welch Anova testing Means Equal, allowing Std Devs Not Equal

F Ratio DFNum DFDen Prob $>$ F

$\begin{array}{llll}30.9536 & 1 & 19.652 & <.0001\end{array}$

t Test
5.5636
Oneway Analysis of Measurement (wt \%) By Sampler Type of Material=SME Simulant, Analytical Block $=2$, Analyte $=$ K2O (wt \%), Target $(w t \%)=0.1591$

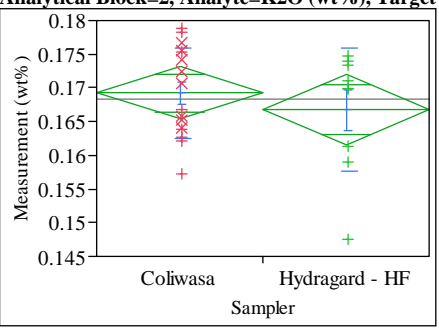

Excluded Rows

Oneway Anova

Summary of Fit

Rsquare $\quad 0.025959$

Adj Rsquare $\quad-0.01639$

Root Mean Square Error $\quad 0.00759$

Mean of Response $\quad 0.168355$

Observations (or Sum Wgts) 25

t Test

Hydragard - HF-Coliwasa

Assuming equal variances

Difference $\quad-0.00248$ t Ratio $\quad-0.78292$

$\begin{array}{lrr}\text { Std Err Dif } & 0.00316 \text { DF } & 23\end{array}$

Upper CL Dif $\quad 0.00407$ Prob $>|t| \quad 0.4417$

$\begin{array}{lll}\text { Lower CL Dif }-0.00902 \text { Prob }>t & 0.7792\end{array}$

$\begin{array}{lll}\text { Confidence } & 0.95 \text { Prob }<\mathrm{t} \quad 0.2208\end{array}$

Analysis of Variance

Source DF Sum of Squares Mean Square F Ratio Prob $>$ F

$\begin{array}{lrrrrr}\text { Sampler } & 1 & 0.00003532 & 0.000035 & 0.6130 & 0.4417\end{array}$

$\begin{array}{llll}\text { Error } & 23 & 0.00132514 & 0.000058\end{array}$

$\begin{array}{lll}\text { C. Total } & 24 & 0.00136046\end{array}$

Means for Oneway Anova

Level Number Mean Std Error Lower 95\% Upper 95\%

$\begin{array}{lrrrrr}\text { Coliwasa } & 16 & 0.169246 & 0.00190 & 0.16532 & 0.17317 \\ \text { Hydragard - HF } & 9 & 0.166770 & 0.00253 & 0.16154 & 0.17200\end{array}$

Std Error uses a pooled estimate of error variance

$\begin{array}{llllll}\text { Means and Std Deviations } & & & & \\ \text { Level } & \text { Number } & \text { Mean } & \text { Std Dev Std Err Mean Lower 95\% Upper 95\% }\end{array}$

$\begin{array}{lrrrrrr}\text { Level } & \text { Number } & \text { Mean } & \text { Std Dev } & \text { Std Err Mean } & \text { Lower 95\% } & \text { Upper 95\% } \\ \text { Coliwasa } & 16 & 0.169246 & 0.006682 & 0.00167 & 0.16569 & 0.17281\end{array}$

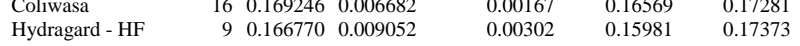

t Test

Hydragard - HF-Coliwasa

Assuming unequal variances

$\begin{array}{lrlr}\text { Difference } & -0.00248 & \text { t Ratio } & -0.71797 \\ \text { Std Err Dif } & 0.00345 & \text { DF } & 13.0039\end{array}$

Std Er Dif $\quad 0.00345$ DF 13.0039

$\begin{array}{lll}\text { Lower CL Dif }-0.00993 \text { Prob }>t & 0.7573\end{array}$

$\begin{array}{lll}\text { Confidence } & 0.95 \text { Prob }<\mathrm{t} \quad 0.2427\end{array}$

Tests that the Variances are Equal

Level Count Std Dev MeanAbsDif to Mean MeanAbsDif to Median

$\begin{array}{lrrrr}\text { Coliwasa } & 16 & 0.0066816 & 0.0058724 & 0.0058724\end{array}$

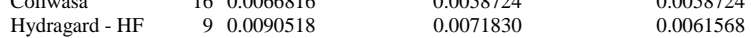

Test F Ratio DFNum DFDen p-Value

$\begin{array}{lrrrr}\text { O'Brien[.5] } & 1.0580 & 1 & 23 & 0.3144\end{array}$

$\begin{array}{lllll}\text { Brown-Forsythe } & 0.0205 & 1 & 23 & 0.8875 \\ & 0.7363 & 1 & 23 & 0.3997\end{array}$

$\begin{array}{lllll}\text { Levene } & 0.7363 & 1 & 23 & 0.3997\end{array}$

$\begin{array}{lllll}\text { F Test 2-sided } & 1.8353 & 8 & 15 & 0.2959\end{array}$

Welch Anova testing Means Equal, allowing Std Devs Not Equal

F Ratio DFNum DFDen Prob $>$ F

$\begin{array}{llll}0.5155 & 1 & 13.004 & 0.4855\end{array}$

t Test

0.7180 


\section{Exhibit B3. Statistical Comparisons for High-Rheology (Phase 2) Simulant Testing (screened data)}

Oneway Analysis of Measurement (wt\%) By Sampler Type of Material=SME Simulant, Analytical Block=2, Analyte $=\mathrm{Li} 20$ (wt \%), Target $(w t \%)=4.674$

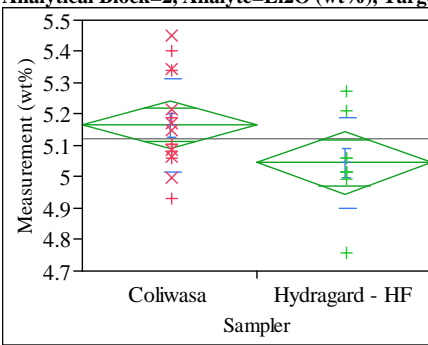

Excher

Oneway Anova

Summary of Fit

Rsquare $\quad 0.144509$

Adj Rsquare $\quad 0.107314$

Root Mean Square Error $\quad 0.146907$

Mean of Response $\quad 5.12218$

Observations (or Sum Wgts)

Test

Hydragard - HF-Coliwasa

Assuming equal variances

Difference $\quad-0.12065$ t Ratio $\quad-1.97108$

Std Err Dif $\quad 0.06121$ DF $\quad 23$

Upper CL Dif 0.00597 Prob $>|t| \quad 0.0609$

$\begin{array}{lll}\text { Lower CL Dif }-0.24728 \text { Prob }>t & 0.9696\end{array}$

$\begin{array}{lrl}\text { Confidence } & 0.95 \text { Prob }<\mathrm{t} & 0.0304\end{array}$

Analysis of Variance

Source DF Sum of Squares Mean Square F Ratio Prob > F

$\begin{array}{llllll}\text { Sampler } & 1 & 0.08384792 & 0.083848 & 3.8852 & 0.0609\end{array}$

$\begin{array}{llll}\text { Error } & 23 & 0.49637722 & 0.021582\end{array}$

C. Total $24 \quad 0.58022514$

Means for Oneway Anova

Level Number Mean Std Error Lower 95\% Upper 95\%

$\begin{array}{lrrrrr}\text { Coliwasa } & 16 & 5.16561 & 0.03673 & 5.0896 & 5.2416 \\ \text { Hydragard - HF } & 9 & 5.04496 & 0.04897 & 4.9437 & 5.1463\end{array}$

Std Error uses a pooled estimate of error variance

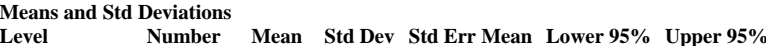

$\begin{array}{lrrrrrr}\text { Level } & \text { Number } & \text { Mean } & \text { Std Dev } & \text { Std Err Mean } & \text { Lower 95\% } & \text { Upper 95\% } \\ \text { Coliwasa } & 16 & 5.16561 & 0.148007 & 0.03700 & 5.0867 & 5.2445\end{array}$

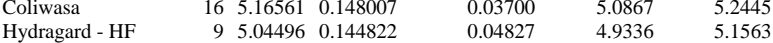

Test

Hydragard - HF-Coliwasa

Assuming unequal variances

$\begin{array}{lrlr}\text { Difference } & -0.12065 & \mathrm{t} \text { Ratio } & -1.98364 \\ \text { Std Err Dif } & 0.06082 & \text { DF } & 17.0271 \\ \text { Upper CL Dif } & 0.00766 \text { Prob }>|\mathrm{t}| & 0.0637 \\ \text { Lower CL Dif } & -0.24896 \text { Prob }>\mathrm{t} & 0.9682 \\ \text { Confidence } & 0.95 & \text { Prob }<\mathrm{t} & 0.0318\end{array}$

Tests that the Variances are Equal

Level Count Std Dev MeanAbsDif to Mean MeanAbsDif to Median

$\begin{array}{lrrrr}\text { Level } & \text { Count } & \text { Std Dev } & \text { MeanAbsDif to Mean } & \text { MeanAbsDif to Median } \\ \text { Coliwasa } & 16 & 0.1480070 & 0.1168957 & 0.1143728\end{array}$

$\begin{array}{lrrrrr}\text { Coliwasa } & 16 & 0.1480070 & & 0.1168957 & 0.1143728 \\ \text { Hydragard - HF } & 9 & 0.1448215 & 0.0940897 & 0.0909002\end{array}$

Test F Ratio DFNum DFDen p-Value

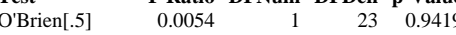

$\begin{array}{lllll}\text { Brown-Forsythe } & 0.2944 & 1 & 23 & 0.5927\end{array}$

$\begin{array}{lllll}\text { Levene } & 0.3479 & 1 & 23 & 0.5611\end{array}$

$\begin{array}{lrrrr}\text { Bartlett } & 0.0047 & 1 & \text {. } & 0.9454\end{array}$

$\begin{array}{lllll}\text { F Test 2-sided } & 1.0445 & 15 & 8 & 0.9960\end{array}$

Welch Anova testing Means Equal, allowing Std Devs Not Equal

F Ratio DFNum DFDen Prob $>$ F

$\begin{array}{rrrr}3.9348 & 1 & 17.027 & 0.0637\end{array}$

t Test

1.9836
Oneway Analysis of Measurement (wt \%) By Sampler Type of Material=SME Simulant, Analytical Block=2, Analyte=Na2O (wt \%), Target (wt \%) $=11.659$

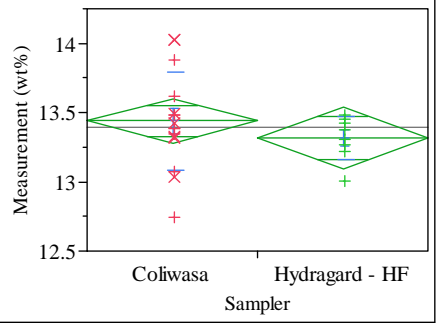

Excluded Rows

Oneway Anova

Summary of Fit

Adj Rsquare $\quad-0.00675$

Root Mean Square Error $\quad 0.302079$

Mean of Response

t Test

Hydragard - HF-Coliwasa

Assuming equal variances

Difference $\quad-0.12211$ t Ratio $\quad-0.9233$

Std Err Dif $\quad 0.13225$ DF 21

Upper CL Dif 0.15292 Prob $>|t| 0.3663$

Lower CL Dif -0.39713 Prob $>t \quad 0.8168$

Confidence $\quad 0.95$ Prob $<\mathrm{t} \quad 0.1832$

Analysis of Variance

Source DF Sum of Squares Mean Square F Ratio Prob $>$ F

$\begin{array}{llllll}\text { Sampler } & 1 & 0.0777911 & 0.077791 & 0.8525 & 0.3663\end{array}$

$\begin{array}{lll}\text { Error } & 21 & 1.9162831 \\ \text { C. Total } & 22 & 1.9940741\end{array}$

Means for Oneway Anova

Level Number Mean Std Error Lower 95\% Upper 95\%

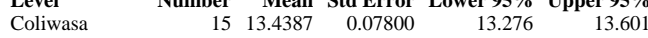

$\begin{array}{lrrrrr}\text { Hydragard - HF } & 8 & 13.3166 & 0.10680 & 13.094 & 13.539\end{array}$

Std Error uses a pooled estimate of error variance

Means and Std Deviations

Level Number Mean Std Dev Std Err Mean Lower 95\% Upper 95\%

$\begin{array}{lllllll}\text { Coliwasa } & 15 & 13.4387 & 0.353484 & 0.09127 & 13.243 & 13.634\end{array}$

$\begin{array}{llllll}\text { Hydragard - HF } & 8 & 13.3166 & 0.154443 & 0.05460 & 13.187\end{array}$

t Test

Hydragard - HF-Coliwasa

Assuming unequal variances

$\begin{array}{lrlr}\text { Difference } & -0.12211 & \mathrm{t} \text { Ratio } & -1.14809 \\ \text { Std Err Dif } & 0.10636 & \mathrm{DF} & 20.55013 \\ \text { Upper CL Dif } & 0.09937 & \text { Prob }>|\mathrm{t}| & 0.2641 \\ \text { Lower CL Dif } & -0.34358 & \text { Prob }>\mathrm{t} & 0.8679 \\ \text { Confidence } & 0.95 & \text { Prob }<\mathrm{t} & 0.1321\end{array}$

Tests that the Variances are Equal

Level Count Std Dev MeanAbsDif to Mean MeanAbsDif to Median

$\begin{array}{lrrrr}\text { Coliwasa } & 15 & 0.3534841 & 0.2542628 & 0.2543227 \\ \text { Hydragard - HF } & 8 & 0.1544433 & 0.1162650 & 0.1162650\end{array}$

Test F Ratio DFNum DFDen p-Value

$\begin{array}{lrrrr}\text { O'Brien[.5] } & 2.5093 & 1 & 21 & 0.1281\end{array}$

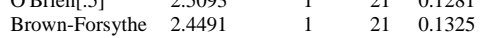

$\begin{array}{lllll}\text { Brown-Forsythe } & 2.4491 & 1 & 21 & 0.1325 \\ \text { Levene } & 2.4887 & 1 & 21 & 0.1296\end{array}$

$\begin{array}{lllll}\text { Levene } & 2.4887 & 1 & 21 & 0.1296 \\ \text { Bartlett } & 4.7291 & 1 & & 0.0297\end{array}$

$\begin{array}{lllll}\text { F Test 2-sided } & 5.2384 & 14 & 7 & 0.0348\end{array}$

Welch Anova testing Means Equal, allowing Std Devs Not Equal

F Ratio DFNum DFDen Prob $>$ F

$\begin{array}{llll}1.3181 & 1 & 20.55 & 0.2641\end{array}$

t Test
1.1481 


\section{Exhibit B3. Statistical Comparisons for High-Rheology (Phase 2) Simulant Testing (screened data)}

Oneway Analysis of Measurement (wt \%) By Sampler Type of Material=SME Simulant, Analytical Block $=2$, Analyte $=\mathrm{SiO} 2(w t \%)$, Target $(w t \%)=50.985$

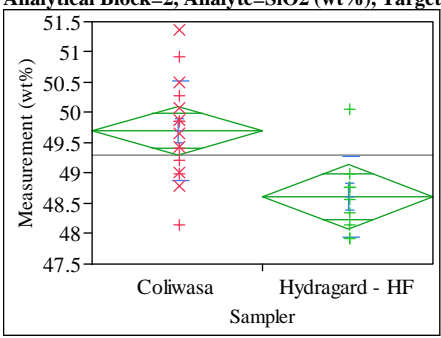

Excluded Rows

Oneway Anova

Summary of Fit

Rsquare

Root Mean Square Error $\quad 0.768743$

Mean of Response 49.30659

Observations (or Sum Wgs)

Test

Hydragard - HF-Coliwasa

Assuming equal variances

Difference $\quad-1.0890$ t Ratio $\quad-3.39972$

$\begin{array}{lrlr}\text { Std Err Dif } & 0.3203 \text { DF } & 23 \\ \text { Upper } & & -3.002\end{array}$

Upper CL Dif -0.4264 Prob $>|t| \quad 0.0025$

$\begin{array}{rrr}\text { Lower CL Dif }-1.7516 & \text { Prob }>t & 0.9988\end{array}$

Confidence $\quad 0.95$ Prob $<\mathrm{t} \quad 0.001$

\section{Analysis of Variance}

Source DF Sum of Squares Mean Square F Ratio Prob $>$ F

$\begin{array}{lrrrrr}\text { Sampler } & 1 & 6.830442 & 6.83044 & 11.5581 & 0.0025\end{array}$

$\begin{array}{lll}\text { Error } & 23 & 13.592198 \\ \text { C. Total } & 24 & 20.422640\end{array}$

Means for Oneway Anova

Level Number Mean Std Error Lower 95\% Upper 95\%

$\begin{array}{lrrrrr}\text { Coliwasa } & 16 & 49.6986 & 0.19219 & 49.301 & 50.096\end{array}$

$\begin{array}{lrrrrr}\text { Coliwasa } & 16 & 49.6986 & 0.19219 & 49.301 & 50.096 \\ \text { Hydragard - HF } & 9 & 48.6097 & 0.25625 & 48.080 & 49.140\end{array}$

Std Error uses a pooled estimate of error variance

Means and Std Deviations

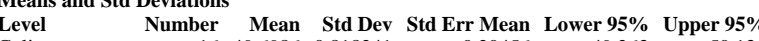

$\begin{array}{lrrrrrr}\text { Coliwasa } & 16 & 49.6986 & 0.818241 & 0.20456 & 49.263 & 50.135 \\ \text { Hydragard - HF } & 9 & 48.6097 & 0.666090 & 0.22203 & 48.098 & 49.122\end{array}$

t Test

Hydragard - HF-Coliwasa

Assuming unequal variances

Difference $\quad-1.0890$ t Ratio $\quad-3.60706$

Std Err Dif $\quad 0.3019$ DF 19.7543

Upper CL Dif -0.4587 Prob $>|t| \quad 0.0018$

Lower CL Dif -1.7192 Prob $>$ t 0.9991

Confidence $\quad 0.95$ Prob $<\mathrm{t} \quad 0.0009$

Tests that the Variances are Equal

$\begin{array}{lrrrr}\text { Level } & \text { Count } & \text { Std Dev } & \text { MeanAbsDif to Mean } & \text { MeanAbsDif to Median } \\ \text { Coliwasa } & 16 & 0.8182415 & 0.6284194 & 0.6284194\end{array}$

$\begin{array}{lrrrr}\text { Hydragard - HF } & 9 & 0.6660904 & 0.4806822 & 0.4991700\end{array}$

Test F Ratio DFNum DFDen p-Value

$\begin{array}{lrrrr}\text { O'Brien[.5] } & 0.3634 & 1 & 23 & 0.5525\end{array}$

$\begin{array}{lllll}\text { O'Brien[.5] } & 0.3634 & 1 & 23 & 0.5525 \\ \text { Brown-Forsythe } & 0.4320 & 1 & 23 & 0.5175 \\ \text { Levene }\end{array}$

$\begin{array}{lllll}\text { Levene } & 0.5567 & 1 & 23 & 0.4632 \\ \text { Bartlett } & 0.4014 & 1 & & 0.5264\end{array}$

$\begin{array}{lrrrr}\text { F Test 2-sided } & 1.5090 & 15 & 8 & 0.5264 \\ \end{array}$

Welch Anova testing Means Equal, allowing Std Devs Not Equal

F Ratio DFNum DFDen Prob $>$ F

$\begin{array}{llll}13.0109 & 1 & 19.754 & 0.0018\end{array}$

t Test
3.6071
Oneway Analysis of Measurement (wt \%) By Sampler Type of Material=SME Simulant, Analytical Block $=2$, Analyte $=$ Sum of Oxides $(w t \%)$, Target $(w t \%)=99.553$

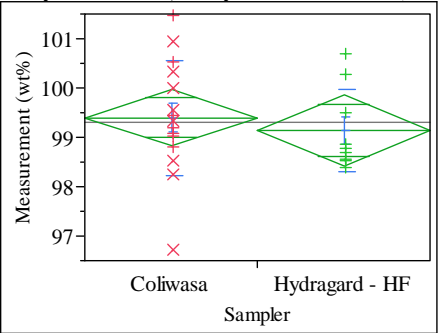

Excluded Rows

2

Oneway Anova

Summary of Fit

$\begin{array}{lr}\text { Rsquare } & 0.015167 \\ \text { Adj Rsquare } & -0.0296 \\ \text { Root Mean Square Error } & 1.056739 \\ \text { Mean of Response } & 99.30299 \\ \text { Observations (or Sum Wgts) } & 24\end{array}$

t Test

Hydragard - HF-Coliwasa

Assuming equal variances

$\begin{array}{lrlr}\text { Difference } & -0.2593 & \text { t Ratio } & -0.58207 \\ \text { Std Err Dif } & 0.4456 & \text { DF } & 22 \\ \text { Upper CL Dif } & 0.6647 & \text { Prob }>|t| & 0.5664 \\ \text { Lower CL Dif } & -1.1834 & \text { Prob }>\mathrm{t} & 0.7168 \\ \text { Confidence } & 0.95 & \text { Prob }<\mathrm{t} & 0.2832\end{array}$

Analysis of Variance

Source DF Sum of Squares Mean Square F Ratio Prob $>$ F

$\begin{array}{lrrrrr}\text { Sampler } & 1 & 0.378340 & 0.37834 & 0.3388 & 0.5664\end{array}$

$\begin{array}{llll}\text { Error } & 22 & 24.567364 & 1.11670\end{array}$

\section{Means for Oneway Anova}

Level Number Mean Std Error Lower 95\% Upper 95\%

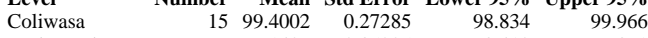

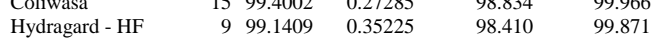

Std Error uses a pooled estimate of error variance

Means and Std Deviations

Level Number Mean Std Dev Std Err Mean Lower 95\% Upper 95\%

$\begin{array}{lrrrrrr}\text { Coliwasa } & 15 & 99.4002 & 1.16733 & 0.30140 & 98.754 & 100.05 \\ \text { Hydragard - HF } & 9 & 99.1409 & 0.82842 & 0.27614 & 98.504 & 99.78\end{array}$

t Test

Hydragard - HF-Coliwasa

Assuming unequal variances

$\begin{array}{lrlr}\text { Difference } & -0.2593 & \mathrm{t} \text { Ratio } & -0.63445 \\ \text { Std Err Dif } & 0.4088 \text { DF } & 21.21213 \\ \text { Upper CL Dif } & 0.5902 \text { Prob }>|\mathrm{t}| & 0.5326 \\ \text { Lower CL Dif } & -1.1089 & \text { Prob }>\mathrm{t} & 0.7337 \\ \text { Confidence } & 0.95 \text { Prob }<\mathrm{t} & 0.2663\end{array}$

Tests that the Variances are Equal

Level Count Std Dev MeanAbsDif to Mean MeanAbsDif to Median

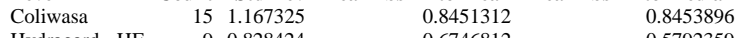

$\begin{array}{lrrrr}\text { Hydragard - HF } & 9 & 0.828424 & 0.6746812 & 0.5792359\end{array}$

Test F Ratio DFNum DFDen p-Value

$\begin{array}{lrrrr}\text { O'Brien[.5] } & 0.7264 & 1 & 22 & 0.4032\end{array}$

$\begin{array}{lllll}\text { Brown-Forsythe } & 0.7216 & 1 & 22 & 0.4048\end{array}$

$\begin{array}{lllll}\text { Levene } & 0.3685 & 1 & 22 & 0.5500\end{array}$

$\begin{array}{lrrrr}\text { Bartlett } & 1.0549 & 1 & \text {. } & 0.3044 \\ \text { F Test 2-sided } & 1.9855 & 14 & 8 & 0.3315\end{array}$

Welch Anova testing Means Equal, allowing Std Devs Not Equal

F Ratio DFNum DFDen Prob $>$ F

$\begin{array}{rrrr}0.4025 & 1 & 21.212 & 0.5326\end{array}$

t Test

0.6344 


\section{Exhibit B3. Statistical Comparisons for High-Rheology (Phase 2) Simulant Testing (screened data)}

Oneway Analysis of Measurement (wt \%) By Sampler Type of Material=SME Simulant, Analytical Block=2, Analyte $=\mathrm{TiO} 2(\mathrm{wt} \%)$, Target $(\mathrm{wt} \%)=0.0711$

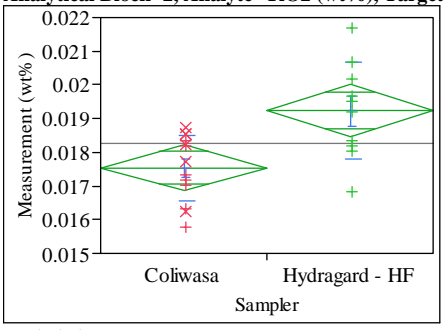

Exc

Oneway Anova

Summary of Fit

Rsquare $\quad 0.352896$

Adj Rsquare $\quad 0.322082$

Root Mean Square Error $\quad 0.001191$

Mean of Response $\quad 0.018274$

Observations (or Sum Wgts)

Test

Hydragard - HF-Coliwasa

Assuming equal variances

Difference $\quad 0.001695$ t Ratio 3.384124

Std Err Dif $\quad 0.000501$ DF $\quad 21$

Upper CL Dif 0.002737 Prob $>|t| \quad 0.0028$

Lower CL Dif 0.000653 Prob $>t \quad 0.0014$

$\begin{array}{lrl}\text { Confidence } & 0.95 \text { Prob }<\mathrm{t} \quad 0.9986\end{array}$

Analysis of Variance

Source DF Sum of Squares Mean Square F Ratio Prob $>$ F

$\begin{array}{lllllll}\text { Sampler } & 1 & 0.00001624 & 0.000016 & 11.4523 & 0.0028\end{array}$

$\begin{array}{lrrr}\text { Sampler } & 1 & 0.00001624 & 0.000016 \\ \text { Error } & 21 & 0.00002978 & 1.418 \mathrm{e}-6\end{array}$

C. Total $22 \quad 0.00004601$

Means for Oneway Anova

Level Number Mean Std Error Lower 95\% Upper 95\%

$\begin{array}{lrrrrr}\text { Coliwasa } & 13 & 0.017537 & 0.00033 & 0.01685 & 0.01822 \\ \text { Hydragard - HF } & 10 & 0.019232 & 0.00038 & 0.01845 & 0.02002\end{array}$

Std Error uses a pooled estimate of error variance

Means and Std Deviations

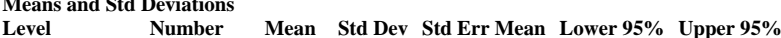

$\begin{array}{lrrrrrr}\text { Level } & \text { Number } & \text { Mean } & \text { Std Dev } & \text { Std Err Mean } & \text { Lower 95\% } & \text { Upper 95\% } \\ \text { Coliwasa } & 13 & 0.017537 & 0.000975 & 0.00027 & 0.01695 & 0.01813\end{array}$

$\begin{array}{lllllll}\text { Coliwasa } & 13 & 0.017537 & 0.000975 & 0.00027 & 0.01695 & 0.01813 \\ \text { Hydragard - HF } & 10 & 0.019232 & 0.001428 & 0.00045 & 0.01821 & 0.02025\end{array}$

t Test

Hydragard - HF-Coliwasa

Assuming unequal variances

Difference $\quad 0.001695$ t Ratio 3.219284

Std Err Dif $\quad 0.000526$ DF 15.14818

Upper CL Dif 0.002816 Prob $>|t| r 0.0057$

Lower CL Dif 0.000574 Prob $>t \quad 0.0028$

$\begin{array}{lrl}\text { Confidence } & 0.95 \text { Prob }<\mathrm{t} & 0.9972\end{array}$

Tests that the Variances are Equal

$\begin{array}{lrrrr}\text { Level } & \text { Count } & \text { Std Dev } & \text { MeanAbsDif to Mean } & \text { MeanAbsDif to Median } \\ \text { Coliwasa } & 13 & 0.0009751 & 0.0008220 & 0.0008366\end{array}$

$\begin{array}{lllll}\text { Coliwasa } & 13 & 0.0009751 & 0.0008220 & 0.0008366 \\ \text { Hydragard - HF } & 10 & 0.0014285 & 0.0011176 & 0.0011176\end{array}$

Test F Ratio DFNum DFDen p-Value

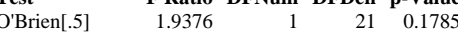

Brown-Forsythe $\quad 1.0768 \quad 11 \quad 21 \quad 0.3112$

$\begin{array}{lllll}\text { Levene } & 1.2198 & 1 & 21 & 0.2819\end{array}$

$\begin{array}{lllll}\text { F Test 2-sided } & 2.1462 & 9 & 12 & 0.2175\end{array}$

Welch Anova testing Means Equal, allowing Std Devs Not Equal

F Ratio DFNum DFDen Prob $>$ F

$\begin{array}{rrrr}10.3638 & 1 & 15.148 & 0.0057\end{array}$

t Test

3.2193
Oneway Analysis of Measurement (wt \%) By Sampler Type of Material=SME Simulant, Analytical Block $=2$, Analyte $=\mathrm{ZrO} 2$ (wt \%), Target $(\mathrm{wt} \%)=0.3547$

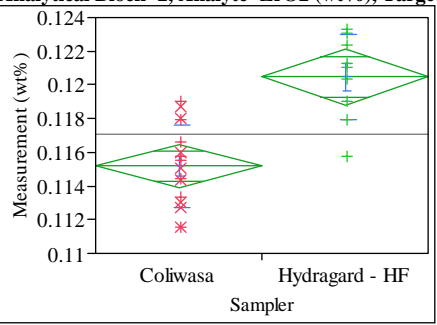

Excluded Rows

1

Oneway Anova

Summary of Fit

Rsquare $\quad 0.538824$

Adj Rsquare $\quad 0.518773$

Root Mean Square Error $\quad 0.002449$

Mean of Response $\quad 0.117077$

Observations (or Sum Wgts) 25

t Test

Hydragard - HF-Coliwasa

Assuming equal variances

Difference $\quad 0.005289$ t Ratio 5.183872

Std Err Dif $\quad 0.001020$ DF 23

Upper CL Dif 0.007399 Prob $>|t|<.0001$

$\begin{array}{llll}\text { Lower CL Dif } & 0.003178 \text { Prob }>t & <.0001\end{array}$

$\begin{array}{lrl}\text { Confidence } & 0.95 \text { Prob }<\mathrm{t} \quad 1.0000\end{array}$

Analysis of Variance

Source DF Sum of Squares Mean Square F Ratio Prob $>$ F

$\begin{array}{lrrrrr}\text { Sampler } & 1 & 0.00016111 & 0.000161 & 26.8725 & <.0001\end{array}$

$\begin{array}{llll}\text { Error } & 23 & 0.00013790 & 0.000006\end{array}$

C. Total $24 \quad 0.00029901$

Means for Oneway Anova

Level Number Mean Std Error Lower 95\% Upper 95\%

$\begin{array}{lrrrrr}\text { Coliwasa } & 16 & 0.115173 & 0.00061 & 0.11391 & 0.11644 \\ \text { Hydragard - HF } & 9 & 0.120461 & 0.00082 & 0.11877 & 0.12215\end{array}$

Std Error uses a pooled estimate of error variance

Means and Std Deviations

$\begin{array}{lccccc}\text { Means and Std Deviations } & & & & & \\ \text { Level } & \text { Number } & \text { Mean } & \text { Std Dev } & \text { Std Err Mean Lower 95\% } & \text { Upper 95\% }\end{array}$

$\begin{array}{lrrrrrr}\text { Level } & \text { Number } & \text { Mean } & \text { Std Dev } & \text { Std Err Mean } & \text { Lower 95\% } & \text { Upper 95\% } \\ \text { Coliwasa } & 16 & 0.115173 & 0.002416 & 0.00060 & 0.11389 & 0.11646\end{array}$

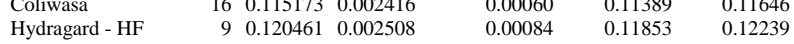

t Test

Hydragard - HF-Coliwasa

Assuming unequal variances

Difference $\quad 0.005289$ t Ratio $\quad 5.12784$

Std Err Dif $\quad 0.001031$ DF 16.18279

Upper CL Dif 0.007473 Prob $>|t| r .0001$

Lower CL Dif 0.003104 Prob $>t \quad<.0001$

$\begin{array}{lrl}\text { Confidence } & 0.95 \text { Prob }<\mathrm{t} \quad 1.0000\end{array}$

Tests that the Variances are Equal

Level Count Std Dev MeanAbsDif to Mean MeanAbsDif to Median

$\begin{array}{lrrrr}\text { Coliwasa } & 16 & 0.0024163 & 0.0019755 & 0.0019755\end{array}$

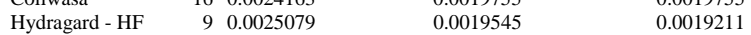

Test F Ratio DFNum DFDen p-Value

$\begin{array}{lrrrr}\text { O'Brien[.5] } & 0.0250 & 1 & 23 & 0.8758\end{array}$

$\begin{array}{lllll}\text { Brown-Forsythe } & 0.0087 & 1 & 23 & 0.9267\end{array}$

$\begin{array}{lllll}\text { Levene } & 0.0014 & 1 & 23 & 0.9702 \\ \text { Batlet } & 0.0139 & 1 & & 0.9063\end{array}$

$\begin{array}{lllll}\text { F Test 2-sided } & 1.0772 & 8 & 15 & 0.8560\end{array}$

Welch Anova testing Means Equal, allowing Std Devs Not Equal

F Ratio DFNum DFDen Prob $>$ F

$26.2947 \quad 1 \quad 16.183<<.0001$

t Test 
SRNL-STI-2011-00693

Revision 0

Exhibit B3. Statistical Comparisons for High-Rheology (Phase 2) Simulant Testing (screened data)

Oneway Analysis of Measurement (wt \%) By Sampler Type of Material=SME Simulant, Analytical Block=3, Analyte=Al2O3 (wt \%), Target $($ wt $\%)=10.869$

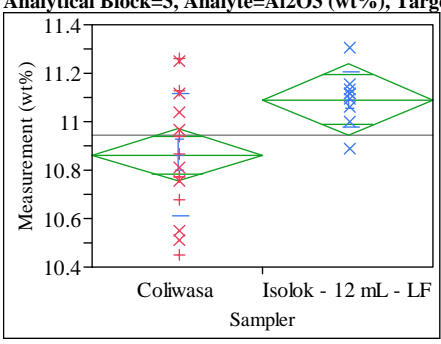

Excluded Rows

Oneway Anova

Summary of Fit

Adj Rsquare

Root Mean Square Error $\quad 0.213734$

Mean of Response $\quad 10.9455$

Observations (or Sum Wgs)

Isolok - $12 \mathrm{~mL}$ - LF-Coliwasa

Assuming equal variances

Difference $\quad 0.227921$ t Ratio 2.559305

\begin{tabular}{lrr} 
Std Err Dif & 0.089056 DF & 23 \\
\hline
\end{tabular}

Upper CL Dif 0.412147 Prob $>|t| \quad 0.0175$

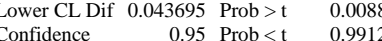

Analysis of Variance

Source DF Sum of Squares Mean Square F Ratio Prob $>$ F

$\begin{array}{lrrrrr}\text { Sampler } & 1 & 0.2992202 & 0.299220 & 6.5500 & 0.0175\end{array}$

$\begin{array}{llll}\text { Error } & 23 & 1.0506906 & 0.045682\end{array}$

C. Total $24 \quad 1.3499108$

Means for Oneway Anova

Level Number Mean Std Error Lower 95\% Upper 95\%

$\begin{array}{lrrrrr}\text { Coliwasa } & 16 & 10.8634 & 0.05343 & 10.753 & 10.974\end{array}$

$\begin{array}{lrrrrr}\text { Coliwasa } & 16 & 10.8634 & 0.05343 & 10.753 & 10.974 \\ \text { Isolok - 12 mL - LF } & 9 & 11.0914 & 0.07124 & 10.944 & 11.239\end{array}$

Std Error uses a pooled estimate of error variance

Means and Std Deviations

$\begin{array}{lrrrrrr}\text { Level } & \text { Number } & \text { Mean } & \text { Std Dev } & \text { Std Err Mean } & \text { Lower 95\% } & \text { Upper 95\% } \\ \text { Coliwasa } & 16 & 10.8634 & 0.251473 & 0.06287 & 10.729 & 10.997\end{array}$

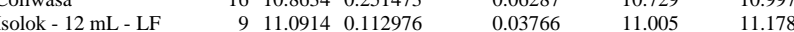

Test

Isolok - $12 \mathrm{~mL}$ - LF-Coliwasa

Assuming unequal variances

Difference $\quad 0.227921$ t Ratio $\quad 3.110091$

Std Err Dif $\quad 0.073284$ DF 22.30993

Upper CL Dif 0.379781 Prob $>|t| \quad 0.0050$

Lower CL Dif 0.076061 Prob $>t \quad 0.0025$

$\begin{array}{lll}\text { Confidence } & 0.95 \text { Prob }<\mathrm{t} \quad 0.9975\end{array}$

Tests that the Variances are Equal

$\begin{array}{lrrrr}\text { Level } & \text { Count } & \text { Std Dev } & \text { MeanAbsDif to Mean } & \text { MeanAbsDif to Median } \\ \text { Coliwasa } & 16 & 0.2514733 & 0.2043022 & 0.2043022\end{array}$

$\begin{array}{lrrrr}\text { Coliwasa } & 16 & 0.2514733 & 0.2043022 & 0.2043022 \\ \text { Isolok - 12 mL - LF } & 9 & 0.1129757 & 0.0755800 & 0.0734806\end{array}$

Test F Ratio DFNum DFDen p-Value

O'Brien[5] - 5.5850

$\begin{array}{lllll}\text { O'Brien[.5] } & 4.5850 & 1 & 23 & 0.043 \\ & 6.4913 & 1 & 23 & 0.0180\end{array}$

Brown-Forsythe $\quad 6.4913 \quad 11230.018$

$\begin{array}{lllll}\text { Levene } & 6.6228 & 1 & 23 & 0.0170 \\ \text { Bartlett } & 5.0723 & 1 & . & 0.0243\end{array}$

$\begin{array}{lllll}\text { F Test 2-sided } & 4.9547 & 15 & 8 & 0.0281\end{array}$

Welch Anova testing Means Equal, allowing Std Devs Not Equal

F Ratio DFNum DFDen Prob > F

$\begin{array}{llll}9.6727 & 1 & 22.31 & 0.0050\end{array}$

t Test
3.1101
Oneway Analysis of Measurement (wt \%) By Sampler Type of Material=SME Simulant, Analytical Block=3, Analyte $=\mathrm{B} 2 \mathrm{O} 3(\mathrm{wt} \%)$, Target $(\mathrm{wt} \%)=4.259$

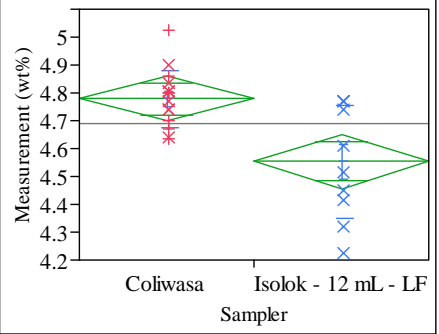

Excluded Rows

1

Oneway Anova

Summary of Fit

Rsquare $\quad 0.369347$

Adj Rsquare $\quad 0.341927$

Root Mean Square Error $\quad 0.150429$

Mean of Response 4.688174

Observations (or Sum Wgts) 25

t Test

Isolok - $12 \mathrm{~mL}$ - LF-Coliwasa

Assuming equal variances

$\begin{array}{lrlr}\text { Difference } & -0.22539 & \mathrm{t} \text { Ratio } & -3.67017 \\ \text { Std Err Dif } & 0.06141 & \text { DF } & 23 \\ \text { Upper CL Dif } & -0.09835 & \text { Prob }>|t| & 0.0013 \\ \text { Lower CL Dif } & -0.35243 & \text { Prob }>t & 0.9994 \\ \text { Confidence } & 0.95 & \text { Prob }<\mathrm{t} & 0.0006\end{array}$

Analysis of Variance

Source DF Sum of Squares Mean Square F Ratio Prob $>$ F

$\begin{array}{llllll}\text { Sampler } & 1 & 0.30481203 & 0.304812 & 13.4701 & 0.0013\end{array}$

$\begin{array}{llll}\text { Error } & 23 & 0.52046135 & 0.022629\end{array}$

C. Total $24 \quad 0.82527338$

Means for Oneway Anova

Level Number Mean Std Error Lower 95\% Upper 95\%

$\begin{array}{lrrrrr}\text { Coliwasa } & 15 & 4.77833 & 0.03884 & 4.6980 & 4.8587\end{array}$

$\begin{array}{lllll}\text { Isolok - } 12 \text { mL - LF } & 10 & 4.55294 & 0.04757 & 4.4545\end{array} 4.6513$

Std Error uses a pooled estimate of error variance

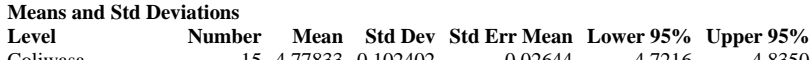

$\begin{array}{lllllll}\text { Coliwasa } & 15 & 4.77833 & 0.102402 & 0.02644 & 4.7216 & 4.8350 \\ \text { Isolok - 12 mL - LF } & 10 & 4.55294 & 0.203757 & 0.06443 & 4.4072 & 4.6987\end{array}$

t Test

Isolok - $12 \mathrm{~mL}$ - LF-Coliwasa

Assuming unequal variances

$\begin{array}{lrlr}\text { Difference } & -0.22539 & \mathrm{t} \text { Ratio } & -3.23619 \\ \text { Std Err Dif } & 0.06965 & \text { DF } & 12.06617 \\ \text { Upper CL Dif } & -0.07374 & \text { Prob }>|t| & 0.0071 \\ \text { Lower CL Dif } & -0.37705 & \text { Prob }>\mathrm{t} & 0.9965 \\ \text { Confidence } & 0.95 & \text { Prob }<\mathrm{t} & 0.0035\end{array}$

Tests that the Variances are Equal

Level Count Std Dev MeanAbsDif to Mean MeanAbsDif to Median

$\begin{array}{lrrrr}\text { Coliwasa } & 15 & 0.1024024 & 0.0764190 & 0.0751310\end{array}$

Isolok - $12 \mathrm{~mL}$ - LF $\quad 10 \quad 0.2037575 \quad 0.1738746 \quad 0.1738746$

Test F Ratio DFNum DFDen p-Value

$\begin{array}{lrrrr}\text { O'Brien[.5] } & 7.5837 & 1 & 23 & 0.0113\end{array}$

Brown-Forsythe $9.6683 \quad 1 \quad 23 \quad 0.0049$

$\begin{array}{lllll}\text { Levene } & 10.0404 & 1 & 23 & 0.0043\end{array}$

$\begin{array}{lllll}\text { Bartlett } & 5.0712 & 1 & 0.0243\end{array}$

$\begin{array}{lllll}\text { F Test 2-sided } & 3.9592 & 9 & 14 & 0.0216\end{array}$

Welch Anova testing Means Equal, allowing Std Devs Not Equal

F Ratio DFNum DFDen Prob $>$ F

$\begin{array}{rrrr}10.4729 & 1 & 12.066 & 0.0071\end{array}$

t Test

3.236 


\section{Exhibit B3. Statistical Comparisons for High-Rheology (Phase 2) Simulant Testing (screened data)}

Oneway Analysis of Measurement (wt \%) By Sampler Type of Material=SME Simulant, Analytical Block=3, Analyte $=\mathrm{CaO}(\mathrm{wt} \%)$, Target $(\mathrm{wt} \%)=1.1462$

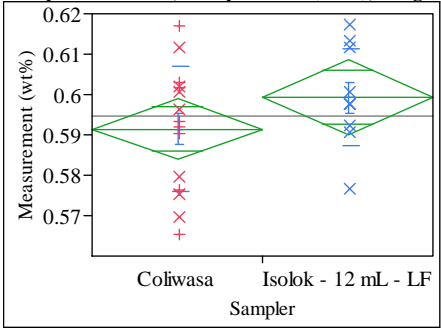

Excluded Rows

Oneway Anova

Summary of Fit

Rsquare

Adj Rsquare

Root Mean Square Error $\quad 0.014176$

Mean of Response $\quad 0.594604$

Test

Isolok - $12 \mathrm{~mL}$ - LF-Coliwasa

Assuming equal variances

Difference $\quad 0.00779$ t Ratio 1.34581

$\begin{array}{lrlr}\text { Std Err Dif } & 0.00579 \text { DF } & 23 \\ & & \end{array}$

Upper CL Dif 0.01976 Prob $>|t| \quad 0.1915$

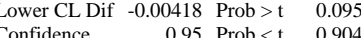

Analysis of Variance

Source DF Sum of Squares Mean Square F Ratio Prob $>$ F

$\begin{array}{lrrrrr}\text { Sampler } & 1 & 0.00036400 & 0.000364 & 1.8112 & 0.1915\end{array}$

$\begin{array}{llll}\text { Error } & 23 & 0.00462234 & 0.000201\end{array}$

C. Total $24 \quad 0.00498634$

Means for Oneway Anova

Level Number Mean Std Error Lower 95\% Upper 95\%

$\begin{array}{lrrrrr}\text { Coliwasa } & 15 & 0.591488 & 0.00366 & 0.58392 & 0.59906\end{array}$

$\begin{array}{llllll}\text { Coliwasa } & 15 & 0.591488 & 0.00366 & 0.58392 & 0.59906 \\ \text { Isolok - 12 mL - LF } & 10 & 0.599277 & 0.00448 & 0.59000 & 0.60855\end{array}$

Std Error uses a pooled estimate of error variance

Means and Std Deviations

$\begin{array}{lrrrrrr}\text { Level } & \text { Number } & \text { Mean } & \text { Std Dev } & \text { Std Err Mean } & \text { Lower 95\% } & \text { Upper 95\% } \\ \text { Coliwasa } & 15 & 0.591488 & 0.015377 & 0.00397 & 0.58297 & 0.60000\end{array}$

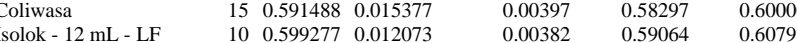

t Test

Isolok - $12 \mathrm{~mL}$ - LF-Coliwasa

Assuming unequal variances

Difference $\quad 0.00779$ t Ratio $\quad 1.414043$

Std Err Dif $\quad 0.00551$ DF 22.25768

Upper CL Dif 0.01920 Prob $>|t| \quad 0.1712$

Lower CL Dif -0.00363 Prob $>t \quad 0.0856$

$\begin{array}{lrl}\text { Confidence } & 0.95 \text { Prob }<\mathrm{t} & 0.9144\end{array}$

Tests that the Variances are Equal

$\begin{array}{lrrrr}\text { Level } & \text { Count } & \text { Std Dev } & \text { MeanAbsDif to Mean } & \text { MeanAbsDif to Median } \\ \text { Coliwasa } & 15 & 0.0153773 & 0.0123876 & 0.0123130 \\ \text { Isolok - 12 mL - LF } & 10 & 0.0120734 & 0.0088989 & 0.0085351\end{array}$

Test F Ratio DFNum DFDen p-Value

O'Brien[.5] $\quad 0.8737 \quad 1 \quad 23 \quad 0.3597$

$\begin{array}{lllll}\text { O'Brien[.5] } & 0.8737 & 1 & 23 & 0.3597\end{array}$

Brown-Forsythe $1.1573 \quad 1 \quad 23 \quad 0.2932$

$\begin{array}{lllll}\text { Levene } & 1.0987 & 1 & 23 & 0.3054 \\ \text { Bartlett } & 0.5866 & 1 & & 0.4437\end{array}$

$\begin{array}{lrrrr}\text { F Test 2-sided } & 1.6222 & 14 & 9 & 0.4737\end{array}$

Welch Anova testing Means Equal, allowing Std Devs Not Equal

F Ratio DFNum DFDen Prob $>$ F

$\begin{array}{llll}1.9995 & 1 & 22.258 & 0.1712\end{array}$

$\mathbf{t}$ Test
1.4140
Oneway Analysis of Measurement (wt \%) By Sampler Type of Material=SME Simulant, Analytical Block $=3$, Analyte $=\mathrm{CuO}(\mathrm{wt} \%)$, Target $(\mathrm{wt} \%)=0.0504$

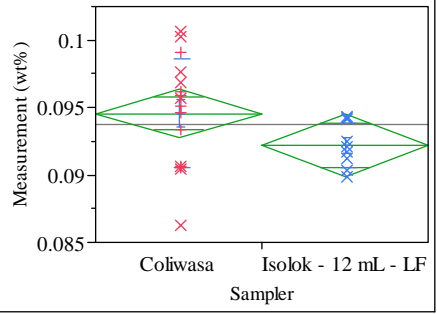

Excluded Rows

Oneway Anova

Summary of Fit

Rsquare $\quad 0.109478$

Adj Rsquare $\quad 0.07076$

Root Mean Square Error $\quad 0.003413$

Mean of Response 0.093705

Observations (or Sum Wgts) 25

Test

Isolok - $12 \mathrm{~mL}$ - LF-Coliwasa

Assuming equal variances

Difference $\quad-0.00239$ t Ratio $\quad-1.68154$

$\begin{array}{lll}\text { Std Err Dif } & 0.00142 \text { DF } & 23\end{array}$

Upper CL Dif 0.00055 Prob $>|t| \quad 0.1062$

$\begin{array}{llll}\text { Lower CL Dif } & -0.00533 & \text { Prob }>t & 0.9469\end{array}$

$\begin{array}{lrl}\text { Confidence } & 0.95 \text { Prob }<\mathrm{t} & 0.0531\end{array}$

Analysis of Variance

Source DF Sum of Squares Mean Square F Ratio Prob $>$ F

$\begin{array}{lrrrrr}\text { Sampler } & 1 & 0.00003294 & 0.000033 & 2.8276 & 0.1062\end{array}$

$\begin{array}{llll}\text { Error } & 23 & 0.00026796 & 0.000012\end{array}$

C. Total $24 \quad 0.00030090$

Means for Oneway Anova

Level Number Mean Std Error Lower 95\% Upper 95\%

$\begin{array}{lrrrrr}\text { Coliwasa } & 16 & 0.094566 & 0.00085 & 0.09280 & 0.09633 \\ \text { Isolok - 12 mL - LF } & 9 & 0.092174 & 0.00114 & 0.08982 & 0.09453\end{array}$

Std Error uses a pooled estimate of error variance

$\begin{array}{lrrrrr}\text { Means and Std Deviations } & & & & & \\ \text { Level } & \text { Number } & \text { Mean } & \text { Std Dev } & \text { Std Err Mean Lower 95\% Upper 95\% }\end{array}$

$\begin{array}{lrrrrrr}\text { Coliwasa } & 16 & 0.094566 & 0.004045 & 0.00101 & 0.09241 & 0.09672 \\ \text { Isolok - 12 mL - LF } & 9 & 0.092174 & 0.001679 & 0.00056 & 0.09088 & 0.09347\end{array}$

t Test

Isolok - $12 \mathrm{~mL}$ - LF-Coliwasa

Assuming unequal variances

Difference $\quad-0.00239$ t Ratio $\quad-2.0691$

Std Err Dif $\quad 0.00116$ DF 21.76978

Upper CL Dif $6.987 \mathrm{e}-6$ Prob $>|\mathrm{t}| \quad 0.0506$

Lower CL Dif -0.00479 Prob $>$ t 0.9747

$\begin{array}{lrl}\text { Confidence } & 0.95 \text { Prob }<\mathrm{t} & 0.0253\end{array}$

Tests that the Variances are Equal

Level Count Std Dev MeanAbsDif to Mean MeanAbsDif to Median

$\begin{array}{lrrrr}\text { Coliwasa } & 16 & 0.0040447 & 0.0032332 & 0.0031530\end{array}$

$\begin{array}{llll} & 16 & 0.0040447 & 0.0032332\end{array}-0.0031530$

Test F Ratio DFNum DFDen p-Value

$\begin{array}{lrrrr}\text { O'Brien[.5] } & 3.8074 & 1 & 23 & 0.0633\end{array}$

$\begin{array}{lllll}\text { Brown-Forsythe } & 4.0561 & 1 & 23 & 0.0559\end{array}$

$\begin{array}{lllll}\text { Levene } & 5.5567 & 1 & 23 & 0.0273 \\ \text { Batle } & 5.9605 & 1 & & 0.0146\end{array}$

F Test 2-sided $\quad 5.8000-15 \quad 8 \quad 0.0170$

Welch Anova testing Means Equal, allowing Std Devs Not Equal

F Ratio DFNum DFDen Prob $>$ F

$\begin{array}{rrrr}4.2812 & 1 & 21.77 & 0.0506\end{array}$

t Test

2.069 
SRNL-STI-2011-00693

Revision 0

Exhibit B3. Statistical Comparisons for High-Rheology (Phase 2) Simulant Testing (screened data)

Oneway Analysis of Measurement (wt \%) By Sampler Type of Material=SME Simulant, Analytical Block $=3$, Analyte $=\mathrm{Fe} 2 \mathrm{O} 3(\mathrm{wt} \%)$, Target $(\mathrm{wt} \%)=11.462$

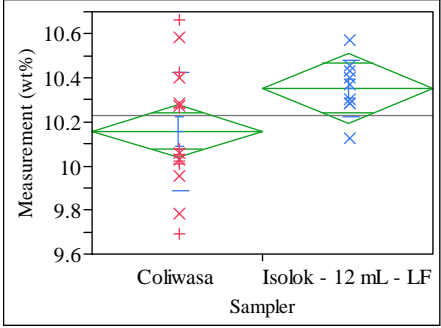

Excluded Rows

Oneway Anova

Summary of Fit

Adj Rsquare

0.114915

Root Mean Square Error $\quad 0.230202$

$\begin{array}{lr}\text { Mean of Response } & 10.22807 \\ \text { Observations (or Sum Wgts) } & 25\end{array}$

t Test

Isolok - $12 \mathrm{~mL}$ - LF-Coliwasa

Assuming equal variances

Difference $\quad 0.19460$ t Ratio 2.028805

$\begin{array}{lrlr}\text { Std Err Dif } & 0.09592 & \text { DF } & 23 \\ \text { Upper CL Dif } & 0.39302 & \text { Prob }>|t| & 0.0542\end{array}$

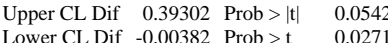

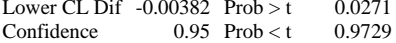

Analysis of Variance

Source DF Sum of Squares Mean Square F Ratio Prob $>$ F

$\begin{array}{lrrrrr}\text { Sampler } & 1 & 0.2181220 & 0.218122 & 4.1161 & 0.0542\end{array}$

$\begin{array}{lll}\text { Error } & 23 & 1.2188396 \\ \text { C. Total } & 24 & 1.4369616\end{array}$

Means for Oneway Anova

Level Number Mean Std Error Lower 95\% Upper 95\%

$\begin{array}{lrrrrr}\text { Coliwasa } & 16 & 10.1580 & 0.05755 & 10.039 & 10.277 \\ \text { Isolok - 12 mL - LF } & 9 & 10.3526 & 0.07673 & 10.194 & 10.511\end{array}$

Std Error uses a pooled estimate of error variance

Means and Std Deviations

$\begin{array}{lrrrrrr}\text { Level } & \text { Number } & \text { Mean } & \text { Std Dev } & \text { Std Err Mean } & \text { Lower 95\% } & \text { Upper 95\% } \\ \text { Coliwasa } & 16 & 10.1580 & 0.269553 & 0.06739 & 10.014 & 10.302\end{array}$

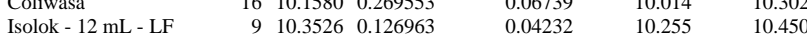

t Test

Isolok - $12 \mathrm{~mL}$ - LF-Coliwasa

Assuming unequal variances

Difference $\quad 0.194598$ t Ratio $\quad 2.445456$

Std Err Dif $\quad 0.079575$ DF 22.57973

Upper CL Dif 0.359382 Prob $>|t| 0.0227$

Lower CL Dif 0.029814 Prob $>\mathrm{t} \quad 0.0113$

$\begin{array}{lll}\text { Confidence } & 0.95 \text { Prob }<\mathrm{t} \quad 0.9887\end{array}$

Tests that the Variances are Equal

$\begin{array}{lrrrr}\text { Level } & \text { Count } & \text { Std Dev } & \text { MeanAbsDif to Mean } & \text { MeanAbsDif to Median } \\ \text { Coliwasa } & 16 & 0.2695531 & 0.2207099 & 0.2108807\end{array}$

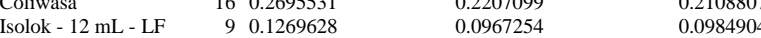

Test F Ratio DFNum DFDen p-Value

O'Brien[5] 3.2547

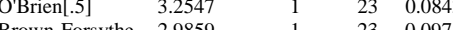

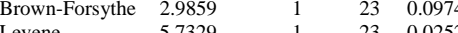

$\begin{array}{lllll}\text { Levene } & 5.7329 & 1 & 23 & 0.0252 \\ \text { Bartlett } & 4.5615 & 1 & & 0.0327\end{array}$

$\begin{array}{lrrrr}\text { F Test 2-sided } & 4.5075 & 15 & 8 & 0.0376\end{array}$

Welch Anova testing Means Equal, allowing Std Devs Not Equal

F Ratio DFNum DFDen Prob $>$ F

$\begin{array}{llll}5.9803 & 1 & 22.58 & 0.0227\end{array}$

t Test
2.4455
Oneway Analysis of Measurement (wt \%) By Sampler Type of Material=SME Simulant, Analytical Block=3, Analyte $=\mathrm{MgO}(\mathrm{wt} \%)$, Target $(\mathrm{wt} \%)=1.014$

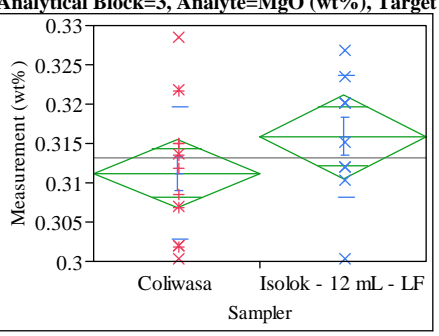

Excluded Rows

1

Oneway Anova

Summary of Fit

Rsquare $\quad 0.079746$

Adj Rsquare $\quad 0.039735$

Root Mean Square Error $\quad 0.008152$

Mean of Response $\quad 0.313087$

Observations (or Sum Wgts)

t Test

Isolok - $12 \mathrm{~mL}$ - LF-Coliwasa

Assuming equal variances

$\begin{array}{lrlr}\text { Difference } & 0.00470 & \text { t Ratio } & 1.411773 \\ \text { Std Err Dif } & 0.00333 \text { DF } & 23 \\ \text { Upper CL Dif } & 0.01158 & \text { Prob }>|t| & 0.1714 \\ \text { Lower CL Dif } & -0.00219 & \text { Prob }>\mathrm{t} & 0.0857 \\ \text { Confidence } & 0.95 & \text { Prob }<\mathrm{t} & 0.9143\end{array}$

Analysis of Variance

Source DF Sum of Squares Mean Square F Ratio Prob $>$ F

$\begin{array}{lrrrrr}\text { Sampler } & 1 & 0.00013246 & 0.000132 & 1.9931 & 0.1714\end{array}$

$\begin{array}{llll}\text { Error } & 23 & 0.00152852 & 0.000066\end{array}$

C. Total $24 \quad 0.00166098$

Means for Oneway Anova

$\begin{array}{lrrrrr}\text { Level } & \text { Number } & \text { Mean } & \text { Std Error } & \text { Lower 95\% } & \text { Upper 95\% } \\ \text { Coliwasa } & 15 & 0.311208 & 0.00210 & 0.30685 & 0.31556\end{array}$

$\begin{array}{llllll}\text { Isolok - } 12 \mathrm{~mL} \text { - LF } & 15 & 0.311208 & 0.00210 & 0.30685 & 0.3155 \\ & & 0.315906 & 0.00258 & 0.31057 & 0.32124\end{array}$

Std Error uses a pooled estimate of error variance

Means and Std Deviations

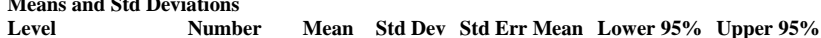

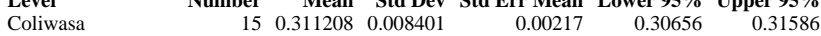

$\begin{array}{lllllll}\text { Isolok - } 12 \mathrm{~mL} \text { - LF } & 10 & 0.315906 & 0.007749 & 0.00245 & 0.31036 & 0.32145\end{array}$

t Test

Isolok - $12 \mathrm{~mL}$ - LF-Coliwasa

Assuming unequal variances

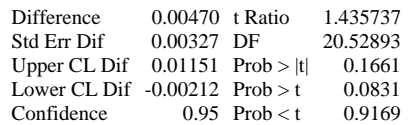

Tests that the Variances are Equal

Level Count Std Dev MeanAbsDif to Mean MeanAbsDif to Median

$\begin{array}{lrrrr}\text { Coliwasa } & 15 & 0.0084013 & 0.0067806 & 0.0068543\end{array}$

$\begin{array}{lllll}\text { Isolok - } 12 \mathrm{~mL} \text { - LF } & 10 & 0.0077486 & 0.0067806 & 0.0068543\end{array}$

Test F Ratio DFNum DFDen p-Value

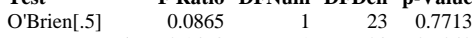

$\begin{array}{lllll}\text { Brown-Forsythe } & 0.1478 & 1 & 23 & 0.7042 \\ & 0.1241 & 1 & 23 & 0.7278\end{array}$

$\begin{array}{lllll}\text { Levene } & 0.1241 & 1 & 23 & 0.7278 \\ \text { Batlett } & 0.0676 & 1 & & 0.7948\end{array}$

$\begin{array}{lllll}\text { F Test 2-sided } & 1.1756 & 14 & 9 & 0.8291\end{array}$

Welch Anova testing Means Equal, allowing Std Devs Not Equal

F Ratio DFNum DFDen Prob $>$ F

$\begin{array}{llll}2.0613 & 1 & 20.529 & 0.1661\end{array}$

t Test 


\section{Exhibit B3. Statistical Comparisons for High-Rheology (Phase 2) Simulant Testing (screened data)}

Oneway Analysis of Measurement (wt \%) By Sampler Type of Material=SME Simulant, Analytical Block $=3$, Analyte $=\mathrm{NiO}(\mathrm{wt} \%)$, Target $(\mathrm{wt} \%)=0.41$

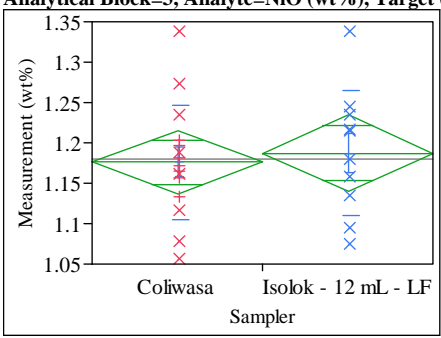

Excluded Rows

Oneway Anova

Summary of Fit

Rsquare

Root Mean Square Error $\quad 0.073779$

Mean of Response 1.180676

Observations (or Sum Wgis)

Test

Isolok - $12 \mathrm{~mL}$ - LF-Coliwasa

Assuming equal variances

Difference $\quad 0.01137$ t Ratio $\quad 0.377408$

$\begin{array}{lrrr}\text { Std Err Dif } & 0.03012 \text { DF } & 23 \\ \text { Upper CL Dif } & 0.07368 & \text { Prob }>|t| & 0.7093\end{array}$

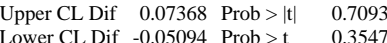

$\begin{array}{lrl} & \\ \text { Confidence } & 0.95 \text { Prob }<\mathrm{t} & 0.6453\end{array}$

Analysis of Variance

Source DF Sum of Squares Mean Square F Ratio Prob $>$ F

$\begin{array}{llllll}\text { Sampler } & 1 & 0.00077534 & 0.000775 & 0.1424 & 0.7093\end{array}$

$\begin{array}{lll}\text { Error } & 23 & 0.12519852 \\ \text { C. Total } & 24 & 0.12597386\end{array}$

Means for Oneway Anova

Level Number Mean Std Error Lower 95\% Upper 95\%

$\begin{array}{lrrrrr}\text { Level } & \text { Number } & \text { Mean } & \text { Std Error } & \text { Lower 95\% } & \text { Upper 95\% } \\ \text { Coliwasa } & 15 & 1.17613 & 0.01905 & 1.1367 & 1.2155 \\ \text { Isolok - 12 mL - LF } & 10 & 1.18750 & 0.02333 & 1.1392 & 1.2358\end{array}$

Std Error uses a pooled estimate of error variance

Means and Std Deviations

$\begin{array}{lrrrrrr}\text { Level } & \text { Number } & \text { Mean } & \text { Std Dev } & \text { Std Err Mean } & \text { Lower 95\% } & \text { Upper 95\% } \\ \text { Coliwasa } & 15 & 1.17613 & 0.070971 & 0.01832 & 1.1368 & 1.2154\end{array}$

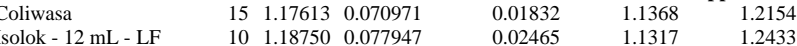

t Test

Isolok - $12 \mathrm{~mL}$ - LF-Coliwasa

Assuming unequal variances

Difference $\quad 0.01137$ t Ratio $\quad 0.37011$

Std Err Dif $\quad 0.03071 \mathrm{DF} \quad 18.13608$

Upper CL Dif 0.07586 Prob $>|t| 0.7156$

Lower CL Dif -0.05313 Prob $>\mathrm{t} \quad 0.3578$

$\begin{array}{lrl}\text { Confidence } & 0.95 \text { Prob }<\mathrm{t} & 0.6422\end{array}$

Tests that the Variances are Equal

$\begin{array}{lrrrr}\text { Level } & \text { Count } & \text { Std Dev } & \text { MeanAbsDif to Mean } & \text { MeanAbsDif to Median } \\ \text { Coliwasa } & 15 & 0.0709713 & 0.0502440 & 0.0503910 \\ \text { Isolok - 12 mL - LF } & 10 & 0.0779470 & 0.0605710 & 0.0605710\end{array}$

Test F Ratio DFNum DFDen p-Value

O'Brien[5] 0.0941

$\begin{array}{lllll} & 0.0941 & 1 & 23 & 0.7618 \\ \text { O'Brien[.5] } & 0.2784 & 1 & 23 & 0.6028\end{array}$

$\begin{array}{lllll}\text { Brown-Forsythe } & 0.2784 & 1 & 23 & 0.6028\end{array}$

$\begin{array}{lllll}\text { Levene } & 0.2906 & 1 & 23 & 0.5950 \\ \text { Bartlett } & 0.0932 & 1 & & 0.7602\end{array}$

$\begin{array}{lllll}\text { F Test 2-sided } & 1.2062 & 9 & 14 & 0.7266\end{array}$

Welch Anova testing Means Equal, allowing Std Devs Not Equal

F Ratio DFNum DFDen Prob $>$ F

$\begin{array}{llll}0.1370 & 1 & 18.136 & 0.7156\end{array}$

t Test
0.3701
Oneway Analysis of Measurement (wt \%) By Sampler Type of Material=SME Simulant, Analytical Block $=3$, Analyte $=\mathrm{SiO} 2(\mathrm{wt} \%)$, Target $(\mathrm{wt} \%)=50.985$

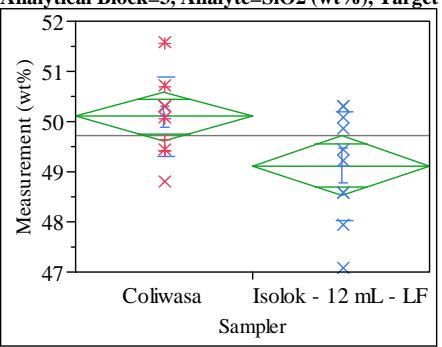

Excluded Rows

1

Oneway Anova

Summary of Fit

Rsquare $\quad 0.230937$

Adj Rsquare $\quad 0.1975$

Root Mean Square Error $\quad 0.917224$

Mean of Response 49.70877

Observations (or Sum Wgts)

t Test

Isolok - $12 \mathrm{~mL}$ - LF-Coliwasa

Assuming equal variances

Difference $\quad-0.9841$ t Ratio $\quad-2.62803$

Std Err Dif $\quad 0.3745$ DF 23

$\begin{array}{lrr}\text { Upper CL Dif } & -0.2095 \text { Prob }>|t| & 0.0150\end{array}$

Lower CL Dif -1.7587 Prob $>t \quad 0.9925$

$\begin{array}{lrl}\text { Confidence } & 0.95 \text { Prob }<\mathrm{t} & 0.0075\end{array}$

Analysis of Variance

Source DF Sum of Squares Mean Square F Ratio Prob > F

$\begin{array}{llllll}\text { Sampler } & 1 & 5.810457 & 5.81046 & 6.9065 & 0.0150\end{array}$

$\begin{array}{llll}\text { Error } & 23 & 19.349884 & 0.84130\end{array}$

$\begin{array}{lll}C \text { Total } 24 & 25.160341\end{array}$

Means for Oneway Anova

Level Number Mean Std Error Lower 95\% Upper 95\%

$\begin{array}{lrrrrr}\text { Coliwasa } & 15 & 50.1024 & 0.23683 & 49.612 & 50.592\end{array}$

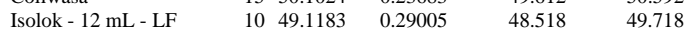

Std Error uses a pooled estimate of error variance

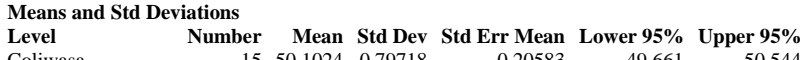

$\begin{array}{llllllr}\text { Coliwasa } & 15 & 50.1024 & 0.79718 & 0.20583 & 49.661 & 50.544 \\ \text { Isolok - 12 mL - LF } & 10 & 49.1183 & 1.07770 & 0.34080 & 48.347 & 49.889\end{array}$

t Test

Isolok - $12 \mathrm{~mL}$ - LF-Coliwasa

Assuming unequal variances

$\begin{array}{lrlr}\text { Difference } & -0.9841 & \text { t Ratio } & -2.47173 \\ \text { Std Err Dif } & 0.3981 & \text { DF } & 15.44253 \\ \text { Upper CL Dif } & -0.1376 & \text { Prob }>|t| & 0.0255 \\ \text { Lower CL Dif } & -1.8306 & \text { Prob }>t & 0.9872 \\ \text { Confidence } & 0.95 & \text { Prob }<\mathrm{t} & 0.0128\end{array}$

Tests that the Variances are Equal

$\begin{array}{lrrrr}\text { Level } & \text { Count } & \text { Std Dev } & \text { MeanAbsDif to Mean } & \text { MeanAbsDif to Median } \\ \text { Coliwasa } & 15 & 0.797179 & 0.6161184 & 0.6132660\end{array}$

$\begin{array}{lllll}\text { Coliwasa } & 15 & 0.797179 & 0.6161184 & 0.6132660 \\ \text { Isolok - 12 mL - LF } & 10 & 1.077701 & 0.8728344 & 0.8557200\end{array}$

Test F Ratio DFNum DFDen p-Value

$\begin{array}{lrrrr}\text { O'Brien[.5] } & 1.3154 & 1 & 23 & 0.2632\end{array}$

$\begin{array}{lllll}\text { Brown-Forsythe } & 1.1958 & 1 & 23 & 0.2855\end{array}$

$\begin{array}{lllll}\text { Levene } & 1.5064 & 1 & 23 & 0.2321 \\ \text { Brtlett } & 0.9800 & 1 & & 0.3222\end{array}$

$\begin{array}{lllll}\text { F Test 2-sided } & 1.8276 & 9 & 14 & 0.3008\end{array}$

Welch Anova testing Means Equal, allowing Std Devs Not Equal

F Ratio DFNum DFDen Prob $>$ F

$\begin{array}{rrrr}6.1094 & 1 & 15.443 & 0.0255\end{array}$

t Test 


\section{Exhibit B3. Statistical Comparisons for High-Rheology (Phase 2) Simulant Testing (screened data)}

Oneway Analysis of Measurement (wt \%) By Sampler Type of Material=SME Simulant, Analytical Block=3, Analyte $=$ Sum of Oxides $(w t \%)$, Target $(w t \%)=99.553$

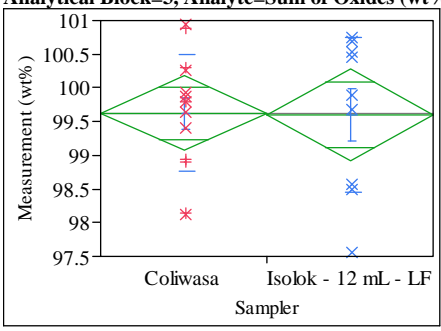

Exc

Oneway Anova

Summary of Fit

Rsquare $\quad 0.00015$

Adj Rsquare $\quad-0.04746$

Root Mean Square Error $\quad 0.987082$

Mean of Response 99.61727

Observations (or Sum Wgts)

Test

Isolok - $12 \mathrm{~mL}$ - LF-Coliwasa

Assuming equal variances

$\begin{array}{lrlr}\text { Difference } & -0.02378 & \mathrm{t} \text { Ratio } & -0.05638 \\ \text { Std Err Dif } & 0.42173 \text { DF } & 21 \\ \text { Upper CL Dif } & 0.85325 & \text { Prob }>|t| & 0.9556 \\ \text { Lower CL Dif } & -0.90081 & \text { Prob }>t & 0.5222 \\ \text { Confidence } & 0.95 \text { Prob }<\mathrm{t} & 0.4778\end{array}$

Analysis of Variance

Source DF Sum of Squares Mean Square F Ratio Prob $>$ F

$\begin{array}{lllllll}\text { Sampler } & 1 & 0.003097 & 0.003097 & 0.0032 & 0.9556\end{array}$

$\begin{array}{lrrr}\text { Sampler } & 1 & 0.003097 & 0.003097 \\ \text { Error } & 21 & 20.460963 & 0.974332\end{array}$

$\begin{array}{lll}\text { C. Total } & 22 & 20.464060\end{array}$

Means for Oneway Anova

$\begin{array}{lrrrrr}\text { Level } & \text { Number } & \text { Mean } & \text { Std Error } & \text { Lower 95\% } & \text { Upper 95\% } \\ \text { Coliwasa } & 14 & 99.6266 & 0.26381 & 99.078 & 100.18\end{array}$

Isolok - 12 mL - LF $\quad 999.6028 \quad 0.32903 \quad 98.919 \quad 100.29$

Std Error uses a pooled estimate of error variance

Means and Std Deviations

Level Number Mean Std Dev Std Err Mean Lower 95\% Upper 95\%

$\begin{array}{lrrrrrr}\text { Coliwasa } & 14 & 99.6266 & 0.86910 & 0.23228 & 99.125 & 100.13 \\ \text { Isolok - 12 mL - LF } & 9 & 99.6028 & 1.15334 & 0.38445 & 98.716 & 100.49\end{array}$

t Test

Isolok - $12 \mathrm{~mL}$ - LF-Coliwasa

Assuming unequal variances

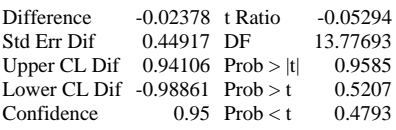

Tests that the Variances are Equal

Level Count Std Dev MeanAbsDif to Mean MeanAbsDif to Median

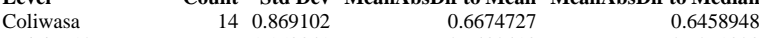

Isolok - $12 \mathrm{~mL}$ - LF $\quad 9 \begin{array}{llll}1.153341 & 0.6674727 & 0.9430413\end{array}$

Test F Ratio DFNum DFDen p-Value

$\begin{array}{lrrrr}\text { O'Brien[.5] } & 1.2584 & 1 & 21 & 0.2746\end{array}$

$\begin{array}{lllll}\text { Brown-Forsythe } & 1.1401 & 1 & 21 & 0.2978\end{array}$

$\begin{array}{lllll}\text { Levene } & 1.4046 & 1 & 21 & 0.2492\end{array}$

$\begin{array}{lllll} & 0.7789 & 1 & \text {. } & 0.3775\end{array}$

Welch Anova testing Means Equal, allowing Std Devs Not Equal

F Ratio DFNum DFDen Prob $>$ F

$\begin{array}{rrrr}0.0028 & 1 & 13.777 & 0.9585\end{array}$

t Test

0.0529
Oneway Analysis of Measurement (wt \%) By Sampler Type of Material=SME Simulant, Analytical Block=3, Analyte $=\mathrm{TiO} 2(w t \%)$, Target $(w t \%)=0.0711$

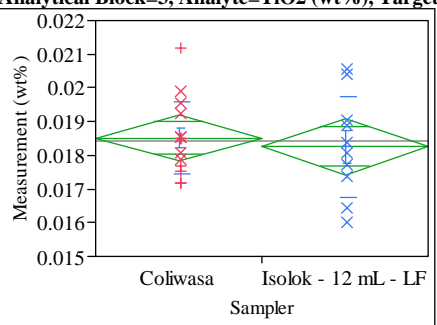

Excluded Rows

Oneway Anova

Summary of Fit

Rsquare $\quad 0.011403$

Adj Rsquare $\quad-0.03158$

Root Mean Square Error $\quad 0.001261$

Mean of Response $\quad 0.018409$

Observations (or Sum Wgts) 25

t Test

Isolok - $12 \mathrm{~mL}$ - LF-Coliwasa

Assuming equal variances

$\begin{array}{lrlr}\text { Difference } & -0.00027 & \text { t Ratio } & -0.51507 \\ \text { Std Err Dif } & 0.00051 & \text { DF } & 23 \\ \text { Upper CL Dif } & 0.00080 & \text { Prob }>|t| & 0.6114 \\ \text { Lower CL Dif } & -0.00133 & \text { Prob }>t & 0.6943 \\ \text { Confidence } & 0.95 & \text { Prob }<\mathrm{t} & 0.3057\end{array}$

Analysis of Variance

Source DF Sum of Squares Mean Square F Ratio Prob $>$ F

$\begin{array}{llllll}\text { Sampler } & 1 & 0.00000042 & 4.2202 \mathrm{e}-7 & 0.2653 & 0.6114\end{array}$

$\begin{array}{llll}\text { Error } & 23 & 0.00003659 & 1.5908 \mathrm{e}-6\end{array}$

C. Total $24 \quad 0.00003701$

Means for Oneway Anova

Level Number Mean Std Error Lower 95\% Upper 95\%

$\begin{array}{lrrrrr}\text { Coliwasa } & 15 & 0.018515 & 0.00033 & 0.01784 & 0.01919 \\ \text { Isolok - 12 mL - LF } & 10 & 0.018250 & 0.00040 & 0.01742 & 0.01907\end{array}$

Std Error uses a pooled estimate of error variance

Means and Std Deviations

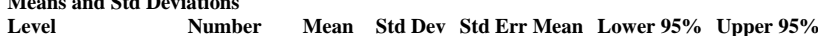

$\begin{array}{lrrrrrr}\text { Coliwasa } & 15 & 0.018515 & 0.001079 & 0.00028 & 0.01792 & 0.01911\end{array}$

$\begin{array}{lllllll}\text { Isolok - } 12 \mathrm{~mL} \text { - LF } & 10 & 0.018250 & 0.001501 & 0.00047 & 0.01718 & 0.01932\end{array}$

t Test

Isolok - $12 \mathrm{~mL}$ - LF-Coliwasa

Assuming unequal variances

$\begin{array}{lrlr}\text { Difference } & -0.00027 & \text { t Ratio } & -0.4818 \\ \text { Std Err Dif } & 0.00055 & \text { DF } & 15.11554 \\ \text { Upper CL Dif } & 0.00091 & \text { Prob }>|t| & 0.6369 \\ \text { Lower CL Dif } & -0.00144 & \text { Prob }>\text { t } & 0.6816 \\ \text { Confidence } & 0.95 & \text { Prob }<\mathrm{t} & 0.3184\end{array}$

Tests that the Variances are Equal

Level Count Std Dev MeanAbsDif to Mean MeanAbsDif to Median

$\begin{array}{lrrrr}\text { Coliwasa } & 15 & 0.0010791 & 0.0007562 & 0.0007562\end{array}$

$\begin{array}{lllll}\text { Isolok - } 12 \mathrm{~mL} \text { - LF } & 10 & 0.0015013 & 0.00011659 & 0.00011659\end{array}$

Test F Ratio DFNum DFDen p-Value

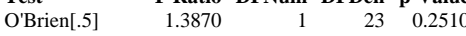

$\begin{array}{lllll}\text { Brown-Forsythe } & 1.6021 & 1 & 23 & 0.2183\end{array}$

$\begin{array}{lllll}\text { Levene } & 1.6073 & 1 & 23 & 0.2176\end{array}$

$\begin{array}{lllll}\text { Bartlett } & 1.1763 & 1 & \text {.j } & 0.278\end{array}$

Welch Anova testing Means Equal, allowing Std Devs Not Equal

F Ratio DFNum DFDen Prob $>$ F

$\begin{array}{llll}0.2321 & 1 & 15.116 & 0.6369\end{array}$

t Test

0.4818 


\section{Exhibit B3. Statistical Comparisons for High-Rheology (Phase 2) Simulant Testing (screened data)}

Oneway Analysis of Measurement (wt \%) By Sampler Type of Material=SME Simulant, Analytical Block $=3$, Analyte $=\mathrm{ZnO}(\mathrm{wt} \%)$, Target $(\mathrm{wt} \%)=0.0958$

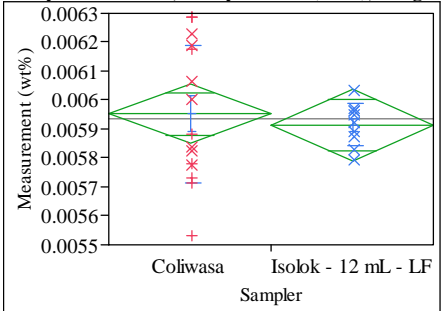

Excluded Rows

Oneway Anova

Summary of Fit

Adj Rsquare

-0.000191
0.005937

Observations (or Sum Wgts)

t Test

Isolok - $12 \mathrm{~mL}$ - LF-Coliwas

Assuming equal variances

Difference $\quad-3.76 \mathrm{e}-5$ t Ratio $\quad-0.48035$

Std Err Dif $7.818 \mathrm{e}-5$ DF 23

Upper CL Dif 0.00012 Prob $>|t| \quad 0.6355$

$\begin{array}{lll}\text { Lower CL Dif }-0.00020 & \text { Prob }>t & 0.6822\end{array}$

$\begin{array}{lrr}\text { Confidence } & 0.95 \text { Prob }<\mathrm{t} & 0.3178\end{array}$

Analysis of Variance

Source DF Sum of Squares Mean Square F Ratio Prob > F

$\begin{array}{llllll}\text { Sampler } & 1 & 8.46068 \mathrm{e}-9 & 8.4607 \mathrm{e}-9 & 0.2307 & 0.6355\end{array}$

$\begin{array}{llll}\text { Error } & 23 & 8.43386 \mathrm{e}-7 & 3.6669 \mathrm{e}-8\end{array}$

C. Total $24 \quad 8.51846 \mathrm{e}-7$

Means for Oneway Anova

$\begin{array}{lrrrrr}\text { Level } & \text { Number } & \text { Mean } & \text { Std Error } & \text { Lower 95\% } & \text { Upper 95\% } \\ \text { Coliwasa } & 15 & 0.005952 & 0.00005 & 0.00585 & 0.00605\end{array}$

$\begin{array}{lllllr}\text { Coliwasa } & 15 & 0.005952 & 0.00005 & 0.00585 & 0.00605 \\ \text { Isolok - 12 mL - LF } & 10 & 0.005915 & 0.00006 & 0.00579 & 0.00604\end{array}$

Std Error uses a pooled estimate of error variance

Means and Std Deviations

Level Number Mean Std Dev Std Err Mean Lower 95\% Upper 95\%

$\begin{array}{lllllll}\text { Coliwasa } & 15 & 0.005952 & 0.000238 & 6.16 \mathrm{e}-5 & 0.00582 & 0.00608\end{array}$

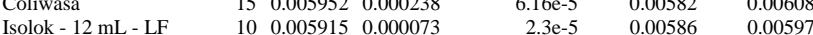

t Test

Isolok - $12 \mathrm{~mL}$ - LF-Coliwas

Assuming unequal variances

$\begin{array}{lrlr}\text { Difference } & -3.76 \mathrm{e}-5 & \mathrm{t} \text { Ratio } & -0.57148 \\ \text { Std Err Dif } & 6.571 \mathrm{e}-5 \text { DF } & 17.63839 \\ \text { Upper CL Dif } & 0.00010 & \text { Prob }>|\mathrm{t}| & 0.5749 \\ \text { Lower CL Dif } & -0.00018 & \text { Prob }>\mathrm{t} & 0.7126 \\ \text { Confidence } & 0.95 & \text { Prob }<\mathrm{t} & 0.2874\end{array}$

Tests that the Variances are Equal

Level Count Std Dev MeanAbsDif to Mean MeanAbsDif to Median

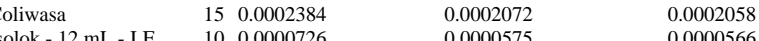

Test $\quad$ F Ratio DFNum DFDen p-Value

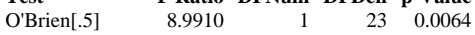

Brown-Forsythe $\begin{array}{rrrr}8.9996 & 1 & 23 & 0.0021\end{array}$

$\begin{array}{lllll}\text { Levene } & 18.5808 & 1 & 23 & 0.0003\end{array}$

$\begin{array}{lrrrr}\text { Bartlett } & 10.8069 & 1 & \text {. } & 0.0010 \\ \text { F Test 2-sided } & 10.7721 & 14 & 9 & 0.0011\end{array}$

Welch Anova testing Means Equal, allowing Std Devs Not Equal

F Ratio DFNum DFDen Prob > F

$\begin{array}{llll}0.3266 & 1 & 17.638 & 0.5749\end{array}$

t Test

0.5715
Oneway Analysis of Measurement (wt \%) By Sampler Type of Material=SME Simulant, Analytical Block=4, Analyte=Al2O3 (wt \%), Target $(w t \%)=10.869$

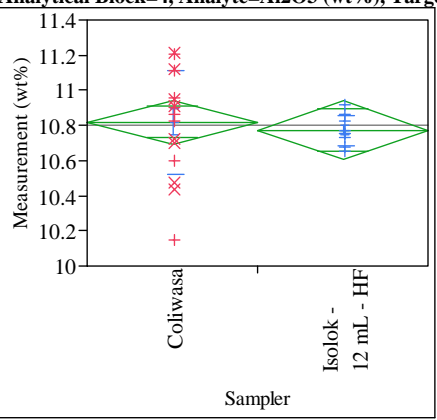

Excluded Rows

Oneway Anova

Summary of Fit

Rsquare

Adj Rsquare

$\begin{array}{lr}\text { Adj Rsquare } & -0.03466 \\ \text { Root Mean Square Error } & 0.244745\end{array}$

Mean of Response

0.244745

10.80114
25

Isolok - $12 \mathrm{~mL}$ - HF-Coliwasa

Assuming equal variances

Difference $\quad-0.04514$ t Ratio $\quad-0.44263$

$\begin{array}{lrrr}\text { Std Err Dif } & 0.10198 \text { DF } & 23 \\ \text { Upper } & \text { D } & \end{array}$

$\begin{array}{lrll}\text { Upper CL Dif } & 0.16582 & \text { Prob }>|t| & 0.6622 \\ \text { Lower CL Dif } & -0.25609 & \text { Prob }>t & 0.6689\end{array}$

$\begin{array}{lrrr}\text { Lower CL Dif } & -0.25609 & \text { Prob }>t & 0.6689 \\ \text { Confidence } & 0.95 & \text { Prob }<t & 0.3311\end{array}$

Analysis of Variance

Source DF Sum of Squares Mean Square F Ratio Prob $>$ F

$\begin{array}{lrrrrr}\text { Sampler } & 1 & 0.0117357 & 0.011736 & 0.1959 & 0.6622\end{array}$

$\begin{array}{llll}\text { Error } & 23 & 1.3777045 & 0.059900\end{array}$

Means for Oneway Anova

Level Number Mean Std Error Lower 95\% Upper 95\%

$\begin{array}{lrrrrr}\text { Coliwasa } & 16 & 10.8174 & 0.06119 & 10.691 & 10.944 \\ \text { Isolok - 12 mL - HF } & 9 & 10.7722 & 0.08158 & 10.603 & 10.941\end{array}$

Std Error uses a pooled estimate of error variance

Means and Std Deviations

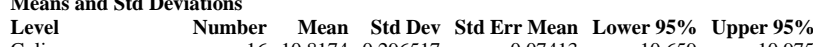

$\begin{array}{lrrrrrr}\text { Coliwasa } & 16 & 10.8174 & 0.296517 & 0.07413 & 10.659 & 10.975 \\ \text { Isolok - 12 mL - HF } & 9 & 10.7722 & 0.085782 & 0.02859 & 10.706 & 10.838\end{array}$

t Test

Isolok - $12 \mathrm{~mL}$ - HF-Coliwasa

Assuming unequal variances

$\begin{array}{lrlr}\text { Difference } & -0.04514 & \text { t Ratio } & -0.56811 \\ \text { Std Err Dif } & 0.07945 & \text { DF } & 19.00681 \\ \text { Upper CL Dif } & 0.12115 & \text { Prob }>|t| & 0.5766 \\ \text { Lower CL Dif } & -0.21143 & \text { Prob }>\text { t } & 0.7117 \\ \text { Confidence } & 0.95 & \text { Prob }<\mathrm{t} & 0.2883\end{array}$

Tests that the Variances are Equal

$\begin{array}{lrrrr}\text { Level } & \text { Count } & \text { Std Dev } & \text { MeanAbsDif to Mean } & \text { MeanAbsDif to Median } \\ \text { Coliwasa } & 16 & 0.2965171 & 0.2314638 & 0.2243781\end{array}$

$\begin{array}{lrlll}\text { Coliwasa } & 16 & 0.2965171 & 0.2314638 & 0.224378 \\ \text { Isolok - } 12 \mathrm{~mL}-\mathrm{HF} & 9 & 0.0857823 & 0.0657826 & 0.0629833\end{array}$

Test F Ratio DFNum DFDen p-Value

$\begin{array}{lllll}\text { O'Brien[.5] } & 3.6171 & 1 & 23 & 0.0698\end{array}$

$\begin{array}{lllll}\text { Brown-Forsythe } & 5.6533 & 1 & 23 & 0.0261\end{array}$

$\begin{array}{lll}1 & 23 & 0.026 \\ -1 & 23 & 0.0115\end{array}$

$\begin{array}{lrrrr}\text { Levene } & 7.5519 & 1 & 23 & 0.0115 \\ \text { Bartlett } & 10.4992 & 1 & 8 & 0.0012\end{array}$

$\begin{array}{lrrrr}\text { Bartlett } & 10.4992 & 1 & \dot{0} & 0.0012 \\ \text { F Test 2-sided } & 11.9482 & 15 & 8 & 0.0014\end{array}$

Welch Anova testing Means Equal, allowing Std Devs Not Equal

F Ratio DFNum DFDen Prob $>$ F

$\begin{array}{llll}0.3227 & 1 & 19.007 & 0.5766\end{array}$

t Test 


\section{Exhibit B3. Statistical Comparisons for High-Rheology (Phase 2) Simulant Testing (screened data)}

Oneway Analysis of Measurement (wt \%) By Sampler Type of Material=SME Simulant, Analytical Block=4, Analyte $=\mathrm{BaO}(\mathrm{wt} \%)$, Target $(\mathrm{wt} \%)=0.0919$

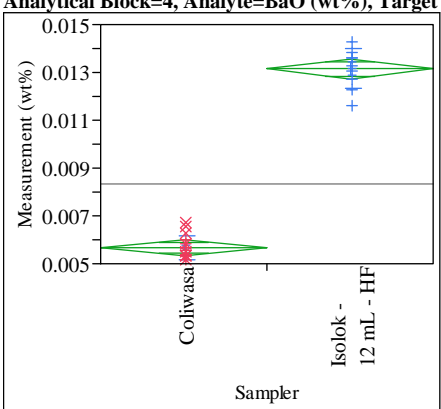

Exch

Oneway Anova

Summary of Fit

Adj Rsquare

0.971775

Error $\quad 0.000625$

0.008359

Observations (or Sum Wgts)

t Test

Isolok - $12 \mathrm{~mL}$ - HF-Coliwasa

Assuming equal variances

Difference $\quad 0.007485$ t Ratio $\quad 28.76321$

Std Err Dif $\quad 0.000260$ DF 23

Upper CL Dif 0.008023 Prob $>|t|<.0001$

Lower CL Dif 0.006947 Prob $>\mathrm{t} \quad<.0001$

$\begin{array}{lrl}\text { Confidence } & 0.95 \text { Prob }<\mathrm{t} & 1.0000\end{array}$

Analysis of Variance

Source DF Sum of Squares Mean Square F Ratio Prob $>$ F

$\begin{array}{lllllll}\text { Sampler } & 1 & 0.00032271 & 0.000323 & 827.3221 & <.0001\end{array}$

$\begin{array}{llll}\text { Error } & 23 & 0.00000897 & 3.901 \mathrm{e}-7\end{array}$

C. Total $24 \quad 0.00033168$

Means for Oneway Anova

$\begin{array}{lrrrrr}\text { Level } & \text { Number } & \text { Mean } & \text { Std Error } & \text { Lower 95\% } & \text { Upper 95\% } \\ \text { Coliwasa } & 16 & 0.005665 & 0.00016 & 0.00534 & 0.00599\end{array}$

$\begin{array}{lrrrrr}\text { Coliwasa } & 16 & 0.005665 & 0.00016 & 0.00534 & 0.00599 \\ \text { Isolok - 12 mL - HF } & 9 & 0.013150 & 0.00021 & 0.01272 & 0.01358\end{array}$

Std Error uses a pooled estimate of error variance

Means and Std Deviations

Level Number Mean Std Dev Std Err Mean Lower 95\% Upper 95\%

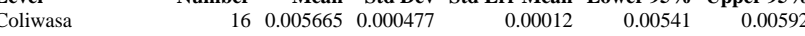

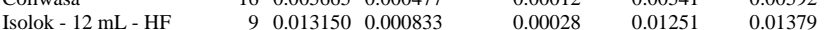

tTest

Isolok - $12 \mathrm{~mL}$ - HF-Coliwasa

Assuming unequal variances

Difference $\quad 0.007485$ t Ratio 24.76103

Std Err Dif $\quad 0.000302$ DF 11.02552

Upper CL Dif 0.008150 Prob $>|t| r .0001$

Lower CL Dif 0.006820 Prob $>\mathrm{t} \quad<.000$

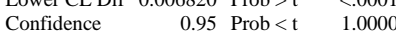

Tests that the Variances are Equal

Level Count Std Dev MeanAbsDif to Mean MeanAbsDif to Median

$\begin{array}{lllll}\text { Coliwasa } & 16 & 0.0004773 & 0.0003843 & 0.0003712\end{array}$

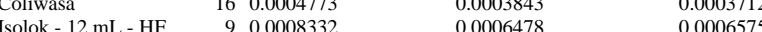

Test F Ratio DFNum DFDen p-Value

$\begin{array}{lrrrr}\text { Test } & \text { F Ratio } & \text { DFNum } & \text { DFDen } & \text { p-Value } \\ \text { O'Brien[.5] } & 3.4242 & 1 & 23 & 0.0771 \\ \text { Brown-Forsythe } & 3.0970 & 1 & 23 & 0.0917 \\ \text { Levene } & 3.2499 & 1 & 23 & 0.0845 \\ \text { Bartlett } & 3.2913 & 1 & . & 0.0696 \\ \text { F Test 2-sided } & 3.0474 & 8 & 15 & 0.0600\end{array}$

Welch Anova testing Means Equal, allowing Std Devs Not Equal

F Ratio DFNum DFDen Prob $>$ F

$\begin{array}{rrrr}613.1085 & 1 & 11.026 & <.0001\end{array}$

t Test

24.7610
Oneway Analysis of Measurement (wt \%) By Sampler Type of Material=SME Simulant, Analytical Block $=4$, Analyte $=\mathrm{Cr} 2 \mathrm{O} 3(\mathrm{wt} \%)$, Target $(\mathrm{wt} \%)=\mathbf{0 . 0 9 9 8}$

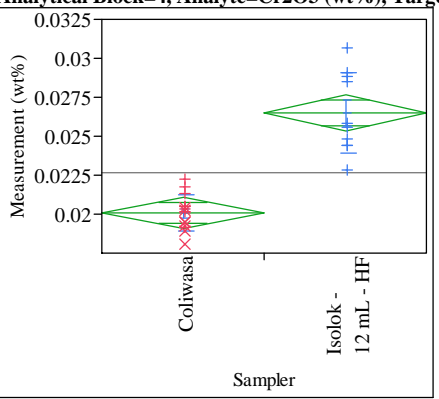

Excluded Rows

Summary of Fit

Rsquare $\quad 0.760293$

Adj Rsquare $\quad 0.74987$

Root Mean Square Error $\quad 0.00184$

$\begin{array}{lr}\text { Mean of Response } & 0.022649 \\ \text { Observations (or Sum Wgts) } & 25\end{array}$

t Test

Isolok - $12 \mathrm{~mL}$ - HF-Coliwasa

Assuming equal variances

Difference $\quad 0.006416$ t Ratio 8.541096

$\begin{array}{lrlr}\text { Std Err Dif } & 0.000751 \text { DF } & 23 \\ \text { Upper CL } & 0.007970 \text { Prob }>|t| & <.0001\end{array}$

$\begin{array}{llll}\text { Upper CL Dif } & 0.007970 & \text { Prob }>|t| & <.0001 \\ \text { Lower CL Dif } & 0.004862 & \text { Prob }>t & <.0001\end{array}$

$\begin{array}{lrlr}\text { Lower CL Dif } & 0.004862 & \text { Prob }>t & <.0001 \\ \text { Confidence } & 0.95 & \text { Prob }<t & 1.0000\end{array}$

Analysis of Variance

Source DF Sum of Squares Mean Square F Ratio Prob $>$ F

$\begin{array}{lrrrrr}\text { Sampler } & 1 & 0.00024702 & 0.000247 & 72.9503 & <.0001\end{array}$

$\begin{array}{llll}\text { Error } & 23 & 0.00007788 & 3.386 \mathrm{e}-6\end{array}$

C. Total $24 \quad 0.00032491$

Means for Oneway Anova

Level Number Mean Std Error Lower 95\% Upper 95\%

$\begin{array}{llllrr}\text { Coliwasa } & 15 & 0.020082 & 0.00048 & 0.01910 & 0.02107\end{array}$

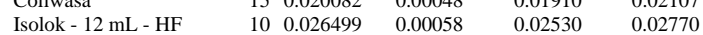

Std Error uses a pooled estimate of error variance

Means and Std Deviations

Level Number Mean Std Dev Std Err Mean Lower 95\% Upper 95\%

$\begin{array}{llllllr}\text { Coliwasa } & 15 & 0.020082 & 0.001132 & 0.00029 & 0.01946 & 0.02071 \\ \text { Isolok - 12 mL - HF } & 10 & 0.026499 & 0.002581 & 0.00082 & 0.02465 & 0.02835\end{array}$

t Test

Isolok - $12 \mathrm{~mL}$ - HF-Coliwasa

Assuming unequal variances

Difference $\quad 0.006416$ t Ratio 7.401764

Std Err Dif $\quad 0.000867$ DF 11.33631

Upper CL Dif 0.008318 Prob $>|t| \quad<.0001$

Lower CL Dif 0.004515 Prob $>t \quad<.0001$

$\begin{array}{lll}\text { Confidence } & 0.95 \text { Prob }<\mathrm{t} \quad 1.0000\end{array}$

Tests that the Variances are Equal

Level Count Std Dev MeanAbsDif to Mean MeanAbsDif to Median

$\begin{array}{lrrrr}\text { Coliwasa } & 15 & 0.0011319 & 0.0008653 & 0.0008477 \\ \text { Isolok - 12 mL - HF } & 10 & 0.0025808 & 0.0022158 & 0.0020901\end{array}$

Test F Ratio DFNum DFDen p-Value

$\begin{array}{lrrrr}\text { O'Brien[.5] } & 9.4751 & 1 & 23 & 0.0053\end{array}$

\begin{tabular}{lrrrr} 
O'Brien[.5] & 9.4751 & 1 & 23 & 0.0053 \\
Brown-Forsythe & 7.2119 & 1 & 23 & 0.0132 \\
\hline & 14.3379 & 1 & 23 & 0.0010
\end{tabular}

$\begin{array}{lrrrr}\text { Levene } & 14.3379 & 1 & 23 & 0.0010 \\ \text { Bartlett } & 7.1855 & 1 & . & 0.0073\end{array}$

$\begin{array}{lllll}\text { F Test 2-sided } \quad 5.1990 & 9 & 14 & 0.0064\end{array}$

Welch Anova testing Means Equal, allowing Std Devs Not Equal

F Ratio DFNum DFDen Prob $>$ F

$\begin{array}{llll}54.7861 & 1 & 11.336 & <.0001\end{array}$

t Test

7.4018 


\section{Exhibit B3. Statistical Comparisons for High-Rheology (Phase 2) Simulant Testing (screened data)}

Oneway Analysis of Measurement (wt \%) By Sampler Type of Material=SME Simulant, Analytical Block=4, Analyte $=\mathrm{CuO}(w t \%)$, Target $(w t \%)=0.0504$

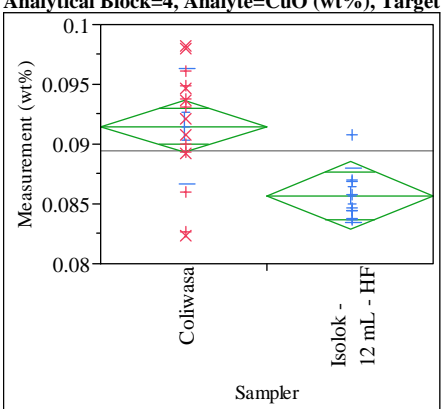

Excluded Rows

Oneway Anova

Summary of Fit

Adj Rsquare $\quad 0.332554$

Root Mean Square Error $\quad 0.004106$

Mean of Response

0.089414

t Test

Isolok - $12 \mathrm{~mL}$ - HF-Coliwasa

Assuming equal variances

Difference $\quad-0.00579$ t Ratio $\quad-3.38522$

Std Err Dif $\quad 0.00171$ DF 23

Upper CL Dif -0.00225 Prob $>|t| \quad 0.0025$

Lower CL Dif -0.00933 Prob $>t \quad 0.9987$

$\begin{array}{lrl}\text { Confidence } & 0.95 \text { Prob }<\mathrm{t} \quad 0.0013\end{array}$

Analysis of Variance

Source DF Sum of Squares Mean Square F Ratio Prob $>$ F

$\begin{array}{lllllll}\text { Sampler } & 1 & 0.00019324 & 0.000193 & 11.4597 & 0.0025\end{array}$

$\begin{array}{llll}\text { Error } & 23 & 0.00038785 & 0.000017\end{array}$

C. Total $24 \quad 0.00058109$

Means for Oneway Anova

$\begin{array}{lrrrrr}\text { Level } & \text { Number } & \text { Mean } & \text { Std Error } & \text { Lower 95\% } & \text { Upper 95\% } \\ \text { Coliwasa } & 16 & 0.091499 & 0.00103 & 0.08938 & 0.09362\end{array}$

$\begin{array}{lrrrrr}\text { Coliwasa } & 16 & 0.091499 & 0.00103 & 0.08938 & 0.09362 \\ \text { Isolok - 12 mL - HF } & 9 & 0.085707 & 0.00137 & 0.08287 & 0.08854\end{array}$

Std Error uses a pooled estimate of error variance

Means and Std Deviations

Level Number Mean Std Dev Std Err Mean Lower 95\% Upper 95\%

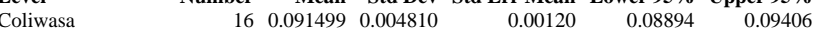

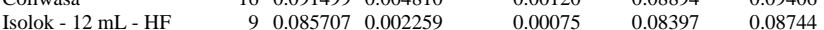

Test

Isolok - $12 \mathrm{~mL}$ - HF-Coliwasa

Assuming unequal variances

Difference $\quad-0.00579$ t Ratio $\quad-4.08239$

Std Err Dif $\quad 0.00142$ DF $\quad 22.56562$

Upper CL Dif -0.00285 Prob $>|t| r \mid r .0005$

Lower CL Dif -0.00873 Prob $>\mathrm{t} \quad 0.9998$

$\begin{array}{lrl}\text { Confidence } & 0.95 \text { Prob }<\mathrm{t} & 0.0002\end{array}$

Tests that the Variances are Equal

$\begin{array}{lrrrr}\text { Level } & \text { Count } & \text { Std Dev } & \text { MeanAbsDif to Mean } & \text { MeanAbsDif to Median } \\ \text { Coliwasa } & 16 & 0.0048098 & 0.0038111 & 0.0037476 \\ \text { Isolok - } 12 \mathrm{~mL} \text { - HF } & 9 & 0.0022593 & 0.0016783 & 0.0015717\end{array}$

Test F Ratio DFNum DFDen p-Value

$\begin{array}{lrrrr}\text { O'Brien[.5] } & 2.9524 & 1 & 23 & 0.0992 \\ \text { Brown-Forsythe } & 3.6917 & 1 & 23 & 0.0672\end{array}$

$\begin{array}{lllll}\text { Levene } & 4.6321 & 1 & 23 & 0.0421\end{array}$

$\begin{array}{lrrrr}\text { Bartlett } & 4.5904 & 1 & \text {. } & 0.0322 \\ \text { F Test 2-sided } & 4.5321 & 15 & 8 & 0.0370\end{array}$

Welch Anova testing Means Equal, allowing Std Devs Not Equal

F Ratio DFNum DFDen Prob $>$ F

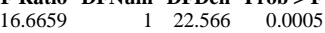

t Test

4.0824
Oneway Analysis of Measurement ( $w t \%)$ By Sampler Type of Material=SME Simulant, Analytical Block=4, Analyte $=$ Na2O (wt $\%)$, Target $(w t \%)=11.659$

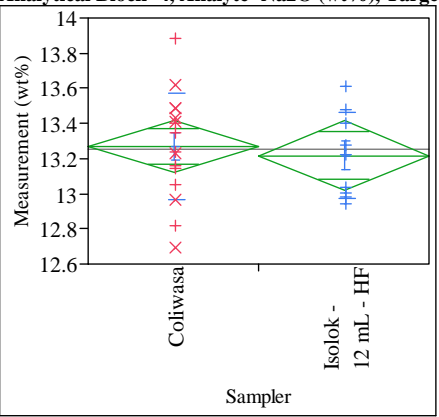

Excluded Rows

Oneway Anova

ummary of Fi

Rsquare

Adj Rsquare

Root Mean Square Error $\quad \begin{array}{r}-0.0352 \\ 0.283396\end{array}$

Mean of Response

Observations (or Sum Wgts) r $\begin{array}{r}13.25 \\ \hline\end{array}$

t Test

Isolok - $12 \mathrm{~mL}$ - HF-Coliwasa

Assuming equal variances

$\begin{array}{lrlr}\text { Difference } & -0.05064 & \text { t Ratio } & -0.42889 \\ \text { Std Err Dif } & 0.11808 & \text { DF } & 23 \\ \text { Upper CL Dif } & 0.19363 & \text { Prob }>|t| & 0.6720 \\ \text { Lower CL Dif } & -0.29491 & \text { Prob }>t & 0.6640 \\ \text { Confidence } & 0.95 & \text { Prob }<\mathrm{t} & 0.3360\end{array}$

Analysis of Variance

Source DF Sum of Squares Mean Square F Ratio Prob > F

$\begin{array}{lrrrrr}\text { Sampler } & 1 & 0.0147731 & 0.014773 & 0.1839 & 0.6720\end{array}$

$\begin{array}{llll}\text { Error } & 23 & 1.8472061 & 0.080313\end{array}$

C. Total $24 \quad 1.8619792$

Means for Oneway Anova

Level Number Mean Std Error Lower 95\% Upper 95\%

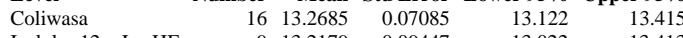

$\begin{array}{lrrrrr}\text { Isolok - } 12 \text { mL - HF } & 9 & 13.2179 & 0.09447 & 13.022 & 13.413\end{array}$

Std Error uses a pooled estimate of error variance

Means and Std Deviations

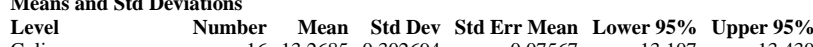

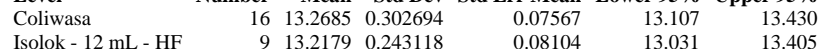

t Test

Isolok - $12 \mathrm{~mL}$ - HF-Coliwasa

Assuming unequal variances

$\begin{array}{lrlr}\text { Difference } & -0.05064 & \text { t Ratio } & -0.45675 \\ \text { Std Err Dif } & 0.11088 & \text { DF } & 19.94582 \\ \text { Upper CL Dif } & 0.18068 & \text { Prob }>|t| & 0.6528 \\ \text { Lower CL Dif } & -0.28197 & \text { Prob }>\mathrm{t} & 0.6736 \\ \text { Confidence } & 0.95 & \text { Prob }<\mathrm{t} & 0.3264\end{array}$

Tests that the Variances are Equal

$\begin{array}{lrrrr}\text { Level } & \text { Count } & \text { Std Dev } & \text { MeanAbsDif to Mean } & \text { MeanAbsDif to Median } \\ \text { Coliwasa } & 16 & 0.3026940 & 0.2350575 & 0.2350575\end{array}$

$\begin{array}{lrlll}\text { Isolok - } 12 \mathrm{~mL}-\mathrm{HF} & 16 & 0.3026940 & 0.2350575 & 0.2350575 \\ & 9 & 0.2431180 & 0.2013679 & 0.2066933\end{array}$

Test F Ratio DFNum DFDen p-Value

$\begin{array}{lllll}\text { O'Brien[.5] } & 0.4862 & 1 & 23 & 0.4926\end{array}$

$\begin{array}{lllll}\text { Brown-Forsythe } & 0.1806 & 1 & 23 & 0.6748\end{array}$

$\begin{array}{llll}0.2513 & 1 & 23 & 0.6748\end{array}$

$\begin{array}{lllll}\text { Levene } & 0.2513 & 1 & 23 & 0.6209\end{array}$

$\begin{array}{lrrrr}\text { Bartlett } & 0.4540 & 1 & \dot{0} & 0.5004 \\ \text { F Test 2-sided } & 1.5501 & 15 & 8 & 0.5409\end{array}$

Welch Anova testing Means Equal, allowing Std Devs Not Equal

F Ratio DFNum DFDen Prob $>$ F

$\begin{array}{llll}0.2086 & 1 & 19.946 & 0.6528\end{array}$

t Test 


\section{Exhibit B3. Statistical Comparisons for High-Rheology (Phase 2) Simulant Testing (screened data)}

Oneway Analysis of Measurement (wt \%) By Sampler Type of Material=SME Simulant, Analytical Block=4, Analyte $=\mathrm{SiO} 2(w t \%)$, Target $(w t \%)=50.985$

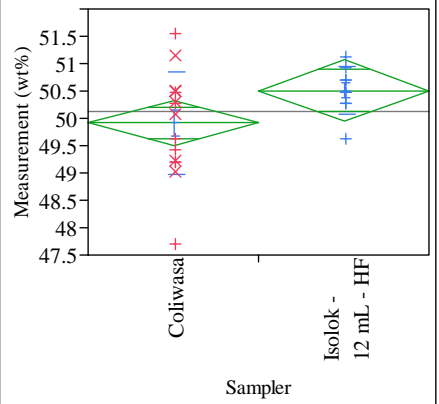

Excluded Rows

Oneway Anova

Summary of Fit

Rsquare

Adj Rsquare

$\begin{array}{lr}\text { Mean of Response } & 50.12808 \\ \text { Observations (or Sum Wgts) } & 25\end{array}$

t Test

Isolok - $12 \mathrm{~mL}$ - HF-Coliwasa

Assuming equal variances

Difference $\quad 0.5987$ t Ratio 1.788655

$\begin{array}{lrlr}\text { Std Err Dif } & 0.3347 & \text { DF } & 23 \\ \text { Upper CL Dif } & 1.2911 & \text { Prob }>|t| & 0.0869\end{array}$

$\begin{array}{rrll}\text { Upper CL Dif } & 1.2911 & \text { Prob }>|t| & 0.0869 \\ \text { Lower CL Dif } & -0.0937 & \text { Prob }>t & 0.0434\end{array}$

$\begin{array}{lrlll} & \text { Lower CL Dif } & -0.0937 & \text { Prob }>t & 0.0434 \\ \text { Confidence } & 0.95 & \text { Prob }<\mathrm{t} & 0.9566\end{array}$

Analysis of Variance

Source DF Sum of Squares Mean Square F Ratio Prob $>$ F

$\begin{array}{lrrrrr}\text { Sampler } & 1 & 2.064672 & 2.06467 & 3.1993 & 0.0869\end{array}$

$\begin{array}{lrrr}\text { Error } & 23 & 14.843136 & 0.64535 \\ \text { C. Total } & 24 & 16.907808 & \end{array}$

Means for Oneway Anova

Level Number Mean Std Error Lower 95\% Upper 95\%

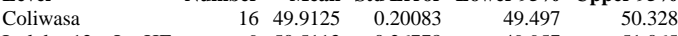

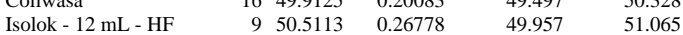

Std Error uses a pooled estimate of error variance

Means and Std Deviations

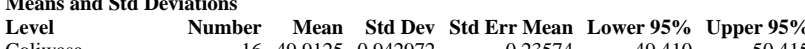

$\begin{array}{lrrrrrr}\text { Coliwasa } & \text { Number } & \text { Mean } & \text { Std Dev } & \text { Std Err Mean } & \text { Lower 95\% } & \text { Upper 95\% } \\ \text { Isolok - 12 mL - HF } & 9 & 49.9125 & 0.942972 & 0.23574 & 49.410 & 50.415 \\ & 9 & 50.5113 & 0.433762 & 0.14459 & 50.178 & 50.845\end{array}$

t Test

Isolok - $12 \mathrm{~mL}$ - HF-Coliwasa

Assuming unequal variances

Difference $\quad 0.59871$ t Ratio 2.164909

Std Err Dif $\quad 0.27655$ DF 22.4509

Upper CL Dif 1.17157 Prob $>|t| \quad 0.0413$

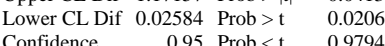

Tests that the Variances are Equal

$\begin{array}{lrrrr}\text { Level } & \text { Count } & \text { Std Dev } & \text { MeanAbsDif to Mean } & \text { MeanAbsDif to Median } \\ \text { Coliwasa } & 16 & 0.9429720 & 0.7537690 & 0.7353844 \\ \text { Isolok - 12 mL - HF } & 9 & 0.4337618 & 0.3116511 & 0.3090100\end{array}$

Test F Ratio DFNum DFDen p-Value

$\begin{array}{lrrrr}\text { O'Brien[.5] } & 2.1577 & 1 & 23 & 0.1554\end{array}$

$\begin{array}{lllll}\text { Brown-Forsythe } & 3.7816 & 1 & 23 & 0.0641\end{array}$

$\begin{array}{lll}1 & 23 & 0.0641 \\ -1 & 23 & 0.0306\end{array}$

$\begin{array}{lllll}\text { Levene } & 5.3083 & 1 & 23 & 0.0306\end{array}$

$\begin{array}{lrrrr}\text { Bartlett } & 4.8149 & 1 & \text {. } & 0.0282 \\ \text { F Test 2-sided } & 4.7260 & 15 & 8 & 0.0325\end{array}$

Welch Anova testing Means Equal, allowing Std Devs Not Equal

F Ratio DFNum DFDen Prob $>$ F

$\begin{array}{llll}4.6868 & 1 & 22.451 & 0.0413\end{array}$

t Test
Oneway Analysis of Measurement (wt \%) By Sampler Type of Material=SME Simulant, Analytical Block $=4$, Analyte=Sum of Oxides $(w t \%)$, Target $(w t \%)=99.553$

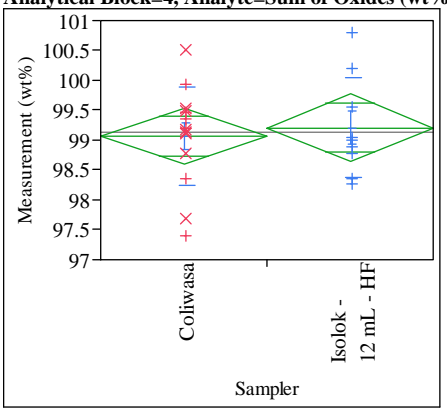

Excluded Rows

3

Oneway Anova

Summary of Fit

$\begin{array}{ll}\text { Adj Rsquare } & -0.03959 \\ \text { Root Mean Square Error } & 0.826709\end{array}$

Mean of Response

Observations (or Sum Wgts)

t Test

Isolok - $12 \mathrm{~mL}$ - HF-Coliwasa

Assuming equal variances

Difference $\quad 0.14228$ t Ratio $\quad 0.402819$

Std Err Dif $\quad 0.35321$ DF 21

Upper CL Dif 0.87682 Prob $>|t| \quad 0.6912$

$\begin{array}{lrl}\text { Lower CL Dif }-0.59226 & \text { Prob }>t & 0.3456\end{array}$

$\begin{array}{lrl}\text { Confidence } & 0.95 \text { Prob }<\mathrm{t} & 0.6544\end{array}$

Analysis of Variance

Source DF Sum of Squares Mean Square F Ratio Prob $>$ F

$\begin{array}{lrrrrr}\text { Sampler } & 1 & 0.110898 & 0.110898 & 0.1623 & 0.6912\end{array}$

$\begin{array}{llll}\text { Error } & 21 & 14.352390 & 0.683447\end{array}$

C. Total $22 \quad 14.463288$

Means for Oneway Anova

Level Number Mean Std Error Lower 95\% Upper 95\%

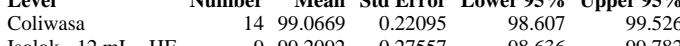

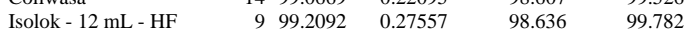

Std Error uses a pooled estimate of error variance

Means and Std Deviations

Level Number Mean Std Dev Std Err Mean Lower 95\% Upper 95\%

$\begin{array}{lrrrrr}\text { Coliwasa } & 1499.0669 & 0.819638 & 0.21906 & 98.594 & 99.540\end{array}$

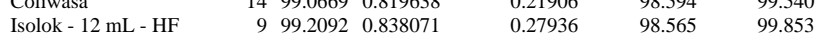

t Test

Isolok - $12 \mathrm{~mL}$ - HF-Coliwasa

Assuming unequal variances

$\begin{array}{lrlr}\text { Difference } & 0.14228 & \mathrm{t} \text { Ratio } & 0.400784 \\ \text { Std Err Dif } & 0.35500 & \text { DF } & 16.925 \\ \text { Upper CL Dif } & 0.89152 & \text { Prob }>|t| & 0.6936 \\ \text { Lower CL Dif } & -0.60696 & \text { Prob }>t & 0.3468 \\ \text { Confidence } & 0.95 & \text { Prob }<\mathrm{t} & 0.6532\end{array}$

Tests that the Variances are Equal

Level Count Std Dev MeanAbsDif to Mean MeanAbsDif to Median

$\begin{array}{lllll}\text { Coliwasa } & 14 & 0.8196380 & 0.5842977 & 0.5630615\end{array}$

Isolok - $12 \mathrm{~mL}$ - HF $\quad 90.8380712 \quad 0.6483275$

Test F Ratio DFNum DFDen p-Value

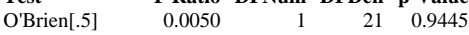

Brown-Forsythe $0.0173 \quad 1 \quad 21 \quad 0.8966$

$\begin{array}{lllll}\text { Levene } & 0.0815 & 1 & 21 & 0.7781\end{array}$

$\begin{array}{lllll}\text { Bartlett } & 0.0047 & 1 & 0.9455 \\ \text { F Test 2-sided } & 1.0455 & 8 & 13 & 0.9049\end{array}$

Welch Anova testing Means Equal, allowing Std Devs Not Equal

F Ratio DFNum DFDen Prob $>$ F

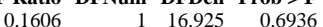

t Test

0.4008 


\section{Exhibit B3. Statistical Comparisons for High-Rheology (Phase 2) Simulant Testing (screened data)}

Oneway Analysis of Measurement (wt \%) By Sampler Type of Material=SME Simulant, Analytical Block=4, Analyte $=\mathrm{TiO} 2(\mathrm{wt} \%)$, Target $(\mathrm{wt} \%)=0.0711$

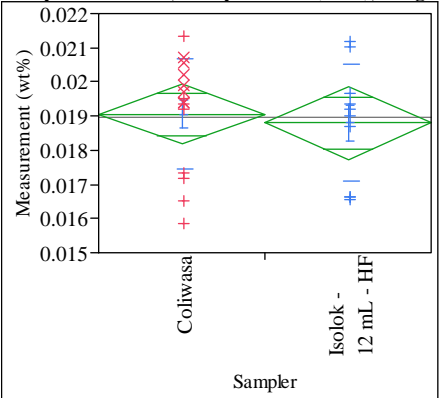

Exchich

Oneway Anova

Summary of Fit

Adj Rsquare

-0.001644
0.018954

Observations (or Sum Wgts) $\quad 25$

Test

Isolok - $12 \mathrm{~mL}$ - HF-Coliwasa

Assuming equal variances

Difference $\quad-0.00027$ t Ratio $\quad-0.40019$

$\begin{array}{lrr}\text { Std Err Dif } \quad 0.00067 \text { DF } & 23\end{array}$

Upper CL Dif 0.00112 Prob $>|t| \quad 0.6927$

Lower CL Dif -0.00166 Prob $>t \quad 0.6536$

$\begin{array}{lrl}\text { Confidence } & 0.95 \text { Prob }<\mathrm{t} & 0.3464\end{array}$

Analysis of Variance

Source DF Sum of Squares Mean Square F Ratio Prob $>$ F

$\begin{array}{llllll}\text { Sampler } & 1 & 0.00000043 & 4.3271 \mathrm{e}-7 & 0.1602 & 0.6927\end{array}$

$\begin{array}{llll}\text { Error } 23 & 0.00006214 & 2.7018 \mathrm{e}-6\end{array}$

C. Total $24 \quad 0.00006257$

Means for Oneway Anova

$\begin{array}{lrrrrr}\text { Level } & \text { Number } & \text { Mean } & \text { Std Error } & \text { Lower 95\% } & \text { Upper 95\% } \\ \text { Coliwasa } & 15 & 0.019062 & 0.00042 & 0.01818 & 0.01994\end{array}$

$\begin{array}{lrrrrr}\text { Coliwasa } & 15 & 0.019062 & 0.00042 & 0.01818 & 0.01994 \\ \text { Isolok - 12 mL - HF } & 10 & 0.018793 & 0.00052 & 0.01772 & 0.01987\end{array}$

Std Error uses a pooled estimate of error variance

Means and Std Deviations

Level Number Mean Std Dev Std Err Mean Lower 95\% Upper 95\%

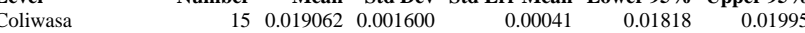

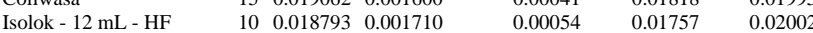

Test

Isolok - $12 \mathrm{~mL}$ - HF-Coliwasa

Assuming unequal variances

$\begin{array}{lrlr}\text { Difference } & -0.00027 & \mathrm{t} \text { Ratio } & -0.3947 \\ \text { Std Err Dif } & 0.00068 & \text { DF } & 18.51901 \\ \text { Upper CL Dif } & 0.00116 & \text { Prob }>|t| & 0.6976 \\ \text { Lower CL Dif } & -0.00170 & \text { Prob }>\mathrm{t} & 0.6512 \\ \text { Confidence } & 0.95 & \text { Prob }<\mathrm{t} & 0.3488\end{array}$

Tests that the Variances are Equal

Level Count Std Dev MeanAbsDif to Mean MeanAbsDif to Median

$\begin{array}{lllll}\text { Coliwasa } & 15 & 0.0015999 & 0.0012437 & 0.0011320\end{array}$

Isolok - $12 \mathrm{~mL}$ - HF $\quad 10 \quad 0.0017096 \quad 0.0013337 \quad 0.0012894$

Test F Ratio DFNum DFDen p-Value

$\begin{array}{lrrrr}\text { O'Brien[.5] } & 0.0786 & 1 & 23 & 0.7816\end{array}$

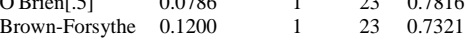

$\begin{array}{lllll}\text { Levene } & 0.0529 & 1 & 23 & 0.8201\end{array}$

$\begin{array}{lllll}\text { Bartlett } & 0.0465 & 1 & & 0.8293 \\ \text { F Test 2-sided } & 1.1418 & 9 & 14 & 0.7949\end{array}$

Welch Anova testing Means Equal, allowing Std Devs Not Equal

F Ratio DFNum DFDen Prob $>$ F

$\begin{array}{lrrr}0.1558 & 1 & 18.519 & 0.6976\end{array}$

t Test

0.3947
Oneway Analysis of Measurement (wt \%) By Sampler Type of Material=SME Simulant, Analytical Block=5, Analyte=Al2O3 $(w t \%)$, Target $(w t \%)=10.869$

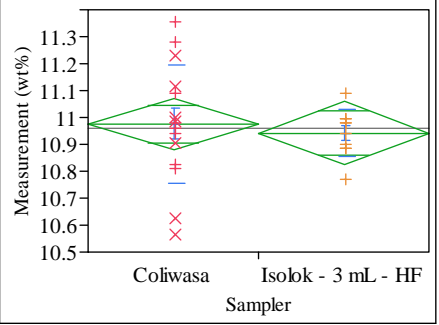

Excluded Rows

Oneway Anova

Summary of Fit

Rsquare $\quad 0.008778$

Adj Rsquare $\quad-0.03432$

Root Mean Square Error $\quad 0.181176$

Mean of Response 10.96212

Observations (or Sum Wgs)

t Test

Isolok - $3 \mathrm{~mL}$ - HF-Coliwasa

Assuming equal variances

Difference $\quad-0.03338$ t Ratio $\quad-0.45131$

$\begin{array}{lrr}\text { Std Err Dif } & 0.07396 \text { DF } & 23 \\ \text { Up } & & \end{array}$

$\begin{array}{lll}\text { Upper CL Dif } & 0.11963 \text { Prob }>|t| & 0.6560 \\ \text { Low } & & 0.6720\end{array}$

$\begin{array}{lrll}\text { Lower CL Dif } & -0.18639 & \text { Prob }>t & 0.6720 \\ \text { Confidence } & 0.95 & \text { Prob }<\mathrm{t} & 0.3280\end{array}$

Analysis of Variance

Source DF Sum of Squares Mean Square F Ratio Prob $>$ F

$\begin{array}{llllll}\text { Sampler } & 1 & 0.00668581 & 0.006686 & 0.2037 & 0.6560\end{array}$

$\begin{array}{llll}\text { Error } & 23 & 0.75496856 & 0.032825 \\ \text { C. Total } & 24 & 0.76165437 & \end{array}$

Means for Oneway Anova

Level Number Mean Std Error Lower 95\% Upper 95\%

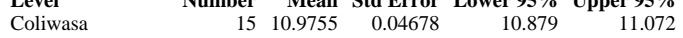

$\begin{array}{llllll}\text { Isolok - } 3 \mathrm{~mL} \text { - HF } & 10 & 10.9421 & 0.05729 & 10.824 & 11.061\end{array}$

Std Error uses a pooled estimate of error variance

Means and Std Deviations

Level Number Mean Std Dev Std Err Mean Lower 95\% Upper 95\%

$\begin{array}{lllllll}\text { Coliwasa } & 15 & 10.9755 & 0.221375 & 0.05716 & 10.853 & 11.098\end{array}$

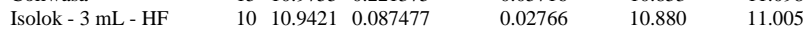

t Test

Isolok - $3 \mathrm{~mL}$ - HF-Coliwasa

Assuming unequal variances

Difference $\quad-0.03338$ t Ratio $\quad-0.52568$

Std Err Dif $\quad 0.06350$ DF 19.6493

Upper CL Dif 0.09923 Prob $>|\mathrm{t}| \quad 0.6050$

Lower CL Dif -0.16599 Prob $>t \quad 0.6975$

$\begin{array}{lll}\text { Confidence } & 0.95 \text { Prob }<\mathrm{t} \quad 0.3025\end{array}$

Tests that the Variances are Equal

$\begin{array}{lrrrr}\text { Level } & \text { Count } & \text { Std Dev } & \text { MeanAbsDif to Mean } & \text { MeanAbsDif to Median } \\ \text { Coliwasa } & 15 & 0.2213754 & 0.1614053 & 0.1612373 \\ \text { Isolok - 3 mL - HF } & 10 & 0.0874766 & 0.0661325 & 0.0661325\end{array}$

Test F Ratio DFNum DFDen p-Value

$\begin{array}{lrrrr}\text { O'Brien[.5] } & 3.8008 & 1 & 23 & 0.0635\end{array}$

$\begin{array}{lllll}\text { OBrien[.5] } & 3.8008 & 1 & 23 & 0.0635 \\ \text { Brown-Forsythe } & 3.8494 & 1 & 23 & 0.0620\end{array}$

\begin{tabular}{lllll} 
Levene & 3.9088 & 1 & 23 & 0.0601 \\
Bartlett & 7.1628 & 1 & & 0.0074 \\
\hline
\end{tabular}

$\begin{array}{lrrrr}\text { F Test 2-sided } & 6.4044 & 14 & 9 & 0.0083\end{array}$

Welch Anova testing Means Equal, allowing Std Devs Not Equal

F Ratio DFNum DFDen Prob $>$ F

$\begin{array}{llll}0.2763 & 1 & 19.649 & 0.6050\end{array}$

t Test 


\section{Exhibit B3. Statistical Comparisons for High-Rheology (Phase 2) Simulant Testing (screened data)}

Oneway Analysis of Measurement (wt \%) By Sampler Type of Material=SME Simulant, Analytical Block=5, Analyte $=$ B2O3 (wt $\%)$, Target $(w t \%)=4.259$

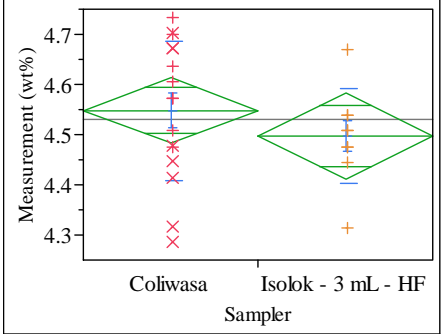

Exc

Excluded Rows

Oneway Anova
Summary of Fit

Rsquare $\quad 0.039951$

Adj Rsquare $\quad-0.00179$

Root Mean Square Error $\quad 0.125067$

Mean of Response 4.529755

Observations (or Sum Wgts) 25

t Test

Isolok - $3 \mathrm{~mL}$ - HF-Coliwasa

Assuming equal variances

$\begin{array}{lrlr}\text { Difference } & -0.05098 & \mathrm{t} \text { Ratio } & -0.97832 \\ \text { Std Err Dif } & 0.05211 & \text { DF } & 23 \\ \text { Upper CL Dif } & 0.05682 & \text { Prob }>|t| & 0.3381 \\ \text { Lower CL Dif } & -0.15878 & \text { Prob }>t & 0.8310 \\ \text { Confidence } & 0.95 & \text { Prob }<t & 0.1690\end{array}$

Analysis of Variance

Source DF Sum of Squares Mean Square F Ratio Prob $>$ F

$\begin{array}{llllll}\text { Sampler } & 1 & 0.01497104 & 0.014971 & 0.9571 & 0.3381\end{array}$

$\begin{array}{llll}\text { Error } & 23 & 0.35976113 & 0.015642\end{array}$

C. Total $24 \quad 0.37473217$

$\begin{array}{lrrrrr}\begin{array}{l}\text { Means for Oneway Anova } \\ \text { Level }\end{array} & & & & \\ \text { Number } & \text { Mean } & \text { Std Error } & \text { Lower 95\% } & \text { Upper 95\% } \\ \text { Coliwasa } & 16 & 4.54811 & 0.03127 & 4.4834 & 4.6128 \\ \text { Isolok - 3 mL - HF } & 9 & 4.49713 & 0.04169 & 4.4109 & 4.5834\end{array}$

Std Error uses a pooled estimate of error variance

Means and Std Deviations

Level $\quad$ Number Mean Std Dev Std Err Mean Lower 95\% Upper 95\%

$\begin{array}{lrrrrrr}\text { Level } & \text { Number } & \text { Mean } & \text { Std Dev } & \text { Std Err Mean } & \text { Lower 95\% } & \text { Upper 95\% } \\ \text { Coliwasa } & 16 & 4.54811 & 0.138867 & 0.03472 & 4.4741 & 4.6221\end{array}$

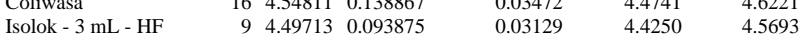

t Test

Isolok - $3 \mathrm{~mL}$ - HF-Coliwasa

Assuming unequal variances

$\begin{array}{lrlr}\text { Difference } & -0.05098 & \mathrm{t} \text { Ratio } & -1.0908 \\ \text { Std Err Dif } & 0.04674 & \text { DF } & 22.02093 \\ \text { Upper CL Dif } & 0.04594 & \text { Prob }>|t| & 0.2872 \\ \text { Lower CL Dif } & -0.14790 & \text { Prob }>t & 0.8564 \\ \text { Confidence } & 0.95 & \text { Prob }<\mathrm{t} & 0.1436\end{array}$

Tests that the Variances are Equal

Level Count Std Dev MeanAbsDif to Mean MeanAbsDif to Median

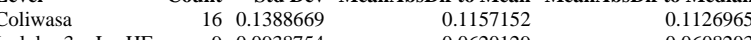

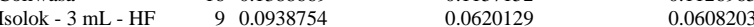

Test F Ratio DFNum DFDen p-Value

$\begin{array}{lrrrr}\text { O'Brien[.5] } & 1.5396 & 1 & 23 & 0.2272\end{array}$

$\begin{array}{lllll}\text { Brown-Forsythe } & 2.6680 & 1 & 23 & 0.1160\end{array}$

$\begin{array}{lllll}\text { Levene } & 3.4448 & 1 & 23 & 0.0763\end{array}$

$\begin{array}{lrrrr} & 1.3819 & 1 & \text {. } & 0.2398 \\ \text { Test 2-sided } & 2.1882 & 15 & 8 & 0.2651\end{array}$

Welch Anova testing Means Equal, allowing Std Devs Not Equal

F Ratio DFNum DFDen Prob $>$ F

$\begin{array}{rrrr}1.1898 & 1 & 22.021 & 0.2872\end{array}$

t Test

1.0908
Oneway Analysis of Measurement (wt \%) By Sampler Type of Material=SME Simulant, Analytical Block=5, Analyte $=\mathrm{CaO}(\mathrm{wt} \%)$, Target $(\mathrm{wt} \%)=1.1462$

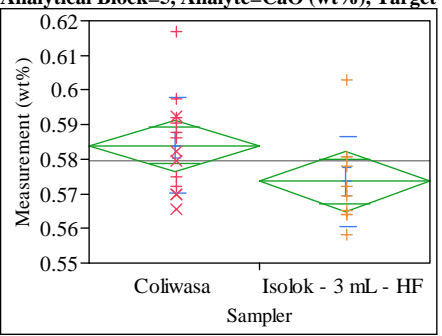

Excluded Rows

Oneway Anova

Summary of Fit

Root 0.098703

0.013434

Observations (or Sum Wgts) \begin{tabular}{rr}
0.579619 \\
\hline
\end{tabular}

t Test

Isolok - $3 \mathrm{~mL}$ - HF-Coliwasa

Assuming equal variances

Difference $\quad-0.01043$ t Ratio -1.8758

$\begin{array}{lrr}\text { Std Err Dif } & 0.00556 \text { DF } & 22 \\ \text { Upper CL } & 0.00110 \text { Prob }>|t| & 0.0740\end{array}$

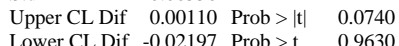

$\begin{array}{lrll}\text { Lower CL Dif } & -0.02197 & \text { Prob }>t & 0.9630 \\ \text { Confidence } & 0.95 & \text { Prob }<\mathrm{t} & 0.0370\end{array}$

Analysis of Variance

Source DF Sum of Squares Mean Square F Ratio Prob $>$ F

$\begin{array}{lrrrrr}\text { Sampler } & 1 & 0.00063507 & 0.000635 & 3.5188 & 0.0740\end{array}$

$\begin{array}{lll}\text { Error } & 22 & 0.00397056 \\ \text { C. Total } & 23 & 0.00460563\end{array}$

Means for Oneway Anova

Level Number Mean Std Error Lower 95\% Upper 95\%

$\begin{array}{lrrrrr}\text { Coliwasa } & 14 & 0.583966 & 0.00359 & 0.57652 & 0.59141\end{array}$

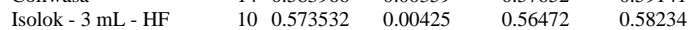

Std Error uses a pooled estimate of error variance

Means and Std Deviations

Level Number Mean Std Dev Std Err Mean Lower 95\% Upper 95\%

$\begin{array}{lllllll}\text { Coliwasa } & 14 & 0.583966 & 0.013793 & 0.00369 & 0.57600 & 0.59193\end{array}$

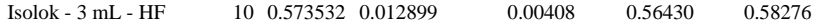

t Test

Isolok - $3 \mathrm{~mL}$ - HF-Coliwasa

Assuming unequal variances

Difference $\quad-0.01043$ t Ratio -1.89782

Std Err Dif $\quad 0.00550$ DF 20.32007

Upper CL Dif 0.00102 Prob $>|t| \quad 0.0720$

Lower CL Dif -0.02189 Prob $>t \quad 0.9640$

$\begin{array}{lrl}\text { Confidence } & 0.95 \text { Prob }<\mathrm{t} \quad 0.0360\end{array}$

Tests that the Variances are Equal

$\begin{array}{lrrrr}\text { Level } & \text { Count } & \text { Std Dev } & \text { MeanAbsDif to Mean } & \text { MeanAbsDif to Median } \\ \text { Coliwasa } & 14 & 0.0137926 & 0.0106939 & 0.0106939\end{array}$

Isolok - $3 \mathrm{~mL}$ - HF $\quad 100.0128991 \quad 0.0096265 \quad 0.0093746$

Test F Ratio DFNum DFDen p-Value

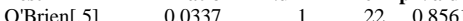

$\begin{array}{lllll}\text { Brown-Forsythe } & 0.1430 & 1 & 22 & 0.7089\end{array}$

Brown-Forsythe $0.1430 \quad 1 \quad 220.7089$

$\begin{array}{lllll}\text { Levene } & 0.1013 & 1 & 22 & 0.7532 \\ \text { Bartlett } & 0.0451 & 1 & & 0.8317\end{array}$

$\begin{array}{llrrr}\text { F Test 2-sided } & 1.1433 & 13 & 9 & 0.8612\end{array}$

Welch Anova testing Means Equal, allowing Std Devs Not Equal

F Ratio DFNum DFDen Prob $>$ F

$\begin{array}{llll}3.6017 & 1 & 20.32 & 0.0720\end{array}$

t Test
1.8978 


\section{Exhibit B3. Statistical Comparisons for High-Rheology (Phase 2) Simulant Testing (screened data)}

Oneway Analysis of Measurement (wt \%) By Sampler Type of Material=SME Simulant, Analytical Block=5, Analyte=Fe2O3 (wt \%), Target $(w t \%)=11.462$

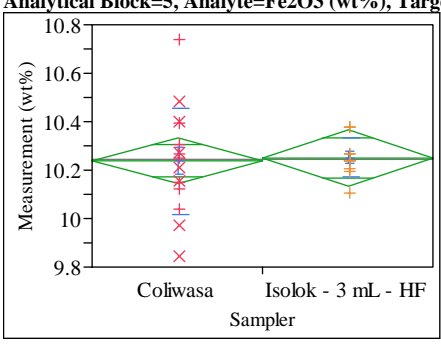

Excluded Rows

Oneway Anova

Summary of Fit

Rsquare

Root Mean Square Error $\quad 0.177945$

Mean of Response $\quad 10.24294$

t Test

Isolok - $3 \mathrm{~mL}$ - HF-Coliwasa

Assuming equal variances

Difference $\quad 0.01334$ t Ratio $\quad 0.183684$

$\begin{array}{lrrr}\text { Std Err Dif } & 0.07265 & \text { DF } & 23 \\ \text { Upper CL Dif } & 0.16362 & \text { Prob }>|t| & 0.8559\end{array}$

Upper CL Dif 0.16362 Prob $>|t| \quad 0.8559$

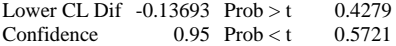

Analysis of Variance

Source DF Sum of Squares Mean Square F Ratio Prob $>$ F

$\begin{array}{lrrrrr}\text { Sampler } & 1 & 0.00106835 & 0.001068 & 0.0337 & 0.8559\end{array}$

$\begin{array}{lll}\text { Error } & 23 & 0.72827857 \\ \text { C. Total } & 24 & 0.72934692\end{array}$

Means for Oneway Anova

Level Number Mean Std Error Lower 95\% Upper 95\%

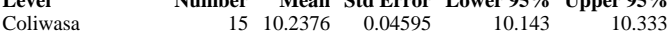

$\begin{array}{llllll}\text { Coliwasa } & 15 & 10.2376 & 0.04595 & 10.143 & 10.333 \\ \text { Isolok - 3 mL - HF } & 10 & 10.2509 & 0.05627 & 10.135 & 10.367\end{array}$

Std Error uses a pooled estimate of error variance

Means and Std Deviations

Level Number Mean Std Dev Std Err Mean Lower 95\% Upper 95\%

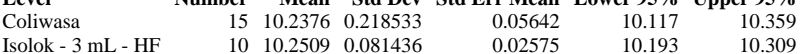

solok - $3 \mathrm{~mL}-\mathrm{HF}$

Test

Isolok - $3 \mathrm{~mL}$ - HF-Coliwasa

Assuming unequal variances

Difference $\quad 0.01334$ t Ratio $\quad 0.215141$

Std Err Dif $\quad 0.06202$ DF 19.14749

Upper CL Dif 0.14309 Prob $>|t| \quad 0.8319$

Lower CL Dif -0.11641 Prob $>t \quad 0.4160$

$\begin{array}{lrl}\text { Confidence } & 0.95 \text { Prob }<\mathrm{t} & 0.5840\end{array}$

Tests that the Variances are Equal

$\begin{array}{lrrrr}\text { Level } & \text { Count } & \text { Std Dev } & \text { MeanAbsDif to Mean } & \text { MeanAbsDif to Median } \\ \text { Coliwasa } & 15 & 0.2185329 & 0.1591097 & 0.1591733 \\ \text { Isolok - 3 mL - HF } & 10 & 0.0814357 & 0.0571880 & 0.0543286\end{array}$

Test F Ratio DFNum DFDen p-Value

O'Brien[5] 2.6496

$\begin{array}{lllll}\text { O'Brien[.5] } & 2.6496 & 1 & 23 & 0.1172\end{array}$

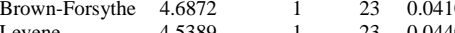

$\begin{array}{lllll}\text { Levene } & 4.5389 & 1 & 23 & 0.0440 \\ \text { Bartlett } & 7.9484 & 1 & & 0.0048\end{array}$

$\begin{array}{lrrrr}\text { F Test 2-sided } & 7.2012 & 1 & \dot{9} & 0.0048 \\ \end{array}$

Welch Anova testing Means Equal, allowing Std Devs Not Equal

F Ratio DFNum DFDen Prob $>$ F

$\begin{array}{llll}0.0463 & 1 & 19.147 & 0.8319\end{array}$

t Test
0.2151
Oneway Analysis of Measurement (wt \%) By Sampler Type of Material=SME Simulant, Analytical Block=5, Analyte $=$ K2O (wt \%), Target $(w t \%)=0.1591$

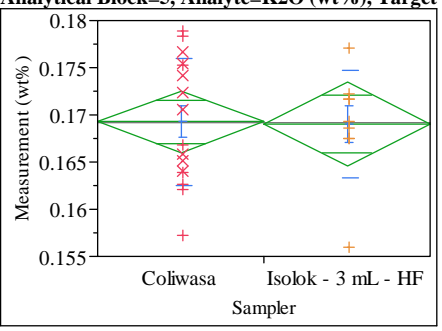

Excluded Rows

1

Oneway Anova

Summary of Fit

Rsquare $\quad 0.000249$

Adj Rsquare $\quad-0.04322$

Root Mean Square Error $\quad 0.006372$

Mean of Response $\quad 0.169174$

Observations (or Sum Wgts) 25

t Test

Isolok - $3 \mathrm{~mL}$ - HF-Coliwasa

Assuming equal variances

$\begin{array}{lrlr}\text { Difference } & -0.00020 & \mathrm{t} \text { Ratio } & -0.07562 \\ \text { Std Err Dif } & 0.00265 & \text { DF } & 23 \\ \text { Upper CL Dif } & 0.00529 & \text { Prob }>|t| & 0.9404 \\ \text { Lower CL Dif } & -0.00569 & \text { Prob }>\mathrm{t} & 0.5298 \\ \text { Confidence } & 0.95 & \text { Prob }<\mathrm{t} & 0.4702\end{array}$

Analysis of Variance

Source DF Sum of Squares Mean Square F Ratio Prob $>$ F

$\begin{array}{lllllll}\text { Sampler } & 1 & 0.00000023 & 2.322 \mathrm{e}-7 & 0.0057 & 0.9404\end{array}$

$\begin{array}{lrrr}\text { Sampler } & 1 & 0.00000023 & 2.322 \mathrm{e}-7 \\ \text { Error } & 23 & 0.00093376 & 0.000041\end{array}$

C. Total $24 \quad 0.00093399$

Means for Oneway Anova

Level Number Mean Std Error Lower 95\% Upper 95\%

$\begin{array}{lrrrrr}\text { Coliwasa } & 16 & 0.169246 & 0.00159 & 0.16595 & 0.17254 \\ \text { Isolok - 3 mL - HF } & 9 & 0.169046 & 0.00212 & 0.16465 & 0.17344\end{array}$

Std Error uses a pooled estimate of error variance

Means and Std Deviations

$\begin{array}{llllll}\text { Means and Std Deviations } & & & & \\ \text { Level } & \text { Number } & \text { Mean } & \text { Std Dev Std Err Mean Lower 95\% Upper 95\% }\end{array}$

$\begin{array}{lrrrrrr}\text { Level } & \text { Number } & \text { Mean } & \text { Std Dev } & \text { Std Err Mean } & \text { Lower 95\% } & \text { Upper 95\% } \\ \text { Coliwasa } & 16 & 0.169246 & 0.006682 & 0.00167 & 0.16569 & 0.17281\end{array}$

$\begin{array}{lrrrrrr}\text { Isolok - 3 mL - HF } & 16 & 0.169246 & 0.006682 & 0.00167 & 0.16569 & 0.17281 \\ & 9 & 0.169046 & 0.005746 & 0.00192 & 0.16463 & 0.17346\end{array}$

t Test

Isolok - $3 \mathrm{~mL}$ - HF-Coliwasa

Assuming unequal variances

Difference $\quad-0.00020$ t Ratio $\quad-0.079$

Std Err Dif $\quad 0.00254$ DF 18.95178

Upper CL Dif 0.00512 Prob $>|\mathrm{t}| \quad 0.9379$

Lower CL Dif -0.00552 Prob $>t \quad 0.5311$

$\begin{array}{lll}\text { Confidence } & 0.95 \text { Prob }<\mathrm{t} \quad 0.4689\end{array}$

Tests that the Variances are Equal

$\begin{array}{lrrrr}\text { Level } & \text { Count } & \text { Std Dev } & \text { MeanAbsDif to Mean } & \text { MeanAbsDif to Median } \\ \text { Coliwasa } & 16 & 0.0066816 & 0.0058724 & 0.0058724\end{array}$

$\begin{array}{lrrrr} & 16 & 0.0066816 & 0.0058724 & 0.0058724 \\ \text { Isolok - } 3 \mathrm{~mL}-\mathrm{HF} & 9 & 0.0057456 & 0.0037030 & 0.0037476\end{array}$

Test F Ratio DFNum DFDen p-Value

$\begin{array}{lrrrr}\text { O'Brien[.5] } & 0.2841 & 1 & 23 & 0.5991\end{array}$

$\begin{array}{lllll}\text { Brown-Forsythe } & 2.2818 & 1 & 23 & 0.1445 \\ & 2.4112 & 1 & 23 & 0.1341\end{array}$

$\begin{array}{lllll}\text { Levene } & 2.4112 & 1 & 23 & 0.1341 \\ \text { Batlett } & 0.2190 & 1 & & 0.6398\end{array}$

$\begin{array}{lllll}\text { F Test 2-sided } & 1.3524 & 15 & 8 & 0.6853\end{array}$

Welch Anova testing Means Equal, allowing Std Devs Not Equal

F Ratio DFNum DFDen Prob $>$ F

$\begin{array}{rrrr}0.0062 & 1 & 18.952 & 0.9379\end{array}$

t Test

0.0790 
SRNL-STI-2011-00693

Revision 0

Exhibit B3. Statistical Comparisons for High-Rheology (Phase 2) Simulant Testing (screened data)

Oneway Analysis of Measurement (wt \%) By Sampler Type of Material=SME Simulant, Analytical Block=5, Analyte $=\mathrm{MgO}(\mathrm{wt} \%)$, Target $(\mathrm{wt} \%)=1.014$

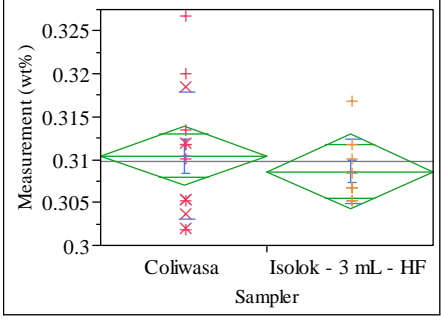

Excluded Rows

3

Oneway Anova

Summary of Fit

Rsquare $\quad 0.021652$

Adj Rsquare $\quad-0.02494$

Root Mean Square Error $\quad 0.006281$

Mean of Response $\quad 0.309742$

Observations (or Sum Wgts)

Test

Isolok - $3 \mathrm{~mL}$ - HF-Coliwasa

Assuming equal variances

Difference $\quad-0.00183$ t Ratio -0.68173

$\begin{array}{llr}\text { Std Err Dif } & 0.00268 \text { DF } & 21\end{array}$

Upper CL Dif 0.00375 Prob $>|t| \quad 0.5029$

Lower CL Dif -0.00741 Prob $>t \quad 0.7486$

$\begin{array}{lrl}\text { Confidence } & 0.95 \text { Prob }<\mathrm{t} & 0.2514\end{array}$

Analysis of Variance

Source DF Sum of Squares Mean Square F Ratio Prob $>$ F

$\begin{array}{llllll}\text { Sampler } & 1 & 0.00001833 & 0.000018 & 0.4648 & 0.5029\end{array}$

$\begin{array}{lrrr}\text { Sampler } & 1 & 0.00001833 & 0.000018 \\ \text { Error } & 21 & 0.00082841 & 0.000039\end{array}$

$\begin{array}{lll}\text { C. Total } & 22 & 0.00084675\end{array}$

Means for Oneway Anova

$\begin{array}{lrrrrr}\text { Level } & \text { Number } & \text { Mean } & \text { Std Error } & \text { Lower 95\% } & \text { Upper 95\% } \\ \text { Coliwasa } & 14 & 0.310457 & 0.00168 & 0.30697 & 0.31395\end{array}$

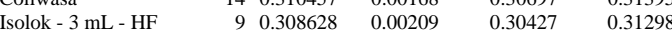

Std Error uses a pooled estimate of error variance

Means and Std Deviations

Level $\quad$ Number Mean Std Dev Std Err Mean Lower 95\% Upper 95\%

$\begin{array}{lrrrrrr}\text { Coliwasa } & 14 & 0.310457 & 0.007421 & 0.00198 & 0.30617 & 0.31474\end{array}$

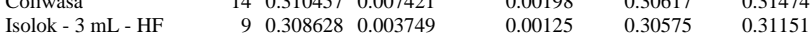

t Test

Isolok - $3 \mathrm{~mL}$ - HF-Coliwasa

Assuming unequal variances

$\begin{array}{lrlr}\text { Difference } & -0.00183 & \mathrm{t} \text { Ratio } & -0.78037 \\ \text { Std Err Dif } & 0.00234 & \text { DF } & 20.19781 \\ \text { Upper CL Dif } & 0.00306 & \text { Prob }>|t| & 0.4442 \\ \text { Lower CL Dif } & -0.00672 & \text { Prob }>t & 0.7779 \\ \text { Confidence } & 0.95 & \text { Prob }<\mathrm{t} & 0.2221\end{array}$

Tests that the Variances are Equal

Level Count Std Dev MeanAbsDif to Mean MeanAbsDif to Median

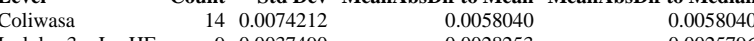

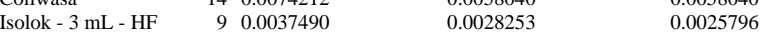

Test F Ratio DFNum DFDen p-Value

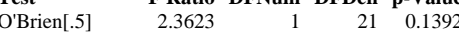

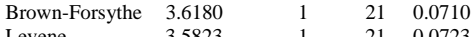

$\begin{array}{lllll}\text { Levene } & 3.5823 & 1 & 21 & 0.0723 \\ \text { Bartlett } & 3.7262 & 1 & .0 .0536\end{array}$

$\begin{array}{lllll}\text { F Test 2-sided } & 3.9184 & 13 & 8 & 0.0596\end{array}$

Welch Anova testing Means Equal, allowing Std Devs Not Equal

F Ratio DFNum DFDen Prob $>$ F

$\begin{array}{rrrr}0.6090 & 1 & 20.198 & 0.4442\end{array}$

t Test
0.7804
Oneway Analysis of Measurement (wt \%) By Sampler Type of Material=SME Simulant, Analytical Block $=5$, Analyte $=\mathbf{M n O}(\mathrm{wt} \%)$, Target $(\mathrm{wt} \%)=1.779$

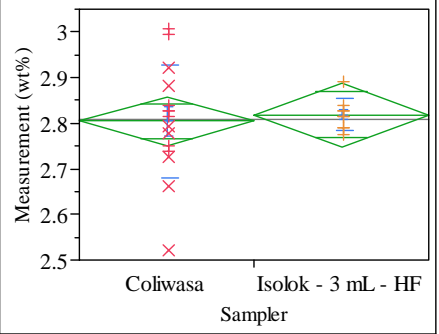

Excluded Rows

2

Oneway Anova

Summary of Fit

Adj Rsquare

0.005283

Root Mean Square Error $\quad 0.101535$

Mean of Response

Observations (or Sum Wgts) $\quad 24$

t Test

Isolok - $3 \mathrm{~mL}$ - HF-Coliwasa

Assuming equal variances

Difference $\quad 0.01463$ t Ratio 0.34182

$\begin{array}{lll}\text { Std Err Dif } \quad 0.04281 \text { DF } & 22\end{array}$

$\begin{array}{lrr}\text { Upper CL Dif } & 0.10342 \text { Prob }>|t| & 0.7357\end{array}$

Lower CL Dif -0.07415 Prob $>t \quad 0.3679$

$\begin{array}{lrl}\text { Confidence } & 0.95 \text { Prob }<\mathrm{t} & 0.6321\end{array}$

Analysis of Variance

Source DF Sum of Squares Mean Square F Ratio Prob > F

$\begin{array}{llllll}\text { Sampler } & 1 & 0.00120455 & 0.001205 & 0.1168 & 0.7357\end{array}$

$\begin{array}{llll}\text { Error } & 22 & 0.22680554 & 0.010309\end{array}$

C. Total $23-0.22801009$

Means for Oneway Anova

Level Number Mean Std Error Lower 95\% Upper 95\%

$\begin{array}{lrrrrr}\text { Coliwasa } & 15 & 2.80449 & 0.02622 & 2.7501 & 2.8589 \\ \text { Isolok - 3 mL - HF } & 9 & 2.81912 & 0.03384 & 2.7489 & 2.8893\end{array}$

Std Error uses a pooled estimate of error variance

Means and Std Deviations $\quad$ Mean Std Dev Std Err Mean Lower 95\% Upper 95\%

$\begin{array}{lrrrrrr}\text { Level } & \text { Number } & \text { Mean } & \text { Std Dev } & \text { Std Err Mean } & \text { Lower 95\% } & \text { Upper 95\% } \\ \text { Coliwasa } & 15 & 2.80449 & 0.124538 & 0.03216 & 2.7355 & 2.8735\end{array}$

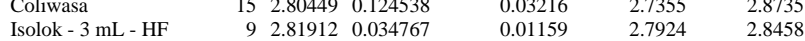

t Test

Isolok - $3 \mathrm{~mL}$ - HF-Coliwasa

Assuming unequal variances

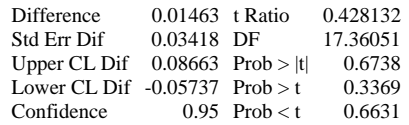

Tests that the Variances are Equal

$\begin{array}{lrrrr}\text { Level } & \text { Count } & \text { Std Dev } & \text { MeanAbsDif to Mean } & \text { MeanAbsDif to Median } \\ \text { Coliwasa } & 15 & 0.1245379 & 0.0902118 & 0.0903840\end{array}$

$\begin{array}{lrrrr}\text { Coliwasa } & 15 & 0.1245379 & 0.0902118 & 0.0903840 \\ \text { Isolok - 3 mL - HF } & 9 & 0.0347666 & 0.0248676 & 0.0243893\end{array}$

Test F Ratio DFNum DFDen p-Value

$\begin{array}{lrrrr}\text { O'Brien[.5] } & 2.7590 & 1 & 22 & 0.1109\end{array}$

$\begin{array}{lllll}\text { Brown-Forsythe } & 5.3444 & 1 & 22 & 0.0305 \\ \text { Levene } & 5.3283 & 1 & 22 & 0.0308\end{array}$

$\begin{array}{lrrrr}\text { Levene } & 5.3283 & 1 & 22 & 0.0308\end{array}$

$\begin{array}{lllll}\text { F Test 2-sided } & 12.8315 & 14 & 8 & 0.0011\end{array}$

Welch Anova testing Means Equal, allowing Std Devs Not Equal

F Ratio DFNum DFDen Prob $>$ F

$\begin{array}{lrrr}0.1833 & 1 & 17.361 & 0.6738\end{array}$

t Tes

0.428 


\section{Exhibit B3. Statistical Comparisons for High-Rheology (Phase 2) Simulant Testing (screened data)}

Oneway Analysis of Measurement (wt \%) By Sampler Type of Material=SME Simulant, Analytical Block $=5$, Analyte $=\mathrm{SiO} 2(w t \%)$, Target $(w t \%)=50.985$

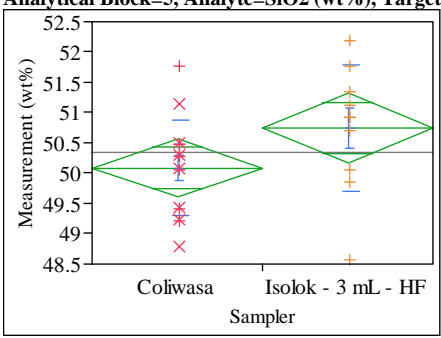

Excluded Rows

Oneway Anova

Summary of Fit

Root Mean Square Error $\quad 0.895859$

Mean of Response $\quad 50.35056$

Observations (or Sum Wgs)

Test

Isolok - $3 \mathrm{~mL}$ - HF-Coliwasa

Assuming equal variances

Difference $\quad 0.6561$ t Ratio 1.7938

Std Err Dif $\quad 0.3657$ DF $\quad 23$

Upper CL Dif 1.4126 Prob $>|t| 0.0860$

Lower CL Dif -0.1005 Prob $>$ t $\quad 0.0430$

Confidence $\quad 0.95$ Prob $<\mathrm{t} \quad 0.9570$

Analysis of Variance

$\begin{array}{lrrrrr}\text { Source } & \text { DF } & \text { Sum of Squares } & \text { Mean Square } & \text { F Ratio } & \text { Prob }>\text { F } \\ \text { Sampler } & 1 & 2.582425 & 2.58243 & 3.2177 & 0.0860\end{array}$

$\begin{array}{lll}\text { Error } & 23 & 18.458971 \\ \text { C. Total } & 24 & 21.041397\end{array}$

Means for Oneway Anova

Level Number Mean Std Error Lower 95\% Upper 95\%

$\begin{array}{llllll}\text { Coliwasa } & 15 & 50.0881 & 0.23131 & 49.610 & 50.567\end{array}$

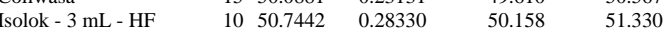

Std Error uses a pooled estimate of error variance

Means and Std Deviations

Level Number Mean Std Dev Std Err Mean Lower 95\% Upper 95\%

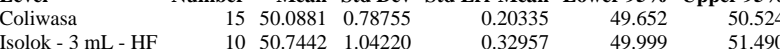

t Test

Isolok - $3 \mathrm{~mL}$ - HF-Coliwasa

Assuming unequal variances

Difference $\quad 0.6561$ t Ratio 1.694103

Std Err Dif $\quad 0.3873$ DF 15.69447

Upper CL Dif 1.4783 Prob $>|t| \quad 0.1100$

Lower CL Dif -0.1662 Prob $>t \quad 0.0550$

Confidence $\quad 0.95$ Prob $<\mathrm{t} \quad 0.9450$

Tests that the Variances are Equal

$\begin{array}{lrrrr}\text { Level } & \text { Count } & \text { Std Dev } & \text { MeanAbsDif to Mean } & \text { MeanAbsDif to Median } \\ \text { Coliwasa } & 15 & 0.787552 & 0.5971024 & 0.5847420 \\ \text { Isolok - 3 mL - HF } & 10 & 1.042200 & 0.7615908 & 0.7273620\end{array}$

\begin{tabular}{|c|c|}
\hline Isolok - $3 \mathrm{~mL}$ - & \\
\hline
\end{tabular}

Test F Ratio DFNum DFDen p-Value

O'Brien[.5] $0.7794 \quad 1 \quad 230.3865$

$\begin{array}{lllll}\text { O'Brien[.5] } & 0.7794 & 1 & 23 & 0.3865 \\ \text { Brown-Forsythe } & 0.3172 & 1 & 23 & 0.5787 \\ \text { Levene } & 0.5107 & 1 & 23 & 0.4820\end{array}$

$\begin{array}{lllll}\text { Levene } & 0.5107 & 1 & 23 & 0.4820 \\ \text { Bartlett } & 0.8452 & 1 & . & 0.3579\end{array}$

$\begin{array}{lllll}\text { F Test 2-sided } & 1.7512 & 9 & 14 & 0.3579\end{array}$

Welch Anova testing Means Equal, allowing Std Devs Not Equal

F Ratio DFNum DFDen Prob > F

$\begin{array}{llll}2.8700 & 1 & 15.694 & 0.1100\end{array}$

t Test
1.6941
Oneway Analysis of Measurement (wt \%) By Sampler Type of Material=SME Simulant, Analytical Block $=5$, Analyte $=$ Sum of Oxides $(w t \%)$, Target $(w t \%)=99.553$

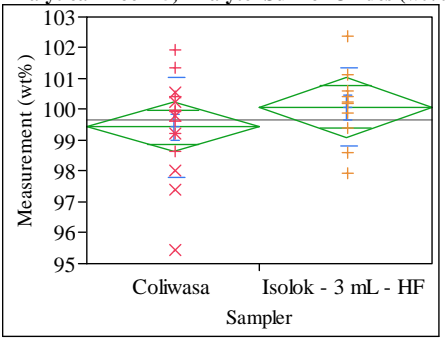

Excluded Rows

1

Oneway Anova

Summary of Fit

Rsquare

Adj Rsquare

0.005506

Mean of Response $\quad 1.489953$

\section{t Test}

Isolok - $3 \mathrm{~mL}$ - HF-Coliwasa

Assuming equal variances

$\begin{array}{lrlr}\text { Difference } & 0.6474 & \text { t Ratio } & 1.064366 \\ \text { Std Err Dif } & 0.6083 \text { DF } & 23 \\ \text { Upper CL Dif } & 1.9057 \text { Prob }>|t| & 0.2982 \\ \text { Lower CL Dif } & -0.6109 & \text { Prob }>t & 0.1491 \\ \text { Confidence } & 0.95 & \text { Prob }<\mathrm{t} & 0.8509\end{array}$

Analysis of Variance

Source DF Sum of Squares Mean Square F Ratio Prob $>$ F

$\begin{array}{lrrrrr}\text { Sampler } & 1 & 2.514938 & 2.51494 & 1.1329 & 0.2982\end{array}$

$\begin{array}{llll}\text { Error } & 23 & 51.059087 & 2.21996\end{array}$

C. Total $24 \quad 53.574025$

Means for Oneway Anova

Level Number Mean Std Error Lower 95\% Upper 95\%

$\begin{array}{lrrrrr}\text { Coliwasa } & 15 & 99.430 & 0.38470 & 98.634 & 100.23\end{array}$

$\begin{array}{lrrrrr}\text { Isolok - 3 mL - HF } & 10 & 100.077 & 0.47116 & 99.102 & 101.05\end{array}$

Std Error uses a pooled estimate of error variance

Means and Std Deviations

Level Number Mean Std Dev Std Err Mean Lower 95\% Upper 95\%

$\begin{array}{lllllll}\text { Coliwasa } & 15 & 99.430 & 1.62118 & 0.41859 & 98.532 & 100.33\end{array}$

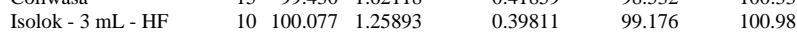

t Test

Isolok - $3 \mathrm{~mL}$ - HF-Coliwasa

Assuming unequal variances

$\begin{array}{lrlr}\text { Difference } & 0.6474 & \mathrm{t} \text { Ratio } & 1.120745 \\ \text { Std Err Dif } & 0.5777 & \text { DF } & 22.34377 \\ \text { Upper CL Dif } & 1.8444 & \text { Prob }>|t| & 0.2743 \\ \text { Lower CL Dif } & -0.5495 & \text { Prob }>\mathrm{t} & 0.1371 \\ \text { Confidence } & 0.95 & \text { Prob }<\mathrm{t} & 0.8629\end{array}$

Tests that the Variances are Equal

Level Count Std Dev MeanAbsDif to Mean MeanAbsDif to Median

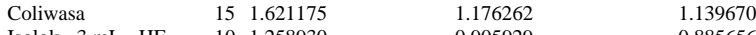

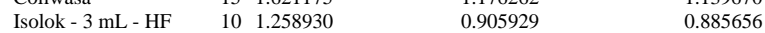

$\begin{array}{lrrrr}\text { Test } & \text { F Ratio } & \text { DFNum } & \text { DFDen } & \text { p-Value } \\ \text { O'Brien[.5] } & 0.4114 & 1 & 23 & 0.5276 \\ \text { Brown-Forsythe } & 0.3464 & 1 & 23 & 0.5619 \\ \text { Levene } & 0.4564 & 1 & 23 & 0.5061 \\ \text { Bartlett } & 0.6396 & 1 & . & 0.4238 \\ \text { F Test 2-sided } & 1.6583 & 14 & 9 & 0.4496\end{array}$

Welch Anova testing Means Equal, allowing Std Devs Not Equal

F Ratio DFNum DFDen Prob $>$ F

$\begin{array}{rrrr}1.2561 & 1 & 22.344 & 0.2743\end{array}$

t Test

1.1207 


\section{Exhibit B3. Statistical Comparisons for High-Rheology (Phase 2) Simulant Testing (screened data)}

Oneway Analysis of Measurement (wt \%) By Sampler Type of Material=SME Simulant, Analytical Block=5, Analyte $=\mathrm{TiO} 2(\mathrm{wt} \%)$, Target $(\mathrm{wt} \%)=0.0711$

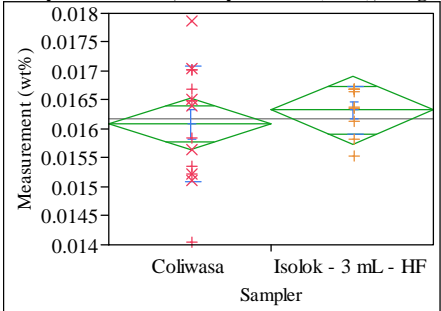

Excluded Rows

Oneway Anova

Summary of Fit

Adj Rsquare

$\quad 0.000836$

of Response $\quad 0.016174$

Observations (or Sum Wgts)

Test

Isolok - $3 \mathrm{~mL}$ - HF-Coliwasa

Assuming equal variances

Difference $\quad 0.00024$ t Ratio $\quad 0.672802$

Std Err Dif $\quad 0.00035$ DF 22

Upper CL Dif 0.00097 Prob $>|t| \quad 0.5081$

Lower CL Dif -0.00049 Prob $>t \quad 0.2540$

$\begin{array}{lrl}\text { Confidence } & 0.95 \text { Prob }<\mathrm{t} \quad 0.7460\end{array}$

Analysis of Varianc

Source DF Sum of Squares Mean Square F Ratio Prob $>$ F

$\begin{array}{llllll}\text { Sampler } & 1 & 0.00000032 & 3.1656 \mathrm{e}-7 & 0.4527 & 0.5081\end{array}$

Error $22 \quad 0.00001539 \quad 6.9932 \mathrm{e}-7$

C. Total $23 \quad 0.00001570$

Means for Oneway Anova

$\begin{array}{lrrrrr}\text { Level } & \text { Number } & \text { Mean } & \text { Std Error } & \text { Lower 95\% } & \text { Upper 95\% } \\ \text { Coliwasa } & 15 & 0.016085 & 0.00022 & 0.01564 & 0.01653\end{array}$

$\begin{array}{lrrrrr}\text { Coliwasa } & 15 & 0.016085 & 0.00022 & 0.01564 & 0.01653 \\ \text { Isolok - 3 mL - HF } & 9 & 0.016322 & 0.00028 & 0.01574 & 0.01690\end{array}$

Std Error uses a pooled estimate of error variance

Means and Std Deviations

Level Number Mean Std Dev Std Err Mean Lower 95\% Upper 95\%

$\begin{array}{lllllll}\text { Coliwasa } & 15 & 0.016085 & 0.000999 & 0.00026 & 0.01553 & 0.01664\end{array}$

$\begin{array}{lrrrrrr}\text { Coliwasa } & 15 & 0.016085 & 0.000999 & 0.00026 & 0.01553 & 0.01664 \\ \text { Isolok - 3 mL - HF } & 9 & 0.016322 & 0.000419 & 0.00014 & 0.01600 & 0.01664\end{array}$

t Test

Isolok - $3 \mathrm{~mL}$ - HF-Coliwas

Assuming unequal variances

Difference $\quad 0.00024$ t Ratio 0.80865

Std Err Dif $\quad 0.00029$ DF 20.3422

Upper CL Dif 0.00085 Prob $>|t| 0.4281$

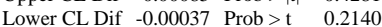

$\begin{array}{lrl}\text { Confidence } & 0.95 \text { Prob }<t \quad 0.7860\end{array}$

Tests that the Variances are Equal

Level Count Std Dev MeanAbsDif to Mean MeanAbsDif to Median

$\begin{array}{lrrrr}15 & 0.0009994 & 0.0008325 & 0.0008195\end{array}$

Test F Ratio DFNum DFDen p-Value

$\begin{array}{lrrrr}\text { O'Brien[.5] } & 3.5239 & 1 & 22 & 0.0738\end{array}$

$\begin{array}{lllll}\text { Brown-Forsythe } & 5.5239 & 1 & 22 & 0.0738 \\ & 5.3774 & 1 & 22 & 0.0301\end{array}$

$\begin{array}{lllll}\text { Levene } & 7.8218 & 1 & 22 & 0.0105\end{array}$

$\begin{array}{lrrrr}\text { Bartlett } & 5.7883 & 1 & \text {. } & 0.0161 \\ \text { F Test 2-sided } & 5.6979 & 14 & 8 & 0.0185\end{array}$

Welch Anova testing Means Equal, allowing Std Devs Not Equal

F Ratio DFNum DFDen Prob $>$ F

$\begin{array}{llll}0.6539 & 1 & 20.342 & 0.4281\end{array}$

t Test

0.8087
Oneway Analysis of Measurement (wt \%) By Sampler Type of Material=SME Simulant, Analytical Block=6, Analyte=Al2O3 $(w t \%)$, Target $(w t \%)=10.869$

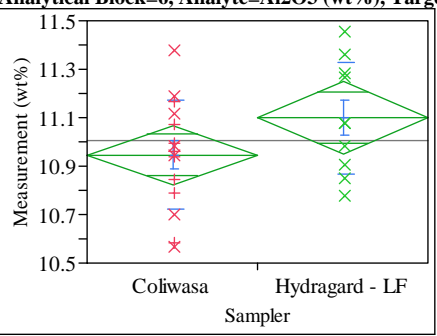

Excluded Rows

Oneway Anova

Summary of Fit

\begin{tabular}{ll} 
Adj Rsquare & 0.065857 \\
\hline
\end{tabular}

Root Mean Square Error $\quad 0.227551$

Mean of Response $\quad 11.00747$

Observations (or Sum Wgts) 25

t Test

Hydragard - LF-Coliwasa

Assuming equal variances

Difference $\quad 0.15242$ t Ratio 1.640733

\begin{tabular}{lrlr} 
Std Err Dif & 0.09290 & DF & 23 \\
\hline
\end{tabular}

Upper CL Dif 0.34459 Prob $>|t| \quad 0.1145$

Lower CL Dif -0.03975 Prob $>t \quad 0.0572$

Confidence $\quad 0.95$ Prob $<\mathrm{t} \quad 0.9428$

Analysis of Variance

Source DF Sum of Squares Mean Square F Ratio Prob $>$ F

$\begin{array}{lrrrrr}\text { Sampler } & 1 & 0.1393905 & 0.139391 & 2.6920 & 0.1145\end{array}$

$\begin{array}{lll}\text { Error } & 23 & 1.1909269 \\ \text { C. Total } & 24 & 1.3303175\end{array}$

Means for Oneway Anova

Level Number Mean Std Error Lower 95\% Upper 95\%

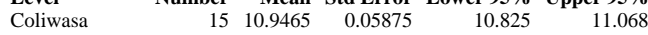

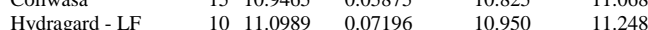

Std Error uses a pooled estimate of error variance

Means and Std Deviation

Level Number Mean Std Dev Std Err Mean Lower 95\% Upper 95\%

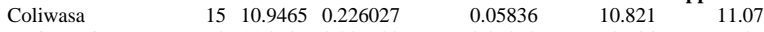

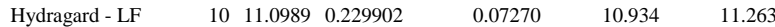

t Test

Hydragard - LF-Coliwasa

Assuming unequal variances

Difference $\quad 0.15242$ t Ratio 1.634922

Std Err Dif $\quad 0.09323$ DF 19.20852

Upper CL Dif 0.34740 Prob $>|\mathrm{t}| \quad 0.1183$

Lower CL Dif -0.04256 Prob $>t \quad 0.0592$

$\begin{array}{lll}\text { Confidence } & 0.95 \text { Prob }<\mathrm{t} \quad 0.9408\end{array}$

Tests that the Variances are Equal

$\begin{array}{lrrrr}\text { Level } & \text { Count } & \text { Std Dev } & \text { MeanAbsDif to Mean } & \text { MeanAbsDif to Median } \\ \text { Coliwasa } & 15 & 0.2260266 & 0.1696351 & 0.1700550\end{array}$

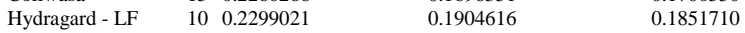

Test F Ratio DFNum DFDen p-Value

$\begin{array}{lllll}\text { O'Brien[.5] } & 0.0051 & 1 & 23 & 0.9439\end{array}$

$\begin{array}{lllll}\text { Brown-Forsythe } & 0.0744 & 1 & 23 & 0.7875\end{array}$

$\begin{array}{lllll}\text { Levene } & 0.1510 & 1 & 23 & 0.7012\end{array}$

$\begin{array}{lllll}\text { Bartlett } & 0.0030 & 1 & \text {. } & 0.9561\end{array}$

$\begin{array}{lllll}\text { F Test 2-sided } & 1.0346 & 9 & 14 & 0.9208\end{array}$

Welch Anova testing Means Equal, allowing Std Devs Not Equal

F Ratio DFNum DFDen Prob $>$ F

$\begin{array}{llll}2.6730 & 1 & 19.209 & 0.1183\end{array}$

t Test 


\section{Exhibit B3. Statistical Comparisons for High-Rheology (Phase 2) Simulant Testing (screened data)}

Oneway Analysis of Measurement (wt \%) By Sampler Type of Material=SME Simulant, Analytical Block=6, Analyte $=\mathrm{CaO}(\mathrm{wt} \%)$, Target $(\mathrm{wt} \%)=1.1462$

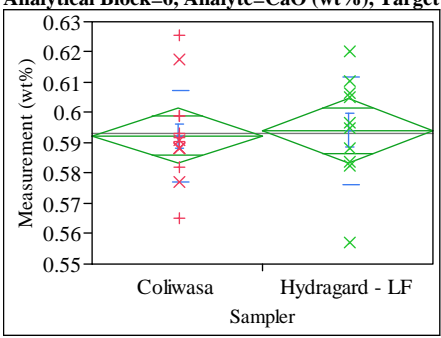

Excluded Rows

Oneway Anova

Summary of Fit

Rsquare

$-0.04229$

Root Mean Square Error $\quad 0.016245$

Mean of Response $\quad 0.593086$

t Test

Hydragard - LF-Coliwasa

Assuming equal variances

Difference $\quad 0.00174$ t Ratio $\quad 0.258543$

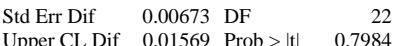

Upper CL Dif 0.01569 Prob $>|t| \quad 0.7984$

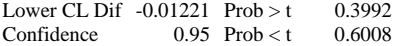

Analysis of Variance

Source DF Sum of Squares Mean Square F Ratio Prob $>$ F

$\begin{array}{lrrrrr}\text { Sampler } & 1 & 0.00001764 & 0.000018 & 0.0668 & 0.7984\end{array}$

$\begin{array}{llll}\text { Error } & 22 & 0.00580596 & 0.000264\end{array}$

$\begin{array}{lll}\text { C. Total } & 23 & 0.00582360\end{array}$

Means for Oneway Anova

Level Number Mean Std Error Lower 95\% Upper 95\%

$\begin{array}{lrrrrr}\text { Level } & \text { Number } & \text { Mean } & \text { Std Error } & \text { Lower } 95 \% & \text { Upper 95\% } \\ \text { Coliwasa } & 14 & 0.592361 & 0.00434 & 0.58336 & 0.60137\end{array}$

$\begin{array}{llllll}\text { Coliwasa } & 14 & 0.592361 & 0.00434 & 0.58336 & 0.60137 \\ \text { Hydragard - LF } & 10 & 0.594100 & 0.00514 & 0.58345 & 0.60475\end{array}$

Std Error uses a pooled estimate of error variance

Means and Std Deviations

Level Number Mean Std Dev Std Err Mean Lower 95\% Upper 95\%

$\begin{array}{lllllll}\text { Coliwasa } & 14 & 0.592361 & 0.015076 & 0.00403 & 0.58366 & 0.60107 \\ \text { Hydragard - LF } & 10 & 0.594100 & 0.017799 & 0.00563 & 0.58137 & 0.60683\end{array}$

t Test

Hydragard - LF-Coliwasa

Assuming unequal variances

Difference $\quad 0.00174$ t Ratio $\quad 0.251225$

Std Err Dif $\quad 0.00692$ DF 17.4203

Upper CL Dif 0.01632 Prob $>|t| \quad 0.8046$

Lower CL Dif -0.01284 Prob $>\mathrm{t} \quad 0.4023$

$\begin{array}{lll}\text { Confidence } \quad 0.95 \text { Prob }<\mathrm{t} \quad 0.5977 & \end{array}$

Tests that the Variances are Equal

$\begin{array}{lrrrr}\text { Level } & \text { Count } & \text { Std Dev } & \text { MeanAbsDif to Mean } & \text { MeanAbsDif to Median } \\ \text { Coliwasa } & 14 & 0.0150759 & 0.0102370 & 0.0098943\end{array}$

$\begin{array}{lrrrr}\text { Coliwasa } & 14 & 0.0150759 & 0.0102370 & 0.0098943 \\ \text { Hydragard - LF } & 10 & 0.0177991 & 0.0132644 & 0.0131525\end{array}$

Test F Ratio DFNum DFDen p-Value

$\begin{array}{lrrrr}\text { O'Brien[.5] } & 0.2390 & 1 & 22 & 0.6298\end{array}$

$\begin{array}{lllll}\text { O'Brien[.5] } & 0.2390 & 1 & 22 & 0.6298 \\ \text { Brown-Forsythe } & 0.4915 & 1 & 22 & 0.4906\end{array}$

$\begin{array}{lllll}\text { Levene } & 0.4560 & 1 & 22 & 0.5065 \\ \text { Bartlett } & 0.2844 & 1 & & 0.5938\end{array}$

$\begin{array}{lllll}\text { F Test 2-sided } & 1.3939 & 9 & 13 & 0.5680\end{array}$

Welch Anova testing Means Equal, allowing Std Devs Not Equal

F Ratio DFNum DFDen Prob $>$ F

$\begin{array}{llll}0.0631 & 1 & 17.42 & 0.8046\end{array}$

t Test
Oneway Analysis of Measurement (wt \%) By Sampler Type of Material=SME Simulant, Analytical Block $=6$, Analyte $=\mathrm{Cr} 2 \mathrm{O} 3(\mathrm{wt} \%)$, Target $(\mathrm{wt} \%)=0.0998$

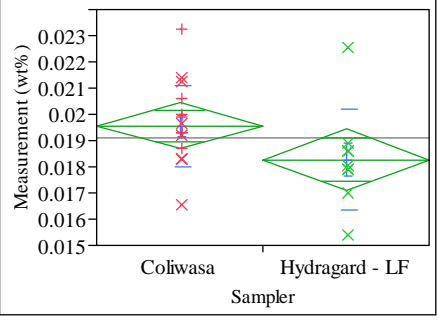

Excluded Rows

1

Oneway Anova

Summary of Fit

Rsquare $\quad 0.127524$

Adj Rsquare $\quad 0.08959$

Root Mean Square Error $\quad 0.001686$

Mean of Response $\quad 0.019094$

Observations (or Sum Wgts) 25

t Test

Hydragard - LF-Coliwasa

Assuming equal variances

Difference $\quad-0.00129$ t Ratio -1.83351

$\begin{array}{llr}\text { Std Err Dif } \quad 0.00070 \text { DF } & 23\end{array}$

Upper CL Dif 0.00017 Prob $>|t| \quad 0.0797$

Lower CL Dif -0.00274 Prob $>t \quad 0.9601$

$\begin{array}{lrl}\text { Confidence } & 0.95 \text { Prob }<\mathrm{t} & 0.0399\end{array}$

Analysis of Variance

Source DF Sum of Squares Mean Square F Ratio Prob $>$ F

$\begin{array}{lrrrrr}\text { Sampler } & 1 & 0.00000956 & 9.556 \mathrm{e}-6 & 3.3617 & 0.0797\end{array}$

$\begin{array}{llll}\text { Error } & 23 & 0.00006538 & 2.8426 \mathrm{e}-6\end{array}$

C. Total $24 \quad 0.00007494$

Means for Oneway Anova

Level Number Mean Std Error Lower 95\% Upper 95\%

$\begin{array}{lrrrrr}\text { Coliwasa } & 16 & 0.019558 & 0.00042 & 0.01869 & 0.02043 \\ \text { Hydragard - LF } & 9 & 0.018270 & 0.00056 & 0.01711 & 0.01943\end{array}$

Std Error uses a pooled estimate of error variance

$\begin{array}{llllll}\begin{array}{l}\text { Means and Std Deviations } \\ \text { Level }\end{array} \text { Number } & \text { Mean } & \text { Std Dev Std Err Mean Lower 95\% } & \text { Upper 95\% }\end{array}$

$\begin{array}{lrrrrrr}\text { Level } & \text { Number } & \text { Mean } & \text { Std Dev } & \text { Std Err Mean } & \text { Lower 95\% } & \text { Upper 95\% } \\ \text { Coliwasa } & 16 & 0.019558 & 0.001553 & 0.00039 & 0.01873 & 0.02039\end{array}$

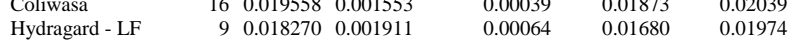

t Test

Hydragard - LF-Coliwasa

Assuming unequal variances

Difference $\quad-0.00129$ t Ratio $\quad-1.72655$

Std Err Dif $\quad 0.00075$ DF 14.01103

Upper CL Dif 0.00031 Prob $>|\mathrm{t}| \quad 0.1062$

Lower CL Dif -0.00289 Prob $>t \quad 0.9469$

$\begin{array}{lrl}\text { Confidence } & 0.95 \text { Prob }<\mathrm{t} & 0.0531\end{array}$

Tests that the Variances are Equal

Level Count Std Dev MeanAbsDif to Mean MeanAbsDif to Median

$\begin{array}{lrrrr}\text { Coliwasa } & 16 & 0.0015525 & 0.0011202 & 0.0010871\end{array}$

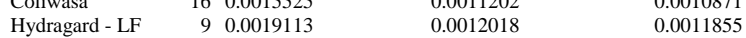

Test F Ratio DFNum DFDen p-Value

$\begin{array}{lrrrr}\text { O'Brien[.5] } & 0.2905 & 1 & 23 & 0.5951\end{array}$

$\begin{array}{lllll}\text { Brown-Forsythe } & 0.0359 & 1 & 23 & 0.8513\end{array}$

$\begin{array}{lllll}\text { Levene } & 0.0273 & 1 & 23 & 0.8702 \\ \text { Batlt } & 0.4454 & 1 & & 0.5045\end{array}$

$\begin{array}{lllll}\text { F Test 2-sided } & 1.5156 & 8 & 15 & 0.4643\end{array}$

Welch Anova testing Means Equal, allowing Std Devs Not Equal

F Ratio DFNum DFDen Prob $>$ F

$\begin{array}{lrrr}2.9810 & 1 & 14.011 & 0.1062\end{array}$

t Test 


\section{Exhibit B3. Statistical Comparisons for High-Rheology (Phase 2) Simulant Testing (screened data)}

Oneway Analysis of Measurement (wt \%) By Sampler Type of Material=SME Simulant, Analytical Block=6, Analyte $=\mathrm{CuO}(\mathrm{wt} \%)$, Target $(\mathrm{wt} \%)=0.0504$

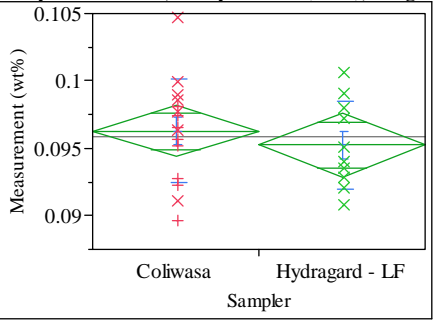

Excl
1

Oneway Anova

Summary of Fit

Rsquare $\quad 0.021725$

Adj Rsquare $\quad-0.02081$

Root Mean Square Error $\quad 0.003604$

Mean of Response $\quad 0.095868$

Observations (or Sum Wgts)

Test

Hydragard - LF-Coliwasa

Assuming equal variances

Difference $\quad-0.00105$ t Ratio $\quad-0.71468$

$\begin{array}{lll}\text { Std Err Dif } & 0.00147 \text { DF } & 23\end{array}$

Upper CL Dif $\quad 0.00199$ Prob $>|t| \quad 0.4820$

Lower CL Dif -0.00410 Prob $>t \quad 0.7590$

$\begin{array}{lll}\text { Confidence } & 0.95 \text { Prob }<\mathrm{t} \quad 0.2410\end{array}$

Analysis of Variance

Source DF Sum of Squares Mean Square F Ratio Prob $>$ F

$\begin{array}{lrrrrr}\text { Sampler } & 1 & 0.00000663 & 6.634 \mathrm{e}-6 & 0.5108 & 0.4820\end{array}$

$\begin{array}{lrrr}\text { Sampler } & 1 & 0.00000663 & 6.634 \mathrm{e}-6 \\ \text { Error } & 23 & 0.00029873 & 0.000013\end{array}$

$\begin{array}{lll}\text { C. Total } & 24 & 0.00029873 \\ & & \end{array}$

Means for Oneway Anova

Level Number Mean Std Error Lower 95\% Upper 95\%

$\begin{array}{lrrrrr}\text { Coliwasa } & 15 & 0.096288 & 0.00093 & 0.09436 & 0.09821\end{array}$

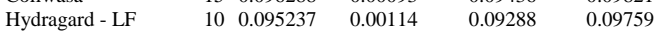

Std Error uses a pooled estimate of error variance

Means and Std Deviations

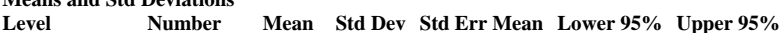

$\begin{array}{lrrrrrr}\text { Coliwasa } & 15 & 0.096288 & 0.003816 & 0.00099 & 0.09418 & 0.09840\end{array}$

$\begin{array}{lllllll}\text { Hydragard - LF } & 15 & 0.096288 & 0.003816 & 0.00099 & 0.09418 & 0.09840 \\ & 10 & 0.003246 & 0.00103 & 0.09291 & 0.09756\end{array}$

t Tes

Hydragard - LF-Coliwasa

Assuming unequal variances

Difference $\quad-0.00105$ t Ratio -0.739

Std Err Dif $\quad 0.00142$ DF 21.49555

Upper CL Dif 0.00190 Prob $>|t| r \mid r .4679$

Lower CL Dif -0.00401 Prob $>\mathrm{t} \quad 0.7660$

$\begin{array}{lll}\text { Confidence } & 0.95 \text { Prob }<\mathrm{t} \quad 0.2340\end{array}$

Tests that the Variances are Equal

$\begin{array}{lrrrr}\text { Level } & \text { Count } & \text { Std Dev } & \text { MeanAbsDif to Mean } & \text { MeanAbsDif to Median } \\ \text { Coliwasa } & 15 & 0.0038164 & 0.0028441 & 0.0028458\end{array}$

$\begin{array}{lllll}\text { Coliwasa } & 15 & 0.0038164 & 0.0028441 & 0.0028458 \\ \text { Hydragard - LF } & 10 & 0.0032460 & 0.0027239 & 0.0026789\end{array}$

Test F Ratio DFNum DFDen p-Value

O'Brien[.5] $\quad 0.2852 \quad 11230.5984$

$\begin{array}{lllll}\text { Brown-Forsythe } & 0.0342 & 1 & 23 & 0.8550\end{array}$

$\begin{array}{lllll}\text { Levene } & 0.0193 & 1 & 23 & 0.8907\end{array}$

$\begin{array}{lllll}\text { Bartlett } & 0.2670 & 1 & 0.6054\end{array}$

$\begin{array}{lllll}\text { F Test 2-sided } & 1.3823 & 14 & 9 & 0.6364\end{array}$

Welch Anova testing Means Equal, allowing Std Devs Not Equal

F Ratio DFNum DFDen Prob $>$ F

$\begin{array}{rrrr}0.5461 & 1 & 21.496 & 0.4679\end{array}$

t Test
0.7390
Oneway Analysis of Measurement (wt \%) By Sampler Type of Material=SME Simulant, Analytical Block $=6$, Analyte $=\mathrm{Fe} / \mathrm{Li}$, Target $(\mathrm{wt} \%)=2.452$

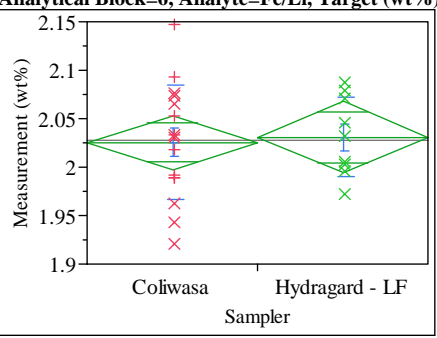

Excluded Rows

Oneway Anova

Summary of Fit

Rsquare

Adj Rsquare

Root Mean Square Error $\quad 0.053661$

Mean of Response 2.027543

Observations (or Sum Wgts) 25

t Test

Hydragard - LF-Coliwasa

Assuming equal variances

Difference $\quad 0.00532$ t Ratio $\quad 0.23798$

$\begin{array}{lrlr}\text { Std Err Dif } & 0.02236 \text { DF } & 23 \\ \text { Upper CL } & 0.05157 & \text { Prob }>|t| & 0.8140\end{array}$

$\begin{array}{lll}\text { Upper CL Dif } & 0.05157 \quad \text { Prob }>|t| & 0.8140\end{array}$

$\begin{array}{lrll}\text { Lower CL Dif } & -0.04093 & \text { Prob }>t & 0.4070 \\ \text { Confidence } & 0.95 & \text { Prob }<\mathrm{t} & 0.5930\end{array}$

Analysis of Variance

Source DF Sum of Squares Mean Square F Ratio Prob > F

$\begin{array}{llllll}\text { Sampler } & 1 & 0.00016309 & 0.000163 & 0.0566 & 0.8140\end{array}$

$\begin{array}{lll}\text { Error } & 23 & 0.06622922 \\ \text { C. Total } & 24 & 0.06639231\end{array}$

Means for Oneway Anova

Level Number Mean Std Error Lower 95\% Upper 95\%

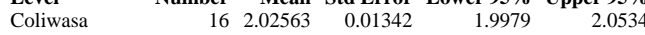

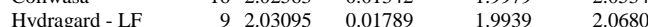

Std Error uses a pooled estimate of error variance

Means and Std Deviations

Level Number Mean Std Dev Std Err Mean Lower 95\% Upper 95\%

$\begin{array}{lllllll}\text { Coliwasa } & 16 & 2.02563 & 0.059283 & 0.01482 & 1.9940 & 2.0572\end{array}$

$\begin{array}{llllll}\text { Hydragard - LF } & 9 & 2.03095 & 0.041098 & 0.01370 & 1.9994\end{array}$

t Test

Hydragard - LF-Coliwasa

Assuming unequal variances

Difference $\quad 0.00532$ t Ratio $\quad 0.263652$

\begin{tabular}{lll} 
Std Err Dif $\quad 0.02018$ DF 21.77607 \\
\hline
\end{tabular}

Upper CL Dif 0.04720 Prob $>|t| \quad 0.7945$

Lower CL Dif -0.03656 Prob $>\mathrm{t} \quad 0.3973$

Confidence $\quad 0.95$ Prob $<\mathrm{t} \quad 0.6027$

Tests that the Variances are Equal

$\begin{array}{lrrrr}\text { Level } & \text { Count } & \text { Std Dev } & \text { MeanAbsDif to Mean } & \text { MeanAbsDif to Median } \\ \text { Coliwasa } & 16 & 0.0592829 & 0.0460802 & 0.0456218\end{array}$

$\begin{array}{lllll}\text { Hydragard - LF } & 9 & 0.0410980 & 0.0342428 & 0.0357353\end{array}$

Test F Ratio DFNum DFDen p-Value

$\begin{array}{lllll}\text { O'Brien[.5] } & 1.2139 & 1 & 23 & 0.2820\end{array}$

$\begin{array}{lllll}\text { Brown-Forsythe } & 0.5884 & 1 & 23 & 0.4508\end{array}$

$\begin{array}{lllll}\text { Brown-Forsythe } & 0.5884 & 1 & 23 & 0.4508 \\ \text { Levene } & 0.8554 & 1 & 23 & 0.3646\end{array}$

$\begin{array}{lllll}\text { Levene } & 0.8554 & 1 & 23 & 0.3646 \\ \text { Bartlett } & 1.2187 & 1 & & 0.2696\end{array}$

$\begin{array}{lllll}\text { F Test 2-sided } & 2.0807 & 15 & 8 & 0.2971\end{array}$

Welch Anova testing Means Equal, allowing Std Devs Not Equal

F Ratio DFNum DFDen Prob $>$ F

t Test 
SRNL-STI-2011-00693

Revision 0

Exhibit B3. Statistical Comparisons for High-Rheology (Phase 2) Simulant Testing (screened data)

Oneway Analysis of Measurement (wt \%) By Sampler Type of Material=SME Simulant, Analytical Block=6, Analyte $=$ Fe2O3 $(w t \%)$, Target $(w t \%)=11.462$

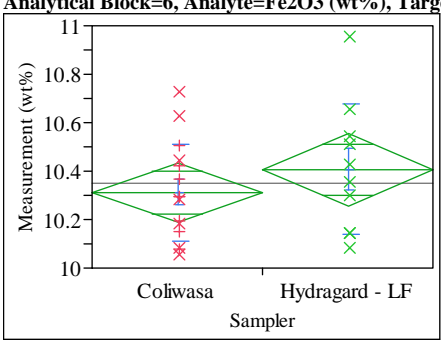

Excluded Rows

Oneway Anova

Summary of Fit

Rsquare

Adj Rsquare

0.000615

Root Mean Square Error $\quad 0.230606$

Mean of Response 10.34988

Observations (or Sum Wgts) 25

t Test

Hydragard - LF-Coliwasa

Assuming equal variances

Difference $\quad 0.09484$ t Ratio 1.007353

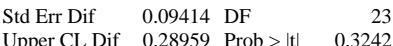

Upper CL Dif 0.28959 Prob $>|t| \quad 0.3242$

$\begin{array}{lrrr}\text { Lower CL Dif } & -0.09992 & \text { Prob }>t & 0.1621 \\ \text { Confidence } & 0.95 & \text { Prob }<\mathrm{t} & 0.8379\end{array}$

Analysis of Variance

Source DF Sum of Squares Mean Square F Ratio Prob $>$ F

$\begin{array}{lrrrrr}\text { Sampler } & 1 & 0.0539641 & 0.053964 & 1.0148 & 0.3242\end{array}$

$\begin{array}{llll}\text { Error } & 23 & 1.2231207 & 0.053179\end{array}$

C. Total $24 \quad 1.2770848$

Means for Oneway Anova

Level Number Mean Std Error Lower 95\% Upper 95\%

$\begin{array}{lrrrrr}\text { Coliwasa } & 15 & 10.3119 & 0.05954 & 10.189 & 10.435\end{array}$

$\begin{array}{llllll}\text { Coliwasa } & 15 & 10.3119 & 0.05954 & 10.189 & 10.435 \\ \text { Hydragard - LF } & 10 & 10.4068 & 0.07292 & 10.256 & 10.558\end{array}$

Std Error uses a pooled estimate of error variance

Means and Std Deviations

Level $\quad$ Number Mean Std Dev Std Err Mean Lower 95\% Upper 95\%

$\begin{array}{lllllll}\text { Coliwasa } & 15 & 10.3119 & 0.201790 & 0.05210 & 10.200 & 10.424\end{array}$

$\begin{array}{lllllll}\text { Hydragard - LF } & 10 & 10.4068 & 0.269372 & 0.08518 & 10.214 & 10.599\end{array}$

t Test

Hydragard - LF-Coliwasa

Assuming unequal variances

Difference $\quad 0.09484$ t Ratio $\quad 0.949758$

Std Err Dif $\quad 0.09985$ DF 15.59091

Upper CL Dif 0.30697 Prob $>|t| \quad 0.3567$

Lower CL Dif -0.11730 Prob $>\mathrm{t} \quad 0.1784$

$\begin{array}{lr}\text { Confidence } & 0.95 \text { Prob }<\mathrm{t} \quad 0.8216\end{array}$

Tests that the Variances are Equal

$\begin{array}{lrrrr}\text { Level } & \text { Count } & \text { Std Dev } & \text { MeanAbsDif to Mean } & \text { MeanAbsDif to Median } \\ \text { Coliwasa } & 15 & 0.2017902 & 0.1608889 & 0.1572670\end{array}$

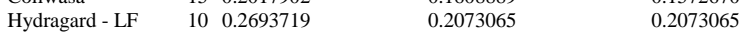

Test F Ratio DFNum DFDen p-Value

$\begin{array}{lrrrr}\text { O'Brien[.5] } & 1.0237 & 1 & 23 & 0.3222\end{array}$

$\begin{array}{lllll}\text { O'Brien[.5] } & 1.0237 & 1 & 23 & 0.3222 \\ \text { Brown-Forsythe } & 0.8012 & 1 & 23 & 0.3800\end{array}$

$\begin{array}{lllll}\text { Levene } & 0.7340 & 1 & 23 & 0.4004 \\ \text { Bartlett } & 0.8990 & 1 & & 0.3431\end{array}$

$\begin{array}{lllll}\text { F Test 2-sided } & 1.7820 & 9 & 14 & 0.3431 \\ \end{array}$

Welch Anova testing Means Equal, allowing Std Devs Not Equal

F Ratio DFNum DFDen Prob > F

$\begin{array}{llll}0.9020 & 1 & 15.591 & 0.3567\end{array}$

t Test
0.9498
Oneway Analysis of Measurement (wt \%) By Sampler Type of Material=SME Simulant, Analytical Block $=6$, Analyte $=\mathrm{Li} 2 \mathrm{O}(\mathrm{wt} \%)$, Target $(\mathrm{wt} \%)=4.674$

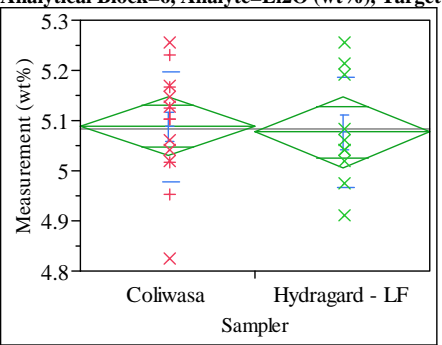

Excluded Rows

1

Oneway Anova

Summary of Fit

Rsquare $\quad 0.002851$

Adj Rsquare $\quad-0.0405$

Root Mean Square Error $\quad 0.109679$

Mean of Response 5.083427

Observations (or Sum Wgts) 25

t Test

Hydragard - LF-Coliwasa

Assuming equal variances

$\begin{array}{lrlr}\text { Difference } & -0.01148 & \mathrm{t} \text { Ratio } & -0.25643 \\ \text { Std Err Dif } & 0.04478 & \text { DF } & 23 \\ \text { Upper CL Dif } & 0.08114 & \text { Prob }>|t| & 0.7999 \\ \text { Lower CL Dif } & -0.10411 & \text { Prob }>t & 0.6001 \\ \text { Confidence } & 0.95 & \text { Prob }<\mathrm{t} & 0.3999\end{array}$

Analysis of Variance

Source DF Sum of Squares Mean Square F Ratio Prob > F

$\begin{array}{llllll}\text { Sampler } & 1 & 0.00079104 & 0.000791 & 0.0658 & 0.7999\end{array}$

$\begin{array}{llll}\text { Error } & 23 & 0.27667731 & 0.012029\end{array}$

C. Total $24 \quad 0.27746835$

Means for Oneway Anova

Level Number Mean Std Error Lower 95\% Upper 95\%

$\begin{array}{lrrrrr}\text { Coliwasa } & 15 & 5.08802 & 0.02832 & 5.0294 & 5.1466\end{array}$

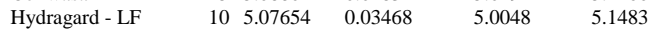

Std Error uses a pooled estimate of error variance

Means and Std Deviations

Level Number Mean Std Dev Std Err Mean Lower 95\% Upper 95\%

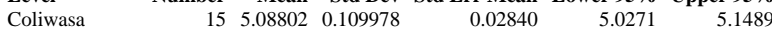

$\begin{array}{lllllll}\text { Hydragard - LF } & 10 & 5.07654 & 0.109212 & 0.03454 & 4.9984 & 5.1547\end{array}$

t Test

Hydragard - LF-Coliwasa

Assuming unequal variances

$\begin{array}{lrlr}\text { Difference } & -0.01148 & \mathrm{t} \text { Ratio } & -0.25681 \\ \text { Std Err Dif } & 0.04471 & \text { DF } & 19.54083 \\ \text { Upper CL Dif } & 0.08192 & \text { Prob }>|t| & 0.8000 \\ \text { Lower CL Dif } & -0.10489 & \text { Prob }>\mathrm{t} & 0.6000 \\ \text { Confidence } & 0.95 & \text { Prob }<\mathrm{t} & 0.4000\end{array}$

Tests that the Variances are Equal

Level Count Std Dev MeanAbsDif to Mean MeanAbsDif to Median

$\begin{array}{lllll}\text { Coliwasa } & 15 & 0.1099777 & 0.0832455 & 0.0803749\end{array}$

$\begin{array}{lllll}\text { Hydragard - LF } & 10 & 0.1092124 & 0.0852548 & 0.0818102\end{array}$

Test F Ratio DFNum DFDen p-Value

$\begin{array}{lrrrr}\text { O'Brien[.5] } & 0.0005 & 1 & 23 & 0.9821\end{array}$

Brown-Forsythe $0.0023 \quad 1 \quad 23 \quad 0.9622$

$\begin{array}{lllll}\text { Levene } & 0.0056 & 1 & 23 & 0.9412\end{array}$

$\begin{array}{lllll}\text { F Test 2-sided } & 1.0141 & 14 & 9 & 1.0000\end{array}$

Welch Anova testing Means Equal, allowing Std Devs Not Equal

F Ratio DFNum DFDen Prob $>$ F

$\begin{array}{lrrr}0.0660 & 1 & 19.541 & 0.8000\end{array}$

t Test 


\section{Exhibit B3. Statistical Comparisons for High-Rheology (Phase 2) Simulant Testing (screened data)}

Oneway Analysis of Measurement (wt \%) By Sampler Type of Material=SME Simulant, Analytical Block=6, Analyte $=\mathrm{MgO}(\mathrm{wt} \%)$, Target $(\mathrm{wt} \%)=1.014$

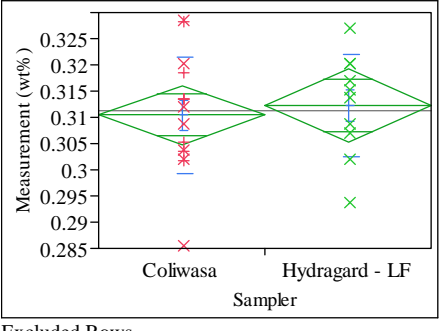

Excl
1

Oneway Anova

Summary of Fit

Adj Rsquare 0.007612

Reot Mean Square Error 0.010638

Mean of Respons Eis 0.010638

Observations (or Sum Wgts) $\quad 25$

Test

Hydragard - LF-Coliwasa

Assuming equal variances
Oneway Analysis of Measurement (wt \%) By Sampler Type of Material=SME Simulant, Analytical Block $=6$, Analyte $=\mathrm{MnO}(\mathrm{wt} \%)$, Target $(\mathrm{wt} \%)=1.779$

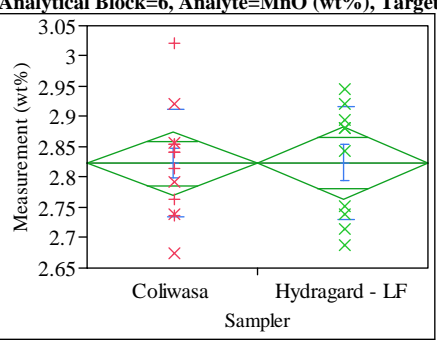

Excluded Rows

Oneway Anova

Summary of Fit

Rsquare

Adj Rsquare

Mean of Response

Observations (or Sum Wgts)

Test

Hydragard - LF-Coliwasa

Assuming equal variances

$\begin{array}{lrlr}\text { Difference } & 0.00109 & \text { t Ratio } & 0.028625 \\ \text { Std Err Dif } & 0.03817 & \text { DF } & 21 \\ \text { Upper CL Dif } & 0.08047 & \text { Prob }>|t| & 0.9774 \\ \text { Lower CL Dif } & -0.07828 & \text { Prob }>\text { t } & 0.4887 \\ \text { Confidence } & 0.95 & \text { Prob }<\text { t } & 0.5113\end{array}$

Analysis of Variance

Source DF Sum of Squares Mean Square F Ratio Prob $>$ F

$\begin{array}{lrrrrr}\text { Sampler } & 1 & 0.00000675 & 6.747 \mathrm{e}-6 & 0.0008 & 0.9774\end{array}$

$\begin{array}{lll}\text { Error } & 21 & 0.17291787 \\ \text { C. Total } & 22 & 0.17292462\end{array}$

Means for Oneway Anova

Level Number Mean Std Error Lower 95\% Upper 95\%

$\begin{array}{lrrrrr}\text { Coliwasa } & 13 & 2.82276 & 0.02517 & 2.7704 & 2.8751\end{array}$

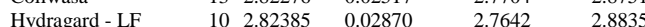

Std Error uses a pooled estimate of error variance

Means and Std Deviation

Level Number Mean Std Dev Std Err Mean Lower 95\% Upper 95\%

$\begin{array}{lllllll}\text { Coliwasa } & 13 & 2.82276 & 0.088448 & 0.02453 & 2.7693 & 2.8762\end{array}$

$\begin{array}{llllll}\text { Hydragard - LF } & 10 & 2.82385 & 0.093715 & 0.02964 & 2.7568\end{array}$

\section{t Test}

Hydragard - LF-Coliwasa

Assuming unequal variances

$\begin{array}{lrlr}\text { Difference } & 0.00109 & \mathrm{t} \text { Ratio } & 0.028399 \\ \text { Std Err Dif } & 0.03847 & \text { DF } & 18.90289 \\ \text { Upper CL Dif } & 0.08164 & \text { Prob }>|t| & 0.9776 \\ \text { Lower CL Dif } & -0.07946 & \text { Prob }>t & 0.4888\end{array}$

Lower CL Dif -0.07946 Prob $>t \quad 0.4888$

Tests that the Variances are Equal

$\begin{array}{lrrrr}\text { Level } & \text { Count } & \text { Std Dev } & \text { MeanAbsDif to Mean } & \text { MeanAbsDif to Median } \\ \text { Coliwasa } & 13 & 0.0884477 & 0.0649420 & 0.0635668\end{array}$

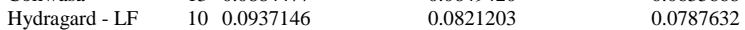

Test F Ratio DFNum DFDen p-Value

$\begin{array}{lllll}\text { O'Brien[.5] } & 0.0441 & 1 & 21 & 0.8356\end{array}$

$\begin{array}{lllll}\text { Brown-Forsythe } & 0.3627 & 1 & 21 & 0.5535\end{array}$

Brown-Forsythe $\begin{array}{llll}0.3627 & 1 & 21 & 0.5535\end{array}$

$\begin{array}{lllll}\text { Levene } & 0.6915 & 1 & 21 & 0.4150 \\ \text { Bartlett } & 0.0330 & 1 & & 0.8559\end{array}$

$\begin{array}{lllll}\text { F Test 2-sided } & 1.1226 & 9 & 12 & 0.8324\end{array}$

Welch Anova testing Means Equal, allowing Std Devs Not Equal

F Ratio DFNum DFDen Prob $>$ F

t Test 


\section{Exhibit B3. Statistical Comparisons for High-Rheology (Phase 2) Simulant Testing (screened data)}

Oneway Analysis of Measurement (wt \%) By Sampler Type of Material=SME Simulant, Analytical Block=6, Analyte $=\mathrm{Na2O}(\mathrm{wt} \%)$, Target $(\mathrm{wt} \%)=11.659$

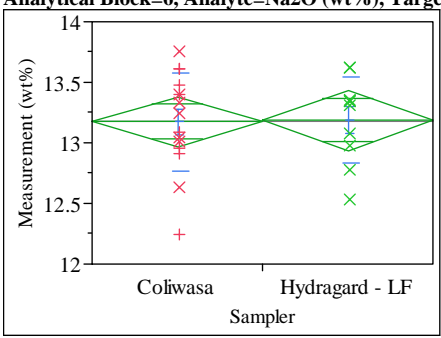

Excluded Rows

Oneway Anova

Summary of Fit

Rsquare

Adj Rsquare

$-0.0433$

0.385613

13.1802
Observations (or Sum Wgts)

t Test

Hydragard - LF-Coliwasa

Assuming equal variances

Difference $\quad 0.00989$ t Ratio 0.062794

$\begin{array}{lrr}\text { Std Err Dif } & 0.15743 \text { DF } & 23 \\ \text { Dper } & \end{array}$

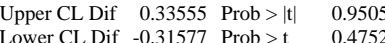

$\begin{array}{lrrr}\text { Lower CL Dif } & -0.31577 & \text { Prob }>t & 0.4752 \\ \text { Confidence } & 0.95 & \text { Prob }<\mathrm{t} & 0.5248\end{array}$

Analysis of Variance

Source DF Sum of Squares Mean Square F Ratio Prob $>$ F

$\begin{array}{lrrrrr}\text { Sampler } & 1 & 0.0005863 & 0.000586 & 0.0039 & 0.9505\end{array}$

$\begin{array}{llll}\text { Error } & 23 & 3.4200320 & 0.148697\end{array}$

C. Total $24 \quad 3.4206183$

Means for Oneway Anova

Level Number Mean Std Error Lower 95\% Upper 95\%

$\begin{array}{lrrrrr}\text { Coliwasa } & 15 & 13.1763 & 0.09956 & 12.970 & 13.382\end{array}$

$\begin{array}{llllll}\text { Hydragard - LF } & 10 & 13.1861 & 0.12194 & 12.934 & 13.438\end{array}$

Std Error uses a pooled estimate of error variance

Means and Std Deviations

Level Number Mean Std Dev Std Err Mean Lower 95\% Upper 95\%

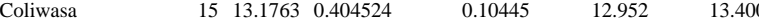

$\begin{array}{lllllll}\text { Hydragard - LF } & 10 & 13.1861 & 0.354193 & 0.11201 & 12.933 & 13.440\end{array}$

t Test

Hydragard - LF-Coliwasa

Assuming unequal variances

Difference $\quad 0.00989$ t Ratio $\quad 0.064547$

Std Err Dif $\quad 0.15315$ DF 21.16811

Upper CL Dif 0.32822 Prob $>|t| 0.9491$

Lower CL Dif -0.30845 Prob $>t \quad 0.4746$

$\begin{array}{lll}\text { Confidence } & 0.95 \text { Prob }<\mathrm{t} \quad 0.5254\end{array}$

Tests that the Variances are Equal

$\begin{array}{lrrrr}\text { Level } & \text { Count } & \text { Std Dev } & \text { MeanAbsDif to Mean } & \text { MeanAbsDif to Median } \\ \text { Coliwasa } & 15 & 0.4045241 & 0.3186073 & 0.3199253\end{array}$

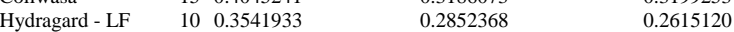

Test F Ratio DFNum DFDen p-Value

O'Brien[5] 0.1784

$\begin{array}{lllll}\text { O'Brien[.5] } & 0.1784 & 1 & 23 & 0.6766 \\ \text { Brown-Forsythe } & 0.3275 & 1 & 23 & 0.5727 \\ \text { Levene }\end{array}$

$\begin{array}{lllll}\text { Levene } & 0.1418 & 1 & 23 & 0.7100 \\ \text { Bartlett } & 0.1809 & 1 & & 0.6706\end{array}$

$\begin{array}{lrrrr}\text { F Test 2-sided } & 1.3044 & 1 & \dot{9} & 0.6706 \\ \end{array}$

Welch Anova testing Means Equal, allowing Std Devs Not Equal

F Ratio DFNum DFDen Prob $>$ F

$\begin{array}{llll}0.0042 & 1 & 21.168 & 0.9491\end{array}$

t Test
0.0645
Oneway Analysis of Measurement (wt \%) By Sampler Type of Material=SME Simulant, Analytical Block=6, Analyte $=\mathrm{SiO} 2(\mathrm{wt} \%)$, Target $(\mathrm{wt} \%)=50.985$

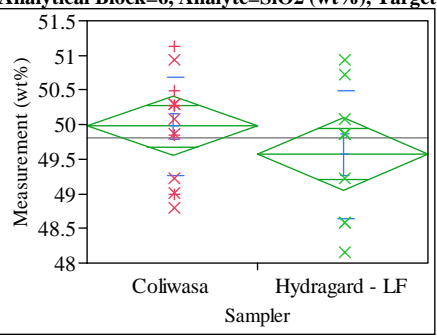

Excluded Rows

Oneway Anova

Summary of Fit

Rsquare

Adj Rsquare

$\quad 0.80008$

49.81146
Observations (or Sum Wgts)

t Test

Hydragard - LF-Coliwasa

Assuming equal variances

Difference $\quad-0.4065$ t Ratio $\quad-1.24442$

$\begin{array}{lrlr}\text { Std Err Dif } & 0.3266 & \mathrm{DF} & 23 \\ \end{array}$

Upper CL Dif 0.2692 Prob $>|t| \quad 0.2259$

Lower CL Dif -1.0822 Prob $>\mathrm{t} \quad 0.8871$

$\begin{array}{lll}\text { Confidence } & 0.95 \text { Prob }<\mathrm{t} \quad 0.1129\end{array}$

Analysis of Variance

Source DF Sum of Squares Mean Square F Ratio Prob > F

$\begin{array}{lrrrrr}\text { Sampler } & 1 & 0.991293 & 0.991293 & 1.5486 & 0.2259\end{array}$

$\begin{array}{llrl}\text { Error } & 23 & 14.722937 & 0.640128\end{array}$

C. Total $24 \quad 15.714229$

Means for Oneway Anova

Level Number Mean Std Error Lower 95\% Upper 95\%

$\begin{array}{lrrrrr}\text { Coliwasa } & 15 & 49.9740 & 0.20658 & 49.547 & 50.401\end{array}$

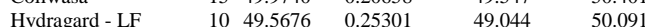

Std Error uses a pooled estimate of error variance

Means and Std Deviations

Level Number Mean Std Dev Std Err Mean Lower 95\% Upper 95\%

$\begin{array}{lllllll}\text { Coliwasa } & 15 & 49.9740 & 0.703975 & 0.18177 & 49.584 & 50.364\end{array}$

$\begin{array}{lllllll}\text { Hydragard - LF } & 10 & 49.5676 & 0.930042 & 0.29411 & 48.902 & 50.233\end{array}$

t Test

Hydragard - LF-Coliwasa

Assuming unequal variances

Difference $\quad-0.4065$ t Ratio $\quad-1.17564$

Std Err Dif $\quad 0.3457$ DF $\quad 15.71448$

Upper CL Dif 0.3276 Prob $>|t| \quad 0.2572$

Lower CL Dif -1.1405 Prob $>t \quad 0.8714$

Confidence $\quad 0.95$ Prob $<\mathrm{t} \quad 0.1286$

Tests that the Variances are Equal

$\begin{array}{lrrrr}\text { Level } & \text { Count } & \text { Std Dev } & \text { MeanAbsDif to Mean } & \text { MeanAbsDif to Median } \\ \text { Coliwasa } & 15 & 0.7039751 & 0.5590704 & 0.5276940\end{array}$

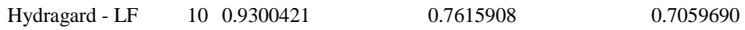

Test F Ratio DFNum DFDen p-Value

$\begin{array}{lllll}\text { O'Brien[.5] } & 1.5737 & 1 & 23 & 0.2223\end{array}$

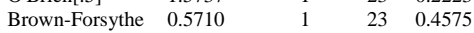

$\begin{array}{lllll}\text { Levene } & 1.3367 & 1 & 23 & 0.2595\end{array}$

$\begin{array}{lllll}\text { Bartlett } & 0.8351 & 1 & . & 0.3608\end{array}$

$\begin{array}{lllll}\text { F Test 2-sided } & 1.7454 & 9 & 14 & 0.3378\end{array}$

Welch Anova testing Means Equal, allowing Std Devs Not Equal

F Ratio DFNum DFDen Prob $>$ F

$\begin{array}{llll}1.3821 & 1 & 15.714 & 0.2572\end{array}$

t Test 


\section{Exhibit B3. Statistical Comparisons for High-Rheology (Phase 2) Simulant Testing (screened data)}

Oneway Analysis of Measurement (wt \%) By Sampler Type of Material=SME Simulant, Analytical Block=6, Analyte=Sum of Oxides (wt \%), Target $(w t \%)=99.553$

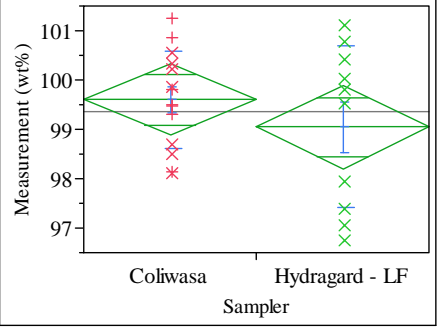

Excluded Rows

Oneway Anova

Summary of Fit

Rsquare

Adj Rsquare

0.002424

\begin{tabular}{ll}
0.294031 \\
\hline
\end{tabular}

99.37305

Observations (or Sum Wgts) 24

Test

Hydragard - LF-Coliwasa

Assuming equal variances

Difference $\quad-0.5505$ t Ratio -1.02756

Std Err Dif $\quad 0.5358$ DF 22

Upper CL Dif 0.5606 Prob $>|t| 0.3153$

Lower CL Dif -1.6617 Prob $>\mathrm{t} \quad 0.8423$

$\begin{array}{lll}\text { Confidence } & 0.95 \text { Prob }<\mathrm{t} \quad 0.1577\end{array}$

Analysis of Variance

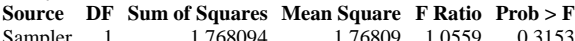

$\begin{array}{lrrrrr}\text { Sampler } & 1 & 1.768094 & 1.76809 & 1.0559 & 0.3153\end{array}$

$\begin{array}{llll}\text { Error } & 22 & 36.839350 & 1.67452\end{array}$

C. Total $23 \quad 38.607444$

Means for Oneway Anova

Level Number Mean Std Error Lower 95\% Upper 95\%

Coliwase 14 99.6024 0.34584 Lower 95\% Upper 95\%

$\begin{array}{llllrr}\text { Hydragard - LF } & 14 & 99.6024 & 0.34584 & 98.885 & 100.32 \\ & 10 & 99.0519 & 0.40921 & 98.203 & 99.90\end{array}$

Std Error uses a pooled estimate of error variance

Means and Std Deviations

Level Number Mean Std Dev Std Err Mean Lower 95\% Upper 95\%

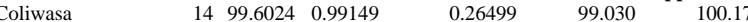

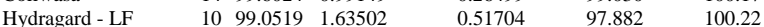

t Test

Hydragard - LF-Coliwasa

Assuming unequal variances

Difference $\quad-0.5505$ t Ratio $\quad-0.9476$

Std Err Dif $\quad 0.5810$ DF $\quad 13.69479$

Upper CL Dif 0.6982 Prob $>|t| \quad 0.3598$

Lower CL Dif -1.7993 Prob $>t \quad 0.8201$

$\begin{array}{lrl}\text { Confidence } & 0.95 \text { Prob }<\mathrm{t} \quad 0.1799\end{array}$

Tests that the Variances are Equal

Level Count Std Dev MeanAbsDif to Mean MeanAbsDif to Median

$\begin{array}{lllll} & 14 & 0.991491 & 0.794837 & 0.794837\end{array}$

Test F Ratio DFNum DFDen p-Value

$\begin{array}{lrrrr}\text { O'Brien[.5] } & 6.9422 & 1 & 22 & 0.0151 \\ \text { Brown-Forsythe } & 2.8796 & 1 & 22 & 0.1038 \\ \text { Levene } & 7.2173 & 1 & 22 & 0.0135 \\ \text { Bartlett } & 2.5908 & 1 & . & 0.1075 \\ \text { F Test 2-sided } & 2.7194 & 9 & 13 & 0.0994\end{array}$

Welch Anova testing Means Equal, allowing Std Devs Not Equal

F Ratio DFNum DFDen Prob > F

$\begin{array}{llrr}0.8980 & 1 & 13.695 & 0.3598\end{array}$

t Test

0.9476
Oneway Analysis of Measurement (wt \%) By Sampler Type of Material=SME Simulant, Analytical Block=6, Analyte $=\mathrm{ZnO}(\mathrm{wt} \%)$, Target $(\mathrm{wt} \%)=\mathbf{0 . 0 9 5 8}$

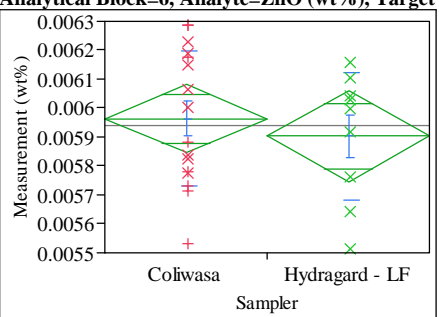

Excluded Rows

1

Oneway Anova

Summary of Fit

Adj Rsquare

Mean of Response

(or Sum Wgts)

Test

Hydragard - LF-Coliwasa

Assuming equal variances

Difference $\quad-6.1 \mathrm{e}-5$ t Ratio $\quad-0.63585$

Std Err Dif $\quad 0.000096$ DF 23

Upper CL Dif 0.00014 Prob $>|t| \quad 0.5311$

$\begin{array}{lll}\text { Lower CL Dif }-0.00026 & \text { Prob }>t \quad 0.7344\end{array}$

$\begin{array}{lrl}\text { Confidence } & 0.95 \mathrm{Prob}<\mathrm{t} & 0.2656\end{array}$

Analysis of Variance

Source DF Sum of Squares Mean Square F Ratio Prob $>$ F

$\begin{array}{lrrrrr}\text { Sampler } & 1 & 2.14539 \mathrm{e}-8 & 2.1454 \mathrm{e}-8 & 0.4043 & 0.5311\end{array}$

Error $23 \quad 1.22045 \mathrm{e}-6 \quad 5.3063 \mathrm{e}-8$

C. Total $24 \quad 1.24191 \mathrm{e}-6$

Means for Oneway Anova

Level Number Mean Std Error Lower 95\% Upper 95\%

$\begin{array}{lrrrrr}\text { Coliwasa } & 16 & 0.005964 & 5.76 \mathrm{e}-5 & 0.00585 & 0.00608 \\ \text { Hydragard - LF } & 9 & 0.005903 & 7.68 \mathrm{e}-5 & 0.00574 & 0.00606\end{array}$

Std Error uses a pooled estimate of error variance

Means and Std Deviations

Level Number Mean Std Dev Std Err Mean Lower 95\% Upper 95\%

$\begin{array}{lllllll}\text { Coliwasa } & 16 & 0.005964 & 0.000235 & 5.88 \mathrm{e}-5 & 0.00584 & 0.00609\end{array}$

$\begin{array}{lrrrrrr}\text { Hydragard - LF } & 9 & 0.005903 & 0.000221 & 7.36 \mathrm{e}-5 & 0.00573 & 0.00607\end{array}$

t Test

Hydragard - LF-Coliwasa

Assuming unequal variances

$\begin{array}{lrlr}\text { Difference } & -6.1 \mathrm{e}-5 & \text { t Ratio } & -0.64761 \\ \text { Std Err Dif } & 9.424 \mathrm{e}-5 & \text { DF } & 17.6337 \\ \text { Upper CL Dif } & 0.00014 & \text { Prob }>|\mathrm{t}| & 0.5256 \\ \text { Lower CL Dif } & -0.00026 & \text { Prob }>\mathrm{t} & 0.7372 \\ \text { Confidence } & 0.95 & \text { Prob }<\mathrm{t} & 0.2628\end{array}$

Tests that the Variances are Equal

Level Count Std Dev MeanAbsDif to Mean MeanAbsDif to Median

$\begin{array}{lllll}\text { Coliwasa } & 16 & 0.0002352 & 0.0002062 & 0.0002062\end{array}$

$\begin{array}{lrrrr}\text { Hydragard - LF } & 9 & 0.0002209 & 0.0001789 & 0.0001701\end{array}$

Test F Ratio DFNum DFDen p-Value

$\begin{array}{lrrrr}\text { O'Brien[.5] } & 0.0833 & 1 & 23 & 0.7755\end{array}$

$\begin{array}{lllll}\text { Brown-Forsythe } & 0.4737 & 1 & 23 & 0.4982\end{array}$

$\begin{array}{lllll}\text { Levene } & 0.3909 & 1 & 23 & 0.5380\end{array}$

$\begin{array}{lrrrr}\text { Bartlett } & 0.0387 & 1 & \text {. } & 0.8440 \\ \text { F Test 2-sided } & 1.1339 & 15 & 8 & 0.8938\end{array}$

Welch Anova testing Means Equal, allowing Std Devs Not Equal

F Ratio DFNum DFDen Prob $>$ F

$0.4194 \quad 1 \quad 17.634 \quad 0.5256$

t Test

0.6476 


\section{Appendix C.}

Supplemental Tables and Exhibits for Low-Rheology (Phase 3) Simulant Testing 
Table C1. Density Measurements for Phase 3

\begin{tabular}{|c|c|c|c|c|}
\hline Sampling Mechanism & Flow Rate (LF/HF) & Sampler w Location & Sample ID & Sample Density (g/mL) \\
\hline Coliwasa & LF & Coliwasa Low & DCL01 & 1.2453 \\
\hline Coliwasa & LF & Coliwasa Low & DCL02 & 1.2620 \\
\hline Coliwasa & LF & Coliwasa Low & DCL03 & 1.2604 \\
\hline Coliwasa & LF & Coliwasa Low & DCL04 & 1.2621 \\
\hline Coliwasa & $\mathrm{HF}$ & Coliwasa Low & DCL05 & 1.2453 \\
\hline Coliwasa & $\mathrm{HF}$ & Coliwasa Low & DCL06 & 1.2643 \\
\hline Coliwasa & $\mathrm{HF}$ & Coliwasa Low & DCL07 & 1.2655 \\
\hline Coliwasa & $\mathrm{HF}$ & Coliwasa Low & DCL08 & 1.2683 \\
\hline Coliwasa & LF & Coliwasa High & DCH01 & 1.2608 \\
\hline Coliwasa & LF & Coliwasa High & DCH02 & 1.2610 \\
\hline Coliwasa & LF & Coliwasa High & DCH03 & 1.2666 \\
\hline Coliwasa & LF & Coliwasa High & DCH04 & 1.2445 \\
\hline Coliwasa & $\mathrm{HF}$ & Coliwasa High & DCH05 & 1.2366 \\
\hline Coliwasa & $\mathrm{HF}$ & Coliwasa High & DCH06 & 1.2429 \\
\hline Coliwasa & $\mathrm{HF}$ & Coliwasa High & DCH07 & 1.2428 \\
\hline Coliwasa & $\mathrm{HF}$ & Coliwasa High & DCH08 & 1.2486 \\
\hline Hydragard & LF & Hydragard & DLHY01 & 1.2456 \\
\hline Hydragard & LF & Hydragard & DLHY02 & 1.2582 \\
\hline Hydragard & LF & Hydragard & DLHY03 & 1.2394 \\
\hline Hydragard & LF & Hydragard & DLHY04 & 1.2510 \\
\hline Hydragard & LF & Hydragard & DLHY05 & 1.2466 \\
\hline Hydragard & LF & Hydragard & DLHY06 & 1.2399 \\
\hline Hydragard & LF & Hydragard & DLHY07 & 1.2456 \\
\hline Hydragard & LF & Hydragard & DLHY08 & 1.2453 \\
\hline Hydragard & LF & Hydragard & DLHY09 & 1.2576 \\
\hline Hydragard & LF & Hydragard & DLHY10 & 1.2720 \\
\hline Hydragard & $\mathrm{HF}$ & Hydragard & DHHY01 & 1.2659 \\
\hline Hydragard & HF & Hydragard & DHHY02 & 1.2552 \\
\hline Hydragard & $\mathrm{HF}$ & Hydragard & DHHY03 & 1.2629 \\
\hline Hydragard & $\mathrm{HF}$ & Hydragard & DHHY04 & 1.2505 \\
\hline Hydragard & $\mathrm{HF}$ & Hydragard & DHHY05 & 1.2400 \\
\hline Hydragard & $\mathrm{HF}$ & Hydragard & DHHY06 & 1.2476 \\
\hline Hydragard & $\mathrm{HF}$ & Hydragard & DHHY07 & 1.2550 \\
\hline Hydragard & $\mathrm{HF}$ & Hydragard & DHHY08 & 1.2545 \\
\hline Hydragard & $\mathrm{HF}$ & Hydragard & DHHY09 & 1.2544 \\
\hline Hydragard & $\mathrm{HF}$ & Hydragard & DHHY10 & 1.2586 \\
\hline Isolok & LF & Isolok & DLIB01 & 1.2596 \\
\hline Isolok & LF & Isolok & DLIB02 & 1.2585 \\
\hline Isolok & LF & Isolok & DLIB03 & 1.2512 \\
\hline Isolok & LF & Isolok & DLIB04 & 1.2754 \\
\hline Isolok & LF & Isolok & DLIB05 & 1.2616 \\
\hline Isolok & LF & Isolok & DLIB06 & 1.2680 \\
\hline Isolok & LF & Isolok & DLIB07 & 1.2446 \\
\hline Isolok & LF & Isolok & DLIB08 & 1.2439 \\
\hline Isolok & LF & Isolok & DLIB09 & 1.2521 \\
\hline Isolok & LF & Isolok & DLIB10 & 1.2566 \\
\hline Isolok & $\mathrm{HF}$ & Isolok & DHIB01 & 1.2510 \\
\hline Isolok & $\mathrm{HF}$ & Isolok & DHIB02 & 1.2578 \\
\hline Isolok & $\mathrm{HF}$ & Isolok & DHIB03 & 1.2478 \\
\hline Isolok & $\mathrm{HF}$ & Isolok & DHIB04 & 1.2524 \\
\hline Isolok & $\mathrm{HF}$ & Isolok & DHIB05 & 1.2441 \\
\hline Isolok & $\mathrm{HF}$ & Isolok & DHIB06 & 1.2555 \\
\hline Isolok & $\mathrm{HF}$ & Isolok & DHIB07 & 1.2510 \\
\hline Isolok & $\mathrm{HF}$ & Isolok & DHIB08 & 1.2351 \\
\hline Isolok & $\mathrm{HF}$ & Isolok & DHIB09 & 1.2496 \\
\hline Isolok & HF & Isolok & DHIB10 & 1.2588 \\
\hline
\end{tabular}


Table C2. Solids Measurements for Phase 3

\begin{tabular}{|c|c|c|c|c|c|}
\hline $\begin{array}{l}\text { Sampling } \\
\text { Mechanism }\end{array}$ & \begin{tabular}{|l} 
Flow Rate \\
(LF/HF)
\end{tabular} & $\begin{array}{l}\text { Sampler } \\
\text { w Location }\end{array}$ & \begin{tabular}{|l|} 
Sample \\
ID
\end{tabular} & \begin{tabular}{|r|} 
wt \% dried \\
solids
\end{tabular} & $\begin{array}{r}\text { wt\% vitrified } \\
\text { solids }\end{array}$ \\
\hline Coliwasa & LF & Coliwasa Low & CL01 & 31.45 & 26.65 \\
\hline Coliwasa & LF & Coliwasa Low & CL02 & 31.55 & 26.75 \\
\hline Coliwasa & LF & Coliwasa Low & CL03 & 31.57 & 26.78 \\
\hline Coliwasa & LF & Coliwasa Low & CL04 & 31.48 & 26.67 \\
\hline Coliwasa & $\mathrm{HF}$ & Coliwasa Low & CL05 & 31.77 & 27.00 \\
\hline Coliwasa & $\mathrm{HF}$ & Coliwasa Low & CL06 & 31.51 & 26.71 \\
\hline Coliwasa & $\mathrm{HF}$ & Coliwasa Low & CL07 & 31.71 & 26.92 \\
\hline Coliwasa & $\mathrm{HF}$ & Coliwasa Low & CL08 & 31.70 & 26.90 \\
\hline Coliwasa & LF & Coliwasa High & $\mathrm{CH} 01$ & 31.52 & 26.72 \\
\hline Coliwasa & LF & Coliwasa High & $\mathrm{CH} 02$ & 31.54 & 26.74 \\
\hline Coliwasa & LF & Coliwasa High & $\mathrm{CH} 03$ & 31.59 & 26.82 \\
\hline Coliwasa & LF & Coliwasa High & $\mathrm{CH} 04$ & 31.40 & 26.60 \\
\hline Coliwasa & $\mathrm{HF}$ & Coliwasa High & CH05 & 31.41 & 26.62 \\
\hline Coliwasa & $\mathrm{HF}$ & Coliwasa High & CH06 & 31.62 & 26.82 \\
\hline Coliwasa & $\mathrm{HF}$ & Coliwasa High & $\mathrm{CH} 07$ & 31.61 & 26.82 \\
\hline Coliwasa & $\mathrm{HF}$ & Coliwasa High & CH08 & 31.67 & 26.86 \\
\hline Hydragard & LF & Hydragard & LHY01 & 30.83 & 25.98 \\
\hline Hydragard & LF & Hydragard & LHY02 & 31.02 & 26.16 \\
\hline Hydragard & LF & Hydragard & LHY03 & 30.91 & 26.01 \\
\hline Hydragard & LF & Hydragard & LHY04 & 30.74 & 25.85 \\
\hline Hydragard & LF & Hydragard & LHY05 & 30.85 & 25.99 \\
\hline Hydragard & LF & Hydragard & LHY06 & 30.83 & 25.98 \\
\hline Hydragard & LF & Hydragard & LHY07 & 31.03 & 26.14 \\
\hline Hydragard & LF & Hydragard & LHY08 & 30.87 & 25.92 \\
\hline Hydragard & LF & Hydragard & LHY09 & 30.68 & 25.77 \\
\hline Hydragard & LF & Hydragard & LHY10 & 30.90 & 26.00 \\
\hline Hydragard & $\mathrm{HF}$ & Hydragard & HHY01 & 30.94 & 26.17 \\
\hline Hydragard & $\mathrm{HF}$ & Hydragard & HHY02 & 30.67 & 25.81 \\
\hline Hydragard & $\mathrm{HF}$ & Hydragard & HHY03 & 30.76 & 25.91 \\
\hline Hydragard & $\mathrm{HF}$ & Hydragard & HHY04 & 30.85 & 26.04 \\
\hline Hydragard & $\mathrm{HF}$ & Hydragard & HHY05 & 30.70 & 25.88 \\
\hline Hydragard & $\mathrm{HF}$ & Hydragard & HHY06 & 30.78 & 25.91 \\
\hline Hydragard & $\mathrm{HF}$ & Hydragard & HHY07 & 30.75 & 25.88 \\
\hline Hydragard & $\mathrm{HF}$ & Hydragard & HHY08 & 30.87 & 26.03 \\
\hline Hydragard & $\mathrm{HF}$ & Hydragard & HHY09 & 30.60 & 25.72 \\
\hline Hydragard & $\mathrm{HF}$ & Hydragard & HHY10 & 30.59 & 25.73 \\
\hline Isolok (3 mL) & LF & Isolok (3 mL) & LIS01 & 31.73 & 26.83 \\
\hline Isolok (3 mL) & LF & Isolok (3 mL) & LIS02 & 31.43 & 26.73 \\
\hline Isolok (3 mL) & LF & Isolok (3 mL) & LIS03 & 31.56 & 26.88 \\
\hline Isolok (3 mL) & LF & Isolok (3 mL) & LIS04 & 31.51 & 26.74 \\
\hline Isolok (3 mL) & LF & Isolok (3 mL) & LIS05 & 31.58 & 27.00 \\
\hline Isolok (3 mL) & LF & Isolok (3 mL) & LIS06 & 31.61 & 26.83 \\
\hline Isolok (3 mL) & LF & Isolok (3 mL) & LIS07 & 31.92 & 27.05 \\
\hline Isolok (3 mL) & LF & Isolok (3 mL) & LIS08 & 31.50 & 26.61 \\
\hline Isolok (3 mL) & LF & Isolok (3 mL) & LIS09 & 31.64 & 26.80 \\
\hline Isolok (3 mL) & LF & Isolok (3 mL) & LIS10 & 31.69 & 26.88 \\
\hline Isolok (3 mL) & $\mathrm{HF}$ & Isolok (3 mL) & HIS01 & 31.62 & 27.23 \\
\hline Isolok (3 mL) & $\mathrm{HF}$ & Isolok (3 mL) & HIS02 & 31.35 & 26.65 \\
\hline Isolok (3 mL) & HF & Isolok (3 mL) & HIS03 & 31.37 & 26.76 \\
\hline Isolok (3 mL) & $\mathrm{HF}$ & Isolok (3 mL) & HIS04 & 31.70 & 27.09 \\
\hline Isolok (3 mL) & $\mathrm{HF}$ & Isolok (3 mL) & HIS05 & 31.37 & 26.74 \\
\hline Isolok (3 mL) & $\mathrm{HF}$ & Isolok (3 mL) & HIS06 & 31.66 & 26.97 \\
\hline Isolok (3 mL) & $\mathrm{HF}$ & Isolok (3 mL) & HIS07 & 31.47 & 26.85 \\
\hline Isolok (3 mL) & $\mathrm{HF}$ & Isolok (3 mL) & HIS08 & 31.58 & 26.87 \\
\hline Isolok (3 mL) & $\mathrm{HF}$ & Isolok (3 mL) & HIS09 & 32.00 & 27.26 \\
\hline Isolok (3 mL) & HF & Isolok (3 mL) & HIS10 & 31.70 & 26.97 \\
\hline Isolok $(12 \mathrm{~mL})$ & LF & Isolok $(12 \mathrm{~mL})$ & LIB01 & 31.50 & 26.75 \\
\hline Isolok $(12 \mathrm{~mL})$ & LF & Isolok $(12 \mathrm{~mL})$ & LIB02 & 31.70 & 26.92 \\
\hline Isolok $(12 \mathrm{~mL})$ & LF & Isolok $(12 \mathrm{~mL})$ & LIB03 & 31.45 & 26.71 \\
\hline Isolok $(12 \mathrm{~mL})$ & LF & Isolok $(12 \mathrm{~mL})$ & LIB04 & 31.34 & 26.55 \\
\hline Isolok (12 mL) & LF & Isolok $(12 \mathrm{~mL})$ & LIB05 & 31.57 & 26.77 \\
\hline Isolok (12 mL) & LF & Isolok (12 mL) & LIB06 & 31.56 & 26.73 \\
\hline Isolok (12 mL) & LF & Isolok (12 mL) & LIB07 & 31.62 & 26.77 \\
\hline
\end{tabular}


Table C2. Solids Measurements for Phase 3

\begin{tabular}{|c|c|c|c|c|c|}
\hline $\begin{array}{l}\text { Sampling } \\
\text { Mechanism }\end{array}$ & $\begin{array}{l}\text { Flow Rate } \\
\text { (LF/HF) }\end{array}$ & \begin{tabular}{|l|} 
Sampler \\
w Location \\
\end{tabular} & \begin{tabular}{|l|} 
Sample \\
ID
\end{tabular} & $\begin{array}{r}\text { wt\% dried } \\
\text { solids } \\
\end{array}$ & $\begin{array}{r}\text { wt \% vitrified } \\
\text { solids } \\
\end{array}$ \\
\hline Isolok (12 mL) & LF & Isolok (12 mL) & LIB08 & 31.68 & 26.80 \\
\hline Isolok (12 mL) & LF & Isolok (12 mL) & LIB09 & 31.70 & 26.74 \\
\hline Isolok (12 mL) & LF & Isolok (12 mL) & LIB10 & 31.73 & 26.83 \\
\hline Isolok (12 mL) & HF & Isolok (12 mL) & HIB01 & 31.40 & 26.69 \\
\hline Isolok (12 mL) & $\mathrm{HF}$ & Isolok $(12 \mathrm{~mL})$ & HIB02 & 31.51 & 26.69 \\
\hline Isolok (12 mL) & $\mathrm{HF}$ & Isolok $(12 \mathrm{~mL})$ & HIB03 & 31.47 & 26.68 \\
\hline Isolok (12 mL) & $\mathrm{HF}$ & Isolok (12 mL) & HIB04 & 31.57 & 26.83 \\
\hline Isolok (12 mL) & $\mathrm{HF}$ & Isolok (12 mL) & HIB05 & 31.54 & 26.80 \\
\hline Isolok (12 mL) & $\mathrm{HF}$ & Isolok $(12 \mathrm{~mL})$ & HIB06 & 31.57 & 26.77 \\
\hline Isolok (12 mL) & $\mathrm{HF}$ & Isolok (12 mL) & HIB07 & 31.57 & 26.78 \\
\hline Isolok (12 mL) & $\mathrm{HF}$ & Isolok $(12 \mathrm{~mL})$ & HIB08 & 31.68 & 26.89 \\
\hline Isolok (12 mL) & $\mathrm{HF}$ & Isolok $(12 \mathrm{~mL})$ & HIB09 & 31.46 & 26.65 \\
\hline Isolok (12 mL) & HF & Isolok (12 mL) & HIB10 & 31.70 & 26.88 \\
\hline
\end{tabular}


Table C3. Phase 3 Elemental Concentration Measurements (part 1)

\begin{tabular}{|c|c|c|c|c|c|c|c|c|c|c|c|c|c|c|c|c|c|c|c|}
\hline \begin{tabular}{|c|} 
Type of \\
Material \\
\end{tabular} & Sampler & $\begin{array}{l}\text { Flow } \\
\text { Rate }\end{array}$ & $\begin{array}{c}\text { Sample } \\
\text { Order }\end{array}$ & \begin{tabular}{|c|}
$\begin{array}{c}\text { Preparation } \\
\text { Block }\end{array}$ \\
\end{tabular} & $\begin{array}{c}\begin{array}{c}\text { Preparation } \\
\text { Sequence }\end{array} \\
\end{array}$ & \begin{tabular}{|c|}
$\begin{array}{c}\text { Analytical } \\
\text { Block }\end{array}$ \\
\end{tabular} & \begin{tabular}{|c|} 
Analytical \\
Sequence \\
\end{tabular} & \begin{tabular}{|l} 
Sample ID \\
\end{tabular} & $\begin{array}{c}\text { Al } \\
(\mathrm{ug} / \mathrm{g})\end{array}$ & $\begin{array}{c}\begin{array}{c}\text { B } \\
(\mathrm{ug} / \mathrm{g})\end{array} \\
\end{array}$ & $\begin{array}{c}\begin{array}{c}\text { Ba } \\
(\mathbf{u g} / \mathrm{g})\end{array} \\
\end{array}$ & $\begin{array}{c}\begin{array}{c}\text { Ca } \\
(\mathrm{ug} / \mathrm{g})\end{array} \\
\end{array}$ & $\begin{array}{c}\begin{array}{c}\mathrm{Ce} \\
(\mathrm{ug} / \mathrm{g})\end{array} \\
\end{array}$ & $\begin{array}{c}\begin{array}{c}\mathbf{C r} \\
(\mathrm{ug} / \mathrm{g})\end{array} \\
\end{array}$ & $\left.\begin{array}{c}\mathbf{C u} \\
(\mathbf{u g} / \mathrm{g})\end{array}\right)$ & $\begin{array}{c}\begin{array}{c}F e \\
(\mathrm{ug} / \mathrm{g})\end{array} \\
\end{array}$ & $\begin{array}{c}\mathbf{K} \\
(\mathbf{u g} / \mathrm{g})\end{array}$ & $\begin{array}{c}\mathrm{La} \\
\text { (ug/g) }\end{array}$ & $\begin{array}{c}\mathbf{L i} \\
(\mathbf{u g} / \mathrm{g})\end{array}$ \\
\hline ARG-1 & None & 0 & 0 & 1 & 1 & \begin{tabular}{|l|}
1 \\
\end{tabular} & \begin{tabular}{|l|}
1 \\
\end{tabular} & \begin{tabular}{|l|} 
ARG-1B11 \\
\end{tabular} & 23600 & 24100 & 792 & 10000 & $<712$ & 652 & 77.7 & 94600 & 23700 & $<148$ & 14200 \\
\hline SME Simulant & Isolok - $3 \mathrm{~mL}$ & LF & 18 & 1 & 9 & 1 & 2 & LIS03 & 54400 & 13000 & 907 & 8260 & $<559$ & 621 & 270 & 76700 & $<2750$ & $<116$ & 22200 \\
\hline SME Simulant & Isolok - $3 \mathrm{~mL}$ & LF & 8 & 1 & 27 & 1 & 3 & LIS01 & 54500 & 12600 & 920 & 8170 & $<611$ & 724 & 273 & 76600 & $<3000$ & $<127$ & 21300 \\
\hline SME Simulant & Coliwasa & LF & 20 & 1 & 2 & 1 & 4 & $\begin{array}{l}\text { CL02 } \\
\end{array}$ & 53800 & 12800 & 921 & 8440 & $<649$ & 771 & 303 & 76800 & $<3190$ & $<135$ & 20900 \\
\hline SME Simulant & Coliwasa & LF & 38 & 1 & 16 & 1 & 5 & CH03 & 55300 & 12800 & 939 & 8350 & $<548$ & 747 & 252 & 77800 & $<2690$ & $<114$ & 21300 \\
\hline SME Simulant & Coliwasa & LF & 63 & 1 & 13 & 1 & 6 & CH04 & 55700 & 12700 & 952 & 8480 & $<581$ & 757 & 292 & 78500 & $<2860$ & $<121$ & 21100 \\
\hline SME Simulant & Isolok - $3 \mathrm{~mL}$ & LF & 44 & 1 & 3 & 1 & 7 & LIS07 & 53000 & 12600 & 883 & 7820 & $<609$ & 601 & 245 & 74100 & $<2990$ & $<127$ & 21400 \\
\hline SME Simulant & Coliwasa & $\mathrm{HF}$ & 23 & 1 & 28 & 1 & 8 & CH06 & 53700 & 12700 & 878 & 7960 & $<607$ & 698 & 202 & 75700 & $<2980$ & $<126$ & 20800 \\
\hline SME Simulant & Coliwasa & LF & 21 & 1 & 14 & 1 & 9 & $\mathrm{CH} 02$ & 54900 & 13000 & 918 & 8170 & $<615$ & 727 & 289 & 77700 & $<3020$ & $<128$ & 21200 \\
\hline SME Simulant & Coliwasa & $\mathrm{HF}$ & 3 & 1 & 20 & 1 & 10 & CL05 & 54400 & 12900 & 924 & 8400 & $<610$ & 812 & 301 & 77400 & $<3000$ & $<127$ & 21800 \\
\hline SME Simulant & Isolok - $3 \mathrm{~mL}$ & LF & 12 & 1 & 25 & 1 & 11 & LIS02 & 53200 & 11500 & 899 & 8040 & $<617$ & 619 & 272 & 76000 & $<3030$ & $<128$ & 20600 \\
\hline SME Simulant & Coliwasa & LF & 40 & 1 & 19 & 1 & 12 & CL03 & 55200 & 12000 & 922 & 8220 & $<576$ & 759 & 240 & 77600 & $<2830$ & $<120$ & 20700 \\
\hline SME Simulant & Coliwasa & $\mathrm{HF}$ & 18 & 1 & 26 & 1 & 13 & CL06 & 54400 & 12900 & 959 & 8500 & $<644$ & 854 & 233 & 77500 & $<3160$ & $<134$ & 20500 \\
\hline SME Simulant & Coliwasa & $\mathrm{HF}$ & 1 & 1 & 22 & 1 & 14 & CH05 & 54800 & 12000 & 914 & 8230 & $<614$ & 869 & 301 & 78000 & $<3020$ & $<128$ & 21200 \\
\hline ARG-1 & None & 0 & 0 & 1 & 15 & 1 & 15 & ARG-1B12 & 23600 & 24700 & 810 & 10100 & $<573$ & 665 & $<41.6$ & 97200 & 21500 & $<119$ & 14200 \\
\hline SME Simulant & $\begin{array}{l}\text { Coliwasa } \\
\end{array}$ & $\mathrm{HF}$ & 36 & 1 & 21 & 1 & 16 & CL07 & 51100 & 12000 & 867 & 7640 & $<582$ & 739 & 208 & 72500 & $<2860$ & $<121$ & 20000 \\
\hline SME Simulant & Isolok - $3 \mathrm{~mL}$ & $\mathrm{LF}$ & 31 & 1 & 24 & 1 & 17 & LIS05 & 52300 & 12500 & 853 & 7540 & $<545$ & 746 & 301 & 74500 & $<2680$ & $<113$ & 20100 \\
\hline SME Simulant & Isolok - $3 \mathrm{~mL}$ & LF & 58 & 1 & 4 & 1 & 18 & LIS10 & 54500 & 12600 & 907 & 8110 & $<639$ & 656 & 318 & 76600 & $<3140$ & $<133$ & 20800 \\
\hline SME Simulant & Coliwasa & $\mathrm{HF}$ & 63 & 1 & 5 & 1 & 19 & CL08 & 55000 & 12500 & 930 & 8270 & $<624$ & 837 & 281 & 78000 & $<3070$ & $<130$ & 20500 \\
\hline SME Simulant & Isolok - $3 \mathrm{~mL}$ & LF & 25 & 1 & 7 & 1 & 20 & LIS04 & 54500 & 13000 & 911 & 8200 & $<581$ & 649 & 302 & 77700 & $<2860$ & $<121$ & 20800 \\
\hline SME Simulant & Isolok - $3 \mathrm{~mL}$ & LF & 42 & 1 & 11 & 1 & 21 & LIS06 & 55200 & 12200 & 892 & 8050 & $<605$ & 603 & 325 & 77600 & $<2970$ & $<126$ & 20900 \\
\hline SME Simulant & Coliwasa & $\mathrm{HF}$ & 65 & 1 & 6 & 1 & 22 & CH08 & 54500 & 12800 & 907 & 8120 & $<580$ & 716 & 261 & 77500 & $<2850$ & $<121$ & 21000 \\
\hline SME Simulant & Coliwasa & $\mathrm{HF}$ & 34 & 1 & 18 & 1 & 23 & CH07 & 54500 & 12900 & 917 & 8230 & $<595$ & 711 & 302 & 77400 & $<2930$ & $<124$ & 20800 \\
\hline SME Simulant & Coliwasa & LF & 1 & 1 & 12 & 1 & 24 & CH01 & 53700 & 13100 & 902 & 8460 & $<629$ & 714 & 257 & 76600 & $<3090$ & $<131$ & 21600 \\
\hline SME Simulant & Isolok - $3 \mathrm{~mL}$ & LF & 54 & 1 & 17 & 1 & 25 & LIS09 & 54200 & 13200 & 898 & 7980 & $<622$ & 646 & 247 & 76400 & $<3060$ & $<129$ & 20900 \\
\hline SME Simulant & $\begin{array}{l}\text { Coliwasa } \\
\end{array}$ & LF & 4 & 1 & 10 & 1 & 26 & $\begin{array}{ll}\text { CL01 } \\
\end{array}$ & 54800 & 13100 & 937 & 8100 & $<537$ & 698 & 250 & 77300 & $<2640$ & $<112$ & 20800 \\
\hline SME Simulant & Isolok - $3 \mathrm{~mL}$ & LF & 49 & 1 & 8 & 1 & 27 & LIS08 & 54200 & 12800 & 887 & 7960 & $<680$ & 638 & 228 & 75900 & $<3340$ & $<141$ & 20500 \\
\hline SME Simulant & Coliwasa & LF & 65 & 1 & 23 & 1 & 28 & CL04 & 55200 & 12700 & 913 & 8350 & $<569$ & 735 & 253 & 77800 & $<2800$ & $<118$ & 21500 \\
\hline ARG-1 & None & 0 & 0 & 1 & 29 & 1 & 29 & ARG-1B13 & 24000 & 24800 & 800 & 10200 & $<650$ & 659 & $<47.3$ & 96900 & 22600 & $<135$ & 14300 \\
\hline ARG-1 & None & 0 & 0 & 2 & 1 & 2 & 1 & ARG-1B21 & 24900 & 25800 & 842 & 10400 & $<598$ & 689 & 62.8 & 99100 & 23100 & $<124$ & 14500 \\
\hline SME Simulant & Hydragard & $\mathrm{HF}$ & 11 & 2 & 3 & 2 & 2 & HHY02 & 60200 & 13700 & 1030 & 9600 & $<601$ & 888 & 286 & 85500 & $<2960$ & $<125$ & 21000 \\
\hline SME Simulant & $\begin{array}{l}\text { Coliwasa } \\
\end{array}$ & $\mathrm{HF}$ & 36 & 1 & 21 & 2 & 3 & $\begin{array}{l}\text { CL07 } \\
\end{array}$ & 55400 & 13000 & 927 & 8330 & $<582$ & 762 & 293 & 77600 & $<2860$ & $<121$ & 21900 \\
\hline SME Simulant & Hydragard & $\mathrm{HF}$ & 24 & 2 & 2 & 2 & 4 & HHY04 & 58400 & 12400 & 954 & 8680 & $<553$ & 872 & 287 & 82100 & $<2720$ & $<115$ & 21000 \\
\hline SME Simulant & Coliwasa & $\mathrm{HF}$ & 1 & 1 & 22 & 2 & 5 & CH05 & 56600 & 12400 & 937 & 8360 & $<614$ & 867 & 261 & 79800 & $<3020$ & $<128$ & 21400 \\
\hline SME Simulant & Coliwasa & $\mathrm{HF}$ & 3 & 1 & 20 & 2 & 6 & CL05 & 56100 & 13400 & 934 & 8400 & $<610$ & 805 & 298 & 79400 & $<3000$ & $<127$ & 21700 \\
\hline SME Simulant & Coliwasa & $\mathrm{HF}$ & 23 & 1 & 28 & 2 & 7 & CH06 & 55200 & 13200 & 897 & 8370 & $<607$ & 686 & 276 & 78200 & $<2980$ & $<126$ & 21800 \\
\hline SME Simulant & Coliwasa & LF & 1 & 1 & 12 & 2 & 8 & CH01 & 55100 & 13100 & 914 & 8670 & $<629$ & 718 & 331 & 78400 & $<3090$ & $<131$ & 21600 \\
\hline SME Simulant & Coliwasa & LF & 20 & 1 & 2 & 2 & 9 & CL02 & 55000 & 12800 & 933 & 8670 & $<649$ & 752 & 355 & 79800 & $<3190$ & $<135$ & 21000 \\
\hline SME Simulant & Coliwasa & $\mathrm{HF}$ & 63 & 1 & 5 & 2 & 10 & $\begin{array}{ll}\text { CL08 } \\
\end{array}$ & 55900 & 12400 & 924 & 8140 & $<624$ & 812 & 285 & 79300 & $<3070$ & $<130$ & 20400 \\
\hline SME Simulant & $\begin{array}{l}\text { Coliwasa } \\
\end{array}$ & HF & 65 & 1 & 6 & 2 & 11 & CH08 & 56000 & 12900 & 924 & 8670 & $<580$ & 711 & 289 & 79800 & $<2850$ & $<121$ & 21000 \\
\hline SME Simulant & Hydragard & $\mathrm{HF}$ & 45 & 2 & 7 & 2 & 12 & HHY07 & 58100 & 12300 & 960 & 8900 & $<624$ & 752 & 373 & 83500 & $<3070$ & $<130$ & 20100 \\
\hline SME Simulant & Coliwasa & LF & 4 & 1 & 10 & 2 & 13 & CL01 & 56300 & 13000 & 955 & 8600 & $<537$ & 713 & 244 & 80100 & $<2640$ & $<112$ & 20700 \\
\hline SME Simulant & Coliwasa & $\mathrm{HF}$ & 18 & 1 & 26 & 2 & 14 & CL06 & 56200 & 12600 & 938 & 8590 & $<644$ & 816 & 285 & 80300 & $<3160$ & $<134$ & 21000 \\
\hline ARG-1 & None & 0 & 0 & 2 & 12 & 2 & 15 & ARG-1B22 & 23900 & 25000 & 812 & 10500 & $<657$ & 665 & $<47.8$ & 98400 & 21700 & $<137$ & 14200 \\
\hline SME Simulant & Coliwasa & LF & 65 & 1 & 23 & 2 & 16 & CL04 & 55800 & 12200 & 910 & 8390 & $<569$ & 699 & 304 & 79200 & $<2800$ & $<118$ & 20600 \\
\hline SME Simulant & Coliwasa & LF & 40 & 1 & 19 & 2 & 17 & CL03 & 56300 & 12500 & 923 & 8490 & $<576$ & 758 & 325 & 79800 & $<2830$ & $<120$ & 21000 \\
\hline SME Simulant & $\begin{array}{l}\text { Coliwasa } \\
\end{array}$ & LF & 38 & 1 & 16 & 2 & 18 & CH03 & 55300 & 12600 & 933 & 8260 & $<548$ & 737 & 293 & 79100 & $<2690$ & $<114$ & 20600 \\
\hline SME Simulant & Hydragard & $\mathrm{HF}$ & 57 & 2 & 6 & 2 & 19 & HHY09 & 58700 & 12300 & 1000 & 8930 & $<588$ & 864 & 348 & 84500 & $<2890$ & $<122$ & 20700 \\
\hline
\end{tabular}


Table C3. Phase 3 Elemental Concentration Measurements (part 1)

\begin{tabular}{|c|c|c|c|c|c|c|c|c|c|c|c|c|c|c|c|c|c|c|c|}
\hline $\begin{array}{c}\text { Type of } \\
\text { Material } \\
\end{array}$ & Sampler & $\begin{array}{l}\text { Flow } \\
\text { Rate }\end{array}$ & $\begin{array}{c}\text { Sample } \\
\text { Order }\end{array}$ & \begin{tabular}{|c|}
$\begin{array}{c}\text { Preparation } \\
\text { Block }\end{array}$ \\
\end{tabular} & $\begin{array}{c}\begin{array}{c}\text { Preparation } \\
\text { Sequence }\end{array} \\
\end{array}$ & \begin{tabular}{|c|}
$\begin{array}{c}\text { Analytical } \\
\text { Block }\end{array}$ \\
\end{tabular} & \begin{tabular}{|c|} 
Analytical \\
Sequence \\
\end{tabular} & Sample ID & $\begin{array}{c}\mathrm{Al} \\
(\mathrm{ug} / \mathrm{g})\end{array}$ & $\begin{array}{c}\text { B } \\
(\mathrm{ug} / \mathrm{g})\end{array}$ & $\begin{array}{c}\begin{array}{c}\text { Ba } \\
(\mathrm{ug} / \mathrm{g})\end{array} \\
\end{array}$ & $\begin{array}{c}\begin{array}{c}\text { Ca } \\
\text { (ug/g) }\end{array} \\
\end{array}$ & $\begin{array}{c}\begin{array}{c}\mathrm{Ce} \\
(\mathrm{ug} / \mathrm{g})\end{array} \\
\end{array}$ & $\begin{array}{c}\mathbf{C r} \\
(\mathrm{ug} / \mathrm{g})\end{array}$ & $\left.\begin{array}{c}\mathrm{Cu} \\
(\mathbf{u g} / \mathrm{g})\end{array}\right)$ & $\begin{array}{c}\begin{array}{c}F e \\
(\mathrm{ug} / \mathrm{g})\end{array} \\
\end{array}$ & $\begin{array}{c}\mathbf{K} \\
(\mathrm{ug} / \mathrm{g})\end{array}$ & $\begin{array}{c}\mathrm{La} \\
\text { (ug/g) }\end{array}$ & $\begin{array}{c}\mathbf{L i} \\
\text { (ug/g) }\end{array}$ \\
\hline SME Simulant & $\begin{array}{l}\text { Coliwasa } \\
\end{array}$ & LF & 21 & 1 & 14 & \begin{tabular}{|l|}
2 \\
\end{tabular} & \begin{tabular}{|l|}
20 \\
\end{tabular} & $\mathrm{CH} 02$ & 54900 & 12900 & 923 & 8560 & $<615$ & 718 & 263 & 78400 & $<3020$ & $<128$ & 20800 \\
\hline SME Simulant & Hydragard & $\mathrm{HF}$ & 6 & 2 & 4 & 2 & 21 & HHY01 & 56800 & 12500 & 951 & 9090 & $<582$ & 866 & 242 & 81800 & $<2860$ & $<121$ & 20800 \\
\hline SME Simulant & Hydragard & $\mathrm{HF}$ & 19 & 2 & 10 & 2 & 22 & HHY03 & 58500 & 12000 & 975 & 8560 & $<576$ & 847 & 299 & 83200 & $<2830$ & $<120$ & 19500 \\
\hline SME Simulant & Hydragard & $\mathrm{HF}$ & 50 & 2 & 5 & 2 & 23 & $\begin{array}{l}\text { HHY08 } \\
\end{array}$ & 57600 & 11900 & 925 & 8460 & $<592$ & 716 & 299 & 81100 & $<2910$ & $<123$ & 20900 \\
\hline SME Simulant & Hydragard & $\mathrm{HF}$ & 37 & 2 & 9 & 2 & 24 & HHY06 & 57300 & 12400 & 962 & 8950 & $<618$ & 750 & 279 & 82900 & $<3040$ & $<129$ & 21200 \\
\hline SME Simulant & $\begin{array}{l}\text { Coliwasa } \\
\end{array}$ & $\mathrm{HF}$ & 34 & 1 & 18 & 2 & 25 & CH07 & 56300 & 13500 & 945 & 8860 & $<595$ & 733 & 314 & 80100 & $<2930$ & $<124$ & 21300 \\
\hline SME Simulant & $\begin{array}{l}\text { Coliwasa } \\
\end{array}$ & $\mathrm{LF}$ & 63 & 1 & 13 & 2 & 26 & CH04 & 55400 & 11700 & 916 & 8490 & $<581$ & 706 & 325 & 78700 & $<2860$ & $<121$ & 20900 \\
\hline SME Simulant & Hydragard & $\mathrm{HF}$ & 32 & 2 & 8 & 2 & 27 & HHY05 & 58200 & 10200 & 913 & 10400 & $<584$ & 788 & 328 & 82400 & $<2870$ & $<121$ & 20800 \\
\hline SME Simulant & Hydragard & $\mathrm{HF}$ & 61 & 2 & 11 & 2 & 28 & HHY10 & 59700 & 11100 & 994 & 9130 & $<590$ & 773 & 279 & 85700 & $<2900$ & $<123$ & 20400 \\
\hline ARG-1 & None & 0 & 0 & 2 & 23 & 2 & 29 & ARG-1B23 & 24500 & 23900 & 799 & 10500 & $<647$ & 726 & $<47.1$ & 98100 & 24100 & $<135$ & 14400 \\
\hline ARG-1 & None & 0 & 0 & 3 & 1 & 3 & 1 & ARG-1B31 & 24900 & 24700 & 808 & 10200 & $<625$ & 659 & $<45.4$ & 98500 & 22500 & $<130$ & 14700 \\
\hline SME Simulant & Isolok - $12 \mathrm{~mL}$ & LF & 11 & 3 & 4 & 3 & 2 & LIB02 & 53400 & 13400 & 871 & 7980 & $<600$ & 712 & 372 & 74000 & $<2950$ & $<125$ & 23600 \\
\hline SME Simulant & \begin{tabular}{|l|} 
Coliwasa \\
\end{tabular} & $\mathrm{HF}$ & 36 & 1 & 21 & 3 & 3 & CL07 & 55600 & 13200 & 912 & 8240 & $<582$ & 771 & 322 & 77500 & $<2860$ & $<121$ & 21800 \\
\hline SME Simulant & Isolok - $12 \mathrm{~mL}$ & LF & 26 & 3 & 8 & 3 & 4 & LIB04 & 57200 & 12100 & 924 & 8610 & $<604$ & 704 & 418 & 79500 & $<2970$ & $<126$ & 21300 \\
\hline SME Simulant & \begin{tabular}{|l} 
Coliwasa \\
\end{tabular} & $\mathrm{HF}$ & 1 & 1 & 22 & 3 & 5 & CH05 & 57200 & 12100 & 932 & 8520 & $<614$ & 865 & 412 & 80300 & $<3020$ & $<128$ & 22100 \\
\hline SME Simulant & Coliwasa & $\mathrm{HF}$ & 3 & 1 & 20 & 3 & 6 & CL05 & 56500 & 12400 & 919 & 8490 & $<610$ & 791 & 359 & 80000 & $<3000$ & $<127$ & 22300 \\
\hline SME Simulant & Coliwasa & $\mathrm{HF}$ & 23 & 1 & 28 & 3 & 7 & CH06 & 55000 & 13000 & 898 & 8320 & $<607$ & 701 & 266 & 77300 & $<2980$ & $<126$ & 21500 \\
\hline SME Simulant & Coliwasa & LF & 1 & 1 & 12 & 3 & 8 & CH01 & 56200 & 12200 & 900 & 8900 & $<629$ & 699 & 352 & 79300 & $<3090$ & $<131$ & 22900 \\
\hline SME Simulant & Coliwasa & LF & 20 & 1 & 2 & 3 & 9 & CL02 & 56200 & 13100 & 947 & 8760 & $<649$ & 807 & 325 & 80400 & $<3190$ & $<135$ & 21900 \\
\hline SME Simulant & Coliwasa & $\mathrm{HF}$ & 63 & 1 & 5 & 3 & 10 & CL08 & 57400 & 11900 & 930 & 8420 & $<624$ & 819 & 286 & 81000 & $<3070$ & $<130$ & 21500 \\
\hline SME Simulant & Coliwasa & $\mathrm{HF}$ & 65 & 1 & 6 & 3 & 11 & CH08 & 56500 & 12500 & 914 & 8440 & $<580$ & 715 & 303 & 79700 & $<2850$ & $<121$ & 21900 \\
\hline SME Simulant & Isolok - $12 \mathrm{~mL}$ & LF & 45 & 3 & 7 & 3 & 12 & LIB07 & 55400 & 11800 & 908 & 8360 & $<628$ & 719 & 296 & 77900 & $<3090$ & $<131$ & 22200 \\
\hline SME Simulant & \begin{tabular}{|l|} 
Coliwasa \\
\end{tabular} & LF & 4 & 1 & 10 & 3 & 13 & CL01 & 57100 & 12800 & 959 & 8450 & $<537$ & 714 & 291 & 79700 & $<2640$ & $<112$ & 21300 \\
\hline SME Simulant & Coliwasa & $\mathrm{HF}$ & 18 & 1 & 26 & 3 & 14 & CL06 & 57300 & 12800 & 957 & 8880 & $<644$ & 863 & 376 & 80600 & $<3160$ & $<134$ & 21700 \\
\hline ARG-1 & None & 0 & 0 & 3 & 12 & 3 & 15 & ARG-1B32 & 24800 & 24300 & 826 & 10400 & $<637$ & 707 & $<46.3$ & 99500 & 23800 & $<133$ & 14600 \\
\hline SME Simulant & Coliwasa & LF & 65 & 1 & 23 & 3 & 16 & CL04 & 56300 & 12000 & 916 & 8500 & $<569$ & 727 & 347 & 78400 & $<2800$ & $<118$ & 21100 \\
\hline SME Simulant & Coliwasa & LF & 40 & 1 & 19 & 3 & 17 & $\begin{array}{ll}\text { CL03 } \\
\end{array}$ & 56000 & 12300 & 934 & 8400 & $<576$ & 769 & 329 & 78800 & $<2830$ & $<120$ & 21600 \\
\hline SME Simulant & Coliwasa & LF & 38 & 1 & 16 & 3 & 18 & $\mathrm{CH} 03$ & 55900 & 11700 & 901 & 8380 & $<548$ & 707 & 291 & 78100 & $<2690$ & $<114$ & 22000 \\
\hline SME Simulant & \begin{tabular}{|l} 
Isolok $-12 \mathrm{~mL}$ \\
\end{tabular} & LF & 56 & 3 & 11 & 3 & 19 & LIB09 & 56800 & 11500 & 937 & 8420 & $<605$ & 877 & 328 & 79100 & $<2970$ & $<126$ & 23500 \\
\hline SME Simulant & \begin{tabular}{|l|} 
Coliwasa \\
\end{tabular} & LF & 21 & 1 & 14 & 3 & 20 & CH02 & 56800 & 12700 & 924 & 8320 & $<615$ & 722 & 289 & 78900 & $<3020$ & $<128$ & 22000 \\
\hline SME Simulant & Isolok - $12 \mathrm{~mL}$ & LF & 5 & 3 & 5 & 3 & 21 & LIB01 & 56300 & 12800 & 920 & 8420 & $<583$ & 702 & 341 & 78900 & $<2870$ & $<121$ & 22400 \\
\hline SME Simulant & Isolok - $12 \mathrm{~mL}$ & LF & 23 & 3 & 10 & 3 & 22 & LIB03 & 57200 & 12300 & 880 & 9750 & $<606$ & 841 & 317 & 79000 & $<2980$ & $<126$ & 22100 \\
\hline SME Simulant & Isolok - $12 \mathrm{~mL}$ & $\mathrm{LF}$ & 50 & 3 & 2 & 3 & 23 & LIB08 & 53000 & 12100 & 843 & 7670 & $<559$ & 678 & 343 & 71800 & $<2750$ & $<116$ & 22300 \\
\hline SME Simulant & \begin{tabular}{|l|l} 
Isolok - $12 \mathrm{~mL}$ \\
\end{tabular} & LF & 41 & 3 & 9 & 3 & 24 & LIB06 & 50700 & 12700 & 801 & 6810 & $<607$ & 658 & 323 & 69000 & $<2980$ & $<126$ & 24500 \\
\hline SME Simulant & \begin{tabular}{|l|} 
Coliwasa \\
\end{tabular} & $\mathrm{HF}$ & 34 & 1 & 18 & 3 & 25 & CH07 & 53600 & 11600 & 875 & 8180 & $<595$ & 653 & 301 & 74700 & $<2930$ & $<124$ & 20600 \\
\hline SME Simulant & \begin{tabular}{|l|} 
Coliwasa \\
\end{tabular} & LF & 63 & 1 & 13 & 3 & 26 & CH04 & 57600 & 12300 & 954 & 8730 & $<581$ & 774 & 332 & 80800 & $<2860$ & $<121$ & 22400 \\
\hline SME Simulant & Isolok - $12 \mathrm{~mL}$ & LF & 32 & 3 & 3 & 3 & 27 & LIB05 & 56500 & 12300 & 912 & 8350 & $<621$ & 732 & 404 & 78500 & $<3050$ & $<129$ & 22300 \\
\hline SME Simulant & Isolok - $12 \mathrm{~mL}$ & LF & 60 & 3 & 6 & 3 & 28 & LIB10 & 52100 & 12100 & 865 & 8040 & $<596$ & 650 & 289 & 71200 & $<2930$ & $<124$ & 23500 \\
\hline ARG-1 & \begin{tabular}{|l|} 
None \\
\end{tabular} & 0 & 0 & 3 & 23 & 3 & 29 & ARG-1B33 & 24200 & 23600 & 811 & 10300 & $<674$ & 652 & $<49$ & 97000 & 22400 & $<140$ & 14800 \\
\hline ARG-1 & \begin{tabular}{|l|} 
None \\
\end{tabular} & 0 & 0 & 4 & 1 & 4 & 1 & ARG-1B41 & 24100 & 24600 & 854 & 10500 & $<679$ & 671 & $<49.4$ & 98500 & 23000 & $<141$ & 14800 \\
\hline SME Simulant & Isolok - $12 \mathrm{~mL}$ & $\mathrm{HF}$ & 13 & 4 & 11 & 4 & 2 & HIB02 & 55000 & 13600 & 944 & 8400 & $<588$ & 732 & 254 & 77300 & $<2890$ & $<122$ & 22200 \\
\hline SME Simulant & \begin{tabular}{|l|} 
Coliwasa \\
\end{tabular} & $\mathrm{HF}$ & 36 & 1 & 21 & 4 & 3 & CL07 & 52200 & 12000 & 886 & 7950 & $<582$ & 717 & 269 & 74200 & $<2860$ & $<121$ & 21200 \\
\hline SME Simulant & Isolok - $12 \mathrm{~mL}$ & $\mathrm{HF}$ & 25 & 4 & 4 & 4 & 4 & HIB04 & 55500 & 12400 & 924 & 8270 & $<615$ & 812 & 354 & 77500 & $<3020$ & $<128$ & 21800 \\
\hline SME Simulant & \begin{tabular}{|l|} 
Coliwasa \\
\end{tabular} & $\mathrm{HF}$ & 1 & 1 & 22 & 4 & 5 & CH05 & 56700 & 12200 & 949 & 8500 & $<614$ & 892 & 319 & 79600 & $<3020$ & $<128$ & 22500 \\
\hline SME Simulant & Coliwasa & $\mathrm{HF}$ & 3 & 1 & 20 & 4 & 6 & CL05 & 55500 & 13100 & 944 & 8550 & $<610$ & 797 & 329 & 78800 & $<3000$ & $<127$ & 23200 \\
\hline SME Simulant & Coliwasa & $\mathrm{HF}$ & 23 & 1 & 28 & 4 & 7 & CH06 & 56300 & 13400 & 960 & 8350 & $<607$ & 716 & 211 & 79400 & $<2980$ & $<126$ & 22000 \\
\hline SME Simulant & Coliwasa & LF & 1 & 1 & 12 & 4 & 8 & CH01 & 57000 & 13300 & 967 & 9000 & $<629$ & 745 & 217 & 80600 & $<3090$ & $<131$ & 22800 \\
\hline SME Simulant & Coliwasa & LF & 20 & 1 & 2 & 4 & 9 & CL02 & 54800 & 12600 & 956 & 8530 & $<649$ & 757 & 261 & 78900 & $<3190$ & $<135$ & 21500 \\
\hline
\end{tabular}


Table C3. Phase 3 Elemental Concentration Measurements (part 1)

\begin{tabular}{|c|c|c|c|c|c|c|c|c|c|c|c|c|c|c|c|c|c|c|c|}
\hline $\begin{array}{c}\text { Type of } \\
\text { Material } \\
\end{array}$ & Sampler & $\begin{array}{l}\text { Flow } \\
\text { Rate }\end{array}$ & $\begin{array}{c}\text { Sample } \\
\text { Order }\end{array}$ & \begin{tabular}{|c|}
$\begin{array}{c}\text { Preparation } \\
\text { Block }\end{array}$ \\
\end{tabular} & $\begin{array}{c}\begin{array}{c}\text { Preparation } \\
\text { Sequence }\end{array} \\
\end{array}$ & \begin{tabular}{|c|}
$\begin{array}{c}\text { Analytical } \\
\text { Block }\end{array}$ \\
\end{tabular} & \begin{tabular}{|c|} 
Analytical \\
Sequence \\
\end{tabular} & Sample ID & $\begin{array}{c}\mathrm{Al} \\
(\mathrm{ug} / \mathrm{g})\end{array}$ & $\begin{array}{c}\text { B } \\
(\mathrm{ug} / \mathrm{g})\end{array}$ & $\begin{array}{c}\begin{array}{c}\text { Ba } \\
(\mathbf{u g} / \mathrm{g})\end{array} \\
\end{array}$ & $\begin{array}{c}\begin{array}{c}\text { Ca } \\
\text { (ug/g) }\end{array} \\
\end{array}$ & $\begin{array}{c}\mathrm{Ce} \\
(\mathrm{ug} / \mathrm{g})\end{array}$ & $\begin{array}{c}\begin{array}{c}\mathbf{C r} \\
(\mathrm{ug} / \mathrm{g})\end{array} \\
\end{array}$ & $\left.\begin{array}{c}\mathrm{Cu} \\
(\mathbf{u g} / \mathrm{g})\end{array}\right)$ & $\begin{array}{c}\begin{array}{c}F e \\
(\mathrm{ug} / \mathrm{g})\end{array} \\
\end{array}$ & $\begin{array}{c}\mathbf{K} \\
(\mathrm{ug} / \mathrm{g})\end{array}$ & $\begin{array}{c}\mathrm{La} \\
\text { (ug/g) }\end{array}$ & $\begin{array}{c}\mathbf{L i} \\
(\mathbf{u g} / \mathrm{g})\end{array}$ \\
\hline SME Simulant & Coliwasa & $\mathrm{HF}$ & 63 & 1 & 5 & \begin{tabular}{|l|}
4 \\
\end{tabular} & \begin{tabular}{|l|}
10 \\
\end{tabular} & CL08 & 57200 & 11900 & 964 & 8490 & $<624$ & 798 & 265 & 79900 & $<3070$ & $<130$ & 21400 \\
\hline SME Simulant & \begin{tabular}{|l|l} 
Isolok - $12 \mathrm{~mL}$ \\
\end{tabular} & $\mathrm{HF}$ & 46 & 4 & 2 & 4 & 11 & HIB07 & 56100 & 12700 & 943 & 8320 & $<610$ & 852 & 276 & 79100 & $<3000$ & $<127$ & 21400 \\
\hline SME Simulant & \begin{tabular}{|l} 
Coliwasa \\
\end{tabular} & LF & 4 & 1 & 10 & 4 & 12 & CL01 & 57200 & 13000 & 998 & 8520 & $<537$ & 705 & 302 & 80800 & $<2640$ & $<112$ & 21700 \\
\hline SME Simulant & Coliwasa & $\mathrm{HF}$ & 18 & 1 & 26 & 4 & 13 & CL06 & 56400 & 12600 & 966 & 8590 & $<644$ & 826 & 267 & 80300 & $<3160$ & $<134$ & 21600 \\
\hline ARG-1 & None & 0 & 0 & 4 & 7 & 4 & 14 & ARG-1B42 & 24300 & 24400 & 847 & 10200 & $<691$ & 670 & $<50.2$ & 98000 & 22200 & $<144$ & 14600 \\
\hline SME Simulant & Coliwasa & LF & 65 & 1 & 23 & 4 & 15 & CL04 & 56300 & 12200 & 943 & 8490 & $<569$ & 714 & 293 & 79200 & $<2800$ & $<118$ & 21400 \\
\hline SME Simulant & Coliwasa & $\mathrm{LF}$ & 40 & 1 & 19 & 4 & 16 & $\begin{array}{ll}\text { CL03 } \\
\end{array}$ & 56600 & 12500 & 965 & 8400 & $<576$ & 758 & 252 & 80200 & $<2830$ & $<120$ & 21900 \\
\hline SME Simulant & Coliwasa & LF & 21 & 1 & 14 & 4 & 17 & CH02 & 55700 & 13200 & 965 & 8310 & $<615$ & 732 & 210 & 78900 & $<3020$ & $<128$ & 21700 \\
\hline SME Simulant & Isolok - $12 \mathrm{~mL}$ & $\mathrm{HF}$ & 7 & 4 & 12 & 4 & 18 & HIB01 & 56800 & 12900 & 957 & 8490 & $<582$ & 760 & 224 & 80100 & $<2860$ & $<121$ & 22100 \\
\hline SME Simulant & \begin{tabular}{|l} 
Isolok - $12 \mathrm{~mL}$ \\
\end{tabular} & $\mathrm{HF}$ & 21 & 4 & 6 & 4 & 19 & HIB03 & 57300 & 12200 & 960 & 8490 & $<537$ & 780 & 261 & 81200 & $<2640$ & $<112$ & 22100 \\
\hline SME Simulant & \begin{tabular}{|l} 
Isolok $-12 \mathrm{~mL}$ \\
\end{tabular} & $\mathrm{HF}$ & 49 & 4 & 5 & 4 & 20 & HIB08 & 53700 & 11300 & 944 & 8360 & $<595$ & 1060 & 250 & 76400 & $<2920$ & $<124$ & 19800 \\
\hline SME Simulant & \begin{tabular}{|l} 
Isolok - $12 \mathrm{~mL}$ \\
\end{tabular} & $\mathrm{HF}$ & 38 & 4 & 8 & 4 & 21 & HIB06 & 54600 & 12400 & 911 & 8090 & $<573$ & 865 & 301 & 76800 & $<2820$ & $<119$ & 22200 \\
\hline SME Simulant & \begin{tabular}{|l|} 
Coliwasa \\
\end{tabular} & $\mathrm{HF}$ & 34 & 1 & 18 & 4 & 22 & CH07 & 57200 & 12800 & 963 & 8730 & $<595$ & 718 & 237 & 81300 & $<2930$ & $<124$ & 22000 \\
\hline SME Simulant & Coliwasa & LF & 63 & 1 & 13 & 4 & 23 & CH04 & 56300 & 12200 & 971 & 8590 & $<581$ & 734 & 342 & 80200 & $<2860$ & $<121$ & 21800 \\
\hline SME Simulant & Isolok - $12 \mathrm{~mL}$ & $\mathrm{HF}$ & 33 & 4 & 10 & 4 & 24 & HIB05 & 54800 & 12100 & 921 & 7950 & $<600$ & 729 & 331 & 76500 & $<2950$ & $<125$ & 22200 \\
\hline SME Simulant & \begin{tabular}{|l} 
Isolok - $12 \mathrm{~mL}$ \\
\end{tabular} & $\mathrm{HF}$ & 58 & 4 & 3 & 4 & 25 & HIB10 & 52700 & 12800 & 903 & 7780 & $<601$ & 739 & 191 & 73900 & $<2950$ & $<125$ & 22600 \\
\hline ARG-1 & \begin{tabular}{|l|} 
None \\
\end{tabular} & 0 & 0 & 4 & 13 & 4 & 26 & ARG-1B43 & 24000 & 23400 & 834 & 10200 & $<645$ & 652 & $<46.9$ & 97700 & 23600 & $<134$ & 14500 \\
\hline SME Simulant & Coliwasa & $\mathrm{HF}$ & 65 & 1 & 6 & 4 & 27 & CH08 & 57200 & 12600 & 962 & 8560 & $<580$ & 723 & 286 & 80500 & $<2850$ & $<121$ & 21800 \\
\hline SME Simulant & \begin{tabular}{|l|} 
Coliwasa \\
\end{tabular} & LF & 38 & 1 & 16 & 4 & 28 & CH03 & 56600 & 12500 & 965 & 8450 & $<548$ & 731 & 247 & 80000 & $<2690$ & $<114$ & 21700 \\
\hline SME Simulant & Isolok - $12 \mathrm{~mL}$ & $\mathrm{HF}$ & 56 & 4 & 9 & 4 & 29 & HIB09 & 60800 & 12200 & 1080 & 9230 & $<623$ & 988 & 357 & 87700 & $<3060$ & $<130$ & 20900 \\
\hline ARG-1 & None & 0 & 0 & 2 & 1 & 5 & 1 & ARG-1B21 & 25100 & 25000 & 832 & 10500 & $<598$ & 706 & 87 & 98000 & 21200 & $<124$ & 15000 \\
\hline SME Simulant & Isolok - $3 \mathrm{~mL}$ & $\mathrm{HF}$ & 20 & 2 & 16 & 5 & 2 & HIS03 & 57100 & 12100 & 926 & 8600 & $<608$ & 780 & 448 & 79000 & $<2990$ & $<127$ & 22500 \\
\hline SME Simulant & Isolok - $3 \mathrm{~mL}$ & $\mathrm{HF}$ & 9 & 2 & 21 & 5 & 3 & HIS01 & 57700 & 13300 & 1020 & 8920 & $<617$ & 744 & 365 & 79600 & $<3030$ & $<128$ & 23400 \\
\hline SME Simulant & Coliwasa & LF & 20 & 1 & 2 & 5 & 4 & CL02 & 56100 & 13400 & 942 & 8690 & $<649$ & 797 & 409 & 79400 & 3210 & $<135$ & 22200 \\
\hline SME Simulant & Coliwasa & LF & 38 & 1 & 16 & 5 & 5 & $\mathrm{CH} 03$ & 56600 & 12600 & 927 & 8350 & $<548$ & 745 & 326 & 78700 & $<2690$ & $<114$ & 22200 \\
\hline SME Simulant & $\begin{array}{l}\text { Coliwasa } \\
\end{array}$ & LF & 63 & 1 & 13 & 5 & 6 & CH04 & 57100 & 12700 & 935 & 8700 & $<581$ & 742 & 409 & 79900 & $<2860$ & $<121$ & 22300 \\
\hline SME Simulant & Isolok - $3 \mathrm{~mL}$ & $\mathrm{HF}$ & 44 & 2 & 18 & 5 & 7 & HIS07 & 57800 & 13200 & 961 & 8650 & $<623$ & 743 & 329 & 79200 & $<3060$ & $<130$ & 22200 \\
\hline SME Simulant & Coliwasa & $\mathrm{HF}$ & 23 & 1 & 28 & 5 & 8 & CH06 & 57300 & 13300 & 939 & 8520 & $<607$ & 721 & 312 & 78900 & $<2980$ & $<126$ & 22400 \\
\hline SME Simulant & Coliwasa & LF & 21 & 1 & 14 & 5 & 9 & $\mathrm{CH} 02$ & 57400 & 12700 & 962 & 8710 & $<615$ & 720 & 371 & 79500 & $<3020$ & $<128$ & 23100 \\
\hline SME Simulant & Coliwasa & $\mathrm{HF}$ & 3 & 1 & 20 & 5 & 10 & CL05 & 56500 & 12900 & 902 & 8250 & $<610$ & 788 & 380 & 78400 & $<3000$ & $<127$ & 21400 \\
\hline SME Simulant & Isolok - $3 \mathrm{~mL}$ & $\mathrm{HF}$ & 14 & 2 & 15 & 5 & 11 & HIS02 & 56000 & 12000 & 929 & 8360 & $<620$ & 702 & 384 & 77100 & $<3050$ & $<129$ & 21800 \\
\hline SME Simulant & $\begin{array}{l}\text { Coliwasa } \\
\end{array}$ & LF & 40 & 1 & 19 & 5 & 12 & $\begin{array}{ll}\text { CL03 } \\
\end{array}$ & 57800 & 12700 & 953 & 8540 & $<576$ & 782 & 344 & 80200 & $<2830$ & $<120$ & 21900 \\
\hline SME Simulant & Coliwasa & $\mathrm{HF}$ & 18 & 1 & 26 & 5 & 13 & $\begin{array}{ll}\text { CL06 } \\
\end{array}$ & 57200 & 13000 & 979 & 8780 & $<644$ & 864 & 368 & 79400 & $<3160$ & $<134$ & 22200 \\
\hline SME Simulant & Coliwasa & $\mathrm{HF}$ & 1 & 1 & 22 & 5 & 14 & CH05 & 57200 & 12800 & 980 & 8780 & $<614$ & 908 & 380 & 79700 & $<3020$ & $<128$ & 23100 \\
\hline ARG-1 & None & 0 & 0 & 2 & 12 & 5 & 15 & ARG-1B22 & 25200 & 26200 & 879 & 10800 & $<657$ & 691 & 47.8 & 98500 & 22900 & $<137$ & 15500 \\
\hline SME Simulant & Coliwasa & $\mathrm{HF}$ & 36 & 1 & 21 & 5 & 16 & CL07 & 56000 & 12800 & 920 & 8640 & $<582$ & 775 & 381 & 78000 & $<2860$ & $<121$ & 22400 \\
\hline SME Simulant & Isolok - $3 \mathrm{~mL}$ & $\mathrm{HF}$ & 31 & 2 & 19 & 5 & 17 & HIS05 & 56700 & 12800 & 950 & 8770 & $<624$ & 766 & 382 & 79300 & $<3070$ & $<130$ & 22500 \\
\hline SME Simulant & Isolok - $3 \mathrm{~mL}$ & $\mathrm{HF}$ & 60 & 2 & 22 & 5 & 18 & HIS10 & 55800 & 12900 & 952 & 8480 & $<629$ & 733 & 432 & 77600 & $<3090$ & $<131$ & 23000 \\
\hline SME Simulant & Coliwasa & $\mathrm{HF}$ & 63 & 1 & 5 & 5 & 19 & $\begin{array}{ll}\text { CL08 } \\
\end{array}$ & 56700 & 12900 & 942 & 8730 & $<624$ & 839 & 374 & 79400 & 3290 & $<130$ & 21800 \\
\hline SME Simulant & Isolok - $3 \mathrm{~mL}$ & $\mathrm{HF}$ & 26 & 2 & 14 & 5 & 20 & HIS04 & 55200 & 13100 & 910 & 8420 & $<552$ & 676 & 425 & 76200 & $<2710$ & $<115$ & 22900 \\
\hline SME Simulant & Isolok - $3 \mathrm{~mL}$ & $\mathrm{HF}$ & 35 & 2 & 20 & 5 & 21 & HIS06 & 56500 & 13900 & 920 & 8660 & $<616$ & 730 & 367 & 78500 & $<3030$ & $<128$ & 22400 \\
\hline SME Simulant & Coliwasa & $\mathrm{HF}$ & 65 & 1 & 6 & 5 & 22 & CH08 & 56600 & 13800 & 926 & 8290 & $<580$ & 751 & 377 & 78800 & $<2850$ & $<121$ & 22300 \\
\hline SME Simulant & Coliwasa & $\mathrm{HF}$ & 34 & 1 & 18 & 5 & 23 & CH07 & 56700 & 14300 & 946 & 8930 & $<595$ & 765 & 432 & 79500 & $<2930$ & $<124$ & 22200 \\
\hline SME Simulant & Coliwasa & LF & 1 & 1 & 12 & 5 & 24 & CH01 & 55700 & 14200 & 923 & 9040 & $<629$ & 757 & 371 & 79000 & $<3090$ & $<131$ & 22900 \\
\hline SME Simulant & Isolok - $3 \mathrm{~mL}$ & $\mathrm{HF}$ & 54 & 2 & 17 & 5 & 25 & HIS09 & 54900 & 13300 & 887 & 8350 & $<549$ & 737 & 391 & 75500 & $<2700$ & $<114$ & 23500 \\
\hline SME Simulant & Coliwasa & LF & 4 & 1 & 10 & 5 & 26 & $\begin{array}{ll}\text { CL01 } \\
\end{array}$ & 57100 & 14100 & 963 & 8430 & $<537$ & 734 & 323 & 79400 & $<2640$ & $<112$ & 21900 \\
\hline SME Simulant & Isolok - $3 \mathrm{~mL}$ & $\mathrm{HF}$ & 48 & 2 & 13 & 5 & 27 & HIS08 & 56400 & 12600 & 907 & 8400 & $<593$ & 662 & 339 & 78100 & $<2910$ & $<123$ & 22400 \\
\hline SME Simulant & Coliwasa & LF & 65 & 1 & 23 & 5 & 28 & CL04 & 57000 & 13000 & 917 & 8660 & $<569$ & 727 & 383 & 79100 & $<2800$ & $<118$ & 22000 \\
\hline
\end{tabular}


Table C3. Phase 3 Elemental Concentration Measurements (part 1)

\begin{tabular}{|c|c|c|c|c|c|c|c|c|c|c|c|c|c|c|c|c|c|c|c|}
\hline $\begin{array}{l}\text { Type of } \\
\text { Material }\end{array}$ & Sampler & $\begin{array}{l}\text { Flow } \\
\text { Rate }\end{array}$ & $\begin{array}{l}\text { Sample } \\
\text { Order }\end{array}$ & $\begin{array}{c}\text { Preparation } \\
\text { Block }\end{array}$ & $\begin{array}{c}\text { Preparation } \\
\text { Sequence }\end{array}$ & $\begin{array}{c}\text { Analytical } \\
\text { Block }\end{array}$ & $\begin{array}{c}\text { Analytical } \\
\text { Sequence }\end{array}$ & Sample ID & $\begin{array}{c}\mathrm{Al} \\
\text { (ug/g) }\end{array}$ & $\begin{array}{c}\mathrm{B} \\
(\mathrm{ug} / \mathrm{g})\end{array}$ & $\begin{array}{c}\mathrm{Ba} \\
\text { (ug/g) }\end{array}$ & $\begin{array}{c}\mathrm{Ca} \\
\text { (ug/g) }\end{array}$ & $\begin{array}{c}\mathrm{Ce} \\
(\mathrm{ug} / \mathrm{g})\end{array}$ & $\begin{array}{c}\mathrm{Cr} \\
\text { (ug/g) }\end{array}$ & $\begin{array}{c}\mathrm{Cu} \\
(\mathrm{ug} / \mathrm{g})\end{array}$ & $\begin{array}{c}\mathrm{Fe} \\
\text { (ug/g) }\end{array}$ & $\begin{array}{c}\mathrm{K} \\
\text { (ug/g) }\end{array}$ & $\begin{array}{c}\mathrm{La} \\
\text { (ug/g) }\end{array}$ & $\begin{array}{c}\mathbf{L i} \\
\text { (ug/g) }\end{array}$ \\
\hline ARG-1 & None & 0 & 0 & 2 & 23 & \begin{tabular}{|l|}
5 \\
\end{tabular} & \begin{tabular}{|l|}
29 \\
\end{tabular} & \begin{tabular}{|l|} 
ARG-1B23 \\
\end{tabular} & 25200 & 27200 & 832 & 11000 & $<647$ & 793 & 136 & 98300 & 23500 & $<135$ & 15000 \\
\hline ARG-1 & None & 0 & 0 & 3 & 1 & 6 & 1 & ARG-1B31 & 25100 & 25300 & 823 & 10500 & $<625$ & 697 & $<45.4$ & 98700 & 21600 & $<130$ & 14400 \\
\hline SME Simulant & Hydragard & LF & 15 & 3 & 21 & 6 & 2 & LHY03 & 55700 & 12900 & 906 & 8350 & $<604$ & 732 & 320 & 78200 & $<2970$ & $<126$ & 20800 \\
\hline SME Simulant & Hydragard & LF & 6 & 3 & 18 & 6 & 3 & LHY01 & 57900 & 12600 & 901 & 8380 & $<608$ & 726 & 311 & 80800 & $<2990$ & $<127$ & 21100 \\
\hline SME Simulant & Coliwasa & LF & 20 & 1 & 2 & 6 & 4 & CL02 & 56100 & 13300 & 931 & 8900 & $<649$ & 803 & 311 & 79500 & $<3190$ & $<135$ & 21400 \\
\hline SME Simulant & Coliwasa & LF & 38 & 1 & 16 & 6 & 5 & $\mathrm{CH} 03$ & 56500 & 12600 & 909 & 8290 & $<548$ & 744 & 254 & 79100 & $<2690$ & $<114$ & 21100 \\
\hline SME Simulant & Coliwasa & LF & 63 & 1 & 13 & 6 & 6 & CH04 & 56500 & 12200 & 907 & 8400 & $<581$ & 748 & 343 & 79700 & $<2860$ & $<121$ & 21500 \\
\hline SME Simulant & Hydragard & LF & 47 & 3 & 13 & 6 & 7 & $\begin{array}{l}\text { LHY07 } \\
\end{array}$ & 57000 & 12300 & 932 & 8440 & $<584$ & 836 & 348 & 80300 & $<2870$ & $<122$ & 21500 \\
\hline SME Simulant & Coliwasa & $\mathrm{HF}$ & 23 & 1 & 28 & 6 & 8 & CH06 & 56400 & 13200 & 909 & 8720 & $<607$ & 725 & 289 & 78900 & $<2980$ & $<126$ & 21400 \\
\hline SME Simulant & Coliwasa & LF & 21 & 1 & 14 & 6 & 9 & CH02 & 56500 & 13000 & 904 & 8280 & $<615$ & 721 & 278 & 79200 & $<3020$ & $<128$ & 21400 \\
\hline SME Simulant & $\begin{array}{l}\text { Coliwasa } \\
\end{array}$ & $\mathrm{HF}$ & 3 & 1 & 20 & 6 & 10 & CL05 & 55800 & 12100 & 886 & 8600 & $<610$ & 774 & 346 & 78300 & $<3000$ & $<127$ & 21800 \\
\hline SME Simulant & Hydragard & LF & 10 & 3 & 20 & 6 & 11 & LHY02 & 59700 & 12300 & 969 & 9390 & $<610$ & 778 & 323 & 83800 & $<3000$ & $<127$ & 20500 \\
\hline SME Simulant & Coliwasa & LF & 40 & 1 & 19 & 6 & 12 & CL03 & 57200 & 11600 & 876 & 8480 & $<576$ & 714 & 316 & 79700 & $<2830$ & $<120$ & 21300 \\
\hline SME Simulant & Coliwasa & $\mathrm{HF}$ & 18 & 1 & 26 & 6 & 13 & CL06 & 56400 & 12400 & 920 & 8560 & $<644$ & 824 & 276 & 79400 & $<3160$ & $<134$ & 21100 \\
\hline SME Simulant & Coliwasa & $\mathrm{HF}$ & 1 & 1 & 22 & 6 & 14 & CH05 & 57000 & 12400 & 932 & 8660 & $<614$ & 912 & 319 & 80100 & $<3020$ & $<128$ & 21500 \\
\hline ARG-1 & None & 0 & 0 & 3 & 12 & 6 & 15 & ARG-1B32 & 24500 & 26100 & 830 & 10700 & $<637$ & 757 & $<46.3$ & 99000 & 22500 & $<133$ & 14300 \\
\hline SME Simulant & $\begin{array}{l}\text { Coliwasa } \\
\end{array}$ & $\mathrm{HF}$ & 36 & 1 & 21 & 6 & 16 & CL07 & 55900 & 12500 & 898 & 8590 & $<582$ & 778 & 318 & 78000 & $<2860$ & $<121$ & 21600 \\
\hline SME Simulant & Hydragard & LF & 30 & 3 & 14 & 6 & 17 & LHY05 & 55700 & 12800 & 870 & 8100 & $<583$ & 707 & 225 & 76700 & $<2870$ & $<121$ & 21700 \\
\hline SME Simulant & Hydragard & LF & 59 & 3 & 17 & 6 & 18 & LHY10 & 79100 & 15700 & 1270 & 13000 & $<599$ & 1060 & 479 & 111000 & $<2940$ & $<125$ & 30100 \\
\hline SME Simulant & Coliwasa & $\mathrm{HF}$ & 63 & 1 & 5 & 6 & 19 & CL08 & 57200 & 12400 & 914 & 8650 & $<624$ & 831 & 345 & 79700 & $<3070$ & $<130$ & 21100 \\
\hline SME Simulant & Hydragard & LF & 28 & 3 & 16 & 6 & 20 & LHY04 & 59100 & 12100 & 930 & 8790 & $<620$ & 740 & 307 & 82400 & $<3050$ & $<129$ & 21200 \\
\hline SME Simulant & Hydragard & LF & 37 & 3 & 15 & 6 & 21 & LHY06 & 58600 & 12300 & 961 & 8870 & $<569$ & 897 & 277 & 82000 & $<2800$ & $<118$ & 20700 \\
\hline SME Simulant & $\begin{array}{l}\text { Coliwasa } \\
\end{array}$ & $\mathrm{HF}$ & 65 & 1 & 6 & 6 & 22 & CH08 & 56700 & 12500 & 885 & 8410 & $<580$ & 717 & 310 & 79100 & $<2850$ & $<121$ & 21600 \\
\hline SME Simulant & Coliwasa & $\mathrm{HF}$ & 34 & 1 & 18 & 6 & 23 & CH07 & 56700 & 12900 & 918 & 8690 & $<595$ & 742 & 277 & 79800 & $<2930$ & $<124$ & 21400 \\
\hline SME Simulant & $\begin{array}{l}\text { Coliwasa } \\
\end{array}$ & LF & 1 & 1 & 12 & 6 & 24 & CH01 & 55800 & 13200 & 914 & 8690 & $<629$ & 754 & 310 & 78900 & $<3090$ & $<131$ & 22100 \\
\hline SME Simulant & Hydragard & LF & 53 & 3 & 19 & 6 & 25 & LHY09 & 57300 & 11600 & 913 & 8540 & $<580$ & 766 & 255 & 78600 & $<2850$ & $<121$ & 20400 \\
\hline SME Simulant & Coliwasa & LF & 4 & 1 & 10 & 6 & 26 & CL01 & 57300 & 12900 & 947 & 8330 & $<537$ & 724 & 302 & 79700 & $<2640$ & $<112$ & 21200 \\
\hline SME Simulant & Hydragard & LF & 48 & 3 & 22 & 6 & 27 & LHY08 & 58200 & 12600 & 930 & 9870 & $<590$ & 753 & 266 & 81400 & $<2900$ & $<123$ & 20100 \\
\hline SME Simulant & Coliwasa & LF & 65 & 1 & 23 & 6 & 28 & CL04 & 57000 & 12600 & 925 & 8740 & $<569$ & 760 & 297 & 79600 & $<2800$ & $<118$ & 21200 \\
\hline ARG-1 & None & 0 & 0 & 3 & 23 & 6 & 29 & ARG-1B33 & 25200 & 25400 & 831 & 10500 & $<674$ & 726 & $<49$ & 97900 & 20700 & $<140$ & 14400 \\
\hline
\end{tabular}


Table C3. Phase 3 Elemental Concentration Measurements (part 2)

\begin{tabular}{|c|c|c|c|c|c|c|c|c|c|c|c|c|c|c|c|c|c|c|c|}
\hline $\begin{array}{c}\text { Type of } \\
\text { Material }\end{array}$ & Sampler & $\begin{array}{l}\text { Flow } \\
\text { Rate }\end{array}$ & $\begin{array}{c}\text { Sample } \\
\text { Order }\end{array}$ & \begin{tabular}{|c|}
$\begin{array}{c}\text { Preparation } \\
\text { Block }\end{array}$ \\
\end{tabular} & $\begin{array}{c}\text { Preparation } \\
\text { Sequence }\end{array}$ & \begin{tabular}{|c|}
$\begin{array}{c}\text { Analytical } \\
\text { Block }\end{array}$ \\
\end{tabular} & \begin{tabular}{|c} 
Analytical \\
Sequence
\end{tabular} & Sample ID & $\begin{array}{c}\mathrm{Mg} \\
(\mathrm{ug} / \mathrm{g})\end{array}$ & $\begin{array}{c}\text { Mn } \\
(\text { ug/g) }\end{array}$ & $\begin{array}{c}\mathrm{Na} \\
(\mathrm{ug} / \mathrm{g})\end{array}$ & $\begin{array}{c}\mathbf{N i} \\
(\mathrm{ug} / \mathrm{g})\end{array}$ & $\begin{array}{c}\mathbf{P} \\
(\mathrm{ug} / \mathrm{g})\end{array}$ & $\begin{array}{c}\text { Pb } \\
(\mathrm{ug} / \mathrm{g})\end{array}$ & $\begin{array}{c}\mathrm{S} \\
(\mathrm{ug} / \mathrm{g})\end{array}$ & $\begin{array}{c}\mathrm{Si} \\
\text { (ug/g) }\end{array}$ & $\mathrm{Ti}$ (ug/g) & $\begin{array}{c}\mathrm{Zn} \\
(\mathrm{ug} / \mathrm{g})\end{array}$ & $\begin{array}{c}\mathrm{Zr} \\
(\mathrm{ug} / \mathrm{g})\end{array}$ \\
\hline ARG-1 & None & 0 & 0 & 1 & 1 & 1 & 1 & ARG-1B11 & 4890 & 13600 & 80300 & 7860 & 753 & $<331$ & $<1060$ & 225000 & 6890 & 222 & 980 \\
\hline SME Simulant & Isolok - $3 \mathrm{~mL}$ & LF & 18 & 1 & 9 & 1 & 2 & LIS03 & 5960 & 12800 & 79300 & 3560 & $<207$ & 775 & $<<30$ & 245000 & 487 & 835 & 2780 \\
\hline SME Simulant & Isolok - $3 \mathrm{~mL}$ & LF & 8 & 1 & 27 & 1 & 3 & LIS01 & 5960 & 12700 & 76000 & 3680 & $<226$ & 788 & $<907$ & 245000 & 502 & 827 & 2740 \\
\hline SME Simulant & Coliwasa & LF & 20 & 1 & 2 & 1 & 4 & CL02 & 6120 & 13100 & 75800 & 3540 & $<240$ & 830 & $<964$ & 245000 & 496 & 850 & 2720 \\
\hline SME Simulant & Coliwasa & LF & 38 & 1 & 16 & 1 & 5 & CH03 & 6110 & 13100 & 77400 & 3650 & $<202$ & 864 & 1120 & 249000 & 492 & 844 & 2780 \\
\hline SME Simulant & Coliwasa & LF & 63 & 1 & 13 & 1 & 6 & CH04 & 6130 & 13100 & 78600 & 3740 & $<215$ & 981 & 1190 & 244000 & 497 & 850 & 2740 \\
\hline SME Simulant & Isolok - $3 \mathrm{~mL}$ & LF & 44 & 1 & 3 & 1 & 7 & LIS07 & 5770 & 12300 & 74200 & 3340 & $<225$ & 849 & $<904$ & 251000 & 508 & 774 & 2720 \\
\hline SME Simulant & Coliwasa & $\mathrm{HF}$ & 23 & 1 & 28 & 1 & 8 & CH06 & 5900 & 12600 & 75700 & 3460 & $<224$ & 602 & 1240 & 243000 & 483 & 792 & 2750 \\
\hline SME Simulant & Coliwasa & LF & 21 & 1 & 14 & 1 & 9 & $\mathrm{CH} 02$ & 6020 & 12900 & 79600 & 3570 & $<227$ & 653 & 1010 & 245000 & 482 & 826 & 2770 \\
\hline SME Simulant & Coliwasa & $\mathrm{HF}$ & 3 & 1 & 20 & 1 & 10 & CL05 & 6040 & 12900 & 75300 & 3570 & $<226$ & 635 & 1100 & 249000 & 495 & 829 & 2770 \\
\hline SME Simulant & Isolok - $3 \mathrm{~mL}$ & LF & 12 & 1 & 25 & 1 & 11 & LIS02 & 5880 & 12600 & 79900 & 3420 & $<228$ & 1710 & $<916$ & 242000 & 460 & 806 & 2680 \\
\hline SME Simulant & Coliwasa & LF & 40 & 1 & 19 & 1 & 12 & CL03 & 6020 & 12900 & 77800 & 3560 & $<213$ & 1540 & 1510 & 241000 & 473 & 835 & 2750 \\
\hline SME Simulant & Coliwasa & $\mathrm{HF}$ & 18 & 1 & 26 & 1 & 13 & CL06 & 6070 & 13000 & 70500 & 3720 & $<238$ & 2130 & 1440 & 247000 & 493 & 875 & 2750 \\
\hline SME Simulant & Coliwasa & $\mathrm{HF}$ & 1 & 1 & 22 & 1 & 14 & CH05 & 6030 & 12900 & 77800 & 3950 & $<227$ & 473 & 1220 & 243000 & 478 & 820 & 2740 \\
\hline ARG-1 & None & 0 & 0 & 1 & 15 & 1 & 15 & ARG-1B12 & 4990 & 13900 & 81500 & 8190 & 688 & 341 & $<850$ & 230000 & 7010 & 199 & 1000 \\
\hline SME Simulant & Coliwasa & $\mathrm{HF}$ & 36 & 1 & 21 & 1 & 16 & CL07 & 5650 & 12100 & 72800 & 3350 & $<215$ & 402 & $<864$ & 234000 & 468 & 779 & 2620 \\
\hline SME Simulant & Isolok - $3 \mathrm{~mL}$ & LF & 31 & 1 & 24 & 1 & 17 & LIS05 & 5710 & 12200 & 74400 & 3420 & $<202$ & $<253$ & $<809$ & 243000 & 489 & 757 & 2690 \\
\hline SME Simulant & Isolok - $3 \mathrm{~mL}$ & LF & 58 & 1 & 4 & 1 & 18 & LIS10 & 5890 & 12600 & 73500 & 3580 & $<236$ & 337 & 1090 & 247000 & 508 & 812 & 2760 \\
\hline SME Simulant & Coliwasa & $\mathrm{HF}$ & 63 & 1 & 5 & 1 & 19 & CL08 & 5970 & 12800 & 75800 & 3710 & $<231$ & 500 & 1330 & 243000 & 461 & 834 & 2760 \\
\hline SME Simulant & Isolok - $3 \mathrm{~mL}$ & LF & 25 & 1 & 7 & 1 & 20 & LIS04 & 5980 & 12800 & 80800 & 3610 & $<215$ & $<270$ & $<863$ & 243000 & 498 & 819 & 2770 \\
\hline SME Simulant & Isolok - $3 \mathrm{~mL}$ & LF & 42 & 1 & 11 & 1 & 21 & LIS06 & 6000 & 12800 & 74800 & 3420 & $<224$ & $<281$ & $<898$ & 252000 & 511 & 789 & 2770 \\
\hline SME Simulant & Coliwasa & $\mathrm{HF}$ & 65 & 1 & 6 & 1 & 22 & CH08 & 6020 & 12800 & 76200 & 3530 & $<214$ & $<269$ & 1070 & 242000 & 498 & 795 & 2770 \\
\hline SME Simulant & Coliwasa & $\mathrm{HF}$ & 34 & 1 & 18 & 1 & 23 & CH07 & 6080 & 13000 & 73400 & 3490 & $<220$ & $<277$ & 1050 & 244000 & 487 & 812 & 2790 \\
\hline SME Simulant & Coliwasa & LF & 1 & 1 & 12 & 1 & 24 & CH01 & 6090 & 13100 & 75600 & 3520 & $<232$ & $<292$ & 1250 & 242000 & 493 & 817 & 2730 \\
\hline SME Simulant & Isolok - $3 \mathrm{~mL}$ & LF & 54 & 1 & 17 & 1 & 25 & LIS09 & 5880 & 12600 & 74400 & 3570 & $<230$ & $<289$ & $<923$ & 246000 & 511 & 805 & 2770 \\
\hline SME Simulant & $\begin{array}{l}\text { Coliwasa } \\
\end{array}$ & LF & 4 & 1 & 10 & 1 & 26 & CL01 & 5940 & 12700 & 81300 & 3520 & $<199$ & $<250$ & 919 & 238000 & 486 & 792 & 2750 \\
\hline SME Simulant & Isolok - $3 \mathrm{~mL}$ & LF & 49 & 1 & 8 & 1 & 27 & LIS08 & 5860 & 12600 & 76500 & 3450 & $<251$ & $<316$ & $<<1010$ & 239000 & 487 & 775 & 2730 \\
\hline SME Simulant & Coliwasa & LF & 65 & 1 & 23 & 1 & 28 & CL04 & 6020 & 12900 & 78200 & 3570 & $<210$ & $<265$ & 1080 & 244000 & 503 & 813 & 2790 \\
\hline ARG-1 & None & 0 & 0 & 1 & 29 & 1 & 29 & ARG-1B13 & 4970 & 13900 & 81500 & 7920 & 786 & $<302$ & $<966$ & 229000 & 7050 & 189 & 1020 \\
\hline ARG-1 & None & 0 & 0 & 2 & 1 & 2 & 1 & ARG-1B21 & 5010 & 14000 & 86800 & 8260 & 779 & $<278$ & $<888$ & 230000 & 7100 & 198 & 1020 \\
\hline SME Simulant & Hydragard & $\mathrm{HF}$ & 11 & 2 & 3 & 2 & 2 & $\begin{array}{l}\text { HHY02 } \\
\end{array}$ & 6620 & 14200 & 76200 & 4040 & $<222$ & 670 & 1670 & 239000 & 500 & 938 & 2830 \\
\hline SME Simulant & Coliwasa & $\mathrm{HF}$ & 36 & 1 & 21 & 2 & 3 & CL07 & 5970 & 12700 & 81100 & 3520 & $<215$ & 774 & 1450 & 248000 & 489 & 806 & 2770 \\
\hline SME Simulant & Hydragard & $\mathrm{HF}$ & 24 & 2 & 2 & 2 & 4 & HHY04 & 6200 & 13300 & 80900 & 3680 & $<204$ & 701 & 1500 & 245000 & 499 & 811 & 2870 \\
\hline SME Simulant & Coliwasa & $\mathrm{HF}$ & 1 & 1 & 22 & 2 & 5 & $\mathrm{CH} 05$ & 6080 & 13000 & 83400 & 3970 & $<227$ & 738 & 1410 & 245000 & 502 & 797 & 2800 \\
\hline SME Simulant & Coliwasa & $\mathrm{HF}$ & 3 & 1 & 20 & 2 & 6 & CL05 & 6120 & 13100 & 79800 & 3570 & $<226$ & $<284$ & 1330 & 250000 & 519 & 815 & 2820 \\
\hline SME Simulant & Coliwasa & $\mathrm{HF}$ & 23 & 1 & 28 & 2 & 7 & CH06 & 5970 & 12800 & 80900 & 3450 & $<224$ & $<282$ & 1120 & 244000 & 498 & 805 & 2810 \\
\hline SME Simulant & Coliwasa & LF & 1 & 1 & 12 & 2 & 8 & CH01 & 6120 & 13100 & 81500 & 3500 & $<232$ & $<292$ & 1190 & 243000 & 500 & 813 & 2750 \\
\hline SME Simulant & Coliwasa & LF & 20 & 1 & 2 & 2 & 9 & CL02 & 6240 & 13300 & 80800 & 3570 & $<240$ & 1100 & 1410 & 247000 & 503 & 828 & 2750 \\
\hline SME Simulant & Coliwasa & $\mathrm{HF}$ & 63 & 1 & 5 & 2 & 10 & CL08 & 5950 & 12800 & 80300 & 3670 & $<231$ & 727 & 1760 & 240000 & 483 & 820 & 2760 \\
\hline SME Simulant & Coliwasa & $\mathrm{HF}$ & 65 & 1 & 6 & 2 & 11 & CH08 & 6070 & 13000 & 82000 & 3540 & $<214$ & 1320 & 1490 & 243000 & 476 & 819 & 2720 \\
\hline SME Simulant & Hydragard & $\mathrm{HF}$ & 45 & 2 & 7 & 2 & 12 & HHY07 & 6320 & 13500 & 81100 & 3770 & $<231$ & 1450 & 1250 & 239000 & 476 & 865 & 2790 \\
\hline SME Simulant & Coliwasa & LF & 4 & 1 & 10 & 2 & 13 & CL01 & 6060 & 12900 & 87800 & 3610 & $<199$ & 1200 & 1900 & 240000 & 476 & 816 & 2780 \\
\hline SME Simulant & Coliwasa & $\mathrm{HF}$ & 18 & 1 & 26 & 2 & 14 & CL06 & 6160 & 13200 & 75900 & 3520 & $<238$ & 607 & 1500 & 249000 & 512 & 824 & 2830 \\
\hline ARG-1 & None & 0 & 0 & 2 & 12 & 2 & 15 & ARG-1B22 & 4960 & 13900 & 86400 & 8090 & 774 & $<305$ & $<976$ & 227000 & 7000 & 190 & 1030 \\
\hline SME Simulant & Coliwasa & LF & 65 & 1 & 23 & 2 & 16 & CL04 & 6030 & 12800 & 82500 & 3480 & $<210$ & 363 & 1230 & 241000 & 494 & 789 & 2780 \\
\hline SME Simulant & Coliwasa & LF & 40 & $\frac{x}{1}$ & 19 & 2 & 17 & CL03 & 6060 & 12900 & 83500 & 3570 & $<213$ & 426 & 1620 & 241000 & 488 & 811 & 2790 \\
\hline SME Simulant & Coliwasa & LF & 38 & 1 & 16 & 2 & 18 & $\mathrm{CH} 03$ & 6070 & 12900 & 80900 & 3560 & $<202$ & 794 & 1600 & 246000 & 493 & 821 & 2770 \\
\hline SME Simulant & Hydragard & $\mathrm{HF}$ & 57 & 2 & 6 & 2 & 19 & HHY09 & 6500 & 13800 & 83300 & 3900 & $<217$ & 872 & 1380 & 235000 & 490 & 892 & 2800 \\
\hline
\end{tabular}


Table C3. Phase 3 Elemental Concentration Measurements (part 2)

Revision 0

\begin{tabular}{|c|c|c|c|c|c|c|c|c|c|c|c|c|c|c|c|c|c|c|c|}
\hline $\begin{array}{c}\text { Type of } \\
\text { Material }\end{array}$ & Sampler & $\begin{array}{l}\text { Flow } \\
\text { Rate }\end{array}$ & $\begin{array}{c}\text { Sample } \\
\text { Order }\end{array}$ & $\begin{array}{c}\text { Preparation } \\
\text { Block }\end{array}$ & $\begin{array}{c}\text { Preparation } \\
\text { Sequence }\end{array}$ & $\begin{array}{c}\text { Analytical } \\
\text { Block }\end{array}$ & $\begin{array}{c}\text { Analytical } \\
\text { Sequence }\end{array}$ & Sample ID & $\begin{array}{c}\mathbf{M g} \\
\text { (ug/g) }\end{array}$ & $\begin{array}{c}\text { Mn } \\
\text { (ug/g) }\end{array}$ & $\begin{array}{c}\mathrm{Na} \\
(\mathrm{ug} / \mathrm{g})\end{array}$ & $\begin{array}{c}\mathrm{Ni} \\
(\mathrm{ug} / \mathrm{g})\end{array}$ & $\begin{array}{c}\mathbf{P} \\
(\mathrm{ug} / \mathrm{g})\end{array}$ & $\begin{array}{c}\mathbf{P b} \\
(\mathbf{u g} / \mathbf{g})\end{array}$ & $\underset{(\mathrm{ug} / \mathrm{g})}{\mathrm{S}}$ & $\begin{array}{c}\mathrm{Si} \\
\text { (ug/g) }\end{array}$ & Ti (ug/g) & $\begin{array}{c}\text { Zn } \\
\text { (ug/g) }\end{array}$ & $\begin{array}{c}\mathrm{Zr} \\
(\mathbf{u g} / \mathrm{g})\end{array}$ \\
\hline SME Simulant & Coliwasa & LF & 21 & 1 & 14 & 2 & 20 & CH02 & 5960 & 12700 & 83200 & 3590 & $<227$ & 1240 & 1310 & 242000 & 466 & 822 & 2720 \\
\hline SME Simulant & Hydragard & $\mathrm{HF}$ & 6 & 2 & 4 & 2 & 21 & HHY01 & 6340 & 13500 & 81000 & 3640 & $<215$ & 1120 & 1500 & 242000 & 490 & 847 & 2750 \\
\hline SME Simulant & Hydragard & $\mathrm{HF}$ & 19 & 2 & 10 & 2 & 22 & HHY03 & 6410 & 13500 & 79400 & 3660 & $<213$ & 575 & 1420 & 246000 & 504 & 851 & 2880 \\
\hline SME Simulant & Hydragard & $\mathrm{HF}$ & 50 & 2 & 5 & 2 & 23 & HHY08 & 6120 & 13300 & 81000 & 3600 & $<219$ & $<275$ & 1460 & 241000 & 498 & 782 & 2770 \\
\hline SME Simulant & Hydragard & $\mathrm{HF}$ & 37 & 2 & 9 & 2 & 24 & HHY06 & 6420 & 13800 & 82400 & 3780 & $<229$ & 1740 & 2300 & 237000 & 462 & 867 & 2730 \\
\hline SME Simulant & Coliwasa & $\mathrm{HF}$ & 34 & 1 & 18 & 2 & 25 & CH07 & 6180 & 13200 & 79800 & 3590 & $<220$ & $<277$ & 1480 & 247000 & 508 & 829 & 2810 \\
\hline SME Simulant & Coliwasa & LF & 63 & 1 & 13 & 2 & 26 & CH04 & 6020 & 12900 & 82400 & 3470 & $<215$ & 1010 & 1690 & 241000 & 473 & 794 & 2700 \\
\hline SME Simulant & Hydragard & $\mathrm{HF}$ & 32 & 2 & 8 & 2 & 27 & HHY05 & 6480 & 13300 & 90800 & 3380 & $<216$ & 1530 & 2070 & 238000 & 466 & 775 & 2590 \\
\hline SME Simulant & Hydragard & $\mathrm{HF}$ & 61 & 2 & 11 & 2 & 28 & HHY10 & 6580 & 14100 & 83500 & 3790 & $<218$ & 2150 & 1670 & 234000 & 456 & 897 & 2820 \\
\hline ARG-1 & None & 0 & 0 & 2 & 23 & 2 & 29 & ARG-1B23 & 4960 & 13800 & 87400 & 7740 & 721 & $<301$ & $<961$ & 228000 & 7030 & 171 & 1000 \\
\hline ARG-1 & None & 0 & 0 & 3 & 1 & 3 & 1 & ARG-1B31 & 5110 & 14200 & 85500 & 7940 & 800 & $<290$ & $<927$ & 230000 & 7010 & 199 & 987 \\
\hline SME Simulant & Isolok - $12 \mathrm{~mL}$ & LF & 11 & 3 & 4 & 3 & 2 & LIB02 & 5740 & 12300 & 82100 & 3470 & $<222$ & $<279$ & 1300 & 249000 & 499 & 785 & 2620 \\
\hline SME Simulant & Coliwasa & $\mathrm{HF}$ & 36 & 1 & 21 & 3 & 3 & CL07 & 6110 & 12900 & 80600 & 3500 & $<215$ & $<270$ & 1160 & 247000 & 510 & 804 & 2790 \\
\hline SME Simulant & Isolok - $12 \mathrm{~mL}$ & LF & 26 & 3 & 8 & 3 & 4 & LIB04 & 6210 & 13200 & 87400 & 3450 & $<223$ & $<280$ & 1020 & 238000 & 499 & 796 & 2740 \\
\hline SME Simulant & Coliwasa & $\mathrm{HF}$ & 1 & 1 & 22 & 3 & 5 & CH05 & 6280 & 13400 & 82200 & 3990 & $<227$ & 419 & 1380 & 246000 & 500 & 815 & 2770 \\
\hline SME Simulant & Coliwasa & $\mathrm{HF}$ & 3 & 1 & 20 & 3 & 6 & CL05 & 6330 & 13400 & 79200 & 3500 & $<226$ & 973 & 1690 & 254000 & 512 & 822 & 2770 \\
\hline SME Simulant & Coliwasa & $\mathrm{HF}$ & 23 & 1 & 28 & 3 & 7 & CH06 & 6070 & 12800 & 78700 & 3550 & $<224$ & 450 & 1480 & 242000 & 486 & 809 & 2720 \\
\hline SME Simulant & Coliwasa & LF & 1 & 1 & 12 & 3 & 8 & CH01 & 6360 & 13500 & 80700 & 3400 & $<232$ & 423 & 1250 & 245000 & 493 & 809 & 2710 \\
\hline SME Simulant & Coliwasa & LF & 20 & 1 & 2 & 3 & 9 & CL02 & 6450 & 13700 & 80200 & 3680 & $<240$ & 890 & $<964$ & 250000 & 487 & 881 & 2750 \\
\hline SME Simulant & Coliwasa & $\mathrm{HF}$ & 63 & 1 & 5 & 3 & 10 & CL08 & 6260 & 13400 & 81100 & 3630 & $<231$ & 1450 & 1700 & 246000 & 481 & 847 & 2750 \\
\hline SME Simulant & Coliwasa & $\mathrm{HF}$ & 65 & 1 & 6 & 3 & 11 & CH08 & 6230 & 13200 & 80800 & 3560 & $<214$ & 1240 & 1480 & 244000 & 478 & 855 & 2700 \\
\hline SME Simulant & Isolok - $12 \mathrm{~mL}$ & LF & 45 & 3 & 7 & 3 & 12 & LIB07 & 6010 & 12800 & 79300 & 3600 & $<232$ & 1250 & 1460 & 249000 & 478 & 796 & 2650 \\
\hline SME Simulant & Coliwasa & LF & 4 & 1 & 10 & 3 & 13 & CL01 & 6190 & 13200 & 86200 & 3580 & $<199$ & 1200 & 1600 & 242000 & 473 & 817 & 2750 \\
\hline SME Simulant & Coliwasa & $\mathrm{HF}$ & 18 & $\frac{x}{1}$ & 26 & 3 & 14 & CL06 & 6370 & 13600 & 75400 & 3660 & $<238$ & 1490 & 1930 & 252000 & 494 & 873 & 2790 \\
\hline ARG-1 & None & 0 & 0 & 3 & 12 & 3 & 15 & ARG-1B32 & 5170 & 14400 & 86000 & 8120 & 752 & 929 & $<946$ & 230000 & 7000 & 210 & 974 \\
\hline SME Simulant & Coliwasa & LF & 65 & 1 & 23 & 3 & 16 & CL04 & 6160 & 13100 & 81500 & 3570 & $<210$ & 1570 & 1570 & 241000 & 481 & 827 & 2680 \\
\hline SME Simulant & Coliwasa & LF & 40 & 1 & 19 & 3 & 17 & CL03 & 6180 & 13100 & 81800 & 3650 & $<213$ & 941 & 1650 & 239000 & 482 & 842 & 2730 \\
\hline SME Simulant & Coliwasa & LF & 38 & 1 & 16 & 3 & 18 & CH03 & 6180 & 13100 & 80000 & 3400 & $<202$ & 843 & 1300 & 245000 & 485 & 802 & 2710 \\
\hline SME Simulant & Isolok - $12 \mathrm{~mL}$ & LF & 56 & 3 & 11 & 3 & 19 & LIB09 & 6100 & 13100 & 79900 & 3750 & $<224$ & 1240 & 1550 & 244000 & 491 & 834 & 2710 \\
\hline SME Simulant & $\begin{array}{l}\text { Coliwasa } \\
\end{array}$ & LF & 21 & 1 & 14 & 3 & 20 & $\mathrm{CH} 02$ & 6180 & 13200 & 83500 & 3570 & $<227$ & 1040 & 1580 & 245000 & 477 & 830 & 2740 \\
\hline SME Simulant & Isolok - $12 \mathrm{~mL}$ & LF & 5 & 3 & 5 & 3 & 21 & LIB01 & 6210 & 13200 & 82500 & 3580 & $<216$ & 944 & 1250 & 249000 & 502 & 836 & 2730 \\
\hline SME Simulant & Isolok - $12 \mathrm{~mL}$ & LF & 23 & 3 & 10 & 3 & 22 & LIB03 & 6140 & 13100 & 84700 & 3600 & $<224$ & 1900 & 1820 & 248000 & 499 & 883 & 2640 \\
\hline SME Simulant & Isolok - $12 \mathrm{~mL}$ & LF & 50 & 3 & 2 & 3 & 23 & LIB08 & 5390 & 11500 & 81700 & 3250 & $<207$ & 1550 & 1790 & 250000 & 486 & 720 & 2590 \\
\hline SME Simulant & Isolok - $12 \mathrm{~mL}$ & LF & 41 & 3 & 9 & 3 & 24 & LIB06 & 5030 & 10700 & 80800 & 3170 & $<224$ & 1520 & 1550 & 257000 & 497 & 697 & 2530 \\
\hline SME Simulant & Coliwasa & $\mathrm{HF}$ & 34 & 1 & 18 & 3 & 25 & CH07 & 5960 & 12600 & 74600 & 3250 & $<220$ & 2020 & 1260 & 231000 & 436 & 799 & 2530 \\
\hline SME Simulant & $\begin{array}{l}\text { Coliwasa } \\
\end{array}$ & LF & 63 & 1 & 13 & 3 & 26 & $\mathrm{CH} 04$ & 6400 & 13600 & 84400 & 3680 & $<215$ & 1330 & 1670 & 249000 & 514 & 854 & 2770 \\
\hline SME Simulant & Isolok - $12 \mathrm{~mL}$ & LF & 32 & 3 & 3 & 3 & 27 & LIB05 & 6150 & 13100 & 83200 & 3580 & $<229$ & 1120 & 1410 & 249000 & 492 & 846 & 2670 \\
\hline SME Simulant & Isolok - $12 \mathrm{~mL}$ & LF & 60 & 3 & 6 & 3 & 28 & LIB10 & 5530 & 11700 & 81100 & 3220 & $<220$ & 859 & 1430 & 247000 & 484 & 732 & 2580 \\
\hline ARG-1 & None & 0 & 0 & 3 & 23 & 3 & 29 & ARG-1B33 & 5060 & 14100 & 86000 & 7910 & 668 & 569 & $<1000$ & 227000 & 6930 & 192 & 984 \\
\hline ARG-1 & None & 0 & 0 & 4 & 1 & 4 & 1 & ARG-1B41 & 5170 & 14500 & 86000 & 8090 & 661 & 453 & $<1010$ & 227000 & 6960 & 191 & 946 \\
\hline SME Simulant & Isolok - $12 \mathrm{~mL}$ & $\mathrm{HF}$ & 13 & 4 & 11 & 4 & 2 & HIB02 & 6200 & 13200 & 76700 & 3580 & $<217$ & 637 & 1190 & 252000 & 512 & 830 & 2680 \\
\hline SME Simulant & Coliwasa & $\mathrm{HF}$ & 36 & 1 & 21 & 4 & 3 & $\begin{array}{l}\text { CL07 } \\
\end{array}$ & 5880 & 12500 & 77300 & 3260 & $<215$ & $<270$ & $<864$ & 234000 & 472 & 757 & 2620 \\
\hline SME Simulant & Isolok - $12 \mathrm{~mL}$ & $\mathrm{HF}$ & 25 & 4 & 4 & 4 & 4 & HIB04 & 5910 & 12700 & 76000 & 3560 & $<227$ & 648 & 969 & 246000 & 487 & 783 & 2670 \\
\hline SME Simulant & Coliwasa & $\mathrm{HF}$ & 1 & 1 & 22 & 4 & 5 & $\mathrm{CH} 05$ & 6270 & 13400 & 82500 & 3960 & $<227$ & 486 & 1140 & 243000 & 480 & 825 & 2720 \\
\hline SME Simulant & Coliwasa & $\mathrm{HF}$ & 3 & 1 & 20 & 4 & 6 & CL05 & 6250 & 13300 & 79300 & 3510 & $<226$ & 485 & 1300 & 247000 & 500 & 813 & 2730 \\
\hline SME Simulant & Coliwasa & $\mathrm{HF}$ & 23 & 1 & 28 & 4 & 7 & CH06 & 6260 & 13400 & 81200 & 3630 & $<224$ & 2170 & 1660 & 246000 & 469 & 849 & 2720 \\
\hline SME Simulant & Coliwasa & LF & 1 & 1 & 12 & 4 & 8 & CH01 & 6520 & 13900 & 82800 & 3660 & $<232$ & 1400 & 1370 & 248000 & 498 & 852 & 2700 \\
\hline SME Simulant & Coliwasa & LF & 20 & 1 & 2 & 4 & 9 & CL02 & 6390 & 13600 & 79900 & 3520 & $<240$ & 2250 & 1120 & 243000 & 467 & 843 & 2630 \\
\hline
\end{tabular}


Table C3. Phase 3 Elemental Concentration Measurements (part 2)

Revision 0

\begin{tabular}{|c|c|c|c|c|c|c|c|c|c|c|c|c|c|c|c|c|c|c|c|}
\hline $\begin{array}{c}\text { Type of } \\
\text { Material }\end{array}$ & Sampler & $\begin{array}{l}\text { Flow } \\
\text { Rate }\end{array}$ & $\begin{array}{c}\text { Sample } \\
\text { Order }\end{array}$ & \begin{tabular}{|c|}
$\begin{array}{c}\text { Preparation } \\
\text { Block }\end{array}$ \\
\end{tabular} & $\begin{array}{c}\text { Preparation } \\
\text { Sequence }\end{array}$ & \begin{tabular}{|c|}
$\begin{array}{c}\text { Analytical } \\
\text { Block }\end{array}$ \\
\end{tabular} & \begin{tabular}{|c|} 
Analytical \\
Sequence \\
\end{tabular} & Sample ID & $\begin{array}{c}\mathrm{Mg} \\
(\mathrm{ug} / \mathrm{g})\end{array}$ & $\begin{array}{c}\text { Mn } \\
(\text { (ug/g) }\end{array}$ & $\begin{array}{c}\mathrm{Na} \\
\text { (ug/g) }\end{array}$ & $\begin{array}{c}\mathrm{Ni} \\
(\mathrm{ug} / \mathrm{g})\end{array}$ & $\begin{array}{c}\mathbf{P} \\
(\mathrm{ug} / \mathrm{g})\end{array}$ & $\begin{array}{c}\mathbf{P b} \\
(\mathrm{ug} / \mathrm{g})\end{array}$ & $\begin{array}{c}\mathrm{S} \\
(\mathrm{ug} / \mathrm{g})\end{array}$ & $\begin{array}{c}\mathrm{Si} \\
\text { (ug/g) }\end{array}$ & $\mathrm{Ti}(\mathrm{ug} / \mathrm{g})$ & $\begin{array}{c}\mathrm{Zn} \\
(\mathrm{ug} / \mathrm{g})\end{array}$ & $\begin{array}{c}\mathrm{Zr} \\
(\mathrm{ug} / \mathrm{g})\end{array}$ \\
\hline SME Simulant & Coliwasa & $\mathrm{HF}$ & 63 & 1 & 5 & 4 & \begin{tabular}{|l|}
10 \\
\end{tabular} & CL08 & 6240 & 13400 & 81700 & 3620 & $<231$ & 3170 & 1720 & 243000 & 444 & 839 & 2690 \\
\hline SME Simulant & Isolok - $12 \mathrm{~mL}$ & $\mathrm{HF}$ & 46 & 4 & 2 & 4 & 11 & HIB07 & 6220 & 13200 & 86300 & 3650 & $<226$ & 1960 & 1520 & 241000 & 481 & 822 & 2640 \\
\hline SME Simulant & Coliwasa & LF & 4 & 1 & 10 & 4 & 12 & CL01 & 6310 & 13500 & 88300 & 3570 & $<199$ & 1550 & 1230 & 243000 & 477 & 820 & 2730 \\
\hline SME Simulant & Coliwasa & $\mathrm{HF}$ & 18 & 1 & 26 & 4 & 13 & CL06 & 6360 & 13600 & 75400 & 3580 & $<238$ & 2210 & 1560 & 248000 & 463 & 835 & 2750 \\
\hline ARG-1 & None & 0 & 0 & 4 & 7 & 4 & 14 & ARG-1B42 & 5110 & 14300 & 85900 & 8170 & 512 & 1550 & $<1030$ & 226000 & 6860 & 205 & 937 \\
\hline SME Simulant & Coliwasa & LF & 65 & 1 & 23 & 4 & 15 & CL04 & 6260 & 13300 & 82700 & 3550 & $<210$ & 1440 & 1160 & 242000 & 482 & 813 & 2700 \\
\hline SME Simulant & Coliwasa & LF & 40 & 1 & 19 & 4 & 16 & CL03 & 6310 & 13500 & 83500 & 3670 & $<213$ & 1240 & 1300 & 242000 & 472 & 844 & 2720 \\
\hline SME Simulant & Coliwasa & LF & 21 & 1 & 14 & 4 & 17 & $\mathrm{CH} 02$ & 6210 & 13200 & 83400 & 3660 & $<227$ & 2650 & 1780 & 242000 & 482 & 850 & 2690 \\
\hline SME Simulant & Isolok - $12 \mathrm{~mL}$ & $\mathrm{HF}$ & 7 & 4 & 12 & 4 & 18 & HIB01 & 6300 & 13400 & 81000 & 3710 & $<215$ & 2370 & 1650 & 248000 & 471 & 831 & 2640 \\
\hline SME Simulant & Isolok - $12 \mathrm{~mL}$ & $\mathrm{HF}$ & 21 & 4 & 6 & 4 & 19 & HIB03 & 6430 & 13800 & 83900 & 3790 & $<198$ & 2110 & 1490 & 245000 & 474 & 861 & 2730 \\
\hline SME Simulant & Isolok - $12 \mathrm{~mL}$ & $\mathrm{HF}$ & 49 & 4 & 5 & 4 & 20 & HIB08 & 6060 & 12900 & 75400 & 3580 & $<220$ & 1310 & 907 & 227000 & 438 & 799 & 2530 \\
\hline SME Simulant & Isolok - $12 \mathrm{~mL}$ & $\mathrm{HF}$ & 38 & 4 & 8 & 4 & 21 & HIB06 & 6020 & 12900 & 79000 & 3480 & $<212$ & 1320 & 1340 & 243000 & 483 & 789 & 2590 \\
\hline SME Simulant & Coliwasa & $\mathrm{HF}$ & 34 & 1 & 18 & 4 & 22 & $\mathrm{CH} 07$ & 6480 & 13800 & 79900 & 3530 & $<220$ & 2020 & 1180 & 249000 & 502 & 838 & 2760 \\
\hline SME Simulant & Coliwasa & LF & 63 & 1 & 13 & 4 & 23 & $\mathrm{CH} 04$ & 6340 & 13600 & 83200 & 3610 & $<215$ & 2070 & 1520 & 244000 & 464 & 841 & 2680 \\
\hline SME Simulant & Isolok - $12 \mathrm{~mL}$ & $\mathrm{HF}$ & 33 & 4 & 10 & 4 & 24 & HIB05 & 5950 & 12700 & 85400 & 3420 & $<222$ & 1410 & 1210 & 247000 & 479 & 790 & 2600 \\
\hline SME Simulant & Isolok - $12 \mathrm{~mL}$ & $\mathrm{HF}$ & 58 & 4 & 3 & 4 & 25 & HIB10 & 5670 & 12100 & 79500 & 3540 & $<222$ & 1500 & 1530 & 250000 & 483 & 771 & 2530 \\
\hline ARG-1 & None & 0 & 0 & 4 & 13 & 4 & 26 & ARG-1B43 & 5080 & 14200 & 85000 & 7910 & 515 & 1300 & $\begin{array}{l}<958 \\
\end{array}$ & 225000 & 6810 & 202 & 944 \\
\hline SME Simulant & Coliwasa & $\mathrm{HF}$ & 65 & 1 & 6 & 4 & 27 & CH08 & 6320 & 13500 & 81500 & 3640 & $<214$ & 2590 & 1970 & 243000 & 465 & 842 & 2610 \\
\hline SME Simulant & Coliwasa & LF & 38 & 1 & 16 & 4 & 28 & $\mathrm{CH} 03$ & 6330 & 13400 & 81200 & 3590 & $<202$ & 2590 & 1470 & 247000 & 455 & 825 & 2720 \\
\hline SME Simulant & Isolok - $12 \mathrm{~mL}$ & $\mathrm{HF}$ & 56 & 4 & 9 & 4 & 29 & HIB09 & 6970 & 14900 & 84500 & 4210 & $<230$ & 2820 & 2420 & 233000 & 466 & 992 & 2680 \\
\hline ARG-1 & None & 0 & 0 & 2 & 1 & 5 & 1 & ARG-1B21 & 5220 & 14600 & 84500 & 8090 & 792 & 547 & $<888$ & 226000 & 6900 & 203 & 971 \\
\hline SME Simulant & Isolok - $3 \mathrm{~mL}$ & $\mathrm{HF}$ & 20 & 2 & 16 & 5 & 2 & HIS03 & 6310 & 13500 & 80700 & 3490 & $<225$ & $<283$ & $<903$ & 248000 & 527 & 776 & 2730 \\
\hline SME Simulant & Isolok - $3 \mathrm{~mL}$ & $\mathrm{HF}$ & 9 & 2 & 21 & 5 & 3 & HIS01 & 6440 & 13700 & 89400 & 3840 & $<228$ & 1180 & $<916$ & 245000 & 503 & 871 & 2740 \\
\hline SME Simulant & Coliwasa & LF & 20 & 1 & 2 & 5 & 4 & CL02 & 6570 & 13900 & 79300 & 3530 & $<240$ & 1060 & 1130 & 247000 & 503 & 838 & 2670 \\
\hline SME Simulant & $\begin{array}{l}\text { Coliwasa } \\
\end{array}$ & LF & 38 & 1 & 16 & 5 & 5 & $\mathrm{CH} 03$ & 6400 & 13600 & 79700 & 3480 & $<202$ & 663 & 1170 & 248000 & 505 & 798 & 2750 \\
\hline SME Simulant & $\begin{array}{l}\text { Coliwasa } \\
\end{array}$ & LF & 63 & 1 & 13 & 5 & 6 & $\mathrm{CH} 04$ & 6450 & 13800 & 81700 & 3510 & $<215$ & $<270$ & 1110 & 245000 & 479 & 810 & 2730 \\
\hline SME Simulant & Isolok - $3 \mathrm{~mL}$ & $\mathrm{HF}$ & 44 & 2 & 18 & 5 & 7 & HIS07 & 6380 & 13600 & 82600 & 3600 & $<230$ & 1550 & $<926$ & 249000 & 496 & 819 & 2900 \\
\hline SME Simulant & Coliwasa & $\mathrm{HF}$ & 23 & 1 & 28 & 5 & 8 & $\mathrm{CH} 06$ & 6390 & 13600 & 80900 & 3520 & $<224$ & 1850 & 1300 & 248000 & 499 & 795 & 2760 \\
\hline SME Simulant & Coliwasa & LF & 21 & 1 & 14 & 5 & 9 & $\mathrm{CH} 02$ & 6400 & 13700 & 84000 & 3520 & $<227$ & 2420 & 1240 & 246000 & 481 & 799 & 2700 \\
\hline SME Simulant & Coliwasa & $\mathrm{HF}$ & 3 & 1 & 20 & 5 & 10 & CL05 & 6380 & 13500 & 78800 & 3450 & $<226$ & 1370 & 1680 & 249000 & 493 & 816 & 2720 \\
\hline SME Simulant & Isolok - $3 \mathrm{~mL}$ & $\mathrm{HF}$ & 14 & 2 & 15 & 5 & 11 & HISO2 & 6230 & 13300 & 86500 & 3440 & $<229$ & 1820 & 969 & 247000 & 474 & 790 & 2700 \\
\hline SME Simulant & $\begin{array}{l}\text { Coliwasa } \\
\end{array}$ & LF & 40 & 1 & 19 & 5 & 12 & CL03 & 6470 & 13700 & 80200 & 3610 & $<213$ & 880 & 1440 & 244000 & 485 & 813 & 2730 \\
\hline SME Simulant & Coliwasa & $\mathrm{HF}$ & 18 & 1 & 26 & 5 & 13 & CL06 & 6460 & 13800 & 74200 & 3620 & $<238$ & 1510 & 1260 & 248000 & 485 & 822 & 2750 \\
\hline SME Simulant & Coliwasa & $\mathrm{HF}$ & 1 & 1 & 22 & 5 & 14 & CH05 & 6440 & 13700 & 82700 & 4030 & $<227$ & 1690 & 1260 & 245000 & 487 & 877 & 2700 \\
\hline ARG-1 & None & 0 & 0 & 2 & 12 & 5 & 15 & ARG-1B22 & 5260 & 14600 & 88100 & 8240 & 745 & 1570 & $<976$ & 229000 & 6930 & 203 & 980 \\
\hline SME Simulant & $\begin{array}{l}\text { Coliwasa } \\
\end{array}$ & $\mathrm{HF}$ & 36 & 1 & 21 & 5 & 16 & CL07 & 6300 & 13400 & 80400 & 3410 & $<215$ & 449 & 1090 & 247000 & 499 & 785 & 2730 \\
\hline SME Simulant & Isolok - $3 \mathrm{~mL}$ & $\mathrm{HF}$ & 31 & 2 & 19 & 5 & 17 & HIS05 & 6370 & 13500 & 80800 & 3460 & $<231$ & $<290$ & $\begin{array}{l}<926 \\
\end{array}$ & 248000 & 524 & 809 & 2770 \\
\hline SME Simulant & Isolok - $3 \mathrm{~mL}$ & $\mathrm{HF}$ & 60 & 2 & 22 & 5 & 18 & HIS10 & 6290 & 13300 & 82300 & 3520 & $<233$ & $<292$ & $<934$ & 249000 & 518 & 814 & 2640 \\
\hline SME Simulant & Coliwasa & $\mathrm{HF}$ & 63 & 1 & 5 & 5 & 19 & CL08 & 6310 & 13600 & 79900 & 3620 & $<231$ & $<290$ & 1280 & 242000 & 502 & 810 & 2760 \\
\hline SME Simulant & Isolok - $3 \mathrm{~mL}$ & $\mathrm{HF}$ & 26 & 2 & 14 & 5 & 20 & HIS04 & 6130 & 13100 & 79700 & 3420 & $<204$ & $<256$ & $<819$ & 249000 & 506 & 783 & 2720 \\
\hline SME Simulant & Isolok - $3 \mathrm{~mL}$ & $\mathrm{HF}$ & 35 & 2 & 20 & 5 & 21 & HIS06 & 6280 & 13500 & 82700 & 3540 & $<228$ & $<286$ & $<914$ & 244000 & 527 & 797 & 2780 \\
\hline SME Simulant & Coliwasa & $\mathrm{HF}$ & 65 & 1 & 6 & 5 & 22 & CH08 & 6320 & 13500 & 79700 & 3550 & $<214$ & $<269$ & 1000 & 241000 & 519 & 792 & 2700 \\
\hline SME Simulant & Coliwasa & $\mathrm{HF}$ & 34 & 1 & 18 & 5 & 23 & CH07 & 6450 & 13800 & 76800 & 3590 & $<220$ & $<277$ & $<884$ & 245000 & 533 & 832 & 2790 \\
\hline SME Simulant & Coliwasa & LF & 1 & 1 & 12 & 5 & 24 & CH01 & 6490 & 13900 & 80000 & 3530 & $<232$ & $<292$ & $<933$ & 244000 & 523 & 813 & 2720 \\
\hline SME Simulant & Isolok - $3 \mathrm{~mL}$ & $\mathrm{HF}$ & 54 & 2 & 17 & 5 & 25 & HIS09 & 6040 & 12900 & 78400 & 3350 & $<203$ & $<255$ & $<815$ & 251000 & 512 & 756 & 2730 \\
\hline SME Simulant & Coliwasa & LF & 4 & 1 & 10 & 5 & 26 & CL01 & 6320 & 13500 & 85800 & 3590 & $<199$ & $<250$ & 1090 & 239000 & 497 & 789 & 2730 \\
\hline SME Simulant & Isolok - $3 \mathrm{~mL}$ & $\mathrm{HF}$ & 48 & 2 & 13 & 5 & 27 & HIS08 & 6260 & 13400 & 80600 & 3370 & $<219$ & $<275$ & $<880$ & 247000 & 513 & 759 & 2800 \\
\hline SME Simulant & Coliwasa & LF & 65 & 1 & 23 & 5 & 28 & CL04 & 6360 & 13600 & 81800 & 3460 & $<210$ & $<265$ & 1030 & 243000 & 509 & 760 & 2780 \\
\hline
\end{tabular}


SRNL-STI-2011-00693

Revision 0

Table C3. Phase 3 Elemental Concentration Measurements (part 2)

\begin{tabular}{|c|c|c|c|c|c|c|c|c|c|c|c|c|c|c|c|c|c|c|c|}
\hline $\begin{array}{c}\text { Type of } \\
\text { Material }\end{array}$ & Sampler & $\begin{array}{l}\text { Flow } \\
\text { Rate }\end{array}$ & $\begin{array}{c}\text { Sample } \\
\text { Order }\end{array}$ & \begin{tabular}{|c|}
$\begin{array}{c}\text { Preparation } \\
\text { Block }\end{array}$ \\
\end{tabular} & $\begin{array}{c}\text { Preparation } \\
\text { Sequence }\end{array}$ & $\begin{array}{c}\begin{array}{c}\text { Analytical } \\
\text { Block }\end{array} \\
\end{array}$ & \begin{tabular}{|c|} 
Analytical \\
Sequence \\
\end{tabular} & Sample ID & $\begin{array}{c}\text { Mg } \\
\text { (ug/g) }\end{array}$ & $\begin{array}{c}\begin{array}{c}\text { Mn } \\
\text { (ug/g) }\end{array} \\
\end{array}$ & $\begin{array}{c}\mathrm{Na} \\
(\mathrm{ug} / \mathrm{g})\end{array}$ & $\begin{array}{c}\mathrm{Ni} \\
(\mathrm{ug} / \mathrm{g})\end{array}$ & $\begin{array}{c}\mathbf{P} \\
(\mathbf{u g} / \mathrm{g})\end{array}$ & $\begin{array}{c}\mathbf{P b} \\
(\mathbf{u g} / \mathrm{g})\end{array}$ & $\begin{array}{c}\mathrm{S} \\
(\mathrm{ug} / \mathrm{g})\end{array}$ & $\begin{array}{c}\mathrm{Si} \\
(\mathrm{ug} / \mathrm{g})\end{array}$ & $\mathrm{Ti}(\mathrm{ug} / \mathrm{g})$ & $\begin{array}{c}\mathrm{Zn} \\
(\mathrm{ug} / \mathrm{g})\end{array}$ & $\begin{array}{c}\mathrm{Zr} \\
(\mathrm{ug} / \mathrm{g})\end{array}$ \\
\hline ARG-1 & None & 0 & 0 & 2 & 23 & 5 & \begin{tabular}{|l|}
29 \\
\end{tabular} & ARG-1B23 & 5220 & 14600 & 84400 & 8250 & 784 & $<301$ & $<961$ & 227000 & 6970 & 182 & 952 \\
\hline ARG-1 & None & 0 & 0 & 3 & 1 & 6 & 1 & ARG-1B31 & 5060 & 14100 & 85600 & 8270 & 685 & 1160 & 983 & 228000 & 6980 & 203 & 943 \\
\hline SME Simulant & Hydragard & LF & 15 & 3 & 21 & 6 & 2 & LHY03 & 6080 & 12900 & 83700 & 3520 & $<223$ & 700 & 1970 & 237000 & 469 & 806 & 2640 \\
\hline SME Simulant & Hydragard & LF & 6 & 3 & 18 & 6 & 3 & LHY01 & 6230 & 13300 & 85800 & 3480 & $<225$ & 521 & 1620 & 237000 & 478 & 814 & 2710 \\
\hline SME Simulant & Coliwasa & LF & 20 & 1 & 2 & 6 & 4 & CL02 & 6300 & 13400 & 79900 & 3650 & $<240$ & $<302$ & 1390 & 247000 & 508 & 852 & 2790 \\
\hline SME Simulant & Coliwasa & LF & 38 & 1 & 16 & 6 & 5 & CH03 & 6170 & 13100 & 80100 & 3530 & $<202$ & $<254$ & 1250 & 247000 & 501 & 799 & 2770 \\
\hline SME Simulant & Coliwasa & LF & 63 & 1 & 13 & 6 & 6 & CH04 & 6180 & 13200 & 81700 & 3540 & $<215$ & $<270$ & 1610 & 244000 & 500 & 802 & 2760 \\
\hline SME Simulant & Hydragard & LF & 47 & 3 & 13 & 6 & 7 & LHY07 & 6190 & 13300 & 82800 & 3830 & $<216$ & 292 & 1450 & 238000 & 485 & 851 & 2560 \\
\hline SME Simulant & Coliwasa & $\mathrm{HF}$ & 23 & 1 & 28 & 6 & 8 & CH06 & 6110 & 13000 & 80700 & 3580 & $<224$ & 988 & 1870 & 247000 & 485 & 814 & 2770 \\
\hline SME Simulant & Coliwasa & LF & 21 & 1 & 14 & 6 & 9 & $\mathrm{CH} 02$ & 6100 & 13100 & 82800 & 3520 & $<227$ & 647 & 1500 & 244000 & 483 & 810 & 2740 \\
\hline SME Simulant & Coliwasa & $\mathrm{HF}$ & 3 & 1 & 20 & 6 & 10 & CL05 & 6120 & 13000 & 78200 & 3380 & $<226$ & 899 & 1440 & 247000 & 492 & 782 & 2720 \\
\hline SME Simulant & Hydragard & LF & 10 & 3 & 20 & 6 & 11 & LHY02 & 6550 & 13900 & 85500 & 3670 & $<226$ & 1110 & 1830 & 233000 & 475 & 898 & 2750 \\
\hline SME Simulant & Coliwasa & LF & 40 & 1 & 19 & 6 & 12 & CL03 & 6150 & 13100 & 82400 & 3310 & $<213$ & $<268$ & 1190 & 242000 & 506 & 755 & 2800 \\
\hline SME Simulant & Coliwasa & $\mathrm{HF}$ & 18 & 1 & 26 & 6 & 13 & CL06 & 6220 & 13200 & 74300 & 3500 & $<238$ & 1180 & 1480 & 248000 & 496 & 817 & 2780 \\
\hline SME Simulant & Coliwasa & $\mathrm{HF}$ & 1 & 1 & 22 & 6 & 14 & CH05 & 6190 & 13200 & 82700 & 4080 & $<227$ & 1240 & 1930 & 245000 & 501 & 829 & 2720 \\
\hline ARG-1 & None & 0 & 0 & 3 & 12 & 6 & 15 & ARG-1B32 & 5050 & 14100 & 85900 & 8520 & 663 & 490 & $<946$ & 229000 & 7010 & 194 & 1010 \\
\hline SME Simulant & Coliwasa & $\mathrm{HF}$ & 36 & 1 & 21 & 6 & 16 & CL07 & 6080 & 12900 & 81300 & 3400 & $<215$ & 412 & 1250 & 248000 & 507 & 807 & 2750 \\
\hline SME Simulant & Hydragard & LF & 30 & 3 & 14 & 6 & 17 & LHY05 & 5840 & 12600 & 79400 & 3500 & $<216$ & $<271$ & 1250 & 250000 & 521 & 766 & 2660 \\
\hline SME Simulant & Hydragard & LF & 59 & 3 & 17 & 6 & 18 & LHY10 & 8690 & 18300 & 104000 & 5200 & $<221$ & 414 & 2210 & 323000 & 667 & 1140 & 3380 \\
\hline SME Simulant & Coliwasa & $\mathrm{HF}$ & 63 & 1 & 5 & 6 & 19 & CL08 & 6090 & 13100 & 80600 & 3610 & $<231$ & $<290$ & 1300 & 242000 & 504 & 803 & 2810 \\
\hline SME Simulant & Hydragard & LF & 28 & 3 & 16 & 6 & 20 & LHY04 & 6360 & 13600 & 81500 & 3600 & $<229$ & $<288$ & 1180 & 234000 & 497 & 812 & 2710 \\
\hline SME Simulant & Hydragard & LF & 37 & 3 & 15 & 6 & 21 & LHY06 & 6300 & 13400 & 82600 & 3800 & $<210$ & 1120 & 1610 & 240000 & 486 & 888 & 2730 \\
\hline SME Simulant & Coliwasa & $\mathrm{HF}$ & 65 & 1 & 6 & 6 & 22 & CH08 & 6100 & 13000 & 81100 & 3420 & $<214$ & $<269$ & 1330 & 242000 & 507 & 760 & 2700 \\
\hline SME Simulant & Coliwasa & $\mathrm{HF}$ & 34 & 1 & 18 & 6 & 23 & CH07 & 6250 & 13300 & 78500 & 3500 & $<220$ & 1010 & 1490 & 247000 & 489 & 824 & 2790 \\
\hline SME Simulant & Coliwasa & LF & 1 & 1 & 12 & 6 & 24 & $\mathrm{CH} 01$ & 6260 & 13400 & 81400 & 3570 & $<232$ & 606 & 1260 & 244000 & 488 & 846 & 2730 \\
\hline SME Simulant & Hydragard & LF & 53 & 3 & 19 & 6 & 25 & LHY09 & 5990 & 12700 & 87900 & 3570 & $<214$ & 836 & 1720 & 241000 & 481 & 807 & 2460 \\
\hline SME Simulant & Coliwasa & LF & 4 & 1 & 10 & 6 & 26 & CL01 & 6120 & 13100 & 87300 & 3570 & $<199$ & 918 & 1670 & 240000 & 476 & 807 & 2740 \\
\hline SME Simulant & Hydragard & LF & 48 & 3 & 22 & 6 & 27 & LHY08 & 6650 & 13700 & 82500 & 3550 & $<218$ & 890 & 1440 & 244000 & 489 & 830 & 2820 \\
\hline SME Simulant & Coliwasa & LF & 65 & 1 & 23 & 6 & 28 & CL04 & 6180 & 13200 & 84000 & 3640 & $<210$ & 1360 & 1820 & 244000 & 473 & 836 & 2790 \\
\hline ARG-1 & None & 0 & 0 & 3 & 23 & 6 & 29 & ARG-1B33 & 5040 & 14000 & 86400 & 8400 & 527 & 895 & $<1000$ & 227000 & 6970 & 192 & 947 \\
\hline
\end{tabular}


Table C4. Phase 3 Oxide Concentration Measurements (part 1)

Revision 0

\begin{tabular}{|c|c|c|c|c|c|c|c|c|c|c|c|c|c|c|c|c|c|}
\hline $\begin{array}{l}\text { Type of } \\
\text { Material }\end{array}$ & Sampler Details & $\begin{array}{l}\text { Flow } \\
\text { Rate }\end{array}$ & $\begin{array}{r}\text { Sample } \\
\text { Order }\end{array}$ & $\begin{array}{r}\text { Preparation } \\
\text { Block }\end{array}$ & $\begin{array}{r}\text { Preparation } \\
\text { Sequence }\end{array}$ & $\begin{array}{r}\text { Analytical } \\
\text { Block }\end{array}$ & $\begin{array}{r}\text { Analytical } \\
\text { Sequence }\end{array}$ & Sample ID & $\begin{array}{l}\text { Al2O3 } \\
\text { (wt\%) }\end{array}$ & $\begin{array}{r}\text { B2O3 } \\
\text { (wt\%) }\end{array}$ & \begin{tabular}{r|} 
BaO \\
(wt\%)
\end{tabular} & $\begin{array}{r}\mathrm{CaO} \\
\text { (wt\%) }\end{array}$ & $\begin{array}{l}\text { Cr2O3 } \\
\text { (wt\%) }\end{array}$ & $\begin{array}{r}\mathrm{CuO} \\
\text { (wt\%) }\end{array}$ & $\begin{array}{l}\text { Fe2O3 } \\
\text { (wt\%) }\end{array}$ & $\begin{array}{r}\mathrm{K} 2 \mathrm{O} \\
\text { (wt\%) }\end{array}$ & $\begin{array}{r}\text { Li2O } \\
\text { (wt\%) }\end{array}$ \\
\hline ARG-1 & None & 0 & 0 & \begin{tabular}{|r|}
1 \\
\end{tabular} & $\begin{array}{r}1 \\
\end{array}$ & $\begin{array}{r}1 \\
\end{array}$ & 1 & \begin{tabular}{|l|} 
ARG-1B11 \\
\end{tabular} & 4.459 & 7.760 & 0.088 & 1.399 & 0.095 & 0.010 & 13.525 & 2.855 & 3.057 \\
\hline SME Simulant & Isolok - $3 \mathrm{~mL}$ & LF & 18 & 1 & 9 & 1 & 2 & \begin{tabular}{|l|} 
LISO3 \\
\end{tabular} & 10.279 & 4.186 & 0.101 & 1.156 & 0.091 & 0.034 & 10.966 & 0.166 & 4.779 \\
\hline SME Simulant & Isolok - $3 \mathrm{~mL}$ & LF & 8 & 1 & 27 & 1 & 3 & LIS01 & 10.298 & 4.057 & 0.103 & 1.143 & 0.106 & 0.034 & 10.952 & 0.181 & 4.586 \\
\hline SME Simulant & Coliwasa - low & LF & 20 & 1 & 2 & 1 & 4 & CL02 & 10.166 & 4.121 & 0.103 & 1.181 & 0.113 & 0.038 & 10.980 & 0.192 & 4.500 \\
\hline SME Simulant & Coliwasa - high & LF & 38 & 1 & 16 & 1 & 5 & $\mathrm{CH} 03$ & 10.449 & 4.121 & 0.105 & \begin{tabular}{l|l|}
1.168 \\
\end{tabular} & 0.109 & 0.032 & 11.123 & 0.162 & 4.586 \\
\hline SME Simulant & \begin{tabular}{|l|} 
Coliwasa - high \\
\end{tabular} & LF & 63 & 1 & 13 & 1 & 6 & CH04 & 10.525 & 4.089 & 0.106 & 1.187 & 0.111 & 0.037 & 11.223 & 0.172 & 4.543 \\
\hline SME Simulant & \begin{tabular}{|l|l} 
Isolok - $3 \mathrm{~mL}$ \\
\end{tabular} & LF & 44 & 1 & 3 & 1 & 7 & LIS07 & 10.014 & 4.057 & 0.099 & 1.094 & 0.088 & 0.031 & 10.594 & 0.180 & 4.607 \\
\hline SME Simulant & \begin{tabular}{|l|} 
Coliwasa - high \\
\end{tabular} & $\mathrm{HF}$ & 23 & 1 & 28 & 1 & 8 & CH06 & $\begin{array}{l}10.147 \\
\end{array}$ & 4.089 & 0.098 & 1.114 & 0.102 & 0.025 & 10.823 & 0.179 & 4.478 \\
\hline SME Simulant & \begin{tabular}{|l|} 
Coliwasa - high \\
\end{tabular} & LF & 21 & 1 & 14 & 1 & 9 & $\mathrm{CH} 02$ & 10.373 & 4.186 & 0.102 & 1.143 & 0.106 & 0.036 & 11.109 & 0.182 & 4.564 \\
\hline SME Simulant & \begin{tabular}{|l|} 
Coliwasa - low \\
\end{tabular} & HF & 3 & 1 & 20 & 1 & 10 & CL05 & 10.279 & 4.154 & 0.103 & 1.175 & 0.119 & 0.038 & 11.066 & 0.181 & 4.693 \\
\hline SME Simulant & Isolok - $3 \mathrm{~mL}$ & LF & 12 & 1 & 25 & 1 & 11 & LIS02 & 10.052 & 3.703 & 0.100 & 1.125 & 0.090 & 0.034 & 10.866 & 0.182 & 4.435 \\
\hline SME Simulant & Coliwasa - low & LF & 40 & 1 & 19 & 1 & 12 & CL03 & 10.430 & 3.864 & 0.103 & 1.150 & 0.111 & 0.030 & 11.094 & 0.170 & 4.457 \\
\hline SME Simulant & Coliwasa - low & $\mathrm{HF}$ & 18 & 1 & 26 & 1 & 13 & CL06 & 10.279 & 4.154 & 0.107 & 1.189 & 0.125 & 0.029 & 11.080 & 0.190 & 4.413 \\
\hline SME Simulant & \begin{tabular}{|l|} 
Coliwasa - high \\
\end{tabular} & $\mathrm{HF}$ & 1 & 1 & 22 & 1 & 14 & \begin{tabular}{|l|}
$\mathrm{CH} 05$ \\
\end{tabular} & 10.354 & 3.864 & 0.102 & 1.152 & 0.127 & 0.038 & 11.152 & 0.182 & 4.564 \\
\hline ARG-1 & \begin{tabular}{|l|} 
None \\
\end{tabular} & 0 & 0 & 1 & 15 & 1 & 15 & \begin{tabular}{|l|} 
ARG-1B12 \\
\end{tabular} & 4.459 & 7.953 & 0.090 & 1.413 & 0.097 & 0.003 & 13.897 & 2.590 & 3.057 \\
\hline SME Simulant & Coliwasa - low & $\mathrm{HF}$ & 36 & 1 & 21 & 1 & 16 & \begin{tabular}{|l|} 
CL07 \\
\end{tabular} & 9.655 & 3.864 & 0.097 & 1.069 & 0.108 & 0.026 & 10.365 & 0.172 & 4.306 \\
\hline SME Simulant & Isolok - 3 mL & LF & 31 & 1 & 24 & 1 & 17 & LIS05 & 9.882 & 4.025 & 0.095 & $\begin{array}{ll}1.055 \\
\end{array}$ & 0.109 & 0.038 & 10.651 & 0.161 & 4.327 \\
\hline SME Simulant & Isolok - $3 \mathrm{~mL}$ & LF & 58 & 1 & 4 & 1 & 18 & LIS10 & 10.298 & 4.057 & $\begin{array}{ll}0.101 \\
\end{array}$ & 1.135 & 0.096 & 0.040 & 10.952 & 0.189 & 4.478 \\
\hline SME Simulant & Coliwasa - low & $\mathrm{HF}$ & 63 & 1 & 5 & 1 & 19 & CL08 & 10.392 & 4.025 & 0.104 & 1.157 & 0.122 & 0.035 & 11.152 & 0.185 & 4.413 \\
\hline SME Simulant & Isolok - $3 \mathrm{~mL}$ & LF & 25 & 1 & 7 & 1 & 20 & LIS04 & 10.298 & 4.186 & 0.102 & 1.147 & 0.095 & 0.038 & 11.109 & 0.172 & 4.478 \\
\hline SME Simulant & Isolok - $3 \mathrm{~mL}$ & LF & 42 & 1 & 11 & 1 & 21 & LIS06 & 10.430 & 3.928 & 0.100 & 1.126 & 0.088 & 0.041 & 11.094 & 0.179 & 4.500 \\
\hline SME Simulant & \begin{tabular}{|l|} 
Coliwasa - high \\
\end{tabular} & $\mathrm{HF}$ & 65 & 1 & 6 & 1 & 22 & CH08 & 10.298 & 4.121 & 0.101 & 1.136 & 0.105 & 0.033 & 11.080 & 0.172 & 4.521 \\
\hline SME Simulant & \begin{tabular}{|l|} 
Coliwasa - high \\
\end{tabular} & HF & 34 & 1 & 18 & 1 & 23 & CH07 & 10.298 & 4.154 & 0.102 & 1.152 & 0.104 & 0.038 & 11.066 & 0.176 & 4.478 \\
\hline SME Simulant & \begin{tabular}{|l} 
Coliwasa - high \\
\end{tabular} & LF & 1 & 1 & 12 & 1 & 24 & CH01 & 10.147 & 4.218 & 0.101 & 1.184 & 0.104 & 0.032 & 10.952 & 0.186 & 4.650 \\
\hline SME Simulant & \begin{tabular}{|l} 
Isolok - $3 \mathrm{~mL}$ \\
\end{tabular} & LF & 54 & 1 & 17 & 1 & 25 & LIS09 & 10.241 & 4.250 & 0.100 & 1.117 & 0.094 & 0.031 & 10.923 & 0.184 & 4.500 \\
\hline SME Simulant & Coliwasa - low & LF & 4 & 1 & 10 & 1 & 26 & CL01 & 10.354 & 4.218 & 0.105 & 1.133 & 0.102 & 0.031 & 11.052 & 0.159 & 4.478 \\
\hline SME Simulant & Isolok - $3 \mathrm{~mL}$ & LF & 49 & 1 & 8 & 1 & 27 & LIS08 & 10.241 & 4.121 & 0.099 & 1.114 & 0.093 & 0.029 & 10.851 & 0.201 & 4.413 \\
\hline SME Simulant & Coliwasa - low & LF & 65 & 1 & 23 & 1 & 28 & CL04 & 10.430 & 4.089 & 0.102 & 1.168 & 0.107 & 0.032 & 11.123 & 0.169 & 4.629 \\
\hline ARG-1 & None & 0 & 0 & 1 & 29 & 1 & 29 & ARG-1B13 & 4.535 & 7.985 & 0.089 & 1.427 & 0.096 & 0.003 & 13.854 & 2.722 & 3.079 \\
\hline ARG-1 & None & 0 & 0 & 2 & 1 & 2 & 1 & ARG-1B21 & 4.705 & 8.307 & 0.094 & 1.455 & \begin{tabular}{|c|}
0.101 \\
\end{tabular} & \begin{tabular}{ll|}
0.008 \\
\end{tabular} & |14.168 & 2.783 & 3.122 \\
\hline SME Simulant & Hydragard & $\mathrm{HF}$ & 11 & 2 & 3 & 2 & 2 & \begin{tabular}{|l|} 
HHY02 \\
\end{tabular} & 11.375 & 4.411 & 0.115 & 1.343 & 0.130 & 0.036 & 12.224 & 0.178 & 4.521 \\
\hline SME Simulant & Coliwasa - low & $\mathrm{HF}$ & 36 & 1 & 21 & 2 & 3 & CL07 & 10.468 & 4.186 & 0.103 & 1.166 & \begin{tabular}{l|l|}
0.111 \\
\end{tabular} & 0.037 & 11.094 & 0.172 & 4.715 \\
\hline SME Simulant & Hydragard & $\mathrm{HF}$ & 24 & 2 & 2 & 2 & 4 & HHY04 & 11.035 & 3.993 & 0.107 & 1.215 & 0.127 & 0.036 & 11.738 & 0.164 & 4.521 \\
\hline SME Simulant & \begin{tabular}{|l|} 
Coliwasa - high \\
\end{tabular} & $\mathrm{HF}$ & 1 & 1 & 22 & 2 & 5 & CH05 & 10.695 & 3.993 & 0.105 & 1.170 & $\begin{array}{ll}0.127 \\
\end{array}$ & 0.033 & 11.409 & 0.182 & 4.607 \\
\hline SME Simulant & \begin{tabular}{|l|} 
Coliwasa - low \\
\end{tabular} & $\mathrm{HF}$ & 3 & 1 & 20 & 2 & 6 & CL05 & 10.600 & 4.315 & 0.104 & 1.175 & 0.118 & 0.037 & 11.352 & 0.181 & 4.672 \\
\hline SME Simulant & \begin{tabular}{|l|} 
Coliwasa - high \\
\end{tabular} & HF & 23 & 1 & 28 & 2 & 7 & CH06 & 10.430 & 4.250 & 0.100 & 1.171 & 0.100 & 0.035 & 11.180 & 0.179 & 4.693 \\
\hline SME Simulant & \begin{tabular}{|l} 
Coliwasa - high \\
\end{tabular} & LF & 1 & 1 & 12 & 2 & 8 & CH01 & 10.411 & 4.218 & 0.102 & 1.213 & 0.105 & 0.041 & 11.209 & 0.186 & 4.650 \\
\hline SME Simulant & \begin{tabular}{|l|} 
Coliwasa - low \\
\end{tabular} & LF & 20 & 1 & 2 & 2 & 9 & CL02 & 10.392 & 4.121 & 0.104 & 1.213 & 0.110 & 0.044 & 11.409 & 0.192 & 4.521 \\
\hline SME Simulant & Coliwasa - low & $\mathrm{HF}$ & 63 & 1 & 5 & 2 & 10 & CL08 & 10.562 & 3.993 & 0.103 & 1.139 & 0.119 & 0.036 & 11.338 & 0.185 & 4.392 \\
\hline SME Simulant & \begin{tabular}{|l|} 
Coliwasa - high \\
\end{tabular} & $\mathrm{HF}$ & 65 & 1 & 6 & 2 & 11 & CH08 & 10.581 & 4.154 & 0.103 & 1.213 & 0.104 & 0.036 & 11.409 & 0.172 & 4.521 \\
\hline SME Simulant & \begin{tabular}{|l} 
Hydragard \\
\end{tabular} & $\mathrm{HF}$ & 45 & 2 & 7 & 2 & 12 & HHY07 & 10.978 & 3.960 & 0.107 & 1.245 & 0.110 & 0.047 & 11.938 & 0.185 & 4.327 \\
\hline SME Simulant & Coliwasa - low & LF & 4 & 1 & 10 & 2 & 13 & \begin{tabular}{|l|} 
CL01 \\
\end{tabular} & 10.638 & 4.186 & 0.107 & 1.203 & 0.104 & 0.031 & 11.452 & 0.159 & 4.457 \\
\hline SME Simulant & Coliwasa - low & $\mathrm{HF}$ & 18 & 1 & 26 & 2 & 14 & \begin{tabular}{|l|} 
CL06 \\
\end{tabular} & 10.619 & 4.057 & 0.105 & 1.202 & 0.119 & 0.036 & 11.480 & 0.190 & 4.521 \\
\hline ARG-1 & None & 0 & 0 & 2 & 12 & 2 & 15 & ARG-1B22 & 4.516 & 8.050 & 0.091 & 1.469 & 0.097 & 0.003 & 14.068 & 2.614 & 3.057 \\
\hline SME Simulant & Coliwasa - low & LF & 65 & 1 & 23 & 2 & 16 & \begin{tabular}{|l|} 
CL04 \\
\end{tabular} & 10.543 & 3.928 & 0.102 & 1.174 & 0.102 & 0.038 & 11.323 & 0.169 & 4.435 \\
\hline SME Simulant & Coliwasa - low & LF & 40 & 1 & 19 & 2 & 17 & CL03 & 10.638 & 4.025 & 0.103 & 1.188 & 0.111 & 0.041 & 11.409 & 0.170 & 4.521 \\
\hline SME Simulant & \begin{tabular}{|l|} 
Coliwasa - high \\
\end{tabular} & LF & 38 & 1 & 16 & 2 & 18 & $\mathrm{CH} 03$ & 10.449 & 4.057 & 0.104 & 1.156 & 0.108 & 0.037 & 11.309 & 0.162 & 4.435 \\
\hline SME Simulant & \begin{tabular}{|l|} 
Hydragard \\
\end{tabular} & $\mathrm{HF}$ & 57 & 2 & 6 & 2 & 19 & HHY09 & 11.091 & 3.960 & 0.112 & 1.249 & 0.126 & 0.044 & 12.081 & 0.174 & 4.457 \\
\hline
\end{tabular}


Table C4. Phase 3 Oxide Concentration Measurements (part 1)

Revision 0

\begin{tabular}{|c|c|c|c|c|c|c|c|c|c|c|c|c|c|c|c|c|c|}
\hline $\begin{array}{l}\text { Type of } \\
\text { Material }\end{array}$ & Sampler Details & $\begin{array}{l}\text { Flow } \\
\text { Rate }\end{array}$ & $\begin{array}{r}\text { Sample } \\
\text { Order }\end{array}$ & $\begin{array}{r}\text { Preparation } \\
\text { Block }\end{array}$ & $\begin{array}{r}\text { Preparation } \\
\text { Sequence }\end{array}$ & $\begin{array}{r}\text { Analytical } \\
\text { Block }\end{array}$ & $\begin{array}{r}\text { Analytical } \\
\text { Sequence }\end{array}$ & Sample ID & $\begin{array}{l}\text { Al2O3 } \\
\text { (wt\%) }\end{array}$ & $\begin{array}{r}\text { B2O3 } \\
\text { (wt\%) }\end{array}$ & $\begin{array}{r}\text { BaO } \\
\text { (wt\%) }\end{array}$ & $\begin{array}{r}\mathrm{CaO} \\
\text { (wt\%) }\end{array}$ & $\begin{array}{l}\text { Cr2O3 } \\
\text { (wt\%) }\end{array}$ & $\begin{array}{r}\mathrm{CuO} \\
\text { (wt\%) }\end{array}$ & $\begin{array}{l}\text { Fe2O3 } \\
\text { (wt\%) }\end{array}$ & $\begin{array}{r}\mathrm{K} 2 \mathrm{O} \\
\text { (wt\%) }\end{array}$ & $\begin{array}{r}\text { Li2O } \\
\text { (wt\%) }\end{array}$ \\
\hline SME Simulant & Coliwasa - high & LF & 21 & \begin{tabular}{|r|}
1 \\
\end{tabular} & $\begin{array}{r}14 \\
\end{array}$ & \begin{tabular}{|r|}
2 \\
\end{tabular} & 20 & CH02 & 10.373 & 4.154 & 0.103 & 1.198 & 0.105 & 0.033 & 11.209 & 0.182 & 4.478 \\
\hline SME Simulant & Hydragard & $\mathrm{HF}$ & 6 & 2 & 4 & 2 & 21 & HHY01 & $\begin{array}{ll}10.732 \\
\end{array}$ & 4.025 & 0.106 & 1.272 & \begin{tabular}{ll|}
0.127 \\
\end{tabular} & 0.030 & 11.695 & 0.172 & 4.478 \\
\hline SME Simulant & Hydragard & $\mathrm{HF}$ & 19 & 2 & 10 & 2 & 22 & HHY03 & 11.054 & 3.864 & 0.109 & 1.198 & 0.124 & 0.037 & 11.895 & 0.170 & 4.198 \\
\hline SME Simulant & Hydragard & $\mathrm{HF}$ & 50 & 2 & 5 & 2 & 23 & HHY08 & 10.884 & 3.832 & 0.103 & 1.184 & 0.105 & 0.037 & 11.595 & 0.175 & 4.500 \\
\hline SME Simulant & Hydragard & $\mathrm{HF}$ & 37 & 2 & 9 & 2 & 24 & HHY06 & 10.827 & 3.993 & 0.107 & 1.252 & 0.110 & 0.035 & 11.852 & 0.183 & 4.564 \\
\hline SME Simulant & Coliwasa - high & $\mathrm{HF}$ & 34 & 1 & 18 & 2 & 25 & CH07 & 10.638 & 4.347 & 0.106 & 1.240 & 0.107 & 0.039 & 11.452 & 0.176 & 4.586 \\
\hline SME Simulant & \begin{tabular}{|l|} 
Coliwasa - high \\
\end{tabular} & LF & 63 & 1 & 13 & 2 & 26 & CH04 & 10.468 & 3.767 & 0.102 & 1.188 & 0.103 & 0.041 & 11.252 & 0.172 & 4.500 \\
\hline SME Simulant & \begin{tabular}{|l|} 
Hydragard \\
\end{tabular} & $\mathrm{HF}$ & 32 & 2 & 8 & 2 & 27 & HHY05 & 10.997 & 3.284 & 0.102 & 1.455 & 0.115 & 0.041 & 11.781 & 0.173 & 4.478 \\
\hline SME Simulant & Hydragard & $\mathrm{HF}$ & 61 & 2 & 11 & 2 & 28 & HHY10 & 11.280 & 3.574 & 0.111 & 1.277 & 0.113 & 0.035 & 12.253 & 0.175 & 4.392 \\
\hline ARG-1 & \begin{tabular}{|l|} 
None \\
\end{tabular} & 0 & 0 & 2 & 23 & 2 & 29 & ARG-1B23 & $\begin{array}{ll}4.629 \\
\end{array}$ & 7.696 & 0.089 & 1.469 & 0.106 & 0.003 & 14.025 & 2.903 & 3.100 \\
\hline ARG-1 & None & 0 & 0 & 3 & 1 & 3 & 1 & ARG-1B31 & 4.705 & 7.953 & 0.090 & 1.427 & 0.096 & 0.003 & 14.083 & 2.710 & 3.165 \\
\hline SME Simulant & Isolok - $12 \mathrm{~mL}$ & LF & 11 & 3 & 4 & 3 & 2 & LIB02 & 10.090 & 4.315 & 0.097 & 1.117 & 0.104 & 0.047 & 10.580 & 0.178 & 5.081 \\
\hline SME Simulant & Coliwasa - low & $\mathrm{HF}$ & 36 & 1 & 21 & 3 & 3 & CL07 & 10.506 & 4.250 & 0.102 & 1.153 & 0.113 & 0.040 & 11.080 & 0.172 & 4.693 \\
\hline SME Simulant & Isolok - $12 \mathrm{~mL}$ & LF & 26 & 3 & 8 & 3 & 4 & \begin{tabular}{|l} 
LIB04 \\
\end{tabular} & 10.808 & 3.896 & 0.103 & 1.205 & 0.103 & 0.052 & 11.366 & 0.179 & 4.586 \\
\hline SME Simulant & \begin{tabular}{|l|} 
Coliwasa - high \\
\end{tabular} & $\mathrm{HF}$ & 1 & 1 & 22 & 3 & 5 & CH05 & 10.808 & 3.896 & 0.104 & 1.192 & 0.126 & 0.052 & 11.480 & 0.182 & 4.758 \\
\hline SME Simulant & \begin{tabular}{|l|} 
Coliwasa - low \\
\end{tabular} & $\mathrm{HF}$ & 3 & 1 & 20 & 3 & 6 & CL05 & 10.676 & 3.993 & 0.103 & 1.188 & 0.116 & 0.045 & 11.438 & 0.181 & 4.801 \\
\hline SME Simulant & Coliwasa - high & $\mathrm{HF}$ & 23 & 1 & 28 & 3 & 7 & CH06 & 10.392 & 4.186 & 0.100 & 1.164 & 0.102 & 0.033 & 11.052 & 0.179 & 4.629 \\
\hline SME Simulant & Coliwasa - high & LF & 1 & 1 & 12 & 3 & 8 & $\mathrm{CH} 01$ & 10.619 & 3.928 & 0.100 & 1.245 & 0.102 & 0.044 & 11.338 & 0.186 & 4.930 \\
\hline SME Simulant & \begin{tabular}{|l|} 
Coliwasa - low \\
\end{tabular} & LF & 20 & 1 & 2 & 3 & 9 & CL02 & 10.619 & 4.218 & 0.106 & 1.226 & 0.118 & 0.041 & 11.495 & 0.192 & 4.715 \\
\hline SME Simulant & Coliwasa - low & $\mathrm{HF}$ & 63 & 1 & 5 & 3 & 10 & \begin{tabular}{|l|} 
CL08 \\
\end{tabular} & 10.846 & 3.832 & 0.104 & 1.178 & 0.120 & 0.036 & 11.581 & 0.185 & 4.629 \\
\hline SME Simulant & \begin{tabular}{|l|} 
Coliwasa - high \\
\end{tabular} & $\mathrm{HF}$ & 65 & 1 & 6 & 3 & 11 & CH08 & 10.676 & 4.025 & 0.102 & 1.181 & 0.105 & 0.038 & 11.395 & 0.172 & 4.715 \\
\hline SME Simulant & \begin{tabular}{|l|} 
Isolok - $12 \mathrm{~mL}$ \\
\end{tabular} & LF & 45 & 3 & 7 & 3 & 12 & LIB07 & 10.468 & 3.799 & 0.101 & 1.170 & 0.105 & 0.037 & 11.137 & 0.186 & 4.779 \\
\hline SME Simulant & Coliwasa - low & LF & 4 & 1 & 10 & 3 & 13 & CL01 & 10.789 & 4.121 & 0.107 & 1.182 & 0.104 & 0.036 & 11.395 & 0.159 & 4.586 \\
\hline SME Simulant & Coliwasa - low & $\mathrm{HF}$ & 18 & 1 & 26 & 3 & 14 & CL06 & 10.827 & 4.121 & 0.107 & 1.242 & 0.126 & 0.047 & 11.523 & 0.190 & 4.672 \\
\hline ARG-1 & None & 0 & 0 & 3 & 12 & 3 & 15 & ARG-1B32 & 4.686 & 7.824 & 0.092 & 1.455 & 0.103 & 0.003 & 14.226 & 2.867 & 3.143 \\
\hline SME Simulant & Coliwasa - low & LF & 65 & 1 & 23 & 3 & 16 & CL04 & 10.638 & 3.864 & 0.102 & 1.189 & 0.106 & 0.043 & 11.209 & 0.169 & 4.543 \\
\hline SME Simulant & Coliwasa - low & LF & 40 & 1 & 19 & 3 & 17 & CL03 & 10.581 & 3.960 & 0.104 & 1.175 & 0.112 & 0.041 & 11.266 & 0.170 & 4.650 \\
\hline SME Simulant & \begin{tabular}{|l|} 
Coliwasa - high \\
\end{tabular} & LF & 38 & 1 & 16 & 3 & 18 & $\mathrm{CH} 03$ & 10.562 & 3.767 & 0.101 & 1.173 & 0.103 & 0.036 & 11.166 & 0.162 & 4.736 \\
\hline SME Simulant & \begin{tabular}{|l|} 
Isolok - $12 \mathrm{~mL}$ \\
\end{tabular} & LF & 56 & 3 & 11 & 3 & 19 & LIB09 & 10.732 & 3.703 & 0.105 & 1.178 & 0.128 & 0.041 & 11.309 & 0.179 & 5.059 \\
\hline SME Simulant & Coliwasa - high & LF & 21 & 1 & 14 & 3 & 20 & \begin{tabular}{|l|}
$\mathrm{CH} 02$ \\
\end{tabular} & 10.732 & 4.089 & 0.103 & 1.164 & 0.106 & $\begin{array}{ll}0.036 \\
\end{array}$ & 11.280 & 0.182 & 4.736 \\
\hline SME Simulant & \begin{tabular}{|l|l|l} 
Isolok - $12 \mathrm{~mL}$ \\
\end{tabular} & LF & 5 & 3 & 5 & 3 & 21 & LIB01 & 10.638 & 4.121 & 0.103 & 1.178 & $\begin{array}{ll}0.103 \\
\end{array}$ & 0.043 & 11.280 & 0.173 & 4.822 \\
\hline SME Simulant & Isolok - $12 \mathrm{~mL}$ & LF & 23 & 3 & 10 & 3 & 22 & LIB03 & 10.808 & 3.960 & 0.098 & 1.364 & 0.123 & 0.040 & 11.295 & 0.179 & 4.758 \\
\hline SME Simulant & Isolok - $12 \mathrm{~mL}$ & LF & 50 & 3 & 2 & 3 & 23 & LIB08 & 10.014 & 3.896 & 0.094 & 1.073 & 0.099 & 0.043 & 10.265 & 0.166 & 4.801 \\
\hline SME Simulant & Isolok - $12 \mathrm{~mL}$ & LF & 41 & 3 & 9 & 3 & 24 & \begin{tabular}{|l|l|} 
LIB06 \\
\end{tabular} & 9.580 & 4.089 & 0.089 & 0.953 & 0.096 & 0.040 & 9.865 & 0.179 & 5.275 \\
\hline SME Simulant & \begin{tabular}{|l|} 
Coliwasa - high \\
\end{tabular} & $\mathrm{HF}$ & 34 & 1 & 18 & 3 & 25 & CH07 & 10.128 & 3.735 & 0.098 & 1.145 & 0.095 & 0.038 & 10.680 & 0.176 & 4.435 \\
\hline SME Simulant & \begin{tabular}{|l|} 
Coliwasa - high \\
\end{tabular} & LF & 63 & 1 & 13 & 3 & 26 & CH04 & 10.884 & 3.960 & 0.107 & 1.222 & 0.113 & 0.042 & 11.552 & 0.172 & 4.822 \\
\hline SME Simulant & \begin{tabular}{|l|l} 
Isolok - $12 \mathrm{~mL}$ \\
\end{tabular} & LF & 32 & 3 & 3 & 3 & 27 & LIB05 & 10.676 & 3.960 & 0.102 & 1.168 & 0.107 & 0.051 & 11.223 & 0.184 & 4.801 \\
\hline SME Simulant & Isolok - $12 \mathrm{~mL}$ & LF & 60 & 3 & 6 & 3 & 28 & LIB10 & 9.844 & 3.896 & 0.097 & 1.125 & 0.095 & 0.036 & 10.179 & 0.176 & 5.059 \\
\hline ARG-1 & None & 0 & 0 & 3 & 23 & 3 & 29 & ARG-1B33 & 4.573 & 7.599 & 0.091 & 1.441 & 0.095 & 0.003 & 13.868 & 2.698 & 3.186 \\
\hline ARG-1 & None & 0 & 0 & 4 & 1 & 4 & 1 & ARG-1B41 & 4.554 & 7.921 & 0.095 & 1.469 & 0.098 & 0.003 & 14.083 & 2.771 & 3.186 \\
\hline SME Simulant & Isolok - $12 \mathrm{~mL}$ & HF & 13 & 4 & 11 & 4 & 2 & HIB02 & 10.392 & 4.379 & 0.105 & 1.175 & 0.107 & 0.032 & 11.052 & 0.174 & 4.779 \\
\hline SME Simulant & Coliwasa - low & $\mathrm{HF}$ & 36 & 1 & 21 & 4 & 3 & \begin{tabular}{|l|} 
CL07 \\
\end{tabular} & \begin{tabular}{r|}
9.863 \\
\end{tabular} & 3.864 & 0.099 & 1.112 & 0.105 & 0.034 & 10.608 & 0.172 & 4.564 \\
\hline \begin{tabular}{|l|} 
SME Simulant \\
\end{tabular} & Isolok - $12 \mathrm{~mL}$ & $\mathrm{HF}$ & 25 & 4 & 4 & 4 & 4 & \begin{tabular}{|l|l|} 
HIB04 \\
\end{tabular} & \begin{tabular}{l|l|}
10.487 \\
\end{tabular} & 3.993 & 0.103 & \begin{tabular}{|l|}
1.157 \\
\end{tabular} & 0.119 & 0.044 & 11.080 & 0.182 & 4.693 \\
\hline SME Simulant & \begin{tabular}{|l|} 
Coliwasa - high \\
\end{tabular} & $\mathrm{HF}$ & 1 & 1 & 22 & 4 & 5 & CH05 & 10.713 & 3.928 & 0.106 & 1.189 & 0.130 & 0.040 & 11.380 & 0.182 & 4.844 \\
\hline SME Simulant & \begin{tabular}{|l|} 
Coliwasa - low \\
\end{tabular} & $\mathrm{HF}$ & 3 & 1 & 20 & 4 & 6 & CL05 & 10.487 & 4.218 & 0.105 & 1.196 & 0.116 & 0.041 & 11.266 & 0.181 & 4.995 \\
\hline SME Simulant & \begin{tabular}{|l|} 
Coliwasa - high \\
\end{tabular} & $\mathrm{HF}$ & 23 & 1 & 28 & 4 & 7 & $\mathrm{CH} 06$ & 10.638 & 4.315 & 0.107 & 1.168 & 0.105 & 0.026 & 11.352 & 0.179 & 4.736 \\
\hline SME Simulant & \begin{tabular}{|l|} 
Coliwasa - high \\
\end{tabular} & LF & 1 & 1 & 12 & 4 & 8 & $\mathrm{CH} 01$ & 10.770 & 4.282 & 0.108 & 1.259 & 0.109 & \begin{tabular}{l|l}
0.027 \\
\end{tabular} & 11.523 & 0.186 & 4.909 \\
\hline SME Simulant & \begin{tabular}{|l|} 
Coliwasa - low \\
\end{tabular} & LF & 20 & 1 & 2 & 4 & 9 & CL02 & 10.354 & 4.057 & 0.107 & 1.194 & 0.111 & 0.033 & 11.280 & 0.192 & 4.629 \\
\hline
\end{tabular}


Table C4. Phase 3 Oxide Concentration Measurements (part 1)

\begin{tabular}{|c|c|c|c|c|c|c|c|c|c|c|c|c|c|c|c|c|c|}
\hline $\begin{array}{l}\text { Type of } \\
\text { Material }\end{array}$ & Sampler Details & \begin{tabular}{|l|} 
Flow \\
Rate
\end{tabular} & $\begin{array}{r}\text { Sample } \\
\text { Order }\end{array}$ & $\begin{array}{r}\text { Preparation } \\
\text { Block }\end{array}$ & $\begin{array}{r}\text { Preparation } \\
\text { Sequence }\end{array}$ & $\begin{array}{r}\text { Analytical } \\
\text { Block }\end{array}$ & $\begin{array}{r}\text { Analytical } \\
\text { Sequence }\end{array}$ & Sample ID & $\begin{array}{l}\text { Al2O3 } \\
\text { (wt\%) }\end{array}$ & $\begin{array}{r}\text { B2O3 } \\
\text { (wt\%) }\end{array}$ & $\begin{array}{r}\text { BaO } \\
\text { (wt\%) }\end{array}$ & $\begin{array}{r}\mathrm{CaO} \\
\text { (wt\%) }\end{array}$ & $\begin{array}{r}\text { Cr2O3 } \\
\text { (wt\%) }\end{array}$ & $\begin{array}{r}\mathrm{CuO} \\
\text { (wt\%) }\end{array}$ & $\begin{array}{l}\text { Fe2O3 } \\
\text { (wt\%) }\end{array}$ & \begin{tabular}{r|}
$\mathrm{K} 2 \mathrm{O}$ \\
(wt\%)
\end{tabular} & $\begin{array}{r}\mathbf{L i 2 O} \\
\text { (wt\%) }\end{array}$ \\
\hline SME Simulant & Coliwasa - low & $\mathrm{HF}$ & 63 & 1 & 5 & 4 & 10 & CL08 & 10.808 & 3.832 & 0.108 & 1.188 & 0.117 & 0.033 & 11.423 & 0.185 & 4.607 \\
\hline SME Simulant & Coliwasa - high & $\mathrm{HF}$ & 46 & 4 & 2 & 4 & 11 & HIB07 & 10.600 & 4.089 & 0.105 & 1.164 & 0.125 & 0.035 & 11.309 & 0.181 & 4.607 \\
\hline SME Simulant & Isolok - $12 \mathrm{~mL}$ & LF & 4 & 1 & 10 & 4 & 12 & CL01 & 10.808 & 4.186 & 0.111 & 1.192 & 0.103 & 0.038 & 11.552 & 0.159 & 4.672 \\
\hline SME Simulant & Coliwasa - low & HF & 18 & 1 & 26 & 4 & 13 & CL06 & 10.657 & 4.057 & 0.108 & 1.202 & 0.121 & 0.033 & 11.480 & 0.190 & 4.650 \\
\hline \begin{tabular}{|l|} 
ARG-1 \\
\end{tabular} & Coliwasa - low & 0 & 0 & 4 & 7 & 4 & 14 & ARG-1B42 & 4.591 & 7.857 & 0.095 & 1.427 & 0.098 & 0.003 & 14.011 & 2.674 & 3.143 \\
\hline SME Simulant & None & LF & 65 & 1 & 23 & 4 & 15 & CL04 & 10.638 & 3.928 & 0.105 & 1.188 & 0.104 & 0.037 & 11.323 & 0.169 & 4.607 \\
\hline SME Simulant & Coliwasa - low & LF & 40 & 1 & 19 & 4 & 16 & CL03 & 10.695 & 4.025 & 0.108 & 1.175 & 0.111 & 0.032 & 11.466 & 0.170 & 4.715 \\
\hline SME Simulant & Coliwasa - low & LF & 21 & 1 & 14 & 4 & 17 & $\mathrm{CH} 02$ & 10.525 & 4.250 & 0.108 & 1.163 & 0.107 & 0.026 & 11.280 & 0.182 & 4.672 \\
\hline SME Simulant & Coliwasa - high & $\mathrm{HF}$ & 7 & 4 & 12 & 4 & 18 & HIB01 & 10.732 & 4.154 & 0.107 & 1.188 & 0.111 & 0.028 & 11.452 & 0.172 & 4.758 \\
\hline SME Simulant & Isolok - $12 \mathrm{~mL}$ & HF & 21 & 4 & 6 & 4 & 19 & HIB03 & 10.827 & 3.928 & 0.107 & 1.188 & 0.114 & 0.033 & 11.609 & 0.159 & 4.758 \\
\hline SME Simulant & \begin{tabular}{|l|} 
Coliwasa - high \\
\end{tabular} & $\mathrm{HF}$ & 49 & 4 & 5 & 4 & 20 & HIB08 & 10.147 & 3.638 & 0.105 & 1.170 & 0.155 & 0.031 & 10.923 & 0.176 & 4.263 \\
\hline SME Simulant & \begin{tabular}{|l|l} 
Isolok - $12 \mathrm{~mL}$ \\
\end{tabular} & $\mathrm{HF}$ & 38 & 4 & 8 & 4 & 21 & HIB06 & 10.317 & 3.993 & 0.102 & 1.132 & 0.126 & 0.038 & 10.980 & 0.170 & 4.779 \\
\hline SME Simulant & Isolok - $12 \mathrm{~mL}$ & $\mathrm{HF}$ & 34 & 1 & 18 & 4 & 22 & CH07 & 10.808 & 4.121 & 0.108 & 1.222 & 0.105 & 0.030 & 11.623 & 0.176 & 4.736 \\
\hline SME Simulant & Isolok - $12 \mathrm{~mL}$ & LF & 63 & 1 & 13 & 4 & 23 & CH04 & 10.638 & 3.928 & 0.108 & 1.202 & 0.107 & 0.043 & 11.466 & 0.172 & 4.693 \\
\hline SME Simulant & Isolok - $12 \mathrm{~mL}$ & $\mathrm{HF}$ & 33 & 4 & 10 & 4 & 24 & HIB05 & 10.354 & 3.896 & 0.103 & 1.112 & 0.107 & 0.041 & 10.937 & 0.178 & 4.779 \\
\hline SME Simulant & Coliwasa - high & HF & 58 & 4 & 3 & 4 & 25 & HIB10 & 9.958 & 4.121 & 0.101 & 1.089 & 0.108 & 0.024 & 10.565 & 0.178 & 4.866 \\
\hline \begin{tabular}{|l|} 
ARG-1 \\
\end{tabular} & Coliwasa - high & 0 & 0 & 4 & 13 & 4 & 26 & ARG-1B43 & 4.535 & 7.535 & 0.093 & 1.427 & 0.095 & 0.003 & 13.968 & 2.843 & 3.122 \\
\hline SME Simulant & Isolok - $12 \mathrm{~mL}$ & $\mathrm{HF}$ & 65 & 1 & 6 & 4 & 27 & CH08 & 10.808 & 4.057 & 0.107 & 1.198 & 0.106 & 0.036 & 11.509 & 0.172 & 4.693 \\
\hline SME Simulant & Isolok - $12 \mathrm{~mL}$ & LF & 38 & 1 & 16 & 4 & 28 & CH03 & 10.695 & 4.025 & 0.108 & 1.182 & 0.107 & 0.031 & 11.438 & 0.162 & 4.672 \\
\hline SME Simulant & None & HF & 56 & 4 & 9 & 4 & 29 & HIB09 & $\begin{array}{l}11.488 \\
\end{array}$ & 3.928 & 0.121 & 1.291 & 0.144 & 0.045 & 12.538 & 0.184 & 4.500 \\
\hline \begin{tabular}{|l|} 
ARG-1 \\
\end{tabular} & \begin{tabular}{|l|} 
None \\
\end{tabular} & 0 & 0 & 2 & 1 & 5 & 1 & ARG-1B21 & \begin{tabular}{|l|}
4.743 \\
\end{tabular} & 8.050 & 0.093 & 1.469 & 0.103 & 0.011 & 14.011 & 2.554 & 3.229 \\
\hline SME Simulant & Isolok - $3 \mathrm{~mL}$ & $\mathrm{HF}$ & 20 & 2 & 16 & 5 & 2 & HIS03 & 10.789 & 3.896 & 0.103 & 1.203 & 0.114 & 0.056 & 11.295 & 0.180 & 4.844 \\
\hline \begin{tabular}{|l|} 
SME Simulant \\
\end{tabular} & Isolok - $3 \mathrm{~mL}$ & HF & 9 & 2 & 21 & 5 & 3 & \begin{tabular}{|l|} 
HIS01 \\
\end{tabular} & 10.902 & 4.282 & 0.114 & 1.248 & 0.109 & 0.046 & 11.380 & 0.182 & 5.038 \\
\hline SME Simulant & Coliwasa - low & LF & 20 & 1 & 2 & 5 & 4 & CL02 & 10.600 & 4.315 & 0.105 & 1.216 & 0.116 & 0.051 & 11.352 & 0.387 & 4.779 \\
\hline SME Simulant & Coliwasa - high & LF & 38 & 1 & 16 & 5 & 5 & $\mathrm{CH} 03$ & 10.695 & 4.057 & 0.103 & 1.168 & 0.109 & 0.041 & 11.252 & 0.162 & 4.779 \\
\hline SME Simulant & Coliwasa - high & LF & 63 & 1 & 13 & 5 & 6 & CH04 & 10.789 & 4.089 & 0.104 & 1.217 & 0.108 & 0.051 & 11.423 & 0.172 & 4.801 \\
\hline SME Simulant & Isolok - 3 mL & $\mathrm{HF}$ & 44 & 2 & 18 & 5 & 7 & \begin{tabular}{|l|} 
HIS07 \\
\end{tabular} & $\begin{array}{l}10.921 \\
\end{array}$ & 4.250 & 0.107 & 1.210 & 0.109 & 0.041 & 11.323 & 0.184 & 4.779 \\
\hline SME Simulant & Coliwasa - high & $\mathrm{HF}$ & 23 & 1 & 28 & 5 & 8 & CH06 & 10.827 & 4.282 & 0.105 & 1.192 & 0.105 & 0.039 & 11.280 & 0.179 & 4.822 \\
\hline SME Simulant & Coliwasa - high & LF & 21 & 1 & 14 & 5 & 9 & $\mathrm{CH} 02$ & 10.846 & 4.089 & 0.107 & 1.219 & 0.105 & 0.046 & 11.366 & 0.182 & 4.973 \\
\hline SME Simulant & Coliwasa - low & $\mathrm{HF}$ & 3 & 1 & 20 & 5 & 10 & CL05 & 10.676 & 4.154 & 0.101 & 1.154 & 0.115 & 0.048 & 11.209 & $\begin{array}{ll}0.181 \\
\end{array}$ & 4.607 \\
\hline SME Simulant & Isolok - $3 \mathrm{~mL}$ & $\mathrm{HF}$ & 14 & 2 & 15 & 5 & 11 & HISO2 & 10.581 & 3.864 & 0.104 & 1.170 & $\begin{array}{ll}0.103 \\
\end{array}$ & 0.048 & 11.023 & $\begin{array}{ll}0.184 \\
\end{array}$ & 4.693 \\
\hline SME Simulant & Coliwasa - low & LF & 40 & 1 & 19 & 5 & 12 & CL03 & 10.921 & 4.089 & 0.106 & 1.195 & 0.114 & 0.043 & 11.466 & 0.170 & 4.715 \\
\hline SME Simulant & Coliwasa - low & $\mathrm{HF}$ & 18 & 1 & 26 & 5 & 13 & CL06 & 10.808 & 4.186 & 0.109 & 1.228 & 0.126 & 0.046 & 11.352 & 0.190 & 4.779 \\
\hline SME Simulant & \begin{tabular}{|l|} 
Coliwasa - high \\
\end{tabular} & $\mathrm{HF}$ & 1 & 1 & 22 & 5 & 14 & \begin{tabular}{|l|} 
CH05 \\
\end{tabular} & 10.808 & 4.121 & 0.109 & 1.228 & 0.133 & 0.048 & 11.395 & 0.182 & 4.973 \\
\hline ARG-1 & \begin{tabular}{|l|} 
None \\
\end{tabular} & 0 & 0 & 2 & 12 & 5 & 15 & ARG-1B22 & 4.762 & 8.436 & 0.098 & 1.511 & 0.101 & 0.006 & 14.083 & 2.759 & 3.337 \\
\hline SME Simulant & Coliwasa - low & HF & 36 & 1 & 21 & 5 & 16 & \begin{tabular}{|l|l|} 
CL07 \\
\end{tabular} & 10.581 & 4.121 & 0.103 & 1.209 & 0.113 & 0.048 & 11.152 & 0.172 & 4.822 \\
\hline \begin{tabular}{|l|} 
SME Simulant \\
\end{tabular} & Isolok - $3 \mathrm{~mL}$ & $\mathrm{HF}$ & 31 & 2 & 19 & 5 & 17 & \begin{tabular}{|l|} 
HIS05 \\
\end{tabular} & $\begin{array}{ll}10.713 \\
\end{array}$ & 4.121 & 0.106 & 1.227 & 0.112 & 0.048 & 11.338 & 0.185 & 4.844 \\
\hline SME Simulant & Isolok - 3 mL & $\mathrm{HF}$ & 60 & 2 & 22 & 5 & 18 & HIS10 & 10.543 & 4.154 & 0.106 & 1.187 & 0.107 & 0.054 & 11.094 & 0.186 & 4.952 \\
\hline SME Simulant & Coliwasa - low & $\mathrm{HF}$ & 63 & 1 & 5 & 5 & 19 & CL08 & 10.713 & 4.154 & 0.105 & 1.222 & 0.123 & 0.047 & 11.352 & 0.396 & 4.693 \\
\hline SME Simulant & Isolok - $3 \mathrm{~mL}$ & $\mathrm{HF}$ & 26 & 2 & 14 & 5 & 20 & HIS04 & 10.430 & 4.218 & 0.102 & 1.178 & 0.099 & 0.053 & 10.894 & 0.163 & 4.930 \\
\hline SME Simulant & Isolok - $3 \mathrm{~mL}$ & HF & 35 & 2 & 20 & 5 & 21 & HIS06 & 10.676 & 4.476 & 0.103 & 1.212 & 0.107 & 0.046 & 11.223 & 0.182 & 4.822 \\
\hline \begin{tabular}{|l|} 
SME Simulant \\
\end{tabular} & \begin{tabular}{|l|} 
Coliwasa - high \\
\end{tabular} & $\mathrm{HF}$ & 65 & 1 & 6 & 5 & 22 & \begin{tabular}{|l|} 
CH08 \\
\end{tabular} & $\begin{array}{l}10.695 \\
\end{array}$ & 4.443 & 0.103 & 1.160 & 0.110 & 0.047 & 11.266 & 0.172 & 4.801 \\
\hline \begin{tabular}{|l|} 
SME Simulant \\
\end{tabular} & \begin{tabular}{|l|} 
Coliwasa - high \\
\end{tabular} & $\mathrm{HF}$ & 34 & 1 & 18 & 5 & 23 & CH07 & 10.713 & 4.604 & 0.106 & 1.249 & 0.112 & 0.054 & 11.366 & 0.176 & 4.779 \\
\hline SME Simulant & Coliwasa - high & LF & 1 & 1 & 12 & 5 & 24 & CH01 & 10.525 & 4.572 & 0.103 & 1.265 & 0.111 & 0.046 & 11.295 & 0.186 & 4.930 \\
\hline SME Simulant & Isolok - $3 \mathrm{~mL}$ & HF & 54 & 2 & 17 & 5 & 25 & HIS09 & 10.373 & 4.282 & 0.099 & 1.168 & 0.108 & 0.049 & 10.794 & 0.163 & 5.059 \\
\hline SME Simulant & Coliwasa - low & LF & 4 & 1 & 10 & 5 & 26 & CL01 & $\begin{array}{l}10.789 \\
\end{array}$ & 4.540 & 0.108 & 1.180 & 0.107 & 0.040 & 11.352 & 0.159 & 4.715 \\
\hline SME Simulant & Isolok - 3 mL & $\mathrm{HF}$ & 48 & 2 & 13 & 5 & 27 & \begin{tabular}{|l|} 
HIS08 \\
\end{tabular} & 10.657 & 4.057 & 0.101 & 1.175 & 0.097 & 0.042 & 11.166 & 0.175 & 4.822 \\
\hline SME Simulant & Coliwasa - low & LF & 65 & 1 & 23 & 5 & 28 & \begin{tabular}{|l|} 
CL04 \\
\end{tabular} & 10.770 & 4.186 & 0.102 & 1.212 & 0.106 & 0.048 & 11.309 & 0.169 & 4.736 \\
\hline
\end{tabular}


Table C4. Phase 3 Oxide Concentration Measurements (part 1)

Revision 0

\begin{tabular}{|c|c|c|c|c|c|c|c|c|c|c|c|c|c|c|c|c|c|}
\hline $\begin{array}{l}\text { Type of } \\
\text { Material }\end{array}$ & Sampler Details & \begin{tabular}{|l|} 
Flow \\
Rate
\end{tabular} & $\begin{array}{r}\text { Sample } \\
\text { Order }\end{array}$ & $\begin{array}{r}\text { Preparation } \\
\text { Block }\end{array}$ & $\begin{array}{r}\text { Preparation } \\
\text { Sequence }\end{array}$ & \begin{tabular}{|r|} 
Analytical \\
Block
\end{tabular} & $\begin{array}{r}\text { Analytical } \\
\text { Sequence }\end{array}$ & Sample ID & $\begin{array}{l}\text { Al2O3 } \\
\text { (wt\%) }\end{array}$ & $\begin{array}{r}\text { B2O3 } \\
\text { (wt\%) }\end{array}$ & $\begin{array}{r}\mathrm{BaO} \\
\text { (wt\%) }\end{array}$ & $\begin{array}{r}\mathrm{CaO} \\
(\mathrm{wt} \%)\end{array}$ & $\begin{array}{r}\text { Cr2O3 } \\
\text { (wt\%) }\end{array}$ & $\begin{array}{r}\mathrm{CuO} \\
\text { (wt\%) }\end{array}$ & $\begin{array}{l}\text { Fe2O3 } \\
\text { (wt\%) }\end{array}$ & \begin{tabular}{r|}
$\mathrm{K} 2 \mathrm{O}$ \\
(wt\%)
\end{tabular} & $\begin{array}{r}\mathbf{L i 2 O} \\
\text { (wt\%) }\end{array}$ \\
\hline ARG-1 & None & 0 & 0 & 2 & 23 & \begin{tabular}{|l|}
5 \\
\end{tabular} & 29 & ARG-1B23 & $\begin{array}{l}4.762 \\
\end{array}$ & 8.758 & 0.093 & 1.539 & 0.116 & 0.017 & 14.054 & 2.831 & 3.229 \\
\hline ARG-1 & \begin{tabular}{|l|} 
None \\
\end{tabular} & 0 & 0 & 3 & 1 & 6 & 1 & ARG-1B31 & 4.743 & 8.146 & 0.092 & 1.469 & 0.102 & 0.003 & 14.111 & 2.602 & 3.100 \\
\hline SME Simulant & Hydragard & LF & 15 & 3 & 21 & 6 & 2 & LHY03 & 10.525 & 4.154 & 0.101 & 1.168 & 0.107 & 0.040 & 11.180 & 0.179 & 4.478 \\
\hline SME Simulant & Hydragard & LF & 6 & 3 & 18 & 6 & 3 & LHY01 & 10.940 & 4.057 & 0.101 & 1.173 & 0.106 & 0.039 & 11.552 & 0.180 & 4.543 \\
\hline SME Simulant & Coliwasa - low & LF & 20 & 1 & 2 & 6 & 4 & CL02 & 10.600 & 4.282 & 0.104 & 1.245 & 0.117 & 0.039 & 11.366 & 0.192 & 4.607 \\
\hline SME Simulant & Coliwasa - high & LF & 38 & 1 & 16 & 6 & 5 & $\mathrm{CH} 03$ & 10.676 & 4.057 & 0.101 & 1.160 & 0.109 & 0.032 & 11.309 & 0.162 & 4.543 \\
\hline SME Simulant & Coliwasa - high & LF & 63 & 1 & 13 & 6 & 6 & CH04 & 10.676 & 3.928 & 0.101 & 1.175 & 0.109 & 0.043 & 11.395 & 0.172 & 4.629 \\
\hline SME Simulant & Hydragard & LF & 47 & 3 & 13 & 6 & 7 & LHY07 & 10.770 & 3.960 & 0.104 & 1.181 & 0.122 & 0.044 & 11.480 & 0.173 & 4.629 \\
\hline SME Simulant & Coliwasa - high & $\mathrm{HF}$ & 23 & 1 & 28 & 6 & 8 & CH06 & 10.657 & 4.250 & 0.101 & 1.220 & 0.106 & 0.036 & 11.280 & 0.179 & 4.607 \\
\hline SME Simulant & Coliwasa - high & LF & 21 & 1 & 14 & 6 & 9 & CH02 & 10.676 & 4.186 & 0.101 & 1.159 & 0.105 & 0.035 & 11.323 & 0.182 & 4.607 \\
\hline SME Simulant & Coliwasa - low & HF & 3 & 1 & 20 & 6 & 10 & CL05 & 10.543 & 3.896 & 0.099 & 1.203 & 0.113 & 0.043 & 11.195 & 0.181 & 4.693 \\
\hline SME Simulant & Hydragard & LF & 10 & 3 & 20 & 6 & 11 & LHY02 & 11.280 & 3.960 & 0.108 & 1.314 & 0.114 & 0.040 & 11.981 & 0.181 & 4.413 \\
\hline SME Simulant & Coliwasa - low & LF & 40 & 1 & 19 & 6 & 12 & CL03 & 10.808 & 3.735 & 0.098 & 1.187 & 0.104 & 0.040 & 11.395 & 0.170 & 4.586 \\
\hline SME Simulant & Coliwasa - low & HF & 18 & 1 & 26 & 6 & 13 & CL06 & 10.657 & 3.993 & 0.103 & 1.198 & 0.120 & 0.035 & 11.352 & 0.190 & 4.543 \\
\hline SME Simulant & \begin{tabular}{|l|} 
Coliwasa - high \\
\end{tabular} & $\mathrm{HF}$ & 1 & 1 & 22 & 6 & 14 & CH05 & 10.770 & 3.993 & 0.104 & 1.212 & $\begin{array}{ll}0.133 \\
\end{array}$ & 0.040 & 11.452 & 0.182 & 4.629 \\
\hline ARG-1 & \begin{tabular}{|l|} 
None \\
\end{tabular} & 0 & 0 & 3 & 12 & 6 & 15 & ARG-1B32 & 4.629 & 8.404 & 0.093 & 1.497 & $\begin{array}{ll}0.111 \\
\end{array}$ & 0.003 & 14.154 & 2.710 & 3.079 \\
\hline SME Simulant & Coliwasa - low & $\mathrm{HF}$ & 36 & 1 & 21 & 6 & 16 & CL07 & 10.562 & 4.025 & 0.100 & 1.202 & 0.114 & 0.040 & 11.152 & 0.172 & 4.650 \\
\hline SME Simulant & Hydragard & LF & 30 & 3 & 14 & 6 & 17 & LHY05 & 10.525 & 4.121 & 0.097 & 1.133 & 0.103 & 0.028 & 10.966 & 0.173 & 4.672 \\
\hline SME Simulant & Hydragard & LF & 59 & 3 & 17 & 6 & 18 & LHY10 & 14.946 & 5.055 & 0.142 & 1.819 & 0.155 & 0.060 & 15.870 & 0.177 & 6.480 \\
\hline SME Simulant & Coliwasa - low & $\mathrm{HF}$ & 63 & 1 & 5 & 6 & 19 & \begin{tabular}{|l|} 
CL08 \\
\end{tabular} & 10.808 & 3.993 & 0.102 & 1.210 & 0.121 & 0.043 & 11.395 & 0.185 & 4.543 \\
\hline SME Simulant & Hydragard & LF & 28 & 3 & 16 & 6 & 20 & LHY04 & $\begin{array}{l}11.167 \\
\end{array}$ & 3.896 & 0.104 & 1.230 & 0.108 & 0.038 & 11.781 & 0.184 & 4.564 \\
\hline SME Simulant & Hydragard & LF & 37 & 3 & 15 & 6 & 21 & LHY06 & 11.072 & 3.960 & 0.107 & 1.241 & 0.131 & 0.035 & 11.724 & 0.169 & 4.457 \\
\hline SME Simulant & Coliwasa - high & HF & 65 & 1 & 6 & 6 & 22 & \begin{tabular}{|l|} 
CH08 \\
\end{tabular} & 10.713 & 4.025 & 0.099 & 1.177 & 0.105 & 0.039 & 11.309 & 0.172 & 4.650 \\
\hline SME Simulant & Coliwasa - high & HF & 34 & 1 & 18 & 6 & 23 & CH07 & 10.713 & 4.154 & 0.102 & 1.216 & 0.108 & 0.035 & 11.409 & 0.176 & 4.607 \\
\hline SME Simulant & Coliwasa - high & LF & 1 & 1 & 12 & 6 & 24 & CH01 & 10.543 & 4.250 & 0.102 & 1.216 & 0.110 & 0.039 & 11.280 & 0.186 & 4.758 \\
\hline SME Simulant & Hydragard & LF & 53 & 3 & 19 & 6 & 25 & LHY09 & $\begin{array}{l}10.827 \\
\end{array}$ & 3.735 & 0.102 & 1.195 & 0.112 & 0.032 & 11.237 & 0.172 & 4.392 \\
\hline SME Simulant & Coliwasa - low & LF & 4 & 1 & 10 & 6 & 26 & \begin{tabular}{|l|} 
CL01 \\
\end{tabular} & $\begin{array}{l}10.827 \\
\end{array}$ & 4.154 & 0.106 & 1.166 & 0.106 & 0.038 & 11.395 & 0.159 & 4.564 \\
\hline SME Simulant & Hydragard & LF & 48 & 3 & 22 & 6 & 27 & LHY08 & 10.997 & 4.057 & 0.104 & 1.381 & 0.110 & 0.033 & 11.638 & 0.175 & 4.327 \\
\hline SME Simulant & Coliwasa - low & LF & 65 & 1 & 23 & 6 & 28 & CL04 & 10.770 & 4.057 & 0.103 & 1.223 & 0.111 & 0.037 & 11.380 & 0.169 & 4.564 \\
\hline ARG-1 & None & 0 & 0 & 3 & 23 & 6 & 29 & ARG-1B33 & $\begin{array}{l}4.762 \\
\end{array}$ & 8.179 & 0.093 & 1.469 & 0.106 & 0.003 & 13.997 & 2.494 & 3.100 \\
\hline
\end{tabular}


Table C4. Phase 3 Oxide Concentration Measurements (part 2)

\begin{tabular}{|c|c|c|c|c|c|c|c|c|c|c|c|c|c|c|c|c|c|}
\hline $\begin{array}{l}\text { Type of } \\
\text { Material }\end{array}$ & \begin{tabular}{|l|} 
Sampler \\
Details
\end{tabular} & \begin{tabular}{|l|} 
Flow \\
Rate
\end{tabular} & $\begin{array}{r}\text { Sample } \\
\text { Order }\end{array}$ & $\begin{array}{r}\text { Preparation } \\
\text { Block }\end{array}$ & $\begin{array}{r}\text { Preparation } \\
\text { Sequence }\end{array}$ & $\begin{array}{r}\text { Analytical } \\
\text { Block }\end{array}$ & $\begin{array}{r}\text { Analytical } \\
\text { Sequence }\end{array}$ & Sample ID & $\begin{array}{r}\text { MgO } \\
\text { (wt\%) }\end{array}$ & $\begin{array}{r}\text { MnO } \\
\text { (wt\%) }\end{array}$ & $\begin{array}{r}\text { Na2O } \\
\text { (wt\%) }\end{array}$ & $\begin{array}{r}\mathrm{NiO} \\
\text { (wt\%) }\end{array}$ & $\begin{array}{r}\text { SiO2 } \\
\text { (wt\%) }\end{array}$ & $\begin{array}{r}\text { TiO2 } \\
\text { (wt\%) }\end{array}$ & $\begin{array}{r}\mathrm{ZnO} \\
\text { (wt\%) }\end{array}$ & $\begin{array}{r}\text { ZrO2 } \\
\text { (wt\%) }\end{array}$ & $\begin{array}{r}\text { Sum } \\
\text { (wt\%) }\end{array}$ \\
\hline ARG-1 & \begin{tabular}{|l|} 
None \\
\end{tabular} & 0 & 0 & 1 & 1 & 1 & 1 & ARG-1B11 & 0.811 & 1.756 & 10.824 & 1.000 & 48.134 & 1.149 & 0.028 & 0.132 & 97.084 \\
\hline SME Simulant & Isolok - $3 \mathrm{~mL}$ & LF & 18 & 1 & 9 & 1 & 2 & LIS03 & 0.988 & 1.653 & 10.690 & 0.453 & 52.413 & 0.081 & 0.104 & 0.376 & 98.514 \\
\hline SME Simulant & Isolok - $3 \mathrm{~mL}$ & LF & 8 & 1 & 27 & 1 & 3 & LIS01 & 0.988 & 1.640 & 10.245 & 0.468 & 52.413 & 0.084 & 0.103 & 0.370 & 97.769 \\
\hline SME Simulant & Coliwasa - low & LF & 20 & 1 & 2 & 1 & 4 & CL02 & 1.015 & 1.691 & 10.218 & 0.450 & 52.413 & $\begin{array}{ll}0.083 \\
\end{array}$ & 0.106 & 0.367 & 97.737 \\
\hline SME Simulant & Coliwasa - high & LF & 38 & 1 & 16 & 1 & 5 & $\mathrm{CH} 03$ & 1.013 & 1.691 & 10.434 & 0.464 & 53.269 & 0.082 & 0.105 & 0.376 & 99.289 \\
\hline SME Simulant & Coliwasa - high & LF & 63 & 1 & 13 & 1 & 6 & \begin{tabular}{|l|l} 
CHO4 \\
\end{tabular} & 1.017 & 1.691 & 10.595 & 0.476 & 52.199 & 0.083 & 0.106 & 0.370 & 98.529 \\
\hline SME Simulant & Isolok - $3 \mathrm{~mL}$ & LF & 44 & 1 & 3 & 1 & 7 & $\begin{array}{ll}\text { LIS07 } \\
\end{array}$ & 0.957 & 1.588 & 10.002 & 0.425 & 53.696 & 0.085 & 0.096 & 0.367 & 97.981 \\
\hline SME Simulant & Coliwasa - high & $\mathrm{HF}$ & 23 & 1 & 28 & 1 & 8 & CH06 & 0.978 & 1.627 & 10.204 & 0.440 & 51.985 & 0.081 & 0.099 & 0.371 & 96.841 \\
\hline SME Simulant & Coliwasa - high & LF & 21 & 1 & 14 & 1 & 9 & $\mathrm{CH} 02$ & 0.998 & 1.666 & 10.730 & 0.454 & 52.413 & 0.080 & 0.103 & 0.374 & 98.621 \\
\hline SME Simulant & Coliwasa - low & HF & 3 & 1 & 20 & 1 & 10 & CL05 & 1.002 & 1.666 & 10.150 & 0.454 & 53.269 & 0.083 & 0.103 & 0.374 & 98.908 \\
\hline SME Simulant & Isolok - 3 mL & LF & 12 & 1 & 25 & 1 & 11 & LIS02 & 0.975 & 1.627 & 10.771 & 0.435 & 51.771 & 0.077 & 0.100 & 0.362 & 96.706 \\
\hline SME Simulant & Coliwasa - low & LF & 40 & 1 & 19 & 1 & 12 & CL03 & 0.998 & 1.666 & 10.487 & 0.453 & 51.557 & 0.079 & 0.104 & 0.371 & 97.125 \\
\hline SME Simulant & Coliwasa - low & $\mathrm{HF}$ & 18 & 1 & 26 & 1 & 13 & CL06 & 1.007 & 1.679 & 9.503 & 0.473 & 52.841 & 0.082 & 0.109 & 0.371 & 97.632 \\
\hline SME Simulant & Coliwasa - high & $\mathrm{HF}$ & 1 & 1 & 22 & 1 & 14 & CH05 & 1.000 & 1.666 & 10.487 & 0.503 & 51.985 & 0.080 & 0.102 & 0.370 & 97.727 \\
\hline \begin{tabular}{|l|} 
ARG-1 \\
\end{tabular} & None & 0 & 0 & 1 & 15 & 1 & 15 & ARG-1B12 & 0.827 & 1.795 & 10.986 & 1.042 & 49.204 & 1.169 & 0.025 & 0.135 & 98.743 \\
\hline SME Simulant & Coliwasa - low & $\mathrm{HF}$ & 36 & 1 & 21 & 1 & 16 & CL07 & 0.937 & 1.562 & 9.813 & 0.426 & 50.060 & $\begin{array}{ll}0.078 \\
\end{array}$ & 0.097 & 0.354 & 92.990 \\
\hline SME Simulant & Isolok - $3 \mathrm{~mL}$ & LF & 31 & 1 & 24 & 1 & 17 & LIS05 & 0.947 & 1.575 & 10.029 & 0.435 & 51.985 & 0.082 & 0.094 & 0.363 & 95.855 \\
\hline SME Simulant & Isolok - 3 mL & LF & 58 & 1 & 4 & 1 & 18 & LIS10 & 0.977 & 1.627 & 9.908 & 0.456 & 52.841 & 0.085 & 0.101 & 0.373 & 97.712 \\
\hline SME Simulant & Coliwasa - low & $\mathrm{HF}$ & 63 & 1 & 5 & 1 & 19 & CL08 & 0.990 & 1.653 & 10.218 & 0.472 & 51.985 & 0.077 & 0.104 & 0.373 & 97.457 \\
\hline SME Simulant & Isolok - 3 mL & LF & 25 & 1 & 7 & 1 & 20 & LIS04 & 0.992 & 1.653 & 10.892 & 0.459 & 51.985 & 0.083 & 0.102 & 0.374 & 98.164 \\
\hline SME Simulant & Isolok - $3 \mathrm{~mL}$ & LF & 42 & 1 & 11 & 1 & 21 & LIS06 & 0.995 & 1.653 & 10.083 & 0.435 & 53.910 & 0.085 & 0.098 & 0.374 & 99.120 \\
\hline SME Simulant & Coliwasa - high & $\mathrm{HF}$ & 65 & 1 & 6 & 1 & 22 & $\mathrm{CH} 08$ & 0.998 & 1.653 & 10.272 & 0.449 & 51.771 & 0.083 & 0.099 & 0.374 & 97.266 \\
\hline SME Simulant & Coliwasa - high & $\mathrm{HF}$ & 34 & 1 & 18 & 1 & 23 & CH07 & 1.008 & 1.679 & 9.894 & 0.444 & 52.199 & 0.081 & 0.101 & 0.377 & 97.351 \\
\hline SME Simulant & Coliwasa - high & LF & 1 & 1 & 12 & 1 & 24 & CH01 & 1.010 & 1.691 & 10.191 & $\begin{array}{ll}0.448 \\
\end{array}$ & 51.771 & 0.082 & 0.102 & 0.369 & 97.237 \\
\hline SME Simulant & Isolok - $3 \mathrm{~mL}$ & LF & 54 & 1 & 17 & 1 & 25 & LIS09 & 0.975 & 1.627 & 10.029 & 0.454 & 52.627 & 0.085 & 0.100 & 0.374 & 97.712 \\
\hline SME Simulant & Coliwasa - low & LF & 4 & 1 & 10 & 1 & 26 & CL01 & 0.985 & 1.640 & 10.959 & 0.448 & 50.915 & 0.081 & 0.099 & 0.371 & 97.131 \\
\hline SME Simulant & Isolok - 3 mL & LF & 49 & 1 & 8 & 1 & 27 & LIS08 & 0.972 & 1.627 & 10.312 & 0.439 & 51.129 & 0.081 & 0.096 & 0.369 & 96.189 \\
\hline SME Simulant & Coliwasa - low & LF & 65 & 1 & 23 & 1 & 28 & CL04 & 0.998 & 1.666 & 10.541 & 0.454 & 52.199 & 0.084 & 0.101 & 0.377 & 98.270 \\
\hline ARG-1 & \begin{tabular}{|l|} 
None \\
\end{tabular} & 0 & 0 & 1 & 29 & 1 & 29 & ARG-1B13 & 0.824 & 1.795 & 10.986 & 1.008 & 48.990 & 1.176 & 0.024 & 0.138 & 98.731 \\
\hline \begin{tabular}{|l|} 
ARG-1 \\
\end{tabular} & \begin{tabular}{|l|} 
None \\
\end{tabular} & 0 & 0 & 2 & 1 & 2 & 1 & ARG-1B21 & 0.831 & 1.808 & 11.701 & 1.051 & 49.204 & 1.184 & 0.025 & $\begin{array}{ll}0.138 \\
\end{array}$ & 100.683 \\
\hline SME Simulant & Hydragard & $\mathrm{HF}$ & 11 & 2 & 3 & 2 & 2 & HHY02 & 1.098 & 1.834 & 10.272 & 0.514 & 51.129 & $\begin{array}{ll}0.083 \\
\end{array}$ & 0.117 & 0.382 & 99.762 \\
\hline SME Simulant & Coliwasa - low & $\mathrm{HF}$ & 36 & 1 & 21 & 2 & 3 & CL07 & 0.990 & 1.640 & 10.932 & 0.448 & 53.055 & 0.082 & 0.100 & 0.374 & 99.673 \\
\hline SME Simulant & Hydragard & HF & 24 & 2 & 2 & 2 & 4 & HHY04 & 1.028 & 1.717 & 10.905 & 0.468 & 52.413 & 0.083 & 0.101 & 0.388 & 100.038 \\
\hline SME Simulant & Coliwasa - high & $\mathrm{HF}$ & 1 & 1 & 22 & 2 & 5 & CH05 & 1.008 & 1.679 & 11.242 & 0.505 & 52.413 & 0.084 & 0.099 & 0.378 & 99.727 \\
\hline SME Simulant & Coliwasa - low & $\mathrm{HF}$ & 3 & 1 & 20 & 2 & 6 & CL05 & 1.015 & 1.691 & 10.757 & 0.454 & 53.483 & 0.087 & 0.101 & 0.381 & 100.523 \\
\hline SME Simulant & Coliwasa - high & $\mathrm{HF}$ & 23 & 1 & 28 & 2 & 7 & CH06 & 0.990 & 1.653 & 10.905 & 0.439 & 52.199 & 0.083 & 0.100 & 0.380 & 98.888 \\
\hline SME Simulant & Coliwasa - high & LF & 1 & 1 & 12 & 2 & 8 & $\mathrm{CH} 01$ & 1.015 & 1.691 & 10.986 & 0.445 & 51.985 & 0.083 & 0.101 & 0.371 & 98.815 \\
\hline SME Simulant & Coliwasa - low & LF & 20 & 1 & 2 & 2 & 9 & CL02 & 1.035 & 1.717 & 10.892 & 0.454 & 52.841 & 0.084 & 0.103 & 0.371 & 99.605 \\
\hline SME Simulant & Coliwasa - low & $\mathrm{HF}$ & 63 & 1 & 5 & 2 & 10 & CL08 & 0.987 & 1.653 & 10.824 & 0.467 & 51.343 & 0.081 & 0.102 & 0.373 & 97.695 \\
\hline SME Simulant & Coliwasa - high & $\mathrm{HF}$ & 65 & 1 & 6 & 2 & 11 & CH08 & 1.007 & 1.679 & 11.054 & 0.450 & 51.985 & 0.079 & 0.102 & 0.367 & 99.016 \\
\hline SME Simulant & Hydragard & $\mathrm{HF}$ & 45 & 2 & 7 & 2 & 12 & HHY07 & 1.048 & 1.743 & 10.932 & 0.480 & 51.129 & 0.079 & 0.108 & 0.377 & 98.794 \\
\hline SME Simulant & Coliwasa - low & LF & 4 & 1 & 10 & 2 & 13 & CL01 & 1.005 & 1.666 & 11.835 & 0.459 & 51.343 & 0.079 & 0.102 & 0.376 & 99.201 \\
\hline \begin{tabular}{|l|l|} 
SME Simulant \\
\end{tabular} & Coliwasa - low & $\mathrm{HF}$ & 18 & 1 & 26 & 2 & 14 & CL06 & 1.022 & 1.704 & 10.231 & 0.448 & 53.269 & 0.085 & 0.103 & 0.382 & 99.574 \\
\hline ARG-1 & None & 0 & 0 & 2 & 12 & 2 & 15 & ARG-1B22 & 0.823 & 1.795 & 11.647 & 1.029 & 48.562 & 1.168 & 0.024 & 0.139 & 99.151 \\
\hline SME Simulant & Coliwasa - low & LF & 65 & 1 & 23 & 2 & 16 & CL04 & 1.000 & 1.653 & 11.121 & 0.443 & 51.557 & 0.082 & 0.098 & 0.376 & 98.144 \\
\hline SME Simulant & Coliwasa - low & LF & 40 & 1 & 19 & 2 & 17 & CL03 & 1.005 & 1.666 & 11.256 & 0.454 & 51.557 & 0.081 & 0.101 & 0.377 & 98.703 \\
\hline SME Simulant & Coliwasa - high & LF & 38 & 1 & 16 & 2 & 18 & $\mathrm{CH} 03$ & 1.007 & 1.666 & 10.905 & 0.453 & 52.627 & 0.082 & 0.102 & 0.374 & 99.032 \\
\hline SME Simulant & Hydragard & $\mathrm{HF}$ & 57 & 2 & 6 & 2 & 19 & HHY09 & 1.078 & 1.782 & 11.229 & 0.496 & 50.274 & 0.082 & 0.111 & 0.378 & 98.724 \\
\hline
\end{tabular}


Table C4. Phase 3 Oxide Concentration Measurements (part 2)

\begin{tabular}{|c|c|c|c|c|c|c|c|c|c|c|c|c|c|c|c|c|c|}
\hline $\begin{array}{l}\text { Type of } \\
\text { Material }\end{array}$ & \begin{tabular}{|l|} 
Sampler \\
Details
\end{tabular} & \begin{tabular}{|l|} 
Flow \\
Rate
\end{tabular} & $\begin{array}{r}\text { Sample } \\
\text { Order }\end{array}$ & $\begin{array}{r}\text { Preparation } \\
\text { Block }\end{array}$ & $\begin{array}{r}\text { Preparation } \\
\text { Sequence }\end{array}$ & $\begin{array}{r}\text { Analytical } \\
\text { Block }\end{array}$ & $\begin{array}{r}\text { Analytical } \\
\text { Sequence }\end{array}$ & Sample ID & $\begin{array}{r}\text { MgO } \\
\text { (wt\%) }\end{array}$ & $\begin{array}{r}\text { MnO } \\
\text { (wt\%) }\end{array}$ & $\begin{array}{r}\text { Na2O } \\
\text { (wt\%) }\end{array}$ & $\begin{array}{r}\mathrm{NiO} \\
\text { (wt\%) }\end{array}$ & $\begin{array}{r}\text { SiO2 } \\
\text { (wt\%) }\end{array}$ & $\begin{array}{r}\text { TiO2 } \\
\text { (wt\%) }\end{array}$ & $\begin{array}{r}\mathrm{ZnO} \\
\text { (wt\%) }\end{array}$ & $\begin{array}{r}\text { ZrO2 } \\
\text { (wt\%) }\end{array}$ & $\begin{array}{r}\text { Sum } \\
\text { (wt\%) }\end{array}$ \\
\hline SME Simulant & Coliwasa - high & LF & 21 & 1 & 14 & 2 & 20 & CH02 & 0.988 & 1.640 & 11.215 & 0.457 & \begin{tabular}{|l|}
51.771 \\
\end{tabular} & 0.078 & 0.102 & 0.367 & 98.453 \\
\hline SME Simulant & Hydragard & $\mathrm{HF}$ & 6 & 2 & 4 & 2 & 21 & HHY01 & 1.051 & 1.743 & 10.919 & 0.463 & \begin{tabular}{|l}
51.771 \\
\end{tabular} & 0.082 & 0.105 & 0.371 & 99.144 \\
\hline SME Simulant & Hydragard & $\mathrm{HF}$ & 19 & 2 & 10 & 2 & 22 & HHY03 & 1.063 & 1.743 & 10.703 & 0.466 & 52.627 & 0.084 & 0.106 & 0.389 & 99.830 \\
\hline SME Simulant & \begin{tabular}{|l|} 
Hydragard \\
\end{tabular} & $\mathrm{HF}$ & 50 & 2 & 5 & 2 & 23 & HHY08 & 1.015 & 1.717 & 10.919 & 0.458 & 51.557 & $\begin{array}{ll}0.083 \\
\end{array}$ & 0.097 & 0.374 & 98.635 \\
\hline SME Simulant & Hydragard & $\mathrm{HF}$ & 37 & 2 & 9 & 2 & 24 & HHY06 & 1.065 & 1.782 & 11.108 & 0.481 & 50.701 & 0.077 & 0.108 & 0.369 & 98.613 \\
\hline SME Simulant & Coliwasa - high & $\mathrm{HF}$ & 34 & 1 & 18 & 2 & 25 & CH07 & 1.025 & 1.704 & 10.757 & 0.457 & 52.841 & 0.085 & 0.103 & 0.380 & 100.042 \\
\hline SME Simulant & Coliwasa - high & LF & 63 & 1 & 13 & 2 & 26 & CH04 & 0.998 & 1.666 & 11.108 & 0.442 & 51.557 & 0.079 & 0.099 & 0.365 & 97.905 \\
\hline SME Simulant & Hydragard & $\mathrm{HF}$ & 32 & 2 & 8 & 2 & 27 & HHY05 & 1.075 & \begin{tabular}{|l|l|}
1.717 \\
\end{tabular} & 12.240 & 0.430 & 50.915 & 0.078 & 0.096 & 0.350 & 99.327 \\
\hline SME Simulant & Hydragard & $\mathrm{HF}$ & 61 & 2 & 11 & 2 & 28 & HHY10 & 1.091 & 1.821 & 11.256 & 0.482 & 50.060 & 0.076 & 0.112 & 0.381 & 98.488 \\
\hline \begin{tabular}{|l|} 
ARG-1 \\
\end{tabular} & \begin{tabular}{|l|} 
None \\
\end{tabular} & 0 & 0 & 2 & 23 & 2 & 29 & ARG-1B23 & 0.823 & 1.782 & 11.782 & 0.985 & 48.776 & 1.173 & 0.021 & 0.135 & 99.497 \\
\hline ARG-1 & None & 0 & 0 & 3 & 1 & 3 & 1 & ARG-1B31 & 0.847 & 1.834 & 11.525 & 1.010 & 49.204 & 1.169 & 0.025 & 0.133 & 99.980 \\
\hline SME Simulant & Isolok - $12 \mathrm{~mL}$ & LF & 11 & 3 & 4 & 3 & 2 & LIB02 & 0.952 & 1.588 & 11.067 & 0.442 & 53.269 & 0.083 & 0.098 & 0.354 & 99.459 \\
\hline SME Simulant & Coliwasa - low & $\mathrm{HF}$ & 36 & 1 & 21 & 3 & 3 & \begin{tabular}{|l|l|} 
CL07 \\
\end{tabular} & 1.013 & 1.666 & 10.865 & 0.445 & 52.841 & 0.085 & 0.100 & 0.377 & 99.501 \\
\hline SME Simulant & Isolok - $12 \mathrm{~mL}$ & LF & 26 & 3 & 8 & 3 & 4 & LIB04 & 1.030 & 1.704 & 11.782 & 0.439 & 50.915 & 0.083 & 0.099 & 0.370 & 98.720 \\
\hline SME Simulant & Coliwasa - high & $\mathrm{HF}$ & 1 & 1 & 22 & 3 & 5 & CH05 & 1.041 & 1.730 & 11.081 & 0.508 & 52.627 & 0.083 & 0.101 & 0.374 & 100.144 \\
\hline SME Simulant & Coliwasa - low & HF & 3 & 1 & 20 & 3 & 6 & CL05 & 1.050 & 1.730 & 10.676 & 0.445 & 54.338 & 0.085 & 0.102 & 0.374 & 101.340 \\
\hline SME Simulant & Coliwasa - high & $\mathrm{HF}$ & 23 & 1 & 28 & 3 & 7 & $\mathrm{CH} 06$ & 1.007 & 1.653 & 10.609 & 0.452 & 51.771 & 0.081 & 0.101 & 0.367 & 97.878 \\
\hline SME Simulant & Coliwasa - high & LF & 1 & 1 & 12 & 3 & 8 & $\mathrm{CH} 01$ & 1.055 & 1.743 & 10.878 & 0.433 & 52.413 & 0.082 & 0.101 & 0.366 & 99.564 \\
\hline SME Simulant & Coliwasa - low & LF & 20 & 1 & 2 & 3 & 9 & CL02 & 1.070 & 1.769 & 10.811 & 0.468 & 53.483 & 0.081 & 0.110 & 0.371 & 100.892 \\
\hline SME Simulant & Coliwasa - low & $\mathrm{HF}$ & 63 & 1 & 5 & 3 & 10 & CL08 & 1.038 & 1.730 & 10.932 & 0.462 & 52.627 & 0.080 & 0.105 & 0.371 & 99.856 \\
\hline SME Simulant & Coliwasa - high & $\mathrm{HF}$ & 65 & 1 & 6 & 3 & 11 & $\mathrm{CH} 08$ & 1.033 & 1.704 & 10.892 & 0.453 & 52.199 & 0.080 & 0.106 & 0.365 & 99.239 \\
\hline SME Simulant & Isolok - $12 \mathrm{~mL}$ & LF & 45 & 3 & 7 & 3 & 12 & LIB07 & 0.997 & 1.653 & 10.690 & 0.458 & 53.269 & 0.080 & 0.099 & 0.358 & 99.386 \\
\hline SME Simulant & Coliwasa - low & LF & 4 & 1 & 10 & 3 & 13 & CL01 & 1.026 & 1.704 & 11.620 & 0.456 & 51.771 & 0.079 & 0.102 & 0.371 & 99.609 \\
\hline SME Simulant & Coliwasa - low & $\mathrm{HF}$ & 18 & 1 & 26 & 3 & 14 & \begin{tabular}{|l|l|} 
CL06 \\
\end{tabular} & 1.056 & 1.756 & 10.164 & 0.466 & 53.910 & 0.082 & 0.109 & 0.377 & 100.777 \\
\hline ARG-1 & None & 0 & 0 & 3 & 12 & 3 & 15 & ARG-1B32 & 0.857 & 1.859 & 11.593 & 1.033 & 49.204 & 1.168 & 0.026 & 0.132 & 100.272 \\
\hline SME Simulant & Coliwasa - low & LF & 65 & 1 & 23 & 3 & 16 & CL04 & 1.022 & 1.691 & 10.986 & 0.454 & 51.557 & 0.080 & 0.103 & 0.362 & 98.119 \\
\hline SME Simulant & Coliwasa - low & LF & 40 & 1 & 19 & 3 & 17 & CL03 & 1.025 & 1.691 & 11.027 & 0.464 & 51.129 & 0.080 & 0.105 & 0.369 & 97.952 \\
\hline SME Simulant & Coliwasa - high & LF & 38 & 1 & 16 & 3 & 18 & CH03 & 1.025 & 1.691 & 10.784 & 0.433 & 52.413 & 0.081 & 0.100 & 0.366 & 98.699 \\
\hline SME Simulant & Isolok - $12 \mathrm{~mL}$ & LF & 56 & 3 & 11 & 3 & 19 & $\begin{array}{l}\text { LIB09 } \\
\end{array}$ & 1.012 & 1.691 & 10.771 & 0.477 & 52.199 & 0.082 & 0.104 & 0.366 & 99.136 \\
\hline SME Simulant & Coliwasa - high & LF & 21 & 1 & 14 & 3 & 20 & $\mathrm{CH} 02$ & 1.025 & 1.704 & 11.256 & 0.454 & 52.413 & 0.080 & 0.103 & 0.370 & 99.834 \\
\hline SME Simulant & Isolok - $12 \mathrm{~mL}$ & LF & 5 & 3 & 5 & 3 & 21 & LIB01 & 1.030 & 1.704 & 11.121 & 0.456 & 53.269 & 0.084 & 0.104 & 0.369 & 100.597 \\
\hline SME Simulant & Isolok - $12 \mathrm{~mL}$ & LF & 23 & 3 & 10 & 3 & 22 & LIB03 & 1.018 & 1.691 & 11.418 & 0.458 & 53.055 & 0.083 & 0.110 & 0.357 & 100.815 \\
\hline SME Simulant & Isolok - $12 \mathrm{~mL}$ & LF & 50 & 3 & 2 & 3 & 23 & LIB08 & 0.894 & 1.485 & 11.013 & 0.414 & 53.483 & 0.081 & 0.090 & 0.350 & 98.260 \\
\hline SME Simulant & Isolok - $12 \mathrm{~mL}$ & LF & 41 & 3 & 9 & 3 & 24 & $\begin{array}{ll}\text { LIB06 } \\
\end{array}$ & 0.834 & 1.382 & 10.892 & 0.403 & 54.980 & 0.083 & 0.087 & 0.342 & 99.169 \\
\hline SME Simulant & Coliwasa - high & $\mathrm{HF}$ & 34 & 1 & 18 & 3 & 25 & \begin{tabular}{|l|l} 
CH07 \\
\end{tabular} & 0.988 & 1.627 & 10.056 & 0.414 & 49.418 & 0.073 & 0.099 & 0.342 & 93.546 \\
\hline SME Simulant & Coliwasa - high & LF & 63 & 1 & 13 & 3 & 26 & CH04 & 1.061 & 1.756 & 11.377 & 0.468 & 53.269 & 0.086 & 0.106 & 0.374 & 101.371 \\
\hline \begin{tabular}{|l|} 
SME Simulant \\
\end{tabular} & Isolok - $12 \mathrm{~mL}$ & LF & 32 & 3 & 3 & 3 & 27 & LIB05 & 1.020 & 1.691 & 11.215 & 0.456 & 53.269 & 0.082 & 0.105 & 0.361 & 100.471 \\
\hline SME Simulant & Isolok - $12 \mathrm{~mL}$ & LF & 60 & 3 & 6 & 3 & 28 & LIB10 & 0.917 & 1.511 & 10.932 & 0.410 & 52.841 & 0.081 & 0.091 & 0.349 & 97.639 \\
\hline \begin{tabular}{|l|} 
ARG-1 \\
\end{tabular} & None & 0 & 0 & 3 & 23 & 3 & 29 & ARG-1B33 & 0.839 & 1.821 & 11.593 & 1.007 & 48.562 & 1.156 & 0.024 & 0.133 & 98.688 \\
\hline \begin{tabular}{|l|} 
ARG-1 \\
\end{tabular} & None & 0 & 0 & 4 & 1 & 4 & 1 & ARG-1B41 & 0.857 & 1.872 & 11.593 & 1.029 & 48.562 & 1.161 & 0.024 & 0.128 & 99.406 \\
\hline SME Simulant & Isolok - $12 \mathrm{~mL}$ & $\mathrm{HF}$ & 13 & 4 & 11 & 4 & 2 & HIB02 & 1.028 & 1.704 & 10.339 & 0.456 & 53.910 & 0.085 & 0.103 & 0.362 & 100.184 \\
\hline SME Simulant & Coliwasa - low & $\mathrm{HF}$ & 36 & 1 & 21 & 4 & 3 & CL07 & 0.975 & 1.614 & 10.420 & 0.415 & 50.060 & 0.079 & 0.094 & 0.354 & 94.432 \\
\hline \begin{tabular}{|l|l|} 
SME Simulant \\
\end{tabular} & Isolok - $12 \mathrm{~mL}$ & $\mathrm{HF}$ & 25 & 4 & 4 & 4 & 4 & \begin{tabular}{|l|} 
HIB04 \\
\end{tabular} & 0.980 & 1.640 & 10.245 & 0.453 & 52.627 & 0.081 & 0.097 & 0.361 & 98.342 \\
\hline SME Simulant & Coliwasa - high & $\mathrm{HF}$ & 1 & 1 & 22 & 4 & 5 & \begin{tabular}{|l|l|} 
CH05 \\
\end{tabular} & 1.040 & 1.730 & 11.121 & 0.504 & 51.985 & 0.080 & 0.103 & 0.367 & 99.444 \\
\hline SME Simulant & Coliwasa - low & $\mathrm{HF}$ & 3 & 1 & 20 & 4 & 6 & CL05 & 1.036 & 1.717 & 10.690 & 0.447 & 52.841 & 0.083 & 0.101 & 0.369 & 99.890 \\
\hline SME Simulant & Coliwasa - high & $\mathrm{HF}$ & 23 & 1 & 28 & 4 & 7 & CH06 & 1.038 & 1.730 & 10.946 & 0.462 & 52.627 & 0.078 & 0.106 & 0.367 & 99.981 \\
\hline SME Simulant & Coliwasa - high & LF & 1 & 1 & 12 & 4 & 8 & $\mathrm{CH} 01$ & 1.081 & 1.795 & 11.161 & 0.466 & 53.055 & 0.083 & 0.106 & 0.365 & 101.286 \\
\hline SME Simulant & Coliwasa - low & LF & 20 & 1 & 2 & 4 & 9 & CL02 & 1.060 & 1.756 & 10.771 & 0.448 & 51.985 & 0.078 & 0.105 & 0.355 & 98.514 \\
\hline
\end{tabular}


Table C4. Phase 3 Oxide Concentration Measurements (part 2)

\begin{tabular}{|c|c|c|c|c|c|c|c|c|c|c|c|c|c|c|c|c|c|}
\hline $\begin{array}{l}\text { Type of } \\
\text { Material }\end{array}$ & \begin{tabular}{|l|} 
Sampler \\
Details
\end{tabular} & \begin{tabular}{|l|} 
Flow \\
Rate
\end{tabular} & $\begin{array}{r}\text { Sample } \\
\text { Order }\end{array}$ & $\begin{array}{r}\text { Preparation } \\
\text { Block }\end{array}$ & $\begin{array}{r}\text { Preparation } \\
\text { Sequence }\end{array}$ & $\begin{array}{r}\text { Analytical } \\
\text { Block }\end{array}$ & $\begin{array}{r}\text { Analytical } \\
\text { Sequence }\end{array}$ & Sample ID & $\begin{array}{r}\text { MgO } \\
\text { (wt\%) }\end{array}$ & $\begin{array}{r}\text { MnO } \\
\text { (wt\%) }\end{array}$ & $\begin{array}{r}\text { Na2O } \\
\text { (wt\%) }\end{array}$ & $\begin{array}{r}\mathrm{NiO} \\
\text { (wt\%) }\end{array}$ & $\begin{array}{r}\text { SiO2 } \\
\text { (wt\%) }\end{array}$ & $\begin{array}{r}\text { TiO2 } \\
\text { (wt\%) }\end{array}$ & $\begin{array}{r}\mathrm{ZnO} \\
\text { (wt\%) }\end{array}$ & $\begin{array}{r}\text { ZrO2 } \\
\text { (wt\%) }\end{array}$ & $\begin{array}{r}\text { Sum } \\
\text { (wt\%) }\end{array}$ \\
\hline SME Simulant & Coliwasa - low & HF & 63 & 1 & 5 & 4 & 10 & CL08 & 1.035 & 1.730 & 11.013 & 0.461 & 51.985 & 0.074 & 0.104 & 0.363 & 99.066 \\
\hline SME Simulant & Coliwasa - high & $\mathrm{HF}$ & 46 & 4 & 2 & 4 & 11 & HIB07 & 1.031 & 1.704 & 11.633 & 0.464 & 51.557 & 0.080 & 0.102 & 0.357 & 99.145 \\
\hline SME Simulant & Isolok - $12 \mathrm{~mL}$ & LF & 4 & 1 & 10 & 4 & 12 & CL01 & 1.046 & 1.743 & 11.903 & 0.454 & 51.985 & 0.080 & 0.102 & 0.369 & 100.503 \\
\hline SME Simulant & Coliwasa - low & $\mathrm{HF}$ & 18 & 1 & 26 & 4 & 13 & CL06 & 1.055 & 1.756 & 10.164 & 0.456 & 53.055 & 0.077 & 0.104 & $\begin{array}{ll}0.371 \\
\end{array}$ & 99.536 \\
\hline ARG-1 & Coliwasa - low & 0 & 0 & 4 & 7 & 4 & 14 & ARG-1B42 & 0.847 & 1.846 & 11.579 & 1.040 & 48.348 & 1.144 & 0.026 & 0.127 & 98.857 \\
\hline SME Simulant & None & LF & 65 & 1 & 23 & 4 & 15 & CL04 & 1.038 & 1.717 & 11.148 & 0.452 & 51.771 & 0.080 & 0.101 & 0.365 & 98.772 \\
\hline SME Simulant & Coliwasa - low & LF & 40 & 1 & 19 & 4 & 16 & CL03 & 1.046 & 1.743 & 11.256 & 0.467 & 51.771 & 0.079 & 0.105 & 0.367 & 99.331 \\
\hline SME Simulant & Coliwasa - low & LF & 21 & 1 & 14 & 4 & 17 & $\mathrm{CH} 02$ & 1.030 & 1.704 & 11.242 & 0.466 & 51.771 & 0.080 & 0.106 & 0.363 & 99.075 \\
\hline SME Simulant & Coliwasa - high & $\mathrm{HF}$ & 7 & 4 & 12 & 4 & 18 & HIB01 & 1.045 & 1.730 & 10.919 & 0.472 & 53.055 & 0.079 & 0.103 & 0.357 & 100.461 \\
\hline SME Simulant & Isolok - $12 \mathrm{~mL}$ & $\mathrm{HF}$ & 21 & 4 & 6 & 4 & 19 & HIB03 & 1.066 & 1.782 & 11.310 & 0.482 & 52.413 & 0.079 & 0.107 & 0.369 & 100.331 \\
\hline SME Simulant & Coliwasa - high & $\mathrm{HF}$ & 49 & 4 & 5 & 4 & 20 & HIB08 & 1.005 & 1.666 & 10.164 & 0.456 & 48.562 & 0.073 & 0.099 & 0.342 & 92.974 \\
\hline SME Simulant & Isolok - $12 \mathrm{~mL}$ & $\mathrm{HF}$ & 38 & 4 & 8 & 4 & 21 & HIB06 & 0.998 & 1.666 & 10.649 & 0.443 & 51.985 & 0.081 & 0.098 & 0.350 & 97.906 \\
\hline SME Simulant & Isolok - $12 \mathrm{~mL}$ & $\mathrm{HF}$ & 34 & 1 & 18 & 4 & 22 & CH07 & 1.075 & 1.782 & 10.771 & 0.449 & 53.269 & 0.084 & 0.104 & 0.373 & 100.835 \\
\hline SME Simulant & Isolok - $12 \mathrm{~mL}$ & LF & 63 & 1 & 13 & 4 & 23 & CH04 & 1.051 & 1.756 & 11.215 & 0.459 & 52.199 & 0.077 & 0.105 & 0.362 & 99.583 \\
\hline SME Simulant & Isolok - $12 \mathrm{~mL}$ & $\mathrm{HF}$ & 33 & 4 & 10 & 4 & 24 & HIB05 & 0.987 & 1.640 & 11.512 & 0.435 & 52.841 & 0.080 & 0.098 & 0.351 & 99.452 \\
\hline SME Simulant & Coliwasa - high & $\mathrm{HF}$ & 58 & 4 & 3 & 4 & 25 & HIB10 & 0.940 & 1.562 & 10.717 & 0.450 & 53.483 & 0.081 & 0.096 & 0.342 & 98.680 \\
\hline \begin{tabular}{|l|} 
ARG-1 \\
\end{tabular} & Coliwasa - high & 0 & 0 & 4 & 13 & 4 & 26 & ARG-1B43 & 0.842 & 1.834 & 11.458 & 1.007 & 48.134 & 1.136 & 0.025 & 0.128 & 98.184 \\
\hline SME Simulant & Isolok - $12 \mathrm{~mL}$ & $\mathrm{HF}$ & 65 & 1 & 6 & 4 & 27 & CH08 & 1.048 & 1.743 & 10.986 & 0.463 & 51.985 & 0.078 & 0.105 & 0.353 & 99.446 \\
\hline SME Simulant & Isolok - $12 \mathrm{~mL}$ & LF & 38 & 1 & 16 & 4 & 28 & CH03 & 1.050 & 1.730 & 10.946 & 0.457 & 52.841 & 0.076 & 0.103 & 0.367 & 99.988 \\
\hline SME Simulant & None & $\mathrm{HF}$ & 56 & 4 & 9 & 4 & 29 & \begin{tabular}{|l|} 
HIB09 \\
\end{tabular} & 1.156 & 1.924 & 11.391 & 0.536 & 49.846 & 0.078 & 0.123 & 0.362 & 99.655 \\
\hline ARG-1 & \begin{tabular}{|l|} 
None \\
\end{tabular} & 0 & 0 & 2 & 1 & 5 & 1 & \begin{tabular}{|l|} 
ARG-1B21 \\
\end{tabular} & 0.866 & 1.885 & 11.391 & 1.029 & 48.348 & 1.151 & 0.025 & 0.131 & 99.089 \\
\hline SME Simulant & Isolok - $3 \mathrm{~mL}$ & $\mathrm{HF}$ & 20 & 2 & 16 & 5 & 2 & \begin{tabular}{|l|} 
HIS03 \\
\end{tabular} & 1.046 & 1.743 & 10.878 & 0.444 & 53.055 & 0.088 & 0.097 & 0.369 & 100.201 \\
\hline SME Simulant & Isolok - $3 \mathrm{~mL}$ & $\mathrm{HF}$ & 9 & 2 & 21 & 5 & 3 & HIS01 & 1.068 & 1.769 & 12.051 & 0.489 & 52.413 & 0.084 & 0.108 & 0.370 & 101.654 \\
\hline SME Simulant & Coliwasa - low & LF & 20 & 1 & 2 & 5 & 4 & CL02 & 1.090 & 1.795 & 10.690 & 0.449 & 52.841 & 0.084 & 0.104 & 0.361 & 100.334 \\
\hline SME Simulant & Coliwasa - high & LF & 38 & 1 & 16 & 5 & 5 & $\mathrm{CH} 03$ & 1.061 & 1.756 & 10.744 & 0.443 & 53.055 & 0.084 & 0.099 & 0.371 & 99.980 \\
\hline SME Simulant & Coliwasa - high & LF & 63 & 1 & 13 & 5 & 6 & CH04 & 1.070 & 1.782 & 11.013 & 0.447 & 52.413 & 0.080 & 0.101 & 0.369 & 100.030 \\
\hline SME Simulant & Isolok - 3 mL & $\mathrm{HF}$ & 44 & 2 & 18 & 5 & 7 & \begin{tabular}{|l|} 
HIS07 \\
\end{tabular} & 1.058 & 1.756 & 11.134 & 0.458 & 53.269 & 0.083 & 0.102 & 0.392 & 101.178 \\
\hline SME Simulant & Coliwasa - high & $\mathrm{HF}$ & 23 & 1 & 28 & 5 & 8 & CH06 & 1.060 & 1.756 & 10.905 & 0.448 & 53.055 & 0.083 & 0.099 & 0.373 & 100.612 \\
\hline SME Simulant & Coliwasa - high & LF & 21 & 1 & 14 & 5 & 9 & $\mathrm{CH} 02$ & 1.061 & 1.769 & 11.323 & 0.448 & 52.627 & 0.080 & 0.099 & 0.365 & 100.707 \\
\hline SME Simulant & Coliwasa - low & $\mathrm{HF}$ & 3 & 1 & 20 & 5 & 10 & CL05 & 1.058 & 1.743 & 10.622 & 0.439 & 53.269 & 0.082 & 0.102 & 0.367 & 99.926 \\
\hline SME Simulant & Isolok - $3 \mathrm{~mL}$ & $\mathrm{HF}$ & 14 & 2 & 15 & 5 & 11 & HISO2 & 1.033 & 1.717 & 11.660 & 0.438 & 52.841 & 0.079 & 0.098 & 0.365 & 100.000 \\
\hline SME Simulant & Coliwasa - low & LF & 40 & 1 & 19 & 5 & 12 & CL03 & 1.073 & 1.769 & 10.811 & 0.459 & 52.199 & 0.081 & 0.101 & 0.369 & 99.683 \\
\hline SME Simulant & Coliwasa - low & $\mathrm{HF}$ & 18 & 1 & 26 & 5 & 13 & CL06 & 1.071 & 1.782 & 10.002 & 0.461 & 53.055 & 0.081 & 0.102 & 0.371 & 99.751 \\
\hline SME Simulant & Coliwasa - high & $\mathrm{HF}$ & 1 & 1 & 22 & 5 & 14 & \begin{tabular}{|l|l|} 
CH05 \\
\end{tabular} & 1.068 & 1.769 & 11.148 & 0.513 & 52.413 & 0.081 & 0.109 & 0.365 & 100.463 \\
\hline ARG-1 & None & 0 & 0 & 2 & 12 & 5 & 15 & \begin{tabular}{|l} 
ARG-1B22 \\
\end{tabular} & 0.872 & 1.885 & 11.876 & 1.049 & 48.990 & 1.156 & 0.025 & 0.132 & 101.077 \\
\hline SME Simulant & Coliwasa - low & $\mathrm{HF}$ & 36 & 1 & 21 & 5 & 16 & \begin{tabular}{|l} 
CL07 \\
\end{tabular} & 1.045 & 1.730 & 10.838 & 0.434 & 52.841 & 0.083 & 0.098 & 0.369 & 99.759 \\
\hline \begin{tabular}{|l|} 
SME Simulant \\
\end{tabular} & Isolok - $3 \mathrm{~mL}$ & $\mathrm{HF}$ & 31 & 2 & 19 & 5 & 17 & HIS05 & 1.056 & 1.743 & 10.892 & 0.440 & 53.055 & $\begin{array}{l}0.087 \\
\end{array}$ & 0.101 & 0.374 & 100.443 \\
\hline SME Simulant & Isolok - $3 \mathrm{~mL}$ & $\mathrm{HF}$ & 60 & 2 & 22 & 5 & 18 & HIS10 & 1.043 & 1.717 & 11.094 & 0.448 & 53.269 & 0.086 & 0.101 & 0.357 & 100.499 \\
\hline SME Simulant & Coliwasa - low & $\mathrm{HF}$ & 63 & 1 & 5 & 5 & 19 & CL08 & 1.046 & 1.756 & 10.771 & 0.461 & 51.771 & 0.084 & 0.101 & 0.373 & 99.167 \\
\hline SME Simulant & Isolok - 3 mL & HF & 26 & 2 & 14 & 5 & 20 & HIS04 & 1.017 & 1.691 & 10.744 & 0.435 & 53.269 & 0.084 & 0.097 & 0.367 & 99.772 \\
\hline SME Simulant & Isolok - $3 \mathrm{~mL}$ & $\mathrm{HF}$ & 35 & 2 & 20 & 5 & 21 & HIS06 & 1.041 & 1.743 & 11.148 & 0.450 & 52.199 & 0.088 & 0.099 & 0.376 & 99.991 \\
\hline SME Simulant & Coliwasa - high & $\mathrm{HF}$ & 65 & 1 & 6 & 5 & 22 & CH08 & 1.048 & 1.743 & 10.744 & 0.452 & 51.557 & 0.087 & 0.099 & 0.365 & 98.890 \\
\hline \begin{tabular}{|l|l|} 
SME Simulant \\
\end{tabular} & Coliwasa - high & $\mathrm{HF}$ & 34 & 1 & 18 & 5 & 23 & CH07 & 1.070 & 1.782 & 10.353 & 0.457 & 52.413 & 0.089 & 0.104 & 0.377 & 99.804 \\
\hline SME Simulant & Coliwasa - high & LF & 1 & 1 & 12 & 5 & 24 & $\mathrm{CH} 01$ & 1.076 & 1.795 & 10.784 & 0.449 & 52.199 & 0.087 & 0.101 & 0.367 & 99.892 \\
\hline SME Simulant & Isolok - $3 \mathrm{~mL}$ & $\mathrm{HF}$ & 54 & 2 & 17 & 5 & 25 & HIS09 & 1.002 & 1.666 & 10.568 & 0.426 & 53.696 & 0.085 & 0.094 & 0.369 & 100.003 \\
\hline SME Simulant & Coliwasa - low & LF & 4 & 1 & 10 & 5 & 26 & CL01 & 1.048 & 1.743 & 11.566 & 0.457 & 51.129 & 0.083 & 0.098 & 0.369 & 99.483 \\
\hline SME Simulant & Isolok - $3 \mathrm{~mL}$ & $\mathrm{HF}$ & 48 & 2 & 13 & 5 & 27 & HIS08 & 1.038 & 1.730 & 10.865 & 0.429 & 52.841 & 0.086 & 0.094 & 0.378 & 99.754 \\
\hline SME Simulant & Coliwasa - low & LF & 65 & 1 & 23 & 5 & 28 & CL04 & 1.055 & 1.756 & 11.027 & 0.440 & 51.985 & 0.085 & 0.095 & 0.376 & 99.456 \\
\hline
\end{tabular}


Table C4. Phase 3 Oxide Concentration Measurements (part 2)

\begin{tabular}{|c|c|c|c|c|c|c|c|c|c|c|c|c|c|c|c|c|c|}
\hline $\begin{array}{l}\text { Type of } \\
\text { Material }\end{array}$ & \begin{tabular}{|l|} 
Sampler \\
Details
\end{tabular} & $\begin{array}{l}\text { Flow } \\
\text { Rate }\end{array}$ & $\begin{array}{r}\text { Sample } \\
\text { Order } \\
\end{array}$ & $\begin{array}{r}\text { Preparation } \\
\text { Block } \\
\end{array}$ & $\begin{array}{r}\text { Preparation } \\
\text { Sequence } \\
\end{array}$ & $\begin{array}{r}\text { Analytical } \\
\text { Block } \\
\end{array}$ & $\begin{array}{r}\text { Analytical } \\
\text { Sequence } \\
\end{array}$ & Sample ID & $\begin{array}{r}\text { MgO } \\
(w t \%)\end{array}$ & $\begin{array}{r}\mathrm{MnO} \\
(\mathrm{wt} \%) \\
\end{array}$ & $\begin{array}{r}\text { Na2O } \\
(w t \%)\end{array}$ & $\begin{array}{r}\mathrm{NiO} \\
\text { (wt\%) }\end{array}$ & $\begin{array}{r}\mathrm{SiO2} \\
(\mathrm{wt} \%) \\
\end{array}$ & $\begin{array}{r}\text { TiO2 } \\
\text { (wt\%) } \\
\end{array}$ & $\begin{array}{r}\mathrm{ZnO} \\
(\mathrm{wt} \%) \\
\end{array}$ & $\begin{array}{r}\mathrm{ZrO2} \\
(\mathrm{wt} \%) \\
\end{array}$ & $\begin{array}{r}\text { Sum } \\
\text { (wt\%) }\end{array}$ \\
\hline ARG-1 & None & 0 & 0 & 2 & 23 & 5 & 29 & ARG-1B23 & 0.866 & 1.885 & 11.377 & 1.050 & 48.562 & 1.163 & $\begin{array}{r}0.023 \\
\end{array}$ & 0.129 & 100.452 \\
\hline ARG-1 & None & 0 & 0 & 3 & 1 & 6 & 1 & ARG-1B31 & 0.839 & 1.821 & 11.539 & 1.052 & 48.776 & 1.164 & 0.025 & 0.127 & 99.712 \\
\hline SME Simulant & Hydragard & LF & 15 & 3 & 21 & 6 & 2 & LHY03 & 1.008 & 1.666 & 11.283 & 0.448 & 50.701 & 0.078 & 0.100 & 0.357 & 97.573 \\
\hline SME Simulant & \begin{tabular}{|l|} 
Hydragard \\
\end{tabular} & LF & 6 & 3 & 18 & 6 & 3 & LHY01 & 1.033 & 1.717 & 11.566 & 0.443 & 50.701 & 0.080 & 0.101 & 0.366 & 98.698 \\
\hline SME Simulant & \begin{tabular}{|l|} 
Coliwasa \\
\end{tabular} & LF & 20 & 1 & 2 & 6 & 4 & CL02 & 1.045 & 1.730 & 10.771 & 0.464 & 52.841 & 0.085 & 0.106 & 0.377 & 99.972 \\
\hline SME Simulant & Coliwasa & LF & 38 & 1 & 16 & 6 & 5 & $\mathrm{CH} 03$ & 1.023 & 1.691 & 10.797 & 0.449 & 52.841 & 0.084 & 0.099 & 0.374 & 99.508 \\
\hline SME Simulant & \begin{tabular}{|l|} 
Coliwasa \\
\end{tabular} & LF & 63 & 1 & 13 & 6 & 6 & CH04 & 1.025 & 1.704 & 11.013 & 0.450 & 52.199 & 0.083 & 0.100 & 0.373 & 99.176 \\
\hline SME Simulant & Hydragard & LF & 47 & 3 & 13 & 6 & 7 & LHY07 & 1.026 & 1.717 & 11.161 & 0.487 & 50.915 & 0.081 & 0.106 & 0.346 & 98.304 \\
\hline SME Simulant & \begin{tabular}{|l|} 
Coliwasa \\
\end{tabular} & $\mathrm{HF}$ & 23 & 1 & 28 & 6 & 8 & CH06 & 1.013 & 1.679 & 10.878 & 0.456 & 52.841 & 0.081 & 0.101 & 0.374 & 99.861 \\
\hline SME Simulant & Coliwasa & LF & 21 & 1 & 14 & 6 & 9 & $\mathrm{CH} 02$ & 1.012 & 1.691 & 11.161 & 0.448 & 52.199 & 0.081 & 0.101 & 0.370 & 99.436 \\
\hline SME Simulant & \begin{tabular}{|l|} 
Coliwasa \\
\end{tabular} & $\mathrm{HF}$ & 3 & 1 & 20 & 6 & 10 & CL05 & 1.015 & 1.679 & 10.541 & 0.430 & 52.841 & 0.082 & 0.097 & 0.367 & 99.019 \\
\hline SME Simulant & Hydragard & LF & 10 & 3 & 20 & 6 & 11 & LHY02 & 1.086 & 1.795 & 11.525 & 0.467 & 49.846 & 0.079 & 0.112 & 0.371 & 98.674 \\
\hline SME Simulant & \begin{tabular}{|l|} 
Coliwasa \\
\end{tabular} & LF & 40 & 1 & 19 & 6 & 12 & CL03 & 1.020 & 1.691 & 11.108 & 0.421 & 51.771 & 0.084 & 0.094 & 0.378 & 98.690 \\
\hline SME Simulant & Coliwasa & $\mathrm{HF}$ & 18 & 1 & 26 & 6 & 13 & CL06 & 1.031 & 1.704 & 10.016 & 0.445 & 53.055 & $\begin{array}{ll}0.083 \\
\end{array}$ & 0.102 & 0.376 & 99.001 \\
\hline SME Simulant & Coliwasa & $\mathrm{HF}$ & 1 & 1 & 22 & 6 & 14 & CH05 & 1.026 & 1.704 & 11.148 & 0.519 & 52.413 & 0.084 & 0.103 & 0.367 & 99.879 \\
\hline \begin{tabular}{|l|} 
ARG-1 \\
\end{tabular} & None & 0 & 0 & 3 & 12 & 6 & 15 & ARG-1B32 & 0.837 & 1.821 & 11.579 & 1.084 & 48.990 & 1.169 & 0.024 & 0.136 & 100.321 \\
\hline SME Simulant & \begin{tabular}{|l|} 
Coliwasa \\
\end{tabular} & $\mathrm{HF}$ & 36 & 1 & 21 & 6 & 16 & CL07 & 1.008 & 1.666 & 10.959 & 0.433 & 53.055 & 0.085 & 0.100 & 0.371 & 99.694 \\
\hline SME Simulant & Hydragard & LF & 30 & 3 & 14 & 6 & 17 & LHY05 & 0.968 & 1.627 & 10.703 & 0.445 & 53.483 & 0.087 & 0.095 & 0.359 & 99.586 \\
\hline SME Simulant & Hydragard & LF & 59 & 3 & 17 & 6 & 18 & LHY10 & 1.441 & 2.363 & 14.019 & 0.662 & 69.099 & 0.111 & 0.142 & 0.457 & 132.998 \\
\hline SME Simulant & \begin{tabular}{|l|} 
Coliwasa \\
\end{tabular} & $\mathrm{HF}$ & 63 & 1 & 5 & 6 & 19 & $\begin{array}{l}\text { CL08 } \\
\end{array}$ & 1.010 & 1.691 & 10.865 & 0.459 & 51.771 & 0.084 & 0.100 & 0.380 & 98.760 \\
\hline SME Simulant & Hydragard & LF & 28 & 3 & 16 & 6 & 20 & LHY04 & 1.055 & 1.756 & 10.986 & 0.458 & 50.060 & 0.083 & 0.101 & 0.366 & 97.937 \\
\hline SME Simulant & Hydragard & LF & 37 & 3 & 15 & 6 & 21 & LHY06 & 1.045 & 1.730 & 11.134 & 0.484 & 51.343 & 0.081 & 0.111 & 0.369 & 99.192 \\
\hline SME Simulant & \begin{tabular}{|l|} 
Coliwasa \\
\end{tabular} & $\mathrm{HF}$ & 65 & 1 & 6 & 6 & 22 & \begin{tabular}{|l|l|} 
CH088 \\
\end{tabular} & 1.012 & 1.679 & 10.932 & 0.435 & 51.771 & $\begin{array}{ll}0.085 \\
\end{array}$ & 0.095 & 0.365 & 98.661 \\
\hline SME Simulant & Coliwasa & $\mathrm{HF}$ & 34 & 1 & 18 & 6 & 23 & CH07 & 1.036 & 1.717 & 10.582 & 0.445 & 52.841 & 0.082 & 0.103 & 0.377 & 99.704 \\
\hline SME Simulant & \begin{tabular}{|l|} 
Coliwasa \\
\end{tabular} & LF & 1 & 1 & 12 & 6 & 24 & CH01 & 1.038 & 1.730 & 10.973 & 0.454 & 52.199 & 0.081 & 0.105 & 0.369 & 99.435 \\
\hline SME Simulant & Hydragard & LF & 53 & 3 & 19 & 6 & 25 & LHY09 & 0.993 & 1.640 & 11.849 & $\begin{array}{ll}0.454 \\
\end{array}$ & 51.557 & 0.080 & 0.100 & 0.332 & 98.810 \\
\hline SME Simulant & \begin{tabular}{|l|} 
Coliwasa \\
\end{tabular} & LF & 4 & 1 & 10 & 6 & 26 & CL01 & 1.015 & $\begin{array}{ll}1.691 \\
\end{array}$ & 11.768 & 0.454 & 51.343 & 0.079 & 0.100 & 0.370 & 99.335 \\
\hline SME Simulant & Hydragard & LF & 48 & 3 & 22 & 6 & 27 & LHY08 & 1.103 & 1.769 & 11.121 & 0.452 & 52.199 & 0.082 & 0.103 & 0.381 & 100.031 \\
\hline SME Simulant & \begin{tabular}{|l|} 
Coliwasa \\
\end{tabular} & LF & 65 & 1 & 23 & 6 & 28 & CL04 & 1.025 & 1.704 & 11.323 & 0.463 & 52.199 & 0.079 & 0.104 & 0.377 & 99.689 \\
\hline \begin{tabular}{|l|} 
ARG-1 \\
\end{tabular} & None & 0 & 0 & 3 & 23 & 6 & 29 & ARG-1B33 & 0.836 & 1.808 & 11.647 & 1.069 & 48.562 & 1.163 & 0.024 & 0.128 & 99.437 \\
\hline
\end{tabular}


Table C5. Reference Calcine Oxide Concentrations for Low-Rheology SME Simulant (Phase 3 Testing)

\begin{tabular}{|c|c|}
\hline Oxide & Targeted wt \% \\
\hline $\mathrm{Al} 2 \mathrm{O} 3$ (wt\%) & 10.869 \\
\hline B2O3 (wt\%) & 4.259 \\
\hline $\mathrm{BaO}(\mathrm{wt} \%)$ & 0.0919 \\
\hline $\mathrm{CaO}(\mathrm{wt} \%)$ & 1.1462 \\
\hline Cr2O3 (wt\%) & 0.0998 \\
\hline $\mathrm{CuO}(\mathrm{wt} \%)$ & 0.0504 \\
\hline $\mathrm{Fe} 2 \mathrm{O} 3(\mathrm{wt} \%)$ & 11.462 \\
\hline K2O (wt\%) & 0.1591 \\
\hline Li2O (wt\%) & 4.674 \\
\hline $\mathrm{MgO}(\mathrm{wt} \%)$ & 1.014 \\
\hline $\mathrm{MnO}(\mathrm{wt} \%)$ & 1.779 \\
\hline $\mathrm{Na} 2 \mathrm{O}(\mathrm{wt} \%)$ & 11.659 \\
\hline $\mathrm{NiO}(\mathrm{wt} \%)$ & 0.41 \\
\hline $\mathrm{SiO} 2(\mathrm{wt} \%)$ & 50.985 \\
\hline TiO2 (wt\%) & 0.0711 \\
\hline $\mathrm{ZnO}(\mathrm{wt} \%)$ & 0.0958 \\
\hline ZrO2 (wt\%) & 0.3547 \\
\hline Sum of Oxides (wt\%) & 99.553 \\
\hline
\end{tabular}

Shaded rows indicate oxides that are at reportable concentrations and that are considered critical in the comparisons conducted as part of this study. 


\section{Table C6. Comparisons for Density and Solids from Low-Rheology (Phase 3) Testing}

Density Measurements for Low Rheology Simulant

\begin{tabular}{|c|c|c|c|c|c|c|c|c|c|c|c|c|}
\hline & & & & & & & & Statistically & $\mathrm{g} / \mathrm{mL}$ & $\%$ of BL & & \\
\hline & Sampler Mean & BL Mean & Difference & Difference & Statistically & & & Different & Bound on & Bound on & & \\
\hline Comparisons & $(\mathrm{g} / \mathrm{mL})$ & $(\mathrm{g} / \mathrm{mL})$ & $(\mathrm{g} / \mathrm{mL})$ & (\%) & Significant Bias & Sampler Std Dev & BL Std Dev & Variances & Bias (95\%) & Bias (95\%) & $\mathrm{n}$ sampler & $\mathrm{nBL}$ \\
\hline Coliwasa High Location vs Coliwasa Low Location (BL) & 1.2505 & 1.2591 & -0.009 & $-0.7 \%$ & no & 0.01080 & 0.00888 & \begin{tabular}{|l|} 
no \\
\end{tabular} & \begin{tabular}{|c|c|}
0.0193 \\
\end{tabular} & $1.5 \%$ & 8 & 8 \\
\hline Hydragard (LF) vs Coliwasa & 1.2501 & 1.2548 & -0.005 & $-0.4 \%$ & no & 0.00997 & 0.01057 & no & 0.0133 & $1.1 \%$ & 10 & 16 \\
\hline Isolok - $12 \mathrm{~mL}$ (LF) vs Coliwasa & 1.2571 & 1.2548 & 0.002 & $0.2 \%$ & no & 0.00985 & 0.01057 & no & 0.0191 & $1.5 \%$ & 10 & 16 \\
\hline Isolok - $12 \mathrm{~mL}(\mathrm{HF})$ vs Coliwasa & 1.2503 & 1.2548 & -0.004 & $-0.4 \%$ & no & 0.00697 & 0.01057 & yes & 0.0116 & $0.9 \%$ & 10 & 16 \\
\hline
\end{tabular}

Weight Percent (wt\%) Dried Solids for Low Rheology Simulant

\begin{tabular}{|c|c|c|c|c|c|c|c|c|c|c|c|c|}
\hline & & & & & & & & & & & & \\
\hline & & & & & & & & Statistically & wt\% & $\%$ of BL & & \\
\hline & Sampler Mean & BL Mean & Difference & Difference & Statistically & & & Different & Bound on & Bound on & & \\
\hline Comparisons & $(\mathrm{wt} \%)$ & $(\mathrm{wt} \%)$ & (wt\%) & $(\%)$ & Significant Bias & Sampler Std Dev & BL Std Dev & Variances & Bias $(95 \%)$ & Bias (95\%) & n sampler & $\mathrm{nBL}$ \\
\hline Coliwasa High Location vs Coliwasa Low Location (BL) & 31.545 & 31.593 & -0.047 & $-0.2 \%$ & no & 0.09813 & 0.11889 & no & 0.1644 & $0.5 \%$ & 8 & 8 \\
\hline Hydragard (LF) vs Coliwasa & 30.866 & 31.569 & -0.703 & $-2.2 \%$ & yes & 0.10906 & 0.10813 & no & 0.7930 & $2.5 \%$ & 10 & 16 \\
\hline Hydragard (HF) vs Coliwasa & 30.751 & 31.569 & -0.818 & $-2.6 \%$ & yes & 0.11474 & 0.10813 & no & 0.9098 & $2.9 \%$ & 10 & 16 \\
\hline Isolok - 12mL (LF) vs Coliwasa & 31.585 & 31.569 & 0.016 & $0.1 \%$ & no & 0.12651 & 0.10813 & no & 0.1122 & $0.4 \%$ & 10 & 16 \\
\hline Isolok - $12 \mathrm{~mL}$ (HF) vs Coliwasa & 31.547 & 31.569 & -0.022 & $-0.1 \%$ & no & 0.09381 & 0.10813 & no & 0.1074 & $0.3 \%$ & 10 & 16 \\
\hline Isolok - $3 \mathrm{~mL}$ (LF) vs Coliwasa & 31.617 & 31.569 & 0.048 & $0.2 \%$ & no & 0.13953 & 0.10813 & no & 0.1488 & $0.5 \%$ & 10 & 16 \\
\hline Isolok - $3 \mathrm{~mL}$ (HF) vs Coliwasa & 31.582 & 31.569 & 0.013 & $0.0 \%$ & no & 0.20176 & 0.10813 & no & 0.1383 & $0.4 \%$ & 10 & 16 \\
\hline
\end{tabular}

Wt\% Vitrified Solids for Low Rheology Simulant

\begin{tabular}{|c|c|c|c|c|c|c|c|c|c|c|c|c|}
\hline & & & & & & & & Statistically & wt\% & $\%$ of BL & & \\
\hline & Sampler Mean & BL Mean & Difference & Difference & Statistically & & & Different & Bound on & Bound on & & \\
\hline Comparisons & $(w t \%)$ & $(w t \%)$ & $(w t \%)$ & $(\%)$ & Significant Bias & Sampler Std Dev I & BL Std Dev & Variances & Bias $(95 \%)$ & Bias $(95 \%)$ & n sampler & $\mathrm{nBL}$ \\
\hline Coliwasa High Location vs Coliwasa Low Location (BL) & 26.750 & 26.798 & -0.047 & $-0.2 \%$ & no & \begin{tabular}{|l|l|}
0.09798 \\
\end{tabular} & 0.12803 & no & 0.1698 & $0.6 \%$ & \begin{tabular}{|l|l|}
8 \\
\end{tabular} & 8 \\
\hline Hydragard (LF) vs Coliwasa & 25.980 & 26.774 & -0.794 & $-3.0 \%$ & yes & 0.11738 & 0.11283 & no & 0.8891 & $3.3 \%$ & 10 & 16 \\
\hline Hydragard (HF) vs Coliwasa & 25.908 & 26.774 & -0.866 & $-3.2 \%$ & yes & 0.14109 & 0.11283 & no & 0.9691 & $3.6 \%$ & 10 & 16 \\
\hline Isolok - 12mL (LF) vs Coliwasa & 26.757 & 26.774 & -0.017 & $-0.1 \%$ & no & 0.09440 & 0.11283 & no & 0.1052 & $0.4 \%$ & 10 & 16 \\
\hline Isolok - 12mL (LF) scrnd vs Coliwasa & 26.780 & 26.774 & 0.006 & $0.0 \%$ & no & 0.06384 & 0.11283 & no & 0.0912 & $0.3 \%$ & 9 & 16 \\
\hline Isolok - 12mL (HF) vs Coliwasa & 26.766 & 26.774 & -0.008 & $0.0 \%$ & no & 0.08579 & 0.11283 & no & 0.0939 & $0.4 \%$ & 10 & 16 \\
\hline Isolok - $3 \mathrm{~mL}$ (LF) vs Coliwasa & 26.835 & 26.774 & 0.061 & $0.2 \%$ & no & 0.12886 & 0.11283 & no & 0.1603 & $0.6 \%$ & 10 & 16 \\
\hline Isolok - $3 \mathrm{~mL}(\mathrm{HF})$ vs Coliwasa & 26.939 & 26.774 & 0.165 & $0.6 \%$ & yes & 0.20556 & 0.11283 & yes & 0.3190 & $1.2 \%$ & 10 & 16 \\
\hline
\end{tabular}


Table C7. Oxide Comparisons between the Low and High Coliwasa Samples for the Low-Rheology (Phase 3) Testing

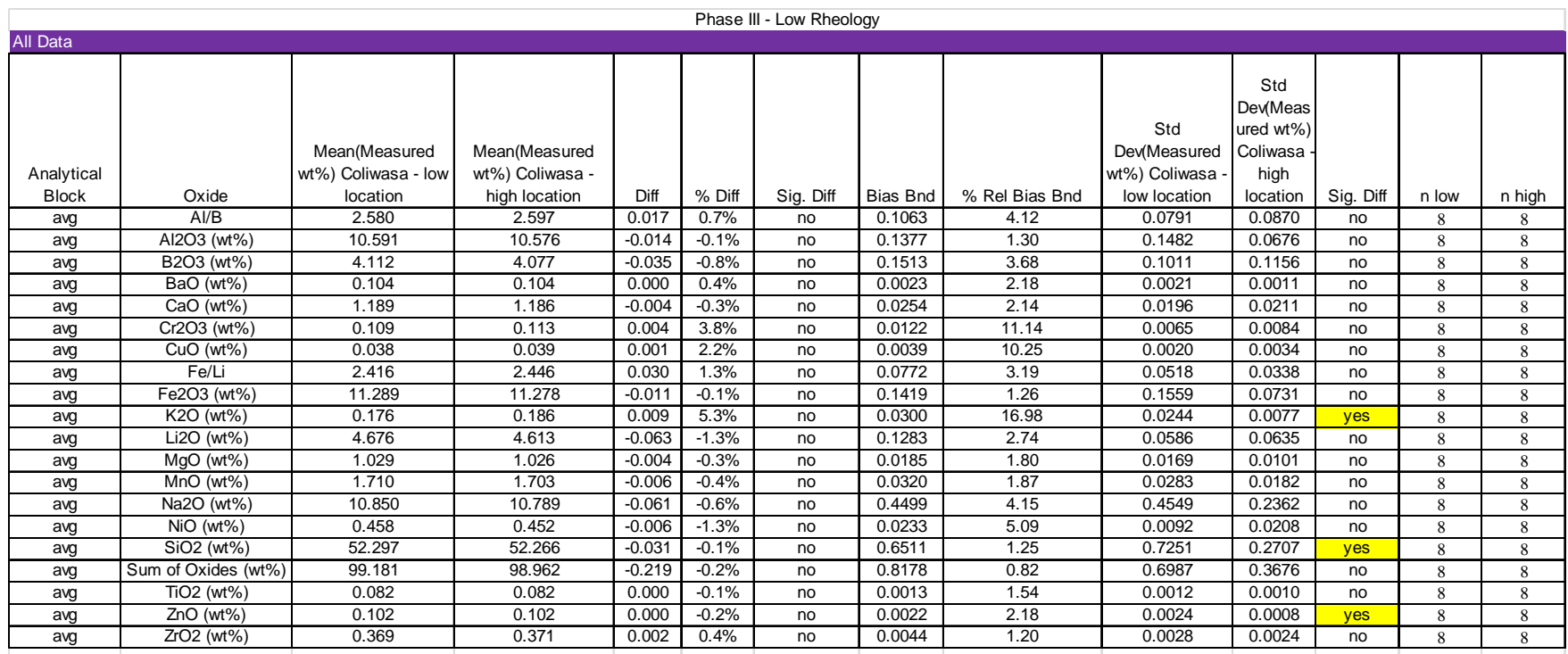

Phase III - Low Rheology

\begin{tabular}{|c|c|c|c|c|c|c|c|c|c|c|c|c|c|}
\hline \\
\hline $\begin{array}{l}\text { Analytical } \\
\text { Block }\end{array}$ & Oxide & \begin{tabular}{|} 
Mean(Measured \\
wt\%) Coliwasa - low \\
location
\end{tabular} & $\begin{array}{l}\text { Mean(Measured } \\
\text { wt\%) Coliwasa - } \\
\text { high location }\end{array}$ & Diff & $\%$ Diff & Sig. Diff & Bias Bnd & $\%$ Rel Bias Bnd & $\begin{array}{c}\text { Std } \\
\text { Dev(Measured } \\
\text { wt\%) Coliwasa } \\
\text { low location }\end{array}$ & $\begin{array}{c}\text { Std } \\
\text { Dev(Meas } \\
\text { ured wt\%) } \\
\text { Coliwasa } \\
\text { high } \\
\text { location }\end{array}$ & Siq. Diff & n low & $n$ high \\
\hline avg & $\mathrm{Al} / \mathrm{B}$ & \begin{tabular}{|c|}
2.597 \\
\end{tabular} & 2.580 & -0.017 & $-0.7 \%$ & no & \begin{tabular}{|l|}
0.1063 \\
\end{tabular} & 4.09 & 0.0791 & \begin{tabular}{|l|}
0.0870 \\
\end{tabular} & no & 8 & 8 \\
\hline avg & Al2O3 (wt\%) & 10.576 & 10.591 & 0.014 & $0.1 \%$ & no & 0.1377 & 1.30 & 0.1482 & 0.0676 & no & 8 & 8 \\
\hline avg & $\mathrm{B} 2 \mathrm{O} 3(\mathrm{wt} \%)$ & 4.077 & 4.112 & 0.035 & $0.9 \%$ & no & 0.1513 & 3.71 & 0.1011 & 0.1156 & no & $\frac{0}{8}$ & $\frac{0}{8}$ \\
\hline avg & $\mathrm{BaO}(\mathrm{wt} \%)$ & 0.104 & 0.104 & 0.000 & $-0.4 \%$ & no & 0.0023 & 2.17 & 0.0021 & 0.0011 & no & $\frac{8}{8}$ & 8 \\
\hline avg & $\mathrm{CaO}(\mathrm{wt} \%)$ & 1.186 & 1.189 & 0.004 & $0.3 \%$ & no & 0.0254 & 2.14 & 0.0196 & 0.0211 & no & 8 & 8 \\
\hline avg & $\mathrm{Cr} 2 \mathrm{O} 3(\mathrm{wt} \%)$ & 0.113 & 0.106 & -0.007 & $-6.2 \%$ & yes & 0.0125 & 11.04 & 0.0065 & 0.0017 & yes & 8 & 7 \\
\hline avg & $\mathrm{CuO}(\mathrm{wt} \%)$ & 0.039 & 0.038 & -0.001 & $-2.2 \%$ & no & 0.0039 & 10.02 & 0.0020 & 0.0034 & no & 8 & 8 \\
\hline avg & $\mathrm{Fe} / \mathrm{Li}$ & 2.446 & 2.416 & -0.030 & $-1.2 \%$ & no & 0.0772 & 3.15 & 0.0518 & 0.0338 & no & 8 & 8 \\
\hline$\frac{a v g}{\text { avg }}$ & $\mathrm{Fe} 2 \mathrm{O} 3(\mathrm{wt} \%)$ & 11.330 & $\frac{2.409}{11.289}$ & -0.041 & $-0.4 \%$ & no & 0.1121 & 0.99 & 0.0493 & 0.0731 & no & $\frac{0}{7}$ & $\frac{0}{8}$ \\
\hline avg & $\mathrm{K} 2 \mathrm{O}(\mathrm{wt} \%)$ & 0.186 & 0.176 & -0.009 & $-5.0 \%$ & no & 0.0300 & 16.13 & 0.0244 & 0.0077 & yes & 8 & 8 \\
\hline avg & $\mathrm{Li} 2 \mathrm{O}(\mathrm{wt} \%)$ & 4.595 & 4.676 & 0.081 & $1.8 \%$ & yes & 0.1376 & 3.00 & 0.0280 & 0.0635 & no & 7 & 8 \\
\hline avg & $\mathrm{MgO}(\mathrm{wt} \%)$ & 1.026 & 1.029 & 0.004 & $0.4 \%$ & $\frac{y_{05}}{\text { no }}$ & 0.0185 & $\frac{1.00}{1.80}$ & 0.0169 & 0.0101 & no & $\frac{1}{8}$ & $\frac{0}{8}$ \\
\hline avg & $\mathrm{MnO}(\mathrm{wt} \%)$ & 1.711 & 1.710 & -0.002 & $-0.4 \%$ & no & $\begin{array}{l}0.0218 \\
0.0218\end{array}$ & 1.28 & 0.0178 & 0.0182 & no & $\frac{8}{7}$ & 8 \\
\hline avg & $\mathrm{Na} 2 \mathrm{O}(\mathrm{wt} \%)$ & 10.789 & 10.850 & 0.061 & $0.6 \%$ & no & 0.4499 & 4.17 & 0.4549 & 0.2362 & no & 8 & 8 \\
\hline avg & $\mathrm{NiO}(\mathrm{wt} \%)$ & 0.452 & 0.451 & -0.001 & $-0.3 \%$ & no & 0.0093 & 2.06 & 0.0092 & 0.0041 & no & 8 & 7 \\
\hline avg & $\mathrm{SiO} 2(\mathrm{wt} \%)$ & 52.266 & 52.219 & -0.046 & $-0.1 \%$ & no & 0.6577 & 1.26 & 0.7251 & 0.1708 & yes & 8 & 7 \\
\hline avg & Sum of Oxides (wt\%) & 98.962 & 99.181 & 0.219 & $0.2 \%$ & no & 0.8178 & 0.83 & 0.6987 & 0.3676 & no & 8 & 8 \\
\hline avg & \begin{tabular}{|l|}
$\mathrm{TiO} 2(\mathrm{wt} \%)$ \\
\end{tabular} & 0.082 & 0.082 & 0.000 & $0.4 \%$ & no & 0.0015 & 1.80 & 0.0012 & 0.0007 & no & $\frac{0}{8}$ & $\frac{0}{7}$ \\
\hline avg & $\mathrm{ZnO}(\mathrm{wt} \%)$ & 0.102 & 0.102 & 0.000 & $0.2 \%$ & no & 0.0022 & 2.18 & 0.0024 & 0.0008 & yes & $\frac{0}{8}$ & $\frac{7}{8}$ \\
\hline avg & ZrO2 (wt\%) & 0.371 & 0.369 & -0.002 & $-0.4 \%$ & no & 0.0044 & 1.20 & 0.0028 & 0.0024 & no & $\frac{0}{8}$ & 8 \\
\hline
\end{tabular}


SRNL-STI-2011-00693

Revision 0

Table C8. Oxide Comparisons between the Samplers for the Low-Rheology (Phase 3) Testing

\begin{tabular}{|c|c|c|c|c|c|c|c|c|c|c|c|c|c|c|}
\hline $\begin{array}{c}\text { Analytical } \\
\text { Block }\end{array}$ & Sampler & Oxide & $\begin{array}{l}\text { Mean(Measured } \\
\text { wt\%) Coliwasa } \\
\text { (low+high) }\end{array}$ & $\begin{array}{l}\text { Mean(Measured } \\
\text { wt\%) Other } \\
\text { Sampler }\end{array}$ & Diff & \% Diff & $\begin{array}{l}\text { Sig. } \\
\text { Diff }\end{array}$ & $\begin{array}{l}\text { Bias } \\
\text { Bnd }\end{array}$ & $\begin{array}{c}\% \\
\text { Rel } \\
\text { Bias } \\
\text { Bnd }\end{array}$ & $\begin{array}{c}\text { Std } \\
\text { Dev(Measured } \\
\text { wt\%) Coliwasa } \\
\text { (low+high) }\end{array}$ & $\begin{array}{c}\text { Std } \\
\text { Dev(Measured } \\
\text { wt\%) Other } \\
\text { Sampler }\end{array}$ & $\begin{array}{l}\text { Sig. } \\
\text { Diff }\end{array}$ & $\stackrel{\text { n }}{\text { Col }}$ & $\begin{array}{c}\text { n } \\
\text { Other }\end{array}$ \\
\hline 1 & Isolok-LFR-3mL & $\mathrm{Al} / \mathrm{B}$ & 2.521 & 2.518 & -0.003 & $-0.1 \%$ & no & 0.075 & 2.97 & 0.080 & 0.097 & no & 16 & 10 \\
\hline 1 & Isolok-LFR-3mL & Al2O3 (wt\%) & 10.286 & 10.203 & -0.083 & $-0.8 \%$ & no & 0.240 & 2.33 & 0.201 & 0.166 & no & 16 & 10 \\
\hline 1 & Isolok-LFR-3mL & B2O3 (wt\%) & 4.083 & 4.057 & -0.026 & $-0.6 \%$ & no & 0.138 & 3.37 & 0.119 & 0.156 & no & 16 & 10 \\
\hline 1 & Isolok-LFR-3mL & $\mathrm{BaO}(\mathrm{wt} \%)$ & 0.103 & 0.100 & -0.003 & $-2.5 \%$ & yes & 0.005 & 4.50 & 0.003 & 0.002 & no & 16 & 10 \\
\hline 1 & Isolok-LFR-3mL & $\mathrm{CaO}(\mathrm{wt} \%)$ & 1.154 & 1.121 & -0.032 & $-2.8 \%$ & yes & 0.058 & 5.01 & 0.031 & 0.029 & no & 16 & 10 \\
\hline 1 & Isolok-LFR-3mL & Cr2O3 (wt\%) & 0.111 & 0.095 & -0.016 & $-14.3 \%$ & yes & 0.022 & 20.12 & 0.008 & 0.007 & no & 16 & 10 \\
\hline 1 & Isolok-LFR-3mL & CuO (wt\%) & 0.033 & 0.035 & 0.002 & $5.3 \%$ & no & 0.005 & 15.70 & 0.004 & 0.004 & no & 16 & 10 \\
\hline 1 & Isolok-LFR-3mL & $\mathrm{Fe} / \mathrm{Li}$ & 2.442 & 2.417 & -0.025 & $-1.0 \%$ & no & 0.072 & 2.94 & 0.048 & 0.068 & no & 16 & 10 \\
\hline 1 & Isolok-LFR-3mL & Fe2O3 (wt\%) & 11.027 & 10.896 & -0.132 & $-1.2 \%$ & no & 0.288 & 2.61 & 0.199 & 0.167 & no & 16 & 10 \\
\hline 1 & Isolok-LFR-3mL & K2O (wt\%) & 0.177 & 0.180 & 0.003 & $1.5 \%$ & no & 0.011 & 6.36 & 0.010 & 0.011 & no & 16 & 10 \\
\hline 1 & Isolok-LFR-3mL & Li2O (wt\%) & 4.517 & 4.510 & -0.007 & $-0.1 \%$ & no & 0.097 & 2.15 & 0.099 & 0.124 & no & 16 & 10 \\
\hline 1 & Isolok-LFR-3mL & $\mathrm{MgO}(\mathrm{wt} \%)$ & 0.997 & 0.977 & -0.021 & $-2.1 \%$ & yes & 0.035 & 3.56 & 0.019 & 0.015 & no & 16 & 10 \\
\hline 1 & Isolok-LFR-3mL & $\mathrm{MnO}(\mathrm{wt} \%)$ & 1.662 & 1.627 & -0.035 & $-2.1 \%$ & yes & 0.060 & 3.61 & 0.033 & 0.027 & no & 16 & 10 \\
\hline 1 & Isolok-LFR-3mL & Na2O (wt\%) & 10.294 & 10.296 & 0.002 & $0.0 \%$ & no & 0.302 & 2.93 & 0.360 & 0.359 & no & 16 & 10 \\
\hline 1 & Isolok-LFR-3mL & $\mathrm{NiO}$ (wt\%) & 0.457 & 0.446 & -0.011 & $-2.4 \%$ & no & 0.024 & 5.36 & 0.018 & 0.014 & no & 16 & 10 \\
\hline 1 & Isolok-LFR-3mL & $\mathrm{SiO} 2$ (wt\%) & 52.052 & 52.477 & 0.425 & $0.8 \%$ & no & 1.106 & 2.13 & 0.799 & 0.850 & no & 16 & 10 \\
\hline 1 & Isolok-LFR-3mL & Sum (wt\%) & 97.507 & 97.572 & 0.065 & $0.1 \%$ & no & 1.125 & 1.15 & 1.400 & 1.027 & no & 16 & 10 \\
\hline 1 & Isolok-LFR-3mL & TiO2 (wt\%) & 0.081 & 0.083 & 0.002 & $2.0 \%$ & no & 0.003 & 4.26 & 0.002 & 0.003 & no & 16 & 10 \\
\hline 1 & Isolok-LFR-3mL & $\mathrm{ZnO}(\mathrm{wt} \%)$ & 0.102 & 0.100 & -0.003 & $-2.8 \%$ & yes & 0.005 & 5.35 & 0.003 & 0.003 & no & 16 & 10 \\
\hline 1 & Isolok-LFR-3mL & ZrO2 (wt\%) & 0.371 & 0.370 & -0.001 & $-0.3 \%$ & no & 0.005 & 1.44 & 0.005 & 0.005 & no & 16 & 10 \\
\hline 2 & Hydragard - HFR & $\mathrm{Al} / \mathrm{B}$ & 2.566 & 2.850 & 0.284 & $11.1 \%$ & yes & 0.419 & 16.32 & 0.099 & 0.232 & no & 16 & 10 \\
\hline 2 & Hydragard - HFR & Al2O3 (wt\%) & 10.532 & 11.025 & 0.494 & $4.7 \%$ & yes & 0.614 & 5.83 & 0.103 & 0.195 & no & 16 & 10 \\
\hline 2 & Hydragard - HFR & B2O3 (wt\%) & 4.109 & 3.890 & -0.220 & $-5.3 \%$ & yes & 0.400 & 9.74 & 0.150 & 0.297 & no & 16 & 10 \\
\hline 2 & Hydragard - HFR & $\mathrm{BaO}(\mathrm{wt} \%)$ & 0.104 & 0.108 & 0.004 & $4.2 \%$ & yes & 0.007 & 7.01 & 0.002 & 0.004 & yes & 16 & 10 \\
\hline 2 & Hydragard - HFR & $\mathrm{CaO}$ (wt\%) & 1.188 & 1.269 & 0.081 & $6.8 \%$ & yes & 0.139 & 11.68 & 0.026 & 0.079 & yes & 16 & 10 \\
\hline 2 & Hydragard - HFR & Cr2O3 (wt\%) & 0.110 & 0.119 & 0.009 & $8.3 \%$ & yes & 0.016 & 14.45 & 0.007 & 0.009 & no & 16 & 10 \\
\hline 2 & Hydragard - HFR & $\mathrm{CuO}$ (wt\%) & 0.037 & 0.038 & 0.001 & $1.9 \%$ & no & 0.004 & 11.08 & 0.004 & 0.005 & no & 16 & 10 \\
\hline 2 & Hydragard - HFR & $\mathrm{Fe} / \mathrm{Li}$ & 2.495 & 2.681 & 0.186 & $7.5 \%$ & yes & 0.250 & 10.04 & 0.068 & 0.091 & no & 16 & 10 \\
\hline 2 & Hydragard - HFR & Fe2O3 (wt\%) & 11.330 & 11.905 & 0.575 & $5.1 \%$ & yes & 0.739 & 6.52 & 0.113 & 0.221 & yes & 16 & 10 \\
\hline 2 & Hydragard - HFR & K2O (wt\%) & 0.177 & 0.175 & -0.002 & $-1.1 \%$ & no & 0.009 & 5.03 & 0.010 & 0.006 & no & 16 & 10 \\
\hline 2 & Hydragard - HFR & Li2O (wt\%) & 4.544 & 4.444 & -0.100 & $-2.2 \%$ & yes & 0.186 & 4.10 & 0.099 & 0.110 & no & 16 & 10 \\
\hline 2 & Hydragard - HFR & $\mathrm{MgO}(\mathrm{wt} \%)$ & 1.006 & 1.061 & 0.055 & $5.5 \%$ & yes & 0.075 & 7.43 & 0.014 & 0.026 & yes & 16 & 10 \\
\hline 2 & Hydragard - HFR & $\mathrm{MnO}(\mathrm{wt} \%)$ & 1.673 & 1.760 & 0.087 & $5.2 \%$ & yes & 0.119 & 7.11 & 0.024 & 0.043 & yes & 16 & 10 \\
\hline 2 & Hydragard - HFR & $\mathrm{Na} 2 \mathrm{O}(\mathrm{wt} \%)$ & 11.001 & 11.048 & 0.047 & $0.4 \%$ & no & 0.385 & 3.50 & 0.334 & 0.504 & no & 16 & 10 \\
\hline 2 & Hydragard - HFR & $\mathrm{NiO}$ (wt\%) & 0.455 & 0.474 & 0.019 & $4.2 \%$ & yes & 0.034 & 7.57 & 0.015 & 0.023 & no & 16 & 10 \\
\hline 2 & Hydragard - HFR & $\mathrm{SiO} 2(\mathrm{wt} \%)$ & 52.239 & 51.258 & -0.981 & $-1.9 \%$ & yes & 1.616 & 3.09 & 0.708 & 0.845 & no & 16 & 10 \\
\hline 2 & Hydragard - HFR & Sum (wt\%) & 99.062 & 99.135 & 0.073 & $0.1 \%$ & no & 0.664 & 0.67 & 0.780 & 0.573 & no & 16 & 10 \\
\hline 2 & Hydragard - HFR & TiO2 (wt\%) & 0.082 & 0.081 & -0.001 & $-1.7 \%$ & no & 0.004 & 4.42 & 0.002 & 0.003 & no & 16 & 10 \\
\hline 2 & Hydragard - HFR & $\mathrm{ZnO}$ (wt\%) & 0.101 & 0.106 & 0.005 & $4.9 \%$ & yes & 0.010 & 9.42 & 0.001 & 0.006 & yes & 16 & 10 \\
\hline 2 & Hydragard - HFR & ZrO2 (wt\%) & 0.375 & 0.376 & 0.001 & $0.4 \%$ & no & 0.008 & 2.15 & 0.005 & 0.011 & no & 16 & 10 \\
\hline
\end{tabular}


SRNL-STI-2011-00693

Revision 0

Table C8. Oxide Comparisons between the Samplers for the Low-Rheology (Phase 3) Testing

\begin{tabular}{|c|c|c|c|c|c|c|c|c|c|c|c|c|c|c|}
\hline $\begin{array}{c}\text { Analytical } \\
\text { Block }\end{array}$ & Sampler & Oxide & $\begin{array}{c}\text { Mean(Measured } \\
\text { wt\%) Coliwasa } \\
\text { (low+high) }\end{array}$ & $\begin{array}{l}\text { Mean(Measured } \\
\text { wt\%) Other } \\
\text { Sampler }\end{array}$ & Diff & $\%$ Diff & $\begin{array}{l}\text { Sig. } \\
\text { Diff }\end{array}$ & $\begin{array}{l}\text { Bias } \\
\text { Bnd }\end{array}$ & $\begin{array}{c}\% \\
\text { Rel } \\
\text { Bias } \\
\text { Bnd }\end{array}$ & $\begin{array}{c}\text { Std } \\
\text { Dev(Measured } \\
\text { wt\%) Coliwasa } \\
\text { (low+high) }\end{array}$ & $\begin{array}{c}\text { Std } \\
\text { Dev(Measured } \\
\text { wt\%) Other } \\
\text { Sampler }\end{array}$ & $\begin{array}{l}\text { Sig. } \\
\text { Diff }\end{array}$ & $\stackrel{\text { n }}{\text { Col }}$ & $\begin{array}{c}\text { n } \\
\text { Other }\end{array}$ \\
\hline 3 & Isolok-LFR-12mL & $\mathrm{Al} / \mathrm{B}$ & 2.666 & 2.621 & -0.045 & $-1.7 \%$ & no & 0.184 & 6.91 & 0.108 & 0.184 & yes & 16 & 10 \\
\hline 3 & Isolok-LFR-12mL & Al2O3 (wt\%) & 10.643 & 10.366 & -0.277 & $-2.6 \%$ & yes & 0.605 & 5.68 & 0.192 & 0.447 & yes & 16 & 10 \\
\hline 3 & $\begin{array}{l}\text { Isolok-LFR-12mL } \\
\end{array}$ & B2O3 (wt\%) & 3.997 & 3.964 & -0.033 & $-0.8 \%$ & no & 0.169 & 4.24 & 0.158 & 0.174 & no & 16 & 10 \\
\hline 3 & Isolok-LFR-12mL & $\mathrm{BaO}(\mathrm{wt} \%)$ & 0.103 & 0.099 & -0.004 & $-4.0 \%$ & yes & 0.008 & 7.46 & 0.003 & 0.005 & yes & 16 & 10 \\
\hline 3 & Isolok-LFR-12mL & $\mathrm{CaO}(\mathrm{wt} \%)$ & 1.189 & 1.153 & -0.036 & $-3.0 \%$ & no & 0.111 & 9.33 & 0.030 & 0.104 & yes & 16 & 10 \\
\hline 3 & Isolok-LFR-12mL & Cr2O3 (wt\%) & 0.111 & 0.106 & -0.004 & $-3.8 \%$ & no & 0.012 & 11.14 & 0.009 & 0.011 & no & 16 & 10 \\
\hline 3 & Isolok-LFR-12mL & $\mathrm{CuO}(\mathrm{wt} \%)$ & 0.041 & 0.043 & 0.002 & $6.0 \%$ & no & 0.007 & 16.31 & 0.005 & 0.005 & no & 16 & 10 \\
\hline 3 & $\begin{array}{l}\text { Isolok-LFR-12mL } \\
\end{array}$ & $\mathrm{Fe} / \mathrm{Li}$ & 2.412 & 2.220 & -0.192 & $-8.0 \%$ & yes & 0.328 & 13.62 & 0.052 & 0.189 & yes & 16 & 10 \\
\hline 3 & Isolok-LFR-12mL & $\mathrm{Fe} 2 \mathrm{O} 3(\mathrm{wt} \%)$ & 11.308 & 10.850 & -0.458 & $-4.1 \%$ & yes & 0.875 & 7.74 & 0.234 & 0.569 & yes & 16 & 10 \\
\hline 3 & Isolok-LFR-12mL & K2O (wt\%) & 0.177 & 0.178 & 0.001 & $0.6 \%$ & no & 0.007 & 4.05 & 0.010 & 0.006 & yes & 16 & 10 \\
\hline 3 & Isolok-LFR-12mL & $\mathrm{Li} 2 \mathrm{O}$ (wt\%) & 4.691 & 4.902 & 0.212 & $4.5 \%$ & yes & 0.366 & 7.81 & 0.117 & 0.206 & yes & 16 & 10 \\
\hline 3 & Isolok-LFR-12mL & $\mathrm{MgO}(\mathrm{wt} \%)$ & 1.033 & 0.970 & -0.063 & $-6.1 \%$ & yes & 0.113 & 10.89 & 0.022 & 0.068 & yes & 16 & 10 \\
\hline 3 & Isolok-LFR-12mL & $\mathrm{MnO}(\mathrm{wt} \%)$ & 1.709 & 1.610 & -0.099 & $-5.8 \%$ & yes & 0.182 & 10.66 & 0.039 & 0.114 & yes & 16 & 10 \\
\hline 3 & Isolok-LFR-12mL & Na2O (wt\%) & 10.876 & 11.090 & 0.214 & $2.0 \%$ & no & 0.521 & 4.79 & 0.394 & 0.322 & no & 16 & 10 \\
\hline 3 & Isolok-LFR-12mL & $\mathrm{NiO}$ (wt\%) & 0.455 & 0.441 & -0.014 & $-3.0 \%$ & no & 0.032 & 7.02 & 0.020 & 0.025 & no & 16 & 10 \\
\hline 3 & $\begin{array}{l}\text { Isolok-LFR-12mL } \\
\end{array}$ & $\mathrm{SiO} 2$ (wt\%) & 52.386 & 53.055 & 0.669 & $1.3 \%$ & no & 1.595 & 3.04 & 1.164 & 1.023 & no & 16 & 10 \\
\hline 3 & Isolok-LFR-12mL & Sum (wt\%) & 99.270 & 99.365 & 0.095 & $0.1 \%$ & no & 1.442 & 1.45 & 1.886 & 1.031 & no & 16 & 10 \\
\hline 3 & Isolok-LFR-12mL & TiO2 (wt\%) & 0.081 & 0.082 & 0.001 & $1.2 \%$ & no & 0.003 & 3.87 & 0.003 & 0.001 & no & 16 & 10 \\
\hline 3 & Isolok-LFR-12mL & $\mathrm{ZnO}(\mathrm{wt} \%)$ & 0.103 & 0.099 & -0.005 & $-4.6 \%$ & no & 0.010 & 9.90 & 0.003 & 0.008 & yes & 16 & 10 \\
\hline 3 & $\begin{array}{l}\text { Isolok-LFR-12mL } \\
\end{array}$ & $\mathrm{ZrO} 2$ (wt\%) & 0.369 & 0.357 & -0.011 & $-3.0 \%$ & yes & 0.018 & 5.00 & 0.008 & 0.009 & no & 16 & 10 \\
\hline 4 & Isolok-HFR-12mL & $\mathrm{Al} / \mathrm{B}$ & 2.614 & 2.630 & 0.016 & $0.6 \%$ & no & 0.124 & 4.75 & 0.102 & 0.165 & no & 16 & 10 \\
\hline 4 & Isolok-HFR-12mL & Al2O3 (wt\%) & 10.619 & 10.530 & -0.089 & $-0.8 \%$ & no & 0.356 & 3.35 & 0.239 & 0.425 & no & 16 & 10 \\
\hline 4 & Isolok-HFR-12mL & B2O3 (wt\%) & 4.067 & 4.012 & -0.055 & $-1.4 \%$ & no & 0.195 & 4.80 & 0.150 & 0.195 & no & 16 & 10 \\
\hline 4 & Isolok-HFR-12mL & $\mathrm{BaO}(\mathrm{wt} \%)$ & 0.107 & 0.106 & -0.001 & $-0.9 \%$ & no & 0.004 & 4.03 & 0.003 & 0.006 & no & 16 & 10 \\
\hline 4 & Isolok-HFR-12mL & $\mathrm{CaO}$ (wt\%) & 1.189 & 1.167 & -0.023 & $-1.9 \%$ & no & 0.057 & 4.79 & 0.030 & 0.055 & no & 16 & 10 \\
\hline 4 & Isolok-HFR-12mL & Cr2O3 (wt\%) & 0.110 & 0.122 & 0.011 & $10.3 \%$ & no & 0.024 & 21.37 & 0.007 & 0.017 & yes & 16 & 10 \\
\hline 4 & Isolok-HFR-12mL & $\mathrm{CuO}(\mathrm{wt} \%)$ & 0.034 & 0.035 & 0.001 & $4.0 \%$ & no & 0.006 & 18.40 & 0.005 & 0.007 & no & 16 & 10 \\
\hline 4 & Isolok-HFR-12mL & $\mathrm{Fe} / \mathrm{Li}$ & 2.415 & 2.408 & -0.006 & $-0.3 \%$ & no & 0.131 & 5.44 & 0.064 & 0.171 & yes & 16 & 10 \\
\hline 4 & Isolok-HFR-12mL & Fe2O3 (wt\%) & 11.373 & 11.245 & -0.129 & $-1.1 \%$ & no & 0.527 & 4.63 & 0.229 & 0.542 & yes & 16 & 10 \\
\hline 4 & Isolok-HFR-12mL & K2O (wt\%) & 0.177 & 0.175 & -0.002 & $-0.9 \%$ & no & 0.009 & 4.98 & 0.010 & 0.007 & no & 16 & 10 \\
\hline 4 & Isolok-HFR-12mL & Li2O (wt\%) & 4.712 & 4.678 & -0.034 & $-0.7 \%$ & no & 0.152 & 3.23 & 0.115 & 0.179 & no & 16 & 10 \\
\hline 4 & $\begin{array}{l}\text { Isolok-HFR-12mL } \\
\end{array}$ & $\mathrm{MgO}(\mathrm{wt} \%)$ & 1.044 & 1.024 & -0.020 & $-1.9 \%$ & no & 0.063 & 6.06 & 0.023 & 0.059 & yes & 16 & 10 \\
\hline 4 & Isolok-HFR-12mL & $\mathrm{MnO}(\mathrm{wt} \%)$ & 1.734 & 1.702 & -0.032 & $-1.9 \%$ & no & 0.104 & 6.00 & 0.040 & 0.098 & yes & 16 & 10 \\
\hline 4 & Isolok-HFR-12mL & $\mathrm{Na} 2 \mathrm{O}$ (wt\%) & 10.985 & 10.888 & -0.097 & $-0.9 \%$ & no & 0.476 & 4.33 & 0.391 & 0.548 & no & 16 & 10 \\
\hline 4 & Isolok-HFR-12mL & $\mathrm{NiO}(\mathrm{wt} \%)$ & 0.458 & 0.465 & 0.007 & $1.5 \%$ & no & 0.025 & 5.56 & 0.017 & 0.028 & no & 16 & 10 \\
\hline 4 & Isolok-HFR-12mL & $\mathrm{SiO} 2(\mathrm{wt} \%)$ & 52.199 & 52.028 & -0.171 & $-0.3 \%$ & no & 1.398 & 2.68 & 0.773 & 1.663 & yes & 16 & 10 \\
\hline 4 & Isolok-HFR-12mL & Sum (wt\%) & 99.355 & 98.713 & -0.642 & $-0.6 \%$ & no & 2.133 & 2.15 & 1.502 & 2.190 & no & 16 & 10 \\
\hline 4 & Isolok-HFR-12mL & TiO2 (wt\%) & 0.079 & 0.080 & 0.000 & $0.6 \%$ & no & 0.003 & 3.60 & 0.003 & 0.003 & no & 16 & 10 \\
\hline 4 & Isolok-HFR-12mL & $\mathrm{ZnO}$ (wt\%) & 0.103 & 0.103 & 0.000 & $-0.4 \%$ & no & 0.005 & 4.78 & 0.003 & 0.008 & no & 16 & 10 \\
\hline 4 & Isolok-HFR-12mL & ZrO2 (wt\%) & 0.364 & 0.355 & -0.009 & $-2.6 \%$ & yes & 0.015 & 4.21 & 0.006 & 0.009 & no & 16 & 10 \\
\hline
\end{tabular}


SRNL-STI-2011-00693

Revision 0

Table C8. Oxide Comparisons between the Samplers for the Low-Rheology (Phase 3) Testing

\begin{tabular}{|c|c|c|c|c|c|c|c|c|c|c|c|c|c|c|}
\hline $\begin{array}{c}\text { Analytical } \\
\text { Block }\end{array}$ & Sampler & Oxide & $\begin{array}{l}\text { Mean(Measured } \\
\text { wt\%) Coliwasa } \\
\text { (low+high) }\end{array}$ & $\begin{array}{l}\text { Mean(Measured } \\
\text { wt\%) Other } \\
\text { Sampler }\end{array}$ & Diff & \% Diff & $\begin{array}{l}\text { Sig. } \\
\text { Diff }\end{array}$ & $\begin{array}{l}\text { Bias } \\
\text { Bnd }\end{array}$ & $\begin{array}{c}\% \\
\text { Rel } \\
\text { Bias } \\
\text { Bnd }\end{array}$ & $\begin{array}{c}\text { Std } \\
\text { Dev(Measured } \\
\text { wt\%) Coliwasa } \\
\text { (low+high) }\end{array}$ & $\begin{array}{c}\text { Std } \\
\text { Dev(Measured } \\
\text { wt\%) Other } \\
\text { Sampler }\end{array}$ & $\begin{array}{l}\text { Sig. } \\
\text { Diff }\end{array}$ & $\stackrel{\text { n }}{\text { Col }}$ & $\begin{array}{c}\text { n } \\
\text { Other }\end{array}$ \\
\hline 5 & Isolok-HFR-3mL & $\mathrm{Al} / \mathrm{B}$ & 2.531 & 2.567 & 0.036 & $1.4 \%$ & no & 0.137 & 5.42 & 0.119 & 0.124 & no & 16 & 10 \\
\hline 5 & Isolok-HFR-3mL & Al2O3 (wt\%) & 10.735 & 10.659 & -0.076 & $-0.7 \%$ & no & 0.192 & 1.79 & 0.106 & 0.183 & no & 16 & 10 \\
\hline 5 & Isolok-HFR-3mL & B2O3 (wt\%) & 4.250 & 4.160 & -0.090 & $-2.1 \%$ & no & 0.246 & 5.79 & 0.188 & 0.186 & no & 16 & 10 \\
\hline 5 & Isolok-HFR-3mL & $\mathrm{BaO}(\mathrm{wt} \%)$ & 0.105 & 0.105 & -0.001 & $-0.5 \%$ & no & 0.003 & 3.06 & 0.002 & 0.004 & no & 16 & 10 \\
\hline 5 & Isolok-HFR-3mL & $\mathrm{CaO}(\mathrm{wt} \%)$ & 1.207 & 1.198 & -0.009 & $-0.8 \%$ & no & 0.034 & 2.80 & 0.031 & 0.027 & no & 16 & 10 \\
\hline 5 & Isolok-HFR-3mL & Cr2O3 (wt\%) & 0.113 & 0.106 & -0.007 & $-6.3 \%$ & yes & 0.013 & 11.44 & 0.008 & 0.005 & no & 16 & 10 \\
\hline 5 & Isolok-HFR-3mL & CuO (wt\%) & 0.046 & 0.048 & 0.002 & $4.0 \%$ & no & 0.006 & 11.88 & 0.004 & 0.005 & no & 16 & 10 \\
\hline 5 & Isolok-HFR-3mL & $\mathrm{Fe} / \mathrm{Li}$ & 2.363 & 2.288 & -0.075 & $-3.2 \%$ & yes & 0.125 & 5.30 & 0.049 & 0.075 & no & 16 & 10 \\
\hline 5 & Isolok-HFR-3mL & $\mathrm{Fe} 2 \mathrm{O} 3(\mathrm{wt} \%)$ & 11.324 & 11.153 & -0.171 & $-1.5 \%$ & yes & 0.317 & 2.80 & 0.080 & 0.199 & yes & 16 & 10 \\
\hline 5 & Isolok-HFR-3mL & K2O (wt\%) & 0.202 & 0.179 & -0.024 & $-11.7 \%$ & no & 0.064 & 31.47 & 0.074 & 0.009 & yes & 16 & 10 \\
\hline 5 & Isolok-HFR-3mL & $\mathrm{Li} 2 \mathrm{O}$ (wt\%) & 4.794 & 4.878 & 0.084 & $1.8 \%$ & no & 0.172 & 3.58 & 0.099 & 0.115 & no & 16 & 10 \\
\hline 5 & Isolok-HFR-3mL & $\mathrm{MgO}(\mathrm{wt} \%)$ & 1.062 & 1.040 & -0.022 & $-2.1 \%$ & yes & 0.035 & 3.31 & 0.012 & 0.020 & no & 16 & 10 \\
\hline 5 & Isolok-HFR-3mL & $\mathrm{MnO}(\mathrm{wt} \%)$ & 1.764 & 1.728 & -0.036 & $-2.1 \%$ & yes & 0.057 & 3.22 & 0.019 & 0.031 & no & 16 & 10 \\
\hline 5 & Isolok-HFR-3mL & Na2O (wt\%) & 10.834 & 11.103 & 0.270 & $2.5 \%$ & no & 0.598 & 5.52 & 0.362 & 0.445 & no & 16 & 10 \\
\hline 5 & Isolok-HFR-3mL & $\mathrm{NiO}$ (wt\%) & 0.453 & 0.446 & -0.008 & $-1.7 \%$ & no & 0.023 & 4.97 & 0.018 & 0.018 & no & 16 & 10 \\
\hline 5 & Isolok-HFR-3mL & $\mathrm{SiO} 2$ (wt\%) & 52.426 & 52.990 & 0.564 & $1.1 \%$ & yes & 1.020 & 1.94 & 0.602 & 0.440 & no & 16 & 10 \\
\hline 5 & Isolok-HFR-3mL & Sum (wt\%) & 99.871 & 100.349 & 0.478 & $0.5 \%$ & yes & 0.934 & 0.94 & 0.497 & 0.623 & no & 16 & 10 \\
\hline 5 & Isolok-HFR-3mL & TiO2 (wt\%) & 0.083 & 0.085 & 0.002 & $2.0 \%$ & no & 0.004 & 4.64 & 0.003 & 0.003 & no & 16 & 10 \\
\hline 5 & Isolok-HFR-3mL & $\mathrm{ZnO}$ (wt\%) & 0.101 & 0.099 & -0.001 & $-1.5 \%$ & no & 0.005 & 4.48 & 0.003 & 0.004 & no & 16 & 10 \\
\hline 5 & Isolok-HFR-3mL & $\mathrm{ZrO} 2$ (wt\%) & 0.369 & 0.372 & 0.002 & $0.7 \%$ & no & 0.008 & 2.17 & 0.004 & 0.009 & no & 16 & 10 \\
\hline 6 & Hydragard-LFR & $\mathrm{Al} / \mathrm{B}$ & 2.635 & 2.736 & 0.101 & $3.8 \%$ & yes & 0.200 & 7.58 & 0.106 & 0.130 & no & 16 & 9 \\
\hline 6 & Hydragard-LFR & Al2O3 (wt\%) & 10.687 & 10.900 & 0.213 & $2.0 \%$ & yes & 0.419 & 3.92 & 0.093 & 0.265 & yes & 16 & 9 \\
\hline 6 & Hydragard-LFR & B2O3 (wt\%) & 4.061 & 3.989 & -0.072 & $-1.8 \%$ & no & 0.193 & 4.74 & 0.146 & 0.127 & no & 16 & 9 \\
\hline 6 & Hydragard-LFR & $\mathrm{BaO}(\mathrm{wt} \%)$ & 0.102 & 0.103 & 0.001 & $1.4 \%$ & no & 0.004 & 3.61 & 0.002 & 0.003 & no & 16 & 9 \\
\hline 6 & Hydragard-LFR & $\mathrm{CaO}$ (wt\%) & 1.198 & 1.224 & 0.026 & $2.2 \%$ & no & 0.087 & 7.28 & 0.025 & 0.079 & yes & 16 & 9 \\
\hline 6 & Hydragard-LFR & Cr2O3 (wt\%) & 0.112 & 0.113 & 0.001 & $0.5 \%$ & no & 0.008 & 6.74 & 0.008 & 0.009 & no & 16 & 9 \\
\hline 6 & Hydragard-LFR & $\mathrm{CuO}(\mathrm{wt} \%)$ & 0.038 & 0.037 & -0.002 & $-4.3 \%$ & no & 0.005 & 13.20 & 0.003 & 0.005 & no & 16 & 9 \\
\hline 6 & Hydragard-LFR & $\mathrm{Fe} / \mathrm{Li}$ & 2.459 & 2.560 & 0.101 & $4.1 \%$ & yes & 0.189 & 7.70 & 0.042 & 0.113 & yes & 16 & 9 \\
\hline 6 & Hydragard-LFR & Fe2O3 (wt\%) & 11.337 & 11.504 & 0.168 & $1.5 \%$ & no & 0.418 & 3.69 & 0.081 & 0.324 & yes & 16 & 9 \\
\hline 6 & Hydragard-LFR & K2O (wt\%) & 0.177 & 0.176 & -0.001 & $-0.5 \%$ & no & 0.008 & 4.51 & 0.010 & 0.005 & no & 16 & 9 \\
\hline 6 & Hydragard-LFR & Li2O (wt\%) & 4.611 & 4.497 & -0.114 & $-2.5 \%$ & yes & 0.204 & 4.42 & 0.058 & 0.114 & yes & 16 & 9 \\
\hline 6 & Hydragard-LFR & $\mathrm{MgO}(\mathrm{wt} \%)$ & 1.022 & 1.035 & 0.013 & $1.3 \%$ & no & 0.046 & 4.53 & 0.011 & 0.043 & yes & 16 & 9 \\
\hline 6 & Hydragard-LFR & $\mathrm{MnO}(\mathrm{wt} \%)$ & 1.697 & 1.713 & 0.016 & $0.9 \%$ & no & 0.061 & 3.59 & 0.018 & 0.058 & yes & 16 & 9 \\
\hline 6 & Hydragard-LFR & $\mathrm{Na} 2 \mathrm{O}(\mathrm{wt} \%)$ & 10.927 & 11.259 & 0.332 & $3.0 \%$ & yes & 0.648 & 5.93 & 0.379 & 0.343 & no & 16 & 9 \\
\hline 6 & Hydragard-LFR & $\mathrm{NiO}$ (wt\%) & 0.452 & 0.460 & 0.008 & $1.8 \%$ & no & 0.025 & 5.60 & 0.022 & 0.016 & no & 16 & 9 \\
\hline 6 & Hydragard-LFR & $\mathrm{SiO} 2(\mathrm{wt} \%)$ & 52.386 & 51.201 & -1.186 & $-2.3 \%$ & yes & 1.867 & 3.56 & 0.535 & 1.122 & no & 16 & 9 \\
\hline 6 & Hydragard-LFR & Sum (wt\%) & 99.364 & 98.756 & -0.608 & $-0.6 \%$ & yes & 1.104 & 1.11 & 0.435 & 0.774 & no & 16 & 9 \\
\hline 6 & $\begin{array}{l}\text { Hydragard-LFR } \\
\end{array}$ & TiO2 (wt\%) & 0.083 & 0.081 & -0.001 & $-1.6 \%$ & no & 0.003 & 3.84 & 0.002 & 0.003 & no & 16 & 9 \\
\hline 6 & Hydragard-LFR & $\mathrm{ZnO}$ (wt\%) & 0.101 & 0.103 & 0.003 & $2.6 \%$ & no & 0.006 & 6.15 & 0.003 & 0.005 & no & 16 & 9 \\
\hline 6 & Hydragard-LFR & ZrO2 (wt\%) & 0.373 & 0.361 & -0.012 & $-3.2 \%$ & yes & 0.023 & 6.25 & 0.004 & 0.015 & yes & 16 & 9 \\
\hline
\end{tabular}


SRNL-STI-2011-00693

Revision 0

Table C9. Oxide Comparisons between the Samplers for the Low-Rheology (Phase 3) Testing (screened data)

\begin{tabular}{|c|c|c|c|c|c|c|c|c|c|c|c|c|c|c|}
\hline $\begin{array}{c}\text { Analytical } \\
\text { Block }\end{array}$ & Sampler & Oxide & $\begin{array}{c}\text { Mean } \\
\text { (Measured } \\
\text { wt\%) Coliwasa } \\
\text { (low+high) }\end{array}$ & $\begin{array}{l}\text { Mean(Measured } \\
\text { wt\%) Other } \\
\text { Sampler }\end{array}$ & Diff & \% Diff & $\begin{array}{l}\text { Sig. } \\
\text { Diff }\end{array}$ & $\begin{array}{l}\text { Bias } \\
\text { Bnd }\end{array}$ & $\begin{array}{l}\% \\
\text { Rel } \\
\text { Bias } \\
\text { Bnd } \\
\end{array}$ & $\begin{array}{c}\text { Std } \\
\text { Dev(Measured } \\
\text { wt\%) Coliwasa } \\
\text { (low+high) }\end{array}$ & $\begin{array}{c}\text { Std } \\
\text { Dev(Measured } \\
\text { wt\%) Other } \\
\text { Sampler }\end{array}$ & $\begin{array}{l}\text { Sig. } \\
\text { Diff }\end{array}$ & $\underset{\text { Col }}{\text { Col }}$ & $\begin{array}{c}\text { n } \\
\text { Other }\end{array}$ \\
\hline 1 & Isolok-LFR-3mL & $\mathrm{Al} / \mathrm{B}$ & 2.521 & 2.518 & -0.003 & $-0.1 \%$ & no & 0.075 & 2.97 & 0.0799 & 0.0971 & no & 16 & 10 \\
\hline 1 & Isolok-LFR-3mL & Al2O3 (wt\%) & 10.328 & 10.203 & -0.125 & $-1.2 \%$ & yes & 0.240 & 2.32 & 0.1135 & 0.1662 & no & 15 & 10 \\
\hline 1 & Isolok-LFR-3mL & B2O3 (wt\%) & 4.134 & 4.096 & -0.037 & $-0.9 \%$ & no & 0.106 & 2.56 & 0.0551 & 0.0990 & no & 13 & 9 \\
\hline 1 & Isolok-LFR-3mL & $\mathrm{BaO}$ (wt\%) & 0.103 & 0.101 & -0.002 & $-2.4 \%$ & yes & 0.004 & 4.02 & 0.0022 & 0.0013 & no & 15 & 9 \\
\hline 1 & Isolok-LFR-3mL & $\mathrm{CaO}(\mathrm{wt} \%)$ & 1.159 & 1.129 & -0.031 & $-2.7 \%$ & yes & 0.049 & 4.24 & 0.0222 & 0.0191 & no & 15 & 9 \\
\hline 1 & Isolok-LFR-3mL & Cr2O3 (wt\%) & 0.111 & 0.095 & -0.016 & $-14.3 \%$ & yes & 0.022 & 20.12 & 0.0081 & 0.0071 & no & 16 & 10 \\
\hline 1 & Isolok-LFR-3mL & $\mathrm{CuO}(\mathrm{wt} \%)$ & 0.033 & 0.035 & 0.002 & $5.3 \%$ & no & 0.005 & 15.70 & 0.0041 & 0.0041 & no & 16 & 10 \\
\hline 1 & Isolok-LFR-3mL & $\mathrm{Fe} / \mathrm{Li}$ & 2.442 & 2.417 & -0.025 & $-1.0 \%$ & no & 0.072 & 2.94 & 0.0482 & 0.0682 & no & 16 & 10 \\
\hline 1 & Isolok-LFR-3mL & Fe2O3 (wt\%) & 11.072 & 10.896 & -0.176 & $-1.6 \%$ & yes & 0.284 & 2.57 & 0.0957 & 0.1668 & no & 15 & 10 \\
\hline 1 & Isolok-LFR-3mL & K2O (wt\%) & 0.177 & 0.180 & 0.003 & $1.5 \%$ & no & 0.011 & 6.36 & 0.0095 & 0.0114 & no & 16 & 10 \\
\hline 1 & Isolok-LFR-3mL & Li2O (wt\%) & 4.517 & 4.510 & -0.007 & $-0.1 \%$ & no & 0.097 & 2.15 & 0.0986 & 0.1240 & no & 16 & 10 \\
\hline 1 & Isolok-LFR-3mL & $\mathrm{MgO}(\mathrm{wt} \%)$ & 1.001 & 0.977 & -0.025 & $-2.5 \%$ & yes & 0.035 & 3.54 & 0.0109 & 0.0154 & no & 15 & 10 \\
\hline 1 & Isolok-LFR-3mL & $\mathrm{MnO}(\mathrm{wt} \%)$ & 1.668 & 1.627 & -0.041 & $-2.5 \%$ & yes & 0.060 & 3.62 & 0.0196 & 0.0265 & no & 15 & 10 \\
\hline 1 & Isolok-LFR-3mL & Na2O (wt\%) & 10.346 & 10.296 & -0.050 & $-0.5 \%$ & no & 0.326 & 3.15 & 0.3024 & 0.3595 & no & 15 & 10 \\
\hline 1 & Isolok-LFR-3mL & $\mathrm{NiO}$ (wt\%) & 0.457 & 0.446 & -0.011 & $-2.4 \%$ & no & 0.024 & 5.36 & 0.0177 & 0.0138 & no & 16 & 10 \\
\hline 1 & Isolok-LFR-3mL & $\mathrm{SiO} 2(\mathrm{wt} \%)$ & 52.185 & 52.477 & 0.292 & $0.6 \%$ & no & 0.899 & 1.72 & 0.6183 & 0.8501 & no & 15 & 10 \\
\hline 1 & Isolok-LFR-3mL & Sum (wt\%) & 97.808 & 97.572 & -0.236 & $-0.2 \%$ & no & 0.965 & 0.99 & 0.7392 & 1.0271 & no & 15 & 10 \\
\hline 1 & Isolok-LFR-3mL & TiO2 (wt\%) & 0.081 & 0.083 & 0.002 & $2.0 \%$ & no & 0.003 & 4.26 & 0.0020 & 0.0027 & no & 16 & 10 \\
\hline 1 & Isolok-LFR-3mL & $\mathrm{ZnO}$ (wt\%) & 0.102 & 0.100 & -0.003 & $-2.8 \%$ & yes & 0.005 & 5.35 & 0.0032 & 0.0032 & no & 16 & 10 \\
\hline 1 & Isolok-LFR-3mL & $\mathrm{ZrO} 2$ (wt\%) & 0.372 & 0.370 & -0.002 & $-0.6 \%$ & no & 0.005 & 1.43 & 0.0028 & 0.0048 & no & 15 & 10 \\
\hline 2 & Hydragard - HFR & $\mathrm{Al} / \mathrm{B}$ & 2.566 & 2.794 & 0.228 & $8.9 \%$ & yes & 0.336 & 13.08 & 0.0989 & 0.1615 & no & 16 & 9 \\
\hline 2 & Hydragard - HFR & $\mathrm{Al2O}$ (wt\%) & 10.532 & 11.025 & 0.494 & $4.7 \%$ & yes & 0.614 & 5.83 & 0.1035 & 0.1946 & no & 16 & 10 \\
\hline 2 & Hydragard - HFR & B2O3 (wt\%) & 4.109 & 3.900 & -0.209 & $-5.1 \%$ & yes & 0.343 & 8.35 & 0.1496 & 0.1475 & no & 16 & 8 \\
\hline 2 & Hydragard - HFR & $\mathrm{BaO}(\mathrm{wt} \%)$ & 0.104 & 0.108 & 0.004 & $4.2 \%$ & yes & 0.007 & 7.01 & 0.0016 & 0.0039 & yes & 16 & 10 \\
\hline 2 & Hydragard - HFR & $\mathrm{CaO}$ (wt\%) & 1.188 & 1.248 & 0.060 & $5.1 \%$ & yes & 0.091 & 7.63 & 0.0258 & 0.0478 & no & 16 & 9 \\
\hline 2 & Hydragard - HFR & Cr2O3 (wt\%) & 0.110 & 0.119 & 0.009 & $8.3 \%$ & yes & 0.016 & 14.45 & 0.0075 & 0.0091 & no & 16 & 10 \\
\hline 2 & Hydragard - HFR & $\mathrm{CuO}(\mathrm{wt} \%)$ & 0.037 & 0.038 & 0.001 & $1.9 \%$ & no & 0.004 & 11.08 & 0.0036 & 0.0048 & no & 16 & 10 \\
\hline 2 & Hydragard - HFR & $\mathrm{Fe} / \mathrm{Li}$ & 2.495 & 2.681 & 0.186 & $7.5 \%$ & yes & 0.250 & 10.04 & 0.0676 & 0.0913 & no & 16 & 10 \\
\hline 2 & Hydragard - HFR & Fe2O3 (wt\%) & 11.330 & 11.905 & 0.575 & $5.1 \%$ & yes & 0.739 & 6.52 & 0.1131 & 0.2214 & yes & 16 & 10 \\
\hline 2 & Hydragard - HFR & K2O (wt\%) & 0.177 & 0.175 & -0.002 & $-1.1 \%$ & no & 0.009 & 5.03 & 0.0095 & 0.0061 & no & 16 & 10 \\
\hline 2 & Hydragard - HFR & Li2O (wt\%) & 4.544 & 4.444 & -0.100 & $-2.2 \%$ & yes & 0.186 & 4.10 & 0.0993 & 0.1099 & no & 16 & 10 \\
\hline 2 & Hydragard - HFR & $\mathrm{MgO}(\mathrm{wt} \%)$ & 1.006 & 1.061 & 0.055 & $5.5 \%$ & yes & 0.075 & 7.43 & 0.0139 & 0.0263 & yes & 16 & 10 \\
\hline 2 & Hydragard - HFR & $\mathrm{MnO}(\mathrm{wt} \%)$ & 1.673 & 1.760 & 0.087 & $5.2 \%$ & yes & 0.119 & 7.11 & 0.0236 & 0.0426 & yes & 16 & 10 \\
\hline 2 & Hydragard - HFR & $\mathrm{Na} 2 \mathrm{O}$ (wt\%) & 10.997 & 10.996 & 0.000 & $0.0 \%$ & no & 0.164 & 1.49 & 0.1716 & 0.1867 & no & 14 & 8 \\
\hline 2 & Hydragard - HFR & $\mathrm{NiO}$ (wt\%) & 0.451 & 0.474 & 0.022 & $5.0 \%$ & yes & 0.039 & 8.64 & 0.0075 & 0.0227 & yes & 15 & 10 \\
\hline 2 & Hydragard - HFR & $\mathrm{SiO} 2(\mathrm{wt} \%)$ & 52.239 & 51.258 & -0.981 & $-1.9 \%$ & yes & 1.616 & 3.09 & 0.7083 & 0.8450 & no & 16 & 10 \\
\hline 2 & Hydragard - HFR & Sum (wt\%) & 99.062 & 99.135 & 0.073 & $0.1 \%$ & no & 0.664 & 0.67 & 0.7801 & 0.5730 & no & 16 & 10 \\
\hline 2 & Hydragard - HFR & TiO2 (wt\%) & 0.082 & 0.081 & -0.001 & $-1.7 \%$ & no & 0.004 & 4.42 & 0.0025 & 0.0029 & no & 16 & 10 \\
\hline 2 & Hydragard - HFR & $\mathrm{ZnO}(\mathrm{wt} \%)$ & 0.101 & 0.106 & 0.005 & $4.9 \%$ & yes & 0.010 & 9.42 & 0.0015 & 0.0064 & yes & 16 & 10 \\
\hline 2 & Hydragard - HFR & $\mathrm{ZrO} 2$ (wt\%) & 0.375 & 0.379 & 0.004 & $1.2 \%$ & no & 0.009 & 2.49 & 0.0051 & 0.0069 & no & 16 & 9 \\
\hline
\end{tabular}


SRNL-STI-2011-00693

Revision 0

Table C9. Oxide Comparisons between the Samplers for the Low-Rheology (Phase 3) Testing (screened data)

\begin{tabular}{|c|c|c|c|c|c|c|c|c|c|c|c|c|c|c|}
\hline $\begin{array}{c}\text { Analytical } \\
\text { Block }\end{array}$ & Sampler & Oxide & $\begin{array}{c}\text { Mean } \\
\text { (Measured } \\
\text { wt\%) Coliwasa } \\
\text { (low+high) }\end{array}$ & $\begin{array}{l}\text { Mean(Measured } \\
\text { wt\%) Other } \\
\text { Sampler }\end{array}$ & Diff & \% Diff & $\begin{array}{l}\text { Sig. } \\
\text { Diff }\end{array}$ & $\begin{array}{l}\text { Bias } \\
\text { Bnd }\end{array}$ & $\begin{array}{l}\% \\
\text { Rel } \\
\text { Bias } \\
\text { Bnd } \\
\end{array}$ & $\begin{array}{c}\text { Std } \\
\text { Dev(Measured } \\
\text { wt\%) Coliwasa } \\
\text { (low+high) }\end{array}$ & $\begin{array}{c}\text { Std } \\
\text { Dev(Measured } \\
\text { wt\%) Other } \\
\text { Sampler }\end{array}$ & $\begin{array}{l}\text { Sig. } \\
\text { Diff }\end{array}$ & $\underset{\text { Col }}{\text { Col }}$ & $\begin{array}{c}\text { n } \\
\text { Other }\end{array}$ \\
\hline 3 & Isolok-LFR-12mL & $\mathrm{Al} / \mathrm{B}$ & 2.666 & 2.621 & -0.045 & $-1.7 \%$ & no & 0.184 & 6.91 & 0.1079 & 0.1844 & yes & 16 & 10 \\
\hline 3 & Isolok-LFR-12mL & Al2O3 (wt\%) & 10.677 & 10.366 & -0.311 & $-2.9 \%$ & no & 0.635 & 5.95 & 0.1382 & 0.4466 & yes & 15 & 10 \\
\hline 3 & Isolok-LFR-12mL & B2O3 (wt\%) & 3.997 & 3.964 & -0.033 & $-0.8 \%$ & no & 0.169 & 4.24 & 0.1577 & 0.1740 & no & 16 & 10 \\
\hline 3 & Isolok-LFR-12mL & $\mathrm{BaO}$ (wt\%) & 0.103 & 0.099 & -0.004 & $-4.0 \%$ & yes & 0.008 & 7.46 & 0.0026 & 0.0047 & yes & 16 & 10 \\
\hline 3 & Isolok-LFR-12mL & $\mathrm{CaO}(\mathrm{wt} \%)$ & 1.189 & 1.152 & -0.037 & $-3.1 \%$ & yes & 0.068 & 5.74 & 0.0302 & 0.0430 & no & 16 & 8 \\
\hline 3 & Isolok-LFR-12mL & Cr2O3 (wt\%) & 0.111 & 0.106 & -0.004 & $-3.8 \%$ & no & 0.012 & 11.14 & 0.0090 & 0.0109 & no & 16 & 10 \\
\hline 3 & Isolok-LFR-12mL & $\mathrm{CuO}(\mathrm{wt} \%)$ & 0.041 & 0.043 & 0.002 & $6.0 \%$ & no & 0.007 & 16.31 & 0.0048 & 0.0054 & no & 16 & 10 \\
\hline 3 & Isolok-LFR-12mL & $\mathrm{Fe} / \mathrm{Li}$ & 2.412 & 2.220 & -0.192 & $-8.0 \%$ & yes & 0.328 & 13.62 & 0.0524 & 0.1892 & yes & 16 & 10 \\
\hline 3 & Isolok-LFR-12mL & Fe2O3 (wt\%) & 11.350 & 10.850 & -0.500 & $-4.4 \%$ & yes & 0.912 & 8.04 & 0.1693 & 0.5693 & yes & 15 & 10 \\
\hline 3 & Isolok-LFR-12mL & K2O (wt\%) & 0.177 & 0.179 & 0.002 & $1.4 \%$ & no & 0.008 & 4.52 & 0.0095 & 0.0038 & yes & 16 & 9 \\
\hline 3 & Isolok-LFR-12mL & Li2O (wt\%) & 4.708 & 4.902 & 0.194 & $4.1 \%$ & yes & 0.347 & 7.38 & 0.0982 & 0.2063 & yes & 15 & 10 \\
\hline 3 & Isolok-LFR-12mL & $\mathrm{MgO}(\mathrm{wt} \%)$ & 1.033 & 0.970 & -0.063 & $-6.1 \%$ & yes & 0.113 & 10.89 & 0.0216 & 0.0681 & yes & 16 & 10 \\
\hline 3 & Isolok-LFR-12mL & $\mathrm{MnO}(\mathrm{wt} \%)$ & 1.709 & 1.610 & -0.099 & $-5.8 \%$ & yes & 0.182 & 10.66 & 0.0394 & 0.1144 & yes & 16 & 10 \\
\hline 3 & Isolok-LFR-12mL & Na2O (wt\%) & 10.881 & 11.090 & 0.209 & $1.9 \%$ & no & 0.470 & 4.32 & 0.2917 & 0.3223 & no & 14 & 10 \\
\hline 3 & Isolok-LFR-12mL & $\mathrm{NiO}$ (wt\%) & 0.454 & 0.441 & -0.013 & $-2.8 \%$ & no & 0.031 & 6.82 & 0.0117 & 0.0246 & yes & 14 & 10 \\
\hline 3 & Isolok-LFR-12mL & $\mathrm{SiO} 2(\mathrm{wt} \%)$ & 52.584 & 53.081 & 0.497 & $0.9 \%$ & no & 1.187 & 2.26 & 0.8828 & 0.4033 & no & 15 & 8 \\
\hline 3 & Isolok-LFR-12mL & Sum (wt\%) & 99.652 & 99.365 & -0.286 & $-0.3 \%$ & no & 1.218 & 1.22 & 1.1470 & 1.0306 & no & 15 & 10 \\
\hline 3 & Isolok-LFR-12mL & TiO2 (wt\%) & 0.082 & 0.082 & 0.000 & $0.5 \%$ & no & 0.002 & 2.48 & 0.0022 & 0.0013 & no & 15 & 10 \\
\hline 3 & Isolok-LFR-12mL & $\mathrm{ZnO}(\mathrm{wt} \%)$ & 0.103 & 0.099 & -0.005 & $-4.6 \%$ & no & 0.010 & 9.90 & 0.0032 & 0.0075 & yes & 16 & 10 \\
\hline 3 & Isolok-LFR-12mL & $\mathrm{ZrO} 2$ (wt\%) & 0.370 & 0.357 & -0.013 & $-3.5 \%$ & yes & 0.020 & 5.35 & 0.0045 & 0.0093 & yes & 15 & 10 \\
\hline 4 & Isolok-HFR-12mL & $\mathrm{Al} / \mathrm{B}$ & 2.614 & 2.630 & 0.016 & $0.6 \%$ & no & 0.124 & 4.75 & 0.1018 & 0.1653 & no & 16 & 10 \\
\hline 4 & Isolok-HFR-12mL & $\mathrm{Al2O} 3(\mathrm{wt} \%)$ & 10.669 & 10.424 & -0.246 & $-2.3 \%$ & yes & 0.463 & 4.34 & 0.1328 & 0.2748 & yes & 15 & 9 \\
\hline 4 & Isolok-HFR-12mL & B2O3 (wt\%) & 4.067 & 4.012 & -0.055 & $-1.4 \%$ & no & 0.195 & 4.80 & 0.1504 & 0.1945 & no & 16 & 10 \\
\hline 4 & Isolok-HFR-12mL & $\mathrm{BaO}(\mathrm{wt} \%)$ & 0.107 & 0.104 & -0.003 & $-2.7 \%$ & yes & 0.005 & 4.35 & 0.0010 & 0.0022 & yes & 14 & 9 \\
\hline 4 & Isolok-HFR-12mL & $\mathrm{CaO}$ (wt\%) & 1.190 & 1.153 & -0.037 & $-3.1 \%$ & yes & 0.064 & 5.41 & 0.0149 & 0.0346 & yes & 14 & 9 \\
\hline 4 & Isolok-HFR-12mL & Cr2O3 (wt\%) & 0.110 & 0.122 & 0.011 & $10.3 \%$ & no & 0.024 & 21.37 & 0.0074 & 0.0165 & yes & 16 & 10 \\
\hline 4 & Isolok-HFR-12mL & $\mathrm{CuO}(\mathrm{wt} \%)$ & 0.034 & 0.035 & 0.001 & $4.0 \%$ & no & 0.006 & 18.40 & 0.0051 & 0.0069 & no & 16 & 10 \\
\hline 4 & Isolok-HFR-12mL & $\mathrm{Fe} / \mathrm{Li}$ & 2.415 & 2.366 & -0.049 & $-2.0 \%$ & no & 0.122 & 5.04 & 0.0636 & 0.1146 & no & 16 & 9 \\
\hline 4 & Isolok-HFR-12mL & Fe2O3 (wt\%) & 11.424 & 11.101 & -0.323 & $-2.8 \%$ & yes & 0.568 & 4.97 & 0.1081 & 0.3137 & yes & 15 & 9 \\
\hline 4 & Isolok-HFR-12mL & K2O (wt\%) & 0.177 & 0.175 & -0.002 & $-0.9 \%$ & no & 0.009 & 4.98 & 0.0095 & 0.0072 & no & 16 & 10 \\
\hline 4 & Isolok-HFR-12mL & Li2O (wt\%) & 4.678 & 4.724 & 0.046 & $1.0 \%$ & no & 0.124 & 2.65 & 0.0693 & 0.1098 & no & 14 & 9 \\
\hline 4 & Isolok-HFR-12mL & $\mathrm{MgO}(\mathrm{wt} \%)$ & 1.046 & 1.009 & -0.037 & $-3.6 \%$ & yes & 0.067 & 6.39 & 0.0117 & 0.0381 & yes & 14 & 9 \\
\hline 4 & Isolok-HFR-12mL & $\mathrm{MnO}(\mathrm{wt} \%)$ & 1.742 & 1.677 & -0.065 & $-3.7 \%$ & yes & 0.114 & 6.55 & 0.0241 & 0.0627 & yes & 15 & 9 \\
\hline 4 & Isolok-HFR-12mL & Na2O (wt\%) & 10.923 & 10.888 & -0.035 & $-0.3 \%$ & no & 0.449 & 4.11 & 0.3149 & 0.5479 & yes & 15 & 10 \\
\hline 4 & Isolok-HFR-12mL & $\mathrm{NiO}$ (wt\%) & 0.458 & 0.457 & -0.001 & $-0.2 \%$ & no & 0.010 & 2.18 & 0.0069 & 0.0144 & no & 14 & 9 \\
\hline 4 & Isolok-HFR-12mL & $\mathrm{SiO} 2(\mathrm{wt} \%)$ & 52.342 & 52.028 & -0.314 & $-0.6 \%$ & no & 1.521 & 2.91 & 0.5404 & 1.6626 & yes & 15 & 10 \\
\hline 4 & Isolok-HFR-12mL & Sum (wt\%) & 99.683 & 99.351 & -0.333 & $-0.3 \%$ & no & 1.045 & 1.05 & 0.7563 & 0.9075 & no & 15 & 9 \\
\hline 4 & Isolok-HFR-12mL & TiO2 (wt\%) & 0.079 & 0.080 & 0.001 & $0.7 \%$ & no & 0.003 & 3.35 & 0.0027 & 0.0012 & no & 16 & 8 \\
\hline 4 & Isolok-HFR-12mL & $\mathrm{ZnO}(\mathrm{wt} \%)$ & 0.104 & 0.101 & -0.003 & $-3.2 \%$ & yes & 0.006 & 5.95 & 0.0016 & 0.0036 & yes & 15 & 9 \\
\hline 4 & Isolok-HFR-12mL & $\mathrm{ZrO} 2$ (wt\%) & 0.365 & 0.355 & -0.010 & $-2.8 \%$ & yes & 0.016 & 4.38 & 0.0053 & 0.0089 & no & 15 & 10 \\
\hline
\end{tabular}


SRNL-STI-2011-00693

Revision 0

Table C9. Oxide Comparisons between the Samplers for the Low-Rheology (Phase 3) Testing (screened data)

\begin{tabular}{|c|c|c|c|c|c|c|c|c|c|c|c|c|c|c|}
\hline $\begin{array}{c}\text { Analytical } \\
\text { Block }\end{array}$ & Sampler & Oxide & $\begin{array}{c}\text { Mean } \\
\text { (Measured } \\
\text { wt\%) Coliwasa } \\
\text { (low+high) }\end{array}$ & $\begin{array}{l}\text { Mean(Measured } \\
\text { wt\%) Other } \\
\text { Sampler }\end{array}$ & Diff & \% Diff & $\begin{array}{l}\text { Sig. } \\
\text { Diff }\end{array}$ & $\begin{array}{l}\text { Bias } \\
\text { Bnd }\end{array}$ & $\begin{array}{l}\% \\
\text { Rel } \\
\text { Bias } \\
\text { Bnd } \\
\end{array}$ & $\begin{array}{c}\text { Std } \\
\text { Dev(Measured } \\
\text { wt\%) Coliwasa } \\
\text { (low+high) }\end{array}$ & $\begin{array}{c}\text { Std } \\
\text { Dev(Measured } \\
\text { wt\%) Other } \\
\text { Sampler }\end{array}$ & $\begin{array}{l}\text { Sig. } \\
\text { Diff }\end{array}$ & $\begin{array}{c}\text { n } \\
\text { Col }\end{array}$ & $\begin{array}{c}\text { n } \\
\text { Other }\end{array}$ \\
\hline 5 & Isolok-HFR-3mL & $\mathrm{Al} / \mathrm{B}$ & 2.531 & 2.567 & 0.036 & $1.4 \%$ & no & 0.137 & 5.42 & 0.1194 & 0.1243 & no & 16 & 10 \\
\hline 5 & Isolok-HFR-3mL & Al2O3 (wt\%) & 10.735 & 10.659 & -0.076 & $-0.7 \%$ & no & 0.192 & 1.79 & 0.1055 & 0.1831 & no & 16 & 10 \\
\hline 5 & Isolok-HFR-3mL & B2O3 (wt\%) & 4.250 & 4.160 & -0.090 & $-2.1 \%$ & no & 0.246 & 5.79 & 0.1881 & 0.1858 & no & 16 & 10 \\
\hline 5 & Isolok-HFR-3mL & $\mathrm{BaO}(\mathrm{wt} \%)$ & 0.105 & 0.105 & -0.001 & $-0.5 \%$ & no & 0.003 & 3.06 & 0.0025 & 0.0042 & no & 16 & 10 \\
\hline 5 & Isolok-HFR-3mL & $\mathrm{CaO}(\mathrm{wt} \%)$ & 1.207 & 1.198 & -0.009 & $-0.8 \%$ & no & 0.034 & 2.79 & 0.0308 & 0.0268 & no & 16 & 10 \\
\hline 5 & Isolok-HFR-3mL & Cr2O3 (wt\%) & 0.112 & 0.106 & -0.006 & $-5.2 \%$ & yes & 0.011 & 9.61 & 0.0061 & 0.0054 & no & 15 & 10 \\
\hline 5 & Isolok-HFR-3mL & $\mathrm{CuO}(\mathrm{wt} \%)$ & 0.046 & 0.048 & 0.002 & $5.2 \%$ & no & 0.006 & 12.84 & 0.0036 & 0.0049 & no & 15 & 10 \\
\hline 5 & Isolok-HFR-3mL & $\mathrm{Fe} / \mathrm{Li}$ & 2.363 & 2.288 & -0.075 & $-3.2 \%$ & yes & 0.125 & 5.30 & 0.0490 & 0.0748 & no & 16 & 10 \\
\hline 5 & Isolok-HFR-3mL & Fe2O3 (wt\%) & 11.324 & 11.153 & -0.171 & $-1.5 \%$ & yes & 0.317 & 2.80 & 0.0801 & 0.1988 & yes & 16 & 10 \\
\hline 5 & Isolok-HFR-3mL & K2O (wt\%) & 0.175 & 0.179 & 0.003 & $1.9 \%$ & no & 0.011 & 6.20 & 0.0089 & 0.0088 & no & 14 & 10 \\
\hline 5 & Isolok-HFR-3mL & Li2O (wt\%) & 4.794 & 4.878 & 0.084 & $1.8 \%$ & no & 0.172 & 3.58 & 0.0987 & 0.1149 & no & 16 & 10 \\
\hline 5 & Isolok-HFR-3mL & $\mathrm{MgO}(\mathrm{wt} \%)$ & 1.062 & 1.040 & -0.022 & $-2.1 \%$ & yes & 0.035 & 3.31 & 0.0124 & 0.0197 & no & 16 & 10 \\
\hline 5 & Isolok-HFR-3mL & $\mathrm{MnO}(\mathrm{wt} \%)$ & 1.764 & 1.728 & -0.036 & $-2.1 \%$ & yes & 0.057 & 3.22 & 0.0194 & 0.0309 & no & 16 & 10 \\
\hline 5 & Isolok-HFR-3mL & Na2O (wt\%) & 10.841 & 10.998 & 0.157 & $1.5 \%$ & no & 0.396 & 3.65 & 0.2374 & 0.3126 & no & 14 & 9 \\
\hline 5 & Isolok-HFR-3mL & $\mathrm{NiO}$ (wt\%) & 0.450 & 0.441 & -0.009 & $-1.9 \%$ & yes & 0.016 & 3.66 & 0.0083 & 0.0103 & no & 15 & 9 \\
\hline 5 & Isolok-HFR-3mL & $\mathrm{SiO} 2(\mathrm{wt} \%)$ & 52.426 & 52.990 & 0.564 & $1.1 \%$ & yes & 1.020 & 1.94 & 0.6024 & 0.4402 & no & 16 & 10 \\
\hline 5 & Isolok-HFR-3mL & Sum (wt\%) & 99.871 & 100.349 & 0.478 & $0.5 \%$ & yes & 0.934 & 0.94 & 0.4966 & 0.6235 & no & 16 & 10 \\
\hline 5 & Isolok-HFR-3mL & TiO2 (wt\%) & 0.083 & 0.085 & 0.002 & $2.0 \%$ & no & 0.004 & 4.64 & 0.0026 & 0.0027 & no & 16 & 10 \\
\hline 5 & Isolok-HFR-3mL & $\mathrm{ZnO}$ (wt\%) & 0.100 & 0.099 & -0.001 & $-0.9 \%$ & no & 0.004 & 3.65 & 0.0025 & 0.0042 & no & 15 & 10 \\
\hline 5 & Isolok-HFR-3mL & $\mathrm{ZrO} 2$ (wt\%) & 0.369 & 0.369 & 0.000 & $0.1 \%$ & no & 0.005 & 1.27 & 0.0043 & 0.0064 & no & 16 & 9 \\
\hline 6 & Hydragard-LFR & $\mathrm{Al} / \mathrm{B}$ & 2.635 & 2.736 & 0.101 & $3.8 \%$ & yes & 0.200 & 7.58 & 0.1055 & 0.1305 & no & 16 & 9 \\
\hline 6 & Hydragard-LFR & Al2O3 (wt\%) & 10.687 & 10.900 & 0.213 & $2.0 \%$ & yes & 0.419 & 3.92 & 0.0933 & 0.2648 & yes & 16 & 9 \\
\hline 6 & Hydragard-LFR & B2O3 (wt\%) & 4.061 & 3.989 & -0.072 & $-1.8 \%$ & no & 0.193 & 4.74 & 0.1463 & 0.1272 & no & 16 & 9 \\
\hline 6 & Hydragard-LFR & $\mathrm{BaO}(\mathrm{wt} \%)$ & 0.102 & 0.103 & 0.001 & $1.4 \%$ & no & 0.004 & 3.61 & 0.0021 & 0.0034 & no & 16 & 9 \\
\hline 6 & Hydragard-LFR & $\mathrm{CaO}$ (wt\%) & 1.198 & 1.224 & 0.026 & $2.2 \%$ & no & 0.070 & 5.82 & 0.0252 & 0.0788 & no & 16 & 9 \\
\hline 6 & Hydragard-LFR & Cr2O3 (wt\%) & 0.111 & 0.113 & 0.002 & $1.8 \%$ & no & 0.009 & 8.19 & 0.0055 & 0.0088 & yes & 15 & 9 \\
\hline 6 & Hydragard-LFR & $\mathrm{CuO}(\mathrm{wt} \%)$ & 0.038 & 0.037 & -0.002 & $-4.3 \%$ & no & 0.005 & 13.20 & 0.0033 & 0.0049 & no & 16 & 9 \\
\hline 6 & Hydragard-LFR & $\mathrm{Fe} / \mathrm{Li}$ & 2.459 & 2.560 & 0.101 & $4.1 \%$ & yes & 0.189 & 7.70 & 0.0421 & 0.1129 & yes & 16 & 9 \\
\hline 6 & Hydragard-LFR & $\mathrm{Fe} 2 \mathrm{O} 3(\mathrm{wt} \%)$ & 11.337 & 11.504 & 0.168 & $1.5 \%$ & no & 0.418 & 3.69 & 0.0806 & 0.3237 & yes & 16 & 9 \\
\hline 6 & Hydragard-LFR & K2O (wt\%) & 0.177 & 0.176 & -0.001 & $-0.5 \%$ & no & 0.008 & 4.51 & 0.0095 & 0.0050 & no & 16 & 9 \\
\hline 6 & Hydragard-LFR & $\mathrm{Li} 2 \mathrm{O}$ (wt\%) & 4.611 & 4.497 & -0.114 & $-2.5 \%$ & yes & 0.204 & 4.42 & 0.0584 & 0.1136 & yes & 16 & 9 \\
\hline 6 & Hydragard-LFR & $\mathrm{MgO}$ (wt\%) & 1.022 & 1.035 & 0.013 & $1.3 \%$ & no & 0.046 & 4.53 & 0.0111 & 0.0428 & yes & 16 & 9 \\
\hline 6 & Hydragard-LFR & $\mathrm{MnO}(\mathrm{wt} \%)$ & 1.697 & 1.713 & 0.016 & $0.9 \%$ & no & 0.061 & 3.59 & 0.0182 & 0.0581 & yes & 16 & 9 \\
\hline 6 & Hydragard-LFR & $\mathrm{Na} 2 \mathrm{O}$ (wt\%) & 10.932 & 11.259 & 0.327 & $3.0 \%$ & yes & 0.569 & 5.20 & 0.2182 & 0.3430 & no & 14 & 9 \\
\hline 6 & Hydragard-LFR & $\mathrm{NiO}$ (wt\%) & 0.447 & 0.460 & 0.013 & $2.8 \%$ & yes & 0.025 & 5.55 & 0.0126 & 0.0162 & no & 15 & 9 \\
\hline 6 & Hydragard-LFR & $\mathrm{SiO} 2(\mathrm{wt} \%)$ & 52.386 & 51.201 & -1.186 & $-2.3 \%$ & yes & 1.867 & 3.56 & 0.5348 & 1.1219 & no & 16 & 9 \\
\hline 6 & Hydragard-LFR & Sum (wt\%) & 99.364 & 98.756 & -0.608 & $-0.6 \%$ & yes & 1.104 & 1.11 & 0.4354 & 0.7741 & no & 16 & 9 \\
\hline 6 & Hydragard-LFR & TiO2 (wt\%) & 0.083 & 0.080 & -0.002 & $-2.5 \%$ & yes & 0.004 & 4.40 & 0.0019 & 0.0015 & no & 16 & 8 \\
\hline 6 & Hydragard-LFR & $\mathrm{ZnO}(\mathrm{wt} \%)$ & 0.101 & 0.103 & 0.002 & $2.2 \%$ & no & 0.006 & 5.58 & 0.0029 & 0.0052 & no & 15 & 9 \\
\hline 6 & Hydragard-LFR & $\mathrm{ZrO} 2$ (wt\%) & 0.373 & 0.361 & -0.012 & $-3.2 \%$ & yes & 0.023 & 6.25 & 0.0044 & 0.0146 & yes & 16 & 9 \\
\hline
\end{tabular}


SRNL-STI-2011-00693

Revision 0

Table C10. Phase 3 (Low-Rheology) Sampler Comparisons Grouped by Analytical Block (screened data)

\begin{tabular}{|c|c|c|c|c|c|c|c|c|c|c|c|c|c|c|}
\hline $\begin{array}{l}\text { Analytical } \\
\text { Block }\end{array}$ & Sampler & Oxide & $\begin{array}{l}\text { Mean(Measured } \\
\text { wt\%) Coliwasa } \\
\text { (low+high) }\end{array}$ & $\begin{array}{l}\text { Mean(Measured } \\
\text { wt\%) Other } \\
\text { Sampler }\end{array}$ & Diff & $\%$ Diff & $\begin{array}{l}\text { Sig. } \\
\text { Diff }\end{array}$ & $\begin{array}{l}\text { Bias } \\
\text { Bnd }\end{array}$ & $\begin{array}{c}\% \\
\text { Rel } \\
\text { Bias } \\
\text { Bnd }\end{array}$ & $\begin{array}{c}\text { Std } \\
\text { Dev(Measured } \\
\text { wt\%) } \\
\text { Coliwasa } \\
\text { (low+high) }\end{array}$ & $\begin{array}{c}\text { Std } \\
\text { Dev(Measured } \\
\text { wt\%) Other } \\
\text { Sampler }\end{array}$ & $\begin{array}{l}\text { Sig. } \\
\text { Diff }\end{array}$ & $\begin{array}{c}\mathrm{n} \\
\mathrm{Col}\end{array}$ & $\begin{array}{c}\mathrm{n} \\
\text { Other }\end{array}$ \\
\hline 2 & Hydragard - HFR & $\mathrm{Al} / \mathrm{B}$ & 2.566 & 2.794 & 0.228 & $8.9 \%$ & yes & 0.336 & 13.08 & 0.0989 & 0.1615 & no & 16 & 9 \\
\hline 6 & Hydragard-LFR & $\mathrm{Al} / \mathrm{B}$ & 2.635 & 2.736 & 0.101 & $3.8 \%$ & yes & 0.200 & 7.58 & 0.1055 & 0.1305 & no & 16 & 9 \\
\hline 3 & Isolok-LFR-12mL & $\mathrm{Al} / \mathrm{B}$ & 2.666 & 2.621 & -0.045 & $-1.7 \%$ & no & 0.184 & 6.91 & 0.1079 & 0.1844 & yes & 16 & 10 \\
\hline 5 & Isolok-HFR-3mL & $\mathrm{Al} / \mathrm{B}$ & 2.531 & 2.567 & 0.036 & $1.4 \%$ & no & 0.137 & 5.42 & 0.1194 & 0.1243 & no & 16 & 10 \\
\hline 4 & Isolok-HFR-12mL & $\mathrm{Al} / \mathrm{B}$ & 2.614 & 2.630 & 0.016 & $0.6 \%$ & no & 0.124 & 4.75 & 0.1018 & 0.1653 & no & 16 & 10 \\
\hline 1 & Isolok-LFR-3mL & $\mathrm{Al} / \mathrm{B}$ & 2.521 & 2.518 & -0.003 & $-0.1 \%$ & no & 0.075 & 2.97 & 0.0799 & 0.0971 & no & 16 & 10 \\
\hline 3 & Isolok-LFR-12mL & Al2O3 (wt\%) & 10.677 & 10.366 & -0.311 & $-2.9 \%$ & no & 0.635 & 5.95 & 0.1382 & 0.4466 & yes & 15 & 10 \\
\hline 2 & Hydragard - HFR & $\mathrm{Al} 2 \mathrm{O} 3(\mathrm{wt} \%)$ & 10.532 & 11.025 & 0.494 & $4.7 \%$ & yes & 0.614 & 5.83 & 0.1035 & 0.1946 & no & 16 & 10 \\
\hline 4 & Isolok-HFR-12mL & $\mathrm{Al} 2 \mathrm{O} 3(\mathrm{wt} \%)$ & 10.669 & 10.424 & -0.246 & $-2.3 \%$ & yes & 0.463 & 4.34 & 0.1328 & 0.2748 & yes & 15 & 9 \\
\hline 6 & Hydragard-LFR & Al2O3 (wt\%) & 10.687 & 10.900 & 0.213 & $2.0 \%$ & yes & 0.419 & 3.92 & 0.0933 & 0.2648 & yes & 16 & 9 \\
\hline 1 & Isolok-LFR-3mL & Al2O3 (wt\%) & 10.328 & 10.203 & -0.125 & $-1.2 \%$ & yes & 0.240 & 2.32 & 0.1135 & 0.1662 & no & 15 & 10 \\
\hline 5 & Isolok-HFR-3mL & Al2O3 (wt\%) & 10.735 & 10.659 & -0.076 & $-0.7 \%$ & no & 0.192 & 1.79 & 0.1055 & 0.1831 & no & 16 & 10 \\
\hline 2 & Hydragard - HFR & $\mathrm{B} 2 \mathrm{O} 3(\mathrm{wt} \%)$ & 4.109 & 3.900 & -0.209 & $-5.1 \%$ & yes & 0.343 & 8.35 & 0.1496 & 0.1475 & no & 16 & 8 \\
\hline 5 & Isolok-HFR-3mL & B2O3 (wt\%) & 4.250 & 4.160 & -0.090 & $-2.1 \%$ & no & 0.246 & 5.79 & 0.1881 & 0.1858 & no & 16 & 10 \\
\hline 4 & Isolok-HFR-12mL & B2O3 (wt\%) & 4.067 & 4.012 & -0.055 & $-1.4 \%$ & no & 0.195 & 4.80 & 0.1504 & 0.1945 & no & 16 & 10 \\
\hline 6 & Hydragard-LFR & B2O3 (wt\%) & 4.061 & 3.989 & -0.072 & $-1.8 \%$ & no & 0.193 & 4.74 & 0.1463 & 0.1272 & no & 16 & 9 \\
\hline 3 & Isolok-LFR-12mL & B2O3 (wt\%) & 3.997 & 3.964 & -0.033 & $-0.8 \%$ & no & 0.169 & 4.24 & 0.1577 & 0.1740 & no & 16 & 10 \\
\hline 1 & Isolok-LFR-3mL & B2O3 (wt\%) & 4.134 & 4.096 & -0.037 & $-0.9 \%$ & no & 0.106 & 2.56 & 0.0551 & 0.0990 & no & 13 & 9 \\
\hline 3 & Isolok-LFR-12mL & $\mathrm{BaO}(\mathrm{wt} \%)$ & 0.103 & 0.099 & -0.004 & $-4.0 \%$ & yes & 0.008 & 7.46 & 0.0026 & 0.0047 & yes & 16 & 10 \\
\hline 2 & Hydragard - HFR & $\mathrm{BaO}$ (wt\%) & 0.104 & 0.108 & 0.004 & $4.2 \%$ & yes & 0.007 & 7.01 & 0.0016 & 0.0039 & yes & 16 & 10 \\
\hline 4 & Isolok-HFR-12mL & $\mathrm{BaO}$ (wt\%) & 0.107 & 0.104 & -0.003 & $-2.7 \%$ & yes & 0.005 & 4.35 & 0.0010 & 0.0022 & yes & 14 & 9 \\
\hline 1 & Isolok-LFR-3mL & $\mathrm{BaO}(\mathrm{wt} \%)$ & 0.103 & 0.101 & -0.002 & $-2.4 \%$ & yes & 0.004 & 4.02 & 0.0022 & 0.0013 & no & 15 & 9 \\
\hline 6 & Hydragard-LFR & $\mathrm{BaO}(\mathrm{wt} \%)$ & 0.102 & 0.103 & 0.001 & $1.4 \%$ & no & 0.004 & 3.61 & 0.0021 & 0.0034 & no & 16 & 9 \\
\hline 5 & Isolok-HFR-3mL & $\mathrm{BaO}(\mathrm{wt} \%)$ & 0.105 & 0.105 & -0.001 & $-0.5 \%$ & no & 0.003 & 3.06 & 0.0025 & 0.0042 & no & 16 & 10 \\
\hline 2 & Hydragard - HFR & $\mathrm{CaO}$ (wt\%) & 1.188 & 1.248 & 0.060 & $5.1 \%$ & yes & 0.091 & 7.63 & 0.0258 & 0.0478 & no & 16 & 9 \\
\hline 6 & Hydragard-LFR & $\mathrm{CaO}$ (wt\%) & 1.198 & 1.224 & 0.026 & $2.2 \%$ & no & 0.070 & 5.82 & 0.0252 & 0.0788 & no & 16 & 9 \\
\hline 3 & Isolok-LFR-12mL & $\mathrm{CaO}(\mathrm{wt} \%)$ & 1.189 & 1.152 & -0.037 & $-3.1 \%$ & yes & 0.068 & 5.74 & 0.0302 & 0.0430 & no & 16 & 8 \\
\hline 4 & Isolok-HFR-12mL & $\mathrm{CaO}(\mathrm{wt} \%)$ & 1.190 & 1.153 & -0.037 & $-3.1 \%$ & yes & 0.064 & 5.41 & 0.0149 & 0.0346 & yes & 14 & 9 \\
\hline 1 & Isolok-LFR-3mL & $\mathrm{CaO}$ (wt\%) & 1.159 & 1.129 & -0.031 & $-2.7 \%$ & yes & 0.049 & 4.24 & 0.0222 & 0.0191 & no & 15 & 9 \\
\hline 5 & Isolok-HFR-3mL & $\mathrm{CaO}(\mathrm{wt} \%)$ & 1.207 & 1.198 & -0.009 & $-0.8 \%$ & no & 0.034 & 2.79 & 0.0308 & 0.0268 & no & 16 & 10 \\
\hline 4 & Isolok-HFR-12mL & Cr2O3 (wt\%) & 0.110 & 0.122 & 0.011 & $10.3 \%$ & no & 0.024 & 21.37 & 0.0074 & 0.0165 & yes & 16 & 10 \\
\hline 1 & Isolok-LFR-3mL & Cr2O3 (wt\%) & 0.111 & 0.095 & -0.016 & $-14.3 \%$ & yes & 0.022 & 20.12 & 0.0081 & 0.0071 & no & 16 & 10 \\
\hline 2 & Hydragard - HFR & Cr2O3 (wt\%) & 0.110 & 0.119 & 0.009 & $8.3 \%$ & yes & 0.016 & 14.45 & 0.0075 & 0.0091 & no & 16 & 10 \\
\hline 3 & Isolok-LFR-12mL & Cr2O3 (wt\%) & 0.111 & 0.106 & -0.004 & $-3.8 \%$ & no & 0.012 & 11.14 & 0.0090 & 0.0109 & no & 16 & 10 \\
\hline 5 & Isolok-HFR-3mL & Cr2O3 (wt\%) & 0.112 & 0.106 & -0.006 & $-5.2 \%$ & yes & 0.011 & 9.61 & 0.0061 & 0.0054 & no & 15 & 10 \\
\hline 6 & Hydragard-LFR & Cr2O3 (wt\%) & 0.111 & 0.113 & 0.002 & $1.8 \%$ & no & 0.009 & 8.19 & 0.0055 & 0.0088 & yes & 15 & 9 \\
\hline 4 & Isolok-HFR-12mL & $\mathrm{CuO}(\mathrm{wt} \%)$ & 0.034 & 0.035 & 0.001 & $4.0 \%$ & no & 0.006 & 18.40 & 0.0051 & 0.0069 & no & 16 & 10 \\
\hline
\end{tabular}


SRNL-STI-2011-00693

Revision 0

Table C10. Phase 3 (Low-Rheology) Sampler Comparisons Grouped by Analytical Block (screened data)

\begin{tabular}{|c|c|c|c|c|c|c|c|c|c|c|c|c|c|c|}
\hline $\begin{array}{l}\text { Analytical } \\
\text { Block }\end{array}$ & Sampler & Oxide & $\begin{array}{c}\text { Mean(Measured } \\
\text { wt\%) Coliwasa } \\
\text { (low+high) }\end{array}$ & $\begin{array}{l}\text { Mean(Measured } \\
\text { wt\%) Other } \\
\text { Sampler }\end{array}$ & Diff & $\%$ Diff & $\begin{array}{l}\text { Sig. } \\
\text { Diff }\end{array}$ & $\begin{array}{l}\text { Bias } \\
\text { Bnd }\end{array}$ & $\begin{array}{c}\% \\
\text { Rel } \\
\text { Bias } \\
\text { Bnd }\end{array}$ & $\begin{array}{c}\text { Std } \\
\text { Dev(Measured } \\
\text { wt\%) } \\
\text { Coliwasa } \\
\text { (low+high) }\end{array}$ & $\begin{array}{c}\text { Std } \\
\text { Dev(Measured } \\
\text { wt\%) Other } \\
\text { Sampler }\end{array}$ & $\begin{array}{l}\text { Sig. } \\
\text { Diff }\end{array}$ & $\begin{array}{c}\mathrm{n} \\
\mathrm{Col}\end{array}$ & $\begin{array}{c}\mathrm{n} \\
\text { Other }\end{array}$ \\
\hline 3 & Isolok-LFR-12mL & $\mathrm{CuO}$ (wt\%) & 0.041 & 0.043 & 0.002 & $6.0 \%$ & no & 0.007 & 16.31 & 0.0048 & 0.0054 & no & 16 & 10 \\
\hline 1 & Isolok-LFR-3mL & $\mathrm{CuO}(\mathrm{wt} \%)$ & 0.033 & 0.035 & 0.002 & $5.3 \%$ & no & 0.005 & 15.70 & 0.0041 & 0.0041 & no & 16 & 10 \\
\hline 6 & Hydragard-LFR & $\mathrm{CuO}(\mathrm{wt} \%)$ & 0.038 & 0.037 & -0.002 & $-4.3 \%$ & no & 0.005 & 13.20 & 0.0033 & 0.0049 & no & 16 & 9 \\
\hline 5 & Isolok-HFR-3mL & $\mathrm{CuO}(\mathrm{wt} \%)$ & 0.046 & 0.048 & 0.002 & $5.2 \%$ & no & 0.006 & 12.84 & 0.0036 & 0.0049 & no & 15 & 10 \\
\hline 2 & Hydragard - HFR & $\mathrm{CuO}(\mathrm{wt} \%)$ & 0.037 & 0.038 & 0.001 & $1.9 \%$ & no & 0.004 & 11.08 & 0.0036 & 0.0048 & no & 16 & 10 \\
\hline 3 & Isolok-LFR-12mL & $\mathrm{Fe} / \mathrm{Li}$ & 2.412 & 2.220 & -0.192 & $-8.0 \%$ & yes & 0.328 & 13.62 & 0.0524 & 0.1892 & yes & 16 & 10 \\
\hline 2 & Hydragard - HFR & $\mathrm{Fe} / \mathrm{Li}$ & 2.495 & 2.681 & 0.186 & $7.5 \%$ & yes & 0.250 & 10.04 & 0.0676 & 0.0913 & no & 16 & 10 \\
\hline 6 & Hydragard-LFR & $\mathrm{Fe} / \mathrm{Li}$ & 2.459 & 2.560 & 0.101 & $4.1 \%$ & yes & 0.189 & 7.70 & 0.0421 & 0.1129 & yes & 16 & 9 \\
\hline 5 & Isolok-HFR-3mL & $\mathrm{Fe} / \mathrm{Li}$ & 2.363 & 2.288 & -0.075 & $-3.2 \%$ & yes & 0.125 & 5.30 & 0.0490 & 0.0748 & no & 16 & 10 \\
\hline 4 & Isolok-HFR-12mL & $\mathrm{Fe} / \mathrm{Li}$ & 2.415 & 2.366 & -0.049 & $-2.0 \%$ & no & 0.122 & 5.04 & 0.0636 & 0.1146 & no & 16 & 9 \\
\hline 1 & Isolok-LFR-3mL & $\mathrm{Fe} / \mathrm{Li}$ & 2.442 & 2.417 & -0.025 & $-1.0 \%$ & no & 0.072 & 2.94 & 0.0482 & 0.0682 & no & 16 & 10 \\
\hline 3 & Isolok-LFR-12mL & Fe2O3 (wt\%) & 11.350 & 10.850 & -0.500 & $-4.4 \%$ & yes & 0.912 & 8.04 & 0.1693 & 0.5693 & yes & 15 & 10 \\
\hline 2 & Hydragard - HFR & Fe2O3 (wt\%) & 11.330 & 11.905 & 0.575 & $5.1 \%$ & yes & 0.739 & 6.52 & 0.1131 & 0.2214 & yes & 16 & 10 \\
\hline 4 & Isolok-HFR-12mL & Fe2O3 (wt\%) & 11.424 & 11.101 & -0.323 & $-2.8 \%$ & yes & 0.568 & 4.97 & 0.1081 & 0.3137 & yes & 15 & 9 \\
\hline 6 & Hydragard-LFR & Fe2O3 (wt\%) & 11.337 & 11.504 & 0.168 & $1.5 \%$ & no & 0.418 & 3.69 & 0.0806 & 0.3237 & yes & 16 & 9 \\
\hline 5 & Isolok-HFR-3mL & Fe2O3 (wt\%) & 11.324 & 11.153 & -0.171 & $-1.5 \%$ & yes & 0.317 & 2.80 & 0.0801 & 0.1988 & yes & 16 & 10 \\
\hline 1 & Isolok-LFR-3mL & Fe2O3 (wt\%) & 11.072 & 10.896 & -0.176 & $-1.6 \%$ & yes & 0.284 & 2.57 & 0.0957 & 0.1668 & no & 15 & 10 \\
\hline 1 & Isolok-LFR-3mL & K2O (wt\%) & 0.177 & 0.180 & 0.003 & $1.5 \%$ & no & 0.011 & 6.36 & 0.0095 & 0.0114 & no & 16 & 10 \\
\hline 5 & Isolok-HFR-3mL & K2O (wt\%) & 0.175 & 0.179 & 0.003 & $1.9 \%$ & no & 0.011 & 6.20 & 0.0089 & 0.0088 & no & 14 & 10 \\
\hline 2 & Hydragard - HFR & K2O (wt\%) & 0.177 & 0.175 & -0.002 & $-1.1 \%$ & no & 0.009 & 5.03 & 0.0095 & 0.0061 & no & 16 & 10 \\
\hline 4 & Isolok-HFR-12mL & K2O (wt\%) & 0.177 & 0.175 & -0.002 & $-0.9 \%$ & no & 0.009 & 4.98 & 0.0095 & 0.0072 & no & 16 & 10 \\
\hline 3 & Isolok-LFR-12mL & K2O (wt\%) & 0.177 & 0.179 & 0.002 & $1.4 \%$ & no & 0.008 & 4.52 & 0.0095 & 0.0038 & yes & 16 & 9 \\
\hline 6 & Hydragard-LFR & K2O (wt\%) & 0.177 & 0.176 & -0.001 & $-0.5 \%$ & no & 0.008 & 4.51 & 0.0095 & 0.0050 & no & 16 & 9 \\
\hline 3 & Isolok-LFR-12mL & $\mathrm{Li} 2 \mathrm{O}$ (wt\%) & 4.708 & 4.902 & 0.194 & $4.1 \%$ & yes & 0.347 & 7.38 & 0.0982 & 0.2063 & yes & 15 & 10 \\
\hline 6 & Hydragard-LFR & $\mathrm{Li} 2 \mathrm{O}$ (wt\%) & 4.611 & 4.497 & -0.114 & $-2.5 \%$ & yes & 0.204 & 4.42 & 0.0584 & 0.1136 & yes & 16 & 9 \\
\hline 2 & Hydragard - HFR & $\mathrm{Li} 2 \mathrm{O}$ (wt\%) & 4.544 & 4.444 & -0.100 & $-2.2 \%$ & yes & 0.186 & 4.10 & 0.0993 & 0.1099 & no & 16 & 10 \\
\hline 5 & Isolok-HFR-3mL & Li2O (wt\%) & 4.794 & 4.878 & 0.084 & $1.8 \%$ & no & 0.172 & 3.58 & 0.0987 & 0.1149 & no & 16 & 10 \\
\hline 4 & Isolok-HFR-12mL & Li2O (wt\%) & 4.678 & 4.724 & 0.046 & $1.0 \%$ & no & 0.124 & 2.65 & 0.0693 & 0.1098 & no & 14 & 9 \\
\hline 1 & Isolok-LFR-3mL & $\mathrm{Li} 2 \mathrm{O}$ (wt\%) & 4.517 & 4.510 & -0.007 & $-0.1 \%$ & no & 0.097 & 2.15 & 0.0986 & 0.1240 & no & 16 & 10 \\
\hline 3 & Isolok-LFR-12mL & $\mathrm{MgO}(\mathrm{wt} \%)$ & 1.033 & 0.970 & -0.063 & $-6.1 \%$ & yes & 0.113 & 10.89 & 0.0216 & 0.0681 & yes & 16 & 10 \\
\hline 2 & Hydragard - HFR & $\mathrm{MgO}(\mathrm{wt} \%)$ & 1.006 & 1.061 & 0.055 & $5.5 \%$ & yes & 0.075 & 7.43 & 0.0139 & 0.0263 & yes & 16 & 10 \\
\hline 4 & Isolok-HFR-12mL & $\mathrm{MgO}(\mathrm{wt} \%)$ & 1.046 & 1.009 & -0.037 & $-3.6 \%$ & yes & 0.067 & 6.39 & 0.0117 & 0.0381 & yes & 14 & 9 \\
\hline 6 & Hydragard-LFR & $\mathrm{MgO}$ (wt\%) & 1.022 & 1.035 & 0.013 & $1.3 \%$ & no & 0.046 & 4.53 & 0.0111 & 0.0428 & yes & 16 & 9 \\
\hline 1 & Isolok-LFR-3mL & $\mathrm{MgO}$ (wt\%) & 1.001 & 0.977 & -0.025 & $-2.5 \%$ & yes & 0.035 & 3.54 & 0.0109 & 0.0154 & no & 15 & 10 \\
\hline 5 & Isolok-HFR-3mL & $\mathrm{MgO}(\mathrm{wt} \%)$ & 1.062 & 1.040 & -0.022 & $-2.1 \%$ & yes & 0.035 & 3.31 & 0.0124 & 0.0197 & no & 16 & 10 \\
\hline 3 & Isolok-LFR-12mL & $\mathrm{MnO}(\mathrm{wt} \%)$ & 1.709 & 1.610 & -0.099 & $-5.8 \%$ & yes & 0.182 & 10.66 & 0.0394 & 0.1144 & yes & 16 & 10 \\
\hline 2 & Hydragard - HFR & $\mathrm{MnO}$ (wt\%) & 1.673 & 1.760 & 0.087 & $5.2 \%$ & yes & 0.119 & 7.11 & 0.0236 & 0.0426 & yes & 16 & 10 \\
\hline
\end{tabular}


SRNL-STI-2011-00693

Revision 0

Table C10. Phase 3 (Low-Rheology) Sampler Comparisons Grouped by Analytical Block (screened data)

\begin{tabular}{|c|c|c|c|c|c|c|c|c|c|c|c|c|c|c|}
\hline $\begin{array}{c}\text { Analytical } \\
\text { Block }\end{array}$ & Sampler & Oxide & $\begin{array}{c}\text { Mean(Measured } \\
\text { wt\%) Coliwasa } \\
\text { (low+high) }\end{array}$ & $\begin{array}{l}\text { Mean(Measured } \\
\text { wt\%) Other } \\
\text { Sampler }\end{array}$ & Diff & \% Diff & $\begin{array}{l}\text { Sig. } \\
\text { Diff }\end{array}$ & $\begin{array}{l}\text { Bias } \\
\text { Bnd }\end{array}$ & $\begin{array}{c}\% \\
\text { Rel } \\
\text { Bias } \\
\text { Bnd } \\
\end{array}$ & $\begin{array}{c}\text { Std } \\
\text { Dev(Measured } \\
\text { wt\%) } \\
\text { Coliwasa } \\
\text { (low+high) } \\
\end{array}$ & $\begin{array}{c}\text { Std } \\
\text { Dev(Measured } \\
\text { wt\%) Other } \\
\text { Sampler }\end{array}$ & $\begin{array}{l}\text { Sig. } \\
\text { Diff }\end{array}$ & $\begin{array}{c}\mathrm{n} \\
\mathrm{Col} \\
\end{array}$ & $\begin{array}{c}n \\
\text { Other }\end{array}$ \\
\hline 4 & Isolok-HFR-12mL & $\mathrm{MnO}(\mathrm{wt} \%)$ & 1.742 & 1.677 & -0.065 & $-3.7 \%$ & yes & 0.114 & 6.55 & 0.0241 & 0.0627 & yes & 15 & 9 \\
\hline 1 & Isolok-LFR-3mL & $\mathrm{MnO}(\mathrm{wt} \%)$ & 1.668 & 1.627 & -0.041 & $-2.5 \%$ & yes & 0.060 & 3.62 & 0.0196 & 0.0265 & no & 15 & 10 \\
\hline 6 & Hydragard-LFR & $\mathrm{MnO}(\mathrm{wt} \%)$ & 1.697 & 1.713 & 0.016 & $0.9 \%$ & no & 0.061 & 3.59 & 0.0182 & 0.0581 & yes & 16 & 9 \\
\hline 5 & Isolok-HFR-3mL & $\mathrm{MnO}(\mathrm{wt} \%)$ & 1.764 & 1.728 & -0.036 & $-2.1 \%$ & yes & 0.057 & 3.22 & 0.0194 & 0.0309 & no & 16 & 10 \\
\hline 6 & Hydragard-LFR & $\mathrm{Na} 2 \mathrm{O}$ (wt\%) & 10.932 & 11.259 & 0.327 & $3.0 \%$ & yes & 0.569 & 5.20 & 0.2182 & 0.3430 & no & 14 & 9 \\
\hline 3 & Isolok-LFR-12mL & $\mathrm{Na} 2 \mathrm{O}$ (wt\%) & 10.881 & 11.090 & 0.209 & $1.9 \%$ & no & 0.470 & 4.32 & 0.2917 & 0.3223 & no & 14 & 10 \\
\hline 4 & Isolok-HFR-12mL & $\mathrm{Na} 2 \mathrm{O}(\mathrm{wt} \%)$ & 10.923 & 10.888 & -0.035 & $-0.3 \%$ & no & 0.449 & 4.11 & 0.3149 & 0.5479 & yes & 15 & 10 \\
\hline 5 & Isolok-HFR-3mL & $\mathrm{Na} 2 \mathrm{O}(\mathrm{wt} \%)$ & 10.841 & 10.998 & 0.157 & $1.5 \%$ & no & 0.396 & 3.65 & 0.2374 & 0.3126 & no & 14 & 9 \\
\hline 1 & Isolok-LFR-3mL & $\mathrm{Na} 2 \mathrm{O}$ (wt\%) & 10.346 & 10.296 & -0.050 & $-0.5 \%$ & no & 0.326 & 3.15 & 0.3024 & 0.3595 & no & 15 & 10 \\
\hline 2 & Hydragard - HFR & $\mathrm{Na2O}$ (wt\%) & 10.997 & 10.996 & 0.000 & $0.0 \%$ & no & 0.164 & 1.49 & 0.1716 & 0.1867 & no & 14 & 8 \\
\hline 2 & Hydragard - HFR & $\mathrm{NiO}$ (wt\%) & 0.451 & 0.474 & 0.022 & $5.0 \%$ & yes & 0.039 & 8.64 & 0.0075 & 0.0227 & yes & 15 & 10 \\
\hline 3 & Isolok-LFR-12mL & $\mathrm{NiO}(\mathrm{wt} \%)$ & 0.454 & 0.441 & -0.013 & $-2.8 \%$ & no & 0.031 & 6.82 & 0.0117 & 0.0246 & yes & 14 & 10 \\
\hline 6 & Hydragard-LFR & $\mathrm{NiO}(\mathrm{wt} \%)$ & 0.447 & 0.460 & 0.013 & $2.8 \%$ & yes & 0.025 & 5.55 & 0.0126 & 0.0162 & no & 15 & 9 \\
\hline 1 & Isolok-LFR-3mL & $\mathrm{NiO}$ (wt\%) & 0.457 & 0.446 & -0.011 & $-2.4 \%$ & no & 0.024 & 5.36 & 0.0177 & 0.0138 & no & 16 & 10 \\
\hline 5 & Isolok-HFR-3mL & $\mathrm{NiO}$ (wt\%) & 0.450 & 0.441 & -0.009 & $-1.9 \%$ & yes & 0.016 & 3.66 & 0.0083 & 0.0103 & no & 15 & 9 \\
\hline 4 & Isolok-HFR-12mL & $\mathrm{NiO}$ (wt\%) & 0.458 & 0.457 & -0.001 & $-0.2 \%$ & no & 0.010 & 2.18 & 0.0069 & 0.0144 & no & 14 & 9 \\
\hline 6 & Hydragard-LFR & $\mathrm{SiO} 2(\mathrm{wt} \%)$ & 52.386 & 51.201 & -1.186 & $-2.3 \%$ & yes & 1.867 & 3.56 & 0.5348 & 1.1219 & no & 16 & 9 \\
\hline 2 & Hydragard - HFR & $\mathrm{SiO} 2(\mathrm{wt} \%)$ & 52.239 & 51.258 & -0.981 & $-1.9 \%$ & yes & 1.616 & 3.09 & 0.7083 & 0.8450 & no & 16 & 10 \\
\hline 4 & Isolok-HFR-12mL & $\mathrm{SiO} 2(\mathrm{wt} \%)$ & 52.342 & 52.028 & -0.314 & $-0.6 \%$ & no & 1.521 & 2.91 & 0.5404 & 1.6626 & yes & 15 & 10 \\
\hline 3 & Isolok-LFR-12mL & $\mathrm{SiO} 2(\mathrm{wt} \%)$ & 52.584 & 53.081 & 0.497 & $0.9 \%$ & no & 1.187 & 2.26 & 0.8828 & 0.4033 & no & 15 & 8 \\
\hline 5 & Isolok-HFR-3mL & $\mathrm{SiO} 2(\mathrm{wt} \%)$ & 52.426 & 52.990 & 0.564 & $1.1 \%$ & yes & 1.020 & 1.94 & 0.6024 & 0.4402 & no & 16 & 10 \\
\hline 1 & Isolok-LFR-3mL & $\mathrm{SiO} 2(w \mathrm{t} \%)$ & 52.185 & 52.477 & 0.292 & $0.6 \%$ & no & 0.899 & 1.72 & 0.6183 & 0.8501 & no & 15 & 10 \\
\hline 3 & Isolok-LFR-12mL & Sum of Oxides (wt\%) & 99.652 & 99.365 & -0.286 & $-0.3 \%$ & no & 1.218 & 1.22 & 1.1470 & 1.0306 & no & 15 & 10 \\
\hline 6 & Hydragard-LFR & Sum of Oxides (wt\%) & 99.364 & 98.756 & -0.608 & $-0.6 \%$ & yes & 1.104 & 1.11 & 0.4354 & 0.7741 & no & 16 & 9 \\
\hline 4 & Isolok-HFR-12mL & Sum of Oxides (wt\%) & 99.683 & 99.351 & -0.333 & $-0.3 \%$ & no & 1.045 & 1.05 & 0.7563 & 0.9075 & no & 15 & 9 \\
\hline 1 & Isolok-LFR-3mL & Sum of Oxides (wt\%) & 97.808 & 97.572 & -0.236 & $-0.2 \%$ & no & 0.965 & 0.99 & 0.7392 & 1.0271 & no & 15 & 10 \\
\hline 5 & Isolok-HFR-3mL & Sum of Oxides (wt\%) & 99.871 & 100.349 & 0.478 & $0.5 \%$ & yes & 0.934 & 0.94 & 0.4966 & 0.6235 & no & 16 & 10 \\
\hline 2 & Hydragard - HFR & Sum of Oxides (wt\%) & 99.062 & 99.135 & 0.073 & $0.1 \%$ & no & 0.664 & 0.67 & 0.7801 & 0.5730 & no & 16 & 10 \\
\hline 5 & Isolok-HFR-3mL & $\mathrm{TiO} 2(\mathrm{wt} \%)$ & 0.083 & 0.085 & 0.002 & $2.0 \%$ & no & 0.004 & 4.64 & 0.0026 & 0.0027 & no & 16 & 10 \\
\hline 2 & Hydragard - HFR & $\mathrm{TiO} 2(\mathrm{wt} \%)$ & 0.082 & 0.081 & -0.001 & $-1.7 \%$ & no & 0.004 & 4.42 & 0.0025 & 0.0029 & no & 16 & 10 \\
\hline 6 & Hydragard-LFR & TiO2 (wt\%) & 0.083 & 0.080 & -0.002 & $-2.5 \%$ & yes & 0.004 & 4.40 & 0.0019 & 0.0015 & no & 16 & 8 \\
\hline 1 & Isolok-LFR-3mL & TiO2 (wt\%) & 0.081 & 0.083 & 0.002 & $2.0 \%$ & no & 0.003 & 4.26 & 0.0020 & 0.0027 & no & 16 & 10 \\
\hline 4 & Isolok-HFR-12mL & TiO2 (wt\%) & 0.079 & 0.080 & 0.001 & $0.7 \%$ & no & 0.003 & 3.35 & 0.0027 & 0.0012 & no & 16 & 8 \\
\hline 3 & Isolok-LFR-12mL & $\mathrm{TiO} 2(\mathrm{wt} \%)$ & 0.082 & 0.082 & 0.000 & $0.5 \%$ & no & 0.002 & 2.48 & 0.0022 & 0.0013 & no & 15 & 10 \\
\hline 3 & Isolok-LFR-12mL & $\mathrm{ZnO}(\mathrm{wt} \%)$ & 0.103 & 0.099 & -0.005 & $-4.6 \%$ & no & 0.010 & 9.90 & 0.0032 & 0.0075 & yes & 16 & 10 \\
\hline 2 & Hydragard - HFR & $\mathrm{ZnO}(\mathrm{wt} \%)$ & 0.101 & 0.106 & 0.005 & $4.9 \%$ & yes & 0.010 & 9.42 & 0.0015 & 0.0064 & yes & 16 & 10 \\
\hline 4 & Isolok-HFR-12mL & $\mathrm{ZnO}$ (wt\%) & 0.104 & 0.101 & -0.003 & $-3.2 \%$ & yes & 0.006 & 5.95 & 0.0016 & 0.0036 & yes & 15 & 9 \\
\hline
\end{tabular}


SRNL-STI-2011-00693

Revision 0

Table C10. Phase 3 (Low-Rheology) Sampler Comparisons Grouped by Analytical Block (screened data)

\begin{tabular}{|c|c|c|c|c|c|c|c|c|c|c|c|c|c|c|}
\hline $\begin{array}{c}\text { Analytical } \\
\text { Block }\end{array}$ & Sampler & Oxide & $\begin{array}{c}\text { Mean(Measured } \\
\text { wt\%) Coliwasa } \\
\text { (low+high) }\end{array}$ & $\begin{array}{l}\text { Mean(Measured } \\
\text { wt\%) Other } \\
\text { Sampler }\end{array}$ & Diff & $\%$ Diff & $\begin{array}{l}\text { Sig. } \\
\text { Diff }\end{array}$ & $\begin{array}{l}\text { Bias } \\
\text { Bnd }\end{array}$ & $\begin{array}{c}\% \\
\text { Rel } \\
\text { Bias } \\
\text { Bnd } \\
\end{array}$ & $\begin{array}{c}\text { Std } \\
\text { Dev(Measured } \\
\text { wt\%) } \\
\text { Coliwasa } \\
\text { (low+high) } \\
\end{array}$ & $\begin{array}{c}\text { Std } \\
\text { Dev(Measured } \\
\text { wt\%) Other } \\
\text { Sampler }\end{array}$ & $\begin{array}{l}\text { Sig. } \\
\text { Diff }\end{array}$ & $\begin{array}{c}\mathrm{n} \\
\mathrm{Col}\end{array}$ & $\begin{array}{c}\mathrm{n} \\
\text { Other }\end{array}$ \\
\hline 6 & Hydragard-LFR & $\mathrm{ZnO}$ (wt\%) & 0.101 & 0.103 & 0.002 & $2.2 \%$ & no & 0.006 & 5.58 & 0.0029 & 0.0052 & no & 15 & 9 \\
\hline 1 & Isolok-LFR-3mL & $\mathrm{ZnO}(\mathrm{wt} \%)$ & 0.102 & 0.100 & -0.003 & $-2.8 \%$ & yes & 0.005 & 5.35 & 0.0032 & 0.0032 & no & 16 & 10 \\
\hline 5 & Isolok-HFR-3mL & $\mathrm{ZnO}(\mathrm{wt} \%)$ & 0.100 & 0.099 & -0.001 & $-0.9 \%$ & no & 0.004 & 3.65 & 0.0025 & 0.0042 & no & 15 & 10 \\
\hline 6 & Hydragard-LFR & $\mathrm{ZrO} 2$ (wt\%) & 0.373 & 0.361 & -0.012 & $-3.2 \%$ & yes & 0.023 & 6.25 & 0.0044 & 0.0146 & yes & 16 & 9 \\
\hline 3 & Isolok-LFR-12mL & $\mathrm{ZrO} 2(\mathrm{wt} \%)$ & 0.370 & 0.357 & -0.013 & $-3.5 \%$ & yes & 0.020 & 5.35 & 0.0045 & 0.0093 & yes & 15 & 10 \\
\hline 4 & Isolok-HFR-12mL & $\mathrm{ZrO} 2(\mathrm{wt} \%)$ & 0.365 & 0.355 & -0.010 & $-2.8 \%$ & yes & 0.016 & 4.38 & 0.0053 & 0.0089 & no & 15 & 10 \\
\hline 2 & Hydragard - HFR & $\mathrm{ZrO} 2$ (wt\%) & 0.375 & 0.379 & 0.004 & $1.2 \%$ & no & 0.009 & 2.49 & 0.0051 & 0.0069 & no & 16 & 9 \\
\hline 1 & Isolok-LFR-3mL & $\mathrm{ZrO} 2$ (wt\%) & 0.372 & 0.370 & -0.002 & $-0.6 \%$ & no & 0.005 & 1.43 & 0.0028 & 0.0048 & no & 15 & 10 \\
\hline 5 & Isolok-HFR-3mL & $\mathrm{ZrO} 2$ (wt\%) & 0.369 & 0.369 & 0.000 & $0.1 \%$ & no & 0.005 & 1.27 & 0.0043 & 0.0064 & no & 16 & 9 \\
\hline
\end{tabular}


SRNL-STI-2011-00693

Revision 0

Exhibit C1. Density, Weight Percent Solids, and Oxide Measurements from the Low-Rheology (Phase 3) Testing

Analyte $=$ density $(\mathrm{g} / \mathrm{mL})$

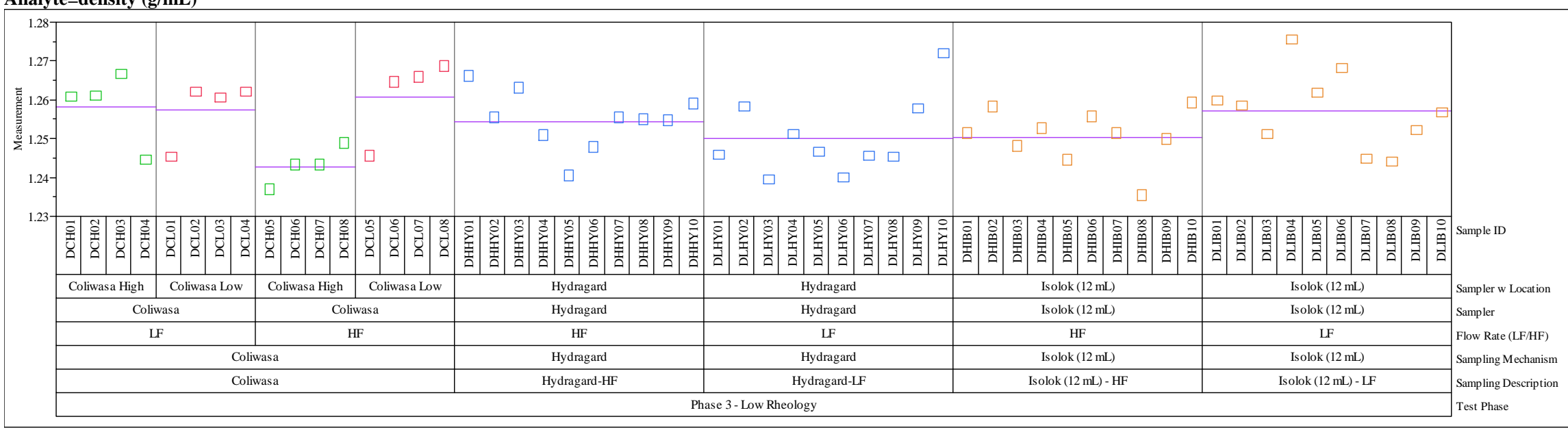

Analyte $=\mathbf{w t} \%$ dried solids

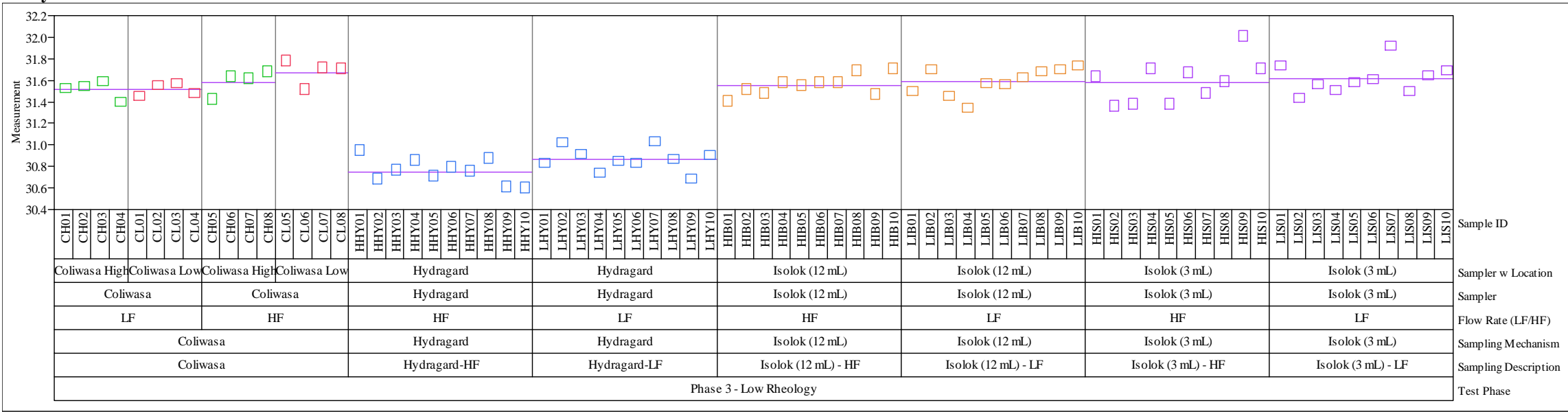


SRNL-STI-2011-00693

Revision 0

Exhibit C1. Density, Weight Percent Solids, and Oxide Measurements from the Low-Rheology (Phase 3) Testing

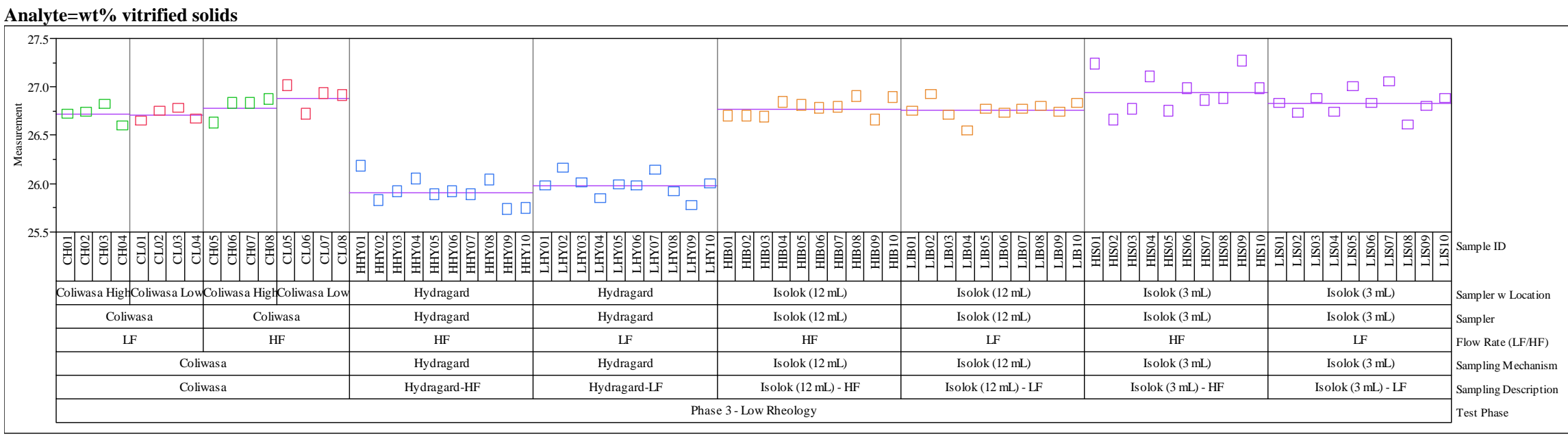

Variability Gauge Type of Material=SME Simulant, Oxide/Analyte $=$ Al/B

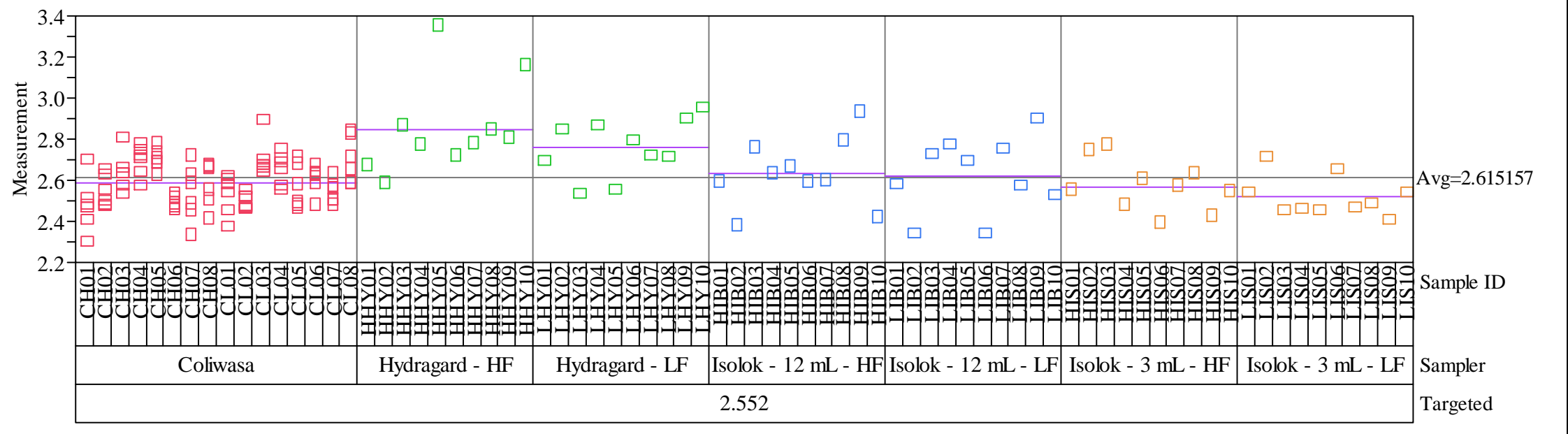


Exhibit C1. Density, Weight Percent Solids, and Oxide Measurements from the Low-Rheology (Phase 3) Testing

Variability Gauge Type of Material=SME Simulant, Oxide/Analyte=Al2O3 (wt\%)

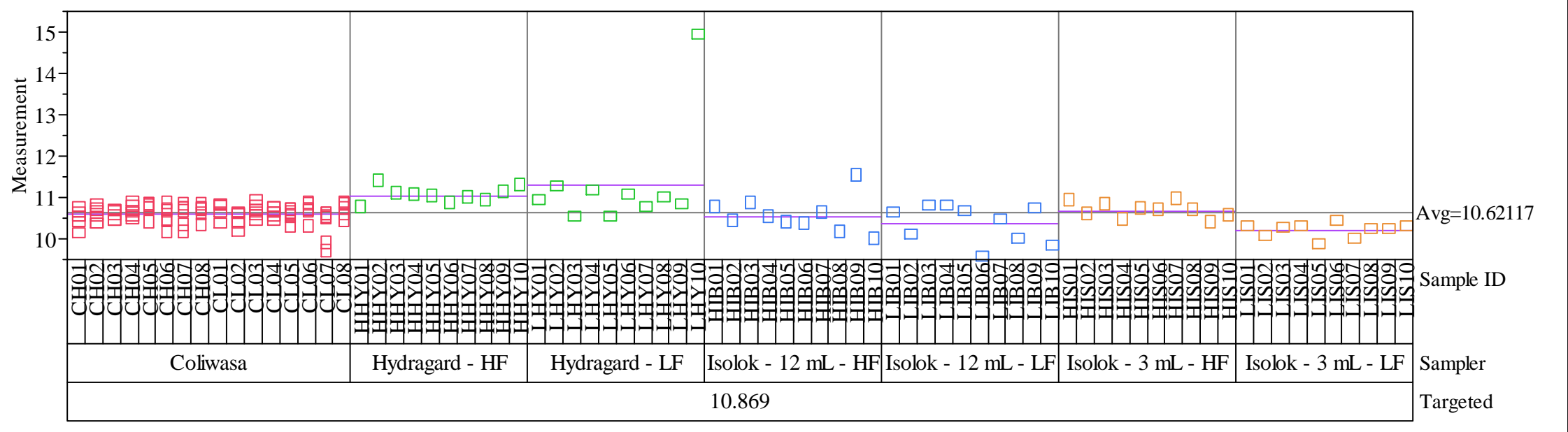

Variability Gauge Type of Material=SME Simulant, Oxide/Analyte=B2O3 (wt\%)

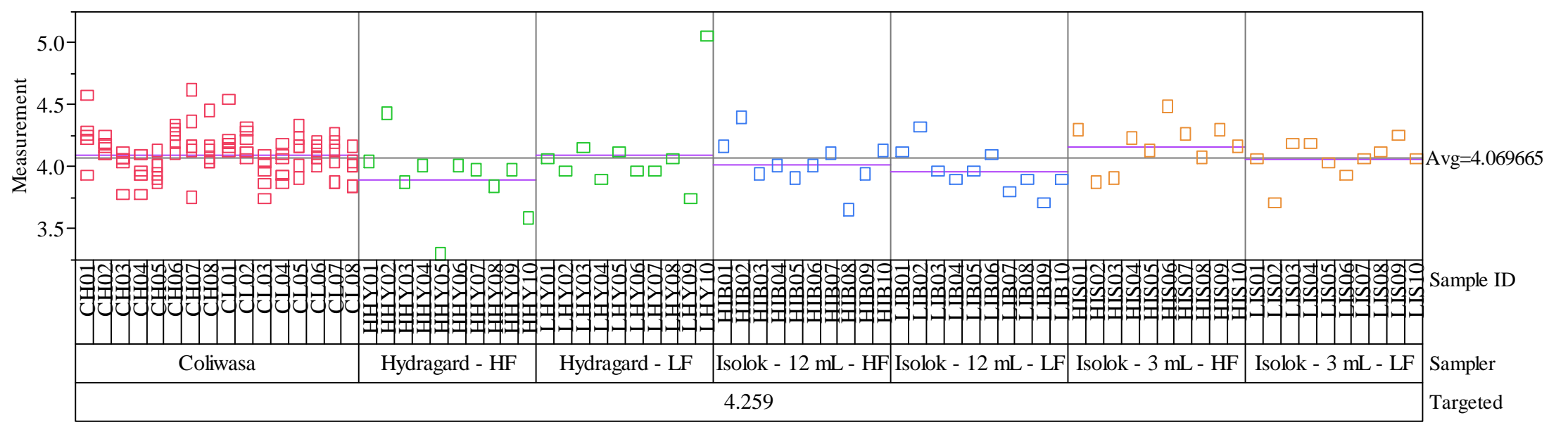


Exhibit C1. Density, Weight Percent Solids, and Oxide Measurements from the Low-Rheology (Phase 3) Testing

Revision 0

Variability Gauge Type of Material=SME Simulant, Oxide/Analyte=BaO (wt\%)

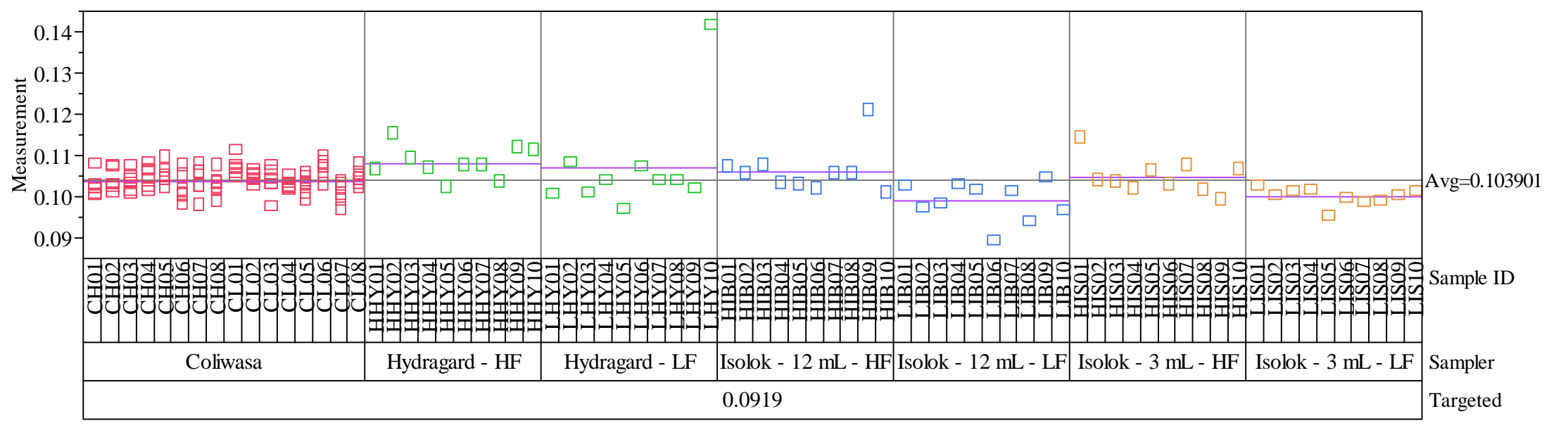

Variability Gauge Type of Material=SME Simulant, Oxide/Analyte=CaO (wt\%)

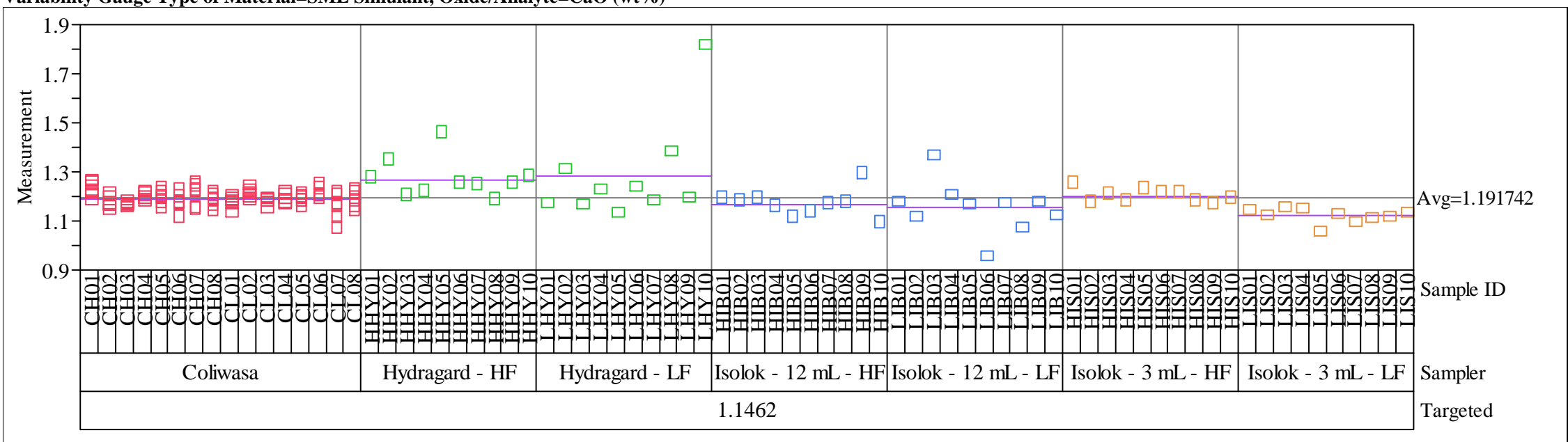


Exhibit C1. Density, Weight Percent Solids, and Oxide Measurements from the Low-Rheology (Phase 3) Testing

Variability Gauge Type of Material=SME Simulant, Oxide/Analyte=Cr2O3 (wt\%)

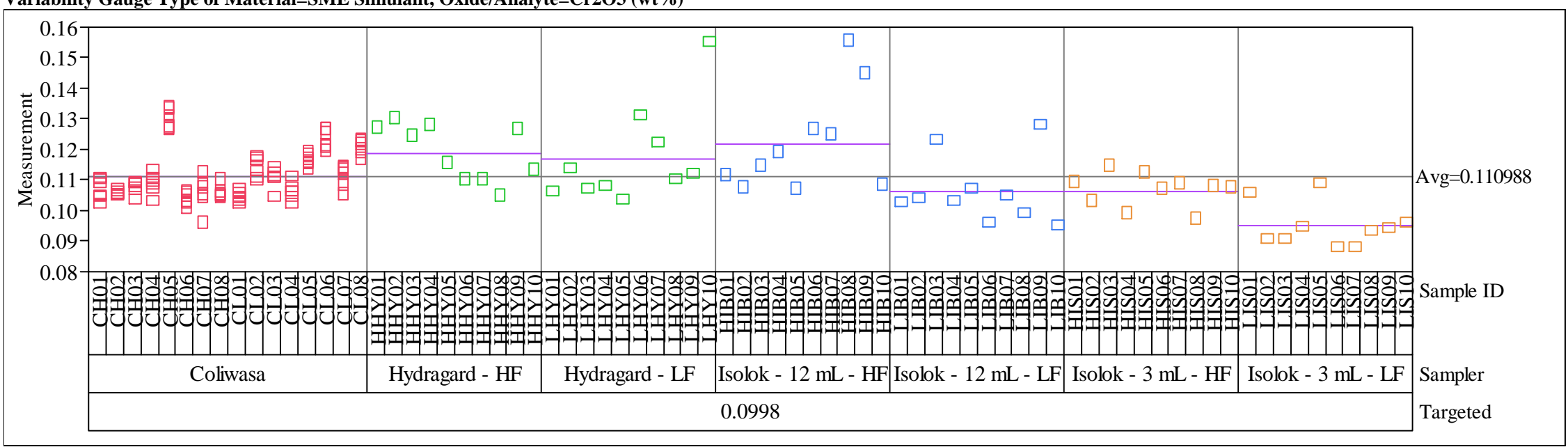

Variability Gauge Type of Material=SME Simulant, Oxide/Analyte=CuO (wt\%)

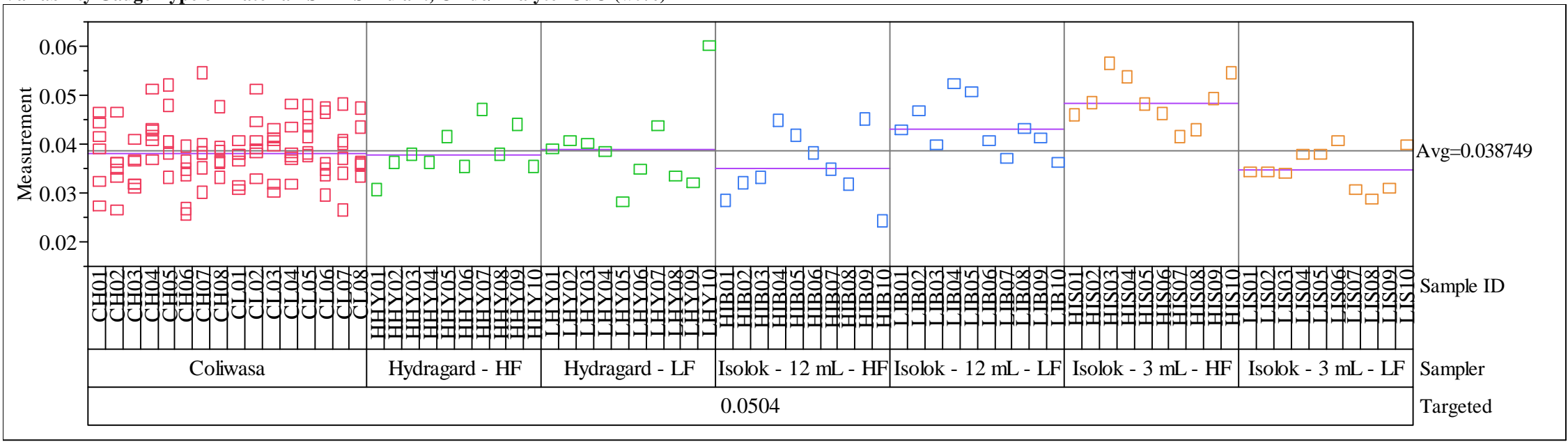


Exhibit C1. Density, Weight Percent Solids, and Oxide Measurements from the Low-Rheology (Phase 3) Testing

Variability Gauge Type of Material=SME Simulant, Oxide/Analyte=Fe/Li

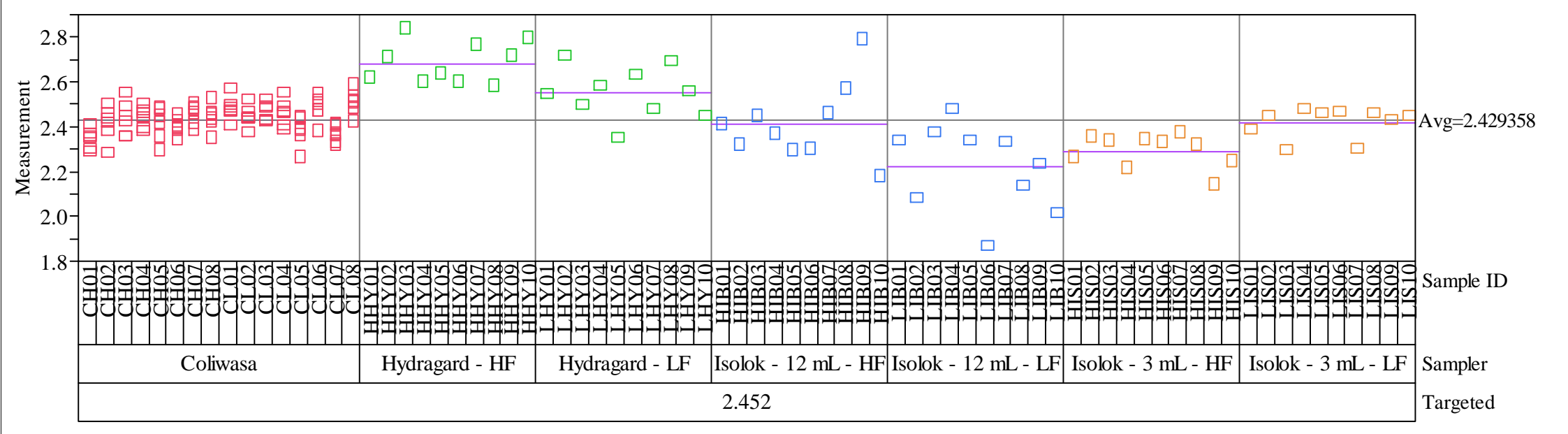

Variability Gauge Type of Material=SME Simulant, Oxide/Analyte=Fe2O3 (wt\%)

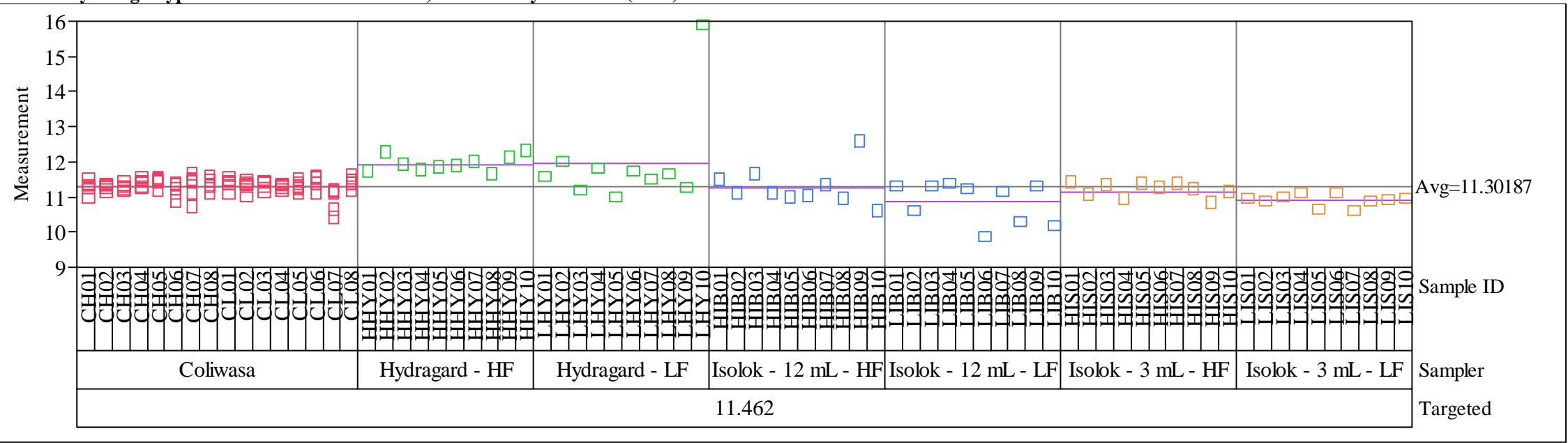


Exhibit C1. Density, Weight Percent Solids, and Oxide Measurements from the Low-Rheology (Phase 3) Testing

Variability Gauge Type of Material=SME Simulant, Oxide/Analyte=K2O (wt \%)

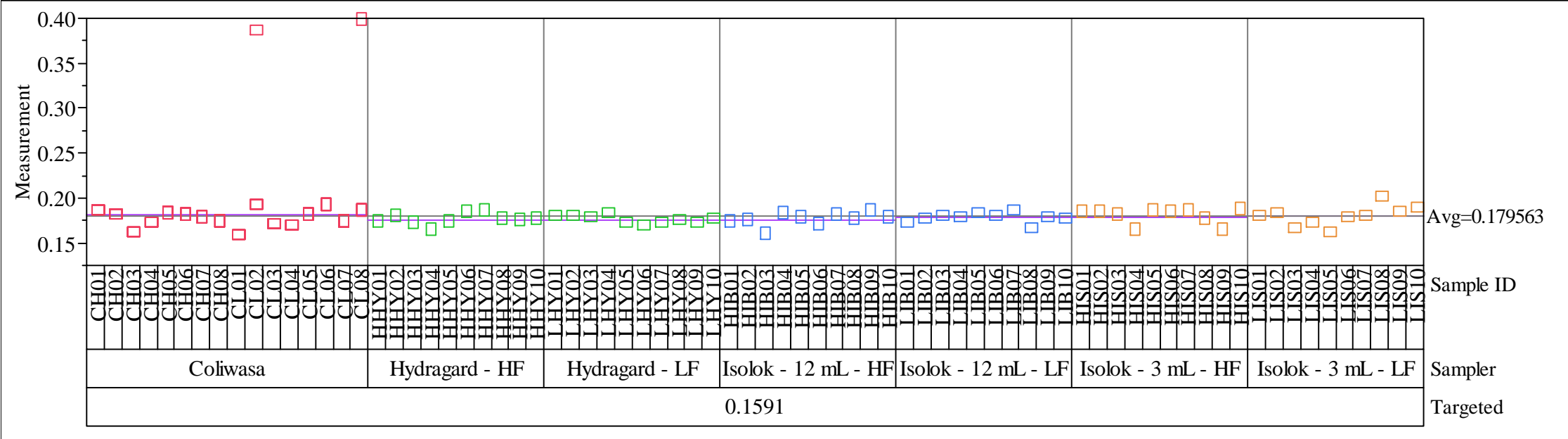

Variability Gauge Type of Material=SME Simulant, Oxide/Analyte=Li2O (wt\%)

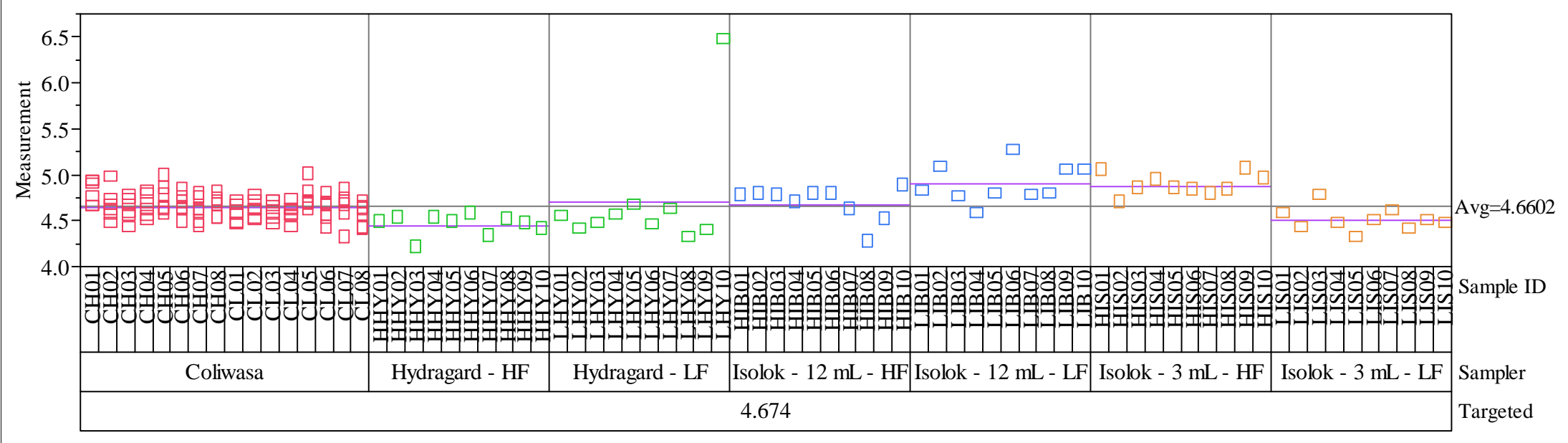


Exhibit C1. Density, Weight Percent Solids, and Oxide Measurements from the Low-Rheology (Phase 3) Testing

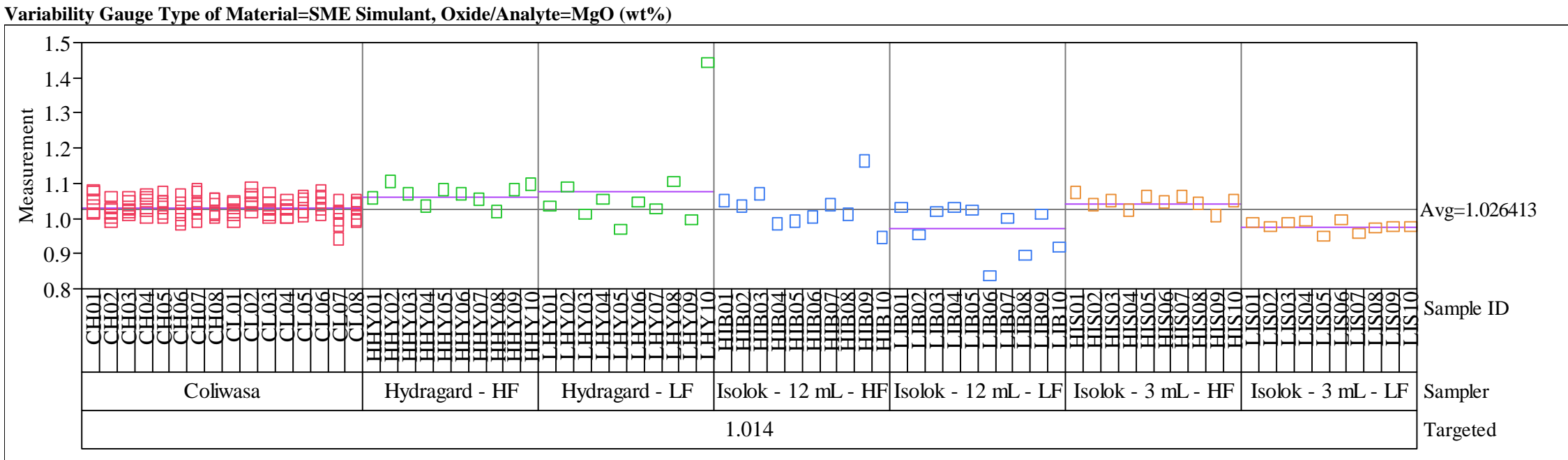

Variability Gauge Type of Material=SME Simulant, Oxide/Analyte=MnO (wt\%)

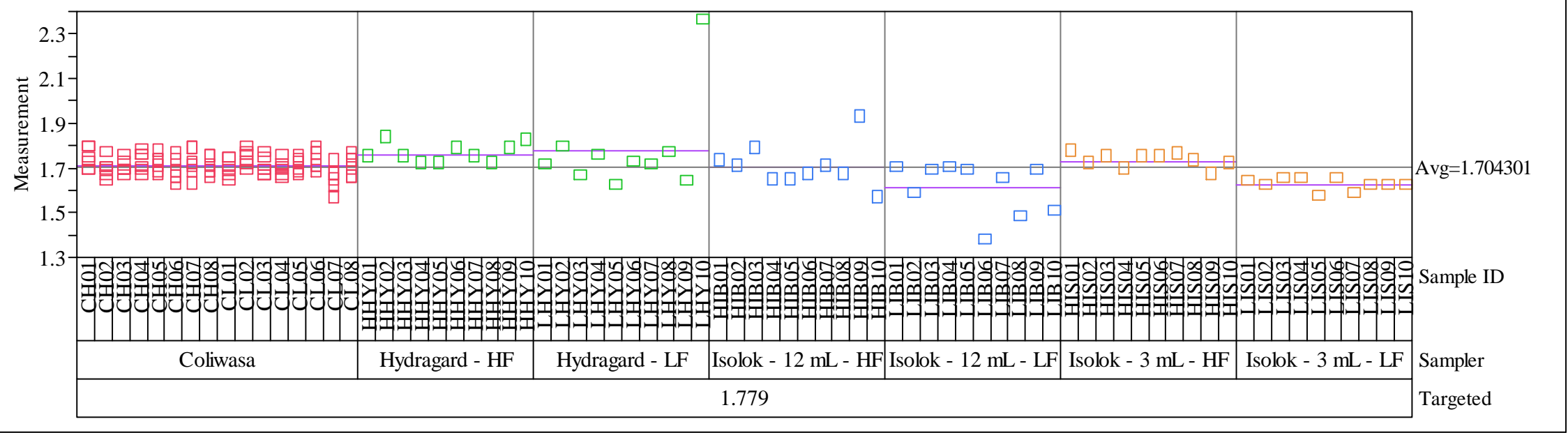


Exhibit C1. Density, Weight Percent Solids, and Oxide Measurements from the Low-Rheology (Phase 3) Testing

Variability Gauge Type of Material=SME Simulant, Oxide/Analyte=Na2O (wt\%)

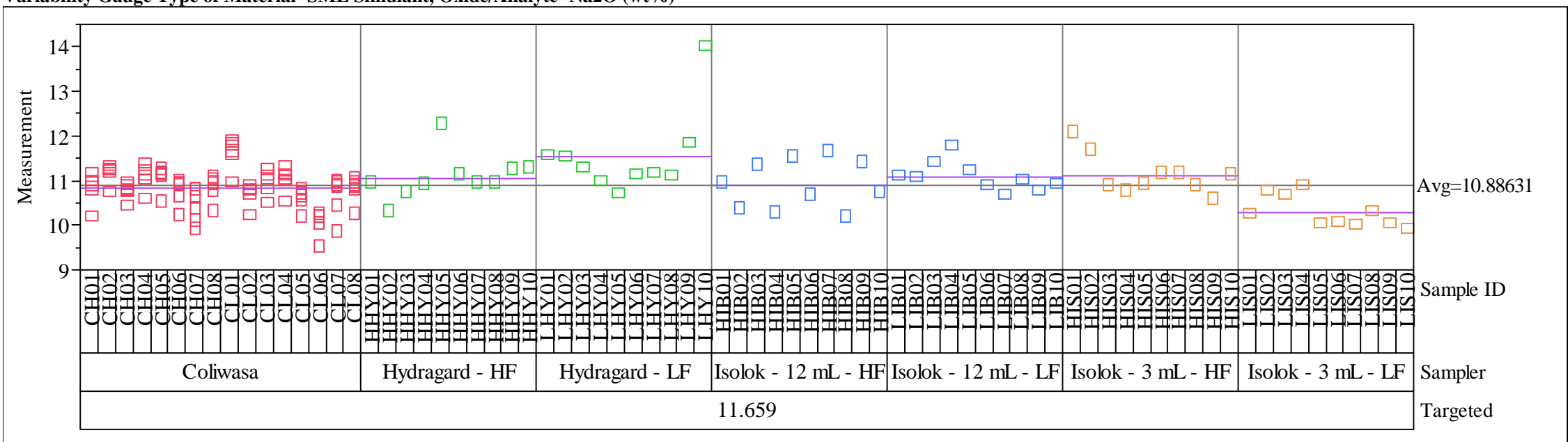

Variability Gauge Type of Material=SME Simulant, Oxide/Analyte=NiO (wt\%)

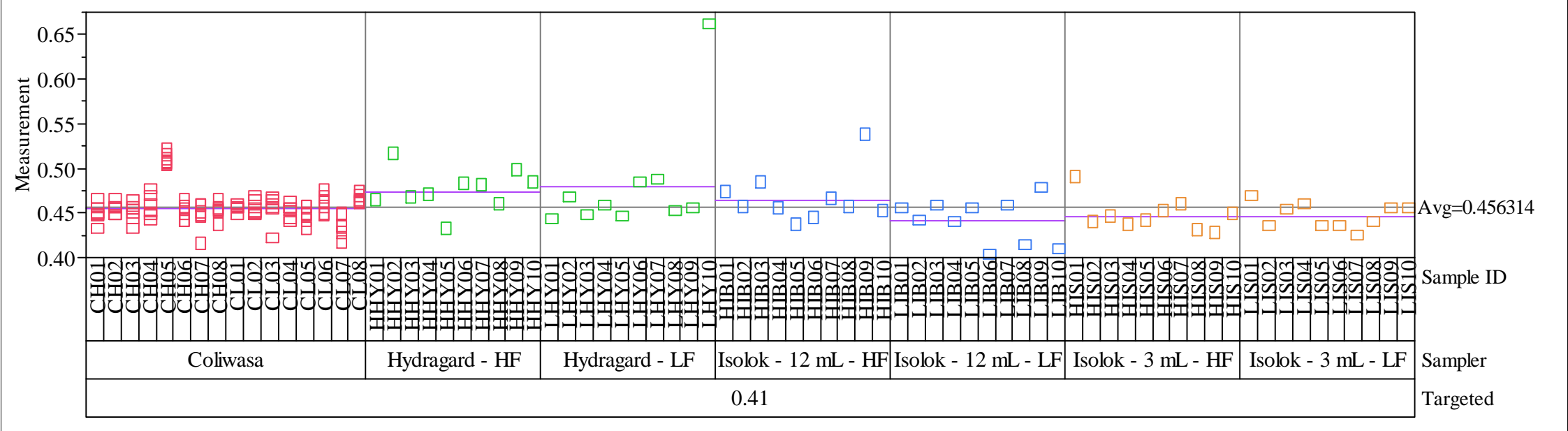


Exhibit C1. Density, Weight Percent Solids, and Oxide Measurements from the Low-Rheology (Phase 3) Testing

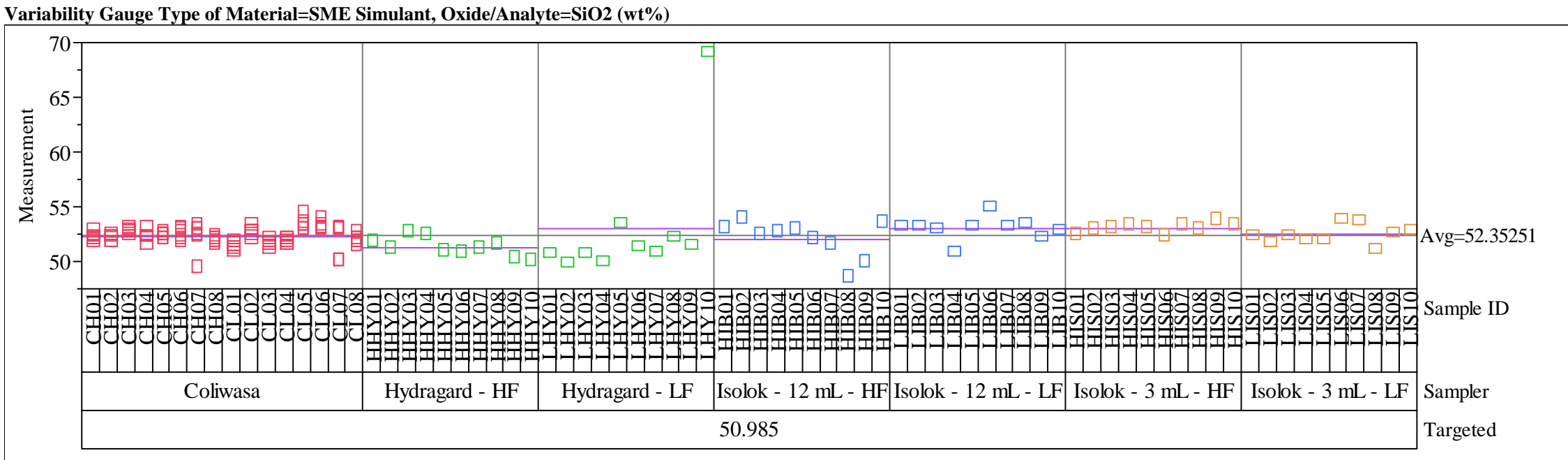

Variability Gauge Type of Material=SME Simulant, Oxide/Analyte=Sum of Oxides (wt \%)

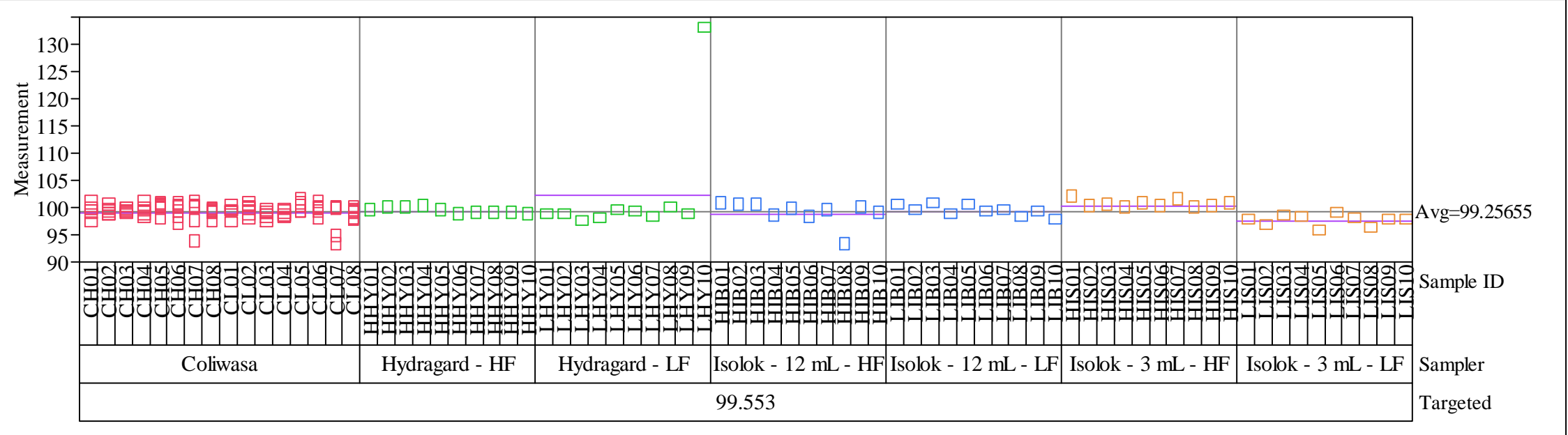


Exhibit C1. Density, Weight Percent Solids, and Oxide Measurements from the Low-Rheology (Phase 3) Testing

Variability Gauge Type of Material=SME Simulant, Oxide/Analyte=TiO2 (wt\%)

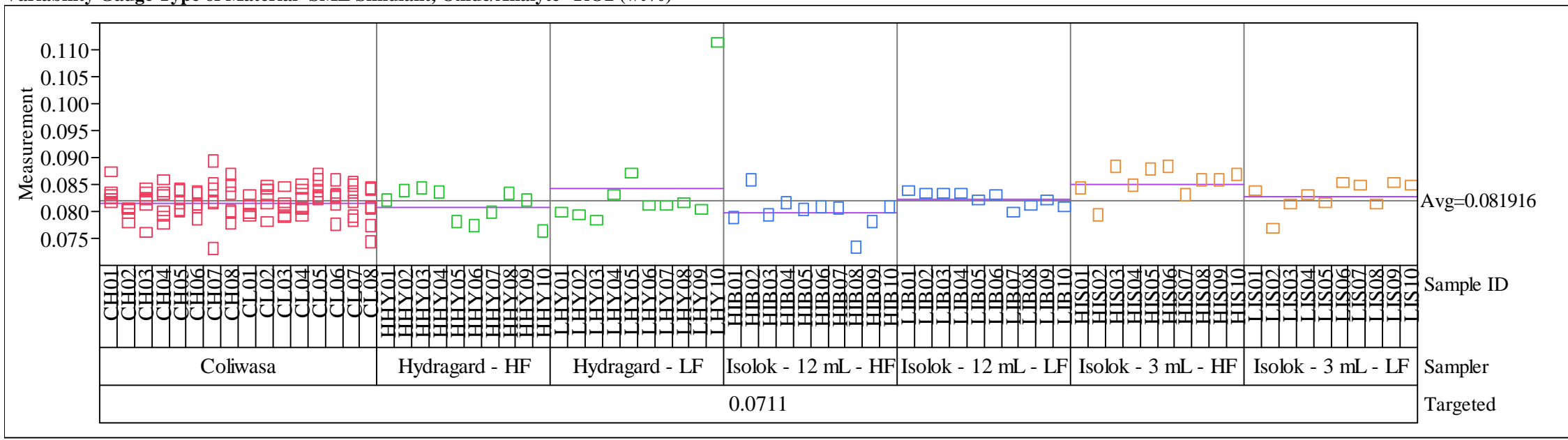

Variability Gauge Type of Material=SME Simulant, Oxide/Analyte=ZnO (wt\%)

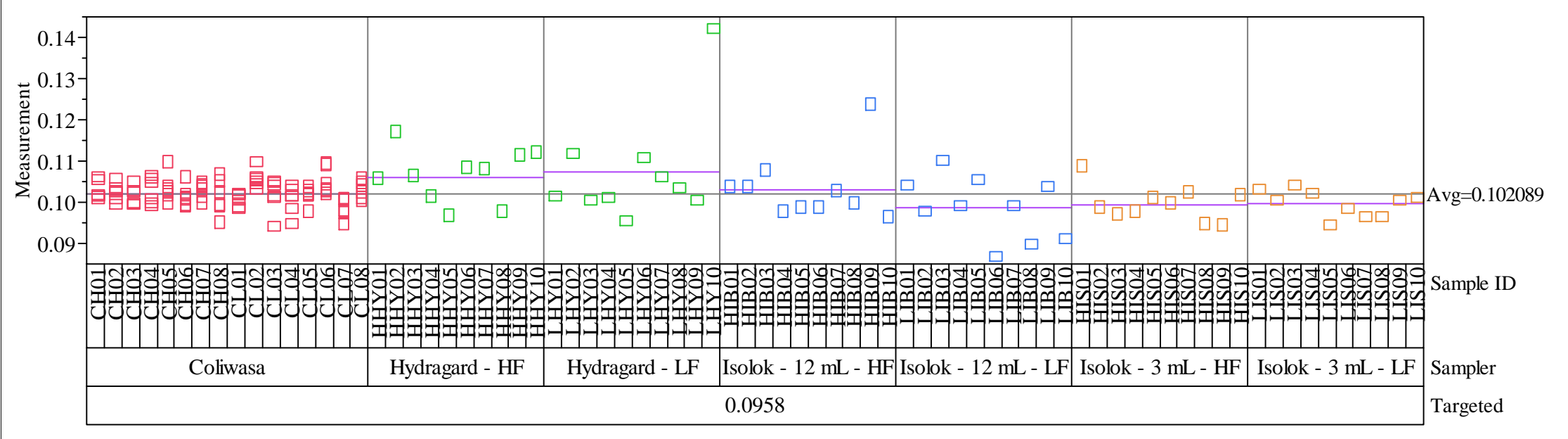


Exhibit C1. Density, Weight Percent Solids, and Oxide Measurements from the Low-Rheology (Phase 3) Testing

Variability Gauge Type of Material=SME Simulant, Oxide/Analyte=ZrO2 (wt\%)

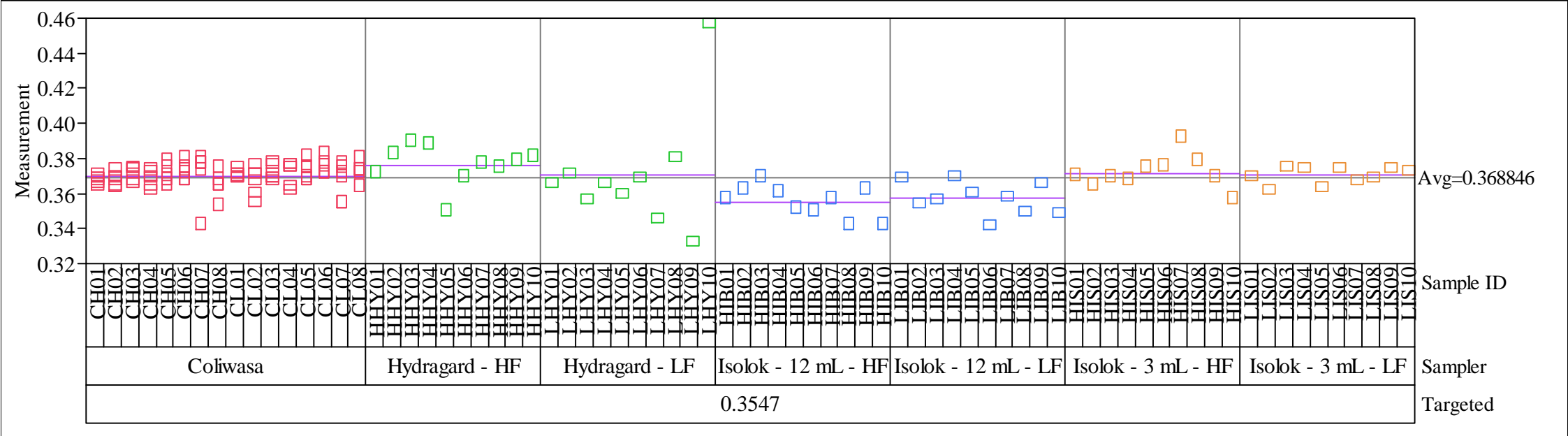


SRNL-STI-2011-00693

Revision 0

\section{Exhibit C2. Statistical Comparisons for Low-Rheology (Phase 3) Simulant Testing}

Oneway Analysis of Measurement By Sampler w Location Test Phase=Phase 3 - Low Rheology, Analyte=sample density $(\mathrm{g} / \mathrm{mL})$

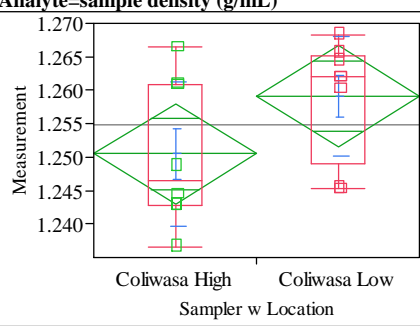

Oneway Anova

Summary of Fit

Adj Rsquare

0.179

0.120357

1.254805

t Test

Coliwasa Low-Coliwasa High

Assuming equal variances

Difference $\quad 0.00866$ t Ratio $\quad 1.747107$

Std Err Dif $\quad 0.00496$ DF $\quad 14$

Upper CL Dif 0.01929 Prob $>|t| \quad 0.1025$

Lower CL Dif -0.00197 Prob $>$ t $\quad 0.0513$

$\begin{array}{lrl}\text { Confidence } & 0.95 \text { Prob }<\mathrm{t} & 0.9487\end{array}$

Analysis of Variance

$\begin{array}{lrrrrr}\text { Source } & \text { DF } & \text { Sum of Squares } & \text { Mean Square } & \text { F Ratio } & \text { Prob }>\text { F } \\ \text { Sampler w Location } & 1 & 0.00029991 & 0.000300 & 3.0524 & 0.1025\end{array}$

$\begin{array}{llll}\text { Error } & 14 & 0.00137554 & 0.000098\end{array}$

$\begin{array}{lll}\text { C. Total } & 15 & 0.00167545\end{array}$

Means for Oneway Anova

Level Number Mean Std Error Lower 95\% Upper 95\%

$\begin{array}{lrrrrr}\text { Coliwasa High } & 8 & 1.25048 & 0.00350 & 1.2430 & 1.2580\end{array}$

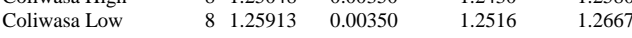

Std Error uses a pooled estimate of error variance

Means and Std Deviations

Level Number Mean Std Dev Std Err Mean Lower 95\% Upper 95\%

Level Hum 8 , $1.25048 \quad 0.010849$

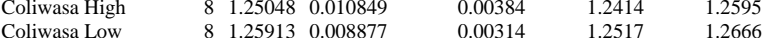

Test

Coliwasa Low-Coliwasa High

Assuming unequal variances

Difference $\quad 0.00866$ t Ratio $\quad 1.747107$

Std Err Dif $\quad 0.00496$ DF $\quad 13.47219$

Upper CL Dif 0.01933 Prob $>|t| \quad 0.1034$

Lower CL Dif -0.00201 Prob $>t \quad 0.0517$

$\begin{array}{lrl}\text { Confidence } & 0.95 \text { Prob }<\mathrm{t} & 0.9483\end{array}$

Tests that the Variances are Equal

$\begin{array}{lrrrr}\text { Level } & \text { Count } & \text { Std Dev } & \text { MeanAbsDif to Mean } & \text { MeanAbsDif to Median } \\ \text { Coliwasa High } & 8 & 0.0108490 & 0.0092351 & 0.0087784\end{array}$

$\begin{array}{lllll}\text { Coliwasa High } & 8 & 0.0108490 & 0.0092351 & 0.0087784 \\ \text { Coliwasa Low } & 8 & 0.0088772 & 0.0069162 & 0.0058942\end{array}$

Test F Ratio DFNum DFDen p-Value

$\begin{array}{lrrrr}\text { O'Brien[.5] } & 0.5789 & 1 & 14 & 0.4594\end{array}$

$\begin{array}{lllll}\text { Brown-Forsythe } & 0.6941 & 1 & 14 & 0.4187\end{array}$

$\begin{array}{lllll}\text { Levene } & 0.9696 & 1 & 14 & 0.3415\end{array}$

$\begin{array}{lllll}\text { Bartlett } & 0.2611 & 1 & \text {. } & 0.6094\end{array}$

Welch Anova testing Means Equal, allowing Std Devs Not Equal

F Ratio DFNum DFDen Prob $>$ F

$\begin{array}{rrrr}3.0524 & 1 & 13.472 & 0.1034\end{array}$

t Test
1.7471
Oneway Analysis of Measurement By Sampler w Location Test Phase=Phase 3 - Low Rheology, Analyte $=\mathbf{w t} \%$ dried solids

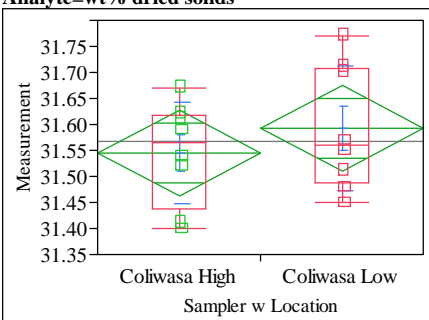

Oneway Anova

Summary of Fit

Adj Rsquare

ror $\quad 0.109005$

Mean of Response 31.56875

Observations (or Sum Wgts) $\quad 16$

Test

Coliwasa Low-Coliwasa High

Assuming equal variances

Difference $\quad 0.04750$ t Ratio $\quad 0.871518$

Std Err Dif $\quad 0.05450$ DF 14

Upper CL Dif 0.16440 Prob $>|t| \quad 0.3982$

Lower CL Dif -0.06940 Prob $>t \quad 0.1991$

$\begin{array}{lrl}\text { Confidence } & 0.95 \text { Prob }<\mathrm{t} & 0.8009\end{array}$

Analysis of Variance

$\begin{array}{lrrrrr}\text { Source } & \text { DF } & \text { Sum of Squares } & \text { Mean Square } & \text { F Ratio } & \text { Prob > F } \\ \text { Sampler w Location } & 1 & 0.00902500 & 0.009025 & 0.7595 & 0.3982 \\ \text { Error } & 14 & 0.16635000 & 0.011882 & & \\ \text { C. Total } & 15 & 0.17537500 & & & \end{array}$

Means for Oneway Anova

Level Number Mean Std Error Lower 95\% Upper 95\%

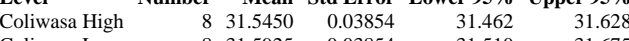

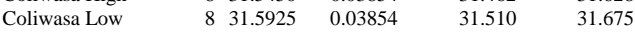

Std Error uses a pooled estimate of error variance

Means and Std Deviations

Level Number Mean Std Dev Std Err Mean Lower 95\% Upper 95\%

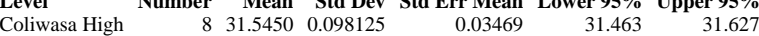

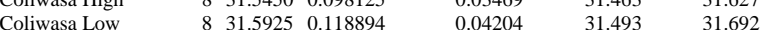

Test

Coliwasa Low-Coliwasa High

Assuming unequal variances

$\begin{array}{lrlr}\text { Difference } & 0.04750 & \mathrm{t} \text { Ratio } & 0.871518 \\ \text { Std Err Dif } & 0.05450 & \mathrm{DF} & 13.51389 \\ \text { Upper CL Dif } & 0.16479 & \text { Prob }>|\mathrm{t}| & 0.3987 \\ \text { Lower CL Dif } & -0.06979 & \text { Prob }>\mathrm{t} & 0.1993 \\ \text { Confidence } & 0.95 & \text { Prob }<\mathrm{t} & 0.8007\end{array}$

Tests that the Variances are Equal

$\begin{array}{lrrrr}\text { Level } & \text { Count } & \text { Std Dev } & \text { MeanAbsDif to Mean } & \text { MeanAbsDif to Median } \\ \text { Coliwasa High } & 8 & 0.0981253 & 0.0775000 & 0.0775000\end{array}$

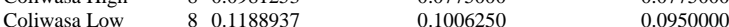

Test F Ratio DFNum DFDen p-Value

$\begin{array}{llrrr}\text { O'Brien[.5] } & 0.6084 & 1 & 14 & 0.4484\end{array}$

Brown-Forsythe $\quad 0.2970 \quad 11 \quad 14 \quad 0.5944$

$\begin{array}{lllll}\text { Levene } & 0.8029 & 1 & 14 & 0.3854\end{array}$

$\begin{array}{lllll}\text { Bartlett } & 0.2393 & 1 & \text {. } & 0.6247\end{array}$

F Test 2-sided 1.4681

Welch Anova testing Means Equal, allowing Std Devs Not Equal

F Ratio DFNum DFDen Prob $>$ F

$\begin{array}{lrrr}0.7595 & 1 & 13.514 & 0.3987\end{array}$

t Test 


\section{Exhibit C2. Statistical Comparisons for Low-Rheology (Phase 3) Simulant Testing}

Oneway Analysis of Measurement By Sampler w Location Test Phase=Phase 3 - Low Rheology, Analyte $=$ wt $\%$ vitrified solids

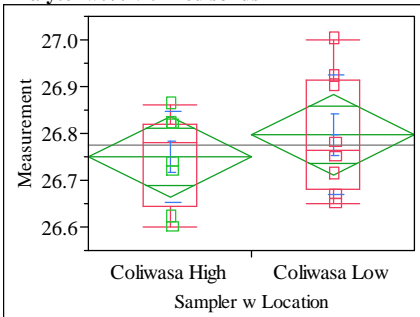

Oneway Anova

Summary of Fit

Adj Rsquare

0.047257

$-0.0208$

Root Mean Square Error $\quad 0.114002$

Mean of Response

26.77375

Observations (or Sum Wgts) 16

t Test

Coliwasa Low-Coliwasa High

Assuming equal variances

Difference $\quad 0.04750$ t Ratio 0.83332

Std Err Dif $\quad 0.05700$ DF $\quad 14$

Upper CL Dif 0.16975 Prob $>|t| \quad 0.4187$

Lower CL Dif -0.07475 Prob $>\mathrm{t} \quad 0.2093$

$\begin{array}{lr}\text { Confidence } & 0.95 \text { Prob }<\mathrm{t} \quad 0.7907\end{array}$

Analysis of Variance

$\begin{array}{lrrrrr}\text { Source } & \text { DF } & \text { Sum of Squares } & \text { Mean Square } & \text { F Ratio } & \text { Prob }>\text { F } \\ \text { Sampler w Location } & 1 & 0.00902500 & 0.009025 & 0.6944 & 0.4187\end{array}$

$\begin{array}{llll}\text { Error } & 14 & 0.18195000 & 0.012996\end{array}$

$\begin{array}{lll}\text { C. Total } & 15 & 0.19097500\end{array}$

Means for Oneway Anova

Level Number Mean Std Error Lower 95\% Upper 95\%

$\begin{array}{lrrrrr}\text { Coliwasa High } & 8 & 26.7500 & 0.04031 & 26.664 & 26.836\end{array}$

$\begin{array}{llllll}\text { Coliwasa Low } & 8 & 26.7975 & 0.04031 & 26.711 & 26.884\end{array}$

Std Error uses a pooled estimate of error variance

Means and Std Deviations

Level Number Mean Std Dev Std Err Mean Lower 95\% Upper 95\%

Coliwasa High Number 26.7500 0.097980

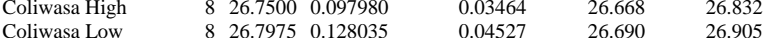

t Test

Coliwasa Low-Coliwasa High

Assuming unequal variances

$\begin{array}{lrlr}\text { Difference } & 0.04750 & \text { t Ratio } & 0.83332 \\ \text { Std Err Dif } & 0.05700 & \text { DF } & 13.10498 \\ \text { Upper CL Dif } & 0.17054 & \text { Prob }>|t| & 0.4196 \\ \text { Lower CL Dif } & -0.07554 & \text { Prob }>\text { t } & 0.2098 \\ \text { Confidence } & 0.95 & \text { Prob }<\mathrm{t} & 0.7902\end{array}$

Tests that the Variances are Equal

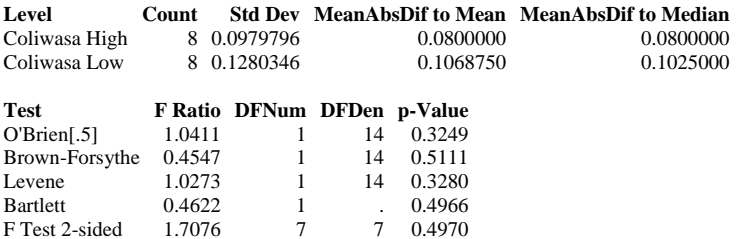

Welch Anova testing Means Equal, allowing Std Devs Not Equal

F Ratio DFNum DFDen Prob $>$ F

$0.6944 \quad \begin{array}{rrr}1 & 13.105 & 0.4196\end{array}$

t Test
Oneway Analysis of Measurement By Sampling Description Test Phase=Phase 3 - Low Rheology, Analyte=sample density $(\mathrm{g} / \mathrm{mL})$

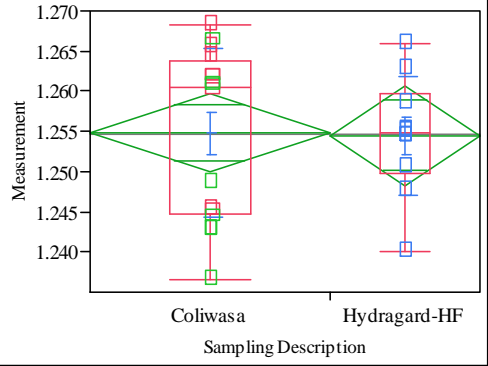

Oneway Anova

Summary of Fit

Rsquare

Adj Rsquare

Root Mean Square Error $\quad-0.04134$

Observations (or Sum Wgts) $\quad 26$

t Test

Hydragard-HF-Coliwasa

Assuming equal variances

Difference $\quad-0.00033$ t Ratio -0.0863

Std Err Dif $\quad 0.00383$ DF 24

Upper CL Dif 0.00757 Prob $>|t| \quad 0.9319$

Lower CL Dif -0.00823 Prob $>t \quad 0.5340$

Confidence $\quad 0.95$ Prob $<\mathrm{t} \quad 0.4660$

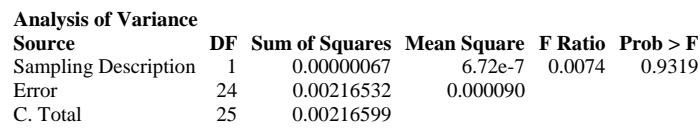

C. Total

Means for Oneway Anova

Level Number Mean Std Error Lower 95\% Upper 95\%

$\begin{array}{llllll}\text { Coliwasa } & 16 & 1.25481 & 0.00237 & 1.2499 & 1.2597\end{array}$

$\begin{array}{llllll}\text { Hydragard-HF } & 10 & 1.25447 & 0.00300 & 1.2483 & 1.2607\end{array}$

Std Error uses a pooled estimate of error variance

Means and Std Deviation

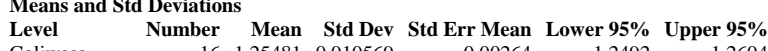

$\begin{array}{lrrrrrr}\text { Coliwasa } & 16 & 1.25481 & 0.010569 & 0.00264 & 1.2492 & 1.2604 \\ \text { Hydragard-HF } & 10 & 1.25447 & 0.007378 & 0.00233 & 1.2492 & 1.2598\end{array}$

\section{t Test}

Hydragard-HF-Coliwasa

Assuming unequal variances

$\begin{array}{lrlr}\text { Difference } & -0.00033 & \text { t Ratio } & -0.09375 \\ \text { Std Err Dif } & 0.00352 & \text { DF } & 23.59903 \\ \text { Upper CL Dif } & 0.00695 & \text { Prob }>|t| & 0.9261 \\ \text { Lower CL Dif } & -0.00761 & \text { Prob }>\text { t } & 0.5370 \\ \text { Confidence } & 0.95 & \text { Prob }<\mathrm{t} & 0.4630\end{array}$

Tests that the Variances are Equal

$\begin{array}{lrrrr}\text { Level } & \text { Count } & \text { Std Dev } & \text { MeanAbsDif to Mean } & \text { MeanAbsDif to Median } \\ \text { Coliwasa } & 16 & 0.0105687 & 0.0096992 & 0.0090052\end{array}$

$\begin{array}{lrrrr}\text { Coliwasa } & 16 & 0.0105687 & 0.0096992 & 0.0090052 \\ \text { Hydragard-HF } & 10 & 0.0073777 & 0.0050673 & 0.0050540\end{array}$

Test F Ratio DFNum DFDen p-Value

$\begin{array}{lllll}\text { O'Brien[.5] } & 2.9068 & 1 & 24 & 0.1011\end{array}$

$\begin{array}{lllll}\text { Brown-Forsythe } & 2.0149 & 1 & 24 & 0.1686\end{array}$

$\begin{array}{lllll}\text { Levene } & 7.8558 & 1 & 24 & 0.0099\end{array}$

$\begin{array}{lllll}\text { Bartlett } & 1.2870 & 1 & \text {. } & 0.2566\end{array}$

$\begin{array}{lllll}\text { F Test 2-sided } & 2.0521 & 15 & 9 & 0.2776\end{array}$

Welch Anova testing Means Equal, allowing Std Devs Not Equal

F Ratio DFNum DFDen Prob $>$ F

$\begin{array}{llll}0.0088 & 1 & 23.599 & 0.9261\end{array}$

$\mathbf{t}$ Test
0.0938 


\section{Exhibit C2. Statistical Comparisons for Low-Rheology (Phase 3) Simulant Testing}

Oneway Analysis of Measurement By Sampling Description Test Phase=Phase 3 - Low Rheology, Analyte $=$ wt $\%$ dried solids

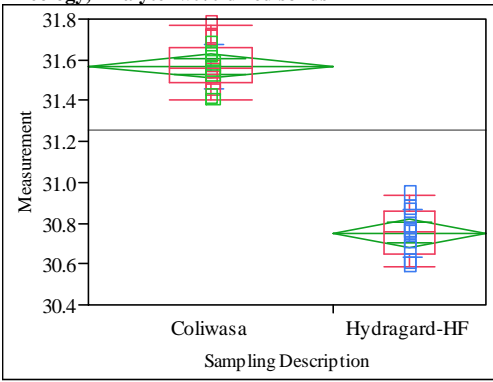

Oneway Anova

Summary of Fit

0.933349

Root Mean Square Error $\quad 0.930572$

Mean of Response 31.25423

Observations (or Sum Wgts) $\quad 26$

t Test

Hydragard-HF-Coliwasa

Assuming equal variances

Difference $\quad-0.81775$ t Ratio $\quad-18.3327$

Std Err Dif $\quad 0.04461$ DF 24

Upper CL Dif -0.72569 Prob $>|t| \quad<.0001$

Lower CL Dif -0.90981 Prob $>\mathrm{t} \quad 1.0000$

$\begin{array}{lrl}\text { Confidence } & 0.95 \text { Prob }<\mathrm{t} & <.0001\end{array}$

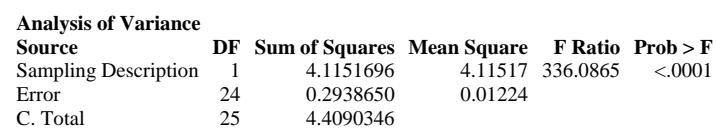

C. Total 25

4.4090346

Means for Oneway Anova

Level Number Mean Std Error Lower 95\% Upper 95\%

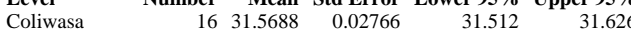

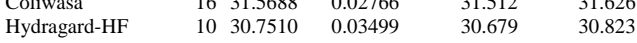

Std Error uses a pooled estimate of error variance

Means and Std Deviations

Level Number Mean Std Dev Std Err Mean Lower 95\% Upper 95\%

$\begin{array}{lllllll}\text { Coliwasa } & 16 & 31.5688 & 0.108128 & 0.02703 & 31.511 & 31.626\end{array}$

Hydragard-HF

t Test

Hydragard-HF-Coliwasa

Assuming unequal variances

Difference $\quad-0.81775$ t Ratio $\quad-18.073$

Std Err Dif $\quad 0.04525$ DF $\quad 18.36803$

Upper CL Dif -0.72283 Prob $>|t| \quad<.0001$

Lower CL Dif -0.91267 Prob $>t \quad 1.0000$

$\begin{array}{lrr}\text { Confidence } & 0.95 \text { Prob }<\mathrm{t} & <.0001\end{array}$

Tests that the Variances are Equal

Level Count Std Dev MeanAbsDif to Mean MeanAbsDif to Median

$\begin{array}{lllll}\text { Coliwasa } & 16 & 0.1081280 & 0.0862500 & 0.0862500\end{array}$

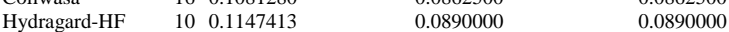

Test F Ratio DFNum DFDen p-Value

$\begin{array}{lrrrr}\text { O'Brien[.5] } & 0.0680 & 1 & 24 & 0.7965\end{array}$

Brown-Forsythe $\quad 0.0115 \quad 1 \quad 24 \quad 0.915$

$\begin{array}{lllll}\text { Levene } & 0.0117 & 1 & 24 & 0.9148\end{array}$

$\begin{array}{lllll}\text { Bartlett } & 0.0383 & 1 & & 0.8449 \\ \text { F Test 2-sided } & 1.1261 & 9 & 15 & 0.8056\end{array}$

Welch Anova testing Means Equal, allowing Std Devs Not Equal

F Ratio DFNum DFDen Prob $>$ F

$326.6351 \quad 1 \quad 18.368<.0001$

$\mathbf{t}$ Test
18.0730
Oneway Analysis of Measurement By Sampling Description Test Phase=Phase 3 - Low Rheology, Analyte $=w t \%$ vitrified solids

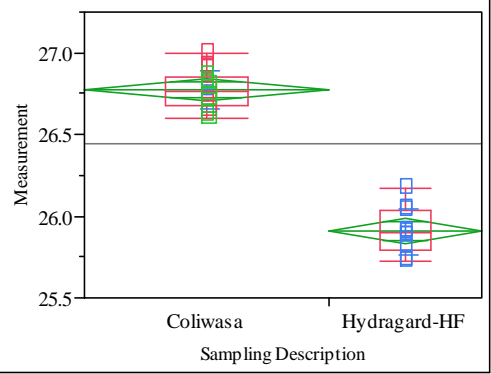

Oneway Anova

Summary of Fit

Adj Rsquare

0.124187

Observations (or Sum Wgts) 26.4407

Test

Hydragard-HF-Coliwasa

Assuming equal variances

Difference $\quad-0.86575$ t Ratio $\quad-17.2938$

Std Err Dif $\quad 0.05006$ DF 24

Upper CL Dif -0.76243 Prob $>|t| \quad<.0001$

Lower CL Dif -0.96907 Prob $>t \quad 1.0000$

$\begin{array}{lrl}\text { Confidence } & 0.95 \text { Prob }<\mathrm{t} & <.0001\end{array}$

$\begin{array}{lrrrrr}\begin{array}{lrrrr}\text { Analysis of Variance } \\ \text { Source }\end{array} & \text { DF } & \text { Sum of Squares } & \text { Mean Square } & \text { F Ratio } & \text { Prob > F } \\ \text { Sampling Description } & 1 & 4.6124496 & 4.61245 & 299.0768 & <.0001 \\ \text { Error } & 24 & 0.3701350 & 0.01542 & & \\ \text { C. Total } & 25 & 4.9825846 & & & \end{array}$

Means for Oneway Anova

Level Number Mean Std Error Lower 95\% Upper 95\%

$\begin{array}{lrrrrr}\text { Coliwasa } & 16 & 26.7738 & 0.03105 & 26.710 & 26.838\end{array}$

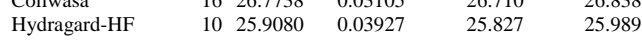

Std Error uses a pooled estimate of error variance

Means and Std Deviations

Level Number Mean Std Dev Std Err Mean Lower 95\% Upper 95\%

$\begin{array}{lllllll}\text { Coliwasa } & 16 & 26.7738 & 0.112835 & 0.02821 & 26.714 & 26.834\end{array}$

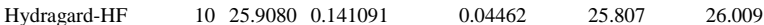

Test

Hydragard-HF-Coliwasa

Assuming unequal variances

Difference $\quad-0.86575$ t Ratio $\quad-16.401$

Std Err Dif $\quad 0.05279$ DF $\quad 16.09058$

Upper CL Dif -0.75390 Prob $>|t|<.0001$

Lower CL Dif -0.97760 Prob $>t \quad 1.0000$

$\begin{array}{lrl}\text { Confidence } & 0.95 \text { Prob }<\mathrm{t} & <.0001\end{array}$

Tests that the Variances are Equal

Level Count Std Dev MeanAbsDif to Mean MeanAbsDif to Median

$\begin{array}{lllll}\text { Coliwasa } & 16 & 0.1128347 & 0.0912500 & 0.0912500\end{array}$

Hydragard-HF $\quad 10 \quad 0.1410910$

Test F Ratio DFNum DFDen p-Value

$\begin{array}{lrrrr}\text { O'Brien[.5] } & 0.7823 & 1 & 24 & 0.3852\end{array}$

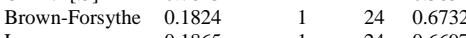

$\begin{array}{lllll}\text { Levene } & 0.1865 & 1 & 24 & 0.6697\end{array}$

$\begin{array}{lllll}\text { Bartlett } & 0.5535 & 1 & & 0.4569 \\ \text { F Test 2-sided } & 1.5636 & 9 & 15 & 0.4267\end{array}$

Welch Anova testing Means Equal, allowing Std Devs Not Equal

F Ratio DFNum DFDen Prob $>$ F

$268.9937<1<16.091<0001$

t Test

16.4010 


\section{Exhibit C2. Statistical Comparisons for Low-Rheology (Phase 3) Simulant Testing}

Oneway Analysis of Measurement By Sampling Description Test Phase=Phase 3 - Low Rheology, Analyte=sample density $(\mathrm{g} / \mathrm{mL})$

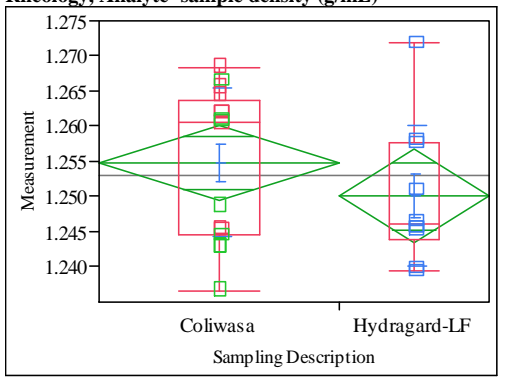

Oneway Anova

Summary of Fit

Rsquare

Adj Rsquare

Reot 0.010349

$\begin{array}{lr}\text { Mean of Response } & 1.252998 \\ \text { Observations (or Sum Wgts) } & 26\end{array}$

Test

Hydragard-LF-Coliwasa

Assuming equal variances

Difference $\quad-0.00470$ t Ratio $\quad-1.12624$

$\begin{array}{lrrr}\text { Std Err Dif } & 0.00417 & \text { DF } & 24 \\ \text { Upper CL Dif } & 0.00391 & \text { Prob }>|t| & 0.2712\end{array}$

$\begin{array}{lrll}\text { Upper CL Dif } & 0.00391 & \text { Prob }>|t| & 0.2712 \\ \text { Lower CL Dif } & -0.01331 & \text { Prob }>t & 0.8644\end{array}$

$\begin{array}{lrll}\text { Lower CL Dif } & -0.01331 & \text { Prob }>t & 0.8644 \\ \text { Confidence } & 0.95 & \text { Prob }<\mathrm{t} & 0.1356\end{array}$

$\begin{array}{lrrrrr}\text { Analysis of Variance } & & & & & \\ \text { Source } & \text { DF } & \text { Sum of Squares } & \text { Mean Square } & \text { F Ratio } & \text { Prob }>\text { F } \\ \text { Sampling Description } & 1 & 0.00013584 & 0.000136 & 1.2684 & 0.2712 \\ \text { Error } & 24 & 0.00257034 & 0.000107 & & \\ \text { C. Total } & 25 & 0.00270618 & & & \end{array}$

Means for Oneway Anova

Level Number Mean Std Error Lower 95\% Upper 95\%

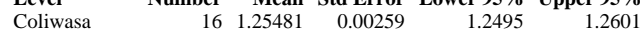

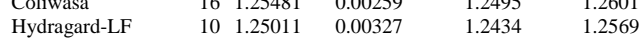

Std Error uses a pooled estimate of error variance

Means and Std Deviations

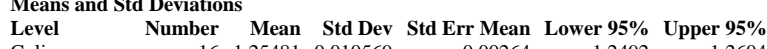

$\begin{array}{lrrrrrr}\text { Coliwasa } & \text { 16 } & 1.25481 & 0.010569 & 0.00264 & 1.2492 & 1.2604 \\ \text { Hydragard-LF } & 10 & 1.25011 & 0.009972 & 0.00315 & 1.2430 & 1.2572\end{array}$

Test

Hydragard-LF-Coliwasa

Hydragard-LF-Coliwasa
Assuming unequal variances

$\begin{array}{lrlr}\text { Difference } & -0.00470 & \text { t Ratio } & -1.14207 \\ \text { Std Err Dif } & 0.00411 & \text { DF } & 20.12254 \\ \text { Upper CL Dif } & 0.00388 & \text { Prob }>|t| & 0.2668 \\ \text { Lower CL Dif } & -0.01328 & \text { Prob }>\text { t } & 0.8666 \\ \text { Confidence } & 0.95 & \text { Prob }<\mathrm{t} & 0.1334\end{array}$

Tests that the Variances are Equal

$\begin{array}{lrrrr}\text { Level } & \text { Count } & \text { Std Dev } & \text { MeanAbsDif to Mean } & \text { MeanAbsDif to Median } \\ \text { Coliwasa } & 16 & 0.0105687 & 0.0096992 & 0.0090052\end{array}$

$\begin{array}{lllll}\text { Coliwasa } & 16 & 0.0105687 & 0.0096992 & 0.0090052 \\ \text { Hydragard-LF } & 10 & 0.0099716 & 0.0076602 & 0.0069505\end{array}$

Test F Ratio DFNum DFDen p-Value

$\begin{array}{lllll}\text { O'Brien[.5] } & 0.0622 & 1 & 24 & 0.8052 \\ \text { Brown-Fosyth }\end{array}$

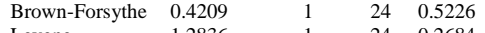

$\begin{array}{lllll}\text { Levene } & 1.2836 & 1 & 24 & 0.2684\end{array}$

$\begin{array}{lrrrr}\text { Bartlett } & 0.0360 & 1 & & 0.8495 \\ \text { F Test 2-sided } & 1.1233 & 15 & 9 & 0.8889\end{array}$

Welch Anova testing Means Equal, allowing Std Devs Not Equal

F Ratio DFNum DFDen Prob $>$ F

$\begin{array}{rrrr}1.3043 & 1 & 20.123 & 0.2668\end{array}$

t Test
1.1421
Oneway Analysis of Measurement By Sampling Description Test Phase=Phase 3 - Low Rheology, Analyte $=$ wt $\%$ dried solids

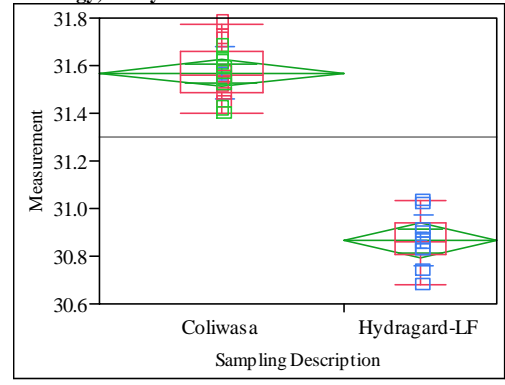

Oneway Anova

Summary of Fit

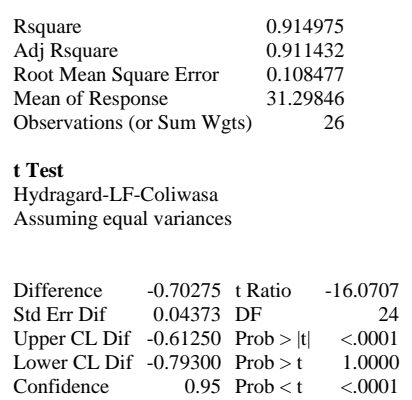

$\begin{array}{lrrrrr}\begin{array}{lrrr}\text { Analysis of Variance } \\ \text { Source }\end{array} & \text { DF } & \text { Sum of Squares } & \text { Mean Square } & \text { F Ratio } & \text { Prob > F } \\ \text { Sampling Description } & 1 & 3.0391235 & 3.03912 & 258.2687 & <.0001 \\ \text { Error } & 24 & 0.2824150 & 0.01177 & & \\ \text { C. Total } & 25 & 3.3215385 & & & \end{array}$

Means for Oneway Anova

$\begin{array}{lrrrrr}\text { Level } & \text { Number } & \text { Mean } & \text { Std Error } & \text { Lower 95\% } & \text { Upper 95\% } \\ \text { Coliwasa } & 16 & 31.5688 & 0.02712 & 31.513 & 31.625\end{array}$

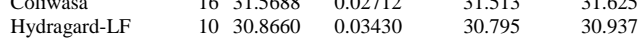

Std Error uses a pooled estimate of error variance

Means and Std Deviations

Level Number Mean Std Dev Std Err Mean Lower 95\% Upper 95\%

$\begin{array}{lllllll}\text { Coliwasa } & 16 & 31.5688 & 0.108128 & 0.02703 & 31.511 & 31.626\end{array}$

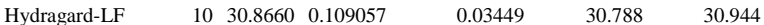

t Test

Hydragard-LF-Coliwasa

Assuming unequal variances

$\begin{array}{lll}\text { Difference } & -0.70275 \text { t Ratio } \quad-16.0377\end{array}$

Std Err Dif $\quad 0.04382$ DF $\quad 19.12496$

Upper CL Dif -0.61108 Prob $>|t| r .0001$

$\begin{array}{lll}\text { Lower CL Dif }-0.79442 \text { Prob }>t & 1.0000\end{array}$

$\begin{array}{lrl}\text { Confidence } & 0.95 \text { Prob }<\mathrm{t} & <.0001\end{array}$

Tests that the Variances are Equal

Level Count Std Dev MeanAbsDif to Mean MeanAbsDif to Median

$\begin{array}{lllll}\text { Coliwasa } & 16 & 0.1081280 & 0.0862500 & 0.0862500\end{array}$

$\begin{array}{lllll}\text { Hydragard-LF } & 10 & 0.1090566 & 0.0800000 & 0.0800000\end{array}$

Test F Ratio DFNum DFDen p-Value

$\begin{array}{lrrrr}\text { O'Brien[.5] } & 0.0012 & 1 & 24 & 0.9726\end{array}$

$\begin{array}{lllll}\text { Brown-Forsythe } & 0.0571 & 1 & 24 & 0.8131\end{array}$

$\begin{array}{lllll}\text { Levene } & 0.0580 & 1 & 24 & 0.8117\end{array}$

$\begin{array}{lllrl}\text { Bartlett } & 0.0008 & 1 & \text {. } & 0.9776 \\ \text { F Test 2-sided } & 1.0172 & 9 & 15 & 0.9375\end{array}$

Welch Anova testing Means Equal, allowing Std Devs Not Equal

F Ratio DFNum DFDen Prob $>$ F

$\begin{array}{rrrr}257.2091 & 1 & 19.125 & <.0001\end{array}$

t Test

16.0377 


\section{Exhibit C2. Statistical Comparisons for Low-Rheology (Phase 3) Simulant Testing}

Oneway Analysis of Measurement By Sampling Description Test Phase=Phase 3 - Low Rheology, Analyte $=\mathrm{wt} \%$ vitrified solids

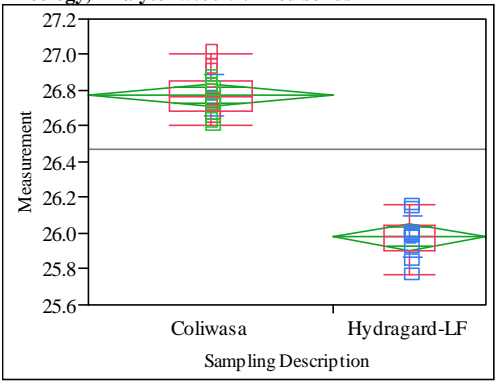

Oneway Anova

Summary of Fit

Adj Rsquare

0.921735

0.11456
$\quad 6.46846$

Observations (or Sum Wgts) $\quad 26$

t Test

Hydragard-LF-Coliwasa

Assuming equal variances

Difference $\quad-0.79375$ t Ratio $\quad-17.188$

Std Err Dif $\quad 0.04618$ DF 24

Upper CL Dif -0.69844 Prob $>|t|<.0001$

Lower CL Dif -0.88906 Prob $>t \quad 1.0000$

$\begin{array}{lll}\text { Confidence } & 0.95 \text { Prob }<\mathrm{t} \quad<.0001\end{array}$

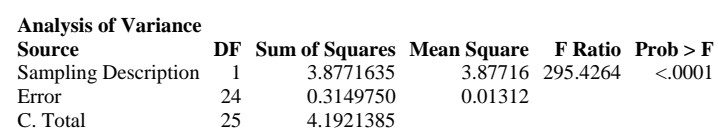

C. Total 25

4.1921385

Means for Oneway Anova

Level Number Mean Std Error Lower 95\% Upper 95\%

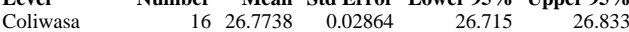

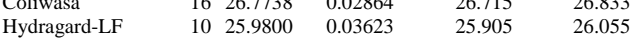

Std Error uses a pooled estimate of error variance

Means and Std Deviations

Level Number Mean Std Dev Std Err Mean Lower 95\% Upper 95\%

$\begin{array}{lllllll}\text { Coliwasa } & 16 & 26.7738 & 0.112835 & 0.02821 & 26.714 & 26.834\end{array}$

$\begin{array}{lllllll}\text { Hydragard-LF } & 10 & 25.9800 & 0.117379 & 0.03712 & 25.896 & 26.064\end{array}$

t Test

Hydragard-LF-Coliwas

Assuming unequal variances

Difference $\quad-0.79375$ t Ratio $\quad-17.0256$

Std Err Dif $\quad 0.04662$ DF $\quad 18.66277$

Upper CL Dif -0.69605 Prob $>|t| r .0001$

Lower CL Dif -0.89145 Prob $>t \quad 1.0000$

$\begin{array}{lrr}\text { Confidence } & 0.95 \text { Prob }<\mathrm{t} & <.0001\end{array}$

Tests that the Variances are Equal

Level Count Std Dev MeanAbsDif to Mean MeanAbsDif to Median

$\begin{array}{lllll}\text { Coliwasa } & 16 & 0.1128347 & 0.0912500 & 0.0912500\end{array}$

$\begin{array}{lllll}\text { Hydragard-LF } & 10 & 0.1173788 & 0.0800000 & 0.0800000\end{array}$

Test F Ratio DFNum DFDen p-Value

$\begin{array}{lrrrr}\text { O'Brien[.5] } & 0.0231 & 1 & 24 & 0.8804\end{array}$

$\begin{array}{lllll}\text { Brown-Forsythe } & 0.1568 & 1 & 24 & 0.8804 \\ & 0.1587 & 1 & 24 & 0.6938\end{array}$

$\begin{array}{lllll}\text { Levene } & 0.1587 & 1 & 24 & 0.6938\end{array}$

$\begin{array}{lllll}\text { Bartlett } & 0.0169 & 1 & & 0.8966 \\ \text { F Test 2-sided } & 1.0822 & 9 & 15 & 0.8569\end{array}$

Welch Anova testing Means Equal, allowing Std Devs Not Equal

F Ratio DFNum DFDen Prob $>$ F

$\begin{array}{rrrr}289.8721 & 1 & 18.663 & <.0001\end{array}$

t Test

17.0256
Oneway Analysis of Measurement By Sampling Description Test Phase=Phase 3 - Low Rheology, Analyte=sample density $(\mathrm{g} / \mathrm{mL})$

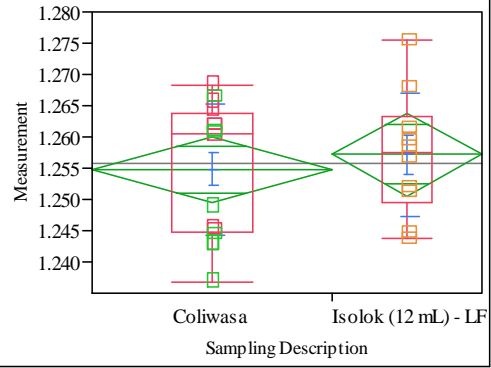

Oneway Anova

Summary of Fit

Rsquare

Adj Rsquare

Root Mean Square Error $\quad 0.010304$

Mean of Response

Observations (or Sum Wgts) $\quad 26$

t Test

Isolok (12 mL) - LF-Coliwasa

Assuming equal variances

Difference $\quad 0.00234$ t Ratio $\quad 0.562292$

$\begin{array}{lrlr}\text { Std Err Dif } & 0.00415 & \text { DF } & 24 \\ \text { Upper CL Dif } & 0.01091 & \text { Prob }>|t| & 0.5791\end{array}$

$\begin{array}{lrll}\text { Upper CL Dif } & 0.01091 & \text { Prob }>|t| & 0.5791 \\ \text { Lower CL Dif } & -0.00624 & \text { Prob }>t & 0.2896\end{array}$

$\begin{array}{lrrr}\text { Lower CL Dif } & -0.00624 & \text { Prob }>t & 0.2896 \\ \text { Confidence } & 0.95 & \text { Prob }<t & 0.7104\end{array}$

Analysis of Variance

$\begin{array}{lrrrrr}\text { Analysis of Variance } & & & & & \\ \text { Source } & \text { DF } & \text { Sum of Squares } & \text { Mean Square } & \text { F Ratio } & \text { Prob }>\text { F } \\ \text { Sampling Description } & 1 & 0.00003357 & 0.000034 & 0.3162 & 0.5791\end{array}$

Error

$0.00254836 \quad 0.00010$

C. Total

$25 \quad 0.00258194$

Means for Oneway Anova

Level Number Mean Std Error Lower 95\% Upper 95\%

$\begin{array}{llllll}\text { Coliwasa } & 16 & 1.25481 & 0.00258 & 1.2495 & 1.2601\end{array}$

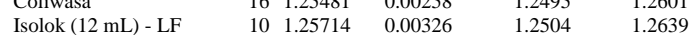

Std Error uses a pooled estimate of error variance

Means and Std Deviations

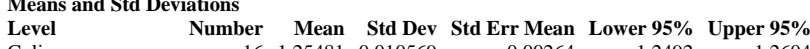

$\begin{array}{lrrrrrr}\text { Coliwasa } & 16 & 1.25481 & 0.010569 & 0.00264 & 1.2492 & 1.2604 \\ \text { Isolok (12 mL) - LF } & 10 & 1.25714 & 0.009848 & 0.00311 & 1.2501 & 1.2642\end{array}$

t Test

Isolok $(12 \mathrm{~mL})$ - LF-Coliwasa

Assuming unequal variances

Difference $\quad 0.00234$ t Ratio 0.571893

Std Err Dif $\quad 0.00408$ DF 20.30632

$\begin{array}{lll}\text { Upper CL Dif } \quad 0.01085 \text { Prob }>|t| & 0.5737\end{array}$

Lower CL Dif -0.00618 Prob $>t \quad 0.2868$

$\begin{array}{lrl}\text { Confidence } & 0.95 \text { Prob }<\mathrm{t} \quad 0.7132\end{array}$

Tests that the Variances are Equal

$\begin{array}{lrrrr}\text { Level } & \text { Count } & \text { Std Dev } & \text { MeanAbsDif to Mean } & \text { MeanAbsDif to Median } \\ \text { Coliwasa } & 16 & 0.0105687 & 0.0096992 & 0.0090052\end{array}$

$\begin{array}{lllll} & 16 & 0.0105687 & 0.0096992 & 0.0090052 \\ \text { Isolok (12 mL) - LF } & 10 & 0.0098484 & 0.0074677 & 0.0074677\end{array}$

Test F Ratio DFNum DFDen p-Value

$\begin{array}{lllll}\text { O'Brien[.5] } & 0.1258 & 1 & 24 & 0.7260\end{array}$

$\begin{array}{lllll}\text { Brown-Forsythe } & 0.2848 & 1 & 24 & 0.5985\end{array}$

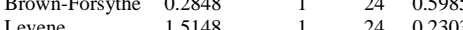

$\begin{array}{lllll}\text { Levene } & 1.5148 & 1 & 24 & 0.2303\end{array}$

$\begin{array}{lrrrr}\text { Bartlett } & 0.0530 & 1 & & 0.8180 \\ \text { F Test 2-sided } & 1.1516 & 15 & 9 & 0.8571\end{array}$

Welch Anova testing Means Equal, allowing Std Devs Not Equal

F Ratio DFNum DFDen Prob $>$ F

$\begin{array}{llll}0.3271 & 1 & 20.306 & 0.5737\end{array}$

t Test 


\section{Exhibit C2. Statistical Comparisons for Low-Rheology (Phase 3) Simulant Testing}

Oneway Analysis of Measurement By Sampling Description Test Phase=Phase 3 - Low Rheology, Analyte $=$ wt $\%$ dried solids

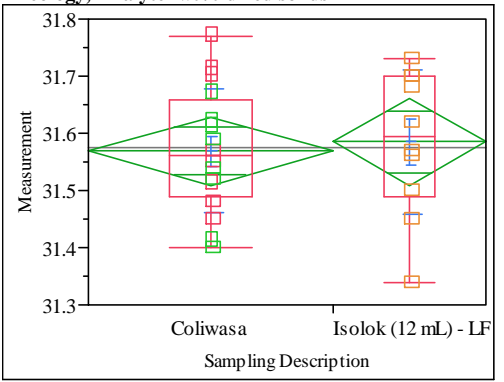

Oneway Anova

Summary of Fit

Adj Rsquare

$\quad 0.115366$

31.575

t Test

Isolok (12 mL) - LF-Coliwasa

Assuming equal variances

Difference $\quad 0.01625$ t Ratio $\quad 0.34942$

Std Err Dif $\quad 0.04651$ DF 24

Upper CL Dif 0.11223 Prob $>|t| \quad 0.7298$

Lower CL Dif -0.07973 Prob $>t \quad 0.3649$

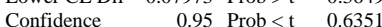

\section{Analysis of Variance}

$\begin{array}{lrrrrr}\text { Source } & \text { DF } & \text { Sum of Squares } & \text { Mean Square } & \text { F Ratio } & \text { Prob > F } \\ \text { Sampling Description } & 1 & 0.00162500 & 0.001625 & 0.1221 & 0.7298 \\ \text { Error } & 24 & 0.31942500 & 0.013309 & & \end{array}$

\section{Means for Oneway Anova}

$\begin{array}{lrrrrr}\text { Level } & \text { Number } & \text { Mean } & \text { Std Error } & \text { Lower 95\% } & \text { Upper 95\% } \\ \text { Coliwasa } & 16 & 31.5688 & 0.02884 & 31.509 & 31.628\end{array}$

$\begin{array}{lrrrrr}\text { Coliwasa } & 16 & 31.5688 & 0.02884 & 31.509 & 31.628 \\ \text { Isolok (12 mL) - LF } & 10 & 31.5850 & 0.03648 & 31.510 & 31.660\end{array}$

Std Error uses a pooled estimate of error variance

Means and Std Deviations

Level Number Mean Std Dev Std Err Mean Lower 95\% Upper 95\%

$\begin{array}{llllll}\text { Coliwasa } & 16 & 31.5688 & 0.108128 & 0.02703 & 31.511\end{array}$

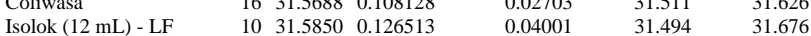

t Test

Isolok (12 mL) - LF-Coliwasa

Assuming unequal variances

Difference $\quad 0.01625$ t Ratio $\quad 0.336555$

Std Err Dif $\quad 0.04828$ DF $\quad 16.97131$

Upper CL Dif 0.11813 Prob $>|t| \quad 0.7406$

Lower CL Dif -0.08563 Prob $>t \quad 0.3703$

$\begin{array}{lrl}\text { Confidence } & 0.95 \text { Prob }<\mathrm{t} & 0.6297\end{array}$

Tests that the Variances are Equal

Level Count Std Dev MeanAbsDif to Mean MeanAbsDif to Median

$\begin{array}{lllll}\text { Coliwasa } & 16 & 0.1081280 & 0.0862500 & 0.0862500\end{array}$

$\begin{array}{lllll}\text { Isolok }(12 \mathrm{~mL}) \text { - LF } & 10 & 0.1265131 & 0.1010000 & 0.1010000\end{array}$

Test F Ratio DFNum DFDen p-Value

$\begin{array}{lrrrr}\text { O'Brien[.5] } & 0.4142 & 1 & 24 & 0.5259\end{array}$

$\begin{array}{lllll}\text { Brown-Forsythe } & 0.3194 & 1 & 24 & 0.5772\end{array}$

$\begin{array}{lllll}\text { Levene } & 0.3266 & 1 & 24 & 0.5730\end{array}$

$\begin{array}{lllll}\text { Bartlett } & 0.2714 & 1 & & 0.6024 \\ \text { F Test 2-sided } & 1.3690 & 9 & 15 & 0.5676\end{array}$

Welch Anova testing Means Equal, allowing Std Devs Not Equal

F Ratio DFNum DFDen Prob > F

$\begin{array}{rrrr}0.1133 & 1 & 16.971 & 0.7406\end{array}$

t Test

0.3366

Oneway Analysis of Measurement By Sampling Description Test Phase=Phase 3 - Low Rheology, Analyte $=w t \%$ vitrified solids

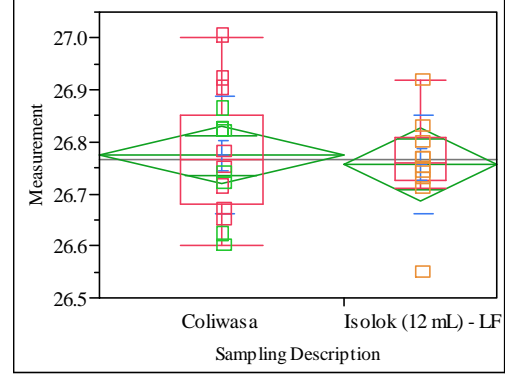

Oneway Anova

Summary of Fit

Adj Rsquare

Root Mean Square Error

Mean of Response

Observations (or Sum Wgts)

0.006326

$-0.03508$

0.106299

t Test

Isolok (12 mL) - LF-Coliwasa

Assuming equal variances

Difference $\quad-0.01675$ t Ratio $\quad-0.3909$

Std Err Dif $\quad 0.04285$ DF 24

Upper CL Dif 0.07169 Prob $>|t| 0.6993$

Lower CL Dif -0.10519 Prob $>t \quad 0.6503$

$\begin{array}{lll}\text { Confidence } & 0.95 \text { Prob }<\mathrm{t} \quad 0.3497\end{array}$

\section{Analysis of Variance}

$\begin{array}{lrrrrr}\text { Source } & \text { DF } & \text { Sum of Squares } & \text { Mean Square } & \text { F Ratio } & \text { Prob }>\text { F } \\ \text { Sampling Description } & 1 & 0.00172654 & 0.001727 & 0.1528 & 0.6993\end{array}$

$\begin{array}{llll}\text { Error } & 24 & 0.27118500 & 0.011299\end{array}$

$\begin{array}{lll}\text { C. Total } & 25 & 0.27291154\end{array}$

Means for Oneway Anova

Level Number Mean Std Error Lower 95\% Upper 95\%

$\begin{array}{lrrrrr}\text { Coliwasa } & 16 & 26.7738 & 0.02657 & 26.719 & 26.829\end{array}$

$\begin{array}{llllll}\text { Isolok }(12 \mathrm{~mL}) \text { - LF } & 10 & 26.7570 & 0.03361 & 26.688 & 26.829 \\ & & & \end{array}$

Std Error uses a pooled estimate of error variance

Means and Std Deviations

Level Number Mean Std Dev Std Err Mean Lower 95\% Upper 95\%

$\begin{array}{lllllll}\text { Coliwasa } & 16 & 26.7738 & 0.112835 & 0.02821 & 26.714 & 26.834\end{array}$

Isolok (12 mL) - LF $\quad 1026.7570 \quad 0.094405-0.02985-26.689-26.825$

Test

Isolok (12 mL) - LF-Coliwas

Assuming unequal variances

$\begin{array}{lrlr}\text { Difference } & -0.01675 & \text { t Ratio } & -0.40782 \\ \text { Std Err Dif } & 0.04107 & \text { DF } & 21.81273 \\ \text { Upper CL Dif } & 0.06847 & \text { Prob }>|t| & 0.6874 \\ \text { Lower CL Dif } & -0.10197 & \text { Prob }>\text { t } & 0.6563 \\ \text { Confidence } & 0.95 & \text { Prob }<\mathrm{t} & 0.3437\end{array}$

Tests that the Variances are Equal

Level Count Std Dev MeanAbsDif to Mean MeanAbsDif to Median

$\begin{array}{lllll}\text { Coliwasa } & 16 & 0.1128347 & 0.0912500 & 0.0912500\end{array}$

Isolok $(12 \mathrm{~mL})$ - LF $\quad 10 \quad 0.0944046$

$\begin{array}{lrrrr}\text { Test } & \text { F Ratio } & \text { DFNum } & \text { DFDen } & \text { p-Value } \\ \text { O'Brien[.5] } & 0.3408 & 1 & 24 & 0.5648 \\ \text { Brown-Forsythe } & 1.3241 & 1 & 24 & 0.2612 \\ \text { Levene } & 1.3414 & 1 & 24 & 0.2582 \\ \text { Bartlett } & 0.3307 & 1 & \text {. } & 0.5652 \\ \text { F Test 2-sided } & 1.4286 & 15 & 9 & 0.5993\end{array}$

Welch Anova testing Means Equal, allowing Std Devs Not Equal

F Ratio DFNum DFDen Prob > F

$\begin{array}{llll}0.1663 & 1 & 21.813 & 0.6874\end{array}$

t Test

0.4078 


\section{Exhibit C2. Statistical Comparisons for Low-Rheology (Phase 3) Simulant Testing}

Oneway Analysis of Measurement By Sampling Description Test Phase=Phase 3 - Low Rheology, Analyte=sample density $(\mathrm{g} / \mathrm{mL})$

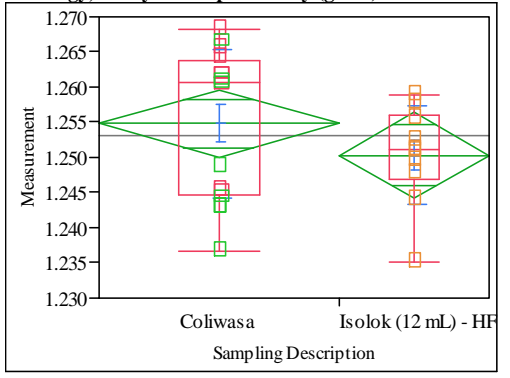

Oneway Anova

Summary of Fit

Rsquare

Adj Rsquare

0.01604

Reot Mean Square Eror 0.009383

1.253079
Observations (or Sum Wgts)

t Test

Isolok (12 mL) - HF-Coliwasa

Assuming equal variances

Difference $\quad-0.00449$ t Ratio -1.18644

$\begin{array}{lrlr}\text { Std Err Dif } & 0.00378 \text { DF } & 24 \\ \text { Uper CL Dif } & 0.00332 & \text { Prob }>|t| & 0.2471\end{array}$

$\begin{array}{lrlr}\text { Upper CL Dif } & 0.00332 & \text { Prob }>|t| & 0.2471 \\ \text { Lower CL Dif } & -0.01229 & \text { Prob }>t & 0.8765\end{array}$

$\begin{array}{lrl}\text { Confidence } & 0.95 \text { Prob }<\mathrm{t} \quad 0.1235\end{array}$

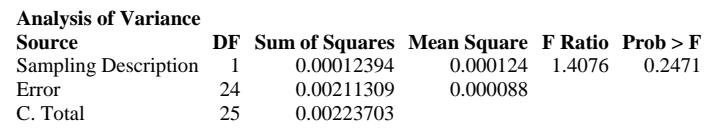

Means for Oneway Anova

Level Number Mean Std Error Lower 95\% Upper 95\%

$\begin{array}{lllllr}\text { Coliwasa } & 16 & 1.25481 & 0.00235 & 1.2500 & 1.2596\end{array}$

$\begin{array}{llllll}\text { Coliwasa } & 16 & 1.25481 & 0.00235 & 1.2500 & 1.2596 \\ \text { Isolok }(12 \mathrm{~mL}) \text { - HF } & 10 & 1.25032 & 0.00297 & 1.2442 & 1.2564\end{array}$

Std Error uses a pooled estimate of error variance

Means and Std Deviations

$\begin{array}{lrrrrrr}\text { Level } & \text { Number } & \text { Mean } & \text { Std Dev } & \text { Std Err Mean } & \text { Lower 95\% } & \text { Upper 95\% } \\ \text { Coliwasa } & 16 & 1.25481 & 0.010569 & 0.00264 & 1.2492 & 1.2604\end{array}$

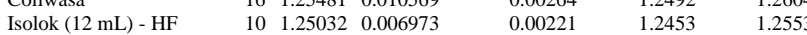

t Test

Isolok (12 mL) - HF-Coliwasa

Assuming unequal variances

Difference $\quad-0.00449$ t Ratio -1.30401

Std Err Dif $\quad 0.00344$ DF 23.87111

Upper CL Dif 0.00262 Prob $>|t| \quad 0.2047$

Lower CL Dif -0.01159 Prob $>t \quad 0.8977$

$\begin{array}{lrl}\text { Confidence } & 0.95 \text { Prob }<\mathrm{t} \quad 0.1023\end{array}$

Tests that the Variances are Equal

$\begin{array}{lrrrr}\text { Level } & \text { Count } & \text { Std Dev } & \text { MeanAbsDif to Mean } & \text { MeanAbsDif to Median } \\ \text { Coliwasa } & 16 & 0.0105687 & 0.0096992 & 0.0090052 \\ \text { Isolok (12 mL) - HF } & 10 & 0.0069733 & 0.0049263 & 0.0047917\end{array}$

$\begin{array}{lrrrr}\text { Test } & \text { F Ratio } & \text { DFNum } & \text { DFDen } & \text { p-Value } \\ \text { O'Brien[.5] } & 3.4932 & 1 & 24 & 0.0739 \\ \text { Brown-Forsythe } & 2.3378 & 1 & 24 & 0.1393 \\ \text { Levene } & 9.2124 & 1 & 24 & 0.0057 \\ \text { Bartlett } & 1.6970 & 1 & . & 0.1927 \\ \text { F Test 2-sided } & 2.2970 & 15 & 9 & 0.2097\end{array}$

Welch Anova testing Means Equal, allowing Std Devs Not Equal

F Ratio DFNum DFDen Prob $>$ F

$\begin{array}{llll}1.7004 & 1 & 23.871 & 0.2047\end{array}$

t Test
1.3040
Oneway Analysis of Measurement By Sampling Description Test Phase=Phase 3 - Low Rheology, Analyte $=w t \%$ dried solids

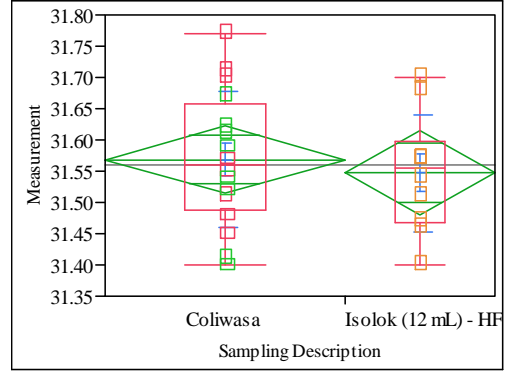

Oneway Anova

Summary of Fit

Rsquare

Adj Rsquare

$-0.0294$

Root Mean Square Error $\quad 0.102994$

Mean of Response

31.56038

Observations (or Sum Wgts) 26

t Test

Isolok (12 mL) - HF-Coliwasa

Assuming equal variances

Difference $\quad-0.02175$ t Ratio $\quad-0.52387$

$\begin{array}{lrlr}\text { Std Err Dif } & 0.04152 \text { DF } & 24 \\ \text { Upper CL Dif } & 0.06394 \text { Prob }>|t| & 0.6052\end{array}$

$\begin{array}{lrll}\text { Upper CL Dif } & 0.06394 & \text { Prob }>|t| & 0.6052 \\ \text { Lower CL Dif } & -0.10744 & \text { Prob }>t & 0.6974\end{array}$

$\begin{array}{lrrr}\text { Lower CL Dif } & -0.10744 & \text { Prob }>t & 0.6974 \\ \text { Confidence } & 0.95 & \text { Prob }<t & 0.3026\end{array}$

$\begin{array}{lrrrrr}\begin{array}{lrrrr}\text { Analysis of Variance } \\ \text { Source }\end{array} & \text { DF } & \text { Sum of Squares } & \text { Mean Square } & \text { F Ratio } & \text { Prob > F } \\ \text { Sampling Description } & 1 & 0.00291115 & 0.002911 & 0.2744 & 0.6052 \\ \text { Error } & 24 & 0.25458500 & 0.010608 & & \\ \text { C. Total } & 25 & 0.25749615 & & & \end{array}$

$\begin{array}{lll}\text { C. Total } & 25 & 0.25749615\end{array}$

Means for Oneway Anova

Level Number Mean Std Error Lower 95\% Upper 95\%

$\begin{array}{llllll}\text { Coliwasa } & 16 & 31.5688 & 0.02575 & 31.516 & 31.622\end{array}$

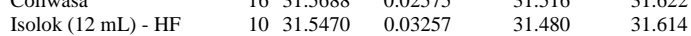

Std Error uses a pooled estimate of error variance

Means and Std Deviations

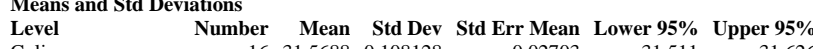

$\begin{array}{lrrrrrr}\text { Coliwasa } & 16 & 31.5688 & 0.108128 & 0.02703 & 31.511 & 31.626 \\ \text { Isolok }(12 \mathrm{~mL}) \text { - HF } & 10 & 31.5470 & 0.093814 & 0.02967 & 31.480 & 31.614\end{array}$

t Test

Isolok (12 mL) - HF-Coliwas

Assuming unequal variances

Difference $\quad-0.02175$ t Ratio $\quad-0.54192$

Std Err Dif $\quad 0.04014$ DF 21.32767

Upper CL Dif 0.06164 Prob $>|t| \quad 0.5935$

Lower CL Dif -0.10514 Prob $>t \quad 0.7033$

$\begin{array}{lll}\text { Confidence } & 0.95 \text { Prob }<\mathrm{t} \quad 0.2967\end{array}$

Tests that the Variances are Equal

$\begin{array}{lrrrr}\text { Level } & \text { Count } & \text { Std Dev } & \text { MeanAbsDif to Mean } & \text { MeanAbsDif to Median } \\ \text { Coliwasa } & 16 & 0.1081280 & 0.0862500 & 0.0862500\end{array}$

$\begin{array}{lrrrr}\text { Coliwasa } & 16 & 0.1081280 & 0.0862500 & 0.0862500 \\ \text { Isolok (12 mL) - HF } & 10 & 0.0938142 & 0.0710000 & 0.0710000\end{array}$

Test F Ratio DFNum DFDen p-Value

$\begin{array}{lrrrr}\text { O'Brien[.5] } & 0.3198 & 1 & 24 & 0.5770\end{array}$

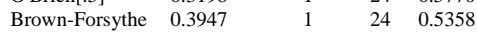

$\begin{array}{lllll}\text { Levene } & 0.4034 & 1 & 24 & 0.5314\end{array}$

$\begin{array}{lllll}\text { Levene } & 0.4034 & 1 & 24 & 0.5314\end{array}$

$\begin{array}{lrrrr}\text { Bartlett } & 0.2113 & 1 & & 0.6457 \\ \text { F Test 2-sided } & 1.3284 & 15 & 9 & 0.6819\end{array}$

Welch Anova testing Means Equal, allowing Std Devs Not Equal

F Ratio DFNum DFDen Prob $>$ F

$\begin{array}{llll}0.2937 & 1 & 21.328 & 0.5935\end{array}$

t Test 


\section{Exhibit C2. Statistical Comparisons for Low-Rheology (Phase 3) Simulant Testing}

Oneway Analysis of Measurement By Sampling Description Test Phase=Phase 3 - Low Rheology, Analyte $=$ wt $\%$ vitrified solids

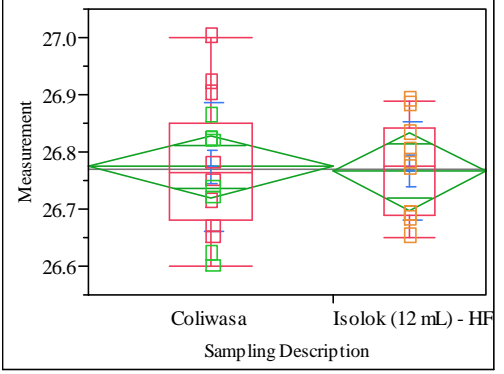

Oneway Anova

Summary of Fit

Adj Rsquare

Root Mean Square Error $\quad-0.04017$

0.103524

Observations (or Sum Wgts) 26

t Test

Isolok (12 mL) - HF-Coliwasa

Assuming equal variances

Difference $\quad-0.00775$ t Ratio $\quad-0.18571$

$\begin{array}{lrr}\text { Std Err Dif } & 0.04173 \text { DF } & 24 \\ & \end{array}$

Upper CL Dif 0.07838 Prob $>|\mathrm{t}| \quad 0.8542$

Lower CL Dif -0.09388 Prob $>\mathrm{t} \quad 0.5729$

$\begin{array}{lll}\text { Confidence } & 0.95 \text { Prob }<\mathrm{t} \quad 0.4271\end{array}$

\section{Analysis of Variance}

Source

Sampling

Error

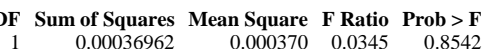
0.00036962 0.010717

Means for Oneway Anova

$\begin{array}{lrrrrr}\text { Level } & \text { Number } & \text { Mean } & \text { Std Error } & \text { Lower 95\% } & \text { Upper 95\% } \\ \text { Coliwasa } & 16 & 26.7738 & 0.02588 & 26.720 & 26.827\end{array}$

$\begin{array}{llrrrr}\text { Coliwasa } & 16 & 26.7738 & 0.02588 & 26.720 & 26.827 \\ \text { Isolok (12 mL) - HF } & 10 & 26.7660 & 0.03274 & 26.698 & 26.834\end{array}$

Std Error uses a pooled estimate of error variance

Means and Std Deviations

Level Number Mean Std Dev Std Err Mean Lower 95\% Upper 95\%

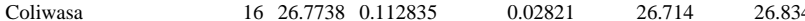

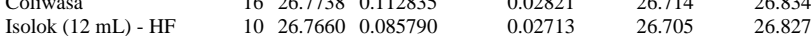

t Test

Isolok (12 mL) - HF-Coliwasa

Assuming unequal variances

Difference $\quad-0.00775$ t Ratio $\quad-0.19802$

Std Err Dif $\quad 0.03914$ DF 22.91188

Upper CL Dif 0.07323 Prob $>|t| \quad 0.8448$

Lower CL Dif -0.08873 Prob $>\mathrm{t} \quad 0.5776$

$\begin{array}{lrl}\text { Confidence } & 0.95 \text { Prob }<\mathrm{t} & 0.4224\end{array}$

Tests that the Variances are Equal

Level Count Std Dev MeanAbsDif to Mean MeanAbsDif to Median

$\begin{array}{lllll}\text { Coliwasa } & 16 & 0.1128347 & 0.0912500 & 0.0912500\end{array}$

Isolok (12 mL) - HF $\quad 100.0857904 \quad 0.0708000 \quad 0.0700000$

Test F Ratio DFNum DFDen p-Value

$\begin{array}{lrrrr}\text { O'Brien[.5] } & 1.0592 & 1 & 24 & 0.3137\end{array}$

$\begin{array}{lllll}\text { Brown-Forsythe } & 0.8659 & 1 & 24 & 0.3614\end{array}$

$\begin{array}{lllll}\text { Levene } & 0.8362 & 1 & 24 & 0.3696\end{array}$

$\begin{array}{lrrrr}\text { Bartlett } & 0.7640 & 1 & \text {. } & 0.3821 \\ \text { F Test 2-sided } & 1.7298 & 15 & 9 & 0.4095\end{array}$

Welch Anova testing Means Equal, allowing Std Devs Not Equal

F Ratio DFNum DFDen Prob $>$ F

$\begin{array}{llll}0.0392 & 1 & 22.912 & 0.8448\end{array}$

t Test

0.1980
Oneway Analysis of Measurement By Sampling Description Test Phase=Phase 3 - Low Rheology, Analyte $=\mathrm{wt} \%$ dried solids

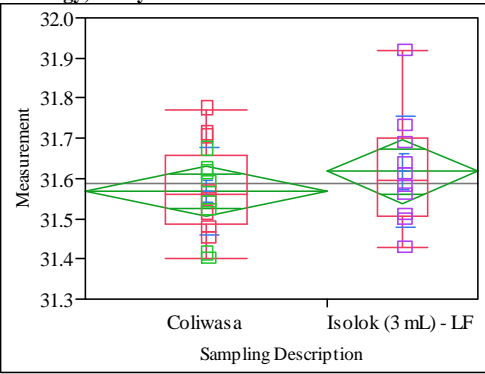

Oneway Anova

Summary of Fit

Rsquare

Adj Rsquare

Mean of Response 31.58731

Observations (or Sum Wgts) 26

t Test

Isolok (3 mL) - LF-Coliwasa

Assuming equal variances

Difference $\quad 0.04825$ t Ratio 0.990329

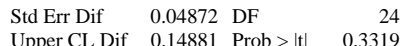

$\begin{array}{lrll}\text { Upper CL Dif } & 0.14881 & \text { Prob }>|t| & 0.3319 \\ \text { Lower CL Dif } & -0.05231 & \text { Prob }>t & 0.1659\end{array}$

$\begin{array}{lrrr}\text { Lower CL Dif } & -0.05231 & \text { Prob }>t & 0.1659 \\ \text { Confidence } & 0.95 & \text { Prob }<t & 0.8341\end{array}$

Analysis of Variance

$\begin{array}{lrrrrr}\text { Analysis of Variance } & & & & & \\ \text { Source } & \text { DF } & \text { Sum of Squares } & \text { Mean Square } & \text { F Ratio } & \text { Prob }>\text { F } \\ \text { Sampling Description } & 1 & 0.01432654 & 0.014327 & 0.9808 & 0.3319\end{array}$

Error

$0.35058500 \quad 0.01460$

C. Total

$25 \quad 0.3649115$

Means for Oneway Anova

Level Number Mean Std Error Lower 95\% Upper 95\%

$\begin{array}{lllllr}\text { Coliwasa } & 16 & 31.5688 & 0.03022 & 31.506 & 31.631\end{array}$

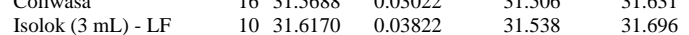

Std Error uses a pooled estimate of error variance

Means and Std Deviations

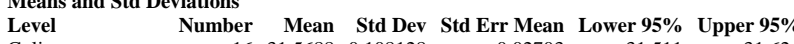

$\begin{array}{lrrrrrr}\text { Coliwasa } & 16 & 31.5688 & 0.108128 & 0.02703 & 31.511 & 31.626 \\ \text { Isolok (3 mL) - LF } & 10 & 31.6170 & 0.139527 & 0.04412 & 31.517 & 31.717\end{array}$

t Test

Isolok (3 mL) - LF-Coliwasa

Assuming unequal variances

Difference $\quad 0.04825$ t Ratio $\quad 0.932464$

Std Err Dif $\quad 0.05174$ DF 15.6974

Upper CL Dif 0.15812 Prob $>|t| \quad 0.3652$

Lower CL Dif -0.06162 Prob $>\mathrm{t} \quad 0.1826$

$\begin{array}{lrl}\text { Confidence } & 0.95 \text { Prob }<\mathrm{t} \quad 0.8174\end{array}$

Tests that the Variances are Equal

$\begin{array}{lrrrr}\text { Level } & \text { Count } & \text { Std Dev } & \text { MeanAbsDif to Mean } & \text { MeanAbsDif to Median } \\ \text { Coliwasa } & 16 & 0.1081280 & 0.0862500 & 0.0862500\end{array}$

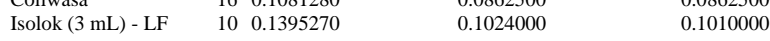

Test F Ratio DFNum DFDen p-Value

$\begin{array}{lllll}\text { O'Brien[.5] } & 0.7059 & 1 & 24 & 0.4091\end{array}$

Brown-Forsythe $\quad 0.2369 \quad 1 \quad 24 \quad 0.6308$

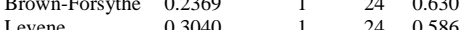

$\begin{array}{lllll}\text { Levene } & 0.3040 & 1 & 24 & 0.5865 \\ \text { Bartlett } & 0.7224 & 1 & & 0.3954\end{array}$

$\begin{array}{lllll}\text { Bartlett } & 0.7224 & 1 & & 0.3954 \\ \text { F Test 2-sided } & 1.6651 & 9 & 15 & 0.3677\end{array}$

Welch Anova testing Means Equal, allowing Std Devs Not Equal

F Ratio DFNum DFDen Prob $>$ F

$\begin{array}{llll}0.8695 & 1 & 15.697 & 0.3652\end{array}$

t Test

0.9325 


\section{Exhibit C2. Statistical Comparisons for Low-Rheology (Phase 3) Simulant Testing}

Oneway Analysis of Measurement By Sampling Description Test Phase=Phase 3 - Low Rheology, Analyte $=$ wt $\%$ vitrified solids

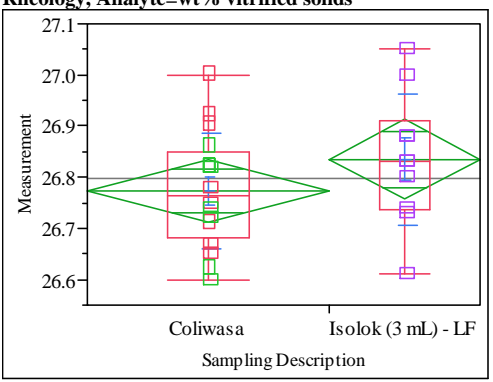

Oneway Anova

Summary of Fit

Rsquare

Adj Rsquare $\quad 0.024489$

Root Mean Square Error $\quad 0.119098$

$\begin{array}{lr}\text { Mean of Response } & 26.79731 \\ \text { Observations (or Sum Wgts) } & 26\end{array}$

t Test

Isolok (3 mL) - LF-Coliwasa

Assuming equal variances

Difference $\quad 0.06125$ t Ratio $\quad 1.275776$

$\begin{array}{lrlr}\text { Std Err Dif } & 0.04801 & \text { DF } & 24 \\ \text { Upper CL Dif } & 0.16034 & \text { Prob }>|t| & 0.2142\end{array}$

$\begin{array}{rrrr}\text { Upper CL Dif } & 0.16034 & \text { Prob }>|t| & 0.2142 \\ \text { Lower CL Dif } & -0.03784 & \text { Prob }>t & 0.1071\end{array}$

$\begin{array}{lrll}\text { Lower CL Dif } & -0.03784 & \text { Prob }>t & 0.1071 \\ \text { Confidence } & 0.95 & \text { Prob }<t & 0.8929\end{array}$

$\begin{array}{lrrrrr}\text { Analysis of Variance } & & & & & \\ \text { Source } & \text { DF } & \text { Sum of Squares } & \text { Mean Square } & \text { F Ratio } & \text { Prob > F } \\ \text { Sampling Description } & 1 & 0.02308654 & 0.023087 & 1.6276 & 0.2142 \\ \text { Error } & 24 & 0.34042500 & 0.014184 & & \\ \text { C. Total } & 25 & 0.36351154 & & & \end{array}$

Means for Oneway Anova

Level Number Mean Std Error Lower 95\% Upper 95\%

$\begin{array}{llrrrr}\text { Coliwasa } & 16 & 26.7738 & 0.02977 & 26.712 & 26.835\end{array}$

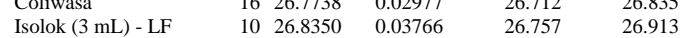

Std Error uses a pooled estimate of error variance

Means and Std Deviations

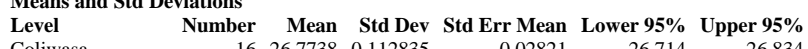

$\begin{array}{lrrrrrr}\text { Coliwasa } & 16 & 26.7738 & 0.112835 & 0.02821 & 26.714 & 26.834 \\ \text { Isolok (3 mL) - LF } & 10 & 26.8350 & 0.128863 & 0.04075 & 26.743 & 26.927\end{array}$

t Test

Isolok (3 mL) - LF-Coliwasa

Assuming unequal variances

Difference $\quad 0.06125$ t Ratio 1.235853

Std Err Dif $\quad 0.04956$ DF 17.30757

Upper CL Dif 0.16567 Prob $>|t| \quad 0.2330$

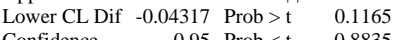

$\begin{array}{lll}\text { Confidence } & 0.95 \text { Prob }<\mathrm{t} & 0.8835\end{array}$

Tests that the Variances are Equal

$\begin{array}{lrrrr}\text { Level } & \text { Count } & \text { Std Dev } & \text { MeanAbsDif to Mean } & \text { MeanAbsDif to Median } \\ \text { Coliwasa } & 16 & 0.1128347 & 0.0912500 & 0.0912500 \\ \text { Isolok (3 mL) - LF } & 10 & 0.1288625 & 0.0940000 & 0.0930000\end{array}$

Test F Ratio DFNum DFDen p-Value

$\begin{array}{lllll}\text { O'Brien[.5] } & 0.2642 & 1 & 24 & 0.6120\end{array}$

$\begin{array}{lllll}\text { Brown-Forsythe } & 0.0037 & 1 & 24 & 0.9520\end{array}$

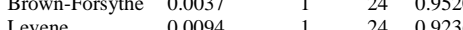

$\begin{array}{lllrr}\text { Levene } & 0.0094 & 1 & 24 & 0.9236 \\ \text { Bartlett } & 0.1936 & 1 & . & 0.6600\end{array}$

$\begin{array}{lllrl}\text { F Test 2-sided } & 1.3043 & 9 & 15 & 0.6237\end{array}$

Welch Anova testing Means Equal, allowing Std Devs Not Equal

F Ratio DFNum DFDen Prob $>$ F

$\begin{array}{llll}1.5273 & 1 & 17.308 & 0.2330\end{array}$

$\mathbf{t}$ Test
1.2359
Oneway Analysis of Measurement By Sampling Description Test Phase=Phase 3 - Low Rheology, Analyte $=\mathrm{wt} \%$ dried solids

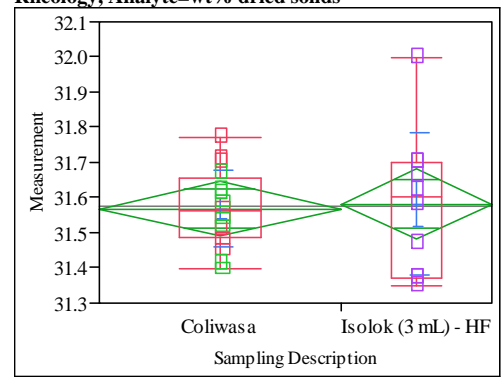

Oneway Anova

Summary of Fit

Rsquare

Adj Rsquare

Mean of Response 31.57385

Observations (or Sum Wgts) 26

t Test

Isolok (3 mL) - HF-Coliwasa

Assuming equal variances

$\begin{array}{lrlr}\text { Difference } & 0.01325 & \text { t Ratio } & 0.218777 \\ \text { Std Err Dif } & 0.06056 & \text { DF } & 24 \\ \text { Upper CL Dif } & 0.13825 & \text { Prob }>|t| & 0.8287 \\ \text { Lower CL Dif } & -0.11175 & \text { Prob }>\text { t } & 0.4143 \\ \text { Confidence } & 0.95 & \text { Prob }<\mathrm{t} & 0.5857\end{array}$

Analysis of Variance

Source DF Sum of Squares Mean Square F Ratio Prob $>$ F $\begin{array}{lrrrrr}\text { Sampling Description } & 1 & 0.00108038 & 0.001080 & 0.0479 & 0.8287 \\ \text { Error } & 24 & 0.54173500 & 0.022572 & & \end{array}$

Error

C. Total

$25 \quad 0.54281538$

Means for Oneway Anova

Level Number Mean Std Error Lower 95\% Upper 95\%

$\begin{array}{llllll}\text { Coliwasa } & 16 & 31.5688 & 0.03756 & 31.491 & 31.646\end{array}$

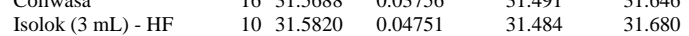

Std Error uses a pooled estimate of error variance

Means and Std Deviations

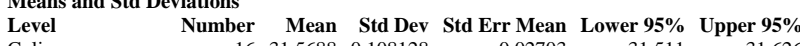

$\begin{array}{lrrrrrr}\text { Coliwasa } & 16 & 31.5688 & 0.108128 & 0.02703 & 31.511 & 31.626 \\ \text { Isolok }(3 \mathrm{~mL}) \text { - HF } & 10 & 31.5820 & 0.201759 & 0.06380 & 31.438 & 31.726\end{array}$

t Test

Isolok (3 mL) - HF-Coliwasa

Assuming unequal variances

Difference $\quad 0.01325$ t Ratio 0.191219

Std Err Dif $\quad 0.06929$ DF 12.28371

Upper CL Dif 0.16384 Prob $>|t| \quad 0.8515$

Lower CL Dif -0.13734 Prob $>t \quad 0.4257$

$\begin{array}{lll}\text { Confidence } \quad 0.95 \text { Prob }<\mathrm{t} \quad 0.5743 & \end{array}$

Tests that the Variances are Equal

$\begin{array}{lrrrr}\text { Level } & \text { Count } & \text { Std Dev } & \text { MeanAbsDif to Mean } & \text { MeanAbsDif to Median } \\ \text { Coliwasa } & 16 & 0.1081280 & 0.0862500 & 0.0862500 \\ \text { Isolok (3 mL) - HF } & 10 & 0.2017589 & 0.1540000 & 0.1540000\end{array}$

Test F Ratio DFNum DFDen p-Value

$\begin{array}{lllll}\text { O'Brien[.5] } & 3.3538 & 1 & 24 & 0.0795\end{array}$

$\begin{array}{lllll}\text { Brown-Forsythe } & 3.5677 & 1 & 24 & 0.0711\end{array}$

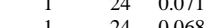

$\begin{array}{lllll}\text { Levene } & 3.6536 & 1 & 24 & 0.0680\end{array}$

$\begin{array}{lllrl}\text { Bartlett } & 4.3627 & 1 & \text {. } & 0.0367 \\ \text { F Test 2-sided } & 3.4817 & 9 & 15 & 0.0323\end{array}$

Welch Anova testing Means Equal, allowing Std Devs Not Equal

F Ratio DFNum DFDen Prob $>$ F

$\begin{array}{llll}0.0366 & 1 & 12.284 & 0.8515\end{array}$

t Test 


\section{Exhibit C2. Statistical Comparisons for Low-Rheology (Phase 3) Simulant Testing}

Oneway Analysis of Measurement By Sampling Description Test Phase=Phase 3 - Low Rheology, Analyte $=$ wt $\%$ vitrified solids

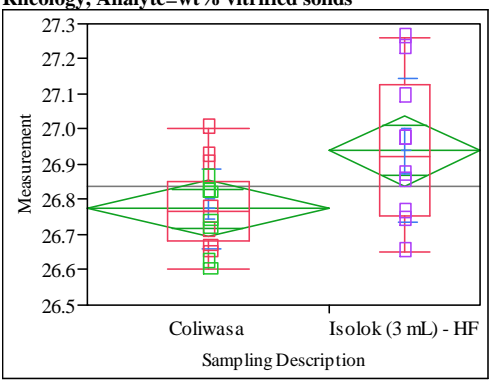

Oneway Anova

Summary of Fit

Rsquare

Root Mean Square Error $\quad 0.15428$

$\begin{array}{lr}\text { Mean of Response } & 26.83731 \\ \text { Observations (or Sum Wgts) } & 26\end{array}$

t Test

Isolok (3 mL) - HF-Coliwasa

Assuming equal variances

Difference $\quad 0.165250$ t Ratio 2.657061

Std Err Dif $\quad 0.062193$ DF 24

Upper CL Dif 0.293610 Prob $>|t| \quad 0.0138$

Lower CL Dif 0.036890 Prob $>t \quad 0.0069$

$\begin{array}{lll}\text { Confidence } & 0.95 \text { Prob }<\mathrm{t} \quad 0.9931\end{array}$

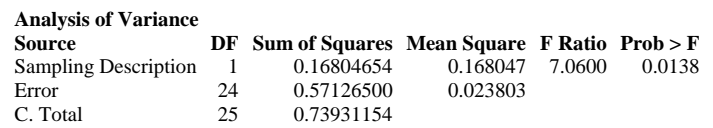

Means for Oneway Anova

Level Number Mean Std Error Lower 95\% Upper 95\%

$\begin{array}{lllllr}\text { Coliwasa } & 16 & 26.7738 & 0.03857 & 26.694 & 26.853\end{array}$

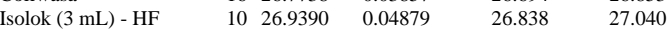

Std Error uses a pooled estimate of error variance

Means and Std Deviations

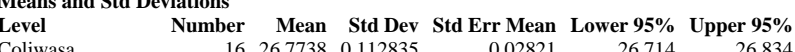

$\begin{array}{lrrrrrr}\text { Coliwasa } & 16 & 26.7738 & 0.112835 & 0.02821 & 26.714 & 26.834 \\ \text { Isolok (3 mL) - HF } & 10 & 26.9390 & 0.205559 & 0.06500 & 26.792 & 27.086\end{array}$

t Test

Isolok (3 mL) - HF-Coliwasa

Assuming unequal variances

Difference $\quad 0.165250$ t Ratio 2.332055

Std Err Dif $\quad 0.070860$ DF 12.44412

Upper CL Dif 0.319032 Prob $>|t| r \mid \begin{aligned} & 12.4442 \\ & 0.0372\end{aligned}$

Lower CL Dif 0.011468 Prob $>t \quad 0.0186$

$\begin{array}{lrl}\text { Confidence } & 0.95 \text { Prob }<\mathrm{t} & 0.9814\end{array}$

Tests that the Variances are Equal

$\begin{array}{lrrrr}\text { Level } & \text { Count } & \text { Std Dev } & \text { MeanAbsDif to Mean } & \text { MeanAbsDif to Median } \\ \text { Coliwasa } & 16 & 0.1128347 & 0.0912500 & 0.0912500 \\ \text { Isolok (3 mL) - HF } & 10 & 0.2055589 & 0.1650000 & 0.1650000\end{array}$

Test F Ratio DFNum DFDen p-Value

$\begin{array}{lllll}\text { O'Brien[.5] } & 5.7356 & 1 & 24 & 0.0248\end{array}$

$\begin{array}{lllll}\text { Brown-Forsythe } & 4.7080 & 1 & 24 & 0.0401\end{array}$

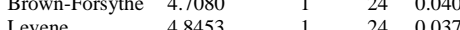

$\begin{array}{llrrr}\text { Levene } & 4.8453 & 1 & 24 & 0.0376 \\ \text { Bartlett } & 4.0372 & 1 & . & 0.0445\end{array}$

$\begin{array}{lllll}\text { F Test 2-sided } & 3.3188 & 9 & 15 & 0.0393\end{array}$

Welch Anova testing Means Equal, allowing Std Devs Not Equal

$\begin{array}{rrrrr}\text { F Ratio } & \text { DFNum } & \text { DFDen } & \text { Prob > F } \\ 5.4385 & 1 & 12.444 & 0.0372\end{array}$

t Test

2.3321
Oneway Analysis of Mean(Measurement) By Sampler Details Oxide/Analyte=Al/B

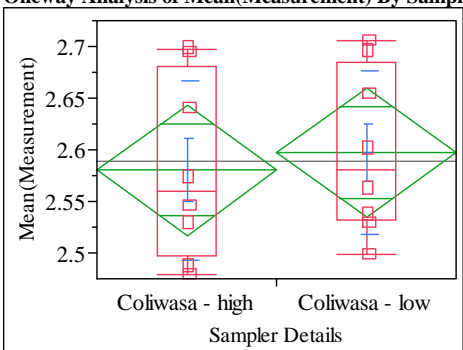

Oneway Anova

Summary of Fit

$\begin{array}{lr}\text { Rsquare } & 0.011912 \\ \text { Adj Rsquare } & -0.05867 \\ \text { Root Mean Square Error } & 0.083171 \\ \text { Mean of Response } & 2.588802 \\ \text { Observations (or Sum Wgts) } & 16\end{array}$

Observations (or Sum Wgts)

t Test

Coliwasa - low-Coliwasa - high

Assuming equal variances

Difference $\quad 0.01708$ t Ratio 0.410827

$\begin{array}{lll}\text { Std Err Dif } \quad 0.04159 \text { DF } & 14\end{array}$

Upper CL Dif 0.10628 Prob $>|t| \quad 0.6874$

Lower CL Dif -0.07211 Prob $>\mathrm{t} \quad 0.3437$

$\begin{array}{lll}\text { Confidence } & 0.95 \text { Prob }<\mathrm{t} \quad 0.6563\end{array}$

Analysis of Variance

Source DF Sum of Squares Mean Square F Ratio Prob $>$ F

$\begin{array}{lrrrrr}\text { Source } & \text { DF } & \text { Sum of Squares } & \text { Mean Square } & \text { F Ratio } & \text { Prob }>\text { F } \\ \text { Sampler Details } & 1 & 0.00116751 & 0.001168 & 0.1688 & 0.6874\end{array}$

$\begin{array}{lrll}\text { Error } & 14 & 0.09684407 & 0.006917\end{array}$

C. Total $\quad 15-0.09801158$

Means for Oneway Anova

Level Number Mean Std Error Lower 95\% Upper 95\%

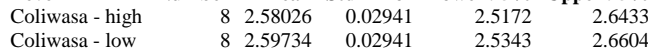

Std Error uses a pooled estimate of error variance

Means and Std Deviations

Level $\quad$ Number Mean Std Dev Std Err Mean Lower 95\% Upper 95\%

$\begin{array}{lrrrrrr}\text { Coliwasa - high } & 8 & 2.58026 & 0.087043 & 0.03077 & 2.5075 & 2.6530\end{array}$

$\begin{array}{lllllll}\text { Coliwasa - low } & 8 & 2.59734 & 0.079110 & 0.02797 & 2.5312 & 2.6635\end{array}$

t Test

Coliwasa - low-Coliwasa - high

Assuming unequal variances

Difference $\quad 0.01708$ t Ratio 0.410827

Std Err Dif $\quad 0.04159$ DF $\quad 13.87405$

Upper CL Dif 0.10635 Prob $>|t| r \mid r 675$

Lower CL Dif -0.07218 Prob $>t \quad 0.3437$

$\begin{array}{lll}\text { Confidence } & 0.95 \text { Prob }<\mathrm{t} \quad 0.6563\end{array}$

Tests that the Variances are Equal

Level Count Std Dev MeanAbsDif to Mean MeanAbsDif to Median

$\begin{array}{lrrrr}\text { Coliwasa - high } & 8 & 0.0870432 & 0.0724234 & 0.0703177\end{array}$

$\begin{array}{lllll}\text { Coliwasa - low } & 8 & 0.0791098 & 0.0724234 & 0.0703177 \\ & & & 0.0660145 & 0.0660145\end{array}$

Test F Ratio DFNum DFDen p-Value

$\begin{array}{lrrrr}\text { O'Brien[.5] } & 0.1906 & 1 & 14 & 0.6691\end{array}$

\begin{tabular}{lllll} 
Brown-Forsythe & 0.0367 & 1 & 14 & 0.8509 \\
\hline & 0.1149 & 1 & 14 & 0.7397
\end{tabular}

$\begin{array}{lllll}\text { Levene } & 0.1149 & 1 & 14 & 0.7397\end{array}$

$\begin{array}{lllll}\text { F Test 2-sided } & 1.2106 & 7 & 7 & 0.8074\end{array}$

Welch Anova testing Means Equal, allowing Std Devs Not Equal

F Ratio DFNum DFDen Prob $>$ F

$\begin{array}{llll}0.1688 & 1 & 13.874 & 0.6875\end{array}$

t Test
0.4108 
SRNL-STI-2011-00693

Revision 0

\section{Exhibit C2. Statistical Comparisons for Low-Rheology (Phase 3) Simulant Testing}

Oneway Analysis of Mean(Measurement) By Sampler Details Oxide/Analyte=Al2O3 (wt\%)

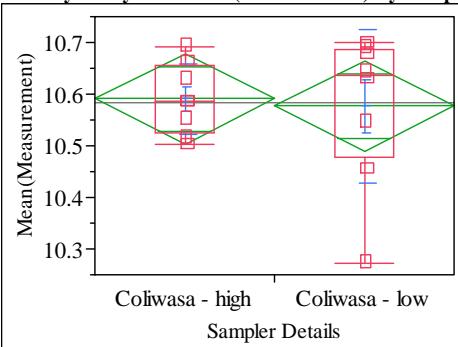

Oneway Anova

Summary of Fi

$\begin{array}{lr}\text { Rsquare } & 0.004305 \\ \text { Adj Rsquare } & -0.06682 \\ \text { Root Mean Square Error } & 0.115205 \\ \text { Mean of Response } & 10.58356 \\ \text { Observations (or Sum Wgts) } & 16\end{array}$

t Test

Coliwasa - low-Coliwasa - high

Assuming equal variances

Difference $\quad-0.01417$ t Ratio $\quad-0.24602$

Std Err Dif $\quad 0.05760$ DF $\quad 14$

Upper CL Dif 0.10937 Prob $>|t| \quad 0.8092$

Lower CL Dif -0.13772 Prob $>$ t 0.5954

$\begin{array}{lrl}\text { Confidence } & 0.95 \text { Prob }<\mathrm{t} \quad 0.4046\end{array}$

Analysis of Variance

Source DF Sum of Squares Mean Square F Ratio Prob $>$ F

$\begin{array}{lrrrrr}\text { Source } & \text { DF } & \text { Sum of Squares } & \text { Mean Square } & \text { F Ratio } & \text { Prob }>\text { F } \\ \text { Sampler Details } & 1 & 0.00080330 & 0.000803 & 0.0605 & 0.8092\end{array}$

$\begin{array}{lrll}\text { Error } & 14 & 0.18580961 & 0.013272\end{array}$

$\begin{array}{lll}\text { C. Total } & 15 & 0.1866129\end{array}$

Means for Oneway Anova

$\begin{array}{lrrrrr}\text { Level } & \text { Number Mean } & \text { Std Error } & \text { Lower 95\% } & \text { Upper 95\% }\end{array}$

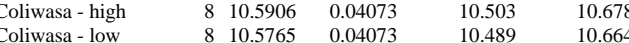

Std Error uses a pooled estimate of error variance

Means and Std Deviations

Level Number Mean Std Dev Std Err Mean Lower 95\% Upper 95\%

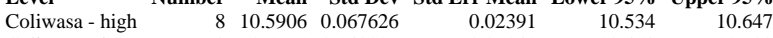

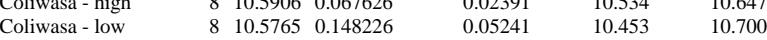

Test

Coliwasa - low-Coliwasa - high

Assuming unequal variances

Difference $\quad-0.01417$ t Ratio $\quad-0.24602$

Std Err Dif $\quad 0.05760$ DF 9.793093

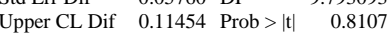

$\begin{array}{lll}\text { Lower CL Dif }-0.14289 & \text { Prob }>t & 0.5946\end{array}$

$\begin{array}{lrl}\text { Confidence } & 0.95 \text { Prob }<\mathrm{t} \quad 0.4054\end{array}$

Tests that the Variances are Equal

Level Count Std Dev MeanAbsDif to Mean MeanAbsDif to Median

$\begin{array}{lrrrr}\text { Coliwasa - high } & 8 & 0.0676260 & 0.0527485 & 0.0519613\end{array}$

$\begin{array}{lllll}\text { Coliwasa - low } & 8 & 0.1482260 & 0.1145509 & 0.1007733\end{array}$

Test F Ratio DFNum DFDen p-Value

$\begin{array}{lrrrr}\text { O'Brien[.5] } & 1.7219 & 1 & 14 & 0.2106\end{array}$

Brown-Forsythe $\quad 1.1946 \quad 110.14 \quad 0.2928$

$\begin{array}{lllll}\text { Levene } & 3.6516 & 1 & 14 & 0.0767\end{array}$

$\begin{array}{lllll}\text { Bartlett } & 3.6677 & 1 & \text {. } & 0.0555\end{array}$

$\begin{array}{lllll}\text { F Test 2-sided } & 4.8042 & 7 & 7 & 0.0553\end{array}$

Welch Anova testing Means Equal, allowing Std Devs Not Equal

F Ratio DFNum DFDen Prob > F

$\begin{array}{rrrr}0.0605 & 1 & 9.7931 & 0.8107\end{array}$

t Test

0.2460
Oneway Analysis of Mean(Measurement) By Sampler Details Oxide/Analyte=B2O3 (wt \%)

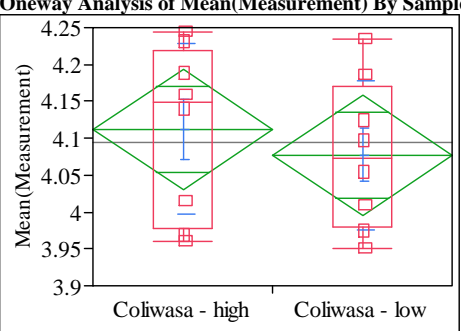

Sampler Details

\section{Oneway Anova}

Summary of Fit

Rsqua

Adj Rsquare

0.028644
-0.04074

oot Mean Square Error $\quad 0.108578$

Mean of Response

Observations (or Sum Wgts) 16

t Test

Coliwasa - low-Coliwasa - high

Assuming equal variances

Difference $\quad-0.03488$ t Ratio $\quad-0.64253$

Std Err Dif $\quad 0.05429$ DF 14

Upper CL Dif 0.08156 Prob $>|t| \quad 0.5309$

Lower CL Dif -0.15132 Prob $>\mathrm{t} \quad 0.7345$

$\begin{array}{lrl}\text { Confidence } & 0.95 \text { Prob }<\mathrm{t} & 0.2655\end{array}$

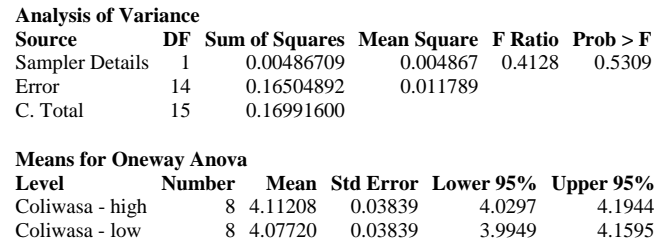

Std Error uses a pooled estimate of error variance

Means and Std Deviations

Level Number Mean Std Dev Std Err Mean Lower 95\% Upper 95\%

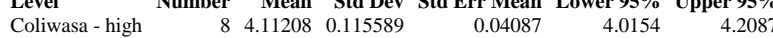

$\begin{array}{lllllll}\text { Coliwasa - low } & 8 & 4.07720 & 0.101082 & 0.03574 & 3.9927 & 4.1617\end{array}$

t Test

Coliwasa - low-Coliwasa - high

Assuming unequal variances

Difference $\quad-0.03488$ t Ratio $\quad-0.64253$

$\begin{array}{lll}\text { Std Err Dif } & 0.05429 \text { DF } & 13.75554\end{array}$

Upper CL Dif $\quad 0.08175$ Prob $>|t| r 0.5311$

Lower CL Dif -0.15152 Prob $>t \quad 0.7345$

$\begin{array}{lrl}\text { Confidence } & 0.95 \text { Prob }<\mathrm{t} & 0.2655\end{array}$

Tests that the Variances are Equal

Level Count Std Dev MeanAbsDif to Mean MeanAbsDif to Median

$\begin{array}{lrrrr}\text { Coliwasa - high } & 8 & 0.1155890 & 0.0989448 & 0.0925721\end{array}$

$\begin{array}{lllll}\text { Coliwasa - low } & 8 & 0.1010821 & 0.0989448 & 0.0925721 \\ & & & \end{array}$

Test F Ratio DFNum DFDen p-Value

$\begin{array}{lrrrr}\text { O'Brien[.5] } & 0.3472 & 1 & 14 & 0.5651\end{array}$

\begin{tabular}{lllll} 
Brown-Forsythe & 0.1205 & 1 & 14 & 0.7337 \\
\hline & 0.4943 & 1 & 14 & 0.4935
\end{tabular}

$\begin{array}{lllll}\text { Levene } & 0.4943 & 1 & 14 & 0.4935\end{array}$

$\begin{array}{lllll}0.1172 & 1 & \text { B } & 0.7321\end{array}$

Welch Anova testing Means Equal, allowing Std Devs Not Equal

F Ratio DFNum DFDen Prob $>$ F

$\begin{array}{rrrr}0.4128 & 1 & 13.756 & 0.5311\end{array}$

t Test

0.6425 
SRNL-STI-2011-00693

Revision 0

\section{Exhibit C2. Statistical Comparisons for Low-Rheology (Phase 3) Simulant Testing}

Oneway Analysis of Mean(Measurement) By Sampler Details Oxide/Analyte=BaO (wt $\%$ )

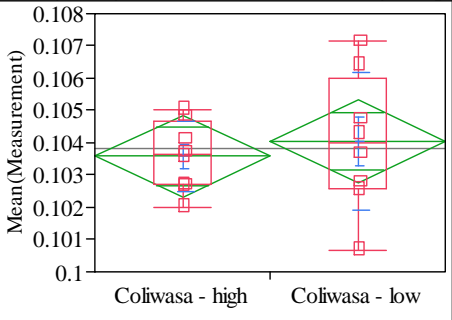

Sampler Details

\section{Oneway Anova}

Summary of Fit

$\begin{array}{lr}\text { Rsquare } & 0.020618 \\ \text { Adj Rsquare } & -0.04934 \\ \text { Root Mean Square Error } & 0.00168 \\ \text { Mean of Response } & 0.103811 \\ \text { Observations (or Sum Wgts) } & 16 \\ & \\ \text { t Test } & \\ \text { Coliwasa - low-Coliwasa - high } \\ \text { Assuming equal variances }\end{array}$

Difference $\quad 0.00046$ t Ratio $\quad 0.542886$

Std Err Dif $\quad 0.00084$ DF $\quad 14$

Upper CL Dif 0.00226 Prob $>|t| \quad 0.5957$

Lower CL Dif -0.00135 Prob $>$ t 0.2979

$\begin{array}{lrl}\text { Confidence } & 0.95 \text { Prob }<\mathrm{t} & 0.7021\end{array}$

\begin{tabular}{|c|c|c|c|c|c|}
\hline \multicolumn{6}{|c|}{ Analysis of Variance } \\
\hline Source & DF & Sum of Squares & s Mean Square & F Ratio & Prob $>$ F \\
\hline Sampler Details & 1 & 0.00000083 & $3 \quad 8.3139 \mathrm{e}-7$ & 0.2947 & $\quad 0.5957$ \\
\hline Error & 14 & 0.00003949 & $2.8209 \mathrm{e}-6$ & & \\
\hline C. Total & 15 & 0.00004032 & & & \\
\hline \multicolumn{6}{|c|}{ Means for Oneway Anova } \\
\hline Level & Num & Mean & Std Error Low & ver $95 \%$ & Upper 9 \\
\hline Coliwasa - high & & 80.103583 & 0.00059 & 0.10231 & \\
\hline Coliwasa - low & & $8 \quad 0.104039$ & 0.00059 & 0.10277 & 0.1053 \\
\hline
\end{tabular}

Std Error uses a pooled estimate of error variance

Means and Std Deviations

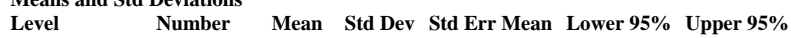

$\begin{array}{lrrrrrr}\text { Coliwasa - high } & 8 & 0.103583 & 0.001077 & 0.00038 & 0.10268 & 0.10448\end{array}$

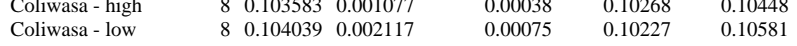

t Test

Coliwasa - low-Coliwasa - high

Assuming unequal variances
Oneway Analysis of Mean(Measurement) By Sampler Details Oxide/Analyte=CaO (wt \%)

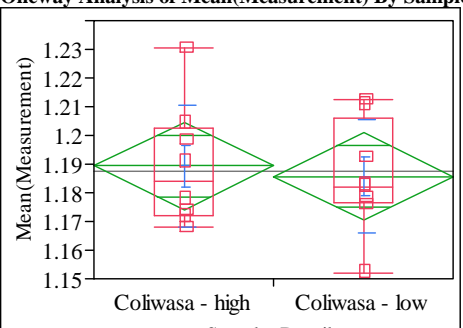

Sampler Details

\section{Oneway Anova}

Summary of Fit

$\begin{array}{lr}\text { Rsquare } & 0.008633 \\ \text { Adj Rsquare } & -0.06218 \\ \text { Root Mean Square Error } & 0.02037 \\ \text { Mean of Response } & 1.187484 \\ \text { Observations (or Sum Wgts) } & 16\end{array}$

t Test

Coliwasa - low-Coliwasa - high

Assuming equal variances

Difference $\quad-0.00356$ t Ratio $\quad-0.34917$

$\begin{array}{lll}\text { Std Err Dif } \quad 0.01018 \text { DF } & 14\end{array}$

Upper CL Dif $\quad 0.01829$ Prob $>|t| \quad 0.7322$

Lower CL Dif -0.02540 Prob $>t \quad 0.6339$

$\begin{array}{lrl}\text { Confidence } & 0.95 \text { Prob }<\mathrm{t} & 0.3661\end{array}$

$\begin{array}{lrrrrr}\begin{array}{l}\text { Analysis of Variance } \\ \text { Source }\end{array} & \text { DF } & \text { Sum of Squares } & \text { Mean Square } & \text { F Ratio } & \text { Prob > F } \\ \text { Sampler Details } & 1 & 0.00005059 & 0.000051 & 0.1219 & 0.7322 \\ \text { Error } & 14 & 0.00580908 & 0.000415 & & \\ \text { C. Total } & 15 & 0.00585967 & & & \end{array}$

Means for Oneway Anova

Level Number Mean Std Error Lower 95\% Upper 95\%

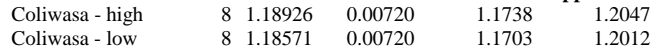

Std Error uses a pooled estimate of error variance

Means and Std Deviations

Level Number Mean Std Dev Std Err Mean Lower 95\% Upper 95\%

$\begin{array}{lrrrrrr} & \text { Number } & \text { Mean } & \text { Std Dev } & \text { Std Err Mean } & \text { Lower 95\% } & \text { Upper 95\% } \\ \text { Coliwasa - high } & 8 & 1.18926 & 0.021123 & 0.00747 & 1.1716 & 1.2069\end{array}$

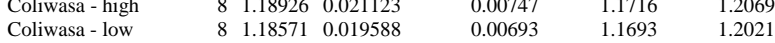

t Test

Coliwasa - low-Coliwasa - high

Assuming unequal variances

Difference $\quad-0.00356$ t Ratio $\quad-0.34917$

$\begin{array}{lll}\text { Std Err Dif } \quad 0.01018 \text { DF } & 13.92107\end{array}$

Upper CL Dif 0.01830 Prob $>|t| r .7322$

Lower CL Dif -0.02541 Prob $>t \quad 0.6339$

Confidence $\quad 0.95$ Prob $<\mathrm{t} \quad 0.3661$

Tests that the Variances are Equal

Level Count Std Dev MeanAbsDif to Mean MeanAbsDif to Median

$\begin{array}{lrrrr}\text { Coliwasa - high } & 8 & 0.0211230 & 0.0164989 & 0.0164989\end{array}$

$\begin{array}{lllll}\text { Coliwasa - low } & 8 & 0.0195880 & 0.0164989 & 0.0164989 \\ & & & \end{array}$

$\begin{array}{llll}\text { Test } & \text { F Ratio DFNum DFDen p-Value }\end{array}$

$\begin{array}{lllll}\text { O'Brien[.5] } & 0.0422 & 1 & 14 & 0.8401\end{array}$

\begin{tabular}{lllll} 
Brown-Forsythe & 0.1854 & 1 & 14 & 0.6734 \\
\hline & 0.1160 & 1 & 14 & 0.7384
\end{tabular}

$\begin{array}{lllll}\text { Levene } & 0.1160 & 1 & 14 & 0.7384\end{array}$

$\begin{array}{lllll}0.0371 & 1 & \text {. } & 0.8472\end{array}$

Welch Anova testing Means Equal, allowing Std Devs Not Equal

F Ratio DFNum DFDen Prob $>$ F

$\begin{array}{rrrr}0.1219 & 1 & 13.921 & 0.7322\end{array}$

t Test

0.3492

t Test

0.5429 
SRNL-STI-2011-00693

Revision 0

\section{Exhibit C2. Statistical Comparisons for Low-Rheology (Phase 3) Simulant Testing}

Oneway Analysis of Mean(Measurement) By Sampler Details Oxide/Analyte=Cr2O3 (wt\%)

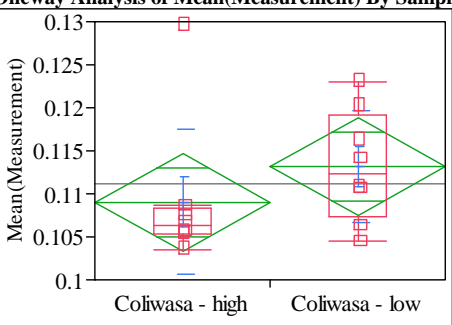

Sampler Details

\section{Oneway Anova}

Summary of Fit

\subsection{3 \\ Adj Rsquare $\quad 0.014256$ \\ Root Mean Square Error $\quad 0.00748$ \\ $\begin{array}{lr}\text { Mean of Response } & 0.111117 \\ \text { Observations (or Sum Wgts) } & 16\end{array}$ \\ t Test \\ Coliwasa - low-Coliwasa - high}

Assuming equal variances

Difference $\quad 0.00413$ t Ratio $\quad 1.10315$

Std Err Dif $\quad 0.00374$ DF 14

Upper CL Dif 0.01215 Prob $>|t| \quad 0.2886$

Lower CL Dif -0.00390 Prob $>t \quad 0.1443$

$\begin{array}{lll}\text { Confidence } & 0.95 \text { Prob }<\mathrm{t} \quad 0.8557\end{array}$

\begin{tabular}{|c|c|c|c|c|c|}
\hline \multicolumn{6}{|c|}{ Analysis of Variance } \\
\hline Source & DF & Sum of Squares & Mean Square & F Ratio & Prob $>$ F \\
\hline Sampler Details & 1 & 0.00006809 & 0.000068 & 1.2169 & 0.2886 \\
\hline Error & 14 & 0.00078338 & 0.000056 & & \\
\hline C. Total & 15 & 0.00085147 & & & \\
\hline \multicolumn{6}{|c|}{ Means for Oneway Anova } \\
\hline Level & Num & Mean & Std Error Low & er $95 \%$ & Upper 95\% \\
\hline Coliwasa - high & & $\begin{array}{ll}8 & 0.109054\end{array}$ & 0.00264 & 0.10338 & 0.11473 \\
\hline Coliwasa - low & & $8 \quad 0.113180$ & 0.00264 & 0.10751 & 0.11885 \\
\hline
\end{tabular}

$\begin{array}{llllll}\text { Coliwasa - low } & 8 & 0.113180 & 0.00264 & 0.10751 & 0.11885\end{array}$

Std Error uses a pooled estimate of error variance

Means and Std Deviations

Level $\quad$ Number Mean Std Dev Std Err Mean Lower 95\% Upper 95\%

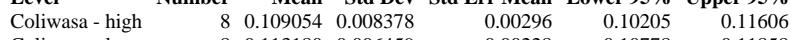

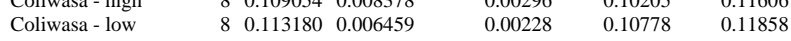

t Test

Coliwasa - low-Coliwasa - high

Assuming unequal variances
Difference $\quad 0.00413$ t Ratio $\quad 1.10315$

Std Err Dif $\quad 0.00374$ DF 13.14852

Upper CL Dif $\quad 0.01220$ Prob $>|t| \quad 0.2897$

Lower CL Dif -0.00394 Prob $>t \quad 0.1449$

Confidence $\quad 0.95$ Prob $<\mathrm{t} \quad 0.8551$

Tests that the Variances are Equal

Level Count Std Dev MeanAbsDif to Mean MeanAbsDif to Median

$\begin{array}{lrrrr}\text { Coliwasa - high } & 8 & 0.0083782 & 0.0050928 & 0.0040499\end{array}$

$\begin{array}{lllll}\text { Coliwasa - low } & 8 & 0.0064588 & 0.0051917 & 0.0051917\end{array}$

Test F Ratio DFNum DFDen p-Value

$\begin{array}{lrrrr}\text { O'Brien[.5] } & 0.1941 & 1 & 14 & 0.6663\end{array}$

$\begin{array}{lllll}\text { Brown-Forsythe } & 0.1454 & 1 & 14 & 0.7087\end{array}$

$\begin{array}{lllll}\text { Levene } & 0.0015 & 1 & 14 & 0.9694\end{array}$

$\begin{array}{lllll}\text { Bartlett } & 0.4374 & 1 & \text {. } & 0.5084\end{array}$

Welch Anova testing Means Equal, allowing Std Devs Not Equal

F Ratio DFNum DFDen Prob $>$ F

$\begin{array}{rrrr}1.2169 & 1 & 13.149 & 0.2897\end{array}$

$\mathbf{t}$ Test
1.1032
Oneway Analysis of Mean(Measurement) By Sampler Details Oxide/Analyte=CuO (wt \%)

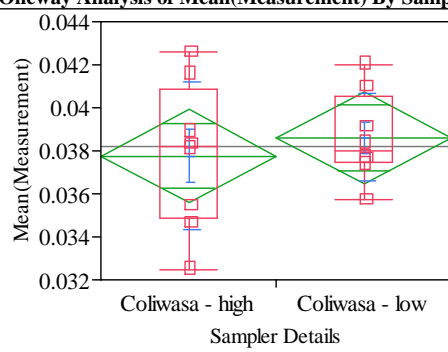

Oneway Anova

Summary of Fit

Rsquare

$-0.04486$

Root Mean Squar

Mean of Response

0.038186
16

t Test

Coliwasa - low-Coliwasa - high

Assuming equal variances

Difference $\quad 0.00084$ t Ratio 0.596618

$\begin{array}{lll}\text { Std Err Dif } \quad 0.00141 \text { DF } & 14\end{array}$

Upper CL Dif 0.00387 Prob $>|t| \quad 0.5603$

Lower CL Dif -0.00219 Prob $>$ t 0.2801

$\begin{array}{lll}\text { Confidence } & 0.95 \text { Prob }<\mathrm{t} \quad 0.7199\end{array}$

$\begin{array}{lrrrrr}\begin{array}{l}\text { Analysis of Variance } \\ \text { Source }\end{array} & \text { DF } & \text { Sum of Squares } & \text { Mean Square } & \text { F Ratio } & \text { Prob > F } \\ \text { Sampler Details } & 1 & 0.00000284 & 2.8383 \mathrm{e}-6 & 0.3560 & 0.5603 \\ \text { Error } & 14 & 0.00011163 & 7.9737 \mathrm{e}-6 & & \\ \text { C. Total } & 15 & 0.00011447 & & & \end{array}$

Means for Oneway Anova

Level Number Mean Std Error Lower 95\% Upper 95\%

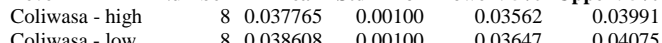

Std Error uses a pooled estimate of error variance

Means and Std Deviations

$\begin{array}{lcccc}\text { Means and Std Deviations } & \text { Number } & \text { Mean } & \text { Std Dev Std Err Mean Lower 95\% } & \text { Upper 95\% }\end{array}$

$\begin{array}{lrrrrrr}\text { Level } & \text { Number } & \text { Mean } & \text { Std Dev } & \text { Std Err Mean } & \text { Lower 95\% } & \text { Upper 95\% } \\ \text { Coliwasa - high } & 8 & 0.037765 & 0.003433 & 0.00121 & 0.03490 & 0.04063\end{array}$

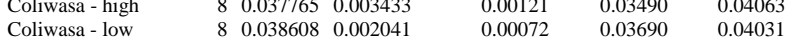

t Test

Coliwasa - low-Coliwasa - high

Assuming unequal variances

$\begin{array}{lrlr}\text { Difference } & 0.00084 & \text { t Ratio } & 0.596618 \\ \text { Std Err Dif } & 0.00141 & \text { DF } & 11.39877 \\ \text { Upper CL Dif } & 0.00394 & \text { Prob }>|t| & 0.5624 \\ \text { Lower CL Dif } & -0.00225 & \text { Prob }>\text { t } & 0.2812 \\ \text { Confidence } & 0.95 & \text { Prob }<\mathrm{t} & 0.7188\end{array}$

Tests that the Variances are Equal

Level Count Std Dev MeanAbsDif to Mean MeanAbsDif to Median

$\begin{array}{lrrrr}\text { Coliwasa - high } & 8 & 0.0034326 & 0.0026672 & 0.0025844\end{array}$

$\begin{array}{lllll}\text { Coliwasa - low } & 8 & 0.0020407 & 0.0026672 & 0.0025844 \\ & & & \end{array}$

Test F Ratio DFNum DFDen p-Value

$\begin{array}{lrrrr}\text { O'Brien[.5] } & 2.2232 & 1 & 14 & 0.1581\end{array}$

$\begin{array}{lllll}\text { Brown-Forsythe } & 1.4628 & 1 & 14 & 0.2465 \\ & 1.9098 & 1 & 14 & 0.1886\end{array}$

$\begin{array}{lllll}\text { Levene } & 1.9098 & 1 & 14 & 0.1886\end{array}$

$\begin{array}{lllll}\text { F Test 2-sided } & 2.8293 & 7 & 7 & 0.1934\end{array}$

Welch Anova testing Means Equal, allowing Std Devs Not Equal

F Ratio DFNum DFDen Prob $>$ F

$\begin{array}{rrrr}0.3560 & 1 & 11.399 & 0.5624\end{array}$

t Test 
SRNL-STI-2011-00693

Revision 0

\section{Exhibit C2. Statistical Comparisons for Low-Rheology (Phase 3) Simulant Testing}

Oneway Analysis of Mean(Measurement) By Sampler Details Oxide/Analyte=Fe/Li

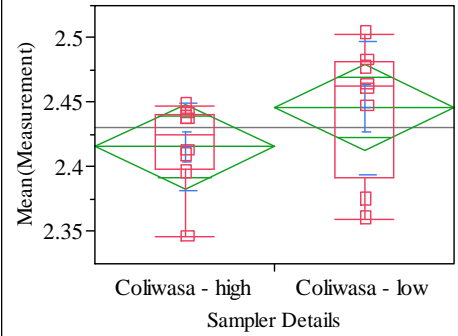

Oneway Anova

Summary of Fi

\begin{tabular}{|c|c|c|c|}
\hline \multicolumn{4}{|c|}{0.120098} \\
\hline \multicolumn{2}{|l|}{ Adj Rsquare } & \multicolumn{2}{|c|}{$\begin{array}{l}0.120098 \\
0.057248\end{array}$} \\
\hline \multicolumn{2}{|c|}{ Root Mean Square Error } & \multicolumn{2}{|c|}{$0.05 / 246$} \\
\hline \multicolumn{2}{|c|}{ Mean of Response } & \multicolumn{2}{|c|}{2.430778} \\
\hline \multicolumn{2}{|c|}{ Observations (or Sum Wgts) } & & 16 \\
\hline \multicolumn{4}{|c|}{$\begin{array}{l}\text { t Test } \\
\text { Coliwasa - low-Coliwasa - high }\end{array}$} \\
\hline Difference & $0.03024 \mathrm{t}$ & t Ratio & 1.382341 \\
\hline Std Err Dif & $0.02187 \mathrm{D}$ & DF & 14 \\
\hline Upper CL Dif & $0.07715 \mathrm{P}$ & Prob $>|t|$ & 0.1885 \\
\hline Lower CL Dif & $-0.01668 \mathrm{P}$ & Prob $>t$ & 0.0943 \\
\hline Confidence & $0.95 \mathrm{P}$ & Prob $<\mathrm{t}$ & 0.9057 \\
\hline
\end{tabular}

Analysis of Variance

$\begin{array}{lrrrrr}\text { Source } & \text { DF } & \text { Sum of Squares } & \text { Mean Square } & \text { F Ratio } & \text { Prob }>\text { F } \\ \text { Sampler Details } & 1 & 0.00365665 & 0.003657 & 1.9109 & 0.1885 \\ \text { Error } & 14 & 0.02679049 & 0.001914 & & \end{array}$

Means for Oneway Anova

$\begin{array}{lrrrr}\text { Level Number Mean } & \text { Std Error } & \text { Lower 95\% } & \text { Upper 95\% }\end{array}$

$\begin{array}{llllll}\text { Coliwasa - low } & 8 & 2.41566 & 0.01547 & 2.3825 & 2.4488 \\ & 8 & 2.44590 & 0.01547 & 2.4127 & 2.4791\end{array}$

Std Error uses a pooled estimate of error variance

Means and Std Deviations

Level Number Mean Std Dev Std Err Mean Lower 95\% Upper 95\%

$\begin{array}{lrrrrrr}\text { Coliwasa - high } & 8 & 2.41566 & 0.033826 & 0.01196 & 2.3874 & 2.4439\end{array}$

$\begin{array}{lllllll}\text { Coliwasa - low } & 8 & 2.44590 & 0.051798 & 0.01831 & 2.4026 & 2.4892\end{array}$

t Test

Coliwasa - low-Coliwasa - high

Assuming unequal variances
Difference $\quad 0.03024$ t Ratio 1.382341

Std Err Dif $\quad 0.02187$ DF 12.05163

Upper CL Dif $\quad 0.07787$ Prob $>|t| \quad 0.1919$

Lower CL Dif -0.01740 Prob $>\mathrm{t} \quad 0.0960$

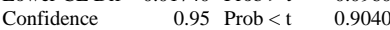

Tests that the Variances are Equal

Level Count Std Dev MeanAbsDif to Mean MeanAbsDif to Median

$\begin{array}{lrrrr}\text { Coliwasa - high } & 8 & 0.0338258 & 0.0252881 & 0.0252881\end{array}$

$\begin{array}{lllll}\text { Coliwasa - low } & 8 & 0.0517980 & 0.0396271 & 0.0357634\end{array}$

Test F Ratio DFNum DFDen p-Value

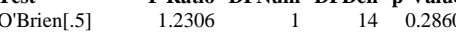

Brown-Forsythe $\quad 0.4311 \quad 11 \quad 14 \quad 0.5221$

$\begin{array}{lllll}\text { Levene } & 1.2636 & 1 & 14 & 0.2799\end{array}$

$\begin{array}{lllll}\text { Bartlett } & 1.1521 & 1 & \text {. } & 0.2831\end{array}$

Welch Anova testing Means Equal, allowing Std Devs Not Equal

F Ratio DFNum DFDen Prob $>$ F

$\begin{array}{rrrr}1.9109 & 1 & 12.052 & 0.1919\end{array}$

t Test
Oneway Analysis of Mean(Measurement) By Sampler Details Oxide/Analyte=Fe2O3 (wt \%)

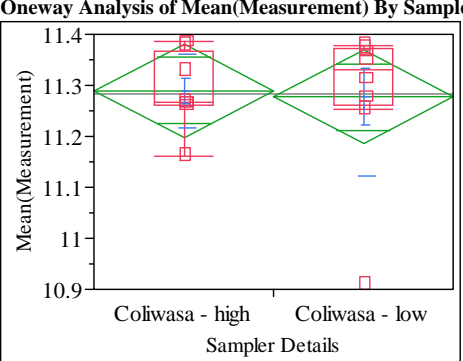

Oneway Anova

Summary of Fit

$\begin{array}{lr}\text { Rsquare } & 0.002462 \\ \text { Adj Rsquare } & -0.06879 \\ \text { Root Mean Square Error } & 0.121779 \\ \text { Mean of Response } & 11.28331 \\ \text { Observations (or Sum Wgts) } & 16\end{array}$

Observations (or Sum Wgts)

t Test

Coliwasa - low-Coliwasa - high

Assuming equal variances

Difference $\quad-0.01132$ t Ratio $\quad-0.18589$

$\begin{array}{lll}\text { Std Err Dif } \quad 0.06089 \text { DF } & 14\end{array}$

Upper CL Dif 0.11928 Prob $>|t| \quad 0.8552$

Lower CL Dif -0.14191 Prob $>t \quad 0.5724$

$\begin{array}{lrl}\text { Confidence } & 0.95 \text { Prob }<\mathrm{t} \quad 0.4276\end{array}$

$\begin{array}{lrrrrr}\begin{array}{l}\text { Analysis of Variance } \\ \text { Source }\end{array} & \text { DF } & \text { Sum of Squares } & \text { Mean Square } & \text { F Ratio } & \text { Prob > F } \\ \text { Sampler Details } & 1 & 0.00051243 & 0.000512 & 0.0346 & 0.8552 \\ \text { Error } & 14 & 0.20762216 & 0.014830 & & \\ \text { C. Total } & 15 & 0.20813459 & & & \end{array}$

Means for Oneway Anova

Level Number Mean Std Error Lower 95\% Upper 95\%

$\begin{array}{llllll}\text { Coliwasa - high } & 8 & 11.2890 & 0.04306 & 11.197 & 11.381 \\ \text { Coliwasa - low } & 8 & 11.2777 & 0.04306 & 11.185 & 11.370\end{array}$

Std Error uses a pooled estimate of error variance

Means and Std Deviations

Level $\quad$ Number Mean Std Dev Std Err Mean Lower 95\% Upper 95\%

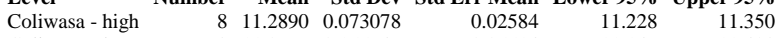

$\begin{array}{lllllll}\text { Coliwasa - low } & 8 & 11.2777 & 0.155949 & 0.05514 & 11.228 & 11.350 \\ & & & & & & \end{array}$

t Test

Coliwasa - low-Coliwasa - high

Assuming unequal variances

Difference $\quad-0.01132$ t Ratio $\quad-0.18589$

Std Err Dif $\quad 0.06089$ DF 9.932813

Upper CL Dif 0.12448 Prob $>|t| r 0.8563$

Lower CL Dif -0.14711 Prob $>t \quad 0.5719$

Confidence $\quad 0.95$ Prob $<\mathrm{t} \quad 0.4281$

Tests that the Variances are Equal

Level Count Std Dev MeanAbsDif to Mean MeanAbsDif to Median

$\begin{array}{lrrrr}\text { Coliwasa - high } & 8 & 0.0730778 & 0.0560710 & 0.0503374\end{array}$

$\begin{array}{lllll}\text { Coliwasa - low } & 8 & 0.1559485 & 0.0560710 & 0.0503374 \\ & & & 0.0981429 & 0.0890584\end{array}$

Test F Ratio DFNum DFDen p-Value

$\begin{array}{lrrrr}\text { O'Brien[.5] } & 0.8521 & 1 & 14 & 0.3716\end{array}$

\begin{tabular}{lllll} 
Brown-Forsythe & 0.5547 & 1 & 14 & 0.4687 \\
\hline & 0.9403 & 1 & 14 & 0.3487
\end{tabular}

$\begin{array}{lllll}\text { Levene } & 0.9403 & 1 & 14 & 0.3487\end{array}$

$\begin{array}{lllll}\text { Bartlett } & 3.4413 & 1 & & 0.0636\end{array}$

Welch Anova testing Means Equal, allowing Std Devs Not Equal

F Ratio DFNum DFDen Prob $>$ F

$\begin{array}{rrrr}0.0346 & 1 & 9.9328 & 0.8563\end{array}$

t Test

0.1859 
SRNL-STI-2011-00693

Revision 0

\section{Exhibit C2. Statistical Comparisons for Low-Rheology (Phase 3) Simulant Testing}

Oneway Analysis of Mean(Measurement) By Sampler Details Oxide/Analyte=K2O (wt\%)

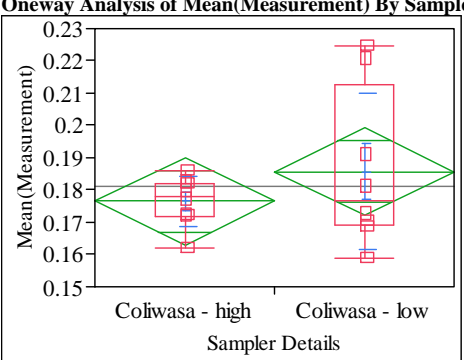

Oneway Anova

Summary of Fi

\section{Rsquare $\quad 0.070173$ \\ Adj Rsquare $\quad 0.003757$ \\ Root Mean Square Error $\quad 0.018067$ \\ Mean of Response 0.181117 \\ Observations (or Sum Wgts) 16 \\ t Test \\ Coliwasa - low-Coliwasa - high}

Assuming equal variances

$\begin{array}{lrlr}\text { Difference } & 0.00929 & \text { t Ratio } & 1.027891 \\ \text { Std Err Dif } & 0.00903 & \text { DF } & 14 \\ \text { Upper CL Dif } & 0.02866 & \text { Prob }>|t| & 0.3214\end{array}$

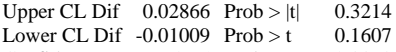

$\begin{array}{lrl}\text { Confidence } & 0.95 \text { Prob }<\mathrm{t} \quad 0.8393\end{array}$

\begin{tabular}{|c|c|c|c|c|c|c|c|}
\hline \multicolumn{8}{|c|}{ Analysis of Variance } \\
\hline Source & DF & \multicolumn{2}{|c|}{ Sum of Squares } & s Mean Square & F Ratio & \multicolumn{2}{|l|}{ Prob $>$ F } \\
\hline Sampler Details & 1 & & 0.00034488 & \multirow{2}{*}{\multicolumn{2}{|c|}{$\begin{array}{ll}0.000345 & 1.0566 \\
0.000326 & \end{array}$}} & \multirow[t]{2}{*}{0.3214} & \\
\hline Error & 14 & & 0.00456983 & & & & \\
\hline C. Total & 15 & & 0.00491471 & & & & \\
\hline \multicolumn{8}{|c|}{ Means for Oneway Anova } \\
\hline Level & Num & & Mean & Std Error Lowe & er $95 \% \quad \mathrm{~L}$ & \multicolumn{2}{|l|}{ Upper 95\% } \\
\hline Coliwasa - high & & 8 & 0.176474 & $0.00639 \quad 0$ & 0.16277 & & \\
\hline Coliwasa - low & & 8 & 0.185759 & 0.00639 & 0.17206 & \multicolumn{2}{|l|}{0.19946} \\
\hline \multicolumn{8}{|c|}{ Std Error uses a pooled estimate of error variance } \\
\hline \multicolumn{8}{|c|}{ Means and Std Deviations } \\
\hline Level & Num & ber & Mean & Std Dev Std Er & rr Mean & Lower 95\% & Upper $95 \%$ \\
\hline Coliwasa - high & & 8 & 0.176474 & 0.007659 & 0.00271 & 0.17007 & 0.18288 \\
\hline Coliwasa - low & & 8 & 0.185759 & 0.024376 & 0.00862 & 0.16538 & 0.20614 \\
\hline
\end{tabular}

t Test

Coliwasa - low-Coliwasa - high

Assuming unequal variances

$\begin{array}{lrlr}\text { Difference } & 0.00929 & \text { t Ratio } & 1.027891 \\ \text { Std Err Dif } & 0.00903 \text { DF } & 8.368923 \\ \text { Upper CL Dif } & 0.02996 & \text { Prob }>|t| & 0.3328 \\ \text { Lower CL Dif } & -0.01139 & \text { Prob }>t & 0.1664 \\ \text { Confidence } & 0.95 \text { Prob }<\mathrm{t} & 0.8336\end{array}$

Tests that the Variances are Equal

$\begin{array}{lrrrr}\text { Level } & \text { Count } & \text { Std Dev } & \text { MeanAbsDif to Mean } & \text { MeanAbsDif to Median } \\ \text { Coliwasa - high } & 8 & 0.0076593 & 0.0058724 & 0.0058724\end{array}$

$\begin{array}{lllll}\text { Coliwasa - high } & 8 & 0.0076593 & 0.0058724 & 0.0058724 \\ \text { Coliwasa - low } & 8 & 0.0243756 & 0.0194367 & 0.0181694\end{array}$

Test F Ratio DFNum DFDen p-Value

O'Brien[.5] $\quad 4.7148 \quad 11 \quad 14 r 0.0476$

$\begin{array}{lllll}\text { Brown-Forsythe } & 3.5869 & 1 & 14 & 0.0791\end{array}$

$\begin{array}{lllll}\text { Levene } & 8.1023 & 1 & 14 & 0.0129\end{array}$

$\begin{array}{lllll} & 7.3000 & 1 & \text { Bartlett } & 0.0069\end{array}$

Welch Anova testing Means Equal, allowing Std Devs Not Equal

F Ratio DFNum DFDen Prob $>$ F

$\begin{array}{rrrr}1.0566 & 1 & 8.3689 & 0.3328\end{array}$

$\mathbf{t}$ Test
1.0279

Oneway Analysis of Mean(Measurement) By Sampler Details Oxide/Analyte=Li2O (wt\%)

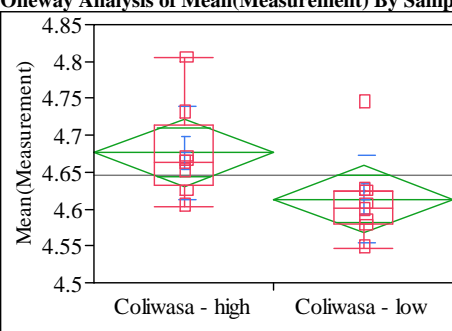

Sampler Details

Oneway Anova

Summary of Fit

Rsquare

0.176963

Root 0.061097

Mean of Response 4.644882
16

t Test

Coliwasa - low-Coliwasa - high

Assuming equal variances

Difference $\quad-0.06279$ t Ratio $\quad-2.05552$

$\begin{array}{llr}\text { Std Err Dif } & 0.03055 \text { DF } & 14\end{array}$

Upper CL Dif 0.00273 Prob $>|t| \quad 0.0590$

Lower CL Dif -0.12831 Prob $>\mathrm{t} \quad 0.9705$

$\begin{array}{lrl}\text { Confidence } & 0.95 \text { Prob }<\mathrm{t} & 0.0295\end{array}$

$\begin{array}{lrrrrr}\begin{array}{l}\text { Analysis of Variance } \\ \text { Source }\end{array} & \text { DF } & \text { Sum of Squares } & \text { Mean Square } & \text { F Ratio } & \text { Prob > F } \\ \text { Sampler Details } & 1 & 0.01577180 & 0.015772 & 4.2252 & 0.0590 \\ \text { Error } & 14 & 0.05225938 & 0.003733 & & \\ \text { C. Total } & 15 & 0.06803118 & & & \end{array}$

Means for Oneway Anova

Level Number Mean Std Error Lower 95\% Upper 95\%

$\begin{array}{llrrrr}\text { Coliwasa - high } & 8 & 4.67628 & 0.02160 & 4.6299 & 4.7226\end{array}$

Std Error uses a pooled estimate of error variance

Means and Std Deviations

Means and Std Deviations
Level Number Mean Std Dev Std Err Mean Lower 95\% Upper 95\%

$\begin{array}{lrrrrrr}\text { Level } & \text { Number } & \text { Mean } & \text { Std Dev } & \text { Std Err Mean } & \text { Lower 95\% } & \text { Upper 95\% } \\ \text { Coliwasa - high } & 8 & 4.67628 & 0.063488 & 0.02245 & 4.6232 & 4.7294\end{array}$

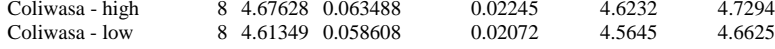

t Test

Coliwasa - low-Coliwasa - high

Assuming unequal variances

Difference $\quad-0.06279$ t Ratio $\quad-2.05552$

Std Err Dif $\quad 0.03055$ DF 13.91136

Upper CL Dif 0.00277 Prob $>|t| \quad 0.0591$

Lower CL Dif -0.12835 Prob $>\mathrm{t} \quad 0.9704$

Confidence $\quad 0.95$ Prob $<\mathrm{t} \quad 0.0296$

Tests that the Variances are Equal

Level Count Std Dev MeanAbsDif to Mean MeanAbsDif to Median

$\begin{array}{lrrrr}\text { Coliwasa - high } & 8 & 0.0634884 & 0.0453006 & 0.0412639\end{array}$

$\begin{array}{lllll}\text { Coliwasa - low } & 8 & 0.0586076 & 0.0453006 & 0.0412639 \\ & & & 0.0383485 & 0.0367787\end{array}$

Test F Ratio DFNum DFDen p-Value

$\begin{array}{lllll}\text { O'Brien[.5] } & 0.0288 & 1 & 14 & 0.8677\end{array}$

$\begin{array}{lllll}\text { Brown-Forsythe } & 0.0371 & 1 & 14 & 0.8500 \\ & 0.1124 & 1 & 14 & 0.7424\end{array}$

$\begin{array}{lllll}\text { Levene } & 0.1124 & 1 & 14 & 0.7424\end{array}$

$\begin{array}{lllll}\text { Bartlett } & 0.0418 & 1 & \text { T } & 0.8381 \\ \text { F Test 2-sided } & 1.1735 & 7 & 7 & 0.8382\end{array}$

Welch Anova testing Means Equal, allowing Std Devs Not Equal

F Ratio DFNum DFDen Prob $>$ F

$\begin{array}{rrrr}4.2252 & 1 & 13.911 & 0.0591\end{array}$

t Test

2.0555 
SRNL-STI-2011-00693

Revision 0

\section{Exhibit C2. Statistical Comparisons for Low-Rheology (Phase 3) Simulant Testing}

Oneway Analysis of Mean(Measurement) By Sampler Details Oxide/Analyte=MgO (wt\%)

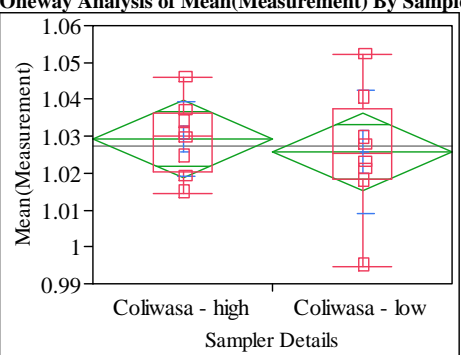

Oneway Anova

Summary of Fi

$\begin{array}{lr}\text { Rsquare } & 0.018786 \\ \text { Adj Rsquare } & -0.0513 \\ \text { Root Mean Square Error } & 0.01388 \\ \text { Mean of Response } & 1.027524 \\ \text { Observations (or Sum Wgts) } & 16 \\ & \\ \text { t Test } & \\ \text { Coliwasa - low-Coliwasa - high } \\ \text { Assuming equal variances }\end{array}$

Difference $\quad-0.00359$ t Ratio $\quad-0.51772$

$\begin{array}{lll}\text { Std Err Dif } \quad 0.00694 \text { DF } & 14\end{array}$

Upper CL Dif 0.01129 Prob $>|t| \quad 0.6127$

Lower CL Dif -0.01848 Prob $>\mathrm{t} \quad 0.6936$

$\begin{array}{lrl}\text { Confidence } & 0.95 \text { Prob }<\mathrm{t} \quad 0.3064\end{array}$

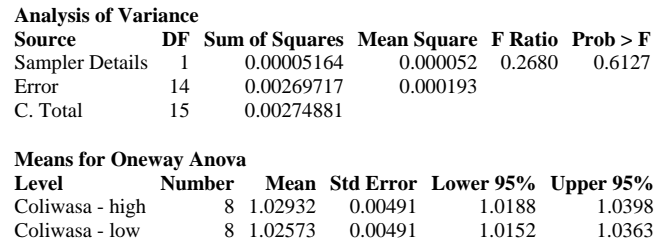

Std Error uses a pooled estimate of error variance

Means and Std Deviations

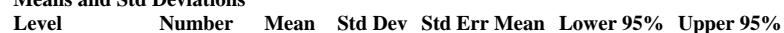

$\begin{array}{lrrrrr}\text { Coliwasa - high } & 8 & 1.02932 & 0.010062 & 0.00356 & 1.0209\end{array}$

$\begin{array}{lllllll}\text { Coliwasa - low } & 8 & 1.02573 & 0.016854 & 0.00596 & 1.0116 & 1.0398\end{array}$

t Test

Coliwasa - low-Coliwasa - high

Assuming unequal variances
Difference $\quad-0.00359$ t Ratio -0.51772

Std Err Dif $\quad 0.00694$ DF 11.4272

Upper CL Dif $\quad 0.01161$ Prob $>|t| \quad 0.6145$

$\begin{array}{lll}\text { Lower CL Dif }-0.01880 & \text { Prob }>t & 0.6927\end{array}$

Confidence $\quad 0.95$ Prob $<\mathrm{t} \quad 0.3073$

Tests that the Variances are Equal

Level Count Std Dev MeanAbsDif to Mean MeanAbsDif to Median

$\begin{array}{lrrrr}\text { Level } & \text { Count } & \text { Std Dev } & \text { MeanAbsDif to Mean } & \text { MeanAbsDif to Median } \\ \text { Coliwasa - high } & 8 & 0.0100619 & 0.0075833 & 0.0074623\end{array}$

$\begin{array}{lllll}\text { Coliwasa - low } & 8 & 0.0168544 & 0.0075833 & 0.0074623 \\ & & \end{array}$

Test F Ratio DFNum DFDen p-Value

$\begin{array}{lrrrr}\text { O'Brien[.5] } & 1.1623 & 1 & 14 & 0.2992\end{array}$

$\begin{array}{lllll}\text { Brown-Forsythe } & 0.8516 & 1 & 14 & 0.3717\end{array}$

$\begin{array}{lllll}\text { Levene } & 0.8188 & 1 & 14 & 0.3808\end{array}$

$\begin{array}{llll}\text { Bartlett } & 1.6665 & 1 & 0.1967\end{array}$

$\begin{array}{lllll}\text { F Test 2-sided } & 2.8059 & 7 & 7 & 0.1969\end{array}$

Welch Anova testing Means Equal, allowing Std Devs Not Equal

F Ratio DFNum DFDen Prob > F

$\begin{array}{lrrr}0.2680 & 1 & 11.427 & 0.6145\end{array}$

t Test
Oneway Analysis of Mean(Measurement) By Sampler Details Oxide/Analyte=MnO (wt\%)

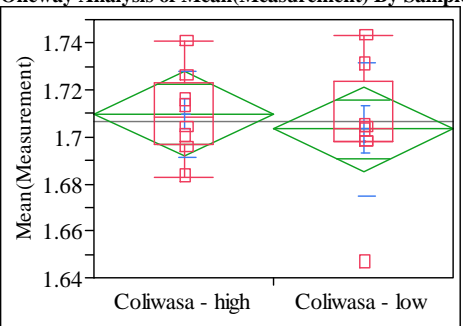

Sampler Details

\section{Oneway Anova}

Summary of Fit

Rsqua

$\begin{array}{ll}\text { Rdj Rsquare } & 0.020571 \\ \text { Rere } & -0.04939\end{array}$

Root Mean Square Error $\quad-0.04939$

Mean of Response 0.023811

$\begin{array}{lr}\text { Observations (or Sum Wgts) } & 16\end{array}$

t Test

Coliwasa - low-Coliwasa - high

Assuming equal variances

Difference $\quad-0.00646$ t Ratio -0.54226

$\begin{array}{lll}\text { Std Err Dif } \quad 0.01191 \text { DF } & 14\end{array}$

Upper CL Dif 0.01908 Prob $>|t| \quad 0.5962$

Lower CL Dif -0.03199 Prob $>$ t 0.7019

$\begin{array}{lll}\text { Confidence } & 0.95 \text { Prob }<\mathrm{t} \quad 0.2981\end{array}$

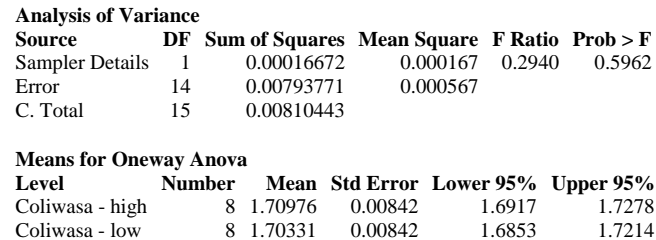

Std Error uses a pooled estimate of error variance

Means and Std Deviations

Level Number Mean Std Dev Std Err Mean Lower 95\% Upper 95\%

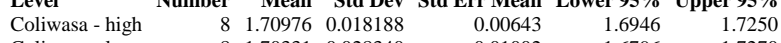

$\begin{array}{lllllll}\text { Coliwasa - low } & 8 & 1.70331 & 0.028340 & 0.01002 & 1.6796 & 1.7250 \\ & & & & & & \end{array}$

t Test

Coliwasa - low-Coliwasa - high

Assuming unequal variances

$\begin{array}{lrlr}\text { Difference } & -0.00646 & \text { t Ratio } & -0.54226 \\ \text { Std Err Dif } & 0.01191 \text { DF } & 11.92982 \\ \text { Upper CL Dif } & 0.01950 & \text { Prob }>|t| & 0.5976 \\ \text { Lower CL Dif } & -0.03241 & \text { Prob }>t & 0.7012 \\ \text { Confidence } & 0.95 & \text { Prob }<\mathrm{t} & 0.2988\end{array}$

Tests that the Variances are Equal

Level Count Std Dev MeanAbsDif to Mean MeanAbsDif to Median

$\begin{array}{lrrrr}\text { Coliwasa - high } & 8 & 0.0181877 & 0.0139880 & 0.0139880\end{array}$

$\begin{array}{lllll}\text { Coliwasa - low } & 8 & 0.0283402 & 0.0139880 & 0.0139880 \\ & & & 0.0172160 & 0.0172160\end{array}$

Test F Ratio DFNum DFDen p-Value

$\begin{array}{lllll}\text { O'Brien[.5] } & 0.7704 & 1 & 14 & 0.3949\end{array}$

$\begin{array}{lllll}\text { Brown-Forsythe } & 0.1455 & 1 & 14 & 0.7086\end{array}$

$\begin{array}{lllll}\text { Levene } & 0.1458 & 1 & 14 & 0.7083\end{array}$

$\begin{array}{lllll}\text { Bartlett } & 1.2452 & 1 & & 0.2645\end{array}$

Welch Anova testing Means Equal, allowing Std Devs Not Equal

F Ratio DFNum DFDen Prob $>$ F

$\begin{array}{llrr}0.2940 & 1 & 11.93 & 0.5976\end{array}$

t Test

0.5423 
SRNL-STI-2011-00693

Revision 0

\section{Exhibit C2. Statistical Comparisons for Low-Rheology (Phase 3) Simulant Testing}

Oneway Analysis of Mean(Measurement) By Sampler Details Oxide/Analyte=Na2O (wt \%)

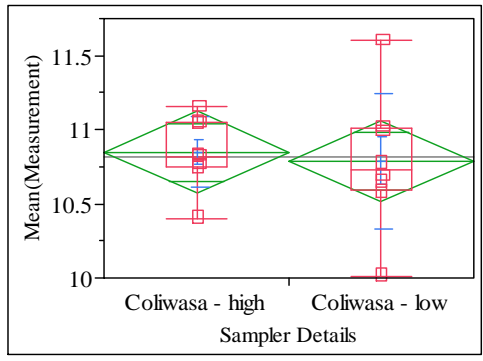

Oneway Anova

Summary of Fit

Rsquare

Adj Rsquare

$\begin{array}{ll}0.362445 \\ \text { Mean of Response } & 10.81939\end{array}$

Observations (or Sum Wgts)

Test

Coliwasa - low-Coliwasa - high

Assuming equal variances

Difference $\quad-0.06122$ t Ratio -0.3378

$\begin{array}{lrlr}\text { Std Err Dif } & 0.18122 & \text { DF } & 14 \\ \text { Upper CL Dif } & 0.32746 & \text { Prob }>|t| & 0.7405\end{array}$

$\begin{array}{rrrr}\text { Upper CL Dif } & 0.32746 & \text { Prob }>|t| & 0.7405 \\ \text { Lower CL Dif } & -0.44990 & \text { Prob }>t & 0.6297\end{array}$

$\begin{array}{lll}\text { Confidence } & 0.95 \text { Prob }<\mathrm{t} \quad 0.3703\end{array}$

Analysis of Variance

$\begin{array}{lrrrrr}\text { Analysis of Variance } & & & & \\ \text { Source } & \text { DF } & \text { Sum of Squares } & \text { Mean Square } & \text { F Ratio } & \text { Prob }>\text { F } \\ \text { Sampler Details } & 1 & 0.0149924 & 0.014992 & 0.1141 & 0.7405 \\ \text { Error } & 14 & 1.8391250 & 0.131366 & & \\ \text { C. Total } & 15 & 1.8541174 & & & \end{array}$

Means for Oneway Anova

$\begin{array}{lrrrrr}\text { Level } & \text { Number } & \text { Mean } & \text { Std Error } & \text { Lower 95\% } & \text { Upper 95\% } \\ \text { Coliwasa - high } & 8 & 10.8500 & 0.12814 & 10.575 & 11.125\end{array}$

$\begin{array}{llllll}\text { Coliwasa - low } & 8 & 10.7888 & 0.12814 & 10.514 & 11.064\end{array}$

Std Error uses a pooled estimate of error variance

Means and Std Deviations

Level Number Mean Std Dev Std Err Mean Lower 95\% Upper 95\%

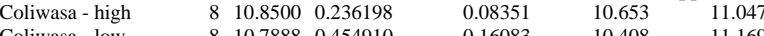

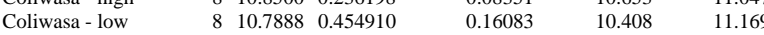

t Test

Coliwasa - low-Coliwasa - high

Assuming unequal variances

Difference $\quad-0.06122$ t Ratio $\quad-0.33783$

Std Err Dif $\quad 0.18122$ DF $\quad 10.51852$

Upper CL Dif 0.33988 Prob $>|t| 0.7421$

Lower CL Dif -0.46233 Prob $>t \quad 0.6289$

$\begin{array}{lll}\text { Confidence } & 0.95 \text { Prob }<\mathrm{t} \quad 0.3711\end{array}$

Tests that the Variances are Equal

$\begin{array}{lrrrr}\text { Level } & \text { Count } & \text { Std Dev } & \text { MeanAbsDif to Mean } & \text { MeanAbsDif to Median } \\ \text { Coliwasa - high } & 8 & 0.2361976 & 0.1740465 & 0.1687808 \\ \text { Coliwasa - low } & 8 & 0.4549097 & 0.3143227 & 0.3097592\end{array}$

F Ratio DFNum DFDen p-Value

Test F Ratio DFNum DFDen p-Value

$\begin{array}{lllll}\text { O'Brien[.5] } & 1.4118 & 1 & 14 & 0.2545 \\ \text { Brown-Forsythe } & 1.2693 & 1 & 14 & 0.2788\end{array}$

$\begin{array}{lllll}\text { Brown-Forsythe } & 1.2693 & 1 & 14 & 0.2788 \\ \text { Levene } & 1.3665 & 1 & 14 & 0.2619\end{array}$

$\begin{array}{lllll}\text { Levene } & 1.3665 & 1 & 14 & 0.2619 \\ \text { Bartlett } & 2.6261 & 1 & & 0.1051\end{array}$

$\begin{array}{lllll}\text { F Test 2-sided } & 3.7094 & 7 & 7 & 0.1051\end{array}$

Welch Anova testing Means Equal, allowing Std Devs Not Equal

F Ratio DFNum DFDen Prob $>$ F

$\begin{array}{llll}0.1141 & 1 & 10.519 & 0.7421\end{array}$

t Test
0.3378
Oneway Analysis of Mean(Measurement) By Sampler Details Oxide/Analyte=NiO (wt\%)

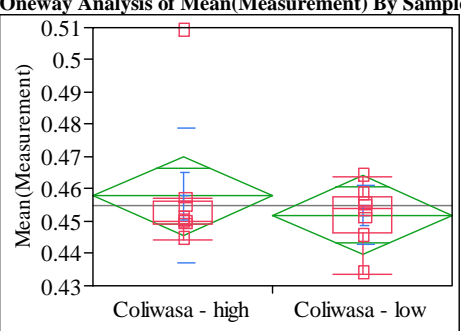

Sampler Details

Oneway Anova

Summary of Fit

Rsquare

$\begin{array}{ll}\text { Rsquare } & 0.038791 \\ \text { Adj Rsquare } & -0.02987\end{array}$

$-0.02987$

Error $\quad 0.016083$

Mean of Response

Observations (or Sum Wgts)

0.454892
16

Test

Coliwasa - low-Coliwasa - high

Assuming equal variances

Difference $\quad-0.00604$ t Ratio $\quad-0.75166$

$\begin{array}{lll}\text { Std Err Dif } & 0.00804 \text { DF } & 14\end{array}$

Upper CL Dif 0.01120 Prob $>|t| \quad 0.4647$

Lower CL Dif -0.02329 Prob $>\mathrm{t} \quad 0.7676$

$\begin{array}{lll}\text { Confidence } & 0.95 \text { Prob }<\mathrm{t} & 0.2324\end{array}$

$\begin{array}{lrrrrr}\begin{array}{l}\text { Analysis of Variance } \\ \text { Source }\end{array} & \text { DF } & \text { Sum of Squares } & \text { Mean Square } & \text { F Ratio } & \text { Prob > F } \\ \text { Sampler Details } & 1 & 0.00014614 & 0.000146 & 0.5650 & 0.4647 \\ \text { Error } & 14 & 0.00362114 & 0.000259 & & \\ \text { C. Total } & 15 & 0.00376728 & & & \end{array}$

Means for Oneway Anova

Level Number Mean Std Error Lower 95\% Upper 95\%

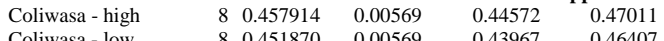

$\begin{array}{llll}8 & 0.451870=0.00569 & 0.43967 & 0.46407\end{array}$

Std Error uses a pooled estimate of error variance

Means and Std Deviations

Level Number Mean Std Dev Std Err Mean Lower 95\% Upper 95\%

$\begin{array}{lrrrrrr}\text { Coliwasa - high } & 8 & 0.457914 & 0.020817 & 0.00736 & 0.44051 & 0.47532\end{array}$

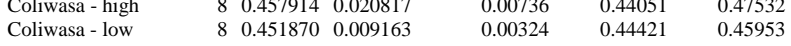

t Test

Coliwasa - low-Coliwasa - high

Assuming unequal variances

$\begin{array}{lrlr}\text { Difference } & -0.00604 & \text { t Ratio } & -0.75166 \\ \text { Std Err Dif } & 0.00804 & \text { DF } & 9.614655 \\ \text { Upper CL Dif } & 0.01197 & \text { Prob }>|t| & 0.4703 \\ \text { Lower CL Dif } & -0.02406 & \text { Prob }>t & 0.7649 \\ \text { Confidence } & 0.95 & \text { Prob }<\mathrm{t} & 0.2351\end{array}$

Tests that the Variances are Equal

Level Count Std Dev MeanAbsDif to Mean MeanAbsDif to Median

$\begin{array}{lrrrr}\text { Coliwasa - high } & 8 & 0.0208167 & 0.0126654 & 0.0097293\end{array}$

$\begin{array}{lllll}\text { Coliwasa - low } & 8 & 0.0091635 & 0.0065149 & 0.0097293 \\ & & & \end{array}$

Test F Ratio DFNum DFDen p-Value

$\begin{array}{lllll}\text { O'Brien[.5] } & 0.7986 & 1 & 14 & 0.3866\end{array}$

Brown-Forsythe $\quad 0.2297 \quad 11 \quad 14 \quad 0.6391$

$\begin{array}{lllll}\text { Levene } & 1.0601 & 1 & 14 & 0.3207\end{array}$

$\begin{array}{lllll}3.9789 & 1 & - & 0.0461\end{array}$

Welch Anova testing Means Equal, allowing Std Devs Not Equal

F Ratio DFNum DFDen Prob $>$ F

$\begin{array}{rrrr}0.5650 & 1 & 9.6147 & 0.4703\end{array}$

t Test 
SRNL-STI-2011-00693

Revision 0

\section{Exhibit C2. Statistical Comparisons for Low-Rheology (Phase 3) Simulant Testing}

Oneway Analysis of Mean(Measurement) By Sampler Details Oxide/Analyte=SiO2 (wt\%)

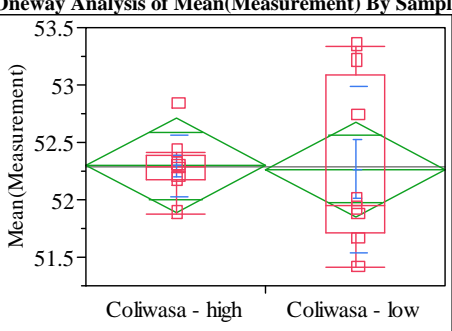

Sampler Details

\section{Oneway Anova}

Summary of Fi

$\begin{array}{lr}\text { Rsquare } & 0.000928 \\ \text { Adj Rsquare } & -0.07043 \\ \text { Root Mean Square Error } & 0.547276 \\ \text { Mean of Response } & 52.28137 \\ \text { Observations (or Sum Wgts) } & 16 \\ & \\ \text { t Test } & \\ \text { Coliwasa - low-Coliwasa - high } \\ \text { Assuming equal variances }\end{array}$

Difference $\quad-0.03120$ t Ratio $\quad-0.11401$

$\begin{array}{lll}\text { Std Err Dif } \quad 0.27364 \text { DF } & 14\end{array}$

Upper CL Dif 0.55570 Prob $>|t| \quad 0.9108$

Lower CL Dif -0.61809 Prob $>\mathrm{t} \quad 0.5446$

$\begin{array}{lrl}\text { Confidence } & 0.95 \text { Prob }<\mathrm{t} \quad 0.4554\end{array}$

\begin{tabular}{|c|c|c|c|c|c|c|c|}
\hline \multicolumn{8}{|c|}{ Analysis of Variance } \\
\hline Source & DF & Sum & of Square & es Mean S & Square & F Ratio & o Prob $>F$ \\
\hline Sampler Details & 1 & & 0.003893 & & 003893 & 0.0130 & $0 \quad 0.9108$ \\
\hline Error & 14 & & 4.1931550 & & 299511 & & \\
\hline C. Total & 15 & & 4.1970482 & & & & \\
\hline Mean & way $A$ & & & & & & \\
\hline Level & Num & nber & Mean S & Std Error & Lowe & 295\% U & Upper 95\% \\
\hline Coliwasa - high & & 8 & 52.2970 & 0.19349 & & 51.882 & 52.712 \\
\hline Coliwasa - low & & 8 & 52.2658 & 0.19349 & & 51.851 & 52.681 \\
\hline
\end{tabular}

Std Error uses a pooled estimate of error variance

Means and Std Deviations

Level Number Mean Std Dev Std Err Mean Lower 95\% Upper 95\%

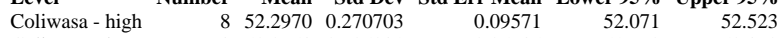

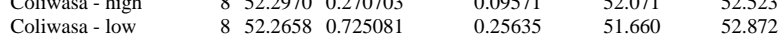

t Test

Coliwasa - low-Coliwasa - high

Assuming unequal variances

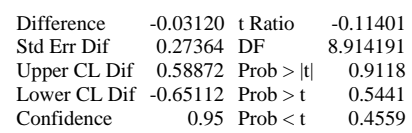

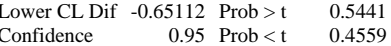

Tests that the Variances are Equal

Level Count Std Dev MeanAbsDif to Mean MeanAbsDif to Median

$\begin{array}{lrrrr}\text { Coliwasa - high } & 8 & 0.2707031 & 0.1693613 & 0.1693613\end{array}$

$\begin{array}{lllll}\text { Coliwasa- low } & 8 & 0.7250807 & 0.6183914 & 0.5481956\end{array}$

Test F Ratio DFNum DFDen p-Value

O'Brien[.5] $\quad 6.1046 \quad 110.0269$

$\begin{array}{lrrrr}\text { Brown-Forsythe } & 3.4037 & 1 & 14 & 0.0863\end{array}$

$\begin{array}{lllll}\text { Levene } & 12.4844 & 1 & 14 & 0.0033\end{array}$

$\begin{array}{lllll}\text { Bartlett } & 5.5220 & 1 & & 0.0188\end{array}$

Welch Anova testing Means Equal, allowing Std Devs Not Equal

F Ratio DFNum DFDen Prob $>$ F

$\begin{array}{lrrr}0.0130 & 1 & 8.9142 & 0.9118\end{array}$

t Test
0.1140
Oneway Analysis of Mean(Measurement) By Sampler Details Oxide/Analyte=Sum of Oxides (wt\%)

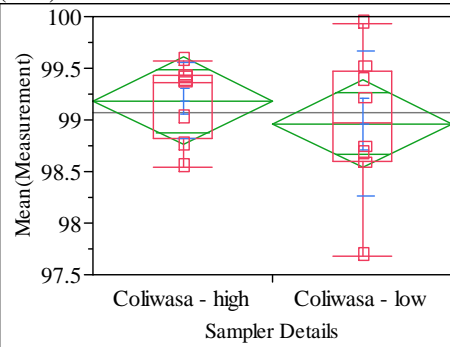

\section{Oneway Anova}

Summary of Fit

\section{Rsquare}

Adj Rsquare

0.558252

Mean of Response 99.07151

t Test

Coliwasa - low-Coliwasa - high

Assuming equal variances

Difference $\quad-0.21910$ t Ratio $\quad-0.78496$

Std Err Dif $\quad 0.27913$ DF $\quad 14$

Upper CL Dif 0.37956 Prob $>|t| \quad 0.4456$

$\begin{array}{lll}\text { Lower CL Dif }-0.81777 \text { Prob }>t & 0.7772\end{array}$

$\begin{array}{lll}\text { Confidence } & 0.95 \text { Prob }<\mathrm{t} \quad 0.2228\end{array}$

Analysis of Variance
Source DF Sum of Squares Mean Square F Ratio Prob $>$ F

$\begin{array}{lrrrrr}\text { Source } & \text { DF } & \text { Sum of Squares } & \text { Mean Square } & \text { F Ratio } & \text { Prob }>\text { F } \\ \text { Sampler Details } & 1 & 0.1920218 & 0.192022 & 0.6162 & 0.4456\end{array}$

$\begin{array}{lrrr}\text { Error } & 14 & 4.3630350 & 0.311645 \\ \text { C. Total } & 15 & 4.5550568 & \end{array}$

Means for Oneway Anova

Level Number Mean Std Error Lower 95\% Upper 95\%

$\begin{array}{llllll}\text { Coliwasa - high } & 8 & 99.1811 & 0.19737 & 98.758 & 99.604\end{array}$

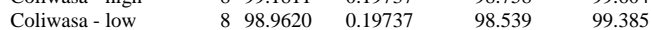

Std Error uses a pooled estimate of error variance

$\begin{array}{llllll}\begin{array}{l}\text { Means and Std Deviations } \\ \text { Level }\end{array} \text { Number } & \text { Mean } & \text { Std Dev Std Err Mean Lower 95\% } & \text { Upper 95\% }\end{array}$

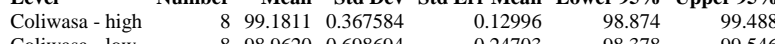

$\begin{array}{lllllll}\text { Coliwasa - low } & 8 & 98.9620 & 0.698694 & 0.24703 & 98.378 & 99.546\end{array}$

t Test

Coliwasa - low-Coliwasa - high

Assuming unequal variances

$\begin{array}{lrlr}\text { Difference } & -0.21910 & \text { t Ratio } & -0.78496 \\ \text { Std Err Dif } & 0.27913 & \text { DF } & 10.59922 \\ \text { Upper CL Dif } & 0.39810 & \text { Prob }>|t| & 0.4497 \\ \text { Lower CL Dif } & -0.83630 & \text { Prob }>\mathrm{t} & 0.7752 \\ \text { Confidence } & 0.95 & \text { Prob }<\mathrm{t} & 0.2248\end{array}$

Tests that the Variances are Equal

Level Count Std Dev MeanAbsDif to Mean MeanAbsDif to Median

$\begin{array}{lrrrr}\text { Coliwasa - high } & 8 & 0.3675835 & 0.3082538 & 0.2649063\end{array}$

$\begin{array}{lllll}\text { Coliwasa - low } & 8 & 0.6986938 & 0.3082538 & 0.2649063 \\ & & & \end{array}$

Test F Ratio DFNum DFDen p-Value

$\begin{array}{lllll}\text { O'Brien[.5] } & 1.8603 & 1 & 14 & 0.1941\end{array}$

Brown-Forsythe $\quad 2.6290 \quad 1 \quad 14 \quad 0.1272$

$\begin{array}{lllll}\text { Levene } & 2.5984 & 1 & 14 & 0.1293\end{array}$

$\begin{array}{lllll}\text { Levertlett } & 2.5278 & 1 & 14 & 0.1293 \\ & & 3.1119\end{array}$

$\begin{array}{lllll}\text { Bartlett } & 2.5278 & 1 & \text {. } & 0.1119 \\ \text { F Test 2-sided } & 3.6129 & 7 & 7 & 0.1118\end{array}$

Welch Anova testing Means Equal, allowing Std Devs Not Equal

F Ratio DFNum DFDen Prob $>$ F

t Test
0.7850 
SRNL-STI-2011-00693

Revision 0

\section{Exhibit C2. Statistical Comparisons for Low-Rheology (Phase 3) Simulant Testing}

Oneway Analysis of Mean(Measurement) By Sampler Details Oxide/Analyte=TiO2 (wt\%)

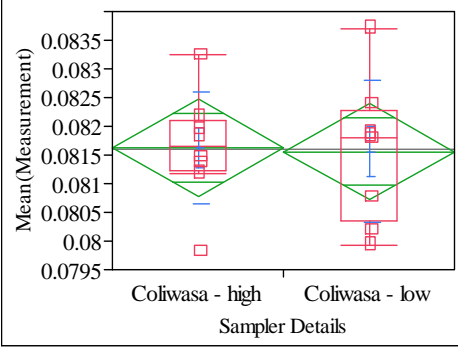

Oneway Anova

Rsquare $\quad 0.001007$

Adj Rsquare

Root Mean Square Error $\quad 0.001112$

Mean of Response $\quad 0.081595$

Observations (or Sum Wgts) 16

t Test

Coliwasa - low-Coliwasa - high

Assuming equal variances

Difference $\quad-6.6 \mathrm{e}-5$ t Ratio $\quad-0.1188$

Std Err Dif $\quad 0.00056$ DF 14

Upper CL Dif 0.00113 Prob $>|t| 0.9071$

Lower CL Dif -0.00126 Prob $>t \quad 0.5464$

$\begin{array}{lll}\text { Confidence } & 0.95 \text { Prob }<\mathrm{t} \quad 0.4536\end{array}$

Analysis of Variance

$\begin{array}{lrrrrr}\text { Source } & \text { DF } & \text { Sum of Squares } & \text { Mean Square } & \text { F Ratio } & \text { Prob }>\text { F } \\ \text { Sampler Details } & 1 & 1.74372 \mathrm{e}-8 & 1.7437 \mathrm{e}-8 & 0.0141 & 0.9071\end{array}$

$\begin{array}{lrrr}\text { Sampler Details } & 1 & 1.74372 \mathrm{e}-8 & 1.7437 \mathrm{e}-8 \\ \text { Error } & 14 & 0.00001730 & 1.2356 \mathrm{e}-6\end{array}$ 0.00001732

Means for Oneway Anova

$\begin{array}{lrrrrr}\text { Level } & \text { Number } & \text { Mean } & \text { Std Error } & \text { Lower 95\% } & \text { Upper 95\% } \\ \text { Coliwasa - high } & 8 & 0.081628 & 0.00039 & 0.08078 & 0.08247\end{array}$

$\begin{array}{llllll}\text { Coliwasa - low } & 8 & 0.081562 & 0.00039 & 0.08072 & 0.08240\end{array}$

Std Error uses a pooled estimate of error variance

Means and Std Deviations

Level Number Mean Std Dev Std Err Mean Lower 95\% Upper 95\%

$\begin{array}{lrrrrrr}\text { Coliwasa - high } & 8 & 0.081628 & 0.000976 & 0.00034 & 0.08081 & 0.08244\end{array}$

$\begin{array}{lllllll}\text { Coliwasa - low } & 8 & 0.081562 & 0.001233 & 0.00044 & 0.08053 & 0.08259\end{array}$

t Test

Coliwasa - low-Coliwasa - high

Assuming unequal variances

Difference $\quad-6.6 \mathrm{e}-5$ t Ratio $\quad-0.1188$

Std Err Dif $\quad 0.00056$ DF 13.29883

Upper CL Dif 0.00113 Prob $>|t| r \mid r .9072$

Lower CL Dif -0.00126 Prob $>t \quad 0.5464$

$\begin{array}{lrl}\text { Confidence } & 0.95 \text { Prob }<\mathrm{t} \quad 0.4536\end{array}$

Tests that the Variances are Equal

Level Count Std Dev MeanAbsDif to Mean MeanAbsDif to Median

$\begin{array}{lrrrr}\text { Coliwasa - high } & 8 & 0.0009756 & 0.0006672 & 0.0006672\end{array}$

$\begin{array}{lllll}\text { Coliwasa - low } & 8 & 0.0012326 & 0.0009426 & 0.0008861\end{array}$

Test F Ratio DFNum DFDen p-Value

$\begin{array}{lrrrr}\text { O'Brien[.5] } & 0.3797 & 1 & 14 & 0.5476\end{array}$

Brown-Forsythe $\quad 0.3390 \quad 11 \quad 14 \quad 0.5697$

$\begin{array}{lllll}\text { Levene } & 0.6407 & 1 & 14 & 0.4368\end{array}$

$\begin{array}{lllll} & 0.3539 & 1 & \text { Bartlett } & 0.5519\end{array}$

Welch Anova testing Means Equal, allowing Std Devs Not Equal

F Ratio DFNum DFDen Prob $>$ F

$\begin{array}{rrrr}0.0141 & 1 & 13.299 & 0.9072\end{array}$

t Test

0.1188
Oneway Analysis of Mean(Measurement) By Sampler Details Oxide/Analyte=ZnO (wt \%)

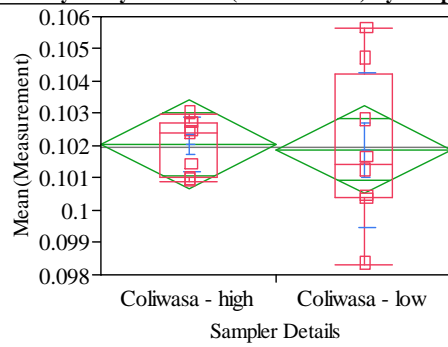

Oneway Anova

Summary of Fit

Rsquare

Adj Rsure $\quad-0.06868$

Error $\quad 0.001803$

Mean of Response

0.101965
16

t Test

Coliwasa - low-Coliwasa - high

Assuming equal variances

Difference $\quad-0.00017$ t Ratio $\quad-0.18988$

Std Err Dif $\quad 0.00090$ DF 14

Upper CL Dif 0.00176 Prob $>|t| \quad 0.8521$

Lower CL Dif -0.00210 Prob $>t \quad 0.5739$

$\begin{array}{lll}\text { Confidence } & 0.95 \text { Prob }<\mathrm{t} \quad 0.4261\end{array}$

$\begin{array}{lrrrrr}\begin{array}{l}\text { Analysis of Variance } \\ \text { Source }\end{array} & \text { DF } & \text { Sum of Squares } & \text { Mean Square } & \text { F Ratio } & \text { Prob > F } \\ \text { Sampler Details } & 1 & 0.00000012 & 1.1718 \mathrm{e}-7 & 0.0361 & 0.8521 \\ \text { Error } & 14 & 0.00004550 & 3.2502 \mathrm{e}-6 & & \\ \text { C. Total } & 15 & 0.00004562 & & & \end{array}$

Means for Oneway Anova

Level Number Mean Std Error Lower 95\% Upper 95\%

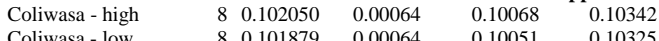

Std Error uses a pooled estimate of error variance

Means and Std Deviations

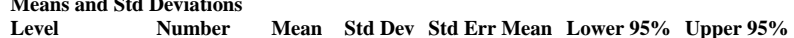

$\begin{array}{lrrrrrr}\text { Level } & \text { Number } & \text { Mean } & \text { Std Dev } & \text { Std Err Mean } & \text { Lower 95\% } & \text { Upper 95\% } \\ \text { Coliwasa - high } & 8 & 0.102050 & 0.000842 & 0.00030 & 0.10135 & 0.10275\end{array}$

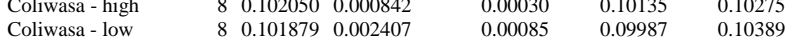

t Test

Coliwasa - low-Coliwasa - high

Assuming unequal variances

Difference $\quad-0.00017$ t Ratio $\quad-0.18988$

$\begin{array}{lll}\text { Std Err Dif } \quad 0.00090 \text { DF } & 8.6887\end{array}$

Upper CL Dif 0.00188 Prob $>|t| \quad 0.8538$

Lower CL Dif -0.00222 Prob $>$ t 0.5731

Confidence $\quad 0.95$ Prob $<\mathrm{t} \quad 0.4269$

Tests that the Variances are Equal

Level Count Std Dev MeanAbsDif to Mean MeanAbsDif to Median

$\begin{array}{lrrrr}\text { Coliwasa - high } & 8 & 0.0008420 & 0.0007294 & 0.0006509\end{array}$

$\begin{array}{lllll}\text { Coliwasa - low } & 8 & 0.0024065 & 0.0007294 & 0.0006509 \\ & & & \end{array}$

Test F Ratio DFNum DFDen p-Value

$\begin{array}{lrrrr}\text { O'Brien[.5] } & 4.0358 & 1 & 14 & 0.0642\end{array}$

$\begin{array}{lllll}\text { Brown-Forsythe } & 4.0252 & 1 & 14 & 0.0645 \\ & 5.3315 & 1 & 14 & 0.0367\end{array}$

$\begin{array}{lllll}\text { Levene } & 5.3315 & 1 & 14 & 0.0367\end{array}$

$\begin{array}{lllll}\text { Bartlett } & 6.1735 & 1 & & 0.0130\end{array}$

Welch Anova testing Means Equal, allowing Std Devs Not Equal

F Ratio DFNum DFDen Prob $>$ F

$\begin{array}{lrrr}0.0361 & 1 & 8.6887 & 0.8538\end{array}$

t Test

0.1899 


\section{Exhibit C2. Statistical Comparisons for Low-Rheology (Phase 3) Simulant Testing}

Oneway Analysis of Mean(Measurement) By Sampler Details Oxide/Analyte=ZrO2 (wt\%)

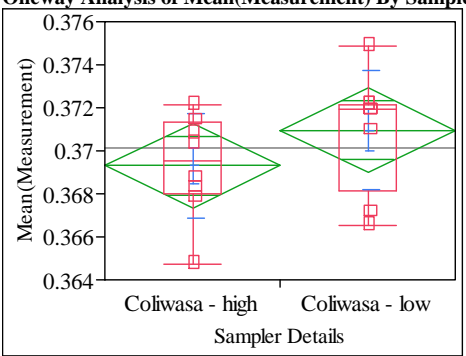

Oneway Anova

Summary of Fi

\begin{tabular}{|c|c|c|c|}
\hline \multicolumn{4}{|l|}{ Rsquare } \\
\hline \multicolumn{2}{|l|}{ Adj Rsquare } & 0.040 & 913 \\
\hline \multicolumn{2}{|c|}{ Root Mean Square Error } & 0.002 & 593 \\
\hline \multicolumn{2}{|c|}{ Mean of Response } & 0.370 & 133 \\
\hline \multicolumn{2}{|c|}{ Observations (or Sum Wgts } & & 16 \\
\hline \multicolumn{4}{|c|}{ Coliwasa - low-Coliwasa - high } \\
\hline Difference & $0.00166 \mathrm{t}$ & t Ratio & 1.280579 \\
\hline Std Err Dif & $0.00130 \mathrm{I}$ & DF & 14 \\
\hline Upper CL Dif & $0.00444 \mathrm{~F}$ & Prob $>|t|$ & 0.2212 \\
\hline Lower CL Dif & $-0.00112 \mathrm{~F}$ & Prob $>t$ & 0.1106 \\
\hline Confidence & $0.95 \mathrm{~F}$ & $\operatorname{Prob}<\mathrm{t}$ & 0.8894 \\
\hline
\end{tabular}

Analysis of Variance

$\begin{array}{lrrrrr}\text { Source } & \text { DF } & \text { Sum of Squares } & \text { Mean Square } & \text { F Ratio } & \text { Prob }>\text { F } \\ \text { Sampler Details } & 1 & 0.00001103 & 0.000011 & 1.6399 & 0.2212\end{array}$

$\begin{array}{lrrrrr}\text { Sampler Details } & 1 & 0.00001103 & 0.000011 & 1.6399 & 0.2212 \\ \text { Error } & 14 & 0.00009414 & 6.724 \mathrm{e}-6 & & \end{array}$

$\begin{array}{lll}\text { Error } & 14 & 0.00009414 \\ \text { C. Total } & 15 & 0.00010517\end{array}$

Means for Oneway Anova

$\begin{array}{lrrrrr}\text { Level } & \text { Number } & \text { Mean } & \text { Std Error } & \text { Lower 95\% } & \text { Upper 95\% } \\ \text { Coliwasa - high } & 8 & 0.369303 & 0.00092 & 0.36734 & 0.37127\end{array}$

$\begin{array}{llllll}\text { Coliwasa- low } & 8 & 0.370963 & 0.00092 & 0.36900 & 0.37293\end{array}$

Std Error uses a pooled estimate of error variance

Means and Std Deviations

Level $\quad$ Number Mean Std Dev Std Err Mean Lower 95\% Upper 95\%

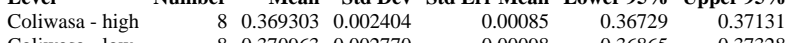

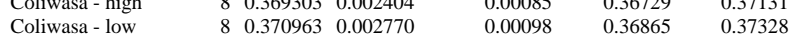

t Test

Coliwasa - low-Coliwasa - high

Assuming unequal variances

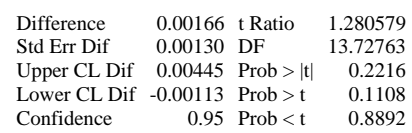

Tests that the Variances are Equal

Level Count Std Dev MeanAbsDif to Mean MeanAbsDif to Median

$\begin{array}{lrrrr}\text { Coliwasa - high } & 8 & 0.0024036 & 0.0018855 & 0.0018855\end{array}$

$\begin{array}{lllll}\text { Coliwasa - low } & 8 & 0.0027698 & 0.0018855 & 0.0018855 \\ & & & 0.0020543 & 0.0018011\end{array}$

$\begin{array}{lrrrr}\text { Test } & \text { F Ratio } & \text { DFNum } & \text { DFDen } & \text { p-Value } \\ \text { O'Brien[.5] } & 0.1633 & 1 & 14 & 0.6922\end{array}$

Brown-Forsythe $\quad 0.0084 \quad 1 \quad 14 \quad 0.9283$

$\begin{array}{lllll}\text { Levene } & 0.0500 & 1 & 14 & 0.8263\end{array}$

$\begin{array}{lllll}\text { Bartlett } & 0.1309 & 1 & 0.7175\end{array}$

$\begin{array}{lllll}\text { F Test 2-sided } & 1.3279 & 7 & 7 & 0.7177\end{array}$

Welch Anova testing Means Equal, allowing Std Devs Not Equal

F Ratio DFNum DFDen Prob > F

$\begin{array}{llll}1.6399 & 1 & 13.728 & 0.2216\end{array}$

$\mathbf{t}$ Test
1.2806
Oneway Analysis of Measurement By Sampling Description Test Phase=Phase 3 - Low Rheology, Analyte $=$ sample density $(\mathrm{g} / \mathrm{mL})$

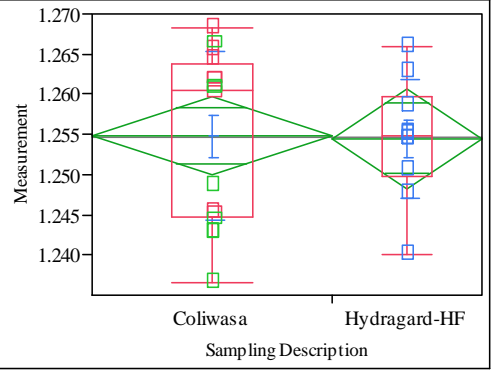

Oneway Anova

Summary of Fit

Rsquare

Root Mean Square Error $\quad 0.009499$

Mean of Response $\quad 1.254678$

Observations (or Sum Wgts) 26

t Test

Hydragard-HF-Coliwasa

Assuming equal variances

Difference $\quad-0.00033$ t Ratio -0.0863

$\begin{array}{lrlr}\text { Std Err Dif } & 0.00383 & \text { DF } & 24 \\ \text { Upper CL Dif } & 0.00757 & \text { Prob }>|t| & 0.9319\end{array}$

$\begin{array}{lrll}\text { Upper CL Dif } & 0.00757 & \text { Prob }>|t| & 0.9319 \\ \text { Lower CL Dif } & -0.00823 & \text { Prob }>t & 0.5340\end{array}$

$\begin{array}{lrll}\text { Lower CL Dif } & -0.00823 & \text { Prob }>t & 0.5340 \\ \text { Confidence } & 0.95 & \text { Prob }<t & 0.4660\end{array}$

$\begin{array}{lrrrrr}\text { Analysis of Variance } & & & & & \\ \text { Source } & \text { DF } & \text { Sum of Squares } & \text { Mean Square } & \text { F Ratio } & \text { Prob }>\text { F } \\ \text { Sampling Description } & 1 & 0.00000067 & 6.72 \mathrm{e}-7 & 0.0074 & 0.9319 \\ \text { Error } & 24 & 0.00216532 & 0.000090 & & \\ \text { C. Total } & 25 & 0.00216599 & & & \end{array}$

$\begin{array}{lll}\text { C. Total } & 25 & 0.00216599\end{array}$

Means for Oneway Anova

Level Number Mean Std Error Lower 95\% Upper 95\%

$\begin{array}{llllll}\text { Coliwasa } & 16 & 1.25481 & 0.00237 & 1.2499 & 1.2597\end{array}$

$\begin{array}{llllll}\text { Hydragard-HF } & 10 & 1.25447 & 0.00300 & 1.2483 & 1.2607\end{array}$

Std Error uses a pooled estimate of error variance

Means and Std Deviations

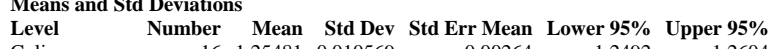

$\begin{array}{lrrrrrr}\text { Coliwasa } & 16 & 1.25481 & 0.010569 & 0.00264 & 1.2492 & 1.2604 \\ \text { Hydragard-HF } & 10 & 1.25447 & 0.007378 & 0.00233 & 1.2492 & 1.2598\end{array}$

\section{t Test}

Hydragard-HF-Coliwasa

Assuming unequal variances

$\begin{array}{lrlr}\text { Difference } & -0.00033 & \text { t Ratio } & -0.09375 \\ \text { Std Err Dif } & 0.00352 & \text { DF } & 23.59903 \\ \text { Upper CL Dif } & 0.00695 \text { Prob }>|t| & 0.9261 \\ \text { Lower CL Dif } & -0.00761 & \text { Prob }>\mathrm{t} & 0.5370 \\ \text { Confidence } & 0.95 & \text { Prob }<\mathrm{t} & 0.4630\end{array}$

Tests that the Variances are Equal

$\begin{array}{lrrrr}\text { Level } & \text { Count } & \text { Std Dev } & \text { MeanAbsDif to M } \\ \text { Coliwasa } & 16 & 0.0105687 & & 0.0096 \\ \text { Hydragard-HF } & 10 & 0.0073777 & & 0.0050 \\ & & & & \\ \text { Test } & \text { F Ratio } & \text { DFNum } & \text { DFDen } & \text { p-Value } \\ \text { O'Brien[.5] } & 2.9068 & 1 & 24 & 0.1011 \\ \text { Brown-Forsythe } & 2.0149 & 1 & 24 & 0.1686 \\ \text { Levene } & 7.8558 & 1 & 24 & 0.0099 \\ \text { Bartlett } & 1.2870 & 1 & . & 0.2566 \\ \text { F Test 2-sided } & 2.0521 & 15 & 9 & 0.2776\end{array}$

Welch Anova testing Means Equal, allowing Std Devs Not Equal

F Ratio DFNum DFDen Prob $>$ F

$\begin{array}{llll}0.0088 & 1 & 23.599 & 0.9261\end{array}$

$\mathbf{t}$ Test
0.0938 


\section{Exhibit C2. Statistical Comparisons for Low-Rheology (Phase 3) Simulant Testing}

Oneway Analysis of Measurement By Sampling Description Test Phase=Phase 3 - Low Rheology, Analyte $=$ wt $\%$ dried solids

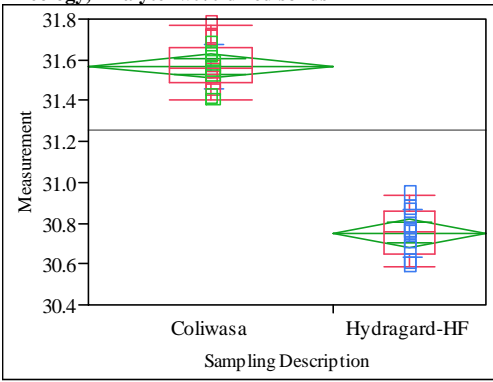

Oneway Anova

Summary of Fit

0.933349

Root Mean Square Error $\quad 0.930572$

Mean of Response 31.25423

Observations (or Sum Wgts) $\quad 26$

t Test

Hydragard-HF-Coliwasa

Assuming equal variances

Difference $\quad-0.81775$ t Ratio $\quad-18.3327$

Std Err Dif $\quad 0.04461$ DF 24

Upper CL Dif -0.72569 Prob $>|t| \quad<.0001$

Lower CL Dif -0.90981 Prob $>\mathrm{t} \quad 1.0000$

$\begin{array}{lrl}\text { Confidence } & 0.95 \text { Prob }<\mathrm{t} & <.0001\end{array}$

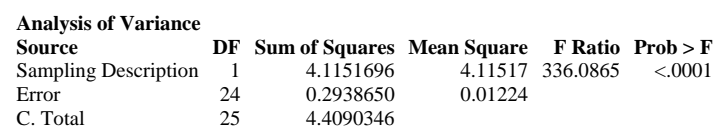

C. Total 25

4.4090346

Means for Oneway Anova

Level Number Mean Std Error Lower 95\% Upper 95\%

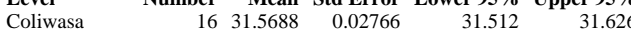

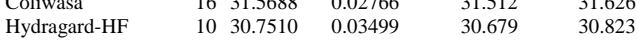

Std Error uses a pooled estimate of error variance

Means and Std Deviations

Level Number Mean Std Dev Std Err Mean Lower 95\% Upper 95\%

$\begin{array}{lllllll}\text { Coliwasa } & 16 & 31.5688 & 0.108128 & 0.02703 & 31.511 & 31.626\end{array}$

Hydragard-HF

t Test

Hydragard-HF-Coliwasa

Assuming unequal variances

Difference $\quad-0.81775$ t Ratio $\quad-18.073$

Std Err Dif $\quad 0.04525$ DF 18.36803

Upper CL Dif -0.72283 Prob $>|t| \quad<.0001$

Lower CL Dif -0.91267 Prob $>t \quad 1.0000$

$\begin{array}{lrr}\text { Confidence } & 0.95 \text { Prob }<\mathrm{t} & <.0001\end{array}$

Tests that the Variances are Equal

Level Count Std Dev MeanAbsDif to Mean MeanAbsDif to Median

$\begin{array}{lllll}\text { Coliwasa } & 16 & 0.1081280 & 0.0862500 & 0.0862500\end{array}$

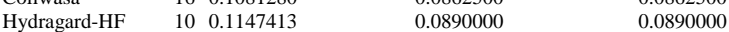

Test F Ratio DFNum DFDen p-Value

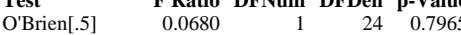

$\begin{array}{lllll}\text { Brown-Forsythe } & 0.0115 & 1 & 24 & 0.9154\end{array}$

$\begin{array}{lllll}\text { Levene } & 0.0117 & 1 & 24 & 0.9148\end{array}$

$\begin{array}{lllll}\text { Bartlett } & 0.0383 & 1 & & 0.8449 \\ \text { F Test 2-sided } & 1.1261 & 9 & 15 & 0.8056\end{array}$

Welch Anova testing Means Equal, allowing Std Devs Not Equal

F Ratio DFNum DFDen Prob $>$ F

$326.6351 \quad 1 \quad 18.368<.0001$

$\mathbf{t}$ Test
18.0730
Oneway Analysis of Measurement By Sampling Description Test Phase=Phase 3 - Low Rheology, Analyte $=w t \%$ vitrified solids

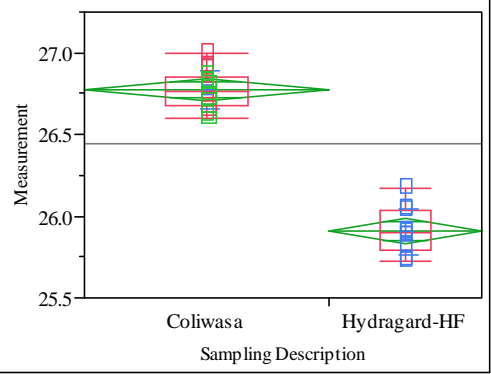

Oneway Anova

Summary of Fit

Adj Rsquare

0.124187

Observations (or Sum Wgts) 26.4407

Test

Hydragard-HF-Coliwasa

Assuming equal variances

$\begin{array}{rrr}\text { Difference } & -0.86575 \text { t Ratio } & -17.2938 \\ \text { Std Err Dif } & 0.05006 & \text { DF }\end{array}$

Upper CL Dif -0.76243 Prob $>|t|<.0001$

$\begin{array}{llll}\text { Lower CL Dif }-0.96907 \text { Prob }>t & 1.0000\end{array}$

$\begin{array}{lrl}\text { Confidence } & 0.95 \text { Prob }<\mathrm{t} & <.0001\end{array}$

$\begin{array}{lrrrrr}\begin{array}{lrrrr}\text { Analysis of Variance } \\ \text { Source }\end{array} & \text { DF } & \text { Sum of Squares } & \text { Mean Square } & \text { F Ratio } & \text { Prob > F } \\ \text { Sampling Description } & 1 & 4.6124496 & 4.61245 & 299.0768 & <.0001 \\ \text { Error } & 24 & 0.3701350 & 0.01542 & & \\ \text { C. Total } & 25 & 4.9825846 & & & \end{array}$

Means for Oneway Anova

Level Number Mean Std Error Lower 95\% Upper 95\%

$\begin{array}{lrrrrr}\text { Coliwasa } & 16 & 26.7738 & 0.03105 & 26.710 & 26.838\end{array}$

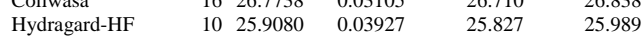

Std Error uses a pooled estimate of error variance

Means and Std Deviations

Level Number Mean Std Dev Std Err Mean Lower 95\% Upper 95\%

$\begin{array}{lllllll}\text { Coliwasa } & 16 & 26.7738 & 0.112835 & 0.02821 & 26.714 & 26.834\end{array}$

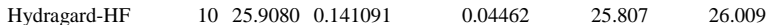

Test

Hydragard-HF-Coliwasa

Assuming unequal variances

Difference $\quad-0.86575$ t Ratio $\quad-16.401$

Std Err Dif $\quad 0.05279$ DF $\quad 16.09058$

Upper CL Dif -0.75390 Prob $>|t|<.0001$

Lower CL Dif -0.97760 Prob $>t \quad 1.0000$

$\begin{array}{lrl}\text { Confidence } & 0.95 \text { Prob }<\mathrm{t} & <.0001\end{array}$

Tests that the Variances are Equal

Level Count Std Dev MeanAbsDif to Mean MeanAbsDif to Median

$\begin{array}{lllll}\text { Coliwasa } & 16 & 0.1128347 & 0.0912500 & 0.0912500\end{array}$

Hydragard-HF $\quad 10 \quad 0.1410910$

Test F Ratio DFNum DFDen p-Value

$\begin{array}{lrrrr}\text { O'Brien[.5] } & 0.7823 & 1 & 24 & 0.3852\end{array}$

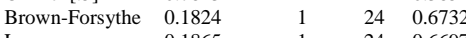

$\begin{array}{lllll}\text { Levene } & 0.1865 & 1 & 24 & 0.6697\end{array}$

$\begin{array}{lllll}\text { Bartlett } & 0.5535 & 1 & & 0.4569 \\ \text { F Test 2-sided } & 1.5636 & 9 & 15 & 0.4267\end{array}$

Welch Anova testing Means Equal, allowing Std Devs Not Equal

F Ratio DFNum DFDen Prob $>$ F

$268.9937<1<.091<0001$

t Test

16.4010 


\section{Exhibit C2. Statistical Comparisons for Low-Rheology (Phase 3) Simulant Testing}

Oneway Analysis of Measurement By Sampling Description Test Phase=Phase 3 - Low Rheology, Analyte=sample density $(\mathrm{g} / \mathrm{mL})$

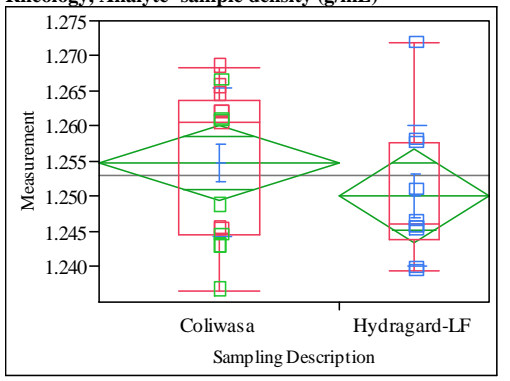

Oneway Anova

Summary of Fit

Rsquare

Adj Rsquare

Reot 0.010349

$\begin{array}{lr}\text { Mean of Response } & 1.252998 \\ \text { Observations (or Sum Wgts) } & 26\end{array}$

Test

Hydragard-LF-Coliwasa

Assuming equal variances

Difference $\quad-0.00470$ t Ratio $\quad-1.12624$

$\begin{array}{lrrr}\text { Std Err Dif } & 0.00417 & \text { DF } & 24 \\ \text { Upper CL Dif } & 0.00391 & \text { Prob }>|t| & 0.2712\end{array}$

$\begin{array}{lrll}\text { Upper CL Dif } & 0.00391 & \text { Prob }>|t| & 0.2712 \\ \text { Lower CL Dif } & -0.01331 & \text { Prob }>t & 0.8644\end{array}$

$\begin{array}{lrll}\text { Lower CL Dif } & -0.01331 & \text { Prob }>t & 0.8644 \\ \text { Confidence } & 0.95 & \text { Prob }<\mathrm{t} & 0.1356\end{array}$

$\begin{array}{lrrrrr}\text { Analysis of Variance } & & & & & \\ \text { Source } & \text { DF } & \text { Sum of Squares } & \text { Mean Square } & \text { F Ratio } & \text { Prob > F } \\ \text { Sampling Description } & 1 & 0.00013584 & 0.000136 & 1.2684 & 0.2712 \\ \text { Error } & 24 & 0.00257034 & 0.000107 & & \\ \text { C. Total } & 25 & 0.00270618 & & & \end{array}$

Means for Oneway Anova

Level Number Mean Std Error Lower 95\% Upper 95\%

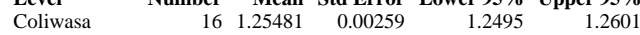

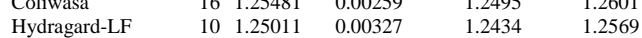

Std Error uses a pooled estimate of error variance

Means and Std Deviations

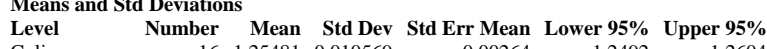

$\begin{array}{lrrrrrr}\text { Coliwasa } & \text { Num } & 1.25481 & 0.010569 & 0.00264 & 1.2492 & 1.2604 \\ \text { Hydragard-LF } & 10 & 1.25011 & 0.009972 & 0.00315 & 1.2430 & 1.2572\end{array}$

Test

Hydragard-LF-Coliwasa

Hydragard-LF-Coliwasa
Assuming unequal variances

$\begin{array}{lrlr}\text { Difference } & -0.00470 & \text { t Ratio } & -1.14207 \\ \text { Std Err Dif } & 0.00411 & \text { DF } & 20.12254 \\ \text { Upper CL Dif } & 0.00388 & \text { Prob }>|t| & 0.2668 \\ \text { Lower CL Dif } & -0.01328 & \text { Prob }>\text { t } & 0.8666 \\ \text { Confidence } & 0.95 & \text { Prob }<\mathrm{t} & 0.1334\end{array}$

Tests that the Variances are Equal

$\begin{array}{lrrrr}\text { Level } & \text { Count } & \text { Std Dev } & \text { MeanAbsDif to Mean } & \text { MeanAbsDif to Median } \\ \text { Coliwasa } & 16 & 0.0105687 & 0.0096992 & 0.0090052\end{array}$

$\begin{array}{lllll}\text { Coliwasa } & 16 & 0.0105687 & 0.0096992 & 0.0090052 \\ \text { Hydragard-LF } & 10 & 0.0099716 & 0.0076602 & 0.0069505\end{array}$

Test F Ratio DFNum DFDen p-Value

$\begin{array}{lllll}\text { O'Brien[.5] } & 0.0622 & 1 & 24 & 0.8052 \\ \text { Brown-Fosyte } & 0.4289 & 1 & 24 & 0.5226\end{array}$

$\begin{array}{lllll}\text { Brown-Forsythe } & 0.4209 & 1 & 24 & 0.5226\end{array}$

$\begin{array}{lllll}\text { Levene } & 1.2836 & 1 & 24 & 0.2684\end{array}$

$\begin{array}{lrrrr}\text { Bartlett } & 0.0360 & 1 & & 0.8495 \\ \text { F Test 2-sided } & 1.1233 & 15 & 9 & 0.8889\end{array}$

Welch Anova testing Means Equal, allowing Std Devs Not Equal

F Ratio DFNum DFDen Prob $>$ F

$\begin{array}{rrrr}1.3043 & 1 & 20.123 & 0.2668\end{array}$

$\mathbf{t}$ Test
1.1421
Oneway Analysis of Measurement By Sampling Description Test Phase=Phase 3 - Low Rheology, Analyte $=$ wt $\%$ dried solids

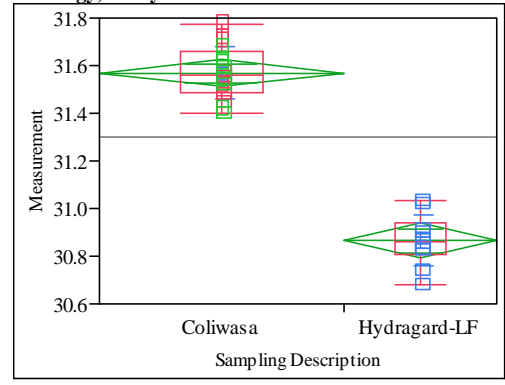

Oneway Anova

Summary of Fit

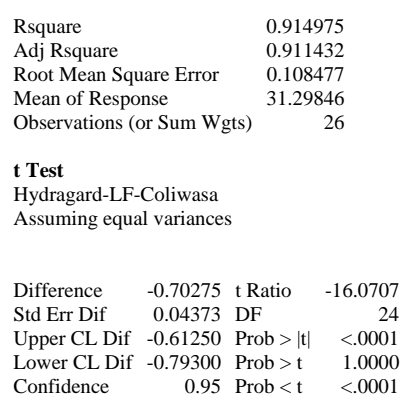

$\begin{array}{lrrrrr}\begin{array}{lrrr}\text { Analysis of Variance } \\ \text { Source }\end{array} & \text { DF } & \text { Sum of Squares } & \text { Mean Square } & \text { F Ratio } & \text { Prob > F } \\ \text { Sampling Description } & 1 & 3.0391235 & 3.03912 & 258.2687 & <.0001 \\ \text { Error } & 24 & 0.2824150 & 0.01177 & & \\ \text { C. Total } & 25 & 3.3215385 & & & \end{array}$

Means for Oneway Anova

$\begin{array}{lrrrrr}\text { Level } & \text { Number } & \text { Mean } & \text { Std Error } & \text { Lower 95\% } & \text { Upper 95\% } \\ \text { Coliwasa } & 16 & 31.5688 & 0.02712 & 31.513 & 31.625\end{array}$

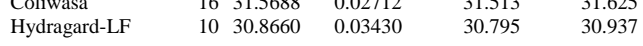

Std Error uses a pooled estimate of error variance

Means and Std Deviations

Level Number Mean Std Dev Std Err Mean Lower 95\% Upper 95\%

$\begin{array}{lllllll}\text { Coliwasa } & 16 & 31.5688 & 0.108128 & 0.02703 & 31.511 & 31.626\end{array}$

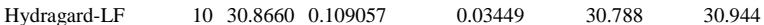

t Test

Hydragard-LF-Coliwasa

Assuming unequal variances

$\begin{array}{lll}\text { Difference } & -0.70275 \text { t Ratio } \quad-16.0377\end{array}$

Std Err Dif $\quad 0.04382$ DF $\quad 19.12496$

Upper CL Dif -0.61108 Prob $>|t| r .0001$

$\begin{array}{lll}\text { Lower CL Dif }-0.79442 \text { Prob }>t & 1.0000\end{array}$

$\begin{array}{lrl}\text { Confidence } & 0.95 \text { Prob }<\mathrm{t} & <.0001\end{array}$

Tests that the Variances are Equal

Level Count Std Dev MeanAbsDif to Mean MeanAbsDif to Median

$\begin{array}{lllll}\text { Coliwasa } & 16 & 0.1081280 & 0.0862500 & 0.0862500\end{array}$

$\begin{array}{lllll}\text { Hydragard-LF } & 10 & 0.1090566 & 0.0800000 & 0.0800000\end{array}$

Test F Ratio DFNum DFDen p-Value

$\begin{array}{lrrrr}\text { O'Brien[.5] } & 0.0012 & 1 & 24 & 0.9726\end{array}$

$\begin{array}{lllll}\text { Brown-Forsythe } & 0.0571 & 1 & 24 & 0.8131\end{array}$

$\begin{array}{lllll}\text { Levene } & 0.0580 & 1 & 24 & 0.8117\end{array}$

$\begin{array}{lllrl}\text { Bartlett } & 0.0008 & 1 & \text {. } & 0.9776 \\ \text { F Test 2-sided } & 1.0172 & 9 & 15 & 0.9375\end{array}$

Welch Anova testing Means Equal, allowing Std Devs Not Equal

F Ratio DFNum DFDen Prob $>$ F

$\begin{array}{rrrr}257.2091 & 1 & 19.125 & <.0001\end{array}$

t Test

16.0377 


\section{Exhibit C2. Statistical Comparisons for Low-Rheology (Phase 3) Simulant Testing}

Oneway Analysis of Measurement By Sampling Description Test Phase=Phase 3 - Low Rheology, Analyte $=\mathrm{wt} \%$ vitrified solids

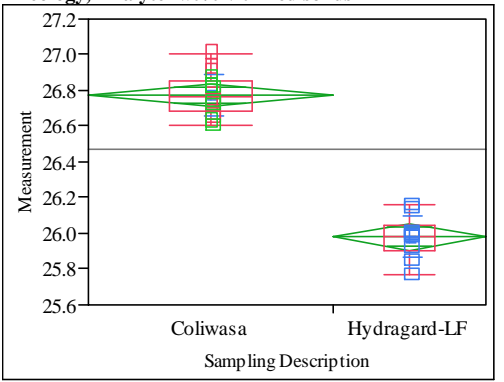

Oneway Anova

Summary of Fit

Adj Rsquare

0.921735

0.11456
$\quad 6.46846$

Observations (or Sum Wgts) $\quad 26$

t Test

Hydragard-LF-Coliwasa

Assuming equal variances

Difference $\quad-0.79375$ t Ratio $\quad-17.188$

Std Err Dif $\quad 0.04618$ DF 24

Upper CL Dif -0.69844 Prob $>|t|<.0001$

Lower CL Dif -0.88906 Prob $>t \quad 1.0000$

$\begin{array}{lll}\text { Confidence } & 0.95 \text { Prob }<\mathrm{t} \quad<.0001\end{array}$

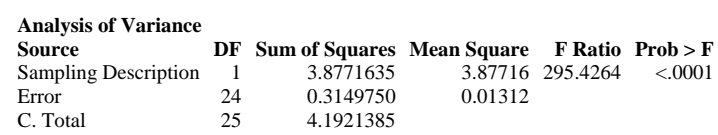

C. Total 25

4.1921385

Means for Oneway Anova

Level Number Mean Std Error Lower 95\% Upper 95\%

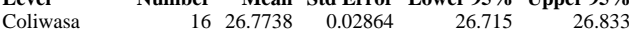

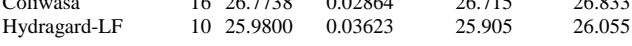

Std Error uses a pooled estimate of error variance

Means and Std Deviations

Level Number Mean Std Dev Std Err Mean Lower 95\% Upper 95\%

$\begin{array}{lllllll}\text { Coliwasa } & 16 & 26.7738 & 0.112835 & 0.02821 & 26.714 & 26.834\end{array}$

$\begin{array}{lllllll}\text { Hydragard-LF } & 10 & 25.9800 & 0.117379 & 0.03712 & 25.896 & 26.064\end{array}$

t Test

Hydragard-LF-Coliwas

Assuming unequal variances

Difference $\quad-0.79375$ t Ratio $\quad-17.0256$

Std Err Dif $\quad 0.04662$ DF $\quad 18.66277$

Upper CL Dif -0.69605 Prob $>|t| \quad<.0001$

Lower CL Dif -0.89145 Prob $>t \quad 1.0000$

$\begin{array}{lrr}\text { Confidence } & 0.95 \text { Prob }<\mathrm{t} & <.0001\end{array}$

Tests that the Variances are Equal

Level Count Std Dev MeanAbsDif to Mean MeanAbsDif to Median

$\begin{array}{lllll}\text { Coliwasa } & 16 & 0.1128347 & 0.0912500 & 0.0912500\end{array}$

$\begin{array}{lllll}\text { Hydragard-LF } & 10 & 0.1173788 & 0.0800000 & 0.0800000\end{array}$

Test F Ratio DFNum DFDen p-Value

$\begin{array}{lrrrr}\text { O'Brien[.5] } & 0.0231 & 1 & 24 & 0.8804\end{array}$

$\begin{array}{lllll}\text { Brown-Forsythe } & 0.1568 & 1 & 24 & 0.8804 \\ & 0.1587 & 1 & 24 & 0.6938\end{array}$

$\begin{array}{lllll}\text { Levene } & 0.1587 & 1 & 24 & 0.6938\end{array}$

$\begin{array}{lllll}\text { Bartlett } & 0.0169 & 1 & & 0.8966 \\ \text { F Test 2-sided } & 1.0822 & 9 & 15 & 0.8569\end{array}$

Welch Anova testing Means Equal, allowing Std Devs Not Equal

F Ratio DFNum DFDen Prob $>$ F

$\begin{array}{rrrr}289.8721 & 1 & 18.663 & <.0001\end{array}$

t Test

17.0256
Oneway Analysis of Measurement By Sampling Description Test Phase=Phase 3 - Low Rheology, Analyte=sample density $(\mathrm{g} / \mathrm{mL})$

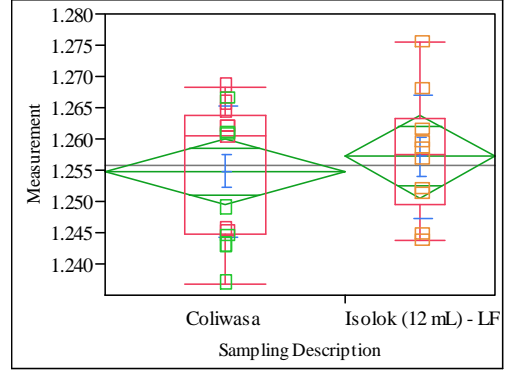

Oneway Anova

Summary of Fit

Rsquare

Adj Rsquare

0.010304

$\begin{array}{lr}\text { Mean of Response } & 1.255703 \\ \text { Observations (or Sum Wgts) } & 26\end{array}$

t Test

Isolok (12 mL) - LF-Coliwasa

Assuming equal variances

Difference $\quad 0.00234$ t Ratio $\quad 0.562292$

$\begin{array}{lrlr}\text { Std Err Dif } & 0.00415 & \text { DF } & 24 \\ \text { Upper CL Dif } & 0.01091 & \text { Prob }>|t| & 0.5791\end{array}$

$\begin{array}{lrll}\text { Upper CL Dif } & 0.01091 & \text { Prob }>|t| & 0.5791 \\ \text { Lower CL Dif } & -0.00624 & \text { Prob }>t & 0.2896\end{array}$

$\begin{array}{lrrr}\text { Lower CL Dif } & -0.00624 & \text { Prob }>t & 0.2896 \\ \text { Confidence } & 0.95 & \text { Prob }<t & 0.7104\end{array}$

Analysis of Variance

$\begin{array}{lrrrrr}\text { Analysis of Variance } & & & & & \\ \text { Source } & \text { DF } & \text { Sum of Squares } & \text { Mean Square } & \text { F Ratio } & \text { Prob }>\text { F } \\ \text { Sampling Description } & 1 & 0.00003357 & 0.000034 & 0.3162 & 0.5791\end{array}$

Error

$0.00254836 \quad 0.00010$

C. Total

$25 \quad 0.00258194$

Means for Oneway Anova

Level Number Mean Std Error Lower 95\% Upper 95\%

$\begin{array}{llllll}\text { Coliwasa } & 16 & 1.25481 & 0.00258 & 1.2495 & 1.2601\end{array}$

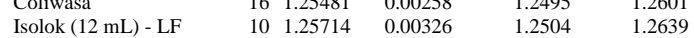

Std Error uses a pooled estimate of error variance

Means and Std Deviations

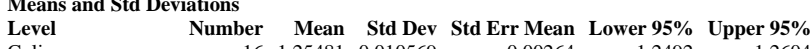

$\begin{array}{lrrrrrr}\text { Coliwasa } & 16 & 1.25481 & 0.010569 & 0.00264 & 1.2492 & 1.2604 \\ \text { Isolok (12 mL) - LF } & 10 & 1.25714 & 0.009848 & 0.00311 & 1.2501 & 1.2642\end{array}$

t Test

Isolok (12 mL) - LF-Coliwasa

Assuming unequal variances

Difference $\quad 0.00234$ t Ratio 0.571893

Std Err Dif $\quad 0.00408$ DF 20.30632

$\begin{array}{lll}\text { Upper CL Dif } \quad 0.01085 \text { Prob }>|t| & 0.5737\end{array}$

Lower CL Dif -0.00618 Prob $>t \quad 0.2868$

$\begin{array}{lll}\text { Confidence } & 0.95 \text { Prob }<\mathrm{t} \quad 0.7132\end{array}$

Tests that the Variances are Equal

$\begin{array}{lrrrr}\text { Level } & \text { Count } & \text { Std Dev } & \text { MeanAbsDif to Mean } & \text { MeanAbsDif to Median } \\ \text { Coliwasa } & 16 & 0.0105687 & 0.0096992 & 0.0090052\end{array}$

$\begin{array}{lrrrr}\text { Coliwasa } & 16 & 0.0105687 & 0.0096992 & 0.0090052 \\ \text { Isolok (12 mL) - LF } & 10 & 0.0098484 & 0.0074677 & 0.0074677\end{array}$

Test F Ratio DFNum DFDen p-Value

$\begin{array}{lllll} & 0.1258 & 1 & 24 & 0.7260 \\ \text { Brown-Forsythe } & 0.2848 & 1 & 24 & 0.5985\end{array}$

$\begin{array}{lllll}\text { Brown-Forsythe } & 0.2848 & 1 & 24 & 0.5985\end{array}$

$\begin{array}{lllll}\text { Levene } & 1.5148 & 1 & 24 & 0.2303\end{array}$

$\begin{array}{lrrrr}\text { Bartlett } & 0.0530 & 1 & & 0.8180 \\ \text { F Test 2-sided } & 1.1516 & 15 & 9 & 0.8571\end{array}$

Welch Anova testing Means Equal, allowing Std Devs Not Equal

F Ratio DFNum DFDen Prob $>$ F

$\begin{array}{llll}0.3271 & 1 & 20.306 & 0.5737\end{array}$

t Test
0.5719 


\section{Exhibit C2. Statistical Comparisons for Low-Rheology (Phase 3) Simulant Testing}

Oneway Analysis of Measurement By Sampling Description Test Phase=Phase 3 - Low Rheology, Analyte $=$ wt $\%$ dried solids

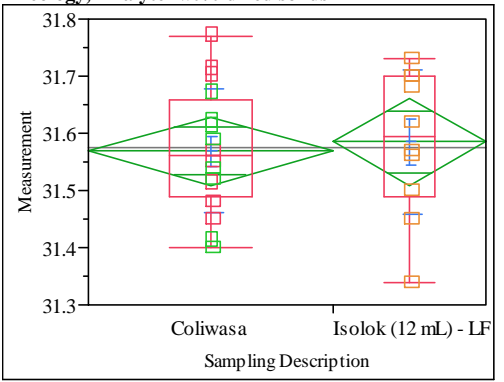

Oneway Anova

Summary of Fit

Adj Rsquare

$\quad 0.115366$

31.575

t Test

Isolok (12 mL) - LF-Coliwasa

Assuming equal variances

Difference $\quad 0.01625$ t Ratio $\quad 0.34942$

Std Err Dif $\quad 0.04651$ DF 24

Upper CL Dif 0.11223 Prob $>|t| \quad 0.7298$

Lower CL Dif -0.07973 Prob $>t \quad 0.3649$

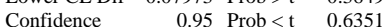

\section{Analysis of Variance}

$\begin{array}{lrrrrr}\text { Source } & \text { DF } & \text { Sum of Squares } & \text { Mean Square } & \text { F Ratio } & \text { Prob > F } \\ \text { Sampling Description } & 1 & 0.00162500 & 0.001625 & 0.1221 & 0.7298 \\ \text { Error } & 24 & 0.31942500 & 0.013309 & & \end{array}$

\section{Means for Oneway Anova}

$\begin{array}{lrrrrr}\text { Level } & \text { Number } & \text { Mean } & \text { Std Error } & \text { Lower 95\% } & \text { Upper 95\% } \\ \text { Coliwasa } & 16 & 31.5688 & 0.02884 & 31.509 & 31.628\end{array}$

$\begin{array}{lrrrrr}\text { Coliwasa } & 16 & 31.5688 & 0.02884 & 31.509 & 31.628 \\ \text { Isolok (12 mL) - LF } & 10 & 31.5850 & 0.03648 & 31.510 & 31.660\end{array}$

Std Error uses a pooled estimate of error variance

Means and Std Deviations

Level Number Mean Std Dev Std Err Mean Lower 95\% Upper 95\%

$\begin{array}{llllll}\text { Coliwasa } & 16 & 31.5688 & 0.108128 & 0.02703 & 31.511\end{array}$

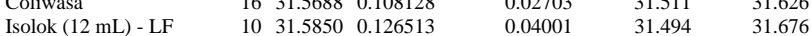

t Test

Isolok (12 mL) - LF-Coliwasa

Assuming unequal variances

Difference $\quad 0.01625$ t Ratio $\quad 0.336555$

Std Err Dif $\quad 0.04828$ DF $\quad 16.97131$

Upper CL Dif 0.11813 Prob $>|t| \quad 0.7406$

Lower CL Dif -0.08563 Prob $>t \quad 0.3703$

$\begin{array}{lrl}\text { Confidence } & 0.95 \text { Prob }<\mathrm{t} & 0.6297\end{array}$

Tests that the Variances are Equal

Level Count Std Dev MeanAbsDif to Mean MeanAbsDif to Median

$\begin{array}{lllll}\text { Coliwasa } & 16 & 0.1081280 & 0.0862500 & 0.0862500\end{array}$

$\begin{array}{lllll}\text { Isolok }(12 \mathrm{~mL}) \text { - LF } & 10 & 0.1265131 & 0.1010000 & 0.1010000\end{array}$

Test F Ratio DFNum DFDen p-Value

$\begin{array}{lrrrr}\text { O'Brien[.5] } & 0.4142 & 1 & 24 & 0.5259\end{array}$

$\begin{array}{lllll}\text { Brown-Forsythe } & 0.3194 & 1 & 24 & 0.5772\end{array}$

$\begin{array}{lllll}\text { Levene } & 0.3266 & 1 & 24 & 0.5730\end{array}$

$\begin{array}{lllll}\text { Bartlett } & 0.2714 & 1 & & 0.6024 \\ \text { F Test 2-sided } & 1.3690 & 9 & 15 & 0.5676\end{array}$

Welch Anova testing Means Equal, allowing Std Devs Not Equal

F Ratio DFNum DFDen Prob > F

$\begin{array}{rrrr}0.1133 & 1 & 16.971 & 0.7406\end{array}$

t Test

0.3366

Oneway Analysis of Measurement By Sampling Description Test Phase=Phase 3 - Low Rheology, Analyte $=w t \%$ vitrified solids

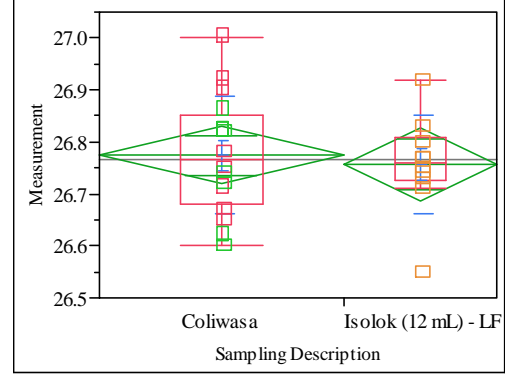

Oneway Anova

Summary of Fit

Adj Rsquare

Root Mean Square Error

Mean of Response

Observations (or Sum Wgts)

0.006326

$-0.03508$

0.106299

t Test

Isolok (12 mL) - LF-Coliwasa

Assuming equal variances

Difference $\quad-0.01675$ t Ratio $\quad-0.3909$

Std Err Dif $\quad 0.04285$ DF 24

Upper CL Dif 0.07169 Prob $>|t| 0.6993$

Lower CL Dif -0.10519 Prob $>t \quad 0.6503$

$\begin{array}{lll}\text { Confidence } & 0.95 \text { Prob }<\mathrm{t} \quad 0.3497\end{array}$

\section{Analysis of Variance}

$\begin{array}{lrrrrr}\text { Source } & \text { DF } & \text { Sum of Squares } & \text { Mean Square } & \text { F Ratio } & \text { Prob }>\text { F } \\ \text { Sampling Description } & 1 & 0.00172654 & 0.001727 & 0.1528 & 0.6993\end{array}$

$\begin{array}{llll}\text { Error } & 24 & 0.27118500 & 0.011299\end{array}$

$\begin{array}{lll}\text { C. Total } & 25 & 0.27291154\end{array}$

Means for Oneway Anova

Level Number Mean Std Error Lower 95\% Upper 95\%

$\begin{array}{lrrrrr}\text { Coliwasa } & 16 & 26.7738 & 0.02657 & 26.719 & 26.829\end{array}$

$\begin{array}{llllll}\text { Isolok }(12 \mathrm{~mL}) \text { - LF } & 10 & 26.7570 & 0.03361 & 26.688 & 26.829 \\ & & & \end{array}$

Std Error uses a pooled estimate of error variance

Means and Std Deviations

Level Number Mean Std Dev Std Err Mean Lower 95\% Upper 95\%

$\begin{array}{lllllll}\text { Coliwasa } & 16 & 26.7738 & 0.112835 & 0.02821 & 26.714 & 26.834\end{array}$

Isolok (12 mL) - LF $\quad 1026.7570 \quad 0.094405-0.02985-26.689-26.825$

Test

Isolok (12 mL) - LF-Coliwas

Assuming unequal variances

$\begin{array}{lrlr}\text { Difference } & -0.01675 & \text { t Ratio } & -0.40782 \\ \text { Std Err Dif } & 0.04107 & \text { DF } & 21.81273 \\ \text { Upper CL Dif } & 0.06847 & \text { Prob }>|t| & 0.6874 \\ \text { Lower CL Dif } & -0.10197 & \text { Prob }>\text { t } & 0.6563 \\ \text { Confidence } & 0.95 & \text { Prob }<\mathrm{t} & 0.3437\end{array}$

Tests that the Variances are Equal

Level Count Std Dev MeanAbsDif to Mean MeanAbsDif to Median

$\begin{array}{lllll}\text { Coliwasa } & 16 & 0.1128347 & 0.0912500 & 0.0912500\end{array}$

Isolok $(12 \mathrm{~mL})$ - LF $\quad 10 \quad 0.0944046$

$\begin{array}{lrrrr}\text { Test } & \text { F Ratio } & \text { DFNum } & \text { DFDen } & \text { p-Value } \\ \text { O'Brien[.5] } & 0.3408 & 1 & 24 & 0.5648 \\ \text { Brown-Forsythe } & 1.3241 & 1 & 24 & 0.2612 \\ \text { Levene } & 1.3414 & 1 & 24 & 0.2582 \\ \text { Bartlett } & 0.3307 & 1 & \text {. } & 0.5652 \\ \text { F Test 2-sided } & 1.4286 & 15 & 9 & 0.5993\end{array}$

Welch Anova testing Means Equal, allowing Std Devs Not Equal

F Ratio DFNum DFDen Prob > F

$\begin{array}{llll}0.1663 & 1 & 21.813 & 0.6874\end{array}$

t Test

0.4078 


\section{Exhibit C2. Statistical Comparisons for Low-Rheology (Phase 3) Simulant Testing}

Oneway Analysis of Measurement By Sampling Description Test Phase=Phase 3 - Low Rheology, Analyte=sample density $(\mathrm{g} / \mathrm{mL})$

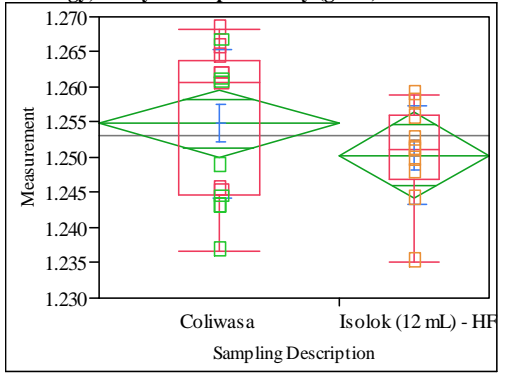

Oneway Anova

Summary of Fit

Rsquare

Adj Rsquare

0.01604

Reot Mean Square Eror 0.009383

1.253079
Observations (or Sum Wgts)

t Test

Isolok (12 mL) - HF-Coliwasa

Assuming equal variances

Difference $\quad-0.00449$ t Ratio -1.18644

$\begin{array}{lrlr}\text { Std Err Dif } & 0.00378 \text { DF } & 24 \\ \text { Uper CL Dif } & 0.00332 & \text { Prob }>|t| & 0.2471\end{array}$

$\begin{array}{lrlr}\text { Upper CL Dif } & 0.00332 & \text { Prob }>|t| & 0.2471 \\ \text { Lower CL Dif } & -0.01229 & \text { Prob }>t & 0.8765\end{array}$

$\begin{array}{lrl}\text { Confidence } & 0.95 \text { Prob }<\mathrm{t} \quad 0.1235\end{array}$

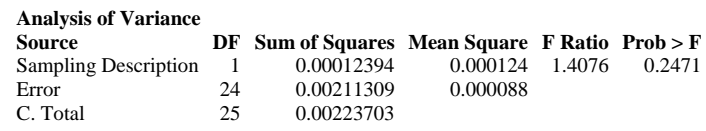

Means for Oneway Anova

Level Number Mean Std Error Lower 95\% Upper 95\%

$\begin{array}{lllllr}\text { Coliwasa } & 16 & 1.25481 & 0.00235 & 1.2500 & 1.2596\end{array}$

$\begin{array}{llllll}\text { Coliwasa } & 16 & 1.25481 & 0.00235 & 1.2500 & 1.2596 \\ \text { Isolok }(12 \mathrm{~mL}) \text { - HF } & 10 & 1.25032 & 0.00297 & 1.2442 & 1.2564\end{array}$

Std Error uses a pooled estimate of error variance

Means and Std Deviations

$\begin{array}{lrrrrrr}\text { Level } & \text { Number } & \text { Mean } & \text { Std Dev } & \text { Std Err Mean } & \text { Lower 95\% } & \text { Upper 95\% } \\ \text { Coliwasa } & 16 & 1.25481 & 0.010569 & 0.00264 & 1.2492 & 1.2604\end{array}$

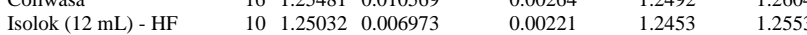

t Test

Isolok (12 mL) - HF-Coliwasa

Assuming unequal variances

Difference $\quad-0.00449$ t Ratio -1.30401

Std Err Dif $\quad 0.00344$ DF 23.87111

Upper CL Dif 0.00262 Prob $>|t| \quad 0.2047$

Lower CL Dif -0.01159 Prob $>t \quad 0.8977$

$\begin{array}{lrl}\text { Confidence } & 0.95 \text { Prob }<\mathrm{t} \quad 0.1023\end{array}$

Tests that the Variances are Equal

$\begin{array}{lrrrr}\text { Level } & \text { Count } & \text { Std Dev } & \text { MeanAbsDif to Mean } & \text { MeanAbsDif to Median } \\ \text { Coliwasa } & 16 & 0.0105687 & 0.0096992 & 0.0090052 \\ \text { Isolok (12 mL) - HF } & 10 & 0.0069733 & 0.0049263 & 0.0047917\end{array}$

$\begin{array}{lrrrr}\text { Test } & \text { F Ratio } & \text { DFNum } & \text { DFDen } & \text { p-Value } \\ \text { O'Brien[.5] } & 3.4932 & 1 & 24 & 0.0739 \\ \text { Brown-Forsythe } & 2.3378 & 1 & 24 & 0.1393 \\ \text { Levene } & 9.2124 & 1 & 24 & 0.0057 \\ \text { Bartlett } & 1.6970 & 1 & . & 0.1927 \\ \text { F Test 2-sided } & 2.2970 & 15 & 9 & 0.2097\end{array}$

Welch Anova testing Means Equal, allowing Std Devs Not Equal

F Ratio DFNum DFDen Prob $>$ F

$\begin{array}{llll}1.7004 & 1 & 23.871 & 0.2047\end{array}$

t Test
1.3040
Oneway Analysis of Measurement By Sampling Description Test Phase=Phase 3 - Low Rheology, Analyte $=w t \%$ dried solids

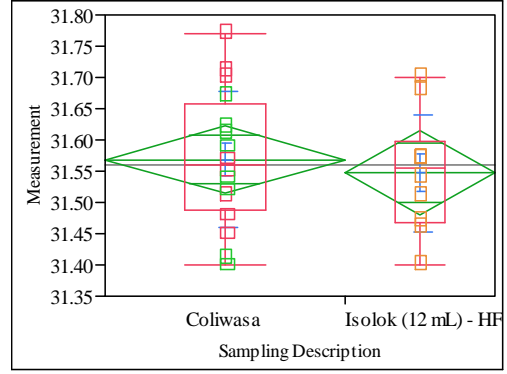

Oneway Anova

Summary of Fit

Rsquare

Adj Rsquare

0.029

Root Mean Square Error $\quad 0.102994$

Mean of Response

31.56038

Observations (or Sum Wgts) 26

t Test

Isolok (12 mL) - HF-Coliwasa

Assuming equal variances

Difference $\quad-0.02175$ t Ratio $\quad-0.52387$

$\begin{array}{lrlr}\text { Std Err Dif } & 0.04152 \text { DF } & 24 \\ \text { Upper CL Dif } & 0.06394 \text { Prob }>|t| & 0.6052\end{array}$

$\begin{array}{lrll}\text { Upper CL Dif } & 0.06394 & \text { Prob }>|t| & 0.6052 \\ \text { Lower CL Dif } & -0.10744 & \text { Prob }>t & 0.6974\end{array}$

$\begin{array}{lrrr}\text { Lower CL Dif } & -0.10744 & \text { Prob }>t & 0.6974 \\ \text { Confidence } & 0.95 & \text { Prob }<t & 0.3026\end{array}$

$\begin{array}{lrrrrr}\begin{array}{lrrrr}\text { Analysis of Variance } \\ \text { Source }\end{array} & \text { DF } & \text { Sum of Squares } & \text { Mean Square } & \text { F Ratio } & \text { Prob > F } \\ \text { Sampling Description } & 1 & 0.00291115 & 0.002911 & 0.2744 & 0.6052 \\ \text { Error } & 24 & 0.25458500 & 0.010608 & & \\ \text { C. Total } & 25 & 0.25749615 & & & \end{array}$

$\begin{array}{lll}\text { C. Total } & 25 & 0.25749615\end{array}$

Means for Oneway Anova

Level Number Mean Std Error Lower 95\% Upper 95\%

$\begin{array}{llllll}\text { Coliwasa } & 16 & 31.5688 & 0.02575 & 31.516 & 31.622\end{array}$

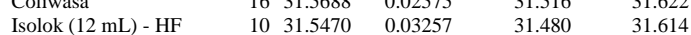

Std Error uses a pooled estimate of error variance

Means and Std Deviations

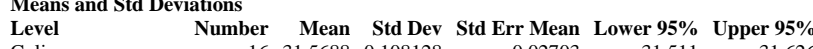

$\begin{array}{lrrrrrr}\text { Coliwasa } & 16 & 31.5688 & 0.108128 & 0.02703 & 31.511 & 31.626 \\ \text { Isolok }(12 \mathrm{~mL}) \text { - HF } & 10 & 31.5470 & 0.093814 & 0.02967 & 31.480 & 31.614\end{array}$

t Test

Isolok (12 mL) - HF-Coliwas

Assuming unequal variances

Difference $\quad-0.02175$ t Ratio $\quad-0.54192$

Std Err Dif $\quad 0.04014$ DF 21.32767

Upper CL Dif 0.06164 Prob $>|t| \quad 0.5935$

Lower CL Dif -0.10514 Prob $>t \quad 0.7033$

$\begin{array}{lll}\text { Confidence } & 0.95 \text { Prob }<\mathrm{t} \quad 0.2967\end{array}$

Tests that the Variances are Equal

$\begin{array}{lrrrr}\text { Level } & \text { Count } & \text { Std Dev } & \text { MeanAbsDif to Mean } & \text { MeanAbsDif to Median } \\ \text { Coliwasa } & 16 & 0.1081280 & 0.0862500 & 0.0862500\end{array}$

$\begin{array}{lrrrr}\text { Coliwasa } & 16 & 0.1081280 & 0.0862500 & 0.0862500 \\ \text { Isolok }(12 \mathrm{~mL})-\mathrm{HF} & 10 & 0.0938142 & 0.0710000 & 0.0710000\end{array}$

Test F Ratio DFNum DFDen p-Value

$\begin{array}{lrrrr}\text { O'Brien[.5] } & 0.3198 & 1 & 24 & 0.5770\end{array}$

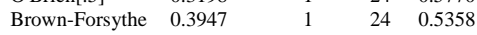

$\begin{array}{lllll}\text { Levene } & 0.4034 & 1 & 24 & 0.5314\end{array}$

$\begin{array}{llrrr}\text { Levene } & 0.4034 & 1 & 24 & 0.5314 \\ \text { Bartlett } & 0.2113 & 1 & . & 0.6457\end{array}$

$\begin{array}{lllll}\text { F Test 2-sided } & 1.3284 & 15 & 9 & 0.6819\end{array}$

Welch Anova testing Means Equal, allowing Std Devs Not Equal

F Ratio DFNum DFDen Prob $>$ F

$\begin{array}{llll}0.2937 & 1 & 21.328 & 0.5935\end{array}$

t Test 


\section{Exhibit C2. Statistical Comparisons for Low-Rheology (Phase 3) Simulant Testing}

Oneway Analysis of Measurement By Sampling Description Test Phase=Phase 3 - Low Rheology, Analyte $=$ wt $\%$ vitrified solids

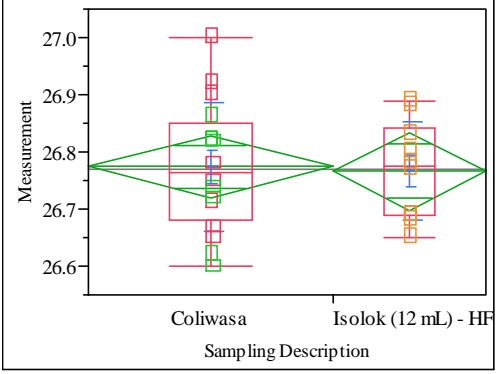

Oneway Anova

Summary of Fit

Adj Rsquare

Root Mean Square Error $\quad-0.04017$

0.103524

Observations (or Sum Wgts) 26

t Test

Isolok (12 mL) - HF-Coliwasa

Assuming equal variances

Difference $\quad-0.00775$ t Ratio $\quad-0.18571$

$\begin{array}{lrr}\text { Std Err Dif } & 0.04173 \text { DF } & 24 \\ & \end{array}$

Upper CL Dif 0.07838 Prob $>|\mathrm{t}| \quad 0.8542$

Lower CL Dif -0.09388 Prob $>\mathrm{t} \quad 0.5729$

$\begin{array}{lll}\text { Confidence } & 0.95 \text { Prob }<\mathrm{t} \quad 0.4271\end{array}$

\section{Analysis of Variance}

Source

Sampling

Error

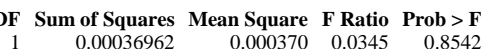
0.00036962 0.010717

Means for Oneway Anova

$\begin{array}{lrrrrr}\text { Level } & \text { Number } & \text { Mean } & \text { Std Error } & \text { Lower 95\% } & \text { Upper 95\% } \\ \text { Coliwasa } & 16 & 26.7738 & 0.02588 & 26.720 & 26.827\end{array}$

$\begin{array}{llrrrr}\text { Coliwasa } & 16 & 26.7738 & 0.02588 & 26.720 & 26.827 \\ \text { Isolok (12 mL) - HF } & 10 & 26.7660 & 0.03274 & 26.698 & 26.834\end{array}$

Std Error uses a pooled estimate of error variance

Means and Std Deviations

Level Number Mean Std Dev Std Err Mean Lower 95\% Upper 95\%

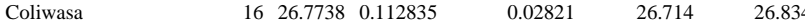

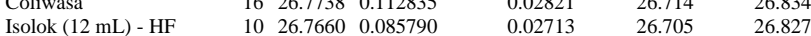

t Test

Isolok (12 mL) - HF-Coliwasa

Assuming unequal variances

Difference $\quad-0.00775$ t Ratio $\quad-0.19802$

Std Err Dif $\quad 0.03914$ DF 22.91188

Upper CL Dif 0.07323 Prob $>|t| \quad 0.8448$

Lower CL Dif -0.08873 Prob $>\mathrm{t} \quad 0.5776$

$\begin{array}{lrl}\text { Confidence } & 0.95 \text { Prob }<\mathrm{t} & 0.4224\end{array}$

Tests that the Variances are Equal

Level Count Std Dev MeanAbsDif to Mean MeanAbsDif to Median

$\begin{array}{lllll}\text { Coliwasa } & 16 & 0.1128347 & 0.0912500 & 0.0912500\end{array}$

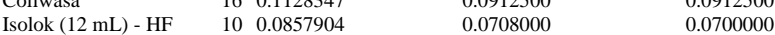

$\begin{array}{lrrrr}\text { Test } & \text { F Ratio } & \text { DFNum } & \text { DFDen } & \text { p-Value } \\ \text { O'Brien[.5] } & 1.0592 & 1 & 24 & 0.3137 \\ \text { Brown-Forsythe } & 0.8659 & 1 & 24 & 0.3614 \\ \text { Levene } & 0.8362 & 1 & 24 & 0.3696 \\ \text { Bartlett } & 0.7640 & 1 & \text {. } & 0.3821 \\ \text { F Test 2-sided } & 1.7298 & 15 & 9 & 0.4095\end{array}$

Welch Anova testing Means Equal, allowing Std Devs Not Equal

F Ratio DFNum DFDen Prob $>$ F

$\begin{array}{llll}0.0392 & 1 & 22.912 & 0.8448\end{array}$

t Test

0.1980
Oneway Analysis of Measurement By Sampling Description Test Phase=Phase 3 - Low Rheology, Analyte $=w t \%$ dried solids

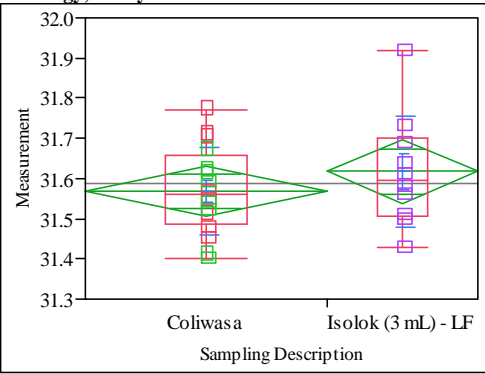

Oneway Anova

Summary of Fit

Rsquare

Adj Rsquare

Mean of Response 31.58731

Observations (or Sum Wgts) 26

t Test

Isolok (3 mL) - LF-Coliwasa

Assuming equal variances

Difference $\quad 0.04825$ t Ratio 0.990329

$\begin{array}{lrlr}\text { Std Err Dif } & 0.04872 & \text { DF } & 24 \\ \text { Upper CL Dif } & 0.14881 & \text { Prob }>|t| & 0.3319\end{array}$

$\begin{array}{lrll}\text { Upper CL Dif } & 0.14881 & \text { Prob }>|t| & 0.3319 \\ \text { Lower CL Dif } & -0.05231 & \text { Prob }>t & 0.1659\end{array}$

$\begin{array}{lrrr}\text { Lower CL Dif } & -0.05231 & \text { Prob }>t & 0.1659 \\ \text { Confidence } & 0.95 & \text { Prob }<t & 0.8341\end{array}$

Analysis of Variance

$\begin{array}{lrrrrr}\text { Analysis of Variance } & & & & & \\ \text { Source } & \text { DF } & \text { Sum of Squares } & \text { Mean Square } & \text { F Ratio } & \text { Prob }>\text { F } \\ \text { Sampling Description } & 1 & 0.01432654 & 0.014327 & 0.9808 & 0.3319\end{array}$

Error

$0.35058500 \quad 0.01460$

C. Total

$25 \quad 0.3649115$

Means for Oneway Anova

Level Number Mean Std Error Lower 95\% Upper 95\%

$\begin{array}{llllrr}\text { Coliwasa } & 16 & 31.5688 & 0.03022 & 31.506 & 31.631\end{array}$

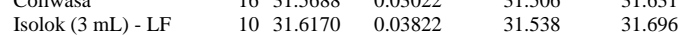

Std Error uses a pooled estimate of error variance

Means and Std Deviations

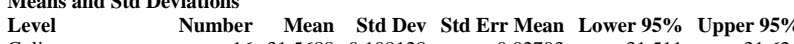

$\begin{array}{lrrrrrr}\text { Coliwasa } & 16 & 31.5688 & 0.108128 & 0.02703 & 31.511 & 31.626 \\ \text { Isolok (3 mL) - LF } & 10 & 31.6170 & 0.139527 & 0.04412 & 31.517 & 31.717\end{array}$

t Test

Isolok (3 mL) - LF-Coliwasa

Assuming unequal variances

Difference $\quad 0.04825$ t Ratio $\quad 0.932464$

Std Err Dif $\quad 0.05174$ DF 15.6974

Upper CL Dif 0.15812 Prob $>|t| \quad 0.3652$

Lower CL Dif -0.06162 Prob $>t \quad 0.1826$

$\begin{array}{lll}\text { Confidence } & 0.95 \text { Prob }<\mathrm{t} \quad 0.8174\end{array}$

Tests that the Variances are Equal

$\begin{array}{lrrrr}\text { Level } & \text { Count } & \text { Std Dev } & \text { MeanAbsDif to Mean } & \text { MeanAbsDif to Median } \\ \text { Coliwasa } & 16 & 0.1081280 & 0.0862500 & 0.0862500\end{array}$

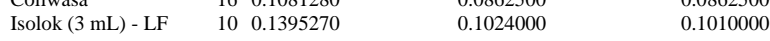

Test F Ratio DFNum DFDen p-Value

$\begin{array}{lllll}\text { O'Brien[.5] } & 0.7059 & 1 & 24 & 0.4091\end{array}$

$\begin{array}{lllll}\text { Brown-Forsythe } & 0.2369 & 1 & 24 & 0.6308\end{array}$

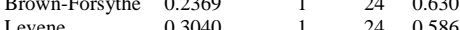

$\begin{array}{lllll}\text { Levene } & 0.3040 & 1 & 24 & 0.5865 \\ \text { Bartlett } & 0.7224 & 1 & & 0.3954\end{array}$

$\begin{array}{lllrrr}\text { Bartlett } & 0.7224 & 1 & & 0.3954 \\ \text { F Test 2-sided } & 1.6651 & 9 & 15 & 0.3677\end{array}$

Welch Anova testing Means Equal, allowing Std Devs Not Equal

F Ratio DFNum DFDen Prob $>$ F

$\begin{array}{llll}0.8695 & 1 & 15.697 & 0.3652\end{array}$

t Test

0.9325 


\section{Exhibit C2. Statistical Comparisons for Low-Rheology (Phase 3) Simulant Testing}

Oneway Analysis of Measurement By Sampling Description Test Phase=Phase 3 - Low Rheology, Analyte $=$ wt $\%$ vitrified solids

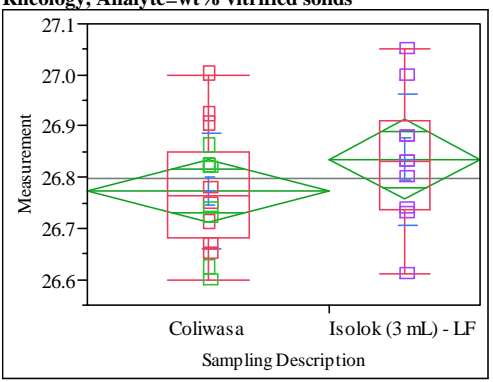

Oneway Anova

Summary of Fit

Rsquare

Adj Rsquare $\quad 0.024489$

Root Mean Square Error $\quad 0.119098$

$\begin{array}{lr}\text { Mean of Response } & 26.79731 \\ \text { Observations (or Sum Wgts) } & 26\end{array}$

t Test

Isolok (3 mL) - LF-Coliwasa

Assuming equal variances

Difference $\quad 0.06125$ t Ratio $\quad 1.275776$

$\begin{array}{lrlr}\text { Std Err Dif } & 0.04801 & \text { DF } & 24 \\ \text { Upper CL Dif } & 0.16034 & \text { Prob }>|t| & 0.2142\end{array}$

$\begin{array}{rrrr}\text { Upper CL Dif } & 0.16034 & \text { Prob }>|t| & 0.2142 \\ \text { Lower CL Dif } & -0.03784 & \text { Prob }>t & 0.1071\end{array}$

$\begin{array}{lrll}\text { Lower CL Dif } & -0.03784 & \text { Prob }>t & 0.1071 \\ \text { Confidence } & 0.95 & \text { Prob }<t & 0.8929\end{array}$

$\begin{array}{lrrrrr}\text { Analysis of Variance } & & & & & \\ \text { Source } & \text { DF } & \text { Sum of Squares } & \text { Mean Square } & \text { F Ratio } & \text { Prob > F } \\ \text { Sampling Description } & 1 & 0.02308654 & 0.023087 & 1.6276 & 0.2142 \\ \text { Error } & 24 & 0.34042500 & 0.014184 & & \\ \text { C. Total } & 25 & 0.36351154 & & & \end{array}$

Means for Oneway Anova

Level Number Mean Std Error Lower 95\% Upper 95\%

$\begin{array}{llrrrr}\text { Coliwasa } & 16 & 26.7738 & 0.02977 & 26.712 & 26.835\end{array}$

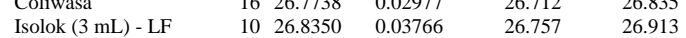

Std Error uses a pooled estimate of error variance

Means and Std Deviations

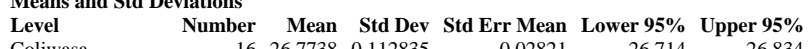

$\begin{array}{lrrrrrr}\text { Coliwasa } & 16 & 26.7738 & 0.112835 & 0.02821 & 26.714 & 26.834 \\ \text { Isolok (3 mL) - LF } & 10 & 26.8350 & 0.128863 & 0.04075 & 26.743 & 26.927\end{array}$

t Test

Isolok (3 mL) - LF-Coliwasa

Assuming unequal variances

Difference $\quad 0.06125$ t Ratio 1.235853

Std Err Dif $\quad 0.04956$ DF 17.30757

Upper CL Dif 0.16567 Prob $>|t| \quad 0.2330$

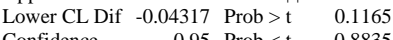

$\begin{array}{lll}\text { Confidence } & 0.95 \text { Prob }<\mathrm{t} & 0.8835\end{array}$

Tests that the Variances are Equal

$\begin{array}{lrrrr}\text { Level } & \text { Count } & \text { Std Dev } & \text { MeanAbsDif to Mean } & \text { MeanAbsDif to Median } \\ \text { Coliwasa } & 16 & 0.1128347 & 0.0912500 & 0.0912500 \\ \text { Isolok (3 mL) - LF } & 10 & 0.1288625 & 0.0940000 & 0.0930000\end{array}$

Test F Ratio DFNum DFDen p-Value

$\begin{array}{lllll}\text { O'Brien[.5] } & 0.2642 & 1 & 24 & 0.6120\end{array}$

Brown-Forsythe $0.0037 \quad 1 \quad 240.952$

$\begin{array}{lll}1 & 24 & 0.9520 \\ -1 & 24 & 0.9236\end{array}$

$\begin{array}{lllll}\text { Levene } & 0.0094 & 1 & 24 & 0.9236 \\ \text { Bartlett } & 0.1936 & 1 & & 0.6600\end{array}$

$\begin{array}{lllll}\text { F Test 2-sided } & 1.3043 & 9 & 15 & 0.6237\end{array}$

Welch Anova testing Means Equal, allowing Std Devs Not Equal

F Ratio DFNum DFDen Prob $>$ F

$\begin{array}{llll}1.5273 & 1 & 17.308 & 0.2330\end{array}$

t Test
1.2359
Oneway Analysis of Measurement By Sampling Description Test Phase=Phase 3 - Low Rheology, Analyte $=w t \%$ dried solids

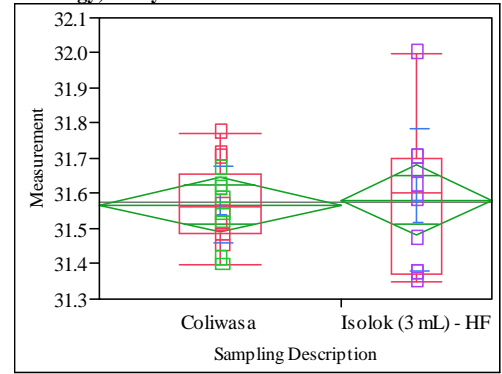

Oneway Anova

Summary of Fit

Rsquare

Adj Rsquare

$-0.039$

Root Mean Square Error $\quad 0.150241$

Mean of Response 31.57385

Observations (or Sum Wgts) 26

t Test

Isolok (3 mL) - HF-Coliwasa

Assuming equal variances

Difference $\quad 0.01325$ t Ratio $\quad 0.218777$

$\begin{array}{lrlr}\text { Std Err Dif } & 0.06056 & \text { DF } & 24 \\ \text { Upper CL Dif } & 0.13825 & \text { Prob }>|t| & 0.8287\end{array}$

$\begin{array}{lrll}\text { Upper CL Dif } & 0.13825 & \text { Prob }>|t| & 0.8287 \\ \text { Cower CL Dif } & -0.11175 & \text { Prob }>t & 0.4143\end{array}$

$\begin{array}{lrll}\text { Lower CL Dif } & -0.11175 & \text { Prob }>t & 0.4143 \\ \text { Confidence } & 0.95 & \text { Prob }<t & 0.5857\end{array}$

$\begin{array}{lrrrrr}\text { Analysis of Variance } & & & & & \\ \text { Source } & \text { DF } & \text { Sum of Squares } & \text { Mean Square } & \text { F Ratio } & \text { Prob }>\text { F } \\ \text { Sampling Description } & 1 & 0.00108038 & 0.001080 & 0.0479 & 0.8287 \\ \text { Error } & 24 & 0.54173500 & 0.022572 & & \\ \text { C. Total } & 25 & 0.54281538 & & & \end{array}$

C. Tota

Means for Oneway Anova

Level Number Mean Std Error Lower 95\% Upper 95\%

$\begin{array}{llllll}\text { Coliwasa } & 16 & 31.5688 & 0.03756 & 31.491 & 31.646\end{array}$

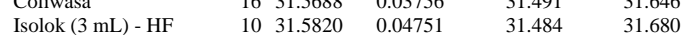

Std Error uses a pooled estimate of error variance

Means and Std Deviations

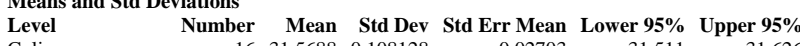

$\begin{array}{lrrrrrr}\text { Coliwasa } & 16 & 31.5688 & 0.108128 & 0.02703 & 31.511 & 31.626 \\ \text { Isolok }(3 \mathrm{~mL}) \text { - HF } & 10 & 31.5820 & 0.201759 & 0.06380 & 31.438 & 31.726\end{array}$

t Test

Isolok (3 mL) - HF-Coliwasa

Assuming unequal variances

Difference $\quad 0.01325$ t Ratio 0.191219

Std Err Dif $\quad 0.06929$ DF 12.28371

Upper CL Dif $\quad 0.16384$ Prob $>|t| \quad 0.8515$

Lower CL Dif -0.13734 Prob $>t \quad 0.4257$

$\begin{array}{lll}\text { Confidence } & 0.95 \text { Prob }<\mathrm{t} \quad 0.5743\end{array}$

Tests that the Variances are Equal

$\begin{array}{lrrrr}\text { Level } & \text { Count } & \text { Std Dev } & \text { MeanAbsDif to Mean } & \text { MeanAbsDif to Median } \\ \text { Coliwasa } & 16 & 0.1081280 & 0.0862500 & 0.0862500 \\ \text { Isolok (3 mL) - HF } & 10 & 0.2017589 & 0.1540000 & 0.1540000\end{array}$

Test F Ratio DFNum DFDen p-Value

$\begin{array}{lllll}\text { O'Brien[.5] } & 3.3538 & 1 & 24 & 0.0795\end{array}$

$\begin{array}{lllll}\text { Brown-Forsythe } & 3.5677 & 1 & 24 & 0.0711\end{array}$

$\begin{array}{lllll}\text { Levene } & 3.6536 & 1 & 24 & 0.0680\end{array}$

$\begin{array}{lllrl}\text { Bartlett } & 4.3627 & 1 & \text {. } & 0.0367 \\ \text { F Test 2-sided } & 3.4817 & 9 & 15 & 0.0323\end{array}$

Welch Anova testing Means Equal, allowing Std Devs Not Equal

F Ratio DFNum DFDen Prob $>$ F

$\begin{array}{llll}0.0366 & 1 & 12.284 & 0.8515\end{array}$

t Test 


\section{Exhibit C2. Statistical Comparisons for Low-Rheology (Phase 3) Simulant Testing}

Oneway Analysis of Measurement By Sampling Description Test Phase=Phase 3 - Low Rheology, Analyte $=$ wt $\%$ vitrified solids

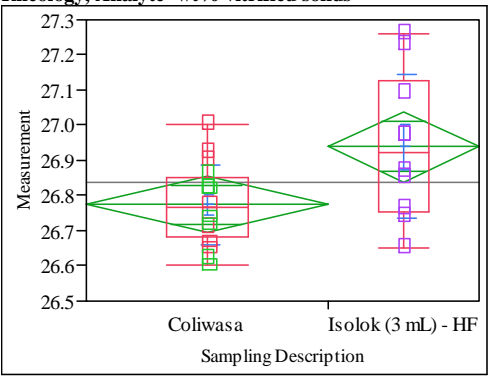

Oneway Anova

Summary of Fit

Rsquare

Adj Rsquare

Merror $\quad 0.15428$

Observations (or Sum Wgts) 26.8373

t Test

Isolok (3 mL) - HF-Coliwasa

Assuming equal variances

Difference $\quad 0.165250$ t Ratio 2.657061

$\begin{array}{lrlr}\text { Std Err Dif } & 0.062193 \text { DF } & 24 \\ \text { Upper CL Dif } & 0.293610 \text { Prob }>|t| & 0.0138\end{array}$

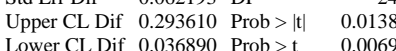

$\begin{array}{lrll}\text { Lower CL Dif } & 0.036890 & \text { Prob }>t & 0.0069 \\ \text { Confidence } & 0.95 & \text { Prob }<t & 0.9931\end{array}$

$\begin{array}{lrrrrr}\begin{array}{l}\text { Analysis of Variance } \\ \text { Source }\end{array} & \text { DF } & \text { Sum of Squares } & \text { Mean Square } & \text { F Ratio } & \text { Prob > F } \\ \text { Sampling Description } & 1 & 0.16804654 & 0.168047 & 7.0600 & 0.0138 \\ \text { Error } & 24 & 0.57126500 & 0.023803 & & \\ \text { C. Total } & 25 & 0.73931154 & & & \end{array}$

Means for Oneway Anova

Level Number Mean Std Error Lower 95\% Upper 95\%

$\begin{array}{llrrrr}\text { Coliwasa } & 16 & 26.7738 & 0.03857 & 26.694 & 26.853\end{array}$

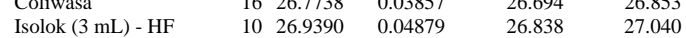

Std Error uses a pooled estimate of error variance

Means and Std Deviations

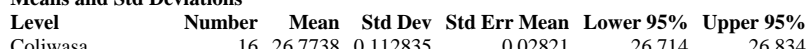

$\begin{array}{lrrrrrr}\text { Coliwasa } & 16 & 26.7738 & 0.112835 & 0.02821 & 26.714 & 26.834 \\ \text { Isolok (3 mL) - HF } & 10 & 26.9390 & 0.205559 & 0.06500 & 26.792 & 27.086\end{array}$

t Test

Isolok (3 mL) - HF-Coliwasa

Assuming unequal variances

Difference $\quad 0.165250$ t Ratio $\quad 2.332055$

Std Err Dif $\quad 0.070860$ DF 12.44412

Upper CL Dif 0.319032 Prob $>|t| \quad 0.0372$

Lower CL Dif 0.011468 Prob $>t \quad 0.0186$

$\begin{array}{lll}\text { Confidence } & 0.95 \text { Prob }<\mathrm{t} \quad 0.9814\end{array}$

Tests that the Variances are Equal

$\begin{array}{lrrrr}\text { Level } & \text { Count } & \text { Std Dev } & \text { MeanAbsDif to Mean } & \text { MeanAbsDif to Median } \\ \text { Coliwasa } & 16 & 0.1128347 & 0.0912500 & 0.0912500 \\ \text { Isolok (3 mL) - HF } & 10 & 0.2055589 & 0.1650000 & 0.1650000\end{array}$

$\begin{array}{lrrrr}\text { Test } & \text { F Ratio } & \text { DFNum } & \text { DFDen } & \text { p-Value } \\ \text { O'Brien[.5] } & 5.7356 & 1 & 24 & 0.0248 \\ \text { Brown-Forsythe } & 4.7080 & 1 & 24 & 0.0401 \\ \text { Levene } & 4.8453 & 1 & 24 & 0.0376 \\ \text { Bartlett } & 4.0372 & 1 & . & 0.0445 \\ \text { F Test 2-sided } & 3.3188 & 9 & 15 & 0.0393\end{array}$

Welch Anova testing Means Equal, allowing Std Devs Not Equal

F Ratio DFNum DFDen Prob $>$ F

$\begin{array}{llll}5.4385 & 1 & 12.444 & 0.0372\end{array}$

t Test
Oneway Analysis of Measurement By Sampler Type of Material=SME Simulant, Analytical Block $=1$, Oxide $/$ Analyte $=A \mathrm{l} / \mathrm{B}$, Targeted $=2.552$

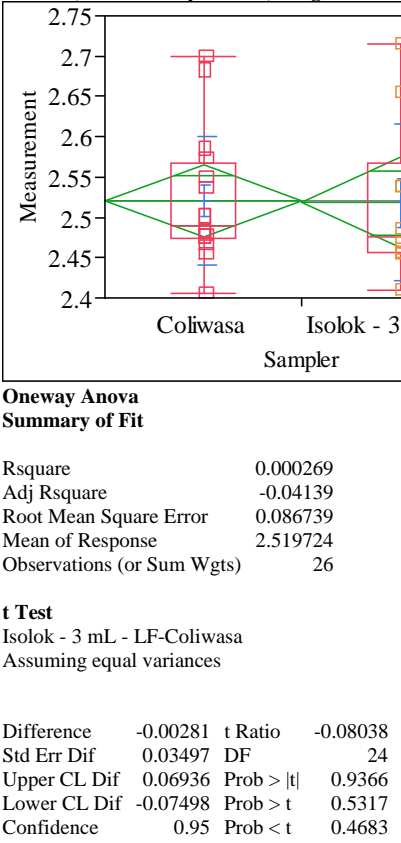

Analysis of Variance

Source DF Sum of Squares Mean Square F Ratio Prob $>$ F

$\begin{array}{llllll}\text { Sampler } & 1 & 0.00004861 & 0.000049 & 0.0065 & 0.9366\end{array}$

$\begin{array}{llll}\text { Error } & 24 & 0.18056764 & 0.007524\end{array}$

C. Total $25 \quad 0.18061625$

Means for Oneway Anova

Level Number Mean Std Error Lower 95\% Upper 95\%

$\begin{array}{lrrrr}\text { Coliwasa } & 16 & 2.52080 & 0.02168 & 2.4760\end{array}$

$\begin{array}{llllll}\text { Isolok - 3 mL - LF } & 16 & 2.52080 & 0.02168 & 2.4760 & 2.5656 \\ & 10 & 2.51799 & 0.02743 & 2.4614 & 2.5746\end{array}$

Std Error uses a pooled estimate of error variance

Means and Std Deviations

Level $\quad$ Number Mean Std Dev Std Err Mean Lower 95\% Upper 95\%

$\begin{array}{lllllll}\text { Coliwasa } & 16 & 2.52080 & 0.079897 & 0.01997 & 2.4782 & 2.5634\end{array}$

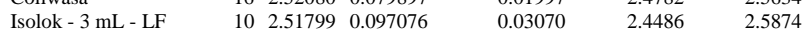

Test

Isolok - $3 \mathrm{~mL}$ - LF-Coliwasa

Assuming unequal variances

$\begin{array}{lll}\text { Difference } & -0.00281 \text { t Ratio } & -0.07674 \\ \text { Std } & & \end{array}$

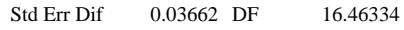

Upper CL Dif 0.07465 Prob $>|t| 0.9398$

Lower CL Dif -0.08027 Prob $>\mathrm{t} \quad 0.5301$

$\begin{array}{lrr}\text { Confidence } & 0.95 \text { Prob }<\mathrm{t} & 0.4699\end{array}$

Tests that the Variances are Equal

Level Count Std Dev MeanAbsDif to Mean MeanAbsDif to Median

$\begin{array}{lllll}\text { Coliwasa } & 16 & 0.0798975 & 0.0619843 & 0.0564631\end{array}$

Test F Ratio DFNum DFDen p-Value

$\begin{array}{lrrrr}\text { O'Brien[.5] } & 0.3946 & 1 & 24 & 0.5358\end{array}$

$\begin{array}{lllll}\text { Brown-Forsythe } & 0.1768 & 1 & 24 & 0.6779\end{array}$

$\begin{array}{lllll}\text { Levene } & 0.3879 & 1 & 24 & 0.5393\end{array}$

$\begin{array}{lllrl}\text { Bartlett } & 0.4191 & 1 & \text {. } & 0.5174 \\ \text { F Test 2-sided } & 1.4762 & 9 & 15 & 0.4851\end{array}$

Welch Anova testing Means Equal, allowing Std Devs Not Equal

F Ratio DFNum DFDen Prob $>$ F

$\begin{array}{llrr}0.0059 & 1 & 16.463 & 0.9398\end{array}$

t Test

0.0767 


\section{Exhibit C2. Statistical Comparisons for Low-Rheology (Phase 3) Simulant Testing}

Oneway Analysis of Measurement By Sampler Type of Material=SME Simulant, Analytical Block=1, Oxide/Analyte=Al2O3 (wt \%), Targeted=10.869

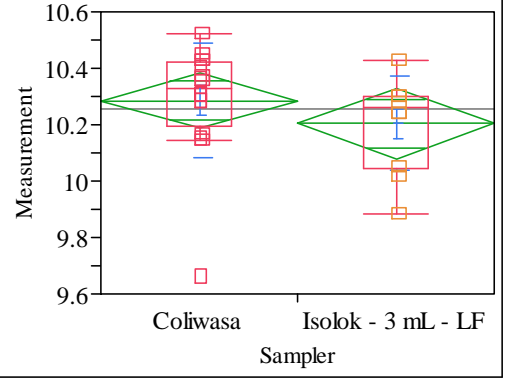

Oneway Anova

Summary of Fit

Adj Rsquare

0.188546
0.25417

10.25417

t Test

Isolok - $3 \mathrm{~mL}$ - LF-Coliwasa

Assuming equal variances

Difference $\quad-0.08267$ t Ratio $\quad-1.08763$

Std Err Dif $\quad 0.07601$ DF 24

Upper CL Dif $\quad 0.07420$ Prob $>|t| \quad 0.2876$

Lower CL Dif -0.23953 Prob $>\mathrm{t} \quad 0.8562$

$\begin{array}{lrr}\text { Confidence } & 0.95 \text { Prob }<\mathrm{t} & 0.1438\end{array}$

Analysis of Variance

Source DF Sum of Squares Mean Square F Ratio Prob $>$ F

$\begin{array}{lrllll}\text { Sampler } & 1 & 0.04205296 & 0.042053 & 1.1829 & 0.2876\end{array}$

$\begin{array}{llll}\text { Error } & 24 & 0.85319099 & 0.035550\end{array}$

C. Total $25 \quad 0.89524395$

Means for Oneway Anova

Level Number Mean Std Error Lower 95\% Upper 95\%

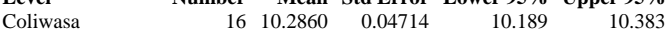

$\begin{array}{llllll}\text { Isolok - 3 mL - LF } & 10 & 10.2860 & 0.04714 & 10.189 & 10.383 \\ & 10 & 10.2033 & 0.05962 & 10.080 & 10.326\end{array}$

Std Error uses a pooled estimate of error variance

Means and Std Deviations

Level Number Mean Std Dev Std Err Mean Lower 95\% Upper 95\%

$\begin{array}{llllll}\text { Coliwasa } & 16 & 10.2860 & 0.200783 & 0.05020 & 10.179\end{array}$

$\begin{array}{llllllr}\text { Isolok - 3 mL - LF } & 10 & 10.2033 & 0.166161 & 0.05254 & 10.084 & 10.322\end{array}$

t Test

Isolok - $3 \mathrm{~mL}$ - LF-Coliwasa

Assuming unequal variances

Difference $\quad-0.08267$ t Ratio $\quad-1.13759$

Std Err Dif $\quad 0.07267$ DF 21.95244

Upper CL Dif 0.06806 Prob $>|t| \quad 0.2676$

Lower CL Dif -0.23339 Prob $>t \quad 0.8662$

$\begin{array}{lrl}\text { Confidence } & 0.95 \text { Prob }<\mathrm{t} & 0.1338\end{array}$

Tests that the Variances are Equal

Level Count Std Dev MeanAbsDif to Mean MeanAbsDif to Median

$\begin{array}{lllll}16 & 0.2007825 & 0.1304936 & 0.1275412\end{array}$

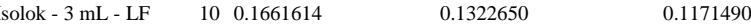

$\begin{array}{lrrrr}\text { Test } & \text { F Ratio } & \text { DFNum } & \text { DFDen } & \text { p-Value } \\ \text { O'Brien[.5] } & 0.1287 & 1 & 24 & 0.7230 \\ \text { Brown-Forsythe } & 0.0311 & 1 & 24 & 0.8616 \\ \text { Levene } & 0.0011 & 1 & 24 & 0.9733 \\ \text { Bartlett } & 0.3716 & 1 & . & 0.5421 \\ \text { F Test 2-sided } & 1.4601 & 15 & 9 & 0.5755\end{array}$

Welch Anova testing Means Equal, allowing Std Devs Not Equal

F Ratio DFNum DFDen Prob $>$ F

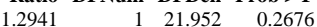

t Test

1.1376
Oneway Analysis of Measurement By Sampler Type of Material=SME Simulant, Analytical Block=1, Oxide/Analyte=B2O3 (wt $\%$ ), Targeted $=4.259$

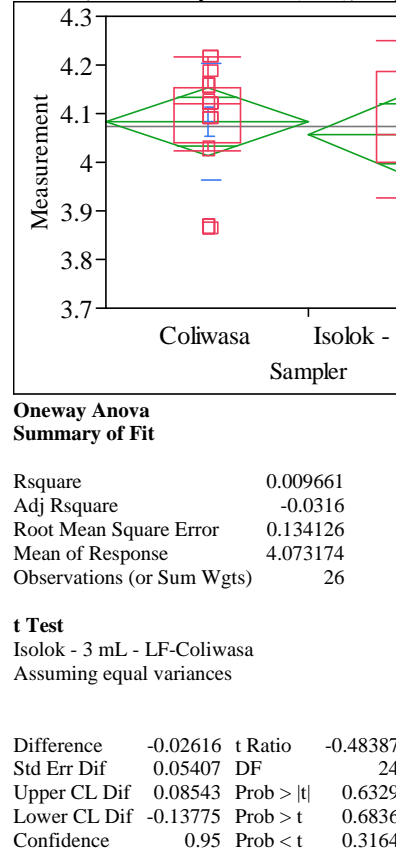

Analysis of Variance

Source DF Sum of Squares Mean Square F Ratio Prob $>$ F

$\begin{array}{lrrrrr}\text { Sampler } & 1 & 0.00421190 & 0.004212 & 0.2341 & 0.6329\end{array}$

$\begin{array}{llll}\text { Error } & 24 & 0.43175224 & 0.017990\end{array}$

C. Total $25 \quad 0.43596414$

Means for Oneway Anova

Level Number Mean Std Error Lower 95\% Upper 95\%

$\begin{array}{llllrr}\text { Coliwasa } & 16 & 4.08324 & 0.03353 & 4.0140 & 4.1524\end{array}$

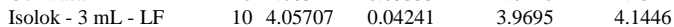

Std Error uses a pooled estimate of error variance

Means and Std Deviations

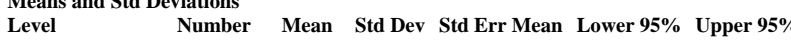

$\begin{array}{lrrrrrr}\text { Coliwasa } & 16 & 4.08324 & 0.119451 & 0.02986 & 4.0196 & 4.1469\end{array}$

$\begin{array}{llllll}\text { Isolok - } 3 \text { mL - LF } & 10 & 4.05707 & 0.155536 & 0.04918 & 3.9458\end{array}$

t Test

Isolok - $3 \mathrm{~mL}$ - LF-Coliwasa

Assuming unequal variances

Difference $\quad-0.02616$ t Ratio $\quad-0.45466$

Std Err Dif $\quad 0.05754$ DF 15.58758

Upper CL Dif 0.09608 Prob $>|t| 0.6556$

Lower CL Dif -0.14841 Prob $>\mathrm{t} \quad 0.6722$

$\begin{array}{lll}\text { Confidence } & 0.95 \text { Prob }<\mathrm{t} \quad 0.3278\end{array}$

Tests that the Variances are Equal

$\begin{array}{lrrrr}\text { Level } & \text { Count } & \text { Std Dev } & \text { MeanAbsDif to Mean } & \text { MeanAbsDif to Median } \\ \text { Coliwasa } & 16 & 0.1194513 & 0.0895535 & 0.0825099\end{array}$

$\begin{array}{lllll}\text { Coliwasa } & 16 & 0.1194513 & 0.0895535 & 0.0825099 \\ \text { Isolok - 3 mL - LF } & 10 & 0.1555359 & 0.1030368 & 0.1030368\end{array}$

Test F Ratio DFNum DFDen p-Value

$\begin{array}{lllll}\text { O'Brien[.5] } & 0.5940 & 1 & 24 & 0.4484\end{array}$

Brown-Forsythe $0.2592 \quad-1 \quad 24 \quad 0.6153$

$\begin{array}{lllll}0 & 1 & 24 & 0.6153\end{array}$

$\begin{array}{lllll}\text { Levene } & 0.1361 & 1 & 24 & 0.7154 \\ \text { Bartlett } & 0.7751 & 1 & & 0.3787\end{array}$

$\begin{array}{lllll}\text { F Test 2-sided } & 1.6954 & 9 & 15 & 0.3517\end{array}$

Welch Anova testing Means Equal, allowing Std Devs Not Equal

F Ratio DFNum DFDen Prob $>$ F

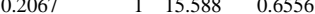

t Test 
SRNL-STI-2011-00693

Revision 0

\section{Exhibit C2. Statistical Comparisons for Low-Rheology (Phase 3) Simulant Testing}

Oneway Analysis of Measurement By Sampler Type of Material=SME Simulant, Analytical Block $=1$, Oxide $/$ Analyte $=\mathbf{B a O}(w t \%)$, Targeted $=0.0919$

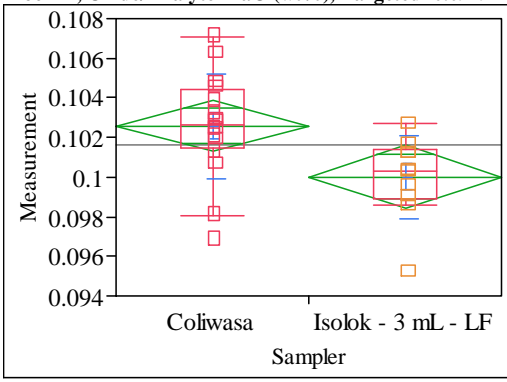

Oneway Anova

Rsquare $\quad 0.219512$

Adj Rsquare $\quad 0.18699$

Root Mean Square Error $\quad 0.002457$

Mean of Response 0.101589

t Test

Isolok - $3 \mathrm{~mL}$ - LF-Coliwasa

Assuming equal variances

Difference $\quad-0.00257$ t Ratio -2.59807

$\begin{array}{lrr}\text { Std Err Dif } & 0.00099 \text { DF } & 24 \\ \text { Upper CL Dif } & -0.00053 \text { Prob }>|t| & 0.0158\end{array}$

$\begin{array}{rrrr}\text { Upper CL Dif } & -0.00053 & \text { Prob }>|t| & 0.0158 \\ \text { Lower CL Dif } & -0.00462 & \text { Prob }>t & 0.9921\end{array}$

$\begin{array}{lrl}\text { Confidence } & 0.95 \text { Prob }<\mathrm{t} & 0.0079\end{array}$

Analysis of Variance

Source DF Sum of Squares Mean Square F Ratio Prob $>$ F

$\begin{array}{lrrrrr}\text { Sampler } & 1 & 0.00004076 & 0.000041 & 6.7500 & 0.0158\end{array}$

$\begin{array}{llll}\text { Error } & 24 & 0.00014492 & 6.038 \mathrm{e}-6\end{array}$

C. Total $25 \quad 0.0001856$

Means for Oneway Anova

Level Number Mean Std Error Lower 95\% Upper 95\%

$\begin{array}{lllrrr}\text { Coliwasa } & 16 & 0.102578 & 0.00061 & 0.10131 & 0.10385\end{array}$

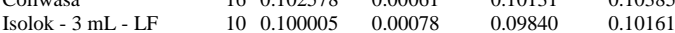

Std Error uses a pooled estimate of error variance

Means and Std Deviations

$\begin{array}{lrrrrrr}\text { Level } & \text { Number } & \text { Mean } & \text { Std Dev } & \text { Std Err Mean } & \text { Lower 95\% } & \text { Upper 95\% } \\ \text { Coliwasa } & 16 & 0.102578 & 0.002651 & 0.00066 & 0.10117 & 0.10399\end{array}$

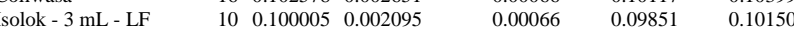

t Test

Isolok - $3 \mathrm{~mL}$ - LF-Coliwasa

Assuming unequal variances

Difference $\quad-0.00257$ t Ratio $\quad-2.74614$

Std Err Dif $\quad 0.00094$ DF 22.50093

Upper CL Dif -0.00063 Prob $>|t| \quad 0.0116$

Lower CL Dif -0.00451 Prob $>t \quad 0.9942$

$\begin{array}{lrl}\text { Confidence } & 0.95 \text { Prob }<\mathrm{t} & 0.0058\end{array}$

Tests that the Variances are Equal

$\begin{array}{lrrrr}\text { Level } & \text { Count } & \text { Std Dev } & \text { MeanAbsDif to Mean } & \text { MeanAbsDif to Median } \\ \text { Coliwasa } & 16 & 0.0026508 & 0.0018701 & 0.0018701 \\ \text { Isolok - 3 mL - LF } & 10 & 0.0020954 & 0.0015140 & 0.0014626\end{array}$

Test F Ratio DFNum DFDen p-Value

O'Brien[.5] $\quad 0.4198 \quad 1 \quad 24 \quad 0.5232$

O'Brien[.5] 0.4198

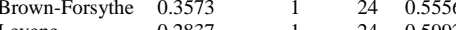

$\begin{array}{lllll}\text { Levene } & 0.2837 & 1 & 24 & 0.5992 \\ \text { Bartlett } & 0.5675 & 1 & . & 0.4513\end{array}$

$\begin{array}{lrrrr}\text { F Test 2-sided } & 1.6003 & 15 & \dot{9} & 0.4815\end{array}$

Welch Anova testing Means Equal, allowing Std Devs Not Equal

F Ratio DFNum DFDen Prob $>$ F

$\begin{array}{llll}7.5413 & 1 & 22.501 & 0.0116\end{array}$

t Test

2.7461
Oneway Analysis of Measurement By Sampler Type of Material=SME Simulant, Analytical Block $=1$, Oxide/Analyte $=\mathrm{CaO}(\mathrm{wt} \%)$, Targeted $=\mathbf{1 . 1 4 6 2}$

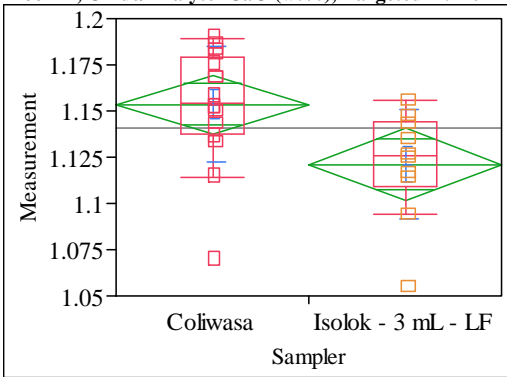

Oneway Anova

Summary of Fit

Rsquare

Adj Rsquare

Root Mean Square Error $\quad 0.030479$

Mean of Response 1.141155

Test

Isolok - $3 \mathrm{~mL}$ - LF-Coliwasa

Assuming equal variances

Difference $\quad-0.03246$ t Ratio $\quad-2.64205$

$\begin{array}{lrrr}\text { Std Err Dif } & 0.01229 \text { DF } & 24 \\ \text { Upper CL Dif } & -0.00710 \text { Prob }>|t| & 0.0143\end{array}$

$\begin{array}{llll}\text { Upper CL Dif } & -0.00710 & \text { Prob }>|t| & 0.0143 \\ \text { Lower CL Dif }-0.05782 & \text { Prob }>t & 0.9929\end{array}$

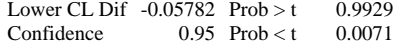

Analysis of Variance

Source DF Sum of Squares Mean Square F Ratio Prob $>$ F

$\begin{array}{llllll}\text { Sampler } & 1 & 0.00648459 & 0.006485 & 6.9804 & 0.0143\end{array}$

$\begin{array}{lll}\text { Error } & 24 & 0.02229517 \\ \text { C. Total } & 25 & 0.02877976\end{array}$

Means for Oneway Anova

Level Number Mean Std Error Lower 95\% Upper 95\%

$\begin{array}{lrrrrr}\text { Coliwasa } & 16 & 1.15364 & 0.00762 & 1.1379 & 1.1694\end{array}$

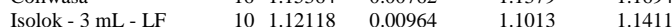

Std Error uses a pooled estimate of error variance

Means and Std Deviations

Level Number Mean Std Dev Std Err Mean Lower 95\% Upper 95\%

$\begin{array}{lllllll}\text { Coliwasa } & 16 & 1.15364 & 0.031116 & 0.00778 & 1.1371 & 1.1702\end{array}$

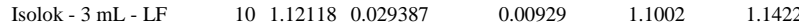

t Test

Isolok - $3 \mathrm{~mL}$ - LF-Coliwasa

Assuming unequal variances

Difference $\quad-0.03246$ t Ratio $\quad-2.67854$

Std Err Dif $\quad 0.01212$ DF 20.10761

Upper CL Dif -0.00719 Prob $>|t| \quad 0.0144$

Lower CL Dif -0.05773 Prob $>\mathrm{t} \quad 0.9928$

$\begin{array}{lrl}\text { Confidence } & 0.95 \text { Prob }<\mathrm{t} \quad 0.0072\end{array}$

Tests that the Variances are Equal

$\begin{array}{lrrrr}\text { Level } & \text { Count } & \text { Std Dev } & \text { MeanAbsDif to Mean } & \text { MeanAbsDif to Median } \\ \text { Coliwasa } & 16 & 0.0311156 & 0.0225621 & 0.0225621 \\ \text { Isolok - 3 mL - LF } & 10 & 0.0293873 & 0.0210440 & 0.0202884\end{array}$

Test F Ratio DFNum DFDen p-Value

$\begin{array}{lllll}\text { O'Brien[.5] } & 0.0208 & 1 & 24 & 0.8865\end{array}$

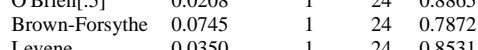

$\begin{array}{lllll}\text { Levene } & 0.0350 & 1 & 24 & 0.8531 \\ \text { Bartlett } & 0.0348 & 1 & & 0.8520\end{array}$

$\begin{array}{lrrrr}\text { Bartlett } & 0.0348 & 1 & \dot{0} & 0.8520 \\ \text { F Test 2-sided } & 1.1211 & 15 & 9 & 0.8915\end{array}$

Welch Anova testing Means Equal, allowing Std Devs Not Equal

F Ratio DFNum DFDen Prob $>$ F

$\begin{array}{llll}7.1746 & 1 & 20.108 & 0.0144\end{array}$

t Test

2.6785 
SRNL-STI-2011-00693

Revision 0

\section{Exhibit C2. Statistical Comparisons for Low-Rheology (Phase 3) Simulant Testing}

Oneway Analysis of Measurement By Sampler Type of Material=SME Simulant, Analytica Block $=1$, Oxide $/$ Analyte $=$ Cr2O3 $($ wt $\%)$, Targeted $=0.0998$

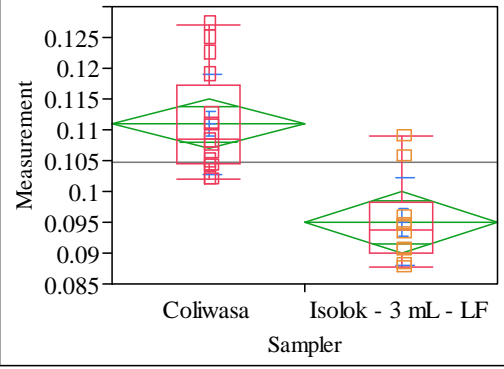

Oneway Anova

Rsquare $\quad 0.519586$

Adj Rsquare $\quad 0.49956$

Root Mean Square Error $\quad 0.007736$

Mean of Response 0.104825

Observations (or Sum Wgs)

Isolok - $3 \mathrm{~mL}$ - LF-Coliwasa

Assuming equal variances

Difference $\quad-0.01589$ t Ratio $\quad-5.09479$

$\begin{array}{lrr}\text { Std Err Dif } & 0.00312 \text { DF } & 24 \\ \text { Upper CL Dif } & -0.00945 \text { Prob }>|t| & <.0001\end{array}$

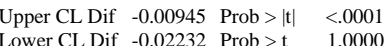

$\begin{array}{lrl}\text { Confidence } & 0.95 \text { Prob }<\mathrm{t} & <.0001\end{array}$

Analysis of Variance

Source DF Sum of Squares Mean Square F Ratio Prob $>$ F

$\begin{array}{lllllll}\text { Sampler } & 1 & 0.00155333 & 0.001553 & 25.9569 & <.0001\end{array}$

Error $24 \quad 0.00143622$

C. Total $25 \quad 0.00298955$

Means for Oneway Anova

Level Number Mean Std Error Lower 95\% Upper 95\%

$\begin{array}{llrrrr}\text { Coliwasa } & 16 & 0.110935 & 0.00193 & 0.10694 & 0.11493\end{array}$

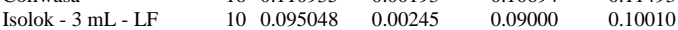

Std Error uses a pooled estimate of error variance

Means and Std Deviations

$\begin{array}{lrrrrrr}\text { Level } & \text { Number } & \text { Mean } & \text { Std Dev } & \text { Std Err Mean } & \text { Lower 95\% } & \text { Upper 95\% } \\ \text { Coliwasa } & 16 & 0.110935 & 0.008088 & 0.00202 & 0.10663 & 0.11525\end{array}$

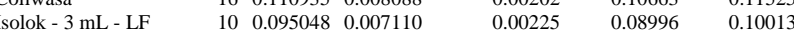

Test

Isolok - $3 \mathrm{~mL}$ - LF-Coliwasa

Assuming unequal variances

Difference $\quad-0.01589$ t Ratio $\quad-5.25395$

Std Err Dif $\quad 0.00302$ DF 21.14456

Upper CL Dif -0.00960 Prob $>|t| \quad<.0001$

Lower CL Dif -0.02217 Prob $>t \quad 1.0000$

$\begin{array}{lll}\text { Confidence } & 0.95 \text { Prob }<\mathrm{t} \quad<.0001\end{array}$

Tests that the Variances are Equal

$\begin{array}{lrrrr}\text { Level } & \text { Count } & \text { Std Dev } & \text { MeanAbsDif to Mean } & \text { MeanAbsDif to Median } \\ \text { Coliwasa } & 16 & 0.0080878 & 0.0063580 & 0.0061022 \\ \text { Isolok - 3 mL - LF } & 10 & 0.0071105 & 0.0051185 & 0.0049548\end{array}$

Test F Ratio DFNum DFDen p-Value

O'Brien[.5] 0.2127

$\begin{array}{lllll} & 0.2127 & 1 & 24 & 0.6488 \\ \text { O'Brien[.5] } & 0.2789 & 1 & 24 & 0.6023\end{array}$

$\begin{array}{lllll}\text { Brown-Forsythe } & 0.2789 & 1 & 24 & 0.6023\end{array}$

$\begin{array}{lllll}\text { Levene } & 0.4302 & 1 & 24 & 0.5182 \\ \text { Bartlett } & 0.1743 & 1 & . & 0.6763\end{array}$

$\begin{array}{llrrr}\text { F Test 2-sided } & 1.2938 & 15 & 9 & 0.7132\end{array}$

Welch Anova testing Means Equal, allowing Std Devs Not Equal

F Ratio DFNum DFDen Prob $>$ F

$\begin{array}{llll}27.6040 & 1 & 21.145 & <.0001\end{array}$

t Test

5.2540
Oneway Analysis of Measurement By Sampler Type of Material=SME Simulant, Analytical Block $=1$, Oxide/Analyte $=\mathrm{CuO}(\mathrm{wt} \%)$, Targeted $=\mathbf{0 . 0 5 0 4}$

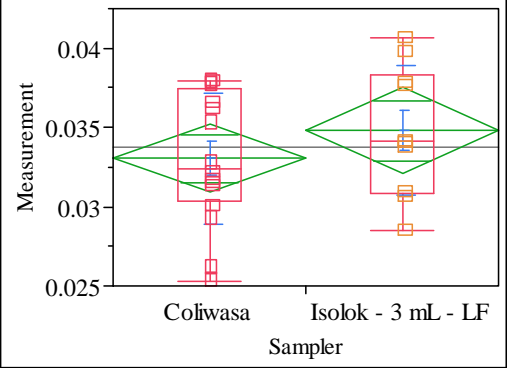

Oneway Anova

Summary of Fit

Rsquare

Adj Rsquare

Root Mean Square Error $\quad 0.004122$

$\begin{array}{lr}\text { Mean of Response } & 0.033731 \\ \text { Observations (or Sum Wgts) } & 26\end{array}$

t Test

Isolok - $3 \mathrm{~mL}$ - LF-Coliwasa

Assuming equal variances

Difference $\quad 0.00176$ t Ratio 1.057466

$\begin{array}{lrlr}\text { Std Err Dif } & 0.00166 & \text { DF } & 24 \\ \text { Upper } & & 24\end{array}$

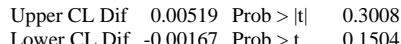

$\begin{array}{lrll}\text { Lower CL Dif } & -0.00167 & \text { Prob }>t & 0.1504 \\ \text { Confidence } & 0.95 & \text { Prob }<t & 0.8496\end{array}$

Analysis of Variance

Source DF Sum of Squares Mean Square F Ratio Prob $>$ F

$\begin{array}{llllll}\text { Sampler } & 1 & 0.00001900 & 0.000019 & 1.1182 & 0.3008\end{array}$

$\begin{array}{llll}\text { Error } & 24 & 0.00040783 & 0.000017\end{array}$

C. Total $25 \quad 0.00042683$

Means for Oneway Anova

Level Number Mean Std Error Lower 95\% Upper 95\%

$\begin{array}{lrrrrr}\text { Coliwasa } & 16 & 0.033055 & 0.00103 & 0.03093 & 0.03518\end{array}$

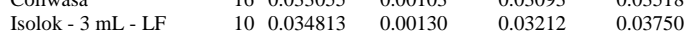

Std Error uses a pooled estimate of error variance

Means and Std Deviations

Level Number Mean Std Dev Std Err Mean Lower 95\% Upper 95\%

$\begin{array}{llllrrr}\text { Coliwasa } & 16 & 0.033055 & 0.004144 & 0.00104 & 0.03085 & 0.03526 \\ \text { Isolok - } 3 \mathrm{~mL}-\mathrm{LF} & 10 & 0.034813 & 0.004086 & 0.00129 & 0.03189 & 0.03774\end{array}$

t Test

Isolok - $3 \mathrm{~mL}$ - LF-Coliwasa

Assuming unequal variances

Difference $\quad 0.00176$ t Ratio 1.061043

Std Err Dif $\quad 0.00166$ DF 19.46389

Upper CL Dif 0.00522 Prob $>|t| \quad 0.3017$

Lower CL Dif -0.00170 Prob $>t \quad 0.1508$

$\begin{array}{lll}\text { Confidence } & 0.95 \text { Prob }<\mathrm{t} \quad 0.8492\end{array}$

Tests that the Variances are Equal

$\begin{array}{lrrrr}\text { Level } & \text { Count } & \text { Std Dev } & \text { MeanAbsDif to Mean } & \text { MeanAbsDif to Median } \\ \text { Coliwasa } & 16 & 0.0041438 & 0.0034513 & 0.0034033 \\ \text { Isolok - 3 mL - LF } & 10 & 0.0040860 & 0.0033448 & 0.0032171\end{array}$

Test F Ratio DFNum DFDen p-Value

$\begin{array}{lllll}\text { O'Brien[.5] } & 0.0042 & 1 & 24 & 0.9489\end{array}$

$\begin{array}{lllll}\text { Brown-Forsythe } & 0.0393 & 1 & 24 & 0.8446\end{array}$

$\begin{array}{lllll}\text { Brown-Forsythe } & 0.0393 & 1 & 24 & 0.8446 \\ \text { Levene } & 0.0159 & 1 & 24 & 0.9008\end{array}$

$\begin{array}{lllll}\text { Levene } & 0.0159 & 1 & 24 & 0.9008 \\ \text { Bartlett } & 0.0021 & 1 & . & 0.9633\end{array}$

$\begin{array}{lllll}\text { F Test 2-sided } & 1.0285 & 15 & 9 & 1.0000\end{array}$

Welch Anova testing Means Equal, allowing Std Devs Not Equal

F Ratio DFNum DFDen Prob > F

$\begin{array}{llll}1.1258 & 1 & 19.464 & 0.3017\end{array}$

t Test

1.0610 


\section{Exhibit C2. Statistical Comparisons for Low-Rheology (Phase 3) Simulant Testing}

Oneway Analysis of Measurement By Sampler Type of Material=SME Simulant, Analytical Block $=1$, Oxide/Analyte $=\mathrm{Fe} / \mathrm{Li}$, Targeted $=2.452$

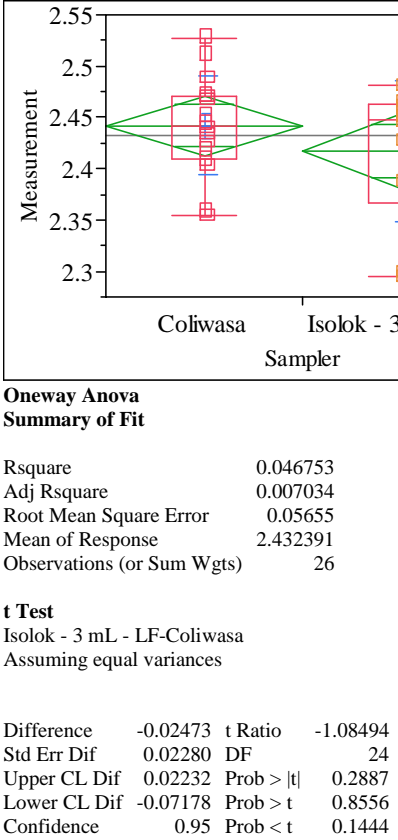

Analysis of Variance

Source DF Sum of Squares Mean Square F Ratio Prob $>$ F

$\begin{array}{lrllll}\text { Sampler } & 1 & 0.00376422 & 0.003764 & 1.1771 & 0.2887\end{array}$

$\begin{array}{llll}\text { Error } & 24 & 0.07674929 & 0.003198\end{array}$

C. Total $25 \quad 0.08051351$

Means for Oneway Anova

Level Number Mean Std Error Lower 95\% Upper 95\%

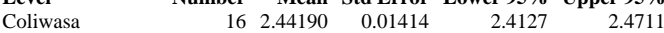

$\begin{array}{llllll}\text { Coliwasa } & 16 & 2.44190 & 0.01414 & 2.4127 & 2.4711 \\ \text { Isolok - 3 mL - LF } & 10 & 2.41717 & 0.01788 & 2.3803 & 2.4541\end{array}$

Std Error uses a pooled estimate of error variance

Means and Std Deviations

Level Number Mean Std Dev Std Err Mean Lower 95\% Upper 95\%

$\begin{array}{llllll}\text { Coliwasa } & 16 & 2.44190 & 0.048239 & 0.01206 & 2.4162\end{array}$

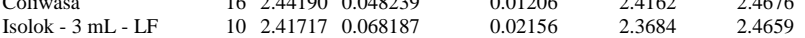

t Test

Isolok - $3 \mathrm{~mL}$ - LF-Coliwasa

Assuming unequal variances

Difference $\quad-0.02473$ t Ratio $\quad-1.00107$

Std Err Dif $\quad 0.02471$ DF 14.651

Upper CL Dif 0.02804 Prob $>|t| \quad 0.3330$

Lower CL Dif -0.07750 Prob $>\mathrm{t} \quad 0.8335$

$\begin{array}{lll}\text { Confidence } & 0.95 \text { Prob }<\mathrm{t} \quad 0.1665\end{array}$

Tests that the Variances are Equal

Level Count Std Dev MeanAbsDif to Mean MeanAbsDif to Median

$\begin{array}{lllll} & 16 & 0.0482388 & 0.0369253 & 0.0369253\end{array}$

Test F Ratio DFNum DFDen p-Value

$\begin{array}{lrrrr} & \text { F Ratio } & \text { DFNum } & \text { DFen } & \text { p-Value } \\ \text { O'Brien[.5] } & 1.5155 & 1 & 24 & 0.2302 \\ \text { Brown-Forsythe } & 0.2911 & 1 & 24 & 0.5945 \\ \text { Levene } & 1.6436 & 1 & 24 & 0.2121 \\ \text { Bartlett } & 1.3398 & 1 & . & 0.2471 \\ \text { F Test 2-sided } & 1.9980 & 9 & 15 & 0.2268\end{array}$

Welch Anova testing Means Equal, allowing Std Devs Not Equal

F Ratio DFNum DFDen Prob $>$ F

$\begin{array}{llll}1.0021 & 1 & 14.651 & 0.3330\end{array}$

t Test

1.0011
Oneway Analysis of Measurement By Sampler Type of Material=SME Simulant, Analytica Block $=1$, Oxide $/$ Analyte $=\mathrm{Fe} 2 \mathrm{O} 3$ ( $w$ t $\%)$, Targeted $=11.462$

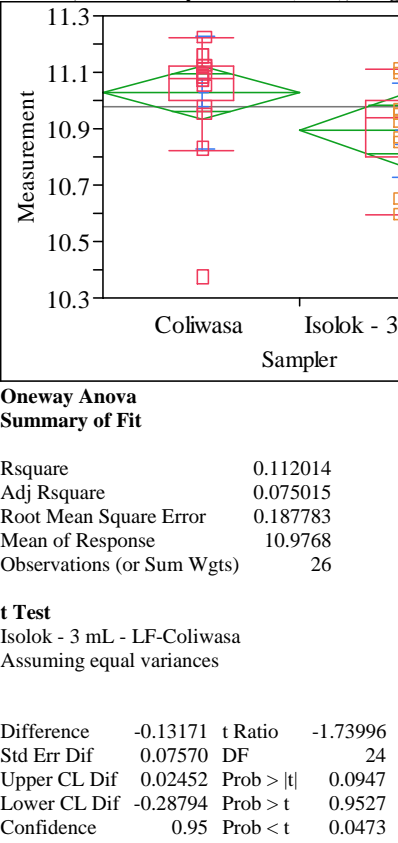

Analysis of Variance

Source DF Sum of Squares Mean Square F Ratio Prob $>$ F

$\begin{array}{llllll}\text { Sampler } & 1 & 0.10675580 & 0.106756 & 3.0275 & 0.0947\end{array}$

$\begin{array}{llll}\text { Error } & 24 & 0.84630241 & 0.035263\end{array}$

C. Total $25 \quad 0.95305821$

Means for Oneway Anova

Level Number Mean Std Error Lower 95\% Upper 95\%

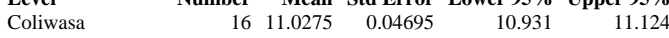

$\begin{array}{llllll}\text { Isolok - 3 mL - LF } & 16 & 11.0275 & 0.04695 & 10.931 & 11.124 \\ & 10 & 10.8957 & 0.05938 & 10.773 & 11.018\end{array}$

Std Error uses a pooled estimate of error variance

Means and Std Deviations

Level Number Mean Std Dev Std Err Mean Lower 95\% Upper 95\%

$\begin{array}{lllllll}\text { Coliwasa } & 16 & 11.0275 & 0.199320 & 0.04983 & 10.921 & 11.134\end{array}$

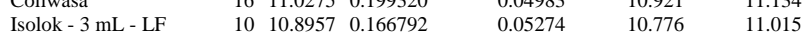

Test

Isolok - $3 \mathrm{~mL}$ - LF-Coliwasa

Assuming unequal variances

$\begin{array}{lrlr}\text { Difference } & -0.13171 & \text { t Ratio } & -1.8152 \\ \text { Std Err Dif } & 0.07256 & \text { DF } & 21.81057 \\ \text { Upper CL Dif } & 0.01885 & \text { Prob }>|t| & 0.0833 \\ \text { Lower CL Dif } & -0.28227 & \text { Prob }>\text { t } & 0.9584 \\ \text { Confidence } & 0.95 & \text { Prob }<\mathrm{t} & 0.0416\end{array}$

Tests that the Variances are Equal

Level Count Std Dev MeanAbsDif to Mean MeanAbsDif to Median

$\begin{array}{lllll}\text { Coliwasa } & 16 & 0.1993201 & 0.1237584 & 0.1045468\end{array}$

Test F Ratio DFNum DFDen p-Value

$\begin{array}{lrrrr}\text { O'Brien[.5] } & 0.0930 & 1 & 24 & 0.7630\end{array}$

Brown-Forsythe $\quad 0.0497 \quad 1 \quad 24 \quad 0.8255$

$\begin{array}{lllll}\text { Levene } & 0.0000 & 1 & 24 & 0.9951\end{array}$

$\begin{array}{lrrrr}\text { Bartlett } & 0.3301 & 1 & \text {. } & 0.5656 \\ \text { F Test 2-sided } & 1.4281 & 15 & 9 & 0.5997\end{array}$

Welch Anova testing Means Equal, allowing Std Devs Not Equal

F Ratio DFNum DFDen Prob $>$ F

$3.2949 \quad 1 \quad 21.811 \quad 0.0833$

t Test

1.8152 


\section{Exhibit C2. Statistical Comparisons for Low-Rheology (Phase 3) Simulant Testing}

Oneway Analysis of Measurement By Sampler Type of Material=SME Simulant, Analytical Block $=1$, Oxide/Analyte $=$ K2O ( wt $\%$ ), Targeted $=0.1591$

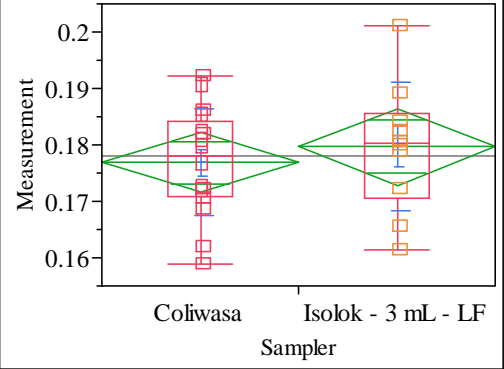

Oneway Anova

Summary of Fit

Adj Rsquare

$-0.02323$

0.010252

0.177933

Observations (or Sum Wgts)

Test

Isolok - $3 \mathrm{~mL}$ - LF-Coliwasa

Assuming equal variances

Difference $\quad 0.00272$ t Ratio 0.657671

Std Err Dif $\quad 0.00413$ DF 24

Upper CL Dif 0.01125 Prob $>|t| \quad 0.5170$

Lower CL Dif -0.00581 Prob $>t \quad 0.2585$

$\begin{array}{lrl}\text { Confidence } & 0.95 \text { Prob }<\mathrm{t} \quad 0.7415\end{array}$

Analysis of Variance

Source DF Sum of Squares Mean Square F Ratio Prob $>$ F

$\begin{array}{llllll}\text { Sampler } & 1 & 0.00004546 & 0.000045 & 0.4325 & 0.5170\end{array}$

$\begin{array}{llll}\text { Error } & 24 & 0.00252232 & 0.000105\end{array}$

C. Total $25 \quad 0.00256778$

Means for Oneway Anova

\begin{tabular}{lrrrrr} 
Level & Number & Mean & Std Error & Lower 95\% & Upper 95\% \\
\hline & 16 & 0.176888 & 0.00256 & 0.17160 & 0.18218
\end{tabular}

$\begin{array}{lllrrr}\text { Coliwasa } & 16 & 0.176888 & 0.00256 & 0.17160 & 0.18218 \\ \text { Isolok - 3 mL - LF } & 10 & 0.179606 & 0.00324 & 0.17291 & 0.18630\end{array}$

Std Error uses a pooled estimate of error variance

Means and Std Deviations

Level Number Mean Std Dev Std Err Mean Lower 95\% Upper 95\%

Coliwasa $\quad 16 \quad 0.1768880 .009502$

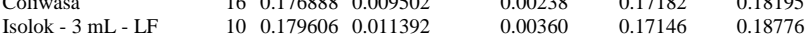

t Test

Isolok - $3 \mathrm{~mL}$ - LF-Coliwasa

Assuming unequal variances

Difference $\quad 0.00272$ t Ratio $\quad 0.629846$

Std Err Dif $\quad 0.00432$ DF $\quad 16.64109$

Upper CL Dif 0.01184 Prob $>|t| \quad 0.5373$

Lower CL Dif -0.00640 Prob $>t \quad 0.2687$

$\begin{array}{lrl}\text { Confidence } & 0.95 \text { Prob }<\mathrm{t} & 0.7313\end{array}$

Tests that the Variances are Equa

Level Count Std Dev MeanAbsDif to Mean MeanAbsDif to Median

$\begin{array}{lllll}16 & 0.0095021 & 0.0077923 & 0.0077923\end{array}$

$\begin{array}{lllll}\text { Isolok - } 3 \mathrm{~mL} \text { - LF } & 10 & 0.0113918 & 0.0080467 & 0.0079504\end{array}$

$\begin{array}{lrrrr}\text { Test } & \text { F Ratio } & \text { DFNum } & \text { DFDen } & \text { p-Value } \\ \text { O'Brien[.5] } & 0.4524 & 1 & 24 & 0.5076 \\ \text { Brown-Forsythe } & 0.0039 & 1 & 24 & 0.9507 \\ \text { Levene } & 0.0106 & 1 & 24 & 0.9189 \\ \text { Bartlett } & 0.3630 & 1 & & 0.5468 \\ \text { F Test 2-sided } & 1.4373 & 9 & 15 & 0.5136\end{array}$

Welch Anova testing Means Equal, allowing Std Devs Not Equal

F Ratio DFNum DFDen Prob $>$ F

$\begin{array}{rrrr}0.3967 & 1 & 16.641 & 0.5373\end{array}$

t Test

0.6298
Oneway Analysis of Measurement By Sampler Type of Material=SME Simulant, Analytical Block=1, Oxide/Analyte $=$ Li2O (wt $\%$ ), Targeted $=4.674$

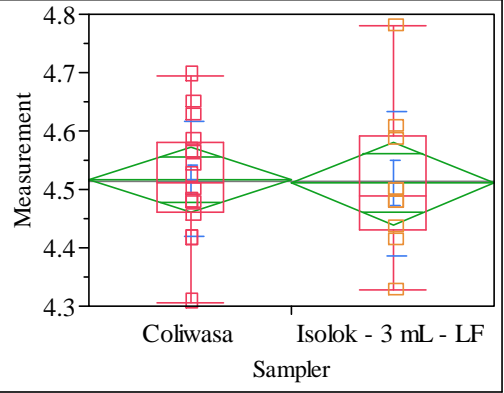

Oneway Anova

Summary of Fit

Rsquare

Adj Rsquare

Mean of Response 4.514466

Observations (or Sum Wgts)

t Test

Isolok - $3 \mathrm{~mL}$ - LF-Coliwasa

Assuming equal variances

Difference $\quad-0.00673$ t Ratio -0.1534

$\begin{array}{lrrr}\text { Std Err Dif } & 0.04386 & \text { DF } & 24 \\ \text { Upper CL Dif } & 0.08379 & \text { Prob }>|t| & 0.8794\end{array}$

$\begin{array}{lrll}\text { Upper CL Dif } & 0.08379 & \text { Prob }>|t| & 0.8794 \\ \text { Lower CL Dif } & -0.09725 & \text { Prob }>t & 0.5603\end{array}$

$\begin{array}{lrll}\text { Lower CL Dif } & -0.09725 & \text { Prob }>t & 0.5603 \\ \text { Confidence } & 0.95 & \text { Prob }<t & 0.4397\end{array}$

Analysis of Variance

Source DF Sum of Squares Mean Square F Ratio Prob $>$ F

$\begin{array}{lrrrrr}\text { Sampler } & 1 & 0.00027854 & 0.000279 & 0.0235 & 0.8794\end{array}$

$\begin{array}{lll}\text { Error } & 24 & 0.28409521\end{array}$

C. Total $25 \quad 0.28437375$

Means for Oneway Anova

Level Number Mean Std Error Lower 95\% Upper 95\%

$\begin{array}{llllrr}\text { Coliwasa } & 16 & 4.51705 & 0.02720 & 4.4609 & 4.5732\end{array}$

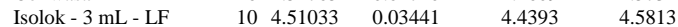

Std Error uses a pooled estimate of error variance

Means and Std Deviations

Level Number Mean Std Dev Std Err Mean Lower 95\% Upper 95\%

$\begin{array}{lrrrrrr}\text { Coliwasa } & 16 & 4.51705 & 0.098570 & 0.02464 & 4.4645 & 4.5696\end{array}$

$\begin{array}{llllll}\text { Isolok - } 3 \mathrm{~mL} \text { - LF } & 10 & 4.51033 & 0.123987 & 0.03921 & 4.4216\end{array}$

t Test

Isolok - $3 \mathrm{~mL}$ - LF-Coliwasa

Assuming unequal variances

Difference $\quad-0.00673$ t Ratio -0.14528

Std Err Dif $\quad 0.04631$ DF 16.01533

Upper CL Dif 0.09144 Prob $>|t| 0.8863$

Lower CL Dif -0.10489 Prob $>t \quad 0.5568$

$\begin{array}{lll}\text { Confidence } & 0.95 \text { Prob }<\mathrm{t} & 0.4432\end{array}$

Tests that the Variances are Equal

$\begin{array}{lrrrr}\text { Level } & \text { Count } & \text { Std Dev } & \text { MeanAbsDif to Mean } & \text { MeanAbsDif to Median } \\ \text { Coliwasa } & 16 & 0.0985701 & 0.0766971 & 0.0766971\end{array}$

$\begin{array}{lllll}\text { Isolok - 3 mL - LF } & 10 & 0.1239866 & 0.0882689 & 0.0766971 \\ & & \end{array}$

Test F Ratio DFNum DFDen p-Value

$\begin{array}{lllll}\text { O'Brien[.5] } & 0.5000 & 1 & 24 & 0.4863\end{array}$

$\begin{array}{lllll}\text { Brown-Forsythe } & 0.0625 & 1 & 24 & 0.8048\end{array}$

$\begin{array}{llll} & 1 & 24 & 0.8048\end{array}$

$\begin{array}{lllll}\text { Levene } & 0.1765 & 1 & 24 & 0.6782\end{array}$

$\begin{array}{lllrl}\text { B Test 2-sided } & 0.5836 & 1 & . & 0.4449 \\ \text { F Test } & 1.5822 & 9 & 15 & 0.4152\end{array}$

Welch Anova testing Means Equal, allowing Std Devs Not Equal

F Ratio DFNum DFDen Prob $>$ F

$\begin{array}{llll}0.0211 & 1 & 16.015 & 0.8863\end{array}$

t Test 
SRNL-STI-2011-00693

Revision 0

\section{Exhibit C2. Statistical Comparisons for Low-Rheology (Phase 3) Simulant Testing}

Oneway Analysis of Measurement By Sampler Type of Material=SME Simulant, Analytical Block=1, Oxide/Analyte $=\mathrm{MgO}$ (wt \%), Targeted $=1.014$

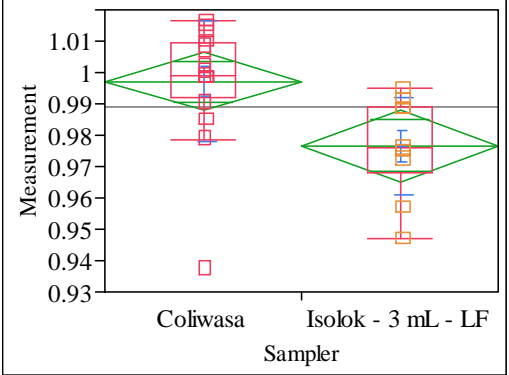

Oneway Anova

Summary of Fi

Rsquare

Adj Rsquare

Root Mean Square Error $\quad 0.01788$

Mean of Response

Observations (or Sum Wgts)

Test

Isolok - $3 \mathrm{~mL}$ - LF-Coliwasa

Assuming equal variances

Difference $\quad-0.02058$ t Ratio $\quad-2.85568$

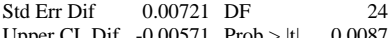

Upper CL Dif -0.00571 Prob $>|t| 0.0087$

Lower CL Dif -0.03546 Prob $>t \quad 0.9956$

$\begin{array}{lrl}\text { Confidence } & 0.95 \text { Prob }<\mathrm{t} \quad 0.0044\end{array}$

Analysis of Variance

Source DF Sum of Squares Mean Square F Ratio Prob $>$ F

$\begin{array}{lllllll}\text { Sampler } & 1 & 0.00260730 & 0.002607 & 8.1549 & 0.0087\end{array}$

$\begin{array}{llll}\text { Error } & 24 & 0.00767331 & 0.000320\end{array}$

C. Total $25 \quad 0.01028062$

Means for Oneway Anova

Level Number Mean Std Error Lower 95\% Upper 95\%

Coliwasa 160.997157

$\begin{array}{llllll}\text { Isolok - 3 mL - LF } & 16 & 0.997157 & 0.00447 & 0.98793 & 1.0064 \\ & 10 & 0.976573 & 0.00565 & 0.96490 & 0.9882\end{array}$

Std Error uses a pooled estimate of error variance

Means and Std Deviations

Level Number Mean Std Dev Std Err Mean Lower 95\% Upper 95\%

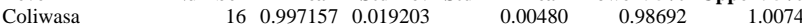

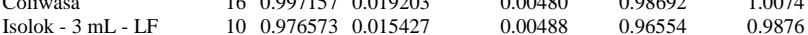

t Test

Isolok - $3 \mathrm{~mL}$ - LF-Coliwasa

Assuming unequal variances

Difference $\quad-0.02058$ t Ratio -3.00734

Std Err Dif $\quad 0.00684$ DF 22.31515

Upper CL Dif -0.00640 Prob $>|t| \quad 0.0064$

Lower CL Dif -0.03477 Prob $>t \quad 0.9968$

$\begin{array}{lrl}\text { Confidence } & 0.95 \text { Prob }<\mathrm{t} & 0.0032\end{array}$

Tests that the Variances are Equal

Level Count Std Dev MeanAbsDif to Mean MeanAbsDif to Median

$\begin{array}{llll}16 & 0.0192031 & 0.0122818 & 0.0117117\end{array}$

$\begin{array}{lllll}\text { Isolok - } 3 \mathrm{~mL} \text { - LF } & 10 & 0.0154270 & 0.0114423 & 0.0114423\end{array}$

Test F Ratio DFNum DFDen p-Value

$\begin{array}{lrrrr}\text { O'Brien[.5] } & 0.1654 & 1 & 24 & 0.6878\end{array}$

$\begin{array}{lllll}\text { Brown-Forsythe } & 0.0025 & 1 & 24 & 0.9603\end{array}$

$\begin{array}{lllll}\text { Levene } & 0.0263 & 1 & 24 & 0.8724\end{array}$

$\begin{array}{lrrrr}\text { Bartlett } & 0.4941 & 1 & \text {. } & 0.4821 \\ \text { F Test 2-sided } & 1.5494 & 15 & 9 & 0.5135\end{array}$

Welch Anova testing Means Equal, allowing Std Devs Not Equal

F Ratio DFNum DFDen Prob $>$ F

$\begin{array}{llll}9.0441 & 1 & 22.315 & 0.0064\end{array}$

t Test
Oneway Analysis of Measurement By Sampler Type of Material=SME Simulant, Analytical Block=1, Oxide/Analyte=MnO (wt \%), Targeted $=1.779$

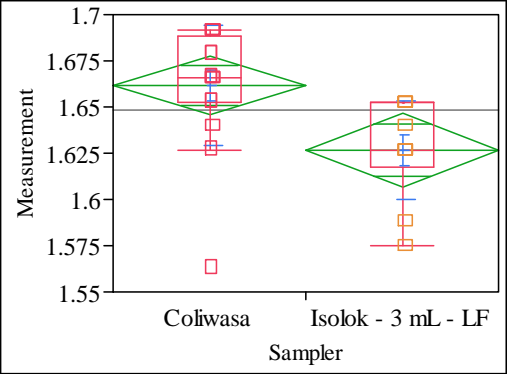

Oneway Anova

Summary of Fit

Rsquare

Adj Rsquare

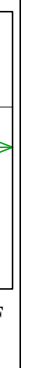

Root Mean Square Error $\quad 0.030446$

$\begin{array}{lr}\text { Mean of Response } & 1.648266 \\ \text { Observations (or Sum Wgts) } & 26\end{array}$

t Test

Isolok - $3 \mathrm{~mL}$ - LF-Coliwasa

Assuming equal variances

Difference $\quad-0.03470$ t Ratio $\quad-2.82741$

$\begin{array}{lrlr}\text { Std Err Dif } & 0.01227 \text { DF } & 24 \\ \text { Upper CL } & \end{array}$

$\begin{array}{llll}\text { Upper CL Dif }-0.00937 & \text { Prob }>|t| & 0.0093 \\ \text { Lower CL Dif }-0.06003 & \text { Prob }>t & 0.9953\end{array}$

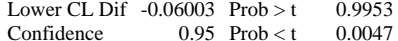

Analysis of Variance

Source DF Sum of Squares Mean Square F Ratio Prob $>$ F

$\begin{array}{llllll}\text { Sampler } & 1 & 0.00741021 & 0.007410 & 7.9942 & 0.0093\end{array}$

$\begin{array}{llll}\text { Error } & 24 & 0.02224667 & 0.000927\end{array}$

C. Total $25 \quad 0.02965688$

Means for Oneway Anova

Level Number Mean Std Error Lower 95\% Upper 95\%

$\begin{array}{llrrrr}\text { Coliwasa } & 16 & 1.66161 & 0.00761 & 1.6459 & 1.6773\end{array}$

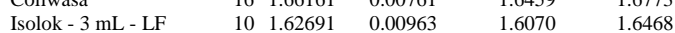

Std Error uses a pooled estimate of error variance

Means and Std Deviations

Level Number Mean Std Dev Std Err Mean Lower 95\% Upper 95\%

$\begin{array}{lllllll}\text { Coliwasa } & 16 & 1.66161 & 0.032569 & 0.00814 & 1.6443 & 1.6790\end{array}$

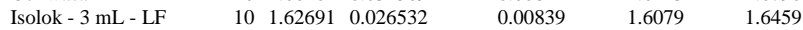

t Test

Isolok - $3 \mathrm{~mL}$ - LF-Coliwasa

Assuming unequal variances

Difference $\quad-0.03470$ t Ratio $\quad-2.96807$

Std Err Dif $\quad 0.01169$ DF 22.1483

Upper CL Dif -0.01046 Prob $>|t| 0.0071$

Lower CL Dif -0.05894 Prob $>t \quad 0.9965$

Confidence $\quad 0.95$ Prob $<\mathrm{t} \quad 0.0035$

Tests that the Variances are Equal

$\begin{array}{lrrrr}\text { Level } & \text { Count } & \text { Std Dev } & \text { MeanAbsDif to Mean } & \text { MeanAbsDif to Median } \\ \text { Coliwasa } & 16 & 0.0325692 & 0.0216881 & 0.0201750\end{array}$

Isolok - $3 \mathrm{~mL}$ - LF $\quad 10 \quad 0.0265316 \quad 0.0180768 \quad 0.0180768$

Test F Ratio DFNum DFDen p-Value

O'Brien[.5] $\quad 0.1640 \quad 1 \quad 240.6891$

$\begin{array}{llll} & 1 & 24 & 0.689 \\ \text { Brown-Forsythe } 0.1640 & 1 & 24 & 0.8231\end{array}$

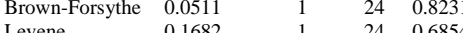

$\begin{array}{lllll}\text { Levene } & 0.1682 & 1 & 24 & 0.6854 \\ \text { Bartlett } & 0.4346 & 1 & \cdot & 0.5097\end{array}$

$\begin{array}{lrrrr}\text { Bartlett } & 0.4346 & 1 & & 0.5097 \\ \text { F Test 2-sided } & 1.5069 & 15 & 9 & 0.5421\end{array}$

Welch Anova testing Means Equal, allowing Std Devs Not Equal

F Ratio DFNum DFDen Prob > F

$\begin{array}{llll}8.8094 & 1 & 22.148 & 0.0071\end{array}$

t Test

2.9681 
SRNL-STI-2011-00693

Revision 0

\section{Exhibit C2. Statistical Comparisons for Low-Rheology (Phase 3) Simulant Testing}

Oneway Analysis of Measurement By Sampler Type of Material=SME Simulant, Analytical Block=1, Oxide/Analyte=Na2O (wt \%), Targeted $=11.659$

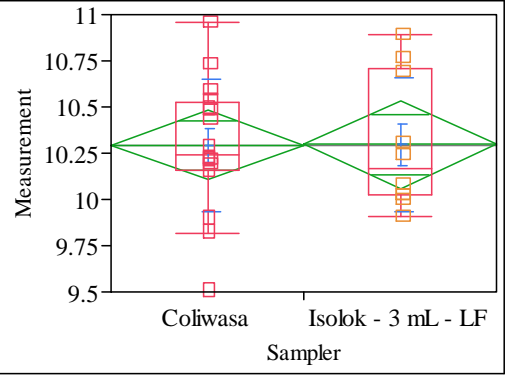

Oneway Anova

Rsquare $\quad 0.000011$

Adj Rsquare $\quad-0.04166$

Root Mean Square Error $\quad 0.359916$

Mean of Response 10.29457

(or Sum Wgts)

Test

Isolok - $3 \mathrm{~mL}$ - LF-Coliwasa

Assuming equal variances

Difference $\quad 0.00236$ t Ratio $\quad 0.016259$

$\begin{array}{lrrr}\text { Std Err Dif } & 0.14509 \text { DF } & 24 \\ \text { Uper } & \text { Dr } & 0.9872\end{array}$

$\begin{array}{rrrr}\text { Upper CL Dif } & 0.30180 & \text { Prob }>|t| & 0.9872 \\ \text { Lower CL Dif } & -0.29709 & \text { Prob }>t & 0.4936\end{array}$

$\begin{array}{lrl}\text { Confidence } & 0.95 \text { Prob }<\mathrm{t} & 0.5064\end{array}$

Analysis of Variance

Source DF Sum of Squares Mean Square F Ratio Prob $>$ F

$\begin{array}{lrrrrr}\text { Sampler } & 1 & 0.0000342 & 0.000034 & 0.0003 & 0.9872\end{array}$

$\begin{array}{llll}\text { Error } & 24 & 3.1089468 & 0.129539 \\ \text { C. Total } & 25 & 3.1089811 & \end{array}$

Means for Oneway Anova

Level Number Mean Std Error Lower 95\% Upper 95\%

$\begin{array}{lrrrrr}\text { Coliwasa } & 16 & 10.2937 & 0.08998 & 10.108 & 10.479\end{array}$

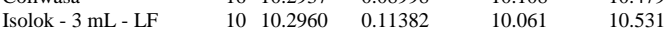

Std Error uses a pooled estimate of error variance

Means and Std Deviations

$\begin{array}{lrrrrrr}\text { Level } & \text { Number } & \text { Mean } & \text { Std Dev } & \text { Std Err Mean } & \text { Lower 95\% } & \text { Upper 95\% } \\ \text { Coliwasa } & 16 & 10.2937 & 0.360192 & 0.09005 & 10.102 & 10.486\end{array}$

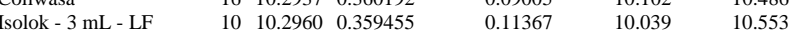

t Test

Isolok - $3 \mathrm{~mL}$ - LF-Coliwasa

Assuming unequal variances

Difference $\quad 0.00236$ t Ratio $\quad 0.016267$

Std Err Dif $\quad 0.14502$ DF 19.28386

Upper CL Dif 0.30558 Prob $>|t| 0.9872$

Lower CL Dif -0.30086 Prob $>\mathrm{t} \quad 0.4936$

$\begin{array}{lrl}\text { Confidence } & 0.95 \text { Prob }<\mathrm{t} & 0.5064\end{array}$

Tests that the Variances are Equal

$\begin{array}{lrrrr}\text { Level } & \text { Count } & \text { Std Dev } & \text { MeanAbsDif to Mean } & \text { MeanAbsDif to Median } \\ \text { Coliwasa } & 16 & 0.3601919 & 0.2723381 & 0.2696000 \\ \text { Isolok - 3 mL - LF } & 10 & 0.3594554 & 0.2960208 & 0.2857760\end{array}$

Test F Ratio DFNum DFDen p-Value

$\begin{array}{lrrrr}\text { O'Brien[.5] } & 0.0001 & 1 & 24 & 0.9941\end{array}$

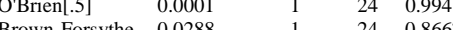

$\begin{array}{lllll}\text { Brown-Forsythe } & 0.0288 & 1 & 24 & 0.8667\end{array}$

$\begin{array}{llrrr}\text { Levene } & 0.0792 & 1 & 24 & 0.7808 \\ \text { Bartlett } & 0.0000 & 1 & . & 0.9946\end{array}$

$\begin{array}{lllll}\text { F Test 2-sided } & 1.0041 & 15 & 9 & 0.9946\end{array}$

Welch Anova testing Means Equal, allowing Std Devs Not Equal

F Ratio DFNum DFDen Prob > F

$\begin{array}{llll}0.0003 & 1 & 19.284 & 0.9872\end{array}$

t Test

0.0163
Oneway Analysis of Measurement By Sampler Type of Material=SME Simulant, Analytica Block $=1$, Oxide/Analyte $=\mathrm{NiO}$ ( $\mathrm{wt} \%$ ), Targeted $=\mathbf{0 . 4 1}$

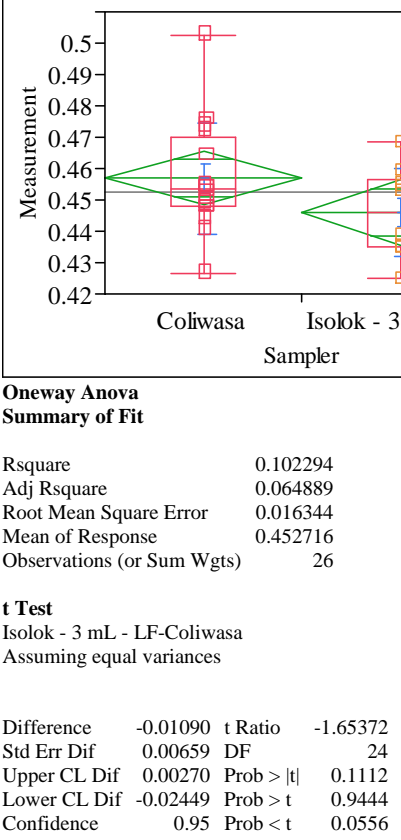

Analysis of Variance

Source DF Sum of Squares Mean Square F Ratio Prob $>$ F

$\begin{array}{lrrrrr}\text { Sampler } & 1 & 0.00073057 & 0.000731 & 2.7348 & 0.1112\end{array}$

$\begin{array}{llll}\text { Error } & 24 & 0.00641134 & 0.000267\end{array}$

C. Total $25 \quad 0.00714192$

Means for Oneway Anova

Level Number Mean Std Error Lower 95\% Upper 95\%

$\begin{array}{lrrrrr}\text { Coliwasa } & 16 & 0.456907 & 0.00409 & 0.44847 & 0.46534\end{array}$

$\begin{array}{llllll}\text { Isolok - 3 mL - LF } & 16 & 0.456907 & 0.00409 & 0.44847 & 0.46534 \\ & 10 & 0.446011 & 0.00517 & 0.43534 & 0.45668\end{array}$

Std Error uses a pooled estimate of error variance

Means and Std Deviations

Level Number Mean Std Dev Std Err Mean Lower 95\% Upper 95\%

$\begin{array}{lllllll}\text { Coliwasa } & 16 & 0.456907 & 0.017678 & 0.00442 & 0.44749 & 0.46633\end{array}$

$\begin{array}{lllllll}\text { Isolok - 3 mL - LF } & 10 & 0.446011 & 0.013839 & 0.00438 & 0.43611 & 0.45591\end{array}$

Test

Isolok - $3 \mathrm{~mL}$ - LF-Coliwasa

Assuming unequal variances

$\begin{array}{lrlr}\text { Difference } & -0.01090 & \text { t Ratio } & -1.75183 \\ \text { Std Err Dif } & 0.00622 & \text { DF } & 22.60877 \\ \text { Upper CL Dif } & 0.00198 & \text { Prob }>|t| & 0.0934 \\ \text { Lower CL Dif } & -0.02377 & \text { Prob }>\text { t } & 0.9533 \\ \text { Confidence } & 0.95 & \text { Prob }<\mathrm{t} & 0.0467\end{array}$

Tests that the Variances are Equal

Level Count Std Dev MeanAbsDif to Mean MeanAbsDif to Median

$\begin{array}{lllll}\text { Coliwasa } & 16 & 0.0176779 & 0.0129934 & 0.0120092\end{array}$

Test F Ratio DFNum DFDen p-Value

$\begin{array}{lrrrr}\text { O'Brien[.5] } & 0.3912 & 1 & 24 & 0.5376\end{array}$

Brown-Forsythe $\quad 0.0003 \quad 1 \quad 24 \quad 0.9856$

$\begin{array}{lllll}\text { Levene } & 0.0538 & 1 & 24 & 0.8186\end{array}$

$\begin{array}{lrrrr}\text { Bartlett } & 0.6141 & 1 & \text {. } & 0.4333 \\ \text { F Test 2-sided } & 1.6317 & 15 & 9 & 0.4628\end{array}$

Welch Anova testing Means Equal, allowing Std Devs Not Equal

F Ratio DFNum DFDen Prob $>$ F

$\begin{array}{llrr}3.0689 & 1 & 22.609 & 0.0934\end{array}$

t Tes

1.7518 
SRNL-STI-2011-00693

Revision 0

\section{Exhibit C2. Statistical Comparisons for Low-Rheology (Phase 3) Simulant Testing}

Oneway Analysis of Measurement By Sampler Type of Material=SME Simulant, Analytica Block=1, Oxide $/$ Analyte $=\mathrm{SiO} 2(\mathrm{wt} \%$ ), Targeted $=\mathbf{5 0 . 9 8 5}$

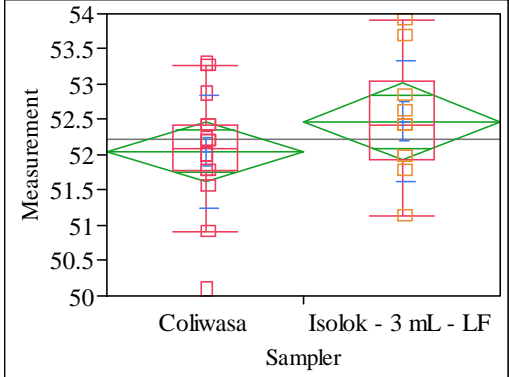

Oneway Anova

Summary of Fit

Rsquare $\quad 0.064677$

Adj Rsquare $\quad 0.025705$

Root Mean Square Error $\quad 0.81875$

Mean of Response $\quad 52.21538$

Observations (or Sum Wgts) 26

Test

Isolok - $3 \mathrm{~mL}$ - LF-Coliwasa

Assuming equal variances

Difference $\quad 0.4252$ t Ratio 1.28825

Std Err Dif $\quad 0.3300$ DF 24

Upper CL Dif 1.1064 Prob $>|t| \quad 0.2099$

Lower CL Dif -0.2560 Prob $>t \quad 0.1050$

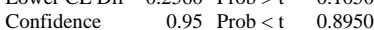

Analysis of Variance

Source DF Sum of Squares Mean Square F Ratio Prob $>$ F

$\begin{array}{lrrrrr}\text { Sampler } & 1 & 1.112511 & 1.11251 & 1.6596 & 0.2099\end{array}$

$\begin{array}{llll}\text { Error } & 24 & 16.088481 & 0.67035\end{array}$

C. Total $25 \quad 17.200992$

Means for Oneway Anova

Level Number Mean Std Error Lower 95\% Upper 95\%

$\begin{array}{lrrrrr}\text { Coliwasa } & 16 & 52.0518 & 0.20469 & 51.629 & 52.474\end{array}$

$\begin{array}{llllll}\text { Coliwasa } & 16 & 52.0518 & 0.20469 & 51.629 & 52.474 \\ \text { Isolok - 3 mL - LF } & 10 & 52.4770 & 0.25891 & 51.943 & 53.011\end{array}$

Std Error uses a pooled estimate of error variance

Means and Std Deviations

Level Number Mean Std Dev Std Err Mean Lower 95\% Upper 95\%

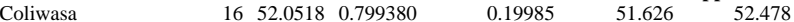

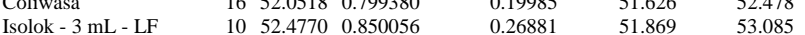

t Test

Isolok - $3 \mathrm{~mL}$ - LF-Coliwasa

Assuming unequal variances

Difference $\quad 0.4252$ t Ratio 1.269367

Std Err Dif $\quad 0.3350$ DF $\quad 18.33701$

Upper CL Dif 1.1280 Prob $>|t| \quad 0.2202$

Lower CL Dif -0.2776 Prob $>t \quad 0.1101$

$\begin{array}{lrr}\text { Confidence } & 0.95 \text { Prob }<\mathrm{t} \quad 0.8899\end{array}$

Tests that the Variances are Equal

Level Count Std Dev MeanAbsDif to Mean MeanAbsDif to Median

$\begin{array}{lllll} & 16 & 0.7993800 & 0.5481956 & 0.5481956\end{array}$

Test F Ratio DFNum DFDen p-Value

\begin{tabular}{lrrrr} 
O'Brien[.5] & 0.0363 & 1 & 24 & 0.8505 \\
\hline
\end{tabular}

$\begin{array}{lllll}\text { Brown-Forsythe } & 0.1027 & 1 & 24 & 0.7514\end{array}$

$\begin{array}{lllll}\text { Levene } & 0.1469 & 1 & 24 & 0.7049\end{array}$

$\begin{array}{lllll}\text { Bartlett } & 0.0411 & 1 & & 0.8394 \\ \text { F Test 2-sided } & 1.1308 & 9 & 15 & 0.8002\end{array}$

Welch Anova testing Means Equal, allowing Std Devs Not Equal

F Ratio DFNum DFDen Prob $>$ F

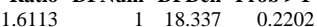

t Test

1.2694
Oneway Analysis of Measurement By Sampler Type of Material=SME Simulant, Analytical Block=1, Oxide/Analyte $=$ Sum of Oxides (wt \%), Targeted $=99.553$

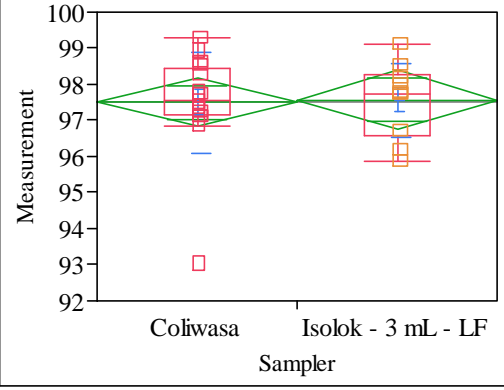

Oneway Anova

Summary of Fit

Rsqua

$\quad-0.04096$

$\begin{array}{lr}\text { Root Mean Square Error } & 1.273204 \\ \text { Mean of Response } & 97.532\end{array}$

97.532
26

t Test

Isolok - $3 \mathrm{~mL}$ - LF-Coliwasa

Assuming equal variances

Difference $\quad 0.0654$ t Ratio $\quad 0.127343$

Std Err Dif $\quad 0.5132$ DF $\quad 24$

Upper CL Dif 1.1246 Prob $>|t| \quad 0.8997$

Lower CL Dif -0.9939 Prob $>\mathrm{t} \quad 0.4499$

$\begin{array}{lll}\text { Confidence } & 0.95 \text { Prob }<\mathrm{t} \quad 0.5501\end{array}$

Analysis of Variance

Source DF Sum of Squares Mean Square F Ratio Prob $>$ F

$\begin{array}{lrrrrr}\text { Source } & \text { DF } & \text { Sum of Squares } & \text { Mean Square } & \text { F Ratio } & \text { Prob }>\text { F } \\ \text { Sampler } & 1 & 0.026287 & 0.02629 & 0.0162 & 0.8997\end{array}$

$\begin{array}{lrrr}\text { Error } & 24 & 38.905187 & 1.62105\end{array}$

$\begin{array}{lll}\text { C. Total } & 25 & 38.931475\end{array}$

Means for Oneway Anova

Level Number Mean Std Error Lower 95\% Upper 95\%

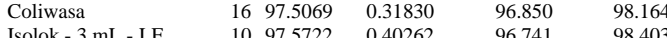

Std Error uses a pooled estimate of error variance

Means and Std Deviations

Level Number Mean Std Dev Std Err Mean Lower 95\% Upper 95\%

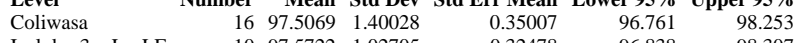

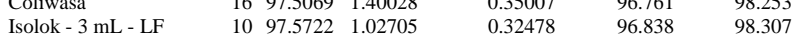

t Test

Isolok - $3 \mathrm{~mL}$ - LF-Coliwasa

Assuming unequal variances

$\begin{array}{lrlr}\text { Difference } & 0.0654 & \text { t Ratio } & 0.136868 \\ \text { Std Err Dif } & 0.4775 \text { DF } & 23.2394 \\ \text { Upper CL Dif } & 1.0526 \text { Prob }>|t| & 0.8923 \\ \text { Lower CL Dif } & -0.9219 & \text { Prob }>\text { t } & 0.4462 \\ \text { Confidence } & 0.95 & \text { Prob }<\mathrm{t} & 0.5538\end{array}$

Tests that the Variances are Equal

Level Count Std Dev MeanAbsDif to Mean MeanAbsDif to Median

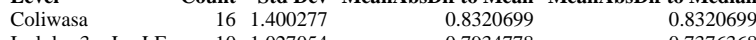

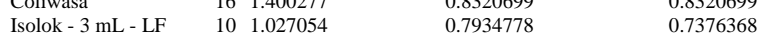

Test F Ratio DFNum DFDen p-Value

$\begin{array}{lllll}\text { O'Brien[.5] } & 0.2537 & 1 & 24 & 0.6191\end{array}$

$\begin{array}{lllll}\text { Brown-Forsythe } & 0.0580 & 1 & 24 & 0.8117\end{array}$

$\begin{array}{lllll}\text { Levene } & 0.0102 & 1 & 24 & 0.9203\end{array}$

$\begin{array}{lrrrr}\text { Bartlett } & 0.9692 & 1 & \dot{0} & 0.3249 \\ \text { F Test 2-sided } & 1.8588 & 15 & 9 & 0.3496\end{array}$

Welch Anova testing Means Equal, allowing Std Devs Not Equal

$\begin{array}{rrrr}\text { F Ratio } & \text { DFNum } & \text { DFDen } & \text { Prob }>\text { F } \\ 0.0187 & 1 & 23.239 & 0.8923\end{array}$

$\mathbf{t}$ Test
0.1369 
SRNL-STI-2011-00693

Revision 0

\section{Exhibit C2. Statistical Comparisons for Low-Rheology (Phase 3) Simulant Testing}

Oneway Analysis of Measurement By Sampler Type of Material=SME Simulant, Analytical Block=1, Oxide/Analyte=TiO2 ( $w$ t $\%$ ), Targeted $=\mathbf{0 . 0 7 1 1}$

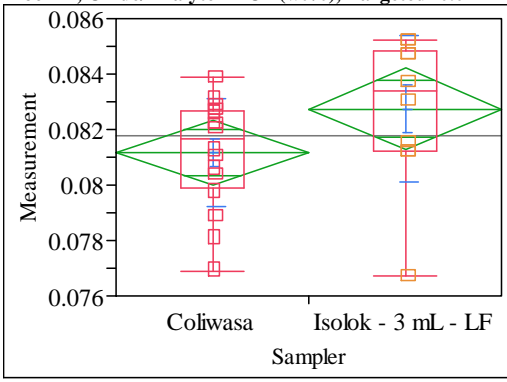

Oneway Anova

Rsquare $\quad 0.113854$

Adj Rsquare $\quad 0.076932$

Root Mean Square Error $\quad 0.002247$

Mean of Response $\quad 0.08177$

t Test

Isolok - $3 \mathrm{~mL}$ - LF-Coliwasa

Assuming equal variances

Difference $\quad 0.00159$ t Ratio 1.756013

$\begin{array}{lrlr}\text { Std Err Dif } & 0.00091 & \text { DF } & 24 \\ \text { Upper CL Dif } & 0.00346 & \text { Prob }>|t| & 0.0918\end{array}$

$\begin{array}{rrrr}\text { Upper CL Dif } & 0.00346 & \text { Prob }>|t| & 0.0918 \\ \text { Lower CL Dif } & -0.00028 & \text { Prob }>t & 0.0459\end{array}$

$\begin{array}{lrll}\text { Lower CL Dif } & -0.00028 & \text { Prob }>t & 0.0459 \\ \text { Confidence } & 0.95 & \text { Prob }<\mathrm{t} & 0.9541\end{array}$

Analysis of Variance

Source DF Sum of Squares Mean Square F Ratio Prob $>$ F

$\begin{array}{llllll}\text { Sampler } & 1 & 0.00001557 & 0.000016 & 3.0836 & 0.0918\end{array}$

$\begin{array}{llll}\text { Error } & 24 & 0.00012122 & 5.051 \mathrm{e}-6\end{array}$

C. Total $25 \quad 0.00013679$

Means for Oneway Anova

Level Number Mean Std Error Lower 95\% Upper 95\%

$\begin{array}{lllllr}\text { Coliwasa } & 16 & 0.081159 & 0.00056 & 0.08000 & 0.08232\end{array}$

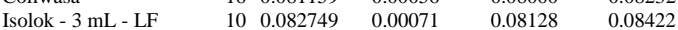

Std Error uses a pooled estimate of error variance

Means and Std Deviations

$\begin{array}{lrrrrrr}\text { Level } & \text { Number } & \text { Mean } & \text { Std Dev } & \text { Std Err Mean } & \text { Lower 95\% } & \text { Upper 95\% } \\ \text { Coliwasa } & 16 & 0.081159 & 0.001960 & 0.00049 & 0.08011 & 0.08220\end{array}$

$\begin{array}{lllllll}\text { Isolok - } 3 \mathrm{~mL} \text { - LF } & 10 & 0.082749 & 0.002658 & 0.00084 & 0.08085 & 0.08465\end{array}$

t Test

Isolok - $3 \mathrm{~mL}$ - LF-Coliwasa

Assuming unequal variances

Difference $\quad 0.00159$ t Ratio $\quad 1.635166$

Std Err Dif $\quad 0.00097$ DF 15.11303

Upper CL Dif 0.00366 Prob $>|t| \quad 0.1227$

Lower CL Dif -0.00048 Prob $>t \quad 0.0613$

$\begin{array}{lrl}\text { Confidence } & 0.95 \text { Prob }<\mathrm{t} & 0.9387\end{array}$

Tests that the Variances are Equal

$\begin{array}{lrrrr}\text { Level } & \text { Count } & \text { Std Dev } & \text { MeanAbsDif to Mean } & \text { MeanAbsDif to Median } \\ \text { Coliwasa } & 16 & 0.0019604 & 0.0015624 & 0.0015533 \\ \text { Isolok - 3 mL - LF } & 10 & 0.0026577 & 0.0020483 & 0.0019849\end{array}$

Test F Ratio DFNum DFDen p-Value

$\begin{array}{ll}1 & 24 \\ 1 & 0.3719\end{array}$

$\begin{array}{lllll} & 0.8280 & 1 & 24 & 0.3719 \\ \text { O'Brien[.5] } & 0.5364 & 1 & 24 & 0.4710\end{array}$

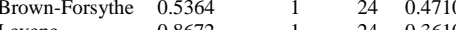

$\begin{array}{lllll}\text { Levene } & 0.8672 & 1 & 24 & 0.3610 \\ \text { Bartlett } & 1.0334 & 1 & . & 0.3094\end{array}$

$\begin{array}{lllll}\text { F Test 2-sided } & 1.8380 & 9 & 15 & 0.2857\end{array}$

Welch Anova testing Means Equal, allowing Std Devs Not Equal

F Ratio DFNum DFDen Prob $>$ F

$\begin{array}{llll}2.6738 & 1 & 15.113 & 0.1227\end{array}$

t Test

1.6352
Oneway Analysis of Measurement By Sampler Type of Material=SME Simulant, Analytical Block $=1$, Oxide/Analyte $=\mathrm{ZnO}($ wt $\%)$, Targeted $=\mathbf{0 . 0 9 5 8}$

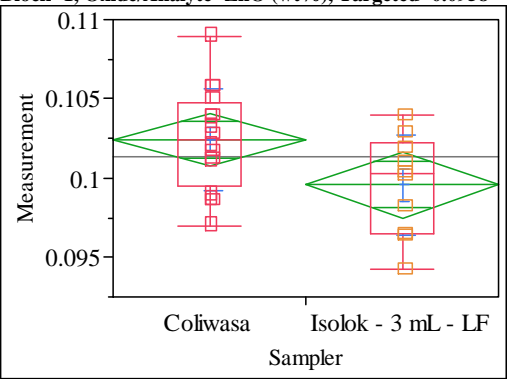

Oneway Anova

Summary of Fit

Rsquare

Adj Rsquare

Root Mean Square Error $\quad 0.003175$

Mean of Response

Observations (or Sum Wgts) $\quad 26$

Isolok - $3 \mathrm{~mL}$ - LF-Coliwas

Assuming equal variances

Difference $\quad-0.00284$ t Ratio -2.21651

$\begin{array}{lrrr}\text { Std Err Dif } & 0.00128 \text { DF } & 24 \\ \text { Upper CL } & -0.00020 & \text { Prob }>|t| & 0.036\end{array}$

$\begin{array}{llll}\text { Upper CL Dif } & -0.00020 & \text { Prob }>|t| & 0.0364 \\ \text { Lower CL Dif }-0.00548 & \text { Prob }>t & 0.9818\end{array}$

$\begin{array}{lll}\text { Confidence } & 0.95 \text { Prob }<\mathrm{t} & 0.0182\end{array}$

Analysis of Variance

Source DF Sum of Squares Mean Square F Ratio Prob $>$ F

$\begin{array}{llllll}\text { Sampler } & 1 & 0.00004952 & 0.000050 & 4.9129 & 0.0364\end{array}$

$\begin{array}{lll}\text { Error } & 24 & 0.00024189\end{array}$ 0.000010

Means for Oneway Anova

Level Number Mean Std Error Lower 95\% Upper 95\%

$\begin{array}{lrrrrr}\text { Coliwasa } & 16 & 0.102408 & 0.00079 & 0.10077 & 0.10405\end{array}$

$\begin{array}{llllll}\text { Isolok - } 3 \mathrm{~mL} \text { - LF } & 10 & 0.099572 & 0.00100 & 0.09750 & 0.10164\end{array}$

Std Error uses a pooled estimate of error variance

Means and Std Deviations

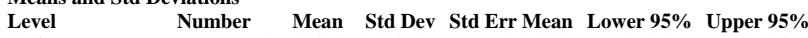

$\begin{array}{lrrrrrr}\text { Coliwasa } & 16 & 0.102408 & 0.003185 & 0.00080 & 0.10071 & 0.10411 \\ \text { Isolok - 3 mL - LF } & 10 & 0.099572 & 0.003158 & 0.00100 & 0.09731 & 0.10183\end{array}$

t Test

Isolok - $3 \mathrm{~mL}$ - LF-Coliwasa

Assuming unequal variances

Difference $\quad-0.00284$ t Ratio -2.2211

Std Err Dif $\quad 0.00128$ DF 19.38254

Upper CL Dif -0.00017 Prob $>|t| \quad 0.0384$

Lower CL Dif -0.00551 Prob $>t \quad 0.9808$

$\begin{array}{lrl}\text { Confidence } & 0.95 \text { Prob }<\mathrm{t} & 0.0192\end{array}$

Tests that the Variances are Equal

$\begin{array}{lrrrr}\text { Level } & \text { Count } & \text { Std Dev } & \text { MeanAbsDif to Mean } & \text { MeanAbsDif to Median } \\ \text { Coliwasa } & 16 & 0.0031849 & 0.0025129 & 0.0025129 \\ \text { Isolok - 3 mL - LF } & 10 & 0.0031576 & 0.0026041 & 0.0024772\end{array}$

Test F Ratio DFNum DFDen p-Value

$\begin{array}{lllll}\text { O'Brien[.5] } & 0.0012 & 1 & 24 & 0.9724\end{array}$

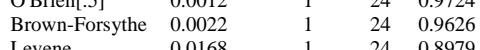

$\begin{array}{lllll}\text { Levene } & 0.0168 & 1 & 24 & 0.8979\end{array}$

$\begin{array}{lrrrr}\text { Bartlett } & 0.0008 & 1 & \dot{0} & 0.9774 \\ \text { F Test 2-sided } & 1.0174 & 15 & 9 & 1.0000\end{array}$

Welch Anova testing Means Equal, allowing Std Devs Not Equal

F Ratio DFNum DFDen Prob > F

$\begin{array}{llll}4.9333 & 1 & 19.383 & 0.0384\end{array}$

t Test 


\section{Exhibit C2. Statistical Comparisons for Low-Rheology (Phase 3) Simulant Testing}

Oneway Analysis of Measurement By Sampler Type of Material=SME Simulant, Analytical Block=1, Oxide/Analyte $=\mathrm{ZrO} 2$ (wt \%), Targeted $=0.3547$

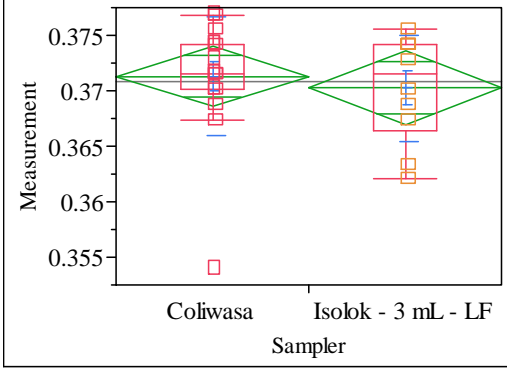

Oneway Anova

Rsquare $\quad 0.010431$

Adj Rsquare

$-0.0308$

0.005163

Mean of Response 0.370899

Observations (or Sum Wgts) 26

Isolok - $3 \mathrm{~mL}$ - LF-Coliwas

Assuming equal variances

Difference $\quad-0.00105$ t Ratio -0.50297

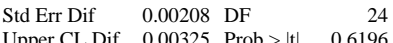

$\begin{array}{rrrr}\text { Upper CL Dif } & 0.00325 & \text { Prob }>|t| & 0.6196 \\ \text { Lower CL Dif } & -0.00534 & \text { Prob }>t & 0.6902\end{array}$

$\begin{array}{lrl}\text { Confidence } & 0.95 \text { Prob }<\mathrm{t} \quad 0.3098\end{array}$

Analysis of Variance

Source DF Sum of Squares Mean Square F Ratio Prob $>$ F

$\begin{array}{lrrrrr}\text { Sampler } & 1 & 0.00000674 & 6.744 \mathrm{e}-6 & 0.2530 & 0.6196\end{array}$

Error $24 \quad 0.0006398$ 0.000027

Means for Oneway Anova

Level Number Mean Std Error Lower 95\% Upper 95\%

$\begin{array}{lllllr}\text { Coliwasa } & 16 & 0.371301 & 0.00129 & 0.36864 & 0.37397\end{array}$

$\begin{array}{llllll} & 10 & 0.370254 & 0.00163 & 0.36688 & 0.37362\end{array}$

Std Error uses a pooled estimate of error variance

Means and Std Deviations

Level Number Mean Std Dev Std Err Mean Lower 95\% Upper 95\%

\begin{tabular}{lrrrrrr} 
Coliwasa & 16 & 0.371301 & 0.005378 & 0.00134 & 0.36844 & 0.37417 \\
\hline
\end{tabular}

t Test

Isolok - $3 \mathrm{~mL}$ - LF-Coliwasa

Assuming unequal variances

Difference $\quad-0.00105$ t Ratio $\quad-0.51723$

Std Err Dif $\quad 0.00202$ DF 20.97777

Upper CL Dif 0.00316 Prob $>|t| \quad 0.6104$

Lower CL Dif -0.00526 Prob $>t \quad 0.6948$

$\begin{array}{lrl}\text { Confidence } & 0.95 \text { Prob }<\mathrm{t} & 0.3052\end{array}$

Tests that the Variances are Equal

$\begin{array}{lrrrr}\text { Level } & \text { Count } & \text { Std Dev } & \text { MeanAbsDif to Mean } & \text { MeanAbsDif to Median } \\ \text { Coliwasa } & 16 & 0.0053778 & 0.0032715 & 0.0032082 \\ \text { Isolok - 3 mL - LF } & 10 & 0.0047843 & 0.0039173 & 0.0039173\end{array}$

Test F Ratio DFNum DFDen p-Value

O'Brien[.5] 0.0505

$\begin{array}{lll}1 & 24 & 0.8242 \\ 1 & 24 & 0.6429\end{array}$

Brown-Forsythe $\begin{array}{llll}0.2205 & 1 & 24 & 0.6429\end{array}$

$\begin{array}{lllll}\text { Levene } & 0.1955 & 1 & 24 & 0.6623 \\ \text { Bartlett } & 0.1441 & 1 & . & 0.7043\end{array}$

$\begin{array}{llrrr}\text { F Test 2-sided } & 1.2635 & 15 & 9 & 0.7417\end{array}$

Welch Anova testing Means Equal, allowing Std Devs Not Equal

F Ratio DFNum DFDen Prob > F

$\begin{array}{llll}0.2675 & 1 & 20.978 & 0.6104\end{array}$

t Test
Oneway Analysis of Measurement By Sampler Type of Material=SME Simulant, Analytical Block $=2$, Oxide $/$ Analyte $=\mathrm{Al} / \mathbf{B}$, Targeted $=2.552$

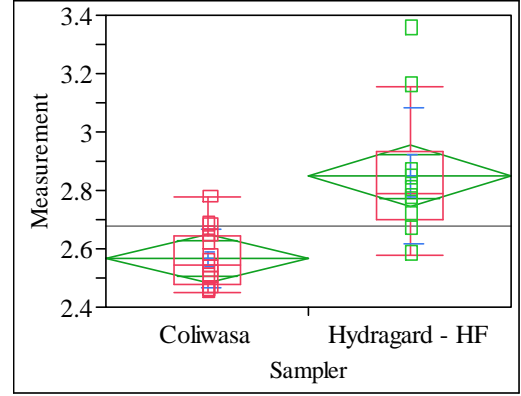

Oneway Anova

Summary of Fit

Rsquare

Adj Rsquare

Observations (or Sum Wgts) $\quad 26$

t Test

Hydragard - HF-Coliwasa

Assuming equal variances

Difference $\quad 0.283778$ t Ratio 4.339738

$\begin{array}{lrlr}\text { Std Err Dif } & 0.065391 & \text { DF } & 24 \\ \text { Upper CL Dif } & 0.418738 & \text { Prob }>|t| & 0.0002\end{array}$

$\begin{array}{llll}\text { Upper CL Dif } & 0.418738 & \text { Prob }>|t| & 0.0002 \\ \text { Lower CL Dif } & 0.148819 & \text { Prob }>t & 0.0001\end{array}$

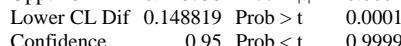

Analysis of Variance

Source DF Sum of Squares Mean Square F Ratio Prob $>$ F

$\begin{array}{lrrrrr}\text { Sampler } & 1 & 0.4955703 & 0.495570 & 18.8333 & 0.0002\end{array}$

$\begin{array}{llll}\text { Error } & 24 & 0.6315236 & 0.026313\end{array}$

C. Total $25 \quad 1.1270939$

Means for Oneway Anova

Level Number Mean Std Error Lower 95\% Upper 95\%

$\begin{array}{llllll}\text { Coliwasa } & 16 & 2.56607 & 0.04055 & 2.4824 & 2.6498\end{array}$

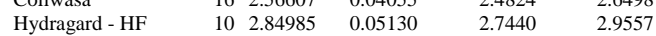

Std Error uses a pooled estimate of error variance

Means and Std Deviations

Level Number Mean Std Dev Std Err Mean Lower 95\% Upper 95\%

$\begin{array}{lrrrrrr}\text { Coliwasa } & 16 & 2.56607 & 0.098929 & 0.02473 & 2.5134 & 2.6188 \\ \text { Hydragard - HF } & 10 & 2.84985 & 0.232073 & 0.07339 & 2.6838 & 3.0159\end{array}$

t Test

Hydragard - HF-Coliwasa

Assuming unequal variances

$\begin{array}{lrlr}\text { Difference } & 0.283778 & \text { t Ratio } & 3.664341 \\ \text { Std Err Dif } & 0.077443 & \text { DF } & 11.07472 \\ \text { Upper CL Dif } & 0.454090 & \text { Prob }>|t| & 0.0037 \\ \text { Lower CL Dif } & 0.113467 & \text { Prob }>\text { t } & 0.0018 \\ \text { Confidence } & 0.95 & \text { Prob }<\mathrm{t} & 0.9982\end{array}$

Tests that the Variances are Equal

$\begin{array}{lrrrr}\text { Level } & \text { Count } & \text { Std Dev } & \text { MeanAbsDif to Mean } & \text { MeanAbsDif to Median } \\ \text { Coliwasa } & 16 & 0.0989291 & 0.0824976 & 0.0801678\end{array}$

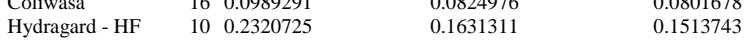

Test F Ratio DFNum DFDen p-Value

$\begin{array}{rrrr}3.7019 & 1 & 24 & 0.0663\end{array}$

$\begin{array}{lllll}\text { Brown-Forsythe } & 2.1545 & 1 & 24 & 0.1551\end{array}$

$\begin{array}{lllll}\text { Levene } & 3.7435 & 1 & 24 & 0.0649\end{array}$

$\begin{array}{lllrl}\text { Bartlett } & 8.0250 & 1 & & 0.0046 \\ \text { F Test 2-sided } & 5.5030 & 9 & 15 & 0.0039\end{array}$

Welch Anova testing Means Equal, allowing Std Devs Not Equal

F Ratio DFNum DFDen Prob $>$ F

$\begin{array}{llll}13.4274 & 1 & 11.075 & 0.0037\end{array}$

t Test 
SRNL-STI-2011-00693

Revision 0

\section{Exhibit C2. Statistical Comparisons for Low-Rheology (Phase 3) Simulant Testing}

Oneway Analysis of Measurement By Sampler Type of Material=SME Simulant, Analytical Block =2, Oxide/Analyte=Al2O3 (wt \%), Targeted $=10.869$

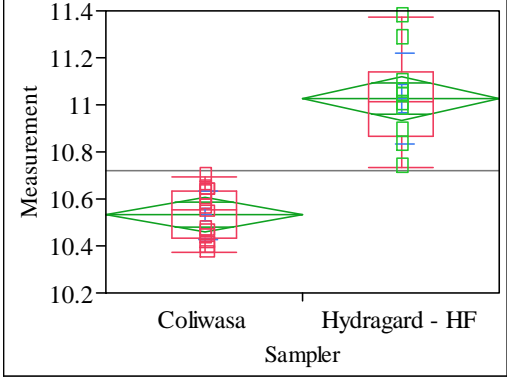

Oneway Anova

Summary of Fit

Adj Rsquare

0.738995

Mean of Response $\quad 0.144532$

10.72146

t Test

Hydragard - HF-Coliwasa

Assuming equal variances

Difference $\quad 0.493632$ t Ratio 8.472528

Std Err Dif 0.058263 DF 24

Upper CL Dif 0.613880 Prob $>|t|<.0001$

Lower CL Dif 0.373384 Prob $>t \quad<.0001$

$\begin{array}{lrl}\text { Confidence } & 0.95 \text { Prob }<\mathrm{t} \quad 1.0000\end{array}$

Analysis of Variance

Source DF Sum of Squares Mean Square F Ratio Prob $>$ F

$\begin{array}{llllll}\text { Sampler } & 1 & 1.4995226 & 1.49952 & 71.7837 & <.0001\end{array}$

$\begin{array}{llll}\text { Error } & 24 & 0.5013468 & 0.02089\end{array}$

C. Total $25 \quad 2.0008694$

Means for Oneway Anova

Level Number Mean Std Error Lower 95\% Upper 95\%

Coliwasa 16 . $10.53160 .03613-10.457$

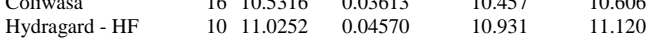

Std Error uses a pooled estimate of error variance

Means and Std Deviations

Level Number Mean Std Dev Std Err Mean Lower 95\% Upper 95\%

$\begin{array}{lllllll}\text { Coliwasa } & 16 & 10.5316 & 0.103463 & 0.02587 & 10.476 & 10.587\end{array}$

$\begin{array}{lllllll}\text { Hydragard - HF } & 10 & 11.0252 & 0.194587 & 0.06153 & 10.886 & 11.164\end{array}$

Test

Hydragard - HF-Coliwasa

Assuming unequal variances

Difference $\quad 0.493632$ t Ratio 7.395328

Std Err Dif $\quad 0.066749$ DF $\quad 12.23237$

Upper CL Dif 0.638760 Prob $>|t| \quad<.0001$

Lower CL Dif 0.348504 Prob $>t \quad<.0001$

$\begin{array}{lrl}\text { Confidence } & 0.95 \text { Prob }<\mathrm{t} \quad 1.0000\end{array}$

Tests that the Variances are Equal

Level Count Std Dev MeanAbsDif to Mean MeanAbsDif to Median

$\begin{array}{lllll}16 & 0.1034634 & 0.0912274 & 0.0897512\end{array}$

$\begin{array}{lllll}\text { Cydragard - HF } & 10 & 0.1945869 & 0.1417125 & 0.1417125\end{array}$

$\begin{array}{lrrrr}\text { Test } & \text { F Ratio } & \text { DFNum } & \text { DFDen } & \text { p-Value } \\ \text { O'Brien[.5] } & 4.4083 & 1 & 24 & 0.0465 \\ \text { Brown-Forsythe } & 2.2183 & 1 & 24 & 0.1494 \\ \text { Levene } & 2.2492 & 1 & 24 & 0.1467 \\ \text { Bartlett } & 4.4727 & 1 & & 0.0344 \\ \text { F Test 2-sided } & 3.5372 & 9 & 15 & 0.0302\end{array}$

Welch Anova testing Means Equal, allowing Std Devs Not Equal

F Ratio DFNum DFDen Prob $>$ F

$\begin{array}{rrrr}54.6909 & 1 & 12.232 & <.0001\end{array}$

t Test

7.3953
Oneway Analysis of Measurement By Sampler Type of Material=SME Simulant, Analytica Block=2, Oxide/Analyte=B2O3 (wt \%), Targeted $=4.259$

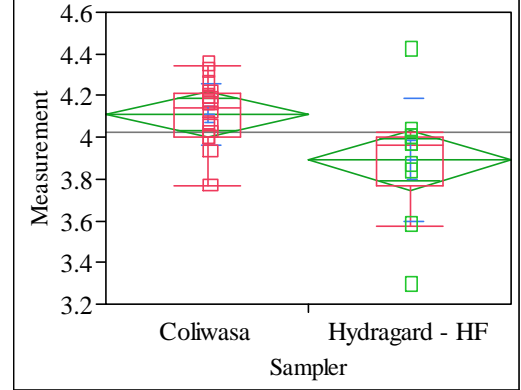

Oneway Anova

Summary of Fit

Rsquare

Adj Rsquare

Mean of Responser $\quad 0.21673$

Observations (or Sum Wgts) $\quad 26$

t Test

Hydragard - HF-Coliwasa

Assuming equal variances

Difference $\quad-0.21976$ t Ratio $\quad-2.51534$

$\begin{array}{lrlr}\text { Std Err Dif } & 0.08737 & \text { DF } & 24 \\ \text { Upper CL Dif } & -0.03944 & \text { Prob }>|t| & 0.0190\end{array}$

$\begin{array}{llll}\text { Upper CL Dif } & -0.03944 & \text { Prob }>|t| & 0.0190 \\ \text { Lower CL Dif } & -0.40008 & \text { Prob }>t & 0.9905\end{array}$

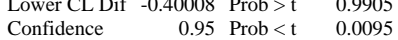

Analysis of Variance

Source DF Sum of Squares Mean Square F Ratio Prob $>$ F

$\begin{array}{lrrrrr}\text { Sampler } & 1 & 0.2971917 & 0.297192 & 6.3269 & 0.0190\end{array}$

$\begin{array}{llll}\text { Error } & 24 & 1.1273379 & 0.046972\end{array}$

C. Total $25 \quad 1.4245297$

Means for Oneway Anova

Level Number Mean Std Error Lower 95\% Upper 95\%

$\begin{array}{llllrr}\text { Coliwasa } & 16 & 4.10940 & 0.05418 & 3.9976 & 4.2212\end{array}$

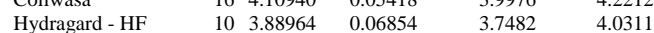

Std Error uses a pooled estimate of error variance

Means and Std Deviations

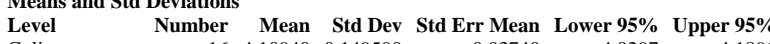

$\begin{array}{lrrrrrr}\text { Coliwasa } & 16 & 4.10940 & 0.149590 & 0.03740 & 4.0297 & 4.1891\end{array}$

$\begin{array}{lllllll}\text { Hydragard - HF } & 10 & 3.88964 & 0.296588 & 0.09379 & 3.6775 & 4.1018\end{array}$

t Test

Hydragard - HF-Coliwasa

Assuming unequal variances

Difference $\quad-0.21976$ t Ratio $\quad-2.17646$

Std Err Dif $\quad 0.10097$ DF 11.90874

Upper CL Dif 0.00042 Prob $>|t| \quad 0.0504$

Lower CL Dif -0.43994 Prob $>t \quad 0.9748$

$\begin{array}{lll}\text { Confidence } \quad 0.95 \text { Prob }<\mathrm{t} \quad 0.0252 & \end{array}$

Tests that the Variances are Equal

$\begin{array}{lrrrr}\text { Level } & \text { Count } & \text { Std Dev } & \text { MeanAbsDif to Mean } & \text { MeanAbsDif to Median } \\ \text { Coliwasa } & 16 & 0.1495897 & 0.1182307 & 0.1167214\end{array}$

$\begin{array}{lrrrr}\text { Coliwasa } & 16 & 0.1495897 & 0.1182307 & 0.1167214 \\ \text { Hydragard - HF } & 10 & 0.2965884 & 0.2009218 & 0.1867542\end{array}$

Test F Ratio DFNum DFDen p-Value

$\begin{array}{lllll}2.7102 & 1 & 24 & 0.1127\end{array}$

$\begin{array}{lllll}\text { Brown-Forsythe } 1.1618 & 1 & 24 & 0.2918\end{array}$

$\begin{array}{lllll}\text { Levene } & 2.0198 & 1 & 24 & 0.168 \\ \text { Bartlett } & 5.2388 & 1 & & 0.0221\end{array}$

$\begin{array}{lllrl}\text { Bartlett } & 5.2388 & 1 & & 0.0221 \\ \text { F Test 2-sided } & 3.9310 & 9 & 15 & 0.0192\end{array}$

Welch Anova testing Means Equal, allowing Std Devs Not Equal

F Ratio DFNum DFDen Prob $>$ F

$\begin{array}{llll}4.7370 & 1 & 11.909 & 0.0504\end{array}$

t Test
2.1765 
SRNL-STI-2011-00693

Revision 0

\section{Exhibit C2. Statistical Comparisons for Low-Rheology (Phase 3) Simulant Testing}

Oneway Analysis of Measurement By Sampler Type of Material=SME Simulant, Analytical Block $=2$, Oxide $/$ Analyte $=\mathrm{BaO}(\mathrm{wt} \%)$, Targeted $=\mathbf{0 . 0 9 1 9}$

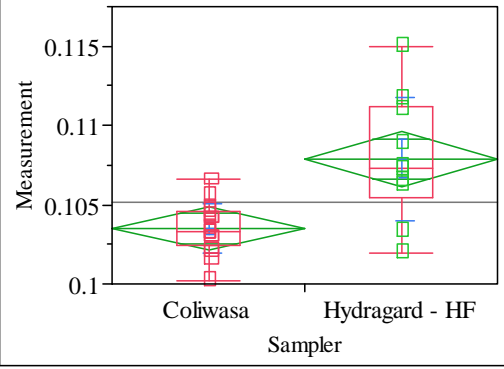

Oneway Anova

Rsquare $\quad 0.404738$

Adj Rsquare $\quad 0.37993$

Root Mean Square Error $\quad 0.002697$

Mean of Response 0.105196

(orservations (or Sum Wgis)

Test

Hydragard - HF-Coliwasa

Assuming equal variances

Difference $\quad 0.004392$ t Ratio 4.039605

$\begin{array}{lrlr}\text { Std Err Dif } & 0.001087 & \text { DF } & 24 \\ \text { Upper CL Dif } & 0.006636 & \text { Prob }>|t| & 0.0005\end{array}$

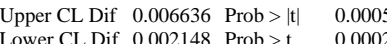

$\begin{array}{lrll}\text { Lower CL Dif } & 0.002148 & \text { Prob }>t & 0.0002 \\ \text { Confidence } & 0.95 & \text { Prob }<\mathrm{t} & 0.9998\end{array}$

Analysis of Variance

Source DF Sum of Squares Mean Square F Ratio Prob $>$ F

$\begin{array}{lrrrrr}\text { Sampler } & 1 & 0.00011871 & 0.000119 & 16.3184 & 0.0005\end{array}$

$\begin{array}{llll}\text { Error } & 24 & 0.00017459 & 7.274 \mathrm{e}-6\end{array}$

C. Total $25 \quad 0.00029329$

Means for Oneway Anova

Level Number Mean Std Error Lower 95\% Upper 95\%

$\begin{array}{lrrrrr}\text { Coliwasa } & 16 & 0.103507 & 0.00067 & 0.10211 & 0.10490\end{array}$

$\begin{array}{llllll}\text { Coliwasa } & 16 & 0.103507 & 0.00067 & 0.10211 & 0.10490 \\ \text { Hydragard - HF } & 10 & 0.107899 & 0.00085 & 0.10614 & 0.10966\end{array}$

Std Error uses a pooled estimate of error variance

Means and Std Deviations

Level Number Mean Std Dev Std Err Mean Lower 95\% Upper 95\%

$\begin{array}{llllllr}\text { Coliwasa } & 16 & 0.103507 & 0.001573 & 0.00039 & 0.10267 & 0.10434\end{array}$

$\begin{array}{lllllll}\text { Hydragard - HF } & 10 & 0.107899 & 0.003908 & 0.00124 & 0.10510 & 0.11069\end{array}$

t Test

Hydragard - HF-Coliwasa

Assuming unequal variances

Difference $\quad 0.004392$ t Ratio $\quad 3.386539$

Std Err Dif $\quad 0.001297$ DF 10.84917

Upper CL Dif 0.007251 Prob $>|t| 0.0062$

Lower CL Dif 0.001533 Prob $>t \quad 0.0031$

Confidence $\quad 0.95$ Prob $<\mathrm{t} \quad 0.9969$

Tests that the Variances are Equal

Level Count Std Dev MeanAbsDif to Mean MeanAbsDif to Median

$\begin{array}{lrrrr}\text { Coliwasa } & 16 & 0.0015734 & 0.0011941 & 0.0011933 \\ \text { Hydragard - HF } & 10 & 0.0039080 & 0.0029788 & 0.0028806\end{array}$

Test F Ratio DFNum DFDen p-Value

$\begin{array}{lrrrr}\text { O'Brien[.5] } & \text { F Ratio } & \text { DFNum } & \text { DFDen } & \text { p-Value } \\ \text { O'1335 } & 1 & 24 & 0.0207\end{array}$

$\begin{array}{lllll}\text { O'Brien[.5] } & 6.1335 & 1 & 24 & 0.0207 \\ \text { Brown-Forsythe } & 5.7640 & 1 & 24 & 0.0245 \\ \text { Levene } & 7.4625 & 1 & 24 & 0.0116\end{array}$

$\begin{array}{lllll}\text { Levene } & 7.4625 & 1 & 24 & 0.0116 \\ \text { Bartlett } & 9.0809 & 1 & . & 0.0026\end{array}$

$\begin{array}{lllll}\text { F Test 2-sided } & 6.1691 & 9 & 15 & 0.0022\end{array}$

Welch Anova testing Means Equal, allowing Std Devs Not Equal

F Ratio DFNum DFDen Prob $>$ F

$\begin{array}{llll}11.4686 & 1 & 10.849 & 0.0062\end{array}$

t Test
3.3865
Oneway Analysis of Measurement By Sampler Type of Material=SME Simulant, Analytical Block $=2$, Oxide/Analyte $=\mathrm{CaO}(\mathrm{wt} \%)$, Targeted $=\mathbf{1 . 1 4 6 2}$

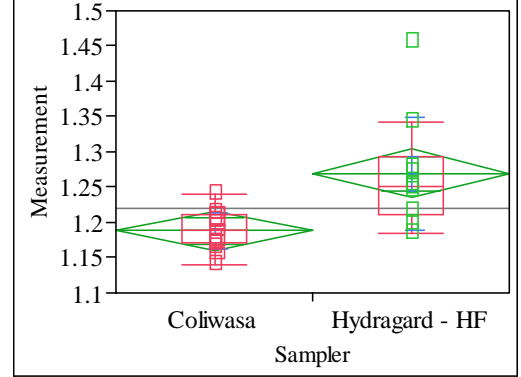

Oneway Anova

Summary of Fi

Adj Rsquare

0.377076

Root Mean Square Error $\quad 0.052761$

Mean of Response 1.219188

Observations (or Sum Wgts) $\quad 26$

t Test

Hydragard - HF-Coliwasa

Assuming equal variances

Difference $\quad 0.081066$ t Ratio 3.81156

Std Err Dif $\quad 0.021268$ DF 24

Upper CL Dif 0.124962 Prob $>|t| \quad 0.0008$

Lower CL Dif 0.037170 Prob $>$ t 0.0004

$\begin{array}{lrl}\text { Confidence } & 0.95 \text { Prob }<\mathrm{t} & 0.9996\end{array}$

Analysis of Variance

Source DF Sum of Squares Mean Square F Ratio Prob $>$ F

$\begin{array}{lllllll}\text { Sampler } & 1 & 0.04044136 & 0.040441 & 14.5280 & 0.0008\end{array}$

$\begin{array}{llll}\text { Error } & 24 & 0.06680846 & 0.002784 \\ \text { C. Total } & 25 & 0.10724982 & \end{array}$

Means for Oneway Anova

Level Number Mean Std Error Lower 95\% Upper 95\%

$\begin{array}{lrrrrr}\text { Coliwasa } & 16 & 1.18801 & 0.01319 & 1.1608 & 1.2152\end{array}$

$\begin{array}{llllll}\text { Coliwasa } & 16 & 1.18801 & 0.01319 & 1.1608 & 1.2152 \\ \text { Hydragard - HF } & 10 & 1.26907 & 0.01668 & 1.2346 & 1.3035\end{array}$

Std Error uses a pooled estimate of error variance

Means and Std Deviations

Level Number Mean Std Dev Std Err Mean Lower 95\% Upper 95\%

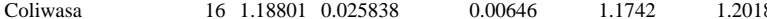

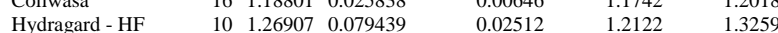

Test

Hydragard - HF-Coliwasa

Assuming unequal variances

Difference $\quad 0.081066$ t Ratio 3.125391

Std Err Dif $\quad 0.025938$ DF $\quad 10.20272$

Upper CL Dif 0.138704 Prob $>|t| \quad 0.0105$

Lower CL Dif 0.023428 Prob $>t \quad 0.0053$

$\begin{array}{lrl}\text { Confidence } & 0.95 \text { Prob }<\mathrm{t} & 0.9947\end{array}$

Tests that the Variances are Equal

Level Count Std Dev MeanAbsDif to Mean MeanAbsDif to Median

$\begin{array}{lllll}\text { Coliwasa } & 16 & 0.0258377 & 0.0207366 & 0.0207257\end{array}$

Test F Ratio DFNum DFDen p-Value

$\begin{array}{lrrrr}\text { O'Brien[.5] } & 3.3618 & 1 & 24 & 0.0792\end{array}$

$\begin{array}{lllll}\text { Brown-Forsythe } & 3.6121 & 1 & 24 & 0.0694\end{array}$

$\begin{array}{lrlll}\text { Levene } & 5.4593 & 1 & 24 & 0.0281\end{array}$

$\begin{array}{lrrrr}\text { Bartlett } & 13.4421 & 1 & \text {. } & 0.0002 \\ \text { F Test 2-sided } & 9.4527 & 9 & 15 & 0.0002\end{array}$

Welch Anova testing Means Equal, allowing Std Devs Not Equal

F Ratio DFNum DFDen Prob $>$ F

$9.7681 \quad 1 \quad 10.203 \quad 0.0105$

t Test

3.1254 
SRNL-STI-2011-00693

Revision 0

\section{Exhibit C2. Statistical Comparisons for Low-Rheology (Phase 3) Simulant Testing}

Oneway Analysis of Measurement By Sampler Type of Material=SME Simulant, Analytical Block $=2$, Oxide $/$ Analyte $=\mathrm{Cr} 2 \mathrm{O} 3(\mathrm{wt} \%)$, Targeted $=\mathbf{0 . 0 9 9 8}$

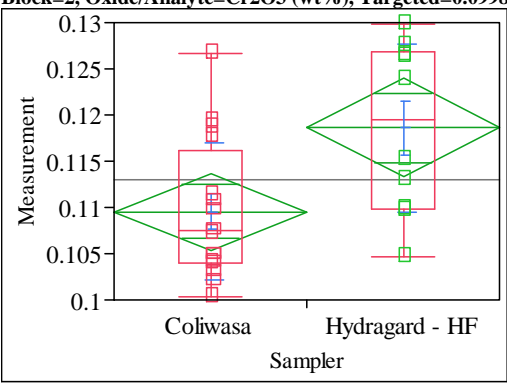

Oneway Anova

Rsquare $\quad 0.242137$

Adj Rsquare $\quad 0.21056$

Root Mean Square Error $\quad 0.008123$

Mean of Response $\quad 0.113044$

t Tes

Hydragard - HF-Coliwasa

Assuming equal variances

Difference $\quad 0.009067$ t Ratio 2.769116

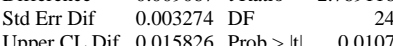

$\begin{array}{lll}\text { Upper CL Dif } 0.015826 & \text { Prob }>|t| & 0.0107 \\ \text { Con } & & 0.0053\end{array}$

$\begin{array}{lrll}\text { Lower CL Dif } & 0.002309 & \text { Prob }>t & 0.0053 \\ \text { Confidence } & 0.95 & \text { Prob }<\mathrm{t} & 0.9947\end{array}$

Analysis of Variance

Source DF Sum of Squares Mean Square F Ratio Prob $>$ F

$\begin{array}{llllll}\text { Sampler } & 1 & 0.00050596 & 0.000506 & 7.6680 & 0.0107\end{array}$

Error $24 \quad 0.0015835$

0.000066

Means for Oneway Anova

$\begin{array}{lrrrrr}\text { Level } & \text { Number } & \text { Mean } & \text { Std Error } & \text { Lower 95\% } & \text { Upper 95\% } \\ \text { Coliwasa } & 16 & 0.109556 & 0.00203 & 0.10536 & 0.11375\end{array}$

$\begin{array}{llllll}\text { Coliwasa } & 16 & 0.109556 & 0.00203 & 0.10536 & 0.11375 \\ \text { Hydragard - HF } & 10 & 0.118623 & 0.00257 & 0.11332 & 0.12393\end{array}$

Std Error uses a pooled estimate of error variance

Means and Std Deviations

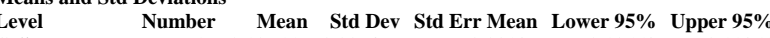

$\begin{array}{lrrrrrr}\text { Coliwasa } & 16 & 0.109556 & 0.007467 & 0.00187 & 0.10558 & 0.11354\end{array}$

$\begin{array}{lllllll}\text { Hydragard - HF } & 10 & 0.118623 & 0.009112 & 0.00288 & 0.11211 & 0.12514\end{array}$

t Test

Hydragard - HF-Coliwasa

Assuming unequal variances

Difference $\quad 0.009067$ t Ratio $\quad 2.641066$

Std Err Dif $\quad 0.003433 \mathrm{DF} \quad 16.40707$

Upper CL Dif 0.016331 Prob $>|t| \quad 0.0175$

Lower CL Dif 0.001804 Prob $>t \quad 0.0088$

$\begin{array}{lll}\text { Confidence } & 0.95 \text { Prob }<\mathrm{t} \quad 0.9912\end{array}$

Tests that the Variances are Equal

$\begin{array}{lrrrr}\text { Level } & \text { Count } & \text { Std Dev } & \text { MeanAbsDif to Mean } & \text { MeanAbsDif to Median } \\ \text { Coliwasa } & 16 & 0.0074672 & 0.0059389 & 0.0057094\end{array}$

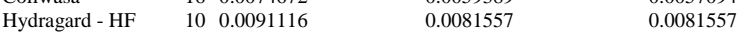

Test F Ratio DFNum DFDen p-Value

$\begin{array}{lrrrr}\text { O'Brien[.5] } & 0.8385 & 1 & 24 & 0.3689\end{array}$

$\begin{array}{lllll}\text { O'Brien[.5] } & 0.8385 & 1 & 24 & 0.3689 \\ \text { Brown-Forsythe } & 1.8538 & 1 & 24 & 0.1860\end{array}$

$\begin{array}{lllll}\text { Levene } & 2.0497 & 1 & 24 & 0.1651 \\ \text { Bartlett } & 0.4380 & 1 & . & 0.5081\end{array}$

$\begin{array}{lllll}\text { F Test 2-sided } & 1.4889 & 9 & 15 & 0.4761\end{array}$

Welch Anova testing Means Equal, allowing Std Devs Not Equal

F Ratio DFNum DFDen Prob $>$ F

$\begin{array}{llll}6.9752 & 1 & 16.407 & 0.0175\end{array}$

t Test

2.6411
Oneway Analysis of Measurement By Sampler Type of Material=SME Simulant, Analytical Block $=2$, Oxide $/$ Analyte $=\mathbf{C u O}(w t \%)$, Targeted $=\mathbf{0 . 0 5 0 4}$

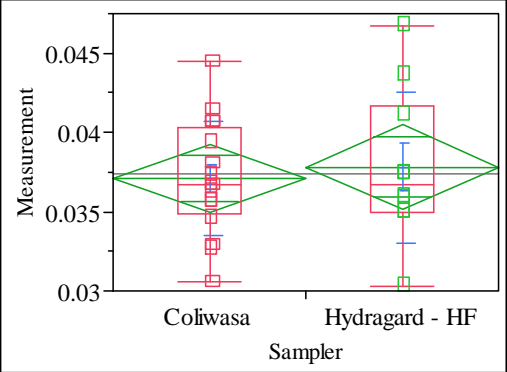

Oneway Anova

Summary of Fit

Rsquare

Adj Rsquare

0.00408

Observations (or Sum Wgts) $\quad 26$

t Test

Hydragard - HF-Coliwasa

Assuming equal variances

Difference $\quad 0.00071$ t Ratio $\quad 0.432882$

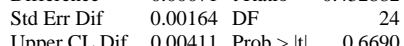

$\begin{array}{rrr}\text { Upper CL Dif } & 0.00411 \text { Prob }>|t| & 0.6690 \\ \text { Lower CL Dif }-0.00268 & \text { Prob }>t & 0.3345\end{array}$

$\begin{array}{lrrr}\text { Lower CL Dif } & -0.00268 & \text { Prob }>t & 0.3345 \\ \text { Confidence } & 0.95 & \text { Prob }<\mathrm{t} & 0.6655\end{array}$

Analysis of Variance

Source DF Sum of Squares Mean Square F Ratio Prob $>$ F

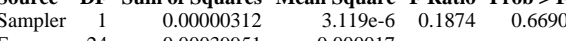

$\begin{array}{lll}\text { Error } & 24 & 0.00039951 \\ \text { C. Total } & 25 & 0.00040263\end{array}$

Means for Oneway Anova

Level Number Mean Std Error Lower 95\% Upper 95\%

$\begin{array}{lrrrrr}\text { Coliwasa } & 16 & 0.037092 & 0.00102 & 0.03499 & 0.03920\end{array}$

$\begin{array}{llllll}\text { Coliwasa } & 16 & 0.037092 & 0.00102 & 0.03499 & 0.03920 \\ \text { Hydragard - HF } & 10 & 0.037804 & 0.00129 & 0.03514 & 0.04047\end{array}$

Std Error uses a pooled estimate of error variance

Means and Std Deviation

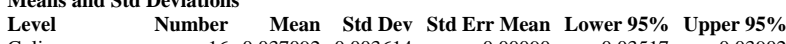

$\begin{array}{llllllr}\text { Coliwasa } & 16 & 0.037092 & 0.003614 & 0.00090 & 0.03517 & 0.03902\end{array}$

$\begin{array}{lllllll}\text { Hydragard - HF } & 10 & 0.037804 & 0.004756 & 0.00150 & 0.03440 & 0.04121\end{array}$

t Test

Hydragard - HF-Coliwasa

Assuming unequal variances

Difference $\quad 0.00071$ t Ratio 0.405795

Std Err Dif $\quad 0.00175$ DF 15.46207

Upper CL Dif 0.00444 Prob $>|t| 0.6905$

Lower CL Dif -0.00302 Prob $>\mathrm{t} \quad 0.3452$

$\begin{array}{lll}\text { Confidence } \quad 0.95 \text { Prob }<\mathrm{t} \quad 0.6548 & \end{array}$

Tests that the Variances are Equal

$\begin{array}{lrrrr}\text { Level } & \text { Count } & \text { Std Dev } & \text { MeanAbsDif to Mean } & \text { MeanAbsDif to Median } \\ \text { Coliwasa } & 16 & 0.0036144 & 0.0027823 & 0.0027305\end{array}$

$\begin{array}{lrrrr} & 16 & 0.0036144 & 0.0027823 & 0.0027305 \\ \text { Hydragard - HF } & 10 & 0.0047557 & 0.0035801 & 0.0034299\end{array}$

Test F Ratio DFNum DFDen p-Value

$\begin{array}{lrrrr}\text { O'Brien[.5] } & 0.9687 & 1 & 24 & 0.3348\end{array}$

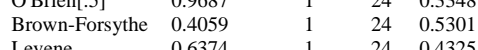

$\begin{array}{lllll}\text { Levene } & 0.6374 & 1 & 24 & 0.4325 \\ \text { Bartlett } & 0.8384 & 1 & & 0.3599\end{array}$

$\begin{array}{lllll}\text { F Test 2-sided } & 1.7312 & 9 & 15 & 0.3338\end{array}$

Welch Anova testing Means Equal, allowing Std Devs Not Equal

F Ratio DFNum DFDen Prob > F

$\begin{array}{llll}0.1647 & 1 & 15.462 & 0.6905\end{array}$

t Test

0.4058 


\section{Exhibit C2. Statistical Comparisons for Low-Rheology (Phase 3) Simulant Testing}

Oneway Analysis of Measurement By Sampler Type of Material=SME Simulant, Analytica Block=2, Oxide/Analyte $=\mathrm{Fe} / \mathrm{Li}$, Targeted $=2.452$

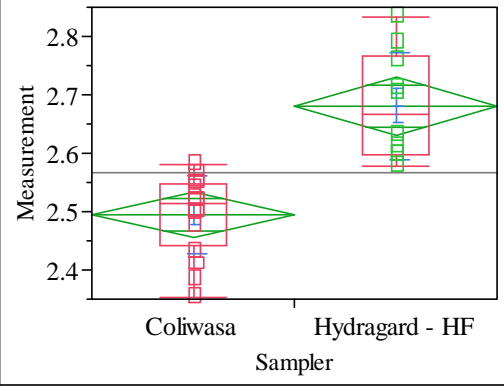

\section{Oneway Anova}

Summary of Fit

Rsquare

Adj Rsquare

$\begin{array}{lr}\text { Mean of Response } & 2.56638 \\ \text { Observations (or Sum Wgts) } & 26\end{array}$

t Test

Hydragard - HF-Coliwasa

Assuming equal variances

Difference $\quad 0.186082$ t Ratio 5.968717

$\begin{array}{lrlr}\text { Std Err Dif } & 0.031176 & \text { DF } & 24 \\ \text { Upper CL Dif } & 0.250426 & \text { Prob }>|t| & <.0001\end{array}$

$\begin{array}{llll}\text { Upper CL Dif } & 0.250426 & \text { Prob }>|t| & <.0001 \\ \text { Lower CL Dif } & 0.121737 & \text { Prob }>t & <.0001\end{array}$

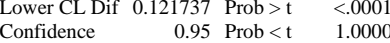

Analysis of Variance

Source DF Sum of Squares Mean Square F Ratio Prob $>$ F

$\begin{array}{lllllll}\text { Sampler } & 1 & 0.21308548 & 0.213085 & 35.6256 & <.0001\end{array}$

$\begin{array}{llll}\text { Error } & 24 & 0.14354999 & 0.005981\end{array}$

C. Total $25 \quad 0.35663547$

Means for Oneway Anova

Level Number Mean Std Error Lower 95\% Upper 95\%

$\begin{array}{lllllr}\text { Coliwasa } & 16 & 2.49481 & 0.01933 & 2.4549 & 2.5347\end{array}$

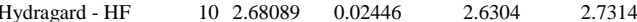

Std Error uses a pooled estimate of error variance

Means and Std Deviations

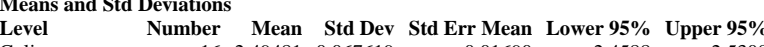

$\begin{array}{lrrrrrr}\text { Coliwasa } & 16 & 2.49481 & 0.067619 & 0.01690 & 2.4588 & 2.5308 \\ \text { Hydragard - HF } & 10 & 2.68089 & 0.091266 & 0.02886 & 2.6156 & 2.7462\end{array}$

Test

Hydragard - HF-Coliwasa

Assuming unequal variances

Difference $\quad 0.186082$ t Ratio 5.563443

Std Err Dif $\quad 0.033447$ DF 15.16393

Upper CL Dif 0.257306 Prob $>|t| r .0001$

$\begin{array}{lrl}\text { Lower CL Dif } & 0.114858 \text { Prob }>t & <.0001\end{array}$

Confidence $\quad 0.95$ Prob $<\mathrm{t} \quad 1.0000$

Tests that the Variances are Equal

$\begin{array}{lrrrr}\text { Level } & \text { Count } & \text { Std Dev } & \text { MeanAbsDif to Mean } & \text { MeanAbsDif to Median } \\ \text { Coliwasa } & 16 & 0.0676190 & 0.0527781 & 0.0507038 \\ \text { Hydragard - HF } & 10 & 0.0912659 & 0.0784223 & 0.0784223\end{array}$

Test F Ratio DFNum DFDen p-Value

$\begin{array}{lllll}\text { O'Brien[.5] } & 1.8636 & 1 & 24 & 0.1849\end{array}$

$\begin{array}{lllll}\text { Brown-Forsythe } & 2.3525 & 1 & 24 & 0.1382\end{array}$

$\begin{array}{lllll}\text { Levene } & 2.5914 & 1 & 24 & 0.1205\end{array}$

$\begin{array}{lllll}\text { Bartlett } & 1.0031 & 1 & . & 0.3166\end{array}$

$\begin{array}{lllll}\text { F Test 2-sided } & 1.8217 & 9 & 15 & 0.2926\end{array}$

Welch Anova testing Means Equal, allowing Std Devs Not Equal

F Ratio DFNum DFDen Prob $>$ F

$\begin{array}{llll}30.9519 & 1 & 15.164 & <.0001\end{array}$

t Test
Oneway Analysis of Measurement By Sampler Type of Material=SME Simulant, Analytical Block $=2$, Oxide $/$ Analyte $=\mathrm{Fe} 2 \mathrm{O} 3$ ( $w$ t $\%)$, Targeted $=11.462$

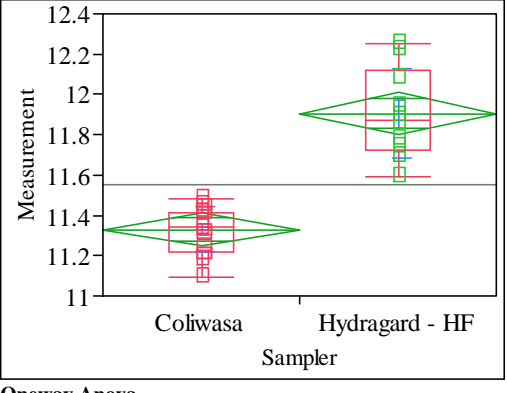

\section{Oneway Anova}

Summary of Fit

Adj Rsquare

0.762587

0.752694
Root Mean Square Error $\quad 0.162385$

Mean of Response $\quad 11.55143$

Observations (or Sum Wgts)

t Test

Hydragard - HF-Coliwasa

Assuming equal variances

Difference $\quad 0.574739$ t Ratio 8.78006

Std Err Dif $\quad 0.065460$ DF 24

Upper CL Dif 0.709841 Prob $>|t|<.0001$

Lower CL Dif 0.439637 Prob $>t \quad<.0001$

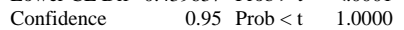

Analysis of Variance

Source DF Sum of Squares Mean Square F Ratio Prob $>$ F

$\begin{array}{lllllll}\text { Sampler } & 1 & 2.0327716 & 2.03277 & 77.0895 & <.0001\end{array}$

$\begin{array}{llll}\text { Error } & 24 & 0.6328559 & 0.02637 \\ \text { C. Total } & 25 & 2.6656274 & \end{array}$

Means for Oneway Anova

Level Number Mean Std Error Lower 95\% Upper 95\%

$\begin{array}{llrrrr}\text { Coliwasa } & 16 & 11.3304 & 0.04060 & 11.247 & 11.414 \\ \text { Hydragard - HF } & 10 & 11.9051 & 0.05135 & 11.799 & 12.011\end{array}$

Std Error uses a pooled estimate of error variance

Means and Std Deviations

Level Number Mean Std Dev Std Err Mean Lower 95\% Upper 95\%

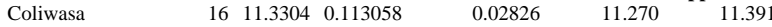

$\begin{array}{lllllll}\text { Hydragard - HF } & 10 & 11.9051 & 0.221391 & 0.07001 & 11.747 & 12.063\end{array}$

t Test

Hydragard - HF-Coliwasa

Assuming unequal variances

Difference $\quad 0.574739$ t Ratio 7.612432

Std Err Dif $\quad 0.075500$ DF $\quad 11.98194$

Upper CL Dif 0.739268 Prob $>|t| \quad<.0001$

Lower CL Dif 0.410211 Prob $>$ t $\quad<.0001$

$\begin{array}{lrl}\text { Confidence } & 0.95 \text { Prob }<\mathrm{t} & 1.0000\end{array}$

Tests that the Variances are Equal

Level Count Std Dev MeanAbsDif to Mean MeanAbsDif to Median

$\begin{array}{lllll}\text { Coliwasa } & 16 & 0.1130578 & 0.0920369 & 0.0911434\end{array}$

$\begin{array}{lllll}\text { Hydragard - HF } & 10 & 0.2213907 & 0.1749953 & 0.1729937\end{array}$

Test F Ratio DFNum DFDen p-Value

$\begin{array}{lrrrr}\text { O'Brien[.5] } & 6.5726 & 1 & 24 & 0.0170\end{array}$

$\begin{array}{lllll}\text { Brown-Forsythe } & 4.6272 & 1 & 24 & 0.0417\end{array}$

$\begin{array}{lllll}\text { Levene } & 5.3191 & 1 & 24 & 0.0300\end{array}$

$\begin{array}{lllll}\text { Bartlett } & 5.0537 & 1 & \dot{0} & 0.0246 \\ \text { F Test 2-sided } & 3.8346 & 9 & 15 & 0.0214\end{array}$

Welch Anova testing Means Equal, allowing Std Devs Not Equal

F Ratio DFNum DFDen Prob $>$ F

$\begin{array}{llll}57.9491 & 1 & 11.982 & <0001\end{array}$

t Test

7.6124 
SRNL-STI-2011-00693

Revision 0

\section{Exhibit C2. Statistical Comparisons for Low-Rheology (Phase 3) Simulant Testing}

Oneway Analysis of Measurement By Sampler Type of Material=SME Simulant, Analytical Block=2, Oxide/Analyte=K2O (wt \%), Targeted $=\mathbf{0 . 1 5 9 1}$

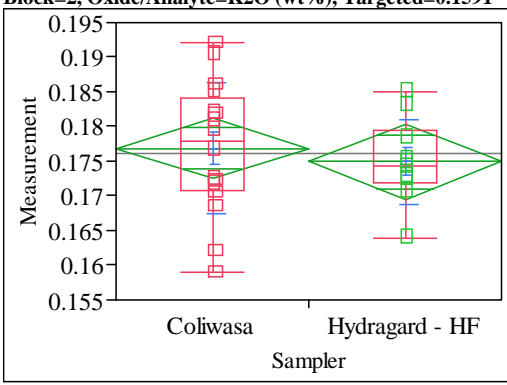

Oneway Anova

Rsquare $\quad 0.013262$

Adj Rsquare $\quad-0.0278$

Root Mean Square Error $\quad 0.008385$

Mean of Response 0.17615

Observations (or Sum Wgts)

Test

Hydragard - HF-Coliwasa

Assuming equal variances

Difference $\quad-0.00192$ t Ratio -0.56795

$\begin{array}{lrrr}\text { Std Err Dif } & 0.00338 \text { DF } & 24 \\ \text { Upper CL Dif } & 0.00506 & \text { Prob }>|t| & 0.5753\end{array}$

$\begin{array}{lrlr}\text { Upper CL Dif } & 0.00506 & \text { Prob }>|t| & 0.5753 \\ \text { Lower CL Dif } & -0.00890 & \text { Prob }>t & 0.7123\end{array}$

$\begin{array}{lrl}\text { Confidence } & 0.95 \text { Prob }<\mathrm{t} & 0.2877\end{array}$

Analysis of Variance

Source DF Sum of Squares Mean Square F Ratio Prob $>$ F

$\begin{array}{lrllll}\text { Sampler } & 1 & 0.00002268 & 0.000023 & 0.3226 & 0.5753\end{array}$

$\begin{array}{lll}\text { Error } & 24 & 0.00168756 \\ \text { C. Total } & 25 & 0.00171024\end{array}$

Means for Oneway Anova

Level Number Mean Std Error Lower 95\% Upper 95\%

$\begin{array}{lrrrrr}\text { Coliwasa } & 16 & 0.176888 & 0.00210 & 0.17256 & 0.18121\end{array}$

$\begin{array}{llllll}\text { Coliwasa } & 16 & 0.176888 & 0.00210 & 0.17256 & 0.18121 \\ \text { Hydragard - HF } & 10 & 0.174968 & 0.00265 & 0.16950 & 0.18044\end{array}$

Std Error uses a pooled estimate of error variance

Means and Std Deviations

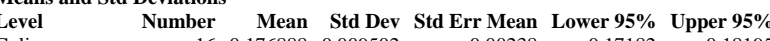

$\begin{array}{lrrrrrr}\text { Coliwasa } & 16 & 0.176888 & 0.009502 & 0.00238 & 0.17182 & 0.18195\end{array}$

$\begin{array}{lllllll}\text { Hydragard - HF } & 10 & 0.174968 & 0.006085 & 0.00192 & 0.17062 & 0.17932\end{array}$

t Test

Hydragard - HF-Coliwasa

Assuming unequal variances

Difference $\quad-0.00192$ t Ratio $\quad-0.62801$

Std Err Dif $\quad 0.00306$ DF 23.95426

Upper CL Dif 0.00439 Prob $>|t| \quad 0.5359$

Lower CL Dif -0.00823 Prob $>t \quad 0.7320$

$\begin{array}{lll}\text { Confidence } & 0.95 \text { Prob }<\mathrm{t} \quad 0.2680\end{array}$

Tests that the Variances are Equal

$\begin{array}{lrrrr}\text { Level } & \text { Count } & \text { Std Dev } & \text { MeanAbsDif to Mean } & \text { MeanAbsDif to Median } \\ \text { Coliwasa } & 16 & 0.0095021 & 0.0077923 & 0.0077923\end{array}$

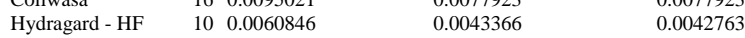

Test F Ratio DFNum DFDen p-Value

$\begin{array}{lllll}\text { O'Brien[.5] } & 2.0717 & 1 & 24 & 0.1630\end{array}$

$\begin{array}{lllll}\text { Brown-Forsythe } & 3.2844 & 1 & 24 & 0.1630 \\ & 3.3404 & 1 & 24 & 0.0825\end{array}$

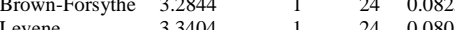

$\begin{array}{lrrrr}\text { Levene } & 3.3404 & 1 & 24 & 0.0801 \\ \text { Bartlett } & 1.9347 & 1 & . & 0.1642\end{array}$

$\begin{array}{llrrr}\text { F Test 2-sided } & 2.4388 & 15 & 9 & 0.1794\end{array}$

Welch Anova testing Means Equal, allowing Std Devs Not Equal

F Ratio DFNum DFDen Prob $>$ F

$\begin{array}{llll}0.3944 & 1 & 23.954 & 0.5359\end{array}$

t Test

0.6280
Oneway Analysis of Measurement By Sampler Type of Material=SME Simulant, Analytica Block=2, Oxide/Analyte $=$ Li2O (wt\%), Targeted $=4.674$

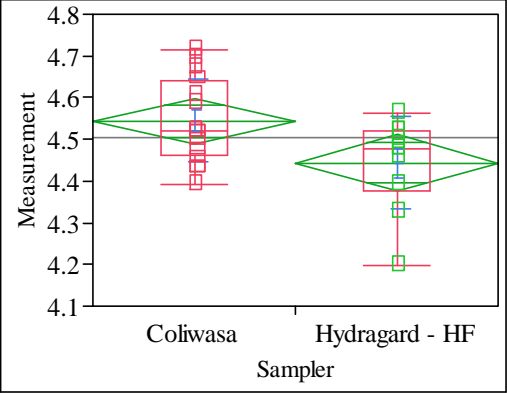

Oneway Anova

Summary of Fit

Rsquare

Adj Rsquare

Mean of Response 4.505357
26

Test

Hydragard - HF-Coliwasa

Assuming equal variances

Difference $\quad-0.10038$ t Ratio $\quad-2.40881$

$\begin{array}{lrlr}\text { Std Err Dif } & 0.04167 & \text { DF } & 24 \\ \text { Upper CL Dif } & -0.01437 & \text { Prob }>|t| & 0.0240\end{array}$

$\begin{array}{lrll}\text { Upper CL Dif } & -0.01437 & \text { Prob }>|t| & 0.0240 \\ \text { Lower CL Dif } & -0.18638 & \text { Prob }>t & 0.9880\end{array}$

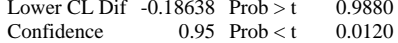

Analysis of Variance

Source DF Sum of Squares Mean Square F Ratio Prob $>$ F

$\begin{array}{lrrrrr}\text { Sampler } & 1 & 0.06200576 & 0.062006 & 5.8024 & 0.0240\end{array}$

$\begin{array}{lll}\text { Error } & 24 & 0.25647074\end{array}$

C. Total $25 \quad 0.31847650$

Means for Oneway Anova

Level Number Mean Std Error Lower 95\% Upper 95\%

$\begin{array}{lllllr}\text { Coliwasa } & 16 & 4.54396 & 0.02584 & 4.4906 & 4.5973\end{array}$

$\begin{array}{llllll}\text { Hydragard - HF } & 10 & 4.44359 & 0.03269 & 4.3761 & 4.5111\end{array}$

Std Error uses a pooled estimate of error variance

Means and Std Deviation

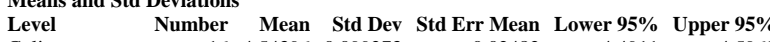

$\begin{array}{lrrrrrr}\text { Coliwasa } & 16 & 4.54396 & 0.099273 & 0.02482 & 4.4911 & 4.5969 \\ \text { Hydragard - HF } & 10 & 4.44359 & 0.109871 & 0.03474 & 4.3650 & 4.5222\end{array}$

\section{t Test}

Hydragard - HF-Coliwasa

Assuming unequal variances

$\begin{array}{lrlr}\text { Difference } & -0.10038 & \text { t Ratio } & -2.35092 \\ \text { Std Err Dif } & 0.04270 & \text { DF } & 17.75419 \\ \text { Upper CL Dif } & -0.01059 & \text { Prob }>|t| & 0.0305 \\ \text { Lower CL Dif } & -0.19017 & \text { Prob }>\mathrm{t} & 0.9848 \\ \text { Confidence } & 0.95 & \text { Prob }<\mathrm{t} & 0.0152\end{array}$

Tests that the Variances are Equal

$\begin{array}{lrrrr}\text { Level } & \text { Count } & \text { Std Dev } & \text { MeanAbsDif to Mean } & \text { MeanAbsDif to Median } \\ \text { Coliwasa } & 16 & 0.0992730 & 0.0824157 & 0.0766971\end{array}$

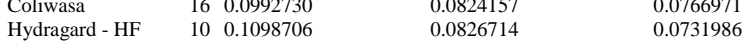

$\begin{array}{llll}\text { Test } & \text { F Ratio DFNum DFDen p-Value }\end{array}$

$\begin{array}{llll}0.1279 & 1 & 24 & 0.7237\end{array}$

$\begin{array}{lllll}\text { Brown-Forsythe } 0.0140 & 1 & 24 & 0.9068\end{array}$

$\begin{array}{lllll}\text { Levene } & 0.0001 & 1 & 24 & 0.9913 \\ \text { Bartlett } & 0.1124 & 1 & & 0.7374\end{array}$

$\begin{array}{lllrl}\text { Bartlett } & 0.1124 & 1 & & 0.7374 \\ \text { F Test 2-sided } & 1.2249 & 9 & 15 & 0.6996\end{array}$

Welch Anova testing Means Equal, allowing Std Devs Not Equal

F Ratio DFNum DFDen Prob $>$ F

$\begin{array}{llll}5.5268 & 1 & 17.754 & 0.0305\end{array}$

t Test 


\section{Exhibit C2. Statistical Comparisons for Low-Rheology (Phase 3) Simulant Testing}

Oneway Analysis of Measurement By Sampler Type of Material=SME Simulant, Analytical Block=2, Oxide/Analyte=MgO (wt \%), Targeted $=1.014$

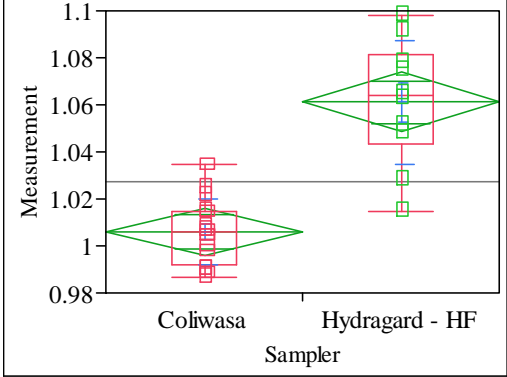

Oneway Anova

Summary of Fit

Adj Rsquare

0.019471

t Test

Hydragard - HF-Coliwasa

Assuming equal variances

Difference $\quad 0.055180$ t Ratio $\quad 7.030263$

Std Err Dif $\quad 0.007849$ DF 24

Upper CL Dif 0.071379 Prob $>|t|<.0001$

Lower CL Dif 0.038981 Prob $>t \quad<.0001$

$\begin{array}{lrl}\text { Confidence } & 0.95 \text { Prob }<\mathrm{t} \quad 1.0000\end{array}$

Analysis of Variance

Source DF Sum of Squares Mean Square F Ratio Prob $>$ F

$\begin{array}{lllllll}\text { Sampler } & 1 & 0.01873738 & 0.018737 & 49.4246 & <.0001\end{array}$

$\begin{array}{llll}\text { Error } & 24 & 0.00909865 & 0.000379\end{array}$

C. Total $25 \quad 0.02783604$

Means for Oneway Anova

Level Number Mean Std Error Lower 95\% Upper 95\%

Coliwasa $161.00597>0.00487-0.9959$

$\begin{array}{llllll}\text { Hydragard - HF } & 16 & 1.00597 & 0.00487 & 0.9959 & 1.0160 \\ & 10 & 1.06115 & 0.00616 & 1.0484 & 1.0739\end{array}$

Std Error uses a pooled estimate of error variance

Means and Std Deviations

Level Number Mean Std Dev Std Err Mean Lower 95\% Upper 95\%

Coliwasa $\quad 16 \quad 1.00597 \quad 0.013859$ S 0.00346

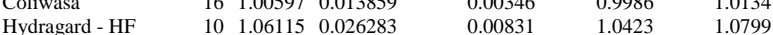

Test

Hydragard - HF-Coliwasa

Assuming unequal variances

Difference $\quad 0.055180$ t Ratio 6.127802

Std Err Dif $\quad 0.009005$ DF 12.17921

Upper CL Dif 0.074768 Prob $>|t| \quad<.0001$

Lower CL Dif 0.035592 Prob $>t \quad<.0001$

$\begin{array}{lrl}\text { Confidence } & 0.95 \text { Prob }<\mathrm{t} \quad 1.0000\end{array}$

Tests that the Variances are Equal

Level Count Std Dev MeanAbsDif to Mean MeanAbsDif to Median

$\begin{array}{lllll}\text { Coliwasa } & 16 & 0.0138595 & 0.0105717 & 0.0105717\end{array}$

$\begin{array}{lllll}\text { Hydragard - HF } & 10 & 0.0262835 & 0.0204303 & 0.0200654\end{array}$

Test F Ratio DFNum DFDen p-Value

$\begin{array}{lrrrr}\text { O'Brien[.5] } & 4.9606 & 1 & 24 & 0.0356\end{array}$

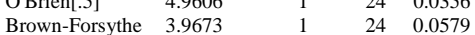

$\begin{array}{lllll}\text { Levene } & 4.5765 & 1 & 24 & 0.0428\end{array}$

$\begin{array}{lllll}\text { Bartlett } & 4.5897 & 1 & & 0.0322 \\ \text { F Test 2-sided } & 3.5964 & 9 & 15 & 0.0282\end{array}$

Welch Anova testing Means Equal, allowing Std Devs Not Equal

F Ratio DFNum DFDen Prob $>$ F

$\begin{array}{rrrr}37.5500 & 1 & 12.179 & <.0001\end{array}$

t Test

6.1278
Oneway Analysis of Measurement By Sampler Type of Material=SME Simulant, Analytical Block $=2$, Oxide/Analyte $=\mathrm{MnO}(\mathrm{wt} \%)$, Targeted $=1.779$

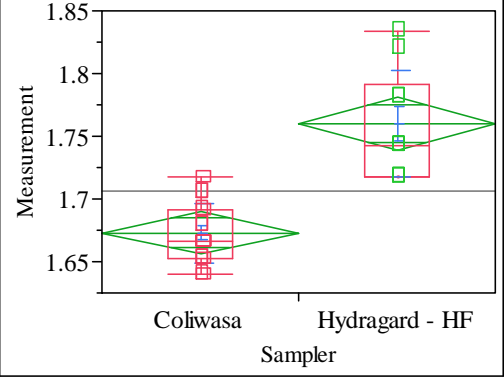

Oneway Anova

Summary of Fit

Adj Rsquare

0.65362

0.65362
Root Mean Square Error $\quad 0.032068$

Mean of Response $\quad 0.032068$

Observations (or Sum Wgts)

t Test

Hydragard - HF-Coliwasa

Assuming equal variances

Difference $\quad 0.086995$ t Ratio 6.72964

Std Err Dif $\quad 0.012927$ DF 24

Upper CL Dif 0.113675 Prob $>|t|<.0001$

Lower CL Dif 0.060314 Prob $>t \quad<.0001$

$\begin{array}{lrl}\text { Confidence } & 0.95 \text { Prob }<\mathrm{t} \quad 1.0000\end{array}$

Analysis of Variance

Source DF Sum of Squares Mean Square F Ratio Prob $>$ F

$\begin{array}{lllllll}\text { Sampler } & 1 & 0.04657268 & 0.046573 & 45.2881 & <.0001\end{array}$

$\begin{array}{llll}\text { Error } & 24 & 0.02468077 & 0.001028\end{array}$

C. Total $25 \quad 0.07125345$

Means for Oneway Anova

Level Number Mean Std Error Lower 95\% Upper 95\%

$\begin{array}{lrrrrr}\text { Coliwasa } & 16 & 1.67291 & 0.00802 & 1.6564 & 1.6895\end{array}$

$\begin{array}{llllll}\text { Hydragard - HF } & 10 & 1.75991 & 0.01014 & 1.7390 & 1.7808\end{array}$

Std Error uses a pooled estimate of error variance

Means and Std Deviations

Level Number Mean Std Dev Std Err Mean Lower 95\% Upper 95\%

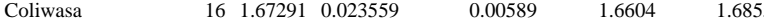

Hydragard - HF 101.759910 .042629

t Test

Hydragard - HF-Coliwasa

Assuming unequal variances

$\begin{array}{lrlr}\text { Difference } & 0.086995 & \text { t Ratio } & 5.913563 \\ \text { Std Err Dif } & 0.014711 & \text { DF } & 12.49092 \\ \text { Upper CL Dif } & 0.118908 & \text { Prob }>|t| & <.0001 \\ \text { Lower CL Dif } & 0.055081 & \text { Prob }>t & <.0001 \\ \text { Confidence } & 0.95 & \text { Prob }<t & 1.0000\end{array}$

Tests that the Variances are Equal

Level Count Std Dev MeanAbsDif to Mean MeanAbsDif to Median

$\begin{array}{lllll}\text { Coliwasa } & 16 & 0.0235592 & 0.0194689 & 0.0185610\end{array}$

$\begin{array}{lllll}\text { Hydragard - HF } & 10 & 0.0426292 & 0.0356371 & 0.0322800\end{array}$

Test F Ratio DFNum DFDen p-Value

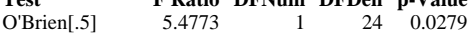

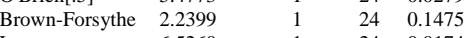

$\begin{array}{lllll}\text { Levene } & 6.5260 & 1 & 24 & 0.0174\end{array}$

$\begin{array}{lllrl}\text { Bartlett } & 3.9471 & 1 & \text {. } & 0.0470 \\ \text { F Test 2-sided } & 3.2741 & 9 & 15 & 0.0415\end{array}$

Welch Anova testing Means Equal, allowing Std Devs Not Equal

F Ratio DFNum DFDen Prob $>$ F

$34.9702 \quad 1 \quad 12.491<0001$

t Test

5.9136 
SRNL-STI-2011-00693

Revision 0

\section{Exhibit C2. Statistical Comparisons for Low-Rheology (Phase 3) Simulant Testing}

Oneway Analysis of Measurement By Sampler Type of Material=SME Simulant, Analytical Block=2, Oxide/Analyte=Na2O (wt \%), Targeted=11.659

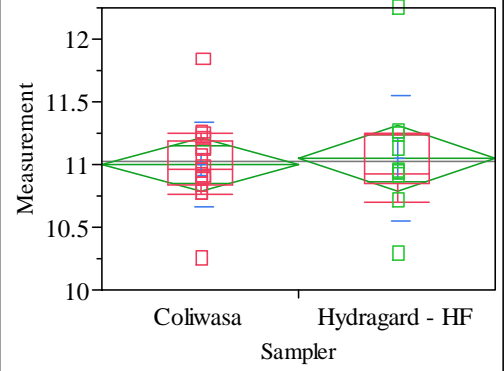

Oneway Anova

Summary of Fit

Adj Rsquare

Root Mean Square Error $\quad-0.03813$

Mean of Response $\quad 0.406213$

Observations (or Sum Wgts) 11.01938

t Test

Hydragard - HF-Coliwasa

Assuming equal variances

Difference $\quad 0.04684$ t Ratio $\quad 0.286064$

Std Err Dif $\quad 0.16375$ DF 24

Upper CL Dif 0.38481 Prob $>|t| \quad 0.7773$

Lower CL Dif -0.29112 Prob $>t \quad 0.3886$

$\begin{array}{lrl}\text { Confidence } & 0.95 \text { Prob }<\mathrm{t} & 0.6114\end{array}$

Analysis of Variance

Source DF Sum of Squares Mean Square F Ratio Prob $>$ F

$\begin{array}{llllll}\text { Sampler } & 1 & 0.0135032 & 0.013503 & 0.0818 & 0.7773\end{array}$

$\begin{array}{llll}\text { Error } & 24 & 3.9602237 & 0.165009\end{array}$

C. Total $25 \quad 3.9737269$

Means for Oneway Anova

Level Number Mean Std Error Lower 95\% Upper 95\%

$\begin{array}{lrrrrr}\text { Coliwasa } & 16 & 11.0014 & 0.10155 & 10.792 & 11.211\end{array}$

$\begin{array}{llllll}\text { Coliwasa } & 16 & 11.0014 & 0.10155 & 10.792 & 11.211 \\ \text { Hydragard - HF } & 10 & 11.0482 & 0.12846 & 10.783 & 11.313\end{array}$

Std Error uses a pooled estimate of error variance

Means and Std Deviations

Level Number Mean Std Dev Std Err Mean Lower 95\% Upper 95\%

$\begin{array}{llllll}\text { Coliwasa } & 16 & 11.0014 & 0.333835 & 0.08346\end{array}$

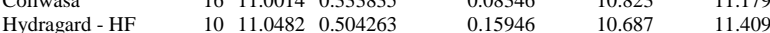

Test

Hydragard - HF-Coliwasa

Assuming unequal variances

Difference $\quad 0.04684$ t Ratio $\quad 0.260265$

Std Err Dif $\quad 0.17998$ DF $\quad 13.9767$

Upper CL Dif 0.43293 Prob $>|t| \quad 0.7985$

Lower CL Dif -0.33924 Prob $>t \quad 0.3992$

$\begin{array}{lrr}\text { Confidence } & 0.95 \text { Prob }<\mathrm{t} & 0.6008\end{array}$

Tests that the Variances are Equal

Level Count Std Dev MeanAbsDif to Mean MeanAbsDif to Median

$\begin{array}{lllll}16 & 0.3338353 & 0.2276856 & 0.2257900\end{array}$

$\begin{array}{lllll}\text { Hydragard - HF } & 10 & 0.5042633 & 0.3278336 & 0.3046480\end{array}$

$\begin{array}{lrrrr}\text { Test } & \text { F Ratio } & \text { DFNum } & \text { DFDen } & \text { p-Value } \\ \text { O'Brien[.5] } & 0.8756 & 1 & 24 & 0.3587 \\ \text { Brown-Forsythe } & 0.3834 & 1 & 24 & 0.5416 \\ \text { Levene } & 0.7205 & 1 & 24 & 0.4044 \\ \text { Bartlett } & 1.9084 & 1 & & 0.1671 \\ \text { F Test 2-sided } & 2.2817 & 9 & 15 & 0.1519\end{array}$

Welch Anova testing Means Equal, allowing Std Devs Not Equal

F Ratio DFNum DFDen Prob > F

$\begin{array}{rrrr}0.0677 & 1 & 13.977 & 0.7985\end{array}$

t Test

0.2603
Oneway Analysis of Measurement By Sampler Type of Material=SME Simulant, Analytica Block $=2$, Oxide/Analyte $=\mathrm{NiO}(\mathrm{wt} \%)$, Targeted $=\mathbf{0 . 4 1}$

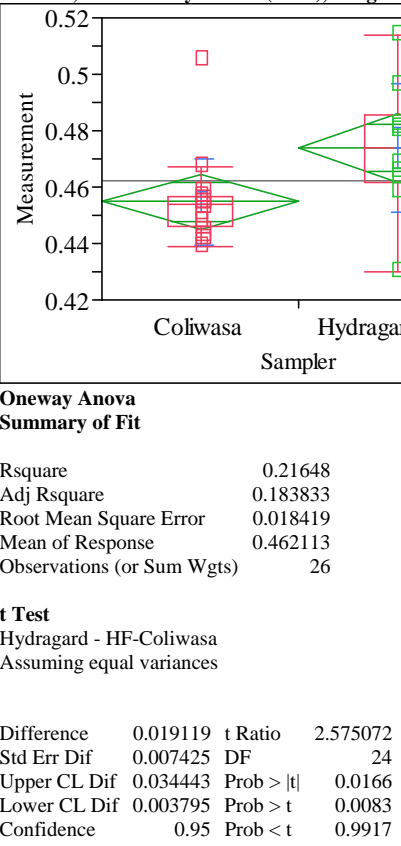

Analysis of Variance

Source DF Sum of Squares Mean Square F Ratio Prob $>$ F

$\begin{array}{lrrrrr}\text { Sampler } & 1 & 0.00224953 & 0.002250 & 6.6310 & 0.0166\end{array}$

$\begin{array}{llll}\text { Error } & 24 & 0.00814186 & 0.000339 \\ \text { C. Total } & 25 & 0.01039139 & \end{array}$

Means for Oneway Anova

Level Number Mean Std Error Lower 95\% Upper 95\%

$\begin{array}{llrrrr}\text { Coliwasa } & 16 & 0.454760 & 0.00460 & 0.44526 & 0.46426\end{array}$

$\begin{array}{llllll}\text { Hydragard - HF } & 10 & 0.473879 & 0.00582 & 0.46186 & 0.48590\end{array}$

Std Error uses a pooled estimate of error variance

Means and Std Deviations

Level Number Mean Std Dev Std Err Mean Lower 95\% Upper 95\%

$\begin{array}{lllllll}\text { Coliwasa } & 16 & 0.454760 & 0.015255 & 0.00381 & 0.44663 & 0.46289\end{array}$

$\begin{array}{lllllll}\text { Hydragard - HF } & 10 & 0.473879 & 0.022733 & 0.00719 & 0.45762 & 0.49014\end{array}$

Test

Hydragard - HF-Coliwasa

Assuming unequal variances

$\begin{array}{lrlr}\text { Difference } & 0.019119 & \text { t Ratio } & 2.349439 \\ \text { Std Err Dif } & 0.008138 & \text { DF } & 14.10829 \\ \text { Upper CL Dif } & 0.036561 & \text { Prob }>|t| & 0.0339 \\ \text { Lower CL Dif } & 0.001678 & \text { Prob }>t & 0.0169 \\ \text { Confidence } & 0.95 & \text { Prob }<t & 0.9831\end{array}$

Tests that the Variances are Equal

Level Count Std Dev MeanAbsDif to Mean MeanAbsDif to Median

$\begin{array}{lllll} & 16 & 0.0152550 & 0.0089274 & 0.0087484 \\ \text { Hydragard - HF } & 10 & 0.027331 & 0.0167970 & 0.0167970\end{array}$

Test F Ratio DFNum DFDen p-Value

$\begin{array}{lrrrr}\text { O'Brien[.5] } & 0.8921 & 1 & 24 & 0.3543\end{array}$

$\begin{array}{lllll}\text { Brown-Forsythe } & 2.3245 & 1 & 24 & 0.1404\end{array}$

$\begin{array}{lllll}\text { Levene } & 2.2611 & 1 & 24 & 0.1457\end{array}$

$\begin{array}{lllll}\text { Bartlett } & 1.7844 & 1 & \text { · } & 0.1816 \\ \text { F Test 2-sided } & 2.2207 & 9 & 15 & 0.1654\end{array}$

Welch Anova testing Means Equal, allowing Std Devs Not Equal

F Ratio DFNum DFDen Prob $>$ F

$\begin{array}{llrr}5.5199 & 1 & 14.108 & 0.0339\end{array}$

t Test

2.3494 
SRNL-STI-2011-00693

Revision 0

\section{Exhibit C2. Statistical Comparisons for Low-Rheology (Phase 3) Simulant Testing}

Oneway Analysis of Measurement By Sampler Type of Material=SME Simulant, Analytica Block $=2$, Oxide $/$ Analyte $=\mathrm{SiO} 2($ wt $\%)$, Targeted $=\mathbf{5 0 . 9 8 5}$

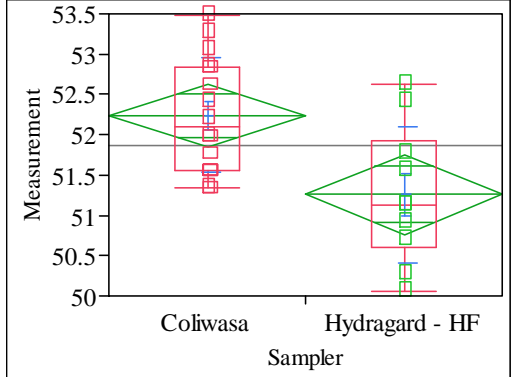

Oneway Anova

Summary of Fi

Adj Rsquare

0.762431

51.8615

t Test

Hydragard - HF-Coliwasa

Assuming equal variances

Difference $\quad-0.9814$ t Ratio $\quad-3.19316$

Std Err Dif $\quad 0.3073$ DF 24

Upper CL Dif -0.3471 Prob $>|t| \quad 0.0039$

Lower CL Dif -1.6157 Prob $>\mathrm{t} \quad 0.9980$

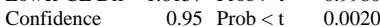

Analysis of Variance

Source DF Sum of Squares Mean Square F Ratio Prob $>$ F

$\begin{array}{llllll}\text { Sampler } & 1 & 5.927099 & 5.92710 & 10.1963 & 0.0039\end{array}$

$\begin{array}{llrr}\text { Error } & 24 & 13.951207 & 0.58130\end{array}$

C. Total $25 \quad 19.878306$

Means for Oneway Anova

Level Number Mean Std Error Lower 95\% Upper 95\%

Coliwasa $16 \quad 52.2390 \quad 0.19061-51.8460$

$\begin{array}{llllll}\text { Hydragard - HF } & 16 & 52.2390 & 0.19061 & 51.846 & 52.632 \\ & 10 & 51.2576 & 0.24110 & 50.760 & 51.755\end{array}$

Std Error uses a pooled estimate of error variance

Means and Std Deviations

Level Number Mean Std Dev Std Err Mean Lower 95\% Upper 95\%

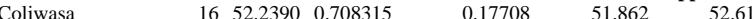

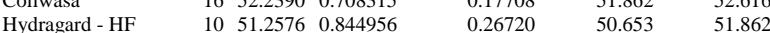

Test

Hydragard - HF-Coliwasa

Assuming unequal variances

Difference $\quad-0.9814$ t Ratio $\quad-3.06163$

Std Err Dif $\quad 0.3205$ DF $\quad 16.70797$

Upper CL Dif -0.3042 Prob $>|t| \quad 0.0072$

Lower CL Dif -1.6586 Prob $>t \quad 0.9964$

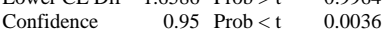

Tests that the Variances are Equal

$\begin{array}{lrrrr}\text { Level } & \text { Count } & \text { Std Dev } & \text { MeanAbsDif to Mean } & \text { MeanAbsDif to Median } \\ \text { Coliwasa } & 16 & 0.7083151 & 0.6066921 & 0.6016781\end{array}$

$\begin{array}{lllll}\text { Hydragard - HF } & 10 & 0.8449558 & 0.6674616 & 0.6417900\end{array}$

$\begin{array}{lrrrr}\text { Test } & \text { F Ratio } & \text { DFNum } & \text { DFDen } & \text { p-Value } \\ \text { O'Brien[.5] } & 0.7112 & 1 & 24 & 0.4074 \\ \text { Brown-Forsythe } & 0.0522 & 1 & 24 & 0.8212 \\ \text { Levene } & 0.1512 & 1 & 24 & 0.7008 \\ \text { Bartlett } & 0.3432 & 1 & . & 0.5580 \\ \text { F Test 2-sided } & 1.4230 & 9 & 15 & 0.5245\end{array}$

Welch Anova testing Means Equal, allowing Std Devs Not Equal

F Ratio DFNum DFDen Prob $>$ F

$\begin{array}{rrrr}9.3736 & 1 & 16.708 & 0.0072\end{array}$

t Test

3.0616
Oneway Analysis of Measurement By Sampler Type of Material=SME Simulant, Analytical Block=2, Oxide/Analyte=Sum of Oxides (wt \%), Targeted $=99.553$

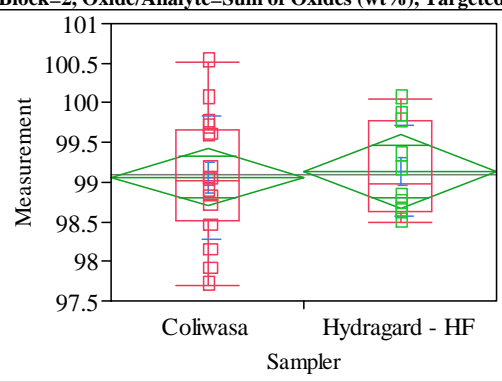

Oneway Anova

Summary of Fit

Rsquare

Adj Rsquare

$-0.03883$

Square Error $\quad 0.709538$

Mean of Response

Observations (or Sum Wgts) $\quad 26$

Hydragard - HF-Coliwasa

Assuming equal variances

Difference $\quad 0.07321$ t Ratio 0.255962

$\begin{array}{lrlr}\text { Std Err Dif } & 0.28602 \text { DF } & 24 \\ \text { Upper CL Dif } & 0.66354 & \text { Prob }>|t| & 0.8002\end{array}$

$\begin{array}{lrl}\text { Upper CL Dif } 0.66354 \text { Prob }>|t| & 0.8002 \\ \text { Lower CL Dif }-0.51711 & \text { Prob }>t & 0.4001\end{array}$

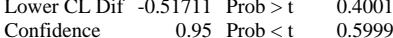

Analysis of Variance

Source DF Sum of Squares Mean Square F Ratio Prob $>$ F

$\begin{array}{lrrrrr}\text { Sampler } & 1 & 0.032984 & 0.032984 & 0.0655 & 0.8002\end{array}$

$\begin{array}{llll}\text { Error } & 24 & 12.082663 & 0.503444\end{array}$

C. Total $25 \quad 12.115647$

Means for Oneway Anova

Level Number Mean Std Error Lower 95\% Upper 95\%

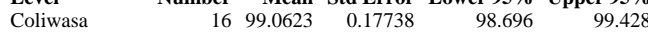

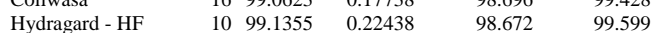

Std Error uses a pooled estimate of error variance

Means and Std Deviation

Level Number Mean Std Dev Std Err Mean Lower 95\% Upper 95\%

$\begin{array}{lllllll}\text { Coliwasa } & 16 & 99.0623 & 0.780070 & 0.19502 & 98.647 & 99.478\end{array}$

$\begin{array}{llllll}\text { Hydragard - HF } & 10 & 99.1355 & 0.573006 & 0.18120 & 98.726\end{array}$

t Test

Hydragard - HF-Coliwasa

Assuming unequal variances

$\begin{array}{lrlr}\text { Difference } & 0.07321 & \mathrm{t} \text { Ratio } & 0.275018 \\ \text { Std Err Dif } & 0.26621 & \text { DF } & 23.2269 \\ \text { Upper CL Dif } & 0.62360 \text { Prob }>|t| & 0.7857 \\ \text { Lower CL Dif } & -0.47718 & \text { Prob }>\mathrm{t} & 0.3929 \\ \text { Confidence } & 0.95 & \text { Prob }<\mathrm{t} & 0.6071\end{array}$

Tests that the Variances are Equal

$\begin{array}{lrrrr}\text { Level } & \text { Count } & \text { Std Dev } & \text { MeanAbsDif to Mean } & \text { MeanAbsDif to Median } \\ \text { Coliwasa } & 16 & 0.7800699 & 0.6135462 & 0.6097831\end{array}$

$\begin{array}{lrrrr} & 16 & 0.7800699 & 0.6135462 & 0.4846898\end{array}$

Test F Ratio DFNum DFDen p-Value

$\begin{array}{lllll}\text { O'Brien[.5] } & 1.2639 & 1 & 24 & 0.2720\end{array}$

$\begin{array}{lllll}\text { Brown-Forsythe } & 0.5657 & 1 & 24 & 0.4593\end{array}$

$\begin{array}{lllll}\text { Levene } & 0.6609 & 1 & 24 & 0.4242\end{array}$

$\begin{array}{lllll}\text { Levene } & 0.6609 & 1 & 24 & 0.4242 \\ \text { Bartlett } & 0.9603 & 1 & . & 0.3271\end{array}$

$\begin{array}{lllll}\text { F Test 2-sided } & 1.8533 & 15 & 9 & 0.3519\end{array}$

Welch Anova testing Means Equal, allowing Std Devs Not Equal

F Ratio DFNum DFDen Prob $>$ F

$\begin{array}{llll}0.0756 & 1 & 23.227 & 0.7857\end{array}$

t Test

0.2750 
SRNL-STI-2011-00693

Revision 0

\section{Exhibit C2. Statistical Comparisons for Low-Rheology (Phase 3) Simulant Testing}

Oneway Analysis of Measurement By Sampler Type of Material=SME Simulant, Analytical Block $=2$, Oxide $/$ Analyte $=\mathrm{TiO} 2(\mathrm{wt} \%)$, Targeted $=\mathbf{0 . 0 7 1 1}$

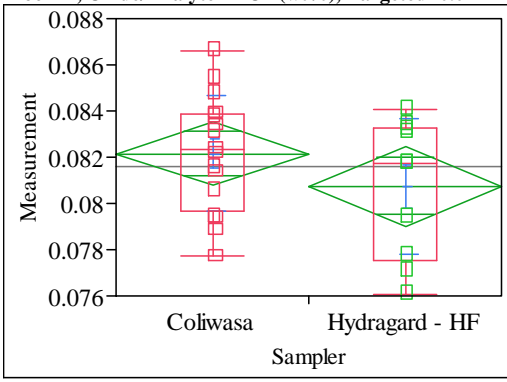

Oneway Anova

Rsquare $\quad 0.065779$

Adj Rsquare $\quad 0.026853$

Root Mean Square Error $\quad 0.002674$

Mean of Response $\quad 0.0816$

Observations (or Sum Wgs)

Test

Hydragard - HF-Coliwasa

Assuming equal variances

Difference $\quad-0.00140$ t Ratio $\quad-1.29994$

$\begin{array}{lrlr}\text { Std Err Dif } & 0.00108 \text { DF } & 24 \\ \text { Upper CL Dif } & 0.00082 & \text { Prob }>|t| & 0.2060\end{array}$

$\begin{array}{rrrr}\text { Upper CL Dif } & 0.00082 & \text { Prob }>|t| & 0.2060 \\ \text { Lower CL Dif } & -0.00363 & \text { Prob }>t & 0.8970\end{array}$

$\begin{array}{lrl}\text { Confidence } & 0.95 \text { Prob }<\mathrm{t} & 0.1030\end{array}$

Analysis of Variance

Source DF Sum of Squares Mean Square F Ratio Prob $>$ F

$\begin{array}{llllll}\text { Sampler } & 1 & 0.00001208 & 0.000012 & 1.6899 & 0.2060\end{array}$

$\begin{array}{llll}\text { Error } & 24 & 0.00017158 & 7.149 \mathrm{e}-6\end{array}$

C. Total $25 \quad 0.00018366$

Means for Oneway Anova

Level Number Mean Std Error Lower 95\% Upper 95\%

$\begin{array}{lrrrrr}\text { Coliwasa } & 16 & 0.082149 & 0.00067 & 0.08077 & 0.08353\end{array}$

$\begin{array}{llllll}\text { Coliwasa } & 16 & 0.082149 & 0.00067 & 0.08077 & 0.08353 \\ \text { Hydragard - HF } & 10 & 0.080748 & 0.00085 & 0.07900 & 0.08249\end{array}$

Std Error uses a pooled estimate of error variance

Means and Std Deviations

Level Number Mean Std Dev Std Err Mean Lower 95\% Upper 95\%

$\begin{array}{lllllll}\text { Coliwasa } & 16 & 0.082149 & 0.002497 & 0.00062 & 0.08082 & 0.08348\end{array}$

$\begin{array}{lllllll}\text { Hydragard - HF } & 10 & 0.080748 & 0.002945 & 0.00093 & 0.07864 & 0.08285\end{array}$

t Test

Hydragard - HF-Coliwasa

Assuming unequal variances

Difference $\quad-0.00140$ t Ratio $\quad-1.24979$

Std Err Dif $\quad 0.00112$ DF 16.86463

Upper CL Dif 0.00097 Prob $>|t| \quad 0.2284$

Lower CL Dif -0.00377 Prob $>t \quad 0.8858$

$\begin{array}{lll}\text { Confidence } & 0.95 \text { Prob }<\mathrm{t} \quad 0.1142\end{array}$

Tests that the Variances are Equal

$\begin{array}{lrrrr}\text { Level } & \text { Count } & \text { Std Dev } & \text { MeanAbsDif to Mean } & \text { MeanAbsDif to Median } \\ \text { Coliwasa } & 16 & 0.0024972 & 0.0020120 & 0.0020016\end{array}$

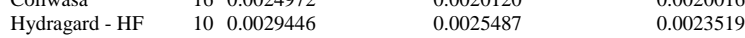

Test F Ratio DFNum DFDen p-Value

$\begin{array}{lllll}\text { O'Brien[.5] } & 0.6740 & 1 & 24 & 0.4197\end{array}$

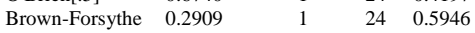

$\begin{array}{lllll}\text { Levene } & 1.0166 & 1 & 24 & 0.3234\end{array}$

$\begin{array}{lllll}\text { Bartlett } & 0.2993 & 1 & & 0.5843 \\ \text { F Test 2-sided } & 1.3905 & 9 & 15 & 0.5500\end{array}$

Welch Anova testing Means Equal, allowing Std Devs Not Equal

F Ratio DFNum DFDen Prob $>$ F

$\begin{array}{llll}1.5620 & 1 & 16.865 & 0.2284\end{array}$

t Test

1.2498
Oneway Analysis of Measurement By Sampler Type of Material=SME Simulant, Analytical Block $=2$, Oxide $/$ Analyte $=\mathrm{ZnO}$ ( $w$ t $\%$ ), Targeted $=\mathbf{0 . 0 9 5 8}$

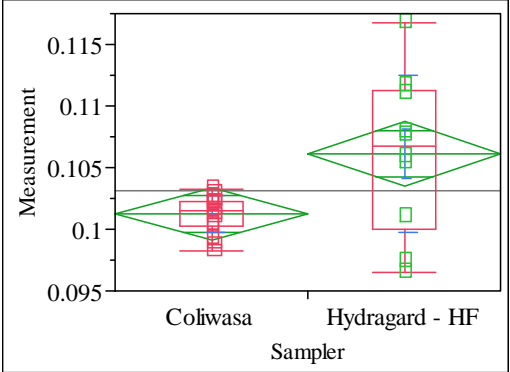

Oneway Anova

Summary of Fit

Rsquare

Adj Rsquare

Root Mean Square Error $\quad 0.004105$

Mean of Response

Observations (or Sum Wgts) $\quad 26$

t Test

Hydragard - HF-Coliwasa

Assuming equal variances

Difference $\quad 0.004909$ t Ratio 2.966467

$\begin{array}{lrr}\text { Std Err Dif } & 0.001655 \text { DF } & 24 \\ \text { Upper } & \end{array}$

$\begin{array}{llll}\text { Upper CL Dif } & 0.008325 & \text { Prob }>|t| & 0.0067 \\ \text { Lower } & & \end{array}$

$\begin{array}{lrl}\text { Lower CL Dif } & 0.001494 \text { Prob }>t & 0.0034 \\ \text { Confidence } & 0.95 \text { Prob }<\mathrm{t} & 0.9966\end{array}$

Analysis of Variance

Source DF Sum of Squares Mean Square F Ratio Prob $>$ F

$\begin{array}{lrrrrr}\text { Sampler } & 1 & 0.00014831 & 0.000148 & 8.7999 & 0.0067\end{array}$

$\begin{array}{llll}\text { Error } & 24 & 0.00040448 & 0.000017\end{array}$

C. Total $25 \quad 0.00055279$

Means for Oneway Anova

Level Number Mean Std Error Lower 95\% Upper 95\%

$\begin{array}{lrrrrr}\text { Coliwasa } & 16 & 0.101210 & 0.00103 & 0.09909 & 0.10333\end{array}$

$\begin{array}{llllll}\text { Hydragard - HF } & 10 & 0.106119 & 0.00130 & 0.10344 & 0.10880\end{array}$

Std Error uses a pooled estimate of error variance

Means and Std Deviations

Level Number Mean Std Dev Std Err Mean Lower 95\% Upper 95\%

$\begin{array}{lllllll}\text { Coliwasa } & 16 & 0.101210 & 0.001492 & 0.00037 & 0.10041 & 0.10201\end{array}$

$\begin{array}{lllllll}\text { Hydragard - HF } & 10 & 0.106119 & 0.006421 & 0.00203 & 0.10153 & 0.11071\end{array}$

t Test

Hydragard - HF-Coliwasa

Assuming unequal variances

Difference $\quad 0.004909$ t Ratio 2.377854

Std Err Dif $\quad 0.002065$ DF 9.611061

Upper CL Dif 0.009535 Prob $>|t| 0.0397$

Lower CL Dif 0.000284 Prob $>t \quad 0.0199$

$\begin{array}{lll}\text { Confidence } & 0.95 \text { Prob }<\mathrm{t} \quad 0.9801\end{array}$

Tests that the Variances are Equal

$\begin{array}{lrrrr}\text { Level } & \text { Count } & \text { Std Dev } & \text { MeanAbsDif to Mean } & \text { MeanAbsDif to Median } \\ \text { Coliwasa } & 16 & 0.0014920 & 0.0011894 & 0.0011592\end{array}$

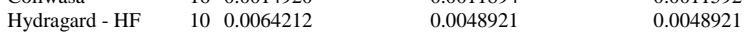

Test F Ratio DFNum DFDen p-Value

$\begin{array}{lllll}\text { O'Brien[.5] } & 10.0825 & 1 & 24 & 0.0041\end{array}$

Brown-Forsythe $13.7365 \quad 1124 \quad 0.0011$

$\begin{array}{lllll}\text { Brown-Forsythe } & 13.7365 & 1 & 24 & 0.0011 \\ \text { Levene } & 14.2072 & 1 & 24 & 0.0009\end{array}$

$\begin{array}{lllll}\text { Levene } & 14.2072 & 1 & 24 & 0.0009 \\ \text { Bartlett } & 21.3441 & 1 & . & <.0001\end{array}$

$\begin{array}{lllll}\text { F Test 2-sided } & 18.5222 & 9 & 15 & <.0001\end{array}$

Welch Anova testing Means Equal, allowing Std Devs Not Equal

F Ratio DFNum DFDen Prob $>$ F

$\begin{array}{llll}5.6542 & 1 & 9.6111 & 0.0397\end{array}$

t Test

2.3779 


\section{Exhibit C2. Statistical Comparisons for Low-Rheology (Phase 3) Simulant Testing}

Oneway Analysis of Measurement By Sampler Type of Material=SME Simulant, Analytical Block $=2$, Oxide $/$ Analyte $=\mathrm{ZrO2}$ ( $w$ t \%), Targeted $=0.3547$

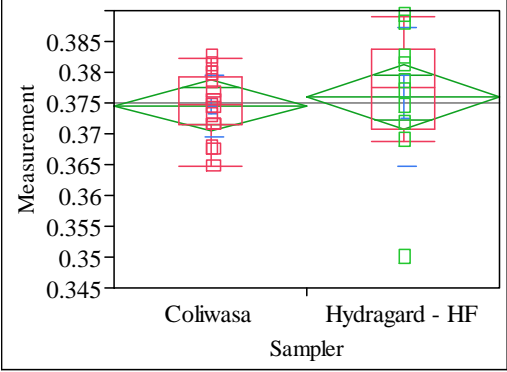

Oneway Anova

Rsquare $\quad 0.00804$

Adj Rsquare $\quad-0.0332$

Root Mean Square Error $\quad 0.007978$

Mean of Response 0.375055

Observations (or Sum Wgs)

Test

Hydragard - HF-Coliwasa

Assuming equal variances

Difference $\quad 0.00142$ t Ratio $\quad 0.441042$

$\begin{array}{lrlr}\text { Std Err Dif } & 0.00322 & \text { DF } & 24 \\ \text { Upper CL Dif } & 0.00806 & \text { Prob }>|t| & 0.6631\end{array}$

$\begin{array}{lrll}\text { Upper CL Dif } & 0.00806 & \text { Prob }>|t| & 0.6631 \\ \text { Lower CL Dif } & -0.00522 & \text { Prob }>t & 0.3316\end{array}$

$\begin{array}{lrll} & \\ \text { Confidence } & 0.95 & \text { Prob }<\mathrm{t} & 0.6684\end{array}$

Analysis of Variance

Source DF Sum of Squares Mean Square F Ratio Prob $>$ F

$\begin{array}{lrrrrr}\text { Sampler } & 1 & 0.00001238 & 0.000012 & 0.1945 & 0.6631\end{array}$

$\begin{array}{llll}\text { Error } & 24 & 0.00152742 & 0.000064\end{array}$

C. Total $25 \quad 0.00153980$

Means for Oneway Anova

Level Number Mean Std Error Lower 95\% Upper 95\%

\begin{tabular}{lrrrrr} 
Coliwasa & 16 & 0.374509 & 0.00199 & 0.37039 & 0.37863 \\
\hline
\end{tabular}

$\begin{array}{llllll}\text { Coliwasa } & 16 & 0.374509 & 0.00199 & 0.37039 & 0.37863 \\ \text { Hydragard - HF } & 10 & 0.375928 & 0.00252 & 0.37072 & 0.38113\end{array}$

Std Error uses a pooled estimate of error variance

Means and Std Deviations

Level Number Mean Std Dev Std Err Mean Lower 95\% Upper 95\%

$\begin{array}{lrrrrrr}\text { Coliwasa } & 16 & 0.374509 & 0.005114 & 0.00128 & 0.37178 & 0.37723\end{array}$

$\begin{array}{lllllll}\text { Hydragard - HF } & 10 & 0.375928 & 0.011231 & 0.00355 & 0.36789 & 0.38396\end{array}$

t Test

Hydragard - HF-Coliwasa

Assuming unequal variances

Difference $\quad 0.00142$ t Ratio 0.375766

Std Err Dif $\quad 0.00377$ DF 11.36941

Upper CL Dif 0.00969 Prob $>|t| \quad 0.7140$

Lower CL Dif -0.00686 Prob $>\mathrm{t} \quad 0.3570$

$\begin{array}{lll}\text { Confidence } & 0.95 \text { Prob }<\mathrm{t} \quad 0.6430\end{array}$

Tests that the Variances are Equal

$\begin{array}{lrrrr}\text { Level } & \text { Count } & \text { Std Dev } & \text { MeanAbsDif to Mean } & \text { MeanAbsDif to Median } \\ \text { Coliwasa } & 16 & 0.0051140 & 0.0040524 & 0.0040524\end{array}$

$\begin{array}{lllll}\text { Hydragard - HF } & 10 & 0.0112305 & 0.0078887 & 0.0076996\end{array}$

Test F Ratio DFNum DFDen p-Value

$\begin{array}{lrrrr}\text { O'Brien[.5] } & 2.6664 & 1 & 24 & 0.1155\end{array}$

\begin{tabular}{lllll} 
Brown-Forsythe & 2.8068 & 1 & 24 & 0.1155 \\
\hline & 3.3837 & 1 & 24 & 0.1068
\end{tabular}

$\begin{array}{lllll}\text { Brown-Forsythe } & 2.8068 & 1 & 24 & 0.1068\end{array}$

$\begin{array}{lllll}\text { Levene } & 3.3837 & 1 & 24 & 0.0782 \\ \text { Bartlett } & 6.8719 & 1 & . & 0.0088\end{array}$

$\begin{array}{lllll}\text { F Test 2-sided } & 4.8225 & 9 & 15 & 0.0075\end{array}$

Welch Anova testing Means Equal, allowing Std Devs Not Equal

F Ratio DFNum DFDen Prob $>$ F

$\begin{array}{llll}0.1412 & 1 & 11.369 & 0.7140\end{array}$

t Test
0.3758
Oneway Analysis of Measurement By Sampler Type of Material=SME Simulant, Analytica Block $=3$, Oxide $/$ Analyte $=\mathrm{Al} / \mathrm{B}$, Targeted $=2.552$

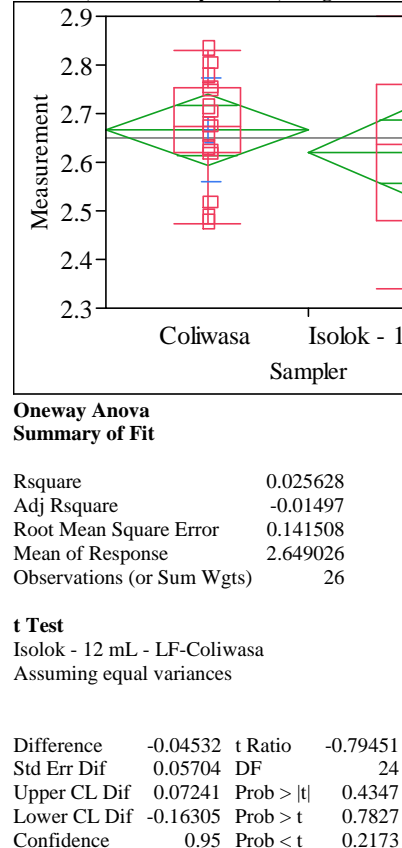

Analysis of Variance

Source DF Sum of Squares Mean Square F Ratio Prob > F

$\begin{array}{lrrrrr}\text { Sampler } & 1 & 0.01264047 & 0.012640 & 0.6312 & 0.4347\end{array}$

$\begin{array}{llll}\text { Error } & 24 & 0.48058856 & 0.020025\end{array}$

C. Total $25 \quad 0.49322903$

Means for Oneway Anova

Level Number Mean Std Error Lower 95\% Upper 95\%

$\begin{array}{llllrr}\text { Coliwasa } & 16 & 2.66646 & 0.03538 & 2.5934 & 2.7395\end{array}$

$\begin{array}{llllll}\text { Isolok - } 12 \mathrm{~mL} \text { - LF } & 10 & 2.62114 & 0.04475 & 2.5288 & 2.7135\end{array}$

Std Error uses a pooled estimate of error variance

Means and Std Deviations

Level Number Mean Std Dev Std Err Mean Lower 95\% Upper 95\%

$\begin{array}{lrrrrrr}\text { Coliwasa } & 16 & 2.66646 & 0.107927 & 0.02698 & 2.6089 & 2.7240 \\ \text { Isolok - 12 mL - LF } & 10 & 2.62114 & 0.184350 & 0.05830 & 2.4893 & 2.7530\end{array}$

t Test

Isolok - $12 \mathrm{~mL}$ - LF-Coliwas

Assuming unequal variances

Difference $\quad-0.04532$ t Ratio $\quad-0.70553$

Std Err Dif $\quad 0.06424$ DF 12.91334

Upper CL Dif 0.09355 Prob $>|t| \quad 0.4930$

Lower CL Dif -0.18419 Prob $>t \quad 0.7535$

$\begin{array}{lrl}\text { Confidence } & 0.95 \text { Prob }<\mathrm{t} \quad 0.2465\end{array}$

Tests that the Variances are Equal

$\begin{array}{lrrrr}\text { Level } & \text { Count } & \text { Std Dev } & \text { MeanAbsDif to Mean } & \text { MeanAbsDif to Median } \\ \text { Coliwasa } & 16 & 0.1079269 & 0.0839512 & 0.0832960\end{array}$

$\begin{array}{lllll}\text { Coliwasa } & 16 & 0.1079269 & 0.0839512 & 0.0832960 \\ \text { Isolok - 12 mL - LF } & 10 & 0.1843503 & 0.1492653 & 0.1492653\end{array}$

Test F Ratio DFNum DFDen p-Value

$\begin{array}{lllll}\text { O'Brien[.5] } & 4.3152 & 1 & 24 & 0.0486\end{array}$

$\begin{array}{lllll}\text { Brown-Forsythe } & 4.2746 & 1 & 24 & 0.0496\end{array}$

Bevene $4.3445-11240.0496$

$\begin{array}{lllll}\text { Levene } & 4.3445 & 1 & 24 & 0.0479\end{array}$

$\begin{array}{lllll}\text { Bartlett } & 3.2203 & 1 & & 0.0727 \\ \text { F Test 2-sided } & 2.9176 & 9 & 15 & 0.0648\end{array}$

Welch Anova testing Means Equal, allowing Std Devs Not Equal

F Ratio DFNum DFDen Prob $>$ F

$\begin{array}{llll}0.4978 & 1 & 12.913 & 0.4930\end{array}$

t Test 


\section{Exhibit C2. Statistical Comparisons for Low-Rheology (Phase 3) Simulant Testing}

Oneway Analysis of Measurement By Sampler Type of Material=SME Simulant, Analytical Block $=3$, Oxide/Analyte $=\mathrm{Al} 2 \mathrm{O} 3$ ( wt $\%$ ), Targeted $=10.869$

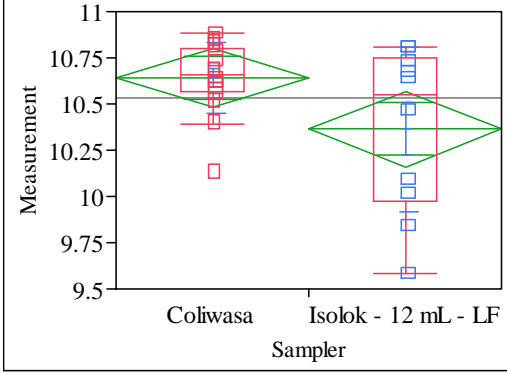

Oneway Anova

Rsquare $\quad 0.167393$

Adj Rsquare $\quad 0.13270$

Root Mean Square Error $\quad 0.312611$

Mean of Response $\quad 10.53614$

(orervations (or Sum Wgs)

Test

Isolok - $12 \mathrm{~mL}$ - LF-Coliwasa

Assuming equal variances

Difference $\quad-0.27681$ t Ratio $\quad-2.19661$

$\begin{array}{lrlr}\text { Std Err Dif } & 0.12602 & \text { DF } & 24 \\ \text { Upper CL Dif } & -0.01672 & \text { Prob }>|t| & 0.0379\end{array}$

$\begin{array}{rrrr}\text { Upper CL Dif } & -0.01672 & \text { Prob }>|t| & 0.0379 \\ \text { Lower CL Dif } & -0.53690 & \text { Prob }>t & 0.9810\end{array}$

$\begin{array}{lrl}\text { Confidence } & 0.95 \text { Prob }<\mathrm{t} \quad 0.0190\end{array}$

Analysis of Variance

Source DF Sum of Squares Mean Square F Ratio Prob $>$ F

$\begin{array}{llllll}\text { Sampler } & 1 & 0.4715369 & 0.471537 & 4.8251 & 0.0379\end{array}$

$\begin{array}{llll}\text { Error } & 24 & 2.3454139 & 0.097726\end{array}$

C. Total $25 \quad 2.8169508$

Means for Oneway Anova

Level Number Mean Std Error Lower 95\% Upper 95\%

$\begin{array}{lrrrrr}\text { Coliwasa } & 16 & 10.6426 & 0.07815 & 10.481 & 10.804\end{array}$

$\begin{array}{llllll}\text { Coliwasa } & 16 & 10.6426 & 0.07815 & 10.481 & 10.804 \\ \text { Isolok - 12 mL - LF } & 10 & 10.3658 & 0.09886 & 10.162 & 10.570\end{array}$

Std Error uses a pooled estimate of error variance

Means and Std Deviations

$\begin{array}{lrrrrrr}\text { Level } & \text { Number } & \text { Mean } & \text { Std Dev } & \text { Std Err Mean } & \text { Lower 95\% } & \text { Upper 95\% } \\ \text { Coliwasa } & 16 & 10.6426 & 0.191515 & 0.04788 & 10.541 & 10.745\end{array}$

$\begin{array}{lllllll}\text { Isolok - } 12 \mathrm{~mL} \text { - LF } & 10 & 10.3658 & 0.446622 & 0.14123 & 10.046 & 10.685\end{array}$

Test

Isolok - $12 \mathrm{~mL}$ - LF-Coliwasa

Assuming unequal variances

Difference $\quad-0.27681$ t Ratio $\quad-1.85619$

Std Err Dif $\quad 0.14913$ DF 11.09951

Upper CL Dif 0.05106 Prob $>|t| \quad 0.0901$

Lower CL Dif -0.60468 Prob $>\mathrm{t} \quad 0.9549$

$\begin{array}{lrl}\text { Confidence } & 0.95 \text { Prob }<\mathrm{t} & 0.0451\end{array}$

Tests that the Variances are Equal

$\begin{array}{lrrrr}\text { Level } & \text { Count } & \text { Std Dev } & \text { MeanAbsDif to Mean } & \text { MeanAbsDif to Median } \\ \text { Coliwasa } & 16 & 0.1915149 & 0.1369887 & 0.1369887\end{array}$

$\begin{array}{lrrrr}\text { Coliwasa } & 16 & 0.1915149 & 0.1369887 & 0.1369887 \\ \text { Isolok - 12 mL - LF } & 10 & 0.4466225 & 0.3869696 & 0.3665630\end{array}$

Test F Ratio DFNum DFDen p-Value

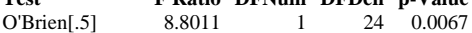

$\begin{array}{lrrrr}\text { O'Brien[.5] } & 8.8011 & 1 & 24 & 0.0067 \\ \text { Brown-Forsythe } & 7.3810 & 1 & 24 & 0.0120 \\ \text { Levene } & 16.8510 & 1 & 24 & 0.0004\end{array}$

$\begin{array}{lrrrr}\text { Levene } & 16.8510 & 1 & 24 & 0.0004 \\ \text { Bartlett } & 7.9189 & 1 & . & 0.0049\end{array}$

$\begin{array}{lllll}\text { F Test 2-sided } & 5.4385 & 9 & 15 & 0.0041\end{array}$

Welch Anova testing Means Equal, allowing Std Devs Not Equal

F Ratio DFNum DFDen Prob $>$ F

$\begin{array}{llll}3.4454 & 1 & 11.1 & 0.0901\end{array}$

t Test

1.8562
Oneway Analysis of Measurement By Sampler Type of Material=SME Simulant, Analytica Block $=3$, Oxide/Analyte $=$ B2O3 $($ wt $\%$ ), Targeted $=4.259$

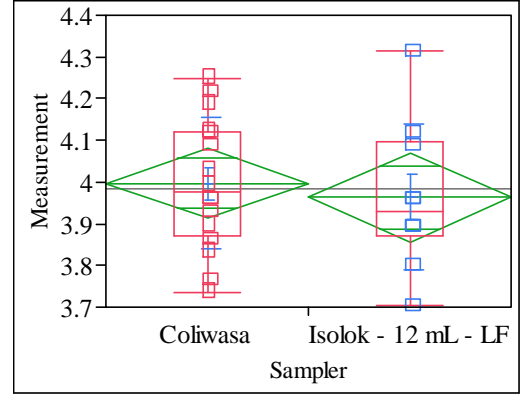

Oneway Anova

Summary of Fit

Rsquare

Adj Rsquare

Root Mean Square Error $\quad 0.164006$

Mean of Response 3.984007
26

Test

Isolok - $12 \mathrm{~mL}$ - LF-Coliwasa

Assuming equal variances

Difference $\quad-0.03300$ t Ratio $\quad-0.49921$

$\begin{array}{lrlr}\text { Std Err Dif } & 0.06611 & \text { DF } & 24 \\ \text { Upper CL Dif } & 0.10345 & \text { Prob }>|t| & 0.6222\end{array}$

$\begin{array}{lrll}\text { Upper CL Dif } & 0.10345 & \text { Prob }>|t| & 0.6222 \\ \text { Lower CL Dif } & -0.16945 & \text { Prob }>t & 0.6889\end{array}$

$\begin{array}{lrrr}\text { Lower CL Dif } & -0.16945 & \text { Prob }>t & 0.6889 \\ \text { Confidence } & 0.95 & \text { Prob }<t & 0.3111\end{array}$

Analysis of Variance

Source DF Sum of Squares Mean Square F Ratio Prob $>$ F

$\begin{array}{lrrrrr}\text { Sampler } & 1 & 0.00670315 & 0.006703 & 0.2492 & 0.6222\end{array}$

$\begin{array}{llll}\text { Error } & 24 & 0.64554833 & 0.026898\end{array}$

$\begin{array}{lll}\text { C. Total } 25 & 0.65225148\end{array}$

Means for Oneway Anova

Level Number Mean Std Error Lower 95\% Upper 95\%

$\begin{array}{lrrrrr}\text { Coliwasa } & 16 & 3.99670 & 0.04100 & 3.9121 & 4.0813 \\ \text { Isolok - 12 mL - LF } & 10 & 3.96370 & 0.05186 & 3.8567 & 4.0707\end{array}$

Std Error uses a pooled estimate of error variance

Means and Std Deviations

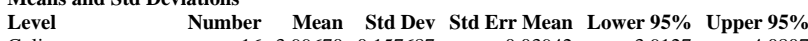

$\begin{array}{lrrrrrr}\text { Coliwasa } & 16 & 3.99670 & 0.157687 & 0.03942 & 3.9127 & 4.0807 \\ \text { Isolok - 12 mL - LF } & 10 & 3.96370 & 0.174027 & 0.05503 & 3.8392 & 4.0882\end{array}$

t Test

Isolok - $12 \mathrm{~mL}$ - LF-Coliwasa

Assuming unequal variances

$\begin{array}{lrlr}\text { Difference } & -0.03300 & \text { t Ratio } & -0.48754 \\ \text { Std Err Dif } & 0.06770 & \text { DF } & 17.79505 \\ \text { Upper CL Dif } & 0.10934 & \text { Prob }>|t| & 0.6318 \\ \text { Lower CL Dif } & -0.17534 & \text { Prob }>\mathrm{t} & 0.6841 \\ \text { Confidence } & 0.95 & \text { Prob }<\mathrm{t} & 0.3159\end{array}$

Tests that the Variances are Equal

$\begin{array}{lrrrr}\text { Level } & \text { Count } & \text { Std Dev } & \text { MeanAbsDif to Mean } & \text { MeanAbsDif to Median } \\ \text { Coliwasa } & 16 & 0.1576875 & 0.1292991 & 0.1287960\end{array}$

$\begin{array}{lllll}\text { Coliwasa } & 16 & 0.1576875 & 0.1292991 & 0.1287960 \\ \text { Isolok - 12 mL - LF } & 10 & 0.1740269 & 0.1268641 & 0.1255761\end{array}$

Test F Ratio DFNum DFDen p-Value

$\begin{array}{lllll}\text { O'Brien[.5] } & 0.1461 & 1 & 24 & 0.7057\end{array}$

$\begin{array}{lllll}\text { Brown-Forsythe } & 0.0063 & 1 & 24 & 0.9372\end{array}$

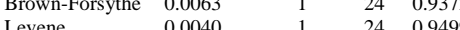

$\begin{array}{lllrr}\text { Levene } & 0.0040 & 1 & 24 & 0.9499 \\ \text { Bartlett } & 0.1062 & 1 & . & 0.7445\end{array}$

$\begin{array}{llll}\text { F Test 2-sided } \quad 1.2180 & 9 & 15 & 0.7066\end{array}$

Welch Anova testing Means Equal, allowing Std Devs Not Equal

F Ratio DFNum DFDen Prob $>$ F

$\begin{array}{llll}0.2377 & 1 & 17.795 & 0.6318\end{array}$

t Test 
SRNL-STI-2011-00693

Revision 0

\section{Exhibit C2. Statistical Comparisons for Low-Rheology (Phase 3) Simulant Testing}

Oneway Analysis of Measurement By Sampler Type of Material=SME Simulant, Analytical Block=3, Oxide/Analyte=BaO (wt \%), Targeted $=0.0919$

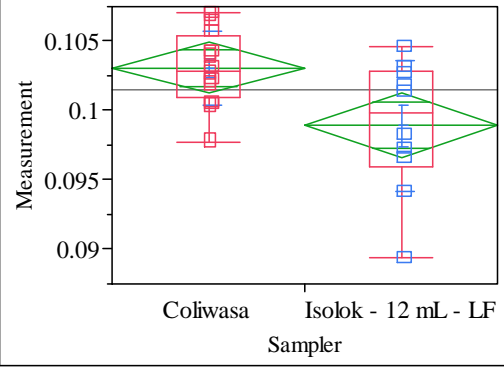

Oneway Anova

Rsquare $\quad 0.256751$

Adj Rsquare $\quad 0.225782$

Root Mean Square Error $\quad 0.003574$

Mean of Response $\quad 0.101486$

t Test

Isolok - $12 \mathrm{~mL}$ - LF-Coliwasa

Assuming equal variances

Difference $\quad-0.00415$ t Ratio -2.87935

$\begin{array}{lrlr}\text { Std Err Dif } & 0.00144 \text { DF } & 24 \\ \text { Upper CL Dif } & -0.00117 \text { Prob }>|t| & 0.0083\end{array}$

$\begin{array}{rrrr}\text { Upper CL Dif } & -0.00117 & \text { Prob }>|t| & 0.0083 \\ \text { Lower CL Dif }-0.00712 & \text { Prob }>t & 0.9959\end{array}$

$\begin{array}{lrl}\text { Confidence } & 0.95 \text { Prob }<\mathrm{t} \quad 0.0041\end{array}$

Analysis of Variance

Source DF Sum of Squares Mean Square F Ratio Prob $>$ F

$\begin{array}{llllll}\text { Sampler } & 1 & 0.00010587 & 0.000106 & 8.2907 & 0.0083\end{array}$

$\begin{array}{llll}\text { Error } & 24 & 0.00030648 & 0.000013\end{array}$

C. Total $25 \quad 0.00041235$

Means for Oneway Anova

Level Number Mean Std Error Lower 95\% Upper 95\%

$\begin{array}{lrrrrr}\text { Coliwasa } & \text { Num } & 0.103081 & 0.00089 & 0.10124 & 0.10492\end{array}$

$\begin{array}{llllll}\text { Coliwasa } & 16 & 0.103081 & 0.00089 & 0.10124 & 0.10492 \\ \text { Isolok - 12 mL - LF } & 10 & 0.098933 & 0.00113 & 0.09660 & 0.10127\end{array}$

Std Error uses a pooled estimate of error variance

Means and Std Deviations

$\begin{array}{lrrrrrr}\text { Level } & \text { Number } & \text { Mean } & \text { Std Dev } & \text { Std Err Mean } & \text { Lower 95\% } & \text { Upper 95\% } \\ \text { Coliwasa } & 16 & 0.103081 & 0.002650 & 0.00066 & 0.10167 & 0.10449\end{array}$

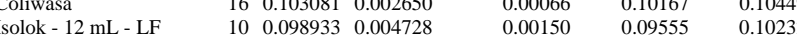

Test

Isolok - $12 \mathrm{~mL}$ - LF-Coliwasa

Assuming unequal variances

Difference $\quad-0.00415$ t Ratio $\quad-2.53654$

Std Err Dif $\quad 0.00164$ DF 12.58993

Upper CL Dif -0.00060 Prob $>|t| \quad 0.0253$

Lower CL Dif -0.00769 Prob $>\mathrm{t} \quad 0.9873$

$\begin{array}{lll}\text { Confidence } & 0.95 \text { Prob }<\mathrm{t} \quad 0.0127\end{array}$

Tests that the Variances are Equal

$\begin{array}{lrrrr}\text { Level } & \text { Count } & \text { Std Dev } & \text { MeanAbsDif to Mean } & \text { MeanAbsDif to Median } \\ \text { Coliwasa } & 16 & 0.0026498 & 0.0021074 & 0.0021074\end{array}$

$\begin{array}{lrrrr}\text { Coliwasa } & 16 & 0.0026498 & 0.0021074 & 0.0021074 \\ \text { Isolok - 12 mL - LF } & 10 & 0.0047277 & 0.0038073 & 0.0038073\end{array}$

Test F Ratio DFNum DFDen p-Value

$\begin{array}{lrrrr}\text { O'Brien[.5] } & 3.4670 & 1 & 24 & 0.0749\end{array}$

\begin{tabular}{lllll} 
Brown-Forsythe & 4.3168 & 1 & 24 & 0.0749 \\
\hline & 4 & 24 & 0.0486
\end{tabular}

Brown-Forsythe $4.3168 \quad 11 \quad 24 \quad 0.0486$

$\begin{array}{lrrrr}\text { Levene } & 4.7172 & 1 & 24 & 0.0400 \\ \text { Bartlett } & 3.7632 & 1 & . & 0.0524\end{array}$

$\begin{array}{lllll}\text { F Test 2-sided } & 3.1832 & 9 & 15 & 0.0464\end{array}$

Welch Anova testing Means Equal, allowing Std Devs Not Equal

F Ratio DFNum DFDen Prob $>$ F

$\begin{array}{llll}6.4340 & 1 & 12.59 & 0.0253\end{array}$

t Test

2.5365
Oneway Analysis of Measurement By Sampler Type of Material=SME Simulant, Analytical Block $=3$, Oxide $/$ Analyte $=\mathrm{CaO}($ wt $\%)$, Targeted $=1.1462$

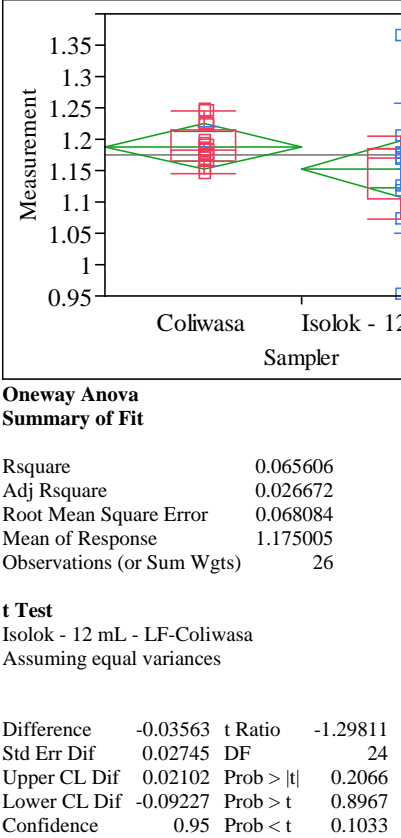

Analysis of Variance

Source DF Sum of Squares Mean Square F Ratio Prob $>$ F

$\begin{array}{llllll}\text { Sampler } & 1 & 0.00781103 & 0.007811 & 1.6851 & 0.2066\end{array}$

$\begin{array}{llll}\text { Error } & 24 & 0.11124943 & 0.004635\end{array}$

C. Total $25 \quad 0.11906046$

Means for Oneway Anova

Level Number Mean Std Error Lower 95\% Upper 95\%

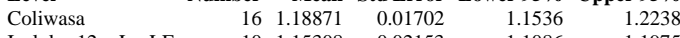

$\begin{array}{llllll}\text { Isolok - 12 mL - LF } & 10 & 1.18871 & 0.01702 & 1.1536 & 1.2238 \\ & & & 0.02153 & 1.1086 & 1.1975\end{array}$

Std Error uses a pooled estimate of error variance

Means and Std Deviations

Level Number Mean Std Dev Std Err Mean Lower 95\% Upper 95\%

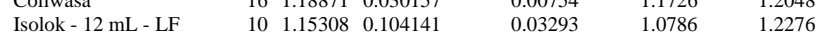

Test

Isolok - $12 \mathrm{~mL}$ - LF-Coliwasa

Assuming unequal variances

Difference $\quad-0.03563$ t Ratio $\quad-1.05455$

Std Err Dif $\quad 0.03378$ DF $\quad 9.951691$

Upper CL Dif 0.03970 Prob $>|t| \quad 0.3166$

Lower CL Dif -0.11095 Prob $>t \quad 0.8417$

$\begin{array}{lrl}\text { Confidence } & 0.95 \text { Prob }<\mathrm{t} & 0.1583\end{array}$

Tests that the Variances are Equal

Level Count Std Dev MeanAbsDif to Mean MeanAbsDif to Median

$\begin{array}{lllll}\text { Coliwasa } & 16 & 0.0301569 & 0.0230212 & 0.0221249\end{array}$

Isolok - 12 mL - LF $\quad 10 \quad 0.1041409 \quad 0.0689526$

Test F Ratio DFNum DFDen p-Value

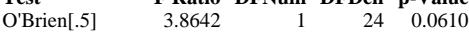

Brown-Forsythe $4.4692 \quad 1 \quad 24 \quad 0.0451$

$\begin{array}{lrrrr}\text { Levene } & 5.6421 & 1 & 24 & 0.0259\end{array}$

$\begin{array}{lllll}\text { Bartlett } & 16.0514 & 1 & \dot{0} & <.0001 \\ \text { F Test 2-sided } & 11.9253 & 9 & 15 & <.0001\end{array}$

Welch Anova testing Means Equal, allowing Std Devs Not Equal

F Ratio DFNum DFDen Prob $>$ F

$\begin{array}{llr}1.1121 & 9.9517 & 0.3166\end{array}$

t Test

1.0546 
SRNL-STI-2011-00693

Revision 0

\section{Exhibit C2. Statistical Comparisons for Low-Rheology (Phase 3) Simulant Testing}

Oneway Analysis of Measurement By Sampler Type of Material=SME Simulant, Analytical Block $=3$, Oxide $/$ Analyte $=$ Cr2O3 $($ wt $\%$ ), Targeted $=0.0998$

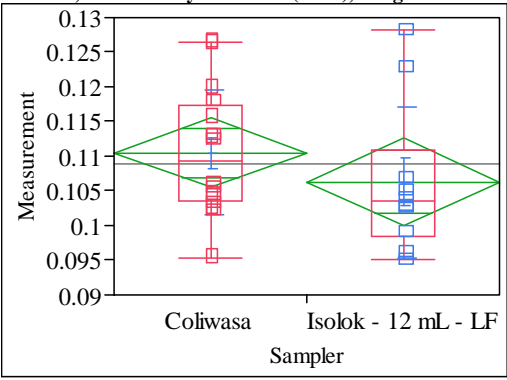

Oneway Anova

Rsquare $\quad 0.045571$

Adj Rsquare $\quad 0.005804$

Root Mean Square Error $\quad 0.009742$

Mean of Response 0.108889

(or Sum Wgs)

Tes

Isolok - $12 \mathrm{~mL}$ - LF-Coliwasa

Assuming equal variances

Difference $\quad-0.00420$ t Ratio $\quad-1.07048$

$\begin{array}{lrlr}\text { Std Err Dif } & 0.00393 \text { DF } & 24 \\ \text { Upper CL Dif } & 0.00390 & \text { Prob }>|t| & 0.2950\end{array}$

$\begin{array}{rrrr}\text { Upper CL Dif } & 0.00390 & \text { Prob }>|t| & 0.2950 \\ \text { Lower CL Dif } & -0.01231 & \text { Prob }>t & 0.8525\end{array}$

$\begin{array}{lrl}\text { Confidence } & 0.95 \text { Prob }<\mathrm{t} & 0.1475\end{array}$

Analysis of Variance

Source DF Sum of Squares Mean Square F Ratio Prob $>$ F

$\begin{array}{lrrrrr}\text { Sampler } & 1 & 0.00010876 & 0.000109 & 1.1459 & 0.2950\end{array}$

$\begin{array}{llll}\text { Error } & 24 & 0.00227776 & 0.000095\end{array}$

C. Total $25 \quad 0.00238652$

Means for Oneway Anova

Level Number Mean Std Error Lower 95\% Upper 95\%

\begin{tabular}{lrrrrr} 
Coliwasa & Nut & 0.110506 & 0.00244 & 0.10548 & 0.11553 \\
\hline
\end{tabular}

$\begin{array}{llllll}\text { Coliwasa } & 16 & 0.110506 & 0.00244 & 0.10548 & 0.11553 \\ \text { Isolok - 12 mL - LF } & 10 & 0.106302 & 0.00308 & 0.09994 & 0.11266\end{array}$

Std Error uses a pooled estimate of error variance

Means and Std Deviations

$\begin{array}{lrrrrrr}\text { Level } & \text { Number } & \text { Mean } & \text { Std Dev } & \text { Std Err Mean } & \text { Lower 95\% } & \text { Upper 95\% } \\ \text { Coliwasa } & 16 & 0.110506 & 0.008976 & 0.00224 & 0.10572 & 0.11529\end{array}$

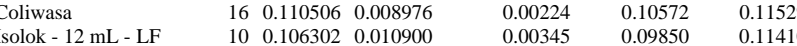

t Test

Isolok - $12 \mathrm{~mL}$ - LF-Coliwas

Assuming unequal variances

Difference $\quad-0.00420$ t Ratio $\quad-1.02212$

Std Err Dif $\quad 0.00411$ DF 16.47012

Upper CL Dif 0.00449 Prob $>|t| \quad 0.3215$

Lower CL Dif -0.01290 Prob $>t \quad 0.8392$

$\begin{array}{lrl}\text { Confidence } & 0.95 \text { Prob }<\mathrm{t} & 0.1608\end{array}$

Tests that the Variances are Equal

$\begin{array}{lrrrr}\text { Level } & \text { Count } & \text { Std Dev } & \text { MeanAbsDif to Mean } & \text { MeanAbsDif to Median } \\ \text { Coliwasa } & 16 & 0.0089758 & 0.0074998 & 0.0074998 \\ \text { Isolok - 12 mL - LF } & 10 & 0.0109000 & 0.0078371 & 0.0071472\end{array}$

Test F Ratio DFNum DFDen p-Value

$\begin{array}{lrrrr}\text { O'Brien[.5] } & 0.4878 & 1 & 24 & 0.4916\end{array}$

\begin{tabular}{lllll} 
Brown-Forsythe & 0.0190 & 1 & 24 & 0.4916 \\
\hline & 0.0220 & 1 & 24 & 0.8916
\end{tabular}

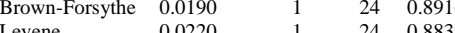

$\begin{array}{lllrr}\text { Levene } & 0.0220 & 1 & 24 & 0.8833 \\ \text { Bartlett } & 0.4169 & 1 & . & 0.5185\end{array}$

$\begin{array}{lllll}\text { F Test 2-sided } & 1.4747 & 9 & 15 & 0.4862\end{array}$

Welch Anova testing Means Equal, allowing Std Devs Not Equal

F Ratio DFNum DFDen Prob $>$ F

$\begin{array}{llll}1.0447 & 1 & 16.47 & 0.3215\end{array}$

t Test

1.0221
Oneway Analysis of Measurement By Sampler Type of Material=SME Simulant, Analytical Block $=3$, Oxide/Analyte $=\mathrm{CuO}(\mathrm{wt} \%)$, Targeted $=\mathbf{0 . 0 5 0 4}$

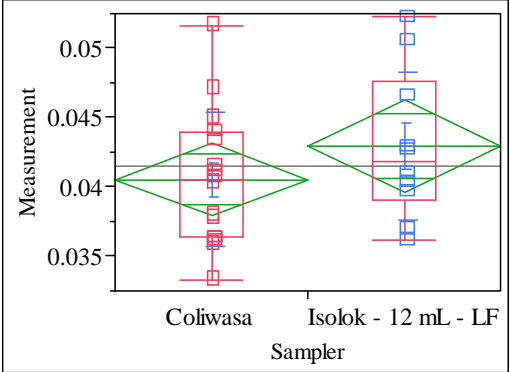

Oneway Anova

Summary of Fit

Rsquare

Adj Rsquare

Root Mean Square Error $\quad 0.005038$

Mean of Response $\quad 0.041463$

Observations (or Sum Wgts) 26

Isolok - $12 \mathrm{~mL}$ - LF-Coliwasa

Assuming equal variances

Difference $\quad 0.00241$ t Ratio 1.188794

$\begin{array}{lrlr}\text { Std Err Dif } & 0.00203 & \text { DF } & 24 \\ \text { Upper CL Dif } & 0.00661 & \text { Prob }>|t| & 0.2462\end{array}$

$\begin{array}{lrlr}\text { Upper CL Dif } & 0.00661 & \text { Prob }>|t| & 0.2462 \\ \text { Lower CL Dif } & -0.00178 & \text { Prob }>t & 0.1231\end{array}$

$\begin{array}{lll}\text { Confidence } & 0.95 \text { Prob }<\mathrm{t} & 0.8769\end{array}$

Analysis of Variance

Source DF Sum of Squares Mean Square F Ratio Prob $>$ F

$\begin{array}{llllll}\text { Sampler } & 1 & 0.00003587 & 0.000036 & 1.4132 & 0.2462\end{array}$

$\begin{array}{llll}\text { Error } & 24 & 0.00060921 & 0.000025\end{array}$

C. Total $25 \quad 0.00064508$

Means for Oneway Anova

Level Number Mean Std Error Lower 95\% Upper 95\%

$\begin{array}{lrrrrr}\text { Coliwasa } & 16 & 0.040535 & 0.00126 & 0.03794 & 0.04313\end{array}$

Isolok - $12 \mathrm{~mL}$ - LF $\quad 10 \quad 0.042949 \quad 0.00159 \quad 0.03966 \quad 0.04624$

Std Error uses a pooled estimate of error variance

Means and Std Deviations

Level Number Mean Std Dev Std Err Mean Lower 95\% Upper 95\%

$\begin{array}{llllllr}\text { Coliwasa } & 16 & 0.040535 & 0.004822 & 0.00121 & 0.03797 & 0.04310\end{array}$

$\begin{array}{lllllll}\text { Isolok - } 12 \mathrm{~mL} \text { - LF } & 10 & 0.042949 & 0.005379 & 0.00170 & 0.03910 & 0.04680\end{array}$

t Test

Isolok - $12 \mathrm{~mL}$ - LF-Coliwasa

Assuming unequal variances

Difference $\quad 0.00241$ t Ratio 1.158071

Std Err Dif $\quad 0.00208$ DF 17.64237

Upper CL Dif 0.00680 Prob $>|t| \quad 0.2623$

Lower CL Dif -0.00197 Prob $>t \quad 0.1311$

$\begin{array}{lll}\text { Confidence } & 0.95 \text { Prob }<\mathrm{t} \quad 0.8689\end{array}$

Tests that the Variances are Equal

$\begin{array}{lrrrr}\text { Level } & \text { Count } & \text { Std Dev } & \text { MeanAbsDif to Mean } & \text { MeanAbsDif to Median } \\ \text { Coliwasa } & 16 & 0.0048223 & 0.0037789 & 0.0037789\end{array}$

Isolok - 12 mL - LF $10 \quad 0.0053788 \quad 0.0041234 \quad 0.0040684$

Test F Ratio DFNum DFDen p-Value

$\begin{array}{lllll}\text { O'Brien[.5] } & 0.1691 & 1 & 24 & 0.6846\end{array}$

Brown-Forsythe $0.0546 \quad 1124 \quad 0.6846$

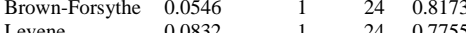

$\begin{array}{lllll}\text { Levene } & 0.0832 & 1 & 24 & 0.7755 \\ \text { Bartlett } & 0.1305 & 1 & . & 0.7179\end{array}$

$\begin{array}{lllll}\text { F Test 2-sided } & 1.2441 & 9 & 15 & 0.6805\end{array}$

Welch Anova testing Means Equal, allowing Std Devs Not Equal

F Ratio DFNum DFDen Prob $>$ F

$\begin{array}{llll}1.3411 & 1 & 17.642 & 0.2623\end{array}$

t Test

1.1581 


\section{Exhibit C2. Statistical Comparisons for Low-Rheology (Phase 3) Simulant Testing}

Oneway Analysis of Measurement By Sampler Type of Material=SME Simulant, Analytical Block $=3$, Oxide $/$ Analyte $=\mathrm{Fe} / \mathrm{Li}$, Targeted $=2.452$

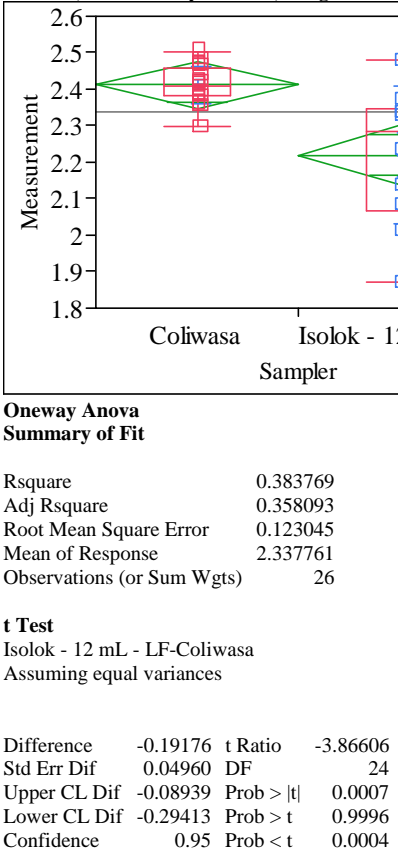

Analysis of Variance

Source DF Sum of Squares Mean Square F Ratio Prob $>$ F

$\begin{array}{lrrrrr}\text { Sampler } & 1 & 0.22629167 & 0.226292 & 14.9464 & 0.0007\end{array}$

$\begin{array}{llll}\text { Error } & 24 & 0.36336453 & 0.015140\end{array}$

C. Total $25 \quad 0.58965620$

Means for Oneway Anova

Level Number Mean Std Error Lower 95\% Upper 95\%

$\begin{array}{llrrrr}\text { Coliwasa } & 16 & 2.41152 & 0.03076 & 2.3480 & 2.4750\end{array}$

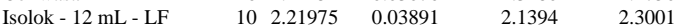

Std Error uses a pooled estimate of error variance

Means and Std Deviations

Level $\quad$ Number Mean Std Dev Std Err Mean Lower 95\% Upper 95\%

$\begin{array}{lrrrrrr}\text { Coliwasa } & 16 & 2.41152 & 0.052380 & 0.01310 & 2.3836 & 2.4394 \\ \text { Isolok - 12 mL - LF } & 10 & 2.21975 & 0.189212 & 0.05983 & 2.0844 & 2.3551\end{array}$

t Test

Isolok - $12 \mathrm{~mL}$ - LF-Coliwas

Assuming unequal variances

Difference $\quad-0.19176$ t Ratio $\quad-3.13079$

Std Err Dif $\quad 0.06125$ DF 9.869225

Upper CL Dif -0.05504 Prob $>|t| \quad 0.0108$

$\begin{array}{lrll}\text { Lower CL Dif } & -0.32848 & \text { Prob }>t & 0.9946 \\ \text { Confidence } & 0.95 & \text { Prob }<t & 0.0054\end{array}$

Tests that the Variances are Equal

$\begin{array}{lrrrr}\text { Level } & \text { Count } & \text { Std Dev } & \text { MeanAbsDif to Mean } & \text { MeanAbsDif to Median } \\ \text { Coliwasa } & 16 & 0.0523801 & 0.0398799 & 0.0398799 \\ \text { Isolok - 12 mL - LF } & 10 & 0.1892117 & 0.1552550 & 0.1521522\end{array}$

$\begin{array}{lrrrr}\text { Test } & \text { F Ratio } & \text { DFNum } & \text { DFDen } & \text { p-Value } \\ \text { O'Brien[.5] } & 9.2203 & 1 & 24 & 0.0057 \\ \text { Brown-Forsythe } & 12.7392 & 1 & 24 & 0.0016 \\ \text { Levene } & 20.2937 & 1 & 24 & 0.0001 \\ \text { Bartlett } & 17.0994 & 1 & . & <.0001 \\ \text { F Test 2-sided } & 13.0486 & 9 & 15 & <.0001\end{array}$

Welch Anova testing Means Equal, allowing Std Devs Not Equal

F Ratio DFNum DFDen Prob > F

$\begin{array}{llll}9.8018 & 1 & 9.8692 & 0.0108\end{array}$

t Test
Oneway Analysis of Measurement By Sampler Type of Material=SME Simulant, Analytical Block=3, Oxide $/$ Analyte $=$ Fe2O3 $($ wt $\%$ ), Targeted $=11.462$

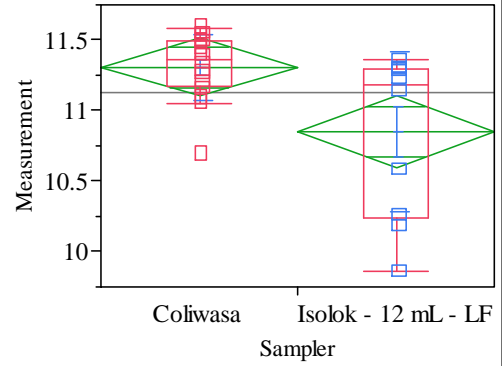

Oneway Anova

Summary of Fi

$\begin{array}{ll}\text { Adj Rsquare } & 0.225704 \\ \text { Root Mean Square Error } & 0.394701\end{array}$

0.256676

Mean of Response 11.13186

Observations (or Sum Wgts)

t Test

Isolok - $12 \mathrm{~mL}$ - LF-Coliwas

Assuming equal variances

Difference $\quad-0.45804$ t Ratio $\quad-2.87878$

$\begin{array}{lll}\text { Std Err Dif } \quad 0.15911 \text { DF } & 24\end{array}$

Upper CL Dif -0.12966 Prob $>|t| 0.0083$

Lower CL Dif -0.78642 Prob $>\mathrm{t} \quad 0.9959$

$\begin{array}{lrl}\text { Confidence } & 0.95 \text { Prob }<\mathrm{t} \quad 0.0041\end{array}$

Analysis of Variance

Source DF Sum of Squares Mean Square F Ratio Prob $>$ F

$\begin{array}{llllll}\text { Sampler } & 1 & 1.2910816 & 1.29108 & 8.2874 & 0.0083\end{array}$

$\begin{array}{llll}\text { Error } & 24 & 3.7389286 & 0.15579 \\ \text { C. Total } & 25 & 5.0300102 & \end{array}$

Means for Oneway Anova

Level Number Mean Std Error Lower 95\% Upper 95\%

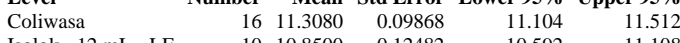

$\begin{array}{llllll}\text { Isolok - 12 mL - LF } & 10 & 10.8500 & 0.12482 & 10.592 & 11.108\end{array}$

Std Error uses a pooled estimate of error variance

Means and Std Deviations

Level Number Mean Std Dev Std Err Mean Lower 95\% Upper 95\%

$\begin{array}{lllllll}\text { Coliwasa } & 16 & 11.3080 & 0.234137 & 0.05853 & 11.183 & 11.433\end{array}$

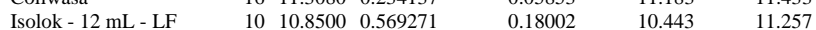

Test

Isolok - $12 \mathrm{~mL}$ - LF-Coliwas

Assuming unequal variances

$\begin{array}{lrr}\text { Difference } & -0.45804 \text { t Ratio } \quad-2.4197\end{array}$

Std Err Dif $\quad 0.18930$ DF $\quad 10.93037$

Upper CL Dif -0.04108 Prob $>|t| 0.0342$

Lower CL Dif -0.87500 Prob $>t \quad 0.9829$

$\begin{array}{lrl}\text { Confidence } & 0.95 \text { Prob }<\mathrm{t} & 0.0171\end{array}$

Tests that the Variances are Equal

Level Count Std Dev MeanAbsDif to Mean MeanAbsDif to Median

$\begin{array}{lllll}\text { Coliwasa } & 16 & 0.2341375 & 0.1779306 & 0.1742447\end{array}$

Isolok - 12 mL - LF $\quad 10 \quad 0.5692708 \quad 0.5021106$

Test F Ratio DFNum DFDen p-Value

$\begin{array}{lllrr}\text { O'Brien[.5] } & 10.2683 & 1 & 24 & 0.0038\end{array}$

$\begin{array}{llll}\text { Brown-Forsythe } \quad 4.4605 & 1 & 24 & 0.0453\end{array}$

$\begin{array}{lllll}\text { Levene } & 21.8233 & 1 & 24 & <.0001\end{array}$

$\begin{array}{lrrrr}\text { Bartlett } & 8.6806 & 1 & \dot{0} & 0.0032 \\ \text { F Test 2-sided } & 5.9115 & 9 & 15 & 0.0027\end{array}$

Welch Anova testing Means Equal, allowing Std Devs Not Equal

F Ratio DFNum DFDen Prob $>$ F

$\begin{array}{llrr}5.8549 & 1 & 10.93 & 0.0342\end{array}$

t Test

2.419 


\section{Exhibit C2. Statistical Comparisons for Low-Rheology (Phase 3) Simulant Testing}

Oneway Analysis of Measurement By Sampler Type of Material=SME Simulant, Analytical Block=3, Oxide/Analyte=K2O (wt \%), Targeted $=\mathbf{0 . 1 5 9 1}$

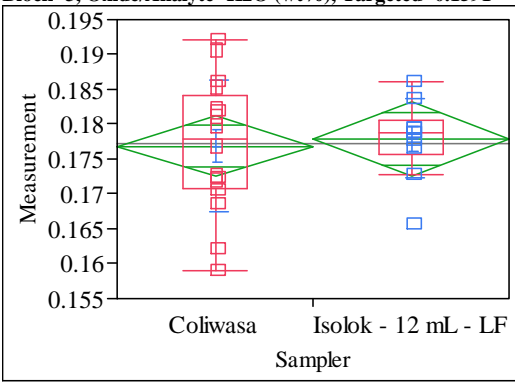

Oneway Anova

Summary of Fit

Rsquare

Root Mean Square Error $\quad 0.008267$

0.177285

Observations (or Sum Wgts)

t Test

Isolok - $12 \mathrm{~mL}$ - LF-Coliwas

Assuming equal variances

Difference $\quad 0.00103$ t Ratio $\quad 0.309494$

Std Err Dif $\quad 0.00333$ DF $\quad 24$

Upper CL Dif 0.00791 Prob $>|t| \quad 0.7596$

Lower CL Dif -0.00585 Prob $>\mathrm{t} \quad 0.3798$

$\begin{array}{lll}\text { Confidence } & 0.95 \text { Prob }<\mathrm{t} \quad 0.6202\end{array}$

Analysis of Variance

Source DF Sum of Squares Mean Square F Ratio Prob $>$ F

$\begin{array}{lrrrrr}\text { Sampler } & 1 & 0.00000655 & 6.547 \mathrm{e}-6 & 0.0958 & 0.7596\end{array}$

Error $\quad 24 \quad 0.00164037 \quad 0.000068$

C. Total $25 \quad 0.0016469$

Means for Oneway Anova

Level Number Mean Std Error Lower 95\% Upper 95\%

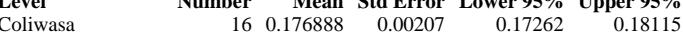

$\begin{array}{llllll}\text { Isolok - 12 mL - LF } & 16 & 0.176888 & 0.00207 & 0.17262 & 0.18115 \\ & 10 & 0.177919 & 0.00261 & 0.17252 & 0.18332\end{array}$

Std Error uses a pooled estimate of error variance

Means and Std Deviations

Level Number Mean Std Dev Std Err Mean Lower 95\% Upper 95\%

$\begin{array}{lllllll} & 16 & 0.176888 & 0.009502 & 0.00238 & 0.17182 & 0.18195\end{array}$

$\begin{array}{lllllll}\text { Colok - } 12 \mathrm{~mL} \text { - LF } & 10 & 0.177919 & 0.005637 & 0.00178 & 0.17389 & 0.18195\end{array}$

t Test

Isolok - $12 \mathrm{~mL}$ - LF-Coliwas

Assuming unequal variances

Difference $\quad 0.00103$ t Ratio $\quad 0.347284$

Std Err Dif $\quad 0.00297$ DF 23.97776

Upper CL Dif 0.00716 Prob $>|t| \quad 0.7314$

Lower CL Dif -0.00510 Prob $>\mathrm{t} \quad 0.3657$

$\begin{array}{lrl}\text { Confidence } & 0.95 \text { Prob }<\mathrm{t} \quad 0.6343\end{array}$

Tests that the Variances are Equal

Level Count Std Dev MeanAbsDif to Mean MeanAbsDif to Median

$\begin{array}{lllll}\text { Coliwasa } & 16 & 0.0095021 & 0.0077923 & 0.0077923 \\ \text { Isolok - 12 mL - LF } & 10 & 0.0056372 & 0.0038065 & 0.0036138\end{array}$

$\begin{array}{lrrrr}\text { Test } & \text { F Ratio } & \text { DFNum } & \text { DFDen } & \text { p-Value } \\ \text { O'Brien[.5] } & 2.4682 & 1 & 24 & 0.1293 \\ \text { Brown-Forsythe } & 4.5509 & 1 & 24 & 0.0433 \\ \text { Levene } & 4.4779 & 1 & 24 & 0.0449 \\ \text { Bartlett } & 2.5984 & 1 & . & 0.1070 \\ \text { F Test 2-sided } & 2.8413 & 15 & 9 & 0.1177\end{array}$

Welch Anova testing Means Equal, allowing Std Devs Not Equal

F Ratio DFNum DFDen Prob $>$ F

$\begin{array}{rrrr}0.1206 & 1 & 23.978 & 0.7314\end{array}$

t Test

0.3473
Oneway Analysis of Measurement By Sampler Type of Material=SME Simulant, Analytical Block=3, Oxide/Analyte $=$ Li2O (wt $\%$, Targeted $=4.674$

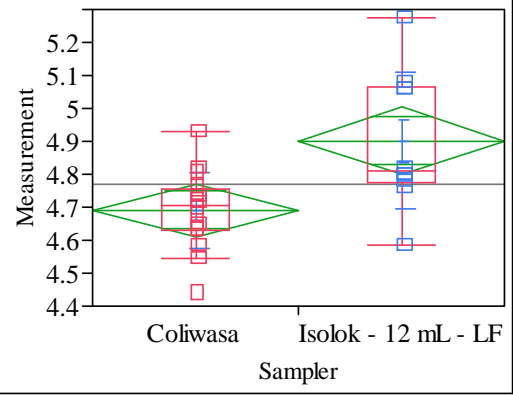

Oneway Anova

Summary of Fit

Rsquare

Adj Rsquare $\quad 0.31905$

Root Mean Square Error $\quad 0.156478$

Mean of Response 4.771986

Observations (or Sum Wgts)

\section{Test}

Isolok - $12 \mathrm{~mL}$ - LF-Coliwasa

Assuming equal variances

Difference $\quad 0.211522$ t Ratio $\quad 3.35334$

Std Err Dif $\quad 0.063078$ DF 24

Upper CL Dif 0.341709 Prob $>|t| \quad 0.0026$

Lower CL Dif 0.081336 Prob $>t \quad 0.0013$

Confidence $\quad 0.95$ Prob $<\mathrm{t} \quad 0.9987$

Analysis of Variance

Source DF Sum of Squares Mean Square F Ratio Prob > F

$\begin{array}{lrrrrr}\text { Sampler } & 1 & 0.27533376 & 0.275334 & 11.2449 & 0.0026\end{array}$

$\begin{array}{llll}\text { Error } & 24 & 0.58764574 & 0.024485\end{array}$

$\begin{array}{lllll}\text { Means for Oneway Anova } & & & \\ \text { Level } & \text { Number } & \text { Mean Std Error } & \text { Lower 95\% } & \text { Upper 95\% }\end{array}$

$\begin{array}{lrrrrr}\text { Level } & \text { Number } & \text { Mean } & \text { Std Error } & \text { Lower 95\% } & \text { Upper 95\% } \\ \text { Coliwasa } & 16 & 4.69063 & 0.03912 & 4.6099 & 4.7714\end{array}$

$\begin{array}{llllll}\text { Isolok - 12 mL - LF } & 16 & 4.99063 & 0.03912 & 4.6099 & 4.7714 \\ & & 0.04948 & 4.8000 & 5.0043\end{array}$

Std Error uses a pooled estimate of error variance

Means and Std Deviations

Level Number Mean Std Dev Std Err Mean Lower 95\% Upper 95\%

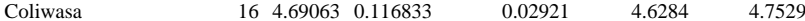

Isolok - 12 mL - LF $\quad 104.90215 \quad 0.206262 \quad 0.06523 \quad 4.7546 \quad 5.0497$

t Test

Isolok - $12 \mathrm{~mL}$ - LF-Coliwas

Assuming unequal variances

Difference $\quad 0.211522$ t Ratio 2.959725

Std Err Dif $\quad 0.071467$ DF 12.66582

Upper CL Dif 0.366332 Prob $>|t| \quad 0.0113$

Lower CL Dif 0.056712 Prob $>t \quad 0.0057$

$\begin{array}{lrl}\text { Confidence } & 0.95 \text { Prob }<\mathrm{t} \quad 0.9943\end{array}$

Tests that the Variances are Equal

Level Count Std Dev MeanAbsDif to Mean MeanAbsDif to Median

$\begin{array}{lllll} & 16 & 0.1168333 & 0.0864524 & 0.0861160\end{array}$

$\begin{array}{lrrrr}\text { Test } & \text { F Ratio } & \text { DFNum } & \text { DFDen } & \text { p-Value } \\ \text { O'Brien[.5] } & 3.8354 & 1 & 24 & 0.0619 \\ \text { Brown-Forsythe } & 2.4310 & 1 & 24 & 0.1320 \\ \text { Levene } & 6.5816 & 1 & 24 & 0.0170 \\ \text { Bartlett } & 3.6281 & 1 & . & 0.0568 \\ \text { F Test 2-sided } & 3.1168 & 9 & 15 & 0.0504\end{array}$

Welch Anova testing Means Equal, allowing Std Devs Not Equal

F Ratio DFNum DFDen Prob $>$ F

$\begin{array}{llr}8.7600 \quad 12.666 & 0.0113\end{array}$

t Test

2.9597 
SRNL-STI-2011-00693

Revision 0

\section{Exhibit C2. Statistical Comparisons for Low-Rheology (Phase 3) Simulant Testing}

Oneway Analysis of Measurement By Sampler Type of Material=SME Simulant, Analytical Block $=3$, Oxide $/$ Analyte $=\mathrm{MgO}$ (wt $\%$ ), Targeted $=1.014$

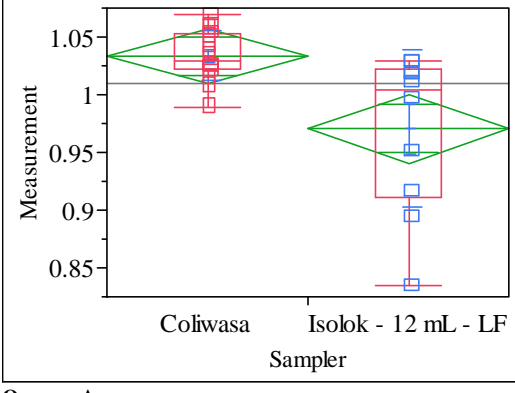

Oneway Anova

Rsquare $\quad 0.33477$

Adj Rsquare $\quad 0.307052$

Root Mean Square Error $\quad 0.045084$

Mean of Response $\quad 1.009139$

Observations (or Sum Wgts) 26

t Test

Isolok - $12 \mathrm{~mL}$ - LF-Coliwasa

Assuming equal variances

Difference $\quad-0.06316$ t Ratio $\quad-3.4753$

Std Err Dif $\quad 0.01817$ DF 24

Upper CL Dif -0.02565 Prob $>|t| \quad 0.0020$

Lower CL Dif -0.10067 Prob $>t \quad 0.9990$

$\begin{array}{llll}\text { Confidence } & 0.95 \text { Prob }<\mathrm{t} \quad 0.0010\end{array}$

Analysis of Variance

Source DF Sum of Squares Mean Square F Ratio Prob $>$ F

$\begin{array}{lrrrrr}\text { Source } & \text { DF } & \text { Sum of Squares } & \text { Mean Square } & \text { F Ratio } & \text { Prob }>\mathbf{F} \\ \text { Sampler } & 1 & 0.02454922 & 0.024549 & 12.0777 & 0.0020\end{array}$

$\begin{array}{llll}\text { Error } & 24 & 0.04878245 & 0.002033\end{array}$

$\begin{array}{lll}\text { C. Total } & 25 & 0.07333167\end{array}$

Means for Oneway Anova

$\begin{array}{lrrrrr}\text { Level } & \text { Number } & \text { Mean } & \text { Std Error } & \text { Lower 95\% } & \text { Upper 95\% } \\ \text { Coliwasa } & 16 & 1.03343 & 0.01127 & 1.0102 & 1.0567\end{array}$

$\begin{array}{llllll}\text { Isolok - } 12 \mathrm{~mL} \text { - LF } & 10 & 0.97027 & 0.01426 & 0.9408 & 0.9997\end{array}$

Std Error uses a pooled estimate of error variance

Means and Std Deviations

Level Number Mean Std Dev Std Err Mean Lower 95\% Upper 95\%

$\begin{array}{lrrrrrr}\text { Coliwasa } & 16 & 1.03343 & 0.021581 & 0.00540 & 1.0219 & 1.0449\end{array}$

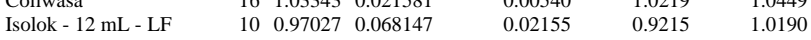

t Test

Isolok - $12 \mathrm{~mL}$ - LF-Coliwasa

Assuming unequal variances
Difference $\quad-0.06316$ t Ratio $\quad-2.84313$

Std Err Dif $\quad 0.02222$ DF $\quad 10.13969$

Upper CL Dif -0.01375 Prob $>|t| \quad 0.0172$

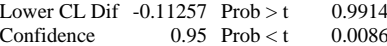

Tests that the Variances are Equal

Level Count Std Dev MeanAbsDif to Mean MeanAbsDif to Median

$\begin{array}{lrrrr}\text { Coliwasa } & 16 & 0.0215810 & 0.0171401 & 0.0171012\end{array}$

$\begin{array}{lllll} & 16 & 0.0215810 & 0.0171401 & 0.0171012 \\ \text { Isolok - } 12 \mathrm{~mL} \text { - LF } & 10 & 0.0681472 & 0.0568465 & 0.0515731\end{array}$

Test F Ratio DFNum DFDen p-Value

$\begin{array}{lrrrr}\text { O'Brien[.5] } & 7.2520 & 1 & 24 & 0.0127\end{array}$

Brown-Forsythe $\quad 6.0187 \quad 1 \quad 24 \quad 0.0218$

$\begin{array}{lllll}\text { Levene } & 19.7891 & 1 & 24 & 0.0002\end{array}$

$\begin{array}{lrrrr}\text { Bartlett } & 14.0288 & 1 & & 0.0002 \\ \text { F Test 2-sided } & 9.9713 & 9 & 15 & 0.0001\end{array}$

Welch Anova testing Means Equal, allowing Std Devs Not Equal

$\begin{array}{rrrr}\text { F Ratio } & \text { DFNum } & \text { DFDen } & \text { Prob }>\text { F } \\ 8.0834 & 1 & 10.14 & 0.0172\end{array}$

t Test

2.8431
Oneway Analysis of Measurement By Sampler Type of Material=SME Simulant, Analytical Block $=3$, Oxide $/$ Analyte $=\mathrm{MnO}($ wt $\%)$, Targeted $=1.779$

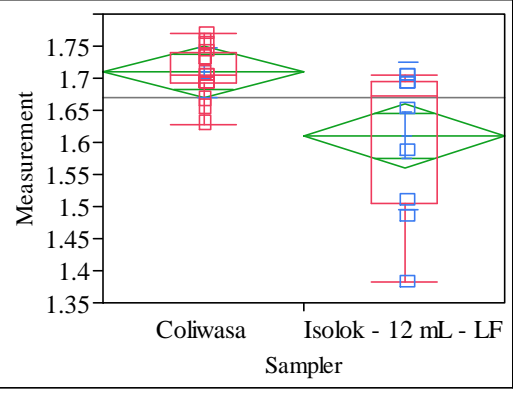

Oneway Anova

Summary of Fit

Rsqua

0.270827

Root Mean Square Error $\quad 0.076654$

$\begin{array}{lr}\text { Mean of Response } & 1.671111 \\ \text { Observations (or Sum Wgts) } & 26\end{array}$

t Test

Isolok - $12 \mathrm{~mL}$ - LF-Coliwasa

Assuming equal variances

Difference $\quad-0.09910$ t Ratio $\quad-3.20709$

Std Err Dif $\quad 0.03090$ DF 24

Upper CL Dif -0.03532 Prob $>|t| \quad 0.0038$

Lower CL Dif -0.16287 Prob $>\mathrm{t} \quad 0.9981$

$\begin{array}{lll}\text { Confidence } & 0.95 \text { Prob }<\mathrm{t} \quad 0.0019\end{array}$

Analysis of Variance

Source DF Sum of Squares Mean Square F Ratio Prob $>$ F

$\begin{array}{lrrrrr}\text { Source } & \text { DF } & \text { Sum of Squares } & \text { Mean Square } & \text { F Ratio } & \text { Prob }>\mathbf{F} \\ \text { Sampler } & 1 & 0.06043527 & 0.060435 & 10.2854 & 0.0038\end{array}$

$\begin{array}{llll}\text { Error } & 24 & 0.14101990 & 0.005876\end{array}$

C. Total $25 \quad 0.20145516$

Means for Oneway Anova

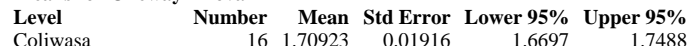

$\begin{array}{llllll}\text { Isolok - 12 mL - LF } & 16 & 1.70923 & 0.01916 & 1.6697 & 1.7488 \\ & 10 & 1.61013 & 0.02424 & 1.5601 & 1.6602\end{array}$

Std Error uses a pooled estimate of error variance

Means and Std Deviations

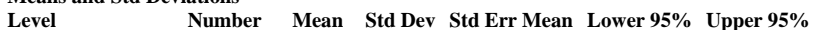

$\begin{array}{lrrrrrr}\text { Coliwasa } & 16 & 1.70923 & 0.039412 & 0.00985 & 1.6882 & 1.7302\end{array}$

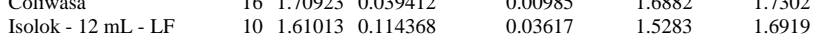

t Test

Isolok - $12 \mathrm{~mL}$ - LF-Coliwasa

Assuming unequal variances

Difference $\quad-0.09910$ t Ratio $\quad-2.64375$

Std Err Dif $\quad 0.03748$ DF $\quad 10.35131$

Upper CL Dif -0.01596 Prob $>|t| r 0.0239$

Lower CL Dif -0.18224 Prob $>t \quad 0.9880$

$\begin{array}{lrl}\text { Confidence } & 0.95 \text { Prob }<\mathrm{t} \quad 0.0120\end{array}$

Tests that the Variances are Equal

Level Count Std Dev MeanAbsDif to Mean MeanAbsDif to Median

$\begin{array}{lrrrr}\text { Coliwasa } & 16 & 0.0394116 & 0.0312713 & 0.0306660\end{array}$

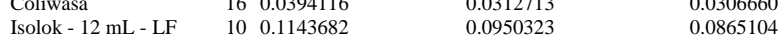

Test F Ratio DFNum DFDen p-Value

$\begin{array}{lllll}\text { O'Brien[.5] } & 6.9603 & 1 & 24 & 0.0144\end{array}$

Brown-Forsythe $5.1222 \quad 1 \quad 24 \quad 0.0329$

$\begin{array}{lllll}\text { Levene } & 17.1250 & 1 & 24 & 0.0004\end{array}$

$\begin{array}{lllll}\text { Bartlett } & 12.125015 & 1 & 0.0005\end{array}$

F Test 2-sided $\quad 8.4210 \quad-9 \quad 150.000$

Welch Anova testing Means Equal, allowing Std Devs Not Equal

F Ratio DFNum DFDen Prob $>$ F

$\begin{array}{lllll}6.9894 & 1 & 10.351 & 0.0239\end{array}$

t Test

2.6437 
SRNL-STI-2011-00693

Revision 0

\section{Exhibit C2. Statistical Comparisons for Low-Rheology (Phase 3) Simulant Testing}

Oneway Analysis of Measurement By Sampler Type of Material=SME Simulant, Analytical Block $=3$, Oxide/Analyte $=\mathrm{Na2O}(\mathrm{wt} \%)$, Targeted $=11.659$

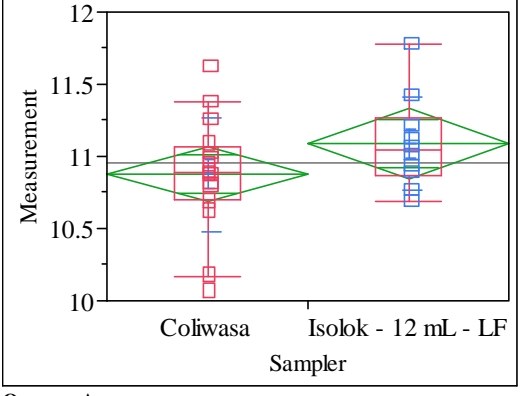

Oneway Anova

Summary of Fit

Rsquare $\quad 0.079519$

Adj Rsquare $\quad 0.041165$

Root Mean Square Error $\quad 0.368965$

Mean of Response 10.9582

Observations (or Sum Wgts) $\quad 26$

t Test

Isolok - $12 \mathrm{~mL}$ - LF-Coliwasa

Assuming equal variances

Difference $\quad 0.21416$ t Ratio 1.439903

Std Err Dif $\quad 0.14873$ DF 24

Upper CL Dif 0.52114 Prob $>|t| \quad 0.1628$

Lower CL Dif -0.09281 Prob $>\mathrm{t} \quad 0.0814$

$\begin{array}{lrl}\text { Confidence } & 0.95 \text { Prob }<\mathrm{t} \quad 0.9186\end{array}$

Analysis of Variance

Source DF Sum of Squares Mean Square F Ratio Prob $>$ F

$\begin{array}{lrrrrr}\text { Source } & \text { DF } & \text { Sum of Squares } & \text { Mean Square } & \text { F Ratio } & \text { Prob }>\text { F } \\ \text { Sampler } & 1 & 0.2822523 & 0.282252 & 2.0733 & 0.1628\end{array}$

$\begin{array}{lrrr}\text { Sampler } & 1 & 0.2822523 & 0.282252 \\ \text { Error } & 24 & 3.2672507 & 0.136135\end{array}$

C. Total $25 \quad 3.5495030$

Means for Oneway Anova

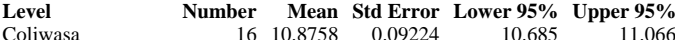

$\begin{array}{llllll} & 16 & 10.8758 & 0.09224 & 10.685 & 11.066 \\ \text { Isolok - 12 mL - LF } & 10 & 11.0900 & 0.11668 & 10.849 & 11.331\end{array}$

Std Error uses a pooled estimate of error variance

Means and Std Deviations

Level $\quad$ Number Mean Std Dev Std Err Mean Lower 95\% Upper 95\%

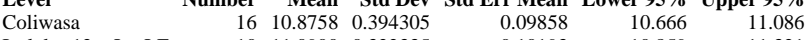

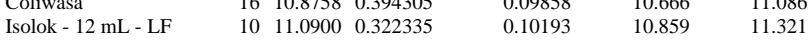

t Test

Isolok - $12 \mathrm{~mL}$ - LF-Coliwasa

Assuming unequal variances

Difference $\quad 0.21416$ t Ratio $\quad 1.510319$

Std Err Dif $\quad 0.14180$ DF 22.10548

Upper CL Dif 0.50816 Prob $>|t| r|r| r \mid$

Lower CL Dif -0.07983 Prob $>$ t $\quad 0.0726$

$\begin{array}{lrl}\text { Confidence } & 0.95 \text { Prob }<\mathrm{t} \quad 0.9274\end{array}$

Tests that the Variances are Equal

Level Count Std Dev MeanAbsDif to Mean MeanAbsDif to Median

$\begin{array}{lrrrr}\text { Coliwasa } & 16 & 0.3943054 & 0.2707584 & 0.2704425 \\ \text { Isolok - 12 mL - LF } & 10 & 0.3223352 & 0.2350912 & 0.2305080\end{array}$

Test F Ratio DFNum DFDen p-Value

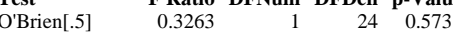

$\begin{array}{lllll}\text { Brown-Forsythe } & 0.1480 & 1 & 24 & 0.7039\end{array}$

$\begin{array}{lllll}\text { Levene } & 0.1219 & 1 & 24 & 0.7301\end{array}$

$\begin{array}{lrrrr}\text { Bartlett } & 0.4202 & 1 & \text {. } & 0.5168\end{array}$

Welch Anova testing Means Equal, allowing Std Devs Not Equal

F Ratio DFNum DFDen Prob $>$ F

$\begin{array}{rrrr}2.2811 & 1 & 22.105 & 0.1451\end{array}$

t Test
Oneway Analysis of Measurement By Sampler Type of Material=SME Simulant, Analytica Block $=3$, Oxide $/$ Analyte $=\mathrm{NiO}($ wt $\%$ ), Targeted $=0.41$

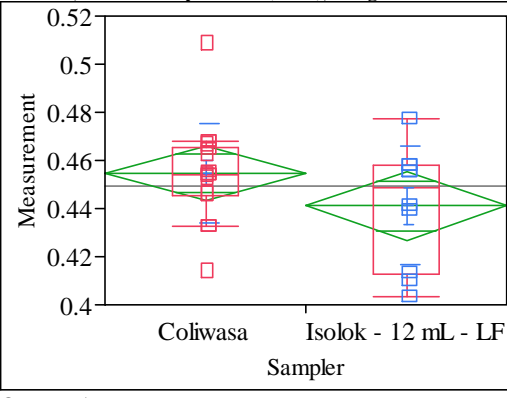

Oneway Anova

Summary of Fit

Rsqua

0.049024

are Error $\quad 0.022143$

Mean of Response 0.449486

t Test

Isolok - $12 \mathrm{~mL}$ - LF-Coliwasa

Assuming equal variances

Difference $\quad-0.01350$ t Ratio $\quad-1.51287$

Std Err Dif $\quad 0.00893$ DF 24

Upper CL Dif 0.00492 Prob $>|t| \quad 0.1434$

Lower CL Dif -0.03193 Prob $>t \quad 0.9283$

$\begin{array}{lrl}\text { Confidence } & 0.95 \text { Prob }<\mathrm{t} \quad 0.0717\end{array}$

Analysis of Variance

Source DF Sum of Squares Mean Square F Ratio Prob $>$ F

$\begin{array}{lrrrrr}\text { Source } & \text { DF } & \text { Sum of Squares } & \text { Mean Square } & \text { F Ratio } & \text { Prob }>\mathbf{F} \\ \text { Sampler } & 1 & 0.00112227 & 0.001122 & 2.2888 & 0.1434\end{array}$

$\begin{array}{lrrr}\text { Sampler } & 1 & 0.00112227 & 0.001122 \\ \text { Error } & 24 & 0.01176801 & 0.000490\end{array}$

$\begin{array}{lll}\text { Error } & 24 & 0.01176801 \\ \text { C. Total } & 25 & 0.01289028\end{array}$

Means for Oneway Anova

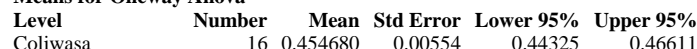

$\begin{array}{llllll}\text { Isolok - } 12 \mathrm{~mL} \text { - LF } & 16 & 0.454680 & 0.00554 & 0.44325 & 0.4661 \\ & 10 & 0.441176 & 0.00700 & 0.42672 & 0.45563\end{array}$

Std Error uses a pooled estimate of error variance

Means and Std Deviations

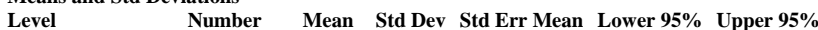

$\begin{array}{lrrrrrr}\text { Coliwasa } & 16 & 0.454680 & 0.020496 & 0.00512 & 0.44376 & 0.46560\end{array}$

$\begin{array}{lllllll}\text { Coliwasa } & 16 & 0.454680 & 0.020496 & 0.00512 & 0.44376 & 0.46560 \\ \text { Isolok - 12 mL - LF } & 10 & 0.441176 & 0.024646 & 0.00779 & 0.42355 & 0.45881\end{array}$

t Test

Isolok - $12 \mathrm{~mL}$ - LF-Coliwasa

Assuming unequal variances

Difference $\quad-0.01350$ t Ratio $\quad-1.44785$

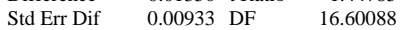

Upper CL Dif 0.00621 Prob $>|t| r 0.1663$

$\begin{array}{lll}\text { Lower CL Dif }-0.03322 & \text { Prob }>t \quad 0.9169\end{array}$

$\begin{array}{lrl}\text { Confidence } & 0.95 \text { Prob }<\mathrm{t} \quad 0.0831\end{array}$

Tests that the Variances are Equal

Level Count Std Dev MeanAbsDif to Mean MeanAbsDif to Median

$\begin{array}{lrrrr}\text { Coliwasa } & 16 & 0.0204959 & 0.0136496 & 0.0135998 \\ \text { Isolok - 12 mL - LF } & 10 & 0.0246459 & 0.0198001 & 0.0197238\end{array}$

Test F Ratio DFNum DFDen p-Value

$\begin{array}{lrlll}\text { O'Brien[.5] } & 0.3606 & 1 & 24 & 0.5538\end{array}$

Brown-Forsythe $1.0134 \quad 11 \quad 24 \quad 0.3241$

$\begin{array}{lllll}\text { Levene } & 1.1480 & 1 & 24 & 0.2946\end{array}$

$\begin{array}{lllll}\text { Bartlett } & 0.3753 & 1 & 0.5401\end{array}$

F Test 2-sided $\quad 1.4460 \quad 9 \quad 150.54071$

Welch Anova testing Means Equal, allowing Std Devs Not Equal

F Ratio DFNum DFDen Prob $>$ F

$\begin{array}{llll}2.0963 & 1 & 16.601 & 0.1663\end{array}$

t Test 


\section{Exhibit C2. Statistical Comparisons for Low-Rheology (Phase 3) Simulant Testing}

Oneway Analysis of Measurement By Sampler Type of Material=SME Simulant, Analytica Block $=3$, Oxide/Analyte $=\mathrm{SiO} 2$ ( $\mathbf{w t} \%$ ), Targeted $=\mathbf{5 0 . 9 8 5}$

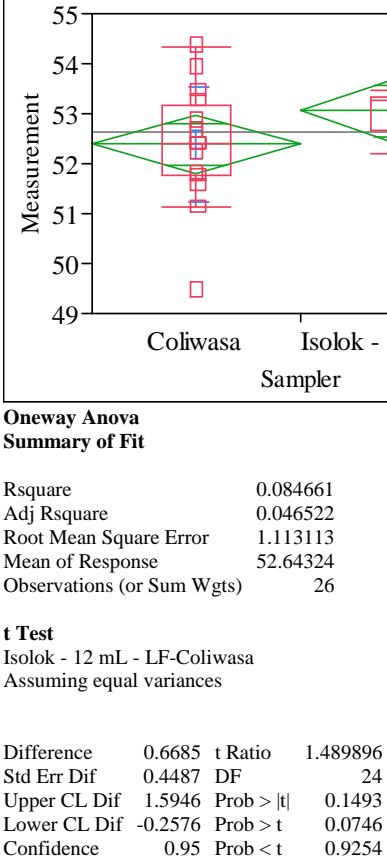

Analysis of Variance

Source DF Sum of Squares Mean Square F Ratio Prob $>$ F

$\begin{array}{llllll}\text { Sampler } & 1 & 2.750363 & 2.75036 & 2.2198 & 0.1493\end{array}$

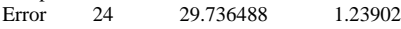

C. Total $25 \quad 32.486851$

Means for Oneway Anova

Level Number Mean Std Error Lower 95\% Upper 95\%

$\begin{array}{lrrrrr}\text { Coliwasa } & 16 & 52.3861 & 0.27828 & 51.812 & 52.960 \\ \text { Isolok - 12 mL - LF } & 10 & 53.0546 & 0.35200 & 52.328 & 53.781\end{array}$

Std Error uses a pooled estimate of error variance

Means and Std Deviations

Level Number Mean Std Dev Std Err Mean Lower 95\% Upper 95\%

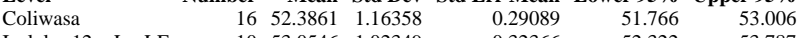

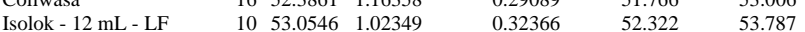

TTest

Isolok - $12 \mathrm{~mL}$ - LF-Coliwas

Assuming unequal variances

$\begin{array}{lrlr}\text { Difference } & 0.6685 & \text { t Ratio } & 1.536253 \\ \text { Std Err Dif } & 0.4352 & \text { DF } & 21.13742 \\ \text { Upper CL Dif } & 1.5732 & \text { Prob }>|t| & 0.1393 \\ \text { Lower CL Dif } & -0.2361 & \text { Prob }>\text { t } & 0.0697 \\ \text { Confidence } & 0.95 & \text { Prob }<\mathrm{t} & 0.9303\end{array}$

Tests that the Variances are Equal

Level Count Std Dev MeanAbsDif to Mean MeanAbsDif to Median

Coliwasa $\quad 16 \quad 1.163577$

Isolok - 12 mL - LF $\quad 101.023491 \quad 0.6417900 \quad 0.5990040$

Test F Ratio DFNum DFDen p-Value

$\begin{array}{lrrrr}\text { O'Brien[.5] } & 0.1059 & 1 & 24 & 0.7477\end{array}$

$\begin{array}{lllll}\text { Brown-Forsythe } & 0.3738 & 1 & 24 & 0.5467\end{array}$

$\begin{array}{lllll}\text { Levene } & 0.2724 & 1 & 24 & 0.6065\end{array}$

$\begin{array}{lrrrr}\text { Bartlett } & 0.1729 & 1 & \text {. } & 0.6775 \\ & 1.2925 & 15 & 9 & 0.7144\end{array}$

Welch Anova testing Means Equal, allowing Std Devs Not Equal

F Ratio DFNum DFDen Prob $>$ F

$\begin{array}{llll}2.3601 & 1 & 21.137 & 0.1393\end{array}$

t Test

1.5363
Oneway Analysis of Measurement By Sampler Type of Material=SME Simulant, Analytical Block=3, Oxide/Analyte=Sum of Oxides (wt $\%$ ), Targeted $=99.553$

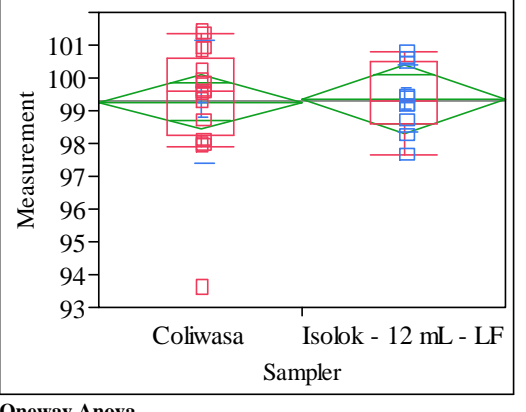

Oneway Anova

Rsquare

Adj Rsquare

Root Mean Square Error $\quad 1.619236$

Mean of Response 99.30673

Observations (or Sum Wgts)

\section{Test}

Isolok - $12 \mathrm{~mL}$ - LF-Coliwas

Assuming equal variances

$\begin{array}{lrlr}\text { Difference } & 0.0952 & \mathrm{t} \text { Ratio } & 0.145776 \\ \text { Std Err Dif } & 0.6527 & \text { DF } & 24 \\ \text { Upper CL Dif } & 1.4423 & \text { Prob }>|t| & 0.8853 \\ \text { Lower CL Dif } & -1.2520 & \text { Prob }>\text { t } & 0.4427 \\ \text { Confidence } & 0.95 & \text { Prob }<\text { t } & 0.5573\end{array}$

Analysis of Variance

Source DF Sum of Squares Mean Square F Ratio Prob $>$ F

$\begin{array}{lrrrrr}\text { Sampler } & 1 & 0.055718 & 0.05572 & 0.0213 & 0.8853\end{array}$

$\begin{array}{llll}\text { Error } & 24 & 62.926179 & 2.62192\end{array}$

Means for Oneway Anova

Level Number Mean Std Error Lower 95\% Upper 95\%

$\begin{array}{lrrrrr}\text { Level } & \text { Number } & \text { Mean } & \text { Std Error } & \text { Lower 95\% } & \text { Upper 95\% } \\ \text { Coliwasa } & 16 & 99.2701 & 0.40481 & 98.435 & 100.11\end{array}$

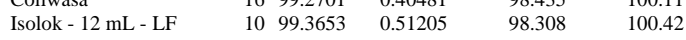

Std Error uses a pooled estimate of error variance

Means and Std Deviations

Level Number Mean Std Dev Std Err Mean Lower 95\% Upper 95\%

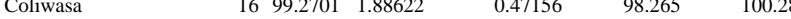

$\begin{array}{llllll}\text { Isolok - } 12 \mathrm{~mL} \text { - LF } & 10 & 99.3653 & 1.03056 & 0.32589 & 98.628\end{array}$

t Test

Isolok - $12 \mathrm{~mL}$ - LF-Coliwas

Assuming unequal variances

$\begin{array}{lrlr}\text { Difference } & 0.0952 & \text { t Ratio } & 0.166 \\ \text { Std Err Dif } & 0.5732 & \text { DF } & 23.72871 \\ \text { Upper CL Dif } & 1.2789 & \text { Prob }>|t| & 0.8696 \\ \text { Lower CL Dif } & -1.0886 & \text { Prob }>\mathrm{t} & 0.4348 \\ \text { Confidence } & 0.95 & \text { Prob }<\mathrm{t} & 0.5652\end{array}$

Tests that the Variances are Equal

Level Count Std Dev MeanAbsDif to Mean MeanAbsDif to Median

$\begin{array}{lllll} & 16 & 1.886225 & 1.273320 & 1.207732 \\ \text { Isolok - } 12 \mathrm{~mL} \text { - LF } & 10 & 1.030562 & 0.780356 & 0.780356\end{array}$

Test F Ratio DFNum DFDen p-Value

$\begin{array}{lrrrr}\text { O'Brien[.5] } & 0.7804 & 1 & 24 & 0.3858\end{array}$

$\begin{array}{lllll}\text { Brown-Forsythe } & 0.7669 & 1 & 24 & 0.3899\end{array}$

$\begin{array}{lllll}\text { Levene } & 1.1617 & 1 & 24 & 0.2918\end{array}$

$\begin{array}{llrrr}\text { Bartlett } & 3.4003 & 1 & . & 0.0652\end{array}$

$\begin{array}{lllll}\text { F Test 2-sided } & 3.3500 & 15 & 9 & 0.0723\end{array}$

Welch Anova testing Means Equal, allowing Std Devs Not Equal

F Ratio DFNum DFDen Prob $>$ F

$\begin{array}{llll}0.0276 & 1 & 23.729 & 0.8696\end{array}$

t Test

0.1660 


\section{Exhibit C2. Statistical Comparisons for Low-Rheology (Phase 3) Simulant Testing}

Oneway Analysis of Measurement By Sampler Type of Material=SME Simulant, Analytical Block $=3$, Oxide/Analyte $=$ TiO2 $($ wt $\%$ ), Targeted $=\mathbf{0 . 0 7 1 1}$

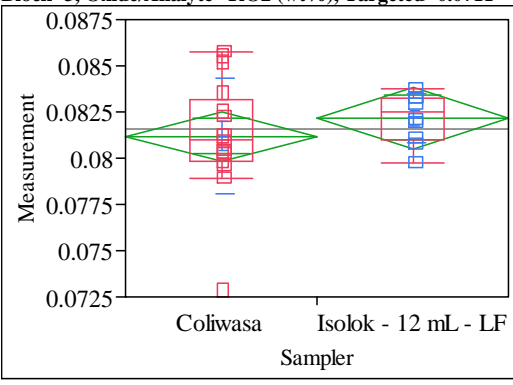

Oneway Anova

Rsquare $\quad 0.035588$

$\begin{array}{lr}\text { Adj Rsquare } & -0.0046 \\ \text { Root Mean Square Error } & 0.002589\end{array}$

$\begin{array}{ll}\text { Root Mean Square Error } & 0.002589 \\ \text { Mean of Response } & 0.081578\end{array}$

Observations (or Sum Wgts) 26

Isolok - $12 \mathrm{~mL}$ - LF-Coliwas

Assuming equal variances

Difference $\quad 0.00098$ t Ratio $\quad 0.941078$

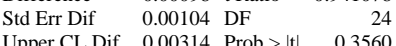

$\begin{array}{rrrr}\text { Upper CL Dif } & 0.00314 \text { Prob }>|t| & 0.3560 \\ & -0.00117 & \text { Prob }>t & 0.1780\end{array}$

$\begin{array}{lrl}\text { Confidence } & 0.95 \text { Prob }<\mathrm{t} \quad 0.8220\end{array}$

Analysis of Variance

Source DF Sum of Squares Mean Square F Ratio Prob $>$ F

$\begin{array}{llllll}\text { Sampler } & 1 & 0.00000593 & 5.9347 \mathrm{e}-6 & 0.8856 & 0.3560\end{array}$

$\begin{array}{llll}\text { Error } & 24 & 0.00016083 & 6.7011 \mathrm{e}-6\end{array}$

C. Total $25 \quad 0.00016676$

Means for Oneway Anova

Level Number Mean Std Error Lower 95\% Upper 95\%

$\begin{array}{lrrrrr}\text { Coliwasa } & \text { Numer } & 0.081200 & 0.00065 & 0.07986 & 0.08254\end{array}$

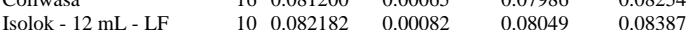

Std Error uses a pooled estimate of error variance

Means and Std Deviations

$\begin{array}{lrrrrr}\text { Level } & \text { Number } & \text { Mean } & \text { Std Dev Std Err Mean Lower 95\% Upper 95\% }\end{array}$

$\begin{array}{lllllll} & 16 & 0.081200 & 0.003111 & 0.00078 & 0.07954 & 0.08286 \\ \text { Isolok - } 12 \mathrm{~mL}-\mathrm{LF} & 10 & 0.082182 & 0.001321 & 0.00042 & 0.08124 & 0.08313\end{array}$

Test

Isolok - $12 \mathrm{~mL}$ - LF-Coliwasa

Assuming unequal variances

Difference $\quad 0.00098$ t Ratio 1.112575

Std Err Dif $\quad 0.00088$ DF 21.8677

Upper CL Dif 0.00281 Prob $>|t| \quad 0.2780$

Lower CL Dif -0.00085 Prob $>t \quad 0.1390$

$\begin{array}{lrl}\text { Confidence } & 0.95 \text { Prob }<\mathrm{t} & 0.8610\end{array}$

Tests that the Variances are Equal

$\begin{array}{lrrrr}\text { Level } & \text { Count } & \text { Std Dev } & \text { MeanAbsDif to Mean } & \text { MeanAbsDif to Median } \\ \text { Coliwasa } & 16 & 0.0031106 & 0.0021332 & 0.0021163\end{array}$

$\begin{array}{lrrrr}\text { Coliwasa } & 16 & 0.0031106 & 0.0021332 & 0.0021163 \\ \text { Isolok - 12 mL - LF } & 10 & 0.0013205 & 0.0010842 & 0.0010842\end{array}$

$\begin{array}{lrrrr}\text { Test } & \text { F Ratio } & \text { DFNum } & \text { DFDen } & \text { p-Value } \\ \text { O'Brien[.5] } & 1.5531 & 1 & 24 & 0.2247\end{array}$

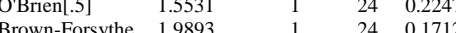

Brown-Forsythe $1.9893 \quad 1124 \quad 0.1712$

$\begin{array}{lllll}\text { Levene } & 2.1311 & 1 & 24 & 0.1573 \\ \text { Bartlett } & 6.3192 & 1 & . & 0.0119\end{array}$

$\begin{array}{lllll}\text { F Test 2-sided } & 5.5484 & 15 & 9 & 0.0135\end{array}$

Welch Anova testing Means Equal, allowing Std Devs Not Equal

F Ratio DFNum DFDen Prob $>$ F

$\begin{array}{llll}1.2378 & 1 & 21.868 & 0.2780\end{array}$

t Test
Oneway Analysis of Measurement By Sampler Type of Material=SME Simulant, Analytical Block $=3$, Oxide $/$ Analyte $=\mathrm{ZnO}($ wt $\%)$, Targeted $=\mathbf{0 . 0 9 5 8}$

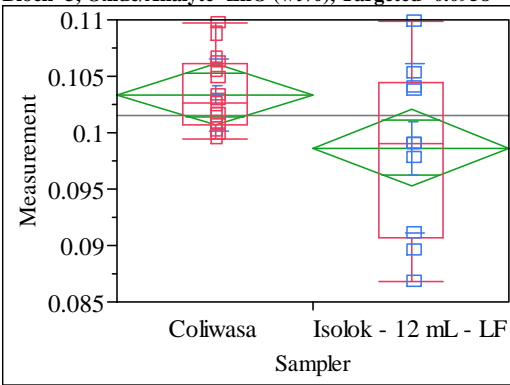

Oneway Anova

Summary of Fit

Adj Rsquare

Mean of Response $\quad 0.005247$

Observations (or Sum Wgts)

t Test

Isolok - $12 \mathrm{~mL}$ - LF-Coliwas

Assuming equal variances

$\begin{array}{lrr}\text { Difference } & -0.00471 \text { t Ratio } & -2.22916 \\ \text { Std Err Dif } & 0.00211 & 24\end{array}$

$\begin{array}{rrr}0.00211 & \text { DF } & 24 \\ \text { Upper CL Dif } & -0.00035 \text { Prob }>|t| r 0.0354\end{array}$

Lower CL Dif -0.00908 Prob $>$ t 0.9823

$\begin{array}{lrl}\text { Confidence } & 0.95 \text { Prob }<\mathrm{t} & 0.0177\end{array}$

Analysis of Variance

Source DF Sum of Squares Mean Square F Ratio Prob $>$ F

$\begin{array}{lllllll}\text { Sampler } & 1 & 0.00013679 & 0.000137 & 4.9692 & 0.0354\end{array}$

$\begin{array}{llll}\text { Error } & 24 & 0.00066066 & 0.000028\end{array}$

C. Total $25 \quad 0.00079745$

Means for Oneway Anova

Level Number Mean Std Error Lower 95\% Upper 95\%

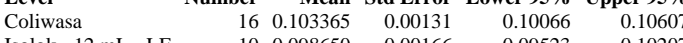

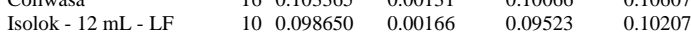

Std Error uses a pooled estimate of error variance

Means and Std Deviations

Level Number Mean Std Dev Std Err Mean Lower 95\% Upper 95\%

$\begin{array}{lllllll}\text { Coliwasa } & 16 & 0.103365 & 0.003190 & 0.00080 & 0.10167 & 0.10506\end{array}$

Isolok - $12 \mathrm{~mL}$ - LF $\quad 100.098650-0.007513-0.00238-0.09328-0.10402$

t Test

Isolok - $12 \mathrm{~mL}$ - LF-Coliwas

Assuming unequal variances

$\begin{array}{lrlr}\text { Difference } & -0.00471 & \text { t Ratio } & -1.88128 \\ \text { Std Err Dif } & 0.00251 & \text { DF } & 11.05841 \\ \text { Upper CL Dif } & 0.00080 & \text { Prob }>|t| & 0.0865 \\ \text { Lower CL Dif } & -0.01023 & \text { Prob }>\text { t } & 0.9567 \\ \text { Confidence } & 0.95 & \text { Prob }<\mathrm{t} & 0.0433\end{array}$

Tests that the Variances are Equal

Level Count Std Dev MeanAbsDif to Mean MeanAbsDif to Median

$\begin{array}{lllll}\text { Coliwasa } & 16 & 0.0031901 & 0.0026413 & 0.0025830\end{array}$

Isolok - $12 \mathrm{~mL}$ - LF $\quad 100.0075130 \quad 0.0058755$

Test F Ratio DFNum DFDen p-Value

$\begin{array}{lrrrr}\text { O'Brien[.5] } & 9.0119 & 1 & 24 & 0.0062\end{array}$

$\begin{array}{lllll}\text { Brown-Forsythe } & 6.6171 & 1 & 24 & 0.0167\end{array}$

$\begin{array}{lllll}\text { Levene } & 7.5791 & 1 & 24 & 0.0111\end{array}$

$\begin{array}{lllrl}\text { Bartlett } & 8.0958 & 1 & \text {. } & 0.0044 \\ \text { F Test 2-sided } & 5.5463 & 9 & 15 & 0.0037\end{array}$

Welch Anova testing Means Equal, allowing Std Devs Not Equal

F Ratio DFNum DFDen Prob $>$ F

$3.5392 \quad 11.058 \quad 0.0865$

t Test

1.8813 


\section{Exhibit C2. Statistical Comparisons for Low-Rheology (Phase 3) Simulant Testing}

Oneway Analysis of Measurement By Sampler Type of Material=SME Simulant, Analytical Block $=3$, Oxide/Analyte $=\mathrm{ZrO} 2$ ( $w$ t $\%$ ), Targeted $=0.3547$

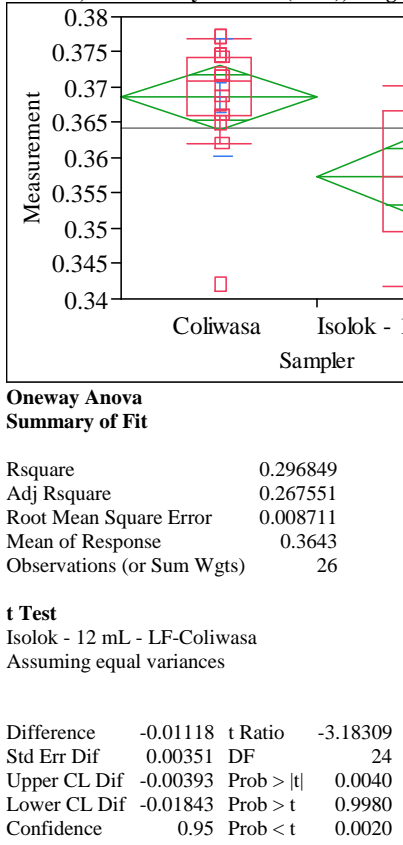

Analysis of Variance

Source DF Sum of Squares Mean Square F Ratio Prob $>$ F

$\begin{array}{lllllll}\text { Sampler } & 1 & 0.00076889 & 0.000769 & 10.1321 & 0.0040\end{array}$

$\begin{array}{llll}\text { Error } & 24 & 0.00182129 & 0.000076\end{array}$

C. Total $25 \quad 0.00259018$

Means for Oneway Anova

Level Number Mean Std Error Lower 95\% Upper 95\%

$\begin{array}{lrrrrr}\text { Coliwasa } & 16 & 0.368600 & 0.00218 & 0.36410 & 0.37309\end{array}$

$\begin{array}{llllll}\text { Coliwasa } & 16 & 0.368600 & 0.00218 & 0.36410 & 0.37309 \\ \text { Isolok - 12 mL - LF } & 10 & 0.357422 & 0.00275 & 0.35174 & 0.36311\end{array}$

Std Error uses a pooled estimate of error variance

Means and Std Deviations

Level Number Mean Std Dev Std Err Mean Lower 95\% Upper 95\%

Coliwasa $\quad 16 \quad 0.368600 \quad 0.008369$

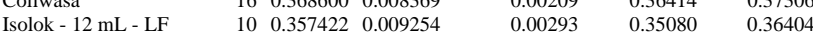

Test

Isolok - $12 \mathrm{~mL}$ - LF-Coliwas

Assuming unequal variances

Difference $\quad-0.01118$ t Ratio -3.10724

Std Err Dif $\quad 0.00360$ DF $\quad 17.76682$

Upper CL Dif -0.00361 Prob $>|t| \quad 0.0062$

Lower CL Dif -0.01874 Prob $>t \quad 0.9969$

$\begin{array}{lrl}\text { Confidence } & 0.95 \text { Prob }<\mathrm{t} & 0.0031\end{array}$

Tests that the Variances are Equa

Level Count Std Dev MeanAbsDif to Mean MeanAbsDif to Median

$\begin{array}{lllll}\text { Coliwasa } & 16 & 0.0083688 & 0.0054454 & 0.0052343\end{array}$

Isolok - $12 \mathrm{~mL}$ - LF $\quad 10 \quad 0.0092540-0.0072943$

Test F Ratio DFNum DFDen p-Value

$\begin{array}{lrrrr}\text { O'Brien[.5] } & 0.0553 & 1 & 24 & 0.816\end{array}$

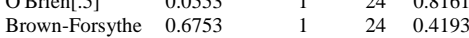

$\begin{array}{lllll}\text { Levene } & 0.6197 & 1 & 24 & 0.4389\end{array}$

$\begin{array}{lllll}\text { Bartlett } & 0.1105 & 1 & & 0.7396 \\ \text { F Test 2-sided } & 1.2228 & 9 & 15 & 0.7018\end{array}$

Welch Anova testing Means Equal, allowing Std Devs Not Equal

F Ratio DFNum DFDen Prob > F

$\begin{array}{rrrr}9.6549 & 1 & 17.767 & 0.0062\end{array}$

t Test
3.1072
Oneway Analysis of Measurement By Sampler Type of Material=SME Simulant, Analytica Block $=4$, Oxide $/$ Analyte $=A \mathrm{I} / \mathrm{B}$, Targeted $=\mathbf{2 . 5 5 2}$

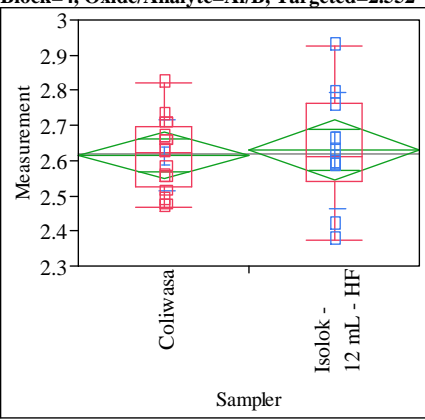

Oneway Anova

Summary of Fit

$\begin{array}{lr}\text { Rsquare } & 0.004129 \\ \text { Adj Rsquare } & -0.03737 \\ \text { Root Mean Square Error } & 0.129339 \\ \text { Mean of Response } & 2.620135 \\ \text { Observations (or Sum Wgts) } & 26\end{array}$

t Test

Isolok - $12 \mathrm{~mL}$ - HF-Coliwasa

Assuming equal variances

Difference $\quad 0.01645$ t Ratio 0.315434

$\begin{array}{lll}\text { Std Err Dif } & 0.05214 \text { DF } & 24\end{array}$

Upper CL Dif 0.12405 Prob $>|t| \quad 0.7552$

Lower CL Dif -0.09116 Prob $>t \quad 0.3776$

Confidence $\quad 0.95$ Prob $<\mathrm{t} \quad 0.6224$

Analysis of Variance

Source DF Sum of Squares Mean Square F Ratio Prob $>$ F

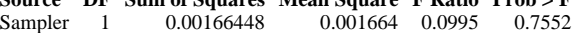

$\begin{array}{lrrr}\text { Sampler } & 1 & 0.00166448 & 0.001664 \\ \text { Error } & 24 & 0.40148809 & 0.016729\end{array}$

C. Total $25 \quad 0.40315258$

Means for Oneway Anova

$\begin{array}{lrrrrr}\text { Level } & \text { Number } & \text { Mean } & \text { Std Error } & \text { Lower 95\% } & \text { Upper 95\% } \\ \text { Coliwasa } & 16 & 2.61381 & 0.03233 & 2.5471 & 2.6805\end{array}$

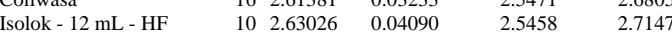

Std Error uses a pooled estimate of error variance

Means and Std Deviations

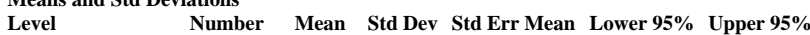

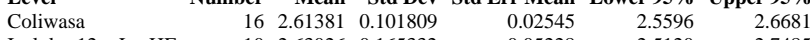

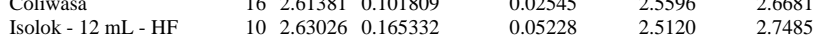

t Test

Isolok - $12 \mathrm{~mL}$ - HF-Coliwasa

Assuming unequal variances

Difference $\quad 0.01645 \mathrm{t}$ Ratio $\quad 0.28283$

Std Err Dif $\quad 0.05815$ DF $\quad 13.32241$

$\begin{array}{lrr}\text { Upper CL Dif } & 0.14176 \text { Prob }>|t| & 0.7817\end{array}$

$\begin{array}{lll}\text { Lower CL Dif }-0.10887 & \text { Prob }>t \quad 0.3908\end{array}$

$\begin{array}{lll}\text { Confidence } & 0.95 \text { Prob }<\mathrm{t} \quad 0.6092\end{array}$

Tests that the Variances are Equal

Level Count Std Dev MeanAbsDif to Mean MeanAbsDif to Median

$\begin{array}{lrrrr}\text { Coliwasa } & 16 & 0.1018088 & 0.0833742 & 0.0823067\end{array}$

$\begin{array}{lllll}\text { Isolok - } 12 \mathrm{~mL} \text { - HF } & 10 & 0.1653322 & 0.1211870 & 0.1204340\end{array}$

Test F Ratio DFNum DFDen p-Value

$\begin{array}{lllll}\text { O'Brien[.5] } & 2.9164 & 1 & 24 & 0.1006\end{array}$

$\begin{array}{lllll}\text { Brown-Forsythe } & 1.3917 & 1 & 24 & 0.2497\end{array}$

$\begin{array}{lllll}\text { Levene } & 1.4726 & 1 & 24 & 0.2368\end{array}$

$\begin{array}{lllll}\text { Bartlett } & 2.6412 & 1 & . & 0.1041\end{array}$

Welch Anova testing Means Equal, allowing Std Devs Not Equal

F Ratio DFNum DFDen Prob $>$ F

$\begin{array}{llll}0.0800 & 1 & 13.322 & 0.7817\end{array}$

t Test

0.2828 


\section{Exhibit C2. Statistical Comparisons for Low-Rheology (Phase 3) Simulant Testing}

Oneway Analysis of Measurement By Sampler Type of Material=SME Simulant, Analytical Block $=4$, Oxide/Analyte=Al2O3 $(w t \%)$, Targeted $=10.869$

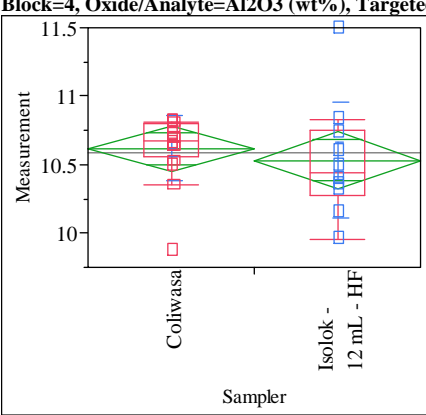

Oneway Anova

Summary of Fit

Adj Rsquare

Root Mean Square Error $\quad 0.321449$

10.58483

Observations (or Sum Wgts)

26

t Test

Isolok - $12 \mathrm{~mL}$ - HF-Coliwasa

Assuming equal variances

$\begin{array}{lrr}\text { Difference } & -0.08881 \text { t Ratio } & -0.68534 \\ \text { Std Err Dif } & 0.12958 & \text { DF }\end{array}$

$\begin{array}{lrlr}\text { Std Err Dif } & 0.12958 \text { DF } & 24 \\ \text { Upper CL Dif } & 0.17863 & \text { Prob }>|t| & 0.4997\end{array}$

Lower CL Dif -0.35625 Prob $>t \quad 0.7502$

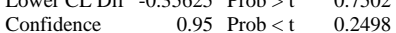

Analysis of Variance

Source DF Sum of Squares Mean Square F Ratio Prob $>$ F

$\begin{array}{lrrrrr}\text { Sampler } & 1 & 0.0485329 & 0.048533 & 0.4697 & 0.4997\end{array}$

Error $\quad 24 \quad 2.4799037 \quad 0.103329$

C. Total $25 \quad 2.5284366$

Means for Oneway Anova

Level Number Mean Std Error Lower 95\% Upper 95\%

$\begin{array}{lrrrrr}\text { Coliwasa } & 16 & 10.6190 & 0.08036 & 10.453 & 10.785\end{array}$

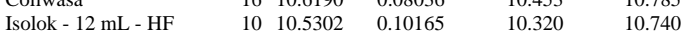

Std Error uses a pooled estimate of error variance

Means and Std Deviations

Level Number Mean Std Dev Std Err Mean Lower 95\% Upper 95\%

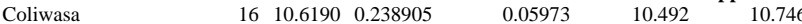

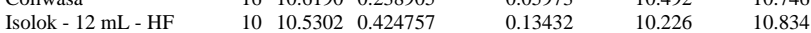

t Test

Isolok - $12 \mathrm{~mL}$ - HF-Coliwasa

Assuming unequal variances

Difference $\quad-0.08881$ t Ratio $\quad-0.60412$

Std Err Dif $\quad 0.14700$ DF $\quad 12.6149$

Upper CL Dif 0.22976 Prob $>|t| \quad 0.5565$

Lower CL Dif -0.40737 Prob $>t \quad 0.7218$

$\begin{array}{lrl}\text { Confidence } & 0.95 \text { Prob }<\mathrm{t} & 0.2782\end{array}$

Tests that the Variances are Equal

Level Count Std Dev MeanAbsDif to Mean MeanAbsDif to Median

$\begin{array}{lllll} & 16 & 0.2389053 & 0.1558837 & 0.1440744 \\ \text { Isolok - } 12 \mathrm{~mL} \text { - HF } & 10 & 0.4247571 & 0.3053432 & 0.2966515\end{array}$

$\begin{array}{lrrrr}\text { Test } & \text { F Ratio } & \text { DFNum } & \text { DFDen } & \text { p-Value } \\ \text { O'Brien[.5] } & 1.6522 & 1 & 24 & 0.2109 \\ \text { Brown-Forsythe } & 2.4534 & 1 & 24 & 0.1304 \\ \text { Levene } & 2.8471 & 1 & 24 & 0.1045 \\ \text { Bartlett } & 3.7182 & 1 & . & 0.0538 \\ \text { F Test 2-sided } & 3.1610 & 9 & 15 & 0.0477\end{array}$

Welch Anova testing Means Equal, allowing Std Devs Not Equal

F Ratio DFNum DFDen Prob $>$ F

$\begin{array}{llll}0.3650 & 1 & 12.615 & 0.5565\end{array}$

t Test

0.6041
Oneway Analysis of Measurement By Sampler Type of Material=SME Simulant, Analytical Block $=4$, Oxide/Analyte $=\mathrm{B} 2 \mathrm{O} 3(\mathrm{wt} \%)$, Targeted $=4.259$

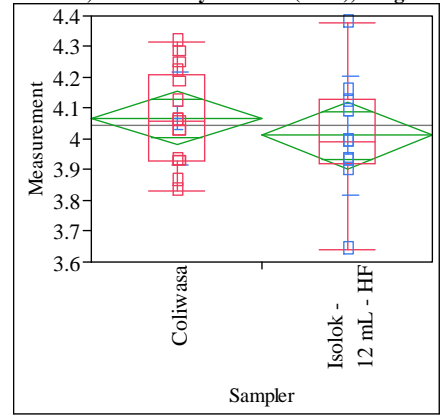

Oneway Anova

Summary of Fi

Rsqua

$\begin{array}{ll}\text { Rsquare } & 0.026781 \\ \text { Adj Rsquare } & -0.1687\end{array}$

$-0.01377$

$\quad 0.16832$

Mean of Response 4.045928
26

t Test

Isolok - $12 \mathrm{~mL}$ - HF-Coliwasa

Assuming equal variances

Difference $\quad-0.05514$ t Ratio $\quad-0.81266$

$\begin{array}{lll}\text { Std Err Dif } & 0.06785 \text { DF } & 24\end{array}$

Upper CL Dif $\quad 0.08490$ Prob $>|t| \quad 0.4244$

Lower CL Dif -0.19518 Prob $>$ t $\quad 0.7878$

$\begin{array}{lll}\text { Confidence } & 0.95 \text { Prob }<\mathrm{t} \quad 0.2122\end{array}$

Analysis of Variance

Source DF Sum of Squares Mean Square F Ratio Prob $>$ F

$\begin{array}{lrrrrr}\text { Sampler } & 1 & 0.01871081 & 0.018711 & 0.6604 & 0.4244\end{array}$

$\begin{array}{lrrr}\text { Sampler } & 1 & 0.01871081 & 0.018711 \\ \text { Error } & 24 & 0.67995632 & 0.028332\end{array}$

$\begin{array}{lll}\text { Error } & 24 & 0.67995632 \\ \text { C. Total } & 25 & 0.69866713\end{array}$

Means for Oneway Anova

$\begin{array}{lrrrrr}\text { Level } & \text { Number } & \text { Mean } & \text { Std Error } & \text { Lower 95\% } & \text { Upper 95\% } \\ \text { Coliwasa } & 16 & 4.06714 & 0.04208 & 3.9803 & 4.1540\end{array}$

Isolok - 12 mL - HF $\quad \begin{array}{lllll}10 & 4.01200 & 0.05323 & 3.9021 & 4.1219\end{array}$

Std Error uses a pooled estimate of error variance

Means and Std Deviations

Level Number Mean Std Dev Std Err Mean Lower 95\% Upper 95\%

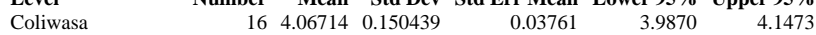

$\begin{array}{lllllll}\text { Isolok - } 12 \mathrm{~mL} \text { - HF } & 16 & 4.06714 & 0.150439 & 0.03761 & 3.9870 & 4.1473 \\ & 10 & 4.01200 & 0.194501 & 0.06151 & 3.8729 & 4.1511\end{array}$

t Test

Isolok - $12 \mathrm{~mL}$ - HF-Coliwasa

Assuming unequal variances

Difference $\quad-0.05514$ t Ratio $\quad-0.76484$

Std Err Dif $\quad 0.07209$ DF 15.67371

Upper CL Dif $\quad 0.09795$ Prob $>|t| r 0.4557$

$\begin{array}{lll}\text { Lower CL Dif }-0.20823 & \text { Prob }>t & 0.7721\end{array}$

$\begin{array}{lll}\text { Confidence } & 0.95 \text { Prob }<\mathrm{t} & 0.2279\end{array}$

Tests that the Variances are Equal

Level Count Std Dev MeanAbsDif to Mean MeanAbsDif to Median

$\begin{array}{lrrrr}\text { Coliwasa } & 16 & 0.1504392 & 0.1212494 & 0.1187338\end{array}$

$\begin{array}{lllll}\text { Isolok - } 12 \mathrm{~mL} \text { - HF } & 10 & 0.1945014 & 0.1390997 & 0.1352358\end{array}$

Test F Ratio DFNum DFDen p-Value

$\begin{array}{lllll}\text { O'Brien[.5] } & 0.7523 & 1 & 24 & 0.3943 \\ \end{array}$

$\begin{array}{lllll}\text { Brown-Forsythe } & 0.1453 & 1 & 24 & 0.7065\end{array}$

$\begin{array}{lllll}\text { Levene } & 0.1873 & 1 & 24 & 0.6690\end{array}$

$\begin{array}{lllll}\text { Bartlett } & 0.7336 & 1 & 0.3917\end{array}$

Welch Anova testing Means Equal, allowing Std Devs Not Equal

F Ratio DFNum DFDen Prob $>$ F

$\begin{array}{llll}0.5850 & 1 & 15.674 & 0.4557\end{array}$

t Test 


\section{Exhibit C2. Statistical Comparisons for Low-Rheology (Phase 3) Simulant Testing}

Oneway Analysis of Measurement By Sampler Type of Material=SME Simulant, Analytical Block $=4$, Oxide $/$ Analyte $=\mathrm{BaO}($ wt $\%)$, Targeted $=\mathbf{0 . 0 9 1 9}$

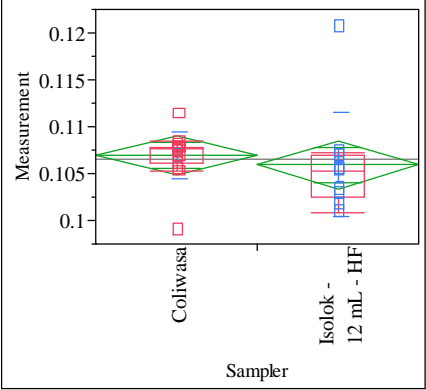

Oneway Anova

Rsquare $\quad 0.016399$

Adj Rsquare

$-0.02458$

are Error $\quad 0.003963$

$\begin{array}{lr}\text { Mean of Response } & 0.106544 \\ \text { Observations (or Sum Wgts) } & 26\end{array}$

t Test

Isolok - $12 \mathrm{~mL}$ - HF-Coliwasa

Assuming equal variances

Difference $\quad-0.00101$ t Ratio $\quad-0.6325$

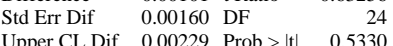

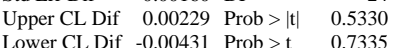

$\begin{array}{lrll}\text { Lower CL Dif } & -0.00431 & \text { Prob }>t & 0.7335 \\ \text { Confidence } & 0.95 & \text { Prob }<\mathrm{t} & 0.2665\end{array}$

Analysis of Variance

Source DF Sum of Squares Mean Square F Ratio Prob $>$ F

$\begin{array}{lrrrrr}\text { Sampler } & 1 & 0.00000628 & 6.283 \mathrm{e}-6 & 0.4001 & 0.5330\end{array}$

Error $24 \quad 0.00037685$

C. Total $25 \quad 0.00038314$

Means for Oneway Anova

Level Number Mean Std Error Lower 95\% Upper 95\%

$\begin{array}{lllllr}\text { Coliwasa } & 16 & 0.106933 & 0.00099 & 0.10489 & 0.10898\end{array}$

$\begin{array}{llllll}\text { Isolok - } 12 \mathrm{~mL} \text { - HF } & 10 & 0.105922 & 0.00125 & 0.10334 & 0.10851\end{array}$

Std Error uses a pooled estimate of error variance

Means and Std Deviations

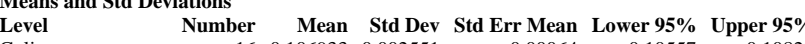

$\begin{array}{lrllrrr}\text { Coliwasa } & 16 & 0.106933 & 0.002551 & 0.00064 & 0.10557 & 0.10829 \\ \text { Isolok - 12 mL - HF } & 10 & 0.105922 & 0.005570 & 0.00176 & 0.10194 & 0.10991\end{array}$

t Test

Isolok - $12 \mathrm{~mL}$ - HF-Coliwasa

Assuming unequal variances

Difference $\quad-0.00101$ t Ratio $\quad-0.53938$

Std Err Dif $\quad 0.00187$ DF 11.39693

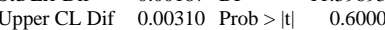

$\begin{array}{lll}\text { Lower CL Dif }-0.00512 & \text { Prob }>t & 0.7000\end{array}$

Confidence $\quad 0.95$ Prob $<\mathrm{t} \quad 0.3000$

Tests that the Variances are Equal

$\begin{array}{lrrrr}\text { Level } & \text { Count } & \text { Std Dev } & \text { MeanAbsDif to Mean } & \text { MeanAbsDif to Median } \\ \text { Coliwasa } & 16 & 0.0025511 & 0.0015457 & 0.0013817 \\ \text { Isolok - 12 mL - HF } & 10 & 0.0055701 & 0.0033696 & 0.0031597\end{array}$

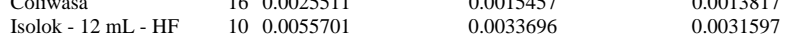

Test F Ratio DFNum DFDen p-Value

$\begin{array}{lrrrr}\text { O'Brien[.5] } & 1.4817 & 1 & 24 & 0.2353\end{array}$

Brown-Forsythe $\begin{array}{llll}1.8208 & 1 & 24 & 0.1898\end{array}$

$\begin{array}{llll}1 & 24 & 0.1898 \\ 1 & 24 & 0.1526 & 0 \\ 1 & 0 & 0\end{array}$

$\begin{array}{lllll}\text { Levene } & 2.1829 & 1 & 24 & 0.1526 \\ \text { Bartlett } & 6.7751 & 1 & . & 0.0092\end{array}$

$\begin{array}{lllll}\text { F Test 2-sided } & 4.7674 & 9 & 15 & 0.0079\end{array}$

Welch Anova testing Means Equal, allowing Std Devs Not Equal

F Ratio DFNum DFDen Prob $>$ F

$\begin{array}{llll}0.2909 & 1 & 11.397 & 0.6000\end{array}$

t Test
Oneway Analysis of Measurement By Sampler Type of Material=SME Simulant, Analytica Block $=4$, Oxide $/$ Analyte $=\mathrm{CaO}(\mathrm{wt} \%)$, Targeted $=\mathbf{1 . 1 4 6 2}$

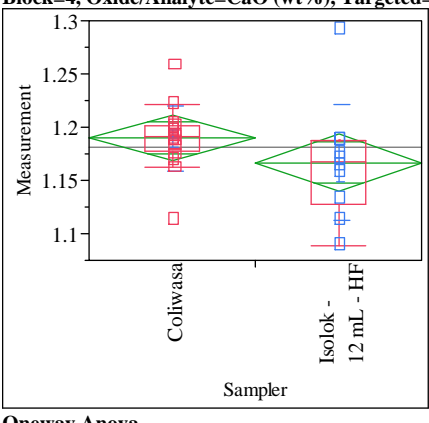

Oneway Anova

Summary of Fit

Adj Rsquare

Root Mean Square Error $\quad 0.034098$

Mean of Response $\quad 1.180656$

Observations (or Sum Wgts)

\section{Test}

Isolok - $12 \mathrm{~mL}$ - HF-Coliwas

Assuming equal variances

$\begin{array}{lrlr}\text { Difference } & -0.02275 & \text { t Ratio } & -1.37206 \\ \text { Std Err Dif } & 0.01658 & \text { DF } & 24 \\ \text { Upper CL Dif } & 0.01147 & \text { Prob }>|t| & 0.1827 \\ \text { Lower CL Dif } & -0.05698 & \text { Prob }>t & 0.9086 \\ \text { Confidence } & 0.95 & \text { Prob }<\mathrm{t} & 0.0914\end{array}$

Analysis of Variance

Source DF Sum of Squares Mean Square F Ratio Prob $>$ F

$\begin{array}{lrrrrr}\text { Sampler } & 1 & 0.00318626 & 0.003186 & 1.8825 & 0.1827\end{array}$

$\begin{array}{llll}\text { Error } & 24 & 0.04062067 & 0.001693\end{array}$

Means for Oneway Anova

$\begin{array}{lllll}\text { Means for Oneway Anova } & & & \\ \text { Level Number } & \text { Mean Std Error } & \text { Lower 95\% Upper 95\% }\end{array}$

$\begin{array}{lrrrrr}\text { Level } & \text { Number } & \text { Mean } & \text { Std Error } & \text { Lower 95\% } & \text { Upper 95\% } \\ \text { Coliwasa } & 16 & 1.18941 & 0.01029 & 1.1682 & 1.2106\end{array}$

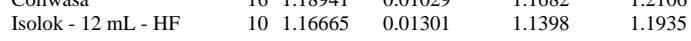

Std Error uses a pooled estimate of error variance

Means and Std Deviations

Level Number Mean Std Dev Std Err Mean Lower 95\% Upper 95\%

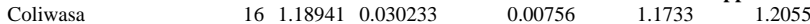

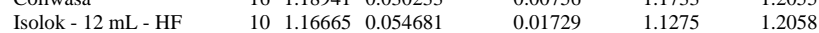

t Test

Isolok - $12 \mathrm{~mL}$ - HF-Coliwas

Assuming unequal variances

$\begin{array}{lrlr}\text { Difference } & -0.02275 & \text { t Ratio } & -1.20576 \\ \text { Std Err Dif } & 0.01887 \text { DF } & 12.49379 \\ \text { Upper CL Dif } & 0.01818 \text { Prob }>|t| & 0.2502 \\ \text { Lower CL Dif } & -0.06369 & \text { Prob }>\text { t } & 0.8749 \\ \text { Confidence } & 0.95 & \text { Prob }<\mathrm{t} & 0.1251\end{array}$

Tests that the Variances are Equal

$\begin{array}{lrrr}\text { Level Count } & \text { Std Dev MeanAbsDif to Mean MeanAbsDif to Median }\end{array}$

$\begin{array}{lllll}\text { Coliwasa } & 16 & 0.0302325 & 0.0186268 & 0.0186268 \\ \text { Isolok - } 12 \mathrm{~mL} \text { - HF } & 10 & 0.0546815 & 0.0358195 & 0.0358195\end{array}$

$\begin{array}{lrrrr}\text { Test } & \text { F Ratio } & \text { DFNum } & \text { DFDen } & \text { p-Value } \\ \text { O'Brien[.5] } & 1.7498 & 1 & 24 & 0.1984 \\ \text { Brown-Forsythe } & 1.9605 & 1 & 24 & 0.1743 \\ \text { Levene } & 1.9630 & 1 & 24 & 0.1740 \\ \text { Bartlett } & 3.9417 & 1 & . & 0.0471 \\ \text { F Test 2-sided } & 3.2714 & 9 & 15 & 0.0416\end{array}$

Welch Anova testing Means Equal, allowing Std Devs Not Equal

F Ratio DFNum DFDen Prob $>$ F

$\begin{array}{llll}1.4539 & 1 & 12.494 & 0.2502\end{array}$

t Test

1.2058 


\section{Exhibit C2. Statistical Comparisons for Low-Rheology (Phase 3) Simulant Testing}

Oneway Analysis of Measurement By Sampler Type of Material=SME Simulant, Analytical Block $=4$, Oxide $/$ Analyte $=\mathrm{Cr} 2 \mathrm{O} 3(\mathrm{wt} \%)$, Targeted $=\mathbf{0 . 0 9 9 8}$

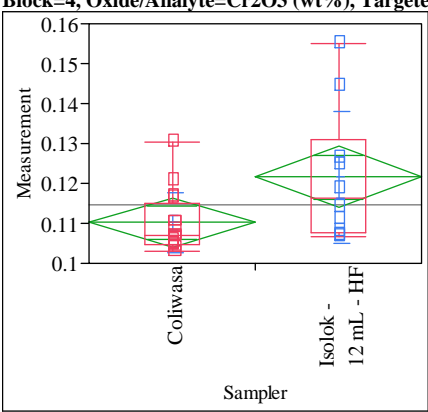

Oneway Anova

Summary of Fit

Adj Rsquare

Root Mean Square Erro

0.194656

0.1611

Mean of Response

0.114567

Observations (or Sum Wgts)

t Test

Isolok - $12 \mathrm{~mL}$ - HF-Coliwasa

Assuming equal variances

Difference $\quad 0.011366$ t Ratio $\quad 2.408515$

Std Err Dif 0.004719 DF

Upper CL Dif 0.021105 Prob $>|t| \quad 0.0241$

Lower CL Dif 0.001626 Prob $>t \quad 0.0120$

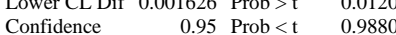

Analysis of Variance

Source DF Sum of Squares Mean Square F Ratio Prob $>$ F

$\begin{array}{llllll}\text { Sampler } & 1 & 0.00079496 & 0.000795 & 5.8009 & 0.0241\end{array}$

Error $\quad \begin{array}{lll}24 & 0.00328895 & 0.000137\end{array}$

C. Total $25 \quad 0.00408390$

Means for Oneway Anova

Level Number Mean Std Error Lower 95\% Upper 95\%

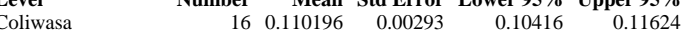

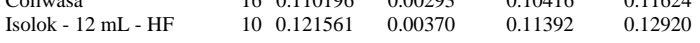

Std Error uses a pooled estimate of error variance

Means and Std Deviations

Level Number Mean Std Dev Std Err Mean Lower 95\% Upper 95\%

$\begin{array}{lllllll}\text { Coliwasa } & 16 & 0.110196 & 0.007415 & 0.00185 & 0.10624 & 0.11415\end{array}$

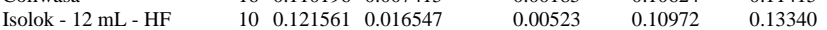

t Test

Isolok - $12 \mathrm{~mL}$ - HF-Coliwasa

Assuming unequal variances

Difference $\quad 0.01137$ t Ratio $\quad 2.047404$

Std Err Dif $\quad 0.00555$ DF $\quad 11.29389$

Upper CL Dif 0.02355 Prob $>|t| \quad 0.0646$

Lower CL Dif -0.00081 Prob $>\mathrm{t} \quad 0.0323$

$\begin{array}{lrl}\text { Confidence } & 0.95 \text { Prob }<\mathrm{t} \quad 0.9677\end{array}$

Tests that the Variances are Equal

Level Count Std Dev MeanAbsDif to Mean MeanAbsDif to Median

$\begin{array}{lllll}\text { Coliwasa } & 16 & 0.0074147 & 0.0055609 & 0.0050334\end{array}$

Isolok - $12 \mathrm{~mL}$ - HF $\quad 100.0165472 \quad 0.0128095 \quad 0.0122336$

$\begin{array}{lrrrr}\text { Test } & \text { F Ratio } & \text { DFNum } & \text { DFDen } & \text { p-Value } \\ \text { O'Brien[.5] } & 4.3068 & 1 & 24 & 0.0488 \\ \text { Brown-Forsythe } & 4.2324 & 1 & 24 & 0.0507 \\ \text { Levene } & 6.7282 & 1 & 24 & 0.0159 \\ \text { Bartlett } & 7.1464 & 1 & . & 0.0075 \\ \text { F Test 2-sided } & 4.9804 & 9 & 15 & 0.0064\end{array}$

Welch Anova testing Means Equal, allowing Std Devs Not Equal

F Ratio DFNum DFDen Prob > F

$\begin{array}{llrr}4.1919 & 1 & 11.294 & 0.0646\end{array}$

t Test

2.0474
Oneway Analysis of Measurement By Sampler Type of Material=SME Simulant, Analytical Block $=4$, Oxide $/$ Analyte $=\mathrm{CuO}(\mathrm{wt} \%)$, Targeted $=\mathbf{0 . 0 5 0 4}$

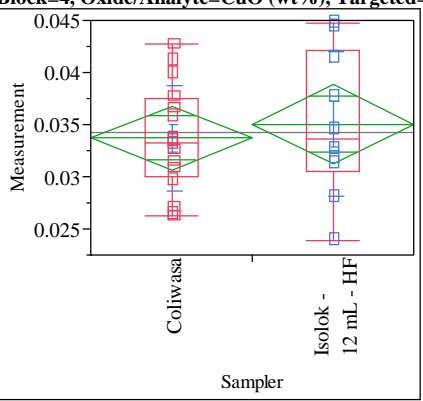

Oneway Anova

Summary of Fit

Rsquare

Adj Rsquare

$\begin{array}{lr}\text { Mean of Response } & 0.034213 \\ \text { Observations (or Sum Wgts) } & 26\end{array}$

t Test

Isolok - $12 \mathrm{~mL}$ - HF-Coliwasa

Assuming equal variances

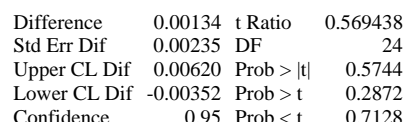

$\begin{array}{lrl}\text { Confidence } & 0.95 \text { Prob }<t \quad 0.7128\end{array}$

Analysis of Variance

Source DF Sum of Squares Mean Square F Ratio Prob $>$ F

$\begin{array}{lrrrrr}\text { Sampler } & 1 & 0.00001107 & 0.000011 & 0.3243 & 0.5744\end{array}$

$\begin{array}{llll}\text { Error } & 24 & 0.00081906 & 0.0000034\end{array}$

C. Total $25 \quad 0.00083013$

Means for Oneway Anova

Level Number Mean Std Error Lower 95\% Upper 95\%

$\begin{array}{llllrr}\text { Coliwasa } & 16 & 0.033697 & 0.00146 & 0.03068 & 0.03671\end{array}$

$\begin{array}{llllll}\text { Isolok - } 12 \mathrm{~mL} \text { - HF } & 10 & 0.035038 & 0.00185 & 0.03123 & 0.03885\end{array}$

Std Error uses a pooled estimate of error variance

Means and Std Deviations

$\begin{array}{llllll}\text { Level } & \text { Number } & \text { Mean } & \text { Std Dev Std Err Mean Lower 95\% Upper 95\% }\end{array}$

$\begin{array}{lllllll}\text { Coliwasa } & 16 & 0.033697 & 0.005091 & 0.00127 & 0.03098 & 0.03641\end{array}$

$\begin{array}{lllllll}\text { Isolok - } 12 \mathrm{~mL} \text { - HF } & 10 & 0.035038 & 0.006914 & 0.00219 & 0.03009 & 0.03998\end{array}$

t Test

Isolok - $12 \mathrm{~mL}$ - HF-Coliwasa

Assuming unequal variances

$\begin{array}{lrlr}\text { Difference } & 0.00134 & \text { t Ratio } & 0.530048 \\ \text { Std Err Dif } & 0.00253 \text { DF } & 15.09345 \\ \text { Upper CL Dif } & 0.00673 \text { Prob }>|t| & 0.6038 \\ \text { Lower CL Dif } & -0.00405 & \text { Prob }>\mathrm{t} & 0.3019 \\ \text { Confidence } & 0.95 & \text { Prob }<\mathrm{t} & 0.6981\end{array}$

Tests that the Variances are Equal

$\begin{array}{lrrrr}\text { Level } & \text { Count } & \text { Std Dev } & \text { MeanAbsDif to Mean } & \text { MeanAbsDif to Median } \\ \text { Coliwasa } & 16 & 0.0050912 & 0.0040038 & 0.0039666\end{array}$

$\begin{array}{lllll}\text { Coliwasa } & 16 & 0.0050912 & 0.0040038 & 0.0039666 \\ \text { Isolok - 12 mL - HF } & 10 & 0.0069142 & 0.0055930 & 0.0054954\end{array}$

Test F Ratio DFNum DFDen p-Value

$\begin{array}{lllll}\text { O'Brien[.5] } & 1.9118 & 1 & 24 & 0.1795\end{array}$

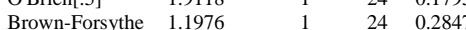

$\begin{array}{lll}1 & 24 & 0.284 \\ 1 & 24 & 0.2335\end{array}$

$\begin{array}{lllll}\text { Levene } & 1.4936 & 1 & 24 & 0.2335 \\ \text { Batlett } & 1.0452 & 1 & & 0.3066\end{array}$

$\begin{array}{lllll}\text { Bartlett } & 1.0452 & 1 & & 0.3066 \\ \text { F Test 2-sided } & 1.8443 & 9 & 15 & 0.2831\end{array}$

Welch Anova testing Means Equal, allowing Std Devs Not Equal

F Ratio DFNum DFDen Prob $>$ F

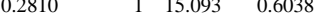

t Test 
SRNL-STI-2011-00693

Revision 0

\section{Exhibit C2. Statistical Comparisons for Low-Rheology (Phase 3) Simulant Testing}

Oneway Analysis of Measurement By Sampler Type of Material=SME Simulant, Analytica Block $=4$, Oxide/Analyte $=\mathrm{Fe} / \mathrm{Li}$, Targeted $=2.452$

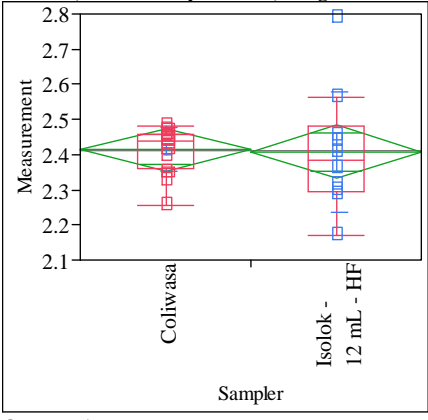

Oneway Anova

Summary of Fit

Rsquare 0.000798

$-0.04084$

Root Mean Square Error $\quad 0.11634$

Mean of Response 2.412087

Observations (or Sum Wgts) $\quad 26$

t Test

Isolok - $12 \mathrm{~mL}$ - HF-Coliwasa

Assuming equal variances

Difference $\quad-0.00649$ t Ratio $\quad-0.13844$

Std Err Dif $\quad 0.04690$ DF $\quad 24$

Upper CL Dif 0.09030 Prob $>|t| \quad 0.8910$

Lower CL Dif -0.10329 Prob $>$ t 0.5545

$\begin{array}{lll}\text { Confidence } & 0.95 \text { Prob }<\mathrm{t} \quad 0.4455\end{array}$

Analysis of Variance

Source DF Sum of Squares Mean Square F Ratio Prob $>$ F

$\begin{array}{lrrrrr}\text { Source } & \text { DF } & \text { Sum of Squares } & \text { Mean Square } & \text { F Ratio } & \text { Prob }>\text { F } \\ \text { Sampler } & 1 & 0.00025942 & 0.000259 & 0.0192 & 0.8910\end{array}$

$\begin{array}{lrrr}\text { Sampler } & 1 & 0.00025942 & 0.000259 \\ \text { Error } & 24 & 0.32484669 & 0.013535\end{array}$

$\begin{array}{lll}\text { C. Total } & 25 & 0.32510611\end{array}$

Means for Oneway Anov

$\begin{array}{lrrrrr}\text { Level } & \text { Number } & \text { Mean } & \text { Std Error } & \text { Lower 95\% } & \text { Upper 95\% } \\ \text { Coliwasa } & 16 & 2.41458 & 0.02909 & 2.3546 & 2.4746\end{array}$

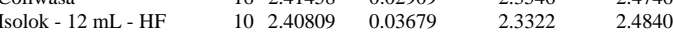

Std Error uses a pooled estimate of error variance

Means and Std Deviations

Level Number Mean Std Dev Std Err Mean Lower 95\% Upper 95\%

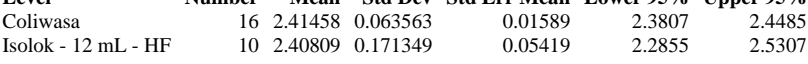

t Test

Isolok - $12 \mathrm{~mL}$ - HF-Coliwasa

Assuming unequal variances
Difference $\quad-0.00649$ t Ratio $\quad-0.11498$

Std Err Dif $\quad 0.05647$ DF 10.56778

Upper CL Dif 0.11841 Prob $>|t| \quad 0.9106$

Lower CL Dif -0.13140 Prob $>t \quad 0.5447$
Con

$\begin{array}{lrl}\text { Confidence } & 0.95 \text { Prob }<\mathrm{t} \quad 0.4553\end{array}$

Tests that the Variances are Equal

Level Count Std Dev MeanAbsDif to Mean MeanAbsDif to Median

$\begin{array}{lrrrr}\text { Coliwasa } & 16 & 0.0635630 & 0.0499235 & 0.0449501\end{array}$

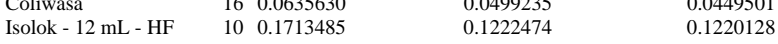

$\begin{array}{lrrrr}\text { Test } & \text { F Ratio } & \text { DFNum } & \text { DFDen } & \text { p-Value } \\ \text { O'Brien[.5] } & 3.7355 & 1 & 24 & 0.0652\end{array}$

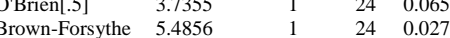

$\begin{array}{lllll}\text { Levene } & 5.7004 & 1 & 24 & 0.0252\end{array}$

$\begin{array}{lllll}\text { Bartlett } & 10.6812 & 1 & 0.0011\end{array}$

$\begin{array}{lrrrr}\text { F Test 2-sided } & 7.2669 & 9 & 15 & 0.0009\end{array}$

Welch Anova testing Means Equal, allowing Std Devs Not Equal

F Ratio DFNum DFDen Prob $>$ F

$\begin{array}{llll}0.0132 & 1 & 10.568 & 0.9106\end{array}$

t Test

T Test
Oneway Analysis of Measurement By Sampler Type of Material=SME Simulant, Analytica Block $=4$, Oxide/Analyte=Fe2O3 (wt\%), Targeted $=11.462$

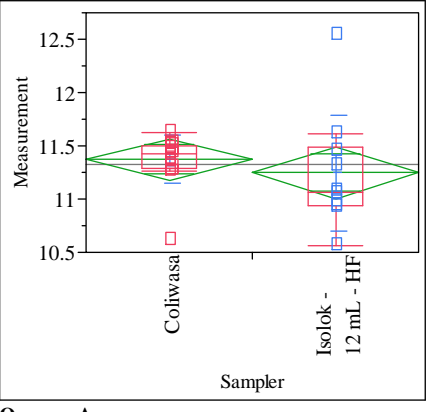

Summary of Fit

Adj Rsquare

Root Mean Square Error $\quad-0.01166$

Mean of Response $\quad 11.32377$

Observations (or Sum Wgts)

26

\section{Test}

Isolok - $12 \mathrm{~mL}$ - HF-Coliwas

Assuming equal variances

$\begin{array}{lrlr}\text { Difference } & -0.12867 & \text { t Ratio } & -0.84369 \\ \text { Std Err Dif } & 0.15251 & \text { DF } & 24 \\ \text { Upper CL Dif } & 0.18610 & \text { Prob }>|t| & 0.4072 \\ \text { Lower CL Dif } & -0.44344 & \text { Prob }>t & 0.7964 \\ \text { Confidence } & 0.95 & \text { Prob }<\mathrm{t} & 0.2036\end{array}$

Analysis of Variance

Source DF Sum of Squares Mean Square F Ratio Prob $>$ F

$\begin{array}{lrrrrr}\text { Sampler } & 1 & 0.1018876 & 0.101888 & 0.7118 & 0.4072\end{array}$

$\begin{array}{llll}\text { Error } & 24 & 3.4353193 & 0.143138\end{array}$

Means for Oneway Anova

$\begin{array}{lllll}\text { Level } & \text { Number } & \text { Mean Std Error Lower 95\% Upper 95\% }\end{array}$

$\begin{array}{lrrrrr}\text { Coliwasa } & 16 & 11.3733 & 0.09458 & 11.178 & 11.568\end{array}$

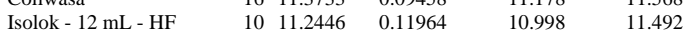

Std Error uses a pooled estimate of error variance

Means and Std Deviations

Level Number Mean Std Dev Std Err Mean Lower 95\% Upper 95\%

$\begin{array}{lllllll}\text { Coliwasa } & 16 & 11.3733 & 0.229169 & 0.05729 & 11.251 & 11.495\end{array}$

Isolok - $12 \mathrm{~mL}$ - HF $\quad 10 \quad 11.2446 \quad 0.542376 \quad 0.17151 \quad 10.857$

t Test

Isolok - $12 \mathrm{~mL}$ - HF-Coliwas

Assuming unequal variances

Difference $\quad-0.12867$ t Ratio $\quad-0.71157$

Std Err Dif $\quad 0.18083$ DF $\quad 11.03805$

Upper CL Dif 0.26916 Prob $>|t| \quad 0.4915$

Lower CL Dif -0.52651 Prob $>\mathrm{t} \quad 0.7543$

$\begin{array}{lrl}\text { Confidence } & 0.95 \text { Prob }<\mathrm{t} & 0.2457\end{array}$

Tests that the Variances are Equal

Level Count Std Dev MeanAbsDif to Mean MeanAbsDif to Median

$\begin{array}{lllll}\text { Coliwasa } & 16 & 0.2291686 & 0.1411829 & 0.1340344 \\ \text { Isolok - 12 mL - HF } & 10 & 0.5423760 & 0.3860190 & 0.3531359\end{array}$

$\begin{array}{lrrrr}\text { Test } & \text { F Ratio } & \text { DFNum } & \text { DFDen } & \text { p-Value } \\ \text { O'Brien[.5] } & 2.3512 & 1 & 24 & 0.1383 \\ \text { Brown-Forsythe } & 3.1199 & 1 & 24 & 0.0901 \\ \text { Levene } & 5.4439 & 1 & 24 & 0.0283 \\ \text { Bartlett } & 8.1852 & 1 & & 0.0042 \\ \text { F Test 2-sided } & 5.6013 & 9 & 15 & 0.0036\end{array}$

Welch Anova testing Means Equal, allowing Std Devs Not Equal

F Ratio DFNum DFDen Prob $>$ F

$\begin{array}{rrrr}0.5063 & 1 & 11.038 & 0.4915\end{array}$

t Test

0.7116 


\section{Exhibit C2. Statistical Comparisons for Low-Rheology (Phase 3) Simulant Testing}

Oneway Analysis of Measurement By Sampler Type of Material=SME Simulant, Analytical Block=4, Oxide/Analyte $=$ K2O $($ wt $\%)$, Targeted $=0.1591$

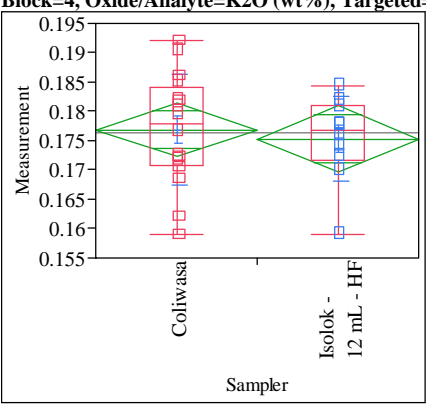

Oneway Anova

Rsquare $\quad 0.008121$

Adj Rsquare $\quad-0.0332$

Root Mean Square Error $\quad 0.008722$

$\begin{array}{lr}\text { Mean of Response } & 0.176289 \\ \text { Observations (or Sum Wgts) } & 26\end{array}$

t Test

Isolok - $12 \mathrm{~mL}$ - HF-Coliwasa

Assuming equal variances

Difference $\quad-0.00156$ t Ratio $\quad-0.44328$

$\begin{array}{lrlr}\text { Std Err Dif } & 0.00352 \text { DF } & 24 \\ \text { Upper CL Dif } & 0.00570 & \text { Prob }>|t| & 0.6615\end{array}$

$\begin{array}{rrrr}\text { Upper CL Dif } & 0.00570 & \text { Prob }>|t| & 0.6615 \\ \text { Lower CL Dif } & -0.00881 & \text { Prob }>t & 0.6692\end{array}$

$\begin{array}{lrll}\text { Lower CL Dif } & -0.00881 & \text { Prob }>t & 0.6692 \\ \text { Confidence } & 0.95 & \text { Prob }<t & 0.3308\end{array}$

Analysis of Variance

Source DF Sum of Squares Mean Square F Ratio Prob $>$ F

$\begin{array}{lrrrrr}\text { Sampler } & 1 & 0.00001495 & 0.000015 & 0.1965 & 0.6615\end{array}$

$\begin{array}{llll}\text { Error } & 24 & 0.00182556 & 0.000076\end{array}$

C. Total $25 \quad 0.00184050$

Means for Oneway Anova

Level Number Mean Std Error Lower 95\% Upper 95\%

$\begin{array}{llllll}\text { Coliwasa } & 16 & 0.176888 & 0.00218 & 0.17239 & 0.18139\end{array}$

$\begin{array}{llllll}\text { Isolok - } 12 \mathrm{~mL} \text { - HF } & 10 & 0.175330 & 0.00276 & 0.16964 & 0.18102\end{array}$

Std Error uses a pooled estimate of error variance

Means and Std Deviations

$\begin{array}{llllll}\text { Level } & \text { Number } & \text { Mean } & \text { Std Dev Std Err Mean Lower 95\% Upper 95\% }\end{array}$

$\begin{array}{lrrrrrr}\text { Coliwasa } & 16 & 0.176888 & 0.009502 & 0.00238 & 0.17182 & 0.18195 \\ \text { Isolok - 12 mL - HF } & 10 & 0.175330 & 0.007236 & 0.00229 & 0.17015 & 0.1805\end{array}$

t Test

Isolok - $12 \mathrm{~mL}$ - HF-Coliwasa

Assuming unequal variances

Difference $\quad-0.00156$ t Ratio $\quad-0.4725$

Std Err Dif $\quad 0.00330$ DF 22.89686

Upper CL Dif $\quad 0.00527$ Prob $>|t| \quad 0.6410$

$\begin{array}{lrlr}\text { Lower CL Dif } & -0.00838 & \text { Prob }>t & 0.6795 \\ \text { Confidence } & 0.95 & \text { Prob }<\mathrm{t} & 0.3205\end{array}$

Tests that the Variances are Equal

$\begin{array}{lrrrr}\text { Level } & \text { Count } & \text { Std Dev } & \text { MeanAbsDif to Mean } & \text { MeanAbsDif to Median } \\ \text { Coliwasa } & 16 & 0.0095021 & 0.0077923 & 0.0077923 \\ \text { Isolok - 12 mL - HF } & 10 & 0.0072357 & 0.0052280 & 0.0051196\end{array}$

Test F Ratio DFNum DFDen p-Value

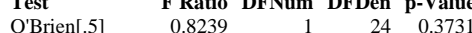

$\begin{array}{lllll}\text { Brown-Forsythe } & 1.6696 & 1 & 24 & 0.2086\end{array}$

$\begin{array}{lllll}\text { Brown-Forsythe } & 1.6696 & 1 & 24 & 0.2086\end{array}$

$\begin{array}{lrrrr}\text { Levene } & 1.6723 & 1 & 24 & 0.2083 \\ \text { Bartlett } & 0.7558 & 1 & . & 0.3846\end{array}$

$\begin{array}{lllll}\text { F Test 2-sided } & 1.7246 & 15 & 9 & 0.4121\end{array}$

Welch Anova testing Means Equal, allowing Std Devs Not Equal

F Ratio DFNum DFDen Prob $>$ F

$\begin{array}{llll}0.2233 & 1 & 22.897 & 0.6410\end{array}$

t Test
Oneway Analysis of Measurement By Sampler Type of Material=SME Simulant, Analytica Block $=4$, Oxide/Analyte $=\mathrm{Li} 2 \mathrm{O}(\mathrm{wt} \%)$, Targeted $=4.674$

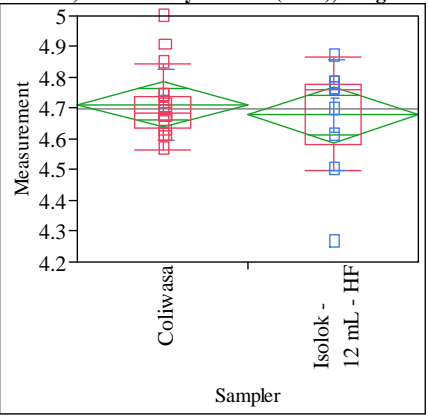

Oneway Anova
Summary of Fit

Rsquare

Adj Rsquare $\quad-0.0267$

Root Mean Square Error $\quad 0.142255$

Mean of Response 4.699118
26

t Test

Isolok - $12 \mathrm{~mL}$ - HF-Coliwasa

Assuming equal variances

Difference $\quad-0.03391$ t Ratio $\quad-0.5913$

Std Err Dif $\quad 0.05734$ DF 24

Upper CL Dif 0.08445 Prob $>|t| 0.5598$

Lower CL Dif -0.15226 Prob $>t \quad 0.7201$

$\begin{array}{lll}\text { Confidence } & 0.95 \text { Prob }<\mathrm{t} \quad 0.2799\end{array}$

Analysis of Variance

Source DF Sum of Squares Mean Square F Ratio Prob $>$ F

$\begin{array}{lrrrrr}\text { Source } & \text { DF } & \text { Sum of Squares } & \text { Mean Square } & \text { Fatio } & \text { Prob }>\text { F } \\ \text { Sampler } & 1 & 0.00707547 & 0.007075 & 0.3496 & 0.5598\end{array}$

$\begin{array}{lrrr}\text { Sampler } & 1 & 0.00707547 & 0.007075 \\ \text { Error } & 24 & 0.48567621 & 0.020237\end{array}$

$\begin{array}{lll}\text { Error } & 24 & 0.48567621 \\ \text { C. Total } & 25 & 0.49275169\end{array}$

Means for Oneway Anova

$\begin{array}{lrrrrr}\text { Level } & \text { Number } & \text { Mean } & \text { Std Error } & \text { Lower 95\% } & \text { Upper 95\% } \\ \text { Coliwasa } & 16 & 4.71216 & 0.03556 & 4.6388 & 4.7856\end{array}$

$\begin{array}{llllll}\text { Isolok - } 12 \text { mL - HF } & 10 & 4.67825 & 0.04499 & 4.5854 & 4.7711\end{array}$

Std Error uses a pooled estimate of error variance

Means and Std Deviations

Level Number Mean Std Dev Std Err Mean Lower 95\% Upper 95\%

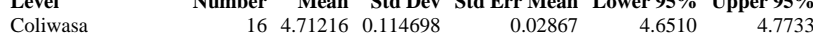

$\begin{array}{lllllll}\text { Isolok - } 12 \mathrm{~mL} \text { - HF } & 16 & 4.71216 & 0.114698 & 0.02867 & 4.6510 & 4.7733 \\ & 10 & 4.67825 & 0.178992 & 0.05660 & 4.5502 & 4.8063\end{array}$

t Test

Isolok - $12 \mathrm{~mL}$ - HF-Coliwasa

Assuming unequal variances

Difference $\quad-0.03391$ t Ratio $\quad-0.5344$

Std Err Dif $\quad 0.06345$ DF $\quad 13.67201$

Upper CL Dif 0.10249 Prob $>|t| r 0.6016$

Lower CL Dif -0.17030 Prob $>t \quad 0.6992$
Cont

Confidence $\quad 0.95$ Prob $<\mathrm{t} \quad 0.3008$

Tests that the Variances are Equal

$\begin{array}{lrrrr}\text { Level } & \text { Count } & \text { Std Dev } & \text { MeanAbsDif to Mean } & \text { MeanAbsDif to Median } \\ \text { Coliwasa } & 16 & 0.1146979 & 0.0827521 & 0.0780426\end{array}$

$\begin{array}{lllll}\text { Coliwasa } & 16 & 0.1146979 & 0.0827521 & 0.0780426 \\ \text { Isolok - } 12 \mathrm{~mL} \text { - HF } & 10 & 0.1789916 & 0.1330492 & 0.1141037\end{array}$

Test F Ratio DFNum DFDen p-Value

$\begin{array}{lrrrr}\text { O'Brien[.5] } & 1.2645 & 1 & 24 & 0.2719\end{array}$

$\begin{array}{lllll}\text { Brown-Forsythe } & 0.5725 & 1 & 24 & 0.4566\end{array}$

$\begin{array}{lllll}\text { Levene } & 1.8767 & 1 & 24 & 0.1834\end{array}$

$\begin{array}{lllll}\text { Bartlett } & 2.2238 & 1 & 0.1359\end{array}$

$\begin{array}{lllll}\text { F Test 2-sided } & 2.4353 & 9 & 15 & 0.1229\end{array}$

Welch Anova testing Means Equal, allowing Std Devs Not Equal

F Ratio DFNum DFDen Prob $>$ F

$\begin{array}{llll}0.2856 & 1 & 13.672 & 0.6016\end{array}$

t Test

0.5344 
SRNL-STI-2011-00693

Revision 0

\section{Exhibit C2. Statistical Comparisons for Low-Rheology (Phase 3) Simulant Testing}

Oneway Analysis of Measurement By Sampler Type of Material=SME Simulant, Analytical Block $=4$, Oxide/Analyte $=\mathrm{MgO}$ (wt $\%$ ), Targeted $=1.014$

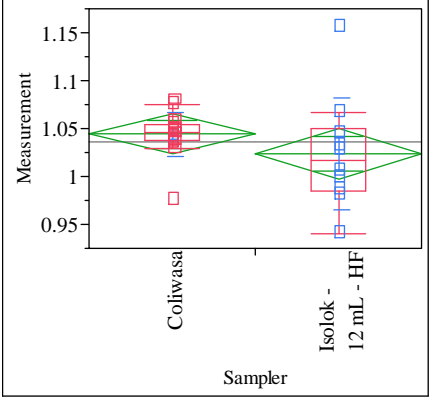

Oneway Anova

Summary of Fit

Root Mean Square Error $\quad 0.040314$

1.036182

Observations (or Sum Wgts) 26

t Test

Isolok - $12 \mathrm{~mL}$ - HF-Coliwasa

Assuming equal variances

Difference $\quad-0.02033$ t Ratio -1.25129

$\begin{array}{lrr}\text { Std Err Dif } & 0.01625 \text { DF } & 24 \\ & \end{array}$

Upper CL Dif 0.01321 Prob $>|t| \quad 0.2229$

$\begin{array}{lrrr}\text { Lower CL Dif } & -0.05388 & \text { Prob }>t & 0.8886 \\ \text { Confidence } & 0.95 & \text { Prob }<\mathrm{t} & 0.1114\end{array}$

Analysis of Variance

Source DF Sum of Squares Mean Square F Ratio Prob $>$ F

$\begin{array}{llllll}\text { Sampler } & 1 & 0.00254467 & 0.002545 & 1.5657 & 0.2229\end{array}$

Error $\quad 24 \quad 0.03900552 \quad 0.001625$

C. Total $25 \quad 0.04155019$

Means for Oneway Anova

Level Number Mean Std Error Lower 95\% Upper 95\%

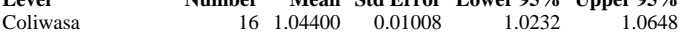

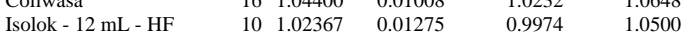

Std Error uses a pooled estimate of error variance

Means and Std Deviations

Level Number Mean Std Dev Std Err Mean Lower 95\% Upper 95\%

$\begin{array}{lllllll}\text { Coliwasa } & 16 & 1.04400 & 0.023057 & 0.00576 & 1.0317 & 1.0563\end{array}$

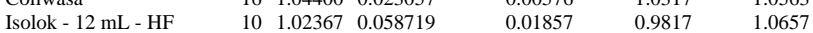

t Test

Isolok - $12 \mathrm{~mL}$ - HF-Coliwasa

Assuming unequal variances

Difference $\quad-0.02033$ t Ratio $\quad-1.04589$

Std Err Dif $\quad 0.01944$ DF 10.75834

Upper CL Dif 0.02258 Prob $>|t| \quad 0.3185$

Lower CL Dif -0.06325 Prob $>t \quad 0.8407$

$\begin{array}{lrl}\text { Confidence } & 0.95 \text { Prob }<\mathrm{t} \quad 0.1593\end{array}$

Tests that the Variances are Equal

Level Count Std Dev MeanAbsDif to Mean MeanAbsDif to Median

$\begin{array}{lllll} & 16 & 0.0230575 & 0.0144972 & 0.0141992 \\ \text { Isolok - } 12 \mathrm{~mL}-\mathrm{HF} & 10 & 0.0587185 & 0.0416233 & 0.0416233\end{array}$

$\begin{array}{lrrrr}\text { Test } & \text { F Ratio } & \text { DFNum } & \text { DFDen } & \text { p-Value } \\ \text { O'Brien[.5] } & 3.1553 & 1 & 24 & 0.0884 \\ \text { Brown-Forsythe } & 5.8296 & 1 & 24 & 0.0237 \\ \text { Levene } & 5.9328 & 1 & 24 & 0.0227 \\ \text { Bartlett } & 9.5587 & 1 & . & 0.0020 \\ \text { F Test 2-sided } & 6.4853 & 9 & 15 & 0.0017\end{array}$

Welch Anova testing Means Equal, allowing Std Devs Not Equal

F Ratio DFNum DFDen Prob $>$ F

$\begin{array}{llll}1.0939 & 1 & 10.758 & 0.3185\end{array}$

t Test

1.0459
Oneway Analysis of Measurement By Sampler Type of Material=SME Simulant, Analytical Block=4, Oxide/Analyte=MnO (wt\%), Targeted $=1.779$

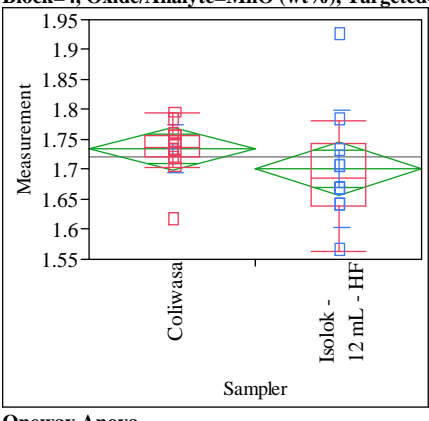

Summary of Fit

Root Mean Square Error $\quad 0.067662$

Mean of Response 1.721766

Observations (or Sum Wgts) 26

\section{Test}

Isolok - $12 \mathrm{~mL}$ - HF-Coliwasa

Assuming equal variances

$\begin{array}{lrlr}\text { Difference } & -0.03244 & \text { t Ratio } & -1.18941 \\ \text { Std Err Dif } & 0.02728 & \text { DF } & 24 \\ \text { Upper CL Dif } & 0.02385 & \text { Prob }>|t| & 0.2459 \\ \text { Lower CL Dif } & -0.08873 & \text { Prob }>t & 0.8770 \\ \text { Confidence } & 0.95 & \text { Prob }<\mathrm{t} & 0.1230\end{array}$

Analysis of Variance

Source DF Sum of Squares Mean Square F Ratio Prob $>$ F

$\begin{array}{lrrrrr}\text { Sampler } & 1 & 0.00647658 & 0.006477 & 1.4147 & 0.2459\end{array}$

$\begin{array}{llll}\text { Error } & 24 & 0.10987456 & 0.004578\end{array}$

Means for Oneway Anova

Level Number Mean Std Error Lower 95\% Upper 95\%

$\begin{array}{lrrrrr}\text { Coliwasa } & 16 & 1.73424 & 0.01692 & 1.6993 & 1.7692\end{array}$

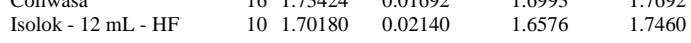

Std Error uses a pooled estimate of error variance

Means and Std Deviations

Level Number Mean Std Dev Std Err Mean Lower 95\% Upper 95\%

$\begin{array}{lllllll}\text { Coliwasa } & 16 & 1.73424 & 0.039649 & 0.00991 & 1.7131 & 1.7554\end{array}$

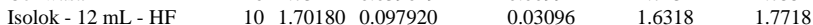

t Test

Isolok - $12 \mathrm{~mL}$ - HF-Coliwas

Assuming unequal variances

$\begin{array}{lrlr}\text { Difference } & -0.03244 & \text { t Ratio } & -0.99781 \\ \text { Std Err Dif } & 0.03251 & \text { DF } & 10.8705 \\ \text { Upper CL Dif } & 0.03922 & \text { Prob }>|t| & 0.3401 \\ \text { Lower CL Dif } & -0.10411 & \text { Prob }>\text { t } & 0.8300 \\ \text { Confidence } & 0.95 & \text { Prob }<\mathrm{t} & 0.1700\end{array}$

Tests that the Variances are Equal

Level Count Std Dev MeanAbsDif to Mean MeanAbsDif to Median

$\begin{array}{lllll}\text { Coliwasa } & 16 & 0.0396488 & 0.0250170 & 0.0250170 \\ \text { Isolok - } 12 \mathrm{~mL} \text { - HF } & 10 & 0.0979195 & 0.0671424 & 0.0671424\end{array}$

$\begin{array}{lrrrr}\text { Test } & \text { F Ratio } & \text { DFNum } & \text { DFDen } & \text { p-Value } \\ \text { O'Brien[.5] } & 2.9464 & 1 & 24 & 0.0990 \\ \text { Brown-Forsythe } & 4.5429 & 1 & 24 & 0.0435 \\ \text { Levene } & 4.7844 & 1 & 24 & 0.0387 \\ \text { Bartlett } & 8.9734 & 1 & . & 0.0027 \\ \text { F Test 2-sided } & 6.0993 & 9 & 15 & 0.0023\end{array}$

Welch Anova testing Means Equal, allowing Std Devs Not Equal

F Ratio DFNum DFDen Prob $>$ F

$\begin{array}{llrr}0.9956 & 1 & 10.87 & 0.3401\end{array}$

t Test

0.9978 


\section{Exhibit C2. Statistical Comparisons for Low-Rheology (Phase 3) Simulant Testing}

Oneway Analysis of Measurement By Sampler Type of Material=SME Simulant, Analytical Block=4, Oxide/Analyte $=\mathrm{Na2O}(\mathrm{wt} \%)$, Targeted $=11.659$

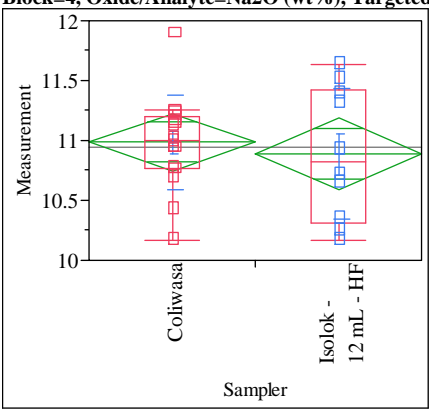

Oneway Anova

Summary of Fit

Adj Rsquare

10.94732

t Test

Isolok - $12 \mathrm{~mL}$ - HF-Coliwasa

Assuming equal variances

$\begin{array}{lrr}\text { Difference } & -0.09672 \text { t Ratio } \quad-0.52622 \\ \text { Std Err Dif } & 0.18380 & -24\end{array}$

$\begin{array}{lrr}\text { Std Err Dif } & 0.18380 \text { DF } & 24 \\ \text { Upper CL } & 0.28262 \text { Prob }>|t| & 0.6036\end{array}$

Lower CL Dif -0.47606 Prob $>\mathrm{t} \quad 0.6982$

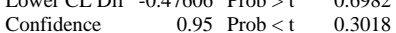

Analysis of Variance

Source DF Sum of Squares Mean Square F Ratio Prob $>$ F

$\begin{array}{lrrrrr}\text { Sampler } & 1 & 0.0575666 & 0.057567 & 0.2769 & 0.6036\end{array}$

$\begin{array}{llll}\text { Error } & 24 & 4.9893769 & 0.207891 \\ \text { C. Total } & 25 & 5.0469435 & \end{array}$

Means for Oneway Anova

Level Number Mean Std Error Lower 95\% Upper 95\%

$\begin{array}{lrrrrr}\text { Coliwasa } & 16 & 10.9845 & 0.11399 & 10.749 & 11.220\end{array}$

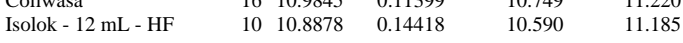

Std Error uses a pooled estimate of error variance

Means and Std Deviations

Level Number Mean Std Dev Std Err Mean Lower 95\% Upper 95\%

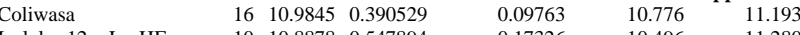

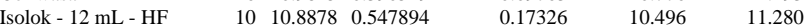

t Test

Isolok - $12 \mathrm{~mL}$ - HF-Coliwasa

Assuming unequal variances

Difference $\quad-0.09672$ t Ratio $\quad-0.48633$

Std Err Dif $\quad 0.19887$ DF $\quad 14.73187$

Upper CL Dif 0.32784 Prob $>|t| \quad 0.6339$

Lower CL Dif -0.52128 Prob $>\mathrm{t} \quad 0.6831$

$\begin{array}{lrl}\text { Confidence } & 0.95 \text { Prob }<\mathrm{t} \quad 0.3169\end{array}$

Tests that the Variances are Equal

Level Count Std Dev MeanAbsDif to Mean MeanAbsDif to Median

$\begin{array}{lllll} & 16 & 0.3905286 & 0.2731806 & 0.2729700 \\ \text { Isolok - } 12 \mathrm{~mL} \text { - HF } & 10 & 0.5478938 & 0.4650600 & 0.4650600\end{array}$

$\begin{array}{lrrrr}\text { Test } & \text { F Ratio } & \text { DFNum } & \text { DFDen } & \text { p-Value } \\ \text { O'Brien[.5] } & 1.9068 & 1 & 24 & 0.1800 \\ \text { Brown-Forsythe } & 3.2300 & 1 & 24 & 0.0849 \\ \text { Levene } & 3.3308 & 1 & 24 & 0.0805 \\ \text { Bartlett } & 1.2818 & 1 & . & 0.2576 \\ \text { F Test 2-sided } & 1.9683 & 9 & 15 & 0.2367\end{array}$

Welch Anova testing Means Equal, allowing Std Devs Not Equal

F Ratio DFNum DFDen Prob $>$ F

$\begin{array}{llrr}0.2365 & 1 & 14.732 & 0.6339\end{array}$

t Test

0.4863
Oneway Analysis of Measurement By Sampler Type of Material=SME Simulant, Analytical Block=4, Oxide/Analyte $=\mathrm{NiO}$ (wt $\%$ ), Targeted $=0.41$

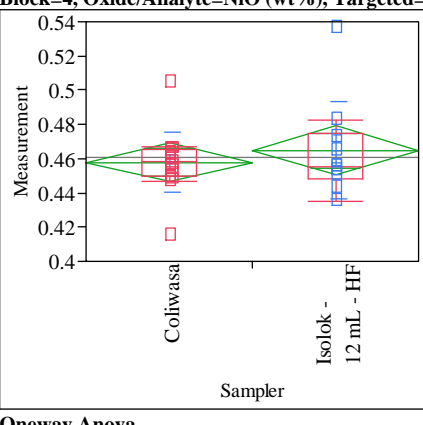

neway Anova

Summary of Fit

Adj Rsquare

-0.022224
$0.00 t$ Mean Square Error $\quad 0.460449$

Observations (or Sum Wgts) 26

\section{Test}

Isolok - $12 \mathrm{~mL}$ - HF-Coliwas

Assuming equal variances

$\begin{array}{lrlr}\text { Difference } & 0.00694 & \text { t Ratio } & 0.774109 \\ \text { Std Err Dif } & 0.00896 & \text { DF } & 24 \\ \text { Upper CL Dif } & 0.02543 & \text { Prob }>|t| & 0.4464 \\ \text { Lower CL Dif } & -0.01156 & \text { Prob }>t & 0.2232 \\ \text { Confidence } & 0.95 & \text { Prob }<\mathrm{t} & 0.7768\end{array}$

$\begin{array}{lll} & \\ \text { Cower CL Dif }-0.01156 \text { Prob }>t & 0.2232\end{array}$

Analysis of Variance

Source DF Sum of Squares Mean Square F Ratio Prob $>$ F

$\begin{array}{lrrrrr}\text { Sampler } & 1 & 0.00029598 & 0.000296 & 0.5992 & 0.4464\end{array}$

$\begin{array}{llll}\text { Error } & 24 & 0.01185393 & 0.000494\end{array}$

C. Total $25 \quad 0.01214990$

Means for Oneway Anova

Level Number Mean Std Error Lower 95\% Upper 95\%

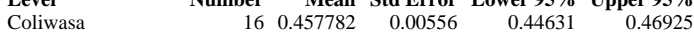

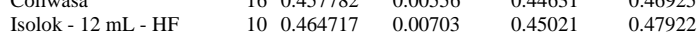

Std Error uses a pooled estimate of error variance

Means and Std Deviations

Level Number Mean Std Dev Std Err Mean Lower 95\% Upper 95\%

$\begin{array}{lllllll}\text { Coliwasa } & 16 & 0.457782 & 0.017494 & 0.00437 & 0.44846 & 0.46710\end{array}$

$\begin{array}{llllll}\text { Isolok - } 12 \mathrm{~mL} \text { - HF } & 10 & 0.464717 & 0.028408 & 0.00898 & 0.44439\end{array}$

Test

Isolok - $12 \mathrm{~mL}$ - HF-Coliwas

Assuming unequal variances

$\begin{array}{lrlr}\text { Difference } & 0.00694 & \text { t Ratio } & 0.694099 \\ \text { Std Err Dif } & 0.00999 & \text { DF } & 13.32268 \\ \text { Upper CL Dif } & 0.02847 & \text { Prob }>|t| & 0.4995 \\ \text { Lower CL Dif } & -0.01460 & \text { Prob }>\text { t } & 0.2498 \\ \text { Confidence } & 0.95 & \text { Prob }<\mathrm{t} & 0.7502\end{array}$

Tests that the Variances are Equal

Level Count Std Dev MeanAbsDif to Mean MeanAbsDif to Median

$\begin{array}{lllll}\text { Coliwasa } & 16 & 0.0174940 & 0.0106572 & 0.0106572\end{array}$

Isolok - $12 \mathrm{~mL}$ - HF $\quad 10 \quad 0.0284084 \quad 0.0191893 \quad 0.0173060$

$\begin{array}{lrrrr}\text { Test } & \text { F Ratio } & \text { DFNum } & \text { DFDen } & \text { p-Value } \\ \text { O'Brien[.5] } & 0.9801 & 1 & 24 & 0.3320 \\ \text { Brown-Forsythe } & 0.8283 & 1 & 24 & 0.3718 \\ \text { Levene } & 1.6920 & 1 & 24 & 0.2057 \\ \text { Bartlett } & 2.6408 & 1 & . & 0.1042 \\ \text { F Test 2-sided } & 2.6370 & 9 & 15 & 0.0936\end{array}$

Welch Anova testing Means Equal, allowing Std Devs Not Equal

F Ratio DFNum DFDen Prob $>$ F

$\begin{array}{lllr}0.4818 & 1 & 13.323 & 0.4995\end{array}$

t Tes

0.6941 


\section{Exhibit C2. Statistical Comparisons for Low-Rheology (Phase 3) Simulant Testing}

Oneway Analysis of Measurement By Sampler Type of Material=SME Simulant, Analytical Block=4, Oxide/Analyte=SiO2 (wt\%), Targeted $=50.985$

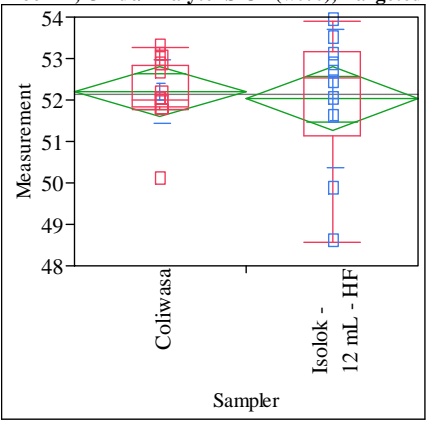

Oneway Anova

Rsquare $\quad 0.005297$

Adj Rsquare $\quad-0.03615$

Root Mean Square Error $\quad 1.187585$

$\begin{array}{lr}\text { Mean of Response } & 52.1331 \\ \text { Observations (or Sum Wgts) } & 26\end{array}$

t Test

Isolok - $12 \mathrm{~mL}$ - HF-Coliwasa

Assuming equal variances

Difference $\quad-0.1711$ t Ratio $\quad-0.3575$

Std Err Dif $\quad 0.4787$ DF $\quad 24$

Upper CL Dif 0.8169 Prob $>|t| 0.7238$

Lower CL Dif -1.1592 Prob $>\mathrm{t} \quad 0.6381$

Confidence $\quad 0.95$ Prob $<\mathrm{t} \quad 0.3619$

Analysis of Variance

Source DF Sum of Squares Mean Square F Ratio Prob $>$ F

$\begin{array}{lrrrrr}\text { Sampler } & 1 & 0.180248 & 0.18025 & 0.1278 & 0.7238\end{array}$

$\begin{array}{llrr}\text { Error } & 24 & 33.848567 & 1.41036\end{array}$

C. Total $25 \quad 34.028815$

Means for Oneway Anova

Level Number Mean Std Error Lower 95\% Upper 95\%

$\begin{array}{llllrr}\text { Coliwasa } & 16 & 52.1989 & 0.29690 & 51.586 & 52.812\end{array}$

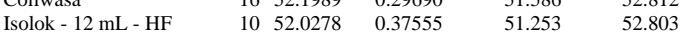

Std Error uses a pooled estimate of error variance

Means and Std Deviations

Level Number Mean Std Dev Std Err Mean Lower 95\% Upper 95\%

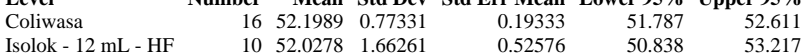

t Test

Isolok - $12 \mathrm{~mL}$ - HF-Coliwasa

Assuming unequal variances

Difference $\quad-0.1711$ t Ratio -0.30552

Std Err Dif $\quad 0.5602$ DF 11.47247

Upper CL Dif 1.0556 Prob $>|t| r \mid 0.7654$

$\begin{array}{lrll}\text { Lower CL Dif } & -1.3979 & \text { Prob }>t & 0.6173 \\ \text { Confidence } & 0.95 & \text { Prob }<\mathrm{t} & 0.3827\end{array}$

Tests that the Variances are Equal

$\begin{array}{lrrrr}\text { Level } & \text { Count } & \text { Std Dev } & \text { MeanAbsDif to Mean } & \text { MeanAbsDif to Median } \\ \text { Coliwasa } & 16 & 0.773311 & 0.561566 & 0.534825 \\ \text { Isolok - 12 mL - HF } & 10 & 1.662609 & 1.232237 & 1.155222\end{array}$

Test F Ratio DFNum DFDen p-Value

$\begin{array}{lllll}\text { O'Brien[.5] } & 3.5451 & 1 & 24 & 0.0719\end{array}$

Brown-Forsythe $2.9794 \quad-1 \quad 24 \quad 0.0972$

$\begin{array}{lll}1 & 24 & 0.0972 \\ 1 & 24 & 0.0370\end{array}$

$\begin{array}{lllll}\text { Levene } & 4.8780 & 1 & 24 & 0.0370 \\ \text { Bartlett } & 6.5178 & 1 & & 0.0107\end{array}$

$\begin{array}{lllll}\text { Bartlett } & 6.5178 & 1 & & 0.0107 \\ \text { F Test 2-sided } & 4.6224 & 9 & 15 & 0.0092\end{array}$

Welch Anova testing Means Equal, allowing Std Devs Not Equal

F Ratio DFNum DFDen Prob $>$ F

$\begin{array}{llll}0.0933 & 1 & 11.472 & 0.7654\end{array}$

t Test
Oneway Analysis of Measurement By Sampler Type of Material=SME Simulant, Analytical Block $=4$, Oxide/Analyte $=$ Sum of Oxides ( $w$ t $\%$ ), Targeted $=99.553$

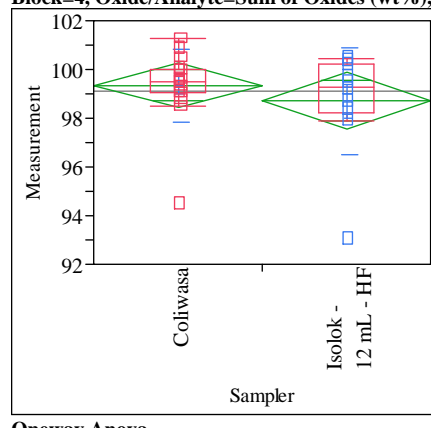

\section{Summary of Fi}

Rsquare

Adj Rsquare 0.031888

Root Mean Square Error $\quad 1.791642$

Mean of Response Ere 1.791642

Observations (or Sum Wgts) $\quad 26$

Isolok - $12 \mathrm{~mL}$ - HF-Coliwasa

Assuming equal variances

$\begin{array}{lrlr}\text { Difference } & -0.6421 & \mathrm{t} \text { Ratio } & -0.88911 \\ \text { Std Err Dif } & 0.7222 & \text { DF } & 24 \\ \text { Upper CL Dif } & 0.8485 & \text { Prob }>|t| & 0.3828 \\ \text { Lower CL Dif } & -2.1328 & \text { Prob }>\mathrm{t} & 0.8086 \\ \text { Confidence } & 0.95 & \text { Prob }<\mathrm{t} & 0.1914\end{array}$

Analysis of Variance

Source DF Sum of Squares Mean Square F Ratio Prob $>$ F

$\begin{array}{llllll}\text { Sampler } & 1 & 2.537563 & 2.53756 & 0.7905 & 0.3828\end{array}$

$\begin{array}{lrrr}\text { Error } & 24 & 77.039506 & 3.20998\end{array}$

C. Total $25 \quad 79.577069$

Means for Oneway Anova

Level $\quad$ Number Mean Std Error Lower 95\% Upper 95\%

$\begin{array}{lrrrrr}\text { Coliwasa } & 16 & 99.3551 & 0.44791 & 98.431 & 100.28\end{array}$

$\begin{array}{llllr}\text { Isolok - } 12 \text { mL - HF } & 10 & 98.7130 & 0.56657 & 97.544\end{array}$

Std Error uses a pooled estimate of error variance

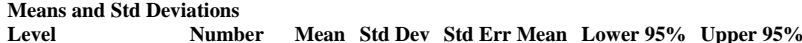

$\begin{array}{lrrrrrr}\text { Level } & \text { Number } & \text { Mean } & \text { Std Dev } & \text { Std Err Mean } & \text { Lower 95\% } & \text { Upper 95\% } \\ \text { Coliwasa } & 16 & 99.3551 & 1.50246 & 0.37562 & 98.555 & 100.16\end{array}$

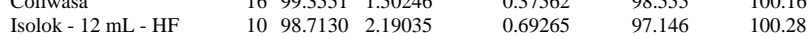

Isolok - $12 \mathrm{~mL}$ - HF-Coliwasa

Assuming unequal variances

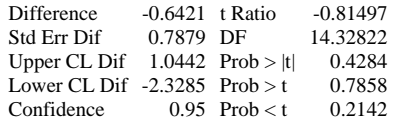

Tests that the Variances are Equal

Level Count Std Dev MeanAbsDif to Mean MeanAbsDif to Median

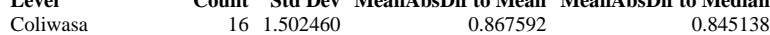

Isolok - $12 \mathrm{~mL}$ - HF 10 2.

Test F Ratio DFNum DFDen p-Value

$\begin{array}{lrrrr}\text { O'Brien[.5] } & 0.4916 & 1 & 24 & 0.4899\end{array}$

$\begin{array}{lllll}\text { Brown-Forsythe } & 0.5932 & 1 & 24 & 0.4487\end{array}$

$\begin{array}{lllll}\text { Levene } & 0.8823 & 1 & 24 & 0.3569\end{array}$

$\begin{array}{lllll}\text { Bartlett } & 1.5919 & 1 & 0.2071\end{array}$

F Test 2-sided $2.1253 \quad-9$

Welch Anova testing Means Equal, allowing Std Devs Not Equal

F Ratio DFNum DFDen Prob $>$ F

$\begin{array}{rrrr}0.6642 & 1 & 14.328 & 0.4284\end{array}$

t Test 


\section{Exhibit C2. Statistical Comparisons for Low-Rheology (Phase 3) Simulant Testing}

Oneway Analysis of Measurement By Sampler Type of Material=SME Simulant, Analytical Block =4, Oxide/Analyte $=\mathrm{TiO} 2$ (wt $\%$ ), Targeted $=\mathbf{0 . 0 7 1 1}$

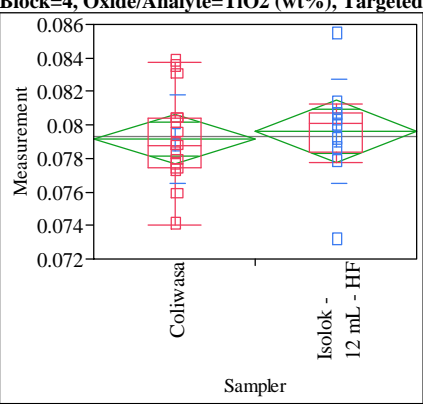

Oneway Anova

Rsquare $\quad 0.007389$

Adj Rsquare $\quad-0.03397$

Root Mean Square Error $\quad 0.002839$

$\begin{array}{lr}\text { Mean of Response } & 0.079333 \\ \text { Observations (or Sum Wgts) } & 26\end{array}$

t Test

Isolok - $12 \mathrm{~mL}$ - HF-Coliwasa

Assuming equal variances

Difference $\quad 0.00048$ t Ratio $\quad 0.422678$

$\begin{array}{lrrr}\text { Std Err Dif } & 0.00114 & \text { DF } & 24 \\ \text { Upper CL } & 0.00285 & \text { Prob }>|t| & 0.6763\end{array}$

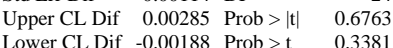

$\begin{array}{lrrr}\text { Lower CL Dif } & -0.00188 & \text { Prob }>t & 0.3381 \\ \text { Confidence } & 0.95 & \text { Prob }<\mathrm{t} & 0.6619\end{array}$

Analysis of Variance

Source DF Sum of Squares Mean Square F Ratio Prob $>$ F

$\begin{array}{lrrrrr}\text { Sampler } & 1 & 0.00000144 & 1.4399 \mathrm{e}-6 & 0.1787 & 0.6763\end{array}$

$\begin{array}{llll}\text { Error } & 24 & 0.00019343 & 8.0596 \mathrm{e}-6\end{array}$

C. Total $25 \quad 0.00019487$

Means for Oneway Anova

Level Number Mean Std Error Lower 95\% Upper 95\%

$\begin{array}{lllrrr}\text { Coliwasa } & 16 & 0.079147 & 0.00071 & 0.07768 & 0.08061\end{array}$

$\begin{array}{llllll}\text { Isolok - } 12 \mathrm{~mL} \text { - HF } & 10 & 0.079630 & 0.00090 & 0.07778 & 0.08148\end{array}$

Std Error uses a pooled estimate of error variance

Means and Std Deviations

$\begin{array}{lrrrrrr}\text { Level } & \text { Number } & \text { Mean } & \text { Std Dev } & \text { Std Err Mean } & \text { Lower 95\% } & \text { Upper 95\% } \\ \text { Coliwasa } & 16 & 0.079147 & 0.002672 & 0.00067 & 0.07772 & 0.08057\end{array}$

$\begin{array}{lllllll}\text { Isolok - } 12 \mathrm{~mL} \text { - HF } & 10 & 0.079630 & 0.003097 & 0.00098 & 0.07741 & 0.08185\end{array}$

t Test

Isolok - $12 \mathrm{~mL}$ - HF-Coliwas

Assuming unequal variances

Difference $\quad 0.00048$ t Ratio 0.408035

Std Err Dif $\quad 0.00119$ DF $\quad 17.10328$

Upper CL Dif 0.00298 Prob $>|t| \quad 0.6883$

$\begin{array}{lrll}\text { Lower CL Dif } & -0.00202 & \text { Prob }>t & 0.3442 \\ \text { Confidence } & 0.95 & \text { Prob }<\mathrm{t} & 0.6558\end{array}$

Tests that the Variances are Equal

$\begin{array}{lrrrr}\text { Level } & \text { Count } & \text { Std Dev } & \text { MeanAbsDif to Mean } & \text { MeanAbsDif to Median } \\ \text { Coliwasa } & 16 & 0.0026723 & 0.0020746 & 0.0020225 \\ \text { Isolok - 12 mL - HF } & 10 & 0.0030969 & 0.0020216 & 0.0019682\end{array}$

Test F Ratio DFNum DFDen p-Value

O'Brien[.5] $\quad 0.1973 \quad 1 \quad 240.6609$

$\begin{array}{lllll}\text { Brown-Forsythe } & 0.0046 & 1 & 24 & 0.9464\end{array}$

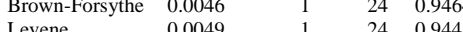

$\begin{array}{lllll}\text { Levene } & 0.0049 & 1 & 24 & 0.9445 \\ \text { Bartlett } & 0.2390 & 1 & . & 0.6249\end{array}$

$\begin{array}{lllll}\text { F Test 2-sided } & 1.3430 & 9 & 15 & 0.5895\end{array}$

Welch Anova testing Means Equal, allowing Std Devs Not Equal

F Ratio DFNum DFDen Prob $>$ F

$\begin{array}{llll}0.1665 & 1 & 17.103 & 0.6883\end{array}$

t Test
Oneway Analysis of Measurement By Sampler Type of Material=SME Simulant, Analytical Block $=4$, Oxide/Analyte $=\mathrm{ZnO}($ wt $\%)$, Targeted $=0.0958$

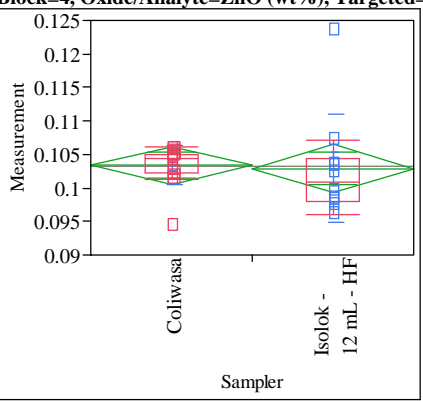

Oneway Anova

Summary of Fit

Rsquare

Adj Rsquare

Root Mean Square Error $\quad 0.005402$

Mean of Response

0.103194
26

Isolok - $12 \mathrm{~mL}$ - HF-Coliwasa

Assuming equal variances

$\begin{array}{lrlr}\text { Difference } & -0.00045 & \text { t Ratio } & -0.20436 \\ \text { Std Err Dif } & 0.00218 & \text { DF } & 24 \\ \text { Upper CL Dif } & 0.00405 & \text { Prob }>|t| & 0.8398 \\ \text { Lower CL Dif } & -0.00494 & \text { Prob }>t & 0.5801 \\ \text { Confidence } & 0.95 & \text { Prob }<t & 0.4199\end{array}$

Analysis of Variance

Source DF Sum of Squares Mean Square F Ratio Prob $>$ F

$\begin{array}{lrrrrr}\text { Sampler } & 1 & 0.00000122 & 1.219 \mathrm{e}-6 & 0.0418 & 0.8398\end{array}$

$\begin{array}{lll}\text { Error } & 24 & 0.00070038\end{array}$

$\begin{array}{rrr}1.219 \mathrm{e}-6 & 0.0418 & 0.8398 \\ 0.000029 & & \end{array}$

C. Total $25 \quad 0.00070159$

Means for Oneway Anova

Level Number Mean Std Error Lower 95\% Upper 95\%

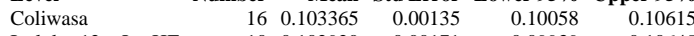

$\begin{array}{llllll}\text { Isolok - } 12 \mathrm{~mL} \text { - HF } & 10 & 0.102920 & 0.00171 & 0.09939 & 0.10645\end{array}$

Std Error uses a pooled estimate of error variance

Means and Std Deviations

$\begin{array}{llllll}\text { Level } & \text { Number } & \text { Mean } & \text { Std Dev Std Err Mean Lower 95\% Upper 95\% }\end{array}$

$\begin{array}{lrrrrrr}\text { Coliwasa } & 16 & 0.103365 & 0.002893 & 0.00072 & 0.10182 & 0.10491 \\ \text { Isolok - 12 mL - HF } & 10 & 0.102920 & 0.007992 & 0.00253 & 0.09720 & 0.10864\end{array}$

t Test

Isolok - $12 \mathrm{~mL}$ - HF-Coliwasa

Assuming unequal variances

Difference $\quad-0.00045$ t Ratio $\quad-0.16929$

Std Err Dif $\quad 0.00263$ DF 10.49182

Upper CL Dif 0.00538 Prob $>|t| 0.8688$

Lower CL Dif -0.00627 Prob $>t \quad 0.5656$

$\begin{array}{lll}\text { Confidence } & 0.95 \text { Prob }<\mathrm{t} \quad 0.4344\end{array}$

Tests that the Variances are Equal

$\begin{array}{lrrrr}\text { Level } & \text { Count } & \text { Std Dev } & \text { MeanAbsDif to Mean } & \text { MeanAbsDif to Median } \\ \text { Coliwasa } & 16 & 0.0028926 & 0.0020111 & 0.0018205\end{array}$

$\begin{array}{lrrrr}\text { Coliwasa } & 16 & 0.0028926 & 0.0020111 & 0.0018205 \\ \text { Isolok - 12 mL - HF } & 10 & 0.0079921 & 0.0051485 & 0.0050290\end{array}$

Test F Ratio DFNum DFDen p-Value

$\begin{array}{lrrrr}\text { O'Brien[.5] } & 2.0957 & 1 & 24 & 0.1606\end{array}$

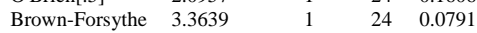

$\begin{array}{lll}1 & 24 & 0.0791 \\ 1 & 24 & 0.0592\end{array}$

$\begin{array}{lrrrr}\text { Levene } & 3.9228 & 1 & 24 & 0.0592\end{array}$

$\begin{array}{lrrrr}\text { B Test 2-sided } & 11.1812 & 1 & & 0.0008 \\ & & 9 & 15 & 0.0007\end{array}$

Welch Anova testing Means Equal, allowing Std Devs Not Equal

F Ratio DFNum DFDen Prob $>$ F

$\begin{array}{llll}0.0287 & 1 & 10.492 & 0.8688\end{array}$

t Test 


\section{Exhibit C2. Statistical Comparisons for Low-Rheology (Phase 3) Simulant Testing}

Oneway Analysis of Measurement By Sampler Type of Material=SME Simulant, Analytical Block $=4$, Oxide/Analyte $=\mathrm{ZrO} 2$ ( $w$ t $\%$ ), Targeted $=\mathbf{0 . 3 5 4 7}$

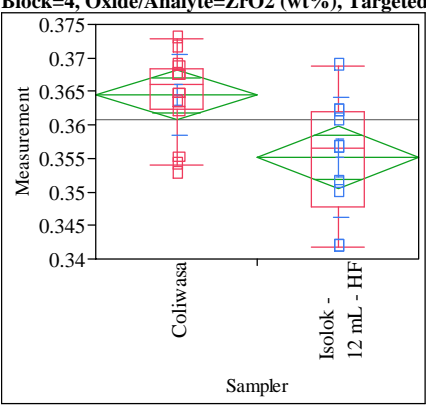

Oneway Anova

Rsquare $\quad 0.299625$

Adj Rsquare $\quad 0.270442$

Root Mean Square Error $\quad 0.007229$

Mean of Response $\quad 0.360871$

Observations (or Sum Wgts) 26

Test

Isolok - $12 \mathrm{~mL}$ - HF-Coliwasa

Assuming equal variances

Difference $\quad-0.00934$ t Ratio -3.20427

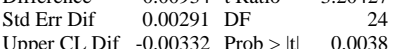

$\begin{array}{rrrr}\text { Upper CL Dif } & -0.00332 & \text { Prob }>|t| & 0.0038 \\ \text { Lower CL Dif } & -0.01535 & \text { Prob }>t & 0.9981\end{array}$

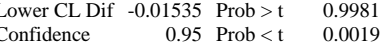

Analysis of Variance

Source DF Sum of Squares Mean Square F Ratio Prob $>$ F

$\begin{array}{lrrrrr}\text { Sampler } & 1 & 0.00053654 & 0.000537 & 10.2673 & 0.0038\end{array}$

Error $24 \quad 0.00125416$

C. Total $25 \quad 0.00179069$

Means for Oneway Anova

Level Number Mean Std Error Lower 95\% Upper 95\%

$\begin{array}{lllrrr}\text { Coliwasa } & 16 & 0.364463 & 0.00181 & 0.36073 & 0.36819\end{array}$

$\begin{array}{llllll}\text { Isolok - } 12 \mathrm{~mL} \text { - HF } & 10 & 0.355125 & 0.00229 & 0.35041 & 0.35984\end{array}$

Std Error uses a pooled estimate of error variance

Means and Std Deviations

$\begin{array}{lrrrrrr}\text { Level } & \text { Number } & \text { Mean } & \text { Std Dev } & \text { Std Err Mean } & \text { Lower 95\% } & \text { Upper 95\% } \\ \text { Coliwasa } & 16 & 0.364463 & 0.005985 & 0.00150 & 0.36127 & 0.36765\end{array}$

$\begin{array}{lllllll}\text { Isolok - } 12 \mathrm{~mL} \text { - HF } & 10 & 0.355125 & 0.008925 & 0.00282 & 0.34874 & 0.3615\end{array}$

t Test

Isolok - $12 \mathrm{~mL}$ - HF-Coliwasa

Assuming unequal variances

Difference $\quad-0.00934$ t Ratio -2.92305

Std Err Dif $\quad 0.00319$ DF 14.10102

Upper CL Dif -0.00249 Prob $>|t| \quad 0.0111$

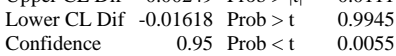

Tests that the Variances are Equal

$\begin{array}{lrrrr}\text { Level } & \text { Count } & \text { Std Dev } & \text { MeanAbsDif to Mean } & \text { MeanAbsDif to Median } \\ \text { Coliwasa } & 16 & 0.0059847 & 0.0045378 & 0.0044745 \\ \text { Isolok - 12 mL - HF } & 10 & 0.0089251 & 0.0071863 & 0.0068891\end{array}$

Test F Ratio DFNum DFDen p-Value

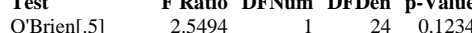

$\begin{array}{lllll}\text { Brown-Forsythe } & 1.6481 & 1 & 24 & 0.1234 \\ \text { Levene } & 2.5374 & 1 & 24 & 0.1243\end{array}$

$\begin{array}{lllll}\text { Levene } & 2.5374 & 1 & 24 & 0.1243 \\ \text { Bartlett } & 1.7910 & 1 & . & 0.1808\end{array}$

$\begin{array}{llll}\text { F Test 2-sided } \quad 2.2240 & 9 & 15 & 0.1647\end{array}$

Welch Anova testing Means Equal, allowing Std Devs Not Equal

F Ratio DFNum DFDen Prob $>$ F

$\begin{array}{llll}8.5442 & 1 & 14.101 & 0.0111\end{array}$

t Test
Oneway Analysis of Measurement By Sampler Type of Material=SME Simulant, Analytical Block $=5$, Oxide $/$ Analyte $=\mathrm{Al} / \mathrm{B}$, Targeted $=\mathbf{2 . 5 5 2}$

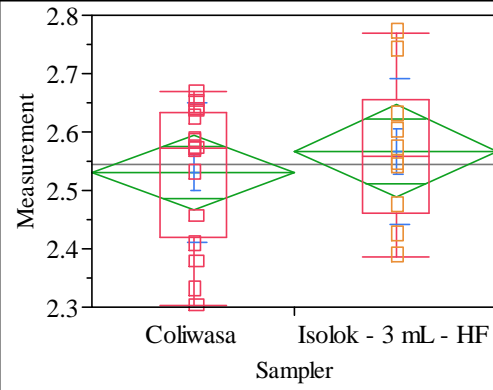

Oneway Anova

Summary of Fit

Rsquare

Adj Rsquare

$-0.0183$

0.121243

Observations (or Sum Wgts) $\quad 2.544463$

t Test

Isolok - $3 \mathrm{~mL}$ - HF-Coliwasa

Assuming equal variances

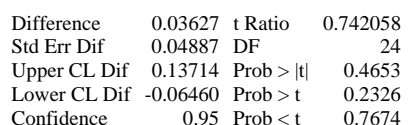

$\begin{array}{lrr}\text { Confidence } & -0.95 \text { Prob }<\mathrm{t} & 0.7674\end{array}$

Analysis of Variance

Source DF Sum of Squares Mean Square F Ratio Prob $>$ F

$\begin{array}{lrrrrr}\text { Sampler } & 1 & 0.00809454 & 0.008095 & 0.5506 & 0.4653\end{array}$

$\begin{array}{lrrr}\text { Error } & 24 & 0.35279941 & 0.014700\end{array}$

C. Total $25 \quad 0.36089394$

Means for Oneway Anova

Level Number Mean Std Error Lower 95\% Upper 95\%

$\begin{array}{llllrr}\text { Coliwasa } & 16 & 2.53051 & 0.03031 & 2.4680 & 2.5931\end{array}$

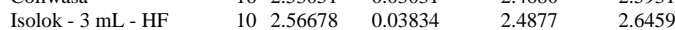

Std Error uses a pooled estimate of error variance

Means and Std Deviation

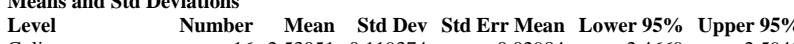

$\begin{array}{lrrrrrr}\text { Coliwasa } & 16 & 2.53051 & 0.119374 & 0.02984 & 2.4669 & 2.5941 \\ \text { Isolok - 3 mL - HF } & 10 & 2.56678 & 0.124297 & 0.03931 & 2.4779 & 2.6557\end{array}$

t Test

Isolok - $3 \mathrm{~mL}$ - HF-Coliwasa

Assuming unequal variances

Difference $\quad 0.03627$ t Ratio 0.734884

Std Err Dif $\quad 0.04935$ DF 18.64884

Upper CL Dif 0.13969 Prob $>|t| \quad 0.4716$

Lower CL Dif -0.06716 Prob $>t \quad 0.2358$

$\begin{array}{lll}\text { Confidence } & 0.95 \text { Prob }<\mathrm{t} \quad 0.7642\end{array}$

Tests that the Variances are Equal

$\begin{array}{lrrrr}\text { Level } & \text { Count } & \text { Std Dev } & \text { MeanAbsDif to Mean } & \text { MeanAbsDif to Median } \\ \text { Coliwasa } & 16 & 0.1193736 & 0.0982936 & 0.0887329\end{array}$

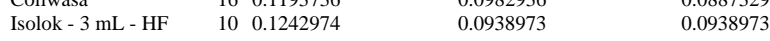

Test F Ratio DFNum DFDen p-Value

$\begin{array}{lllll}\text { O'Brien[.5] } & 0.0294 & 1 & 24 & 0.8654\end{array}$

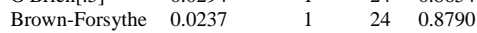

$0.0259-1$ - 240.8734

$\begin{array}{lllll}\text { Levene } & 0.0259 & 1 & 24 & 0.8734\end{array}$

$\begin{array}{lllll}\text { Bartlett } & 0.0177 & 1 & \text {. } & 0.8942 \\ \text { F Test 2-sided } & 1.0842 & 9 & 15 & 0.8545\end{array}$

Welch Anova testing Means Equal, allowing Std Devs Not Equal

F Ratio DFNum DFDen Prob $>$ F

$\begin{array}{llll}0.5401 & 1 & 18.649 & 0.4716\end{array}$

t Test 


\section{Exhibit C2. Statistical Comparisons for Low-Rheology (Phase 3) Simulant Testing}

Oneway Analysis of Measurement By Sampler Type of Material=SME Simulant, Analytical Block=5, Oxide/Analyte=Al2O3 (wt \%), Targeted $=10.869$

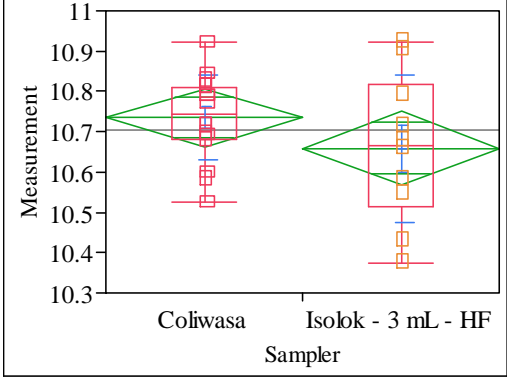

\section{Oneway Anova}

Summary of Fit

Adj Rsquare

0.031864

0.139738

10.70547

t Test

Isolok - $3 \mathrm{~mL}$ - HF-Coliwasa

Assuming equal variances

Difference $\quad-0.07605$ t Ratio $\quad-1.35011$

$\begin{array}{lrr}\text { Std Err Dif } & 0.05633 \text { DF } & 24 \\ & \text { DF }\end{array}$

Upper CL Dif 0.04021 Prob $>|t| \quad 0.1896$

Lower CL Dif -0.19231 Prob $>t \quad 0.9052$

$\begin{array}{lrl}\text { Confidence } & 0.95 \text { Prob }<\mathrm{t} \quad 0.0948\end{array}$

Analysis of Variance

Source DF Sum of Squares Mean Square F Ratio Prob $>$ F

$\begin{array}{lrllll}\text { Sampler } & 1 & 0.03559362 & 0.035594 & 1.8228 & 0.1896\end{array}$

$\begin{array}{llll}\text { Error } & 24 & 0.46864365 & 0.019527\end{array}$

C. Total $25 \quad 0.50423727$

Means for Oneway Anova

Level Number Mean Std Error Lower 95\% Upper 95\%

$\begin{array}{lrrrrr}\text { Coliwasa } & 16 & 10.7347 & 0.03493 & 10.663 & 10.807\end{array}$

$\begin{array}{llllll}\text { Isolok - 3 mL - HF } & 10 & 10.6587 & 0.04419 & 10.567 & 10.807 \\ & & & \end{array}$

Std Error uses a pooled estimate of error variance

Means and Std Deviations

Level Number Mean Std Dev Std Err Mean Lower 95\% Upper 95\%

$\begin{array}{lllllll}\text { Coliwasa } & 16 & 10.7347 & 0.105514 & 0.02638 & 10.678 & 10.791\end{array}$

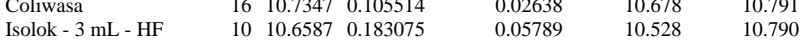

t Test

Isolok - $3 \mathrm{~mL}$ - HF-Coliwasa

Assuming unequal variances

Difference $\quad-0.07605$ t Ratio $\quad-1.19542$

Std Err Dif $\quad 0.06362$ DF $\quad 12.79396$

Upper CL Dif 0.06161 Prob $>|t| \quad 0.2536$

Lower CL Dif -0.21372 Prob $>t \quad 0.8732$

$\begin{array}{lrr}\text { Confidence } & 0.95 \text { Prob }<\mathrm{t} & 0.1268\end{array}$

Tests that the Variances are Equa

Level Count Std Dev MeanAbsDif to Mean MeanAbsDif to Median

$\begin{array}{lllll} & 16 & 0.1055135 & 0.0850275 & 0.0850275\end{array}$

Test F Ratio DFNum DFDen p-Value

$\begin{array}{lrrrr}\text { O'Brien[.5] } & 4.6753 & 1 & 24 & 0.0408\end{array}$

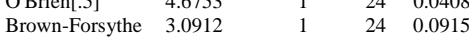

$\begin{array}{lllll}\text { Levene } & 3.1192 & 1 & 24 & 0.0901\end{array}$

$\begin{array}{lllll}\text { Bartlett } & 3.4110 & 1 & \dot{0} & 0.0648 \\ \text { F Test 2-sided } & 3.0105 & 9 & 15 & 0.0576\end{array}$

Welch Anova testing Means Equal, allowing Std Devs Not Equal

F Ratio DFNum DFDen Prob $>$ F

$\begin{array}{lrrr}1.4290 & 1 & 12.794 & 0.2536\end{array}$

t Test
1.1954
Oneway Analysis of Measurement By Sampler Type of Material=SME Simulant, Analytical Block=5, Oxide/Analyte=B2O3 (wt $\%$ ), Targeted $=4.259$

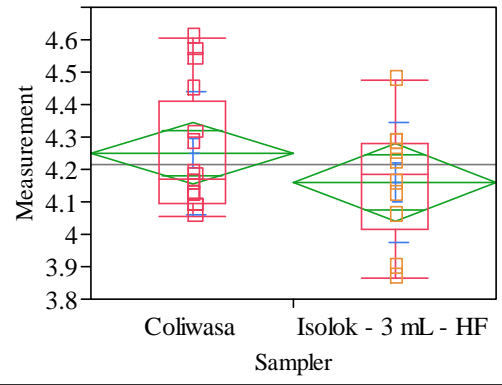

Oneway Anova

Summary of Fit

Rsquare

Adj Rsquare

Root Mean Square Error 0.01678

Mean of Response 0.187244

Observations (or Sum Wgts) $\quad 26$

t Test

Isolok - $3 \mathrm{~mL}$ - HF-Coliwasa

Assuming equal variances

Difference $\quad-0.09016$ t Ratio $\quad-1.19444$

$\begin{array}{lrlr}\text { Std Err Dif } & 0.07548 \text { DF } & 24 \\ \text { Upper CL } & 0.06563 \text { Prob }>|t| & 0.2440\end{array}$

$\begin{array}{rrrr}\text { Upper CL Dif } & 0.06563 & \text { Prob }>|t| & 0.2440 \\ \text { Lower CL Dif } & -0.24594 & \text { Prob }>t & 0.8780\end{array}$

$\begin{array}{lrrr}\text { Lower CL Dif } & -0.24594 & \text { Prob }>t & 0.8780 \\ \text { Confidence } & 0.95 & \text { Prob }<t & 0.1220\end{array}$

Analysis of Variance

Source DF Sum of Squares Mean Square F Ratio Prob > F

$\begin{array}{lrrrrr}\text { Sampler } & 1 & 0.05002044 & 0.050020 & 1.4267 & 0.2440\end{array}$

$\begin{array}{lll}\text { Error } & 24 & 0.84144708\end{array}$

C. Total $25 \quad 0.89146751$

Means for Oneway Anova

Level Number Mean Std Error Lower 95\% Upper 95\%

$\begin{array}{llllrr}\text { Coliwasa } & 16 & 4.25027 & 0.04681 & 4.1537 & 4.3469\end{array}$

$\begin{array}{llllll}\text { Isolok - } 3 \text { mL - HF } & 10 & 4.16011 & 0.05921 & 4.0379 & 4.2823\end{array}$

Std Error uses a pooled estimate of error variance

Means and Std Deviations

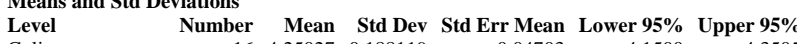

$\begin{array}{lrrrrrr}\text { Coliwasa } & 16 & 4.25027 & 0.188119 & 0.04703 & 4.1500 & 4.3505 \\ \text { Isolok - 3 mL - HF } & 10 & 4.16011 & 0.185777 & 0.05875 & 4.0272 & 4.2930\end{array}$

t Test

Isolok - $3 \mathrm{~mL}$ - HF-Coliwasa

Assuming unequal variances

Difference $\quad-0.09016$ t Ratio -1.19805

Std Err Dif $\quad 0.07525$ DF 19.44104

$\begin{array}{lll}\text { Upper CL Dif } & 0.06711 \quad \text { Prob }>|t| & 0.2453\end{array}$

$\begin{array}{lll}\text { Lower CL Dif } & -0.24742 \text { Prob }>t & 0.8773\end{array}$

$\begin{array}{lll}\text { Confidence } & 0.95 \text { Prob }<\mathrm{t} \quad 0.1227\end{array}$

Tests that the Variances are Equal

$\begin{array}{lrrrr}\text { Level } & \text { Count } & \text { Std Dev } & \text { MeanAbsDif to Mean } & \text { MeanAbsDif to Median } \\ \text { Coliwasa } & 16 & 0.1881186 & 0.1569701 & 0.1408706 \\ \text { Isolok - 3 mL - HF } & 10 & 0.1857770 & 0.1416756 & 0.1416756\end{array}$

Test F Ratio DFNum DFDen p-Value

$\begin{array}{lllll}\text { O'Brien[.5] } & 0.0025 & 1 & 24 & 0.9604\end{array}$

$\begin{array}{lllll}\text { Brown-Forsythe } & 0.0002 & 1 & 24 & 0.9883\end{array}$

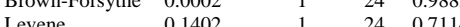

$\begin{array}{lllll}\text { Levene } & 0.1402 & 1 & 24 & 0.7114\end{array}$

$\begin{array}{lrrrr}\text { Bartlett } & 0.0017 & 1 & \dot{0} & 0.9673 \\ \text { F Test 2-sided } & 1.0254 & 15 & 9 & 1.0000\end{array}$

Welch Anova testing Means Equal, allowing Std Devs Not Equal

F Ratio DFNum DFDen Prob $>$ F

$\begin{array}{llll}1.4353 & 1 & 19.441 & 0.2453\end{array}$

t Test 


\section{Exhibit C2. Statistical Comparisons for Low-Rheology (Phase 3) Simulant Testing}

Oneway Analysis of Measurement By Sampler Type of Material=SME Simulant, Analytical Block=5, Oxide/Analyte=BaO (wt \%), Targeted $=0.0919$

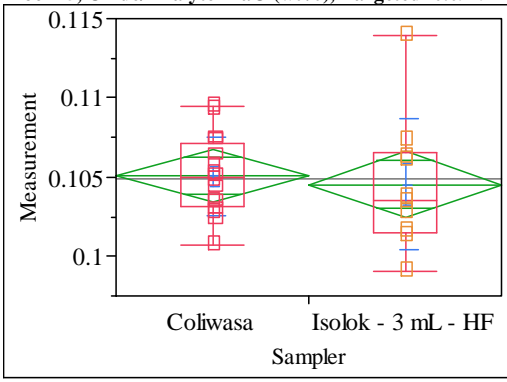

Oneway Anova

Rsquare $\quad 0.007096$

Adj Rsquare $\quad-0.03428$

Root Mean Square Error $\quad 0.0032$

Mean of Response $\quad 0.104857$

t Test

Isolok - $3 \mathrm{~mL}$ - HF-Coliwasa

Assuming equal variances

Difference $\quad-0.00054$ t Ratio -0.41415

$\begin{array}{lrrr}\text { Std Err Dif } & 0.00129 \text { DF } & 24 \\ \text { Upper CL Dif } & 0.00213 & \text { Prob }>|t| & 0.6824\end{array}$

$\begin{array}{lrlr}\text { Upper CL Dif } & 0.00213 & \text { Prob }>|t| & 0.6824 \\ \text { Lower CL Dif } & -0.00321 & \text { Prob }>t & 0.6588\end{array}$

$\begin{array}{lrl} & \\ \text { Confidence } & 0.95 \text { Prob }<\mathrm{t} & 0.3412\end{array}$

Analysis of Variance

Source DF Sum of Squares Mean Square F Ratio Prob > F

$\begin{array}{llllll}\text { Sampler } & 1 & 0.00000177 & 1.767 \mathrm{e}-6 & 0.1715 & 0.6824\end{array}$

Error $24 \quad 0.0002473$

0.000010

Means for Oneway Anova

Level Number Mean Std Error Lower 95\% Upper 95\%

$\begin{array}{lllllr}\text { Coliwasa } & 16 & 0.105063 & 0.00080 & 0.10341 & 0.10672\end{array}$

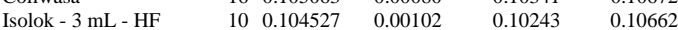

Std Error uses a pooled estimate of error variance

Means and Std Deviations

$\begin{array}{lrrrrrr}\text { Level } & \text { Number } & \text { Mean } & \text { Std Dev } & \text { Std Err Mean } & \text { Lower 95\% } & \text { Upper 95\% } \\ \text { Coliwasa } & 16 & 0.105063 & 0.002477 & 0.00062 & 0.10374 & 0.10638\end{array}$

$\begin{array}{lllllll}\text { Isolok - } 3 \mathrm{~mL}-\mathrm{HF} & 10 & 0.104527 & 0.004154 & 0.00131 & 0.10156 & 0.10750\end{array}$

t Test

Isolok - $3 \mathrm{~mL}$ - HF-Coliwasa

Assuming unequal variances

Difference $\quad-0.00054$ t Ratio -0.36905

Std Err Dif $\quad 0.00145$ DF 13.05913

Upper CL Dif 0.00260 Prob $>|t| \quad 0.7180$

Lower CL Dif -0.00367 Prob $>t \quad 0.6410$

$\begin{array}{lrl}\text { Confidence } & 0.95 \text { Prob }<\mathrm{t} & 0.3590\end{array}$

Tests that the Variances are Equal

$\begin{array}{lrrrr}\text { Level } & \text { Count } & \text { Std Dev } & \text { MeanAbsDif to Mean } & \text { MeanAbsDif to Median } \\ \text { Coliwasa } & 16 & 0.0024772 & 0.0019399 & 0.0019399 \\ \text { Isolok - 3 mL - HF } & 10 & 0.0041536 & 0.0030860 & 0.0029252\end{array}$

Test F Ratio DFNum DFDen p-Value

O'Brien[.5] 1.8585

$\begin{array}{lllll}\text { O'Brien[.5] } & 1.8585 & 1 & 24 & 0.1855 \\ & & 1 & 24 & 0.2670\end{array}$

Brown-Forsythe $1.2916 \quad 11 \quad 24 \quad 0.2670$

$\begin{array}{lllll}\text { Levene } & 2.1117 & 1 & 24 & 0.1591 \\ \text { Bartlett } & 3.0014 & 1 & . & 0.0832\end{array}$

$\begin{array}{lllll}\text { F Test 2-sided } & 2.8114 & 9 & 15 & 0.0744\end{array}$

Welch Anova testing Means Equal, allowing Std Devs Not Equal

F Ratio DFNum DFDen Prob $>$ F

$\begin{array}{llll}0.1362 & 1 & 13.059 & 0.7180\end{array}$

t Test

0.3691
Oneway Analysis of Measurement By Sampler Type of Material=SME Simulant, Analytical Block $=5$, Oxide $/$ Analyte $=\mathrm{CaO}(\mathrm{wt} \%)$, Targeted $=\mathbf{1 . 1 4 6 2}$

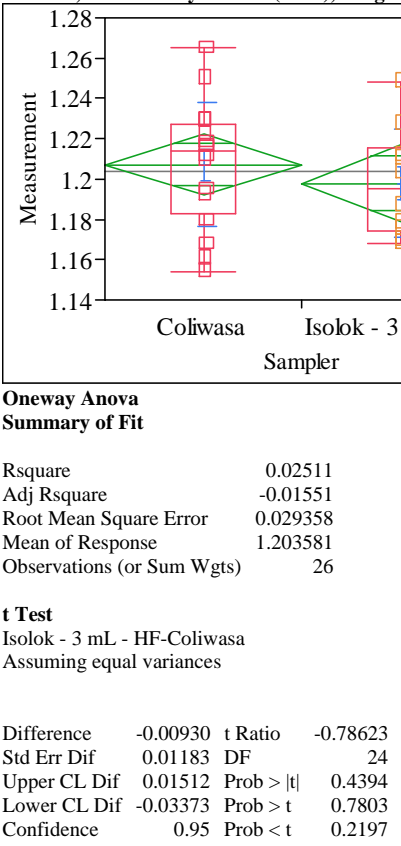

Analysis of Variance

Source DF Sum of Squares Mean Square F Ratio Prob $>$ F

$\begin{array}{llllll}\text { Sampler } & 1 & 0.00053278 & 0.000533 & 0.6182 & 0.4394\end{array}$

$\begin{array}{llll}\text { Error } & 24 & 0.02068550 & 0.000862\end{array}$

C. Total $25 \quad 0.02121829$

Means for Oneway Anova

Level Number Mean Std Error Lower 95\% Upper 95\%

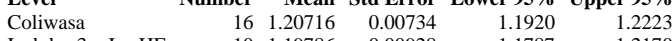

$\begin{array}{llllll}\text { Coliwasa } & 16 & 1.20716 & 0.00734 & 1.1920 & 1.2223 \\ \text { Isolok - 3 mL - HF } & 10 & 1.19786 & 0.00928 & 1.1787 & 1.2170\end{array}$

Std Error uses a pooled estimate of error variance

Means and Std Deviations

Level Number Mean Std Dev Std Err Mean Lower 95\% Upper 95\%

$\begin{array}{lllllll}\text { Coliwasa } & 16 & 1.20716 & 0.030814 & 0.00770 & 1.1907 & 1.2236\end{array}$

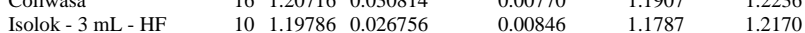

t Test

Isolok - $3 \mathrm{~mL}$ - HF-Coliwasa

Assuming unequal variances

$\begin{array}{lrlr}\text { Difference } & -0.00930 & \text { t Ratio } & -0.81317 \\ \text { Std Err Dif } & 0.01144 & \text { DF } & 21.31708 \\ \text { Upper CL Dif } & 0.01447 & \text { Prob }>|t| & 0.4251 \\ \text { Lower CL Dif } & -0.03308 & \text { Prob }>t & 0.7874 \\ \text { Confidence } & 0.95 & \text { Prob }<\mathrm{t} & 0.2126\end{array}$

Tests that the Variances are Equal

Level Count Std Dev MeanAbsDif to Mean MeanAbsDif to Median

$\begin{array}{lllll}\text { Coliwasa } & 16 & 0.0308142 & 0.0242237 & 0.0234366\end{array}$

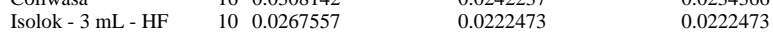

Test F Ratio DFNum DFDen p-Value

$\begin{array}{lrrrr}\text { O'Brien[.5] } & 0.2834 & 1 & 24 & 0.5994\end{array}$

Brown-Forsythe $\quad 0.0270 \quad 1 \quad 24 \quad 0.8709$

$\begin{array}{lllll}\text { Levene } & 0.0909 & 1 & 24 & 0.7657\end{array}$

$\begin{array}{lrrrr}\text { Bartlett } & 0.2091 & 1 & \text {. } & 0.6475 \\ \text { F Test 2-sided } & 1.3264 & 15 & 9 & 0.6837\end{array}$

Welch Anova testing Means Equal, allowing Std Devs Not Equal

F Ratio DFNum DFDen Prob $>$ F

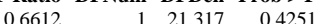

t Test

0.8132 
SRNL-STI-2011-00693

Revision 0

\section{Exhibit C2. Statistical Comparisons for Low-Rheology (Phase 3) Simulant Testing}

Oneway Analysis of Measurement By Sampler Type of Material=SME Simulant, Analytical Block $=5$, Oxide $/$ Analyte $=\mathrm{Cr} 2 \mathrm{O} 3(\mathrm{wt} \%)$, Targeted $=0.0998$

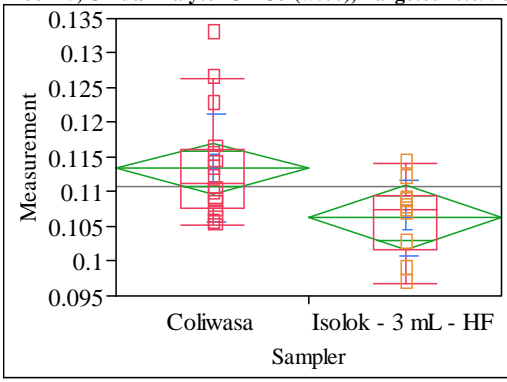

Oneway Anova

Rsquare $\quad 0.20697$

Adj Rsquare $\quad 0.17392$

Root Mean Square Error $\quad 0.007046$

Mean of Response $\quad 0.110677$

(onservations (or Sum Wges)

Test

Isolok - $3 \mathrm{~mL}$ - HF-Coliwas

Assuming equal variances

Difference $\quad-0.00711$ t Ratio $\quad-2.50273$

$\begin{array}{lrlr}\text { Std Err Dif } & 0.00284 & \text { DF } & 24 \\ \text { Upper CL Dif } & -0.00125 & \text { Prob }>|t| & 0.0195\end{array}$

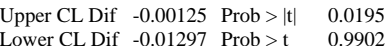

$\begin{array}{lrl}\text { Confidence } & 0.95 \text { Prob }<\mathrm{t} \quad 0.0098\end{array}$

Analysis of Variance

Source DF Sum of Squares Mean Square F Ratio Prob $>$ F

$\begin{array}{lrrrrr}\text { Sampler } & 1 & 0.00031099 & 0.000311 & 6.2637 & 0.0195\end{array}$

$\begin{array}{lll}\text { Error } & 24 & 0.00119159 \\ \text { C. Total } & 25 & 0.00150258\end{array}$

Means for Oneway Anova

Level Number Mean Std Error Lower 95\% Upper 95\%

$\begin{array}{lrrrrr}\text { Coliwasa } & 16 & 0.113411 & 0.00176 & 0.10978 & 0.11705\end{array}$

$\begin{array}{llllll}\text { Coliwasa } & 16 & 0.113411 & 0.00176 & 0.10978 & 0.11705 \\ \text { Isolok - 3 mL - HF } & 10 & 0.106302 & 0.00223 & 0.10170 & 0.11090\end{array}$

Std Error uses a pooled estimate of error variance

Means and Std Deviations

$\begin{array}{lrrrrrr}\text { Level } & \text { Number } & \text { Mean } & \text { Std Dev } & \text { Std Err Mean } & \text { Lower 95\% } & \text { Upper 95\% } \\ \text { Coliwasa } & 16 & 0.113411 & 0.007854 & 0.00196 & 0.10923 & 0.11760\end{array}$

$\begin{array}{lllllll}\text { Isolok - } 3 \mathrm{~mL} \text { - HF } & 10 & 0.106302 & 0.005441 & 0.00172 & 0.10241 & 0.11019\end{array}$

Test

Isolok - $3 \mathrm{~mL}$ - HF-Coliwasa

Assuming unequal variances

Difference $\quad-0.00711$ t Ratio -2.72313

Std Err Dif $\quad 0.00261$ DF 23.64485

Upper CL Dif -0.00172 Prob $>|t| \quad 0.0120$

Lower CL Dif -0.01250 Prob $>t \quad 0.9940$

$\begin{array}{lrl}\text { Confidence } & 0.95 \text { Prob }<\mathrm{t} & 0.0060\end{array}$

Tests that the Variances are Equal

$\begin{array}{lrrrr}\text { Level } & \text { Count } & \text { Std Dev } & \text { MeanAbsDif to Mean } & \text { MeanAbsDif to Median } \\ \text { Coliwasa } & 16 & 0.0078537 & 0.0058898 & 0.0056728 \\ \text { Isolok - 3 mL - HF } & 10 & 0.0054405 & 0.0041480 & 0.0039025\end{array}$

Test F Ratio DFNum DFDen p-Value

O'Brien[.5] 0.8621

$\begin{array}{lllll}\text { O'Brien[.5] } & 0.8621 & 1 & 24 & 0.3624 \\ \text { Brown-Forsythe } & 0.7547 & 1 & 24 & 0.3936\end{array}$

$\begin{array}{lllll}\text { Brown-Forsythe } & 0.7547 & 1 & 24 & 0.3936\end{array}$

$\begin{array}{lllll}\text { Levene } & 0.9646 & 1 & 24 & 0.3358 \\ \text { Bartlett } & 1.3399 & 1 & . & 0.2471\end{array}$

$\begin{array}{lrrrr}\text { F Test 2-sided } & 2.0838 & 15 & 9 & 0.2675\end{array}$

Welch Anova testing Means Equal, allowing Std Devs Not Equal

F Ratio DFNum DFDen Prob $>$ F

$\begin{array}{llll}7.4154 & 1 & 23.645 & 0.0120\end{array}$

t Test
Oneway Analysis of Measurement By Sampler Type of Material=SME Simulant, Analytica Block $=5$, Oxide $/$ Analyte $=\mathbf{C u O}(w t \%)$, Targeted $=\mathbf{0 . 0 5 0 4}$

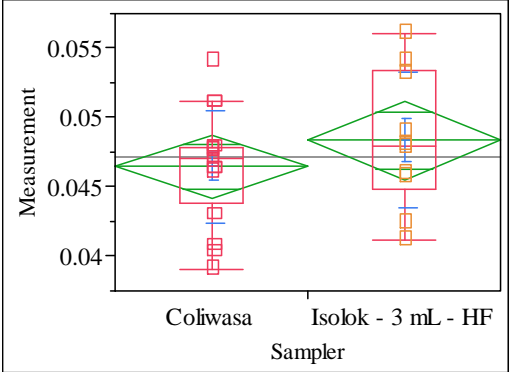

Oneway Anova

Summary of Fit

Rsquare

Adj Rsquare

0.004898

Square Error $\quad 0.004381$

Mean of Response

Observations (or Sum Wgts) $\quad 26$

Isolok - $3 \mathrm{~mL}$ - HF-Coliwasa

Assuming equal variances

Difference $\quad 0.00187$ t Ratio $\quad 1.059744$

$\begin{array}{lrlr}\text { Std Err Dif } & 0.00177 & \text { DF } & 24 \\ \text { Upper CL } & 0.00552 & \text { Prob }>|t| & 0.2998\end{array}$

$\begin{array}{lrll}\text { Upper CL Dif } & 0.00552 & \text { Prob }>|t| & 0.2998 \\ \text { Lower CL Dif } & -0.00177 & \text { Prob }>t & 0.1499\end{array}$

$\begin{array}{lll}\text { Confidence } & 0.95 \text { Prob }<\mathrm{t} & 0.8501\end{array}$

Analysis of Variance

Source DF Sum of Squares Mean Square F Ratio Prob $>$ F

$\begin{array}{llllll}\text { Sampler } & 1 & 0.00002155 & 0.000022 & 1.1231 & 0.2998\end{array}$

$\begin{array}{lll}\text { Error } & 24 & 0.00046058 \\ \text { C. Total } & 25 & 0.00048214\end{array}$

Means for Oneway Anova

Level Number Mean Std Error Lower 95\% Upper 95\%

$\begin{array}{lrrrrr}\text { Coliwasa } & 16 & 0.046473 & 0.00110 & 0.04421 & 0.04873\end{array}$

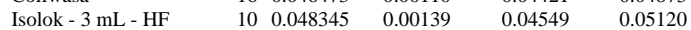

Std Error uses a pooled estimate of error variance

Means and Std Deviations

Level Number Mean Std Dev Std Err Mean Lower 95\% Upper 95\%

$\begin{array}{lllllll}\text { Coliwasa } & 16 & 0.046473 & 0.004038 & 0.00101 & 0.04432 & 0.04862\end{array}$

$\begin{array}{lllllll}\text { Isolok - } 3 \mathrm{~mL} \text { - HF } & 10 & 0.048345 & 0.004899 & 0.00155 & 0.04484 & 0.05185\end{array}$

\section{t Test}

Isolok - $3 \mathrm{~mL}$ - HF-Coliwasa

Assuming unequal variances

$\begin{array}{lrlr}\text { Difference } & 0.00187 & \text { t Ratio } & 1.012072 \\ \text { Std Err Dif } & 0.00185 & \text { DF } & 16.48161 \\ \text { Upper CL Dif } & 0.00578 & \text { Prob }>|t| & 0.3261 \\ \text { Lower CL Dif } & -0.00204 & \text { Prob }>t & 0.1631 \\ \text { Confidence } & 0.95 & \text { Prob }<t & 0.8369\end{array}$

Tests that the Variances are Equal

$\begin{array}{lrrrr}\text { Level } & \text { Count } & \text { Std Dev } & \text { MeanAbsDif to Mean } & \text { MeanAbsDif to Median } \\ \text { Coliwasa } & 16 & 0.0040378 & 0.0028752 & 0.0028322 \\ \text { Isolok - 3 mL - HF } & 10 & 0.0048992 & 0.0037854 & 0.0037304\end{array}$

Test Ratio DFNum DFDen p-Value

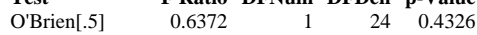

$\begin{array}{lllll}\text { Brown-Forsythe } & 0.5981 & 1 & 24 & 0.4469\end{array}$

Brown-Forsythe $0.5981-12 \quad 240.4469$

$\begin{array}{lllll}\text { Levene } & 0.6614 & 1 & 24 & 0.4241 \\ \text { Bartlett } & 0.4132 & 1 & . & 0.5204\end{array}$

$\begin{array}{lllll}\text { F Test 2-sided } & 1.4722 & 9 & 15 & 0.4880\end{array}$

Welch Anova testing Means Equal, allowing Std Devs Not Equal

F Ratio DFNum DFDen Prob > F

$\begin{array}{llll}1.0243 & 1 & 16.482 & 0.3261\end{array}$

t Test 


\section{Exhibit C2. Statistical Comparisons for Low-Rheology (Phase 3) Simulant Testing}

Oneway Analysis of Measurement By Sampler Type of Material=SME Simulant, Analytical Block $=5$, Oxide/Analyte $=\mathrm{Fe} / \mathrm{Li}$, Targeted $=2.452$

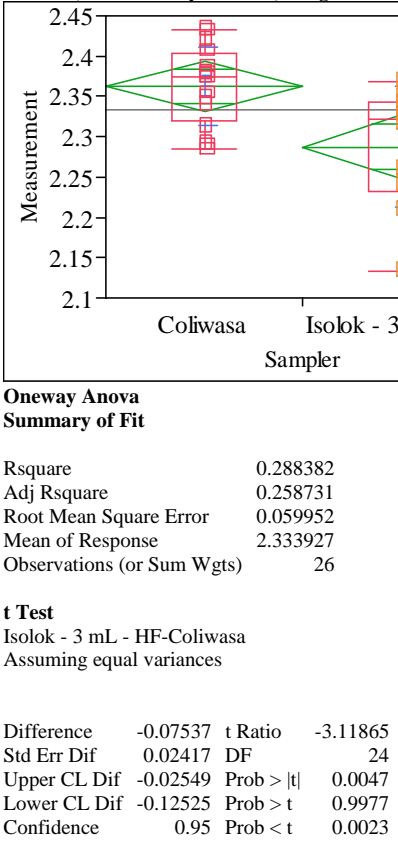

Analysis of Variance

Source DF Sum of Squares Mean Square F Ratio Prob $>$ F

$\begin{array}{llllll}\text { Sampler } & 1 & 0.03495708 & 0.034957 & 9.7260 & 0.0047\end{array}$

$\begin{array}{llll}\text { Error } & 24 & 0.08626084 & 0.003594\end{array}$

C. Total $25 \quad 0.12121793$

Means for Oneway Anova

Level Number Mean Std Error Lower 95\% Upper 95\%

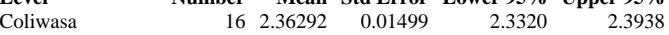

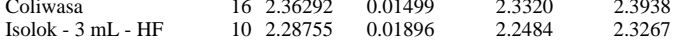

Std Error uses a pooled estimate of error variance

Means and Std Deviations

Level Number Mean Std Dev Std Err Mean Lower 95\% Upper 95\%

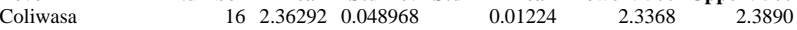

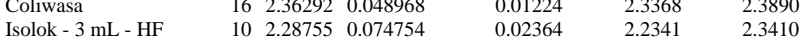

t Test

Isolok - $3 \mathrm{~mL}$ - HF-Coliwasa

Assuming unequal variances

Difference $\quad-0.07537$ t Ratio $\quad-2.83119$

Std Err Dif $\quad 0.02662$ DF $\quad 13.87578$

Upper CL Dif -0.01822 Prob $>|t| \quad 0.0134$

Lower CL Dif -0.13251 Prob $>t \quad 0.9933$

$\begin{array}{lrl}\text { Confidence } & 0.95 \text { Prob }<\mathrm{t} & 0.0067\end{array}$

Tests that the Variances are Equal

Level Count Std Dev MeanAbsDif to Mean MeanAbsDif to Median

$\begin{array}{lllll} & 16 & 0.0489676 & 0.0400536 & 0.0385259\end{array}$

Test F Ratio DFNum DFDen p-Value

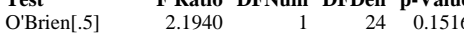

$\begin{array}{lllll}\text { Brown-Forsythe } & 0.9895 & 1 & 24 & 0.3298\end{array}$

$\begin{array}{lllll}\text { Levene } & 2.9701 & 1 & 24 & 0.0977\end{array}$

$\begin{array}{lllll}\text { Bartlett } & 2.0083 & 1 & & 0.1564 \\ \text { F Test 2-sided } & 2.3305 & 9 & 15 & 0.1420\end{array}$

Welch Anova testing Means Equal, allowing Std Devs Not Equal

F Ratio DFNum DFDen Prob $>$ F

$\begin{array}{rrrr}8.0156 & 1 & 13.876 & 0.0134\end{array}$

t Test

2.8312
Oneway Analysis of Measurement By Sampler Type of Material=SME Simulant, Analytical Block $=5$, Oxide $/$ Analyte $=\mathrm{Fe} 2 \mathrm{O} 3$ ( $w$ t $\%)$, Targeted $=11.462$

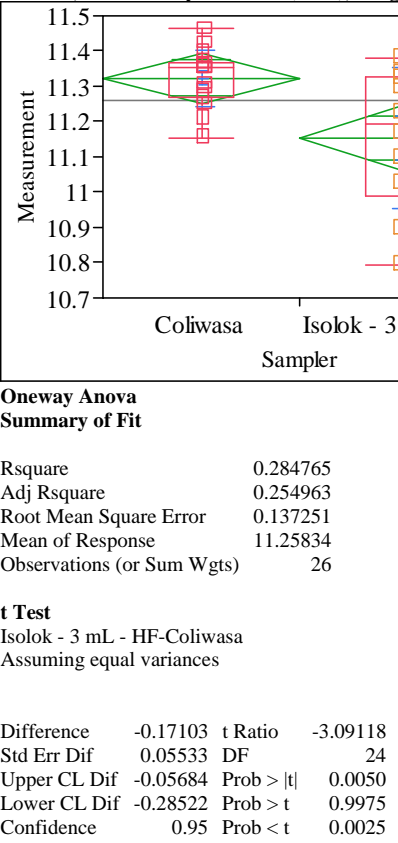

Analysis of Variance

Source DF Sum of Squares Mean Square F Ratio Prob $>$ F

$\begin{array}{llllll}\text { Sampler } & 1 & 0.18000326 & 0.180003 & 9.5554 & 0.0050\end{array}$

$\begin{array}{llll}\text { Error } & 24 & 0.45210889 & 0.018838\end{array}$

C. Total $25 \quad 0.63211215$

Means for Oneway Anova

Level Number Mean Std Error Lower 95\% Upper 95\%

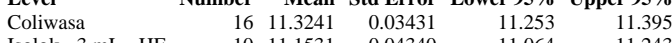

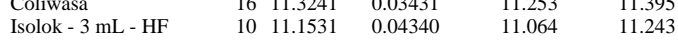

Std Error uses a pooled estimate of error variance

Means and Std Deviations

Level Number Mean Std Dev Std Err Mean Lower 95\% Upper 95\%

$\begin{array}{lllllll}\text { Coliwasa } & 16 & 11.3241 & 0.080109 & 0.02003 & 11.281 & 11.367\end{array}$

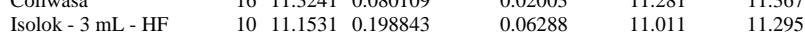

Test

Isolok - $3 \mathrm{~mL}$ - HF-Coliwasa

Assuming unequal variances

Difference $\quad-0.17103$ t Ratio $\quad-2.59164$

Std Err Dif $\quad 0.06599$ DF $\quad 10.85158$

Upper CL Dif -0.02554 Prob $>|t| 0.0253$

Lower CL Dif -0.31652 Prob $>t \quad 0.9873$

$\begin{array}{lrl}\text { Confidence } & 0.95 \text { Prob }<\mathrm{t} & 0.0127\end{array}$

Tests that the Variances are Equal

Level Count Std Dev MeanAbsDif to Mean MeanAbsDif to Median

$\begin{array}{lllll}\text { Coliwasa } & 16 & 0.0801089 & 0.0633312 & 0.0598687\end{array}$

Test F Ratio DFNum DFDen p-Value

$\begin{array}{lrrrr}\text { O'Brien[.5] } & 7.8949 & 1 & 24 & 0.0097\end{array}$

$\begin{array}{lllll}\text { Brown-Forsythe } & 8.3681 & 1 & 24 & 0.0080\end{array}$

$\begin{array}{lrrrr}\text { Levene } & 11.0803 & 1 & 24 & 0.0028\end{array}$

$\begin{array}{lllrl}\text { Bartlett } & 9.0686 & 1 & \text {. } & 0.0026 \\ \text { F Test 2-sided } & 6.1611 & 9 & 15 & 0.0022\end{array}$

Welch Anova testing Means Equal, allowing Std Devs Not Equal

F Ratio DFNum DFDen Prob $>$ F

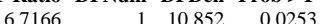

t Test

2.5916 


\section{Exhibit C2. Statistical Comparisons for Low-Rheology (Phase 3) Simulant Testing}

Oneway Analysis of Measurement By Sampler Type of Material=SME Simulant, Analytical Block $=5$, Oxide/Analyte $=$ K2O (wt $\%$ ), Targeted $=\mathbf{0 . 1 5 9 1}$

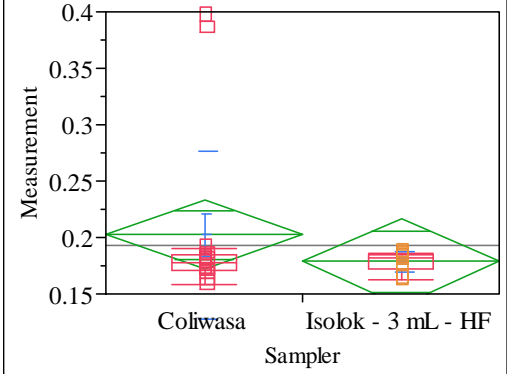

Oneway Anova

Summary of Fit

Adj Rsquare

Root Mean Square Error $\quad-0.00018$

Mean of Respons

t Test

Isolok - $3 \mathrm{~mL}$ - HF-Coliwasa

Assuming equal variances

Difference $\quad-0.02374$ t Ratio $\quad-0.99771$

Std Err Dif $\quad 0.02379$ DF 24

Upper CL Dif 0.02537 Prob $>|t| \quad 0.3284$

Lower CL Dif -0.07284 Prob $>t \quad 0.8358$

$\begin{array}{lrr}\text { Confidence } & 0.95 \mathrm{Prob}<\mathrm{t} & 0.1642\end{array}$

Analysis of Variance

Source DF Sum of Squares Mean Square F Ratio Prob $>$ F

$\begin{array}{llllll}\text { Sampler } & 1 & 0.00346769 & 0.003468 & 0.9954 & 0.3284\end{array}$

$\begin{array}{llll}\text { Error } & 24 & 0.08360682 & 0.003484\end{array}$

C. Total $25 \quad 0.08707451$

Means for Oneway Anova

Level Number Mean Std Error Lower 95\% Upper 95\%

$\begin{array}{lrrrrr}\text { Coliwasa } & 16 & 0.202260 & 0.01476 & 0.17181 & 0.23271\end{array}$

$\begin{array}{llllll}\text { Coliwasa } & 16 & 0.202260 & 0.01476 & 0.17181 & 0.23271 \\ \text { Isolok - 3 mL - HF } & 10 & 0.178522 & 0.01866 & 0.14000 & 0.21704\end{array}$

Std Error uses a pooled estimate of error variance

Means and Std Deviations

Level Number Mean Std Dev Std Err Mean Lower 95\% Upper 95\%

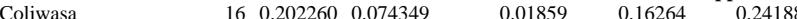

$\begin{array}{lllllll} & \\ \text { Isolok - } 3 \mathrm{~mL} \text { - HF } & 10 & 0.178522 & 0.008757 & 0.00277 & 0.17226 & 0.18479\end{array}$

Test

Isolok - $3 \mathrm{~mL}$ - HF-Coliwasa

Assuming unequal variances

Difference $\quad-0.02374$ t Ratio $\quad-1.26318$

Std Err Dif $\quad 0.01879$ DF $\quad 15.66038$

Upper CL Dif 0.01617 Prob $>|t| \quad 0.2250$

Lower CL Dif -0.06365 Prob $>t \quad 0.8875$

$\begin{array}{lrr}\text { Confidence } & 0.95 \text { Prob }<\mathrm{t} & 0.1125\end{array}$

Tests that the Variances are Equa

Level Count Std Dev MeanAbsDif to Mean MeanAbsDif to Median

$\begin{array}{lllll} & 16 & 0.0743490 & 0.0473088 & 0.0331641 \\ \text { Isolok - } 3 \mathrm{~mL}-\mathrm{HF} & 10 & 0.0087567 & 0.0068903 & 0.0057821\end{array}$

Test F Ratio DFNum DFDen p-Value

$\begin{array}{lrrrr}\text { O'Brien[.5] } & 1.6667 & 1 & 24 & 0.2090\end{array}$

$\begin{array}{lllll}\text { Brown-Forsythe } & 1.4713 & 1 & 24 & 0.2369\end{array}$

$\begin{array}{lllll}\text { Levene } & 5.0986 & 1 & 24 & 0.0333\end{array}$

$\begin{array}{lrrrr}\text { Bartlett } & 26.2298 & 1 & \dot{ } & <.0001 \\ \text { F Test 2-sided } & 72.0884 & 15 & 9 & <.0001\end{array}$

Welch Anova testing Means Equal, allowing Std Devs Not Equal

F Ratio DFNum DFDen Prob > F

$1.5956 \quad 1 \quad 15.66 \quad 0.2250$

t Test
1.2632
Oneway Analysis of Measurement By Sampler Type of Material=SME Simulant, Analytical Block=5, Oxide/Analyte=Li2O (wt \%), Targeted $=4.674$

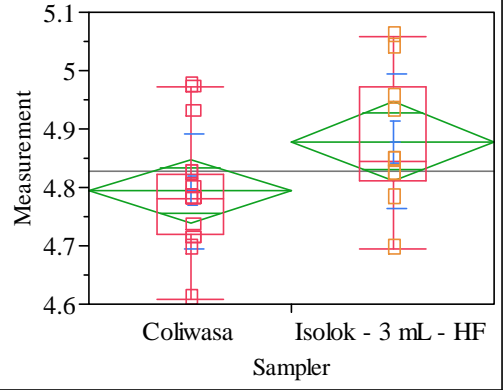

Oneway Anova

Summary of Fit

Rsquare

Adj Rsquare

0.10566

0.105088

$\begin{array}{lr} & 4.826636 \\ \text { Observations (or Sum Wgts) } & 26\end{array}$

t Test

Isolok - $3 \mathrm{~mL}$ - HF-Coliwasa

Assuming equal variances

$\begin{array}{lrlr}\text { Difference } & 0.08423 & \text { t Ratio } & 1.988369 \\ \text { Std Err Dif } & 0.04236 & \text { DF } & 24 \\ \text { Upper CL Dif } & 0.17166 & \text { Prob }>|t| & 0.0583 \\ \text { Lower CL Dif } & -0.00320 & \text { Prob }>t & 0.0291 \\ \text { Confidence } & 0.95 & \text { Prob }<t & 0.9709\end{array}$

Analysis of Variance

Source DF Sum of Squares Mean Square F Ratio Prob $>$ F

$\begin{array}{lrrrrr}\text { Sampler } & 1 & 0.04366194 & 0.043662 & 3.9536 & 0.0583 \\ \text { Error } & 24 & 0.26504545 & 0.011044 & & \end{array}$

Error $24 \quad 0.26504545$

C. Total $25 \quad 0.30870739$

Means for Oneway Anova

Level Number Mean Std Error Lower 95\% Upper 95\%

$\begin{array}{llrrrr}\text { Coliwasa } & 16 & 4.79424 & 0.02627 & 4.7400 & 4.8485\end{array}$

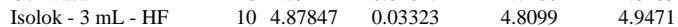

Std Error uses a pooled estimate of error variance

Means and Std Deviations

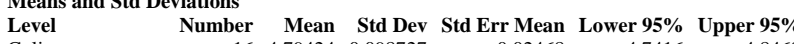

$\begin{array}{lrrrrrr}\text { Coliwasa } & 16 & 4.79424 & 0.098727 & 0.02468 & 4.7416 & 4.8468 \\ \text { Isolok - 3 mL - HF } & 10 & 4.87847 & 0.114911 & 0.03634 & 4.7963 & 4.9607\end{array}$

t Test

Isolok - $3 \mathrm{~mL}$ - HF-Coliwasa

Assuming unequal variances

Difference $\quad 0.08423$ t Ratio 1.917521

Std Err Dif $\quad 0.04393$ DF 17.04329

Upper CL Dif 0.17689 Prob $>|t| \quad 0.0721$

Lower CL Dif -0.00843 Prob $>t \quad 0.0360$

$\begin{array}{lll}\text { Confidence } \quad 0.95 \text { Prob }<\mathrm{t} \quad 0.9640 & \end{array}$

Tests that the Variances are Equal

$\begin{array}{lrrrr}\text { Level } & \text { Count } & \text { Std Dev } & \text { MeanAbsDif to Mean } & \text { MeanAbsDif to Median } \\ \text { Coliwasa } & 16 & 0.0987268 & 0.0704738 & 0.0686237\end{array}$

$\begin{array}{lllll}\text { Isolok - } 3 \mathrm{~mL} \text { - HF } & 10 & 0.1149110 & 0.0704738 & 0.0630053\end{array}$

Test F Ratio DFNum DFDen p-Value

$\begin{array}{lllll}\text { O'Brien[.5] } & 0.3334 & 1 & 24 & 0.5690\end{array}$

Brown-Forsythe $\begin{array}{lllll}0.3452 & 1 & 24 & 0.5623\end{array}$

$\begin{array}{lll}1 & 24 & 0.5623 \\ 1 & 24 & 0.3930\end{array}$

$\begin{array}{lllll}\text { Levene } & 0.7567 & 1 & 24 & 0.3930\end{array}$

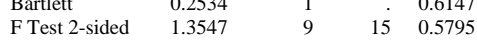

Welch Anova testing Means Equal, allowing Std Devs Not Equal

F Ratio DFNum DFDen Prob $>$ F

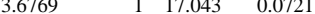

t Test 
SRNL-STI-2011-00693

Revision 0

\section{Exhibit C2. Statistical Comparisons for Low-Rheology (Phase 3) Simulant Testing}

Oneway Analysis of Measurement By Sampler Type of Material=SME Simulant, Analytical Block=5, Oxide/Analyte $=\mathrm{MgO}$ (wt \%), Targeted $=1.014$

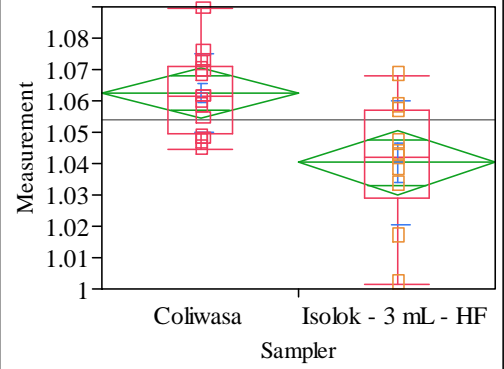

Oneway Anova

Summary of Fit

Adj Rsquare

0.315299

0.015569
0

1.053913

t Test

Isolok - $3 \mathrm{~mL}$ - HF-Coliwasa

Assuming equal variances

Difference $\quad-0.02220$ t Ratio $\quad-3.53727$

Std Err Dif $\quad 0.00628$ DF 24

Upper CL Dif -0.00925 Prob $>|t| \quad 0.0017$

Lower CL Dif -0.03515 Prob $>t \quad 0.9992$

$\begin{array}{lrl}\text { Confidence } & 0.95 \text { Prob }<\mathrm{t} \quad 0.0008\end{array}$

Analysis of Variance

Source DF Sum of Squares Mean Square F Ratio Prob $>$ F

$\begin{array}{lrllll}\text { Sampler } & 1 & 0.00303300 & 0.003033 & 12.5123 & 0.0017\end{array}$

$\begin{array}{llll}\text { Error } & 24 & 0.00581764 & 0.000242\end{array}$

C. Total $25 \quad 0.00885064$

Means for Oneway Anova

Number Mean Std Error Lower 95\% Upper 95\%

$16 \quad 1.06245 \quad 0.00389-1.0544$ Pper $1.07 \%$

$\begin{array}{llllll}\text { Coliwasa } & 16 & 1.06245 & 0.00389 & 1.0544 & 1.0705 \\ \text { Isolok - 3 mL - HF } & 10 & 1.04025 & 0.00492 & 1.0301 & 1.0504\end{array}$

Std Error uses a pooled estimate of error variance

Means and Std Deviations

Level Number Mean Std Dev Std Err Mean Lower 95\% Upper 95\%

$\begin{array}{llllllr}\text { Coliwasa } & 16 & 1.06245 & 0.012435 & 0.00311 & 1.0558 & 1.0691\end{array}$

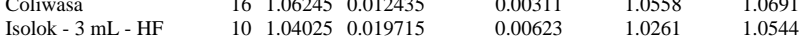

t Test

Isolok - $3 \mathrm{~mL}$ - HF-Coliwasa

Assuming unequal variances

Difference $\quad-0.02220$ t Ratio $\quad-3.18671$

Std Err Dif $\quad 0.00697$ DF $\quad 13.52996$

Upper CL Dif -0.00721 Prob $>|t| \quad 0.0068$

Lower CL Dif -0.03719 Prob $>t \quad 0.9966$

$\begin{array}{lrl}\text { Confidence } & 0.95 \text { Prob }<\mathrm{t} & 0.0034\end{array}$

Tests that the Variances are Equal

Level Count Std Dev MeanAbsDif to Mean MeanAbsDif to Median

$\begin{array}{lllll} & 16 & 0.0124349 & 0.0099887 & 0.0098462 \\ \text { Isolok - } 3 \mathrm{~mL} \text { - HF } & 10 & 0.0197153 & 0.0143277 & 0.0140956\end{array}$

$\begin{array}{lrrrr}\text { Test } & \text { F Ratio } & \text { DFNum } & \text { DFDen } & \text { p-Value } \\ \text { O'Brien[.5] } & 2.2941 & 1 & 24 & 0.1429 \\ \text { Brown-Forsythe } & 1.1397 & 1 & 24 & 0.2963 \\ \text { Levene } & 1.2823 & 1 & 24 & 0.2687 \\ \text { Bartlett } & 2.3857 & 1 & . & 0.1225 \\ \text { F Test 2-sided } & 2.5137 & 9 & 15 & 0.1105\end{array}$

Welch Anova testing Means Equal, allowing Std Devs Not Equal

F Ratio DFNum DFDen Prob $>$ F

$\begin{array}{llll}10.1551 & 1 & 13.53 & 0.0068\end{array}$

t Test

3.1867
Oneway Analysis of Measurement By Sampler Type of Material=SME Simulant, Analytical Block $=5$, Oxide/Analyte $=\mathbf{M n O}($ wt $\%)$, Targeted $=1.779$

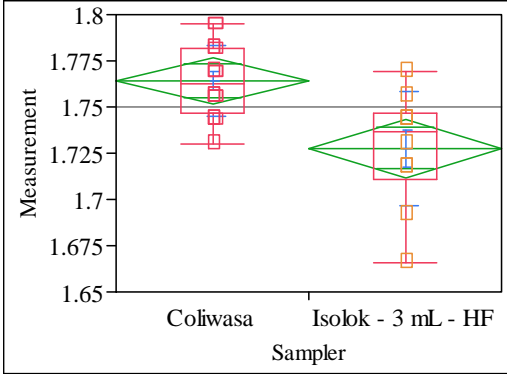

Oneway Anova

Summary of Fit

Rsquare

Adj Rsquare

365246

Root Mean Square Error $\quad 0.024349$

$\begin{array}{lr}\text { Mean of Response } & 1.750073 \\ \text { Observations (or Sum Wgts) } & 26\end{array}$

Test

Isolok - $3 \mathrm{~mL}$ - HF-Coliwasa

Assuming equal variances

Difference $\quad-0.03648$ t Ratio -3.71617

$\begin{array}{lrlr}\text { Std Err Dif } & 0.00982 \text { DF } & 24 \\ \text { Upper CL Dif } & -0.01622 \text { Prob }>|t| & 0.0011\end{array}$

$\begin{array}{llll}\text { Upper CL Dif } & -0.01622 & \text { Prob }>|t| & 0.0011 \\ \text { Lower CL Dif } & -0.05673 & \text { Prob }>t & 0.9995\end{array}$

$\begin{array}{lll}\text { Confidence } & 0.95 \text { Prob }<\mathrm{t} \quad 0.0005\end{array}$

Analysis of Variance

Source DF Sum of Squares Mean Square F Ratio Prob $>$ F

$\begin{array}{llllll}\text { Sampler } & 1 & 0.00818786 & 0.008188 & 13.8099 & 0.0011\end{array}$

$\begin{array}{llll}\text { Error } & 24 & 0.01422953 & 0.000593\end{array}$

C. Total $25 \quad 0.02241739$

Means for Oneway Anova

Level Number Mean Std Error Lower 95\% Upper 95\%

$\begin{array}{llrrrr}\text { Coliwasa } & 16 & 1.76410 & 0.00609 & 1.7515 & 1.7767\end{array}$

$\begin{array}{llllll}\text { Isolok - } 3 \mathrm{~mL} \text { - HF } & 10 & 1.72763 & 0.00770 & 1.7117 & 1.7435\end{array}$

Std Error uses a pooled estimate of error variance

Means and Std Deviations

Level Number Mean Std Dev Std Err Mean Lower 95\% Upper 95\%

$\begin{array}{llllllr}\text { Coliwasa } & 16 & 1.76410 & 0.019368 & 0.00484 & 1.7538 & 1.7744\end{array}$

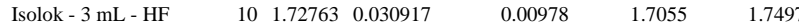

Isolok - $3 \mathrm{~mL}$ - HF-Coliwasa

Assuming unequal variances

Difference $\quad-0.03648$ t Ratio -3.34335

Std Err Dif $\quad 0.01091$ DF $\quad 13.47019$

Upper CL Dif -0.01299 Prob $>|t| \quad 0.0051$

Lower CL Dif -0.05996 Prob $>t \quad 0.9975$

Confidence $\quad 0.95$ Prob $<\mathrm{t} \quad 0.0025$

Tests that the Variances are Equal

$\begin{array}{lrrrr}\text { Level } & \text { Count } & \text { Std Dev } & \text { MeanAbsDif to Mean } & \text { MeanAbsDif to Median } \\ \text { Coliwasa } & 16 & 0.0193680 & 0.0161400 & 0.0161400\end{array}$

Isolok - $3 \mathrm{~mL}$ - HF $\quad 10 \quad 0.0309170 \quad 0.0237581 \quad 0.0232416$

Test F Ratio DFNum DFDen p-Value

$\begin{array}{lllll}\text { O'Brien[.5] } & 2.4509 & 1 & 24 & 0.1306\end{array}$

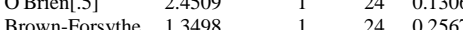

Brown-Forsythe $1.3498 \quad 1024 \quad 0.2567$

$\begin{array}{lllll}\text { Levene } & 1.9405 & 1 & 24 & 0.1764\end{array}$

$\begin{array}{lllll}\text { Bartlett } & 2.4569 & 1 & & 0.1170 \\ \text { F Test 2-sided } & 2.5481 & 9 & 15 & 0.1055\end{array}$

Welch Anova testing Means Equal, allowing Std Devs Not Equal

F Ratio DFNum DFDen Prob $>$ F

$\begin{array}{llll}11.1780 & 1 & 13.47 & 0.0051\end{array}$

t Test

3.3434 


\section{Exhibit C2. Statistical Comparisons for Low-Rheology (Phase 3) Simulant Testing}

Oneway Analysis of Measurement By Sampler Type of Material=SME Simulant, Analytica Block=5, Oxide/Analyte=Na2O (wt \%), Targeted=11.659

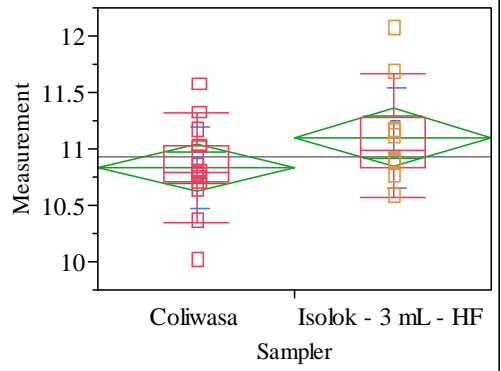

Oneway Anova

Summary of Fit

Adj Rsquare

Root Mean Square Error $\quad 0.069732$

Observations (or Sum Wgts)

t Test

Isolok - $3 \mathrm{~mL}$ - HF-Coliwasa

Assuming equal variances

Difference $\quad 0.26977$ t Ratio 1.69528

Std Err Dif $\quad 0.15913$ DF 24

Upper CL Dif 0.59820 Prob $>|t| 0.1030$

Lower CL Dif -0.05866 Prob $>t \quad 0.0515$

$\begin{array}{lrl}\text { Confidence } & 0.95 \text { Prob }<\mathrm{t} \quad 0.9485\end{array}$

Analysis of Variance

Source DF Sum of Squares Mean Square F Ratio Prob $>$ F

$\begin{array}{llllll}\text { Sampler } & 1 & 0.4478464 & 0.447846 & 2.8740 & 0.1030\end{array}$

$\begin{array}{llll}\text { Error } & 24 & 3.7398794 & 0.155828\end{array}$

C. Total $25 \quad 4.1877258$

Means for Oneway Anova

Level Number Mean Std Error Lower 95\% Upper 95\%

Coliwas 16 10.8337 $0.09869-10.630 \quad 11.037$

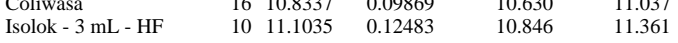

Std Error uses a pooled estimate of error variance

Means and Std Deviations

Level Number Mean Std Dev Std Err Mean Lower 95\% Upper 95\%

$\begin{array}{llllllr}\text { Coliwasa } & 16 & 10.8337 & 0.361529 & 0.09038 & 10.641 & 11.026 \\ \text { Isolok - 3 mL - HF } & 10 & 11.1035 & 0.444638 & 0.14061 & 10.785 & 11.422\end{array}$

t Test

Isolok - $3 \mathrm{~mL}$ - HF-Coliwasa

Assuming unequal variances

Difference $\quad 0.26977$ t Ratio 1.613927

Std Err Dif $\quad 0.16715$ DF $\quad 16.30392$

Upper CL Dif 0.62358 Prob $>|t| \quad 0.1257$

Lower CL Dif -0.08404 Prob $>\mathrm{t} \quad 0.0629$

$\begin{array}{lrr}\text { Confidence } & 0.95 \text { Prob }<\mathrm{t} & 0.9371\end{array}$

Tests that the Variances are Equal

Level Count Std Dev MeanAbsDif to Mean MeanAbsDif to Median

$\begin{array}{lllll}16 & 0.3615294 & 0.2480109 & 0.2451675\end{array}$

Test F Ratio DFNum DFDen p-Value

$\begin{array}{lrrrr}\text { O'Brien[.5] } & 0.3768 & 1 & 24 & 0.545\end{array}$

Brown-Forsythe $\begin{array}{llll}0.3625 & 1 & 24 & 0.5527\end{array}$

$\begin{array}{lllll}\text { Levene } & 0.3882 & 1 & 24 & 0.539\end{array}$

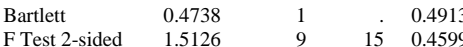

Welch Anova testing Means Equal, allowing Std Devs Not Equal

F Ratio DFNum DFDen Prob $>$ F

$\begin{array}{llll}2.6048 & 1 & 16.304 & 0.1257\end{array}$

t Test

1.6139
Oneway Analysis of Measurement By Sampler Type of Material=SME Simulant, Analytica Block $=5$, Oxide/Analyte $=\mathrm{NiO}(\mathrm{wt} \%)$, Targeted $=\mathbf{0 . 4 1}$

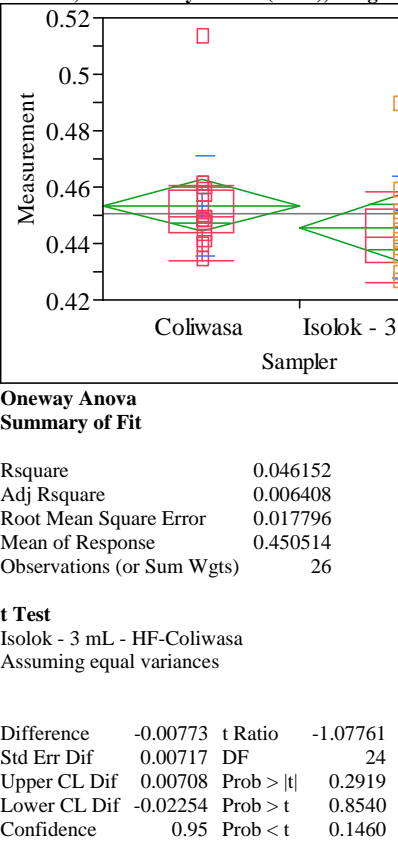

Analysis of Variance

Source DF Sum of Squares Mean Square F Ratio Prob $>$ F

$\begin{array}{lrrrrr}\text { Sampler } & 1 & 0.00036775 & 0.000368 & 1.1612 & 0.2919\end{array}$

$\begin{array}{llll}\text { Error } & 24 & 0.00760055 & 0.000317\end{array}$

C. Total $25 \quad 0.00796830$

Means for Oneway Anova

Level Number Mean Std Error Lower 95\% Upper 95\%

$\begin{array}{lrrrrr}\text { Coliwasa } & 16 & 0.453487 & 0.00445 & 0.44431 & 0.46267\end{array}$

$\begin{array}{llllll}\text { Isolok - 3 mL - HF } & 16 & 0.453487 & 0.00445 & 0.44431 & 0.46267 \\ & 10 & 0.445757 & 0.00563 & 0.43414 & 0.45737\end{array}$

Std Error uses a pooled estimate of error variance

Means and Std Deviations

Level Number Mean Std Dev Std Err Mean Lower 95\% Upper 95\%

$\begin{array}{lllllll}\text { Coliwasa } & 16 & 0.453487 & 0.017717 & 0.00443 & 0.44405 & 0.46293\end{array}$

$\begin{array}{lllllll}\text { Isolok - } 3 \mathrm{~mL} \text { - HF } & 10 & 0.445757 & 0.017926 & 0.00567 & 0.43293 & 0.45858\end{array}$

Test

Isolok - $3 \mathrm{~mL}$ - HF-Coliwasa

Assuming unequal variances

$\begin{array}{lrlr}\text { Difference } & -0.00773 & \text { t Ratio } & -1.07457 \\ \text { Std Err Dif } & 0.00719 & \text { DF } & 19.07717 \\ \text { Upper CL Dif } & 0.00732 & \text { Prob }>|t| & 0.2960 \\ \text { Lower CL Dif } & -0.02278 & \text { Prob }>\text { t } & 0.8520 \\ \text { Confidence } & 0.95 & \text { Prob }<\mathrm{t} & 0.1480\end{array}$

Tests that the Variances are Equal

Level Count Std Dev MeanAbsDif to Mean MeanAbsDif to Median

$\begin{array}{lllll}\text { Coliwasa } & 16 & 0.0177170 & 0.0107765 & 0.0100209\end{array}$

$\begin{array}{llll}\text { Isolok - } 3 \mathrm{~mL} \text { - HF } & 10 & 0.0179262 & 0.0124196\end{array}$

$\begin{array}{lrrrr}\text { Test } & \text { F Ratio } & \text { DFNum } & \text { DFDen } & \text { p-Value } \\ \text { O'Brien[.5] } & 0.0005 & 1 & 24 & 0.9830 \\ \text { Brown-Forsythe } & 0.1275 & 1 & 24 & 0.7242 \\ \text { Levene } & 0.0949 & 1 & 24 & 0.7606 \\ \text { Bartlett } & 0.0015 & 1 & . & 0.9692 \\ \text { F Test 2-sided } & 1.0238 & 9 & 15 & 0.9292\end{array}$

Welch Anova testing Means Equal, allowing Std Devs Not Equal

F Ratio DFNum DFDen Prob $>$ F

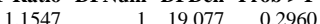

t Test

1.0746 
SRNL-STI-2011-00693

Revision 0

\section{Exhibit C2. Statistical Comparisons for Low-Rheology (Phase 3) Simulant Testing}

Oneway Analysis of Measurement By Sampler Type of Material=SME Simulant, Analytical Block $=5$, Oxide $/$ Analyte $=\mathrm{SiO} 2(\mathrm{wt} \%)$, Targeted $=50.985$

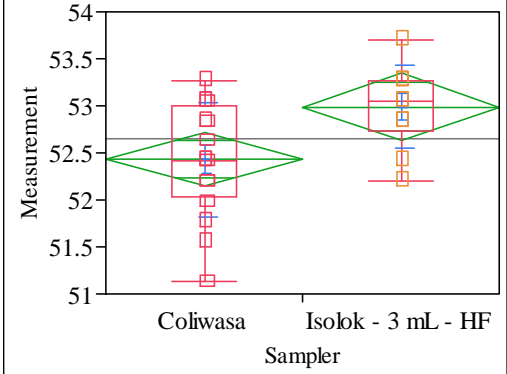

Oneway Anova

Summary of Fi

Adj Rsquare

Root Mean Square Enor 0.181467

0.547227

Observations (or Sum Wgts) 52.64324

t Test

Isolok - $3 \mathrm{~mL}$ - HF-Coliwasa

Assuming equal variances

Difference $\quad 0.56424$ t Ratio 2.557818

Std Err Dif $\quad 0.22059$ DF 24

Upper CL Dif 1.01952 Prob $>|t| 0.0173$

Lower CL Dif 0.10896 Prob $>t \quad 0.0086$

$\begin{array}{lrl}\text { Confidence } & 0.95 \text { Prob }<\mathrm{t} \quad 0.9914\end{array}$

Analysis of Variance

Source DF Sum of Squares Mean Square F Ratio Prob $>$ F

$\begin{array}{lrrrrr}\text { Sampler } & 1 & 1.9591828 & 1.95918 & 6.5424 & 0.0173\end{array}$

$\begin{array}{llll}\text { Error } & 24 & 7.1869853 & 0.29946\end{array}$

C. Total $25 \quad 9.1461681$

Means for Oneway Anova

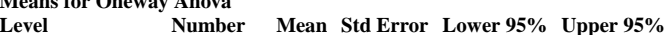

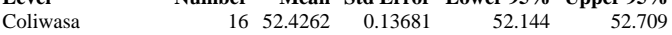

$\begin{array}{llllll}\text { Coliwasa } & 16 & 52.4262 & 0.13681 & 52.144 & 52.709 \\ \text { Isolok - 3 mL - HF } & 10 & 52.9905 & 0.17305 & 52.633 & 53.348\end{array}$

Std Error uses a pooled estimate of error variance

Means and Std Deviations

Level Number Mean Std Dev Std Err Mean Lower 95\% Upper 95\%

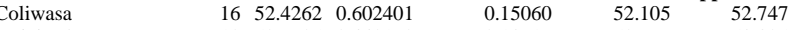

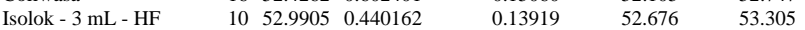

Test

Isolok - $3 \mathrm{~mL}$ - HF-Coliwasa

Assuming unequal variances

Difference $\quad 0.564240$ t Ratio 2.75142

Std Err Dif $\quad 0.205072$ DF 23.2709

Upper CL Dif 0.988192 Prob $>|t| 0.0113$

Lower CL Dif 0.140289 Prob $>t \quad 0.0056$

$\begin{array}{lrl}\text { Confidence } & 0.95 \text { Prob }<\mathrm{t} & 0.9944\end{array}$

Tests that the Variances are Equal

Level Count Std Dev MeanAbsDif to Mean MeanAbsDif to Median

$\begin{array}{lllll}16 & 0.6024007 & 0.4696432 & 0.4679719\end{array}$

Test F Ratio DFNum DFDen p-Value

$\begin{array}{lrrrr}\text { O'Brien[.5] } & 1.0362 & 1 & 24 & 0.3189\end{array}$

$\begin{array}{lllll}\text { Brown-Forsythe } & 1.1844 & 1 & 24 & 0.2873\end{array}$

$\begin{array}{lllll}\text { Levene } & 1.0723 & 1 & 24 & 0.3107\end{array}$

$\begin{array}{lrrrr}\text { Bartlett } & 0.9922 & 1 & \text {. } & 0.3192 \\ \text { F Test 2-sided } & 1.8730 & 15 & 9 & 0.3436\end{array}$

Welch Anova testing Means Equal, allowing Std Devs Not Equal

F Ratio DFNum DFDen Prob $>$ F

$\begin{array}{rrrr}7.5703 & 1 & 23.271 & 0.0113\end{array}$

t Test

2.7514
Oneway Analysis of Measurement By Sampler Type of Material=SME Simulant, Analytical Block=5, Oxide/Analyte=Sum of Oxides (wt \%), Targeted $=99.553$

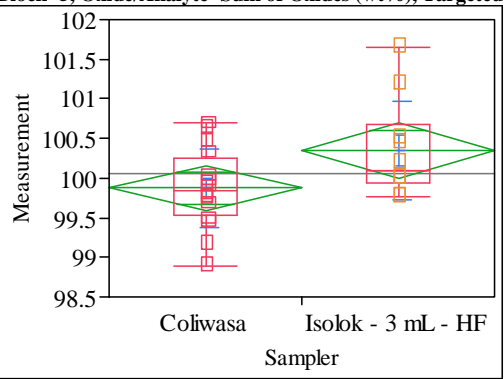

Oneway Anova

Summary of Fit

Rsquare

Root Mean Square Error $\quad 0.547657$

Mean of Response

100.055
26

Isolok - $3 \mathrm{~mL}$ - HF-Coliwasa

Assuming equal variances

Difference $\quad 0.478475$ t Ratio 2.167321

$\begin{array}{lrlr}\text { Std Err Dif } & 0.220768 \text { DF } & 24 \\ \text { Upper CL } & 0.934117 \text { Prob }>|t| & 0.0404\end{array}$

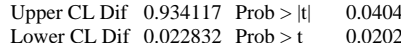

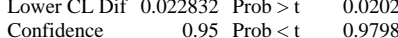

Analysis of Variance

Source DF Sum of Squares Mean Square F Ratio Prob $>$ F

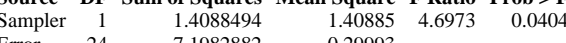

$\begin{array}{llll}\text { Error } & 24 & 7.1982882 & 0.29993\end{array}$

C. Total $25 \quad 8.6071376$

Means for Oneway Anova

Level Number Mean Std Error Lower 95\% Upper 95\%

\begin{tabular}{lrrrrr} 
Coliwasa & 16 & 99.871 & 0.13691 & 99.588 & 100.15 \\
\hline
\end{tabular}

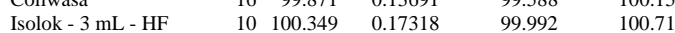

Std Error uses a pooled estimate of error variance

Means and Std Deviations

Level Number Mean Std Dev Std Err Mean Lower 95\% Upper 95\%

$\begin{array}{llllllr}\text { Coliwasa } & 16 & 99.871 & 0.496642 & 0.12416 & 99.606 & 100.14\end{array}$

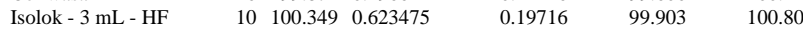

Isolok - $3 \mathrm{~mL}$ - HF-Coliwasa

Assuming unequal variances

Difference $\quad 0.47847$ t Ratio $\quad 2.05356$

Std Err Dif $\quad 0.23300$ DF 16.04025

Upper CL Dif 0.97231 Prob $>|t| 0.0567$

Lower CL Dif -0.01536 Prob $>t \quad 0.0283$

$\begin{array}{lrl}\text { Confidence } & 0.95 \text { Prob }<\mathrm{t} \quad 0.9717\end{array}$

Tests that the Variances are Equal

$\begin{array}{lrrrr}\text { Level } & \text { Count } & \text { Std Dev } & \text { MeanAbsDif to Mean } & \text { MeanAbsDif to Median } \\ \text { Coliwasa } & 16 & 0.4966421 & 0.3719066 & 0.3719066\end{array}$

Isolok - $3 \mathrm{~mL}$ - HF $\quad 100.6234748 \quad 0.4750573 \quad 0.4452841$

Test F Ratio DFNum DFDen p-Value

$\begin{array}{lllll}\text { O'Brien[.5] } & 0.5962 & 1 & 24 & 0.4476\end{array}$

$\begin{array}{lllll}\text { Brown-Forsythe } & 0.2194 & 1 & 24 & 0.6438\end{array}$

$\begin{array}{lllll}\text { Brown-Forsythe } & 0.2194 & 1 & 24 & 0.6438 \\ \text { Levene } & 0.5759 & 1 & 24 & 0.4553\end{array}$

$\begin{array}{lllll}\text { Levene } & 0.5759 & 1 & 24 & 0.4553 \\ \text { Bartlett } & 0.5735 & 1 & . & 0.4489\end{array}$

$\begin{array}{lllll}\text { F Test 2-sided } & 1.5760 & 9 & 15 & 0.4190\end{array}$

Welch Anova testing Means Equal, allowing Std Devs Not Equal

F Ratio DFNum DFDen Prob $>$ F

$\begin{array}{llll}4.2171 & 1 & 16.04 & 0.0567\end{array}$

t Test

2.0536 
SRNL-STI-2011-00693

Revision 0

\section{Exhibit C2. Statistical Comparisons for Low-Rheology (Phase 3) Simulant Testing}

Oneway Analysis of Measurement By Sampler Type of Material=SME Simulant, Analytical Block $=5$, Oxide/Analyte $=$ TiO2 $($ wt $\%$ ), Targeted $=\mathbf{0 . 0 7 1 1}$

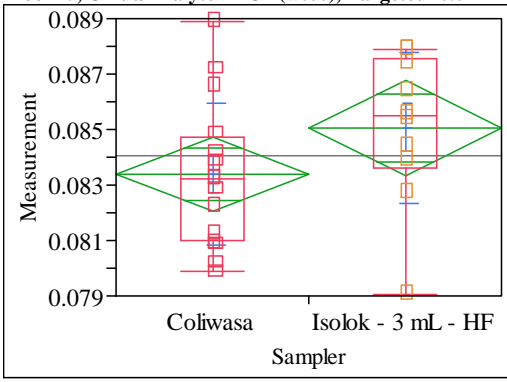

Oneway Anova

Rsquare $\quad 0.094266$

Adj Rsquare $\quad 0.05652$

Root Mean Square Error $\quad 0.002634$

$\begin{array}{lr}\text { Mean of Response } & 0.084035 \\ \text { Observations (or Sum Wgts) } & 26\end{array}$

Test

Isolok - $3 \mathrm{~mL}$ - HF-Coliwasa

Assuming equal variances

Difference $\quad 0.00168$ t Ratio 1.580456

$\begin{array}{lrlr}\text { Std Err Dif } & 0.00106 \text { DF } & 24 \\ \text { Upper CL Dif } & 0.00387 \text { Prob }>|t| & 0.1271\end{array}$

$\begin{array}{rrll}\text { Upper CL Dif } & 0.00387 & \text { Prob }>|t| & 0.1271 \\ \text { Lower CL Dif } & -0.00051 & \text { Prob }>t & 0.0635\end{array}$

$\begin{array}{lll}\text { Confidence } & 0.95 \text { Prob }<\mathrm{t} \quad 0.9365\end{array}$

Analysis of Variance

Source DF Sum of Squares Mean Square F Ratio Prob $>$ F

$\begin{array}{llllll}\text { Sampler } & 1 & 0.00001734 & 0.000017 & 2.4978 & 0.1271\end{array}$

$\begin{array}{llll}\text { Error } & 24 & 0.00016657 & 6.94 \mathrm{e}-6\end{array}$

C. Total $25 \quad 0.0001839$

Means for Oneway Anova

Level Number Mean Std Error Lower 95\% Upper 95\%

$\begin{array}{llllll}\text { Coliwasa } & 16 & 0.083390 & 0.00066 & 0.08203 & 0.08475\end{array}$

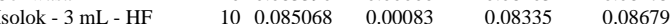

Std Error uses a pooled estimate of error variance

Means and Std Deviations

$\begin{array}{lrrrrrr}\text { Level } & \text { Number } & \text { Mean } & \text { Std Dev } & \text { Std Err Mean } & \text { Lower 95\% } & \text { Upper 95\% } \\ \text { Coliwasa } & 16 & 0.083390 & 0.002576 & 0.00064 & 0.08202 & 0.08476\end{array}$

$\begin{array}{lllllll}\text { Isolok - } 3 \mathrm{~mL} \text { - HF } & 10 & 0.085068 & 0.002728 & 0.00086 & 0.08312 & 0.08702\end{array}$

t Test

Isolok - $3 \mathrm{~mL}$ - HF-Coliwasa

Assuming unequal variances

Difference $\quad 0.00168$ t Ratio 1.558855

Std Err Dif $\quad 0.00108$ DF 18.39883

Upper CL Dif 0.00394 Prob $>|t| 0.1361$

Lower CL Dif -0.00058 Prob $>\mathrm{t} \quad 0.0680$

$\begin{array}{lll}\text { Confidence } & 0.95 \text { Prob }<\mathrm{t} & 0.9320\end{array}$

Tests that the Variances are Equal

$\begin{array}{lrrrr}\text { Level } & \text { Count } & \text { Std Dev } & \text { MeanAbsDif to Mean } & \text { MeanAbsDif to Median } \\ \text { Coliwasa } & 16 & 0.0025765 & 0.0019690 & 0.0019495 \\ \text { Isolok - 3 mL - HF } & 10 & 0.0027284 & 0.0020350 & 0.0019682\end{array}$

Test F Ratio DFNum DFDen p-Value

O'Brien[.5] $\quad 0.0361 \quad-1 \quad 24 \quad 0.8509$

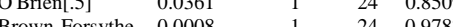

$\begin{array}{lll}1 & 24 & 0.9783 \\ -1 & 24 & 0.9205\end{array}$

$\begin{array}{lllll}\text { Levene } & 0.0102 & 1 & 24 & 0.9205 \\ \text { Bartlett } & 0.0356 & 1 & . & 0.8503\end{array}$

$\begin{array}{lllll}\text { F Test 2-sided } & 1.1214 & 9 & 15 & 0.8110\end{array}$

Welch Anova testing Means Equal, allowing Std Devs Not Equal

F Ratio DFNum DFDen Prob $>$ F

$\begin{array}{llll}2.4300 & 1 & 18.399 & 0.1361\end{array}$

t Test

1.5589
Oneway Analysis of Measurement By Sampler Type of Material=SME Simulant, Analytical Block $=5$, Oxide/Analyte $=\mathrm{ZnO}(\mathrm{wt} \%)$, Targeted $=\mathbf{0 . 0 9 5 8}$

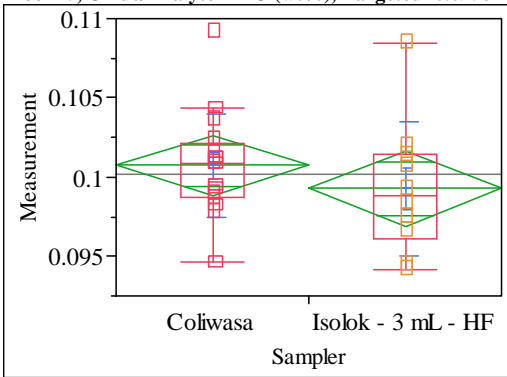

Oneway Anova

Summary of Fit

Rsquare

Adj Rsquare

0.000835

0.003641

Observations (or Sum Wgts) $\begin{array}{rr}0.10017 \\ 26\end{array}$

Isolok - $3 \mathrm{~mL}$ - HF-Coliwasa

Assuming equal variances

Difference $\quad-0.00148$ t Ratio -1.01039

$\begin{array}{lrrr}\text { Std Err Dif } & 0.00147 \text { DF } & 24 \\ \text { Upper CL } & 0.00155 \text { Prob }>|t| & 0.322\end{array}$

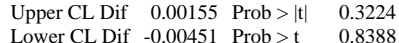

$\begin{array}{lrl}\text { Confidence } & 0.95 \text { Prob }<\mathrm{t} \quad 0.1612\end{array}$

Analysis of Variance

Source DF Sum of Squares Mean Square F Ratio Prob $>$ F

$\begin{array}{llllll}\text { Sampler } & 1 & 0.00001353 & 0.000014 & 1.0209 & 0.3224\end{array}$

$\begin{array}{lll}\text { Error } & 24 & 0.00031812 \\ \text { C. Total } & 25 & 0.00033165\end{array}$

Means for Oneway Anova

Level Number Mean Std Error Lower 95\% Upper 95\%

$\begin{array}{lrrrrr}\text { Coliwasa } & 16 & 0.100743 & 0.00091 & 0.09886 & 0.10262\end{array}$

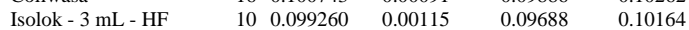

Std Error uses a pooled estimate of error variance

Means and Std Deviations

Level Number Mean Std Dev Std Err Mean Lower 95\% Upper 95\%

$\begin{array}{lllllll}\text { Coliwasa } & 16 & 0.100743 & 0.003266 & 0.00082 & 0.09900 & 0.10248\end{array}$

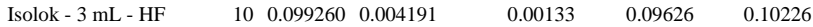

\section{t Test}

Isolok - $3 \mathrm{~mL}$ - HF-Coliwasa

Assuming unequal variances

Difference $\quad-0.00148$ t Ratio $\quad-0.95257$

Std Err Dif $\quad 0.00156$ DF $\quad 15.76646$

Upper CL Dif 0.00182 Prob $>|t| 0.3552$

Lower CL Dif -0.00479 Prob $>t \quad 0.8224$

$\begin{array}{lll}\text { Confidence } & 0.95 \text { Prob }<\mathrm{t} \quad 0.1776\end{array}$

Tests that the Variances are Equal

$\begin{array}{lrrrr}\text { Level } & \text { Count } & \text { Std Dev } & \text { MeanAbsDif to Mean } & \text { MeanAbsDif to Median } \\ \text { Coliwasa } & 16 & 0.0032663 & 0.0022902 & 0.0022795 \\ \text { Isolok - 3 mL - HF } & 10 & 0.0041911 & 0.0030722 & 0.0030622\end{array}$

Test F Ratio DFNum DFDen p-Value

$\begin{array}{llllll}\text { O'Brien[.5] } & 0.4795 & 1 & 24 & 0.4953\end{array}$

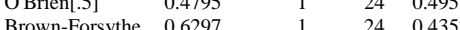

$\begin{array}{lllll}\text { Brown-Forsythe } & 0.6297 & 1 & 24 & 0.4352 \\ \text { Levene } & 0.6459 & 1 & 24 & 0.4295\end{array}$

$\begin{array}{lllll}\text { Levene } & 0.6459 & 1 & 24 & 0.4295 \\ \text { Bartlett } & 0.6906 & 1 & & 0.4060\end{array}$

$\begin{array}{lllll}\text { Bartlett } & 0.6906 & 1 & & 0.4060 \\ \text { F Test 2-sided } & 1.6465 & 9 & 15 & 0.3779\end{array}$

Welch Anova testing Means Equal, allowing Std Devs Not Equal

F Ratio DFNum DFDen Prob $>$ F

$\begin{array}{llll}0.9074 & 1 & 15.766 & 0.3552\end{array}$

t Test 


\section{Exhibit C2. Statistical Comparisons for Low-Rheology (Phase 3) Simulant Testing}

Oneway Analysis of Measurement By Sampler Type of Material=SME Simulant, Analytical Block $=5$, Oxide/Analyte $=\mathrm{ZrO} 2$ (wt \%), Targeted $=\mathbf{0 . 3 5 4 7}$

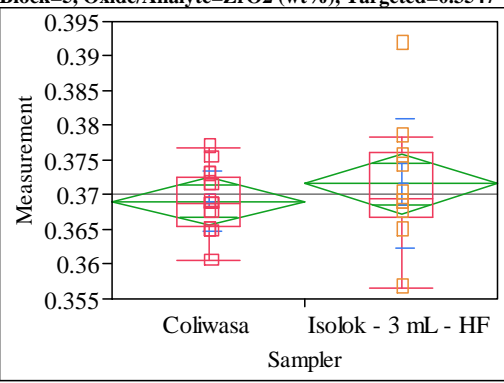

Oneway Anova

Rsquare $\quad 0.035163$

Adj Rsquare $\quad-0.00504$

Root Mean Square Error $\quad 0.006628$

Mean of Response $\quad 0.370067$

t Test

Isolok - $3 \mathrm{~mL}$ - HF-Coliwasa

Assuming equal variances

Difference $\quad 0.00250$ t Ratio 0.935243

$\begin{array}{lrlr}\text { Std Err Dif } & 0.00267 & \text { DF } & 24 \\ \text { Upper CL Dif } & 0.00801 & \text { Prob }>|t| & 0.3590\end{array}$

$\begin{array}{rrrr}\text { Upper CL Dif } & 0.00801 & \text { Prob }>|t| & 0.3590 \\ \text { Lower CL Dif } & -0.00302 & \text { Prob }>t & 0.1795\end{array}$

$\begin{array}{lrl}\text { Confidence } & 0.95 \text { Prob }<\mathrm{t} \quad 0.8205\end{array}$

Analysis of Variance

Source DF Sum of Squares Mean Square F Ratio Prob $>$ F

$\begin{array}{lrrrrr}\text { Sampler } & 1 & 0.00003843 & 0.000038 & 0.8747 & 0.3590\end{array}$

Error $24 \quad 0.00105447$

0.000044

Means for Oneway Anova

Level Number Mean Std Error Lower 95\% Upper 95\%

$\begin{array}{llllrr}\text { Coliwasa } & 16 & 0.369106 & 0.00166 & 0.36569 & 0.37253\end{array}$

$\begin{array}{llllll}\text { Isolok - } 3 \mathrm{~mL}-\mathrm{HF} & 10 & 0.371605 & 0.00210 & 0.36728 & 0.37593\end{array}$

Std Error uses a pooled estimate of error variance

Means and Std Deviations

$\begin{array}{lrrrrrr}\text { Level } & \text { Number } & \text { Mean } & \text { Std Dev } & \text { Std Err Mean } & \text { Lower 95\% } & \text { Upper 95\% } \\ \text { Coliwasa } & 16 & 0.369106 & 0.004286 & 0.00107 & 0.36682 & 0.37139\end{array}$

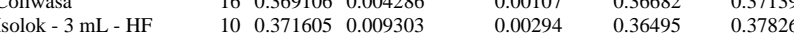

t Test

Isolok - $3 \mathrm{~mL}$ - HF-Coliwasa

Assuming unequal variances

Difference $\quad 0.00250$ t Ratio $\quad 0.798148$

Std Err Dif $\quad 0.00313$ DF 11.4253

Upper CL Dif 0.00936 Prob $>|t| \quad 0.4411$

Lower CL Dif -0.00436 Prob $>t \quad 0.2205$

$\begin{array}{lrl}\text { Confidence } & 0.95 \text { Prob }<\mathrm{t} & 0.7795\end{array}$

Tests that the Variances are Equal

$\begin{array}{lrrrr}\text { Level } & \text { Count } & \text { Std Dev } & \text { MeanAbsDif to Mean } & \text { MeanAbsDif to Median } \\ \text { Coliwasa } & 16 & 0.0042858 & 0.0032926 & 0.0032082 \\ \text { Isolok - 3 mL - HF } & 10 & 0.0093032 & 0.0066459 & 0.0063488\end{array}$

Test F Ratio DFNum DFDen p-Value

O'Brien[.5] $2.9820 \quad 1 \quad 240.0970$

OBrien[.5] 2.9820

Brown-Forsythe $2.7216 \quad 11 \quad 24 \quad 0.1120$

$\begin{array}{lllll}\text { Levene } & 3.7803 & 1 & 24 & 0.0637 \\ \text { Bartlett } & 6.6770 & 1 & . & 0.0098\end{array}$

$\begin{array}{lllll}\text { F Test 2-sided } & 4.7119 & 9 & 15 & 0.0084\end{array}$

Welch Anova testing Means Equal, allowing Std Devs Not Equal

F Ratio DFNum DFDen Prob $>$ F

$\begin{array}{llll}0.6370 & 1 & 11.425 & 0.4411\end{array}$

t Test
Oneway Analysis of Measurement By Sampler Type of Material=SME Simulant, Analytical Block $=6$, Oxide $/$ Analyte $=A \mathrm{l} / \mathrm{B}$, Targeted $=2.552$

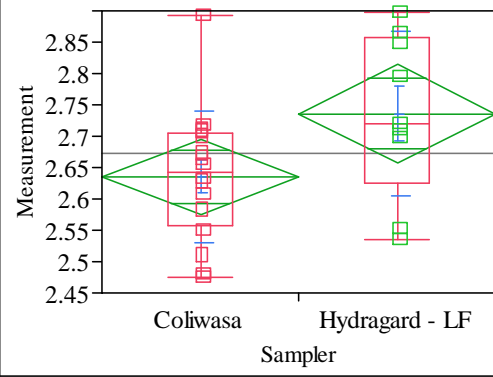

Excluded Rows

1

Oneway Anova

Summary of Fit

Adj Rsquare $\quad 0.161538$

Root Mean Square Error $\quad 0.114816$

Reon 0.114816

2.671409
Observations (or Sum Wgts)

t Test

Hydragard - LF-Coliwasa

Assuming equal variances

Difference $\quad 0.100705$ t Ratio 2.105037

Std Err Dif $\quad 0.047840$ DF 23

Upper CL Dif 0.199670 Prob $>|t| \quad 0.0464$

$\begin{array}{lll}\text { Lower CL Dif } 0.001740 & \text { Prob }>t & 0.0232\end{array}$

$\begin{array}{lrl}\text { Confidence } & 0.95 \text { Prob }<\mathrm{t} & 0.9768\end{array}$

Analysis of Variance

Source DF Sum of Squares Mean Square F Ratio Prob $>$ F

$\begin{array}{llllll}\text { Sampler } & 1 & 0.05841544 & 0.058415 & 4.4312 & 0.0464\end{array}$

$\begin{array}{llll}\text { Error } & 23 & 0.30320491 & 0.013183\end{array}$

$\begin{array}{lll}\text { C. Total } & 24 & 0.36162035\end{array}$

Means for Oneway Anova

Level Number Mean Std Error Lower 95\% Upper 95\%

$\begin{array}{lrrrrr}\text { Coliwasa } & 16 & 2.63516 & 0.02870 & 2.5758 & 2.6945 \\ \text { Hydragard - LF } & 9 & 2.73586 & 0.03827 & 2.6567 & 2.8150\end{array}$

Std Error uses a pooled estimate of error variance

Means and Std Deviations

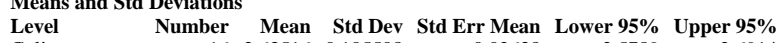

$\begin{array}{lrrrrrr}\text { Coliwasa } & 16 & 2.63516 & 0.105508 & 0.02638 & 2.5789 & 2.6914\end{array}$

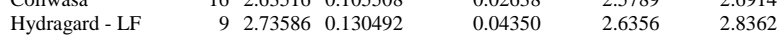

t Test

Hydragard - LF-Coliwasa

Assuming unequal variances

$\begin{array}{lrlr}\text { Difference } & 0.10071 & \mathrm{t} \text { Ratio } & 1.979656 \\ \text { Std Err Dif } & 0.05087 & \text { DF } & 13.95871 \\ \text { Upper CL Dif } & 0.20984 & \text { Prob }>|t| & 0.0678 \\ \text { Lower CL Dif } & -0.00843 & \text { Prob }>\text { t } & 0.0339 \\ \text { Confidence } & 0.95 & \text { Prob }<\mathrm{t} & 0.9661\end{array}$

Tests that the Variances are Equal

Level Count Std Dev MeanAbsDif to Mean MeanAbsDif to Median

$\begin{array}{lrrrr}\text { Coliwasa } & 16 & 0.1055080 & 0.0782704 & 0.0782704\end{array}$

$\begin{array}{rrrrr} & & & & \\ \text { Hydragard - LF } & 9 & 0.1304922 & 0.0782704 & 0.0782704 \\ & & & 0.1034236 & 0.1025799\end{array}$

Test F Ratio DFNum DFDen p-Value

$\begin{array}{lrrrr}\text { O'Brien[.5] } & 0.5698 & 1 & 23 & 0.4580\end{array}$

Brown-Forsythe $0.6868 \quad 1 \quad 23 \quad 0.4158$

$\begin{array}{lllll}\text { Levene } & 0.7696 & 1 & 23 & 0.3894\end{array}$

$\begin{array}{lllll}\text { Bartlett } & 0.4658 & 1 & \text {. } & 0.4949\end{array}$

F Test 2-sided 1.5297

Welch Anova testing Means Equal, allowing Std Devs Not Equal

F Ratio DFNum DFDen Prob $>$ F

$\begin{array}{rrrr}3.9190 & 1 & 13.959 & 0.0678\end{array}$

t Test
1.9797 


\section{Exhibit C2. Statistical Comparisons for Low-Rheology (Phase 3) Simulant Testing}

Oneway Analysis of Measurement By Sampler Type of Material=SME Simulant, Analytical Block $=6$, Oxide/Analyte $=\mathrm{Al} 2 \mathrm{O} 3$ ( wt $\%$ ), Targeted $=10.869$

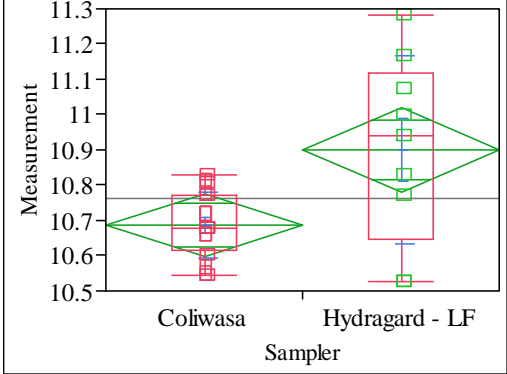

Exc

Exclided Rows

Oneway Anova

Summary of Fit

Rsquare $\quad 0.27397$

Adj Rsquare $\quad 0.242404$

Root Mean Square Error $\quad 0.173383$

Mean of Response $\quad 10.764$

Observations (or Sum Wgts) $\quad 25$

Test

Hydragard - LF-Coliwasa

Assuming equal variances

Difference $\quad 0.212831$ t Ratio $\quad 2.946044$

Std Err Dif $\quad 0.072243$ DF $\quad 23$

Upper CL Dif 0.362277 Prob $>|t| \quad 0.0073$

Lower CL Dif 0.063385 Prob $>t \quad 0.0036$

$\begin{array}{lrl}\text { Confidence } & 0.95 \text { Prob }<\mathrm{t} & 0.9964\end{array}$

Analysis of Variance

Source DF Sum of Squares Mean Square F Ratio Prob $>$ F

$\begin{array}{llllll}\text { Sampler } & 1 & 0.26091136 & 0.260911 & 8.6792 & 0.0073\end{array}$

$\begin{array}{llll}\text { Error } & 23 & 0.69142080 & 0.030062\end{array}$

C. Total $24 \quad 0.95233216$

Means for Oneway Anova

Level Number Mean Std Error Lower 95\% Upper 95\%

$\begin{array}{lrrrrr}\text { Coliwasa } & 16 & 10.6875 & 0.04335 & 10.598 & 10.777\end{array}$

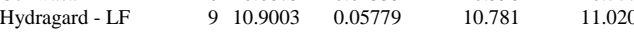

Std Error uses a pooled estimate of error variance

$\begin{array}{llllll}\text { Means and Std Deviations } & & & & \\ \text { Level } & \text { Number } & \text { Mean } & \text { Std Dev Std Err Mean Lower 95\% Upper 95\% }\end{array}$

$\begin{array}{lrrrrrr}\text { Level } & \text { Number } & \text { Mean } & \text { Std Dev } & \text { Std Err Mean } & \text { Lower 95\% } & \text { Upper 95\% } \\ \text { Coliwasa } & 16 & 10.6875 & 0.093303 & 0.02333 & 10.638 & 10.737\end{array}$

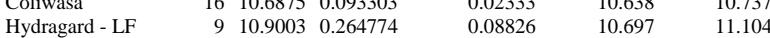

Tes

Hydragard - LF-Coliwasa

Assuming unequal variances

Difference $\quad 0.212831$ t Ratio 2.331421

Std Err Dif $\quad 0.091288$ DF $\quad 9.132851$

Upper CL Dif 0.418882 Prob $>|t| \quad 0.0442$

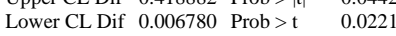

$\begin{array}{lrl}\text { Confidence } & 0.95 \text { Prob }<\mathrm{t} & 0.9779\end{array}$

Tests that the Variances are Equal

$\begin{array}{lrrrr}\text { Level } & \text { Count } & \text { Std Dev } & \text { MeanAbsDif to Mean } & \text { MeanAbsDif to Median } \\ \text { Coliwasa } & 16 & 0.0933025 & 0.0746943 & 0.0732181\end{array}$

$\begin{array}{lrrrr}\text { Coliwasa } & 16 & 0.0933025 & 0.0746943 & 0.0732181 \\ \text { Hydragard - LF } & 9 & 0.2647736 & 0.2122772 & 0.2141433\end{array}$

Test F Ratio DFNum DFDen p-Value

$\begin{array}{lllll}\text { O'Brien[.5] } & 10.4279 & 1 & 23 & 0.0037\end{array}$

Brown-Forsythe $12.3700 \quad 1 \quad 23 \quad 0.0018$

$\begin{array}{lllll}\text { Levene } & 12.7555 & 1 & 23 & 0.0016\end{array}$

$\begin{array}{lrrrr}\text { Bartlett } \quad 11.2596 & 1 & 0.0008\end{array}$

$\begin{array}{lllll}\text { F Test 2-sided } & 8.0531 & 8 & 15 & 0.0006\end{array}$

Welch Anova testing Means Equal, allowing Std Devs Not Equal

F Ratio DFNum DFDen Prob $>$ F

$5.4355 \quad \begin{array}{lrr}0.1329 & 0.0442\end{array}$

t Test
2.3314
Oneway Analysis of Measurement By Sampler Type of Material=SME Simulant, Analytical Block=6, Oxide/Analyte $=$ B2O3 (wt \%), Targeted $=4.259$

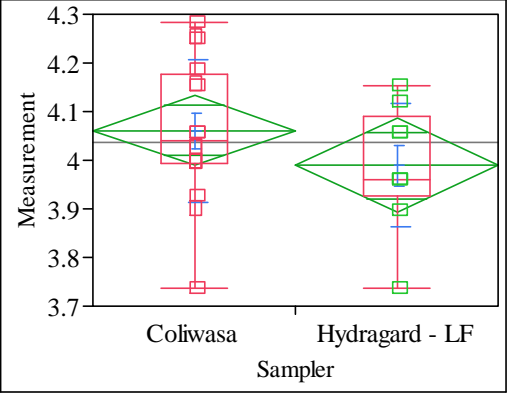

Excluded Rows

Oneway Anova

Summary of Fit

Rsquare $\quad 0.062146$

Adj Rsquare $\quad 0.02137$

Root Mean Square Error $\quad 0.139973$

Mean of Response $\quad 4.035179$

t Test

Hydragard - LF-Coliwasa

Assuming equal variances

$\begin{array}{lrr}\text { Difference } & -0.07200 \text { t Ratio } & -1.23454 \\ \text { Std } & \end{array}$

$\begin{array}{lrlr}\text { Std Err Dif } & 0.05832 & \text { DF } & 23 \\ \text { Upper CL Dif } & 0.04865 & \text { Prob }>|t| & 0.2295\end{array}$

Lower CL Dif -0.19265 Prob $>t \quad 0.8853$

$\begin{array}{lll}\text { Confidence } & 0.95 \text { Prob }<\mathrm{t} \quad 0.1147\end{array}$

Analysis of Variance

Source DF Sum of Squares Mean Square F Ratio Prob $>$ F

$\begin{array}{lrrrrr}\text { Sampler } & 1 & 0.02986029 & 0.029860 & 1.5241 & 0.2295\end{array}$

$\begin{array}{llll}\text { Error } & 23 & 0.45062300 & 0.019592\end{array}$

C. Total $24 \quad 0.48048328$

Means for Oneway Anova

Level Number Mean Std Error Lower 95\% Upper 95\%

$\begin{array}{lrrrrr}\text { Coliwasa } & 16 & 4.06110 & 0.03499 & 3.9887 & 4.1335\end{array}$

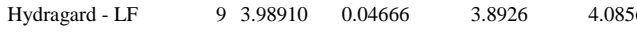

Std Error uses a pooled estimate of error variance

Means and Std Deviations

Level Number Mean Std Dev Std Err Mean Lower 95\% Upper 95\%

$\begin{array}{lllllll}\text { Coliwasa } & 16 & 4.06110 & 0.146320 & 0.03658 & 3.9831 & 4.1391\end{array}$

$\begin{array}{llllll}\text { Hydragard - LF } & 9 & 3.98910 & 0.127221 & 0.04241 & 3.8913\end{array}$

t Test

Hydragard - LF-Coliwasa

Assuming unequal variances

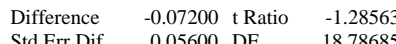

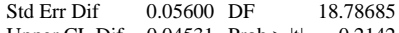

Upper CL Dif 0.04531 Prob $>|t| \quad 0.2142$

Lower CL Dif -0.18931 Prob $>t \quad 0.8929$

$\begin{array}{lll}\text { Confidence } & 0.95 \text { Prob }<\mathrm{t} \quad 0.1071\end{array}$

Tests that the Variances are Equal

$\begin{array}{lrrrr}\text { Level } & \text { Count } & \text { Std Dev } & \text { MeanAbsDif to Mean } & \text { MeanAbsDif to Median } \\ \text { Coliwasa } & 16 & 0.1463196 & 0.1137027 & 0.1126965\end{array}$

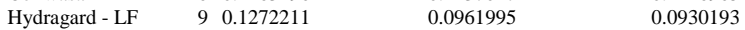

Test F Ratio DFNum DFDen p-Value

$\begin{array}{lllll}\text { O'Brien[.5] } & 0.1918 & 1 & 23 & 0.6655\end{array}$

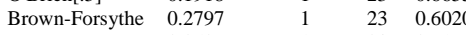

$\begin{array}{lllll}\text { Levene } & 0.2529 & 1 & 23 & 0.6198\end{array}$

$\begin{array}{lrrrr}\text { Bartlett } & 0.1886 & 1 & & 0.6641 \\ \text { F Test 2-sided } & 1.3228 & 15 & 8 & 0.7103\end{array}$

Welch Anova testing Means Equal, allowing Std Devs Not Equal

F Ratio DFNum DFDen Prob $>$ F

$\begin{array}{llll}1.6529 & 1 & 18.787 & 0.2142\end{array}$

t Tes

1.2856 
SRNL-STI-2011-00693

Revision 0

\section{Exhibit C2. Statistical Comparisons for Low-Rheology (Phase 3) Simulant Testing}

Oneway Analysis of Measurement By Sampler Type of Material=SME Simulant, Analytical Block $=6$, Oxide $/$ Analyte $=\mathrm{BaO}(\mathrm{wt} \%)$, Targeted $=\mathbf{0 . 0 9 1 9}$

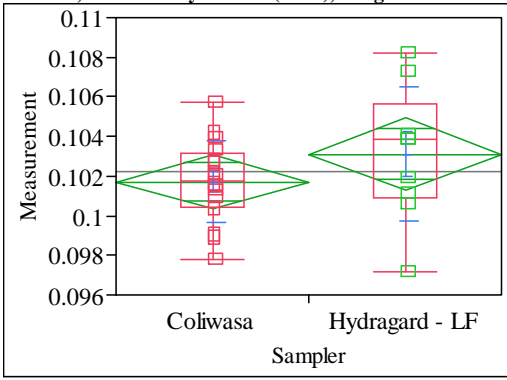

Excluded Rows

Oneway Anova

Summary of Fi

Adj Rsquare $\quad 0.06751$

Root Mean Square Error $\quad 0.026968$

Mean of Response $\quad 0.102213$

Observations (or Sum Wgts) 25

t Test

Hydragard - LF-Coliwasa

Assuming equal variances

Difference $\quad 0.00141$ t Ratio 1.290411

\begin{tabular}{lrr} 
Std Err Dif $\quad 0.00109$ DF & 23 \\
\hline & 0.00367 Prob $>|t|$ & 0.2097
\end{tabular}

Upper CL Dif 0.00367 Prob $>$

Lower CL Dif -0.00085 Prob $>\mathrm{t} \quad 0.1049$

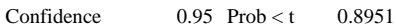

Analysis of Variance

Source DF Sum of Squares Mean Square F Ratio Prob > F

$\begin{array}{lrrrrr}\text { Sampler } & 1 & 0.00001143 & 0.000011 & 1.6652 & 0.2097\end{array}$

C. Total $24 \quad 0.0001693$

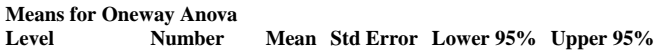

$\begin{array}{lrrrrr}\text { Coliwasa } & 16 & 0.101706 & 0.00066 & 0.10035 & 0.10306\end{array}$

$\begin{array}{lrrrrr}\text { Hydragard - LF } & 16 & 0.101706 & 0.00066 & 0.10035 & 0.10306 \\ & 9 & 0.103115 & 0.00087 & 0.10131 & 0.10492\end{array}$

Std Error uses a pooled estimate of error variance

Means and Std Deviations

$\begin{array}{lllllll}\text { Level Number Mean } & \text { Std Dev } & \text { Std Err Mean } & \text { Lower 95\% } & \text { Upper 95\% }\end{array}$

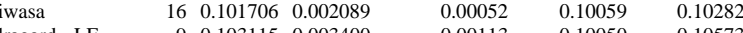

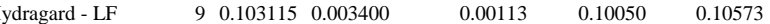

t Test

Hydragard - LF-Coliwasa

Assuming unequal variances

Difference $\quad 0.00141$ t Ratio 1.129038

Std Err Dif $\quad 0.00125$ DF 11.48092

Upper CL Dif 0.00414 Prob $>|t| \quad 0.2819$

Lower CL Dif -0.00132 Prob $>\mathrm{t} \quad 0.1410$

$\begin{array}{lrl}\text { Confidence } & 0.95 \text { Prob }<\mathrm{t} & 0.8590\end{array}$

Tests that the Variances are Equal

Level Count Std Dev MeanAbsDif to Mean MeanAbsDif to Median

$\begin{array}{lrrrr}\text { Coliwasa } & 16 & 0.0020886 & 0.0015840 & 0.0015840 \\ \text { Hydragard - LF } & 9 & 0.0033999 & 0.0025859 & 0.0025059\end{array}$

Test F Ratio DFNum DFDen p-Value

$\begin{array}{lrrrr}\text { O'Brien[.5] } & 2.7590 & 1 & 23 & 0.1103\end{array}$

Brown-Forsythe $\begin{array}{llll}1.7069 & 1 & 23 & 0.2043\end{array}$

$\begin{array}{lllll}\text { Levene } & 2.3093 & 1 & 23 & 0.1422\end{array}$

$\begin{array}{lllll}\text { Bartlett } & 2.5113 & 1 & & 0.1130 \\ \text { F Test 2-sided } & 2.6499 & 8 & 15 & 0.0988\end{array}$

Welch Anova testing Means Equal, allowing Std Devs Not Equal

F Ratio DFNum DFDen Prob $>$ F

$\begin{array}{llll}1.2747 & 1 & 11.481 & 0.2819\end{array}$

t Test

1.1290
Oneway Analysis of Measurement By Sampler Type of Material=SME Simulant, Analytical Block $=6$, Oxide/Analyte $=\mathrm{CaO}($ wt $\%)$, Targeted $=\mathbf{1 . 1 4 6 2}$

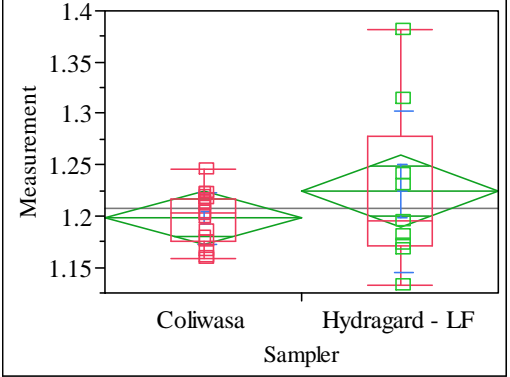

Excluded Rows

Oneway Anova

Summary of Fit

Adj Rsquare

Root Mean Square Error $\quad 0.050753$

Mean of Response Er. 1.207342

$\begin{array}{lr} & 1.207342 \\ \text { Observations (or Sum Wgts) } & 25\end{array}$

Test

Hydragard - LF-Coliwasa

Assuming equal variances

Difference $\quad 0.02601$ t Ratio 1.23002

Std Err Dif $\quad 0.02115$ DF $\quad 23$

$\begin{array}{rrrr}\text { Upper CL Dif } & 0.06976 & \text { Prob }>|t| & 0.2311\end{array}$

Lower CL Dif -0.01773 Prob $>t \quad 0.1156$

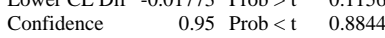

Analysis of Variance

Source DF Sum of Squares Mean Square F Ratio Prob $>$ F

$\begin{array}{llllll}\text { Sampler } & 1 & 0.00389721 & 0.003897 & 1.5129 & 0.2311\end{array}$

$\begin{array}{llll}\text { Error } & 23 & 0.05924574 & 0.002576\end{array}$

C. Total $24 \quad 0.06314295$

Means for Oneway Anova

Level Number Mean Std Error Lower 95\% Upper 95\%

$\begin{array}{lrrrrr}\text { Coliwasa } & 16 & 1.19798 & 0.01269 & 1.1717 & 1.2242 \\ \text { Hydragard - LF } & 9 & 1.22399 & 0.01692 & 1.1890 & 1.2590\end{array}$

Std Error uses a pooled estimate of error variance

Means and Std Deviations

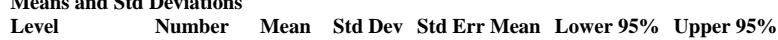

$\begin{array}{lrrrrrr}\text { Level } & \text { Number } & \text { Mean } & \text { Std Dev } & \text { Std Err Mean } & \text { Lower 95\% } & \text { Upper 95\% } \\ \text { Coliwasa } & 16 & 1.19798 & 0.025202 & 0.00630 & 1.1845 & 1.2114\end{array}$

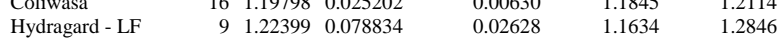

t Test

Hydragard - LF-Coliwasa

Assuming unequal variances

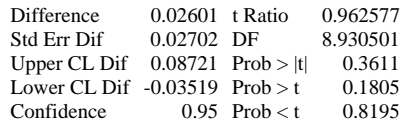

Tests that the Variances are Equal

Level Count Std Dev MeanAbsDif to Mean MeanAbsDif to Median

$\begin{array}{lrrrr}\text { Coliwasa } & 16 & 0.0252023 & 0.0206929 & 0.0202009\end{array}$

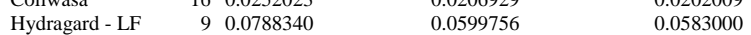

Test F Ratio DFNum DFDen p-Value

$\begin{array}{lrrrr}\text { O'Brien[.5] } & 5.7687 & 1 & 23 & 0.0248\end{array}$

$\begin{array}{lllll}\text { Brown-Forsythe } & 6.3522 & 1 & 23 & 0.0191\end{array}$

$\begin{array}{lllll}\text { Levene } & 10.2110 & 1 & 23 & 0.0040\end{array}$

$\begin{array}{lrrrr}\text { Bartlett } & 13.2985 & 1 & & 0.0003 \\ \text { F Test 2-sided } & 9.7847 & 8 & 15 & 0.0002\end{array}$

Welch Anova testing Means Equal, allowing Std Devs Not Equal

F Ratio DFNum DFDen Prob $>$ F

$\begin{array}{rrrr}0.9266 & 1 & 8.9305 & 0.3611\end{array}$

t Test 
SRNL-STI-2011-00693

Revision 0

\section{Exhibit C2. Statistical Comparisons for Low-Rheology (Phase 3) Simulant Testing}

Oneway Analysis of Measurement By Sampler Type of Material=SME Simulant, Analytica Block $=6$, Oxide $/$ Analyte $=\mathrm{Cr} 2 \mathrm{O} 3(\mathrm{wt} \%)$, Targeted $=\mathbf{0 . 0 9 9 8}$

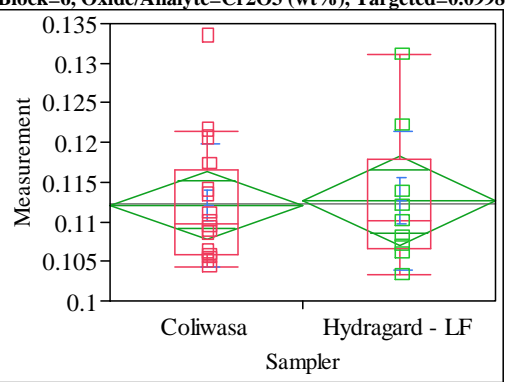

Excluded Rows

Oneway Anova

Summary of Fit

Adj Rsquare

Root Mean Square Error $\quad-0.04238$

Mean of Response $\quad 0.112286$

Observations (or Sum Wgts) 25

t Test

Hydragard - LF-Coliwasa

Assuming equal variances

Difference $\quad 0.00053$ t Ratio 0.155514

\begin{tabular}{lrr} 
Std Err Dif $\quad 0.00340$ DF & 23 \\
\hline
\end{tabular}

Upper CL Dif 0.00756 Prob $>|t| \quad 0.8778$

Lower CL Dif -0.00651 Prob $>t \quad 0.4389$

Confidence $\quad 0.95$ Prob $<\mathrm{t} \quad 0.5611$

Analysis of Variance

Source DF Sum of Squares Mean Square F Ratio Prob $>$ F

$\begin{array}{lrrrrr}\text { Sampler } & 1 & 0.00000161 & 1.611 \mathrm{e}-6 & 0.0242 & 0.8778\end{array}$

$\begin{array}{llll}\text { Error } & 23 & 0.00153186 & 0.000067\end{array}$

C. Total $24 \quad 0.00153347$

$\begin{array}{lcrrrr}\text { Means for Oneway Anova } & & & & \\ \text { Level } & \text { Number } & \text { Mean } & \text { Std Error } & \text { Lower 95\% } & \text { Upper 95\% }\end{array}$

$\begin{array}{lrrrrr}\text { Coliwasa } & 16 & 0.112096 & 0.00204 & 0.10787 & 0.11632 \\ \text { Hydragard - LF } & 9 & 0.112624 & 0.00272 & 0.10700 & 0.11825\end{array}$

Std Error uses a pooled estimate of error variance

Means and Std Deviations

$\begin{array}{lrrrrrr}\text { Level } & \text { Number } & \text { Mean } & \text { Std Dev } & \text { Std Err Mean } & \text { Lower 95\% } & \text { Upper 95\% } \\ \text { Coliwasa } & 16 & 0.112096 & 0.007790 & 0.00195 & 0.10794 & 0.11625\end{array}$

$\begin{array}{lrrrrrr}\text { Hydragard - LF } & 9 & 0.112624 & 0.008815 & 0.00294 & 0.10585 & 0.11940\end{array}$

t Test

Hydragard - LF-Coliwasa

Assuming unequal variances

Difference $\quad 0.00053$ t Ratio $\quad 0.150012$

Std Err Dif $\quad 0.00353$ DF $\quad 15.02529$

Upper CL Dif 0.00804 Prob $>|t| \quad 0.8828$

Lower CL Dif -0.00698 Prob $>t \quad 0.4414$

$\begin{array}{lrr}\text { Confidence } & 0.95 \text { Prob }<\mathrm{t} & 0.5586\end{array}$

Tests that the Variances are Equal

Level Count Std Dev MeanAbsDif to Mean MeanAbsDif to Median

$\begin{array}{lrrrr}\text { Coliwasa } & 16 & 0.0077897 & 0.0058533 & 0.0054901 \\ \text { Hydragard - LF } & 9 & 0.0088152 & 0.0064743 & 0.0062524\end{array}$

$\begin{array}{lrrrr}\text { Test } & \text { F Ratio } & \text { DFNum } & \text { DFDen } & \text { p-Value } \\ \text { O'Brien[.5] } & 0.1101 & 1 & 23 & 0.7430 \\ \text { Brown-Forsythe } & 0.0908 & 1 & 23 & 0.7658 \\ \text { Levene } & 0.0843 & 1 & 23 & 0.7742 \\ \text { Bartlett } & 0.1556 & 1 & & 0.6932 \\ \text { F Test 2-sided } & 1.2806 & 8 & 15 & 0.6465\end{array}$

$\begin{array}{lllll}\text { F Test 2-sided } & 1.2806 & 8 & 15 & 0.6465\end{array}$

Welch Anova testing Means Equal, allowing Std Devs Not Equal

F Ratio DFNum DFDen Prob $>$ F

$\begin{array}{llll}0.0225 & 1 & 15.025 & 0.8828\end{array}$

t Test

0.1500
Oneway Analysis of Measurement By Sampler Type of Material=SME Simulant, Analytica Block $=6$, Oxide $/$ Analyte $=\mathbf{C u O}($ wt $\%)$, Targeted $=\mathbf{0 . 0 5 0 4}$

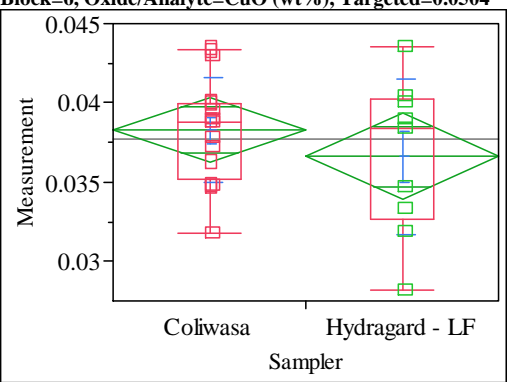

Excluded Rows

Oneway Anova

Summary of Fit

Adj Rsquare

Root Mean Square Error $\quad 0.000797$

Mean of Response $\quad 0.037669$

Observations (or Sum Wgts)

t Test

Hydragard - LF-Coliwasa

Assuming equal variances

$\begin{array}{lrr}\text { Difference } & -0.00166 \text { t Ratio } & -1.00953 \\ \text { Std Err Dif } & 0.00164 & \text { DF }\end{array}$

$\begin{array}{lrlr}\text { Std Err Dif } & 0.00164 \text { DF } & 23 \\ \text { Upper CL Dif } & 0.00174 & \text { Prob }>|t| & 0.3232\end{array}$

Lower CL Dif -0.00505 Prob $>t \quad 0.8384$

$\begin{array}{lrr}\text { Confidence } & 0.95 \text { Prob }<\mathrm{t} & 0.1616\end{array}$

Analysis of Variance

Source DF Sum of Squares Mean Square F Ratio Prob $>$ F

$\begin{array}{llllll}\text { Sampler } & 1 & 0.00001583 & 0.000016 & 1.0191 & 0.3232\end{array}$

$\begin{array}{llll}\text { Error } & 23 & 0.00035724 & 0.000016 \\ \text { C. Total } & 24 & 0.00037307 & \end{array}$

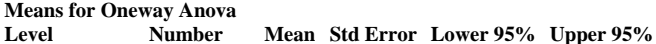

$\begin{array}{lrrrrr}\text { Level } & \text { Number } & \text { Mean } & \text { Std Error } & \text { Lower 95\% } & \text { Upper 95\% } \\ \text { Coliwasa } & 16 & 0.038266 & 0.00099 & 0.03623 & 0.04030\end{array}$

$\begin{array}{lrrrrr}\text { Hydragard - LF } & 9 & 0.038266 & 0.00099 & 0.03623 & 0.04030 \\ & 9 & 0.036608 & 0.00131 & 0.03389 & 0.03933\end{array}$

Std Error uses a pooled estimate of error variance

Means and Std Deviations

Level Number Mean Std Dev Std Err Mean Lower 95\% Upper 95\%

$\begin{array}{lllllll}\text { Coliwasa } & 16 & 0.038266 & 0.003324 & 0.00083 & 0.03649 & 0.04004\end{array}$

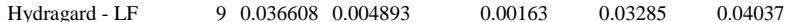

t Test

Hydragard - LF-Coliwasa

Assuming unequal variances

$\begin{array}{lrlr}\text { Difference } & -0.00166 \text { t Ratio } & -0.90562 \\ \text { Std Err Dif } & 0.00183 \text { DF } & 12.25053\end{array}$

Std Err Dif $\quad 0.00183$ DF 12.25053

Upper CL Dif 0.00232 Prob $>|t| 0.3826$

Lower CL Dif -0.00564 Prob $>\mathrm{t} \quad 0.8087$

$\begin{array}{lrl}\text { Confidence } & 0.95 \text { Prob }<\mathrm{t} & 0.1913\end{array}$

Tests that the Variances are Equal

Level Count Std Dev MeanAbsDif to Mean MeanAbsDif to Median

$\begin{array}{lrrrr} & 16 & 0.0033235 & 0.0026102 & 0.0025427 \\ \text { Hydragard - LF } & 9 & 0.0048933 & 0.0040830 & 0.0039362\end{array}$

$\begin{array}{lrrrr}\text { Test } & \text { F Ratio } & \text { DFNum } & \text { DFDen } & \text { p-Value } \\ \text { O'Brien[.5] } & 2.4173 & 1 & 23 & 0.1337 \\ \text { Brown-Forsythe } & 1.7249 & 1 & 23 & 0.2020 \\ \text { Levene } & 2.9268 & 1 & 23 & 0.1006 \\ \text { Bartlett } & 1.5728 & 1 & . & 0.2098 \\ \text { F Test 2-sided } & 2.1677 & 8 & 15 & 0.1870\end{array}$

Welch Anova testing Means Equal, allowing Std Devs Not Equal

F Ratio DFNum DFDen Prob $>$ F

$\begin{array}{llll}0.8202 & 1 & 12.251 & 0.3826\end{array}$

t Test

0.9056 


\section{Exhibit C2. Statistical Comparisons for Low-Rheology (Phase 3) Simulant Testing}

Oneway Analysis of Measurement By Sampler Type of Material=SME Simulant, Analytical Block $=6$, Oxide $/$ Analyte $=\mathrm{Fe} / \mathrm{Li}$, Targeted $=2.452$

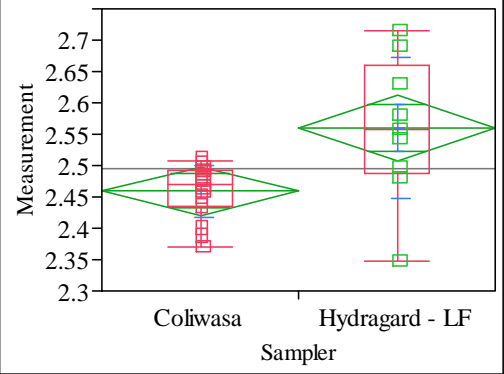

Exc

Oneway Anova

Summary of Fit

Rsquare $\quad 0.314619$

Adj Rsquare $\quad 0.28482$

Root Mean Square Error $\quad 0.074781$

Mean of Response 2.49539

Observations (or Sum Wgts) $\quad 25$

t Test

Hydragard - LF-Coliwasa

Assuming equal variances

Difference $\quad 0.101244$ t Ratio $\quad 3.249307$

Std Err Dif $\quad 0.031159$ DF 23

Upper CL Dif 0.165701 Prob $>|t| \quad 0.0035$

$\begin{array}{lll}\text { Lower CL Dif } 0.036787 & \text { Prob }>t \quad 0.0018\end{array}$

$\begin{array}{lrl} & \\ \text { Confidence } & 0.95 \text { Prob }<\mathrm{t} & 0.9982\end{array}$

Analysis of Variance

Source DF Sum of Squares Mean Square F Ratio Prob $>$ F

$\begin{array}{llllll}\text { Sampler } & 1 & 0.05904196 & 0.059042 & 10.5580 & 0.0035\end{array}$

$\begin{array}{llll}\text { Error } & 23 & 0.12861958 & 0.005592\end{array}$

C. Total $24 \quad 0.18766154$

Means for Oneway Anova

Level Number Mean Std Error Lower 95\% Upper 95\%

$\begin{array}{lrrrrr}\text { Coliwasa } & 16 & 2.45894 & 0.01870 & 2.4203 & 2.4976\end{array}$

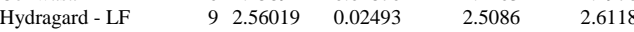

Std Error uses a pooled estimate of error variance

Means and Std Deviations

Level Number Mean Std Dev Std Err Mean Lower 95\% Upper 95\%

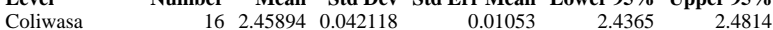

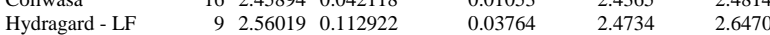

Test

Hydragard - LF-Coliwasa

Assuming unequal variances

Difference $\quad 0.101244$ t Ratio 2.590315

Std Err Dif $\quad 0.039086$ DF $\quad 9.270779$

Upper CL Dif 0.189270 Prob $>|t| r \mid 0.0285$

Lower CL Dif 0.013218 Prob $>t \quad 0.0143$

$\begin{array}{lrl}\text { Confidence } & 0.95 \text { Prob }<\mathrm{t} & 0.9857\end{array}$

Tests that the Variances are Equal

Level Count Std Dev MeanAbsDif to Mean MeanAbsDif to Median

Coliwasa $\quad 16 \quad 0.0421182$

$\begin{array}{lrrrr}\text { Coliwasa } & 16 & 0.0421182 & 0.0326870 & 0.0313272 \\ \text { Hydragard - LF } & 9 & 0.1129217 & 0.0833456 & 0.0849148\end{array}$

Test F Ratio DFNum DFDen p-Value

$\begin{array}{lrrrr}\text { O'Brien[.5] } & 5.8101 & 1 & 23 & 0.0243\end{array}$

$\begin{array}{lllll}\text { Brown-Forsythe } & 7.5412 & 1 & 23 & 0.0115\end{array}$

$\begin{array}{lllll}\text { Levene } & 6.9376 & 1 & 23 & 0.0148\end{array}$

$\begin{array}{lrrrr}\text { Bartlett } & 10.1280 & 1 & & 0.0015 \\ \text { F Test 2-sided } & 7.1881 & 8 & 15 & 0.0011\end{array}$

Welch Anova testing Means Equal, allowing Std Devs Not Equal

F Ratio DFNum DFDen Prob $>$ F

$\begin{array}{rrrr}6.7097 & 1 & 9.2708 & 0.0285\end{array}$

t Test
2.5903
Oneway Analysis of Measurement By Sampler Type of Material=SME Simulant, Analytical Block $=6$, Oxide/Analyte $=\mathrm{Fe} 2 \mathrm{O} 3$ (wt\%), Targeted $=11.462$

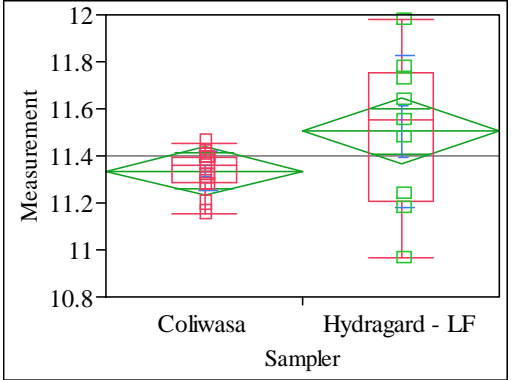

Excluded Rows

Oneway Anova

Summary of Fit

Adj Rsquare $\quad 0.110463$

Root Mean Square Error $\quad 0.201727$

Root Mean Square Eror -0.110427

$\begin{array}{lr}\text { Mean of Response } & 11.397 \\ \text { Observations (or Sum Wgts) } & 25\end{array}$

t Test

Hydragard - LF-Coliwasa

Assuming equal variances

$\begin{array}{lrlr}\text { Difference } & 0.16769 & \text { t Ratio } & 1.995078 \\ \text { Std Err Dif } & 0.08405 & \text { DF } & 23 \\ \text { Upper CL Dif } & 0.34157 & \text { Prob }>|t| & 0.0580 \\ \text { Lower CL Dif } & -0.00618 & \text { Prob }>\text { t } & 0.0290 \\ \text { Confidence } & 0.95 & \text { Prob }<\mathrm{t} & 0.9710\end{array}$

Analysis of Variance

Source DF Sum of Squares Mean Square F Ratio Prob > F

$\begin{array}{llllll}\text { Sampler } & 1 & 0.1619745 & 0.161974 & 3.9803 & 0.0580\end{array}$

$\begin{array}{llll}\text { Error } & 23 & 0.9359541 & 0.040694\end{array}$

$\begin{array}{lll}\text { C. Total } & 24 & 1.0979286\end{array}$

Means for Oneway Anova

Level Number Mean Std Error Lower 95\% Upper 95\%

$\begin{array}{lrrrrr}\text { Coliwasa } & 16 & 11.3366 & 0.05043 & 11.232 & 11.441 \\ \text { Hydragard - LF } & 9 & 11.5043 & 0.06724 & 11.365 & 11.643\end{array}$

Std Error uses a pooled estimate of error variance

Means and Std Deviations

Level Number Mean Std Dev Std Err Mean Lower 95\% Upper 95\%

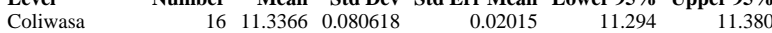

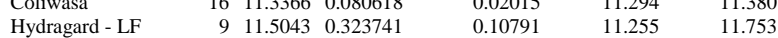

t Test

Hydragard - LF-Coliwasa

Assuming unequal variances

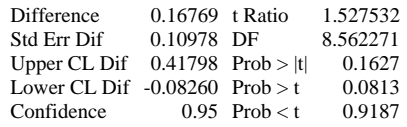

Tests that the Variances are Equal

Level Count Std Dev MeanAbsDif to Mean MeanAbsDif to Median

$\begin{array}{lrrrr}\text { Coliwasa } & 16 & 0.0806176 & 0.0635546 & 0.0616558\end{array}$

$\begin{array}{lrrrr}\text { Hydragard - LF } & 9 & 0.3237410 & 0.2562870 & 0.2589346\end{array}$

Test F Ratio DFNum DFDen p-Value

$\begin{array}{lllll}\text { O'Brien[.5] } & 10.5923 & 1 & 23 & 0.0035\end{array}$

Brown-Forsythe $16.8914 \quad 1 \quad 23 \quad 0.0004$

$\begin{array}{lllll}\text { Levene } & 17.5640 & 1 & 23 & 0.0003\end{array}$

$\begin{array}{lllll}\text { Bartlett } & 19.0085 & 1 & <.0001\end{array}$

Welch Anova testing Means Equal, allowing Std Devs Not Equal

F Ratio DFNum DFDen Prob $>$ F

$\begin{array}{rrrr}2.3334 & 1 & 8.5623 & 0.1627\end{array}$

t Test 
SRNL-STI-2011-00693

Revision 0

\section{Exhibit C2. Statistical Comparisons for Low-Rheology (Phase 3) Simulant Testing}

Oneway Analysis of Measurement By Sampler Type of Material=SME Simulant, Analytical Block=6, Oxide/Analyte $=$ K2O (wt \%), Targeted $=\mathbf{0 . 1 5 9 1}$

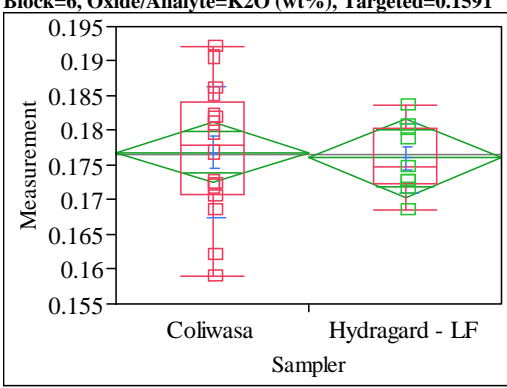

Excluded Rows

Oneway Anova

Summary of Fit

Adj Rsquare

Root Mean Square Error $\quad-0.04048$

Mean of Response $\quad 0.008223$

Observations (or Sum Wgts) 25

t Test

Hydragard - LF-Coliwasa

Assuming equal variances

Difference $\quad-0.00088$ t Ratio $\quad-0.25758$

$\begin{array}{lrr}\text { Std Err Dif } & 0.00343 \text { DF } & 23 \\ & \text { DF }\end{array}$

Upper CL Dif 0.00621 Prob $>|t| \quad 0.7990$

Lower CL Dif -0.00797 Prob $>\mathrm{t} \quad 0.6005$

Confidence $\quad 0.95$ Prob $<\mathrm{t} \quad 0.3995$

Analysis of Varianc

Source DF Sum of Squares Mean Square F Ratio Prob > F

$\begin{array}{llllll}\text { Sampler } & 1 & 0.00000449 & 4.486 \mathrm{e}-6 & 0.0663 & 0.7990\end{array}$

$\begin{array}{llll}\text { Error } & 23 & 0.00155517 & 0.000068\end{array}$

C. Total $24 \quad 0.00155966$

Means for Oneway Anova
Number

$\begin{array}{lrrrrr}\text { Level } & \text { Number } & \text { Mean } & \text { Std Error } & \text { Lower 95\% } & \text { Upper 95\% } \\ \text { Coliwasa } & 16 & 0.176888 & 0.00206 & 0.17264 & 0.18114\end{array}$

$\begin{array}{lrrrrr}\text { Coliwasa } & 16 & 0.176888 & 0.00206 & 0.17264 & 0.18114 \\ \text { Hydragard - LF } & 9 & 0.176005 & 0.00274 & 0.17034 & 0.18168\end{array}$

Std Error uses a pooled estimate of error variance

Means and Std Deviations

Level Number Mean Std Dev Std Err Mean Lower 95\% Upper 95\%

$\begin{array}{lllllll}\text { Coliwasa } & 16 & 0.176888 & 0.009502 & 0.00238 & 0.17182 & 0.18195\end{array}$

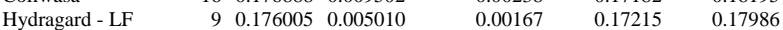

t Test

Hydragard - LF-Coliwasa

Assuming unequal variances

Difference $\quad-0.00088$ t Ratio $\quad-0.30392$

Std Err Dif $\quad 0.00290$ DF 22.97051

Upper CL Dif 0.00512 Prob $>|t| \quad 0.7639$

Lower CL Dif -0.00689 Prob $>t \quad 0.6180$

$\begin{array}{lrl}\text { Confidence } & 0.95 \text { Prob }<\mathrm{t} & 0.3820\end{array}$

Tests that the Variances are Equal

Level Count Std Dev MeanAbsDif to Mean MeanAbsDif to Median

$\begin{array}{lrrrr}\text { Coliwasa } & 16 & 0.0095021 & 0.0077923 & 0.0077923 \\ \text { Hydragard - LF } & 9 & 0.0050101 & 0.0042979 & 0.0043499\end{array}$

$\begin{array}{lrrrr}\text { Test } & \text { F Ratio } & \text { DFNum } & \text { DFDen } & \text { p-Value } \\ \text { O'Brien[.5] } & 3.1173 & 1 & 23 & 0.0907 \\ \text { Brown-Forsythe } & 3.4744 & 1 & 23 & 0.0751 \\ \text { Levene } & 3.8754 & 1 & 23 & 0.0612 \\ \text { Bartlett } & 3.4206 & 1 & . & 0.0644 \\ \text { F Test 2-sided } & 3.5970 & 15 & 8 & 0.0733\end{array}$

$\begin{array}{lllll}\text { F Test 2-sided } & 3.5970 & 15 & 8 & 0.0733\end{array}$

Welch Anova testing Means Equal, allowing Std Devs Not Equal

F Ratio DFNum DFDen Prob $>$ F

$\begin{array}{llll}0.0924 & 1 & 22.971 & 0.7639\end{array}$

t Test

0.3039
Oneway Analysis of Measurement By Sampler Type of Material=SME Simulant, Analytical Block=6, Oxide/Analyte $=$ Li2O (wt\%), Targeted $=4.674$

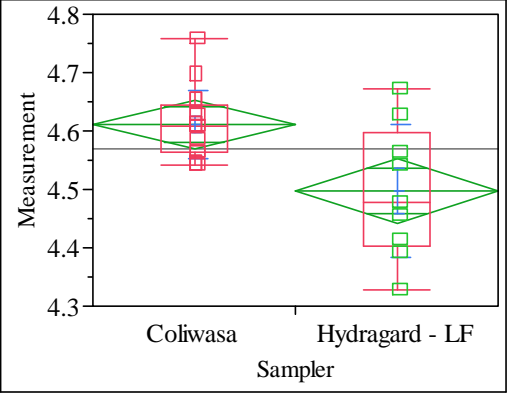

Excluded Rows

Oneway Anova

Summary of Fit

Rsquare

Adj Rsquare

Root Mean Square Error $\quad 0.081959$

Mean of Response 4.570176

t Test

Hydragard - LF-Coliwasa

Assuming equal variances

$\begin{array}{lrr}\text { Difference } & -0.11407 \text { t Ratio } & -3.34043 \\ \text { Std Err Dif } & 0.03415 \text { DF } & 23\end{array}$

$\begin{array}{lrlr}\text { Std Err Dif } & 0.03415 & \text { DF } & 23 \\ \text { Upper CL Dif } & -0.04343 & \text { Prob }>|t| & 0.0028\end{array}$

Lower CL Dif -0.18472 Prob $>t \quad 0.9986$

$\begin{array}{lrl}\text { Confidence } & 0.95 \text { Prob }<\mathrm{t} & 0.0014\end{array}$

Analysis of Variance

Source DF Sum of Squares Mean Square F Ratio Prob $>$ F

$\begin{array}{lrrrrr}\text { Sampler } & 1 & 0.07495391 & 0.074954 & 11.1585 & 0.0028\end{array}$

$\begin{array}{llll}\text { Error } & 23 & 0.15449606 & 0.006717 \\ \text { C. Total } & 24 & 0.22944997 & \end{array}$

Means for Oneway Anova

Level $\quad$ Number Mean Std Error Lower 95\% Upper 95\%

$\begin{array}{lrrrrr}\text { Level } & \text { Number } & \text { Mean } & \text { Std Error } & \text { Lower 95\% } & \text { Upper 95\% } \\ \text { Coliwasa } & 16 & 4.61124 & 0.02049 & 4.5689 & 4.6536\end{array}$

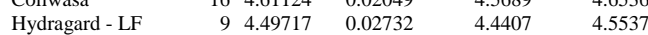

Std Error uses a pooled estimate of error variance

Means and Std Deviations

Level Number Mean Std Dev Std Err Mean Lower 95\% Upper 95\%

$\begin{array}{lllllll}\text { Coliwasa } & 16 & 4.61124 & 0.058417 & 0.01460 & 4.5801 & 4.6424\end{array}$

$\begin{array}{lllllll}\text { Hydragard - LF } & 9 & 4.49717 & 0.113638 & 0.03788 & 4.4098 & 4.5845\end{array}$

t Test

Hydragard - LF-Coliwasa

Assuming unequal variances

$\begin{array}{lrr}\text { Difference } & -0.11407 \text { t Ratio } \quad-2.8099\end{array}$

$\begin{array}{lll}\text { Std Err Dif } \quad 0.04060 \text { DF } & 10.43214\end{array}$

Upper CL Dif -0.02412 Prob $>|t| \quad 0.0178$

Lower CL Dif -0.20402 Prob $>\mathrm{t} \quad 0.9911$

$\begin{array}{lll}\text { Confidence } & 0.95 \text { Prob }<\mathrm{t} \quad 0.0089\end{array}$

Tests that the Variances are Equal

$\begin{array}{lrrrr}\text { Level } & \text { Count } & \text { Std Dev } & \text { MeanAbsDif to Mean } & \text { MeanAbsDif to Median } \\ \text { Coliwasa } & 16 & 0.0584166 & 0.0427216 & 0.0417124\end{array}$

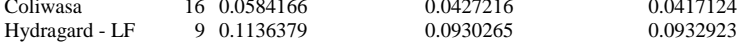

Test F Ratio DFNum DFDen p-Value

$\begin{array}{lrrrr}\text { O'Brien[.5] } & 5.7454 & 1 & 23 & 0.0250\end{array}$

$\begin{array}{lllll}\text { Brown-Forsythe } & 6.7333 & 1 & 23 & 0.0162\end{array}$

$\begin{array}{lllll}\text { Levene } & 7.0716 & 1 & 23 & 0.0140\end{array}$

$\begin{array}{lllrr}\text { Bartlett } & 4.6976 & 1 & 23 & 0.0140 \\ \text { F Test 2-sided } & 3.7842 & 8 & 15 & 0.0302 \\ \end{array}$

Welch Anova testing Means Equal, allowing Std Devs Not Equal

F Ratio DFNum DFDen Prob $>$ F

$\begin{array}{llll}7.8956 & 1 & 10.432 & 0.0178\end{array}$

t Test

2.8099 


\section{Exhibit C2. Statistical Comparisons for Low-Rheology (Phase 3) Simulant Testing}

Oneway Analysis of Measurement By Sampler Type of Material=SME Simulant, Analytical Block=6, Oxide/Analyte=MgO (wt \%), Targeted $=1.014$

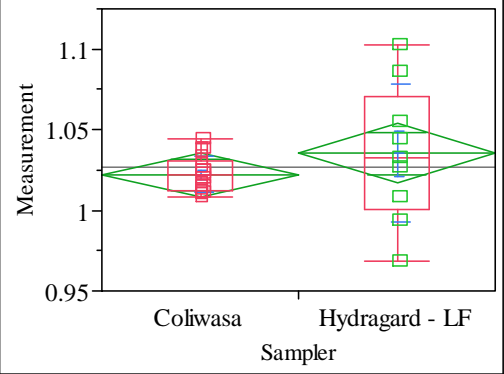

Exc

Oneway Anova

Summary of Fit

Adj Rsquare

Root Mean Square Error $\quad 0.026783$

Mean of Respons Eror 1.026886

\begin{tabular}{lr} 
Observations (or Sum Wgts) & 1.026886 \\
\hline
\end{tabular}

t Test

Hydragard - LF-Coliwasa

Assuming equal variances

Difference $\quad 0.01320$ t Ratio 1.18258

Std Err Dif $\quad 0.01116$ DF 23

Upper CL Dif 0.03628 Prob $>|t| 0.2491$

Lower CL Dif -0.00989 Prob $>t \quad 0.1245$

$\begin{array}{lrl}\text { Confidence } & 0.95 \text { Prob }<\mathrm{t} & 0.8755\end{array}$

Analysis of Variance

Source DF Sum of Squares Mean Square F Ratio Prob $>$ F

$\begin{array}{llllll}\text { Sampler } & 1 & 0.00100321 & 0.001003 & 1.3985 & 0.2491\end{array}$

$\begin{array}{llll}\text { Error } & 23 & 0.01649907 & 0.000717\end{array}$

$\begin{array}{lll}\text { C. Total } & 24 & 0.01750228\end{array}$

Means for Oneway Anova

Level $\begin{gathered}\text { Number } \\ \text { Mean Std Error }\end{gathered}$ Lower 95\% Upper 95\%

$\begin{array}{lrrrrr}\text { Coliwasa } & 16 & 1.02213 & 0.00670 & 1.0083 & 1.0360 \\ \text { Hydragard - LF } & 9 & 1.03533 & 0.00893 & 1.0169 & 1.0538\end{array}$

Std Error uses a pooled estimate of error variance

$\begin{array}{llllll}\begin{array}{l}\text { Means and Std Deviations } \\ \text { Level }\end{array} \text { Number } & \text { Mean } & \text { Std Dev Std Err Mean Lower 95\% Upper 95\% }\end{array}$

$\begin{array}{lrrrrrr}\text { Level } & \text { Number } & \text { Mean } & \text { Std Dev } & \text { Std Err Mean } & \text { Lower 95\% } & \text { Upper 95\% } \\ \text { Coliwasa } & 16 & 1.02213 & 0.011097 & 0.00277 & 1.0162 & 1.0280\end{array}$

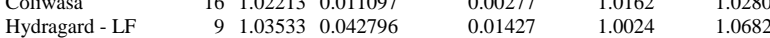

Test

Hydragard - LF-Coliwasa

Assuming unequal variances

$\begin{array}{lrlr}\text { Difference } & 0.01320 & \mathrm{t} \text { Ratio } & 0.908123 \\ \text { Std Err Dif } & 0.01453 & \text { DF } & 8.610056 \\ \text { Upper CL Dif } & 0.04630 & \text { Prob }>|\mathrm{t}| & 0.3885 \\ \text { Lower CL Dif } & -0.01991 & \text { Prob }>\mathrm{t} & 0.1943 \\ \text { Confidence } & 0.95 & \text { Prob }<\mathrm{t} & 0.8057\end{array}$

Tests that the Variances are Equal

Level Count Std Dev MeanAbsDif to Mean MeanAbsDif to Median

Coliwasa $\quad 16 \quad 0.0110974$

$\begin{array}{lrrrr}\text { Coliwasa } & 16 & 0.0110974 & 0.0091206 & 0.0091206 \\ \text { Hydragard - LF } & 9 & 0.0427957 & 0.0326747 & 0.0331660\end{array}$

Test F Ratio DFNum DFDen p-Value

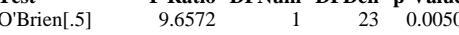

$\begin{array}{lllll}\text { Brown-Forsythe } & 14.2841 & 1 & 23 & 0.0010\end{array}$

Levene $\quad \begin{array}{llll}13.2206 & 1 & 23 & 0.0014\end{array}$

$\begin{array}{lllll}\text { Bartlett } & 18.0424 & 1 & & <.0001 \\ \text { F Test 2-sided } & 14.8716 & 8 & 15 & <.0001\end{array}$

Welch Anova testing Means Equal, allowing Std Devs Not Equal

F Ratio DFNum DFDen Prob $>$ F

$\begin{array}{rrrr}0.8247 & 1 & 8.6101 & 0.3885\end{array}$

t Test
0.9081
Oneway Analysis of Measurement By Sampler Type of Material=SME Simulant, Analytical Block $=6$, Oxide/Analyte $=\mathrm{MnO}(\mathrm{wt} \%)$, Targeted $=1.779$

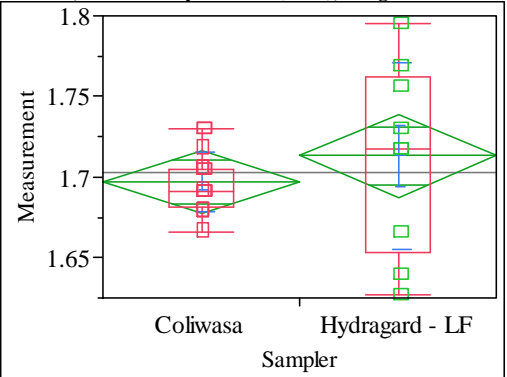

Excluded Rows

1

Oneway Anova

Summary of Fit

Rsquare $\quad 0.043374$

Adj Rsquare $\quad 0.001781$

Root Mean Square Error $\quad 0.0373$

Mean of Response 1.702835

Observations (or Sum Wgts) $\quad 25$

t Test

Hydragard - LF-Coliwasa

Assuming equal variances

$\begin{array}{lrlr}\text { Difference } & 0.01587 & \mathrm{t} \text { Ratio } & 1.021189 \\ \text { Std Err Dif } & 0.01554 & \text { DF } & 23 \\ \text { Upper CL Dif } & 0.04802 & \text { Prob }>|\mathrm{t}| & 0.3178 \\ \text { Lower CL Dif } & -0.01628 & \text { Prob }>\mathrm{t} & 0.1589 \\ \text { Confidence } & 0.95 & \text { Prob }<\mathrm{t} & 0.8411\end{array}$

Analysis of Variance

Source DF Sum of Squares Mean Square F Ratio Prob $>$ F

$\begin{array}{llllll}\text { Sampler } & 1 & 0.00145088 & 0.001451 & 1.0428 & 0.3178\end{array}$

$\begin{array}{llll}\text { Error } & 23 & 0.03199977 & 0.001391\end{array}$

C. Total $24 \quad 0.03345065$

Means for Oneway Anova

Level Number Mean Std Error Lower 95\% Upper 95\%

$\begin{array}{lrrrrr}\text { Coliwasa } & 16 & 1.69712 & 0.00933 & 1.6778 & 1.7164 \\ \text { Hydragard - LF } & 9 & 1.71299 & 0.01243 & 1.6873 & 1.7387\end{array}$

Std Error uses a pooled estimate of error variance

Means and Std Deviations

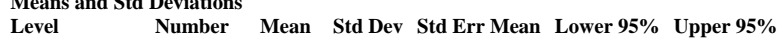

$\begin{array}{lrrrrrr}\text { Level } & \text { Number } & \text { Mean } & \text { Std Dev } & \text { Std Err Mean } & \text { Lower 95\% } & \text { Upper 95\% } \\ \text { Coliwasa } & 16 & 1.69712 & 0.018241 & 0.00456 & 1.6874 & 1.7068\end{array}$

$\begin{array}{lrrrrrr}\text { Hydragard - LF } & 9 & 1.71299 & 0.058104 & 0.01937 & 1.6683 & 1.7577\end{array}$

t Test

Hydragard - LF-Coliwasa

Assuming unequal variances

$\begin{array}{lrlr}\text { Difference } & 0.01587 & \mathrm{t} \text { Ratio } & 0.797632 \\ \text { Std Err Dif } & 0.01990 & \text { DF } & 8.897041 \\ \text { Upper CL Dif } & 0.06096 & \text { Prob }>|t| & 0.4458 \\ \text { Lower CL Dif } & -0.02922 & \text { Prob }>\text { t } & 0.2229 \\ \text { Confidence } & 0.95 & \text { Prob }<\mathrm{t} & 0.7771\end{array}$

Tests that the Variances are Equal

Level Count Std Dev MeanAbsDif to Mean MeanAbsDif to Median

$\begin{array}{lrrrr}\text { Coliwasa } & 16 & 0.0182413 & 0.0144251 & 0.0137190\end{array}$

$\begin{array}{lrrrr}\text { Hydragard - LF } & 9 & 0.0581040 & 0.0459093 & 0.0444747\end{array}$

Test F Ratio DFNum DFDen p-Value

$\begin{array}{lllll}\text { O'Brien[.5] } & 12.5004 & 1 & 23 & 0.0018\end{array}$

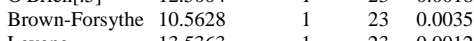

$\begin{array}{lllll}\text { Levene } & 13.5363 & 1 & 23 & 0.0012\end{array}$

$\begin{array}{lllll}\text { Bartlett } & 13.6911 & 1 & 0.0002\end{array}$

F Test 2-sided 10.1461

Welch Anova testing Means Equal, allowing Std Devs Not Equal

F Ratio DFNum DFDen Prob $>$ F

$\begin{array}{rrrr}0.6362 & 1 & 8.897 & 0.4458\end{array}$

t Test
0.7976 


\section{Exhibit C2. Statistical Comparisons for Low-Rheology (Phase 3) Simulant Testing}

Oneway Analysis of Measurement By Sampler Type of Material=SME Simulant, Analytical Block $=6$, Oxide $/$ Analyte $=\mathrm{Na2O}(\mathrm{wt} \%)$, Targeted $=11.659$

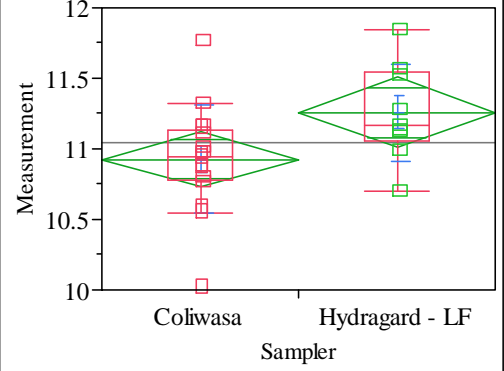

Exc

Oneway Anova

Summary of Fit

Adj Rsquare $\quad 0.133597$

Root Mean Square Error $\quad 0.367033$

Mean of Respons Eror 11.04659

$\begin{array}{lr} & 11.04659 \\ \text { Observations (or Sum Wgts) } & 25\end{array}$

t Test

Hydragard - LF-Coliwasa

Assuming equal variances

Difference $\quad 0.331571$ t Ratio $\quad 2.168115$

Std Err Dif $\quad 0.152930$ DF $\quad 23$

Upper CL Dif 0.647931 Prob $>|t| \quad 0.0407$

Lower CL Dif 0.015210 Prob $>$ t $\quad 0.0204$

$\begin{array}{lrl}\text { Confidence } & 0.95 \text { Prob }<\mathrm{t} & 0.9796\end{array}$

Analysis of Variance

Source DF Sum of Squares Mean Square F Ratio Prob $>$ F

$\begin{array}{llllll}\text { Sampler } & 1 & 0.6332488 & 0.633249 & 4.7007 & 0.0407\end{array}$

$\begin{array}{llll}\text { Error } & 23 & 3.0983996 & 0.134713\end{array}$

C. Total $24 \quad 3.7316484$

Means for Oneway Anova

Level Number Mean Std Error Lower 95\% Upper 95\%

$\begin{array}{lrrrrr}\text { Coliwasa } & 16 & 10.9272 & 0.09176 & 10.737 & 11.117\end{array}$

$\begin{array}{lrrrr}\text { Hydragard - LF } \quad 9 & 11.2588 & 0.12234 & 11.006 & 11.512\end{array}$

Std Error uses a pooled estimate of error variance

Means and Std Deviations

Level Number Mean Std Dev Std Err Mean Lower 95\% Upper 95\%

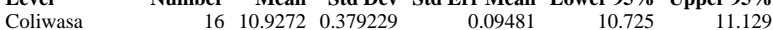

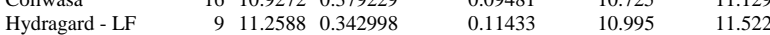

Tes

Hydragard - LF-Coliwasa

Assuming unequal variances

Difference $\quad 0.331571$ t Ratio 2.232388

Std Err Dif $\quad 0.148527$ DF $\quad 18.1959$

Upper CL Dif 0.643374 Prob $>|t| r \mid r .0384$

Lower CL Dif 0.019767 Prob $>t \quad 0.0192$

$\begin{array}{lrl}\text { Confidence } & 0.95 \text { Prob }<\mathrm{t} & 0.9808\end{array}$

Tests that the Variances are Equal

Level Count Std Dev MeanAbsDif to Mean MeanAbsDif to Median

Coliwasa $16 \quad 0.3792291$

$\begin{array}{lrrrr}\text { Coliwasa } & 16 & 0.3792291 & 0.2550669 & 0.2544350 \\ \text { Hydragard - LF } & 9 & 0.3429977 & 0.2639417 & 0.2561200\end{array}$

Test F Ratio DFNum DFDen p-Value

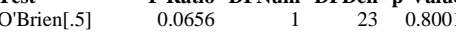

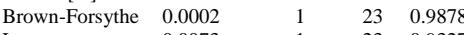

$\begin{array}{lllll}\text { Levene } & 0.0073 & 1 & 23 & 0.9327\end{array}$

$\begin{array}{lrrrr}\text { Bartlett } & 0.0981 & 1 & 0.7541\end{array}$

F Test 2-sided 1.2224

Welch Anova testing Means Equal, allowing Std Devs Not Equal

F Ratio DFNum DFDen Prob $>$ F

$\begin{array}{rrrr}4.9836 & 1 & 18.196 & 0.0384\end{array}$

t Test
2.2324
Oneway Analysis of Measurement By Sampler Type of Material=SME Simulant, Analytical Block $=6$, Oxide $/$ Analyte $=\mathrm{NiO}(\mathrm{wt} \%)$, Targeted $=\mathbf{0 . 4 1}$

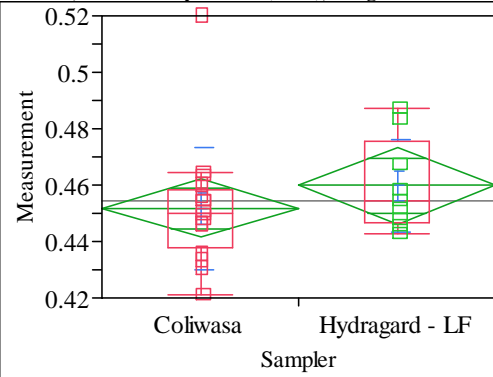

Excluded Rows

Oneway Anova

Summary of Fit

Rsquare $\quad 0.039152$

Adj Rsquare $\quad-0.00262$

Root Mean Square Error $\quad 0.01998$

Mean of Response $\quad 0.454639$

Observations (or Sum Wgts) 25

t Test

Hydragard - LF-Coliwasa

Assuming equal variances

$\begin{array}{lrlr}\text { Difference } & 0.00806 & \mathrm{t} \text { Ratio } & 0.968091 \\ \text { Std Err Dif } & 0.00832 & \text { DF } & 23 \\ \text { Upper CL Dif } & 0.02528 & \text { Prob }>|\mathrm{t}| & 0.3431 \\ \text { Lower CL Dif } & -0.00916 & \text { Prob }>\mathrm{t} & 0.1715 \\ \text { Confidence } & 0.95 & \text { Prob }<\mathrm{t} & 0.8285\end{array}$

Analysis of Variance

Source DF Sum of Squares Mean Square F Ratio Prob $>$ F

$\begin{array}{llllll}\text { Sampler } & 1 & 0.00037411 & 0.000374 & 0.9372 & 0.3431\end{array}$

$\begin{array}{llll}\text { Error } & 23 & 0.00918118 & 0.000399\end{array}$

C. Total $24 \quad 0.00955530$

Means for Oneway Anova

Level Number Mean Std Error Lower 95\% Upper 95\%

$\begin{array}{lrrrrr}\text { Coliwasa } & 16 & 0.451738 & 0.00499 & 0.44140 & 0.46207\end{array}$

$\begin{array}{llllll}\text { Hydragard - LF } & 9 & 0.459797 & 0.00666 & 0.44602 & 0.47357\end{array}$

Std Error uses a pooled estimate of error variance

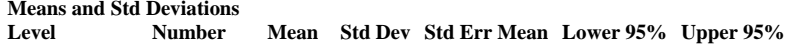

$\begin{array}{lrrrrrr}\text { Level } & \text { Number } & \text { Mean } & \text { Std Dev } & \text { Std Err Mean } & \text { Lower 95\% } & \text { Upper 95\% } \\ \text { Coliwasa } & 16 & 0.451738 & 0.021710 & 0.00543 & 0.44017 & 0.46331\end{array}$

$\begin{array}{lrrrrrr}\text { Hydragard - LF } & 9 & 0.459797 & 0.016246 & 0.00542 & 0.44731 & 0.47228\end{array}$

t Test

Hydragard - LF-Coliwasa

Assuming unequal variances

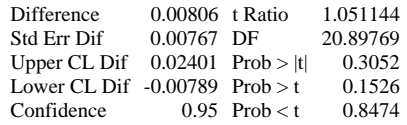

Tests that the Variances are Equal

Level Count Std Dev MeanAbsDif to Mean MeanAbsDif to Median

\begin{tabular}{lr|rrr} 
Coliwasa & 16 & 0.0217097 & 0.0135203 & 0.0133612
\end{tabular}

$\begin{array}{lrrrr}\text { Hydragard - LF } & 9 & 0.0162462 & 0.0130078 & 0.0123008\end{array}$

Test F Ratio DFNum DFDen p-Value

$\begin{array}{lrrrr}\text { O'Brien[.5] } & 0.2385 & 1 & 23 & 0.6299\end{array}$

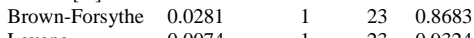

$\begin{array}{lllll}\text { Levene } & 0.0074 & 1 & 23 & 0.9324\end{array}$

$\begin{array}{llrll}\text { Bartlett } & 0.7796 & 1 & 0.3773\end{array}$

$\begin{array}{lllll}\text { F Test 2-sided } & 1.7857 & 15 & 8 & 0.4116\end{array}$

Welch Anova testing Means Equal, allowing Std Devs Not Equal

F Ratio DFNum DFDen Prob $>$ F

$\begin{array}{rrrr}1.1049 & 1 & 20.898 & 0.3052\end{array}$

t Test 


\section{Exhibit C2. Statistical Comparisons for Low-Rheology (Phase 3) Simulant Testing}

Oneway Analysis of Measurement By Sampler Type of Material=SME Simulant, Analytical Block $=6$, Oxide $/$ Analyte $=\mathrm{SiO} 2$ ( wt $\%$ ), Targeted $=\mathbf{5 0 . 9 8 5}$

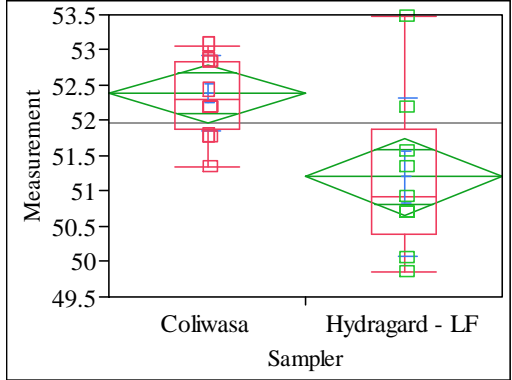

Exc

Oneway Anova

Summary of Fit

Root Mean Square Error $\quad 0.332726$

(a)

$\begin{array}{lr} & 51.95932 \\ \text { Observations (or Sum Wgts) } & 25\end{array}$

t Test

Hydragard - LF-Coliwasa

Assuming equal variances

Difference $\quad-1.1855$ t Ratio $\quad-3.601$

Std Err Dif $\quad 0.3292$ DF 23

Upper CL Dif -0.5045 Prob $>|t| 0.0015$

Lower CL Dif -1.8666 Prob $>t \quad 0.9992$

$\begin{array}{lrl}\text { Confidence } & 0.95 \text { Prob }<\mathrm{t} & 0.0008\end{array}$

Analysis of Variance

Source DF Sum of Squares Mean Square F Ratio Prob $>$ F

$\begin{array}{lllllll}\text { Sampler } & 1 & 8.095556 & 8.09556 & 12.9672 & 0.0015\end{array}$

$\begin{array}{llll}\text { Error } & 23 & 14.359097 & 0.62431\end{array}$

C. Total $24 \quad 22.454652$

Means for Oneway Anova

Level Number Mean Std Error Lower 95\% Upper 95\%

$\begin{array}{lrrrrr}\text { Coliwasa } & 16 & 52.3861 & 0.19753 & 51.977 & 52.795\end{array}$

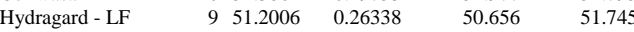

Std Error uses a pooled estimate of error variance

Means and Std Deviations

$\begin{array}{lrrrrrr}\text { Level } & \text { Number } & \text { Mean } & \text { Std Dev } & \text { Std Err Mean } & \text { Lower 95\% } & \text { Upper 95\% } \\ \text { Coliwasa } & 16 & 52.3861 & 0.53482 & 0.13371 & 52.101 & 52.671\end{array}$

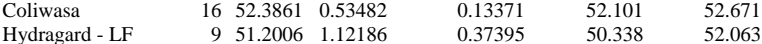

Tes

Hydragard - LF-Coliwasa

Assuming unequal variances

$\begin{array}{lrlr}\text { Difference } & -1.1855 & \text { t Ratio } & -2.98519 \\ \text { Std Err Dif } & 0.3971 & \text { DF } & 10.08827 \\ \text { Upper CL Dif } & -0.3017 & \text { Prob }>|t| & 0.0136 \\ \text { Lower CL Dif } & -2.0694 & \text { Prob }>\text { t } & 0.9932 \\ \text { Confidence } & 0.95 & \text { Prob }<\mathrm{t} & 0.0068\end{array}$

Tests that the Variances are Equal

Level Count Std Dev MeanAbsDif to Mean MeanAbsDif to Median

$\begin{array}{lrrrr}\text { Coliwasa } & 16 & 0.534825 & 0.4546012 & 0.4546012\end{array}$

$\begin{array}{rrrrr}\text { Hydragard - LF } & 9 & 1.121858 & 0.8398733 & 0.8319500\end{array}$

Test F Ratio DFNum DFDen p-Value

$\begin{array}{lrrrr}\text { O'Brien[.5] } & 3.7558 & 1 & 23 & 0.0650\end{array}$

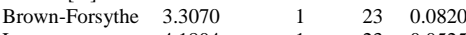

$\begin{array}{lllll}\text { Levene } & 4.1804 & 1 & 23 & 0.0525 \\ & 5.8120 & 1 & & 0.0159\end{array}$

$\begin{array}{lllll}5.8120 & 1 & & 0.0159\end{array}$

Welch Anova testing Means Equal, allowing Std Devs Not Equal

F Ratio DFNum DFDen Prob $>$ F

$\begin{array}{rrrr}8.9113 & 1 & 10.088 & 0.0136\end{array}$

t Test
2.9852
Oneway Analysis of Measurement By Sampler Type of Material=SME Simulant, Analytical Block=6, Oxide/Analyte=Sum of Oxides (wt\%), Targeted $=99.553$

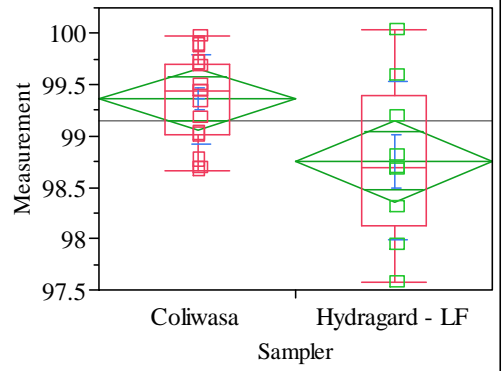

Excluded Rows

Oneway Anova

Summary of Fit

Rsquare $\quad 0.217833$

Adj Rsquare $\quad 0.183825$

Root Mean Square Error $\quad 0.576231$

Mean of Response 99.145

Observations (or Sum Wgts) 25

Hydragard - LF-Coliwasa

Assuming equal variances

Difference $\quad-0.6077$ t Ratio $\quad-2.5309$

Std Err Dif $\quad 0.2401$ DF 23

Upper CL Dif -0.1110 Prob $>|t| \quad 0.0187$

Lower CL Dif -1.1043 Prob $>t \quad 0.9907$

$\begin{array}{lrl}\text { Confidence } & 0.95 \text { Prob }<\mathrm{t} \quad 0.0093\end{array}$

Analysis of Variance

Source DF Sum of Squares Mean Square F Ratio Prob $>$ F

$\begin{array}{llllll}\text { Sampler } & 1 & 2.1268874 & 2.12689 & 6.4055 & 0.0187\end{array}$

$\begin{array}{llll}\text { Error } & 23 & 7.6369747 & 0.33204\end{array}$

C. Total $24 \quad 9.7638621$

Means for Oneway Anova

Level Number Mean Std Error Lower 95\% Upper 95\%

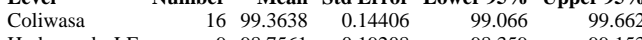

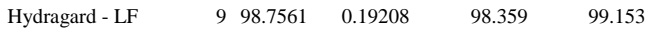

Std Error uses a pooled estimate of error variance

Means and Std Deviations

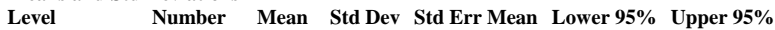

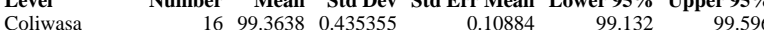

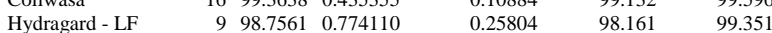

t Test

Hydragard - LF-Coliwasa

Assuming unequal variances

$\begin{array}{lrlr}\text { Difference } & -0.6077 & \mathrm{t} \text { Ratio } & -2.16982 \\ \text { Std Err Dif } & 0.2801 & \text { DF } & 10.91553 \\ \text { Upper CL Dif } & 0.0093 & \text { Prob }>|t| & 0.0530 \\ \text { Lower CL Dif } & -1.2246 & \text { Prob }>\mathrm{t} & 0.9735 \\ \text { Confidence } & 0.95 & \text { Prob }<\mathrm{t} & 0.0265\end{array}$

Tests that the Variances are Equal

Level Count Std Dev MeanAbsDif to Mean MeanAbsDif to Median

\begin{tabular}{ll|lll} 
Coliwasa & 16 & 0.4353546 & 0.3629705 & 0.3541027
\end{tabular}

$\begin{array}{lrrrr}\text { Hydragard - LF } & 9 & 0.7741099 & 0.5767855 & 0.5729941\end{array}$

Test F Ratio DFNum DFDen p-Value

$\begin{array}{lllll}\text { O'Brien[.5] } & 4.4997 & 1 & 23 & 0.0449\end{array}$

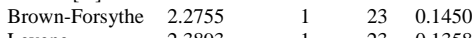

$\begin{array}{lllll}\text { Levene } & 2.3893 & 1 & 23 & 0.1358\end{array}$

$\begin{array}{lllll}\text { Bartlett } & 3.5136 & 1 & 0.0609\end{array}$

F Test 2-sided $\quad 3.1617$

Welch Anova testing Means Equal, allowing Std Devs Not Equal

F Ratio DFNum DFDen Prob $>$ F

$\begin{array}{rrrr}4.7081 & 1 & 10.916 & 0.0530\end{array}$

t Test 
SRNL-STI-2011-00693

Revision 0

\section{Exhibit C2. Statistical Comparisons for Low-Rheology (Phase 3) Simulant Testing}

Oneway Analysis of Measurement By Sampler Type of Material=SME Simulant, Analytical Block=6, Oxide/Analyte $=\mathrm{TiO} 2$ ( $w$ t $\%$ ), Targeted $=0.0711$

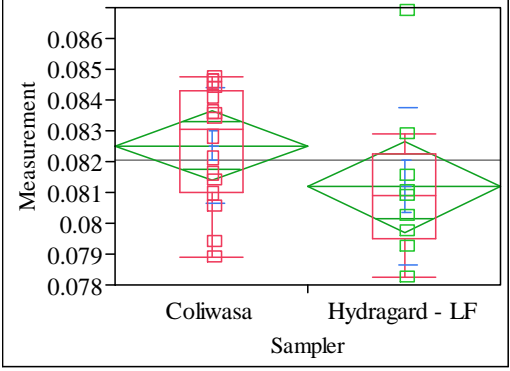

Excluded Rows

Oneway Anova

Summary of Fit

Adj Rsquare

Root Mean Square Error 0.048467

Mean of Response $\quad 0.082046$

Observations (or Sum Wgts) 25

t Test

Hydragard - LF-Coliwasa

Assuming equal variances

Difference $\quad-0.00133$ t Ratio $\quad-1.49079$

$\begin{array}{lrr}\text { Std Err Dif } & 0.00089 \text { DF } & 23 \\ & & \end{array}$

Upper CL Dif 0.00052 Prob $>|t| \quad 0.1496$

$\begin{array}{lrrr}\text { Lower CL Dif } & -0.00317 & \text { Prob }>t & 0.9252 \\ \text { Confidence } & 0.95 & \text { Prob }<\mathrm{t} & 0.0748\end{array}$

Analysis of Variance

Source DF Sum of Squares Mean Square F Ratio Prob > F

$\begin{array}{llllll}\text { Sampler } & 1 & 0.00001019 & 0.000010 & 2.2225 & 0.1496\end{array}$

$\begin{array}{llll}\text { Error } & 23 & 0.00010541 & 4.583 \mathrm{e}-6\end{array}$

C. Total $24 \quad 0.00011559$

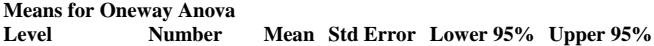

$\begin{array}{lrrrrr}\text { Coliwasa } & 16 & 0.082524 & 0.00054 & 0.08142 & 0.08363\end{array}$

$\begin{array}{lrrrrr}\text { Coliwasa } & 16 & 0.082524 & 0.00054 & 0.08142 & 0.08363 \\ \text { Hydragard - LF } & 9 & 0.081195 & 0.00071 & 0.07972 & 0.08267\end{array}$

Std Error uses a pooled estimate of error variance

Means and Std Deviations

$\begin{array}{lrrrrrr}\text { Level } & \text { Number } & \text { Mean } & \text { Std Dev } & \text { Std Err Mean } & \text { Lower 95\% } & \text { Upper 95\% } \\ \text { Coliwasa } & 16 & 0.082524 & 0.001896 & 0.00047 & 0.08151 & 0.08353\end{array}$

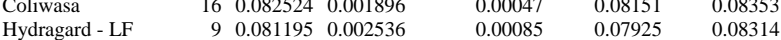

t Test

Hydragard - LF-Coliwasa

Assuming unequal variances

Difference $\quad-0.00133$ t Ratio -1.37192

Std Err Dif $\quad 0.00097$ DF 13.13074

Upper CL Dif 0.00076 Prob $>|t| \quad 0.1931$

Lower CL Dif -0.00342 Prob $>t \quad 0.9035$

$\begin{array}{lrl}\text { Confidence } & 0.95 \text { Prob }<\mathrm{t} & 0.0965\end{array}$

Tests that the Variances are Equal

Level Count Std Dev MeanAbsDif to Mean MeanAbsDif to Median

$\begin{array}{lrrrr}\text { Coliwasa } & 16 & 0.0018964 & 0.0016107 & 0.0015846 \\ \text { Hydragard - LF } & 9 & 0.0025362 & 0.0017298 & 0.0016865\end{array}$

Test F Ratio DFNum DFDen p-Value

$\begin{array}{lrrrr}\text { O'Brien[.5] } & 0.7091 & 1 & 23 & 0.4084\end{array}$

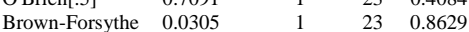

$\begin{array}{lllll}\text { Levene } & 0.0508 & 1 & 23 & 0.8236\end{array}$

$\begin{array}{lllll}\text { Bartlett } & 0.8800 & 1 & & 0.3482 \\ \text { F Test 2-sided } & 1.7885 & 8 & 15 & 0.3159\end{array}$

Welch Anova testing Means Equal, allowing Std Devs Not Equal

F Ratio DFNum DFDen Prob $>$ F

$\begin{array}{llll}1.8822 & 1 & 13.131 & 0.1931\end{array}$

t Test

1.3719
Oneway Analysis of Measurement By Sampler Type of Material=SME Simulant, Analytical Block $=6$, Oxide $/$ Analyte $=\mathrm{ZnO}$ ( $w \mathrm{t} \%$ ), Targeted $=\mathbf{0 . 0 9 5 8}$

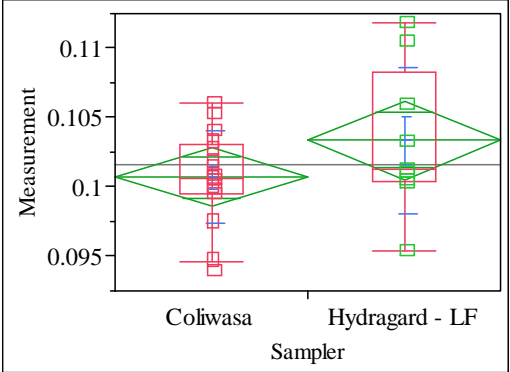

Excluded Rows

Oneway Anova

Summary of Fit

$\begin{array}{lr}\text { Rsquare } & 0.094138 \\ \text { Adj Rsquare } & 0.054753 \\ \text { Root Mean Square Error } & 0.004113 \\ \text { Mean of Response } & 0.10165 \\ \text { Observations (or Sum Wgts) } & 25\end{array}$

Test

Hydragard - LF-Coliwasa

Assuming equal variances

$\begin{array}{lrlr}\text { Difference } & 0.00265 & \text { t Ratio } & 1.546021 \\ \text { Std Err Dif } & 0.00171 & \text { DF } & 23 \\ \text { Upper CL Dif } & 0.00619 & \text { Prob }>|t| & 0.1357 \\ \text { Lower CL Dif } & -0.00090 & \text { Prob }>t & 0.0679 \\ \text { Confidence } & 0.95 & \text { Prob }<t & 0.9321\end{array}$

Analysis of Variance

Source DF Sum of Squares Mean Square F Ratio Prob $>$ F

$\begin{array}{llllll}\text { Sampler } & 1 & 0.00004044 & 0.000040 & 2.3902 & 0.1357\end{array}$

$\begin{array}{llll}\text { Error } & 23 & 0.00038909 & 0.000017\end{array}$

0.00042953

Means for Oneway Anova

$\begin{array}{lrrrrr}\text { Level } & \text { Number } & \text { Mean } & \text { Std Error } & \text { Lower 95\% } & \text { Upper 95\% } \\ \text { Coliwasa } & 16 & 0.100697 & 0.00103 & 0.09857 & 0.10282\end{array}$

$\begin{array}{lrrrrr}\text { Coliwasa } & 16 & 0.100697 & 0.00103 & 0.09857 & 0.10282 \\ \text { Hydragard - LF } & 9 & 0.103346 & 0.00137 & 0.10051 & 0.10618\end{array}$

Std Error uses a pooled estimate of error variance

Means and Std Deviations

$\begin{array}{llrrr}\text { Level Number Mean } & \text { Std Dev } & \text { Std Err Mean } & \text { Lower 95\% } & \text { Upper 95\% }\end{array}$

$\begin{array}{lllllll}\text { Coliwasa } & 16 & 0.100697 & 0.003356 & 0.00084 & 0.09891 & 0.10248\end{array}$

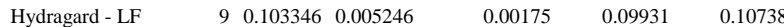

t Test

Hydragard - LF-Coliwasa

Assuming unequal variances

$\begin{array}{llll}\text { Difference } & 0.00265 \text { t Ratio } & 1.366047 \\ \text { Std Err Dif } & 0.00194 & \text { DF } & 11.77349\end{array}$

Std Err Dif $\quad 0.00194$ DF 11.77349

Upper CL Dif 0.00688 Prob $>|t| 0.1975$

Lower CL Dif -0.00159 Prob $>t \quad 0.0987$

$\begin{array}{lrl}\text { Confidence } & 0.95 \text { Prob }<\mathrm{t} \quad 0.9013\end{array}$

Tests that the Variances are Equal

Level Count Std Dev MeanAbsDif to Mean MeanAbsDif to Median

$\begin{array}{lrrrr}\text { Hydragard - LF } & 16 & 0.0033557 & 0.0024351 & 0.0024351 \\ & 9 & 0.0052462 & 0.0040479 & 0.0038450\end{array}$

$\begin{array}{lrrrr}\text { Test } & \text { F Ratio } & \text { DFNum } & \text { DFDen } & \text { p-Value } \\ \text { O'Brien[.5] } & 2.5701 & 1 & 23 & 0.1225 \\ \text { Brown-Forsythe } & 1.3303 & 1 & 23 & 0.2606 \\ \text { Levene } & 2.3482 & 1 & 23 & 0.1391 \\ \text { Bartlett } & 2.1073 & 1 & . & 0.1466 \\ \text { F Test 2-sided } & 2.4441 & 8 & 15 & 0.1292\end{array}$

Welch Anova testing Means Equal, allowing Std Devs Not Equal

F Ratio DFNum DFDen Prob $>$ F

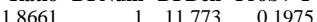

t Test

1.3660 


\section{Exhibit C2. Statistical Comparisons for Low-Rheology (Phase 3) Simulant Testing}

Oneway Analysis of Measurement By Sampler Type of Material=SME Simulant, Analytical Block $=6$, Oxide $/$ Analyte $=\mathrm{ZrO} 2$ ( $w$ t $\%$ ), Targeted $=0.3547$

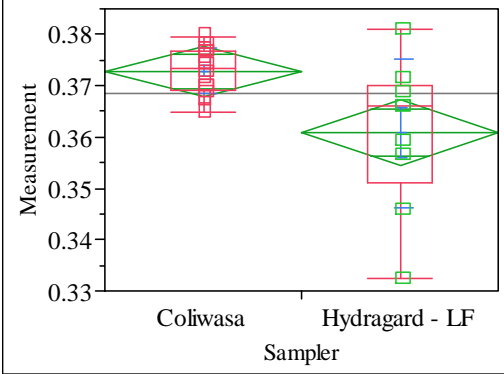

Excluded Rows

Oneway Anova

Summary of Fit

Adj Rsquare

Root Mean Square Error $\quad 0.009294$

Mean of Response

$\begin{array}{lr}\text { Mean of Response } & 0.368498 \\ \text { Observations (or Sum Wgts) } & 25\end{array}$

t Test

Hydragard - LF-Coliwasa

Assuming equal variances

Difference $\quad-0.01201$ t Ratio $\quad-3.10049$

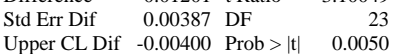

$\begin{array}{rll}\text { Lower CL Dif }-0.02002 \text { Prob }>t & 0.9975\end{array}$

$\begin{array}{lrl}\text { Confidence } & 0.95 \text { Prob }<\mathrm{t} & 0.0025\end{array}$

Analysis of Variance

Source DF Sum of Squares Mean Square F Ratio Prob $>$ F

$\begin{array}{llllll}\text { Sampler } & 1 & 0.00083042 & 0.000830 & 9.6131 & 0.0050\end{array}$

$\begin{array}{llll}\text { Error } & 23 & 0.00198685 & 0.000086\end{array}$

$\begin{array}{lll}\text { C. Total } & 24 & 0.00281728\end{array}$

Means for Oneway Anova

Level $\quad$ Number Mean Std Error Lower 95\% Upper 95\%

$\begin{array}{lrrrrr}\text { Coliwasa } & 16 & 0.372821 & 0.00232 & 0.36801 & 0.37763\end{array}$

$\begin{array}{llllll}\text { Hydragard - LF } & 9 & 0.360814 & 0.00310 & 0.35440 & 0.36722\end{array}$

Std Error uses a pooled estimate of error variance

Means and Std Deviations

Level Number Mean Std Dev Std Err Mean Lower 95\% Upper 95\%

$\begin{array}{lrrrrrr}\text { Coliwasa } & 16 & 0.372821 & 0.004412 & 0.00110 & 0.37047 & 0.37517\end{array}$

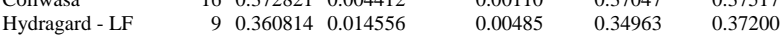

Test

Hydragard - LF-Coliwasa

Assuming unequal variances

Difference $\quad-0.01201$ t Ratio $\quad-2.41319$

Std Err Dif $\quad 0.00498$ DF 8.835573

Upper CL Dif -0.00072 Prob $>|t| r \begin{array}{r}8.035573 \\ 0.0395\end{array}$

$\begin{array}{lll}\text { Lower CL Dif }-0.02329 & \text { Prob }>t & 0.9802\end{array}$

$\begin{array}{lrl}\text { Confidence } & 0.95 \text { Prob }<\mathrm{t} & 0.0198\end{array}$

Tests that the Variances are Equal

Level Count Std Dev MeanAbsDif to Mean MeanAbsDif to Median

$\begin{array}{lrrrr}\text { Coliwasa } & 16 & 0.0044117 & 0.0037147 & 0.0037147\end{array}$

$\begin{array}{lrlll}\text { Hydragard - LF } & 9 & 0.0145555 & 0.0109398 & 0.0103561\end{array}$

Test F Ratio DFNum DFDen p-Value

$\begin{array}{lrrrr}\text { O'Brien[.5] } & 5.8266 & 1 & 23 & 0.0241\end{array}$

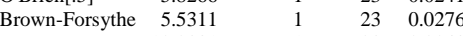

$\begin{array}{lllll}\text { Levene } & 10.0381 & 1 & 23 & 0.0043\end{array}$

$\begin{array}{lllll}\text { Bartlett } & 14.4630 & 1 & 0.0001\end{array}$

$\begin{array}{lllll}\text { F Test 2-sided } & 10.8854 & 8 & 15 & 0.0001\end{array}$

Welch Anova testing Means Equal, allowing Std Devs Not Equal

F Ratio DFNum DFDen Prob $>$ F

$\begin{array}{rrrr}5.8235 & 1 & 8.8356 & 0.0395\end{array}$

t Test
2.4132 
SRNL-STI-2011-00693

Revision 0

\section{Exhibit C3. Statistical Comparisons for Low-Rheology (Phase 3) Simulant Testing (screened data)}

Oneway Analysis of Measurement By Sampling Description Test Phase=Phase 3 - Low Rheology, Analyte $=$ wt $\%$ vitrified solids

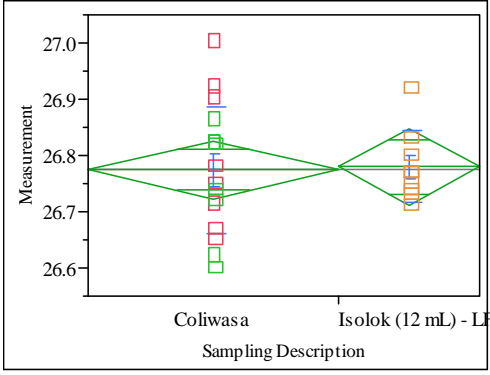

Oneway Anova

Summary of Fit

Rsquare

Adj Rsquare

Root Mean Square Error $\quad 0.098593$

$\begin{array}{lr}\text { Mean of Response } & 26.776 \\ \text { Observations (or Sum Wgts) } & 25\end{array}$

t Test

Isolok (12 mL) - LF-Coliwasa

Assuming equal variances

Difference $\quad 0.00625$ t Ratio $\quad 0.15214$

Std Err Dif 0.04108 DF

Upper CL Dif 0.09123 Prob $>|t| \quad 0.8804$

Lower CL Dif -0.07873 Prob $>t \quad 0.4402$

Confidence $\quad 0.95$ Prob $<\mathrm{t} \quad 0.5598$

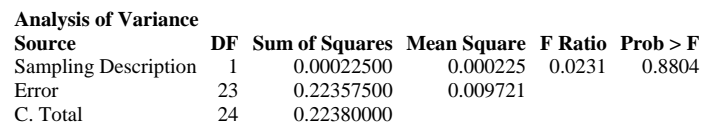

Means for Oneway Anova

Level Number Mean Std Error Lower 95\% Upper 95\%

$\begin{array}{lllllr}\text { Coliwasa } & 16 & 26.7738 & 0.02465 & 26.723 & 26.825\end{array}$

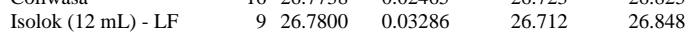

Std Error uses a pooled estimate of error variance

Means and Std Deviations

$\begin{array}{lrrrrrr}\text { Level } & \text { Number } & \text { Mean } & \text { Std Dev } & \text { Std Err Mean } & \text { Lower 95\% } & \text { Upper 95\% } \\ \text { Coliwasa } & 16 & 26.7738 & 0.112835 & 0.02821 & 26.714 & 26.834\end{array}$

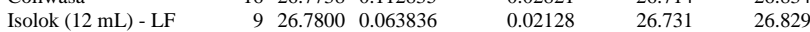

t Test

Isolok (12 mL) - LF-Coliwasa

Assuming unequal variances

Difference $\quad 0.00625$ t Ratio $\quad 0.176882$

Std Err Dif $\quad 0.03533$ DF 22.97772

Upper CL Dif 0.07935 Prob $>|t| \quad 0.8612$

Lower CL Dif -0.06685 Prob $>\mathrm{t} \quad 0.4306$

Confidence $\quad 0.95$ Prob $<\mathrm{t} \quad 0.5694$

Tests that the Variances are Equal

$\begin{array}{lrrrr}\text { Level } & \text { Count } & \text { Std Dev } & \text { MeanAbsDif to Mean } & \text { MeanAbsDif to Median } \\ \text { Coliwasa } & 16 & 0.1128347 & 0.0912500 & 0.0912500 \\ \text { Isolok (12 mL) - LF } & 9 & 0.0638357 & 0.0466667 & 0.0433333\end{array}$

\section{Test F Ratio DFNum DFDen p-Value}

$\begin{array}{lrrrr}\text { O'Brien[.5] } & 2.4405 & 1 & 23 & 0.1319\end{array}$

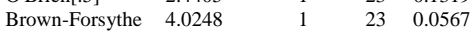

$\begin{array}{lll}1 & 23 & 0.0567 \\ 1 & 23 & 0.0661\end{array}$

$\begin{array}{lllll}\text { Levene } & 3.7219 & 1 & 23 & 0.066\end{array}$

$\begin{array}{lrrrr}\text { Bartlett } & 2.7706 & 1 & \dot{0} & 0.0960 \\ \text { F Test 2-sided } & 3.1243 & 15 & 8 & 0.1084\end{array}$

Welch Anova testing Means Equal, allowing Std Devs Not Equal

F Ratio DFNum DFDen Prob > F

$\begin{array}{llll}0.0313 & 1 & 22.978 & 0.8612\end{array}$

t Test
Oneway Analysis of Mean(Measurement) By Sampler Details Oxide/Analyte=Cr2O3 (wt\%)

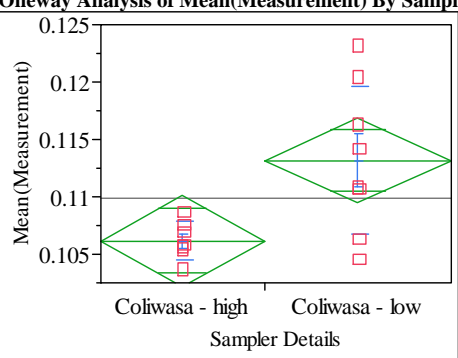

Excluded Rows

Oneway Anova

Summary of Fit

Rsquare

Adj Rsquare

$\begin{array}{ll}\text { Adj Rsquare } & 0.326046 \\ \text { Root Mean Square Error } & 0.004876\end{array}$

Mean of Response

0.004876

Observations (or Sum Wgts)

Test

Coliwasa - low-Coliwasa - high

Assuming equal variances

Difference $\quad 0.007036$ t Ratio 2.787997

$\begin{array}{lllr}\text { Std Err Dif } & 0.002524 & \text { DF } & 13 \\ \text { Upper CL Dif } & 0.012488 & \text { Prob }>|t| & 0.0154\end{array}$

$\begin{array}{llll}\text { Upper CL Dif } & 0.012488 & \text { Prob }>|t| & 0.0154 \\ \text { Lower CL Dif } & 0.001584 & \text { Prob }>t & 0.0077\end{array}$

$\begin{array}{lrll}\text { Lower CL Dif } & 0.001584 & \text { Prob }>t & 0.0077 \\ \text { Confidence } & 0.95 & \text { Prob }<t & 0.9923\end{array}$

$\begin{array}{lrrrrr}\begin{array}{l}\text { Analysis of Variance } \\ \text { Source }\end{array} & \text { DF } & \text { Sum of Squares } & \text { Mean Square } & \text { F Ratio } & \text { Prob }>\text { F } \\ \text { Sampler Details } & 1 & 0.00018483 & 0.000185 & 7.7729 & 0.0154 \\ \text { Error } & 13 & 0.00030912 & 0.000024 & & \\ \text { C. Total } & 14 & 0.00049394 & & & \end{array}$

ay Anova

Level Number Mean Std Error Lower 95\% Upper 95\%

$\begin{array}{llllll}\text { Coliwasa - high } & 7 & 0.106143 & 0.00184 & 0.10216 & 0.11013\end{array}$

$\begin{array}{llllll}\text { Coliwasa - low } & 8 & 0.113180 & 0.00172 & 0.10946 & 0.11690\end{array}$

Std Error uses a pooled estimate of error variance

Means and Std Deviations

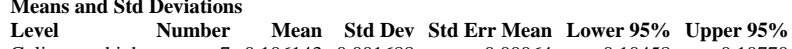

$\begin{array}{lrrrrrr}\text { Coliwasa - high } & 7 & 0.106143 & 0.001688 & 0.00064 & 0.10458 & 0.10770 \\ \text { Coliwasa - low } & 8 & 0.113180 & 0.006459 & 0.00228 & 0.10778 & 0.11858\end{array}$

t Test

Coliwasa - low-Coliwasa - high

Assuming unequal variances

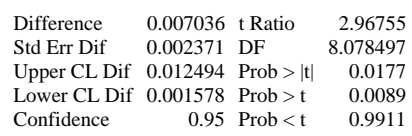

Tests that the Variances are Equal

Level Count Std Dev MeanAbsDif to Mean MeanAbsDif to Median

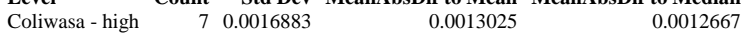

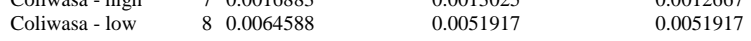

Test $\quad$ F Ratio DFNum DFDen p-Value

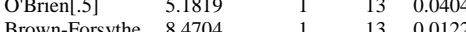

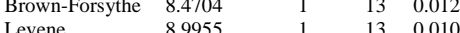

$\begin{array}{lllll}\text { Levene } & 8.9955 & 1 & 13 & 0.0103 \\ \text { Bartlett } & 8.1601 & 1 & & 0.0043\end{array}$

$\begin{array}{lrrrrr}\text { F Test 2-sided } & 14.6348 & & 7 & \dot{6} & 0.0043 \\ \end{array}$

Welch Anova testing Means Equal, allowing Std Devs Not Equal

F Ratio DFNum DFDen Prob $>$ F

$\begin{array}{llll}8.8064 & 1 & 8.0785 & 0.0177\end{array}$

t Test

2.9675 
SRNL-STI-2011-00693

Revision 0

Exhibit C3. Statistical Comparisons for Low-Rheology (Phase 3) Simulant Testing (screened data)

Oneway Analysis of Mean(Measurement) By Sampler Details Oxide/Analyte=Fe2O3 (wt\%)

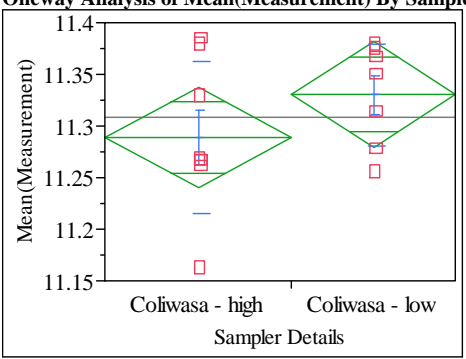

Excluded Rows

neway Anova

Summary of Fit

Rsquare

0.041136
Root Mean Square Error $\quad 0.06323$

$\begin{array}{lr}\text { Mean of Response } & 11.30829 \\ \text { Observations (or Sum Wgts) } & 15\end{array}$

t Test

Coliwasa - low-Coliwasa - high

Assuming equal variances

Difference $\quad 0.04140$ t Ratio 1.265151

$\begin{array}{lrrr}\text { Std Err Dif } & 0.03272 & \text { DF } & 13 \\ \text { Upper CL Dif } & 0.11210 & \text { Prob }>|t| & 0.2280\end{array}$

$\begin{array}{lrl}\text { Lower CL Dif } & -0.02930 \text { Prob }>t & 0.1140\end{array}$

Confidence $\quad 0.95$ Prob $<\mathrm{t} \quad 0.8860$

\begin{tabular}{lrrrrr}
\multicolumn{7}{l}{$\begin{array}{l}\text { Analysis of Variance } \\
\text { Source }\end{array}$} & DF & Sum of Squares & Mean Square & F Ratio & Prob > F \\
Sampler Details & 1 & 0.00639932 & 0.006399 & 1.6006 & 0.2280 \\
Error & 13 & 0.05197474 & 0.003998 & & \\
C. Total & 14 & 0.05837406 & & &
\end{tabular}

Means for Oneway Anova

Level Number Mean Std Error Lower 95\% Upper 95\%

$\begin{array}{llllrr}\text { Coliwasa - high } & 8 & 11.2890 & 0.02236 & 11.241 & 11.337\end{array}$

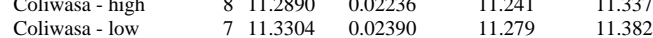

Std Error uses a pooled estimate of error variance

Means and Std Deviations

Level Number Mean Std Dev Std Err Mean Lower 95\% Upper 95\%

$\begin{array}{lrrrrrr}\text { Coliwasa - high } & 8 & 11.2890 & 0.073078 & 0.02584 & 11.228 & 11.350 \\ \text { Coliwasa - low } & 7 & 11.3304 & 0.049316 & 0.01864 & 11.285 & 11.376\end{array}$

\section{t Test}

Coliwasa - low-Coliwasa - high

Assuming unequal variances

Difference $\quad 0.04140$ t Ratio 1.29954

Std Err Dif $\quad 0.03186$ DF 12.29658

Upper CL Dif 0.11063 Prob $>|t| r \mid r .2176$

Lower CL Dif -0.02783 Prob $>t \quad 0.1088$

Confidence $\quad 0.95$ Prob $<\mathrm{t} \quad 0.8912$

Tests that the Variances are Equal

$\begin{array}{lrrrr}\text { Level } & \text { Count } & \text { Std Dev } & \text { MeanAbsDif to Mean } & \text { MeanAbsDif to Median } \\ \text { Coliwasa - high } & 8 & 0.0730778 & 0.0560710 & 0.0503374 \\ \text { Coliwasa - low } & 7 & 0.0493156 & 0.0415294 & 0.0411890\end{array}$

$\begin{array}{llll}\text { Test } & \text { F Ratio DFNum DFDen p-Value }\end{array}$

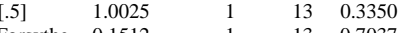

$\begin{array}{lllll}\text { Brown-Forsythe } & 0.1512 & 1 & 13 & 0.7037\end{array}$

$\begin{array}{lllll}\text { Levene } & 0.6958 & 1 & 13 & 0.4193\end{array}$

$\begin{array}{lllll}\text { Bartlett } & 0.8873 & 1 & . & 0.3462\end{array}$

Welch Anova testing Means Equal, allowing Std Devs Not Equal

F Ratio DFNum DFDen Prob $>$ F

$\begin{array}{llll}1.6888 & 1 & 12.297 & 0.2176\end{array}$

t Test
Oneway Analysis of Mean(Measurement) By Sampler Details Oxide/Analyte=Li2O (wt\%)

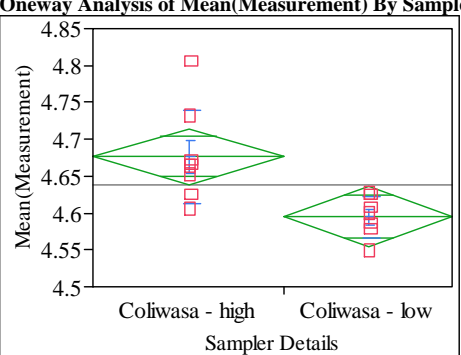

Excluded Rows

neway Anova

Summary of Fit

Rsquare

Adj Rsquare

$\begin{array}{ll}\text { Adj Rsquare } & 0.384919 \\ \text { Root Mean Square Error } & 0.050325\end{array}$

Mean of Response

0.050325

Observations (or Sum Wgts)

15

Coliwasa - low-Coliwasa - high

Assuming equal variances

Difference $\quad-0.08137$ t Ratio $\quad-3.1243$

$\begin{array}{lrlr}\text { Std Err Dif } & 0.02605 & \text { DF } & 13 \\ \text { Upper CL Dif } & -0.02511 & \text { Prob }>|t| & 0.0081 \\ \text { Lower CL Dif } & -0.13764 & \text { Prob }>t & 0.9960\end{array}$

$\begin{array}{lrll}\text { Lower CL Dif } & -0.13764 & \text { Prob }>t & 0.9960 \\ \text { Confidence } & 0.95 & \text { Prob }<t & 0.0040\end{array}$

$\begin{array}{lrrrrr}\begin{array}{l}\text { Analysis of Variance } \\ \text { Source }\end{array} & \text { DF } & \text { Sum of Squares } & \text { Mean Square } & \text { F Ratio } & \text { Prob }>\text { F } \\ \text { Sampler Details } & 1 & 0.02472142 & 0.024721 & 9.7612 & 0.0081 \\ \text { Error } & 13 & 0.03292398 & 0.002533 & & \\ \text { C. Total } & 14 & 0.05764540 & & & \end{array}$

C. Tota

$14 \quad 0.05764540$

Means for Oneway Anova

Level Number Mean Std Error Lower 95\% Upper 95\%

$\begin{array}{llllll}\text { Coliwasa - high } & 8 & 4.67628 & 0.01779 & 4.6378 & 4.7147\end{array}$

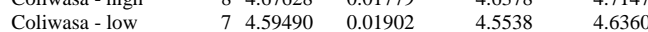

Std Error uses a pooled estimate of error variance

Means and Std Deviations

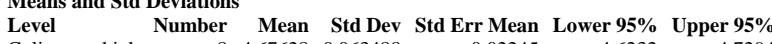

$\begin{array}{lrrrrrr}\text { Coliwasa - high } & 8 & 4.67628 & 0.063488 & 0.02245 & 4.6232 & 4.7294 \\ \text { Coliwasa - low } & 7 & 4.59490 & 0.028014 & 0.01059 & 4.5690 & 4.6208\end{array}$

t Test

Coliwasa - low-Coliwasa - high

Assuming unequal variances

Difference $\quad-0.08137$ t Ratio $\quad-3.27879$

Std Err Dif $\quad 0.02482$ DF $\quad 9.89036$

$\begin{array}{lll}\text { Upper CL Dif }-0.02599 & \text { Prob }>|t| & 0.0084\end{array}$

Lower CL Dif -0.13676 Prob $>t \quad 0.9958$

Confidence $\quad 0.95$ Prob $<\mathrm{t} \quad 0.0042$

Tests that the Variances are Equal

$\begin{array}{lrrrr}\text { Level } & \text { Count } & \text { Std Dev } & \text { MeanAbsDif to Mean } & \text { MeanAbsDif to Median } \\ \text { Coliwasa - high } & 8 & 0.0634884 & 0.0453006 & 0.0412639\end{array}$

$\begin{array}{lllll}\text { Coliwasa - low } & 7 & 0.0280135 & 0.0453006 & 0.0412639 \\ & & & 0.0212361 & 0.0225542\end{array}$

Test $\quad$ F Ratio DFNum DFDen p-Value

$\begin{array}{llll}1.5241 & 1 & 13 & 0.2388 \\ \text { Brown-Forsythe } 0.9808 & 1 & 13 & 0.3401\end{array}$

$\begin{array}{lllll}\text { Bevene } & 0.9808 & 1 & 13 & 0.340 \\ & 2.1053 & 1 & 13 & 0.1705\end{array}$

$\begin{array}{lllll}\text { Levene } & 2.1053 & 1 & 13 & 0.1705 \\ \text { Bartlett } & 3.5051 & 1 & & 0.0612\end{array}$

$\begin{array}{lllll}\text { Bartlett } & 3.5051 & 1 & & 0.0612 \\ \text { F Test 2-sided } & 5.1363 & 7 & 6 & 0.0637\end{array}$

Welch Anova testing Means Equal, allowing Std Devs Not Equal

F Ratio DFNum DFDen Prob $>$ F

$\begin{array}{llll}10.7505 & 1 & 9.8904 & 0.0084\end{array}$

t Test 
Revision 0

Exhibit C3. Statistical Comparisons for Low-Rheology (Phase 3) Simulant Testing (screened data)

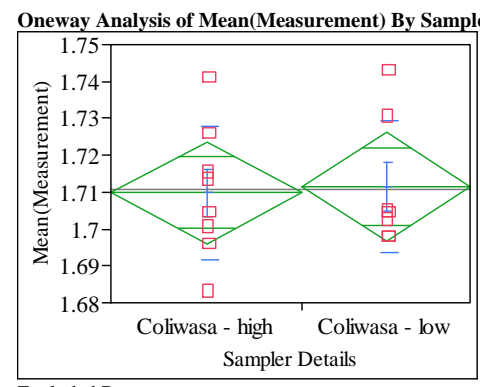

Excluded Rows

Oneway Anova

Summary of Fit

Adj Rsquare $\quad 0.002522$

Root Mean Square Error $\quad 0.018019$

Mean of Response 1.710553

Observations (or Sum Wgts) 15

Coliwasa - low-Coliwasa - high

Assuming equal variances

Difference $\quad 0.00169$ t Ratio $\quad 0.181311$

$\begin{array}{lrlr}\text { Std Err Dif } & 0.00933 & \text { DF } & 13 \\ \text { Upper CL Dif } & 0.02184 & \text { Prob }>|t| & 0.8589\end{array}$

$\begin{array}{lrll}\text { Upper CL Dif } & 0.02184 & \text { Prob }>|t| & 0.8589 \\ \text { Lower CL Dif } & -0.01846 & \text { Prob }>t & 0.4295\end{array}$

$\begin{array}{lrl}\text { Confidence } & 0.95 \text { Prob }<\mathrm{t} & 0.5705\end{array}$

$\begin{array}{lrrrrr}\begin{array}{l}\text { Analysis of Variance } \\ \text { Source }\end{array} & \text { DF } & \text { Sum of Squares } & \text { Mean Square } & \text { F Ratio } & \text { Prob }>\text { F } \\ \text { Sampler Details } & 1 & 0.00001067 & 0.000011 & 0.0329 & 0.8589 \\ \text { Error } & 13 & 0.00422092 & 0.000325 & & \\ \text { C. Total } & 14 & 0.00423159 & & & \end{array}$

Means for Oneway Anova

Level Number Mean Std Error Lower 95\% Upper 95\%

$\begin{array}{lrrrrr}\text { Coliwasa - high } & 8 & 1.70976 & 0.00637 & 1.6960 & 1.7235 \\ \text { Coliwasa - low } & 7 & 1.71145 & 0.00681 & 1.6967 & 1.7262\end{array}$

Std Error uses a pooled estimate of error variance

Means and Std Deviations

Level Number Mean Std Dev Std Err Mean Lower 95\% Upper 95\%

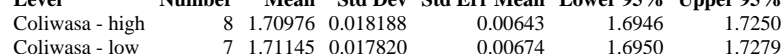

t Test

Coliwasa - low-Coliwasa - high

Assuming unequal variances

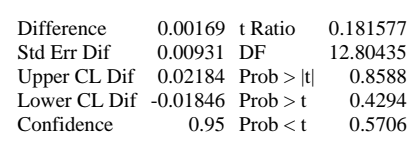

Tests that the Variances are Equal

$\begin{array}{lrrrr}\text { Level } & \text { Count } & \text { Std Dev } & \text { MeanAbsDif to Mean } & \text { MeanAbsDif to Median } \\ \text { Coliwasa - high } & 8 & 0.0181877 & 0.0139880 & 0.0139880 \\ \text { Coliwasa - low } & 7 & 0.0178203 & 0.0144052 & 0.0113749\end{array}$

$\begin{array}{llll}\text { Test } & \text { F Ratio DFNum DFDen p-Value }\end{array}$

\begin{tabular}{lllll}
0.0033 & 1 & 13 & 0.9548 \\
\hline & 0.1571 & 1 & 13 & 0.6982
\end{tabular}

Brown-Forsythe $0.1571 \quad 1 \quad 13 \quad 0.6982$

$\begin{array}{lllll}\text { Levene } & 0.0070 & 1 & 13 & 0.9345 \\ \text { Bartlett } & 0.0025 & 1 & & 0.9602\end{array}$

$\begin{array}{lllll}\text { Bartlett } & 0.0025 & 1 & \dot{0} & 0.9602 \\ \text { F Test 2-sided } & 1.0417 & 7 & 6 & 0.9766\end{array}$

Welch Anova testing Means Equal, allowing Std Devs Not Equal

F Ratio DFNum DFDen Prob $>$ F

$\begin{array}{llll}0.0330 & 1 & 12.804 & 0.8588\end{array}$

t Test

Oneway Analysis of Mean(Measurement) By Sampler Details Oxide/Analyte=NiO (wt\%)

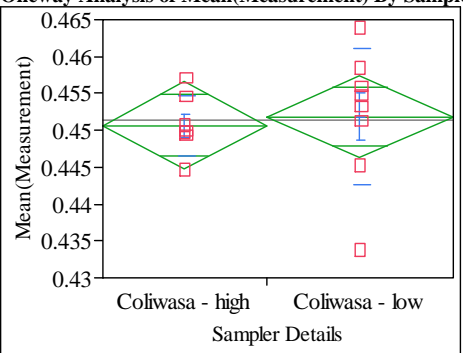

Excluded Rows

Oneway Anova

Summary of Fit

Rsquare $\quad 0.007664$

Adj Rsquare $\quad-0.06867$

Root Mean Square Error $\quad 0.007274$

$\begin{array}{lr}\text { Mean of Response } & 0.451313 \\ \text { Observations (or Sum Wgts) } & 15\end{array}$

t Test

Coliwasa - low-Coliwasa - high

Assuming equal variances

Difference $\quad 0.00119$ t Ratio 0.316872

$\begin{array}{lrrr}\text { Std Err Dif } & 0.00376 \text { DF } & 13 \\ \text { Upper CL Dif } & 0.00933 \text { Prob }>|t| & 0.7564\end{array}$

$\begin{array}{rrrr}\text { Upper CL Dif } & 0.00933 & \text { Prob }>|t| & 0.7564 \\ \text { Lower CL Dif } & -0.00694 & \text { Prob }>t & 0.3782\end{array}$

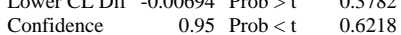

$\begin{array}{lrrrrr}\begin{array}{l}\text { Analysis of Variance } \\ \text { Source }\end{array} & \text { DF } & \text { Sum of Squares } & \text { Mean Square } & \text { F Ratio } & \text { Prob > F } \\ \text { Sampler Details } & 1 & 0.00000531 & 5.313 e-6 & 0.1004 & 0.7564 \\ \text { Error } & 13 & 0.00068791 & 0.000053 & & \\ \text { C. Total } & 14 & 0.00069322 & & & \end{array}$

C. Tota

$14 \quad 0.00069322$

Means for Oneway Anova

Level Number Mean Std Error Lower 95\% Upper 95\%

$\begin{array}{lllllll}\text { Coliwasa - high } & & 7 & 0.450677 & 0.00275 & 0.44474 & 0.45662\end{array}$

$\begin{array}{llllll}\text { Coliwasa - low } & 8 & 0.451870 & 0.00257 & 0.44631 & 0.45743\end{array}$

Std Error uses a pooled estimate of error variance

Means and Std Deviations

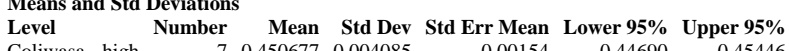

$\begin{array}{lrrrrrr}\text { Coliwasa - high } & 7 & 0.450677 & 0.004085 & 0.00154 & 0.44690 & 0.45446 \\ \text { Coliwasa - low } & 8 & 0.451870 & 0.009163 & 0.00324 & 0.44421 & 0.45953\end{array}$

t Test

Coliwasa - low-Coliwasa - high

Assuming unequal variances

$\begin{array}{lrlr}\text { Difference } & 0.00119 & \text { t Ratio } & 0.332407 \\ \text { Std Err Dif } & 0.00359 & \text { DF } & 9.942443 \\ \text { Upper CL Dif } & 0.00920 & \text { Prob }>|t| & 0.7465 \\ \text { Lower CL Dif } & -0.00681 & \text { Prob }>\text { t } & 0.3732 \\ \text { Confidence } & 0.95 & \text { Prob }<\mathrm{t} & 0.6268\end{array}$

Tests that the Variances are Equal

$\begin{array}{lrrrr}\text { Level } & \text { Count } & \text { Std Dev } & \text { MeanAbsDif to Mean } & \text { MeanAbsDif to Median } \\ \text { Coliwasa - high } & 7 & 0.0040850 & 0.0029086 & 0.0027874\end{array}$

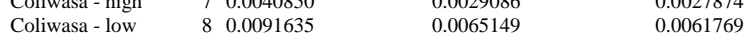

Test F Ratio DFNum DFDen p-Value

$\begin{array}{rrrr}1.4918 & 1 & 13 & 0.2436 \\ \text { Brown-Forsythe } 1.5128 & 1 & 13 & 0.2405\end{array}$

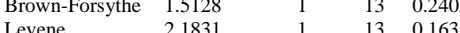

$\begin{array}{lllll}\text { Levene } & 2.1831 & 1 & 13 & 0.1633 \\ \text { Bartlett } & 3.4265 & 1 & & 0.0642\end{array}$

$\begin{array}{lllll}\text { Bartlett } & 3.4265 & 1 & & 0.0642 \\ \text { F Test 2-sided } & 5.0319 & 7 & 6 & 0.0668\end{array}$

Welch Anova testing Means Equal, allowing Std Devs Not Equal

F Ratio DFNum DFDen Prob $>$ F

$\begin{array}{llll}0.1105 & 1 & 9.9424 & 0.7465\end{array}$

t Test 
SRNL-STI-2011-00693

Revision 0

Exhibit C3. Statistical Comparisons for Low-Rheology (Phase 3) Simulant Testing (screened data)

Oneway Analysis of Mean(Measurement) By Sampler Details Oxide/Analyte=SiO2 (wt\%)

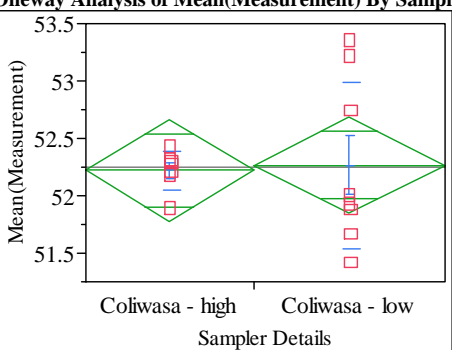

Excluded Rows

neway Anova

Summary of Fit

Rsquare

Adj Rsquare

$-0.07467$

$\begin{array}{lr}\text { Mean of Response } & 52.24408 \\ \text { Observations (or Sum Wgts) } & 15\end{array}$

t Test

Coliwasa - low-Coliwasa - high

Assuming equal variances

Difference $\quad 0.04648$ t Ratio $\quad 0.16491$

Std Err Dif $\quad 0.28184$ DF 13

Upper CL Dif $\quad 0.65536$ Prob $>|t| \quad 0.8716$

Lower CL Dif -0.56241 Prob $>t \quad 0.4358$

Confidence $\quad 0.95$ Prob $<\mathrm{t} \quad 0.5642$

$\begin{array}{lrrrrr}\begin{array}{l}\text { Analysis of Variance } \\ \text { Source }\end{array} & \text { DF } & \text { Sum of Squares } & \text { Mean Square } & \text { F Ratio } & \text { Prob }>\text { F } \\ \text { Sampler Details } & 1 & 0.0080651 & 0.008065 & 0.0272 & 0.8716 \\ \text { Error } & 13 & 3.8552672 & 0.296559 & & \\ \text { C. Total } & 14 & 3.8633322 & & & \end{array}$

Means for Oneway Anova

Level Number Mean Std Error Lower 95\% Upper 95\%

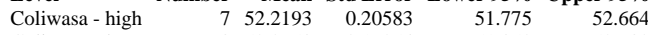

$\begin{array}{llllll}\text { Coliwasa - low } & 8 & 52.2658 & 0.19254 & 51.850 & 52.682\end{array}$

Std Error uses a pooled estimate of error variance

Means and Std Deviations

Level Number Mean Std Dev Std Err Mean Lower 95\% Upper 95\%

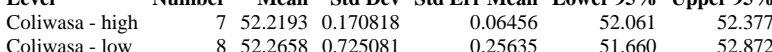

t Test

Coliwasa - low-Coliwasa - high

Assuming unequal variances

Difference $\quad 0.04648$ t Ratio $\quad 0.175817$

Std Err Dif $\quad 0.26436$ DF $\quad 7.879186$

Upper CL Dif 0.65772 Prob $>|t| \quad 0.8649$

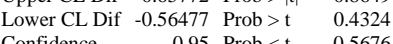

Confidence $\quad 0.95$ Prob $<\mathrm{t} \quad 0.5676$

Tests that the Variances are Equal

$\begin{array}{lrrrr}\text { Level } & \text { Count } & \text { Std Dev } & \text { MeanAbsDif to Mean } & \text { MeanAbsDif to Median } \\ \text { Coliwasa - high } & 7 & 0.1708183 & 0.1193351 & 0.1171521\end{array}$

$\begin{array}{lllll}\text { Coliwasa - high } & 7 & 0.1708183 & 0.1193351 & 0.1171521 \\ \text { Coliwasa - low } & 8 & 0.7250807 & 0.6183914 & 0.5481956\end{array}$

Test $\quad$ F Ratio DFNum DFDen p-Value

$\begin{array}{rrrr}6.7843 & 1 & 13 & 0.0218\end{array}$

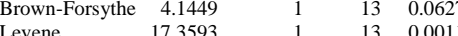

$\begin{array}{lrrrr}\text { Levene } & 17.3593 & 1 & 13 & 0.001 \\ \text { Bartlett } & 9.1922 & 1 & & 0.0024\end{array}$

$\begin{array}{lrrrr}\text { Bartlett } & 9.1922 & 1 & & 0.0024 \\ \text { F Test 2-sided } & 18.0179 & 7 & 6 & 0.0025\end{array}$

Welch Anova testing Means Equal, allowing Std Devs Not Equal

F Ratio DFNum DFDen Prob $>$ F

$\begin{array}{llll}0.0309 & 1 & 7.8792 & 0.8649\end{array}$

t Test
Oneway Analysis of Mean(Measurement) By Sampler Details Oxide/Analyte=TiO2 (wt\%)

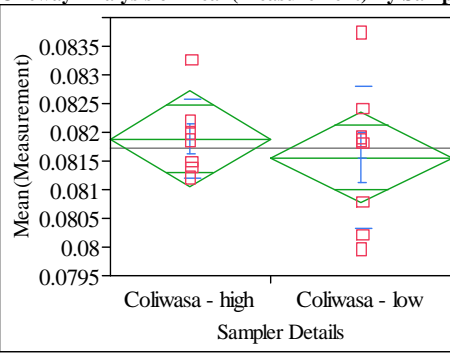

Excluded Rows

Summary of Fi

Rsquare

Adj Rsquare

Root Mean Square Error

Mean of Response

0.028332

$-0.04641$

0.00102

0.081713
$\quad 15$

Coliwasa - low-Coliwasa - high

Assuming equal variances

Difference $\quad-0.00033$ t Ratio $\quad-0.61567$

$\begin{array}{lrrr}\text { Std Err Dif } & 0.00053 \text { DF } & 13 \\ \text { Upper CL Dif } & 0.00082 & \text { Prob }>|t| & 0.5487\end{array}$

$\begin{array}{lrlr}\text { Upper CL Dif } & 0.00082 & \text { Prob }>|t| & 0.5487 \\ \text { Lower CL Dif } & -0.00147 & \text { Prob }>t & 0.7256\end{array}$

$\begin{array}{lll}\text { Confidence } & 0.95 \text { Prob }<\mathrm{t} \quad 0.2744\end{array}$

Analysis of Variance

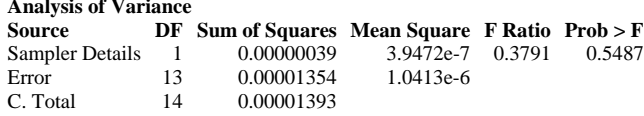

ay Anova

Level Number Mean Std Error Lower 95\% Upper 95\%

$\begin{array}{llllll}\text { Coliwasa - high } & 7 & 0.081887 & 0.00039 & 0.08105 & 0.08272\end{array}$

$\begin{array}{llllll}\text { Coliwasa - low } & 8 & 0.081562 & 0.00036 & 0.08078 & 0.08234\end{array}$

Std Error uses a pooled estimate of error variance

Means and Std Deviations

$\begin{array}{llllll}\text { Level } & \text { Number } & \text { Mean } & \text { Std Dev Std Err Mean Lower 95\% } & \text { Upper 95\% }\end{array}$

$\begin{array}{lrrrrrr}\text { Coliwasa - high } & 7 & 0.081887 & 0.000696 & 0.00026 & 0.08124 & 0.08253 \\ \text { Coliwasa - low } & 8 & 0.081562 & 0.001233 & 0.00044 & 0.08053 & 0.08259\end{array}$

t Test

Coliwasa - low-Coliwasa - high

Assuming unequal variances

$\begin{array}{lrlr}\text { Difference } & -0.00033 & \text { t Ratio } & -0.6389 \\ \text { Std Err Dif } & 0.00051 & \text { DF } & 11.27905 \\ \text { Upper CL Dif } & 0.00079 & \text { Prob }>|t| & 0.5356 \\ \text { Lower CL Dif } & -0.00144 & \text { Prob }>\text { t } & 0.7322 \\ \text { Confidence } & 0.95 \text { Prob }<\mathrm{t} & 0.2678\end{array}$

Tests that the Variances are Equal

$\begin{array}{lrrrr}\text { Level } & \text { Count } & \text { Std Dev } & \text { MeanAbsDif to Mean } & \text { MeanAbsDif to Median } \\ \text { Coliwasa - high } & 7 & 0.0006955 & 0.0004868 & 0.0004964\end{array}$

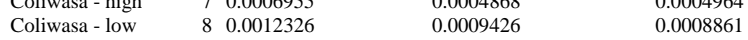

Test $\quad$ F Ratio DFNum DFDen p-Value

$\begin{array}{rrrr}1.5887 & 1 & 13 & 0.2297 \\ \text { Brown-Forsythe } 1.2191 & 1 & 13 & 0.2896\end{array}$

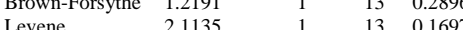

$\begin{array}{lllll}\text { Levene } & 2.1135 & 1 & 13 & 0.1697 \\ \text { Bartlett } & 1.8153 & 1 & & 0.1779\end{array}$

$\begin{array}{lllll}\text { F Test 2-sided } & 3.1406 & 7 & 6 & 0.1845\end{array}$

Welch Anova testing Means Equal, allowing Std Devs Not Equal

F Ratio DFNum DFDen Prob $>$ F

$\begin{array}{llll}0.4082 & 1 & 11.279 & 0.5356\end{array}$

t Test 
Revision 0

\section{Exhibit C3. Statistical Comparisons for Low-Rheology (Phase 3) Simulant Testing (screened data)}

Oneway Analysis of Measurement By Sampler Type of Material=SME Simulant, Analytical Block=1, Oxide/Analyte=Al2O3 ( $w$ t \%), Targeted $=10.869$

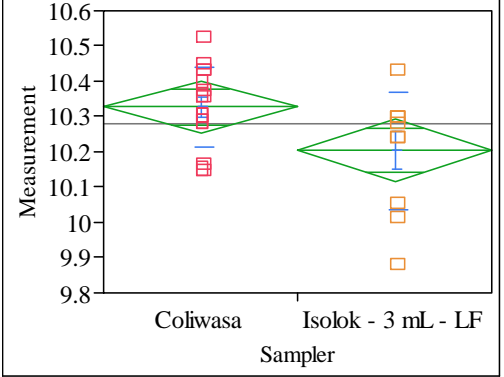

Exc

Exclided Rows

Oneway Anova

Summary of Fit

Root Mean Square Error $\quad 0.142941$

0.136572

$\begin{array}{lr}\text { Mean of Response } & 10.27812 \\ \text { Observations (or Sum Wgts) } & 25\end{array}$

Test

Isolok - $3 \mathrm{~mL}$ - LF-Coliwasa

Assuming equal variances

$\begin{array}{lrlr}\text { Difference } & -0.12471 & \text { t Ratio } & -2.23668 \\ \text { Std Err Dif } & 0.05576 & \text { DF } & 23 \\ \text { Upper CL Dif } & -0.00937 & \text { Prob }>|t| & 0.0353 \\ \text { Lower CL Dif } & -0.24005 & \text { Prob }>t & 0.9824 \\ \text { Confidence } & 0.95 & \text { Prob }<\mathrm{t} & 0.0176\end{array}$

Analysis of Variance

Source DF Sum of Squares Mean Square F Ratio Prob $>$ F

$\begin{array}{llllll}\text { Sampler } & 1 & 0.09331102 & 0.093311 & 5.0027 & 0.0353\end{array}$

$\begin{array}{llll}\text { Error } & 23 & 0.42899646 & 0.018652\end{array}$

C. Total $24 \quad 0.52230748$

Means for Oneway Anova

Level Number Mean Std Error Lower 95\% Upper 95\%

$\begin{array}{llrrrr}\text { Coliwasa } & 15 & 10.3280 & 0.03526 & 10.255 & 10.401\end{array}$

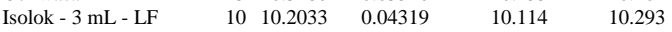

Std Error uses a pooled estimate of error variance

Means and Std Deviations

Level Number Mean Std Dev Std Err Mean Lower 95\% Upper 95\%

$\begin{array}{lllllll}\text { Coliwasa } & 15 & 10.3280 & 0.113550 & 0.02932 & 10.265 & 10.391 \\ \text { Isolok - 3 mL - LF } & 10 & 10.2033 & 0.166161 & 0.05254 & 10.084 & 10.322\end{array}$

Test

Isolok - $3 \mathrm{~mL}$ - LF-Coliwasa

Assuming unequal variances

$\begin{array}{lrlr}\text { Difference } & -0.12471 & \mathrm{t} \text { Ratio } & -2.07255 \\ \text { Std Err Dif } & 0.06017 & \text { DF } & 14.56852 \\ \text { Upper CL Dif } & 0.00388 & \text { Prob }>|t| & 0.0564 \\ \text { Lower CL Dif } & -0.25329 & \text { Prob }>\mathrm{t} & 0.9718 \\ \text { Confidence } & 0.95 & \text { Prob }<\mathrm{t} & 0.0282\end{array}$

Tests that the Variances are Equal

Level Count Std Dev MeanAbsDif to Mean MeanAbsDif to Median

$\begin{array}{lrrrr}\text { Coliwasa } & 15 & 0.1135498 & 0.0911999 & 0.0894363\end{array}$

$\begin{array}{lllll}\text { Isolok - } 3 \mathrm{~mL} \text { - LF } & 10 & 0.1661614 & 0.1322650 & 0.1171490\end{array}$

Test F Ratio DFNum DFDen p-Value

$\begin{array}{lrrrr}\text { O'Brien[.5] } & 1.8584 & 1 & 23 & 0.1860\end{array}$

Brown-Forsythe $\quad 0.4943 \quad 1 \quad 23 \quad 0.4891$

$\begin{array}{lllll}\text { Levene } & 1.7999 & 1 & 23 & 0.1928\end{array}$

$\begin{array}{lllll}\text { F Test 2-sided } \quad 2.1414 & 9 & 14 & 0.1948\end{array}$

Welch Anova testing Means Equal, allowing Std Devs Not Equal

F Ratio DFNum DFDen Prob > F

$\begin{array}{rrrr}4.2955 & 1 & 14.569 & 0.0564\end{array}$

t Test
2.0725
Oneway Analysis of Measurement By Sampler Type of Material=SME Simulant, Analytica Block=1, Oxide/Analyte=B2O3 (wt \%), Targeted $=4.259$

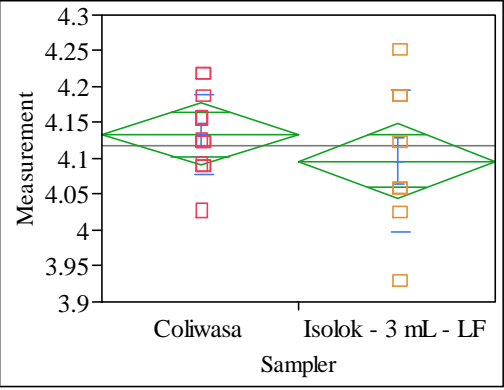

Excluded Rows

neway Anova

Summary of Fit

Rsquare

Adj Rsquare

Root Mean Square Error $\quad 0.014039$

Mean of Response 0.07573

Observations (or Sum Wgts) $\quad 22$

t Test

Isolok - $3 \mathrm{~mL}$ - LF-Coliwasa

Assuming equal variances

$\begin{array}{lrlr}\text { Difference } & -0.03743 & \text { t Ratio } & -1.13975 \\ \text { Std Err Dif } & 0.03284 & \text { DF } & 20 \\ \text { Upper CL Dif } & 0.03107 & \text { Prob }>|t| & 0.2679 \\ \text { Lower CL Dif } & -0.10593 & \text { Prob }>t & 0.8661 \\ \text { Confidence } & 0.95 & \text { Prob }<\mathrm{t} & 0.1339\end{array}$

Analysis of Variance

Source DF Sum of Squares Mean Square F Ratio Prob > F

$\begin{array}{lrrrrr}\text { Sampler } & 1 & 0.00744996 & 0.007450 & 1.2990 & 0.2679\end{array}$

$\begin{array}{llll}\text { Error } & 20 & 0.11470105 & 0.005735\end{array}$

C. Total $21 \quad 0.12215102$

Means for Oneway Anova

Level Number Mean Std Error Lower 95\% Upper 95\%

$\begin{array}{llrrrr}\text { Coliwasa } & 13 & 4.13386 & 0.02100 & 4.0900 & 4.1777\end{array}$

$\begin{array}{lllll}\text { Isolok - } 3 \text { mL - LF } & 9 & 4.09643 & 0.02524 & 4.0438\end{array}$

Std Error uses a pooled estimate of error variance

Means and Std Deviations

Level Number Mean Std Dev Std Err Mean Lower 95\% Upper 95\%

$\begin{array}{lrrrrrr}\text { Coliwasa } & 13 & 4.13386 & 0.055051 & 0.01527 & 4.1006 & 4.1671 \\ \text { Isolok - 3 mL - LF } & 9 & 4.09643 & 0.098953 & 0.03298 & 4.0204 & 4.1725\end{array}$

t Test

Isolok - $3 \mathrm{~mL}$ - LF-Coliwasa

Assuming unequal variances

Difference $\quad-0.03743$ t Ratio -1.02974

Std Err Dif $\quad 0.03635$ DF 11.44531

Upper CL Dif 0.04219 Prob $>|t| \quad 0.3244$

Lower CL Dif -0.11705 Prob $>\mathrm{t} \quad 0.8378$

$\begin{array}{lll}\text { Confidence } & 0.95 \text { Prob }<\mathrm{t} \quad 0.1622\end{array}$

Tests that the Variances are Equal

$\begin{array}{lrrrr}\text { Level } & \text { Count } & \text { Std Dev } & \text { MeanAbsDif to Mean } & \text { MeanAbsDif to Median } \\ \text { Coliwasa } & 13 & 0.0550507 & 0.0430590 & 0.0421064\end{array}$

$\begin{array}{lrrrr}\text { Coliwasa } & 13 & 0.0550507 & 0.0430590 & 0.0421064 \\ \text { Isolok - 3 mL - LF } & 9 & 0.0989534 & 0.0795037 & 0.0751310\end{array}$

Test F Ratio DFNum DFDen p-Value

$\begin{array}{lllll}\text { O'Brien[.5] } & 3.4133 & 1 & 20 & 0.0795\end{array}$

$\begin{array}{lllll}\text { Brown-Forsythe } & 2.0429 & 1 & 20 & 0.1684\end{array}$

Levene

$\begin{array}{lllll}\text { Levene } & 4.1908 & 1 & 20 & 0.0540\end{array}$

$\begin{array}{lllll}\text { F Test 2-sided } & 3.2054 & 1 & . & 0.0734 \\ & 3.2310 & 8 & 12 & 0.0665\end{array}$

Welch Anova testing Means Equal, allowing Std Devs Not Equal

F Ratio DFNum DFDen Prob $>$ F

$\begin{array}{llll}1.0604 & 1 & 11.445 & 0.3244\end{array}$

t Test 
SRNL-STI-2011-00693

Revision 0

\section{Exhibit C3. Statistical Comparisons for Low-Rheology (Phase 3) Simulant Testing (screened data)}

Oneway Analysis of Measurement By Sampler Type of Material=SME Simulant, Analytical Block =1, Oxide/Analyte=BaO (wt \%), Targeted $=0.0919$

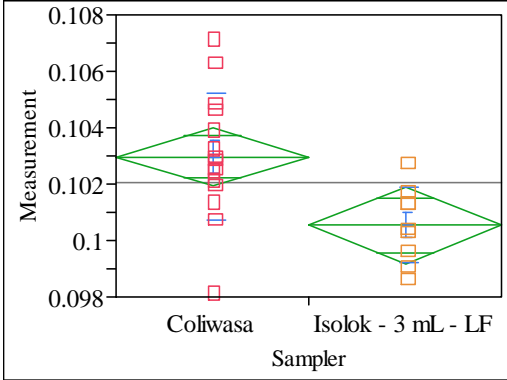

Exclude

Exch

Oneway Anova

Summary of Fit

Rsquare $\quad 0.283078$

Adj Rsquare $\quad 0.25049$

Root Mean Square Error $\quad 0.001955$

Mean of Response $\quad 0.102053$

Observations (or Sum Wgts) $\quad 24$

t Test

Isolok - $3 \mathrm{~mL}$ - LF-Coliwasa

Assuming equal variances

Difference $\quad-0.00243$ t Ratio -2.94733

$\begin{array}{lr}\text { Std Err Dif } \quad 0.00082 \text { DF } & 22\end{array}$

Upper CL Dif -0.00072 Prob $>|t| \quad 0.0074$

Lower CL Dif -0.00414 Prob $>t \quad 0.9963$

$\begin{array}{lrl}\text { Confidence } & 0.95 \text { Prob }<\mathrm{t} & 0.0037\end{array}$

Analysis of Variance

Source DF Sum of Squares Mean Square F Ratio Prob $>$ F

$\begin{array}{llllll}\text { Sampler } & 1 & 0.00003319 & 0.000033 & 8.6867 & 0.0074\end{array}$

$\begin{array}{llll}\text { Error } & 22 & 0.00008405 & 3.821 \mathrm{e}-6\end{array}$

C. Total $23 \quad 0.00011724$

Means for Oneway Anova

Level Number Mean Std Error Lower 95\% Upper 95\%

$\begin{array}{lrrrrr}\text { Coliwasa } & 15 & 0.102964 & 0.00050 & 0.10192 & 0.10401\end{array}$

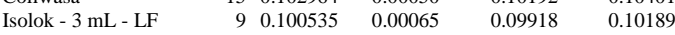

Std Error uses a pooled estimate of error variance

Means and Std Deviations

Level Number Mean Std Dev Std Err Mean Lower 95\% Upper 95\%

$\begin{array}{lllllrr}\text { Coliwasa } & 15 & 0.102964 & 0.002233 & 0.00058 & 0.10173 & 0.10420\end{array}$

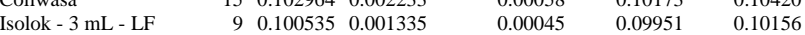

Isolok - $3 \mathrm{~mL}$ - LF-Coliwasa

Assuming unequal variances

$\begin{array}{lrlr}\text { Difference } & -0.00243 & \mathrm{t} \text { Ratio } & -3.33515 \\ \text { Std Err Dif } & 0.00073 & \text { DF } & 21.99079 \\ \text { Upper CL Dif } & -0.00092 & \text { Prob }>|t| & 0.0030 \\ \text { Lower CL Dif } & -0.00394 & \text { Prob }>\text { t } & 0.9985 \\ \text { Confidence } & 0.95 & \text { Prob }<\mathrm{t} & 0.0015\end{array}$

Tests that the Variances are Equal

Level Count Std Dev MeanAbsDif to Mean MeanAbsDif to Median

$\begin{array}{lrrrr}\text { Coliwasa } & 15 & 0.0022327 & 0.0016048 & 0.0016003\end{array}$

Isolok - 3 mL - LF $\quad 9 \quad 0.0013353 \quad 0.0010724 \quad 0.0010669$

Test F Ratio DFNum DFDen p-Value

$\begin{array}{lllll}\text { O'Brien[.5] } & 1.3496 & 1 & 22 & 0.2578\end{array}$

$\begin{array}{lllll}\text { Brown-Forsythe } & 0.9803 & 1 & 22 & 0.3329\end{array}$

$\begin{array}{lllll}\text { Levene } & 1.0001 & 1 & 22 & 0.3282\end{array}$

$\begin{array}{lrrrr}\text { Bartlett } & 2.2591 & 1 & 0.1328\end{array}$

$\begin{array}{lllll}\text { F Test 2-sided } & 2.7959 & 14 & 8 & 0.1475\end{array}$

Welch Anova testing Means Equal, allowing Std Devs Not Equal

F Ratio DFNum DFDen Prob $>$ F

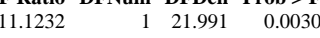

t Test
Oneway Analysis of Measurement By Sampler Type of Material=SME Simulant, Analytical Block $=1$, Oxide/Analyte $=\mathrm{CaO}($ wt $\%)$, Targeted $=1.1462$

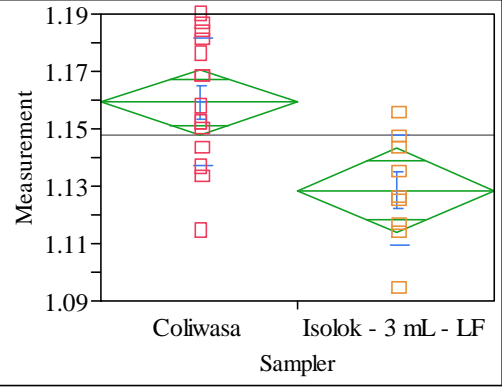

Excluded Rows

neway Anova

Summary of Fit

Rsquare

Adj Rsquare

Root Mean Square Error $\quad 0.021089$

Mean of Response

Observations (or Sum Wgts) $\quad 24$

t Test

Isolok - $3 \mathrm{~mL}$ - LF-Coliwasa

Assuming equal variances

Difference $\quad-0.03075$ t Ratio $\quad-3.45828$

$\begin{array}{lrlr}\text { Std Err Dif } & 0.00889 \text { DF } & 22 \\ \text { Upper CL Dif } & -0.01231 \text { Prob }>|t| & 0.0022\end{array}$

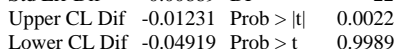

$\begin{array}{lrl}\text { Confidence } & 0.95 \text { Prob }<\mathrm{t} & 0.0011\end{array}$

Analysis of Variance

Analysis of Variance
Source DF Sum of Squares Mean Square F Ratio Prob $>$ F

$\begin{array}{lrrrrr}\text { Sampler } & 1 & 0.00531924 & 0.005319 & 11.9597 & 0.0022\end{array}$

$\begin{array}{llll}\text { Error } & 22 & 0.00978480 & 0.000445\end{array}$

C. Total $23 \quad 0.01510404$

Means for Oneway Anova

Level Number Mean Std Error Lower 95\% Upper 95\%

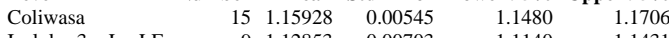

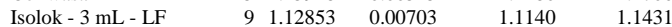

Std Error uses a pooled estimate of error variance

Means and Std Deviations

Level Number Mean Std Dev Std Err Mean Lower 95\% Upper 95\%

$\begin{array}{lrrrrrr}\text { Coliwasa } & 15 & 1.15928 & 0.022167 & 0.00572 & 1.1470 & 1.1716\end{array}$

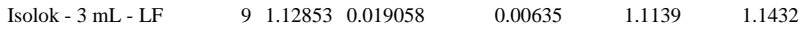

t Test

Isolok - $3 \mathrm{~mL}$ - LF-Coliwasa

Assuming unequal variances

$\begin{array}{lrlr}\text { Difference } & -0.03075 & \text { t Ratio } & -3.59634 \\ \text { Std Err Dif } & 0.00855 \text { DF } & 19.07591 \\ \text { Upper CL Dif } & -0.01286 & \text { Prob }>|t| & 0.0019 \\ \text { Lower CL Dif } & -0.04864 & \text { Prob }>t & 0.9990 \\ \text { Confidence } & 0.95 & \text { Prob }<\mathrm{t} & 0.0010\end{array}$

Tests that the Variances are Equal

$\begin{array}{lrrrr}\text { Level } & \text { Count } & \text { Std Dev } & \text { MeanAbsDif to Mean } & \text { MeanAbsDif to Median } \\ \text { Coliwasa } & 15 & 0.0221667 & 0.0183326 & 0.0185627\end{array}$

$\begin{array}{lrlll}\text { Coliwasa } & 15 & 0.0221667 & 0.0183326 & 0.0185627 \\ \text { Isolok - 3 mL - LF } & 9 & 0.0190583 & 0.0148557 & 0.0147693\end{array}$

Test F Ratio DFNum DFDen p-Value

$\begin{array}{lllll}\text { O'Brien[.5] } & 0.3018 & 1 & 22 & 0.5883\end{array}$

$\begin{array}{lllll}\text { Brown-Forsythe } & 0.6364 & 1 & 22 & 0.4335\end{array}$

Bevene $\quad 0.5426 \quad-120.220 .4335$

$\begin{array}{lllll}\text { Levene } & 0.5426 & 1 & 22 & 0.4692\end{array}$

$\begin{array}{lrrrr}\text { F Test 2-sided } & 1.3528 & 14 & 8 & 0.6432 \\ \end{array}$

Welch Anova testing Means Equal, allowing Std Devs Not Equal

F Ratio DFNum DFDen Prob $>$ F

$\begin{array}{llll}12.9336 & 1 & 19.076 & 0.0019\end{array}$

t Test 


\section{Exhibit C3. Statistical Comparisons for Low-Rheology (Phase 3) Simulant Testing (screened data)}

Oneway Analysis of Measurement By Sampler Type of Material=SME Simulant, Analytical Block=1, Oxide/Analyte=Fe2O3 (wt $\%$ ), Targeted $=11.462$

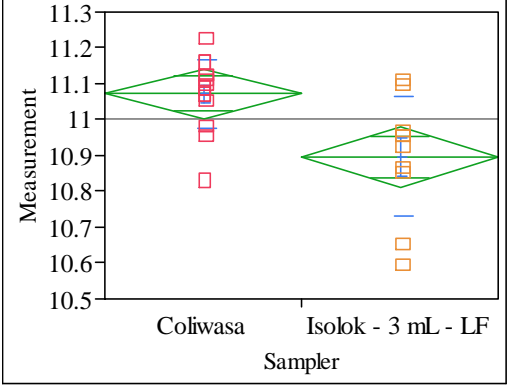

Excluded Rows

Oneway Anova

Summary of Fit

Rsquare

Adj Rsquare

$\begin{array}{lr}\text { Mean of Response } & 11.00126 \\ \text { Observations (or Sum Wgts) } & 25\end{array}$

t Test

Isolok - $3 \mathrm{~mL}$ - LF-Coliwas

Assuming equal variances

Difference $\quad-0.17585$ t Ratio -3.35711

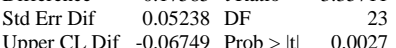

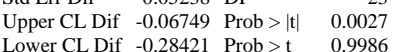

$\begin{array}{lrll}\text { Lower CL Dif } & -0.28421 & \text { Prob }>\mathrm{t} & 0.9986 \\ \text { Confidence } & 0.95 & \text { Prob }<\mathrm{t} & 0.0014\end{array}$

Analysis of Variance
Source DF Sum of Squares Mean Square F Ratio Prob $>$ F

$\begin{array}{lrrrrr}\text { Sampler } & 1 & 0.18554588 & 0.185546 & 11.2702 & 0.0027\end{array}$

\begin{tabular}{llll} 
Error & 23 & 0.37865880 & 0.185546 \\
\hline
\end{tabular}

$\begin{array}{lll}\text { C. Total } 24 & 24 & 0.56420467\end{array}$

Means for Oneway Anova

Level Number Mean Std Error Lower 95\% Upper 95\%

$\begin{array}{llllll}\text { Coliwasa } & 15 & 11.0716 & 0.03313 & 11.003 & 11.140\end{array}$

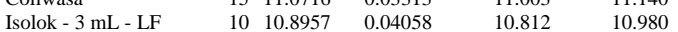

Std Error uses a pooled estimate of error variance

Means and Std Deviations

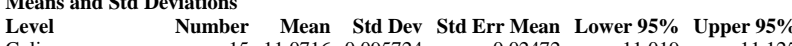

$\begin{array}{lrrrrrr}\text { Coliwasa } & 15 & 11.0716 & 0.095724 & 0.02472 & 11.019 & 11.125 \\ \text { Isolok - 3 mL - LF } & 10 & 10.8957 & 0.166792 & 0.05274 & 10.776 & 11.015\end{array}$

t Test

Isolok - $3 \mathrm{~mL}$ - LF-Coliwasa

Assuming unequal variances

Difference $\quad-0.17585$ t Ratio $\quad-3.01905$

Std Err Dif $\quad 0.05825$ DF 12.98405

Upper CL Dif -0.05000 Prob $>|t| r 0.0099$

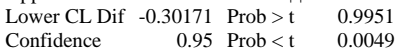

Tests that the Variances are Equal

$\begin{array}{lrrrr}\text { Level } & \text { Count } & \text { Std Dev } & \text { MeanAbsDif to Mean } & \text { MeanAbsDif to Median } \\ \text { Coliwasa } & 15 & 0.0957243 & 0.0655756 & 0.0638599 \\ \text { Isolok - 3 mL - LF } & 10 & 0.1667915 & 0.1240980 & 0.1186651\end{array}$

Test F Ratio DFNum DFDen p-Value

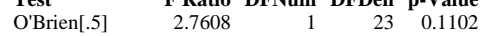

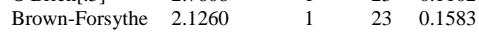

$\begin{array}{llll}2.9511 & 1 & 23 & 0.1583\end{array}$

$\begin{array}{lllll}\text { Levene } & 2.9511 & 1 & 23 & 0.0993 \\ \text { Bartlett } & 3.3277 & 1 & . & 0.0681\end{array}$

$\begin{array}{lllll}\text { F Test 2-sided } \quad 3.0360 & 9 & 14 & 0.0615\end{array}$

Welch Anova testing Means Equal, allowing Std Devs Not Equal

$\begin{array}{rrrr}\text { F Ratio } & \text { DFNum } & \text { DFDen } & \text { Prob > F } \\ 9.1146 & 1 & 12.984 & 0.0099\end{array}$

t Test
Oneway Analysis of Measurement By Sampler Type of Material=SME Simulant, Analytical Block=1, Oxide/Analyte=MgO (wt\%), Targeted $=1.014$

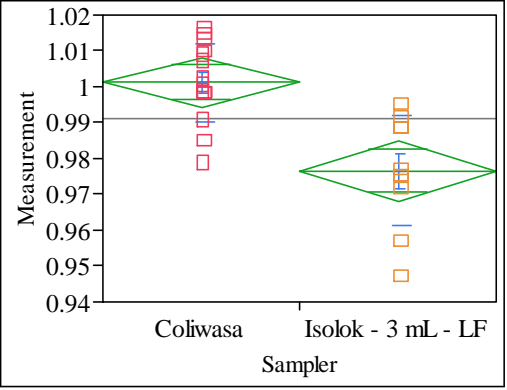

Excluded Rows

Oneway Anova

Summary of Fit

Rsquare

Adj Rsquare

Root Mean Squere

0.012863

$\begin{array}{lr} & 0.991332 \\ \text { Observations (or Sum Wgts) } & 25\end{array}$

t Test

Isolok - $3 \mathrm{~mL}$ - LF-Coliwasa

Assuming equal variances

Difference $\quad-0.02460$ t Ratio $\quad-4.68421$

$\begin{array}{lrlr}\text { Std Err Dif } & 0.00525 & \text { DF } & 23 \\ \text { Upper CL Dif } & -0.01374 & \text { Prob }>|t| & 0.0001\end{array}$

$\begin{array}{lrlr}\text { Upper CL Dif } & -0.01374 & \text { Prob }>|t| & 0.0001 \\ \text { Lower CL Dif }-0.03546 & \text { Prob }>t & 0.9999\end{array}$

$\begin{array}{lrl}\text { Confidence } & 0.95 \text { Prob }<\mathrm{t} & <.0001\end{array}$

Analysis of Variance
Source DF Sum of Squares Mean Square F Ratio Prob $>$ F

$\begin{array}{lrrrrr}\text { Source } & \text { DF } & \text { Sum of Squares } & \text { Mean Square } & \text { F Ratio } & \text { Prob }>\text { F } \\ \text { Sampler } & 1 & 0.00363040 & 0.003630 & 21.9418 & 0.0001\end{array}$

$\begin{array}{lrrr}\text { Sampler } & 1 & 0.00363040 & 0.003630 \\ \text { Error } & 23 & 0.00380548 & 0.000165\end{array}$

$\begin{array}{lll}\text { C. Total } & 24 & 0.00743589\end{array}$

Means for Oneway Anova

Level Number Mean Std Error Lower 95\% Upper 95\%

$\begin{array}{llrrrr}\text { Coliwasa } & 15 & 1.00117 & 0.00332 & 0.99430 & 1.0080\end{array}$

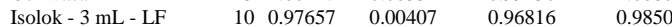

Std Error uses a pooled estimate of error variance

Means and Std Deviations

Level Number Mean Std Dev Std Err Mean Lower 95\% Upper 95\%

$\begin{array}{lrrrrrr}\text { Coliwasa } & 15 & 1.00117 & 0.010901 & 0.00281 & 0.99513 & 1.0072\end{array}$

$\begin{array}{lllllll}\text { Isolok - } 3 \mathrm{~mL} \text { - LF } & 10 & 0.97657 & 0.015427 & 0.00488 & 0.96554 & 0.9876\end{array}$

t Test

Isolok - $3 \mathrm{~mL}$ - LF-Coliwasa

Assuming unequal variances

$\begin{array}{lrlr}\text { Difference } & -0.02460 & \text { t Ratio } & -4.36746 \\ \text { Std Err Dif } & 0.00563 & \text { DF } & 14.9254 \\ \text { Upper CL Dif } & -0.01259 & \text { Prob }>|t| & 0.0006 \\ \text { Lower CL Dif } & -0.03661 & \text { Prob }>t & 0.9997 \\ \text { Confidence } & 0.95 & \text { Prob }<\mathrm{t} & 0.0003\end{array}$

Tests that the Variances are Equal

$\begin{array}{lrrrr}\text { Level } & \text { Count } & \text { Std Dev } & \text { MeanAbsDif to Mean } & \text { MeanAbsDif to Median } \\ \text { Coliwasa } & 15 & 0.0109007 & 0.0083726 & 0.0084021 \\ \text { Isolok - 3 mL - LF } & 10 & 0.0154270 & 0.0114423 & 0.0114423\end{array}$

Test F Ratio DFNum DFDen p-Value

$\begin{array}{lllll}\text { O'Brien[.5] } & 1.5087 & 1 & 23 & 0.2318\end{array}$

$\begin{array}{lllll}\text { Brown-Forsythe } & 0.8697 & 1 & 23 & 0.3607\end{array}$

Bevene $0.9001-1 \quad 230.3607$

$\begin{array}{lllll}\text { Levene } & 0.9001 & 1 & 23 & 0.3526\end{array}$

$\begin{array}{lrrrr}\text { Bartlett } & 1.3026 & 1 & \text {. } & 0.2537 \\ \text { F Test 2-sided } & 2.0029 & 9 & 14 & 0.2356\end{array}$

Welch Anova testing Means Equal, allowing Std Devs Not Equal

F Ratio DFNum DFDen Prob $>$ F

$\begin{array}{llll}19.0747 & 1 & 14.925 & 0.0006\end{array}$

t Test 
Revision 0

\section{Exhibit C3. Statistical Comparisons for Low-Rheology (Phase 3) Simulant Testing (screened data)}

Oneway Analysis of Measurement By Sampler Type of Material=SME Simulant, Analytical Block $=1$, Oxide/Analyte $=\mathrm{MnO}(\mathrm{wt} \%)$, Targeted $=1.779$

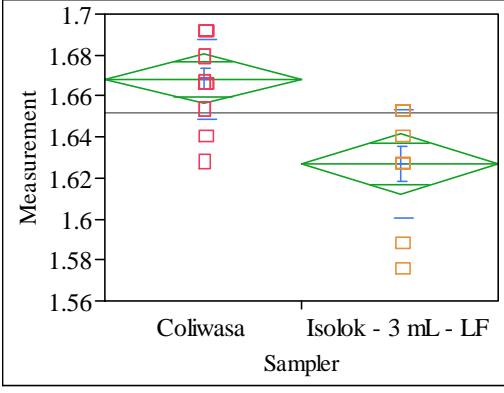

Excluded Rows

Oneway Anova

Summary of Fit

Rsquare

Adj Rsquare

Root Mean Square Error $\quad \begin{array}{rr}0.442803 \\ 0.02259\end{array}$

Mean of Response

Observations (or Sum Wgts) r $\begin{array}{r}1.651703 \\ \hline\end{array}$

Test

Isolok - $3 \mathrm{~mL}$ - LF-Coliwasa

Assuming equal variances

Difference $\quad-0.04132$ t Ratio -4.48026

Std Err Dif $\quad 0.00922$ DF $r .43$

$\begin{array}{rrr}\text { Upper CL Dif } & -0.02224 & \text { Prob }>|t| \\ 0.0002\end{array}$

$\begin{array}{lll}\text { Lower CL Dif } & -0.06040 \text { Prob }>t & 0.9999\end{array}$

$\begin{array}{lll}\text { Confidence } & 0.95 \text { Prob }<\mathrm{t} \quad<.0001\end{array}$

Analysis of Variance

Source DF Sum of Squares Mean Square F Ratio Prob $>$ F

$\begin{array}{lrrrrr}\text { Sampler } & 1 & 0.01024326 & 0.010243 & 20.0727 & 0.0002\end{array}$

Error $23 \quad 0.01024326$

$\begin{array}{lll}\text { Error } & 23 & 0.01173707 \\ \text { C. Total } & 24 & 0.02198033\end{array}$

Means for Oneway Anova

Level Number Mean Std Error Lower 95\% Upper 95\%

$\begin{array}{llllrr}\text { Coliwasa } & 15 & 1.66823 & 0.00583 & 1.6562 & 1.6803\end{array}$

$\begin{array}{llllll}\text { Isolok - } 3 \mathrm{~mL} \text { - LF } & 10 & 1.62691 & 0.00714 & 1.6121 & 1.6417\end{array}$

Std Error uses a pooled estimate of error variance

Means and Std Deviations

Level Number Mean Std Dev Std Err Mean Lower 95\% Upper 95\%

$\begin{array}{lrrrrrr}\text { Coliwasa } & 15 & 1.66823 & 0.019643 & 0.00507 & 1.6574 & 1.6791 \\ \text { Isolok - 3 mL - LF } & 10 & 1.62691 & 0.026532 & 0.00839 & 1.6079 & 1.6459\end{array}$

t Test

Isolok - $3 \mathrm{~mL}$ - LF-Coliwasa

Assuming unequal variances

Difference $\quad-0.04132$ t Ratio -4.21451

Std Err Dif $\quad 0.00980$ DF 15.45274

Upper CL Dif -0.02048 Prob $>|t| \quad 0.0007$

Lower CL Dif -0.06216 Prob $>t \quad 0.9996$

$\begin{array}{lrl}\text { Confidence } & 0.95 \text { Prob }<\mathrm{t} \quad 0.0004\end{array}$

Tests that the Variances are Equal

$\begin{array}{lrrrr}\text { Level } & \text { Count } & \text { Std Dev } & \text { MeanAbsDif to Mean } & \text { MeanAbsDif to Median } \\ \text { Coliwasa } & 15 & 0.0196427 & 0.0151501 & 0.0146336 \\ \text { Isolok - 3 mL - LF } & 10 & 0.0265316 & 0.0180768 & 0.0180768\end{array}$

Test F Ratio DFNum DFDen p-Value

$\begin{array}{lllll}\text { O'Brien[.5] } & 1.0756 & 1 & 23 & 0.3105\end{array}$

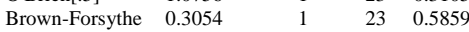

Levene

$\begin{array}{lllll}\text { Levene } & 0.2352 & 1 & 23 & 0.6323 \\ \text { Bartlett } & 0.9742 & 1 & & 0.3236\end{array}$

$\begin{array}{lllrr}\text { Bartlett } & 0.9742 & 1 & & 0.3236 \\ \text { F Test 2-sided } & 1.8244 & 9 & 14 & 0.3022\end{array}$

Welch Anova testing Means Equal, allowing Std Devs Not Equal

F Ratio DFNum DFDen Prob $>$ F

t Test
4.2145
Oneway Analysis of Measurement By Sampler Type of Material=SME Simulant, Analytical Block=1, Oxide/Analyte=Na2O (wt \%), Targeted $=11.659$

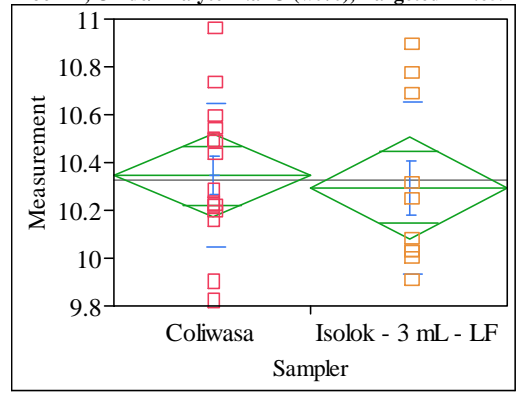

Excluded Rows

Oneway Anova

Summary of Fit

Rsquare

Adj Rsquare

Mean of Response 10.32622
25

Isolok - $3 \mathrm{~mL}$ - LF-Coliwasa

Assuming equal variances

Difference $\quad-0.05033$ t Ratio $\quad-0.37825$

$\begin{array}{lrlr}\text { Std Err Dif } & 0.13305 \text { DF } & 23 \\ \text { Upper CL Dif } & 0.22490 & \text { Prob }>|t| & 0.7087\end{array}$

$\begin{array}{lrll}\text { Upper CL Dif } & 0.22490 & \text { Prob }>|t| & 0.7087 \\ \text { Lower CL Dif } & -0.32555 & \text { Prob }>t & 0.6456\end{array}$

$\begin{array}{lrll}\text { Lower CL Dif } & -0.32555 & \text { Prob }>t & 0.6456 \\ \text { Confidence } & 0.95 & \text { Prob }<t & 0.3544\end{array}$

Analysis of Variance
Source DF Sum of Squares Mean Square F Ratio Prob $>$ F

$\begin{array}{lrrrrr}\text { Sampler } & 1 & 0.0151958 & 0.015196 & 0.1431 & 0.7087\end{array}$

$\begin{array}{lrrr}\text { Error } & 23 & 2.4427935 & 0.106208\end{array}$

C. Total $24 \quad 2.4579893$

Means for Oneway Anova

Level Number Mean Std Error Lower 95\% Upper 95\%

$\begin{array}{llllrr}\text { Coliwasa } & 15 & 10.3463 & 0.08415 & 10.172 & 10.520\end{array}$

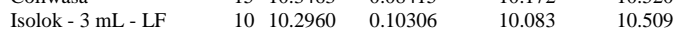

Std Error uses a pooled estimate of error variance

Means and Std Deviations

Level Number Mean Std Dev Std Err Mean Lower 95\% Upper 95\%

$\begin{array}{lrrrrrr}\text { Coliwasa } & 15 & 10.3463 & 0.302362 & 0.07807 & 10.179 & 10.514\end{array}$

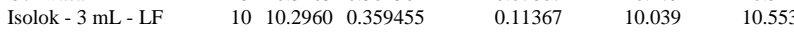

t Test

Isolok - $3 \mathrm{~mL}$ - LF-Coliwasa

Assuming unequal variances

$\begin{array}{lrlr}\text { Difference } & -0.05033 & \text { t Ratio } & -0.36495 \\ \text { Std Err Dif } & 0.13790 \text { DF } & 17.05391 \\ \text { Upper CL Dif } & 0.24054 & \text { Prob }>|t| & 0.7196 \\ \text { Lower CL Dif } & -0.34119 & \text { Prob }>\text { t } & 0.6402 \\ \text { Confidence } & 0.95 & \text { Prob }<\mathrm{t} & 0.3598\end{array}$

Tests that the Variances are Equal

$\begin{array}{lrrrr}\text { Level } & \text { Count } & \text { Std Dev } & \text { MeanAbsDif to Mean } & \text { MeanAbsDif to Median } \\ \text { Coliwasa } & 15 & 0.3023621 & 0.2413220 & 0.2399440 \\ \text { Isolok - 3 mL - LF } & 10 & 0.3594554 & 0.2960208 & 0.2857760\end{array}$

Test F Ratio DFNum DFDen p-Value

$\begin{array}{lllll}\text { O'Brien[.5] } & 0.5191 & 1 & 23 & 0.4785\end{array}$

$\begin{array}{lllll}\text { Brown-Forsythe } & 0.2833 & 1 & 23 & 0.5997\end{array}$

Bevene 0.5958

$\begin{array}{lllll}\text { Levene } & 0.5958 & 1 & 23 & 0.4480 \\ \text { Bartlett } & 0.3197 & 1 & & 0.5718\end{array}$

$\begin{array}{lllll}\text { Bartlett } & 0.3197 & 1 & & 0.5718 \\ \text { F Test 2-sided } & 1.4133 & 9 & 14 & 0.5419\end{array}$

Welch Anova testing Means Equal, allowing Std Devs Not Equal

F Ratio DFNum DFDen Prob $>$ F

$\begin{array}{llll}0.1332 & 1 & 17.054 & 0.7196\end{array}$

t Test 
SRNL-STI-2011-00693

Revision 0

\section{Exhibit C3. Statistical Comparisons for Low-Rheology (Phase 3) Simulant Testing (screened data)}

Oneway Analysis of Measurement By Sampler Type of Material=SME Simulant, Analytical Block $=1$, Oxide/Analyte $=\mathrm{SiO} 2(\mathrm{wt} \%)$, Targeted $=\mathbf{5 0 . 9 8 5}$

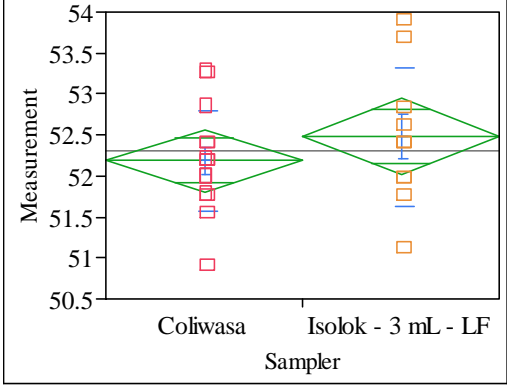

Excluded Rows

Oneway Anova

Summary of Fit

Rsquare

Adj Rsquare

Root Mean Square Error $\quad 0.717936$

Mean of Response

Observations (or Sum Wgts) $r 2.30161$

Test

Isolok - $3 \mathrm{~mL}$ - LF-Coliwas

Assuming equal variances

Difference $\quad 0.29237$ t Ratio 0.997526

$\begin{array}{lrlr}\text { Std Err Dif } & 0.29310 & \text { DF } & 23 \\ \text { Upper CL Dif } & 0.89869 & \text { Prob }>|t| & 0.3289\end{array}$

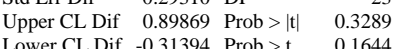

$\begin{array}{lrll}\text { Lower CL Dif } & -0.31394 & \text { Prob }>t & 0.1644 \\ \text { Confidence } & 0.95 & \text { Prob }<\mathrm{t} & 0.8356\end{array}$

Analysis of Variance

Source DF Sum of Squares Mean Square F Ratio Prob $>$ F

$\begin{array}{lrrrrr}\text { Sampler } & 1 & 0.512885 & 0.512885 & 0.9951 & 0.3289\end{array}$

$\begin{array}{llll}\text { Error } & 23 & 11.854931 & 0.515432\end{array}$

C. Total $24 \quad 12.367816$

Means for Oneway Anova

Level Number Mean Std Error Lower 95\% Upper 95\%

\begin{tabular}{llllll} 
& 15 & 52.1847 & 0.18537 & 51.801 & 52.568 \\
\hline
\end{tabular}

Std Error uses a pooled estimate of error variance

Means and Std Deviations

$\begin{array}{llllll}\text { Level } & \text { Number } & \text { Mean Std Dev Std Err Mean Lower 95\% Upper 95\% }\end{array}$

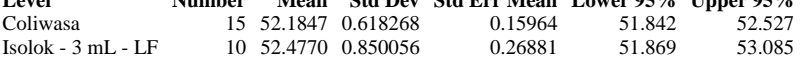

Test

solok - $3 \mathrm{~mL}$ - LF-Coliwasa

Assuming unequal variances

Difference $\quad 0.29237$ t Ratio $\quad 0.935171$

Std Err Dif $\quad 0.31264$ DF 15.24824

Upper CL Dif 0.95780 Prob $>|t| \quad 0.3643$

$\begin{array}{rrr}\text { Lower CL Dif } & -0.37306 \text { Prob }>t & 0.1821 \\ \text { Confidence } & 0.95 \text { Prob }<\text { t } & 0.8179\end{array}$

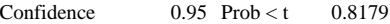

Tests that the Variances are Equal

$\begin{array}{lrrrr}\text { Level } & \text { Count } & \text { Std Dev } & \text { MeanAbsDif to Mean } & \text { MeanAbsDif to Median } \\ \text { Coliwasa } & 15 & 0.6182681 & 0.4430728 & 0.4421220 \\ \text { Isolok - 3 mL - LF } & 10 & 0.8500559 & 0.6332328 & 0.6203970\end{array}$

Test F Ratio DFNum DFDen p-Value

$\begin{array}{lllll}\text { O'Brien[.5] } & 1.2318 & 1 & 23 & 0.2785\end{array}$

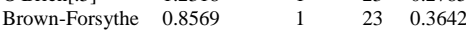

Levene $1.0183 \quad 1-23-0.3234$

$\begin{array}{lllll}\text { Levene } & 1.0183 & 1 & 23 & 0.3234 \\ \text { Bartlett } & 1.0935 & 1 & & 0.2957\end{array}$

$\begin{array}{lllll}\text { F Test 2-sided } & 1.0935 & 1 & & 0.2957 \\ & 1.8903 & 9 & 14 & 0.2755\end{array}$

Welch Anova testing Means Equal, allowing Std Devs Not Equal

F Ratio DFNum DFDen Prob $>$ F

t Test
Oneway Analysis of Measurement By Sampler Type of Material=SME Simulant, Analytical Block=1, Oxide/Analyte $=$ Sum of Oxides (wt \%), Targeted $=99.553$

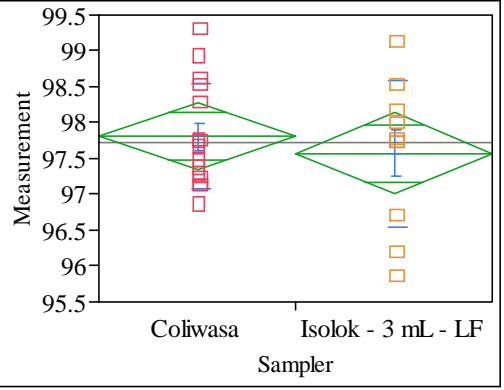

Excluded Rows

Oneway Anova

Summary of Fit

Rsquare

Adj Rsquare

Root Mean Square Error $\quad 0.863343$

Mean of Response

Observations (or Sum Wgts)

t Test

Isolok - $3 \mathrm{~mL}$ - LF-Coliwasa

Assuming equal variances

Difference $\quad-0.23576$ t Ratio $\quad-0.66891$

$\begin{array}{lrlr}\text { Std Err Dif } & 0.35246 \text { DF } & 23 \\ \text { Upper CL } & 0.49335 \text { Prob }>|t| & 0.5102\end{array}$

$\begin{array}{lrlr}\text { Upper CL Dif } & 0.49335 & \text { Prob }>|t| & 0.5102 \\ \text { Lower CL Dif } & -0.96488 & \text { Prob }>t & 0.7449\end{array}$

$\begin{array}{lrr}\text { Confidence } & 0.95 \text { Prob }<\mathrm{t} & 0.2551\end{array}$

Analysis of Variance

Source DF Sum of Squares Mean Square F Ratio Prob > F

$\begin{array}{lrrrrr}\text { Sampler } & 1 & 0.333508 & 0.333508 & 0.4474 & 0.5102\end{array}$

$\begin{array}{llrr}\text { Error } & 23 & 17.143299 & 0.745361\end{array}$

C. Total $24 \quad 17.476807$

Means for Oneway Anova

Level Number Mean Std Error Lower 95\% Upper 95\%

$\begin{array}{llllrr}\text { Coliwasa } & 15 & 97.8080 & 0.22291 & 97.347 & 98.269\end{array}$

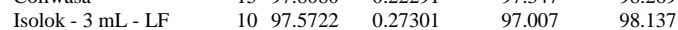

Std Error uses a pooled estimate of error variance

Means and Std Deviations

Level Number Mean Std Dev Std Err Mean Lower 95\% Upper 95\%

$\begin{array}{lrrrrrr}\text { Coliwasa } & 15 & 97.8080 & 0.73920 & 0.19086 & 97.399 & 98.217 \\ \text { Isolok - 3 mL - LF } & 10 & 97.5722 & 1.02705 & 0.32478 & 96.838 & 98.307\end{array}$

t Test

Isolok - $3 \mathrm{~mL}$ - LF-Coliwasa

Assuming unequal variances

Difference $\quad-0.2358$ t Ratio $\quad-0.62585$

Std Err Dif $\quad 0.3767$ DF 15.12944

Upper CL Dif 0.5666 Prob $>|t| \quad 0.5407$

Lower CL Dif -1.0381 Prob $>$ t 0.7296

$\begin{array}{lll}\text { Confidence } \quad 0.95 \text { Prob }<\mathrm{t} \quad 0.2704 & \end{array}$

Tests that the Variances are Equal

$\begin{array}{lrrrr}\text { Level } & \text { Count } & \text { Std Dev } & \text { MeanAbsDif to Mean } & \text { MeanAbsDif to Median } \\ \text { Coliwasa } & 15 & 0.739195 & 0.6101160 & 0.5843872\end{array}$

$\begin{array}{lrrrr}\text { Coliwasa } & 15 & 0.739195 & 0.6101160 & 0.5843872 \\ \text { Isolok - 3 mL - LF } & 10 & 1.027054 & 0.7934778 & 0.7376368\end{array}$

Test F Ratio DFNum DFDen p-Value

$\begin{array}{lllll}\text { O'Brien[.5] } & 1.7350 & 1 & 23 & 0.2007\end{array}$

$\begin{array}{lllll}\text { Brown-Forsythe } & 0.4418 & 1 & 23 & 0.5129\end{array}$

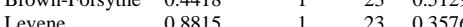

$\begin{array}{lllll}\text { Levene } & 0.8815 & 1 & 23 & 0.3576 \\ \text { Bartlett } & 1.1674 & 1 & . & 0.2799\end{array}$

$\begin{array}{lllll}\text { F Test 2-sided } & 1.9305 & 9 & 14 & 0.2605\end{array}$

Welch Anova testing Means Equal, allowing Std Devs Not Equal

F Ratio DFNum DFDen Prob $>$ F

$\begin{array}{llll}0.3917 & 1 & 15.129 & 0.5407\end{array}$

t Test 


\section{Exhibit C3. Statistical Comparisons for Low-Rheology (Phase 3) Simulant Testing (screened data)}

Oneway Analysis of Measurement By Sampler Type of Material=SME Simulant, Analytical Block=1, Oxide/Analyte $=\mathrm{ZrO2}$ ( wt \%), Targeted $=0.3547$

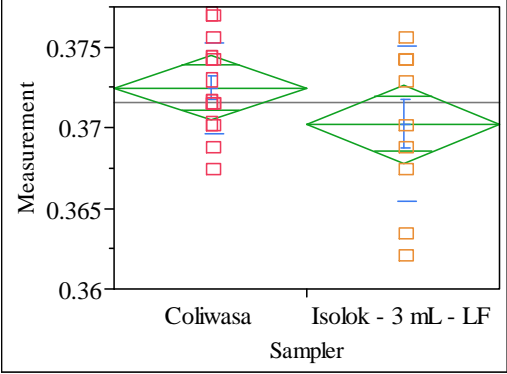

Exc

Oxcluded Rows

Oneway Anova

Summary of Fit

Adj Rsquare $\quad 0.044504$

Root Mean Square Error $\quad 0.003714$

(ot

$\begin{array}{lr} & 0.371578 \\ \text { Observations (or Sum Wgts) } & 25\end{array}$

t Test

Isolok - $3 \mathrm{~mL}$ - LF-Coliwasa

Assuming equal variances

$\begin{array}{lrlr}\text { Difference } & -0.00221 & \text { t Ratio } & -1.45528 \\ \text { Std Err Dif } & 0.00152 & \text { DF } & 23 \\ \text { Upper CL Dif } & 0.00093 & \text { Prob }>|t| & 0.1591 \\ \text { Lower CL Dif } & -0.00534 & \text { Prob }>t & 0.9204 \\ \text { Confidence } & 0.95 & \text { Prob }<t & 0.0796\end{array}$

Analysis of Variance

Source DF Sum of Squares Mean Square F Ratio Prob $>$ F

$\begin{array}{llllll}\text { Sampler } & 1 & 0.00002921 & 0.000029 & 2.1179 & 0.1591\end{array}$

$\begin{array}{llll}\text { Error } & 23 & 0.00031719 & 0.000014\end{array}$

C. Total $24 \quad 0.00034639$

Means for Oneway Anova

Level $\quad$ Number Mean Std Error Lower 95\% Upper 95\%

$\begin{array}{lrrrrr}\text { Coliwasa } & 15 & 0.372461 & 0.00096 & 0.37048 & 0.37444\end{array}$

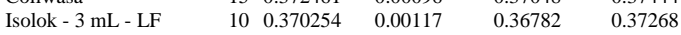

Std Error uses a pooled estimate of error variance

$\begin{array}{llllll}\text { Means and Std Deviations } & & & & & \\ \text { Level } & \text { Number } & \text { Mean } & \text { Std Dev Std Err Mean Lower 95\% Upper 95\% }\end{array}$

$\begin{array}{lrrrrrr}\text { Level } & \text { Number } & \text { Mean } & \text { Std Dev } & \text { Std Err Mean } & \text { Lower 95\% } & \text { Upper 95\% } \\ \text { Coliwasa } & 15 & 0.372461 & 0.002818 & 0.00073 & 0.37090 & 0.37402\end{array}$

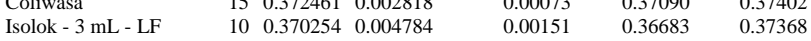

Isolok - $3 \mathrm{~mL}$ - LF-Coliwasa

Assuming unequal variances

$\begin{array}{lrlr}\text { Difference } & -0.00221 & \mathrm{t} \text { Ratio } & -1.31421 \\ \text { Std Err Dif } & 0.00168 & \text { DF } & 13.1913 \\ \text { Upper CL Dif } & 0.00142 & \text { Prob }>|t| & 0.2112 \\ \text { Lower CL Dif } & -0.00583 & \text { Prob }>\text { t } & 0.8944 \\ \text { Confidence } & 0.95 & \text { Prob }<\mathrm{t} & 0.1056\end{array}$

Tests that the Variances are Equal

Level Count Std Dev MeanAbsDif to Mean MeanAbsDif to Median

$\begin{array}{lrrrr}\text { Coliwasa } & 15 & 0.0028181 & 0.0023174 & 0.0022513\end{array}$

Isolok - 3 mL - LF $\quad 10 \quad 0.0047843 \quad 0.0039173 \quad 0.0039173$

Test F Ratio DFNum DFDen p-Value

$\begin{array}{lrrrr}\text { O'Brien[.5] } & 4.3836 & 1 & 23 & 0.0475\end{array}$

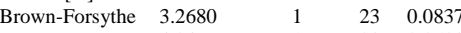

$\begin{array}{lllll}\text { Levene } & 4.2469 & 1 & 23 & 0.0508\end{array}$

$\begin{array}{lllll} & \text { F Test 2-sided } \quad 2.8822 & 9 & 14 & 0.0819\end{array}$

Welch Anova testing Means Equal, allowing Std Devs Not Equal

F Ratio DFNum DFDen Prob $>$ F

$\begin{array}{rrrr}1.7272 & 1 & 13.191 & 0.2112\end{array}$

t Test
1.3142
Oneway Analysis of Measurement By Sampler Type of Material=SME Simulant, Analytical Block $=2$, Oxide $/$ Analyte $=A \mathrm{I} / \mathrm{B}$, Targeted $=2.552$

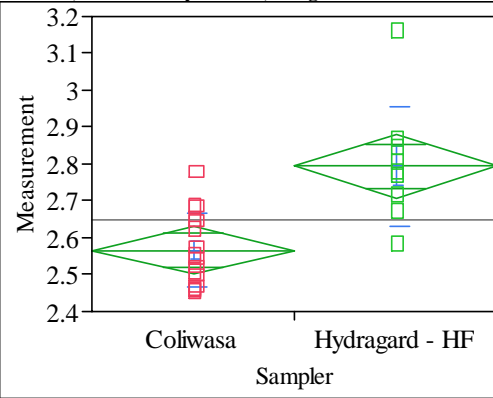

Excluded Rows

Oneway Anova

Summary of Fit

Adj Rsquare

0.434527

0.124314

2.648293

Observations (or Sum Wgts)

Test

Hydragard - HF-Coliwasa

Assuming equal variances

Difference $\quad 0.228393$ t Ratio 4.409343

Std Err Dif $\quad 0.051797$ DF 23

Upper CL Dif 0.335544 Prob $>|t| \quad 0.0002$

Lower CL Dif 0.121241 Prob $>$ t 0.0001

$\begin{array}{lrl}\text { Confidence } & 0.95 \text { Prob }<\mathrm{t} & 0.9999\end{array}$

Analysis of Variance

Source DF Sum of Squares Mean Square F Ratio Prob $>$ F

$\begin{array}{lllllll}\text { Sampler } & 1 & 0.30045966 & 0.300460 & 19.4423 & 0.0002\end{array}$

$\begin{array}{llll}\text { Error } & 23 & 0.35543989 & 0.015454\end{array}$

C. Total $24 \quad 0.65589955$

Means for Oneway Anova

Level Number Mean Std Error Lower 95\% Upper 95\%

$\begin{array}{lrrrrr}\text { Coliwasa } & 16 & 2.56607 & 0.03108 & 2.5018 & 2.6304 \\ \text { Hydragard - HF } & 9 & 2.79446 & 0.04144 & 2.7087 & 2.8802\end{array}$

Std Error uses a pooled estimate of error variance

Means and Std Deviations

Level Number Mean Std Dev Std Err Mean Lower 95\% Upper 95\%

$\begin{array}{lllllrr}\text { Coliwasa } & 16 & 2.56607 & 0.098929 & 0.02473 & 2.5134 & 2.6188\end{array}$

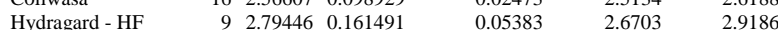

t Test

Hydragard - HF-Coliwasa

Assuming unequal variances

$\begin{array}{lrlr}\text { Difference } & 0.228393 & \text { t Ratio } & 3.855365 \\ \text { Std Err Dif } & 0.059240 & \text { DF } & 11.46157 \\ \text { Upper CL Dif } & 0.358141 & \text { Prob }>|t| & 0.0025 \\ \text { Lower CL Dif } & 0.098644 & \text { Prob }>t & 0.0012 \\ \text { Confidence } & 0.95 & \text { Prob }<t & 0.9988\end{array}$

Tests that the Variances are Equal

Level Count Std Dev MeanAbsDif to Mean MeanAbsDif to Median

$\begin{array}{lllll}\text { Coliwasa } & 16 & 0.0989291 & 0.0824976 & 0.0801678\end{array}$

$\begin{array}{lrrrr}\text { Hydragard - HF } & 9 & 0.1614912 & 0.1066538 & 0.1050515\end{array}$

Test F Ratio DFNum DFDen p-Value

$\begin{array}{lrrrr}\text { O'Brien[.5] } & 1.4877 & 1 & 23 & 0.2349\end{array}$

$\begin{array}{lllll}\text { Brown-Forsythe } & 0.4958 & 1 & 23 & 0.4884\end{array}$

$\begin{array}{lllll}\text { Levene } & 0.5362 & 1 & 23 & 0.4714\end{array}$

$\begin{array}{lllll}\text { Bartlett } & 2.5404 & 1 & \text {. } & 0.1110 \\ \text { F Test 2-sided } & 2.6647 & 8 & 15 & 0.0970\end{array}$

Welch Anova testing Means Equal, allowing Std Devs Not Equal

F Ratio DFNum DFDen Prob $>$ F

$\begin{array}{llll}14.8638 & 1 & 11.462 & 0.0025\end{array}$

t Test

3.8554 
Revision 0

\section{Exhibit C3. Statistical Comparisons for Low-Rheology (Phase 3) Simulant Testing (screened data)}

Oneway Analysis of Measurement By Sampler Type of Material=SME Simulant, Analytical Block=2, Oxide/Analyte $=$ B2O3 (wt $\%$ ), Targeted $=4.259$

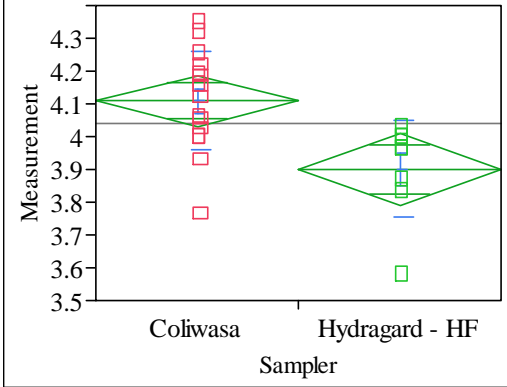

Excluded Rows

Oneway Anova

Summary of Fit

Adj Rsquare

0.323774

Root Mean Squ

0.293037

Mean of Respons

4.039633

t Test

Hydragard - HF-Coliwasa

Assuming equal variances

Difference $\quad-0.20929$ t Ratio -3.24554

Std Err Dif $\quad 0.06449$ DF 22

Upper CL Dif -0.07556 Prob $>|t| 0.0037$

Lower CL Dif -0.34303 Prob $>\mathrm{t} \quad 0.9981$

$\begin{array}{lrl}\text { Confidence } & 0.95 \text { Prob }<\mathrm{t} & 0.0019\end{array}$

Analysis of Variance

Source DF Sum of Squares Mean Square F Ratio Prob $>$ F

$\begin{array}{lllllll}\text { Sampler } & 1 & 0.23362010 & 0.233620 & 10.5335 & 0.0037\end{array}$

$\begin{array}{llll}\text { Error } & 22 & 0.48793252 & 0.022179\end{array}$

C. Total $23 \quad 0.72155262$

Means for Oneway Anova

Level Number Mean Std Error Lower 95\% Upper 95\%

Coliwasa $\quad \begin{array}{llrrr}\text { N } & 4.10940 & 0.03723 & 4.0322 & 4.1866\end{array}$

$\begin{array}{lrrrrr}\text { Hydragard - HF } & 16 & 4.10940 & 0.03723 & 4.0322 & 4.1866 \\ & 8 & 3.90010 & 0.05265 & 3.7909 & 4.0093\end{array}$

Std Error uses a pooled estimate of error variance

Means and Std Deviations

Level Number Mean Std Dev Std Err Mean Lower 95\% Upper 95\%

$\begin{array}{llllll} & 16 & 4.10940 & 0.149590 & 0.03740 & 4.0297\end{array}$

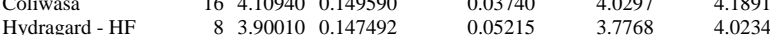

t Test

Hydragard - HF-Coliwasa

Assuming unequal variances

Difference $\quad-0.20929$ t Ratio $\quad-3.26155$

Std Err Dif $\quad 0.06417$ DF $\quad 14.28841$

Upper CL Dif -0.07192 Prob $>|t| r \mid \begin{aligned} 14.28841 \\ 0.0056\end{aligned}$

Lower CL Dif -0.34666 Prob $>t \quad 0.9972$

$\begin{array}{lrl}\text { Confidence } & 0.95 \text { Prob }<\mathrm{t} \quad 0.0028\end{array}$

Tests that the Variances are Equal

Level Count Std Dev MeanAbsDif to Mean MeanAbsDif to Median

$\begin{array}{lllll}\text { Coliwasa } & 16 & 0.1495897 & 0.1182307 & 0.1167214\end{array}$

$\begin{array}{lrlll}\text { Hydragard - HF } & 8 & 0.1474916 & 0.1182307 & 0.1167214 \\ & & & 0.1076654 & 0.0925721\end{array}$

Test F Ratio DFNum DFDen p-Value

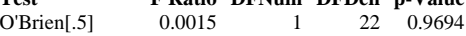

Brown-Forsythe $\quad 0.2813 \quad 1 \quad 22 \quad 0.6012$

$\begin{array}{lllll}\text { Levene } & 0.0764 & 1 & 22 & 0.7849\end{array}$

$\begin{array}{lrrrr}\text { Bartlett } & 0.0018 & 1 & \text {. } & 0.9662 \\ \text { F Test 2-sided } & 1.0287 & 15 & 7 & 1.0000\end{array}$

Welch Anova testing Means Equal, allowing Std Devs Not Equal

F Ratio DFNum DFDen Prob $>$ F

$\begin{array}{llrr}10.6377 & 1 & 14.288 & 0.0056\end{array}$

t Test

$$
1 \quad 14.288 \quad 0.0056
$$

3.2615
Oneway Analysis of Measurement By Sampler Type of Material=SME Simulant, Analytical Block $=2$, Oxide/Analyte $=\mathrm{CaO}(\mathrm{wt} \%)$, Targeted $=1.1462$

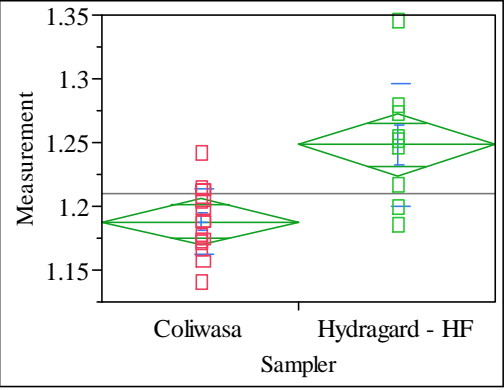

Excluded Rows

neway Anova

Summary of Fit

Rsquare

Adj Rsquare

Root Mean Square Error $\quad 0.400808$

Observations (or Sum Wgts) \begin{tabular}{rr}
1.209748 \\
\hline
\end{tabular}

t Test

Hydragard - HF-Coliwasa

Assuming equal variances

Difference $\quad 0.060389$ t Ratio 4.129639

$\begin{array}{lllr}\text { Std Err Dif } & 0.014623 & \text { DF } & 23 \\ \text { Upper CL Dif } & 0.090640 & \text { Prob }>|t| & 0.0004\end{array}$

$\begin{array}{llll}\text { Upper CL Dif } & 0.090640 & \text { Prob }>|t| & 0.0004 \\ \text { Lower CL Dif } & 0.030138 & \text { Prob }>t & 0.0002\end{array}$

$\begin{array}{lrrr}\text { Lower CL Dif } & 0.030138 & \text { Prob }>t & 0.0002 \\ \text { Confidence } & 0.95 & \text { Prob }<t & 0.9998\end{array}$

Analysis of Variance

Source DF Sum of Squares Mean Square F Ratio Prob > F

$\begin{array}{lrrrrr}\text { Sampler } & 1 & 0.02100581 & 0.021006 & 17.0539 & 0.0004\end{array}$

$\begin{array}{llll}\text { Error } & 23 & 0.02832976 & 0.001232\end{array}$

C. Total $24 \quad 0.04933557$

Means for Oneway Anova

Level Number Mean Std Error Lower 95\% Upper 95\%

$\begin{array}{llllll}\text { Coliwasa } & 16 & 1.18801 & 0.00877 & 1.1699 & 1.2062\end{array}$

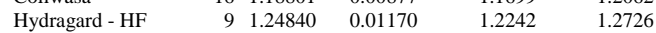

Std Error uses a pooled estimate of error variance

Means and Std Deviations

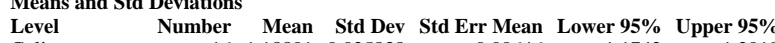

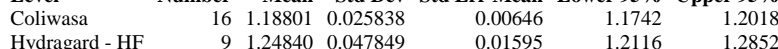

\section{t Test}

Hydragard - HF-Coliwasa

Assuming unequal variances

$\begin{array}{lrlr}\text { Difference } & 0.060389 & \text { t Ratio } & 3.509379 \\ \text { Std Err Dif } & 0.017208 & \text { DF } & 10.68618 \\ \text { Upper CL Dif } & 0.098400 & \text { Prob }>|t| & 0.0051 \\ \text { Lower CL Dif } & 0.022379 & \text { Prob }>t & 0.0026 \\ \text { Confidence } & 0.95 & \text { Prob }<\mathrm{t} & 0.9974\end{array}$

Tests that the Variances are Equal

$\begin{array}{lrrr}\text { Level } & \text { Count } & \text { Std Dev MeanAbsDif to Mean MeanAbsDif to Median } \\ \text { Coliwasa } & 16 & 0.0258377 & 0.0207366\end{array}$

$\begin{array}{lrrrr}\text { Coliwasa } & 16 & 0.0258377 & 0.0207366 & 0.0207257 \\ \text { Hydragard - HF } & 9 & 0.0478486 & 0.0338572 & 0.0340472\end{array}$

Test F Ratio DFNum DFDen p-Value

$\begin{array}{lllll}\text { O'Brien[.5] } & 3.0727 & 1 & 23 & 0.0929\end{array}$

$\begin{array}{lllll}\text { Brown-Forsythe } & 2.1291 & 1 & 23 & 0.1581\end{array}$

2.0486

$\begin{array}{lllll}\text { Levene } & 2.0486 & 1 & 23 & 0.1658 \\ \text { Bartlett } & 4.0292 & 1 & & 0.0447\end{array}$

$\begin{array}{lllll}\text { F Test 2-sided } & 3.4295 & 8 & 15 & 0.0381\end{array}$

Welch Anova testing Means Equal, allowing Std Devs Not Equal

F Ratio DFNum DFDen Prob > F

$\begin{array}{llll}12.3157 & 1 & 10.686 & 0.0051\end{array}$

t Test 
Revision 0

\section{Exhibit C3. Statistical Comparisons for Low-Rheology (Phase 3) Simulant Testing (screened data)}

Oneway Analysis of Measurement By Sampler Type of Material=SME Simulant, Analytical Block $=2$, Oxide $/$ Analyte $=$ Na2O $($ wt $\%$ ), Targeted $=11.659$

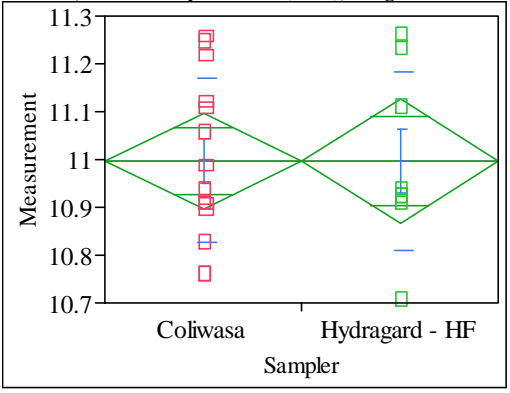

Excluded Rows

Rsquare

Adj Rsquare

$\begin{array}{lr}\text { Adj Rsquare } & -0.05 \\ \text { Root Mean Square Error } \quad 0.177042\end{array}$

Mean of Response

Observations (or Sum Wgts) $\quad 22$

t Test

Hydragard - HF-Coliwasa

Assuming equal variances

Difference $\quad-0.00048$ t Ratio -0.00614

$\begin{array}{lrlr}\text { Std Err Dif } & 0.07847 & \text { DF } & 20 \\ \text { Upper CL Dif } & 0.16319 & \text { Prob }>|t| & 0.9952\end{array}$

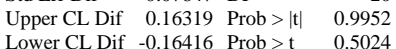

$\begin{array}{lrll}\text { Lower CL Dif } & -0.16416 & \text { Prob }>\mathrm{t} & 0.5024 \\ \text { Confidence } & 0.95 & \text { Prob }<\mathrm{t} & 0.4976\end{array}$

Analysis of Variance
Source DF Sum of Squares Mean Square F Ratio Prob $>$ F

$\begin{array}{lrrrrr}\text { Sampler } & 1 & 0.00000118 & 1.18 \mathrm{e}-6 & 0.0000 & 0.9952\end{array}$

$\begin{array}{lrrr}\text { Error } & 20 & 0.62687492 & 0.031344\end{array}$

$\begin{array}{lll}\text { C. Total } 21 & 21 & 0.62687610\end{array}$

Means for Oneway Anova

Level Number Mean Std Error Lower 95\% Upper 95\%

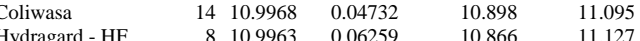

Std Error uses a pooled estimate of error variance

Means and Std Deviations

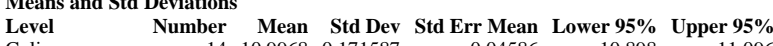

$\begin{array}{lrrrrrr}\text { Coliwasa } & 14 & 10.9968 & 0.171587 & 0.04586 & 10.898 & 11.096 \\ \text { Hydragard - HF } & 8 & 10.9963 & 0.186750 & 0.06603 & 10.840 & 11.152\end{array}$

t Test

Hydragard - HF-Coliwasa

Assuming unequal variances

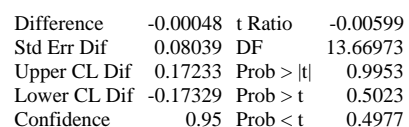

Tests that the Variances are Equal

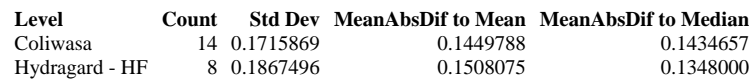

Test F Ratio DFNum DFDen p-Value

$\begin{array}{lllll}\text { O'Brien[.5] } & 0.1364 & 1 & 20 & 0.7158\end{array}$

$\begin{array}{lllll}\text { Brown-Forsythe } & 0.0302 & 1 & 20 & 0.8638\end{array}$

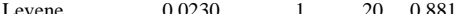

$\begin{array}{lllll}\text { Bartlett } & 0.0627 & 1 & \text {. } & 0.8022\end{array}$

$\begin{array}{lllll}\text { F Test 2-sided } & 1.1845 & 7 & 13 & 0.7504\end{array}$

Welch Anova testing Means Equal, allowing Std Devs Not Equal

F Ratio DFNum DFDen Prob $>$ F

t Test
0.0060
Oneway Analysis of Measurement By Sampler Type of Material=SME Simulant, Analytica Block $=2$, Oxide/Analyte $=\mathrm{NiO}(\mathrm{wt} \%)$, Targeted $=\mathbf{0 . 4 1}$

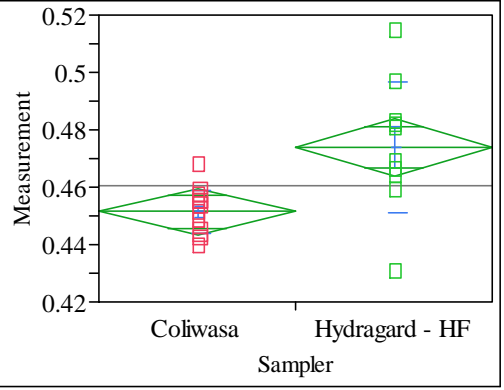

Excluded Rows

Oneway Anova

Summary of Fit

Rsquare

Adj Rsquare

Root Mean Square Error $\quad 0.015365$

Mean of Response

0.358337

Observations (or Sum Wgts)

0.460391
25

t Test

Hydragard - HF-Coliwasa

Assuming equal variances

Difference $\quad 0.022481$ t Ratio 3.583899

$\begin{array}{lllr}\text { Std Err Dif } & 0.006273 & \text { DF } & 23 \\ \text { Upper CL Dif } & 0.035457 & \text { Prob }>|t| & 0.0016 \\ \text { Lower CL Dif } & 0.009505 & \text { Prob }>t & 0.0008\end{array}$

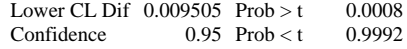

Analysis of Variance
Source DF Sum of Squares Mean Square F Ratio Prob $>$ F

$\begin{array}{lrrrrr}\text { Source } & \text { DF } & \text { Sum of Squares } & \text { Mean Square } & \text { F Ratio } & \text { Prob > F } \\ \text { Sampler } & 1 & 0.00303233 & 0.003032 & 12.8443 & 0.0016\end{array}$

$\begin{array}{lrrr}\text { Sampler } & 1 & 0.00303233 & 0.003032 \\ \text { Error } & 23 & 0.00542991 & 0.000236\end{array}$

C. Total $24 \quad 0.00846223$

Means for Oneway Anova

Level Number Mean Std Error Lower 95\% Upper 95\%

$\begin{array}{llllll}\text { Coliwasa } & 15 & 0.451398 & 0.00397 & 0.44319 & 0.45960\end{array}$

$\begin{array}{llllll}\text { Hydragard - HF } & 10 & 0.473879 & 0.00486 & 0.46383 & 0.48393\end{array}$

Std Error uses a pooled estimate of error variance

Means and Std Deviations

$\begin{array}{lllllll}\text { Level } & \text { Number } & \text { Mean } & \text { Std Dev Std Err Mean Lower 95\% Upper 95\% }\end{array}$

$\begin{array}{lrrrrrr}\text { Coliwasa } & 15 & 0.451398 & 0.007458 & 0.00193 & 0.44727 & 0.45553\end{array}$

$\begin{array}{lllllll}\text { Hydragard - HF } & 10 & 0.473879 & 0.022733 & 0.00719 & 0.45762 & 0.49014\end{array}$

t Test

Hydragard - HF-Coliwasa

Assuming unequal variances

Difference $\quad 0.022481$ t Ratio 3.020684

Std Err Dif $\quad 0.007442$ DF 10.30386

Upper CL Dif 0.038997 Prob $>|t| r 0.0125$

Lower CL Dif 0.005964 Prob $>t \quad 0.0062$

$\begin{array}{lll}\text { Confidence } & 0.95 \text { Prob }<\mathrm{t} \quad 0.9938\end{array}$

Tests that the Variances are Equal

$\begin{array}{lrrrr}\text { Level } & \text { Count } & \text { Std Dev } & \text { MeanAbsDif to Mean } & \text { MeanAbsDif to Median } \\ \text { Coliwasa } & 15 & 0.0074582 & 0.0059610 & 0.0059383\end{array}$

$\begin{array}{lllll}\text { Hydragard - HF } & 10 & 0.0227331 & 0.0059610 & 0.0059383 \\ & & 0.0167970 & 0.0167970\end{array}$

Test F Ratio DFNum DFDen p-Value

$\begin{array}{lllll}\text { OBnown-Forsythe } & 7.6578 & 1 & 23 & 0.0416\end{array}$

$\begin{array}{llll}7.6742 & 1 & 23 & 0.0109\end{array}$

$\begin{array}{lrrrr}\text { Levene } & 7.8072 & 1 & 23 & 0.0103\end{array}$

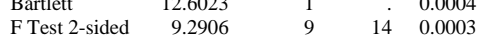

Welch Anova testing Means Equal, allowing Std Devs Not Equal

F Ratio DFNum DFDen Prob $>$ F

$\begin{array}{llll}9.1245 & 1 & 10.304 & 0.0125\end{array}$

t Test 
SRNL-STI-2011-00693

Revision 0

\section{Exhibit C3. Statistical Comparisons for Low-Rheology (Phase 3) Simulant Testing (screened data)}

Oneway Analysis of Measurement By Sampler Type of Material=SME Simulant, Analytical Block $=2$, Oxide/Analyte $=\mathrm{ZrO} 2$ ( $w$ t $\%$ ), Targeted $=0.3547$

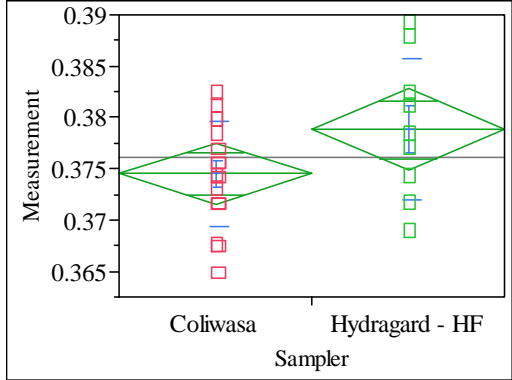

Exclude
1

Oneway Anova

Summary of Fit

Adj Rsquare

Root Mean Square Error 0.005794

Mean 0.376063

0.376063
Observations (or Sum Wgts)

t Test

Hydragard - HF-Coliwasa

Assuming equal variances

$\begin{array}{lrlr}\text { Difference } & 0.00432 & \text { t Ratio } & 1.787254 \\ \text { Std Err Dif } & 0.00241 & \text { DF } & 23 \\ \text { Upper CL Dif } & 0.00931 & \text { Prob }>|t| & 0.0871 \\ \text { Lower CL Dif } & -0.00068 & \text { Prob }>t & 0.0435 \\ \text { Confidence } & 0.95 & \text { Prob }<t & 0.9565\end{array}$

Analysis of Variance

Source DF Sum of Squares Mean Square F Ratio Prob $>$ F

$\begin{array}{llllll}\text { Sampler } & 1 & 0.00010725 & 0.000107 & 3.1943 & 0.0871\end{array}$

$\begin{array}{llll}\text { Error } & 23 & 0.00077224 & 0.000034\end{array}$

C. Total $24 \quad 0.00087949$

Means for Oneway Anova

Level $\quad$ Number $\quad$ Mean Std Error Lower 95\% Upper 95\%

$\begin{array}{lrrrrr}\text { Coliwasa } & 16 & 0.374509 & 0.00145 & 0.37151 & 0.37751\end{array}$

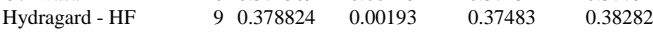

Std Error uses a pooled estimate of error variance

Means and Std Deviations

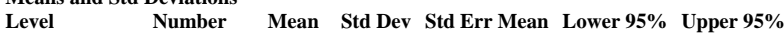

$\begin{array}{lrrrrrr}\text { Coliwasa } & 16 & 0.374509 & 0.005114 & 0.00128 & 0.37178 & 0.37723\end{array}$

$\begin{array}{lrrrrrr}\text { Hydragard - HF } & 9 & 0.378824 & 0.006891 & 0.00230 & 0.37353 & 0.38412\end{array}$

Test

Hydragard - HF-Coliwasa

Assuming unequal variances

$\begin{array}{lrlr}\text { Difference } & 0.00432 & \mathrm{t} \text { Ratio } & 1.64135 \\ \text { Std Err Dif } & 0.00263 & \text { DF } & 13.05574 \\ \text { Upper CL Dif } & 0.00999 & \text { Prob }>|\mathrm{t}| & 0.1246 \\ \text { Lower CL Dif } & -0.00136 & \text { Prob }>\mathrm{t} & 0.0623 \\ \text { Confidence } & 0.95 & \text { Prob }<\mathrm{t} & 0.9377\end{array}$

Tests that the Variances are Equal

Level Count Std Dev MeanAbsDif to Mean MeanAbsDif to Median

Coliwasa 160.0051140

$\begin{array}{lrrrrr}\text { Coliwasa } & 16 & 0.0051140 & 0.0040524 & 0.0040524 \\ \text { Hydragard - HF } & 9 & 0.0068914 & 0.0054699 & 0.0055533\end{array}$

Test F Ratio DFNum DFDen p-Value

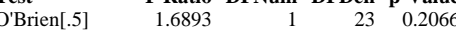

$\begin{array}{lllll}\text { Brown-Forsythe } & 1.2496 & 1 & 23 & 0.2752\end{array}$

$\begin{array}{lllll}\text { Levene } & 1.1082 & 1 & 23 & 0.3034\end{array}$

$\begin{array}{lrrrr} & 0.9273 & 1 & 0.3356\end{array}$

Welch Anova testing Means Equal, allowing Std Devs Not Equal

F Ratio DFNum DFDen Prob $>$ F

$\begin{array}{rrrr}2.6940 & 1 & 13.056 & 0.1246\end{array}$

t Test
1.6414
Oneway Analysis of Measurement By Sampler Type of Material=SME Simulant, Analytical Block $=3$, Oxide/Analyte $=$ Al2O3 (wt $\%$ ), Targeted $=10.869$

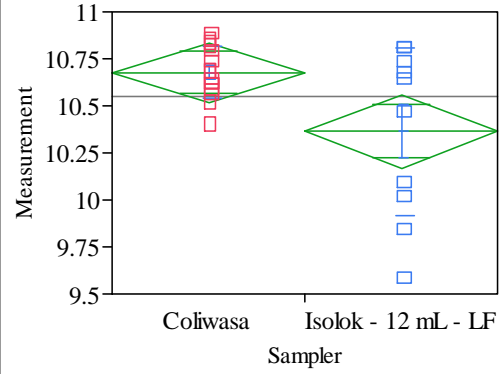

Excluded Rows

Oneway Anova

Summary of Fit

Rsquare $\quad 0.219726$

Adj Rsquare $\quad 0.185801$

Root Mean Square Error $\quad 0.299465$

Mean of Response $\quad 10.55248$

Observations (or Sum Wgts) $\quad 25$

Isolok - $12 \mathrm{~mL}$ - LF-Coliwasa

Assuming equal variances

$\begin{array}{lrlr}\text { Difference } & -0.31114 & \mathrm{t} \text { Ratio } & -2.54496 \\ \text { Std Err Dif } & 0.12226 & \text { DF } & 23 \\ \text { Upper CL Dif } & -0.05823 & \text { Prob }>|\mathrm{t}| & 0.0181 \\ \text { Lower CL Dif } & -0.56404 & \text { Prob }>\mathrm{t} & 0.9909 \\ \text { Confidence } & 0.95 & \text { Prob }<\mathrm{t} & 0.0091\end{array}$

Analysis of Variance

Source DF Sum of Squares Mean Square F Ratio Prob > F

$\begin{array}{llllll}\text { Sampler } & 1 & 0.5808399 & 0.580840 & 6.4768 & 0.0181\end{array}$

$\begin{array}{llll}\text { Error } & 23 & 2.0626295 & 0.089680\end{array}$

C. Total $24 \quad 2.6434694$

Means for Oneway Anova

Level Number Mean Std Error Lower 95\% Upper 95\%

$\begin{array}{lrrrrr}\text { Coliwasa } & 15 & 10.6769 & 0.07732 & 10.517 & 10.837\end{array}$

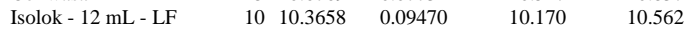

Std Error uses a pooled estimate of error variance

Means and Std Deviations
Level

$\begin{array}{lrrrrrr}\text { Level } & \text { Number } & \text { Mean } & \text { Std Dev } & \text { Std Err Mean } & \text { Lower 95\% } & \text { Upper 95\% } \\ \text { Coliwasa } & 15 & 10.6769 & 0.138199 & 0.03568 & 10.600 & 10.753\end{array}$

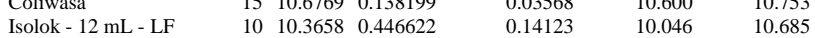

t Test

Isolok - $12 \mathrm{~mL}$ - LF-Coliwasa

Assuming unequal variances

$\begin{array}{lrlr}\text { Difference } & -0.31114 & \text { t Ratio } & -2.13587 \\ \text { Std Err Dif } & 0.14567 & \text { DF } & 10.15903 \\ \text { Upper CL Dif } & 0.01275 & \text { Prob }>|t| & 0.0580 \\ \text { Lower CL Dif } & -0.63503 & \text { Prob }>\text { t } & 0.9710 \\ \text { Confidence } & 0.95 & \text { Prob }<\text { t } & 0.0290\end{array}$

Tests that the Variances are Equal

Level Count Std Dev MeanAbsDif to Mean MeanAbsDif to Median

$\begin{array}{lrrrr}\text { Coliwasa } & 15 & 0.1381989 & 0.1098429 & 0.1095910\end{array}$

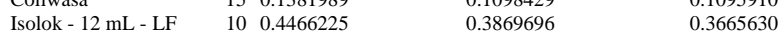

Test F Ratio DFNum DFDen p-Value

$\begin{array}{llrrr}\text { O'Brien[.5] } & 11.9504 & 1 & 23 & 0.0021\end{array}$

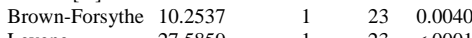

$\begin{array}{lllll}\text { Levene } & 27.5859 & 1 & 23 & <.0001\end{array}$

$\begin{array}{lllll}\text { Bartlett } & 13.8172 & 1 & \text {. } & 0.0002\end{array}$

$\begin{array}{lllll}\text { F Test 2-sided } & 10.4441 & 9 & 14 & 0.0002\end{array}$

Welch Anova testing Means Equal, allowing Std Devs Not Equal

F Ratio DFNum DFDen Prob $>$ F

$\begin{array}{rrrr}4.5620 & 1 & 10.159 & 0.0580\end{array}$

t Test 


\section{Exhibit C3. Statistical Comparisons for Low-Rheology (Phase 3) Simulant Testing (screened data)}

Oneway Analysis of Measurement By Sampler Type of Material=SME Simulant, Analytical Block $=3$, Oxide $/$ Analyte $=\mathrm{CaO}(\mathrm{wt} \%)$, Targeted $=1.1462$

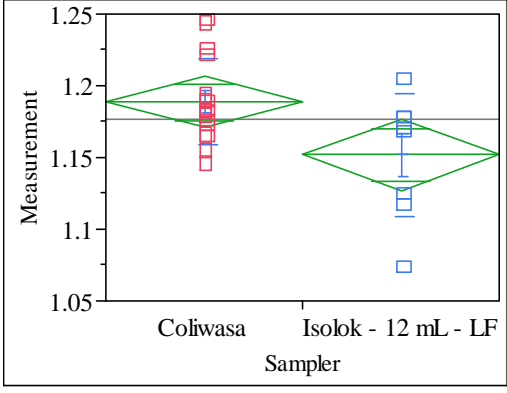

Excluded Rows

Oneway Anova

Summary of Fit

Rsquare

Root Mean Square Error $\quad 0.034749$

Mean of Response 1.176377

Observations (or Sum Wgts) $\quad 24$

t Test

Isolok - $12 \mathrm{~mL}$ - LF-Coliwasa

Assuming equal variances

Difference $\quad-0.03699$ t Ratio $\quad-2.45844$

Std Err Dif $\quad 0.01505$ DF $r .458$

Upper CL Dif -0.00579 Prob $>|t| \quad 0.0223$

$\begin{array}{lll}\text { Lower CL Dif } & -0.06820 \text { Prob }>t & 0.9889\end{array}$

$\begin{array}{lrl}\text { Confidence } & 0.95 \text { Prob }<\mathrm{t} \quad 0.0111\end{array}$

Analysis of Variance

Source DF Sum of Squares Mean Square F Ratio Prob $>$ F

$\begin{array}{lrrrrr}\text { Sampler } & 1 & 0.00729792 & 0.007298 & 6.0439 & 0.0223\end{array}$

$\begin{array}{llll}\text { Error } & 22 & 0.02656449 & 0.007298 \\ \text { C. } & & 0.001207\end{array}$

C. Total $23 \quad 0.03386241$

Means for Oneway Anova

Level Number Mean Std Error Lower 95\% Upper 95\%

$\begin{array}{llllll} & 16 & 1.18871 & 0.00869 & 1.1707 & 1.2067\end{array}$

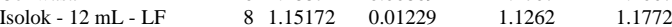

Std Error uses a pooled estimate of error variance

Means and Std Deviations

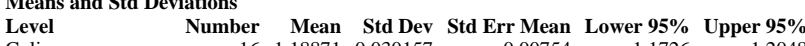

$\begin{array}{lrrrrrr}\text { Coliwasa } & 16 & 1.18871 & 0.030157 & 0.00754 & 1.1726 & 1.2048 \\ \text { Isolok - 12 mL - LF } & 8 & 1.15172 & 0.042967 & 0.01519 & 1.1158 & 1.1876\end{array}$

t Test

solok - $12 \mathrm{~mL}$ - LF-Coliwas

Assuming unequal variances

Difference $\quad-0.03699$ t Ratio -2.18123

Std Err Dif $\quad 0.01696$ DF $\quad 10.57364$

Upper CL Dif 0.00052 Prob $>|t| \quad 0.0527$

Lower CL Dif -0.07450 Prob $>t \quad 0.9736$

$\begin{array}{lll}\text { Confidence } & 0.95 \text { Prob }<\mathrm{t} \quad 0.0264\end{array}$

Tests that the Variances are Equal

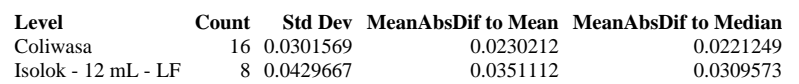

$\begin{array}{lrrrr}\text { Test } & \text { F Ratio } & \text { DFNum } & \text { DFDen } & \text { p-Value } \\ \text { O'Brien[.5] } & 1.6294 & 1 & 22 & 0.2151 \\ \text { Brown-Forsythe } & 0.6416 & 1 & 22 & 0.4317 \\ \text { Levene } & 2.0858 & 1 & 22 & 0.1628 \\ \text { Bartlett } & 1.2136 & 1 & . & 0.2706 \\ \text { F Test 2-sided } & 2.0300 & 7 & 15 & 0.2366\end{array}$

Welch Anova testing Means Equal, allowing Std Devs Not Equal

F Ratio DFNum DFDen Prob $>$ F

t Test
Oneway Analysis of Measurement By Sampler Type of Material=SME Simulant, Analytical Block=3, Oxide $/$ Analyte $=$ Fe2O3 $($ wt $\%$ ), Targeted $=11.462$

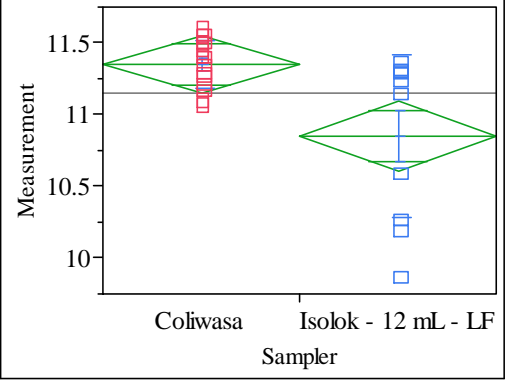

Excluded Rows

Oneway Anova

Summary of Fit

Rsquare

Adj Rsquare

Root Mean Square Error $\quad 0.379818$

Mean of Response

Observations (or Sum Wgts)

t Test

Isolok - $12 \mathrm{~mL}$ - LF-Coliwasa

Assuming equal variances

Difference $\quad-0.49992$ t Ratio $\quad-3.22403$

$\begin{array}{lrlr}\text { Std Err Dif } & 0.15506 & \text { DF } & 23 \\ \text { Upper CL Dif } & -0.17915 & \text { Prob }>|t| & 0.0038\end{array}$

Lower CL Dif -0.82068 Prob $>t \quad 0.9981$

$\begin{array}{lll}\text { Confidence } & 0.95 \text { Prob }<\mathrm{t} \quad 0.0019\end{array}$

Analysis of Variance

Source DF Sum of Squares Mean Square F Ratio Prob $>$ F

$\begin{array}{lrrrrr}\text { Sampler } & 1 & 1.4995106 & 1.49951 & 10.3944 & 0.0038\end{array}$

$\begin{array}{llll}\text { Error } & 23 & 3.3180186 & 0.14426\end{array}$

C. Total $24 \quad 4.8175292$

Means for Oneway Anova

Level Number Mean Std Error Lower 95\% Upper 95\%

$\begin{array}{llllll}\text { Coliwasa } & 15 & 11.3499 & 0.09807 & 11.147 & 11.553\end{array}$

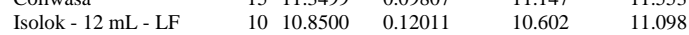

Std Error uses a pooled estimate of error variance

Means and Std Deviations

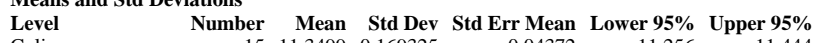

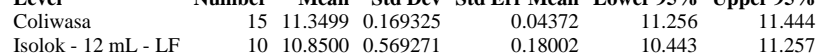

t Test

Isolok - $12 \mathrm{~mL}$ - LF-Coliwasa

Assuming unequal variances

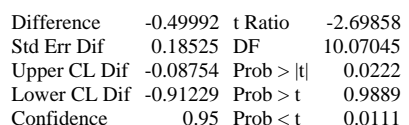

Tests that the Variances are Equal

$\begin{array}{lrrrr}\text { Level } & \text { Count } & \text { Std Dev } & \text { MeanAbsDif to Mean } & \text { MeanAbsDif to Median } \\ \text { Coliwasa } & 15 & 0.1693254 & 0.1411908 & 0.1382043\end{array}$

$\begin{array}{lrrrr}\text { Coliwasa } & 15 & 0.1693254 & 0.1411908 & 0.1382043 \\ \text { Isolok - 12 mL - LF } & 10 & 0.5692708 & 0.5021106 & 0.4446367\end{array}$

Test F Ratio DFNum DFDen p-Value

$\begin{array}{lllll}\text { O'Brien[.5] } & 13.5139 & 1 & 23 & 0.0013\end{array}$

$\begin{array}{lllll}\text { Brown-Forsythe } & 5.9570 & 1 & 23 & 0.0228\end{array}$

Browene $36.1083 \quad 1123<0.0228$

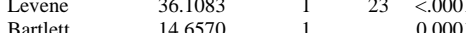

$\begin{array}{lllll}\text { F Test 2-sided } & 11.3030 & 9 & 14 & 0.0001\end{array}$

Welch Anova testing Means Equal, allowing Std Devs Not Equal

F Ratio DFNum DFDen Prob $>$ F

$\begin{array}{llll}7.2824 & 1 & 10.07 & 0.0222\end{array}$

t Test
2.6986 
SRNL-STI-2011-00693

Revision 0

\section{Exhibit C3. Statistical Comparisons for Low-Rheology (Phase 3) Simulant Testing (screened data)}

Oneway Analysis of Measurement By Sampler Type of Material=SME Simulant, Analytical Block=3, Oxide/Analyte $=$ K2O (wt \%), Targeted $=\mathbf{0 . 1 5 9 1}$

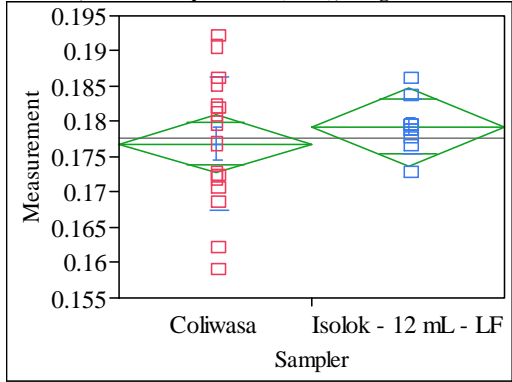

Exc

Oneway Anova

Summary of Fit

Rsquare $\quad 0.021973$

Adj Rsquare $\quad-0.02055$

Root Mean Square Error $\quad 0.008002$

Mean of Response $\quad 0.177751$

Observations (or Sum Wgts) $\quad 25$

t Test

Isolok - $12 \mathrm{~mL}$ - LF-Coliwasa

Assuming equal variances

$\begin{array}{lrlr}\text { Difference } & 0.00240 & \text { t Ratio } & 0.718844 \\ \text { Std Err Dif } & 0.00333 & \text { DF } & 23 \\ \text { Upper CL Dif } & 0.00929 & \text { Prob }>|t| & 0.4795 \\ \text { Lower CL Dif } & -0.00450 & \text { Prob }>\text { t } & 0.2397 \\ \text { Confidence } & 0.95 & \text { Prob }<\mathrm{t} & 0.7603\end{array}$

Analysis of Variance

Source DF Sum of Squares Mean Square F Ratio Prob $>$ F

$\begin{array}{llllll}\text { Sampler } & 1 & 0.00003309 & 0.000033 & 0.5167 & 0.4795\end{array}$

$\begin{array}{llll}\text { Error } & 23 & 0.00147262 & 0.000064\end{array}$

C. Total $24 \quad 0.00150571$

Means for Oneway Anova

$\begin{array}{lrrrrr}\text { Level } & \text { Number } & \text { Mean } & \text { Std Error } & \text { Lower 95\% } & \text { Upper 95\% } \\ \text { Coliwasa } & 16 & 0.176888 & 0.00200 & 0.17275 & 0.18103\end{array}$

$\begin{array}{lrrrrr} & 16 & 0.176888 & 0.00200 & 0.17275 & 0.18103 \\ \text { Isolok - } 12 \mathrm{~mL} \text { - LF } & 9 & 0.179285 & 0.00267 & 0.17377 & 0.18480\end{array}$

Std Error uses a pooled estimate of error variance

$\begin{array}{lllll}\text { Means and Std Deviations } & \text { Number } & \text { Mean Std Dev Std Err Mean Lower 95\% Upper 95\% }\end{array}$

$\begin{array}{lrrrrrr}\text { Level } & \text { Number } & \text { Mean } & \text { Std Dev } & \text { Std Err Mean } & \text { Lower 95\% } & \text { Upper 95\% } \\ \text { Coliwasa } & 16 & 0.176888 & 0.009502 & 0.00238 & 0.17182 & 0.18195\end{array}$

$\begin{array}{lrrrrrr}\text { Coliwasa } & 16 & 0.176888 & 0.009502 & 0.00238 & 0.17182 & 0.18195 \\ \text { Isolok - 12 mL - LF } & 9 & 0.179285 & 0.003845 & 0.00128 & 0.17633 & 0.18224\end{array}$

Isolok - $12 \mathrm{~mL}$ - LF-Coliwasa

Assuming unequal variances

$\begin{array}{lrlr}\text { Difference } & 0.00240 & \text { t Ratio } & 0.887911 \\ \text { Std Err Dif } & 0.00270 & \text { DF } & 21.57549 \\ \text { Upper CL Dif } & 0.00800 & \text { Prob }>|t| & 0.3844 \\ \text { Lower CL Dif } & -0.00321 & \text { Prob }>\text { t } & 0.1922 \\ \text { Confidence } & 0.95 & \text { Prob }<\text { t } & 0.8078\end{array}$

Tests that the Variances are Equal

Level Count Std Dev MeanAbsDif to Mean MeanAbsDif to Median

$\begin{array}{lrrrr}\text { Coliwasa } & 16 & 0.0095021 & 0.0077923 & 0.0077923 \\ \text { Isolok - } 12 \mathrm{~mL} \text { - LF } & 9 & 0.0038448 & 0.0025877 & 0.0025430\end{array}$

Test F Ratio DFNum DFDen p-Value

$\begin{array}{lrrrr}\text { O'Brien[.5] } & 4.2018 & 1 & 23 & 0.0519\end{array}$

Brown-Forsythe $7.8769 \quad 1 \quad 23 \quad 0.0100$

$\begin{array}{lllll}\text { Levene } & 8.1402 & 1 & 23 & 0.0090\end{array}$

$\begin{array}{lrrrr}\text { Bartlett } & 6.2616 & 1 & \text {. } & 0.0123 \\ \text { F Test 2-sided } & 6.1079 & 15 & 8 & 0.0144\end{array}$

Welch Anova testing Means Equal, allowing Std Devs Not Equal

F Ratio DFNum DFDen Prob $>$ F

$\begin{array}{rrrr}0.7884 & 1 & 21.575 & 0.3844\end{array}$

t Test
0.8879
Oneway Analysis of Measurement By Sampler Type of Material=SME Simulant, Analytical Block=3, Oxide/Analyte=Li2O (wt \%), Targeted $=4.674$

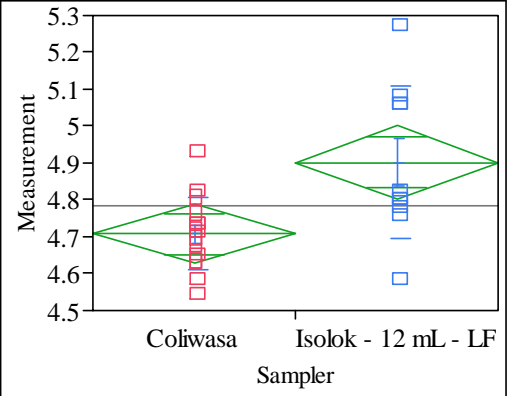

Excluded Rows

Oneway Anova

Summary of Fit

Adj Rsquare

0.304664

Root Mean Square Error $\quad 0.274432$

Mean of Response

Observations (or Sum Wgts)

t Test

Isolok - $12 \mathrm{~mL}$ - LF-Coliwas

Assuming equal variances

Difference $\quad 0.194479$ t Ratio $\quad 3.174511$

Std Err Dif $\quad 0.061263$ DF 23

Upper CL Dif 0.321210 Prob $>|t| \quad 0.0042$

Lower CL Dif 0.067747 Prob $>t \quad 0.0021$

$\begin{array}{lrl}\text { Confidence } & 0.95 \text { Prob }<\mathrm{t} & 0.9979\end{array}$

Analysis of Variance

Source DF Sum of Squares Mean Square F Ratio Prob $>$ F

$\begin{array}{llllll}\text { Sampler } & 1 & 0.22693163 & 0.226932 & 10.0775 & 0.0042\end{array}$

$\begin{array}{llll}\text { Error } & 23 & 0.51792794 & 0.022519\end{array}$

C. Total $24 \quad 0.74485957$

Means for Oneway Anova

Level Number Mean Std Error Lower 95\% Upper 95\%

$\begin{array}{lrrrrr}\text { Coliwasa } & 15 & 4.70767 & 0.03875 & 4.6275 & 4.7878\end{array}$

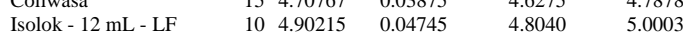

Std Error uses a pooled estimate of error variance

Means and Std Deviations

Level Number Mean Std Dev Std Err Mean Lower 95\% Upper 95\%

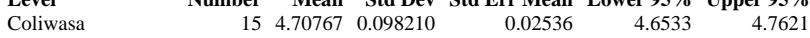

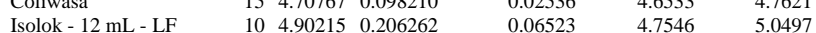

t Test

Isolok - $12 \mathrm{~mL}$ - LF-Coliwas

Assuming unequal variances

$\begin{array}{lrlr}\text { Difference } & 0.194479 & \text { t Ratio } & 2.779002 \\ \text { Std Err Dif } & 0.069981 & \text { DF } & 11.75352 \\ \text { Upper CL Dif } & 0.347311 & \text { Prob }>|t| & 0.0170 \\ \text { Lower CL Dif } & 0.041647 & \text { Prob }>t & 0.0085 \\ \text { Confidence } & 0.95 & \text { Prob }<t & 0.9915\end{array}$

Tests that the Variances are Equal

Level Count Std Dev MeanAbsDif to Mean MeanAbsDif to Median

$\begin{array}{lllll}\text { Coliwasa } & 15 & 0.0982098 & 0.0736770 & 0.0731986\end{array}$

Isolok - $12 \mathrm{~mL}$ - LF $\quad 10 \quad 0.2062619 \quad 0.1730932 \quad 0.1571617$

Test F Ratio DFNum DFDen p-Value

$\begin{array}{lrrrr}\text { O'Brien[.5] } & 5.3520 & 1 & 23 & 0.0300\end{array}$

$\begin{array}{lllll}\text { Brown-Forsythe } & 3.5624 & 1 & 23 & 0.0718\end{array}$

$\begin{array}{lllll}\text { Levene } & 9.9636 & 1 & 23 & 0.0044\end{array}$

$\begin{array}{lllll}\text { Bartlett } & 5.8724 & 1 & \text {. } & 0.0154 \\ \text { F Test 2-sided } & 4.4109 & 9 & 14 & 0.0135\end{array}$

Welch Anova testing Means Equal, allowing Std Devs Not Equal

F Ratio DFNum DFDen Prob $>$ F

$\begin{array}{rrrr}7.7229 & 1 & 11.754 & 0.0170\end{array}$

t Test

2.7790 
SRNL-STI-2011-00693

Revision 0

\section{Exhibit C3. Statistical Comparisons for Low-Rheology (Phase 3) Simulant Testing (screened data)}

Oneway Analysis of Measurement By Sampler Type of Material=SME Simulant, Analytical Block=3, Oxide/Analyte=Na2O (wt \%), Targeted=11.659

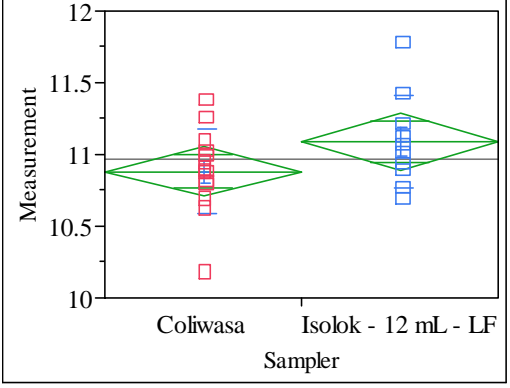

Excluded Rows

Oneway Anova

Summary of Fit

Rsquare

0.110729

Root Mean Square Error $\quad 0.304617$

Mean of Response 10.96823

Observations (or Sum Wgts) $\quad 24$

t Test

Isolok - $12 \mathrm{~mL}$ - LF-Coliwasa

Assuming equal variances

Difference $\quad 0.20875$ t Ratio 1.655103

$\begin{array}{lrl}\text { Std Err Dif } & 0.12612 \text { DF } & 22 \\ & & 0.1121\end{array}$

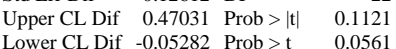

$\begin{array}{lrll}\text { Lower CL Dif } & -0.05282 & \text { Prob }>t & 0.0561 \\ \text { Confidence } & 0.95 & \text { Prob }<\mathrm{t} & 0.9439\end{array}$

Analysis of Variance

Source DF Sum of Squares Mean Square F Ratio Prob $>$ F

$\begin{array}{lrrrrr}\text { Sampler } & 1 & 0.2541904 & 0.254190 & 2.7394 & 0.1121\end{array}$

$\begin{array}{llll}\text { Error } & 22 & 2.0414177 & 0.092792\end{array}$

C. Total $23 \quad 2.2956081$

Means for Oneway Anova

Level Number Mean Std Error Lower 95\% Upper 95\%

$\begin{array}{llllll}\text { Coliwasa } & 14 & 10.8812 & 0.08141 & 10.712 & 11.050\end{array}$

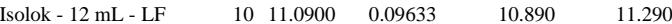

Std Error uses a pooled estimate of error variance

Means and Std Deviations

$\begin{array}{llllll}\text { Level } & \text { Number } & \text { Mean Std Dev Std Err Mean Lower 95\% Upper 95\% }\end{array}$

$\begin{array}{lrrrrrr}\text { Coliwasa } & 14 & 10.8812 & 0.291721 & 0.07797 & 10.713 & 11.050 \\ \text { Isolok - 12 mL - LF } & 10 & 11.0900 & 0.322335 & 0.10193 & 10.859 & 11.321\end{array}$

t Test

solok - $12 \mathrm{~mL}$ - LF-Coliwas

Assuming unequal variances

Difference $\quad 0.20875$ t Ratio 1.626642

Std Err Dif $\quad 0.12833 \mathrm{DF} \quad 18.27978$

Upper CL Dif 0.47806 Prob $>|t| \quad 0.1209$

Lower CL Dif -0.06057 Prob $>t \quad 0.0605$

$\begin{array}{lll}\text { Confidence } & 0.95 \text { Prob }<\mathrm{t} \quad 0.9395\end{array}$

Tests that the Variances are Equal

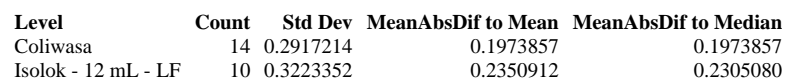

$\begin{array}{lrrrr}\text { Test } & \text { F Ratio } & \text { DFNum } & \text { DFDen } & \text { p-Value } \\ \text { O'Brien[.5] } & 0.0753 & 1 & 22 & 0.7864 \\ \text { Brown-Forsythe } & 0.1423 & 1 & 22 & 0.7097 \\ \text { Levene } & 0.1934 & 1 & 22 & 0.6644 \\ \text { Bartlett } & 0.1022 & 1 & . & 0.7492 \\ \text { F Test 2-sided } & 1.2209 & 9 & 13 & 0.7208\end{array}$

Welch Anova testing Means Equal, allowing Std Devs Not Equal

F Ratio DFNum DFDen Prob > F

$\begin{array}{llll}2.6460 & 1 & 18.28 & 0.1209\end{array}$

t Test
1.6266
Oneway Analysis of Measurement By Sampler Type of Material=SME Simulant, Analytical Block $=3$, Oxide $/$ Analyte $=\mathrm{NiO}(\mathrm{wt} \%)$, Targeted $=\mathbf{0 . 4 1}$

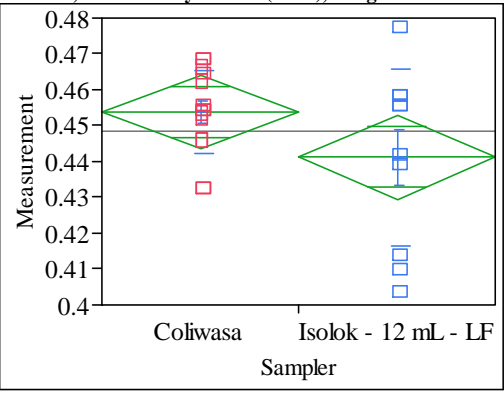

Excluded Rows

Oneway Anova

Summary of Fit

Rsquare

Adj Rsquare

Root Mean Square Error $\quad 0.07379$

Observations (or Sum Wgts) $\quad 24$

t Test

Isolok - $12 \mathrm{~mL}$ - LF-Coliwasa

Assuming equal variances

$\begin{array}{lrlr}\text { Difference } & -0.01265 & \text { t Ratio } & -1.68297 \\ \text { Std Err Dif } & 0.00752 & \text { DF } & 22 \\ \text { Upper CL Dif } & 0.00294 & \text { Prob }>|t| & 0.1065 \\ \text { Lower CL Dif } & -0.02824 & \text { Prob }>\text { t } & 0.9467 \\ \text { Confidence } & 0.95 & \text { Prob }<\mathrm{t} & 0.0533\end{array}$

Analysis of Variance

Source DF Sum of Squares Mean Square F Ratio Prob > F

$\begin{array}{lrrrrr}\text { Sampler } & 1 & 0.00093380 & 0.000934 & 2.8324 & 0.1065\end{array}$

$\begin{array}{llll}\text { Error } & 22 & 0.00725316 & 0.000330\end{array}$

C. Total $23 \quad 0.00818696$

Means for Oneway Anova

Level Number Mean Std Error Lower 95\% Upper 95\%

$\begin{array}{lllllr}\text { Coliwasa } & 14 & 0.453828 & 0.00485 & 0.44376 & 0.46389\end{array}$

$\begin{array}{llllll}\text { Isolok - } 12 \mathrm{~mL} \text { - LF } & 10 & 0.441176 & 0.00574 & 0.42927 & 0.45308\end{array}$

Std Error uses a pooled estimate of error variance

Means and Std Deviations

$\begin{array}{llllll}\text { Level } & \text { Number } & \text { Mean } & \text { Std Dev Std Err Mean Lower 95\% Upper 95\% }\end{array}$

$\begin{array}{lrrrrrr}\text { Coliwasa } & 14 & 0.453828 & 0.011722 & 0.00313 & 0.44706 & 0.46060 \\ \text { Isolok - 12 mL - LF } & 10 & 0.441176 & 0.024646 & 0.00779 & 0.42355 & 0.45881\end{array}$

t Test

Isolok - $12 \mathrm{~mL}$ - LF-Coliwasa

Assuming unequal variances

$\begin{array}{lrlr}\text { Difference } & -0.01265 & \text { t Ratio } & -1.50625 \\ \text { Std Err Dif } & 0.00840 \text { DF } & 11.92801 \\ \text { Upper CL Dif } & 0.00566 & \text { Prob }>|t| & 0.1580 \\ \text { Lower CL Dif } & -0.03097 & \text { Prob }>\text { t } & 0.9210 \\ \text { Confidence } & 0.95 & \text { Prob }<\mathrm{t} & 0.0790\end{array}$

Tests that the Variances are Equal

$\begin{array}{lrrrr}\text { Level } & \text { Count } & \text { Std Dev } & \text { MeanAbsDif to Mean } & \text { MeanAbsDif to Median } \\ \text { Coliwasa } & 14 & 0.0117224 & 0.0088815 & 0.0088166\end{array}$

$\begin{array}{lrrrr}\text { Coliwasa } & 14 & 0.0117224 & 0.0088815 & 0.0088166 \\ \text { Isolok - 12 mL - LF } & 10 & 0.0246459 & 0.0198001 & 0.0197238\end{array}$

Test F Ratio DFNum DFDen p-Value

$\begin{array}{lllll}\text { O'Brien[.5] } & 7.2148 & 1 & 22 & 0.0135\end{array}$

$\begin{array}{lllll}\text { Brown-Forsythe } & 5.4096 & 1 & 22 & 0.0296\end{array}$

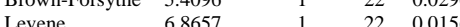

$\begin{array}{llrrr}\text { Levene } & 6.8657 & 1 & 22 & 0.0156 \\ \text { Bartlett } & 5.6107 & 1 & . & 0.0179\end{array}$

$\begin{array}{lllll}\text { F Test 2-sided } & 4.4203 & 9 & 13 & 0.0160\end{array}$

Welch Anova testing Means Equal, allowing Std Devs Not Equal

F Ratio DFNum DFDen Prob $>$ F

$\begin{array}{llll}2.2688 & 1 & 11.928 & 0.1580\end{array}$

t Test 
SRNL-STI-2011-00693

Revision 0

\section{Exhibit C3. Statistical Comparisons for Low-Rheology (Phase 3) Simulant Testing (screened data)}

Oneway Analysis of Measurement By Sampler Type of Material=SME Simulant, Analytical Block $=3$, Oxide/Analyte $=\mathrm{SiO} 2(\mathrm{wt} \%)$, Targeted $=\mathbf{5 0 . 9 8 5}$

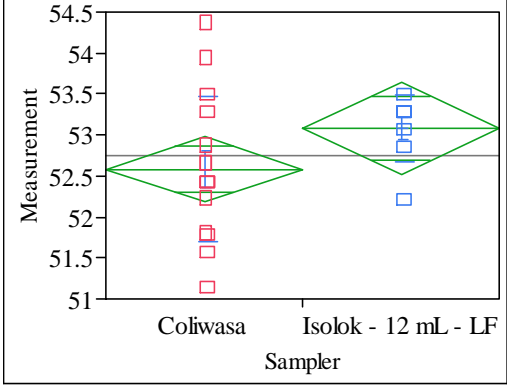

Excluded Rows

Oneway Anova

Summary of Fit

Rsquare $\quad 0.096759$

Adj Rsquare $\quad 0.053748$

Root Mean Square Error $\quad 0.757472$

Mean of Response

52.757
23

t Test

Isolok - $12 \mathrm{~mL}$ - LF-Coliwasa

Assuming equal variances

Difference $\quad 0.4974$ t Ratio 1.499873

$\begin{array}{lrlr}\text { Std Err Dif } & 0.3316 \text { DF } & 21 \\ \text { Upper CL Dif } & 1.1870 & \text { Prob }>|t| & 0.1485\end{array}$

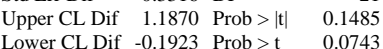

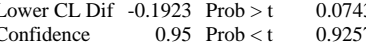

Analysis of Variance

Source DF Sum of Squares Mean Square F Ratio Prob $>$ F

$\begin{array}{lrrrrr}\text { Sampler } & 1 & 1.290752 & 1.29075 & 2.2496 & 0.1485\end{array}$

\begin{tabular}{lrrr} 
Error & 21 & 1.290752 & 1.29075 \\
\hline
\end{tabular}

C. Total $22 \quad 13.339807$

Means for Oneway Anova

Level Number Mean Std Error Lower 95\% Upper 95\%

$\begin{array}{llllll}\text { Coliwasa } & 15 & 52.5840 & 0.19558 & 52.177 & 52.991\end{array}$

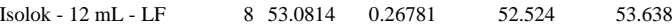

Std Error uses a pooled estimate of error variance

Means and Std Deviations

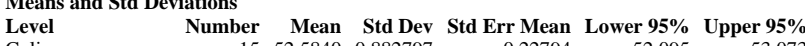

$\begin{array}{lrrrrrr}\text { Coliwasa } & 15 & 52.5840 & 0.882797 & 0.22794 & 52.095 & 53.073 \\ \text { Isolok - 12 mL - LF } & 8 & 53.0814 & 0.403278 & 0.14258 & 52.744 & 53.419\end{array}$

t Test

Isolok - $12 \mathrm{~mL}$ - LF-Coliwas

Assuming unequal variances

Difference $\quad 0.4974$ t Ratio 1.850002

Std Err Dif $\quad 0.2689$ DF 20.74663

Upper CL Dif 1.0569 Prob $>|t| \quad 0.0786$

Lower CL Dif -0.0621 Prob $>t \quad 0.0393$

Confidence $\quad 0.95$ Prob $<\mathrm{t} \quad 0.9607$

Tests that the Variances are Equal

$\begin{array}{lrrrr}\text { Level } & \text { Count } & \text { Std Dev } & \text { MeanAbsDif to Mean } & \text { MeanAbsDif to Median } \\ \text { Coliwasa } & 15 & 0.8827969 & 0.6674616 & 0.6560520 \\ \text { Isolok - 12 mL - LF } & 8 & 0.4032777 & 0.2874684 & 0.2406713\end{array}$

Test F Ratio DFNum DFDen p-Value

$\begin{array}{lllll}\text { O'Brien[.5] } & 2.6745 & 1 & 21 & 0.1169\end{array}$

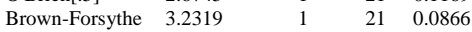

$\begin{array}{lllll} & 3.3622 & 1 & 21 & 0.0809\end{array}$

$\begin{array}{lllll}\text { Levene } & 3.3622 & 1 & 21 & 0.0809\end{array}$

$\begin{array}{lrrrr}\text { Bartlett } & 4.2992 & 1 & \text {; } & 0.0381 \\ \text { F Test 2-sided } & 4.7920 & 14 & 7 & 0.0446\end{array}$

Welch Anova testing Means Equal, allowing Std Devs Not Equal

F Ratio DFNum DFDen Prob $>$ F

$\begin{array}{llll}3.4225 & 1 & 20.747 & 0.0786\end{array}$

t Test
Oneway Analysis of Measurement By Sampler Type of Material=SME Simulant, Analytical Block=3, Oxide/Analyte=Sum of Oxides (wt \%), Targeted $=99.553$

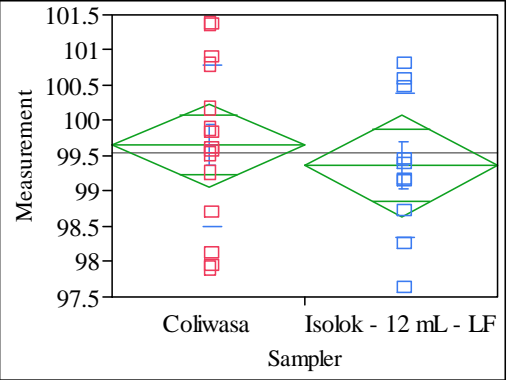

Excluded Rows

1

Oneway Anova

Summary of Fit

Rsquare $\quad 0.017292$

Adj Rsquare $\quad-0.02543$

Root Mean Square Error $\quad 1.102917$

Mean of Response 99.53716

Observations (or Sum Wgts) 25

Isolok - $12 \mathrm{~mL}$ - LF-Coliwasa

Assuming equal variances

$\begin{array}{lrlr}\text { Difference } & -0.2864 & \mathrm{t} \text { Ratio } & -0.63617 \\ \text { Std Err Dif } & 0.4503 & \text { DF } & 23 \\ \text { Upper CL Dif } & 0.6450 & \text { Prob }>|t| & 0.5309 \\ \text { Lower CL Dif } & -1.2179 & \text { Prob }>\mathrm{t} & 0.7345 \\ \text { Confidence } & 0.95 & \text { Prob }<\mathrm{t} & 0.2655\end{array}$

Analysis of Variance

Source DF Sum of Squares Mean Square F Ratio Prob $>$ F

$\begin{array}{llllll}\text { Sampler } & 1 & 0.492309 & 0.49231 & 0.4047 & 0.5309\end{array}$

$\begin{array}{llll}\text { Error } & 23 & 27.977775 & 1.21642\end{array}$

C. Total $24 \quad 28.470084$

Means for Oneway Anova

Level Number Mean Std Error Lower 95\% Upper 95\%

$\begin{array}{lrrrrr}\text { Coliwasa } & 15 & 99.6517 & 0.28477 & 99.063 & 100.24\end{array}$

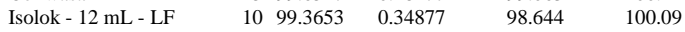

Std Error uses a pooled estimate of error variance

Means and Std Deviations $\quad$ Mean Std Dev Std Err Mean Lower 95\% Upper 95\%

Level Number Mean Std Dev Std Err Mean Lower 95\% Upper 95\%

$\begin{array}{lllllll}\text { Coliwasa } & 15 & 99.6517 & 1.14702 & 0.29616 & 99.017 & 100.29 \\ \text { Isolok - 12 mL - LF } & 10 & 99.3653 & 1.03056 & 0.32589 & 98.628 & 100.10\end{array}$

t Test

Isolok - $12 \mathrm{~mL}$ - LF-Coliwasa

Assuming unequal variances

$\begin{array}{lrlr}\text { Difference } & -0.2864 & \mathrm{t} \text { Ratio } & -0.65048 \\ \text { Std Err Dif } & 0.4404 & \text { DF } & 20.85835 \\ \text { Upper CL Dif } & 0.6297 & \text { Prob }>|t| & 0.5225 \\ \text { Lower CL Dif } & -1.2026 & \text { Prob }>\mathrm{t} & 0.7388 \\ \text { Confidence } & 0.95 & \text { Prob }<\mathrm{t} & 0.2612\end{array}$

Tests that the Variances are Equal

Level Count Std Dev MeanAbsDif to Mean MeanAbsDif to Median

$\begin{array}{lrrrr}\text { Coliwasa } & 15 & 1.147023 & 0.8868522 & 0.8870769\end{array}$

Isolok - $12 \mathrm{~mL}$ - LF $\quad 10 \begin{array}{lll}1.030562 & 0.7803563 & 0.7803563\end{array}$

Test F Ratio DFNum DFDen p-Value

$\begin{array}{lrrrr}\text { O'Brien[.5] } & 0.2126 & 1 & 23 & 0.6490\end{array}$

Brown-Forsythe $\quad 0.1542 \quad 1123 \quad 0.6981$

$\begin{array}{lllll}\text { Levene } & 0.1551 & 1 & 23 & 0.6973 \\ \text { Bartlett } & 0.1180 & 1 & & 0.7312\end{array}$

F Test 2-sided $1.2388 \quad 14 \quad 9 \quad 0.7646$

Welch Anova testing Means Equal, allowing Std Devs Not Equal

F Ratio DFNum DFDen Prob $>$ F

$\begin{array}{rrrr}0.4231 & 1 & 20.858 & 0.5225\end{array}$

t Test 
SRNL-STI-2011-00693

Revision 0

\section{Exhibit C3. Statistical Comparisons for Low-Rheology (Phase 3) Simulant Testing (screened data)}

Oneway Analysis of Measurement By Sampler Type of Material=SME Simulant, Analytical Block $=3$, Oxide $/$ Analyte $=$ TiO2 $($ wt $\%$ ), Targeted $=\mathbf{0 . 0 7 1 1}$

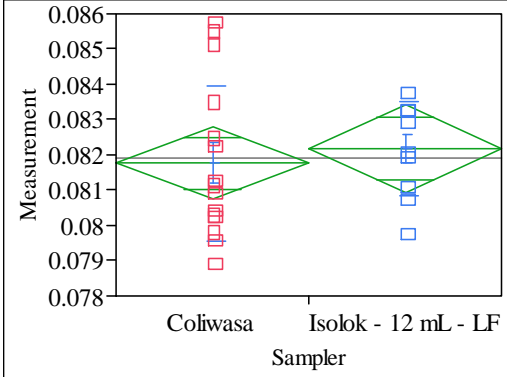

Exc

Oneway Anova

Summary of Fit

Rsquare $\quad 0.012239$

Adj Rsquare $\quad-0.0307$

Root Mean Square Error $\quad 0.001913$

Mean of Response $\quad 0.081932$

Observations (or Sum Wgts) $\quad 25$

t Tes

Isolok - $12 \mathrm{~mL}$ - LF-Coliwasa

Assuming equal variances

Difference $\quad 0.00042$ t Ratio $\quad 0.533838$

Std Err Dif $\quad 0.00078$ DF 23

Upper CL Dif 0.00203 Prob $>|t| \quad 0.5986$

Lower CL Dif -0.00120 Prob $>\mathrm{t} \quad 0.2993$

$\begin{array}{lrr}\text { Confidence } & 0.95 \text { Prob }<\mathrm{t} & 0.7007\end{array}$

Analysis of Variance

Source DF Sum of Squares Mean Square F Ratio Prob $>$ F

$\begin{array}{llllll}\text { Sampler } & 1 & 0.00000104 & 1.0433 \mathrm{e}-6 & 0.2850 & 0.5986\end{array}$

$\begin{array}{llll}\text { Error } & 23 & 0.00008420 & 3.661 \mathrm{e}-6\end{array}$

C. Total $24 \quad 0.00008525$

Means for Oneway Anova

Level Number Mean Std Error Lower 95\% Upper 95\%

$\begin{array}{lrrrrr}\text { Coliwasa } & 15 & 0.081765 & 0.00049 & 0.08074 & 0.08279\end{array}$

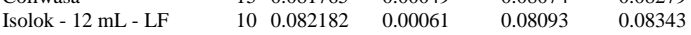

Std Error uses a pooled estimate of error variance

Means and Std Deviations

Level Number Mean Std Dev Std Err Mean Lower 95\% Upper 95\%

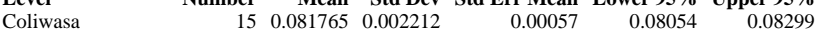

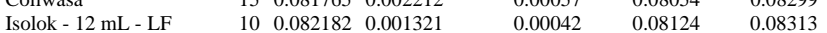

Isolok - $12 \mathrm{~mL}$ - LF-Coliwasa

Assuming unequal variances

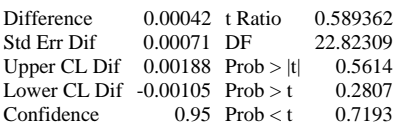

Tests that the Variances are Equal

Level Count Std Dev MeanAbsDif to Mean MeanAbsDif to Median

$\begin{array}{lrrrr}\text { Coliwasa } & 15 & 0.0022121 & 0.0018192 & 0.0017125 \\ \text { Isolok - } 12 \mathrm{~mL} \text { - LF } & 10 & 0.0013205 & 0.0010842 & 0.0010842\end{array}$

Test F Ratio DFNum DFDen p-Value

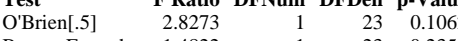

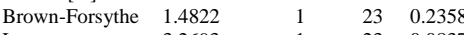

$\begin{array}{lllll}\text { Levene } & 3.2693 & 1 & 23 & 0.0837\end{array}$

$\begin{array}{lrrrr}\text { Bartlett } & 2.4969 & 1 & 0.1141\end{array}$

$\begin{array}{lllll}\text { F Test 2-sided } & 2.8062 & 14 & 9 & 0.1241\end{array}$

Welch Anova testing Means Equal, allowing Std Devs Not Equal

F Ratio DFNum DFDen Prob $>$ F

$\begin{array}{rrrr}\text { F Ratio } & \text { DFNum } & \text { DFDen } & \text { Prob }>\text { F } \\ 0.3473 & 1 & 22.823 & 0.5614\end{array}$

t Test
0.5894
Oneway Analysis of Measurement By Sampler Type of Material=SME Simulant, Analytical Block=3, Oxide/Analyte $=\mathrm{ZrO} 2$ ( wt $\%$ ), Targeted $=\mathbf{0 . 3 5 4 7}$

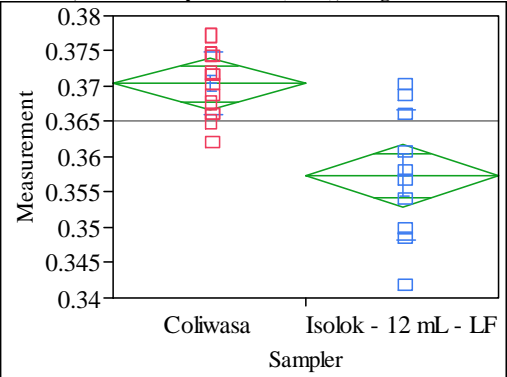

Excluded Rows

Oneway Anova

Summary of Fit

Rsquare $\quad 0.489449$

Adj Rsquare $\quad 0.467251$

Root Mean Square Error $\quad 0.006765$

Mean of Response $\quad 0.365202$

Observations (or Sum Wgts) $\quad 25$

t Test

Isolok - $12 \mathrm{~mL}$ - LF-Coliwasa

Assuming equal variances

$\begin{array}{lrlr}\text { Difference } & -0.01297 & \text { t Ratio } & -4.69568 \\ \text { Std Err Dif } & 0.00276 & \text { DF } & 23 \\ \text { Upper CL Dif } & -0.00725 & \text { Prob }>|t| & <.0001 \\ \text { Lower CL Dif } & -0.01868 & \text { Prob }>t & 1.0000 \\ \text { Confidence } & 0.95 & \text { Prob }<t & <.0001\end{array}$

Analysis of Variance

Source DF Sum of Squares Mean Square F Ratio Prob $>$ F

$\begin{array}{lllllll}\text { Sampler } & 1 & 0.00100896 & 0.001009 & 22.0494 & <.0001\end{array}$

$\begin{array}{llll}\text { Error } & 23 & 0.00105246 & 0.000046\end{array}$

C. Total $24 \quad 0.00206143$

Means for Oneway Anova

Level Number Mean Std Error Lower 95\% Upper 95\%

$\begin{array}{lrrrrr}\text { Coliwasa } & 15 & 0.370389 & 0.00175 & 0.36678 & 0.37400\end{array}$

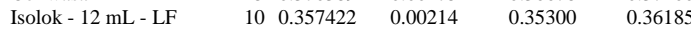

Std Error uses a pooled estimate of error variance

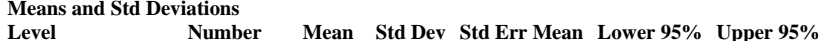

$\begin{array}{lrrrrrr}\text { Level } & \text { Number } & \text { Mean } & \text { Std Dev } & \text { Std Err Mean } & \text { Lower 95\% } & \text { Upper 95\% } \\ \text { Coliwasa } & 15 & 0.370389 & 0.004486 & 0.00116 & 0.36791 & 0.37287\end{array}$

$\begin{array}{lllllll}\text { Isolok - } 12 \mathrm{~mL} \text { - LF } & 10 & 0.357422 & 0.009254 & 0.00293 & 0.35080 & 0.36404\end{array}$

Isolok - $12 \mathrm{~mL}$ - LF-Coliwasa

Assuming unequal variances

$\begin{array}{lrlr}\text { Difference } & -0.01297 & \text { t Ratio } & -4.1203 \\ \text { Std Err Dif } & 0.00315 & \text { DF } & 11.85367 \\ \text { Upper CL Dif } & -0.00610 & \text { Prob }>|t| & 0.0015 \\ \text { Lower CL Dif } & -0.01983 & \text { Prob }>\text { t } & 0.9993 \\ \text { Confidence } & 0.95 & \text { Prob }<\mathrm{t} & 0.0007\end{array}$

Tests that the Variances are Equal

Level Count Std Dev MeanAbsDif to Mean MeanAbsDif to Median

$\begin{array}{lrrrr}\text { Coliwasa } & 15 & 0.0044859 & 0.0036742 & 0.0036021\end{array}$

Isolok - $12 \mathrm{~mL}$ - LF $\quad 10 \quad 0.0092540 \quad 0.0072943 \quad 0.0072943$

Test F Ratio DFNum DFDen p-Value

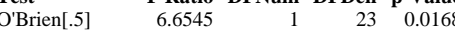

$\begin{array}{lllll}\text { Brown-Forsythe } & 5.4773 & 1 & 23 & 0.0283\end{array}$

$\begin{array}{lllll}\text { Levene } & 5.6891 & 1 & 23 & 0.0257 \\ \text { Bartlett } & 5.6010 & 1 & & 0.0180\end{array}$

$\begin{array}{lllll}\text { F Test 2-sided } & 4.2556 & 9 & 14 & 0.0158\end{array}$

Welch Anova testing Means Equal, allowing Std Devs Not Equal

F Ratio DFNum DFDen Prob $>$ F

$\begin{array}{lrrr}16.9768 & 1 & 11.854 & 0.0015\end{array}$

t Test 


\section{Exhibit C3. Statistical Comparisons for Low-Rheology (Phase 3) Simulant Testing (screened data)}

Oneway Analysis of Measurement By Sampler Type of Material=SME Simulant, Analytical Block=4, Oxide/Analyte=Al2O3 $(\mathrm{wt} \%)$, Targeted $=10.869$

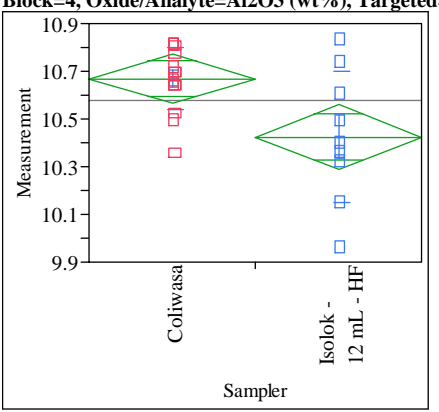

Exc

Oneway Anova

Summary of Fit

Adj Rsquare

0.25264

0.196665

10.57726

24

t Test

Isolok - $12 \mathrm{~mL}$ - HF-Coliwasa

Assuming equal variances

Difference $\quad-0.24564$ t Ratio -2.96226

Std Err Dif $\quad 0.08292$ DF 22

Upper CL Dif -0.07367 Prob $>|t| 0.0072$

Lower CL Dif -0.41760 Prob $>t \quad 0.9964$

$\begin{array}{lrl}\text { Confidence } & 0.95 \text { Prob }<\mathrm{t} \quad 0.0036\end{array}$

Analysis of Variance

Source DF Sum of Squares Mean Square F Ratio Prob $>$ F

$\begin{array}{lllllll}\text { Sampler } & 1 & 0.3393931 & 0.339393 & 8.7750 & 0.0072\end{array}$

$\begin{array}{llll}\text { Error } & 22 & 0.8509001 & 0.038677\end{array}$

C. Total $23 \quad 1.1902932$

Means for Oneway Anova

Level Number Mean Std Error Lower 95\% Upper 95\%

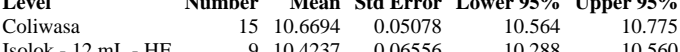

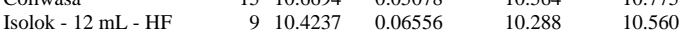

Std Error uses a pooled estimate of error variance

Means and Std Deviations

Level Number Mean Std Dev Std Err Mean Lower 95\% Upper 95\%

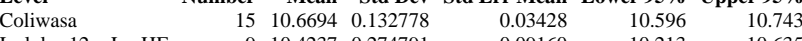

$\begin{array}{lrrrrrr}\text { Isolok - } 12 \mathrm{~mL} \text { - HF } & 9 & 10.4237 & 0.274791 & 0.09160 & 10.213 & 10.635\end{array}$

t Test

Isolok - $12 \mathrm{~mL}$ - HF-Coliwasa

Assuming unequal variances

Difference $\quad-0.24564$ t Ratio $\quad-2.51154$

Std Err Dif $\quad 0.09780$ DF $\quad 10.28309$

Upper CL Dif -0.02853 Prob $>|t| r \mid r .0302$

Lower CL Dif -0.46274 Prob $>t \quad 0.9849$

$\begin{array}{lrl} & \\ \text { Confidence } & 0.95 \text { Prob }<\mathrm{t} & 0.0151\end{array}$

Tests that the Variances are Equal

Level Count Std Dev MeanAbsDif to Mean MeanAbsDif to Median

$\begin{array}{lrrrr}\text { Coliwasa } & 15 & 0.1327782 & 0.0999336 & 0.0982540\end{array}$

$\begin{array}{lrrrr}\text { Coliwasa } & 15 & 0.1327782 & 0.0999336 & 0.0982540 \\ \text { Isolok - } 12 \mathrm{~mL}-\mathrm{HF} & 9 & 0.2747907 & 0.2113441 & 0.2120439\end{array}$

Test F Ratio DFNum DFDen p-Value

\begin{tabular}{lrrrr} 
O'Brien[.5] & F Ratio & DFNum & DFDen & p-Value \\
\hline & 5.0285 & 1 & 22 & 0.0353
\end{tabular}

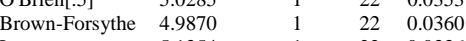

$\begin{array}{lllll}\text { Levene } & 5.1354 & 1 & 22 & 0.0336\end{array}$

$\begin{array}{lllrr}\text { Bartlett } & 5.3764 & 1 & . & 0.0204 \\ \text { F Test 2-sided } & 4.2830 & 8 & 14 & 0.0173\end{array}$

Welch Anova testing Means Equal, allowing Std Devs Not Equal

F Ratio DFNum DFDen Prob > F

$\begin{array}{rrrr}6.3078 & 1 & 10.283 & 0.0302\end{array}$

t Test

2.5115
Oneway Analysis of Measurement By Sampler Type of Material=SME Simulant, Analytical Block $=4$, Oxide/Analyte $=\mathrm{BaO}(\mathrm{wt} \%)$, Targeted $=0.0919$

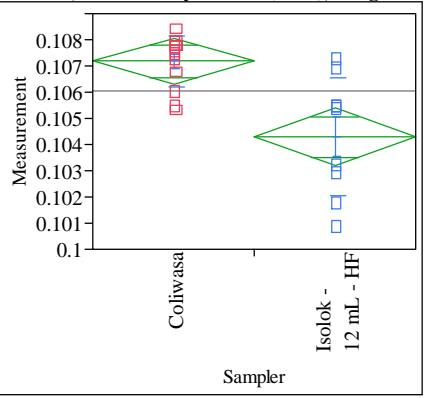

Excluded Rows

Oneway Anova

Summary of Fit

$\begin{array}{ll}\text { Adj Rsquare } & 0.438758 \\ \text { Root Mean Square Error } & 0.001586\end{array}$

0.464269

Mean of Response 0.106053

Observations (or Sum Wgts) 23

\section{Test}

Isolok - $12 \mathrm{~mL}$ - HF-Coliwasa

Assuming equal variances

$\begin{array}{lrr}\text { Difference } & -0.00289 \text { t Ratio } & -4.266 \\ \text { Std Err Dif } & 0.00068 \text { DF } & 21\end{array}$

$\begin{array}{lrlr}\text { Std Err Dif } & 0.00068 & \text { DF } & 21 \\ \text { Upper CL Dif } & -0.00148 & \text { Prob }>|t| & 0.0003\end{array}$

Lower CL Dif -0.00430 Prob $>t \quad 0.9998$

$\begin{array}{lll}\text { Confidence } & 0.95 \text { Prob }<\mathrm{t} \quad 0.0002\end{array}$

Analysis of Variance

Source DF Sum of Squares Mean Square F Ratio Prob $>$ F

$\begin{array}{lrrrrr}\text { Sampler } & 1 & 0.00004577 & 0.000046 & 18.1988 & 0.0003\end{array}$

$\begin{array}{llll}\text { Error } & 21 & 0.00005282 & 2.515 \mathrm{e}-6\end{array}$

C. Total $22 \quad 0.00009859$

Means for Oneway Anova

Level Number Mean Std Error Lower 95\% Upper 95\%

$\begin{array}{lrrrrr}\text { Level } & \text { Number } & \text { Mean } & \text { Std Error } & \text { Lower 95\% } & \text { Upper 95\% } \\ \text { Coliwasa } & 14 & 0.107184 & 0.00042 & 0.10630 & 0.10807\end{array}$

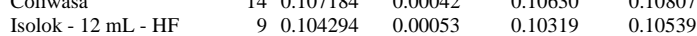

Std Error uses a pooled estimate of error variance

Means and Std Deviations

Level Number Mean Std Dev Std Err Mean Lower 95\% Upper 95\%

$\begin{array}{lrrrrrrr} & 14 & 0.107184 & 0.000975 & 0.00026 & 0.10662 & 0.10775 \\ \text { Isolok - } 12 \mathrm{~mL} \text { - HF } & 9 & 0.104294 & 0.002249 & 0.00075 & 0.10257 & 0.10602\end{array}$

t Test

Isolok - $12 \mathrm{~mL}$ - HF-Coliwas

Assuming unequal variances

$\begin{array}{lrl}\text { Difference } & -0.00289 \text { t Ratio } & -3.64241 \\ \text { Std Err Dif } & 0.00079 & \end{array}$

$\begin{array}{lll}\text { Std Err Dif } \quad 0.00079 & \text { DF } & 9.962395\end{array}$

Upper CL Dif -0.00112 Prob $>|t| \quad 0.0045$

Lower CL Dif -0.00466 Prob $>\mathrm{t} \quad 0.9977$

$\begin{array}{lll}\text { Confidence } & 0.95 \text { Prob }<\mathrm{t} \quad 0.0023\end{array}$

Tests that the Variances are Equal

Level Count Std Dev MeanAbsDif to Mean MeanAbsDif to Median

\begin{tabular}{lrrrr} 
Coliwasa & 14 & 0.0009753 & 0.0007656 & 0.0006859 \\
\hline
\end{tabular}

F Ratio DFNum DFDen p-Value

$\begin{array}{lrrrr}\text { Test } & \text { F Ratio } & \text { DFNum } & \text { DFDen } & \text { p-Value } \\ \text { O'Brien[.5] } & 9.1328 & 1 & 21 & 0.0065 \\ \text { Brown-Forsythe } & 5.4521 & 1 & 21 & 0.0295 \\ \text { Levene } & 13.5054 & 1 & 21 & 0.0014 \\ \text { Bartlett } & 6.7081 & 1 & . & 0.0096 \\ \text { F Test 2-sided } & 5.3155 & 8 & 13 & 0.0082\end{array}$

Welch Anova testing Means Equal, allowing Std Devs Not Equal

F Ratio DFNum DFDen Prob $>$ F

$\begin{array}{llll}13.2671 & 1 & 9.9624 & 0.0045\end{array}$

t Test

3.6424 
Revision 0

\section{Exhibit C3. Statistical Comparisons for Low-Rheology (Phase 3) Simulant Testing (screened data)}

Oneway Analysis of Measurement By Sampler Type of Material=SME Simulant, Analytical Block $=4$, Oxide $/$ Analyte $=\mathrm{CaO}(\mathrm{wt} \%)$, Targeted $=1.1462$

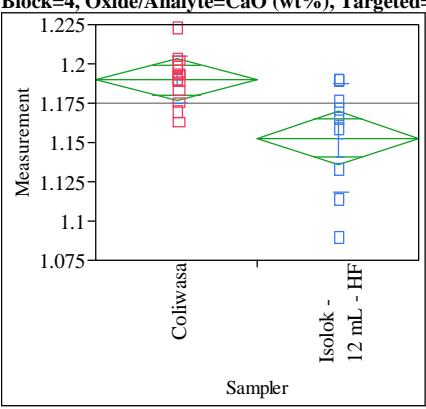

Excluded Rows

Oneway Anova

Summary of Fit

Rsquare

Adj Rsquare

0.376859

0.347185

Root Mean Square Error $\quad 0.024389$

Mean of Response 1.175389

Observations (or Sum Wgts)

Test

Isolok - $12 \mathrm{~mL}$ - HF-Coliwasa

Assuming equal variances

Difference $\quad-0.03713$ t Ratio -3.56374

$\begin{array}{lrr}\text { Std Err Dif } & 0.01042 \text { DF } & 21 \\ \text { Dpper } & 0.0018\end{array}$

$\begin{array}{rrr}\text { Upper CL Dif }-0.01546 \text { Prob }>|t| & 0.0018 \\ \text { Cower CL Dif }-0.05880 & \text { Prob }>t & 0.9991\end{array}$

$\begin{array}{lrl} & \\ \text { Confidence } & 0.95 \text { Prob }<\mathrm{t} & 0.0009\end{array}$

Analysis of Variance

Source DF Sum of Squares Mean Square F Ratio Prob > F

$\begin{array}{lrrrrr}\text { Sampler } & 1 & 0.00755429 & 0.007554 & 12.7002 & 0.0018\end{array}$

$\begin{array}{llll}\text { Error } & 21 & 0.01249113 & 0.000595\end{array}$

C. Total $22 \quad 0.0200454$

Means for Oneway Anova

Level Number Mean Std Error Lower 95\% Upper 95\%

Coliwasa $141.18992 \quad 0.00652 \quad 1.1764$ Pper 95\%

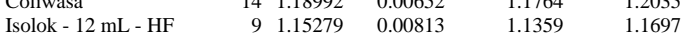

Std Error uses a pooled estimate of error variance

Means and Std Deviations

Level Number Mean Std Dev Std Err Mean Lower 95\% Upper 95\%

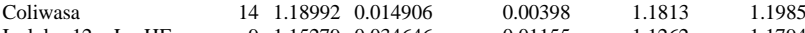

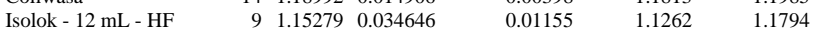

t Test

Isolok - $12 \mathrm{~mL}$ - HF-Coliwasa

Assuming unequal variances

Difference $\quad-0.03713$ t Ratio $\quad-3.03971$

Std Err Dif $\quad 0.01222$ DF 9.930761

Upper CL Dif -0.00989 Prob $>|t| \quad 0.0126$

Lower CL Dif -0.06438 Prob $>\mathrm{t} \quad 0.9937$

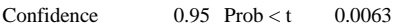

Tests that the Variances are Equal

$\begin{array}{lrrrr}\text { Level } & \text { Count } & \text { Std Dev } & \text { MeanAbsDif to Mean } & \text { MeanAbsDif to Median } \\ \text { Coliwasa } & 14 & 0.0149062 & 0.0107938 & 0.0107938\end{array}$

$\begin{array}{lrrrr} & 14 & 0.0149062 & 0.0107938 & 0.0107938 \\ \text { Isolok - } 12 \mathrm{~mL}-\mathrm{HF} & 9 & 0.0346457 & 0.0278804 & 0.0262739\end{array}$

$\begin{array}{lrrrr}\text { Test } & \text { F Ratio } & \text { DFNum } & \text { DFDen } & \text { p-Value } \\ \text { O'Brien[.5] } & 5.3715 & 1 & 21 & 0.0306 \\ \text { Brown-Forsythe } & 4.7152 & 1 & 21 & 0.0415 \\ \text { Levene } & 8.6918 & 1 & 21 & 0.0077 \\ \text { Bartlett } & 6.8328 & 1 & . & 0.0089 \\ \text { F Test 2-sided } & 5.4021 & 8 & 13 & 0.0076\end{array}$

Welch Anova testing Means Equal, allowing Std Devs Not Equal

F Ratio DFNum DFDen Prob $>$ F

$\begin{array}{llll}9.2398 & 1 & 9.9308 & 0.0126\end{array}$

t Test

3.0397
Oneway Analysis of Measurement By Sampler Type of Material=SME Simulant, Analytical Block $=4$, Oxide $/$ Analyte $=\mathrm{Fe} / \mathrm{Li}$, Targeted $=2.452$

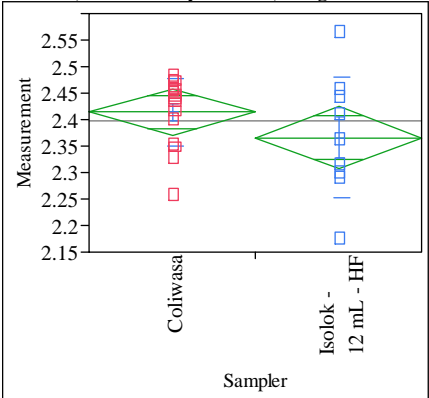

Excluded Rows

1

Oneway Anova

Summary of Fit

Rsquare $\quad 0.075746$

Adj Rsquare $\quad 0.03556$

Root Mean Square Error $\quad 0.084868$

Mean of Response $\quad 2.397106$

Observations (or Sum Wgts) 25

Test

Isolok - $12 \mathrm{~mL}$ - HF-Coliwasa

Assuming equal variances

Difference $\quad-0.04855$ t Ratio $\quad-1.37293$

$\begin{array}{rlr}0.03536 \mathrm{DF} & 23 \\ & \end{array}$

Pre Dif 0.02460 Prob $>|t| \quad 0.1830$

$\begin{array}{lrrr}\text { Lower CL Dif } & -0.12170 & \text { Prob }>t & 0.9085 \\ \text { Confidence } & 0.95 & \text { Prob }<\mathrm{t} & 0.0915\end{array}$

Analysis of Variance

Source DF Sum of Squares Mean Square F Ratio Prob $>$ F

$\begin{array}{llllll}\text { Sampler } & 1 & 0.01357644 & 0.013576 & 1.8849 & 0.1830\end{array}$

$\begin{array}{llll}\text { Error } & 23 & 0.16566029 & 0.007203\end{array}$

C. Total $24 \quad 0.17923673$

Means for Oneway Anova

Level Number Mean Std Error Lower 95\% Upper 95\%

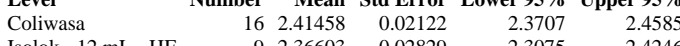

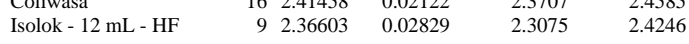

Std Error uses a pooled estimate of error variance

Means and Std Deviations

Level Number Mean Std Dev Std Err Mean Lower 95\% Upper 95\%

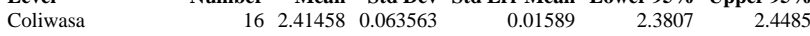

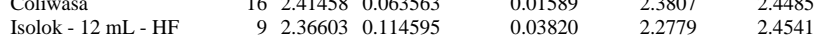

t Test

Isolok - $12 \mathrm{~mL}$ - HF-Coliwasa

Assuming unequal variances

$\begin{array}{lrlr}\text { Difference } & -0.04855 & \mathrm{t} \text { Ratio } & -1.17348 \\ \text { Std Err Dif } & 0.04137 \text { DF } & 10.83549 \\ \text { Upper CL Dif } & 0.04268 \text { Prob }>|\mathrm{t}| & 0.2658 \\ \text { Lower CL Dif } & -0.13978 \text { Prob }>\mathrm{t} & 0.8671 \\ \text { Confidence } & 0.95 & \text { Prob }<\mathrm{t} & 0.1329\end{array}$

Tests that the Variances are Equal

Level Count Std Dev MeanAbsDif to Mean MeanAbsDif to Median

$\begin{array}{lllll}\text { Coliwasa } & 16 & 0.0635630 & 0.0499235 & 0.0449501\end{array}$

$\begin{array}{lrrr}\text { Isolok - } 12 \mathrm{~mL} \text { - HF } & 9 & 0.1145952 & 0.0888405\end{array}$

Test F Ratio DFNum DFDen p-Value

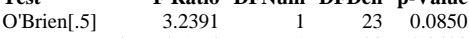

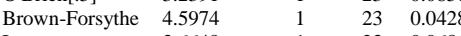

$\begin{array}{lllll}\text { Levene } & 3.6648 & 1 & 23 & 0.0681\end{array}$

$\begin{array}{lllll}\text { Bartlett } & 3.6851 & 1 & 0.0549 \\ \text { F Test 2-sided } & 3.2503 & 8 & 15 & 0.0470\end{array}$

Welch Anova testing Means Equal, allowing Std Devs Not Equal

F Ratio DFNum DFDen Prob $>$ F

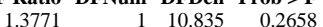

t Test

1.1735 


\section{Exhibit C3. Statistical Comparisons for Low-Rheology (Phase 3) Simulant Testing (screened data)}

Oneway Analysis of Measurement By Sampler Type of Material=SME Simulant, Analytical Block $=4$, Oxide/Analyte $=\mathrm{Fe} 2 \mathrm{O} 3(\mathrm{wt} \%)$, Targeted $=11.462$

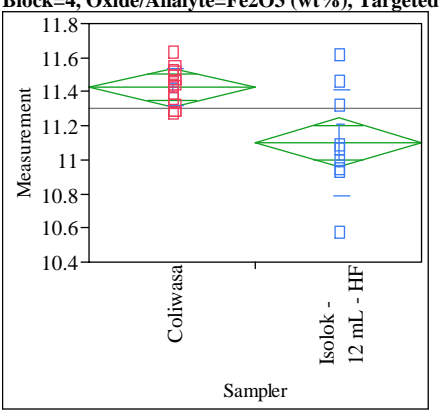

Excluded Rows

Oneway Anova

Summary of Fit

Adj Rsquare

0.35412

0.207925

11.30297

Observations (or Sum Wgts)

t Test

Isolok - $12 \mathrm{~mL}$ - HF-Coliwasa

Assuming equal variances

Difference $\quad-0.32343$ t Ratio $\quad-3.68922$

Std Err Dif $\quad 0.08767$ DF 22

Upper CL Dif -0.14162 Prob $>|t| \quad 0.0013$

Lower CL Dif -0.50524 Prob $>t \quad 0.9994$

$\begin{array}{lrl}\text { Confidence } & 0.95 \text { Prob }<\mathrm{t} \quad 0.0006\end{array}$

Analysis of Variance

Source DF Sum of Squares Mean Square F Ratio Prob $>$ F

$\begin{array}{lllllll}\text { Sampler } & 1 & 0.5884139 & 0.588414 & 13.6103 & 0.0013\end{array}$

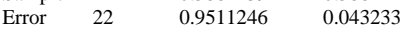

C. Total $23 \quad 1.539538$

Means for Oneway Anova

Level Number Mean Std Error Lower 95\% Upper 95\%

$\begin{array}{lrrrrr}\text { Coliwasa } & 15 & 11.4243 & 0.05369 & 11.313 & 11.536 \\ \text { Isolok - 12 mL - HF } & 9 & 11.1008 & 0.06931 & 10.957 & 11.245\end{array}$

Std Error uses a pooled estimate of error variance

Means and Std Deviations

Level Number Mean Std Dev Std Err Mean Lower 95\% Upper 95\%

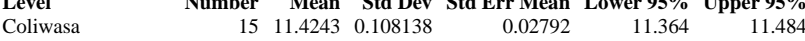

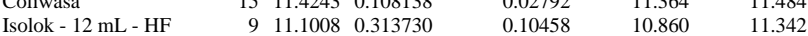

tTest

Isolok - $12 \mathrm{~mL}$ - HF-Coliwasa

Assuming unequal variances

$\begin{array}{lrlr}\text { Difference } & -0.32343 & \mathrm{t} \text { Ratio } & -2.98809 \\ \text { Std Err Dif } & 0.10824 & \text { DF } & 9.15463 \\ \text { Upper CL Dif } & -0.07920 & \text { Prob }>|t| & 0.0150 \\ \text { Lower CL Dif } & -0.56766 & \text { Prob }>\mathrm{t} & 0.9925 \\ \text { Confidence } & 0.95 & \text { Prob }<\mathrm{t} & 0.0075\end{array}$

Tests that the Variances are Equal

Level Count Std Dev MeanAbsDif to Mean MeanAbsDif to Median

$\begin{array}{lrrrr}\text { Coliwasa } & 15 & 0.1081382 & 0.0885779 & 0.0886414\end{array}$

$\begin{array}{lrlll}\text { Coliwasa } & 15 & 0.1081382 & 0.0885779 & 0.0886414 \\ \text { Isolok - } 12 \mathrm{~mL} \text { - HF } & 9 & 0.3137297 & 0.2372243 & 0.2303406\end{array}$

Test F Ratio DFNum DFDen p-Value

$\begin{array}{lrrrr}\text { O'Brien[.5] } & 6.3698 & 1 & 22 & 0.0193 \\ \text { Brown-Forsythe } & 6.5169 & 1 & 22 & 0.0181\end{array}$

$\begin{array}{lrlll}\text { Levene } & 8.3631 & 1 & 22 & 0.0085\end{array}$

$\begin{array}{lrrrr}\text { Bartlett } & 11.1622 & 1 & & 0.0008 \\ \text { F Test 2-sided } & 8.4169 & 8 & 14 & 0.0007\end{array}$

Welch Anova testing Means Equal, allowing Std Devs Not Equal

F Ratio DFNum DFDen Prob $>$ F

$\begin{array}{rrrrr}8.9287 & 1 & 9.1546 & 0.0150\end{array}$

t Test

2.9881
Oneway Analysis of Measurement By Sampler Type of Material=SME Simulant, Analytica Block $=4$, Oxide/Analyte $=$ Li2O $($ wt $\%)$, Targeted $=4.674$

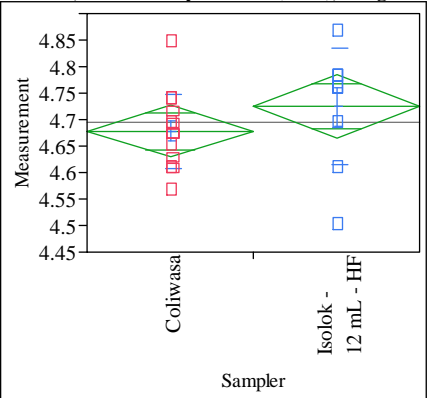

Excluded Rows

3

Oneway Anova

Summary of Fit

Adj Rsquare

Root Mean Square Error 0.024938

0.087018
4.69613

Observations (or Sum Wgts)

t Test

Isolok - $12 \mathrm{~mL}$ - HF-Coliwasa

Assuming equal variances

$\begin{array}{lrlr}\text { Difference } & 0.04648 & \mathrm{t} \text { Ratio } & 1.250063 \\ \text { Std Err Dif } & 0.03718 \text { DF } & 21 \\ \text { Upper CL Dif } & 0.12379 & \text { Prob }>|\mathrm{t}| & 0.2250 \\ \text { Lower CL Dif } & -0.03084 & \text { Prob }>\mathrm{t} & 0.1125 \\ \text { Confidence } & 0.95 & \text { Prob }<\mathrm{t} & 0.8875\end{array}$

Analysis of Variance

Source DF Sum of Squares Mean Square F Ratio Prob $>$ F

$\begin{array}{lrllll}\text { Sampler } & 1 & 0.01183279 & 0.011833 & 1.5627 & 0.2250\end{array}$

$\begin{array}{llll}\text { Error } & 21 & 0.15901655 & 0.007572\end{array}$

C. Total $22 \quad 0.17084933$

Means for Oneway Anova

Level Number Mean Std Error Lower 95\% Upper 95\%

$\begin{array}{lrrrrr}\text { Coliwasa } & 14 & 4.67794 & 0.02326 & 4.6296 & 4.7263\end{array}$

$\begin{array}{lrrrrr}\text { Isolok - 12 mL - HF } & 9 & 4.72442 & 0.02901 & 4.6641 & 4.7847\end{array}$

Std Error uses a pooled estimate of error variance

Means and Std Deviations

Level Number Mean Std Dev Std Err Mean Lower 95\% Upper 95\%

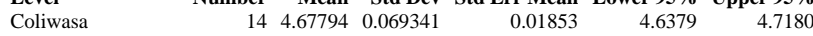

$\begin{array}{lrrrrrr}\text { Isolok - } 12 \mathrm{~mL} \text { - HF } & 14 & 4.67794 & 0.069341 & 0.01853 & 4.6379 & 4.7180 \\ & 9 & 4.72442 & 0.109835 & 0.03661 & 4.6400 & 4.8088\end{array}$

t Test

Isolok - $12 \mathrm{~mL}$ - HF-Coliwasa

Assuming unequal variances

$\begin{array}{lrlr}\text { Difference } & 0.04648 & \mathrm{t} \text { Ratio } & 1.13258 \\ \text { Std Err Dif } & 0.04103 & \text { DF } & 12.13444 \\ \text { Upper CL Dif } & 0.13577 \text { Prob }>|\mathrm{t}| & 0.2793 \\ \text { Lower CL Dif } & -0.04282 & \text { Prob }>\mathrm{t} & 0.1396 \\ \text { Confidence } & 0.95 & \text { Prob }<\mathrm{t} & 0.8604\end{array}$

Tests that the Variances are Equal

Level Count Std Dev MeanAbsDif to Mean MeanAbsDif to Median

$\begin{array}{lllll}\text { Coliwasa } & 14 & 0.0693408 & 0.0500879 & 0.0492091\end{array}$

$\begin{array}{lrrrr}\text { Isolok - } 12 \mathrm{~mL} \text { - HF } & 9 & 0.1098354 & 0.0829265 & 0.0717633\end{array}$

Test F Ratio DFNum DFDen p-Value

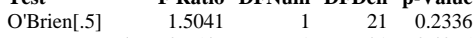

Brown-Forsythe $\quad 0.6547 \quad 1 \quad 21 \quad 0.4275$

$\begin{array}{lllll}\text { Levene } & 2.0009 & 1 & 21 & 0.1719\end{array}$

$\begin{array}{lllll}\text { Bartlett } & 2.0719 & 1 & \text {. } & 0.1500 \\ \text { F Test 2-sided } & 2.5090 & 8 & 13 & 0.1358\end{array}$

Welch Anova testing Means Equal, allowing Std Devs Not Equal

F Ratio DFNum DFDen Prob $>$ F

$\begin{array}{rrrr}1.2827 & 1 & 12.134 & 0.2793\end{array}$

t Tes

1.1326 
Revision 0

\section{Exhibit C3. Statistical Comparisons for Low-Rheology (Phase 3) Simulant Testing (screened data)}

Oneway Analysis of Measurement By Sampler Type of Material=SME Simulant, Analytical Block=4, Oxide/Analyte=MgO (wt\%), Targeted $=1.014$

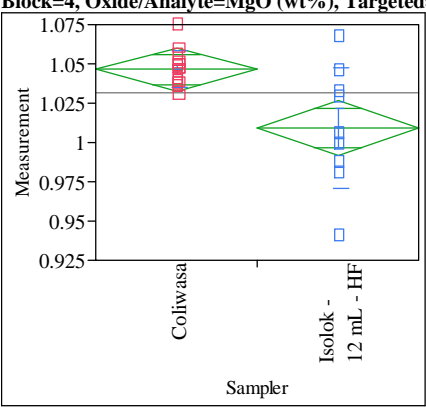

Excluded Rows

Oneway Anova

Summary of Fit

Rsquare

Adj Rsquare

0.362561

0.332206

Root Mean Square Error $\quad 0.02525$

Mean of Response 1.031679

Observations (or Sum Wgts) 23

Test

Isolok - $12 \mathrm{~mL}$ - HF-Coliwasa

Assuming equal variances

Difference $\quad-0.03729$ t Ratio $\quad-3.45605$

$\begin{array}{lrr}\text { Std Err Dif } & 0.01079 \text { DF } & 21 \\ & & \end{array}$

$\begin{array}{lll}\text { Upper CL Dif }-0.01485 \text { Prob }>|t| & 0.0024 \\ \text { Cower CL Dif }-0.05972 \text { Prob }>t & 0.9988\end{array}$

$\begin{array}{lrl}\text { Confidence } & 0.95 \text { Prob }<\mathrm{t} \quad 0.0012\end{array}$

Analysis of Variance

Source DF Sum of Squares Mean Square F Ratio Prob $>$ F

$\begin{array}{lrrrrr}\text { Sampler } & 1 & 0.00761590 & 0.007616 & 11.9443 & 0.0024\end{array}$

$\begin{array}{llll}\text { Error } & 21 & 0.01338996 & 0.000638\end{array}$

C. Total $22 \quad 0.02100586$

Means for Oneway Anova

Level Number Mean Std Error Lower 95\% Upper 95\%

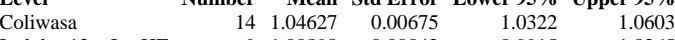

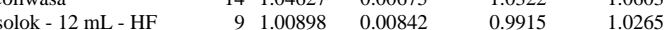

Std Error uses a pooled estimate of error variance

Means and Std Deviations

Level Number Mean Std Dev Std Err Mean Lower 95\% Upper 95\%

$\begin{array}{lrrrrrr}\text { Coliwasa } & 14 & 1.04627 & 0.011662 & 0.00312 & 1.0395 & 1.0530 \\ \text { Isolok - 12 mL - HF } & 9 & 1.00898 & 0.038115 & 0.01270 & 0.9797 & 1.0383\end{array}$

t Test

Isolok - $12 \mathrm{~mL}$ - HF-Coliwasa

Assuming unequal variances

Difference $\quad-0.03729$ t Ratio $\quad-2.8502$

Std Err Dif $\quad 0.01308$ DF 8.971901

Upper CL Dif -0.00768 Prob $>|t| 0.0191$

Lower CL Dif -0.06689 Prob $>\mathrm{t} \quad 0.9904$

Confidence $\quad 0.95$ Prob $<\mathrm{t} \quad 0.0096$

Tests that the Variances are Equal

$\begin{array}{lrrr}\text { Level Count } & \text { Std Dev MeanAbsDif to Mean } & \text { MeanAbsDif to Median } \\ \text { Coliwasa } & 14 & 0.0116620 & 0.0086638\end{array}$

$\begin{array}{lrrrrr} & 14 & 0.0116620 & & 0.0086638 & 0.0086468 \\ \text { Isolok - } 12 \mathrm{~mL} \text { - HF } & 9 & 0.0381149 & 0.0299313 & 0.0302179\end{array}$

$\begin{array}{lrrrr}\text { Test } & \text { F Ratio } & \text { DFNum } & \text { DFDen } & \text { p-Value } \\ \text { O'Brien[.5] } & 6.3673 & 1 & 21 & 0.0198 \\ \text { Brown-Forsythe } & 12.3995 & 1 & 21 & 0.0020 \\ \text { Levene } & 12.1686 & 1 & 21 & 0.0022 \\ \text { Bartlett } & 12.8378 & 1 & . & 0.0003 \\ \text { F Test 2-sided } & 10.6818 & 8 & 13 & 0.0003\end{array}$

Welch Anova testing Means Equal, allowing Std Devs Not Equal

F Ratio DFNum DFDen Prob $>$ F

$\begin{array}{rrrrr}8.1237 & 1 & 8.9719 & 0.0191\end{array}$

t Test

2.8502
Oneway Analysis of Measurement By Sampler Type of Material=SME Simulant, Analytical Block $=4$, Oxide $/$ Analyte $=\mathrm{MnO}($ wt $\%)$, Targeted $=1.779$

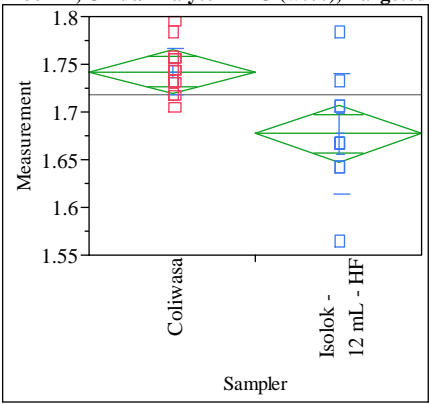

Excluded Rows

2

Oneway Anova

Summary of Fit

Adj Rsquare

Root Mean Square Error $\quad 0.34735$

0.042453

1.717834

t Test

Isolok - $12 \mathrm{~mL}$ - HF-Coliwasa

Assuming equal variances

Difference $\quad-0.06513$ t Ratio $\quad-3.63881$

Std Err Dif $\quad 0.01790$ DF 22

Upper CL Dif -0.02801 Prob $>|t| \quad 0.0014$

Lower CL Dif -0.10226 Prob $>t \quad 0.9993$

$\begin{array}{lrl}\text { Confidence } & 0.95 \text { Prob }<\mathrm{t} \quad 0.0007\end{array}$

Analysis of Variance

Source DF Sum of Squares Mean Square F Ratio Prob $>$ F

$\begin{array}{lllllll}\text { Sampler } & 1 & 0.02386362 & 0.023864 & 13.2410 & 0.0014\end{array}$

$\begin{array}{llll}\text { Error } & 22 & 0.03964966 & 0.001802\end{array}$

C. Total $23 \quad 0.06351328$

Means for Oneway Anova

Level Number Mean Std Error Lower 95\% Upper 95\%

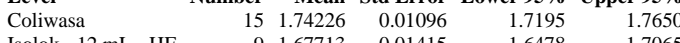

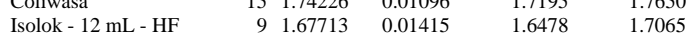

Std Error uses a pooled estimate of error variance

Means and Std Deviations

Level Number Mean Std Dev Std Err Mean Lower 95\% Upper 95\%

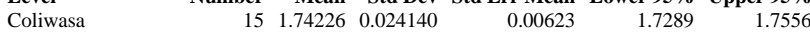

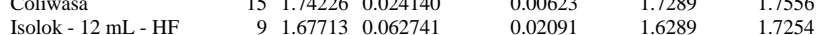

t Test

Isolok - $12 \mathrm{~mL}$ - HF-Coliwasa

Assuming unequal variances

$\begin{array}{lrlr}\text { Difference } & -0.06513 & \mathrm{t} \text { Ratio } & -2.98468 \\ \text { Std Err Dif } & 0.02182 & \text { DF } & 9.441672 \\ \text { Upper CL Dif } & -0.01612 & \text { Prob }>|t| & 0.0146 \\ \text { Lower CL Dif } & -0.11415 & \text { Prob }>\mathrm{t} & 0.9927 \\ \text { Confidence } & 0.95 & \text { Prob }<\mathrm{t} & 0.0073\end{array}$

Tests that the Variances are Equal

Level Count Std Dev MeanAbsDif to Mean MeanAbsDif to Median

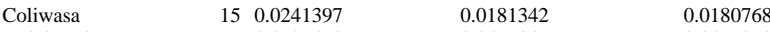

$\begin{array}{lrll}\text { Isolok - } 12 \mathrm{~mL} \text { - HF } & 9 & 0.0627410 & 0.0471846\end{array}$

Test F Ratio DFNum DFDen p-Value

$\begin{array}{lrrrr}\text { O'Brien[.5] } & 4.6969 & 1 & 22 & 0.0413\end{array}$

$\begin{array}{lllll}\text { Brown-Forsythe } & 5.6470 & 1 & 22 & 0.0266\end{array}$

$\begin{array}{lllll}\text { Levene } & 7.1144 & 1 & 22 & 0.0141\end{array}$

$\begin{array}{lllll}\text { Bartlett } & 9.0993 & 1 & \text {. } & 0.0026 \\ \text { F Test 2-sided } & 6.7552 & 8 & 14 & 0.0021\end{array}$

Welch Anova testing Means Equal, allowing Std Devs Not Equal

F Ratio DFNum DFDen Prob $>$ F

$8.9083 \quad 1 \quad 9.4417 \quad 0.0146$

t Test

2.9847 
Revision 0

\section{Exhibit C3. Statistical Comparisons for Low-Rheology (Phase 3) Simulant Testing (screened data)}

Oneway Analysis of Measurement By Sampler Type of Material=SME Simulant, Analytical Block=4, Oxide/Analyte=Na2O $($ wt $\%)$, Targeted $=11.659$

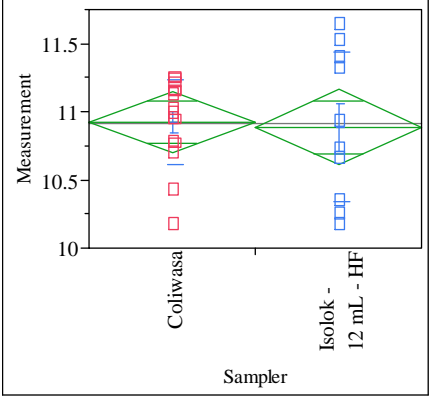

Excluded Rows

Oneway Anova

Summary of Fit

Adj Rsquare

$-0.04155$

0.421686

Observations (or Sum Wgts)

tTest

Isolok - $12 \mathrm{~mL}$ - HF-Coliwasa

Assuming equal variances

Difference $\quad-0.03550$ t Ratio $\quad-0.2062$

Std Err Dif $\quad 0.17215$ DF 23

Upper CL Dif 0.32063 Prob $>|t| \quad 0.8385$

Lower CL Dif -0.39162 Prob $>t \quad 0.5808$

$\begin{array}{lrl}\text { Confidence } & 0.95 \text { Prob }<\mathrm{t} \quad 0.4192\end{array}$

Analysis of Variance

Source DF Sum of Squares Mean Square F Ratio Prob $>$ F

$\begin{array}{llllll}\text { Sampler } & 1 & 0.0075604 & 0.007560 & 0.0425 & 0.8385\end{array}$

$\begin{array}{llll}\text { Error } & 23 & 4.0898347 & 0.177819\end{array}$

C. Total $24 \quad 4.0973951$

Means for Oneway Anova

Level Number Mean Std Error Lower 95\% Upper 95\%

$\begin{array}{lrrrrr}\text { Coliwasa } & 15 & 10.9233 & 0.10888 & 10.698 & 11.149\end{array}$

$\begin{array}{llllll}\text { Isolok - 12 mL - HF } & 15 & 10.9233 & 0.10888 & 10.698 & 11.149 \\ & 10 & 10.8878 & 0.13335 & 10.612 & 11.164\end{array}$

Std Error uses a pooled estimate of error variance

Means and Std Deviations

Level Number Mean Std Dev Std Err Mean Lower 95\% Upper 95\%

$\begin{array}{lrrrrr}\text { Coliwasa } & \text { Number } & \text { Mean } & \text { Std Dev } & \text { Std Err Mean } & \text { Lower 95\% } \\ \text { Upper 95\% }\end{array}$

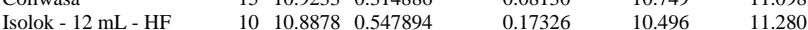

Test

Isolok - $12 \mathrm{~mL}$ - HF-Coliwasa

Assuming unequal variances

Difference $\quad-0.03550$ t Ratio $\quad-0.1854$

Std Err Dif $\quad 0.19139$ DF $\quad 12.99498$

Upper CL Dif 0.37799 Prob $>|t| 0.8557$

$\begin{array}{lll}\text { Lower CL Dif }-0.44898 \text { Prob }>t & 0.5721\end{array}$

$\begin{array}{lrl}\text { Confidence } & 0.95 \text { Prob }<\mathrm{t} & 0.4279\end{array}$

Tests that the Variances are Equal

Level Count Std Dev MeanAbsDif to Mean MeanAbsDif to Median

$\begin{array}{lllll}\text { Coliwasa } & 15 & 0.3148862 & 0.2402436 & 0.2318560\end{array}$

Isolok - $12 \mathrm{~mL}$ - HF $\quad 10 \quad 0.5478938 \quad 0.4650600 \quad 0.4650600$

Test F Ratio DFNum DFDen p-Value

\begin{tabular}{lrrrr} 
O'Brien[.5] & 6.2396 & 1 & 23 & 0.0201 \\
\hline & 6.1022 & 1 & 23 & 0.0213
\end{tabular}

$\begin{array}{lllll}\text { Brown-Forsythe } & 6.1022 & 1 & 23 & 0.0201 \\ & 6.5722 & 1 & 23 & 0.0213\end{array}$

$\begin{array}{lllll}\text { Levene } & 6.5722 & 1 & 23 & 0.0174\end{array}$

$\begin{array}{lllll}\text { Bartlett } & 3.3111 & 1 & \text {. } & 0.0688 \\ \text { F Test 2-sided } & 3.0275 & 9 & 14 & 0.0622\end{array}$

Welch Anova testing Means Equal, allowing Std Devs Not Equal

F Ratio DFNum DFDen Prob $>$ F

$\begin{array}{lrrr}0.0344 & 1 & 12.995 & 0.8557\end{array}$

t Test

0.1855
Oneway Analysis of Measurement By Sampler Type of Material=SME Simulant, Analytical Block $=4$, Oxide $/$ Analyte $=\mathrm{NiO}(\mathrm{wt} \%)$, Targeted $=0.41$

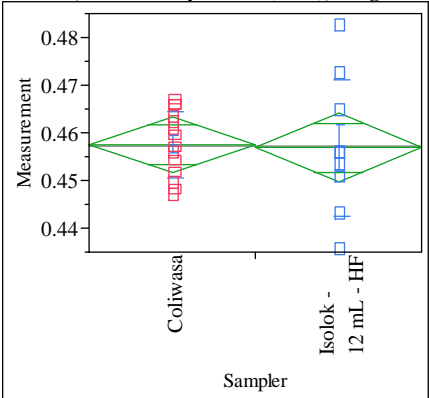

Excluded Rows

3

Oneway Anova

Summary of Fit

Adj Rsquare

Mean of Response

Observations (or Sum Wgts)

0.010417

0.45727

t Test

Isolok - $12 \mathrm{~mL}$ - HF-Coliwasa

Assuming equal variances

Difference $\quad-0.00073$ t Ratio $\quad-0.16337$

Std Err Dif $\quad 0.00445$ DF 21

Upper CL Dif 0.00853 Prob $>|t| \quad 0.8718$

Lower CL Dif -0.00998 Prob $>t \quad 0.5641$

$\begin{array}{lrl}\text { Confidence } & 0.95 \text { Prob }<\mathrm{t} & 0.4359\end{array}$

Analysis of Variance

Source DF Sum of Squares Mean Square F Ratio Prob $>$ F

$\begin{array}{llllll}\text { Sampler } & 1 & 0.00000290 & 2.897 \mathrm{e}-6 & 0.0267 & 0.8718\end{array}$

$\begin{array}{llll}\text { Error } & 21 & 0.00227899 & 0.000109\end{array}$

C. Total $22 \quad 0.00228188$

Means for Oneway Anova

Level Number Mean Std Error Lower 95\% Upper 95\%

$\begin{array}{lrrrrr}\text { Coliwasa } & 14 & 0.457555 & 0.00278 & 0.45176 & 0.46334\end{array}$

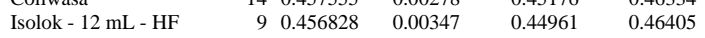

Std Error uses a pooled estimate of error variance

Means and Std Deviations

Level Number Mean Std Dev Std Err Mean Lower 95\% Upper 95\%

$\begin{array}{lllllll} & 14 & 0.457555 & 0.006893 & 0.00184 & 0.45357 & 0.46153\end{array}$

$\begin{array}{lrrrrrr}\text { Isolok - } 12 \mathrm{~mL} \text { - HF } & 9 & 0.456828 & 0.014411 & 0.00480 & 0.44575 & 0.46790\end{array}$

Test

Isolok - $12 \mathrm{~mL}$ - HF-Coliwasa

Assuming unequal variances

$\begin{array}{lrlr}\text { Difference } & -0.00073 & \mathrm{t} \text { Ratio } & -0.14134 \\ \text { Std Err Dif } & 0.00514 & \text { DF } & 10.3879 \\ \text { Upper CL Dif } & 0.01068 & \text { Prob }>|t| & 0.8903 \\ \text { Lower CL Dif } & -0.01213 & \text { Prob }>\mathrm{t} & 0.5548 \\ \text { Confidence } & 0.95 & \text { Prob }<\mathrm{t} & 0.4452\end{array}$

Tests that the Variances are Equal

Level Count Std Dev MeanAbsDif to Mean MeanAbsDif to Median

$\begin{array}{lllll}\text { Coliwasa } & 14 & 0.0068928 & 0.0058171 & 0.0058171\end{array}$

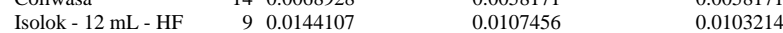

Test F Ratio DFNum DFDen p-Value

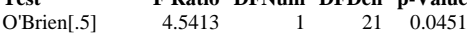

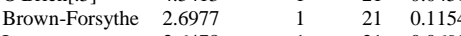

$\begin{array}{lllll}\text { Levene } & 3.6478 & 1 & 21 & 0.0699\end{array}$

$\begin{array}{lllll}\text { Bartlett } & 5.2751 & 1 & 0.0216 \\ \text { F Test 2-sided } & 4.3711 & 8 & 13 & 0.0187\end{array}$

Welch Anova testing Means Equal, allowing Std Devs Not Equal

F Ratio DFNum DFDen Prob $>$ F

$\begin{array}{llll}0.0200 & 1 & 10.388 & 0.8903\end{array}$

t Test

0.1413 
SRNL-STI-2011-00693

Revision 0

Exhibit C3. Statistical Comparisons for Low-Rheology (Phase 3) Simulant Testing (screened data)

Oneway Analysis of Measurement By Sampler Type of Material=SME Simulant, Analytical Block=4, Oxide/Analyte=SiO2 (wt\%), Targeted $=\mathbf{5 0 . 9 8 5}$

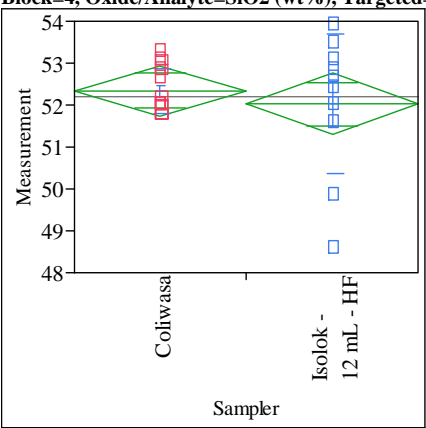

Excluded Rows

Oneway Anova

Summary of Fit

Adj Rsquare

-0.122243
52.21603

Observations (or Sum Wgts)

t Test

Isolok - $12 \mathrm{~mL}$ - HF-Coliwasa

Assuming equal variances

Difference $\quad-0.3138$ t Ratio $\quad-0.68484$

Std Err Dif $\quad 0.4582$ DF 23

Upper CL Dif 0.6340 Prob $>|t| \quad 0.5003$

Lower CL Dif -1.2615 Prob $>t \quad 0.7499$

$\begin{array}{lrl}\text { Confidence } & 0.95 \text { Prob }<\mathrm{t} \quad 0.2501\end{array}$

Analysis of Variance

Source DF Sum of Squares Mean Square F Ratio Prob $>$ F

$\begin{array}{llllll}\text { Sampler } & 1 & 0.590687 & 0.59069 & 0.4690 & 0.5003\end{array}$

$\begin{array}{llll}\text { Error } & 23 & 28.966855 & 1.25943\end{array}$

C. Total $24 \quad 29.557542$

Means for Oneway Anova

$\begin{array}{lrrrrr}\text { Level } & \text { Number } & \text { Mean } & \text { Std Error } & \text { Lower 95\% } & \text { Upper 95\% } \\ \text { Coliwasa } & 15 & 52.3415 & 0.28976 & 51.742 & 52.941\end{array}$

$\begin{array}{lrrrrr}\text { Coliwasa } & 15 & 52.3415 & 0.28976 & 51.742 & 52.941 \\ \text { Isolok - 12 mL - HF } & 10 & 52.0278 & 0.35488 & 51.294 & 52.762\end{array}$

Std Error uses a pooled estimate of error variance

Means and Std Deviations

Level Number Mean Std Dev Std Err Mean Lower 95\% Upper 95\%

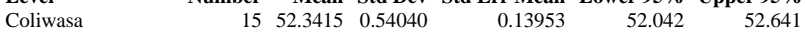

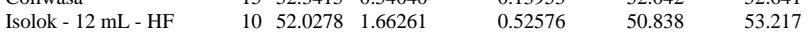

Test

Isolok - $12 \mathrm{~mL}$ - HF-Coliwasa

Assuming unequal variances

Difference $\quad-0.3138$ t Ratio $\quad-0.5768$

Std Err Dif $\quad 0.5440$ DF $\quad 10.2796$

Upper CL Dif 0.8938 Prob $>|t| r \mid \begin{aligned} & 10.2765 \\ & \text { L }\end{aligned}$

Lower CL Dif -1.5213 Prob $>t \quad 0.7118$

$\begin{array}{lrl}\text { Confidence } & 0.95 \text { Prob }<\mathrm{t} \quad 0.2882\end{array}$

Tests that the Variances are Equal

Level Count Std Dev MeanAbsDif to Mean MeanAbsDif to Median

$\begin{array}{llll}15 & 0.540399 & 0.484908 & 0.442122\end{array}$

Test F Ratio DFNum DFDen p-Value

$\begin{array}{lrrrr}\text { O'Brien[.5] } & 4.8639 & 1 & 23 & 0.0377\end{array}$

Brown-Forsythe $\quad 4.1237 \quad 1 \quad 23 \quad 0.0540$

$\begin{array}{lrlll}\text { Levene } & 7.5151 & 1 & 23 & 0.0116\end{array}$

$\begin{array}{lrrrr}\text { Bartlett } & 12.7937 & 1 & \text {. } & 0.0003 \\ \text { F Test 2-sided } & 9.4657 & 9 & 14 & 0.0003\end{array}$

Welch Anova testing Means Equal, allowing Std Devs Not Equal

F Ratio DFNum DFDen Prob $>$ F

$\begin{array}{llll}0.3327 & 1 & 10.28 & 0.5765\end{array}$

t Test

0.5768
Oneway Analysis of Measurement By Sampler Type of Material=SME Simulant, Analytical Block $=4$, Oxide/Analyte $=$ Sum of Oxides ( $w$ t $\%$ ), Targeted $=99.553$

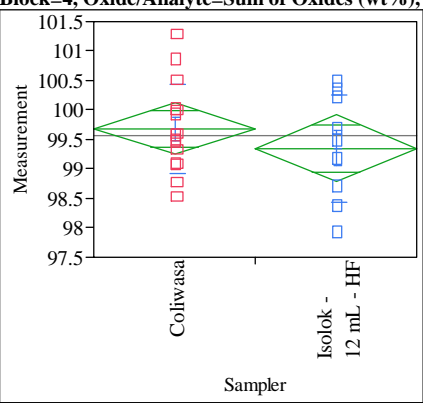

Excluded Rows

Oneway Anova

Summary of Fit

Rsquare

Adj Rsquare

Root Mean Square Error $\quad 0.81456$

$\begin{array}{lr}\text { Mean of Response } & 99.55854 \\ \text { Observations (or Sum Wgts) } & 24\end{array}$

t Test

Isolok - $12 \mathrm{~mL}$ - HF-Coliwasa

Assuming equal variances

Difference $\quad-0.3327$ t Ratio $\quad-0.9688$

$\begin{array}{lrr}\text { Std Err Dif } & 0.3435 \text { DF } & 22 \\ & \end{array}$

Upper CL Dif 0.3795 Prob $>|t| \quad 0.3432$

Lower CL Dif -1.0450 Prob $>t \quad 0.8284$

Confidence $\quad 0.95$ Prob $<\mathrm{t} \quad 0.1716$

Analysis of Variance

Source DF Sum of Squares Mean Square F Ratio Prob $>$ F

$\begin{array}{lrrrrr}\text { Sampler } & 1 & 0.622758 & 0.622758 & 0.9386 & 0.3432\end{array}$

$\begin{array}{llll}\text { Error } & 22 & 14.597309 & 0.663514\end{array}$

C. Total $23 \quad 15.220067$

Means for Oneway Anova

Level Number Mean Std Error Lower 95\% Upper 95\%

$\begin{array}{lrrrrr}\text { Level } & \text { Number } & \text { Mean } & \text { Std Error } & \text { Lower 95\% } & \text { Upper 95\% } \\ \text { Coliwasa } & 15 & 99.6833 & 0.21032 & 99.247 & 100.12\end{array}$

$\begin{array}{lrrrr}\text { Isolok - } 12 \mathrm{~mL} \text { - HF } & 9 & 99.3506 & 0.27152 & 98.787\end{array}$

Std Error uses a pooled estimate of error variance

Means and Std Deviations

Level Number Mean Std Dev Std Err Mean Lower 95\% Upper 95\%

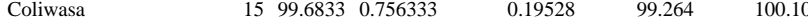

$\begin{array}{llllll}\text { Isolok - } 12 \mathrm{~mL} \text { - HF } & 9 & 99.3506 & 0.907520 & 0.30251 & 98.653\end{array}$

t Test

Isolok - $12 \mathrm{~mL}$ - HF-Coliwas

Assuming unequal variances

$\begin{array}{lrlr}\text { Difference } & -0.3327 & \text { t Ratio } & -0.9241 \\ \text { Std Err Dif } & 0.3601 & \text { DF } & 14.60754 \\ \text { Upper CL Dif } & 0.4365 \text { Prob }>|t| & 0.3704 \\ \text { Lower CL Dif } & -1.1020 & \text { Prob }>\text { t } & 0.8148 \\ \text { Confidence } & 0.95 & \text { Prob }<\mathrm{t} & 0.1852\end{array}$

Tests that the Variances are Equal

Level Count Std Dev MeanAbsDif to Mean MeanAbsDif to Median

$\begin{array}{lrrrr}\text { Coliwasa } & 15 & 0.7563333 & 0.5843035 & 0.5643410 \\ \text { Isolok - } 12 \mathrm{~mL} \text { - HF } & 9 & 0.9075205 & 0.7400247 & 0.7513367\end{array}$

Test F Ratio DFNum DFDen p-Value

Test $\quad$ F Ratio DFNum DFDen p-Value

$\begin{array}{lllll}\text { O'Brien[.5] } & 0.5378 & 1 & 22 & 0.4711 \\ \text { Brown-Forsythe } & 0.8323 & 1 & 22 & 0.3715\end{array}$

$\begin{array}{lllll}\text { Brown-Forsythe } & 0.8323 & 1 & 22 & 0.3715 \\ \text { Levene } & 0.6599 & 1 & 22 & 0.4253\end{array}$

$\begin{array}{lllll}\text { Levene } & 0.6599 & 1 & 22 & 0.4253 \\ \text { Bartlett } & 0.3311 & 1 & . & 0.5650\end{array}$

$\begin{array}{lllll}\text { F Test 2-sided } & 1.4397 & 8 & 14 & 0.5263\end{array}$

Welch Anova testing Means Equal, allowing Std Devs Not Equal

F Ratio DFNum DFDen Prob $>$ F

$\begin{array}{llll}0.8540 & 1 & 14.608 & 0.3704\end{array}$

t Test

0.9241 


\section{Exhibit C3. Statistical Comparisons for Low-Rheology (Phase 3) Simulant Testing (screened data)}

Oneway Analysis of Measurement By Sampler Type of Material=SME Simulant, Analytical Block=4, Oxide/Analyte=TiO2 (wt $\%$ ), Targeted $=0.0711$

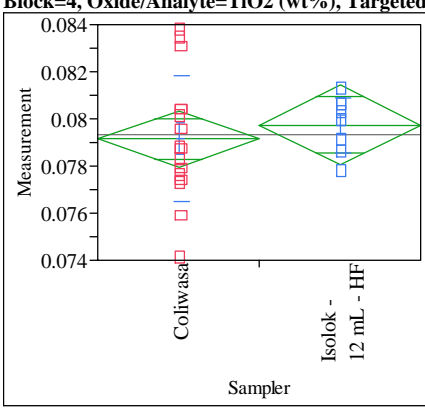

Excluded Rows

Oneway Anova

Summary of Fit

Rsquare

Adj Rsquare

0.015317

$-0.02944$

Root Mean Square Error $\quad 0.002305$

Mean of Response 0.079341

Observations (or Sum Wgts) 24

Test

Isolok - $12 \mathrm{~mL}$ - HF-Coliwasa

Assuming equal variances

Difference $\quad 0.00058$ t Ratio 0.584998

$\begin{array}{lrr}\text { Std Err Dif } & 0.00100 \text { DF } & 22 \\ \text { Upper CL Dif } & 0.00265 \text { Prob }>|t| & 0.5645\end{array}$

Upper CL Dif 0.00265 Prob $>|t| r 0.5645$

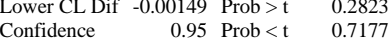

Analysis of Variance

Source DF Sum of Squares Mean Square F Ratio Prob $>$ F

$\begin{array}{lrrrrr}\text { Sampler } & 1 & 0.00000182 & 1.8177 \mathrm{e}-6 & 0.3422 & 0.5645\end{array}$

Error $22 \quad 0.00011685 \quad 5.3115 \mathrm{e}-6$

C. Total $23 \quad 0.00011867$

Means for Oneway Anova

Level Number Mean Std Error Lower 95\% Upper 95\%

$\begin{array}{llllll}\text { Coliwasa } & 16 & 0.079147 & 0.00058 & 0.07795 & 0.08034\end{array}$

Std Error uses a pooled estimate of error variance

Means and Std Deviations

Level Number Mean Std Dev Std Err Mean Lower 95\% Upper 95\%

$\begin{array}{lllllll}\text { Coliwasa } & 16 & 0.079147 & 0.002672 & 0.00067 & 0.07772 & 0.08057\end{array}$

$\begin{array}{lllllll}\text { Isolok - } 12 \mathrm{~mL} \text { - HF } & 8 & 0.079730 & 0.001179 & 0.00042 & 0.07874 & 0.08072\end{array}$

t Test

Isolok - $12 \mathrm{~mL}$ - HF-Coliwasa

Assuming unequal variances

Difference $\quad 0.00058$ t Ratio $\quad 0.741305$

Std Err Dif $\quad 0.00079$ DF 21.85599

Upper CL Dif 0.00222 Prob $>|t| \quad 0.4664$

Lower CL Dif -0.00105 Prob $>\mathrm{t} \quad 0.2332$

$\begin{array}{lll}\text { Confidence } & 0.95 \text { Prob }<\mathrm{t} \quad 0.7668\end{array}$

Tests that the Variances are Equal

Level Count Std Dev MeanAbsDif to Mean MeanAbsDif to Median

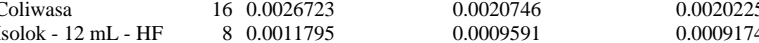

$\begin{array}{lrrrr}\text { Test } & \text { F Ratio } & \text { DFNum } & \text { DFDen } & \text { p-Value } \\ \text { O'Brien[.5] } & 2.8268 & 1 & 22 & 0.1068 \\ \text { Brown-Forsythe } & 2.9635 & 1 & 22 & 0.0992 \\ \text { Levene } & 3.5931 & 1 & 22 & 0.0712 \\ \text { Bartlett } & 4.6826 & 1 & & 0.0305 \\ \text { F Test 2-sided } & 5.1333 & 15 & 7 & 0.0362\end{array}$

Welch Anova testing Means Equal, allowing Std Devs Not Equa

F Ratio DFNum DFDen Prob $>$ F

$\begin{array}{rrrr}0.5495 & 1 & 21.856 & 0.4664\end{array}$

t Test

0.7413
Oneway Analysis of Measurement By Sampler Type of Material=SME Simulant, Analytical Block $=4$, Oxide/Analyte $=\mathrm{ZnO}(\mathrm{wt} \%)$, Targeted $=\mathbf{0 . 0 9 5 8}$

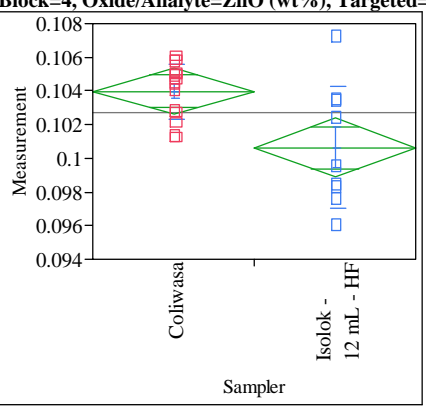

Excluded Rows

Oneway Anova

Summary of Fit

Rsquare

Adj Rsquare

0.307042

0.275544

Root Mean Square Error $\quad 0.002536$

\begin{tabular}{lr} 
Mean of Response & 0.102722 \\
\hline
\end{tabular}

t Test

Isolok - $12 \mathrm{~mL}$ - HF-Coliwasa

Assuming equal variances

$\begin{array}{lrr}\text { Difference } & -0.00334 \text { t Ratio } & -3.12217 \\ \text { Std Err Dif } & 0.00107 \text { DF } & 22\end{array}$

$\begin{array}{lrlr}\text { Std Err Dif } & 0.00107 & \text { DF } & 22 \\ \text { Upper CL } & -0.00112 & \text { Prob }>|t| & 0.0050\end{array}$

$\begin{array}{lrl}\text { Upper CL Dif }-0.00112 \text { Prob }>|t| & 0.0050 \\ \text { Lower CL } & & 0.9975\end{array}$

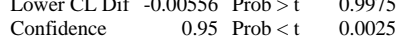

Analysis of Variance

Source DF Sum of Squares Mean Square F Ratio Prob $>$ F

$\begin{array}{lrrrrr}\text { Sampler } & 1 & 0.00006271 & 0.000063 & 9.7480 & 0.0050\end{array}$

Error $22 \quad 0.00014152 \quad 6.433 \mathrm{e}-6$

C. Total $23 \quad 0.00020423$

Means for Oneway Anova

Level Number Mean Std Error Lower 95\% Upper 95\%

$\begin{array}{lrrrrr}\text { Level } & \text { Number } & \text { Mean } & \text { Std Error } & \text { Lower 95\% } & \text { Upper 95\% } \\ \text { Coliwasa } & 15 & 0.103974 & 0.00065 & 0.10262 & 0.10533\end{array}$

$\begin{array}{lrrrrr}\text { Isolok - } 12 \mathrm{~mL} \text { - HF } & 9 & 0.100635 & 0.00085 & 0.09888 & 0.10239\end{array}$

Std Error uses a pooled estimate of error variance

Means and Std Deviations

Level Number Mean Std Dev Std Err Mean Lower 95\% Upper 95\%

$\begin{array}{lllllll}\text { Coliwasa } & 15 & 0.103974 & 0.001615 & 0.00042 & 0.10308 & 0.10487\end{array}$

$\begin{array}{llllll}\text { Isolok - } 12 \mathrm{~mL}-\mathrm{HF} & 9 & 0.100635 & 0.003623 & 0.00121 & 0.09785\end{array}$

t Test

Isolok - $12 \mathrm{~mL}$ - HF-Coliwas

Assuming unequal variances

$\begin{array}{lrlr}\text { Difference } & -0.00334 & \text { t Ratio } & -2.61338 \\ \text { Std Err Dif } & 0.00128 & \text { DF } & 9.941049\end{array}$

Upper CL Dif $-0.00128 \mathrm{DF}>\mid \mathrm{t} r \begin{array}{r}9.941049 \\ 0.0260\end{array}$

Lower CL Dif -0.00619 Prob $>$ t $\quad 0.9870$

0.95 Prob $<t \quad 0.0130$

Tests that the Variances are Equal

$\begin{array}{lrrr}\text { Level } & \text { Count } & \text { Std Dev MeanAbsDif to Mean MeanAbsDif to Median } \\ \text { Coliwasa } & 15 & 0.0016151 & 0.0012697\end{array}$

$\begin{array}{lrrrr}\text { Isolok - } 12 \mathrm{~mL} \text { - HF } & 15 & 0.0016151 & 0.0013377 & 0.0012697 \\ & 9 & 0.0036228 & 0.0030490 & 0.0030428\end{array}$

Test F Ratio DFNum DFDen p-Value

$\begin{array}{lrrrr}\text { Test } & \text { F Ratio } & \text { DFNum } & \text { DFDen } & \text { p-Value } \\ \text { O'Brien[.5] } & 6.5342 & 1 & 22 & 0.0180\end{array}$

$\begin{array}{lllll}\text { Brown-Forsythe } & 7.6352 & 1 & 22 & 0.0113\end{array}$

$\begin{array}{lllll}\text { Levene } & 11.6860 & 1 & 22 & 0.0025\end{array}$

$\begin{array}{lrrrr}\text { Bartlett } & 6.5989 & 1 & . & 0.0102\end{array}$

$\begin{array}{lllll}\text { F Test 2-sided } & 5.0313 & 8 & 14 & 0.0085\end{array}$

Welch Anova testing Means Equal, allowing Std Devs Not Equal

F Ratio DFNum DFDen Prob > F

$\begin{array}{llll}6.8298 & 1 & 9.941 & 0.0260\end{array}$

t Test

2.6134 


\section{Exhibit C3. Statistical Comparisons for Low-Rheology (Phase 3) Simulant Testing (screened data)}

Oneway Analysis of Measurement By Sampler Type of Material=SME Simulant, Analytical Block $=4$, Oxide/Analyte $=\mathrm{ZrO} 2$ ( $w \mathrm{t} \%$ ), Targeted $=\mathbf{0 . 3 5 4 7}$

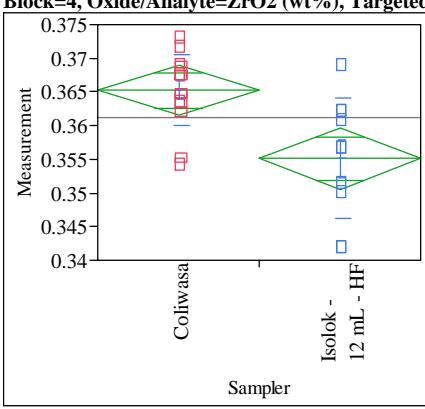

Excluded Rows

Oneway Anova

Summary of Fit

Rsquare

Adj Rsquare

0.35828

0.330379

Root Mean Square Error $\quad 0.006925$

Mean of Response 0.361204

Observations (or Sum Wgts) 25

Test

Isolok - $12 \mathrm{~mL}$ - HF-Coliwasa

Assuming equal variances

Difference $\quad-0.01013$ t Ratio -3.58346

$\begin{array}{lrr}\text { Std Err Dif } & 0.00283 \text { DF } & 23 \\ \text { Dif } & 0.0016\end{array}$

$\begin{array}{lrr}\text { Upper CL Dif }-0.00428 \text { Prob }>|t| & 0.0016 \\ & & 0.9992\end{array}$

$\begin{array}{lrl}\text { Confidence } & 0.95 \text { Prob }<\mathrm{t} \quad 0.0008\end{array}$

Analysis of Variance

Source DF Sum of Squares Mean Square F Ratio Prob > F

$\begin{array}{lrrrrr}\text { Sampler } & 1 & 0.00061582 & 0.000616 & 12.8412 & 0.0016\end{array}$

$\begin{array}{llll}\text { Error } & 23 & 0.00110301 & 0.000048\end{array}$

C. Total $24 \quad 0.00171883$

Means for Oneway Anova

Level Number Mean Std Error Lower 95\% Upper 95\%

$\begin{array}{llllll}\text { Coliwasa } & 15 & 0.365256 & 0.00179 & 0.36156 & 0.36896 \\ \text { Isolok - 12 mL - HF } & 10 & 0.355125 & 0.00219 & 0.35060 & 0.35966\end{array}$

Std Error uses a pooled estimate of error variance

Means and Std Deviations

Level Number Mean Std Dev Std Err Mean Lower 95\% Upper 95\%

$\begin{array}{lllllll} & 15 & 0.365256 & 0.005252 & 0.00136 & 0.36235 & 0.36816\end{array}$

t Test

Isolok - $12 \mathrm{~mL}$ - HF-Coliwasa

Assuming unequal variances

Difference $\quad-0.01013$ t Ratio -3.23553

Std Err Dif $\quad 0.00313$ DF $\quad 13.1826$

Upper CL Dif -0.00338 Prob $>|t| \quad 0.0064$

Lower CL Dif -0.01689 Prob $>\mathrm{t} \quad 0.9968$

$\begin{array}{lll}\text { Confidence } & 0.95 \text { Prob }<\mathrm{t} \quad 0.0032\end{array}$

Tests that the Variances are Equal

$\begin{array}{lrrrr}\text { Level } & \text { Count } & \text { Std Dev } & \text { MeanAbsDif to Mean } & \text { MeanAbsDif to Median } \\ \text { Coliwasa } & 15 & 0.0052515 & 0.0039263\end{array}$

$\begin{array}{lllll} & 15 & 0.0052515 & 0.0039263 & 0.0037822 \\ \text { Isolok - } 12 \mathrm{~mL} \text { - HF } & 10 & 0.0089251 & 0.0071863 & 0.0068891\end{array}$

$\begin{array}{lrrr}\text { Test } & \text { F Ratio DFNum DFDen } & \text { p-Value } \\ \text { Ond } & \text { D.6716 } & \end{array}$

$\begin{array}{lllll}\text { O'Brien[.5] } & 3.6716 & 1 & 23 & 0.0679 \\ \text { Brown-Forsythe } & 2.6326 & 1 & 23 & 0.1183\end{array}$

$\begin{array}{lllll}\text { Brown-Forsythe } & 2.6326 & 1 & 23 & 0.1183 \\ \text { Levene } & 4.1271 & 1 & 23 & 0.0539\end{array}$

$\begin{array}{lllll}\text { Bartlett } & 3.0381 & 1 & . & 0.0813\end{array}$

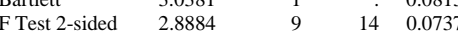

Welch Anova testing Means Equal, allowing Std Devs Not Equal

F Ratio DFNum DFDen Prob $>$ F

$\begin{array}{llll}10.4687 & 1 & 13.183 & 0.0064\end{array}$

t Test
3.2355
Oneway Analysis of Measurement By Sampler Type of Material=SME Simulant, Analytical Block $=5$, Oxide $/$ Analyte $=\mathrm{Cr} 2 \mathrm{O} 3(\mathrm{wt} \%)$, Targeted $=0.0998$

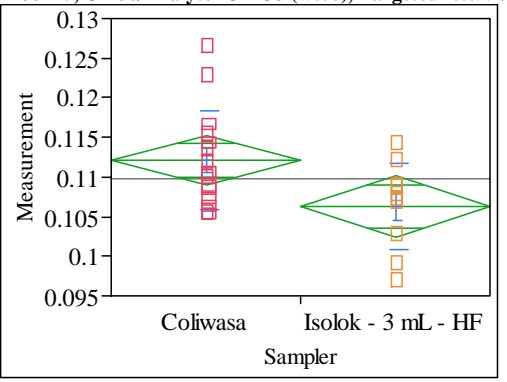

Excluded Rows

Oneway Anova

Summary of Fit

$\begin{array}{lr}\text { Rsquare } & 0.203875 \\ \text { Adj Rsquare } & 0.169261 \\ \text { Root Mean Square Error } & 0.005876 \\ \text { Mean of Response } & 0.109795 \\ \text { Observations (or Sum Wgts) } & 25\end{array}$

\section{Test}

Isolok - $3 \mathrm{~mL}$ - HF-Coliwasa

Assuming equal variances

$\begin{array}{lr}\text { Difference } & -0.00582 \text { t Ratio } \quad-2.42692\end{array}$

$\begin{array}{lrlr}\text { Std Err Dif } & 0.00240 \text { DF } & 23 \\ \text { Upper CL } & 0.0235\end{array}$

Lower CL Dif -0.01078 Prob $>t-0.0235$

$\begin{array}{lrl}\text { Confidence } & 0.95 \text { Prob }<\mathrm{t} & 0.0117\end{array}$

Analysis of Variance

Source DF Sum of Squares Mean Square F Ratio Prob $>$ F

$\begin{array}{lllllll}\text { Sampler } & 1 & 0.00020338 & 0.000203 & 5.8900 & 0.0235\end{array}$

$\begin{array}{llll}\text { Error } & 23 & 0.00079418 & 0.000035 \\ \text { C. Total } & 24 & 0.00099755 & \end{array}$

Means for Oneway Anova

Level Number Mean Std Error Lower 95\% Upper 95\%

$\begin{array}{lrrrrr}\text { Coliwasa } & 15 & 0.112124 & 0.00152 & 0.10899 & 0.11526\end{array}$

$\begin{array}{llllll}\text { Isolok - 3 mL - HF } & 10 & 0.106302 & 0.00186 & 0.10246 & 0.11015\end{array}$

Std Error uses a pooled estimate of error variance

Means and Std Deviations

Level Number Mean Std Dev Std Err Mean Lower 95\% Upper 95\%

$\begin{array}{lllllll}\text { Coliwasa } & 15 & 0.112124 & 0.006140 & 0.00159 & 0.10872 & 0.11552\end{array}$

$\begin{array}{llllll}\text { Isolok - } 3 \mathrm{~mL} \text { - HF } & 10 & 0.106302 & 0.005441 & 0.00172 & 0.10241\end{array}$

t Test

Isolok - $3 \mathrm{~mL}$ - HF-Coliwasa

Assuming unequal variances

$\begin{array}{lrlr}\text { Difference } & -0.00582 \text { t Ratio } & -2.4886 \\ \text { Std Err Dif } & 0.00234 & \text { DF } & 21.02684\end{array}$

Std Err Dif $\quad 0.00234$ DF 21.02684

Upper CL Dif -0.00096 Prob $>|t| 0.0213$

Lower CL Dif -0.01069 Prob $>t \quad 0.9894$

$\begin{array}{lrl}\text { Confidence } & 0.95 \text { Prob }<\mathrm{t} & 0.0106\end{array}$

Tests that the Variances are Equal

Level Count Std Dev MeanAbsDif to Mean MeanAbsDif to Median

$\begin{array}{lllll}\text { Coliwasa } & 15 & 0.0061399 & 0.0047200 & 0.0046381 \\ \text { Isolok - 3 mL - HF } & 10 & 0.0054405 & 0.0041480 & 0.0039025\end{array}$

Test F Ratio DFNum DFDen p-Value

$\begin{array}{lrrrr}\text { Test } & \text { F Ratio } & \text { DFNum } & \text { DFDen } & \text { p-Value } \\ \text { O'Brien[.5] } & 0.1435 & 1 & 23 & 0.7083 \\ \text { Brown-Forsythe } & 0.2043 & 1 & 23 & 0.6555 \\ \text { Levene } & 0.1568 & 1 & 23 & 0.6958 \\ \text { Bartlett } & 0.1502 & 1 & . & 0.6984 \\ \text { F Test 2-sided } & 1.2736 & 14 & 9 & 0.7313\end{array}$

Welch Anova testing Means Equal, allowing Std Devs Not Equal

F Ratio DFNum DFDen Prob $>$ F

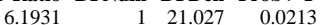

t Test

2.4886 


\section{Exhibit C3. Statistical Comparisons for Low-Rheology (Phase 3) Simulant Testing (screened data)}

Oneway Analysis of Measurement By Sampler Type of Material=SME Simulant, Analytical Block $=5$, Oxide $/$ Analyte $=\mathrm{CuO}($ wt $\%)$, Targeted $=\mathbf{0 . 0 5 0 4}$

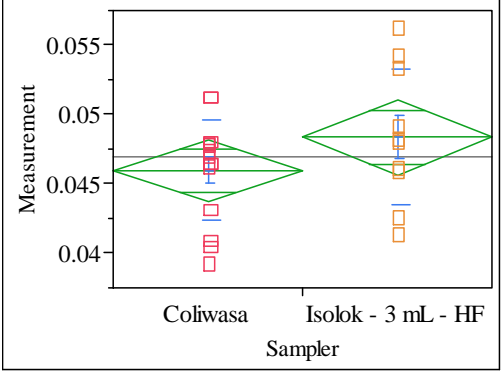

Excluded Rows

Oneway Anova

Summary of Fit

Adj Rsquare

Root Mean Square Error 0.038347

Mean of Response $\quad 0.004165$

Observations (or Sum Wgts) 25

t Test

Isolok - $3 \mathrm{~mL}$ - HF-Coliwasa

Assuming equal variances

Difference $\quad 0.00238$ t Ratio 1.398937

$\begin{array}{lrr}\text { Std Err Dif } & 0.00170 \text { DF } & 23 \\ \text { Upper CL Dif } & 0.00590 \text { Prob }>|t| & 0.1752\end{array}$

$\begin{array}{lrll} & \text { Confidence } & 0.95 \text { Prob }<\mathrm{t} & 0.9124\end{array}$

Analysis of Variance

Source DF Sum of Squares Mean Square F Ratio Prob $>$ F

$\begin{array}{llllll}\text { Sampler } & 1 & 0.00003394 & 0.000034 & 1.9570 & 0.1752\end{array}$

Error $23 \quad 0.00039890 \quad 0.000017$

C. Total $24 \quad 0.0004328$

Means for Oneway Anova

$\begin{array}{lllll}\text { Level } & \text { Number } & \text { Mean Std Error Lower 95\% Upper 95\% }\end{array}$

Coliwas $15 \quad 0.045966 \quad 0.00108 \quad 0.04374 \quad 0.04819$

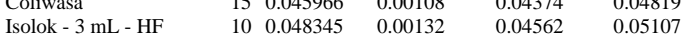

Std Error uses a pooled estimate of error variance

Means and Std Deviations

Level Number Mean Std Dev Std Err Mean Lower 95\% Upper 95\%

$\begin{array}{llllll}15 & 0.045966 & 0.003614 & 0.00093 & 0.04396 & 0.04797\end{array}$

t Test

Isolok - $3 \mathrm{~mL}$ - HF-Coliwasa

Assuming unequal variances

Difference $\quad 0.00238$ t Ratio 1.315055

Std Err Dif $\quad 0.00181$ DF 15.41122

Upper CL Dif 0.00622 Prob $>|t| \quad 0.2077$

Lower CL Dif -0.00147 Prob $>t \quad 0.1039$

$\begin{array}{lrl}\text { Confidence } & 0.95 \text { Prob }<\mathrm{t} & 0.8961\end{array}$

Tests that the Variances are Equal

Level Count Std Dev MeanAbsDif to Mean MeanAbsDif to Median $\begin{array}{lllll}\text { Coliwasa } & 15 & 0.0036142 & 0.0027339 & 0.0025620\end{array}$

$\begin{array}{lrrrr}\text { Test } & \text { F Ratio } & \text { DFNum } & \text { DFDen } & \text { p-Value } \\ \text { O'Brien[.5] } & 1.5973 & 1 & 23 & 0.2189 \\ \text { Brown-Forsythe } & 1.0833 & 1 & 23 & 0.3088 \\ \text { Levene } & 1.0634 & 1 & 23 & 0.3132 \\ \text { Bartlett } & 0.9977 & 1 & . & 0.3179 \\ \text { F Test 2-sided } & 1.8375 & 9 & 14 & 0.2967\end{array}$

$\begin{array}{lllll}\text { F Test 2-sided } & 1.8375 & 9 & 14 & 0.2967\end{array}$

Welch Anova testing Means Equal, allowing Std Devs Not Equal

F Ratio DFNum DFDen Prob $>$ F

$\begin{array}{llll}1.7294 & 1 & 15.411 & 0.2077\end{array}$

t Test

1.3151
Oneway Analysis of Measurement By Sampler Type of Material=SME Simulant, Analytical Block $=5$, Oxide/Analyte $=$ K2O $(\mathrm{wt} \%)$, Targeted $=\mathbf{0 . 1 5 9 1}$

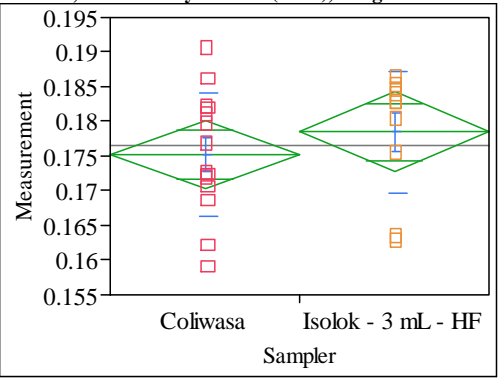

Excluded Rows

2

Oneway Anova

Summary of Fit

$\begin{array}{lr}\text { Rsquare } & 0.035741 \\ \text { Adj Rsquare } & -0.00809 \\ \text { Root Mean Square Error } & 0.008814 \\ \text { Mean of Response } & 0.176599 \\ \text { Observations (or Sum Wgts) } & 24\end{array}$

t Test

Isolok - $3 \mathrm{~mL}$ - HF-Coliwasa

Assuming equal variances

$\begin{array}{lrlr}\text { Difference } & 0.00330 & \text { t Ratio } & 0.903023 \\ \text { Std Err Dif } & 0.00365 & \text { DF } & 22 \\ \text { Upper CL Dif } & 0.01086 & \text { Prob }>|t| & 0.3763 \\ \text { Lower CL Dif } & -0.00427 & \text { Prob }>t & 0.1881 \\ \text { Confidence } & 0.95 & \text { Prob }<\mathrm{t} & 0.8119\end{array}$

Analysis of Variance

Source DF Sum of Squares Mean Square F Ratio Prob $>$ F

$\begin{array}{lrrrrr}\text { Sampler } & 1 & 0.00006335 & 0.000063 & 0.8155 & 0.3763\end{array}$

$\begin{array}{llll}\text { Error } & 22 & 0.00170911 & 0.000078\end{array}$

C. Total $23-0.00177246$

Means for Oneway Anova

Level Number Mean Std Error Lower 95\% Upper 95\%

$\begin{array}{lrrrrr}\text { Coliwasa } & 14 & 0.175226 & 0.00236 & 0.17034 & 0.18011\end{array}$

$\begin{array}{llllll}\text { Isolok - 3 mL - HF } & 10 & 0.178522 & 0.00279 & 0.17274 & 0.18430\end{array}$

Std Error uses a pooled estimate of error variance

Means and Std Deviation

Level Number Mean Std Dev Std Err Mean Lower 95\% Upper 95\%

$\begin{array}{lllllll}\text { Coliwasa } & 14 & 0.175226 & 0.008853 & 0.00237 & 0.17011 & 0.18034\end{array}$

$\begin{array}{lllllll}\text { Isolok - } 3 \mathrm{~mL}-\mathrm{HF} & 10 & 0.178522 & 0.008757 & 0.00277 & 0.17226 & 0.18479\end{array}$

Test

Isolok - $3 \mathrm{~mL}$ - HF-Coliwasa

Assuming unequal variances

$\begin{array}{lll}\text { Difference } & 0.00330 \text { t Ratio } \quad 0.904752\end{array}$

Std Err Dif $\quad 0.00364$ DF 19.67795

Upper CL Dif 0.01090 Prob $>|t| \quad 0.3765$

Lower CL Dif -0.00431 Prob $>t \quad 0.1883$

$\begin{array}{lrl}\text { Confidence } & 0.95 \text { Prob }<\mathrm{t} & 0.8117\end{array}$

Tests that the Variances are Equal

Level Count Std Dev MeanAbsDif to Mean MeanAbsDif to Median

$\begin{array}{lllll}\text { Coliwasa } & 14 & 0.0088534 & 0.0071846 & 0.0071846 \\ \text { Isolok - } 3 \mathrm{~mL} \text { - HF } & 10 & 0.0087567 & 0.0068903 & 0.0057821\end{array}$

$\begin{array}{lrrrr}\text { Test } & \text { F Ratio } & \text { DFNum } & \text { DFDen } & \text { p-Value } \\ \text { O'Brien[.5] } & 0.0016 & 1 & 22 & 0.9686 \\ \text { Brown-Forsythe } & 0.3077 & 1 & 22 & 0.5847 \\ \text { Levene } & 0.0217 & 1 & 22 & 0.8842 \\ \text { Bartlett } & 0.0012 & 1 & . & 0.9721 \\ \text { F Test 2-sided } & 1.0222 & 13 & 9 & 1.0000\end{array}$

$\begin{array}{lrrrr}\text { Bartlett } & 0.0012 & 1 & \text {. } & 0.9721 \\ \text { F Test 2-sided } & 1.0222 & 13 & 9 & 1.0000\end{array}$

Welch Anova testing Means Equal, allowing Std Devs Not Equal

F Ratio DFNum DFDen Prob $>$ F

$\begin{array}{llll}0.8186 & 1 & 19.678 & 0.3765\end{array}$

t Test

0.9048 


\section{Exhibit C3. Statistical Comparisons for Low-Rheology (Phase 3) Simulant Testing (screened data)}

Oneway Analysis of Measurement By Sampler Type of Material=SME Simulant, Analytical Block $=5$, Oxide $/$ Analyte $=$ Na2O $(\mathrm{wt} \%)$, Targeted $=11.659$

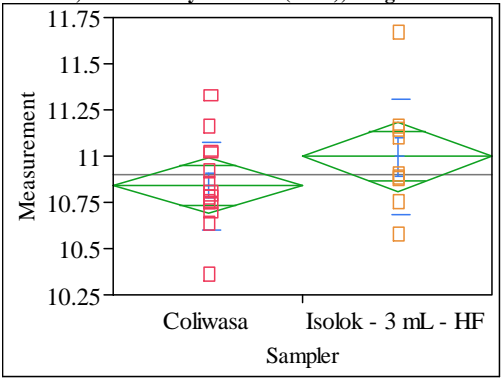

Excluded Rows

Oneway Anova

Summary of Fit

$\begin{array}{ll}0.082251 \\ \text { Adj Rsquare } & 0.038549\end{array}$

Root Mean Square Error $\quad 0.268494$

Mean of Response 10.90239

Observations (or Sum Wgts) 23

t Test

Isolok - $3 \mathrm{~mL}$ - HF-Coliwasa

Assuming equal variances

Difference $\quad 0.15737$ t Ratio $\quad 1.371886$

$\begin{array}{lrr}\text { Std Err Dif } & 0.11471 \text { DF } & 21\end{array}$

Upper CL Dif 0.39593 Prob $>|t| \quad 0.1846$

Lower CL Dif -0.08119 Prob $>t \quad 0.0923$

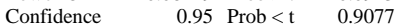

Analysis of Variance

Source DF Sum of Squares Mean Square F Ratio Prob > F

$\begin{array}{lrrrrr}\text { Sampler } & 1 & 0.1356772 & 0.135677 & 1.8821 & 0.1846\end{array}$

$\begin{array}{llll}\text { Error } & 21 & 1.5138740 & 0.072089\end{array}$

C. Total $22 \quad 1.6495512$

Means for Oneway Anova

Level Number Mean Std Error Lower 95\% Upper 95\%

$\begin{array}{lrrrrr}\text { Coliwasa } & 14 & 10.8408 & 0.07176 & 10.692 & 10.990\end{array}$

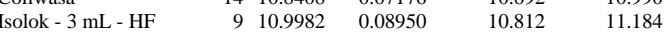

Std Error uses a pooled estimate of error variance

Means and Std Deviations

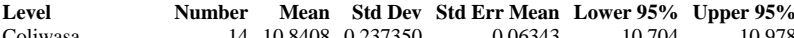

$\begin{array}{lrrrrrr} & 14 & 10.8408 & 0.237350 & 0.06343 & 10.704 & 10.978\end{array}$

t Test

Isolok - $3 \mathrm{~mL}$ - HF-Coliwasa

Assuming unequal variances

Difference $\quad 0.15737$ t Ratio 1.290193

Std Err Dif $\quad 0.12198$ DF 13.85889

Upper CL Dif 0.41924 Prob $>|t| \quad 0.2181$

Lower CL Dif -0.10449 Prob $>t \quad 0.1090$

$\begin{array}{lrl}\text { Confidence } & 0.95 \text { Prob }<\mathrm{t} & 0.8910\end{array}$

Tests that the Variances are Equal

Level Count Std Dev MeanAbsDif to Mean MeanAbsDif to Median

$\begin{array}{lrrrr} & 14 & 0.2373504 & 0.1731767 & 0.1685000 \\ \text { Isolok - } 3 \mathrm{~mL}-\mathrm{HF} & 9 & 0.3125532 & 0.2319891 & 0.2216711\end{array}$

$\begin{array}{lrrrr}\text { Test } & \text { F Ratio } & \text { DFNum } & \text { DFDen } & \text { p-Value } \\ \text { O'Brien[.5] } & 0.5661 & 1 & 21 & 0.4601 \\ \text { Brown-Forsythe } & 0.4054 & 1 & 21 & 0.5312 \\ \text { Levene } & 0.6527 & 1 & 21 & 0.4282 \\ \text { Bartlett } & 0.7366 & 1 & . & 0.3908 \\ \text { F Test 2-sided } & 1.7341 & 8 & 13 & 0.3629\end{array}$

$\begin{array}{lllll}\text { F Test 2-sided } & 1.7341 & 8 & 13 & 0.3629\end{array}$

Welch Anova testing Means Equal, allowing Std Devs Not Equal

F Ratio DFNum DFDen Prob $>$ F

$\begin{array}{llll}1.6646 & 1 & 13.859 & 0.2181\end{array}$

t Test

1.2902
Oneway Analysis of Measurement By Sampler Type of Material=SME Simulant, Analytical Block $=5$, Oxide $/$ Analyte $=\mathrm{NiO}$ (wt $\%$ ), Targeted $=\mathbf{0 . 4 1}$

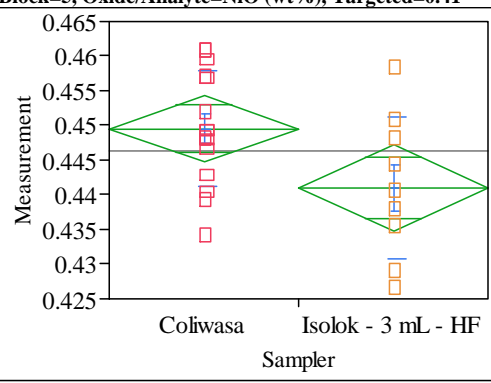

Excluded Rows

2

Oneway Anova

Summary of Fit

$\begin{array}{lr}\text { Rsquare } & 0.185397 \\ \text { Adj Rsquare } & 0.148369 \\ \text { Root Mean Square Error } & 0.009052 \\ \text { Mean of Response } & 0.446329 \\ \text { Observations (or Sum Wgts) } & 24\end{array}$

\section{Test}

Isolok - $3 \mathrm{~mL}$ - HF-Coliwasa

Assuming equal variances

$\begin{array}{lrlr}\text { Difference } & -0.00854 & \mathrm{t} \text { Ratio } & -2.23763 \\ \text { Std Err Dif } & 0.00382 & \text { DF } & 22 \\ \text { Upper CL Dif } & -0.00062 & \text { Prob }>|t| & 0.0357 \\ \text { Lower CL Dif } & -0.01645 & \text { Prob }>t & 0.9822\end{array}$

Analysis of Variance

Source DF Sum of Squares Mean Square F Ratio Prob $>$ F

$\begin{array}{lrrrrr}\text { Sampler } & 1 & 0.00041023 & 0.000410 & 5.0070 & 0.0357\end{array}$

$\begin{array}{llll}\text { Error } & 22 & 0.00180248 & 0.000082\end{array}$

C. Total $23 \quad 0.00221271$

Means for Oneway Anova

Level Number Mean Std Error Lower 95\% Upper 95\%

$\begin{array}{lrrrrr}\text { Coliwasa } & 15 & 0.449532 & 0.00234 & 0.44468 & 0.45438\end{array}$

$\begin{array}{lrrrrr}\text { Isolok - 3 mL - HF } & 9 & 0.440992 & 0.00302 & 0.43473 & 0.44725\end{array}$

Std Error uses a pooled estimate of error variance

Means and Std Deviations

Level Number Mean Std Dev Std Err Mean Lower 95\% Upper 95\%

$\begin{array}{lllllll}\text { Coliwasa } & 15 & 0.449532 & 0.008253 & 0.00213 & 0.44496 & 0.45410\end{array}$

$\begin{array}{llllll}\text { Isolok - } 3 \mathrm{~mL}-\mathrm{HF} & 9 & 0.440992 & 0.010301 & 0.00343 & 0.43307\end{array}$

Test

Isolok - $3 \mathrm{~mL}$ - HF-Coliwasa

Assuming unequal variances

Difference $\quad-0.00854$ t Ratio $\quad-2.11324$

Std Err Dif $\quad 0.00404$ DF 14.15015

Upper CL Dif 0.00012 Prob $>|t| 0.0528$

Lower CL Dif -0.01720 Prob $>\mathrm{t} \quad 0.9736$

$\begin{array}{lrl}\text { Confidence } & 0.95 \text { Prob }<\mathrm{t} & 0.0264\end{array}$

Tests that the Variances are Equal

Level Count Std Dev MeanAbsDif to Mean MeanAbsDif to Median

$\begin{array}{lrrrr} & 15 & 0.0082533 & 0.0065152 & 0.0064473 \\ \text { Isolok - } 3 \mathrm{~mL} \text { - HF } & 9 & 0.0103008 & 0.0081377 & 0.0083419\end{array}$

$\begin{array}{lrrrr}\text { Test } & \text { F Ratio } & \text { DFNum } & \text { DFDen } & \text { p-Value } \\ \text { O'Brien[.5] } & 0.8650 & 1 & 22 & 0.3624 \\ \text { Brown-Forsythe } & 0.7857 & 1 & 22 & 0.3850 \\ \text { Levene } & 0.5718 & 1 & 22 & 0.4576 \\ \text { Bartlett } & 0.4919 & 1 & \mathbf{1} & 0.4831 \\ \text { F Test 2-sided } & 1.5577 & 8 & 14 & 0.4474\end{array}$

Welch Anova testing Means Equal, allowing Std Devs Not Equal

F Ratio DFNum DFDen Prob $>$ F

$\begin{array}{llll}4.4658 & 1 & 14.15 & 0.0528\end{array}$

t Test

2.1132 


\section{Exhibit C3. Statistical Comparisons for Low-Rheology (Phase 3) Simulant Testing (screened data)}

Oneway Analysis of Measurement By Sampler Type of Material=SME Simulant, Analytical Block=5, Oxide/Analyte $=\mathrm{ZnO}$ (wt \%), Targeted $=\mathbf{0 . 0 9 5 8}$

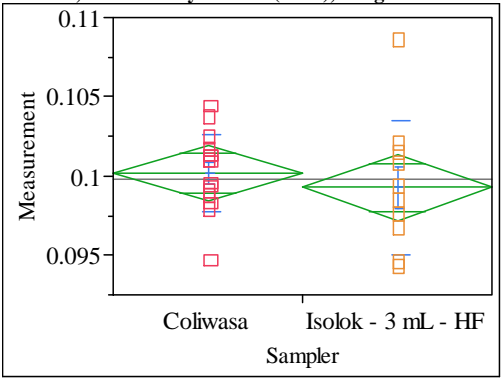

Excluded Rows

Oneway Anova

Summary of Fit

Adj Rsquare

Root Mean Square Error $\quad-0.02201$

Mean of Response $\quad 0.003246$

Observations (or Sum Wgts) 25

t Test

Isolok - $3 \mathrm{~mL}$ - HF-Coliwasa

Assuming equal variances

Difference $\quad-0.00092$ t Ratio $\quad-0.69505$

$\begin{array}{lrr}\text { Std Err Dif } & 0.00133 \text { DF } & 23 \\ & & \end{array}$

Upper CL Dif 0.00182 Prob $>|\mathrm{t}| \quad 0.4940$

Lower CL Dif -0.00366 Prob $>t \quad 0.7530$

$\begin{array}{lll}\text { Confidence } & 0.95 \text { Prob }<\mathrm{t} \quad 0.2470\end{array}$

Analysis of Variance

Source DF Sum of Squares Mean Square F Ratio Prob > F

$\begin{array}{llllll}\text { Sampler } & 1 & 0.00000509 & 5.091 \mathrm{e}-6 & 0.4831 & 0.4940\end{array}$

Error $23 \quad 0.00024239 \quad 0.000011$

C. Total $24 \quad 0.00024748$

Means for Oneway Anova

Level $\quad$ Number Mean Std Error Lower 95\% Upper 95\%

$\begin{array}{lrrrrr}\text { Coliwasa } & 15 & 0.100182 & 0.00084 & 0.09845 & 0.10192\end{array}$

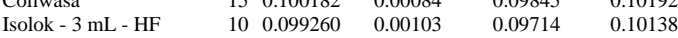

Std Error uses a pooled estimate of error variance

Means and Std Deviations

Level Number Mean Std Dev Std Err Mean Lower 95\% Upper 95\%

$\begin{array}{llllll}15 & 0.100182 & 0.002454 & 0.00063 & 0.09882 & 0.10154\end{array}$

t Test

Isolok - $3 \mathrm{~mL}$ - HF-Coliwasa

Assuming unequal variances

Difference $\quad-0.00092$ t Ratio $\quad-0.62706$

Std Err Dif $\quad 0.00147$ DF 13.14241

Upper CL Dif 0.00225 Prob $>|t| \quad 0.5414$

Lower CL Dif -0.00409 Prob $>t \quad 0.7293$

$\begin{array}{lrl}\text { Confidence } & 0.95 \text { Prob }<\mathrm{t} & 0.2707\end{array}$

Tests that the Variances are Equal

Level Count Std Dev MeanAbsDif to Mean MeanAbsDif to Median

$\begin{array}{lllll} & 15 & 0.0024539 & 0.0019187 & 0.0018755 \\ \text { Isolok - } 3 \mathrm{~mL}-\mathrm{HF} & 10 & 0.0041911 & 0.0030722 & 0.0030622\end{array}$

$\begin{array}{lrrrr}\text { Test } & \text { F Ratio } & \text { DFNum } & \text { DFDen } & \text { p-Value } \\ \text { O'Brien[.5] } & 1.9397 & 1 & 23 & 0.1770 \\ \text { Brown-Forsythe } & 1.8598 & 1 & 23 & 0.1858 \\ \text { Levene } & 1.9789 & 1 & 23 & 0.1729 \\ \text { Bartlett } & 3.0947 & 1 & . & 0.0785 \\ \text { F Test 2-sided } & 2.9171 & 9 & 14 & 0.0711\end{array}$

Welch Anova testing Means Equal, allowing Std Devs Not Equal

F Ratio DFNum DFDen Prob $>$ F

$\begin{array}{llll}0.3932 & 1 & 13.142 & 0.5414\end{array}$

t Test

0.6271
Oneway Analysis of Measurement By Sampler Type of Material=SME Simulant, Analytical Block $=5$, Oxide/Analyte $=\mathrm{ZrO} 2(\mathrm{wt} \%)$, Targeted $=0.3547$

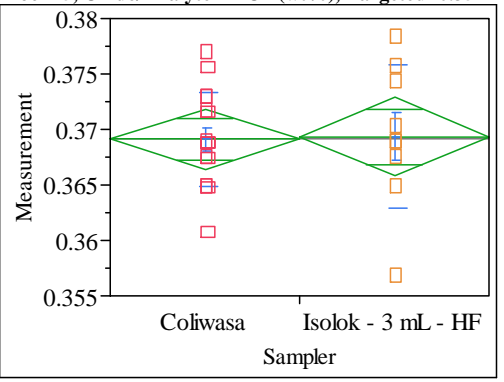

Excluded Rows

Oneway Anova

Summary of Fit

$\begin{array}{lr}\text { Rsquare } & 0.000657 \\ \text { Adj Rsquare } & -0.04279 \\ \text { Root Mean Square Error } & 0.005126 \\ \text { Mean of Response } & 0.369201 \\ \text { Observations (or Sum Wgts) } & 25\end{array}$

\section{Test}

Isolok - $3 \mathrm{~mL}$ - HF-Coliwasa

Assuming equal variances

$\begin{array}{lrlr}\text { Difference } & 0.00026 & \text { t Ratio } & 0.122973 \\ \text { Std Err Dif } & 0.00214 & \text { DF } & 23 \\ \text { Upper CL Dif } & 0.00468 & \text { Prob }>|t| & 0.9032 \\ \text { Lower CL Dif } & -0.00416 & \text { Prob }>t & 0.4516 \\ \text { Confidence } & 0.95 & \text { Prob }<\mathrm{t} & 0.5484\end{array}$

Analysis of Variance

Source DF Sum of Squares Mean Square F Ratio Prob $>$ F

$\begin{array}{lrrrrr}\text { Sampler } & 1 & 0.00000040 & 3.974 \mathrm{e}-7 & 0.0151 & 0.9032\end{array}$

$\begin{array}{llll}\text { Error } & 23 & 0.00060437 & 0.000026\end{array}$

Means for Oneway Anova

$\begin{array}{lllll}\text { Means for Oneway Anova } & & & & \\ \text { Level } & \text { Number } & \text { Mean Std Error } & \text { Lower 95\% } & \text { Upper 95\% }\end{array}$

$\begin{array}{lrrrrr}\text { Level } & \text { Number } & \text { Mean } & \text { Std Error } & \text { Lower 95\% } & \text { Upper 95\% } \\ \text { Coliwasa } & 16 & 0.369106 & 0.00128 & 0.36646 & 0.37176\end{array}$

$\begin{array}{lrllll}\text { Coliwasa } & 16 & 0.369106 & 0.00128 & 0.36646 & 0.37176 \\ \text { Isolok - 3 mL - HF } & 9 & 0.369369 & 0.00171 & 0.36583 & 0.37290\end{array}$

Std Error uses a pooled estimate of error variance

Means and Std Deviations

Level Number Mean Std Dev Std Err Mean Lower 95\% Upper 95\%

$\begin{array}{lllllll}\text { Coliwasa } & 16 & 0.369106 & 0.004286 & 0.00107 & 0.36682 & 0.37139\end{array}$

$\begin{array}{llllll}\text { Isolok - } 3 \mathrm{~mL}-\mathrm{HF} & 9 & 0.369369 & 0.006411 & 0.00214 & 0.36444\end{array}$

t Test

Isolok - $3 \mathrm{~mL}$ - HF-Coliwasa

Assuming unequal variances

$\begin{array}{lll}\text { Difference } & 0.00026 \text { t Ratio } \quad 0.109867\end{array}$

Std Err Dif $\quad 0.00239$ DF $\quad 12.11879$

Upper CL Dif 0.00547 Prob $>|t| \quad 0.9143$

Lower CL Dif -0.00494 Prob $>t \quad 0.4572$

$\begin{array}{lll}\text { Confidence } & 0.95 \text { Prob }<\mathrm{t} & 0.5428\end{array}$

Tests that the Variances are Equal

Level Count Std Dev MeanAbsDif to Mean MeanAbsDif to Median

$\begin{array}{lrrrr}\text { Coliwasa } & 16 & 0.0042858 & 0.0032926 & 0.0032082 \\ \text { Isolok - 3 mL - HF } & 9 & 0.0064114 & 0.0045694 & 0.0045027\end{array}$

$\begin{array}{lrrrr}\text { Test } & \text { F Ratio } & \text { DFNum } & \text { DFDen } & \text { p-Value } \\ \text { O'Brien[.5] } & 1.6167 & 1 & 23 & 0.2163 \\ \text { Brown-Forsythe } & 0.8464 & 1 & 23 & 0.3671 \\ \text { Levene } & 0.8888 & 1 & 23 & 0.3556 \\ \text { Bartlett } & 1.7070 & 1 & . & 0.1914 \\ \text { F Test 2-sided } & 2.2379 & 8 & 15 & 0.1701\end{array}$

Welch Anova testing Means Equal, allowing Std Devs Not Equal

F Ratio DFNum DFDen Prob $>$ F

$\begin{array}{llll}0.0121 & 1 & 12.119 & 0.9143\end{array}$

t Test

0.1099 
Revision 0

\section{Exhibit C3. Statistical Comparisons for Low-Rheology (Phase 3) Simulant Testing (screened data)}

Oneway Analysis of Measurement By Sampler Type of Material=SME Simulant, Analytical Block=6, Oxide/Analyte $=$ Al/B, Targeted $=2.552$

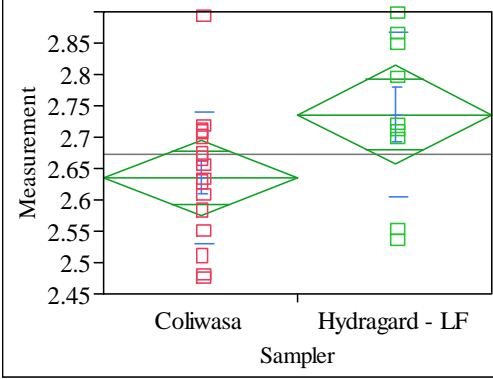

Exc

Excluded Rows

Oneway Anova

Summary of Fit

Adj Rsquare $\quad 0.125083$

Root Mean Square Error 0.114816

Mean of Response

$\begin{array}{lr}\text { Mean of Response } & 2.671409 \\ \text { Observations (or Sum Wgts) } & 25\end{array}$

t Tes

Hydragard - LF-Coliwasa

Assuming equal variances

Difference $\quad 0.100705$ t Ratio 2.105037

Std Err Dif $\quad 0.047840$ DF 23

Upper CL Dif 0.199670 Prob $>|t| \quad 0.0464$

$\begin{array}{lll}\text { Lower CL Dif } 0.001740 \text { Prob }>t & 0.0232\end{array}$

$\begin{array}{lrl}\text { Confidence } & 0.95 \text { Prob }<\mathrm{t} & 0.9768\end{array}$

Analysis of Variance

Source DF Sum of Squares Mean Square F Ratio Prob $>$ F

$\begin{array}{llllll}\text { Sampler } & 1 & 0.05841544 & 0.058415 & 4.4312 & 0.0464\end{array}$

$\begin{array}{llll}\text { Error } & 23 & 0.30320491 & 0.013183\end{array}$

$\begin{array}{lll}\text { C. Total } & 24 & 0.36162035\end{array}$

Means for Oneway Anova

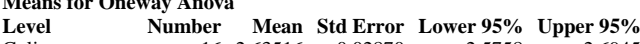

$\begin{array}{lrrrrr}\text { Coliwasa } & 16 & 2.63516 & 0.02870 & 2.5758 & 2.6945\end{array}$

$\begin{array}{llllll}\text { Hydragard - LF } & 9 & 2.73586 & 0.03827 & 2.6567 & 2.8150\end{array}$

Std Error uses a pooled estimate of error variance

Means and Std Deviations

Level Number Mean Std Dev Std Err Mean Lower 95\% Upper 95\%

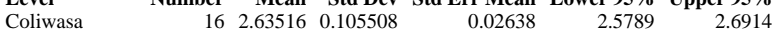

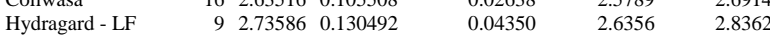

Tes

Hydragard - LF-Coliwasa

Assuming unequal variances

$\begin{array}{lrlr}\text { Difference } & 0.10071 & \text { t Ratio } & 1.979656 \\ \text { Std Err Dif } & 0.05087 & \text { DF } & 13.95871 \\ \text { Upper CL Dif } & 0.20984 & \text { Prob }>|t| & 0.0678 \\ \text { Lower CL Dif } & -0.00843 \text { Prob }>\text { t } & 0.0339 \\ \text { Confidence } & 0.95 & \text { Prob }<\mathrm{t} & 0.9661\end{array}$

Tests that the Variances are Equal

Level Count Std Dev MeanAbsDif to Mean MeanAbsDif to Median

Coliwasa $\quad 16 \quad 0.1055080$

$\begin{array}{lrrrr}\text { Coliwasa } & 16 & 0.1055080 & 0.0782704 & 0.0782704 \\ \text { Hydragard - LF } & 9 & 0.1304922 & 0.1034236 & 0.1025799\end{array}$

Test F Ratio DFNum DFDen p-Value

$\begin{array}{lrrrr}\text { O'Brien[.5] } & 0.5698 & 1 & 23 & 0.4580\end{array}$

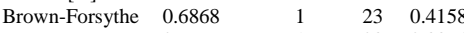

$\begin{array}{lllll}\text { Levene } & 0.7696 & 1 & 23 & 0.3894\end{array}$

$\begin{array}{lllll}\text { Bartlett } & 0.4658 & 1 & 0.4949\end{array}$

$\begin{array}{lllll}\text { F Test 2-sided } & 1.5297 & 8 & 15 & 0.4551\end{array}$

Welch Anova testing Means Equal, allowing Std Devs Not Equal

F Ratio DFNum DFDen Prob $>$ F

$\begin{array}{rrrr}3.9190 & 1 & 13.959 & 0.0678\end{array}$

t Test
1.9797
Oneway Analysis of Measurement By Sampler Type of Material=SME Simulant, Analytical Block =6, Oxide/Analyte $=\mathrm{Al} 2 \mathrm{O} 3$ (wt\%), Targeted $=10.869$

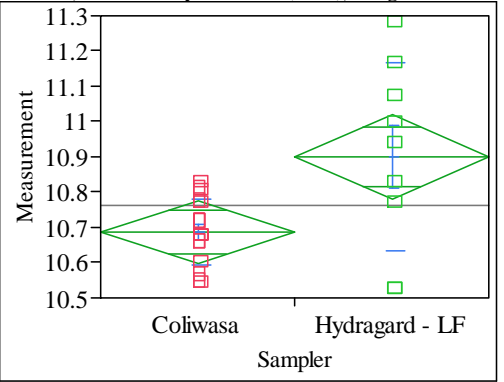

Excluded Rows

Oneway Anova

Summary of Fit

Rsquare $\quad 0.273971$

Adj Rsquare $\quad 0.242404$

Root Mean Square Error $\quad 0.173383$

Mean of Response 10.7641

Observations (or Sum Wgts) $\quad 25$

t Test

Hydragard - LF-Coliwasa

Assuming equal variances

Difference $\quad 0.212831$ t Ratio 2.946044

$\begin{array}{lrlr}\text { Std Err Dif } & 0.072243 & \text { DF } & 23 \\ \text { Upper CL Dif } & 0.362277 & \text { Prob }>|t| & 0.0073\end{array}$

Lower CL Dif 0.063385 Prob $>t \quad 0.0036$

$\begin{array}{lrll} & & \\ \text { Confidence } & 0.95 & \text { Prob }<\mathrm{t} & 0.9964\end{array}$

Analysis of Variance

Source DF Sum of Squares Mean Square F Ratio Prob $>$ F

$\begin{array}{llllll}\text { Sampler } & 1 & 0.26091136 & 0.260911 & 8.6792 & 0.0073\end{array}$

$\begin{array}{llll}\text { Error } & 23 & 0.69142080 & 0.030062\end{array}$

C. Total $24 \quad 0.95233216$

Means for Oneway Anova

Level Number Mean Std Error Lower 95\% Upper 95\%

$\begin{array}{lrrrrr}\text { Coliwasa } & 16 & 10.6875 & 0.04335 & 10.598 & 10.777\end{array}$

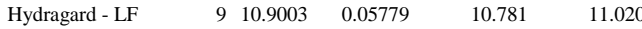

Std Error uses a pooled estimate of error variance

Means and Std Deviations

Level Number Mean Std Dev Std Err Mean Lower 95\% Upper 95\%

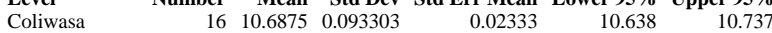

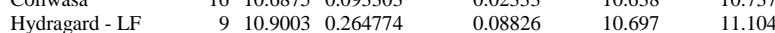

t Test

Hydragard - LF-Coliwasa

Assuming unequal variances

$\begin{array}{lrlr}\text { Difference } & 0.212831 & \mathrm{t} \text { Ratio } & 2.331421 \\ \text { Std Err Dif } & 0.091288 & \mathrm{DF} & 9.132851 \\ \text { Upper CL Dif } & 0.418882 & \text { Prob }>|t| & 0.0442 \\ \text { Lower CL Dif } & 0.006780 & \text { Prob }>\mathrm{t} & 0.0221 \\ \text { Confidence } & 0.95 & \text { Prob }<\mathrm{t} & 0.9779\end{array}$

Tests that the Variances are Equal

Level Count Std Dev MeanAbsDif to Mean MeanAbsDif to Median

\begin{tabular}{lr|rrr} 
Coliwasa & 16 & 0.0933025 & 0.0746943 & 0.0732181
\end{tabular}

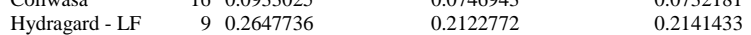

Test F Ratio DFNum DFDen p-Value

$\begin{array}{lllll}\text { O'Brien[.5] } & 10.4279 & 1 & 23 & 0.0037\end{array}$

Brown-Forsythe $12.3700 \quad 1 \quad 23 \quad 0.0018$

$\begin{array}{lllll}\text { Levene } & 12.7555 & 1 & 23 & 0.0016\end{array}$

$\begin{array}{lrrrr}\text { Bartlett } & 11.2596 & 1 & & 0.0008 \\ \text { F Test 2-sided } & 8.0531 & 8 & 15 & 0.0006\end{array}$

Welch Anova testing Means Equal, allowing Std Devs Not Equal

F Ratio DFNum DFDen Prob $>$ F

$\begin{array}{rrrr}5.4355 & 1 & 9.1329 & 0.0442\end{array}$

t Test 
Revision 0

\section{Exhibit C3. Statistical Comparisons for Low-Rheology (Phase 3) Simulant Testing (screened data)}

Oneway Analysis of Measurement By Sampler Type of Material=SME Simulant, Analytical Block=6, Oxide/Analyte $=$ B2O3 $(w t \%)$, Targeted $=4.259$

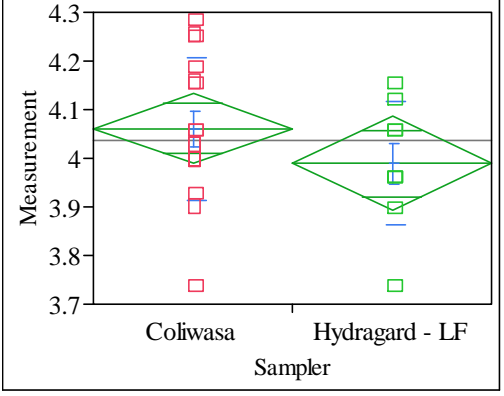

Excluded Rows

Oneway Anova

Summary of Fit

Root Mean Square Error $\quad 0.139973$

Mean of Response 4.035179

Observations (or Sum Wgts)

Test

Hydragard - LF-Coliwasa

Assuming equal variances

Difference $\quad-0.07200$ t Ratio $\quad-1.23454$

$\begin{array}{lrr}\text { Std Err Dif } & 0.05832 \text { DF } & 23 \\ \text { Upper CL Dif } & 0.04865 \text { Prob }>|t| & 0.2295\end{array}$

Upper CL Dif 0.04865 Prob $>|t|-0.2295$

$\begin{array}{lrrr}\text { Lower CL Dif } & -0.19265 & \text { Prob }>t & 0.8853 \\ \text { Confidence } & 0.95 & \text { Prob }<t & 0.1147\end{array}$

Analysis of Variance

Source DF Sum of Squares Mean Square F Ratio Prob $>$ F

$\begin{array}{lrrrrr}\text { Sampler } & 1 & 0.02986029 & 0.029860 & 1.5241 & 0.2295\end{array}$

Error $\begin{array}{lll}23 & 0.45062300 & 0.019592\end{array}$

C. Total $24 \quad 0.48048328$

Means for Oneway Anova

Level Number Mean Std Error Lower 95\% Upper 95\%

\begin{tabular}{lrrrrr} 
Coliwasa & 16 & 4.06110 & 0.03499 & 3.9887 & 4.1335 \\
\hline
\end{tabular}

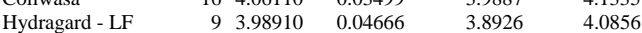

Std Error uses a pooled estimate of error variance

Means and Std Deviations

Level Number Mean Std Dev Std Err Mean Lower 95\% Upper 95\%

$\begin{array}{lllllll}\text { Coliwasa } & 16 & 4.06110 & 0.146320 & 0.03658 & 3.9831 & 4.1391\end{array}$

$\begin{array}{lllllll}\text { Hydragard - LF } & 9 & 3.98910 & 0.127221 & 0.04241 & 3.8913 & 4.0869\end{array}$

t Test

Hydragard - LF-Coliwasa

Assuming unequal variances

Difference $\quad-0.07200$ t Ratio $\quad-1.28563$

Std Err Dif $\quad 0.05600$ DF $\quad 18.78685$

Upper CL Dif 0.04531 Prob $>|\mathrm{t}| \quad 0.2142$

Lower CL Dif -0.18931 Prob $>t$

$\begin{array}{lrl} & & \\ \text { Confidence } & 0.95 \text { Prob }<\mathrm{t} & 0.1071\end{array}$

Tests that the Variances are Equal

$\begin{array}{lrrrr}\text { Level } & \text { Count } & \text { Std Dev } & \text { MeanAbsDif to Mean } & \text { MeanAbsDif to Median } \\ \text { Coliwasa } & 16 & 0.1463196 & 0.1137027 & 0.1126965\end{array}$

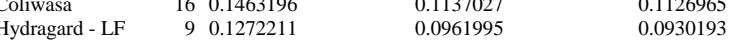

$\begin{array}{lrrr}\text { Test } & \text { F Ratio DFNum DFDen p-Value } \\ \text { O'Brien[5] } & 0.1918 & 1 & \text { p }\end{array}$

$\begin{array}{lllll}\text { O'Brien[.5] } & 0.1918 & 1 & 23 & 0.6655 \\ \text { Brown-Forsythe } & 0.2797 & 1 & 23 & 0.6020\end{array}$

$\begin{array}{lllll}\text { Brown-Forsythe } & 0.2797 & 1 & 23 & 0.6020 \\ \text { Levene } & 0.2529 & 1 & 23 & 0.6198\end{array}$

$\begin{array}{lrrrr}\text { Levene } & 0.2529 & 1 & 23 & 0.6198 \\ \text { Bartlett } & 0.1886 & 1 & . & 0.6641\end{array}$

$\begin{array}{lllll}\text { F Test 2-sided } & 1.3228 & 15 & 8 & 0.7103\end{array}$

Welch Anova testing Means Equal, allowing Std Devs Not Equal

F Ratio DFNum DFDen Prob $>$ F

$\begin{array}{llll}1.6529 & 1 & 18.787 & 0.2142\end{array}$

t Test

1.2856
Oneway Analysis of Measurement By Sampler Type of Material=SME Simulant, Analytical Block $=6$, Oxide $/$ Analyte $=\mathbf{B a O}(\mathrm{wt} \%)$, Targeted $=\mathbf{0 . 0 9 1 9}$

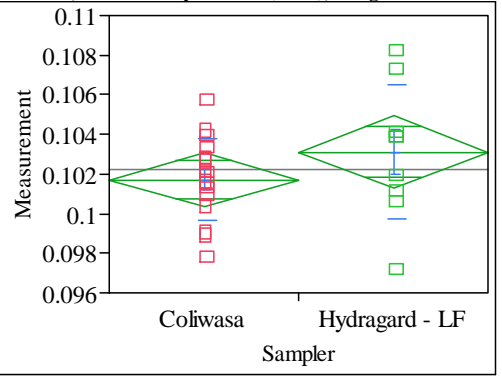

Excluded Rows

Oneway Anova

Summary of Fit

$\begin{array}{lr}\text { Rsquare } & 0.067511 \\ \text { Adj Rsquare } & 0.026968 \\ \text { Root Mean Square Error } & 0.00262 \\ \text { Mean of Response } & 0.102213 \\ \text { Observations (or Sum Wgts) } & 25\end{array}$

Test

Hydragard - LF-Coliwasa

Assuming equal variances

$\begin{array}{lrr}\text { Difference } & 0.00141 \text { t Ratio } & 1.290411 \\ \text { Std Err Dif } & 0.00109 & 23\end{array}$

Upper CL Dif 0.00367 Prob $>|t| \quad 0.2097$

Lower CL Dif -0.00085 Prob $>t$

0.95 Prob $<\mathrm{t} \quad 0.8951$

Analysis of Variance

Source DF Sum of Squares Mean Square F Ratio Prob $>$ F

$\begin{array}{llllll}\text { Sampler } & 1 & 0.00001143 & 0.000011 & 1.6652 & 0.2097\end{array}$

$\begin{array}{llll}\text { Error } & 23 & 0.00015791 & 6.865 \mathrm{e}-6\end{array}$

0.00016934

Means for Oneway Anova

$\begin{array}{lrrrrr}\text { Level } & \text { Number } & \text { Mean } & \text { Std Error } & \text { Lower 95\% } & \text { Upper 95\% } \\ \text { Coliwasa } & 16 & 0.101706 & 0.00066 & 0.10035 & 0.10306\end{array}$

$\begin{array}{lrrrrr}\text { Hydragard - LF } & 9 & 0.101706 & 0.00066 & 0.10035 & 0.10306 \\ & 9 & 0.103115 & 0.00087 & 0.10131 & 0.10492\end{array}$

Std Error uses a pooled estimate of error variance

Means and Std Deviations

Level Number Mean Std Dev Std Err Mean Lower 95\% Upper 95\%

$\begin{array}{lllllll}\text { Coliwasa } & 16 & 0.101706 & 0.002089 & 0.00052 & 0.10059 & 0.10282\end{array}$

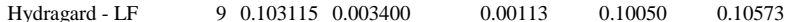

t Test

Hydragard - LF-Coliwasa

Assuming unequal variances

Difference $\quad 0.00141$ t Ratio 1.129038

Std Err Dif $\quad 0.00125$ DF $\quad 11.48092$

Upper CL Dif 0.00414 Prob $>|t| \quad 0.2819$

Lower CL Dif -0.00132 Prob $>\mathrm{t} \quad 0.1410$

$\begin{array}{lrl}\text { Confidence } & 0.95 \text { Prob }<\mathrm{t} & 0.8590\end{array}$

Tests that the Variances are Equal

Level Count Std Dev MeanAbsDif to Mean MeanAbsDif to Median

$\begin{array}{lrrrr} & 16 & 0.0020886 & 0.0015840 & 0.0015840 \\ \text { Hydragard - LF } & 9 & 0.0033999 & 0.0025859 & 0.0025059\end{array}$

Test F Ratio DFNum DFDen p-Value

$\begin{array}{lrrrr}\text { O'Brien[.5] } & 2.7590 & 1 & 23 & 0.1103\end{array}$

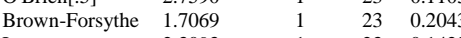

$\begin{array}{lllll}\text { Levene } & 2.3093 & 1 & 23 & 0.1422\end{array}$

$\begin{array}{lrrrr}\text { Bartlett } & 2.5113 & 1 & & 0.1130 \\ \text { F Test 2-sided } & 2.6499 & 8 & 15 & 0.0988\end{array}$

Welch Anova testing Means Equal, allowing Std Devs Not Equal

F Ratio DFNum DFDen Prob $>$ F

$1.2747 \quad 1 \quad 11.481 \quad 0.2819$

t Test

1.1290 
Revision 0

\section{Exhibit C3. Statistical Comparisons for Low-Rheology (Phase 3) Simulant Testing (screened data)}

Oneway Analysis of Measurement By Sampler Type of Material=SME Simulant, Analytical Block=6, Oxide $/$ Analyte $=\mathbf{C a O}($ wt $\%)$, Targeted $=1.1462$

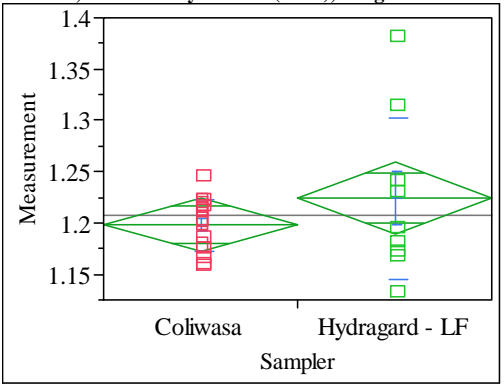

Exc

Exclue Rows

Oneway Anova

Summary of Fit

Adj Rsquare

Root Mean Square Error $\quad 0.050753$

Mean of Response Eror 1.207342

Observations (or Sum Wgts) $\quad 25$

t Test

Hydragard - LF-Coliwasa

Assuming equal variances

Difference $\quad 0.02601$ t Ratio 1.23002

Std Err Dif $\quad 0.02115$ DF 23

Upper CL Dif 0.06976 Prob $>|t| \quad 0.2311$

Lower CL Dif -0.01773 Prob $>\mathrm{t} \quad 0.1156$

$\begin{array}{lrl}\text { Confidence } & 0.95 \text { Prob }<\mathrm{t} & 0.8844\end{array}$

Analysis of Variance

Source DF Sum of Squares Mean Square F Ratio Prob $>$ F

$\begin{array}{llllll}\text { Sampler } & 1 & 0.00389721 & 0.003897 & 1.5129 & 0.2311\end{array}$

$\begin{array}{llll}\text { Error } & 23 & 0.05924574 & 0.002576\end{array}$

C. Total $24 \quad 0.06314295$

Means for Oneway Anova

Level Number Mean Std Error Lower 95\% Upper 95\%

$\begin{array}{lrrrrr}\text { Coliwasa } & 16 & 1.19798 & 0.01269 & 1.1717 & 1.2242 \\ \text { Hydragard - LF } & 9 & 1.22399 & 0.01692 & 1.1890 & 1.2590\end{array}$

Std Error uses a pooled estimate of error variance

$\begin{array}{llllll}\begin{array}{l}\text { Means and Std Deviations } \\ \text { Level }\end{array} \text { Number } & \text { Mean } & \text { Std Dev Std Err Mean Lower 95\% Upper 95\% }\end{array}$

$\begin{array}{lrrrrrr}\text { Level } & \text { Number } & \text { Mean } & \text { Std Dev } & \text { Std Err Mean } & \text { Lower 95\% } & \text { Upper 95\% } \\ \text { Coliwasa } & 16 & 1.19798 & 0.025202 & 0.00630 & 1.1845 & 1.2114\end{array}$

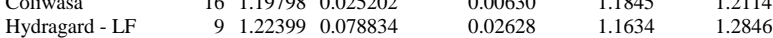

Tes

Hydragard - LF-Coliwasa

Assuming unequal variances

$\begin{array}{lrlr}\text { Difference } & 0.02601 & \text { t Ratio } & 0.962577 \\ \text { Std Err Dif } & 0.02702 & \text { DF } & 8.930501 \\ \text { Upper CL Dif } & 0.08721 & \text { Prob }>|t| & 0.3611 \\ \text { Lower CL Dif } & -0.03519 & \text { Prob }>\text { t } & 0.1805 \\ \text { Confidence } & 0.95 & \text { Prob }<\mathrm{t} & 0.8195\end{array}$

Tests that the Variances are Equal

Level Count Std Dev MeanAbsDif to Mean MeanAbsDif to Median

Coliwasa $\quad 160.0252023$

$\begin{array}{lrrrr}\text { Coliwasa } & 16 & 0.0252023 & 0.0206929 & 0.0202009 \\ \text { Hydragard - LF } & 9 & 0.0788340 & 0.0599756 & 0.0583000\end{array}$

Test F Ratio DFNum DFDen p-Value

$\begin{array}{lllll}\text { O'Brien[.5] } & 5.7687 & 1 & 23 & 0.0248\end{array}$

$\begin{array}{lllll}\text { Brown-Forsythe } & 6.3522 & 1 & 23 & 0.0191\end{array}$

Levene $\quad \begin{array}{llll}10.2110 & 1 & 23 & 0.0040\end{array}$

$\begin{array}{lrrrr}\text { Bartlett } & 13.2985 & 1 & & 0.0003 \\ \text { F Test 2-sided } & 9.7847 & 8 & 15 & 0.0002\end{array}$

Welch Anova testing Means Equal, allowing Std Devs Not Equal

F Ratio DFNum DFDen Prob > F

$\begin{array}{rrrr}0.9266 & 1 & 8.9305 & 0.3611\end{array}$

t Test
0.9626
Oneway Analysis of Measurement By Sampler Type of Material=SME Simulant, Analytical Block $=6$, Oxide $/$ Analyte $=\mathrm{Cr} 2 \mathrm{O} 3(\mathrm{wt} \%)$, Targeted $=0.0998$

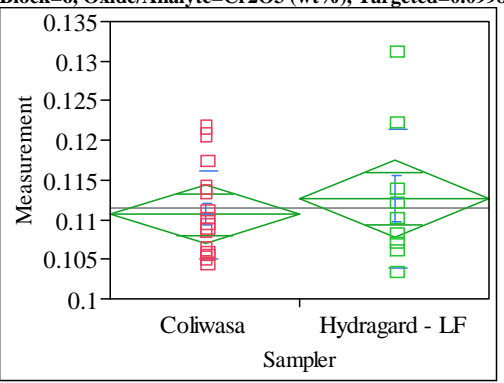

Excluded Rows

Oneway Anova

Summary of Fit

$\begin{array}{lr}\text { Adj Rsquare } & -0.02479 \\ \text { Root Mean Square Error } & 0.006916\end{array}$

Mean of Response

Observations (or Sum Wgts)

t Test

Hydragard - LF-Coliwasa

Assuming equal variances

Difference $\quad 0.00194$ t Ratio 0.666053

$\begin{array}{lrlr}\text { Std Err Dif } & 0.00292 & \text { DF } & 22 \\ \text { Upper CL } & 0.00799 & \text { Prob }>|t| & 0.5123\end{array}$

\begin{tabular}{lll}
$0.95 \quad$ Prob $<\mathrm{t}$ & 0.2562 \\
\hline
\end{tabular}

Analysis of Variance

Source DF Sum of Squares Mean Square F Ratio Prob $>$ F

$\begin{array}{llllll}\text { Sampler } & 1 & 0.00002122 & 0.000021 & 0.4436 & 0.5123\end{array}$

$\begin{array}{llll}\text { Error } & 22 & 0.00105235 & 0.000048 \\ \text { C. Total } & 23 & 0.00107357 & \end{array}$

Means for Oneway Anova

$\begin{array}{lrrrrr}\text { Level } & \text { Number } & \text { Mean } & \text { Std Error } & \text { Lower 95\% } & \text { Upper 95\% } \\ \text { Coliwasa } & 15 & 0.110682 & 0.00179 & 0.10698 & 0.11439\end{array}$

$\begin{array}{lrrrrr}\text { Hydragard - LF } & 15 & 0.110682 & 0.00179 & 0.10698 & 0.11439 \\ & 9 & 0.112624 & 0.00231 & 0.10784 & 0.11741\end{array}$

Std Error uses a pooled estimate of error variance

Means and Std Deviations

Level Number Mean Std Dev Std Err Mean Lower 95\% Upper 95\%

$\begin{array}{lllllll}\text { Coliwasa } & 15 & 0.110682 & 0.005547 & 0.00143 & 0.10761 & 0.11375\end{array}$

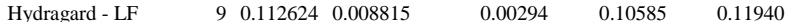

t Test

Hydragard - LF-Coliwasa

Assuming unequal variances

Difference $\quad 0.00194$ t Ratio 0.594194

Std Err Dif $\quad 0.00327$ DF 11.86926

Upper CL Dif 0.00907 Prob $>|t| \quad 0.5635$

Lower CL Dif -0.00519 Prob $>t \quad 0.2818$

$\begin{array}{lrl}\text { Confidence } & 0.95 \text { Prob }<\mathrm{t} & 0.7182\end{array}$

Tests that the Variances are Equal

Level Count Std Dev MeanAbsDif to Mean MeanAbsDif to Median

$\begin{array}{lrrrr} & 15 & 0.0055465 & 0.0044121 & 0.0042971 \\ \text { Hydragard - LF } & 9 & 0.0088152 & 0.0064743 & 0.0062524\end{array}$

$\begin{array}{lrrrr}\text { Test } & \text { F Ratio } & \text { DFNum } & \text { DFDen } & \text { p-Value } \\ \text { O'Brien[.5] } & 1.7502 & 1 & 22 & 0.1994 \\ \text { Brown-Forsythe } & 0.9200 & 1 & 22 & 0.3479 \\ \text { Levene } & 1.3738 & 1 & 22 & 0.2537 \\ \text { Bartlett } & 2.1879 & 1 & & 0.1391 \\ \text { F Test 2-sided } & 2.5259 & 8 & 14 & 0.1239\end{array}$

Welch Anova testing Means Equal, allowing Std Devs Not Equal

F Ratio DFNum DFDen Prob $>$ F

$\begin{array}{llll}0.3531 & 1 & 11.869 & 0.5635\end{array}$

t Test

0.5942 
SRNL-STI-2011-00693

Revision 0

\section{Exhibit C3. Statistical Comparisons for Low-Rheology (Phase 3) Simulant Testing (screened data)}

Oneway Analysis of Measurement By Sampler Type of Material=SME Simulant, Analytical Block $=6$, Oxide $/$ Analyte $=\mathrm{CuO}(\mathrm{wt} \%)$, Targeted $=\mathbf{0 . 0 5 0 4}$

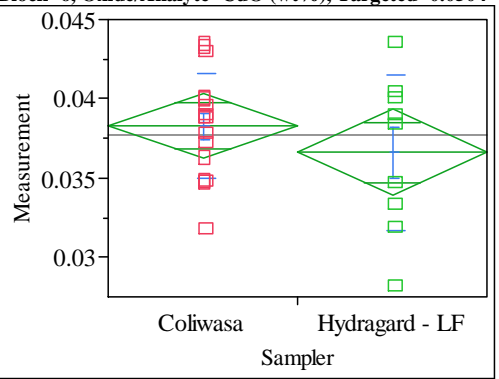

Excluded Rows

Oneway Anova

Summary of Fit

Adj Rsquare

Root Mean Square Error $\quad 0.000797$

Mean of Response $\quad 0.003941$

Observations (or Sum Wgts) 25

t Test

Hydragard - LF-Coliwasa

Assuming equal variances

Difference $\quad-0.00166$ t Ratio -1.00953

$\begin{array}{lrr}\text { Std Err Dif } & 0.00164 \text { DF } & 23 \\ & \end{array}$

Upper CL Dif 0.00174 Prob $>|t|-0.3232$

Lower CL Dif -0.00505 Prob $>\mathrm{t} \quad 0.8384$

Confidence $\quad 0.95$ Prob $<\mathrm{t} \quad 0.1616$

Analysis of Variance

Source DF Sum of Squares Mean Square F Ratio Prob $>$ F

$\begin{array}{llllll}\text { Sampler } & 1 & 0.00001583 & 0.000016 & 1.0191 & 0.3232\end{array}$

Error $\begin{array}{lll}23 & 0.00035724 & 0.000016\end{array}$

C. Total $24 \quad 0.00037307$

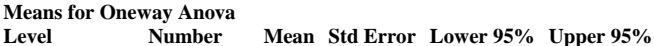

$\begin{array}{lrrrrr}\text { Coliwasa } & 16 & 0.038266 & 0.00099 & 0.03623 & 0.04030\end{array}$

$\begin{array}{lrrrrr}\text { Hydragard - LF } & 16 & 0.038266 & 0.00099 & 0.03623 & 0.04030 \\ & 9 & 0.036608 & 0.00131 & 0.03389 & 0.03933\end{array}$

Std Error uses a pooled estimate of error variance

Means and Std Deviations

$\begin{array}{lllllll}\text { Level Number Mean } & \text { Std Dev } & \text { Std Err Mean Lower 95\% Upper 95\% }\end{array}$

$\begin{array}{llllll}16 & 0.038266 & 0.003324 & 0.00083 & 0.03649 & 0.04004\end{array}$

$\begin{array}{lllllll} & 9 & 0.036608 & 0.004893 & 0.00163 & 0.03285 & 0.04037\end{array}$

t Test

Hydragard - LF-Coliwasa

Assuming unequal variances

Difference $\quad-0.00166$ t Ratio $\quad-0.90562$

Std Err Dif $\quad 0.00183$ DF 12.25053

Upper CL Dif 0.00232 Prob $>|t| \quad 0.3826$

Lower CL Dif -0.00564 Prob $>\mathrm{t} \quad 0.8087$

$\begin{array}{lrl}\text { Confidence } & 0.95 \mathrm{Prob}<\mathrm{t} & 0.1913\end{array}$

Tests that the Variances are Equal

Level Count Std Dev MeanAbsDif to Mean MeanAbsDif to Median

$\begin{array}{lrrrr}\text { Coliwasa } & 16 & 0.0033235 & 0.0026102 & 0.0025427 \\ \text { Hydragard - LF } & 9 & 0.0048933 & 0.0040830 & 0.0039362\end{array}$

$\begin{array}{lrrrr}\text { Test } & \text { F Ratio } & \text { DFNum } & \text { DFDen } & \text { p-Value } \\ \text { O'Brien[.5] } & 2.4173 & 1 & 23 & 0.1337 \\ \text { Brown-Forsythe } & 1.7249 & 1 & 23 & 0.2020 \\ \text { Levene } & 2.9268 & 1 & 23 & 0.1006 \\ \text { Bartlett } & 1.5728 & 1 & & 0.2098 \\ \text { F Test 2-sided } & 2.1677 & 8 & 15 & 0.1870\end{array}$

Welch Anova testing Means Equal, allowing Std Devs Not Equal

F Ratio DFNum DFDen Prob $>$ F

$\begin{array}{llll}0.8202 & 1 & 12.251 & 0.3826\end{array}$

t Test

0.9056
Oneway Analysis of Measurement By Sampler Type of Material=SME Simulant, Analytical Block $=6$, Oxide $/$ Analyte $=\mathrm{Fe} / \mathrm{Li}$, Targeted $=2.452$

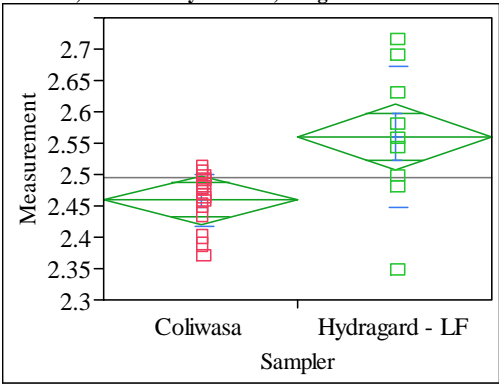

Excluded Rows

1

Oneway Anova

Summary of Fit

Rsquare $\quad 0.314619$

Adj Rsquare $\quad 0.28482$

Root Mean Square Error $\quad 0.074781$

Mean of Response 2.49539

Observations (or Sum Wgts) $\quad 25$

t Test

Hydragard - LF-Coliwasa

Assuming equal variances

Difference $\quad 0.101244$ t Ratio 3.249307

Std Err Dif $\quad 0.031159$ DF 23

Upper CL Dif 0.165701 Prob $>|t| \quad 0.0035$

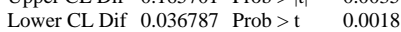

$\begin{array}{lrl}\text { Confidence } & 0.95 \text { Prob }<\mathrm{t} & 0.9982\end{array}$

Analysis of Variance

Source DF Sum of Squares Mean Square F Ratio Prob $>$ F

$\begin{array}{llllll}\text { Sampler } & 1 & 0.05904196 & 0.059042 & 10.5580 & 0.0035\end{array}$

$\begin{array}{llll}\text { Error } & 23 & 0.12861958 & 0.005592\end{array}$

C. Total $24 \quad 0.18766154$

Means for Oneway Anova

Level $\quad$ Number Mean Std Error Lower 95\% Upper 95\%

$\begin{array}{lrrrrr}\text { Coliwasa } & 16 & 2.45894 & 0.01870 & 2.4203 & 2.4976 \\ \text { Hydragard - LF } & 9 & 2.56019 & 0.02493 & 2.5086 & 2.6118\end{array}$

Std Error uses a pooled estimate of error variance

Means and Std Deviations

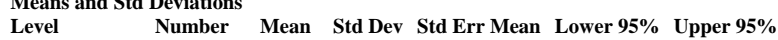

$\begin{array}{lrrrrrr}\text { Level } & \text { Number } & \text { Mean } & \text { Std Dev } & \text { Std Err Mean } & \text { Lower 95\% } & \text { Upper 95\% } \\ \text { Coliwasa } & 16 & 2.45894 & 0.042118 & 0.01053 & 2.4365 & 2.4814\end{array}$

$\begin{array}{lrrrrrr}\text { Hydragard - LF } & 9 & 2.56019 & 0.112922 & 0.03764 & 2.4734 & 2.6470\end{array}$

t Test

Hydragard - LF-Coliwasa

Assuming unequal variances

$\begin{array}{lrlr}\text { Difference } & 0.101244 & \text { t Ratio } & 2.590315 \\ \text { Std Err Dif } & 0.039086 & \text { DF } & 9.270779 \\ \text { Upper CL Dif } & 0.189270 & \text { Prob }>|t| & 0.0285 \\ \text { Lower CL Dif } & 0.013218 & \text { Prob }>t & 0.0143 \\ \text { Confidence } & 0.95 & \text { Prob }<t & 0.9857\end{array}$

Tests that the Variances are Equal

Level Count Std Dev MeanAbsDif to Mean MeanAbsDif to Median

$\begin{array}{lrrrr}\text { Coliwasa } & 16 & 0.0421182 & 0.0326870 & 0.0313272\end{array}$

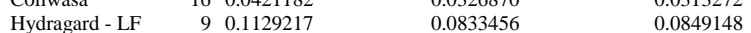

Test F Ratio DFNum DFDen p-Value

$\begin{array}{lllll}\text { O'Brien[.5] } & 5.8101 & 1 & 23 & 0.0243\end{array}$

Brown-Forsythe $7.5412 \quad 1 \quad 23 \quad 0.0115$

$\begin{array}{lllll}\text { Levene } & 6.9376 & 1 & 23 & 0.0148\end{array}$

$\begin{array}{lrrrr}\text { Bartlett } & 10.1280 & 1 & & 0.0015 \\ \text { F Test 2-sided } & 7.1881 & 8 & 15 & 0.0011\end{array}$

Welch Anova testing Means Equal, allowing Std Devs Not Equal

F Ratio DFNum DFDen Prob $>$ F

$\begin{array}{rrrr}6.7097 & 1 & 9.2708 & 0.0285\end{array}$

t Test 
Revision 0

\section{Exhibit C3. Statistical Comparisons for Low-Rheology (Phase 3) Simulant Testing (screened data)}

Oneway Analysis of Measurement By Sampler Type of Material=SME Simulant, Analytical Block=6, Oxide/Analyte=Fe2O3 (wt $\%$ ), Targeted $=11.462$

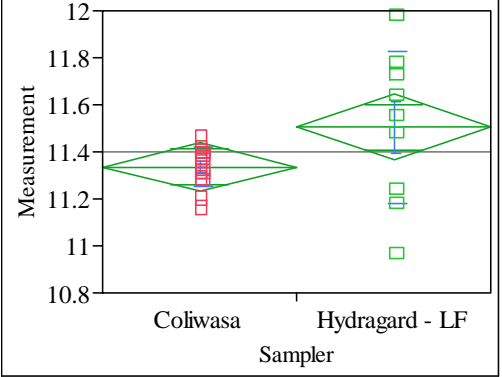

Exc

Oneway Anova

Summary of Fit

Adj Rsquare 0.110463

Root Mean Square Error $\quad 0.201727$

(O)

$\begin{array}{lr} & 11.397 \\ \text { Observations (or Sum Wgts) } & 25\end{array}$

t Test

Hydragard - LF-Coliwasa

Assuming equal variances

Difference $\quad 0.16769$ t Ratio 1.995078

Std Err Dif $\quad 0.08405$ DF 23

Upper CL Dif 0.34157 Prob $>|t| \quad 0.0580$

$\begin{array}{rrr}\text { Lower CL Dif }-0.00618 \text { Prob }>t & 0.0290\end{array}$

$\begin{array}{lrl} & \\ \text { Confidence } & 0.95 \text { Prob }<\mathrm{t} & 0.9710\end{array}$

Analysis of Variance

Source DF Sum of Squares Mean Square F Ratio Prob $>$ F

$\begin{array}{llllll}\text { Sampler } & 1 & 0.1619745 & 0.161974 & 3.9803 & 0.0580\end{array}$

Error $23 \quad 0.9359541-0.040694$

C. Total $24 \quad 1.0979286$

Means for Oneway Anova

Level Number Mean Std Error Lower 95\% Upper 95\%

$\begin{array}{lrrrrr}\text { Coliwasa } & 16 & 11.3366 & 0.05043 & 11.232 & 11.441 \\ \text { Hydragard - LF } & 9 & 11.5043 & 0.06724 & 11.365 & 11.643\end{array}$

Std Error uses a pooled estimate of error variance

Means and Std Deviations

Level N Number Mean Std Dev Std Err Mean Lower 95\% Upper 95\%

$\begin{array}{lrrrrrr}\text { Level } & \text { Number } & \text { Mean } & \text { Std Dev } & \text { Std Err Mean } & \text { Lower 95\% } & \text { Upper 95\% } \\ \text { Coliwasa } & 16 & 11.3366 & 0.080618 & 0.02015 & 11.294 & 11.380\end{array}$

$\begin{array}{lrrrrrr}\text { Hydragard - LF } & 9 & 11.5043 & 0.323741 & 0.10791 & 11.255 & 11.753\end{array}$

Tes

Hydragard - LF-Coliwasa

Assuming unequal variances

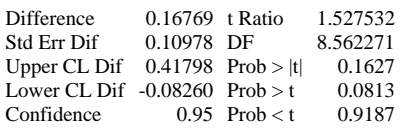

Tests that the Variances are Equal

Level Count Std Dev MeanAbsDif to Mean MeanAbsDif to Median

Coliwasa $\quad 16 \quad 0.0806176$

$\begin{array}{lrrrr}\text { Coliwasa } & 16 & 0.0806176 & 0.0635546 & 0.0616558 \\ \text { Hydragard - LF } & 9 & 0.3237410 & 0.2562870 & 0.2589346\end{array}$

Test F Ratio DFNum DFDen p-Value

O'Brien[.5] $\quad 10.5923 \quad 1 \quad 23 \quad 0.0035$

Brown-Forsythe $16.8914 \quad 1 \quad 23 \quad 0.0004$

$\begin{array}{lllll}\text { Levene } & 17.5640 & 1 & 23 & 0.0003\end{array}$

$\begin{array}{lllll}\text { F Test 2-sided } & 16.1263 & 8 & 15 & <.0001 \\ & & & & \end{array}$

Welch Anova testing Means Equal, allowing Std Devs Not Equal

F Ratio DFNum DFDen Prob $>$ F

$\begin{array}{rrrr}2.3334 & 1 & 8.5623 & 0.1627\end{array}$

t Test
1.5275
Oneway Analysis of Measurement By Sampler Type of Material=SME Simulant, Analytical Block $=6$, Oxide/Analyte $=K 2 O(w t \%)$, Targeted $=\mathbf{0 . 1 5 9 1}$

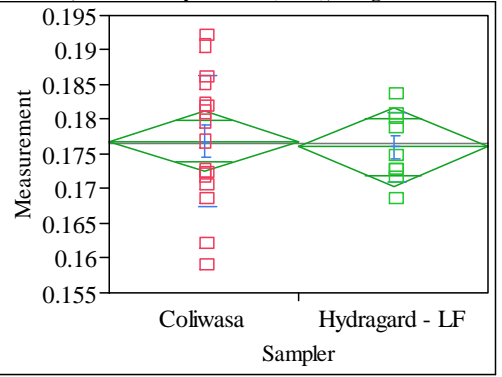

Excluded Rows

Oneway Anova

Summary of Fit

\begin{tabular}{lr} 
Rsquare & 0.002876 \\
Adj Rsquare & -0.04048 \\
Root Mean Square Error & 0.008223 \\
Mean of Response & 0.17657 \\
\hline
\end{tabular}

Observations (or Sum Wgts)

Test

Hydragard - LF-Coliwasa

Assuming equal variances

$\begin{array}{lrr}\text { Difference } & -0.00088 \text { t Ratio } & -0.25758 \\ \text { Std Err Dif } & 0.00343 \text { DF } & 23\end{array}$

$\begin{array}{lrlr}\text { Std Err Dif } & 0.00343 \text { DF } & 23 \\ \text { Upper CL } & 0.7990\end{array}$

Lower CL Dif -0.00797 Prob $>\mathrm{t} \quad 0.6005$

$\begin{array}{lrr}\text { Confidence } & 0.95 \text { Prob }<\mathrm{t} & 0.3995\end{array}$

Analysis of Variance

Source DF Sum of Squares Mean Square F Ratio Prob $>$ F

$\begin{array}{llllll}\text { Sampler } & 1 & 0.00000449 & 4.486 \mathrm{e}-6 & 0.0663 & 0.7990\end{array}$

$\begin{array}{llll}\text { Error } & 23 & 0.00155517 & 0.000068\end{array}$

C. Total $24 \quad 0.00155966$

Means for Oneway Anova $\quad$ Mean Std Error Lower 95\% Upper 95\%

$\begin{array}{lrrrrr}\text { Level } & \text { Number } & \text { Mean } & \text { Std Error } & \text { Lower 95\% } & \text { Upper 95\% } \\ \text { Coliwasa } & 16 & 0.176888 & 0.00206 & 0.17264 & 0.18114\end{array}$

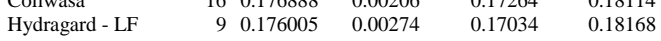

Std Error uses a pooled estimate of error variance

Means and Std Deviations

Level Number Mean Std Dev Std Err Mean Lower 95\% Upper 95\%

$\begin{array}{lllllll}\text { Coliwasa } & 16 & 0.176888 & 0.009502 & 0.00238 & 0.17182 & 0.18195\end{array}$

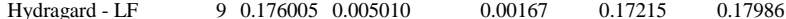

t Test

Hydragard - LF-Coliwasa

Assuming unequal variances

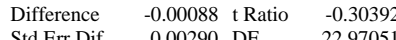

Std Err Dif $\quad 0.00290$ DF 22.97051

Upper CL Dif 0.00512 Prob $>|t| 0.7639$

Lower CL Dif -0.00689 Prob $>\mathrm{t} \quad 0.6180$

$\begin{array}{lrl}\text { Confidence } & 0.95 \text { Prob }<\mathrm{t} & 0.3820\end{array}$

Tests that the Variances are Equal

Level Count Std Dev MeanAbsDif to Mean MeanAbsDif to Median

$\begin{array}{lrrrr} & 16 & 0.0095021 & 0.0077923 & 0.0077923 \\ \text { Hydragard - LF } & 9 & 0.0050101 & 0.0042979 & 0.0043499\end{array}$

$\begin{array}{lrrrr}\text { Test } & \text { F Ratio } & \text { DFNum } & \text { DFDen } & \text { p-Value } \\ \text { O'Brien[.5] } & 3.1173 & 1 & 23 & 0.0907 \\ \text { Brown-Forsythe } & 3.4744 & 1 & 23 & 0.0751 \\ \text { Levene } & 3.8754 & 1 & 23 & 0.0612 \\ \text { Bartlett } & 3.4206 & 1 & . & 0.0644 \\ \text { F Test 2-sided } & 3.5970 & 15 & 8 & 0.0733\end{array}$

Welch Anova testing Means Equal, allowing Std Devs Not Equal

F Ratio DFNum DFDen Prob $>$ F

$\begin{array}{llll}0.0924 & 1 & 22.971 & 0.7639\end{array}$

t Test

0.3039 
SRNL-STI-2011-00693

Revision 0

\section{Exhibit C3. Statistical Comparisons for Low-Rheology (Phase 3) Simulant Testing (screened data)}

Oneway Analysis of Measurement By Sampler Type of Material=SME Simulant, Analytical Block=6, Oxide/Analyte $=\mathrm{Li} 2 \mathrm{O}(\mathrm{wt} \%)$, Targeted $=4.674$

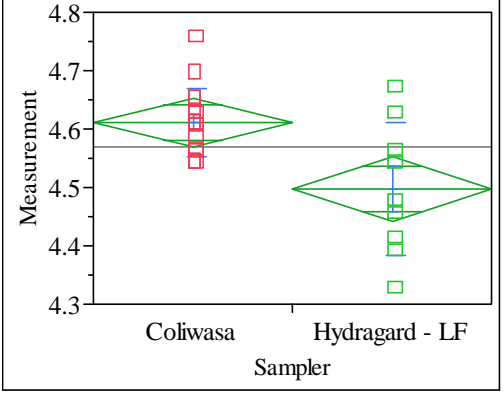

Excluded Rows

Oneway Anova

Summary of Fit

Rsquare

Ad Rs

Root Mean Square Error $\quad 0.081959$

Mean of Response 4.570176

Observations (or Sum Wgts)

Test

Hydragard - LF-Coliwasa

Assuming equal variances

Difference $\quad-0.11407$ t Ratio $\quad-3.34043$

$\begin{array}{lrr}\text { Std Err Dif } & 0.03415 \text { DF } & 23 \\ \text { Dif } & 0.0028\end{array}$

$\begin{array}{rrr}\text { Upper CL Dif }-0.04343 \text { Prob }>|t| & 0.0028 \\ \text { Cower CL Dif }-0.18472 & \text { Prob }>t & 0.9986\end{array}$

$\begin{array}{lrl} & \\ \text { Confidence } & 0.95 \text { Prob }<\mathrm{t} & 0.0014\end{array}$

Analysis of Variance

Source DF Sum of Squares Mean Square F Ratio Prob > F

$\begin{array}{lrrrrr}\text { Sampler } & 1 & 0.07495391 & 0.074954 & 11.1585 & 0.0028\end{array}$

Error $23 \quad 0.15449606 \quad 0.006717$

C. Total $24 \quad 0.22944997$

Means for Oneway Anova

Level Number Mean Std Error Lower 95\% Upper 95\%

$\begin{array}{lrrrrr}\text { Coliwasa } & 16 & 4.61124 & 0.02049 & 4.5689 & 4.6536\end{array}$

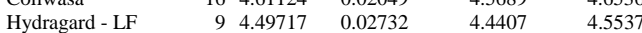

Std Error uses a pooled estimate of error variance

Means and Std Deviations

Level Number Mean Std Dev Std Err Mean Lower 95\% Upper 95\%

$\begin{array}{lllllll}\text { Coliwasa } & 16 & 4.61124 & 0.058417 & 0.01460 & 4.5801 & 4.6424\end{array}$

$\begin{array}{lrrrrrr}\text { Hydragard - LF } & 9 & 4.49717 & 0.113638 & 0.03788 & 4.4098 & 4.5845\end{array}$

t Test

Hydragard - LF-Coliwasa

Assuming unequal variances

Difference $\quad-0.11407$ t Ratio $\quad-2.8099$

Std Err Dif $\quad 0.04060$ DF $\quad 10.43214$

Upper CL Dif -0.02412 Prob $>|t| \quad 0.0178$

Lower CL Dif -0.20402 Prob $>t \quad 0.9911$

$\begin{array}{lrl} & & \\ \text { Confidence } & 0.95 \text { Prob }<\mathrm{t} & 0.0089\end{array}$

Tests that the Variances are Equal

$\begin{array}{lrrrr}\text { Level } & \text { Count } & \text { Std Dev } & \text { MeanAbsDif to Mean } & \text { MeanAbsDif to Median } \\ \text { Coliwasa } & 16 & 0.0584166 & 0.0427216 & 0.0417124\end{array}$

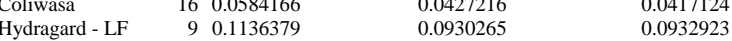

$\begin{array}{lrrrr}\text { Test } & \text { F Ratio } & \text { DFNum } & \text { DFDen } & \text { p-Value } \\ \text { O'Brien[.5] } & 5.7454 & 1 & 23 & 0.0250 \\ \text { Brown-Forsythe } & 6.7333 & 1 & 23 & 0.0162 \\ \text { Levene } & 7.0716 & 1 & 23 & 0.0140 \\ \text { Bartlett } & 4.6976 & 1 & . & 0.0302 \\ \text { F Test 2-sided } & 3.7842 & 8 & 15 & 0.0255\end{array}$

Welch Anova testing Means Equal, allowing Std Devs Not Equal

F Ratio DFNum DFDen Prob $>$ F

$\begin{array}{llll}7.8956 & 1 & 10.432 & 0.0178\end{array}$

t Test

2.8099
Oneway Analysis of Measurement By Sampler Type of Material=SME Simulant, Analytical Block=6, Oxide/Analyte $=\mathrm{MgO}$ (wt $\%$ ), Targeted $=1.014$

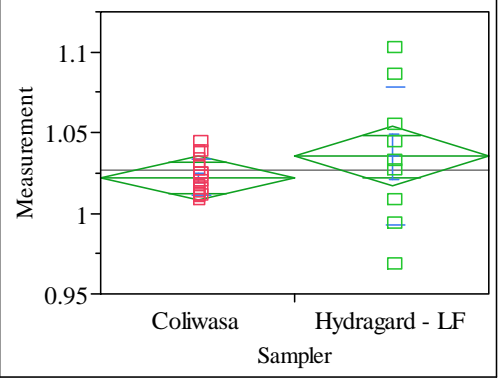

Excluded Rows

1

Oneway Anova

Summary of Fit

Rsquare $\quad 0.057319$

Adj Rsquare $\quad 0.016333$

Root Mean Square Error $\quad 0.026783$

Mean of Response 1.026886

Observations (or Sum Wgts) $\quad 25$

Hydragard - LF-Coliwasa

Assuming equal variances

Difference $\quad 0.01320$ t Ratio $\quad 1.18258$

Std Err Dif $\quad 0.01116$ DF 23

Upper CL Dif 0.03628 Prob $>|t| \quad 0.2491$

Lower CL Dif -0.00989 Prob $>t \quad 0.1245$

$\begin{array}{lrl}\text { Confidence } & 0.95 \text { Prob }<\mathrm{t} & 0.8755\end{array}$

Analysis of Variance

Source DF Sum of Squares Mean Square F Ratio Prob $>$ F

$\begin{array}{llllll}\text { Sampler } & 1 & 0.00100321 & 0.001003 & 1.3985 & 0.2491\end{array}$

$\begin{array}{llll}\text { Error } & 23 & 0.01649907 & 0.000717\end{array}$

C. Total $24 \quad 0.01750228$

Means for Oneway Anova

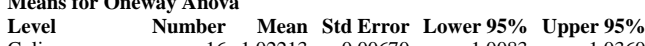

$\begin{array}{lrrrrr}\text { Coliwasa } & 16 & 1.02213 & 0.00670 & 1.0083 & 1.0360 \\ \text { Hydragard - LF } & 9 & 1.03533 & 0.00893 & 1.0169 & 1.0538\end{array}$

Std Error uses a pooled estimate of error variance

Means and Std Deviations

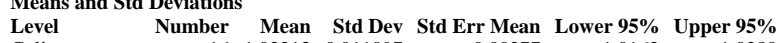

$\begin{array}{lrrrrrr}\text { Level } & \text { Number } & \text { Mean } & \text { Std Dev } & \text { Std Err Mean } & \text { Lower 95\% } & \text { Upper 95\% } \\ \text { Coliwasa } & 16 & 1.02213 & 0.011097 & 0.00277 & 1.0162 & 1.0280\end{array}$

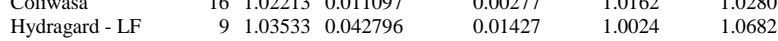

t Test

Hydragard - LF-Coliwasa

Assuming unequal variances

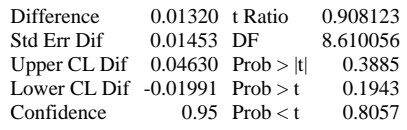

Tests that the Variances are Equal

Level Count Std Dev MeanAbsDif to Mean MeanAbsDif to Median

$\begin{array}{lrrrr}\text { Coliwasa } & 16 & 0.0110974 & 0.0091206 & 0.0091206\end{array}$

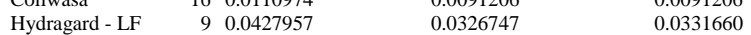

Test F Ratio DFNum DFDen p-Value

$\begin{array}{lrrrr}\text { O'Brien[.5] } & 9.6572 & 1 & 23 & 0.0050\end{array}$

Brown-Forsythe $\begin{array}{llll}14.2841 & 1 & 23 & 0.0010\end{array}$

$\begin{array}{lllll}\text { Levene } & 13.2206 & 1 & 23 & 0.0014\end{array}$

$\begin{array}{lllrr}\text { Bartlett } & 18.0424 & 1 & < & <.0001 \\ \text { F Test 2-sided } & 14.8716 & 8 & 15 & <.0001\end{array}$

Welch Anova testing Means Equal, allowing Std Devs Not Equal

F Ratio DFNum DFDen Prob $>$ F

$\begin{array}{rrrr}0.8247 & 1 & 8.6101 & 0.3885\end{array}$

t Test 
Revision 0

\section{Exhibit C3. Statistical Comparisons for Low-Rheology (Phase 3) Simulant Testing (screened data)}

Oneway Analysis of Measurement By Sampler Type of Material=SME Simulant, Analytical Block=6, Oxide/Analyte=MnO (wt \%), Targeted=1.779

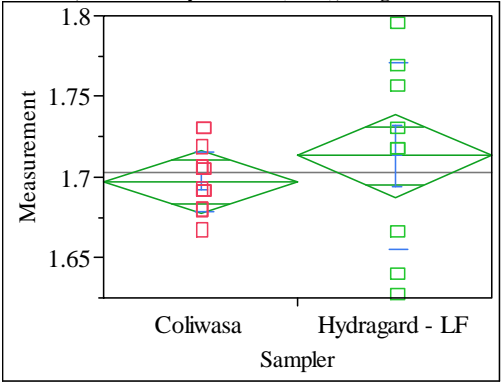

Exc

Oneway Anova

Summary of Fit

Rsquare $\quad 0.043374$

Adj Rsquare $\quad 0.00178$

Root Mean Square Error $\quad 0.0373$

Mean of Response 1.702835

Observations (or Sum Wgts) $\quad 25$

t Test

Hydragard - LF-Coliwasa

Assuming equal variances

Difference $\quad 0.01587$ t Ratio $\quad 1.021189$

Std Err Dif $\quad 0.01554$ DF 23

Upper CL Dif 0.04802 Prob $>|t| \quad 0.3178$

Lower CL Dif -0.01628 Prob $>t \quad 0.1589$
0.95

$\begin{array}{lrl}\text { Confidence } & 0.95 \text { Prob }<\mathrm{t} & 0.8411\end{array}$

Analysis of Variance

Source DF Sum of Squares Mean Square F Ratio Prob $>$ F

$\begin{array}{llllll}\text { Sampler } & 1 & 0.00145088 & 0.001451 & 1.0428 & 0.3178\end{array}$

$\begin{array}{llll}\text { Error } & 23 & 0.03199977 & 0.001391\end{array}$

C. Total $24 \quad 0.03345065$

Means for Oneway Anova

Level Number Mean Std Error Lower 95\% Upper 95\%

$\begin{array}{lrrrrr}\text { Coliwasa } & 16 & 1.69712 & 0.00933 & 1.6778 & 1.7164 \\ \text { Hydragard - LF } & 9 & 1.71299 & 0.01243 & 1.6873 & 1.7387\end{array}$

Std Error uses a pooled estimate of error variance

$\begin{array}{llllll}\begin{array}{l}\text { Means and Std Deviations } \\ \text { Level }\end{array} \text { Number } & \text { Mean } & \text { Std Dev Std Err Mean Lower 95\% } & \text { Upper 95\% }\end{array}$

$\begin{array}{lrrrrrr}\text { Level } & \text { Number } & \text { Mean } & \text { Std Dev } & \text { Std Err Mean } & \text { Lower 95\% } & \text { Upper 95\% } \\ \text { Coliwasa } & 16 & 1.69712 & 0.018241 & 0.00456 & 1.6874 & 1.7068\end{array}$

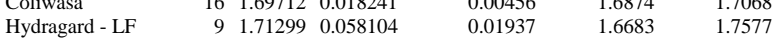

Test

Hydragard - LF-Coliwasa

Assuming unequal variances

$\begin{array}{lrlr}\text { Difference } & 0.01587 & \mathrm{t} \text { Ratio } & 0.797632 \\ \text { Std Err Dif } & 0.01990 & \text { DF } & 8.897041 \\ \text { Upper CL Dif } & 0.06096 & \text { Prob }>|\mathrm{t}| & 0.4458 \\ \text { Lower CL Dif } & -0.02922 & \text { Prob }>\mathrm{t} & 0.2229 \\ \text { Confidence } & 0.95 & \text { Prob }<\mathrm{t} & 0.7771\end{array}$

Tests that the Variances are Equal

Level Count Std Dev MeanAbsDif to Mean MeanAbsDif to Median

Coliwasa $\quad 16 \quad 0.0182413$

$\begin{array}{lrrrr}\text { Coliwasa } & 16 & 0.0182413 & 0.0144251 & 0.0137190 \\ \text { Hydragard - LF } & 9 & 0.0581040 & 0.0459093 & 0.0444747\end{array}$

Test F Ratio DFNum DFDen p-Value

$\begin{array}{lllll}\text { O'Brien[.5] } & 12.5004 & 1 & 23 & 0.0018\end{array}$

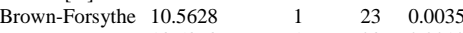

$\begin{array}{lllll}\text { Levene } & 13.5363 & 1 & 23 & 0.0012\end{array}$

$\begin{array}{lllll}\text { Bartlett } & 13.6911 & 1 & 0.0002\end{array}$

$\begin{array}{lllll}\text { F Test 2-sided } 10.1461 & 8 & 15 & 0.0002\end{array}$

Welch Anova testing Means Equal, allowing Std Devs Not Equal

F Ratio DFNum DFDen Prob $>$ F

$\begin{array}{rrrr}0.6362 & 1 & 8.897 & 0.4458\end{array}$

t Test
0.7976
Oneway Analysis of Measurement By Sampler Type of Material=SME Simulant, Analytical Block $=6$, Oxide $/$ Analyte $=\mathrm{Na2O}(\mathrm{wt} \%)$, Targeted $=\mathbf{1 1 . 6 5 9}$

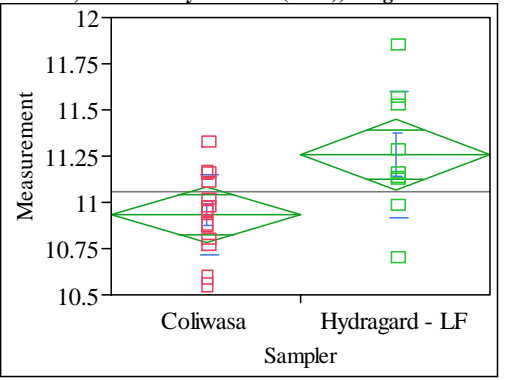

Excluded Rows

Oneway Anova

Summary of Fit

Adj Rsquare

Root Mean Square Error $\quad 0.272561$

Mean of Response 11.06005

Observations (or Sum Wgts) 23

t Test

Hydragard - LF-Coliwasa

Assuming equal variances

Difference $\quad 0.326516$ t Ratio 2.803889

$\begin{array}{lrlr}\text { Std Err Dif } & 0.116451 \text { DF } & 21 \\ \text { Upper CL } & 0.568689 & \text { Prob }>|t| & 0.0106\end{array}$

Upper CL Dif 0.568689 Prob $>$ t 0.0106

Lower CL Dif 0.084343 Prob $>\mathrm{t} \quad 0.0053$

Analysis of Variance

Source DF Sum of Squares Mean Square F Ratio Prob $>$ F

$\begin{array}{llllll}\text { Sampler } & 1 & 0.5840506 & 0.584051 & 7.8618 & 0.0106\end{array}$

$\begin{array}{llll}\text { Error } & 21 & 1.5600847 & 0.074290\end{array}$

Means for Oneway Anov

$\begin{array}{ccccc}\text { Means for } & \text { Oneway Anova } \\ \text { Level } & \text { Number } & \text { Mean Std Error } & \text { Lower 95\% Upper 95\% }\end{array}$

$\begin{array}{lrrrrr}\text { Level } & \text { Number } & \text { Mean } & \text { Std Error } & \text { Lower 95\% } & \text { Upper 95\% } \\ \text { Coliwasa } & 14 & 10.9323 & 0.07285 & 10.781 & 11.084\end{array}$

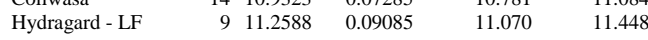

Std Error uses a pooled estimate of error variance

Means and Std Deviations

Level Number Mean Std Dev Std Err Mean Lower 95\% Upper 95\%

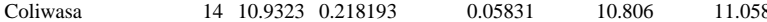

$\begin{array}{lllll}\text { Hydragard - LF } & 9 & 11.2588 & 0.342998 & 0.11433\end{array}$

Test

Hydragard - LF-Coliwasa

Assuming unequal variances

Difference $\quad 0.326516$ t Ratio 2.54404

Std Err Dif $\quad 0.128345$ DF $\quad 12.19579$

Upper CL Dif 0.605659 Prob $>|t| 0.0255$

Lower CL Dif 0.047372 Prob $>$ t 0.0127

$\begin{array}{lll}\text { Confidence } & 0.95 \text { Prob }<\mathrm{t} \quad 0.9873\end{array}$

Tests that the Variances are Equal

Level Count Std Dev MeanAbsDif to Mean MeanAbsDif to Median

$\begin{array}{lrrrrr}\text { Hydragard - LF } & 14 & 0.2181929 & & 0.1656114 & 0.1656114 \\ & 9 & 0.3429977 & 0.2639417 & 0.2561200\end{array}$

Test F Ratio DFNum DFDen p-Value

$\begin{array}{lllll} & 2.2299 & 1 & 21 & 0.1502\end{array}$

$\begin{array}{lllll}\text { Brown-Forsythe } & 1.3976 & 1 & 21 & 0.2503 \\ \text { Levene } & 2.0257 & 1 & 21 & 0.1693\end{array}$

$\begin{array}{llrrr}\text { Levene } & 2.0257 & 1 & 21 & 0.1693 \\ \text { Bartlett } & 2.0038 & 1 & . & 0.1569\end{array}$

$\begin{array}{lllll}\text { F Test 2-sided } & 2.4712 & 8 & 13 & 0.1422\end{array}$

Welch Anova testing Means Equal, allowing Std Devs Not Equal

F Ratio DFNum DFDen Prob $>$ F

$\begin{array}{llll}6.4721 & 1 & 12.196 & 0.0255\end{array}$

t Test

2.5440 
Revision 0

\section{Exhibit C3. Statistical Comparisons for Low-Rheology (Phase 3) Simulant Testing (screened data)}

Oneway Analysis of Measurement By Sampler Type of Material=SME Simulant, Analytical Block=6, Oxide/Analyte $=\mathrm{NiO}$ ( $w \mathrm{t} \%$ ), Targeted $=0.41$

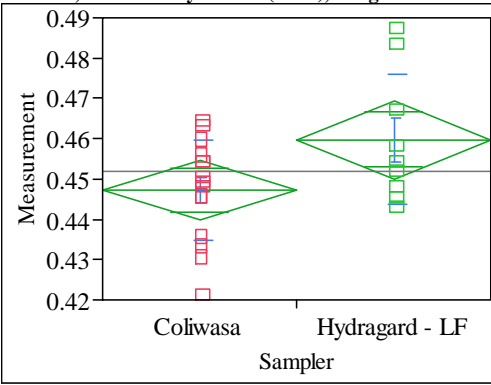

Excl

Oxcluded Rows

Oneway Anova

Summary of Fit

Adj Rsquare

Root Mean Square Error $\quad 0.014028$

0.014028

$\begin{array}{lr}\text { Mean of Response } & 0.45195 \\ \text { Observations (or Sum Wgts) } & 24\end{array}$

t Test

Hydragard - LF-Coliwasa

Assuming equal variances

Difference $\quad 0.012555$ t Ratio 2.122677

Std Err Dif $\quad 0.005915$ DF 22

Upper CL Dif 0.024822 Prob $>|t| 0.0453$

Lower CL Dif 0.000289 Prob $>t \quad 0.0226$

$\begin{array}{lrl}\text { Confidence } & 0.95 \text { Prob }<\mathrm{t} & 0.9774\end{array}$

Analysis of Variance

Source DF Sum of Squares Mean Square F Ratio Prob $>$ F

$\begin{array}{lllllll}\text { Sampler } & 1 & 0.00088670 & 0.000887 & 4.5058 & 0.0453\end{array}$

$\begin{array}{llll}\text { Error } & 22 & 0.00432946 & 0.000197\end{array}$

C. Total $23 \quad 0.00521616$

Means for Oneway Anova

Level Number Mean Std Error Lower 95\% Upper 95\%

$\begin{array}{lrrrrr}\text { Coliwasa } & 15 & 0.447241 & 0.00362 & 0.43973 & 0.45475\end{array}$

$\begin{array}{lrrrrr}\text { Hydragard - LF } & 9 & 0.459797 & 0.00468 & 0.45010 & 0.46949\end{array}$

Std Error uses a pooled estimate of error variance

Means and Std Deviations

Level Number Mean Std Dev Std Err Mean Lower 95\% Upper 95\%

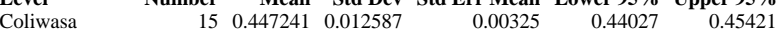

$\begin{array}{lrlllll}\text { Hydragard - LF } & 9 & 0.459797 & 0.016246 & 0.00542 & 0.44731 & 0.47228\end{array}$

Test

Hydragard - LF-Coliwasa

Assuming unequal variances

$\begin{array}{lrlr}\text { Difference } & 0.01256 & \mathrm{t} \text { Ratio } & 1.987953 \\ \text { Std Err Dif } & 0.00632 & \text { DF } & 13.77865 \\ \text { Upper CL Dif } & 0.02612 & \text { Prob }>|t| & 0.0671 \\ \text { Lower CL Dif } & -0.00101 & \text { Prob }>\text { t } & 0.0335 \\ \text { Confidence } & 0.95 & \text { Prob }<\mathrm{t} & 0.9665\end{array}$

Tests that the Variances are Equal

Level Count Std Dev MeanAbsDif to Mean MeanAbsDif to Median

Coliwasa $\quad 15 \quad 0.0125867-0.0098067-0.0096710$

$\begin{array}{lrrrr}\text { Coliwasa } & 15 & 0.0125867 & 0.0098067 & 0.0096710 \\ \text { Hydragard - LF } & 9 & 0.0162462 & 0.0130078 & 0.0123008\end{array}$

Test F Ratio DFNum DFDen p-Value

$\begin{array}{lllll}\text { O'Brien[.5] } & 0.9938 & 1 & 22 & 0.3297\end{array}$

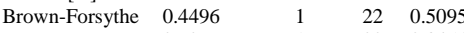

$\begin{array}{lllll}\text { Levene } & 0.9296 & 1 & 22 & 0.3454\end{array}$

$\begin{array}{lllll}\text { Bartlett } & 0.6548 & 1 & 0.4184\end{array}$

$\begin{array}{lllll}\text { F Test 2-sided } & 1.6660 & 8 & 14 & 0.3856\end{array}$

Welch Anova testing Means Equal, allowing Std Devs Not Equal

F Ratio DFNum DFDen Prob > F

$\begin{array}{rrrr}3.9520 & 1 & 13.779 & 0.0671\end{array}$

t Test
1.9880
Oneway Analysis of Measurement By Sampler Type of Material=SME Simulant, Analytica Block $=6$, Oxide $/$ Analyte $=\mathrm{SiO} 2(\mathrm{wt} \%)$, Targeted $=50.985$

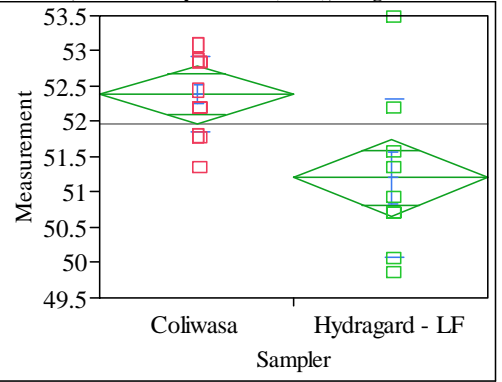

Excluded Rows

Oneway Anova

Summary of Fit

Rsquare $\quad 0.360529$

Adj Rsquare $\quad 0.332726$

Root Mean Square Error $\quad 0.790132$

Mean of Response 51.95932

Observations (or Sum Wgts) 25

Hydragard - LF-Coliwasa

Assuming equal variances

Difference $\quad-1.1855$ t Ratio $\quad-3.601$

Std Err Dif $\quad 0.3292$ DF 23

Upper CL Dif -0.5045 Prob $>|t| \begin{array}{rr}0.0015 \\ 0.04\end{array}$

Lower CL Dif -1.8666 Prob $>t \quad 0.9992$

$\begin{array}{lrl}\text { Confidence } & 0.95 \text { Prob }<\mathrm{t} & 0.0008\end{array}$

Analysis of Variance

Source DF Sum of Squares Mean Square F Ratio Prob $>$ F

$\begin{array}{lllllll}\text { Sampler } & 1 & 8.095556 & 8.09556 & 12.9672 & 0.0015\end{array}$

$\begin{array}{llll}\text { Error } & 23 & 14.359097 & 0.62431\end{array}$

C. Total $24 \quad 22.454652$

Means for Oneway Anova

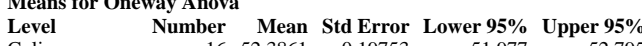

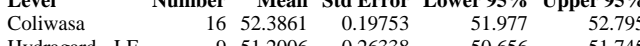

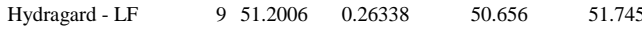

Std Error uses a pooled estimate of error variance

Means and Std Deviations

Level Number Mean Std Dev Std Err Mean Lower 95\% Upper 95\%

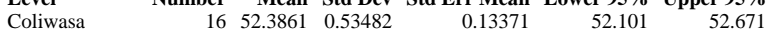

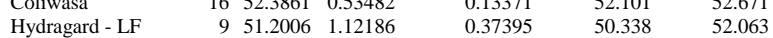

t Test

Hydragard - LF-Coliwasa

Assuming unequal variances

$\begin{array}{lrlr}\text { Difference } & -1.1855 & \text { t Ratio } & -2.98519 \\ \text { Std Err Dif } & 0.3971 & \text { DF } & 10.08827 \\ \text { Upper CL Dif } & -0.3017 & \text { Prob }>|t| & 0.0136 \\ \text { Lower CL Dif } & -2.0694 & \text { Prob }>\text { t } & 0.9932 \\ \text { Confidence } & 0.95 & \text { Prob }<\mathrm{t} & 0.0068\end{array}$

Tests that the Variances are Equal

Level Count Std Dev MeanAbsDif to Mean MeanAbsDif to Median

$\begin{array}{lrrrr}\text { Coliwasa } & 16 & 0.534825 & 0.4546012 & 0.4546012\end{array}$

$\begin{array}{lrrrr}\text { Hydragard - LF } & 9 & 1.121858 & 0.8398733 & 0.8319500\end{array}$

Test F Ratio DFNum DFDen p-Value

$\begin{array}{lrrrr}\text { O'Brien[.5] } & 3.7558 & 1 & 23 & 0.0650\end{array}$

Brown-Forsythe $3.3070 \quad 1 \quad 23 \quad 0.0820$

$\begin{array}{lllll}\text { Levene } & 4.1804 & 1 & 23 & 0.0525\end{array}$

$\begin{array}{lllll}\text { Bartlett } & 5.8120 & 1 & & 0.0159 \\ \text { F Test 2-sided } & 4.4000 & 8 & 15 & 0.0132\end{array}$

Welch Anova testing Means Equal, allowing Std Devs Not Equal

F Ratio DFNum DFDen Prob $>$ F

$\begin{array}{rrrr}8.9113 & 1 & 10.088 & 0.0136\end{array}$

t Test 
Revision 0

\section{Exhibit C3. Statistical Comparisons for Low-Rheology (Phase 3) Simulant Testing (screened data)}

Oneway Analysis of Measurement By Sampler Type of Material=SME Simulant, Analytical Block=6, Oxide/Analyte=Sum of Oxides (wt\%), Targeted=99.553

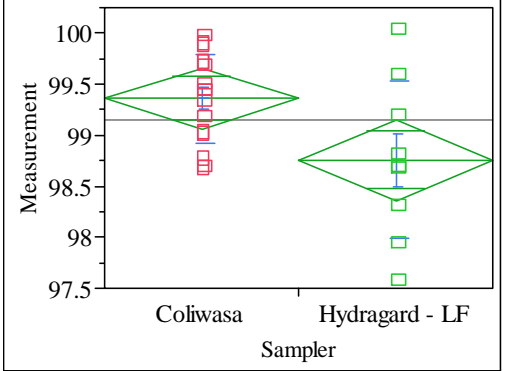

Excluded Rows

Oneway Anova

Summary of Fit

Adj Rsquare

0.217833

0.183825

Root Mean Square Error $\quad 0.576231$

99.145

t Test

Hydragard - LF-Coliwasa

Assuming equal variances

Difference $\quad-0.6077$ t Ratio $\quad-2.5309$

Std Err Dif $\quad 0.2401$ DF 23

Upper CL Dif -0.1110 Prob $>|t| \quad 0.0187$

$\begin{array}{rll}\text { Lower CL Dif }-1.1043 \text { Prob }>t & 0.9907\end{array}$

$\begin{array}{lrl}\text { Confidence } & 0.95 \text { Prob }<\mathrm{t} \quad 0.0093\end{array}$

Analysis of Variance

Source DF Sum of Squares Mean Square F Ratio Prob $>$ F

$\begin{array}{llllll}\text { Sampler } & 1 & 2.1268874 & 2.12689 & 6.4055 & 0.0187\end{array}$

$\begin{array}{llll}\text { Error } & 23 & 7.6369747 & 0.33204\end{array}$

C. Total $24 \quad 9.7638621$

Means for Oneway Anova

Level Number Mean Std Error Lower 95\% Upper 95\%

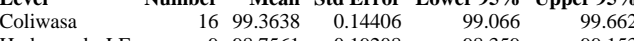

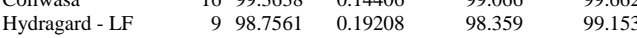

Std Error uses a pooled estimate of error variance

Means and Std Deviations

Level Number Mean Std Dev Std Err Mean Lower 95\% Upper 95\%

$\begin{array}{lrrrrrr}\text { Level } & \text { Number } & \text { Mean } & \text { Std Dev } & \text { Std Err Mean } & \text { Lower 95\% } & \text { Upper 95\% } \\ \text { Coliwasa } & 16 & 99.3638 & 0.435355 & 0.10884 & 99.132 & 99.596\end{array}$

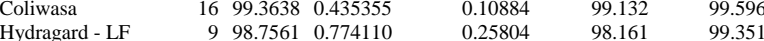

t Test

Hydragard - LF-Coliwasa

Assuming unequal variances

Difference $\quad-0.6077$ t Ratio $\quad-2.16982$

Std Err Dif $\quad 0.2801$ DF 10.91553

Upper CL Dif 0.0093 Prob $>|t| 0.0530$

Lower CL Dif -1.2246 Prob $>$ t 0.9735

$\begin{array}{lrl}\text { Confidence } & 0.95 \text { Prob }<\mathrm{t} & 0.0265\end{array}$

Tests that the Variances are Equal

Level Count Std Dev MeanAbsDif to Mean MeanAbsDif to Median

Coliwasa $\quad 16 \quad 0.4353546$

$\begin{array}{lrrrr}\text { Coliwasa } & 16 & 0.4353546 & 0.3629705 & 0.3541027 \\ \text { Hydragard - LF } & 9 & 0.7741099 & 0.5767855 & 0.5729941\end{array}$

Test F Ratio DFNum DFDen p-Value

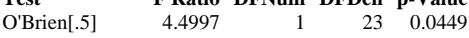

Brown-Forsythe $2.2755 \quad 1 \quad 23 \quad 0.145$

$\begin{array}{lllll}\text { Levene } & 2.3893 & 1 & 23 & 0.1358\end{array}$

\begin{tabular}{lllll} 
Bartlett & 3.5136 & 1 & & 0.0609 \\
\hline & 3.1617 & 8 & 15 & 0.0523
\end{tabular}

Welch Anova testing Means Equal, allowing Std Devs Not Equal

F Ratio DFNum DFDen Prob $>$ F

$\begin{array}{rrrr}4.7081 & 1 & 10.916 & 0.0530\end{array}$

t Test

2.1698
Oneway Analysis of Measurement By Sampler Type of Material=SME Simulant, Analytical Block $=6$, Oxide $/$ Analyte $=$ TiO2 $(w t \%)$, Targeted $=0.0711$

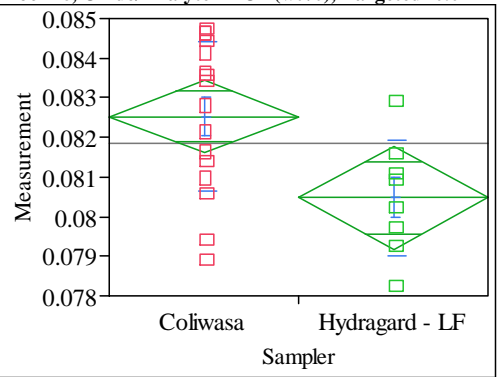

Ex

Oneway Anova

Summary of Fit

Adj Rsquare

0.24465
Root Mean Square Error $\quad 0.210316$

Mean of Response $\quad 0.081843$

Observations (or Sum Wgts)

Test

Hydragard - LF-Coliwasa

Assuming equal variances

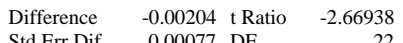

$\begin{array}{lrlr}\text { Std Err Dif } & 0.00077 & \text { DF } & 22 \\ \text { Upper CL Dif } & -0.00046 & \text { Prob }>|t| & 0.0140\end{array}$

Lower CL Dif -0.00363 Prob

Lonfidence -0.95 Prob $<\mathrm{t} \quad 0.0070$

Analysis of Variance

Source DF Sum of Squares Mean Square F Ratio Prob $>$ F

$\begin{array}{lrrrrr}\text { Sampler } & 1 & 0.00002227 & 0.000022 & 7.1256 & 0.0140\end{array}$

$\begin{array}{llll}\text { Error } & 22 & 0.00006875 & 3.125 \mathrm{e}-6\end{array}$

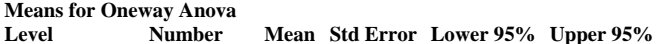

$\begin{array}{lrrrrr}\text { Level } & \text { Number } & \text { Mean } & \text { Std Error } & \text { Lower 95\% } & \text { Upper 95\% } \\ \text { Coliwasa } & 16 & 0.082524 & 0.00044 & 0.08161 & 0.08344\end{array}$

$\begin{array}{lrrrrr}\text { Hydragard - LF } & 16 & 0.082524 & 0.00044 & 0.08161 & 0.08344 \\ & 8 & 0.080481 & 0.00062 & 0.07918 & 0.08178\end{array}$

Std Error uses a pooled estimate of error variance

Means and Std Deviations

Level Number Mean Std Dev Std Err Mean Lower 95\% Upper 95\%

$\begin{array}{lllllll}\text { Coliwasa } & 16 & 0.082524 & 0.001896 & 0.00047 & 0.08151 & 0.08353\end{array}$

$\begin{array}{llllll}\text { Hydragard - LF } & 8 & 0.080481 & 0.001454 & 0.00051 & 0.07927\end{array}$

Test

Hydragard - LF-Coliwasa

Assuming unequal variances

$\begin{array}{lrr}\text { Difference } & -0.00204 \text { t Ratio } & -2.92171 \\ \text { Std Err Dif } & 0.00070 & \end{array}$

Std Err Dif $\quad 0.00070$ DF $\quad 17.92045$

Upper CL Dif -0.00057 Prob $>|t| \quad 0.0091$

Lower CL Dif -0.00351 Prob $>t \quad 0.9954$

$\begin{array}{lrl}\text { Confidence } & 0.95 \text { Prob }<\mathrm{t} \quad 0.0046\end{array}$

Tests that the Variances are Equal

Level Count Std Dev MeanAbsDif to Mean MeanAbsDif to Median

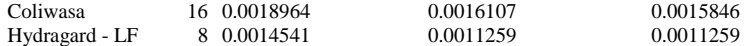

Test F Ratio DFNum DFDen p-Value

$\begin{array}{lrrrr}\text { O'Brien[.5] } & 0.8899 & 1 & 22 & 0.3557\end{array}$

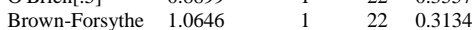

$\begin{array}{lllll}\text { Levene } & 1.6125 & 1 & 22 & 0.2174\end{array}$

$\begin{array}{lrrrr}\text { Bartlett } & 0.5936 & 1 & \text {. } & 0.4410\end{array}$

$\begin{array}{lllll}\text { F Test 2-sided } & 1.7009 & 15 & 7 & 0.4881\end{array}$

Welch Anova testing Means Equal, allowing Std Devs Not Equal

F Ratio DFNum DFDen Prob $>$ F

$\begin{array}{llll}8.5364 & 1 & 17.92 & 0.0091\end{array}$

t Test

2.9217 
Revision 0

\section{Exhibit C3. Statistical Comparisons for Low-Rheology (Phase 3) Simulant Testing (screened data)}

Oneway Analysis of Measurement By Sampler Type of Material=SME Simulant, Analytica Block $=6$, Oxide/Analyte $=\mathrm{ZnO}$ ( $w \mathrm{t} \%)$, Targeted $=\mathbf{0 . 0 9 5 8}$

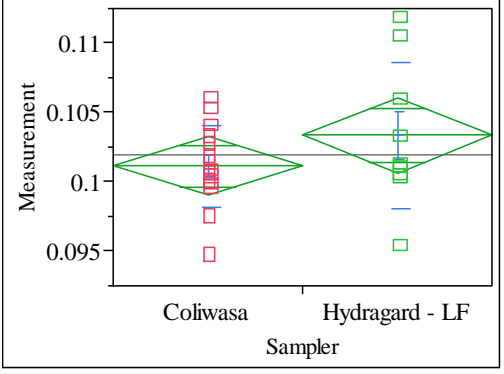

Excluded Rows

Oneway Anova

Summary of Fit

0.074053
0.031965

Root Mean Square Error $\quad 0.003937$

0.10197

t Test

Hydragard - LF-Coliwasa

Assuming equal variances

Difference $\quad 0.00220$ t Ratio 1.326447

Std Err Dif $\quad 0.00166$ DF 22

Upper CL Dif 0.00564 Prob $>|t| \quad 0.1983$

Lower CL Dif -0.00124 Prob $>t \quad 0.0991$

$\begin{array}{lll}\text { Confidence } & 0.95 \text { Prob }<\mathrm{t} \quad 0.9009\end{array}$

Analysis of Variance

Source DF Sum of Squares Mean Square F Ratio Prob > F

$\begin{array}{llllll}\text { Sampler } & 1 & 0.00002727 & 0.000027 & 1.7595 & 0.1983\end{array}$

$\begin{array}{llll}\text { Error } & 22 & 0.00034101 & 0.000016\end{array}$

C. Total $23 \quad 0.00036828$

$\begin{array}{lccc}\text { Means for Oneway Anova } \\ \text { Level } & \text { Number } & \text { Mean Std Error } & \text { Lower 95\% Upper 95\% }\end{array}$

$\begin{array}{lrrrrr}\text { Coliwasa } & 15 & 0.101144 & 0.00102 & 0.09904 & 0.10325\end{array}$

$\begin{array}{lrrrrr}\text { Hydragard - LF } & 15 & 0.101144 & 0.00102 & 0.09904 & 0.10325 \\ & 9 & 0.103346 & 0.00131 & 0.10062 & 0.10607\end{array}$

Std Error uses a pooled estimate of error variance

Means and Std Deviations

$\begin{array}{llllll}\text { Level Number Mean } & \text { Std Dev } & \text { Std Err Mean Lower 95\% Upper 95\% }\end{array}$

$\begin{array}{lllllll} & 15 & 0.101144 & 0.002938 & 0.00076 & 0.09952 & 0.10277\end{array}$

t Test

Hydragard - LF-Coliwasa

Assuming unequal variances

Difference $\quad 0.00220$ t Ratio $\quad 1.155157$

Std Err Dif $\quad 0.00191$ DF 11.06965

Upper CL Dif 0.00639 Prob $>|t| \quad 0.2724$

Lower CL Dif -0.00199 Prob $>t \quad 0.1362$

$\begin{array}{lrl}\text { Confidence } & 0.95 \text { Prob }<\mathrm{t} & 0.8638\end{array}$

Tests that the Variances are Equal

Level Count Std Dev MeanAbsDif to Mean MeanAbsDif to Median

$\begin{array}{lrrrr}\text { Coliwasa } & 15 & 0.0029378 & 0.0021621 & 0.0021660\end{array}$

$\begin{array}{lrrrr}\text { Test } & \text { F Ratio } & \text { DFNum } & \text { DFDen } & \text { p-Value } \\ \text { O'Brien[.5] } & 3.6050 & 1 & 22 & 0.0708 \\ \text { Brown-Forsythe } & 1.9827 & 1 & 22 & 0.1731 \\ \text { Levene } & 3.5658 & 1 & 22 & 0.0722 \\ \text { Bartlett } & 3.4319 & 1 & & 0.0639 \\ \text { F Test 2-sided } & 3.1890 & 8 & 14 & 0.0558\end{array}$

$\begin{array}{lllll}\text { Bartlett } & 3.4319 & 1 & \text {. } & 0.0639 \\ \text { F Test 2-sided } & 3.1890 & 8 & 14 & 0.0558\end{array}$

Welch Anova testing Means Equal, allowing Std Devs Not Equal

F Ratio DFNum DFDen Prob $>$ F

$\begin{array}{llll}1.3344 & 1 & 11.07 & 0.2724\end{array}$

t Test

1.1552
Oneway Analysis of Measurement By Sampler Type of Material=SME Simulant, Analytical Block=6, Oxide/Analyte $=$ ZrO2 (wt $\%)$, Targeted $=0.3547$

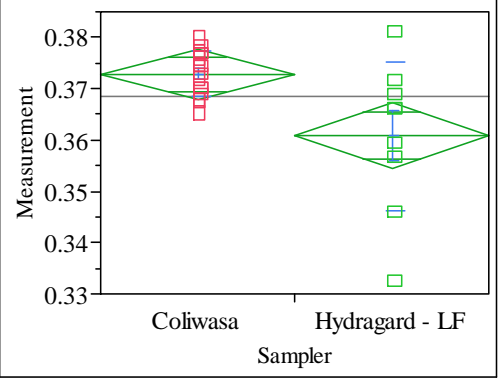

Excluded Rows

1

Oneway Anova

Summary of Fit

Rsquare $\quad 0.294761$

Adj Rsquare $\quad 0.264099$

Root Mean Square Error $\quad 0.009294$

Mean of Response 0.368498

Observations (or Sum Wgts) $\quad 25$

t Test

Hydragard - LF-Coliwasa

Assuming equal variances

Difference $\quad-0.01201$ t Ratio $\quad-3.10049$

$\begin{array}{lll}\text { Std Err Dif } \quad 0.00387 \text { DF } & 23\end{array}$

Upper CL Dif -0.00400 Prob $>|t| \quad 0.0050$

Lower CL Dif -0.02002 Prob $>t \quad 0.9975$

$\begin{array}{lrl}\text { Confidence } & 0.95 \text { Prob }<\mathrm{t} & 0.0025\end{array}$

Analysis of Variance

Source DF Sum of Squares Mean Square F Ratio Prob $>$ F

$\begin{array}{llllll}\text { Sampler } & 1 & 0.00083042 & 0.000830 & 9.6131 & 0.0050\end{array}$

$\begin{array}{llll}\text { Error } & 23 & 0.00198685 & 0.000086\end{array}$

C. Total $24 \quad 0.00281728$

Means for Oneway Anova

Level $\quad$ Number Mean Std Error Lower 95\% Upper 95\%

$\begin{array}{lrrrrr}\text { Coliwasa } & 16 & 0.372821 & 0.00232 & 0.36801 & 0.37763\end{array}$

$\begin{array}{llllll}\text { Hydragard - LF } & 9 & 0.360814 & 0.00310 & 0.35440 & 0.36722\end{array}$

Std Error uses a pooled estimate of error variance

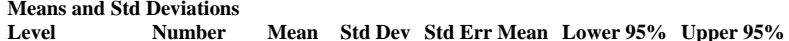

$\begin{array}{lrrrrrr}\text { Level } & \text { Number } & \text { Mean } & \text { Std Dev } & \text { Std Err Mean } & \text { Lower 95\% } & \text { Upper 95\% } \\ \text { Coliwasa } & 16 & 0.372821 & 0.004412 & 0.00110 & 0.37047 & 0.37517\end{array}$

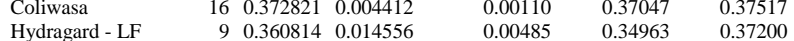

t Test

Hydragard - LF-Coliwasa

Assuming unequal variances

$\begin{array}{lrlr}\text { Difference } & -0.01201 & \mathrm{t} \text { Ratio } & -2.41319 \\ \text { Std Err Dif } & 0.00498 & \text { DF } & 8.835573 \\ \text { Upper CL Dif } & -0.00072 & \text { Prob }>|\mathrm{t}| & 0.0395 \\ \text { Lower CL Dif } & -0.02329 & \text { Prob }>\mathrm{t} & 0.9802 \\ \text { Confidence } & 0.95 & \text { Prob }<\mathrm{t} & 0.0198\end{array}$

Tests that the Variances are Equal

Level Count Std Dev MeanAbsDif to Mean MeanAbsDif to Median

\begin{tabular}{lr|lll} 
Coliwasa & 16 & 0.0044117 & 0.0037147 & 0.0037147
\end{tabular}

$\begin{array}{lrlll}\text { Hydragard - LF } & 9 & 0.0145555 & 0.0109398 & 0.0103561\end{array}$

Test F Ratio DFNum DFDen p-Value

$\begin{array}{lllll}\text { O'Brien[.5] } & 5.8266 & 1 & 23 & 0.0241\end{array}$

$\begin{array}{llll}\text { Brown-Forsythe } 5.5311 & 1 & 23 & 0.0276\end{array}$

$\begin{array}{lllll}\text { Levene } & 10.0381 & 1 & 23 & 0.0043\end{array}$

$\begin{array}{lrrrr}\text { Bartlett } & 14.4630 & 1 & \text {. } & 0.0001\end{array}$

F Test 2-sided 10.8854

Welch Anova testing Means Equal, allowing Std Devs Not Equal

F Ratio DFNum DFDen Prob $>$ F

$\begin{array}{rrrr}5.8235 & 1 & 8.8356 & 0.0395\end{array}$

t Test 


\section{Distribution:}

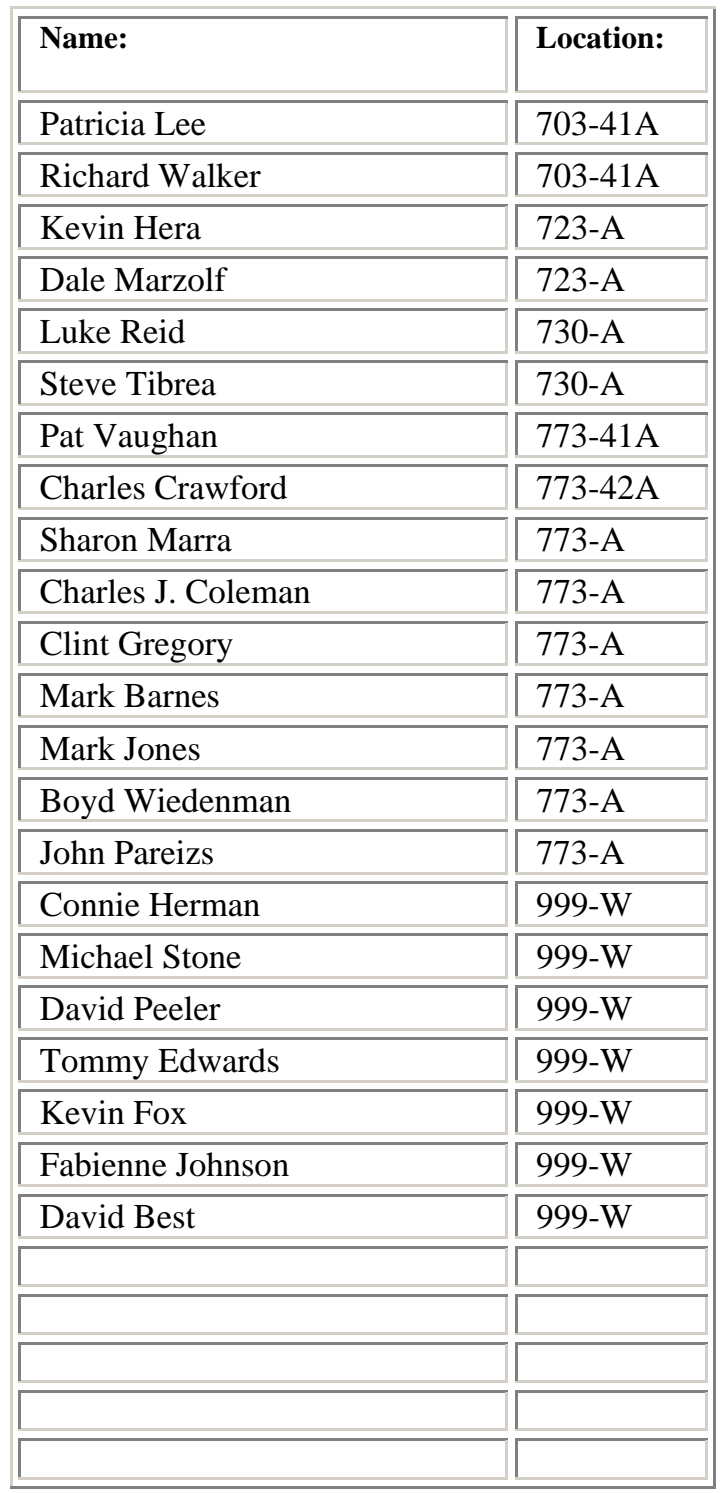

\begin{tabular}{|l|l|}
\hline Name: & Location: \\
\hline Michael T. Hart & $210-S$ \\
\hline Bill Holtzscheiter & $704-15 S$ \\
\hline Omar Cardona-Quiles & $704-24 S$ \\
\hline Hank Elder & $704-24 S$ \\
\hline Terri Fellinger & $704-26 S$ \\
\hline Jonathan Bricker & $704-27 S$ \\
\hline Aaron Staub & $704-27 S$ \\
\hline Perry Bovan & $704-27 S$ \\
\hline Amanda Shafer & $704-27 S$ \\
\hline Mason Clark & $704-27 S$ \\
\hline Helen P. Boyd & $704-27 S$ \\
\hline Roger N. Mahannah & $704-28 S$ \\
\hline Michael T. Feller & $704-28 S$ \\
\hline John Iaukea & $704-30 S$ \\
\hline \hline John Occhipinti & $704-S$ \\
\hline Jeff Ray & $704-S$ \\
\hline Robert Hinds & \\
\hline \hline Ryan McNew & \\
\hline \hline
\end{tabular}

